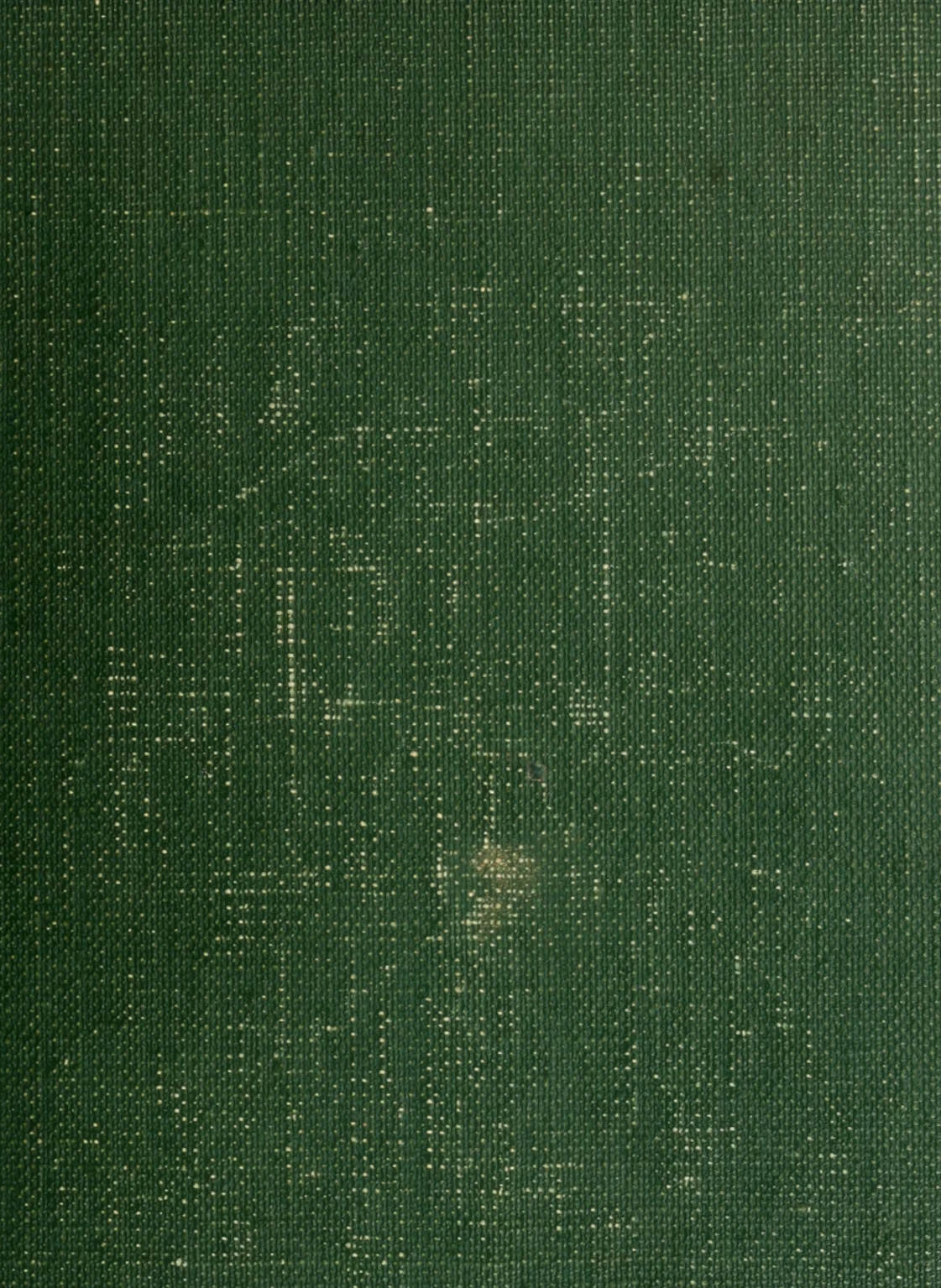





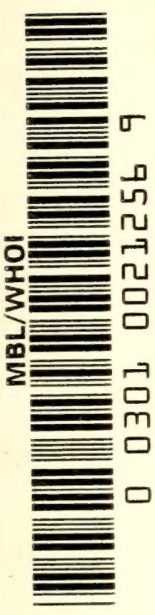





\section{BERGEY'S MANUAL}

\section{$\mathrm{OF}$ \\ DETERMINATIVE \\ BACTERIOLOGY}

BY

ROBERT S. BREED

New York State Experiment Station (Cornell University), Geneva, New York

E. G. D. MURRAY

McGill University, Montreal, Province Quebec, Canada

A. PARKER HITCHENS

University of Pennsylvania, Philadelphia, Pennsylvania

\section{Assisted by}

Sixty Contributors Whose Names and Contributions Appear in the Pages Immediately Following

\section{S I X TH E I T ION}

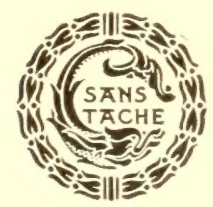

BALTIMORE

THE WILLIAMS \& WILKINS COMPANY 
First Edition, August, 1923

Second Edition, December, 1925

Third Edition, January, 1930

Fourth Edition, March, 1934

Preprint of pages ix +79 of Fifth Edition, October, 1938

Fifth Edition, April, 1939

Copyright, 1948

The Williams \& Wilkins Company

Made in United States of America

Published January, 1948

\author{
Composed and Printed at the \\ WAVERLY PRESS, INC. \\ TOR \\ The Wrllass \& Wrisins Compayy \\ Mt. Royal and Guilford Aves. \\ Baltimore, Md., U. S. A.
}




\section{LIST OF CONTRIBUTORS}

Allen, O. N.
Baldwin, I. L.
Barker, H. A.

Bengtson, Ida A

Bergey, D. H. $\dagger$

Borman, Earl L.

Branham, Sara E.

Breed, Robert S.

Robert S. and Margaret

E. Breed

Buchanan, R. E.

Burkholder, Walter $\mathrm{H}$.

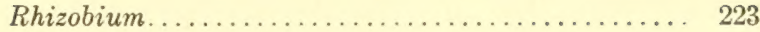

Rhizobium........................... 223

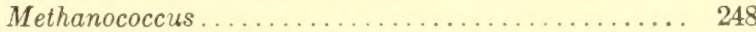

Butyribacterium........................ 380

Methanobacterium......................... 645

Rickettsiaceae............................... 1083

Bartonellaceae ........................... 1100

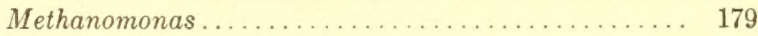

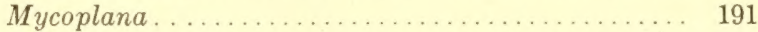

Thiospira.......................... 212

Achromobacter........................... 417

Flavobacterium.............................. 427

Dialister. . . . . . . . . . . . . . . . . . . . . . . . . 594

Paracolobactrum........................... 460

Neisseriaceae. . . . . . . . . . . . . . . . . . . . . . 295

Survey of Classifications................. . 5-38

Rules of Nomenclature . . . . . . . . . . . . . . . . . . .49-64

Nitrobacteriaceae............................. 69

Pseudomonadaceae.......................62,171

Chromobacterium.......................... 231

Methanococcus.......................... 248

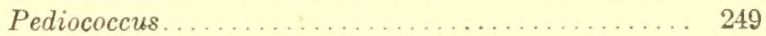

Sarcina............................. 285

Leptotrichia............................ 264

Corynebacteriaceae....................... 381

Achromobacteriaceae........................ 412

App. 2. Eschericheae........................ 461

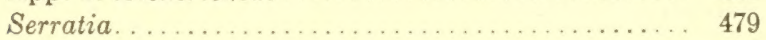

Malleomyces........................... 554

Fusobacterium, 581 and Fusiformis........... 583

Bacteriaceae............................. 596

Caulobacteriineae........................ 827

Chlamydobacteriales....................... 981

Indexes. . . . . . . . . . . . . . . . . . . . . . . . 1297

How Bacteria Are Named and Identified . . . . . . . . 39-48

Etymology. . . ......................... $64 \mathrm{ff}$.

Myxobacteriales............................ 1005

Pseudomonadaceae...................82 and 150

Corynebacterium........................... 381

Erwinia ............................. 463

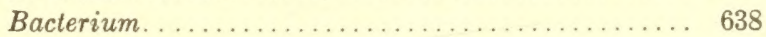

† Deceased, September, 1937. 
Chapman, Orren D.

Chester, F. D.†

Clise, Eleanore $\mathrm{H}$.

Conn, H. J.

Davis, Gordon E.

Dienes, Louis

Edwards, P. R.

Evans, Alice C.

Fred, E. B.

Hagan, W. A.

Hall, Ivan C.

Hanks, John H.

Harvey, Philip

Haynes, Wm. C.

Henrici, A. T. $\ddagger$

Hitchens, A. Parker

Hitchner, E. R.

Hofer, A. W.

Holmes, Francis O.

Hucker, G. J.

Huddleson, I. F. Johnson, Frank H.

Kauffmann, F.

Kelly, C. D.

Kirby, Harold Meyer, K. F.
Klebsiella....................... 457

Donovania............................ 559

Erwinia .............................. 463

Bacillus ............................... 704

App. 3. Micrococcus. . . .................... 252

Pasteurella............................. 546

App. 1. Bacteroides, 575; App. Eubacteriineae, 692;

App. Nocardia, 915; App. Streptomyces, 967; and

App. Spirochaetales, 1051

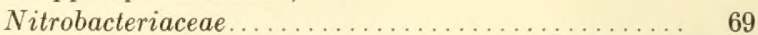

Agrobacterium............................ 227

App. 3. Corynebacterium .................. 407

Alcaligenes......................... 412

Borrelia................................ 1058

Borrelomycetaceae. . . . . . . . . . . . . . . . . . . . 1287

Salmonella............................... 492

Parvobacteriaceae.......................... 545

Rhizobium............................ 223

Parvobacteriaceae ........................ 545

Anaerobic section Micrococcus, 246; Neisseria, 255;

Veillonella, 302; Diplococcus, 308; and Streptococcus, 328

Mycobacterium...................... 876

Pasteurella .............................. 546

Vibrio................................. 192

Spirillum............................ 216

Caulobacteriineae...................... 827

Nocardia . . . . . . . . . . . . . . . . . . . . . . 892

Actinomyces. ........................... 925

Streptomyces........................... 929

Chlamydobacteriales........................ 981

Vibrio . . . . . . . . . . . . . . . . . . . . . . . . . . 192

Mimeae................................. 595

Editor Supplement I, 1082, II, 1125 and III, 1287

Aeromonas .............................. 101

Azotobacteriaceae....................... 219

Virales.................................. 1200

Micrococcus............................. 235

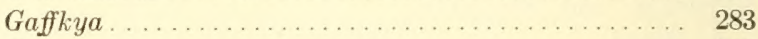

Streptoccccus.......................... 312

Leuconostoc............................. 346

Brucella ........................... 560

Phosphorescent Bacteria.................... 633

Salmonella................................. 492

Acetobacter............................. 179

Bacteroides. ......................... 564

Noguchia ............................... 592

App. 1. Rickettsiales..................... 1121

Pasteurella......................... 545 
Murray, E. G. D.

Neisseriaceae. ........................... 295

Diplococcus.............................. 305

Streptococcus......................... 312

Corynebacteriaceae...................... 381

Parvobacteriaceae .......................... 545

Spirochaetales......................... 1051

Pederson, C. S. $\quad$ Leuconostoc........................ 346

Lactobacillus............................. 349

Leptotrichia. . ......................... 364

Microbacterium...................... 370

Butyribacterium . . .................... 380

Peshkoff, Michael A. Caryophanales......................... 1002

Pittman, Margaret $\quad$ Hemophilus......................... 584

Rahn, Otto Characterizations of Groups. . . . . . . . . . . $64 \mathrm{ff}$.

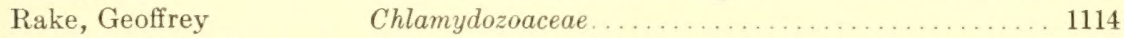

Reed, Guilford B. $\quad$ Mycobacteriaceae ..................... 875

Rettger, L. F. Lactobacillus........................ 349

Robinson, George H. $\dagger \quad$ Spirochaetales ......................... 1051

Roy, T. E.

Rustigian, Robert

Sherman, J. M.

Smith, Frederick

Smith, N. R.

Speck, M. L.

Spray, R. S.

Stanier, R. Y.

Bacteroides. ........................... 564

Proteus............................ 486

Streptococcus. ........................ 312

Lactobacillus. ........................... 349

Salmonella.............................. 492

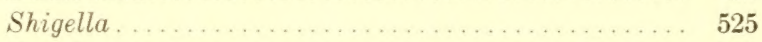

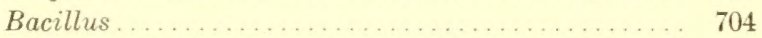

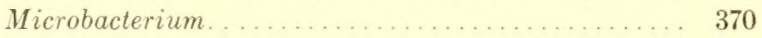

Clostridium........................... 763

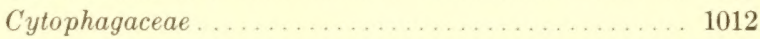

Sporocytophagaceae........................ 1048

Starkey, R. L. Nitrobacteriaceae....................... 69

Steinhaus, Edward A. Insect Microbiology . . . . . . . . . . . 417, 742 ff.

Rickettsiaceae............................. 1083

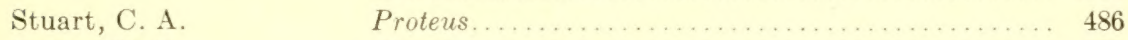

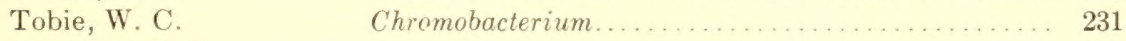

Van Niel, C. B. Propionibacterium ..................... 372

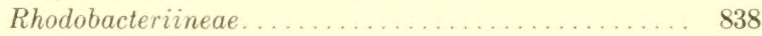

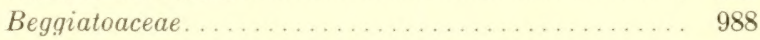

Achromatiaceae. .......................... 997

Acetobacter. . . . . . . . . . . . . . . . . . . . . 179

Actinomycetaceae . . . . . . . . . . . . . . . . . . 892

Streptomycetaceae . . . . . . . . . . . . . . . . . . . . 929

Bartonellaceae ........................... 1100

Pasteurella ........................... 546

Escherichia........................... 444

Aerobacter............................. 453

Proteus................................ 486

ZoBell, Claude E. $\quad$ Desulfovibrio . . . . . . . . . . . . . . . . 209

Marine Microbiology..............107, 418, $431 \mathrm{ff}$.

$\dagger$ Deceased, October, 1945. 



\section{PREFACE TO SIXTH EDITION}

More than the usual amount of time and effort has been given toward making this new edition of Bergey's Manual useful. The volume has been completely revised and is reset in double column format so that each page carries about 20 per cent more type than the pages in the fifth edition. Those who are interested in special groups of bacteria will find something new in the presentation of the relationships in every genus. Because of our rapidly expanding knowledge, changes in the outline classification and text were made necessary. These changes have in every case been made by specialists in consultation with the Editorial Board. Every sperialist possesses first hand knowledge of the species in the group that he or she has reviewed.

Because increasing knowledge has shown the fission fungi to be a larger and more diversified group than previously realized, the number of species described has increased from 1335 in the fifth edition to 1630 in the present edition of the Manual. This number does not cover all of the descriptions found in the literature for, as in all other fields of biology, many of the descriptions are so inadequate that the species described cannot now be identified. Many descriptions are obvious or probable duplications of previous descriptions while still others are based on nothing more substantial than the author's belicf that he had something new, he having made but little effort to compare his cultures with those found by previous investigators. An indication of the large number of inadequate descriptions will be found by referring to the material in the appendixes to the various groups, and to the index where synonyms and incompletely described species are shown in italies.

The large number of these poorly described species suggests that there has been much unsatisfactory work done in the field of bacteriological taxonomy. Progress in this inad quately developed field is needed as it would help to clarify the approach to desirable research in many fields of bacteriology.

It is believed that both teachers and investigators will find the new Source and Habitat index useful. It is important to know what organisms have been described from any giren habitat in determining the identity of a described species or whether a given species is new.

The future development of taxonomic work holds several interesting possibilities of increased international cooperation such as between the various National Type Culture Collections and within the International 
Association of Microbiologists. The Trust Funds provided through the generosity of Dr. Bergey before his death have been used in developing the present edition of the Manual and future funds are to be used in the same way under the management of a self-perpetuating Board of Editor-Trustees.

We are all under obligation to those who have given so freely of their time and special knowledge in preparing this edition of the Manual. Moreover the Editor-in-Chief is under special obligation to his wife, Margaret Edson Breed who has carried the burden of the indexing; to Mrs. Eleanore Heist Clise who has given invaluable service in bibliographical research, in proof reading and other ways; and to his secretary, Miss Maude Hogan, who has cared for many difficult manuscripts and a voluminous technical correspondence.

Many binomials not previously mentioned in the Manual will be found in the Index of Genus and Species Names. Each new name means that there is a new bibliographic reference in the text. Practically all of the incomplete references of previous editions and all new references have been examined in the original, something that is essential in all accurate taxonomic work. The index of names is the most complete list that has appeared in the literature and should always be consulted before new genus or species names are proposed.

This edition of the Manual has been more than four years in press, thanks to the care that has been taken to make it complete and useful. Throughout, the Editorial Board has had the cooperation and understanding help of the publishers of the book who themselves have been forced to meet and overcome the trying difficulties of the war years.

The plan of the present book is such that it will be found useful both to teachers and research workers.

Robert S. Breed, Chairman

E. G. D. Murray

A. Parier Hitchens

Board of Editor-Trustees.

April, 1947. 


\title{
PREFACE OF FIRST EDITION
}

The elaborate system of classification of the bacteria into families, tribes and genera by a Committee on Characterization and Classification of the Society of American Bacteriologists $(1917,1920)$ has made it very desirable to be able to place in the hands of students a more detailed key for the identification of species than any that is available at present. The valuable book on "Determinative Bacteriology" by Professor F. D. Chester, published in 1901, is now of very little assistance to the student, and all previous classifications are of still less value, especially as earlier systems of classification were based entirely on morphologic characters.

It is hoped that this manual will serve to stimulate efforts to perfect the classitication of bacteria, especially by emphasizing the valuable features as well as the weaker points in the new system which the Committee of the Society of American Bacteriologists has promulgated. The Committee does not regard the classification of species offered here as in any sense final, but merely a progress report leading to more satisfactory classification in the future.

The Committee desires to express its appreciation and thanks to those members of the society who gave valuable aid in the compilation of material and the classification of certain species.

The assistance of all bacteriologists is earnestly solicited in the correction of possible errors in the text; in the collection of descriptions of all bacteria that may have been omitted from the text; in supplying more detailed descriptions of such organisms as are described incompletely; and in furnishing complete descriptions of new organisms that may be discovered, or in directing the attention of the Committee to publications of such newly described bacteria.

\author{
David H. Bergey, Chairman \\ Francis C. Harrison \\ ROBERT S. BREED \\ Bernard W. HaMmer \\ Frank M. Huntoon \\ Committee on Manual.
}

August, 1923. 



\section{CONTENTS}

Introduction .......................................... I

Historical Survey of Classifications ............................ 5

How Bacteria are Named and Identified ......................... 39

Rules of Nomenclature .................................... 49

Class Schizomycetes Nägeli .................................... 65

Order I. Eubacteriales Buchanan ............................ 66

Suborder I. Eubacteriineae Breed, Murray and Hitchens............ 67

Family I. Nitrobacteriaceae Buchanan ...................... 69

Tribe I. Nitrobacterieae Winslow et al ..................... 70

Genus I. Nitrosomonas Winogradsky .................. 70

Genus II. Nitrosococcus Winogradsky .................. 71

Genus III. Nitrosospira Winogradsky ................... 71

Genus IV. Nitrosocystis Winogradsky.................. 72

Genus V. Nitrosogloea H. Winogradsky ................. 73

Genus VI. Nitrobacter Winogradsky ................... 74

Genus VII. Nitrocystis H. Winogradsky ................ 75

Tribe II. Hydrogenomonadeae Pribram.................... 76

Genus I. Hydrogenomonas Orla-Jensen .................. $\quad 76$

Tribe III. Thiobacillcae Bergey, Breed and Murray ............. 78

Genus I. Thiobacillus Beijerinck ...................... 78

Family II. Pseudomonadaceae Winslow et \&l................... 82

Tribe I. Pseudomonadeae Kluyver and Van Niel ............... 82

Genus I. Pseudomonas Migula.......................... 82

Genus II. Yanthomonas Dowson ...................... 150

Genus III. Methanomonas Orla-Jensen ................... 179

Genus IV. Acetobacter Beijerinck ..................... 179

Genus V. Protaminobacter den Dooren de Jong ............... 189

Genus VI. Mycoplana Gray and Thornton................ 192

Tribe II. Spirilleae Kluyver and Van Niel ................... 192

Genus I. Vibrio Müller............................ 192

Genus II. Desulfovibrio Kluyver and Van Niel............. 207

Genus III. Cellvibrio Winogradsky ....................... 209

Genus IV. Cellfalcicula Winogradsky .................... 211

Genus V. Thiospira Vislouch ........................ 212

Genus VI. Spirillum Ehrenberg....................... 212

Family III. Azotobacteriaceae Bergey, Breed and Murray ............ 219

Genus I. Azotobacter Beijerinck ....................... 219

Appendix: Genus A. Azotomonas Stapp ..................... 221

Family IV. Rhizobiaceae Conn ........................... 223

Genus I. Rhizobium Frank.......................... 223

Genus II. Agrobacterium Conn ....................... 227

Genus III. Chromobacterium Bergonzini................... 231

Family V. Micrococcaceae Pribram.......................... 235

Genus I. Micrococcus Cohn.......................... 235

Appendix: Genus A. Methanococcus Kluyver and Van Niel ....... 248

Genus B. Pediococcus Balcke ................... 249 
Genus II. Gaffkya Trevisan........................ 283

Genus III. Sarcina Goodsir. . . . . . . . . . . . . . . . . . . 285 Subgenera:

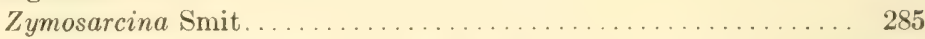

Methanosarcina Kluyver and Van Niel............... 285

Sarcinococcus Breed........................... 285

Sporosarcina Orla-Jensen........................... 285

Family VI. Neisseriaceae Prévot........................... 295

Genus I. Neisseria Trevisan.......................... 295

Genus II. Veillonella Prévot......................... 302

Family VII. Lactobacteriaceae Orla-Jensen. . . . . . . . . . . . . . 305

Tribe I. Streptococceae Trevisan........................ 305

Genus I. Diplococcus Weichselbaum.................. 305

Genus II. Streptococcus Rosenbach..................... 312

Genus III. Leuconostoc Van Tieghem..................... 346

Tribe II. Lactobacilleae Winslow et al..................... 349

Genus I. Lactobacillus Beijerinck........................ 349

Sub-genera:

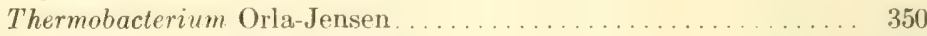

Streptobacterium Orla-Jensen..................... 350

Betabacterium Orla-Jensen........................ 350

Appendix: Genus A. Leptotrichia Trevisan................. 365

Genus II. Microbacterium Orla-Jensen.................. 370

Genus III. Propionibacterium Orla-Jensen................ 372

Genus IV. Butyribacterium Barker.................... 379

Family VIII. Corynebacteriaceae Lehmann and Neumann........... 381

Genus I. Corynebacterium Lehmann and Neumann. ........... 381

Genus II. Listeria Pirie.............................. 408

Genus III. Erysipelothrix Rosenbach.................... 410

Family IX. Achromobacteriaceae ....................... 412

Genus I. Alkaligenes Castellani and Chalmers............. 412

Genus II. Achromobacter Bergey et al................... 417

Genus III. Flavobacterium Bergey et al................ 427

Family X. Enterobacteriaceae Rahn..................... 443

Tribe I. Eschericheae Bergey, Breed and Murray................ 444

Genus I. Escherichia Castellani and Chalmers............. 444

Genus II. Aerobacter Beijerinck.................... 453

Genus III. Klebsiclla Trevisan. . . . . . . . . . . . . . . . 457

Appendix: Genus A. Paracolobactrum Borman, Stuart and

Wheeler .................................. 459

Tribe II. Erwineae Winslow et al.................... 463

Genus I. Erwinia Winslow et al...................... 463

Tribe III. Serrateae Bergey, Breed and Murray ................ 479

Genus I. Serratia Bizio smend. Breed and Breed............. 479

Tribe IV. Proteae Castellani and Chalmers................ 486

Genus I. Proteus Hauser. . . . . . . . . . . . . . . . . . . . . . . 486

Tribe V. Salmonelleae Bergey, Breed and Murray............. 492

Genus I. Salmonella Lignières........................ 492

Genus II. Shigella Castellani and Chalmers.............. 535 
Family XI. Parvobacteriaceae Rahn.................... 545

Tribe I. Pasteurelleae Castellani and Chalmers.............. 545

Genus I. Pasteurella Trevisan ..................... 546

Genus II. Malleomyces Hallier...................... $5 \boldsymbol{b}^{2}$

Genus III. Actinobacillus Brumpt................... 556

Appendix: Genus A. Donovania Anderson et al.............. 558

Tribe II. Brucelleae Bergey, Breed and Murray.............. 560

Genus I. Brucella Meyer and Shaw.................... 560

Tribe III. Bacteroideae Breed, Murray and Hitchens............. 564

Genus I. Bacteroides Castellani and Chalmers............... 564

Genus II. Fusobacterium Knorr...................... 581

Appendix: Genus A. Fusiformis Hoelling................ 583

Tribe IV. Hemophileae Winslow et al.................... 584

Genus I. Hemophilus Winslow et al................... 584

Genus II. Moraxella Lwoff. . . . . . . . . . . . . . . . . . . . . . 590

Genus III. Noguchia Olitsky, Syverton and Tyler . . . . . . . . . . 592

Genus IV. Dialister Bergey et al..................... 594

Appendix: Tribe Vimeae DeBord .................... 595

Family XII. Bacteriaceae Cohn....................... 596

Genus I. Bacterium Ehrenberg . . . . . . . . . . . . . . . . . . 596

Subgenera:

Kurthia Trevisan. . . . . . . . . . . . . . . . . . . . . . . . . . 600

Cellulomonas Bergey et al......................... 613

Saccharobacterium Sickles and Shaw . ............... 623

Agarbacterium Angst............................ 624

Photobacterium Beijerinck...................... 633

Methanobacterium Kluyver and Van Niel............... 645

Appendix: Suborder Eubacteriineae: Overlooked species and syn-

onvms........................................ 692

Family XIII. Bacillaceae Fisher ...................... 704

Genus I. Bacillus Cohn........................... 705

Genus II. Clostridium Prazmowski.................... 763

Suborder II. Caulobacteriineae Breed, Murray and Hitchens........... 828

Family I. Nevskiaceae Henrici and Johnson.................. 830

Genus I. Nevskia Famintzin...................... 830

Family II. Gallionellaceae Henrici and Johnson. ............... 830

Genus I. Gallionella Ehrenberg........................ 831

Family III. Caulobacteriaceae Henrici and Johnson............... 832

Genus I. Caulobacter Henrici and Johnson................. 832

Family IV. Siderocapsaceae Pribram....................... 833

Genus I. Siderocapsa Molisch........................ 833

Genus II. Sideromonas Cholodny ..................... 834

Appendix: Family Pasteuriaceae Laurent. . . . . . . . . . . . . . . . 836

Genus I. Pasteuria Metchnikoff...................... 836

Genus II. Blastocaulis Henrici and Johnson................ 836

- Suborder III. Rhodobacteriineae Breed, Murray and Hitchens......... 838

Family I. Thiorhodaceae Molisch........................ 841

Genus I. Thiosarcina Winogradsky................. 842

Genus II. Thiopedia Winogradsky .................. 843

Genus III. Thiocapsa Winogradsky. ................... 844

Genus IV. Thiodictyon Winogradsky.................. 845 
Genus V. Thiothece Winogradsky................... 846

Genus VI. Thiocystis Winogradsky.................... 846

Genus VII. Lamprocystis Schroeter..................... 847

Genus VIII. Amoebobacter Winogradsky. .................. 848

Genus IX. Thiopolycoccus Winogradsky................ 850

Genus X. Thiospirillum Winogradsky................ 850

Genus XI. Rhabdomonas Cohn..................... 853

Genus XII. Rhodothece Molisch....................... 855

Genus XIII. Chromatium Perty........................ 856

Family II. Athiorhodaceae Molisch....................... 861

Genus I. Rhodopseudomonas Kluyver and Van Niel emend. Van Niel............................... 861

Genus II. Rhodospirillum Molisch.................. 866

Family III. Chlorobacteriaceae Geitler and Pascher............... 869

Genus I. Chlorobium Nadson......................... 869

Genus II. Pelodictyon Lauterborn......................... 870

Genus III. Clathrochloris Geitler...................... 872

Genus IV. Chlorobacterium Lauterborn..................... 872

Genus V. Chlorochromatium Lauterborn.................. 873

Genus VI. Cylindrogloea Perfiliew..................... 873

Order II. Actinomycetales Buchanan........................ 875

Family I. Mycobacteriaceae Chester....................... 875

Genus I. Mycobacterium Lehmann and Neumann.............. 876

Family II. Actinomycetaceae Buchanan..................... 892

Genus I. Nocardia Trevisan. . . . . . . . . . . . . . . . . . . . . . 892

Genus II. Actinomyces Harz....................... 925

Family III. Streptomycetaceae Waksman and Henrici............ 929

Genus I. Streptomyces Waksman and Henrici............... 929

Genus II. Micromonospora Ørskov..................... 978

Order III. Chlamydobacteriales Buchanan...................... 981

Family I. Chlamydobacteriaceae Migula..................... 981

Genus I. Sphaerotilus Kützing........................ 982

Genus II. Clonothrix Roze........................... 983

Genus III. Leptothrix Kützing. . . . . . . . . . . . . . . . . . . . 983

Family II. Crenothricaceae Hansgirg........................ 987

Genus I. Crenothrix Cohn............................... 987

Family III. Beggiatoaceae Migula....................... 988

Genus I. Thiothrix Winogradsky ...................... 988

Genus II. Beggiatoa Trevisan......................... 990

Genus III. Thiospirillopsis Uphof .................... 993

Genus IV. Thioploca Lauterborn..................... 993

Appendix: Family Achromatiaceae Massart...................... 997

Genus I. Achromatium Schewiakoff................... 997

Genus II. Thiovulum Hinze. . . . . . . . . . . . . . . . . . . . . . . 999

Genus III. Macromonas Utermöhl and Koppe................ 1000

Appendix: Order Caryophanales Peshkoff ....................... 1002

Family I. Pontothricaceae Peshkoff . . . . . . . . . . . . . . . . . . . . 1002

Genus I. Pontothrix Nadson and Krassilnikow. .............. 1002

Family II. Arthromitaceae Peshkoff... . . . . . . . . . . . . . . . . . . . . 1002

Genus I. Arthromitus Leidy . . . . . . . . . . . . . . . . . . . . . . . 1002

Genus II. Coleomitus Duboseq and Grassé................ 1003 
Family III. Oscillospiraceae Peshkoff ....................... 1003

Genus I. Oscillospira Chatton and Perard ................... 1004

Family IV. Caryophanaceae Peshkoff... . . . . . . . . . . . . . . . . . . . 1004

Genus I. Caryophanon Peshkoff........................... 1004

Order IV. Myxobacteriales Jahn............................ 1005

Family I. Cytophagaceae Stanier............................ 1012

Genus I. Cytophaga Stanier.......................... 1012

Family II. Archangiaceae Jahn. ....................... 1017

Genus I. Archangium Jahn. . . . . . . . . . . . . . . . . . . . . 1017

Genus II. Stelangium Jahn......................... 1020

Family III. Sorangiaceae Jahn. . . . . . . . . . . . . . . . . . . 1021

Genus I. Sorangium Jahn. ............................ 1021

Family IV. Polyangiaceae Jahn........................... 1025

Genus I. Polyangium Jahn......................... 1025

Genus II. Synangium Jahn............................ 1032

Genus III. Melittangium Jahn. ......................... 1033

Genus IV. Podangium Jahn.......................... 1034

Genus V. Chondromyces Berkeley and Curtis. ............... 1036

Family V. Hycococcaceae Jahn........................... 1040

Genus I. Myxococcus Thaxter........................ 1010

Genus II. Chrondrococcus Jahn. . . . . . . . . . . . . . . . . . 1044

Genus III. Angiococcus Jahn. . . . . . . . . . . . . . . . . . . . 1047

Genus IV. Sporocytophaga Stanier....................... 1048

Order V. Spirochaetales Buchanan........................... 1051

Family I. Spirochaetaceue Swellengrebel.................... . 1051

Genus I. Spirochaeta Ehrenberg...................... 1051

Genus II. Saprospira Gross......................... 105t

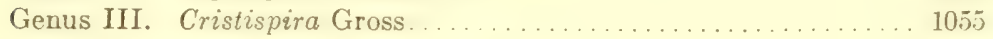

Family II. Treponemataceae Schaudinn...................... 1058

Genus I. Borrelia Swellengrebel....................... 1058

Genus II. Treponema Swellengrebel...................... 1071

Genus III. Leptospira Noguchi......................... 1076

Supplement I . . . . . . . . . . . . . . . . . . . . . . . . . . . . . . . 1081

Order Rickettsiales Gieszcykiewicz. . . . . . . . . . . . . . . . . . . . . . . . 1083

Family I. Rickettsiaceae Pinkerton........................ 1083

Genus I. Rickettsia da Rocha-Lima..................... 1084

Genus II. Coxiella Bengtson. . . . . . . . . . . . . . . . . . . . . 1092

Genus III. Cowdria Bengtson. . . . . . . . . . . . . . . . . . . . . . . . . 1094

Family II. Bartonellaceae Gieszeykiewicz..................... 1100

Genus I. Bartonella Strong, Tyzzer and Sellards............. 1100

Genus II. Haemobartonella Tyzzer and Weinman. ............. 1102

Genus III. Grahamella Brumpt.......................... 1109

Genus IV. Eperythrozoön Schilling.................... 1111

Family III. Chlamydozoaceae Moshkovsky .................... 1114

Genus I. Chlamydozoðn Halberstaedter and von Prowazek....... 1114

Genus II. Miyagawanella Brumpt..................... 1115

Genus III. Colesiota Rake............................ 1119

Appendix: Genus A. Caryococcus Dangeard.................. 1121

Genus B. Drepanospira Petschenko.................. 1122

Genus C. Holospora Haffkine..................... 1122 
Supplement II. . . . . . . . . . . . . . . . . . . . . . . . . . . . . 1125

Order Virales Breed, Murray and Hitchens...................... 1128

Suborder I. Phagineae Holmes........................... 1128

Family I. Phagaceae Holmes.......................... 1128

Genus I. Phagus Holmes............................ 1128

Suborder II. Phytophagineae Holmes.......................... 1145

Family I. Chlorogenaceae Holmes. . . . . . . . . . . . . . . . . . . . 1145

Genus I. Chlorogenus Holmes.......................... 1146

Genus II. Carpophthora McKinney.................... 1151

Genus III. Morsus Holmes. . . . . . . . . . . . . . . . . . . . 1153

Genus IV. Aureogenus Black........................ 1154

Genus V. Galla Holmes........................... 1157

Genus VI. Fractilinae McKinney.................... 1159

Family II. Marmoraceae Holmes............................ 1163

Genus I. Marmor Holmes.......................... 1163

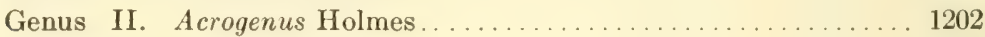

Genus III. Corium Holmes. . . . . . . . . . . . . . . . . . . . . . . . 1203

Genus IV. Nanus Holmes......................... 1206

Genus V. Rimocortius Milbrath and Zeller............... 1208

Genus VI. Adelonosus Brierley and Smith................ 1211

Family III. Annulaceae Holmes. . . . . . . . . . . . . . . . . . . . . . . . 1212

Genus I. Annulus Holmes. . . . . . . . . . . . . . . . . . . . . . 1212

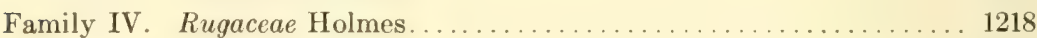

Genus I. Ruga Holmes. . . . . . . . . . . . . . . . . . . . . . . . 1218

Family V. Savoiaceae Holmes. . . . . . . . . . . . . . . . . . . . . . . 1221

Genus I. Savoia Holmes. . . . . . . . . . . . . . . . . . . . . . . . 1221

Family VI. Lethaceae Holmes. . . . . . . . . . . . . . . . . . . . . . 1223

Genus I. Lethum Holmes. . . . . . . . . . . . . . . . . . . . . . . . . 1223

Suborder III. Zoophagineae Holmes. . . . . . . . . . . . . . . . . . . 1225

Family I. Borrelinaceae Holmes.......................... 1225

Genus I. Borrelina Holmes.......................... 1225

Genus II. Morator Holmes. . . . . . . . . . . . . . . . . . . . . 1227

Family II. Borreliotaceae Holmes......................... 1229

Genus I. Borreliota Goodpasture...................... 1229

Genus II. Briareus Holmes.......................... 1233

Genus III. Scelus Holmes. . . . . . . . . . . . . . . . . . . . . . . . . 1234

Genus IV. Hostis Holmes. . . . . . . . . . . . . . . . . . . . . 1239

Genus V. Moliter Holmes............................ 1241

Family III. Erronaceae Holmes.......................... 1248

Genus 1. Erro Holmes............................. 1248

Genus II. Legio Holmes. . . . . . . . . . . . . . . . . . . . . . 1257

Genus III. Formido Holmes. . . . . . . . . . . . . . . . . . . . . 1263

Family IV. Charonaceae Holmes. .......................... 1265

Genus I. Charon Holmes........................... 1265

Genus II. Tarpeia Holmes.......................... 1268

Genus III. Tortor Holmes. . . . . . . . . . . . . . . . . . . 1275

Family V. Trifuriaceae Holmes. . . . . . . . . . . . . . . . . . . . . . 1282

Genus I. Trifur Holmes. . . . . . . . . . . . . . . . . . . . . . . . . 1282

Family VI. Rabulaceae Holmes. . . . . . . . . . . . . . . . . . . . . 1284

Genus I. Rabula Holmes. . . . . . . . . . . . . . . . . . . . . . . . 1284

Supplement III . . . . . . . . . . . . . . . . . . . . . . . . . . . . 1287

Family Borrelomycetaceae Turner......................... 1291

Genus I. Ascococcus Borrel et al...................... 1291 


\section{INTRODUCTION}

\section{Suggestions for the USE of the Manual in Classifying UnKNown ORganisMS}

No organism can be classified before we have determined, through detailed study, its morphological, cultural, physiological and pathogenic characters.

The characters used in the keys to orders, families and genera may ordinarily be determined by the use of a dozen or more of the procedures described in the Manual of Pure Culture Study issued by the Committee on Bacteriological Technic (H. J. Conn, Chairman, Geneva, New York) of the Society of American Bacteriologists. Nore complete examinations must be made as indicated in the Manual of Pure Culture Study, and in the Descriptive Charts which accompany this Manual where it is desired to identify individual species. These tests must be made if bacteria are to be accurately identified and described.

It is urged that beginning students be taught the technics necessary for the identification of species in the hope that the taxonomic work of the future may be placed on a more satisfactory basis.

After a complete study of the characters of the organism has been made, turn to page 65 and ascertain first in which order the organism belongs. When the order and suborder (if necessary) have been ascertained, turn to the page of the MANEAL on which the key to that order or suborder is given. In this key ascertain the family or subfamily to which the organism belongs.

When the family or subfamily has been decided on, again refer to the page of the MANCAL on which the key to that family or subfamily is given. In this key ascertain the tribe to which the organism belongs.

When the tribe has been decided on, again find the page of the ManUaL on which the key to the tribe is given. In this key ascertain the genus to which the organism belongs.

When the genus has been decided on, again refer to the page of the Maxual on which the key to that genus is given. In this key, trace out the species under investigation.

For example, if one wishes to trace a short, peritrichous, Gram-negative, non-spore-forming rod that grows well on ordinary culture media at $37^{\circ} \mathrm{C}$, fermenting glucose and lactose with production of acid and gas, not liquefying gelatin, producing no pigment on any culture medium, with negative reaction for acetylmethylcarbinol, producing indole and reducing nitrates, consult the key to the orders on page 65 . 
In this key examine A. Cells rigid, not flexuous. This indicates our organism as its cells remain constant in form.

We next examine 1. Cells single, in chains or masses. Not branching and mycelial in character. Not arranged in filaments. Not acid fast. As the organism in question occurs as single cells or at most as short chains and is not acid fast, this indicates that it belongs to the Order Eubacteriales.

We now examine a. Do not possess photosynthetic pigments. Cells do not contain free salfur. As our organism is umpigmented and the cells do not contain free sulfur, this indicates that our organism belongs to the Sub-order Eubacteriineae. We note that the key to this suborder is on page 67.

We next attempt to ascertain the family to which the organism belongs by tracing it through the key to the families of the Sub-order Eubacteriineae, p. 67.

I. No endospores indicates our organism. We proceed to A. Can develop on inorganic media. As the organism cannot grow without organic carbon, we turn to B. Cannot develop on inorganic media.

This corresponds with the physiology of our organism; so we turn to 1. Polar flagellate, ete. As our organism is peritrichous, we proceed to 2. Large oval, pleomorphic cells sometimes almost yeast-like in appearance. Free living in soil. Fix free nitrogen. As this does not correspond with the morphology or physiology of our organism, we next examine 3. Peritrichous or non-motile rods, and cocci. This corresponds with the characteristics of our organism.

We turn to a. Heterotrophic rods which may not require organic nitrogen for growth. Usually motile with one to six or more flagella. Usually form nodules or tubercles on roots of plants, or show violet chromogenesis.

This again does not indicate our organism; so we turn next to aa. Heterotrophic rods or cocci which utilize organic nitrogen and usually carbohydrates. As our rod-shaped organism prefers a medium containing organic nitrogen, we proceed to b. Spherical cells in masses, tetrads and packets.

This does not correspond to the morphology of our organism, and we now proceed to bb. Spherical cells which grow in pairs and chains; and rods. This includes our rod-shaped organism; so we turn to c. Gram-positive cocci and rods. Non-motile. Since these are not the characteristics of our organism, we turn to ec. Gram-negative rods. When motile, from four to many peritrichous flagella.

Our organism is Gram-negative and peritrichous; so we proceed to d. Grow well on ordinary media containing peptone. Aerobic to facultative anaerobic.

This corresponds with the characteristics of the organism we have studied; so we turn next to e. Gram-negative, straight rods which ferment 
sugars with the formation of organic acids. This again corresponds with our organism. We turn next to f. Produce little or no acid from litmus milk. This does not correspond with the characters we have determined for our organism. We proceed to ff. Produce $\mathrm{CO}_{2}$ and frequently visizle gas $\left(\mathrm{CO}_{2}+\right.$ $\mathrm{H}_{2}$ ) from glucose. Reduce nitrates, etc.

Our organism produces visible gas from glucose and reduces nitrates. This indicates that it belongs to Family $X$. Enterobacteriaceae, p. 443.

This appears to fit our unknown organism. We now refer to page 443 on which the key to the Family Enterobacteriaceae is found. In this key we ascertain the Tribe to which our organism belongs. 1. Ferment glucose and lactose with the formation of acid and visible gas. Usually do not liquefy gelatin. Tribe $I$. Eschericheae.

This corresponds with the characters exhibited by our organism. We refer to the key for Tribe I. Eschericheae on the same page. 1. Methyl red test positive. Voges-Prostiauer test negative. Salts of citric acid may or may not be used as sole source of carbon. Genus I. Escherichia, p. 444.

This description appears to correspond with that of our unknown organism. We find the key to the species of Genus Escherichia follows the key to the Tribe Eschericheae. On tracing our organism in this key we find that it corresponds to Escherichia coli. A brief description of this organism is found on the same page.

* In the use of keys for identifying bacteria, the student is confronted with two difficulties, both based primarily on lack of knowledge and experience. The first is insufficient knowledge concerning the morphology, physiology, possible pathogenicity and habitat of the microorganisms that are to be identified. This may be due to careless observations or to poor training in the special techniques that must be used in determining the identity of a given bacterium.

The second difficulty in the use of a key comes from inexperience in the use of technical terms; that is, the student may not thoroughly understand the meaning of the statement in the key and so cannot follow a route through the key with certainty. For example in the keys used here, the student must know the difference (1) between chains of cells which are composed of dividing cells which do not separate at once, and (2) filaments which are composed of dividing cells which remain more permanently together and are normally flattened against each other on adjacent sides. They may show some differentiation into hold fast cells and reproductive cells (conidia), (3) Both chains of cells and filaments are to be distinguished from the mycelial threads found in Actinomycetaceae. These are unseptate and branching with a true branching.

* Condensed and paraphrased from Hitchcock's Descriptive Systematic Botany, New York, 1935. 
The student should be warned not to take descriptions in the MANUAL too literally or too rigidly. Descriptions are usually drawn to represent average findings. Especially among bacteria, characters such as sugar fermentations, gelatin liquefaction, presence or absence of flagella and other things will vary. Sometimes these variations are due to slight, possibly unrecognized variations in the techniques used in determining these characters. Real knowledge of the characteristics of species may also be very incomplete. This is true not only of the physiological activities of these microorganisms; but also in regard to such detectable structural features as the number and position of flagella. Dark field movies of motile cells and photographs taken with the recently developed electron microscope are revealing new and heretofore unsuspected facts regarding structural features.

Source and habitat data are frequently helpful in aiding the student to recognize species of bacteria and may indicate that the pathogenicity of the culture in question may need to be tried on some specific animal or plant. By habitat is meant the kind of a place in which the organism normally grows; by source, the particular material and place from which the culture was obtained. This source may or may not indicate the natural habitat. The source of cultures is invariably more limited in scope than the habitat as bacteria normally occur wherever their particular habitat may be found in a world wide distribution.

The student is also reminded that it is impracticable to note all exceptions in keys. Bacteria like other living things are classified according to a combination of characters, not according to some single character, and exceptions to the characters noted in the keys will occur in nature. These may not be known to or may have been overlooked by the author of the key. On the other hand, the importance of such exceptions should not be overemphasized and the student would do well to use the key as if there were no exceptions. 


\section{HISTORICAL SURVEY OF CLASSIFICATIONS OF BACTERIA, WITH EMIPHASIS ON OUTLINES PROPOSED SINCE $1923^{*}$}

There have been numerous attempts to arrange the species of bacteria in natural systems of classification. The first simple system of Müller (Vermium terrestrium et fluviatilium, 1773) which he developed further a few years later (Animalcula infusoria fluviatilia et marina, 1786) listed but two genera (Vibrio and Monas) that included organisms that would today probably be accepted as bacteria. Polyangium Link (Mag. d. Ges. Naturforsch. Freunde zu Berlin, 3, 1809, 12) is apparently the oldest of the generic terms retained in its original meaning for a bacterial genus while Serratia Bizio (Biblioteca italiana o sia giornale de lettera, scienze ed arti, 30, 1823, 288) was proposed only fourteen years later.

Systems of classification developed after 1773 are given in complete outline form in the first edition of the MANUAL (1923) and this section of the MANUAL was reprinted without material change in the second (1925) and third (1930) editions. While it is not felt to be necessary to repeat these outlines in their entirety, sufficient reference is made below to permit the student to trace the origin of generic terms that are no longer commonly found in classification outlines. No attempt has been made to include reference to other little used generic terms except as they appear as synonyms in the descriptive portion of the MANLAL. For the origin of generic terms proposed before 1925, see Enlows (The Generic Names of Bacteria, Bul. No. 121, Hygienic Laboratory, Washington, D. C., 1920) and Buchanan (General Systematic Bacteriology, Baltimore, 1925).

Bory St. Vincent (Microscopiques, Dictionnaire classique d'histoire naturelle, 10, 1826, 533) introduced the generic terms Spiritina, Melanella, Lactrimatoria and Pupella and accepted Tibrio for microorganisms, some of which must have been bacteria. None of these terms, except Tibrio, are in current use for bacterial groups.

Three of the terms accepted or proposed by Ehrenberg (Die Infusionstierchen als volkommene Organismen, Leipzig, 1838); namely, Vibrio, Spirillum and Spirochacta, are still used. The generic term Bacterium proposed first by Ehrenberg in 1828 (Symbolae Physicae seu Icones et Descriptiones Animalium Evertebratorum Separasitis Insectis quae ex Itinere per Africam Borealem et Asiam Occidentalem, IV. Evertebrata, Berlin) to include but a single species Bacterium triloculare from an oasis

* Contributed by Prof. R. S. Breed, New York State Experiment Station, Geneva, New York, July, 1938; revised, September, 1943. 
in North Africa, has had a varied history because this type species (monotypy) is no longer identifiable. It was reintroduced into the classification employed in the fifth edition of the MaNUaL to cover species of non-sporeforming rods whose positions in the outline given in the ManUaL have not yet been satisfactorily determined (Breed and Conn, Jour. Bact., 31, 1936, 517 ) and is used in the present edition with the same meaning. The term Spirodiscus was applied by Ehrenberg to a single organism that he found in a mountain stream. It has never been reidentified and subsequent authors have discarded this term.

Two new generic terms (Mctallacter, Sporonema) were introduced by Perty (Zur Kenntniss kleinster Lebensformen, 1852). Neither Metallacter nor Sporonema is in common use at the present time.

Davaine (Dictionaire encyclop. des sciences méd., Art. bactéries, 1868) introduced one new generic term, Bacteridium, for straight motionless rods like the anthrax bacillus.

The generic terms employed by Cohn in his first classification (Untersuchungen über Bakterien. I. Beiträge z. Biol. d. Pflanzen, 1, Heft 2, $1872,146)$ are all in current use. Only one (Bacillus) was new. Other generic terms were introduced into his second paper (Untersuchungen über Bakterien. II. ibid., 1, Heft 3, 1875, 141) which contained his more complete classification. For various reasons, six of these, Merismopedia, Clathrocystis, Ascococcus, Myconostoc, Cladothrix and Streptothrix are not found in recent bacteriological classifications.

Mangin (Les Bactéries, Paris, 1878) recognized three subgenera of the genus Monas, the first of which Rhabdomonas Cohn, 1875 is still used as a generic term, while the other two, Ophidomonas Ehrenberg, 1838 and Spiromonas Perty, 1852 have been dropped.

The bacterial species that had been placed in the genus Clathrocystis by Cohn (1875) were separated and placed in a new genus Cohnia by Winter (Die Pilze in Rabenhorst's Kryptogamen Flora, 1880), and this name is also used by Burrill (The Bacteria, Springfield, Ill., 1882). Because this name had previously been proposed for a genus of lilies, it was soon dropped.

Zopf (Die Spaltpilze, Leipzig, 1883) accepts Phragmidiothrix, a generic name suggested by Engler in 1882 for a single species found on the body of a crustacean (Gammarus locusta). Later authors generally either merge this genus with Crenothrix Cohn or disregard it because of the indefinite description of the one species included in it.

Baumgarten (Lehrbuch der pathologischen Mykologie, Braunschweig, 1890) following Hueppe accepts the term, Spirulina, for a genus of pleomorphic bacteria, disregarding the previous use of the term by algologists.

The generic terms found in Migula's first outline (Bakterienkunde für 
Landwirte, Berlin, 1890) were those in conventional use at the time and many of them continue in use. Two new terms were introduced for motile types in his second outline (Arb. Bact. Inst. Karlsruhe, 1, 1894, 235) and are also found in his later outlines (Engler and Prantl, Die natürlichen Pflanzenfamilien, 1, 1a, 1895, 29, and System der Bakterien, 1, 1897, 46, and 2, 1900, 269 and 275) which have not been generally felt to be necessary by subsequent authors. These are Planococcus and Planosarcina. Spirosoma introduced by Migula in 1894 and Rhabochromatium Winogradsky accepted by Migula in 1900 are likewise no longer generally used. Newskia (original spelling Nevskia Famintzen, Bull. Acad. Imp. Sci., St. Petersburg, 34 (N.S. 2), 1892, 484) has recently been revived by Henrici and Johnson (Jour. Bact., 29, 1935, 3 and 30, 1935, 83). The generic term Microspira Schroeter, accepted by Migula in 1894, is still frequently accepted in place of Vibrio as many regard it as having a better status than the later term.

The term $P$ seudomonas was first proposed for polar flagellate bacteria by Migula in his 1894 outline with reference to but a single species, Pseudomonas violacea, an organism which later investigators have shown to be peritrichous (Cruess-Callaghan and Gorman, Sci. Proc. Roy. Dublin Soc., 21, 1935, 213). Pseudomonas was repeated in the 1895 outline with descriptions of Pseudomonas pyocyanea and other species. Later authors have generally accepted the term Pseudomonas as valid.

Fischer (Jahrb. f. wissensch. Bot., Berlin, 27, 1895, 1) introduced a logical outline classification in which he proposed various new terms which have never come into general use. These are Paracloster, Paraplectrum, Arthrobacter, Bactrinium, Clostrinium, Plectrinium, Arthrobactrinium, Bactrillum, Clostrillium, Plectrillium, Arthrobactrillium, Bactridium, Plectridium, Diplectridium, and Arthrobactridium. In his modified classification (Vorlesungen über Bakterien, 1897), he also accepts Pediococcus Balcke, a term that has fallen into disuse except in the brewing industry,

In the conservative classification proposed by Lehmann and Neumann (Atlas und Grundriss der Bakteriologie, 2 vols., 1896, München), internationally accepted rules of nomenclature were followed. All of the generic terms employed by them are still in current use, their most important contribution being their acceptance of the suggestion that the genus Bacillus be separated from the genus Bacterium on the basis of endospore formation by the rods included in Bacillus. Two new genera were proposed (Corynebacterium and Mycobacterium) that have been generally accepted by later workers.

No new generic terms are proposed by Chester either in his preliminary reports (Delaware College of Agriculture, 9th Ann. Rept., 1897, 53 and 62; 
11th Ann. Rept., 1899, 36), or in his complete outline (Manual Determ. Bact., 1901). Almost all of the generic terms found in his outlines are still in current use.

The term A planobacter suggested by Erwin F. Smith (Bacteria in Relation to Plant Diseases, $1,1905,171$, Washington) was accepted by certain American phytopathologists for a time but has never come into general use.

Because other differences between the non-chromogenic and chromogenic micrococci are unimportant, two generic terms, Albococcus and A urococcus, suggested by the Winslows (Science, 21, 1905, 669; Systematic Relationships of the Coccaceae, New York, 1908) have not come into general use. They also suggested Rhodococcus to include Rhodococcus roseus and $R$. fulvus apparently without realizing that Zopf (Ber. d. deutsch. bot. Gesellsch. Berlin, 9, 1891, 28) had previously used the same term for Rhodococcus erythromyxa and R. rhodochrous. Hansgirg (Engler and Prantl, Die natürlichen Pflanzenfamilien, 1, 1a, 1895, 52) had also used it previously to designate a sub-genus of the green algae, and later Molisch (Die Purpurbakterien, Jena, 1907, 20) used Rhodococcus for a genus of the purple bacteria to include Rhodococcus capsulatus.

In his complete outline of the classification of bacteria presented in 1909, Orla-Jensen (Cent. f. Bakt., II Abt., 22, 1909, 305) introduced many new generic terms in an effort to create a nomenclature that appeared to him to express the natural relationships of bacteria more satisfactorily than names previously suggested had done. Thus he used the suffixes coccus and sarcina for spherical bacteria and monas for all genera known to be lophotrichous or so related to these types that they were regarded as essentially lophotrichous in nature. In the same way the suffix bacterium was used for genera of non-spore-forming rods that were regarded as essentially peritrichous in nature, and the suffix bacillus for similar spore-forming rods. As, however, subsequent investigators have (1) accepted the priority rule, (2) felt that it was impossible to recognize the type of motility found in the ancestry of truly non-motile groups, or (3) felt that other characters were more fundamental than those selected by Orla-Jensen, many of these terms have not been generally used by later workers.

Among the little used terms suggested or accepted by Orla-Jensen are: Acetimonas, Nitromonas, Azotomonas, Rhizomonas, Corynemonas, Mycomonas, Sulfomonas, Thiomonas, Thiococcus, Rhodomonas, Rhododictyon, Amoebomonas, Rhodopolycoccus, Rhodosarcina, Spirophyllum, Denitromonas, Liquidomonas, Liquidovibrio, Liquidococcus, Solidococcus, Solidovibrio, Sporosarcina, Denitrobacterium, Caseobacterium, Liquidobacterium, Urobacillus, Butyribacillus, Pectobacillus, Cellulobacillus, Putribacillus and Botulobacillus. 
While Nitromonas is not new, it is redefined as a synonym of Nitrobacter Winogradsky (Arch. Sci. Biol. St. Petersburg, 1, 1892, 87), rather than as a synonym of Nitromonas Winogradsky (Ann. Inst. Past., 3, 1890, 258). Spirophyllum is from Ellis (Cent. f. Bakt., II Abt., 19, 1907, 507).

In a later monograph on The Lactic Acid Bacteria ( Iém. d. Acad. Roy. Sci. et Lettres de Danemark, Sect. Sci., 8 Sér., 5, 1919, No. 2) Orla-Jensen proposes the following additional generic terms: Betacoccus, Betabacterium, Streptobacterium, Thermobacterium and Microbacterium. The term Tetracoccus is introduced with a meaning different from that given the term previously by v. Klecki (Cent. f. Bakt., 15, 1894, 354).

Buchanan prepared an outline classification in 1916 (Jour. Bact., 1, $1916,591$; $2,1917,155,347,603 ; 3,1918,27,175,301,403,461,591)$ which was utilized in part by the group of which he was a member (Winslow, Broadhurst, Buchanan, Krumwiede and Smith) in their preliminary Report to the Society of American Bacteriologists (Jour. Bact., 2, 1917, 552) and in the final report by Winslow, Broadhurst, Buchanan, Krumwiede, Rogers and Smith (Jour. Bact., 5, 1920, 191).

Although prepared earlier, some parts of the Buchanan outline were not published until after the first Winslow et al. report. As these reports formed the most important basis for the classification used in the first edition of the MANUAL, it is natural that the generic terms utilized are, in general, the same as those used in the MANUAL.

Generic and subgeneric terms included by Buchanan that are not used in the present edition of the MANual are: Paraspirillum Dobell (Arch. f. Protistenk., 24, 1911, 97), Eubacillus Hansgirg (Osterr. Bot. Ztschr., 38, 1888, 264; not Eubacillus Dangeard, Le Botaniste, 2, 1891, 151) and Metabacterium Chatton and Perard (Comp. rend. Soc. Biol., Paris, 65, 1913, 1232). Siderocapsa Molisch (Ann. Jard. Bot. Buitenzorg, Ser. 2, Supp. 3, 1909, 29) used by Buchanan but dropped by Winslow et al (Jour. Bact., 2, 1917, 549) does not appear in the MANTAL classification outline until the present (6th) edition. The term Mycoderma recognized both by Buchanan (Jour. Bact., 3, 1918, 45) and in the preliminary Winslow et al. report (Jour. Bact., 2, 1917, 551) was replaced by the later and more valid term Acetobacter in the final report by Winslow et al. (Jour. Bact., 5, 1920, 201). Pfeifferella Buchanan (Jour. Bact., 3, 1918, 54) which is used in the three outline classifications under discussion and also in the first, second and third editions of the MANUAL, appeared in the literature through a clerical error (Buchanan, General Systematic Bacteriology, 1925, 420). It was combined in the fourth edition of the MAXUAL with the genus Actinobacillus under the latter name. Nocardia Trevisan (1889) used by Buchanan and in the preliminary report by Winslow et al. (1917) was merged with Actinomyces 
Harz (Jahresber. München. Thierarzneisch. for 1877-78, 125) in the final report by Winslow et al. Erythrobacillus Fortineau (Compt. rend. Soc. Biol. Paris, 58, 1905, 104) is used by Winslow et al. (1920) but was not accepted in the first and following editions of the MANUAL as it is a synonym of the older Serratia Bizio (1823). Moreover, the species which must be accepted as type for the genus (Erythrobacillus pyosepticus Fortineau (monotypy)) is a species which has been reported by Breed (Manual, 3rd ed., 1930, 117) to be a variant of the older Serratia marcescens.

One of the most unsatisfactory portions of recent classifications, such as those outlined by Buchanan (1917-18) and by Winslow et al. (1917), is the treatment given the organisms of the coliform-dysentery-typhoid group in that the term Bacterium is retained for these as suggested by OrlaJensen (1909). A strict limitation of Bacterium to this groupgives it a still different meaning from that which it had had in previous and current classifications, and makes it necessary to find some other place for many other species of Gram-negative, non-spore-forming rods, some of which are well known and well described. The relationships of these miscellaneous species to other non-spore-forming rods is frequently poorly understood. In some cases, further study will probably show that they should be placed in well known and currently recognized genera. In others, further study will probably show that some of these species of non-spore-forming rods should be grouped in new genera.

Winslow et al. (1920) recognized this situation and broadened their definition of Bacterium thereby placing such well known species as are included in the colon-dysentery-typhoid group with other species of non-spore-forming rods of quite a different character. For this reason, partial use was made in the first edition of the MANUAL of the numerous generic terms newly proposed by Castellani and Chalmers (Manual of Tropical Medicine, 3rd ed., 1919). Thus the following new terms were introduced: Alcaligenes, Salmonella, Escherichia and Encapsulatus; and the earlier terms Aerobacter Beijerinck (1900) and Eberthella Buchanan (1918). Later it was found that Encapsulatus was a synonym of Klcbsiella Trevisan (1887), so that the latter term was accepted in the second and subsequent editions of the MANUAL. Shigella Castellani and Chalmers was recognized as distinct from Eberthella in the third and subsequent editions.

Many of the new terms suggested by Castellani and Chalmers were, however, synonyms of earlier valid terms or have not been considered necessary, and so they have not come into general use. These are Nigrococcus, Graciloides, Cloaca, Eberthus, Dysenteroides, Lankoides, Wesenbergus, Balkanella and Enteroides. No new generic terms are given by Castellani and Chalmers in their later report (Ann. Inst. Past., 34, 1920, 600). 
Orla-Jensen (Jour. Bact., 6, 1921, 263), in a paper published after the manuscript of the first edition of the Manual was prepared, suggested the use of Colibacterium and Aerogenesbacterium for the two genera in the coliform group and adds quite a number of other new terms formed in accordance with his system of nomenclature. These are, in most cases, synonyms of earlier valid names. The new terms are Coccomonas, Spiromonas (used in a new, different sense from that of earlier authors), Fluoromonas, Photomonas, Propionicoccus, Butyriclostridium and Putriclostridium.

Many new terms are proposed in the classification drawn up by Heller (Jour. Bact., 6, 1921, 521; and 7, 1922, 1). Details are given in the group of anaerobic spore-formers only. Here each of the new generic terms is based on a single species. The following outline is given in the first of these papers, two new genera (Rivoltillus and Metchnikovillus) being made the type genera for two new subfamilies Clostridioideae and Putrificoideae, respectively.

Phylum I. Bacteria

Class I. Eubacterieae

Order 1. Eubacteriales

Family 6 (?). Clostridiaceae

Subfamily 1. Clostridioideae

Subfamily 2. Putrificoideae

Order 2. Thiobacteriales

Order 3. Chlamydobacteriales

Class II. Myxobacterieae

In the more complete outline in the second paper, one generic term (Clostridium) is old, although it is used in a new and restricted sense, while with the exception of the type genera mentioned above, the other terms are new. In the subfamily Clostridioideae, the new terms are Omelianskillus, Macintoshillus, Douglasillus, Henrillus, Flemingillus, Vallorillus, Multifermentans, Hiblerillus, Welchillus, Stoddardillus, Arloingillus, Meyerillus and Novillus. Ten new generic terms are used in the subfamily Putrificoideae as follows: Seqninillus, Reglillus, Robertsonillus, Nicollaierillus, Martellillus, Recordillus, Tissierillus, Putrificus, Ermengemillus, and Weinbergillus. As there does not seem to be any good reason for sub-dividing the genus Clostridium in this way, the latter term has been used to cover anaerobic spore-forming rods in all previous editions of the MANUAL, and is again used in the present edition in this sense rather than with the restricted meaning proposed by Heller.

Enderlein (Sitzber. Gesell. Naturf. Freunde, Berlin, 1917, 309) proposed an outline classification covering the Kingdom of Mychota, or bacteria, which was based on comparative morphology with special emphasis on life cycles. This was as follows: 
Phylum I. Dimychota

Kreis A. Holocyclomorpha

Class I. Gonascola

Order a. Synascota

Family 1. Schaudinnidae

Genus a. Schaudinnum

b. Theciobactrum

Family 2. Sphaerotilidae

Genus a. Phragmidiothrix

b. Newskia

c. Chlamydothrix

d. Sphaerotilus

e. Clonothrix

Family 3. Syncrotidae

Genus a. Crenothrix

b. Beggiatoa

c. Syncrotis

d. Zygostasis

Family 4. Spirillidae

Genus a. Gallionella

b. Spirillum

c. Dicrospirillum

Family 5. Spirochaetidae

Genus a. Cristispira

b. Treponema

c. Entomospira

d. Spirochaeta

e. Cacospira

Family 6. Microspiridae

Genus a. Spirobacillus

b. Spirosoma

c. Photobacterium

d. Microspira

e. Dicrospira

Family 7. Corynobacteriidae

Subfamily 1. Actinomycinae

Genus a. Actinomyces

Subfamily 2. Eisenberginae

Genus a. Eisenbergia

Subfamily 3. Sclerotrichinae.

Genus a. Zettnowia

b. Schlerothrix

Subfamily 4. Corynobacteriinat

Genus a. Corynobacterium

b. Heterocystia

c. Cladascus

d. Zygoplagia

Subfamily 5. Pseudostrepinae

Genus a. Pseudostreplus

Order b. Ascola

Family 8. Bacteriidae 


\author{
Genus a. Atremis \\ b. Bacterium \\ c. Lamprella \\ d. Eucystia \\ e. Dicrobactrum \\ f. Acystia \\ Family 9. Fusiformidae \\ Genus a. Fusiformis \\ Class II. Sporascota \\ Order a. Parasynascota \\ Family 10. Migulanidae \\ Genus a. Migulanum \\ Order b. Parascota \\ Family 11. Bacillidae \\ Genus a. Rhagadascia \\ b. Plectridium \\ c. Bacillus \\ d. Bactrillum \\ e. Kochella \\ f. Fischerinum \\ Kreis B. Hemicyclomorpha \\ Class I. Anascola \\ Family 12. Hemallosidae \\ Genus a. Hemallosis \\ Phylum II. Monomychota \\ Kreis A. Acyclomorpha \\ Family 1. Mogallidae \\ Genus a. Mogallia \\ Family 2. Sarcinidae \\ Genus a. Diplococcus \\ b. Sarcina \\ c. Paulosarcina \\ Family 3. Micrococcidae \\ Genus a. Micrococcus \\ b. Planococcus \\ c. Streptococcus \\ d. Phacelium
}

Three of the new generic terms, Cladascus type species $C$. furcabilis Enderlein, Zygoplagia type species Z. alternans Enderlein and Heterocystia type species $H$. multiformiis Enderlein, had been proposed in an earlier paper (Sitzber. Gesell. Naturf. Freunde, Berlin, 1916, 395). The following generic terms in the 1917 outline are new: Schaudinnum, Theciobactrum, Syncrotis, Zygostasis, Dicrospirillum, Entomospira, Cacospira, Dicrospira, Eisenbergia, Zettnowia, Pseudostreptus, Atremis, Lamprella, Eucystia, Dicrobactrum, Acystia, Migulanum, Rhagadascia, Kochella, Fischerinum, Hemallosis, Mogallia, Paulosarcina and Phacelium. Note that Corynobacterium is spelled with an o instead of an e.

Terms accepted from earlier workers that have not previously been 
mentioned are: Spirobacillus Metschnikoff (Ann. Inst. Past., 3, 1889, 62), Photobacterium Beijerinck, Maanblad voor Natuurwetenschappen Amsterdam, 16, 1889, 1 and Arch. Neérl. d. Sci. Exactes, 23, 1889, 401), and Sclerothrix Metschnikoff (Arch. f. Path. Anat. u. Physiol., 113, 1888, 63-94; not Sclerothrix Kuetzing, Species Algarum, 1849, 319).

The above outline was changed in 1925, p. $235 \mathrm{ff}$. (Bakterien-Cyclogenie, Berlin, 390 pp.) by the addition of one new family, Chondromycidae, to include the genus Newskia, formerly included in Sphaerotilidae, and nine genera not previously given as follows: Chondromyces, Cystodesmia, Monocystia, Ophiocystia, Apelmocoena, Polyangium, Cystoecemia, Myxococcus and Dactylocoena. All except Chondromyces, Polyangium and Myxococcus are taken from Enderlein (Bemerkungen zur Systematik der Chondromyciden, Berlin, 1924, 6 pp.).

The new genus Löhnisium is added in the Family Eisenbergiinae to include the acetic acid and legume bacteria, and he also proposes the generic term Macrocystita (p. 278) for certain bacteria described by Peklo (O mšici krváve (Study of the blood louse). Zemědelskěho Archivu (Agricultural Archives), 1, 1916) from aphids. According to Enderlein it is not clear whether this genus should be included in the Family Bacteriidae or in Corynobacteriidae.

Two genera proposed by others are also accepted. These are Calymmatobacterium Aragão and Vianna (Mem. Inst. Oswaldo Cruz, 6, 1912, 211) placed in the family Migulanidae, and Leuconostoc Van Tieghem placed in the family Micrococcidae.

Later Enderlein (Sitzber. Gesell. Naturf. Freunde Berlin, 1930, 104-105) accepts Serratia Bizio in place of Dicrobactrum and Leptotrichia Trevisan in place of Syncrotis. Streptus with Streptus scarlatinae as type species, is proposed to cover the streptococci not included in Pseudostreptus.

The outline suggested by Pringsheim (Lotos, 71, 1923, 357) is similar to that used by Lehmann and Neumann (Atlas und Grundriss der Bakteriologie, 2 vols., 1896, München). It is a conventional division into spherical, rod-shaped and curved forms so far as the true bacteria are concerned except that the pseudomonads are included in the same family as the vibrios and spirilla. Rhodobacteriales is recognized as an order to include the sulfur purple bacteria and the nonsulfur purple bacteria. Few details are given in regard to the other orders. His outline follows:

Schizomycetes

Order I. Eubacteriales

Family 1. Coccaceae

Genus a. Streptococcus

b. Micrococcus

c. Sarcina 
Family 2. Bacteriaceae

Genus a. Bacterium

b. Bacillus

Family 3. Spirillaceae

Genus a. Pseudomonas

b. Vibrio

c. Spirillum

Order II. Rhodobacteriales

Family 1. Rhodobacterinae

2. Thiorhodinae

Order III. Myxobacteriales

Family 1. Myxobacteriaccae

Order IV. Mycobacteriales

Family 1. Corynebacteriaceae

2. Mycobacteriaceae

3. Actinomycetaceae

(Also possibly the long rod, lactic acid bacteria.)

Order V. Desmobacteriales

Family 1. Chlamydobacteriaceae

2. Beggiatoaceae

The first outline classification drawn up by Janke (Allgemeine Technische Mikrobiologie, I Teil, Dresden, 1924, p. 63) is an adaptation and expansion of that drann up by Migula (System der Bakterien, 1900). The new genera recognized by Janke are Planostreptococcus A. Meyer (Die Zelle der Bakterien, Jena, 1912), Thioploca Lauterborn (Ber. dtsch. Bot. Gesell., 25, 1907, 238), Thiobacterium Molisch (Cent. f. Bakt., II Abt., 33, 1912, 55), Thiobacillus Beijerinck (Cent. f. Bakt., II Abt., 11, 1904, 593), Thiovibrio Janke (loc. cit.), Thiospirillum Winogradsky (Beiträge zu Morphol. u. Physiol. d. Bakterien. Heft I. Schwefelbakterien. Leipzig, 1888), Thiosphaerella Nadson (Bull. Jar. bot. Petersburg, 19, 1913, 106; ref. in Cent. f. Bakt., II Abt., 43, 1915, 469), Thiooulum Hintze (Ber. Dtsch. Bot. Gesell., 31, 1913, 189), Spirophyllum Ellis (Proc. Roy. Soc. Edinburgh, 27, I, 1907, 21; ref. in Cent. f. Bakt., II Abt., 19, 1907, 502), Nodofolium Ellis (Cent. f. Bakt., II Abt., 26, 1910, 321), and Actinococcus Beijerinck (Fol. Microbiol., 2, 1914, 185).

Janke's outline classification is given below:

Order I. Eubacteria

Family 1. Coccaceae

Genus a. Streptococcus

b. Micrococcus

c. Sarcina

d. Planostreptococcus

e. Planococcus

f. Planosarcina

Family 2. Bacteriaceae

Genus a. Bacillus

b. Bacterium 
Family 3. Spirillaceae

Genus a. Microspira

b. Spirillum

c. Spirosoma

Order II. Rhodobacteria

Family 1. Thiorhodaceae

Subfamily 1a. Thiocysteae

Genus a. Thiocystis

b. Thiocapsa

c. Thiosphaera

d. Thiosphaerion

e. Thiosarcina

Subfamily 2b. Lamprocysteae

Genus a. Lamprocystis

Subfamily 3c. Thiopedieae

Genus a. Thiopedia

b. Thioderma

Subfamily 4d. Amoebobacterieae

Genus a. Amoebobacter

b. Thiothece

c. Thiodictyon

d. Thiopolycoccus

Subfamily 5e. Chromatieae

Genus a. Chromatium

b. Rhabdochromatium

c. Thiorhodospirillum

Subfamily 6f. Rhodocapseae

Genus a. Rhodocapsa

b. Rhodothece

Family 2. Athiorhodaceae

Subfamily 1a. Rhodocysteae

Genus a. Rhodocystis

b. Rhodonostoc

c. Rhodococcus

d. Rhodobacterium

e. Rhodobacillus

f. Rhodovibrio

g. Rhodospirillum

Order III. Thiobacteria

Family 1. Beggiatoaceae

Genus a. Thiothrix

b. Beggiatoa

c. Thioploca

Family 2. Thiobacteriaceae

Genus a. Thiophysa

b. Thiobacterium

c. Thiobacillus

d. Thiovibrio

e. Thiospirillum

f. Thiosphaerella

g. Thiovulum

h. Achromatium 
Order IV. Phycobacteria

Genus a. Leptothrix

b. Clonothrix

c. Cladothrix

d. Crenothrix

e. Phragmidiothrix

Appendix Genera Gallionella, Spirophyllum, Nodofolium

Order V. Mycobacteria

Family 1. Mycobacteriaceae

Genus a. Corynebacterium

b. Mycobacterium

Family 2. Actinomycetaceae

Genus a. Actinomyces

b. Actinococcus

Order VI. Myxobacteria

Family 1. Myxobacteriacene

Genus a. Myxococcus

b. Chondromyces

c. Polyangium

Lehmann and Neumann (Bakt. Diag., 2 vols., 7th ed., München, 1926-27; Breed, Eng. trans., New York, 1931) developed their first simple and much used outline classification, drawn up in 1896, in later editions of their Determinative Bacteriology. The 1927 Lehmann and Neumann outline is as follows:

Class I. Schizomycetes

Order I. Schizomycetales

Family 1. Coccaceae

Genus a. Streptococcus

b. Surcina

c. Micrococcus

Sub-genus a. Diplococcus

b. (Gram-positive group)

Family 2. Bacteriaceae

Genus a. Bacterium

Sub-genus a. Nitrosomonas

b. Nitrobacter

c. Rhizobium

d. Haemophilus

e. Brucella

f. Pasteurella

g. (Glanders and dysentery group)

h. (Photogenic group)

i. (Aerogenes group)

j. Encapsulatus

k. (Typhoid group)*

1. Salmonella

m. (Coli group)*

* In a footnote under these groups, the authors refer to the names given by Castellani and Chalmers. 
n. Acetobacterium

o. (Cloacae group)

p. (Red chromogens)

q. (Blue and violet chromogens)

r. Pseudomonas

s. Proteus

App. Erysipelothrix

Genus b. Fusobacterium

c. Plocamobacterium

Family 3. Desmobacteriaceae

Genus a. Beggiatoa

b. Leptothrix

Sub-genus a. Leptothrix

b. Chlamydothrix

Genus c. Crenothrix

d. Cladothrix

e. Thiothrix

Family 4. Spirillaceae

Genus a. Vibrio

b. Spirillum

Family 5. Spirochaetaceae

Genus a. Spirochaeta

Family 6. Bacillaceae

Genus a. Bacillus

Sub-genus a. (Aerobic group)

b. (Anaerobic group)

Order II. Actinomycetales

Family 1. Proactinomycelaceae

Genus a. Corynebacterium

b. Mycobacterium

Family 2. Actinomycetaceae

Genus a. Actinomyces

The generic term Bacterium is retained in this outline to cover those groups of the true bacteria that are Gram-negative, non-spore-forming, motile and non-motile rods. Lehmann and Neumann recognize 20 subgroups in this genus, many of which correspond with the genera recognized in the Manual. In an effort to develop a rational nomenclature the term Acetobacterium (occurs first in review by Ludwig, Cent. f. Bakt., II Abt., $4,1898,870$ ) is used in place of Acetobacter, Plocamobacterium (Loewi, Wien. klin. Wchnschr., 33, 1920, 730) in place of Lactobacillus, and Fusobacterium (Knorr, Cent. f. Bakt., I Abt., Orig., 89, 1922, 4) in place of Fusiformis without regard to priority. Encapsulatus Castellani and Chalmers (Manual Tropical Med., 3rd ed., 1919, 934) is used in place of Klebsiella Trevisan (Atti Accad. Fis.-Med.-Stat. Milano, Ser. 4, 3, 1885, 107).

Janke (Cent. f. Bakt., II Abt., 66, 1926, 481) reprints the classification developed in the first edition of the present Manual and compares it with that proposed by Orla-Jensen and Enderlein. 
The second complete outline drawn up by Janke (Oesterr. Bot. Zeitschr., $78,1929,108)$ is similar to the classification employed by Lehmann and Neumann (Bakt. Diag., 2 vols., 7 th ed., München, 1926-27). He follows Enderlein in placing $A$ zotobacter in close association with the spore-forming rods. No new generic terms are suggested. His sub-groups of the genus Bacterium are even more closely similar to the genera used in the present edition of the MANCAL than are the sub-groups of Lehmann and Neumann.

Family 1. Coccaceae

Genus a. Micrococcus

b. Neisseria

c. Streptococcus

Divided into 4 groups.

d. Sarcina

Divided into 2 groups.

Family 2. Bacillaceae

Genus a. Bacillus

Divided into 16 groups.

b. Azotobacter

Family 3. Bacteriaceae

Genus a. Bacterium

Divided into 27 groups.

b. Fusiformis

Family 4, Corynobacteriaceae

Genus a. Mycobacterium

b. Corynobacterium

c. Actinomyces

Family 5. Spirillaceae

Genus a. Microspira

Divided into 2 groups.

b. Spirillum

Divided into 2 groups.

Family 6. Spirochaetaceae

Genus a. Spirochaeta

b. Borrelia

c. Treponema

d. Cristispira

e. Saprospira

f. Leplospira

Family 7. Desmobacteriaceae

Genus a. Beggiatoa

b. Thioploca

c. Thiothrix

d. Leptotrichia

e. Crenothrix

f. Sphaerotilus

g. Clonothrix

h. Leptothrix

i. Phragmidiolhrix 


\section{Family 8. Myxobacteriaceae \\ Genus a. Myxococcus \\ b. Polyangium \\ c. Chondromyces}

Pribram (Jour. Bact., 18, 1929, 361) has rearranged some groups and combined others (e.g., Rhizobium, Diplococcus, Leuconostoc, Serratia, Flavobacterium, Chromobacterium, Achromobacter, Cellulomonas) recognized in the first edition of the MANUAL with little change in the nomenclature except among the anaerobic non-spore-forming rods and among the sporeforming rods. Unfortunately, he has sometimes used family and species names as generic names, thus in the latter case introducing adjectives and adjectival terms as substantives. New generic terms suggested are: Dialisterea, Bacteroidea, Centrosporus, Fusibacillus, Pseudobacillus, Megatherium, Flexus, Anthrax, Botulinus, Chavvoea, Botulinea, Putrificus, Welchia, Phleobacterium, Distasoa, Tissieria, and Actinoidomyces. Astasia as it appears in this outline does not appear to be the same as Astasia .Meyer (Flora, 84, 1897, 185). Aerobacillus is not synonymous with Aerobacillus Donker (Inaug. Diss., Delft, 1926). Sideromonas is accepted from Cholodny (Ber. Deutsch. Bot. Ges., 40, 1922, 326).

Pribram's complete outline follows:

Class Schizomycetes

Subclass A. Protozoobacteria

Order I. Spirochaetales

Family 1. Spirochaetaceae

Genus a. Spirochaeta

b. Treponema

c. Spironema

Family 2. Cristispiraceae

Genus a. Saprospira

b. Cristispira

c. Leptospira

Subclass B. Eubacteria

Order I. Protobacteriales

Family 1. Nitrobacteriaceae

Related to Pseudomonas

Tribe A. Hydrogenomonadae

Genus a. Hydrogenomonas

b. Methanomonas

c. Carboxyodomonas

Tribe B. Nitrobactereae

Genus a. Nitrosomonas

b. Nitrobacter

Family 2. Thiobacillaceae

Tribe A. Thiobacilleae

Genus a. Thiobacillus 
Order II. Metabacteriales

Family 1. Pseudomonadaceae

Tribe A. Spirilleae

Genus a. Spirillum

Tribe B. Vibrioneae

Genus a. Vibrio

Tribe C. Pseudomonadeae

Genus a. Pseudomonas

b. Azotobacter

Connects with Polyangiaceae and Nitrobacteriaceae

Family 2. Bacteriaceae

Tribe A. Aerobactereae

Genus a. Aerobacter

b. Escherichia

c. Salmonella

d. Eberthella

e. Proteus

Tribe B. Pasteurelleae

Genus a. Alcaligenes

b. Pasteurella

Connects with Pfeifferella

c. Hemophilus

Connects with Dialister

Family 3. Micrococcaceae

Tribe A. Streptococceae

Genus a. Neisseria

b. Streptococcus

Tribe B. Micrococceae

Genus a. Micrococcus

b. Siaphylococcus

c. Sarcina

Subclass C. Mycobacteria

Connects with Algobacteria

Order I. Bacteriomycetales

Family 1. Leptotrichaceae

Tribe A. Acetobactereae

Genus a. Acetobacter

Connects with Salmonella and Tissieria

Tribe B. Leptotricheae

Genus a. Kurthia

b. Lactobacillus

Connects with Corynebacterium

c. Leptotrichia

Connects with Erysipelothrix

Family 2. Bacteroidaceae

Tribe A. Dialistereae

Genus a. - Type species Dialisterea variegata

Connects with Distasoa

b. - Type species Dialisterea variabilis

c. Dialister 
Connects with Hemophilus

Tribe B. Bacteroideae

Genus a. - Type species Bacteroidea multiformis

b. Bacteroides

Connects with Tissieria

c. Type species Bacteroidea fusiformis

Order II. Bacillomycetales

Family 1. Bacillaceae

Sub-family 1a. Aerobacilloideae

Tribe A. Aerobacilleae

Sub-tribe A1. Centrosporineae

Genus a. Centrosporus

b. Fusibacillus

Sub-tribe A2. Aerobacillineae

Genus a. Aerobacillus

Tribe B. Pseudobacilleae

Genus a. Pseudobacillus

Sub-family 1b. Bacilloideae

Tribe A. Bacilleae

Sub-tribe A1. Bacillineae

Genus a. Bacillus

b. Megatherium

Sub-tribe A2. Astasineae

Genus a. Astasia

b. Flexus

Tribe B. Anthraceae

Genus a. Anthrax

Family 2. Clostridiaceae

Sub-family 2a. Botulinoideae

Tribe A. Botulineae

Genus a. Botulinus

b. Chauvoea

c. Type species Botulinea saccharolytica

d. __ Type species Botulinea butyrica

Tribe B. Putrificeae

Genus a. Putrificus

Sub-family 2b. Clostridioideae

Tribe A. Welchieae

Genus a. Welchia

Tribe B. Clostridieae

Genus a. Clostridium

Order III. Actinomycetales

Family 1. Mycobacteriaceae

Tribe A. Actinobacilleae

Genus a. Pfeifferella

Connects with Pasteurella

b. Actinobacillus

c. Corynebacterium

d. Erysipelothrix

Connects with Leptotrichia 
Tribe B. Mycobactereae

Genus a. Phleobacterium

b. Mycobacterium

Tribe C. Tissierieae

Genus a. Distasoa

b. Tissieria

Connects with Bacteroides, Corynebacterium and Acetobacter

Family 2. Actinomycetaceae

Tribe A. Actinoidomycetaceae

Genus a. Actinoidomyces

Tribe B. Actinomycetaceae

Genus a. Actinomyces

Subclass D. Algobacteria

Order I. Desmobacteriales

Family 1. Sphaerotilaceae

Genus a. Sphaerotilus

Order II. Siderobacteriales

Family 1. Chlamydotrichaceae

Tribe A. Chlamydotricheae

Genus a. Leptothrix

b. Crenothrix

Family 2. Siderocapsaceae

Genus a. Didymohelix

b. Siderocapsa

c. Sideromonas

Order III. Thiobacteriales

Family 1. Rhodobacteriaceae

Sub-family 1a. Chromatoideae

Tribe A. Thiocapseae

Genus a. Thiocystis

b. Thiosphaera

c. Thiosphaerion

d. Thiocapsa

e. Thiosarcina

f. Lamprocystis

Tribe B. Thiopedieae

Genus a. Lampropedia

b. Thioderma

Tribe C. Amoebobacteriae

Genus a. Amoebobacter

b. Thiodictyon

c. Thiothece

d. Thiopolycoccus

Tribe D. Chromatieae

Genus a. Chromatium

b. Rhabdomonas

c. Thiospirillum

d. Rhodocapsa

e. Rhodcthece 
Sub-family 1b. Rhodobacteroideae

Tribe A. Rhodobacteriaceae

Genus a. Rhodobacterium

b. Rhodobacillus

c. Rhodovibrio

d. Rhodospirillum

e. Rhodosphaera

Tribe B. Rhodocysteae

Genus a. Rhodocystis

b. Rhodonostoc

Connects with Leuconostoc

Family 2. Beggiatoaceae

Genus a. Thiothrix

b. Beggiatoa

c. Thioploca

Family 3. Achromatiaceae

Genus a. Achromatium

b. Thiophysa

c. Thiospira

d. Hillhousia

Order IV. Myxobacteriales

Family 1. Polyangiaceae

Genus a. Chondromyces

b. Polyangium

Family 2. Myxococcaceae

Genus a. Myxососсиs

Later Pribram (Klassification der Schizomyceten (Bakterien), Leipzig and Wien, 1933, 143 pp.) developed this classification into a suggestive outline based on his experience in caring for the cultures of the Kral Collection. His most interesting contribution is the separation of the class of Schizomycetes into three subclasses which are based on differences in fundamental biological and nutritional relationships. The fourth sub-class of his earlier outline (the Protozoobacteria with its single order Spirochaetales) is omitted from this outline. The first class, Algobacteria, includes the bacteria that are primarily free-living in water, usually motile with polar flagellation and live on easily soluble foodstuffs. They are frequently surrounded by insoluble secretions such as capsules, sheaths, etc., and form insoluble products in their protoplasm, such as calcium, sulfur and iron compounds, and pigments. The class Eubacteria includes those bacteria whose normal habitat is the animal body or complex waste products of plant or animal origin. Because of adaptation to environment, these organisms are motile or non-motile and can utilize compounds of complex molecular structure. The third sub-class, Mycobacteria, is adapted to life in soil, and shows a distinct tendency to differentiation in morphology and spore formation.

Internationally accepted rules of nomenclature are generally followed, and the generic terms proposed in his earlier outline that were not formed 
in accordance with recommended practices are discarded. He has revived Ulvina Kützing, 1837 (status explained by Buchanan, General Systematic Bacteriology, 1925, p. 518) in place of Acetobacter Beijerinck and accepted Plocamobacterium (Loewi, Wien. Hin. Wchschr., 33, 1920, 730) in place of Lactobacillus Beijerinck, 1901. Among the spore-forming rods, he has accepted Bactrillum Fischer and Welchillus Heller, 1921. Malleomyces Hallier (Bot. Ztg., 24, 1866, 383) is used for the glanders bacillus. Anthracillus is apparently new.

The new outline has the following form:

\section{Class. Schizomycetes}

Subclass A. Algobacteria

Order 1. Micrococcales

Family 1. Micrococcaceae

Genus a. Micrococcus

b. Rhodococcus

c. Rhodocapsa

d. Thiocapsa

e. Thiosphaera

f. Thiosphaerion

g. Thiocystis

h. Lamprocystis

i. Sarcina

j. Thiosarcina

Family 2. Pediococcaceae

Genus a. Pediococcus

b. Lampropedia

c. Thiothece

d. Thiopolycoccus

e. Thioderma

f. Amoebomonas

g. Rhodothece

h. Rhodonostoc

i. Thiophysa

Order 2. Pseudomonadales

Family 1. Pseudomonadaceae

Genus a. Pseudomonas

b. Rhodobacillus

c. Chromalium

d. Nitrosomonas

e. Vibrio

f. Rhodovibrio

g. Myxococcus

h. Spirillum

i. Rhodospirillum

j. Thiospira

k. Thiospirillum

Family 2. Serratiaceae

Genus a. Serratia

b. Hillhousza 
Family 3. Nitrobacteriaceae

Genus a. Nitrobacter

b. Rhodobacterium

c. Rhodocystis

d. Didymohelix

e. Sideromonas

f. Siderocapsa

g. Chondromyces

h. Polyangium

i. Amoebobacter

j. Thiodictyon

Family 4. Azotobacteriaceae

Genus a. Rhizobium

b. Azotobacter

Order 3. Leptotrichales

Family 1. Leptotrichaceae

Genus a. Leptothrix

b. Sphaerotilus

c. Crenothrix

Family 2. Clonothrichaceae

Genus a. Clonothrix

Order 4. Rhabdomonadales

Family 1. Rhabdomonadaceae

Genus a. Beggiatoa

b. Rhabdomonas

c. Thioploca

d. Thiothrix

Family 2. Spirochaetaceae

Genus a. Spirochaeta

b. Treponema

c. Leptospira

d. Cristispira

e. Saprospira

Subclass B. Eubacteria

Order 1. Aerobacteriales

Family 1. Aerobacteriaceae

Genus a. Aerobacter

b. Escherichia

c. Salmonella

d. Eberthella

e. Shigella

Family 2. Pasteurellaceae

Genus a. Pasteurella

b. Brucella

c. Haemophilus

d. Neisseria

Order 2. Plocamobacteriales

Family 1. Streptococcaceae

Genus a. Streptococcus

Family 2. Ulvinaceae

Genus a. Proteus

b. Kurthia 


\author{
c. Ulvina \\ d. Plocamobacterium \\ e. Leptotrichia \\ Family 3. Bacteroidaceae \\ Genus a. Dialister \\ b. Aerobacteroides \\ c. Bacteroides \\ d. Fusobacterium \\ Subclass C. Mycobacteria \\ Order 1. Bacillales \\ Family 1. Bacillaceae \\ Genus a. Bactrillum \\ b. Aerobacillus \\ c. Bacillus \\ d. Anthracillus \\ Family 2. Clostridiaceae \\ Genus a. Clostridium \\ b. Welchillus \\ Order 2. Mycobacteriales \\ Family 1. Mycobacteriaceae \\ Genus a. Malleomyces \\ b. Actinobacillus \\ c. Corynebacterium \\ d. Erysipelothrix \\ e. Mycobacterium \\ f. Distasoa \\ g. Tissieria \\ Family 2. Actinomycetaceae \\ Genus a. Actinomycoides \\ b. Actinomyces
}

Janke (Cent. f. Bakt., II Abt., 80, 1930, 481) reprints the earlier outline prepared by Pribram (1929) and, after commenting on Lehmann and Neumann's (1927) outline, proposes an outline which is slightly modified from his own previous (1929) outline. Two new subgeneric terms are used, Anaerobacillus and Eubacterium. The sub-genus Aerobacillus is apparently not the same as Aerobacillus Donker (Inaug. Diss., Delft, 1926), nor as Aerobacillus Pribram (Jour. Bact., 18, 1929, 361).

Family I. Micrococcaceae

Genus 1. Micrococcus

Divided into 2 sections.

2. Nersseria

3. Streptococcus

Divided into 4 sections.

4. Sarcina

Divided into 2 sections.

Family II. Bacillaceae

Genus 1. Bacillus

Sub-genus a. Anaerobacillus or better Clostridium

Divided in to 6 sections. 
Family III. Bacteriaceae

b. Aerobacillus or better Eubacillus

Divided into 10 sections.

Genus 1. Bacterium

Sub-genus a. Pseudomonas

Divided into 6 sections.

b. Eubacterium

Divided into 11 sections.

c. Trichobacterium

Divided into 6 sections.

Genus 2. Fusiformis

Family IV. Corynobacteriaceae

Genus 1. Mycobacterium

2. Pfeifferella

3. Erysipelothrix

4. Corynobacterium

5. Actinomyces

Family V. Spirillaceae

Genus 1. Microspira or Vibrio

Sub-genus a. Microspira

Genus 2. Spirillum

b. Spirosoma

Sub-genus a. Spirella

b. Dicrospirillum

Family VI. Spirochaetaceae

Genus 1. Spirochaeta.

2. Cacospira

3. Entomospira

4. Treponema

5. Cristispira

6. Saprospira

7. Leptospira

Family VII. Desmobacteriaceae

As in 1929 outline.

Family VIII. Myxobacteriaceae

As in 1929 outline.

Kluyver and Van Niel (Cent. f. Bakt., II Abt., 94, 1936, 369) have developed an outline classification in which they indicate four lines of development from the simplest form of cell that is existent and conceivable, the sphere. They assign family rank to each of these four groups of bacteria, placing the lophotrichous (and related non-motile) rod-shaped bacteria first (Pseudomonadaceae). This is followed by the family of spherical bacteria (Micrococcaceae) and the family of permanently non-motile, rodshaped bacteria (Mycobacteriaceae). The final family includes the peritrichous (and related non-motile) rod-shaped bacteria, the Bacteriaceae. These are grouped in the tribes of each family in accordance with their fundamental metabolism as photo-autotrophic, photo-heterotrophic, chemoautotrophic and chemo-heterotrophic. Their outline follows: 
Family A. Pseudomonadaceae

I. Tribe Spirilleae

Genus 1. Thiospirillum

2. Phaeospirillum

3. Rhodospirillum

4. Sulfospirillum

5. Spirillum

II. Tribe Vibrioneae

Genus 1. Chromatium

2. Rhodovibrio

3. Didymohelix

4. Vibrio

5. Desulfovibrio

III. Tribe Pseudomonadeae

Genus 1. Thiothece

2. Phaeomonas

3. Rhodomonas

4. Sulfomonas

5. Sideromonas

6. Nitrosomonas

7. Nitrobacter

8. Acetobacter

9. Pseudomonas

10. Rhizobium

11. Azotobacter

12. Listerella

13. Aeromonas

14. Zymomonas

15. Methanobacterium

Family B. Micrococcacene

IV. Tribe Micrococceae

Genus 1. Chlorobium

2. Thiopolycoccus

3. Rhodococcus

4. Achromatium

5. Siderocapsa

6. Nitrosococcus

7. Neisseria

8. Micrococcus

9. Veillonella

10. Peptococcus

11. Methanococcus

V. Tribe Sarcineae

Genus 1. Thiopedia

2. Thiosarcina

3. Gaffkya

4. Sarcina

5. Zymosarcina

6. Butyrisarcina

7. Methanosarcina 
VI. Tribe Sporosarcineae

Genus 1. Sporosarcina

VII. Tribe Streptococceae

Genus 1. Peptostreptococcus

2. Streptococcus

3. Betacoccus

Family C. Mycobacteriaceae

VIII. Tribe Corynebacterieae

Genus 1. Corynebacterium

2. Fusiformis

3. Propionibacterium

4. Streptobacterium

5. Betabacterium

IX. Tribe Mycobacterieae

Genus 1. Mycobacterium

2. Thermobacierium

Family D. Bacteriaceae

X. Tribe Bacterieae

Genus 1. Kurthia

2. Alcaligenes

3. Bacterium

4. Aerobacter

XI. Tribe Bacilleae

Genus 1. Bacillus

2. Aerobacillus

3. Zymobacillus

4. Clostridium

5. Peptoclostridium

Some old names are displaced by new descriptive terms: Phaeospirillum Sulfospirillum, Desulfovibrio, Phaeomonas, Aeromonas, Zymomonas, Methanobacterium, Methanococcus, Methanosarcina, Butyrisarcina, Peptococcus, Peptostreptococcus, Zymobacillus. Rhodomonas is not used in the same sense as Rhodomonas Orla-Jensen (Cent. f. Bakt., II Abt., 22, 1909, 331 and 334), the latter being a synonym of Chromatium Perty (Zur Kenntniss kleinster Lebensformen, 1852). Sulfomonas is indicated as new and as a synonym of Thiobacillus Beijerinck (Cent. f. Bakt., II Abt., 11, 1904, 598) although the same term is used by Orla-Jensen (loc. cit.). Three new terms are accepted:Chlorobium Nadson (Bull. Jard. Bot. St. Petersburg, 6, 1906, 184), Zymosarcina Smit (Die Gärungssarcinen, Jena, 1930) and Peptoclostridium (Donker, Inaug. Diss., Delft, 1926).

Rahn (Cent. f. Bakt., II Abt., 96, 1937, 273) has reviewed the characters of the species of Eubacteriales included in the fourth edition of this MandaL. He places 146 of the spore-forming species in a Sub-order A. Endosporales with a single family, and 536 of the species of non-spore-forming rods in a Sub-order B. Asporales in seven families. Unclassifiable species (total 224) are placed in a temporary eighth family Bacteriaceae. His outline follows: 


\section{Order Eubacteriales}

Suborder A. Endosporales

Family I. Endosporaceae

Genus 1. Bacillus

2. Aerobacillus

3. Clostridium

Suborder B. Asporales

Family I. Gramoxidaceae

Genus 1. Micrococcus (including Staphylococcus, Gaffkya, Rhodococcus and most of the species of Sarcina)

2. Kurthia

Family II. Gramanoxidaceae

Tribe a. Streptococceae

Genus 1. Streptococcus (including Diplococcus)

2. Leuconostoc

3. Peptostreptococcus

Tribe b. Lactobacilleae

Genus 4. Lactobacillus (including part of Bacteroides)

5. Propionibacterium

Tribe c. Sarcineae

Genus 6. Zymosarcina

7. Butyrisarcina

8. Methanosarcina

Family III. Neissereaceae

Genus 1. Neisseria

2. Veillonella

Family IV. Protobacteriaceae

Tribe a. Protobacterieae

Genus 1. Carboxydomonas

2. Methanomonas

Tribe b. Nitrobacterieae

Genus 1. Nitrosomonas

2. Nitrobacter

3. Nitrosococcus

Family V. Enterobacteriaceae

Genus 1. Enterobacter (including Escherichia, Salmonella, Aerobacter, Klebsiella, Proteus, Erwinia, Eberthella, Shigella, and parts of Serratia, Pseudomonas, Flavobacterium and Achromobacter)

Family VI. Pseudomonadaceae

Genus 1. Pseudomonas (includes Phytomonas and other lophotrichous types only)

2. Vibrio

3. Spirillum

4. Acetobacter

5. Azotobacter

6. Rhizobium 


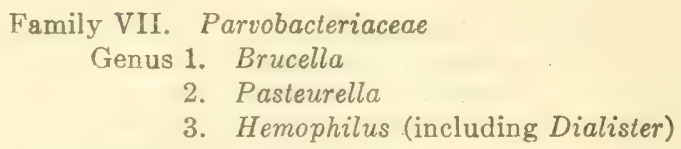

Family VIII. Bacteriaceae

Unclassifiable genera including Alcaligenes and Protaminobacter; some species from each of the following genera, Achromobacter, Chromobacterium, Cellulomonas, Bacteroides, Flavobacterium, Phytomonas, Pseudomonas, Serratia; and three species from the Family Nitrobacteriaceae.

One of the generic terms used in this outline is new, i.e., Enterobacter. Two other generic terms, Fluorescens and Erythrobacterium, are proposed incidentally ( $p .284$ ). The first includes the peritrichous forms included in the Mandal under Pseudomonas and the second includes those red, nonspore-forming rods that are not included in Serratia. In another footnote (p. 281) a substitute, Virgula, is suggested for Enterobacter. Emphasis is placed on sporulation, Gram stain, and oxygen demand as the most important characters aside from cell form and flagellation.

Prévot, as an outgrowth of his studies on anaerobes with Weinberg (Weinberg, Nativelle and Prévot, Les microbes anaérobies, 1937, 1186 pp., Paris), has written a series of papers in which he has developed a classification of anaerobic bacteria (Ann. Sci. Nat., 10 Sér., 15, 1933, 23-260; Ann. Inst. Past., 60, 1938, 285-307; 61, 1938, 72-91; 64, 1940, 117-125). The conclusions reached in these studies are summarized in his Manual de Classification et de Détermination des Bactéries Anaérobies, Monographie de l'Institut Pasteur, Paris, 1940, 223 pp. He regards the bacteria as comprising a kingdom, Schizomycetes, intermediate between the animal and plant kingdoms and notes the presence of strict anaerobes in at least three of the seven orders recognized in the 5th edition of the MaNual. These orders he regards as classes. The genus Bacteroides Castellani and Chalmers (Manual of Trop. Med., 3rd ed., 1919, 959) type species, Bacteroides fragilis, is dropped (Ann. Inst. Past., 60, 1938, 288), and several new terms are proposed for the organisms included by Castellani and Chalmers and later investigators in the genus. Among the new generic names is Ristella which is based on Ristella fragitis, the species used by Castellani and Chalmers as the type species for Bacteroides.

The complete outline classification developed by Prévot in his Monograph (loc. cit., p. 17) is given below:

Kingdom. Schizomycetes Nägeli

Class I. Eubacteriales

Sub-Class I. Non sporogenous Eubacteriales

Order I. Micrococcales 
Family I. Neisseriaceae

Tribe I. Neisserieae

Genus a. Neisseria

Tribe 2. Veillonelleae

Genus a. Veillonella

Family 2. Micrococcaceae

Tribe 1. Streptococceae

Genus a. Diplococcus

b. Streptococcus

Tribe 2. Staphylococceae

Genus a. Gaffira

b. Staphylococcus

Tribe 3. Micrococceae

Genus a. Sarcina

b. Micrococcus

Order II. Bacteriales

Family 1. Ristellaceae

Genus a. Ristella

b. Pasteurella

c. Dialister

d. Zuberella

e. Capsularis

Family 2. Bacteriaceae

Genus a. Eubacterium

b. Calenabacterium

c. Ramibacterium

d. Cillobacterium

Order III. Spirillales

Family 1. Vibrionaceae

Genus a. Vibrio

Sub-class II. Sporogenous Eubacteriales

Order I. Clostridiales

Family 1. Endosporaceae

Genus a. Endosporus

b. Paraplectrum

Family 2. Closiridiaceae

Genus a. Inflabilis

b. Welchia

c. Clostridium

Order IX. Plectridiales

Family 1. Terminosporaceae

Genus a. Terminosporus

b. Caduceus

Family 2. Plectridiaceae

Genus a. Plectridium

b. Acuformis

Order III. Sporovibrionales

Family 1. Sporovibrionaceae

Genus a. Sporovibrio 
Class II. Actinomycetales

Family 1. Spherophoraceae

Genus a. Spherophorus

b. Spherocillus

c. Fusiformis

d. Fusocillus

e. Leptotrichia

Family 2. Actinomycetaceae

Genus a. Actinobacterium

b. Bifidibacterium

c. Corynebacterium

Class III. Spirochetales

Family 1. Spirochaetaceae

Genus a. Treponema

b. Borrelia

In this outline, there are minor modifications in the names and in endings given to the orders and tribes as compared with those given in his preliminary papers. In the Order Hicrococcales, Leuconostoc has been dropped as a genus of the tribe Streptococceac and Rhodococcus has been dropped as a genus of the Tribe Staphylococceae. Teillonella proposed by Prévot as a new genus in 1933 (loc. cit., p. 70) is included as a genus in the Family Neisseriaceae. The spelling of Gaffkya is changed to Gaffkia. In the first of Prévot's papers published in 1938 (loc. cit.), he proposes the following new genera in the Order Bacteriales: Ristella, Zuberella, Capsularis, Eubacterium, Catenabacterium, Ramibacterium and Cillobacterium. In the same paper he also proposes the following new genera in the Order Actinomycetales: Spherophorus, Spherocillus, Fusocillus, Pseudoleptothrix (withdrawn in 1940 in favor of Leptotrichia Trevisan). He also accepts one genus Actinobacterium (Haas, Cent. f. Bakt., I Abt., Orig., 40, 1906, 180) not previously mentioned in this discussion. With the single change noted (Pseudoleptothrix to Lepototrichia), the outlines of the genera in the orders Bacteriales and 1ctinomycetales remains in the 1940 outline as it was given in 1938 .

In the outline given in Prévot's Monograph (loc. cit., p. 17) one change is made in the generic terms recognized in the Order Clostridiales from those recognized in his second paper published in 1938. The genus name Palmula proposed in 1938, having been found to be invalid because of prior use for a genus of Protozoa, is changed to Acuformis. Other generic names which appeared for the first time in the 1938 outline are Endosporus, Inflabitis, Terminosporus and Caduceus. Welchia proposed by Prévot in 933 (loc. cit., p. 44) was previously proposed by Pribram (Jour. Bact., 18, 1929, 374) for the same group of anaerobic spore-forming rods. A third order, Sporovibrionales, is proposed by Prévot in his Monograph (loc. cit., p. 15) to include the family Sporovibrionaccae (Ann. Inst. Past., 64, 1940, 
119). This order and family include a single genus Sporovibrio Starkey (Arch. f. Microb., 9, 1938, 300) syn. Desulfovibrio Kluyver and Tan Niel (Cent. f. Bakt., II Abt., 94, 1936, 389). Two genera (Treponema and Borrelia) of Spirochaetales are listed by Prévot in his Monograph (loc.cit., p. 16) as including anaerobic species.

Stanier and Van Niel (Jour. Bact., 42, 1941, 437-466) have proposed a rearrangement of the classification outline as indicated below:

$\begin{array}{cc}\text { Kingdom Monera } & \\ \text { Division I } & \text { Myxophyta (Blue-green algae) } \\ \text { Division II } & \text { Schizomycetae (Bacteria) } \\ \text { Class I } & \text { Eubacteriae } \\ \text { Order I } & \text { Rhodobacteriales } \\ \text { Order II } & \text { Eubacteriales } \\ \text { Order III } & \text { Actinomycetales } \\ \text { Class II } & \text { Myxobacteriae } \\ \text { Order I } & \text { Myxobacteriales } \\ \text { Class III } & \text { Spirochaetae } \\ \text { Order I } & \text { Spirochaelales }\end{array}$

Appendix to Division Schizomycetae

Group I Includes two families, Leptotrichaccae and Crenothricaceae

Group II Achromatiaceae

Group III Pasteuriaceae (Includes three genera, Pasteuria, Hyphomicrobium and Blastocaulis)

The genera Mycobacterium, Corynebacterium, Erysipelothrix, Leptotrichia, Nevskia, Gallionella, Caulobacter, Thiospira, Siderocapsa and Sideromonas are placed in Eubacteriales. Two genera not previously discussed in this review whose relationships to other bacteria have recently been clarified are Sporocytophaga Stanier (Jour. Bact., 40, 1940, 629) and Cytophaga Winogradsky (Ann. Inst. Past., 43, 1929, 578).

This rearrangement has been carried out by including the organisms placed in the Order Caulobacteriales Henrici and Johnson (Jour. Bact., S0, 1935, 61-93) in the Order Eubacteriales (Buchanan, Jour. Bact., 2, 1917, 162). The genera of the Order Chlamydobacteriales Buchanan (loc. cit.) are transferred to an appendix or are dropped (Clonothrix) as belonging to the blue-green algae. Three of the remaining five orders are raised to the rank of classes, one of which (Eubacteriae) includes three orders Rhodobacteriales (Pringsheim, Lotos, 71 , 1923, 351), Eubacteriales (Buchanan, loc. cit.) and Actinomycetales (Buchanan, loc. cit.). Rhodobacteriales includes the sulfur purple, the non-sulfur purple and the green bacteria, the colorless sulfur bacteria (Beggiatoaceae) being transferred to the Myxophyta with the change of the name of the Order from Thiohacteriales Buchanan (loc. cit.) to Rhodobacteriales Pringsheim (loc. cit.). 
The outline classification below is proposed by the Editorial Board of the Manual for use in the present (6th) edition of the Mandal. It is based on those developed by Bergey et al. in earlier editions. These, in turn, were based on the outline classifications developed by Buchanan (Jour. Bact., 1, 1916, 591 ; 2, 1917, 155 ff.; 3, 1918, 27 ff.) and Winslow et al. (Jour. Bact., 5, $1920,191)$.

Phylum Schizophyta

Class I. Schizophyceae

Class II. Schizomycetes

Order I. Eubacteriales

Sub-Order I. Eubacteriineae (includes Corynebacteriaceae)

Sub-Order II. Caulobacteriineae

Sub-Order III. Rhodobacteriineae

Order II. Actinomycelales (includes Mycobacterium, Actinomyces, and related genera)

Order III. Chlamydobacteriales

Family I. Leptotrichaceae

Family II. Crenothrichaceae

Family III. Beggiatoaceae

Appendix Achromatiaceae

Order IV. Myxobacteriales

Order V. Spirochaetales

Supplement: Groups whose relationships are uncertain.

Group I. Order Rickettsiales. Group II. Order Virales. Group III.

Family Borrelomycelaceae.

In this, the arrangement of Schizomycetes as a class coordinate with Schizophyceae, both belonging to a phylum Schizophyta of the plant kingdom, is maintained as before. The number of orders is reduced from seven as given in the fifth edition of the MANUAL to five, through recognition of the fact that the rigid, unicellular, sometimes branching but never truly mycelial nor filamentous organisms belonging to three of the previously recognized orders are presumably more closely related to each other than they are to the organisms in the four remaining orders. The family Corynebacteriaceae has been transferred from the order Actinomycetales to Eubacteriales.

The colorless, filamentous, sulfur bacteria (Beggiatoaceae) have been placed in the order Chlamydobacteriales with the other filamentous bacteria that are clearly related to the blue-green algae. While this marks the greatest deviation from the outline previously used, and separates these colorless sulfur bacteria from the purple sulfur bacteria placed in Rhodobacteriineae, it is in accordance with the arrangement accepted by Lehmann and Neumann (Bakt. Diag., 4 Aufl., 2, 1907, 598), Pringsheim (Lotos, 71, 1923, 307) and others. Rhodobacteriineac is also limited to the purple and green bacteria as suggested by Pringsheim (loc. cit.) and accepted by Kluyver and Van Niel (loc. cit.), by Stanier and Van Niel (loc. cit.) and others. 
The Rickettsiales and Borrelomycetaceue are placed in a supplement as their relationships are still obscure. Several authors would place them near some of the organisms now placed in Pasteurella and Haemophitus. The viruses (Tirales) whose nature and relationships are still more obscure are also placed in a supplemental group.

Although this outline maintains the simplicity that distinguished its predecessors, and provides places for all types of microorganisms thus far described that may properly be grouped under the fission fungi, it should not be regarded in any sense as final. An attempt has been made to express natural relationships, but these are so frequently obscure or unknown that in many places utilitarian considerations have prevailed. In some places, groups of known doubtful significance have been allowed to stand as they are out of a desire not to make unnecessary changes. It has appeared desirable to be conservative in making changes in the outline as used previously.

Addenda: After the above was in page proof, it was discovered that reference to the outline classification of Gieszezykiewicz (Bull. Adad. Polonaise d. Sci. et d. Lettres, Cl. Sci. Math. et Nat., Sér. B., 1939, 27 pp.) had inadvertently been omitted. This outline has some features like the outline that Lehmann and Neumann used in 1927 (see p. 17) and some like the outline used in the 4 th ed. of the MANUAL.

The genus Bacterium is retained as in the Lehmann and Neumann outline for Gram-negative, non-spore-forming, peritrichous or polar flagellate rods. Trrelve sub-genera are recognized and these bear subgeneric scientific names that are much the same as those used for genera in the 4 th ed. of the Maxual. A new subgeneric name Enterobacterium (see Enterobacter Rahn) is proposed to cover the genera Escherichia, Aerobacter, Klebsiella, Salmonella, Eberthella and Shigclla. Loefferella previously used by Gay et al. (Agents of Disease and Heat Resistance, Indianapolis, 1935, 782) is here also used as a subgeneric name for the glanders bacilus; and Chromobacterium is used for the organisms more properly placed in Serratia Bizio.

Corynebacterium is transferred from the order Actinomycetales to Eubacteriales and the family Corynebacteriaceas is made to include Lactobacillus, Erysipelothix and Fusobacterium. Among the Spirochactales, the genus name Spirochacta is displaced by a new generic term, Ehrenbergia, and is itself used to displace Borrelia.

A seventh order Rickettsiales is proposed to include two families: Rickettsiaceae with one genus Rickettsio da Rocha Lima (Berl. klin. Wchnschr., 1916, 567); and Bartonellaceae with the genera, Bartonella Strong. Tyzzer, Brues, Sellards and Gastiaburí (Jour. Amer. Med. Assoc., 61, 1913, 1713), and Grahamella Brumpt (Bull. Soc. Path. Exot., 4, 1911, 514). 
During 1945, Soriano (Ciencia e Investigación, 1, 1945, 92-94 and 146147; Rev. Argentina de Agronomia, 12, 1945, 120) proposed an arrangement of the Class Schizomycetes in which he recognizes a new Order, Flexibacteriales, to include the ffamilies Cytophagaceae and Beggiatoaceae and an entirely new Family Flexibacteriaceae containing a single genus Flexibacter. The latter includes five newly recognized species of flexuous bacteria as follows: Flexibacter flexilis, type species, $F$. elegans, $F$. giganteus, $F$. albuminosus and $F$. aureus.

The outline given below shows how this new order and new family are fitted by Soriano into the classification used in the fifth edition of the MANUAL.

Class Schizomycetes

Subclass Eubacteria. Rigid cells. Subclass Flexibacteria. Flexuous cells.

Order I. Eubacteriales

Order II. Caulobacteriales

Order III. Rhodobacteriales

Order IV. Actinomycetales

Order V. Chlamydobacteriales
Order VI. Flexibacteriales

Family I. Cytophagaceae

Family II. Beggiatoaceae

Family III. Flexibacteriaceae

Order VII. Myxobacteriales

Order VIII. Spirochaetales

Prévot (Ann. Inst. Past. 72, 1946, 1) has developed his classification of Class Actinomycetales, subdividing it into orders and including several genera not recognized in his 1940 outline. This classification is as follows:

Class Actinomycetales

Order I. Actinobucteriales. New order. Not acid-fast
Family I. Spherophoraceae.
Family II. Actinomycetaceae.
Genus I. Spherophorus
Genus I. Actinomyces
Genus II. Harerhillia
Genus II. Proactinomyces
Genus III. Spherocillus
Genus III. Corynebacterium
Genus IV. Fusiformis
Genus IV. Actinobacterium
Genus V. Fusocillus
Genus V. Bifidibacterium
Genus VI. Leptotrichia
Genus VI. Erysipelothrix

Order II. Mycobacteriales. New order. Acid-fast

Genus I. Mycobacterium

This classification differs from that used in this edition of the Manual in that it places several genera of Gram-negative organisms in Actinomycetales. These are Spherophorus, Haverhillia, Spherocillus, Fusiformis and Fusocillus, all of which are included here under Parvobacteriaceae. Leptotrichia which Prévot regards as Gram-negative is generally accepted as being a Gram-positive group. It is discussed in this edition of the ManUaL in connection with the genus Lactobacillus. 


\section{HOW BACTERIA ARE NATIED AND IDENTIFIED*}

Some principles of taxonomy and nomenclature. "Taxonomy is that branch of biology that deals with the orderly arrangement of plants and animals" (Johnson, Taxonomy of the Flowering Plants, New York, 1931, p. 3).

The necessity for applying names to species or kinds of bacteria is selfevident. It is highly desirable that the name applied to an organism by one person should be understood by others. It is further desirable that as far as practicable all individuals use the same name for the same kind of organism. It is helpful, therefore, if there can be an agreement regarding the method of naming organisms, and as to the correct name for each organism. The term nomenclature is applied to the naming of plants and animals, and under this term may be included all discussions as to methods of naming and correctness of particular names.

It is not enough that bacteria be named. Some method of classification of the bacteria is essential if the names are to be rendered accessible and available, and identification of unknown forms be made possible. Taxonomy is that branch of biology which treats of classification in aceordance with a convention or law. It is apparent that taxonomy must be dependent in part for its satisfactory development upon nomenclature. Exen though there may not be agreement among bacteriologists as to the exact classification that is to be used, nevertheless it is highly desirable that there be agreement as to some of the fundamental characteristics of satisfactory biological classifications in general.

What kinds of names are used. Two kinds of names are commonly given to the different kinds of plants and animals, the common, provincial, vernacular or casual names on the one hand and the international or scientific names on the other. These should be carefully differentiated, and their respective advantages and disadvantages noted.

It is inevitable, and on the whole probably desirable, that for each kind of familiar animal or plant in each language there will be coined a name. Usually the name for the same organism will be different in each language. For example, we have in English Oak, in German Eiche, in Latin Quercus, etc. For many uncommon kinds, however, there may be no such vernacular names developed. There have been, of course, many casual or vernacular names given to kinds of bacteria. In English we speak of the tubercle bacillus, the typhoid germ, the gonococcus, the Welch bacillus, the golden

* Contributed by Prof. R. E. Buchanan, Iowa State College, Ames, Iowa, January, 1934; revised, March, 1943. 
pus coccus, and many others. Similarly, we find in German Typhusbazillen and in French bacille typhique, enterococcus, etc. The use of these common names offers certain advantages. It does away frequently with the necessity of repeating longer and more formal scientific names. Not infrequently scientific names may be adopted into a language, and converted into vernacular names. For example, the English name aster and the scientific generic name $A$ ster are applied to the same group. This is frequently a convenience, but there are also some difficulties, which will be emphasized below.

In contrast to common, vernacular or casual names, the scientific name for each kind of organism (each plant or animal) is supposed to be the same in all countries and in all languages. When such a scientific name is used, ro question should arise in any language as to what organism is intended. The names thus applied are supposed to conform to certain general rules that have been formulated by international agreement. Obviously the use of such names is advantageous whenever one is desirous of accuracy, and of being definitely understood in all languages. It is further evident that in ill questions relating to taxonomy and classification it is highly desirable that the scientific names be used.

International rules for nomenclature. In order that there be an international set of scientific names, it is essential that there be an international agreement as to the rules which should govern their creation. Both of the great groups of biologists, the botanists and the zoologists, have met in numerous international congresses in which delegates were accredited by the great botanical and zoological societies, museums, and educational institutions of the world. Codes of nomenclature designed to tell how names shall be manufactured and used, and how to tell which of two or more names that have been used is correct, have been developed by each of these groups. These codes or lists of rules and recommendations are quite similar in essentials for botany and zoology, although they differ in some details.

The question arises: Are either or both of these codes satisfactory or adaptable to the use of bacteriologists. Three views have been expressed by various writers. Some few have suggested that the naming of bacteria cannot well conform to the approved international rules as their classification involves considerations not familiar to botanists and zoologists generally. The second group, also a very small one, has insisted that unicellular forms of life are neither plants or animals, but protista, and that taxonomic rules, etc., should be distinct for this group and coördinate with the corresponding rules for plants and for animals.

The third view, more commonly expressed, is that the bacteria are sufficiently closely related to the plants and animals, so that (in so far as 
they apply) the international agreements of the botanists (or zoologists) should be used as a basis for naming them.

International opinion on this topic was finally crystallized by resolutions adopted by the First International Congress of the International Society for Microbiology held in Paris in 1930 and by the Fifth International Botanical Congress held in Cambridge, England in the same year.

The resolutions unanimously adopted by the plenary session of the International Society for Microbiology were in part as follows:

"It is clearly recognized that the living forms with which the microbiologists concern themselves are in part plants, in part animals, and in part primitive. It is further recognized that in so far as they may be applicable and appropriate the nomenclatural codes agreed upon by International Congresses of Botany and Zoology should be followed in the naming of micro-organisms. Bearing in mind, however, the peculiarly independent course of development that bacteriology has taken in the past fifty years, and the elaboration of special descriptive criteria which bacteriologists have of necessity developed, it is the opinion of the International Society for Microbiology that the bacteria constitute a group for which special arrangements are necessary. Therefore the International Society for Microbiology has decided to consider the subject of bacterial nomenclature as a part of its permanent program."

The International Society of Microbiologists established a permanent Nomenclature Committee to pass upon suggestions and to make recommendations. This committee is composed of members from all participating nations. Two secretaries were named, one (Dr. St. John-Brooks of the Lister Institute, London, England) to represent primarily medical and veterinary bacteriology, and one (Dr. R. S. Breed, New York State Agricultural Experiment Station, Geneva, New York, U. S. A.) to represent other phases of bacteriology.

The coöperation of the International Botanical Congress was solicited in the naming of this committee. The resolutions were approved by the Section on Bacteriology of the Botanical Congress and the Congress itself incorporated into the Botanical Code certain special provisions relating to the bacteria. It also specifically recognized the International Committee as the body to prepare recommendations relating to bacterial nomenclature.

It is apparent, therefore, that there has been international agreement (in so far as this can be achieved) that bacteriologists should follow the botanical or zoological codes in the naming of bacteria to the extent they are applicable, and that exceptions or new problems should be presented to the International Committee.

These rules are so important in determining the validity of bacterial names that the rules of the Botanical Code are included in somewhat 
abridged form in the section that follows this introduction. Any student who has occasion to name a new species or a new genus or determine the validity of a name should familiarize himself with these rules and recommendations.

An effort has been made in the present volume to use nomenclature in conformity with these rules.

Some general principles of nomenclature. Every student of bacteriology should be familiar with certain rules of nomenclature if he is to use names intelligently. If he wishes to correct names improperly used or if he desires to name new species, there are additional rules which he must observe.

1. Each distinct kind of bacterium is called a species.

2. To each distinct species a name is given consisting usually of two Latin words, as Bacillus subtilis.

3. The first word is the name of the genus or group to which the organism belongs. It is always written with a capital letter. It is a Latin or Greek word, or a new word compounded from Latin or Greek roots, or it may be derived from some other language; but this is important, whatever its origin when used as a generic name it must be regarded and treated as a Latin noun. If it is a word not found in classic Latin, it is regarded as modern Latin. Some generic names in bacteriology which are Latin or formed from Latin roots are Bacillus (masculine) a small rod; Cristispira (feminine) a crested spiral; Lactobacillus (masculine) a milk small rod; Sarcina (feminine) a packet or bundle. Many others are words from the Greek or compounded from Greek roots, with the words transliterated into Latin letters and endings in conformity with Latin usage; words of Greek origin are Micrococcus (masculine) a small grain (sphere); Bacterium (neuter) a small rod; Clostridium (neuter) a small spindle; Corynebacterium (neuter) clubbed small rod; Actinomyces (masculine) ray fungus. Other generic names have been given in honor of persons or places as Beggiatoa (feminine), Borrelia (feminine), Eberthella (feminine), Pasteurella (feminine), Erwinia (feminine), Zopfius (masculine).

4. The second word in the scientific name is a specific epithet. It is not capitalized except that certain authors capitalize species names derived from proper nouns.

It may be:

(a) An adjective modifying the noun, and indicating by its ending agreement with the generic name in gender, as Bacterium album (white Bacterium), Bacillus albus (white Bacillus), Sarcina alba (white Sarcina), Eberthella dispar (the different Eberthella), Bacterium variabile (the variable Bacterium), Brucella melitensis (the maltese Brucella), Bacillus teres (the rounded Bacillus), Bacillus graveolens (sweet-smelling Bacillus). 


$\begin{array}{lcl} & \text { Typical adjectives } & \\ \text { Mssculine } & \text { Feminine } & \text { Nouter } \\ \text { albus } & \text { alba } & \text { album } \\ \text { niger } & \text { nigra } & \text { nigrum } \\ \text { tener } & \text { tenera } & \text { tenerum } \\ \text { acer } & \text { acris } & \text { acre } \\ \text { variabilis } & \text { variabilis } & \text { variabile } \\ \text { dispar } & \text { dispar } & \text { dispor } \\ \text { coccoides } & \text { coccoides } & \text { coccoides } \\ \text { aerogenes } & \text { aerogenes } & \text { aerogenes }\end{array}$

(b) An adjective in the form of the present participle of a verb, as Clostridium dissolvens (the dissolving Clostridium, in the sense of the Clostridium which is able to dissolve), Bacillus adhaerens (the adhering Bacillus), Acetobacter ascendens (the climbing Acetobacter), Bacillus esterificans (the ester-producing Bacillus). The endings for present participles used as adjectives are the same for all genders. The past participle is used occasionally, as in Pseudomonas aptata (the adapted Psendomonas), Spiritlum attenuatum (the attenuated Spirillum).

(c) A noun in the genitive (possessive) modifying the generic name. There is no necessary agreement in gender or number. Examples, Clostridium welchii (Welch's Clostridium), Salmonella pullowum the Salmonella of chicks), Streptococcus lactis (the Streptococcus of milk), Brucella abortus (the Brucella of abortion), Clostridium tetani (the Clostridium of tetanus), Diplococcus pneumoniae (the Diplococcus of pneumonia), Salmonella anatum (the Salmonella of ducks).

(d) A noun in apposition, that is, an explanatory noun. This does not agree necessarily with the generic name in gender. This method of naming is relatively not common in bacteriology. Examples are Actinomyces scabies (the scurf or scab Actinomyces), Bacillus lacticola (the milk-dweller bacillus), Bacillus radicicola (the root-dweller bacillus).

5. The author of the name is often indicated following the name of the species, as Bacillus subtitis Cohn. Sometimes a name is indicated also in parenthesis, as Microcuccus luteus (Schroeter) Cohn. This means that Schroeter first named the species, giving it the name luters, but placed it in another genus (Bacteridium). Cohn placed it in a new genus. It should be noted that the name of a person, following the name of an organism is frequently not the person who first discovered or described it, but the person who first gave it the name used. For example, Clostridium welchii (Migula) Holland was first described by Dr. Wm. H. Welch, but not named by him. It was named by Migula in honor of Dr. Welch and later it was placed in the genus Clostridium by Holland.

6. Sometimes species of bacteria are subdivided into varieties. These are likewise given Latin designations, and the entire name written as: 
Streptococcus lactis var. maltigenes (the Streptococcus of milk producing malt flavor).

Some principles of taxonomy. It is important further that the student of bacteriology recognize the meaning of certain terms used regularly in classifications.

(1) Species (plural species). A species of plant (or animal) is assumed above to be one kind of plant. But how much difference must exist between two cultures of bacteria before one is justified in regarding the organisms in them as being of distinct kinds or species? No rule can be laid down. It depends largely upon convenience and a more or less arbitrary decision. As stated by Hitchcock (Descriptive Systematic Botany, New York, 1925, p. 8): "The unit of classification is a coherent group of like individuals, called a species. The term is difficult to define with precision because a species is not a definite entity, but a taxonomic concept." Hucker and Pederson (New York Agric. Exper. Sta. Tech. Bull. 167, 1930, p. 39) state: "The difficulty met with among these lower forms in dividing them into well-defined groups has led many to question whether these small groups or 'species' are natural groups and whether such groups can be considered to be similar to 'species' among higher forms. However this may be, it is necessary to arrange bacteria as well as possible into groups or so-called 'species' for convenience in classification," and again (Hucker, New York Agric. Exper. Sta. Tech. Bull. 100, 1924, 29), "characters applicable to the differentiation of species must evidence a certain amount of constancy when studied over a large series of tests. Furthermore, characters adapted to the differentiation of larger natural groups or genera should, in addition to constancy, show some correlation with other constant characteristics. The presence of this relationship or correlation between characters for the division of genera indicates that the groupings are being made along natural rather than artificial lines."

Type culture. It is quite evident that when a new species of bacterium is described, it must include the particular culture from which the species description was made. This original culture is termed the type culture. We may develop a definition as follows:-A species of bacterium is the type culture or specimen together with all other cultures or specimens regarded by an investigator as sufficiently like the type (or sufficiently closely related to it) to be grouped with it. It is self-evident that different investigators may not draw the same boundaries for a given species. This leads to some practical difficulties, but no better definition has been evolved.

There are certain special cases which require brief discussion.

(a) How should one designate the different stages in an organism that exhibits a growth cycle? There seems to be increasing evidence that certain bacteria show cycles in morphology which parallel to some degree those well 
known among the fungi. Such, for example, may well be the rough ( $R$ ) and smooth (S) types described for many bacteria, possibly the filterable stages noted by many authors, the so-called G types, etc. It is evident that an adequate description of any species of bacterium should include a description of each of these stages in the cyclical development wherever such is proved to exist. In all other cases in botany and in zoology which involve growth stages or cycles one stage has been chosen and designated as the mature or adult or perfect stage. In ferns, for example, names and classifications are based largely upon the sporophytic generation, in insects upon the adult or imago, in the rusts upon the stage in which the teleutospores are produced. There has been no international agreement as to what stage should be thus designated for the bacteria. Beyond doubt, it would be the stage which is most easily cultured and studied in the laboratory, the stage with which we are best acquainted in the laboratory. It might easily happen in bacteria (as it has with fungi) that two different stages of the life cycle of single species have been described and named as separate species. When the mistake has been discovered, the name given to the mature or perfect stage is the one that is accepted. In general the descriptions given in the present volume are those which may be regarded as belonging to the perfect stage. Unfortunately it is not yet possible accurately to group the stages in many of the bacteria that have definite growth cycles.

It is desirable frequently to designate the stage with which one is working. This may be done by some conventional symbol, as S (smooth type), G (filterable stage), etc.

(b) How should one designate variants which differ in some minor respects from the type, but which do not constitute growth stages? For example, the species Bacillus subtitis normally produces endospores. Suppose that an asporogenous race is derived from such, agreeing with the parent culture in all respects, but showing no tendency to revert to spore production. What such an organism should be called is a matter of judgment. It might frequently be designated as an asporogenous strain, or more technically if one desires as a variety. It might be termed, for example, Bacillus subtitis var. asporus. In other cases such expressions as Diplococcus pneumoniae Type I, or the Rawlings strain of the typhoid bacillus may be used.

Unfortunately there is no general agreement upon the exact significance which the word "strain" should have in bacteriology. It is recommended that it refer merely to source, e.g. the Rawlings strain of Eberthella typhosa, and that it be never used to connote a biological character. This would not prevent such expressions as "a non-motile strain of Salmonclla suipestifer". but it would make erroneous a statement to the effect that the A 
strain of influenza virus differs from the $B$ strain in certain ways. In other words, "strain" is not a synonym of "type" or "variety". We may have as many yellow strains of the typhoid bacillus as we have of cultures of it, from different sources or specimens.

(2) Genus (plural genera). A genus is a group of related species. In some cases a genus may include only a single species (is said to be monotypic) in most cases several to many species are included in a genus. The question asked above may be paraphrased. How close must be the resemblances (how close the relationships) among the species of a group to entitle them to inclusion in the same genus? In other words, how is it possible to delimit accurately the boundaries of a genus? This is a matter on which there is no agreement, and probably can be none. Much of the confusion in modern bacteriological terminology is to be attributed to this fact. Nevertheless, in course of time experience tends to delimit many genera with reasonable accuracy. As stated by Hitcheock (Descriptive Systematic Botany, New York, 1925, p. 9): "Convenience may play a rôle in determining generic lines. Extremely large groups may be broken up on the basis of differences of smaller degree not common to a group of closely allied species, than if the group consisted of a few species. In general, the botanist, in delimiting genera, keeps in mind two important requirements, that of showing natural affinities and that of aiding correct identification." However, a genus may be defined helpfully in another way. One of the species described as belonging to a genus is designated as the type species. A genus may be defined then, as including this type species together with such other species as the investigator (or taxonomist) regards as sufficiently closely related. It is apparent that some authors may draw the lines narrowly, others broadly. Some authors, for example, recognize only two genera of rod-shaped bacteria, one for those without endospores (Bacterium), and one for those producing endospores (Bacillus). These genera thus defined are very large, each containing hundreds, perhaps thousands, of species. Other students break up these large genera into many smaller ones. There is not much point to the question as to which is right and which is wrong. A better question is, which is the more convenient, better represents relationships, better facilitates diagnosis and proves most useful. As organisms become better known, it may be possible through the agency of the International Committee on Nomenclature to reach agreements where lack of agreement leads to serious confusion or misunderstanding.

(3) Family. A family in taxonomy is a group of related genera. In general the name of the family is made from the name or former name of one of their genera by affixing the suffix -aceae to the root. The word is regarded as plural. Among bacterial families commonly recognized are Bacillaceae, Bacteriaceae, Micrococcaceae, Spirochaetaceae, Actinomycetaceae 
(4) Order. An order is a group of related families. It is named usually (not always) by substituting the suffix -ales for -aceae in the name of the type family. Among ordinal names that have been used in bacteriology are Actinomycetales, spirochaetales, Eubacteriales.

(5) Class. A class is a group of related orders. In this treatise it is considered that the bacteria constitute a class of the plant kingdom, and this is named Schizomycetes.

(6) Other categories. Other categories or rantis of names are used for higher groups. Sometimes families are divided into sub-families, these into tribes, these into subtribes, and these finally into genera.

How to identify an organism by name. One of the main purposes of a manual of determinative bacteriology is to facilitate the finding of the correct scientific name of a bacterium. Such is the purpose of this volume. It is well, however, to note some of the reasons why this result, the identification of an unknown culture, may not eventuate. Among these reasons the following may be listed:

(1) The unknown organism araiting identification by the investigator may easily be one which has never been named, or perhaps adequately described. For the most part there has been little effort on the part of bacteriologists to describe or name bacteria except as they have been found to have some economic significance or possess some striking or unusual characteristics. It is quite probable that there are many times as many species of bacteria undescribed and named as have been described. Such undescribed species are all about us. It is not surprising, therefore, if one frequently encounters undescribed species. When such unnamed species are encountered, particularly if they are of economic importance or are related to such forms, it is highly desirable that they should be described, named and the results published and made accessible.

(2) The unknown organism may have been described and named in some publication, but the description and name have been over-looked in the preparation of the MANrat. Perhaps the description has been so inadequate or incomplete that it has not been possible to place it in the classification. It should be noted that the number of species that have been described is so great that no one individual can know them all. Progress in classification comes about largely as the result of the work of specialists in particular groups. For example, Ford made a study of all of the aerobic spore-bearing bacteria which he had secured from various sources. He studied also the descriptions of such bacteria in the literature, and then monographed the group. Similar studies on other groups have resulted in more or less complete monographs. Such, for example, are the monographs on the intestinal group by Welden and Levine, of the acetic bacteria by Hoyer, and Visser 't Hooft, of the cocci by Hucker, of the 
pathogenic spore-bearing anaerobes by the English Commission, by Weinberg, and by others, of the red, rod-shaped bacteria by Hefferan and by Breed, of the actinomycetes by Waksman and by Lieske, of the root nodule bacteria of legumes by Fred and his co-workers, etc. Unfortunately most groups of bacteria have not thus been monographed. It is evidently the function of a manual such as this to draw largely upon the work of the monographers, and to supplement their achievements as far as possible by less satisfactory consideration of the unmonographed groups.

It is clear that because an organism cannot be identified from this text is not proof that it has not been described and named. The species most closely related may be determined, then the literature searched carefully for species described still more closely related or perhaps one identical.

(3) It is possible, of course, that an error has been made in the selection of the correct name. It is desirable that users of these keys and descriptions should be familiar with the rules governing the correct choice of names, and make suitable corrections where needed.

Steps in determining the name of an organism. The steps in the identification of an unknown organism are usually the following:

(1) Preparation of an adequate description of the organism.

(2) Knowledge of construction and use of keys.

(3) Determination of order, family and genus by use of key.

Preparation of description of organism. Before attempting to determine the name of an "unknown" organism an adequate description is essential. Just what characteristics must be emphasized depends upon the group in which the organism falls. It is desirable that the knowledge of the characters of the unknown be as complete as possible.

Use and construction of keys. An exceptionally clear and satisfactory discussion of the making and use of keys and synopses is given by Hitchcock (Descriptive Systematic Botany, New York, 1925, p. 104). Anyone planning to monograph a group is advised to read this. He states: "A key is an orderly arrangement of a series of contrasting or directly comparable statements, by which groups of the same category may be distinguished and indicated or identified," and "A key is primarily a mechanical device by which one may arrive at the name of the ultimate member of the group." In general the keys used in this MANUAL are dichotomous, that is, the successive divisions are in twos, differentiation being into two contrasted groups.

Determination of order, family and genus by use of keys. The method of doing this is discussed in the introduction beginning on page 1. 


\section{RULES OF NOMENCLATURE*}

In Paris in 1930, the First International Mlicrobiological Congress roted to follow the rules of nomenclature agreed upon by International Congresses of Botany and Zoology "in so far as they may be applicable and appropriate." The adoption of the date of the publication of Species Plantarum by Linnaeus in 1753 as the point of departure for bacteriological nomenclature was recommended. This recommendation was approved by the plenary session of the Microbiological Congress (Proc. $1^{\text {er }}$ Cong. Internat. Microbiol., Paris, 1930, 2, 1932, 519) and by the plenary session of the Botanical Congress (Rept. Proc. 5th Internat. Bot. Cong., 1930, Cambridge, 1931, p. 16 and 28$)$.

This Congress also provided for the organization of an International Committee on Bacteriological Nomenclature with two permanent secretaries:

1. To represent primarily medical and reterinary bacteriology,-Dr. R. St. John-Brooks, Lister Institute, London, England.

2. To represent primarily other phases of bacteriology,-Dr. R. S. Breed, Experiment Station, Geneva, New York, U. S. A.

During the years that have elapsed since its appointment, this Committee has organized and has taken various actions in the interest of a more stable nomenclature and classification. Some of these have been completed and accepted by the Second International Congress of Microbiology held in London, 1936. These completed actions are quoted below, and are incorporated into the classification used in the descriptive portion of the MANUAL.

The International Rules of Botanical Nomenclature were originally adopted by the International Botanical Congresses of Vienna (1903) and Brussels (1910). They were modified by the Cambridge Congress (1930) so as to accept the type method, and validate species descriptions of bacteria unaccompanied by a Latin diagnosis. Some further but less important modifications were made at the Amsterdam Congress (1935) (See Sprague, Science, 83, 1936, 416).

The following are the most important of the rules that are of interest to bacteriologists taken from the latest available edition of the Botanical Code (Gustav Fischer, Jena, 1935). Sections that were newly adopted or amended by the Amsterdam Botanical Congress (1935) are indicated in the text.

* Contributed by Prof. R. S. Breed, New York State Experiment Station, Geneva, New York, September, 1938; revised, October, 1943. 


\section{INTERNATIONAL RULES OF BOTANICAL NOMENCLATURE, 1930-1935}

\section{Chapter 1. General Considerations and Guiding Principles (Art. 1-9)}

Art. 1. Botany cannot make satisfactory progress without a precise system of nomenclature, which is used by the great majority of botanists in all countries.

Art. 2. The precepts on which this precise system of botanical nomenclature is based are divided into principles, rules, and recommendations. The principles (Art. 1-9, 10-14, 15-19) form the basis of the rules and recommendations. The object of the rules (Art, 19-74) is to put the nomenclature of the past into order and to provide for that of the future. They are always retroactive; names or forms of nomenclature contrary to a rule (illegitimate names or forms) cannot be maintained. The recommendations deal with subsidiary points, their object being to bring about greater uniformity and clearness in future nomenclature : names or forms contrary to a recommendation cannot on that account be rejected, but they are not examples to be followed.

Art. 3. The rules of nomenclature should be simple and founded on considerations sufficiently clear and forcible for everyone to comprehend and be disposed to accept.

Art. 4. The essential points in nomenclature are: (1) to aim at fixity of names; (2) to avoid or to reject the use of forms and names which may cause error or ambiguity or throw science into confusion.

Next in importance is the avoidance of all useless creation of names.

Other considerations, such as absolute grammatical correctness, regularity or euphony of names, more or less prevailing custom, regard for persons, etc., notwithstanding their undeniable importance, are relatively accessory.

Art. 5. In the absence of a relevant rule, or where the consequences of rules are doubtful, established custom must be followed.

Art. 7. Scientific names of all groups are usually taken from Latin or Greek. When taken from any language other than Latin, or formed in an arbitrary manner, they are treated as if they were Latin. Latin terminations should be used so far as possible for new names.

Art. 8. Nomenclature deals with: (1) the terms which denote the rank of taxonomic groups (Art. 10-14); (2) the names which are applied to the individual groups (Art. 15-72).

Art. 9. The rules and recommendations of botanical nomenclature apply to all groups of the plant kingdom, recent and fossil, with certain distinctly specified exceptions.

\section{Chapter II. Categories of Taxonomic Groups, and the Terms Denoting Them (Art. 10-14, Rec. I, II)}

Art. 10. Every individual plant belongs to a species (species), every species to a genus (genus), every genus to a family (familia), every family to an order (orda) every order to a class (classis), every class to a division (diviso).

Chapter III. Names of Taxonomic Groups (Art. 15-72, Rec. III-L)

Section 1. General Principles: Priority (Art. 15-17, Rec. III)

Art. 15. The purpose of giving a name to a taxonomic group is not to indicate the characters or the history of the group, but to supply a means of referring to it.

Art. 16. Each group with a given circumscription, position, and rank can bear only one valid name, the earliest that is in accordance with the Rules of Nomenclature. 
Section 2. The Type Method (Art. 18, Rec. IV-VII)

Art. 18. The application of names of taxonomic groups is determined by means of nomenclatural types. A nomenclatural type is that constituent element of a group to which the name of the group is permanently attached, whether as an accepted name or as a synonym. The name of a group must be changed if the type of that name is excluded (see Art. 66).

\section{Section 3. Limitation of the Principle of Priority: Publication, Starting-points, Conservation of Names (Art. 19-22)}

Art. 19: A name of a taxonomic group has no status under the Rules, and has no claim to recognition by botanists, unless it is validly published (see Art. 37).

Art. 20. Legitimate botanical nomenclature begins for the different groups of plants at the following dates:

(h) Myxomycetes, 1753 (Linnaeus, Species Plantarum, ed. 1).*

Art. 21. However, to avoid disadvantageous changes in the nomenclature of genera by the strict application of the Rules of Nomenelature, and especially of the principle of priority in starting from the dates given in Art. 20, the Rules provide a list of names which must be retained as exceptions. These names are by preference those which have come into general use in the fifty years following their publication, or which have been used in monographs and important floristic works up to the year 1890.

Section 4. Nomenclature of the Taxonomic Groups According to Their Categories (Art.23-35, Rec. VII-XX)

1. Names of Groups above the Rank of Family.

Rec. $I X$. Orders are designated preferably by the name of one of their prineipal families with the ending -ales.

2. Names of Families and Subfamities, Tribes, and Sub-tribes.

Art. 23. Names of families are taken from the name or former name of one of their genera and end in -aceae.

Art. 24. Names of subfamilies (subfamiliae) are taken from the name of one of the genera in the group, with the ending -oideae, similarly for tribes (tribus), with the ending -eae, and for subtribes (subtribus) with the ending-inae.

3. Names of Genera and Subdivisions of Genera.

Art. 25. Names of genera are substantives (or adjectives used as substantives), in the singular number and written with an initial capital, which may be compared with our family names. These names may be taken from any source whatever, and may even be composed in an absolutely arbitrary manner.

Recommendation $X$. Botanists who are forming generic names show judgment and taste by attending to the following recommendations:

(a) Not to make names long or difficult to pronounce.

(b) Not to dedicate genera to persons quite unconnected with botany or at least with natural science, nor to persons quite unknown.

(c) Not to take names from barbarous languages, unless those names are frequently cited in books of travel, and have an agreeable form that is readily adaptable to the Latin tongue and to the tongues of civilized countries.

* See page 48 for action on date for Schizomyceles. 
(d) To indicate, if possible, by the formation or ending of the name the affinities or analogies of the genus.

(e) To avoid adjectives used as nouns.

(f) Not to give a genus a name whose form is rather that of a subgenus or section (e.g. Eusideroxylon, a name given to a genus of Lauraceae. This, however, being legitimate, cannot be altered).

(g) Not to make names by combining words from different languages (nomina hybrida).

4. Names of Species (binary names).

Art. 27. Names of species are binary combinations consisting of the name of the genus followed by a single specific epithet. If an epithet consists of two or more words, these must either be united into one or joined by a hyphen. Symbols forming part of specific epithets proposed by Linnaeus must be transeribed.

The specific epithet, when adjectival in form and not used as a substantive, agrees with the generic names.

Recommendations:

XIII. The specific epithet should, in general, give some indication of the appearance, the characters, the origin, the history or the properties of the species. If taken from the name of a person it usually recalls the name of the one who discovered or described it, or was in some way concerned with it.

XIV. Names of men and women, and also of countries and localities used as specific epithets, may be substantives in the genitive (Clusii, saharae) or adjectives (Clusianus, dahuricus). It will be well, in the future, to avoid the use of the genitive and the adjectival form of the same epithet to designate two different species of the same genus: e.g. Lysimachia Hemsleyana Maximum. (1891), and L. Hesısleyi Franch. (1895).

XV. In forming specific epithets botanists will do well to have regard also to the following recommendations:

(a) To avoid those which are very long and difficult to pronounce.

(b) To avoid those which express a character common to all, or nearly all, the species of a genus.

(c) To avoid using the names of little-known or very restricted localities, unless the species is quite local.

(d) To avoid, in the same genus, epithets which are very much alike, especially those which differ only in their last letters.

(e) Not to adopt unpublished names found in travellers' notes or in herbaria, attributing them to their authors, unless these have approved publication.

(f) Not to name a species after a person who has neither discovered, nor described, nor figured, nor in any way studied it.

(g) To avoid epithets which have been used before in any closely-allied genus.

(h) To avoid specific epithets formed of two or more (hyphened) words.

(i) To avoid epithets which have the same meaning as the generic name (pleonasm).

\section{Section 5. Conditions of Effective Publication (Art.36)}

Art. 36. Publication is effected, under these Rules, either by sale or distribution of printed matter or indelible autographs to the general public, or to specified representative botanical institutions.

No other kind of publication is accepted as effective: communication of new names at a public meeting, or the placing of names in collections or gardens open to the public, does not constitute effective publication. 


\section{Section 6. Conditions and Dates of Valid Publication of Names (Art. 37-45, Rec. XXI-XXIX)}

Art. 37. A name of a taxonomic group is not validly published unless it is both (1) effectively published (see Art. 36), and (2) accompanied by a description of the group or by a reference to a previously and effectively published description of it.

Art. 38. From January 1, 1935, names of new groups of recent plants, the Bacteria excepted, are considered as validly published only when they are accompanied by a Latin diagnosis.

Art. 40. A name of a taxonomic group is not validly published when it is merely cited as a synonym.

Art.42. A name of a genus is not validly published unless it is accompanied (1) by a description of the genus, or (2) by the citation of a previously and effectively published description of the genus under another name, or (3) by a reference to a previously and effectively published description of the genus as a subgenus, section or other subdivision of a genus.

Art. 43. The name of a monotypic new genus based on a new species is validated (1) by the provision of a combined generic and specific description, (2) by the provision of a plate with analyses showing essential characters; but this applies only to plates and generic names published before January 1, 1908.

Art. 44. The name of a species or of a subdivision of a species is not validly published unless it is accompanied (1) by a description of the group, or (2) by the citation of a previously and effectively published description of the group under another name, or (3) by a plate or figure with analyses showing essential characters; but this applies only to plates or figures published before January 1,190 S.

Art. 45. The date of a name or of an epithet is that of its valid publication (see Art 19,36$)$. For purposes of priority, however, only legitimate names and epithets published in legitimate combinations are taken into consideration (see Art.60). In the absence of proof to the contrary, the date given in the work containing the name or epithet must be regarded as correct.

Botanists will do well in publishing to conform to the following recommendations:

XXI. Not to publish a new name without clearly indicating whether it is the name of a family or a tribe, a genus or a section, a species or a variety; briefly, without expressing an opinion as to the rank of the group to which the name is given.

Not to publish the name of a new group without indicating its type (see Recommendation $\mathrm{IV}$ ).

XXII. To avoid publishing or mentioning in their publications unpublished names which they do not accept, especially if the persons responsible for these names have not formally authorized their publication (see Recommendation XT (e).

XXYI. To give the etymology of new generic names and also of new epithets when the meaning of these is not obvious.

XXVII. To indicate precisely the date of publication of their works and that of the placing on sale or the distribution of named and numbered plants when these are accompanied by printed diagnoses. In the case of a work appearing in parts, the last published sheet of the volume should indicate the precise dates at which the different fascicles or parts of the volumes were published as well as the number of pages in each.

XIVIII. When works are published in periodicals, to require the publisher to indicate on the separate copies the date (year and month) of publication and also the title of the periodical from which the work is extracted. 
XXIX. Separate copies should always bear the pagination of the periodical of which they form a part; if desired they may also bear a special pagination.

\section{Section 7. Citation of Authors' Names for Purposes of Precision (Art. 46-49, Rec. $X X X-X X X I I$ )}

Art. 46. For the indication of the name (unitary, binary, or ternary) of a group to be accurate and complete, and in order that the date may be readily verified it is necessary to cite the author who first published the name in question.

Art. 47. An alteration of the diagnostic characters or of the circumscription of a group does not warrant the citation of an author other than the one who first published its name.

When the changes have been considerable, an indication of their nature and of the author responsible for the change is added, the words mutatis charact, or pro parte, or excl.gen., excl. sp., excl. var., or some other abridged indication being employed.

Art. 48. When a name of a taxonomic group has been proposed but not published by one author, and is subsequently validly published and ascribed to him (or her) by another author who supplied the description, the name of the latter author must be appended to the citation with the connecting word " $e x . "$

If it is desirable or necessary to abbreviate such a citation, the name of the publishing author, being the more important, must be retained.

When a name and description by one author are published by another author, the word apud is used to connect the names of the two authors, except where the name of the second author forms part of the title of a book or periodical in which case the connecting word in is used instead.

Art. 49. When a genus or a group of lower rank is altered in rank but retains its name or epithet, the original author must be cited in parenthesis, followed by the name of the author who effected the alteration. The same holds when a subdivision of a genus, a species, or a group of lower rank is transferred to another genus or species with or without alteration of rank.

\section{Section 8. Retention of Names or Epithets of Groups which are Remodelled or Divided (Art. 50-52)}

Art. 50. An alteration of the diagnostic characters, or of the circumscription of a group, does not warrant a change in its name, except in so far as this may be necessitated (1) by transference of the group (Art. 53-55), or (2) by its union with another group of the same rank (Art. 56-57), or (3) by a change of its rank (Art. 58).

Art. 51. When a genus is divided into two or more genera, the generic name must be retained for one of them, or (if it has not been retained) must be re-established. When a particular species was originally designated as the type, the generic name must be retained for the genus including that species. When no type was designated, a type must be chosen according to the regulations which will be given (Appendix I).*

Art. 52. When a species is divided into two or more species, the specific epithet must be retained for one of them, or (if it has not been retained) must be re-established. When a particular specimen was originally designated as the type, the specific epithet must be retained for the species including that specimen. When no type was designated, a type must be chosen according to the regulations to be given (Appendix I).

* Appendix I has not been published as yet. See Type Basis Code, p. 61. 


\section{Section 9. Retention of Names or Epithets of Groups Below the Rank of Genus on Transforence to Another Genus or Species (Art. 53-55)}

Art. 53. When a subdivision of a genus is transferred to another genus (or placed under another generic name for the same genus) without change of rank, its subdivisional name must be retained, or (if it has not been retained) must be re-established unless one of the following obstacles exists: (1) that the resulting association of names has been previously published validly for a different subdivision, or (2) that there is available an earlier validly published sub-divisional name of the same rank.

Art. 54. When a species is transferred to another genus (or placed under another generic name for the same genus), without change of rank, the specific epithet must be retained or (if it has not been retained) must be re-established, unless one of the following obstacles exists: (1) that the resulting binary name has been previously and validly published for a different species, (2) that there is available an earlier validly published specific epithet.

"When, on transference to another genus, the specific epithet has been applied erroneously in its new position to a different plant, the new combination must be retained for the plant on which the epithet was originally based, and must be attributed to the author who first published it." (Accepted in this revised form at the Amsterdam Botanical Congress, 1935.)

Art. 55. When a variety or other subdivision of a species is transferred, without change of rank, to another genus or species (or placed under another generic or sperific name for the same genus or species), the original subdivisional epithet must be retained or (if it has not been retained) must be re-established, unless one of the following obstacles exists: (1) that the resulting ternary combination has been previously and validly published for a subdivision based on a different type, even if that subdivision is of a different rank; (2) that there is an earlier validly published subdivisional epithet available.

When the epithet of a subdivision of a species, on transference to another species, has been applied erroneously in its new position to a different plant, the epithet must be retained for the plant on which the group was originally based.

Example: The variety micranthum Gren. \& Godf. (Fl. France, i, 171:1817) of Helianthemum italicum Pers., when transferred, as a variety to $H$. penicillatum Thib., retains its varietal epithet, becoming $H$. penicillatum var. micranthum (Gren. \& Godr.) Grosser (in Engl. Pfanzenreich, Heft 14, 115: 1903).

\section{Section 10. Choice of Names when Two Groups of the Same Rank are United, or in Fungi with a Pleomorphic Life-cycle

$$
\text { (Art. 56, 57, Rec. XXXIII-XXXI) }
$$

Art.56. When two or more groups of the same rank are united, the oldest legitimate name or (in species and their subdivisions) the oldest legitimate epithet is retained. If the names or epithets are of the same date, the author who unites the groups has the right of choosing one of them. The author who first adopts one of them, definitely treating another as a synonym or referring it to a subordinate group, must be followed.

Art. 57. Among Fungi with a pleomorphic life-cycle the different successive states of the same species (anamorphoses, status) can bear only one generic and specific name (binary), that is the earliest which has been given, starting from Fries, Systema, or Fries, Synopsis, to the state containing the form which it has been agreed to call the perfect form, provided that the name is otherwise in conformity with the Rules. The perfect state is that which ends in the ascus stage in the Ascomycetes, 
in the basidium, in the Basidiomycetes, in the teleutospore or its equivalent in the Uredinales, and in the spore in the Ustilaginales.

Generic and specific names given to other states have only a temporary value. They cannot replace a generic name already existing and applying to one or more species, any one of which contains the "perfect" form.

The nomenclature of Fungi which have not a pleomorphic life-cycle follows the ordinary rules.

\section{Section 11. Choice of Names when the Rank of a Group is Changed}

Art. 58. When a tribe becomes a family, when a subgenus or section becomes a genus, when a subdivision of a species becomes a species, or when the reverse of these changes takes place, and in general when a group changes its rank, the earliest legitimate epithet given to the group in its new rank is valid, unless that name or the resulting association or combination is a later homonym (see Art.60,61).

\section{Section 12. Rejection of Names (Art. 59-69, Rec. XXXVII)}

Art. 59. A name or epithet must not be rejected, changed, or modified merely because it is badly chosen, or disagreeable, or because another is preferable or better known (see also Art. 69).

Art. 60. A name must be rejected if it is illegitimate (see Art. 2). The publication of an epithet in an illegitimate combination must not be taken into consideration for purposes of priority, "except as indicated in Art. 61." (Added at the Amsterdam Botanical Congress, 1935.)

A name is illegitimate in the following cases:

(1) If it was superfluous when published, i.e., if there was a valid name (see Art. 16) for the group to which it was applied, with its particular circumscription, position and rank.

(2) If it is a binary or ternary name published in contravention of Art. 16, 50, 52, or 54, i.e., if its author did not adopt the earliest legitimate epithet available for the group with its particular circumscription, position, and rank.

(3) If it is a later homonym (see Art.61) (except as regards Art. 54 and 55).

(4) If it is a generic name which must be rejected under Art. 67.

(5) If its specific epithet must be rejected under Art. 68.

Art. 61. A name of a taxonomic group is illegitimate and must be rejected if it is a later homonym, that is, if it duplicates a name previously and validly published for a group of the same rank based on a different type. Even if the earlier homonym is illegitimate, or is generally treated as a synonym on taxonomic grounds, the later homonym must be rejected. "When an author simultaneously publishes the same new name for more than one group, the first author who adopts one of them, or substitutes another name for one of them, must be followed." (Added at the Amsterdam Botanical Congress, 1935.)

Art. 62. A name of a taxonomic group must be rejected if, owing to its use with different meanings, it becomes a permanent source of confusion or error. A list of names to be abandoned for this reason (Nomina ambigua) will form Appendix IV.*

Art. 63. A name of a taxonomic group must be rejected when its application is uncertain (Nomen dubium): e.g., Ervum soloniense L. (Cent. II. Pl. 28:1756) is a name the application of which is uncertain; it must, therefore, be rejected (see Schinz and Thell in Vierteljahrsschr. Nat. Ges. Zürich, viii, 71: 1913).

* Appendix IV has not been published as yet. 
Art. 64. A name of a taxonomic group must be rejected if the characters of that group were derived from two or more entirely discordant elements, especially if those elements were erroneously supposed to form part of the same individual.

A list of names to be abandoned for this reason (Nomina confusa) will form Appendix VI.*

Art. 65. A name or epithet of a taxonomic group must be rejected when it is based on a monstrosity

Art. 66. The name of an order, suborder, family or subfamily, tribe or subtribe must be changed when it is taken from the name of a genus which is known not to belong to the group in question-e.g. if the genus Portulaca were excluded from the family now known as Portulacaceae, the residual group could no longer bear the name Portulacaceae, and would have to be renamed.

Art. 67. Names of genera are illegitimate in the following special cases and must be rejected:

(1) When they are merely words not intended as names: e.g. Anonymous Walt. (Fl. Carol. 2, 4, 9, etc.: 1788) must be rejected as being a word applied to 28 different genera by Walter to indicate that they were without names.

(2) When they coincide with a technical term currently used in morphology unless they were accompanied, when originally published, by specific names in accordance with the binary method of Linnaeus. On and after Jan. 1, 1912 , all new generic names coinciding with such technical terms are unconditionally rejected.

(3) When they are unitary designations of species:e.g. Ehrhart (Phytophylacium: 1780; and Beitr. iv, 145-150: 1798) proposed unitary names for various species known at that time under binary names: e.g. Phaeocephalum for Schoenus fuscus, and Leptostachys for Carex leptostachys. These names, which resemble generic names, should not be confused with them, and must be rejected, unless they have been published as generic names by a subsequent author.

(4) When they consist of two words, unless these words were from the first combined into one, or joined by a hyphen.

Art. 68. Specific epithets are illegitimate in the following cases and must be rejected:

(1) When they are merely words not intended as names.

(2) When they are merely ordinal adjectives being used for enumeration.

(3) When they exactly repeat the generic name with or without the addition of a transcribed symbol.

(4) When they were published in works in which the Linnean system of binary nomenclature for species was not consistently employed.

Art. 69. In cases foreseen in Art. 60-68 the name or epithet to be rejected is replaced by the oldest legitimate name, or (in a combination) by the oldest legitimate epithet. If none exists, a new name or epithet must be chosen. Where a new epithet is required, an author may, if he wishes, adopt an epithet previously given to the group in an illegitimate combination, if there is no obstacle to its employment in the new position or sense.

\section{Section 13. Orthography of Names (Art. 70-71, Rec. XXXVIII-XLIV)}

Art. 70. The original spelling of a name or epithet must be retained, except in the case of a typographic error, or of a clearly unintentional orthographic error. When the difference between two generic names lies in the termination, these names must

* Appendix VI has not been published as yet. 
be regarded as distinct, even though differing by one letter only. This does not apply to mere orthographic variants of the same name.

Note 1. The words "original spelling" in this Article mean the spelling employed when the name was validly published.

2. The use of a wrong connecting vowel or vowels (or the omission of a connecting vowel in a specific epithet, or in that of a subdivision of a species) is treated as an unintentional orthographic error which may be corrected (see Rec. XLIV). "The liberty of correcting a name must be used with reserve, especially if the change affects the first syllable, and above all the first letter of the name." (Added at the Amsterdam Botanical Congress, 1935.)

3. In deciding whether two or more slightly different names should be treated as distinct or as orthographical variants, the essential consideration is whether they may be confused with one another or not: if there is serious risk of confusion, they should be treated as orthographic variants. Doubtful cases should be referred to the Executive Committee.

4. Specific and other epithets of Greek origin differing merely by having Greek and Latin terminations respectively are orthographic variants. Epithets bearing the same meaning and differing only slightly in form are (considered as) orthographic variants. The genitive and adjectival forms of a personal name are, however, treated as different

Recommendations: epithets (e.g. Lysimachia Hemsleyana and L. Hemsleyi).

XXXVIII. When a new name is derived from a Greek word containing the spiritus asper (rough breathing), this should be transcribed as the letter $h$.

XXXIX. When a new name for a genus, subgenus or section is taken from the name of a person, it should be formed in the following manner:-

(a) When the name of the person ends in a vowel the letter $a$ is added (thus Bouteloua after Boutelou; Ottoa after Otto; Sloanea after Sloane), except when the name already ends in $a$, when $e a$ is added (e.g. Collaea after Colla).

(b) When the name of the person ends in a consonant, the letters $i a$ are added (e.g. Magnusia after Magnus, Ramondia after Ramond), except when the name ends in er, when $a$ is added (e.g. Kernera after Kerner).

(c) The syllables which are not modified by these endings, retain their original spelling, even with the consonants $k$ and $w$ or with groupings of vowels which were not used in classical Latin. Letters foreign to botanical Latin should be transcribed, and diacritic signs suppressed. The Germanic $\ddot{a}, \ddot{o}, \ddot{u}$ become $a e, o e, u e$; the French $e, \grave{e}, \hat{e}$ become generally $e$. In works in which diphthongs are not represented by special type, the diaeresis sign should be used where required, e.g., Cephaëlis, not Cephaelis.

(d) Names may be accompanied by a prefix or a suffix, or modified by anagram or abbreviation. In these cases they count as different words from the original name.

Examples: Durvillea and Urvillea; Lapeyrousea and Peyrousea; Englera, Englerastrum and Englerella; Bouchea and Ubochea; Gerardia and Graderia.

XL. When a new specific or other epithet is taken from the name of a man, it should be formed in the following manner:-

(a) When the name of the person ends in a vowel, the letter $i$ is added (thus Glazioui from Glaziou, Bureaui from Bureau), except when the name ends in $a$, when $e$ is added (thus balansae from Balansa). 
(b) When the name ends in a consonant, the letters $i i$ are added (thus Magnusii from Magnus, Ramondii from Ramond), except when the name ends in -er when $i$ is added (thus Kerneri from Kerner).

(c) The syllables which are not modified by these endings retain their original speliing, even with the consonants $k$ or $w$ or with groupings of vowels which were not used in classical Latin. Letters foreign to botanical Latin should be transcribed and diacritic signs suppressed. The Germanic $\ddot{a}, \ddot{o}$, $\ddot{u}$ become $a e, o e, o u$, the French $e, \grave{e}, \hat{e}$ become generally $e$. The diaeresis sign should be used where required.

(d) When epithets taken from the name of a person have an adjectival form they are formed in a similar way (e.g. Geranium Robertianum, Verbena Hasslerana).

XLI. The same provisions apply to epithets formed from the names of women. When these have a substantival form they are given a feminine termination (e.g. Cypripedium Hookerae, Rosa Beatricis, Scabiosa Olgae, Omphalodes luciliae).

XLII. The specific (or other) epithets should be written in conformity with the original spelling of the words from which they are derived and in accordance with the rules of Latin and latinization.

\section{Examples: silvestris (not sylvestris) sinensis (not chinensis).}

XLIII. Specific (or other) epithets should be written with a small initial letter, except those which are derived from names of persons (substantives or adjectives), or are taken from generic "or vernacular" names (substantives or adjectives). (Emended Amsterdam Botanical Congress, 1935. See page 61 for actions taken by Second International Microbiological Congress, London, 1936 governing Bacteriological Nomenclature.)

XLIV. In the formation of specific (or other) epithets composed of two or several roots taken from Latin or Greek, the vowel placed between the two roots becomes a connecting vowel, in Latin $i$, in Greek o; thus menthifolia, salvifolia, not menthaefolia, salviafolia. When the second root begins with a vowel and euphony requires, the connecting vowel should be eliminated (e.g. lepidantha). The connecting vowels ae should be retained only where this is required for etymological reasons (e.g. caricaeformis from Carica, in order to avoid confusion with cariciformis from Carex). In certain compounds of Greek words no connecting vowel is required, e.g. brachycarpus and glycylphyllus.

\section{Section 14. Gender of Generic Names}

Art. 72. The gender of generic names is governed by the following regulations:-

(1) "A Greek or a Latin word adopted as a generic name retains its classical gender. In cases where the classical gender varies, the author has the right of choice between the alternative genders. In doubtful cases, general usage should be followed." "The following names, however, whose classical gender is masculine, are treated as feminine in accordance with historic usage: Adonis, Orchis, Stachys, Diospyros, Strychnos. Hemerocallis (m. in Sp. Pl. : Lat. and Gr. hemercalles $\mathrm{n}$.) is also treated as feminine to bring it into conformity with all other generic names ending in is." (Emended Amsterdam Botanical Congress, 1935.) See Van Eseltine, Jour. Bact., 26, 1933,569 , for discussion of the gender of generic names used for bacteria.

(2) Generic names which are modern compounds formed from two or more Greek or Latin words take the gender from the last. If the ending is altered, however, the gender will follow it.

(3) Arbitrarily formed generic names or vernacular names used as generic names take the gender assigned to them by their authors. Where the original 
author has failed to indicate the gender, the next subsequent author has the right of choice.

\section{Section 15. Various Recommendations (Rec. XLV-L)}

XLV. When writing in modern languages botanists should use Latin scientific names or those immediately derived from them, in preference to names of another kind or origin (popular names). They should avoid the use of the latter unless these are very clear and in common use.

XLVII. Only the metric system should be used in botany for reckoning weights and measures. The foot, inch, line, pound, ounce, etc., should be rigorously excluded from scientific language.

Altitude, depth, rapidity, etc., should be measured in meters. Fathoms, knots, miles, etc, are terms which should disappear from scientific language.

XLVIII. Very minute dimensions should be reckoned in $\mu$ (micromillimeters, microns, or thousandths of a millimeter) and not in fractions of millimeters or of lines, etc.: fractions encumbered with ciphers and commas easily give rise to mistakes.

XLIX. Authors should indicate clearly and precisely the scale of the figures which they publish.

L. Temperatures should be expressed in degrees of the centigrade thermometer of Celsius.

Chapter IV. Interpretation and Modification of the Rules (Art.73, 74)

Art. 73. A small permanent International Executive Committee is established with functions including the following:

(1) Interpreting the Rules in doubtful cases, and issuing considered "Opinions" on the basis of the evidence submitted.

(2) Considering Nomina conservanda, Nomina ambigua, Nomina dubia and Nomina confusa, and making recommendations thereon to the next International Botanical Congress.

(3) Considering all proposals for the modification of the Rules and reporting thereon to the next Congress.

(4) Reporting on the effects of modifications of the Rules accepted at the preceding Congress.

Art. 74. These Rules can be modified only by competent persons at an International Botanical Congress convened for the express purpose. Modifications accepted at one Congress remain on trial until the next Congress, at which they will receive sanetion unless undesirable consequences, reported to the Executive Committee, show need for further amendment or rejection.

Eight appendices have been or are to be prepared for this Code as follows: (1) †Regulations for determining types, (2) † Nomina conservanda familiarum, (3) *Nomina generica conservanda, (4) †Nomina ambigua, (5) †Nomina dubia, (6) †Nomina confusa, (7) *Representative botanical institutions recognized under Art. 34, (8) †Nomenclature of garden plants.

Unfortunately the first appendix which is of greatest interest to bacteriologists has not been prepared. As many bacteriologists, especially those in other countries, have not caught the significance of the type species

* These appendixes have been prepared.

$\dagger$ These appendixes have not been published as yet. 
concept as a means of defining bacterial genera, the reader is referred to the writings of Hitchcock (Amer. Jour. Bot., 8, 1921, 251; Descriptive Systematic Botany, New York, 1925) for an excellent exposition of the value of this idea to systematists.

Hitchcock (1921, p. 252) explains this concept briefly as follows: "The old concept was that a genus was a group of species having a given combination of characters; a species, similarly, a group of specimens. The new type concept is that, from the nomenclatural standpoint, a genus is a group of species allied to the type species; a species, a group of individuals similar to the type specimen."

Rules for determining types taken from the Type Basis Code of Nomenclature (Science, $49,1919,333 ; 53,1921,312$ ) drawn up by a Committee of which Hitcheock was Chairman are quoted as these are the most authoritative rules thus far available.

\section{Type Basis Code of Nomenclature (Hitchcock et al.)}

Article 4. The nomenclatural type species of a genus is the species or one of the species included when the genus was originally published.

If a genus included but one species when originally published, this species is the type.

When more than one species is included in the original publication of the genus, the type is determined by the following rules:

(a) When, in the original publication of a genus, one of the species is definitely designated as type, this species shall be accepted as the type regardless of other considerations.

If typicus or typus is used as a new sperific name for one of the species, this species shall be accepted as the type as if it were definitely designated.

(b) The publication of a new generic name as an avowed substitute for an earlier one does not change the type of the genus.

(c) If a genus, without an originally designated type, contains among its original species one with the generic name used as a specific name, either as a valid name or synonym, that species is to be accepted as the type.

(d) If a genus, when originally published, includes more than one species, and no species is definitely designated as type, nor indicated according to (c), the choice of the type should accord with the following principles:

1. Species inquirendae or species doubtfully referred to the genus, or mentioned as in any way exceptional are to be excluded from consideration in selecting the type.

2. Genera of the first edition of Linnaeus's "Species Plantarum" (1753) are usually typified through the citations given in the fifth edition of his "Genera Plantarum" (175t) except when inconsistent with the preceding articles.

3. Species which definitely disagree with the generic description (provided others agree), or which possess characters stated in the generic description as rare or unusual, are to be excluded from consideration in selecting the type. 


\section{RECOMMENDATIONS}

Article 5. In the future it is recommended that authors of generic names definitely designate type species; and that in the selection of types of genera previously published, but of which the type would not be indicated by the preceding rules, the following points be taken into consideration:

(a) The type species should usually be the species or one of the species which the author had chiefly in mind. This is often indicated by

1. A closer agreement with the generic description.

2. Certain species being figured (in the same work).

3. The specific name, such as vulgaris, communis, medicinalis or officinalis.

(b) The type species should usually be the one best known to the author. It may be assumed that an indigenous species (from the standpoint of the author), or an economic species, or one grown in a botanical garden and examined by the author, would usually represent an author's idea of a genus.

(c) In Linnaean genera the type should usually be chosen from those species included in the first technical use of the genus in pre-Linnaean literature.

(d) The types of genera adopted through citations of non-binomial literature (with or without change of name) should usually be selected from those of the original species which received names in the first binomial publication.

(e) The preceding conditions having been met, preference should be shown for a species which will retain the generic name in its most widely used sense, or for one which belongs to a division of the genus containing a larger number of species, or, especially in Linnaean genera, for the historically oldest species.

(f) Among species equally eligible, the preference should be given to the first known to have been designated as the type.

(g) If it is impossible to select a type under the conditions mentioned above, the first of equally eligible species should be chosen.

While the rules and recommendations of the above botanical codes are applicable in general to bacteria and related microorganisms, the fact that these are not infallible is evident because the rules developed independently by zoologists (see Proc. Biol. Soc. Washington, 39, 1926, 75, for the latest Code of Zoological Nomenclature) frequently follow a quite different course. In some cases at least the zoological rules will appeal to microbiologists as more likely to produce uniformity of usage than the botanical rules.

For example, microbiologists assembled at the Second International Microbiological Congress in London, 1936 accepted (Jour. Bact., 33, 1937, 445) Art. 13 of the International Rules of Zoological Nomenclature as preferable to Rec. 43 of the Botanical Rules to govern bacteriological practice. This reads as follows: "While specific substantive names derived from names of persons may be written with a capital initial letter, all other specific names are to be written with a small initial letter. Some examples taken from bacteriological literature are: Salmonella Schottmuelleri or Salmonella schottmuelleri, Bacillus Welchii or Bacillus welchii, Acetobacter Pasteurianum or Acetobacter pasteurianum, Corynebacterium ovis, Nitrosomonas javanensis, Rhizobium japonicum." 
In the MANUAL all species names are written with a small letter. It is felt that the value of a name as a name is lessened if capitals or other marks are used to indicate etymology. The derivation of generic and specific names is given separately in the descriptive material.

Likewise for obvious reasons, microbiologists refused (Jour. Bact., 38, $1937,445)$ to follow the botanical and zoological practice which permits the use of duplicate generic names, one for an animal and the other for a plant group; and accepted the following rules to govern their practice.

"a. Generic homonyms are not permitted in the group Protista.

b. It is advisable to avoid homonyms amongst Protista on the one hand, a plant or animal on the other."

The following actions of the International Committee on Bacteriological Nomenclature (Cent. f. Bact., II Abt., 92, 1935, 481) were confirmed (Jour. Bact., 33, 1937, 445).

Bacillus Cohn 1872 was accepted as a genus conservandum with Bacillus subtilis Cohn emend. Prazmowski 1880 as type species. It was agreed that Bacillus should be defined so as to exclude bacterial species which do not form endospores; and that the so-called Marburg strain found in type culture collections should be accepted as the type or standard strain.

At the Third International Congress of Microbiology held in New York City in September, 1939, a series of recommendations of the Permanent International Committees on Bacteriological Nomenclature were accepted at the plenary session of the Congress. The third and fourth recommendations were:

3. That the Nomenclature Committee, as at present constituted, shall continue to function under the auspices of the International Association of Microbiologists as it did under the International Society for Microbiology.

4. That the International Committee shall select from its membership a Judicial Commission consisting of twelve members, exclusive of members ex officio, and shall designate a Chairman from the membership of the Commission. The two Permanent Secretaries of the International Committee on Bacteriological Nomenclature shall be members ex officio of the Judicial Commission. The Commissioners shall serve in three classes of four commissioners each for nine years, so that one class of four commissioners shall retire at every International Congress. In case of the resignation or death of any Commissioner, his place shall be filled for the unexpired term by the International Committee at its next meeting.

By prompt action at and subsequent to the Congress ballots were cast in spite of war conditions by 26 of the 62 members of the Permanent Committee on Nomenclature. These ballots when examined by the joint Secretaries of the Permanent Committee in November, 1912 were found to have resulted in the selection of the persons whose names appear below. These 
are grouped in the three classes specified by the Permanent Committee, those receiving the highest number of votes being placed in the nine year class, those receiving the next highest in the six year class, etc. Names in the classes are arranged alphabetically.

Elected for nine years.-(The term normally expires in 1948.) R. E. Buchanan (U.S.A.), A. J. Kluyver (The Netherlands), E. G. D. Murray (Canada), S. Orla Jensen (Denmark): Elected for six years.-(Term normally expires in 1945.) J. Howard Brown (U.S.A.), A.-R. Prévot (France), J. Ramsbottom (Great Britain), Th. Thjötta (Norway); Elected for three years.-(Term normally would have expired in 1942.) A. Lwoff (France), R. Renaux (Belgium), A. Sordelli (Argentine), C. Stapp (Germany).

This announcement was made (Sci., 97, 1943, 370) in the hope that some plan for taking tentative action on questions of nomenclature could be developed by those members of the Commission who could be reached under war conditions.

While no provision was made in 1939 for the contingencies that have arisen, it is felt that those elected should serve until successors are elected. Professor R. E. Buchanan has been asked to act as Chairman pro tem of the Judicial Commission as there is no possibility of securing an election under the rules as adopted.

Tentative International Rules of Bacteriological Nomenclature were presented to the Third International Congress of Microbiology by a U.S.A.Canadian Committee on Compilation of Proposals on Bacteriological Nomenclature. As it proved impossible to give adequate consideration to these proposals during the Congress, the following recommendations of the Permanent Committee on Nomenclature were accepted:

1. That a recognized Bacteriological Code be developed.

2. That publication of such a proposed Code, when developed, be authorized with the proviso that it shall be regarded as wholly tentative, but in the hope that it shall be widely tested so that it may be brought up for further consideration and final disposition at the next Microbiological Congress which should normally take place in 1942 .

Copies of this tentative Code have been issued in mimeographed form by Prof. R. E. Buchanan, Iowa State College, Ames, Iowa, U.S.A., Chairman of the U.S.A.-Canadian Committee and may be obtained from him. 


\section{CLASS SCHIZOMYCETES NÖGELI}

(Bericht Verhandl. d. bot. Section d. 33 Versammling deutsch. Naturforsch.u. Arzt. Bot. Ztg., 1857, 760.)

Synonyms: Bacteria Cohn, Beitr. Biol. d. Pflanzen, 1, Heft 1, 1872, 136; Bacteriaceae Cohn, ibid., 237; Bacteriales Clements (as an ordinal name), The Genera of Fungi, Minneapolis, 1909, 8; Schizomycetaceae De Toni and Trevisan, in Saccardo, Sylloge Fungorum, 8, 1889, 923; Schizomycetacea Castellani and Chalmers, Manual of Tropical Medicine, 3rd ed., 1919, 924; Mychota Enderlein, Eakteriencyclogenie, 1924, 236; Schizomycetae Stanier and Van Niel, Jour. Bact., 42, 1941, 458.

Typically unicellular plants. Cells usually small, sometimes ultramicroscopic. Frequently motile. As in the closely related blue-green algae (Class Schizophyceae), the cells lack the definitely organized nucleus found in the cells of higher plants and animals. However, bodies containing chromatin which may represent simple nuclei are demonstrable in some cases. Individual cells may be spherical; or straight, curved or spiral rods. These cells may occur in regular or irregular masses or even in cysts. Where they remain attached to each other after cell division, they may form chains or even definite filaments. The latter may show some differentiation into holdfast cells, and into motile or non-motile reproductive cells (conidia). Some grow as branching mycelial threads whose diameter is not greater than that of ordinary bacterial cells, i.e., about one micron. Some species produce pigments. The true purple and green bacteria possess pigments much like or related to the true chlorophylls of higher plants. These pigments have photosynthetic properties. The phycocyanin found in the blue green algae does not occur in the Schizomycetes. Multiplication is typically by cell division. Endospores are formed by some species included in Eubacteriales. Sporocysts are found in Myxobacteriales. Liltramicroscopic reproductive bodies are found in Borrelomycetaceae. The bacteria are freeliving, saprophytic, parasitic or even pathogenic. The latter types catuse diseases of either plants or animals. Seren orders are recognized.

\section{Key to the Orders and Sub-()iders of the Class Schizomycetes.}

A. Cells rigid, not flexuous. Motility by means of flagella or by a gliding movement. 1. Cells single, in chains or masses. Not branching and myeclial in character. Not arranged in filaments. Not acid-fast. Motility when present by means of flagella.

Order I. Eubacteriales, p. 66.

a. Do not possess photosynthetic pigments. Cells do not contain free sulfur.

b. Not attached by a stalk. Do not deposit ferric hydroxide.

Sub-Order I. Eubacteriineae, p. 67.

bb. Attached to substrate, usually by a stalk. Some deposit ferric hydroxide.

Sub-Order II. Caulobacteriineae, p. 827.

aa. Possesses photosynthetic chlorophyll-like pigments. Some cells contain free sulfur.

Sub-Order III. Rhodobacteriineae, p. 838. 
2. Organisms forming elongated usually branching and mycelial cells. Multiply by cell division, special spores, oidiospores and conidia. Sometimes acid-fast. Non-motile.

Order II. Actinomycetales, p. 895.

3. Cells in filaments frequently enclosed in a tubular sheath with or without a deposit of ferric hydroxide. Sometimes attached. Motile flagellate and nonmotile conidia. Filaments sometimes motile with a gliding movement. Cells sometimes contain free sulfur.

Order III. Chlamydobacteriales, p. 981.

B. Cells flexuous, not rigid.

1. Cells elongate. Motility, by creeping on substrate

Order IV. Myxobacteriales, p. 1005.

2. Cells spiral. Motility, free swimming by flexion of cells.

Order V. Spirochaetales, p. 1051.

Supplements: Groups whose relationships are uncertain.

1. Obligate intracellular parasites or dependent directly on living cells.

a. Not ultramicroscopic and only rarely filterable. More than 0.1 micron in diameter.

Group I. Order Rickettsiales, p. 1083.

aa. Usually ultramieroscopic and filterable. Except for certain pox viruses of animals and a few plant viruses, less than 0.1 micron in diameter.

Group II. Order Virales, p. 1128.

2. Grow in cell-free culture media with the development of polymorphic structures including rings, globules, filaments and minute reproductive bodies (less than 0.3 micron in diameter).

Group III. Family Borrelomycetaceae, p. 1291.

\section{ORDER I. EUBACTERIALES BUCHANAN.}

(Jour. Bact., 2, 1917, 162.)

Simple and undifferentiated rigid cells which are either spherical or rod-shaped. The rods may be short or long, straight or curved or spiral. Some groups or species are non-motile, others show locomotion by means of flagella. Elongated cells divide by transverse fission and may remain attached to each other in chains. Spherical organisms divide either by parallel fission producing chains, or by fission alternating in two or three planes producing thus either tetrads or cubes of 8 and multiples of 8 cells. Many spherical cells form irregular masses in which the plane of division cannot be ascertained. Endospores occur in some species. Some species are chromogenic, but only in a few is the pigment photosynthetic (bacteriochlorophyll or other chlorophyll-like pigments).

A group of rather large, spherical to short rod-shaped, colorless sulfur bacteria, which some feel should be included in the order Eubacteriales, has been attached as an Appendix to the order Chlamydobacteriales on account of the physiological similarity between the former organisms and the Beggiatoaceae. These are in Family Achromatiaceae, p. 997. 


\section{Sub-Order I. Eubacterineae Breed, Murray and Hitchens.}

(Jour. Bact., 47, 1944, 421.)

These are, as the name Eubacteriinae implies, the true bacteria in the narrower sense of the word. The cells are rigid and free. Branching occurs only under abnormal conditions of life. They are not attached by holdfasts nor stalks. They form no sheaths. One-third of the species form pigments, but these have no photosynthetic properties. Endospores occur in one family (Bacillaceae), rarely in others.

\section{Key to the Families of the Sub-Order Eubacterineae.}

I. No endospores (except Sporosarcina).

A. Can develop on inorganic media. Autotrophic and facultative autotrophic. Family I. Nitrobacteriaceae, p. 69.

B. Cannot develop on inorganic media (exceptions, see Family XII. Bacteriaceae). Heterotrophic.

1. Polar flagellate, straight, curved or spiral rods. Gram-negative. (Some species with a single flagellum will be found under Family IV. Rhizobiaceae, Family V. Micrococcaceae and Family VIII. Corynebacteriaceae).

Family II. Pseudomonadaceae, p. 82.

2. Large, oval, pleomorphic cells sometimes almost yeast-like in appearance. Free living in soil. Fix free nitrogen. Peritrichous flagellation.

Family III. Azotobacteriaceae, p. 219.

3. Peritrichous or non-motile rods, and cocci.

a. Heterotrophic rods which may not require organic nitrogen for growth. Usually motile with one to six or more flagella. Usually form nodules or tubercles on roots of plants, or show violet chromogenesis.

Family IV. Rhizobiaceae, p. 223.

aa. Heterotrophic rods or cocci which utilize organic nitrogen and usually carbohydrates.

b. Spherical cells in masses, tetrads, and packets. A few species are motile with one or two flagella.

c. Gram-positive to Gram-negative cocci. Not obligate parasites.

\section{Family V. Micrococcaceae, p. 235.}

cc. Gram-negative, and sometimes anacrobic cocci. Obligate parasites.

Family VI. Neisseriaceae, p. 295.

bb. Spherical cells which grow in pairs and chains; and rods.

c. Gram-positive cocci and rods. Non-motile (some species of Streptococcaceae or Corynebacteriaceae may show motility).

d. Microaerophilic to anaerobic cocci and rods. Frequently in chains. Active in the fermentation of sugars. Never reduce nitrates.

Family VII. Lactobacteriaceae, p. 305.

dd. Usually aerobic, but sometimes anaerobic rods. Less active in the fermentation of sugars. May or may not reduce nitrates.

Family VIII. Corynebacteriaceae, p. 381. 
cc. Gram-negative rods. When motile, from four to many peritrichous flagella.

d. Grow well on ordinary media containing peptone. Aerobic to facultative anaerobic.

e. Gram-negative, straight rods which ferment sugars with the formation of organic acids.

f. Produce little or no acid in litmus milk. May or may not reduce nitrates. Many yellow chromogens. Borderline between this and following family indistinct. Some species anaerobic.

Family IX. Achromobacteriaceae, p. 412.

ff. Produce $\mathrm{CO}_{2}$ and frequently visible gas $\left(\mathrm{CO}_{2}+\right.$ $\mathrm{H}_{2}$ ) from glucose. Reduce nitrates. Usually from the alimentary, respiratory or urinary tract of vertebrates, though some are free-living or even plant parasites.

Family X. Enterobacteriaceae, p. 443.

dd. Small Gram-negative rods. Obligate parasites which usually require body fluids for growth. Do not grow well on ordinary media. Some are anaerobic.

Family XI. Parvobacteriaceae, p. 545.

cce. Rods of varied types not included in above families.

Aerobic to facultative anaerobic.

Family XII. Bacleriaceae, p. 596.

II. Form endospores. Large rods, sometimes in chains. Acrobic to anaerobic.

Family XIII. Bacillaceae, p. 704. 


\section{*FAMILY I. NITROBACTERIACEAE BLCHANAN}

(Jour. Bact., 2, 1917, 349 and Jour. Bact., 3, 1918, 179.)

Cells without endospores. Rod-shaped or ellipsoidal except for one spherical species (Nitrosococcus nitrosus). Spiral rods in Nitrosospira and in one species of Thiobacillus. Flagella either polar (so far as known), or absent. Gram stain uncertain, but presumably Gram-negative for all of the polar flagellate, rod-shaped species except for Nitrosomonas monocella which is reported to be Gram-positive. Capable of growing without organic compounds, using $\mathrm{CO}_{2}$ as the source of carbon, and obtaining their energy by oxidation of ammonia, nitrite, hydrogen, sulfur, or thiosulfate. Some species can also utilize organic compounds. Non-parasitic, usually soil or water forms.

\section{Kcy to the tribes and genera of family Nitrobacteriaceae.}

A. Organisms oxidize ammonia to nitrite, or nitrite to nitrate. Growth on standard media very poor or absent.

Tribe I. Nitrobacterieae, p. 70 .

a. Cells oxidize ammonia to nitrite.

b. Cells are separate, free or in dense aggregates. Do not form zoogloea. c. Cells ellipsoidal.

cc. Cells spherical.

$$
\text { Genus I. Nitrosomonas, p. } 70 .
$$

Genus II. Nitrosococcus, p. 71 .

cec. Cells spiral.

bb. Cells form a zoogloea.

Genus III. Nitrosospira, p. 71 .

c. The zoogloea is surrounded by a common membrane forming a cyst.

Genus IV. Nitrosocystis, p. 72.

cc. The massed cells are embedded in slime. No common membrane surrounds the cells.

Genus V. Nitrosogloea, p. 73.

aa. Cells oxidize nitrite to nitrate.

b. Cells form no zoogloea.

Genus VI. Nitrobacter, p. 74.

bb. Cells form a zoogloea.

Genus VII. Nitrocystis, p. 75.

B. Organisms oxidize hydrogen.

Tribe II. Hydrogenomonadeae, p. 76 .

a. Aerobic, non-spore-forming rods with single polar flagellum, or non-motile.

Genus I. Hydrogenomonas, p. 76.

* Text revised by Prof. R. S. Breed and Prof. H. J. Conn, Geneva, N. Y., Dec., 1937. Completely revised by Dr. R. L. Starkey, New Jersey Agricultural Experiment Station, New Brunswick, N. J., March, 1943. 
C. Organisms oxidize sulfur or thiosulfate and similar inorganic compounds of sulfur.

Tribe III. Thiobacilleae, p. 78.

a. Aerobic to anaerobic, non-spore-forming rods with a single polar flagellum on each (so far as known), or non-motile.

Genus I. Thiobacillus, p. 78.

TRIBE I. NITROBACTERIEAE WINSLOW ET AL.

(Jour. Bact., 5, 1920, 201.)

Organisms deriving energy from the oxidation of ammonia to nitrite or from nitrite to nitrate and depend on this oxidation for growth. Fail to grow on media containing organic matter in the absence of the specific inorganic materials used as sources of energy. Many organic compounds commonly used in standard culture media are toxic to this group.

\section{Genus I. Nitrosomonas Winogradsky.}

(Nitromonas Winogradsky, Ann. Inst. Past., 4, 1890, 257; Arch. Sci. biol., St. Peters ${ }^{-}$ burg, 1, 1892, 127; emend. S. and H. Winogradsky, Ann. Inst. Past. 50, 1933, 350.)

Cells ellipsoidal, non-motile or with a single polar flagellum, occurring singly, in pairs, short chains or irregular masses, which are not enclosed in a common membrane. Oxidize ammonia to nitrite more rapidly than the other genera of this tribe. From Latin, nitrosus, full of soda; M.L. nitrous; and Greek monas, a unit; M.L. a monad.

The type species is Nitrosomonas europaea Winogradsky.

1. Nitrosomonas europaea Winogradsky. (Arch. Sci. biol., St. Petersburg, 1, 1892, 127; Bacterium nitrosomonas Lehmann and Neumann, Bakt. Diag., 2nd ed., 2, 1899, 187; Pseudomonas europaea Migula, in Engler and Prantl, Die natürl. Pflanzenfam., 1, 1a, 1895, 29; Planococcus europaeus Vuillemin, Ann. Mycologie, Berlin, 11, 1913, 525.) From Latin, europacus, of Europe.

Rods : 0.9 to 1.0 by 1.1 to 1.8 microns occurring singly, rarely in chains of three to four. Possess a single polar flagellum 3 to 4 times the length of the rods, or rarely one at either end.

Grow readily in aqueous media without organic matter, and containing ammonium sulfate, potassium phosphate, and magnesium carbonate. The cells accumulate in soft masses around the particles of magnesium carbonate at the bottom of the flask. The liquid is oceasionally turbid through development of motile swarmer cells or monads.
Small, compact, sharply defined colonies brownish in color on silica gel.

Aerobic.

Strictly autotrophic.

Source: Soils of Zurich, Switzerland; of Gennevilliers, France; and Kazan, Russia.

Habitat: Presumably widely distributed in soil.

1a. Nitrosomonas europaea var. italica Perotti (Rendic. d. Accad. d. Lincei Roma, 15, 1906, 516; Abs. in Cent. f. Bakt., II Abt., 19, 1907, 337). Also see Engel and Skallau (Cent. f. Bakt., II Abt., 97, 305, 1937).

2. Nitrosomonas monocella Nelson. (Cent. f. Bakt., II Abt., 83, 1931, 287.) From Greek monos, single and Latin cella, room; M.L. single cell.

Ovoid rods: 0.6 to 0.9 micron, often occurring in pairs. Young cells nearly spherical. Motile by means of a single 
polar flagellum 3 to 5 times as long as the rod. Gram-positive (Nelson). Found negative by H. J. Conn (personal communication).

No growth in nutrient broth, nutrient agar, nutrient or plain gelatin, plain or litmus milk, glucose or plain yeast water, or on potato.

Silica gel or agar plates of inorganic medium: No typical colonies, but yellowish brown masses of growth around particles of $\mathrm{CaCO}_{3}$ in the medium.

Inorganic liquid medium containing ammonium salts: Uniform development throughout the liquid as well as in the carbonate sediment.

Even low concentrations of organic matter retard or completely inhibit the initiation of growth. Plant extracts are toxic.
Free $\mathrm{CO}_{2}$ and $\mathrm{O}_{2}$ necessary for growth.

Optimum pH 8.0 to 9.0. Poor growth below $\mathrm{pH}$ 7.0. Some growth above $\mathrm{pH}$ 9.0 .

Optimum temperature for growth and oxidation $28^{\circ} \mathrm{C}$.

Aerobic.

Strictly autotrophic.

Source: Isolated from field soil.

Habitat: Presumably widely distributed in soil.

S. Winogradsky and H. Winogradsky (Ann. Inst. Pasteur, 50, 1933, 394) have described 5 cultures of Nitrosomonas which were obtained from soils of France. An additional culture has been described by H. Winogradsky (Ann. Inst. Pasteur, $58,1937,394)$ from activated sludge.

\section{Genus II. Nitrosococcus Winogradsky.}

(Arch. Sci. biol., St. Petersburg, 1, 1892, 127.)

Cells large spheres, non-motile, not producing zoogloea. Oxidize ammonia to nitrite. From Latin, nitrosus, full of soda; and Greek kokkos, grain; M.L. nitrous coccus.

The type species is Nitrosococcus nitrosus (Migula) Bergey et al.

1. Nitrosococcus nitrosus (Migula) Bergey et al. (Nitrosococcus Winogradsky, Ann. Inst. Pasteur, 5, 1891, 577; Arch. Sci. biol., St. Petersburg, 1, 1892, 127 ; Micrococcus nitrosus Migula, Syst. d. Bakt., 2, 1900, 194; Nitrosococcus americanus Buchanan, Jour. Bact., 3, 1918, 180; Manual, 2nd ed., 1925, 35.) From Latin, nitrous, full of soda; M.L. nitrous.

Large spheres, 1.5 to 1.7 microns in size, with thick cell membrane. Motility could not be demonstrated. Stains readily with aniline dyes. Observed no zoogloea formation. Gram-positive
(Omelianski, Cent. f. Bakt., II Abt., 19, 1907, 263).

Liquid medium: Turbidity.

Silica gel : Both dark and light colonies. Surface colonies look like small drops of a turbid yellowish liquid.

Aerobic.

Optimum temperature $20^{\circ}$ to $25^{\circ} \mathrm{C}$.

Source: Isolated from soil from (Quito, Ecuador; Companias, Brazil; Melbourne, Australia.

Habitat: Presumably widely distributed in soil.

\section{Genus III. Nitrosospira Winogradsky.}

(Compt. rend. Acad. Sci., Paris, 192, 1931, 1004; Ann. Inst. Pasteur, 50, 1933, 406.)

Cells spiral-shaped. Oxidize ammonia to nitrite very slowly. From Latin, nitrosus, full of soda; and spira, coil, spiral; M.L. nitrous spiral.

The type species is Nitrosospira briensis Winogradsky. 
1. Nitrosospira briensis Winogradsky. (Ann. Inst. Pasteur, 50, 1933, 407.) From French, Brie, a place name; M.L. of Brie.

Spirals wound tightly to form very small cylinders as long as 15 to $20 \mathrm{mi}$ crons. Short spirals have the appearance of short rods and ellipsoidal cells. Small pseudo-cocei were observed in old cultures.

Colonies on silica gel: Small colonies which occasionally contain eyst-like aggregates of cells. The cysts are more poorly developed than in Nitrosocystis.

Aerobic.

Reaction optimum: $\mathrm{pH} 7.0$ to 7.2 .
Source: Uncultivated pasture soil of Brie, France.

Habitat: Presumably widely distributed in soil.

2. Nitrosospira antarctica Winogradsky. (Ann. Inst. Pasteur, 50, 1933, 407.) From Greek, antarkitos, southern, antarctic.

Cells and colonies similar to $N$. briensis except that the cells are generally wound together to form more compact spirals.

Aerobic.

Reaction optimum: $\mathrm{pH} 7.0$ to 7.2

Source: Soil from the Antarctic.

Habitat: Presumably widely distributed in soil.

\section{Genus IV. Nitrosocystis Winogradsky.}

(Compt. rend. Acad. Sci., Paris, 192, 1931, 1003; Ann. Inst. Pasteur, 50, 1933, 399.)

Cells ellipsoidal or elongated, uniting in compact, rounded aggregates surrounded by a common membrane to form cysts. The cysts disintegrate to free the cells, particularly when transferred to fresh media. Within the cyst, the cells are embedded in slime. Oxidize ammonia to nitrite at a rate intermediate between Nitrosomonas and Nitrosospira. From Latin, nitrosus, full of soda; and Greek, kystis, bladder; M.L. nitrous cyst.

The type species is Nitrosocystis javanensis comb. nov.

1. Nitrosocystis javanensis comb. nov. (Nitrosomonas javanensis Winogradsky, Arch. Sci. biol., St. Petersburg, 1, 1892, 127; Pseudomonas javanensis Migula, in Engler and Prantl, Die natürl. Pflanzenfam., 1, 1a, 1895, 30; Compt. rend. Acad. Sci., Paris, 192, 1931, 1003.) From Latin, of Java.

Small ellipsoidal cells having a diameter of 0.5 to 0.6 micron. Possess a polar flagellum 20 times as long as the rods.

In liquid medium produces very compact zoogloeal masses of cells and motile swarmers. The large zoogloea are themselves composed of smaller compact aggregates of cells.

On silica gel the colonies are circular to elliptical becoming clear or light brown.

Aerobic.

Strictly autotrophic.

Source: Soil of Buitenzorg, Java; Tokyo, Japan; La Reghaia, Tunisia.
Habitat: Presumably widely distributed in soil.

2. Nitrosocystis coccoides nom. nov. (Nitrosocystis $a, \mathrm{~S}$. Winogradsky and $\mathrm{H}$. Winogradsky, Ann. Inst. Pasteur, 50, 1933, 401.) From Greek, kokkos, a grain; eidos, form, shape; M.L. coccus-like.

Ellipsoidal cells about 1.5 microns in diameter. Occur as compact aggregates of cells imbedded in mucus and surrounded by a thickened capsule to form cyst-like bodies. Cells rarely solitary but more often in pairs and in small groups of four or more. Probably motile. The mucus which surrounds the cells is not readily stained, whereas the outside coating stains more easily.

Colonies on silica gel: As colonies develop, the coating of $\mathrm{CaCO}_{3}$ on the gel becomes yellowish and dissolves and the 
colony appears as a bulbous, angular, brown body which may become $0.5 \mathrm{~mm}$. in diameter. The cells are held firmly together in these irregularly shaped bulbous aggregates.

Aerobic.

Source: Poor soils of Brie and elsewhere in France.

Habitat: Presumably widely distributed in forest and manured soils.

A similar culture called Nitrosocystis $B . A$. Was isolated from activated sludge by H. Winogradsky (Compt, rend. Acad. Sei., Paris, 200, 1935, 1888; Ann. Inst. Pasteur, 58, 1937, 326). It produced compact, bulbous, dented cyst-like aggregates of cells having a yellow color. The colonies produced clear zones on silica gel coated with $\mathrm{CaCO}_{3}$. These cysts were composed of oval or elongated coccoid cells imbedded in mucus and surrounded by a thickened capsule, com- posed of two layers. The cells become dispersed from the cysts as motile cells and form new colonies. This culture differs from $\mathbf{N}$. coccoides in that the colonies have a pale reddish yellow color and the oval cells are 0.5 by 1.5 microns in size.

Cultures of Nitrosocystis were obtained by Rommell (Svensk. botan. Tidskrift, $26,1932,303)$ from forest soils. Kingma Boltjes (Arch. f. Mikrobiol., 6, 1935, 79) ubtained cultures which produced masses of cells, some of which were loose and others compact. They were not believed to be true zoogloea since no capsule or slimy substance was noted. The development of true cysts by nitrifying bacteria was questioned. Winogradsky (Bull. d. l'Inst. Pasteur, 33, 1935, 1074) concluded that Kingma Boltjes worked with a culture of Nitrosocystis and not of Nitrosomonas as was believed.

\section{Genus V. Nitrosogloea $H$. Winogradstiy.}

(Compt. rend. Acad. Sci., Paris, 200, 1935, 1857; Ann. Inst. Pasteur, 58, 1937, 335.)

Cells ellipsoidal or rod-shaped. Embedded in slime to form zoogloea. No common membrane surrounds the cells aggregates. Oxidize ammonia to nitrite. From Latin, nitrosus, full of soda; and Greek, gloea, glue, jelly; M.L. nitrous jelly.

The type species is Nitrosogloea merismoides H. Winogradsky.

1. Nitrosogloea merismoides H. Winogradsky. (Nitrosocystis "I", H. Winogradsky, Trans. Third Intern. Cong. Soil Sci., Oxford, 1, 1935, 139; Compt. rend. Acad. Sci., Paris, 200, 1935, 1887; Ann. Inst. Pasteur, 58, 1937, 333.) From Greek, merismos, a dividing, division; eidos, form, shape; M.L. division-like.

Ellipsoidal cells: 0.5 by 1.5 microns. Oval cells or short rods forming tetrads or chains, each group with its own sheath. The groups vary in shape to produce branched chains, irregular or compact aggregates.

Colonies on silica gel: Cells encased in a pale yellow mucilage giving the colony a dull appearance. Colony surface studded with little humps.
Aerobic.

Source: Activated sludge.

Habitat: Unknown.

2. Nitrosogloea schizobacteroides $\mathrm{H}$. Winogradsky. (Vitrosocystis "II", H. Winogradsky, Trans. Third Intern. Cong. Soil Sci., Oxford, 1, 1935, 139; Compt. rend. Acad. Sci., Paris, 200, 1935, 1887 ; Ann. Inst. Pasteur, 58, 1937, 333.) From Greek, schizo, to split; balterion, a small rod; eidos, form, shape; M.L. Iike a dividing rod.

Rods: Elongated rods or short filaments 3 to 4 microns long.

Colonies on silica gel: Flat groups of cells are produced which are united in a common sheath. The aggregates form a 
pseudo-tissue of interwoven filaments suggestive of a fungus pad. The pad can be removed as a unit from the medium.

Aerobic.

Source: Activated sludge.

Habitat: Unknown.

3. Nitrosogloea membranacea $H$. Winogradsky. (Nitrosocystis "III", H. Winogradsky, Trans. Third Intern. Cong. Soil Sci., Oxford, 1, 1935, 139; Compt. rend. Acad. Sci., Paris, 200, 1935, 1887; Ann. Inst. Pasteur, 58, 1937, 333.) From
Latin, membranaceus, of skin or membrane.

Ellipsoidal cells commonly in pairs and also solitary.

Colonies on silica gel: Appear as dull mucoid material with a pale straw color. The cells are held firmly together so that the entire colony is easily picked up with the transfer needle. No structural units within the colony.

Aerobic.

Source: Activated sludge.

Habitat: Unknown.

\section{Genus VI. Nitrobacter Winogradsky.}

(Winogradsky, Arch. Sei. biol., St. Petersburg, 1, 1892, 127; Vitromonas OrlaJensen, Cent. f. Bakt., II Abt., 22, 1909, 334; not Vitromonas Winogradsky, Ann. Inst. Past., 4, 1890, 257; Nitrobacterium Castellani and Chalmers, Manual Trop. Med., $1919,933$.

Cells rod-shaped. Oxidize nitrite to nitrate. From Latin, nitrum, soda; M.L. nitre; and Greek baktron, a small rod.

The type species is Nitrobacter winogradskyi Buchanan.

1. Nitrobacter winogradskyi Buchanan. (Nitrobacter Winogradsky, Arch. Sci. biol., St. Petersburg, 1, 1892, 127; Bacterium nitrobacter Lehmann and Neumann, Bakt. Diag., 2nd ed., 2, 1899, 187; Bacillus nitrobacter Löhnis, Vorlesungen landw. Bakt., Berlin, 1913, 152; Buchanan, Jour. Bact., 3, 1918, 180; Nitrobacterium nitrobacter Castellani and Chalmers, Manual Trop. Med., 1919, 933.) Named for S. Winogradsky, 1856- , the Russian microbiologist, who first isolated these bacteria.

Description taken from Gibbs, Soil Sci., 8, 1919, 448.

Short, non-motile rods with gelatinous membrane, 0.6 to 0.8 by 1.0 to $1.2 \mathrm{mi}$ crons. Does not stain readily. Gramnegative (Omelianski, Cent. f. Bakt., II Abt., 19, 1907, 263.)

Can be cultivated on media free of organic matter. Sensitive to certain organic compounds.

Washed agar colonies: In 7 to 10 days very small, light brown, circular to irregular colonies, becoming darker.
Silica gel: Colonies smaller but more dense than on washed agar.

Washed agar slant: In 7 to 10 days scanty, grayish streak.

Inorganic solution medium: After 10 days flocculent sediment. Sensitive to ammonium salts under alkaline conditions.

Nitrite is oxidized to nitrate.

Aerobic.

Strictly autotrophic.

Optimum temperature $25^{\circ}$ to $28^{\circ} \mathrm{C}$.

Source: Soil.

Habitat: Presumably widely distributed in soil.

2. Nitrobacter agile Nelson. (Cent.f. Bakt., II Abt., 83, 1931, 287.) From Latin agile, quick, agile, motile.

Rods : 0.5 by 0.8 to 0.9 micron, occurring singly, sometimes in pairs or larger aggregates. Rapidly motile with a long. thin, polar flagellum often 7 to 10 times as long as the rod. (Non-motile culture obtained by Kingma Boltjes, Arch. f. Mikrobiol., 6, 1935, 79.) Gram-negative. 
No growth in nutrient broth, nutrient agar, nutrient or plain gelatin, litmus or plain milk, glucose or plain yeast water, or on potato.

Nitrite agar: After two weeks, produces semi-spherical, minute, nearly transparent colonies. Oxidation usually complete in 10 to 14 days.

Inorganic liquid medium containing nitrite: Produces uniformly dispersed growth.

Optimum $\mathrm{pH} 7.6$ to 8.6 . Limits of growth 6.6 to 10.0 .
Temperature relations: Optimum for growth $25^{\circ}$ to $30^{\circ} \mathrm{C}$. Optimum for oxidation $28^{\circ} \mathrm{C}$. No oxidation at $37^{\circ} \mathrm{C}$. Thermal death point $60^{\circ} \mathrm{C}$. for five minutes.

Strictly autotrophic.

Aerobic.

Source: Isolated from greenhouse soils and from sewage effluents in Madison, Wisconsin.

Habitat: Presumably widely distributed in soil.

\section{Genus VII. Nitrocystis $H$. Winogradsky.}

(Trans. Third Intern. Cong. Soil Sci., Oxford, 1, 1935, 139; Nitrogloea H. Winogradsky, Comp. rend. Acad. Sci., Paris, 200, 1935, 1888.)

Cells ellipsoidal or rod-shaped. Embedded in slime and united into compact zoogloeal aggregates. Oxidize nitrite to nitrate. From Latin, nitrum, soda; M.L. nitre; and Greek, kystis, bladder; M.L. nitric cyst.

The type species is Nitrocystis sarcinoides.

1. Nitrocystis sarcinoides $H$. Winogradsky. (Nitrocystis B. A., Winogradsky, H., Compt. rend. Acad. Sci., Paris, 200, 1935, 1888; Nitrocystis " $I$ " and "II", Winogradsky, H., Trans. Third Intern. Cong. Soil Sci., Oxford, 1, 1935, 139; Ann. Inst. Pasteur, 58, 1937, 336.) From Latin, sarcina, a packet; M.L. Sarcina, a genus; Greek, eidos, form; M.L. Sarcina-like.

Rods: Small rods 0.5 by 1.0 micron. Cells ellipsoidal or wedge-shaped and grouped in sarcina-like packets.

Colonies on silica gel: On the surface of gel coated with kaolin the colonies appear as small raised amber warts. The colonies grow up to $5 \mathrm{~mm}$, in diameter. The colonies are viscous and sticky when young and they become brown with age, shrink, and look like scales and become hard like grains of sand. Each colony is enveloped in several layers of a thick slime which bolds the cells together so that the entire colony can be removed with a transfer needle.

Aerobic.

Source: Activated sludge.

Habitat: Unknown.
2. Nitrocystis micropunctata $\mathrm{H}$. Winogradsky. (Nitrocystis "III", Winogradsky, H., Trans. Third Intern. Cong. Soil Sci., Oxford, 1, 1935, 139; Nitrogloea micropunctata Winogradsky, H., Compt. rend. Acad. Sci., Paris, 200, 1935, 1888; Ann. Inst. Pasteur, 58, 1937, 326.) From Greek, mikros, small, little; and Latin, punctatus, spotted; M.L. with small spots.

Cells are ellipsoidal rods about 0.5 micron in diameter which stain poorly except at the ends. Encased in a viscous slime.

Colonies on silica gel: Like $N$. sarcinoides except that the colonies are more clear and they have a more plastic consistency. The cells are not held together by the slime in the colony as with $N$. sarcinoides. The capsule is more readily differentiated in old colonies.

Aerobic.

Source: Activated sludge.

Habitat: Unknown.

Appendix: The following have been placed in the Tribe Nitrobacterieae, sometimes incorrectly so:

Bactoderma alba Winogradsky. (Ann. 
Inst. Pasteur, 50, 1933, 41Ł.) From soil. This is the type species of genus Bactoderma Winogradsky.

Bactoderma rosea Winogradsky (loc. cit., p. 415). Isolated from soil.

Bacterium nitrificans Chester. (Nitratbildner aus Northeim, Burri and Stutzer, Cent. f. Bakt., II Abt., 1, 1895, 735; Chester, Ann. Rept. Del. Col. Agr. Exp. Sta., 9, 1897, 94; Bacillus nitrificans Chester Man. Determ. Bact., 1901, 239; Achromobacter nitrificans Bergey et al., Manual, 1st ed.. 1923, 137.) From soil. Deseription of this organism was shown by Winogradsky (Cent. f. Bakt., II Abt., 2, 1896, 415 and 449) to have been based on impure cultures.

Microderma minutissima Winogradsky. From soil. This is the type species of genus Microderma Winogradsky.

Microderma vacuolata Winogradsky (loc. cit.). Isolated from soil.

Ni'rosobacillus thermophilus Campbell. See Bacillus appendix.

Nitrobacter flavum Sack. (Cent. f. Bakt., II Abt., 62, 1924, 20.) Isolated from garden earth. See description,
Manual, 5th ed., 1939, 74. Heterotrophic and does not belong here (Kingma Boltjes, Arch. f. Mikrobiol., 6, 1935, 83). Vitrobacter oligotrophum Beijerinck. (Folia Microbiol., 3, 1914, 91; Verzamelde Geschriften van M. W. Beijerinck, 5, 1922,190 .) Isolated from soil. On cultivation this species lost its autotrophic habit and became heterotrophic. The organism was then called Nitrobacter polytrophum Beijerinck.

Nitrobacter opacum Sack (loc. cit. p. 21). Source and relationships as above. See Manual, 5th ed., 1939, 75.

Nitrobacter punctatum Sack (loc. cit., p. 20). Source and relationships as above. See Manual, 5th ed., 1939, 75.

Nitrobacter roseo-album Sack (loc. cit., p. 17 ; Scrratia roseo-alba Bergey et al., Manual, 3rd ed., 1930, 125.) Source and relationships as above. See description, Manual, 5th ed., 1939, 74.

Nitrosomonas groningensis Sack. (Cent. f. Bakt., II Abt., 64, 1925, 34.) Source and relationships as above. See description, Manual, 5th ed., 1939, 77.

TRIBE II. HYDROGENOMONADEAE PRIBRAM.

(Jour. Bact., 18, 1929, 370.)

Short rods, non-motile or with lophotrichous flagella. Organisms capable of deriving energy from oxidation of hydrogen. They probably grow well on organic media without hydrogen, although this has not been shown to be true for all species.

\section{Genus I. Hydrogenomonas Orla-Jensen.*}

$$
\text { (Cent. f. Bakt., II Abt., 22, 1909, 311.) }
$$

As the only genus of the tribe, its definition is identical with the definition of the tribe. From Greek hydōr, water; genos, producing and monas, a unit.

The type species is Hydrogenomonas pantotropha (Kaserer) Orla-Jensen.

* This group of bacteria is characterized by the ability to grow in substrates containing no organic matter and to utilize elemental hydrogen as the source of energy for growth. Under these conditions $\mathrm{CO}_{3}$ is used as the source of carbon. Bacteria with similar physiological characteristics but differing in morphology are placed in the genera Bacterium, Bacillus and Clostridium. Although other bacteria and even certain algae have enzyme systems which can activate hydrogen and reduce $\mathrm{CO}_{2}$ in the process, there is no evidence that these organisms are able to grow in inorganic media with hydrogen as the exclusive source of energy (See: Stephenson and Stickland, Biochem. Jour., 25, 1931, 205, 215; Woods, Biochem. Jour., 30, 1936, 515; Lee and Umbreit, Cent. f. Bakt., II Abt., 101, 1940, 354; Gaffron, Amer. Jour. Bot., 27, 1940, 273). 


\section{Key to the species of genus Hydrogenomonas.}

A. Not sensitive to high $\mathrm{O}_{2}$ concentrations. Growth in solution media under autotrophic conditions characterized by turbidity without pellicle formation.

1. Hydrogenomonas pantotropha.

B. Sensitive to high $\mathrm{O}_{2}$ concentrations. Growth in solution media under autotrophic conditions characterized by pellicle adhering to walls of container.

2. Hydrogenomonas vitrea.

C. Sensitive to high $\mathrm{O}_{2}$ concentrations. Growth in solution media under autotrophic conditions without pellicle formation.

3. Hydrogenomonas flava.

\section{Hydrogenomonas pantotropha} (Kaserer) Orla-Jensen. (Bacillus pantotrophus Kaserer. Cent. f. Bakt., II Abt., 16, 1906, 688; Orla-Jensen, Cent. f. Bakt., II Abt., 22, 1909, 311.) From Greek pantos, everything and trophos, feeds on; M.L. omnivorous.

Rods: 0.4 to 0.5 by 1.2 to 1.5 microns with rounded ends. Occur singly, in pairs, and in chains. Encapsulated. Actively motile by means of a single long polar flagellum. Gram stain not recorded. Bipolar staining in old cultures.

Inorganic solution: When cultivated under an atmosphere of $\mathrm{O}_{2}, \mathrm{CO}_{2}$ and $\mathrm{H}_{2}$, the liquid becomes turbid without pellicle formation.

Inorganic solid media: When cultivated under an atmosphere of $\mathrm{O}_{2}, \mathrm{CO}_{2}$ and $\mathrm{H}_{2}$, the colonies are yellow and slimy, and the agar plates have an odor resembling hot soapy water.

Gelatin colonies: Yellow, smooth, rarely concentrically ringed or greenish.

Gelatin stab: Growth only at surface. As a rule no liquefaction.

Agar colonies: Same as on gelatin, greenish, often slimy.

Broth: Turbid, somewhat slimy, and occasional pellicle.

Milk: No coagulation. A yellow pellicle forms. Medium becomes slimy and assumes a dirty flesh color.

Potato: Moist, yellow, glistening.

Indole is not formed.

Hydrogen sulfide is not formed.

Nitrite is not produced from nitrate.

Does not act on carbohydrates.

Aerobic.
Optimum temperature $28^{\circ}$ to $30^{\circ} \mathrm{C}$.

Facultative autotroph.

Distinctive characters: Develops autotrophically in inorganic medium under an atmosphere of $\mathrm{H}_{2}, \mathrm{O}_{2}$ and $\mathrm{CO}_{2}$. Oxidizes hydrogen to water and uses $\mathrm{C}_{2} \mathrm{O}_{2}$ as the source of carbon for growth.

Source: Isolated from soil near Vienna.

Habitat: Probably widely distributed in soil.

2. Hydrogenomonas vitrea Niklewski. (Jahrb. f. wissensch. Botanik, 48, 1910, 113). From Latin vitreus, of glass, transparent.

Rods : 2.0 microns in length, cells adhering to each other as by slime. Notility not observed.

Agar colonies on inorganic medium in presence of $\mathrm{H}_{2}, \mathrm{O}_{2}$ and $\mathrm{CO}_{2}$ : Delicate, transparent, with slight fluorescence, and yellow center. Surface folded. Do not develop readily beneath the surface of medium.

Agar streak on inorganic substrate: Same as agar colonies except that growth is spreading.

Inorganic liquid medium in presence of $\mathrm{H}_{2}, \mathrm{O}_{2}$ and $\mathrm{CO}_{2}$ : Pellicle, adherent to wall of tube. Cood development when there is from 2 to $\&$ per cent oxygen in the gas. At higher $O$. concentrations good growth occurs only in association with $H$. flave or other bacteria.

Oxidizes hydrogen to water.

Microaerophilic, growing in an atmosphere of low oxygen tension, not exceeding 8 per cent.

Facultative autotroph. 
Distinctive characters: Grows in substrates containing no organic matter and produces a pellicle.

Source: Isolated from mud, garden soil, pasture land, vegetable mold, and peat.

Habitat: Presumably widely distributed in soil.

3. Hydrogenomonas flava Niklewski. (Jahrb. f. wissensch. Botanik., 48, 1910 , 113; emend. Kluyver and Manten, Antonie v. Leuwenhoek, 8, 1942, 71.) From Latin flavus, yellow.

Rods: 1.5 microns in length. Motility by polar flagella. Gram-negative.

Agar colonies on inorganic medium in presence of $\mathrm{H}_{2}, \mathrm{O}_{2}$ and $\mathrm{CO}_{2}$ : Small, smooth, yellow, shining, adhering to medium. Develop well below surface of medium, but growth is paler.

Gelatin not liquefied.

Inorganic liquid medium in presence of $\mathrm{H}_{2}, \mathrm{O}_{2}$, and $\mathrm{CO}_{2}$ : No pellicle formation. Good development when there is from 2 to 8 per cent oxygen in the gas. At higher $\mathrm{O}_{2}$ concentrations good growth occurs only in association with $H$. vitrea or other bacteria.

Oxidizes hydrogen to water.

Microaerophilic, growing in an atmosphere of low oxygen tension, not exceeding 8 per cent.

Facultative autotroph.

Distinctive characters: Found singly on slides whereas the rod-shaped cells of Hydrogenomonas vitrea tend to cling together in masses. Colonies on agar opaque, not transparent.

Source: Same as $H$. vitrea.

Habitat: Presumably widely distributed in soil.

Appendix: Incompletely described species are found in the literature as follows :

Hydrogenomonas agilis Niklewski. (Jubliäumsschrift f. Prof. E. Godlewski. Kosmos, Lemberg, 1913; See Cent. f. Bakt., II Abt., 40, 1914, 430.) From soil.

Hydrogenomonas minor Niklewski. (Jubliäumsschrift f. Prof. E. Godlewski. Kosmos, Lemberg, 1913; See Cent. f. Bakt., II Abt., 40, 1914, 431.) From soil.

TRIBE III. THIOBACILLEAE BERGEY, BREED AND MURRAY.

$$
\text { (Preprint, Manual, 5th ed., Oct., 1938, v.) }
$$

Organisms capable of deriving their energy from oxidation of sulfur or sulfur compounds. Most species do not grow on organic media.

\section{Genus I. Thiobacillus Beijerinck.}

(Beijerinck, Cent.f. Bakt., II Abt., 11, 1904, 593; Sulfomonas Orla-Jensen, Cent. f. Bakt., II Abt., 22, 1909, 314; not Thiobacillus Ellis, Sulphur bacteria, London, 1932, 130; Thiobacterium Lehmann and Neumann, Bakt. Diag., 7 Aufl., 2, 1927, 517; not Thiobacterium Janke, Allgemeine Tech. Mikrobiol., 1, 1924, 68, Leipzig.)

Small Gram-negative, rod-shaped cells. Non-motile or motile by means of a single polar flagellum. Derive their energy from the oxidation of incompletely oxidized sulfur compounds, principally from elemental sulfur and thiosulfate but in some cases also from sulfide, sulfite, and polythionates. The principal product of oxidation is sulfate, but sulfur is sometimes formed. They grow under acid or alkaline conditions and derive their carbon from carbon dioxide or from bicarbonates in solution; some are obligate and some facultative autotrophic. One species is facultative anaerobic. From Greek theion, sulfur and Latin bacillus, a small rod.

The type species is Thiobacillus thioparus Beijerinck. 


\section{Key to the species of genus Thiobacillus.}

\section{Aerobic.}

A. Strictly autotrophic.

1. Optimum reaction for growth close to neutrality.

1. Thiobacillus thioparus.

2. Optimum reaction for growth $\mathrm{pH} 2.0$ to 3.5 .

2. Thiobacillus thiooxidans.

B. Facultative autotrophic.

3. Thiobacillus novellus.

4. Thiobacillus coproliticus.

II. Anaerobic in presence of nitrate.

\section{Thiobacillus denitrificans.}

1. Thiobacillus thioparus Beijerinck. (Cent. f. Bakt., II Abt., 11, 1904, 593; Nathanson, Mitt. Zool. Station Neapel, 15, 1902, 655; Sulfomonas thioparus OrlaJensen, Cent. f. Bakt., II Abt., 22, 1909, 326). From Greek theion, sulfur and paro, to make.

Thin, short rods, 0.5 by 1 to $3.0 \mathrm{mi}$ crons. Motile (non-motile culture reported. See Starkey, Soil Sci., 39, 1935, 197.) Gram-negative.

Thiosulfate medium (liquid): Pellicle consisting of cells and free sulfur.

Thiosulfate agar: Colonies small, circular, whitish yellow due to precipitated sulfur.

Optimum reaction: Close to neutrality.

Strictly autotrophic. Derives its energy by the oxidation of thiosulfate to sulfate and sulfur; also oxidizes sulfur to sulfate.

Aerobic.

Source: Sea water, river water, mud, sewage, and soil.

Habitat: Presumably widely distributed.

2. Thiobacillus thiooxidans Waksman and Joffe. (Jour. Bact., 7, 1922, 239; Sulfomonas thiooxidans Waksman, Jour. Bact., 7, 1922, 616; Thiobacterium thiooxydans Lehmann and Neumann, Bakt. Diag., 7 Aufl., 2, 1927, 517.) From Greek theion, sulfur and M.L. to oxidize.

Short rods: 0.5 by 1.0 micron with rounded ends. Occur singly, in pairs, or in chains. Motile by means of a single polar flagellum. Gram-negative (Starkey, Soil Sci., 39, 1935, 210).

Thiosulfate agar: Scant growth. Nearly transparent colonies.

Sulfur medium (liquid): Uniform turbidity. No sediment or surface growth. Medium becomes very acid (below $\mathrm{pH}$ 1.0).

Thiosulfate medium (liquid): Uniform turbidity. Medium becomes acid and sulfur is precipitated.

Nitrogen sources: Utilizes ammonia nitrogen but not nitrate nitrogen which is toxic. Asparagin, urea and peptone not utilized.

Temperature relations: Optimum $28^{\circ}$ to $30^{\circ} \mathrm{C}$. Slow growth at $18^{\circ}$ and $37^{\circ} \mathrm{C}$. Death occurs at $55^{\circ}$ to $60^{\circ} \mathrm{C}$.

Optimum reaction: $\mathrm{pH}$ 2.0-3.5. (Limiting reactions, $\mathrm{pH} 6.0$ to less than $\mathrm{pH}$ 0.5.)

Strictly autotrophic, deriving its energy from the oxidation of elementary sulfur and thiosulfate, oxidizing these to sulfuric acid. It utilizes the $\mathrm{CO}_{2}$ of the atmosphere as a source of carbon.

Strictly aerobic.

Distinctive characters: This species produces more acid, from oxidation of sulfur, and continues to live in a more acid medium, than any other living organism yet reported, the hydrogen-ion concentration of the medium increasing to a pH 0.6 and less.

Source: Isolated from composts of soil, sulfur, and rock phosphate and soils containing incompletely oxidized sulfur compounds.

Habitat: Soil. 
3. Thiobacillus novellus Starkey. (Jour. Bact., 28, 1934, 365; Jour. Gen. Physiol., 18, 1935, 325; Soil Sci., 39, 1935, 207, 210.) From Latin novellus, new

Short rods or ellipsoidal cells: 0.4 to 0.8 by 0.6 to 1.8 microns. Non-motile. Gram-negative.

Gelatin stab: Mucoid growth at point of inoculation. Sub-surface growth meager. Slow liquefaction.

Agar plate: Growth slow, colorless, moist, raised, circular, $1 \mathrm{~mm}$ in diameter. Deep colonies tiny, lens-shaped.

Thiosulfate agar plate: Growth slow, becoming white from precipitated sulfur. Surface colonies small, circular, moist. Crystals of $\mathrm{CaSO}_{4}$ appear throughout the agar.

Agar slant: Growth fairly abundant, soft, somewhat ropy, raised, shining, moderately spreading; whitish in reflected light, brownish opalescence in transmitted light.

Thiosulfate agar slant: Growth very thin, practically colorless. No sub-surface growth. Sulfur usually precipitated as white frosty film on the surface.

Agar stab: White to cream-colored growth confined close to point of inoculation. Penetrates to bottom of tube.

Thiosulfate agar stab: No appreciable surface growth.

Broth: Slightly turbid. Gelatinous pellicle. Forms long streamer-like network extending from surface to the bottom. Some sediment.

Thiosulfate solution medium: Uniform turbidity. No pellicle. Whitish sediment with thin incomplete membrane on the bottom of the flask. Reaction acid in a few days, changes $\mathrm{pH} 7.8$ to 5.8 with decomposition of a small quantity of thiosulfate.

Sulfur solution medium of slightly alkaline reaction: No growth.

Potato slant: Growth limited, creamcolored, moist, shining, slightly brown.

Litmus milk: Slow development of slight alkalinity.

Facultative autotrophic.
Optimum reaction: Close to neutrality (limiting reactions $\mathrm{pH} 5.0$ to 9.0 ).

Aerobic.

Distinctive characters: Oxidizes thiosulfate to sulfate and sulfuric acid. Does not oxidize free sulfur.

Source: Isolated from soils.

Habitat: Soils.

4. Thiobacillus coproliticus Lipman and MeLees. (Soil Sci., 50, 1940, 432.) Latinized form of the English word coprolite, fossil dung.

Long thin rods: 0.1 to 0.2 by 6 to 8 (may measure 3 to 40 ) microns. Straight, S-shaped, and curved cells. Motile by means of a single polar flagellum.

Peptone soil extract agar: Slight growth.

Nutrient solution: Little or no growth.

Thiosulfate agar: Slow development. Produces small watery colonies raised above the agar surface. Colonies have been noted which were white from precipitated sulfur.

Thiosulfate solution: Thiosulfate is oxidized. Little or no turbidity. No pellicle. No sediment. Change in reaction from $\mathrm{pH} 7.6$ to 6.1 .

Sulfur medium: Sulfur is oxidized. No turbidity.

Facultative autotrophic.

Aerobic.

Distinctive characters: Develops in inorganic media and oxidizes thiosulfate and sulfur to sulfate. Media with slightly alkaline reactions most favorable for growth.

Source: Coprolite rock material from Triassic period (Arizona).

Habitat: Unknown.

5. Thiobacillus denitrificans Beijerinck. (Cent. f. Bakt., II Abt., 11, 190t, 597 ; Sulfomonas denitrificans Orla-Jensen, Cent. f. Bakt., II Abt., 22, 1909, 314.) From Latin, de, from; and M.L. nitrifico, to nitrify.

Short rods, 0.5 by 1 to 3.0 microns long. Motile by means of a single polar flagel- 
lum (Tjulpanova-Mosseviteh, Arch. d. Sci. Biol., U.S.S.R., 30, 1930, 203).

Inorganic liquid medium: Growth with production of gas, predominantly nitrugen.

Thiosulfate agar medium: Colonies thin, clear, or weakly opalescent.

Optimum reaction: Neutral or slightly: alkaline.

Autotrophic, utilizing carbon from $\mathrm{CO}_{2}$, carbonates and bicarbonates. Considered to be strictly autotrophic by Lieske (Ber. d. deutsch. botan. Gesell., 30, 1912, 12.) and facultative by TjulpanovaMossevitch (loc. cit.). Beijerinck stated (Kon. Akad. v. Wetenschappen Amsterdam, 42, 1920, 899) that whereas the organism developed initially in an inorganic medium, it lost the autotrophic habit by cultivation in an organic medium.

Facultative anderobic or even microaerophilic. Can live in the absence of free $\mathrm{O}_{2}$ in the presence of nitrate.

Distinctive characters: Oxidizes thiosulfate to sulfate under anaerobic conditions using nitrate as the hydrogen acceptor which is reduced to $\aleph_{2}$. Also oxidizes sulfide, elemental sulfur, and dithionate:

Habitat: Canal and river water, salt water, soil, peat, composts and mud.

Appendix: The following species have been placed in Thiobacillus or are regarded as belonging to the genus:

Thiobacillus concretivorus Parker. (Austral. Jour. Exper. Biol. and Med. Aci., 23, 1945, 81.) From corroded concrete sewers. Similar to or identical with Thiobacillus thiooxidans Waksman and Joffe.

Thiobacillus crenatus Emoto. (Proc. Imp. Acad. Tokyo, 5, 1929, 149.) Isolated from mud of hot springs in Japan. See description, Manual, 5th ed., 1939, 84. Almost identical with Thiobacillus thiooxidans Waksman and Joffe.

Thiobacillus lobatus Emoto (loc. cit., p. 148). Source and relationships as above. See description, Manual, 5th ed., $1939,83$.

Thiobacillus thermitanus Emoto. (Bot. Mag. Tokyo, 42, 1928, 422.) Source and relationships as above. See deseription, Manual, 5th ed., 1939, 83.

Thiobacillus trautweinii Bergey et al. see Flavobacterium appendix.

Thiobacillus umbonatus Emoto (loc. cit., p. 150). Source and relationships as above. See description, Manual, 5th ed., 1939,84 .

Thiobacterium beijerinckii Issatchenko and Salimowskaja. (Zur Morphologie u. Physiol. der Thionsäurebakterien (Russian with German abstract), Izyiestia Gosud. Gidrobiol. Inst., No. 21, 1928, 61.) From salt seas in Russia. Similar to or identical with Thiobacillus thiopurus Beijerinck.

Thiobacterium beijerinckii var. jacobsenii Issatschenko and Salimowskaja (loc. cit.). Variety of previously mentioned species.

Thiobacterium nathansonii Issatchenko and Salimowskaja (loc. cit.). From salt seas in Russia. Similar to or identical with Thiobacillus thioparus Beijerinck. 


\title{
FAMILY II. PSEUDOMONADACEAE WINSLOW ET AL.
}

\author{
(Jour. Bact., 2, 1917, 555.)
}

Cells without endospores, elongate rods, straight or more or less spirally curved. One genus (Mycoplana) has branched cells. Usually motile by polar flagella which are either single or in small or large tufts. A few species are non-motile. Gram-negative (a few doubtful Gram-positive tests are recorded in Pseudomonas). Grow well and fairly rapidly on the surface of ordinary culture media excepting Methanomonas and some vibrios that attack cellulose. They are preferably aerobic, only certain vibrios including Desulfovibrio being anaerobic. Either water or soil forms, or plant or animal pathogens.

1. Straight rods.

Key to the tribes of family Pseudomonadaceae.

2. Cells more or less spirally curved.

Tribe I. Pseudomonadeae, p. 82.

Tribe II. Spirilleae, p. 192.

TRIBE I. PSEUDOMONADEAE KLUYVER AND VAN NIEL.

(Cent. f. Bakt., II Abt., 94, 1936, 397.)

This tribe includes all of the straight and branching rods of the family.

Key to the genera of tribe Pseudomonadeae.

I. Soil and water bacteria. Few animal and many plant pathogens. Usually produce a water-soluble pigment which diffuses through the medium as a bluish-green or yellowish-green pigment.

Genus I. Pseudomonas, p. 82.

II. Cells usually monotrichous with yellow non-water-soluble pigment. Mostly plant pathogens causing necrosis.

Genus II. Xanthomonas, p. 150.

III. Soil bacteria which oxidize methane.

Genus III. Methanomonas, p. 179.

IV. Bacteria which oxidize alcohol to acetic acid.

Genus IV. Acetobacter, p. 179.

V. Soil and water bacteria known to attack protamines.

Genus V. Protaminobacter, p. 189.

VI. Soil bacteria with branching cells. Capable of using aromatic compounds, as phenol, etc., as a source of energy.

Genus VI. Mycoplana, p. 191.

\section{Genus I. Pseudomonas Migula.*}

(Migula Arb. bakt. Inst. Karlsruhe, 1, 1894, 237; Bacterium Ehrenberg emend. Cohn, Beitr. z. Biol. d. Pflanzen, 1, Heft 1, 1872, 167; Bactrillum Fischer, Jahrb. f. wissensch. Bot., 27, 1895, 139; Bactrinium Fischer, ibid., 41; Arthrobactrinium Fischer, ibid., 139; Arthrobactrillum Fischer, ibid., 139; Bactrinius Kendall, Public Health, 28, 1902, 481; Bactrillius Kendall, ibid.; Bacterium Ehrenberg emend. Smith, Bacteria

* Revised for the 5th ed. of the Manual by Prof. D. H. Bergey, Philadelphia, Pennsylvania, 1937. Further revision for the 6th ed. by Prof. R. S. Breed, New York State Experiment Station, Geneva, New York, with incorporation of the plant pathogenic species by Prof. Walter H, Burkholder, Cornell University, Ithaca, New York, April, 1943. 
in Relation to Plant Disease, 1, 1905, 171; Denitromonas Orla-Jensen, Cent. f. Bakt., II Abt., 22, 1909, 314; Liquidomonas Orla-Jensen, ibid., 332; Lamprella Enderlein, Sitzber. Gesell. naturf. Freunde, Berlin, 1917, 317; Fluoromonas Orla-Jensen, Jour. Bact.. 6, 1921, 271.)

Cells monotrichous, lophotrichous or non-motile. If pigments are produced, they are of greenish hue, flurescent, and water-soluble.* Gram-negative except Nos. 88, 122 and 128. Frequently ferment glucose, sometimes with the formation of visible gas. Inactive in the fermentation of lactose. Nitrates are frequently reduced either to nitrites or ammonia, or to free nitrogen. Some species split fat and attack hydrocarbons. Soil, water, and plant pathogens; very few animal pathogens. Certain salt water species (Nos. 58-61) some of which live in heavy brine are temporarily retained in this genus although they produce non-water-soluble pigments or phosphorescence. From Gr. pseudes, false; monas, a unit; M. L. monad.

The type species is Pseudomonas aeruginosa (Schroeter) Migula.

\section{Key to the species of genus Pseudomonas.}

I. Soil and fresh water forms with a few that are pathogenic on cold or warm blooded animals.

1. Green fluorescent pigment produced.

a. Gelatin liquefied.

b. Polar flagellate.

c. Grow readily at $37^{\circ} \mathrm{C}$. Usually bluish-green.

1. Pseudomonas aeruginosa.

2. Pscudomonas jaegeri.

ce. Grow poorly or not at all it $37^{\circ} \mathrm{C}$.

d. Milk not coagulated becoming alkaline.

e. Soil and water organisms. Not known to digest cellulose.

3. Pseudomonas fluorescens.

4. Pseudomonas viscosa.

5. Pseudomonas fairmountensis.

6. Pseudomonas ureae.

7. Pseudomonas pavonacea.

ee. Soil forms that attack cellulose.

8. Pseudomonas effusa.

eee. Pathogenic for lizards.

9. Pseudomonas reptilivorous.

da. Milk unchanged becoming blue in association with lactic acid bacteria.

10. Pseudomonas syncyanea.

ddd. Milk coagulated.

11. Pseudomonas schuylkilliensis.

12. Pseudomonas chlororaphis.

13. Pseudomonas myxogenes.

14. Pseudomonas septica.

addd. Soil form. Action on milk not recorded.

15. Pseudomonas boreopolis.

* See Tobie, Jour. Boct., 49, 1945, 45!) for a discussion of the nature of these pigments. 
bb. Non-motile.

c. Cirows readily at $37^{\circ} \mathrm{C}$.

16. Pscudomonas smaragdina.

cc. Grows poorly or not at all at $37^{\circ} \mathrm{C}$.

17. Pseudomonas chlorina.

aa. Gelatin not liquefied.

b. Polar flagellate.

c. Grow readily at $37^{\circ} \mathrm{C}$. Usually bluish-green.

18. Pseudomonas oleovorans.

19. Pseudomonas incognita.

20. Pseudomonas convexa.

21. Pseudomonas mildenbergii.

cc. Grow poorly or not at all at $37^{\circ} \mathrm{C}$.

d. Milk not coagulated.

22. Pseudomonas putida.

23. Pseudomonas scissa.

24. Psendomonas ovalis.

25. Pseudomonas striata.

26. Pseudomonas denitrificans.

dd. Milk coagulated.

27. Pseudomonas solaniolens.

bb. Non-motile.

c. Grows poorly or not at all at $37^{\circ} \mathrm{C}$.

d. Milk not coagulated.

28. Pseudomonas eisenbergii.

2. Green fluorescent pigment not produced or not reported.

a. Gelatin liquefied.

b. Polar flagellate.

c. Grow poorly or not at all at $37^{\circ} \mathrm{C}$. No visible gas from sugars.

d. Rapid reduction litmus. Putrid odor.

29. Pseudomonas putrefaciens.

dd. Slow reduction litmus. Alkaline.

30. Pseudomonas mephitica.

31. Pseudomonas geniculala.

ddd. Acid coagulated.

32. Pseudomonas fragi.

cc. Acid and visible gas from glucose. Optimum temperature variable.

d. Litmus milk reduced and alkaline.

33. Pseudomonas nebulosa.

dd. Litmus milk acid coagulaterl.

34. Pseudomonas coadunata.

35. Pseudomonas multistriata.

36. Pseudomonas punclata.

37. Pseudomonas hydrophila.

38. Pseudomonas ichthyosmia.

aa. Gelatin not liquefied.

b. Polar flagellate.

c. Grow at $37^{\circ} \mathrm{C}$. 
39. Pseudomonas ambigua.

40. Pseudomonas sinuosa.

41. Pseudomonas cruciviae.

cc. Grow poorly or not at all at $37^{\circ} \mathrm{C}$.

d. Action on hydrocarbons and cellulose unknown.

42. Pseudomonas rugosa.

dd. Utilize hydrocarbons.

43. Pseudomonas desmolyticum.

44. Pseudomonas rathonis.

45. Pseudomonas dacunhae.

46. Pseudomonas arvilla.

47. Pseudomonas salopium.

ddd. Titilize cellulose.

48. Pseudomonas minuscula.

49. Pseudomonas tralucida.

50. Pseudomonas mira.

aaa. Action on gelatin not recorded. Produces alcoholic fermentation of glucose.

51. Pseudomonas lindneri.

II. Sea water to brine species. Some species phosphorescent.

a. Gelatin liquefied.

b. Polar flagellate.

c. From sea water. Not deeply pigmented.

d. Nitrites not produced from nitrates.

52. Pseudomonas membranoformis.

53. Pseudomonas marinoglutinosa.

dd. Nitrites produced from nitrates so far as known.

e. Digest agar.

54. Pseudomonas gelatica.

ce. Deposit calcium carbonate in sea water gelatin and agar media in old cultures.

55. Pseudomonas calcis.

56. Pseudomonas calciprecipitans.

eee. Ciuses skin lesions in marine fish.

57. Pseudomonas ichthyodermis.

re. Produce highly colored pigments in media containing salt or in heavy brines.

d. Blackens salted butter.

58. Pseudomonas nigrificans.

dd. Causes purple discoloration of salted beans.

59. Pseudomonas beijerinckii.

ddd. Reddens heavy brines (more than 18 per cent salt).

60. Pseudomonas salinaria.

61. Pseudomonas cutirubra.

rce. Phosphorescent bacteria from decaying fish and crustaceans, and phosphorescent organs of sea animals.

d. Gelatin liquefied.

62. Pseudomonas harveyi.

dd. Gelatin not liquefied.

63. Pseudomonas phosphorescens.

64. Pseudomonas pierantonii. 
III. Plant pathogens, causing leaf spot, leaf stripe and similar diseases. 1. Green fluorescent pigment produced.

a. Gelatin liquefied.

b. Acid from sucrose.

c. Nitrites produced from uitrates.

65. Pseudomonas martyniae.

66. Pseudomonas striafaciens.

67. Pseudomonas tomato.

cc. Nitrites not produced from nitrates.

d. Growth in 5 per cent salt.

68. Pseudomonas aceris.

69. Pseudomonas angulata.

70. Pseudomonas aptata.

71. Pseudomonas primulae.

72. Pseudomonas viridilivida.

dd. No growth in 5 per cent salt.

e. Beef peptone agar turns brown.

73. Pseudomonas delphinii.

ee. Beef peptone agar uncolored.

74. Pseudomonas berberidis.

75. Pseudomonas coronafaciens.

75a. Pseudomonas coronafaciens var. alropupurea.

76. Pseudomonas lachrymans.

77. Pseudomonas maculicola.

78. Pseudomonas marginata.

79. Pseudomonas medicaginis.

79a. Pseudomonas phaseolicola.

80. Pseudomonas pisi.

S1. Pseudomonas syringae.

Adl. Growth in salt solutions not recorded.

82. Pseudomonas atrofaciens.

83. Pseudomonas cumini.

84. Pseudomonas desaiana.

85. Pseudomonas crodii.

86. Pscudomonas apii.

87. Pseudomonas matthiolae.

88. Pseudomonas mors-prunorum.

89. Pseudomonas rimaefaciens.

90. Pseudomonas papulans.

91. Pseudomonas pseudozoogloeue.

92. Pseudomonas tabaci.

ccc. Nitrite production not reported.

93. Pseudomonas lapsa.

bb. No acid from sucrose.

c. Nitrites produced from nitrates.

94. Pseudomonas bowlesiae.

95. Pseudomonas intybi.

96. Pseudomonas marginalis.

97. Pseudomonas setariae. 
cc. Nitrites not produced from nitrates.

d. Lipolytic.

98. Pseudomonas polycolor.

dd. Not lipolytic.

99. Pseudomonas viridiflava.

99a. Pseudomonas viridiflava var. concentrica.

ddd. Lipolytic action not reported.

100. Pseudomonas ananas.

101. Pseudomonas ligustri.

102. Pseudomonas sesami.

103. Pseudomonas tolaasii.

bbb. Acid from sucrose not reported.

c. Nitrites produced from nitrates.

d. Motile.

101. Pseudomonas xanthochlora.

dd. Non-motile.

105. Pseudomonas rhiroctonia.

ce. Nitrites not produced from nitrates.

106. Pseudomonas barkeri.

107. Pseudomonas gladioli.

108. Pseudomonas mellea.

cce. Nitrite production not reported.

109. Pseudomonas betlis.

110. Pseudomonas panacis.

a. Gelatin not liquefied.

b. Acid from sucrose.

c. Nitrites produced from nitrates.

111. Pseudomonas alcuritidis.

ce. Nitrites not produced from nitrates.

112. Pseudomonas glycinea.

112n. Pseudomonas glycinea var. japonica.

113. Pseudomonas savastanoi.

113a. Pseudomonas savastanoi var. fraxini.

114. Pseudomonas tonclliana.

bb. No acid from sucrose.

c. Nitrites not produced from nitrates.

115. Pseudomonas calendulae.

116. Pseudomonas cichorii.

117. Pseudomonas cissicola.

118. Pseudomonas nectarophila.

119. Pseudomonas viburni.

bbb. Acid from sucrose not reported.

c. Nitrites not produced from nitrates.

120. Pseudomonas mori.

121. Pseudomonas stizolobii.

122. Pseudomonas viciae.

2. Green fluorescent pigment not produced or not reported.

a. Gelatin liquefied.

b. Acid from sucrose. 
c. Nitrites produced from nitrates.

d. Beef-peptone agar turns dark brown.

123. Pseudomonas alliicola.

124. Pseudomonas gardeniae.

dd. Beef-peptone agar remains uncolored or light discoloration after several weeks.

e. Colonies tan to brown.

125. Pseudomonas caryophylli.

126. Pseudomonas solanacearum.

126a. Pseudomonas solanacearnm var. asiatica.

ee. Colonies white or colorless.

127. Pseudomonas castaneae.

128. Pseudomonas seminum.

cc. Nitrites not produced from nitrates.

129. Pseudomonas passiftoriae.

bb. No acid from sucrose.

130. Pseudomonas fabac.

bbb. Acid from sucrose not reported.

c. Nitrites not produced from nitrates.

131. Pseudomonas astragali.

132. Pseudomonas colurnae.

133. Pseudomonas maublancii.

134. Pseudomonas polygoni.

cc. Nitrate production not reported.

135. Pseudomonas iridicola.

136. Pseudomonas levistici.

137. Pseudomonas radiciperda.

aa. Gelatin not liquefied.

b. Acid from sucrose.

c. Nitrites not produced from nitrates.

138. Pseudomonas melaphthora.

cc. Gas from nitrates.

139. Pseudomonas helianthi.

bh. No acid from sucrose.

c. Nitrites produced from nitrates.

140. Pseudomonas alboprecipitans.

141. Pseudomonas petasitis.

142. Pseudomonas lignicola.

ce. Nitrites not produced from nitrates.

143. Pseudomonas andropogoni.

144. Pseudomonas woodsii.

bbb. Acid from sucrose not reported.

c. Nitrites produced from nitrates.

145. Pseudomonas panici-miliacei.

146. Pseudomonas saliciperda.

cc. Nitrites not produced from nitrates.

147. Pseudomonas eriobotryae.

aar. Gelatin liquefaction not reported.

b. Nitrites not produced from nitrates.

148. Pseudomonas wieringae. 
1. Pseudomonas aeruginosa (Sehroeter) Migula. (Bacterium aeruginosum Schroeter, in Cohn, Beitrage z. Biologie, 1. Heft 2, 1872, 126; Bacillus pyocyaneus Gessard, Compt. rend. Acad. Sci., Paris, 94, 1882, 536; Micrococcus pyocyaneus Zopf, Spaltpilze, 2 Aufl., 1884, 83; Bacillua aeruginosus Trevisan, Atti Accad. Fis.-Med. Stat., Milano, Ser. 4, 3, 1885, 11; Bacillus fluorescens Crookshank, Man. of Bact., 3rd ed., 1890, 247; not Bacillus fluorescens Bergey et al., Manual, 1st ed., 1923, 287; Pscudomonas pyocyanea Migula, in Engler and Prantl, Die natürl. Pflanzenfam, 1, 1a, 1895, 29; Bacterium pyocyaneum Lehmann and Neumann, Bakt. Diag., 1 Aufl, 2, 1896, 267; Migula, Syst. Bakt., 2, 1900, 884.) From Latin, full of copper rust. or verdigris; green.

Rods: 0.5 to 0.6 by 1.5 microns, occurring singly, in pairs and short chains. Motile, possessing one to three polar flagella. Monotrichous (Reid, Naghski, Farrell and Haley, Penn. Agr. Exp. Sta., Bull. 422, 1942, 6). Gram-negative.

Gelatin colonies : Yellowish or greenishyellow, fringed, irregular, skein-like, granular, rapidly liquefying.

Gelatin stab: Rapid liquefaction. The fluid assumes a yellowish-green or bluish-green color.

Agar colonies: Large, spreading, grayish with dark center and translucent edge, irregular. Medium greenish.

Agar slant: Abundant, thin, white, glistening, the medium turning green to dark brown or black, fluorescent.

Broth: Marked turbidity with thick pellicle and heavy sediment. Medium vellowish-yreen to blue, with fluoreswince, later brownish. Produces pyocyanin, fluorescein and pyrorubrin (Am. Jour. Hyg., 5, 1925, 707).

Litmus milk: A soft coagulum is formed, with rapid peptonization and reduction of litmus. Reaction alkaline.

Potato: Luxuriant, dirty-brown, the medium becoming dark green.

Indole usually not formed (Sandiford, Jour. Path. and Bact., 44, 1937, 567).
Nitrates are reduced to nitrites and nitrogen.

Glucose, fructose, galactose, arabinose, maltose, lactose, sucrose, dextrin, inulin, glycerol, mannitol and dulcitol are not attacked. Acid from glucose (Sandiford, loc. cit.).

Blood serum: Liquefied. Yellow liquid, greenish on surface.

Blood hemolyzed.

Cultures have marked odor of trimethylamine.

Aerobic, facultative.

Optimum temperature $37^{\circ} \mathrm{C}$.

Pathogenic for rabbits, guinea pigs, rats and mice.

Common name: Blue pus organism.

Source: Pus from wounds. Regarded as identical with one of the plant pathogens (Pseudomonas polycolor) by Elrod and Braun (Jour. Bact., 44, 1912, 633).

Habitat: Cause of various human and animal lesions. Found in polluted water and sewage.

2. Pseudomonas jaegeri Migula. (Bacillus proteus fluorescens H. Jaeger, Ztschr. f. Hyg., 12, 1892, 593; Migula, Syst. d. Bakt., 2, 1900, 885; Bacillus proteus-fuorescens Holland, Jour. Bact., 5, 1920, 220; Proteus fluorescens Holland, ibid., 224; Pseudomonas protea-fluorescens Holland, ibid., 224.) Named for H. Jaeger who first described the species.

Short, thick rods, with rounded ends, occurring singly and in pairs. Motile with a tuft of polar flagella which may be pushed to one side where cells remain in a chain. Gram-negative.

Gelatin colonies: Small, transparent, becoming proteus-like.

Gelatin stab: Marked surface growth. Saccate to infundibuliform liquefaction. Liquefied portion green fluorescent.

Agar slant: Thick, yellowish-white layer, the medium becoming greenishfluorescent. At times gas is formed.

Broth: Turbid, with greenish-gray pel. licle and sediment.

Litmus milk: Not coagulated. 
Potato: Thick, pale yellow becoming dark brown layer, slimy. The medium becomes bluish-gray.

Indole not formed.

Nitrites not produced from nitrates.

Aerobic, facultative.

Optimum temperature $37^{\circ} \mathrm{C}$.

Pathogenic for mice.

Source: Regarded by Jaeger as the cause of Weil's disease (infectious jaundice) as it was found repeatedly in patients suffering from this disease. See Leptospira icterohaemorrhagiae.

Habitat: Water.

3. Pseudomonas fluorescens Migula. (Bacillus fluorescens liquefaciens Flügge, Die Mikroorganismen, 1856, 289; Migula, in Engler and Prantl, Die natürl. Pflanzenfamilien, 1, 1a, 1895, 29; Bacterium fuorescens Lehmann and Neumann, Bakt. Diag., 1 Aufl., 2, 1896, 272.) From Latin, fluor, flowing; M.L. fluoresco, to fluoresce.

Rods: 0.3 to 0.5 by 1.0 to 1.8 microns, occurring singly and in pairs. Motile, possessing a polar flagellum. Gramnegative.

Gelatin colonies: Circular, with greenish center, lobular, liquefying quickly.

Gelatin stab: Infundibuliform liquefaction, with whitish to reddish-gray sediment.

Agar slant: Abundant, reddish layer, becoming reddish-gray. The medium shows greenish to olive-brown coloration.

Broth: Turbid, flocculent, with yellowish-green pellicle and grayish sediment.

Litmus milk: No coagulation; becoming alkaline.

Potato: Thick, grayish-yellow, spreading, becoming light sepia-brown in color.

Indole is not formed.

Nitrates reduced to nitrites and anmonia.

Acid from glucose.

Blond serum liquefied.

Aerobic.

Optimum temperature $20^{\circ}$ to $25^{\circ} \mathrm{C}$.

Not pathogenic.

Source: Water, sewage, feces.

Habitat: Soil and water.
4. Pseudomonas viscosa (Frankland and Frankland) Migula. (Bacillus viscosus G. and P. Frankland, Ztschr. f. Hyg., 6, 1889, 391 ; Migula, Syst. d. Bakt., 2, 1900, 900.) From M. L. viscidus, sticky, viscid.

Small rods : 0.5 by 1.5 to 2.0 microns, occurring singly. Motile and presumably polar flagellate. Gram-negative.

Gelatin colonies: Grayish, granular, with fimbriate margin. Medium assumes a green fluorescent color around each colony.

Gelatin stab: Infundibuliform liquefaction. Liquefied portion green fluorescent with greenish-white pellicle.

Agar slant: Thin, greenish-white, the medium becoming greenish.

Broth: Turbid, with greenish pellicle.

Litmus milk: Not coagulated.

Potato: Moist, chocolate-brown, viscid.

Indole not formed.

Nitrites not produced from nitrates. Destroys nitrate with the production of ammonia.

Aerobic, facultative.

Distinctive characters: Resembles Pseudomonas fluorescens except that growth on agar, gelatin and potato is viscid.

Optimum temperature $20^{\circ} \mathrm{C}$.

Source: Unfiltered water from Kent, England. Common.

Habitat: Water.

\section{Pseudomonas}

fairmountensis

(Wright) Chester. (Bacillus fairmountensis Wright, Memoirs Nat. Acad. Sci., \%, 1895, 458; Chester, Man. Determ. Bact., 1901, 311; Achromobacter fairmountense Bergey et al., Manual, 1st ed., 1923, 146.) From M. L. of Fairmount Park (Philadelphia.)

Medium-sized rods, occurring singly, in pairs and in chains. Motile, possessing polar flagella. Gram-negative.

Gelatin colonies: Circular, white, translucent. Dark centers with a greenish shimmer, thinner edges and faint radial lines.

Gelatin stab: Crateriform liquefaction. 
Agar slant: Grayish-white, glistening. Broth: Turbid.

Litmus milk: Alkaline, litmus reduced.

Potato: Raised, granular, spreading, viscid.

Indole is formed.

Nitrites not produced from nitrates.

Aerobic, facultative.

Optimum temperature $20^{\circ}$ to $25^{\circ} \mathrm{C}$.

Habitat: Water.

6. Pseudomonas ureae Bergey et al. (Culture No. 3 of Rubentschick, Cent. f. Bakt., II Abt., 72, 1927, 101; Bergey et al., Manual, 3rd ed., 1930, 173.) From Greek, urum, urine; M. L., urea, urea.

Rods : 0.6 to 0.7 by 1.7 to 2.0 microns, occurring singly and in pairs. Motile. Gram-positive.

Gelatin stab: Infundibuliform liquefaction.

Agar colonies: Circular, grayish-white.

Agar slant: Grayish-white layer becoming greenish-fluorescent.

Broth: Turbid.

Litmus milk: Peptonized.

Potato: Yellowish-brown streak.

Indole not formed.

Nitrates reduced with gas formation. Ammonia formed.

Urea attacked.

Hydrogen sulfide formed.

Methylene blue reduced.

Aerobic, facultative.

Can grow at $0^{\circ} \mathrm{C}$.

Optimum temperature $20^{\circ} \mathrm{C}$.

Habitat: Sewage filter beds.

This species is included here through an oversight. It should have been placed in the Appendix to the genus Pseudomonas as the original description is too incomplete to determine its real nature. It is reported to be Cram-positive and motile; but the number and arrangement of flagella are not given. If it really is (iram-positive, the species is probably peritrichous and does not be long in Pseudomonas.

7. Pseudomonas pavonacea Levine and Soppeland. (Bul. No. 77, Iowa
State Agricultural College, 1926, 41.) From Latin, paro, peacock.

Rods : 0.5 by 4.5 microns, with truncate ends, occurring singly and in chains. Motile. Gram-negative.

Gelatin stab: Crateriform liquefaction. Medium becoming brown.

Agar colonies: Circular, raised, becoming green, amorphous, entire.

Agar slant: Greenish, smooth, glistening, viscid, medium becoming slightly brown.

Broth: Turbid, with viscid sediment. Medium turned dark brown.

Litmus milk: Slightly alkaline. Litmus reduced. Peptonized after 10 days.

Potato: No growth.

Indole not formed.

Nitrites not produced from nitrates.

Starch not hydrolyzed.

Blood serum liquefied in 5 days.

No acid or gas from carbohydrate media.

Aerobic, facultative.

Optimum temperature $22^{\circ} \mathrm{C}$.

Source: Isolated from activated sludge.

8. Pseudomonas effusa Kellerman et al. (Kellerman, McBeth, Scales and Smith, Cent. f. Bakt., II Abt., 39, 1913, 515; also soil Science, 1, 1916, 472; Cellulomonas effusa Bergey et al., Manual, 1 st ed., 1923, 162; Bacillus effusus appears first as a synonym in Bergey at al., ibid.; later used as name of species 5th (d., 1939, 616.) From Latin, cffusus, effuse, spread out.

Rods: 0.4 by 1.7 microns. Motile with one to three polar flagella. Gramnegative.

Gelatin stab: Liquefaction.

Agar slant: Luxuriant, glistening, moist, creamy growth. Greenish fluorescence.

Peptone starch agar slant: Abundant, flat, moist rich creamy growth. Medium shows greenish fluorescence.

Broth: Turbid.

Litmus milk: Alkaline. Coagulation and digestion. 
Potato: Rich, creamy spreading growth.

Indole not formed.

Nitrites not produced from nitrates. Ammonia is produced.

Acid from glucose, maltose, starch, glycerol and mannitol. No acid from lactose or sucrose.

Cellulose is attacked.

Aerobic, facultative.

Optimum temperature $20^{\circ} \mathrm{C}$.

Source: Isolated from soils in Utah.

Habitat: Soil.

8a. Pseudomonas effusa var, non-liquefaciens Kellerman et al. (loc. cit.). I non-liquefying variety that acts more slowly on litmus milk.

Source: Soils from Utah.

9. Pseudomonas reptilivorous Caldwell and Ryerson. (Jour. Bact., 39, 1940, 335.) From Latin, reptile, a reptile and voro, to devour, destroy.

Rods: 0.5 by 1.5 and 2.0 microns, oc curring singly, in pairs and in short chains and having rounded ends. Actively motile with two to six polar flagella. Gram-negative.

Gelatin colonies: After 24 hours, small, circular, smooth, entire. Liquefaction with a yellowish-green fluorescence.

Gelatin stab: Infundibuliform liquefaction becoming stratiform. Putrid odor present.

Agar cultures: Circular, smooth, glistening, slightly raised, butyrous, translurent, $2 \mathrm{~mm}$ in diameter.

Agar slant: Growth abundant, smooth, filiform, glistening, butyrous and translucent.

Broth: Turbid with pellicle and sediment. Putrid odor.

Litmus milk: Alkaline, peptonization, complete reduction. Disagreeable odor.

Potato: Growth moderate, spreading, glistening, yellowish-gray to creamy. Disagreeable odor. Medium becomes brownish-gray .

Indole not formed.

Nitrates not produced from nitrates.
Hydrogen sulfide not produced.

Slightly acid, becoming alkaline in glucose. No acid from arabinose, xylose, lactose, sucrose, maltose, trehalose, raffinose, mannitol, dulcitol, inositol and salicin.

Starch not hydrolyzed.

Pathogenic for guinea pigs and rabbits, horned lizards, Gila monsters and chuckwallas.

Temperature relations: Optimum $20^{\circ}$ to $25^{\circ} \mathrm{C}$. Maximum $37^{\circ} \mathrm{C}$.

Distinctive characters: Yellowishgreen fluorescence present in meat infusion media. Pathogenic.

Source: Isolated in a bacterial disease of horned lizards and Gila monsters.

Habitat: Pathogenic for lizards.

10. Pseudomonas syncyanea (Ehrenberg) Migula. (Vibrio syncyaneus Ehrenberg, Berichte ü.d. Verh. d. k. Preuss. Akad. d. Wissensch. z. Berlin, 5, 1840, 202 ; Vibrio cyanogenes Fuchs, Magazin für die gesamte Tierheilkunde, 7, 1841, 190; Bacillus syncyaneus Schroeter, Kryptogam. Flora von Schlesien, 3, 1, 1886, 157; Bacillus cyanogenus Zopf, Die Spaltpilze, 3 Aufl., 1885, 86; Migula, in Engler and Prantl, Die natürl. Pflanzenfam., 1, 1a, 1895, 29; Bacterium syncyaneum Lehmann and Neumann, Bakt. Diag., 1 Aufl., 2, 1896, 275; Pseudomonas cyanogenes Holland, Jour. Bact., 5, 1920, 224.) From Greek, syn, with; kyaneos, dark blue, dark.

Rods with rounded ends, occurring singly, occasionally in chains, 0.7 by 2.0 to 4.0 microns. Motile with two to four polar flagella. Gram-negative.

Gelatin colonies: Flat, bluish, translucent.

Gelatin stab: Surface growth shiny, grayish blue. The medium is colored steel-blue with greenish fluorescence. Gelatin is liquefied. Some strains do not liquefy.

Agar slant: Grayish-white streak. The medium takes on a bluish-gray color with slight fluorescence. 
Broth: Turbid with marked fluorescence.

Litmus milk: Unchanged. In association with lactic acid bacteria the milk takes on a deep blue color.

Potato: Yellowish-gray, shiny layer, becoming bluish-gray. The medium becomes bluish-gray.

Indole not formed.

Nitrites not produced from nitrates.

Aerobic, facultative.

Optimum temperature $25^{\circ} \mathrm{C}$.

Habitat: The cause of blue milk.

\section{Pseudomonas schuylkilliensis} Chester. (Bacillus fuorescens schuylkilliensis Wright, Memoirs, Natl. Acad. Sei., 7, 1895, 448; Chester, Determinative Bact., 1901, 320.) From MI. I. of the Sehuylkill (River).

Synonyms: Pseudomonas capsulata Chester, Man. Determ. Bact., 1901, 322 (Bacillus fluorescens capsulatus Pottien. Ztschr. f. Hyg., 11, 1896, 140); Pscudomonas dermatogenes Fuhrmann, Cont. f. Bakt., II Abt., 17, 1906, 356 .

Short rods, with rounded ends, occurring singly, in pairs and in chains. Motile, possessing a polar flagelhum. Cram negative.

Gelatin colonies: Grayish-white, translucent, with brownish center, radiate margin, becoming bluish-green.

Gelatin stab: Slow crateriform liquefaction, with blue-green fluorescence

Agar slant: Grayish, translueent growth. Medium shows greenish fluorescent.

Broth: Turbirl, with slight pellicle and blue-green fluorescence. Stringy sediment.

Litmus milk: Coagulated, with slow reduction of litmus; peptonized.

Potato: Brownish, spreading, viseid. thick.

Indole is formed (trace).

Nitrites not produced from nitrates.

Aerobic, facultative.

Does not grow at $35^{\circ}$ to $36^{\circ} \mathrm{C}$,
Source: Isolated from Schuylkill River water.

Habitat: Water.

12. Pseudomonas chlororaphis (Guignard and Sauvageau) Bergey et al. ( $B a-$ cillus chlororaphis Guignard and Sauvageau, Compt. rend. Soc. Biol. Paris, 1, 10 sér., 1894, \$ł1; Bergey et al., Manual, 3d ed., 1930, 163; also see Lasseur and Dupaix-Lasseur, Trav. Lab. Microbiol. Fac. Pharm. Nancy, Fasc. 9, 1936, 35.) From Greek, chlorros, greenish yellow; raphis, needle.

Rods : 0.8 by 1.5 microns, with rounded ends, occurring singly and in pairs. Motile with polar flagella. Gram-negative.

Gelatin colonies: Circular, viscid, transparent, glistening, lobate margin, with fluorescent corona. Dissociates readily (Iasseur and Dupaix-Lasseur. loc. cit.)

Gelatin stab: Stratiform liquefaction

Broth: Turbid, fluorescent, with crystals of green, water-soluble chlororaphine.

Litmus milk: Coagulation. Peptonization. Crystals of chlororaphine form in the central part of the culture.

Potato: Citron-yellow layer. Crystals of chlororaphine are formed.

Nitrates reduced to nitrites.

Indole not formed.

Pigment formation: Asvaragine, potassium phosphate, glycerol, sulfate of magnesium and sulfate of iron are indispensable to the formation of crystals of chlororaphine.

Aerobic, facultative. Optimum temperature $25^{\circ}$ to $30^{\circ} \mathrm{C}$.

Pathogenic for laboratory animals. Exotoxin formed.

Habitat: Water.

13. Pseudomonas myxogenes Fuhrmann. (Cent. f. Bakt., II Abt., 17, 1907, 356.) From Greek, myxa, mucus; gennā, to beget; M. L. slime producing.

Rods : 0.4 to 0.5 by 1.0 to 1.5 microns, occurring singly and in pairs. Motile, possessing a bundle of five to seven polar flagella. Gram-negative. 
Gelatin colonies: Smooth, soft, flat, spreading, entire, yellowish-green.

Gelatin stab: Growth along stab. Liquefaction with yellowish-white sediment.

Agar colonies : Circular, raised, smooth, amorphous, entire.

Agar slant: Yellowish-white, moist, glistening, becoming light green-fluorescent.

Broth: Turbid, with yellowish-white sediment.

Litmus milk: Floceulent precipitation. Slow peptonization with yellow serum. Alkaline.

Potato: Dirty yellow to olive, moist, glistening, entire.

Indole is formed.

Nitrates reduced to nitrites and ammonia. No gas formed.

Aerobic, facultative.

Optimum temperature $22^{\circ} \mathrm{C}$.

Source: Isolated from beer.

14. Pseudomonas septica Bergey et al. (Bacillus fuorescens septicus Stutzer and Wsorow, Cent. f. Bakt., II Abt., 71, 1927, 113; Bergey et al., Manual, 3rd ed., 1930,169.) From Greek, septikos, putrefactive, septic.

Rods : 0.6 to 0.8 by 0.8 to 2.0 microns, occurring singly. Motile with a polar flagellum. Gram-negative.

Gelatin stab: Infundibuliform licpuefaction.

Agar colonies: Circular with opalescent center and transparent periphery.

Agar slant : Moderate, undulate margin.

Broth: Turbid with fragile pellicle, greenish in upper portion.

Litmus milk: Alkaline, coagulated.

Blood serum not liquefied.

Acid from glueose.

Aerobic, facultative.

Optimum temperature $20^{\circ} \mathrm{C}$.

Habitat: Disease of caterpillars.

15. Pseudomonas boreopolis Gray and Thornton. (Gray and Thornton, Cent. f. Bakt., II Abt., 73, 1928, 74.) From
Greek, boreas, the North wind; polis, city; M. L. North City.

Rods : 0.5 to 1.0 by 2.0 to 3.0 microns, occurring singly and in pairs. Motile with one to five polar flagella. Gramnegative.

Gelatin colonies: Liquefied.

Gelatin stab: Liquefied. Medium reddened.

Agar colonies: Circular or amoeboid, white to buff, flat to convex, smooth, glistening, translucent border.

Agar slant: Filiform, whitish, raised, smooth, glistening, fluorescent.

Broth: Turbid.

Nitrates reduced to nitrites.

Starch not hydrolyzed.

Acid produced from glueose.

Attacks naphthalene.

Aerobic, facultative.

Optimum temperature $20^{\circ}$ to $25^{\circ} \mathrm{C}$.

Habitat: Soil.

16. Pseudomonas smaragdina Migula. (Bacillus smaragdinus foetidus Reiman, Inaug. Dissertation, Würzburg, 1887; Migula, Syst. d. Bakt., 2, 1900, 890.) From Greek, smaragdinas, green like the smaragdus, the emerald.

Small rods, occurring singly. Nonmotile. Gram-negative

Gelatin colonies: Small, convex, irregular, whitish with greenish shimmer.

Gelatin stab: Slight surface growth. Infundibuliform liquefaction. The liquefied medium becomes light emerald green in color.

Agar colonies: Small, brownish-yellow, convex.

Agar slant: Abundant growth with greenish fluorescence.

Broth: Turbid.

Litmus milk: Not coagulated.

Potato: Dark brown, becoming chocolate brown.

Indole not formed.

Nitrates not reduced.

The cultures give off an odor resembling jasmine.

Aerobic, facultative. 
Optimum temperature $37^{\circ} \mathrm{C}$.

Subcutaneous and intravenous inoculations into rabbits cause death in 36 to 48 hours.

Source: Isolated from nasal secretions in ozena.

17. Pseudomonas chlorina (Frankland and Frankland) Levine and Soppeland. (Bacillus chlorinus G. and P. Frankland, Philos. Trans. Roy. Soc. London, 178, 1887, 27t; Bacterium chlorinum Migula, Syst. d. Bakt., 2, 1900, 471; Levine and Soppeland, Bul. No. 77, Iora State Agricultural College, 1926.) From Greek, chloros, greenish yellow.

Pods : 0.5 by 1.5 micron, occurring singly and in short chains. Non-motile. Gramnegative.

Gelatin stab: Crateriform liquefaction with green fluorescence. Lemon yellow sediment.

Agar colonies: Circular, raised, smooth, amorphous, entire, becoming greenish yellow.

Agar slant: Slightly raised, glistening, the medium becoming light greenish yellow.

Broth: Moderate turbidity. Dirty yellow sediment. To pellicle.

Litmus milk: Peptonized. Litmus reduced.

Potato: Scant, olive green growth.

Indole formed.

Nitrites produced from nitrates.

Starch hydrolyzed.

Blood serum liquefied in 5 days.

Acid from glucose.

Aerobic, facultative.

Optimum temperature $22^{\circ} \mathrm{C}$.

Source: Air.

18. Pseudomonas oleovorans Lee and Chandler. (Jour. Bact., 41, 1941, 378.) From M. L. oil destroying.

Short rods : 0.5 by 0.8 to 1.5 microns, occurring singly and in pairs. Motile. Gram-negative.

Gelatin stab: No liquefaction after 6 weeks.
Gelatin colonies: Up to $1 \mathrm{~mm}$. in diameter, fluorescent; similar to agar colonies.

Surface agar colonies: After $2 \pm$ hours 1 to $2 \mathrm{~mm}$. in diameter, smooth, convex, shiny, opaque, creamy, fluorescent by transmitted light. Edge entire in young colonies.

Deep agar colonies: 0.5 by 1.0 to 1.5 $\mathrm{mm}$., lens-shaped, buff-colored, not fluorescent.

Agar slant: Growth raised, smooth, fluorescent, edge erose.

Broth: After 24 hours moderate turbidity with slight yellowish viscid sediment.

No pellicle or ring.

Litmus milk: No change.

Indole not formed.

Potato: Good growth.

Nitrites are produced from nitrates.

Starch is hydrolyzed.

To acid from glucose, lactose, sucrose, galactose, xylose, mannitol, salicin and glycerol.

Equally good growth at $25^{\circ}$ and $37^{\circ} \mathrm{C}$. Aerobic.

Distinctive character: The fluorescent quality of the colonies is not imparted to any of the artificial media used.

Source: Isolated from cutting compound (oil-water emulsion) circulating in a machine shop. The oil in this compound may be utilized as a sole source of energy.

Habitat: Probably oil-soaked soils. Abundant in cutting compounds.

19. Pseudomonas incognita Chester. (Bacillus fuorescens incognitus Wright, Memoirs Nat. Acad. Sci., 7, 1895, 436; Chester, Determinative Bacteriology, 1901, 323.) From Latin, in, not; cogito, to think; M. I. unknown.

Short rods, with rounded ends, occurring singly, in pairs and in chains. Motile, possessing a polar flagellum. Gramnegative.

Gelatin colonies: Thin, transparent, slightly granular, becoming greenish. Margin undulate. The medium assumes a blue-green fluorescence. 
Gelatin stab: No liquefaction.

Agar slant: Thin, moist, translucent, becoming greenish.

Broth: Turbid, with pellicle, becoming greenish.

Litmus milk: Slightly acid in a month. The litmus is slowly reduced.

Potato: Moist, glistening, spreading, brown.

Indole is formed (trace).

Nitrites are produced from nitrates.

Aerobic, facultative.

Optimum temperature $35^{\circ} \mathrm{C}$.

Habitat: Water.

20. Pseudomonas convexa Chester. (Bacillus fluorescens convexus Wright, Memoirs Nat. Acad. Sci., 7, 1895, 438; Chester, Determinative Bacteriology, 1901, 325.) From Latin, convexus, convex, arched.

Short, thick rods, with rounded ends. Motile, possessing a polar flagellum. Gram-negative.

Gelatin colonies: Circular, convex, glistening, bright greenish, translucent. The medium becomes blue-green, fluorescent.

Gelatin stab: Light green, raised, glistening surface growth. No liquefaction.

Agar slant: Moist, translucent, glistening, light greenish. The medium assumes a greenish color.

Broth: Turbid, becoming greenish.

Litmus milk: No coagulation; alkaline.

Potato: Pale brown, spreading.

Indole not formed.

Nitrites not produced from nitrates.

Aerobic, facultative.

Optimum temperature $30^{\circ} \mathrm{C}$.

Habitat: Water.

21. Pseudomonas mildenbergii Bergey et al. (Der blaue bacillus, Mildenberg, Cent. f. Bakt., II Abt., 56, 1922, 309; Pseudomonas cyanogena Bergey et al., Manual, 1st ed., 1923, 129; not Bacillus cyanogenes Flügge, Die Mikroorgan- ismen, 1886, 201; not Pseudomonas cyanogenes Hammer, Dairy Bact., 1928, 70; Bergey et al., Manual, 3rd ed., 1930, 172.) Named for Mildenberg who first isolated this species.

Rods : 0.3 to 0.5 by 1.0 to 3.5 microns, with rounded ends, occurring singly. Motile, possessing polar flagella. Gramnegative.

Gelatin colonies: Circular, lobed, smooth, glistening, slightly raised, steelblue, entire.

Gelatin stab: No liquefaction.

Agar colonies: Small, circular, yellowish or reddish-yellow, entire, becoming lobed, grayish-green, iridescent. The medium becomes dirty grayish-green.

Agar slant: Smooth, spreading, slimy, glistening, grayish-green to dark green, fluorescent.

Broth: Turbid green, iridescent to opalescent with slimy sediment.

Litmus milk: Not coagulated, blue ring.

Potato: Slimy, glistening, spreading, steel blue.

Indole not formed.

Nitrites not produced from nitrates.

Aerobic, facultative.

Optimum temperature $25^{\circ} \mathrm{C}$.

Source: Isolated from air.

2. Pseudomonas putida (Trevisan) Migula. (Bacillus fluorescens putidus Flügge, Die Miliroorganismen, 2 Aufl., 1886, 288; Bacillus putidus Trevisan, I gen. e le specie d. Batteriacee, 1889, 18; Migula, in Engler and Prantl, Die natür. Pflanzenfam., 1, 1a, 1895, 29; Bacillus fluorescens putridus (sic) Kruse, in Flügge, Die Mikroorganismen, 2, 1896, 292; Bacterium putidum Lehmann and Neumann, Bakt. Diag., 1 Aufl., 2, 1896, 271; Pseudomonas putrida (sic) Migula, Syst. d. Bakt., 2, 1900, 912.) It is not clear which spelling should be used. Fither is correct. From Iatin putida or putrida, rotten, stinking.

Rods, with rounded ends. Motile, 
possessing polar flagella. Gram-negative.

Gelatin colonies: Small, finely granular, fluorescent with dark center, surrounded by a yellow zone, with pale gray margin.

Gelatin stab: Dirty-white surface growth, becoming greenish, fluorescent. No liquefaction.

Agar colonies : Circular, raised, smooth, amorphous, entire, with fluorescent zone around the periphery.

Agar slant: Yellowish-green layer, becoming fluorescent.

Broth: Turbid, fluorescent.

Litmus milk: Unchanged.

Potato: Thin, gray to brownish, slimy layer.

Cultures give off odor of trimethylamine.

Indole not formed.

Nitrites are produced from nitrates.

Aerobic, facultative.

Optimum temperature $25^{\circ} \mathrm{C}$. Will grow at $37^{\circ} \mathrm{C}$. (Reid et al., Penn. Agr. Exp. Sta., Bull. 422, 1942, 9).

Distinctive features: Identical with Pseudomonas fuorescens Migula according to Lehmann and Neumann (loc. cit.) except that it does not liquefy gelatin. See Pseudomonas eisenbergii Migula.

Habitat: Putrefying materials; water.

23. Pseudomonas scissa (Frankland and Frankland) Migula. (Bacillus scissus G. and P. Frankland, Ztschr. f. Hyg., 6, 1889, 398; Migula, Syst. d. Bakt., 2, 1900, 927.) From Latin, scissus, p.p. of scindo, to cut.

Rods: 0.5 by 0.5 to 1.0 mieron, with rounded ends, occurring singly, in pairs and in chains; on gelatin, coccus-like. Motile with presumably polar flagella. Gram-negative.

Gelatin colonies: Small, greenish.

Gelatin stab: Thin, smooth, glistening surface growth, irregular, serrate margin. No liquefaction. The medium becomes light green in color.

Agar slant: Smooth, glistening, lobed. The medium assumes a greenish color.

Broth: Turbid, with whitish sediment.
Litmus milk: Not coagulated.

Potato: Glistening, reddish-brown growth.

Indole not formed.

Nitrites produced from nitrates.

Aerobic, facultative.

Optimum temperature $20^{\circ} \mathrm{C}$

Distinctive characters: Resembles Pseudomonas viscosa Migula.

Source: Found in water and soils in Kent, England.

Habitat: Water and soil.

24. Pseudomonas ovalis Chester. (Bacillus fluorescens ovalis Ravenel, Memoirs Nat. Acad. Sci., 8, 1896, 9; Chester, Determinative Bacteriology, 1901, 325; not Bacillus ovalis Wright, Memoirs Nat. Acad. Sci., $\%, 1895,435$. ) From ovum, egg; M. I. oval.

Rods : 0.3 to 0.7 by 0.7 to 1.3 microns, occurring singly. Motile, possessing a single polar flagellum. Gram-negative.

Gelatin colonies: Irregular, lobate, slightly granular.

Gelatin stab : No liquefaction.

Agar colonies : Circular, opaque, entire, «reenish fluorescence.

Agar slant: Thick, white, becoming greenish, fluorescent.

Broth: Turbid, with pellicle.

Litmus milk: No coagulation; alkaline.

Potato: Luxuriant, dirty-brown.

Indole not formed.

Nitrites not produced from nitrates.

Starch not hydrolyzed.

Blood serum not liquefied.

Acid from glucose.

Aerobic, facultative.

Optimum temperature $25^{\circ} \mathrm{C}$.

Habitat: Soil. Has been found in intestinal canal.

25. Pseudomonas striata Chester. (Bacillus striatus viridis Ravenel, Nemoirs Nat. Acad. Sci., 8, 1896, 22; Chester, Determinative Bacteriology, 1901, 325.) From Iatin, strio, streak, groove.

Slender rods, of variable lengths, stain- 
ing irregularly, occurring singly and in pairs. Motile, possessing polar flagella. Gram-negative.

Gelatin colonies: Circular, yellowish, with filamentous border.

Gelatin stab: Raised, white surface growth. No liquefaction.

Agar slant: Thin, yellowish-green, smooth, glistening.

Broth: Turbid, becoming slightly greenish.

Litmus milk: No coagulation; becoming alkaline; litmus reduced.

Potato: Moist, glistening, becoming chocolate-brown.

Indole is formed.

Nitrites are produced from nitrates.

Aerobic.

Optimum temperature $25^{\circ} \mathrm{C}$.

Habitat: Soil.

26. Pseudomonas denitrificans Bergey et al. (Bacillus denitrificans fluorescens Christensen, Cent. f. Bakt., II Abt., 11, 1903, 190; Bergey et al., Manual, 1st ed., 1923, 131.) From Latin, de, from, out of; nitrum, soda, nitre; M. L. denitrifying.

Rods : 0.5 to 0.7 by 0.5 to 1.25 microns, occurring singly and in pairs in large, slimy masses. Motile. Gram-negative.

Gelatin colonies: Small, circular, contoured, raised, moist, pearly-gray, glistening.

Gelatin stab: Whitish, lobed surface growth. Yellowish-green growth in stab. No liquefaction.

Agar colonies: Pearly white, circular, entire.

Agar slant: Broad, whitish, contoured, moist, entire.

Broth: Turbid, with thick, wrinkled pellicle.

Litmus milk: Not coagulated.

Potato: Reddish-gray layer.

Indole not formed.

Nitrates reduced with production of nitrogen.

Aerobic, facultative.

Optimum temperature $25^{\circ} \mathrm{C}$.

Habitat: Soil.
27. Pseudomonas solaniolens Paine. (Rept. Int. Conf. Phytopath. and Econ. Ent. Holland, 1923, 77; Phytomonas solaniolens Bergey et al., Manual, 3rd ed., 1930, 274.) From M. L. Solanum, a generic name.

Small oval rods: Motile with a polar flagellum. Gram-negative.

Produces an iridescence in gelatin.

Gelatin: No liquefaction.

Gelatin colonies: Round. Iridescence in medium.

Agar colonies: Pale buff.

Litmus milk: Curd, with no sign of digestion.

Potato: Pale buff-colored growth, no change in medium.

Nitrites not produced from nitrates.

Acid but not gas from glucose. No acid or gas from lactose, sucrose, mannitol or glycerol.

Starch: Action feeble.

Optimum temperature $20^{\circ}$ to $30^{\circ} \mathrm{C}$.

Aerobic, facultative.

Source: Isolated from potato showing internal rust spots.

28. Pseudomonas eisenbergii Migula. (Bacillus fluorescens non liquefaciens Eisenberg, Bakt. Diag., 3 Aufl., 1891, 145; Bacillus fluorescens immobilis Kruse, in Flügge, Die Mikroorganismen, 2, 1896, 294; Migula, Syst. d. Bakt., 2, 1900, 913; Bacterium immobile Chester, Man. Determ. Bact., 1901, 180; Pseudomonas nonliquefaciens Bergey et al., Manual, 1st ed., 1923, 132.) Named for Eisenberg, the bacteriologist who first reported this species.

Short, slender rods, with rounded ends, occurring singly. Non-motile. Kruse (loc. cit., p. 293) lists the motile form of this organism as Bacillus fluorescens non liquefaciens. Gram-negative.

Gelatin colonies: Fern-like surface colonies. Medium around colonies has a pearly luster.

Gelatin stab: Surface growth has fluorescent shimmer. No liquefaction.

Agar slant: Greenish layer.

Broth: Turbid, fluorescent. 
Litmus milk: Unchanged.

Potato: Diffuse, brownish layer. Medium acquires a grayish-blue color.

Indole is not formed.

Nitrites produced from nitrates.

Acid from glucose.

Blood serum liquefied.

Aerobic, facultative.

Optimum temperature $25^{\circ} \mathrm{C}$.

Not pathogenic.

Habitat: Water.

29. Pseudomonas putrefaciens (Derby and Hammer) Long and Hammer. (Achromobacter putrefaciens Derby and Hammer, Iowa Agr. Exp. Sta. Res. Bul. 145, 1931, 401; Long and Hammer, Jour. Bact., 41, 1941, 100.) From Latin, putrida, rotten, stinking; faciens, making.

Rods : 0.5 to 1.0 by 1.1 to 4.0 microns, occurring singly and in pairs. Motile, with a single flagellum. Gram-negative.

Gelatin stab: Rapid, saccate to stratiform liquefaction, with reddish-brown sediment in the liquefied portion.

Agar colony : Circular, smooth, glistening, slightly raised, somewhat transparent, with brownish tinge.

Agar slant: Echinulate, slightly reddish-brown, viscous.

Broth: Turbid, with thin, gray pellicle, and reddish-brown sediment.

Litmus milk: Rapid reduction and proteolysis with odor of putrefaction.

Potato: Echinulate, smooth, glistening, viscous, reddish-brown.

Indole not produced.

Nitrites are produced from nitrates.

Acid from maltose and sucrose. No action on glucose, fructose, galactose, arabinose, lactose, raffinose, dextrin, inulin, salicin, amygdalin, glycerol.

Ammonia is formed.

Aerobic, facultative.

Optimum temperature $21^{\circ} \mathrm{C}$.

Source: Isolated from tainted butter.

Habitat: Milk, cream, butter, water, soil, creamery equipment (Long and Hammer, loc. cit.; Claydon and Hammer,
Iowa Agr. Exp. Sta., Res. Bull. 267, 1939.)

30. Pseudomonas mephitica Claydon and Hammer. (Jour. Bact., 37, 1939, 254.) From Latin, mephitis, pestilential, malodorous; M.L. Mephitis, a generic name.

Rods : 0.5 to 1.0 by 1.5 to 14.0 microns, occurring singly, in pairs and in chains. Actively motile with a polar flagellum. Gram-negative.

Gelatin: Slow liquefaction.

Agar colonies: Convex, circular, about $3 \mathrm{~mm}$. in diameter, shiny, grayish white, edge entire, of the consistency of bread dough.

Agar slant: Growth grayish-white, wrinkled, echinulate. After 1 or 2 days, a skunk-like odor develops.

Broth: Turbid. Sediment. White pellicle.

Potato: Growth echinulate, shiny, brownish.

Litmus milk: A skunk-like odor develops in 1 to 2 days. Grayish blue surface ring in about 3 days. Alkaline in 7 to 10 days. In two weeks complete reduction. Slight proteolysis and viscosity.

Hydrogen sulfide not produced.

Indole not formed.

Nitrites produced from nitrates.

Acid but not gas produced slowly from glucose, fructose, maltose, and sucrose. No acid from arabinose, dextrin, galactose, glycerol, lactose, mannitol, raffinose or salicin.

Aerobic, facultative.

Optimum temperature $21^{\circ} \mathrm{C}$. Growth slight at $5^{\circ}$ and $30^{\circ} \mathrm{C}$. No growth at $37^{\circ} \mathrm{C}$.

Source: Several cultures isolated from butter having a skunk-like odor.

Habitat: Probably from water.

31. Pseudomonas geniculata (Wright) Chester. (Bacillus geniculatus Wright, Memoirs Nat. Acad. Sci., 7, 1895, 459; Chester, Man. Determ. Bact., 1901, 313; Achromobacter geniculatum Bergey et al., Manual, 1st ed., 1923, 146.) From Latin, 
geniculatus, p.p. of geniculo, knotted, jointed.

Medium-sized rods, occurring singly, in pairs and chains, motile, possessing polar flagella. Gram-negative.

Gelatin colonies: Circular, whitish, translucent. Deep colonies yellowish.

Gelatin stab : Infundibuliform liquefaction. Sediment light pink.

Agar slant: Grayish, glistening, translucent, limited, becoming brownish-gray.

Broth: Turbid, with slight gray pellicle and sediment.

Litmus milk: Alkaline; reduction of litmus; slight coagulation.

Potato: Thin, brownish, moist, glistening, viscid.

Indole not formed.

Nitrites not produced from nitrates.

Aerobic, facultative.

Optimum temperature $20^{\circ}$ to $25^{\circ} \mathrm{C}$.

Habitat: Water.

\section{Pseudomonas fragi (Eichholz)}

Huss emend. Hussong, Long and Hammer (Bacterium fragi Eichholz, Cent. f. Bakt., II Abt., 9, 1902, 425; Huss, Cent. f. Bakt., II Abt., 19, 1907, 661; Hussong, Long and Hammer, Iowa Agr. Exp. Sta. Res. Bull. 225, 1937, 122; also see Long and Hammer, Jour. Dairy Sci., 20, 1937, 448.) From Latin fragum, strawberry.

Description from Hussong, Long and Hammer, loc. cit.

Rods : 0.5 to 1.0 by 0.75 to 4.0 mierons, occurring singly, in pairs and in chains. Motile with a polar flagellum. Gramnegative.

Gelatin: Crateriform to stratiform liquefaction in 3 to 4 days.

Agar colonies: Convex, glistening, generally butyrous, occasionally viscid. Rough, smooth and intermediate forms are recognized in the description quoted. The rough forms are less proteolytic, and less active in the hydrolysis of fats.

Agar slant: Growth abundant, spreading, raised, white, shiny, generally butyrous. Sweet ester-like odor resembling that of the flower of the May apple.

Broth: Turbidity and sediment with a thin pellicle.
Litmus milk: Acid ring followed by acid coagulum at surface. Complete coagulation in 2 to 3 weeks, some digestion. Characteristic May apple or strawberry odor.

Potato: Growth echinulate to arborescent, raised, glistening, white, becoming brownish.

Indole not produced.

Nitrites not produced from nitrates.

Ammonia produced from peptone.

Hydrogen sulfide not produced.

Acid from glucose and galactose, sometimes arabinose. No acid from glycerol, inulin, lactose, fructose, maltose, mannitol, raffinose, salicin and sucrose.

No acetylmethylcarbinol produced.

Fat is generally hydrolyzed.

Aerobic.

Grows from $10^{\circ}$ to $30^{\circ} \mathrm{C}$. No growth at $37^{\circ} \mathrm{C}$. Very sensitive to heat.

Source: Isolated from milk and other dairy products, dairy utensils, water, ete.

Habitat: Soil and water. Widely distributed (Morrison and Hammer, Jour. Dairy Sci., 24, 1941, 9).

Hussong (Thesis, Iowa State College, 1932) regards Bacterium fragi Eichholz (loc. cit.) as the $\mathrm{R}$ type, Pseudomonas jragariae I Gruber (Cent. f. Bakt., II Abt., 9, 1902, 705) as the $\mathrm{O}$ form, and Pseudumonas fragariae Il Gruber (Cent. f. Bakt., II Abt., 14, 1905, 122) as the S form of the same organism. He makes no mention of Pseudomonas fragaroidea Huss (loc. cit.) which from its description would belong to the smooth type. A brief characterization of each of these organisms follows: (1) Bacterium fragi came from milk as drawn from an individual cow; it does not liquefy gelatin, exhibits no fluorescence, is strongly alkaline in litmus milk, and does not grow at $37^{\circ} \mathrm{C}$, (2) Pseudomonas fragariae I came from fodder beets; it does not liquefy gelatin, has weak blue-greenish fluorescence, is weakly alkaline in milk, and grows at $37^{\circ} \mathrm{C}$, (3) Pseudomonas fragariae $I I$ came from pasteurized milk; it liquefies gelatin, coagulates milk, and does not grow at $37^{\circ} \mathrm{C}$, (4) Pseudomonas 
fragaroidea came from butter; it liquefies gelatin, coagulates milk, and grows at $37^{\circ} \mathrm{C}$.

* 33. Pseudomonas nebulosa (Wright) Chester. (Bacillus nebulosus Wright, Memoirs Nat. Acad. Sci., \%, 1895, 465; Chester, Man. Determ. Bact., 1901, 311; Achromobacter nebulosum Bergey et al., Manual, 1st ed., 1923, 145; not Bacillus nebulosus Hallé, Thèse de Paris, 1898; not Bacillus nebulosus Vincent, Ann. Inst. Past., 21, 1907, 69; not Bacillus nebulosus Migula, Syst. d. Bakt., 2, 1900, 844; not Bacillus nebulosus Goresline, Jour. Bact., 27, 1934, 52.) From Latin, nebula, mist.

Medium-sized rods, occurring singly. Motile, possessing polar flagella. Gramnegative.

Gelatin colonies: Thin, circular, gray, translucent, hazy, with white center.

Gelatin stab: Crateriform liquefaction.

Agar slant: Thin, transparent streak.

Broth: Turbid, with gray sediment.

Litmus milk: Alkaline; reduction of litmus.

Potato: Scanty growth.

Indole not formed.

Nitrites not produced from nitrates.

Sugar gelatin in deep stab: Fair growth, with some gas formation.

Aerobic, facultative.

Optimum temperature $30^{\circ}$ to $35^{\circ} \mathrm{C}$.

Habitat: Water.

Probable synonym: Psendomonas centrifugans Chester. (Man. Determ. Bact., 1901, 312; Bacillus centrifugans Wright, Mem. Nat. Acad. Sci., 7, 1895, 462.)

34. Pseudomonas coadunata (Wright) Chester. (Bacillus coadunatus Wright, Memoirs Nat. Acad. Sci., 7, 1895, 460; Chester, Man. Determ. Bact., 1901, 310; Achromobacter coadunatum Bergey et al., Manual, 1923, 147.) 1st ed., From Latin. coadunatus, to unite closely.
Medium-sized rods, with rounded ends, occurring singly, in pairs and in chains. Motile, possessing a polar flagellum. Gram-negative.

Gelạtin colonies: Circular, brownish, dense.

Gelatin stab : Crateriform to stratiform liquefaction.

Agar slant: Gray, translucent, spreading.

Broth: Turbid, with gray pellicle and sediment. The medium has a slight greenish tint.

Litmus milk: Acid; coagulated.

Indole is formed.

Nitrites not produced from nitrates,

Sugar gelatin in deep stab: Good growth of discreet and confluent whitish colonies. Marked gas production; no liquefaction.

Aerobic, facultative.

Optimum temperature $20^{\circ}$ to $25^{\circ} \mathrm{C}$.

Habitat: Water.

35. Pseudomonas multistriata (Wright) Chester. (Bacillus multistriatus Wright, Memoirs Nat. Acad. Sci., 7, 1895, 462; Chester, Man. Determ. Bact., 1901, 310; Achromobacter multistriatum Bergey et al., Manual, 1st ed., 1923, 147.) From Latin, multus, many, much; striatus, grooved.

Medium-sized rods, with rounded ends, occurring singly and in pairs. Motile, possessing polar flagella. Gram-negative.

Gelatin colonies: Circular, grayishwhite, translucent.

Gelatin stab: Crateriform liquefaction.

Agar slant: Narrow, translucent, grayish streak.

Broth: Turbid.

Litmus milk : Slightly acid; coagulated.

Potato: Grayish to creamy, thick, glistening, viscid, spreading.

Indole not formed.

Nitrites not produced from nitrates.

Sugar gelatin in deep stab: Vigorous growth with marked gas production; also liquefaction.

* Prof. E. R. Hitchner, Univ of Maine, Orono, Maine assisted in rearranging the (lescriptions of the acid and gas producing pseudomonads (Aeromonas), April, 1943. 
Aerobic, facultative.

Optimum temperature $20^{\circ}$ to $25^{\circ} \mathrm{C}$.

Habitat: Water.

36. Pseudomonas punctata (Zimmermann) Chester. (Bacillus punctatus Zimmermann, Bakt. unserer Trink-und Nutzwässer, Chemnitz, 1, 1890, 38; Bacillus aquatilis communis Kruse, in Flügge, Die Mikroorganismen, 2, 1896 , 315; Bacterium punctatum Lehmann and Neumann, Bakt. Diag., 1 Aufl., 2, 1896, 238; Chester, Man. Determ. Bact., 1901, 313; Achromobacter punctatum Bergey et al., Manual, 1st ed., 1923, 147.) From Iatin, punctus, a puncture, point; M. I. punctate, dotted.

Rods : 0.7 by 1.0 to 1.5 micron, occurring singly, in pairs and in chains. Motile with a single polar flagellum. Gramnegative.

Gelatin colonies: Small, circular, gray, erose to filamentous, punctiform.

Gelatin stab: Crateriform liquefaction. No pellicle.

Agar slant: Gray, smooth, filamentous.

Broth: Turbid with delicate pellicle.

Litmus milk: Acid; coagulated; peptonized.

Potato: Brownish-yellow to brownishred color.

Indole is formed.

Nitrites not produced from nitrates.

Hydrogen sulfide is formed.

Acid and gas from glucose.

Aerobic, facultative.

Optimum temperature $25^{\circ}$ to $30^{\circ} \mathrm{C}$.

Source: Common in the Chemnitz. tap water.

Habitat: Cause of a hemorrhagic septicemia in carp (Cyprinus) (Schäperclaus, Ztschr. f. Fischerei, 28, 1930; Cent. f. Bakt., II Abt., 105, 1942, 19).

37. Pseudomonas hydrophila (Chester) comb. nov. (Bacillus hydrophilus fuscus Sanarelli, Cent. f. Bakt.. 9, 1891, 222 ; Bacterium hydrophilus fuscus Chester, Delaware College Agr. Expt. Sta., 9th Ann. Rept., 1897, 92; Bacillus hydro- philus Chester, Manual Determ. Bact., 1901, 235; Bacterium hydrophilum Weldin and Levine, Bact. Abs., 7, 1923, 14; Proteus hydrophilus Bergey et al., Manual, 1st ed., 1923, 211; Aeromonas hydrophila Stanicr, Jour. Bact., 46, 1943, 213.) From Greek, hydōr, water, philus, loving; II. L. water-loving.

It was reported by Russell, Jour. Amer. Med. Assoc., 30, 1898, 1442 and later by Emerson and Norris, Jour. Exper. Med., $7,1905,32$ who made a complete study of its properties and its pathogenic action.

Weldin (Iowa State College Jour. Sci., 1, 1927, 151) considers Bacillus ranicida Ernst (Beiträge $z$. path. Anat. u. z. Allgemein. Pathol., 8, 1890, 204; Bacterium ranicida Chester, Ann. Rept. Del. Col. Agr. Exp. Sta., 9, 1897, 141) a possible synonym of Proteus hydrophilus.

Rods: 0.6 by 1.3 microns, occurring singly and in chains. Motile, with a single polar flagellum (Kulp and Borden, Jour. of Bact., 44, 1942, 673). Gramnegative.

Gelatin colonies: Small, circular, gray, translucent, stippled.

Gelatin stab: Napiform liquefaction.

Agar colonies: Whitish, raised, moist, stippled.

Agar slant: Thin, whitish, glassy, spreading, becoming yellowish.

Broth: Turbid, with heavy pellicle.

Litmus milk: Acid; coagulated; peptonized.

Potato: Yellowish-brown, moist slightly raised.

Indole is formed.

Nitrites produced from nitrates.

Acid and gas from glucose, maltose, sucrose and mannitol. No action on lactose.

Gas ratio $\mathrm{H}_{2}: \mathrm{CO}_{2}=1: 4.71$. Methyl red negative, acetylmethylcarbinol positive, indol negative, citrate positive (Speck and Stark, Jour. Bact., 44, 1942, 697).

Aerobic, facultative.

Optimum temperature $37^{\circ} \mathrm{C}$.

Pathogenic for frogs, salamanders, fish, 
mice, guinea pigs and rabbits, causing hemorrhagic septicemia.

Distinctive characters: Much like Pseudomonas punctata (Guthrie and Hitchner, Jour. Bact., 45, 1943, 52).

Source: Isolated from frogs dead of septicemia (red leg).

Habitat: Water and infected fresh water animals.

38. Pseudomonas ichthyosmia (Hammer) comb. nov. (Bacillus ichthyosmius Hammer, Iowa Agr. Sta. Res. Bul. 38, 1917; Escherichia ichthyosmia Bergey et al., Manual, 1st ed., 1923, 201; Proteus ichthyosmius Bergey et al., Manual, th ed., 1934, 364.) From Greek, ichthys, a fish; osmē, an odor.

Rods : 0.6 to 0.8 by 1.0 to $\therefore 1$ microns, occur singly. Notile with a single polar flagellum (Breed). Gram-negative.

Gelatin stab: Liquefaction.

Agar colonies: Small, white, becoming darker with age.

Agar slant: Dirty white, viscid growth

Broth: Turbid with gray sediment.

Litmus milk: Acid. Litmus reduced. Cultures have fishy odor.

Potato: Thin, glistening layer.

Indole is formed.

Nitrites produced from nitrates.

Acid and gas from glucose, fructose, galactose, maltose, sucrose, glycerol, salicin and mannitol. Lactose, dulcitol, raffinose and inulin not fermented.

Aerobic, facultative.

Optimum temperature $20^{\circ} \mathrm{C}$.

Source: Isolated from can of evaporated milk having a fishy odor.

Habitat: Not known.

\section{Pseudomonas ambigua (Wright)} Chester. (Bacillus ambiguus Wright, Memoirs Nat. Acad. Sci., 7, 1895, 439; Chester, Man. Determ. Bact., 1901, 308; Achromobacter ambiguum Bergey et al., Manual, 1st ed., 1923, 148.) From Latin, ambiguus, doubtful, uncertain.

Small rods, with rounded ends, occurring singly, in pairs and in chains. Mo- tile, possessing a polar flagellum. Gramnegative.

Gelatin colonies: Gray, translucent, slightly raised, irregular, radiate, with transparent margin.

Gelatin stab: No liquefaction.

Agar slant: Gray, limited, entire.

Broth: Turbid, with gray sediment.

Litmus milk: Acid, slowly coagulated.

Potato: Gray to creamy, viscid, spreading.

Indole is furmed.

Nitrites not produced from nitrates.

Aerobic, facultative.

Optimum temperature $30^{\circ}$ to $35^{\circ} \mathrm{C}$.

Habitat: Water.

\section{Pseudomonas sinuosa (Wright)}

Chester. (Bacillus sinuosus Wright, Memoirs Nat. Acad. Sci., 7, 1895, 440 ; Chester, Man. Determ. Bact., 1901, 307; Achromobacter sinosum (sic) Bergey et al., Manual, 1st ed., 1923, 148.) From Latin, sinuosus, full of bends, sinuous.

Medium-sized rods, with rounded ends, orcurring singly, in pairs and in chains. Iotile, possessing two to four polar flagella. Gram-negative.

Gelatin colonies: Thin, translucent, irregular, center brownish.

Gelatin stab: Grayish-white, glistening, translucent. No liquefaction.

Agar slant: Scanty, grayish growth.

Broth: Turbid, with gray sediment.

Litmus milk: Unchanged.

Potato: Grayish-white, moist, spread. ing.

Indole is formed.

Nitrites not produced from nitrates.

Aerobic, facultative.

Optimum temperature $30^{\circ}$ to $35^{\circ} \mathrm{C}$.

Habitat: Water.

41. Pseudomonas cruciviae Gray and Thornton. (Gray and Thornton, Cent. f. Bakt., II Abt., 78, 1928, 91; Achromobacter cruciviae Bergey et al., Manual, 3rd ed., 1930, 215.) From Latin, crux, a cross, via, way, road; from Wayeross, a place name. 
Rods : 1.0 by 1.0 to 3.0 microns, occurring singly and in pairs. Motile with one to five polar flagella. Gram-negative.

Gelatin colonies: Circular, white with buff center, convex, smooth, undulate.

Gelatin stab: No liquefaction.

Agar colonies: Circular or amoeboid, white to buff, flat to convex, smooth, entire.

Agar slant: Filiform, pale buff, raised, smooth, undulate.

Broth: Turbid.

Nitrites not produced from nitrates.

Starch not hydrolyzed.

No acid in carbohydrate media.

Attack phenol and $m$-cresol.

Aerobic, facultative.

Optimum temperature 30 to $35^{\circ} \mathrm{C}$.

Habitat: Soil.

\section{Pseudomonas rugosa (Wright)}

Chester. (Bacillus rugosus Wright, Memoirs Nat. Acad. Sci., 7, 1895, 438; not Bacillus rugosus Henrici, Arb. Bakt. Inst. 'Tech. Hochsch. Karlsruhe, 1, 1894, 28; not Bacillus rugosus Chester, Determinative Bacteriology, 1901, 220; Chester, Determinative Bacteriology, 1901, 323.) From Latin, rugosus, wrinkled.

Small rods, with rounded ends, occurring singly, in pairs and in chains. Motile, possessing one to four polar flagella. Gram-negative.

Gelatin colonies: Grayish, translucent, slightly raised, irregular, sinuous, radiately erose to entire.

Gelatin stab: Dense, grayish-green, limited, wrinkled, reticulate surface growth. No liquefaction.

Agar slant: Grayish-white, limited, slightly wrinkled, translucent.

Broth: Turbid, with grayish pellicle and sediment.

Litmus milk: Acid, coagulated.

Potato: Moist, glistening, brown.

Indole is formed.

Nitrites not produced from nitrates.

Aerobic.

Optimum temperature $30^{\circ} \mathrm{C}$.

Habitat: Water.
43. Pseudomonas desmolyticum Gray and Thornton. (Gray and Thornton, Cent. f. Bakt., II Abt., 73, 1928, 90; Achromobacter desmolyticum Bergey et al., Manual, 3rd ed., 1930, 217.) From Greek desmos, bond, band; lytikos, able to dissolve.

Rods : 0.7 to 0.8 by 2.0 to 3.0 microns, occurring singly and in pairs. Motile, with one to five polar flagella. Gramnegative.

Gelatin colonies: Circular, gray to buff, raised or umbonate. Smooth, glistening, entire.

Gelatin stab: No liquefaction.

Agar colonies: Circular or amoeboid, whitish, flat or convex, smooth, translucent to opaque, entire.

Agar slant: Filiform, pale buff, raised, smooth, undulate.

Broth: Turbid.

Nitrites produced from nitrates.

Starch not hydrolyzed.

Acid from glucose.

Attack phenol and naphthalene.

Aerobic, facultative.

Optimum temperature $25^{\circ} \mathrm{C}$.

Habitat: Soil.

44. Pseudomonas rathonis Gray and Thornton. (Gray and Thornton, Cent. f. Bakt., II Abt., 73, 1928, 90; Achromobacter rathonis Bergey et al., Manual, 3rd ed., 1930, 216.) From M. L. of Ratho Park (Edinburgh).

Small rods: 0.5 to 1.0 by 1.0 to 3.0 microns. Motile, with polar flagella. Gram-negative.

Gelatin colonies: Circular, white, raised, smooth, glistening, undulate.

Gelatin stab: No liquefaction.

Agar colonies: Circular, buff, flat, smooth, glistening, entire.

Agar slant: Filiform, pale buff, convex, smooth, glistening, undulate.

Broth: Turbid, with pellicle.

Nitrites produced from nitrates.

Starch hydrolyzed.

Acid from glucose and glycerol. 
Attack phenol and cresol at times, also naphthalene.

Aerobic, facultative.

Optimum temperature $25^{\circ} \mathrm{C}$

Habitat: Manure and soil.

45. Pseudomonas dacunhae Gray and Thornton. (Gray and Thornton, Cent. f. Bakt., II Abt., 73, 1928, 90; Achromobacter dacunhae Bergey et al., Manual, 3rd ed., 1930, 217.) From M. I. from the Island of d'Acunha.

Rods: 0.5 to 0.8 by 1.5 to 3.0 microns. Motile with one to six polar flagella. Gram-negative.

Gelatin colonies: Circular, whitish, raised, smooth, glistening, undulate.

Gelatin stab: No liquefaction.

Agar colonies: Circular to amoeboid white, flat, glistening, opaque, entire.

Agar slant: Filiform, pale buff, raised, smooth, glistening, undulate.

Broth: Turbid.

Nitrites produced from nitrates.

Starch not hydrolyzed.

No acid from carbohydrate medin.

Attack phenol.

Aerobic, facultative.

Optimum temperature $25^{\circ} \mathrm{C}$.

Habitat: Soil.

46. Pseudomonas arvilla Gray and Thornton. (Gray and Thornton, Cent. f. Bakt., II Abt., 73, 1928, 90; Achromobacter arvillum Bergey et al., Manual, 3rd ed., 1930, 217.) From Latin, arvum, an arable field; M. L. dim. a little field.

Rods : 0.5 to 0.7 by 2.0 to 3.0 microns. Motile with one to five polar flagella. Gram-negative.

Gelatin colonies: Circular, whitish, convex, smooth, glistening, lobate.

Gelatin stab: No liquefaction.

Agar colonies: Circular or amoeboid, white to buff, flat to convex, smooth, glistening, opaque, entire.

Agar slant: Filiform, whitish, concave, smooth, ringed, entire.

Broth: Turbid.

Nitrites not produced from nitrates.
Starch not hydrolyzed.

Acid from glucose.

Attacks naphthalene.

Aerobic, facultative.

Optimum temperature $25^{\circ} \mathrm{C}$.

Habitat: Soil.

47. Pseudomonas salopium Gray and Thornton. (Gray and Thornton, Cent. f. Bakt., II Abt., 73, 1928, 91 ; Achromobacter salopium Bergey et al., Manual, 3rd ed., 1930, 219.) From Latin, Salop, Shropshire.

Rods : 0.7 to 1.0 by 1.0 to 3.0 microns, occurring singly and in pairs. Notile with one to six polar flagella. Ciramnegative.

Gelatin colonies: Cireular, grayishbuff, flat, rugose or ringed, translucent border.

Gelatin stab: No liquefaction.

Agar colonies: Circular or amoeboid, white to buff, flat to convex, smooth, glistening, translucent border, entire.

Agar slant: Filiform, whitish, raised, smooth, glistening, lobate.

Broth: Turbid with pellicle.

Nitrites not produced from nitrates.

Starch not hydrolyzed.

Acid from glucose and sucrose.

Attacks naphthalene.

Aerobic, facultative.

Optimum temperature $25^{\circ} \mathrm{C}$.

Habitat: Soil.

48. Pseudomonas minuscula MeBeth. (MeBeth, Soil Science, 1, 1916, 437; Cellulomonas minuscula Bergey et al., Manual, 1st ed., 1923, 162.) From Latin dim. rather small.

Rods : 0.5 by 0.9 micron. Motile with one to two polar flagella. Gram-negative.

Gelatin stab: Moderate growth. Slight napiform liquefaction.

Agar colonies: Small, circular, slightly convex, butyrous becoming brittle, grayish-rhite, finely granular, entire.

Agar slant: Moderate, flat, grayishwhite.

Broth: Turbid. 
Litmus milk: Acid, not digested.

Potato: No apparent growth.

Indole is formed.

Nitrites produced from nitrates.

Ammonia is produced.

Acid from glucose, lactose, maltose, sucrose and starch.

Aerobie, facultative.

Optimum temperature $20^{\circ} \mathrm{C}$.

Habitat : Soil.

49. Pseudomonas tralucida Kellerman et al. (Kellerman, McBeth Scales and Smith, Cent. f. Bakt., II Abt., 39, 1913, 37 ; Cellulomonas tralucida Bergey et al., Manual 1st ed., 1923, 163.) From Latin, clear, transparent.

Rods : 0.6 by 1.2 microns. Motile with one or two polar flagella. Gram-negative.

Gelatin stab: No liquefaction.

Agar slant: Scant, grayish growth.

Broth : Turbid.

Litmus milk: Acid.

Potato: No growth.

Indole not formed.

Nitrites produced from nitrates.

Ammonia not produced.

Acid from glucose, maltose, lactose, sucrose, starch, glycerol and mannitol.

Aerobie, facultative.

Optimum temperature $20^{\circ} \mathrm{C}$.

Habitat: Soil.

50. Pseudomonas mira MeBeth. (Mc. Beth, Soil Science, 1, 1916, 437; Cellulomonas mira Bergey et al., Manual, 1st ed., 1923, 165.) From Latin, mirus, wonderful, extraordinary.

Rods : 0.4 by 1.6 microns. Motile with a single polar flagellum. Gram-negative.

Gelatin stab: Good growth. No liquefaction.

Agar colonies: Circular, convex, grayish-white, granular, lacerate.

Agar slant: Moderate, flat, grayishwhite, somewhat iridescent.

Broth: Turbid.

Litmus milk: Alkaline.

Potato: Moderate, grayish-white.

Indole not formed.
Nitrites produced from nitrates.

Ammonia is produced.

Acid from glucose, maltose, lactose, sucrose, starch, glycerol and mannitol.

Aerobic, facultative.

Optimum temperature $20^{\circ} \mathrm{C}$.

Habitat: Soil.

51. Pseudomonas lindneri Ḱluyver and Hoppenbrouwers. (Lindner, $50 \mathrm{Ju}$ biläumsber. Westpreuss. Bot.-Zool. Vereins, 1928, 253; Termobacterium mobile Lindner, Atlas d. Mikrosk. GrundI. d. Gärungsk., 3 Aufl., 2, 1928, Taf. 68; Kluyver and Hoppenbrouwers, Arch. f. Mikrobiol., 2, 1931, 259; Achromobacter mobile Kluyver and Hoppenbrouwers, ibid., 258; not Pseudomonas mobilis Migula, Syst. d. Bakt., 2, 1900, 923.) Named for Lindner, the German bacteriologist who first studied this organism.

Short rods 1.4 to 2.0 by 4.0 to $5.0 \mathrm{mi}$ crons. Occurring singly, in pairs and short chains. Motile with a single polar flagellum. Gram-negative.

Peptone gelatin: Poor growth.

Peptone agar: Poor growth.

Wort agar: White, round, raised colonies, $1 \mathrm{~mm}$. in diameter. Good growth. Still better where 2 per cent sucrose, or yeast extract with sucrose is added. Chalk added to neutralize acid.

Broth: Poor growth in peptone or yeast extract broth unless sugars are added.

Carbon dioxide, ethyl alcohol and some lactic acid produced from glucose and fructose, but not from mannose. May or may not ferment sucrose. May produce as much as 10 per cent alcohol.

Catalase produced.

Anaerobic, facultative.

Optimum temperature $30^{\circ} \mathrm{C}$.

Distinctive character: The fermentation resembles the alcoholic fermentation produced by yeasts.

Source: Isolated from the fermenting sap (pulque) of Agave americana in Mexico.

Habitat: Fermenting plant juices in tropical countries (Mexico). 
52. Pseudomonas membranoformis (Zobell and Allen) Zobell. (Achromobacter membranoformis Zobell and Allen, Jour. Bact., 29, 1935, 246; Zobell, Jour. Bact., 46, 1943, 45). From Latin membrana, membrane, and forma having the form of.

Rods: 0.9 to 1.2 by 3.5 to 4.8 microns, occurring singly and in pairs. Motile with lophotrichous flagella. Eneapsulated. Gram-negative.

Gelatin stab: Growth filiform, best at top, with slow crateriform liquefaction.

Agar colonies: Circular, 1.0 to $2.5 \mathrm{~mm}$. with crinkled surface.

Agar slant: Moderate, beaded, raised growth. Membranous consistency. Becomes browned with age.

Broth : Slight turbidity, flocculent sediment, film of growth on walls of test tube.

Milk: No growth.

Potato: No growth.

Indole not formed.

Nitrites not produced from nitrates.

No $\mathrm{H}_{2 \mathrm{~S}} \mathrm{~S}$ produced.

Acid but not gas from glueose, sucrose, dextrin and mannitol. No acid from lactose or xylose.

No diastatic action.

Optimum temperature $20^{\circ}$ to $25^{\circ} \mathrm{C}$.

Aerobic.

Source: Sea water.

Habitat: Sea water.

\section{Pseudomonas marinoglutinosa}

(Zobell and Allen) Zobell. (Achromobacter marinoglutinosus Zobell and Allen, Jour. Bact,, 29, 1935, 246; Zobell, Jour. Bact., 46, 1943, 45). From Latin marinus, pertaining to the sea, and glutinosus, full of glue, sticky.

short rods: 0.7 to 1.0 by 1.8 to $2.4 \mathrm{mi}$ crons, with rounded ends, occurring singly, in pairs and in clumps. Motile with polar flagella. Staining granular. Encapsulated. Gram-negative.

Gelatin stab: Moderate filiform growth with slight napiform liquefaction. No pigment.

Agar colonies: Round with concentric circles and crinkled radial lines, 1.5 to $5.0 \mathrm{~mm}$. in diameter. No pigment.

Agar slant: Moderate, filiform, flat. Butyrous consistency.

Broth: Moderate clouding, marked ring, adherent film of growth on test tube wall, and flaky sediment.

Milk: No growth.

Potato: No growth.

Indole not formed.

Nitrites not produced from nitrates.

Hydrogen sulfide and ammonia produced from Bacto-tryptone.

Acid but not gas from xylose and dextrin. No acid from glucose, lactose, surrose and mannitol.

Starch is hydrolyzed.

Optimum temperature $20^{\circ}$ to $25^{\circ} \mathrm{C}$

Acrobie, facultative.

Source: Sea water.

Habitat: Sea water.

54. Pseudomonas gelatica (Gran) Bergey et al. (Bacillus gelaticus Giran, Bergens Museums Aarbog., 1902, 14; Bacterium gelaticum Lundestad, Cent. f. Bakt., II Abt., 75, 1928, 328; Bergey et al., Manual, 3rd ed., 1930, 175.) From French, like gelatin.

Rods, with rounded ends, 0.6 to 1.2 by 1.2 to 2.6 microns, occurring singly, in pairs, and sometimes in short chains. Iotile. Gram-negative.

Fish-gelatin colonies: Circular, transparent, glistening, becoming brownish in color.

Fish-gelatin stab: Liquefaction infundibuliform, with greenish color.

Sea-weed agar colonies: Circular, flat, entire, glistening, reddish-brown center with grayish-white periphery. Liquefied.

Fish-agar slant: Flat, transparent streak, with undulate margin, reddishbrown.

Broth: Turbid with flocculent pellicle, and greenish-yellow sediment.

Indole not formed.

Nitrites are produced from nitrates.

Starch hydrolyzed. 
No action on sugars.

Anaerobic, facultative.

Optimum temperature 20 to $25^{\circ} \mathrm{C}$.

Habitat: Sea water of Norwegian coast.

\section{Pseudomonas calcis (Drew)}

Kellerman and Smith. (Bacterium calcis Drew, Yearbook Carnegie Inst. Wash., 11, 1912, 136-144; Kellerman and Smith, Proc. Nat. Acad. Sci., 4, 1914, 400.) From Latin calx (calc-), lime.

Ovoid rods, 1.1 by 1.5 to 3 microns, usually single but may form long chains. Actively motile with one polar flagellum. Gram-negative.

Grows best in sea water or 3 per cent salt media. Deposits $\mathrm{CaCO}_{3}$.

Agar colonies: Circular, with finely irregular outline, granular appearance, elevated, spreading; old colonies having brownish tinge in center.

Gelatin stab: Infundibuliform lique. faction.

Gelatin colonies: Small, with liquefaction.

Broth: Good growth especially in presence of potassium nitrate, peptone or calcium malate.

Acid from glucose, mannite and sucrose but not from lactose.

Nitrates reduced to nitrites and ammonia.

Aerobic, facultative.

Optimum temperature 20 to $28^{\circ} \mathrm{C}$.

Habitat: Sea water and marine mud.

Bavendamm (Arch. f. Mikrobiol., 3, 1932, 214) states that Pseudomonas calcis is probably synonymous with Bacterium brandti, Bacterium bauri and Bacterium feiteli described by Parlandt (Bull. Jard. imp. Bot. St. Petersburg, 11, 1911, 97-105).

56. Pseudomonas calciprecipitans Molisch. (Cent. f. Bakt., II Abt., 65, 1925, 130.) From Latin, calx (calc-), lime; praecipito, to cast down headlong, to precipitate.

Thin rods: 0.5 to 0.8 by 1.5 to $3.6 \mathrm{mi}$ erons, with rounded ends, often staining irregularly. Motile, with one polar flagellum. Gram-negative.

Gelatin colonies: Circular, light brown in color (large colonies show $\mathrm{CaCO}_{3}$ crystals).

Gelatin stab: Surface growth with filiform growth in depth. Liquefaction starts at bottom.

Agar colonies (sea water). Grayishwhite, glistening. In two to three weeks crystals of calcium carbonate form in the agar.

Agar slant: Slight, whitish, surface growth, becoming thick, spreading, glistening, with abundant $\mathrm{CaCO}_{3}$ crystals in medium.

Ammonia formed.

Aerobic, facultative.

Optimum temperature $20^{\circ} \mathrm{C}$.

Habitat: Sea water.

57. Pseudomonas ichthyodermis (Wells Zobell) Zobell and Upham. (Achromobacter ichthyodermis Wells and Zobell, Proc. Nat. Acad. Sci., 20, 123, 1934; Zobell and Upham, Bull. Scripps Inst. Oceanography, 5, 1944, 246 and 253.) From Greek, ichthys, a fish; derma, skin.

Small rods, 0.9 to 1.3 by 3 to 5 microns, occurring singly and in pairs. No spores. Encapsulated. Polar flagella. Pleomorphic forms predominate in old cultures. Gram-negative.

Requires sea water following initial isolation. The following differential media are prepared with sea water.

Agar colonies: Glistening, colorless, convex, eircular colonies 2 to $4 \mathrm{~mm}$. in diameter.

Agar slants : Abundant, filiform, raised, smooth, opalescent growth.

Gelatin tube: Rapid erateriform liquefaction complete in 5 days at $18^{\circ} \mathrm{C}$.

Sea water broth: Turbidity, with pellicle, little granular sediment and no odor.

Milk: No growth. Casein digested when 3 per cent salt is added.

Potato: No growth unless dialyzed in sea water. Then fair growth with nc pigment. 
Acid from glucose, maltose, sucrose and mannitol but not from lactose or glycerol.

Starch hydrolyzed.

Ammonia liberated from peptone but .no hydrogen sulfide produced.

Indole formed in tryptophane sea water broth.

Nitrites produced from nitrates.

Optimum temperature 20 to $25^{\circ} \mathrm{C}$.; $30^{\circ} \mathrm{C}$. incubation will kill recently isolated organisms.

Aerobic, facultative.

Source: Isolated from diseased kilifish (Fundulus parvipinnis).

Habitat: Skin lesions and muscle tissue of infected marine fish.

58. Pseudomonas nigrifaciens White (Scientific Agriculture, 20, 1940, 643.) From Latin niger, black and faciens. making.

Rods: 0.3 to 0.7 by 1 to 5 microns, occurring singly or in pairs, and having rounded ends. Actively motile, with a single polar flagellum. Gram-negative.

Gelatin stab : Pigmented surface growth after 24 hours. Slight erateriform liquefaction changing to saccate.

Agar colonies: Circular. convex. smooth, glistening, entire, 2 to $4 \mathrm{~mm}$ in diameter. Slight fluorescence in early stages. The medium assumes a brownish color.

Agar slant: Growth filiform, smooth, moist, glistening, with blackish pigmentation at $4^{\circ}$ and $15^{\circ} \mathrm{C}$. in $48 \mathrm{hrs}$, the medium turning bromnish. Slight fluorescence in early stages.

Broth: Turbid after 24 hours. After 5 to 6 days a black ring and then a pellicle forms, later a black sediment. Medium turns brown.

Litmus milk: A black ring appears aiter 3 days at $15^{\circ} \mathrm{C}$. followed by a pellicle. Litmus is reduced. Alkaline reaction. No coagulation. Digested with a putrid odor.

Indole not formed.
Nitrites not produced from nitrates in 7 days. No gas produced.

starch is hydrolyzed. Natural fats not hydrolyzed.

Alkaline reaction produced in sucrose, maltose, lactose, glucose, mannitol and raffinose broth ( $\mathrm{pH} 8.2$ ). No gas produced.

Ammonia produced in peptone broth. Aerobic.

Optimum pH 6.8 to 8.4 .

Temperature relations: Minimum $4^{\circ} \mathrm{C}$. Optimum $25^{\circ} \mathrm{C}$. Maximum $33-35^{\circ} \mathrm{C}$.

Distinctive characters: $\mathrm{No}$ or slow yrowth in culture media in the absence of salt. Maximum growth and pigmentation appeared with 1.5 and 2.5 per cent salt. Optimum pigmentation occurs at $4^{\circ}$ and $15^{\circ} \mathrm{C}$.

Source: Several cultures isolated from samples of discolored butter.

Habitat: Causes a black to reddishbrown discoloration of print butter. Evirlently widely distributed in nature.

59. Pseudomonas beijerinckii Hof. (Travaux botaniques néerlandais, 32, 1935, 152.) Named for M. W. Beijerinck, Dutch bacteriologist

Small rods: Motile with polar flagella. Gelatin: No liquefaction.

Indole not formed.

Nitrites produced from nitrates by four out of six strains.

Cellulose not decomposed.

Acid from glucose. In yeast-water with 2 per cent glucose and 12 per cent NaCl no gas is produced.

Pigment production: Insoluble purple pigment produced but not in all media; is localized markedly; reduced oxygen tension necessary; optimum pH 8.0; not produced in yeast-water or in peptonewater; produced only when grown in extracts of heans or some other vegetable.

\section{Aerobic}

Source: Six strains isolated from beans preserved with salt.

Habitat: Causes purple discoloration of salted beans. 
60. Pseudomonas salinaria Harrison and Kennedy. (Harrison and Kennedy, Trans. Royal Soc. of Canada, 16, 1922, 121; Serratia salinaria Bergey et al., Manual, 1st ed., 1923, 93; Flavobacterium (Halobacterium) salinarium Elazari-Volcani, Studies on the Microflora of the Dead Sea, Thesis, Hebrew Univ., Jerusalem, 1940, 59.) From Latin, salinae, saltworks.

Probable synonym: Serratia sambharianus Dixit and Vachna, Current Sci., 11, 1942, 107 (see Biol. Abs., 17, 1943, 793). Halophilic growing in 30 per cent salt. From salt lake in India.

Occurs as spheres and rods, 2.0 to 3.0 microns in diameter, 1.0 to 1.6 by 3.0 to 15.0 microns, occurring singly, as ovoid, amoeboid, clavate, cuneate, truncate, spindle, club, pear-shape, and irregular forms. Motile, frequently with a flagellum at each pole. Gram-negative.

Does not grow on ordinary culture media. Grows well on salted fish.

Codfish agar (16 to 30 per cent salt): Growth slow, smooth, raised, coarsely granular, entire, pale pink to scarlet (Ridgway chart).

No acid from carbohydrate media.

Indole not formed.

Nitrites not produced from nitrates.

Aerobic, facultative.

Optimum temperature $42^{\circ} \mathrm{C}$.

Source: Isolated from cured codfish (Harrison and Kennedy, loc. cit.). Isolated from salted fish by Browne (Absts. Bact., 6, 1922, 25 and Proc. Soc. Exper. Biol. and Med., 19, 1922, 321) who regarded this pleomorphic bacterium as two organisms-Spirochaeta halophilica and Bacterium halophilica.

Habitat: Produees reddening of dried codfish and causes rusty herring. In sea salt, and salt ponds containing not less than 16 per cent salt.

61. Pseudomonas cutirubra Lochhead. (Serratia cutirubra Lochhead, Can. Jour. of Research, 10, 1934, 275; Bacterium cutirubrum Lochhead, Jour. Bact., 27, 1934, 62; ibid., 45, 1943, 575; Flavobacterium (Halobacterium cutirubrum Elazari-Volcani, loc. cit., 59.) From Latin, cutis, slin, hide; ruber, red.

Occurs as rods and spheres. Spheres 1 to 1.5 microns in diameter. Rods 1.5 to 8.0 by $0.7-1.4$ microns. Rod forms motile with single polar flagellum. Coccoid forms motile when young. Gramnegative.

No growth on ordinary media.

Milk agar (20 per cent salt to saturation; optimum 28-32 per cent): Colonies 3-4 mm. in diameter, round and slightly convex. Pink to rose dorée (Ridgway chart).

Milk agar slants: Growth filiform, slightly spreading, rather flat with smooth, glistening surface and membranous consistency. Proteolytic action.

Liquid media: No or slight growth.

Gelatin (salt) : Pronounced liquefaction.

Indole not formed, Lochhead (loc. cit.), Faint test, Gibbons (Jour. Biol. Board Canada, 3, 1936, 75).

Nitrites not produced from nitrates.

Diastatic action negative.

No carbohydrate fermentation.

Aerobic, obligate.

Optimum temperature $37^{\circ} \mathrm{C}$.

Halophilic.

Source: Isolated from salted hides.

Habitat: Sea water and sea salt.

62. Pseudomonas harveyi Johnson comb. nov. (Achromobacter harveyi Johnson and Shunk, Jour. Bact., 31, 1936, 587.) Named for E. N. Harvey, who studied luminous bacteria.

Rods : 0.5 to 1.0 by 1.2 to 2.5 microns, oceurring singly or in pairs, with rounded ends. Occasionally slightly curved; ends occasionally slightly pointed. Non-sporeforming. Capsules absent. Motile with a single polar flagellum, 2 to 3 times the length of the cell. Gram-negative. 
Sea water gelatin colonies: After 24 hours at $20^{\circ} \mathrm{C}$, circular, about $1.5 \mathrm{~mm}$. in diameter or larger, margin slightly undulate, sunken due to the beginning of liquefaction, interior somewhat zonate; colonies surrounded by a halo of numerous small secondary colonies, circular and finely granular. In crowded plates a large number of gas bubbles are formed. Luminescent.

Sea water gelatin stab: Rapid saccate liquefaction complete in 5 days at $22^{\circ} \mathrm{C}$. Abundant flocculent sediment.

Sea water agar colonies: Mostly very large, 6 to $8 \mathrm{~cm}$. in diameter in 24 hours, flat, highly iridescent, circular with undulate margin, or composed of narrow and close or wide filamentous growth. Occasionally small colonies appear that are circular, with entire or slightly undulate margin, often producing irregular secondary growth, surface always smooth. Luminescent.

Sea water agar slant: Growth abundant, spreading, grayishly viscous, homogeneous, iridescent, the medium becoming rapidly alkaline when inoculated at an initial $\mathrm{pH}$ of 7.0 . With fish decoctions added to the medium, luminescence is much brighter and growth becomes brownish after several days.

Growth on autoclaved fish: Abundant, smooth, glistening, yellowish, becoming dirty brown after several days. Mild putrefactive odor. Luminescence very brilliant.

Sea water containing 0.2 per cent peptone: Abundant uniform turbidity, thin pellicle, sediment accumulating over a period of several days. Luminescence at surface only unless the tube is shaken.

Milk, with or without the addition of 2.8 per cent salt: No growth.

Potato plugs resting on cotton saturated with sea water: Growth slight, somewhat spreading, slightly brownish. Luminous.

Indole is formed (Gore's method).

Nitrites are produced from nitrates.

Ammonia is produced in peptone media (Hansen method).
Fixed acid from glucose, fructose, mannose, galactose, sucrose, maltose, mannitol, dextrin, glycogen, trehalose, cellobiose; slowly from salicin. Nonfixed acid from melezitose; slight acid from sorbitol, disappearing in 24 hours. No acid from glycerol, xylose, arabinose, dulcitol, inositol, adonitol, erythritol, arabitol, lactose, raffinose, rhamnose, fucose or alpha methyl glucoside.

Starch agar: Wide zone of hydrolysis.

Hydrogen sulfide is produced (Zobell and Fantham method).

Temperature relations: Optimum $35^{\circ}$ to $39^{\circ} \mathrm{C}$. Abundant growth at $22^{\circ}$ to $25^{\circ} \mathrm{C}$. Optimum luminescence at $20^{\circ}$ to $40^{\circ} \mathrm{C}$.

Not pathogenic for white rats or amphipods.

Aerobic, facultative anaerobe.

Source: Isolated from a dead amphipod (Talorchestia $s p$.) at Woods Hole, Massachusetts.

Habitat: Sea water.

63. Pseudomonas phosphorescens (Fischer) Bergey et al. (Bacillus phosphorescens Fischer, Zeitschr. f. Hyg., 2, 1857, 5s; Photobacterium indicum Beijerinck, Arch. Néerl. d. Sci. Exactes, 23, 1889, 401; Bacterium phosphorescens Lehmann, Cent. f. Bakt., 5, 1889, 785; not Bacterium phosphorescens Fischer, Cent. f. Bakt., 3, 1885, 107; Bergey et al., Manual,3rd ed., 1930, 177.) From Greek phosphore $\overline{0}$, to bear or bring light.

See page 699 for additional synonyms.

Description taken from Fischer (loc. cit.).

Small, thick rods: 2 to 3 times as long as wide, with rounded ends. Motile. Stain lightly with aniline dyes.

Gelatin colonies: After 36 hours, small, circular, gray-white, punctiform. Liquefaction. Bluish to green phosphorescence in 4 to 5 days.

Blood serum: Gray-white, slimy growth.

Potato: Thin white Iayer in 2 to 3 days. 
Cooked fish: Abundant growth. Entire surface covered with a gray-white, slimy growth. Bluish-white phosphorescence.

Alkaline broth: Slight turbidity in 24 hours. Pellicle in 3 days.

Acid broth: No turbidity. No phosphorescence.

Milk: No growth.

No gas formed.

Not pathogenic for laboratory animals.

Aerobic.

Optimum temperature $20^{\circ}$ to $30^{\circ} \mathrm{C}$.

Source: From sea water of the West Indies.

Habitat: Sea water.

64. Pseudomonas pierantonii (Zirpolo) Bergey et al. (Micrococcus pierantonii Zirpolo, Boll. del. Societa dei Natural. in Napoli, 31, 1918, 75; Cocco-bacillus pierantonii Meissner, Cent. f. Bakt., II Abt., 67, 1926, 204; Bergey et al., Manual, 3rd ed., 1930, 176.) Named for Pierantoni, an Italian.

Oval rods: 0.8 by 1.0 to 2.0 microns. Polymorphic rods, sometimes vacuolated. Motile. Gram-negative.

Gelatin colonies: Circular, luminous. Gelatin stab: Not liquefied.

Sepia agar colonies: Circular, white, convex, smooth, serrate edge. Intense greenish luminescence.

Egg-glycerol agar slant: Yellowishgreen, luminous streak.

Broth: Turbid.

Indole not formed.

Acid from glucose and maltose, some strains also produce acid from lactose and sucrose.

Best growth in alkaline media.

Aerobic.

Optimum temperature $33^{\circ} \mathrm{C}$.

Source: Isolated from the photogenic organ of the cephalopod Rondeletia minor.
*65. Pseudomonas martyniae (Elliott) Stapp. (Bacterium martyniae Elliott, Jour. Agr. Res., 29, 1924, 490; Stapp, in Sorauer, Handbuch der Pflanzenkr., 2, 5 Auf., 1928, 278; Phytomonas martyniae Bergey et al., Manual, 3rd ed., 1930, 262.) From M. L. Martynia, a generic name.

Rods : 0.59 to 1.68 microns. Capsules. Chains. Motile with one to several bipolar flagella. Gram-negative.

Green fluorescent pigment produced.

Gelatin : Liquefied.

Beef agar colonies: White, round, smooth, glistening, raised.

Broth: Clouding in bands. Thin pellicle. Small crystals.

Milk: Soft acid curd with peptonization.

Nitrites produced from nitrates after 2 weeks.

Indole not produced.

Hydrogen sulfide production slight.

Acid but not gas from glucose, galactosce, arabinose and sucrose. No acid from rhamnose, lactose, maltose, raffinose, mannitol and glycerol.

Starch hydrolysis none or feeble.

Optimum temperature $26^{\circ} \mathrm{C}$. Maximum $37^{\circ} \mathrm{C}$. Minimum $1.5^{\circ} \mathrm{C}$.

Optimum $\mathrm{pH} 6.0$ to 6.7 . $\mathrm{pH}$ range 5.4 to 8.9 .

Aerobic.

Source: Isolated from diseased leaves of the unicorn plant from Kansas.

Habitat: Pathogenic on Martynia louisiana.

66. Pseudomonas striafaciens (Elliott) Burkholder. (Bacterium striajuciens Elliott, Jour. Agr. Res., 35, 1927, 823; Phytomonas striafaciens Bergey et al., Manual, 3rd ed., 1930, 268; Burkholder. Phytopath., 32, 1942, 601.) From L. stria, stripe; faciens, making,

* The section covering the pseudomonads that cause plant diseases has been revised by Prof. Walter H. Burkholder, Cornell Univ., Ithaca, New York, April, 1943. 
referring to the type of lesion caused on the blades of oats.

Rods: 0.66 by 1.76 microns. Motile with one to several flagella. Capsules. Gram-negative.

Green fluorescent pigment produced.

Gelatin: Liquefied.

Beef-peptone agar colonies: White, raised, margins entire or slightly undulating.

Broth: Clouding in layers. Ring and slight pellicle.

Milk: Alkaline, sometimes a soft curd which digests or clears.

Slight production of nitrites from nitrates.

Indole not produced.

Acid but not gas from glucose, fructose and sucrose. No acid from lactose, maltose, glycerol and mannitol.

Starch: Hydrolysis slight.

Optimum temperature $22^{\circ} \mathrm{C}$.

Optimum pH 6.5 to 7.0 .

Aerobic.

Distinctive characters: Differs from Pseudomonas coronafaciens in that the cells are somewhat smaller and the pathogen produces a streak on oat blades instead of a halo spot.

Source: Forty cultures isolated from oats gathered in various parts of America.

Habitat: Pathogenic on cultivated oats, and to a slight degree, on barley .

67. Pseudomonas tomato (Okabe) comb. nov. (Bacterium tomato Okabe, Jour. Soc. Trop. Agr. Formosa, 5, 1933, 32; Phytomonas tomato Magrou, in Hauduroy et al., Dict. d. Bact. Path., Paris, 1937, 422.) Named for the host plant, tomato.

Probable synonym: Bacterium punclulans Bryan, Phytopath., 23, 1933, 897.

Rods : 0.69 to 0.97 by 1.8 to 6.8 microns. Motile with 1 to 3 polar flagella. Gramnegative.

Green fluorescent pigment produced in culture.

Gelatin: Slow liquefaction.

Beef-extract agar colonies: White, circular, flat and glistening.

Broth: Turbid in 24 hours. Pellicle.
Milk: Becomes alkaline and clears.

Nitrites are usually produced from nitrates.

Indole not produced.

No $\mathrm{H}_{2} \mathrm{~S}$ produced.

Acid but not gas from glucose, sucrose and lactose. No acid from maltose and glycerol.

Starch hydrolysis feeble.

Slight grow th in 3 per cent salt.

Optimum temperature $20^{\circ}$ to $25^{\circ} \mathrm{C}$. Maximum $33^{\circ} \mathrm{C}$.

Aerobic.

Source: Isolated from diseased tomato leaves.

Habitat: Pathogenic on tomato, Lycopersicon esculentum.

68. Pseudomonas aceris (Ark) Burkholder. (Phytomonas aceris Ark, Phytopath., 29, 1939, 969; Burkholder, Phytopath., 32, 1942, 601.) From Latin acer, maple; M.L. Acer, generic name.

Rods : 0.3 to 0.8 by 0.8 to 2.5 microns. Motile with 1 to 2 polar flagella. Gramnegative.

Green fluorescent pigment produced.

Gelatin: Liquificd.

Beef-extract-peptone agar: Colonies are grayish-white. Appearing in 24 hours.

Broth: Turbid.

Milk: Clearing with no coagulation.

Nitrites not produced from nitrates.

Indole not produced.

Hydrogen sulfide not produced.

Acid from glucose, fructose, galactose, arabinose, xylose, sucrose, maltose, lactose, raffinose, mannitol, glycerol and dulcitol.

Slight growth in broth plus 6 per cent salt (Burkholder).

Temperature: $13^{\circ}$ to $31^{\circ} \mathrm{C}$.

Source: From diseased leaves of the large leaf maple, Acer macrophillum.

Habitat: Causes a disease of Acer spp.

69. Pseudomonas angulata (Fromme and Murray) Holland. (Bacterium angulatum Fromme and Murray, Jour. Agr. Res., 16, 1919, 219; Holland, Jour. Bact., 5, 1920, 22t; Phytomonas angulata Bergey 
et al., Manual, 3rd ed., 1930, 267.) From L. angulatus, referring to the type of lesion produced on the tobacco leaf.

Description taken from Clara (Cornel] Agr. Exp. Sta. Mem. 159, 1934, 24).

Rods : 0.75 to 1.5 by 1.5 to 3.0 microns. Motile by 1 to 6 polar flagella. Gramnegative.

Gelatin: Liquefaction.

Green fluorescent pigment produced.

Beef-extract agar colonies: Dull white, circular, raised, smooth and glistening.

Broth: Turbid in 36 hours and greenish.

Milk: Alkaline.

Nitrites not produced from nitrates.

Indole not produced.

No $\mathrm{H}_{2} \mathrm{~S}$ produced.

Lipolytic action negative (Starr and Burkholder, Phytopath., 32, 1942, 601).

Acid but not gas from glucose, galactose, fructose, mannose, arabinose, $x y-$ lose, sucrose and mannitol. Alkaline reaction from salts of citric, malic, succinic and tartaric acid. Rhamnose, maltose, lactose, raffinose, glycerol, salicin, and acetic, lactic and formic acids are not fermented. Starch not hydrolyzed.

Slight growth in broth plus 5 to 6 per cent salt (Burkholder).

Facultative anaerobe.

Distinctive characters: Braun (Phytopath., 27, 1937, 283) considers this species to be identical in culture with Pseudomonas tabaci, but they differ in the type of disease they produce.

Sources: Isolated by Fromme and Murray from small angular leaf spots on tobacco.

Habitat: Causes the angular leaf spot of tobaceo (Nicotiana tabacum).

70. Pseudomonas aptata (Brown and Jamieson) Stevens. (Bacterium aptatum Brown and Jamieson, Jour. Agr. Res., 1, 1913, 206; Phytomonas aptata Bergey et al., Manual, 1st ed., 1923, 184; Stevens, Plant Disease Fungi, New York, 1925, 22.) From Latin aptatus adapted.

Rods : 0.6 to 1.2 microns. Motile with bipolar flagella. Gram-negative.
Green fluorescent pigment produced in culture.

Gelatin: Liquefaction.

Agar slants: Moderate growth along streak, filiform, whitish, glistening.

Broth: Turbid: A pellicle formed.

Milk: Becomes alkaline and clears.

Nitrites not produced from nitrates.

Indole not produced in 10 days. Slight amount found later.

No $\mathrm{H}_{2} \mathrm{~S}$ produced.

Acid from glucose, galactose and sucrose. No acid from lactose, maltose and mannitol (Paine and Banfoot, Ann. Appl. Biol., 11, 1924, 312).

Starch not hydrolyzed.

Slight growth in broth plus 7 per cent salt (Burkholder).

Optimum temperature $27^{\circ}$ to $28^{\circ} \mathrm{C}$. Maximum $34^{\circ}$ to $35^{\circ} \mathrm{C}$. Minimum below $1^{\circ} \mathrm{C}$.

Aerobic.

Source: Isolated from diseased nasturtium leaves from Virginia and diseased beet leaves from Utah.

Habitat: Pathogenic on sugar beets, nasturtiums, and lettuce.

71. Pseudomonas primulae (Ark and Gardner) Starr and Burkholder. (Phytomonas primulae Ark and Gardner, Phytopath., 26, 1936, 1053; Starr and Burkholder, Phytopath., 32, 1942, 601.) From L. primulus, first; M.L. Primula, a generic name.

Rods: 0.51 to 0.73 by 1.0 to 3.16 microns. Motile with a polar flagellum. Gram-negative.

Green fluorescent pigment produced in culture.

Gelatin: Liquefaction.

Agar colonies: Round, convex, smooth, glistening, yellowish.

Milk: Coagulated.

Nitrites not produced from nitrates.

Indole not produced. No $\mathrm{H}_{2} \mathrm{~S}$ produced.

Not lipolytic (Starr and Burkholder, Phytopath., 32, 1942, 601).

Acid but not gas from glucose, lactose, sucrose, maltose, galactose, arabinose, 
glycerol, dulcitol and mannitol. Starch not hydrolyzed.

Growth in broth plus 5 per cent salt.

Optimum temperature $19^{\circ}$ to $22^{\circ} \mathrm{C}$. Maximum $34^{\circ} \mathrm{C}$. Minimum $10^{\circ} \mathrm{C}$.

Optimum pH 6.8 to 7.0. Minimum 4.5 to 5.0 .

Facultative anaerobe.

Source: Isolated from leaf-spot of Primula polyantha.

Habitat: Pathogenic on Primula sp $\boldsymbol{p}$.

72. Pseudomonas viridilivida (Brown) Holland. (Bacterium viridilividum Brown, Jour. Agr. Res., 4, 1915. 475; Holland, Jour. Bact., 5, 1920, 225; Phytomonas viridilivida Bergey et al., Manual, 1st ed., 1923, 187.) From Latin, viridis, green; lividus, blue.

Rods : 1.0 to 1.25 by 1.25 to 3.0 microns. Motile with 1 to 3 polar flagella. (iramnegative.

Green fluorescent pigment produced in culture.

Gelatin: Slow liquefaction.

Beef agar colonies: Cream white, round, smooth, translucent, edges entire.

Broth: Turbid, becomes lime green.

Milk: Alkaline and clears.

Nitrites not produced from nitrates.

Indole is produced.

Not lipolytic (Starr and Burkholder, Phytopath., 32, 1912, 601).

Acid from glucose and sucrose (Burkholder).

Grows well in 4.5 per cent salt. Grows in 7 per cent salt (Burkholder).

Maximum temperature $34.5^{\circ} \mathrm{C}$. Minimum $1.5^{\circ} \mathrm{C}$

Aerobic.

Source: Isolated from diseased Iettuce from Louisiana.

Habitat: Pathogenic on lettuce, Lactuca sativa.

\section{Pseudomonas delphinii (Smith)} Stapp. (Bacillus delphini Smith, Science, 19, 1904, 417; Bacterium delphinii Bryan, Jour. Agr. Res., 28, 1924, 261; Stapp, in Sorauer, Handbuch der Pflanzenkrankheiten, 2, 5 Auf., 1928, 106;
Phytomonas delphinii Bergey et al., Manual, 3rd ed., 1930, 261.) From Latin, delphin, a dolphin; M.I. Delphinium, a generic name.

Rods : 0.6 to 0.8 by 1.5 to 2.0 microns. Chains present. Notile with 1 to 6 polar flagella. Capsules. Gram-negative.

Green fluorescent pigment produced in culture.

Gelatin: Liquified.

Beef agar slants: Growth thin, smooth, shining, transparent, margins entire, crystals. Agar becomes dark brown.

Broth: Turbid in 24 hours with delicate pellicle.

Milk: Becomes alkaline and clears.

Nitrites not produced from nitrates.

Indole not produced.

No $\mathrm{H}_{2} \mathrm{~S}$ produced.

Lipolytic action negative (Starr and Burkholder, Phytopath., 32, 1942, 601).

Acid from glucose, galactose and fructose; slightly acid from sucrose. No acid from lactose, maltose, glycerol and mannitol.

Starch: Hydrolysis feeble.

Weak growth in broth plus 4 per cent salt.

Optimum pH 6.7 to 7.1 . $\mathrm{pH}$ range 5.6 to 8.6 .

Optimum temperature $25^{\circ} \mathrm{C}$. Maximum $30^{\circ} \mathrm{C}$. Minimum $1{ }^{\circ} \mathrm{C}$. or less.

Source: Isolated from black spot of delphinium.

Habitat: Pathogenic on delphinium causing a black spot in the leaves.

74. Pseudomonas berberidis (Thornberry and Anderson) Stapp. (Phytomonas berberidis Thornberry and Anderson, Jour. Agr. Res., 43, 1931, 36; Stapp, Bot. Rev., 1, 1935, 407; Bacterium berberidis Burgwitz, Phytopathogenic Bacteria, Leningrad, 1935, 153.) From M.L. Berberis, barberry, generic name.

Rods: 0.5 to 1.0 by 1.5 to 2.5 microns, occurring singly or in pairs. Motile with 2 to 4 polar flagella. Capsules present. Gram-negative (Burkholder); not Gram-positive as stated in original description. 
Green fluorescent pigment produced in culture (Burkholder).

Gelatin: Not liquefied.

Glucose agar slants: Growth moderate, filiform at first, later beaded, raised, smooth, white. Butyrous in consistency.

Milk: Becomes alkaline. No other change.

Nitrites not produced from nitrates.

Indole not produced.

No $\mathrm{H}_{2} \mathrm{~S}$ produced.

Not lipolytic (Starr and Burkholder, Phytopath., 32, 1942, 601).

Acid from glucose, galactose, and sucrose. Maltose and rhamnose not utilized (Burkholder).

No gas from carbohydrates.

Starch not hydrolyzed.

Optimum temperature $18^{\circ} \mathrm{C}$. Maximum $30^{\circ} \mathrm{C}$. Minimum $7^{\circ} \mathrm{C}$.

Aerobic.

Sources: Repeated isolations from leaves and twigs of barberry.

Habitat: Pathogenic on barberry, Berberis thunbergerii and $B$. vulgaris.

75. Pseudomonas coronafaciens (Elliott) Stapp. (Bacterium coronafaciens Elliott, Jour. Agr. Res., 19, 1920, 153; Phytomonas coronafaciens Bergey et al., Manual, 1st ed., 1923, 180; Stapp, in Sorauer, Handbuch der Pflanzenkrankheiten, 2, 5 Auf., 1928, 20.) From L. corona, halo; faciens, producing, referring to the lesions on oat blades.

Probable synonyms: Elliott (Bact. Plant Pathogens, 1930, 122) lists as synonyms, Bacillus avenae (Russell, Johns Hopkins Univ. Thesis, 1892) and Bacillus avenae Manns and Pseudomonas avenae Manns (Ohio Agr. Exp. Sta. Bul. 210, 1909, 133; Phytomonas avenae Bergey et al., Manual, 3rd ed., 1930, 263).

Rods: 0.65 by 2.3 microns, occurring in chains. Motile with polar flagella. Capsules. Gram-negative.

Green fluorescent pigment produced in culture.

Gelatin: Slow liquefaction.

Nutrient agar colonies: White, becom- ing irregularly circular, flat with raised margins.

Broth: Slight turbidity in 24 hours. Heavy pellicle formed.

Milk: Alkaline. A soft curd formed followed by clearing. Curd sometimes absent.

Nitrites not produced from nitrates.

Indole not formed.

No $\mathrm{H}_{2} \mathrm{~S}$ formed.

Not lipolytic (Starr and Burkholder, Phytopath., 32, 1942, 601).

Acid but no gas from glucose and sucrose. Starch hydrolysis slight.

Slight growth in broth plus 2 per cent salt.

Optimum temperature $24^{\circ}$ to $25^{\circ} \mathrm{C}$. Maximum $31^{\circ} \mathrm{C}$. Minimum $1^{\circ} \mathrm{C}$.

Source: Numerous isolations from blighted blades of oats.

Habitat: Causes a halo spot on oats (Avena sativa). Artificial inoculations show barley (Hordeum vulgare), rye (Secale cereale) and wheat (T'riticum aestivum) to be susceptible.

75a. Pseudomonas coronafaciens var. atropurpurea (Reddy and Godkin) Stapp. (Bacterium coronafaciens var. atropurpureum Reddy and Godkin, Phytopath., 13, 1923, 81 ; Stapp, in Sorauer, Handbuch der Pflanzenkrankheiten, 2, 5 Auf., 1928, 39 ; Phytomonas coronafaciens var. atropurpurea Magrou, in Hauduroy et al., Dict. d. Bact. Path., Paris, 1937, 371.) From L. ater, black, dark; purpureus, purple, referring to the color of the lesion on brome grass.

Distinctive characters: This variety differs from Pseudomonas coronafaciens in that it infects the brome-grass, Bromus inermis, where it produces a water soaked spot which is dark purple in color.

Source: Numerous isolations from diseased brome-grass.

Habitat: Pathogenic on Bromus inermis and Agropyron repens. Has been artificially inoculated on oats, Avena sativa. 
76. Pseudomonas lachrymans (Smith and Bryan) Carsner. (Bacterium lachrymans Smith and Bryan, Jour. Agr. Res., 5, 1915, 466; Carsner, Jour. Agr. Res., 15, 1918, 15; Bacillus lachrymans Holland, Jour. Bact., 5, 1920, 218; Phytomonas lachrymans Bergey et al., Manual, 1st ed., 1923, 184.) From Latin, causing tears, probably referring to the opaque drops formed on the lesion caused by this pathogen.

Synonym: Elliott (Man. Bact. Plant Pathogens, 1930, 147) lists the following as a synonym: Bacillus burgeri Potebnia, Khartov Prov. Agr. Exp. Sta., 1, 1915, 37.

Description from Smith and Bryan (loc. cit.) and Clara (Cornell Agr. Exp. Sta. Mem. 159, 1934, 26).

Rods: 0.5 by 1 to 2 microns. Motile with 1 to 5 polar flagella. Capsules. Gram-negative.

Green fluorescent pigment produced in culture

Gelatin: Liquefied.

Beef-peptone agar colonies: Circular, smooth, glistening, transparent, whitish, entire margins.

Broth: Turbid in 24 hours. White precipitate with crystals.

Milk: Turns alkaline and clears.

Nitrites not produced from nitrates.

Indole reaction weak.

No $\mathrm{H}_{2} \mathrm{~S}$ produced.

Not lipolytic (Starr and Burkholder, Phytopath., 32, 1942, 601).

Acid but not gas from glucose, fructose, mannose, arabinose, xylose, sucrose and mannitol. Alkaline reaction from salts of citric, malic and succinic acid. Maltose, rhamnose, lactose, raffinose, glycerol and salicin not fermented (Clara, loc. cit.).

Starch partially digested. Not digested (Clara, loc. cit.).

Growth in 3 per cent salt after 12 days. No growth in 4 per cent salt.

Optimum temperature $25^{\circ}$ to $27^{\circ} \mathrm{C}$. Maximum $35^{\circ} \mathrm{C}$. Minimum $1^{\circ} \mathrm{C}$.

Aerobic. Facultative anaerobe (Clara, loc. cit.).
Source: Isolated from diseased cucumber leaves collected in New York, Wisconsin, Indiana and in Ontario, Canada.

Habitat: Pathogenic on cucumber, Cucumis sativus, and related plants.

77. Pseudomonas maculicola (McCulloch) Stevens. (Bacterium maculicolum MeCulloch, L. S. Dept. Agr., Bur. Plant Ind. Bul., 225, 1911, 14; Stevens, The Fungi which cause Plant Diseases, 1913, 28 ; Phytomonas maculicola Bergey et al., Manual, 1st ed., 1923, 189; Bacterium maccullochianum Burgwitz, Phytopathogenic Bacteria, Leningrad, 1935, 77.) From L. maculus, spot; -cola, dweller.

Rods: 0.9 by 1.5 to 3 microns. Filaments present. Motile with 1 to 5 polar flagella. Gram-negative.

Green fluorescent pigment produced in culture.

Gelatin: Liquefied.

Beef-peptone agar colonies: Whitish, circular, shining, translucent, edges entire.

Broth: Turbid. No ring or pellicle

Milk: Becomes alkaline and clears.

Nitrites not produced from nitrates.

Indole production feeble.

No H.S formed.

Not lipolytic (Starr and Burkholder, Phytopath., 32, 1942, 601).

Acid from glucose, galactose, xylose, sucrose, glycerol, and mannitol. Alkaline reaction from salts of citric, malic, malonic, and succinic acid. Salicin, maltose, and salts of hippuric and tartaric not utilized (Burkholder).

Slight growth in broth plus 4 per cent salt (Erw. Smith, Bact. Plant Diseases, 1920,306 ).

Aerobic.

Optimum temperature $24^{\circ}$ to $25^{\circ} \mathrm{C}$. Maximum $29^{\circ} \mathrm{C}$. Minimum $0^{\circ} \mathrm{C}$.

Source: Isolated from diseased cauliflower leaves from Virginia.

Habitat: Pathogenic on cauliflower and cabbage.

Note: Bacterium maculicola var. japonicum Takimoto, Bul. Sci. Fak. Terkult 
Kjusu Imp. Univ., 4, 1931, 545 has not been seen.

\section{Pseudomonas marginata (McCul-} loch) Stapp. (Bacterium marginatum McCulloch, Science, 54, 1921, 115; Jour. Agr. Res., 29, 1924, 174; Phytomonas marginata Bergey et al., Manual, 1st ed., 1923, 188; Stapp, in Sorauer, Handbuch der Pflanzenkrankheiten, 2, 5 Auf., 1928, 56.) From L. marginatus, having a border, probably refers to the definite margin of the colony.

Rods : 0.5 to 0.6 by 0.8 to 1.8 microns. Motile with 1 to 4 bipolar flagella. Capsules. Gram-negative.

Green fluorescent pigment produced in Uschinsky's and Fermi's solutions.

Gelatin: Liquefied.

Agar colonies: White, circular, smooth, translucent, viscid, with definite margins at first thin but later thick and contoured. Surface wrinkled.

Milk: At first slightly acid, then alkaline. Casein digested.

Nitrites not produced from nitrates.

Indole production slight.

Hydrogen sulfide production slight.

Lipolytic (Starr and Burkholder, Phytopath., 32, 1942, 601).

Acid but not gas from glucose, lactose, sucrose and glycerol.

Starch hydrolysis feeble.

Growth in 3.5 per cent salt. No growth in 4 per cent salt. pH range, 4.6 to 9.1 .

Optimum temperature $30^{\circ}$ to $32^{\circ} \mathrm{C}$. Maximum $40^{\circ} \mathrm{C}$. Minimum $8^{\circ}$ to $9^{\circ} \mathrm{C}$.

Source: Repeatedly isolated from diseased gladiolus.

Habitat: Pathogenic on Gladiolus spp. and Iris spp.

79. Pseudomonas medicaginis Sackett. (Sackett, Science, 31, 1910, 553; also Colorado Agr. Exp. Sta., Bull. 158, 1910, 11; Bacillus medicaginis Holland, Jour. Bact., 5, 1920, 219; Phytomonas medicaginis Bergey et al., Manual, 1st ed., 1923, 179; Bacterium medicaginis Elliott, Bact. Plant Path., 1930, 162.) From L. medica, ancient Media; M.L. Medicago, a generic name.

Rods : 0.7 by 1.2 microns. Motile with 1 to 4 flagella. Filaments present. Gram-negative.

Green fluorescent pigment produced in culture.

Gelatin: Not liquefied.

Nutrient agar colonies: Growth in 24 hours whitish, glistening.

Broth: Turbid in 24 hours. Pellicle formed. Viscid sediment.

Milk: Becomes alkaline. No change.

Nitrites not produced from nitrates.

Indole not produced.

No $\mathrm{H}_{2} \mathrm{~S}$ produced.

Not lipolytic (Starr and Burkholder, Phytopath., 32, 1942, 601).

Starch not hydrolyzed.

No gas from carbohydrates. Acid from sucrose.

Slight grow th in broth plus 3.75 per cent salt.

Optimum temperature $28^{\circ}$ to $30^{\circ}$. Maximum $37.5^{\circ} \mathrm{C}$.

Aerobic.

Source: Isolated from brown lesions on leaves and stems of alfalfa.

Habitat: Pathogenic on alfalfa, Medicago sp.

79a. Pseudomonas phaseolicola (Burkholder) Dowson. (Phytomonas medi. caginis var. phaseolicola Burkholder, Phytopath., 16, 1926, 915; Bacterium medicaginis var. phaseolicola Link and Hull, Bot. Gaz., 83, 1927, 413; Pseudomonas medicaginis var. phaseolicola Stapp and Kotte, Nachrichtenb. f. d. Deutschen Pflanzenschutzdienst, 9, 1929, 35; Dowson, Brit. Mycol. Soc. Trans., 26, 1943, 10.) From L. phaseolus, bean; M.L. Phaseolus, a generic name;-cola, dwoller.

Synonym: Bacterium puerariae Hedges. (Phytopath., 17, 1927, 48 and 20, 1930, 140; Phytomonas puerariae Bergey et al., Manual, 3rd ed., 1930, 267.)

Description from Burkholder and Zaleski (Phytopath., 22, 1932, 85). 
Rods: 1 by 2 microns, sometimes slightly curved, filaments present. Motile with polar flagellum. Gramnegative.

Green fluorescent pigment produced in culture.

Gelatin stab: Slow liquefaction.

Beef extract agar: Whitish, circular colonies, $2 \mathrm{~mm}$. in diameter. Edges entire.

Broth: Turbid.

Milk: Alkaline.

Nitrites not produced from nitrates.

Indole not formed.

Hydrogen sulfide not formed.

Not lipolytic (Starr and Burhholder, Photopath., 32, 1912, 601).

Acid but no gas from glucose, fructose, mannose, arabinose, xylose, sucrose and glycerol. To acid from rhamnose, lactose, maltose, mannitol and salicin. Alkali from salts of citric and malic acids, but not from acetic, formic, lactic or tartaric acids. Starch and cellulose not hydrolyzed.

Slight growth in broth plus 4 per cent salt.

Optimum temperature $20^{\circ}$ to $23^{\circ} \mathrm{C}$. Maximum $33^{\circ} \mathrm{C}$. Minimum $2.5^{\circ} \mathrm{C}$. (Hedges, loc. cit.).

Optimum pH 6.7 to 7.3. Maximum 8.8 to 9.2. Minimum 5.0 to 5.3. (Kiotte, Phyt. Zeitsch., 2, 1930, 453.)

Microaerophilic.

Source: Isolated from leaves, pod and stem of beans showing halo blight.

Habitat: Pathogenic on beans (Phaseulus vulgaris), the kudzu vine (Pueraria hirsuta) and related plants.

80. Pseudomonas pisi Sackett. (Sackett, Colorado Agr. Exp. Sta., Bull. 21s, 1916, 19; Bacterium pisi Erw. Smith, An Introduction to Bacterial Diseases of Plants, 1920, 474 ; Phytomonas pisi Bergey et al., Manual, 1st ed., 1923, 181.) From Gr. pisum, the pea; M.L. Pisum, a generic name.

Rods: 0.68 to 2.26 microns. Motile with a polar flagellum. Gram-negative.
Green fluorescent pigment produced in culture.

Gelatin: Liquefaction.

Agar slants: Moderate growth in 24 hours, filiform, glistening, grayish-white. Broth: Turbid with a scum in 5 days. Milk: Alkaline, soft curd, clears.

Nitrites not produced from nitrates. Indole not produced:

No $\mathrm{H}_{2} \mathrm{~S}$ produced.

Not lipolytic (Starr and Burkholder, Phytopath., 32, 1942, 601).

Acid but not gas from glucose, galactose and sucrose.

Starch not hydrolyzed.

Optimum temperature $27^{\circ}$ to $25^{\circ} \mathrm{C}$. Maximum $37.5^{\circ} \mathrm{C}$. Minimum $7^{\circ} \mathrm{C}$.

Aerobic.

Source: Ten cultures isolated from 5 collections of diseased peas showing water soaked lesions on stems and petioles.

Habitat: Pathogenic on garden peas, $P$ isum sativum and field peas, $P$. sativum var. arvense.

81. Pseudomonas syringae van Hall. (Kennis der Bakter. Pflanzenziekte, Inaug. Diss., Amsterdam, 1902, 191 ; Bacterium syringae Erw. Smith, Bacteria in Relation to Plant Diseases, 1, 1905, 63; Phytomonas syringae Bergey et al., Manual, 3rd ed., 1930, 257.) From Latin, syringa, a nymph that was changed into a reed; II.L. Syringa, a generic name.

Synonyms: Bryan (Jour. Agr. Res., 36, 1928, 225) lists Bacterinm citriputeale C. O. Smith, Phytopath., 3, 1913, 69, and Bacterium citrarefaciens Lee, Jour. Agr. Res., 9, 1917, 1 (Pseudomonas citrarefaciens Stapp, in Sorauer, Handb. d. Pflanzenkrankheiten, 2, 5 Aufl., 1928, 190). Clara (Cornell Agr. Exp. Sta. Mem. 159, 1931, 29) lists Bacterium vignae (Pseudomonas vignae) Gardner and Kiendrick, Science, 57, 1923, 275 (Phytomonas vignae Bergey et al., Manual, 1st ed., 1923, 189), Pseudomonas viridifaciens Tisdale and Williamson, Jour. Agr. Res., 25, 1923, 141 .(Bacterium viridifaciens Tisdale and Williams, ibid.; Phytomonas 
viridifaciens Bergey et al., Manual, 2nd ed., 1925, 208), and Phytomonas vignae var. leguminophila Burkholder, Cornell Agr. Exp. Sta. Mem. 127, 1930, 51. Wilson (Phytopath., 30, 1940, 27) lists Phytomonas cerasi (Griffin) Bergey et al. (Pseudomonas cerasus Griffin, Science, 34, 1911, 615; Bacillus cerasus Holland, Jour. Bact., 5, 1920, 217; Bergey et al., Manual, 3rd ed., 1930, 262; Bacterium cerasi Elliott, Bact. Plant Pathogens, 1930, 109.) This would include, therefore the following synonyms which have been listed for Phytomonas cerasi. Clara (Cornell Agr. Exp. Sta. Mem. $159,1934,25$ ) lists Bacterium trifoliorum Jones et al. (Jour. Agr. Res., 25, 1923, 471; Phytomonas trifoliorum Burkholder, Phytopath., 16, 1926, 922; Pseudomonas trifoliorum Stapp, in Sorauer, Handb. d. Pflanzenkrankheiten, 2, 5 Aufl., 1928, 177) and Bacterium holci Kendrick (Phytopath., 16, 1926, 236; Pseudomonas holci Kendrick, ibid.; Phytomonas holci Bergey et al., Manual, 3rd ed., 1930, 258). Wilson (Hilgardia, 10, 1936, 213) lists Pscudomonas prunicola Wormald (Ann. Appl. Biol., 17, 1930, 725), Pseudomonas cerasi var. prunicola Wilson (Hilgardia, 8, 1933, 83), Bacterium citriputcale C. O. Smith (Phytopath., 4, 1913, 69; Pseudomonas citriputealis Stapp, in Sorauer, Handb. d. Pflanzenkrankheiten, 2, 5 Aufl., 1928, 190; Phytomonas citriputealis Bergey et al., Manual, 3rd ed., 1930, 278) and Pseudomonas utiformica Clara, Science, 75, 1932, 111 (Phytomonas utiformica Clara, Cornell Agr. Exp. Sta. Mem. 159, 1934, 29; Bacterium utiformica Burgwitz, Phytopathogenic Bacteria, Leningrad, 1935, 444). A probable synonym is Phytomonas spongiosa (Aderhold and Ruhland) Magrou (Bacillus spongiosus Aderhold and Ruhland, Cent. f. Bakt., II Abt., 15, 1905, 376; Pseudomonas spongiosa Braun, Die Landwirtschaft, 41, 42, 1927, 2 pp.; Bacterium spongiosum Elliott, Man. Bact. Plant Pathogens, 1930, 214; Magrou, in Hauduroy et al., Dict. d. Bact. Path., Paris, 1937, 414). C. O. Smith
(Phytopath., 33, 1943, 82) lists the following as a synonym: Pseudomonas hibisci (Nakata and Takimoto) Stapp (Bacterium hibisci Nakata and Takimoto, Ann. Phytopath. Soc. Japan, 1, 5, 1923, 18; Stapp, in Sorauer, Handbuch der Pflanzenkrankheiten, 5 Aufl., 2, 1928, 203; Phytomonas hibisci Bergey et al., Manuals 3rd ed., 1930, 264).

Description from Clara (loc. cit.).

Rods : 0.75 to 1.5 by 1.5 to 3.0 microns. Motile with 1 or 2 polar flagella. Gramnegative.

Green fluorescent pigment produced in culture.

Gelatin: Liquefaction.

Beef-extract agar colonies: Circular, grayish-white with bluish tinge. Surface smooth. Edges entire or irregular.

Broth: Turbid in 36 hours. No pellicle.

Milk: Alkaline.

Nitrites not produced from nitrates.

Indole not produced.

No $\mathrm{H}_{2} \mathrm{~S}$ produced.

Not lipolytic (Starr and Burkholder, Phytopath., 32, 1942, 601).

Slight growth in broth plus 4 per cent salt.

Acid but not gas from glucose, galactose, mannose, arabinose, xylose, sucrose, mannitol and glycerol. Alkaline reaction from salts of citric, malic, succinic and lactic acid. Rhamnose, maltose, lactose, raffinose, salicin, and acetic, formic and tartaric acid not fermented. Starch not hydrolyzed.

Facultative anaerobe.

Source: Van Hall originally isolated the pathogen from lilac.

Habitat: Pathogenic on lilac, citrus, cow peas, beans, lemons, cherries and many unrelated plants.

81a. Orsini reports the following as a variety-Bacterium syringae var. capsici Orsini. (Intern. Bull. Plant Prot., 33, 1942 , 33.) Pathogenic on the pepper plant (Capsicum).

82. Pseudomonas atrofaciens (McCulloch) Stevens. (Bacterium atrofaciens 
MeCulloch, Jour. Agr. Res., 18, 1920, 519; Phytomonas atrofaciens Bergey et al., Manual, 1st ed., 1923, 185; Stevens, Plant Disease Fungi, New York, 1925, 22.) From Latin, ater, black; faciens, making, referring to the color of the lesion on wheat.

Rods: 0.6 by 1 to 2.7 microns. Long chains formed in culture. Capsules present. Motile with 1 to 4 polar or bipolar flagella. Gram-negative.

Green fluorescent pigment produced in culture.

Gelatin: Liquefied.

Beef-peptone-agar colonies: Circular, shining, translucent, white.

Broth: Growth never heavy, slight rim, and a delicate pellicle.

Milk: Becomes alkaline and clears. Nitrites not produced from nitrates.

Indole: Slight production.

Hydrogen sulfide: Slight production.

Acid and no gas from glucose, galartose and sucrose.

Starch is slightly hydrolyzed.

Optimum temperature $25^{\circ}$ to $28^{\circ} \mathrm{C}$. Maximum $36^{\circ}$ to $37^{\circ} \mathrm{C}$ and minimum below $2^{\circ} \mathrm{C}$.

Aerobic.

Sources: Isolated from diseased wheat grains collected throughout Lnited States and Canada.

Habitat: Causes a basal glume-rot of wheat.

83. Pseudomonas cumini (Kovacevski) Dowson. (Phytomonas cumini Kovacevski, Bull. soc. Bot. Bulgarie, 7, 1936, 27 ; Dowson, Trans. Brit. Mycol. Soc., 26, 1943, 10.) From Greek cuminum, rumin; M.L. Cuminum, a generic name.

Rods : 0.5 to 0.7 by 1 to 3 microns, occurring in chains and filaments. Motile with 1 to 3 polar flagella. Gram-negative.

Green fluorescent pigment formed in culture.

Gelatin: Rapidly liquefied.

Potato agar colonies: Grayish-white, circular, glistening, smooth, butyrous.

Broth: Moderate turbidity. Pseudozoogloea.
Milk: Not coagulated. Casein peptonized.

Nitrites not produced from nitrates. Indole not formed.

No $\mathrm{H}_{2} \mathrm{~S}$ formed.

Acid but not gas from glucose and sucrose. No acid from lactose or glycerol. Starch not hydrolyzed.

Temperature range $5^{\circ} \mathrm{C}$ to $31^{\circ} \mathrm{C}$.

Aerobic.

Source: Isolated from blighted cumin (C'uminum).

Habitat: Pathogenic on cumin and dill.

84. Pseudomonas desaiana (Burkholder) comb. nov. (B. pyocyaneus saccharum Desai, Ind. Jour. Agr. Sci., 5, 1935, 391; Phytomomas desaiana Burkholder, in Bergey et al., Manual, 5th ed., 1939, 174.) Named for Desai who first isolated the species.

Rods : 0.6 to 1.2 by 1.2 to 2.2 microns. Motile with a polar flagellum. Gramnegative.

Green fluorescent pigment produced in culture.

Gelatin: Liquefaction.

Agar colonies: Grayish-blue. Raised.

Broth: Light clouding. Pellicle.

Milk: Peptonized without coagulation. Nitrites not produced from nitrates,

Indole not formed.

Glucose, sucrose, lactose and glycerol fermented without gas.

Stareh: Hydrolysis present.

Optimum temperature $30^{\circ} \mathrm{C}$.

Aerobic.

Source: Isolated from stinking rot of sugar cane in India and associated with a white non-pathogenic bacterium.

Habitat: Pathogenic on sugar cane, Saccharum officinarum.

85. Pseudomonas erodii Lewis. (Phy topath., 4, 1914, 231; Bacterium erodii Lewis, ibid.; Phytomonas erodii Bergey et al., Manual, 3rd ed., 1930, 256.) From Greek, erodius, heron; M.L. Erodium, a generic name.

Rods : 0.6 to 0.8 by 1.2 to 1.8 microns. 
Motile with 1 to 3 polar flagella. Gramnegative.

Green fluorescent pigment produced in culture.

Gelatin: Liquefaction.

Agar stroke: Heavy, smooth, creamcolored growth in 24 hours.

Broth: Dense clouding in 24 hours.

Milk: Turns alkaline and clears, litmus reduced.

Nitrites not produced from nitrates.

Indole produced in 14 days.

No $\mathrm{H}_{2} \mathrm{~S}$ produced.

Acid but not gas from glucose, sucrose, lactose and glycerol.

Temperature: No growth at $35^{\circ} \mathrm{C}$.

Aerobic, obligate.

Source: Isolations from Erodium texanum and 4 varieties of Pelargonium.

Habitat: Causes a leaf spot of Erodium texanum and Pelargonium spp.

86. Pseudomonas apii Jagger. (Jagger, Jour. Agr. Res., 21, 1921, 186; Phytomonas apii Bergey et al., Manual, 1st ed., 1923, 184; Pseudomonas jaggeri Stapp, in Sorauer, Handbuch der Pflanzenkrankheiten, 2, 5 Aufl., 1928, 210; Bacterium jaggeri Elliott, Bacterial Plant Pathogens, 1930, 142; Phytomonas jaggeri Magrou, in Handuroy et al., Dict. d. Bact. Path., Paris, 1937, 371.) From Latin, apium, parsley, M.L. Apium, a generic name.

Description from Clara (Cornell Agr. Exp. Sta. Mem. 159, 1934, 24).

Rods : 0.75 to 1.5 by 1.5 to 3.0 microns. Motile with a polar flagellum. Gramnegative.

Green fluorescent pigment produced in various media.

Gelatin: Liquefaction.

Beef-extract agar colonies: Circular, glistening, smooth, edges entire. Grayish-white with bluish tinge.

Broth: Turbid in 36 hours. Pellicle formed.

Milk: Becomes alkaline. No curd.

Nitrites not produced from nitrates. Indole not formed.
No $\mathrm{H}_{2} \mathrm{~S}$ formed.

Acid but not gas from glucose, galactose, fructose, mannose, arabinose, xylose, sucrose, mannitol and glycerol. Alkaline reaction from salts of acetic, citric, malic and succinic acids. Rhamnose, maltose, lactose, raffinose, salicin, and formic, lactic and tartaric acid are not utilized.

Starch not hydrolyzed.

Facultative anaerobe.

Distinctive characters: Pathogenicity appears limited to celery.

Source: Jagger isolated this repeatedly from diseased celery leaves.

Habitat: Pathogenic on celery, Apium graveolens.

\section{Pseudomonas matthiolae (Briosi} and Pavarino) Dowson. (Bacterium matthiolae Briosi and Pavarino, Atti della Reale Accad. dei Lincei Rend., 21, 1912, 216; Phytomonas matthiolae, Bergey et al., Manual, 3rd ed., 1930, 266; Mushin, Proc. Roy. Soc. Victoria, 53, 1941, 201 ; Dowson, Trans. Brit. Mycol. Soc., 26, 1943, 10.) From M.L. Matthiola, a generic name.

Rods: 0.4 to 0.6 by 2 to 4 microns. Gram-positive. Gram-negative (Mushin, loc. cit.).

Green fluorescent pigment produced in culture.

Gelatin: Liquefied.

Beef agar colonies: White, circular colonies, slightly elevated, margins smooth.

Broth: Slightly turbid. Becomes pale green.

Milk: Coagulation with acid reaction.

Nitrites produced from nitrates

(Mushin).

Hydrogen sulfide not formed.

Acid from glucose, galactose, fructose, mannose, rhamnose, glycerol, mannitol, acetic acid, citric acid, formic acid, lactic acid, malic acid, and succinic acid. Feeble acid in maltose. No acid, no gas in lactose, sucrose, raffinose, starch, salicin, and tartaric acid (Mushin).

Optimum temperature 20 to $24^{\circ} \mathrm{C}$. 
Maximum temperature 38.5 C. Minimum below $0^{\circ} \mathrm{C}$. (Mushin).

Limits of growth in broth are $\mathrm{pH} 4.4$ to $\mathrm{pH} 9.5$ (Mushin).

Aerobic.

Source: Isolated from vascular and parenchymatic disease of stocks, Matthiola incana var. annua.

Habitat: Pathogenic on stocks.

Nоте: Burkholder (Phytopath., 28, 1938, 936) and Santarelli (Rev. di Pat. Veg., 29, 1939, 364) consider this species a synonym of Pseudomonas syringae. Adam and Pugsley (Jour. Dept. Agric. Victoria, 32, 1934, 306) give a description of a green fluorescent pathogen on stocks which is similar to Pseudomonas syringae. Mushin (loc. cit.) considers Pseudomonas matthiolae to be a distinct species.

88. Pseudomonas mors-prunorum Wormald. (Jour. Pom. and Hort. Sci., 9, 1931, 251; Phytomonas mors-prunorum Wormald, Trans. Brit. Mycol. Soc., 17, 1932, 169; Bacterium mors-prunorum, ibid.) From L. mors, death; prunus, plums.

Rods: Motile with a polar flagellum. Gram-positive (1931). Gram-negative (1932).

Note: Possibly a green fluorescent organism since it produces a faint yellow color in Uschinsky's solution.

Gelatin: Liquefaction.

Agar colonies: White.

Broth plus 5 per cent sucrose: White and cloudy.

Nitrites not produced from nitrates.

Acid but not gas from glucose, lactose, sucrose and glycerol.

Starch not hydrolyzed.

Strict aerobe.

Distinctive characters: Differs from Pseudomonas prunicola (Pseudomonas syringae) in that it produces a white cloudy growth in broth plus 5 per cent sucrose; a rapid acid production in nutrient agar plus 5 per cent sucrose, and a faint yellow or no color in Uschinsky's solution.
Source: Isolated from cankers on plum trees in England.

Habitat: Pathogenic on Prunus spp.

89. Pseudomonas rimaefaciens Koning. (Chron. Bot., 4, 1938, 11; Meded. Phytop. Labor, Willie Comm. Scholt., 1'́, 1938, 24.) From L. rima, fissure; faciens, producing.

Rods: 0.6 to 2.4 microns in length. Motile with 1 to 3 polar flagella. Gramnegative.

Yellow-green fluorescent water-soluble pigment produced in culture.

Gelatin : Liquified.

Agar colonies: Round, convex, smooth, somewhat granular with hyaline edge.

Broth: Turbid. Surface growth with a sediment in a few days.

Milk: Alkaline and clears.

Nitrites not produced from nitrates. Peptone, asparagin, urea, gelatin, nitrates and ammonia salts are sources of nitrogen.

Hydrogen sulfide not produced.

Indole production slight.

Growth with the following carbon sources plus $\mathrm{NO}_{3}$, glucose, sucrose, glycerol, succinates, malates, citrates and oxalates. Less growth with mannitol, fructose, galactose, lactose, salicylate. Acid is produced from the sugars. No growth with dextrin, inulin, maltose, lactose, rhamnose, salicin, tartrates, acetates, formates.

Starch not hydrolyzed.

Aerobic.

Optimum temperature $25^{\circ} \mathrm{C}$. Maximum about $37^{\circ} \mathrm{C}$. Very slow growth at $14^{\circ} \mathrm{C}$. Thermal death point $42^{\circ}$ to $48^{\circ} \mathrm{C}$.

Source: Strains of the pathogen isoated from poplar cankers in France and in the Netherlands.

Habitat: Pathogenic on Populus brabantica, $P$. trichocarpa and $P$. candicans.

This may be Pseudomonas syringae since the characters are the same and both organisms can infect Impatiens $s p$. Pseudomonas syringae infects poplars (Elliott, Bacterial Plant Pathogens, 1930, 218). 
90. Pseudomonas papulans Rose. (Rose, Phytopath., 7, 1917, 198; Phytomonas papulans Bergey et al., Manual, 3rd ed., 1930, 267; Bacterium papulans Elliott, Bacterial Plant Pathogens, 1930, 175; Phytomonas syringae var. papulans Smith, Jour. Agr. Res., 68, 1944, 294.) From I. papulans, forming blisters.

Rods : 0.6 by 0.9 to 2.3 microns. Motile with 1 to 6 polar flagella. Gram-negative.

Green fluorescent pigment produced in culture.

Gelatin: Liquefied.

Broth: Moderate turbidity in 24 hours.

Milk: Alkaline and at times a soft coagulum.

Nitrites not produced from nitrates. Indole: May or may not be produced.

Acid but not gas formed from glucose and sucrose.

Optimum temperature $25^{\circ}$ to $28^{\circ} \mathrm{C}$. Maximum $37^{\circ} \mathrm{C}$.

Source: Twenty-five cultures isolated from blisters on apples and from rough bark.

Habitat: Pathogenic on apple trees.

91. Pseudomonas pseudozoogloeae (Honing) Stapp. (Bacterium pseudozoogloeae Honing, Bull. van Het. Deli Proefstation, Medan, 1, 1914, 7; Stapp, in Sorauer, Handbuch der Pflanzenkrankheiten, 2, 5 Auf., 1928, 274; Phytomonas pseudozooglocae Bergey et al., 3rd ed., 1930, 261.) From Gr., pseudo, false; M. L. zoogloea, zooglea.

Rods : 0.7 to 1.5 by 0.9 to 2.5 microns. Chains. Motile with 1 or 2 polar flagella. Gram-negative.

Green fluorescent pigment produced in culture.

Gelatin: Liqueíaction.

Agar colonies: Round, flat, yellowgray.

Broth: Moderate turbidity with pseudozoogloea in the pellicle.

Milk: Coagulation. No clearing.

Nitrites not produced from nitrates.

Indole not formed.

Hydrogen sulfide produced.
Acid but not gas from glucose, lactose, maltose, sucrose and mannitol.

Facultative anaerobe.

Source: Isolated from the black rust of tobacco.

Habitat: Pathogenic on tobaceo, Nicotiana tabacum.

92. Pseudomonas tabaci (Wolf and Foster) Stevens. (Bacterium tabacum Wolf and Foster, Science, 46, 1917, 362; also Jour. Agr. Res., 12, 1918, 449; Phytomonas tabaci Bergey et al., Manual, 1st ed., 1923, 185; Stevens, Plant Disease Fungi, New York, 1925, 34.) From Nicotiana tabacum, tobacco.

Rods : 1.2 by 3.3 microns. Motile with a polar flagellum. Gram-negative.

Gelatin: Liquefaction.

Potato agar colonies: Grayish-white, circular, raised, wet-shining, smooth.

Milk: Alkaline; clears.

Nitrites not produced from nitrates. Indole not formed.

Acid from glucose, galactose, fructose, 1 -arabinose, xylose, sucrose, pectin, mannitol and glycerol (Braun, Phytopath., 27, 1937, 289).

Ammonium sulfate, potassium nitrate, cystine, glutamic acid, glycine, succinimide, oxamide, acetamide, and urea can be used as nitrogen source (Braun).

Starch not hydrolyzed. Aerobic.

Distinctive character: Braun (loc. cit.) states that Pseudomonas tabaci and Pseudomonas angulata are identical in culture.

Source: Isolated from wildfire lesions on tobacco leaves in North Carolina.

Habitat: Pathogenic on tobacco, Nicotiana tabacum.

93. Pseudomonas lapsa (Ark) Burkholder. (Phylomonas lapsa Ark, Phytopath., 30, 1940, 1; Burkholder, ibid., 32, 1942, 601.) From Latin, lapsus, falling, referring to a symptom of the disease.

Rods: 0.56 by 1.55 microns. Motile with 1 to 4 polar flagella.

Produces fluorescence in Uschinsky's, Fermi's, and Cohn's solutions. 
Gelatin: Liquefied (Burkholder).

Acid but no gas is produced from glucose, sucrose, maltose, lactose, glycerine, arabinose, xylose, galactose, raffinose and mannitol.

Slight growth in broth plus 5 per cent salt (Burkholder).

Source: Isolated from stalk rot of field corn in California; also from Diabrotica beetles.

Habitat: Pathogenic on corn and sugar cane,

Note: Like Pseudomonas desiana.

94. Pseudomonas bowlesiae (Lewis and Watson) Dowson. (Phytomonas bowlesii Lewis and Watson, Phytopath.. 17, 1927, 511; Bacterium bowlesii Elliott, Bacterial Plant Pathogens, 1930, 96; Dowson, Trans. Brit. Mycol. Soc., 26, 1943, 9.) From M. L. Boulesia, a generic name.

Rods : 0.5 to 0.7 by 1.2 to 1.6 microns, occurring singly, in pairs or in short chains. Motile with bipolar flagella. Gram-negative.

Green fluorescent pigment produced in culture.

Gelatin: Liquefied.

Agar slants: Yellowish, moist, glistening and viscid.

Broth: Cniform turbidity throughout. Heavy viscous sediment in old cultures.

Milk: Alkaline; coagulation, with a slow peptonization.

Nitrites are produced from nitrates.

Indole is produced.

Hydrogen sulfide is produced.

Acid from glucose, maltose and xylose. No acid from sucrose.

Optimum temperature $27^{\circ} \mathrm{C}$. Maximum $37^{\circ} \mathrm{C}$. Minimum $-1^{\circ} \mathrm{C}$.

Optimum pH 7.2. $\mathrm{pH}$ range 4.5 to 8.6.

Aerobic.

Source: Isolated from diseased, water soaked spots of bowlesia.

Habitat: Pathogenic on Boulesia septentrionalis.

95. Pseudomonas intybi (Swingle) Stapp. (Phytomonas intybi Swingle,
Phytopath., 15, 1925, 730; Stapp, in Soraurer, Handbuch der Pflanzenkrankheiten, 2, 5 Auf., 1928, 291; Bacterium intybi Elliott, Bacterial Plant Pathogens, 1930, 142.) From Latin, intibus, endive.

Description from Stapp, Cent. f. Bakt., II Abt., 91, 1935, 232.

Rods : 0.4 to 0.5 by 1.4 to 2.8 microns. Motile with one to several polar flagella. Gram-negative.

Green fluorescent pigment formed in culture.

Gelatin: Liquefaction.

Agar colonies: White, glistening, transparent.

Broth: Turbid with fragile pellicle, and good sediment.

Milk: Coagulated. Casein not peptonized.

Nitrites are produced from nitrates with the formation of gas.

Indole not formed.

Acid but not gas from arabinose, xylose and glucose. No acid from sucrose.

Optimum temperature $23^{\circ}$ to $28^{\circ} \mathrm{C}$. Maximum $40^{\circ}$ to $42^{\circ} \mathrm{C}$. Minimum $0^{\circ} \mathrm{C}$.

Distinctive character: Differs from Pseudomonas cichorii in that it liquefies gelatin and produces nitrites from nitrates.

Source: Isolated from French endive, Cichorium intybus by Swingle, from $C$. endiva and lettuce, Lactuca sativa by Stapp.

Habitat: Pathogenic on endive and lettuce, causing a rot.

96. Pseudomonas marginalis (Brown) Stevens. (Bacterium marginale Brown, Jour. Agr. Res., 13, 1918, 386; Phytomonas marginalis Bergey et al., Manual, 1st ed., 1923, 182; Stevens, Plant Disease Fungi, New York, 1925, 30.) From Latin, margo (marginis), edge, margin; M.L. marginalis, on the margin, a character of the disease.

Description from Brown (loc. cit.) and Clara (Cornell Agr. Exp. Sta. Mem. 159, 1934, 27).

Rods: Motile with 1 to 3 polar flagella. Gram-negative. 
Green fluorescent pigment produced in culture.

Gelatin: Liquefaction.

Agar colonies: Cream-colored to yellowish.

Broth: Turbid, with pellicle.

Milk: Alkaline. Soft curd at times.

Nitrites are produced from nitrates. Not produced (Clara, loc. cit.).

Indole not produced.

No $\mathrm{H}_{2} \mathrm{~S}$ produced.

Acid but not gas from glucose, galactose, fructose, mannose, arabinose, xylose, rhamnose, mannitol and glycerol. Alkali from salts of acetic, citric, malic, formic, lactic, succinic and tartaric acid. Sucrose, maltose, lactose, raffinose and salicin not fermented (Clara, loc. cit.).

Starch hydrolysis feeble. None (Clara, loc. cit.).

Optimum temperature $25^{\circ}$ to $26^{\circ} \mathrm{C}$. Maximum $38^{\circ} \mathrm{C}$. Minimum $0^{\circ} \mathrm{C}$.

Aerobic.

Source: Isolated from marginal lesion on lettuce from Kansas.

Habitat: Pathogenic on lettuce and related plants.

97. Pseudomonas setariae (Okabe) comb. nov. (Bacterium setariae Okabe, Jour. Soc. Trop. Agr. Formosa, 6, 1934, 63; Phytomonas setariae Burkholder, in Bergey, Manual, 5th ed., 1939, 183.) From L. seta, bristle; -arius, like; M. L. Setaria, a generic name.

Rods : 0.4 to 0.8 by 1.8 to 4.4 microns. Motile with a polar, seldom bipolar, flagellum. Gram-negative.

Yellowish water-soluble pigment produced in culture.

Gelatin: Slow liquefaction.

Beef-extract agar colonies: Circular, white, opalescent, smooth, glistening.

Broth: Turbid after 18 hours. Pellicle.

Milk: Alkaline; clears.

Nitrites are produced from nitrates.

Indole is produced.

$\mathrm{No} \mathrm{H}_{2} \mathrm{~S}$ produced.

Acid but not gas from glucose, galac- tose and glycerol. No acid from lactose, maltose or sucrose.

Starch: Feeble hydrolysis.

Grows in 3 per cent salt.

Optimum temperature $31^{\circ}$ to $34^{\circ} \mathrm{C}$. Maximum $42^{\circ} \mathrm{C}$.

Aerobic.

Source: Isolated from brown stripe of Italian millet.

Habitat: Pathogenic on Italian millet, Setaria italica.

98. Pseudomonas polycolor Clara. (Clara, Phytopath., 20, 1930, 704; Phytomonas polycolor Clara, ibid., Bacterium polycolor Burgwitz, Phytopathogenic Bacteria, Leningrad, 1935, 148.) From Gr. poly, many; L. color, color.

Note: Delacroix (Comp. rend. Acad. Sci., Paris, 137, 1903, 454) describes Bacillus aerogenosus as being a tobacco pathogen. The organism described by Delacroix might be the same as Pseudomonas polycolor. Braun and Elrod (Jour. Bact., 43, 1942, 40) are of the opinion that Clara's pathogen is Pseudomonas aeruginosa.

Description taken from Clara (Cornell Agr. Exp. Sta. Mem. 159, 1934, 28).

Rods : 0.75 to 1.2 by 1.05 to 3.0 microns. Motile with 1 or 2 polar flagella. Gramnegative.

Green fluorescent pigment produced in culture.

Gelatin: Liquefaction.

Beef-extract agar colonies: Grayishwhite, circular, raised, thin transparent margins.

Broth: Turbid in 36 hours with thin pellicle.

Milk: Alkaline; no curd.

Nitrites not produced from nitrates.

Indole not produced.

No $\mathrm{H}_{2} \mathrm{~S}$ produced.

Lipolytic (Starr and Burkholder, Phytopath., 32, 1942, 601).

Acid but not gas from glucose, galactose, fructose, mannose, arabinose, xylose, mannitol and glycerol. Alkaline reaction from salts of acetic, citric, malic, 
lactic and formic acid. Rhamnose, sucrose, maltose, lactose, raffinose and salicin not fermented.

Starch not hydrolyzed.

Facultative anaerobe.

Good growth in broth plus 7 per cent salt.

Optimum temperature $25^{\circ}$ to $30^{\circ} \mathrm{C}$. Maximum $37^{\circ}$ to $39^{\circ} \mathrm{C}$.

Distinctive character: Differs from Pseudomonas mellea in type of lesion produced, does not digest starch, nor reduce nitrates and does not form acid from lactose nor sucrose. Pathogenic for laboratory animals (Elrod and Braun, Sci. 94, 1941, 520).

Source: Repeatedly isolated from leaf spot of tobacco in the Philippines.

Habitat: Pathogenic on tobacco.

99. Pseudomonas viridiflava (Burkholder) Clara. (Phytomonas viridiflava Burkh., Cornell Agr. Exp. Sta. Mem. 127, 1930, 63; Clara, Science, 75, 1934, 111; Bacterium viridiflavum Burgwitz, Phytopathogenic Bacteria, Leningrad, 1935, 127.) From Latin viridis, green; flavus, vellow.

Description from Clara (Cornell Agr. Exp. Sta. Mem. 139, 1934, 30).

Rods : 0.75 to 1.5 by 1.5 to 3.15 microns . Motile with 1 or 2 polar flagella. Gramnegative.

Green fluorescent pigment produced in culture.

Gelatin: Liquefaction.

Beef-extract agar colonies: Grayishwhite, margins corrugated, edges irregular.

Broth: Turbid in 36 hours.

Milk: Becomes alkaline and clears.

Nitrites not produced from nitrates.

Indole not formed.

No $\mathrm{H}_{2} \mathrm{~S}$ produced.

Not lipolytic (Starr and Burkholder, Phytopath., 32, 1942, 601).

Acid but not gas from glucose, fructose, mannose, arabinose, xylose, mannitol and glycerol. Alkaline reaction from salts of acetic, citric, malic, lactic and succinic acids. Sucrose, lactose, maltose, raffinose, salicin, and salts of formic and tartaric acids not fermented.

Starch: No hydrolysis.

Growth in broth plus 5 per cent $\mathrm{NaCl}$. Facultative anaerobe.

Source: Two cultures isolated from spotted beans, one from England and one from Switzerland.

Habitat: Pathogenic on bean, Phaseolus vulgaris.

99a. Pseudomonas viridiflava var. concentrica (Petersen) comb. nov. (Phytomonas viridiflava var. concentrica Petersen, Tridsskr. f. Planteavl., 38, 1932, 851; Bacterium viridiflavum var. concentricum Burgwitz, Phytopathogenic Bacteria, Leningrad, 1935, 127.) From M. L. concentricus, concentric, referring to the rings on the colonies.

Distinctive characters: Differs from Pseudomonas viridiflava in that it does not grow in Uschinsky's solution, and also in the shape of the colonies.

Source: Isolated from the stems and leaves of blighted beans in Denmark.

Habitat: Pathogenic on the bean, Phaseolus vulgaris.

100. Pseudomonas ananas Serrano. (Serrano, Philipp. Jour. Sci., 55, 1934, 355 ; Phytomonas ananas and Bacterium ananas Serrano, ibid. (not to be confused with Erwinia ananas Serrano, ibid., 36, 1925, 271); Bacterium serranoi Burgwitz, Bact. Dis. of Plants, Leningrad, 1936.) From Brazilian Indian, ananas, pincapple; M.L. Ananas, generic name.

Rods : 0.6 by 1.8 microns. Motile with 1 to 4 polar flagella. Gram-negative.

Green fluorescent pigment produced in certain media.

Gelatin: Liquefied.

Beef-extract glucose agar colonies: White, with undulating edges, smooth to rugose, glistening to dull.

Beef-extract agar: Growth scant.

Broth: Feeble growth. 
Milk: Becomes alkaline with curd.

Nitrites not produced from nitrates.

Indole not formed.

$\mathrm{No} \mathrm{H}_{2} \mathrm{~S}$ formed.

Acid but not gas from glucose, xylose and mannitol. Feeble with laetose. No acid with sucrose.

Starch not hydrolyzed.

Optimum temperature $30^{\circ}$ to $31^{\circ} \mathrm{C}$. Maximum $45^{\circ} \mathrm{C}$. Minimum $7^{\circ}$ to $10^{\circ} \mathrm{C}$. Aerobic.

Habitat: Causes a rot of pineapples, Ananas comosus.

101. Pseudomonas ligustri (d'Oliveira) comb. nov. (Bacterium ligustri d'Oliveira, Revista Agron., 24, 1936, 434.) From L. ligustrum, privet; M. L. Ligustrum, a generic name.

Rods: 0.5 to 0.7 by 1.3 to 3 microns. No chains. No capsules. Motile with 2 to 5 polar flagella. Gram-negative.

Green pigment produced on Dox agar, and in broth.

Gelatin: Liquefied.

Beef-extract agar colonies: Growth moderate. Milky white, circular, convex.

Broth: Turbid in 24 hours. No pellicle.

Milk: Coagulated in 6 days, and later digested. Litmus slightly acid.

Nitrites not produced from nitrates. Indole not produced.

Ammonia not produced.

No gas from carbohydrates. Acid from glucose, galactose, arabinose and mannose. No acid from sucrose, maltose, lactose, raffinose, mannitol and salicin.

Source: From diseased Japanese privet in Lisbon, Portugal.

Habitat: Pathogenic on privet, Ligustrum japonicum.

102. Pseudomonas sesami Malkoff. (Malkoff, Cent. f. Bakt., II Abt., 16, 1906, 665; Bacterium sesami Nakata, Ann. Phyt. Soc. Japan, 2, 1930, 242; Phytomonas sesami Kovachersky, Ann. Univ. de Sofia, Fac. Agron., 8, 1930, 464.) From Gr. sesamum, sesame; M. L. Sesamum, a generic name.
Synonym: Nakata (loc. cit.) lists Bacterium sesamicola Takimoto, Jour. Plant Protect. Tokyo, 8, 1927, 433 (Phytomonas sesamicola Magrou, in Hauduroy et al., Dict. d. Bact. Path., 1937, 412).

Description from Nakata (loc, cit.).

Rods : 0.6 to 0.8 by 1.2 to 3.8 microns. Motile with 2 to 5 polar flagella. Gramnegative.

Green fluorescent pigment produced in culture.

Gelatin: Liquefaction rapid.

Beef-agar colonies: Circular, flat, striate, smooth, entire margins, white.

Broth: Growth rapid. No pellicle.

Milk: Alkaline. No coagulation.

Nitrites not produced from nitrates.

Indole not produced.

No $\mathrm{H}_{2} \mathrm{~S}$ produced.

Acid but not gas from glucose. No acid from lactose, sucrose or glycerol.

Starch not hydrolyzed.

Optimum temperature $30^{\circ} \mathrm{C}$. Maximum $35^{\circ} \mathrm{C}$. Minimum $0^{\circ} \mathrm{C}$.

Facultative anaerobe.

Source: Isolated from brown spots on leaves and stems of sesame.

Habitat: Pathogenic on sesame.

103. Pseudomonas tolaasii Paine. (Paine, Ann. Appl. Biol., 5, 1919, 210; Phytomonas tolaasi Bergey et al., Man ual, 3rd ed., 1930, 259; Bacterium tolaasi Elliott, Bacterial Plant Pathogens, 1930, 226.) Named for A. G. Tolaas who first reported the species.

Rods : 0.4 to 0.5 by 0.9 to 1.7 microns. Motile with 1 to 5 polar flagella. Gram. negative.

Green fluorescent pigment produced in culture.

Gelatin: Liquefaction.

Bouillon agar: Streak develops in 24 hours, dirty bluish-white, wet-shining and slightly raised.

Broth: Turbid in 24 hours. Pellicle. Milk: Becomes alkaline and clears. Nitrites not produced from nitrates.

Indole production slight.

Acid but not gas from glucose. No acid from lactose or sucrose. 
Gtarch hydrolysis feeble.

Optimum temperature $25 \mathrm{C}$

source: Isolated in England from brown-spot of cultivated mushrooms.

Habitat: Pathogenic on cultivated mushrooms.

104. Pseudomonas xanthochlora (Schuster) Stapp. (Bacterium xanthochlorum Schuster, Arbeit. a. d. Kaiserl. Biolog. Anstalt. f. Land. u. Forstw., 8, 1912, 452; Phytomonas xanthochlora Bergey et al., Manual, 1st ed., 1923, 180; Stapp, in Sorauer, Handbuch der Pflanzenkrankheiten, 2, 5 Auf., 1928, 213.) From Gr. xanthus, yellow; chlorus, green.

Description from Erw. Smith, Bacteria in Rel. to Plant Dis., 3, 1914, 272.

Rods : 0.75 to 1.5 by 3.0 microns. Motile with 1 to 3 flagella. Gram-negative.

Green fluorescent pigment produced in culture.

Gelatin: Slow liquefaction.

Agar colonies: Circular, slightly raised, vellow-white.

Broth: Strong clouding in 24 hours. A white pellicle.

Milk: slow coagulation and clearing.

Nitrites are produced from nitrates.

Indole is produced after 10 days.

Hydrogen sulfide produced slowly.

Acid but not gas from glucose and galactose.

Optimum temperature $27^{\circ}$ (. Masimum $44^{\circ} \mathrm{C}$. Minimum $2^{\circ} \mathrm{C}$.

Source: Isolated from rotting potato tubers in Germany.

Habitat: Pathogenic on potato tubers and a number of unrelated plants.

105. Pseudomonas rhizoctonia (Thomas) comb. nov. (Aplanobacter rhizoctonia Thomas, Ohio Agr. Exp. Sta. Bull. 359, 1922, 211; Bacterium rhizoctonia stapp, in Sorauer, Handbuch der Pflanzenkrankheiten, 2, 5 Auf., 1928, 290; Phytomonas rhizoctonia Burkholder, Phytopath., 20, 1930, 7.) From (ir. rhizo, root; ctonus, murder.

Rods : 0.5 to 0.85 by 1.4 to 1.9 microns. Non-motile. Gram-negative.
Green fluorescent pigment produced in culture.

Gelatin: Liquefaction.

Nutrient agar colonies: Greenish-yellow, later olive-buff, circular, raised, slightly viscid.

Broth: Turbid, prrite yellow.

Milk: Alkaline; clears.

Nitrites are produced from nitrates. Indole reaction very slight.

No $\mathrm{H}_{2} \mathrm{~S}$ formed.

Starch: Potato starch slightly hydrolyzed.

Growth in 8 per cent salt.

Optimum temperature $25^{\circ}$ to $27^{\circ} \mathrm{C}$. Maximum $38^{\circ} \mathrm{C}$. Ninimum $0^{\circ} \mathrm{C}$.

Source: Isolated from roots of lettuce showing the rosette disease.

Habitat: Pathogenic on roots of lettuce.

106. Pseudomonas barkeri (Berridge) Clara. (Bacillus of pear blossom disease, Barker and Grove, Ann. Appl. Biol., 1, 1914, 94; Barker and Grove's organism, Doidge, Ann. Appl. Biol., 4, 1917, 50; B. barkeri Berridge, Ann. Appl. Biol., 11, 1924, 73; Phytomonas barkeri Bergey et al., Manual, 3rd ed., 1930, 265; Bacterium barkeri Elliott, Bacterial Plant Pathogens, 1930, 95; Clara, Science, 75, 1934 , 11.) Named for B. T. P. Barker who first reported the species.

Description from Doidge (loc. cit.).

Rods: 0.5 to 0.8 by 2 to 4 microns. Motile with 1 to 4 polar flagella. Gramnegative (Burkholder), not Gram-positive as stated.

Green fluorescent pigment produced in culture.

Gelatin: Liquefaction.

Agar: Growth is white, feeble, flat, glistening, smooth edged.

Broth: Slightly turbid in 24 hours.

Milk: Slowly cleared.

Nitrites not produced from nitrates.

Indole not formed unless culture warmed.

Starch slowly digested.

Source: Barker made many cultures 
from blighted pear blossoms. received a culture from Barker.

Habitat: Causes a blossom blight of pear.

107. Pseudomonas gladioli Severini. (Severini, Annali d. Bot., Rome, 11, 1913, 420; Bacterium gladioli Elliott, Bact. Plant Pathogens, 1930, 132; Phytomonas gladioli Nagrou, in Hauduroy et al., Dict. d. Bact. Path., Paris, 1937, 356.) From L. gladiolus, a little sword; M. L. Gladiolus, a generic name.

Rods : 0.6 by 2.3 to 2.8 microns. Motile with one or more polar flagella. Gram-negative.

A pale yellow water-soluble pigment found, later orange.

Gelatin colonies: Cream-colored, wartlike. Rapid liquefaction.

Milk: Coagulated and slowly peptonized.

Nitrites not produced from nitrates.

Indole not formed.

No gas.

Aerobic.

Optimum temperature $28^{\circ}$ to $30^{\circ} \mathrm{C}$.

Habitat: Causes a corm rot of gladiolus and other tubers.

108. Pseudomonas mellea Johnson. (Bacterium melleum Johnson, Jour. Agr. Res., 23, 1923, 489; Johnson, loc. cit., 489; Phytomonas mellea Bergey et al., Manual, 3rd ed., 1930, 254.) From L. melleus, of or belonging to honey, the color of the colonies.

Rods: 0.6 by 1.8 microns. Capsules. Motile with 1 to 7 polar flagella. Gramnegative.

Green fluorescent pigment produced in culture.

Gelatin: Liquefied.

Potato - glucose agar: Abundant growth, smooth, glistening, viscid, honeycolored.

Broth: Turbid in 24 hours. Pellicle. Milk: Alkaline; clears.

Nitrites not produced from nitrates. Indole not formed.
No $\mathrm{H}_{2} \mathrm{~S}$ formed.

Starch hydrolysis feeble.

Growth inhibited by 4 per cent salt. Optimum temperature, $26^{\circ}$ to $28^{\circ} \mathrm{C}$. Maximum $36^{\circ} \mathrm{C}$.

Facultative anaerobe.

Distinctive character: Differs from Pseudomonas pseudozoogloeae in that it produces on tobacco a brown instead of a black spot with a halo, is orange-yellow in culture, and turns milk alkaline.

Source: Isolated from brown rusty spots on tobacco in Wisconsin.

Habitat: Pathogenic on leaves of tobacco, Nicotiana tabacum.

109. Pseudomonas betlis (Ragunathan) comb. nov. (Bacterium betle Ragunathan, Ann. Roy. Card., Peradeniya, Ceylon, 11, 1928, 51; A planobacter betle Elliott, Bact. Plant Pathogens, 1930, 4; Phytomonas betlis Magrou, in Hauduroy et al., Dict. d. Bact. Path., Paris, 1937, 337.) From Malayan, betle, betel, a kind of pepper, Piper betle.

Rods : 0.5 by 1.5 to 2.5 microns, oceurring singly or in short chains. Nonmotile. Gram-negative.

Green pigment formed in nutrient gelatin and in broth.

Gelatin: Liquefaction.

Bovril agar colonies: Honey-yellow, circular at first, later echinulate. Raised, smooth and shiny.

Broth: Surface becomes cloudy in 2 days. Pellicle.

No gas from lactose, maltose or sucrose. Starch is reduced.

Aerobic.

Source: Five cultures isolated from leaf spots on the betel vine.

Habitat: Pathogenic on the betel vine, Piper betle.

110. Pseudomonas panacis (Takimoto) Dowson. (Bacterium panaxi Nakata and Takimoto, Bul. Agr. Sta. Chosen, 5, 1922, 1; Phytomonas panaxi Magrou, in Hauduroy et al., Dict. d. Bact. Path., Paris, 1937, 389; Dowson, Trans. Brit. 
Mycol. Soc., 26, 1943, 10.) From Gr. panax (panicis), a plant heal-all; M. L. $P$ anax, a generic name.

Description from Elliott, Bact. Plant Pathogens, 1930, 173.

Rods : 0.5 by 1.3 to 1.5 microns. Chains. Motile with 4 to 6 polar flagella. Gramnegative.

Green fluorescent pigment produced in culture.

Gelatin: Slight liquefaction.

Agar colonies: White.

Milk: Coagulated.

No gas from sugars.

Habitat: Causes a root rot of ginseng; Panax quinquefolium.

111. Pseudomonas aleuritidis (MICCulloch and Demaree) Stapp. (Bacterium aleuritidis McCulloch and Demaree, Jour. Agr. Res., 45, 1932, 339; Stapp, Bot. Rev., 1, 1935, 408; Phytomonas aleuritidis Magrou, in Hauduroy et al., Dict. d. Bact. Path., Paris, 1937, 328.) From Gr. aleurites, of wheaten flour; M. L. Aleurites, generic name.

Rods: 0.6 to 0.7 by 1.1 to 3 microns. Motile with 1 to 5 polar, rarely bipolar, flagella. Capsules present. Gram-negative.

Green fluorescent pigment produced in certain media.

Gelatin: Not liquefied.

Beef agar slants: Growth is thin, white and viscid.

Broth: A heavy white surface growth in 24 hours. Sediment.

Milk: Becomes alkaline, but no separation.

Nitrites are produced from nitrates.

Indole test feebly positive.

Hydrogen sulfide test feebly positive.

Acid but no gas from glucose, galactose and glycerol. Slow acid production from sucrose, maltose and lactose.

Starch hydrolysis feeble.

Optimum temperature $27^{\circ}$ to $28^{\circ} \mathrm{C}$. Maximum temperature $37^{\circ} \mathrm{C}$.

Optimum pH 6.2 to 6.8 . pH range $5 . \frac{4}{x}$ to 8.9 .
Source: Isolations from naturally infected tung oil trees in Georgia.

Habitat: Pathogenic on the tung oil tree (Aleurites fordi), on the bean (Phaseolus vulgaris) and the castor bean (Ricinus communis).

112. Pseudomonas gíycinea Coerper. (Bacterium glycineum Coerper, Jour. Agric. Research, 18, 1919, 188; Coerper, loc. cit., 188; Phytomonas glycinea Burkholder, Phytopath., 16, 1926, 922.) From glycys, sweet; ine, like; M.L. Glycine, generic name.

Synonym: Bacterium sojae Wolf, Phytopath., 10, 1920, 132 (Phytomonas sojae Burkholder, Phytopath., 16, 1926, 922; Pseudomonas sojae Stapp, in Sorauer, Handb. d. Pflanzenkrankheiten, 2, 5 Aufl., 1928, 174). See Elliott, Bact. Plant Pathogens, 1930, 134; and Shunk and Wolf, Phytopath., 11, 1921, 18.

Rods: 1.2 to 1.5 by 2.3 to 3 microns. Motile with polar flagella. Gram-negative.

Green fluorescent pigment produced in culture.

Gelatin: Jot liquefied.

Beef-peptone agar colonies: Appear in 24 hours. Circular, creamy white, smooth, shining and convex. Margins entire. Butyrous in consistency.

Milk: Litmus turns blue and later a separation of the milk occurs. Casein not digested.

Nitrites not produced from nitrates.

Indole test feebly positive.

Not lipolytic (Starr and Burkholder, Phytopath., 82, 1942, 601).

Starch not hydrolyzed.

Acid from glucose and sucrose.

Optimum temperature $24^{\circ}$ to $26^{\circ} \mathrm{C}$. Maximum $35^{\circ} \mathrm{C}$. Minimum $2^{\circ} \mathrm{C}$.

Facultative anaerobe.

Source: A number of cultures isolated from soy beans in Wisconsin.

Habitat: Pathogenic on soybean, Glycine $\max ($ Soja $\max$ )

112a. Pseudomonas glycinea var. japonica (Takimoto) comb. nov. (Bac- 
terium soyae var. japonicum Takimoto, Jour. Plant Protect. Tokyo, 14, 1927, 556; Bacterium glycineum var. japonicum Elliott, Bact. Plant Pathogens, 1930, 136; Phytomonas glycinea var. japonica Magrou, in Hauduroy et al., Diet. d. Bact. Path., Paris, 1937, 358.) From M. L. Japonicus, japanese.

Distinctive characters: Differs slightly from Pseudomonas glycinea in size of cell, length of chains, action in milk, and color in media. Okabe (Jour. Soc. Trop. Agr., Formosa, 5, 1933, 162) gives a description of the organism which leads one to believe the differences are not great enough to be varietal.

Source: Isolated from leaf spots on soy bean in Formosa.

Habitat: Pathogenic on soy bean, Glycine $\max$.

\section{Pseudomonas savastanoi (Erw.} Smith) Stevens. (Bacterium sauastanoi Erw. Smith, U. S. Dept. Agr. Plant Ind. Bull. 131, 1908, 31; Stevens, The Fungi which Cause Plant Diseases, 1913, 33; Phytomonas savastanoi Bergey et al., Manual, 1st ed., 1923, 190.) Named for F. Savastano, the Italian plant pathologist.

\оте: Smith (loc. cit.) lists and diseards the following species since they were either mixed cultures or names with no descriptions: Bacterium oleae Areangeli, Istit. Bot. delle R. Univ. di Pisa, Ricerche e Lavori, fasc. 1, 1886, 109; Bacillus oleae tuberculosis Savastano, Atti. R. Accad. Naz. Lincei Rend. Cl. Sci. Fis., Mat. e Nat., 5, 1889, 92; Bacillus prillieuxianus Trevisan, I generi e le specie delle Batteriacee, Milano, 1889, 19; Bacillus oleae De Toni and Trevisan, in Saccardo, Sylloge Fungorum, 8, 1889, 982.

Description from Brown, Jour. Agr. Res., 44, 1932, 711.

Rods: 0.4 to 0.8 by 1.2 to 3.3 microns. Motile with 1 to 4 polar flagella. Gramnegative.

Green fluorescent pigment found in culture.
Gelatin: No liquefaction.

Beef agar colonies: White, smooth, flat, glistening, margins erose or entire.

Broth: Turbid on the second day. No pellicle or ring.

Milk: Becomes alkaline.

Nitrites not produced from nitrates. $\mathrm{No} \mathrm{H}_{2} \mathrm{~S}$ produced.

Acid but not gas from glucose, galactose and sucrose.

Starch is hydrolyzed.

Optimum temperature $23^{\circ}$ to $24^{\circ} \mathrm{C}$. Maximum $32^{\circ} \mathrm{C}$. Minimum $1{ }^{\circ} \mathrm{C}$.

Optimum pH 6.8 to 7.0 . Maximum 8.5. Minimum 5.6.

Aerobic.

Source: Smith isolated his cultures from olive galls collected in California. Habitat: Pathogenic on olive.

113a. Pseudomonas savastanoi var. fraxini (Brown) Dowson. (Bacterium savastanoi var. fraxini Brown, Jour. Agr. Res., 44, 1932, 721; Phytomonas savastanoi var. fraxini Magrou, in Hauduroy et al., Diet. d. Bact. Path., Paris, 1937, 410; Pseudomonas fraxini Skoric, Ann. Exp. For. Zagreb, 6, 1938, 66; Dowson, Trans. Brit. Mycol. Soc., 26, 1943, 11.) From M. I. Fraxinus, a generic name.

Distinctive characters: Differs but slightly from Pseudomonas savastanoi, but is pathogenic on ash and not on olive.

Source: Three cultures isolated from cankers on ash.

Habitat: Pathogenic on ash, Fraxinus excelsior and $F$. americana.

114. Pseudomonas tonelliana (Ferraris) comb. nov. (Bacterium tonellianum Ferraris, Trattato di Patologia e Terapia Vegetale, 3rd ed., 1, 1926, 104; Phytomonas tonelliana Adams and Pugsley, Jour. Dept. Agr. Victoria, 32, 1934, 304.) Named for Tonelli, the Italian botanist.

Synonym: Pseudomonas savastanoi var. nerii C. O. Smith, Phytopath., 18, 1928, 503.

Description from Smith (loc. cit.) unless otherwise noted 
Rods : 0.5 to 0.6 by 1.5 to 2.5 microns. Motile with 1 to 3 polar flagella. Gramnegative (Adams and Pugsley, loc. cit.).

Gelatin: No liquefaction.

Potato glucose agar colonies: Flat, circular, shining, margins somewhat undulated.

Broth: Dense clouding with partial pellicle.

Milk: Alkaline. No separation.

Nitrites not produced from nitrates (Adams and Pugsley).

Indole produced. Not produced (Adams and Pugsley).

Acid but not gas from glucose and sucrose. No acid from lactose (Adams and Pugsley).

Starch not hydrolyzed (Adams and Pugsley).

Distinctive character: Pseudomonas savastanoi is similar in culture but is not pathogenic on oleanders.

Source: Both Ferraris and C. O. Smith isolated the pathogen from galls on oleander.

Habitat: Pathogenic on oleander, Nerium oleander.

115. Pseudomonas calendulae (Takimoto) Dowson. (Bacterium calendulae Takimoto, Ann. Phytopath. Soc. Japan, 5, 1936, 341; Phytomonas calendulae Burkholder, in Manual, 5th ed., 1939, 201 ; Dowson, Trans. Brit. Mycol. Soc., 26, 1943, 9.) From Latin, calendae, throughout the month; M.L. Calendula, a generic name.

Rods: 0.5 by 1 to 2 microns. Motile with 1 to 3 polar flagella. Gram-negative.

Green fluorescent pigment produced in Uschinsky's and in Cohn's solutions.

Gelatin: Not liquefied.

Agar colonies: Circular, smooth, flat, dirty white.

Broth: Turbid.

Milk: No coagulation.

Nitrites not produced from nitrates.

Indole formed in small amount.

No $\mathrm{H}_{2} \mathrm{~S}$ produced.
Acid but not gas from glucose and glycerol. No acid from lactose or sucrose.

Starch not hydrolyzed.

Optimum temperature $27^{\circ}$ to $30^{\circ} \mathrm{C}$. Maximum $37^{\circ} \mathrm{C}$. Minimum $0^{\circ}$ to $7^{\circ} \mathrm{C}$.

Habitat: Pathogenic on marigolds, Calendula officinalis.

116. Pseudomonas cichorii (Swingle) Stapp. (Phytomonas cichorii Swingle, Phytopath., 15, 1925, 730; Stapp, in Sorauer, Handbuch der Pflanzenkrankheiten, 2, 5 Auf., 1928, 291; Bacterium cichorii Elliott, Bact. Plant Pathogens, 1930, 112.) From Gr. cichoria, chicory; M. L. Cichorium, generic name.

Probable synonyms: Pseudomonas endiviae Kotte, Phyt. Ztschr., 1, 1930, 609; Phytomonas endiviae (Kotte) Clara, Cornell Agr. Exp. Sta. Mem. 159, 1934, 26 ; and Bucterium formosanum Okabe, Jour. Soc. Trop. Agr., Formosa, 7, 1935, 65 .

Description from Clara (loc. cit.) which is a description of a culture of Pseudomonas endiviae from Kotte. Swingle's description is very meager.

Rods : 0.75 to 1.5 by 1.5 to 3.75 microns. Motile with 1 or 2 polar flagella. Gramnegative.

Green fluorescent pigment produced in culture.

Gelatin: No liquefaction.

Beef-extract agar colonies: Circular, grayish-white with bluish tinge, raised with slightly irregular edges.

Broth: Turbid in 36 hours with a smooth viscous pellicle.

Milk: Alkaline.

Nitrites not produced from nitrates. Indole not formed.

No $\mathrm{H}_{2} \mathrm{~S}$ formed.

Not lipolytic (Starr and Burkholder, Phytopath., 32, 1942, 601).

Acid but not gas from glucose, galactose, fructose, mannose, arabinose, xylose, mannitol and glycerol. Alkaline production from salts of acetic, citric, lactic, malic, succinic and tartaric acids. 
Rhamnose, maltose, sucrose, lactose, raffinose and salicin not utilized.

Starch not hydrolyzed.

Slight growth in broth plus 6 per cent NaCl.

Optimum pH 6.8 to 7.1. Maximum 9.2 to 9.4 . Minimum 5.0 to 5.3 (Kotte, Phyt. Ztsch., 2, 1930, 453).

Facultative anaerobe.

Distinctive characters: Differs from Pseudomonas intybi in that it does not liquefy gelatin or reduce nitrates to nitrites.

Source: Isolated from rot of French endive, Cichorium intybus by Swingle and by Okabe, and from $C$. endivia by Kotte.

Habitat: Pathogenic on endive, lettuce and larkspur.

117. Pseudomonas cissicola (Takimoto) comb. nov. (A planobacter cissicola Takimoto, Ann. Phytopath. Soc. Japan., 9, 1939, 43.) From Greek, cissus, ivy; M. L. Cissus a generic name.

Rods : 0.5 to 0.9 by 1.0 to 2.0 microns. Non-motile. Capsules. Gram-negative.

Green fluorescent pigment formed in Uschinsky's solution.

Gelatin: No liquefaction.

Potato-extract agar colonies: Circular, convex, smooth, and dirty white.

Broth: Feeble clouding followed by precipitation of pellicle and rim.

Nitrites not produced from nitrates. Indole not formed.

Hydrogen sulfide not produced.

No acid nor gas from sucrose, glucose, lactose and glycerol.

Starch is not digested.

Salt toleration is 3 per cent.

Optimum temperature $30^{\circ} \mathrm{C}$. Maximum $35^{\circ} \mathrm{C}$. Minimum $10^{\circ} \mathrm{C}$. Thermal death point $49^{\circ}$ to $50^{\circ} \mathrm{C}$.

Source: Isolated from black spots on leaves of Japanese ivy, Cissus japonica in Japan.

Habitat: Pathogenic only on Cissus japonica.

118. Pseudomonas nectarophila (Doidge) Rosen and Bleeker. (Bac- terium nectarophila Doidge, Ann. Appl. Biol., 4, 1917, 73; Phytomonas nectarophila Bergey et al., Manual, 3rd ed., 1930, 262; Rosen and Bleeker, Jour. Agr. Res., 46, 1933, 98.) From Gr. nectar, nectar; philus, loving.

Rods: 0.5 to 0.7 by 0.6 to 1.5 microns. Motile with 1 to 5 polar flagella. Capsules. Gram-negative.

Green fluorescent pigment produced in culture.

Gelatin: No liquefaction.

Nutrient agar colonies: Yellowishwhite, wet-shining, smooth, margins irregular.

Broth: Heavy turbidity in 24 hours. Sediment.

Milk: Cleared.

Nitrites not produced from nitrates.

Indole not formed.

Acid from glucose and galactose. No acid from sucrose.

Starch hydrolysis feeble.

Optimum temperature 25 to $30^{\circ} \mathrm{C}$.

Facultative anaerobe.

Distinctive character: Differs from $P$ seudomonas barkeri in that it does not liquefy gelatin, nor produce indole. Produces capsules.

Source: Isolated from blighted pear blossoms in South Africa.

Habitat: Pathogenic on pear blossoms.

119. Pseudomonas viburni (Thornberry and Anderson) Stapp. (Phytomonas viburni Thornberry and Anderson, Phytopath., 21, 1931, 912; Stapp, Bot. Rev., 1, 1935, 407; Bacterium viburni Burgwitz, Phytopathogenic Bacteria, Leningrad, 1935,160 .) From L. viburnum, the wayfaring tree; M. L. Viburnum, a generic name.

Rods: 0.5 to 1.0 by 1 to 2.0 microns. Capsules present. Motile with 2 to 4 polar flagella. Gram-negative (Burkholder); not Gram-positive as stated.

Green fluorescent pigment produced in culture (Burkholder).

Gelatin: No liquefaction.

Glucose beef-extract colonies: Dull gray, circular, edges entire. 
Broth: Turbid with pellicle.

Milk: Alkaline.

Nitrites not produced from nitrates. Indole not formed.

No $\mathrm{H}_{2} \mathrm{~S}$ formed.

Not lipolytic (Starr and Burkholder, Phytopath., 32, 1942, 601).

Acid from glucose and galactose, but not sucrose (Burkholder).

Starch: No hydrolysis.

Slight growth in 3.5 per cent salt (Burkholder).

Optimum temperature $25^{\circ} \mathrm{C}$. Minimum $12^{\circ} \mathrm{C}$. Maximum $35^{\circ} \mathrm{C}$.

Aerobic.

Source: Isolated from angular leaf spots and stem lesions on arrow-wood, Viburnum opulus, etc.

Habitat: Pathogenic on Viburnum spp.

120. Pseudomonas mori (Boyer and Lambert) Stevens. (Bacterium mori Boyer and Lambert, Compt. rend. Acad. Sci. Paris, 117, 1893, 342; Bacterium mori Boyer and Lambert emend. Erw. Smith, Science, 31, 1910, 792; Stevens, The Fungi which Cause Plant Diseases, 1913, 30; Bacillus mori Holland, Jour. Bact., 5, 1920, 222; Phytomonas mori Bergey et al., Manual, 1st ed., 1923, 191.) From Gr. morum, mulberry; M. L. Morus, a generic name.

Synonyms: Elliott (Bact. Plant Pathugens, 1930, 166) lists Bacillus cubonianus Macchiati, Staz. Sperim. Agr. Ital., 23, 1892, 228 (Macchiati described the disease due to Pseudomonas mori, but gave an incorrect description of the pathogen); also Bacterium cubonianum Ferraris, Curiano le Plante, 6, 1928, 180 (Ferraris uses Macchiati's name but the description of Pseudomonas mori).

Description from Smith (loc. cit.).

Rods: 0.9 to 1.3 by 1.8 to 4.5 microns. Motile with a polar flagellum. Gramnegative.

Green fluorescent pigment produced in culture.

Gelatin: Not liquefied.

Agar colonies: White, slow-growing, smooth, flat, edges entire becoming undulate.
Milk: Becomes alkaline and clears.

Nitrites not produced from nitrates. Indole none or feeble production.

Hydrogen sulfide not produced (Okabe, Jour. Soc. Trop. Agr., 5, 1933, 166).

No growth in broth plus 4 per cent salt (Okabe, loc. cit.).

No gas from carbohydrates.

Temperature range $1^{\circ} \mathrm{C}$ to $35^{\circ} \mathrm{C}$.

Source: Smith isolated the pathogen from blighted shoots of mulberry from Georgia. Also received cultures from Arkansas and the Pacific Coast.

Habitat: Pathogenic on mulberry, Morus.

121. Pseudomonas stizolobii (Wolf) comb. nov. (A planobacter stizolobii Wolf, Phytopath., 10, 1920, 79; Bacterium stizolobii McCulloch, Phytopath., 18, 1928, 460; Phytomonas stizolobii Bergey et al., Manual, 3rd ed., 1930, 280.) From Gr. stizo, to prick; lobium, a little lobe; Stizolobium, a generic name.

Rods : 0.6 to 0.7 by 1.0 to 1.6 microns. Non-motile (Wolf). Motile with a short polar flagellum (McCulloch). Capsules. Gram-negative.

Gelatin: No liquefaction.

Agar colonies: Circular, smooth, white, raised and opaque. Margins entire to slightly undulate.

Broth: Slightly turbid throughout. No pellicle or ring.

Milk: Alkaline.

Nitrites not produced from nitrates. Indole not formed.

No acid or gas in peptone broth plus sugars.

Starch not hydrolyzed.

Optimum temperature $25^{\circ}$ to $28^{\circ} \mathrm{C}$.

Distinctive characters: Differs from Pseudomonas sojae (Pseudomonas glycinea) in the smaller size of cell, and absence of pellicle and dense clouding of broth. The pathogen does not infect soy bean.

Source: Isolated from the leaf spot of velvet bean.

Habitat: Pathogenic on velvet bean, Stizolobium deeringianium. 
122. Pseudomonas viciae Cyeda. (Uyeda in Takimoto, Jour. Plant Protect., Japan, 2, 1915, 845; Bacterium viciae Nakata, see Elliott, Bact. Plant Pathogens, 1930, 259; Phytomonas viciae Magrou, in Hauduroy et al., Dict. d. Bact. Path., Paris, 1937, 430.) From L. vicia, vetch; M. L. Vicia, a generic name.

Rods : 0.5 to 0.8 by 1.2 to 2.0 microns. Motile with 2 to 4 polar flagella. Grampositive.

Green fluorescent pigment produced in culture.

Gelatin colonies: Pale white, glistening, finally turning brown. No liquefaction.

Milk: Coagulates and clears.

Nitrites not produced from nitrates.

No $\mathrm{H}_{2} \mathrm{~S}$ produced.

Facultative anaerobe.

Habitat: Pathogenic on the broad bean (Vicia faba), the turnip (Brassica rapa), the carrot (Daucus carota) and the sweet potato (Ipomoca batatas).

123. Pseudomonas alliicola Burkholder. (Burkholder, Phytopath., 32, 1942, 146; Phytomonas alliicola Burkholder, ibid.) From L. allium, onion; -cola, dweller.

Rods : 0.7 to 1.4 by 1.05 to 2.8 microns. Motile with 1 to several polar flagella, at times bi-polar. Gram-negative.

Gelatin: Liquefaction.

Beef-extract peptone agar streaks: Moderate in growth, white at first, later dirty in appearance, edges wavy, consistency viscid. Medium deep brown.

Potato-glucose agar frequently becomes greenish.

Broth: Turbid with light pellicle. Brown.

Milk: Cleared and litmus reduced. Neutral.

Nitrites produced from nitrates.

Indole not produced.

Hydrogen sulfide not produced.

Lipolytic action very strong.

Acid but no gas from l-arabinose, dxylose, rhamnose, glucose, d-galactose, fructose, d-lactose, maltose, sucrose, glycerol, mannitol and salicin. Alkali from salts of acetic, citric, formic, hippuric, lactic, malic, succinic, tartaric acids.

Starch not hydrolysed.

Slight growth in broth plus 4 per cent salt.

Aerobic.

Optimum temperature $30^{\circ} \mathrm{C}$. Maximum $41^{\circ} \mathrm{C}$. Minimum $5{ }^{\circ} \mathrm{C}$.

Source: Seven isolates from storage rot of onion bulbs.

Habitat: Pathogenic on onion bulbs, Allium cepa.

124. Pseudomonas gardeniae Burkholder and Pirone. (Burkholder and Pirone, Phytopath., 31, 1941, 194; Phytomonas gardeniae Burkholder and Pirone, ibid.) From M. L. Gardenia, a generic name.

Rods: 0.75 by 2.4 microns. Motile with 1 to 2 polar flagella. Gram-negative.

Gelatin: Liquefaction.

Beef-extract peptone agar colonies: Growth fair, white to dirty gray and viscid. Medium becoming dark brown.

Potato-glucose agar: No brown color.

Broth: Turbid with pellicle. Dark brown.

Milk: Soft curd with pellicle. Clears in zones. Litmus reduced.

Nitrites produced from nitrates.

Hydrogen sulfide not produced.

Indole not formed.

Acid from glucose, galactose, xylose, rhamnose, sucrose, maltose, mannitol, glycerol, and salicin. Alkali produced from the salts of citric, malic, malonic, succinic, tartaric and hyppuric acids. Good growth in tyrosine and in asparagine broth.

Starch is not hydrolyzed.

Aerobic.

Source: Eight isolates from leaf spots of gardenias in New Jersey.

Habitat: Pathogenic on leaves of Gardenia jasminoides .

125 Pseudomonas caryophylli Burkholder. (Burkholder, Phytopath., 31, 
1941, 143; Phytomonas caryophylli, Burkholder, ibid.) From M. L. Caryophyllus, an old generic name.

Rods : 0.35 to 0.95 by 1.05 to $3.18 \mathrm{mi}-$ crons. At times slightly curved. Motile with 1 to several polar flagella. Frequently bipolar. Gram-negative.

Gelatin: Liquefaction after 3 to 4 weeks.

Potato glucose agar colonies: 3 to 4 $\mathrm{mm}$ in diameter, circular, smooth, glistening, edges entire. Color is tan to gray mauve. Old culture dark brown. Consistency butyrous.

Broth: Turbid with a white sediment.

Milk: Litmus slowly becomes blue. Slight reduction at bottom of tube. No clearing.

Nitrites produced from nitrates. Also ammonia and gas are produced in a synthetic nitrate medium. Asparagine, $\mathrm{K}^{-} \mathrm{NO}_{3}$ and $\mathrm{NH}_{4} \mathrm{H}_{2} \mathrm{PO}_{4}$ can be utilized.

Indole not formed.

Hydrogen sulfide not formed.

Lipolytic action slight to moderate.

Acid from l-arabinose, d-xylose, rhamnose, glucose, d-galactose, fructose, dlactose, maltose, and sucrose, glycerol, mannitol, and salicin. Alkali with sodium salts of acetic, citric, formic, hippuric, lactic, malic, maleic, succinic and tartaric acid.

Starch not hydrolyzed.

Aerobic.

Optimum temperature $30^{\circ}$ to $33^{\circ} \mathrm{C}$. Maximum $46^{\circ} \mathrm{C}$. Minimum $5^{\circ} \mathrm{C}$. or less. Slight growth in broth plus 3.5 per cent salt.

Source: Isolated first by L. K. Jones and later by W. H. Burkholder from dying carnation plants from Spokane, Washington. Twelve isolates used in description.

Habitat: Pathogenic on roots and stalks of the carnation, Dianthus caryophyllus.

126. Pseudomonas solanacearum Erw. Smith. (Bacillus solanacearum Erw. Smith, U.S. Dept. Agr., Div. Teg. Phys. and Path., Bul. 12, 1896, 10; Bacterium solanacearum Chester, Ann. Rept. Del. Col. Agr. Exp. Sta., 9, 1897, 73; Erw. Smith. Bacteria in Relation to Plant Diseases, 3, 1914, 178; Phytomonas solanaccarum Bergey et al., Manual, 1st ed., 1923, 186.) From L. solanum nightshade; M. L. Solanaceae, a plant family.

Probable synonyms: Elliott (Bact. Plant Pathogens, 1930, 203) lists the following: Bacillus nicotianae Uyeda, Cent. f. Bakt., II Abt., 13, 1904, 327; Bacillus sesami Malkoff and Pseudomonas sesami Malkoff, Cent. f. Bakt., II Abt., 16, 1906, 664; Bacillus musae Rorer, Phytopath., 1, 1911, 45; Bacillus musarum Zeman, Rev. Facul. Agr. Univ., La Plat, 1' . 1921, 17; Erwinia nicotianae Bergey et al., Manual, 1st ed., 1923, 172; Phytomonas ricini Archibald, Trop. Agr., Trinidad, 4, 1927, 124.

Description taken from Elliott (loc. cit.),

Rods: 0.5 to 1.5 microns. Motile with a polar flagellum. Gram-negative.

Gelatin: Nakata (Jour. Sci. Agr. Soc. Tokyo, 294, 1927, 216) states there are two forms, one of which shows slight liquefaction. The other shows no liquefaction.

Agar colonies: Small, irregular, roundish, smooth, wet-shining, opalescent, becoming brown.

Broth: Slight pellicle. Broth turns brown.

Milk: Cleared without precipitation of casein.

Nitrites produced from nitrates.

Indole not formed.

Hydrogen sulfide not produced (Burkholder).

Glucose, sucrose, glycerol, sodium citrate, peptone, tyrosine, asparagine and glutanic acid are utilized Mushin, Austral. Jour. Expt. Biol. and Med., 16, 1938, 325).

Nitrogen sources utilized are ammonia, nitrates $\left(\mathrm{K}_{\mathrm{NO}}\right)$ asparagine, tyrosine, peptone and glutamic acid, but not potassium nitrite (Mushin, loc. cit.).

Starch not hydrolyzed. 
Optimum temperature $35^{\circ}$ to $37^{\circ} \mathrm{C}$. Maximum $41^{\circ} \mathrm{C}$. Minimum $10^{\circ} \mathrm{C}$.

Pathogenicity readily lost in culture.

Source: Isolated from brown-rot of solanaceous plants.

Habitat: Soil pathogen in warm moist climates attacking numerous species of plants, especially potato, tobacco, and tomato.

126a. Pseudomonas solanacearum var. asiatica (Erw. Smith) Stapp. (Bacterium solanacearum var. asiaticum Erw. Smith, Bact. in Relation to Plant Diseases, 3, 1914, 282; Stapp, in Sorauer, Handbuch der Pflanzenkrankheiten, 2, 5 Auf., 1928, 253; Phytomonas solanacearum var. asiatica Magrou, in Hauduroy et al., Dict. d. Bact. Path., Paris, 1937, 414.) From Gr. asiaticus, asiatic.

Distinctive characters: Differs from Pseudomonas solanacearum in that it turns litmus milk and cream red.

Source: Isolated by J. A. Honing from diseased tobacco plants in Medan, Sumatra.

127. Pseudomonas castaneae (Kawamura) comb. nov. (Bacterium castaneae Kawamura, Ann. Phytopath. Soc. Japan, 3, 1934, 15; Phytomonas castaneae Magrou, in Hauduroy et al., Dict. d. Bact. Path., Paris, 1937, 343.) From M. L. Castanea, a generic name.

Rods : 0.8 to 1.2 by 1.0 to 1.8 microns. Motile with 1 to 5 polar flagella. Gramnegative.

Gelatin: Liquefied.

Beef agar colonies: White, circular, edges slightly undulate, viscid.

Milk: No coagulation. Peptonized.

Acid but not gas from glucose, sucrose and glycerol. No acid from lactose.

Optimum temperature $25^{\circ}$ to $27^{\circ} \mathrm{C}$. Maximum $35^{\circ} \mathrm{C}$. Minimum $3^{\circ} \mathrm{C}$.

Facultative anaerobe.

Habitat: Causes water-soaked spotting on leaves and shoots of chestnut, Castanea.

128. Pseudomonas seminum Cayley. (Cayley, Jour. Agr. Sci., 8, 1917, 461 ; Bacterium seminum Stevenson, Foreign
Plant Dis., U.S.D.A. Office of Sec'y., 1926, 141; Phytomonas seminum Bergey et al., Manual, 3rd ed., 1930, 272.) From I. semen (seminus) seed.

Rods : 1.0 by 4.0 to 5.0 microns. Sporelike bodies present. Capsules. Motile with a single flagellum. Gram-positive.

Gelatin: Rapid liquefaction.

Agar colonies: White, more or less circular, transparent, spreading.

Broth: Turbid. Pellicle.

Litmus milk: Milk becomes clear and apricot color.

Nitrites produced from nitrates.

Acid but not gas from glucose and sucrose. No acid from lactose.

Starch: No hydrolysis.

Optimum temperature $25^{\circ} \mathrm{C}$.

Facultative anaerobe.

Source: Isolated from seeds, stems and pods of diseased peas in England.

Habitat: Pathogenic on peas.

129. Pseudomonas passiflorae (Reid) comb. nov. (Phytomonas passiflorae Reid, New Zealand Jour. Sei. and Tech., 22, 1939, 264a.) From L. passio, passion; flora, flower; M. L. Passiflora, a generic name.

Rods: 0.2 to 0.5 by 1.2 to 3.2 microns. Motile with 1 to 5 polar flagella. Capsules present. Gram-negative.

Gelatin: Liquefied.

Beef-peptone agar colonies: Small, flat, smooth, dry, shining, translucent, grayish and butyrous.

Broth: Turbid in 4 days. Transient pellicle.

Milk: Slightly alkaline. No coagulation nor clearing.

Nitrites not produced from nitrates. No growth on synthetic nitrate agar.

Indole not formed.

Hydrogen sulfide not formed.

Acid reaction occurs in galactose, starch and sucrose. No gas.

Starch is not hydrolysed.

Source: From diseased leaves and fruit of the passion-fruit in New Zealand.

Habitat: Pathogenic on Passiflora edulis. 
130. Pseudomonas fabae ( $\mathrm{Yu}$ ) comb. nov. (Phytomonas fabae Yu, Bull. of the Chinese Bot. Soc., 2, 1936, 34.) From Latin, faba bean.

Rods: 0.8 to 1.1 by 1.1 to 2.8 microns. Motile with 1 to 4 polar flagella. Gramnegative.

Gelatin: Liquefied.

Nutrient agar colonies: Circular, entire, viscid, glistening, raised, smooth to wrinkled, white to salmon. Medium amber.

Broth: Turbid after 12 hours. Pellicle.

Milk: Growth slow. Clears.

Nitrites produced from nitrates.

Indole production slight.

Hydrogen sulfide not produced.

Acid but not gas from glucose. No acid nor gas developed from arabinose, xylose, fructose, galactose, sucrose, lactose, maltose, raffinose, dextrin, inulin, mannitol or adonitol in a 1 per cent Bacto-peptone broth.

Starch: Very weak diastatic action.

Optimum temperature $35^{\circ} \mathrm{C}$. Maximum 37 to $38^{\circ} \mathrm{C}$. Minimum $4^{\circ} \mathrm{C}$. Thermal death point 52 to $53^{\circ} \mathrm{C}$.

Aerobic.

Growth retarded in 2 per cent salt. Very slight growth in 3 per cent salt.

Source: From diseased broad beans at Nanking, China.

Habitat: Pathogenic on broad or Windsor bean, Vicia faba.

131. Pseudomonas astragali (Takimoto) comb. nov. (Bacterium astragali Takimoto, Jour. Plant Protect., 17, 1930, 732; Phytomonas astragali Burkholder, in Nanual, 5th ed., 1939, 197.) From M. L. Astragalus, a generic name.

Description translated by Dr. K. Togashi.

Rods: 0.7 to 0.5 by 1.2 to 2.2 microns. Notile, with 1 or 2 flagella. Gramnegative.

Gelatin: Liquefied.

Agar plates: Growth somewhat slow, colorless or grayish-white, entire margins, more or less aqueous, butyrous.
Uschinsky's medium: Growth vigorous. turbid, not viscid, ring, and sediment.

Milk: No coagulation of casein, slow digestion. Alkaline.

Nitrites not produced from nitrates. Indole not formed.

Hydrogen sulfide produced in small amount.

No acid or gas from glucose, sucrose, lactose and glycerol in broth.

Starch not hydrolyzed.

Temperature relations: Minimum below $5^{\circ}$ and maximum $33^{\circ} \mathrm{C}$. Thermal death point $50^{\circ}$ to $51^{\circ} \mathrm{C}$.

Aerobic.

Source: Species isolated from Astragalus $s p$.

Habitat: Causes a black leaf-spot of Astragalus sp.

132. Pseudomonas colurnae (Thornberry and Anderson) comb. nov. (Phytomonas colurnae Thornberry and Anderson, Phytopath., 27, 1937, 948.) From the species, Corylus colurna.

Rods : 0.8 to 1.0 by 1.0 to 1.8 microns. Single, in pairs or chains. Capsules. Motile with 1 to 2 polar flagella. Gramnegative.

Gelatin: Liquified.

Glucose agar slants: Growth filiform, raised, dull, smooth, opaque and viscid.

Broth: Moderate turbidity. Ring.

Milk: Peptonization complete with acid production. No reduction of litmus nor coagulation.

Nitrites not produced from nitrates. Indole not produced.

Hydrogen sulfide not produced.

No appreciable amount of gas from xylose, glucose, sucrose or glycerol.

Starch hydrolyzed.

Optimum temperature $21^{\circ} \mathrm{C}$. Minimum $5^{\circ} \mathrm{C}$. Maximum $35^{\circ} \mathrm{C}$. Thermal death point $50^{\circ} \mathrm{C}$.

Aerobic.

Source: From leaves and young stems of the Turkish hazelnut in Illinois.

Habitat: Pathogenic on the Turkish hazelnut, Corylus colurna. 
133. Pseudomonas maublancii (Foex and Lansade) comb. nov. (Bacterium maublancii Foex and Lansade, Comp. rend. Acad. Sci. Paris, 202, 1936, 2174; Phytomonas maublancii Burkholder, in Manual, 5th ed., 1939, 198.) Named for M. Maublanc, French colonial plant pathologist.

Rods : 0.4 by 1.3 microns. Motile with 1 to 3 polar flagella. Gram-negative.

Gelatin: Liquefied.

Gelatin colonies: Round, translucent, margins entire.

Broth: Thin pellicle.

Milk: Not coagulated; clears.

Nitrites not produced from nitrates.

Indole not formed.

No $\mathrm{H}_{2} \mathrm{~S}$ formed.

Carbohydrates not fermented.

Ammonia produced.

Growth in Fermi's solution, not in Uschinsky's solution.

Source: Isolated from rotting vascular and parenchymatic tissue of banana stalks.

Habitat: Causes a disease of the banana plant.

134. Pseudomonas polygoni (Thornberry and Anderson) comb. nov. (Phytomonas polygoni Thornberry and Anderson, Phytopath., 27, 1937, 947.) From Gr. polygonum, knot-weed; M. L. Polygonum, a generic name.

Rods : 0.5 to 1.5 by 1.5 to 2.5 microns. Motile with 2 to 8 bi-polar flagella. Capsules. Gram-positive (?). Other species reported by these investigators as Gram-positive have proved to be Gramnegative on a retest (Burkholder).

Gelatin: Liquified. Brown.

Glucose agar slant: Abundant, filiform, flat, dull, smooth, pale olive-gray, butyrous. Medium turns brown.

Broth: Turbid. Pellicle.

Milk: Alkaline and clears. Litmus not reduced.

Nitrites not produced from nitrates. Indole not formed.

Hydrogen sulfide not produced.
No appreciable amount of gas from carbohydrates.

Starch: No hydrolysis.

Optimum temperatures $18^{\circ} \mathrm{C}$. Minimum $7^{\circ} \mathrm{C}$. Maximum $35^{\circ} \mathrm{C}$.

Aerobic.

Source: From diseased leaves of Polygonum convolvulus in Illinois.

Habitat: Pathogenic on black bindweed, Polygonum convolvulus.

135. Pseudomonas iridicola (Takimoto) Stapp. (Baclerium iridicola Takimoto, Fungi, Nippon Fungological Soc., 1, 1931, 24; Stapp, Bot. Rev., 1, 1935, 408; Phytomonas iridicola Burkholder, in Manual, 5th ed., 1939, 198.) From Gr. iris (iridis), iris, a rainbow; -cola, dweller.

Rods: 0.7 to 0.8 by 1.2 to 2 microns. Motile with 1 to 3 polar flagella. Gramnegative.

Gelatin: Liquefied.

Beef agar colonies: White, circular, raised or convex.

Milk: Clears without coagulation.

No acid or gas from carbohydrates.

Starch digested.

Optimum temperature $38^{\circ} \mathrm{C}$. Minimum $4^{\circ} \mathrm{C}$.

Source: Isolated from a brown leaf spot of iris.

Habitat: Pathogenic on Iris tectorum and Iris japonica.

136. Pseudomonas levistici Osterwalder. (Osterwalder, Cent. f. Bakt., II Abt., 25, 1909, 260; Bacterium levistici Stevenson, Foreign Plant Dis., U. S. Dept. Agr., Office of Sec'y., 1926, 101; Phytomonas levistici Magrou, in Hauduroy et al., Dict. d. Bact. Path., Paris, 1937, 373.) From M. L. Levisticum, a generic name.

Rods : 0.5 to 0.7 by 1.1 to 1.5 microns. Motile with a polar flagellum. Gramnegative.

Gelatin: Colonies greenish-white. Liquefaction.

Nutrient agar: Good growth at room temperature. Yellowish-white. 
Broth: Pellicle.

Indole formed.

No $\mathrm{H}_{2} \mathrm{~S}$ produced.

Source: Isolated from spots on the leaves of lovage.

Habitat: Pathogenic on lovage, Levisticum officinale.

137. Pseudomonas radiciperda (Javoronkova) Stapp. (Bacterium radiciperda Javoronkova, Bull. Plant Protect, Leningrad, Ser. II, 5 , no. 1, 1932, 161 ; Stapp, Bot. Rev., 1, 1935, 408; Phytomonas radiciperda Magrou, in Hauduroy et al., Dict. d. Bact. Path., 1937, 401.) From L. radix (radicis), root; perdo, to destroy.

Deseription from Javoronkova, Rev. App. Myc., 11, 1932, 6.52

Rods: 0.8 by 1 to 2 microns. Capsules. Motile with 1 or 2 polar flagella. Ciramnegative.

Gelatin: Liquefaction.

Beef-peptone agar colonies: Round, smooth, shining, white to pale yellow.

Milk: Peptonized.

Indole not formed.

No $\mathrm{H}_{2} \mathrm{~S}$ formed.

Acid but not gas from carbohydrates. Optimum temperature $23^{\circ}$ to $25^{\circ} \mathrm{C}$.

Aerobic.

Habitat: Causes a root rot of red clover (Trifolium pratense), lentils (Lens esculenta) and lucerne.

138. Pseudomonas melophthora Allen and Riker. (Allen and Riker, Phytopath.. 22, 1932, 557; Bacterium melophthorum Allen and Riker, ibid.; Phytomonas melophthora Allen and Riker, ibid.) From Gr. melum, apple; phthora, destroyer.

Rods: 0.68 by 1.32 microns. Motile with 2 polar flagella. Gram-negative; Gram-positive cells appear in old cultures.

Gclatin: No liquefaction.

Nutrient agar plus 2 per cent glucose: Colonies appear in 36 hours. After 3 days colonies circular, smooth, glistening, convex; edges entire; light pink, but not constant.

Broth: Ciood growth. Pellicle and sediment.

Milk: Little change, if any

Nitrites not produced from nitrates.

Indole not formed.

No $\mathrm{H}_{2} \mathrm{~S}$ produced.

Acid from arabinose, glucose, galactose, fructose, sucrose and glycerol. No acid from lactose, maltose, dextrin and inulin.

Starch not hydrolyzed.

Optimum temperature $21^{\circ}$ to $25^{\circ} \mathrm{C}$.

Source: Description based on 7 cultures isolated from rotting apples and from apple maggots.

Habitat: Pathogenic on apples, and found with the apple maggot, Rhagoletis pomonella.

139. Pseudomonas helianthi (Kawamura) comb. nov. (Bacterium helianthi Kawamura, Ann. Phyt. Soc. Japan, 4, 1934, 27; Phytomonas helianthi Magrou, in Hauduroy et al., Dict. d. Bact. Path., Paris, 1937, 362.) From M. I. Helianthus, a generic name.

Probable synonym: Phytomonas helianthi var. tuberosi Thornberry and Anderson, Phytopath., 27, 1937, 948.

Rods: 1 to 1.4 by 1.6 to 2.4 microns. Motile with a single polar flagellum. Gram-negative.

Gelatin: No liquefaction.

Beef agar colonies: White, circular, edges entire.

Broth: Turbid. Pellicle.

Milk: Peptonized. Litmus reduced.

Nitrates: Gas production.

Indole not produced.

No $\mathrm{H}_{2} \mathrm{~S}$ produced.

Acid but not gas from sucrose and glycerol. No acid from lactose and maltose.

Starch hydrolyzed.

Optimum temperature $27^{\circ}$ to $28^{\circ} \mathrm{C}$. Maximum $35.5^{\circ} \mathrm{C}$. Minimum $12^{\circ} \mathrm{C}$.

Good growth at pH 6.4. No growth pH 5.4 and pH 8.S.

Habitat: Pathogenic on sunflower, Helianthus debilis.

\section{Pseudomonas}

Rosen. (Rosen, Ann. Missouri Bot. 
Garden, 9, 1922, 383; Bacterium alboprecipitans Elliott, Bact. Plant Path., 1930, 89; Phytomonas alboprecipitans Bergey et al., Manual, 3rd ed., 1930, 277.) From Latin albus, white and precipitans, precipitating, referring to the white precipitate produced in culture.

Rods: 0.6 by 1.8 microns, occurring singly or in pairs. Capsules present. Motile with a polar flagellum. Gramnegative.

Gelatin: Not liquefied.

Nutrient agar colonies: White, circular, raised, smooth, sticky, with margins entire. Whitish discoloration of the medium.

Broth: Turbid in 24 hours. Heavy sediment in old cultures.

Milk: Becomes alkaline and slowly clears.

Nitrites produced from nitrates.

Indole not produced.

No $\mathrm{H}_{2} \mathrm{~S}$ produced.

Acid but not gas from glucose, fructose, glycerol and mannitol. No acid from lactose, maltose or sucrose.

Starch is hydrolyzed.

Optimum temperature $30^{\circ}$ to $35^{\circ} \mathrm{C}$. Maximum temperature $40^{\circ} \mathrm{C}$. Minimum $0^{\circ} \mathrm{C}$.

\section{Aerobic.}

Distinctive characteristics: White precipitate in culture media.

Source: Isolated a number of times from foxtail grass.

Mabitat: Pathogenic on foxtail, Chaetochloa lutescens and other grasses.

141. Pseudomonas petasitis (Takimoto) comb. nov. (Bacterium petasitis Takimoto, Ann. Phyt. Soc. Japan, 2, 1927, 55; Phytomonas petasitis Magrou, in Hauduroy et al., Dict. d. Bact. Path., Paris, 1937, 393.) From M. L. Petasites, a generic name.

Rods : 0.8 to 1.1 by 1.1 to 1.7 microns. Motile with a polar flagellum. Gramnegative.

Gelatin: No liquefaction.
Beef agar colonies: White, circular or ameboid, butyrous.

Broth: Strong turbidity. Pellicle.

Milk: Coagulated in 30 days.

Nitrites produced from nitrates with gas formation.

Indole not produced.

No $\mathrm{H}_{2} \mathrm{~S}$ produced.

No evident acid in peptone broth but gas from glucose, lactose and sucrose. Acid but not gas from glycerol.

Weak growth in broth plus 6 percent salt.

Optimum temperature $27^{\circ}$ to $30^{\circ} \mathrm{C}$. Maximum $47^{\circ} \mathrm{C}$. Minimum approximately $5^{\circ} \mathrm{C}$.

Source: Isolated from brown to black lesions on Petasites japonicus in Japan.

Habitat: Pathogenic on leaves of Petasites japonicus.

142. Pseudomonas lignicola Westerdijk and Buisman. (De Iepenziekte, Arnhem, 1929, 51.) From Latin, lignum, wood; -cola, dweller.

Rods: Single or short chains. Motile with 1 to several polar flagella. Gramnegative.

Gelatin: No liquefaction.

Malt agar streaks: Milk white with a colorless edge.

Broth: Turbid with light pellicle.

Milk: No coagulation. No acid.

Nitrites not produced from nitrates.

Indole not formed.

Starch hydrolysis slight.

Optimum temperature $\pm 25^{\circ} \mathrm{C}$.

Source: From vessels of elm wood showing dark discoloration, in Holland.

Habitat: Pathogenic in elm wood.

143. Pseudomonas andropogoni (Erw. Smith) Stapp. (Bacterium andropogoni Erw. Smith, Bacteria in Relation to Plant Diseases, 2, 1911, 63; Elliott and Smith, Jour. Agr. Res., 38, 1929, 4; Stapp, in Sorauer, Handbuch der Pflanzenkrankheiten, 2, 5 Auf., 1928, 27; Phytomonas andropogoni Bergey et al., Manual, 3rd ed., 1930, 276.) From M. 
L. Andropogon, a generic name (a synonym of Holcus).

Description from Elliott and Smith (loc. cit.).

Rods: 0.64 by 1.76 microns. Motile with one to several bipolar flagella. Capsules. Gram-negative.

Crelatin: Fecble liquefaction or none.

Beef-extract agar colonies: Slow-growing, round, smooth, glistening, viscid, white.

Broth: Growth slow with moderate turbidity in 48 hours. A thin pellicle.

Milk: Alkaline and clears.

Nitrites not produced from nitrates.

Indole not formed.

$\mathrm{No}_{2} \mathrm{~S}$ formed.

Not lipolytic (Starr and Burkholder, Phytopath., 32, 1942, 601).

Acid but not gas from glucose, arabinose, fructose and xylose. No acid from sucrose, maltose, lactose, raffinose, glycerol and mannitol.

Starch partially digested.

Optimum temperature $22^{\circ}$ to $30^{\circ} \mathrm{C}$. Maximum $37^{\circ}$ to $38^{\circ} \mathrm{C}$. Minimum $1.5^{\circ} \mathrm{C}$.

Optimum pH 6.0 to 6.6. Maximum 8.3 to 8.6. Minimum 5.0.

Source: Elliott used for her description 4 cultures isolated from lesions on sorgo, sorghum and broom-corn.

Habitat: Pathogenic on sorghum, Holcus sorghum.

144. Pseudomonas woodsil (Smith) Stevens. (Bacterium woodsii Erw. Smith, Bact. in Relation to Plant Diseases, 2, 1911, 62; Stevens, Plant Disease Fungi, New York, 1925, 39; Phytomonas wnodsii Bergey et al., Manual, 3rd ed., 1930 , 256.) Named for A. F. Woods, American plant pathologist.

Description from Burkholder and Guterman, Phytopath., 25, 1935, 118.

Rods: 0.67 by 1.56 microns. Motile with a polar flagellum. Gram-negative.

Gelatin: No liquefaction.

Beef-extract agar slants: Growth slow and scant, filiform, creamy, butyrous.

Broth: Turbid.
Milk: Becomes alkaline but otherwise little changed.

Nitrites not produced from nitrates. Indole not formed.

No $\mathrm{H}_{2} \mathrm{~S}$ formed.

Not lipolytic (Starr and Burkholder, Phytopath., 32, 1942, 601).

Aeid but not gas from glucose, fructose, galactose, arabinose, xylose, rhamnose, lactose, glycerol and mannitol. Alkaline reaction from salts of acetic, citric, malic and succinic acids. Sucrose, maltose, salicin, and lactic and formic acids not fermented. Starch not hydrolyzed.

Slight growth in broth plus 3 per cent salt.

Aerobic.

Source: Isolated from water-soaked lesions on carnation leaves.

Habitat: Pathogenic on carnation, Dianthus caryophyllus.

145. Pseudomonas panici-miliacei (Ikata and Yamauchi) comb. nov. (Bacterium panici-miliacei Ikata and Yamauchi, Jour. Plant Protect., 18, 1931, 35; Phytomonas panici-miliacei Burkholder, in Manual, 5th ed., 1939, 204.) Irom M. L. Panicum miliaceum.

Description translated by Dr. IK. Togashi.

Rods : 0.8 to 1.1 by 1.8 to 2.6 microns. Motile, with a single flagellum. Gramnegative.

Gelatin: Not liquefied.

Potato-agar plates: Growth moderate, whitish, then tinged with light orange, undulating margins.

Broth: Turbid, white pellicle formed.

Milk: No coagulation and slow digestion. Alkaline.

Nitrites are produced from nitrates. Indole not formed.

No $\mathrm{H}_{2} \mathrm{~S}$ produced.

No acid and no gas from sucrose, glucose, lactose, glycerol and sodium nitrate.

Starch not hydrolyzed.

Optimum temperature $30^{\circ}$ to $35^{\circ} \mathrm{C}$. 
Facultative anaerobe.

Source: Species first isolated from millet, Panicum miliaceum.

Habitat: Causes a leaf stripe of Panicum miliaceum.

146. Pseudomonas saliciperda Lindeijer. (Lindeijer, Inaug. Diss., Univ. Amsterdam, 1932; Phytopath. Ztschr., 6, 1933, 373; Bacterium saliciperda Burgwitz, Phytopathogenic Bacteria, Leningrad, 1935, 106; Phytomonas saliciperda Magrou, in Hauduroy et al., Dict. d. Bact. Path., Paris, 1937, 408.) From L. salix (salicis), willow; perdo, to destroy.

Rods: 1.2 to 2.1 microns in length. Motile with a polar flagellum. Gramnegative.

Gelatin: No liquefaction.

Beef wort agar colonies: Gray-white.

Milk: No acid nor coagulation.

Nitrites produced (small amount) from nitrates.

Indole formation slight.

No gas from carbohydrates.

Starch not hydrolyzed.

Facultative anaerobe.

Source: Isolated from wilted branches of willow and pathogenicity proved.

Habitat: Pathogenic on willow, Salix spp.

147. Pseudomonas eriobotryae (Takimoto) Dowson. (Bacterium eriobotryae Takimoto, Jour. Plant Protect., 18, 1931, 354 ; Phytomonas eriobotryae Burkholder, in Manual, 5th ed., 1939, 205; Dowson, Trans. Brit. Myeol. Soe., 26, 1943, 10.) From M. L. Eriobotrya, a generic name.

Translated by Dr. K. Togashi.

Rods : 0.7 to 0.9 by 2.2 to 3.0 microns. Motile, with 1 or 2 flagella. Gramnegative.

Gelatin: Not liquefied.

Agar-plates: Colonies appear after 3 days, white or hyaline, butyrous, margins entire.

Broth: Moderately turbid, pellicle powdery, ring formed.
Milk: No coagulation, peptonized slowly. Alkaline.

Nitrites not produced from nitrates.

Indole not formed.

No $\mathrm{H}_{2} \mathrm{~S}$ produced.

No acid or gas from glucose, sucrose, lactose and glycerol in broth.

Starch not hydrolyzed.

Temperature relations: Minimum below $4^{\circ} \mathrm{C}$, optimum $25^{\circ}$ to $26^{\circ} \mathrm{C}$, and maximum $32^{\circ} \mathrm{C}$. Thermal death point $51^{\circ} \mathrm{C}$.

Aerobic.

Source: Species isolated from loquat, Eriobotrya japonica.

Habitat: Causes a bud rot of Eriobotrya japonica.

148. Pseudomonas wieringae (Elliott) comb. nov. (Phytomonas betae Wieringa, Nederl. Tijdschr. Hyg., Microbiol. en Serol., Leiden, 2, 1927, 148; Bacterium wieringae Elliott, Man. Bact. Plant Pathogens, 1930, 264; Phytomonas wieringae Burkholder, in Manual, 5th ed., 1939, 206.) Named for K. L. Wieringa, plant pathologist of Holland.

Because Bacterium bctae Chester (Ann. Rept. Del. Col. Agr. Exp. Sta., 9, 1897, 53) may be a pseudomonad, the species name proposed by Elliott has been retained.

Description from Elliott (loc, cit.).

Rods : 0.5 to 2.0 microns. Motile with 1 to 5 polar flagella. Gram-negative.

Beef-agar colonies: Smooth, round, white to grayish, fluorescent.

Milk: Cleared in 5 days. Not coagulated.

Nitrites not produced from nitrates.

No gas from sugars.

Optimum temperature $28^{\circ}$ to $30^{\circ} \mathrm{C}$.

Maximum $37^{\circ} \mathrm{C}$. Minimum $4^{\circ} \mathrm{C}$.

Source: Isolated from vascular rot of beets in Holland.

Habitat: Pathogenic on beets, Beta vulgaris. 
Appendix I*: The following species are believed to belong in the genus Pseudomonas although descriptions are frequently incomplete.

Achromobacter pellucidum Harrison (Canadian Jour. Res., 1, 1929, 236.) Isolated from halibut. For a description of this species, see Bergey et al., Manual. 5th ed., 1939, 619.

Bacillus aurantiacus tingitanus Remlinger and Bailly. (Maroc Medical, No. 150, 1935; See Lasseur, Dupaix and Babou, Trav. Lab. Microbiol. Fac. Pharm. Nancy, Fasc. 8, 1935, 41.) From water. Dissociates readily. Related to Pseudomonas fluorescens aureus Zimmermann. See p. 615 .

Bacillus cyaneofluorescens 'Langemeister. (Cent. f. Bakt., I Abt., 18, 1895, 321 ; Pseudomonas cyaneofluorescens .Migula, Syst. d. Bakt., 2, 1900, 906.) From blue milk.

Bacillus fluorescens nivalis Eisenber.. (Eine Gletscherbakterie, Schmelck. Cent. f. Bakt., 4, 1888, 545; Eisenberg, Bakt. Diag., 3 Aufl., 1891, 77.) From the melting snow of a glacier. Probably a synonym of Pseudomonas fluorescens.

Bacillus lactis saponacei Weigmann and Zirn. (Cent. f. Bakt., 15, 1894, 468. From soapy milk.

Bacterium auxinophilum Jacobs. (Ann. Appl. Biol., 22, 1935, 619.) A Gram-negative organism with a polar flagellum which liquefies gelatin rapidly.

Bacterium bosporum Kalninš. (Latvijas Ūniversitätes Raksti, Serija I, To. 11, 1930, 259.) Decomposes cellulose. Single polar flagellum. From soil.

Bacterium briosii Pavarino. (Atti Ist. Bot. d. P. Univ. di Pavia, Ser. 2, 12, $1910,337$.$) The natural host is Lyco-$ persicon esculentum. Pavarino (Rev. di Patol. Veg., 6, 1913, 161) states that this organism and Phytobacter lycopersicum Groenewege (Meded. Pijks. Hoogere Land, Tuin- en Boschbourschool, Dell 5, $5,1912,217$ ) should be considered identi- cal. It closely resembles Bacterium vesicatorium Doidge (Jour. Dept. Agr. So. Africa, 1, 1920, 718) according to Gardner and Kendrick (Jour. Agr. Res., 21, 1921, 140).

Bacterium elaphorum KaIninšs. (Latvijas Üniversitātes Raksti, Serija I, No. 11, 1930, 257.) Decomposes cellulose. Single polar flagellum. From soil.

Bacterium fraentelii Hashimoto. (Zeit. f. Hyg., 31, 1899, 88.) A pleomorphic polar flagellate bacterium. From milk.

Bacterium gummis Comes. (Comes, Napoli, Maggio 18, 1884, 14; see Comes, Atti d. R. Ist. d'incoraggiamento alli Sc., Ser. 3, 3, 1884, 4; Bacillus gummis Trevisan, I generi e le specie delle Batteriacee, Milano, 1889, 17.) Pathogenic on grapes, Vitis spp.

Bacterium kraneriani Pavarino. (Atti R. Accad. Naz. Lincei Rend. Cl. Sci. Fis., Nat. et Nat., 20, 1911, 233.) Pathogenic on the orchid, Oncidium krameriani.

Bacterium pusiolum Kalnin̦s. (Latvijas Ūniversitātes Raksti, Serija I, †o. 11, 1930, 261.) Decomposes celluInse. Single polar flagellum. From manure.

Bacterium protozoides Kalninšs. (Latvijas Universitätes Raksti, Serija I, No. 11, 1930, 263.) Decomposes cellulose. Single polar flagellum. From soil.

Pseudomonas acuta Migula. (Culture Yo. 11, Lembke, Arch. f. Hyg., 29, 1897, 317 ; Migula, Syst. d. Bakt., 2, 1900, 921.) From the intestine.

Pseudomonas alba Migula. (Bacillus fluorescens albus Zimmermann, Bakt. unserer Trink- u. Nutzwässer, I Reihe, 1890, 18; Migula, Syst. d. Bakt., 2, 1900, 909.) From water. Bacillus fuorescens non liquefaciens Eisenberg, Bakt. Diag., 3 Aufl., 1891, 145 may be identical according to Migula (loc. cit.).

Pseudomonas allii (Griffiths) Migula. (Bacterium allium Griffiths, Proc. Roy. Soc. Edinburgh, 51, 1857, 40; Migula.

* Appendixes I and II prepared by Prof. Robert S. Breed, New York State Experiment Station, Geneva, New York, July, 1943. 
Syst. d. Bakt., 2, 1900, 932.) From rotted onions.

Pseudomonas aquatilis Migula. (Tataroff, Inaug. Diss., Dorpat, 1891, 31; Migula, Syst. d. Bakt., 2, 1900, 933.) From water. Said to form spores.

Pseudomonas aromatica Migula. (Bacillus crassus aromaticus Tataroff, Inaug. Diss., Dorpat, 1891, 27; Migula, Syst. d. Bakt., 2, 1900, 880.) From water.

Pseudomonas aromatica var. quercitopyrogallica Kluyver, Hof and Boezaardt. (Enzymologia, 7 1939, 28.)

Pseudomonas articulata Kern. (Arb. bakt. Inst. Karlsruhe, 1, Heft 4, 1896, 462.) From the stomachs of birds.

Pseudomonas aucubicola Trapp. (Phytopath., 26, 1936, 264.) Isolated from Aucuba japonica. Tot pathogenic.

Pseudomonas aurea Migula. (Bacillus fluorescens aureus Zimmermann, Bakt. unserer Trink- u. Nutzwässer, I Reihe, 1890, 14; Migula, Syst. d. Bakt., 2, 1900 , 931.) From water.

Pseudomonas brassicae acidae Gruber. (Cent. f. Bakt., II Abt., 22, 1909, 558). From sauerkraut. Identical with Bacterium brassicae acidae Conrad (Arch. f. Hyg., 29, 1897, 75) according to Lehmann and Neumann (Bakt. Diag., 5 Aufl., 2 1912, 380.)

Pseudomonas butyri Migula. (Bacillus butyri fluorescens Lafar, Arch. f. Hyg., 13, 1891, 19 ; Migula, Syst. d. Bakt., 2, 1900, 894.) From butter.

Pseudomonas calciphila Molisch. (Cent.f. Bakt., II Abt., 65, 1925, 136.) From fresh water. Deposits $\mathrm{CaCO}_{3}$.

Pseudomonas calco-acetica Clifton. (Enzymologia, 4, 1937, 246.)

Pseudomonas capsulata Migula. (Syst. d. Bakt., 2, 1900, 915; not Pseudomonas capsulata Bergey et al., Manual, 1st ed., 1923, 124.) Similar to Pseudomonas macroselmis Migula.

Pseudomonas caryocyanea (Dupaix) Beijerinck. (Bacillus caryocyaneus Dupaix, Thesis, Univ. of Nancy, 1933, 1; Beijerinck, see Dupaix, ibid., 13; Bac- terium caryocyaneum Dupaix, ibid., 246.) Isolated from rotten willow wood, from yeast mash and beer-wort. Name appears first as Bacillus caryocyaneus on a culture sent by Beijerinck from Delft, Holland to the National Collection of Type Cultures, Lister Institute, London. Pegarded by Dupaix as closely related to Bacillus cyaneo-fluorescens Zangemeister (Cent. f. Bakt., I Abt., 18, 1895, 321; Pseudomonas cyaneo-fluorescens Migula, Syst. d. Bakt., 2, 1900, 906); Der blaue Bacillus, Mildenberg (Cent. f. Bakt., II Abt., 56, 1922, 309; Pseudomonas mildenbergii Bergey et al., Manual, 3rd ed., 1930, 172); and Bacillus pyocyaneus Gessard (Compt. rend. Acad. Sci. Paris, 94, 1882, 536).

Pseudomonas catarrhalis Chester. (Bacillus der Hundestaupe, Jess, Cent. f. Bakt., II Abt., 25, 1899, 541; Chester, Man. Determ. Bact., 1901, 308.) Isolated from catarrh in dogs.

Pseudomonas caviae Scherago. (Jour. Bact., 31, 1936, 83; Jour. Inf. Dis., 60, $1937,245$.$) Cause of an epizootic septi-$ cemia in guinea pigs.

Pseudomonas chlorophacna Migula. (Syst. d. Bakt., 2, 1900, 899.)

Pseudomonas coccacea Migula. (Culture No. 10, Lembke, Arch. f. Hyg., 29, 1897, 317; Migula, Syst. d. Bakt., 2, 1900, 924.) From the intestine.

Pseudomonas cohaerea (sic) (Wright) Chester. (Bacillus cohaerens Wright, Mem. Nat. Acad. Sci., 7, 1895, 464; Chester, Man. Determ. Bact., 1901, 312.) From water.

Pseudomonas coli Migula. (Culture No. 8, Lembke, Arch. f. Hyg., 29, 1897, 315; Migula, Syst. d. Bakt., 2, 1900, 920.) From the intestine.

Pseudomonas colloides Migula. (Bacillus fuorescens putidus colloides Tataroff, Inaug. Diss., Dorpat, 1891, 40; Migula, Syst. d. Bakt., 2, 1900, 902.) From water. Said to form spores.

Pscudomonas conradi Lehmann and Neumann. (Bakt. Diag., 5 Aufl., 2, 1912, 394.) Red pigment. 
Pseudomonas delabens (Wright) Chester. Bacillus delabens Wright, Nem. Nat. Acad. Sci., 7, 1895, 456; Chester, Man. Determ. Bact., 1901, 314.) From water.

Pseudomonas duplex Migula. (Culture No. 7, Lembke, Arch. f. Hyg., 29, 1897, 314; Migula, Syst. d. Bakt., 2, 1900, 922.) From the intestine.

Pseudomonas ellipsoidea Migula. (Bacillus oogenes fluorescens $\beta$, Zörkendörfer, Arch. f. Hyg., 16, 1893, 393; Migula, Syst. d. Bakt., 2, 1900, 925.) From hens' eggs.

Pseudomonas ephemerocyanea Fuller and Norman. (Jour. Bact., 46, 1943, 274.) From soil. Decomposes cellulose.

Pseudomonas erythra Fuller and Norman. (Jour. Bact., 46, 1943, 276.) From soil. Decomposes cellulose.

Pseudomonas erythrospora (Cohn) Migula. (Bacillus erythrosporus Cohn, Beitr. z. Biol. d. Pfanzen, 9, Heft 1, 1879, 12s; Migula, in Engler and Prantl, Die naütrl. Pflanzenfam., 1, 1a, 1895, 29.) From air, meat infusion and water. Said to form spores.

Pseudomonas fimbriata (Wright) Chester. (Bacillus fimbriatus Wright, Mem. Nat. Acad. Sci., \%, 1s95, 163; Chester, Man. Determ. Bact., 1901, 313.) From water.

Pseudomonas fluorescens cxitiosus van Hall. (Ztschr. f. Pflanzenkr., 19, 1903, 132.) Causes soft rot of shoots and bulbs of iris (Iris spp.).

Pseudomonas foliacea Chester. (Bacillus fluorescens foliacens Wright, Mem. Nat. Acad. Sci., $\tilde{\gamma}, 1895,439$; Chester, Man. Determ. Bact., 1901, 324; Bacillus fluorescens-foliaccus Chester, ibid.) From water. Very similar to Pseudomonas incognita Chester.

Pseudomonas gasoformans Migula. (Ein neuer gasbildender Bacillus, Gärtner, Cent. f. Bakt., 15, 1894, 1; Migula, Syst. d. Bakt., 2, 1900, 883.) Gas bubbles in gelatin stab.
Pscudomonas gracilis Migula. (Syst. d. Bakt., \&, 1900, 888.) Morphologically like Pseudomonas fluorescens Migula.

Pseudomonas granulata Kern. (Arb. bakt. Inst. Karlsruhe, 1, Heft 4, 1896, 464.) From the stomach and intestine of birds.

Pseudomonas halestorgus ElazariVolcani. (Ph.D. Thesis, Hebrew Univ., Jerusalem, 1940.) A halophilic pseudomonad from the Dead Sea.

Pseudomonas hydrosulfurea Migula. (Bacillus oogenes hydrosulfureus $\beta$, Zörkendörfer, Arch. f. Hyg., 16, 1893, 385; Migula, Syst. d. Bakt., 2, 1900, 898.) From hens' eggs.

Pseudomonas iridis van Hall. (Van Hall, Thesis, Univ. Amsterdam, 1902 and Ztsehr. f. Pflanzenkr., 13, 1903, 129; Bacterium iridis Elliott, Man. Bact. Plant Path., 1930, 142; Pliytomonas iridis Magrou, in Hauduroy et al., Dict. d. Bact. Path., Paris, 1937, 369.) Causes a rot of bulbs and leaves of iris (Iris spp.).

Pseudomonas iris (Frick) Migula. (Bacillus iris Frick, Arch. f. path. Anat., 116, 1889, 292; according to Eisenberg, Bakt. Diag., 3 Aufl., 1891, 148; Migula, Syst. d. Bakt., 2, 1900, 931.)

Pseudomonas italica (Fò and Chiapella) Reinelt. (Quoted from Lehmann and Neumann, Bakt. Diag., 7 Aufl., 2, 1927, 367.) Phosphorescent.

Pseudomonas jaranica (Eijkmann) Migula. (Photobacterium javanense Eijkmann, Gencesk. Tijdschr. v. Nederl.-Indië, 32, 1892, 109; Abst. in Cent. f. Bakt., 12, 1892, 656; Bacillus jaraniensis Dyar, Annals New York Acad. Sci., 8, 1895, 359; Bacterium jaraniensis Chester, Man. Determ. Bact., 1901, 170; Photobacterium javanicum Lehmann and Neumann, Bakt. Diag., 1 Aufl., 2, 1896, 199; Migula, Syst. d. Bakt., Q, 1900, 953.) From sea fish in Java. Blue-green Iuminescence.

Psendomonas lactica Woiss. (Arb. bakt. Inst. Karlsruhe, 2, Heft 3, 1902, 238.) Fronil a vegetable infusion.

Pseudomonas lasia Fuller and Torman. 
(Jour. Bact., 46, 1943, 275.) From soil Decomposes cellulose

Pseudomonas lembkei Migula. (Culture No. 12, Lembke, Arch. f. Hyg., 29 1897, 318; Migula, Syst. d. Bakt., 2, 1900, 896.) From the intestine.

Pseudomonas liquefaciens (Tataroff) Migula. (Bacillus liquefaciens Tataroff, Inaug. Diss., Dorpat, 1891, 29; Migula, Syst. d. Bakt., 2, 1900, 876.) From water

Pseudomonas listeri Weiss. (Arb. bakt. Inst. Karlsruhe, 2, Heft 3, 1902, 260. From a vegetable infusion.

Pseudomonas longa Migula. (Bacillus fluorescens longus Zimmermann, Bakt. unserer Trink- u. Nutzwässer, I Reihe, 1890, 20; Migula, Syst. d. Bakt.. 2, 1900, 907.) From water.

Pseudomonas macroselmis Migula. (Bacillus fuorescens putidus Tataroff, Inaug. Diss., Dorpat, 1891, 42; Migula, in Engler and Prantl, Die natürl. Pflanzenfam., 1, 1a, 1895, 29.) From water.

Pseudomonas maidis (Eisenberg) Migula. (Bacillus maidis Eisenberg, Bakt. Diag., 3 Aufl., 1891, 119; Migula, Syst. d. Bakt., 2, 1900, 877.) From corn grains soaked in water and from feces of pellagra patients.

Pseudomonas maschekii Migula. (Blaugrüner Bacillus, Maschek, Bakt. Untersuch. d. Leitmeritzer Trinkwasser, Jahresber. d. Oberrealschule zu Leitmeritz, 1887; Migula, Syst. d. Bakt. 2, 1900, 916.) From water.

Pseudomonas melochlora (Winkler and Schrötter) Migula. (Bacillus melochloros Winkler and Schrötter, Ein neuer grünen Farbstoff entwickelnder Bacillus, Wien, 1890; Migula, Syst. d. Bakt., 2, 1900, 893.) From caterpillar feces.

Pseudomonas mesenterica Migula. (Bacillus fluorescens mesentericus Tataroff, Inaug. Diss., Dorpat, 1891, 38; Migula, Syst. d. Bakt., 2, 1900, 903.) From water.

Pseudomonas metalloides Migula: (Bacillus rosaceus metalloides Tataroff, Inaug. Diss., Dorpat, 1891, 65; not Bacterium rosaceus metalloides Dowdeswell, Ann. de Microgr., 1, 1888-89, 310, see
Heffernan, C'ent. f. Bakt., II Abt., 8, 1902, 689; Pseudomonas rosacea Migula, in Engler and Prantl, Die natürl. Pflanzenfam., 1, 1a, 1895, 29; Migula, Syst. d. Bakt., 2, 1900, 938.) Single flagellum. Red and yellow-red pigment. From water.

Pseudomonas minutissima Migula. (Bacillus fluorescens liquefaciens minutissimus Unna and Tommasoli, Monatsh. f. prakt. Dermat., 8, 1889, 57; according to Eisenberg, Bakt. Diag., 3 Aufl., 1891, 76; Migula, Syst. d. Bakt., 2, $1900,891$.$) Found on human skin in$ cases of seborrhoic eczema.

Pseudomonas mobilis Migula. (Culture No. 9, Lembke, Arch. f. Hyg., 29, 1897, 316; Migula, Syst. d. Bakt., 2, 1900,923 .) From the intestine.

Pseudomonas monadiformis (Kruse) Chester. (Bacillus coli mobilis Messea, Riv. d'Igiene, Rome, 1890; Bacillus monadiformis Kiruse, in Flügge, Die Mikroorganismen, 2, 1896, 374; Chester, Man. Determ. Bact., 1901, 308.) From typhoid stools.

Pseudomonas mucidolens Levine and Anderson. (Jour. Bact., 23, 1932, 337.) Causes musty odors in eggs. Also milk (Olsen and Hammer, Iowa State Coll Jour. Sci., 9, 1934, 125).

Pseudomonas mucidolens var. tarda Levine and Anderson. (Jour. Bact., 23, $1932,337$.$) Causes musty odors in eggs.$ Pseudomonas nexibilis (Wright) Chester. (Bacillus nexibilis Wright, Mem. Nat. Acad. Sci., 7, 1895, 441; Chester, Man. Determ. Bact., 1901, 309.) From water.

Pseudomonas nivalis Szilvinyi. (Cent. f. Bakt., II Abt., 94, 1936, 216.) A red chromogen isolated from red snow in Austria.

Pseudomonas ochroleuca Migula. (Bacillus $\gamma$, Zörkendörfer, Arch. f. Hyg., 16, 1893, 396; Migula, Syst. d. Bakt., 2, 1900, 897.) From hens' eggs.

Pseudomonas oogenes Migula. (Bacillus oogenes hydrosulfureus $\delta$, Zörkendörfer, Arch. f. Hyg., 16, 1893, 386; 
Migula, Syst. d. Bakt., 2, 1900, 878.) Single flagellum. From hens' eggs.

Pseudomonas ovi Migula. (Bacillu. oogenes fluorescens $\epsilon$, Zörkendörfer, Arch . f. Hyg., 16, 1893, 395; Migula, Syst.d. Bakt., 2, 1900, 924.) From hens' eggs. Pseudomonas ovicola Migula. (Bacillus oogenes fluorescens $\gamma$, Zörkendörfer, Arch. f. Hyg., 16, 1893, 394; Migula, Syst. d. Bact., 2, 1900, 925.) From hens' eggs.

Pseudomonas pallescens Migula. (Bacillus viridis pallescens Frick, in Virchow, Arch. f. path. Anat., 116, 1889, 292 ; according to Eisenberg, Bakt. Diag. 3 Auf., 1891, 154; Migula, Syst. d. Bakt. 2, 1900, 927.) Source not given.

\section{Pseudomonas pansinii Migula.}

(Bacillus fluorescens non liquefaciens Pansini, in Virchow, Arch. f. path. Anat. 122, 1890, 452; Migula, Syst.d. Bakt., 2, $1900,926$.

Pseudomonas pelliculosa Migula. (Bacillus oogenes fluorescens $\delta$, Zörkendörfer, Arch. f. Hyg., 16, 1893, 395 ; Migula, Syst. d. Bakt., 2, 1900, 926.) From hens' eggs.

Pseudomonas pellucida Kern. (Arb). bakt. Inst. Karlsruhe, 1, Heft 4, 1896. 465.) From the intestine of birds.

Pseudomonas plehniae Spieckermann and Thienemann. (Arch. f. Hyg., 74, 1911, 110.) Isolated from carp. Pathogenic for many species of fish.

Pseudomonas plicata (Frankland and Frankland) Migula. (Bacillus plicatus Frankland and Frankland, Phil. Trans. Roy Soc. London, 178, B, 1857, 273: Migula, Syst. d. Bakt., 2, 1900, 881.) From air.

Pseudomonas pseudotyphosa Migula. (Typhusähnlicher Bacillus, Lustig, Diag. d. Bakt. d. Wassers, 1893, 16; Migula, Syst. d. Bakt., 2, 1900, 893.) From water

\section{Pseudomonas pullulans (Wright)}

Chester. (Bacillus pullulans Wright, Mem. Nat. Acad. Sci., 7, 1894, 445; Chester, Man. Determ. Bact,, 1901, 315.) From water.
Pseudomonas protea Frost. (U. S. Public Health Ser., Hyg. Lab. Bull. 66, $1910,27$.$) From filtered river water.$ Can be agglutinated by specific typhoid immune-serum.

Pseudomonas rosea Chester. (Bacillus roseus vini Bordas, Joulin and Rackowski, Compt. rend. Acad. Sci. Paris, 126, 1898, 1550; Chester, Man. Determ. Bact., 1901, 327; not Pseudomonas rosea Migula, in Engler and Prantl, Die natürl. Pfllanzenfam., 1, 1a, 1895, 30.) From wine. Said to form spores.

Pseudomonas (Hydrogenomonas) sac charophila Doudoroff. (Enzymologia, 9, 1940, 50.) From stagnant water.

Pseudomonas sapolactica (Eichholz) De Rossi. (Bacterium sapolacticum Eichholz, Cent. f. Bakt., II Abt., 9, 1902, 631; De Rossi, Microbiologia Agraria e Technica, Torino, 1927, 693.) Isolated from soapy milk.

Pseudomonas sericea Migula. (Seidenglänzender Bacillus, Tataroff, Inaug. Diss., Dorpat, 1891, 26; Migula, Syst. d. Bakt., 2, 1900, 882.)

Pseudomonas tenuis Migula. (Bacillus fluorescens tenuis Zimmermann, Bakt . unserer Trink- u. Nutzwässer, I Reihe. 1890, 16; Migula, Syst. d. Bakt., 2. 1900, 910.) From water.

Pscudomonas trommelschlägel (Ravenel) Chester. (Bacillus trommelschlägel Ravenel, Mem. Nat. Acad. Sci., 8, 1896 , 26; Chester, Man. Determ. Bact., 1901, 327.) From soil. Said to form spores.

Pseudomonas undulala Chester. (Bacillus fluorescens undulatus Parenel, Mem. Nat. Acad. Sci., \&, 1896, 20; Chester, Man. Determ. Bact., 1901, 328.) From soil. Said to form spores.

Pseudomonas virescens (Frick) Migula. (Bacillus virescens Frick, Arch. f. path. Anat., 116, 1889, 292; Migula, Syst.d. Bakt., 2, 1900,916.) From green sputum. Pseudomonas viridans (Symmers) Migula. (Bacillus viridans Symmers, Brit. Med. Jour., No. 1615, 1891, 1252; Abst. in Cent. f. Bakt., 12, 1892, 165; Migula, Syst. d. Bakt., 2, 1900, 890.) From water. 
Pscudomonas viridescens Chester. (Bacillus viridescens liquefaciens Ravenel, Mem. Nat. Acad. Sci., 8, 1896, 24; Chester, Man. Determ. Bact., 1901, 328.) From soil. Said to form spores.

Pseudomonas viridis Migula. (Bacillis der grünen Diarrhöe der Kinder, Lesage, Arch. d. Physiol, norm. et path., 20, 1858, 212; see Eisenberg, Bakt. Diag., 3 Aufl., 1891. 238; Migula, Syst. d. Bakt., 2, 1900, 856.) From intestine of children.

Pseudomonas weigmanni Migula. (Bakterie IV, Weigmann and Zirn, Cent. f. Bakt., 15, 1894, 466; Migula, Syst.d. Bakt,, 2, 1900, 892.) From soapy milk.

Pseudomonas zörkendörferi Migula. (Bacillus oogenes fluorescens $\alpha$, Zörkendörfer, Arch. f. Hyg., 16, 1893, 392; Migula, Syst. d. Bakt., 2, 1900, 897.) From hens' eggs.

Appendix II : The following polar flagellate organism has been described from activated sludge. H. Winogradsky has also described polar flagellate forms from the same source that form zoogloea (Compt. rend. Acad. Sei. Paris, 200,
1035, 1887; Ann. Inst. Pasteur, 58, 1937, $333)$.

Zoogloea ramigera Kruse emend. Butterfield. (Kruse, in Flügge, Die Mikroorganismen, 3 Aufl., 1, 1896, 68; Butterfield, Public Health Reports, 50, 1935, 671; Culture No. 50, Wattie, Pub. Health Reports, 5\%, 1942, 1519.)

Rods: 1 by 2 to 4 microns, with rounded ends. Non-spore-forming. Capsules present. Motile with a single long polar flagellum. Gram-negative.

Gelatin: No liquefaction.

Grows better in aerated liquid media. Agar: Scant growth.

Indole not formed.

No $\mathrm{H}_{2} \mathrm{~S}$ produced.

No acid or gas from curbohydrates.

Nitrites not produced from nitrates.

Optimum pH 7.0 to 7.4 .

Optimum temperature $28^{\circ}$ to $30^{\circ} \mathrm{C}$. Good growth at $20^{\circ}$ and at $37^{\circ} \mathrm{C}$. Minimum temperature $4^{\circ} \mathrm{C}$.

Strict aerobe.

Distinctive character: Oxidizes sewage. Source: Isolated from activated sludge. Habitat: Produces zoogloeal masses in activated sludge.

\section{Genus II. Xanthomonas Dowson*}

(Phytomonas Bergey et al., Manual, 1st ed., 1923, 17t; Dowson, Cent. f. Bakt., II Abt,, $100,1939,187$.

Cells usually monotrichous, with yellow, water-insoluble pigment. Proteins are usually readily digested. Milk usually becomes alkaline. Hydrogen sulfide is pro. duced. Asparagin is not sufficient as an only source of earbon and nitrogen. Acid is produced from mono- and disaceharides. Mostly plant pathogens causing necrosis. From Gr. xanthus, yellow; monas, a unit; M. L. monad.

The type species is Xanthomonas hyacinthi (Wakker) Dowson.

\section{Key to the species of genus Xanthomonas.}

1. Colonies yellow.

a. Gelatin liquefied.

b. Starch hydrolysis feeble.

c. Nitrites not produced from nitrates.

1. Xanthomonas hyacinthi.

2. Xanthomonas pruni.

3. Xanthomonas vitians.

* Prepared by Prof. Walter H. Burkholder, Cornell Univ., Ithaca, N. Y., June, 1943. 
cc. Nitrites produced from nitrates.

4. Xanthomonas beticola.

5. Xanthomonas lactucae-scariolac.

6. Xanthomonas rubrilineans.

bb. Starch hydrolysis strong.

c. Nitrites not produced from nitrates.

d. No brown pigment in beef-extract agar.

7. Xanthomonas barbareae.

8. Xanthomonas begoniac.

9. Tanthomonas campestris.

9a. Xanthomonas campestris var. armoraciae.

10. Xanthomonas citri.

11. Xanthomonas corylina.

12. Xanthomonas cucurbitae.

13. Xanthomonas dieffenbachiae.

14. Xanthomonas holcicola.

15. Xanthomonas incanae.

16. Xanthomonas juglandis.

17. Xanthomonas lespedezae.

18. Xanthomonas malvacearum.

19. Xanihomonas pelargonii.

20. Xanthomonas phaseoli.

20a. Xanthomonas phaseoli var. sojense.

21. Xanthomonas plantaginis.

22. Xanthomonas ricinicola.

23a. Xanthomonas transtucens f. sp. hordci.

23b. Xanthomonas transtucens f. sp. undulosa.

23e. Xanthomonas translucens f. sp. secalis.

23d. Xanthomonas translucens f. sp. hordei-avenae.

23e. Xahthomonas translucens f. sp. cerealis.

24. Xanthomonas vasculornm.

25. Tanthomonas resicatoria.

25a. Xanthomonas vesicatoria var. raphani.

dd. Brown pigment produced in beef-extract media.

26. Xanthomonas nakatac.

20b. Xanthomonas phaseoli var. fuscans.

cc. Nitrites produced from nitrates.

27. Xanthomonas papavcricola.

ccc. Ammonia formed in nitrate media.

28. Xanthomonas alfalfae.

bbb. Starch not hydrolyzed.

c. Nitrites produced from nitrates.

29. Xanthomonas acernae.

cc. Nitrites not produced from nitrates.

30. Xanthomonas carotae.

31. Xanthomonas hederae.

32. Tanthomonas phormicola.

25. Xanthomonas vesicatoria.

ccc. Ammonia formed in nitrate media.

33. Xanthomonas geranii. 
bbbb. Starch hydrolysis not reported.

c. Nitrites produced from nitrates.

34. Xanthomonas antirrhini.

35. Xanthomonas heterocea.

ce. Nitrites not produced from nitrates.

36. Xanihomonas gummisudans.

37. Xanthomonas lactucae.

38. Xanthomonas nigromaculans.

aa. Gelatin not liquefied.

b. Starch not hydrolyzed.

39. Xanthomonas oryzae.

aaa. Gelatin not reported.

b. Starch hydrolyzed.

40. Xanthomonas celebensis.

2. Colonies whitish to cream.

a. Gelatin liquefied.

b. Starch hydrolyzed.

c. Nitrites produced from nitrates.

41. Xanthomonas panici.

42. Xanthomonas proteomaculans.

43. Xanthomonas manihotis.

ce. Nitrites not reported.

44. Xanthomonas rubrisubalbicans.

bb. Starch not reported.

45. Xanthomonas cannae.

46. Xanthomonas zingiberi.

47. Xanthomonas conjaci.

1. Xanthomonas hyacinthi (Wakker) Dowson. (Bacterium hyacinthi Wakker, Botan. Centralblatt, 14, 1883, 315; Bacillus hyacinthi Trevisan, I generi e le specie delle Batteriacee, $1889 ; 19 ;$ P seudomonas hyacinthi Erw. Smith, Bot. Gazette, 24, 1897, 188; Phytomonas hyacinthi Bergey et al., Manual, 1st ed., 1923, 177; Dowson, Cent. f. Bakt., II Abt., 100, 1939, 188.) From Gr. hyacinthus, the hyacinth; M. L. Hyctcinthus, a generic name.

Description from Smith, Div. Veg. Phys. and Path., U. S. D. A. Bul. 26, 1901, 40 .

Rods: 0.4 to 0.6 by 0.8 to 2 microns. Motile with a polar flagellum. Filaments present. Gram-negative.

Gelatin: Slow liquefaction.

Agar colonies: Circular, flat, moist, shining, bright yellow. Media stained brown.
Milk: Casein is precipitated and digested. Tyrosine crystals produced.

Nitrites not produced from nitrates.

Indole: Slight production.

Hydrogen sulfide is produced.

Acid, no gas, from glucose, fructose, galactose, sucrose and maltose.

Starch: Hydrolysis slight.

Optimum temperature $28^{\circ}$ to $30^{\circ} \mathrm{C}$. Maximum $34^{\circ}$ to $35^{\circ} \mathrm{C}$. Minimum $4^{\circ} \mathrm{C}$.

Aerobic, with the exception of maltose, where it is facultative anaerobic.

Habitat: Produces a yellow rot of hyacinth bulbs, Hyacinthus orientalis.

\section{Xanthomonas pruni (Erw. Smith)} Dowson. (Pseudomonas pruni Erw. Smith, Science, N. S. 17, 1903, 456; Bacterium pruni Erw. Smith, Bacteria in Relation to Plant Dis., 1, 1905, 171; Bacillus pruni Holland, Jour. Bact., 5, 1920, 220; Phytomonas pruni Bergey et 
al., Manual, Ist ed., 1923, 179; Dowson, Cent. f. Bakt., II Abt., 100, 1939, 190.) From L. prunus, plum; M. L. Prunus, a generic name.

Probable synonym: Phytomonas cerasi wraggi Sackett, Col. Agr. Exp. Sta. Rept., 38, 1925, 17; Pseudomonas cerasi wraggi, ibid.; Bacterium cerasi wraggi Elliott, Bact. Plant Pathogens, 1930, 111.

Description from Dunegan, U.S. Dept. Agr., Tech. Bull. 273, 1932, 23.

Rods : 0.2 to 0.4 by 0.8 to 1.0 microns. Capsules. Motile with a polar flagellum. Gram-negative.

Gelatin: Liquefaction.

Beef-extract agar colonies: Yellow, circular, smooth, convex, edges entire.

Broth: Turbid becoming viscid.

Milk: Precipitation of casein and digestion.

Nitrites not produced from nitrates. Indole not formed.

Hydrogen sulfide not produced. Hy drogen sulficle produced (Burkholder).

Lipolytic (Starr and Burkholder, Phytopath., 32, 1942, 600).

Acid from arabinose, xylose, glucose, fructose. galactose, mannose, maltose, lactose, sucrose, raffinose, melezitose.

Starch is hydrolyzed (slight).

Aerobic.

Optimum temperature $24^{\circ}$ to $29^{\circ} \mathrm{C}$. Maximum $37^{\circ} \mathrm{C}$.

Source: Smith isolated the pathogen from Japanese plums.

Habitat: Pathogenic on plum (Prunus salicina), peach ( $P$. persica), apricot ( $P$. armenicca), etc.

3. Xanthomonas vitians (Brown) Starr and Weiss. (Bacterium vitians Brown, Jour. Agr. Res., 13, 1918, 379; Phytomonas vitians Bergey et al., Manual, 1st ed., 1923, 183; Pseudomonas vitians Stapp. in Sorauer, Handb. d. Pflanzenkrank., 2, 5 Aufl., 1928, 2S7; Starr and Weiss, Phytopath., 89, 1943, 316.) From Latin, iitians, injuring, infecting:

Rods: Motile with bipolar flagella. Gram-negative.
Gelatin: Slow liquefaction.

Beef-extract agar colonies: Circular, smooth, thin, cream to cream-yellow.

Broth: Turbid with yellow ring.

Milk: Clears and turns alkaline.

Nitrites not produced from nitrates.

Indole: Feeble production.

Hydrogen sulfide: Feeble production.

Acid but not gas from glucose.

Starch: Feeble hydrolysis.

Optimum temperature $26^{\circ}$ to $28^{\circ} \mathrm{C}$. Maximum $35^{\circ} \mathrm{C}$. Minimum $0^{\circ} \mathrm{C}$.

Aerobic.

Source: Isolated from the stem of diseased lettuce plants from South Carolina.

Habitat: Pathogenic on Iettuce, Lactuca sativa.

4. Xanthomonas beticola (Smith, Brown and Townsend) comb. nov. (Bacterium beticolum Smith, Brown and Tornsend, C. S. Dept. Agr., Bur. Plant Ind., Bul. 213, 1911, 194; Pseudomonas beticola Holland, Jour. Bact., 5, 1920, 224; Phytomonas beticola Bergey et al., Manual, 1st ed., 1923, 182.) From Latin, beta, beet; -cola, dweller.

Description from Brown, Jour. Agr. Res., 37, 192S, 167, where the species is referred to as Bacterium beticola (Smith, Brown and Townsend) Potebnia.

Pods : 0.4 to 0.8 by 0.6 to 2.0 microns. Motile with 1 to 4 polar flagella. Capsules. Gram-variable.

Gelatin: Liquefaction.

Beef-agar slants: Moderate filiform growth, flat, glistening, yellow:

Broth: Turbid, yellow ring, abundant sediment.

Milk: Coagulation and peptunization.

Indole not formed.

Hydrogen sulfide formed.

Nitrites produced from nitrates.

Acid from gluc'ose, sucrose, maltose, mannitol. No acid from lactose.

Stareh hydrolysis feeble.

Optimum temperature $29^{\circ} \mathrm{C}$. Maximum $39^{\circ} \mathrm{C}$. Minimum $1.5^{\circ} \mathrm{C}$. 
Optimum pH 6.5. Maximum 9.0 to 9.5. Minimum 4.5 to 4.8 .

Tolerates salt up to 9 per cent.

Aerobic.

Source: Isolated from galls on sugar beets collected in Colorado, Kansas, and Virginia.

Habitat: Produces gall on sugar beets and on garden beets.

Note: It is doubtful whether this species belongs in this geuus.

5. Xanthomonas lactucae-scariolae (Thornberry and Anderson) comb. nov. (Phytomonas lactucae-scariolae Thornberry and Anderson, Phytopath., 27, 1937, 109.) From Lactuca scariola, the host.

Rods : 0.5 to 1.0 by 1.0 to 1.5 microns. Motile with 1 or 2 polar flagella. Chains present. Capsules. Gram-negative.

Gelatin: Slow liquefaction.

Glucose agar colonies: Round, entire, finely granular, amber yellow.

Broth: Turbid. No pellicle. A yellow rim.

Milk: Slight acid, and peptonization.

Nitrites are produced from nitrates.

Hydrogen sulfide not formed.

No gas from carbon sources.

Starch: Slight diastatic activity.

Optimum temperature $25^{\circ} \mathrm{C}$. Maximum $35^{\circ} \mathrm{C}$. Minimum $7^{\circ} \mathrm{C}$.

Aerobic.

Source: Isolated from necrotic lesions on wild lettuce.

Habitat: Pathogenic on wild lettuce, Lactuca scariola, but not on cultivated lettuce, Lactuca sativa.

6. Xanthomonas rubrilineans (Lee et al.) Starr and Burkholder. (Phytomonas rubrilineans Lee, Purdy, Barnum and Martin, Hawaiian Sugar Planters' Assoc. Bull., 1925, 25; Pseudomonas rubrilineans Stapp, in Sorauer, Handb. d. Pflanzenkrank., 2, 5 Aufl., 1928, 35; Bacterium. rubrilineans Elliott, Man. Bact. Plant Path., 1930, 195; Starr and Burkholder, Phytopath., 32, 1942, 600.) From L. ruber, red; lineans, striping.
Rods: 0.7 by 1.67 microns. Motile with 1 or seldom more polar flagella. Gram-negative.

Gelatin: Liquefaction.

Agar (Beef-extract + glucose) colonies: Small, smooth, glistening, buff to yellow.

Broth: Turbid with pellicle. Sediment.

Milk: Casein precipitated and digested.

Nitrites are produced from nitrates.

Indole not produced.

Hydrogen sulfide not formed.

Not lipolytic (Starr and Burkholder, Phytopath., 32, 1942, 600).

Acid from glucose, fructose, arabinose, xylose, lactose, sucrose, raffinose and mannitol.

Starch: Slight hydrolysis.

Growth range, $\mathrm{pH} 5.4$ to $\mathrm{pH} 7.3$.

Facultative anaerobe.

Source: Description from 3 cultures isolated from the red stripe lesions in sugar cane.

Habitat: Pathogenic on sugar cane.

7. Xanthomonas barbareae Burkholdes. (Burkholder, Phytopath., 31, 1941, 348 . Phytomonas barbareae Burkholder, ibid.) From M. L. Barbarea, a generic name.

Rods : 0.4 to 0.95 by 1.0 to 3.15 microns. Motile with a single polar flagellum. Gram-negative.

Gelatin: Liquefaction.

Beef-extract peptone colonies: Circular, yellow, smooth, butyrous, growth moderate.

Potato glucose agar: Growth abundant, pale yellow. Mucoid.

Broth: Turbid, yellow granular ring.

Milk: Soft curd, with clearing and production of tyrosine crystals. Litmus reduced.

Nitrates utilized but no nitrites formed. Asparagine and nitrites not utilized.

Hydrogen sulfide produced.

Indole not formed.

Lipolytic (Starr and Burkholder, loc. cit.).

Acid from glucose, galactose, xylose, maltose, sucrose, and glycerol. Alkali 
produced from salts of malonic, citric, malic, and succinic acids. Rhamnose, salicin and hippuric acid salts not utilized.

Starch hydrolyzed.

Aerobic.

Distinctive characters: Similar to Xanthomonas campestris but does not infect cabbage, cauliflower or horseradish.

Source: From black rot of winter cress, Barbarea vulgaris.

Habitat: Pathogenic on leaves and stems of Barbarea vulgaris.

\section{Xanthomonas begoniae (Talkimoto)} Dowson. (Bacteriumbegoniae Takimoto, Jour. Plant. Protert., 21, 1934. 262; Pseudomonas begoniae Stapp. Arbeiten Biol. Reichsanst. f. Land- und Forstw., 22, 1938, 392 ; Phytomonas begonine Burkholder, in Manual, 5th ed., 1939, 162; Dowson, Cent. f. Bakt., Il Abt., 100, 1939, 190.) From M. L. Begonia, a generic name.

Probable synonyms: Bacterium begoniae Buchwald nom. nud., GartnerTidende, 45, 1933, 1; Phytomonas flava begoniae Wieringa, Tidschr. Plantziekt., 41, 1935, 312; Bacterium flavozonala McCulloch, Jour. Agr. Res., 54, 1937, 859 (Xanthomonas flavozonatum Dowson, loc. cit.).

Translated by Dr. K. Togashi.

Rods : 0.5 to 0.6 by 1.2 to 2.0 microns. Motile with a polar flagellum. Gramnegative.

Gelatin: No liquefaction. Liquefaction (Wieringa, loc. cit., McCulloch, loc. cit., Dowson, loc. cit., and Stapp, loc, cit.).

Potato agar colonies: Circular, convex, smooth, moist, shining, yellow.

Broth: Turbid. Yellow pellicle and precipitation.

Milk: No coagulation. Casein digested Alkaline.

Nitrites not produced from nitrates.

Indole not produced.

Hydrogen sulfide produced.

Lipolytic (Starr and Burliholder, Phy. topath., 32, 1942, 600).
Starch hydrolyzed (Dowson, Jour. Roy. Hort. Soc., 63, 1938, 289).

No acid or gas in peptone broth from glucose, sucrose, lactose or glycerol. Acid from glucose, sucrose, lactose, mannitol and glycerol in peptone-free medium (McCulloch, loc. cit.).

Optimum temperature $27^{\circ} \mathrm{C}$. Maximum $37^{\circ} \mathrm{C}$. Minimum $1^{\circ}$ to $3{ }^{\circ} \mathrm{C}$.

Source: Isolated from leaf spot of begonia.

Habitat: Pathogenic on Begonia spp.

9. Xanthomonas campestris (Pammel) Dowson. (Bacillus campestris Pammel, Iowa Agr. Exp. Sta. Bull. 27, 1895, 130; Pseudomonas campestris Erw. Smith, Cent. f. Bakt., II Abt., 3, 1897, 2st; Bacterium campestris (sic) Chester, Del. Col. Agr. Exp. Sta. Ann. Rept., 9, 1597, 110; Phytomomas campestris Bergey et al., Manual, 1st ed., 1923, 176; Dowson, Cent. f. Bakt., II Abt., 100, 1939, 190.)

From L. campestris of the field.

Description from MeCulloch (Jour. Agr. Res., 38, 1929, 278). Species is probably composed of several varieties. See descriptions by Mekta, Ann. Appl. Biol., 12, 1925, 330; Paine and Nirula, Ann. Appl. Biol., 15, 1928, 46; Wormald and Frampton, Inn. Rept. East. Mall. Res. Sta., 1926 and 1927, II Supplement, 1928, 108; and others.

Rods : 0.3 to 0.5 by 0.7 to 2.0 microns. Motile with a polar flagellum. Capsules. Gram-negative.

Gelatin: Liquefied.

Beef agar colonies: Wax yellow, round, smooth, shining, translucent, margins entire.

Broth: Turbid with vellow rim and sometimes a pellicle.

Milk: Casein digested with the formation of tyrosine erystals. Alkaline.

Nitrites not produced from nitrates.

Indole formation weak.

Hydrogen sulfide produced.

Lipolytic (Starr and Burkholder, Phytopath., 32, 1942, 600).

Acid, no gas, from glucose, suerose, lactose, glycerol and mannitol. 
Starch is hydrolyzed.

Optimum temperature $28^{\circ}$ to $30^{\circ} \mathrm{C}$.

Maximum $36^{\circ} \mathrm{C}$.

Aerobic.

Distinctive characters: Causes a vascular infection in cabbage, eauliflower and rutabagas.

Source: Pammel (loc. cit.) first isolated the pathogen from diseased rutabagas.

Habitat: Pathogenic on cabbage, cauliflower and other related species.

9a. Xanthomonas campestris var. armoraciae (McCulloch) Burkholder. (Baclerium campestre var. armoraciae MeCulloch, Jour. Agr. Res., 38, 1929, 269; Phytomonas campestris var. aimoraciae Bergey et al., Manual, 3rd ed. 1930, 251; Burkholder, Phytopath., 32. 19t2, 601.) From Gr. Armoracia, the horse radish.

Cultural characters same as Xanthomonas campestris.

Distinctive characters: Causes a leaf spot of horse radish. No vascular infection.

Source: Isolated from diseased horseradish leaves collected in Washington, D. C., Virginia, Connecticut, Iowa and Missouri.

Habitat: Pathogenic on horse radish and related species.

10. Xanthomonas citri (Hasse) Dowson (Pseudomonas citri Hasse, Jour. Agr. Res., 4, 1915, 97 ; Bacterium citri Doidge, Union So. Africa, Dept. Agr. Sci. Bul. 8, 1916, 20; Bacillus citri Holland, Jour. Bact., 5, 1920, 218; Phytomonas citri Bergey et al., Manual, 1st ed., 1923, 181 ; Dowson, Cent. f. Bakt., II Abt., 100, 1939, 190.) From M. L. Citrus, a generie name.

Rods : 0.5 to 0.75 by 1.5 to 2 microns, occurring in chains. Motile with a single polar flagellum. Gram-negative.

Gelatin : Liquefied.

Beef agar colonies: Appear in $36-48$ hours, circular, smooth, raised. dull yellow:

Broth: Turbid in 24 hours. A yellow ring formed.
Milk: Casein is precipitated.

Nitrites not produced from nitrates. Hydrogen sulfide produced (Reid, New Zealand Jour. Sei. and Tech., 22, 1938,60).

Indole not formed.

No gas from glucose, lactose or mannitol.

Starch hydrolyzed (Reid, loc. cit.). Aerobic.

Optimum temperature, $25^{\circ}$ to $34^{\circ} \mathrm{C}$. Maximum $38^{\circ} \mathrm{C}$. Minimum $10^{\circ} \mathrm{C}$. (Okabe, Jour. Soc. Trop. Agr., 4, 1932, 476).

Source: Isolated from canker on orange. Habitat: Produces a canker on many species of Citrus and related plants.

11. Xanthomonas corylina Miller, Bollen, Simmons, Gross, and Barss. Miller et al., Phytopath., 30, 1940, 731; Phytomonas corylina Miller et al., ibid.) From Gr. corylus, the hazelnut; M. I. corylina, of the hazel nut.

Rods : 0.5 to 0.7 by 1.1 to 3.8 microns. Motile with a polar flagellum. Capsules. Gram-negative.

Gelatin: Liquefaction.

Nutrient glucose-agar streaks: Abundant growth, fliform, convex, glistening, smooth, opaque, pale lemon yellow, viscid.

Broth: Turbid. Ring formed in 2-5 days.

Milk: Enzymatic curd that is slowly ligested. Litmus reduced. Crystal formation (Burkholder).

Nitrites not produced from nitrates.

Nitrogen sources utilized are peptone, aspartic acid, alanine, leucine, sodium ammonium phosphate, allantoin, tyrosine, uric acid and brucine.

Indole is not produced.

Hydrogen sulfide not produced on lead acetate agar. $\mathrm{H}_{2} \mathrm{~S}$ produced after Zobell and Feltham's method (Burkholder).

Selenium dioxide reduced.

Lipolytic (Starr and Burkholder, Phytoputh., 32, 1942, 600).

Acid, no gas from glucose, fructose, galactose, lactose, sucrose, maltose, $x y$ lose, raffinose, mannitol, glycerol, and starch. Alkali from salts of citric, lactic, 
malic and succinic acids. Arabinose, rhamnose, dulcitol, salicin, inulin, and cellulose not utilized.

Starch is hydrolyzed.

Optimum temperature $28^{\circ}$ to $32^{\circ} \mathrm{C}$. Maximum $37^{\circ} \mathrm{C}$. Minimum $5^{\circ}$ to $7^{\circ} \mathrm{C}$. Thermal death point $53^{\circ}$ to $55^{\circ} \mathrm{C}$.

$\mathrm{pH}$ range for growth: $\mathrm{pH} 5.2$ to 10.5 . Optimum pH 6 to 8 .

Strict aerobe.

Distinctive characters: Cultural char acters the same or similar to Xanthomonas juglandis. The two speries do not cross-infect.

Source: 26 isolates from widely scattered filbert orchards in Oregon and Washington.

Habitat: Pathogenic on filberts (Corylus avellana and $C$. maxima).

12. Xanthomonas cucurbitae (Bryan) Dowson. (Bacterium cucurbitae Bryan. Science, 63, 1926, 165; Bryan, Jour. Agr. Res., 40, 1930, 389; Phylomonas cucurbitae Bergey et al., Manual, 3rd ed. 1930, 251 ; Pseudomonas cucurbitae Stapp, Bot. Rev., 1, 1935, 408; Dowson, Cent. f Bakt., II Abt., 100, 1939, 190.) From I. curcurbita, a gourd; M. I. Cucurbita, it generic name.

Rods : 0.45 to 0.6 by 0.5 to 1.3 microns. Motile, usually with a single polar flagellum. Gram-negative.

Gelatin: Liquefied.

Beef-agar slants: Growth moderate, mustard yellow, undulating margins. viscid to butyrous.

Broth: Moderately turbid. Ring and yellow sediment.

Milk : Precipitation of casein and digestion. Alkaline.

Nitrites not produced from nitrates.

Indole not formed.

Hydrogen sulfide produced.

Acid from glucose, galactose, fructose. lactose, maltose, sucrose and glycerol. No acid from mannitol.

Starch is hydrolyzed.

Optimum temperature $25^{\circ}$ to $30^{\circ} \mathrm{C}$ Maximum $35^{\circ} \mathrm{C}$.
Optimum $\mathrm{pH} 6.5$ to 7.0. Limits of growth $\mathrm{pH} 5.8$ to 9.0 .

Slight growth in 5 per cent salt.

Aerobic.

Source: Species first isolated from squash.

Habitat: Causes a leaf spot of squash and related plants.

13. Xanthomonas dieffenbachiae (McCulloch and Pirone) Dowson. (Phytomonas dieffenbachiae McCulloch and Pirone, Phytopath., 29, 1939, 962; Bacterium dieffenbachiae McCulloch and Pirone, ibid.; Dowson, Trans. Brit. Mreol. Soc., 26, 1943, 12.) From M. L. Dieffenbachia, a generic name.

Rods : 0.3 to 0.4 by 1.0 to 1.5 microns. Iotile with a single polar flagellum. Capsules. Gram-negative.

Gelatin: Liquefied.

Beef-infusion peptone agar colonies: Slow groming, circular, flat, smooth, translucent. Butyrous. Massicot to Naples yellow

Broth: Turbid. Yellow rim or slight pellicle.

Milk: Slow peptonization and formation of tyrosine crystals. Litmus reduced.

Nitrites not formed from nitrates:

Indole not produced.

Hydrogen sulfide produced.

Acid from glucose, sucrose, lactose, galactose, fructose and glycerol. Growth but no acid in maltose and mannitol.

Starch moderately hydrolyzed.

Optimum temperature $30^{\circ}$ to $31^{\circ} \mathrm{C}$ Maximum $37^{\circ}$ to $38^{\circ} \mathrm{C}$. Minimum $5^{\circ} \mathrm{C}$. Aerobe.

Source: Seven isolates from diseased leaves of Dieffenbachia picta.

Habitat: Pathogenic on Dieffenbachia picta. Artificial infection of Dracaena fragrans.

14. Xanthomonas holcicola (Elliott) Starr and Burkholder. (Bacterium hol. ricola Elliott. Jour. Agr. Res., 40, 1930. 972; Phytomonas holcicola Bergey et al. Manual, 4th ed., 1934, 271; Pseudomonas 
holcicola Stapp, Bot. Rev., 1, 1935, 407; Starr and Burkholder, Phytopath., 32, 1942, 600.) From Gr. holcus, sorghum, -cola, dweller; M. L. Holcus, a generic name.

Rods: 0.75 by 1.58 microns. Motile with 1 or 2 polar flagella. Capsules. Gram-negative.

Gelatin: Liquefied.

Beef-infusion peptone agar colonies: Round, umbonate, glistening, smooth, translucent to opaque, wax yellow, butyrous.

Broth: Trace of growth in 24 hours. Later turbid with a slight ring.

Milk: Casein precipitated and peptonized. Alkaline.

Nitrite production doubtful.

Indole not produced.

Hydrogen sulfide is produced.

Lipolytic (Starr and Burkholder, loc. cit.).

Acid, no gas, from sucrose.

Starch is hydrolyzed.

Optimum temperature $28^{\circ}$ to $30^{\circ} \mathrm{C}$. Maximum $36^{\circ}$ to $37^{\circ} \mathrm{C}$. Minimum $4^{\circ} \mathrm{C}$.

Optimum pH 7.0 to 7.5. Growth range $\mathrm{pH} 5.5$ to 9.0 .

Source: Isolated from many collections of sorghum leaves showing a streak disease.

Habitat: Pathogenic on leaves of Holcus sorghum and $H$. halepensis.

15. Xanthomonas incanae (Kendrick and Baker) Starr and Weiss. (Phytomonas incanae Kendrick and Baker, California Bull. 665, 1942, 10; Starr and Weiss, Phytopath., 89, 1943, 316.) From its host plant Matthiola incana; L. incanus, quite gray or hoary.

Rods : 0.4 to 0.8 by 0.6 to 2.5 microns. Motile with a polar flagellum. Gramnegative.

Gelatin: Liquefied.

Beef extract agar colonies: Round, smooth, convex or pulvinate, glistening, margin entire, pieric yellow to a mber color.

Broth: Turbid.

Milk: No coagulation. A clearing of the medium.
Nitrites not produced from nitrates. Indole not formed.

Lipolytic (Starr and Burkholder, Phytopath., 32, 1942, 600).

Acid but no gas from glucose, lactose, sucrose, mannitol, d-galactose, xylose, d-mannose, raffinose, trehalose, and glycerol. No acid from maltose, l-arabinose, or rhamnose.

Starch not hydrolyzed. Starch hydrolyzed (Burkholder).

Tolerates 3 per cent salt.

Growth in beef broth at $\mathrm{pH} 4.4$.

Aerobic.

Distinctive characters: Causes a disease of flowering stock but not of cabbage. Differs from Xanthomonas campestris in that it does not utilize l-arabinose, nor maltose.

Source: Four isolates from diseased plants of Matthiola incana.

Habitat: Pathogenic on flowering stocks.

16. Xanthomonas juglandis (Pierce) Dowson. (Pseudomonas juglandis Pierce, Bot. Gaz., 31, 1901, 272; Bacterium juglandis Erw. Smith, Bacteria in Relation to Plant Dis., 1, 1905, 171; Bacillus juglandis Holland, Jour. Bact., 5, 1920 , 218; Phyfomonas juglandis Bergey et al., Manual, 3rd ed., 1930, 247; Dowson, Cent. f. Bakt., II Abt., 100, 1939, 190.) From I. juglans (-andis), the walnut; M. L. Juglans, a generic name.

Description taken from Miller et al., Phytopath., 30, 1940, 731.

Rods : 0.5 to 0.7 by 1.1 to 3.8 microns. Motile with a polar flagellum. Capsules. Gram-negative.

Gelatin: Liquefaction.

Nutrient glucose-agar streaks: Abundant growth, filiform, convex, glistening, smooth, opaque, pale lemon yellow, viscid.

Broth: Turbid. Ring formed in 2 to 5 days.

Milk: Enzymatic curd that is slowly digested. Litmus reduced. Crystal formation (Burkholder).

Nitrites not produced from nitrates. 
Nitrogen sources utilized are peptone, aspartic acid, alanine, leucine, sodium ammonium phosphate, allantoin, tyrosine uric acid and brucine.

Indole is not produced.

Hydrogen sulfide not produced on lead acetate agar. $\mathrm{H}_{2} \mathrm{~S}$ produced after Zobell and Feltham's method (Burkholder).

Selenium dioxide reduced.

Lipolytic (Starr and Burkholder, Phytopath., 32, 1942, 600).

Acid, no gas from glucose, fructose, galactose, lactose, sucrose, maltose, $\mathrm{xy}$ lose, raffinose, mannitol, glycerol, and starch. Alkali from salts of citric, lactic, malic and succinic acid. Arabinose, rhamnose, dulcitol, salicin, inulin, and cellulose not utilized.

Starch is hydrolyzed.

Optimum temperature $28^{\circ}$ to $32^{\circ} \mathrm{C}$. Maximum $37^{\circ} \mathrm{C}$. Minimum $5^{\circ}$ to $7^{\circ} \mathrm{C}$. Thermal death point $53^{\circ}$ to $55^{\circ} \mathrm{C}$.

$\mathrm{pH}$ range for growth $\mathrm{pH} 5.2$ to 10.5 . Optimum pH 6 to 8 .

Source: Isolated from black spots on the leaves and nuts of English walnuts, Juglans regia.

Habitat: Pathogenic on the walnut, Juglans spp.

17. Xanthomonas lespedezae (Ayers, Lefebvre and Johnson) comb. nov. (Phytomonas lespedezae Ayers, Lefebvre and Johnson, C. S. Dept. Agr. Tech. Bul. 704, 1939, 19.) From M. I. Lespedeza, a generic name.

Rods : 0.56 by 1.62 microns. Single, in pairs or occasional short chain. Motile with 1 polar flagellum. Gramnegative. Capsules.

Gelatin: Liquefied. Also egg albumin and blood serum.

Nutrient agar colonies: Circular, raised, glistening, translucent, and visrid. Yellow.

Broth: Turbid in 48 hours.

Milk: Peptonized and becomes alkaline.

Nitrites not produced from nitrates. Indole is produced after 11 days.

Hydrogen sulfide is produced.
No gas in carbohydrates.

Starch hydrolyzed.

Aerobic.

Optimum temperature near $35^{\circ} \mathrm{C}$. No growth at $5^{\circ} \mathrm{C}$. or at $40^{\circ} \mathrm{C}$.

Source: Isolated from diseased Lespedeza spp. collected in Virginia, New York and Illinois.

Habitat: Pathogenic on Lespedeza spp.

18. Xanthomonas malvacearum (Erw. Smith) Dowson. (Pseudomonas malvacearum Erw. Smith, U. S. Dept. Agr., Div. Teg. Phys. and Path., Bul. 28, 1901, 153 ; Bacterium malvacearum Erw. Smith, Bact. in Rel. to Plant Diseases, 1, 1905, 171; Bacillus malvacearum Holland, Jour. Bact., 5, 1920, 219; Phytomonas malvacearum Bergey et al., Manual, 1st ed., 1923, 178; Dowson, Cent. f. Bakt., II Abt., 100, 1939, 190.) From L. malva, mallows; M. L. Malvaceae, a family name.

Description from Elliott, Man. Bact. Plant Pathogens, 1930, 153; and Lewis, Phytopath., 20, 1930, 723.

Rods: Motile with one polar flagellum. Gram-negative.

Gelatin: Liquefaction.

Agar slants: Moderate growth, convex, smooth, glistening, pale yellow, wavy to irregular margins.

Broth: Slight to moderate turbidity. Sediment.

Milk: Casein precipitated and slowly digested.

Nitrites not produced from nitrates. Hydrogen sulfide is produced (Burkholder).

Not lipolytic (Starr and Burkholder, Phytopath., 32, 1942, 600).

Acid but not gas from glucose, galactuse, fructose, xylose, lactose, maltose, sucrose, raffinose, glycerol, inulin and glycogen. Alkaline reaction from salts of acetic, citric, lactic and succinic acids. No fermentation of arabinose, mannitol, dulcitol, salicin, and salts of formic, oxalic and tartaric acids (Lewis, loc. cit).

Starch hydrolyzed (Lewis, loc. cit.). 
Optimum temperature $25^{\circ}$ to $30^{\circ} \mathrm{C}$ Maximum $36^{\circ}$ to $38^{\circ} \mathrm{C}$. (Elliott, loc. cit.).

Source: Isolated from angular leaf spot of cotton.

Habitat: Pathogenic on cotton whereever it is grown, causing a leaf spot, a stem lesion and a boll lesion.

19. Xanthomonas pelargonii (Brown) Starr and Burkholder. (Bacterium pelargoni Brown, Jour. Agr. Res., 28, 1923, 372 ; Pseudomonas pelargoni Stapp, in Sorauer, Handb. d. Pflanzenkrank, 2, 5 Aufl., 1928, 181; Phytomonas pelargonii Bergey et al., Manual, 3rd ed., 1930, 250 ; Starr and Burkholder, Phytopath., 32, 1942, 600.) From Greek, pelargus, the stork; M. L. Pelargonium, a generic name for the stork's bill geranium.

Rods : 0.67 by 1.02 microns. Capsules. Motile with a polar flagellum. Gramnegative.

Gelatin: Slow liquefaction.

Beef-agar colonies: Cream-colored, glistening, round, with delicate internal markings.

Broth: Turbid in 24 hours. Incom. plete pellicle.

Milk: Alkaline. Clearing in bands.

Nitrites not produced from nitrates.

Indole formation slight.

Hydrogen sulfide produced.

Lipolytic (Starr and Burkholder, Phy topath., 32, 1942, 600).

Slight acid but not gas from glucose, sucrose and glycerol.

Stareh hydrolysis feebly positive.

Optimum temperature $27^{\circ} \mathrm{C}$. Maximum $35^{\circ} \mathrm{C}$.

No growth in broth plus 3.5 per cent salt.

Aerobic.

Source: Isolated from spots un leaves of Pelargonium from District of Columbia, Maryland and New Jersey.

Habitat: Pathogenic on Pelargonium spp. and Geranium spp.

20. Xanthomonas phaseoli (Erw. Smith) Dowson. (Bacillus phaseoli Erw. Smith, Bot. Gaz., 24, 1897, 192; A. A. A.
S. Proc., 46, 1898, 288; Pseudomonas phaseoli Erw. Smith, U. S. Dept. Agr., Div. Veg. Phys, and Path., Bul. 28, 1901, 1; Bacterium phaseoli Erw. Smith, Bact. in Rel. to Plant Dis., 1, 1905, 72; Phytomonas phaseoli Bergey et al., Manual, 1st ed., 1923, 177; Dowson, Cent. f. Bakt., II Abt., 100, 1939, 190.) From Gr. phaseolus, the bean; M. L. Phaseolus, a generic name.

Description from Burkholder, Cornell Agr. Exp. Sta. Mem. 127, 1930, 18; and Phytopath., 22, 1932, 609.

Rods: 0.87 by 1.9 microns. Motile with a polar flagellum. Gram-negative.

Gelatin: Liquefaction.

Beef-extract agar colonies: Circular, amber yellow, smooth, butyrous, edges entire.

Broth: Turbid in 24 hours. Yellow ring.

Milk: Casein precipitated and digested. Alkaline. Tyrosine crystals formed.

Nitrites not produced from nitrates.

Indole not formed.

Hydrogen sulfide produced.

Lipolytic (Starr and Burkholder, Phytopath., 32, 1942, 600).

Acid but not gas from glucose, galactose, fructose, arabinose, xylose, maltose, lactose, sucrose, raffinose and glycerol. Alkaline reaction from salts of acetic, malic, citric and succinic acids. Mannitol, dulcitol, salicin and formic and tartaric acids not fermented.

Starch is hydrolyzed.

Aerobic.

Very slight growth in beef broth plus 4 per cent salt (Hedges, Jour. Agr. Res., 29, 1924, 243).

Distinctive character: Similar in culture to Xanthomonas campestris, $X$. juglandis, $X$. vesicatoria, etc., but they do not eross infect

Habitat: Pathogenic on the bean (Phaseolus vulgaris), the hyacinth bean (Dolichos lablab), the lupine (Lupinus polyphillus), etc. Not pathogenic on the soy bean (Glycine sp.), nor cowpea (Vigna $s p$.). 
20a. Xanthomonas phaseoli var. sojensis (Hedges) Starr and Burkholder. (Bacterium phaseoli var. sojense Hedges, Science, 56, 1922, 11; Jour. Agr. Res., 29, 1924, 229; Phytomonas phaseoli var. sojense Burkholder, Phytopath., 20, 1930, 7; Starr and Burkholder, Phytopath., 32, 1942, 600.) From M. L. the soy bean, Soja, a generic name; M. L. sojensis, of the soybean.

Synonyms: Pseudomonas glycines $\mathrm{Na}$. kano, Jour. Plant Protect. Tokyo, 6, 1919, 39 (Bacterium glycines Elliott, Manual Bact. Plant Path., 1928, 133; Phytomonas glycines Nagrou, in Hauduroy et al., Dict. d. Bact. Path.. 1937, 358). (See Takimoto, Jour. Plant Protect. Tokyo, 18, 1931, 29; and Okabe, Jour. Trop. Agr. Formosa, 4, 1932, 473.)

Distinctive character: Differs from Xanthomonas phaseoli in that it infects the soy bean, Glycine max.

Source: Isolated from pustules on the leaves and pods of soy bean, both in America and in Japan.

Habitat: Pathogenic on the soy bean, Glycine max and the common bean, Phaseolus vulgaris.

20b. Xanthomonas phaseoli var. fus. cans (Burkholder) Starr and Burkholder. (Phytomonas phaseoli var. fuscans Burkholder, Cornell Agr. Exp. Sta. Mem. 126, 1930, 22; Phytopath., 22, 1932, 699; Bacterium phaseoli var. fuscans Oliabe, Jour. Soc. Trop. Agr. Formosa, 5, 1933, 161 ; Pseudomonas phaseoli var. fuscans Stapp, Bot. Rev., 1, 1935, 407; Starr and Burkholder, Phytopath., 32, 1942, 600.) From L. fuscans, producing a brown color.

Distinctive characters: Differs from $X$ anthomonas phaseoli in that it produces a deep brown color in beef-extract-peptone media and in tyrosine media. Action on maltose negative or feeble.

Source: Two cultures isolated; one from a diseased bean leaf (1924) and a diseased pod (1927) collected in Switzerland.
Habitat: Pathogenic on beans, Phaseol.us vulgaris, and related plants.

21. Xanthomonas plantaginis (Thornberry and Anderson) comb. nov. (Phytomonas plantaginis Thornberry and Anderson, Phytopath., 27, 1937, 947.) From Latin, Plantago (-aginis), plantain; M. L. Plantago, a generic name.

Rods : 0.6 to 1.0 by 1.0 to 1.8 microns. Occurring singly or in chains. Capsules. Motile with 1 to 2 polar flagella. Gramnegative.

Gelatin: Slight liquefaction.

Glucose agar slant: Growth moderate, filiform, raised, opaque, yellow and viscid.

Broth: Moderately turbid with ring.

Milk: Slight acidity, no reduction of litmus. Peptonization.

Nitrites not produced from nitrates. Indole not formed.

Hydrogen sulfide not produced.

No appreciable amount of gas from carbohydrates.

Starch is hydrolyzed.

Optimum temperature $25^{\circ} \mathrm{C}$. Minimum $12^{\circ} \mathrm{C}$. Maximum $35^{\circ} \mathrm{C}$. Thermal death point $50^{\circ} \mathrm{C}$.

Aerobic.

Source: From diseased leaves of Plantngo lanceolata in Illinois.

Habitat: Páthogenic on Plantagospp.

22. Xanthomonas ricinicola (Elliott) Dowson. (Bacterium ricini Yoshi and Takimoto, Jour. Plant Protect. Tokyo, 15, 1928, 12; Bacterium ricinicola Elliott, Man. Bact. Plant Path., 1930, 193; Phytomonas ricinicola Burkholder, in Manual, 5th ed., 1939, 152; Dowson, Cent. f. Bakt., II Abt., 100, 1939, 190; Xanthomonas ricini Dowson, ibid.) From L. living on the castor bean; M. L. Ricinus, a generic name.

Rods: 0.4 to 0.9 by 1.3 to 2.6 microns. Capsules. Short chains. Motile with polar flagella. Gram-negative.

Gelatin : Liquefaction.

Nutrient agar colonies: Lemon yellow, changing to brown. 
Milk: Slightly acid. No coagulation. Peptonization.

Nitrites not produced from nitrates. Acid but not gas from lactose.

Starch hydrolyzed.

Optimum temperature $29^{\circ}$ to $30^{\circ} \mathrm{C}$. Maximum $39^{\circ} \mathrm{C}$. Minimum $2.5^{\circ} \mathrm{C}$.

Aerobic.

Source: Isolated from leaf-spot of castor-bean.

Habitat: Pathogenic on Ricinus communis.

23a. Xanthomonas translucens f. sp. hordei Hagborg. (Canadian Jour. of Res., 20, 1942, 317.) From L, translucens, shining through, translucent, referring to the character of the lesion produced by this pathogen. Form name from Hordeum, a generic name.

Synonyms: Bacterium translucens Jones, Johnson and Reddy, Jour. Agr. Res., 11, 1917, 637; Pseudomonas translucens, ibid.; Phytomonas translucens Bergey et al, Manual, 3rd ed., 1930, 252; Xanthomonas translucens Dowson, Cent. f. Bakt., II Abt., 100, 1939, 190.

Rods: 0.5 to 0.8 by 1 to 2.5 microns. Motile with a single polar flagellum. Gram-negative.

Gelatin: Liquefaction.

Beef-peptone agar colonies: Round, smooth, shining, amorphous except for inconspicuous somewhat irregular concentric striations within, wax-yellow tinged with old gold; margin entire.

Broth: Turbidity becomes rather strong. Pellicle.

Milk: Soft coagulum and digestion. Milk clears. Tyrosine crystals produced.

Nitrites not produced from nitrates.

Indole : Slight formation.

Hydrogen sulfide produced.

Lipolytic (Starr and Burkholder, Phytopath., 32, 1942, 600).

Ammonia from peptone.

Acid but not gas from glucose, dfructose, d-mannose, d-galactose, sucrose, lactose, and sometimes salicin. No utilization of l-rhamnose, inositol, maltose, raffinose, inulin, d-mannitol, and dulcitol.

Starch hydrolyzed.

Optimum temperature $26^{\circ} \mathrm{C}$. Maximum $36^{\circ} \mathrm{C}$. Minimum $6{ }^{\circ} \mathrm{C}$.

Aerobic.

Distinctive characters: All forms of Xanthomonas translucens have the same cultural characters. They differ mainly in pathogenicity. This form is pathogenic on barley, Hordeum spp.; but not on oats, Avena spp., rye, Secale cereale nor on wheat, Triticum spp.

Source: Isolated from leaves and seed of barley, Hordeum vulgare.

Habitat: Occurs naturally on barley.

23b. Xanthomonas translucens f. sp. undulosa (Smith, Jones and Reddy) Hagborg. (Bacterium translucens var. undulosum Smith, Jones and Reddy, Science, 50, 1919, 48; Pseudomonas translucens var. undulosa Stapp, in Sorauer, Handb. d. Pflanzenk., 2, 5 Auf., 1928, 17; Phytomonas translucens var. undulosa Hagborg, Canadian Jour. Res., 14, 1936, 347; Hagborg, Canadian Jour. Res., 20, 1942, 317.) From L. unda; M.L. undulosus, undulate, referring to the undulation of the colony.

Distinctive characters: Cultural characters same as all forms of Xanthomonas translucens. Pathogenic on wheat, Triticium spp., barley, Hordeum spp. and rye, Secale cereale but not on oats, Avena spp.

Source: Isolated repeatedly from black chaff of wheat.

Habitat: Usually found on wheat causing the black chaff, and on rye.

23c. Xanthomonas translucens f. sp. secalis (Reddy, Godkin and Johnson) Hagborg. (Bacterium translucens var. secalis Reddy, Godkin and Johnson, Jour. Agr. Res., 28, 1924, 1039; Pseudomona translucens var. secalis Stapp, in Sorauer, Handb. d. Pflanzenkr., 2, 5 Aufl., 1928, 24; Phytomonas translucens var. secalis Burkholder, in Manual, 5th ed., 1939, 160 ;Hagborg, Canadian Jour. 
Pes., 20, 1942, 317.) From M.L. Secale, a generic name. Distinctive characters: Cultural characters same as other forms of Xanthomonas translucens. This form pathogenic on rye Secale cereale, but not on Triticum spp., Hordenm $s p p$. nor Avena spp.

Source: Isolated from leaf spot on rye, Secale cereale.

Habitat: Pathogenic on rye.

23d. Xanthomonas translucens f. sp. hordei-avenae Hagborg. (Canadian Jour. Res., 20, 1942,317.) From M.L. Hordeum and Avena, generic names.

Distinctive characters: Cultural characters same as other forms of Xanthomonas translucens. Pathogenic on barley, Hordeum spp. and oats, Avena spp., but not on wheat, Triticium spp., nor rye, Secale cereale.

Source: Isolated 6 times from barley at various places in Canada.

Habitat: Occurs naturally on barley.

23e. Xanthomonas translucens f. sp. cerealis Hagborg. (Canadian Jour. Res., 20, 1942,317.) From L., of cereal.

Distinctive characters: Cultural characters same as other forms of Tanthomonas translucens. Pathogenic on wheat, Triticum spp.; oats, Arena spp.; barley, Hordeum spp.; and rye, Secale cereale.

Source: Isolated from wheat in Canada. Habitat: Occurs naturally on wheat.

\section{Xanthomonas vasculorum (Cobb)} Dorson. (Bacillus vascularum (sic) Cobb, Agr. Gaz of New South Wales, 4 , 1S93, 777; Abst. in Cent. f. Bakt., II Abt., $1,1895,41$; Bacterium vascularum Migula, Syst. d. Bakt., 2, 1900, 512; Pseudomonas vascularum Erw. Smith, L.S. Dept. Agr., Div. Veg. Phys. and Path., Bul. 28, 1901, 153; Phytomonas vascularum Bergey et al., Manual, 1st ed., 1923, 179; Dowson, Cent. f. Bakt., II Abt., 100, 1939, 190.) From L. vasculum, a small vessel; M. I. the vascular system.

Note: Erw. Smith (Bact. in Piel. to
Plant Dis., 3, 1914, 88) states that probably Spegazzini (El Polville de la Cana de Azucar, June, 1895, La Plata, Supl. Rev. Azuc., Buenos Aires, No. 16, 1895) reported the disease caused by Xanthomonas vasculorum but that Bacillus sacchari Spegazzini which he claimed to be the pathogen, was a saprophyte.

Description from Smith (loc. cit., 54).

Rods : 0.4 by 1.0 microns. Notile with a polar flagellum. Gram-variable.

Gelatin: Liquefaction feeble. Liquefaction good (Burkholder).

Beef-extract agar colonies: Pale yellow. smooth, glistening, not noticeably viscid.

Broth: Good growth.

Milk: Alkaline.

Nitrites not produced from nitrates.

Lipolytic (Starr and Burkholder, Phytopath., 32, 1042, 600).

Acid but not gas from glucose, fructose and glycerol.

Starch hydrolyzed (Burkholder).

Optimum temperature $30^{\circ} \mathrm{C}$. Maximum $35^{\circ}$ to $37.5^{\circ} \mathrm{C}$ (Elliott, loc. cit.).

Habitat: Pathogenic on sugar cane, Saccharum officinarum, causing a bacterial gummosis.

\section{Xanthomonas vesicatoria (Doidge)} Dowson. (Bacterium vesicatorium Doidge, Jour. Dept. Agr., S. Africa, 1, 1920, 718; also Ann. Appl. Biol., 7, 1921, 428; Pseudomonas vesicatoria Stapp, in Sorauer, Handb. d. Pflanzenkrank., 2, 5 Aufl., 1928, 259; Phytomonas vesicatoria Bergey et al., Manual, 3rd ed., 1930, 253; Dowson, Cent. f. Bakt., II Abt., 100, 1939, 190.) From L.vesica, a blister ; M. L. vesicatorius, causing blisters.

Synonyms: Gardner and Kendrick (Phytopath., 13, 1923, 307) list Pseudomonos exitiosa Gardner and Kendrick (Phytopath., 11, 1921, 55; Bacterium exitiosum Gardner and Kendrick, Jour. Agr. Ries., 21, 1921, 141; Phytomonas exitiosa Bergey et al., Manual, 1st ed., 1923,183 ) and an unnamed species, Higgins (Phytopath, 12, 1922, 513).

Rods : 0.6 to 0.7 by 1.0 to 1.5 microns 
Motile with a polar flagellum. Capsules. Gram-positive. Gram-negative (Gardner and Kendrick; and Higgins).

Gelatin: Liquefaction.

Nutrient agar colonies: Good growth Circular, wet-shining, Naples yellow, edges entire.

Milk: Casein precipitated and slowly digested. Tyrosine crystals.

Nitrites not produced from nitrates.

Indole not formed.

Hydrogen sulfide produced (Burkb ilder).

Lipolytic (Starr and Burkholder, Phytopath., 32, 1942, 600).

Acid but not gas from glucose, fructose, sucrose, lactose, galactose, glycerol and dextrin.

Certain strains hydrolyze starch, others do not (Burkholder and Li, Phytopath., 31, 1941, 753).

Optimum temperature $30^{\circ} \mathrm{C}$.

Source: Isolated from spotted tomato fruits in South Africa.

Habitat: Pathogen on tomatoes, Lycopersicon esculentum and peppers, Capsicum annuum.

25a. Xanthomonas vesicatoria var. raphani (White) Starr and Burkholder. (Bacterium vesicatoria var. raphani White, Phytopath., 20, 1930, 653; Phytomonas vesicatoria var. raphani Burkholder, in Manual, 5th ed., 1939, 154; Starr and Burkholder, Phytopath., 32, 1942, 600.) From M. L. Raphanus, the radish, a generic name.

Distinctive characters: Cultural characters similar to Xanthomonas vesicatoria, but differs in that it is able to attack radishes, turnips, and other crucifers. Differs from Xanthomonas campestris in that it does not cause a vascular disease, and differs from Xanthomonas campestris var. armoraciae in that it is not pathogenic on horseradish.

Source: Isolated from leaf spots of radish and turnips in Indiana.

Habitat: Pathogenic on radish, turnips, and other crucifers; and on tomato and pepper.
26. Xanthomonas nakatae (Okabe) Dowson. (Bacterium nakatae Type B, Okabe, Jour. Soc. Trop. Agr., Formosa, 5, 1933, 161; Phylomonas nakatae Burkholder, in Manual, 5th ed., 1939, 154; Dowson, Trans. Brit. Mycol. Soc., 26, 1943, 12.) Named for Nakata, the Japanese plant pathologist.

Rods : 0.3 to 0.4 by 1.1 to 2.5 microns. Capsules. Motile with a polar flagellum. Gram-negative.

Gelatin: Liquefaction. Brown color.

Beef-extract agar colonies: Amber yellow, round, smooth, glistening, margins entire. Brown.

Broth: Moderate turbidity with yellow ring. Medium turns brown.

Milk: Casein is precipitated and digested. Tyrosine crystals. Brown color.

Nitrites not produced from nitrates.

Indole not formed.

Slight amount $\mathrm{H}_{2} \mathrm{~S}$ produced.

Acid but not gas from glucose, sucrose, maltose and lactose.

Starch: Strong diastatic action.

Optimum temperature $30^{\circ}$ to $32^{\circ} \mathrm{C}$. Maximum $39^{\circ} \mathrm{C}$. Minimum $10^{\circ} \mathrm{C}$.

No growth in beef extract broth plus 2 per cent salt.

Aerobic.

Distinctive character: Differs from Type $\mathrm{A}$ in that it produces a brown pigment in culture. (Description of Type A not seen.)

Source: Isolated from water-soaked to brown leaf spots on jute.

Habitat: Pathogenic on jute, Corchorus capsularis.

27. Xanthomonas papavericola (Bryan and MeWhorter) Dowson. (Bacterium papavericola Bryan and McWhorter, Jour. Agr. Res., 40, 1930, 9; Phytomonas papavericola Bergey et al., Manual, 4th ed., 1934, 266; Dowson, Cent. f. Bakt., II Abt., 100, 1939, 190.) From L. papaver, poppy; -cola, dweller; M. L. Papaver, a generic name.

Rods: 0.6 to 0.7 by 1 to 1.7 microns. Chains. Capsules. Motile with a single polar flagellum. Gram-negative. 
Gelatin: Liquefaction.

Beef agar colonies: Mustard yellow to primuline yellow, circular, margins entire.

Broth : Turbidity prompt with a yellow ring and an incomplete pellicle.

Milk: Soft coagulation, peptonization and production of tyrosine crystals.

Nitrates: A weak reaction for nitrites after 10 days.

Indole not formed.

Hydrogen sulfide is produced.

Lipolytic (Starr and Burkholder, Phy topath., 32, 1942, 600).

Acid but not gas from glucose, galactose, fructose, sucrose, lactose, maltose, glycerol and mannitol.

Starch is hydrolyzed.

Optimum temperature $25^{\circ}$ to $30^{\circ} \mathrm{C}$. Maximum $35^{\circ} \mathrm{C}$.

No growth in broth plus 5 per cent salt

Aerobic.

Source: Isolated from black spots on leaves, buds and pods of poppy.

Habitat: Pathogenic on poppy, Paparer rhneas.

\section{Xanthomonas alfalfae (Riker} et al.) Dowson. (Bacterium alfalfae Riker, Jones and Davis, Jour. Agr. Res.. 51, 1935, 175; Phytomonas alfalfae Riker nt al., ibid.; Pseudomonas alfalfae Riker et al., ibid.; Dowson, Trans. Brit. Mycol. Soc., 26, 1943, 11.) From Spanish, of alfalfa.

Rods: 0.45 by 2.4 microns. Motile with a polar flagellum. Gram-negative.

Gelatin : Liquefied.

Nutrient agar stroke: Growth abundant, filiform, smooth, glistening, butyrous, pale yellow.

Broth: Turbid in 24 hours. Light sediment.

Milk: Casein is precipitated and digested.

Ammonia formed slowly in a nitrate medium.

Carbohydrates : No acid in yeast broth plus sugars.

Starch is hydrolyzed.

Aerobic.
Optimum temperature $24^{\circ}$ to $32^{\circ} \mathrm{C}$. Maximum below $36^{\circ} \mathrm{C}$. Minimum below $4^{\circ} \mathrm{C}$.

Source : Six single cell cultures isolated from diseased alfalfa.

Habitat: Pathogenic on the leaves of alfalfa, Medicago sativa.

29. Xanthomonas acernea (Ogawa) comb. nov. (Pseudomonas acernea Ogawa, Ann. Phyt. Soc. Japan, 7, 1937, 123; Phytomonas acernea Ark, Phytopath., 29, 1939, 968.) From L. acerneus, of the maple.

Rods : 0.2 to 0.6 by 0.5 to 1.2 microns. Motile with one polar flagellum. Gram. negative.

Gelatin: Liquified.

Agar colonies: Round, smooth, convex, white to citron yellow, glistening, translucent with amorphous structure.

Broth: Turbid.

Milk: Slowly cleared, slightly acid. No coagulation.

Nitrites produced from nitrates.

Hydrogen sulfide produced.

No gas produced in peptone water plus sugars.

Starch not hydrolyzed.

Optimum temperature about $32^{\circ} \mathrm{C}$. Thermal death point $59^{\circ} \mathrm{C}$.

Aerobic.

Source: From diseased leaves of Acer trifidum in Japan.

Habitat: Causes a disease in Acer spp. and in Aesculus turbinata and Koelrenteria paniculata.

30. Xanthomonas carotae (Kendrick) Dowson. (Phytomonas carotae Kendrick, Jour. Agr. Res., 49, 1934, 504; Pseudomonas carotae Kendrick, ibid.; Dowson, Cent. f. Bakt., II Abt., 100, 1939, 190.) From L. carota, the carrot.

Rods: 0.42 to 0.85 by 1.38 to 2.75 microns. Motile with 1 or 2 polar flagella. Gram-negative.

Gelatin: Liquefied.

Potato glucose agar: Colonies round, smooth, glistening, margins entire, straw yellow in color. 
Milk: Casein precipitated and milk cleared; alkaline.

Nitrites not produced from nitrates.

Indole not formed.

Acid, no gas, from glucose, $d$-galactose, xylose, $d$-mannose, $l$-arabinose, sucrose, lactose, raffinose, trehalose, $d$-mannitol and glycerol. No acid from maltose and rhamnose.

Starch not hydrolyzed.

Optimum temperature $25^{\circ}$ to $30^{\circ} \mathrm{C}$.

Tolerates 4 per cent salt at pH 7 .

Aerobic.

Source: Two original isolations from diseased carrots and a reisolation from inoculated carrots were used for the description.

Habitat: Pathogenic on leaves of Daucus carota var. sativa.

\section{Xanthomonas hederae (Arnaud)} Dowson. (Bacterium hederae Arnaud, Compt. rend. Acad. Sci., Paris, 171, 1920, 121; Phytomonas hederae Burkholder and Guterman, Phytopath., 22, 1932, 783; Dowson, Cent. f. Bakt., II Abt., 100, 1939, 190.) From L. hedera, ivy; M. L. Hedera, a generic name.

Description taken from Burkholder and Guterman (loc. cit.).

Rods: 0.6 by 2.13 microns. Motile with a single polar flagellum. Gramnegative.

Gelatin: Liquefied.

Beef-extract-agar slants: Growth good, filiform, amber yellow, butyrous.

Broth: Turbid.

Milk: Casein is precipitated and digested. Milk becomes alkaline.

Nitrites not produced from nitrates.

Hydrogen sulfide is formed.

Indole not formed.

Not lipolytic (Starr and Burkholder, Phytopath., 32, 1942, 600).

Acid from glucose, fructose, galactose, xylose, sucrose, lactose and glycerol. Alkali from salts of acetic, citric, lactic, malic and succinic acids. The following are not utilized: arabinose, rhamnose, maltose, salicin, starch, cellulose and formic acid.
Aerobic, facultative.

Source: Isolated from discased ivy leaves.

Habitat: Pathogenic on ivy, Hedera helix.

32. Xanthomonas phormicola (Takimoto) Dowson. (Bacterium phormicola Takimoto, Jour. Plant Protect., 20, 1933, 777 ; Phytomonas phormicola Burkholder, in Manual, 5th ed., 1939, 159; Dowson, Trans. Brit. Mycol. Soc., 26, 1943, 12.) From M. L. Phormium, a generic name.

Description translated by Dr. K. Togashi.

Rods: 0.5 to 0.6 by 1 to 2 microns. Motile, with a single flagellum. Gramnegative.

Gelatin: Liquefied.

Agar colonies: Light yellow, then waxy yellow; butyrous, then viscid.

Broth: Turbid, pellicle formed.

Milk: Casein coagulated slowly and precipitated, then digested. Alkaline.

Nitrites not produced from nitrates.

Indole not formed.

Hydrogen sulfide produced.

No gas from sucrose, glucose, lactose and glycerol.

No acid from various sugars in broth.

Optimum temperature about $29^{\circ} \mathrm{C}$. Maximum $39^{\circ} \mathrm{C}$. Minimum about $0^{\circ} \mathrm{C}$. Aerobic.

Source: Species isolated from New Zealand flax, Phormium tenax.

Habitat: Causes a leaf stripe of Phormium tenax.

33. Xanthomonas geranii (Burkholder) Dowson. (Phytomonas geranii Burkholder, Phytopath., 27, 1937, 560; Dowson, Cent. f. Bakt., II Abt., 100, 1939, 190.) From Greek, geranos, crane; M. L. Geranium, a generic name.

Rods: 0.75 to 2.0 microns. Motile with a single polar flagellum. Gramnegative.

Gelatin: Liquefied.

Beef-extract agar slants: Moderate to good filiform growth, glistening, primuline yellow. Develops in $2 t$ hours. 
Broth: Turbid in 24 hours. No pellicle but a moderate sediment.

Milk: Becomes clear with a heavy casein precipitate. Peptonization with crystal formation.

Nitrates reduced to ammonia.

Indole not formed.

Hydrogen sulfide formed.

Lipolytic (Starr and Burkholder, Phytopath., 32, 1942, 600).

Acid from glucose, galactose, fructose, xylose, rhamnose, lactose, sucrose, raffinose and glycerol. Alkaline reaction from salts of eitric, malic, malonic and succinic acid. No growth in arabinose or formic, hippuric, maleic or tartaric acid.

Starch not hydrolyzed.

Aerobe.

Distinctive characters: Pathogenic on Geranium spp., not on the house geranium, Pelargonium hortorum. In culture similar to Xanthomonas pelargonii.

Source: Three cultures isolated from Geranium sanguineum.

Habitat: Pathogenic on Geranium sanguineum, G. maculatum, G. pratense and G. sylvaticum.

34. Xanthomonas antirrhini (Takimoto) Dowson. (Pseudomonas antirrhini Takimoto, Bot. Mag. Tokyo, 34, 1920, 257; Bacterium antirrhini Elliott, Man. Bact. Plant Path., 1930, 93; Phytomonas antirrhini Magrou, in Hauduroy et al., Dict. d. bact. path., Paris, 1937, 331 ; Dowson, Trans. Brit. Mycol. Soc., 26, 1943, 11.) From Gr. antirrhinum, snapdragon; M. L. Antirrhinum, a generic name.

Description from Elliott (loc.cit.).

Rods : 0.3 to 0.4 by 0.8 to 1.2 microns. Motile with polar flagelli. Capsules. Gram-negative.

Gelatin: Liquefied.

Agar colonies: Round, glistening, white, later yellow.

Milk: Coagulated and casein digested.

Nitrites are produced from nitrates. No gas produced.

Aerobic.
Optimum temperature $26^{\circ}$ to $27^{\circ} \mathrm{C}$. Maximum $34^{\circ} \mathrm{C}$.

Habitat: Causes a leaf spot of Antirrhinum majus.

35. Xanthomonas heterocea (Vzoroff) comb. nov. (Phytomonas heterocea Vzoroff, Bull. North Caucasian Plant Prot. Sta. Roztoff-on-Don, 6-7, 1930, 263; Bacterium heteroceum Burgwitz, Phytopathogenic bacteria, Leningrad, $1935,135$.$) From Gr. heterus, another,$ different.

Description taken from Rev. App. Myc., 10, 1931, 628.

Rods : 0.4 to 0.6 by 1.0 to 2.0 microns. Motile. Gram-negative.

Gelatin: Slow liquefaction.

Agar colonies: Round, convex, smooth, semi-transparent, glistening, yellow to amber, $2 \mathrm{~mm}$. in diameter. Pitted surface.

Milk: No congulation. At first acid, later alkaline.

Nitrites produced from nitrates.

Indole not formed.

Hydrogen sulfide produced.

Acid from glucose, galactose, arabinose, xylose, sucrose, maltose, salicin, glycerol and mannitol. Does not ferment lactose, inulin, ethyl alcohol, esculin, adonitol or dulcitol.

Optimum temperature $25^{\circ}$ to $30^{\circ} \mathrm{C}$.

Source: Isolated from diseased tobacco in the North Caucasus.

Habitat: Pathogenic on Nicotiana tabacum.

36. Xanthomonas gummisudans (MeCulloch) Starr and Burkholder. (Bacterium gummisudans MeCulloch, Phytopath., 14, 1924, 63; also Jour. Agr. Res., 27, 1924, 229; Pseudomonas gummisudans Stapp, in Sorauer, Handb. d. Pflanzenkrank., 2, 5 Aufl., 1928, 54; Phytomonas gummisudans Bergey et al., Manual, 2nd ed., 1925, 201; Starr and Burkholder, Phytopath., 32, 1942, 600.) From L. gummi, gum; sudans, sweating, dripping.

Rods : 0.6 to 0.8 by 1 to 2.8 microns. 
Capsules. Motile with a polar flagellum.

Gram-negative.

Gelatin: Liquefied.

Beef-peptone agar colonies: Amber yellow, circular, transparent, smooth, with definite margins.

Broth: Moderately turbid with a yellow ring.

Milk: Soft curd which is digested with formation of tyrosine crystals.

Nitrites not produced from nitrates.

Indole not produced.

Hydrogen sulfide produced.

Lipolytic (Starr and Burkholder, loc. cit.).

Acid from glucose and sucrose.

Optimum temperature $30^{\circ} \mathrm{C}$. Maximum $36^{\circ} \mathrm{C}$. Minimum $2^{\circ} \mathrm{C}$.

Aerobic.

Source : From gummy lesions on gladiolus leaves.

Habitat: Pathogenic on leaves of gladioli.

37. Xanthomonas lactucae (Yamamoto) Dowson. (Bacterium lactucae Yamamoto, Jour. Plant Protect., 21, 1934, 532; Phytomonas lactucae Bergey et al., Manual, 5th ed., 1939, 163; Dowson, Trans. Brit. Mycol. Soc., 26, 1943, 12.) From L. lactuca, lettuce; M. I. Lactuca, a generic name.

Description translated by Dr. K. Togashi.

Rods : 0.6 to 0.8 by 1.75 to 2.8 microns. Motile with a polar flagellum. Gramnegative.

Gelatin: Liquefaction slow.

Agar colonies: Circular, convex, margin entire, surface smooth, wet-shining, yellow.

Broth: Turbid. Ring and pellicle.

Milk: Slow peptonization.

Nitrites not produced from nitrates.

Indole not produced.

Hydrogen sulfide produced.

Acid, no gas, from glucose, sucrose, and lactose in bouillon; no acid from glycerol in bouillon.

Optimum temperature $28^{\circ} \mathrm{C}$. Maximum $35^{\circ} \mathrm{C}$. Minimum below $2^{\circ} \mathrm{C}$.
Aerobic.

Source: Isolated from leaf spot of lettuce.

Habitat: Pathogenic on leaves of asparagus lettuce, Lactuca sativa var. angustata.

38. Xanthomonas nigromaculans (Takimoto) Dowson. (Bacterium nigromaculans Takimoto, Jour. Plant Protect., Tokyo, 14, 1927, 522; Phytomonas nigromaculans Magrou, in Hauduroy et al., Dict. d. Bact. Path., Paris, 1937, 387; Dowson, Trans. Brit. Mycol. Soc., 26, 1943, 12.) From L. niger, black; maculans, spotting.

Description translated by Dr. K. Togashi.

Rods : 0.6 to 0.9 by 1.5 to 2.8 microns. Motile with 1 or 2 polar flagella. Gramnegative.

Gelatin: Liquefaction.

Agar colonies: Yellow, circular, margins entire, smooth, glistening.

Broth: Growth moderate with yellow pellicle.

Milk: Coagulation and digestion of the casein.

Nitrites not produced from nitrates.

Indole not produced.

No acid or gas from glucose, sucrose, lactose, mannitol and glycerol in peptone water.

Optimum temperature $27^{\circ}$ to $28^{\circ} \mathrm{C}$. Maximum $33^{\circ} \mathrm{C}$. Minimum $0^{\circ} \mathrm{C}$.

Aerobic.

Source: Isulated from lesions on leaf and petioles of burdock.

Habitat: Pathogenic on leaves and petioles of Arctium lappa, the burdock.

39. Xanthomonas oryzae (Uyeda and Ishiyama) Dowson. (Pseudomonas oryzae Uyeda and Ishiyama, Proc. Third Pan-Pacific Sci. Congr., Tokyo, 2, 1926, 2112; Bacterium oryzae Nakata, see Elliott, Man. Bact. Plant Path., 1930, 172; Phytomonas oryzae Magrou, in Hauduroy et al., Dict. d. Bact. Path., Paris, 1937, 388; Dowson, Trans. Brit. 
Mycol. Soc., 26, 1943, 12.) From Gr oryza, rice; M. L. Oryza, a generic name. Probable synonym: Pseudomonas ito ana Tochinai, Ann. Phytopath. Soc. Japan, 2, 1932, 456; Bacterium itoanum Burgwitz, Phytopathogenic Bacteria, Leningrad, 1935, 74; Phytomonas itoana Magrou, in Hauduroy et al., Dict. d. Bact. Path., Paris, 1937, 370.

Rods : 0.5 to 0.8 by 1.0 to 2.0 microns. Motile with a polar flagellum. Gramnegative.

Gelatin: No liquefaction.

Nutrient agar colonies: Round, smooth, glistening, wax yellow.

Milk: Slightly acid

Nitrites are not produced from nitrates.

Hydrogen sulfide produced.

Acid but no gas from glucose, lactose and sucrose.

Optimum temperature $26^{\circ}$ to $30^{\circ} \mathrm{C}$.

Strict aerobe.

Source: Isolated from a leaf blight of rice.

Habitat: Pathogenic on rice, Oryza sativa.

40. Xanthomonas celebensis (Gäumann) Dowson. (Pseudomonas celebensis Gäumann, Ztschr. f. Pflanzenkrank., 33, 1923, 11; Meded. Inst. voor Plantenziek., Buitenzorg, 59, 1923, 17 ; Bacterium celebense Elliott, Man. Bact. Plant Path., 1930, 108; Phytomonas celebensis Magrou, in Hauduroy et al., Dict. d. Bact. Path., Paris, 1937, 343; Dowson, Trans. Brit. Mycol. Soc., 26, 1943, 11.) From M. L. of the island Celebes.

Rods : 0.9 by 1.5 microns. Motile by a polar flagellum. Gram-negative.

Agar colonies: Grayish yellow.

Broth: Thin pellicle.

Milk: Coagulated and cleared.

Nitrites not produced from nitrates.

Sodium selenite: Brick red.

Starch is hydrolyzed.

Source: From vascular bundles of diseased bananas in Celebes.

Habitat: Causes the blond disease of banana.
41. Xanthomonas panici (Elliott) comb. nov. (Bacterium panici Elliott, Jour. Agr. Res., 26, 1923, 157; Psendomonas panici Stapp, in Sorauer, Handb. d. Pflanzenkrank., 2, 5 Aufl., 1928, 27; Phytomonas panici Bergey et al., Manual, 3rd ed., 1930, 269.) From M. L. Panicum, a generic name.

Rods : 0.69 by 1.66 microns. Capsules. Motile by 1 or rarely 2 polar flagella. Gram-negative.

Gelatin : Liquefaction slow.

Beef agar colonies: Round, white, smooth, glistening, margins at first entire, later undulate.

Broth : Moderate turbidity in 24 hours. Thin pellicle. Medium brownish.

Milk: Alkaline and clears.

Nitrites are produced from nitrates.

Indole not produced.

Hydrogen sulfide produced.

No gas from carbohydrates.

Starch: Hydrolysis moderate.

Optimum temperature $33^{\circ} \mathrm{C}$. Maximum $45^{\circ} \mathrm{C}$. Minimum $5^{\circ} \mathrm{C}$.

Optimum $\mathrm{pH} 6.15$ to 6.3 . $\mathrm{pH}$ range 5.4 to 10.0 .

Aerobic.

Distinctive characters: Differs from Pseudomonas andropogoni in that it liquefies gelatin, produces nitrites from nitrates, and does not infect sorghum and broom corn.

Source: Isolation from water soaked lesions on leaves, sheaths and culms of millet collected in Wisconsin and in $\mathrm{S}$. Dakota.

Habitat: Pathogenic on proso millet, Panicum miliaceum.

42. Xanthomonas proteamaculans (Paine and Stansfield) comb.nov. (Pseudomonas proteamaculans Paine and Stansfield, Aun. Appl. Biol., 6, 1919, 38; Phytomonas proteamaculans Bergey et al., Manual, 3rd ed., 1930, 217 ; Bacterium proteamaculans Elliott, Man. Bact. Plant Path., 1930, 186.) From M. L. Protea, a generic name; maculans, spotting. Rods : 0.6 to 0.8 by 0.8 to 1.6 microns. 
Motile with 1 to 3 polar flagella. Grampositive.

Gelatin: Liquefaction.

Agar slant: Growth wet-shining, dirty white with a faint yellow tinge.

Broth: Turbid in 24 hours. Slight ring.

Milk: Acid with soft curd after 2 days.

Later a separation of whey.

Nitrites are produced from nitrates.

Acid and gas from glucose, sucrose and mannitol. No acid or gas from lactose.

Starch : Slight hydrolysis.

Source: Repeated isolation from a leafspot of Protea in England.

Habitat: Pathogenic on Protea cynaroides.

43. Xanthomonas manihotis (ArthaudBerthet) comb. nov. (Bacillus manihotus Arthaud-Berthet by Bondar, Chacaras and Quintaes 5(4), 1912, 15; Bacillus manihot Bondar (and Arthaud-Berthet), Bol. Agric., São Paulo, 16, 1915, 513; Bacterium manihotus Drummond and Hipolito, Ceres, 2, 1941, 298; Phytomonas manihotis Viegas, Rev. d. Agr., Pieracicaba, 15, 1940, 475.) From M. L. Manihotus, a generic name

Description from Burkholder, Phytopath., 32, 1942, 147.

Rods : 0.35 to 0.93 by 1.4 to 2.8 microns. Gram-negative and mostly non-motile. One isolate showed a few cells with 1 polar flagellum. Amaral (Instit. Biol., São Paulo, Arq., 13, 1942, 120) states that the species is motile with one polar flagellum.

Gelatin : Liquefaction.

Beef-extract-peptone agar: Streaks raised, ivory-color, smooth, shiny, with edges entire.

Potato-glucose agar: Growth abundant, white to hyaline, very mucoid.

Broth: Turbid with a whitish granular ring.

Litmus milk: Litmus reduced and milk clears. With return of color, litmus is purple.

Indole not formed.

Hydrogen sulfide is formed.
Nitrites produced from nitrates (Drummond and Hipolito, loc. cit.).

Asparagine not used as a nitrogen and carbon source. No growth in nitrate synthetic broth.

Weak growth but slight acid production in synthetic medium plus glucose, d-galactose, d-fructose, d-xylose, maltose and sucrose. No growth in rhamnose, 1-arabinose, d-lactose, glycerol, mannitol and salicin. Good growth with alkaline reaction in same medium plus salts of the following acids: acetic, citric, malic, maleic and succinic. The salts of formic, hippuric, lactic and tartaric acids were not utilized.

Starch not hydrolyzed. Amaral (loc. cit.) finds hydrolysis.

Lipolytic action slight.

Aerobic.

Optimum temperature $30^{\circ} \mathrm{C}$. Maximum $38^{\circ} \mathrm{C}$. Minimum $5^{\circ} \mathrm{C}$.

Source: First isolated from the cassava, Manihotus utilissima in Brazil.

Habitat: Produces a wilt disease on various species of Manihotus.

\section{Xanthomonas rubrisubalbicans} (Christopher and Edgerton) comb. nov. (Phytomonas rubrisubalbicans Christopher and Edgerton, Jour. Agr. Res., 41, 1930, 266; Bacterium rubrisubalbicans Burgwitz, Phytopathogenic Bacteria, Leningrad, 1935, 105.) From L. ruber, red; subalbicans, nearly white.

Rods: Short with polar flagella. Capsules. Gram-negative.

Gelatin: No liquefaction.

Bacto-glucose agar colonies: Circular, glistening, viscid, milky gray to buff. Margins translucent, entire.

Broth: Turbid after 24 hours. Pellicle and a ropy sediment.

Indole produced.

Hydrogen sulfide produced.

No gas from carbohydrates.

Starch hydrolyzed.

Optimum temperature $30^{\circ} \mathrm{C}$.

Optinum pH 6.8 to 8.0 .

Source: Isolated many times from mottled stripe of sugar cane in Louisiana. 
Habitat: Pathogenic on sugar cane, Johnson's grass and sorghum.

\section{Xanthomonas cannae (Bryan)} comb. nov. (Bacterium cannae Bryan, Jour. Agr. Res., 21, 1921, 152; Phytomonas cannae Bergey et al., Manual, 1st ed., 1923, 188; Pseudomonas cannae Stapp, in Sorauer, Handb. d. Pflanzenkrank., 2, 5 Aufl., 1928, 65.) From Gr. canna, a reed; M. L. Canna, a generic name.

Rods : 0.5 to 0.7 by 1.0 to 2.0 microns. Motile with 1 to 3 polar flagella. Capsules. Gram-negative.

Gelatin: Slow liquefaction.

Agar streaks: Filiform, white, moist, with thin margins and granular centers.

Broth: Turbid, heavy sediment.

Milk: Alkaline and clears.

Nitrites are produced from nitrates.

Indole not produced.

Hydrogen sulfide produced.

Optimum temperature $35^{\circ} \mathrm{C}^{\circ}$. Maximum $40^{\circ} \mathrm{C}$. Minimum $5^{\circ} \mathrm{C}$

Aerobic.

Source: Isolated from discased canna leaves collected in Washington, D. C. and in Illinois.

Habitat: Causes disease in Canno indica.

46. Xanthomonas zingiberi (L'yeda) comb. now. (Lyeda, Cent. f. Bakt., II Abt., 17, 1907, 383; Pseudomonas zingiberi Lyeda, Rept. Imp. Agr. Exp. Sta., Japan, No. 35, 1908, 114; Bacterium zingiberi Nakata, see Elliott, Man. Bact. Plant Path., 1930, 266; Phytomonas zingiberi Magrou, in Hauduroy, et al., Dict. d. Bact. Path., Paris, 1937, 437.) From I. zingiberis, ginger; M. I. Zingiber, a generic name.

Description from Stapp, in Sorauer,
Handb. d. Pflanzenkrank., 2, 5 Aufl, 1928,65 .

Rods : 0.5 to 1.1 by 0.75 to 1.8 microns. Non-motile at first, later a polar flagellum. Gram-negative.

Gelatin: Liquefaction.

Agar colonies: White.

Milk: Coagulation and peptonization of the casein.

Nitrites are produced from nitrates.

Indole not formed.

Hydrogen sulfide is formed.

No gas from glucose.

Optimum temperature $28^{\circ} \mathrm{C}$. Maximum $40^{\circ} \mathrm{C}$. Minimum $5^{\circ} \mathrm{C}$.

Source: Isolated from ginger plant showing a rot at the base of the sprouts.

Habitat: Pathogenic on ginger, Zingiber officinale.

47. Xanthomonas conjaci (Lyeda) comb. nov. (Pseudomonas conjac Lyeda, Bot. Mag. Tokyo, 24, 1910, 182; Bacterium conjac Elliott, Man. Bact. Plant Path., 1930, 121; Phytomonas conjac Magrou, in Hauduroy et al., Dict. d. Bact. Path., Paris, 1937, 347.) From II. I. conjac, the specific name of the plant which this species attacks.

Description from Elliott (loc.cit.).

Rods: 0.75 to 1.0 by 1.5 microns. Iotile with 1 to 4 polar Hagella. Grampositive.

Gelatin colonies: Circular to irregular, light yellow.

Broth: Pellicle formed.

Milk: Coagulation.

Conjac: Liquefied.

Nitrites produced from nitrates.

Indole produced.

Hydrogen sulfide produced.

Gas from glueose

Favorable temperature $24^{\circ} \mathrm{C}$.

Habitat: Pathogenic on Amorphophallus konjac.

Appendix I:* The following organisms placed in the genus Pseudomonas apparently belong in Xanthomonas. Some may even be plant pathogens although they were

* Prepared by Prof. Robert S. Breed, New York State Experiment Station, Geneva, New York, July, 1943. 
isolated from water, soil and similar sources. Pigment is usually yellow and is not water-soluble.

Key to yellow and other chromogenic species in genus Pseudomonas.

1. Colonies yellow.

a. Gelatin liquefied.

b. Nitrites produced from nitrates.

c. Acid and gas produced from glucose.

1. Pseudomonas fermentans.

ce. Acid but no gas from glucose.

2. Pseudomonas trifolii.

3. Pseudomonas xanthe.

cec. Action on glucose not recorded.

4. Pseudomonas caudata.

bb. Nitrites not produced from nitrates.

c. Litmus milk acid or ferment lactose.

5. Pseudomonas perlurida.

6. Pseudomonas iridescens.

cc. Litmus milk not coagulated. Yellow sediment.

7. Pseudomonas turcosa.

ccc. Litmus milk slimy, alkaline.

8. Pseudomonas ochracea.

aa. No liquefaction of gelatin.

b. Nitrites produced from nitrates.

c. Litmus milk, slow coagulation.

9. Pseudomonas cerevisiae.

cc. Litmus milk, acid but no digestion.

10. Pseudomonas arguta.

ecc. No growth in litmus milk.

11. Pseudomonas subcreta.

cece. Action on litmus milk, not recorded.

12. Pseudomonas pictorum.

bb. Nitrites not produced from nitrates.

c. Butter colored pellicle on litmus milk.

13. Pseudomonas lacunogenes.

cc. No surface pellicle.

14. Pseudomonas segnis.

2. Colonies on gelatin blue center surrounded by yellow zone with peripheral green zone.

a. Gelatin liquefied.

b. Nitrites produced from nitrates.

15. Pseudomonas lemonnieri.

1. Pseudomonas fermentans von Wolzogen Kühr. (von Wolzogen Kühr, Cent. f. Bakt., II Abt., 85, 1932, 228; Flavobacterium fermentans Bergey et al., Manual, 4th ed., 1934, 155.) From Latin, fermento, to ferment.

Rods : 0.4 to 0.6 by 1.7 to 3.4 microns, with rounded ends, occurring singly and in pairs. Motile, with a single or occasionally 2 or 3 polar flagella. Gramnegative.

Gelatin colonies: Circular, grayish, with rapid liquefaction.

Gelatin stab: Liquefaction crateriform. 
Agar colonies: Circular, slightly convex, opaque, gray by reflected, and light-brown by transmitted light.

Agar slant: Gray, becoming yellowish.

Broth: Turbid with pellicle.

Litmus milk: Acid.

Potato: Gray to yellowish growth.

Indole is formed.

Nitrites produced from nitrates.

Acid and visible gas from glucose, lactose and sucrose.

Acetylmethylcarbinol is formed.

Ammonia is formed from peptone and asparagin.

Hydrogen sulfide is formed.

Starch is hydrolyzed.

Lipase is formed. Catalase positive.

Aerobic, facultative.

Optimum temperature $37^{\circ} \mathrm{C}$.

Distinctive character: Proluecs gas in lactose formentation tubes.

Source: Ten cultures from the larvac of a midge (Chironomus plumosus) anil from filtered water.

Habitat: Unknown.

2. Pseudomonas trifolii Huss. (Huss, Cent. f. Bakt., II Abt., 19, 1907, 68; Flavobacterium trifolii Bergey et al., MFanual, 1st od, 1923, 111.) From Latin, tres (tri-), three; folim, leaf; M. T. Trifolium, clover.

Possible synonym: Bacillus annulatus Wright. Wright, Memoirs Nat. Acad. S.ci., 7, 1895, 443; Pseudomonas annulata Chester, Man. Determ. Bact., 1901, 315; Relationship to Bacillus annulatus Zim. mermann uncertain. Die Bakt. unserer Trink- und Nutzwässer, Chemnitz, II Reihe, 1890, 30; Flavobacterium annulatum Bergey et al., Manual, 1st. ed., 1923. 110 .)

According to Mack (Cent. f. Bakt., II Abt., 95, 1936, 218) the following organism is to be regarded as identical with Pseudomonas trifolii: Bacillus mesentericus aureus Winkler (Cent. f. Bakt., II Abt., $5,1899,577$ ) regarded by Burri (Cent. f. Bakt., II Abt., 10, 1902, 756) and Düggeli (Cent. f. Bakt., II Abt., 12, 1904, 602) as identical with the organism which Düggeli (loc. cit.) names Bacterium herbicola aureum. The organism studied as Bacterium herbicola by Hüttig (Cent. f. Bakt., II Abt., 84, 1931, 231) is not regarded as identical with the Burri and Düggeli organism by Mack. Beijerinck (Cent. f. Bakt., II Abt., 15, 1905, 366) states that Bacillus herbicola of Burri and Düggeli is identical with his Bacillus anglomerans (Botan. Ztg., 1888, 749). If so, this binomial has priority.

Rods : 0.5 to 0.7 by 0.75 to 2.0 microns, occurring singly, in pairs and in chains. Motile, possessing a single polar flagellum. Gram-negative.

Gelatin colonies: Convex, smooth, moist, glistening, grayish-yellow.

Gelatin stab: Napiform liquefaction.

Agar colonies: Small, circular, grayish, hecoming brownish-yellow.

Agar slant : Jellowish, becoming brownish-yelloir streak, lacerate margin.

Broth: Turbid, with grayish-yellow pellicle and sediment.

Litmus milk: Slowly coagulated; alkaline; with yellow ring.

Potato: Thick, yellowish, flat, smonth, glistening.

Hydrogen sulfide produced

Indole is formed.

Acid from glucose, sucrose, xylose. arabinose, and mannitol. No acid from lactose.

Yitrites produced from nitrates.

Cultures have an agreeable odor.

Volutin formed.

Aerobic, facultative

Optimum temperature $33^{\circ}$ to $35^{\circ} \mathrm{C}$

Source: Isolated from clover hay.

Habitat : Evidently a common organism on the leaves of plants

3. Pseudomonas xanthe Zettnow (Zettnow, Cent. f. Bakt., I Abt., Orig., \%7, 1915, 220; Flavobacterium zettnowii Bergey et al., Manual, 1st ed., 1923, 112; Flavobacterium xanthium (sic) Bergey et al., Manual, 3rd ed., 1930, 145.) From Gr. xanthus, yellow. 
Rods : 0.5 to 0.6 by 0.4 to 1.4 microns. Motile, possessing a single or occasionally two or more very long (20 microns) polar flagella. Gram-negative.

Gelatin colonies: Circular, yellow, granular.

Gelatin stab: Pale-yellow surface growth. Brownish yellow under surface colonies. Saccate liquefaction.

Agar slant: Dark yellow, glistening, with dark yellow sediment in water of condensation. Pigment not water-soluble.

Broth: Turbid.

Litmus milk: Slightly acid. Litmus reduced.

Potato: Grayish yellow to brownish growth.

Indole formed.

Nitrites are produced from nitrates.

Acid formed in glucose.

Starch hydrolyzed.

Blood serum not liquefied.

Aerobic, facultative.

Optimum temperature $30^{\circ} \mathrm{C}$.

Source: Air contamination.

\section{Pseudomonas caudata (Wright)} Conn. (Bacillus caudatus Wright, Memoirs Nat. Acad. Sci., 7, 1895, 444; Bacterium caudatus Chester, Annual Rept. Del. Col. Agr. Exp. Sta., 9, 1897, 107; Conn, Jour. Agr. Res., 16, 1919, 313; Flavobacterium caudatum Bergey et al., Manual, 1st ed., 1923, 109.) From Latin, cauda, tail.

Rods : Long, granular, slender, occurring singly, in pairs and in ehains. Appear like cocci in old cultures. Motile, possessing a polar flagellum (Conn). Gramnegative.

Gelatin colonies: Yellow, translucent, smooth, undulate.

Gelatin stab: Villous growth in stab. Crateriform liquefaction.

Agar slant: Yellow to orange, glistening, translucent, slightly spreading. May lose power to form pigment.

Broth: Turbid, with yellow sediment.

Litmus milk: Unchanged.

Potato: Dark yellow, raised, rough, spreading.
Indole not formed.

Nitrites and ammonia produced from nitrates.

Ammonia produced from peptone.

Starch is digested.

Aerobic, facultative.

Optimum temperature $25^{\circ} \mathrm{C}$.

Habitat: Water.

\section{Pseudomonas perlurida Kellerman} et al. (Kellerman, McBeth, Scales and Smith, Cent. f. Bakt., II Abt., 39, 1913, 516 ; also McBeth, Soil Sci., 1, 1916, 472; Cellulomonas perlurida Bergey et al., Manual, 1st ed., 1923, 163.)

Rods : 0.4 by 1.0 micron. Motile with one to three polar flagella. Gramnegative.

Gelatin stab: Liquefaction.

Agar slant: Moderate, flat, faint yellow growth.

Broth: Turbid in 5 days.

Litmus milk: Acid. Peptonization after 16 days.

Potato: Scant yellow growth with bleaching along line of growth.

Indole not formed.

Nitrites not produced from nitrates.

Ammonia is produced.

Acid from glucose, maltose, lactose, sucrose, starch, glycerol and mannitol.

Aerobic, facultative.

Optimum temperature $20^{\circ} \mathrm{C}$.

Source: Soil from Virginia, Louisiana and Missouri.

Habitat : Soil.

5a. Pseudomonas perlurida var. virginiana Kellerman et al. (loc. cit.). Does not grow on potato and liquefies gelatin rapidly.

Source : Soil from Virginia.

6. Pseudomonas iridescens Stanier. (Jour. Bact., 43, 1941, 542.) From Latin, iridescent.

Rods : 0.2 to 0.3 by 1.5 to 7.0 microns, average length 5.0 to 6.0 microns, occurring singly. Non-motile. Gram-negative. 
Sea water gelatin stab: Filiform growth Liquefaction by some strains.

Sea water agar colonies: Concave, 2 to $3 \mathrm{~mm}$ in diameter, smooth, glistening, translucent, pale yellow, edge irregular. After 2 to 3 days a marked iridescence. Later colonies rough, opaque, bright yellow, sunken central portion with translucent periphery.

Sea water agar slant: Growth spreading, smooth, glistening, translucent, pale yellow, iridescent, butyrous.

Sea water broth: Turbid, light yellow, granular pellicle.

Indole not formed.

Nitrites not produced from nitrates.

Hydrogen sulfide not produced.

Catalase positive.

Urease negative.

Acid from xylose, glucose, galactose, lactose, maltose, sucrose and cellobiose. No acid from arabinose. Starch and cellulose are attacked.

Aerobic.

Optimum temperature $23^{\circ} \mathrm{C}$. Minimum $5^{\circ} \mathrm{C}$. Maximum $30^{\circ} \mathrm{C}$.

Salt range: 0.25 to 6.0 per cent. Optimum 1.0 to 4.0 per cent.

Source: Sea water.

Habitat: Common along the coast of the North Pacific.

\section{Pseudomonas turcosa (Zimmer-} mann) Migula. (Bacillus turcosa Zimmermann, Bakt. unserer Trink- und Nutzwässer, Chemnitz, 2, 1894, 32; Migula, Syst. d. Bakt.2, 1900, 937; Flazobacterium turcosum Bergey et al., Manual, 1st ed., 1923, 111.) From M. L. turcois, turquoise.

Rods : 0.5 by 1.05 to 1.82 microns, occurring singly. A short polar flagellum (Migula). Gram-negative.

Gelatin colonies: Small, translucent, yellow.

Gelatin stab: Small, yellow, convex surface growth, with slight brownish tint. Liquefaction, with grayish to greenish color in liquefied portion.

Agar slant: Abundant, glistening, greenish to sulfur yellow streak.
Broth: Slightly turbid with yellow sediment.

Litmus milk: To coagulation. Yellow sediment.

Potato: Clear chromium yellow growth over entire surface.

Indole is not formed.

Nitrites not produced from nitrates.

Acid from glucose. Slight action on sucrose.

Aerobic, facultative.

Optimum temperature $30^{\circ} \mathrm{C}$.

Source: Isolated by Tataroff from a well in Dorpat (Die Dorpaten Wasserbakterien, Inaug. Diss., 1891, 52, No. 24).

Habitat: Water, sea water.

\section{Pseudomonas ochracea (Zimmer-} mann) Chester. (Bacillus ochraceus Zimmermann, Bakt. unserer Trink- und Nutzwässer, Chemnitz, 1, 1890, 60; Chester, Determinative Bacteriology, 1901, 316; Flavobacterium ochraceum Bergey et al., Manual, 1st ed., 1923, 110; Chromobacterium ochraceum Topley and Wilson, Princ. Bact. and Immun., 1, 1931, 405.) From Greek, ochros, pale yellow.

Rods : 0.7 to 0.8 by 1.2 to 4.5 microns, occurring in pairs and longer chains. Slow undulatory motion (Zimmermann). Polar flagella (Lehmann and Neumann, Bakt. Diag., 1 Aufl., 2, 1896, 255). Gramnegative.

Gelatin colonies : Pale yellow to golden, ochre yellow, slightly raised, with slightly fringed margin, granular.

Gelatin stab: Yellowish to yellow-gray surface growth. Infundibuliform liquefaction. Pale yellow to ochre yellow sediment.

Agar colonies: Thin, flat, yellowish, smooth.

Agar slant: Thin, yellowish-gray to ochraceous growth.

Broth: Slightly turbid, with pale yellow sediment.

Litmus milk: Medium becomes slimy; alkaline.

Potato: Ochre-yellow streak.

Indole is formed. 
Nitrites not produced from nitrates. Hydrogen sulfide is formed.

Aerobic, facultative.

Optimum temperature $35^{\circ} \mathrm{C}$.

Source: Chemnitz tap water.

Habitat: Water.

9. Pseudomonas cerevisiae Fuhrmann. (Fuhrmann, Cent. f. Bakt., II Abt., 16, 1906, 309; Flavobacterium cerevisiae Bergey et al., Manual, 1st ed. 1923, 111.) From Latin, cerevisia, beer.

Rods: Straight and slightly curved, 0.6 by 1.5 to 2.0 microns, occurring singly and in chains. Motile, possessing tuft, four to six polar flagella. Gram-negative.

Gelatin colonies: Circular, white, slightly contoured, becoming brownishyellow.

Gelatin stab: Slight yellowish growth in stab. No liquefaction.

Agar colonies: Thin, spreading, contoured.

Agar slant: Moist, glistening, thin, pale yellow, spreading, contoured.

Litmus milk: Slow coagulation

Potato: Yellowish-brown, spreading growth.

Indole not formed.

Nitrites produced from nitrates.

No gas from glucose.

Aerobic, facultative.

Optimum temperature $30^{\circ} \mathrm{C}$.

Source: Isolated from beer.

Habitat: Unknown.

10. Pseudomonas arguta McBeth. (McBeth, Soil Science, 1, 1916, 465; Cellulomonas arguata (sic) Bergey et al., Manual, 1st ed., 1923, 164.) From Latin, arguo, to show.

Rods : 0.3 by 0.8 micron. Motile with one or two polar flagella. Gram-negative.

Gelatin stab: Moderate, yellowish growth. No liquefaction in 30 days.

Agar colonies: Circular, slightly convex, soft, grayish-white, granular, entire.

Agar slant: Scant, grayish-white growth.

Potato agar slant: Moderate, yellowish, glistening.
Broth : Turbid.

Ammonia cellulose agar: Enzymatic zone 2 to $3 \mathrm{~mm}$ in 30 days.

Filter paper broth: Paper is reduced to loose floceulent mass which disintegrates very readily on slight agitation. More rapid decomposition when the broth contains ammonium sulfate, potassium nitrate, peptone or casein as sources of nitrogen.

Litmus milk: Acid, not digested.

Potato: No growth.

Indole not formed.

Nitrites produced from nitrates.

Ammonia not produced.

Acid from glucose, maltose, lactose, starch. No acid from glycerol, mannitol or sucrose.

Aerobic, facultative.

Optimum temperature $20^{\circ} \mathrm{C}$.

Source: Isolated twice from California soils.

Habitat: Soil.

11. Pseudomonas subcreta McBeth and Scales. (McBeth and Scales, Bur. Plant Industry, U. S. Dept. Agr., Bul. 266, 1913, 37; Cellulomonas subcreta Bergey et al., Manual, 1st ed., 1923, 164.) From Latin, sub, under, imperfect; creta chalk.

Rods : 0.3 by 1.4 microns. Motile with one to five polar flagella. Gram-negative.

Gelatin stab : Filiform growth, no liquefaction.

Cellulose agar: No surface growth. Moderate, generally faint yellow growth in medium, area of growth sunken.

Agar slant: Glistening, smooth, moist, vitreous to faint yellow.

Starch agar: Enzymatic zone 2 to $4 \mathrm{~mm}$.

Broth: No growth.

Litmus milk: No growth.

Potato: Growth scanty, concave due to slight liquefaction, white to faint yellow. Bleached around growth.

Indole not formed.

Trace of nitrites produced from nitrates.

Ammonia not produced.

Acid from glucose, lactose, maltose, 
sucrose and starch. No acid from glycerol or mannitol.

Aerobic, facultative.

Optimum temperature $20^{\circ} \mathrm{C}$.

Habitat : Soil

12. Pseudomonas pictorum Gray and Thornton. (Gray and Thornton, Cent. f. Bakt., II Abt., 73, 1928, 89; Achromobacter pictorum Bergey et al., Manual, 3rd ed., 1930, 217.) From Latin, picti, the Picts of Eastern Scotland.

Rods : 0.5 to 0.8 by 1.5 to 5.0 microns. Motile usually with a single polar flagellum. Gram-negative.

Gelatin colonies: Circular, greenishyellow, convex, smooth, glistening, entire.

Gelatin stab: No liquefaction.

Agar colonies : Circular, yellow, convex, smooth, glistening, entire.

Agar slant: Filiform, yellow, convex, smooth, glistening, entire.

Broth : Turbid.

Nitrites produced from nitrates.

Starch not hydrolyzed.

Acid from glueose and maltose.

Attacks phenol.

Aerobic, facultative.

Optimum temperature $25 \mathrm{C}$.

Source: One culture from soil.

Habitat: Soil.

13. Pseudomonas lacunogenes Goresline. (Jour. Bact., 26, 1933, 447.) From Latin lacuno, dimple and genero, to produce.

Short rods: 0.2 to 0.3 by 1.0 to 1.2 microns, with pointed ends, occurring singly or in pairs. Notile with a single polar flagellum from 2 to 15 microns in length. Gram-negative.

Plain gelatin stab: No growth.

Nutrient gelatin stab: Growth brownish-yellow, half-way down stab, heavier at surface. No liquefaction.

Nutrient agar colonies: Small, yellow; surface of the agar pitted or dimpled. After 5 days colonies 5 to $7 \mathrm{~mm}$ in diam- eter, orange-yellow, slightly raised, surrounded by a depression.

Nutrient agar slant: Growth heavy, light orange-yellow; consistency of warm butter; edge entire, slightly raised. Shallow depression formed on each side of streak. Agar softened beneath growth.

Nutrient broth: Turbid in 48 hours. Light orange-yellow pellicle; considerable viscous sediment.

Litmus milk: Alkaline; butter-colored pellicle. Reduction in bottom of tube after 10 days. No curd. No digestion.

Potato: Growth moderate, orange-yellow, smooth. No darkening.

Indole not formed.

Nitrites not produced from nitrates.

Starch agar plates not hydrolyzed.

Utilizes arabinose, galactose, lactose, fructose, maltose, melezitose, raffinose, starch, xylose, glucose, mannose, sucrose, pectin, rhamnose, salicin and dextrin. No growth in dulcitol, erythritol, glycerol, sorbitol, mannitol or inulin.

Limits of pH : 5.4 to 10.0 .

Temperature relations : Optimum $28^{\circ} \mathrm{C}$. Good growth at $25^{\circ} \mathrm{C}$. Moderate growth at $20^{\circ}$ and at $37^{\circ} \mathrm{C}$. No growth at $10^{\circ}$ and at $42^{\circ} \mathrm{C}$.

Facultative anaerobe.

Distinctive characters: Softens agar; considerable change in viscosity of agar due to this digestion; utilization of ammonium sulfate as nitrogen source.

Source: Three cultures isolated from an experimental trickling filter receiving creamery wastes.

Habitat: Probably widely distributed in nature.

14. Pseudomonas segnis Goresline. (Jour. Bact., 26, 1933, 452.) From Latin segne, non-energetic.

Short rods : 0.2 to 0.3 by 1.0 to $1.2 \mathrm{mi}$ crons, with pointed ends, occurring singly or in pairs. Motile with a single polar flagellum. Gram-negative.

Plain gelatin stab: No growth.

Nutrient gelatin stab: Growth yellow, half-way down stab, best at surface. No liquefaction. 
Nutrient agar colonies: Very small, light yellow; surface pitted. After 5 days colonies $5 \mathrm{~mm}$ in diameter.

Nutrient agar slant: Growth heavy, orange-yellow, consistency of warm butter; edge entire, slightly raised; slight depression formed on each side of growth. Agar softened beneath growth.

Nutrient broth: Turbid in 48 hours. No pellicle or surface growth. Moderate amount of sediment. Old cultures with a yellow ring at surface and occasionally a loose membrane.

Litmus milk: Slightly alkaline after 10 days. No reduction. No surface growth.

Potato: Scant yellow-orange growth. No darkening.

Indole not formed.

Nitrites not produced from nitrates.

No $\mathrm{H}_{2} \mathrm{~S}$ produced.

Starch not hydrolyzed.

Utilizes arabinose, glucose, galactose, lactose, fructose, maltose, mannose, xylose, sucrose, melezitose and raffinose.

Limits of $\mathrm{pH}: 5.8$ to 9.0 .

Temperature relations: Optimum $28^{\circ} \mathrm{C}$. Good growth at $25^{\circ} \mathrm{C}$. Noderate growth at $20^{\circ}$ and at $37^{\circ} \mathrm{C}$. No growth at $10^{\circ}$ and at $42^{\circ} \mathrm{C}$.

Facultative anaerobe.

Distinctive characters: Softens agar; considerable change in viscosity of agar due to this digestion.

Source: Isolated from an experimental trickling filter receiving creamery wastes.

Habitat: Probably widely distributed in nature.

15. Pseudomonas lemonnieri (Lasseui) comb. nov. (Bacillus lemonnieri Lasseur, Compt. rend. Soc. Biol. Paris, 74, 1913, 47; Bul. de la Soc. des Sci. de Nancy, 1924; Flavobacterium lasseuri Bergey et al., Manual, 3rd ed., 1930, 144.) Named for Prof. G. le Monnier, a French scientist.

Rods: 0.5 to 0.7 by 1.0 to 2.0 microns, occurring singly and in pairs. Motile with a single polar flagellum. Gramnegative.

Gelatin colonies (glucose): Circular with blue center, a granular, yellow zone and a peripheral blue zone. Rapid liquefaction with blue crystals.

Gelatin stab: Liquefied.

Agar colonies: Circular, yellowish, lobate margin.

Agar slant: Jellowish streak, smooth, glistening.

Broth: Turbid with thin pellicle.

Litmus milk: After 48 hours the surlace of the milk becomes yellow to cream color turning blue. A soft coagulum is formed.

Potato: Raised growth, Prussian blue in color, with variations.

Indole is not formed.

Nitrites produced from nitrates.

Aerobic, facultative.

Optimum temperature $22^{\circ}$ to $25^{\circ} \mathrm{C}$.

Habitat: Water.

Appendix II:* The following inadequatcly described species may belong to the genus Xanthomonas.

Bacterium citri deliciosae Passalacqua. (Rev. Pat. Veg., 24, 1934, 27.) Isolated from Citrus sp.

Bacterium malvacearum var. barbadense Evelyn. (Ann. Rept. Agric. Barbados for 1926-27, 1928, 15.) Isolated from cotton.

Pseudomonas amaranti (sic) Smith. (U. S. Dept. Agr., Div. Veg. Phys. and Path. Bull., 28, 1901, 153; Bacterium amaranthi Smith, Bact, in Relation to Plant Dis., 3, 1914, 148; Phytomonas amaranthi Bergey et al., Manual, 1st ed., 1923, 186.) Isolated from diseased amaranthus. Growth in culture similar to Xanthomonas campestris and Xanthomonas hyacinthi.

Pseudomonas alutacea Migula. (Ledergelber Bacillus, Tataroff, Die Dorpater Wasserbakterien, Inaug. Diss., Dorpat,

* Prepared by Prof. Pobert S. Breed, New York State Experiment Station, Geneva, New York, July, 1943. 
1891, 61; Migula, Syst. d. Bakt., 2, 1900, 936.) Isolated from water.

Pseudomonas graveolans Migula. (Bacillus aquatilis graveolens Tataroff, Die Dorpater Wasserbakterien, Inaug. Diss., Dorpat, 1891, 48; Migula, Syst. d. Bakt., 2, 1900, 934.) Isolated from water. Not Pseudomonas graveolens Levine and Anderson (Jour. Bact., 23, 1932, 343) isolated from musty eggs, and by Olsen and Hammer (Iowa State Coll. Jour. Sci., 9, 1934, 125) from milk.

Pserdomonas resinacea Migula. (Harzfarbener Bacillus, Tataroff, Die Dorpater Wasserbakterien, Inaug. Diss., Dorpat, 1891, 61; Migula, Syst. d. Bakt., 2, 1900, 935.) Isolated from water.

Ianthomonas taraxaci Niederhauser. (Phytopath., 33, 1943, 961.) Pathogenic on Russian dandelion (Taraxacum kok-saghz).

\section{Genus III. Methanomonas Orla-Jensen. * \\ (Cent.f. Bakt., II Abt., 22, 1909, 311.)}

Cells monotrichous, capable of obtaining energy from oxidation of methane to $\mathrm{CO}_{2}$ and water.

The type species is Methanomonas methanica (Söhngen) Orla-Jensen.

1. Methanomonas methanica (Söhngen) Orla-Jensen. (Bacillus methanicus Söhngen, Cent. f. Bakt., II Abt., 15, 1906, 513; Orla-Jensen, Cent. f. Bakt., II Abt., 22, 1909, 311.) From methane.

Short rods : 0.5 to 0.8 by 2.0 to $3.0 \mathrm{mi}$ crons, motile in young cultures by means of a single flagellum. In older cultures nearly spherical. Can be cultivated in an atmosphere composed of one part $\mathrm{CH}$; and two parts air on washed agar containing the necessary inorganic salts. The growth is membranous.
At the end of tro rreeks, the organisms changed an atmosphere containing 225 $\mathrm{ml}$. $\mathrm{CH}_{4}$ and $321 \mathrm{ml}$. O to the followi.te :
$\mathrm{CH}_{4}$
$0 \mathrm{ml}$.
$\mathrm{CO}_{2}$
$78 \mathrm{ml}$.
$\mathrm{O}_{2}$

In addition, $21 \mathrm{ml} . \mathrm{CO}_{2}$ was dissolved in the liquid.

Ilabitat: Presumably widely distrib$11 . \mathrm{d}$ in soil.

\section{Genus $I V$. Acetobacter Beijerinck.}

(Proc. Kon. Akad. v. Wetenschapp., Amsterdam, 2, 1900, 495.)

Acetobacter aceti first appeared (Kral's Sammlung v. Mikroorg., Prague, 1898, 4) as a synonym of Bacterium aceti Hansen. Beijerinck (loc. cit.) mentions Acctobacter aceti in a footnote of a later paper. The genus name Acetobacter was accepted by Fuhrmann (Beiheft Bot. Centralbl.. Orig., 19, 1905, 8) and others. From Latin, acetum, vinegar; bactrum, rod.

Synonyms: ? Ulvina Kützing, Algae aquae dulcis, ete., 11th decade, 1837; 11ycoderma Thompson, Ann. d. Chem. u. Pharmacie, 83, 1852, 89; ? Tmbina Naegeli, Bericht über die Verhandlingen der bot. Section der 33 Tersammlung deutscher Naturforscher. und Arzter. Bot. Ztg., 1857, 760; Bacterium Lanzi, 1. Giorn. bot. ital., 1S76, 257; Tomula Saccardo, Atti Soc. Ven. Trent., 5, 1878, 315; Bacteriopsis (in part) Trevisan, Atti Accad. Fisio-Nedico-Statistica Milano, Ser. 4, 3, 18s.5, 103; Micrococcus Maggi, Jour. Microg., 10, 1886; Bacillus Schroeter, Kryptogamen Flora von Schlesien, 8, 1, 1886, 161; Termobacterium Zeidler, Cent. f. Bakt., II Abt., 2, 1896, 739; Acetobac-

* Prepared by Prof. D. H. Bergey, Philadelphia, Pennsylvania, December, 1922.

† Revised by Dr. C. D. Kelly, McGill Lniv., Montreal, P. Q., Canada, July, 1938; further revision by Dr. Reese H. Vaughn, Lniv. of California, Berkeley, California June, 1943. 
terium Ludwig, in abstract of Hoyer's Inaug. Diss., Cent. f. Bakt., II Abt., 4, 1898, 867; Acetimonas Orla-Jensen, Cent. f. Bakt., II Abt., 22, 1909, 312.

In addition, the sub-generic names Euacetobacter and Acetogluconobacter have been proposed by Asai, Jour. Agr. Soc. Japan, 11, 1935, 502. The genus Gluconobacter and the sub-genera Eugluconobacter and Gluconoacetobacter Asai (loc. cit.) may be synonyms in whole or in part.

Individual cells ellipsoidal to long and rod-shaped, occurring singly, in pairs, or in short or long chains. Motile with polar flagella, or non-motile. Involution forms may be spherical, elongated, filamentous, club-shaped, swollen, curved or even branched. Young cells Gram-negative; old cells often Gram-variable. Obligate aerobes; as a rule strongly catalase positive, sometimes weakly so. Oxidize various organic compounds to organic acids and other oxidation products which may undergo further oxidation. Common oxidation products include acetic acid from ethyl alcohol, gluconic and sometimes ketogluconic acid from glucose, dihydroxyacetone from glycerol, sorbose from sorbitol, etc. Nutritional requirements vary from simple to complex. Development generally best in yeast infusion or yeast autolysate media with added ethyl alcohol or other oxidizable substrate. Optimum temperature variable with the species. Widely distributed in nature where they are particularly abundant in plant materials undergoing alcoholic fermentation; of importance to man for their role in the completion of the carbon cycle and for the production of vinegar.

The type species is Acetobacter aceti (Kützing) Beijerinck.

Key to species of genus Acetobacter.

I. Oxidize acetic acid to carbon dioxide and water.

A. Capable of utilizing ammonium salts as a sole source of nitrogen (Hoyer's solution).*

1. Acetobacter aceti.

B. Do not utilize ammonium salts as a sole source of nitrogen.*

1. Forms a thick, zoogloeal, cellulose membrane on the surface of liquid media.

2. Acetobacter xylinum.

2. Do not form a thick, zoogloeal membrane on the surface of liquid media.

3. Acetobacter rancens.

3a. Acetobacter pasteurianum.

3b. Acetobacter kuetzingianum.

II. Do not oxidize acetic acid.

A. Form pigments in glucose media.

1. Dark brown to blackish pigment.

4. Acetobacter melanogenum.

2. Pink to rose pigment.

5. Acetobacter roseum.

B. Do not form pigments.

1. Optimum temperature $30^{\circ}$ to $35^{\circ} \mathrm{C}$.

6. Acetobacter suboxydans.

2. Optimum temperature $20^{\circ}$ to $25^{\circ} \mathrm{C}$.

7. Acetobacter oxydans.

* It is not known with certainty whether Acetobacter pasteurianum and Acetobacter kuetzingianum are capable of using inorganic nitrogen as a sole source of nitrogen for growth. However, since these two species are among those first described it is advisable to retain them for the present. See Acetobacter rancens Beijerinck. 
1. Acetobacter aceti (Kützing) Beijerinck. (Ulvina aceti Kützing, Algae aquae dulcis etc., 11th decade, 1837; Mycoderma aceti Thompson, Ann. d. Chem. u. Pharmacie, 83, 1852, 89; Umbina aceti Naegeli, Bericht über die Verhandlingen der bot. Section der 33 Versammlung deutscher Naturforscher und Arzter. Bot. Ztg., 1857, 760; Bacterium aceti Lanzi, N. Giorn. bot. ital., 1S76, 257; Torula aceti Saccardo, Atti Soc. Ven. Trent., 5, 1878, 315; Bacteriopsis aceti Trevisan, Atti della Accademia Fisio-Medico-Statistica in Milano, Ser. 4, 3, 1885, 103; Micrococcus aceti Maggi, Jour. Microg., 10, 1886; Bacillus aceti Schroeter, Kryptogamen Flora von Schlesien, 3, 1, 1886, 161; Bacillus aceticus Flügge, Die Mikroorganismen, 1886, 313; Beijerinck, Kral's Sammlung v. Mikroorg., Prague, 1898, 7 ; Beijerinck, Proc. Kon. Akad. v. Wetensch., Amsterdam, 2, 1900, 495; Bacterium hansenianum Chester, Man. Determ. Bact.. 1901, 126.)

From Latin acetum, vinegar.

Rods : 0.4 to 0.8 by 1.0 to 2.0 microns, occurring singly and in long chains, frequently showing large club-shaped forms. Stain yellow with iodine solution. Motility variable. Iotile cells possess a single polar flagellum (Vaughn, Jour. Bact., 46, 1943, 394). Forms large. shiny colonies on beer gelatin containing 10 per cent sucrose.

Forms slimy pellicle on fluid media, or ring or turbidity without pellicle.

Acid from glucose, ethyl alcohol, propyl alcohol and glycol. No acid from arabinose, fructose, galactose, sorbose, sucrose, maltose, lactose, raffinose, dextrin, starch, glycogen, inulin, methyl alcohol, isopropyl alcohol, butyl alcohol, isobutyl alcohol, amyl alcohol, glycerol, erythritol, mannitol, dulcitol and acetaldehyde (Henneberg, Die deutsch. Essigind., 2, 1898, 147).

Aerobic.

Distinctive characters: Marked oxidative power causing rapid and complete oxidation of substrate as glucose or ethyl alcohol; ability to utilize inorganic nitro- gen salts as a sole source of nitrogen (Hoyer, Inaug. Diss., Leiden, 1898, 43; Beijerinck, Cent. f. Bakt., II Abt., 4, 1898, 215); grow th and oxidative activity in association with fermenting yeasts (Vaughn, Jour. Bact., 36, 1938, 360).

Optimum temperature $30^{\circ} \mathrm{C}$. Growth occurs between $10^{\circ}$ and $42^{\circ} \mathrm{C}$.

Habitat: Vinegar; souring fruits, vegetables and beverages.

2. Acetobacter xylinum (Brown) Holland. (Bacterium xylinum Brown, Jour. Chem. Soc., London, 49, 18s6, 439; Holland, Jour. Bact., 5, 1920, 216; Bacillus xylinus Holland, ibid., 221.) From Gr. xylinus, mooden (in reference to the cellulose in the membrane).

Rods, about 2 microns long, occurring singly and in chains. The cells have a slimy envelope which gives the cellulose reaction.

A film forms on the surface of liquids. This film becomes cartilagenous and falls to the bottom. This zoogloeal film forms on all liquid media in which growth occurs; the nature of the medium influences the thickness of the film which may vary from 1 to 250 millimeters.

X-ray pattern studies made by Khouvine, Champetier and Sutra (Compt. rend. Acad. Sci. Paris, 194, 1932, 208) and by Barsha and Hibbert (Can. Jour. Research, 10, 1934, 170) have shown that the cellulose contained in the membranes formed by Acetobacter xylinum is identical with cotton cellulose.

Acid from glucose, ethyl alcohol, propyl alcohol and glyeol. No acid from arabinose, fructose, galactose, maltose, lactose, raffinose, dextrin, stareh, methyl alcohol, isopropyl aleohol, butyl alcohol, isobutyl alcohol, amyl alcohol, minnitol and acetaldehyde (Henneberg, Die deutsch. Essigind., 2, 1898, 147).

Aerobic.

Distinctive character: The production of thick, leathery, zoogloeal cellulosic membranes on the surface of liquids.

Optimum temperature $28^{\circ} \mathrm{C}$. 
Habitat: Vinegar; souring fruits, vegetables and beverages.

\section{Acetobacter rancens Beijerinck.} (Bacterium rancens Beijerinck, Cent. f. Bakt., II Abt., 4, 1898, 211; Beijerinck, Kral's Sammlung v. Microorg., Prague, 1898, 4.) From L. rancens, being rancid.

Beijerinck (loc. cit.) in a footnote stated that "two of the many varieties of $B$. rancens have been described by Henneberg under the names of $B$. oxydans and $B$. acetosum. Hansen erroneously called this species B. aceti as did Brown. Neither Hansen nor Brown knew $B$. aceti Pasteur." No further morphological description is given .

The following description is taken in part from a study of a culture of Acetobacter rancens received from Kiluyver (Vaughn).

Rods with the usual morphological appearance of eultures of acetic acid bacteria. Gram-negative. Motility variable. Motile cells possess a single polar flagellum (Vaughn, Jour. Bact., $46,1943,394)$. Involution forms commonly appear as filaments and enlarged cells.

Wort agar slant: Growth abundant, butyrous, pale-buff in color in one week.

Yeast infusion, glucose, calcium carbonate slant: Growth abundant, butyrous and cream-colored in one week.

With petri dish cultures well isolated colonies are large, smooth and butyrous on either medium.

Broth cultures containing peptone or yeast infusion form a mucilaginous, slimy pellicle. Beijerinck (loc. cit.) called this polysaccharide pellicle, cellulose-like and intimated that the mucilaginous material in the pellicle was somewhat different from that produced by Acetobacter xylinum. The pellicle material stained blue when treated with iodine and hydroiodic acid.

Acid from glucose, ethyl alcohol, propyl alcohol, butyl alcohol, glycol, adonitol, mannitol and sorbitol. No acid from numerous other compounds tested.
Distinctive character: Production of a thin, mucilaginous, slimy, polysaccharide membrane on the surface of liquids as compared with the thick, true cellulose membrane of Acetobacter xylinum grown under the same conditions. Beijerinck (loc. cit.) reported the production of a cellulose-like membrane with some cultures of Acetobacter rancens.

Source: Isolated from shavings in the quick vinegar process.

Habitat: Found in fermented grain mash, malt beverages, mother of vinegar.

Beijerinck (Cent. f. Bakt., II Abt., 4, 1898,211 ) thought that the next two species were hardly more than varieties of Acetobacter rancens.

3a. Acetobacter pasteurianum (Hansen) Beijerinck. (Mycoderma pasteurianum Hansen, Compt. rend. d. Trav. d. Lab. d. Carlsberg, 1. 1879, 96; Bacterium pasteurianum Zopf, Die Spaltpilze, 2 Aufl, 1884, 49; Beijerinck, TKral's Sammlung v. Microörg., Prague, 1898, 7.) Named for Pasteur, the French chemist and bacteriologist.

Rods : 0.4 to 0.8 by 1.0 micron, occurring singly and in chains, at times showing thick, club-shaped forms. Motility variable. Miotile cells possess a single polar flagellum (Vaughn, Jour. Bact., 46, 1943, 394). Stains blue with iodine.

Wort gelatin colonies: Small, circular, entire, gray, slimy.

Forms a dry, wrinkled folded pellicle on double beer with one per cent alcohol.

Meat infusion gelatin: Widespread, later rosette form, toothed.

Acid from glucose, ethyl alcohol, propyl alcohol and glycol. No acid from arabinose, fructose, galactose, sorbose, sucrose, maltose, lactose, raffinose, dextrin, starch, glycogen, inulin, methyl alcohol, isopropyl alcohol, butyl alcohol, isobutyl alcohol, amyl alcohol, glycerol, erythritol, mannitol, dulcitol and acetaldehyde (Henneberg, Die deutsch. Essigind., 2, 1898, 147).

Aerobic. 
Optinum temperature $30^{\circ} \mathrm{C}$. Growth occurs between $5^{\circ}$ and $42^{\circ} \mathrm{C}$.

Habitat: Vinegar; beer and beer wort.

3b. Acetobacter kuetzingianum (Hansen) Bergey et al. (Bacterium kuetzingianum Hansen, Compt. rend. d. Trav. d. Lab. d. Carlsberg, 3, 1894. 191; Bergey et al... Manual, 1sted, 1923,35.) Named for Kuctzing. the German botanist.

Short, thick rods, occurring singly: Rarely forming chains of notable length. Capsule stained blue with iodine and with potassium indide. Yon-motile.

Double beer gelatin colonies: Small, entire, with vermiform surface

Wort gelatin colonies: Small, entire, with surface free of wrinkles.

Double beer: Forms a rather thick, folded pellicle. Distinguished from Acetobacter aceti in showing heavier growth above the surface of the media.

Acid from glucose, ethyl alcohol, propyl alcohol and glycol. To acid from arabinose, fructose, galactose, sorbose, sucrose, maltose, lactose, raffinose, dextrin, starch, glycogen, inulin, methy alcohol, isopropyl alcohol, butyl alcohol, isobutyl alcohol, amyl alcohol, alycerol, erythritol, mannitol, dulcitol and acetaldehyde (Henneberg, Die deutsch. Essigind., 2, 1898, 147).

Aerobic.

Optimum temperature $34^{\circ} \mathrm{C}$, maximum $42^{\circ} \mathrm{C}$, minimum 6 to $7^{\circ} \mathrm{C}$.

Habitat: Beer. Found in double beer.

4. Acetobacter melanogenum Beijerinck. (Cent. f. Bakt., II Abt., 29, 1911, 175.) From Greek melas (melan), black; -genes, producing.

Rods: Non-motile or motile. Notile cells possess a single polar flagellum (Vaughn. Jour. Bact., 46, 1943, 394).

Gelatin: Apparent liquefaction probably caused by acid, not an enzyme. When hcld on artificial media for some time, the power of liquefying gelatin is lost, probably due to a slower production of acid. Deep brown pigment produced; gelatin becomes insoluble in boiling water and in trypsin solution.

Beer- or wort-gelatin plates: Characteristic dark brown, wide-spreading, diffuse areas.

Tap water-ilgar-glucose-peptone-potassium phosphate-iron citrate-chalk medium: In 24 hours at $30^{\circ} \mathrm{C}$, black, spreading, diffuse areas.

Utilizes peptone as a source of nitrogen. Produces the pigment from peptone only if maltose or glucose is present as a source of carbon. When grown in glucrof-peptone brath with $\mathrm{CaCO}_{3}$ at $25^{\circ}$ to $30^{\circ} \mathrm{C}$, black pigment is produced after several weeks, and the carbonate is changed to calcium gluconate.

Pigment: The pigment causing the brown coloration is an aromatic substance which is blackened by iron salts. Reduecs alkaline solutions of silver and mercury, blackening them.

Oxidizes mannitol and surbitol to frucius and sorbose. Does not at tack sucrose and fructose. Nuch gluconic acid is produced. Acid from glueose and maltusc. Acetic acid produced from alcohol.

Distinctive character: The formation of dark brown to black pigment in media containing a suitable substrate; particularly gl ucose.

Source: Isolated from beer.

Habitat: Causes light-colored beer to become darker brown. It is a very strong beer-vinegar bacterium. Also found in souring fruits.

5. Acetobacter roseum Taughn. (Bacterium hoshigaki var. rosea Takahashi and Asai, Cent. f. Bakt., II Abt., 82, 1930, 390; Acetobacter hoshigaki Bergey et al., Manual, 4th ed., 1934, 39; Vaughn, Mallerstein Lab. Communicatin: , E, No. 14, 1942, 20.) From Latin, rosa, rose.

Rods : 0.7 to 0.9 by 1.5 to 1.8 microns, generally occurring singly, at most in pairs, often in chains. Non-motile. Pellicle on fluid media yields no starch or cellulose reaction. 
Koji (a mixture of rice and mold spores used to start fermentation of Japanese bread and saké) extract agar colonies: Small, granular, circular, glistening, umbonate, becoming brownish.

Wort agar colonies: Circular, milkywhite, becoming brownish in center and yellowish at periphery.

Glucose saké agar: Circular, milkywhite, granular, umbonate, entire.

Hoshigaki (dried persimmons) extract agar: Circular, milky-white, granular, becoming yellowish-brown in the center and grayish-white at the periphery.

Koji extract agar streak: Grayishwhite, glistening with ciliate margin, becoming purple brown to brown.

Koji extract: Turbid with thin film, ascending on wall of tube.

Bouillon: Turbid with ring formation.

Yeast infusion glucose agar: Colonies similar to those on wort agar.

Yeast infusion glucose broth: Turbid with thin, ascending film.

Red color produced on saké wort agar and all media containing calcium carbonate.

Acid from glucose, fructose, galactose, arabinose, glycerol, mannitol, ethyl and propyl alcohol. No acid from maltose, sucrose, lactose, raffinose, dextrin, starch, inulin, sorbitol, glycogen, isodulcitol and methyl alcohol.

Forms gluconic acid from glucose.

Aerobic.

Optimum temperatures $30^{\circ}$ to $35^{\circ} \mathrm{C}$; maximum $40^{\circ}$ to $41^{\circ} \mathrm{C}$; minimum $10^{\circ}$ to $15^{\circ} \mathrm{C}$.

Thermal death point $50^{\circ} \mathrm{C}$ for 5 minutes.

Distinctive character: The formation of a rose to red pigment in suitable media; particularly those containing glucose and calcium carbonate.

Source: Isolated from fermenting mash of dried persimmons (hoshigaki), and souring figs and dates.

Note: Vaughn, Wallerstein Lab. Communications, 5, No. 14, 1942, 20, has proposed the name Acetobacter roseum to replace the name Acetobacter hoshigahi.
As originally described, this organism was given the name Bacterium hoshigaki var. rosea by Takahashi and Asai (loc. cit.) without the authors having first named and described the species $\mathrm{Bac}$ terium hoshigaki. The Japanese word "hoshigaki" has been used in a confusing manner viz. Takahashi and Asai, loc. cit. (Bacterium industrium var. hoshigati) and Takahashi and Asai, Jour. Agr. Chem. Soc. Japan, 9, 1933, 351 and Cent. f. Bakt., II Abt., 87, 1933, 385 (Bacterium hoshigahi var. glucuronicum I, II and III). None of these Japanese names are in the form of true binomials.

\section{Acetobacter suboxydans Kluyver} and de Leeuw. (Paper read at the convention of the Dutch Society of Microbiology, Utrecht, December, 1923, see Tijdschrift v. Vergelijkende Geneeskunde, 10, Afl. 2-3, 1924.) From L. sub, under, less; Gr. oxys, sharp, acid; dans, giving, i.e. less acid giving; less oxidizing.

Short rods: Occurring singly or in chains. Non-motile. Morphologically like Acetobacter rancens.

Forms very thin, hardly visible pellicle on fluid media.

Wort agar colonies: Very small, circular, slightly yellow.

Acid from ethyl alcohol, propyl alcohol, glycol, glucose, glycerol and sorbitol.

Optimum temperature $30^{\circ} \mathrm{C}$.

Distinctive character: Partial oxidation of substrates as indicated by the formation of ealcium 5-keto gluconate crystals on the surface of agar slants containing glucose and calcium carbonate.

Source: Isolated from spoiled beer.

Habitat: Beer.

\section{Acetobacter oxydans (Henneberg)} Bergey et al. (Bacterium oxydans Henneberg, Cent. f. Bakt., II Abt., 3, 1897, 223; Bacillus oxydans Migula, Syst. d. Bakt., 2, 1900, 800; Bergey et al., Manual, 1st ed., 1923, 36.) From Gr. oxys, sharp, acid; dans, giving.

Rods : 0.8 to 1.2 by 2.4 to 2.7 microns, occurring singly and in chains. Motile 
cells possess a single polar flagellum (Vaughn, Jour. Bact., 46, 1943, 394). The chains show bud-like swellings.

Gelatin colonies : Circular, becoming irregular in shape with peculiar ramifications.

Acid from arabinose, fructose, glucose, galactose, sucrose, maltose, raffinose, dextrin, ethyl alcohol, propyl alcohol, erythritol, mannitol, glycol and glycerol. No acid from sorbose, lactose, starch, glycogen, inulin, methyl alcohol, isopropyl alcohol, butyl alcohol, isobutyl alcohol, amyl alcohol, dulcitol and acetaldehyde (Henneberg, Die deutsch. Essigind., 2, 1895, 147).

Aerobic.

Optimum temperature $18^{\circ}$ to $21^{\circ} \mathrm{C}$.

Distinctive characters: Low optimum temperature for growth and oxidation of substrates; and the ability to oxidize a large number of substrates.

Habitat: Beer.

Appendix: The following species have been described, but until more comparative studies have been made, no change in nomenclature is recommended or advisable.

1. Acetobacter zeidleri Beijerinck. (Termobacterium aceti Zeidler, Cent. f. Bakt., II Abt., 2, 1896, 739; Baclerium zeidleri Beijerinck, Acetobacter zeidleri Beijerinck, Kral's Sammlung v. Mikroorg., Prague, 1898, 7; Bacillus zeidleri Migula, Syst. d. Bakt., 2, 1900, 801; Acetobacter lindneri Bergey et al., Manual, 1st ed., 1923, 36.) Named for A. Zeidler, who first isolated this species.

Rods, occurring singly and in chains, showing large sausage-shaped involution forms. Motile with a single polar flagellum (Zeidler, Cent. f. Bakt., II Abt., 4, 1898, 669).

Wort gelatin: Small, circular, slightly granular, yellowish-brown, entire colonies. No liquefaction.

Dirty, yellowish-brown pellicle on liquid media.

Wort gelatin slant: Strongly glistening, transparent, whitish in center, smooth, very weakly liquefied.

Potato: Very seant growth.

Acid from glucose, ethyl alcohol, propyl alcohol and glycol. No acid from arabinose, fructose, galactose, maltose, lactose, raffinose, dextrin, glycogen methyl alcohol, isopropyl alcohol. butyl alcohol, isobutyl alcohol, amyl alcohol, glycerol, mannitol and acetaldehyde (Henneberg, Die deutsch. Essigind., 2, 1898, 147).

Aerobic, facultative.

Optimum temperature $25^{\circ} \mathrm{C}$.

Habitat: Beer wort.

2. Acetobacter acetosum (Henneberg) Bergey et al. (Bacterium acetosum Henneberg, Cent. f. Bakt., II Abt., 3, 1897 , 223; Bergey et al., Manual, 1st ed., 1923, 36.) From Latin, acelum, vinegar.

Rods : 0.4 to 0.8 by 1.0 micron, occurring singly and in chains. Non-motile. Stains yellow with iodine.

On beer, yeast water and glucuse solutions a firm, coherent, uniform, smooth, white film that becomes folded (Henneberg, Gärungsbakt., 2, 1926, 201).

Acid from glucose, galactose, ethyl alcohol, and propyl alcohol. No acid from arabinose, fructose, sorbose, sucrose, maltose, lactose, raffinose, dextrin, starch, glycogen, inulin, methyl alcohol, isopropyl alcohol, butyl alcohol, isobutyl alcohol, amyl alcohol, glycerol, erythritol, mannitol, dulcitol and acetaldehyde (Henneberg, Die deutsch. Essigind., 2, 1898, 147).

Optimum temperature $28^{\circ} \mathrm{C}$, maximum $36^{\circ} \mathrm{C}$, minimum $8^{\circ} \mathrm{C}$ (Henneberg, Cent. f. Bakt., II Abt., 4, 1898, 14).

Habitat: Beer.

3. Acetobacter ascendens (Henneberg) Bergey et al. (Bacterium ascendens Henneberg, Zeitschr. f. deutsche Essigind., Berlin, No. 19 to 23, 1898, 145; also see Cent. f. Bakt., II Abt., 4, 1898, 933; Bergey et al., Manual, 1st ed., 1923, 37.) From Latin, ascendo, pp. ascendens, ascending.

Rods, occurring singly, rarely in chains. 
Non-motile. Do not give the cellulose reaction with iodine solution.

Glucose gelatin colonies: Dry, white, with white area surrounding the colony.

Fluid cultures have a tough pellicle rising on the wall of the flask.

Acid from ethyl alcohol, propyl alcohol and glycol. No acid from arabinose, fructose, glucose, galactose, sucrose, maltose, lactose, raffinose, dextrin, starch, methyl alcohol, isopropyl alcohol, butyl alcohol, isobutyl alcohol, amyl alcohol, glycerin, mannitol and acetaldehyde (Henneberg, Die deutsch. Essigind., 2, 1898, 147).

Aerobic.

Optimum temperature $31^{\circ} \mathrm{C}$.

Habitat: Isolated from vinegar and from red wine.

4. Acetobacter plicatum Fuhrmann. (Beibefte z. bot. Centralbl., Orig., 19, 1905, 8.) Description given in Cent. f. Bakt., II Abt., 15, 1906, 377. From plicatus, folded.

Rods : 0.55 to 0.7 by 0.75 to 0.9 microns when grown on agar at $28^{\circ}$ to $30^{\circ} \mathrm{C}$. Young streak cultures 0.4 to 0.6 by 1.4 to 1.6 microns with homogeneous staining when grown on beef-extract-gelatin at $22^{\circ} \mathrm{C}$. 0.5 by 1.5 to 1.7 microns with uneven staining (polar) when grown on wine gelatin. At about $40^{\circ} \mathrm{C}$ the organisms form swollen and greatly elongated forms. Non-motile.

Agar slant: Pale yellowish, translucent growth.

Alcohol-free beer with glucose and sucrose: Turbid with thick pellicles.

Potato: Growth limited.

Ferments alcohol to form acetic acid. Optimum temperature $28^{\circ}$ to $30^{\circ} \mathrm{C}$.

Habitat: Wine.

\section{Acetobacter acetigenum (Henne-} berg) Bergey et al. (Bacterium acetigenum Henneberg, Cent. f. Bakt., II Abt., 4, 1898, 14; Bacillus acetigenum Migula, Syst. d. Bakt., 2, 1900, 801; Bergey et al., Manual, 1st ed., 1923, 35.) From Latin, producing vinegar.
Rods, occurring singly and in pairs. 0.8 to 1.2 by 1.2 to 1.4 microns. Motile. Cells give a cellulose reaction with $\mathrm{H}_{2} \mathrm{SO}_{4}$ and iodine.

Glucose gelatin colonies : Raised, grayish, slimy.

Fluid cultures show a tough, slimy pellicle.

Acid from glucose, ethyl alcohol, propyl alcohol and glycol. No acid from arabinose, fructose, galactose, sorbose, sucrose, maltose, lactose, raffinose, dextrin, starch, glycogen, inulin, methyl alcohol, isopropyl alcohol, butyl alcohol, isobutyl alcohol, amyl alcohol, glycerol, erythritol, mannitol, dulcitol and acetaldehyde (Henneberg, Die deutsch. Fssigind., 2, 1898, 147).

Aerobic.

Optimum temperature $33^{\circ} \mathrm{C}$. Thermal death point $43^{\circ}$ to $45^{\circ} \mathrm{C}$ for 5 minutes.

Habitat: Vinegar.

6. Acetobacter industrium (Henneberg) Bergey et al. (Bacterium industrium Henneberg, Zeitschr. f. deutsche Essigindustrie, Berlin, 1898; Cent. f. Bakt., II Abt., 4, 1898, 933; Bacillus industrius Migula, Syst. d. Bakt., 2, 1900, 801; Bergey et al., Manual, 1st ed., 1923, 36.) From Latin industrius, diligent.

Rods: 0.3 to 0.8 by 2.4 to 20 microns, occurring singly and in chains. No distinet color produced with iodine. Motile.

Forms pellicle on fluid culture media.

Acid from arabinose, fructose, glucose, galactose, sucrose, maltose, lactose, raffinose, starch, dextrin, ethyl alcohol, propyl alcohol, glycol, glycerol and mannitol. No acid from isopropyl alcohol, butyl alcohol, isobutyl alcohol, amyl alcohol and acetaldehyde (Henneberg, Dic deutsch. Essigind., 2, 1898, 147).

Aerobic.

Optimum temperature $23^{\circ} \mathrm{C}$. Maxi mum $35^{\circ} \mathrm{C}$. Minimum $8^{\circ} \mathrm{C}$.

Habitat: Beer wort. 
7. Bacterium schuezenbachii Henneberg. (Die deutsche Essigind., No. 11-18, 1906; also Cent. f. Bakt., II Abt., 17, 1906, 790.) Named for Schüzenbach, the inventor of the German quick vinegar process.

Rods: 0.3 to 0.4 by 1.0 to 3.6 microns, occurring singly, in pairs and chains. The cells are round, oval or elongated, not infrequently sickle-shaped or irregularly bent with rounded or pointed ends. Not stained with iodine. Non-motile.

Wort gelatin colonies: Found, shiny, transparent with yellowish-brown centers.

A non-coherent film produced on the surface of liquid media.

Acid from arabinose, fructose, glucose. galactose, maltose, lactose, dextrin, ethyl alcohol, propyl alcohol, glycerol and erythritol. Small amount of acid from sucrose and raffinose. No acid from mannitol (Henneberg, Handbuch d. Gärungsbakt., 2, 1925, 239).

Temperature relations: Optimum 25 to $27.5^{\circ} \mathrm{C}$. Scant growth at $3 t^{\circ}$ to $35^{\circ} \mathrm{C}$ and $13^{\circ}$ to $15^{\circ} \mathrm{C}$. No growth at $37^{\circ}$ and $7.5^{\circ} \mathrm{C}$.

Source: Isolated from vinegar in the quick vinegar process.

Habitat: Produces acetic acil in quick vinegar process.

S. Bacterium xylinoides Henneberg. (Die deutsche Essigind., No. 11 to $1 \mathrm{~s}$, 1906 ; also Cent. f. Bakt., II Abt., 17 , 1906, 794.) From Greek, woody.

Rods: 0.5 to 0.5 microns (round cells and 0.5 to 1.2 microns (long forms), occurring singly, in pairs or chains, cells round and as short and long rods. The thick membrane like that produced by Acetobacter xylinum gives the reaction for cellulose with iodine and sulfuric acid, but the thin membrane does not.

Wort gelatin: Colonies are produced like drops of water, often with light bromm kernels in the center.

Wort gelatin streak: Growth transparent at first, later whitish.
Three types of membrane on fluid media are formed by this species and all three may even be found on a culture at one time. A thin, firm, coherent membrane like that of Bacterium orleanense may be formed or one that is voluminous, scumlike (like coagulated egg-white), coherent, somewhat slimy and finally thick like that of Bacterium xylinum. Also a membrane may be formed that is intermediate in type.

Acid from arabinose, glucose, galacuse, sucrose, maltose, ethyl alcohol, propyl alcohol, glycerol and erythritol. small amount of acid from fructose and mannitol.

Temperature relations: Optimum $28^{\circ} \mathrm{C}$. slight growth at $14^{\circ} \mathrm{C}$. No growth at $6^{\circ} \mathrm{C}$.

Source: Isolated from wine vinegar from the Rhine and other sections.

Habitat: Found in vinegar made by the Orleans method.

9. Bacterium orleanense Henneberg. (Die deutsche Essigind., No. 11-18, 1906; also (ent. f. Bakt., II Abt., 17, 1906, 792.) Latinized, of Orleans.

Rods : 0.4 to 0.5 by 1.2 to 2.1 microns, occurring singly or in chains. The cells are round, elongated or as involution forms, with straight or curved cells appearing. Not stained with iodine. Non-motile.

Wort gelatin: Colonies irregular in form, whitish in color, about $1 \mathrm{~mm}$. in diameter in 2 days.

Wort gelatin streak: (irowth often slimy, transparent, liquid mass with yellowish-brown sediment.

Wort agar streak: Some strains form isolated, moist, slimy, transparent colonies and on the water of condensation isolated whitish colonies are formed. Other strains form a coherent, transparent coating with a light brown preripitate later and individual, distinct, round colonies of the same color.

Characteristic of this species is the firm coherent film on the surface of liquid media. 
Acid from arabinose, glucose, galactose, maltose, lactose, raffinose, dextrin, ethyl alcohol, propyl alcohol, glycerol, erythritol and mannitol. Small amount of acid from fructose and sucrose (Henneberg, Handbuch d. Gärungsbakt., 2, 1926, 239).

Temperature relations: Optimum $20^{\circ}$ to $30^{\circ} \mathrm{C}$. Slight growth at $35^{\circ}$ to $36^{\circ} \mathrm{C}$ and $14^{\circ}$ to $15^{\circ} \mathrm{C}$. No growth at $39^{\circ}$ and at $7^{\circ}$ to $8^{\circ} \mathrm{C}$.

Source: Isolated from vinegar in the quick vinegar process.

Habitat: Can be used both in the quick or German process and the Orleans method of making vinegar.

10. Bacterium vini acetati Henneberg. (Die deutsche Essigind., No. 11-18, 1906; also Cent. f. Bakt., II Abt., 17, 1906, 797.) From Latin vinum, wine and acetum, vinegar.

Rods : 0.3 to 0.8 by 0.8 to 2.0 microns, occurring singly, in pairs and sometimes as short chains of three; cell round, oval or slightly elongated, and rarely moderately long forms. Streptococcus-like cells are found on older agar cultures and spindle forms in beer gelatin with 10 per cent sucrose.

Wort gelatin: Round, moist, shiny, transparent colonies with whitish sediment in the center.

The film on liquid media is not strongly coherent and the liquid is cloudy.

Acid from arabinose, fructose, glucose, galactose, sucrose, maltose, raffinose, dextrin, ethyl alcohol, propyl alcohol, glycerol and erythritol. No acid from lactose (Henneberg, Handbuch d. Gärungsbakt., 2, 1925, 239).

Optimum temperature $28^{\circ}$ to $33^{\circ} \mathrm{C}$.

Source: Wine vinegar.

Habitat: Found in vinegar made by the Orleans method for wine vinegar.

11. Bacterium curvum Henneberg. (Die deutsche Essigind., No. 11-18, 1906; also Cent. f. Bakt., II Abt., 17, 1906, 791.) From Latin, curvus, bent.

Rods : 0.4 to 0.5 by 2.0 to 2.4 microns, occurring singly or in pairs, cells usually oval or elongated, not inf requently sickleshaped, with rounded or pointed ends. Not stained with iodine solution. Nonmotile.

Wort gelatin: Transparent, round colonies with raised center and edge, frequently whitish and dry.

A non-coherent scanty pellicle is formed on the surface of liquid media which sinks readily and the liquid is quite turbid.

Forms round white islands on the surface of wort with 3 per cent alcohol.

In old cultures on beer are to be found numerous smooth light brown raised colonies about $1 \mathrm{~mm}$ in diameter on the uniform transparent base of the surface membrane.

Acid from arabinose, glucose, raffinose, dextrin, ethyl alcohol, propyl alcohol, glycerol and erythritol. Small amount of acid from fructose, galactose and mannitol. No acid from sucrose, maltose and lactose (Henneberg, Handbuch d. Gärungsbakt., 2, 1925, 239).

Temperature relations: Optimum $25^{\circ}$ to $30^{\circ} \mathrm{C}$. Scant growth at $16^{\circ}$ to $17^{\circ} \mathrm{C}$. No growth at $7^{\circ}$ to $8^{\circ} \mathrm{C}$. Growth at $35^{\circ} \mathrm{C}$. No growth at $39^{\circ} \mathrm{C}$.

Source: Isolated from vinegar in the quick vinegar process.

Habitat: Produces acetic acid in the quick vinegar process.

12. Acetobacter viscosum Shimwell. (Bacterium aceti viscosum Day and Baker, Cent. f. Bakt., II Abt., 36, 1913, 433; Bacillus aceti viscosum Day and Baker, ibid., 437; Also see Baker, Day and Hulton, Jour. Inst. Brewing, -, 1912, 651 ; Shimwell, Jour. Inst. Brewing, 42 (N. S. 32), 1936, 586.) From Latin, viscous or slimy.

Rods : 0.4 by 1.2 microns which produce ropiness in beer. No capsules observed. Non-motile as a rule. Weakly Grampositive.

Source: From ropy beer. 
13. Acetobacter capsulatum Shimwell. (Jour. Inst. Brewing, 42 (N. S. 32), 1936, 585.) From Latin, capsulated.

Coccoid rods, 0.5 to 1.0 micron in malt extract media. 0.6 to 1.5 microns in other media. Produce ropiness in beer. Capsulated. Motile. Gram-negative.

Source: From ropy beer.

14. Acetobacter gluconicum (Hermann).* (Bacterium gluconicum Hermann, Biochem. Zeit., 192, 1928, 198; also see Hermann, Biochem. Zeit., 205, 1929, 297 and Hermann and Neuschul, Biochem. Zeit., 239, 1931, 129.)

It is unfortunate that an organism so well described must be placed with other species of uncertain standing. However, this organism is so closely related to the other organisms described in the literature that further study is necessary .

Source: From kombucha, a mixture of fungi and bacteria from tea infusions.

15. Acetobacter turbidans Cosbie, Tošić and Walker. (Jour. Inst. Brewing, $48,1942,82$.
This beer vinegar bacterium is characterized by the production of intense turbidity in beer and ale. The deseription given does not, at present, warrant recognition of the organism as a new species.

Source: From beer.

\section{Bacterium dihydroxyacetonicum} Virtanen and Bärlund. (Biochem. Zeit., 169, 1926, 170.)

There is no adequate description of this bacterium, and it is doubtful whether it can be properly evaluated since various species of Acetobacter also possess the ability to produce dihydroxyacetone from glycerol. Consideration of this as a nomen nudem was indicated by Virtanen to Vaughn in a personal communication in 1938 .

Source: From beet juice.

17. Acetobacter peroxydans Visser 't Hooft. (Inaug. Diss., Delft, 1925, 98.)

The exact taxonomic position of this bacterium will not be clear until further comparative studies have been made.

Source: From hydrogen peroxide solutions.

\section{Genus $\Gamma^{*}$. Protaminobacter den Dooren de Jong. $\dagger^{\prime}$}

(Bijdrage tot de kennis van het mineralisatieproces. Thesis, Rotterdam, 1926, 159.) From M. L., protamine and Latin, bactrum, rod.

Cells motile or non-motile. Capable of dissimilating alkylamins. Pigmentation frequent. Soil or water forms.

The type species is Protaminobacter alboflavum den Dooren de Jong.

\section{Key to the species of genus Protaminobacter.}

I. Non-motile. Gelatin colonies light yellow to colorless.

1. Protaminobacter alboflavum.

II. Motile. Gelatin colonies red.

\section{Protaminobacter rubrum.}

1. Protaminobacter alboflavum den Dooren de Jong. (Thesis, Rotterdam, 1926, 159; also see Cent. f. Bakt., II
Abt., 71, 1927, 218.) From Latin albus, white; flavus, yellow.

Rods: Non-motile. Gram-negative.

* It is uncertain at present who first used this combination.

† Prepared by Prof. D. H. Bergey, Philadelphia, Pennsylvania, June, 1929; further revision by Prof. Robert S. Breed, New York State Experiment Station, Geneva New York, April, 1913. 
Gelatin colonies: Circular, dry, light yellow or colorless.

Gelatin stab: No liquefaction.

Agar colonies: Circular, opaque, pigment bright red, yellow, light gray or colorless.

Amine agar colonies: Circular, white to dark yellow.

See table below for list of organic substances utilized.
2. Protaminobacter rubrum den Dooren de Jong. (Thesis, Rotterdam, 1926, 159; also see Cent. f. Baht., II Abt., 71, 1927, 218.) From Latin, ruber, red.

Rods: Motile with single polar flagellum (Weaver, Samuels and Sherago, Jour. Bact., 35, 1938, 59). Gram-negative.

Gelatin colonies: Circular, red, dry. Gelatin stab: No liquefaction.

\section{TABLE I.-Organic Substances Utilized as a Source of Carbon by Varieties of} Protaminobacter alboflavum

\begin{tabular}{|c|c|c|c|c|c|c|c|c|c|c|c|c|c|c|}
\hline ORGANIC ACIDS & $\alpha$ & $\beta$ & $\gamma$ & $\delta$ & AMINO COMPOUNDS & $\alpha$ & $\beta$ & $\gamma$ & $\delta$ & AMINES & $\alpha$ & $\boldsymbol{\beta}$ & $\boldsymbol{\gamma}$ & $\delta$ \\
\hline Acetic..... & + & + & + & + & $\alpha$-alanin.......... & 0 & 0 & + & + & Ethyl... & + & + & + & + \\
\hline Valerianic & + & + & 0 & + & $\alpha$ - aminocapronic & & & & & Diethyl.. & + & + & 0 & 0 \\
\hline & & & & & acid ............ & + & 0 & + & 0 & Propyl.... & + & + & + & + \\
\hline$\alpha$-crotonic. & + & + & + & + & Leucin ........ & + & + & 0 & 0 & Isopropyl.. & 0 & + & 0 & 0 \\
\hline Undecyclic & 0 & 0 & 0 & + & Propionamid & + & 0 & + & 0 & Dipropyl.... & + & + & 0 & 0 \\
\hline Lactic..... . & + & 0 & 0 & + & Capronamid. & + & 0 & + & 0 & Tripropyl... & + & 0 & 0 & 0 \\
\hline$\beta$-oxybutyric . . . & + & + & + & + & Uric acid..... & + & 0 & 0 & 0 & Butyl....... & + & 0 & + & 0 \\
\hline Succinic ....... & + & + & + & + & Hippuric acid & + & 0 & 0 & 0 & Isobutyl.... & + & + & + & + \\
\hline Formic . & + & + & + & + & & & & & & Diisobutyl. & + & + & 0 & 0 \\
\hline Glutaric. & 0 & + & 0 & + & ALCOHOL & & & & & Amyl....... & + & + & + & + \\
\hline Adipic... & 0 & 0 & 0 & + & & & & & & Diamyl.... & 0 & + & 0 & 0 \\
\hline Fumaric. & + & + & + & + & Ethyl. & + & + & + & + & Ethanol.... & + & + & + & + \\
\hline Malic.... & + & + & + & + & Etnys. & + & t & + & + & Glucosamin & + & + & + & 0 \\
\hline Tartaric & 0 & + & 0 & 0 & & & & & & Benzyl..... & + & 0 & + & 0 \\
\hline Citric . . ..... & + & + & + & + & SUGAR & & & & & & & & & \\
\hline $\begin{array}{l}\boldsymbol{\beta} \text {-phenylpropi- } \\
\text { onic } \ldots \ldots \ldots\end{array}$ & + & 0 & 0 & 0 & Glucose... & + & + & + & 0 & & & & & \\
\hline Quinic......... & + & + & 0 & 0 & & & & & & & & & & \\
\hline
\end{tabular}

Catalase is formed.

Aerobic, facultative.

Optimum temperature $30^{\circ} \mathrm{C}$.

Habitat: Soil and water.

Note: The author recognizes four varieties of this species which he differentiates on the basis of organic substances attacked (see Table) and pigment produced. Variety $\alpha$ shows light yellow growth on gelatin, bright red on agar and yellow on amine agar. Variety $\beta$ is light yellow on gelatin, yellow on agar and dark yellow on amine agar. Variety $\gamma$ is light yellow on gelatin, light gray on agar and yellow on amine agar. Taricty $\delta$ is colorless on gelatin and agar and white on amine agar.
Agar colonies: Circular, red, opaque. Amine agar colonies: Circular, dark red.

The following organic acids are attacked: Acetic, lactic, $\beta$-oxybutyric, glycerinic, succinic, malonic, formic, methyl formic, glutaric, maleinic, fumarie, malic, tartaric, citric and quinic.

The following amino compounds are attacked: Sarcosin, betain, hippuric acid, asparagine, propionamid, capronamid, lactamid, succinamid, allantoin and uric acid.

Glucose is fermented.

Catalase is formed.

Aerobic, facultative.

Optimum temperature $30^{\circ} \mathrm{C}$.

Habitat: Soil and water. 


\section{Genus VI. Mycoplana Gray and Thornton.*}

(Cent.f. Bakt., II Abt., \%3, 192S, 82.) From Greek, myłēs, fungus; planē, a wanderer or traveller.

Cells branching, especially in young cultures. Frequently banded when stained. Capable of using phenol and similar aromatic compounds as a sole source of energy. Grow well on standard culture media.

Type species Mycoplana dimorpha Gray and Thornton.

\section{Key to the species of genus Mycoplana.}

I. Gelatin not liquefied.

II. Gelatin liquefied.

1. Mycoplana dimorpha.

2. Mycoplana bullata.

1. Mycoplana dimorpha Gray and Thornton. (Cent. f. Bakt., II Abt., 73, 1928, 82.) From Greek, di, two; morphos, forms.

Short, curved and irregular rods, 0.5 to 0.7 by 1.25 to 4.5 microns, showing branching especially in young cultures. † Motile, with long polar flagella. Gramnegative.

Gelatin colonies : Circular, bufi, smooth, resinous, entire.

Gelatin stab: No liquefaction. Growtl? filiform.

Agar colonies: Circular, buff, convex, smooth, glistening, entire.

Agar slant: Filiform, white, convex, glistening, entire.

Broth: Turbid, with surface ring.

Nitrites not produced from nitrates but gas evolved in fermentation tubes.

Starch hydrolyzed.

No acid from carbohydrate media.

Attacks phenol.

Aerobic.

Optimum temperature below $30^{\circ} \mathrm{C}$.

Source: One strain isolated from soil. Habitat: Probably in soil.
2. Mycoplana bullata Gray and Thornton. (Cent. f. Bakt., II Abt., 73, 1928, 83.) From Latin, bullatus, furnished with a boss or knob.

Rods, curved and irregular, branching, 0.8 to 1.0 by 2.25 to 4.5 microns. THotile with polar Hagella. Gram-negative.

Gelatin colonies : Circular, buff, smooth, glistening, edge diffuse. Partially liquefied.

Gelatin stab: Saccate liquefaction.

Agar colonies: Circular, white, convex, smooth, glistening, entire.

Agar slant: Filiform, white, convex, smooth, glistening, entire.

Broth: Turbid.

Nitrites not produced from nitrates. Gas, presumably $\mathbf{N}$, in fermentation tubes.

Starch not hydrolyzed.

No acid from carbohydrate media.

Attacks phenol.

Aerobic.

Optimum temperature below $30^{\circ} \mathrm{C}$.

Source: Two strains isolated from soil. Habitat: Probably in soil.

* Prepared by Prof. D. H. Bergey, Philadelphia, Pennsylvania, June, 1929.

† The original statements regarding the flagellation of these species are contradictory. The first reads "Polar, peritrichous; the second "Polar or peritrichous". Drawings given usually indicate peritrichous rather than polar flagellation. Further study is needed before these species can be properly placed in relation to other known species.-Editors. 
TRIBE II. SPIRILLEAE KLUYVER AND VAN NIEL.

(Cent. f. Bakt., II Abt., 94, 1936, 346.)

More or less spirally curved cells.

\section{Key to the genera of tribe Spirilleae.}

I. Generally motile by means of a single polar flagellum.

A. Short, bent rods occurring singly or united into spirals.

Genus I. Vibrio, p. 192.

B. Slightly curved rods of variable length. Strict anaerobes which reduce sulfates to hydrogen sulfide.

Genus II. Desulfovibrio, p. 207.

C. Cells oxidize cellulose forming oxycellulose. Growth on ordinary culture media is feeble.

1. Long, slightly curved rods with rounded ends.

Genus III. Cellvibrio, p. 209.

2. Short, curved rods with pointed ends.

Genus IV. Cellfalcicula, p. 211.

II. Generally motile by means of a tuft of polar flagella. Cells of varying thickness, and length and pitch of spiral, forming either long curves or portions of a turn.

A. Oxidize inorganic sulfur compounds. Cells contain free sulfur granules.

B. Not as above.

Genus V. Thiospira, p. 212.

Genus VI. Spirillum, p. 212.

\section{Genus I. Vibrio Müller.*}

(Müller, Vermium terrestrium et fluviatilum, 1, 1773, 39; Pacinia Trevisan, Att ${ }^{\mathrm{i}}$ d. Accad. Fisio-Medico-Statistica in Milano, Ser. 4, 3, 1885, 83; Microspira Schroeter, in Cohn, Kryptogamen-Flora von Schlesien, 3, 1, 1886, 168; Pseudospira De Toni and Trevisan, Sylloge Fungorum, 8, 1889, 1018; Photobacterium Beijerinck, Arch. néerl. d. sei. exactes, 23, 1889, 401; Liquidovibrio Orla-Jensen, Cent. f. Bakt. II Abt., 22, 1909, 333; Solidovibrio Orla-Jensen, ibid.; Dicrospira Enderlein, Sitzber. Ges. naturf. Freunde, Berlin, 1917, 313.) From Latin, vibro, vibrate.

Cells short, curved, single or united into spirals. Motile by means of a single polar flagellum which is usually relatively short; rarely, two or three flagella in one tuft. They grow well and rapidly on the surface of standard culture media. Aerobic to anaerobic species. Mostly water forms, a few parasites.

The type species is Vibrio comma (Schroeter) Winslow et al.

\section{Key to the species of genus Vibrio.}

I. Gelatin liquefied.

A. Nitrites produced from nitrates.

1. Indole is formed.

a. Milk not coagulated.

1. Vibrio comma.

2. Vibrio berolinensis.

aa. Milk coagulated.

3. Vibrio metschnikovii.

* Revised by Prof. D. H. Bergey, Philadelphia, Pennsylvania, April, 1937 ; partial revision by Capt. Wm. C. Haynes, Sn.C., Fort Bliss, Texas, July, 1943 and by Lt. Col. A. Parker Hitchens, University of Pennsylvania, Philadelphia, Penna., December, 1943. 
2. Indole not formed.

a. Milk not coagulated.

4. Vibrio tyrogenus.

5. Vibrio xenopus.

B. Nitrites not produced from nitrates.

1. Indole is formed.

a. Milk coagulated, peptonized.

6. Vibrio piscium.

2. Indole not formed.

a. Milk acid, coagulated.

aa. Milk not coagulated.

7. Vibrio proteus.

8. Vibrio wolfi.

9. Vibrio sputigenus.

10. Vibrio liquefaciens.

b. Growth on potato thin, barely visible.

11. Vibrio strictus.

bb. No growth on potato.

12. Vibrio aquatilis.

aaa. Action on milk not reported.

b. Acid from glucose. Attacks naphthalene.

13. Vibrio neocistes.

bb. No acid from carbohydrates. Attacks naphthalene.

14. Vibrio cuneatus.

bbb. No acid from carbohydrates. Liquefies agar.

15. Vibrio granii.

II. Gelatin not liquefied.

A. Nitrites produced from nitrates.

1. Acid and gas from glucose.

16. Vibrio leonardii.

2. Acid but not gas from glucose. Liquefies agar.

17. Vibrio agarliquefaciens.

B. Nitrites not produced from nitrates.

1. Acid from glueose.

18. Vibrio cyclosites.

2. No acid from carbohydrates.

19. Vibrio percolans.

C. Nitrite production not reported.

1. Requires the addition of ammonium sulfate for growth. Ammonium sulfate agar liquefied.

20. Vibrio andoi.

2. Do not require ammonium sulfate for growth,

a. Indole not formed.

b. Microaerophilic, becoming aerobic.

21. Vibrio fetus.

bb. Aerobic, facultative.

22. Vibrio pierantonii.

1. Vibrio comma (Schroeter) Wins- 479; Spirillum cholerae asiaticae Zopf, low et al. (Kommabacillus, Koch, Die Spaltpilze, 3 Aufl., 1885, 69; Pacinia Berliner klin. Wochenschr., 21, 1884, choleraeasiaticae Trevisan, Atti d. Accad. 
Fisio-Med.-Siatistica in Milano, Ser. 4, 3, 1885, 84; Microspira comma Schroeter, in Cohn, Kryptogamen Flora v. Schlesien, 3, 1, 1886, 168; Vibrio cholerae Neisser, Arch. f. Hyg., 19, 1893, 199; Vibrio cholerae asiaticae Pfeiffer, in Flügge, Die Mikroorganismen, 2, 1896, 527; Winslow ct al., Jour. Bact., 5, 1920, 204; Bacillus cholerae Holland, Jour. Bact., 5, 1920 , 217; Bacillus comma Holland, ibid., 218; Spirillum cholerae-asiaticae Holland, ibid., 225; Vibrio cholerae-asiaticae Holland, ibid., 226.) From Latin, comma.

Slightly curved rods, 0.3 to 0.6 by 1.0 to 5.0 microns, occurring singly and in spiral chains. Cells may be long, thin and delicate or short and thick. May lose their curved form on arificial cultivation. Motile, possessing a single polar flagellum. Gram-negative.

Gelatin colonies: Small, yellowishwhite.

Gelatin stab: Rapid napiform liquefaction.

Agar colonies : Circular, whitish-brown, moist, glistening, translucent, slightly raised, entire.

Agar slant: Brownish-gray, moist, glistening.

McConkey's medium: Good growth, colonies colorless when young, soon pinkish, medium becones darker red.

Broth: Slightly turbid, with fragile, wrinkled pellicle and flocculent precipitate.

Peptone water: Characteristic rapid growth, chiefly at surface, where after 6 to 9 hours, a delicate membrane is formed; little turbidity, deposit apparently derived from pellicle (Topley and Wilson, Princip. Bact. and Immun., 2nd ed., 1936, 388). Readily isolated from the surface film of 0.1 per cent peptone water.

Litmus milk: Alkaline at the top and slightly acid at bottom; generally not coagulated; peptonized; reduced.
Potato: Dirty-white to yellowish, moist, glistening, spreading.

Blood serum: Abundant growth, sometimes slow liquefaction.

Blood agar: The blood pigment is digested forming a greenish zone around colonies; a true soluble hemolysin is not formed (the El Tor vibrio also digests blood pigment but in addition produces a soluble hemolysin. Otherwise it is said to be indistinguishable from the typical cholera vibrio).

Indole is formed.

Nitrites produced from nitrates.

Cholera-red reaction, which depends on production of indole and reduction of nitrates is positive.

Hydrogen sulfide is formed.

Acid but not gas from glucose, fructose, galactose, maltose, sucrose and mannitol. Slowly from glycerol. Does not attack lactose, inulin or dulcitol.

Group I of Heiberg (Classification of Vibrio cholerae and Cholera-like Vibrios. Copenhagen, 1935) ferments mannose and sucrose but not arabinose.

Hydrolyzes starch actively in alkaline media.

High alkali but low acid tolerance; optimum pH 7.6 to 8.0 ; for isolation on Dieudonne's medium $\mathrm{pH} 9.0$ to 9.6 .

Aerobic, grows best in abundant oxygen; under strict anaerobiosis may fail to grow altogether.

Optimum temperature $37^{\circ} \mathrm{C}$. Maximum $42^{\circ} \mathrm{C}$. Minimum $14^{\circ} \mathrm{C}$.

Source: From intestinal contents of cholera patients in Egypt and India.

Habitat: Intestinal contents of cholera patients and carriers.

The relationships existing among the cholerigenic and non-pathogenic water vibrios, although studied intensively, have not yet been completely defined. As a working scheme, based on somatic (O) and flagellar (H) antigen studies, Gardner and Vankatraman (Jour. Hyg.: 35, 1935, 262-282) suggest the following 


\section{Cholera group of vibrios.}

(Biochemically similar. Common $\mathrm{H}$ antigen.)

Non-hemolytic

(goat cells).

Cholera vibrios.

Types-original, variant and middle.
Hemolytic (goat cells).
El Tor vibrios.
Types-original
and variant
(?middle).

O sub-groups II, III, IV, V, VI and individual races (mostly hemolytic). Paracholera, cholera-like, and some El Tor vibrios.

(Types within sub-groups underlined.)
Linton (Bact. Rev., 4, 1940, 275) has outlined a classification of the vibrios based upon their protein and polysaccharide structures. Using chemical methods, it was found that one polysaccharide and one protein was commonly obtained from each strain of vibrio; when exceptions occurred, it was invariably noted that the strain was undergoing dissociation. Given a single protein and polysaccharide in each vibrio, it was possible to divide the strains into six groups, which were numbered in the order of their discovery as shown in the table.

A chemical grouping of the cholerigenic and water vibrios.

\begin{tabular}{r|r|r}
\hline Group & Protein Type & $\begin{array}{c}\text { Polysaccharide } \\
\text { Type }\end{array}$ \\
\cline { 1 - 3 } I & I & I \\
II & I & II \\
III & II & II \\
IV & II & I \\
V & II & III \\
VI & I & III \\
\hline
\end{tabular}

The strains of Groups I and II possess the same protein and different polysaccharides. These are derived from cases of cholera and have the serological and biochemical characteristies of $\mathrm{O}$ Group I, Vibrio cholera. Group I strains are more common than those of Group II, which have, however, been isolated from epidemics with a high mortality. The phospholipid fraction is common to both types when isolated in the early part of an epidemic, but is not found in strains of other groups. The harmless water vibrios, which are so heterogeneous serologically (Tatylor and Ahuja, Indian Jour. Med. Res., 26, 1938, 8-32) form a single chemical group with a homogeneous structure. They fall into Group III, which differs in its protein structure from the authentic cholera vibrios, and resembles Group II in its polysaccharide. The vibrios of Group IV, which came from EI Tor and from chronic vibrio carriers are believed on cpidemiological grounds to be harmless, although serological methods have failed to distinguish them from cholerigenic vibrios. Group V, which, like III and IV, contains protein II, consists, like Group IV, of strains from chronic vibrio carriers. Group VI strains are only rarely isolated in nature and representatives of this group are generally found among collections of old laboratory strains. They appear to be the result of polysaccharide variation from Group I 
after long-continued growth on artificial media.

2. Vibrio berolinensis Neisser. (Arch. f. Hyg., 19, 1893, 200; Microspira berolinensis Migula, in Engler and Prantl, Die natürl. Pfanzenfam., 1, 1a, 1895, 33.) From M. L., the genitive of Berolina, the Latin name for Berlin.

Curved rods, somewhat smaller than Vibrio comma. Frequently occurring in pairs. Motile, possessing a polar flagellum. Pleomorphic. Gram-negative.

Gelatin colonies: Small, grayish, slightly granular, fragmented.

Gelatin stab: Slow, napiform liquefaction.

Agar slant: Grayish-yellow, moist, glistening.

Broth: Turbid, with gray pellicle.

Litmus milk: No coagulation, no acid.

Potato: Brownish streak.

Indole is formed.

Nitrites produced from nitrates.

Not pathogenic for mice, pigeons or guinea pigs.

Aerobic, facultative.

Optimum temperature $37^{\circ} \mathrm{C}$. Minimum above $10^{\circ} \mathrm{C}$. Maximum less than $60^{\circ} \mathrm{C}$.

Source: Isolated from filtered Spree river water.

3. Vibrio metschnikovii Gamaléia. (Gamaléia, Ann. Inst. Pasteur, 2, 1888, 482; Pacinia metschnikoff Trevisan, I generi e le specie delle Batteriacee, 1889, 23; Spirillum metschnikori Sternberg, Man. of Bact., 1893, 511; Vibrio nordhafen Pfuhl, Ztschr. f. Hyg., 22, 1894, 234; Microspira metschnikoffii Migula, in Engler and Prantl, Die natürl. Pflanzenfam., 1, 1a, 1895, 33.) Named for Metschnikoff, Russian bacteriologist.

Probable synonyms: Vibrio schuylkiliensis Abbott, Jour. Exp. Med., 1, 1896, 42t (Microspira schuylkiliensis Chester, Manual Determ. Bact., 1901, 334); Vibrio danubicus Ileider, Cent. f.
Bakt., 14, 1893, 341 (Microspira danubica Migula in Engler and Prantl, Die natürl. Pflanzenfam., 1, 1a, 1895, 33; Spirillum danubicum Holland, Jour. Bact., 5, 1920, 225).

Curved rods, somewhat shorter and thicker than Vibrio comma. Motile. Gram-negative.

Gelatin colonies: Like those of Vibrio comma.

Gelatin stab: Rapid, napiform liquefaction.

Agar slant: Yellowish, plumose, moist, glistening.

Broth: Turbid, with thin, white pellicle.

Litmus milk: Acid, coagulated (eighth day); not peptonized.

Potato: Delicate, brownish growth.

Indole is formed.

Nitrites produced from nitrates.

Pathogenic for pigeons, fowls, and guinea pigs.

Aerobic, facultative.

Optimum temperature $37^{\circ} \mathrm{C}$. Maximum less than $45^{\circ} \mathrm{C}$.

Source: Isolated from fowl dead of a choleraic disease.

Habitat: The intestinal contents of chickens, pigeons and other animals suffering from a cholera-like disease.

4. Vibrio tyrogenus (Flügge) Holland. (Käsespirillen, Deneke, Deutsch. med. Wochnschr., 11, 1885, 33; Spirillum tyrogenum Flügge, Die Mikroorganismen, 2 Aufl.. 1886, 386; Pacinia denekei Trevisan, I generi e le specie delle Batteriacee, 1889, 23; Microspira tyrogena Migula, in Engler and Prantl, Die natürl. Pflanzenfam., 1, 1a, 1895, 33; Holland, Jour. Bact., 5, 1920, 225; Vibrio denehei Hauduroy et al., Dict. d. Bact. Path., 1937, 541.) From Greek tyros, cheese; genes, produced from.

Curved rods, rather smaller and more slender than Vibrio comma, often very long, closely wound spirals. Motile, possessing a polar flagellum. Gramnegative. 
Gelatin colonies: Small, gray, granular, entire.

Gelatin stab: Rapid, saccate liquefaction.

Agar slant: Yellowish-white, plumose, glistening.

Broth : Turbid.

Litmus milk: Not coagulated.

Potato: No growth.

Indole not formed.

Slight production of nitrites from nitrates.

Aerobic, facultative.

Optimum temperature $30^{\circ} \mathrm{C}$.

Source: Isolated from cheese.

5. Vibrio xenopus Schrire and Greenfield. (Trans. Royal Soc. So. Africa, 17, 1930, 309.) From Xenopus, a genus of African toads.

Spiral forms, occurring singly and in pairs. Non-motile. Gram-negative.

Gelatin stab: Slow, crateriform liquefaction.

Agar colonies: Small, white, glistening, slimy, entire.

Agar slant: Crrayish-white, slimy, entire.

Broth: Turbid with flocculent sediment.

Litmus milk: Unchanged.

Potato: Not reported.

Indole is not formed.

Nitrites are produced slowly from nitrates.

Blood serum is peptonized.

Starch is not hydrolyzed.

Acid from glucose, fructose, maltose, glycerol and sorbitol.

Aerobic, facultative.

Optimum temperature $37^{\circ} \mathrm{C}$.

Source: Found in abscess of pectoral muscle of African toad.

6. Vibrio piscium David. (Cent. f. Bakt., I Abt., Orig., 102, 1927, 46.) From Latin piscis, fish.

Curved rods : 0.3 to 0.5 by 2.0 microns. Motile with a single polar flagellum. Gram-negative.
Gelatin colonies: Circular, granular, opaque.

Gelatin stab: Napiform liquefaction.

Agar colonies: Yellowish, circular, smooth, entire, iridescent.

Agar slant: Light yellow, transparent streak.

Broth: Slight turbidity, with thin pellicle.

Litmus milk: Soft coagulum. Peptonized, alkaline.

Potato: Brownish-red streak.

Indole is formed.

Nitrites not produced from nitrates.

Hydrogen sulfide formed.

No action in sugar media.

Pathogenic for frogs.

Aerobic, facultative.

Optimum temperature $18^{\circ}$ to $20^{\circ} \mathrm{C}$.

Habitat: Causes epidemic infection in fish.

7. Vibrio proteus Buchner. (Kommabacillus der cholera nostras, Finkler and Prior, Deutsche med. Wochenschr., 1884, 632; Buchner, Sitzungsber. d. Gesel. f. Morph. u. Physiol., München, Heft 1, 1885, 10; Pacinia finkleri Trevisan, Atti d. Acead. Fisio-MedicoStatistica in Milano, Ser. 4, 3, 1885, 84; Microspira finkleri Schroeter, in Cohn, Krytogamen-Flora von Schlesien, 3, 1, 1886, 168; Spirillum finkleri Crookshank, Man. of Bact., 3rd ed., 1890, 282; Microspira protea Chester, Manual of Determinative Bacteriology, 1901, 338; Tibrio finkleri Holland, Jour. Bact., 5, 1920 , 225.) From Greek, Proteus, a marine deity who had the power of assuming any shape he chose.

Curved rods : 0.4 to 0.6 by 2.4 microns, often pointed at both ends. Motile, possessing a polar flagellum. Gramnegative.

Gelatin colonies: Small, gray, circular, granular, entire.

Gelatin stab: Rapid, saccate liquefaction.

Agar slant: Dirty grayish, plumose. Broth: Turbid, with fetid odor. 
Litmus milk : Slightly acid; coagulated; peptonized.

Potato: Grayish, slimy layer.

Indole not formed.

Nitrites not produced from nitrates. Aerobic, facultative.

Optimum temperature $30^{\circ} \mathrm{C}$.

Source: Isolated from feces of patients suffering from cholera nostras.

Habitat: Intestinal contents in cholera nostras and cholera infantum.

8. Vibrio wolfii (Migula) Bergey et al. (Bacillus choleroides Wolf, Münch. med. Wochenschr., 40, 1893, 693; Microspira wolfii Migula, Syst. d. Bakt., 2, 1900, 1001; not Microspira choleroides Migula, loc. cit., 992; Bergey et al., Manual, 1st ed., 1923, 80.) Named for Wolf, who first isolated this organism.

Curved rods and $\mathbf{S}$-shaped forms. Motile. Gram-negative.

Gelatin colonies: Small, grayish-white, spreading.

Gelatin stab: Infundibuliform liquefaction.

Agar slant: Gray, moist layer.

Broth: Turbid, with gray pellicle.

Litmus milk: Acid; coagulated.

Potato: Yellowish-white layer.

Blood serum: Rapid liquefaction.

Indole not formed.

Nitrites not produced from nitrates.

Aerobic, facultative.

Optimum temperature $37^{\circ} \mathrm{C}$.

Source: Isolated from cervical secretions in chronic endometritis.

9. Vibrio sputigenus (Migula) Bergey et al. (Vibrio aus Sputum, Brix, Hyg. Rundschau, 4, 1894, 913; Microspira sputigena Migula, Syst. d. Bakt., 2, 1900, 981; Bergey et al., Manur1, 1st ed., 1923, so.) From Latin, spuo (sputus), sputum;-genes, produced from.

Slightly curved rods, about the same size and form as Tibrio comma, occurring singly, occasionally three or four in a chain. Motile. Possessing a polar flagel lum. Gram-negative.
Gelatin colonies: Small, circular, slightly granular, yellowish, becoming brownish.

Gelatin: Crateriform liquefaction.

Agar slant: Grayish-white, moist.

Broth: Turbid, no pellicle formed.

Litmus milk: Acid; coagulated.

Potato: Thin, gray layer, spreading. Indole not formed.

Nitrites not produced from nitrates. Aerobic, facultative.

Optimum temperature $37^{\circ} \mathrm{C}$.

Source: Isolated from sputum.

10. Vibrio liquefaciens (Migula) Bergey et al. (Bonhoff, Arch. f. Hyg., 19, 1893, 248; Microspira liquefaciens Migula, Syst. d. Bakt., 2, 1900, 990 ; Bergey et al., Manual, 1st ed., 1923, 81.) From Latin, liquefacio, to make liquid.

Comma and S-shaped rods. Motile. Gram-negative.

Gelatin colonies: Circular, with irregular margin, surrounded by a rosecolored zone.

Gelatin stab: Slow, napiform liquefaction.

Agar slant: Smooth, grayish, plumose. Broth: Turbid, with heavy grayish pellicle.

Litmus milk: Acid; coagulated.

Potato: Moist, brownish layer.

Indole is not formed.

Nitrites not produced from nitrates. Acrobic, facultative.

Optimum temperature $37^{\circ} \mathrm{C}$.

IIabitat: Water.

11. Vibrio strictus Kutscher. (Ztschr. f. Hyg., 19, 1895, 469.) From Latin stringo (strictus), constricted.

Markedly curved rods, of about twice the size of Vibrio comma. Motile. Gram-negative.

Gelatin colonies: Small, circular, yellowish, with serrate margin.

Gelatin stab: Slow, napiform to saccate liquefaction.

Agar slant: Growth plumose, moist.

Broth: Turbid, with gray pellicle. 
Litmus milk: Not coagulated.

Potato: Thin, barely visible layer.

Blood serum is slowly liquefied.

Indole is not formed.

Nitrites not produced from nitrates.

Pathogenic for guinea pigs.

Aerobic, facultative.

Optimum temperature $37^{\circ} \mathrm{C}$.

Habitat: Water.

12. Vibrio aquatilis Günther. (Deutsche med. Wochenschr., 1892, 1124; Microspira aquatilis Migula, System der Bakterien, 2, 1900, 993.) From Latin, aquaticus, living in water.

Curved rods, like Vibrio comma. Motile, possessing a polar flagellum. Gram-negative.

Gelatin colonies: Circular, brownish, finely granular, entire.

Gelatin stab: Crateriform liquefaction.

Agar slant: Moist, grayish, glistening.

Broth: Slightly turbid.

Litmus milk: Not coagulated.

Potato: No growth.

Indole not formed.

Nitrites not produced from nitrates. Aerobic, facultative.

Optimum temperature $30^{\circ} \mathrm{C}$.

Habitat: Water.

13. Vibrio neocistes Gray and Thornton. (Gray and Thomton, (ent. f Bakt., II Abt., 73, 192S, 92.) From Greek néos, new and kistē box or ark. Here used as the equivalent of Newark, the name of a city in England.

Curved rods : 0.5 to 1.0 by 1.0 to 4.0 microns. Motile with one to three polar flagella. Gram stain not recorded.

Gelatin colonies: Liquefied.

Gelatin stab: Liquefied. Medium reddened:

Agar colonies: Circular or amoeboid, buff to brownish, convex, smooth, glistening, entire.

Agar slant: Filiform, fluorescent, raised, smooth, glistening, undulate.

Broth: Turbid.

Nitrites not produced from nitrates.

Starch not hydrolyzed.
Acid from glucose.

Attacks naphthalene.

Acrobic, facultative.

Optimum temperature.

Habitat: Soil.

14. Vibrio cuneatus Gray and Thornton. (Gray and Thornton, Cent. f. Bakt., II Abt., 73, 1928, 92.) From Latin, cunco (cuneatus) wedge.

Curved rods : 1.0 by 1.0 to 3.0 microns, the cells tapering at one extremity. Motile with one to five polar flagella. Gram-negative.

Gelatin colonies: Liqueficd.

Gelatin stab: Liquefied.

Agar colonies: Circular to amoeboid, white to buff, flat to convex, smooth, translucent, border entire.

Agar slant: Filiform, whitish, smooth, glistening.

Indole not recorded.

Nitrites not produced from nitrates.

Starch not hydrolyzed.

No acid from arbohydrate media.

Attacks naphthalene.

Aerobic, facultative.

Optimum temperature $30^{\circ}$ to $35^{\circ} \mathrm{C}$.

Source: One strain isolated from soil from Rothamsted, England.

Habitat: Soil.

15. Vibrio granii (Lundestad) Stanier. (Bacterium granii Lundestad, Cent. f. Bakt., II Abt., 75, 1928, 330 ; Achromobacter granii Bergey et al., Manual, 3rd ed., 1930, 222; Stanier, Jour. Bact., 42, 1941, 538.) Named for Prof. H. H. Gran, who first detected agar-liquefying bacteria.

Rods : 0.6 to 0.8 by 1.4 to 2.4 microns, with rounded ends, occurring singly, in pairs, and at times in short chains. Motile. Polar flagellate (Stanier, loc. cit.). Gram-negative.

Fish-gelatin colonies: Punctiform, black, glistening.

Fish-gelatin stab: Slow, erateriform liquefaction.

Sea-weed agar colonies: Circular, flat 
opaque, glistening, white, slimy, entire. Agar is dissolved.

Fish-agar slant: Flat, white, elevated, glistening, undulate. Liquefied.

Broth: Turbid with grayish-white, slimy sediment.

Indole not formed.

Nitrites not produced from nitrates.

Starch usually hydrolyzed.

No action on sugars.

Aerobic, facultative.

Optimum temperature $20^{\circ}$ to $25^{\circ} \mathrm{C}$.

Source: Sea water of Norwegian Coast.

Habitat: Presumably sea water and on sea weeds.

16. Vibrio leonardii Métalnikov and Chorine. Ann. d. l'Inst. Pasteur, 42, 1928, 1647.) Named for Leonard.

Curved rods with rounded ends, 0.5 to 1.0 by 2.0 to 3.0 microns. Motile with 1 to 3 polar flagella. Gram-negative

Gelatin stab: No liquefaction.

Agar colonies: Small, transparent, circular, having a characteristic odor.

Broth: Turbid, with thin pellicle.

Litmus milk: No coagulation, acid, with reduction of litmus.

Potato: Slight, colorless growth.

Indole not formed.

Nitrites produced from nitrates.

Blood serum not liquefied.

Hydrogen sulfide formed.

Acid and gas from glucose, fructose, galactose, lactose, sucrose and mannitol. No acid or gas from maltose or glycerol.

Aerobic, facultative.

Optimum temperature $30^{\circ} \mathrm{C}$.

Habitat: Highly pathogenic for insects as Galleria mellonella I. (bee moth), and Pyrausta nubialis Hübn. (European corn borer).

17. Vibrio agarliquefaciens (Gray and Chalmers) Bergey et al. (Microspira agar-liquefaciens Gray and Chalmers, Ann. Appl. Biol., 11, 1924, 325; Manual, 4th ed., 1934, 119.) From Latin, liquefacio, liquefying; Malay, agar, a jelly from seaweeds.

Short curved rods, usually c-shaped, with occasional S-shaped and coccoid forms. Cells 2.0 microns long by 0.5 to 0.7 micron broad; 3.0 to 5.0 microns long in division stages. Coccoid forms stained, 0.5 to 0.7 micron long. Motile with a single polar flagellum. Gram stain not reported.

Gelatin stab: Very slight surface growth after one month; the streak then shows a beaded line. No liquefaction.

Agar colonies: Surface colonies appear as a whitish growth in a depression, surrounded by a white ring. The colony is later surrounded by a ring of liquid agar. Deep colonies show a clear area and may be irregular, oval or angular.

Agar slant: A deep groove is cut along the inoculation streak, whitish growth along sides. The gel is later much weakened.

Broth: Slightly turbid. No pellicle.

Acid from glucose, lactose and maltose. No acid from sucrose or glycerine.

Utilizes ammonia salts as a source of nitrogen.

Decomposes cellulose and agar. The presence of one per cent glucose prevents the liquefaction of agar.

Nitrites produced from nitrates.

Starch is hydrolyzed.

Aerobic.

Temperature relations: Optimum $25^{\circ}$ C., will grow at $16^{\circ}$ but not at $34^{\circ} \mathrm{C}$.

Habitat: Soil.

18. Vibrio cyclosites Gray and Thornton. (Gray and Thornton, Cent. für Bakt., II Abt., 73, 1928, 92.) From Greek kyklos, circle or ring; sĩléo, to eat: M. L. cyclosites, feeding on rings, i.e. ring compounds.

Curved rods: 0.5 to 1.0 by 1.5 to 4.0 microns. Motile with a single polar flagellum. Gram-negative.

Gelatin colonies: Circular, buff to brown, flat, smooth, glistening, entire.

Gelatin stab: No liquefaction.

Agar colonies: Circular to irregular, pale buff (later greenish), smooth, entire.

Agar stab: Filiform, greenish buff, raised, smooth, undulate. 
Broth: Turbid.

Indole not reported.

Nitrites not produced from nitrates.

Starch not hydrolyzed.

Acid from glucose.

Attacks phenol and $m$-cresol.

Aerobic, facultative.

Optimum temperature $30^{\circ}$ to $35^{\circ} \mathrm{C}$.

Habitat: Soil.

19. Vibrio percolans Mudd and Warren. (Jour. of Bact., 8, 1923, 447.) From Latin, percolo (percolatus), filtering.

Curved rods : 0.3 to 0.4 by 1.5 to 1.8 microns, occurring singly or in short chains. Pleomorphic. Actively motile by means of 1 to 3 polar flagella. Gramnegative.

Gelatin stab: No liquefaction.

Agar colonies: Circular, slightly convex, amorphous, entire.

Agar slant: Bluish-nhite, glistening, streak.

Broth: Turbid. Pellicle, sediment.

Litmus milk: Unchanged.

Potato: White, slimy streak.

Indole not formed.

Nitrites not produced from nitrates.

Blood serum not liquefier.

Starch not hydrolyzed.

No action on carbohydrates.

Passes through bacterial filters.

Aerobic, facultative.

Optimum temperature $30^{\circ} \mathrm{C}$.

Non-pathogenic.

Source: Isolated from hay infusion.

Habitat: Water.

20. Vibrio andoi Aoi and Orikura.

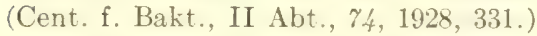
Named for Andoi, a Japanese scientist.

Curved rods, with more or less tapering ends, c-or s-shaped, 0.5 to 0.8 by 1.5 to 2.5 microns. Notile, with a single polar flagellum. Gram-negative.

Gelatin: No growth.

Agar media : No growth.

Broth: No growth.

Litmus milk: No growth.

Potato: No growth.
Ammonium sulfate agar colonies: Punctiform, circular, concave, surrounded with clear zone.

Ammonium sulfate agar slant: Grayish, becoming straw-yellow, sinking into the medium as the agar liquefies.

Cellulose media: No growth.

Starch hydrolyzed.

Glucose, fructose, galactose, mannose, xylose and "honyak" are fermented.

Xylan is decomposed.

Cellobiose is decomposed.

Aerobic, facultative.

Optimum temperature $25^{\circ} \mathrm{C}$. Minimum $8{ }^{\circ} \mathrm{C}$. Maximum $37^{\circ} \mathrm{C}$.

Source: Rotted stable manure.

Habitat: Presumably decomposing organic matter.

21. Vibrio fetus Smith and Taylor. SSpirillum causing abortion in sheep, MacFadyean and Stockman, Rept. Dept. Comm. Ministry Agric. on Epizootic Abortion, London, 1909, 156; ibid., 1913, 111 ; Spirillum associated with infectious abortion, Smith. Jour. Exp. Med., 28, 1918, 701; Smith and Taylor, ibid., 30, 1919, 299; Spirillum fetus Lehmann and Neumann, Bakt. Diag., 7 Aufl., 2, 1927, 552 ; Vibrio foetus ovis Buxton, First Rept. of Director, Lniv. Cambridge Instit. Animal Pathol., 1929-1930, 47.) From I. foetus, fetus.

Curved rods: The smallest forms appear as minute curved $\mathrm{s}$-shaped lines, other forms very long; 0.2 to 0.5 by 1.5 to 5.0 microns. Motile by means of one, rarely two, polar flagella. Occasionally forms capsules. Granules present in older cultures. Gram-negative.

Gelatin: No growth.

Agar slant: No surface growth by freshly isolated strains. Laboratory strains produce a scanty, grayish-white, glistening surface growth.

Subsurface agar colonies: Small, yellow, opaque.

Broth: A viscid ring pellicle may appear, faint clouding of the medium occurs; a filmy, stringy deposit may settle out.

Litmus milk: No growth 
Potato: No growth.

Indole not formed.

Nitrite production not reported.

Blood serum slant: Feeble growth. No liquefaction.

No gas from carbohydrates. No change or slightly acid from glueose, lactose and sucrose.

Optimum temperature $37^{\circ} \mathrm{C}$. Withstands $55^{\circ} \mathrm{C}$ for 5 minutes.

Aerobic or microaerophilic.

Pathogenesis: Causes abortion in cattle.

Source: Twenty-two strains isolated from the placentas or fetuses of cows having abortion.

Habitat: Causes abortion in cattle.

22. Vibrio pierantonii (Zirpolo) Meissner. (Bacillus pierantonii Zirpolo, Boll. Soc. nat. Napoli, 30, 1918, 206; Meissner, Cent. f. Bakt., 11 Abt., 67, 1926, 200.) Named for Pierantoni, an Italian bacteriologist.

Rods : 0.5 by 1.5 microns, with rounded ends. Motile with one to three polar flagella. Gram-negative.

Gelatin colonies: Circular, and irregularly lobulate.

Gelatin stab: No liquefaction.

Agar colonies: Circular, light green, smooth, entire.

Glycerin agar slant: Slightly luminous streak.

Broth: Turbid, with pellicle.

Indole not formed.

Acid from glucose and maltose. Some strains also attack lactose, sucrose and mannitol.

Best growth in alkaline media.

Aerobic, facultative.

Optimum temperature $37^{\circ} \mathrm{C}$.

Source: Isolated from the photogenic organ of the cephalopod Sepiola intermedia Naef.

Appendix:* The following species have also been listed in the literature. Many are inadequately lescribed.
Microspira bonhoffi Migula. (Bonhoff, Arch. f. Hyg., 19, 1893, 252; Migula, Syst. d. Bakt., 2, 1900, 1008.) From water.

Microspira canalis Migula. (Spirillimm saprophiles $\gamma$ and Tibrio saprophiles $\gamma$ Weibel, Cent. f. Bakt., 2, 1887, 469; Migula, Syst. d. Bakt., 2, 1900, 1004; Microspira cloacu Chester, Man. Determ. Bact., 1901, 341.) Possibly identical with Microspira saprophiles Migula, Microspira weibelii Migula, Vibrio surati Ford, Vibrio smithii Ford. From sewage.

ITicros pira coprophila Migula. (Group 3, No. 6, Kutseher, Ztschr. f. Hyg., 19, 1895, 475; Migula, Syst. der Bakt., 2, 1900, 986.) From fecal matter.

ITicrospira maasei (v. Hoff) Migula. (Spirillum maasei v. Hoff, Cent. f. Bakt., I Abt., 21, 1897, 797; Migula, Syst. d. Bakt.. 2, 1900, 978.) Possibly a variety of Tibrio comma Winslow et al. From Potterdam tap water.

Wicrospira milleri Migula. (Miller, Deutsche med. Wchnschr., 11, 1885, 138; Migula, Syst. d. Bakt., 2, 1900, 981; Spirillum milleri Holland, Jour. Bact., 5, 1920, 225; Vibrio milleri Holland, ibid.) Probably identical with Vibrio proteus atcording to Migula. From dental caries.

Microspira murmanensis Issatchenko. (Recherches sur les microbes de l'océan glacial aretique (in Russian), Petrograd, 1914, 240.) From sea water.

Microspira saprophiles Migula. (Heuvibrio $\beta$, Weibel, Cent. f. Bakt., 2, 1887, 469 ; Vibrio saprophiles $\beta$ Weihel, Cent. $\mathrm{f}$. Bakt., 4, 1888, 225; Migula, Syst. d. Bakt., 2, 1900, 1006; Microspira weibeli Chester, Man. Determ. Bact., 1901, 230.) Probably identical with Microspira cloaca Chester and Vibrio surati Ford. From sewage.

Microspira tyrosinalica Beijerinck. (Kon. Akad. Wetenschappen, Amster(lam, 13, 1911, 1068.) From sewage.

IIicrospira weibelii Migula. (Vibrio

* Prepared by Mr. Wm. C. Haynes, New York State Experiment Station, Genera, New Fork, Jan., 1939; Revised by Capt. Wm. C. Haynes, Sn. C., Fort Bliss, Texas, July, 1943. 
saprophiles a Weibel, Cent. f. Bakt., 2, 1887, 465; ibid., 4, 1888, 225; Migula, Syst. d. Bakt., 2, 1900, 1005; Microspira saprophile Chester, Manual Determ. Bact., 1901, 341; T'ibrio saprophiles Ford, Textb. of Bakt., 1927, 356.) Possibly identical with Microspira cloaca Chester, Tibrio surati Ford, I ibrin smithii Ford. From sewage.

Spirillum lipofermm Beijerinck. (Cent.f. Bakt., II Abt.. 63, 1925, 353; Chromatium lipoferum Bergey et al., Manuil. 3rd ed., 1930, 531.) From garden carth and sewage. Giesberger (Beiträgr zur Kenntnis der Gattung Spirillum Ehlog. Inaug. Diss., Delft. 1936, 64) regards this organism as it Vibrio. Has a single polar flagellum.

Spirillum nasicola Trevisan. (Aasenschleimvibrin, Weibel, Cent. f. Bakt., 2, 1857, 465; Trevisan, I genori e le specie delle Batteriace, 1849. 24; Tibrio nasalis Eisenberg, Bakt. Diag.. 3 Aufl., 1891, 213; Spirillum nasale Siternberg, Man. of Bact., 1893, 697; Sipirosoma nasale Migula, in Engler and Prant]. Die natürl. Pflanzenfam.. 1. 1 凡, 1895. 31.) From human nasal mueus.

Spirillum parvum Esmarch. (Cent.f. Bakt., I Abt., Orig., 32, 1902, 565; also see Zettnow, ibir., $78,1916,1$; Tibrin parus Lehmann and Jemmann. Pakt. Diag., \& Aut1., 2, 1907, 494.) From dercaying organic matter.

Vibrio albensis Lehmann and leumann. (Elbe vibrio. Dunbar, Deutsch. med. Wochnschr., 19, 1893, 799; Lehmann and Neumann, Bakt. Diag., I Auft. 2, 1896, 340; Microspira dunbari Migula Syst. d. Bakt., 2, 1900, 1013; Photospiritlum dunbari Miquel and Cambier. Traite de Bact., Paris. 1902, SS1; Photobacterium dunbari Ford, Textb. of Bakt., 1927, 621.) From water of the river Elbe. Phosphorescent.

Vibrio amylocella Gray. (Canad. Jour. Res., 17, 1939, 154.) Decomposes cellulose. Produces glucose from starch. From soil.

Tibrio anguillarum Bergman. (Ber.a. d. k. Bayr. Biolog. Versuchstat., Mün- chen, 2, 1909.) From an infectious disrase of eels.

Tibrio aureus Weibel. (Weibel, Cent. f. Bakt., 4, 1858, 225, 257, 281; spirillum aureum Trevisan, I generi e le specie delle Batteriacee, 1889, 24; Spirillum aurenm stemberg, Man. of Bact., 1893, 700 ; Spirosoma aureum Migula, syst. d. Bakt.. 2. 1900, 958.) Possibly identical with Vibrio flarus $W$ Heibel and Vibrio farcscens Weibel. From sewage.

I ibrio beijerinckii Stanier.

(Jour. Bact., 42, 1941, 527-554.) Narine agardigesting vibrio.

Tibrio buccalis Prévot. (Vibrion B, Repaci, Compt. rend. Soc. Biol., Paris, 1909, 630; Prévot, Man. de Classif. des Bact. Anaér., Paris 1940. \$2.) Anaerobe. From the buecal eavity

Vibrio bulbosa Kalnins. (Latvijas Universitātes Raksti, Serija I, No. 11, 1930, 237.) Decomposes cellulose. From soil.

Tibrio cardii Klein. (Cent, f. Bakt, I Abt., Orig., 38, 1905, 173.) Possibly identical with Tibrio cuneatus Gray and Thornton and Vibrio marimus Ford. From the mussel (Cardium edule).

Vibrio castra Kalnins. (Latvijas Üniveristrs Raksti, Serija I, Jo. 11, 1930, 241.) Decomposes cellulose. From soil.

Tibrio choleroides $\alpha$ and $\beta$ Bujwid. (Cent. f. I3akt., 13, 1893, 120;. Hicrespira choleroides Migula, Syst. d. Bakt., 2, 1900, 992.) Probably a less vigorous strain of Tibrio comma IVinslow et al. according to Chester, Man. Determ. Bact., 1001, 337. From water.

Tiurio chrysanthemoides Lchmann and Teumann. Spirillum-like organism, Jones. Cent. f. Bakt., II Abt.. 14. 1904, 459; Tchmann and Toumann, Bakt. Diag., 4 Aufl., 2, 1907, 493.) From five samples of tap water and sewage.

Tibrio crassus (Veillon and Repaci) Prévot. (Spirillum crassum Veillon and Reprei, Ann. Inst. Past., 26, 1912, 306; Prérot, Man. de Classif. des Bact. Anaér., Paris, 19t0, 85.) Anaerobe. From the buccal carity.

Vibrio crassus var. D, Prévot. (Spirille 
D, Repaci, Ann. Inst. Past., 26, 1912, 550 ; Prévot, Man. de Classif. des Bact. Anaér., Paris, 1940, 86.) Anaerobe. From the buccal cavity.

Vibrio crasteri Hauduroy et al. (Craster, in Violle, Le Choléra, Masson édit., 1919; Hauduroy et al., Dict. d. Bact. Path., 1937, 541.) Isolated from healthy persons. Resembles Vibrio comma.

Vibrio cucumis Kalninš.

(Latvijas Ūniversitātes Raksti, Serija I, No. 11, 1930, 243.) Decomposes cellulose. From soil.

Vibrio devorans Beijerinck. (Cent. f. Bakt., II Abt., 11, 1903, 598.) From water.

Vibrio drennani Chalmers and Waterfield. (Drennan, Jour. Inf. Dis., 14, 1914, 251; Chalmers and Waterfield, Jour. Trop. Med., 19, 1916, 165.) Colonies white, turning dark brown. From feces.

Vibrio Alavescens Weibel. (Cent. f. Bakt., 4, 1888, 225, 257, 281; Spirillum flavescens Trevisan, I generi e le specie delle Batteriacee, 1889, 24; Spirillum flavescens Sternberg, Man. of Bact., 1893. $700 ;$ Spirosoma flavescens Migula, Syst. d. Bakt., 2, 1900, 959.) Possibly identical with Vibrio aureus Weibel and Vibrio flavus Weibel. From sewage.

Vibrio flavus Weibel. (Cent. f. Bakt., 4, 1888, 225, 257, 281; Spirillum flavum Trevisan, I generi e le specie delle Batteriacee, 1889, 24; Spirillum flavum. Sternberg, Man. of Bact., 1893, 700; Spirosoma flavum Migula, Syst. d. Bakt., 2, 1900, 959.) Possibly identical with Vibrio aureus Weibel and Vibrio flavescens Weibel. From sewage.

Vibrio fuscus Stanier. (Jour. Bact., 42, 1941, 540.) Marine agar-digesting vibrio.

Vibrio gauduchcau Hauduroy et al. (Gauducheau, in Violle, Le Choléra, Masson édit., 1919; Hauduroy et al., Dict. d. Bact., 1937, 543.) From the blood of a fever patient. Resembles Vibrio comma.

Vibrio ghinda Pfeiffer. (Pasquale,
Gior. med. d. r. esercito, 1891 ; Pfeiffer, in Flügge, Die Mikroörganismen, 2, 1896, 590 ; Microspira ghinda Migula, Syst. d. Bakt., 2, 1900, 996.) From water.

Vibrio grossus (Migula) Ford. (Vibrio No. 1, Kutscher, Ztsehr. f. Hyg., 20, 1895, 46; Microspira grossa Migula, Syst. d. Bakt., 2, 1900, 1012; Ford, Textb. of Bact., 1927, 343.) From liquid manure.

Vibrio halobicus desulfuricans Horowitz-Wlassowa and Sonntag. (Arb. a. d. Stantl. wissensch. NahrungsmittelInstitut 1931 (Russian); see Ztschr. f. Unters. d. Lebensm., 62, 1931, 597.) A halophilic vibrio found in salted sardines, anchovies and other marine fish.

Vibrio helcogenes Fischer. (Cent. f. Bakt., 14, 1894, 73; Microspira helcogenes Migula, Syst. d. Bakt., 2, 1900, 978.) From descriptions, indistinguishable from Vibrio proteus according to Chester, Man. Determ. Bact., 1901, 339. From feces.

Vibrio hyos Ford. (Vibrio No. 3, Kutscher, Ztschr. f. Hyg., 20, 1895, 46; Spirillum mobile Migula, Syst. d. Bakt., 2, 1900, 1020; Ford, Textb. of Bact., 1927, 342.) Isolated from liquid manure.

Vibrio iners Besson, Ranque and Senez. (Compt. rend. Soc. Biol. Paris, 79, 1918, 1097.) From the feces of persons having dysentery.

Vibrio intermedius (Migula) Ford. (Group V, No. 9 of cholera-like vibrios, Kutscher, Ztschr. f. Hyg., 20, 1895, 481; Microspira intermedia Migula, Syst. d. Bakt., 2, 1900, 967 ; Ford, Textb. of Bact., 1927, 342.) Possibly identical with Vibrio berolinensis Neisser. From water.

Vibrio ivanoff Pfeiffer. (Ivanoff, Ztschr. f. Hyg., 15, 1893, 134; Pfeiffer, in Flügge, Die Mikroörganismen, 2, 1896, 592.) Probably a variety of Vibrio comma Winslow et al. according to Chester, Man. Determ. Bact., 1901, 337. From feces of a cholera patient.

Vibrio jejuni Jones, Orcutt and Little. (Jour. Exp. Med., 53, 1931, 853.) From small intestine of calves suffering from diarrhea.

Tibrio kegallensis Hauduroy et al. 
(Dict. d. Bact. Path., 1937, 54t.) From water.

Vibrio klimenko Hauduroy et al. (Kilimenko, in Violle, Le Choléra, Masson édit., 1919; Hauduroy et al., Diet. d. Bact. Path., 1937, 54t.) Resembles Tibrio comma. From the intestine.

Vibrio leidensis Horst. (Inaug. Diss., Leiden, 1921; abst. in Cent. f. Bakt., I Abt., Ref., 73, 1922, 282.) From a liver abscess.

* Tibrio lingualis Eisenberg. (Zungenbelag-Vibrio. Weibel. Cent. f. Bakt., 4, 18s8, 227; Eisenberg. Bakt. Diag.. 3 Aufl.. 1891, 212; Spirillum linguae Sternberg, Man of Bact., 1893, 697; Spirosoma linguale Migula, in Engler and Prantl, Die natürl. Pflanzenfam., 1, 1 a, 1895, 31.) From deposit on the tongus.

Vibrio lissabonensis Pestana-Bettencourt. (Cent. f. Bakt., 16, 1894, 401.) According to Chantemesse identical, or nearly so, with Vibrio proteus. From deseriptions, indistinguishable from $\mathrm{Vib}$ rio proteus according to Chester, Man. Determ. Bact., 1901, 339. From feces of a cholera patient.

Tibrio malamoria Kalniň. (Latvijas Eniversitutes Raksti, Serija I, No. 11, : 930, 250.) Decomposes cellulose. From soil.

Vibrio marinus (Pussell) Ford. (Spirillum marinum Russell, Ztschr. f. Hyg., 11, 1891, 165; . Kicrospira marina Migula, Syst. d. Bakt., 2, 1900, 1002 ; Ford, Textb. of Bact., 1927, 347.) From sea water. Closely resembles Vibrio cuneatus Gray and Thornton and Vibrio cardii Kilein.

Vibrio massauah Pfeiffer. (Pasquale, Gior. med. d. r. esercito, 1891; Pasquale, Baumgarten's Jahresberichte, 7, 1891,336; Pfeiffer, in Flügge, Die Mikrö̈rganismen, 2, 1896, 589;. Microspira massauah Miæula, Syst. d. Bakt., 2, 1900, 063; Spirillum massauah Chester, Manual Determ. Bact., 1901, 343; Spirillum massowah Holland, Jour. Bact., 5, 1920, 225; Tibrio massowah Holland, ibid.) From feces of a cholera patient.
Vibrio mulieris Prévot. (Man. de Classif. des Bact. Anaér., Paris, 1940, 84.) Anaerobe. From the female genital tract.

Vibrio napi Kalnin:. (Latvijas Universitātes Rakisti, Serija I, No. 11, 1930, 252.) Decomposes cellulose. From soil.

ribrio n'dianka Hauduroy et al. (Thiroux, in Violle, Le Choléra, Masson edit., 1919; Hauduroy et al., Dict. d. Bact. Path., 1937, 5ł6.) Isolated from a patient having a cholera-like disease.

Vibrio pericoma Kalnins. (Latvijas Universitītes Raksti, Serija I, No. 11, 1930, 256.) Decomposes cellulose. From soil.

Vibrio polymorphus Prévot. (Spirochete B, Repaci, Ann. Inst. Past., 26, 1912 , 544; Vibrio pscudospirochacta B, Weinberg, Nativelle and Prévot, Les Microbes Anaérobies, 1936, 852; Prévot, Man. de Classif. des Bact. Anaér., Paris, 1940, 83.) Anaerobe. From the buccal cavity.

Vibrio polymorphus var. peritriche Prévot. SSpirochete C, Repaci, Ann. Inst. Past., 26, 1912, 548; Vibrio pseudospirochaeta $C$, Weinberg. Nativelle and Prévot, Les Microbes Anaérobies, 1936, 854; Prévot, Man. de Classif. des Bact. Anaér., Paris, 1940, 84.) Anaerobe. From the buccal cavity.

Vibrio portuensis (Migula) Ford. (Der portuenser Vibrio, Jorge, Cent. f. Bakt., I Abt., 19, 1896, 277; Microspira portuensis Migula, Syst. d. Bakt., 2, 1900, 1007; Ford, Textb. of Bart., 1927, 353.) F From water.

Vibrio prima Kalnins. (Latvijas Ūni. versitātes Raksti, Serija I, No. 11, 1930, 235.) Decomposes cellulose. From soil.

Tribrio pseudospirochaeta Prévot. (Spirochete A, Repaci, Ann. Inst. Past., 26, 1912, 539; Vilrin pseudospirochaeta A, Weinberg, Nativelle and Prérot, Les Microbes Anaćrobies, 1936, 819; Prévot, Man. de Classif. des Bact. Anaér., Paris, 1940, 83.) Anaerobe. From the buceal cavity.

Vibrio putridus Prévot. (Vibrion C, Repaci, Compt. rend. Soc. Biol. Paris,

* See Nocardia lingualis Chalmers and Christopherson. 
1909, 630; Prévot, Man. de Classif. des Bact. Anaér., Paris, 1940, 83.) Anaerobe. From the buccal cavity.

Vibrio pyogenes (Doerr) Lehmann and Neumann. (Eiterspirillum, Mezinescu, Cent. f. Bakt., I Abt., Orig., 35, 1904, 201; Spirillum pyogenes Doerr, Cent. f. Bakt., I Abt., Orig., 38, 1905, 15; Lehmann and Neumann, Bakt. Diag., 4 Aufl., 2, 1907, 493.) From pus in a case of pyelitis calculosa. Non-motile.

Vibrio ranicula Kalninš. (Latvijas Universitātes Raksti, Serija I, No. 11, 1930, 248.) Decomposes cellulose. From soil.

Vibrio rigensis Kalninš. (Latvijas Universitātes Raksti, Serija I, No. 11, 1930,254.) Decomposes cellulose. From soil.

Vibrio rubicundus Gottron et al. (Gottron, Weaver and Sherago, Jour. Bact., 43, 1942, 61.) From a trickling filter.

Vibrio septicus Kolle. (Kolle and Schümann in Kolle and Wassermann, Handb. d. path. Mikroörg., 2 Aufl., 4, $1912,101$.$) Identical with Vibrio comma$ culturally and morphologically. From a cholera-like disease.

Vibrio smithii (Migula) Ford. (Smith, Cent. f. Bakt., 10, 1891, 177; Microspira smithii Migula, Syst. d. Bakt., 2, 1900, 1006; Ford, Textb. of Bact., 1927, 340.) Possibly identical with Microspira saprophiles Migula, Microspira weibelii Migula, Microspira cloaca Chester and Vibrio surati Ford. From abscesses of large intestine of swine.

Vibrio spermatozoides Löfler. (Cent. f. Bakt., 7, 1890, 638.) From kohlrabi infusions.

Vibrio sputigenus (Miller) Prévot. (Spirillum sputigenum Miller, Dic Mikroorg. d. Mundhöhle, 2nd ed., 1892; Prévot, Man. de Classif. des Bact. Anaér., Paris, 1940, 85; not Tibrio sputigenus Bergey et al., Manual, 1st ed., 1923, 80.) Anaerobe. From the buccal cavity.

Vibrio sputigenus var. minutissimus Prévot. (Muhlens, Cent. f, Bakt., I
Abt., 48, 1909, 523; Prévot, Man. de Classif. des Bact. Anaér., Paris, 1940, 85.) Anaerobe. From the buccal cavity.

Vibrio sputorum Prévot. (Man. de Classif. des Bact. Anaér., Paris, 1940, 85.) Anaerobe. Isolated from a case of bronchitis.

Vibrio stationis Kalniňs. (Latvijas Universitātes Raksti, Serija I, No. 11, 1930,239.) Decomposes cellulose. From soil.

Vibrio stomatitis Prévot. (Vibrion A, Repaci, Compt. rend. Soc. Biol. Paris, 1909, 630; Prévot, Man. de Classif. des Bact. Anaér., Paris, 1940, 82.) Anaerobe. From the buccal cavity.

Vibrio subtilissimus (Migula) Ford. (Spirillum No. 1, Kutscher, Ztschr. f. Hyg., 20, 1895, 46; Spirillum tenerrimum Lehmann and Neuman, Bakt. Diag., 2, 1896,346 ; Spirillum subtilissimum Migula, Syst. d. Bakt., 2, 1900, 1020; Ford, Textb. of Bact., 1927, 341.) Regarded by Kutscher as being probably identical with the organism found by Smith (Cent. f. Bakt., 16, 1894, 324) in swine dung. Resembles Vibrio strictus.

Vibrio suis Ford. (Vibrio No. 2, Kutscher, Ztschr. f. Hyg., 20, 1895, 46; Spirillum coprophilum Migula, Syst. d. Bakt., 2, 1900, 1019; not Microspira coprophila Migula, loc. cit., 986; Ford, Textb. of Bact., 1927, 341.) From liquid manure.

Vibrio surati (Lamb and Paton) Ford. (Spirillum surati Lamb and Paton, Arch. Int. Med., 12, 1913, 259; Treponema surati Brumpt, Nouveau Traité de Médecine, Paris, 4, 1922, 514; Ford, Textb. of Bact., 1927, 337.) Isolated from a case of vegetative endocarditis. Closely resembles Vibrio smithii Ford, Microspira weibelii Migula, Microspira saprophiles Migula and Microspira cloaca Chester.

Vibrio synthetica Kalninš. (Latvijas Ūniversitätes Raksti, Serija I, No. 11, 1930,245.) Decomposes cellulose. From soil.

Vibrio tenuis Veillon and Repaci. 
(Ann. Inst. Past., 26, 1912, 300.) Anaerobe. From the buccal cavity.

Vibrio terrigenus Günther. (Cent. f. Bakt., 16, 1894, 746; Spirillum terrigenum Migula, Syst. d. Bakt., 2, 1900, 1017 ; Microspira terrigena Chester, Man. Determ. Bact., 1901, 341.) Closely related to Vibrio tonsillaris Stephens and Smith. From soil.

Vibrio tonsillaris Stephens and Smith. (Cent. f. Bakt., 19, 1896, 929; Microspira tonsillaris Migula, Syst. d. Bakt., 2,
1900, 1009.) Closely related to Vibrio terrigenus Günther. From buccal cavity.

Vibrio toulonensis Hauduroy et al. (Vibrion, Defressine and Cazeneuve, in Violle, Le Choléra, Masson édit., 1919 Hauduroy et al., Dict. d. Bact. Path. 1937, 547.) From mussel beds in the bay of Toulon.

Vibrio xylitica Kalninš. (Latvijas Universitātes Raksti, Serija I, No. 11, 1930,232.) Decomposes cellulose. From soil.

\section{Genus II. Desulfovibrio Kluyver and van Niel.*}

(Cent. f. Bakt., II Abt., 94, 1936, 369; Sporovibrio Starkey, Arch. f. Mikrobiol., 9, 1938, 300.) From M. L. desulfo, an abbreviation of the poorly constructed word desulfofication, used to indicate reduction of sulfur compounds by bacteria; vibrio, vibrio.

Slightly curved rods of variable length, usually occurring singly but sometimes in short chains which have the appearance of spirilla. Swollen pleomorphic forms are common. Actively motile by means of a single polar flagellum. Strict anaerobes which reduce sulfates to hydrogen sulfide. Found in sea water, marine mud, fresh water, and soil.

The type species is Desulfovibrio desulfuricans (Beijerinck) Iluyver and van Niel.

\section{Desulfovibrio desulfuricans} (Beijerinck) Kluyver and van Niel. (Bacterium hydrosulfureum ponticum Zelinsky, Proc. Russ. Phys, and Chem. Soc., 25, 1893, 298; Spirillum desulfuricans Beijerinck, Cent. f. Bakt., II Abt., 1, 1895, 1: Bacillus desulfuricans Saltet, Cent. f. Bakt., II Abt., 6, 1900, 648; Microspira desulfuricans Migula, Syst. d. Bakt., 2, 1900, 1016; Kluyver and van Niel, Cent. f. Bakt., II Abt., 94, 1936, 369; Vibrio desulfuricans Holland, Jour. Bact., 5, 1920, 225; Sporovibrio desulfuricans Starkey, Tioninkl. Nederland. Akad. v. Wetenschappen, Proc., 41, 1938, 425; also in Arch. f. Microbiol., 9, 1938, 268.) From MI. L. present part. desulfurico, sulfur reducing.

Slightly curved rods, 0.5 to 1.0 by 1 to $\tilde{y}$ microns, usually occurring singly but sometimes in pairs and short chains which cause them to look like spirilla. Swollen pleomorphic forms are common. Older cells appear black due to precipitated ferric sulfide. Actively motile, possessing a polar flagellum. Gramnegative. Stains readily with carbol fuchsin.

Grows best in freshwater media. Fails to develop in sea water upon initial isolation.

Produces opalescent turbidity in absence of oxygen in mineral media enriched with sulfate and peptone.

Media containing iron salts blackened. Bacteria found associated with precipitateu ferrous sulfide.

Peptone-glucose agar colonies (in absence of air): Small, circular, slightly raised, dull, entire, soft in consistency.

Gelatin not liquefied.

Peptone, asparagine, glycine, alanine, aspartic acid, ethanol, propanol, butanol,

* Prepared by Dr. Claude E. ZoBell, Scripps Institution of Oceanography, La Jolla, California, Jan., 1943. 
glycerol, glucose, lactate, succinate and malate known to be utilized as hydrogen donors.

Produces up to $500 \mathrm{ml} . \mathrm{H}_{2} \mathrm{~S}$ per liter.

Nitrites not produced from nitrates.

Reduces sulfate to hydrogen sulfide. Also reduces sulfites, sulfur, thiosulfates and hyposulfites.

Optimum pH 6 to 7.5 , limits pH 5 to 9 .

Optimum temperature 25 to $30^{\circ} \mathrm{C}$. Maximum 35 to $40^{\circ} \mathrm{C}$.

Anaerobic.

Habitat: Soil, sewage, water.

\section{Desulfovibrio aestuarii (van} Delden) comb. nov. (Microspira aestuarii van Delden, Cent. f. Bakt., II Abt., 11, 1904, 81; Vibrio desulfuricans (halophilic strain) Baars, Over Sulfaatreductie door Bakterien, Diss. Delft, 1930, 164 pp.) From Latin, aestuarium, estuary.

Morphologically indistinguishable from Desulfovibrio desulfuricans described above, although it has a greater tendency to pleomorphism, and is slightly larger. Motile, possessing a polar flagellum. Gram-negative.

Grows preferentially in media prepared with sea water or 3 per cent salt mineral solution enriched with sulfate and peptone. According to Baars (loc. cit.) the marine species can be acclimatized to tolerate hypotonic salt solutions but Rittenberg (Studies on Marine SulphateReducing Bacteria, Thesis, Univ. of Calif., 1941, 115 pp.) was unable to confirm this observation. Likewise Rittenberg was unable to acclimatize $D$. aestuarii to tolerate temperatures exceeding $45^{\circ} \mathrm{C}$ or to produce endospores.

Produces faint turbidity in absence of oxygen in sea water enriched with sulfate and peptone. Organisms most abundant in sediment.

Agar colonies: Small, circular, slightly raised, darker centers, entire, soft consistency.

Gelatin not liquefied.

Peptone, asparagine, glycine, alanine, glucose, fructose, ethanol, butanol, glycerol, acetate, lactate and malate known to be utilized in presence of sulfate.

Reduces sulfate to hydrogen sulfide. Also reduces sulfites, sulfur, thiosulfates and hyposulfites.

Produces up to $950 \mathrm{ml} . \mathrm{H}_{2} \mathrm{~S}$ per liter.

Nitrites not produced from nitrates.

Optimum temperature $25^{\circ}$ to $30^{\circ} \mathrm{C}$. Maximum $35^{\circ}$ to $40^{\circ} \mathrm{C}$.

Optimum pH 6 to 8 , limits $\mathrm{pH} 5.5$ to 8.5.

Anaerobic.

Habitat: Sea water, marine mud, brine and oil wells.

\section{Desulfovibrio rubentschickii}

(Baars) comb. nov. (Vibrio rubentschickii Baars, Over Sulfaatreductie door Bakterien, Diss. Delft, 1930, 164 pp.) Named for L. Rubentschick.

Slightly curved rods, 0.5 to 1.0 by 1 to 5 microns, usually occurring singly, sometimes in pairs and short chains. Actively motile, possessing a polar flagellum. Gram-negative. Morphologically indistinguishable from Desulfovibrio desulfuricans.

Reduces sulfate to hydrogen sulfide. Also reduces sulfites, sulfur, thiosulfates and hyposulfites.

Culturally and physiologically like $D$. desulfuricans except that $D$. rubentschickii utilizes propionic acid, butyric acid, valeric acid, palmitic acid, stearic acid, galactose, sucrose, lactose and maltose.

Anaerobic.

Habitat: Soil and ditch water.

Appendix: The following species has also been regarded as belonging in this genus.

Vibrio thermodesulfuricans Elion. (Cent.f. Bakt., II Abt., 63, 1924, 58); Vibrio desulfuricans (thermophilic strain) Baars, Over Sulfaatreductie door Bakterien, Diss. Delft, 1930, 164 pp.; Sporovibrio desulfuricans Starkey (Koninkl. Nederland. Akad. u. Wetenschappen, Proc., 41, 1938, 425, also see Arch. f. 
Microbiol., 9, 1938, 268.) A thermophilic sulfate-reducing anaerobe which grows at 30 to $65^{\circ} \mathrm{C}$. and which, according to Starkey, produces endospores. Elion described Vibrio thermodesulfuricans (Cent. f. Bakt., II Abt., 63, 1924, 58) which grows at temperatures no lower than 30 to $40^{\circ} \mathrm{C}$. and has an optimum of $55^{\circ} \mathrm{C}$. Morphologically it is much like Desulfovibrio desulfuricans and $D$. aestuarii although the thermophilic form is shorter, more rod-like, less motile and more pleomorphic. According to Baars (loc. cit.), Fibrio thermodesulfuricans Elion can be acclimatized to grow at lower temperatures and it is found abundantly in environments where the temperature has never been as high as $30^{\circ} \mathrm{C}$. This observation is confirmed by Starkey (Arch. f. Microbiol., 9, 1938, 268) who found further that the thermophilic form found in nature or developed by acclimatization to higher temperatures produces endospores. However, sporeformation appears to be the exception rather than the rule. The pleomorphic, peritrichous, sporogenous, sulfatereducer is more rod-like than the asporogenous cultures and many cells of the sporogenous eultures are Gram-positive whereas asporogenous cultures of Desulfovibrio desulfuricans are Gram-negative. all of which leaves a question whether the sporogenous sulfate-reducer is a Bacillus or a Desulforibrio. Rittenberg (Studies on Marine Sulfate-reducing Bacteria, Thesis, Univ. Calif., 1941, 115 pp.) was unable to adapt the marine sulfate reducer to grow at low salinities or at high temperatures, nor could it be induced to form spores.

Desulforibrio halohydrocarbonoclastirus Zohell (L. S. Patent No. 2,413,27s; Science Vews Letter, Jan. 11, 1947.) From oil hearing rocks.

\section{Genus III. Cellvibrio Winogradsky.*}

(Ann. Inst. Pasteur, 43, 1929. 577.) From .T. L. cell, an abbreviation for cellulose; vibrio, vibrio.

Long slender rods, slightly curved, with rounded ends, show deeply staining granules which appear to be coneerned in reproduction. Monotrichous. Most species produce a yellow or brown pigment with cellulose. Oxidize cellulose, forming oxycellulose. Growth on ordinary culture media is feeble. Found in soil.

The type species is Cellvibrio ochraceus Winogradsky.

\section{Key to the species of genus Cellvibrio.}

I. No growth on glucose or starch agar.

A. Ochre-yellow pigment produced on filter paper.

1. Cellvibrio ochraceus.

II. Growth on glucose and starch agar.

1. Poor growth on starch agar.

1. Cream-colored pigment which becomes brown with age is produced on filter paper.

\section{Cellvibrio flavescens.}

B. Abumdant growth on starch agar.

1. Scanty growth on glueose agar.

a. Intense yellow pigment produced on filter paper.

3. Cellvibrio fulvus.

2. Abundant growth on glucose agar.

a. No pigment produced on filter paper.

4. Cellvibrio vulgaris.

* Revised by Prof. Robert S. Breed, New York State Fxperiment Station, Geneva, New York, Sept., 1937; no change. July, 1913. 
1. Cellvibrio ochraceus Winogradsky. (Ann. Inst. Pasteur, 43, 1929, 549, 601.) From Greek, ōchra, yellow ochre; M. L. like ochre, yellow.

Plump, curved rods with rounded ends, 2.0 to 4.0 microns long, rarely occurring as spirals. Chromatic granule frequently found in center. Motile with a single flagellum. Gram-negative.

Produces diffuse, light ochre-colored, mucilaginous colonies on cellulose silica gel medium.

No action or growth on plain agar. No growth on peptone, glucose, starch or tragacanth gum agar.

Filter paper streaks: Entire paper colored ochre-yellow in 48 hrs.

Aerobic, facultative.

Optimum temperature $20^{\circ} \mathrm{C}$.

Distinctive character: Rapid ochrecolored growth.

Habitat: Soil. Disintegrates vegetable fibers.

2. Cellvibrio flavescens Winogradsky. (Ann. Inst. Pasteur, 43, 1929, 608.) From Latin, part. adj. of flavesco, to turn yellow or golden.

Plump, curved rods, flexuous, with rounded ends, 0.5 by 2.5 to 5.0 microns. Shows metachromatic granules. Motile with a single flagellum. Gram-negative.

Produces diffuse, cream-colored growth becoming brownish; mucilaginous colonies on cellulose silica gel medium.

Good growth on peptone agar. Colonies $1 \mathrm{~mm}$ in 4 days. Grows poorly on glucose, starch and gum agars.

Filter paper streaks: Almost as rapid in growth as Cellvibrio ochraceus and colors entire paper in 2 to 3 days.

Aerobic, facultative.

Optimum temperature $20^{\circ} \mathrm{C}$.

Distinctive characters: Smaller, less curved rods that grow on a greater variety of media than Cellvibrio ochraceus, but do not attack cellulose as readily.

Source: Isolated from a pile of old damp sawdust.

\section{Habitat: Soil. Disintegrates vegetable} fibers.

3. Cellvibrio fulvus Stapp and Bortels. (Culture Y, Dubos, Jour. Bact., 15, 1928, 230; Stapp and Bortels, Cent. f. Bakt., II Abt., 90, 1934, 42.) From Latin, fulvus, reddish yellow.

Slightly curved rods: 0.3 to 0.4 by 1.5 to 3.0 microns. Show involution forms. Motile by means of a single polar flagellum. Gram-negative.

Cellulose is decomposed. Grows on filter paper with an intense egg-yellow color which in older cultures may deepen to rust brown.

Glucose agar: Very scanty growth.

Sucrose agar: Very slight growth.

Maltose agar: Abundant yellow growth.

Lactose agar: Fairly abundant yellow growth.

Starch agar: Very abundant, bright yellow growth which later turns brown.

Nutrient broth: No growth.

Temperature relations: Optimum $25^{\circ}$ to $30^{\circ} \mathrm{C}$. Minimum $5^{\circ} \mathrm{C}$. Maximum $32^{\circ}$ to $35^{\circ} \mathrm{C}$. No growth at $37^{\circ} \mathrm{C}$. Thermal death point $39^{\circ}$ to $40^{\circ} \mathrm{C}$.

Aerobic.

Source: Isolated from forest soil in Germany and from soil in the United States.

Habitat: Widely distributed in soils.

4. Cellvibrio vulgaris Stapp and Bortels. (Culture Co, Dubos, Jour. Bact., 15, 1928, 230; Stapp and Bortels, Cent. f. Bakt., II Abt., 90, 1934, 44.) From Latin, vulgaris, common.

Curved rods : 0.3 by 2.9 to 4.0 microns. Shows involution forms. Motile by means of a single polar flagellum. Gram. negative.

Cellulose is decomposed. Grows on filter paper without the formation of pigment.

Glucose agar: Abundant growth. No pigment.

Sucrose agar: Abundant slightly yellow growth. 
Maltose agar: Abundant yellorish growth.

Lactose agar: Very heavy growth.

Starch agar: Very abundant yellowish growth.

Nutrient broth: No growth.

Temperature relations: Optimum $25^{\circ}$ to $30^{\circ} \mathrm{C}$. Minimum $5^{\circ} \mathrm{C}$. Maximum $32^{\circ}$ to $35^{\circ} \mathrm{C}$. No growth at $37^{\circ} \mathrm{C}$. Thermal death point $44^{\circ}$ to $45^{\circ} \mathrm{C}$.

Aerobic.

Source: Isolated from forest soil in Germany and from soils in the United States.

Habitat: Widely distributed in soils.

\section{Genus IV. Cellfalcicula Winograilsky.*}

(Ann. Inst. Pasteur, 43, 1929, 616.) From M. L. cell, an abbreviation for cellulose; Latin dim., falcicula, a small sickle.

Short rods or spindles, not exceeding 2.0 microns in length, with pointed ends, containing metachromatic granules. Old cultures show coccoid forms. Monotrichous. Oxidize cellulose, forming oxycellulose. Growth on ordinary culture media is feeble. Soil bacteria.

The type species is Cellfalcicula viridis Winogradsky.

1. Cellfalcicula viridis Winogradsky. (Ann. Inst. Pasteur, 43. 1929, 616.) From Latin, viridis, green.

Plump, small spindles, 0.7 by 2.0 microns, with rounded ends. Notile with a single flagellum. Gram-negative.

Produces diffuse green, mucilaginous colonies on cellulose silica gel medium.

Filter paper streaks: Rapid spreading growth colored green in 3 days at $30^{\circ} \mathrm{C}$.

Hydrocellulose agar: Growth rapid, green; minute yellowish-green, mucous colonies on streaking.

No growth on peptone, glucose, starch or gum agar.

Aerobic, facultative.

Optimum temperature $20^{\circ} \mathrm{C}$.

Habitat : Soil.

2. Cellfalcicula mucosa Winogradsky. (Ann. Inst. Pasteur, 43, 1929, 621.) From Latin, mucosus, mucous.

Plump, curved spindles, with slightly pointed ends. Motile with a single polar flagellum. Contain a single chromatic granule. Cram-negative.

Produces diffuse, cream-colored, muci- laginous colonies on cellulose silica gel medium.

Hydrocellulose agar: Abundant grayish growth.

No growth on peptone, glucose, starch or gum agar.

Aerobic, facultative.

Optimum temperature $20^{\circ} \mathrm{C}$.

Hatbitat : Soil.

3. Cellfalcicula fusca Winogradsky. (Ann. Inst. Pasteur, 43, 1929, 622.) From Latin, fuscus, dark, tawny.

Plump, curved spindles, 0.5 by 1.2 to 2.5 microns, with slightly pointed ends and a central chromatic granule. Notile with a single polar flagellum. Gramnegative.

Produces diffuse, brownish, slightly marbled or veined colonies on cellulose silica gel medium.

Filter paper streak: Paper becomes a partially transparent, dry, non-mucilaginous pellicle adherent to gel.

Aerobic, facultative.

Optimum temperature $20^{\circ} \mathrm{C}$.

Source: Isolated from a pile of old damp sawdust.

Habitat: Probably rotting wood.

* Revised by Prof. Robert s. Breed, New Tork State Experiment Station, Geneva, New York, Sept., 1937; no change, July, 1913. 


\section{Genus V. Thiospira Vislouch.*}

(Jour, de Microbiologie, 1, 1914, 50; Sulfospirillum Kluyver and van Nicl, Cent. f. Bakt., II Abt., 94, 1936, 396.) From Greek, theion, sulfur; speira, coil.

Colorless, motile, slightly bent rods, somewhat pointed at the ends, with granules of sulfur within the cells and a small number of flagella at the ends.

The type species is Thinspira winogradskyi (Omelianski) Vislouch.

\section{Thiospira winogradskyi (Ome-} lianski) Vislouch. (Thiospirillum winogradskyi Omelianski, Cent. f. Bakt., II Abt., 14, 1905, 769; Thiospirillum granulatum Molisch, Cent. f. Bakt., II Abt., 33, 1912, 55; Vislouch, Jour. de Microbiologie (Russian), 1, 1914, 50; Sulfospirillum winogradskyi Kluyver and van Niel, Cent. f. Bakt., II Abt., 94, 1936, 397.) Named for Winogradsky, the Russian bacteriologist.

Large, sulfur spirilla, somewhat pointed at the ends, 2 to 2.5 microns thick, to 50 microns long. Numerous granules of sulfur. Very motile, with one to two polar flagella.

Habitat: Curative mud.
2. Thiospira bipunctata (Molisch) Vislouch. (Spirillum bipunctatum Molisch, Cent. f. Bakt., II Abt., 33, 1912, 55 ; Vislouch, Jour. de Microbiologie (Russian), 1, 1914, 50.) From Latin, bi, two; punctum, points.

Small, slightly bent sulfur spirilla, markedly pointed at the ends, 6.6 by 14 microns long, 1.7 to 2.4 microns wide (in the center of the cell). Both ends are filled more or less with large volutin (metachromatic) granules. Several minute granules of sulfur are present in the clear center and sometimes at the ends. ()ld cells possess one flagellum at each end; young cells have a flagellum at one end.

Habitat: Sea and salt waters.

\section{Genus VT. Spirillum E'hrenberg. $\dagger$}

(Ehrenberg, Abhandlungen d. Berl. Akad., 1830, 38; Spirosoma Migula, Arb. bakt. Inst. Karlsruhe, 1, 1894, 237; Dicrospirillum. Finderlein, Sitzber. Gesell. naturf. Freunde, Berlin, 1917,313.) From Greek, speira, a spire or coil.

Cells form either long screws or portions of a turn. Volutin granules are usually present. Usually motile by means of a tuft of polar flagella (5-20) which may occur at one or both ends of the cells. Aerobic, growing well on ordinary culturemedia, except for one saprophyte and the pathogenic species. These have not yet been cultivated. Usually found in fresh and salt water containing organic matter.

The type species is Spirillum undula (Müller) Ehrenberg.

\section{Key to the species of genus Spirillum.}

I. One micron or less in diameter.

1. Volutin granules present.

a. Slow to rapid liquefaction of gelatin.

b. Grayish to brown growth on potato.

1. Spirillum undula.

* Prepared by Prof. D. H. Bergey, Philadelphia, Penn., October, 1922.

$\dagger$ Revised by Prof. D. H. Bergey, Philadelphia, Pennsylvania, April, 1937; further revision by Prof. Robert S. Breed, New York State Experiment Station, Geneva, New York, June, 1943, based on Monograph by Giesberger, Inaug. Diss., Utrecht, Nov. 30, 1936. 
bb. Light yellowish-orange growth on potato.

\section{Spirillum serpens.}

aa. No liquefaction of gelatin. Of small size ( 0.5 micron in diameter).

b. Colonies on agar white becoming brownish black and slightly wrinkled.

\section{Spirillum itersonii.}

bb. Colonies on agar white and smooth.

2. No volutin granules observed.

4. Spirillum tenue.

b. Single flagellum.

\section{Spirillum virginianum.}

bb. Tuft of flagella.

6. Spirillum minus.

II. Over one micron in diameter.

1. Grows poorly on peptone agar and potato.

7. Spirillum kutscheri.

2. Not positively known to have been cultivated on artificial media. Very evident volutin granules.

8. Spirillum volutans.

3. Cells more or less deformed by fat drops.

9. Spirillum lipoferum.

1. Spirillum undula (Müller) Ehrenberg. (Vibrio undula Müller, Animalcula infusoria et marina, 1786; Ehrenberg, Infusionstierchen, 1838; Spirillum undula minor Kutscher, Cent. f. Bakt., I Abt., 18, 1895, 614.) From Latin, undulatus, wave-like.

Stout threads, 0.9 micron in diameter, with one-half to three turns. The wave lengths are 6 microns. Width of spiral, 3.0 microns. Tufts of three to nine flagella at each pole. Volutin granules present. Gram-negative.

Gelatin colonies: The surface colonies are circular, granular, greenish-yellow; entire.

Gelatin stab: Thick, white, rugose surface growth. Very slow liquefaction.

Agar colonies: Grayish-white, smooth.

Broth: Turbid.

Potato: Grayish-brown growth.

Indole not formed.

Catalase positive.

Nitrites not produced from nitrates.

Aerobic, facultative.

Optimum temperature $25^{\circ} \mathrm{C}$.

Cohn (Beitrage z. Biol. d. Pflanzen, 1, Heft 2, 1875, 132) reports that he could not distinguish this organism from Vibrio prolifer Ehrenberg.

Habitat: Putrid and stagnant water.

2. Spirillum serpens (Müller) Winter. (Vibrio serpens Nüller, Animaleula infusoria et marina, 1786, 43; Winter, in Rabenhorst's Kryptogamen-Flora, 1, Die Pilze, 1851, 63.) From Latin, serpens, serpent.

Long, curved rods with two to three wave-like undulations, 0.8 to 1.0 micron in diameter; wave length, 8 to 9 microns. Width of spiral 1.5 to 1.8 mierons. Volutin granules in cytoplasm. Motile, possessing tuits of flagella at both poles. Gram-negutive.

Gelatin colonies: Yellowish to brownish, granular, entire.

Gelatin stab: Yellowish surface growth. Slow liquefaction.

Agar colonies: Heavy cream-colored growth.

Agar slant: Grayish, with yellowish center, granular, entire.

Broth : Turbid.

Litmus milk: Unchanged.

Potato: Clear orange-yellow growth Indole not formed. 
Catalase positive.

Nitrites not produced from nitrates.

Aerobic, facultative.

Optimum temperature $35^{\circ} \mathrm{C}$.

Habitat: Stagnant water.

3. Spirillum itersonii Giesberger. (Inaug. Diss., Utrecht, 1936, 46 and 57.) Named for van Iterson, the Dutch bacteriologist.

The smallest of the spirilla isolated from water. First observed by van Iterson (Proc. Kon. Akad. v. Wetensch. Amsterdam, 5, 1902, 685).

Small spirals, 0.5 micron in diameter. Wave length, 3 to 3.5 microns. Spiral width, 1 to 1.5 microns. Motile with bipolar tufts of flagella. Gram-negative.

Grows readily on peptone agar. White colonies becoming brownish black, and slightly wrinkled.

Gelatin stab: No liquefaction.

Brownish-orange growth on potato.

Volutin granules may be present.

Catalase is produced.

Acid from glucose, fructose, ethyl alcohol, n-propyl alcohol, n-butyl alcohol, and glycerol. Utilizes acetic, propionie, n-butyrie, tartaric, fumaric, lactic, citric, and succinic acids.

Grows well in peptone broth. Also utilizes ammonia compounds.

Anaerobic growth in the presence of nitrates when organic or ammonia nitrogen is also available.

Optimum temperature : $30^{\circ} \mathrm{C}$.

Source: Isolated from water.

Habitat: Water.

4. Spirillum tenue Ehrenberg. (Infusionstierchen, 1838; see Bonhoff, Arch. f. Hyg., 26, 1896, 162.) From Isatin, tenuis, thin.

Slender spirals. Diameter 0.7 micron. Wave lengths 4.5 to 5.0 microns. Width of spiral 1.5 to 1.8 microns.

Actively motile in peptone water with tufts of flagella at each pole. Volutin granules present. Gram-negative.

Agar colonies: White, smooth.
Peptone agar slant: Heavy growth.

Gelatin stab: No liquefaction.

Catalase positive.

Potato: Light brown growth.

Acid from glucose and fructose. Slight acid from several other sugars and glycerols. Utilizes salts of acetic, propionic, n-butyric, tartaric, lactic, citric, malic, and succinic acids.

Ammonia compounds are used as a source of nitrogen.

Optimum temperature, $30^{\circ} \mathrm{C}$.

Source: Found in putrefying vegetable matter.

Habitat: Putrefying materials.

5. Spirillum virginianum Dimitroff. (Jour. of Bact., 12, 1926, 19.) From M. L. genitive of Virginia.

Spirals consisting of $\frac{1}{2}$ to 3 complete turns in young cultures, older cultures showing 7 turns, 0.6 to 0.9 by 3 to 11 microns. Motile with a single polar flagellum on one or both ends. Gramnegative.

Gelatin colonies: Entire, convex, eircular, moist, colorless.

Gelatin stab: Growth along entire stab. No liquefaction. (Dimitroff, loc. cit.) Active liquefaction. (Giesberger, Inaug. Diss., Utrecht, 1936, 65.)

Agar colonies: Dew drop, convex, entire, moist, colorless.

Agar slant: Dew drop, isolated colonies.

Broth: Cloudy, no flocculation.

Uschinsky's protein-free medium : Abundant growth.

Litmus milk: No growth.

Loeffler's blood serum: Convex, isolated dew drop colonies. No liquefaction.

Lead acetate agar: $\mathrm{No}_{2} \mathrm{H}_{2} \mathrm{~S}$.

Voges-Proskauer and methyl red negative.

No volutin granules observed (Giesberger, loc. cit., p. 60).

Potato: No growth.

Indole not formed.

Nitrites not produced from nitrates.

No acid or gas from carbohydrates. 
(Dimitroff, loc. cit.). Utilizes lactates and citrates (Giesberger, loc. cit.).

Aerobic, facultative.

Optimum temperature $35^{\circ} \mathrm{C}$.

Source: Isolated from mud on an oyster shell.

Habitat: Probably muddy bottom of brackish water.

6. Spirillum minus Carter. (Carter, Sci. Mem. Med. Officers Army India, 3, 1887, 45; Spirillum minor Carter, ibill.; Spirochaeta laverani Breinl and Kinghorn, Mem. Liveronol Sch. Trop. Med. 21, 1906, 55; Spirochaeta muris Wenyon, Jour. Hyg., 6, 1906, 580; Spirochaeta muris var. virginiana MacNeal, Proc. Soc. Exper. Biol. and Merd., 4, 1907, 125; Spirochaeta muris var. galatziana Mezincescu, Compt. rend. Soc. Biol. Paris, 66, 1909, 58; Treponema muris Moore, Principles of Microbiology, 1912, 414; Spirochaeta morsus muris Futaki, Takiki, Taniguchi and Osumi, Jour. Exp. Med., 25, 1917, 33; Spirochaeta petit Row, Ind. Jour. Med. Res., 5, 1917, 386 ; Spironema muris Noguchi, Jour. Exp. Med., 27, 1918, 584; Spirochaeta japonica Dujitrie de la Rivière, Ann. de Méd., 5, 1918, 181 ; Spirochacta morsusmuris Castellani and Chalmers, Man. Trop. Med., 3rd ed., 1919, 447; Spiroschaudinnia morsusmuris Castellani and Chalmers, ibid.; Spirochacta sodoku Troisier, 1920, according to Pettit, Contribution à l'Étude des Spirochétidés, Vanves, II, 1928, 231; Treponema japonicum Brumpt, Nouveau Traité de Médecine, Paris, 4, 1922, 505; Treponema morsus muris Brumpt, ibid., 506; Treponema minor Brumpt, ibid., 507 ; Treponema laverani Brumpt, ibid., 507 ; Treponema sodoku Brumpt, ibid., 514; Spirochata pettiti Row, Jour. Trop. Med. and Hye., 25, 1922, 364; Treponemella muris San Giorgi, Pathologica rivista, 14, 1922, 461; Borrelia muris Bergey et al., Manual, 2nd ed., 1925, 435; Spirillum minus var. morsus muris Ruys, Cent. f. Bakt., I Abt., Orig., 103, 1927, 270; Spirillum minus var. muris Ruys, ibid.; Spironema minor Ford, Textb. of Bact., 1927, 962; Spironema laverani Ford, ibid., 963; Spironema muris var. virginiana Ford, ibid., 963; Spirclla morsusmuris Noguchi, in Jordan and Falk, Newer Knowledge Bact. and Immun., 1928, 497; Spirella muris Noguchi, ibid.) From Latin, minus, less.

Deseription taken from Adachi, Jour. Exp. Med., 33, 1921, 647 and Giesberger, Inaug. Diss., Delft, 1936, 67.

Short thick cells: 0.5 by 3.0 microns, having 2 or 3 windings which are thick, regular and spiral. Actively motile by means of bipolar tufts of flagella. Gramnegative.

Has not been cultivated on artificial media .

Acrobic, facultative.

Pathogenic for man, monkeys, rats. mice and guinea pigs.

This species is regarded by some as a spirochaete. Because of its habitat and wide distribution it has been described under many different names. It is possible that some of these names indirate varieties or even separate species. See Beeson (Jour. Amer. Med. Assoc, 123, 1943, 332) for important literature.

Source: Found in the blood of rats and mice.

Habitat: The cause of rat-bite fever. Widely distributed.

7. Spirillum kutscheri Migula. (Spirillum undula majus Kutscher, Cent. f. Bakt., I Abt., 18, 1895, 614; Migula, Syst. d. Bakt., 2, 1900, 1024.) Named for Kutseher, the German bacteriologist who first isolated the organism.

Stout threads, 1.5 microns in diameter. Wave lengths 10.5 to 12.5 microns. Width of spiral, 3 to 4.5 microns. May lose their spiral form on continued cultivation. Motile with tufts of fagella at the poles. Gram-negative.

Gelatin colonies: Transparent, round, surface colonies. Deep colonies, dark brown. 
Gelatin stab: Slow liquefaction.

Agar colonies grow poorly, granular. Deep colonies yellowish-green to dark brown.

Agar slant: Delicate, transparent growth.

Potato: Limited growth.

Volutin present.

Catalase positive.

Utilizes malic and succinic acids.

Grows well on peptone broth. Also utilizes ammonia compounds.

Optimum temperature, $22^{\circ}$ to $27^{\circ} \mathrm{C}$.

Source: Isolated from putrid materials and liquid manure.

Habitat: Putrefying liquids.

8. Spirillum volutans Ehrenberg. (Prototype, Vibrio spirillum Müller, Animalcula infusoria, 1786; Ehrenberg, Die Infusionstierchen als Volkommene Organismen, 1838.) From M. L. volutin.

Spirals 1.5 microns in diameter. Wave length, 13 to 15 microns, width of spiral, 4 to 5 microns. The largest of the spirilla. Slightly attenuated ends. Motile, possessing a tuft of ten to fifteen flagella at each pole. Dark granules of volutin in the cytoplasm. Gram-negative.

Migula (Syst. d. Bakt., 2, 1900, 1025) reports that this species has not been cultivated on artificial media, and that the cultures so described by Kutseher (Ztschr. f. Hyg., 20, 1895, 58) are of a different species which Migula names Spirillum giganteum. Vahle (Cent. f. Bakt., II Abt., 25, 1910, 237) later describes the cultural characters of an organism which he regards as identical with Kutscher's organism. Giesberger (Inaug. Diss., Delft, 1936, 65) saw what he felt was the true Spirillum volutans but could not cultivate it.

Optimum temperature $35^{\circ} \mathrm{C}$.

Habitat: Stagnant water.
9. Spirillum lipoferum Beijerinck. (Azotobacter spirillum Beijerinck, Kon. Akad. Wetensch. Amsterdam, 30, 1923, 431 quoted from Giesberger, Inaug. Diss., Delft, 1936, 24; Spirillum lipoferum Beijerinck, Cent. f. Bakt., II Abt., 63, 1925, 353; Chromatium lipoferum Bergey et al., Manual, 3rd ed., 1930, 531.) From Greek, lipos, fat; Latin, fero, to bear.

Curved cells with one-half to one spiral turn, containing minute fat droplets. These may deform the cells. Motile with lophotrichous flagella. Gramnegative.

Calcium malate agar colonies: Circular, small, transparent, dry. The malate is oxidized to calcium carbonate. Cells contain fat drops.

Peptone agar colonies: More abundant development. Cells lack fat drops and are typically spirillum in form.

Glucose peptone broth: Cells actively motile with large fat drops.

Fixes atmospheric nitrogen in partially pure cultures, i.e., free from Azotobacter and Clostridium (Beijerinck, loc. cit.). Schröder (Cent. f. Bakt., II Abt., 85, 1932,17 ) failed to find fixation of nitrogen when she used cultures derived from a single cell.

Aerobic.

Optimum temperature $22^{\circ} \mathrm{C}$.

Beijerinck regards this as a transitional form between Spirillum and Azotobacter. Giesberger (loc. cit., p. 64-65) thinks it a Vibrio.

Habitat: Garden soil.

Appendix: * The following additional species have been mentioned in the literature. Many are inadequately deseribed. Sume may not belong here.

* Prepared by Mr. Wm. C. Haynes, New lork State Experiment Station, Geneva, New York, Jan., 1939; Revised by Capt. Wm. C. Haynes, Sn. C., Fort Bliss, Texas, July, 1943 
Spirella canis Duboseq and Lebailly (Compt. rend. Acad. Sci. Paris, 15', 1912, 835.) From the stomach of a dog.

Spirillum amyliferum Van Tieghem. (Bull. Soc. botan. de France, 26, 1879 , 65.) Said to produce spores. Ford (Textb. of Bact., 1927, 364) thinks this organism was probably a spirochacte because of its mode of division. Found in frog spawn fungus of sugar factories.

Spirillum attenuatum Warming. (Om nogle ved Danmarks Kyster levende Bakterier. Kjobenhavn, 1876; Spirosoma attenuatum Migula, Syst. d. Bakt., 2, 1900, 959.) Ford (loc. cit., 363) states that this incompletely described organism would now be regarded either as a spirillum or as a spirochaete. From sen coast of Denmark.

Spirillum cardiopyrogenes Sardjito. (Geneesk. Tijdschr. voor Ned.-Indic, 72, 1932, 1359; ibid., 73, 1933, 822.) From hlood of a patient with periearditis.

Spirillum colossus Errera. (Rec. trav. bot. Bruxelle, 5, 1902; Abst. in Cent. f. Bakt., II Abt., 9, 1902, 608.) A giant form isolated from brackish sea water. Probably the same as Spirillum volutans Ehrenberg.

Spirillum concentricum Kitasato. (Cent. f. Bakt., 3, 1855, 73.) Found in putrefying blood.

Spirillum crassum Veillon and Repaci. (Ann. Inst. Past., 26, 1912, 300.) Described as having peritrichous flagella. From lung lesions in human tuberculosis.

Spirillum endoparagogicum Sorohin. (Cent.f. Balit., 1, 1857, 465.) Described as producing spores in old cultures. From rain water in bark of poplar tree. Spirillum giganteum Migula. (Spiritlum volutans Kutseher, Ztschr. f. Hyg., 20, 1895, 5s; Migula, Syst. d. Bakt., 2, 1900,1025 .) From putrefying liquids.

Spirillum hachaizae Kowalski. (Cent. f. Bakt., 16, 1894, 324; Spirillum hachaizicum Kowalski, ibid., 321; Spirochaete hachaizae Castellani and Chalmers, Man. Trop. Med., 1st ed., 1910, 316 ; Treponema hachaizae Brumpt, Nouveau Traité de Médecine, Paris, 4, 1922, 495.) Found in feces of cholera patients and also of healthy individuals.

Spirillum kollwitzii Vislouch. (Jour. de Microbiol. (Russian), 1, 1914, 50.)

Spirillum leucomelaenum Perty. (Zur Fenntniss kleinster Lebensformen. Berne, 1852. Also see Koch, Mitt. Kais. Gesundheitsamte, 1, 1881, 48.) From stagnant water.

Spirillum monospora Dobell. (Quart. Jour. Micr. Sci., 52, 1908, 121.) Described as producing spores. From large intestine of frogs and toads.

Spirillum nigrum Rist. (Thèse méd., Paris, 1898 ; see Cent. f. Bakt., I Abt., 30, 1901, 299.) Strict anaerobe from pus.

Spirillum ostreae Noguchi. (Jour. Exp. Med., 34, 1921, 295.) From oysters.

Spirillum periplaneticum Kunstler and Gineste. (Compt. rend. Soc. Biol. Paris, 61, 1906, 135.) From the intestine of the cockroach, Periplaneta americana.

Spirillum pyogenes Mezincescu. (Cent. f. Bakt., I Abt., Orig., 35, 1904, 201; Spirochaeta pyogenes Blanchard, Semaine Méd., 26, 1906, 1; Treponema pyogenes Brumpt, Nouveau 'Traité de Médecine, Paris, 4, 1922, 511.) From a case of pyelitis calculosa.

Spirillum rappini De Toni and Trevisan. (Spirochaete, Rappin, Contr. ì l'Etude d. Bactér. de la Bouche à l'État normal, 1881, 68; De Toni and Trevisan, in Saccardo, Sylloge Fungorum, 8, 1889, 1009.) From the stomach of a dog.

Spirillum recti physeteris Beauregard. (Compt. rend. Acad. Sci. Paris, 125, 1897, 255.) From ambergris.

Spirillum rugula (Müller) Winter. (Vibrio rugula Nüller, Animalcula infusoria, 1786; Cohn, Beitrage z. Biol. d. Pflanz., 1, Heft 2, 1872, 178; Bonhoff, Arch. f. Hyg., 26, 1896, 162; Winter, Die Pilze, in Rabenhorst's KryptogamenFlora, 188t.) Prazmowski found spores, but it is not certain his cultures were pure. Bonhoff also observed spores, but 
concluded that they were due to contaminating organisms (Ford, Textb. of Bact., 1927, 360). From water.

Spirillum sporiferum Migula. (Syst. d. Bakt., 2, 1900, 1028.) Produces spores. The spirals in which the spore formation is beginning are like Spirillum leucomelaenum Perty (Ford, loc. cit., 336). Giesberger (loc. cit., p. 60) places this and other so-called spore-forming spirilla in Sporospirillum Orla-Jensen (Cent. f. Bakt., II Abt., 22, 1909, 340). From a bean infusion.

Spirillum sputigenum Miller. (Die Mikroörganismen der Mundhöhle. Leipzig, 1892; Deutsche med. Wchnschr., 32, 1906, 1 and 348.) Hoffman and Prowazek (Cent. f. Bakt., I Abt., Orig., 41, 1906, 741) claim that Spirillum sputigenum has peritrichous flagella. Giesberger (loc. cit., 63) places this in Selenomonas Prowazek (Cent. f. Bakt., I Abt., Orig., 70, 1913, 36). Muhlens (Cent. f. Bakt., I Abt., Orig., 48, 1909, 525) reports 1 to 3 flagella, the majority of the organisms having apparently a single thick flagellum (a bunch of flagella) on the concave side (Ford, loc. cit., 367). Anaerobic. From the buccal cavity.

Spirillum stomachi Lehmann and Neumann. (Spirillum Form $\alpha, \beta, \gamma, \delta$ Salomon, Cent. f. Bakt.. I Abt., 19, 1896 , 433; Lehmann and Neumann. Bakt. Diag., 2 Auf1., 2, 1899, 362.) Found in stomach of dog, eat and rat.

Paraspirillum vejdovskii Dobell. (Arch. f. Protistenk., 24, 1911,97.) Found only once in fresh water containing Oscillatoria. Flagellate flexible spiral cells described as possessing a nucleus. This may be a protozoan.

Sprrobacillus gigas Certes. (Bull. Soc. Zool. France, 14, 1889, 322; abst. in Ann. de Microgr., 2, 1889-1890, 137.) From water.

Tibriothrix tonsillaris Tunnicliff and Jackson. (Organism from Actinomyceslike granules, Tunnicliff, Jour. Inf. Dis., 38, 1926, 366; Tunnicliff and Jackson, ibid., 46, 1930, 12.) From tonsillar granules. May be identical with Leptothrix asteroide Mendel and as a Gramnegative, anaerobe may belong in $\mathrm{Bac}$ teroides according to Rosebury (Bact. Rev., 8, 1944, 202).

Vibriothrix zeylanica (Castellani) Castellani. (Spritlum zeylanicum Castellani Jour. Ceylon Branch Brit. Ned. Assoc., 7, 1910, 5 and Philipp. Jour. Sci., 5, No. 2, Sect. B., Medical Sciences, July, 1910; Tibrio zeylanicus Castellani, 1913, Bacillus zeylanicus Castellani, 1913 and Vibriothrix zeylanica Castellani, 1917, quoted from Castellani and Chalmers, Man. Trop. Med., 3rd ed., 1919, 1069 ; Spirobacillus zeylanicus Castellani, Spagnuolo and Russo, Bull. Soc. Path. Hxot., 11, 1918, 271.) Motile. Gramnegative. From cases of dysenteric enteritis in Ceylon. This is the type species of the genus Vibriothrix Castellani (see Castellani and Chalmers, loc. cit., 106s). 


\section{FAMILY III. AZOTOBACTERIACEAE BERGEY, BREED AND MLRRAY**}

(Preprint, Manual, 5th ed., October, 1938, v and 71.)

Cells without endospores. Relatively large rods or even cocci, sometimes almost yeast-like in appearance. The type of flagellation in this genus has been definitely established as peritrichous. Gram-negative. Obligate aerobes, usually growing in a film on the surface of the culture medium. Capable of fixing atmospheric nitrogen when provided with carbohydrate or other energy source. Crow best on media deficient in nitrogen. Soil and water bacteria.

There is a single genus.

\section{Genus I. Azotobacter Beijerinck.}

(Beijerinck, Cent. f. Bakt., II Abt., 7, 1901, 567; Azotomonas Orla-Jensen, Cent. f. Bakt., II Abt., 24, 1909, 444.)

The definition is identical with that of the family. From Gr. azous, not living. French, azote, nitrogen; Gr. baktron, rod, stick.

The type species is Azotobacter chroococcum Beijerinck.

\section{Azotobacter chroococcum Beijer-} inck. (Cent. f. Bakt., II Abt., \%, 1901, 567 and $9,1902,3$; Bacillus azotobacter Löhnis and Hanzawa, Cent. f. Bakt., II Abt., 42, 1914, 1; Bacillus chroococcus Buchanan, General Syst. Bact., Baltimore, 1925, 194.) From Gr. chroa, eolor; coccos, grain; M. L. sphere.

According to Löhnis and smith (Jour. Agr. Res., 23, 1923, 401) Azotobacter beijerinckii Lipman (New Jersey Agr. Exp. Sta. Rept., 25, 1901, 247), Azotobacter woodstownii Lipman (ibid.), Azotobactersmyrnii Lipman and Burgess (Cent. f. Bakt., II Abt., 44, 1915, 50t) and Azotobacter hilgardii Lipman (Ścience, 29, 1909, 941) are identical with Azotobacter chroococcum. Greene (Soil Sci., 39, 1935, 327) studied Azotobacter chroococcum and Azotobacter beijerinckii by chemical analyses and found the chemical composition of the cells to be practically identical, but different from that of Azotobacter vinelandii and Azotobacter agile. Smith (private communication) feels that Azotobacter beijerinctii is a non-pigmented rough strain of Azotobacter chroococcum. (irows in absence of organic nitrogen. Rods : 2.0 to 3.0 by 3.0 to 6.0 microns, occurring in pairs and packets and ocrasionally in chains. The cells show three or four refractile granules. The organisms are surrounded by a slimy membrane of variable thickness, usually becoming brownish in older cultures, due possibly to the conversion of tyrosine to melanin. The coloring matter is insoluble in water, alcohol, ether and chloroform. Notile by means of numerous peritrichous flagella (Hofer, Jour. Bact., 47, 1944, 415.) Gram-negative.

Gelatin colonies: Very small, circular, yellow, granular, later becoming yellowish-brown.

Gelatin stab: Only slight growth in the stab. No liquefaction.

Mannitol agar stab: Gray, may become brownish.

Nutrient broth: No growth even in the presence of glucose; peptone utilized with difficulty.

Litmus milk: Becoming clearer in 10 to 14 days.

Potato: Glossy, barely visible, slimy

* Revised by Dr. A. W. Hofer, New York State Experiment Station, Geneva, New York, June, 1938; further revision by Dr. A. W. Hofer, July, 1943. 
to wrinkled; may become yellowish, brownish-yellow or chocolate brown.

The organism fixes atmospheric nitrogen and gives off $\mathrm{CO}_{2}$, utilizing glucose and sucrose. Other generally used carbon compounds are fructose, maltose, mannitol, inulin, dextrin, galactose, arabinose, starch, glycerol, ethyl alcohol, acetate, butyrate, citrate, lactate, malate, propionate and succinate.

Nitrate: Improves growth in amounts less than $1 \mathrm{gm}$. per liter; greater amounts are toxic.

Fixes nitrogen moderately actively.

Chemical analysis: Four-day cultures grown upon mannitol agar (Greene, 1935), when dried, are found to contain less than 0.5 per cent of hemicelluloses, less than 20 per cent of crude protein, less than 5 per cent of ash, and more than 30 per cent of lignin-like materials. The nitrogen fraction contains less than 1 per cent of amide nitrogen, less than 1 per cent of humin nitrogen and about 1 per cent of basic nitrogen.

Aerobic.

Optimum temperature $25^{\circ} \mathrm{C}$. to $28^{\circ} \mathrm{C}$.

Distinctive characters: Inability to grow in peptone media, even in the presence of glucose; frequent occurrence of a dark brown or black pigment.

Source: Isolated from soil.

Habitat: Occurs naturally in the majority of neutral or alkaline field soils.

\section{Azotobacter agile Beijermck} (Cent. f. Bakt., II Abt., \%, 1901, 577.) From L. agilis, agile, quick.

In studies on the chemical composition of cells Greene (Soil Sci., 39, 1935, 327) found Azotobacter vinelandii Lipman (New Jersey Agr. Exp. Sta. Rept., 24, $1903,238)$ to be very similar to $A z$ zotobacter agile Beijerinck. Smith and Löhnis (Jour. Agr. Res., 23, 1923, 401) agree and state furthermore that the two are identical; they believe also that $A$ zotobacter vitrcrm Löhnis and Westermann (Cent. f. Bakt., II Abt., 22, 1908, 234) is another synonym of Azotobacter agile.
Smith (private communication) states that Azotobacter vitreum is a very weak growing, smooth strain of Azotobacter agile. Kluyver and van Reenen (Arch. Mikrobiol., 4, 1933, 299) feel that a distinction should be made between Azotobacter agile and Azotobacter vinelandii. In regard to the former, Kluyver and van den Bout (Arch. Mikrobiol., 7, 1936, 263) suggest that it be further subdivided into Azotobacter agile and Azotobacter agile var. atypica, the latter referring to an Azotobacter agile form that fails to produce pigment.

Rods : 4 to 6 microns in length, almost spherical. Actively motile by means of numerous peritrichous flagella (Hofer, loc. cit.). Some strains are reported to be non-motile. Gram-negative.

Grows in absence of organic nitrogen.

Gelatin: No liquefaction.

Mannitol agar colonies: Circular, grayish white, translucent with whitish center.

Washed agar colonies: Show slight bluish-green fluorescence.

Mannitol agar slant: Grayish, translucent, fluorescent.

Plain agar slant: Yellowish-white, smooth, glistening, translucent with opaque center.

Broth: Turbid, with sediment.

Litmus milk: Becoming elear in 10 to 14 days.

Potato: Yellowish-white, slimy, becoming yellowish-brown.

In the presence of organic acids, a greenish or reddish pigment is formed.

The organism fixes atmospheric nitrogen actively, and gives off $\mathrm{CO}_{2}$.

Aerobic.

Chemical analysis: Four-day cultures grown upon mannitol agar (Greene, 1935), when dried, contain more than 4 per cent of hemicelluloses, more than 45 per cent of crude protein, more than 7 per cent of ash, and less than 4 per cent of ligninlike materials. The nitrogen fraction contains more than 1 per cent amide nitrogen, more than 1 per cent humin 
nitrogen, and 2 per cent or more of basic nitrogen.

Optimum temperature $25^{\circ} \mathrm{C}$ to $28^{\circ} \mathrm{C}$.

Distinctive characters: Lack of a brown pigment; occasional fluorescence; growth in peptone broth containing glucose.

Source: Originally isolated from canal water at Delft.

Habitat: Occurs in water and soil.

3. Azotobacter indicum starliey and De. (Soil Sci. 47, 337, 1939.) From L. indicus, of India.

Rods: Ellipsoidal, from 0.5 to 1.2 by 1.7 to 2.7 mierons when grown on nitrogen free glueose agar. One of the distinctive characteristics is the presence of two large, round, highly refractive bodies in the cells, one usually at earh end. Motile by means of numerous peritrichous flagella (Hofer. loc. cit.). Ciram-negative.

The organism grows slowly lut in time produces large amounts of slime.
Has high acid tolerance, since it grows from $\mathrm{pH} 3$ to 9 .

Sucrose or glucose agar plates : Colonies are colorless, round, very much raised, and uniformly turbid, having much the appearance of heavy starch paste. After two weeks, a buff to light brown color develop:

Mannitol agar slant: Grows very poorly.

Peptone agar slant with 0.5 per cent glucose: Limited grayish growth.

Nutrient broth: No growth.

Liquid media generally: Turbidity with some sediment.

Fixes atmospheric nitrogen readily with either glucose or sucrose as source of energy.

Aerobic.

Optimum temperature : $30^{\circ} \mathrm{C}$

Distinctive characters: Tolerance of acidity, wide limits of pH tolerated, abundant slime production, large globules of fat within cells.

source: Soils of India

Habitat : Soils.

Appendix I : The relationship of the following speeies to the speeies placed in $A z o-$ tobacter is not yet entirely clear.

\section{Genus Azotomonas Stapp.}

(Cent. f. Bakt., II Abt., 102, 1940, 18; not Azotomonas Orla-Jensen, Cent. f. Bakt., II Abt., 24, 1909, 484.)

Rod to coccus-shaped aerobic bacteria, motile by means of 1 to 3 polar Hagella. No endospores. No fat-like reserve food granules in the cells. Form acid and gas from glucose, and other sugars and alcohols. Form indole. Chemo-heterotrophic. Many carbon compounds other than sugars used as sources of energy. Active in the fixation of atmospheric nitrogen. Live in soil. From Gr. azous, mô living. French, azote, nitrogen; Greek, monas, a unit; MI. L. monad.

The type species is Azotomonas insolita.

Azotomonas insolita Stapp. (Ab)stracts of Communications, Third Internat. Congr. for Microbiol.. Sect. VIII. 1939. 306; abst. in Proc. Soil Sci. Soc. of America, 4, 1939, 244; Cent. f. Bakt., II Abt., 102, 1940, 1.) From Latin insolitus, unusual.

Cocenid rods: 0.6 to 1.2 hy 0.6 to 1.8 microns. Motile with one to three polar flagella. Gram-negative.

Gelatin: No liquefaction.

Agar slant: Glistening white growth.

Agar colonies: Flat, whitish, edge entire. Weakly fluorescent.

Broth: Strong turbidity. Sediment. Pellicle. 
Milk: No change.

Potato: Growth somewhat dry, not slimy, dirty gray, spreading.

Nitrites produced from nitrates.

Fixes nitrogen.

Ammonium salts utilized.

Acid and gas from adonitol, arabinose, dextrin, glucose, galactose, glycerine, inositol, lactose, fructose, maltose, mannitol, mannose, raffinose, rhamnose, salicin, sorbitol, starch, sucrose and xylose.
Starch is hydrolyzed.

Hydrogen sulfide produced.

Optimum temperature $25^{\circ}$ to $30^{\circ} \mathrm{C}$. Minimum $7^{\circ}$ to $9.5^{\circ} \mathrm{C}$. Maximum $48^{\circ} \mathrm{C}$. Good growth at $37^{\circ} \mathrm{C}$. Thermal death point $60^{\circ} \mathrm{C}$.

Limits of $\mathrm{pH} 3.3$ to 9.5 .

Aerobic.

Source: From a mixture of chopped cotton husks and rice hulls.

Habitat: Soil. 


\section{FAMILY IV. RHIZOBIACEAE COÑ.}

(Jour. Bact., 36, 1938, 321.)

Cells without endospores, rod-shaped, sparsely flagellated (one polar or lateral flagellum, or 2 to 4 peritrichous ones); some species non-motile. Usually Gram-negative. One genus (Chromobacterium) produces a violet pigment. Grow aerobically on ordinary culture media containing glucose. Glucose and sometimes other carbohydrates are utilized, without appreciable acid formation. Saprophytes, symbionts and pathogens. The latter are usually plant pathogens forming abnormal growths on roots and stems.

\section{Key to genera of family Rhizobiaceae.}

I. Cells capable of fixing free nitrogen when growing symbiotically on the roots of Leguminosae.

Genus I. Rhizobium, p. 223.

II. Either plant pathogens which attack roots or produce hypertrophies on stems; or free-living non-chromogenic soil or water forms. Do not fix nitrogen.

Genus II. Agrobacterium, p. 227.

III. Usually free-living soil and water forms which produce a violet chromogenesis.

Genus III. Chromobacterium, p. 231.

\section{Genus I. Rhizobium Frank.*}

(Phytomyxa Schroeter, in Cohn, Kryptogamen-Flora von Schlesien, 3, 1856, 134; Frank, Ber. d. deut. bot. Gesellsch., 7, 1889, 380; Rhizobacterium Kirchner, Beitr. z. Biol. d. Pflanzen, 7, 1895, 221; Rhizomonas Orla-Jensen, Cent. f. Bakt., II Abt., 22, 1909, 328.) From Greek rhiza, root; bios, life.

Rods : $0.5-0.9$ by $1.2-3.0$ microns. Motile when young, commonly changing to bacteroidal forms (a) upon artificial culture media containing alkaloids or glucosides, or in which acidity is increased; or (b) during symbiosis within the nodule. Gramnegative. Aerobic, heterotrophic, growing best with extracts of yeast, malt or other plant materials. Nitrates may be reduced to nitrites. Nitrites are not utilized. Gelatin is not liquefied or is very slightly liquefied after long incubation. Optimum temperature $25^{\circ} \mathrm{C}$. This group is capable of producing nodules on the roots of Leguminosae, and of fixing free nitrogen during this symbiosis.

The type species is Rhizobium leguminosarum Frank.

\section{Key to the species of genus Rhizobium.}

1. Litmus milk alkaline.

a. Formation of serum zone in milk.

b. Moderate growth, slight acid reaction on yeast water agar plus mono-, diand trisaccharides.

c. Causes formation of root nodules on species of the genera Lathyrus, Pisum, Vicia and Lens. Bacteroids irregular with $\mathrm{x}, \mathrm{y}$, star-, and club-shaped forms; rods peritrichous when young.

1. Rhizobium leguminosarum.

ce. Causes formation of root nodules on Phaseolus vulgaris, P. multiflorus and $P$. angustifolius. Bacteroids vacuolated rods, few branched forms; young cells peritrichous.

2. Rhizobium phaseoli.

* The genus Rhizobium was revised by Dr. and Mrs. O. N. Allen under the direction of Prof. E. B. Fred and Prof. I. L. Baldwin, Univ of Wisconsin, Madison, Wis., Jan., 1938; further revision by Dr. O. I. Allen, Jan., 1943. 
ccc. Causes formation of nodules on species of genus Trifolium. Bacteroids pear-shaped, swollen, vacuolated. Pentoses usually not fermented.

aa. No serum zone formed in milk.

\section{Rhizobium trifolii.}

b. Scant grow th, alkaline reaction on yeast water agar plis most carbohydrates.

c. Causes formation of nodules on species of genus Lupinus and on Ornithopus sativus. Bacteroids vacuolated, rods seldom branched.

4. Rhizobium lupini.

cc. Causes formation of nodules on Soja max. Bacteroids long slender rods, seldom vacuolated or branched; young cells monotrichous.

\section{Rhizobium japonicum.*}

2. Litmus milk acid.

a. Formation of serum zone in milk.

b. Moderate growth, slight acid reaction on yeast water agar plus mono-, diand trisaccharides.

c. Causes formation of root nodules on species of the genera Melilotus, Medicago, and Trigonella. Bacteroids club-shaped, branched, young cells peritrichous.

\section{Rhizobium meliloti.}

1. Rhizobium leguminosarum Frank emend. Baldwin and Fred. (Frank, Iandwirtschäf tliche Jahrbücher, 19, 1890, 563 ; Rhizobium polymorphum Dangeard, Rhizobium fabae Dangeard, Le Botaniste, Sér. 16, 1926, 192-194; Baldwin and Fred, Jour. Bact., 17, 1929, 146.) From Latin, of the legume family (Leguminosae).

Note: The following binomials have been used for species of this genus. The names given were used by their authors to cover one or more of the species here recognized as belonging to the genus Rhizobium. Where a question mark (?) is used it indicates that the species was too poorly described to be recognizable today. Schinzia cellulicolo Frank, 1877 (all species) Leunis, synopsis der drei
Naturreiche. 2 Theil, Botanik, III Abt., Kryptogamen, Sec. 914, 1877, 1944; Schinzia leguminosarum Frank (all species), Bot. Ztg., 37, 1879, 377; Phytomyxa leguminosarum Schrocter (all except Rhizobium lupini), in Cohn, Kryptogamen-Flora von Schlesien, 3, I, 1886 , 135; Bacillus radicicola Beijerinck (all species), Bot.Ztg., 46, 1888, 726; Bacillus fabae Beijerinck (from broad bean) and Bacillus ornithopi Beijerinck (from serradlella), Bot. Ztg., 48, 1890, 837; Cladochytrium tuberculorum Vuillemin (all species?), Ann. Sci. Agron. Franc. et Etrang., 5, I, 1888, 193; Bacterium radicicola Prazmowski (all species), Landw. Vers. Sta., 37, 1890, 204; Rhizobium mutabile Schneider (several species) Rhizobium curvum Schneider (?), Rhizo-

* No specific name has been proposed for the organism causing the formation of nodules on plants that are members of the so-called "cowpea" group. Data showing possible inter-relationships of eertain plant species of the soybean and cowpea crossinoculation groups prompted Walker and Brown (Soil Science, 39, 1935, 221-225) to propose a consolidation of the two groups to be recognized as being inoculated by a single species, Rhizobium japonicum. Results obtained recently by Reid and Baldwin (Proc. Soil Sei. Soc. Amer. for 1936, 1, 1937, 219) show these inter-relationships to include the lupine group also. 
bium frankii var. majus and var. minus Schneider (?), Rhizobium nodosum Schneider (?), Rhizobium dubium Schneider (?), Bui. Torrey Bot. Club; 19, 1892, 213; Rhizobium sphaeroides Schneider (?), Ber, deut, bot. Gesell., 12, 1894, 16; Bacillus tuberigenus Gonnermann and Micrococcus tuberigenus Gonnermann, Landw. Jahrb., 23, 1894, 654, 657, are thought by Fred, Baldwin and MeCoy (Tniversity of Wisconsin, Studics in Science, No. 5, 1932, 140) not to be true nodule organisms and to be too poorly described to be recognizable today; Rhyzobium pasteurianum Mazé (all species), Ann. Inst. Pasteur, 19, 1899, 146; Psendorhizobium ramosum Hartleb (?) (Chem. Zeit., 24, 1900, 887) (used for noninfective culture claimed by Stutzer Mitt. Jandw. Inst. Breslau, 1, Heft 3, 1900, 63) to be genuine root nodule organism); Rhizobium radicicola Hiltner and Störmer (several species) and Rhizobium beijerinckii Hiltner and Störmer (from lupine, serradella and soy bean), Arb. Biol. Abt. f. Land-u. Forstwirthschaft a. K. Gerundheitsamte, 3, 1903, 261; Pseudomonas radicicola Noore (all species), L. s. Dept. Agr. Bur. Plant Ind., Bul. 71, 1905, 27; Rhizomonas beijerinckii Orla-Jensen and Rhizomonas radicicola Orla-Jensen (see Hiltner and Störmer). Cent. f. Bakt., II Abt., 22, 1909.328 ; Bacillus or Bacterium radicicola Löhnis and Hansen peritrichous species), Jour. Agr. Rescarch, 20, 1921, 5.51; Phizobium radicicolum Bergey et al., Manual, 1st ed., 1923. to (monotrichous species); Rhizobium loti Dangeard (from lotus), Rhizobium simplex Dangeard (from sainfoin), Rhizobium torulosum Dangeard (from Scotch broom), Le Botaniste, Sér. 16, 1926, 195-197.

Rods: 0.5 to 0.9 by 1.2 to 3.0 microns. Intile with peritrichous flagella. Bacteroids commonly irregular with $\mathrm{x}, \mathrm{y}$, star-and club-shaped forms. Tacuolate forms predominate. Gram-negative.

Growth on mannitol agar is rapid, with tendency to spread. Streak is raised, glistening, semi-transiucent, white, slimy and occasionally viscous. Considerable gum is formed.

Slight acid production from glucose, galactose, mannose, lactose and maltose.

Aerobic.

Optimum temperature $25^{\circ} \mathrm{C}$.

Source: Root nodules on Lathyrus, Pisum (pea), Vicia (vetch) and Lens (lentil).

Habitat: Widely distributed in soils where the above mentioned legumes are grown

2. Rhizobium phaseoli Dangeard. (Le Botaniste, Sér. 16, 1926, 197.) From Latin, phascolus, bean; M. L. Phaseolus, a generic name.

Rods: Notile with peritrichous flawe-1li. Bacteroids are usually rod-shaped, often vacuolatel with few branched forms. Lisually smaller than in Rhizobium leguminosarum and $R$. trifolii. Gram-negative.

Growth on mannitol agar is rapid with tendency to spread. Streak inoculation is raised, glistening, semi-translucent, white, sliniy. Occasionally mucilaginous but this character is not so marked as in Rhizobium trifolii.

Very slight acid formation from glucose, galactose, mannose, sucrose and lactose.

Aerobic.

Optimum temperature $25^{\circ} \mathrm{C}$.

source: Root nodules of Phaseolus vulgaris (kidney bean), $P$. angustifolius (bean) and $P$. multiforus (scarlet runner,. (Burrill and Hansen, Ill. Agr. Exp. Sta. Bul. 202, 1917, 137.)

Habitat: Widely distributed in the soils in which beans are grown.

3. Rhizobium trifolii Dangeard. (Le Botaniste, Sér. 16, 1926, 191.) From M. L. Trifolium, a generic name.

Rods: Motile with peritrichous flagella. Bacteroids from nodules are pear. shaped, swollen and vacuolated. Rarely $\mathrm{x}$ and $\mathrm{y}$ shapes. Gram-negative. 
Growth on mannitol agar is rapid. The colonies are white becoming turbid with age. Frequently mucilaginous. Streak cultures transparent at first. Growth mucilaginous later flowing down the agar slant and accumulating as a slimy mass at the bottom. Produces large amounts of gum.

Slight acid production from glucose, galactose, mannose, lactose and maltose.

Aerobic.

Optimum temperature $25^{\circ} \mathrm{C}$.

Source: Root nodules of species of Trifolium (clover).

Habitat: Widely distributed in the soils where clover grows.

4. Rhizobium lupini (Schroeter) Eckhardt, Baldwin and Fred. (Phytomyxa lupini Schroeter, in Cohn, KryptogamenFlora von Schlesien, 3, I, 1886, 135; Rhizobium minimum Dangeard, Le Botaniste, Sér. 16, 1926, 198; Eckhardt, Baldwin and Fred, Jour. Bact., 21, 1931, 273.) From Latin, Lupinus, lupine.

Rods : Motile with flagella 1 to 4, usually 2 or 3 . Bacteroids are vacuolate rods, seldom if ever branched. Gram-negative.

Growth on yeast water, mannitol agar is scant to moderate with alkaline reaction.

Beef-peptone gelatin: Little growth with extremely slow liquefaction.

On galactose an alkaline reaction serves to differentiate Rhizobium lupini from all fast-growing rhizobia ( $R$. phaseoli, $R$. meliloti, $R$. trifolii, and R. leguminosarum). An initial alkaline reaction followed more quickly by an acid reaction on rhamnose and xylose separates $R$. lupini from slow-growing $R$. japonicum and the Rhizobium sp. from cow pea.

In general Rhizobium lupini produces slight to moderate acidity on pentose sugars and no change or alkaline reaction on hexoses, disaccharides and trisaccharides.

Litmus milk: No serum zone, no reduction, and a slight alkaline reaction.
Meager growth on potato and parsnip slants, and carrot agar.

Aerobic.

Optimum temperature $25^{\circ} \mathrm{C}$.

Source: Root nodules on Lupinus (lupine), Serradella and Ornithopus.

Habitat: Widely distributed in soils in which these legumes grow.

\section{Rhizobium japonicum (Kirchner)}

Buchanan. (Rhizobacterium japonicum Kirehner, Beiträge zur Biol. d. Pflanzen, 7, 1895, 213; Pseudomonas japonica Löhnis and Hansen, Bacterium japonicum Löhnis and Hansen, Jour. Agr. Res., 20, 1921, 551; Rhizobium sojae Dangeard, Le Botaniste, Sér. 16, 1926, 200 ; Buchanan, Proc. Iowa Acad. Sci., 33, 1926, 81.) From M. L., of Japan.

Rods: Motile with monotrichous flagella. Bacteroids of nodules are long and slender with only occasional branched and swollen forms. Gram-negative.

Growth on mannitol agar is slow and scant. The streak is slightly raised, glistening, opaque, white, butyrous, with little gum formation.

Pentose sugars give better grow th than the hexoses.

Little if any acid formed from carbohydrates. Acid slowly formed from xylose and arabinose.

Aerobic.

Optimum temperature $25^{\circ} \mathrm{C}$.

Source: Root nodules on Soja $\max$ (soy bean).

Habitat: Widely distributed in soils where soy beans are grown.

6. Rhizobium meliloti Dangeard. (Le Botaniste, Sér. 16, 1926, 194.) From Greek, melilot, a kind of clover; M. L., Melilotus.

Rods: Motile with peritrichous flagella. Bacteroids club-shaped and branched. Gram-negative.

Growth on mannitol agar is fairly rapid. The streak is raised, glistening, opaque, pearly white, butyrous. Considerable gum is formed. 
Acid from glucose, galactose, mannose and sucrose.

Aerobic.

Optimum temperature $25^{\circ} \mathrm{C}$.

Source: Root nodules of Melitotus (sweet clover), Medicago, and Trigonella.

Habitat: Widely distributed in soils in which these legumes grow.
Note: See Monograph on Root Nodule Bacteria and Leguminous Plants by E. B. Fred, I. I. Baldwin and Elizabeth MeCoy, Lniversity of Wisconsin Studies in Science, Madison, No. 5, 1932, xx + $3+3 \mathrm{pp}$. for a more complete discussion of this group with an extensive bibliography.

\section{Genus II. Agrobacterium Conn.*}

(Jour. Bact., 44, 1942, 359.) From Greek, agrus, a field; M.L., bacterium, a small rod.

Small, short rods which are typically motile with 1 to 4 peritrichous flagella (if only one flagellum, lateral attachment is as common as polar). Ordinarily Gramnegative. On ordinary culture media, they do not produce visible gas nor sufficient acid to be detectable by litmus. In synthetic media, enough $\mathrm{C}^{\mathrm{O}} \mathrm{2}_{2}$ may be produced to show acid with brom thymol blue, or sometimes with brom cresol purple. Gelatin is either very slowly liquefied or not at all. Free nitrogen cannot be fixed; but other inorganic forms of nitrogen (nitrates or ammonium salts) can ordinarily be utilized. Optimum temperature, $25^{\circ}$ to $30^{\circ} \mathrm{C}$. Habitat: Soil, or plant roots in the soil; or the stems of plants where they produce hypertrophies.

The type species is Agrobacterium tumefaciens (Smith and Townsend) Conn.

\section{Key to the species of genus Agrobacterium.}

I. Plant pathogens. Produce browning of mannitol-calcium-glycerophosphate agar. Nitrate reduction weak or none.

A. Nitrite produced from nitrate to a slight extent. Galls produced on plant roots. 1. Agrobacterium tumefaciens.

B. Nitrite not produced from nitrate.

1. Pathogenic to apples.

2. Pathogenic to raspberries and blackberries.

\section{Agrobacterium rhizogenes.}

3. Agrobacterium rubi.

II. Not pathogenic to plants. Produces browning in mannitol-calcium-glycerophos. phate agar. Nitrate reduction vigorous, with disappearance of the nitrate.

4. Agrubacterium radiobacter.

1. Agrobacterium tumefaciens (Smith and Townsend) Conn. (Bacterium tumefaciens Erw. Smith and Townsend, Science, N. S. 25, 1907, 672; Pseudomonas tumefaciens Stevens, The Fungi which Cause Plant Disease, 1913, 35; Bacillus tumefaciens Holland, Jour. Bact., 5, 1920, 220 ; not Bacillus tumefaciens Wilson, Lancet, 1, 1919, 675; Phytomonas tumefaciens Bergey et al., Manual, 1st ed.,
1923, 189; Conn, Jour. Bact., 44, 1942, 359.) From Latin tumefaciens, swelling up, producing a tumor.

Probable synonyms: Bacillus ampelopsorae Trevisan, in Saccardo, Sylloge Fungorum, 8, 1889, 983; Bacillus ampelopsorae Trevisan emend. Cavara, Staz. Sperim. Agara. Ital. Modena, 30, 1897, 483; see Elliott, Bact. Plant Pathogens, 1930, 235.

* Prepared by Prof. H. J. Conn, New Iork State Experiment Station, Geneva, New York, September, 1943. 
Among the synonyms listed in previous editions of the Manual has been Polymonas tumefaciens Lieske, Cent. f. Bakt., I Abt., Orig., 108, 1928, 118. This is only a partial synonym, however, as its author described it as the cause of animal and human eancer, of which he regarded crown-gall of plants as merely a phase: for the origin of this theory, see Smith and Townsend, Sci., N.S. 25, 1907, 671, and Smith, Jour. Cancer Res., 7 , 1922, 1-105.

Description taken from the following: Riker, Banfield, Wright, Keitt and Sagen, Jour. Agr. Res., 41, 1930, 507; Sagen, Riker and Baldwin, Jour.Bact, 28, 1931, 571; Hendrickson, Baldwin and Riker, Jour. Bact., 28, 1934, 597.

Rods : 0.7 to 0.8 by 2.5 to 3.0 microns, occurring singly or in pairs. Capsules. Motile with 1 to 4 flagella. Gramnegative.

Agar colonies: Small, white, circular, smooth, glistening, translucent, entire.

Broth: Slightly turbid, with thin pellicle.

Litmus milk: Slow coagulation. Litmus reduced. Neutral to alkaline.

Nitrites produced from nitrates to a very slight extent.

Indole: Slight amount.

Slight acid from glucose, fructose, arabinose, galactose, mannitol and salicin .

Starch not hydrolyzed.

Optimum temperature $25^{\circ}$ to $28^{\circ} \mathrm{C}$.

Facultative anaerobe.

Distinctive characters: Causes a gall formation parenchymatous in character which because of its soft nature is subject to injury and decay.

Agrobacterium tumefaciens strongly absorbs congo red and aniline blue in contrast to little or no absorption by $A$. rhizogenes. A. tumefaciens makes abundant growth on sodium selenite agar and calcium glycerophosphate medium with mannitol in contrast to no growth or a very slight trace by $A$. rhizogenes (Hendrickson et al., Jour. Bact., 28, 1934, 597 ).
Source: Isolated from galls on plants.

Habitat: Causes galls on Paris daisy and cross-inoculable on over 40 families.

\section{Agrobacterium rhizogenes (Riker} et al.) Conn. (Bacterium rhizogenes Riker, Banfield, Wright, Keitt and Sagen, Jour. Agr. Res., 41, 1930, 536; Phytomonas rhizogenes Riker et al., ibid., 536; Pseudomonas rhizogenes Riker et al., ibid. 536 ; Conn, Jour. Bact., 44, 1942, 359.) From Greek, rhiza, root; genes, producing.

Rods: 0.4 by 1.4 microns, occurring singly. Motile with one to 4 flagella. Encapsulated. Not acid-fast. Gramnegative.

Gelatin: No liquefaction.

Agar colonies: Circular, smooth, convex, finely granular; optical characters, translucent through gray to almost white.

Agar slant: Moderate, filiform, translucent, raised, smooth, slimy.

Broth: Turbid, with heavy pellicle.

Litmus milk: Acid, slow reduction.

Indole not formed.

Nitrites not produced from nitrates.

Acid but not gas from arabinose, $x y$ lose, rhamnose, glucose, galactose, mannose, maltose, lactose, salicin and erythritol. No acid or gas from fructose, sucrose, raffinose, melezitose, starch, dextrin, inulin, aesculin, dulcitol or mannitol.

Starch not hydrolyzed.

Optimum temperature $20^{\circ}$ to $28^{\circ} \mathrm{C}$.

Aerobic.

Distinctive characters: Agrobacterium rhizogenes differs from Agrobacterium tumefaciens by stimulating root formation instead of soft parenchymatous crown galls. A. rhizogenes lacks ability of A. tumefaciens to utilize simple nitrogenous compounds as $\mathrm{KNO}_{3}$. A. rhizogenes absorbs congo red and brom thymol blue slightly and aniline blue not at all. Will not grow on sodium selenite agar (see A. tumefaciens for response to same materials). Does not infect tomato 
Sources: Description made from ten cultures isolated from hairy-root of apple and other plants.

Habitat: Pathogenic on apple, etc.

\section{Agrobacterium rubi (Hildebrand)}

Starr and Weiss. (Phytomonas rubi Hildebrand, Jour. Agr. Res., 61, 1940, 694; Bacterium rubi Hildebrand, ibid., 694; Pseudomonas rubi Hildebrand, ibid., 694; Banfield, Phytopath., 20, 1930, 123; Pinckard, Jour. Agr. Res., 50, 1935, 933; Starr and Weiss, Phytopath., 33, 1943, 316.) From Latin, rubus, blackberry bush; M. L., Rubus, a generic name.

Rods : 0.6 by 1.7 microns. Singly, in pairs or short chains. Notile with 1 to 1 flagella. Gram-negative.

Gelatin: No liquefaction.

Potato-mannitol-agar slants: Growth slow, moderate, filiform, white to creamywhite, with butyrous consistency later becoming leathery .

Broth: Turbid in 36 to 48 hours.

Milk: A slight serum-zone, pink color, acid and curd formed.

Nitrites not produced from nitrates.

Ferric ammonium citrate, uric acid, oxamide, succinimide, l-asparagine, 1tyrosine, l-cystine, d-glutamic acid and yeast extract can be used as a source of nitrogen (Pinckard, loc. cit.).

Hydrogen sulfide not formed.

Indole not formed.

Acid from glucose, d-galactose, dmannose, d-fructose, d-xylose, d-arabinose, sucrose, and maltose. None from lactose (Pinckard, loc. cit.).

Starch not hydrolyzed.

Optimum temperature $28^{\circ} \mathrm{C}$. Minimum $8^{\circ} \mathrm{C}$. and maximum $36^{\circ} \mathrm{C}$. (Pinckard, loc. cit.).

Distinctive characters. Differs from Agrobacterium tumefaciens in that it does not utilize nitrates, and groms much more slowly on ordinary media. Infects only members of the genus Rubus. Starr and Weiss (Phytopath., 33, 1943, 317) state that this species unlike Agrobacterium tumefaciens and Agrobacterium rhizogenes does not utilize asparagin as a sole source of carbon and nitrogen.

Source: Isolated by Banfield (loc. cit.) and by Hildebrand (loc. cit.) from raspberry canes, $R$ ubus spp.

Habitat: Pathogenic on black and purple cane raspberries, and blackberries, and to a lesser extent on red raspberries.

4. Agrobacterium radiobacter (Beijerinck and van Delden) Conn. (Bacillus radiobacter Beijerinck and van Delden, Cent. f. Bakt., II Abt., 9, 1902, 3; Bacterium radiobacter Löhnis, Cent. f. Bakt., Il Abt., 14, 1905, 589; Rhizobium radiobacter Pribram, Klassifikation der Schizomyceten, Leipzig, 1933,53;Achromobacter radiobacter Bergey et al., Manual, 4 th ed., 1931, 230; Alcaligenes radiobacter Conn, in Minual, 5th ed., 1939, 97 ; Conn, Jour. Bact., 44, 1942,359.) From Latin,radius, the spolie of a wheel; I atin, bactrum, a rod.

Small rods, 0.15 to 0.75 by 0.3 to 2.3 microns, oceurring singly, in pairs and under certain conditions, in star-shaped clusters. Notile with one to four flagella. Prevailingly Gram-negative; but an oceasional culture is variable.

Nutrient gelatin stab: No liquefaction.

Agar slant: Flat, whitish slimy layer.

Mannitol-calcium-glycerophosphateagar streak plates: Abundant, raised, slimy growth surrounded by a brown halo with an outer zone of white precipitate (Riker et al., Jour. Agr. Res., 41, $1930,524)$.

Broth: Turbid; with heavy ring or pellicle if veal infusion is present.

Litmus milk: Serum zone with pellicle in one week; usually turns a chocolate brown in 2 weeks; same in plain milk, but with less browning.

Potato: Raised slimy mass becoming brownish; potato may be browned.

Nitrates disappear (assimilated or reduced).

Stareh not hydrolyzer.

No organic acid or visible gas from sugars; nearly all sugars, glycerol and 
mannitol are utilized with the production of $\mathrm{CO}_{2}$.

Optimum temperature $28^{\circ} \mathrm{C}$. Minimum near $1^{\circ} \mathrm{C}$. Maximum $45^{\circ} \mathrm{C}$.

Aerobic.

Media containing $\mathrm{KNO}_{3}, \mathrm{~K}_{2} \mathrm{HPO}_{4}$, and glycerol, ethyl or propyl alcohol become alkaline to phenol red. (Sagen, Riker and Baldwin, Jour. Bact., 28, 1934, 571.)

Growth occurs in special alkaline media of $\mathrm{pH} 11.0$ to 12.0 (Hofer, Jour. Amer. Soc. Agron., 27, 1935, 228).

Hydrogen sulfide produced if grown in ZoBell and Feltham's medium (Jour. Bact., 28, 1934, 169).

Distinctive characters: Browning of mannitol-calcium-glycerophosphate agar. Inability to cause plant disease or to produce nodules on roots of legumes. Complete utilization (disappearance of nitrate) in the peptone-salt medium of Riker et al. (Jour. Agr. Res., 41, 1930, 529) and failure to absorb congo red (ibid., 528).

The species bears at least superficial resemblances to certain Rhizobium spp., but may be distinguished from them by the first two characters listed above, and the following in addition: Growth at a reaction of $\mathrm{pH}$ 11-12. Heavy ring or pellicle formation on veal infusion broth. $\mathrm{H}_{2} \mathrm{~S}$ production in the mannitol-tryptone medium of ZoBell and Feltham (loc. cit.). Production of milky white precipitate on nitrate-glycerol-soil-extract agar.

Source: Isolated from soil.

Habitat: Soil, around the roots of plants, especially legumes.

Note: Palacios and Bari (Proc. Indian Acad. Sci., 3, 1936, 362; Abs. in Cent. f. Bakt., II Abt., 95, 1937, 423) have described Bacillus concomitans as a symbiont from legume nodules that has no power to fix nitrogen although it is very much like legume nodule bacteria (Rhizobium spp.). This organism resembles Agrobacterium radiobacter.
Appendix: The following species probably belong in Agrobacterium, but are not sufficiently well described to make their relationship certain.

1. Agrobacterium gypsophilae (Brown) Starr and Weiss. (Bacterium gypsophilae Brown, Jour. Agr. Res., 48, 1934, 1109; Pseudomonas gypsophilae Stapp, Bot. Rev., 1, 1935, 407; Phytomonas gypsophilae Stapp, ibid., 407; Starr and Weiss, Phytopath., 33, 1943, 316.) From M. L., Gypsophila, a generic name.

Rods : 0.2 to 0.8 by 0.4 to 1.4 microns. Motile with 1 to 4 flagella. Capsules. Gram-negative.

Gelatin: Liquefaction slow, beginning after 1 month.

Beef-infusion agar colonies: Circular, Naples yellow, smooth or rough, butyrous.

Broth: Turbid in 24 hours.

Milk: Coagulation and peptonization. Nitrites are produced from nitrates.

Indole not produced.

Hydrogen sulfide: A trace may be produced.

Acid but not gas from glucose, sucrose, maltose, mannitol and glycerol. No acid from lactose.

Starch not hydrolyzed.

Aerobic, facultative.

Distinctive characters: Differs from Xanthomonas beticola in starch hydrolysis, $\mathrm{H}_{2} \mathrm{~S}$ production, and will not crossinoculate with this species.

Source: Isolated from several galls on Gypsophila.

Habitat: Produces galls in Gypsophila paniculata and related plants.

2. Bacterium pseudotsugae Hansen and Smith. (Hansen and R .E. Smith, Hilgardia, 10, 1937, 576; Phytomonas pseudotsugae Burkholder, in Manual, 5th ed., 1939, 209.) From M. L., Pseudotsuga, a generic name

Rods: 0.5 to 1.5 by 1.9 to 3.9 microns. Probably motile; type of flagellation doubtful. Gram-negative.

Gelatin: Liquefied. 
Nutrient agar slant: Growth scanty, flat, glistening, smooth, translucent, whitish.

Broth: Growth slight. No sediment. Milk: No acid.

Nitrites produced from nitrates.

Hydrogen sulfide production slight.

Acid but not gas from glucose, fruc- tose, galactose and maltose. No acid or gas from lactose, sucrose or glycerol. starch not hydrolyzed.

Facultative aerobe.

Source: Isolated from galls on Douglas fir in California.

Habitat: Pathogenic on Douglas fir, Pseudotsuga taxifolia.

\section{Genus III. Chromobacterium Bergonzini.*}

(Ann. Societa d. Naturalisti in Modena, Ser. 2, 14, 1881, 153.) Greek, chroma, color; M. L., bacterium, a small rod.

Rods, 0.4 to 0.8 by 1.0 to 5.0 microns. Motile with 1 to 4 or more flagella. Gramnegative. A violet pigment is formed which is soluble in alcohol, but not in water or chloroform. Grow on ordinary culture media, usually forming acid from glucose, sometimes from maltose, not from lactose. Gelatin is liquefied. Indole is not produced. Nitrate usually recluced to nitrite. Optimum temperature $20-25^{\circ} \mathrm{C}$. but some grow well at $37^{\circ} \mathrm{C}$. Csually saprophytic soil and water bacteria.

The type species is Chromobacterinm violaceum (Schroeter) Bergonzini.

\section{Key to the species of genus Chromobacterium.}

I. Motile rods. Single flagellum.

A. Acid from glucose and maltose. No acid from sucrose. Nitrites produced from nitrates. No growth at $37^{\circ} \mathrm{C}$.

1. Chromobacterium violaceum.

II. Motile rods. Flagella generally peritrichous.

A. Acid from glucose. Nitrites generally not produced from nitrates. Good growth at $37^{\circ} \mathrm{C}$.

\section{Chromobacterium ianthinum.}

B. Generally no acid from glucose.

Nitrites produced from nitrates. No

\section{Chromobacterium amethystinum.}

\section{Chromobacterium violaceum} (Schroeter) Bergonzini. (Bacteridium violaceum Schroeter, Beiträge z. Biol. d. Pflanzen, 1, Heft 2, 1872, 126; Micrococcus violaceus Cohn, Beiträge z. Biol. d. Pflanzen, 1, Heft 2, 1872, 157 ; Cromobacterium violaceum (sic) Bergonzini, Ann. Societa d. Naturalisti in Modena, Ser. 2, 14, 1851, 153; Bacillus violaceus Schroeter, Kryptogamen-Flora von Schlesien, 3, 1886, 157; Streptococcus violaceus Trevisan, I generi e le specie delle
Batteriacee, 1889, 31; Pseudomonas riolacea Migula, Arb. a. d. Bakt. Inst. Karlsruhe, 1, 1894, 237; Bacterium violaceum Lehmann and Neumann, Bakt. Diag., 1 Aufl., 2, 1896, 58; see 2 Aufl., 2, 1899, 262.) From Latin, violaceus, violet-colored.

Tote: Bacterium ianthinum Zopf (Die Spaltpilze, 1885, 68) has been regarded as identical with the above organism by Schroeter (Kryptogamen-Flora von Schlesien, 3, 1, 1886, 157), and by Leh-

* Adapted by Prof. Robert S. Breed, New York State Experiment Station, Geneva, New York from Cruess-Callaghan and Gorman, Scientific Proc. Royal Dublin Society, 21, 1935, 213 in Jan. 1938; further revision, July, 1945 by Robert S. Breed with the assistance of Capt. W. C. Tobie, Sn. C., Old Greenwich, Conn. 
mann and Neumann (Bakt. Diag., 1 Aufl., 2, 1896, 266; also 7 Aufl., 2, 1927, 463). Lehmann and Neumann (loc. cit.) also consider Bacillus violaceus laurenticus Lustig (Diagnostik der Bakterien des Wassers, 1893, 103) as being identical with Bacterium violaceum.

Slender rods : 0.8 to 1.0 by 2.0 to 5.0 microns, occurring singly and in chains. Motile, with a single flagellum. Gramnegative.

Gelatin colonies : Circular, gray, entire margin, assuming a violet color in the center.

Gelatin stab: Infundibuliform liquefaction with violet sediment in fluid.

Agar colonies: Whitish, flat, glistening, moist, becoming violet.

Agar slant: Deep, violet, moist, shiny spreading growth.

Broth: Slightly turbid, with violet ring and ropy sediment.

Litmus milk: Violet pellicle. Diges. tion. Alkaline.

Potato: Limited, dark violet growth.

Löffler's blood serum : Slowly liquefied.

Indole not formed.

Nitrites produced from nitrates.

Acid from glucose and usually from maltose. No acid from lactose or sucrose.

Aerobic, facultative.

Optimum, temperature $25^{\circ}$ to $30^{\circ} \mathrm{C}$. No growth at $37^{\circ} \mathrm{C}$. Slight growth at $2^{\circ}$ to $4^{\circ} \mathrm{C}$.

Source: Originally grown on slices of cooked potato exposed to air contamination, and incubated at room temperature.

Habitat: Water.

2. Chromobacterium ianthinum (Zopf) Holland. (Bacterium ianthinum Zopf, Die Spaltpilze, 2 Aufl., 1881,62; Bacillus janthinus Flügge, Die Mikroorganismen, 1886, 291 ; Bacteridium ianthinum Schroeter, Kryptogamen Flora von Schlesien, 3, 1, 1886, 157; Pseudomonas ianthina Migula, Syst. d. Bakt., 2, 1900, 941; Pseudomonas janthina Chester, Man. Determ. Bact., 1901, 317; Holland, Jour. Bact., 5, 1920, 222.) From Greek, ianthinus, violet-blue.
Rods : 0.5 to 0.8 by 1.5 to 5.0 microns, occurring singly. Motile with peritrichous flagella. Gram-negative.

Gelatin colonies: Circular, yellow, becoming violet.

Gelatin stab: White to violet surface growth. Infundibuliform liquefaction.

Agar colonies: Creamy center, violet margin.

Agar slant: Yellowish, moist, glistening, becoming deep violet.

Broth: Turbid, with light violet pellicle.

Litmus milk: Slow coagulation with violet cream layer. Litmus decolorized from below.

Potato: Violet to violet-black, spreading growth.

Indole not formed.

Nitrites generally not produced from nitrates.

Acid from glucose. No acid from maltose, lactose and sucrose.

Aerobic, facultative.

Optimum temperature $30^{\circ} \mathrm{C}$. Grows well at $37^{\circ} \mathrm{C}$. No growth at 2 to $4^{\circ} \mathrm{C}$.

Source: Originally grown on pieces of pig's bladder floated on badly contam inated water.

Habitat: Water and soil. This may be the species that causes a fatal septicemia in animals and man. See Chromobacterium violaceum manilae.

\section{Chromobacterium amethystinum} (Chester) Holland. (Bacillus membranaceus amethystinus Eisenberg, Bakt. Diag., 1891, 421; Bacterium amethystinus Chester, Ann. Rept. Del. Col. Agr. Exp. Sta., 9, 1897, 117; Bacterium membranaceus amethystinus Chester, ibid., 13s: Bacillus amethystinus Holland, Jour. Bact., 5, 1920, 217; not Bacillus amethystinus Chester, loc. cit., 262; Holland, loc. cit., 222; Bacterium membranaceum amethystinum Lehmann and Neumann, Bakt. Diag., 7 Aufl., 2, 1927, 463; Bacterium violaceum amethystinum CruessCallaghan and Gorman, Sci. Proc. Royal Dublin Society, 21, 1935, 219.) From Greek, bluish-violet, amethyst. 
Rods : 0.5 to 0.8 by 1.0 to 1.4 microns, oceurring singly. Motile with a single or occasionally with peritrichous flagella. Gram-negative.

Gelatin colonies: Thin, bluish, becoming violet, crumpled.

Gelatin stab: Heavy, violet-black pellicle. Liquefied.

Agar colonies: Deep violet, surface rugose.

Agar slant: Thick, moist, yellowishwhite, becoming violet with metallic luster.

Broth: Pellicle with violet sediment, fluid hecoming violet.

Litmus milk: Violet pellicle. Digestion turning alkaline.

Potato: Deep violet, rugose spreading growth.

Indole not formed.

Nitrites produced from nitrates.

Usually no acid from glueose, maltose and sucrose. To acid from lactose.

Aerobic, facultative.

Optimum temperature $30^{\circ} \mathrm{C}$. No growth at $37^{\circ} \mathrm{C}$. Good growth in 7 days at 2 to $4^{\circ} \mathrm{C}$.

Original source: Found once by Jolles in spring water from Spalato.

Habitat: Water.

Appendix: The following organisms have been assigned to this genus or are believed to belong here. Additional comparutive studies are badly needed.

Bacillus cyaneofuscus Beijerinck. (Beijerinck, Bot. Ztung.. 49, 1891, 704; Bacterium cyanofuscus Chester, Amn. Rept. Del. (isl. Igr. Exp. Sta., 9, 1597, 116 and 132.) From black glue, blue Edam cheese, water and soil.

Bacillus lacmus Schroeter. (Schroeter. in Cohn, Tiryptogamen-Flora ron Schlesien, 3, 1, 1889, 158.) In greenhouse on fresh paint.

Bacillus lilacinus Macé. (Traité Pratiçue Bact., 6e éd., 2, 1913. 416.) From water.

Bacillus membranaceus amethystinus mobilis Germano. (Germano, Cent. f. Bakt., 12, 1892, 516; Bacillus amethysti- nus mobilis Kruse, in Flügge, Die Mikroorganismen, 3 Aufl., 2, 1896, 313; Bacterim amethystinus molitis (hester. Ann. Reput. Del. Col. Igr. Exp. Sta., 9, 1897. 117; Bacterium membranaceus mobilis (hester, ibid. 138.) Psendomonas amethyslina Migula, Syst. d. Bakt., 2, 1900, 94t; Bacillus amethyslinus Chester, Man. Determ. Bact., 1301. 262.) From dust.

Bacillus pavoninus Forster. (Forster, in van der sleen, sur l'examen bactériologicue qualitatif de l'eau. Arch. Teyler, Sér. 2, Tome 4, 3 partic, 1s94, No. 59, Haarlem, Heriticre Loosjes. Also see Godfrin, Thése, Nancy, 1934, 46.) Causes blue discoloration of Edam cheese.

Bacillus polychromogenes (hamot and Thiry. Bacille polychrome, Thiry, Compt. rend. Sor. Biol., Paris, 48, 1596 . \$.5; Chamot and Thiry, Bot. Gaz., 30, $1900,378$.$) From well water at Nancy.$ Probably a Pseudomonas (Tobie, persmal (communication).

Bacillus violaceus Frankland and Frankland. Frankland and Frankland, Ztschr.f. Hyg., 6, 1888, 394; Pscudomonas pseudoianthina Migula, Syst. d. Bakt., 2, 1900, 942.) Isolated from tap water. Said to produce spores.

Bacillus violaceus laurentius Jordan. (Jordan, Mass. State Bd. Health Rept., 1590. S38; Bacterium violaceus lamentius ('hester, Ann. Rept. Del. ('ul. Agr. Exp. Sta.. 9, 1497, 117; Psendomonas Laurentia Migula. Syst. d. Bakt., 2, 1300,947 ; Bacillus violaceus C'hester, Man. Determ. Bact., 1901. 262; Chromobacterium violaceum lanrentium ford. Textb. Bact., 1927, 470.) Isolated from sewage effluent.

Bacillus violaceus luletiensis Irruse. (Irruse, in Flügge, Die Mikroorganismen, 3 Aufl, 2, 1596, 311; Bacillus lutetiensis Chester, Man. Determ. Bact., 1901, 306; Chromobacterium violaceum lutetiense Ford, Textb. Bact., 1927, 170.) From water.

Bacillus violucets surtoryi Witeldele. Thèse, Pharm. Strashourg, 1938. 5.5. 
From dental pus. Said to form spores.

Bacterium cristallino violaceum Cholkevitch. (Cholkevitch, 1922, quoted from Godfrin, Contribution a l'étude des bactéries bleues et violettes. Thése, Nancy, 1934, 93.) From peat.

Chromobacteriumbamptonii Bergey etal. (Bacillus membranaceus amethystinus II, Bampton, Cent. f. Bakt., I Abt., Orig., 71, 1913, 137; Bergey et al., Manual, 1st ed., 1923, 119; Chromobacterium membranaceum amethystinum. II Ford, Textb. Bact., 1927, 473.) From water.

Chromobacterium coeruleum (Voges) Bergey et al. (Bacillus coeruleus Voges, Cent. f. Bakt., 14, 1893, 303; Bacterium coeruleus Chester, Ann. Rept. Del. Col. Agr. Exp. Sta., 9, 1897, 117; Psendomonas coerulea Migula, Syst. d. Bakt., 2, 1900, 945; Bergey et al., Manual, 1st ed., 1923, 120.) From water.

Chromobacterium cohaerens Grimes. (Sci. Proc. Royal Dublin Society, 19, 1930, 381.) From well water.

Chromobacterium hibernicum Grimes. (Sci. Proc. Royal Dublin Society, 19, 1930, 381.) From well water.

Chromobacterium lividum (Voges) Holland. (Plagge and Proskauer, Zeitsch. f. Hyg., 2, 1857, 463; Bacillus lividus Voges, Cent. f. Bakt., 14, 1893, 303; relationship to Bacillus lividus Zimmermann uncertain. Die Bakt. unserer Trink- und Nutzwässer, Chemnitz, 2, 1894, 18; Bacillus violaceus berolinensis Kruse, in Flugge, Die Mikroorganismen, 3 Aufl., 2, 1896, 311; Bacterium lividus Chester, Ann. Rept. Del. Col. Agr. Exp. Sta., 9, 1897, 117; Bacillus berolinensis Chester, Man. Determ. Bact., 1901, 305; Holland, Jour. Bact., 5, 1920, 215.) From water.

Chromobacterium maris-moitui Elazari-Volcani. (Studies on the Microflora of the Dead Sea, Thesis, Hebrew Univ., Jerusalem, 1940, vii and 76.) From the Dead Sea.

Chromobacterium membranaceum Bergey et al. (Bacillus membranaceus amethystinus I, Bampton, Cent. f. Bakt., I Abt., Orig., 71 , 1913, 135; Bergey et al., Manual, 1st ed., 1923, 119; Chromobac- terium membranaceum amethystinum I Ford, Textb. Bact., 1927, 472.) From water.

Chromobacterium membranaceum amethystinum III Ford. (Ford, Textb. Bact., 1927, 474; Bacillus membranaceus amethystinus III Bampton, Cent. f. Bakt., I Abt., Orig., 71, 1913, 138.) From water.

Chromobacterium membranaceum amethystinum IV Ford. (Ford, Textb. Bact., 1927, 474; Bacillus membranaceus amethystinus $I V$ Bampton, Cent. f. Bakt., I Abt., Orig., 71, 1913, 138.) From water.

Chromobacterium smithii (Chester) Bergey et al. (Bacillus coeruleus Smith, Medical News, 2, 1887, 755; Bacterium cocruleus Chester, Ann. Rept. Del. Col. Agr. Exp. Sta., 9, 1897, 118; Pseudomonas smithii Chester, Man. Determ. Bact., 1901, 318; Chromobacterium coeruleum Ford, Textb. Bact., 1927, 475; not Chromobacterium coeruleum Bergey et al., Manual, 1st ed., 1923, 120; Bergey et al., ibid., 121.) From water.

Chromobacterium violaceum manilae Ford. (Bacillus violaceus manilae Woolley, U. S. Dept. Int., Bur. Govt. Labs. Bull. 15, 1904 and Bull. Johns Hopkins Hosp., 16, 1905, 89; Ford, Textb. Bact., 1927,471 .) Isolated from fatal septicemias in water buffalo (Woolley) and man (Schattenberg and Harris, Jour. Bact., $44,1942,509)$. More likely to be a variety of Chromobacterium ianthinum which grows at $37^{\circ} \mathrm{C}$. than of $C$, violaceum which does not grow at $37^{\circ} \mathrm{C}$.

Chromobacterium viscofucatum (Harrison and Barlow) Bergey et al. (Bacterium viscofucatum and Bacillus viscofucatus Harrison and Barlow, Cent. f. Bakt., II Abt., 15, 1905, 517; Trans. Royal Soc. Canada, 2nd Ser., 11, 1905; Bergey et al., Manual, 1st ed., 1923, 119.) From oily butter. Probably a non-motile Pseudomonas (Tobie, personal communication).

Chromobacterium viscosum Grimes. (Cent. f. Bakt., II Abt., 72, 1927, 367.) From butter.

Pseudomonas pseudoviolacea Migula. (Syst. d. Bakt., 2, 1900, 943.) From river water. 


\title{
FAMIIT Y. MICROCOCCACEAL PRIBRAM.*
}

\author{
(Jour. Bact., 18, 1929, 385.)
}

Cells without endospores except in Sporosarcina. Cells in their free condition spherical; during division somewhat elliptical. Division in two or three planes. If the cells remain in contact after division, they are frequently flattened in the plane of last division. They occur singly, in pairs, tetrads, packets or irregular masses. Motility rare. Generally Gram-positive. Many species form a yellow, orange, pink or red pigment. Most species are preferably acrobic, producing abundant growth on ordinary culture media, but capable of slight anaerobic growth. A few species are strictly anaerobic. Metabolism heterotrophic. Carbohydrates are frequently fermented to acid. Gelatin is often liquefied. Facultative parasites and saprophytes. Frequently live on the skin, in skin glands or skin gland secretions of Vertcbrata.

\section{Key to the genera of family Micrococcaceae.}

1. Cells occur in plates, groups or in irregular packets and masses, never in chains. Pigment, when present, is yellow, orange or red. Gram-positive to Gramnegative.

Genus I. Micrococcus, p. 235.

II. On the animal body and in special media cells occur as tetrads. In ordinary media cells may necur in pairs and irregular masses. White to pale yellow.

Genus II. Gaffkya, p. $2 \$ 3$.

III. Cells occur in regular packets. Yellow or orange pigment usually formed.

Genus III. Sarcina, p. 285.

\section{Genus I. Micrococcus Cohn.*}

(Cohn, Beiträge z. Biol. d. Pflanzen, 1, Heft 2, 1s72, 153; Microsphaera Cohn, Arch. f. path. Anat., 55, 1872, 237; not Microsphaera Léveillé, Ann. Sici. Nat. Bot., Sér. 3, 16, 1851, 3s1; A scocuccus Cohn, Beiträge z. Biol. d. Pflanzen, 1, Heft 3, 1875, 154; Pediococcus Balcke, Wchnschr. f. Brauerei, 1, 1s8t, 183; Merista Van Tieghem, Traité de Botanique, Paris, 1854, 1114; Staphylococcus Rosenbach. Mikroorganismen bei den Wundinfektions-krankheiten des Menschen, 1884, 27; Wonococcus Miller, Deutsch. med. Wehnschr., 12, 1886, No. 8, 117; Botryomyces Bollinger, Deutsch. Ztschr. f. Tiermed., 13, 1ss7, 77; Urococcus Miquel, Ann. Microg., 1, 1888, 51s; Galactococcus Guillebeau, Jahrb. d. Schweiz, 4, 1890, 32; Rhodococcus Zopf, Ber. d. deutsch. Bot. Gesellsch., Berlin, 9, 1891, 2S; Pyococeus Ludwin, Lehrb. d. niederen Kryptog., 1892, 27; Planococcus Migula, Arb. Bakt. Inst. Karlsruhe, 1, 1894, 236; Carphococcus Hohl, C'ent. f. Bakt., II Abt., 9, 1902, 338; Albococcus Winslow and Rogers, Jour. Inf. Dis.. 3, 1906, 5t1; Aurococeus Winslow and Rogers, ibid., 5t0; Pedioplana Wolff, C'ent. f. Bakt., II Abt., 18, 1907, 9; Melococens Nedrigailov, Charkov Med. Zurnal, 4, 1907, 301; Solidococcus, Liquidococcus, Indolococeus and Peptonococcus Orla-Jensen, Cent. f. Bakt., II Aht., 22, 1909, 332; Planomerista Vuillemin, Ann. Mycol., 11, 1913, 525; Tetracoceus Orla-Jensen (in part), The Isactic Acid Bacteria, 1919, 76.) From Greek micrus, small; coccus, a grain; M. L., a sphere.

Cells in plates or irregular masses (never in long chains or packets). Gram-positive to Gram-negative. Crowth on agar usually abundant, some species form no

* The genera Micrococcus and Staphylococcus have been combined and completely revised by Prof. C. J. Hucker, New York State Experiment Station, Geneva, New York, March, 1943 so far as the aerobic species are concerned. Dr. Ivan C. Hall, Presbyterian Hospital, New York City, revised the anaerobic section, January, 1944. 
pigment but others form yeliow or less commonly oringe, or red pigment. Glucose broth slightly acid, lactose broth generally neutral. Gelatin frequently liquefied, but not rapidly. Facultative parasites and saprophytes.

The type species is Micrococcus luteus (Schroeter) Cohn.

\section{Key to the species of genus Micrococcus.}

1. Aerobic to facultative anaerobic species.

I. No pink or red pigment on agar media.

A. Nitrites not produced from nitrates.

1. Utilize $\mathrm{NH}_{4} \mathrm{H}_{2} \mathrm{PO}_{4}$ as sole source of nitrogen.*

a. Yellow pigment on agar media. Not acido-proteolytic.

1. Micrococcus luteus.

aa. No pigment produced. Not acido-proteolytic.

b. Utilizes urea as a sole source of nitrogen.**

2. Micrococcus ureae.

bb. Does not utilize urea.

3. Micrococcus freudenreichii.

aaa. Acido-proteolytic in litmus milk.

8. Micrococcus caseolyticus.

2. Do not utilize $\mathrm{NH}_{4} \mathrm{H}_{2} \mathrm{PO}_{4}$ as sole source of nitrogen.

a. Yellow pigment produced.

4. Micrococcus flavus.

aa. No pigment produced.

5. Micrococcus candidus.

B. Nitrites produced from nitrates.

1. Utilize $\mathrm{NH}_{4} \mathrm{H}_{2} \mathrm{PO}_{4}$ as sole souree of nitrogen.

a. Yellow pigment on agar media. Not acido-proteolytic.

b. Celatin liquefied.

bb. Felatin not liquefied.

6. Micrococcus conglomeratus.

7. Micrococcus varians.

aa. Usually not chromogenic. Actively acido-proteoly tic in litmus milk.

8. Micrococcus caseolyticus.

2. Do not utilize $\mathrm{NH}_{4} \mathrm{H}_{2} \mathrm{PO}_{4}$ as sole source of nitrogen.

a. Gelatin liquefied. Ferment mannitol.

b. Abundant orange growth on agar media.

9a. Micrococcus pyogenes var. aureus.

bb. Abundant white growth on agar media.

9b. Micrococcus pyogenes var. albus.

bbb. Yellow growth on agar media.

10. Micrococcus citreus.

aa. Gelatin not liquefied or very slowly liquefied.

b. Abundant orange to white growth on agar media. Ferments mannitol.

11. Micrococcus aurantiacus.

bb. Seant white translucent growth on agar media. Does not ferment mannitol.

12. Micrococcus epidermidis.

* That is, will grow and produce acid (sometimes slowly) on slants containing 1.5 per cent washed agar, 0.1 per cent ammonium phosphate, 1.0 per cent glucose. 0.02 per cent potassium chloride, 0.02 per cent magnesium sulfate. Add brom-cresolpurple as an indicator (Hucker, N. Y. State Exper. Sta., Tech. Bul. 100, 1924, 25; Tech. Bul. 101, 1924, 36-40); Manual Pure Culture Study of Bacteria. Soc. Amer. Bact., Geneva, N. Y., Leaflet II, 9th ed., 1944, 14.) 
II. Pink or red pigment on agar media.

A. Gelatin liquefied, slowly. Produces rose-colored pigment.

13. Micrococcus roseus.

B. Gelatin not liquefied.

1. Non-motile.

a. Produces cinnabar-colored pigment on gelatin.

14. Micrococcus cinnabareus.

aa. Produces light, flesh-colored pigment on agar slant. Ferments glycerol and mannitol.

15. Micrococcus rubens.

aaa. Produces brick-colored pigment on agar slant. Does not ferment glycerol and mannitol.

16. Micrococcus rhodochrous.

2. Motile. Produces red pigment.

17. Micrococcus agilis.

2. Anaerobic species.

I. Forms gas from nitrogenous media.

A. Acid from glucose.

18. Micrococcus aerogenes.

B. No acid from glucose

1. No blackening of colonies in deep agar.

19. Micrococcus asaccharolyticus.

2. Hydrogen sulfide formed. Deep agar colonies become black.

20. Micrococcus niger.

II. No gas formed from nitrogenous media.

A. Acid from glucose.

1. Acid from lactose.

2. No acid from lactose.

21. Micrococcus grigoroff.

22. Micrococcus anderobius.

1. Micrococcus luteus (Schroeter)

Cohn. (Bactoridium luteum Schroeter, Beitr. z. Biol. d. Pflan., 1, Heft 2, 1872, 119; Cohn, ibid., 153.) From Latin, luceus golden-yellom.

Spheres: 1.0 to 1.2 microns, occurring in pairs and fours. Non-motile. Grampositive.

Gelatin colonies: Jellowish-white to yellow, raised, with undulate margin.

Gelatin stab: No liquefaction.

Agar colonies: Small, yellowish, glistening, raised.

Agar slant: Citron-yellow, smooth.

Broth: Clear, with yellowish sediment.

Litmus milk: Usually slightly acid, not coagulated.

Potato: Thin, glistening, citron-yellow growth.

Indole not formed.

Nitrites not produced from nitrates.
Acid from glucose, sucrose and mannitul. No acid from lactose.

Starch not hydrolyzed.

Ammoria produced from peptone.

Ltilizes $\mathrm{NH}_{4} \mathrm{H}_{2} \mathrm{PO}_{4}$ as a source of nitrogen.

Saprophytic.

Aerobic.

Optimum temperature $25^{\circ} \mathrm{C}$.

Source: Isolated by Schroeter from dust contaminations on cooked potato.

Habitat : Found in skim milk and dairy products, and on dust particles.

2. Micrococcus ureae Cohn. (Cohn, Beitr. z. Biol. d. Pflanzen, 1, Heft 2, 1872, 158; not Micrococcus ureae Flügge, Die Mikroorganismen, 2 Aufl., 1886, 169 ; Merisla ureae Prazmowski, Biol. Cent., 8, 1888, 301; Streptococcus ureae Trevisan, I generi e le specie delle Bat- 
teriacee, Milan, 1889, 31; Urococcus ureae Beijerinck, Cent. f. Bakt., II Abt., 7, 1901, 52; Albococcus ureae Kligler, Jour. Infect. Dis., 12, 1943, 442; Staphylococcus ureae Holland, Jour. Bact., 5, 1920, 225.) From Greek, urum, urine; M. L., urea, urea.

See Micrococcus liquefaciens Migula in the appendix for references to the gelatin-liquefying form of the species.

Spheres: 0.8 to 1.0 micron, occurring singly, in pairs and in clumps. Never in chains. Non-motile. Gram-variable.

Gelatin colonies: Small, white, translucent, slimy, becoming fissured.

Gelatin stab: Slight, white growth. Very slow or no liquefaction.

Agar colonies: White, slightly raised.

Agar slant: Grayish-white, raised, glistening, butyrous.

Broth: Turbid, with viseid sediment.

Litmus milk: Slightly alkaline; litmus slowly reduced.

Milk: Acid.

Potato: Slight, grayish to pale olive growth.

Indole not formed.

Nitrites not produced from nitrates.

Urea fermented to ammonium carbonate.

Acid produced from glucose, lactose, sucrose and mannitol.

Starch not hydrolyzed.

Ammonium salts are utilized.

Ammonia produced from peptone.

Saprophytic.

Aerobic.

Optimum temperature $25^{\circ} \mathrm{C}$.

Source: Isolated from fermenting urine.

Habitat: Found in stale urine and in soil containing urine.

3. Micrococcus freudenreichii Guillebeau. (Landwirtsch. Jahrb. d. Schweiz, 5, 1891, 135.) Named for E. v. Freudenreich, Swiss bacteriologist.

Synonyms: Micrococcus acidi lactis Krueger, Cent. f. Bakt., 7, 1890, 464 (Micrococcus acidi lactis liquefaciens Eisenberg, Bakt. Diag., 3 Aufl., 1891, 409; Micrococcus acidi lactici liquefaciens
Stemberg, Man. of Bact., 1893, 601; Micrococcus acidilactis Migula, Syst.d. Bakt., 2, 1900, 112; Micrococcus acidifcans Migula, ibid.); Micrococcus lactis viscosus Sternberg, Man. of Bact., 1893, 604; Micrococcus amarifaciens Migula, Syst. d. Bakt., 2, 1900, 100; Coccus lactis viscosi Gruber, Cent. f. Bakt., II Abt., 9, 1902, 790 (Micrococcus lactis viscosi Löhnis, Cent. f. Bakt., II Abt., 18, 1907, 144); Micrococcus lactis albidus Conn, Esten and Stocking, Storrs Agr. Exp. Sta. 18th Ann. Rept., 1906, 91.

Spheres: 2.0 microns in diameter, occuring singly and in clumps, rarely in short chains. Non-motile. Gram-positive.

Milk gelatin colonies: Small, white, opaque.

Milk gelatin stab: Infundibuliform liquefaction.

Agar colonies: White, slimy.

Agar streak: White, smooth.

Broth: Turbid, with white sediment.

Litmus milk: Acid; coagulated; peptonized.

Potato: Moderate white to yellow streak.

Indole not formed.

Nitrites not produced from nitrates.

Starch not hydrolyzed.

Ammonia produced from peptone.

Does not utilize urea as a source of nitrogen.

Acid from glucose, lactose and sucrose. Some strains form acid from mannitol; others from glycerol.

Saprophytic.

Aerobic.

Optimum temperature $20^{\circ} \mathrm{C}$.

Habitat: Milk and dairy utensils.

4. Micrococcus flavus Trevisan. (Micrococcus flavus liquefaciens Flügge, Die Mikroorganismen, 2 Aufl., 1886, 174; Trevisan, I generi e le specie delle Batteriacee, Milan, 1889, 34; Micrococcus flawus-liquefaciens Chester, Man. Determ. Bact., 1901, 99.) From Latin, flavus, yellow.

Spheres: 0.8 to 0.9 micron, occurring 
singly, in clumps, and occasionally in fours. Occasionally cultures are found that are motile with a single flagellum. Otherwise non-motile. Gram-variable.

Gelatin colonies: Small, circular, yellowish to yellowish-brown, somewhat serrate margin, granulated, sharply contoured.

Gelatin stab: Yellow, wrinkled surface growth with slow, crateriform liquefaction.

Agar colonies: Small, pale yellowish, homogeneous, entire.

Agar slant: Canary-yellow, somewhat dry, wrinkled, raised, entire.

Broth: Turbid with yellowish ring and sediment.

Litmus milk: Slightly acid, soft coagulum formed, with slight reduction; slowly peptonized.

Potato: Slight, canary-yellow growth.

Indole is not formed.

Nitrites not produced from nitrates.

Starch not hydrolyzed.

Acid is generally formed from glucose and lactose. Sucrose, glycerol and mannitol generally not fermented.

Ammonium salts are utilized

Ammonia produced from peptone.

Non-pathogenic.

Aerobic.

Optimum temperature $25^{\circ} \mathrm{C}$.

Source: Original source not given.

Habitat: Found in skin gland secretions, milk, dairy products, and dairy utensils.

\section{Micrococcus candidus Cohn} (Cohn, Beitr. z. Biol. d. Pflanzen, 1, Heft 2, 1872, 160 ; Staphylococcus candidus Holland, Jour. Bact., 5, 1920, 223.) From Latin candidus, shining white.

Spheres: 0.5 to 0.7 micron, occurring singly. Non-motile. Gram-positive.

Gelatin colonies: White, granular, with irregular or entire margin.

Gelatin stab: White surface growth. Filiform. No liquefaction.

Agar colonies: Punctiform, white, smooth, entire, iridescent.
Agar slant: Smooth, white, glistening, iridescent.

Broth: Turbid, with pellicle.

Litmus milk: Slightly acid; not coagulated.

Potato: Thick, porcelain white, glistening.

Indole not produced.

Nitrites not produced from nitrates.

Starch not hydrolyzed.

Ammonia produced from peptone.

Ammonium salts not utilized.

Acid from glucose, sucrose, lactose and glycerol.

Non-pathogenic.

Aerobic.

Optimum temperature $25^{\circ} \mathrm{C}$.

Source: Originally appeared as white colonies on cooked potato exposed to dust contaminations.

Habitat: Found in skin secretions, milk and dairy products.

6. Micrococcus conglomeratus Migula. (Citronengelber Diplococeus, Bumm, Der Mikroorganismen der gonorrhoischen Schleimhauterkrankungen, 1 Aufl., 1S85, 17; Hicrococcus citreus conglomeratus Flügge, Die Mikroorganismen, 2 Aufl., 1886, 182; Diplococcus citreus conglomeratus Bumm, ibid., 2.Aufl., 1887; Neisseria citrea Trevisan, I generi e le specie delle Batteriacee, Milan, 1889, 32; Merismopedia citreus conglomeratus Dyar, Ann, N. Y. Acad. Sci., 8, 1895, 352; Migula, Syst. d. Bakt., 2, 1900, 146; not Micrococcus conglomeratus Weichselbaum, 1887, see Trevisan, loc. cit., 33; Micrococcus citreus Winslow and Winslow, Systematic Relationships of the Coccaceae, 1908, 218.) From Latin, conglomeratus, rolled together, crowded.

Spheres: 0.8 to 1.2 microns, occurring singly, in pairs, in fours, and in large clumps. Non-motile. Gram-variable.

Gelatin colonies: Small, circular, yellow with radiate margin.

Gelatin stab: Slow crateriform liquefaction. 
Agar colonies : Luxuriant, moist, sulfur yellow.

Agar slant: Light yellow, plumose, slightly rugose, somewhat dull, raised center and transparent margin.

Broth: Turbid, with light orange ring and sediment.

Milk: Generally acid but not sufficient to curdle.

Potato: No growth.

Indole not formed.

Nitrites produced from nitrates.

Blood not hemolyzed.

Starch not hydrolyzed.

Acid from glucose and lactose generally, sometimes from sucrose. Mannitol and glycerol generally not fermented.

Ammonia produced from peptone.

Utilizes $\mathrm{NH}_{4} \mathrm{H}_{2} \mathrm{PO}_{4}$ as a source of nitrogen.

Resistant to drying and heat.

Non-pathogenic.

Aerobic.

Optimum temperature $25^{\circ} \mathrm{C}$.

Source: Found in gonorrhoeal pus and dust.

Habitat : Infections, milk, dairy products, dairy utensils, water, common.

\section{Micrococcus varians Migula.}

(Merismopedia flava varians Dyar, Ann. N. Y. Acad. Sci., 8, 1895, 346; Migula, Syst. d. Bakt., 2, 1900, 135̃ Merismopedia flava-varians Chester, Man. Determ. Bact., 1901, 103; Micrococcus lactis varians Conn, Esten and Stocking, Storrs Agr. Exp. Sta. Rept. for 1906, 121.) From Latin, varians varying.

Spheres: 0.8 to 1.0 micron, occurring singly, in pairs and in fours. Occasionally cultures are found that are motile with a single flagellum. Otherwise nonmotile. Gram-variable.

Gelatin colonies : Small, circular, whitish to yellow, capitate, moruloid.

Gelatin stab: Scant growth. No liquefaction.

Agar colonies: Small, yellow, raised, glistening.
Agar slant: Plumose, yellow, variegated.

Broth: Turbid, with yellow, granular sediment.

Litmus milk : Acid; coagulated on boiling.

Potato: Raised, dry, bright-yellow, glistening.

Indole not formed.

Nitrites produced from nitrates.

Acid from glucose, lactose, sucrose, raffinose and frequently from glycerol and mannitol. No acid from salicin or inulin.

Starch not hydrolyzed.

Ammonia produced from peptone.

Utilizes $\mathrm{NH}_{4} \mathrm{H}_{2} \mathrm{PO}_{4}$ as a source of nitrogen.

Saprophytic.

Aerobic.

Optimum temperature $25^{\circ} \mathrm{C}$.

Source: Original strains found in a contaminated jar of sterilized milk.

Habitat: Has been found in body secretions, dairy products, dairy utensils, dust and water, including sea water.

8. Micrococcus caseolyticus Evans. (Evans, Jour. Inf. Dis., 18, 1916, 455; Micrococcus casei Hucker, N. Y. Agr. Exp. Sta. Tech. Bull. 102, 1924, 17 ; Probably Micrococcus casei Holland, Jour. Bact., 5, 1920, 223.)

Identical in part with Micrococcus casei acidoproteolyticus $I$ and II Gorini, Rev. Gén. du Lait, 8, 1910, 337; Tetracoccus liquefaciens Orla-Jensen, The Lactic Acid Bacteria, 1919, 80 (Micrococcus casei liquefaciens Orla-Jensen, Doktordisputats, 1904; Tetracoccus casei liquefaciens Orla-Jensen, The Lactic Acid Bacteria, 1919, 80 ; Micrococcus liquefaciens Holland, Jour. Bact., 5, 1920, 224. Also see references under Sireptococcus liquefaciens.) From Latin, caseus, cheese, casein; and Greek, lyticus, able to dissolve; M. L., dissolving, digesting.

Spheres, variable in size, occurring in clumps. Non-motile. Gram-positive.

Gelatin stab: Liquefaction generally 
begins after first day and continues rapidly.

Agar colonies: Iellow to orange (Evans, loc. cit.), pearly white (Hucker, loc. cit.).

Agar stroke: Yellow to orange (Evans, loc. cit.), pearly white (Hucker, loc.cit.), luxuriant growth.

Broth: Generally grows with smooth turbidity although certain strains give heavy precipitate with clear supernatant fluid.

Litmus milk: Acid, peptonized. Whey generally clear.

Potato: Scanty white growth. Certain strains may show yellow pigment.

Indole not formed.

Nitrites usually produced from nitrates.

Acid from glucose, lactose, maltose, mannitol and glycerol. No action on raffinose.

Forms dextrorotary lactic acid (OrlaJensen, 1919, loc. cit.).

Asparagin and urea decomposed by some strains.

Utilizes $\mathrm{NH}_{4} \mathrm{H}_{2} \mathrm{PO}$, as a source of nitrogen.

Optimum temperature $22^{\circ} \mathrm{C}$.

Aerobic.

Saprophytic.

Source: Eight cultures from bovine udder.

Habitat: Milk and dairy products, especially cheese, dairy utensils.

\section{9a. Micrococcus pyogenes var. aureus} (Rosenbach) Zopf. (Staphylococcus pyogenes aureus Rosenbach, Mikroorganismen bei den Wundinfectionskrankheiten des Menschen. Wiesbaden, 1884, 19; Staphylococcus aureus Rosenbach, ibid. 27; Hicrococeus pyogenes var. aureus Zopf. Die Spaltpilze, 3 Aufl., 1855, 56; Micrococcus aureus Zopf, ibid., 57; Micrococcus pyogenes Lehmann and Xeumann, Bakt. Diag., 1 Aufl., 2, 1896, 165 ; Aurococcus aureus Winslow and Rogers. Jour. Inf. Dis.. 3, 1906, 55t; . Hicrococcus lactis varians Conn, Esten and Stocking, Storrs Agr. Exp. Sta. Rept. for 1906.
121; Staphylococcus pyogenes Andremes and Gordon, Rept. (35th) Med. Officer Local Govt. Board, London, 1907, 549; (Tetracoccus) Micrococcus pyogenes aureus Orla-Jensen, The Lactic Acid Bacteria, 1919, 81; Staphylococcus pyogenes-aureus Holland, Jour. Bact., 5, 1920, 225.) From Greek, pyon, pus; II. L.,-genes, producing. From Latin, aureus, golden.

Spheres: 0.8 to 1.0 micron, occurring singly, in pairs, in short chains, and in irregular clumps. Non-motile. Grampositive.

Gelatin stab: Saccate liquefaction with yellowish pellicle and yellow to orange sediment.

Agar colonies: Circular, smooth, yellowish to orange, glistening, butyrous, entire.

Agar slant: Abundant, opaque, smooth, flat, moist, yellowish to orange.

Broth: Turhid with yellowish ring and sediment, becoming clear.

Litmus milk: Acid; coagulated.

Potato: Abundant, orange, glistening Indole not formed.

Nitrites produced from nitrates.

Acid from glucose, lactose, sucrose, mannitol and glycerol, but not from raffinose, salicin or inulin.

Forms inactive or levorotary lactic acid (Orla-Jensen, loc cit.).

Slight H.s formation.

Stareh not hydrolyzed.

Does not utilize $\mathrm{NH}_{4} \mathrm{H}_{2} \mathrm{PO}_{4}$, as a source of nitrogen.

Ammonia produced from peptone.

Pathogenic. Individual strains vary in their ability to produce hemolysin, coagulase and other metabolic products.

Certain strains, under favorable conditions. produce not only exotoxins (hematoxin, dermatoxin, lethal toxin, etc.) but also a potent enterotoxin which is a significant cause of food poisoning (Dolman and Wilson, Jour. Immunology, 35, 1938, 13).

Aerobic, facultative.

Optimum temperature $37^{\circ} \mathrm{C}$.

Source: Isolated from pus in wounds. 
Habitat: Skin and mucous membranes. The cause of boils, abscesses, furuncles suppuration in wounds, etc.

9b. Micrococcus pyogenes var. albus (Rosenbach) Schroeter. (Staphylococcus pyogenes albus Rosenbach, Mikroorganismen bei den Wundinfektionskrankheiten des Menschen, Wiesbaden, 188t, 2; Staphylococcus albus Iiosenbach, ibid., 27; Micrococcus pyogenes var. albus Schroeter, in Cohn, Irryptog. Flora v. Schlesien, 3, 1, 1886, 147; Micrococcus pyogenes Migula, Syst. d. Bakt., 2, 1900, s7; Albococcus pyogenes Winslow and Rogers, Jour. Inf. Dis., 3, 1906, 544; Micrococcus albus Buchanan, Veterinary Bacteriology 1911, 196; (Tetracoccus) Microccocus pyogenes albus Orla-Jensen, The Lactic Acid Bacteria, 1919, 81; Staphylococcus pyogenes-albus Holland, Jour. Bact., 5, 1920, 225.) From Latin, albus, white.

Spheres: 0.6 to 0.8 micron, occurring singly, in pairs and in irregular groups. Non-motile. Gram-positive.

Gelatin stab: Saccate liquefaction with heavy white sediment.

Agar colonies: Circular, white, smooth, glistening, entire.

Ten per cent cvaporated milk agar: Growth at $20^{\circ} \mathrm{C}$ frequently orange (Chapman, Jour. Bact., 45, 1943, 405).

Agar slant: Abundant, white, smooth, glistening.

Broth: Turbid, with delicate pellicle and white sediment.

Litmus milk: Acid; coagulated. Little or no visible peptonization.

Potato: Thick, smooth, white, glistening.

Indole not formed.

Nitrites produced from nitrates.

Hydrogen sulfide is formed.

Acid formed from glucose, lactose, sucrose, glycerol and mannitol, but not from raffinose, salicin and inulin.

Forms inactive or levorotary lactic acid (Orla-Jensen, loc. cit.).

Starch not hydrolyzed.

Ammonia produced from peptone.

Does not utilize $\mathrm{NH}_{4} \mathrm{H}_{2} \mathrm{PO}_{4}$ as a source of nitrogen.
Pathogenic. Production of toxins, coagulase and hemolysin as in Micrococcus aureus.

Aerobic, facultative.

Optimum temperature $37^{\circ} \mathrm{C}$.

Source: Originally isolated from pus.

Habitat: Skin and mucous membranes. Occurs in wounds, boils, abscesses, etc.

10. Micrococcus citreus Migula. (Staphylococcus pyogenes citreus Passet, Aetiologie der eiterigen phlegmone des Menschen, Berlin, 1885, 9; Micrococcus p)yogenes citreus Schroeter, in Cohn, Kiryptog. Flora v. Schlesien, 3, 1, 1886, 147; Migula, Syst. d. Bakt., 2, 1900, 147 ; Staphylococcus citreus Bergey et al., Manual, 1st ed., 1923,55.) From Latin, citreus, of or relating to the citrus tree; M. L., lemon yellow.

Spheres: 0.9 micron, occurring singly. Gram-positive.

Gelatin colonies: Circular, pale yellow, granular, entire, liquefying in 6 days.

Gelatin stab: Lemon yellow surface growth sinking into the medium. Grayish-white growth in stab. Complete liquefaction in 43 days.

Agar colonies: Small, yellow, smooth, entire.

Agar slant: Broad, lemon yellow, glistening, elastic.

Broth: Turbid, with yellow sediment and pellicle.

Litmus milk: Acid, with slow coagulation.

Potato: Thin, grayish streak, becoming citron yellow.

Indole not formed.

Nitrites produced from nitrates.

starch not hydrolyzed.

Acid from glucose, lactose, sucrose, raffinose, inulin, salicin, glycerol and mannitol.

Does not utilize $\mathrm{NH}_{4} \mathrm{H}_{2} \mathrm{PO}_{4}$ as a source of nitrogen.

Ammonia produced from peptone.

Aerobic, facultative.

Pathogenic.

Optimum temperature $37^{\circ} \mathrm{C}$.

Source: Originally isolated from pus. 
Habitat: Skin and mucous membranes of vertebrates.

11. Micrococcus aurantiacus (Schroeter) Cohn. (Bacteridium aurantiacum. Schroeter, Beitr. z. Biol., 1, Heft 2, 1872, 126; Cohn, Beitr. z. Biol., 1, Heft 2, 1872, 154; Pediococcus aurantiacus De Toni and Trevisan, in Saccardo, sylloge Fungorum, 8, 1839, 1051; Micrococcus aurantiacussorghi Bruyning, Arch. Néer. Sci. Exact. et Nat., 1, 1898, 297; Streptococcus aurantiacus Chester, Man. Determ. Bact., 1901, 69; Aurococcus aurantiacus Winslow and Winslow, Systematic Relationships of the Coccaceae, 1908, 186; Sarcina aurantiaca Holland, Jour. Bact., 5, 1920, 225 (not Sarcina aurantiaca Flügge, Die Mikroorganismen, 2 Aufl., 1886, 180); Staphylococcus aurantiacus Holland, ibid.) From Latin, aurum, gold; M. L., aurantium, the orange; M.L.. aurantiacus, orange-colored.

Spheres: Slightly ellipsoidal, 1.3 to 1.5 microns, occurring singly, in short chains and in small clumps. Yon-motile. Gram-positive.

Gelatin colonies: Circular to oval, smooth, glistening with yellow to orange center.

Gelatin stab: Yellow surface growth. No liquefaction.

Agar colonies: Circular, smooth, glistening, yellow to orange, entire.

Agar slant : Buff to scant orange-yellow, beaded growth, raised, glistening.

Broth: Turbid, with pellicle.

Litmus milk: Faintly acid, no coagulation.

Potato: Slimy, yellow growth. Pigment is insoluble in alcohol and ether.

Indole not produced.

Nitrites generally produced from nitrates.

Slight acidity from glucose, fructose, sucrose, lactose and mannitol. No acid from raffinose, salicin, inulin.

Starch not hydrolyzed.

Ammonia produced from peptone.

No growth in ammonium media.

May be pathogenic.

Optimum temperature $25^{\circ} \mathrm{C}$.
Aerobic.

Source: First isolated from colonies that grew on boiled egg exposed to dust contamination.

Habitat: [sually isolated from infections but also found in milk, cheese and dust.

Note: Albococcus epidermidis (var. A) K̈ligler (Jour. Infect. Dis., 12, 1913, 44t) which was based on a white culture received from Kral under the name Micrococcus aurantiacus mas apparently a white strain of this organism as it grew luxuriantly on ordinary agar.

12. Micrococcus epidermidis (Winslow and Winslow) Hucker. (Staphylococcus epidermidis albus Welch, Amer. Jour. of Med. Nèi., Phila., N. S., 102, 1891, 441; Micrococcus epidermidis albus Randolph, Jour. Amer. Med. Assoc., 31, 1898, 706; Albococcus epidermidis IVinslow and Winslow. Sist. Relationships Coccaceae, New York, 1908, 201; Staphylococcus epidermidis Evans, Jour. Inf. Dis., 15, 1916, 449; Hucker, X. Y. Agr. Exp. Sta. Tech. Bull. 102, 1924, 21.) From Greek, epidermis, the outer skin.

Spheres: 0.5 to 0.6 micron, occurring singly, in pairs and in irregular groups. Ton-motile. Gram-positive.

Gelatin stab: White surface growth with slow saceate liquefaction.

Agar colonies: Rather scant, white, translucent.

Broth: Turbid, with white ring and sediment.

Litmus milk: Acid.

Potato: Limited growth, white.

Indole not formed.

Nitrites are produced from nitrates. Usually does not utilize $\mathrm{NH}_{4} \mathrm{H}_{2} \mathrm{PO}_{4}$ as a source of nitrogen.

Acid formed from glucose, fructose, maltose, lactose and sucrose, but not from mannitol, raffinose, salicin or inulin.

Usually fails to hemolyze blood. No coagulase produced.

Parasitic rather than pathogenic.

Aerobic, facultative. 
Optimumtemperature $37^{\circ} \mathrm{C}$.

Source: Originally isolated from small stitch abscesses and other skin wounds.

Habitat: Skin and mucous membranes.

\section{Micrococcus roseus Flügge.} (Rosafarbiger Diplococcus, Bumm, Der Mikroorganismen der gonorrhoischen Schleimhauterkrankungen, 1 Aufl., 1885, 25; Flügge, Die Mikroorganismen, 2 Aufl., 1886, 183; Neisseria rosea Trevisan, I generi e le specie delle Batteriacee, Milan, 1889, 32; Diplococcus roseus Eisenberg, Bakt. Diag., 3 Aufl., 1891, 12 ; Merismopedia rosea Dyar, Ann. N. Y. Acad. Sci., 8, 1895, 354; Rhodococcus roseus Winslow and Rogers, Jour. Inf. Dis., 3, 1906, 545.) From Latin, roseus, rosecolored.

Spheres : 1.0 to 1.5 microns, occurring singly and in pairs. Non-motile. Gramvariable.

Gelatin colonies: Rose surface growth usually with slow liquefaction.

Agar colonies: Circular, entire, rosered surface colonies.

Agar slant: Thick, rose-red, smooth, glistening streak.

Broth: Slightly turhid with rosecolored sediment.

Litmus milk: Unchanged to alkaline, usually reddish sediment after 14 days.

Usually produce nitrites from nitrates.

Potato: Raised, rose-red, smooth, glistening.

Stareh not hydrolyzed.

Acid from glycerol and mannitol.

Utilizes $\mathrm{NH}_{4} \mathrm{H}_{2} \mathrm{PO}_{4}$ as a source of nitrogen.

Saprophytic.

Aerobic.

Optimum temperature $25^{\circ} \mathrm{C}$

Source: Dust contamination.

Habitat: Widespread, as it occurs in dust.

14. Micrococcus cinnabareus Flügge. (Flügge, Die Mikroorganismen, 2 Aufl., 1886, 174; Rhodococcus cinnabareus Winslow and Rogers, Jour. Inf. Dis., 3, 1906, 545.) From M. L., cinnabar-colored.
Spheres: 1.0 micron, occurring singly and in pairs. Non-motile. Gram-variable.

Gelatin colonies : Small, circular, bright red, becoming cinnabar red.

Gelatin stab: Thick, raised, rose to cinnabar red growth on surface. No liquefaction. White colonies along stab.

Agar slant: A rarmine-red streak. Slow growth.

Broth: Turbid.

Litmus milk: Slightly alkaline to slightly acid.

Potato: Slowly developing vermillion red streak.

Small amount of acid from test sugars. Indole not formed.

Does not utilize $\mathrm{NH}_{4} \mathrm{H}_{2} \mathrm{PO}_{4}$ as a source of nitrogen.

Nitrites produced from nitrates.

Starch not hydrolyzed.

Saprophytic.

Aerobic.

Optimum temperature $25^{\circ} \mathrm{C}$.

Source: Found as contamination of cultures.

Habitat: Csually found as a dust contamination.

15. Micrococcus rubens Migula. (Micrococcus tetragenus ruber Bujwid, in Schneider, Arb. bakt. Inst. Karlsruhe, 1, Heft 2, 1894, 215; Migula, Syst. d. Bakt., 2, 1900, 177; Micrococcus ruber and Rhodococcus ruber Holland, Jour. Bact., $6,1920,224$; Hicrococcus roseofulvus Hucker, N. Y. S. Agr. Exp. Sta. Tech. Bull. 135, 1928, 27 ; not Hicrococcus roseofulvus Lehmann and Neumann, Bakt. Diag., 1 Aufl., 2, 1896, 177 and 439; Rhodococeus roseofuleus Pribram, Klassifikation der Schizomyceten, 1933, 44). From Latin, rubens, ruddy.

The following description is taken from Migula (loc. cit.) and from Hucker (loc. cit.) supplemented from unpublished notes of the latter. Also see Breed (Jour. Bact., 45, 1943, 455).

Spheres: 1.3 to 4.0 microns, average size 2.1 microns, occurring in fours and 
in irregular masses, generally not singly or in pairs. Non-motile. Gram-negative to Gram-variable

Gelatin colonies: After several days, small, pink or flesh-colored, shiny, butyrous, 0.5 to several $\mathrm{mm}$. in diameter. Smaller colonies have regular edges; larger colonies have lobate edges.

Gelatin streak: Thick, shiny, fleshcolored to carmine-red growth, generally spreading.

Gelatin stab: Scant, whitish growth along line of stab; surface growth fleshred. No liquefaction after several weeks, but a slight softening of the medium underneath the growth.

Agar slant: Luxuriant, thick, spreading, slimy, flesh-colored growth.

Broth: Bright red, slimy sediment. No pellicle.

Milk: Generally arid curd followed by slight peptonization.

Nitrites produced from nitrates.

Acid from glucose, sucrose, mannitol and glycerol. To action on lactose or starch.

Pigment soluble in ether, benzol, carbom bisulfide, chloroform and alcohol. Not soluble in water (Schneider, loc. cit.).

Saprophytic.

Grows well at $26^{\circ}$ to $37^{\circ} \mathrm{C}^{\circ}$.

Aerobic.

Source: Original culture isolated by Bujwid in Bern, Switzerland and sent to Migula at Karlsruhe, (iermany.

Habitat: Unknown.

16. Micrococcus rhodochrous (Zopf) Migula. (Rhodococeus rhodochrous Zopf, Berichte d. deutsch. bot. Gesellsch., 9, 1891, 22; Migula, Syst. d. Bakt., 2, 1900, 162.) From Greek, rhodum, rose; chros, color.

Spheres: 0.5 to 1.0 micron, occurring singly. Ton-motile. (iram-variable.

Gelatin colonies: Small, circular, glistening, raised, entire, dark, reddishbrown,
Gelatin stab: Dark, carmine-red, dry surface growth. Slight growth in stab. No liquefaction.

Agar slant: Carmine-red streak, becoming brick-red in color.

Broth: Thick rose-red pellicle with red, flocculent sediment.

Litmus milk: Slightly alkaline.

Potato: Carmine-red streak.

Does not ferment glycerol and mannitol.

Aerobic.

Saprophytic.

Optimum temperature $25^{\circ} \mathrm{C}$.

Habitat: Water.

17. Micrococcus agilis Ali-Cohen. (Ali-Cohen, Cent. f. Bakt., 6, 1889, 36; Planosarcina agilis Migula, in Engler and Prantl, Die natürl. Pflanzenfam., 1, 1a, 189.5, 20; Micrococcus agilis ruber Peppler, Cent. f. Batkt., I Abt., 29, 1901, 352; Planococcus agilis Chester, Man. Ieterm. Bact., 1901, 115; Rhodococcus agilis Winslow and Rogers, Jour. Inf. Dis., 3, 1906, 5t5̄; Sarcina agilis Enderlein, Sitzber. Giesell. Naturf. Freunde Berlin, 1930, 1s2; not Sarcina agilis Matzuschita, Zeit. f. Hyg., 35, 1900, 496 ; not Sarcina agil is Saito, Jour. Coll. Sci. Imp. 'niv. Tokyo, 23, 1908, .) From Latin, agilis, agile.

spheres, 1.0 micron, oceurring singly, in pairs and in fours. Intile by means of one or two flagella. Gram-variable.

Celatin "olonies: Small, gray, beeming distinctly rose-colored.

Gelatin stab: Thin, whitish growth in stab. On surface thick, rose-red, glistening growth. Generally no liquefaction.

Agar slant: Glistening, dark rose-red, lobed, much variation in color.

Broth: Slightly turbid, with slight, rose-colored ring and pink sediment.

Litmus milk: Slightly acid, pink sediment.

Potato: slow growth as small, rosecolored colonies.

Loeffler's blood serum: Pink, spread- 
ing, shiny, abundant. Slow liquefaction.

Indole not formed.

Nitrites produced (trace).

Ammonia formed (trace).

Does not utilize $\mathrm{NH}_{4} \mathrm{H}_{2} \mathrm{PO}_{4}$ as source of nitrogen.

Acid from glucose, sucrose, inulin, glycerol and mannitol. No acid from raffinose.

Aerobic.

Saprophytic.

Optimum temperature $25^{\circ} \mathrm{C}$.

Source: Isolated from water.

Habitat: Water, sea water, on sea fish.

*18. Micrococcus aerogenes (Schottmüller) Bergey et al. (Staphylococcus aerogenes Schottmüller, Cent. f. Bakt., I Abt., Orig., 64, 1912, 270; Bergey et al., Manual, 1st ed., 1923, 70; not Micrococcus aerogenes Miller, Deutsch. med. Wchnschr., 12, 1886, 119.) From Greek, forming air or gas.

Description according to Prévot, Ann. Sci. Nat., Sér. Bot. et Zool., 15, 1933, 212.

Spheres : 0.6 to 0.8 micron, occurring in clusters, sometimes in pairs or short chains. Gram-positive.

Gelatin : No liquefaction.

Deep agar colonies: Small, lenticular, nearly spherical, yellowish white. Some gas bubbles produced, not fetid.

Blood agar colonies: Very small, grayish. No true hemolysis, but a narrow clear zone is formed.

Serum agar: Colonies lenticular. Gas not fetid.

Neutral red serum agar: Colonies lenticular. Gas produced. Neutral red changed to greenish yellow.

Glucose broth with blood: Turbid. Gas produced. Hydrogen sulfide not produced. Slight hemolysis.

Glucose serum broth: Turbid. Gas produced.

Peptone water with serum: Gas. Indole produced.
Milk: Growth feeble. Neither acid nor coagulated.

Proteins not attacked.

Glucose and fructose attacked slightly by two out of three strains.

Does not plasmolyse readily.

Neutral red broth: Changed to yellowish green.

Nitrites not produced from nitrates. Optimum pH 6.5 to 8.0 .

Optimum temperature $37^{\circ} \mathrm{C}$.

Pathogenic.

Strict anaerobe.

Distinctive character: Fermentation of glucose and gas production from peptones.

Source: Isolated (Schottmüller) from cases of puerperal fever. Three strains from infected tonsils studied by Prévot.

Habitat: Natural cavities, especially the tonsils and female genital organs.

19. Micrococcus asaccharolyticus (Distaso) comb. nov. (Staphylococcus asaccharolyticus Distaso, Cent. f. Bakt.. I Abt., Orig., 62, 1912, 445.) From Greek, not dissolving sugar.

Deseription according to Prévot, Ann. Sci. Nat., Sér. Bot., 15, 1933, 211.

Large spheres: 1.0 to 1.2 microns, occurring in very large clusters, also in pairs and short chains. Gram-positive.

Gelatin: At $37^{\circ} \mathrm{C}$, growth resembles tufts of cotton which precipitate. No liquefaction.

Deep agar colonies: Very delicate, pin-point, transparent. A few bubbles of gas produced.

Broth: Turbid. Growth settles at the bottom of the tube as a sort of viscous zooglea. Unpleasant odor produced.

Peptone water: Turbid. Indole prnduced.

Milk: Feebly acidified, but not coagulated.

Egg white not attacked.

Carbohydrates not attacked.

Strict anaerobe.

* Anaerobic section revised by Dr. Ivan C. Hall, New York, N. Y. 
Distinctive characters: Large size; unpleasant odor; production of indole; production of gas.

Souree: Isolated from the large intestine of a man with intestinal intoxication.

Habitat: Intestine. Tot common.

Note: Weinberg, Nativelle and Prévot (Les Microbes Anaérobies, 1937, 1023) regard Micrococcus indolicus Christiansen (Ac. Pat. Micr. Seand., 18, 1934, 12) as a variety of this species giving it the name staphylococcus asaccharolyticus var. indolicus. This variety differs from the species by forming opaque lens-shaped colonies and by a more abundant production of gas from peptone.

20. Micrococcus niger Hall. (Jour. Bact., 20, 1930, 409.) From Latin, niger, black.

Small spheres : 0.6 micron in diameter, occurring in irregular masses, occasionally in pairs. Gram-positive.

Gelatin: After 5 days a dark sediment is produeed which gradually gets more and more intensely black. No liquefaction.

Deep agar colonies: Slow growth. It first very tiny, colorless, irregularly globular, smooth, dense. Small bubbles of gas sometimes produced. After several days colonies become brown, then black. If exposed to air, colonies facte to a dull gray. Medium not discolored.

Blood agar slant: After 4 or 5 clays, minute, black colonies, round, smooth, glistening, $0.5 \mathrm{~mm}$. in diameter. Nonhemolytie.

Broth: After $t$ or 5 days uniform turbidity and slight production of gas which contains $\mathrm{H}_{2} \mathrm{~S}$. Black sediment.

Coagulated serum: Minutr, brown colonies appor on the 8th day. No liquefaction.

Milk: No change.

Brain medium : Turbid after 4 or 5 days at $37^{\circ} \mathrm{C}$. Yniform gas production about the 6th day. Discoloration of the medium not marked.
No acid from carbohydrates. Black sediment produced.

Non-pathogenic for guinea-pigs and rabbits.

Optimum temperature $37^{\circ} \mathrm{C}$. No growth below $30^{\circ} \mathrm{C}$.

Strict anaerobe.

Distinctive characters: Formation of a water-insoluble, black pigment. Growth slow, visible after 2 to 4 days.

Source: Isolated from urine of an aged woman.

Hahitat: Unknown.

21. Micrococcus grigoroffi Prévot. (Wicrococcus A, Grigoroff, Thèse de Cieneve, 1905; Prévot, Ann. Sci. Nat., Sér. Bot. et Zcool., 15, 1933, 219.) Named for Grigoroff, who first isolated this organism.

Small spheres: Average size 0.7 micron, oceurring singly or in irregular masses. Gram-positive.

Gelatin: Colonies appear in four days. No liquefaction.

Deep agar colonies: After three days, round, lenticular, yellowish.

Glucose broth: Turbid after 2 days with whitish sediment. Neither gas nor fetid odor produced. The medium is acidified.

Milk: Crood growth. Acid. Coagulation.

Acid from glucose, maltose, lactose, fructose and sorbitol.

One strain slightly pathogenic.

Optimum temperature $37^{\circ} \mathrm{C}$.

ritrict anacrobe.

Distinctive characters: This is the only anaerobic coccus growing in irregular masses that coagulates milk. Lactose is fermented.

Source: Five strains isolated from the uppendix by Grigoroff. One strain isolated from an appendix by Prévot.

Habitat: Human digestive tract. Not common.

22. Micrococcus anaerobius (Hamm) comb. nov. (Anaerobic staphylococcus, Jungano, Cumpt. rend. Soe. Biol. Paris, 59, 1907, 707; Staphylococcus anaerobius 
Hamm, Die puerperale Wundinfektion, Berlin, 1912; not Staphylococcus anaerobius Heurlin, Bakt. Unters. d. Keimgehaltes im Genitalkanale d. fiebernden Wöchnerinnen, Helsingfors, 1910, 120.) From Greek, living without air.

Description according to Prévot, Ann. Sci. Nat., Sér. Bot., 15, 1933, 209.

Small spheres: 0.5 to 0.6 micron, occurring in masses. Gram-positive.

Gelatin: No liquefaction.

Deep agar colonies: Lenticular, thick. No gas produced.

Broth: Turbid, later clearing. Sediment.

Glucose broth: Good growth. Neither acid nor gas produced.

Peptone water: No turbidity. No gas. Indole not produced.

Milk: Neither coagulated nor acidified.

Coagulated serum not attacked.

Egg white not attacked.
Carbohydrates not attacked by the strains of Jungano. Acid feebly produced from glucose and galactose by Prévot's strain.

Does not plasmolyse.

Temperature relations: Optimum $36^{\circ}$ to $38^{\circ} \mathrm{C}$. At $22^{\circ} \mathrm{C}$ growth slow, poor. No growth below $22^{\circ} \mathrm{C}$. Killed in ten minutes at $80^{\circ} \mathrm{C}$ or in half an hour at $60^{\circ} \mathrm{C}$.

Optimum pH 6.0 to 8.0 .

Pathogenic for guinea-pigs and rabbits.

Strict anaerobe.

Distinctive characters: Neutral red broth remains unchanged. No gas produced.

Source: First isolated by Jungano from a case of cystitis. Found by Prévot in the pus from a suppurated tonsil.

Habitat: Urinary tract, urethra, intestine, buecal eavity and conjunctiva.

Appendix I*: The following genus is organized on a physiological basis. Because of this no attempt is made to fit it into the classification outline. A single species has been described.

Genus A. Methanococcus Kluyver and van Niel.

(Cent. f. Bakt., II Abt., 94, 1936, 400.)

Spherical cells, occurring singly or in masses. Motility not observed. No endospores formed. Gram-variable. Chemo-heterotrophic, anaerobic, fermenting various organic compounds with the formation of methane. Saprophytes.

The type species is Methanococcus mazei Barker.

1. Methanococcus mazei Barker. (Pseudosareina, Mazé, Compt. rend. Soc. Biol., Paris, 78, 1915, 398; Barker, Arch.f. Mikrobiol., 7, 1936, 430.) Named for Mazé, the French bacteriologist who first gave a clearly recognizable description of this type of methane organism.

Small spherical cells, occurring singly, in large, irregular masses, or in regular cysts of various sizes and forms. Tonmotile. Stains readily with erythrosine. Gram-variable.

Grows on calcium acetate enrichment media and ferments the acetate vigorously.
Grows slowly on agar containing 2 per cent clcar mud extract.

Ferments acetic and butyric acids with production of methane in the presence of $\mathrm{CO}_{2}$. Ethyl and butyl alcohols not attacked.

Does not utilize organic nitrogen.

Obligate anaerobe.

Grows best at $30^{\circ}$ to $37^{\circ} \mathrm{C}$.

Sources: Garden soil, black mud containing $\mathrm{H}_{2} \mathrm{~s}$, feces of herbivorous animals.

Habitat: One of the most active methane-producing organisms found in nature.

* Appendixes I and II prepared by Prof. Robert S. Breed, New York State Experiment Station. (Geneva, New Tork, December. 1943. 
Appendix II: The following genus is recognized by workers in the brewing industry. It includes species that present characters intermediate between. Hicrococcus, Sarcina and Streptococcus. Many students prefer to regard these as species of Micrococcus (Hucker, X. Y. State Exper. Sta., Tech. Bul. 102, 1924, 5), of Sarcina (Macé, Traité pratique d. Bact., th ed., 1901, 460) or of Streptococcus (Shimwell, Sect. 670 in Hind, Brewing Science and Practice. Tew York, 19t0). Others (Mees, Thesis, Delft, 1934) would include in the genus, the species described as Tetracoccus by Orla-Jensen (The Iactic Acid Bacteria, Copenhagen, 1919, 76).

\section{Genus B. Pediococcus Balctie.}

(Wchnschr. f. Bratuerei, 1, 1884, 257.)

Cocci occurring singly, in pairs and tetrads. Non-motile. No endospores. Grampositive. Facultative anaerobes under favorable conditions, especially in acid media. Nitrites not produced from nitrates. Produce acidification and more or less clouding of wort and beer. Saprophytes.

The type species is Pedincoccus cerevisiae Balcke.

\section{Pediococcus cerevisiae Balcke.} (Ferment No. 7, Pasteur, Etudes sur la bière, Paris, 1876. t; Sarcinu Balcke, Wehnschr. f. Brauerei, 1, 185t, 183; ibid., 1, 18st, 257; Merismopedia cerevisiae Dyar, Ann. X. Y. Acad. Sei., 8, 1895. 348; Micrococcus cerevisiae Migula, Syst. d. Bakt., 2, 1900, 77; Sarcina cerevisiat Macé, Traité Pratique d. Bact., th ed.. 1901, 460.) From Latin, cerevisia, heer.

Spheres: 1 to 1.3 microns, occurring singly, in pairs or tetrads. In acid media the latter prevail. Catalase negative. Non-motile. Gram-positive.

No growth in alkaline media,

Peptone, meat-extract gelatin: Whit becoming yellowish to yellowish brown. No liquefaction.

Wort gelatin with Ca-carbonate: White colonies, 2 to $3 \mathrm{~mm}$; carbonate dissolved.

Meat extract gelatin stab: Growth along stab, white raised surface growth. No liquefaction.

Litmus milk: No growth.

Potato: Scanty growth.

Acid from glucose, fructose, maltose, sucrose.

Wort and beer: Slight to moderately turbid growth, strong development on bottom of the flask. Hop sensitive, but may develop in heavily hopped beers under special conditions.
Does not utilize urea.

Nitrites not produced from nitrates. Facultative anaerobic.

Killed at $60^{\circ} \mathrm{C}$. in 8 minutes. Optimum temperature: $25^{\circ} \mathrm{C}$.

Source: Sarcina-sick beer.

Habitat: Wort, beer and beer yeast.

Additional species have been described from spoiled wort and beer which vary but slightly from the species first named and described by Balcke. These are listed below together with other species that have been placed in the genus.

Pediococcus acidilactici Lindner. (Lindner, Wchnschr. f. Brauerei, 3, No. 23, 1887, see Cent. f. Bakt., 2, 1887, 342; also see Die Sarcina-Organismen der Gährungsgewerbe, Lindner, Inaug. Diss., Berlin, 1888, 26, and Cent. f. Bakt., 4, 1885, 429; Micrococcus pseudocerevisiae Migula, Syst. d. Bakt., 2, 1900, 77; . Iicrucoccus acidi-lactici Chester, Man. Determ. Bact., 1901, 88.) From spoiled mash.

Pediococcus albus Lindner. (Die Sarcina-Organismen der Gährungsgewerbe, Lindner, Inaug. Diss., Berlin, 1887, 39; see Cent.f. Bakt., 4, 1888, 429; Wicrococcus pseudosarcina Migula, Syst. d. Bakt., 2, 1900, 92; Micrococcus albus Chester, Man. Determ. Bact., 1901, 97.) From spoiled beer.

Pediococcus damnosus Claussen. 
(Compt. rend. Trav. Labor. de Carlsberg, $6,1906,68$; Streptococcus damnosus Shimwell and Kirkpatrick, Jour. Inst. Brewing, 45, 1939, 137.) From clear, spoiled beer.

Pediococcus halophilus Mees. (Tetracoccus No. 1, Orla-Jensen, The Lactic Acid Bacteria, 1919, 77; Mees, Thesis, Delft, 1934, 94.) From anchovy pickle.

Pediococcus hennebergi Sollied. (Ztschr. Spiritusindus., 26, 1903, 491.) From spoiled beer.

Pediococcus kochii Trevisan. (Mikrokokkus in Wundsecreten bei Menschen, Kioch; Trevisan, I generi e le specie delle Batteriacee, Milan, 1889, 28.)

Pediococcus pentosaceus Mees. (Tetracoccus No. 2, Orla-Jensen, The Lactic Acid Bacteria, 1919, 78; Mees, Thesis, Delft, 1934, 94.) From yeast.

Pediococcus perniciosus Claussen (loc. cit.). From clouded, spoiled beer.

Pediococcus sarcinaeformis Reichard. (Ztschr. f. d. ges. Brauwesen, 17, 1894, 257.) From spoiled beer.

Pediococcus urinae equi Mees. (Pferdeurinsarcina, von Huth, Alg. Ztg. f. Bierber. u. Malzfabr., 185, 968 and 981, 1885; ibid., 1886, 141; Mees, Thesis, Delft, 1934, 95.) From horse urine.

Pediococcus violaceus (Kützing) Trevisan. Merismopedia violacea Kü̈tzing; Trevisan, I generi e le specic delle Batteriacee, Milan, 1889, 28.)

Pedioplana haeckeli Wolff. (Cent. f. Bakt., II Abt., 18, 1907, 9.) Motile. From rotting beets. Placed in a new genus Pedioplana Wolff (loc. cit., 9).

Streptococcus damnosus var. mucosus Shimwell. (Shimwell, Sect. 670, Hind, Brewing Science and Practice, New York, 1940.) From ropy beer.

Appendix III*. The following species have been found in the literature and are listed here chiefly for their historical interest. Many are incompletely described, while many others are identical with previously described species. See Monographs by Winslow and Winslow, Systematic Relationships of the Coccaceae, 1908 and Hucker, N. Y. State Exper. Sta., Tech. Buls. Nos. 99-103. References are to Tech. Bul. 102.

Ascococcus cantabridgensis Hankin. (Quoted from Lehmann and Neumann, Bakt. Diag., 2 Aufl., 2, 1899, 165.) Migula (Syst. d. Bakt., 2, 1900, 195) reports he is unable to find further reference to this organism and we likewise are unable to trace it. From the human mouth.

Ascococcus gangrenosus Bevan. (Med. News, No. 1003, 1892, 375; Abst. in Cent. f. Bakt., 13, 1893, 796.) From a gangrenous foot.

Ascococcus vibrans van Tieghem. (Bul. Soc. Bot. France, 27, 1880, 150.) From water.

Aurococcus tropicus Chalmers and O'Farrell. (1913, quoted from Castellani and Chalmers, Man. Trop. Med., 3rd ed., 1919, 931.) Found in Ceylon in granulating ulcers of skin.

Coccus carduus Heurlin. (Bakt. Unters. d. Keimgehaltes im Genitalkanale d. fiebernden Wöchnerinnen. Helsingfors, 1910, 136.) Anaerobic. From genital canal.

Coccus caudatus Heurlin (loc. cit., 84). From genital canal.

Coccus vaginalis Heurlin (loc. cit., 79). From genital canal.

Galactococcus albus Guillebeau. (Landwirtsch. Jahrb. d. Schweiz, 4, 1892, 27; Abst. in Cent. f. Bakt., 12, 1892, 101.) From milk from an inflamed udder.

Galactococcus fulvus Guillebeau (loc. cit.). From milk from an inflamed udder.

Galactococcus versicolor Guillebeau (loc. cit.). From milk from an inflamed udder.

Gyrococcus flaccidifex Glaser and Chapman. (Science, 36, 1912, 219.) Isolated from the gypsy moth, Porthetria dispar. Jodococcus vaginatus Miller. (Miller,

* Prepared for Prof. G. J. Hucker by Mrs. Eleanore Heist Clise, New York State Experiment Station, Geneva, New York, March, 1943. 
Mikroorganismen der Mundhöhle, 1889, 54; Bacterium iogenum Baumgartner, Ergebnisse d. ges. Zahnheilk., Heft 2, 1910, 729; Abst. in Cent. f. Bakt., I Abt., Ref., 48, 1911, 621.) From the oral cavity.

Merismopedia aurantiaca Maggiora. (Giorn. Soc. Ital. d'Igiene, 11, 1889, 354; Abst. in Cent. f. Balt., 8, 1890, 13.) From the normal skin of the human foot.

Micrococcus achrous Migula. (No. 16, Lembke, Arch. f. Hyg., 26, 1896, 310; Migula, Syst. 1. Bakt., 2, 1900, 201.) From feces. Winslow and Winslow (Systematic Relationships of the Coccaceae, $1908,224)$ state that this species is apparently a synonym of Micrococcus candicans Flügge.

Micrococcus acidi lactici Marpmann. (Ergänzungsheft d. Cent. f. allg. Gesundheitspflege, 2, 1886, 22.) Found in fresh milk.

Hicrococcus acidovorux Müller-Thurgau and Osterwalder. (Cent. f. Bakt., II Abt., 36, 1913, 236.) From wine. Hucker (loc, cit., 6) considers this a synonym of Micrococcus luteus Cohn or Micrococcus varians Migula.

Micrococcus acne Holland, (Jour. Bact., 5, 1920, 223; Staphylococcus acne Holland, ibid., 225; see . Hicrococeus cutis communis Sibouraud.

Wicrococcus (Staphylococcus) acridicida Kufferath. (Ann. de Ciembloux, 27, 1921, 253.) Isolated from diseased locusts from Greece. Resembles Micrococcus aureus Zopf.

Micrococcus aerius Chester. (No, 4t), Conn, Storrs Agr. Exp. Sta. 7th Ann. Rept., 1895, 81; Chester, Man. Determ. Bact., 1901, 104.) From rlust. Hucker (loc. cit., 12) states that this species appears to be identical with Micrococcus aureus Zopf.

Micrococcus aerogenes Miller. Miller, Deutsche med. Wehnschr., 12, 18\$6, 119; not Micrococcus aerogenes Bergey ot al., Manual, 1st ed., 1923, 70.) From the alimentary canal.
Micrococcus agilis albus Catterina. (Cent. f. Bakt., I Abt., Orig., 34, 1903, 108.) Found in septicemia of rabbits. Motile with one or two flagella.

Micrococcus albatus Kern. (Arb. bakt. Inst. Karlsruhe, 1, Heft 4, 1897, 479.) From the intestine of a woodpecker (Picus major). Winslow and Winslow (Systematic Relationships of the Coccacere, 1908, 199) state that this species appears to be a synonym of Micrococcus albus Schroeter; while Hucker (N. Y. Agr. Exper. Sta., Tech. Bull. 102, 19) regards it as a synonym of Micrococcus frendenreichii Guillebeau or .Micrococcus ureae Cohn.

Micrococcus albescens Henrici. (Arb. bakt. Inst. Karlsruhe, 1, Heft 1, 1894, 76.) From cheese. Winslow and Winslow (loc. cit., 199) state that this species appears to be a synonym of Micrococcus albus Schroeter; while Hucker (loc. cit., 19) regards it as a synonym of Micrococcus freudenrcichii (ruillebeau or of $\mathrm{Mi}$ crococcus ureae Cohn.

Wicrococcus albidus Losski. (Losski, Inaug. Diss., Dorpat, 1893, 55̃; not Micrococcus albidus Henrici, see Microcuccus subniveus below; not Hicrococcus albidus Roze. Compt, rend. Acad. Sci. Paris, 122, 1896, 750.) From soil. Hucker (loc. cit., 19) regards this species as a synonym of . Micrococcus freudenreichii Guillebeau or Micrococens ureae (ohnt

Microcuccus albocereus Migula. (Staphylucoccus cereus albus Passet, Intersuch. i. d. Aetiol. d. eiterigen Phlegmone d. Menschen, Berlin, 1885, 53, and Fortschr. d. Med., 3, 1885; Micrococcus cereus albus Flügge, Die Mikroorganismen, 2 Iufl., 1886, 182; Staphylococcus cereus Trevisan, I generi e le specie delle Batteriacee, Milan, 1889, 32; Migula, Syst. d. Bakt., 2, 1900, 56; Staphylococcus cereus-albus Holland, Jour. Bact., 5, 1920, 225.) From human pus, also from water. Winslow and Winslow (Systematic Relationships of the Coccaceae, 1908, 205) consider this a synonym of . Micrococcus 
candidus Cohn or of Gaffkya tetragena Trevisan.

Micrococcus albus Frankland and Frankland. (Phil. Trans. Roy. Soe, London, 178, B, 1888, 264; not Micrococcus albus Matzuschita, Cent. f. Bakt., I Abt., 29, 1901, 382; not Micrococcus albus Buchanan, Veterinary Bacteriology, 1911, 196; not Micrococcus albus Macé, Traité Pratique de Bact., 6th éd., 1912, 605.) From air. Resembles Micrococcus candicans.

Micrococcus albus II Maggiora. (Cent. f. Bakt., 8, 1890, 13.) See Micrococcus opalescens De Toni and Trevisan. From the skin of the human foot.

Micrococcus amplus Migula. (Grauweisser Diplococcus, Bumm, Der Mikroorg. d. gonorrh. Schleimhauterkrank., 1 Aufl., 1885, 17; Micrococcus albicans amplus Flügge, Dic Mikroorganismen, 2 Aufl., 1886, 183; Neisseria albicans Trevisan, I generi e le specie delle Batteriacee, Milan, 1889, 32; Diplococcus albicans amplus Eisenberg, Bakt. Diag., 3 Auf., 1891, 24; Migula, Syst. d. Bakt., 2,1900,118; Micrococcus albicans Chester, Man. Determ. Bact., 1901, 80.) From vaginal secretions. Hucker (loc. cit., 15) considers this species identical with Micrococcus albus Schroeter.

Micrococcus ampullaceus Kiern. (Arb. bakt. Inst. Karlsruhe, 1 , Heft 4, 1897, 477.) From the intestine of a dove Columba oenas). Hucker (loc. cit., 19) considers this a synonym of Micrococcus freudenreichii Guillebeau or Micrococcus ureae Cohn.

Micrococcus annulatus Fern (loc. cit., 490). From the stomach contents of the hedge sparrow (Passer montanus) and the intestine of the rock dove Columba livia). Winslow and Winslow (Syste. matic Relationships of the Coccaceae, 1908,216 ) consider this species a synonym of Micrococcus flavus Lehmann and Neumann.

Micrococcus aquatilis Bolton. (Zsehr. f. Hyg., 1, 1886, 94; not Micrococcus aquatilis Chester, see below.) From water. Winslow and Winslow (loc. cit., 224 ) state that this species is apparently a synonym of Micrococcus candicans Flügge.

Micrococcus aquatilis Chester. (Micrococcus aquatilis invisibilis Vaughan, Amer. Jour. Med. Sci., 104, 1892, 183; Chester, Man. Determ. Bact., 1901, 88.) From water. Winslow and Winslow (loc. cit., 224) state that this species is apparently a synonym of Micrococcus candicans Flügge.

Micrococcus aquatilis albus. (Quoted from Toporoff, Protok. d. Kaiserl. kaukasisch Mediz. Gesellsch., 1892, No. 21; Abst. in Cent. f. Bakt., 18, 1893, 487.) From water.

Micrococcus aquatilis flavus. (Quoted from Toporoff, loc. cit.) From water.

Micrococcus aqueus Migula. (No. 25, Lembke, Arch. f. Hyg., 26, 1896, 317 ; Migula, Syst. d. Bakt., 2, 1900, 204.) From feces. Winslow and Winslow (loc. cit., 184) state that this species is apparently a synonym of Micrococcus aureus Zopf, while Hucker (loc. cit., 15) regards this as a synonym of Micrococcus albus sichroeter.

Micrococcus arborescens lactis Conn. (Conn, Storrs Agr. Exp. Sta. 12th Ann. Rept., 1900, 46; Micrococcus lactis arborescens Conn, Esten and Stocking, Storrs Agr. Exp. Sta. 18th Ann. Rept., 1907, 110.) From milk. Hucker (loc. cit., 21) regards this as a synonym of Micrococcus candidus Cohn or Micrococcus epidermidis Hucker.

Micrococcus argenteus Migula. (No. 27, Lembke, Arch. f. Hyg., 26, 1896, 317 ; Migula, Syst. 1. Bakt., 2, 1900, 206.) From feces. Winslow and Winslow (loc. cit., 184) state that this species is apparently a synonym of Micrococcus aureus Zopf, while Hucker (loc. cit., 10) considers it a synonym of Micrococcus conglomeratus Migula.

Micrococcus ascoformans Johne. (Zoogloea pulmonis equi Bollinger, Arch. f. path. Anat., 49, 1870, 583; Discomyces equi Rivolta, Giorn. di Anat. e Fisiolog., 
10, 1884; Johne, Ber. u. d. Veterin. im Königr. Sachsen, Jahr 1885, 47; Ascococcus johnei Cohn in letter to Johne, Deutsche Ztschr. f. Thiermed., 12, 1886, 210; Micrococcus botryogenus Rabe, Deut. Ztschr. f. Thiermed., 12, 1886, 137 ; Botryomyces equi Bollinger, Deut. Ztschr. f. Thiermed., 13, 1887, 176; Botryococcus ascoformans Kitt, Cent. f. Bakt., 3, 1888, 247; Bollingera equi Trevisan, I generi e le specie delle Batteriacee, Milan, 1889, 26; Staphylococcus ascoformans Ford, Textb. of Bact., 1927, 424; Staphylococcus aureus var. equi Hauduroy et al., Dict. d. Bact. Path., 1937, 504.) Causes botryomycosis in horses. Lehmann and Neumann (Bakt. Diag., 7 Aufl., 2, 1927, 291) consider this a form of Micrococcus aureus Zopf; while Hucker (loc. cit., 15) regards this as a form of Micrococcus albus Schroeter.

Micrococcus ascoformis Fermi. (Areh. f. Hyg., 10, 1890, 10.) Presumably intended for Micrococcus ascoformans Johne.

Micrococcus asper Migula. (Seibert, Inaug. Diss., Würzburg, 1894, 12; Migula, Syst. d. Bakt., 2, 1900, 82.) From a hairbrush. Winslow and Winslow (loc. cit., 205) consider this species to be a synonym of Micrococcus candidus Cohn or of Gaffkya tetragena Trevisan.

Micrococcus aurantiacus-sorghi Bruyning. (Arch. Néer. Sci. Exact. et Nat., 1, 1898, 297; Streptococcus aurantiacus Chester, Man. Determ. Bact., 1901, 69.) From sorghum.

Micrococcus aureus Chester. (Micrococcus cremoides aureus Dyar, Ann. N. Y. Acad. Sci., 8, 1895, 349; Chester, Manual Determ. Bact., 1901, 99.) From dust. Regarded by Dyar as a varietal form of Micrococcus cremoides Zimmermann. Winslow and Winslow (loc. cit., 184) consider this species a synonym of $\mathrm{M} i$ crococcus aureus Zopf.

Micrococcus aureus lactis Conn. (Storrs Agr. Exp. Sta. 12th Ann. Rept., 1900, 36.) From milk. This seems to be identical with Micrococcus lactis aureus $A$, Conn, Fsten and Stocking, Storrs
Agr. Exper. Sta. 18th Ann. Rept., 1907, 119. Hucker (loc. cit., 9) regards this species as identical in part with Micrococcus flavus Lehmann and Neumann and with Micrococcus conglomeratus Migula.

Micrococcus (Sarcina) baccatus Migula. (No. 18, Lembke, Arch. f. Hyg., 26, 1896, 311; Migula, Syst. d. Bakt., 2, 1900, 202.) From feces. Winslow and Winslow (loc. cit., 232) state that this is a yellow, gelatin-liquefying sareina, apparently a synonym of Sarcina flava De Bary. Hucker (loc. cit., 10) considers this a synonym of Micrococcus conglomeratus Migula.

Micrococcus badius Lehmann and Neumann. (Bakt. Diag., 1 Aufl., 2, 1896, 163.) Received from the Kral collection as Sarcina lutea. Winslow and Winslow (loc. cit., 216) consider this a synonym of Micrococcus flavus Lehmann and Neumann.

Micrococcus baregensis purpureus Robine and Hauduroy. (Compt. rend. Soc. Biol., Paris, 98, 1928, 25.) From hot sulfur springs at Barèges.

Micrococcus beigelii (Rabenhorst) Migula. (Pleurococcus beigelii Küchenmeister and Rabenhorst, Hedwigia, 1867, No. 4; Sclerotium beigelianum Hallier, 1868; Zoogloea beigeliana Eberth, 1873; Hyalococcus beigelii Schroeter, Kryptog.Flora v. Schlesien, 3, 1, 1886, 152; Chlamydatomus beigelii Trevisan, Rendiconti Reale Inst. Lombardo de Sci. e Lett., Ser. II, 12, 1879, 22; Migula, Syst. d. Bakt., 2, 1900, 193; Trichosporum beigelii Vuillemin, 1901.) From human hair.

Micrococcus beri-beri Pekelharing. (Pekelharing, Weekblad v. h. Ned. Tijdschr. v. Geneesk., No. 25; also Pekelharing and Winkler, Deut. med. Wchnschr., No. 39, 1887, 845; Neisseria winkleri Trevisan, I generi e le specie delle Batteriacee, Milan, 1889, 32.) Considered the cause of beri-beri by Pekelharing. Winslow and Winslow (loc. cit., 184) state that this is apparently a synonym of Micrococcus aureus Zopf; while Hucker (loc. cit., 11) considers 
this a synonym of Micrococcus citreus Migula.

Micrococcus bicolor Kern. (Arb. bakt. Inst. Karlsruhe, 1, Heft 4, 1897, 485.) From the intestine of a dove (Columba oenas). Hucker (loc. cit., 21) considers this a synonym of Micrococcus candidus Cohn or of Micrococcus epidermidis Hucker.

Micrococcus billrothii (Cohn) Migula. (Ascococcus billrothii Cohn, Beitr. z. Biol. d. Pflanzen, 1, Heft 3, 1875, 151; Migula, Syst. d. Bakt., 2, 1900, 195.) Found in putrefying meat infusion.

Micrococcus biskra Heydenreich. (Heydenreich, Ausgabe d. Haupt Med.Verhalt., St. Petersburg, 1888; see Cent. f. Bakt., 5, 1889, 163; Staphylococcus biskrae Trevisan, I generi e le specie delle Batteriacee, Milan, 1889, 32; Micrococcus heydenreichii Chester, Man. Determ. Bact., 1901, 91.) Found in ulcers in an Oriental shin disease. Winslow and Winslow (loc. cit., 184) state that this is apparently a synonym of Micrococcus aureus Zopf; while Hucker (loc. cit., 11) considers it a synonym of Micrococcus citreus Migula.

Micrococcus boleti Passerini. (Erbar. crittogam. Italiano, II ser., No. 1199; quoted from Trevisan, I generi e le specic delle Batteriacee, Milan, 1889, 34.) Saprophytic on a fungus (Boletus edulis).

II icrococcus bombycis (Naegeli) Cohn. (Panhistophyton ovatum Lebert, Jahresher. ï. d. Wirksamkeit d. Vereins z. Beförd. d. Seidenbaues f. Brandenburg im Jahre 1856-57, 28; and Berliner Fntomolog. Ztschr., 1858; Nosema bombycis Naegeli, Botan. Seet. d. 33 Versammly. d. Naturf. u. Aerzte in Bonn, 1857, 160; and Botan. Zeitg., 1857, 760; Hicrazyma bombycis Béchamp, Compt. rend. Acad. Sci., Paris. 64, 1867, 1045; Cohn, Beitr. z. Biol. d. Pflanzen, 1, Heft 2, 1872, 165; Micrococcus ovatus Winter, in Rabenhorst, Frypt. Flora v. Deutschl., Oesterr. u. d. Schweiz, 2 Aufl., 1, 1884, 47 ; Streptococcus bombycis Zopf, Die Spalt- pilze, 2 Aufl., 1884, 52.) Found in the blood and organs of diseased silkworms (Bombyx mori).

Micrococcus boreus Issatchenko. (Recherches sur les Microbes de l'Oceán Glacial Arctique, Petrograd, 1914, 144.) From sea water.

Micrococcus bovinus Migula. (Micrococcus der Lungenseuche der Rinder, Poels and Nolen, Fortschr. d. Med., 1886, 217; Migula, Syst. d. Bakt., 2, $1900,67$.$) From the lungs of diseased$ cattle. Hucker (loc. cit., 22) regards this a synonym of Micrococcus candidus Cohn or of Micrococcus epidermidis Hucker.

Micrococcus bovis Migula. (Hematococcus, Babes, Compt. rend. Acad. Sei., Paris, 107, 1888, 692 and $110,1890,800$ and 975 ; also see Arch. f. path. Anat., 115, 1889; Neisseria babesi Trevisan, I generi e le specie delle Batteriacee, Milan, 1889, 32; Haematococcus bovis Eisenberg, Bakt. Diag., 3 Aufl., 1891, 271 ; Migula, Syst. d. Bakt., 2, 1900, 85.) From the blood and organs of cattle.

Micrococcus burchardti Trevisan. (Coccus bei keratitis phlyctaenulosa, Burchardt, Cent. f. Bakt., 1, 1887, 392; Trevisan, I generi e le specie delle Batteriacee, Milan, 1889, 33.) Pathogenic. From the cornea of a rabbit.

Micrococcus butyri (v. Klecki) Migula. (Diplococcus butyri von Klecki, Cent. f. Bakt., 16, 1894, 358; Migula, Syst.d. Bakt., 2, 1900, 216.) From rancid butter. Winslow and Winslow (loc. cit., 220) consider this a synonym of Micrococcus luteus Cohn.

Micrococcus bulyrifuorescens Teichert. (Inaug. Diss., Jena, 1904; Abst. in Cent. f. Bakt., II Abt., 13, 1904, 561.) From milk. Exhibits at green fluorescence.

Micrococcus butyricus (von Klecki) Migula. (Tetracoccus butyricus von Klecki, Cent. f. Bakt., 15, 1894, 360; Migula, Syst. d. Bakt., 2, 1900, 216.) From rancid butter. Winslow and Winslow (loc. cit., 220) consider this a synonym of Micrococcus luteus Cohn. 
Micrococcus calco-aceticus Beijerinck. (Proc. Sect. Sci., Kon. Akad. v. Wetenschappen, 13, 1911, 1066; Abst. in Cent. f. Bakt., II Abt., 31, 1912, 290.) Occurs commonly in soils.

Micrococcus campeneus Orme. (Jour. Trop. Med. and Hyg., 11, 1908, No. 10, May 15 ; Abst. in Cent. f. Bakt., I Abt., Ref., 43, 1909, 299.)

Micrococcus candicans Flügge. (Di€ Mikroorganismen, 2 Aufl., 1886, 173; Albococcus candicans Winslow and Rogers, Jour. Inf. Dis., 3, 1906, 544; Staphylococcus candicans Holland, Jour. Bact., 5, 1920, 225.) From air, water and milk. Hucker (loc. cit., 22) regards this a synonym of Micrococeus candidus Cohn or of Micrococcus epidermidis Hucker. For a description of this species, see Bergey et al., Manual, 5th ed., $1939,255$.

Micrococcus canescens Migula. (Micrococcus No. 4, Adametz, Landwirtsch. Jahrb., 18, 1889, 240 ; Migula, Syst. d. Bakt., 2, 1900, 51; Albococcus canescens Winslow and Rogers, Jour. Inf. Dis., 3, 1906, 544; Staphylococcus canescens Holland, Jour. Bact., 5, 1920, 225.) From Emmenthal cheese. Winslow and Winslow (loc. cit., 224) state that this is apparently a synonym of Micrococcus candicans Flügge.

Micrococcus capillorum (Buhl) Trevisan. (Zoogloea capillorum Buhl, Ztschr. f. ration. Med., II Reihe, 14, 18-, 356; Palmella capillorum Kühn, Abhandl. d. Naturf. Ges. zu Halle, 9, Heft 1, 18-, 62; Palmellina capillorum Rabenhorst, Flor. Eur. Alg., 3, 1856 (?), 35; Trevisan, I generi e le specie delle Batteriacee, Milan, 1889, 33.) From the skin. Considered pathogenic.

Micrococcus capsaformans Jamieson and Edington. (Brit. Med. Jour., 1, June 11, 1887, 1262; Micrococcus capriformis (sic), Abst. in Cent. f. Bakt., 2, 1887, 223.) From the scales and blood of scarlet fever patients. Not pathogenic.

Micrococcus carbo Renault. (Compt. rend. Acad. Sci., Paris, 123, 1896, 935.)
Micrococcus carneus Zimmermann. (Roter Coccus, Maschek. Bakt. Untersuch d. Leitmeritz. Trinkwassers, No. 5, 1887, 60; Zimmermann, Die Bakt. unserer Trink- u. Nutzwässer, Chemnitz, I Reihe, 1890, 78.) From water. Hucker (loc. cit., 25 and 26) regards this species as identical with Micrococcus roseus Flügge or .Hicrococcus cinnabareus Flügge.

Micrococcus carnicolor Frankland and Frankland. (Phil. Trans. Roy. Soc. London, 178, B, 1888, 263; not Micrococcus carnicolor Kern, see Micrococcus subcarneus below.) From air. Hucker (loc. cit., 25) states that this species may be identical with Micrococcus roseus Flügge.

Micrococcus carniphilus Wilhelmy. (Arb. bakt. Inst. Karlsruhe, 3, 1903, 10.) From a meat extract.

Micrococcus casei amari edamicus Raamot. (Inaug. Diss., Königsberg, 1906; Abst. in Cent. f. Bakt., II Abt., 18, 1907, 348.) From pasteurized skim milk:

Micrococcus castellanii Chalmers and O'Farrell. (Ann. Trop. Med. and Parasitol., 7, 1913, 528; Rhodococcus castellanii Castellani and Chalmers, Man. Trop. Med., 3rd ed., 1919, 2102.) Found in the red variety of trichomycosis axillaris, a tropical disease.

Micrococcus cartharinensis Issatchenko. (Recherches sur les Microbes de l'Oceán Glacial Artique, Petrograd, 1914, 148.) From sea water.

Micrococcus cellaris (Schroeter) Migula. (Leucocystis cellaris Schroeter, Kiryptog. Flora v. Schlesien, 3, 1886, 152; Migula, Syst. d. Bakt., 2, 1900, 195.) From a coating on the walls of damp cellars and mines.

Micrococcus centropunctatus Issat. chenko. (Recherches sur les Microbes de l'Oceán Glacial Aretique, Petrograd, 1914, 146.) From sea water.

Micrococcus cerasinus Migula. (Micrococcus aus roter Milch, Keferstein; Cent. f. Bakt., I Abt., 21, 1897, 177; Micrococcus cerasinus lactis Heim, Lehrb. 
d. Bakt., 2 Aufl., 1898, 299; Migula, Syst. d. Bakt., 2, 1900, 170; not Micrococcus cerasinus Lehmann and Neumann, Bakt. Diag., 1 Aufl., 2, 1896, 179; Micrococcus kefersteinii Chester, Man. Determ. Bact., 1901, 107.) From red milk. Hucker (loc. cit., 26) regards this species as identical with Micrococcus cinnabareus Flügge.

Micrococcus cereus Migula. (Staphylococcus cereus flavus Passet, Untersuchungen über die Aetiologie der eiterigen Phlegmone des Menschen, 1885, 53; Micrococcus cereus flavus Flügge, Die Mikroorganismen, 2 Aufl., 1886, 182; Staphylococcus passeti Trevisan, I generi e le specie delle Batteriacee, Milan, 1889, 32; Migula, Syst. d. Bakt., 2, 1900, 126; Staphylococcus cereus-flavus Holland, Jour. Bact., 5, 1920, 225.) From pus. Winslow and Winslow (loc cit., 220) consider this species identical with Micrococcus luteus Migula. For a description of this organism, see Bergey et al., Manual, 5th ed., 1939, 241.

Micrococcus cereus aureus Dyar. (Ann. N. Y. Acad. Sci., 8, 1895, 347. Obtained as Staphylococcus cereus aureus from Kral's laboratory; also found in air.

Micrococcus cerinus Henrici. (Arb. bakt. Inst. Karlsruhe, 1, Heft 1, 1894 , 84.) From Swiss cheese. Winslow and Winslow (loc. cit., 216) consider this a synonym of Micrococcus flavus Trevisan.

Micrococcus chersonesia Corbet. (Quart. Jour. Rubber Research Inst., Malaya, 2, 1930, 150.) From the latex of the rubber tree (Hevea brasiliensis). For a description of this species, set Bergey et al., Manual, 5th ed., 1939, 258.

Micrococcus chinicus Enmerling and Abderhalden. (Cent. f. Bakt., II Abt, 10, 1902, 337.) Putrefying meat.

Micrococcus chlorinus Cohn. (Grüngelber Coccus, Maschek, Jahresber. d. Kom.- Oberrealschule zu Leitmeritz, 1887, 66; Cohn, Beitr. z. Biol. d. Pflanzen, 1, Heft 2, 1872, 155.) From water and dust. Hucker (loc. cit., 10) considers this a synonym of Micrococcus conglomeratus Migula.

Micrococcus chromidrogenus citreus Trommsdorff. (Münch. Med. Wochnschr., 1904, No. 29, 1286 ; Abst. in Cent. f. Bakt., I Abt., Ref., 37, 1905, 60.) Isolated from a case of chromidrosis of the axilla.

Micrococcus chromidrogenus ruber Trommsdorff (loc. cit.). Isolated from a case of chromidrosis of the axilla.

Micrococcus chromoflavus Huss. (Cent. f. Bakt., II Abt., 19, 1907, 520.) From cheese

Micrococcus chryseus Frankland and Frankland. (Phil. Trans. Roy. Soc. London, 178, B, 1888, 268.) From dust. Winslow and Winslow (loc. cit., 184) state that this species is apparently a synonym of Micrococeus aureus Zopf.

Micrococcus cinnabarinus Zimmermann. (Die Bakt. unserer Trink- u. Nutzwässer, Chemnitz, I Reihe, 1890, 76.) From water. Hucker (loc. cit, , 26) regards this species as identical with Micrococcus cinnabareus Flügge.

Micrococcus cirrhiformis Migula. (Ranken Coccus, Maschek, Jahresber. d. Kom.- Oberrealschule in Leitmeritz, 1857, 66; Migula, Syst. d. Bakt., 2, 1900, 53.) From water. Hucker (loc. cit., 22) considers this a synonym of Micrococcus candidus Cohn or of Micrococcus epidermidis Hucker.

Micrococcus citreus $I$ and II, Maggiora. (Giorn. Soc. Ital. d'Igiene, 11, 1889, 354; Abst. in Cent. f. Bakt., 8, 1890, 13; not Micrococcus citreus Eisenberg, Bakt. Diag., 3 Aufl., 1891, 36; not Micrococcus citreus Migula, Syst. d. Bakt., 2, 1900, 147 ; not Micrococcus citreus Winslow and Winslow, Systematic Relationships of the Coccaceae, 1908, 218.) From the normal skin of the foot.

Micrococcus citreus granulatus Freund. (Inaug. Diss., Erlangen, 1893, 27; Abst. in Cent. f. Bakt., 16, 1894, 641; Micrococcus granulatus Bazarewski, Cent. f. Bakt., II Abt., 15, 1905, 7; not Micrococcus granulatus Weiss, Arb. bakt. Inst. 
Karlsruhe, 2, Heft 3, 1902, 197.) From the oral cavity. Hucker (loc. cit., 9) regards this as a synonym of Micrococcus flavus Trevisan.

Micrococcus citreus lactis Conn. (Storrs Agr. Exp. Sta. 12th Ann. Rept., $1900,40$. ) From milk. Hucker (loc. cit., 10) considers this a synonym of Micrococcus conglomeratus Migula.

Micrococcus citreus rigensis Bazarewski. (Cent. f. Bakt., II Abt., 15, 1905, 5.) From dust.

Micrococcus citrinus Migula. (Diplococcus citreus liquefaciens Unna and Tommasoli, Monats. f. prakt. Dermatologie, 9, 1889, 56; Migula, Syst. d. Bakt., 2, 1900, 150; Micrococcus tommasoli Chester, Man. Determ. Bact., 1901, $101 ;$ Micrococcus citreus liquefaciens Winslow and Winslow, Systematic Relationships of the Coccaceae, 1908, 216.) From human skin in a case of eczema. Winslow and Winslow (loc. cit., 216) consider this a synonym of Micrococcus flavus Trevisall.

Micrococcus claviformis von Besser. (Beitr. z. path. Anat. u. z. allgem. Path., $6,1889,340$; see Cent. f. Bakt., 7,1890 , 152.) Found nnce in nasal secretions.

Micrococcus coccineus Migula. (Micrococcus No. VI, Adametz, Landwirtsch. Jahrb., 18, 1889, 242; Migula, Syst.d. Bakt., 2, 1900, 174.) From Emmenthal cheese. Hucker (loc. cit., 26) regards this species identical with Micrococcus cinnabareus Flügge.

Micrococcus coli brevis Lehmann. (Lehmann, Inciug. Diss., München, 1903; Abst. in Cent. f. Bakt., I Abt., Ref., 36, 1905, 688.) From feces of infants.

Micrococcus communis lactis Conn. (Storrs Agr. Exp. Sta. 12th Ann. Rept, 1900 , 48.) From milk. Hucker (loc. cit., 19) considers this a synonym of Micrococcus freudenreichii. Guillebeau or of Micrococcus ureae Cohn.

Micrococcus commensalis (Turró) Migula. (Diplococcus commensalis Turró, Cent. f. Bakt., 16, 1894, 1; Migula, Syst. d. Bakt., 2, 1900, 125.) From sputum. Winslow and Winslow (loc. cit., 220) consider this a synonym of Micrococcus luteus Cohn.

Micrococcus commutatus De Toni and Trevisan. (Micrococcus albus $\mathrm{I}$ or $\mathrm{Mi}$ crococcus albus fluidificans Maggiora, Giorn. Soc. Ital. d'Igiene, 11, 1889, 350; De Toni and Trevisan, in Saccardo, Sylloge Fungorum, 8, 1889, 1079.)

Micrococcus concentricus Zimmermann. (Die Bakt. unserer Trink- u. Nutzwässer, Chemnitz, I Reihe, 1890, 86.) From water. Winslow and Winslow (loc. cit., 224) state that this is apparently a synonym of Micrococcus candicans Flügge.

Micrococcus confluens Kern. (Arb. bakt. Inst. Farlsruhe, 1, Heft 4, 1897, 494.) From the stomach and intestine of the starling (Sturnus vulgaris) and the finch (Fringella carduelis). Winslow and Winslow (loc. cit., 216) consider this a synonym of Micrococcus flavus Trevisan.

Micrococcus conjunctivae Migula. (Micrococcus liquefaciens conjunctivae Gombert, Recherches expérimentales sur les microbes des conjunctives, Montpellier and Paris, 1889; Migula, Syst.d. Bakt., 2, 1900, 115.) From normal human conjunctiva. Hucker (loc. cit., 15) considers this a synonym of Micrococcus albus Schroeter.

Micrococcus conjunctividis Migula. (Micrococcus flavus conjunctivae Gomhert, Recherches expérimentales sur les microbes des conjunctives, Montpellier and Paris, 1889; Migula, Syst.d. Bakt., 2, 1900, 141.) From normal human conjunctiva. Winslow and Winslow (loc. cit., 216) consider this a synonym of Micrococcus flavus Trevisan; while Hucker (loc. cit., 11) regards it as a synonym of Micrococcus citreus Migula.

Micrococcus conoideus Migula. (Staphylococcus salivarius pyogenes Biondi, Ztschr. f. Hyg., 2, 1887, 227 ; Staphylococcus sialopyus Trevisan, I generi e le specie delle Batteriacee, Milan, 1889, 
32; Micrococcus salivarius pyogenes Freire, Mémoire sur la bactériologie, pathogénie, traitement et prophylaxie de la fievre jaune. Rio de Janiero, 1898; Abst. in Cent. f. Bakt., I Abt., 26, 1899, 741 ; Staphylococcus pyogenes salivarius, quoted from Goadby, Trans. of Odontolog. Society, June, 1899, see Abst. in Cent.f. Bakt., I Abt., Ref., 31, 1902, 493; Migula, Syst. d. Bakt., 2, 1900, 102.) From saliva. Hucker (loc. cit,, 12 and 15) regards this as a synonym of Micrococcus aureus Zopf or of Iicrococcus albus Schroeter.

Micrococcus corallinus Cantani. (Cantani, Cent. f. Bakt., I Abt., 23, 1898, 311 ; Rhodococcus coralinus (sic) Levine and Soppeland, Iowa State Coll. Engineering Exp. Sta. Bul. 77, 1926, 22.) From dust. Hucker (loc. cit., 25) considers this a synonym of Micrococcus roseus Flügge. Levine and Soppeland (loc. cit.) regard this as a synonym of Rhodococcus fulvus Winslow and Rogers. For a description of this species, see Bergey et al., Manual, 5th ed., 1939, 253.

Micrococcus coralloides Zimmermann. (Die Bakt. unserer Trink-u. Nutzwässer, Chemnitz, II Reihe, 1894, 72.) From water. Winslow and Winslow (loc. cit., 199) state that this species appears to be a synonym of Micrococcus albus Zopf; while Hucker (loc. cit., 17) considers it a synonym of Micrococcus caseolyticus Evans.

Micrococcus corrugatus Migula. (Merismopedia mesentericus corrugatus Dyar, Ann. N. Y. Acad. Sci., 8, 1895, 355 ; Migula, Syst. d. Bakt., 2, 1900, 161.) From dust. Winslow and Winslow (loc. cit., 216) consider this a synonym of Micrococcus flavus Trevisan.

Micrococcus coryzae (Hajek) Migula. (Diplococcus coryzae Hajek, Berliner klin. Wochnschr., No. 33, 1888; Migula, Syst. d. Bakt., 2, 1900, 63.) From secretions in acute catarrh. Winslow and Winslow (loc. cit., 205) consider this a synonym of Micrococcus candidus Cohn or of Gaffkya tetragena Trevisan.
Micrococcus cremoides Zimmermann. (Die Bakt. unserer Trink-u. Nutzwässer, Chemnitz, I Reihe, 1890, 74.) From water. Winslow and Winslow (loc. cit., 216) consider this a synonym of Micrococcus flavus Trevisan; while Hucker (loc. cit., 10) considers it a synonym of Micrococcus conglomeratus Migula.

Micrococcus cremoides albus Dyar. (Ann. N. I. Acad. Sci., 8, 1895, 350). From dust. Regarded by Dyar as a white form of Micrococcus cremoides Zimmermann.

Micrococcus cremorisviscosi (Hammer and Cordes) Bergey et al. (Staphylococcus cremoris-viscosi Hammer and Cordes, Jour. Dairy Sci., 3, 1920, 291; Bergey et al., Manual, 3rd ed., 1930, 86.) From ropy milk. For a description of this species, see Bergey et al., Manual, 5th ed., 1939, 254.

Micrococcus crepusculum (Ehrenberg) Cohn. (Monas crepusculum Ehrenberg, Abhandl. d. Berliner Akad., 1830, 74 and 1832, 57 ; Cohn, Beitr. z. Biol. d. Pflanzen, 1 , Heft $2,1872,160$.) De Toni and Trevisan (in Saccardo, Sylloge Fungorum, 8, 1889,1082 ) list the following as synonyms of this species; Protococcus nebulosus Kützing, Linneae, 8, 1833, 365; Cryptococcus nebulosus Kützing, Phycol. gener., 1845, 147; Cryptococcus natans Kützing, Spec. Alg., 1849, 146.

Micrococcus cretaceus Henrici. (Arb. bakt. Inst. Karlsruhe, 1, Heft 1, 1894 , 65.) From cheese. Winslow and Winslow (loc. cit., 224) state that this is apparently a synonym of Micrococcus candicans Flügge.

Micrococcus cristatus Glage. (Ztschr. f. Fleisch- u. Milch-hygiene, 10, 1900, 145.) From the surface of wurst and similar meat products.

Micrococcus cruciformis Freire. (Compt. rend. Acad. Sci., Paris, 128, 1899, 1047.) From the stamens and pistils of Hibiscus.

Micrococcus cumulatus Kern. (Arb. bakt. Inst. Karlsruhe, 1, Heft 4, 1897, 497; not Micrococcus cumulatus Chester, 
see Micrococcus tenuissimus Migula.) From the stomach and intestine of the yellow-hammer (Emberiza citrinella) and of the finch (Fringella carduelis). Hucker (loc. cit, 25) regards this as a synonym of Micrococcus roseus Flügge.

Micrococcus cupularis Migula. (No. 29, Lembke, Arch. f. Hyg., 29, 1897, 331; Migula, Syst. d. Bakt., 2, 1900, 211.) From feces. Winslow and Winslow (loc. cit., 216) consider this a synonym of Micrococcus flavus Trevisan.

Micrococcus cupuliformis Migula. (No. 19, Lembke, Arch. f. Hyg., 29, 1897, 325; Migula, Syst. d. Bakt., 2, 1900, 213.) From feces. Winslow and Winslow (loc. cit., 220) consider this a synonym of Micrococcus luteus Cohn.

Micrococcus curtissi Chorine. (Chorine, Internat. Corn Borer Invest. Chicago, 2, 1929, 48.) From diseased larvae of the corn borer (Pyrausta nubilalis). Also virulent to larvae of the flour moth (Ephestia kühniella) and of the bee moth (Galleria mellonella).

Micrococcus cutis communis Sabouraud. (Sabouraud, Ann. d. dermatol. et syphil., 1896, Heft 3; Abst. in Cent. f. Bakt., I Abt., 20, 1896, 249; Staphylococcus cutis communis Sabouraud, Practique Dermatologique, 1, 1903, 714.) From human skin especially in alopecia areata, certain types of eczema and acne. May be the same as Micrococcus epidermidis Hucker.

Micrococcus cyaneus (Schroeter) Cohn. (Bacteridium cyaneum Schroeter, Beitr. z. Biol. d. Pflanzen, 1, Heft 2, 1872, 122 and 126; Cohn, ibid., 156; Nigrococcus cyaneus Castellani and Chalmers, Man. Trop. Med., 3rd ed., 1919, 932.) From dust and water.

Micrococcus cyanogenus Pammel and Combs. (Proc. Iowa Acad. Sci., 3, 1895, 136 ; see Abst. in Cent. f. Bakt., II Abt., 2, 1896, 764.) From milk.

Micrococcus cyclops Henrici. (Arb. bakt. Inst. Karlsruhe, 1, Heft 1, 1894, 69.) From Swiss cheese. Winslow and Winslow (loc. cit., 224) state that this is apparently a synonym of Micrococcus candicans Flügge.

Micrococcus cystiopoeus Müller-Thurgau. (Cent.f. Bakt., II Abt., 20, 1908, 464.) From wine.

Micrococcus cytophagus Merker. (Cent.f. Bakt., II Abt., 31, 1912, 589.) Found on the leaves of Elodea. Utilizes cellulose. Stanier (Bact. Rev., 6, 1942, 150) thinks that these micrococci were microcysts of Sporocytophaga spp.

Micrococcus dantecii Chester. (Coccus du rouge de morue, Le Dantec, Ann. Past. Inst., 5, 1891, 662; Chester, Man. Determ. Bact., 1901, 106.) From red salted codfish. Hucker (loc. cit., 25) considers this a synonym of Micrococeus roseus Flügge.

Micrococcus decalvens (Thin) Schroeter. (Bacterium decalvens Thin, Monats. f. prakt. Dermatol., No. 28, 1885; Schroeter in Cohn, Kryptog.-Flora v. Schlesien, 3, 1, 1886, 149.) From hair follicles in alopecia areata.

Micrococcus decipiens Trevisan. (Bactérie de l'air, Cornil and Babes, Les Bactéries, 1885, 124; Trevisan, I generi e le specie delle Batteriacee, Milan, 1889 , 34.) From dust.

Micrococcus (Streptococcus ?) decolor Migula. (No. 22, Lembke, Arch. f. Hyg., 26, 1896, 314; Migula, Syst. d. Bakt., 2, 1900, 203.) From feces. Hucker (loc. cit., 17) considers this a synonym of Micrococcus caseolyticus Evans.

Micrococcus deformans Crowe. (Brit. Med. Jour., Nov. 27, 1920, 815; Abst. in Cent. f. Bakt., I Abt., Ref., 73, 1922, 8t.) From cases of arthritis. A form of Micrococcus pyogenes albus according to Lehmann and Neumann (Bakt. Diag., 7 Auf., 2, 1927, 293).

Micrococcus delacourianus Roze. (Compt. rend. Acad. Sci., Paris, 123, 1896, 613 and 1323.) From dry rotting potatoes.

Micrococcus dendroporthos Ludwig. (Cent.f. Bakt., 10, 1891, 10.) From the bark of poplar trees (Populus sp.). 
Micrococcus denitrificans Beijerinck. (Cent. f. Bakt., II Abt., 25, 1910, 53.) From Rochelle salts (sodium potassium tartrate).

Micrococcus dermatogenes Fuhrmann. (Cent. f. Bakt., II Abt., 1\%, 1906, 618.) From bottled beer.

Micrococcus difluens schroeter. (In Cohn, Kryptog.-Flora v. Schlesien, 3, 1, 1886, 144.) From dust, feces, etc.

Micrococcus dimorphus Bucherer. (Planta, Arch. f. wissen. Bot., 1934, 98.) A dimorphic bacterium. He reports it as much like Micrococcus melitensis Bruce and Bacterium fraenkelii Hashimoto.

Micrococcus diphtericus (sie) Cohn. (Micrococcus, Ocrtel, Deutsch. Arch. f. klin. Med., 8, 1871; Cohn, Beitr. z. Biol. d. Pflanzen, 1, Heft 2, 1872, 162; Streptococcus diphtheriticus Zopf, Die Spaltpilze, 3 Aufl., 1885, 53.) From throats and nasal passages of diphtheria patients.

Micrococcus dissimilis Dyar. (Sec Sattler, Cent. f. Bakt., 5, 1889, 70; Dyar, Ann. N. Y. Acad. Sci., 8, 1895, 353; Micrococcus trachomatis conjunctivae Sattler in Kral, Die gegenwartigen Bestand der Kral'schen Sammlung von Mikroorganismen, 1900, 19.) From trachoma infections. Hucker (loc. cit., 17 ) considers this a synonym of Micrococcus caseolyticus Evans.

Micrococcus djokjakartensis Zettnow. (Cent. f. Bakt., I Abt., Orig., 75, 1915. 376.) From a sugar factory in Java.

Micrococcus doyeni. De Toni and Trevisan. (Micrococcus urinae albus olearius Doyen, Jour. d. connaiss. médie., No. 14, 1889, 108; De Toni and Trevisan, in Saccardo, Sylloge Fungorum, 8, 1889, 1076.) From urine. Hucker (loc. cit., 16) states that this species is apparently identical with Micrococcus albus Schroeter.

Micrococcus drimophylus Baumgartner. (Baumgartner, Ergebnisse d. ges. Zahnheilk., Heft 2, 1910, 729 ; Abst. in Cent. f. Bakt., I Abt., Ref., 48, 1911, 622.) From the mouth eavity
Micrococcus eatonii Corbet. (Quart. Jour. Rubber Research Inst. Malaya, 2, 1930, 145.) From the latex of the rubber tree (Hevea brasiliensis). For a description of this species, see Bergey et al., Manual, 5th ed., 1939, 244.

. Iicrococcus eburneus Henrici. (Arb. bakt. Inst. Karlsruhe, 1, Heft 1, 1894, 85.) From Camembert cheese. Winslow and Winslow (loc. cit., 224) state that this species is apparently a synonym of Micrococcus candicans Flügge.

Micrococcus ephestiae Mattes. (Sitzungsber. d. Gesellsch. z. Beförd. d. gesamt. Naturwissensch. zu Marburg, 62, 1927, 406.) From the Mediterranean Hour moth (Ephestia kuehniella).

Microcuccus epimetheus Corbet (loc. cit., 148). From the latex of the rubber tree (Hevea brasiliensis). For a description of this species, see Bergey et al., Manual, 5th ed., 1939, 256.

Micrococcus esterificans Beck. (Arb. kaiserl. Gesundheitsamte, 29, Heft 2, 1905; Abst. in Cent. f. Bakt., II Abt., 19. 1907, 594.) Has a characteristic fruity aroma. From butter.

Micrococcus exanthematicus Lewascheff. (Deutsch. med. Wochnschr., No. 13 and 34, 1892; Abst. in Cent. f. Bakt., 12, 1892, 635.) From blood in cases of typhus fever. Motile. Grows anaerobically.

Micrococens excavatus Kern. (Arb. bakt. Inst. Karlsruhe, 1, Heft 4, 1897 , 486.) From the stomach contents of a root (Fulica atra) and a woodpecker (Picus major). Winslow and Winslow (loc. cit., 220) consider this a synonym of Micrococcus luteus Cohn.

Mirrococcus exiguus Kern (loc. cit., $470)$. From the stomach contents of the chaffinch (Fringella coelebs). Winslow and Winslow (loc. cit., 199) state that this appears to be a synonym of Micrococcus albus schroeter; while Hucker (loc. cit., 19) considers it a synonym of Micrococess freudenreichii Guillebeau or of Micrococcus ureae Cohn.

Vicrococeus expositionis ('hester. No. 
34, Conn, Storrs Agr. Exp. Sta. 7th Ann. Rept., 1895, 77; Chester, Man. Determ. Bact., 1901, 92.) From air. Winslow and Winslow (loc.cit. 216) consider this a synonym of Hicrococcus flavus Trevisan; while Hucker (loc. cit., 10) regards it as a synonym of Micrococcus conglomeratus Migula.

Micrococcus expressus Weiss. (Arb. bakt. Inst. Karlsruhe, 2, Heft 3, 1902, 195.) From a carrot infusion. Produces slime. Hucker (loc. cit., 7) considers this species a synonym of Micrococcus luteus Cohn or of Micrococcus varians Migula.

Micrococcus faviformis Migula. (Milchweisser Diplococcus, Bumm, Mikroorg. d. gonorrh. Schleimbautkr., II Ausg., 1887, 18; Micrococcus lacteus faviformis Flügge, Die Mikroorganismen, 2 Aufl., 1886, 182; Neisseria lactea Trevisan, I generi e le specie delle Bat teriacee, Milan, 1889, 32; Migula, Syst. d. Bakt., 2, 1900, 117.) From vaginal and other body secretions. Winslow and Winslow (loc. cit., 199) state that this appears to be a synonym of Micrococcus albus Schroeter.

Micrococcus feddei Herter. (Micrococcus XVI, Choukevitch, Ann. Inst. Past., 25, 1911, 354; Botan. Jahresber., 39, II Abt., Heft 4, 1914, 755; Abst. in Cent. f. Bakt., II Abt., 51, 1920, 367.) From the large intestine of a horse. Resembles Micrococcus roscidur Matzuschita.

Micrococcus fervitosus Adametz and Wichmann (Bakt. d. Trink- u. Nutzwässer, Mitt. Oest. Versuchstat. f. Brauerei u. Mälzerei, Wien, Heft 1, 1888.) From water. Winslow and Winslow (loc. cit., 205) consider this a synonym of Gaffkya tetragena Trevisan.

Micrococcus fickii Trevisan. (Coccus albus non liquefaciens (Coccus candicans) Fick, Ueber Microorg. in Conjunctivalsack, Wiesbaden, 1887 ; Trevisan, I generi e le specie delle Batteriacee, Milan, 1889, 33.) From conjunctiva.

Micrococcus finlayensis Sternberg. (Rept. on Etiolngy and Prevention of
Yellow Fever, Washington, 1891, 219.) Obtained by Finlay in cultures from the liver and spleen of a yellow-fever cadaver. Hucker (loc. cit., 11) considers this a synonym of Micrococcus citreus Migula.

Micrococcus flaccidifex danai Brown. (Amer. Museum Novit., No. 251, 1927, 5.) Causative agent of wilt disease of monarch butterfly larvae (Danais archippus). Considered a sub-species of Gyrococeus flaccidifex Glaser and Chapman (Science, 36, 1912, 219).

Micrococcus flagellatus Klotz. (Jour. Med. Research, 11 (N.S.6), 1904, 493.) Found in an epizootic among rabbits and white rats. Supposedly flagellated.

Micrococcus flavens Henrici. (Arb. bakt. Inst. Karlsruhe, 1, Heft 1, 1894, 80.) From Swiss cheese. Winslow and Winslow (loc. cit., 216) consider this a synonym of Micrococcus flavus Trevisan.

Micrococcus flavescens Henrici. (Arb. bakt. Inst. Karlsruhe, 1, Heft 1, 1894, 79.) From Swiss cheese. Winslow and Winslow (loc. cit., 216) consider this a synonym of Micrococcus flavus Trevisan. For a description of this species, see Bergey et al., Manual, 5th ed., 1939, 246.

Micrococcus flanidus Henrici. (loc. cit., 81; not Micrococcus flavidus Roze, Compt, rend. Acad. Sci., Paris, 122, 1896, 750.) From Swiss and Limburger cheeses. Winslow and Winslow (loc. cit., 216) consider this a synonym of Micrococcus flavus Trevisan.

Micrococcus flavovirens Migula. (Staphylococcus viridis flavescens Guttmann, Arch. f. path. Anat., 107, 1887, 261; Staphylococcus viridi-flavescens Trevisan, I generi e le specie delle Batteriacee, Milan, 1889, 33; Migula, Syst. 1. Bakt., 2, 1900, 124; Micrococcus viridis Chester, Man. Determ. Bact., 1901, 95; Micrococcus viridis-flavescens Winslow and Winslow, Systematic Relationships of the Coceaceae, 1906, 221.) Winslow and Winslow (ibid., 220) consider this a synonym of Wicrococcus luteus Cohn.

Micrococcus flavus non liquefaciens 
Amsler. (Amsler, Korrespondenbl. f. Schweizer Aerzte, 1900, No. 9; Abst. in Cent. f. Bakt., I Abt., 29, 1901, 450.) From thermal springs.

Micrococcus fluorescens Maggiora. (Giorn. Soc. Ital. d'Igiene, 11, 1889, 352; Abst. in Cent. f. Bakt., 8, 1890, 13.) From the skin of the foot.

Micrococcus foetidus Flügge. (Die Mikroorganismen, 2 Aufi., 1886, 172; not Micrococcus foetidus Veillon, Compt. rend. Soc. Biol. Paris, 1893, 867; see Streptococcus foetidus Prévot.) From carious teeth.

Micrococcus foetidus Klamann. (Allgem. med. Centralzeitung, 1887, 1344.) Isolated from the posterior nares of man. Winslow and Winslow (loc. cit., 199) state that this appears to be a synonym of Micrococcus albus Schroeter.

Micrococcus fragilis (Dyar) Migula. (Merismoperlia fragilis Dyar, Ann. N. Y. Acad. Sci., 8, 1895, 351 ; Migula, Syst. d. Bakt., 2, 1900, 186.) From dust. Hucker (loc. cit., 25) states that this species may be identical with Micrococcus roseus Flügge.

Micrococcus franklandiorum Trevisan. (Micrococcus candicans Frankland and Frankland, Phil. Trans. Roy. Soc. London, 178, B, 1888, 270; not Hicrococcus candicans Flügge, Die Mikroorganismen, 2 Aufl., 1886, 173; Trevisan, I generi c le specie delle Batteriacee, Milan, 1889 , 34.) From dust.

Micrococcus fulvus Weiss. (Arb. a. bakt. Inst. Karlsruhe, 2, Heft 3, 1902, 206; not Micrococcus fulvus Cohn, Beitr. z. biol. d. Pflanz., 1, Heft 3, 1875, 181.) From a bean infusion.

Micrococcus fuscus Adametz. (Brauner Coccus, Maschek, Jahresb. d. KommunalOberrealsch. zu Leitmeritz, No. 6, 1867, 60 ; Adametz, Bakt. d. Nutz-u. Trinkwässer, Vienna, 1888; Micrococcus fuscus Castellani and Chalmers, Man. Trop. Med., 3rd ed., 1919, 932.) Hucker (loc. cit., 10) states that this species is probably identical with Micrococcus conglomeratus Migula.
Micrococcus galbanatus Zimmermann. (Bakt. unserer Trink- u. Nutzwässer, Chemnitz, II Reihe, 1894, 68.) From water. Winslow and Winslow (loc. cit., 216) consider this a synonym of Micrococcus flavus Trevisan.

Micrococcus gallicidus Burrill. (Amer. Nat., 17, 1883, 320.) From blood of fowls infected with chicken cholera.

Micrococcus gelatinogenus Bräutigam. (Pharmaceutische Centralhalle, 32, 1891, 427.) From digitalis infusions. See Micrococcus gummosus Happ.

Micrococcus gelatinosus Warrington. (The Lancet, 1, 1888, No. 25; Abst. in Cent. f. Bakt., 4, 1888, 394.) Curdles mill:.

Micrococcus gelatinosus Issatchenko. (Recherches sur les Microbes de l'Océan Glacial Arctique, Petrograd, 1914, 232; not Micrococcus gelatinosus Warrington, The Lancet, 1, 1885, No. 25.) From sea water.

Micrococcus giganteus lactis Conu. (Storrs Agr. Exp. Sta. 12th Ann. Rept., 1900, 46.) From milk.

Wicrococcus gigas Frankland and Frankland. (Philos. Trans. Roy. Sor., Iondon, 178, B, 1888, 268.) From dust. Winslow and Winslow (loc, cit, , 216) consider this a synonym of Micrococcus flaus Trevisan.

Micrococcus gilvus Losski. (Inaug. Diss., Dorpat, 1893, 60.) Winslow and Winslow (loc. cit., 220) consider this a synonym of Micrococcus luteus Cohn.

Micrococcus gingivae Migula. (Micrococcus gingivae pyogenes Miller, Die Mikroorganismen d. Mundhöhle, Leipzig, 1889, 216; Migula, Syst. d. Bakt., 2, 1900, 68.) From alveolar pyorrhoea, also from the mouth of a healthy man.

Micrococcus gingreardi Renault. (Compt. rend. Acad. Sci., Paris, 120, 1895, 217.)

Ificrococcus glandulosus Weiss. (Arb. bakt. Inst. Karlsruhe, $2, \operatorname{Heft} 3,1902,201$.) From an asparagus infusion. Hucker 
(loc. cit., 19) regards this specie as identical with Micrococcus freudenreichii Guillebeau or with Micrococcus ureae Cohn.

Micrococcus globosus Kern.

(Arb. bakt. Inst. Karlsruhe, 1, Heft 4, 1897, 469.) From the stomach contents of a coot (Fulica atra). Winslow and Winslow (loc. cit., 224) state that this is apparently a synonym of Micrococcus candicans Flügge.

Micrococcus granulatus Weiss. (Arb. bakt. Inst. Karlsruhe, 2, Heft 3, 1902, 197.) From a malt infusion.

Micrococcus granulosus Kern (loc. cit., 483). From the stomach contents of the yellow-hammer (Emberiza citrinella) and the starling (Sturnus vulgaris). Winslow and Winslow (loc. cit., 220) consider this a synonym of Micrococcus luteus Cohn.

Micrococcus griseus (Warming) Winter. (Bacterium griseum Warming, Videnskabelige Meddelelser fra den naturhist. Forening i Irjöbenhavn, 1875, 398; Winter, in Rabenhorst, Kryptog.-Flora v. Deutschl., Oesterr. u. d. Schweiz, 2 Aufl., 1, 1884, 47.)

Micrococcus grossus Henrici. (Arb. bakt. Inst. Karlsruhe, 1, Heft 1, 1894, 71.) From Camembert cheese. Winslow and Winslow (loc. cit., 224) state that this is apparently a synonym of Micrococcus candicans Flügge.

Micrococcus gummosus Happ. (Happ, Inaug. Diss., Basel, 1893, 31; not Micrococcus gummosus Weiss, Arb. bakt. Inst. Karlsruhe, 2, Heft 3, 1902, 189.) From snakeroot and digitalis infusions. Presumably Leuconostoc mesenteroides Van Tieghem.

Micrococcus haematodes Zopf. (Microbes de la sueur rouge, Babes, Biol. Centralbl., 2, 1882, No. 8; Zopf, Die Spaltpilze, 3 Aufl., 1885, 60.) The cause of red perspiration. Hucker (loc. cit., 25 ) states that this may be a synonym of Micrococcus roseus Flügge.

Micrococcus haemorrhagicus (Klein) Migula. (Staphylococcus haemorrhagicus
Klein, Cent. f. Bakt., I Abt., 22, 1897, 81; Migula, Syst. d. Bakt., 2, 1900, 88.) Associated with an erythema of the skin resembling anthrax. Winslow and Winslow (loc. cit., 199) state that this appears to be a synonym of Micrococcus albus Schroeter.

Micrococcus halensis Lehmann and Neumann. (Micrococcus acidi paralactici liquefaciens halensis Kozai, Ztschr. f. Hyg., 31, 1899, 374; Lehmann and Neumann, Bakt. Diag., 2 Aufl., 2, 1899 , 210 ; Micrococcus acidi paralactici liquefaciens Thiele, Ztschr. f. Hyg., 46, 1904, 394.) From milk. Hucker (loc. cit., 17) considers this a synonym of Micrococcus caseolyticus Evans.

Micrococcus halophilus Bergey et al. (Culture No. 19, Baranik-Pikowsky, Cent. f. Bakt., II Abt., \%0, 1927, 373; Bergey et al., Manual, 3rd ed., 1930, 89.) From sea water. For a description of this species, see Bergey et al., Manual, 5 th ed., 1939, 25 s.

Micrococcus hauseri (Rosenthal) Migula. (Diplococcus hauseri Rosenthal, Inaug. Diss., Berlin, 1893, 26; Migula, loc. cit., 80.) From the oral cavity. Winslow and Winslow (loc. cit., 224) state that this species is apparently identical with Micrococcus candicans Flügge.

Micrococcus helvolus Henrici. (Arb. bakt. Inst. Karlsruhe, 1, Heft 1, 1894 , 77.) From Swiss cheese. Winslow and Winslow (loc. cit., 220) consider this to be identical with Micrococcus luteus Cohn.

Micrococcus hibiscus Nakahama. (Jour. Agr. Chem. Soc. Japan, 16, 1940, 345, Eng. Abs., Bull. Agr. Chem. Soc., 16, $1940,51$.$) Isolated from retting kenaf$ (Hibiscus).

Micrococcus humidus Migula. (Micrococcus No. 2, Adametz, Landwirtsch. Jahrb., 18, 1889, 239; Migula, Syst. d. Bakt., 2, 1900, 50.) From Emmenthal cheese. Winslow and Winslow (loc. cit., 224) state that this species is apparently identical with Micrococcus candicans Flügge. 
Micrococcus hydrothermicus Cronquist. (Monatsh. f. prakt. Derm., 36, 1903.) Optimum temperature $41^{\circ} \mathrm{C}$.

Micrococcus hymenophagus Renault. (Compt. rend. Acad. Sei., Paris, 120, 1895, 217.)

Micrococcus imperatoris Roze. (Compt. rend. Acad. Sci., Paris, 122, 1896, 545.) Isolated from potatoes. Probably from dust.

Micrococcus inconspicuus Henrici. (Arb. bakt. Inst. Karlsruhe, 1, Heft 1, 1894, 64.) From Swiss cheese. Winslow and Winslow (loc. cit., 224) state that this species is apparently identical with Micrococcus candicans Flügge.

Micrococcus indolicus Christiansen. (Ac. Path. Micr. Scand., 18, 1934, 42; Staphylococcus asaccharolyticus var. indolicus Weinberg, Nativelle and Prévot, Les Microbes Anaérobies, 1937, 1023.) Strict anaerobe. For description see Bergey et al., Manual, 5th ed., 1939, 266.

Micrococcus influenzae Migula. (Mikroorganismus II, Fischel, Ztschr. f. Heilkunde, 12, 1891; Abst. in Cent. f. Bakt., 9, 1891, 611; Migula, Syst. d. Bakt., 2, 1900, 90.) From the blood of an influenza patient. Winslow and Winslow (loc. cit., 199) state that this appears to be identical with Micrococcus albus Schroeter.

Micrococcus insectorum Burrill. (Burrill, Amer. Nat., 17, 1883, 319; Streptococcus insectorum De Toni and Trevisan, in Saceardo. Sylloge Fungorum, 8, 1889, 1061; Bacillus insectorum Smith, 28th Biennial Rept. Kan. State Bd. Agri., 1933, 54.) From the cecal organs of the chinch bug (Blissus leucopterus).

Micrococcus intermedius Stark and Scheib. (Jour. Dairy Sci., 19, 1936, 210.) From butter.

Micrococcus intertriginis Meyer. (N.Y. Med. Jour., $\gamma 0,1900,873$; Abst. in Cent. f. Bakt., I Abt., 30, 1901, 434.) From a case of erythema intertrigo.

Micrococcus iris Henrici. (Arb. bakt. Inst. Karlsruhe, 1, Heft 1, 1894, 67.) From Limburger cheese. Winslow and
Winslow (loc. cit., 224) state that this species is apparently identical with Micrococcus candicans Flügge.

Micrococcus irregularis Weiss. (Arb. bakt. Inst. Karlsruhe, 2, Heft 3, 1902, 184.) From an infusion of beans and carrots.

Micrococcus jongii Chester. (Staphylococcus pyogenes bovis de Jong, Cent. f. Bakt., I Abt., 25, 1899, 64; Staphylococcus bovis de Jong, ibid., 67; Chester, Man. Determ. Bact., 1901, 95.) Associated with suppurative processes in cattle. Winslow and Winslow (loc. cit., 220) consider this a synonym of Micrococcus luteus Cohn.

Micrococcus lactericeus Freund. (Inaug. Diss., Erlangen, 1893, 21; Abst. in Cent.f. Bakt., 16, 1894, 640.) From the human mouth. Hucker (loc. cit., 21) regards this as a synonym of Micrococcus candidus Cohn or of Micrococcus epidermidis Hucker.

Micrococcus lacteus Henrici. (Arb. bakt. Inst. Karlsruhe, 1, Heft 1, 1894, 74.) From cheese. Winslow and Winslow (loc, cit., 199) state that this appears to be a synonym of Micrococcus albus Schroeter; while Hucker (loc. cit., 19) considers it a synonym of Micrococcus freudenreichii Guillebeau or of Micrococcus ureae Cohn.

Micrococcus lactis Chester. (No. 44, Conn, Storrs Agr. Exp. Sta., 7th Ann. Rept., 1895, 79; Chester, Man. Determ. Bact., 1901, 90.) From milk. Winslow and Winslow (loc, cit., 224) state that this is apparently a synonym of Micrococcus candicans Flügge.

Micrococcus lactis II (Hueppe) Scholl. (Quoted from Löhnis, Cent. f. Bakt., II Abt., 18, 1907, 141.) From milk.

Micrococcus lactis acidi Krueger. (Cent. f. Bakt., 7, 1890, 494.) From milk.

Micrococcus lactis albus Conn, Esten and Stocking. (Storrs Agr. Exp. Sta. 18th Ann. Rept., 1907, 120.) From milk. Hucker (loc. cit., 19) considers this a synonym of Micrococcus freudenreichii 
Guillebeau or of Micrococcus ureae Cohn.

Micrococcus lactis amari von Freudenreich. (Bittere Milch Micrococcus, Conn, Cent. f. Bakt., 9, 1891, 6533; von Freudenreich, Cent. f. Bakt., II Abt., 13, 1904, 407.) From the udder and bitter cream.

Micrococcus lactis aureus Conn, Esten and Stocking (loc.cit., 112). From milk, butter, cheese, stable dust. Hucker (loc. cit., 7 and 12) regards this as a synonym of Micrococcus luteus Cohn, of Micrococcus varians Migula or of Micrococcus aureus Zopf.

Micrococcus lactis citreus Conn, Esten and Stocking (loc.cit., 102). From milk. Hucker (loc. cit., 7) considers this species identical with Micrococcus luteus Cohn or with Micrococcus varians Migula.

Micrococcus lactis citronus Conn, Esten and Stocking (loc. cit., 117). From slime on Camembert cheese. Hucker (loc. cit., 12) regards this as a synonym of Micrococcus aureus Zopf.

Micrococcus lactis flaus Conn, Esten and Stocking (loc. cit., 109). May be identical with Micrococcus aurantiacus Cohn. From milk. Hucker (loc. cit., 7 and 12) states that this may be a synonym of Micrococcus luteus Cohn, of Micrococcus varians Migula or of Micrococcus aureus Zopf.

Micrococcus lactis fluorescens Conn, Esten and Stocking (loc.cit., 120). From stable dust. Exhibits a green fluorescence. Hucker (loc. cit., 18) states that this species is very similar to Yicrococcus caseolyticus Evans.

Micrococcus lactis gigas Conn, Esten and Stocking (loc. cit., 116). From milk. Hucker (loc. cit., 22) states that this species is probably identical with Micrococcus candidus Cohn or .Hicrococcus epidermidis Hucker.

Micrococcus lactis giganteus Conn, Esten and Stocking (loc.cit., 122). From milk.

Micrococcus lactis minutissimus Conn, Esten and Stocking (loc.cit., 119). From milk. Hucker (loc. cit., 10) considers this a synonym of Micrococcus conglomeratus Migula.

Wicrococcus lactis rosaceus Conn, Esten and Stocking (loc. cit., 109). From milk. Hucker (loc. cit., 26) states that this is probably identical with Wicrococcus roseus Flügge.

Ificrococens lactis rugosus Conn, Esten and Stocking (loc.cit., 122). From milk.

Micrococcus lactis varians Conn, Esten and Stocking (loc. cit., 121). Commonly found in milk. Nay be identical with Micrococcus aureus Zopf. Hucker (loc. cit., 15) states that this may be identical in part with. Micrococcus albus Schroeter.

Micrococcus lardarius Krassilschtschik. (Memoires Soc. Zool. de France, 9, 1896, 513 ; Compt. rend. Acad. Sci., Paris, 123, 1896 , 428.) From diseased silkworms. Differs from Streptococcus bombycis. Hucker (loc. cit., 22) states that this is probably identical with either Micrococcus candidus Cohn or Micrococcus epidermidis Hucker.

Micrococcus lembliei Migula. (No. 21, Lembke, Arch. f. Hyg., 29, 1897, 327; Migula, Syst. d. Bakt., 2, 1900, 212.) From feces. Winslow and Winslow (loc. cit., 220) consider this a synonym of Micrococcus luteus Cohn, while Hucker (loc. cit., 11) regards it as probably identical with Micrococcus citreus Schroeter.

Micrococcus lentus Migula. (No. 22, Lembke, loc. cit., 328; Migula, loc. cit., 209.) From feces. Winslow and Winslow (loc. cit., 199) state that this appears to be a synonym of Micrococcus albus Schroeter; while Hucker (loc. cit., 19) regards it as probably identical with Iicrococcus freudenreichii Guillebeau or Micrococcus ureae Cohn.

Micrococcus licheniformis Kern. (Arb. bakt. Inst. Karlsruhe, 1, Heft 4, 1897, 482.) From the intestine of the yellowhammer (Emberiza citrinella). Winslow and Winslow (loc. cit., 220) consider this a synonym of Micrococcus luteus Cohn. Hicrococcus lignithum Renault. 
(Compt. rend. Acad. Sci., Paris, 126, 1898, 1828.) A fossil form from lignite.

Micrococcus lipolyticus HorowitzWlassowa. (Ztschr. f. Untersuch. d. Lebensmittel, 62, 1931, 602.) A fat splitting micrococcus from salted fish.

Micrococcus lipolyticus Stark and Scheib. (Jour. Dairy Sci., 19, 1936, 210; not Micrococeus lipolyticus Horowitz-Wlassowa, loc, cil.) From butter.

Micrococcus liquefaciens Migula. (Micrococcus ureae liquefacions Flügge, Die Mikroorganismen, 2 Aufl., 1886, 169; Streptococcus aëthebius Trevisan, I generi e le specie delle Batteriacee, Milan, 18s?) 31; Staphylococcus ureae liquefaciens Lundström, Festschr. d. path. anat. Inst. z. Andenken a.d. 250 jährige Bestehen d. fimnländ. Univ. z. Helsingfors 1890 ; abst. in Cent. f. Bakt., 9, 1891, 672; Migul., Syst. d. Bakt., 2, 1900, 106; Micrococcus aethebius Chester, Man. Determ. Bact., 1901, 7; Crococcus liquefaciens flueggei Mirpuel and Cambier. Traité de Bact., 1902, 627; Hicrococeus liquefaciens flueggei Miquel in Lafits. Handb. d. tech. Mykol., 3, 1904-06, 75; Albococcus ureae Kligler, Jour. Inf. Dis., 12, 1913, 442; not Wicrococcus liquefaciens Bergey et al.. Manual, 1st ed., 1923, 67.) From urine. Hucker (loc. cit., is and 19) states that this species may be identi(al with Micrococcus caseolyticus Evans. Micrococcus freudenreichii Guillebeau or Micrococcus weae Cohn.

Micrococcus liquefaciens acidi $I$ and $I I$, Conn. (Storrs Agr. Exp. Sta. 12th Ann. Rept., 1900, 48.) From milk. Hucker (loc. cit., 18) regards this species as very similar to Micrococens caseolyticus Evans.

.Microsoccus liquidus Migula. (No. 21, Lembke, Areh. f. Hyg., 26, 1896, 313; Migula, Syst. 1. Bakt., 2, 1900, 208.) From feces. Hucker (lof. cit., 15) states that this species appears identical with Micrococcus albus Siehroeter.

Micrococeus lobatus Migulit. (Siebert, Inaug. Diss., Würzburg, 1894, No. 3, 10; Migula, Syst. d. Bakt., 2, 1900, 139.) From the human scalp. Winslow and
Winslow (loc. cit., 18t) state that this is apparently a synonym of Micrococcus aureus Zopf.

Micrococcus locwenbergii Trevisan. (Micrococcus de l'ozène, Löwenberg, Congrès des otulogistes, 1884 and Union médicale, 1884; Trevisan, I generi e le specie delle Batteriacee, Milan, 1889, 33.) From secretions in ozena.

Hicrococcus luridus Liern. (Arb. bakt. Inst. Karlsruhe, 1, Heft, 4, 1897, 480.) From the intestine of the chaffinch (Fringilla coelebs). Winslow and Winslow (loc. cit., 220) consider this a synonym of Hicrococcus luteus Cohn.

Hicrococcus luteolus Henrici. (Arb. balit. Inst. Karlsruhe, 1, Heft 1, 1894, s2; not Micrococcus luteolus Irwin and Harrison, Le Lait, 8, 1928, 881.) From cheese. Winslow and Winslow (loc cit., 216) consider this a synonym of Micrococcus flavus Trevisan. For a description of Irwin and Harrison's organism, see Bergey ct al., Manual, 5th ed., 1939, 24!).)

Hicrococens luteus var. larvae Hauduroy ot al. (Hicrococcus luteus-liquefaciens var. larve 'Toumanoff, Bull. Soc. Centr. (le Méd. Vétér., 80, 1927, 367; Hauduroy et al., Dict. (1. Bact. Path., 1937, 277.) From foulbrood of bees. Pathogenic.

Micrococcus lutosus Kern. (Arb. bakt. Inst. Karlsruhe, 1, Heft 4, 1897, 489.) From the stumach contents of the chatfinch (Fringilla coelebs). Winslow and Winslow (loc. cit., 216) consider this a synonym of Hicrococcus flavus Trevisan.

Micrococcus lysodeititicus Fleming. (Proe. Roy. Soe. London, Ser. B, 93, 1922, 306.) Non-pathogenic.

Micrococcus lyssae (Rivolta) Trevisan. (Cocco-bacterium lyssae Rivolta, 1886; Trevisan, I generi e le specie delle Batteriacer, Milan, 1889, 33.) Spore-bearer. Micrococcus madidus Migula. (No. 19, Lembke, Arch. f. Hyg., 26, 1896, 311; Migula, Syst. 1. Bakt., 2, 1900, 207.) lrom feces. Winslow and Winslow (loc. cit., 18t) state that this is apparently a synonym of Wicrococcus aureus Migula; 
while Hucker (loc. cit., 15) regards it as identical with Micrococeus albus Schroeter.

Micrococcus magnus (Miller) Trevisan. (Jodococcus magnus Miller, Deutsche med. Wochnschr., No. 30, 1888; Trevisan, I generi e le specic delle Batteriacee, Milan, 1889, 33; not Micrococeus magnus Chester, Man. Determ. Bact., 1901, 8.5 (Diplococcus maguus Rosenthal, Cent. f. Bakt., 25, 1899, 1); not . Hicrococcus magnus Stark and Seheih, Jour. Dairy Sci., 19, 1936, 210.)

Mierococens major De Toni and Trevisan. (Micrococcus urinae major Doyen, Jour. d. connaiss. médic., No. 14, 1889 , 108; De Toni and Trevisan, in Saceardo, Sylloge Fungorum, 8, 1889, 1076.) From urine.

Micrococcus major Eckstein. (Ztschr. f. Forst- u. Jagdwesen, 26, 1894, 18; not Micrococcus major DeToni and Trevisan, in Saccardo, Sylloge Fungorum, $8,1889,1076$.) Isolated from the larvae of the nun moth (Lymantria monacha) and Hyponomeuta sp.

Micrococcus manfredii Trevisan. (Micrococcus der progressiven Iymphome im Tierörper, Manfredi, Fortschr. d. Med., 1886, 713; Trevisan. I generi e le specie delle Batteriaces. Milan, 1889 , 33; Streptococcus manfredii DeToni and Trevisan, in Saceardo. Sylloge Fungorum, 8, 1889. 1056; Hicrococcus canus Migula, Syst. d. Bakt., 2, 1900, 63. From sputum. Winslow and Winslow (loc. cit., 206) regard this as at synonym of Micrococcus candidus Cohn or of Gaffkya tetragena. Trevisan.

Micrococcus malolacticus sicifert. (Ztschr.f. d. landwirtsch. Versuchswesen in Oesterreich, 1903, 567; Abst. in Cent. f. Bakt., II Abt., 10, 1903, 664.) From wine. Hucker (loc. cit., 7 ) considers this a synonym of Micrococcus luteus Cohn or of Micrococeus varians Migula.

Micrococcus mammitis Hutchens. (Hutchens in Besson, Pract. Bact. Microbiol. and Serum Therapy, Trans. of 5th ed., 1913, 615; not Streptococcus mammitis bovis Hutchens, ibid., 613.)
From gangrenous mimmitis of milking ewes. This is le microcoque de l'araignée or de la mammite rangréneuse, Nocard, Ann. Inst. Past.. 1, 1אs7, 417 .

Micrococcus marginatus Wilhelmy. (Arb. bakt. Inst. Karlsruhe, 3, 1903, 11.) From meat extrict.

Microroceus marinus Issatchento. (Recherches sur les Microbes de l'Océan Glacial Aretique, Petrograd, 1914, 147.) From sea water.

Micrococcus mastitidis Holland. (Jour. Bact., 5, 1920, 224.) Nomen nudum.

Micrococcus mastobius Trevisan. (Microcoque de la mammite gangréneuse des breois laitières, Nocard, Ann. Inst. Past., 1, 1s87, 417; Trevisan, I generi e le specie delle Batteriacee, Milan, 1889, 33; Micrococcus gangr. ovium, quoted from Freire, Rio de Janciro, 1898; Abst. in Cent. f. Bakt., I Abt., 26, 1899, 841 ; Mierococcus onis Migula, Syst. d. Bakt., 2, 1900, 90; Hicrococcus mastitis Chester, Man. Determ. Bact., 1901, 76; Micrococcus mastidis gangraenosae ovis Pfeiler, Ztschr.f. Infektionskr., parasit. Krankh. u. Hyg. (l. Haustiere, 4, 190s, 132.) Said to be the cause of gangrenous mastitis in sheep. This suecies appears to be Microcuerus myogenes Migula.

Wicrococcus melanocyclus Nerker. (Cent. f. Bakt., II Abt., 41, 1911, 589.) See Actinomyces melanocyclus Irainsky.

Micrococcus melanoglossophorus Spegazzini. (Fung. Arg. Pug., 4, 18--, 316.) From the epithelium of the tongue.

Micrococcus meldensis Roger. (Ber. An. de Soc. d'Agric. de Meaux, 1898.)

Yicrococcus melleus grandinis Harrison. (Bot. Gazette, 26, 1895, 211.)

Micrococcus memelensis Leichmann. (Coccus, Leichmann, Cent. f. Bakt., II Abt., 2, 1896, 780; Leichmann, in Koch, Jahresber., 12, 1901, 25t; Micrococcus acidi laevolactici Weigmann, in Lafar, Handb. d. techn. Mykol., 2, 1905, 62.) From milk.

Micrococcus minimus Weiss. (Arb. bakt. Inst. Karlsruhe, 2, Heft 3, 1902, 
188; not Micrococcus minimus Bergey et al., Manual, 1st ed., 1923, 69.) From a bean infusion. Hucker (loc. cit., 7) considers this a synonym of either Micrococcus luteus Cohn or Micrococcus varians Migula.

Micrococcus minutissimus Issatchenko. (Recherches sur les Microbes de l'Océan Glacial Artique, Petrograd, 1914, 146.) From sea water.

Micrococcus mirificus (Rabenhorst) Trevisan. (Palmella mirifica Rabenhorst, Hedwigia, 1867, 115, and Flor. Europ. Algar., 3, 1856, 35; Trevisan, Rendic. R. Ist. Lombardo, 12, 1879.)

Micrococcus mollis (Dyar) Migula. (Merismopedia mollis Dyar, Ann. N. Y. Acad. Sci., 8, 1895, 352; Migula, Syst. d. Bakt., 2, 1900, 161; A urococcus mollis Winslow and Rogers, Science, 21, 1905, 669 ; Staphylococcus mollis Holland, Jour. Bact., 5, 1920, 225.) From air. A cause of boils in the tropies, according to Castellani and Chalmers (Man. Trop. Med., 3rd ed., 1919, 931). Hucker (loc. cit., 12 ) states that this species is apparently identical with Micrococcus aureus Zopi.

Mierococcus (Diplococcus) morrhuae Klebahn. Mitteil. Inst. Allgm. Botan. Hamburg, 4, 1919, 11-69; Abst. in Cent. f. Bakt., II Abt., 52, 1921, 123.) Halophilic. Associated with spoilage of salted fish.

Micrococcus mucilagineus Weiss. (Arb. bakt. Inst. Kirlsruhe, 2, Heft 3, 1902 , 191.) From bean infusions. Hucker (loc. cit., 11) states that this is probably a synonym of Micrococcus citreus Migula.

Micrococcus mucilaginosus Miguli. (Micrococeus der schleimigen Milch, Ratz, Arch. f. Tierheilkunde, 12, Heft 1 and 2, 1890; Migula, Syst. d. Bakt., 2, 1900, 119; not Micrococcus mucilaginosus Weiss, Arb. bakt. Inst. Karlsruhe, 2, Heft 3, 1902, 205.) From slimy milk. Winslow and Winslow (loc. cit., 199) state that this appears to be a synonym of Micrococcus albus Schroeter; while Hucker (loc. cit., 18) considers it a syn- onym in part of Micrococcus caseolyticus Evans.

Micrococcus mucofaciens Thöni and Thaysen. (Cent. f. Bakt., II Abt., 36, 1913, 359; not Micrococcus mucofaciens Pribran, Klassifikation der Schizomyceten, 1933, 42.) From milk. Hucker (loc. cit., 9) considers this a synonym of Micrococcus flams Trevisan. For a description of this species, see Bergey et al., Manual, 5th ed., 1939, 245.

Iicrococcus myceticus Castellani. (Proc. Soc. Exp. Biol. and Med., 25, 1928, 535-536.) From gummy lesions.

Micrococcus mycodermatus Holland. (Jour. Bact., 5, 1920, 224.)

Micrococcus nacreaceus Migula. (Perlmutterglänzender Diplococcus, Tataroff, Inaug. Diss., Dorpat, 1891, 70; Migula, Syst. d. Bakt., 2, 1900, 62.) Winslow and Winslow (loc. cit., 224) state that this is apparently a synonym of Micrococeus candicans Flügge.

Micrococcus neoformans Doyen. (Doyen, Le Micrococcus neoformans et les néoplasmes, Paris, 1903.) From cancerous tissue. Shown by Andrewes and Gordon (35th Ann. Rept. Local Govt. Board, London, 1905-06, 553) to be identical with Micrococcus epidermidis albus Welch.

Micrococcus neurotomae Paillot. (Compt. rend. Acad. Sei., Paris, 178, 1924, 246.) Gram-negative. From the larvae of Neurotoma nemoralis.

Micrococcus neuvillei Trevisan. (Micrococcus G, Malapert-Neuville, 1887; Trevisan, I generi e le specie delle Batteriacee, Milan, 1889,34.) From mineral water.

Wicrococcus nigrescens Castellani. (Brit. Jour. of Dermatology, 23, 1911, 341; Nigrococcus nigrescens Castellani and Chalmers, Man. Trop. Med., 3rd ed., 1919, 2103.) Produces a black pigment. Found in the black variety of trichomy cosis axillaris, a tropical disease.

Micrococcus nigrofaciens Northrup. Mich. Agr. Exp. Sta. Tech. Bull. No. 18, 1914, 12 ; also in Cent. f. Bakt., II Abt., 
41, 1914, 326.) From diseased larvae of the June beetle (Lachnosterna sp.) and other insects.

Micrococcus nitidus Kern. (Arb. bakt. Inst. Karlsruhe, 1, Heft 4, 1897, 476.) From the stomach and intestine of birds. Winslow and Winslow (loc. cit., 199) state that this appears to be a synonym of Micrococcus albus Schroeter; while Hucker (loc. cit., 19) regards it as a synonym of Micrococcus freudenreichii Guillebeau or of Micrococcus ureae Cohn.

Micrococcus nitrificans Bergey et al. (Micrococcus 6, Rubentschick, Cent. f Bakt., II Abt., 72, 1927, 125; Bergey et al., Manual, 3rd ed., 1930, 88; not Micrococcus nitrificans van Tieghem, Traité de Botanique, Paris, 1883.) From sewage filter beds. For a deseription of this species, see Bergey et al., Manual, 5th ed., 1939, 257.

Micrococcus nivalis Chester. (No. 47, Conn, Storrs Agr. Exp. Sta. 7th Ann. Rept., 1895, 80; Chester, Man. Determ. Bact., 1901, 90.) From dust. Winslow and Winslow (loc. cit., 224) state that this is apparently a synonym of Micrococcus candicans Flügge.

Micrococcus niveus Henrici.

(Arb. bakt. Inst. Karlsruhe, 1, Heft 1, 1894 , 66.) From Swiss cheese. Winslow and Winslow (loc. cit., 224) state that this is apparently a synonym of Hicrococcus candicans Flügge.

Micrococcus nonfermentans Steinhaus. (Jour. Bact., 42, 1941, 779.) From the alimentary tract of the lyreman cicada (Tibicen linnei) and of an unidentified damsel fly (Coenagrionidae).

Micrococcus nubilis Migula. (Coccus B, Foutin, Bakt. Untersuch. von Hagel, Wratsch, No. 49 and 50, 1889 ; see Cent. f. Bakt., 7, 1890, 373; Migula, Syst. d. Bakt., 2, 1900, 60; Micrococcus beta Chester, Man. Determ. Bact., 1901, 87.) Isolated from hail. Winslow and Winslow (loc. cit., 205) consider this to be a synonym of Micrococcus candidus Cohn or of Gaffkya tetragena Trevisan.

Micrococcus nuclei Roze.

(Compt. rend. Acad. Sci., Paris, 122, 1896, 544.) From potatoes.

Micrococcus obscocnus Kern. (Arb. bakt. Inst. Karlsruhe, 1, Heft 4, 1897, 473.) From the stomach contents of a crow (Corvus corone). Winslow and Winslow (loc. cit., 199) state that this appears to be a synonym of Micrococcus albus Schroeter; while Hucker (loc. cit., 19 ) considers it a synonym of Micrococcus freudenreichii Guillebeau or of Micrococcus ureae Cohn.

Micrococcus ochraceus Rosenthal. (Inaug. Diss., Erlangen, 1893, 22 ; Abst. in Cent. f. Bakt., 16, 1894, 1024; not Micrococcus ochraceus Hansgirg, Oestr. Bot. Ztschr., 1885, No. 4.) From the oral cavity. Winslow and Winslow (loc. cit., 220 ) consider this a synonym of Micrococcus luteus Migula. For a description of this species see Bergey et al., Manual, 5th ed., 1939, 242.

Micrococcus ochroleucus Prove. (Prove, Beitr. z. Biol. d. Pflanz., 4, Heft 3, 1887, 409; Streptococcus ochroleucus Trevisan, I generi e le specic delle Batteriacee, Milan, 1889, 31; Planococcus ochroleucus Migula, Syst. d. Bakt., 2, 1900, 272.) From urine. Motile.

Micrococcus odoratus Henrici. (Arb. bakt. Inst. Karlsruhe, 1, Heft 1, 1894, 73.) From cheese. Winslow and Winslow (loc. cit., 22t) state that this is apparently a synonym of Micrococcus candicans Flügge.

Micrococcus odorus Henrici (loc. cit, 72). From cheese. Winslow and Winslow (loc. cit., 224) state that this species is apparently a synonym of Micrococcus candicans Flügge.

Micrococcus olearius DeToni and Trevisan. (Micrococcus urinae flavus olearius Doyen, Jour. d. connaiss. Médic., No. 14, 1889, 108; DeToni and Trevisan, in Saccardo, Sylloge Fungorum, 8, 1889, 1077.) From urine. Hucker (loc. cit, 12) considers this species a synonym of Micrococcus aureus Zopf.

Micrococcus olens Henrici. (Arb. bakt. Inst. Karlsruhe, 1, Heft 1, 1894, 87.) 
From Swiss cheese. Winslow and Winslow (loc. cit., 216) consider this a synonym of Micrococcus flavus Trevisan.

Micrococcus opalescens DeToni and Trevisan. (Micrococcus albus II, Maggiora, Giorn. Soc. Ital. d'Igiene, 11, 1889, 351; DeToni and Trevisan, in Saccardo, Sylloge Fungorum, 8, 1889, 1078.)

Micrococcus orbicularis Chester. ( $\mathrm{Mi}$ crococcus orbicularis flavus Ravenel, Mem. Nat. Acad. Sci., 8, 1896, 8; Chester, Man. Determ. Bact., 1901, 101.) From soil. Winslow and Winslow (loc, cit., 216) consider this a synonym of Micrococcus flavus Trevisan; while Hucker (loc, cit., 10) regards it as a synonym of Micrococcus conglomeratus Migula.

\section{Micrococcus orbiculatus Wright.}

(Mem. Nat. Acad. Sci., 7, 1895, 432.) From Schuylkill River water. Winslow and Winslow (loc, cit., 220) consider this a synonym of Micrococcus luteus Cohn.

Micrococcus ovalis Kern. (Arb. bakt. Inst. Karlsruhe, 1, Heft 4, 1897, 500; not Micrococcus ovalis Escherich, Die Darmbakterien des Säuglings, Stuttgart, 1886, 90.) From the stomach contents of the rock dove (Columba livia). Hucker (loc. cit., 9) regards this as a synonym of Micrococcus flavus Trevisan.

Micrococcus pallens Henrici. (Arb. bakt. Inst. Karlsruhe, 1, Heft 1, 1894, 61.) From cheese. Winslow and Winslow (loc. cit., 205) consider this a synonym of Micrococcus candidus Cohn or of Gaffkya tetragena Trevisan.

Micrococcus pallidus Henrici (loc. cit., 62). From cheese. Hucker (loc. cit., 7) regards this species as identical with either Micrococcus luteus Cohn or Micrococcus varians Migula.

Micrococcus pannosus Kern. (Arb. bakt. Inst. Karlsruhe, 1, Heft 4, 1897, 466.) From the stomach contents of the rock dove (Columba livia) and the intestine of another dove (Columba oenas). Winslow and Winslow (loc. cit., 224) state that this is apparently a synonym of Micrococcus candicans Flügge.

Micrococcus paraffinae Söhngen.
(Cent. f. Bakt., II Abt., 37, 1913, 595.) From garden earth.

Micrococcus parotitidis Korentschewsky. (Cent. f. Bakt., I Abt., Orig., 44, 1907,402 .) Isolated from cases of parotitis epidemica.

Micrococcus parvus (Miller) Trevisan. (Jodococcus parvus Miller, Deutsche med. Wchnschr., No. 30, 1888; Trevisan, I generi e le specie delle Batteriacee, Milan, 1889, 33.)

Micrococcus parvus Migula. (No. 14, Lembke, Arch. f. Hyg., 26, 1896, 309; Migula, Syst. d. Bakt., 2, 1900, 200.) From feces. Winslow and Winslow (loc. cit., 224) state that this species is apparently a synonym of Micrococcus candicans Flügge.

Micrococcus pasteuri Trevisan. (Microbe pyogene de l'eau de Seine, Pasteur, 1877; Trevisan, I generi e le specie delle Batteriacee, Milan, 1889, 34; not Micrococcus pasteuri Sternberg, Trans. Pathol. Soc. of Philadelphia, 12, 1885, 162.) From water.

Micrococcus pellucidus Kern. (Arb. bakt. Inst. Karlsruhe, 1, Heft 4, 1897, 468; not Micrococcus pellucidus Roze, Compt. rend. Acad. Sci., Paris, 122, 1896, 1012.) From the intestine of a sparrow (Passer montanus). Hucker (loc.cit., 23 ) regards this as a synonym of Micrococcus candidus Cohn or of Micrococcus epidermidis Hucker.

Micrococcus pemphigi Migula. (Diplococcus des Pemphigus acutus, Demme, Verhandl. d. Kongr. f. innere Med., Wiesbaden, 1886, 336; Diplococcus pemphigi acuti Lehmann and Neumann, Bakt. Diag., 1 Aufl., 2, 1896, 173; Migula, Syst. d. Bakt., 2, 1900, V and 191; Micrococcus demmei Chester, Man. Determ. Bact., 1901, 74.) Isolated from bullae in a case of pemphigus acutus.

Micrococcus pemphigicontagiosi Castellani and Chalmers. (Micrococcus pemphigi contagiosa Clegg and Wherry, Jour. Inf. Dis., 3, 1906, 171; Castellani and Chalmers, Man. Trop. Med., 3rd ed., 1919, 931.) From bullae in a case of pem- 
phigus contagiosa. This may be a synonym of Micrococcus pemphigineonatorum, see below.

Micrococcus pemphigineonatorum Castellani and Chalmers. (Micrococcus pemphigi neonatorum Almquist, Ztschr. f. Hyg., 10, 1891, 253; Staphylococcus pemphigi neonatorum Lehmann and Neumann, Bakt. Diag., 1 Aufl., 2, 1896, 173; Castellani and Chalmers, Man. Trop. Med., 3rd ed., 1919, 931.) Found in bullae in a case of pemphigus neonatorum. This may be Micrococcus mollis, according to Castellani and Chalmers (loc. cit.). Falls (Jour. Inf. Dis., 20, 1917, 97) identifies this and the previous organism as Micrococcus pyogenes var. aureus Zopf.

Micrococcus percitreus Bergey et al. (Manual, 1st ed., 1923, 63.) From air and water. Hucker (loc. cit., 10) considers this a synonym of Micrococcus conglomeratus Migula. For a deseription of this species, see Bergey et al., Manual, 5th ed., 1939, 248.

Micrococcus perflavus Bergey et al. (Manual, 1st ed., 1923, 62.) From air and water. Hucker (loc. cit., 12) regards this as a synonym of Micrococcus aureus Zopf. For a description of this species, see Bergey et al., Manual, 5th ed., 1939, 247.

Iicrococcus persicus Kern.

(Arb. bakt. Inst. Karlsruhe, 1 , Heft 4, 1897, 499.) From the intestine of a dove ( $\mathrm{Co}$ lumba oenas). Hucker (loc. cit., 25) states that this may be identical with Micrococcus roseus Flügge.

Micrococcus petechialis Trevisan. (Micrococco del dermotifo, Bareggi, 1886; Trevisan, I generi e le specie delle Batteriacee, Milan, 1889, 33.)

Micrococcus petilus Trevisan. (Micrococcus der Pyaemie bei Kaninchen, Kioch, Über d. Aetiolog. d. Wundinfectionskr., Leipzig, 1878; Micrococcus pyaemiae cuniculorum Schroeter, in Cohn, Kryptogam. Flora v. Schlesien, 3, 1, 1886, 148; Trevisan, I generi e le specie delle Batteriacee, Milan, 1889, 33 ; Micro- coccus cuniculorum Migula, Syst. d, Bakt., 2, 1900, 192.) From rabbits.

Micrococcus petrolei Renault. (Compt. rend. Acad. Sci., Paris, 124, 1897, 1315.) A fossil form from oil bearing rocks.

Micrococcus pieridis Burrill. (Quoted from Chittenden, U. S. Dept. Agr., Farmers' Bull. No. 1461, 1926, 6.) From larvae of the cabbage butterfly (Pieris rapae).

Micrococcus pikowskyi Bergey et al. (Culture No. 22, Baranik-Pikowsky, Cent. f. Bakt., II Abt., 70, 1927, 373; Bergey et al., Manual, 3rd ed., 1930, 78.) From sea water. For a description of this species, see Bergey et al., Manual, 5th ed., 1939, 242.

Micrococcus piliformis Weiss. (Arb. bakt. Inst. Karlsruhe, 2, Heft 3, 1902, 194.) From a bean infusion. Hucker (loc. cit., 7) considers this a synonym of Micrococcus luteus Cohn or of Micrococcus varians Migula.

Micrococcus pillonensis Gray and Thornton. (Cent. f. Bakt., II Abt., 73, 1928, 81.) From manure and soil. For a description of this species, see Bergey et al., Manual, 5th ed., 1939, 259.

Hicrococcus pituitoparus (Hohl) Buchanan and Hammer. (Karphococcus (Carphococcus) pituitoparus Hohl, Jahrb. d. Schweiz, 22, 1906, 439; Diplococcus viscosus Sato, Cent. f. Bakt., II Abt., 19, 1907, 27; Buchanan and Hammer, Iowa Agr. Exp. Sta. Res. Bull. 22, 1915, 285.) From slimy milk and from straw. Hucker (loc. cit., 23) states that this species is probably identical with Micrococcus candidus Cohn or with Micrococcus epidermidis Hucker. For a description of this species, see Bergey et al., Manual, 5th ed., 1939, 243 .

Micrococcus plumosus Eisenberg. (Bräutigam, Inaug. Diss., Leipzig, 1886, 18; Federiger Micrococcus, Adametz, Mitteil. d. Oesterr. Versuchssta. f. Brauerei u. Mälzerei, Wien, Heft 1, 1888; Eisenberg, Bakt. Diag., 3 Aufl., 1891, 56.) From feces of cattle and from water. Winslow and Winslow (loc. cit., 
220 ) consider this a synonym of Micrococcus luteus Cohn; while Hucker (loc. cit., 22 and 23 ) regards it as probably identical with Micrococcus candidus Cohn or Micrococcus epidermidis Hucker.

Micrococcus polypus Migula. (Syst. d. Bakt., 2, 1900, 79.) From air. Hucker (loc. cit., 23) states that this species is probably identical with Hicrococcus candidus Cohn or Hicrococcus epidermidis Hucker.

Micrococcus populi Delacroix. (Bul. Mens. Off. Renseig. Agr., Paris, 5, 1906, 1349 and Ann. Inst. Nat. Agron., 2 Ser., 5, 1906, 353.) Parasitic on poplar trees (Populus spp.).

Micrococcus porcellorum Trevisan. (Micrococeus bei Hepatitis enzootica porcellorum, Nonewitsch, Cent. f. Bakt., 3, 1888, 233 ; Trevisan, I generi e le specie delle Batteriacee, Milan, 1889, 33.) From an infected liver.

Micrococcus progrediens sichrocter. (Micrococcus der progressiven Abscessbildung bei Kaninehen, Koch, Über d. Aetiolog. d. Wundinfectionskrankheiten, Leipzig, 1878; Schroeter, in Cohn, Kryptogam.-Flora v. Schlesien, 3, 1, 1886, 148; Micrococcus haematosaprus Trevisan, I generi e le specie delle Batteriacee, Milan, 1889, 33.) From the blood of diseased rabbits.

Micrococcus psalteri Buemann. (Cent. f. Bakt., I Abt., Orig., $71,1913,308$. From the third stomach of cattle.

Micrococcus pseudocyaneus Schroeter. (Kryptogam.- Flora v. Schlesien, 3, 1, 1886, 145.) A synonym of Mierococcus cyaneus Cohn according to Migula, Syst. d. Bakt., 2, 1900, 188.

Micrococcus pseudoinfluenzae Migulit. (Microörganismus I, Fischel, Ztschr. f. Heilkunde, 12, 1891 ; See Cent. f. Bakt.. 9, 1891, 611; Migula, Syst. d. Bakt., 2, 1900,86 .) From the blood of an influenza patient. Hucker (loc. cit., 23) considers this a synonym of Micrococcus candidus Cohn or of Micrococcus epidermidis Hucker.

Micrococcus pulcher Glage. (Zischr. f. Fleisch- u. Milchhyg., 10, 1900, 146; not Micrococcus pulcher Weiss, Arb. bakt. Inst. Karlsruhe, 2, Heft 3, 1902, 182.) From coating on surface of wurst and similar meat products.

Micrococcus pultiformis Kern. (Arb. bakt. Inst. Karlsruhe, 1, Heft 4, 1897, 174.) From stomach contents of the yellow-hammer (Emberiza citrinella) and starling (Sturnus vulgaris) and from the intestine of the woodpecker (Picus $m a$ jor). Winslow and Winslow (loc. cit., 199 ) state that this appears to be a synonym of Micrococcus albus Schroeter; while Hucker (loc. cit., 19) regards it as probably identical with Micrococcus freudenreichii Guillebeau or with Micrococcus ureae Cohn.

Micrococcus punctatus Migula. (No. 18, Lembke, Arch. f. Hyg., 29, 1897, 325; Migula, Syst. d. Bakt., 2, 1900, 213.) From feces. Winslow and Winslow (loc. cit., 199) state that this species appears to be a synonym of Micrococcus albus Sichroeter.

Vicrococcus purpurifaciens Lehmann and Neumann. (Micrococeus, Dudtschenko, ('ent. f. Bakt., II Abt., 42, 1915, 529; Lehmann and Neumann, Bakt. Diag., 6 Aufl., 2, 1920, 755.) From ice. Produces a purple pigment in alkaline gelatin media.

Micrococcus pustulatus Henneberg. (Cent.f. Bakt., II Abt., 55, 1922, 251.) From the human intestine.

Micrococcus putridus Tilanus. (Münch. med. Wchnschr., 34, 1887, 310.) From gelatin, agar, ete., containing iodiform.

Micrococcus pygmacus Henneberg (loc. cit., 252). From the human intestine.

Micrococcus pyocyaneus Francisco. (Revista Valenciana de Ciencias Médicas, 1914, 2; Abst. in Cent. f. Bakt., I Abt., Ref., 63, 1915, 44; not Micrococcus pyocyaneus Gessard, Thesis, Paris, 1882.) From an acne pustule.

Micrococcus pyosepticus (Héricourt and Richet) Solowjew. (Staphylococcus pyosepticus Héricourt and Richet, Compt. 
rend. Acad. Sci., Paris, 107, 1888, 691 ; Solowjew, Abst. in Cent. f. Bakt., I Abt., 18, 1895, 60.) From an abscess in a dog and from dust. Regarded as identical with Micrococcus albus Schroeter.

Micrococcus quadrigeminus Kilebs. (Staphylococcus quadrigeminus Vanselow and Czaplewski, Cent. f. Bakt., I Abt., 25, 1899, 143; see Lehmann and Neumann, Bakt. Diag., 2 Aufl., 2, 1899, 174.) Closely related to Micrococcus albus Schroeter.

Micrococeus quaternus Migula. (Siebert, Inaug. Diss., Würzburg, No. I, 1894, 7; Migula, Syst. d. Bakt., 2, 1900, 92.) Winslow and Winslow (loc. cit., 199) state that this appears to be a synonym of Micrococcus albus Schroeter.

Micrococcus radiatus Flügge. (Die Mikroorganismen, 2 Aufl., 1886, 176; Streptococcus radiatus Crookshank, Man. of Bact., 3rd ed., 1890, 256; not Micrococcus radiatus Kern, see below.) From dust and water. Winslow and Winslow (loc. cit., 199) state that this appears to be a synonym of Micrococcus albus Schroeter; while Hucker (loc. cit., 18) considers it a synonym of Micrococcus caseolyticus Evans.

Micrococcus radiatus Kern. (Kern, Arb. bakt. Inst. Karlsruhe, 1, Heft 4, 1897, 471; Micrococcus radiosus Migula, Syst. d. Bakt., 2, 1900, 114.) From the stomach contents of the starling (Sturnus vulgaris). Winslow and Winslow (loc. cit., 199) state that this appears to be a synonym of Micrococcus albus Schroeter.

Micrococcus reessii Rosenthal. (Inaug. Diss., Berlin, 1893, 19; Abst. in Cent. f. Bakt., 16, 1894, 1024.) From the oral cavity. Winslow and Winslow (loc. cit., 199) state that this appears to be a synonym of Micrococcus albus Schroeter.

Micrococcus regularis Weiss. (Arb. bakt. Inst. Karlsruhe, 2, Heft 3, 1902, 183.) From bean infusions. Hucker (loc. cit., 7) considers this a synonym of Micrococris luteus Cohn or Micrococcus varians Migula.
Micrococcus resinaceus fiern. (Arb. bakt. Inst. Karlsruhe, 1, Heft 4, 1897, 487.) From the stomach contents of the starling (Sturnus vulgaris) and from the intestine of a sparrow (Passer montanus). Winslow and Winslow (loc. cit., 220) regard this as a synonym of Micrococcus luteus Cohn.

Micrococcus rhenanus Migula. (Neuer Mikroroceus aus Rheinwasser, Burri, Arch. f. Hyg., 19, 1893, 34; Migula, Syst. d. Bakt., 2, 1900, 109; Micrococcus rheni Chester, Man. Determ. Bact., 1901, 82; Albococcus rhenanus Winslow and Rogers, Jour. Inf. Dis., 3, 1906, 544.) Fronı Rhine River water. Winslow and Winslow (loc. cit., 199 ) state that this appears to be a synonym of Micrococcus albus Schroeter; while Hucker (loc. cit., 18) considers it a synonym of Micrococcus caseolyticus Evans.

Micrococcus ridleyi Corbet. (Quart. Jour. Rubber Research Inst., Malaya, 2, 1930, 146.) From the latex of the rubber tree (Hevea brasiliensis). For a description of this species, see Bergey et al., Manual, 5th ed., 1939, 244.

Wicrococcus rosaceus Frankland and Frankland. (Trans. Roy. Society, London, 178, B. 188, 269; Rhodococcus rosaceus Holland, Jour. Bact., 5, 1920, 225.) From air. Hucker (loc. cit., 25) states that this species may be identical with Micrococcus roseus Flügge. For a description of this species, see Bergey et al., Manual, 5th ed., 1939, 252.

Micrococcus rosaceus lactis Conn. (Storrs Agr. Exp. Sta. 12th Ann. Rept., 1900, 34; Micrococcus lactis rosaceus Conn, Esten and Stocking, Storrs Agr. Exp. Nta. Rept. for 1906, 108.) From milk.

Micrococcus roscidus Migula. (Micrococcus No. I, Adametz, Landwirtsch. Jahrb., 18, 1889, 238; Migula, Syst. d. Bakt., 2, 1900, 68.) From Emmenthal cheese. Winslow and Winslow (loc. cit, 224 ) state that this is apparently a synonym of Microcccus candicans Flügge.

Micrococcus roseo-persicinus Migula. 
(Rote Kokken von Van Ermengem, Schneider, Arb. bakt. Inst. Karlsruhe, 1. Heft 2, 1894, 216; Migula, Syst. d. Bakt., 2, 1900, 18t.)

Micrococcus rosettaceus Zimmermann. (Bakt. unserer Trink- u. Nutzwässer, Chemnitz, I Reihe, 1890, 72.) From water. Winslow and Winslow (loc, cit., 224) state that this is apparently a synonym of Micrococeus candicans Flügge.

Micrococcus roseus Maggiora. (Giorn. Soe. Ital. d'Igiene, 11, 1889, 356; not Micrococcus roseus Flügge, Die Mikroorganismen, 2 Aufl., 1886, 183; not Micrococcus roseus Gruber, ('ent. f. Bakt., II Abt., 22, 1909, 408.)

Micrococcus rubellus Migula. (Syst.d. Bakt., 2, 1900, 169.) Source not given. Hucker (loc. cit., 27) regards this as identical with Micrococcus cinnabareus Flügge.

Micrococcus rubescens Migula. (No. 20, Lemble, Arch. f. Hyg., 26, 1896, 312; Migula, Syst. d. Bakt., 2, 1900, 208; not Micrococcus rubescens Chester, see Micrococcus subroseus below.) From feces. Hucker (loc. cit., 27) regards this species as identical with Micrococcus cinnabareus Flügge.

Micrococcus rubidus lactis Comn. (Conn, Storrs Agr. Exp. Sta. 12th Ann. Rept., 1900, 34; Micrococcus lactis rubidus Conn, Esten and Stocking, Storrs Agr. Exp. Sta. 18th Ann. Rept., 1907, 117.) From milk. Tesembles Mierococcus cinnabareus Flügge. Hucker (loc. cit., 25) thinks this species may be identical with Micrococcus roseus Flügge.

Micrococcus rubigenosus Kern. (Arb). bakt. Inst. Karlsruhe, 1, Heft 4 , 1897, 492.) From the stomach contents of at dove (Columba oenas). Hucker (loc. cit., 25) states that this species may be identical with Micrococcus roseus Flügge.

Micrococcus rubiginosus Passer. and Beltr. (Fung. Sicil., 18-, no. 35; quoted from DeToni and Trevisan, in Saceardo, Sylloge Fungorum, 8, 1889, 1082.)

Micrnenerus rugatus Migula. (Micro- coccus endocarditidis rugatus Weichselbaum, Beitr. z. path. Anat. u. z. allgm. Pathol., 4, 1889, 164; Migula, Syst. d. Bakt., 2, 1900, 190; Micrococcus endocarditis Chester, Man. Determ. Bact., 1901, 7t.) From ulcerative endocarditis. Winslow and Winslow (loc. cit., 205) consider this a synonym of . Ficrococcus candidus Cohn or of Gaffkya tetragena Trevisan; while Hucker (loc. cit., 15) regards it as a synonym of Micrococcus albus s'chroeter.

Micrococcus rugosus Chester. (No. 2, Conn, Storrs Agr. Exp. Sta. 6th Ann. Rept., 1894, 50; Chester, Man: Determ. Bact., 1901, 101.) From milk and ripened cream. Winslow and Winslow (loc. cit., 216) consider this a synonym of Micrococcus flavus Trevisan.

Micrococcus ruminantium Henneberg. (Cent.f. Bakt., II Abt., 55, 1922, 252.) From the human intestine.

Micrococeus rushmorei Brown. (Amer. Muscum Novit., No. 251, 1927, 4.) Isolated from a fly (Lncilia sericata) which was infected with Bacillus lutzae.

Micrococeus saccatus Migula. (Micrococens albus liquefaciens von Besser, Beitr. z. path. Anat., 6, 18s9, 46; Micrococcus liquefaciens albus, see Cent. f. Bakt., \%, 1890, 152; Migula, Syst. d. Bakt., 2, 1900, 117; Micrococcus liquefaciens Chester, Man. Determ. Bact., 1901, 75; not Microcuccus liquefaciens Holland, Jour. Bact., 5, 1920, 224; Micrococcus alvi ('hester, loc. cit., 81.) From the nasal mucous membrane. Winslow and Winslow (loc. cit., 199) state that this is apparently a synonym of Micrococcus albus schroeter; while Hucker (loc. cit., 19) regards it as probably identiral with. Hicrococcus freudenreichii Guilleheau or with Micrococcus ureae Cohn. For a description of this species, see Bergey et al., Manual, 5th ed., 1939, 254.

Micrococcus salivalis septicus, quoted from Wigura, see Cent. f. Bakt., I Abt., 17, 1895, 899. From the human skin.

Micrococcus sarcinoides Migula. (Syst. d. Bakt., 2, 1900, 168.) Hucker 
(loc. cit., 27 ) considers this identical with Hicrococcus cinnabareus Flügge.

Hicrococcus scariosus Migula. (Siebert, Inaug. Diss., Würzburg, No. II, 1894, 9; Migula, Syst. d. Bakt., 2, 1900 , 91.) From a hairbrush. Winslow and Winslow (loc. cit., 199) state that this appears to be a synonym of Micrococcus albus Schroeter.

Micrococcus scarlatinosus Trevisan. (Trevisan, Batteri italiani, 1879, 19; Streptococcus rubiginosus Edington, Brit. Med. Jour., 1, 1887, 1265; Perroncitoa scarlatinosa Trevisan, I generi e le specie delle Batteriacee, Milan, 1889, 29.) From a scarlet fever patient.

Vicrococcus scarlatinus Migula, (Syst. d. Bakt., 2, 1900, 173.) From feces.

Hicrococcus selenicus Brenner. (Jahrb. f. wissensch. Botan., 57, 1916, 85; Abst. in Cent. f. Bakt., II Abt., 48, 191s, 431.) From mud.

Micrococcus sensibilis Zettnow. (Cent.f. Bakt., I Abt., Orig., 77, 1915, 216.) From dust. Hucker (loc.cit., 19) considers this a synonym of Micrococcus freudenreichii Guillebeau or of .Hicrococcus ureae C'ohn. For a description of this species, see Bergey et al., Mantual, 5th ed., 1939, $2+8$.

Hicrococcus septicus (Kilebs) Cohn. (Microsporon septicum Klebs, Die $\mathrm{Cr}$ sachen der infectiösen Wundkrankheiten, 1871 ; and Zur path. Anat. d. Schusswunden, 1s72; Cohn, Beitr. z. Biol. 1. Pflanzen, 1, Heft 2, 1s72, 16t.) From pus.

Micrococcus serophilus Costa. (Compt. rend. Soc. Biol., Paris, 83, 1920, 931.) From acute articular rheumatism. Micrococcus serratus Migula. (No. 15, Lembke, Arch. f. Hyg., 26, 1896, 309 ; Migula, Syst. d. Bakt., 2, 1900, 200.) From feces. Winslow and Winslow (loc. cit., 205) regard this as a synonym of Wicrococcus candidus Cohn or of Gaffliya tetragena Trevisan.

Micrococcus sialosepticus Trevisan. (Coccus salivarius septicus Biondi, Ztschr. f. Hyg., 2, 1887, 195; C'occus septicus Biondi, ibid., 220; Trevisan, I generi e le specie delle Batteriacee, Milan, 1889, 33; Micrococcus salivarius Migula, Syst. d. Bakt., 2, 1900, 65; Micrococcus salivarius-septicus Chester, Man. Determ. Bact., 1901, 87.) From human saliva. Winslow and Winslow (loc. cit., 205) consider this a synonym of Micrococcus candidus Cohn or of Gaffkya tetragena Trevisan.

Micrococcus siccus Migula. (Micrococcus No. V, Adametz, Landwirtsch. Jahrb., 18, 1889, 241; Migula, Syst.d. Bakt., 2, 1900, 124.) From Emmenthal cheese. Winslow and Winslow (loc. cit., 185 ) state that this is probably a synonym of Micrococcus aurantiacus Cohn; while Hucker (loc. cit., 7 ) considers it a synonym of Micrococcus luteus Cohn or of Micrococcus varians Migula.

Micrococcus similis Dyar. (Ann. X. I. Acad. Sci., 8, 1895, 3\$7.) From dust. Winslow and Winslow (loc. cit., 205) regard this as a synonym of Micrococcus candidus Cohn or of Gaffiya tetragena Trevisan.

Micrococcus simplex Wright. (Mem. Nat. Acad. Sei., 7, 1895, 432.) From Schuylkill River water. Winslow and Winslow (loc. cit., 199) state that this appears to be a synonym of Micrococcus albus Schroeter.

Micrococcus simulans DeToni and Trevisan. (.Micrococcus citreus II, Maggiora, Giorn. Soc. Ital. d'Igiene, 11, 1889, 351; DeToni and Trevisan, in Saccardo, Sylloge Fungorum, 8, 1889, 1079.)

Hicrococcus sordidus Schroeter. (Schroeter in Cohn, Kryptogam.-Flora v. Schlesien, 3, 1, 1886, 145.) Winslow and Winslow (loc. cit., 22t) state that this is apparently a synonym of Micrococcus candicans Flügge.

Micrococcus sphaeroides Gray and Thornton. (Cent. f. Bakt., II Abt., 73, 1928, 74.) From manure and soil. For a description of this species, see Bergey et al., Manual, 5th ed., 1939, 259.

Iicrococcus staphylophagus Serbinov. 
(La Defense des Plantes, Leningrad, 2, 1926, 556; see Rev. Appl. Mycol., 5, 1926, 650.) Considered pathogenic on grapevines.

Micrococcus stellatus (Lustig) Frankland and Frankland. (Stern-Coceus, Maschek. Jahresher. d. Kom.- Oberrealschule, Leitmeritz, Xo. 10, 1887, 62; Coccus slellatus Lustig, Diag. d. Bakt. d. Wassers, 2 Aufl., 1893, 40 ; Frankland and Frankland, Micro-organisms in Water, 1894, 503.) From water. Winsolw and Winslow (loc. cit., 220) regard this as a synonym of Micrococcus luteus Cohn.

Micrococcus strobiliformis Migula. (No. 23, Lembke, Arch. f. Hyg.. 26, 1896 , 315; Migula, Syst. d. Bakt., 2, 1900, 203.) From feces. Winslow and Winslow (loc. cit., 220 ) regard this as a svnonym of Micrococcus lutems Cohn.

Micrococcus subcandicans Lavanchy. (Univ. Genève, Inst. bot. Prof. Chodat. Sér. 8, Fase. 12, 1914, 68; Abst. in C'ent f. Bakt., II Abt., 47, 1917, 611.) From water of Lake Geneva.

Micrococcus subcanus Migula. (Xo. 17, Lembke, Arch. f. Hyg., 26, 1896, 311; Migula, Syst. d. Bakt., 2, 1900, 202. From feces. Winslow and Winslow (loc cit., 224) state that this is apparently a synonym of Hicrococens candicans Flügge.

Micrococcus subcarneus Migula. (Mi crococcus carnicolor Kern, Arb. bakt. Inst. Karlsruhe, 1, Heft 4, 1897, 495 ; not Micrococcus carnicolor Frankland and Frankland, Micro-organisms in water, 1894, 503; Migula, Syst. d. Bakt., 2, 1900, 181.) From the intestines of doves (Columba livia and Columba oenas). Hucker (loc. cit., 26) states that this may be identical with Micrococcus roseus Flügge.

Micrococcus subcitreus Migula. (Citronengelber Micrococcus, Keck, Ueber das Verhalten der Bakterien im Grundwasser, Dorpat Dissertation, 1890, 60; Migula, Syst. d. Bakt., 2, 1900, 147.) From air and water. Winslow and Winslow (loc. cit., 216) consider this a syn- onym of Micrococcus flavus Trevisan. For a description of this species, see Bergey et al., Manual, 5th ed., 1939, 249.

Micrococcus subcretaceus Migula. (Tireideweisserverflüssigender Micrococms, Keck, Inaug. Diss., Dorpat, 1890 , 64; Migula, Syst. d. Bakt., 2, 1900, 107.) Winslow and Winslow (loc. cit., 199) state that this species appears to be a synonym of Micrococcus albus Schroeter.

Micrococcus subflavescens Bergey et al. (Manual, 1st ed., 1923, 61.) From dust and water. Hucker (loc. cit., 9) considers this a synonym of Micrococcus flaves Trevisan. For a description of this species, see Bergey et al., Manual, 5 th ed.. 1939, 246.

Microcuccus subflavidus Migula. (Micrororrus tetragenus subflavus v. Besser, Beitr. z. allgm. Path. u. path. Anat., 6, 1889. 347 ; Migula, Syst. d. Bakt., 2, 1900, 190; Micrococcus subflavus Chester, Man. 1)eterm. Bact., 1901, 96; not Micrococcus subflarus Flügge, Die Mikroorganismen, 2 Auft., 1886, 159.) From normal nasal mucus. Winslow and Winslow (loc. cit. 184) state that this is apparently a synonym of Micrococcus aureus Zopf; while Hucker (loc. cit., 7 and 21 ) considers it probably identical with Vicrococcus $l u$ tens Cohn, Micrococcus varians Migula, or Gaffkya tetragena Trevisan.

Microcuccus subflavus Flügge. (Gelbweisser Diplococeus, Bumm, Die Mikroorg. d. gonorrh. Schleimhauterkr., 1 Aufl., 1485 and 2 Aufl., 1887, 20; Flügge, Die Mikroorganismen, 2 Aufl., 1886, 159; Veisseria subflava Trevisan, I generi e le specie delle Batteriacee, Milan, 1889, 3:; Diplococcus subflavus Fisenberg, Bakt. Diag., 3 Aufl., 1891, 307; not Micrococcus subfavus Chester, Man. Determ. Bact., 1901, 96.) From gonorrheal pus. Winslow and Winslow (loc, cit., 216) consider this a synonym of $\mathrm{Mi}$ crococcus flavus Trevisan. For a description of this species, see Bergey et al., Manual, 5th ed., 1939, 248.

Micrococcus subfuscus Matzuschita. (Cent. f. Bakt., I Abt., 29, 1901, 383.) 
From dust. Similar to Micrococcus juscus Adametz.

Micrococcus subgilvus Migula. (Micrococcus gilvus Henrici, Arb. a bakt. Inst. Karlsruhe, 1, Heft 1, 1894, 78; not Micrococcus gilvus Losski, Inaug. Diss., Dorpat, 1893, 60; Migula, Syst. d. Bakt., 2, 1900, 132.) From cheese. Winslow and Winslow (loc. cit., 220) regard this as a synonym of Micrococcus luteus Cohn.

Micrococcus subgranulatus Migula. (Micrococcus citreus granulatus Freund, Inaug. Diss., Erlangen, 1893, 27 ; Migula, Syst. d. Bakt., 2, 1900, 148; Micrococcus granulatus v. Bagarewski, Cent. f. Bakt., II Abt., 15, 1905, 7.) From the oral cavity. Winslow and Winslow (loc. cit., 216) consider this a synonym of Micrococcus flavus Trevisan. For a description of this species, see Bergey et al., Manual, 5th ed., 1939, 249.

Micrococcus subgriseus Migulą. (Grauer Coccus, Maschek, Jahresb. d. Kom.- Oberrealschule, Leitmeritz, No. 8, 1S87, 61; Migula, Syst. d. Bakt., 2, 1900, 94.) From water. Winslow and Winslow (loc. cit., 199) state that this appears to be a synonym of Micrococcus albus Schroeter; while Hucker (loc. cit., 19) regards it as a synonym of Micrococeus freudenreichii Guillebeau or of Micrococcus ureae Cohn.

Micrococcus sublacteus Migula. (No. 27, Lembke, Arch. f. Hyg., 29, 1897, 329 ; Migula, Syst. d. Bakt., 2, 1900, 210.) From feces. Winslow and Winslow (loc. cit., 199) state that this appears to be a synonym of Micrococcus albus Schroeter; while Hucker (loc. cit., 19) regards it as a synonym of Micrococcus freudenreichii Guillebeau or of Micrococcus ureae Cohn.

Micrococcus sublitacinus Migula. (No. 26, Lembke, Arch. f. Hyg., 26, 1896, 317 ; Migula, Syst. d. Bakt., 2, 1900, 205.) From feces. Hucker (loc. cit., 15) considers this a synonym of Micrococcus albus Schroeter.

Micrncoccus subluteus Weiss. (Arb. bakt. Inst. Karlsruhe, \&, Heft 3, 1902, 198.) From vegetable infusions.

Micrococcus subniveus Migula. (Micrococcus albidus Henrici, Arb. bakt. Inst. Karlsruhe, 1, Heft 1, 1894, 75; not Micrococcus albidus Losski, Inaug. Diss., Dorpat, 1893, 55; Migula, Syst. d. Bakt., 2, 1900, 105.) From Swiss cheese. Winslow and Winslow (loc. cit., 199) state that this appears to be a synonym of Micrococcus albus Schroeter.

Micrococcus subochraceus Migula. (No. 30, Lembke, Arch.f. Hyg., 29, 1897, 332 ; Migula, Syst. d. Bakt., 2, 1900, 215.) From feces. Winslow and Winslow (loc. cit., 216) regard this as a synonym of Micrococcus flavus Trevisan.

Micrococcus subroseus Migula. (Micrococcus roseus Eisenberg, Bakt. Diag., 3 Aufl., 1891, 408; Migula, Syst. d. Bakt., 2, 1900, 176; Nicrococcus rubescens Chester, Man. Determ. Bact., 1901, 105.) From the sputum of an influenza patient. Hucker (loc. cit, 26) states that this may be identical with Micrococcus roseus Flügge.

Micrococcus subterraneus Hansgirg. (Hansgirg, Oest. Bot. Zeitschr., 1888, No. 7-8, 8; Staphylococcus subterraneus DeToni and Trevisan in Saccardo, Sylloge Fungorum, 8, 1889, 1075.) From damp walls of wine cellars in Bohemia.

Micrococcus subtilis Migula. (Diplococcus, Kirchner, Ztschr. f. Hyg., 9, 1890, 528 ; Migula, Syst. d. Bakt., 2, 1300, 192.) Found in the sputum and r'ood of influenza patients.

Micrococcus siculentus Henrici. (Arb. bakt. Inst. Karlsruhe, 1, Heft 1, 1894, 63.) From Swiss cheese. Winslow and Winslow (loc. cit., 224) state that this is apparently a synonym of Micrococcus candicans Flügge.

Micrococcus sulphureus Zimmermann. (Bakt. unserer Trink- u. Nutzwässer, Chemnitz, I Reihe, 1890, 84.) From water. Winslow and Winslow (loc. cit., 220) regard this as a synonym of Micrococcus luteus Cohn.

Micrococcus suis Burrill. (Bacillus 
suis Detmers, Rept. U. S. Dept. Agric. for 1878; Burrill, Amer. Nat., 17, 1883, 320.) From blood of hogs sick with swine plague or hog cholera.

Micrococcus syphiliticus Migula. (Coccen, Disse, Deutsche med. Wchnschr., 13, 1887, 888; Migula, Syst. d. Bakt., 2, 1900 , 218.) This may be synonymous with Micrococcus candicans Flügge.

Micrococcus tardigradus Trevisan. (Micrococcus flavus tardigradus Flügge, Die Mikroorganismen, 2 Aufl., 1886, 175; Trevisan, I generi e le specie delle Batteriacee, Milan, 1889, 34; Micrococcus sulfureus $\beta$-tardigradus Lehmann and Neumann, Bakt. Diag., 1 Aufl., 2, 1896, 163 ; Micrococcus sulfureus var. tardigradus Löhnis and Pillai, Cent, f. Bakt., II Abt., 19, 1907, 92.) From air; also found in water. Winslow and Winslow (loc. cit., 220) regard this as a synonym of Micrococcus luteus Cohn.

Micrococcus tardior Migula. (Diplococcus flavus liquefaciens tardus Lnna and Tommasoli, Monatshefte f. prakt. Dermatol., 9, 1889, 56; Migula, Syst. d. Bakt., 2, 1900, V and 141; Micrococcus epidermis Chester, Man. Determ. Bact., 1901, 97; Diplococcus flavus-liquefaciens Chester, ibid.) From eczema. Winslow and Winslow (loc. cit., 216) regard this as a synonym of Micrococcus flaves Trevisan; while Hucker (loc. cit., 11 ) regards it as a synonym of 1 icrococeus citreus Migula.

Micrococcus tardissimus (Trevisan) Migula. (Milchweisser Micrococeus, Bumm, Mikroorg. d. gonorrh. Schleimhauterkr., 1 Aufl., 1885; Diplococcus albicans tardissimus Flügge, Die Mikroorganismen, 2 Aufl., 1886, 183; Neisseria tardissima Trevisan, I generi e le specie delle Batteriacee, Milan, 1889, 32 ; Micrococcus albicans tardissimus Sternberg, Man. of Bact., 1893, 882; Migula, Syst. d. Bakt., 2, 1900, 49.) Found in vaginal seeretions. Winslow and Winslow (loc. cit., 205) regard this as a synonym of Micrococcus candidus Cohn or of Gafflya tetragena Trevisan; while Hucker (loc. cit., 7 ) considers it a synonym of Micrococcus luteus Cohn or Micrococcus varians Migula .

Micrococcus tardus Migula. (Diplococcus albicans tardus Unna and Tommasoli, Monatshefte f prakt. Dermatol., 9, No. 2, 1889, 49; Micrococcus albicans tardus Sternberg, Man. of Bact., 1893, 882; Migula, Syst. d. Bakt., 2, 1900, 50; Micrococcus eczemae Chester, Man. Determ. Bact., 1901, 86). From eczema. Winslow and Winslow (loc. cit., 216 and 224) regard this as a synonym of Micrococcus flavus Trevisan or of Micrococcus candicans Flügge.

Micrococcus tenacatis Chester. (No. 43, Conn, Storrs Agr. Exp. Sta. 7th Ann. Rept., 1895, 78; Chester, Man. Determ. Bact., 1901, 88.) From milk from Uruguay. Winslow and Winslow (loc. cit., 220 ) state that this is apparently a synonym of Micrococcus candicans Flügge.

Micrococeus tener Weiss. (Arb. bakt. Inst. Karlsruhe, 2, Heft 3, 1902, 200.) From a vegetable infusion.

Micrococeus lenuissimus Migula. (Micrococcus cumulatus tenuis v. Besser, Beitr. z. path. Anat., 6, 1889, 347; Migula, Syst. d. Bakt., 2, 1900, 55; Micrococcus cumulatus Chester, Man. Determ. Bact., 1901, 87.) Frequently found in human nasal mucus. Winslow and Winslow (loc. cit., 205) regard this as a synonym of Micrococcus candidus Cohn or of Gafflya tetragena Trevisan.

Micrococcus tetragenus aureus Boutron. (Thèse, Paris, 1893; Abst. in Cent.f. Bakt., 16, 1894, 971.) Hucker (loc.cit., 21) regards this as a synonym of Gaffkya tetragena Trevisan.

Micrococcus tetragenus concentricus Schenk. (Allg. Wien. med. Zeitung, 1892, 81 and 92 ; Abst. in Cent. f. Bakt., 13, 1893, 720.) From feces. Motile.

Micrococcus tetragenus-pallidus Chester. (Micrococcus tetragenus pallidus, Dyar, Ann. N. Y. Acad. Sci., 8, 1895, 354; (hester, Man. Determ. Bact., 1901, 93.) From dust. Probably a variety of $M i$ crococcus versatilis Chester, see below. 
Micrococcus tetragenus-vividus Chester. (Micrococcus tetragenus vividus Dyar, Ann. X. Y. Acad. Sci., 8, 1895, 354; Chester, Man. Determ. Bact., 1901, 102.) From dust. Probably a variety of Micrococcus versatilis ('hester, see below.

Micrococcus tetras Henrici. (Arb). bakt. Inst. Karlsruhe, 1, Heft 1, 1894, 60; Pediococcus tetras Pribram, Klassifikation der Schizomyceten, 1933, 46. . From cheese. Winslow and Winslow (loc. cit., 22t) state that this species is apparently the same as Micrococcus candicans Flügge.

Micrococcus thermophilus Hansgiry. (Oestr. Bot. Ztschr., No. 3, 1888, 5.) From hot springs.

Micrococcus toxicatus Burrill. (Amer. Nat., 17, 1883, 319.) From poison ivy and other plants in the genus Rhus.

Micrococcus trachomatis Migula. (Trachomococcus, Sattler, in Zehender, Klin. Monatsbl., 1881; Trachomococcus, Michel, Arch. f. Augenheilk., 16, 1886 ; Neisseria micheli Trevisan, I generi e le specie delle Batteriacee, Milan, 1889, 32 ; see Baumgarten, Lehrb. d. path. Mykol., 1, 1890, 421; Migula, Syst. d. Bakt., 2, 1900, 67.) Winslow and Winslow (loc. cit., 205) consider this to be a synonym of Micrococcus candidus Cohn or of Gaffiya tetragena Trevisan.

Micrococcus tributyrus stark and Scheib. (Jour. Dairy Sci., 19, 1936, 210.)

From butter.

Micrococcus tritici Prillieux. Maladies des plantes agricoles, 1, 1895, 7; not Micrococcus tritici Köck, Monatshefte f. Landwirtschaft, 1909, 247, quoted from Lehmann and Neumann, Bakt. Diag., 5 Aufl., 2, 1912, 653.) Considered patho. genic on wheat.

Micrococcus tuberculosus Migula. (No. 23, Lembke, Arch. f. Hyg., 29, 1897. 325 ; Migula, Syst. d. Bakt., 2, 1900, 214.) From feces. Winslow and Winslow (loc. cit., 224) state that this is apparently a synonym of Micrococcus candicans Flügge.
Micrococcus typhoideus Migula. (Coccus A, Foutin, Bakt. Lntersuch. v. Hagel, Wratsch, 1889, No. 49 and 50 ; see Cent. f. Bakt., 7, 1890, 373; Migula, Syst. d. Bakt., \& 1900, 94; Micrococcus alpha Chester, Man. Determ. Bact., 1901, 93.) From hail. Winslow and Winslow (loc. cit., 199) state that this appears to be a synonym of Micrococcus albus Schroeter; while Hucker (loc. cit., 25) states that it may be identical with. Micrococcus roseus Flügge.

Micrococcus ulceris de Luca. (Gazzetta degli Ospitali, 1886; Abst. in Cent. f. Bakt., 1, 1887, 333; Micrococcus ulceris mollis de Luca, ibid.) From the secretion of a venereal ulcer.

IIicrococcus ulmi Brussoff. (Cent. f. Bakt., II Abt., 63, 1925, 261.) Isolated from diseased elm trees.

Micrococcus umbilicatus Weiss. (Arb. bakt. Inst. Karlsruhe, 2, Heft 3, 1902, 186.) From a bean infusion. Hucker (loc. cit., 12) considers this a synonym of Iicrococcus aureus Zopf.

Micrococcus ureae Migula. (Torule ammoniacale, Pasteur, Ann. de Chim. et de Phys., 3 sér., 64, 1862, 52; van Tieghem, Comp. rend. Acad. Sci., Paris, 58, 1864, 210; Torula ureae Lea, Jour. of Physiol., 11, 1890, 226; Migula, in Engler and Prantl. Die natürl. Pflanzenfam., 1, 1a. 1895, 17.) From urine. May not be the same as Micrococcus ureae Cohn.

Micrococcus urinalbus De Toni and Trevisan. (Micrococcus albus urinae Doyen, Jour. d. connaiss. médic., No. 14, 1889, 108; De Toni and Trevisan, in Saccardo, Sylloge Fungorum, 8, 1889, 1076.) From urine. Hucker (loc. cit., 15) considers this a synonym of IIicrococcus albus schroeter.

Micrococcus uruguae Chester. (No. 40, Conn, Storrs Agr. Exp. Sta. 7th Ann. Rept., 1895, 78; Chester, Man. Determ. Bact., 1901, 100.) From milk from Uruguay. Winslow and Winslow (loc. cit., 216) regard this as a synonym of 
Micrococcus flavus Trevisan; while Hucker (loc. cit., 10) regards it as a synonym of Micrococcus conglomeratus Migula.

Micrococcus utriculosus Migula. (No. 20, Lembke, Arch. f. Hyg., 29, 1897, 327 ; Migula, Syst. d. Bakt., 2, 1900, 199.) From feces. Winslow and Winslow (loc. cit., 199) state that this appears to be a synonym of Micrococcus albus Schrocter.

Micrococcus varians lactis Conn. (Storrs Agr. Exp. Sta. 12th Ann. Rept., $1900,37$.$) From milk, cream, dust.$ According to Weigmann (In Lafar, Handb. d. techn. Mykologie, 2, 1905, 13) this is identical with Staphylococcus mastitis albus. Hucker (loc. cit., 11) regards it as a synonym of Micrococcus citreus Migula.

Micrococcus variococcus Müller-Thurgau and Osterwalder. (Cent. f. Bakt., II Abt., 33, 1913, 236.) From wine.

Micrococcus versatilis Chester. (Microcorcus tetragenus febris flavae Finlay; Micrococcus tetragenus versatitis Sternberg, Report un etiology and prevention of yellow fever, Washington, 1891, 164; Chester, Man. Determ. Bact., 1901, 102.) Isolated from the exerement of mosquitoes which had sucked the blood of yellow fever patients; and from dust. Winslow and Winslow (loc. cit.,216) regard this as a synonym of Micrococcus flavus Trevisan.

Micrococcus versicolor Flügge. (Die Mikroorganismen, 2 Aufl., 1886, 177.) From dust. Winslow and Winslow (loc. cit., 220) consider this a synonym of Micrococcus luteus Cohn.

Micrococcus vesicae Heim. (Lehrb. d. Bakt., 2 Aufl., 1898, 297.) From acid urine. Winslow and Winslow (loc cit., 221) state that this is apparently a synonym of Micrococens candicans Flügege.

Micrococcus vesicans Haman. (Tour. Path. and Bact., 9, 1904, 1.) Considered the cause of veld sore, al disense of Africa and tropical Australia.

Hicrococcus vesicosus Weiss. (Arb) bakt. Inst. Karlsruhe, 2, Heft 3, 1902, 203.) From a vegetable infusion.
Hucker (loc. cit., 8) considers this species identical with either Micrococcus luteus Cohn or Micrococcus varians Migula.

Micrococcus vesiculiferus Migula. (No. 28, Lembke, Arch.f. Hyg., 29, 1897, 330 ; Migula, Syst. d. Bakt., 2, 1900, 211.) From feces. Winslow and Winslow (loc. cit., 220) regard this as a synonym of Micrococcus luteus Cohn.

Micrococcus vincenzii Chester. (Micrococcus tetragenus citreus Vincenzi, La Riforma Med., 1897, 758; Chester, Man. Determ. Bact., 1901, 103.) From the submaxillary lymphatic gland of a child. Winslow and Winslow (loc. cit., 220) reg:rrt this as a synonym of Micrococcus luteus (ohn.

Micrococcus vini Migula. (Micrococcus saprogenes vini I, Irramer, Bakt. in Beziehungen z. Landwirtsch. u. d. landwirtsch-techn. Gewerben, II Teil, 1892, 139; Migula, Syst. d. Bakt., 2, 1900, 118.) From wine. Winslow and Winslow (loc. cit., 199) state that this appears to be a synonym of Micrococcus albus Schroeter; while Hucker (loc. cit., 8) considers it identical with Micrococcus luteus Cohn or Mierococcus varians Migula.

Micrococcus viniperda Schroeter. (Schroeter in Cohn, Kryptog.-Flora v. Schlesien, 3, 1, 1886, 144.) From dust, feces, etc.

Micrococcus viscosus Bergey et al. (Micrococcus lactis viscosus $B$, Conn, Esten and Stocking, Storrs Agr. Exp. Sta. Rept. for 1906, 109; Bergey et al., Manual, Ist ed., 1923, 68.) From pasteurizer milk. For a description of this species, see Bergey et al., Manual, 5th al., 1939, 256. See Micrococcus lactis viscosus Sternberg.

Micrococcus viscosus lactis Conn. (Storrs Igr. Lxp. Sta. 12th Ann. Rept., 1900, 44.) From milk.

Micrococcus viticulosus Flügge. (Die Miliroorganismen, 2 Aufl., 1886, 178.) From dust and water. Winslow and Winslow (loc. cit., 205) consider this to 
be a synonym of Micrococcus candidus Cohn or of Gaffkya telragena Trevisan.

Micrococcus vulgaris Echstein (Ztschr. f. Forst- u. Jagdwesen, 26, 1894, 17 ; Abst. in Cent. f. Bakt., I Abt., 18, 1895, 292; not Micrococcus vulgaris Weiss, Arb. bakt. Inst. Karlsruhe, 2, Heft 3, 1902, 193.) From insects.

Micrococcus xanthogenicus (Freire) Trevisan. (Cryptococcus xanthogenicus Freire, Recherches sur la eause de la fièvre jaune, Rio de Janeiro, 1884; Trevisan, I generi e le specie delle Batteriacee, Milan, 1889, 33.) Isolated from yellow fever and supposed by Freire to be the cause of the discase. Winslow and Winslow (loc. cit., 199) state that this appears to be a synonym of Micrococus albus Schroeter.

Micrococcus xenopus Schrire and Greenfield. (Trans. Royal Soc. So. Africa, 17, 1930, 309.) From an abscess in a toad (Xenopus sp.). For a description of this species, see Bergey et al., Manual, 5th ed., 1939, 243.

Iicrococcus xerophilus

Glage. (Ztschr. f. Fleisch-u. Milchhygiene, 10, 1900,145 .) From coating on surface of dry wurst and similar meat products.

lícrococcus zeae Scrbinov. (La Defense des Plantes, 2, 1926, 546.) From flour, grain and seedlings of corn. Was thought to be a cause of pellagra in South Pussia.

Micrococcus zonatus Henrici. (Arb. bakt. Inst. Karlsruhe, 1, Heft 1, 1894, 68.) From cheese. Winslow and Winslow (loc. cit., 224) state that this is apparcntly a synonym of Micrococcus candicans Flügge.

Planococcus casei Migula. Micrococcus No. III, Adametz, Landwirtsch. Jahrb., 18, 1889, 240; Migula, Syst. d. Bakt., 2, 1900, 270.) From Emmenthal cheese.

I lanococcus loeffleri Migula. (Löfler, Cent.f. Bakt., 7, 1890, 637 ; Migula, Syst. d. Bakt., 2, 1900, 273.) From colony on an old gelatin plate.

Planococcus luteus (Adametz) Migula.
(Diplococcus luteus Adametz, Mitteil. d. oesterr. Vers. Station f. Brauerei u. Mälzerei in Wien, Heft I, 1888, 39; Neisseria lutea Trevisan, I generi e le specie delle Batteriacee, Milan, 1889, 32; Migula, Syst. d. Bakt., 2, 1900, 274.) Hucker (loc. cit., 9) considers this species a synonym of Micrococcus flavus Trevisan.

Rhodococcus fulvus Winslow and Rogers. (Jour. Inf. Dis., 8, 1906; 545; not Micrococcus fulvus Cohn, Beitr. z. Biol. d. Pflanzen, 1, Heft 3, 1875, 181.) From soil, air and water.

Staphylococcus albicans Stigell. (Cent. f. Bakt., I Abt., Orig., 45, 190s, 489.) Probably intended for Micrococcus albicans amplus Flügge.

Staphylococcus albus liquefaciens Sternberg. (White liquefying staphylococcus, Escherich, Die Darmbakterien des Säuglings, Stuttgart, 1886, 88; Sternberg, Manual of Bact., 1893, 607.) Found occasionally in the feces of healthy infants.

Staphylococcus albus non liquefaciens Hlava. (Sborník lékařský, II, Prạue, 1887, 12 pp.; see Cent. f. Bakt., 2, 1887, 688.) Probably a synonym of Micrococcus albocereus Migula.

Staphylococcus anaerobius Heurlin. (Bakt. Unters. d. Keimgehaltes im Genitalkanale d. fiebernden Wöchnerinnen, Helsingfors, 1910, 120.) See Tinberg, Nativelle and Prévot, Les Microbes Anzérobies, Paris, 1937, 1027; probably not the same as Staphylococcus anaerobius Hamm, Die puerperale Tundinfektion, Berlin, 1912. Incompletely described. From genital tract.

Staphylococcus anaerobius major Heurlin (loc. cit., 120). From genital tract.

Staphylococcus anaerobius minor Heurlin (loc. cit., 120). From genital tract.

Staphylococcus aureus sarciniformis Rosenhauch. (Klin. Monatsbl.f. Augenheilkunde, Jahrg., 8, 1909, 257 ; Abst. in Cent. f. Bakt., I Abt., Ref., 45, 1910, 787.)

Staphylococcus bovis Ford. (Staphylococcus pyogenes boris Lucet, Ann. Inst. 
Past., 7, 1893, 327 ; Ford, Textb. of Bact., 1927,424 .) Found in suppurative lesions of cattle.

Staphylococcus candidus Warrington. (Lancet, 1, 1888.)

Staphylococcus flavocyaneus Kinaysi. (Jour. Bact., 43, 1942, 368.) Found as a contaminant in dissociation studies.

Staphylococcus flavus non pyogenes Fränkel and Sänger. (Arch. f. path. Anat., 108, 1887, 286; Abst. in Cent. f. Bakt., 3, 1888, 281.) Found in endocarditis ulcerosa.

Staphylococcus griseus Tavel. (Quoted from Lehmann and Neumann, Bakt. Diag., 1 Aufl., 2, 1896, 173.) From pus. Staphylococcus griseus radiatus Viti. (Atti d. R. Acead. d. Fisiocritici di Siena, Ser. IV., 2, 1891, Abst. in Cent. f. Bakt., 11, 1892, 672.) From cases of endocarditis.

Staphylococcus habanensis Gibler. (Quoted from Fernandez, Cronica médico quirúrgica de la Habana, 1891, No. 30; Abst. in Cent. f. Bakt., 11, 1892, 472.) Isolated from the human eye.

Staphylococcus insectorum Krassilstschik. (Quoted from Paillot, Les maladies du ver à soie grasserie et dysenteries, 1928, 171.) From the intestinal tract of the silkworm (Bombyx mori).

Staphylococcus lactis acidi MeDonnell. (Inaug. Diss., Kiel, 1899; Abst. in Cent. f. Bakt., II Abt., 6, 1900, 120.)

Staphylococcus leloirii Trevisan. (Microbe des périfolliculites conglomérées, Leloir, Soc. anatomique, May, 1881; Trevisan, I generi e le specie delle Batteriacee, Milan, 1889, 33.)

Staphylococcus liquefaciens aurantiacus Distaso. (Cent. f. Bakt., I Abt., Orig., 59, 1911, 102.) From feces.

Staphylococcus muscae Glaser. (Amer. Jour. Hyg., 4, 1924, 411.) Causes a fatal infection in house flies (Muscadomestica). For a description of this species, see Bergey et al., Manual, 5th ed., 1939, 264.

Staphylococcus non pyogenes Savor. (Beitr. z. Geburtshilfe u. Gynäkol.v. Hegar, 2, Heft 1, 1898; Abst. in Cent. f.
Bakt., I Abt., 26, 1899, 642.) From urino-genital tract.

Staphylococcus pharyngis Bergey et al. (Manual, 1st ed., 1923, 56.) Found in the human nasopharynx in acute catarrhal inflammation. For a description of this species, see Bergey et al., Manual, 5th ed., 1939, 265.

Staphylococcus putrificus Schottmüller. (Leitfaden f. d. Klinisch-Bakt. KulturMethoden, Berlin, Wien, 1923. Quoted from Weinberg, Nativelle and Prévot, Les Microbes Anaérobies, Paris, 1927, 1027.)

Staphylococcus pyogenes liquefaciens albus Hlava. (Sborník lékařský, II, Prague, 1887, 12 pp.; Abst. in Cent. f. Bakt., 2, 1887, 688.) From small pox pustules.

Staphylococcus pyogenes tenuis Scheibe. (Inaug. Diss., München, 1889; see Cent. f. Bakt., $6,1889,186$.) From middle ear infections.

Staphylococcus roseus Tavel. (Quoted from Lchmann and Neumann, Bakt. Diag., 1 Aufl., 2, 1896, 177.) Evidently identical with Micrococcus roseus Lehmann and Neumann or Micrococcus roseo-fulvus Lehmann and Neumann.

Staphylococcus salivarius Andrewes and Gordon. (35th Ann. Rept. Local Govt. Board London, 1905-06, 558.) From saliva. Probably Micrococcus candidus Cohn.

Staphylococcus ureae candidus Lundström. (Festschr. d. path.-anat. Inst. z. Andenken a. d. 250 jährige Bestehen d. finnländ. Univ. Helsingfors, 1890 ; abst. in (ent. f. Bakt., 9, 1891, 672.) From urine. Probably Micrococcus ureae Cohn.

Staphylococcus ureae non pyogenes Barlow. (Arch. f. Dermat. u. Syph., 1893; Abst. in Cent. f. Bakt., 14, 1893, 456.) From cases of cystitis.

Urococcus dowdeswelli Miquel. (Ann. de Micrographie, 5, 1893, 209.) Ferments urea.

Urococcus van tieghemi Miquel (loc. cit., 161). Ferments urea. 


\section{Genus II. Gaffkya Trevisan.*}

(Trevisan, Atti d. Accad. Fisio-Medico-Statistica in Milano, Ser. 4, 3, 1885, 106; Tetracoccus Klecki, Cent. f. Bakt., 15, 1894, 360; not Tetracoccus Orla-Jensen, The Lactic Acid Bacteria, Mém. Acad. Sci. Danemark, Sec. Sci., 8 sér, 5, 1919, 154; Tetradiplococcus Bartoszewiez and Schwarzwasser, Cent. f. Bakt., II Abt., 21, 1908, 614.) Named for Prof. Georg Gaffky, 1850-1918, Berlin.

Occur in the animal body and in special media as tetrads, while in ordinary culture media they occur in pairs and irregular masses. Aerobic to anaerobic. Gram-positive. Parasitic organisms.

The type species is Gaffkya tetragena (Gaffky) Trevisan.

Key to the species of genus Gaffkya.

I. Facultative aerobe.

II. Strict anaerobe.

1. Gaffkya tetragena (Gaffky) Trevisan. (Micrococcus tetragenus Gaffky, Arch. f. Chirurg., 28, Heft 3, 1883, 500 ; Trevisan, Atti d. Accad. Fisio- MedicoStatistica in Milano, Ser. 4, 3, 1885, 106 ; Micrococcus tetragenus septicus Boutron, Thesis, Paris, 1893; Abst. in Cent. f. Bakt., 16, 1894, 971; Hicrococcus tetragenus albus Boutron, ibid.; Merista septica Hueppe, Principles of Bacteriology (Eng. trans.), 1899, 170; Sarcina septica Hueppe, ibid.; Sarcina tetragena Migula, Syst. d. Bakt., 2, 1900, 225; Merista tetragena Vuillemin, Ann. Mycologie, Berlin, 11, 1913, 525; Staphylococcus tetragenus Holland. Jour. Bact., 5, 1920 , 224; Tetracoccus septicus Neveu-Lemaire, Précis Parasitol. Hum., 5th ed., 1921, 18; Pediococcus tetragenus Pribram, K̈lassifikation der Schizomyceten, 1933, 46.) From Greek, tetra (tetara), four; M. L. genes, producing.

Spheres: 0.6 to 0.8 micron in size, with pseudocapsule (in body fluids) surrounding four of the elements showing typical tetrads. Gram-positive.

\section{Gaffkya tetragena.}

\section{Gafjiya anaerobia.}

Gelatin colonies: Small, 1 to $2 \mathrm{~mm}$, in diameter, white convex.

Gelatin stab: Thick, white surface growth. No liquefaction.

Agar colonies: Circular, white, smooth, glistening, entire. Reimann (Jour. Bact, 31, 1936, 385) has described eleven colony form variants for this species.

Agar slant: White, moist, glistening.

Broth: Clear, with gray viscous sediment.

Litmus milk: Slightly acid.

Potato: White, viscid.

Indole not formed.

Nitrites not produced from nitrates.

Starch not hydrolyzed.

Ammonium salts not utilized.

Acid from glucose, lactose and glycerol.

No $\mathrm{H}_{2} \mathrm{~S}$ formed.

Aerobic, facultative.

Pathogenic for mice and guinea pigs; rabbits less susceptible.

Optimum temperature $37^{\circ} \mathrm{C}$.

Source: Isolated from sputum in tuberculosis; also from air and skin.

Habitat: Mucous membrane of respiratory tract.

* Revised by Prof. G. J. Hucker, N. Y. State Experiment Station, Geneva, New York, March, 1943. 
2. Gaffkya anaerobia (Choukévitch) Prévot. (T'etracoccus anaerobius Choukéviteh, Ann. Inst. Past., 25, 1911, 319; Micrococcus tetragenes anaerobius Hamm, Die puerperale Wundinfektion, Berlin, 1912 ; Prévot, Ann. Sci. Nat., Sér. Bot., 15, 1933, 203.) From Greek, an, without; aer, air; bios, life.

Spheres: About 1.0 to 1.5 microns, occurring in tetrads, sometimes in groups of eight. Gram-positive.

Gelatin: No liquefaction.

Deep agar colonies: After 24 to 48 hours, small, grayish, 2 to $3 \mathrm{~mm}$. in diameter. Abundant production of gas which breaks up the agar.

Broth: Poor growth. Slight sediment.

Milk: Unchanged.

Coagulated proteins not digested.

Optimum temperature $37^{\circ} \mathrm{C}$. growth at $22^{\circ} \mathrm{C}$.

Non-pathogenic for guinca-pigs or rabbits.

Strict anaerobe.

Distinctive characters: Prefers acid media.

Source: Isolated from the female genital tract; isolated from the large intestine of a horse.

Habitat: Probably widely distributed in natural cavities of man and animals.

Appendix: The following species have been placed in the genus Gaffkya or in the genus Tetracoccus.

Gaffkya archeri Trevisan. (A black micrococcus, Archer, Quart. Jour. Microscop. Sei., 1874, 321; Trevisan, I generi e le specie delle Batteriacee, Milan, 1859, 27.)

Gaffkya grandis DeToni and Trevisan. (Mierocoque des reins et des ulcéres syphilitiques de la peau, Babes, in Cornil and Babes, Les Bactér., 2nd ed., 1886, 782; DeToni and Trevisan, in Saccardo, Sylloge Fungorum, 8, 1889, 10.12.)

Gaffiya mendozae DeToni and Trev- isan. (Micrococcus tetragenus and Micrococcus tetragenus mobilis ventriculi Mendoza, Cent. f. Bakt., 6, 1889, 567; DeToni and Trevisan, in Saccardo, Sylloge Fungorum, 8, 1889, 1043; Planococcus tetragenus Migula, Syst. d. Bakt., 2, 1900, 269 ; Micrococcus mendozae Chester, Man. Determ. Bact., 1901, 84; Sarcina tetragena Winslow and Rogers, Jour. Inf. Dis., 3, 1906, 545; Planomerista ventriculi Vuillemin, Ann. Mycolog., Berlin, 11, 1913, 525.) Motile. Isolated from the contents of the stomach. Hucker ( $N$. Y. State Exp. Sta. Tech. Bull. No. 102, 1924,21 ) regards this as a synonym of Gaffkya tetragena Trevisan.

Gafflya tardissima (Altana) Bergey et al. (Tetragenus tardissimus Altana, Cent. f. Bakt., I Abt., Orig., 48, 1909, 42; Bergey et al., Manual, 2nd ed., 1925, 59.) From a natural infection of guinea pigs. See Manual, 5th ed., 1939, 269 for a description of this species.

Gafflya verneti Corbet. (Organism No. 21, Denier and Vernet, La Caoutchouc, 17, 1920, 10491; Corbet, Quart. Jour. Rubber Research Inst., Malaya, 2, 1930, 143.) From the latex of the Para rubber tree (Hevea brasiliensis). For a description of this species, see Manual, 5th ed., 1939, 269.

Tetracoccus carneus halophilus Horowitz-Wlassowa. (Cent.f. Bakt., II Abt., $85,1932,16$.$) Isolated from salted in-$ testines (Wiener skins).

Tetracoccus casei Orla-Jensen. (The Lactic Acid Bacteria, 1919, 80.) From cheese. Probably identical with Micrococens freudenreichii Guillebeau.

Tetracoccus mastitidis Orla-Jensen (loc. cit., 81). From milk of a woman with mastitis. Orla-Jensen thinks this is identical with the staphylococcus that causes mastitis in cows, i.e., Micrococcus pyogenes var. aureus Zopf.

Tetracoccus mycodermatus Orla-Jensen (loc. cit., 81). From Camembert cheese. 


\section{Genus III. Sarcina Goodsir.*}

(Goodsir, Edinborough Med. and Surg. Jour., 1812, 430; Lactosarcina Beijerinck, Arch. néerl. d. sci. exact., Ser. 2, 13, 1908, 359; Urosarcina Miquel, Ann. Microg., 1, 1888, 517; Planosarcina Migula, Arb. Bakt. Inst. Karlsruhe, 1, 1894, 236 ; Pseudosarcina Löhnis, Handb. d. landwirtsch. Bakt., 1910, 449 (Pscudo-sarcina, Mazé, Compt. rend. Acad. Sci. Paris, 187, 1903, 887) ; Sporosarcina Orla-Junsen, C'ent. f. Bakt., II Abt., 22, 1909, 340; Paulosarcina Enderlein, Sitzber. Gesell. Naturf., Berlin, 1917, 319; Phacelium Enderlein, ibid., 319; Zymosarcina Smit, Die Gärungssarcinen. Pflanzenforschung, Heft 14, 1930, 26; Butyrisarcina Kluyver and Van Niel, Cent. f. Bakt., II Abt., 94, 1936, 400; Methanosarcina Kluyver and Van Niel, ibid.) From Latin sarcina, packet, bundle.

Division occurs, under favorable conditions, in three planes, producing regular packets. Usually Gram-positive. Growth on agar abundant, usually with formation of yellow or orange pigment. Glucose broth slightly acid, lactose broth generally neutral. Gelatin frequently liquefied. Nitrites may or may not be produced from nitrates. Saprophytes and facultative parasites.

The type species is Sarcina ventriculi Goodsir.

\section{Key to the species of genus Sarcina.}

I. Microaerophilic to anaerobic.

A. No growth without sugars. Do not produce methane. Sub-genus Zymosarcina Smit (Die Gärungssarcinen. Pflanzenforschung, Heft 14, 1930, 26).

1. Cellulose reaction positive. Slow coagulation in litmus milk.

1. Sarcina ventriculi.

2. Cellulose reaction negative. Litmus milk not coagulated.

2. Sarcina maxima.

B. Does not utilize sugars. Produces methane. Sub-genus Methanosarcina Kluyver and van Niel (Cent. f. Bakt., II Abt., 94, 1936, 400 ).

3. Sarcina methanica.

II. Aerobic.

A. No endospores present. Sub-genus Sarcinococcus subgen. not.

1. Not halophilic.

a. Non-motile.

b. Yellow pigment produced. Nitrites not produced from nitrates. c. Milk alkaline; coagulated.

4. Sarcina lutea.

cc. Milk alkaline; not coagulated.

5. Sarcina flava.

bb. Orange pigment produced. Nitrites produced from nitrates.

6. Sarcina aurantiaca.

aa. Motile.

7. Sarcina citrca.

2. Halophilic red chromogen.

8. Sarcina littoralis.

B. Endospores present. Motile. Sub-genus Sporosarcina Orla-Jensen (Cent.f. Balit., II Abt., 22, 1909, 340).

9. Sarcina ureae.

* Revised by Prof. Robert S. Breed, New York State Fxperiment Station, Geneva, New York, March, 1943. 
1. Sarcina ventriculi Goodsir. (Goodsir, Edinborough Med. and Surg. Jour., 57, 1842, 430; Merismopedia goodsirii Husemann, De anim. et végét., 18-, 13 ; Merismopedia ventriculi Robin, Histoire des végét. parasites, 1853, 331; Anaerobic sarcina, Beijerinck, Proc. of Section of Sciences, Kon. Akad. v. Wetensch., Amsterdam, 7, 1905, 580; Zymosarcina ventriculi Smit, Die Gärungssarcinen. Pflanzenforschung, Jena, Heft 14, 1930, 26; Sarcina beijerincki Prévot, Ann. Sci. Nat., Sér. Bot., 15, 1933, 205.) From Latin, ventriculus, the stomach.

Description taken in part from smit (loc. cit.).

Large spheres: 3.5 to 4.0 microns, occurring in packets of $8,16,32$ or more elements. Non-motile. Gram-positive. Cellulose reaction positive.

Growth occurs only in sugar media, containing peptones.

Gelatin: No liquefaction.

Deep glucose agar colonies: Multilenticular, surrounded by a cloudy zone. Abundant gas.

Glucose agar slant: Round, whitish colonies, several millimeters in diameter.

Glucose broth: Abundiunt, flaky growth. Abundant gas. Acid. No turbidity.

Plain peptone water: No growth.

Sugar peptone water: Abundant growth. Gas. Indole not formed.

Milk: Slow growth. Acid and coagulation.

Coagulated proteins not attacked.

Acid and gas from glucose, fructose, sucrose, maltose, lactose and galactose. No acid from xylose, arabinose, raffinose, mannitol, dulcitol, salicin, starch, glycerin and inulin.

Neutral red broth changed to fluores cent yellow.

Utilizes peptones, wort and yeast water as sourees of nitrogen. ('annot utilize amino acids or inorganic nitrogen.

Principal products of metabolism are carbon dioxide and ethyl alrohol.
Nitrites not produced from nitrates. Non-pathogenic.

Optimum pH 1.5 to 5.0 . Limits of pH 0.9 to 9.8 .

Temperature relations: Optimum $30^{\circ} \mathrm{C}$. Maximum $45^{\circ} \mathrm{C}$. Minimum $10^{\circ} \mathrm{C}$. Killed in ten minutes at $65^{\circ} \mathrm{C}$.

Microaerophilic to anaerobic.

Source: Isolated from a diseased stomach.

Habitat: Garden soil, dust, sand, mud; the stomach.

2. Sarcina maxima Lindner. (Lindner, Die Sarcina-Organismen der Gärungsgewerbe. Inaug. Diss., Berlin, 1888, 5t; Also abstract in Cent. f. Bakt., 4, 1885, 427; Zymosarcina maxima Smit, Die Gärungssarcinen. Pflanzenforschung, Heft 14, 1930, 22; Butyrisarcina maxima liluyver and van Niel, Cent. f. Bakt., II Abt., 94, 1936, 400.) From Itatin maxima, largest.

Deseription from Weinberg, Nativelle and Prévot, Les Microbes Anaérobies, 1937, 1030 and from Smit, loc. cit.

Large spheres: 4.0 to 4.5 microns, occurring in regular packets of $8,16,32$ or more elements. Non-motile. Grampositive.

Growth oecurs only in sugar media, rontaining peptones

Gelatin : No liquefaction.

Deep glucose agar colonies: Multilenticular. Abundant gas produced.

Glucose agar slant: Round, whitish colonies.

Glucose broth: Abundant growth, flaky, gaseous, marked acidification. Disagreeable butyric odor. No turbidity.

Sugar peptone water: Abundant growth, Haky, gaseous, followed by acidification.

Milk: Not coagulated.

Coagulated proteins not attacked.

Cellulose reaction negative.

Aeid and gas from glucose, fructose, galactose, maltose, sucrose and lactose.

Neutral red broth changed to fluorescent yellow. 
Utilizes peptones, yeast water or broth as source of nitrogen. Cannot utilize amino acids or inorganic nitrogen.

Principal products of metabolism are carbon dioxide, butyric and acetic acids.

Non-pathogenic.

Limits of $\mathrm{pH} 1.0$ to 9.5 .

Temperature relations: Optimum $30^{\circ} \mathrm{C}$. Maximum $40^{\circ} \mathrm{C}$. Minimum $15^{\circ} \mathrm{C}$. Filled in twenty minutes at $55^{\circ} \mathrm{C}$.

Microaerophilic to anaerobic.

Source: Isolated from fermenting malt mash.

Iabitat: Acidified flour pastes, wheat bran; seldom in soils. Also intestinal contents of guinea pigs (Crecelius and Rettger, Jour. Bact., 46, 1943, 10 ).

3. Sarcina methanica (Smit) Weinberg et al. (Methaansareine, Söhngen, Inaug. Diss., Delft, 1906, 104; Zymosarcina methanica Smit, Die Gärungssarcinen. Pflanzenforschung, Heft 14, 1930, 25; Methanosarcina methanica Kluyver and Van Niel, Cent. f. Bakt., II Abt., 94, 1936, 400; Barker, Arch. f. Mikrobiol., $\gamma$, 1936, 420; Weinberg, Nativelle and Prévot, Les Microbes Anaérobies, 1937, 1032.) From M. L. methanum, methane; M. L. methanicus, related to methane.

Description from Weinberg, Nativelle and Prévot (loc. cit.) and Smit (loc.cit.).

Spheres: 2.0 to 2.5 microns, occurring in packets of 8 or more cocei. Non-motile. Gram-variable.

Growth in solutions of calcium acctate and possibly butyrate and inorganic ammonium salts. Carbon dioxide is needed for growth.

In acetate-agar (with addition of some $\mathrm{H}_{22} \mathrm{~S}$ and $\mathrm{NaHCO}_{3}$ ): Colonies of 50 to 100 microns are formed, showing gas formation.

Cultural characters as yet unknown.

Peptones not attacked.

Cellulose reaction negative.

Utilizes ammonium salts as source of nitrogen. No organic nitrogen compounds utilized.
Carbohydrates not fermented. Ethyl alcohol is not fermented.

Principal products from the metabolism of calcium acetate and butyrate are methane, carbon dioxide and calcium carbonate.

Non-pathogenic.

Optimum temperature $35^{\circ}$ to $37^{\circ} \mathrm{C}$.

Strict anaerobe. Filled by a short contact with the air.

Distinctive characters: Utilizes ammonium salts and acyclic acids producing methane and carbonic acid.

Source: Sediment in methane fermentation (Weinberg et al.). Isolated from mud (Smit).

Habitat: Swamp waters and mud; fermenting sewage sludge.

4. Sarcina lutea Schroeter. (Kryptog. Flora v. Schlesien, 3, 1, 1886, 154; also see Klein, Microorganisms and Disease, 1885, 43; Eisenberg, Bakt. Diag., 1 Aufl., Taf. 2, 1886; Flügge, Die Mikroorganismen, 2 Aufl., 1886, 179; Frankland and Frankland, Phil. Trans. London, 178, B, 1888, 265.) From Latin luteus, yellow.

Spheres: 1.0 to 1.5 microns, showing packets in all media. Gram-positive.

Gelatin colonies: Circular up to $5 \mathrm{~mm}$. in diameter, sulfur-yellow, sinking into the medium.

Gelatin stab: Slow infundibuliform liquefaction.

Agar colonies: Yellow, coarsely granular, circular, raised, moist, glistening, entire margin.

Agar slant: Sulfur to chrome yellow, smooth, soft.

Broth: Clear with abundant yellow sediment.

Litmus milk: Coagulated, becoming alkaline.

Potato: Sulfur to chrome yellow, raised; sometimes limited growth.

slight indole formation.

Nitrites generally produced from n:trates. 
No acid from glucose, lactose or sucrose.

Hydrogen sulfide is formed.

Aerobic.

Optimum temperature $25^{\circ} \mathrm{C}$.

Habitat: Air, soil and water, skin surfaces.

5. Sarcina flava De Bary. (Vorlesungen über Bakterien, 1887, 151; Sarcina liquefaciens Frankland and Frankland, Philos. Trans. Roy. Soc. London, 178, B, 1888, 267.) From Latin, flavus, yellow.

Spheres: 1.0 to 2.0 microns, occurring in packets of 16 to 32 cells. Gram-positive.

Gelatin colonies: Small, circular, yellowish.

Gelatin stab: Slowly liquefied.

Agar slant: Yellow streak.

Broth: Slowly becoming turbid with whitish, later yellowish sediment.

Litmus milk: Alkaline, not coagulated.

Potato: Yellow streak.

Indole not produced.

Nitrites not produced from nitrates. Aerobic.

Optimum temperature $30^{\circ}$ to $35^{\circ} \mathrm{C}$.

Habitat: Air, water, soil.

6. Sarcina aurantiaca Flügge. (Die Mikxoorganismen, 1886, 180; For description see Frankland and Frankland, Phil. Trans. Roy. Soc. London, 178, B, 1888. 266; Paulosarcina aurantiaca Enderlein, Sitzungsber. Ges. Naturf. Freunde, Berlin, 1917,319.) From M. J., aurantiacus, orange-colored.

Spheres developing packets in all media. Gram-positive.

Gelatin colonies: Small, circular, dark yellow, entire margin, sinking into the medium.

Gelatin stab: Infundibuliform liquefaction.

Agar slant: Slightly raised, orange yellow to orange red, soft, smooth.

Broth: Flocculent turbidity, with abundant sediment.

Litmus milk: Coagulation and digestion.
Potato: Raised, yellow-orange, glistening to dull, granular.

Slight indole formation.

Nitrites not produced from nitrates. No $\mathrm{H}_{2} \mathrm{~S}$ produced.

Aerobic.

Optimuna temperature $30^{\circ} \mathrm{C}$.

Habitat: Air and water.

7. Sarcina citrea (Migula) Bergey et al. (Micrococcus agilis citreus Menge, Cent. f. Bakt., 12, 1892, 52; Planococcus citreus Migula, Arb. bakt. Inst. Karlsruhe, 1, Heft 1, 1894, 236; Micrococcus agiliscitreus Chester, Man. of Bact., 1901, 115; Bergey et al., Manual, 1st ed., 1923, 74.) From M. L. citreus, lemon-yellow.

Spheres: 0.6 to 0.8 micron, occurring singly, in pairs and in packets. Motile, possessing a single flagellum. Grampositive.

Gelatin colonies: Small, circular, yellowish, entire, becoming citron-yellow to orange.

Gelatin stab: No liquefaction.

Agar colonies: Small, yellow, convex, entire, smooth, glistening.

Agar slant: Abundant, yellow, plumose, glistening, taking on an orange color with age.

Broth: 'lurbid.

Potato: Abundant, yellow growth.

Indole not formed.

Nitrites not produced from nitrates. Aerobic.

Optimum temperature $25^{\circ} \mathrm{C}$.

Habitat: Air.

8. Sarcina littoralis Poulsen. (Poulsen, Vidensk. Meddel, naturh. Foren. i Copenhagen, 1879-80, 231-254; Sarcina morrhuae Farlow, L. S. Fish Commission Report for 1878, 1880, 974; Microroccus a, Høye, Bergens Museums Aarbog., No. 7, 2 Hefte, 1901, 39; Microroccus litoralis Kiellerman, Cent. f. Bakt., II Abt., 42, 1915, 399.) From Latin, litus (littus)-toris, the sea shore; -alis, relating to.

The relationships of the following to each other and to Sarcina littoralis are not clear: 
Erythroconis litoralis Oersted. (Naturh. Tidsslirift, 3, 1840-41, 555; Merismopedia litoralis Rabenhorst, Flora Europ. Algarum, 2, 1864-65, 57; Sarcina littoralis Winter in Rabenhorst, Kryptogamen-Flora, 1, I Abt., 1884, 50; Pediococcus litoralis Trevisan, 1 generi e le specie delle Batteriacee, Milano, 1889 , 28; Lampropedia littoralis De Toni and Trevisan, in Saccardo, Sylloge Fungorum $8,1889,1049$. )

Coniothecium bertherandi IÍgnin. (Pevue Mycologique, 6, 188t, 197.) Saccardo and Berlese (Atti. del R. Instituto Veneto, Ser. VI, Tol. 3) consider $C$. bertherandi to be identical with Sarcina littoralis, while Zopf (Die Spaltpilze, 2 Aufl., 1884, 73; ; Aufl., 1885, 102) considers $C$. bertherandi a stage of Beggiatoa roseo-persicina.

Deseription taken from Lochhead (Can. Jour. Res., 10, 1934, 280).

Spheres: 1.2 to 1.6 microns occurring singly, in pairs, in fours, in short chains, and in packets, the arrangement varying with medium, temperature, salt concentration and age of eulture. Non-motile. Gram stain variable, with rather more positive than negative rells.

No growth in ordinary media.

Salt gelatin: Growth slow, with no liquefaction.

Starch media (20 per cent salt) : Colonies usually 1 io $3 \mathrm{~mm}$, round, entire, convex, with a waxy appearance, brick red with a pale horder. inlor appearing gradually.

Starch media slants ( 20 p*r rent salt) : Filiform, slightly raised, entire edge. Coral red in color. Slight derrease in shade as cultures age.

Liquid media: No growth.

Potato: In 20 per rent salt, scanty growth. Slight chalky pink development near the top.

Indole not formed.

Nitrates reduced to nitrites.

Diastatic action negative

Aerobic, obligate.

Halophilic, obligate, 16-32 per cent salt. Optimum 20-24 per cent.
Optimum temperature $37^{\circ} \mathrm{C}$.

Source: Isolated from seashore mud near Copenhagen.

Habitat: Sea water brine, or sea salt. Isolated from salted hides and salted fish.

The following is believed by Kellerman (loc, cit.) to be a variety of Sarcina littoralis:

Diplococcus gadidarum Beckwith. (Beckwith, Cent. f. Bakt., I Abt., Orig., $60,1911,351$; Micrococcus litoralis gadidarum Kellerman, Cent. f. Bakt., II Abt., 42, 1915, 400; Pediococcus gadidarum Pribram, Klassification der Schizomyceten, 1933, 46.) From reddened salted codfish.

9. Sarcina ureae (Beijerinck) Löhnis. (Planosarcina ureae Beijerinck, Cent. f. Bakt., II Abt., 7, 1901, 52; Löhnis, Landwirtsch. bakteriol. Prakticum, 1911, 138; Sporosarcina ureae Kluyver and van Niel, Cent. f. Bakt., II Abt., 94, 1936, 401.) From Greek, urum, urine; M. L., urea, urea.

Probable syuonym: Sarcina psychrocarterica (Rubentschick) Bergey et al. (Urosarcina psychrocarterica Rubentschick, Cent. f. Bakt., II Abt., 64, 1925, 168; ibid., 66, 1926, 161; ibid., 67, 1926, 167; ibid., 68, 1926, 327; Bergey et al., Manual, 3rd ed., 1930, 95.)

Spheres: 0.7 to 1.2 microns, occurring singly, in pairs and in packets. Atypical endospores present. Motile, possessing long peritrichous flagella. Grampositive.

Gelatin colonies: Small, circular, flat, tough, yellowish.

Converts urea into ammonium carbonate.

terobic.

Optimum temperature $20^{\circ} \mathrm{C}$. Resists heating to $80^{\circ} \mathrm{C}$ for 10 minutes.

Source: Isolated from urine.

Appendix: The following names appear in the literature, and are listed here chiefly for their historical interest. 
Many are inadequately described, and probably many are synonyms.

Micrococcus aurantiacus Pagliani, Maggiora and Fratini. (Pagliani et al., 1887; Pediococcus aurantiacus Trevisan, I generi e le specie delle Batteriacee, Milan, 1889, 28; Merismopedia aurantiaca Maggioria, Giorn. Soc. ital. d'Igiene, 11, 1889, 355; Pediococcus maggiorae De Toni and Trevisan, in Saceardo, Sylloge Fungorum, 8, 1889, 1051.) From skin of the human foot.

Planosarcina samesii Migula. (Eine bewegliche Sarcine, Sames, Cent. f. Bakt., II Abt., 4, 1898, 664; Migula, Syst. d. Bakt., 2, 1900, V and 278; Sarcina agilis Matzuschita, Zeit. f. Hyg., 35, 1900, 496.) From liquid manure. Probably identical with Sarcina ureae Löhnis.

Sarcina acidificans Migula. (Sarcina No. VIII, Adametz, Landwirtsch. Jahrb., 18, 1889, 243; Migula, Syst. d. Bakt., 2, 1900, 258.) From cheese. Winslow and Winslow (The Systematic Relationships of the Coccaceae, 1908, 235) regard this species as a variant of Sarcina lutea Schroeter.

Sarcina agilis Saito. (Jour. Coll. Science Imp. Univ. Tokyo, 23, 1908, 68; abst. in Cent. f. Bakt., II Abt., 24, 1909, 228.) From dust.

Sarcina alba Zimmermann. (Weisse Sarcina, Maschek, Bakt. Untersuch. d. Leitmeritzer Trinkwässer, 1887, 64; Zimmermann, Die Bakterien unserer Trinku. Nutzwässer, Chemnitz, I Reihe, 1890 , 90.) From water. Zimmermann reported the presence of spores; subsequent workers failed to observe spores, even when working with original cultures.

Sarcina alba var. incana Appel. (Ber. d. landw. Inst. Königsberg, Heft 5, 1900, 89; quoted from Löhnis, Cent. f. Bakt., II Abt., 18, 1907, 146.) Frequently found in milk. Closely related to Adametz's Sarcinae Nos. VII, VIII and IX

Sarcina albida Gruber. (Arb. bakt. Inst. Karlsruhe, 1, Heft 3, 1895, 256.) From the stomach contents of a man with stomach cancer.
Sarcina alutacea Gruber (loc. cit., 221). From leaven.

Sarcina aurea Macé. (Traité Pratique de Bact., 2nd ed., 1892, 371; not Sarcina aurea Henrici, see below.) From lung exudate. Possesses active oscillary motility, but no flagella.

Sarcina aurea Henrici. (Arb. bakt. Inst. Karlsruhe, 1, Heft 1, 1894, 91; Sarcina aurescens Gruber, ibid., Heft 3, 1895, 263.) From Swiss cheese. Winslow and Winslow (loc. cit., 233) regard this species as a variant of Sarcina flava De Bary which has acquired certain fermentative powers.

Sarcina aurescens var. mucosa Jaiser; quoted from Pribram, Klassifikation der Sehizomyceten, 1933, 14.

Sarcina bicolor Kern. (Arb. bakt. Inst. Karlsruhe, 1, Heft 4, 1897, 505.) From the stomach of a woodpecker (Picus major). Winslow and Winslow (loc. cit., 232) regard this species as identical with Sarcina flava De Bary.

Sarcina butyrica Migula. (Sarcina No. XI, Adametz, Landwirtsch. Jahrb., 18, 1889, 214 ; Migula, Syst. d. Bakt., 2, 1900, 240.) From cheese. Winslow and Winslow (loc. cit., 233) regard this as a variant of Sarcina flava De Bary which has acquired certain fermentative powers.

Sarcina candida Lindner. (Die Sarcina-Organismen der Gährungsgewerbe, Inaug. Diss., Berlin, 1888, 43; Abst. in Cent. f. Bakt., 4, 1888, 427.) From water reservoir of a brewery and from air in the vicinity of the brewery.

Sarcina canescens Stubenrath. (Stubenrath, in Lehmann and Neumann, Bakt. Diag., 1 Aufl., 2, 1896, 143.) Stubenrath considered this a subspecies or variety of his Sarcina equi from which it differed only by its constant gray color on all culture media. Winslow and Winslow (loc. cit., 232) regard this as identical with Sarcina flava De Bary.

Sarcina carnea Gruber. (Arb. bakt. Inst. Karlsruhe, 1, Heft 3, 1895, 278.) From leaven.

Sarcina casei Migula. (Sarcina No. VII, Adametz, Landwirtsch. Jahrb., 18, 
1889, 242; Migula, Syst. d. Bakt., 2, 1900, 239.) From cheese. Winslow and Winslow (loc. cit., 233) regard this species as a variant of Sarcina flava De Bary which has acquired certain fermentative powers.

Sarcina caseolytica Stark and Scheib. (Jour. Dairy Sci., 19, 1936, 212.) From butter.

Sarcina cervina Stubenrath. (Stubenrath, in Lehmann and Neumann, Bakt. Diag., 1 Aufl., 2, 1896, 146.) From the stomach in a case of carcinoma.

Sarcina citrea Winslow and Winslow. (The Systematic Relationships of the Coccacceae, 1908, 234; not Sarcina citrea Bergey et al., Manual, 1st ed., 1923, 74.) This is the name given by Winslow and Winslow to their Type 2, the nitratereducing group of Sarcina.

Sarcina citrina Gruber. (Arb. bakt. Inst. Karlsruhe, 1 , Heft 3, 1895, 269.) From leaven. Winslow and Winslow (loc. cit., 235) regard this species as identical with Sarcina lutea Schroeter.

Sarcina conjunctivae Bergey et al. (Sarcina citrea conjunctivae Verderame, Cent. f. Bakt., I Abt., Orig., 59, 1911, 381; Bergey et al., Manual, 1st ed., 1923, 71.) From the conjunctiva. Gramnegative.

Sarcina devorans Fiern. (Arb. bakt. Inst. Karlsruhe, 1, Heft 4, 1897, 502.) From stomach contents of a sparrow (Passer montanus).

Sarcina equi Stubenrath. (Stubenrath, in Lehmann and Neumann, Bakt. Diag., 1 Aufl., 2, 1896, 143.) Found frequently in the urine of horses. Very similar to Sarcina lutea according to Stubenrath, who names three subspecies or varieties: Sarcina livido-lutescens, $S$. canescens and $S$. variabilis. Winslow and Winslow (loc. cit., 232) regard this species as identical with Sarcina flava De Bary.

Sarcina erythromyxa (Overbeck) Král. (1/icrococcus erythromyxa Overbeck, Nova Acta der Leop.-Carol, 55, No. 7, 1891 ; Krrál, Verzeichnis der abzugebenden Bak.) For a description of this species, see Zimmermann, Die Bakterien unserer Trink- und Nutzwässer, Chemnitz, II, 1894, 70. From water. Produces a red pigment.

Sarcina fimentaria Lehmann and Neumann. (Eine bewegliche Sarcine, Sames, Cent. f. Bakt., II Abt., 4, 1898, 664; Lehmann and Neumann, Bakt. Diag., 2 Aufl., 2, 1899, 146; Planosarcina samesii Migula, Syst. d. Bakt., 2, 1900, V and 278 ; Sarcina samesii Matzuschita, Bakt. Diag., Jena, 1902, 300.) From liquid manure. Exhibits active motility with many long flagella. Pribram (Klassifikation der Schizomyceten, 1933, 45) regards this organism as identical with Sarcina ureae and Sarcina mobilis.

Sarcina flavescens Henrici. (Arb. bakt. Inst. Karlsruhe, 1, Heft 1, 189t, 91.) From Swiss cheese. Winslow and Winslow (loc.cit., 232) regard this species as identical with Sarcina flava De Bary.

Sarcina fulva Stubenrath. (Das Genus Sarcina, München, 1897; see Lehmann and Neumann, Bakt. Diag., 2 Aufl., 2, 1899, 143.) Isolated many times from stomach contents and once from preputial smegma. Similar to Sarcina pulmonum.

Sarcina fusca Gruber. (Arb. bakt. Inst. Karlsruhe, 1, Heft 3, 1895, 282.) From flour.

Sarcina fuscescens De Bary. (Vorlesungen über Bakterien, 2 Aufl., 1887, 181 and Botan. Centralb., 1887, 34. Rr. duced to a synonym of Sarcina ventriculi Goodsir by Migula, Syst. d. Bakt., 2, $1900,259$.$) From the contents of the$ stomach.

Sarcina gasoformans Gruber. (Arb. bakt. Inst. Karlsruhe, 1, Heft 3, 1895, 270.) From leaven. loung cultures produce considerable gas.

Sarcina gigantea Kern. (Arb. bakt. Inst. Karlsruhe, 1, Heft 4, 1897, 508.) From stomach contents of the starling (Sturnus vulgaris). The diameter of a cell is 2.05 to 2.1 microns. Winslow and Winslow (loc. cit., 232) regard this species as identical with Sarcina flava De Bary.

Sarcina gigantea Petter. (Proc. Kon. 
Akad. Wet. Amsterdam, 34, 1931, 1417; Thesis, Utrecht, 1932; Compt. rend. Acarl. Sci., Paris, 196, 1933, 300.) From salted herring. Halophilic.

Sarcina hamaguchiae Saito. (Cent. f. Bakt., II Abt., 17, 1906, 155.) From soy bean mash.

Sarcina incana Gruber (loc. cit., 248). From leaven.

Sarcina incarnata Gruber. (Loc. cit., 279; Rhodococcus incarnatus Winslow and Rogers, Jour. Inf. Dis., 3, 1906, 545.) From!leaven. Produces pink pigment.

Sarcina intermedia Gruber (Loc. cit., 277). From leaven. Winslow and Winslow (loc. cit., 235) regard this species as identical with Sarcina lutea Schroeter.

Sarcina intestinalis Zopf. (Die Spaltpilze, 3 Aufl., 1885, 55.) From the intestines of poultry.

Sarcina lactea Gruber. (Loc. cit., 254; not Sarcina lactea Bergey et al., Manual, 1st ed., 1923, 73.) From leaven.

Sarcina lactis Chester. (No.45, Conn, Storrs Agr. Exp. Sta. Rept., 1894, 79; Chester, Man. Determ. Bact., 1901, 111.) From fermented milk (matzoon). Winslow and Winslow (loc. cit., 232) regard this species as identical with Sarcina flava De Bary.

Sarcina lactis acidi Conn, Esten and Stocking. (Storrs Agr. Exp. Sta. Ann. Rept., 1907, 125.) From milk.

Sarcina lactis albus Conn, Esten and Stocking (loc. cit., 124). From milk.

Sarcina lactis aurantiaca Conn, Esten and Stocking (loc. cit., 125). From milk.

Sarcina lactis lutea Conn, Esten and Stocking (loc. cit, 124). From milk.

Sarcina lembkei Migula. (No. 24, Lembke, Arch. f. Hyg., 26, 1896, 316; Migula, Syst. d. Bakt., 2, 1900, 241.) From the intestine. Winslow and Winslow (loc. cit., 232) regard this species as identical with Sarcina flava De Bary.

Sarcina liquefaciens Frankland and Frankland. (Phil. Trans. Royal Soc.
London, 178, B, 1888, 267.) From dust. Also found in cheese by Henrici (Arb. bakt. Inst. Karlsruhe, 1, Heft 1, 1894, 95). Winslow and Winslow (loc. cit., 232 ) regard this species as identical with Sarcina flava De Bary.

Sarcina livida Gruber (loc. cit., 297). From leaven. Winslow and Winslow (loc, cit., 235) regard this species as identical with Sarcina lutea Schroeter.

Sarcina livido-lutescens Stubenrath. (Stubenrath, in Lehmann and Neumann, Bakt. Diag., 1 Aufl., 2, 1896, 143; Sarcina lutescens Chester, Man. Determ. Bact., 1901,112.) From stools in a case of enteritis. Stubenrath regards this as a subspecies or variety of his Sarcina equi.

Sarcina loewenbergii Macé.

(Une sarcine pathogène, Loewenberg, Ann. Inst. Past., 18, 1899, 358; Macé, Traité Pratique de Bact., 4th ed., 1901, 464.) From the nasal cavity in a case of ozena. Probably a strongly slime-forming variety of Sarcina tetragena, according to Lehmann and Neumann, Bakt. Diag., 5 Aufl., 2, 1912, 206. Also see Galli-Valerio, Cent.f. Bakt., I Abt., Orig., 47, 1908, 177, for discussion.

Sarcina luteola Gruber (loc. cit., 265). From leaven. Winslow and Winslow (loc. cit., 235) regard this species as identical with Sarcina lutea Schroeter.

Sarcina marginata Gruber (loc. cit., 268). From leaven. Winslow and Winslow (loc. cit., 235) regard this as identical with Sarcina lutea Schroeter.

Sarcina meliflava Gruber (loc. cit., 272). From flour. Winslow and Winslow (loc. cit., 235) consider this identical with Sarcina lutea Sehroeter.

Sarcina minuta De Bary. (Vorlesungen über Bakterien, 1 Aufl., 1885; Eng. trans., 2nd ed., 1887, 117 and 185.)

Sarcina mirabilis Kern. (Arb. bakt. Inst. Karlsruhe, 1, Heft 4, 1897, 506.) From intestine of the yellow-hammer (Emberiza citrinella) and a dove (Columba oenas). Winslow and Winslow (loc. cit., 232) consider this species identi(al with Sarcina flava De Bary. 
Sarcina mobilis Maurea. (Cent. f. Bakt., 11, 1892, 228; Planosarcina mobilis Migula, Arb. bakt. Inst. Karlsruhe, 1, 1894, 236; Micrococcus mobilis Dyar, Ann. N. Y. Acad. Sci., 8, 1895, 353.) From old ascitic fluid. Motile, each cell with two short flagella.

Sarcina mucosa Sauerbeck. (Cent. f. Bakt., I Abt., Orig., 50, 1909, 289.) From pulmonary sputum. Probably a slime-forming variety of Sarcina tetragena, according to Lehmann and Neumann, Bakt. Diag., 5 Aufl., 2, 1912, 206.

Sarcina nivea Henrici. (Arb. bakt. Inst. Karlsruhe, 1, Heft 1, 1894, 88.) From cheese.

Sarcina olens Henrici (loc. cit., 94). From Camembert cheese. Winslow and Winslow (loc. cit., 232) regard this species as identical with Sarcina flava De Bary.

Sarcina orangea Cahanesco. (Ann. Inst. Past., 15, 1901, \$56.) From vagina of a dog.

Sarcina paludosa Schroeter. (Schroeter, in Cohn, Kryptog. Flora v. Schlesien, 3, I, 1886, 153.) From the waste water of a sugar factory. Not cultivated.

Sarcina persicina Gruber (loc. cit., 281). From leaven.

Sarcina pseudogonorrhoeae Lehmann and Neumann. (Eine neue Sarcina, Nagano, Cent. f. Bakt., I Abt., Orig., 32, 1902, 327; Lehmann and Neumann, Bakt. Diag., 4 Aufl., 2, 1907, 212 ; Sarcina naganoi Macé, Traité Pratique de Bact., 6th ed., 1, 1912, 631.) From pus.

Sarcina pulchra Henrici (loc. cit., 89). From cheese.

Sarcina pulmonum Hauser. (Lungensarcine, Virchow, Arch. f. path. Anat., 9, 1856, 557; Hauser, Deutsch. Arch. f. klin. Medizin, 42, 1887, 127; Sarcina virchowii Trevisan, Atti dell' Accad. Fisio-Medico-Statistica di Milano, Ser. IV, 3, 1885, 119.) From the sputum of a patient with phthisis. Motile (Lehmann and Neumann, Bakt. Diag., 2 Aufl., 2, 1899, 141). Hauser says this sarcina forms endogenous spores which way be demonstrated by Neisser's method of staining, and which have great resistance to heat. When cultivated in urine, it causes ammoniacal fermentation of the urea. Regarded by Jan Smit (personal communication, 1939) as identical with Sarcina ureae.

Sarcina radiata Kern (loc. cit., 53). From stomach and intestines of the rock dove (Columba livia) and a sparrow (Passer montanus). Winslow and Winslow (loc. cit., 232) regard this as identical with Sarcina flava De Bary.

Sarcina rosacea Migula. (Sarcina rosea (Schroeter) Lindner, Inaug. Diss., Berlin, 1888, 4วّ Migula, Syst. d. Bakt., 2, 1900, 263.) Found frequently in dust and water. Lindner believed his culture to be Sarcina rosea Schroeter.

Sarcina rubra Migula. (Eine rothe Sarcine, Menge, Cent. f. Bakt., 6, 1889, 596; Migula, Syst. d. Bakt., 2, 1900, 261.) From red milk.

Sarcina schaudinni (Wolff) Pribram. (Planosarcina schaudinni Wolff, Cent. f. Bakt., II Abt., 18, 1907, 9; Pribram, Klassifikation der Schizomyceten, 1933, 45.) From rotten places on potatoes. A motile coccus with long flagella.

Sarcina solani Reinke and Berthold. (Die Zersetzung der Kartoffel durch Pilze, Berlin, 1879; see O. Appel in Lafar, Handbuch der Technischen Mykologie, 2, 1905-08, 350.) Found in wet rotting of potatoes.

Sarcina striata Gruber (loc. cit., 271). From flour. Winslow and Winslow (loc. cit., 235) regard this species as identical with Sarcina lutea Schroeter.

Sarcina subflava Ravenel. (Memoirs Nat. Acad. Sci., 8, 1896, 10.) From soil.

Sarcina sulfurea Henrici (loc. cit., 90). From cheese. Winsluw and Winslow (loc. cit., 235) consider this species identical with Sarcina lutea Schroeter.

Sarcina superba Henrici (loc. cit., 93). From cheese. Winslow and Winslow (loc. cit., 232) regard this species as identical with Sarcina flava De Bary.

Sarcina symbiotica Pribram. (Eine gelbe Sarcina, Gropenfiesser, Cent. f. 
Bakt., II Abt., 61, 1925, 495; Pribram, Klassifikation der Schizomyceten, 1933, 45.) Lives symbiotically with cockroaches.

Sarcina thermodurica Wainess and Parfitt. (Jour. Bact., 40, 1940, 157.) From milking machines and other dairy farm utensils. Resists pasteurization temperatures.

Sarcina thermophila Bargagli-Petrucci. (Nuov. Giorn. Bot. Ital., 20, 1913; Abst. in Cent. f. Bakt., II Abt., 43, 1915, 294.) From the borax-yielding waters of Tuscany. Grows at temperatures up to $75^{\circ} \mathrm{C}$.

Sarcina urinae Welcker. (Sarcina renis Hepworth, Microscop. Jour., 5, 1857, 1; Welcker, in Henle and Pfeffer, Ztschr. f. rat. Med., 3 Ser., 5, 1859, 199; Merismopedia urinae Rabenhorst, Flor. europ. algarum, 2, 1865, 59.) Observed in the bladder. See below, Sarcina welckeri.

Sarcina variabilis Stubenrath. (Stubenrath, in Lehmann and Teumann, Bakt. Diag., 1 Aufl., 2, 1896, 143.) From gastric contents. May be considered a subspecies of Sarcina equi Stubenrath. Winslow and Winslow (loc. cit., 232) re- gard this as identical with Sarcina flava De Bary.

Sarcina variegata Pansini. (Arch. f. path. Anat., 122, 1890, 459.) Found in sputum from cases of influenza.

Sarcina velutina Gruber (loc. cit., 275). From leaven. Winslow and Winslow (loc. cit., 235) consider this species identical with Sarcina lutea Schroeter.

Sarcina vermicularis Gruber (loc. cit., 253). From wheat flour.

Sarcina vermiformis Gruber (loc. cit., 266). From leaven. Winslow and Winslow (loc. cit., 235) consider this species identical with Sarcina lutea Schroeter.

Sarcina viridis flavescens Rosenthal. (Inaug. Diss., Erlangen, 1893; Abst. in Cent. f. Bakt., 16, 1894, 1024.) From the oral cavity.

Sarcina welckeri Rossmann. (Rossman, Ceber Urinsarcina, Flora, 40, 1857. 641; Merismopedia welckeri Rabenhorst, Flora europaea, Alg. II, 1865, 58.) From the urinary bladder.

Urosarcina dimorpha Beijerinck. (Cent. f. Bakt., II Abt., 7, 1901, 53.) Reported to form spores. Non-motile. From garden earth. 


\section{FAMILY VI. NEISSERIACEAL PREVO'T. * \\ (Ann. Sci. Nat., Sér. Bot., 15, 1933, 119.)}

Cells spherical, in pairs or in masses. Non-motile. Gram-negative. Pigment formation rare. The family contains aerobic and anaerobiespecies. Somegrow poorly or not at all without mammalian body fluids. Optimum temperature $37^{\circ} \mathrm{C}$. All known species are parasitic.

\section{Key to the genera of family Neisseriaceae.}

I. Occurring in pairs, with adjacent sides usually flattened. Acrobes, facultative anaerobes and anaerobes. Approximately 1 micron in diameter.

Genus I. Neisseria, p. 295.

II. Occurring in masses, rarely in pairs. Anaerobes. Less than .5 micron in diameter.

Genus II. Veillonella, p. 302.

\section{Genus I. Neisseria Trevisan.}

(Trevisan, Atti della Accademia Fisio-Medico-Statistica in Milano, Ser. 4, 3, 1885, 105; Gonococcus Lindau (?), Just's Bot. Jahresber., I Abt., Orig., 26, 1898, 100.) Named for Dr. Albert Neisser who discovered the organism causing gonorrhoea in 1879.

Paired, Gram-negative cocei with adjacent sides flattened. Four of the eleven species produce yellow pigment. Aerobic and anaerobic species occur. Growth on standard media may be poor. Biochemical activities are limited. Few carbohydrates are utilized. Indole is not produced. Nitrates are not reduced. Catalase is produced abundantly. Parasites of mammals so far as known.

The type species is Neisseria gonorrhoeae Trevisan.

\section{Key to the species of genus Neisseria.}

I. Aerobes, facultative anaerobes.

A. Grow best on special culture media or on plain agar containing blood, blood serum or similar enrichment fluids, especially with added glucose. Grow best at $35^{\circ}$ to $37^{\circ} \mathrm{C}$; no growth below $25^{\circ}$ or above $40^{\circ} \mathrm{C}$. Not chromogenic.

1. Acid from glucose, not from maltose. Growth anaerobically.

1. Neisseria gonorrhoeae.

2. Acid from glucose and maltose. No growth anaerobically.

2. Neisseria meningitidis.

B. Grow well on ordinary culture media. Grow well at $22^{\circ} \mathrm{C}$.

1. Non-chromogenic.

a. Moist colonies on agar. No action on glucose, sucrose or mannitol.

3. Neisseria catarrhalis.

aa. Dry erumbly colonies on agar. Acid from glucose and sucrose; but not from mannitol.

4. Neisseria sicca.

* Revised by Prof. E. G. D. Murray, MeGill Lniversity, Montreal, P.Q., Canada in consultation with Dr. Sara E. Branham, United States Public Health Service, Washington, D. C., June, 1938; further revision, August, 1943. Descriptions of anaerobic species reviewed by Dr. Ivan C. Hall, New York City, January, 1944. 
2. Chromogenesis best seen on Löffler's serum.

a. Acid from fructose.

b. Acid from sucrose.

bb. No acid from sucrose.

5. Neisseria perflava.

6. Neisseria flava.

aa. No acid from fructose.

b. Acid from glucose.

7. Neisseria subflava.

bb. No acid from glucose.

8. Neisseria flavescens.

II. Anaerobes.

A. Gas produced from peptone broth.

B. No gas produced.

9. Neisseria discoides.

1. Odor of rancid butter.

2. No rancid odor.

10. Neisseria reniformis.

11. Neisseria orbiculata.

1. Neisseria gonorrhoeae Trevisan. (Micrococcus der Gonorrhoe, Neisser, Vorl. Mitteil., Cent. f. Medicinische Wissenschaft, 17, 1879, 497; Trevisan, Atti della Accademia Fisio-Medico-Statistica in Milano, Ser. 4, 3, 1885, 105.) From Greek, gonorrhoca, flux of semen; M.L. genitive of gonorrhoen.

Synonyms: (ionococcus, Jiplococcus der Gonorrhoe, Bumm, Der Mikroorganismen der gonorrhoischen Schleimhauterkrankung, Weisbaden, 1885, 16; Merismopedia gonorrhoeae Zopf, Die Spaltpilze, 1885,54 ; Micrococcus gonorrhoeae Flügge, Die Mikroorganismen, 1886, 156 ; Micrococcus gonococcus Schroeter, in Cohn, Kryptog. Flora v. Schlesien, 3, I, 1886, 147; Diplococcus gonorrhoeae Lehmann and Neumann, Bakt. Diag., 1 Aufl., 2, 1896, 150 ; Micrococcus gonorrhoeae Lehmann and Neumann, ibid., 4 Aufl., 2, 1907, 212.

Spheres: 0.6 to 1.0 micron, occurring singly and in pairs, the sides Hattened where they are in contact. Gramnegative.

Grows only on media with the addition of body fluids (blood, ascites, etc.), or other specially prepared media.

Colonies are small, transparent, even- tually (2 to 4 days) developing a lobate margin, grayish-white with a pearly opalescence by transmitted light. Larger colonies on special media.

Acid from glucose. No acid from maltose, fructose, sucrose and mannitol.

Optimum temperature $37^{\circ} \mathrm{C}$. Io growth below $25^{\circ}$ or above $40^{\circ} \mathrm{C}$.

Aerobic to facultative anacrobic. Many strains develop more readily with increased $\mathrm{CO}_{2}$ tension.

Common name: Gonococcus.

Source: Originally found in purulent venereal discharges. Also found in blood, conjunctiva, joints and cerebrospinal fluid.

Habitat: The cause of gonorrhoea and other infections of man. Not found in other animals.

2. Neisseria meningitidis (Albrecht and Ghon) Holland. (Diplokokkus intracellularis meningitidis Weichselbaum, Fortschr. d. Med., 5, 1887, 583; Neisseria weichselbaumii Trevisan, I generi e le specie delle Batteriacee, 1889, 32; not Diplococcus intracellularis Jaeger, Ztschr. f. Hyg., 19, 1895, 353; not Tetracoccus intracellularis Jaeger, ibid., 368; not Streptococcus intracellularis Lehmann 
and Neumann, Bakt. Diag., 1 Aufl., 2, 1896, 132; Micrococcus intracellularis Migula, Syst. d Bakt., 2, 1900, 189; Micrococcus meningococcus cerebrospinalis Albrecht and Ghon, Wiener klin. Wochnschr., 14, 1901, 988; not Streptococcus weichselbaumii Chester, Man. Determ. Bact., 1901, 64; not Meningococius intracellularis Jaeger, Cent. f. Bakt., I Abt., Orig., 33, 1903, 23; Micrococcus meningitidis Albrecht and Ghon, Cent. f. Bakt., I Abt., Orig., 33, 1903, 498 ; Diplococcus intracellularis Weichselbaum, Cent. f. Bakt., I Abt., Orig., 33, 1903, 511; Micrococcus intracellularis meningitidis de Bettencourt and França, Ztschr.f. Hyg., 46, 1904, 464; Diplococeus meningitidis, ibid., 495; Holland, Jour. Bact., 5, 1920, 224; Neisseria intracellularis-meningatidis Holland, ibid., 224; Neisseria intracellularis Holland, ibid., 224 ; see also Elser and Huntoon, Jour. Med. Res., 20 (N. S. 15), 1909, 371 and Murray, Med. Res. Council, London, Special Report Series No. 124, 1929 for detailed studies of the group.) From Greek, meninx, meninges, a membrane, a membrane covering the brain; M. I. genitive of meningitis, an inflammation of the meninges.

The binomial, Neisseria intracellularis, used in previous editions of the Manual has proved confusing because the names Micrococcus intracellularis, Diplococcus intracellularis and Streptococcus intracellularis, have been used loosely for unrelated organisms. Veisseria weichselbaumii has been so rarely and loosely used that any attempt to introduce it now is inadvisable despite rights of priority. The equally available name, Neisseria meningitidis, has therefore been adopted to avoid further confusion. It has the obvious advantage of association with the common name, meningococcus, which has been so frequently used in the literature.

In 1898, Councilman, Mallory and Wright (Epidemic Cerebrospinal Meningitis and its Relation to Other Forms of
Meningitis, Boston, 1898) definitely established the Gram-negative coceus as the cause of epidemic meningitis and clarified the confusion created because Jaeger regarded the coccus that he isolated (see Diplococcus crassus von Lingelsheim) as identical with Neisseria meningitidis.

Spheres : 0.6 to 0.8 micron in diameter, occasionally larger, occurring singly, in pairs with adjacent sides flattened, or occasionally in tetrads. Gram-negative.

Good growth is obtained on media containing blood, blood serum and other enrichment fluids with added glucose. Best growth on special media.

Blood agar plates are generally employed to isolate the organism. The colonies are small, slightly convex, transparent, glistening. Colonies large on special media.

Older cultures may show growth on neutral agar or glucose agar, properly prepared. Frequent transplantation is necessary to keep the organism alive in recently isolated strains; older strains survive for one month or longer at $37^{\circ} \mathrm{C}$ and for years on special media.

Acid from glucose and maltose. No acid from fructose, sucrose and mannitol.

Nitrites not produced from nitrates (Branham).

Optimum temperature $37^{\circ} \mathrm{C}$. No growth at $22^{\circ}$ or at $40^{\circ} \mathrm{C}$.

Aerobic, no growth anaerobically.

Common name: Meningococcus.

Source: Originally found in cerebrospinal fluid. Also found in nasopharynx, blood, conjunctiva, pus from joints petechiae in skin, ete.

Habitat: Nasopharynx of man, not found in other animals. Cause of epidemic cerebrospinal fever (meningitis)

Four main varieties or types of Veisseria meningitidis have been differentiated by Gordon and Murray (Jour. Poy. Army Med. Corps, 25 (2), 1915, 423 ) and by others on the basis of agglutination reactions with immune serums. 
3. Neisseria catarrhalis (Frosch and Kolle) Holland. (Micrococcus catarrhalis Frosch and Kiolle, in Flügge, Die Mikroorganismen, 3 Aufl, 2, 1896, 154; Diplococcus pharyngis communis von Lingelsheim, Klin. Jahrb., 15, 1906, 408; Holland, Jour. Bact., 5, 1920, 224.) From Greek, calarrh, a running down.

Spheres : 0.6 to 0.8 micron in diameter, occurring singly or in pairs with adjacent sides flattened, occasionally in fours. Gram-negative.

Agar colonies: Small, circular, grayish white to dirty white, with erose margins.

Broth: Turbid, often with slight pellicle.

No acid from any of the carbohydrates.

Optimum temperature $37^{\circ} \mathrm{C}$. Grows well at $22^{\circ} \mathrm{C}$.

Aerobic, facultative.

Source: Nasopharynx, saliva and respiratory tract.

Habitat: Human mucous membrane of the respiratory tract. Often associated with other organisms in inflammations of the mucous membrane.

Note: Topley and Wilson (Prin. of Bact., 1931, 349) state that Neisseria pharyngis cinerea (Micrococcus pharyngis cinereus von Lingelsheim, Klin. Jahrb., $15,1906,373$ ) resembles Neisseria catarrhalis so closely that it should probably be regarded as a variety of this species.

4. Neisseria sicca (von Lingelsheim) Bergey et al. (Diplococcus pharyngis siccus von Lingelsheim, Klin. Jahrb., 15, 1906, 409; Diplococcus siccus von Lingelsheim, Ztschr. f. Hyg., 59, 1908, 476; Micrococcus pharyngis siccus Kutseher, in Kolle and Wassermann, Handb. d. Path. Mikroorganismen, 2 Aufl., 4, 1912. 603; Micrococcus pharyngis-siccus $\mathrm{Hol}$ land, Jour. of Baet.. 5, 1920, 224; Neisseria pharyngis-sicci (sic) Holland, ibid.; Bergey et al., Manual, 1st ed., 1923, 43.) From Latin, sicca, dry.

Spheres : 0.6 to 0.8 mieron in diameter, occurring singly and in pairs with adjacent sides flattener. (iram-negative.
Blood agar colonies: Grayish, somewhat dry, crumbling when an effort is made to remove them.

Ascitic agar colonies: Small, very firm and adherent to medium, becoming corrugated on the surface.

The organisms precipitate spontaneously when suspended in normal salt solution.

Acid from glucose, fructose, maltose and sucrose. No acid from mannitol.

Optimum temperature $37^{\circ} \mathrm{C}$. Grows at $22^{\circ} \mathrm{C}$.

Aerobic, facultative.

source: Nasopharynx, saliva and sputum.

Habitat: Mucous membrane of the respiratory tract of man.

5. Neisseria perflava Bergey et al. (Chromogenic group I, Elser and Huntoon, Jour. Med. Res., 20 (N. S. 15), 1909, 415; Bergey et al., Manual, 1st ed., 1923, 43.) From Latin per, very and flavus, yellow.

Spheres: 0.6 to 0.8 micron, occurring singly and in pairs with adjacent sides flattened. Gram-negative.

Glucose agar colonies: Small, circular, slightly raised, greenish-gray by reflected light, and greenish-yellow and semiopaque by transmitted light. The surface is smooth, glistening. The growth is adherent to the medium. Chromogenesis best seen on Löfler's blood serum medium.

Ascitic agar colonies: Like those on glueose agar.

Acid from glucose, maltose, fructose, suerose and mannitol.

Optimum temperature $37^{\circ} \mathrm{C}$. Grows at $22^{\circ} \mathrm{C}$.

Aerobic, facultative.

Source: Nasopharynx, saliva and sputum.

Habitat: Mucous membrane of respiratory tract of man.

6. Neisseria flava Bergey et al. (Diplococcus pharyngis flavus $I$ and possibly 
Diplococcus pharyngis flavus II, von Lingelsheim, Klin. Jahrb., 15, 1906, 409; Diplococcus flavus $I$ and possibly Diplococcus flavus II, v. Lingelsheim, Zeitschr. f. Hyg., 59, 1908, 476; Hicrococcus pharyngis flaus $I$ and possibly Mierococcus pharyngis flavus II, Lehmann and Neumann, Bakt. Diag., 7 AuH., 2, 1927, 259; Chromogenic group II, Elser and Huntoon, Jour. Med. Res., 20 (N.S. 15), 1909, 415; Bergey et al., Manual, 1st ed., 1923, 43.) From Latin flavus, yellow.

Spheres: 0.5 to 0.8 micron, occurring singly and in pairs with adjacent sides flattened. Gram-negative.

Glucose agar colonies: Small, circular, slightly raised, greenish-gray by reflected light and greenish-yellow by transmitted light. Growth not adherent to medium. Surface colony is smooth with numerous, rather coarse crumbs in center. Margin entire, or rarely slightly irregular. Chromogenesis best seen on Löffler's blood serum medium.

Ascitic agar colonies: Like those on glucose agar.

Acid from glucose, fructose and maltose. To acid from sucrose or mannitol.

Optimum temperature $37^{\circ} \mathrm{C}$. Grows at $22^{\circ} \mathrm{C}$.

Source: Nasopharynx, cerebro-spinal fluid in cases of meningitis (very rare).

Habitat: Mucous membrane of respiratory tract.

7. Neisseria subflava Bergey et al. (Chromogenic group III, Elser and Huntoon, Jour. Med. Research, 20 (N.S. 15), 1909, 415; Bergey et al., Manual, 1st ed., 1923, 44.) From Latin sub, less and flavus, yellow.

Spheres: 0.6 to 0.8 micron, occurring singly and in pairs with adjacent sides flattened. Gram-negative.

Glucose agar colonies: Small, slightly raised, pale greenish-yellow, especially on primary culture.

Acid from glucose and maltose. No acid from fructose, sucrose or mannitol.
Agglutinates in normal rabbit serum.

Optimum temperature $37^{\circ} \mathrm{C}$. Little or no grow th at $22^{\circ} \mathrm{C}$.

Aerobic, facultative.

Easily confused with Veisseria meningitidis.

Source: Nasopharynx.

Habitat: Mucous membrane of the respiratory tract of man.

\section{Neisseria flavescens Branham.} (C. S. Public Health Service, Pub. Health Repts., 45, 1930, 845.) From Latin flavescens, becoming yellow.

Biscuit-shaped cocei occurring in flattened pairs. Giant forms common. Gram-negative.

Glueose agar: Poor growth.

Blood agar: Good growth, colonies less moist than those of the meningococcus. Golden yellow pigment. Greenish-yellow on Löffler's blood serum medium.

Semisolid agar: Good growth.

No acid from any of the carbohydrates.

Optimum temperature $37^{\circ} \mathrm{C}$.

Aerobic, facultative.

Serologically homogeneous group.

Source: Cerebro-spinal fluid in cases of meningitis.

Habitat: Probably mucous membrane of respiratory tract of man.

Note: Wilson and Smith (Jour. Path. and Bact., 31, 1928, 597) do not regard differences in sugar fermentations, chromogenesis, appearance of colonies, etc. sufficiently constant to warrant the separation of the species Neisseria catarrhalis, N. flava, N. cinerea, N. mucosa and $N$. sicca. They recommend that all be grouped under a single species known as Neisseria pharyngis (Diplococcus pharyngis).

9. Neisseria discoides Prévot. (Ann. Sci. Nat., Sér. Bot., 15, 1933, 106.) From Greek, discoeides, discus shaped; Latin adj., disk-shaped.

Spheres: 0.6 to 0.7 micron, occurring in pairs or tetrads. Gram-negative. 
Gelatin: No liquefaction.

Deep agar colonies: Lenticular, up to $1 \mathrm{~mm}$ in diameter. Grows in a narrow disk about $1 \mathrm{~cm}$ below the surface. Gas produced.

Broth : Turbid. Fine granular precipitate. Slight rancid odor and inflammable, explosive gas produced.

Peptone water: Gas produced.

Indole not formed.

No action on milk.

Coagulated proteins not digested.

Carbohydrates not attacked.

Hydrogen sulfide not produced.

Neutral red glucose broth: Becomes pink, but no further change.

Optimum pH 7.0 to 8.0 .

Temperature relations : Optimum $37^{\circ} \mathrm{C}$. No growth at $28^{\circ} \mathrm{C}$. Filled in half an hour at $60^{\circ} \mathrm{C}$.

Non-pathogenic.

Strict anaerobe.

Distinctive characters: Colonies grow in narrow zone $1 \mathrm{~cm}$ below the surface of an agar stab; gas produced from peptones.

Source: Isolated from bronchial mucus, respiratory system; dental and tonsillary focal infections.

Habitat: Buccal eavity (human) and probably also in other warm-blooded animals.

10. Neisseria reniformis (Cottet) Prévot. (Diplococcus reniformis Cottet, Compt. rend. Soc. Biol., 52, 1900, 421; Micrococcus reniformis Oliver and Wherry, Jour. Inf. Dis., 28, 1921, 341; Prévot, Ann. Sci. Nat., Sér. Bot., 15, 1933, 102.) From Latin, ren (renes), kidney;-formis, form, i.e. kidney-shaped.

Spheres: 0.8 to 1.0 micron, bean-shaped, occurring in pairs. Gram-negative.

Gelatin : No liquefaction.

Deep agar colonies: Appear in 24 to 48 hours; at first punctiform, then lenticular; small, 0.3 to $0.5 \mathrm{~mm}$. No gas produced.

Agar slant : Minute, bluish-white, dewdrop colonies.
Broth: Turbid in 24 hours; flocculent precipitate rapidly formed, clearing the medium. No gas produced, but a rancid odor is present.

Peptone water: Very meagre growth. Traces of indole.

Milk: Unchanged.

Coagulated proteins not digested.

Slight amount of acid from glucose by one strain only.

Optimum pH 7.0. Limits of $\mathrm{pH} 6.0$ to 8.0 .

Temperature relations: Optimum $37^{\circ} \mathrm{C}$. $\mathrm{No}$ growth at $22^{\circ} \mathrm{C}$. Filled in half an hour at $60^{\circ} \mathrm{C}$. or in an hour at $56^{\circ} \mathrm{C}$.

Pathogenic.

Strict anaerobe.

Distinctive character: Odor of rancid butter.

Source: Isolated in several cases from suppurations of the urino-genital system.

Ilabitat: Presumably in bodies of warm-blonded animals.

11. Neisseria orbiculata Prévot. (Diplococcus orbiculus Tissier, Ann. Inst. Past., 22, 1908, 204; Prévot, Ann. Sci. Nat., Sér. Bot., 15, 1933, 109.) From Iatin, orbiculatus, having the form of an orb or sphere.

Spheres: 1.5 to 2.0 microns, occurring in pairs. Gram-negative.

Gelatin: No growth at $22^{\circ} \mathrm{C}$.

Deep agar colonies : After 36 to 48 hours, large, lenticular, very regular, whitish, almost transparent. Gas not produced.

Broth: Turbid. Sediment.

Milk: No coagulation.

Egg white not attacked.

Proteoses attacked without formation of indole.

Acid from glucose. Acid produced feebly from lactose. No acid from sucrose.

Temperature relations : Optimum $37^{\circ} \mathrm{C}$. No growth at $22^{\circ} \mathrm{C}$. Killed at $60^{\circ} \mathrm{C}$.

Non-pathogenic.

Strict anaerobe.

Distinctive characters: Large size; no gas production. 
Source: Isolated from feces of young children.

Habitat: Intestinal canal. Not com. mon.

Appendix I: Additional species have been placed in this genus as given below. Some are undoubtedly identical with previously described species, while some may belong in other genera.

Diplococcus crassus von Lingelsheim. (Diplococcus intracellularis Jaeger, Ztsch. f. Hyg., 19, 1893, 353; Tetracoccus intracellularis, ibid., 318; von Lingelsheim, Ztschr. f. Hyg., 59, 1908, 467; Micrococcus crassus Lehmann and Neumann, Bakt. Diag., 7 Aufl., 2, 1927, 259.) Commonly found in nasopharyngeal secretions, also in the cerebrospinal fluid of suspected cases of meningitis. Also known as Jaeger's coccus or as Jaegersher Modifikation der Meningococcus.

Diplococcus mucosus von Lingelsheim. (von Lingelsheim, Klin. Jahrb., 15, 1906, 373, and Ztschr. f. Hyg., 59, 1908, 457; Neisseria mucosa Murray, in Manual, 5th ed., 1939, 283; not Streptococcus mucosus Howard and Perkins, Jour. Med. Res., 6, (N.S. 1), 1901, 174; not Pneumococcus mucosus Park and Williams, Jour. Exp. Med., 7, 1905, 411.) From nasal secretions. This Gram-negative coccus is said to show similarity to the meningococcus and to be like the diplococcus found by Weichselbaum and Ghon (Weiner Klin. Wehnschr., No. 24, 1905) in nasal secretions of a healthy person. Clearly it is different from the Gram-positive, mucoid type of pneumococeus which is described by Binaghi (Cent. f. Bakt., I Abt., 22, 1897, 273), Howard and Perkins (Jour. Med. Res., 6, 1901, 174), Park and Williams (Jour. Exp. Med., 7, 1905, 411) and others.

Micrococcus pharyngis cinereus von Jingelsheim. (Klin. Jahrb., 15, 1906, 373 ; Micrococcus cinereus v. Lingelsheim, Ztschr. f. Hyg., 59, 1908, 456; Neisseria cinerea Murray, in Manual, 5th ed., 1939,
283.) From mucous membrane of nose and throat.

Neisseria arthritica (Costa) Hauduroy et al. (Micrococcus arthritica Costa, Comp. rend. Soc. Biol., Paris, 85, 1920, 933 ; Hauduroy, Ehringer, Urbain, Guillot and Magrou, Dictionnaire des Bactéries Pathogènes, Paris, 1937, 296.) Isolated from a case of human arthritis.

Neisseria edigtoni (sic) Trevisan. (Diplococcus scarlatinae sanguinis Jamieson and Edington, Brit. Med. Jour., 1, 1887, 1265; Trevisan, I generi e le specie delle Batteriacee, 1889, 32.) From a scarlet fever patient.

Neisseria fulva De Bord. (Jour. Bact., 38, 1939, 119; Iowa State Coll. Jour. Sci., 16, 1942, 471.) From conjunctivitis and vaginitis.

Neisseria gibbonsi Hauduroy et al. (Gram-negative coccus, Gibbons, Jour. Inf. Dis., 45, 1929, 289; Hauduroy et al., Dict. d. Bact. Path., 1937, 300.) Isolated from skin abscesses in rabbits and guinea pigs.

Neisseria gigantea De Bord. (Jour. Bact., 38, 1939, 119; Iowa State Coll. Jour. Sci., 16, 1942, 472.) From a normal vagina.

Neisseria luciliarum Brown. (Amer. Mus. Novit., No. 251, 1927, 3.) A motile, Gram-negative diplococcus that probably should be placed in the genus $M i$ crococcus. From a dead fly, Lucilia sericata killed by Bacillus lutzae.

Neisseria pseudocatarrhalis Huntoon. (Jour. Bact., 27, 1934, 108.) Like Neisseria catarrhalis, shows no action on carbohydrates but is culturally more like Neisseria meningitidis and forms homogeneous suspensions in a salt solution. From nasopharynx.

Neisseria rebellis Trevisan. (Micrococcus in Trachoma folliculare, Kucharksky, 1887 ; Trevisan, I generi e le specie delle Batteriacee, Milan, 1889, 32.) From trachoma.

Neisseria venezuelensis Hauduroy et al. (Riguez, Gaceta Med. de Caracas, 
June 30, 1935; Pedro del Corral, Rev. de Med. y Cir. de la Clinica Macacay, April, 1935; Hauduroy et al., Dict. d. Bact.
Path., 1937, 308.) Found in localized epidemics of cerebrospinal meningitis in Venezuela.

\section{Genus II. Veillonella Prévot.*}

(Ann. Sci. Nat., Sér. Bot., 15, 1933, 118.) Named for A. Veillon, the French bacteriologist, who isolated the type species.

Small, Gram-negative cocci averaging 0.3 micron. Occur in masses, rarely in pairs or short chains. Cells undifferentiated. United by an interstitial substance of ectoplasmic nature. The known species are anaerobic. Good growth on standard culture media. Biochemical activity pronounced. Harmless parasites in mouth and intestine of man and animals.

The type species is Veillonella parvula (Veillon and Zuber) Prévot.

\section{Key to the species of genus Veillonella.}

I. Acid and gas from glucose. Weakly hemolytic.

1. Veillonella parvula.

II. Carbohydrates not attacked. Gas produced from peptone broth. Nonhemolytic.

1. Veillonella parvula (Veillon and Zuber) Prévot. (Staphylococcus parvulus Veillon and Zuber, Arch. méd. Exp., 1898, 5ł2; Micrococcus parvulus Bergey et al., Manual, 3rd ed., 1930, 92; Prévot. Ann. Sci. Nat., Sér. Bot., 15, 1933, 119. From Latin, parvulus, very small.

Description from Prévot (loc. cit.).

Very small spheres : 0.2 to 0.4 micron, occurring in masses, occasionally in very short chains. Gram-negative.

Gelatin : No liquefaction.

Semisolid agar (Veillon) colonies: At first punctiform, becoming lenticular, reaching a diameter of $2 \mathrm{~mm}$. Gas bubbles.

Blood agar colonies: Usually surrounded by a clear halo; weakly hemolytic.

Agar slant: Transparent, bluish, minute colonies.

Peptone broth: Turbid with fine sediment.

Glucose broth: Turbid. Faintly fetid odor. Gas produced contains $\mathrm{CO}_{2}, \mathrm{H}_{2}$ and $\mathrm{H}_{2} \mathrm{~S}$.

Broth serum: Very abundant, rapid growth.

\section{Veillonclla gazogenes.}

Milk: No acid. Not coagulated. Some strains produce gas.

Small amount of indole formed.

Nitrites produced from nitrates.

Acid and gas from glucose. Slight amount of acid from fructose, galactose and sucrose. Some strains feebly attack mannitol, maltose and inulin.

Coagulated protein not attacked.

Ammonia not produced.

Hydrogen sulfide producerl.

Optimum pH 6.5 to 8.0 .

Temperature relations: Optimum $37^{\circ} \mathrm{C}$. Grows feebly at $22^{\circ} \mathrm{C}$. Filled in one hour at $55^{\circ} \mathrm{C}$.

Strict anaerobe.

Distinctive characters: Fermentation of polypeptids to produce hydrogen, carbon dioxide, hydrogen sulfide and indole; fermentation of sugars; hemolysis of blood; production of nitrites from nitrates.

Source: Isolated by Veillon and Zuber from appendices, buccal cavities and lungs. Of the 13 strains studied by Prévot, 3 were isolated from pulmonary gangrene, one from an appendix, one

* Revised by Prof. E. G. D. Murray, McGill University, Montreal, P. Q., Canada, June, 1938. Descriptions reviewed by Dr. Ivan C. Hall, New York City, January, 1944. 
from alveolar pyorrhea, 5 from amniotic fluid, 2 from abscesses and pulmonary congestion and one from the buccal cavity of a normal rabbit. Found in suppurative lesions or pus. It may occasionally be pathogenic and invade the tissues, causing suppurations, alone or in association with other pyogenic organisms.

Habitat: Normally a harmless parasite found in natural cavities of man and animals, especially the mouth and digestive tract.

1a. Veillonella parvula var. minima Prévot. (Staphylococcus minimus Gioelli, Boll. R. Accad. Med. di Genova, 1907; Abst. in Cent. f. Bakt., I Abt., Ref., 42, 1908-09, 595; Micrococcus minimus Bergey et al., Manual, Ist ed., 1923, 69; Prévot, Ann. Sei. Nat., Sér. Bot., 15, 1933, 125.) From Latin, minimus, smallest.

Differs from Teillonella paroula only in its slightly smaller size $(0.2$ to $0.3 \mathrm{mi}$ (ron). Growth only at $37^{\circ} \mathrm{C}$. No growth ongelatin. Growth on the wall of the culture tube in fine flakes, not clouding the medium, and no plasmolysis in a 5 per cent salt solution.

Source: Isolated from a periuterine abscess.

1b. Veillonella parula var.branhamii Prévot. (Anaerobic micrococcus, Branham, Jour. Inf. Dis., 41, 1927, 203; ibid., 42, 1928, 230; Hicrococcus branhamii Bergey et al., Manual, 3rd ed., 1930, 92; Prévot, Ann. Sci. Nat., Sér. Bot., 15, 1933, 126.) Named for Dr. Sara E. Branham, of the National Institute of Health, Washington, D. C.

Serologically distinct from Veillonella parvula. One strain liquefied gelatin slowly,

Source: Isolated from nasal washings in two cases of influenza.

1c. Veillonella parvula var. thomsonii Prévot. (Anaerobic diplococcus, Thomson, Jour. Trop. Med. and Hyg., 26, 1923,
227 and Ann. Pickett-Thomson Res. Lab., 1, 1924-25, 105 and 164; Prévot, Ann. Sci. Nat., Sér. Bot., 15, 1933, 126 ; Micrococcus thomsoni Hauduroy et al., Dict. d. Bact. Path., 1937, 283.) Named for Dr. David Thomson of London, England.

Differs but slightly from Veillonella parvula in that it requires some accessory factor of growth found in serum or similar body fluids, testicular agar and the like.

Source: Found in the throat in measles and scarlet fever.

2. Veillonella gazogenes (Hall and Howitt) Murray. (Micrococcus gazogenes alcalescens anaerobius Lewkowicz, Arch. Méd. Exp., 13, 1901, 633; Micrococcus gazogenes Hall and Howitt, Jour. Inf. Dis., 37, 1925, 112; not Micrococcus gazogenes Choukévitch, Ann. Inst. Pasteur, 25, 1911, 356; Teillonella alcalescens Prévot, Ann. Sci. Nat., Sér. Bot., 15, 1933, 127; Micrococcus alcalescens Hauduroy et al., Dict. d. Bact. Path., 1937, 274; Murray, in Manua!, 5th ed., 1939, 287.) From Latin, the gasproducing Veillonella.

The species name gazogenes as given by $\mathrm{Hall}$ and Howitt is well established in the literature for this organism. It is valid under the rules when the organism is placed in a new genus (Veillonella) in spite of the earlier use of Hicrococcus gazogenes by Choukévitch for a different organism.

Spheres : 0.3 to 0.7 micron, average 0.4 micron, occurring in irregular masses, rarely in pairs, short chains or singly. Gram-negative.

Gelatin: No liquefaction.

Deep agar colonies : At first punctiform, becoming lenticular. Gas bubbles appear after 16 to 18 hours.

Blood agar plate: Minute colonies. Non-hemolytic. Several strains show greenish colonies.

Peptone broth: Gas produced. Broth becomes slightly alkaline.

Indole not formed. 
Milk: Gas, but no acid. No coagulation.

Ammonia and hydrogen produced in small amounts.

Egg-white and coagulated serum not attacked.

Hydrogen sulfide not produced.

Carbohydrates not attacked.

Nitrites not produced from nitrates.

Slowly plasmolysed in 5 per cent $\mathrm{NaCl}$ solution.

Optimum $\mathrm{pH} 6.0$ to 8.0 . Will grow in broth of pH 5.5.

Temperature relations: Optimum $37^{\circ} \mathrm{C}$. Some strains grow at $22^{\circ} \mathrm{C}$. Killed at $56^{\circ} \mathrm{C}$ in one hour, or at $65^{\circ} \mathrm{C}$ in a half nour, or at $80^{\circ} \mathrm{C}$ in 10 minutes.

Non-pathogenic (Lewkowicz's strains). Two strains (Prévot) pathogenic for rabbits.

Strict anaerobe.

Distinctive characters: Differs from Veillonella parvula in that it does not ferment sugars, does not produce $\mathrm{H}_{2} \mathrm{~S}$ nor indole, is not hemolytic, does not produce nitrites from nitrates, and does not develop fetid odors.

Source: Isolated (Lewkowicz) from mouth of a healthy infant. Twenty-four strains (Hall and Howitt) from human saliva. Fifteen strains (Prévot) one from alveolar pyorrhea, one from pulmonary gangrene, 5 from tonsils, one from appendix, 2 from measles, 3 from scarlet fever, and 2 from normal guinea pigs and rabbits.

Habitat: Prevalent in saliva of man and animals.

2a. Veillonella gazogenes var. gingivalis Murray. (Kleiner Micrococcus, Ozaki, Cent. f. Bakt., I Abt., Orig., 62, 1912, 83; Micrococcus gingivalis Bergey et al., Manual, 1st ed., 1923, 69; Veillonella alcalescens var. gingivalis Prévot, Ann. Sci. Nat., Sér. Bot., 15, 1933, 133; Murray in Manual, 5th ed., 1939, 288.) From Latin, pertaining to the gums.
Differs from Veillonella gazogenes by its ability to grow at $22^{\circ} \mathrm{C}$, and by the fact that glucose favors its growth although this carbohydrate is not fermented.

Source: Oral cavity and (Prévot) two strains from the intestine.

2b. Veillonella gazogenes var. minutissima Murray. (Micrococcus minutissimus Oliver and Wherry, Jour. Inf. Dis., 28, 1921, 342; Veillonella alcalescens var. minutissima Prévot, Ann. Sci. Nat., Sér. Bot., 15, 1933, 134; Murray, in Manual, 5th ed., 1939, 288.) From Latin, very tiny.

Differs from Veillonella gazogenes only in that the usual earbohydrates favor growth and that the gas formed is not absorbed by sodium hydroxide and is not inflammable.

Non-pathogenic for rabbits, guinea pigs or white mice (Oliver and Wherry).

Source: Two strains isolated from a mixed infection in aphthous ulcers of the gingival and buceal mucosa of a case of postpoliomyelitic paralysis.

2c. Veillonella gazogenes var. syzygios Murray. (Syzygiococcus scarlatinae Herzberg, Cent. f. Bakt., I Abt., Ref., 90, 1928, 575; Micrococcus syzygios scarlatinae Herzberg, Cent. f. Bakt., I Abt., Orig., 111, 1929, 373; Micrococcus syzygios Bergey et al., Manual, 3rd ed., 1930, 92; Veillonella alcalescens var. syzygios Prévot, Ann. Sci. Nat., Sér. Bot., 15, 1933, 134; Murray, in Manual, 5th ed., 1939, 288.) From Latin, yoked.

Differs from Veillonella gazogenes only by its ability to grow under an atmospheric pressure of $4 \mathrm{~cm}$ mercury, with the formation of $\mathrm{H}_{2} \mathrm{~S}$ in small amounts by some strains, and the production of nitrites from nitrates.

Source: Found by Herzberg in 30 per cent of normal mouths and in 100 per cent of saliva from scarlet fever patients. 


\section{FAMILY VII. LACTOBACTERIACEAE ORLA-JENSEN.}

(Orla-Jensen, Jour. Bact., 6, 1921, 271 ; Streptobacteriaceae Bergey, Breed and Murray, Preprint, Manual, 5th ed., 1938, 71.)

Long or short rods, or cocei which divide like rods in one plane only, producing chains, but never tetrads or packets. Non-motile except for certain cultures of streptococei. Gram-positive. Pigment production is rare; a few species form a yellow, orange, red or rusty brown pigment. Surface growth on all media is poor or absent. Some species are strictly anaerobic. Carbohydrates are essential for good development; they are fermented to lactic acid, sometimes with volatile acids, alcohol and $\mathrm{CO}_{2}$ as by-products (except for the non-fermenting Diplococcus magnus). Gelatin is very rarely liquefied. Nitrate is not reduced to nitrite. Found regularly in the mouth and intestinal tract of man and other animals, dairy products, fermenting vegetahle juices. A few are highly pathogenic.

\section{Key to the tribes of family Lactobacteriaceae.}

I. Cocci occurring singly, in pairs and in chains.

Tribe I. Streptococceae, p. 305.

II. Rods occurring singly, in pairs and in chains. Individual cells may be very long or even filamentous.

Tribe II. Lactobacilleae, p. 349.

TRIBE I. STREPTOCOCCEAE TREVISAN.

(I generi e le specie delle Batteriacee, 1889, 29.)

Cells spherical or elongate, dividing in one plane only, usually occurring in pairs or chains. A few species are strict anaerobes; none grow abundantly on solid media. Carbohydrates and polyalcohols are changed either by homofermentation to lactic acid or by heterofermentation to lactic and acetic acids, alcohol and carbon dioxide. Some pathogenic species grow poorly without blood serum or other enrichment fluids. Catalase negative.

Key to the genera of tribe Streptococceae.

I. Parasites, growing poorly on artificial media. Cells usually in pairs, often elongated. Anaerobic species rarely in tetrads or small clumps.

Genus I. Diplococcus, p. 305.

II. Parasites and saprophytes. Normally forming short or long chains. Ferment glucose to lactic acid with practically no other acids or $\mathrm{CO}_{2}$.

Genus II. Streptococcus, p. 312.

III. Saprophytes. Form chains of eocei to short rods in plant juices and milk. Ferment glucose with the production of $\mathrm{CO}_{2}$, lactic acid, acetic acid and ethyl alcohol. Mannitol is formed from fructose.

Genus III. Leuconostoc, p. 346.

\section{Genus I. Diplococcus Weichselhaum.*}

(Weichselbaum, Wiener med. Jahrb., 82, 1886, 483; Hyalococcus Schroeter, in Cohn, Kryptogamen Flora v. Schlesien, 18\$6, 152; Pseudodiplococcus Bonome, Cent. f. Bakt.,

* Revised by Prof. E. G. D. Murray, MeGill University, Montreal, Canada, September, 1938; anaerobic section adapted from papers by Dr. A. R. Prévot, Institut Pasteur, Paris, France, 1938; further revision by I.t. Col. Elliott S. Robinson, M.C.. Washington, D. C., January, 1944. 
2, 1888, 321; ? Pneumococcus Schmidlechner, Ztschr. f. Geburtshilfe u. Gynäkol., 56, 1905, 291; not Pneumococcus Arloing, Compt. rend. Acad. Sci., Paris, 109, 1889, 430; Mogallia Enderlein, Sitzb. Gesell. Naturf. Freunde, Berlin, 1917, 309.) From Greek diploos, double; kokkos, a grain or berry; M. L., a sphere.

Cells usually in pairs, sometimes in chains or more rarely in tetrads or small clumps. Young cells Gram-positive. Parasites sometimes growing poorly or not at all on artificial media. Fermentative powers usually high, most strains forming acid from glucose, lactose, sucrose and inulin. The aerobic species are bile soluble while the anaerobic species are not bile soluble.

The relationships of the strictly anzerobic diplococci placed in this genus by Prévot (Ann. Sci. Nat., Sér. Bot., 15, 1933, 140) to pneumococei are not yet entirely clear. The anaerobic species are included here in the hope that this arrangement will stimulate research.

The type species is Diplococeus pneumoniae Weichselbaum.

\section{Key to the species of genus Diplococcus.}

I. Aerobic, facultative. Bile soluble.

\section{Diplococcus pneumoniae.}

II. Strictly anaerobic. Tot bile soluble.

A. Greater than 1 micron in diameter.

1. Carbohydrates not attacked.

2. Diplococcus magnus.

B. Not greater than 1 micron in diameter.

1. Acid from glucose and lactose.

a. Capsulated. Pathogenic.

3. Diplococcus palcopneumoniae.

aa. Not capsulated. Non-pathogenic.

4. Diplococcus plagarum-belli.

2. Acid from glucose, not from lactose.

a. Grows on ordinary culture media. Non-pathogenic.

5. Diplococcus constellatus.

aa. No growth on ordinary culture media. Pathogenic.

6. Diplococcus morbillorum.

1. Diplococcus pneumoniae Weichselbaum. (Microbe septicémique du salive, Pasteur, Chamberland and Roux, Compt. rend. Acad. Sci., Paris, 92, 1881, 159; Micrococcus of rabbit septicemia, Sternberg, National Board of Health Bull., Washington, 2, 1881, 781; Coccus lancéolé, Talamon, Communication à la Société anatom. de Paris, 58, 1883, 475; Micrococcus pyogenes tenuis Rosenbach, Mikroorganismen bei den Wundinfektionskrankheiten des Menschen, 1884, 30 (see Neumann, Cent. f. Bakt., $\%, 1890$, 177); Micrococcus pasteuri Sternberg, Trans. Pathol. Soc. of Philadelphia, 12, 1885, 162 (not Micrococcus pasteuri
Trevisan, I generi e le specie delle Batteriacee, 1889, 34); Weichselbaum, Wiener med. Jahrb., 82, 1886, 485; Pneumoniemikrococcus or Pneumococcus, Fränkel, Ztschr. f. klin. Medizin, 10, 1886, 402; Bacillus septicus sputigenus Flügge, Die Mikroorganismen, 2 Aufl., 1886, 262; Bacillus salivarius septicus Biondi, Ztschr. f. Hyg., 2, 1887, 195; Diplococcus lanceolutus sive capsulatus Foà and Bordoni-Uffreduzzi, Archivio per le Sci. Med., 11, 1887, 387; Streptococcus lanceolatus pasteuri Gamaléia, Ann. Inst. Past., 2, 1888, 442; Streptococcus lanceolatus Gamaléia, ibid., 443; Klebsiella salivaris Trevisan, I generi e le specie 
delle Batteriacee, 1889, 26; Micrococcus rosenbachii Trevisan, ibid., 33; Micrococcus pyogenes-tenuis De Toni and Trevisan, in Saccardo, Sylloge Fungorum, 8, 1889, 1031; Micrococcus pneumoniae crouposae Sternberg, Cent. f. Bakt., 12, 1892, 53; Diplococcus lanceolatus capsulatus Kruse and Pansini, Ztschr. f. Hyg., 11, 1892, 335; Diplococcus lanceolatus incorrectly ascribed to Fränkel by Binaghi, Cent. f. Bakt., I Abt., 22, 1897, 278; Micrococcus tenuis Migula, Syst. d. Bakt., 2, 1900, 193; Bacterium pneumoniae Migula, ibid., 347; Bacterium salivarium Migula, ibid., 379; Streptococcus pneumoniae Chester, Man. Determ. Bact., 1901, 63; Micrococcus lanceolatus Longcope, Jour. Med. Res., 7 (N.S. 2), 1902, 220; Pneumococcus lanceolatus Schmidlechner, Ztschr. f. Geburtshilfe u. Gynäkologie, 56, 1905, 291; Pneumococcus pneumoniae Fried, Jour. Exp. Med., 57, 1933, 111.) From Greek pneumonia, inflammation of the lungs.

Monas pulmonale Klebs (Arch. f. exper. Path. u. Pharmakol., 4, 1875, 472) is inadequately described by Klebs and ought not to be regarded as identical with Weichselbaum's organism.

Common name: Pneumococcus.

The organisms occur as oval or spherical forms typically in pairs, occasionally singly or in short chains, 0.5 to 1.25 microns. The distal ends of each pair of organisms tend to be pointed or lancetshaped. Encapsulated. Non-motile. Young cells, Gram-positive.

Gelatin stab: Filiform or beaded growth. No liquefaction.

Infusion agar colonies: Small, transparent, grayish, with entire margin. Elevation high convex, glistening, mucoid to watery.

On blood agar, the colonies are elevated at the center with concentric elevations and depressions. Hemolysis usually slight but often marked in anaerobic culture; methemaglobin formation with green zone around colony.

Beef heart infusion broth: Uniform turbidity with variable amount of sediment.

Addition of glucose, serum, whole blood or ascitic fluid enhances growth.

Meat extract media: Growth irregular, usually poor if any.

Inulin serum water: Usually acid with coagulation .

Litmus milk: Usually acid with coagulation .

Potato: No growth.

Whole bile or 10 per cent solutions of sodium taurocholate or sodium glycocholate added to actively growing broth cultures $\pi$ ill dissolve the organisms. It is customary to use from 0.1 to $0.5 \mathrm{ml}$ of bile for each $0.5 \mathrm{ml}$ of culture.

Aerobic, facultative.

Optimum temperature $37^{\circ} \mathrm{C}$. Usually no growth at $18^{\circ}$ to $22^{\circ} \mathrm{C}$.

Optimum initial pH 7.8 .

Source: Sputum, blood and exudates in pneumonia; cerebrospinal fluid in meningitis; mastoiditis; otitis media; peritonitis; empyema; pericarditis; endoearditis; arthritis; saliva and secretions of respiratory tract in normal persons. Commonest cause of lobar pneumonia.

Habitat: The respiratory tract of man and animals.

At present, thirty-one types of Diplococcus pneumoniae are recognized on the basis of serological reactions, chiefly the Neufeld "Quellung" phenomenon as induced by type-specific immune rabbit serums. Following the description of Pneumococcus 1 by Neufeld and Händel (Arb. a. d. k. Gesundheitsamte, 34, 1910, 293), Dochez and Gillespie (Jour. Amer. Med. Assoc., 61, 1913, 727) divided the species into Types 1, 2, 3 and a heterogenous group 4; Cooper, Edwards and Rosenstein (Jour. Exp. Med., 49, 1929, 461) separated Types 4 to 13 from the strains previously designated as group 4, and later Cooper, Rosenstein, Walter and Peizer (Jour. Exp. Med., 55, 1932, 531) 
continued the classification to Type 32 . Due to marked cross-reactions, it was subsequently decided that Type 6 was identical with Type 26, and that Types 15 and 30 were identical. This resulted in the deletion of the Cooper Types 26 and 30 , thus leaving thirty of the original thirty-two types. Type 33 (Wilder) has been deseribed by Walter, Blount, Beattie and Cotler (Jour. Inf. Dis., 66, 1940, 181) as a distinct type; sufficient recognition has been accorded to justify the acceptance of this type, thereby making a total of thirty-one types of the species. In a still more recent publication, Walter, Guevin, Beattie, Cotler and Bucea (Jour. Immunol., 41, 1941, 279) recommend the addition of nine new types and eight subtypes. These, together with new strains reported by Kaufimann, March and Schmith (Jour. Immunol, $39,1940,397$ ), if eventually recognized, would make a total of fifty-five types. Eddy still more recently, taking into account all known types, raises the number of recognized types to seventy five (U. S. Public Health Repts., 59, 1944, 449-46s).

Note 1. Streptococens mucosus Howard and Perkins. (Howard and Perkins, Jour. Med. Res., 6 (N.S. 1), 1901, 174; Diplococcus capsulatus incorrectly attributed to Fränkel by Binaghi, Cent. f. Bakt., I Aht., 22, 1897, 273; Stre ptoroceus mucosus sichottmüller, Münch. med. Wehnschr., 50, 1903, 909; s'treptococcus lanceolatus var. mucosus Park and Williams, Diplococcus lanceolatus var. mucosus Park and Williams, Diplococcus mucosus Park and Williams, Pneumococcus mucosus Park and Williams, Jour. Exp. Med., $\tau, 1905,411$; Streptococcus mucosus capsulatus Buerger, Cent. f. Bakt., I Abt., 41, 1906, 314.) This organism is no longer recognized as a separate species. Dochez and Gillespic (Jour. Amer. Med. Assoc., 61, 1913, 727),
Wirth (Cent. f. Bakt., I Abt., Orig., 102, $1928,40)$ and others have established the identity of strains of this group as Diplococcus pneumoniae, Type 3.

Buerger (Cent. f. Bakt., I Abt., Orig., $41,1906,314$ ) lists the following capsulated closely related streptococei: Streptococcus involutus Kiurth, Arb. a. d. k. (iesundheitsamte, 8, 1893, 449 (Diplococcus involutus Winslow and Winslow, The Systematic Relationships of the ('occaceae, New York, 1908, 131); Streptococcus aggregalus Seitz, Cent. ‘. Bakt., I tht., 20, 1896, 854; Streptococcus capsulutus Binaghi, Cent. f. Bakt., I Abt., 22, 1\$97, 273; Streptocoque auréole, Le Roy des Barres and Weinberg, Arch. de Méd. expér. et d'anat. pathol., 2, 1899, 399 ; Leuconostoc hominis Hlava, Cent. f. Bakt., I Abt., Orig., 32, 1902, 263.

Note 2. Pneumococei, regardless of serological type, manifest three chief culture phases (or stages): Mucoid, Smooth, and Rough. The Mucoid (M) form corresponds to that previously designated as smooth (S) and represents the typical phase of the species; Smooth (S) supercedes the earlier term Rough (R); and the present Rough ( $R$ ) form is a relatively newly-deseribed variant. The most frequently observed dissociative trend is $\mathrm{M} \rightarrow \mathrm{S} \rightarrow \mathrm{R}$. Serological types are recognizable only in the Mucoid form due to the presence of type-specific polysaccharides in the capsular material; both Smooth and Rough forms are devoid of capsular material, but possess speciesspecific antigens common to all members of the species. Smooth and Rough forms are non-pathogenic, possess distinctive growth characteristics, and require special technic for accurate observations. The cultural characteristics given are those of the mucoid and smooth phases only, e. g., see growth in broth.

* Diplococcus magnus Prévot. (Diplococcus magnus anaerobius Tissier and

* Anaerobic section reviewed by Dr. Ivan C. Hall, New York, N. Y.

$\dagger$ These anaerobic diplococei and streptococci. many of which are putrefactive 
Martelly, Ann. Inst. Past., 16, 1902, 885; Prévot, Ann. Sci. Nat., Sér. Bot., 15, 1933, 140.) From Latin magnus, large.

Large spheres: 1.5 to 1.8 microns, usually in pairs, sometimes occurring singly, in small clumps or very short chains. Gram-positive.

Gelatin: Growth slow, scanty. No liquefaction.

Deep agar colonies: After 24 hours at $37^{\circ} \mathrm{C}$, lenticular, whitish, granular; margin finely cut. No gas produced.

Broth: Turbid, clearing in 4 or 5 days resulting in a viscous mass similar to the zoogloea which Clostridium bifermentans forms.

Peptone water: Slight turbidity. Indole not formed.

Milk: Unchanged.

Fibrin not digested.

Sterilized urine: Turbid in 3 to 4 days The urea is attacked forming $\left(\mathrm{NH}_{4}\right)_{2} \mathrm{CO}_{3}$.

Proteoses: Digested and disintegrated forming $\left(\mathrm{NH}_{4}\right)_{2} \mathrm{CO}_{3}$ with the liberation of $\mathrm{NH}_{3}$.

Carbohydrates not attacked.

Optimum $\mathrm{pH}$ 7.0. Limits of $\mathrm{pH} 5.5$ to 8.5 .

Temperature relations: Optimum $37^{\circ} \mathrm{C}$. Grows from $18^{\circ}$ to $37^{\circ} \mathrm{C}$. Killed in five minutes on boiling or in half an hour at $60^{\circ} \mathrm{C}$.

Non-pathogenie.

Strict anaerobe.

Distinctive characters: Large size; very marked alkalinizing power.

Source: Isolated by Tissier and Martelly (loc. cit.) from putrefying butcher's meat. Isolated by Prévot (loc. cit.) from a case of acute appendicitis.
Habitat: Human digestive tract. Very common on butcher's meat in the process of putrefaction. Probably occurs in household dust.

\section{Diplococcus paleopneumoniae}

Prévot. (An anaerobic pseudopneumococcus, Rist, Thèse de Paris, 1898; Der Fränkelsche Diplococcus, Bolognesi, Cent. f. Bakt., I Abt., Orig., 43, 1907, 113; Prévot, Ann. Sci. Nat., Sér. Bot., 15, 1933, 143.) From Greek paleus, old and pneumonia, inflammation of the lungs.

Spheres: About 0.7 to 1.0 micron, occurring in pairs, rarely occurring singly or in very short chains. Capsulated. Gram-positive.

Gelatin: No liquefaction.

Deep agar colonies: Probably lenticular.

Agar slant colonies: Round, raised, transparent, dew-drop.

Broth: Opalescent turbidity which settles as a rather abundant, powdery, flocculent precipitate. No gas produced.

Glucose or lactose broth: Rapid, abundant growth.

Peptone water (2 per cent): Very slow development. After 4 or 5 days at $37^{\circ} \mathrm{C}$ growth very poor.

Milk: Good growth. Partial coagu lation.

Blood agar: Very rapid, abundant growth.

Acid from glucose and lactose.

Temperature relations : Optimum $37^{\circ} \mathrm{C}$. No growth at $20^{\circ} \mathrm{C}$ nor at $42^{\circ} \mathrm{C}$. Killed at $55^{\circ} \mathrm{C}$.

Pathogenic.

Strict anaerobe.

Distinctive characters: Resembles

and gas-forming, seem to us so different from the fermentative microaerophilic diplococci, streptococci, leuconostocs and lactobacilli that we beleive they should be placed in genera and in a family separate from Lactobacteriaceae. Prévot in a discussion (Ann. Inst. Past., 67, 1941, 87) that has just reached us (Oct., 1945) recognizes this difference in physiology. He would solve the difficulty by returning the fermentative diplococci and streptococei to the family Coccaceae because of resemblances in morphology which do not seem to us to be fundamental-The editors. 
Diplococcus pneumoniae but is a strict anaerobe; highly pathogenic.

Source: Isolated by Rist (loc. cit.) from an osseous abscess; by Bolognesi (loc. cit.) from lesions of pleuropneumonia.

Habitat: Buccal-pharyngeal cavity of man and rodents.

4. Diplococcus plagarum-belli Prévot. (Diplococcus from septic wounds, Adamson, Jour. Path. and Bact., 22, 1919, 393; Prévot, Ann. Sci. Nat., Sér. Bot., 15, 1933, 157.) From Latin plaga, wound; bellum, war.

Spheres: 0.6 to 1.0 micron, occurring in pairs of unequal size or in short chains. Gram-positive.

Gelatin: No liquefaction.

Deep agar colonies: Appear after 24 to 48 hours, gradually increasing in size to $2 \mathrm{~mm}$ in diameter; lenticular, regular, almost transparent. Gas not produced, even in glucose agar.

Broth: Growth precipitates in 5 or 6 days. No gas produced.

Indole not formed.

Milk: Strongly acidified and coagulated in 2 to 3 days.

Serum not digested.

Acid but not gas from glucose, maltose, lactose and sucrose. No acid from mannitol.

Temperature relations: Optimum $37^{\circ} \mathrm{C}$. Not always killed in half an hour at $80^{\circ} \mathrm{C}$.

Non-pathogenic.

Strict anaerobe.

Source: Sixteen strains isolated from fifty-one cases of septic war wounds.

Habitat: Common in septic wounds.

5. Diplococcus constellatus Prévot. (Compt. rend. Soc. Biol. Paris, 91, 1924, 426.) From Latin constellatus, studded with stars.

Description in part from Prévot, Ann. Sci. Nat., Sér. Bot., 15, 1933, 158.

Spheres : 0.5 to 0.6 micron, occurring in pairs and tetrads, rarely in very short chains, never in clusters. Grampositive.

Gelatin: Good growth. No liquefaction.

Deep agar colonies : At first very small, lenticular, biconvex, thick, opaque, yellowish, 0.5 to $1.5 \mathrm{~mm}$ in diameter. Each colony surrounded by many small satellite colonies visible microscopically.

Broth: Growth slow, poor. After 48 hours a slight homogenous turbidity which quickly clears, leaving a slight powdery sediment. Neither gas nor odor produced.

Glucose broth: Growth rapid, abundant.

Proteins not attacked.

Blood broth: Good growth. No hemolysis.

Milk: Poor growth. No change.

Peptone water: Good growth. Not acidified. Indole not formed.

Neutral red broth unchanged.

Acid but not gas from glucose, arabinose. Slightly acid from glycerol. No acid from lactose, inulin, mannitol or dulcitol.

Optimum $\mathrm{pH} 6.0$ to 8.0 .

Optimum temperature $37^{\circ} \mathrm{C}$. Feeble growth at $22^{\circ} \mathrm{C}$. Not thermo-resistant.

Strict anaerobe.

Distinctive character: The microscopic appearance of agar colonies each of which is surrounded by a constellation of satellites.

Source: Isolated from a case of chronic, cryptic tonsillitis. Later isolated from pus in acute appendicitis.

Habitat: Digestive tract, especially the lymphoid tissues, as tonsils and appendix.

6. Diplococcus morbillorum Prévot. (Diplococci from cases of measles, Tunnicliff, Jour. Amer. Med. Assoc., 68, 1917, 1028; Diplococcus rubeolae Tunnicliff, Jour. Inf. Dis., 52, 1933, 39; Prévot, Ann. Sci. Nat., Sér. Bot., 15, 1933, 148; original name withdrawn by Tunnicliff, 
Jour. Inf. Dis., 58, 1936, 1.) From Latin morbus, disease; M. I. morbilli, measles.

Spheres : 0.6 to 0.8 micron, occurring in short chains, rarely in small masses. Gram-positive.

This organism does not develop on ordinary culture media. The addition of fresh serum or ascitic fluid is necessary.

Gelatin: No liquefaction.

Serum agar colonies: Very small, punetiform, appearing after 5 to 22 days. No gas produced.

Glucose agar containing ascitic fluid and blood: Colonies are slightly larger and appear more rapidly; greenish .

Blood agar colonies: Surrounded by a greenish halo. May be large and moist. Gas not produced.

Broth: Very poor growth.

Hemolysed blood broth: Growth flocculent, leaving the liquid clear.
Milk: Unchanged by most strains. Acidified and coagulated by four strains.

Indole not formed.

Bile: Not soluble in bile.

Acid from glucose, sucrose and maltose.

Temperature relations: Optimum $37^{\circ} \mathrm{C}$. Killed in 45 minutes at $57^{\circ} \mathrm{C}$. Withstands $-2^{\circ} \mathrm{C}$ for two weeks.

Strict anaerobe. Most strains become microaerophilic with transfers.

Distinctive characters: Greenish colonies on blood media; poor growth on ordinary media.

Source: Isolated from the throat and blood in measles.

Habitat: Nose, throat, eyes, ears, mucous secretions and blood from cases of measles. 


\section{Genus II. Streptococcus Rosenbach.*}

(Rosenbach, Mikroorganismen bei Wundinfektionskrankheiten des Menschen, 1884, 22 ; Arthrostreptokokkus Hueppe, Wiesbaden, 1886, 144; Sphaerococcus Marpmann, Ergänzungshefte z. Cent. f. allg. Gesundheitspflege, 2, 1889, 121; Perroncitoa, Babesia, Schuetzia Trevisan, I generi e le specie delle Batteriacee, 1889, 29; Lactococcus Beijerinck, Arch. neérl. d. sci. exactes, Sér. 2, 7, 1901, 213; Hypnococcus Bettencourt et al., Cent. f. Bakt., I Abt., Orig., 35, 1904, 55; Myxococcus Gonnermann, Oester. u. Ungar. Ztschr. f. Zuckerind. u. Landwirtsch., 36, 1907, 883; not Myxococcus Thaxter, Bot. Gaz., 17, 1892, 404; Melococcus Amiradzibi, Med. Zurn., 4, 1907, 309; Diplostreptococcus v. Lingelsheim, in Kolle and Wassermann, Handb. d. path. Mikroorg., 2 Aufl., 4, 1912, 494; ? Brachybacterium Troili-Petersson, Cent. f. Bakt., II Abt., 11, 1903, 138; Pseudostreptus Enderlein, Sitzb. Gesell. Naturf. Freunde, Berlin, 1917, 309 ; Planostreptococcus Meyer, Die Zelle der Bakterien, Jena, 1912, 4; Streptus Enderlein, Sitzber. Gesell. Naturf. Freunde, Berlin, 1930, 104; Peptostreptococcus Kluyver and Van Niel, Cent. f. Bakt., II Abt., 94, 1936, 391.) From Greek streptus, flexible or pliant; Greek kokkos, a grain or berry; M. L., a sphere.

Cells spherical or ovoid, rarely elongated into rods, oceurring in pairs, or short or long chains, never in packets or zoogloeal masses. Capsules are not regularly formed, but become conspicuous with some species under certain conditions. Gram-positive, some species decolorizing readily. A few cultures produce a rusty red growth in deep agar stab, or a yellow or orange pigment in starch broth. Growth on artificial media is slight. Agar colonies are small. Surface colonies are translucent. Colonies may be effuse, convex or mucoid. Some species are aided by the addition of native proteins. Mostly facultative anaerobes, with little surface growth in stab cultures. A few are strict anaerobes. Some of the latter attack proteins with production of gas and foul odors. Carbohydrate fermentation by all others is homofermentative, with dextrorotatory lactic acid as the dominant product, while volatile acids, other volatile products and $\mathrm{CO}_{2}$ are either absent or produced in very small amounts. Inulin is rarely attacked. Nitrate is not reduced to nitrite. Not soluble in bile. Common wherever organic matter containing sugars is accumulated. Regularly in the mouth and intestine of man and other animals, dairy products, fermenting plant juices. Some species are highly pathogenic.

The type species is Streptococcus pyogenes Rusenbach.

Note: The classification of streptococci is beset with many difficulties and it seems advisable for the present to accept only such described species about which there is reasonable agreement. With present knowledge, many species which have been separated can justifiably be considered as identical with older species and have been treated as such here. The descriptions of certain other species do not permit their exact identification now and they have been classed as invalid names with no present significance. It is admitted there are grounds for belief that more than one species may be included in certain of the species described here, but the onus of proof lies with the investigators interested in them. It is hoped that the simplification introduced will prove useful as a starting point for the more exact differentiation and description of the species of Streptococcus. The general arrangement used is in

* Revised by Prof. E. G. D. Murray, McGill University, Montreal, Canada, in consultation with Prof. G. J. Hucker, New York State Experiment Station, Geneva, New York and Prof. J. M. Sherman, Cornell University, Ithaca, New York, June, 1938; further revision by Prof. J. M. Sherman, February, 1944. 
harmony with the suggestions made by Hucker (Proc. 2nd Internat. Cong. for Microbiology, London, 1936, 127) and Sherman (Bact. Reviews, 1, 1937, 3).

Serological reactions are included as far as possible in the descriptions but the true significance of these methods is not known and on that account they are not stressed in the primary classification.

Throughout the history of this genus motile streptococei have been reported occasionally (e.g., Streptococcus herbarum Schieblich, Cent. f. Bakt., I Abt., Orig., 124, 1932, 269; Koblmüller, Cent. f. Bakt., I Abt., Orig., 133, 1934, 310; Stölting, Ủber die Streptokokken des normal reifenden Tilsiter Käses. Inaug. Diss., Kiel, 1935, 51; Pownall, Brit. Jour. Exp. Path., 16, 1935, 155) but it is not known whether these constitute definite species or whether (Levenson, Ann. Inst. Past., 60, 1938, 93) motile individuals occasionally appear in ordinarily non-motile species.

The anaerobic streptococci have not been sufficiently studied to be sure whother they should be included in the genus Streptococcus or given separate generic rank. Their metabolic processes seem reason for the latter view. The descriptions given are taken from Prévot (Ann. Sci. Nat., Sér. Bot., 15, 1933, 23).

The material is arranged accordingly in three categories: A key and complete descriptions have been prepared for clearly defined species, species of uncertain taxonomic relationships have been placed in Appendix I $w$ ith their necessarily incomplete descriptions, while even less valid and unidentifiable species are merely listed in Appendix II.

\section{Key to the species of genus Streptococcus.}

I. Facultative anaerobic species.

A. Pyogenic group. No growth at $10^{\circ} \mathrm{C}$. No growth at $45^{\circ} \mathrm{C}$. Generally beta hemolytic. Generally do not curdle litmus milk and reduce litmus slowly if at all. Mannitol and glycerol generally not fermented. Not tolerant of 0.1 per cent methylene blue, 6.5 per cent $\mathrm{NaCl}$ and $\mathrm{pH}$ 9.6. Produce ammonia from peptone.

1. Sodium hippurate not hydrolyzed.

a. Lactose fermented.

b. Sorbitol not fermented but trehalose fermented. Lancefield Group A.

\section{Streptococcus pyogenes.}

bb. Sorbitol fermented and trehalose not fermented. Lancefield Group C.

\section{Streptococcus zooepidemicus.}

aa. Lactose may or may not be fermented. Lancefield Group C.

b. Trehalose not fermented.

bb. Trehalose fermented.

3. Streptococcus equi.

4. Streptococcus equisimilis.

2. Sodium hippurate hydrolyzed. Lancefield Group B.

5. Streptococcus agalactiae.

B. Viridans group. No growth at $10^{\circ} \mathrm{C}$. Growth at $45^{\circ} \mathrm{C}$ (few exceptions in Streptococcus mitis). Reduce litmus after curdling litmus milk; sorbitol and glycerol generally not fermented; mannitol rarely. Not tolerant of 0.1 per cent methylene blue, 6.5 per cent $\mathrm{NaCl}$ or $\mathrm{pH} 9.6$. Not beta 
hemolytic (though they may be under anaerobic conditions) but show varying degrees of greening of blood. Do not produce ammonia from peptone (few exceptions in Streptococcus mitis).

1. Lactose is fermented.

a. Do not grow at $50^{\circ} \mathrm{C}$. Greening or indifferent in blood agar. Raffinose, inulin, salicin and dextrin generally fermented. Esculin generally attacked. Growth with 2 per cent $\mathrm{NaCl}$.

b. Do not survive $60^{\circ} \mathrm{C}$ for 30 minutes. Starch not hydrolyzed. Not tolerant of bile.

c. Mucoid colonies produced on sucrose and raffinose media. 6. Streptococcus salivarius.

cc. Colonies not mucoid on sucrose or raffinose media. Inulin not fermented.

7. Streptococcus mitis.

bb. Survives $60^{\circ} \mathrm{C}$ for 30 minutes. Starch is hydrolyzed except by variety inulinaceus. Tolerant of bile.

8. Streptocorcus bovis (and varieties).

aa. Grows at $50^{\circ} \mathrm{C}$. No action on blood. Esculin not attacked. Raffinose, inulin, salicin and dextrin not fermented. No growth in 2 per cent $\mathrm{NaCl}$.

9. Streptococcus thermophilus.

2. Lactose not fermented. Tolerant of bile.

10. Streptococcus equinus.

C. Lactic group. Growth at $10^{\circ} \mathrm{C}$. No grow th at $45^{\circ} \mathrm{C}$. Reduce litmus prior to curdling of litmus milk. Sorbitol and glycerol not fermented. Not beta hemolytic. Tolerate 0.1 per cent methylene blue, but do not tolerate 6.5 per cent $\mathrm{NaCl}$ or $\mathrm{pH} 9.6$.

1. Maltose and dextrin fermented. Ammonia produced from peptone. Grow th at $40^{\circ} \mathrm{C}$. Group N of Shattock and Mattick.

11. Streptococcus lactis.

2. Maltose and usually dextrin not fermented. Ammonia not produced from peptone. No growth at $40^{\circ} \mathrm{C}$.

12. Streptococcus cremoris.

D. Enterococcus group. Growth at $10^{\circ} \mathrm{C}$. Growth at $45^{\circ} \mathrm{C}$. Usually reduce litmus prior to curdling litmus milk. Sorbitol, glycerol and mannitol generally fermented. May or may not be beta hemolytic. Tolerate 0.1 per cent methylene blue, 6.5 per cent $\mathrm{NaCl}$ and $\mathrm{pH}$ 9.6. Ammonia produced from peptone. Lancefield Group D.

1. Not beta hemolytic.

a. Gelatin not liquefied.

13. Streptococcus faecalis.

aa. Gelatin liquefied.

14. Streplococcus liquefaciens.

2. Beta hemolytic.

a. Mannitol and sorbitol fermented.

15. Streptococcus zymogenes.

aa. Mannitol and sorbitol not fermented.

16. Streptococcus durans.

II. Anaerobic species.

A. Strict anaerobes.

1. Gas and fetid odor produced.

a. No general turbidity in broth. 

b. Acid from maltose.
bb. No acid from maltose.
17. Streptococcus anaerobius.

18. Streptococcus foetidus.

aa. Turbidity in broth.
b. No gas in Veillon's semisolid agar. No gas in peptone water. 19. Streptococcus putridus.
bb. Abundant gas in semisolid agar. Gas in peptone water.
20. Streptococcus lanceolatus.

2. No gas and no fetid odor produced.

a. Milk not coagulated.

aa. Milk coagulated.

21. Streptococcus micros.

b. Viscous sediment in broth. Semisolid agar colonies blacken with age.

22. Streptococcus parvulus.

bb. No viscous sediment in broth. Semisolid agar colonies do not blacken with age.

B. Microaerophilic.

23. Streptococcus intermedius.

1. Strictly anaerobic on isolation, later microaerophilic.

24. Streptococcus evolutus.

1. Streptococcus pyogenes Rosenbach. (Fehleisen, Ueber Erysipel, Deut. Zeit. f. Chir., 16, 1882, 391; Erysipelkokken, Fehleisen, Die Aetiologie des Erysipels, Berlin, 1883; Rosenbach, Mikroorganismen bei Wundinfektionskrankheiten des Menschens, 1884, 22; Streptococcus erysipelatos (sic) Rosenbach, ibid., 22; Micrococcus erysipelatis Zopf, Die Spaltpilze, 2 Aufl., 1881, 86; Streptococcus erysipelatis Zopf, Die Spaltpilze, 3 Aufl., 1885, 51 ; Streptococcus erysipelatosus Klebs, Die Allg. Path., Jena, 1887, 318; Micrococcus scarlatinae and Streptococcus scarlatinae Klein, Report of the Medical Officer of the Local Government Board for 1885-1886, No. 8, 1887, 85; Streptococcus conglomeratus Kurth, Arb. d. k. Gesundheitsamte, 7, 1891, 389; Streptococcus longus von Lingelsheim, Ztschr. f. Hyg., 10, 1891, 331 and 12, 1891, 3C8; Streptococcus puerperalis Arloing, Septicémie puerperale, Paris, 1892 (Jordan, Brit. Med. Jour, 1912, 1); Staphylococcus erysipelatos Hesse, Ztschr. f. Hyg., 34, 1900, 347; Streptococcus longus pathogenes seu erysipelatos Schottmüller, Münch. med. Wehnschr., 50, 1903, 909; Streptococcus longus hemolyticus Sachs, Ztschr.f.Hyg., 63, 1909,466 ; Streptococcus longissimus Thalmann, Cent. f. Bakt., I Abt., Orig., 56, 1910, 248; Streptococcus hemolyticus Rolly, Cent. f. Bakt., I Abt., Orig., 61, 1911, 87; Streptococcus epidemicus Davis, Jour. Am. Med. Assoc., 58, 1912, 1852; Jour. Inf. Dis., 15, 1914, 378; ibid., 19, 1916, 236; Streptococcus hemolysans Blake, Jour. Med. Res., 36 (N.S. 31), 1917, 116; Streptococcus pyogenes haemolyticus Weisenbach, Compt. rend. Soc. Biol. Paris, 81, 1918, 819; Streptus scarlatinae Enderlein, Sitzber. Gesell. Naturf. Freunde Berlin, 1930, 104; Streptococcus pyogenes var. scarlatinae Hauduroy et al., Dict. d. Bact. Path., Paris, 1937, 520.) From Greek pyon, pus; -genes, producing.

Spherical or ovoid cells : 0.6 to 1 micron in diameter in cultures; usually spherical in blood and inflammatory exudates; occurring in chains or pairs. Capsules are variable, sometimes well developed and can be induced. Gram-positive.

Gelatin stab: Growth slight; minute opaque colonies, little surface growth. No liquefaction.

Nutrient agar: Small colonies, translucent, convex, entire, slightly granular; colonies are variable; confluent growth a thin transparent film; tendency for 
colonies to remain discrete. Growth increased by addition of blood or native proteins. Pairs or short chains in surface growth and longer chains in condensation fluid of slants.

Broth: Flocculent sediment of tangled, chains, supernatant broth often clear except in very young cultures. No pellicle.

Potato: Very slight or no visible grow th.

Litmus milk: Acid, seldom curdled, and litmus reduced slowly or not at all.

Acid from glucose, maltose, lactose, sucrose, salicin and trehalose. No acid from inulin, raffinose, arabinose, glycerol, mannitol, sorbitol or dulcitol.

No hydrolysis of sodium hippurate, starch or esculin.

Ammonia is produced from peptone.

Temperature relations: Optimum temperature around $37^{\circ} \mathrm{C}$. No growth at $10^{\circ} \mathrm{C}$ or $45^{\circ} \mathrm{C}$. Does not survive $60^{\circ} \mathrm{C}$ for 30 minutes.

Chemical tolerance: Tolerates 2 per cent $\mathrm{NaCl}$ but not 4 per cent and 6.5 per cent. Final pH in glueose broth 4.8 to 6.0; no growth at $\mathrm{pH} 9.6$. Methylene blue 0.01 per cent and 0.1 per cent not tolerated and not reduced. Inhibited by bile but not soluble.

Action on blood: Superficial and deep colonies cause hemolysis in blood agar, usually with a wide zone surrounding the colony, which may have a well-defined margin circumscribed by a zone of concentrated hemoglobin; the margin of the zone is ill-defined with some strains. Conditions defined by Brown (Rockefeller Inst. Med. Res., Monograph 9, 1919 , 14) known as beta hemolysis. Soluble antigenic hemolysin of more than one kind produced in fluid cultures; influenced by constitution of medium and presence of serum; one is oxygen-sensitive and another is oxygen-stable. Special precautions necessary for its demonstration (F. Smith, Jour. Bact., 34, 1937, 585, 603).

Toxin: An erythrogenic toxin is pro- duced; commonly associated with scarlet fever. Relatively thermostable.

Fibrinolysin: Dissolves human fibrin but not fibrin of rabbit or ox blood. Markedly thermostable.

Serology: Constitutes Group A of Lancefield (C substance; polysaccharide) (Jour. Exp. Med., 57, 1933, 571). Types within the species are distinguishable (M substance; protein); 23 identified by Griffith (Jour. Hyg., 34, 1934, 542). Antigen common to the group ( $\mathrm{P}$ substance; nucleo-protein) also present in other Gram-positive cocci.

Facultative anaerobe. Occasionally in primary culture from lesions, pus, etc. grows only in anaerobic culture.

Source: Human mouth, throat and respiratory tract; inflammatory exudates, blood stream and lesions in human disease of very varied character. Occasionally in milk and udder of cows. Dust in sick rooms, hospital wards and other contaminated sites.

Habitat: In human infections of many varied types. Occasionally in udder infections of cattle and perhaps other animal sources.

\section{Streptococcus zooepidemicus Frost} and Engelbrecht. (Animal pyogenes, Type A of Edwards, Jour. Bact., 27, 1934, 527; Frost and Engelbrecht, A Revision of the Genus Streptococcus, privately published, $1936,3 \mathrm{pp}$. and The Streptococci, 1940, 25 ; Streptococcus pyogenes animalis Seelemann, Deutsche tierarzt. Wehnsehr., 50, 1942, 8 and 48.) From M. L., derived to mean animal epidemicus.

Morphology and general cultural characters resemble Streptococcus pyogenes. Mucoid colonies are common. Capsules are constantly demonstrable and prominent. Gram-positive.

Gelatin stab: No liquefaction.

Litmus milk: May be curdled, litmus not reduced or slowly after curdling.

Acid from glucose, lactose and sorbitol. Acid may be produced from maltose, sucrose and salicin. No acid 
from arabinose, trehalose, raffinose, inulin, glycerol or mannitol.

Does not hydrolyze sodium hippurate, but starch and esculin may be split.

Ammonia is produced from peptone.

Temperature relations: No growth at $10^{\circ} \mathrm{C}$ or at $45^{\circ} \mathrm{C}$. Does not survive $60^{\circ} \mathrm{C}$ for 30 minutes.

Chemical tolerance: Tolerates 2 per cent $\mathrm{NaCl}$ but not 4 per cent and 6.5 per cent. Final pH in glucose broth 4.5 to 5.2. No growth at $\mathrm{pH}$ 9.6. Methylene blue 0.01 per cent and 0.1 per cent not tolerated and not reduced.

Action on blood: Beta hemolysis.

Serology: Group C of Lancefield (loc. cit.). Cross precipitation with Streptococcus equi.

Facultative anaerobe.

Source: Blood stream, inflammatory exudates and lesions of diseased animals. Not known from man.

Habitat: Disease process of domestic and laboratory animals. (Horse: endometritis, foetus. Hog: septicemia. Cow: septicemia, metritis, foetus. Fowls: slipped tendon. Guinea pig: lymphadenitis. Rabbit: septicemia.

Fox: pneumonia.)

3. Streptococcus equi Sand and Jensen. (Bacillus adenitis equi Baruchello, Soc. Veter. de Venetie, Undine, 1886; Giornale di anatomia fisiologica et patologia degli animali domestici, Pisa, Sept., 1887; Sand and Jensen, Deuts. Ztschr. f. Tiermed., 13, 1888, 436, dated December 27, 188 $\mathbf{7}$, Veterinary Congress, Copenhagen, 1887; sometimes incorrectly credited to Schütz, Arch. f. wissens. u. prakt. Tierheilkunde, $14,1888,172$; Streptococcus cappelletti Chester, Manual Determ. Bact.. 1901, 5i; Streptococcus coryzae contagiosae equorum Schütz, in Eisenberg, Bakt. Diag., 3 Aufl., 1891, 270; Streptococcus schütz, Bongert, in Kolle and Wassermann, Handb. d. path. Mikroorg., 2 Aufl., 6, '913, 208.) From Latin equus, horse.

Possible synonyms : Streptococcus peritonitidis equi Hamburger, Cent. f. Bakt., I Abt., 19, 1896, 882 (Streptococcus peri- tonitidis Migula, Syst. d. Bakt., 2, 1900, $21)$; Streptococcus pyogenes equi Hutyra, in Lehmann and Neumann, Bakt. Diag., 7. Aufl., 1927, 221.

Note: Rivolta (Dei parassiti vegetali come introduzione allo studio delle malattie parassitarie e delle alterazione dell' alimento degli animali domestici. Turin, 1873, 161) described chains of cocci in adenitis scrophula equorum, morbus glandulosus.

Holth, reported by C. O. Jensen (Handb. d. Serumtherapie u. Serum diagnostik in d. Veterinar-med. (KlimmerWolff-Eisner), 2, 1911, 223), and Adsersen (Cent.f. Bakt., I Abt., Orig., 76, 1915, 111 ) studied the fermentation reactions of Sand and Jensen's organism. Review of early literature given by Brocq-Rousseau, Forgeot and Lrbain (Le streptocoque gourmeux. Revue de Pathologie Com. parée et d'Hygiene Générale, Paris, 1925)

Ovoid or spherical cells : 0.6 to 1 micron in diameter, sometimes in pus the long axis of the cells are transverse to the chain, and sometimes in the axis of the chain resembling streptobacilli; bacillary forms are not rare; occur in pairs, short or long chains; very long chains common in broth cultures. Capsules often marked in blood of infected mouse and when grown in serum. Gram-positive.

Gelatin stab: Growth uncertain. No liquefaction.

Nutrient agar: Primary aerobic cultures from pus occasionally fail ; growth is poor; small, convex, transparent colonies. Confluent growth is thin, grayish-white or yellowish and more abundant in the condensation water. Growth is increased particularly by horse protein.

Broth: Poor growth even in infusion broth; growth increased by serum (Evans, Jour. Bact., 32, 1936, 541).

Litmus milk: No change. Not curdled and litmus not reduced.

Acid from glucose, maltose, sucrose and salicin. No acid from arabinose, lactose, trehalose, raffinose, inulin, glycerol, mannitol or sorbitol. 
No hydrolysis of sodium hippurate.

Temperature relations: Optimum temperature $37^{\circ} \mathrm{C}$. Growth slow at $20^{\circ} \mathrm{C}$. No growth at $10^{\circ} \mathrm{C}$ or $45^{\circ} \mathrm{C}$. Does not survive $60^{\circ} \mathrm{C}$ for 30 minutes.

Chemical tolerance: Does not tolerate 6.5 per cent $\mathrm{NaCl}$; final $\mathrm{pH}$ in glucose broth 4.8 to 5.5 . Methylene blue is not tolerated 0.01 per cent to 0.1 per cent. Inhibited by bile but not soluble.

Action on blood: On blood-agar, colonies are small and watery, dry out rapidly leaving flat glistening colony. Well-defined wide clear zone of hemolysis (beta hemolysis). Growth in serum broth gives a hemolysin active on horse corpuscles, less so on those of sheep and guinea pig.

Toxin: Subcutaneous injection causes necrosis, other evidence of toxin production is defective.

Fibrinolysin: Usually does not lyse human fibrin; some strains reported to do so.

Serology: A member of Lancefield's Group C (Jour. Exp. Med., 57, 1933, 571); cross precipitation with Species No. 2 (animal pyogenes) of Edwards (Jour. Bact., 27, 1934, 527). Cultures have been poor antigens for production of agglutinating serum and results have been unsatisfactory. Immunized rabbit serum may protect mice from infection, to which mice are very susceptible.

Pathogenicity high for white mice, low or no virulence for rabbits and guinea pigs.

Facultative anaerobe; growth in primary culture often better in depth of medium.

Source: Pus from lesions and mucous membrane of upper respiratory tract of horses. Evidence of occurrence in man is unconvineing.

Habitat: Found only in strangles in horses.

4. Streptococcus equisimilis Frost and Engelbrecht. (Human C, Ogura, Jour. Jap. Soc. Vet. Sci., 8, 1929, 174; Edwards, Jour. Bact., 23, 1932, 259; ibid., 25, 1933, 527; Sherman, Bact. Reviews, 1, 1937, 35;
Frost and Engelbrecht, A Revision of the Genus Streptococcus, privately published, 1936, $3 \mathrm{pp}$. and The Streptococci, 1940, 45.) From M. L., derived to mean similar to equi.

This species is apt to be confused with Streptococcus equi Sand and Jensen, but it is not as fastidious in its growth requirements and shows greater tolerance of methylene blue, lyses human fibrin and ferments glycerol and trehalose. It may or may not ferment lactose.

It is also apt to be confused with Streptococcus pyogenes Rosenbach except for its greater tolerance of methylene blue, glycerol fermentation and especially Lancefield's serological grouping (Jour. Exp. Med., 57, 1933, 371).

Spheres: Gram-positive.

Gelatin: Not liquefied.

Litmus milk: Acid, may be curdled; litmus not reduced before curdling.

Acid from glucose, maltose, sucrose, trehalose and glycerol; may or may not form acid from lactose and salicin. No acid from arabinose, raffinose, inulin, mannitol or sorbitol.

No hydrolysis of sodium hippurate but may hydrolyze starch and esculin.

Ammonia is produced from peptone.

Temperature relations: No growth at $10^{\circ} \mathrm{C}$ and $45^{\circ} \mathrm{C}$. Does not survive $60^{\circ} \mathrm{C}$ for 30 minutes.

Chemical tolerance: Does not tolerate 6.5 per cent $\mathrm{NaCl}$. Final $\mathrm{pH}$ in glucose broth 5.4 to 4.6 ; no growth at pH 9.6. Methylene blue 0.1 per cent not tolerated, but Edwards (Kentucky Agr. Exp. Station Bull. 356, 1935; confirmed by Davis and Guzdar, Jour. Path. and Bact., 43, 1936,197 ) finds resistance to 0.000025 molar methylene blue in infusion-casein digest broth. Rarely grows on 40 per cent bile-blood agar.

Action on blood: Beta hemolysis.

Fibrinolysin: Dissolves human fibrin. Serology : Lancefield (loc. cit) Group C. Facultative anaerobe.

Source: Human nose and throat, vagina and skin; erysipelas and puerperal fever. Uncommon in domestic animals and usu- 
ally associated with other streptococci (Edwards, loc. cit).

Habitat: Human upper respiratory tract and vagina.

Streptococcus dysgalactiae Diernhofer (Milchw. Forsch., 13, 1932, 368), Group II Minett (Proc. 12th Internat. Vet. Cong., $2,1934,511$ ) and Streptococcus pseudoagalactiae Plastridge and Hartsell (Jour. Inf. Dis., 61, 1931, 114) appear to be identical (Little, personal communication). Physiologically these organisms are like Human C types (Streptococcus equisimilis Frost and Engelbrecht) except that they are not hemolytic.

5. Streptococcus agalactiae Lehmann and Neumann. (Streptococcus de la mammite, Nocard and Mollereau, Ann. Inst. Past., 1, 1887, 109; Streptococcus nocardi Trevisan, I generi e le specie delle Batteriacee, 1889, 36 (this name rightly has priority and is valid but has remained unused and it would seem unwise to adopt it in place of a name familiar by usage); Streptococcus mastitis sporadicae Guillebeau and Streptococcus mastitis contagiosae Guillebeau, Landw. Jahrb. d. Schweiz, 4, 1892, 27; abst. in Cent. f. Bakt., 12, 1892, 101 ; Streptococcus agalactiae contagiosae Kitt, Bakterienkunde, Wien, 1893, 322 ; Lehmann and Neumann, Bakt. Diag., 1 Aufl., 2, 1896, 126; Streptococcus mastitidis Migula, Syst. d. Bakt., 2, 1900, 19.) From Greek, want of milk.

According to Hucker and Harrison ( $\mathrm{N}$. Y. Agr. Exp. Sta. Tech. Bul. 246, 1937, 9), Streptococcus agalactice Lehmann and Neumann is identical with Group $I$ of Minett, Stableforth and Edwards (Jour. Comp. Path. and Ther., $46,1933,131$ ) and Group A of Plastridge, Anderson, Brigham and Spaulding (Conn. (Storrs) Agr. Exp. Sta. Bul. 195, 1934).

Deseription largely taken from Hansen, N. Y. Agr. Exp. Sta. Tech. Bul. 232, 1935.

Spherical or ovoid cells : 0.4 to $1.2 \mathrm{mi}$ crons in diameter, occurring in chains of seldom less than four cells and frequently very long; the longer axis of the cells may be in the axis of the chain or may be transverse to it. Chains may appear to be composed of paired cocci. Capsules(?). Gram-positive.

Gelatin stab: Gray, filiform growth. No liquefaction.

Nutrient agar: Small gray colonies.

Broth: Growth is variable in character; most frequently a sticky, flaky deposit which may adhere to the side of the tube but the supernatant fluid is clear; long chains are formed.

Starch broth: May produce yellow to orange sediment.

Litmus milk: Acid followed by curdling. Litmus reduced subsequent to curdling and proceeds from the bottom upwards. Little or no proteolysis.

Indole not formed.

Acid from glucose, maltose, galactose, fructose, lactose, sucrose, mannose, dextrin and trehalose and at times from salicin. No acid from arabinose, raffinose, inulin, xylose, glycerol, mannitol, sorbitol or amygdalin.

Sodium hippurate is hydrolyzed. No hydrolysis of starch and esculin.

Nitrites not produced from nitrates.

Ammonia is produced from peptone.

Temperature relations: Optimum temperature $37^{\circ} \mathrm{C}$. Range of growth tolerance between $15^{\circ} \mathrm{C}$ and $40^{\circ} \mathrm{C}$. No growth at $10^{\circ} \mathrm{C}$ or $45^{\circ} \mathrm{C}$. Does not survive $60^{\circ} \mathrm{C}$ for 30 minutes.

Chemical tolerance: Tolerates 2 per cent $\mathrm{NaCl}$, variable tolerance of 4 per cent $\mathrm{NaCl}$ and does not tolerate 6.5 per cent $\mathrm{NaCl}$. Final $\mathrm{pH}$ in glucose broth 4.2 to 4.6; no growth at $\mathrm{pH}$ 9.6. Methylene blue 0.01 per cent and 0.1 per cent not tolerated and not reduced.

Not soluble in bile and is not inhibited by 10 per cent and usually not by 40 per cent bile.

Action on blood: Variable; between $\frac{1}{3}$ and $\frac{1}{2}$ of the strains produce a narrow clear zone of hemolysis; certain strains described as producing greening. The hemolytic strains produce an oxygenstable, filterable hemolysin. 
Toxin: No evidence of an erythrogenic toxin.

Fibrinolysin : Does not dissolve human fibrin.

Serology : Group B of Lancefield (Jour. Exp. Med., 47, 1933, 571). Three antigenic types have been separated which appear to be associated with the carbohydrate and not the protein fraction.

Facultative anaerobe.

Source: Isolated from milk and tissues from udders infected with mastitis. Occasionally reported from human sources (Lancefield, Jour. Exp. Med., 57, 1933, 571 ; Hare, Jour. Path. Bact., 41, 1935, 499).

Habitat: Udder of cattle with mastitis.

6. Streptococcus salivarius Andrewes and Horder. (Lancet, 2, 1906, 712; Streptococcus cardio-arthritidis Small, Amer. Jour. Med. Sci., 173, 1927, 103.) From Latin salivarius, slimy, clammy; M. L., related to saliva.

Description based on studies by Safford, Sherman and Hodge, Jour. Bact., 33, 1937, 263 and Sherman, Niven and Smiley, Jour. Bact., 45, 1943, 249.

Spherical or ellipsoidal cells, 0.6 to 0.8 micron in diameter, usually in short chains. Long axis of cell lies in axis of chain. Cells are relatively large in liquid media, especially milk. Gram-positive.

Gelatin stab: Filiform growth. No licquefaction.

Plain nutrient agar: Colonies white, small, not more than $0.5 \mathrm{~mm}$ in diameter. Notwithstanding rather vigorous growth on artificial culture media, cultures die out readily.

Nutrient agar containing 5 per cent sucrose or raffinose produces a large, clear, soft, mucoid colony about the diameter of those produced by coliform bacteria and yeasts. This is quite distinctive as no other known species of streptococcus (except occasional strains of Streptococcus bovis) produce colonies of this type on sucrose or raffinose agar. The polysaccharide produced is a soluble levan, some strains producing in addition a smaller amount of insoluble dextran (the polysaccharide in the Streptococcus bovis colonies is a dextran).

Action on blood agar: Indifferent (gamma hemolysis of Brown, Rockefeller Inst. Med. Res., Monograph 9, 1919, 8). No soluble toxin and no hemolysin has been demonstrated

Broth: Variable. Loose, flocculent deposit with clear supernatant fluid and long chains, or uniform or granular turbidity with small deposit and short chains. No pellicle.

Litmus milk: Acidified and curdled promptly by all lactose-fermenting strains. Completely reduced but only after curdling. No digestion.

Potato: Slight growth. Difficult to detect.

Acid from glucose, maltose, sucrose, raffinose, inulin and salicin. No acid from glycerol, mannitol, sorbitol, arabinose or xylose. Trehalose and lactose usually fermented.

To hydrolysis of sodium hippurate and arginine. Splits esculin. Starch is not hydrolyzed.

Ammonia is not produced from peptone.

('hemical tolerance: Tolerates 2 per cent but not 4 per cent $\mathrm{NaCl}$. Final pH in glucose broth between 4.4 and 4.0 . No growth at pH 9.6. Methylene blue 0.01 per cent and 0.1 per cent not tolrated. Not soluble in bile but inhibited by 30 per cent bile in blood agar.

Catalase not produced.

Temperature relations: Optimum growth $37^{\circ}$ to $43^{\circ} \mathrm{C}$. Growth at $45^{\circ} \mathrm{C}$. No grow th at $47^{\circ} \mathrm{C}$. No growth at $10^{\circ} \mathrm{C}$. Does not survive $60^{\circ} \mathrm{C}$ for 30 minutes.

Facultative anaerobe.

Serology: No group antigen has been demonstrated. Contains several serological types.

Source: Saliva and sputum in various pulmonary infections, apical abscesses of teeth, carious lesions of teeth, intestinal tract.

Habitat: Human mouth, throat and nasopharynx. 
7. Streptococcus mitis Andrewes and Horder. (Lancet, 2, 1906, 712.) From Latin mitis, mild.

Synonyms: Streptococcus mitior seu viridans Schottmüller, Münch. med. Wchnschr., 50, 1903, 849 (these names refer to a group of species and they are therefore confused in meaning in medical literature. See Winslow and Winslow. The Systematic Relationships of the Corracene. New York, 1908, and Safiord, Sherman and Hodge, Jour. Bact., 33, 1937, 263). The name Streptococcus mitis was first proposed by Fränkel (Münch. med. Wchnschr., 52, 1904, 548 and 1868). Because others have used this name with varied meanings (Streptococcus mitis seu viridans von Lingelsheim, in Kolle and Wassermann, Handb. d. path. Mikroorg., 2 Aufl., 4, 1912, 453; Streptococcus mitis Holman, Jour. Med. Res., 3ł, 1916.37\%), the more definite emendation of $\mathrm{In}$ drewes and Horder has been used as the basis of the description given here. The relationships of these organisms has been discussed by Brown, Rockefeller Inst. Med. Res., Monograph No. 9, 1919, 86.

Description based on studies by safford, Sherman and Hodge (loc, cit.) and Sherman, Niven and Smiley, Jour. Bact., $45,1943,249$.

Spherical or ellipsoidal cells, 0.6 to 0.8 micron in diameter. Long axis of cell lies in axis of chain. Cells not especially large in liquid media including milk. No capsules. Gram-positive.

Gelatin stab: Filiform growth. No liquefaction.

Nutrient agar: Growth increased when serum or blood is added. Confluent growth, gray and abundant.

Action on blood agar: The colonies are surrounded by a characteristic greening (alpha hemolysis of Brown, Rockefellex Inst. Med. Res., Monograph 9, 1919, 8). This is weak with some strains and is variable under anaerobic conditions. No soluble toxin and no hemolysin has been demonstrated.

Broth: Variable. Loose, flocculent de- posit with clear supernatant fluid and long chains, or granular turbidity with small deposit and short chains. No pellicle.

Litmus milk: Usually acidified and curdled promptly; litmus is completely reduced but only after curdling; no digestion.

Potato: Slight growth which is difficult to detect.

Acid fron glucose, maltose, lactose, sucrose and usually salicin. Variable fermentation of raffinose. No acid from inulin, mannitol, sorbitol, glycerol, arabinose or xylose. Trehalose rarely fermented.

No hydrolysis of sodium hippurate and usually no hydrolysis of arginine. Action on esculin usually negative.

Isually ammonia is not produced from peptone.

Chemical tolerance: Tolerates 2 per cent but not 4 per cent $\mathrm{NaCl}$. Final pH in glueose broth 5.8 to 4.2 , ave. 4.5. No grow th at $\mathrm{pH} 9.6$. Methylene blue 0.01 per cent and 0.1 per cent not tolerated. Not soluble in bile but inhibited by 30 per cent bile in blood agar.

Catalase not produced.

Temperature relations: Optimum grow th $37^{\circ}$ to $40^{\circ} \mathrm{C}$. Many strains do not grow at $45^{\circ} \mathrm{C}$. No growth at $10^{\circ} \mathrm{C}$. Does not survive $60^{\circ} \mathrm{C}$ for 30 minutes.

Facultative anaerobe.

Serology: No group antigen has been demonstrated. Contains several serologica! types.

Source: Saliva and sputum in various pulmonary infections, pus from upper respiratory tract and sinuses, blood and various organs in sub-acute endocarditis.

Habitat: Human mouth, throat and nasopharynx.

8. Streptococcus bovis Orla-Jensen emend. Sherman. (Orla-Jensen, The Lactic Acid Bacteria, 1919, 137; Sherman, Bacteriological Reviews, 1, 1937, 57.) From Latin bos, cow.

The majority of the strains of Strepto- 
coccus inulinaceus may be considered as identical with Streptococcus bovis as described here. The so-called Bargen streptococcus (Bargen, Jour. Amer. Med. Assn., 83, 1921, 332; Arch. Int. Med., 45, 1930, 559) is also considered to be Streptococcus bonis.

Spheres: Occurring in pairs and chains. Capsulated in milk. Gram-positive.

Gelatin stab: No liquefaction.

Iitmus milk: Acid, curdled in 3 to 5 days, followed by reduction of the litmus.

Acid from glucose, fructose, mannose, galactose, maltose, lactose, sucrose, raffinose and salicin; sometimes from mannitol, sorbitol, inulin, arabinose and trehalose. Not from glycerol.

Starch is hydrolyzed by typical strains but not by variety inulinaceus. Esculin is hydrolyzed but not sodium hippurate.

Nitrites not produced from nitrates.

Ammonia not produced from 4 per cent peptone.

Temperature relations: Optimum temperature $35^{\circ} \mathrm{C}$. When freshly isolated, maximum $45^{\circ} \mathrm{C}$. No growth at $22^{\circ} \mathrm{C}$ or below. Survives $60^{\circ} \mathrm{C}$ for 30 minutes, but not $65^{\circ} \mathrm{C}$.

Chemical tolerance: 2 per cent $\mathrm{NaCl}$ growth, 4 per cent $\mathrm{NaCl}$ no growth, 6.5 per cent $\mathrm{NaCl}$ no growth. Final $\mathrm{pH}$ in glucose broth 4.5 to 4.0 . No growth at pH 9.6. May tolerate 0.01 per cent methylene blue but not 0.1 per cent. Tolerant of bile and not soluble.

Action on blood: Not hemolytic; the changes exhibited vary from greening (alpha) to no observable change (gamma).

Soluble hemolysin: Absent.

Toxin: Absent.

Serology: Some cross reaction with Lancefield Group D (Sherman, Jour. Bact., 35, 1938, 81).

Facultative anaerobe.

Distinctive characters: Greening or no change in blood; a higher maximum temperature of growth than Streptococcus salivarius and distinctly higher thermal resistance $\left(60^{\circ} \mathrm{C}\right.$ for 30 minutes); hydrolysis of starch and usually ferments arabinose and sometimes mannitol.
Source : Saliva, feces and intestinal contents of cattle; milk of cows; sometimes abundantly present in human feces (Bargen's coccus) in health and disease. The variety inulinaceus is sometimes abundant in the bovine throat.

Habitat: Bovine mouth and alimentary tract where it is the predominating streptococcus.

\section{Streptococcus thermophilus Orla-} Jensen. (Maelkeri-Bacteriologi, 1916, 37 ; The Lactic Acid Bacteria, 1919, 136.) From Greek thermus, heat; philus, loving.

Spheres : 0.7 to 0.9 micron, with pointed ends, occurring singly and in short chains. Gram-positive.

Gelatin stab: No liquefaction.

Nutrient agar: Small, pin-point, gray, circular colonies. In streak cultures growth is scanty, beaded and gray. Fastidious in nutritive requirements needing appropriate carbohydrates added to peptone-infusion media (especially lactose and sucrose). Viability on laboratory media low.

Broth: Fine granular sediment; usually in very long chains, especially at $45^{\circ} \mathrm{C}$.

Litmus milk: Acid, curdled, followed by partial reduction of the litmus.

Acid from glucose, fructose, lactose, and sucrose; seldom ferments raffinose and arabinose. No acid from maltose, dextrin, inulin, glycerol, mannitol, sorbitol or salicin.

No hydrolysis of sodium hippurate or esculin. Starch may be hydrolyzed on a favorable medium.

Ammonia not formed from 4 per cent peptone.

Temperature relations: Optimum $40^{\circ}$ to $50^{\circ} \mathrm{C}$. Minimum $20^{\circ} \mathrm{C}$. No growth at $53^{\circ} \mathrm{C}$. Survives $60^{\circ}$ and $65^{\circ} \mathrm{C}$ for 30 minutes. Thermal death point $72^{\circ}$ to $74^{\circ} \mathrm{C}$.

Chemical tolerance: Extremely sensitive to salt, no growth with 2 per cent, 4 per cent and 6.5 per cent $\mathrm{NaCl}$. Final $\mathrm{pH}$ in glucose broth 4.5 to 4.0 . No growth 
at $\mathrm{pH}$ 9.6. Not tolerant of 0.01 per cent and 0.1 per cent methylene blue.

No action on blood.

Serology : No cross reaction with Lancefield Group D (Sherman, Jour. Bact., 35, 1938, 81).

Facultative anaerobe.

Distinctive characters: High growth temperature $\left(50^{\circ} \mathrm{C}\right)$ and heat resistance $\left(60^{\circ}\right.$ to $\left.65^{\circ} \mathrm{C}\right)$. Inability to ferment maltose and salicin. Inhibited by 2 per cent $\mathrm{NaCl}$. Nutritive requirements in medium.

Source : Milk and milk products. Used as a starter in making Swiss cheese.

10. Streptococcus equinus Andrewes and Horder. (Lancet, 2, 1906, 712.) From Latin equinus, of horses.

Spheres: Occurring in short chains; the chains are longer in broth than in milk and some cultures give extremely long chains in broth. Gram-positive.

Gelatin stab: Little or no growth at $20^{\circ} \mathrm{C}$. Not liquefied.

Litmus milk: No visible change, grows poorly (with 2 per cent added glucose there is little reduction of litmus).

Acid from glucose, fructose, galactose, maltose and usually from sucrose and salicin; raffinose and inulin are seldom fermented; arabinose, xylose, lactose, mannitol and glycerol are not fermented. The salicin-negative strains correspond to Streptococcus ignavus Holman, Jour. Med. Res., 34, (N. S. 29), 1916, 377.

Starch is not hydrolyzed under ordinary conditions of test (poured plate); it may be hydrolyzed by streak cultures on a very favorable medium. Sodium hippurate is not split. Esculin is hydrolyzed slowly, failure in three days, becomes positive in seven.

Ammonia not produced from 4 per cent peptone.

Temperature relations : Minimum $21^{\circ} \mathrm{C}$. Growth at $45^{\circ} \mathrm{C}$, seldom at $47^{\circ} \mathrm{C}$, and no growth at $48^{\circ} \mathrm{C}$. Sometimes survives $60^{\circ} \mathrm{C}$ for 30 minutes.

Chemical tolerance: Growth in 2 per cent $\mathrm{NaCl}$ but not in 4 per cent and 6.5 per cent. Final pH in glucose broth 4.5 to 4.0 ; no grow th at pH 9.6. Some strains tolerate 0.01 per cent but none tolerate 0.1 per cent methylene blue.

Action on blood: Greening (alpha on horse blood) varying to weak but definite. No hemolysis.

Serology unknown, but no cross reaction with Lancefield Group D (Sherman, Jour. Bact., 35, 1938, 81).

Facultative anaerobe.

Distinctive characters: Minimum temperature of growth $\left(20^{\circ} \mathrm{C}\right)$ and high maximum temperature of growth $\left(47^{\circ} \mathrm{C}\right)$; poor growth in milk, even with added glucose; failure to ferment lactose.

Sources: Human and bovine feces; human mouth, urine and inflammatory exudates (pathogenicity not established). Andrewes and Horder (loc. cit.) failed to find it in feces of fox and stoat.

Habitat: Predominating organism in the intestine of horses.

11. Streptococcus lactis (Lister) Löhnis. (Bacterium lactis Lister, Quart. Jour. Micro. Sci., 13, 1873, 380; 18, 1878, 177 ; Löhnis, Cent. f. Bakt., II Abt., 22, 1909, 553.) From Latin lac, milk.

The following organisms are generally regarded as identical with Streptococcus lactis Löhnis. See Breed, in Jordan and Falk, The Newer Knowledge of Bacteriology and Immunology, Chicago, 1928, 383.

Streptococcus acidi lactici Grotenfelt, Fortschr. d. Med., 7, 1889, 121; Micrococcus acidi paralactici Nencki and Sieber, Monatschr. f. Chem., 10, 1889, 532; Bacillus No. 19, Adametz, Landw. Jahrb., 18, 1889, 227; Eine bestimmte Bacterienart, Günther and Thierfelder, Arch. f. Hyg., 25, 1895, 164; Bacillus acidi. lactici Esten, Storrs Agric. Exper. Sta. Conn., Ann. Rep. for 1896, 1897, 44 (not Milchsäurebacterium, Hueppe, Mitt. d. kais. Gesundheitsamte, 2, 1884, 340, which is Bacillus acidi lactici Zopf, Die Spaltpilze, 3 Aufl., 1885, 87); Bacterium güntheri Lehmann and Neumann, Bakt. Diag., 1 Auf., 2, 1896, 197; Bacterium 
lactis acidi Leichmann, Cent. f. Bakt., II Abt., 2, 1896, 777 (not Bacterium lactis acidi Marpmann, Ergänzungshefte Cent. f. allgem. Gesundheitspflege, 2, 1886. 117); Der ovaler Coccus, Freudenreich, Cent. f. Bakt., II Abt., 1, 1896, 168; Bacillus lacticus Kruse, in Flügge, Die Mikroorganismen, 3 Auft., 2, 1896, 356; Bacterium lacticus Chester, Del. Agr. Exp. Sta., 9th Ann. Rept., 1897, 8s; Bacillus acidi paralactici Kozai, Ztsehr. f. Hyg., 31, 1899, 372; Streptococeus paralacticus Migula, Syst. d. Bakt., 2, 1900 , 18; Bacterium lacticum Migula, Syst. d. Bakt., 2, 1900, 405; Bacterium timncatum Migula, Syst. d. Bakt., 2, 1900, $40 \overline{7}$ (Bacillus No. 19 of Adametz; Bacterium punctatum. Chester, Man. Determ. Bact. 1901, 14ī; not Bactcrium Iruncalum Chester, ibid., 157; not Bacterium truncatum Chester, ibid., 195); Streptococcus grotenfeltii Chester, Manual Determ. Bact., 1901, 67; Lactococcus lactis Beijerinck, Arch. Neérl. d. Sei. Exact. et Nat., Sér. II, 7, 1901, 213; Streptococcu. lacticus Kruse, Cent. f. Bakt., I Abt. Orig., 34, 1903, 737; Streptococcus güntheri Lehmann and Neumann, Bakt. Diag., 4 Aufl., 2, 1907, 242; Bacillus lactis acidi Sewerin, Cent. f. Bakt., II Abt., 22, 1908, s (not Bacillus lactis acidi Marpmann, loc. cit., 120, nor Leichmann. loc. cil., 778); Bacterium leichmanm Wolff, Cent.f. Bakt., II Abt., 24, 1909. 57.

Spheres: Many rells elongated in di rection of ehain; 0.5 to 1 micron; mostly pairs and short chains, with some cultures long chains. Gram-positive.

Gelatin stab: Filiform to beaded growth. No liquefaction.

Nutrient agar colonies: Small, round or oval, gray, entire, slightly raised. Streak culture tends to remain as definite colonies throughout, confluent in parts.

Glucose broth: Turbidity and later sediment.

Potato: No visible growth.

Litmus milk: Acid; complete reduction of litmus before curdling. Young cultures entirely reduced with narrow red band at top which widens with ageing. No digestion and no gas produced, but whey may be expressed.

Acid from glucose, maltose and lactose; variable in arabinose, xylose, maltose, suerose, mannitol and salicin. No acid from raffinose, inulin, glyeerol or sorbitol. Occasional strains have been noted which fail to ferment lactose (Yawger and Sherman, Jour. Dairy Sci., 20, $1937,83)$ and others which do ferment raffinose (Orla-Jensen and Hansen, Cent. f. Bakt., II Abt., 86, 1932, 6).

Starch not hydrolyzed. Sodium hippurate may be hydrolyzed and esculin is split.

Ammonia is produced from 4 per cent peptone.

Temperature relations: No growth at $45^{\circ} \mathrm{C}$. Some strains survive $60^{\circ} \mathrm{C}$ for 30 minutes.

Chemical tolerance: Growth with 2 per cent and 4 per cent $\mathrm{NaCl}$ but not with 6.5 per cent. Final pH in broth 4.5 to 4.0 . No grow th at $\mathrm{pH} 9.6$ but grows at $\mathrm{pH} 9.2$. Tolerates both 0.01 per cent, 0.1 per cent and 0.3 per cent methylene blue. Bile neither lyses nor inhibits growth.

Action on blood: No hemolysis; may show greening or no action.

Serology : Sherman, Smiley and Niven (Jour. Dairy Sci., 23, 1940, 529) have produced a species-specific group serum for this species. Shattock and Mattick (Jour. Hyg., 43, 1943, 173) have desig. nated this group as Group N. The above authors are in agreement in feeling that their studies indicate a close serological relationship between Streptococcus lactis and Streptococcus cremoris. Toxin not known.

Facul tative anaerobe.

Distinctive characters : Grow th at $10^{\circ} \mathrm{C}$ or below and at $40^{\circ} \mathrm{C}$ but not at $45^{\circ} \mathrm{C}$; rapid complete reduction of litmus before curdling milk; growth in presence of 4 per cent but not 6.5 per cent $\mathrm{NaCl}$; ammonia produced from peptone; no growth at pH 9.6 but grows at $\mathrm{pH} 9.2$. 
Source: Isolated from milk by Lister (loc. cit.). Milk and milk products.

Habitat: Not in the udder of cows. Plants may be natural habitat (Stark and Sherman, Jour. Bact., 30, 1935, 639).

Note: The following may be regarded as varieties of Streptococcus lactis: (1) Streptococcus lactis var. maltigenes Hammer and Cordes, Iowa Agr. Exp. Sta. Res. Bull. 68, 1921 ; (2) Streptococcus lactis var. anoxyphilus Hammer and Baker, Iowa Agr. Exp. Sta. Res. Bull. 99, 1926; (3) Streptococcus lactis var. tardus Hammer and Baker, ibid. Also (4) Streptococcus amylolactis, (5) Streptococcus raffinolactis and (6) Streptococcus saccharolactis OrlaJensen and Hansen, Cent. f. Bakt., II Abt., 86, 1932, 6 .

12. Streptococcus cremoris Orla-Jensen. (The Lactic Acid Bacteria, 1919, 132.) From Latin cremor, thick juice; M. L., cream.

Synonyms: ? Streptococcus hollandicus Wiegmann, in Kramer, Die Bakteriologie in ihren Beziehungen zur Landwirtschaft und den Landw. Technischen Gewerben, Wien, 1890 ; Streptococcus lactis B, Ayers, Johnson and Mudge, Jour. Inf. Dis., 34, 1934, 29.

Spheres : 0.6 to 0.7 micron (often larger than Streptococcus lactis); forming long chains, especially in milk, some cultures in pairs. Gram-positive.

Gelatin stab: No liquefaction.

Litmus milk: Acid; complete reduction of litmus before curdling with red line at top broadening with age; clot separates with no digestion of casein; milk becomes slimy.

Acid from glucose and lactose; may ferment maltose, salicin and rarely sucrose, raffinose and mannitol. Arabinose, xylose, sorbitol, inulin and glycerol are not fermented.

No hydrolysis of starch and sodium hippurate but sometimes esculin.

Ammonia not produced from 4 per cent peptone.

Temperature relations: Optimum be- low $30^{\circ} \mathrm{C}$. Minimum $10^{\circ} \mathrm{C}$. Maximum $37^{\circ} \mathrm{C}$. May survive $60^{\circ} \mathrm{C}$ for 30 minutes. Thermal death point $65^{\circ} \mathrm{C}$ to $70^{\circ} \mathrm{C}$.

Chemical tolerance: Grows with 2 per cent but not with 4 per cent and 6.5 per cent $\mathrm{NaCl}$. Final $\mathrm{pH}$ in glucose broth 4.6 to 4.0 . No growth at $\mathrm{pH} 9.6$ and 9.2. Tolerates 0.01 per cent and sometimes 0.1 and seldom 0.3 per cent methylene blue.

Action on blood: No hemolysis.

Facultative anaerobe.

Distinctive characters: Inability to grow at $40^{\circ} \mathrm{C}$; reduction of litmus before curdling milk; no growth in the presence of 4 per cent $\mathrm{NaCl}$ and at $\mathrm{pH} 9.2$; does not grow well on artificial media.

Source: Raw milk and milk products; commercial starters in butter and cheese factories. Not known from human and animal sources.

The following may be regarded as varieties of Streptococcus cremoris: (1) Streptococcus lactis var. hollandicus Buchanan and Hammer, Iowa Agr. Exp. Sta. Res. Bull. 22, 1915; (2) Streptococcus mannitocremoris Orla-Jensen and Hansen, Cent. f. Bakt., II Abt., 86, 1932, 6 .

13. Streptococcus faecalis Andrewes and Horder. (Hicrococcus ovalis Escherich, Die Darmbakterien, Stuttgart, 1886, 89; Entérocoque, Thiercelin, Compt. rend. Soe. Biol., Paris, 54, 1902, 1082; Enterococcus proteiformis Thiercelin and Jouhaud, Compt. rend. Soc. Biol., Paris, 55, 1903, 686; Andrewes and Horder, Lancet, 2, 1906, 708; Streptococcus faecium and Streptococcus glycerinaceus Orla-Jensen, The Lactic Acid Bacteria, 1919, 139 and 140; Diplococcus cnterococcus Neveu-Lemaire, Précis $\mathrm{Pa}$ rasitol. Hum., 5th ed., 1921, 18; Streptococcus ovalis Lehmann and Veumann, Bakt. Diag., 7 Aufl., 2, 1927, 209 and 230; Streptococcus proteiformis Hauduroy et al., Dict. d. Bact. Path., Paris, 1937, 520.) From Latin faex, dregs, residue; II. L., feces, excrement; M. L. faecalis, fecal.

Escherich reclassified his Micrococcus 
ovalis (loc. cit.) as a streptococcus in Jahrb. f. Kinderheilk., 49, 1899, 161.

According to Gorini (Le Lait, 6, 1936, 81) his term Gastrococcus is a synonym of Enterococcus Thiercelin (loc. cit.), Micrococcus ovalis Escherich (loc. cit.) and Streptococcus faecalis Andrewes and Horder (loc. cit.).

Spheres, ovals, of variable size (often large), usually occurring in pairs and sometimes short chains in fluid media. Gram-positive.

Gelatin stab: Filiform growth. No liquefaction.

Nutrient agar: Small, round, raised, milky colonies. Streak culture fairly abundant and confluent.

Broth: Turbid, clearing later with abundant sediment.

Potato: No visible growth.

Litmus milk: Acid, usually reduction of litmus before curdling; no digestion of clot.

Acid from glucose, maltose, lactose, salicin and almost always mannitol; may or may not ferment arabinose, sucrose, raffinose, glycerol, sorbitol. Inulin is seldom fermented.

Starch not hydrolyzed; sodium hippurate may be and esculin is hydrolyzed.

Ammonia is produced from 4 per cent peptone.

Temperature relations : Optimum $37^{\circ} \mathrm{C}$. May grow at $5^{\circ} \mathrm{C}$ and below. Grows at $10^{\circ} \mathrm{C}$ and $45^{\circ} \mathrm{C}$, seldom grows at $50^{\circ} \mathrm{C}$. Survives $62.8^{\circ} \mathrm{C}$ for 30 minutes.

Chemical tolerance: Tolerates 2 per cent, 4 per cent and 6.5 per cent $\mathrm{NaCl}$. Final pH in glucose broth 4.4 to 4.0 . Grows at pH 9.6. Tolerates 0.01 per cent and 0.1 per cent methylene blue. Bile does not lyse or inhibit growth.

Action on blood: Usually greening; sometimes no change.

Toxin unknown.

Serology : Lancefield's Group D (Sherman, Jour. Bact., 35, 1938, 81 ).

Facultative anaerobe.

Distinctive characters: Grow th at $10^{\circ} \mathrm{C}$ and $45^{\circ} \mathrm{C}$; survives $60^{\circ} \mathrm{C}$ for 30 minutes; reduction of litmus before curdling milk; growth at $\mathrm{pH} 9.6$, in the presence of 6.5 per cent $\mathrm{NaCl}$, and 0.1 per cent methylene blue; not hemolytic and does not liquefy gelatin.

Source: Human feces and intestinal contents; inflammatory exudates; blood stream in subacute endocarditis; European foul-brood of bees; milk and milk products, especially cheese; garden plants.

Habitat: Human intestine, milk and milk products.

14. Streptococcus liquefaciens Sternberg emend. Orla-Jensen. (Sternberg, Manual of Bacteriology, 1893, 613; OrlaJensen, The Lactic Acid Bacteria, 1919, 142). From Latin liquefaciens, liquefying.

Synonyms: Micrococcus casei amari Freudenreich, Landw. Jahrb. d. Schweiz, 8, 1894, 136; Streptococcus apis Maassen, Arb. Biol. Abt. f. Land- u. Forstwirtsch. a.d.k. Gesundheitsamte, 6, 1908, 53 (as shown by Hucker, N. Y. Agr. Fxp. Sta. Tech. Bull. 143, 1928, 40 and 190, 1932, 17); Bacillus güntheri Cowan, British Bee-Keepers Guide Book, 20th ed., London, 1911, 171; Streptococcus coli gracilis Escherich, Die Darmbakterien des Säuglings und ihre Beziehungen zur Physiologie der Verdauung, 1856, 77 (Streptococcus gracilis Lchmann and Neumann, Bakt. Diag., 1 Aufl., 2, 1896, 118).

As explained by Hucker (N. Y. Agr. Exp. Sta. Tech. Bull. 144, 1928, 6), some of the acid proteolytic cocei first deseribed by Gorini in 1902 (loc. cit.) are gelatin-liquefying streptococei identical with Streptococcus liquefaciens. Also see Long and Hammer, Iowa Agr. Exp. Sta. Res. Bull. 206, 1936, 219. The following names have been used for these streptococci: Bactéries productrices d'acide et de présure, Gorini, Rev. gén. du Lait, 1, 1902, 173; Micrococcus casci liquefaciens Orla-Jensen, Cent. f. Bakt., II Abt., 13, 1904, 430; Micrococcus casei acido-proteolyticus $I$ (liquefies gelatin) and Micro- 
coccus casei acido-proteolyticus II (does not liquefy gelatin) Gorini, Rev. gén. du Lait, 8, 1910, 337 (Micrococcus casei proteolyticus $I$ and $I I$ Gorini, Rend. Accad. Lincei, Ser. 5, 19, 1910, II Sem., 150); Coccus acido-proteolyticus casei I and Coccus acido-proteolyticus casei II Gorini, Rev. gén. du Lait, 9, 1912, 97. The terms Mammococcus and Caseococcus have also been used for these cocci by Gorini, Le Lait, 6, 1926, 81; Mammococcus acidoproteolylicus Gorini, Act. P. Accad. Sci. Nov. Lync., Vatican City, 88, I Sess., 1934, 42.

Spheres: Usually in pairs, sometimes short chains. Gram-positive.

Gelatin stab: Liquefaction and profuse growth; liquefaction fails in occasional variants but these are nevertheless Lancefield group D and have the other characters of the species. (See Sherman, Stark and Mauer, Jour. Bact., 33, 1937, 492.)

Litmus milk: Acid; curdled and peptonized; the litmus is reduced completely before acidulation and curdling; caseolysis fails in variants not liquefying gelatin (Sherman, Stark and Mauer, loc. cit., 486). Gives milk bitter taste.

Acid from glucose, maltose, sucrose, lactose, trehalose, mannitol, sorbitol, salicin and glycerol (rare failute from sucrose and glycerol); variable fermentation of arabinose and raffinose. Inulin not fermented.

Starch not hydrolyzed, sodium hippurate may be and esculin is hydrolyzed.

Ammonia is produced from 4 per cent peptone.

Temperature relations: Growth at $10^{\circ} \mathrm{C}$ and $45^{\circ} \mathrm{C}$, occasional growth at $50^{\circ} \mathrm{C}$. Survives $60^{\circ} \mathrm{C}$ and $62.8^{\circ} \mathrm{C}$ for 30 minutes.

Chemical tolerance: Tolerates 2, 4 and 6.5 per cent $\mathrm{NaCl}$; final $\mathrm{pH}$ in glucose broth 4.5 to 4.0 ; growth at $\mathrm{pH} 9.6$; tolerates 0.01 per cent and 0.1 per cent methylene blue. Bile tolerant.

Action on blood: No change or greening (alpha). Human fibrin not lysed.

Serology: Lancefield Group D (Sherman, Jour. Bact., 35, 1938, 81).

Facultative anaerobe.
Distinctive characters: Growth at $10^{\circ} \mathrm{C}$ and $45^{\circ} \mathrm{C}$; resistance to $60^{\circ} \mathrm{C}$; growth in presence of 6.5 per cent $\mathrm{NaCl}, 0.1$ per cent methylene blue and at $\mathrm{pH}$ 9.6. Ammonia produced from peptone. Strong reduction of litmus before acidulation of milk, which is afterwards curdled and peptonized; gelatin is liquefied; marked proteolysis. Low final $\mathrm{pH}$ in glucose broth. Fermentation of glycerol and mannitol.

Source: Originally isolated by Sternberg from a cadaver. Dairy and other food products. Foul brood of bees. Plants, feces, human vagina, blood in subacute endocarditis.

Habitat: Human and animal intestine.

15. Streptococcus zymogenes (MacCallum and Hastings) Holland. (Micrococcus zymogenes MacCallum and Hastings, Jour. Exp. Med., 4, 1899, 521; Holland, Jour. Bact., 5, 1920, 226; Staphylococcus zymogenes Ford, Textb. of Bact., 1927, 425.) From Greek zyme, ferment and -genes, producing.

This species shows the same characteristics as Streptococcus liquefaciens except as given below. The two species have sometimes been regarded as identical (Bergey et al., Manual, 3rd ed., 1930, 59).

Gelatin stab: May or may not liquefy gelatin. Otherwise as in Streptococcus liquefaciens.

Action on blood: Beta hemolytic.

Source: Originally isolated from an acute case of endocarditis.

Habitat: Human and animal intestine.

16. Streptococcus durans Sherman and Wing. (Streptococcus hemothermophilus Sherman and Wing, Jour. Dairy Sci., 18, 1935, 657; original name withdrawn by Sherman and Wing, Jour. Dairy Sci., 20, 1937, 165.) From Latin durans, resisting.

Spheres: Occurring in pairs and short chains, more rarely in long chains. Gram-positive.

Gelatin stab: No liquefaction. 
Litmus milk : Acid; curdled, followed by reduction of litmus.

Acid from glucose, maltose, lactose, and usually salicin and trehalose. Raffinose, inulin, sorbitol, arabinose, glycerol not fermented and mannitol and sucrose rarely fermented.

Starch not hydrolyzed. Sodium hippurate and esculin are hydrolyzed.

Ammonia is produced from 4 per cent peptone.

Temperature relations: Growth at $10^{\circ} \mathrm{C}$. Maximum $50^{\circ} \mathrm{C}$. Survives heating for 30 minutes at $62.8^{\circ} \mathrm{C}$ and usually $65.6^{\circ} \mathrm{C}$.

Chemical tolerance: Growth with 2 per cent, 4 per cent and 6.5 per cent $\mathrm{NaCl}$. Final pH in glucose broth 4.5 to 4.0 . Growth at pH 9.6. Tolerates 0.01 per cent and 0.1 per cent methylene blue.

Action on blood: Active hemolysis of beta type (horse, human and rabbit blood); persistent after 5 years culture on media without blood.

Toxin unknown. Non-pathogenic for mice, rabbits and guinea pigs.

Serology : Lancefield's Group D (Sherman, Jour. Bact., 35, 1938, 81 ).

Facultative anaerobe.

Distinctive characters : Growth at $10^{\circ} \mathrm{C}$ and $45^{\circ} \mathrm{C}$; beta hemolysis; failure to ferment sucrose and mannitol; resistance to $60^{\circ} \mathrm{C}$ for 30 minutes; tolerance of 0.1 per cent methylene blue and 6.5 per cent $\mathrm{NaCl}$.

Source: Forty strains were isolated from spray process milk powder.

Habitat: Human intestine, milk and milk products.

*17. Streptococcus anaerobius Krönig emend. Natvig. (Krönig, Zent.f. Gyn., 1895; Natvig, Arch. f. Gyn., 1905, 76.) From Greek an, without; aër, air; bios, life; M. I., anaerobic.

Heurlin (Bakt. Unters. d. Keimgehaltses im Genitalkanale d. fiebernden Wöchnerinnen. Helsingfors, 1910, 122-127) recognizes the following varieties of Streptococcus anaerobius: $S$. anaerobius vulgaris, $S$. anaerobius typ. vulgaris, $S$. anaerobius gonoides, $S$. anaerobius (Wegelius To. 28), S. anaerobius micros (Lewkowicz), and S. anaerobius carduus.

Deseription according to Prévot, Ann. Sei. Nat., Sér. Bot., 15, 1933, 180.

Spheres: Average size 0.8 micron, occurring in chains. Non-motile. Grampositive.

Gelatin: No liquefaction.

Semi-solid agar (Veillon): After 48 hours colonies 1 to $2 \mathrm{~mm}$ in diameter, very regular, lenticular. Gas produced. Igar slightly acidified.

Martin broth: Rapid growth. No turbidity. Sediment in 24 hours. Medium slightly acidified. Feeble production of gas. Slight fetid odor.

Martin glucose broth: Very abundant growth. Gas fetid, inflammable, no $\mathrm{H}_{2} \mathrm{~S}$. Very marked acidification.

Peptone water: Abundant flocculent growth. Gas produced at expense of peptone. Medium not acidified. Neither indole nor $\mathrm{H}_{2} \mathrm{~S}$ produced.

Meat and liver broth: Very abundant growth. Much gas produced which contains $\mathrm{CO}_{2}$ and $\mathrm{H}_{2}$.

Milk: No acid. No coagulation.

Cooked protein (egg white, meat, liver, fibrin and serum) not attacked. Fresh fibrin and fresh organs partially disintegrated with blackening, abundant gas, very fetid odor due in part to $\mathrm{H}_{2} \mathrm{~S}$.

Serum broth: Abundant gas and fetid odor.

Neutral red broth: Changed to fluorescent yellow.

Acid from glucose, fructose, galactose, sucrose and maltose. Mannitol and arabinose sometimes fermented.

Optimum pH 6.0 to 8.0 .

Temperature relations: Optimum $36^{\circ}$ to $38^{\circ} \mathrm{C}$. Grows at $26^{\circ} \mathrm{C}$, but not below $22^{\circ} \mathrm{C}$. Survives 5 minutes at $60^{\circ} \mathrm{C}$ or two minutes at $80^{\circ} \mathrm{C}$. Killed in ten minutes at $80^{\circ} \mathrm{C}$.

Pathogenic.

Strict anaerobe.

Distinctive characters: Very peptolytic; gas produced in peptone water with destruction of the peptone. Differs from

* See footnotes, p. 308. Reviewed by Dr. Ivan C. Hall. 
Streptococcus foetidus by being morphologically like a typical streptococcus. Differs from Streptococcus putridus by its physiology, bread crumb-like growth, and the production of gas in all media.

Source: Isolated in cases of putrefactive gangrene; war wounds; uterus, lochia and blood in puerperal infections; appendicitis; pleurisy; and amniotic fluid.

Habitat: Mouth and intestines. Cavities of man and animals, especially the vagina. Can invade all tissues.

\section{Streptococcus foetidus (Veillon)} Prévot. (Micrococcus foetidus Veillon, Compt. rend. Soc. Biol. Paris, 45, 1893, 867; not Streptococcus foetidus Migula, Syst. d. Bakt., 2, 1900, 38; Prévot, Ann. Sci. Nat., Sér. Bot., 15, 1933, 189.) From Latin foetidus (better fetidus), stinking.

Large spheres: 0.8 to 1.0 micron, occurring normally in short chains, also in tetrads, double or zig-zag chains. Nonmotile. Gram-positive.

Gelatin: No liquefaction.

Semi-solid agar (Veillon): Slow growth. At first punctiform; small colonies $\frac{1}{4}$ to $\frac{1}{2}$ $\mathrm{mm}$ in diameter, growing 1 to $2 \mathrm{~cm}$ below the surface, regular, thick, lenticular, opaque. Gas bubbles produced.

Blood agar: Small brownish hemopeptic zone around the colonies. No true hemolysis.

Martin broth: Poor growth. No turbidity. Flakes form on wall of tube, but rapidly settle to the bottom. Little or no gas. Very faint fetid odor.

Martin glucose broth: Good growth. No turbidity. Gas fetid, inflammable.

Meat and liver broth: Rapid, abundant growth. Abundant gas. Strong fetid odor.

Milk: No acid. No coagulation.

Peptone water: Gas production feeble. Indole not formed.

Neutral red broth changed to fluorescent yellow.

Fresh organs become green, then blacken. Much gas produced containing $\mathrm{H}_{2} \mathrm{~S}$, later the organs are gradually disin- tegrated; partial bioproteolysis and $\mathrm{H}_{2} \mathrm{~S}$ fermentation.

Cooked protein not attacked.

Acid and gas from glucose, fructose, galactose and sucrose. To acid from lactose, maltose, arabinose, glycerol, mannitol, dulcitol or starch.

Temperature relations: Optimum $36^{\circ}$ to $38^{\circ} \mathrm{C}$. Feeble growth at $26^{\circ} \mathrm{C}$. No growth below $22^{\circ} \mathrm{C}$. Filled in one hour at $60^{\circ} \mathrm{C}$ or in ten minutes at $80^{\circ} \mathrm{C}$.

Optimum pH 6.5 to 8.0 .

Pathogenic for guinea pigs and mice. Strict anaerobe.

Common in fetid suppurations and autogenous gangrenous processes.

Source: First isolated from a fatal case of Ludwig's angina. Perinephritic phlegmon; the fetid pus from Bartholin's gland; gangrene of the lung; appendicitis .

Habitat: Mouth, intestine and vagina of man and animals.

18a. Streptococcus foetidus var. buccal is Prévot. (Einen Micrococeus der Mundhöhle, Ozaki, Cent. f. Bakt., I Abt., Orig., 76, 1915, 118; Micrococcus buccalis Bergey et al., Manual, 1st ed., 1923, 69; Prévot, Ann. Sei. Nat., Sér. Bot., 15, 1933, 193.) From mouth.

19. Streptococcus putridus Schottmüller cmend. Prévot. (Schottmüller, Mitteil.a.d. Grenzgeb. d. Med. Chirurg., 21, 1910, 450; Prévot, Ann. Sci. Tat., Sér. Bot., 15, 1933, 170, 181.) From Latin putridus, rotten, decayed.

Synonym: Streptococcus putrificus Schottmüller, Münch. med. Wochnschr., $68,1921,662$.

Spheres: Average size 0.8 micron, occurring in chains. Gram-positive.

Gelatin: No liquefaction.

Semi-solid agar (Veillon): More or less lenticular; colonies 1 to $2 \mathrm{~mm}$ in diameter. No gas produced.

Blood agar: A blackish-brown hemopeptic zone is produced around the colonies, with fetid gas $\left(\mathrm{H}_{2} \mathrm{~S}\right)$. Colonies become brownish, sometimes blackish.

Martin broth: In 6 to 8 hours uniform 
turbidity which does not precipitate completely. No gas. Little odor.

Martin glucose broth: Rapid abundant growth. Uniform turbidity. Sediment. No gas. Slight fetid odor. Black pigment in the sediment.

Meat and liver broth: Very abundant growth, very marked putrid odor. Incomplete sedimentation.

Peptone water: Sparse growth. Neither gas, odor, $\mathrm{H}_{2} \mathrm{~S}$ nor indole.

Milk: No acid. No coagulation.

Cooked protein not attacked.

Deep blood agar: Agar is broken by the gas $\left(\mathrm{H}_{2} \mathrm{~S}\right)$

Fresh blood broth: Abundant gas which contains a large amount of $\mathrm{H}_{2} \mathrm{~S}$. Blood blackens rapidly, has typical putrid odor.

Fresh fibrin broth: The fibrin is broken up and partially digested.

Neutral red changed to fluorescent yellow.

Lead media blackened.

Acid from glucose, fructose and maltose. Acid sometimes produced from sucrose, mannitol and galactose.

Optimum pH 7.0 to 8.5 .

Temperature relations: Optimum $36^{\circ}$ to $38^{\circ} \mathrm{C}$. Growth feeble at $28^{\circ} \mathrm{C}$. No growth below $22^{\circ} \mathrm{C}$. Filled in ten minutes at $80^{\circ} \mathrm{C}$.

Pathogenic when grown in media with fresh tissue and body fluids.

Strict anaerobe.

Distinctive characters: Putrescence but absence of gas in ordinary media; presence of gas and $\mathrm{H}_{2} \mathrm{~S}$ in media with fresh tissue or body fluids.

Source: Normal and fetid lochia, blood in puerperal fever, gangrenous appendicitis, gangrene of the lung, in gas gangrene, gangrenous metastases; war wounds; osteomyelitis; and from amniotic fluid. Found in sea water by Montel and Mousseron (Paris Médical, 1929).

Habitat: Human mouth and intestine and especially the vagina.

20. Streptococcus lanceolatus Prévot. (Coccus lanceolatus anaerobius Tissier,
Compt. rend. Soc. 1: iol. Paris, 94, 1926, 447 ; Prévot, Ann. Sci. Nat., Sér. Bot., 15, 1933, 173 and 193; not Streptococcus lanceolatus pasteuri nor Streptococcus lanceolatus Gamaleïa, Ann. Inst. Past., 2, 1888, 440; not Slreptococcus lanceolatus Saito, Arch. f. Hyg., 75, 1912, 121.) Although Prévot's name is invalid, it is used until further comparative studies have been made. From Latin lanceolatus, having a little lance, pointed.

Large ovoid cells: 1.2 to 1.4 microns with pointed ends, occurring in short chains in culture and in pairs in exudates. Non-motile. Gram-positive.

Gelatin: No liquefaction.

Deep agar colonies: Very large, lenticular. Abundant gas produced which breaks up the medium.

Peptone broth: Uniform turbidity. Granular, glairy sediment.

Peptone water: Good growth. Gas produced.

Milk: No change.

Protein not attacked.

Hydrolyzed albumen reduced to $\mathrm{CO}_{2}$, $\left(\mathrm{NH}_{4}\right)_{2} \mathrm{CO}_{3}$ and $\mathrm{NH}_{3}$.

Acid from sucrose, glucose and starch. No acid from lactose. (Butyric, valerianic and acetic acid are produced, in the proportions $2: 1$ :trace, from glucose and sucrose.)

Non-pathogenic for laboratory animals.

Optimum temperature $37^{\circ} \mathrm{C}$.

Strict anaerobe.

Distinetive characters: Proteolytic and saccharolytic; produces ammonia from hydrolyzed proteins; butyric, valerianic and acetic acid produced from hexoses. No $\mathrm{H}_{2} \mathrm{~S}$ produced.

Source: From human feces in a case of diarrhoea.

Ilabitat: Putrefying materials.

21. Streptococcus micros Prévot. (Streptococcus anaerobius micros Lewkowicz, Arch. Méd. Exp., 13, 1901, 645; Prévot, Ann. Sci. Nat., Sér. Bot., 15, 1933, 193; also see Weiss and Mercado, Jour. Inf. Dis., 62, 1938, 181.) From Greek micrus, small (old spelling, micros). 
Very small spheres : 0.2 to 0.4 micron, occurring in long chains or in pairs. Yonmotile. Gram-positive.

Gelatin: Ponr growth. No liquefaction.

Semi-solid agar (Veillon): Slow growth; colonies at first punctiform, becoming lenticular and later forming processes into the medium. Average size 0.5 to $1.0 \mathrm{~mm}$ in diameter, some reach 2 to $3 \mathrm{~mm}$ growing 2 or $3 \mathrm{~cm}$ below the surface.

Blood agar: No hemolysis. No hemopeptolysis.

Martin broth: Slight particulate turbidity which slowly settles.

Meat and liver broth: Rapid growth. Abundant sediment.

Peptone water: Powdery sediment. Nedium not acidified. No indole formed.

Neutral red broth: Changed to fluorescent yellow.

Milk: Grows with difficulty. No acid. No coagulation.

Acid produced rapidly from glucose, fructose, galactose, sucrose and maltose. No acid from lactose, arabinose, glycerol, mannitol, inulin and starch.

Protein not attacked.

Optimum pH about 7.0.

Optimum temperature $36^{\circ}$ to $38^{\circ} \mathrm{C}$. No growth at $22^{\circ} \mathrm{C}$. Killed in a quarter of an hour at $60^{\circ} \mathrm{C}$.

Non-pathogenie for mice.

No toxin and no hemolysin.

Strict anaerobe.

Distinctive characters: Neither gas nor fetid odor produced. Small size.

Source: Gangrene of the lung; lochia and uterus in puerperal sepsis; appendicitis.

Habitat: Mouth and intestine of man and animals.

22. Streptococcus parvulus Weinberg, Nativelle and Prévot. (Streptococius parvulus non liquefaciens Repaci, Compt. rend. Soc. Biol., Paris, 68, 1910, 528; Weinberg, Nativelle and Prévot, Les Microbes Anaérobies, 1937, 1011; not Streptococcus parvulus Levinthal, Cent. f. Bakt., I Abt., Orig., 106, 1928, 195.)

From Latin pareulus, very small, minute.

Small spheres: Average size 0.3 to 0.4 micron, occurring in short chains. sometimes in pairs. Non-motile. Grampositive.

Gelatin: At $37^{\circ} \mathrm{C}$ slow growth, culture at bottom of the tube; no gas. No liquefaction.

Deep glucose agar colonies: After 48 hours very tiny, lenticular, whitish. Old colonies become blackened. No gas produced.

Broth: Rapid turbidity. Sediment forms in 5 or 6 days as a whitish, mucous mass which clears the fluid. To gas. Faint disagreeable odor.

Indole not formed.

Milk: Coagulation in 24 hours.

Egg white not attacked.

Frebly attacks glucose and lactose. Dues not attack sucrose, galactose and dextrin.

Optinum temperature $37^{\circ} \mathrm{C}$. No grow th at rom temperature. Will grow at $41^{\circ} \mathrm{C}$.

Strict anaerobe.

Distinctive characters: Differs from Streptococcus micros by its black colonies, coagulation of milk and its feeble saccharolytic power. Differs from Streptococcus intermedius by its black colonies, the smallness of its elements, feeble saccharolytic power and the viscous sediment in broth.

Source: Respiratory tract.

Habitat: Unknown.

Veillon and Repaci identified this organism as Streptococcus micros, but Weinberg, Nativelle and Prévot consider it as a distinct species, although rare.

23. Streptococcus intermedius Prévot. (Ann. Inst. Past., 3.9, 1925, 439.) From Latin intermedius, intermediate.

Description taken in part from Prévot, Ann. Sci. Nat., Sér. Bot., 15, 1933, 197.

Spheres: 0.5 to 0.7 micron, very long chains in culture. Non-motile. Grampositive. 
Gelatin: Poor growth. No liquefaction.

Semi-solid agar (Veillon): After 24 hours colonies 1 to $2 \mathrm{~mm}$ in diameter, regular, lenticular; sometimes with complex processes.

Blood agar: No change or slight greening.

Martin broth: Rapid growth. Uniform turbidity which slowly settles.

Martin glucose broth: Abundant growth. Abundant sediment. Medium strongly acidified.

Peptone water: Particulate sediment.

Milk: Very acid. Coagulated in 24 hours, without retraction of clot and not peptonized.

Serum broth (1:2): Rapid growth. Coagulation by acidification.

Proteins not attacked.

Neutral red broth: Changed to fiuorescent yellow.

Acid from glucose, fructose, galactose, maltose and lactose. Acid from sucrose by some strains. The acid produced is lactic acid. No acid from arabinose, glycerol, mannitol, dulcitol, inulin or starch.

Optimum pH 6.0 to 8.5.

Temperature relations: Optimum $36^{\circ}$ to $38^{\circ} \mathrm{C}$. Poor growth at $26^{\circ} \mathrm{C}$. No growth below $22^{\circ} \mathrm{C}$. Filled in half anhour at $70^{\circ} \mathrm{C}$ or in ten minutes at $80^{\circ} \mathrm{C}$.

Pathogenic for guinea pigs and mice. causing small abscesses; sometimes kills in 48 hours.

No toxin and no hemolysin.

Strict anaerobe.

Distinctive characters : Strongly acidifies media. Coagulates milk.

Source: Lochia and uterus in puerperal sepsis; gangrene of the lung; pleurisy; bronchiectasis; appendicitis.

Habitat: Human respiratory and digestive tracts and vagina.

\section{Streptococcus evolutus Prévot.} (Streptococcus Sch., Gräf and Wittneben, Cent. f. Bakt., I Abt., Orig., 39, 1925, 443; Streptococcus Schwarzenbeck, Ford, Textb. of Bact., 1927, 455; also see Weiss and Mercado, Jour. Inf. Dis., 62, 1938, 181.) From Latin evolutus, unrolled.

Description taken in part from Prévot, Ann. Sci. Nat., Sér. Bot., 15, 1933, 199.

Spheres : 0.7 to 1.0 micron, average 0.7 mieron, occurring in pairs or in short and long chains. Pleomorphic. Often appear as short ovoid rods with rounded ends. Gram-positive.

Gelatin: Liquefaction.

Deep agar colonies: Lenticular or rosettes. Growth occurs about $1 \mathrm{~cm}$ beneath the surface; after a transfer the second generation may show a ring of growth in the middle of this sterile zone. This is the characteristic alternate zones appearance. Colonies usually become brownish with age.

Glucose broth: Abundant growth, resembling bread crumbs. Medium strongly acidified (pH 5). A small quantity of lactic acid produced.

Peptone broth: Rapid growth. No general turbidity. Precipitating flocculent growth on the wall of the tube.

Blood agar: No change, sometimes greening.

Peptone water: Flocculent growth. No turbidity. Indole not formed.

Litmus milk: Acid. Curdled in 24 hours, clot retracts and fragments. Slight peptonization with some strains.

Strongly acid in glucose, fructose, galactose, sucrose, lactose and maltose. Arabinose sometimes fermented.

Egg white not attacked.

Pathogenicity: Most strains not pathogenic, some produce slight local swelling subcutaneously with little pus in guinea pigs and mice.

Optimum pH 6.0 to 8.5 .

Optimum temperature $36^{\circ}$ to $38^{\circ} \mathrm{C}$. No growth below $22^{\circ} \mathrm{C}$.

Strict anaerobe at first, becoming facultative with subsequent transfers.

Viability short aerobically and several months anaerobically.

Distinctive characters: Growth in al- 
ternate zones in agar. Strict anaerobe at first, later microaerophilic.

Source: Skin abscess; appendicitis.

Habitat: Respiratory tract, mouth, vagina

Appendix I: Descriptions of poorly defined species, the taxonomic relationships of which are not clear.

1. Streptococcus sp. Long and Bliss. (Minute beta hemolytic streptococcus, Long and Bliss, Jour. Exp. Med., 60, 1934, 619; Long, Bliss and Walcott, ibid. 633.)

Minute cocci, half to two-thirds the size of Streptococcus pyogenes; occurring singly, in pairs, short chains and in small and large masses. Gram-positive, but may decolorize readily.

Blood agar: Very minute colonies is to 30 microns, surrounded by a marked area of hemolysis (beta), easily visible before the colony is seen by naked eye, 4 to 10 times the diameter of the colony. Under the microscope colonies are finely granular, may appear wrinkled and crenated. Colonies become visible after 48 to 96 hours incubation and relative area of hemolysis is 3 to 4 times diameter of colony.

Gelatin: Not liquefied.

Glucose broth: Growth diffuse. abundant.

Litmus milk: Not curdled; litmus not reduced.

Acid from glucose, maltose and sucrose; may or may not attack lactose, trehalose and salicin. No acid from arabinose, raffinose, inulin. glycerol mannitol or sorbitol.

Does not hydrolyze sodium hippurate and starch. Esculin is hydrolyzed.

Ammonia is produced from peptone.

Temperature relations: No growth at $10^{\circ} \mathrm{C}$, very rarely growth at $45^{\circ} \mathrm{C}$. Does not survive $60^{\circ} \mathrm{C}$ for 30 minutes.

Chemical tolerance: Does not tolerate 6.5 per cent $\mathrm{NaCl}$. Final $\mathrm{pH}$ in glucose broth 5.4 to 4.6 ; no 'growth at $\mathrm{pH} 9.6$. Methylene blue 0.1 per cent not tolerated. No grow th on 40 per cent bile-blood ag:ir.
Action on blood: Hemolysis marked before colony is visible. May not produce soluble hemolysin by ordinary methods but does so abundantly by appropriate methods.

Fibrinolysin: No solution of human fibrin.

Serology: Constitutes Group $\mathbf{F}$ of Lancefield and Hare (Jour. Exp. Med., $61,1935,335)$. Four serological types within the group (Bliss, Jour. Bact., 33, 1937,625 ).

Aerobe, facultative anaerobe.

Source: Human throat in health and disease, accessory sinuses, abscesses, vagina, skin and feces.

Hibitat: Human upper respiratory tract.

2. Streptococcus sp. Long and Bliss. (Group II, Long and Bliss, Jour. Exp. Med., 60, 1934, 633; Group G, Lancefield and Hare, Jour. Exp. Med., 61, 1935, 346; Bliss, Jour. Bact., 33, 1937, 625.)

Probably identical with Streptocaccus anginosus Andrewes and Horder (Lancet, 2, 1906, 712) but probably other serological types are included in this group (Sherman. Bacteriological Reviews, 1, 1937,40 ).

Spheres: Gram-positive.

Gelatin: Not liquefied.

Litmus milk: Acid, may be curdled, not reduced.

Acid from glueose, maltose, sucrose, trehalose and salicin; usually acid from lactose, and may or may not from raffinose and glycerol. No acid from arabinose, inulin. mannitol or sorbitol.

follum hippurate usually not hydrolyzed. May hydrolyze stareh and esculin.

Ammonia is produced from peptone.

Temperature relations: No growth at $10^{\circ} \mathrm{C}$ and usually not at $45^{\circ} \mathrm{C}$. Does not survive $60^{\circ} \mathrm{C}$ for 30 minutes.

Chemical tolerance: Does not tolerate 6.5 per cent $\mathrm{XaCl}$. Final $\mathrm{pH}$ in glueose broth 6.0 to 4.6 ; no grow th at pII 9.6 . Methrlene blue 0.1 per cent not tol- 
erated. May grow on 40 per cent bileblood agar, growth in 10 per cent bile.

Action on blood: Hemolytic (beta) with a wider zone than minute beta hemolytic streptococcus. Soluble hemolysin formed.

Fibrinolysis: May dissolve human fibrin, certain strains strongly, others weakly.

Serology : Constitutes Lancefield's and Hare's Group G. Bliss (loc. cit.) has shown serological Types I and II within the group. May include serological Type 16 of Griffith (Jour. Hyg., 34, 1934, 542). Those resembling Streptococcus anginosus seem to form a homogeneous type; others seem unrelated to it.

Aerobic, facultative anaerobe.

Source: Human nose, throat, vagina, skin and feces in health. In human disease in puerperal fever with staphylococcus. Throat of normal domestic animals and in animal infections probably as secondary invaders.

Habitat: Human upper respiratory tract and vagina. Possibly throat of domestic animals.

3. Streptococcus sp. Brown, Frost and Shaw. (Jour. Inf. Dis., 38, 1926, 381; Lancefield, Jour. Exp. Med., 57, 1933, 571.)

Belongs to Lancefield Group E.

Gelatin: Not liquefied.

Litmus milk: Not curdled and not reduced.

Acid from glucose, lactose, trehalose and sorbitol; may form acid from sucrose, glycerol, mannitol and salicin. No acid from arabinose, raffinose or inulin.

No hydrolysis of sodium hippurate; may hydrolyze starch and esculin.

Ammonia is produced from peptone.

Temperature relations: No growth at $10^{\circ} \mathrm{C}$ and $45^{\circ} \mathrm{C}$. Does not survive $60^{\circ} \mathrm{C}$ for 30 minutes.

Chemical tolerance: Does not tolerate 6.5 per cent $\mathrm{NaCl}$. Final $\mathrm{pH}$ in glucose broth 4.8 to 4.2 ; no growth at $\mathrm{pH} 9.6$. Methylene blue 0.1 per cent not tolerated and not reduced. No growth on 40 per cent bile-bloodagar, nor on 10 per cent bile.

Action on blood: Very hemolytic; strains reported by Plastridge and Hartsell (Jour. Inf. Dis., 61, 1937, 110) weakly hemolytic. Streptolysin produced and outstandingly acid stable ('Todd, Jour. Path. and Bact., 39, 1934, 299).

Fibrinolysin: No solution of human fibrin.

Serology: Lancefield Group E, some cross reaction with Group C.

Aerobe, facultative anaerobe.

Source: Certified milk; bovine udder.

Habitat: Probably in udder and dairy products.

4. Streptococcus sp. Hare. (Group H, Hare, Jour. Path. and Bact., 41, 1935, 499.)

Spheres: Gram-positive.

Blood agar: Small colonies, 0.7 to 0.9 $\mathrm{mm}$, smooth surface, greenish color tending to blacken, hard, almost gritty and adherent to the medium. Hemolysis seldom complete except on Brown's horse blood agar. On boiled blood agar definite greening and so different from Groups E, F and $\mathrm{K}$.

Litmus milk: Not curdled and not reduced.

Acid from glucose, maltose, sucrose, raffinose and salicin; acid may be formed from lactose and trehalose. No acid from arabinose, inulin, glycerol, mannitol or sorbitol.

No hydrolysis of sodium hippurate and starch, but may hydrolyze esculin.

Ammonia may or may not be produced from peptone.

Temperature relations: No growth at $10^{\circ} \mathrm{C}$. Growth at $45^{\circ} \mathrm{C}$. May survive $60^{\circ} \mathrm{C}$ for 30 minutes.

Chemical tolerance: Does not tolerate 6.5 per cent $\mathrm{NaCl}$. Final $\mathrm{pH}$ in glucose broth 5.0 to 4.5 ; no growth at $\mathrm{pH} 9.6$. Methylene blue 0.1 per cent not tolerated. No growth on 40 per cent bile-blood agar. Action on blood: Hemolysis incomplete 
and some greening. No soluble hemolysin.

Fibrinolysin: No solution of human fibrin.

Serology: Group H.

Aerobe, facultative anaerobe.

Source: Human throat and feces.

Habitat: Human throat.

5. Streptococcus sp. Hare. (Group K, Hare, Jour. Path. and Bact., 41, 1935, 499.)

Spheres: Gram-positive,

Blood agar: Colonies 0.8 to $1.3 \mathrm{~mm}$, moist and transparent, with crenated edges. Incomplete hemolysis and no alpha-prime appearance.

Acid from glucose, lactose and salicin; may form acid from trehalose (doubtful). No acid from mannitol or sorbitol.

Does not hydrolyze sodium hippurate.

Chemical tolerance: Final $\mathrm{pH}$ in glucose broth 5.1 to 5.4. Does not grow on 10 per cent and 40 per cent bile-blood agar.

Action on blood: Incomplete hemolysis; does not produce soluble hemolysin. Doubtful if truly hemolytic streptococcus.

Fibrinolysin: Does not dissolve human fibrin.

Serology : Group K.

Aerobe, facultative anaerobe.

Source: Human throat.

Habitat: Human throat. No indication of relation to disease.

6. Streptococcus acidominimus Ayers and Mudge. (Ayers and Mudge, Jour. Inf. Dis., 31, 1922, 40; 33, 1923, 155.) From M. L., derived to mean a minimum amount of acid.

Description taken from Smith and Sherman, Jour. Inf. Dis., 65, 1939, 301.

Spheres: Generally occur in short chains. Gram-positive.

Gelatin stab: Filiform, beaded growth. No liquefaction.

Plain nutrient agar: Small round white colonies.
Acid from glucose, lactose and sucrose. May form acid from maltose, trehalose, and mannitol. Sorbitol and salicin usually are not fermented. No acid from arabinose, xylose, raffinose, inulin and glycerol.

Sodium hippurate is hydrolyzed but not starch.

Carbon dioxide is produced from a 4 per cent peptone-infusion broth.

Litmus milk: Little or no visible change.

Ammonia is not produced from peptone.

Temperature relations: No growth at $10^{\circ} \mathrm{C}$. A few cultures grow at $45^{\circ} \mathrm{C}$. Do not survive $60^{\circ} \mathrm{C}$ for 30 minutes.

Chemical tolerance: No growth in .01 per cent methylene blue. Growth in 2 per cent but not in 6.5 per cent $\mathrm{NaCl}$. Final $\mathrm{pH}$ in glucose broth 6.5 to 5.6. No growth at $\mathrm{pH} 9.6$.

Action on blood: No hemolysis, slight greening (alpha).

Serology: Negative reaction with serums representing Lancefield groups A, $\mathrm{B}, \mathrm{C}, \mathrm{D}, \mathrm{E}, \mathrm{F}$ and $\mathrm{G}$.

Facultative anaerobe.

Distinctive character: Small amount of acidity developed in fermentation tests.

Source: Originally 12 cultures were isolated from freshly drawn milk. Also found in bovine vagina, occasionally in the udder, and on the skin of calves.

Habitat: Known to occur abundantly in the bovine vagina.

The relationship between Streptococcus uberis Diernhofer and other similar streptococci is not yet entirely clear. Snith and Sherman (Jour. Inf. Dis., 65, 1939, 301-305) at one time thought that Streptococcus uberis and Streptococcus acidominimus might be identical. Others have regarded Streptococcus uberis as identical with Group III, Minett (Proc. 12th Internat. Vet. Cong., 2, 1934, 511).

Brown (Proc. 3rd Internat. Cong. for Microbiol., 1940, 173) describes a new species, Streptococcus lentus (not Strep- 
tococcus lentus Lehmann, Deutsch. Arch. f. klin. Med., 150, 1926, 144) which belongs to serological group $\mathrm{E}$. He states that a few strains that produced the alphi appearance in blood agar corresponded culturally with Streptococcus uberis.

Later Sherman (personal communication) had an opportunity to determine the serological group of several cultures of Streptococcus uberis carefully identified by R. B. Little and found them tr belong to Group 1). While their characters were not exactly the same as the conventional Streplococcus faccalis, he feels that these cultures of Streptococcus uberis were only a variant type of Streptococcus faecalis.

Appendix II.* The following species of streptococei are listed chiefly because of their historical interest. In many cases the original cultures are lost and their exact taxonomic relationships have not been determined.

Bacterium acetylcholini Habs. (Cent. f. Bakt., II Abt., 97, 1937, 194.) From ensilage. Regarded as a stable type of Enterococcus.

Diplococcus bombycis Paillot. (Annales des Épiphyties, 8, 1922, 131.) From the silkworm (Bombyx mori).

Diplococcus liparis Paillot. (Annales des Epiphyties, 8, 1922, 122.) From larvae of the gypsy moth (Portheria (Lymantria) dispar).

Diplococcus lymantriae Paillot. (Compt. rend. Acad. Sci., Paris, 164, 1917, 526.) From larvae of the gypsy moth (Portheria (Lymantria) dispar).

Diplococcus melolonthae Paillot. (Compt. rend. Soc. Biol., Paris, 69, 1917, 57; Annales des Epiphyties, 8, 1922, 118.) From diseased larvae of cockchafers (Melolontha melolontha).

Diplococcus pieris Paillot. (Annales des Epiphytes, 8, 1922, 128.) From diseased caterpillars of the cabbage butterfly (Pieris brassicae).

Diplococcus scarlatinae sanguinis
Jamieson and Edington. (Brit. Med. Jour., 1, 1887, 1265.) From the desquamation and blood of scarlet fever patients.

Entcrococcus citreus Stutzer and Wsorow. (Cent. f. Bakt., II Abt., 71, 1927, 117.) From normal pupae of a moth (Euxoa segetum).

Lactococcus agglutinans Plevako and Bakushinskaia. (Microbiology (Russian ), 4, 1935, 523; abst. in Cent. f. Bakt., II Abt., 94, 1936, 64.) Agglutinates biker's yeast.

Streptobacillus malae: Goadby. (Jour. State Med. London, 30, 1922, 417 ; Streptocoreus malae Thomson and Thomson, Inn. Pickett-Thomson Res. Lab., 5, 1929, 22.) From the mouth. An aberrant streptococcus.

Streptococcus abortus-equi Hauduroy et al. (Streptococcus abortus equi Ostertag, Monatsh. f. Tierheilk., 12, 1900, 384; Hauduroy et al., Dict. d. Bact. Path., Paris, 1937, 508.) From aborting mares. Streptococcus acidi-lactici Chester. (Sphacrococcus acidi lactici Marpmann, Ergänzungshefte d. allegemeine Gesundheitspflege, 2, 1886, 121 ; not Streptococcus acidi lactici Grotenfeldt, Fortsehr. d. Med., 7, 1889, 124; Micrococcus lacticus Migula, Syst. d. Bakt., 2, 1900, 66; Chester, Man. Determ. Bact., 1901, 65.) From fresh milk.

Streptococcus aerobius Heurlin. (Bakt. Intersuch. d. Keimgehaltes im Genitalkanale der fiebernden Wöchnerinnen, Helsingfors, 1910, 60.) From the genital canal.

Streptococcus aerogenes Wirth. (Cent. f. Bakt., I Abt., Orig., 99, 1926, 290.) From human blood. An aerobic species which produced gas in deep glucose agar.

Streplococcus aerophilus Trevisan. (I generi e le specie delle Batteriacee, Milan, 1889,31 ; not Streptococcus aerophilus Heurlin, Bakt. Untersuch. d. Keimgehaltes im Genitalkanale der fiebernden Wöchnerinnen, Helsingfors, 1910, 62.) From air.

* Prepared by Miss Eleanore Heist, July; 1938; revised by Prof. Robert S. Breed, New York State Experiment Station. Geneva, New York, Fobruary, 1944. 
Streplococcus alactosus Smith and Brown. (Jour. Med. Res., 31, 1915, 455; Rockefeller Inst. for Med. Res., Monograph 9, 1919; Streptococcus haemolyticus II, Holman, Jour. Med. Res., 34, 1916, 377.) From human tonsillitis; peritoneal pus. See Manual, 5th ed., 1939, 352 for description of this species.

Streptococcus albicans Migula. (Schminkeweisser Streptococcus, Tataroff, Inaug. Diss., Dorpat, 1891, 69; Migula, Syst. d. Bakt., 2, 1900, 22.) From water.

Streptococcus albidus Henrici. (Arb. bakt. Inst. Karlsruhe, 1 , Heft 1, 1894, 53.) From Cantal cheese.

Streptococcus albus Sternberg. (Weisser Streptococcus, Maschek, Bakt. Untersuch. d. Leitmeritzer Trinkwasser, Jahresber. d. Oberrealschule zu Leitmeritz, 1887 ; Sternberg, Man. of Bact., 1893, 610; Micrococcus albus Macé, Traité pratique de Bact., 6th ed., 1912, 605.) From water.

Streptococcus allantoicus Barker. (Jour. Bact., 40, 1943, 251.) From black mud, San Francisco Bay.

Streptococcus alvearis (Preuss) Trevisan. (Cryptococcus alvearis Preuss, 1868; Trevisan, I generi e le specie delle Batteriacee, 1889, 31.) From an infection (foulbrood?) in bees.

Streptococcus ambratus Trevisan. (Micrococco ambrato, Perroncito and Ajroldi, Giornale d. r. Accad. d. Med. d. Torino, 48, 1885, 809; Trevisan, I generi e le specie delle Batteriacee, 1889, 30.) From the respiratory tract of a horse.

Streptococcus anhaemolyticus Rolly. (Streptococcus anhaemolyticus vulgaris Zangemeister, Münch. med. Wochnschr., 57, 1910, 1268; Rolly, Cent. f. Bakt., I Abt., Orig., 61, 1912, 90.) Synonym of Streptococcus saprophyticus Mandelbaum (Ztschr. f. Hyg., 58, 1907, 37; see Brown, Monograph No. 9, Rockefeller Inst. Med. Res., 1919,87). From vaginal secretions, milk and saliva .

Streptococcus aphthicola Trevisan. (I generi e le specie delle Batteriacee, 1889,
30.) From the lesions of foot and mouth disease of cattle.

Streptococcus aromaticus van Beynum and Pette. (Directie Landbouw. Verslag. Landbouwk. Onderzoek., 42, 1936, 360 ; also see Hoecker and Hammer, Iowa Agr. Exp. Sta. Res. Bull. 290, 1941, 317.) Produces diacetyl and small amounts of acetylmethylcarbinol in milk. From cream and butter.

Streptococcus articulorum Flügge. (Die Mikroorganismen, 2 Aufl., 1886, 153.) Associated with diphtheria. Trevisan (I generi e le specie delle Batteriacee, 1889, 30) considers this identical with Streptococcus diphteriticus Cohn (Beitr. z. Biol. d. Pflanz., 1, Heft 2, 1872, 162).

Streptococcus asalignus Frost, Gumm and Thomas. (Jour. Inf. Dis., 40, 1927, 703.) From milk.

Streptococcus aurantiacus Killian and Fehér. (Ann. Inst. Past., 55, 1935, 619.) From Sahara Desert soil.

Streptococcus bombycis Sartirana and Paccanaro. (Cent. f. Bakt., I Abt., Orig., 40, 1906, 331; probably not Streptococcus bombycis Zopf, Die Spaltpilze, 2 Aufl., 1881, 52.) From diseased silk worms (Bombyx mori). According to Paillot (Les maladies du ver à soie, Lyon, $1928,171)$ this is the same as Streptococcus pastorianus Krassilstschik.

Streptococcus bonvicini Chester. (Streptococcus della leucaemia, Bonvicini, Cent. f. Bakt., I Abt., 21, 1897, 211; Chester, Man. Determ. Bact., 1901, 59.) From a case of leucaemia in a dog.

Streptococcus bovinus Trevisan. (Micrococcus bovinus Trevisan, Pendiconti Reale Inst. Lombardo di Sci. e Lett., Ser. II, 12, 1879; Trevisan, I generi e le specie delle Batteriacee, 1889, 30; not Micrococcus bovinus Migula, Syst. d. Bakt., 2, 1900, 67; not Streptococcus bovinus Broad. hurst, Jour. Inf. Dis., 17, 1915, 321; not Streptococcus bovinus Frost and Engelbrecht, The Streptococci, 1940, 56.) From liuman throat; bovine, equine, feline and canine feces.

Streptococcus brevis von Lingelsheim. 
(Ztschr. f. Hyg., 10, 1891, 339 and 354.) Not pathogenic. From various human and animal sources.

Streptococcus brevis non hemolyticus Sachs. (Ztschr. f. Hyg., 63, 1909, 466.) From tonsils, vagina and vulva.

Streptococcus brightii DeToni and Trevisan. (Streptococcus bei Morbus Brightii, Mannaberg, Cent. f. klin. Med., 9, 1888, 537; DeToni and Trevisan, in Saceardo, Sylloge Fungorum, 8, 1889, 1057 ; Streptococcus morbi brightii Migula, Syst. d. Bakt., 2, 1900, 28.) From urine of persons suffering from Bright's disease. Probably identical with Streptococcus pyogenes.

Streptococcus buccalis Blake. (Jour. Med. Res., 36, 1917, 124.) From the mouth. Proposed to include both Streptococcus mitis and Streptococcus salivarius.

Streptococcus butyricus (Fitz) DeToni and Trevisan. (Mierococcus butyricus Fitz, Ber. d. deutsch. chem. Gesellsch., Denaeyer Bact. schizom., p. 35; DeToni and Trevisan, in Saccardo, Sylloge Fungorum, 8, 1889, 1064.) From bovine feces. Forms butyric acid from salcium lactate.

Streptococcus cadaveris sternberg. (Man. of Bact., 1893,611.) From liver of yellow fever cadaver.

Streplococcus caprinus Emoto. (Jour. Japan. Soc. Veter. Sci., 3, 1924, 67. From the cerebro-spinal fluid of goats. Pathogenic for goats.

Streptococcus capsulatus Hauduroy et al. (Streptococcus capsulatus gallinarum Dammann and Manegold, Deutsche tierärztl. Wochnschr., 13, 1905, 577 and Arch. f. Tierheilk., 39, 1907, 41; Hauduroy et al., Dict. d. Bact. Path., 1937, 511; not Streptococcus capsulatus Binaghi, Cent. f. Bakt., I Abt., 22, 1897, 273.) Causes a disease of chickens.

Streptococcus carneus Migula. (Micrococcus?, List, Inaug. Diss., Leipzig, 1885, 49; Migula, Syst. d. Bakt., 2, 1900, 36.) From the stomach of a sheep. Probably a micrococcus.

Streptococcus carnis Chester. (Diplo- coccus, Harrevelt, Cent. f. Bakt., I Abt., 26, 1899, 121; Chester, Man. Determ. Bact., 1901, 60.) From meat.

Streptococcus carrosus Lindner. (50 Jubiläumsber. Westpreuss. Bot.-Zool. Vereins, Danzig, 1928, 254.) From Mexican pulque.

Streptococcus casei Burri. (Berichte d. Schweiz. Bot. Gesellsch., 51, 1940, 102.) From Emmenthal cheese.

Streptococcus caucasicus Migula. (Streptococeus a, von Freudenreich, Cent. f. Bakt., II Abt., 3, 1897, 87; Migula, Syst. d. Bakt., 2, 1900, 42.) From kefir. Possibly related to Streptococcus kefir.

Streptococcus cerasinus (Lehmann and Neumann) Migula. (Micrococcus?, List, Inaug. Diss., Leipzig, 1885, 17 ; Kirschroter Micrococcus, Adametz, Mitteil. d. österreich. Versuchssta. f. Brauerei u. Mälzerei in Wien, Heft 1, 1888, 33; Micrococcus cerasinus siccus Eisenberg, Bakt. Diag., 3 Aufl., 1891, 34 ; Micrococcus cerasinus Lehmann and Neumann, Bakt. Diag., 1 Aufl., 2, 1896, 179; Migula, Syst. d. Bakt., 2, 1900, 35; not Micrococcus cerasinus Migula, ibid., 170.) From nasal secretions in at sheep; also from water.

Streptococcus charrini Trevisan. (Microbe de la septicémie consécutive au charbon, Charrin, Compt. rend. Soc, Biol., Paris, 36, 1884, 526; Streptococcus Charrin, Flügge, Die Mikroorganismen, 2 Aufl., 1886, 164; Trevisan, I generi e le specie delle Batteriacee, 1889, 30.) From the organs of a rabbit having anthrax.

Streptococcus cinereus Zimmermann. (Bakt. unserer Trink- u. Nutzwässer, Chemnitz, II Reihe, 1894, 64.) From water.

Streptococcus citreus (Eisenberg) Migula. (Micrococeus?, List, Inaug. Diss., Leipzig, 1885, 60; Crêmefarbiger Micrococcus, Adametz, Mitteil. d. üsterreich. Versuchssta. f. Brauerei u. Mälzerei in Wien, Heft 1, 1888, 31; Micrococcus citreus Eisenberg, Bakt. Diag., 3 Aufl., 1891, 36 ; Migula, Syst. d. Bakt., 2, 1900, 37 ; not Streptococcus citreus Weiss, Arb. bakt. Inst. Karlsruhe, 2, Heft 3, 1902, 179.) 
From the pancreas of a sheep (List); from water (Adametz).

Streptococcus citrophilus van Beynum and Pette. (Directie Landbouw. Verslag. Landbouwk. Onderzoek., 42, 1936, 360 ; also see Hoecker and Hammer, Iowa Agr. Exp. Sta. Res. Bull. 290, 1941, 317.) Produces diacetyl, acetylmethylcarbinol and volatile acids from citric acid. From cream and butter.

Streptococcus coli Migula. (Streptococcus coli brevis Escherich, Die Darmbakterien des Säuglings und ihre Beziehungen zur Physiologie der Verdauung, 1886, 86; Migula, Syst. d. Bakt., 2, 1900, 33.) From stools in cases of infant diarrhoea.

Streptococcus continuosus Black. (Trans. Ill. State Dental Soc., 22, 1886. 189.) From the mouth.

Streptococcus coronatus (Flügge) Trevisan. (Micrococcus coronatus Flügge, Die Mikroorganismen, 2 Aufl., 1886, 175; Trevisan, I generi e le specie delle Bat teriacee, 1889, 31.) From the air.

Streptococcus cuniculi Bergey et al. (Manual, 1st ed., 1923, 50.) From natural infections of rabbits. Morphologically like Streptococcus pyogenes.

Streptococcus cystitidis Migula. (Diplococcus ureae pyogenes Rovsing, Die Blasenentzündungen, ihre Aetiologie, Pathogenese und Behandlung, 1890, 39; Migula, Syst. d. Bakt., 2, 1900, 12.) From a case of cystitis.

Streptococcus debitis Wilhelmy. bokt. Inst. Karleruhe, o, 1903,13 meat extracts.

Streptococcus dentium (Trevisan) Trevisan. (Micrococcus dentium Trevican, Batt. Ital., 1879, 27; Micrococcus foctidus Flügge, Die Mikroorganismen, 2 Aufl., 1886, 172; Trevisan, I generi e le specie delle Batteriacee, 1889, 30.) From carious teeth. Grows anaerobically in nutrient agar with the production of gas and strong odor.

Streptococcus desidens Trevisan. (Micrococcus flarus desidens Flügge, Die Mikroorganismen, 2 Aufl., 1886, 177; Trevisan, I generi e le specie delle Bat- teriacee, 1889, 31; Micrococcus desidens Migula, Syst. d. Bakt., 2, 1900, 143.) From the air.

Streptococcus diacetilactis Matuszewski, Pijanowski and Supinska. (Compt. rend. Inst. Bact. Ecole Cent. Agron., Varsovie, Poland, No. 21, 1936, 1.) From milk. Probably Streptococcus cremoris Orla-Jensen.

Streptococcus diacetyl aromaticus. (Quoted from Karnad, Indian Jour. Vet. Sci., 9, 1939, 349.) From milk. Produces an aroma in milk cultures.

Streptococcus disparis Glaser. (Jour. Agr. Res., 13, 1918, 515.) From the alimentary canal of eaterpillars.

Streptococcus endocarditicus DeToni and Trevisan. (Mikrokokken, Klebs, Arch. f. exper. Pathol., 9, 1878, 52; DeToni and Trevisan, in Saceardo, Sylloge Fungorum, 8, 1889, 1057.) From cases of endocarditis.

Streptococcus enteritidis Chester. (Enteritisstreptokokken, Escherich, Jahrb. f. Kinderheilk., 49, 1899, 161 ; Chester, Man. Determ. Bact., 1901, 59.) From cases of enteritis.

Streptococcus enteritis Chester. (Streptococcus, Hirsh, Cent. f. Bakt., I Abt., 22, 1897, 372 and Libman, ibid., 376: Chester, Man. Determ. Bact., 1901, 56; Streptococcus enteritis var. libmanii Chester, ibid., 66.) From stools in cases of infant diarrhoea.

Streptococcus equarius Frost and Engelbrecht. (A Revision of the Genus Streptococcus, privately published, 1936, 3 pp. and The Streptococci, 1940, 65.) From a throat culture.

Streptococcus equinus Lehmann and Neumann. (Streptokokken die sich zu grossen Konvaluten zusammenballen, Behring, Cent. f. Bakt., 12, 1892, 194; Lehmann and Neumann, Bakt. Diag., 1 Aufl., 2, 1896, 125.) From equine pneumonia. Relation to Streptococcus equinus Andrewes and Horder not clear.

Streptococcus felinus Bergey et al. (Manual, 1st ed., 1923, 50.) From natural infections in eats. Morphologically like Streptococcus pyogenes. 
Streptococcus fermenti (Trevisan) Trevisan. (Micrococcus fermenti Trevisan, Batt. Ital., 1879, 19; Micrococcus viscosus Flügge, Die Mikroorganismen, 2 Aufl., 1886, 172; Trevisan, I generi e le specie delle Batteriacee, 1889,31.) From a slimy growth in wines.

Streptococcus fischeli. Chester. (Microorganismus No. 2, Fischel, Ztschr. f. Heilkunde, 12, 1891, 7 and Cent. f. Bakt., 9, 1891, 611; Chester, Man. Determ. Bact., 1901, 59.) From the blood of influenza patients.

Streptococcus foetidus Migula. (Stinkcoccus, Klamann, Allegem. med. Centralzeitung, 1887, 1347; Diplococcus fluorescens foetidus Eisenberg, Bakt. Diag., 1891, 10; Migula, Syst. d. Bakt., 2, 1900, 38; Streptococcus fluorescens Chester, Man. Determ. Bact., 1901, 70; Streptococcus fuorescens foetidus Miquel and Cambier, Traité de Bact., Paris, 1902, 792.) From cases of ozena.

Streptococcus galleriae ('horine. (Compt. rend. Soc. Biol., Paris, 95, 1926, 201.) From the bee moth (Galleria mellonella).

Streptococcus genitalium Dimock and Edwards. (Kentucky Agr. Exp. Sta. Res. Bull. 286, 1928, 162.) Found commonly in the genital tract of mares.

Streptococcus giganteus Migula. (Streptococcus giganteus urethrae Lustgarten and Mannaberg, Vierteljahrssehr. f. Dermatologie u. Syphilis, 1887, 918; Migula, Syst. d. Bakt., 2, 1900, 39.) From human urethra and from urine.

Streptococcus gingivae. (Quoted from Annals Pickett-Thomson Res. Lab., 3, 1927,154.) From human gums and teeth. Streptococcus granulatus Henrici. (Arb. bakt. Inst. Karlsruhe, 1, Heft 1, 1894, 55.) From cream cheese.

Streptococcus haematosaprus Trevisan. (Mikrokokken der Fäulniss, Flügge, Die Mikroorganismen, 2 Aufl., 1886, 173; Trevisan, I generi e le specie delle Batteriacee, 1889, 31.) From putrefying blood.

Streptococcus halitus Heim and Schlirf.
(Cent. f. Bakt., I Abt., Orig., 100, 1926, 39.) From deposit on the tongue.

Streptococcus havaniensis Sternberg. (Man. of Bact., 1893, 612.) From acid vomit of a yellow-fever patient.

Streptococcus hemolyticus $I, I I$ and $I I I$ Holman. (Jour. Med. Res., 34, 1916, 388.) From various human infections. Streptococcus herbarum Schieblich. (Cent. f. Bakt., I Abt., Orig., 124, 1932, 269.) From green plant material. Motile. Related to Streptococcus lactis except it is flagellated. Kolbmuller (Cent. f. Bakt., I Abt., Orig., 133, 1935, 310 ) identifies this with Enterococcus. Streptococcus hydrophoborum Trevisan. (Streptococcus bei Rabies, Babes, Ztschr. f. Hyg., 5, 1888, 184; Trevisan, I generi e le specie delle Batteriacee, 1889, 30.) From the brain in a case of rabies.

Streptococcus influentiae Trevisan. (I generi e le specie delle Batteriacee, 1889 , 30.) From equine influenza.

Streptococcus influenzae Thomson and Thomson. (Grippestreptokokkus, Seligmann, Cent. f. Bakt., I Abt., Ref., 50, 1911, 81; Thomson and Thomson, Monograph No. 16, Part I, Annals PickettThomson Res. Lab., 1933.) Associated with influenza.

Streptococcus infrequens Holman. (Jour. Med. Res., 34, 1916, 388.) From various human infections.

Streptococcus kirchneri Chester. (Diplococcus, Kirchner, Ztschr. f. Hyg., 9, 1890, 528; Chester, Man. Determ. Bact., 1901, 57.) From sputum in cases of influenza.

Streptococcus kochii Trevisan. (I generi e le specie delle Batteriacee, 1889,30 .) From rabbit septicemia.

Streptococcus lacteus Schröter. (Kryptogam. Flora v. Schlesien, 3, 1, 1886, 149.) From the air and dust.

Streptococcus lactis aromaticus Joshi and Ram Ayyar. (Indian Jour. Vet. Sci., 6, 1936, 141.) Possibly Streptococcus cremoris Orla-Jensen. From cream.

Streptococcus lactis innocuus Stölting. (Inaug. Diss., Kiel, 1935, 16.) From ripening cheese. 
Streptococcus lagerheimii var. subterraneum Migula. (Hansgirg, Oesterr. Zeitung, 1888, No. 7 and 8; Migula, Syst. d. Bakt., 2, 1900, 41.) From the wall of a wine cellar.

Streptococcus (Diplococcus) lanceolatus ovium Gaertner. (Cent. f. Bakt., I Abt., Orig., 54, 1910, 546.) From mastitis in sheep.

Streptococcus lapillus Heim and Schlirf. (Cent. f. Bakt., I Abt., Orig., 100, 1926, 39.) From the oral cavity.

Streptococcus lentus Lehmann. (Lehmann, Deutsch. Arch. f. klin. Med., 150, 1926, 144; Streptococcus pyogenes lentus, ibid., 141 ; not Streptococcus lentus Brown, Rept. Proc. Third Internat. Congr. for Microbiol., New York, 1940, 173.) From urine, cervix, sputum and carious teeth.

Streptococcus libaviensis Flatzek. (Cent. f. Bakt., I Abt., Orig., 82, 1919, 240 ; Bacterium libaviense Flatzek, idem.) From human feces. Motile.

Streptococcus lucae Trevisan. (Micrococcus ulceris mollis de Luca, 1886; Trevisan, I generi e le specie delle Batteriacee, 1889, 30.) From chancroidal ulcers.

Streptococcus luteus Killian and Fchér. (Ann. Inst. Past., 55, 1935, 619.) From Sahara Desert soil.

Streptococcus magnus Henrici. (Arb. bakt. Inst. Farlsruhe, 1, Heft 1, 1894, 54.) From Brie cheese.

Streptococcus malaperti Trevisan. (Micrococcus E, Malapert-Neuville, 1887; Trevisan, I generi e le specie delle Batteriacee, $1889,30$. .) From mineral water of hot springs at schlangenbad.

Streptococcus malignus Trevisan. (Streptococcus pyogenes malignus Flügge, Die Mikroorganismen, 2 Aufl., 1886, 153; Trevisan, I generi e le specie delle Batteriacee, 1889, 30.) From a diseased spleen. Probably identical with Streptococcus pyogenes.

Streptococcus mammitis bovis Hutchens. (Hutchens, in Besson, Pract. Bact. Microbiol. and Serum Therapy. Trans. of 5 th ed., 1913, 613.) From mastitis in cattle.

Streptococcus margaritaceus Schröter.
(In Cohn, Fryptog. Flora v. Schlesien, 3, 1,1886, 149.) From putrefying blood.

Streptococcus mathersi Muslow. (Green producing streptococeus, Tunnicliff, Jour. Amer. Med. Assoc., 11, 1918, 1733; Mather's coccus, Jordan, Jour. Inf. Dis., 25, 1919, 30; Muslow, ibid., 31, 1922, 295.) From sputum in cases of influenza and pneumonia.

Streptococcus maximus Weiss. (Arb. bakt. Inst. Karlsruhe, 2, Heft 3, 1902, 180.) From a bean and carrot infusion.

Streptococcus melanogenes Schlegel. (Berl. tierarztl. Wochnschr., 1906, No. 25, 464.) Produces grayish-yellow pigment in gelatin. Associated with a disease of horses.

Streptococcus meningitidis Bonome. (Cent. f. Bakt., 8, 1890, 172 and 703.) From exudates from cases of cerebrospinal meningitis.

Streptococcus merdarius Trevisan. (Streptococcus des selles, Cornil and Babes, Bactériea, 2nd ed., 1886, 118; Trevisan, I generi e le specie delle Batteriacee, 1889, 31.) From feces.

Streptococcus microapoikia Cooper, Kieller and Johnson. (Amer. Jour. Dis. of Children, 47, 1934, 388 and 596; these authors also use the trinomial Streptococcus micro-apoikia enteritis.) From human throat and feces in enteritis in children. See Manual, 5th ed., 1939. 351 for description of this species.

Streptococcus mirabilis Roscoe and Lunt. (Phil. Trans. Roy. Soc., London, 182, 1892, 648.)

Streptococcus mixtus Bergey et al. (Manual, 1st ed., 1923, 49.) From a variety of pyogenic inflammations.

Streptococcus morbilli Ferry and Fisher. (Jour. Amer. Med. Assoc., 86, 1926 , 933.) From blood of persons in early stages of measles.

Streptococcus morbillosus Trevisan. (Micrococcus morbillosus Trevisan, Rendiconti Reale Inst. Lombardo di Sci. e Lett., Ser. II, 12, 1879; Trevisan, I generi e le specie delle Batteriacee, $1889,30$. From human, canine and porcine measles. Streptococcus murisepticus v. Lingels- 
heim. (Ztschr. f. Hyg., 10, 1891, 331 and 12, 1892, 308.) Migula (Syst. d. Bakt., 2, 1900, 6) considers this a synonym of Streptococcus pyogenes.

Streptococcus mutans Clarke. (Brit. Jour. Exp. Path., 5, 1924, 142.) Shows extreme variability in colony formation. Regarded as the cause of dental caries.

Streptococcus nasalis (Hack) Migula. (Micrococcus nasalis Hack, according to Eisenberg, Bakt. Diag., 3 Aufl., 1891, 55; Migula, Syst. d. Bakt., 2, 1900, 45; Planococcus nasalis Migula, loc. cit., 274.) From nasal secretions. Considered motile.

Streptococcus necroseos Schröter. (Mikrokokkus der progressiven Gewebenekrose bei Mäusen, Koch, Untersuch. über die Ätiologie der Wundinfektionskrankheiten. Leipzig, 1878 or Gesamm. Werke v. Robert Koch, 1, 1912, 87; Schröter, in Cohn, Kryptog. Flora v. Schlesien, 3, 1, 1886, 150; Streptococcus necroticus Trevican, Atti della Acead. Fisio-MedicoStatistica in Milano, Ser. 4, 3, 1885.) From gangrene in mice.

Streptococcus nomae Trevisan. (I generi e le specie delle Batteriacee, 1889, 30.

From gangrene of the mouth.

Streptococcus non-hemolyticus I, II and $I I I$ Holman. (Jour. Med. Res., 3'. 1916, 388.) From various human and animal infections.

Streptococcus odontolyticus Belding and Belding. (Dental Items of Interest, 62, 1940, 308.) From dental caries. Later stated by authors (Jour. Amer. Dent. Assoc., 30, 1943, 713) to be a mucoid variant of Streptococcus salivarius.

Streptococcus opacus Heim and Schlirf. (Cent. f. Bakt., I Abt., Orig., 100, 1926 , 40.) From the oral cavity.

Streptococcus opportunus Brown. (Rept. Proc. Third Internat. Congr. for Microbiol., New York, 1940, 173.) Source not recorded. Belongs to Lancefield's Group B (Sherman, Chase and Niven, Jour. Bact., 41, 1941, 101 ).

Streplococcus ovis Weimann. (Ztschr. f. Infectionskrankh. d. Haustiere, 9, 1911, 255.) From infected sheep.
Streptococcus pallens Henrici. (Arb. bakt. Inst. Karlsruhe, 1, Heft 1, 1894, 57.) From Gouda cheese.

Streptococcus pallidus Henrici. (Arb. bakt. Inst. Karlsruhe, 1 , Heft 1, 1894, 58.) From Neufchâtel cheese.

Streptococcus parmulus Levinthal. (Cent. f. Bakt., I Abt., Orig., 106, 1928, 195.) From the mucous membrane of the human throat.

Streptococcus pastorianus Krassilstschik. (Compt. rend. Acad. Sci. Paris, 123, 1896, 427.) From silkworms.

Streptococcus perniciosus Zopf. (Zopf, Die Spaltpilze, 3 Aufl., 1885, 53; Streptococcus perniciosus psittacorum Flügge, Die Mikroorganismen, 2 Aufl., 1886, 164; Micrococcus perniciosus Crookshank, Man of Bact., 3rd ed., 1890, 252 ; Streptococcus psittaconum Migula, Syst. d. Bakt., 2, 1900, 42.) From an infection of parrots. Found in nodules on the surface of the kidneys, lungs and spleen.

Streptococcus phylophthorus (Frank) Chester. (Micrococcus phytophthorus Frank, Cent. f. Bakt., II Abt., 5, 1899, 134; Chester, Man. Determ. Bact., 1901, 67.) Associnted with blight and rot of potato.

Streptococcus piima Macy. (Jour. Dairy Sei., 6, 1923, 2.) From ropy milk. the Finnish piina.

Sticptococcus pitycumpae a Dufrenoy. (Compt. rend. Soc. Biol., Paris, 71, 1919, 288.) From processionary moth larvae (Cnethocampa pityocampa). Motile. Gram-positive.

Streptococcus pityocampae $\beta$ Dufrenoy (loc. cit.). From processionary moth larvae. Gram-negative.

Streptococcus pleomorphus von Wiesner. (Wien. klin. Wochnschr., 1917, 933 and 1918, 1101; see Lehmann and Neumann, Bakt. Diag., 7 Aufl., 2, 1927, 224.) Occurred frequently during the influenza epidemic of 1918.

Streptococcus pneumosimilis Frost and Engelbrecht. (The Streptococei, 1940, 57.) From milk and from the throats of dairy employees. Not found in bovine feces. 
Streptococcus polymorphus Heim. (Streptococeus, Kraskowska and Nitseh, Cent. f. Bakt., I Abt., Orig., 82, 1918, 264; Heim, see Lehmann and Neumann, Bakt. Diag., 7 Aufl, 2, 1927, 224.) From the throat.

Streptococcus productus Prévot. (Compt. rend. Soc. Biol., Paris, 135, $1941,105$.$) An anaerobic streptococcus$ from a gangrenous lung.

Streptococcus proteiformis var. liquefaciens Hauduroy et al. (Enterococcus proteiformis liquefaciens Hauduroy, Thèse Doctorat Méd., Strasbourg, 1921; Hauduroy et al., Dict. d. Bact. Path., 1937, 521.) From feces.

Streptococcus proteus Chester. (Streptococcus No. 52, Conn, Report Storrs Agr. Exp. Sta., 1894, 81; Chester, Man. Determ. Bact., 1901, 67.) From cream.

Streptococcus pseudohaemolyticus Cumming. (Jour. Path. and Bact., 30, 1927, 279.) From sputum of patients with pulmonary tuberculosis.

Streptococcus putrefaciens Trevisan. (I generi e le specie delle Batteriacee, 1889, 31.) From putrefying blood.

Streptococcus pyogenes bovis Lucet. (Ann. Inst. Past., 7, 1893, 325; also see Crookshank, Textbook of Bact., 4 th ed., Philadelphia, 1900, 188.) From bovine pus.

Streptococcus pyogenes hominis Crookshank. (Textbook of Bact., 4th ed., Philadelphia, 1900, 187.) From human sources.

Streptococcus pyogenes nonhaemolyticus Thomson and Thomson. (Streptocoque pyogené nonhémolytique, Weissenbach, Compt. rend. Soc. Biol., Paris, 81, 1918, 819; Thomson and Thomson, Ann. Pickett-Thomson Res. Lab., 3, 1927, 183.) From feces and various human infections.

Streptococcus radiatus Klein. (Cent. f. Bakt., I Abt., 28, 1900, 417.) From exudate from the udder of a cow.

Streptococcus rheumaticus Poynton and Paine. (Poynton and Paine, Lancet, 2, September 22 and 29, 1900, 861; Diplococcus rheumaticus and Micrococcus rheumaticus Beaton and Walker, Brit. Med.
Jour., January 31, 1903, 237; abst. in Cent. f. Bakt., I Abt., Ref., 39, 1903, 528.) From cases of rheumatism and endocarditis.

Streptococcus rindfleischii Trevisan. (Streptococeus bei Mycosis fungoides, Rindfleisch, Deutsche med. Wchuschr., 11, 1885, 233; Trevisan, I generi e le specie delle Batteriacee, 1889, 30.) From skin infection (mycosis fungoides).

Streptococcus ruber Lundstrom. (Finska Läkaresällskapets Handlingar, 35, 1893.) Red colonies.

Streptococcus rubiginosus Jamieson and Edington. (Brit. Med. Jour., 1, 1887, 1265.) Associated with cases of scarlet fever. Probably identical with Micrococcus pyogenes Klein, according to Macé (Traité Pratique de Bact., Paris, 4 th ed., 1901,425 ).

Streptococcus rugosus Migula. (Streptococcus ureae (non pyogenes) rugosus Rovsing, Die Blasenentzündungen, ihre Aetiologie, Pathogenese und Behandlung, 1890, 44; Migula, Syst. d. Bakt., 2, 1900, 30; Streptococcus rugosus ureae Miquel and Cambier, Traité de Bact., Paris, 1902, 829.) From cases of cystitis.

Streptococcus salivarius brevis and Streptococcus salivarius tenuis Veillon. (Quoted from Thomson and Thomson, Ann. Pickett-Thomson Res. Lab., 3, 1927, 187 and 240.) From the mouth. See Streptococcus tenuis.

Streptococcus sunguineus Migula. (Diplocoecus pyogenes Pasquale, Giorn. med. d. R. esercito e d. R. marina, 1890; Migula, Syst. d. Bakt., 2, 1900, 36.) From a case of bone tuberculusis.

Streptococcus sanguinis Chester. (Streptococcus sanguinis canis Pitfield, Queen's Microscopic Bulletin, Philadelphia, 1897, 44; Chester, Man. Determ. Bact., 1901, 64.) From the blood of dogs.

Streptococcus sanguis White. (Streptococcus s.b.e. Lnewe, Plummer, Niven and Sherman, Jour. Amer. Med. Assoc., 130, 1946, 257; White, Thesis, Cornell Cniv, 1946 quoted from White and Yiven, Jour. Bact., 51, 1946, 721.) From 
blood in cases of subacute bacterial endocarditis.

Streptococcus saprogenes Trevisan. (I generi e le specie delle Batteriacee, 1889 , 31.) From putrefying blood.

Streptococcus saprophyticus Mandelbaum. (Ztschr. f. Hyg., 58, 1908, 37.) See Streptococcus anhaemolyticus vulgaris. From mucous membranes.

Streptococcus schmidti Trevisan. (Coccus bei Fadenziehende Milch, Schmidt-Mülheim, Arch. f. d. ges. Physiol., 27, 1882, 490; Trevisan, I generi e le specie delle Batteriacee, 1889, 31.) From ropy milk.

Streptococcus seiferti DeToni and Trevisan. (Micrococeus bei Influenza, Seifert, in Volkmann, Sammlung Klin. Vorträge, 240; DeToni and Trevisan, in Saccardo, Sylloge Fungorum, 8, 1889 , 1056.) From sputum and nasal secretions of influenza patients.

Streptococcus septicus Miguli. (Streptococcus septicus liquefians Babes, Bakt. Unter. ü. septische Prozesse des Kindesalters, Leipzig, 1889, 22; Streptococcus septicus liquefaciens Babes, according to Eisenberg, Bakt. Diag., 3 Aufl., 1891, 312; Migula, Syst. d. Bakt., 2, 1900, 27; not. Streptococcus septicus Flügge, Die Mikroorganismen, 2 Aufl., 1886, 154.) From the blood and organs of a diseased child.

Streptococcus septopyaemicus Biondi. (Ztschr. f. Hyg., 2, 1887, 194 and 225.) According to Migula (Syst. d. Bakt., 2, 1900,6 ) this is a synonym of Streptococcus pyogenes. From human saliva.

Streptococcus sornthalii (Adametz) Migula. (Nicrococcus sornthalii Adametz, Cent. f. Bakt., II Abt., 1, 1895, 465; Migula, Syst. d. Bakt., 2, 1900, 20.) From milk and hard cheese.

Streptococcus sphagni Miguli. (Syst. d. Bakt., 2, 1900, 40.) From sphagnum in the Black Forest.

Streptococcus sputigenus Migula. (Syst. d. Bakt., 2, 1900, 24.) From sputum.

Streptococcus stenos Bergey et al.
(Manual, 1st ed., 1923, 50.) From a variety of human inflammatory conditions.

Streptococcus stramineus Henrici. (Arb. bakt. Inst. Karlsruhe, 1, Heft 1, 1894, 59.) From Schlosskäse.

Streptococcus subacidus Holman. (Jour. Med. Res., 34, 1916, 388.) From various human infections.

Streptococcus suspectus Trevisan. (Streptococeo dell' ematuria, 'Pisciasangue' dei bovini; Trevisan, I generi e le specie delle Batteriacee, 1889,30.) From blood and spleen in cases of bovine hematuria .

Streptococcus tenuis Veillon. (Arch. Méd. Exp. et Anat., 6, 1894,161.) From human mouth.

Streptococcus terricola van Steenberge. (van Steenberge, Ann. Inst. Past., 34, 1920, 806; not Streptococcus terricola Killian and Fehér, Ann. Inst. Past., 55, 1935, 619.) From garden soil.

Streptococcus toxicatus (Burrill) DeToni and Trevisan. (Micrococcus toxicatus Burrill, The Bacteria. Illinois Industrial Univ., 11 th Ann. Rept., 1882, 42; DeToni and Trevisan, in Saccardo, Sylloge Fungorum, 8, 1889, 1065.) From diseased plant tissue.

Streptococcus trifoliatus Migulat. (Diplococcus ureae (non pyogenes) trifolialus Rovsing, Die Blasenentzündungen, ihre Aetiologie, Pathogenese und Behanglung, 1890, 43; Migula, Syst. d. Bakt., 2, 1900, 29.) From cases of cystitis.

Streptococcus turbidus Lehmann and Neumann. (Bouillon trübende Streptokokken, Behring, Cent. f. Bakt., 12, 1892, 193; Lehmann and Neumann, Bakt, Diag., 1. Lufl., 2, 1896, 125.) From various human infections, especially erysipelas. Presumably a smooth culture of Streptococcus pyogenes.

Streptococcustyrogenus Henrici. (Arb. bakt. Inst. Karlsruhe, 1 , Heft 1, 1894, 50.) From cheeses.

Streptococcus ureae Migula. (Streptococcus pyogenes ureae Rovsing, Die Blas- 
enentzündungen, ihre Aetiologie, Pathogenese und Behandlung, 1890, 45 ; Migula, Syst. d. Bakt., 2, 1900, 28; not Streptococcus ureae Trevisan, I generi e le specie delle Batteriacee, 1889, 31.) From cases of eystitis.

Streptococcus urinae Migula. (Diplococcus ureae (non pyogenes) Rovsing, loc. cit., 45; Migula, Syst. d. Bakt., 2, 1900, 13.) From cases of cystitis.

Streptococcus vaccinae (Cohn) Zopf (Microsphäeren der Vaccine, Cohn, Arch. f. path. Anat., 55, 1872, 237; Microsphaera vaccinae Cohn quoted from Cohn, Beiträge z. Biol, d. Pflanzen, 1, Heft, 2, 1872, 161; Micrococcus vaccinac Cohn, idem; Zopf, Die Spaltpilze, 3 Aufl, 1855, 52.) From lymph of cow pox pustules.

Streptococcus varicellae Trevisan. (Microbio della varicella, Bareggi, 1885, probably in Gazz. Med. ital. Lomb. Milano, 229-242; Trevisan, I generi e le specie delle Batteriacee, 1889, 30.) From chicken-pox pustules.

Streptococcus variolae Trevisan. (Microsphäeren der Variola, Cohn, Arch. f. path. Anat., 55, 1572, 237; Micrococcus variolae Cohn, 1872, quoted from Trevisan. I generi e le specie delle Batteriacee. 1889, 30.) From lymph of small pox pustules. Regarded by Cohn (Beiträge z. Biol. d. Pflanzen, 1, Heft 2, 1872, 161 ? as a variety of Micrococcus raccinae Cohn.

Streptococcus variolae-ovinae (Plaut) DeToni and Trevisan. (Micrococcus variolae ovinae Plaut, Das organisirte Contagium der Schafpocken und die Mitigation desselben, Leipzig, 1882; De'Toni and Trevisan, in Saccardo, Sylloge Fungorum, 8, 1889, 1058.) From the lymph in sheep-pox pustules.

Streptococcus vermiformis Sternberg. (Wurmformiger Streptococcus, Maschek, Bakt. Unters. d. Leitmeritzer Trinkwasser, Jahresb. d. Oberrealschule zu
Leitmeritz, 1887; Sternberg, Man. of Bact., 1893, 611.) From water.

Streptococcus versatilis Broadhurst. (Jour. Inf. Dis., 17, 1915, 323.) From throat of dogs, horse and cattle feces, ete.

Streptococcus vini Migula. (Micrococcus saprogenes vini II, Kramer, Landwirtsch. Versuchsstat., 37, 1890, 325 and Die Bakt. in ihren Beziehungen z. Landwirtsch. u. d. landwirtsch.-technisch. Gewerben, 2, 1892, 140; Migula, Syst. d. Bakt., 2, 1900, 33.) From wine.

Streptococcus viscosus Lehmann and Neumann. (Schleimiger Streptokokken, Behring, Cent. f. Bakt., 12, 1892, 193; Lehmann and Neumann, Bakt. Diag., 1 Aufl., 2, 1896, 125.) From various human infections. Presumably a mucoid culture of Streptococcus pyogenes.

Streptococcus vitulorum Trevisan. (Micrococco della diarrea bianca dei vitel lini, Perroncito, 1886; Trevisan, I generi e le specie delle Batteriacee, 1889, 30.) From white diarrhoea of calves.

Streptococcus vulgaris Loening. (Münch. med. Wochnsehr., 57, 1910, 173 and 247; Streptococcus pyogenes vulgaris Thomson and Thomson, Ann. PickettThomson Res. Lab., 3, 1927, 189.) Names applied to Streptococcus pyogenes.

Streptococcus weissii Trevisan. (Atti d. Accad. Fisio-Medico-Statistica in Milano, Ser. IV, 3, 1885, 119.) From Iung exudate in pleuropneumonia of cattle.

Streptococcus zythi Trevisan. (Torulacée de la bière malade, Pasteur; Trevisan, I generi e le specie delle Batteriacee, 1889, 31.) From spoiled beer.

Streptostaphylococcus parvulus Heurlin. (Heurlin, Bakt. Unters. d. Keimgehaltes im Genitalkanale der fiebernden Wöchnerinnen. Helsingsfors, 1910, 138.) From genital canal. Intermediate between Streptococcus anaerobius Krönig and Staphylococcus parvulus Veillon and Zuber. 


\section{Genus 111. Leuconostoc V'an Tieghem emend. Hucher and Pederson.*}

(Van Tieghem, Ann. Sci. Nat., 6, Sér. 7, 1878, 170; Betacoccus Orla-Jensen, The Lactic Acid Bacteria. Mem. Acad. Sci. Danemark, Sec. d. Sci., 5, Sér. 8, 1919, 146 ; Hucker and Pederson, New York Agr. Exp. Sta. Tech. Bul. 167, 1930, 66.) From Latin leucus, clear, colorless; M. L. Nostoc, a genus of blue-green algae.

Cells normally spherical. Under certain conditions, such as in acid fruits and vegetables, the cells may lengthen and become pointed or even elongated into a rod. Certain types grow with a characteristic slime formation in suerose media. Grow on ordinary culture media, but growth is enhanced by the addition of yeast, tomato or other vegetable extracts. Generally, a limited amount of acid is produced, consisting of lactic and acetic acid; alcohol is also formed, and about one-fourth of the fermented glucose is changed to $\mathrm{CO}_{2}$. Levo lactic acid is always produced, and sometimes dextro lactic acid also. Milk is rarely curdled. Fructose is reduced to mannitol. Habitat: Milk, plant juices.

The type species is Leuconostoc mesenteroides (Cienkowski) Van Tieghem.

\section{Key to the species of genus Leuconostoc.}

I. Acid from sucrose.

A. Acid from pentoses.

1. Leuconostoc mesenteroides.

B. No acid from pentoses.

\section{Leuconostoc dextranicum.}

II. No acid from sucrose.

3. Leuconostoc citrovorum.

1. Leuconostoc mesenteroides (Cienkowski) Van Tieghem. (Ascococcus mesenteroides Cienkowski, Arb. d. Naturf. Gesellsch. a. d. Univ. a. Charkoff, 1878, 12; Van Tieghem, Ann. Sci. Nat., 6, Sér. 7, 1878, 170; Leuconostoc indicum Liesenberg and Zopf, Beitr. z. Physiol. u. Morph. niederer Organis., Heft 1, 1892, 19; Streptococcus mesenterioides Migula, Syst. d. Bakt., 2, 1900, 25; Leuconostoc agglutinans Barendrecht, Cent. f. Bakt., II Abt., 7, 1901, 627; Leuconostoc aller Zettnow, Ztschr. f. Hyg., 57, 1907, 154; Leuconostoc opalanitza Zettnow, loc. cit.; Betacoccus arabinosaceus OrlaJensen, The Lactic Acid Bacteria, 1919, 152 ; Leuconostoc arabinosaceus Holland, Jour. Bact., 5, 1920, 223; Bacillus pleofructi Savage and Hunwicke,
Spec. Rept. Food Investigation Board, London, 1923, 134; Leuconostoc pleofructi Pederson, N. Y. Agr. Exp. Sta. Tech. Bull. 150 and 151, 1929.) From Greek mesenterium, mesentery; eidus, form (like).

Probable synonym: Leuconostoc soyae Belenky, Bull. Sci., Res. Inst. for Leguminous Crops, Moscow (Russian), 5, 1934, 132 .

Spheres: 0.9 to 1.2 microns in diameter, oceurring in pairs and short or long chains. In sucrose solutions the chains are surrounded by a thick, gelatinous, colorless membrane consisting of dextran. Gram-positive.

Glucose gelatin colonies: Small, white to gray ish-white, raised, nodular.

* Revised by Prof. G. J. Hucker and Prof. Carl S. Pederson, New York State Experiment Station, Geneva, New York, September, 1938; further revision, December, 1943. 
Glucose gelatin stab: Growth along entire stab. No liquefaction.

Sucrose broth: Abundant growth with massive formation of slimy material.

Potato: No visible growth.

Indole not formed.

Acid from glucose, fructose, galactose, mannose, xylose, arabinose, sucrose, and generally from lactose, raffinose, salicin and mannitol. Rarely acid from dextrin, starch, inulin, sorbitol, rhamnose or glycerol.

Nitrites not produced from nitrates.

Produces slime from sucrose. Most pronounced in sucrose gelatin stab.

Aerobic, facultative.

Optimum temperature $21^{\circ}$ to $25^{\circ} \mathrm{C}$.

Distinctive characters: Active slime producer in sucrose solutions.

Source: Slime in sugar factory

Habitat: Most active of the genus. Encountered in fermenting vegetable and other plant materials. Frequently isolated from slimy sugar solutions.

\section{Leuconostoc dextranicum (Bei-} jerinck) Hucker and Pederson. (Lactococcus dextranicus Beijerinck, Folia Microbiologica, Delft, 1912, 377; Betacoccus bovis Orla-Jensen, The Lactic Acid Bacteria, Copenhagen, 1919, 152 (Leuconostoc bovis Holland, Jour. Bact., 5, 1920, 223); Streptococcus paracitrovorus Hammer, Research Bul. 63, Iowa Agr. Exp. Sta., 1920; Hucker and Pederson, N. Y. Agr. Exp. Sta. Tech. Bull. 167, 1930, 67.) From Latin dexter, right; M. L. dextranum, dextran; M. L. dextranicus, related to dextran.

Note: The description of Streptococeus b, v. Freudenreich (Cent. f. Bakt., II Abt., 3, 1897, 47) renamed Streptococcus kefir by Migula (Syst. d. Bakt., 2, $1900,44)$ is too indefinite to permit the determination of its exact relationship to the organisms in this genus. It is clear, however, that the Streptococcus kefir of these authors and that of Evans (Jour. Agr. Res., 13, 1918, 235) were very similar to if not identical with Leuconostoc dextranicum. Streptococcus dis- tendens Hammer (Iowa State Coll. Jour. Sci., 2, 1927, 5) may also be identical with Leuconostoc dextranicum.

Spheres : 0.6 to 1.0 micron in diameter, occurring in pairs and in short chains. Gram-positive.

Gelatin stab: Gray filiform growth in stab.

Agar colonies: Small, gray, circular, slightly raised, entire.

Glucose broth: Slight grayish sediment.

Litmus milk: Acid, coagulation. Frequently shows slight reduction of litmus in bottom of tube.

Potato: No visible growth.

Indole not formed.

Nitrites not produced from nitrates.

Produce slime from sucrose in rapidly growing cultures.

Acid from glucose, fructose, galactose, maltose, sucrose, and generally from lactose and mannose. No acid from xylose, arabinose, glycerol, rhamnose, sorbitol, mannitol, starch, rarely raffinose, inulin or dextrin.

Aerobic, facultative.

Optimum temperature of growth $21^{\circ}$ to $25^{\circ} \mathrm{C}$.

Distinctive characters: Produces moderate amount of slime in sucrose solutions.

Source: Dairy starters.

Habitat: Found both in plant materials and in milk products.

\section{Leuconostoc citrovorum (Hammer)} Hucker and Pederson. (Streptococcus citrovorus Hammer, Research Bull. No. 63, Iowa Agr. Exper. Sta., 1920; Hucker and Pederson, N. Y. Agr. Exp. Sta. Tech. Bull. 167, 1930, 67.) From Latin citrus, the citron tree; M. L., lemon or orange, hence eitric acid; voro, devour.

Spheres: 0.6 to 1.0 micron in diameter, occuring in pairs and chains. Grampositive.

Gelatin stab: Filiform growth in stab. No liquefaction.

Agar colonies: Small, gray, entire, slightly raised. 
Agar slant: Small, gray, discrete colonies.

Glucose broth: Slight gray sediment.

Litmus milk: Slightly acid with partial reduction of litmus.

Potato: No visible growth.

Indole not formed.

Nitrites not produced from nitrates.

Grows poorly on ordinary media without the addition of yeast extract or other growth accessory substance.

Acid from glucose, fructose, galactose and lactose. Generally does not form acid from mannose, sucrose, maltose, xylose, arabinose, thamnose, raffinose, glycerol, dextrin, inulin, starch, salicin, mannitol or sorbitol.

Uses citric acid in milk.

Aerobic, facultative.

Optimum temperature $20^{\circ}$ to $25^{\circ} \mathrm{C}$.

Distinctive character: Non-slime producer.

Source: Dairy products.

Habitat: Found in milk and dairy products.

Appendix: This includes species that probably belong in this genus. The descriptions are too meager to permit draw ing any definite conclusion regarding their relationship to the three species recognized above.

Bacterium laevolacticum Migula. (Bacillus acidi laevolactici Schardinger, Monatsh. f. Chemie, 11, 1890, 544; Migula, Syst. d. Bakt., 2, 1900, 406; Bacterium acidi laevolactici Lehmann and Neumann, Bakt. Diag., 4 Aufl., 2, 1907, 178.) From well water.
Leuconostoc lagerheimii Ludwig. (Ludwig, Lehrb. d. niederen Kryptog., 1892, 29; Streptococcus lagerheimii Migula, Syst. d. Bakt., 2, 1900, 41.) From slimy sugar solutions.

Micrococcus gelatinogenus Bräutigam. (Pharmaceutische Centralhalle, 1891, No. 30.) From the air. Forms gum in sucrose media.

Micrococcus gummosus Happ. (Inaug. Diss., Basel, published in Berlin, 1893, 31.) From slimy sugar solutions.

Myxococcus betae Gonnermann. (Oesterr.- Ungar. Ztschr. f. Zuckerind. u. Landw., 136, 1907, 883.) From sugar beet juice.

Streptococcus citrovorus-paracitrovorus Vas and Csiszár. (Milchwirtsch. Fortsch., 18, 1936,68.) From cream and butter.

Streptococcus hornensis Boekhout. (Cent.f. Bakt., II Abt., 6, 1900, 162.) From slimy, sweetened condensed milk. A strong dextran former. Related to Leuconostoc mesenteroides.

Zoogloeatermo Cohn. (Cohn, Nov. Act. Acad. Caes. Leop.-Carol. Nat. Cur., 24, 1854,123 .) The only species in the genus Zoogloea as originally proposed. From running water. Scheibler used this name for a zoogloea-forming organism from slimy sugar solutions in Neue Ztschr. f. Rübenzucker-Ind., 1, 1878, 366 and probbably also in Ztschr. d. Vereins f. Rübenzucker-Ind., 1874, 330. The latter reference apparently is not available in America. See Buchanan, General Syst. Bact., 1925, 530 for a history of the genus Zoogloea. 
TRIBE II. LACTOBACILLEAE WINSLOW ET AL.

(Jour. Bact., 5, 1920, 211.)

Rods, often long and slender. Non-motile. Gram-positive. Pigment formation rare. When present, yellow or orange, to rust or brick-red in color. Poor surface growth (except in genus Microbacterium) because these bacteria are generally microaerophilic or anaerobic. Carbohydrates and polyalcohols are changed either by homofermentation to lactic acid or by heterofermentation to lactic, acetic, propionic or butyric acids, alcohol and carbon dioxide. Growth on potato is poor or absent. Gelatin is not liquefied. Nitrates are not reduced (except in genus Microbacterium). Several species grow at relatively high temperatures. May or may not produce catalase.

\section{Key to the genera of tribe Lactobacilleae.}

I. Always produce lactic acid from carbohydrates.

a. Catalase negative. Microaerophilic.

Genus I. Lactobacillus, p. 349.

aa. Catalase positive. Aerobic.

Genus II. Microbacterium, p. 370.

II. Ferments carbohydrates, polyalcohols and lactic acid with the formation of propionic and acetic acids, and carbon dioxide. Catalase positive.

Genus III. Propionibacterium, p. 372.

III. Ferments carbohydrates, polyalcohols and lactic acid with the formation of butyric and acetic acids, and carbon dioxide. Generally catalase negative.

Genus IV. Butyribacterium, p. 379.

\section{Gems I. Lactobacillus Beijerinck. *}

(? Dispora Kern, Biol. Zent., 2, 1882, 135; ?Tyrothrix Duclaux, Ann. Inst. Nat. Agron., 4, 1882, 79; ?Pacinia Trevisan, Atti della Accad. Fisio-Medico-Statistica in Milano, Ser. 4, 3, 1885, 83; ?Saccharobacillus van Laer, Contributions à l'histoire des ferments des hydrates de carbone. Mém. Acad. Royale de Belgique, 43, 1859; Lactobacter Beijerinck, Cent. f. Bakt., II Abt., 6, 1900, 200; Beijerinck, Arch. néerl.d. sci. exact. et nat. Haárlem, Sér. 2, 7, 1901, 212; Streptobacillus Rist and Khoury, Ann. Inst. Past., 16, 1902, 70; ?Brachybacterium Troili-Petersson, Cent. f. Bakt., II Abt., 11, 1903, 138; C'aseobacterium Orla-Jensen, Cent. f. Bakt., II Abt., 22, 1909, 336; Plocamobacterium Löwi, Wiener klin. Wochnschr., 33, 1920, 730 (in part); Lactobacterium van Steenberge, Ann. Inst. Past., 34, 1920, s06; Bifidobacterium Orla-Jensen, Le Lait. 4, 1924, 469; Acidobacterium Heim, see Schlirf, Cent. f. Bakt., I Aht., Orig., 9\%, 1925. 111: Bifidibacterium Prévot, Inn. Inst. Past., 60, 1933. 303.) From . I. T.., lactire and bacillus, a rod.

Rods, usually fong and slender. Microaerophilie. C'arbohydratess and poly-alcohols are changed by homofermentation to lactic acid, or by heterofermentation to lactic and acetic acids, alcohols and carbon dioxide. Catalase negative. Found in fermenting animal (especially dairy) and plant products.

The type species is Lactobacillus caucasicus Beijerinck.

* Completely revised by Prof. Carl S. Pederson, New York State Experiment Station, Geneva, New York, in consultation with Prof. J. M. Sherman. Cornell Cniversity, Ithaca. New York and Prof. I. F. Rettger, Yale Lniversity, New Haven, Conn., June, 1938; further revision by Prof. Carl S. Pederson. January, 1945. 


\section{Key to the species of genus Lactobacillus.}

I. Produce only traces of by-products other than lactic acid. Homofermentative.

A. Optimum temperature $37^{\circ}$ to $60^{\circ} \mathrm{C}$ or higher. Sub-genus Thermobacterium Orla-Jensen (The Lactic Acid Bacteria, 1919, 160).

1. Acid from lactose.

a. Optimum temperature $37^{\circ}$ to $45^{\circ} \mathrm{C}$.

b. Produce levo lactic acid.

1. Lactobacillus caucasicus.

2. Lactobacillus lactis.

bb. Produce inactive or dextro lactic acid.

c. Microaerophilic.

3. Lactobacillus helveticus.

4. Lactobacillus acidophilus.

ce. Anaerobic in freshly isolated cultures.

5. Lactobacillus bifidus.

aa. Optimum temperature $45^{\circ}$ to $62^{\circ} \mathrm{C}$; usually no acid from maltose.

6. Lactobacillus bulgaricus.

7. Lactobacillus thermophilus.

2. No acid from lactose.

\section{Lactobacillus delbrueckii.}

13. Optimum temperature $28^{\circ}$ to $32^{\circ} \mathrm{C}$. Sub-genus Streptobacterium Orla-Jensen (loc. cit., 166).

1. Acid from lactose.

a. Produces dextro lactic acid. Often prefers lactose to sucrose and maltose.

9. Lactobacillus casei.

aa. Produces inactive lactic acid.

10. Lactobacillus plantarum.

2. No acid from lactose.

11. Lactobacillus leichmannii.

II. Produce considerable amounts of by-products other than lactic acid (carbon dioxide, alcohol and acetic acid; mannitol from fructose). Heterofermentative. Sub-genus Betabacterium Orla-Jensen (loc. cit., 175).*

A. Optimum temperature $28^{\circ}$ to $32^{\circ} \mathrm{C}$. Usually ferment arabinose.

1. Does not ferment rafinose, and usually does not ferment sucrose or lactose.

12. Lactobacillus brevis.

2. Ferment raffinose, sucrose and lactose.

13. Lactobacillus buchneri.

14. Lactobacillus pastorianus.

B. Optimum temperature $35^{\circ}$ to $40^{\circ} \mathrm{C}$ or higher. Usually does not ferment arabinose.

15. Lactobacillus fermenti.

* Also see discussion of Betabacterium caucasicum, p. 358. 
1. Lactobacilus caucasicus Beijerinck. (Bacillus caucasicus Beijerinck, Areh. néerl. d. sci. exact. et nat., 23, 1889, 428; Beijerinck, ibid., Sér. 2, 7, 1901, 212; not Bacillus caucasicus v. Freudenreich, Cent. f. Bakt., II Abt., 3, 1897, 54 and 135 ; Bacterium caucasicus Chester, Ann. Rept. Del. Col. Agr. Exp. Sta., 9, 1897, 130; not Bacterium caucasicum Lehmann and Neumann, Bakt. Diag., 2 Aufl., 2, 1599, 209; not Betabacterium caucasicum Orla-Jensen, The Lactic Acid Bacteria, 1919, 175.) From Greek Caucasia, M. L. caucasicus, of the Caucasus.

The following is a possible or probable synonym: Streptobacillus lebenis Rist and Thoury, Ann. Inst. Past., 16, 1902, 70.

Description taken from the two reports of Beijerinck (loc. cit.).

Rods: Thin and variable in size, occurring singly or in filaments. Non-motile. Non-spore-forming. Gram-positive (not recorded in early descriptions)

Gelatin : No liquefaction.

Wort gelatin: Small, white colonies.

Agar colonies: Small.

Broth: Carbohydrates necessary for growth.

Milk: Rapid acid production with coagulation, no action in casein .

Utilizes animal peptones with difficulty, utilizes vegetable peptones more readily.

Acid from glucose, sucrose, maltose and lactuse. No action on starch. Action on other carbohydrates not studied. Lactose in milk converted to levo lactic acid with little carbon dioxide.

Microaerophilic.

Optimum temperature $40^{\circ}$ to $44^{\circ} \mathrm{C}$. Temperature range $25^{\circ}$ to $45^{\circ} \mathrm{C}$.

Source: From kefir and cheese.

Habitat: Occurs symbiotically with yeast in kefir.

Prototype: Dispora caucasica Kiern. (Kern, Biol. Zent., 2, 18\$2, 135; later in Bull. de la Soc. Imp. des Naturalistes de Moscow, 56, 1882, 168; Bacterium caucasicum Zopf, Die Spaltpilze, 3 Aufl. 1885, 90; Bacillus kaukasicus Flügge, Die Mikroorganismen, 2 Auf., 1856, 270;
Pacinia caucusica Trevisun, I generi e le specie delle Batteriacee, $1889,23$. )

The description by Kern of an organism from kefir grains is confused probably because the organism (a spore former) which he isolated by the use of Cohn's solution was not the same as the presumably granulated lactobacillus he saw in microseopical preparations of kefir. Beijerinck was apparently the first to have isolated a lactobacillus from kefir in pure culture and to have given a sufficiently complete description to make reidentification possible. It should be noted that from the characters given, this could not have been the same species as that isolated later from kefir by $v$. Freudenreich (loc. cit.) and Orla-Jensen (loc. cit.).

2. Lactobacillus lactis (Orla-Jensen) Holland. (Bacillus lactis acidi Leichmann, Cent. f. Bakt., II Abt., 2, 1896, 779 ; Milch. Zeitung, 25, 1896, 67; Thermobacterium lactis Orla-Jensen, The Lactic Acid Bacteria, 1919, 164; Lactobacillus lactis-acidi Holland, Jour. Bact., 5, 1920, 223; Holland, idem.) From Latin lac, milk.

Henneberg (Handb. der Gärungsbakt., 2 Aufl. 2, 1926, 128) regards Bacillus lactis acidi Leichmann as identical with Thermobacterium lactis Orla-Jensen.

Rods: Long forms with a tendeney to grow into threads, of ten strongly curling. Occur singly or in pairs in young vigorous cultures. Generally contain volutin grains. Gram-positive (not recorded in original description).

Milk: Acid produced followed by coagulation in one to four days. 1.7 per cent acid produced.

Acid from fructose, glucose, mannose, galactose, sucrose, maltose, lactose, raffinose and dextrin. Glycerol, xylose, arabinose, rhamnose, sorbitol, mannitol, inulin and starch not fermented. Salicin may or may not be fermented.

Forms levo lactic acid with only a trace of other products

Temperature relations: Optimum $40^{\circ} \mathrm{C}$. 
Minimum $18^{\circ}$ to $22^{\circ} \mathrm{C}$. Maximum $50^{\circ} \mathrm{C}$. Source: From milk and cheese.

Habitat: Lndoubtedly widely distributed in milk or milk products.

3. Lactobacillus helveticus (Orla-Jensen) Holland. (Bacillus $\epsilon$, von Freudenreich, Cent. f. Bakt., II Abt., 1, 1895, 173; also Landw. Jahrb. d. Schweiz, 1895, 211; Bacillus casei $є$, v. Freudenreich and Thöni, Landw. Jahrb. d. Schweiz, 1904, 526; also Orla-Jensen, Cent. f. Bakt., II Abt., 13, 1904, 609; Caseobacterium $\epsilon$, Orla-Jensen, Cent. f. Bakt., II Abt., 22, 1909, 337; Thermobacterium helveticum Orla-Jensen, Maelkeri-Bakteriologie, 1916, 35; also the Lactic Acid Bacteria, 1919, 164; Bacterium casci $\epsilon$, Holland, Jour. Bact., 5, 1920, 221 ; Holland, ibid. 223.) From Latin helveticus, Swiss.

Rods : 0.7 to 0.9 by 2.0 to 6.0 microns, occurring singly and in chains. Nonmotile. Gram-positive.

Whey gelatin colonies: Does not grow readily at temperatures required for incubation of gelatin.

Lactose agar colonies: Small, grayish, viscid.

Milk: Acid, with coagulation; may hecome slimy.

Nitrites not produced from nitrates.

Acid from glucose, fructose, galactose, mannose, maltose, lactose, and smaller amounts from dextrin. The lactic acid is inactive.

Temperature relations: Optimum $40^{\circ}$ to $42^{\circ} \mathrm{C}$. Minimum $20^{\circ}$ to $22^{\circ} \mathrm{C}$. Maximum $50^{\circ} \mathrm{C}$.

Microaerophilic.

Source: From sour milk and cheese.

Habitat: Widely distributed in dairy products.

4. Lactobacillus acidophilus (Moro) Holland. (Bacillus acidophilus Moro, Wiener klin. Wochnschr., 13, 1900, 114; also Jahrb. f. Kinderheilkunde, 52, 1900, 38; Holland, Jour. Bact., 5, 1920, 215; Plocamobacterium acidophilum Lehmann and Neumann, Bakt. Diag., 7 Aufl., 2, 1927, 510; Thermobacterium intestinale
Orla-Jensen, Orla-Jensen and Winther, Cent. f. Bakt., II Abt., 93, 1936, 321.) From Latin acidus, sour; M. L. acidus, acid and Greek phitus, loving.

Possible synonyms: Milchsäurebacillus, Boas and Oppler, Deutsche med. Wochnschr., 21, 1895, 73; Diagnostik und Therapie d. Magenkrankheiten, II Teil, 1907, 265 (Lactobacillus boas-oppleri Bergey et al., Manual, 1st ed. 1923, 243); Bacillus exilis Tissier, La flore intestinale des nourrissons, Paris, 1900, 102; Bacillus gastrophilus Lehmann and Neumann, Bakt. Diag., 4 Aufl., 2, 1907, 424 (Bacterium gastrophilum Lehmann and Neumann, Bakt. Diag., 5 Aufl., 2, 1912, 305); Bacillus acetogenus $\alpha$ Distaso, Cent. f. Bakt., I Abt., Orig., 59, 1911, 49; Bacillus acetogenus $\beta$ Distaso, ibid., 51; Bacillus nectogenus proteiformis Distaso, ibid., 52; Bacillus acetogenus exilis Distaso, ibid., 53 ; Bacillus paraexitis Distaso, ibid., 56; Bacillus dimorphus Distaso, ıbid., 55; Bacillus dimorphus var. longa Distaso, C'ent. f. Bakt., I Abt., Orig., 62, 1912, 440; (Bacleroides dimorphus Bergey et al., Manual, 1st ed., 1923, 258); Streptobacillus longus Distaso, ibid., 439; Thermobacterium acidophilum. Henneberg, Cent. f. Bakt., II Abt., 91, 1934, 102.

Description of Moro supplemented by material from Kulp and Rettger, Jour. Bact., 9, 1924, 357; Curran, Rogers and Whittier, Jour. Bact., 25, 1933, 595; and Rettger, Levy, Weinstein and Weiss, Lactobacillus acidophilus, Tale Univ. Press, New Haven, 1935.

Rods : 0.6 to 0.9 by 1.5 to 6.0 microns, occurring singly, in pairs and in short chains with rounded ends. Non-motile. Dimensions variable (Kulp and Rettger), (Curran, Rogers and Whittier). Grampositive; old cultures often Gram-negative (Moro).

Gelatin : No grow th at $20^{\circ} \mathrm{C}$. No liquefaction.

Wort-agar (Moro) or tomato agar (Kulp) and Rettger) plates: Surface colonies, peripheries a capilliform maze of long, delicate, twisted, fuzzy projections, center appears as a thick, dark, felt-like 
mass. Deep colonies, small, irregularly shaped, with fine radiate or ramified projections.

Wort-agar slants: Growth scanty, limited, dry, veil-like.

Wort-broth: After 48 hours, fine, flocculent sediment. Other acid broths sediment whitish, slight turbidity.

Milk: Slow growth with small inocuIum. Coagulates from the bottom up

Potato: No growth

Acid but no gas from glucose, sucrose and lactose (Moro). Acid from glucose, fructose, galactose, mannose, maltose, lactose and sucrose. Some cultures ferment raffinose and trehalose and have slight action on dextrin. Xylose, arabinose, rhamnose, glycerol, mannitol sorbitol, dulcitol and inositol not fer mented (Tulp and Rettger). Inactive lactic acid and volatile acids formed from sugars (Curran, Rogers and Whittier).

No visible growth in "arbohydrate-fres media (Rettger, Levy, Weinstein and Weiss).

Optimum temperature $37^{\circ} \mathrm{C}$. No growth at $20^{\circ}$ to $22^{\circ} \mathrm{C}$ (Moro). Maximum temperature $43^{\circ}$ to $48^{\circ} \mathrm{C}:$ (Curran, Rogers and Whittier).

Not pathogenic for laboratory animals. Microaerophilic.

Distinctive characters: Grows in acid inedia. Unless frequent transfers are made, organism may become Gram-negative and rapidly develop characteristic degeneration forms (Morn). The socalled original strains of Bacillus acidophilus from the Král collection, described and called Microbacterinm lacticum by Orla-Jensen, do not have the characteristics given by Moro.

Source: From the feces of milk-fed infants. Also from the feces of older persons on high milk or lactose or dext rincontaining diets.

Habitat: As for source.

5. Lactobacillus bifidus (Tissier) $\mathrm{Hol}$ land. Bacillus bifidus communis and Bacillus bifidus Tissier, Recherches sur la flore intestinal des nourrissons, Paris,
1900, 85; Bacteroides bifidus Castellani and Chalmers, Man. Trop. Med., 3rd ed., 1919, 960; Holland, Jour. Bact., 5, 1920, 223 ; Nocardia bifida Vuillemin, Encyclopédie Mycolog., Paris, 2, Champignons Parasites, 1931, 132; Actinomyces bifidus Tannizzi, in Pollacci, Trat. Micopat. Umana, 4, 1934, 13; Cohnistreptothrix bifidus Negroni and Fisher, Rev. Soc. Argentina Biol., 20, 1944, 315.) From Latin bifidus split in two, cleft

Possible synonyms: Coccobacillus oviformis Tissier, Ann. Inst. Past., 22, 1908 , 189 (Bacterium oxiforme Le Blaye and Guggenheim, Manual pratique de diagnostique Bactériologie. Paris, 1914; Bacteroides oriformis Levine and Soppeland, Iowa Finginefring Exp. Sta. Bul. 77, 1926, 35); Bacillus ventriosus Tissier, loc cit. (Berteroides ventriosus Figgerth, Joux. Bact.. 30, 1935, 281); Diplobacillus ueuminatus Distaso, lor cit. Bacteroides ucuminatu: Rergey et al. Manual, 1st ed., 1923, 260 ).

Deseription supplemented from Weiss and Rettger, Jour. Bact., 28, 1934, 501.

Small, slender rods: Average length 4.0 microns, 0.5 to 0.7 by 2 to 8 mierons (Weiss and Rettger), occurring singly or in pairs and short chains, parallel to each wher, very variable in appearance. Branched and club forms develop in some (ultures. Von-motile. Gram-positive but stains irregularly in old cultures (Tissier).

Little or mo grow th in carbohydrate-free agar (Weiss and Rettger).

Deep sugar-agar colonies: After 3 days, solid with slightly irregular edge, whitish. (irow up to $3 \mathrm{~cm}$ from the surface forming : ring. Average diameter $3 \mathrm{~mm}$. No gas.

Sugar broth: (reod growth. Turbid within 3 days. ('Tears with flocculent precipitate

Milk: Cinod growth with large inoculum. No congulation (Tissier). May or may not magulate milk (Weiss and Rettger

Acid but no gas from glucose (Tissier) Acid from glucose, fructose, galactose, 
sucrose, inulin and usually from dextrin, starch, maltose, raffinose and trehalose. A few strains form acid from lactose and salicin. The acid consists of inactive lactic acid and 18 to 25 per cent of volatile acid (Weiss and Rettger).

Optimum temperature $37^{\circ} \mathrm{C}$. May show slight growth at $20^{\circ} \mathrm{C}$. Killed at $60^{\circ} \mathrm{C}$ in 15 minutes.

Non-pathogenic for mice or guinea pigs.

Strict anaerobe (Tissier). Strict anaerobe in primary culture becoming microaerophilic (Weiss and Rettger).

Distinctive characters: Bifurcations and club-shaped forms (Tissier), particularly in infant feces and in primary culture (Weiss and Rettger).

Source: From feces of nursing infants. Habitat: Very common in the feces of infants. May constitute almost the entire intestinal flora of breast-fed infants. Also present in smaller numbers with bottle-fed infants. Possibly more widely distributed than indicated in the intestines of warm-blooded animals.

5a. Lactobacillus parabifidus Weiss and Rettger. (Bacterium bifidum OrlaJensen, The Lactic Acid Bacteria, 1919, 192; Bucteroides bifidus (Group 2) Eggerth, Jour. Bact., 30, 1935, 295; Lactobacillus bifidus $I I$ or Lactobacillus parabifidus Weiss and Rettger, Jour. Bact., 35, 1938, 17; Jour. Inf. Dis., 62, 1938, 115.)

This is the more anaerobic variety of the bifid organisms from feces and seems to be more common in the intestine of adults. In contrast to Lactobacillus bifidus, it produces more volatile acid as well as dextro lactic acid, and ferments arabinose, xylose and melezitose but not mannose.

6. Lactobacillus bulgaricus (Luerssen and Kühn) Holland. (Bacillus A, Grigoroff, Revue Méd. Suisse romande, 25, 1905; Bacillus bulgaricus Luerssen and Kühn, Cent. f. Bakt., II Abt., 20, 1907, 241; Thermobacterium bulgaricum OrlaJensen, The Lactic Acid Bacteria, 1919, 164; Holland, Jour. Bact., 5, 1920, 215;
Acidobacterium bulgaricum Schlirf, Cent. f. Bakt., I Abt., Orig., 97, 1925, 116; Plocamobacterium bulgaricum Lehmann and Neumann, Bakt. Diag., 7 Aufl., 2, 1927, 511.) From Latin bulgaricus, of or related to Bulgaria.

Probable synonyms: Lactobacillus longus Beijerinck, Arch. néerl. d. sci. exact. et nat., Sér. 2, 7, 1901, 212 (not Lactobacillus longus Bergey et al., Manual, 4th ed., 1934, 312); Bacterium casei filans Gorini, Rend. R. Acc. Lincei, 21, 1912, 472; Cent. f. Bakt., II Abt., 3\%, $1913,1$.

Deseription of Luerssen and Kühn supplemented by Grigoroff, loc. cit.; Cohendy, Compt. rend. Soc. Biol. Paris, 58, 1906, 364; Kuntze, Cent. f. Bakt., II Abt., 21, 1908, 737; Bertrand and Duchacek, Ann. Inst. Pist., 23, 1909, 402; White and Avery, Cent. f. Bakt., II Abt., 25, 1910, 161 ; Rahe, Jour. Bact., 3, 1918, 420; Orla-Jensen, The Lactic Acid Bacteria, 1919, 164; Kulp and Rettger, Jour. Bact., 9, 1924, 357; Sherman and Hodge, Jour. Dairy Ści., 19, 1936, 494.

Rods: Slender rods with rounded ends, often in chains. Non-motile. Grampositive, older cultures showing unstained portions (Luerssen and Kühn).

Whey gelatin: No liquefaction (White and Avery).

Colonies: Flat, yellowish-white, 2 to $3 \mathrm{~mm}$. Old cultures have dark centers. Deep colonies globular (Iuerssen and Kühn).

Whey agar colonies: Circular to irregular (White and Avery).

Milk: Coagulation at $37^{\circ} \mathrm{C}$. No gas. No decomposition of casein.

Potato: Yellow-white colonies (Luerssen and Kühn). No growth (Grigoroff), (Coherdy), (White and Avery).

Indole not formed (Grigoroff), (White and Avery).

Nitrites not produced from nitrates.

Results on acid production from sugars vary. Glucose, lactose and galactose are apparently always fermented while xylose, arabinose, sorbose, rhamnose, dulcitol, mannitol, dextrin, inulin and starch 
are never fermented. Early workers (Gigoroff) (Cohendy) noted fermentation of fructose, maltose and sucrose. Later workers (Bertrand and Duchacek), (Orla-Jensen), (Rahe), (Kulp and Rettger), (Sherman and Hodge) noted variable or negative results on sucrose, maltose and unheated fructose.

Forms high acidity in milk. The lactic acid is inactive (Grigoroff), (Bertrand and Duchacek), (White and Avery) or levo (White and Avery), (Orla-Jensen) with small quantities of volatile acid (White and Avery).

Aerobic or anaerobic (Luerssen and Kühn). Microaerophilic (White and Avery). Anaerobic in fresh isolation (Sherman and Hodge).

Optimum temperature $45^{\circ}$ to $50^{\circ} \mathrm{C}$. Minimum $22^{\circ} \mathrm{C}$ (Luerssen and Kühn). Distinctive characters: This species at present is regarded as including the high temperature organisms isolated from milk with difficulty. These ferment glucose, galactose and lactose but usually do not ferment sucrose, maltose or unheated fructose when freshly isolated. hurt.

Source: Originally isolated from yog-

Habitat: Probably present in many milk products if held at high temperature.

7. Lactobacillus thermophilus Ayers and Johnson. (Jour. Bact., 9, 1924, 291.) From Greek thermos, heat and philus, loving.

Description of Ayers and Johnson supplemented by material from Charlton, Jour. Dairy Sei., 15, 1932, 393.

Rods: 0.5 by 3.0 microns. Stains irregularly. Jon-motile (Charlton). Gram-positive

Gelatin stab: No liquefaction.

Agar plate: Small colonies.

Agar slant : Slight, translucent growth (Charlton).

Broth: Turbid (Charlton).

Litmus milk: Acid.
Nitrites not produced from nitrates (Charlton).

Acid from glucose, lactose, sucrose, starch and trace from glycerol. No acid from salicin, mannitol, raffinose or inulin. (Ayers and Johnson). Acid from fructose, galactose, mannose, maltose, raffinose and dextrin. No acid from arabinose, xylose, glycerol, rhamnose, salicin, inulin or mannitol. Dextro lactic acid formed. (Charlton).

This is the thermophilic lactobacillus obtained from pasteurized milk which causes pin-point colonies on agar plates.

Temperature relations: Optimum temperature $50^{\circ}$ to $62.8^{\circ} \mathrm{C}$. Minimum $30^{\circ} \mathrm{C}$. Maximum $65^{\circ} \mathrm{C}$. Thermal death point $71^{\circ} \mathrm{C}$ for 30 minutes or $82^{\circ} \mathrm{C}$ for $2 \frac{1}{2}$ minutes.

Facultative anaerobe. Grows best aerobically.

Source: From pasteurized milk.

Habitat : Known only from pasteurized milk.

8. Lactobacillus delbrueckii (Leichmann) Beijerinck. (Bacillus delbrückii Leichmann, Cent. f. Bakt., II Abt., 2, 1896, 284; Bacillus acidificans longissimus Lafar, Cent. f. Bakt., II Abt., 2, 1896,195 ; Bacillus (?) acidificans Migula, Syst. d. Bakt., 2, 1900, S01; Beijerinek, Arch. néerl. d. sci. exactes et nat., Haárlem, Sér. 2, 7, 1901, 212; Thermobacterium cereale Orla-Jensen, The Lactic Acid Bacteria, 1919, 164; Bacillus acidificans-longissimus Holland, Jour. Bact., $5,1920,216$; Lactobacillus acidificanslongissimus Holland, ibid., 216; Lactobacillus cereale Holland, ibid., 223; Lactobacterium delbrucki (sic) van Steenberge, Ann. Inst. Past., 34, 1920, 820.) Named for Prof. M. Delbrück, German bacteriologist.

Description of Leichmann supplemented by material from Henneberg, Cent. f. Bakt., II Abt., 11, 1903, 154.

Rods : 0.5 to 0.8 by 2.0 to 9.0 microns (Henneberg), occurring singly and in short chains. Non-motile. Gram-positive. 
Gelatin colonies: Small, gray, circular, not liquefied.

Agar colonies: Simall, tlat, crenated.

Agar slant: Narrow, translucent, soft, grayish streak.

Broth : Slightly turbid.

Milk: Unchanged.

Nitrites not produced from nitrates.

Acid from maltose and sucrose (Leichmann) and glucose, fructose, galactose and dextrin. No acid from xylose, arabinose, rhamnose, lactose, raffinose, trehalose, inulin, starch, mannitol or $\alpha$-methyl-glucoside (Henneberg). Levo rotatory lactic acid is formed. Forms 1.6 per cent acid in mash.

This is the high temperature organism of fermenting mashes. In fresh isolations it apparently has a higher optimum temperature than when held in pure culture.

Optimum temperature $15 \mathrm{C}$.

Microaerophilic.

Source: From sour polato mash in a distillery

Habitat: Formenting vegetable and grain mashes.

9. Lactobacillus casei (Orla-Jensen) Holland. (Bacillus $\alpha$, v. Freudenreich, Ann. d. Microg., 2, 1890, 266; also Landw. Jahrb. d. Schweiz, 1891, 20; Bacillus casei $\alpha$, von Freudenreich and Thöni, Landw. Jahrb. d. Schweiz, 1904, 526; also Orla-Jensen, Cent. f. Bakt., II Abt., 13, 1904, 609; Caseobacterium vulgare OrlaJensen, Maelkeri-Bakteriologie, 1916, 35; Streptobacterium casei Orla-Jensen, The Lactic Acid Bacteria, 1919, 166; Bacterium casei $\alpha$, Holland, Jour. Bact., 5 . 1920, 221; Holland, ibid.) From Latin cuseus, cheese.

Rods: Short or long chains of short or long rods. Non-motile. Gram-positive.

Milk: Acid with coagulation in 3 to 5 days or longer, may become slimy. Forms about 1.5 per cent lactic acid.

Utilizes casein and therefore important in cheese ripening.

Acid from glucose, fructose, mannose, galactose, maltose, lactose, mannitol and saliciu. May or may not ferment sucrose. Mostly dextro lactic acid formed though a small amount of levo lactic acid may be formed. Only lactic acid produced with a trace of other by-products.

This is the more common lactic acid rod found in milk and milk products. Orla-Jensen distinguishes it from Lactobacillus plantarum in that it produces dextro lactic acid and usually ferments lactose more readily than sucrose or maltose.

Temperature relations: Optimum $30^{\circ} \mathrm{C}$. Minimum $10^{\circ} \mathrm{C}$. Maximum $37^{\circ}$ to $40^{\circ} \mathrm{C}$ and with some strains $45^{\circ} \mathrm{C}$.

Microaerophilic.

source: From milk and cheese.

Habitat: Probably more widely distributed than indicated by isolations.

10. Lactobacillus plantarum (Orla-Jensen) Holland. (Sireptobacterium plantarum Orla-Jensen, The Lactic Acid Bacteria, Copenhagen, 1919, 174; Holland, Jour. Bact., 5, 1920, 225.) From Latin planta, sprout; M. L.. a plant.

Probable synonyms: Bacillus pabuli acidi II Weiss, Inaug. Diss., Göttingen, 1898; Cent. f. Bakt., II Abt., 5, 1899, 599 (Lactobacillus pabuliacidi Bergey et al., Manual, 1st ed., 1923, 247); $B a$ cillus cucumeris fermentati Heuneberg, Ztschr. f. Spiritusindustire, 26, 1903, 22; Cent. f. Bakt., II Abt.., 11, 1903, 166 (Lactobacillus cucumeris Bergey et al., Manual, 1st ed., 1923, 250); Bacillus wortmannii Henneberg, Cent. f. Bakt., II Abt., 11, 1903, 162 (Lactobacillus wortmannii Bergey et al., Manual, 3rd ed., 1930, 248): Bacillus listeri Henneberg, Ztschr. f. Spiritusindustrie, 26, 1903, 22; ('ent. f. Bakt., II Abt., 11, 1903, 161 (Lactobacterium listeri van Steenberge, Ann. Inst. Past., 34, 1920, 814; Lactobacillus listeri Bergey et al., Manual, 1st ed., 1923, 248); Bacillus maercki Henneberg, loc. cit.; Bacillus leirhmanni II Henneberg, loc. cit.; Bacillus beijerinckii Henneberg, Ztschr. f. Apiritusindustrie, $26,1903,22$; see 
Cent. f. Bakt., II Abt., 11, 1903, 159 (Lactobacillus beijerinckii Bergey et al., Manual, 1st ed., 1923, 248); Lactobacillus pentosus Fred, Peterson and Anderson, Jour. Biol. Chem., 48, 1921, 410; Jour. Biol. Chen., 53, 1922, 111; Lactobacillus arabinosus Fred, Peterson and Anderson, Jour. Biol. Chem., 48, 1921, 410; Bacterium busae asiaticae Tschekan, Cent. f. Bakt., II Abt., $78,1929,89$ (Lactobacillus busaeasiaticus Bergey et al. Manual, 3rd ed., 1930, 288); Bactcrium brassicae Wehmer, Cent. f. Bakt., II Abt., 10, 1903, 628 (Lactobacillus brassicae LeFevre, Abst. Bact., 6, 1922, 25).

Description from Orla-Jensen supplemented by materia! from Pederson, Jour. Bact., 31, 1936, 217.

Rods: Ordinarily 0.7 to 1.0 by 3.0 to 8.0 micrors, occurring singly or in short chains, with rounded ends. Under favorable gromth conditions these organisms tend to be short rods. Under adverse conditions they tend to be longer; for example, in tomato juice agar at $45^{\circ} \mathrm{C}$ (Pederson, N. I. Agr. Exp. Sta. Tech. Bull. 150, 1929). In fermenting vegetables, the organisms tend to become longer as the acidity becomes greater. The organisms are usually longer in milk than in broths. Differences in morphology are well illustrated by OrlaJensen. Non-motile. Gram-positive.

Celatin-yeast extract-glucose stab: Filiform growth. No liquefaction.

Agar slant: Frowth, if any, is very faint.

Broth: Turbid, elearing after a few days. A few strains flocculate.

Litmus milk: Acid, usually coagulated.

Nitrites not produced from nitrates.

The majority of strains form acid from glucose, fructose, mannose, galactose, arabinose, sucrose, maltose, lactose, raffinose and salicin, and to a lesser extent, from sorbitol, mannitol, dextrin, glycerol and xylose. Rhamnose, starch and inulin usually not fermented.

Lactic acid (usually inactive) with only small quantities of acetic acid and carbon dioxide is formerl in the fermenta- tion of hexose sugars. Acetic and lactic acid are produced from the pentoses. Forms up to 1.2 per cent acid in broth.

This species is the inactive lactic acidproducing rod from fermenting materials but is closely related to Lactobacillus casei. It ferments sucrose and maltose as readily as lactose.

Salt tolerance: Usually grows in salt up to 5.5 per cent.

Temperature relations: Optimum temperature $30^{\circ} \mathrm{C}$. Minimum $10^{\circ} \mathrm{C}$. Maximum $40^{\circ} \mathrm{C}$. Thermal death point $65^{\circ}$ to $75^{\circ} \mathrm{C}$ for 15 minutes.

Microaerophilic.

Sources from which isolated: Milk, cheese, butter, kefir, feces, fermenting potatoes, beets, corn, chard, bread dough, sauerkraut, (ncumber pickles, tomato pickles, cauliflower pickles and spoiled tomato products.

Habitat: Widely distributed in nature, particularly in fermenting plant and animal products.

10a. Lactobacillus plantarum var. rudensis Breed and Pederson (not Peterson). (Jour. Bact., 36, 1938, 667.) This chromogenic organism isolated from cheese is one of two species responsible for the development of rusty spots in cheese. It is impossible to determine whether the incompletely deseribed species Bacillus rudensis Connell, Canadian Dept of Agric., Dairying Service, Ottawa, Report for 1897,7 is identical with this variety of Lactobacillus plantarum or with Lactobacillus brevis var. rudensis (see species No. 12a). This chromogenesis is produced in starch media under anaerobic conditions.

11. Lactobacillus leichmannii Bergey et al. (Bacillus leichmanni I, Henneberg, Ztschr. f. Spiritusindustrie, 26, 1903, 22; see C'ent. f. Bakt., II Abt., 11, 1903, 163; Bergey et al., Manual, 2nd ed., 1925, 180.) Named for Prof. G. Leichmann, a German bacteriologist.

Probable synonym: Bacillus leichmanni III, Henneberg, loc. cit. 
Rods : 0.6 by 2.0 to 4.0 microns, oceurring singly and in short chains. The cells show two or more deeply-staining granules. Ton-motile. Gram-positive.

Gelatin stab: To liquefaction.

Agar colonies: Small, clear with white centers.

Agar slant: Limited, grayish streak, better growth in stab.

Broth: Turbid.

Nitrites not produced from nitrates.

Acid from glucose, fructose, maltose, sucrose, trehalose, and slight amounts from galactose, mannitol and $\alpha$-methylglucoside. Lactose, raffinose, arabinose, rhamnose, dextrin and inulin not fermented. Forms 1.3 per cent lactic acid in mash.

Optimum temperature $36^{\circ} \mathrm{C}$. Maximum $40^{\circ}$ to $46^{\circ} \mathrm{C}$.

Microaerophilic.

The species is apparently similar to Lactobacillus delbruectii but has a lower optimum temperature.

Source: From compressed yeast and from fermenting milk.

Habitat: Dairy and plant products.

12. Lactobacillus brevis (Orla-Jensen) Bergey et al. (Bacillus $\gamma, v$. Freudenreich, Landw. Jahrb. d. Schweiz, 1S91, 22; Bacillus casei $\gamma$, v. Freudenreich and Thöni, Landw. Jahrb. d. Schweiz, 1904, 526; also Orla-Jensen, Cent. f. Bakt., II Abt., 13, 1904, 604; Betabacterium. breve Orla-Jensen, The Lactic Acid Bacteria, 1919, 175; Bergey et al., Minual, 4th ed., 1934, 312.) From Latin brevis, short.

Probable synonyms: Bacillus brassicae fermentatac Henneberg, Ztsehr. f. Spiritusindustrie, 26, 1903; Cent. f. Bakt., II Abt., 11, 1903, 167 (Lactobacillus fermentatae Bergey et al., Manual, 1st ed., 1923, 252); Bacillus panis fermentati Henneberg, Ztschr. f. Spiritusindustrie, 25, 1903; Cent. f. Bakt., II Abt., 11, 1903, 168 (Lactobacillus panis Bergey et al., Manual, 1st ed., 1923, 251); Bacillus acidophil-aerogenes Torrey and Tahe. Jour. Inf. Dis., 17, 1915, 437 (Lactobacil- lus acidophil-acrogenes, Holland, Jour. Bact., 5, 1920, 216); Lactobacillus pentoaceticus Fred, Peterson and Davenport, Jour. Biol. Chem., 39, 1919, 357; Peterson and Fred, ibid., 42, 1920, 273; Lactobacillus pcntoaceticus var. magnus Iwasaki, Jour. Agr. Chem. Soe. Japan, 16, 1940, 148; Lactobacillus lycopersici Mickle, Abst. Bact., 8, 1924, 403; Mickle and Breed, New lork Agr. Exp. Sta. Tech. Bull. 110, 1925; Pederson, ibid., Tech. Bull. 150 and 151, 1929; Bacterium soya Saito, Cent. f. Bakt., II Abt., 17, 1907, 20 (Lactobacillus soyae Bergey et al., Manual, 1st ed., 1923, 251).

Bacillus caucasicus von Freudenreich, Cent. f. Bakt., II Abt., 3, 1897, 135 and Betabacterium cancasicum OrlaJensen, The Lactic Acid Bacteria, 1919. 173 were isulated from kefir grains and considered to be the organism Kern isolated in 1852. They are gas-producing lactobacilli but are less active toward sugars than Lactobacillus brevis.

Description supplemented by material from Pederson, Jour. of Bact., 35, 1938, 105.

Rods : 0.7 to 1.0 by 2.0 to 4.0 microns, with rounded ends, oceurring singly and in short chains, and occasionally in long filaments which may show granulation. Ton-motile. Gram-positive.

Gelatin: No liquefaction.

Agar slant: Growth, if any, faint.

Broth: Turbid, clearing after a few days.

Milk : Acid produced but no clot except with some freshly isolated strains.

Does not attack casein as a rule.

Is able to utilize calcium lactate as a source of carbon.

Acid from arabinose, xylose, glucose, fruetose, galictose and maltose. Strains vary in fermentation of lactose, sucrose, mannose and raffinose. Salicin, mannitol, glycerol, rhamnose, dextrin, inulin and starch seldom fermented. Usually shows a particularly vigorous fermentation of arabinose.

Lactic acid usually inactive; acetic acid, ethyl alcohol and carbon dioxidə 
formed in fermentation of aldohexoses. Mannitol produced from fructose. Acetic and lactic acid produced from the pentoses.

This species includes the large group of gas-producing lactic acid rods ordinarily characterized by a marked fermentation of pentoses, particularly arabinose. They usually also ferment fructose more readily than glucose.

Temperature relations: Optimum $30^{\circ} \mathrm{C}$. Growth poor below $15^{\circ}$ and above $37^{\circ} \mathrm{C}$. Maximum $38^{\circ} \mathrm{C}$.

Source: From milk, kefir, cheese, feces, fermenting sauerkraut, ensilage, manure, soils, sour dough, and spoiled tomato products.

Habitat: Widely distributed in nature, particularly in plant and animal products.

12a. Lactobacillus brevis var. rudensis Breed and Pederson. (Lactobacıllus rudensis Davis and Mattick, Proc. Soc Agr. Bact., 1936, 3 (this organism is presumably the same as Bacillus medensis Davis and Mattick, Jour. Dairy Res., 1, 1929, 50); Breed and Pederson (not Peterson), Jour. Bact., 36, 1938, 667.) This chromogenic variety isolated from cheese is a causative agent in the production of rusty spot in cheese. From a study of cultures, it is regarded as a chromogenic variety of Lactobacillus brevis. See also species No. 10a.

\section{Lactobacillus buchneri (Henne-}

berg) Bergey et al. (Bacillus buchneri Henneberg, Cent. f. Bakt., II Abt., 11, 1903, 163; Bergey et al., Manual, 1st ed., 1923, 251.) Named for Prof. E. Buchner, a German bacteriologist.

Probable synonyms: Bacillus wehmeri Henneberg, Cent. f. Bakt., II Abt., 11, 1903, 165 (Lactobacillus wehmeri Bergey et al., Manual, 1st ed., 1923, 249); $B a$ cillus hayducki Henneberg, Cent. f. Bakt., II Abt., 11, 1903, 163 (Lactobacillus hayduckii Bergey et al., Manual, 1st ed., 1923, 253); Bacterium mannitopoeum Müller-Thurgau, Cent. f. Bakt., II Abt., 20, 1908, 396; ibid., 36, 1912, 129; ibid.,
48, 1917 (Lactobacillus mannitopoeus Pederson, Tew Fork Agr. Exp. Sta. Tech. Bull. 150 and 151, 1929; Lactobacillus mannitopoeus var. fermentus Iwasaki, Jour. Agr. Chem. Soc. Japan, 16, 1940, 148).

Description supplemented by material from Pederson, Jour. Bact., 35, 1938, 107.

Rods : 0.35 by 0.7 to 4.0 microns, occurring singly, in pairs and chains or in filaments 25 microns or longer. Non-motile. Gram-positive.

Agar colonies: White to yellowish, adherent.

Agar slant: Growth, if any, faint.

Broth: Turbid, clearing after a few days.

Litmus milk: Usually unchanged but may be slightly acid with no reduction.

Nitrites not produced from nitrates.

Acid usually from arabinose, xylose, glucose, fructose, galactose, mannose, sucrose, lactose, maltose and raffinose. Mannitol, sorbitol, glycerol, rhamnose, salicin, inulin, dextrin and starch fermented by a few strains.

Lactic acid usually inactive. Acetic acid, ethyl alcohol and carbon dioxide formed in the fermentation of aldohexoses. Mannitol produced from fructose. Acetic and lactic acid from pentoses.

Strains of this species might be considered intermediates between Lactobacillus brevis and Lactobacillus fermenti.

Forms 1.3 per cent lactic acid in mash and 2.7 per cent alcohol.

Optimum temperature $32^{\circ}$ to $37^{\circ} \mathrm{C}$. Minimum $10^{\circ}$ to $15^{\circ} \mathrm{C}$. Maximum $44^{\circ}$ to $48^{\circ} \mathrm{C}$.

Source: From sour mash, pressed yeast, molasses, wine, catsup and sauerkraut.

Habitat: Widely distributed in fermenting substances.

14. Lactobacillus pastorianus (Van Laer) Bergey et al. (Saccharobacillus pastorianus Tan Laer, Cont. l'Histoire des Ferments des Hydrates de Carbone, Acad. Roy. de Belge, 1892 ; Bacillus pastorianus Macé, Traité Pratique de Bact., 
4th ed., 1901, 957; Lactobacterium pastorianum van Steenberge, Ann. Inst. Past., \$4, 1920, 816; Bergey et al., Manual, 1st ed., 1923, 246.) Named for Pasteur, French chemist; from Latin pastor, a herdsman.

Probable synonyms: Saccharobacillus pastorianus var. berolinensis Henneberg, Cent. f. Bakt., II Abt., 8, 1902, 186 (Lactobacillus berolinensis Bergey et al.: Manual, 1st ed., 1923, 246); Bacillus lindneri Henneberg, Wochnschr. f. Brauerei, 18, 1901, No. 30; Cent. f. Bakt., II Abt., 8, 1902, 184 (Lactobacillus lindneri Bergey et al., Manual, 1st ed., 1923, 245) ; Bacillus fasciformis Schönfeld and Rommel, Wochnschr. f. Brauerei, 19, 1902, No. 40 ; abst. in Cent. f. Bakt., II Abt., 9, 1902, 807 (Saccharobacillus berolinensis fasciformis Henneberg, Handb. der Gärungsbakteriologic, 2, 1926, 123; Bacillus belorinensis (sic, evidently intended for Bacillus berolinensis) Otani, Cent. f. Bakt., II Abt., 101, 1939, 149).

Description supplemented by material from Henneberg, Cent. f. Bakt., II Abt., 8, 1902, 184; Shimwell, Jour. Inst. Brewing, 41, 1935, 481; and Pederson, Jour. Bact., 35, 1938, 107.

Rods : 0.5 to 1.0 by 7.0 to 35.0 microns, occurring singly and in chains. Nonmotile. Gram-positive.

Gelatin colonies: No growth.

Beer wort gelatin stab: Beaded to arborescent growth.

Beer wort agar colonies: Small, gray, raised, filamentous.

Agar slant: Little or no growth; better in stab.

Broth: Good growth in yeast extract. Turbid.

Litmus milk: Acid.

Nitrites not produced from nitrates.

Acid from arabinose, glucose, fructose, galactose, maltose, sucrose, dextrin, raffinose, trehalose and mannitol and slightly from lactose and starch. No acid from xylose, rhamnose or inulin. Forms 1.5 per cent acid in mash. Also forms $\mathrm{CO}_{2}$ and alcohol, lactic, formic and acetic acid.
The species includes the ordinarily long rod types from spoiled beers. Apparently the same variations in regard to sugar fermentation may be found as are noted for similar species.

Optimum temperature $29^{\circ}$ to $33^{\circ} \mathrm{C}$. Minimum $11^{\circ} \mathrm{C}$. Maximum $37^{\circ} \mathrm{C}$. Microaerophilic.

Source: From sour beer and from distillery yeast.

Habitat: Probably more widely distributed than indicated by isolations.

15. Lactobacillus fermenti Beijerinck. (Beijerinck, Arch. néerl. d. sci. exactes et nat., Sér. 2, 7, 1901, 212; Smit, Ztschr. f. Gärungsphysiol., 5, 1916, 273; Lactobacterium fermentum van Steenberge, Ann. Inst. Past., 34, 1920, 816.) From Iatin fermentum, ferment, yeast.

Probable synonyms: Bacillus $\delta$, von Freudenreich, Cent. f. Bakt., II Abt., 1, 1895, 173; also Landw. Jahrb. d. Schweiz, 1895, 211; Bacillus casei $\delta$, von Freudenreich and Thöni, Landw. Jahrb. d. Schweiz, 1904, 526; also Orla-Jensen, Cent. f. Bakt., II Abt., 13, 1904, 609; Betabacterium longum Orla-Jensen, The Lactic Acid Bacteria, 1919, 174 (Lactobacillus longus Bergey et al., Manual, 4th ed., 1934, 312); Bacterium gayonii Müiler-Thurgau and Osterwalder, Cent. f. Bakt., II Abt., 48, 1917, 1 (Lactobacillus gayonii Pederson, New York Agr. Exp. Sta. Tech. Bull. 150 and 151, 1929); Bacterium intermedium Müller-Thurgau and Osterwalder, Cent. f. Bakt., II Abt., 48, 1917, 1 (Lactobacillus intermedium Bergey et al., Manual, 3rd ed., 1930, 295); Bacillus aderholdi Henneberg, Cent. f. Bakt., II Abt., 11, 1903, 166.

Description supplemented by material from Pederson, Jour. Bact., 35, 1938, 106.

Rods: Variable, usually short (Beijerinck), 0.5 to 1.0 by 3.0 to 15.0 microns (Smit), sometimes in pairs or chains. Non-motile. Gram-positive (Smit).

Yeast extract-glucose-gelatin: Filiform, no liquefaction (Pederson).

Agar colonies: Flat, circular, small, translucent like droplets of water. 
Agar slant: Growth, if any, scant.

Broth: Turbid, clearing after a few dars.

Milk: Unchanged or slightly acid.

Nitrites not produced from nitrates.

Reduction of litmus, methvlene blue, indigo carmine, sodium thiosulfate. $\mathrm{Na}_{2} \mathrm{SO}_{3}$ is reduced to $\mathrm{H}_{2} \mathrm{~S}$ (smit).

Acid usually from glucose, fructose, maltose, suerose and lactose (Beijerinck) and mannose, galactose, and raffinose; some strains ferment xylose; usually does not ferment arabinose, rhamnose, sorbitol, mannitcl, inulin, dextrin, starch or salicin (Pederson).

Lactic acid, usually inactive; acetic acid, ethyl alcohol and carbon dioxide are formed in the fermentation of aldehexoses (Smit), (Pederson). Mannitol is formed in the fermentation of fructose (Beijerinck), (Smit). Acetic acid and lactic acid are produced from pentoses if they are fermented (Pederson).

These are the higher temperature gasproducing rods. They usually do not ferment the pentoses but when they do, the fermentation is seldom as active as that produced by strains of Lactobacillus brevis.

Temperature relations: Optimum $41^{\circ}$ to $42^{\circ} \mathrm{C}$. Minimum $15^{\circ}$ to $18^{\circ} \mathrm{C}$. Maximum $48^{\circ}$ to $50^{\circ} \mathrm{C}$.

Microaerophilic.

Source: From yeast, milk products, fermenting dough, potatoes or vegetables, tomato products and wine.

Habitat: Widely distributed in nature, particularly in fermenting plant or animal products.

Appendix I:* The following species probably should be included in the genus Laclobacillus. Many are duplicates of the species described in full, but the majority are so poorly characterized that they cannot be properly identified.
Acidobacterium aerogenes Schlirf. (Stäbchen Kia, Heim, Cent. f. Bakt., I Abt., Orig., 93, 192t, 252; Schlirf, Cent. f. Bakt., I Abt., Orig., 97, 1925, 114; Plocamobacterium aerogenes Lehmann, in Lehmann and Neumann, Bakt. Diag., 7 Aufl., 2, 1927, 509.) Possibly Lactobacillus brevis Bergey et al. Produces acid and gas from glucose. From dental caries, mouth cavity and intestine.

Acidobacterium lactis Heim. (Heim, quoted from schlirf, Cent. f. Bakt., I Abt., Orig., 97, 1925, 113.) Schlirf says that this species is probably identical with Bacillus necrodentalis Goadby. Regarded by Lehmann (in Lehmann and Neumann, Bakt. Diag., 7 Aufl., 2, 1927, $507-508$ ) as identical with Bacillus acidophilus Moro; or it may be identical with Streptobacterium casei Orla-Jensen. From dental caries, deposit on tongue and in intestine.

Acidubacterium morvi schlirf. (Stäb)chen Ke, Heim, Cent. f. Bakt., I Abt., Orig., 93, 1924, 252; Schlirf, Cent. f Bakt., I Abt., Orig., 9\%, 1925, 114 Plucamobacterium moroi Lehmann, is Lehmann and Neumann, Bakt. Diag.: i Auti., 2, 1927, 508.) From the intestine. Similar to Acidobacterium lactis. Tuchinka (Cent. f. Bakt., I Abt., Orig., 144, 1939, 370) reports this organism as occurring in two cases of meningitis .

Bacillus bifidus aerobius Heurlin. (Bakt. Unters. d. Keimgehaltes im Genitalkanale d. fiebernden Wöchnerinnen. Helsinfors, 1910, 93.) From the genital canal. Resembles Bacillus bifidus communis Tissier.

Bacillus bifidus capitatus Heurlin. (Bakt. Unters. d. Keimgehaltes im Genitalkanale d. fiebernden Wüchnerinnen. Helsingfors, 1910, 175.) From the genital canal.

Bacillus carpathiens Kindraizuk. Oesterr. Molkerei Zcit., 29, 1912, 257.)

* Arranged by Prof. C. S. Pederson, New Fork State Experiment Station, Geneva, New York, March, 1945. 
From the sour milk of the Carpathian region. Presumably Lactobacillus bulgaricus.

Bacillus circularis minor Heurlin. (Bakt. Unters. d. Kieimgehaltes im Genitalkanale d. fieberuden Wöchnerinnen. Helsingfors, 1910, 170.) From the genital canal. Anacrobic.

Bacillus necrodentalis

Goadby .

(Goadby, Microorganisms in dental caries, Dental Cosmos, 42, 1900, 213.) From dental caries.

Bacillus orenburgii Horowitz-Wlassowa. (Cent. f. Bakt., II Abt., 64, 1925, 338.) From kumys (Caucasus). Presumably Lactobacillus bulgaricus.

Bacillus orientale Batchinsky.

(Batchinsky, Areh. d. Gesellsch. d. Naturf. St. Petersburg, 42, 1911 ; quoted from Horowitz-Wlassowa, Cent. f. Bakt., II Abt., 64, 1925, 330.) From kumys in Ufa (U.S.S.R.). Presumably Lactobacillus bulgaricus.

Bacillus sardous (irixoni. (Annali di Med. Navale, 112, 1905, 223 and Cent. f. Bakt., II Abt., 15, 1905, 951; Bacterium sardum miciurati Bianeo, Il Cesilpino, 8, 1912, 33.) From gioddu (Sirrdinia). Presumably Lactobacillus bulgaricus.

Bacillus vaginae Kruse. (Scheidenbacillen, Doederlein, Das Scheidensekrct und seine Bedeutung fiur das Puerperalfieber, Leipzig, 1892, 32; Kruse, in Flügge, Die Mikrcorganismen, 3 Aufl., 2, 1896. 35s; Bacillus vaginalis longus Heurlin, Bakt. Cnters. d. Keingehaltes im Cienitalkinale d. fiebernden Wöchnerimen. Helsingsfors, 1910, 170; Bacillus raginalis Jotten, Arch. f. Hyo.. 91, 1922, 149; Stäbchen Kie, Hein, C'ent. f. Bakt., I Abt., Orig., 93, 1924, 252; Acidobacterium docderleinii Heim, quoted from sichlirf, Cent.f. Bakt., I Alot., Orig., 97, 1925, 104; Plocamobacterium raginae Lehmann, in Lehmann and Neumam, Bakt. Diag., 7 Aufl., 2, 1927, 510; Lactobacillus doederlein Gillespie and Rettger, Jour. Bact., 36, 1938, 623.) Fruse (Allgemeine Mikrobiol., 1910, 287) considers this species n "langen Milchsäurebacillus" all of which he would group under the name
Bacillus lacticus (not Bacillus lacticus Kruse, in Flügge, Die Mikroorganismen, 3.Aufl., 2, 1896, 356 as this is Streptococcus lactis Löhnis). Jotten (loc. cit.) and Thomas (Jour. Inf. Dis., 43, 1928, 218) consider this species identical with Luctobacillus acidophilus (Moro) Holland. From the secretion of the normal vagina. See Bacillus crassus Lipschütz.

Bacterium gracile Müller-T'hurgau. (Cent.f. Bakt., II Abt., 20, 1908, 396; Müller-Thurgau and Osterwalder, ibid., $36,1912,157$; ibid., 48, 1917, 1; Lactobacillus gracile Bergey et al., Manual, 3rd ed., 1930, 297.) This organism which was isolated from wine is probably not a lactobacillus. It may belong to the genus Leuconostoc (subculture examined in 1936, C. S. Pederson).

Bacterium granulosum Lehmann and Jeumann. (Kömchenbacillus, Luerssen and Kühn, Cent. f. Bakt., II Abt., 20, 1907, 241; Lehmann and Neumann, Bakt. Diag., 5 Aufl., 2, 1912, 306.) From yoghurt (Bulgaria). Presumably Lactobacillus bulgaricus.

Bacterium lactis commune Hohennadel. (Arch. f. Hyg., 85, 1916, 237.) From feces. Similar to Lactobacillus acidophilus (Noro) Holland.

Bacterium mazun Weigmann, Gruber and Huss. (Milchsiurebakterium aus Mazun, Düggeli, Cent. f. Bakt., II Abt., 15, 1905, 595; Weigmanm et al., Cent. f. Bakt., II Abt., 19, 1907, 78.) From mazun (Armenia). Presumably Lactobacillus bulgaricus.

Bacterium vermiforme Ward. (Phil. Tran. Roy. Soe. London, 183, 1892, 149; Bacillus ecrmiformis Migula, Syst. d. Bakt., 2, 1900, 652; Btabacterium rermiforme Nayer, Inaug. Diss., Univ. Itrecht, 1938.) Originally isolated from the ginger beer plant fermentation. This is presumably a slime-forming lactobarillus.

Bactoroides acrofaciens Eggerth and Bacteroides biformis Eggerth. (Jour. Bact., 30, 1935. 252-283.) From feces. Possibly lactobacilli but their relationships are not definitely known. 
Lactobacillus betadelbrucckii Kitahara. (Bull. Agr. Chem. Soc. Japan, Tokyo, 16. 1940, 123.) From cereal mash.

Lactobacillus caneus hitahira (loc. cit.). From cereal mash.

Lactobacillus ciliatus Kitahara (loc. (cit.). From cereal mash.

Lactobacillus enzymothermophilus Buck. (Amer. Jour. Pub. Health, 32, 1942, 1230.) A thermophilic growth at $52^{\circ}$ and $62^{\circ} \mathrm{C}$ ) presumably spore-forming bacillus isolated from pasteurized milk.

Lactobacillus fructororans Charlton. Xelson and Werkmin. (Iowa State Coll. Jour. Sci., 9, 1934, 1.) From salad dressing. Similat to Lactobacillus brevis.

Lactobacillus hilgardii Douglus and Cruess. (Food Research, 1, 1936, 113.) This organism was isolated from wine but is not completely described and so cannot be compared with previously described species.

Lactobacillus hyochi Otani, Lactobacillus hyochi var. 1, Otani, Lactobacillus hyochi var. 2, Otani, Lactobacillus filamentosus Otani, Lactobacillus alcoholphilus Otani, and Lactobacillus saprogenes Otani. Jour. Faculty of Agric., Hokkaido Imp. Univ., 39, 1936, 2.) These organisms were isolated from sake. With the possible exception of the last type, they are probably identical with Lactobacillus plantarum or elosely related species.

Lactobacillus odontolyticus Rodriguez. (Bacillus acidophilus odontolyticus I and $I I$, McIntosh, James and LazarusBarlow, Brit. Jour. Exp. Med. and Path., $3,1922,141$; Bacillus acidophitus odontolyticus, ibid., 145; Lactobacillus odontolyticus and Lactobacillus odontolyticus Types I, II and III, Rodriguez, Military Dent. Jour., 5, 1922, 206; Bacillus odontolyticus MeIntosh, James and LazarusBarlow, Brit. Jour. Exp. Med. and Path., 5, 1924, 178; Bacillus acidophilus-odontolyticus Rosebury, Linton and Buchbinder, Jour. Bact., 18, 1929, 395 ; Lactobacillus odontolyticus $I$ and $I I$, Topley and Wilson, Prine. Bact. and Immun., 2nd ed., 1936, 588.) From dental caries.
Type 1 resembles and is possibly identiral with Bacillus acidophilus Moro and Doederlein's bacillus (Bacillus vaginae Kruse). See Rosebury, Bict. Rev., 8, 1944, 189 and Arch. of Path., 38, 1944, 413. Type II shows considerable pleomorphism, short coccal forms appearing in the more alkaline media McIntosh, James and Lazarus-Barlow, Brit. Jour. Exp. Med. and Path., 5. 1924, 183). Types I, II and III of Rodriguez (loc. cit.) do not correspond with Types I and II of McIntosh et al. or with the groupings of Howe and Hatch Jour. Med. Res., 36, 1917, 481).

Lactobacillus panis acidi Nikolaev. (Wiss. Forschungsinst. Bäkerei-indust., [. S. s. R., 5, 1933, 3-11.) Four isolations from bread dough designated by the Cireek letters, $\alpha, \beta, \gamma_{1}$ and $\gamma_{2}$.

Lactobacillus sake Katagiri, Kitahara, Fukami and Sugase. (Bull. Agric. (hem. Soc. Japan, 10, 1934, 153.) From mash used in the manufitcture of sake. Similar to Lactobacillus plantarum.

Lactobacillus xylosus Kitahara. (Bull. Agr. Chem. Soc. Japan, Tokyo, 16, 1940, 123.) From cereal mash.

Lactobacterium cercrisiae van Steenberge. (Ann. Inst. Past., 34, 1920, s06.) From beer.

Lactobacterium conglomeratum van Steenberge (loc. cit., S12). From beerwort,

Lactobacterium filatim van Steenberge (loc. cit., \$12). From beer-wort.

Lactobacterium floccogenum van Steenberge (loc. cit., \$12). From beer-wort.

Lactobacterium grave van Steenberge (loc. cit., S14). From beer-wort.

Lactobactorium multivolaticum ran Steenberge (loc. cit., S14). From beerwort.

Lactobacterium multivolatigenum van Steenberge (loc. cit., S12). From beerwort.

Lactobactcrium oligoacidificans van Steenberge, (loc. cit., S14). From beerwort. 
Lactobacterium parcifermentans van Steenberge (loc. cit., 812). From beerwort.

Lactobacterium terricola van Steenberge (loc. cit., 806). From garden soil.

Lactobacterium viscogenum van Steenberge (loc. cit., 814). From beer-wort Streptohacillus lebenis Rist and Khoury. (Rist and Khoury, Ann. Inst. Past., 16, 1902, 70; Bacillus lebeni Kuntze, C'ent. f. Bakt., II Abt., 21, 1908, 744; Streptobacillus lebensis $\alpha$ and $\beta$ Löhnis, Cent.f. Bakt., II Abt., 22, 1909, 553 ; Streptobacillus lebenis viscosus and Streptobacillus lebenis nonviscosus Severin, Cent. f. Bakt., II Abt., 24,1909 . 488; Bacterium lebenis Lehmann and Neumann, Bakt. Diag., 5 Aufl., 2, 1912, 308.) From leben (Egypt and Vear East). Presumably Lactobacillus bulgaricus.

Streptothix dadhi Chatterjee. (Cent. f. Bakt., I Abt., Orig., 53, 1910, 111. From sour milk (dadhi) of India. Presumably Lactobacillus bulgaricus.

Thermobacterium jugurl Orli1-Jensen. (Yoghurt bakterium, Kuntze, ('ent. f. Bakt., II Abt., 21, 1908, 737; Orla-Jensen. The Lactic Acid Bacteria, 1919, 164; Lactobacillus jugurt Holland, Jour. Bact. 5, 1920, 225.) From jughurt (Bulgaria) Presumably Lactobacillus bulyaricus .

Thermobacterium mathiacolle ('ecilia. (Le Lait, 20, 1910, 385-390.) From sweetened condensed milk. Possibly a spore-former.

Appendix II:* The genus Leptotrichia Trevisan, 1879 is no lunger rerognized as a valid genus. While the eonfusion with Leptothrix Kützing, 1843 was correcterl by Trevisan's work, the ilentity of the type species, Leptotrichia buccalis, is uncertain. Few of the species that have been placed in Leptothrix and Leptotrichia are well enough described to be recognized with certainty.
All descriptions of Leptotrichia buccalis published earlier than 1856 are based on microscopic observations only. This is also true of the three species of Lcptothrix recognized by Miller (Die Mikroorganismen der Mundhöhle, Leipzig, $1889,69-80)$. The species that he distinguished in this way are recognized in the seven editions of Lehmann and Neumann's Bakteriologische Diagnostik published from $1 \mathrm{~S} 96$ to 1927 . Chester (Manual Determ. Bact., 1901, 371) also follows Miller's ideas in regard to the nature of the species of Leptothrix. These authors felt that the identity of the true Leptotrichia bucculis was doubtful.

On the other hand, Vignal (Arch. de Physiol. norm. et path., 8, 1886, 337) isolated what he thought to be this organism, and it is his description that is used with minor changes by Eisenberg (Bakt. Diag., 3 Aufl., 1891, 134), Migula (Nyst. d. Balkit., 2, 1900, 445 ) and in all mlitions of Bergey's Manual (19231939) up to the present edition. A study of V'ignal's work shows, however, that the fil:mmentous organism that he isolated and grew readily in broth, agar and gelatin cultures was in all probability one of the common spore-formers. It grew but rarely on the plates inoculated with material from the mouth. As is clearly shown in his drawing and descriptions, it liquefied gelatin rather quickly with the formation of the characteristic wrinkled pellicle of a spore-former. Soon after, Arustamow (Wratsch, 1889, Nos. 3 and 4 ; abstract in Cent. f. Bakt., $6,1889,349$ ) isolated a similar aerobic, filamentous organism that grew readily at $37^{\circ} \mathrm{C}$ on agar and gelatin, but he also noted large numbers of very tiny colonies of a microaerophilic bacterium which may have been the lactobacilli or lactobacilli-like organisms of later authors. Even recent excelleut reviews of the

* Completely rearranged by Prof. Robert \&. Breed and Prof. Carl S. Pederson, New York State Experiment Station, Geneva, New York, March, 1945. 
early literature (Buchanan, Systematic Bact., 1925, 345-353; Thjøtta, Hartmann and Bøe, Norske Videnkaps Akad. i Oslo, I, Math.-Nat. Klasse, No. 5, $1939,41 \mathrm{pp}$.) or reviews of the voluminous studies of the past thirty years (Rosebury, Bact. Rev., 8, 1944, 198) fail to clear away all of the confusion that has arisen.

Some investigators, such as Heim (Sitzber. d. physik.-med. Soz. in Erlangen for 1922-23, 54, 1925, 121) and his co-workers, grew the mouth streptococci readily and thought them important as the cause of dental caries. Others following the lead of Kiligler (Jour. Allied Dental Soc., 10, 1915, 282 and 445) and Wherry and Oliver (Jour. Inf. Dis., 19, 1916, 299) have found the most important organisms of caries to be the long, Grampositive, granular, non-motile rods that grow like lactobacilli. But even here it is not altogether clear whether the high acid-produeing (Bacillus acidophilus odontolyticus MreIntosh, James and Lazarus-Barlow, British Jour. Exper. Path., 3, 1922, 145) or the low acid-producing type (Leptothrichia buccalis Thjøtta et al., loc. cit., 31) of rods really represents the Leptothrix buccalis of early observers. Some observers, e. g., Balleid (Brit. Dent. Jour., 48, 1925, 289), have even identified a branching organism of the mouth, Actinomyces israeli, as Leptotrichia buccalis according to Rosebury (loc. cit., 200).

As these mouth organisms are apparently better placed in other genera so far as they have been definitely identified, the genus and the species that have been described as belonging in it are merely listed here.

\section{Genus A. Leptotrichia Trevisan.}

(Trevisan, Reale Istituto Lombardo di Scienze e Lettere, Ser. II, 4, 1879, 147 ; not Leptothrix Kützing, Phycologica Generalis, 1843, 198; Bacteriopsis Trevisan, Atti della Accad. Fisio-MedicoStatistica in Milano, Ser. IV, 3, 1885,
103; Rasmussenia De Toni and Trevisan, in Saccardo, Sylloge Fungorum, 8, 1889, 930 ; Syncrotis Enderlein, Sitzber. Gesell. Naturf. Freunde, Berlin, 1917, 312.)

The type species is Leptotrichia buccalis (Robin) Trevisan.

Leptotrichia buccalis (Robin) Trevisan. (Leptothrix buccalis Robin, Histoire naturelle des végétaux parasites, Paris, 1853, 354; Trevisan, Reale Istituto Lombardo di Scienze e Lettere, Ser. II, 4, 1879, 147; Leptothrix III, Rasmussen, Om Dryckning of Microorganismer fra Spyt of sunde Mannesker, 1883; Rasmussenia buccalis De Toni and Trevisan, in Saccardo, Sylloge Fungorum, 8, 1889, 930; Bacterium buccale Migula, Syst. d. Bakt., 2, 1900, 445; not Leptothrix buccalis Chester, Manual Determ. Bakt., 1901, 371; not Bacillus buccalis Chester, ibid., 234; not Bacillus buccalis Trevisan, I generi e le specie delle Batteriacee, 1889, 15; Syncrotis buccalis Enderlein, Sitzungsber. Ges. Naturf. Freunde, Berlin, 1917, 312; Bacillus buccalis Holland, Jour. Bact., 5, 1920, 217.) From the mouth.

Other species that have been associated with this genus are as follows:

Bacillus maximus buccalis Miller. (Deutsche med. Wehnschr., 14, 1888, 612; Bacillus buccalis Trevisan, I generi e le specie delle Batteriacee, 1889, 15.) From the mouth. Regarded by Goadby (The Mycology of the Mouth, London, 1903) and by Kligler (Jour. Allied Dental Soc., $10,1915,152$ ) as a spore-former.

Leptothrix asteroide Mendel. (Compt. rend. Soc. Biol., Paris, 81, 1918, 471-475.) From the mouth. Rosebury (Bact. Rev., $8,1944,202)$ thinks this Gram-negative, anaerobic organism belongs in Bacteroides Castellani and Chalmers.

Leptothrix anaerobius tenuis Lewkowicz. (Arch. méd. exp. anat. path., 13, 1901, 633; Leptothrix tenuis Weinberg et al., Les Microbes Anaérobies, 1937, 814; Pseudoleptothrix tenuis Prévot, Ann. Inst. Past., 60, 1938, 301.) From mouths of infants. 
Leptothrix falciformis Beust. (Dental Cosmos, 50, 1908, 594; Jour. Dent. Res., 16, 1937, 379.) From the mouth.

Leptothrix filiformis Castellani and Chalmers. (Bacillus (Leptothrix?) pyogenes filiformis Flexner, Jour. Exp. Med., 1, 1896, 211; Castellani and Chalmers, Man. Trop. Med., 3rd ed., 1919, 1068.) From the genital tract and thoracic cavities of a rabbit with an acute pleuritis, pericarditis, pneumonia and acute endometritis. Gram-negative. Not regarded as identical with Bacillus piliformis Tyzzer (Jour. Med. Res., 37, 1917,307 ) which is a spore-former.

Leptothrix gagantea Miller. (Miller, Ber. d. deutsch. bot. Gesell., 1, 1883, 221; Leptotrichia gigantea Trevisan, I generi e le specie delle Batteriacee, 1889, 10; Rasmussenia gigantea De Toni and Trevisan, in Saccardo, Sylloge Fungorum, 8, 1889, 930.) From pyorrhoea in dogs, swine and sheep. This name was applied to a mixture of species.

Leptothrix haemoglobinophila sporulens Mackenzie. (In System of Bact., Med. Res. Council, London, 8, 1931,99.) From cerebro-spinal fluid. A Gram-negative spore-former.

Leptothrix innominata Miller. (Die Mikroorganismen der Mundhöhle, 1889, 51, Leipzig; Pseudoleptothrix innominatu Prévot, Ann. Inst. Past., 60, 1938, 301.) Prévot (loc. cit.) regards this species as type for his new genus Pseudoleptothrix. Proposed to include all filamentous forms from the mouth that resemble Leptothrix buccalis Robin.

Leptothrix insectorum Robin. (Histoire naturelle der végétaux parasites, Paris, 1853, 354.) From the rectums of insects.

Leptothrix maxima buccalis Miller. (Miller, Deutsche med. Wchnschr., 14, 1888, 612; Leptotrichia maxima Trevisan, I generi e le specie delle Batteriacee, 1889 , 10; Rasmussenia maxima. De Toni and Trevisan, in Saccardo, Sylloge Fungorum, 8, 1889, 930; Leptothrix buccalis Chester, Man. Determ. Bact., 1901, 371; Bacillus maximus Goadby, Mycology of the Mouth, 1903, 191.) From the mouth. Leptothrix parasitica Kützing. (Kützing, Bot. Zeitg., 1847, 220; quoted from Winter, in Die Pilze, Rabenhorst's Kryptogamen Flora, 2 Aufl., 1, 1880, 57 ; Bacterium parasiticum Billet, Bull. Sci. de la France et de la Belgique, Paris, 21, 1890, 199.) From a brownish deposit on algae.

Leptothrix preputialis Vicentini. (Atti Accad. Med. Chir. di Napoli, 48, 1890-91, quoted from Vicentini, Bacteria of the Sputa. Trans. by Stuter and Saicghi, London, 1897, 89.) From the urethra.

Leptothrix pyogenes cuniculi Muscatello. (Muscatello, 1899, quoted from Nannizzi, in Pollacci, Tratt. Micopat. Umana, 4, 1934, 57; Leptotrichia cunieuli (sic) Nannizzi, ibid., 57.) From spontaneous suppuration in a rabbit.

Leptothrix racemosa Vicentini. (Vicentini, Atti d. r. Accad. Med.-Chir. di Napoli, 46, 1892, 459; Leptotrichia racemosa Nannizzi, in Pollacci, Tratt. Micopat. Umana, 4, 1934, 56.) From the mouth. Conidia-like bodies are described. See Vicentini, Bact. of the Sputa, Eng. Trans. by Stuter and Saieghi, London, 1897 and Williams, Dental Cosmos, 41, 1899, 330.

Leptothrix racemosa vincenti (sic) Mackenzie. (Leptothrix, Matthews, Pract., 74, 1905, 197; Mackenzie, in System of Bact., Med. Res. Council, London, 8, 1931, 94.) From localized empyema. Appears to be the same as Vicentini's organism.

Leptothrix vaginalis Donné. (Donné, quoted from Nannizzi, in Pollacci, Tratt. Micopat. Umana, 4, 1934, 56; Leptotrichia vaginalis Nannizzi, ibid., 56.) Saprophyte from the vagina.

Leptothrix vaginalis von Herff. (Ueber Scheidenmykosen, Samml. klin. Vortr.n. F., 1895, No. 137.) From a case of vaginal mycosis.

Leptothrix variabilis Rasmussen. (Rasmussen, Om Dryckning of Microorganismer fra Spyt of sunde Mannesker, 
1883; Leptothrix II, Zopf, Die Spaltpilze, 3 Aufl., 1885, 107; Leptotrichia variabilis Trevisan, I generi e le specie delle Batteriacae, 1889, 10; Rasmussenia variabilis De Toni and Trevisan, in Saccardo, Sylloge Fungorum, 8, 1889, 931.) From saliva.

Rasmussenia anceps De Toni and Trevisan. (Leptothrix I, Rasmussen, Om Dryckning of Microorganismer fra Spyt of sunde Mannesker, 1853; Bacteriopsis rasmusseni $\mathbf{T}$ evisan, Atti della Accad. Fisio-Med.-Stat. in Milano, Ser. IV, 3, 1885, 103; Bacillus rasmusseni Trevisan, I generi e le specie delle Batteriacee, 1889, 15; De Toni and Trevisan, in Saccardo, Sylloge Fungorum, 8, 1889, 930; Leptotrichia anceps Nannizzi, in Pollacci, Tratt. Micopat. Umana, 'f, 1934, 54.) From saliva.

Appendix III : * Many species of anaerobic, Gram-positive, non-spore-forming, largely parasitic rods have been described. These are similar in many ways to the species included in Lactobacillus. Prévot has arranged these in the following genera. Several are inadequately studied and scarcely deserve recognition. Some, as indicated, may belong in other genera, e.g., spore-formers belonging in genus Clostridium. Some species produce gas in sugar broths or have other characteristies (e.g., motility) that are unusual for the families that include Gram-positive, non-spore-forming rods.

\section{Genus I. Eubacterium Prérot}

(Ann. Inst. Past., 60, 1938, 294.)

Non-motile, straight or curved rods. Usually occurring singly, in pairs or very short chains. Never show branching. Not capsulated. Gram-positive. Anaerobic.

\section{Eubacterium foedans} Prévot. (Bacillus foedans
Lancet, 1, 1908, 1832; Prévot, Ann. Inst. Past., 60, 1938, 294.) From salted ham.

2. Eubacterium niosii (Hauduroy et al.) Prévot. (Anaerobe Bacillus, Niosi, Cent. f. Bakt., I Abt., Orig., 58, 1911, 193; Bacteroides niosii Hauduroy et al., Dict. d. Bact. Path., 1937, 65; Prévot, Ann. Inst. Past., 60, 1938, 294.) From suppurative pleuritis.

3. Eubacterium rectale Prévot. (Un bacille anaérobie, Grooten, Compt. rend. Soc. Biol., Paris, 102, 1929, 43; Bacteroides rectalis Hauduroy et al., Dict. d. Bact. Path., 1927, 72; Prévot, Ann. Inst. Past., 60, 1938, 294.) From rectal ulcer.

4. Eubacterium obsti Prévot. (Bacillus B, Obst, Jour. Inf. Dis., 24, 1919, 159 and 168; Prévot, Ann. Inst. Past., $60,1938,294$.) From stomach of sardines and from their food (small crustaceans).

5. Eubacterium quartum Prévot. (Anaerob No. IV, Rodella, Ztschr. f. Hyg., 41, 1902, 474; Prévot. Ann. Inst. Past.,60, 1938, 294.) From intestine of a child.

6. Eubacterium quintum Prévot. Anaerob Io. I, Rodella, Ztschr. f. Hyg., 41, 1902, 475; Prévot, Man. de Class. et Determ., Monographie Inst. Past.. 1940, 65.) From intestine of a child.

7. Eubacterium ethylicum Prévot. (Bacillus gracilis ethylicus tchalme and Rosenthal, Compt. rend. Soc. Biol., Paris, 57, 1906, 1025; Prévot, Ann. Inst. Past., 60, 1938, 295.) From a human stomach.

8. Eubacterium cadaveris Prévot (see Bacillus cadaveris butyricus Buday). No spores observed.

9. Eubacterium tortuosum (Debono) Prévot. (Bacillus tortuosus Debono, Cent. f. Bakt., I Abt., Orig., 62, 1912, 233 ; Bacteroides tortuosus Bergey et al., Manual, 1st ed., 1923, 529; Prévot, Ann.

* Arranged by Prof. Robert S. Breed, New York State Experiment Station, Geneva, New York, March, 1945. 
Inst. Past., 60, 1938, 295.) From human feces.

10. Eubacterium aerofaciens (Eggerth) Prévot. (Bacteroides aerofaciens Eg. gerth, Jour. Bact., 30, 1935, 282; Prévot, Ann. Inst. Past., 60, 1938, 295.) From human feces.

11. Eubacterium biforme (Eggerth) Prévot. (Bacteroides biformis Eggerth, Jour. Bact., 30, 1935, 283; Prévot, Ann. Inst. Past., 60, 1938, 295.) From human feces.

12. Eubacterium limosum (Eggerth) Prévot. (Bacteroides limosus Eggerth, Jour. Bact., 30, 1935, 290 ; Prévot, Ann. Inst. Past., 60, 1938, 295.) From human feces. Pederson (Jour. Bact., 50, 1945, 478 ) secured a culture of this species from Eggerth, and found that it fermented glucose with the production of higher fatty (presumably butyric) acids and lactic acid. The species should probably be placed in Butyribacterium Barker

13. Eubacterium disciformans (Mas sini) Prévot. (Bacillus disciformans Massini, Ztschr. f. gesammte Exp. Med., 2, 1913, 81; Prévot, Ann. Inst. Past., 60, 1938, 295.) From respiratory system and skin.

14. Eubacterium poeciboides (Roger and Garnier) Prévot. (Bacillus poeciloides Roger and Garnier, Bull. et Mem. Soc. Méd: des Hôpitaux Paris, 2, 1906, 870; Prévot, Ann. Inst. Past., 60, 1938, 295.) From intestine.

15. Eubacterium typhi exanthematici Prévot (see Corynebacterium typhi Topley and Wilson).

17. Eubacterium minutum (Tissier) Prévot. (Bacillus anaerobicus minutus Tissier, Recherches sur la flore intestinale des nourissons, Paris, 1900 ; Bacteroides minutus Hauduroy et al., Dict. d. Bact. Path., 1937, 64; Prévot, Ann. Inst. Past.. $60,1938,295$.$) From intestine of breast -$ fed infant.

18. Eubacterium parvum (Choukévitch) Prévot. (Coccobacillus anaerobicus parvus Choukévitch, Ann. Inst. Past., 25, 1911, 256; Prévot, Ann. Inst. Past., 60, 1938, 295.) From large intestine of a horse.
19. Eubacterium lentum (Eggerth) Prévot. (Bacteroides lentus Eggerth, Jour. Bact., 30, 1935, 280; Prévot, Ann. Inst. Past., 60, 1938, 295.) From human feces.

\section{Genus II. Catenabacterium Prévot.}

(Ann. Inst. Past., 60, 1938, 294.)

Non-motile, straight or curved rods. Esually grow in long chains or filaments. No branching. Not capsulated. Grampositive. Anaerobic.

1. C'atenabactrium helminthoides (Lewkowicz) Prévot. (Bacillus helminthoides Lewkowicz, Arch. de Méd. Exp., 19, 1901, 631; Prévot, Ann. Inst. Past., $60,1938,295$.) From mouth of breastfed infant

2. Catenabacterium filamentosum Prévot. (Jungano, Compt. rend. Soc. Biol., Paris, 60, 1909, 112 and 122; Prévot, Ann. Inst. Past., 60, 1938, 295.) From intestine of a rat.

3. Catcnabacterium lottii Prévot. (Lotti, Ann. Ig. Sper., 19, 1909, 75; Prévot, Ann. Inst. Past., 60, 1938, 296.) From human appendix and intestine.

4. Catcnabacterium catenaforme (Eggerth) Prévot. (Bacteroides catenaformis Eggerth, Jour. Bact., 30, 1935 , 286; Prévot, Ann. Inst. Past., 60, 1938, 296.) From human feces.

5. Catenabaclerium nigrum (Repaci) Prévot. (Streptobacillus gangrenae pulmonaris Repaci, Compt. rend. Soc. Biol., Paris, 61, 1910, 410; Prévot, Ann. Inst. Past., 60, 1938, 296.) From gangrenous tissue found in a lung.

\section{Genus III. Ramibacterium Prévot.}

(Ann. Inst. Past., 60, 1938, 294.)

Non-motile, straight or curved rods with frequent branching. Not capsulated. Gram-positive. Anaerobic.

1. Ramibacterium ramosum (Veillon and Zuber) Prévot. (Bacillus ramosus Veillon and Zuber, Arch. méd, exp. et anat. path., 10, 1898, 542; Nocardia ramosa Vuillemin, Encyclopédie Mycolog., Paris, 2, Champignons Parasites, 1931, 132; Actinomyces ramosus Nan- 
nizzi, in Pollacci, Tratt. Micopat. Umana, 4, 1934, 42; Fusiformis ramosus Topley and Wilson, Princ. Bact. and Immun., 2nd ed., 1936, 358; Hauduroy et al., Dict. d. Bact. Path., 1937, 71 regard Bacillus poeciloides (E'ubacterium poeciloides) as a synonym; Prévot, Ann. Inst. Past., $60,1938,296$.) Commonly found in appendicitis.

2. Ramibacterium ramosoides (Rüneberg) Prévot. (Bacillus ramosoides Rüneberg, Arb. a. d. path. Inst. d. Univ. Helsingfors, 2, 1908, 271, see Cent. f. Bakt., I Abt., Ref., 43, 1909, 665; Prévot, Ann. Inst. Past., 60, 1938, 296.) From peritoneal fluid in appendicitis.

3. Ramibacterium pseudoramosum (Distaso) Prévot. (Bacillus pseudoramosus Distaso, Cent. f. Bakt., I Abt, Orig., 62, 1912, 441; Bacteroides pseudoramosus Bergey et al., Manual, 1st ed., 1923, 259; Prévot, Ann. Inst. Past., 60, 1938, 296.) From human feces.

\section{Genus IV. Cillobacterium Prérot.}

(Ann. Inst. Past., 60, 1938, 294.)

Motile, straight or curved rods. Peritrichous. Not capsulated. Ciram-positive. Anaerobic.

1. Cillobacterium moniliforme (Repaci) Prévot. (Bacillus moniliformis Repaci, Compt. rend. Soc. Biol., Paris, 61, 1910, 216; Prévot, Ann. Inst. Past.. $60,1938,296$.$) From the respiratory$ system.

2. Cillobacterium endocarditis (Routier and Braumberger) Prévot. (Bacille BG, Routier and Braunberger, Compt. rend. Soc. Biol., Paris, 115, 1934, 611; Prévot, Ann. Inst. Past.. 60, 1938, 296.) From febrile endocarditis.

3. Cillobacterium meningitis Prévot. (Stamm S. V., Ghon, Mucha and Müller, Cent. f. Bakt., I Abt., Orig., 41, 1906, 145 and 693; Prévot, Ann. Inst. Past., $60,1938,297$.) From meningitis following chronic otitis.

4. Cillobacterium spatuliforme Prévot (see Bacillus tenuis spatuliformis Distaso). Said to belong to Bacillus welchii group but no spores observed.
5. Cillobacterium mulliforme Prévot (see-Bacillus multiformis Distaso). Said to belong to Bacillus welchii group but no spores observed.

\section{Genus V. Bifidobacterium Orla-Jensen.}

Orla-Jensen, Le Lait, 4, 1924, 469; Bifidibacterium (sic) Prévot, Ann. Inst. Past., 60, 1938, 303.)

Non-motile rods which may be swollen. The ends may be bifurcate or double bifurcate. Gram-positive. Anaerobic. This genus is regarded as one of four genera of lactic acid, rod-shaped bacteria by Orla-Jensen, and he states that the organisms in the genus form dextro rotatory lactic acid. It is placed in the Order Actinomycetales by Prévot.

1. Bifidibacterium bifidum (Tissier) Prévot. (Prévot, Ann. Inst. Past., 60, 1938, 303.) See Lactobacillus bifidus (Tissier) Holland.

2. Bifidibacterium appendicitis Prévot. (Bacillus $\alpha$ Lotti, Ann. Ig. Sper., 19, 1909, 75; Prévot, Ann. Inst. Past., 60, 1938 , 303.) From an infected appendix.

3. Bifidibucterium constellatum (White) Prévot. (Bacillus constellatus White, Jour. Path. and Bact., 24, 1921, 69; Prévot, Ann. Inst. Past., 60, 1938, 303.) From the intestine of bees.

4. Bifidihacterium intestinalis Prévot. (Bacillus intestinalis tuberculiformis Jacobsen. Also uses Bacillus tuberculiformis and Bacillus tuberculiformis inlestimalis, Ann. Inst. Past., 22, 1908, 315; Prévot, Ann. Inst. Past., 60, 1938. 303.) From feces of an infant.

5. Bifidibacterium cornutum (Distaso) Prévot. (Bacillus cornutus Distaso, Cent. f. Bikt., I Abt., Orig., 62, 1912, 443; Bucteroides cormutus Castellani and Chalmers, Man. Trop. Med., 1919, 960; Prévot, Ann. Inst. Past., 60, 1938, 303.) From human mouth and intestine.

6. Bifidibacterium bifurcatum Prévot. (Bacillus bifurcatus gazogenes Choukévitch, Ann. Inst. Past., 22, 1911, 348; Prévot, Ann. Inst. Past., 60, 1938, 303.) From intestine of a horse. 


\section{Genus II. Microbacterium Orla-Jensen.*}

(The Lactic Acid Bacteria, 1919, 179.) From Greek mikros, small and M. L. bacterium, a small rod.

Small rods. Non-motile. Gram-positive. Produce lactic acid but no gas from carbohydrates. Surface growth on media is good. Produce catalase. Usually heatresistant. Found in dairy products and utensils, fecal matter and soil.

The type species is Microbacterium lacticum Orla-Jensen.

\section{Key to the species of genus Microbacterium.}

I. Acid from starch; survives $85^{\circ} \mathrm{C}$ for $2 \frac{1}{2}$ minutes.

\section{Microbacterium lacticum.}

II. No acid from starch; survives $71.6^{\circ} \mathrm{C}$ for $2 \frac{1}{2}$ minutes.

2. Microlacterium flavum.

1. Microbacterium lacticum Orla-Jensen. (The Lactic Acid Bacteria, 1919, 179; Corynebacterium lacticum Jensen, Proc. Limean Soc. of New So. Wales, 59, 1934, 50.) From Latin lac, milk; M. L., pertaining to milk.

Small thin rods: 0.3 by 1.0 micron; may have coccus-like appearance. Tonmotile. Granular. Gram-positive. Angular and pallisade arrangements of cells are characteristic.

Agar slant: White or at times slight greenish-yellow growth; adherent.

Gelatin: No liquefaction.

Milk: Acid, coagulation variable.

Nitrites usually not produced from nitrates.

Indole not formed.

Acid from glucose, fructuse, mannose, galactose, maltose, lactose, dextrin and starch. No acid from xylose, arabinose, rhamnose, or raffinose. Dextro lactic acid formed.

Catalase is produced.

Temperature relations: Minimum $10^{\circ} \mathrm{C}$. Optimum $30^{\circ} \mathrm{C}$. Maximum $35^{\circ} \mathrm{C}$. Survives $85^{\circ} \mathrm{C}$ for $2 \frac{1}{2}$ minutes in skimmilk.

Aerobic to facultative anaerobic.

Source: From cheese, milking equipment, grass, human and bovine feces.
Orla-Jensen (loc. cit., 180-181) identifies the Bacillus acidophilus cultures obtained by him from the Kral collection as belonging to this species. The charac. ters of the Irál cultures deviate from the characters of Bacillus acidophilus as given by Moro.

Habitat: Human and bovine intestinal tract and probably soil.

\section{Microbacterium flavum Orla-Jensen.} (Orla-Jensen, The Lactic Acid Bacteria, 1919, 181 ; Mycobacterium flavum Jensen, Proc. Linnean Soc. of New So. Wales, 59, 1934, 34.) From Latin flavus, yellow.

Rods : 0.5 by 1 to 2 microns. Granular and therefore sometimes confused with micrococci. Non-motile. Gram-positive.

Agar: Surface growth usually yellow and viseid.

Gelatin: No liquefaction.

Broth containing 10 per cent salt: Grows as flaky precipitate.

Milk: Slight acidity with no coagulation.

Nitrites produced from nitrates.

Indole not formed.

Acid from glucose, fructose, mannose, galactose, raffinose and mannitol. No acid from xylose, arabinose, rhamnose,

* Arranged by Prof. C. S. Pederson, New York State Experiment Station, Geneva, New York, June, 1938; further revision by Dr. M. L. Speck, Baltimore, Maryland, Sept., 1943. 
sorbitol, inulin, starch, or salicin. Dextro lactic acid formed.

Catalase is produced.

Temperature relations : Optimum $30^{\circ} \mathrm{C}$. Maximum $35^{\circ} \mathrm{C}$. Minimum $20^{\circ} \mathrm{C}$. Survives $71.6^{\circ} \mathrm{C}$ for $2 \frac{1}{2}$ but not 10 minutes.

Aerobic to facultative anaerobic.

Source: From milk, cheese, butter, milking equipment, bovine feces.

Habitat: Bovine intestinal tract and probably soil.

Appendix: While Orla-Jensen has placed the following species in the genus Microbacterium, the description is incomplete and the organism differs from the other species in the genus in several important characters. Therefore it is placed in this appendix.

Microbacterium liquefaciens Orla-Jensen. (Orla-Jensen, The Lactic Acid Bacteria, 1919, 182; Corynebacterium liquefaciens Jensen, Proc. Linnean Soc. of New So. Wales, 59, 1934, 49.) From Latin liqueo, to be liquid; facio, to make.
Morphologically resembles Microbacterium lacticum.

Agar : Surface growth is faint yellowishgreen.

Gelatin: Liquefied.

Milk: Rennet coagulation in 1 to 3 weeks; the casein is peptonized gradually.

Catalase is produced.

Temperature relations : Optimum $30^{\circ} \mathrm{C}$. Withstands heating to $80^{\circ} \mathrm{C}$.

Action on carbohydrates has not been described; Orla-Jensen states that very little acid is produced.

Source : From milk and more frequently from cheese.

Habitat: Presumably dairy products.

Noтw. The following species may belong here: Bacterium caseolyticum Kitahara, Jour. Agr. Chem. Soc. Japan, Tokyo, 14, 1938, 121 and 1461. A Grampositive, acid-forming and proteolytic rod said by the author to be related to Microbacterium liquefaciens. 


\section{Genus III. Propionibacterium Orla-Jensen.*}

(Cent. f. Bakt., II Abt., 22, 1909, 337.) From MI. L., propionic, and bacterium, a small rod or stick.

Non-motile. Non-spore-forming. Gram-positive bacteria growing under anaerobic conditions in neutral media as short diphtheroid rods, sometimes resembling streptococci; under aerobic conditions with heavy inoculum growing as long, irregular, club-shaped and branched cells. Metachromatic granules demonstrable with Albert's stain. Ferment lactic acid, carbohydrates, and polyalcohols with the formation of propionic and acetic acids and carbon dioxide. As a rule strongly catalase positive, sometimes weakly so. Strong tendency towards anaerobiosis; development very slow, macroscopically visible colonies generally not discernible in less than 5 to 7 days. $\dagger$ Nutritional requirements complex. Development best in yeast extract media with addition of lactates or simple carbohydrates. Optimum temperature $30^{\circ} \mathrm{C}$. Found in dairy products, especially hard cheeses.

The type species is Propionibacterium freudenreichii van Niel.

\section{Key to the species of genus Propionibacterium.}

I. In yeast extract-glucose media growth occurs in the form of small streptococci. Dirty cream-colored growth in stabs, with slight surface growth of same color. Sucrose and maltose not fermented.

A. Not fermenting lactose.

B. Fermenting lactose.

1. Propionibacterium freudenreichii.

\section{Propionibacterium shemanii.}

II. In yeast extract-glucose media growth occurs in the form of typical short rods of diphtheroid appearance. Distinct surface growth in stabs. Sucrose and maltose are fermented.

A. Growth brownish-red.

1. Ferments raffinose and mannitol, but not sorbitol.

3. Propionibacterium rubrum.

2. Ferments sorbitol, but not raffinose and mannitol.

4. Propionibacterium thoenii.

B. Growth in stab ream-colored.

1. Surface growth cream-colored.

a. Ferments l-arabinose and rhamnose.

5. Propionibacterium zeac.

2. Surface growth yellow to orange.

a. Growth in liquid media flocculent, as if agglutinated.

6. Pmpionibacterium peterssonii.

ad. Growth in liquid media dispersed, smooth.

* Revised by Prof. C. B. van Niel, Hopkins Marine Station, Pacific Grove, California, June, 1938; further revision by Prof. Van Niel, January, 1944.

† In an atmosphere containing 5 per cent carhon dioxide. growth is enhanced both aerobically and anaerobically. Contrary to the claim made by Krebs and Eggleston (Biochem. Jour., 35, 1941, 676) a differential effect of earbon dioxide tension on aerobic and anaerobic development has never been ohserved. 
b. Do not ferment dextrin, glyeogen or starch.

7. Propionibacterium jensenii.

8. Propionibacterium rafinosaceum.

bb. Ferments dextrin, glycogen and starch.

9. Propionibacterium technicum.

III. In yeast extract-glucose media growth occurs in the form of highly irregular cells, giving the appearance of involution forms. Distinct surface growth in stabs. Both d-and 1-arabinose are fermented.

A. Involution forms large, swollen spheres. Surface growth orange-yellow. Does not ferment xylose and rhamnose.

10. Propionibacterium arabinosum.

B. Involution forms long, irregular rods. Surface growth cream-colored. Ferments xylose and rhamnose.

11. Propionibacterium pentosaceum.

1. Propionibacterium freudenreichii van Niel. (Bacterium acili propionicia, von Freudenreich and Orla-Jensen, Cent. f. Bakt., II Abt., 17, 1906, 532; Bacterium acidi propionici var. fuscum Thöni and Allemann, Cent. f. Bakt., II Abt., 25, 1910, 29; van Viel, The Propionic Acid Bacteria, Haarlem, 1928, 162 ; Werkman and Brown, Jour. Bact., 26, 1933, 397.) Named for Eduoard von Freudenreich, the Swiss bacteriologist, who isolated this species.

Description taken from van Niel, and Werkman and Brown.

Small spherical cells, 0.5 to 0.6 micron, mostly in pairs and short chains. Little difference in morphology between grow th from anaerobic solid media and neutral or acid liquid media. Aerobic growth irregular, club-shaped and branched, long rods. Non-motile. Show metachromatic granules. Gram-positive.

Yeast gelatin-lactate stab: No liquefaction.

Yeast agar-lactate stab: Dirty grayishcreamy development in stab; very slight surface growth of same color.

Liquid media: Distinctly turbid with grayish-creamy, ropy sediment.

Litmus milk: Slight development, faint reduction. Not coagulated.

Catalase positive.

Indole not formed.

Nitrites not produced from nitrates.

Ferments lactic and pyruvic acids, glycerol, dihydroxyacetone, glucose, fruc- tose, mannose and galactose with the formation chiefly of propionic and acetic acids, and carbon dioxide.

Acid from erythritol, adonitol, inositol and esculin. No acid from amygdalin, d-and l-arabinose, dextrin, dulcitol, glycogen, inulin, lactose, maltose, mannitol, melezitose, melibiose, perseitol, raffinose, rhamnose, sucrose or xylose.

Anaerobic.

Distinctive characters: Inability to ferment any of the disaccharides when inoculated in yeast extract-sugar media.

Source: From dairy products; raw market milk, Swiss cheese.

Habitat: Dairy products.

2. Propionibacterium shermanii van Niel. (Bacterium acidi propionici d, Sherman, Jour. Bact., 6, 1921, 387; van Niel, The Propionic Acid Bacteria, Haarlem, 1928, 163; Werkman and Brown, Jour. Bact., 26, 1933, 400.) Named for J. M. Sherman, the American bacteriologist, who isolated this species.

Description taken from van Niel, and Werkman and Brown.

Small spherical cells, 0.5 to 0.6 micron, mostly in pairs and short chains. Little difference in morphology between growth from anaerobic solid media and neutral or acid liquid media. Aerobic growth irregular, club-shaped and branched rods. Non-motile. Show metachromatic granules. Gram-positive. 
Yeast gelatin-lactate stab: No liquefaction.

Yeast agar-lactate stab : Dirty grayishcreamy development in stab; very slight surface growth of same color.

Liquid media: Distinctly turbid with grayish-creamy, ropy sediment.

Litmus milk: Acid coagulation.

Catalase positive.

Indole not formed.

Nitrites not produced from nitrates.

Ferments lactic and pyruvic acids, glycerol, dihydroxyacetone, glucose, fructose, mannose, galactose and lactose with the formation chiefly of propionic and acetic acids, and carbon dioxide. Occasionally arabinose is fermented.

Acid from erythritol, adonitol, arabitol, inositol and esculin. No acid from amygdalin, dextrin, dulcitol, glycogen, inulin, maltose, mannitol, melezitose, melibiose, perseitol, raffinose, rhamnose, salicin, sorbitol, sucrose, starch, trehalose or xylose

Anaerobic.

Distinctive characters: Resembles Propionibacterium freudenreichii in every respect, but differs in its ability to ferment lactose.

Source: From dairy products; Swiss cheese and buttermilk.

Habitat: Dairy products.

3. Propionibacterium rubrum van Niel. (Bacterium acidi propionici var. rubrum Thöni and Allemann (in part), Cent. f. Bakt., II Abt., 25, 1910, S; van Niel, The Propionic Acid Bacteria, 1928, 164; Werkman and Brown, Jour. Bact., 26, 1933, 414.) From Latin ruber, red.

Medium-sized, stoutish rods to elongated diplococci, 0.8 by 1.2 microns, occurring singly or in pairs, resembling diphtheroids rather than streptococei. Somewhat more slender in media without fermentable carbohydrate. Aerobic growth irregular, club-shaped and branched rods. Non-motile. Show nietachromatic granules. Gram-positive.
Yeast gelatin-lactate stab: No liquefaction.

Yeast agar-lactate stab: Brownish-red development in stab, with appreciable dome-shaped surface growth of same color. (Also see Margolena and Hansen, Cent. f. Bakt., II Abt., 99, 1938, 107.)

Liquid media: Turbidity in early stages, sediment red and smooth.

Litmus milk: Acid coagulation.

Catalase positive; very weak for aerobically grown cells.

Indole not formed.

Nitrites not produced from nitrates.

Ferments lactic and pyruvic acids, glycerol, dihydroxyacetone, glucose, fructose, mannose, galactose, sucrose, maltose, lactose, raffinose and mannitol with the production chicfly of propionic and acetic acids and carbon dioxide.

Acid from erythritol, adonitol, arabitol, amygdalin, esculin, salicin, melezitose and trehalose. No acid from d-and 1arabinose, dextrin, dulcitol, glycogen, inulin, melibiose, perseitol, rhannose, sorbitol, starch or xylose.

Less anaerobic than Propionibacterium freudenreichii and Propionibacterium shermanii.

Distinctive characters: Production of brownish-red pigment under anaerobic and aerobic conditions. Fermentation of raffinose and mannitol, not of sorbitol.

Source: From various dairy products. Habitat: Dairy products.

4. Propionibacterium thoenii van Niel. (Baclerium acidi propionici var. rubrum Thöni and Allemann (in part), Cent.f. Bakt., II Abt., 25, 1910, 8; van Niel, The Propionic Acid Bacteria, 1928, 164; Werkman and Brown, Jour. Bact., 26, 1933, 412.) Named for Thöni, the Swedish bacteriologist, who isolated this organism.

Description taken from van Niel, and Werkman and Brown.

Medium sized, stoutish rods to elongated diplococei, 1.0 by 1.5 microns, occurring singly or in pairs, resembling 
diphtheroids. In media without fermentable carbolydrate, small streptococci. Aerobic growth irregular, clubshaped and branched rods. Non-motile. Shows metachromatic granules. Granpositive.

Yeast gelatin-lactate-stab: No liquefaction.

Yeast agar-lactate-stab: Brownish-red growth throughout stab. with appreciable dome-shaped surface growth of same color.

Liquid media: Turbidity in early stages, sediment smooth and red.

Litmus milk: Mostly acid coagulation.

Catalase positive.

Indole not formed.

Nitrites not produced from nitrates.

Ferments lactic and pyruvic acids, glycerol, dihydroxyacetone, glucose, fructose, mannose, galactose, sucrose, maltose, lactose and sorbitol with the formation of propionic and acetic acids, and carbon dioxide.

Acid from adonitol arabitol, crythritol, esculin, salicin and trehalose. No acid from amygdalin, arabinose, dextrin, dulcitol, glycogen, inulin, mannitol, melezitose, melibiose, perseitol, pectin, raffinose, rhamnose, starch or xylose.

Domke (Milchwirtsch. Forsch., 15, 1933,480 ) reports that this species may or may not ferment lactose and may or may not produce acid from esculin and salicin.

Less anaerobic than Propionibacterium freudenreichii and Propionibacterium shermanii.

Distinctive characters: Closely resembles Propionibacterium rubrum in morphology and in the production of brownish-red pigment under aerobic and anaerobic conditions. Differs from this species in its inability to ferment raffinose and mannitol, whereas fermentation of sorbitol occurs.

The biochemical characteristics of a ten-year-old stock culture have remained unchanged.

Source: From cheese and buttermilk.

Habitat: Dairy products.
5. Propionibacterium zeae Hitchner. (Hitchner. Jour. Bact., 23, 1932, 40; 28, 1935, 473; Werkman and Brown, Jour. Bact., 26, 1933, 411.) From Greek zea, spelt, a kind of grain; II. I. Zea, a generic name.

Description of culture isolated by Hitchner.

Cells in neutral lactate media spherical, 0.8 micron, usually occurring as short streptococci. In carbohydrate media which turn acid during development, distinctly rod-shaped, 0.8 by 2.0 to 3.0 microns, with a slight tendency to the formation of club-shaped cells. Appearance trpically diphtheroid. Aerobic growth irregular, club-shaped and branched rods. Non-motile. Show metachromatic granules. Gram-positive.

Teast gelatin-lactate-stab: No liquefiaction.

Yeast agar-lactate-stab : Cream-colored growth in stab, with distinct surface growth of same color.

Iiquid media: Distinctly turbid; cream-colored; smooth sediment, very ropy.

Iitmus milk: Cuagulated, acid.

Catalase positive, especially when grown in neutral media.

Indole not formed.

Nitrites not produced from nitrates.

Ferments lactic and pyruvic acids, wlycerol, dihydroxyacetone, l-arabinose, rhamnose, glucose, fructose, mannose, galactose, sucrose, cellobiose, maltose, lactose and mamnitol with the formation of propionic and acetic acids and carbon dioxide.

Acid from salicin. No acid from darabinose, dextrin. dulcitol, glycogen, inulin, starch or xylose.

Less anaerohic than Propionibacterium freudenreichii and Propionibacterium shermanii.

Distinctive characters: Cream-colored surface growth, ability to ferment l-arabinose and rhamnose, but not d-arabinose and xylose.

Source: Not definitely recorded. 
Probably from silage.

Habitat: Dairy products.

\section{Propionibacterium peterssonii van} Niel. (Bacterium acidi propionici $c$, Troili-Petersson, Cent. f. Bakt., II Abt., 24, 1909, 333; van Niel, The Propionic Acid Bacteria, 1928, 163; Werkman and Brown, Jour. Bact., 26, 1933, 406. ) Named for Gerda Troili-Petersson, the Swedish bacteriologist, who isolated this organism.

Description taken from van Niel, and Werkman and Brown.

Cells in neutral media spherical. 0.s micron, occurring as short streptococei in clumps. In carbohydrate media which turn acid during development, rod-shaped cells in clumps, 0.8 by 1.5 to 2.0 microns. Aerobic growth, heavily swollen and branched rods. Non-motile. Show metachromatic granules. (iram-positive,

Yeast gelatin-lartate stab: No lique. faction.

Yeast agar-lactate stab: ('ream-colorer) growth, dry and wrinkled, resembling that of Mycobacterium spp.

Liquid media: No turbidity, sedinent a coherent layer, cream-colored.

Litmus milk: Acid, coagulated.

Catalase positive; aerobically developed growth very slightly so.

Indole not formed.

Nitrites not produced from nitrates.

Ferments lactic and pyruvic arids. glycerol, dihydroxyacetone, gluese, frue. tose, mannose, galactose, sucrose, malt ose and lactose with the formation of propionic and acetie acids, and carbon dioxide.

Acid from esculin and salicin. No acid from d-and l-arabinose, cellobiose, dextrin, dulcitol, glyengen, inulin, perseitol, pectin, raffinose, rhamnose, sorbitol. starch or xylose.

Less anaerobic than Propionibacterium freudenreichii and Propionibacterium shermanii.

Distinctive character: Growth in liquid medist in elumps. giving the enl tures the appearance of agglutinated bacteria. So far, the only species among the propionic acid bacteria possessing this characteristic.

Source: From cheese and soil.

Habitat: Dairy products.

\section{Propionibacterium jensenii van} Niel. (Bacterium acidi propionici b, von Freudenreich and Orla-Jensen,Cent. f. Bakt., II Abt., 17, 1906, 532; van Niel, The Propionic Acid Bacteria, 1928, 163; Werkman and Brown, Jour. Bact., 26, 1933, 404.) Named for Prof. S. OrlaJensen, the Danish bacteriologist, who isolated this organism.

Description taken from van Niel, and Werkman and Brown.

In neutral media spherical to short rod-shaped cells, often in pairs or short rhains, 0.8 by 0.8 to 1.5 microns, of typiral diphtheroid appearance. Morphology little influenced by developing acidity. Aerobir growth, irregular long rods, strollen and branched. Non-motile. Metachromatic granules. Gram-positive.

Yeast gelatin-lactate stab: No liquefuction.

Yeast agar-lactate stab: Cream-colored growth in stab, orange-yellow, domeshaped surface growth.

Liquid media: Turbid in early stages; rream-colored, smooth sediment.

Litmus milk: coagulated, acid.

Catalase: Strongly positive.

Indole not formed.

Nitrites not produced from nitrates.

Ferments lactic and pyruvic acids, glycerol, dihydroxyacetone, glucose, fructose, mannose, galactose, sucrose, maltose, lactose and sometimes raffinose and mannitol with the formation of propionic and acetic acids, and carbon dioxide.

Acid from adonitol, arabitol, ery thritol, esculin, inositol and trehalose. No acid from arabinose, cellobiose, dextrin, dulcitol, glycogen, inulin, perseitol, pectin, rhamnose, salicin, sorbitol, starch or xylose. 
Less anaerobic than Propionibacterium freudenreichii.

Distinctive characters: Morphologically similar to Propionibacterium rubrum and Propionibacterium thoenii from which it is chiefly distinguished by the failure to produce a red pigment under anaerobic conditions. The yellow surface growth distinguishes Propionibacterium jensenii from Propionibacterium zeae, as also the inability of the former to ferment I-arabinose and rhamnose.

Source: From cheese and butter.

Habitat: Dairy products.

\section{S. Propionibacterium raffinosaceum}

Werkman and Kendall. (Propionibacterium jensenii var. raffinosaceum van Niel, The Propionic Acid Bacteria, 192s, 162; Werkman and Liendall, Iowa State Coll. Jour. Sci., 6, 1931, 17; Werkman and Brown, Jour. Bact., 26, 1933, 402. From M. L. raffinosum, the sugar raffinose.

Description taken from van Xiel, and Werkman and Brown

Cells in neutral media spherical to short rod-shaped cells, 0.8 by 0.8 to 1.5 microns, of typical diphtheroid appearance. In media in which acid is produced the cells are somewhat longer rodshaped, to 2 microns in length. Aerobic growth irregular, long rods, swollen and branched. Non-motile. Metachromatic granules. Gram-positive.

Yeast gelatin-lactate-stab: No liquefaction.

Yeast agar-lactate-stab: Cream-colored growth in stab; distinct, orange-yellow surface growth.

Liquid media: Turbid in early stages, cream-colored, smooth sediment.

Litmus milk: Coagulated, acid.

Catalase positive; aerobically grown only very slightly so.

Indole not formed.

Nitrites not produced from nitrates.

Ferments lactic and pyruvic acids, glycerol, dihydroxyacetone, glucose, fructose, mannose, galactose, cellobiose, malt- ose, lactose, sucrose, raflinose and mannitol with the production of propionic and acetic acids, and carbon dioxide.

Acid from adonitol, amygdalin, arabitol, erythritol, esculin, inositol, melezitose, salicin and trehalose. Vio acid from d-and l-arabinose, dextrin, dulcitol, glycogen, inulin, melibiose, perseitol, pectin, rhamnose, sorbitol, starch or xylose.

Less anaerobic than Propionibacterium freudenreichii.

Distinctive characters: Differs from Propionibacterium jensenii in its somewhat greater length and the ability to ferment cellobiose and salicin; the behaviour of Propionibacterium jensenii towards raffinose and mannitol is not constant, and hence cannot be used as a differential character. Werkman and Fiendall have reported different agglutination reactions for Propionibacterium jensenii and Propionibacterium raffino. saceum.

Source: From buttermilk.

Habitat: Dairy products.

9. Propionibacterium technicum van Niel. (Van Niel, The Propionic Acid Bacteria, 1928, 164; Werkman and Brown, Jour. Bact., .26, 1933, 401.) From Greek technicus, technical; M. I., of industrial significance.

Description taken from van Niel.

In neutral media spherical cells, 0.8 micron, in pairs and short chains. In acid media short rods, 0.6 by 1.0 to 1.5 microns, often in pairs, with typical diphtheroid appearance. Aerobic growth in the form of irregular long rods, swollen and branched. Non-motile. Metachromatic granules. Gram-positive.

least gelatin-lactate-stab: No liquefaction.

Yeast agar-lactate-stab : Cream-colored development in stab, with distinct yellow surface growth.

Liquid media: Turbid in early stages, cream-colored, somewhat flocculent sediment. 
Litmus milk: Coagulation, acid.

Catalase positive.

Indole not formed.

Nitrites not produced from nitrates.

Ferments lactic and pyruvic acids, glycerol, dihydroxyacetone, arabinose, glucose, galactose, fructose, mannose, lactose, maltose, sucrose, raffinose, dextrin, glycogen and starch with the formation of propionic and acetic acids, and carbon dioxide.

Acid from esculin, salicin and mannitol. No acid from dulcitol, inulin or xylose.

Anaerobic, but less so than Propionibacterium freudenreichii.

Distinctive characters: The ability to ferment the polysaccharides dextrin, glycogen and starch.

Source: From Edam and Tilsit cheese. Habitat: Dairy products.

\section{Propionibacterium arabinosum}

Hitchner. (Hitchner, Jour. Bact., 23, 1932, 40; 28, 1934, 473; Werkman and Brown, Jour. Bact., 26, 1933, 410.) From M. L. arabicum, gum Arabic; M. L. arabinosum, arabinose.

Description of culture isolated by Hitchner.

Cells in neutral lactate media spherical, 0.8 micron, in pairs and short chains. In acid media swollen spheres and ellipsoidal cells occur, mostly 2.0 by 3.0 to 3.5 microns, often in pairs and short chains. Non-motile. Metachromatic granules. Gram-positive.

Yeast gelatin-lactate-stab: No liquefaction.

Yeast agar-lactate-stab: Cream-colored growth in stab, with distinct orangeyellow surface growth.

Liquid cultures: Turbid in early stages, cream-colored, smooth sediment.

Litmus milk: No coagulation.

Catalase very slightly positive.

Indole not formed.

Nitrite production not recorded.

Ferments lactic and pyruvic acids, glycerol, dihydroxyacetone, d- and 1arabinose, glucose, galactose; fructose, mannose, cellobiose, maltose, sucrose, raffinose and mannitol with the production of propionic and acetic acids, and carbon dioxide.

Acid from sorbitol. No acid from dulcitol, xylose, rhamnose, salicin or inulin.

Anaerobic, but less so than Propionibacterium freudenreichii.

Distinctive characters: The development of spherical involution forms in acid media, the almost complete absence of catalase, the ability to ferment both d- and 1-arabinose, but not xylose and rhamnose.

Source: Not definitely stated.

Habitat: Dairy products.

Note: The strain obtained from Dr. E. B. Fred produced only minute amounts of acid from lactose and starch. It is questionable whether these carbohydrates are fermented.

\section{Propionibacterium pentosaceum} van Neil. (Bacillus acidi propionici von Freudenreich and Orla-Jensen, Cent. f. Bakt., II Abt., 17, 1906, 532 ; van Niel, The Propionic Acid Bacteria, 1928, 163; Werkman and Brown, Jour. Bact., 26, 1933, 408.) From M. L. pentosum, a pentose.

Description taken from van Niel, and Werkman and Brown.

In neutral lactate media cells spherical, 0.8 micron, in pairs and short chains. In media developing acidity long, irregular rods. swollen and branched, to 3 to 4 microns in length. Aerobic growth irregular, swollen and branched, long rods. Non-motile. Metachromatic granules. Gram positive.

Yeast gelatin-lactate stab: No lique. faction.

Yeast agar-lactate stab: Cream-colored development in stab, with abundant, cream-colored surface growth.

Liquid media: Turbid in early stages; smooth, creamy sediment, ropy.

Litmus milk: Coagulated, acid.

Catalase: Slightly positive.

Indole not formed. 
Nitrites and free nitrogen produced from nitrates.

Ferments lactic and pyruvic acids, glycerol, dihydroxyacetone, d- and l-arabinose, xylose, rhamnose, glucose, galactose, fructose, mannose, cellobiose, lactose, maltose, sucrose, raffinose, mannitol and sorbitol with the formation of propionic and acetic acids, and carbon dioxide.

Acid from adonitol, arabitol, erythritol, esculin, inositol, salicin and trehalose. No acid from dextrin, dulcitol, glycogen, inulin, perseitol or pectin.

Anaerobic, but less so than any of the other species of the genus.

Distinctive characters: The formation of long, rod-shaped involution forms in acid media; the absence of pigment production, and the ability to ferment $d$ and l-arabinose, rhamnose and xylose.

Source: From Emmental cheese.

Habitat: Dairy products.

Appendix: Cultures of the following species have not been available for study. It is probable that these duplicate previously described species.

Propionibacterium amyloaceum var. au- ranticum Sakaguchi, Swasaki and Yamada. (Bull. Agric. Chem. Soc. Japan, 17, 1941, 13.) From cheese. Resembles Propionibacterium pentosaceum closely.

Propionibacterium coloratum Sakaguchi et al. (loc. cit.). From cheese. Resembles Propionibacterium thoenii.

Propionibacterium globosum Sakaguchi et al. (loc, cit.). From cheese. Resembles Propionibacterium shermanii. Said not to ferment glycerol and erythritol.

Propionibacterium japonicum Sakaguchi et al. (loc. cit.). From cheese. said not to ferment glycerol and erythritol.

Propionibacterium orientum Sakaguchi et al. (loc. cit.). From cheese. Ferments l-arabinose. Resembles Propionibacterium shermanii.

Janoschel (Cent. f. Bakt., II Abt., $106,1944,321$ ) has suggested a key for the identification of the species in this genus. This is based on chromogenesis and cultural characters. He recognizes three additional species: Propionibaclerium casei, Propionibacterium pituitosum and Propionibacterium sanguineum. 


\section{Genus IV. Butyribacterium Barker and Haas,*}

(Jour. Bact., 4\%, 1944, 301.) From the chemical term, butyric and M. L. bacterium, a small rod.

Non-motile, anaerobic to microaerophilic, straight or slightly bent rods. Grampositive. Ferment carbohydrates and lactic acid forming acetic and butyric acids, and carbon dioxide. Generally catalase negative but sometimes weakly positive. Intestinal parasites.

The type species is Butyribacterium rettgeri Barker and Haas.

1. Butyribacterium rettgeri Barker and Haas. (Strain 32, Lewis and Rettger, Jour. Bact., 40, 1940, 298; Barker and Haas, Jour. Bact., 47, 1944, 303.) Named for L. F. Rettger, The American bacteriologist.

Rods: Straight or slightly bent, noncapsulated. 0.7 by 2.3 microns. Occur singly, in pairs and short chains. No branched cells observed but some cells have swollen club-shaped ends. Nonmotile. Gram-positive.

Glucose-cysteine agar: Colonies circular, entire or finely irregular margin, translucent, often with opaque center, grayish-white with yellowish tinge, convex when small, later umbonate, glistening, smooth, finely granular. Develop slowly attaining a diameter of $1.5 \mathrm{~mm}$ in 7 days.

Tryptone-yeast extract-lactate agar: Colonies similar to above except larger $\left(2 \mathrm{~mm}\right.$ in 4 days at $\left.37^{\circ} \mathrm{C}\right)$. Pulvinate rather than umbonate in cross sections.

Glucose-cysteine-broth : Abundant turbidity and sediment. No pellicle.

Agar stab (King and Rettger's medium, Jour. Bact., 44, 1942, 302) : Heavy growth in 2 days. Gas production often causes slight splitting of agar.

Acetic and butyric acid and $\mathrm{CO}_{2}$ pro- duced from glucose and maltose. Oecasionally a small amount of visible gas is produced. Lactic acid fermented readily without visible gas. Arabinose, xylose, lactose, sucrose, trehalose, rhamnose, mannitol, sorbitol, dulcitol and glycerol are not fermented.

Not proteolytic.

Indole and hydrogen sulfide not formed.

Temperature relations : Optimum $37^{\circ} \mathrm{C}$. Maximum 40 to $45^{\circ} \mathrm{C}$. Minimum $15^{\circ} \mathrm{C}$.

Generally catalase negative.

Anaerobic.

Source: From intestinal contents of a white rat.

Habitat: Presumably found generally in the intestine of mammals.

Notks: Pederson (Jour. Bact., 50, 1945, 478) has found that cultures of two species described by Eggerth (Jour. Bact., 30, 1935, 289 and 290) produce higher fatty (presumably butyric) acids and lactic acid from glucose. These are named Bacteroides avidus and B. limosus by Eggerth. Probably these species belong in the genus Butyribacterium.

Bacillus cadaveris butyricus Buday (Cent.f, Bakt., I Abt., 24, 1898, 374) may also belong in this genus.

* Prepared by Prof. C. S. Pederson, New York State Experiment Station, Geneva, New York, January, 1945; reviewed by Dr. H. A. Barker, Berkeley, California. 


\title{
FAMILY VIII. CORYNEBACTERIACEAE LEHMANN AND NEUMANN.
}

\author{
(Bakt. Diag., 4 Aufl., 2, 1907, 500.)
}

Non-motile (motile in Listeria) rods, frequently banded or beaded with metachromatic granules. May show marked diversity of form. Branching cells have been observed in a few species but these are uncommon. Generally Gram-positive but this reaction may vary depending on the nature of the cells. Where pigment is formed, it is grayish-yellow to orange or pink in color. Aerobic to microaerophilic. Anaerobic species have been reported. Gelatin may be liquefied and nitrites may be produced from nitrates. Animal and plant parasites and pathogens. Also from dairy products, soil and water.

\section{Key to the genera of family Corynebacteriaceae.}

I. Aerobic to microaerophilic, non-motile (or questionably motile) rods which are variable in form. Animal and plant parasites and pathogens, with some from dairy products, soil and water.

Genus I. Corynebacterium, p. 381.

II. Small aerobic rods with 1 to 4 flagella. Causes a monocytosis in warm-blooded animals.

Genus II. Listeria, p. 408.

III. Microaerophilic, non-motile rods to long filaments. Pathogenic on warm-blooded animals.

Genus III. Erysipelothrix, p. 410.

\section{Genus I. Corynebacterium Lehmann and Neumann.*}

(Lehmann and Neumann, Bakt. Diag., 1 Auf., 2, 1896, 390; Corynethrix Czaplewski, Deutsche med. Wchns hr., 26, 1909, 723; Corynemonas Orla-Jensen, Cent. f. Bakt., II Abt., 22, 1909, 344; Corynobacterium Enderlein, Sitzber. Gesell. Naturf. Freunde. Berlin, 1917, 309; Plocamobacterium Löwi, Wiener klin. Wrhnsrhr., 33, 1920, 730. From Greek koryne, club and M. L. bacterium, a small rod.

Slender, straight to slightly curved rods, with irregularly stained segments or granules. Frequently show pointed or club-shaped swellings at the ends. Snapping division produces angular and palisade (picket fence) arrangements of cells. Tonmotile with possible exceptions as stated in the text. Gram-positive to variable, sometimes young cells and sometimes old cells being Gram-negative. Granules invariably Gram-positive. Generally quite aerobic, but microaerophilic or even anaerobic species occur. Catalase positive. They may or may not liquefy gelatin, and may or may not produce nitrites from nitrates. They may or may not ferment sugars, but they seldom produce a high acidity. Many species oxidize glucose completely to $\mathrm{CO}_{2}$ and $\mathrm{H}_{2} \mathrm{O}$ without producing visible gas. Some pathogenic species produce a powerful exotoxin. This group is widely distributed in nature. The best known species are parasites and pathogens on man and domestic animals. Other species have been found in birds and insects and the group is probably more widely distributed in the animal kingdom than this. Several species are well known plant pathogens while still other common species are found in dairy products, water and soil.

The type species is Corynebacterium diphtheriae (Flügge) Lehmann and Neumann.

* Rearranged by Prof. E. G. D. Murray, MeGill University, Montreal, P. Q., Canada and Prof. Robert S. Breed, New York State Experiment Station, Geneva, New York, March, 1938; completely revised by Prof. E. G. D. Murray, Montreal, Prof. Robert S. Breed, Geneva and Prof. Walter H. Burkholder, New York State College of Agriculiure, Ithaca. New York, February, 1945. 


\section{Key to the species of genus Corynebacterium.}

I. From human sources.* Non-motile. $f$

A. Aerobic. No liquefaction of gelatin.

1. Acid from glucose and usually maltose and galactose. Usually no acid from sucrose. Causes diphtheria.

\section{Not as in 1 .}

\section{Corynebacterium diphtheriae.}

a. No acid from carbohydrates.

2. Corynebacterium pseudodiphtheriticum.

aa. Acid from glucose and sucrose.

b. Highly pleomorphic, varying from cocci to rods.

3. Corynebacterium enzymicum.

bb. Rods with polar staining with club forms, diphtheroid in appearance.

\section{Corynebacterium xerose.}

bbb. Rods as above but characteristic salmon pink growth on coagulated blood serum.

5. Corynebacterium hoagii.

B. Microaerophilic to anaerobic. Growth feeble or none at all on gelatin.

6. Corynebacterium acnes.

II. From domestic and laboratory animals. Non-motile,

A. Acid from glucose.

1. Grows poorly if at all on ordinary gelatin and agar. Slow liquefaction of serum gelatin and coagulated blood serum. Causes suppurative processes in cattle, swine, and other animals.

7. Corynebacterium pyogenes.

2. No liquefaction of gelatin or blood serum. Grows poorly, if at all, on ordinary gelatin and agar.

a. Cause of pyelonephritis in cattle.

8. Corynebacterium renale.

aa. Found in caseous nodules resembling those of tuberculosis. Found in sheep, horses and some other animals.

9. Corynebacterium pseudotuberculosis.

aaa. From caseous nodules in mice.

10. Corynebacterium kutscheri.

aaaa. Causes a septicemia in mice.

11. Corynebacterium murisepticum.

B. No acid from carbohydrates. No liquefaction of gelatin.

1. From milk and bovine udder.

12. Corynebacterium bovis.

* Habitat relationships are used because comparative studies of the species in the genus are still completely lacking.

$\dagger$ The reports of motile species in this genus present a puzzling problem, particularly as the motile species of plant pathogens placed in the genus are polar flagellate. Some students of the group feel that, if motile species really exist, they should be placed in a separate genus. Others feel that a more careful study of the described polar flagellate species will show that these species really belong elsewhere. Where authors have reported motility, this fact is indicated in the text. It should be noted that similar uncertainties exist in regard to described cases of motility among the streptococci and lactobacilli. 
2. From pneumonia in foals.

III. From insects. Non-motile.

\section{Corynebacterium equi.}

A. No acid from carbohydrates. Slow liquefaction of gelatin.

14. Corynebacterium paurometabolum.

IV. Plant pathogens. Non-motile.

A. Nitrites not produced from nitrates.

1. Colonies cream-colored. Slow liquefaction of gelatin.

a. Bluish granules in growth. Attacks alfalfa.

15. Corynebacterium insidiosum.

aa. No bluish granules, Causes ring rot of potatoes.

16. Corynebacterium sepedonicum.

2. Colonies yellow.

a. No liquefaction of gelatin. Causes a wilt and canker of tomatoes.

17. Corynebacterium michiganense.

B. Nitrites produced from nitrates. Slow or no liquefaction of gelatin.

1. Colonies yellow. Attack members of the grass family.

18. Corynebacterium rathayi.

19. Corynebacterium agropyri.

2. Colonies orange. Parasitic on sweet peas, etc.

20. Corynebacterium fascians.

V. From soil and water. Liquefaction of gelatin in all cases but sometimes very slow (7 weeks).

A. Acid from glucose. Non-motile.

1. Nitrites not produced from nitrates.

2. Nitrites produced from nitrates.

21. Corynebacterium helvolum.

a. Cellulose digested.

aa. Celbulose not digested.

22. Corynebacterium fimi.

23. Corynebacterium tumescens.

B. No acid from glucose. Some indication of motility in No. 25.

1. Cells coccnid to short, straight or curved rods.

24. Corynebacterium simplex.

2. Young cells curved rods in parallel bundles. These may grow out into filaments with branching.

25. Corynebacterium filamentosum.

1. Corynebacterium

diphtheriae

(Flügge) Lehmann and Neumann. (Microsporon diphthericum Klebs (prototype), Verhandl. d. Congr. f. innere Med., 2, 1883, 143; die Klebs'schen Stäbchen, Löffler, Mitteil. a. d. kaiserl. Gesundheitsamte, 1884; Bacillus diphtheriae Flügge, Die Mikroorganismen, 2 Aufl., 1886, 225; Pacinia loefferi Trevisan, I generi e la specie delle Batteriacee, 1889, 23; Lehmann and Neumann, Bakt. Diag., 1 Aufl., 2, 1896, 350;
Bacterium diphtheriae Migula, Syst. d. Bakt., 2, 1900, 499.) From Greek diphthera, a piece of leather; M. L., the disease diphtheria.

Common name: Diphtheria bacillus; Kilebs-Loeffler hacillus.

Rods, varying greatly in dimensions, 0.3 to 0.8 by 1.0 to $\$ .0$ microns, occurring singly. The rods are straight or slightly curved, frequently swollen at one or both ends. The rods do not, as a rule, stain uniformly with methylene blue but 
show alternate bands of stained and unstained material and in addition one or more metachromatic granules which are best shown by special stains. Non-motile. Gram-positive but not intensely so in older cultures.

Gelatin colonies: Slow development. Very small, grayish, lobulate.

Gelatin stab: Slight growth on surface and scant growth in stab. No liquefaction.

Agar slant: Scant, grayish, granular, translucent growth, with irregular margin.

Blood-tellurite media: Produces gray to black colonies.

Colony forms: Smooth (S) colony form: Round and umbonate or convex, with even margin and smooth surface. Opaque when viewed by transmitted light, glistening and somewhat moist in appearance when viewed by reflected light. Colonies about 1 to $3 \mathrm{~mm}$ in diameter. Growth frequently slowed or inhibited by the presenre of potassium tellurite in the medium.

Rough ( $\mathrm{R}$ ) colony form: Flat, margin is very irregular. Surface is pitted and very uneven. Very little light reflected from surface. Translucent when viewed by transmitted light. Colonies about 1 to $5 \mathrm{~mm}$ in diameter.

Intermediate colony forms: Several colony forms are found in this group since the term includes all forms between the pure $\mathrm{S}$ form and the pure $\mathrm{R}$ form. Sr forms very nearly approach the $\mathrm{S}$ colonies and the sR forms nearly approach the pure $\mathbf{R}$ forms. The SR form shows properties distinet frem either the $\mathrm{S}$ or $\mathrm{R}$ forms. The colonies are 3 to $5 \mathrm{~mm}$ in diameter. The margin usually shows indentations. The surface is raised but not convex; it may be nearly level or show a central elevation surrounded by a concentric depression and elevation.

Dwarf (D) colony form: Colonies very small, about $0.2 \mathrm{~mm}$ or less in diameter. Margin round and even. Surface convex.

All of the above colony forms have been isolated from cases of diphtheria (Morton, Jour. Bact., 40, 1940, 768 ff.).

Broth: Uniform turbidity produced by $\mathrm{S}$ form, pellicle produced by $\mathrm{SR}$ form, sediment produced by the $\mathrm{R}$ form.

Litmus milk: Unchanged.

Potato: No visible growth.

Blood serum : Growth grayish to creamcolored, moist, smooth, slightly raised, margin entire. May be bright yellow or occasionally reddish (Hill, Sci., 17, 1903, $375)$.

Indole is not formed.

Nitrites are produced from nitrates.

All strains form acid from glucose and fructose; some strains also ferment galactose, maltose, sucrose, dextrin and glycerol.

Does not hydrolyze urea (Merkel, Cent. f. Bakt., I Abt., Orig., 147, 1941, 398).

A highly poisonous exotoxin is produced in fluid media. This toxin represents the principal disease-producing agency of the organism. Toxin production may fail in otherwise typical strains.

A highly potent antitoxin can be produced by repeated injection of toxin into experimental animals. The antitoxin possesses both curative and protective properties.

Serological types: In a study of 250 strains of Corynebacierium diphtheriae Murray (Jour. Path. and Bact., 41, 1935, 439-45) was able to classify 228 strains into 11 serological types and 22 strains remained unclassified (Morton, Bact. Rev., 4, 1940, 196).

McLeod et al. (Jour. Path. and Bact., 34, 1931, 667; ibid., 36, 1933, 169; Lancet, 1, 1933, 293) describe three types which have been confirmed by other workers; these are distinguishable by colony form on McLeod's blood-tellurite medium, they are antigenically different with subtypes, there is some difference between their toxins (Etris, Jour. Inf. Dis., 50, 1934,220 ) and the severity of disease is associated with the type.

Corynebacterium diphtheriae type gravis 
grows with dark gray, daisy-head colonies; ferments dextrin, starch and glycogen; is not hemolytic; has very few small metachromatic granules; forms a pellicle, granular deposit and there is an early reversal of $\mathrm{pH}$ in broth.

Corynebacterium diphtheriae type mitis yrows in convex, black, shiny, entire colonies; no fermentation of starch and glycogen and is variable with dextrin; hemolytic; metachromatic granules are prominent; diffuse turbidity, infrequent pellicle and there is a late reversal of $\mathrm{pH}$ in broth.

Corynebacterium diphtheriae type intermedius grows a small, flat, umbonate colony with a black center and slightly crenated periphery; not hemolytic; barring of bacilli is accentuated; there is no fermentation of starch and glycogen, and is variable with dextrin; forms no pellicle, a fine granular deposit and there is no reversal of $\mathrm{pH}$ in broth.

Ten years of observations in all parts of the world have shown (McLeod, Bact. Rev., 7, 1943, 1) that a small percentage of strains does not correspond closely to any of these three types. Variant strains are found most frequently in regions where the diphtheria is of mild or moderate severity.

Aerobic, facultative.

Optimum temperature $34^{\circ}$ to $36^{\circ} \mathrm{C}$. Grows well at $37^{\circ} \mathrm{C}$.

Source: Commonly from membranes in the pharynx, larynx, trachea and nose in human diphtheria; from the seemingly healthy pharynx and nose in carriers; oceasionally from the conjunctiva and infected superficial wounds. Found occasionally infecting the nasal passages and wounds in horses. Has been described from natural diseases in fowl.

Habitat: The cause of diphtheria in man. Pathogenic to guinea pigs, kittens and rabbits. For action on other animals see Andrews et al., Diphtheria. London. $1923,170 \mathrm{ff}$.

2. Corynebacterium pseudodiphtherit- icum Lehmann and Neumann. (Bacillus der pseudodiphtherie, Loeffler, Cent. f. Bakt., 2, 1887, 105; G. von HofmannWellenhof, Wien. med. Wochenschr., 38, 1888, 65; Lehmann and Neumann, Bakt. Diag., 1 Aufl., 2, 1896, 361; Bacillus pseudodiphthericus Kruse, in Flügge, Die Mikroorganismen, 3 Aufl., 2, 1896, 476; Bacterium pseudodiphtheriticum Migula, Syst. d. Bakt., 2, 1900, 503; Mycobacterium pseudodiphthericum Chester, Man. Determ. Bact., 1901, 355; Bacillus hoffmanii (sic) Holland, Jour. Bact., 5, 1920, 218; Corynebacterium hoffmanii (sic) Holland, ibid., 220; Corynebacterium pseudodiphtheriae Holland, ibid.; Corynebacterium pseudodiphthericum Bergey et al., Manual, 2nd ed., 1925, 393.) From Greek pseudus, a falsehood; M. L., the disease diphtheria.

Common name: Pseudodiphtheria bacillus or Hofmann's bacillus.

Excellent historical discussions of this and related organisms are given by Bergey, Comparative Studies upon the Pseudo-diphtheria or Hofmann's Bacillus, the Xerosis Bacillus, and the Loeffler Bacillus. Contrib. from Lab. of Hyg., Univ of Penn., No. 2, 1898, 19-54 and by Andrewes et al., Diphtheria. London, 1923, 382-388.

Rods, with rounded ends, 0.3 to 0.5 by 0.8 to 1.5 microns, fairly uniform in size, without swollen ends. Not barred but even staining interrupted by transverse, medial unstained septum; granules usually absent. Non-motile. Grampositive.

Gelatin colonies: Small, grayish to cream-colored, smooth, homogeneous, entire.

Gelatin stab: Slight surface growth with little growth in stab. No liquefaction.

Agar colonies: Opaque, grayish to cream-colored, smooth, homogeneous, entire.

Agar slant: Moist, smooth, white to cream-colored, entire growth.

Loeffler's blood serum : As on agar. 
Broth: Slightly turbid with slight, grayish sediment.

Litmus milk: Unchanged.

Potato: Slight, creamy-white, smooth, entire growth.

Indole not formed.

Nitrites produced from nitrates.

No acid from carbohydrate media.

Hydrolyzes urea (Merkel, Cent. f. Bakt., I Abt., Orig., 147, 1941, 398).

Aerobic, facultative.

Optimum temperature $37^{\circ} \mathrm{C}$.

Not pathogenic.

Source: From oral cavity of 26 out of 45 control cases.

Habitat: Normal throats.

3. Corynebacterium enzymicum (Mellon) Eberson. (An unusual diphtheroid bacillus, Mellon, Med. Record, New York, 81, 1916, 240; Bacillus enzymicus Mellon, Jour. Bact., 2, 1917, 297 ; Eberson, Jour. Inf. Dis., 23, 1918, 29.) From Greek en, inside of ; zyme, leaven; of an enzyme.

Rods, beaded and club-shaped, definitely pleomorphic, showing coccoid forms. Non-motile. Gram-positive.

Gelatin stab: Slight surface growth. No liquefaction.

Glucose agar: Bacillary form shows very small colorless colonies. Coccoid form shows heavy, yellowish-white, moist grcwths.

Blood agar : Same as on glucose agar.

Loeffler's blood serum: Fine, moist, confluent growth.

Glucose broth: Bacillary form shows granular sediment. Coccoid form shows diffuse, luxuriant growth.

Litmus milk: Acid, coagulated.

Potato: No growth.

Indole formation slight.

Slight production of nitrites from nitrates.

Acid from glucose, maltose, sucrose, dextrin and glycerol.

Aerobic, facultative.

Optimum temperature $37^{\circ} \mathrm{C}$.

Pathogenic for rabbits, guinea pigs and mice.
Source: Lungs, blood and joints.

Habitat: From human sources so far as known.

4. Corynebacterium xerose (Neisser and Kuschbert) Lehmann and Neumann. (Bacillus xerosis Neisser and Kuschbert, Breslauer ärtzl. Ztschr., No. 4, 1883; Xerosebacillen, Kuschbert, Deutsche med. Wochnschr., 10, 1884, 321 and 341 ; Pacinia neisseri Trevisan, I generi e le specie delle Batteriacee, 1889, 23; Lehmann and Neumann, Bakt. Diag., 2 Aufl., 2, 1899, 405; Bacterium xerosis Migula, Syst. d. Bakt., 2, 1900, 485.) From Greek xerus, dry.

An excellent historical discussion of this organism is given by Andrewes et al., Diphtheria. London, 1923, 377-382.

Rods, showing polar staining, occasionally club-shaped forms are seen. Non-motile. Gram-positive.

Plain gelatin colonies: Rarely develop.

Serum gelatin stab: No liquefaction.

Agar colonies: Minute, circular, almost transparent, raised, smooth, pearly white.

Agar slant: Thin, grayish, limited growth.

Loeffler's blood serum: Thin, grayish, adherent growth.

Broth: Clear, with slight, granular sediment.

Litmus milk: Unchanged.

Potato: No visible growth.

Indole not formed.

Nitrites not produced from nitrates.

Acid from glucose, fructose, galactose, maltose and sucrose.

Not pathogenic.

Aerobic, facultative.

Optimum temperature $37^{\circ} \mathrm{C}$. Grows very slowly as low as $18^{\circ}$ to $25^{\circ} \mathrm{C}$ (Eberson, Jour. Inf. Dis., 23, 1918, 3).

Source: From normal and diseased conjunctiva.

Habitat: Probably identical with other species described from the skin and other parts of the body. 


\section{Corynebacterium hoagii (Morse)} Eberson. (Bacillus X, Hoag, Boston Med. and Surg. Jour., 157, 1907, 10; Bacillus hoagii Morse, Jour. Inf. Dis., 11, 1912, 284; Eberson, Jour. Inf. Dis., 23, 1918, 10.) Named for Hoag, the bacteriologist who first isolated the species.

Rods : 0.8 to 1.0 by 1.0 to 3.0 microns, occurring singly. Show polar staining in the shorter forms while the longer forms are barred and slightly club-shaped. Non-motile. Gram-positive.

Gelatin colonies : Small, dull, pale pink, entire.

Gelatin stab: Slight pink surface growth. No liquefaction.

Agar colonies: Small, pale pink, dull, granular, entire.

Agar slant: Filiform, dull, pink growth.

Broth: Turbid, with slight pink sediment.

Litmus milk: Slightly alkaline, with pink sediment.

Potato: Dull, filiform streak.

Indole not formed.

Nitrites not produced from nitrates.

Acid from glucose and sucrose but not maltose.

Blood serum: Dull, filiform, pink streak.

Aerobic.

Optimum temperature $30^{\circ} \mathrm{C}$.

Source: From the throat. Air contamination of cultures.

Habitat: Unknown.

\section{Corynebacterium acnes (Gilchrist)}

Eberson. (Bacillus acnes Gilchrist, Johns Hopkins Hosp. Repts., 9, 1901, 425; Actinomyces acnes Gilchrist, ibid., 425; Eberson, Jour. Inf. Dis., 23, 1918, 10; Fusiformis acnes Holland, Jour. Bact., 5, 1920, 233; Propionibacterium acnes Douglas and Gunter, Jour. Bact., 52, 1946, 22.) From M. L. acne, the disease acne.

Rods, vary in dimensions, usually 0.5 by 0.5 to 2.0 microns, sometimes slightly club-shaped. Show alternate bands of stained and unstained material. Nonmotile. Gram-positive.
Growth in culture media very feeble.

Best growth occurs in shake cultures with soft, slightly acid, glucose agar.

Agar slant: Very small, circular transparent colonies which may later become rose-colored.

Loeffler's blood serum: Small, grayish colonies, which may later become rosecolored.

Broth : Clear.

Litmus milk : Soft coagulum.

Potato: No growth in aerobic cultures, but pink streak in anaerobic cultures.

Indole not formed.

Nitrites produced from nitrates.

Acid from glucose, sucrose (slight), maltose, mannitol and inulin. Produces propionic acid (Douglas and Gunter, loc. cit.).

Catalase produced.

Microaerophilic to anaerobic.

Optimum temperature $35^{\circ}$ to $37^{\circ} \mathrm{C}$.

Pathogenic for mice and gives rise to characteristic lesions.

Source: From acne pustules.

Habitat: Sebaceous glands, hair follicles and acne pustules.

Notes: Even before 1901, several authors reported finding bacteria in acne pustules which were evidently diphtheroid in nature. Unna (Monatshefte $\mathrm{f}$. prakt. Derm., 13, 1891, 232) found an organism in acne pustules which he gave the name of Flaschenbacillus. Hodara (Monatshefte f. prakt. Derm., 18, 1894, 586 ) reported the presence of two types of bacteria in acne lesions, the second of which he called Flaschenkugelbacillus. Sabouraud (Ann. Inst. Past., 11, 1897, 134) gave a more accurate description of these diphtheroids which he reported to need an acid medium for growth. He called this bacterium, bacille de séborrhée grasse (Bacillus sabouraudi NeveuLemaire, Précis Parasitol. Hum., 5th ed., 1921, 24).

Additional anaerobic species will be found in the appendix. These are Corynebacterium typhi which Eberson (loc.cit., 19) and Hewlett (Med. Res. Council, Syst. of Bact., London, 5, 1930, 145) regard as practically identical with Corynebac- 
terium acnes, and eleven species listed by Prévot (Manual de Classification et de Détermination des Bactéries Anaérobies. Monographie, Inst. Past., Paris, 1940, 199-204) as follows: Corynebacterium diphtheroides, C.avidum, C. renale cuniculi, C. lymphophilum, C. hepatodystrophicans, C. parvum, $C$. anaerobium, $C^{r}$. granulosum, C.adamsoni, C.liquefaciens, and C. pyogenes bovis.

7. Corynebacterium pyogenes (Glage) Eberson. (Bacillus liquefaciens pyoyenes bovis Lucet, Ann. Inst. Past. 7, 1893, 327; Bucillus liquefaciens pyogenes Lucet, ibid., 327; Bacillus liquefaciens Lucet, ibid.; Bakterium der multipler Abszessbildung der Schweine, Grips. Ztschr. f. Fleisch-u. Wilchhyg., 8, 1s9s. 166; Bacillus pyogenes bovis Kümnemann. Arch. f. wiss. u. prakt. Tierheilk., 29 , 1903, 125; Bucillus pyogenes Cilage, Ztschr. f. Fleiseh- u. Milchhyg., 13, 1903. 166; not Bacillus pyogenes Lucet, Ann. Inst. Past., $\%$, 1893, 327; Bacillus pyo genes suis Lehmann and Neumann, Bakt. Diag., 4 Aufl., 2, 1907, 394; Bacterium hyo pyogenes Lehmann and Neumann, Bakt. Diag., 4 Aufl., 2, 1907, 394; Bacterium pyogenes suis Lehmann and Yeumann. Bakt. Diag., 7 Aufl., 2, 1927, 499; Bac terium pyogenes Ward, Jour. Bact.. 2, 1917, 519; not Bacterium pyogenes Chester, Man. Determ. Bact., 1901, 18t; Eberson, Jour. Inf. Dis., 23, 1918, 5; not Corynebacterium pyogenes Lewand owsky, Cent. f. Bakt., I Abt., Orig. 36, 1904, 473; Corynebacterium pseu dopyogenes Ochi and Zaizen, Jour. Jap. Soc. Tet. Sci., 15, 1936, 12 and 16, 1937, 8.) From Greck pyum, pus; gignomai, producing.

For description see Brown and Orcutt, Jour. Exp. Med., 32, 1920, 214.

Rods : 0.2 by 0.3 to 2 microns in length. Smallest forms appear as scarcely visible points (common in old abscesses). Chains formed. Club forms may be present. Non-motile. Gram-positive.

Serum gelatin: Liquefaction.

No growth on ordinary agar.

Serum agar: Minute colonies after 36 to 48 hours. Surface colonies may increase to $3 \mathrm{~mm}$ in diameter. Colonies smoky brown by transmitted light and bluish-white by reflected light.

Bovine blood serum slants: Pit-like or more general areas of liquefaction.

Serum bouillon: Cloudy with fine flocculent grayish flakes that form a sediment like a streptococcus culture.

Milk: Coagulation after 48 hours at $37^{\circ} \mathrm{C}$, with acid at bottom of tube. Separation of whey and peptonization.

Nitrites not produced from nitrates (Merchant, Jour. Bact., 30, 1935, 108).

Indole not formed.

Acid formed in serum bouillon from glucose, sucrose, lactose, and xylose but not from raffinose, inulin, mannitol and salicin.

Betit hemolytir, not hemoglobinophilic though growth is favored by proteins as egg albumen, serum or blood (Brown and Orcutt, loc. cit.).

Optimum temperature $37^{\circ} \mathrm{C}$. Growth range $20^{\circ}$ to $40^{\circ} \mathrm{C}$.

Intravenous injection of rabbits fatal. Aerobic as well as anaerobic growth. Source: From bovine pus.

Habitat: Found in abscesses in cattle, swine and other domestic animals.

8. Corynebacterium renale (Migula) Ernst. (Bacillus renalis bovis Bollinger, in Enderlen, Zeit. f. Tiermed., 17, 1890, 346; Bacillus pyelonephritis boum (sic) Höflich, Monatsh. f. prakt. Tierheilk., 2, 1891, 356; Bacterium renale Migula, Syst. d. Bakt., 2, 1900, 504; Bacillus renalis Ernst, Cent. f. Bakt., I Abt., Orig., 39, 1905, 550 ; Ernst, ibid., 40, 1905, 80; Corynebacterium renalis bovis Ernst, ibid., 82.) From Latin renalis, kidney.

Description largely taken from Jones and Little, Jour. Exp. Med., 44, 1926, 11.

Rods: 0.7 by 2 to 3 microns. Nonmotile. Usually in masses, rarely single. Bacteria from tissues not as pleomorphic as those from the earlier transfer cultures although many show polar granules or swollen ends. Cultures grown in broth show coccoid forms and beaded rods with swollen ends. Gram-positive. 
Gelatin: Grows poorly if at all. No liquefaction.

Agar: Small punctiform colonies.

Agar slants: Raised, grayish-white, and dry growth (Jones and Little). Other's say cream-colored and moist.

Blood serum slants: Fine gray punctiform colonies in 24 hours at $37^{\circ} \mathrm{C}$ which are a little larger than on agar. Streak searcely $1 \mathrm{~mm}$ in width. Glistening and slimy in fresh cultures. To liquefaction.

Litmus milk: Reduction and coagulation from the bottom. Slow digestion, becoming alkaline.

Broth: Sediment at end of 2 days with clear bouillon above.

Potato: Growth grayish-white; Iater, becoming a dingy yellow, turning the potato brown .

Acid from glucose. No acid from lactose, sucrose, maltose and mannitol. Some strains ferment fructose and mannose (Merchant, Jour. Bact., 30, 1935 109).

Shows a close serological relationship with Corynebacterium pseudotuberculosis (Merchant). Anaerobic

Not pathogenic for laboratory animals. No toxin produced.

Optimum temperature $37^{\circ} \mathrm{C}$.

Source: Found in pyelonephritis in cattle.

Habitat: Occurs in purulent infections of the urinary tract in cattle, sheep, horses and dogs.

9. Corynebacterium pseudotuberculosis (Buchanan) Eberson. (Nocard, Bull. de la Soc. Centr. de méd. Vet., 1885, 207; Pseudotuberculose-Bakterien, Preisz, Cent.f. Bakt., 10, 1891,568; Bacillus pseudotuberculosis ovis Lehmann and Neumann, Bakt. Diag., 1 Aufl., 2, 1896, 362; Bacillus pseudotuberculosis Buchanan, Veter. Bact., Phila., 1911, 238; not Bacillus pseudotuberculosis Eisenberg, Bakt. Diag., 3 Aufl., 1891, 294; Eberson, Jour. Inf. Dis., 23, 1918, 10; Corynebacterium ovis Bergey et al., Manual, 1st ed., 1923, 388; not Corynebacterium pseu- dotuberculosis Bergey et al., Manual, 2nd ed., 1925, 394; Corynebacterium pseudotuberculusis bovis (an evident typographical error) Thomson and Thomson, Ann. Pickett-Thomson Res. Lab., 2, 1926, 132; Corynebacterium pseudotuberculosis ovis Hauduroy et al., Dict. d. Bact. Path., 1937, 159; Corynebacterium preisz-nocardi Hauduroy et al., ioid., 159.) From Greek pseudus, a falsehood; Latin tuberculum, a small nodule; M.L., false tuberculosis.

Common name: Preisz-Nocard bacillus.

Slender rods : 0.5 to 0.6 by 1.0 to 3.0 microns, staining irregularly and showing clubbed forms. Non-motile. Grampositive.

Gelatin colonies: Slight development. Gelatin stab: No liquefaction.

Agar colonies: Thin, cream-colored to orange, folded, serrate, dry.

Loeffler's blood serum: Small, yellow, serrate colonies. No licuuefaction.

Broth: No turbidity. Granular sediment. Pellicle formed (Carne, Jour. Path. and Bact., 49, 1939, 316).

Litmus milk: Lnchanged.

Potato: No growth.

Nitrites not produced from nitrates.

Acid from glucose, fructose, galactose, mannose, sucrose, lactose, maltose and dextrin. Some strains attack xylose.

Causes caseous lymphadenitis in sheep and ulcerative lymphangitis in horses. Forms an exotoxin.

Shows a close serological relationship with Corynebacterium renale (Merchant, Jour. Bact.. 30, 1935, 109).

Acrobic, facultative.

Optimum temperature $37^{\circ} \mathrm{C}$.

Source: From necrotic areas in the kidney of a sheep.

Habitat: Found in caseous lymphadenitis in sheep and ulcerative lesions in horses, cattle and other animals.

10. Corynebacterium kutscheri (Migula) Bergey et al. (Bacillus pseudotuberculosis murium Kutscher, Ztsehr. f. Hyg., 18, 1894, 338; Bacillus pseudotuberculosis murium Lehmann and Neumann, Bakt. Diag., 1 Aufl., 2, 1896, 
362; Bacterium kutscheri Migula, Syst. d. Bakt., 2, 1900, 372; Mycobacterium pseudotuberculosis Chester, Manual Determ. Bact., 1901, 355; Corynebacterium murium Bergey et al., Manual, 1st ed., 1923, 386; Bergey et al., Manual, 2nd ed., 1925, 395.) Named for the bacteriologist Kutscher, who first isolated the species.

Rods with pointed ends, staining irregularly. Non-motile. Gram-positive.

Gelatin colonies: Small, white, translucent.

Gelatin stab: No growth on surface. White, filiform growth in stab. No liquefaction.

Agar colonies: Small, thin, yellowishwhite, translucent, serrate.

Agar slant: Thin, white, translucent

Loeffler's blood serum: Abundant growth. Not peptonized.

Broth: Slight turbidity. Crystals of ammonium magnesium phosphate are formed.

Litmus milk: Unnchanged.

Potato: No growth.

Indole not formed.

Nitrites not produced from nitrates.

Aerobic, facultative.

Optimum temperature $37^{\circ} \mathrm{C}$.

Source: From cheesy mass in lung of mouse.

\section{Corynebacterium murisepticum} v. Holzhausen. (Cent. f. Bakt., I Abt., Orig., 105, 1927-28, 94.) From Latin mus, muris, a mouse; Greek septicus, putrefying, septic.

Slender rods: 1.2 to 1.5 microns in length, with polar granules. Grow out into long filaments. Non-motile. Gram-positive.

Gelatin stab: Feeble growth, with fimbriate outgrowth along line of puncture.

Egg glycerol broth: Good growth.

Loeffler's blood serum: Good growth.

Broth: Turbid.

Litmus milk: Acid. No coagulation.

Potato: Good growth
Indole not formed.

Nitrates not reported.

Acid from glucose, fructose, galactose, maltose, lactose, sucrose, inulin and mannitol. Arabinose and isodulcitol are not attacked.

Hydrogen sulfide formed.

Pathogenic for mice.

Aerobic, facultative.

Optimum temperature $37^{\circ} \mathrm{C}$.

Habitat: Septicemia in mice.

12. Corynebacterium bovis Bergey et al. (B. pseudodiphtheria, Bergey, The Source and Nature of Bacteria in Milk. Penn. Dept. Agr. Bull. 125, 1904, 11; Bergey et al., Manual, 1st ed., 1923, 388.) From Latin bos, bovis, ox; of cattle.

Rods, slender, barred, clubbed, 0.5 to 0.7 by 2.5 to 3.0 microns. Non-motile. Gram-positive.

Gelatin stab: slight, gray, flat surface growth.

Agar colonies: Circular, gray, slightly raised, radiate, undulate, dry.

Agar slant: Thin, gray, filiform, dry growth.

Broth: Slight granular sediment.

Litmus milk: Slowly becoming deeply alkaline.

Potato: No growth.

Indole not formed.

Nitrites not produced from nitrates.

No acid from carbohydrate media.

Blood serum: Thin, gray, filiform growth.

Causes rancidity in cream. Weakly lipolytic on tributyrin agar (Black, Jour. Bact., 41, 1941, 99).

Optimum temperature $37^{\circ} \mathrm{C}$.

Source: In fresh milk drawn directly from the cow's udder.

Note: Miss Alice Evans (personal communication) states that the organism from the udder which she described as Bacterium lipolyticus (sic) (Bacillus abortus var. lipolyticus Evans, Jour. Inf. Dis., 18, 1916, 459; Bacterium abortus var. lipolyticus Evans, Jour. Bact., 2, 
1917, 185; Evans, Jour. Inf. Dis., 22, 191S, 576; not Bacterium lipolyticum Huss, Cent. f. Bakt., II Abt., 20, 1908, 474; Alcaligenes lipolyticus Pacheco, Revista da Sociedade Paulista de Med. Vet., 3, 1933, 9) was probably a Corynebacterium. This is also regarded as probable by Steck (Die latente Infektion der Milchdrüse, Hanover, 1930) and by Bendixen (Ztschr. f. Infektionskrankh. d. Haustier., 43, 1933, 106). Miss Evans also indicates that it is probable that the organism described by Bergey first in 1904 (loc. cit.) and later in the first edition of the Manual as Corynebacterium bovis ras the same organism. This is further confirmed by Black (Jour. Bact., $41,1941,99)$. A description of Bacterium lipolyticum Evans will be found in the Manual, 5th ed., 1939, 803.

13. Corynebacterium equi Magnussen. (Magnusson, Arch. f. Tierheilk. 50, 22 ; Corynebacterium pyogenes (equi) Meissner and Wetzel, Deutsche Tierarztl. Wehnsehr., 31, 1923, 449; Corynebacterium (pyogenes) equi roseum Lütji, ibid., 561; Mycobacterium equi Jensen, Proc. Linn. Soc. New So. Wales, 59, 1931, 33; Corynebacterium magnussonholth Plum, Cornell Vet., 30, 1940, 15; Corynebacterium purulentus Holtman, Jour. Bact., 49, 1945, 161.) From Latin equus, horse.

Description from Dimock and Edmards, Kentucky Agri. Exper. Stat., Bull. 333, 1932; Bruner and Edwards, ibid., Bull. 414, 1941; Merchant, Jour. Bact., 30, 1935, 95; and Brooks and Hucker, Jour. Bact., 48, 1914, 309.

Rods variable according to medium. Coccoid and ellipsoidal cells to rather long curved and sometimes clubbed forms. The latter are especially apt to occur in liquid media. Non-motile. Gram-positive.

Gelatin stab: Good growth. No liquefaction.

Agar colonies: Usually moist, smooth and glistening, tan to yellow (Brooks and Hucker, loc. cit., p. 300) or pink to red chromogenesis (Merchant, loc. cit., p. 107).

Agar slant: Moist heavy growth which may run down the slant (Dimock and Edwards, loc. cit., p. 322).

Broth: Turbid with no pellicle and little sediment (Dimock and Edwards, loc. cit., p. 322). Pellicle and final pH alkaline (Brooks and Hucker, loc. cit., p. 309). Branched cells occur in 6 to 8 hour cultures in broth.

Loeffler's blood serum: Good growth with $\tan$ to yellow chromogenesis. No liquefaction.

Coagulated egg yolk: Vigorous salmonpink growth. Dryer than on agar, resembling wrinkled growth of tubercle bacillus after two weeks.

Litmus milk: No change to slightly alkaline.

Potato: Abundant growth, usually tan, yellow or pink.

Indole not formed.

Hydrogen sulfide produced on appropriate media.

Nitrites produced from nitrates. No ammonia produced.

No acid from carbohydrate media. However, glucose stimulates growth.

Sodium hippurate: Not hydrolyzed.

Esculin: Tot hydrolyzed.

No exotoxin demonstrated in filtrate of broth cultures.

No or slight hemolysis of horse blood.

Not pathogenic for laboratory animals.

Aerobic.

Temperature relations: Optimum $25^{\circ}$ to $37^{\circ} \mathrm{C}$. Maximum $37^{\circ}$ to $45^{\circ} \mathrm{C}$. Minimum $7^{\circ}$ to $18^{\circ} \mathrm{C}$.

Source: Originally isolated from infectious pneumonia of foals.

Habitat: Found in spontaneous pneumonia of foals and other infections in horses. Also in swine, cattle and buffaloes.

Note: Jensen (loc. cit., 33) regards four cultures of soil bacteria isolated in Australia as identical with this organism. 
Because of the acid-fast staining of the cells, especially when grown in milk for 3 to 7 days, he places this species in the genus Mycobacterium. Most cocci retain the stain completely, while the rods take the counterstain. Jensen thinks the organism a widespread soil saprophyte which under certain conditions acquires pathogenic properties. He points out the close relationship of this organism to Bacterium aurantium-roseum Honig (Mededeel. Deli Proefstat. te Medan, 7, 1912 , 223) isolated from fermenting tobacco. He also regards this species as closely related to Mycobacterium coeliacum Gray and Thornton. Red strains seem to be much like Bacillus rubropertinctus Hefferan and Micrococcus (Staphylococcus) erythromyxa Zopf.

\section{Corynebacterium paurometabo-} lum Steinhaus. (Jour. Bact., 41, 1941, 763 and 783.) From Greek paurus, little; metabole, change or little action.

Rods : 0.5 to 0.8 by 1.0 to 2.5 microns, occurring singly, in pairs and in masses. Metachromatic granules present. Nonmotile. Gram-positive.

Gelatin stab: Slow liquefaction at surface.

Agar colonies: White to gray, entire, circular, small, dry, somewhat granular.

Agar slant: Filiform to arborescent, thick, granular growth.

Broth: Abundant granular sediment but no turbidity. Pellicle.

Litmus milk: Alkaline.

Potato: Thick, raised, dry, granular, profuse, gray to light cream-colored growth.

Indole not produced.

Slight production of hydrogen sulfide.

Nitrites not produced from nitrates.

No action on the following carbohydrates: Glucose, lactose, sucrose, maltose, fructose, mannitol, galactose, arabinose, xylose, dextrin, salicin, raffinose, tre- halose, sorbitol, inulin, dulcitol, glycerol, rhamnose, adonitol, mannose, esculin and inositol.

\section{Aerobic.}

Slight alpha hemolysis.

Non-pathogenic for guinea pigs.

A special semi-solid medium, the main nutritive constituents of which were proteose peptone, rabbit serum, gelatin, minced rabbit kidney and carbohydrates, was used for the original isolation. An incubation period of 4 to 7 days at $26^{\circ} \mathrm{C}$ was necessary for the initial isolation. Subsequent transfers to ordinary beefinfusion agar grew out in 24 to 48 hours.

Source: From media inoculated with the mycetome and ovaries of the bedbug, Cimex lectularius L. A very similar diphtheroid strain was isolated from the alimentary tract of the bagworm, Thyridopteryx ephemeraeformis Haw.

Habitat: Distribution in nature unknown.

*15. Corynebacterium insidiosum (Mc(ulloch) Jensen. (Aplanobacter insidiosum McCulloch, Phytopath., 15, 1925, 497; Bacterium insidiosum Stapp, in Sorauer, Handb. der Pflanzenkr., 2, 5 Aufl., 1928, 178; Phytomonas insidiosa Bergey et al., Manual, 3rd ed., 1930, 278; Jensen, Proc. Linnean Soc. of New So. Wales, 59, 1934, 41.) From Latin insidiosus, deceitful, dangerous.

Also see McCulloch, Jour. Agr. Res., 39, 1926, 502.

Rods: 0.4 to 0.5 by 0.7 to 1.0 micron. Capsules present. Non-motile. Grampositive.

Gelatin: Slow liquefaction.

Beef agar colonies: Pale yellow, circular, smooth, shining; edges entire; viscid. Blue granules found on the medium.

Milk: Coagulated after 16 to 20 days. No digestion. An apricot yellow sediment is deposited on the walls of the tube.

* Descriptions of Species nos. 15 to 20 inclusive prepared by Professor Walter H. Burkholder, Ithaca, New York. 
Nitrites not produced from nitrates. Indole not formed.

No $\mathrm{H}_{2} \mathrm{~S}$ produced.

Acid from glucose, sucrose, lactose and glycerol.

Moderate diastatic action.

Grows in 5 per cent salt.

Optimum temperature $23^{\circ} \mathrm{C}$. Maximum $31^{\circ} \mathrm{C}$.

Aerobic.

Distinctive character: Bluish granules produced in culture.

Source: Isolated from diseased alfalfa plants.

Habitat: Vascular pathogen of alfalfa, Medicago sativa.

Note: Jensen (loc, cit.) regards this species as being almost identical with Corynebacterium helvolum Kisskalt and Berend. He isolated one strain from grass soil which he regards as a saprophytic strain of this species. Jensen emphasized the angular arrangement of young cells grown on agar and potato. A faint indication of reduction of nitrates and of diastatic action was obtained. He also reports a weak proteolysis of milk. Optimum reaction is given as $\mathrm{pH}$ 5.6 to 6.8. A slimy variant of the soil strain was isolated from an old culture in glucose broth which seemed to agree better in its characteristics with the organism as described by Jones and McCulloch than did the non-slimy strains

15a. Corynebacterium insidiosum var. saprophyticum Jensen (loc. cit., 42) is based on a non-infectious soil strain. This grew more vigorously with less definite yellow pigment on nutrient agar than the pathogenic strain. Blue-violet, insoluble pigment near edge of growth on glucose agar; no blue pigment on potato; no coagulation of milk; higher temperature maximum and more resistance to acid reaction than the pathogenic strains. From grass soil in Australia. and Burkholder. (Bacterium sepedonicum nomen nudum Spiekermann, Ill. Landw. Zeitung, 33, 1913, 680; Bacterium sepedonicum Spiekermann and Kotthoff, Landw. Jahr., 46, 1914, 674; Aplanobacier sepedonicum Erw. F. Smith, Intro. Bact. Dis. of Plants, 1920, 207; Phytomonas sepedonica Magrou, in Hauduroy et al., Dict. d. Bact. Path., Paris, 1937, 111 ; Skaptason and Burkholder, Phytopath., 32, 1942, 439.) From Greek sepedonicus, putrefactive.

Description from Stapp (Ztschr. f. Par., 5, 1930, 756).

Rods : 0.3 to 0.4 by 0.8 to 1.0 micron. Pleomorphic. Non-motile. Gram-positive.

Gelatin : Liquefaction slight.

Agar colonies: Thin, smooth, translucent, glistening, whitish, 2 to $3 \mathrm{~mm}$ in diameter.

Broth: Weak growth. No pellicle. Light sediment.

Litmus milk : Little change in 6 weeks, after which litmus is reduced.

Indole not formed.

No $\mathrm{H}_{2} \mathrm{~S}$ production or feeble.

Glucose, galactose, fructose, arabinose, xylose, mannitol, glycerol and dulcitol are utilized.

Starch hydrolysis light.

Grows in 4 per cent salt.

Optimum temperature $20^{\circ}$ to $23^{\circ} \mathrm{C}$. Maximum temperature $31^{\circ} \mathrm{C}$. Minimum $4^{\circ} \mathrm{C}$.

Distinctive characters: Differs from Corynebacterium michiganense, in that it is white to cream-colored on various media and has a lower optimum temperature. Corynebacterium michiganense does not infect potatoes.

Source: Stapp used 17 cultures isolated from diseased potatoes.

Habitat: Causes ring rot of potato tubers in Germany.

17. Corynebacterium michiganense (Erw. Smith) Jensen. (Bacterium michiganense Erw. Smith, Science, 31, 1910, 794; Pseudomonas michiganensis Stevens, The Fungi which Cause Plant 
Diseases, 1913, 30; Aplanobacler michiganense Erw. Smith, Bacteria in Tiel. to Plant Dis., 3, 1914, 161; Phytomonas michiganensis Bergey et al., Minual, 1st ed., 1923, 191 ; Jensen, Proc. Linnean Soc. of New So. Wales, 59, 1934, 47 ; Ervinia michiganeae, incorrectly attributed to Bergey by Jensen, loc. cit., 47.) Latinized, of Michigan, where the discase produced by this pathogen was first reported.

Description from Bryan, Jour. $\operatorname{~gr}$. Res., 41, 1930, 825.

Rods : 0.6 to 0.7 by 0.7 to 1.2 microns. Non-motile. Capsules. Ciram-positive. Characteristic angular growth with branching and club-shaped cells (Jensen, loc. cit.).

Beef anar colonics: Growth slow, mustard yellow, smooth, glistening, butyrous.

Chromogenesis: Develops yellowishbrown, light ochre-yellow to sepia brown colors on suitable media (Jensen, loc. cit.).

Gelatin: slow liquefaction.

Broth: Turbidity slow and moderate.

Milk: Slow coagulation. No peptonization.

Nitrites not produced from nitrates.

T tilizes peptone, but not ammonia, nitrite, nitrate, tyrosine, asparagine or glutamic acid Mushin, Austral. Jour. Exp. Biol. and Med., 16, 1938, 326).

Indole not produced.

No $\mathrm{H}_{2} \mathrm{~S}$ produced.

Acid from glucose, sucrose, galactose, fructose, maltose, and slight acid from lactose, glycerol and mannitol.

Starch: Tery weak diastatic action.

No grewth in 3 per cent salt.

() ptimlan femperature $25^{\circ}$ to $27^{\circ}$ (

Maximum, $33^{\circ} \mathrm{C}$. Minimum, $1 \mathrm{C}^{\circ}$

Aerobic.

Source: From the bacterial ranker of tomato.

Habitat: Pathogenic on tomato.

17a. Corynebacterium michiganense var. saprophyticum Jensen (loc. cit., 48). Grows more rapidly and with more moist growth, has a higher temperature maximum and stronger proteolytic activity than the pathogenic strains. From grass soil in Australia.

18. Corynebacterium rathayi (Erw. Snith) Dowson. (Aplanobacter rathayi Erw. Smith, Science, 38, 1913, 926, and Bact. in Rel. to Plant Dis., 3, 191\%, 155; Phytomonas ralhayi Bergey et al., Manual, 1st ed., 1923, 192; Bacterium rathayi Stevens, Fungus Dis. of Plants, 1925, 21 ; Dowson, Brit. Myc. Soc. Trans., $25,1942,313$.) Named for E. Rathay, the Austrian plant pathologist who first isolated the species.

Rods : 0.6 to 0.75 by 0.75 to 1.5 microns. Ton-motile. Not acid-fast. Capsules. Gram-positive.

Gelatin: Slow liquefaction after 7 weeks.

Agar colonies: Small, yellow, slowgrowing.

Milk: (irowth slow. Yellow ring. Litmus milk: Alkaline and reduced.

Nitrites are produced from nitrates.

Potato plugs: Good, yellow, viscid growth.

Acid but no gas from glucose, sucrose and lactose.

Cohn's solution: No growth.

Heavy inoculum necessary in media.

Source: Isolated from slimy heads of Dactylis glomerata by E. Rathay in Austria.

Habitat: Pathogenic on Dactylis glomeratu.

Tote: Bacillus mucilaginosus koeleriae Aujeszky, Botanikai Kiozlemenyek, 13, (Foreign Supl. 41), 1914, 88; Pseudomonas mucilaginosus loeleriae Moesz, Schedis ad Flora Hungarica Exs. Cent. IV, No. 301, Seet. Bot. Mus. Nat. Hung., Budapest. 1915. The deseription of the bacterium is possibly that of the saprophyte, Pseulomonas nuorescens, but the description of the disease is that caused by Corynebacterium rathayi. The specimen in schedis is a head of grain that appears to be infected with Corynebacterium rathayi. 
19. Corynebacterium agropyri (O'Gara) comb. nov. (Aplanobacter agropyri O'Gara, Phytopath., 6, 1916, 343; Phytomonas agropyri Bergey et al., Manual, 1st ed., 1923, 190; Bacterium agropyri Stapp, in Sorauer, Handbuch d. Pflanzenkrankheiten, 5 Auf., 2, 1925, 37.) From Greek agros, field and puros, wheat; M. L. Agropyron, wheat grass.

Rods : 0.4 to 0.6 by 0.6 to 1.1 microns. Capsules. Non-motile. Gram-variable.

Gelatin : No liquefaction.

Nutrient agar slant: Meager, yellow, very viscid growth.

Broth : Light clouding with yellow precipitate.

Milk: Little changed. Jellow sediment formed.

Nitrites are produced from nitrates.

Acid but no gas from glucose, lactose, sucrose and glycerol.

Stareh: Hydrolysis feeble.

Optinum temperature $25^{\circ}$ to $28^{\circ} \mathrm{C}$.

This species is very similar to and may be identical with Corynebacterium rathayi Dowson.

Source: From slimy heads of wheat grass.

Habitat: Found on wheat grass, Agropyron smithii.

20. Corynebacterium fascians (Tilford) Dowson. (Phytomonas fascians Tilford, 5tth Rept. Ohio Agr. Exp. Sta. Bull. 561, 1936, 39; Jour. Agr. Res., 53, 1936, 393; Unnamed pathogen, Lacey, Ann. Appl. Biol., 23, 1936, 308; Dowson, Brit. Myc. Soc. Trans., 25, 19t2, 313.) From Latin fascio, producing a fasciation.

Rods : 0.5 to 0.9 by 1.5 to 4.0 microns. Non-motile. Gram-positive.

Gelatin: No liquefaction.

Potato-glucose agar colonies: Light cream-colored colonies appear after 72 hours. Punctiform, circular, later cadmiun yellow to deep chrome.

Nutrient agar slant: After one reek streak is filiform, flat, dull to glistening, smooth, opaque, cream-colored, and butyrous.
Broth: Slightly turbid. Fragile pellicle with distinct rim.

Milk: Litmus becomes blue. Other changes slight.

Nitrites are produced from nitrates.

Indole not formed.

Hydrogen sulfide is produced.

Acid but no gas from glucose, galactose, fructose, mannose, arabinose, xylose, maltose, sucrose, glycerol, mannitol and dextrin. No acid from rhamnose, lactose, raffinose and inulin.

Starch not hydrolyzed.

Grows in 8 per cent salt.

Optimum temperature $25^{\circ}$ to $28^{\circ} \mathrm{C}$.

Aerobic.

Source: Described from 15 single cell isolates from fasciated growths on sweet peas.

Habitat: Pathogenic on sweet pea, chrysanthemum, geranium, petunia, tobacco, etc.

21. Corynebacterium helvolum (Zimmerman) Kisskalt and Berend. (Bacillus helvolus Zimmermann, Bakt. unserer Trink- u. Nutzwässer, Chemnitz, 1, 1890, 52; Bacterium helvolum Lehmann and Neumann, Bakt. Diag., 1 Aufl., 2, 1S96, 254; Kisskalt and Berend, Cent. f. Bakt., I Abt., Orig., 81, 1918, 446; Flavobacterium helvolum Bergey et al., Manual, 1st ed., 1923, 114.) From Iatin helvus, of a light bay color.

Original description supplemented from Jensen, Proc. Linn. Soc. New So. Wales, 59, 1934, 37.

Rods : 0.5 by 1.0 micron, occurring singly. Show angular arrangement due to snapping division. Variable in morphology. Non-motile. Gram-positive.

Gelatin colonies: Small, circular, yellowish-gray. Liquefaction.

Gelatin stab : Slight development along the stab. Napiform liquefaction.

Agar colonies: Circular, pale yellow, smooth, slightly convex.

Agar slant: Pale yellow, plumose to spreading, moist, undulate.

Milk agar: Growth fair to very abun- 
dant, white to pale yellow. Some strains form a pink pigment. Proteolytic zones clear and broad after 4 days.

Asparagine agar: Seant to good growth, smooth, glistening, white and creamcolored to lemon-yellow or even dull pink.

Broth: Turbid, with gray ring and yellowish sediment. After four days the sediment contains long, curved and branching rods. May resemble small mycelia.

Litmus milk: Slightly acid, with soft coagulum, becoming alkaline; peptonized. Litmus reduced.

Potato: Pale yellow, moist, plumose growth, becoming rough, dull. Slimy variants noted in one strain. A myceloid variant with dry wrinkled growth was found in another strain.

Indole not formed.

Nitrites not produced from nitrates.

Acid from glucose, glycerol and mannitol. Usually from arabinose, sucrose, galactose, fructose.

Aerobic, facultative.

Hydrogen sulfide produced on appro priate media

Optimum temperature $25^{\circ} \mathrm{C}$. T'sually grows at $37^{\circ} \mathrm{C}$.

Source: Originally isolated from water.

Habitat: A common soil Corynebacterium.

22. Corynebacterium fimi (McBeth and Seales) Jensen. (Bacterium fimi. McBeth and Scales, Bur. of Plant Ind., U. S. Dept. Agr., Bull. 266, 1913, 30; Cellulomonas fimi Bergey et al., Manual, 1st ed., 1923, 166; Bacillus fimi Holland, Jour. Bact., 5, 1920, 218; Jensen, Proc. Linn. Soc. New So. Wales, 59, 1934, 48.) From Latin fimus, dung.

Description from Jensen (loc. cit.) who studied an authentic strain.

Rods present typical diphtheroid appearance with angular arrangement, 0.4 to 0.5 by 1.2 to 2.5 microns. Many longer, i rregular, curved, club-shaped and branching cells on Sabouraud's (whey) agar. Non-motile. Gram-negative (Mc-
Beth and Scales). Gram-variable like some other corynebacteria (Jensen).

Crelatin colonies: Small, round, becoming lobate. Slow liquefaction.

Gelatin stab: Granular yellow growth. Infundibuliform liquefaction.

Cellulose agar colonies: Circular, raised, smooth, glistening, gray, entire.

Igar slant: Smooth, glistening, white to lemon-yellow growth.

Glucose and Sabouraud's agar: Growth less abundant and cream-colored.

Asparagine agar: Very scant growth, narrow, thin, glistening, white.

Broth: Uniform turbidity, soft creamcolored to yellow sediment after 3 weeks.

Litmus milk: Coagulated at 3 weeks at $37^{\circ} \mathrm{C}$. Not at $28^{\circ}$ to $30^{\circ} \mathrm{C}$. Faintly acid.

Potato: Slow cream-colored to yellow growth.

Indole is formed.

Nitrites are produced from nitrates.

Ammonia is produced in peptone solutions.

Diastatic action doubtful.

Acid from glucose, fructose, arabinose, xylose, maltose, lactose, sucrose, raffinose, melezitose, dextrin, starch, salicin and glycerol. None or feebly produced from mannitol and dulcitol.

Causes rapid disintegration of cellulose (filter paper) in a 0.5 per cent peptone solution.

Aerobic, facultative.

Optimum temperature $20^{\circ} \mathrm{C}$ (MeBeth and Scales). Better growth at $37^{\circ} \mathrm{C}$ than at $28^{\circ}$ to $30^{\circ} \mathrm{C}$ (Jensen).

Source: Probably isolated from soil. Found in soils of Southern California (McBeth, Soil Sci., 1, 1916, 443).

Habitat: Soil.

Bacterium liquatum McBeth and Scales (McBeth and Scales, Bur. Plant Ind., U. S. Dept. Agr., Bull. 266, 1913, 32 ; Cellulomonas liquata Bergey et al., Manual, 1st ed., 1923, 166) should be regarded as identical with this species as the only signifieant difference reported between the two species by the original authors was that Bacterium liquatum produced a 
yellow chromogenesis more readily. This, however, does not appear to have occurred any more frequently than took place with the authentic culture of $\mathrm{Bac}$ terium fimi when tested by Jensen (loc. cit.).

23. Corynebacterium tumescens Jensen. (Jensen, Proc. Linn. Soc. New So. Wales, $59,1934,45$.) From Latin tumescens, swollen.

Rods show characteristic cytomorphosis in glucose agar, Sabouraud's (whey) agar and milk agar. Cells after 18 to 24 hours at $28^{\circ}$ to $30^{\circ} \mathrm{C}$ are curved, of ten branched, show an angular arrangement. 0.5 to 0.8 by 2.5 to 6.0 microns. After 2 to 3 days many spherical to club-shaped cystites ( 3 microns in diameter) arise as local swellings of the rods. Staining in tensely at first, they gradually change into large, irregular, poorly stained ghost cells which show deeply staining belts and granules. Irregular less swollen deeply stained rods and small cocei $(0 . t$ to 0.5 micron) which resemble the gran ules in the eystites are also present These cocci are living cells. Non-motile. (iram-positive.

Gelatin colonies: Small, opaque, yellow. Liquefaction after 3 to 4 weeks.

Milk agar: Cystites develop in almost pure culture. These sometimes have 2 to $t$ small cocei attached to the wall so that they look like budding yeasts. When transferred to fresh agar, cystites either fail to grow or germinate with 2 to $\frac{4}{ \pm}$ slender germ tubes which regenerate the rods. Crstites are produced most abundantly at $37^{\circ} \mathrm{C}$, sometimes not at all at $16^{\circ}$ to $18^{\circ} \mathrm{C}$

Sabouraud's agar: Cystites sometimes 6 to 8 microns in diameter. Frowth may be cream-colored or even grayish-pink.

Asparagine agar: firowth thin, flat, moist, colorless.

Broth: Faint uniform turbidity; after 2 to 3 weeks, a soft white to eream-colored sediment.

Milk: Thin white ring around surface Soft coagulation after 18 to 20 days. Later, slow digestion. Faintly acid.
Potato: Slow but eventually good growth, restricted, glistening, viscid, cream-colored to grayish-orange.

Acid from glucose, arabinose, galactose. maltose and glycerol; occasionally from sucrose and mannitol.

Nitrites produced from nitrates.

Optimum reaction $\mathrm{pH} 6.2$ to 6.8 .

Slimy variants produced after 172 days growth in lithium solution.

Source : Two strains from grass soils and one from garden soil in Australia.

Habitat: Soil.

24. Corynebacterium simplex Jensen. (Proc. Linn. Soc. New So. Wales, 59, 1934, 43.) From Latin simplex, simple.

Rods : 0.4 to 0.5 by 3.0 to 5.0 microns, curved and in parallel bundles. No branching in older cultures but the cells grow shorter, becoming almost roccoid. Angular arrangement. Nonmotile. Gram-positive.

Gelatin : Colonies very small. Filiform growth along stab. Liquefaction after 4 days.

Asparagine agar: Fair to good growth, becoming moist and glistening. No pigment.

Glucose agar: Abundant growth. Spreading, smooth, glistening, creamcolored to grayish-yellow.

Broth: Uniform turbidity, grayishyellow, viscid sediment.

Milk: Yellowish ring around surface. No coagulation. Complete digestion after 10 to 12 days. Reaction neutral.

Nitrites produced from nitrates.

Starch is not hydrolyzed.

Acid from sucrose. Alkaline reaction in other sugar broths.

Excellent growth at $37^{\circ} \mathrm{C}$

Resembles Corynebacterium filamentosum in cultural characters but does not form long filaments.

Source: From grass soil and red soil from Griffith, Australia.

Habitat: Soil.

25. Corynebacterium filamentosum Jensen. (Proc. Linn. Soc. New So. 
Wales, 59, 1934, 42.) From Latin filamentosus, full of threads.

Rods: Variable in shape. Young cells typically curved, vibrio-like, 0.5 to 0.8 by 2.0 to 7.0 microns, sometimes longer and branched. Always in parallel bundles. Usually non-motile but a few cells exhibit a peculiar oscillatory or rotatory movement. Gram-positive.

Gelatin: Colonies small, spherical, entire. Filiform white growth in stab. Liquefaction slow starting at end of 7 days.

Asparagine agar: Good characteristic growth, widely spreading, central part convex, smooth, glistening, white, sending dendritic projections into the broad marginal part. Usually produces light greenish-yellow soluble pigment.

Glucose agar: Growth less vigorous than on asparagine agar, flat, creamcolored to grayish-yellow, viscid.

Sabouraud (whey) agar: Similar to glucose agar.

Potato: Scant to no growth, flat, glistening, cream-colored to grayish-yellow, surrounded by a white halo.

Broth : Faint uniform turbidity. Soft, flaky, cream-colored sediment.

Milk: White to cream-colored surface ring and sediment. No coagulation. Digestion in 2 to 4 weeks. Neutral to faintly acid.

May produce nitrites from nitrates.

Starch is not hydrolyzed.

Acid from glycerol and arabinose. Strong and rapid alkaline formation in other sugar media.

Optimum reaction $\mathrm{pH} 5.4$ to 5.5 .

Excellent growth at $37^{\circ} \mathrm{C}$.

Aerobic.

Regarded as being much like Vibrio lingualis Eisenberg and Bacterium racemosum Zettnow.

Source: From red soil from Griffith, Australia.

Habitat: Soil.
Appendix I:* The following four species of plant pathogens have an unusual combination of characters in that they are reported to be Gram-positive and polar flagellate. Cultures of two of the four species have been available for study and these and other characters have been rechecked by several persons. Corynebacterium flaccumfaciens shows many wedgeshaped cells and longer cells with a slight curve. It is motile with a single polar flagellum and shows Gram-positive with commonly used procedures for Gram-staining. Corynebacterium poinsettiae shows a straighter form of cell but in other characters is like C. flaccumfaciens. Prof. W. H. Burkholder and Dr. M. P. Starr really feel that these organisms are most closely related to other more typical corynebacteria. They are therefore placed for the present in this appendix, although by the characters used in the keys they would be placed in Pscudomonadaceae.

1. Corynebacterium hypertrophicans (Stahel) comb. nov. (Pseudomonas hypertrophicans Stahel, Phyt. Ztschr., 6, 1933, 445; Phytomonas hypertrophicans Magrou, in Hauduroy et al., Dict. d. Bact. Path., Paris, 1937, 367.) From Greek hyper-trophe, hypertrophy.

Rods : 0.6 to 0.8 by 1.2 to 2.8 microns. Motile with a polar flagellum. Bipolar staining. Gram-positive.

Gelatin: No growth.

Agar colonies: Slow growing, circular, raised, wet-shining, white.

Broth plus sucrose: Growth good. No pellicle.

Milk: No visible change.

Nitrites not produced from nitrates.

Indole not formed.

No $\mathrm{H}_{2} \mathrm{~S}$ produced.

Acid but no gas from glucose, fructose and sucrose. No acid from lactose and glycerol. The acids from sucrose are lactic and formic.

*Prepared by Prof. Walter H. Burkholder, New York State College of Agriculture, Ithaca, New York, May, 1945. 
Aerobic.

Source: From witches' brooms.

Habitat: Pathogenic on Eugenia latifolia.

\section{Corynebacterium flaccumfaciens} (Hedges) Dowson. (Bacterium flaccumfaciens Hedges, Science, 55, 1922, 433; Phytopath., 16, 1926, 20; Phytomonas flaccumfaciens Bergey et al., Manual, lst ed., 1923, 178; Pseudomonas flaccumfaciens Stevens, Plant Diseases of Fungi, 1925, 27; Dowson, Brit. Myc. Soc. Trans., 25, 1942, 313.) From Latin flaccus, flabby or wilted; facio, to make; producing a wilt.

Rods: 0.3 to 0.5 by 0.6 to 3 microns. Motile with a single polar flagellum; also non-motile (Adams and Pugsley, Jour. Dept. Agr. Victoria., 32, 1934, 306). Gram-positive.

Gelatin: Liquefaction feeble.

Beef agar slants: Rather moderate growth, glistening, flat, smooth, viscid and yellow.

Broth : Moderate turbidity in 24 hours. Pellicle formed.

Milk: Acid curd and slow peptonization.

Nitrites not produced from nitrates.

Indole not formed.

No $\mathrm{H}_{2} \mathrm{~S}$ formed.

Acid from glucose, lactose, sucrose and glycerol.

Starch not hydrolyzed.

Slight growth in 5 per cent salt.

Optimum temperature, $31^{\circ} \mathrm{C}$. Maximum temperature $36^{\circ}$ to $40^{\circ} \mathrm{C}$.

Distinctive character: A strict vascular parasite of the bean.

Source: From wilted bean plants from South Dakota.

Habitat: Causes a wilt of beans and related plants.

3. Corynebacterium poinsettiae Starr and Pirone. (Phytopath., 32, 1942, 1080; Phytomonas poinsettiae, ibid.) From M. L., old genus Poinsettia.

Rods: Average cells 0.3 to 0.8 by 1.0 to 3.0 microns. Pleomorphic with some cells $8.5^{\text {microns in length. Granules }}$ and capsules present. Motile with 1 (rarely 2) polar or lateral flagellum. Gram-positive.

Gelatin: Liquefaction.

Loeffler's blood-serum: Liquefaction.

Beef-extract agar colonies: Round, slightly convex, 0.1 to $1.0 \mathrm{~mm}$ in diameter, edges entire, smooth, non-viscid, colorless and almost transparent.

Potato glucose agar slants: Moderate growth, filiform, glistening, non-viscid, salmon to flesh color.

Beef-extract broth: Turbid in 24 hours, abundant pale salmon sediment. No pellicle.

Milk: Slight acidity but no other visible change for 2 weeks, then a soft curd, reduction of litmus, and complete peptonization.

Indole not produced.

Nitrites not produced from nitrates.

Hydrogen sulfide not formed.

Sodium hippurate not hydrolyzed.

Asparagine not utilized as carbonnitrogen source. Uric acid not utilized; urea not hydrolyzed.

No lipolytic activity.

Voges-Proskauer test negative. Methyl red test negative.

Moderate to abundant acid, but no gas, from glucose, fructose, mannose, galactose, sucrose, maltose, cellobiose, melibiose, raffinose, glycerol, erythritol, salicin and amygdalin; weak acid from arabinose, xylose, lactose, trehalose, dextrin and adonitol; no acid from rhamnose, fucose, inulin, glycogen, mannitol, dulcitol, sorbitol and inositol.

Starch hydrolyzed.

No action on cellulose.

Tellurite reduced.

Aerobic.

Growth occurs after 24 hours from $15^{\circ} \mathrm{C}$ to $36^{\circ} \mathrm{C}$; after 48 hours from $7^{\circ} \mathrm{C}$ to $12^{\circ} \mathrm{C}$. No growth above $36^{\circ} \mathrm{C}$ or below $7^{\circ} \mathrm{C}$ at the end of a week.

Source: Fourteen cultures isolated from diseased stems of poinsettia, Euphorbia pulcherrima.

Habitat: Causes a canker of stems and spots on leaves of the poinsettia. 
4. Corynebacterium tritici (Hutchinson) comb. nov. (Pseudomonas tritici Hutchinson, India Dept. of Agr., Bact. Ser., 1, 1917, 174; Phytomonas tritici Bergey et al., Manual, 3rd ed., 1930, 248; Bacterium tritici Elliott, Bacterial Plant Pathogens, 1930, 234.) From Latin triticum, wheat; M. L., from the genus Triticum.

Rods : 0.8 by 2.4 to 3.2 microns. Motile with a polar flagellum. Gram-positive.

Gelatin: No liquefaction.

Agar colonies: Bright yellow becoming orange, glistening, moist, margins entire. Agar brownish.

Broth: Turbid. Thin pellicle.

Milk: Yellow surface and yellow precipitate. Little change.

Nitrites produced from nitrates.

No $\mathrm{H}_{2} \mathrm{~S}$ produced.

Acid but no gas from glucose and lactose.

This species is very similar to and may be identical with Corynebacterium rathayi. Dowson.

Source: From slimy heads of wheat in India.

Habitat: Pathogenic on wheat, Triticum aestivum.

* Appendix II: By the use of names or by the descriptions given, authors have indicated that the following are related to the species placed in Corynebacterium. Many are incompletely described and may be identical with other recognized species.

Bacillus alcalifaciens Kurth. ( $B a$ cillus pseudodiphtheriticus alcalifaciens Kurth, Ztschr. f. Hyg., 28, 1898, 429; ibid., 431.) From patients suspected of having diphtheria.

Bacillus avium Migula. (Bacillus de la diphthérie aviaire, Loir and Ducloux, Ann. Inst. Past., 8, 1894, 599; Bacillus diphtheriae avium Kruse, in Flügge, Die Mikroorganismen, 2 Aufl., 2, 1896 ,
410 ; Bacterium diphtheriae avium (sic) Chester, Ann. Rept. Del. Col. Agr. Exp. Sta., 9, 1897, 75; Migula, Syst.d. Bakt., 2, 1900, 759.) Considered the cause of a diphtheria-like disease of birds in Tunis. Motile. Not now regarded as belonging in Corynebacterium (Andrewes et al., Diphtheria, London, 393).

Bacillus clavatus Kruse and Pasquale. (Kruse and Pasquale, Ztschr. f. Hyg., 16, 1894, 50 and 62 ; not Bacillus clavatus Migula, Syst. d. Bakt., 2, 1900, 597.) From the heart blood, kidney, etc., during autopsy of a person who died with liver abscesses following Egyptian dysentery. This is a pseudodiptheroid (Irruse, in Flügge, Die Mikroorganismen, 3 Aufl., 2, 1896, 477) but is confused by Eberson (Jour. Inf. Dis., 23, 1918, 5) and Thomson and Thomson (Ann. Pickett Thomson Res. Lab., 2, 1926, 65) with anaerobic Bacillus No. III, a spore former isolated by Flügge (Ztschr. f. Hyg., 17, 1894, 290) from boiled milk and named Bacillus clavatus by Migula (loc. cit.) in 1900 .

Bacillus crassus Lipschütz. (Lipschütz, Bakt. Grundriss und Atlas der Geschlechtekrankheiten, Leipzig, 1913, 64; Plocamobacterium crassum Löwi, Wiener klin. Wchnschr., 33, 1920, 733; not Plocamobacterium vaginae Lehmann, in Lehmann and Neumann, Bakt. Diag., 7 Aufl., 2, 1927, 510.) This is the abundant Gram-positive bacillus found in ulcus vulvae acutum. It is the type species (monotypy) of the genus Plocamobacterium Löwi (loc. cit.). According to Löwi this organism liquefies coagulated blood serum and Lipschütz (Cent. f. Bakt., I Abt., Orig., 88, 1922, 5 ) reports that, unlike lactobacilli, this organism will grow on protein media without the addition of sugar. Presumably therefore it is not a lactobacillus and is not identical with Doederlein's bacillus as claimed

* Prepared by Dr. R. F. Brooks, New York State Experiment Station, Geneva, New York, September, 1938; further revision by Prof. Robert S. Breed, New York State Experiment Station, Geneva, New York, March, 1945. 
by Lehmann (loc. cit.) It may belong in Corynebacterium. See Bacillus vaginae Kruse.

Bacillus diphtheriae vitulorum Flügge. (Bacillus der diphtherie beim Kalbe, Löfler, Mitt. a. d. kais. Gesundheitsamte, 2, 18.54, 421; Flügge, Die Mikroorganismen, 2 Aufl., 1886, 265.) From a disease of calves.

Bacillus diphtheroides Klein. (Cent. f. Bakt., I Abt., 28, 1900, 418.) From bovine mastitis. Presumably identical with Corynebacterium pyogenes according to Eberson (Jour. Inf. Dis., 23, 1918, 6).

Bacillus endocarditis griseus Weichselbaum. (Weichselbaum, Beiträge z path. Anat, u. allgem. Path., 4, 1887, 119.) From a case of endocarditis. A motile form. Regarded by Kruse (in Flügge, Die Mikroorganismen, 3 Aufl., 2, 1896, 433 and 479 ) as a diphtheroid. Because of its motility, it is not so regarded by Eberson (Jour. Inf. Dis., 23, 1913, 4).

Bacillus pseudodiphtheriticus acidum aciens Kurth. (Ztschr. f. Hyg., 28, 1898, 431.) From patients suspected of having diphtheria.

Bacillus pseudodiphtheriticus yazogenes Jacobsen. (Ann. Inst. Past., 22, 1908, 308.) From feces. Reported to be a vigorous gas former. Eberson (Jour. Inf. Dis., 23, 1918, 9) thinks this was an impure culture.

Bacillus septatus Gelpke. (Gelpke, in v. Graefe, Arch f. Opthal., 42, 1896, No. 4; Bacterium septatum Gelpke, Arb. bakt. Inst. Karlsruhe, 2, Heft 2, 1898, 73.) From acute epidemic eatarrh. Presumably identical with Corynebacterium xerose according to Eherson (Jour. Inf. Dis., 23, 1918, 3).

Bacillus variabilis lymphae vaccinalis Nakanishi. (Nakanishi, C'ent. f. Bakt., I Abt., Orig., 27, 1900, 641; Corynethrix. bovis Czaplewski, Deutsche med. Wehnschr., 26, 1900, 723.) From calf vaccine lymph. The organisms listed here as Corynebacterium lymphae vaccinalis, Corynebacterium vaccinae and Bacillus variabilis lymphae vacinalis are probably identical.
Bacillus xerosis variolae Klein. (Rept. Local Gov. Board, London, 20, 1890, 219, quoted from Thomson and Thomson, Ann. Pickett-Thomson Res. Lab., 2, 1926, 121.) From vaccine pustules.

Bacterium acnes Migula. (Bacillus der Akne contagiosa des Pferdes, Dieckerhoff, Grawitz, Arch. f. pathol. Anat. u. Physiol., 102, 1886, 148; Bacillus grawitzii Trevisan, I generi e le specie delle Batteriacee, 1889, 13; Bacillus acnes-contagiosae Kruse, in Flügge, Die Mikroorganismen, 3 Aufl., 2, 1896, 445; Migula, Syst. d. Bakt., 2, 1900, 385; Bacterium grawitzii Chester, Manual Determ. Bact., 1901, 154.) From pus and scabs of pustules in acne-contagiosa in horses.

Bacterium candidus Galli-Valerio. (Cent. f. Bakt., I Abt., Orig., 36, 1904, 465.) From infected leg, but not considered causative.

Bacterium coelicolor Müller. (Müller, Cent. f. Bakt., I Abt., Orig., 46, 1908, 195; Bacillus coelicolor Godfrin, Contribution à l'étude des bactéries bleues et violettes, Thèse, Nancy, 1934.) Contaminant on serum agar plate.

Bacterium columbarum Migula. (Bacillus der diphtherie bei der Taube, Löffler, Mitt. a. d. kais. Gesundheitsamte, 2, 1884, 421; Bacillus diphtheriae columbarum Flügge, Die Mikroorganismen, 2 Aufl., 1886, 263; Bacillus diphtheriae-columbarum Trevisan, I generi e le specie delle Batteriacee, 18s9, 13: Baclerium diphtheriac columbarum Chester, Ann. Rept. Del. Col. Agr. Exp. Sta., 9, 1s97, 84; Migula, Syst. 1. Bakt., 2, 1900, 381; not Bacterium columbarum Chester, Manual Determ. Bact.. 14)1, 141; Bacterium diphtheriae Chester, Man. Determ. Bact.. 1901, 141; not Bacterium diphtheriae Migula, Syst. d. Bakt., 2, 1900, 499.) Associated with diphtheria in pigeons. Andrewes et al. (Diphtheria, London, 1923, 393) state that this organism does not belong in Corynebacterium.

Bacterium muris Klein. (Cent. f 
Bakt., I Abt., Orig., 33, 1902, 488; Bacillus muris Mellon, Jour. Bact., 2, 1917, 305.) Causative agent of hepatized lung in white rat.

Bacterium pseudopestis murium GalliValerio. (Cent. f. Bakt., I Abt., Orig., $68,1913,188$.) (ausative agent of thyroid infection in rats. Gram-negative.

Bacterium ribberti Migula. (Bacillus der Darmdiphtherie des Kaninchens, Ribbert, Deutsch. med. Wochnschr., 13, 1887, 141; Bacillus diphtheriae cuniculi Kruse, in Flügge, Die Mikroorganismen, 3 Aufl., 2, 1896, 412; Bacterium diphtheriae cuniculi Chester, Ann. Rept. Del. Col. Agr. Exp. Sta., 9, 1897, 84; Migula, Syst. d. Bakt., 2, 1900, 369; Bacterium cuniculi Chester, Man. Determ. Bact.. 1901, 141.) Associated with a diphtheritic inflammation of the intestines in rabbits.

Coccobacillus diphtheroides Manteufel. (Diphtheroid bacilli, Collis, Sheldon and Hill, Quart. Jour. Med., Ser. 2, 1, 1932, 511; Kokkobacillus diphtheroides Bertrand, Med. Welt, 8, 1934, 150; Manteufel, Cent. f. Bakt., I Abt., Orig., 138, 1937, 308.) From polyarthritis.

Comma variabile Heurlin. (Heurlin, Bakt. Unters. d. Keimgehaltes im Genitalkanale d. fiebernden Wöchnerinnen. Helsingfors, 1910, 145.) From genital canal.

Corynebacterium acidum Eberson.

(Bacillus diphtheroides brevis GrahamSmith, Jour. Hyg., 4, 1904, 258; Eberson, Jour. Inf. Dis., 23, 1918, 9.) From large abscess in mouth and ear.

Corynebacterium adamsoni Prévot. (Bacillus D, Adamson, Jour. Path. and Bact., 22, 1919, 350 and 392; Prévot, Ann. Inst. Past., 60, 1938, 304.) From infected war wounds.

Corynebacterium album Belenky and Popova. (Cent. f. Bakt., I Abt., Orig., $118,1930,444$.) From normal skin of calves and small-pox vaccine.

Corynebacterium anaerobium Prévot. (Bacillus anacrobius diphtheroides Massini, Ztschr. f. gesammte exper.
Med., 2, 1913, 81; Prévot, Ann. Inst. Past., 60, 1938, 304.) From a complicated case of otitis media.

Corynebacterium annamensis Hauduroy et al. (Gillon, Thèse pour le Doctorat Vetérinaire, École Nationale Vetérinaire de Toulouse, France, 1930; Hauduroy et al., Dict. d. Bact. Path., Paris, 1937, 145.) Causative agent of a toxic abdominal infection of sheep in Annam, French Indo-China.

Corynebacterium arthritidis muris Fischl, Koech and Kussat. (Ztschr. f. Hyg., 112, 1931, 421; Corynebacterium arthritidis-muris Hauduroy et al., Dict. d. Bact. Path., 1937, 147.) Causative agent of infected ankle joint in white mouse.

Corynebacterium ascitis Eberson. (Jour. Inf. Dis., 23, 1918, 16.) From ascitic fluid.

Corynebacterium aurantiacum Eberson. (Jour. Inf. Dis., 23, 1918, 14.) Orangered growth. From lymph nodes; one culture from gland in Hodgkin's disease but not specific for the disease.

Corynebacterium auris (GrahamSmith) Eberson. (Bacillus auris GrahamSmith, Jour. Hyg., 4, 1904, 258; Eberson, Jour. Inf. Dis., 23, 1918, 8.) Indole is formed. From pus of ears of scarlet fever patients.

Corynebacterium avidum (Eggerth) Prévot. (Bacteroides avidus Eggerth, Jour. Bact., 30, 1935, 289; Prévot, Ann. Inst. Past., 60, 1938, 304.) Forms gas in some media. From the human intestine. Pederson (Jour. Bact., 50, 1945, 478) secured a culture of this species from Eggerth, and found that it fermented glucose with the production of higher fatty (presumably butyric) acids, and lactic acid. The species should probably be placed in Butyribacterium Barker.

Corynebacterium blattellae Glaser. (Jour. Exp. Med., 51, 1930, 907.) Found in the fat body of the German cockroach (Bluttella germanica). For a more complete description see Manual, 5th ed., $1939,978$. 
Corynebacterium bruneum Kisskalt and Berend. (Bacterium bruneum $\gamma$ arborescens, quoted from Kisskalt and Berend, Cent. f. Bakt., I Abt., Orig., $81,1918,446$; Kisskalt and Berend, idem.) Source not given.

Corynebacterium cerebralis Eberson. (Jour. Inf. Dis., 23, 1918, 17.) From the brain in a case of meningitis.

Corynebacterium ceruminis (GrahamSmith) Eberson. (Bacillus ceruminis Graham-Smith, Jour. Hyg., 4, 1904, 258; Eberson, Jour. Inf. Dis., 23, 1918, 8.) Indole is not formed. From normal and scarlet fever-infected ears.

Corynebacterium commune Martin. (Compt. rend. Soc. Biol., Paris, 81, 1918, 991 and 998.) From the pharynx.

Corynebacterium cremoides (Lehmann and Neumann) Jensen. (Bacterium cremoides Lehmann and Neumann, Bakt Diag., 1 Aufl., 2, 1896, 253; Jensen, Proc. Linn. Soc. New So. Wales, 59, 1934, 40.) From tapwater, Würzburg. Lehmann and Neumann recognize this species as a Corynebacterium in the seventh edition of their determinative bacteriology (Bakt. Diag., 7 Aufl., 2, 1927, 710) but do not use the binomial Corynebacterium cremoides except in the index, page 848 . Jensen has reisolated this organism from soil in Australia. Bacterium cocciforme Migula (Kultur No. 2, Severin, Cent. f. Bakt., II Abt., 1, 1895, 160; Migula, Syst.d. Bakt., 2, 1900, 439) from manure is regarded by Jensen (loc. cit.) as closely related to this species.

Corynebacterium cuculi (GrahamSmith) Bergey et al. (Bacillus cuculi Graham-Smith, Jour of Hyg., 4, 1904, 315; Bergey et al., Manual, 1st ed., 1923, 387.) From the throat of a cuckoo. For a more complete description see Manual, 5th ed., 1939, 802.

Corynebacterium cuniculi Hauduroy et al. (Bacillus pyogenes cuniculi Cominotti, Clinica Veterinaria, 44, 1921, 45; Hauduroy et al., Dict. d. Bact. Path., 1937, 147). Reported as Gram-variable by Cominotti, as Gram-negative by
Hauduroy et al. Causative agent of suppurative infection of rabbit.

Corynebacterium cutis Hauduroy et al. (Bacillus cutis communis Nicolle, quoted from Costa, Troisser and Dauvaugne, Compt. rend. Soc. Biol., Paris, 81, 1918, 1003; Bacillus cutis Costa, Troisser and Dauvaugne, ibid., 1004; Bacterium cutis commune Nicolle, quoted from Debré and Letulle, La Presse Méd., 27, 1919, 515; Hauduroy et al., Diet. d. Bact. Path., 1937, 148). From normal skin and nasal passages.

Corynebacterium delicatum Eberson. (Jour. Inf. Dis., 23, 1918, 16.) From ascitic fluid. Also from blood.

Corynebacterium dermophilum (Rohde) Andrewes et al. (Bacillus dermophilus Rohde, Münch. med. Wchnschr., 68, 1921, 234; Andrewes, Bulloch, Douglas, Dreyer, Fildes, Ledingham and Wolf, Diphtheria, London, H. M. Stationery Office, 1923, 391.) From the skin.

Corynebacterium diphtheroides Prévot. (Bacille diphtheroide, Jungano, Compt . rend. Soe. Biol., Paris, 61, 1909, 112; Prévot, Ann. Inst. Past., 60, 1938, 304.) Forms gas in some media. From the intestines of white rats.

Corynebacterium cpidermidis Eberson. (Jour. Inf. Dis., 23, 1918, 17.) From skin and pus pockets. Resembles Corynebacterium suppuratum Eberson.

Corynebacterium flocculens Eberson. (Jour. Inf. Dis., 23, 1918, 17.) From a case of appendicitis.

Corynebacterium gallinarum Bergey et al. (Bacillus diphtheroides gallinarum Graham-Smith, Jour. of Hyg., 4, 1904, 314; Bergey et al., Manual, 1st ed., 1923,387.) From the throats of ehickens. For a more complete description see Manual, 5th ed., 1939, 802 .

Corynebacterium glandulae Eberson. (Jour. Inf. Dis., 23, 1918, 14.) From lymph glands in Hodgkin's disease but not specific for the disease.

Corynebacterium granulomatis maligni de Negri and Mieremet. (Cent. f. Bakt., I Abt., Orig., 68, 1913, 292.) Causative agent of human malignant granuloma. 
Corynebacterium granulosum Prévot. (Bacille granuleux, Jungano, Compt. rend. Soc. Biol., Paris, 66, 1909, 123; Prévot, Ann. Inst. Past., 60, 1938, 304.) From the intestines of white rats.

Corynebacterium hepatodystrophicans (Kuczinski) Prévot. (Bacillus hepatodystrophicans Tuczinski, Der Erreger des Gelbfiebers-Wesen und Wirkung, Monographie, 1929, Berlin; Prévot, Ann. Inst. Past., 60, 1938, 304.) Manteufel (Cent. f. Bakt., I Abt., Orig., 138, 1937, $309)$ regards this species as identical with Bacillus renale (cuniculi) Manteufel and Herzberg. Common in the organs of monkeys infected with yellow fever virus.

Corynebacterium hodykinii Bunting and Yates. Bunting and Yates, Arch. Internal Med., 12, 1913, 236; Johns Hopkins Hosp. Bull., 25, 1914, 173; Bacillus hodgkini Mellon, Jour. Bact., 2, 1917, 271; Fustormis hodgkini Holland, Jour. Bact., 5, 1920, 223.) From lymph glands in Hodgkin's disease. Not pathogenic. Thought by Fox (Jour. Med. Res., 32, 1915, 309) and Eberson (Jour. Inf. Dis., $23,1918,11$ ) not to represent a definite species. Eberson recognized four separate species isolated from human lymph glands, three being from glands in Hodgkin's disease (Corynebacterium aurantiacum, C. pseudodiphtheriae, C. glandulae and $C$. lymphophilum).

Corynebacterium liquejaciens Prévot. (Bacillus parvus liquefaciens Jungano, Compt. rend. Soc. Biol., Paris, 65, 1908, 618; Prévot, Ann. Inst. Past., 60, 1938 , 304; not Corynebacterium liquefaciens Andrewes et al., Diphtheria, London, 1923, 408; not Corynebacterium liquefaciens Jensen, Proc. Linn. Soc. New So. Wales, 59, 1934, 49.) From human intestine.

Corynebacierium liquefaciens Andrewes et al. (Bacillus diphtheroides liquefaciens Graham-Smith, Jour. Hyg., 4, 1904, 258; Bacillus liquefaciens Mellon, Jour. Bact., 2, 1917, 290; Andrewes, Bulloch, Douglas, Dreyer, Fildes, Ledingham, and Wolf, Diphtheria, London, 1923, 408.) From mouth of a patient. Motile.
Corynebaclerium lymphae vaccinalis Levy and Fickler. (Deutsch. med. Wchnschr., 26, 1900, 418; Corynebacterium pyogenes Lewandowsky, Cent. f. Bakt., I Abt., Orig., 36, 1904, 473.) From animal lymph.

Corynebacterium lymphophilum (Torrey) Eberson. (Bacillus lymphophilus Torrey, Jour. Med. Res., 34, 1916, 79; Eherson, Jour. Inf. Dis., 23, 1918, 23.) Anaerobic. From lymph glands in Hodgkin's disease, but not specifie for the disease.

Corynebacterium maculatum. (GrahamSmith) Ford. (Bacillus maculatus Graham-Smith, Jour. Hyg., 4, 1904, 258; Ford, Textb. Bact., 1927, 277.) From throat. Regarded as a Corynebacterium by Eberson (Jour. Inf. Dis., 23, 1918, 7).

Corynebacterium metritis Hauduroy et al. (Souckin, Sovetskaia Veter., No. 11, 1934; Hauduroy et al., Dict. d. Bact. Path., 1937, 156.) Causative agent of metritis in rabbit.

Corynebacterium miltinum Kisskalt. (Quoted from Kisskalt and Berend, Cent. f. Bakt., I Abt., Orig., 81, 1918, 446). Source not given.

Corynebacterium nodosum (Migula) Eberson. (Bacillus nodosus parvus Lustgarten-Mannaberg, Vierteljahrschrift f. Dermatol. u. Syphilis, 1887, 914; Bacterium nodosum Migula, Syst. d. Bakt., 2, 1900, 416; Eberson, Jour. Inf. Dis., 23, 1918, 4.) Found in the normal human urethra.

Corynebacterium nubilum (Frankland and Franklind) Jensen. (Bacillus nubilus Frankland and Frankland, Ztschr. f. Hyg., 6, 1889, 386; Bacterium nubilum Lehmann and Neumann, Bakt. Diag., 1 Aufl., 2, 1896, 255; Chromobacterium nubile Ford, Textb. of Bact., 1927, 472; Flavobacterium nubilum, incorrectly ascribed to Bergey, by Jensen, Proc. Linn. Soc. New So. Wales, 59, 1934, 44 ; Jensen, idem.) From water and soil. The identity of this species is doubtful. The original description by the Franklands is incomplete. Zimmerman (Bakt. unserer Trink- u. Nutzwässer, Chemnitz, 1, 
$1890,28)$ thought he found the same organism and described it as Gram-negative. Lehmann and Neumann (Bakt. Diag., 1 Aufl., 2, 1896, 255) who studied one of Zimmermann's cultures reported this culture as Gram-positive and nonmotile, while the Franklands and Zimmermann speak of an active, circular motility of the very slender rods. Lehmann and Neumann later (Bakt. Diag., 7 Aufl., 2, 1927, 710) list their Bacterium nubilum (with other Gram-positive, nonmotile rods) as a possible Corynebacterium. Jensen failed to find anything that exactly corresponded to any of these descriptions but describes a small, Gram-positive, poorly-growing, pink to red, slow gelatin-liquefying rod which he says has little in common with corynebacteria as a new variety Corynebacterium nubilum var. nanum. Because the early cultures developed rhizoid growths in stiff gelatin before liquefaction, Zimmermann originally planned to call this species Bacillus nebulosus (loc. cit., 29), a name that has been used by later authors for several different organisms. Attention should be called also to Bacillus caudatus Wright, an organism which Conn found to show occasional motility (polar) and named Pseudomonas cauda datus. This common, slender, gelatinliquef ying, Gram-negative, white to yellow chromogenic rod is much like the Franklands' and Zimmermann's organism (see Conn, New York State Exp. Sta. Tech. Bull. 67, 1919, 38).

Corynebacterium paralyticans (Robertson) Ford. (Bacillus paralyticans Robertson, Rev. Neurol. and Psychiat. Edinburgh, 1, 1903, 470; Ford, Textb. of Bact., 1927, 281.) From cerebrospinal fluid. A diphtheroid. Thought at one time to be the causal agent of general paralysis.

Corynebacterium parvum Prévot. (Corynebacterium parvum infectiosum Mayer, Cent. f. Bakt., I Abt., Orig., 98, 1926, 370; Prévot, Man. de Class. et Déterm. des Bactéries Anaérobies, Mono- graphie, Inst. Past., Paris, 1940, 202.) From blood in a post-natal fever.

Corynebacterium periplanetae Bergey et al. (Corynebacterium periplanetae var. americana Glaser, Jour. Exp. Med., 51, 1930, 59; Bergey et al., Manual, 4th ed., 1934, 550.) Found in the fat body of the American cockroach (Periplaneta americana). For a more complete description see Manual, 5th ed., 1939, 798.

Corynebacterium plumosum (Fox) Ford. (Mycobacterium plumosum Fox, Cent. f. Bakt., I Abt., Orig., 70, 1913, 148; Ford, Textb. Bact., 1927, 281.) From blood of patient with chronic endocarditis.

Corynebacterium pseudodiphtheriae Eberson. (Jour. Inf. Dis., 23, 1918, 14.) Hemoglobinophilic. From tonsils.

Corynebacterium putidum Eberson. (Bacillus diphtheroides liquefaciens Graham-Smith, Jour. Hyg., 4, 1904, 258; Eberson, Jour. Inf. Dis., 23, 1918, 16.) From mouth. Cultures described by Graham-Smith liquefied gelatin and were sluggishly motile.

Corynebacterium pyogenes bovis (Roux) Prérot. (Bacillus pyogenes bovis Roux, Cent. f. Bakt., I Abt., Orig., 34, 1905, 541 ; Eubacterium pyogenes bovis Prévot, Ann. Inst. Past., 60, 1938, 295; Prévot, Man. de Class. et Déterm. des Bactéries Anaérobies, Monographie, Inst. Past., Paris, 1940, 204.) Common in bovine suppuratims. Said by Roux to be identical with Bacillus pyogenes bovis Kunnemann. Prévot says it is probably identical with the pyogenic Corynebacterium of Lucet. See Corynebacterium pyogenes Eberson.

Corynebacterium renale cuniculi Prévot. (Bacterium renale and Bacterium renale (cuniculi) Manteufel and Herzberg, Cent. f. Bakt., I Abt., Orig., $116,1930,266$; Bacillus renale and Bacillus renale (cuniculi) Manteufel, ibid., 138, 1937, 306; Prévot, Ann. Inst. Past., 60, 1938, 304.) Gram-variable. Forms gas in some media. From rabbit kidneys.

Corynebacterium ruedigeri (Mellon) 
Ford. (Virulent pseudodiphtheria bacillus, Hamilton, Jour. Inf. Dis., 1, 1904, 711 ; Ruediger's bacillus, Mellon, Jour. Bact., 2, 1917, 285; Bacillus ruedigeri Mellon, ibid., 290; Ford, Textb. Bact., 1927, 274.) From throats of fatal cases of scarlatina.

Corynebacterium segmentosum Eberson. (Bacillus coryzae segmentosus Cautley, Rept. Med. Officer of Health, Local Govt. Board, London, 1894-95, 455 ; Bacillus septus Benham, Brit. Med. Jour., 1, 1906, 1023; Eberson, Jour. Inf. Dis., 23, 1918, 17; Bacillus segmentosus Holland, Jour. Bact., 5, 1920, 220.) Rods of variable dimensions, mostly resembling Corynebacterium pseudodiphtheriticum Lehmann and Neumann, but occasionally resembling Corynebacterium diphtheriae Lehmann and Neumann. Thomson and Thomson (Ann. PickettThomson Res. Lab., 2, 1926, 65) do not think Cautley's bacillus is recognizable. From nasal secretions.

Corynebacterium squamosum Belenky and Popova. (Cent.f. Bakt., I Abt., Orig., 118,1930 , 444.) From normal skin of calves and small-pox vaceine. Nonhemolytic.

Corynebacterium striatum (Chester) Eberson. (Bacillus striatus flams and Bacillus striatus albus von Besser, Beitr. z. path. Anat. u. Path., 6, 188s, 349; Bacterium striatus flavus Chester, Ann. Rept. Del. Col. Agr. Exp. Sta., 9, 1897, 111; Bacterium striatum Chester, Man. Determ. Bact., 1901, 171; Bacillus flavidus Morse, Jour. Inf. Dis., 11, 1912, 281 ; Corynebacterium flavidum Holland, Jour. Bact., 5, 1920, 218; Eberson, Jour. Inf. Dis., 23, 1918, 5.) Eberson (loc. cit., 7) states that Bacillus diphtheroides citreus Graham-Smith (Jour. Hyg., 4, 1904, 258) corresponds with the yellow variety of this species. From nasal mucus. Resembles Corynebacterium segmentosum.

Corynebacterium suis Hauduroy et al. (Le bacille pyogenes suis, Colin and Rossi, Revue gén. de Méd. vetér., 40, 1931, 137; Hauduroy et al., Dict. d. Bact.
Path., 1937, 167). (ausative agent of caseous suppuration of swine. Gramnegative.

Corynebacterium suppuratum Eberson. (Jour. Inf. Dis., 23, 1918, 17.) From anal pus pocket. Resembles Corynebacterium epidermidis Eberson.

Corynebacterium thermophilus Zavagli. (Amer. Jour. Hyg., 15, 1932, 504.) From raw and pasteurized milk. Grows better at $55^{\circ} \mathrm{C}$ than at $37^{\circ} \mathrm{C}$.

Corynebacterium typhi Topley and Wilson. (Bacillus typhiexanthematici Plotz, Jour. Amer. Med. Assoc., 62, 1913, 1556; La Presse Méd., 43, 1914, 411; Plotz, Olitsky and Baehr, Jour. Inf. Dis., 17, 1915, 17; not Bacillus typhi exanthematici Kilebs, Proc. Internat. Med. Cong., 1, 1881, 323; Corynebacterium typhi-exanthematici Eberson, Jour. Inf. Dis., 23, 1918, 19; Bacterium typhi-exanthematici Holland, Jour. Bact., 5, 1920, 222; Fusiformis typhi-exanthematici Holland, ibid., 221; Topley and Wilson, Prin. of Bact. and Immun., 2nd ed., 1936, 349; Eubacterium typhi-exanthematici Prévot, Ann. Inst. Past., 60, 1938, 295.) From blood of typhus fever patients.

Corynebacterium ulcerogenes Bergey et al. (Corynebacterium diphtheriae ulcerogenes cutaneum. Mrongovius, Cent.f. Bakt., I Abt., Orig., 112, 1929, 51; Bergey et al., Manual, 4th ed., 1934, 550.) From ulcerations of the skin (human). Resembles Corynebacterium epidermidis Eherson and C. suppuratum Eberson.

Corynebacterium vaccinae Galli-Valerio. (Cent. f. Bakt., I Abt., Orig., 36, 1904, 465.) From vaccine pustules in calves.

Corynebacterium xerosis canis (Graham-Smith) Ford. (Bacillus xerosis canis Graham-Smith, Jour. Hyg., 4, 1904, 258; Ford, Textb. Bact., 1927, 271.) From conjunctival saes of dogs.

Corynethrix hominis, C. equi, C. canis, C. anatis, etc. Czaplewski. (Deutsche med. Wchnschr., 26, 1900, 723.) Hypothetical species from the skin of the animals indicated.

Corynethrix pseudotuberculosis murium Bongert. (Ztsehr. f. Hyg., 37, 1901, 
472.) From a multiple, necrotic, caseous pneumonia of mice inoculated with material from equine pneumonia. Regarded by the author as distinct from Bacillus pseudotuberculosis murium Kütscher. Placed in the genus Corynethrix Czaplewski (Deutsche med. Wehnschr., 26, 1900, 723).

Lactobacillus meleagridis Johnson and Pollard. (Diplo-bacillus $\mathrm{P}_{1}$, Johnson and Anderson, Jour. Inf. Dis., 58, 1936, 340; Johnson and Pollard, Jour. Inf. Dis., $66,1940,196$.$) From heart, liver and$ yolk of moribund turkey poults. Presumably a Corynebacterium, not a true Lactobacillus.

Einer sporogenen Pseudo-Diphtheriebazillus, De Simoni. (Cent. f. Bakt., I Abt., Orig., 24, 1898, 294.) From nasal secretion in ozena. Produced spores only in milk and on potato. Thought by Eberson (Jour. Inf. Dis., 23, 1918, 6) to have been a contaminated culture.

Organism in M. H., De Witt. (Jour. Inf. Dis., 10, 1912, 40.) A motile, gasproducing diphtheroid isolated from it generalized diphtheria-like infection.

Appendix III:* The relationships of the following soil organism are not clear, but it apparently should be placed either in Corynebacterium or in a related genus (e.g., Mycobacterium). On agar it is rodshaped and generally Gram-negative in roung cultures, but cocerid and Grampositive in old cultures, a condition noted by Mellon (Jour. Bact., 2, 1917, 278) in connection with Corynebacterium enzymicum. Something similar is noted by Jensen (Proc. Linn. Sor. New So. Wales, 59, 1934, 29-62) in his description of Corynebacterium helvolum. Krassilnikov, on the other hand (Cent.f. Bakt., II Abt., 90, 1934, 432), suggests that this species really belongs to $M$ ycobacterium, and, after seeing a culture furnished him by Conn, has become all the more convinced of this relationship (personal correspondence)
Krassilnikov's studies indicate that there is a group of soil bacteria that grow as rods in young cultures with a tendency to produce branching forms in liquid media and develop coccoid bodies as they grow older. The latter then even divide and multiply like cocci. He considers that practically all so-called micrococei found among soil cultures are really the older stages of Mycobacterium $s p p$. It is very clear that Jensen and Krassilnikov, the two leading students of the saprophytic members of this group found in soil, do not agree as to what constitutes the genus Mycobacterium; their papers appeared almost simultaneously and clearly represent independent work. Krassilnikov's description of this genus comes closest to covering organisms like the following of any of the descriptions in the literature, but it is quite different from Jensen's idea of the genus. In fact, the descriptions given by the former author seem to be more like Jensen's conception of the genus Corynebacterium. Jensen, in his description, takes into account the relative acid-fast staining properties of the groups; but lirassilnikov does not mention either this property or the Gram stain. Inasmuch as the acid-fast propcrty is regarded in the present classification as an important characteristic of Mycobacterium, the following species is included as an appendix, not of that genus, but of Corynebacterium. The relationships of these pleomorphic soil organisms must be regarded as decidedly obscure. Lochhead (Can. Jour. Res., Sec. C $, 16,1938,156)$ speaks of a Bacterium globiforme group and Conn (Jour. Bact., 48, 1945, 359) has recently reported evidence in support of Lochhead's viewpoint. In all probability this group is identical in whole or in part with Krassilnikov's Mycobacterium of soil, although the correctness of his choice of this generic name may be questioned.

*Prepared by Prof. H. J. Conn, New York State Experiment Station, Geneva, New York, July, 1945. 
Bacterium globiforme Conn. (Conn, N. Y. Agr. Exp. Sta. Tech. Bull. 138, 1928 and 172, 1930; Cent. f. Bakt., II Abt., 76, 1928, 77; Achromobactor globiforme Bergey et al., Manual, 3rd ed., 1930, 226.) From Latin, having the form of a globe or sphere.

Short rods: 0.4 to 0.6 by 0.6 to $0.8 \mathrm{mi}$ cron, becoming coceoid in older cultures. In certain liquid synthetic media, branching forms with Gram-positive spherical granules are common. These granules have a tendency to be acid-fast. Non-motile. Rods usually Gram-negative; coceoid forms usually Gram-positive.

Gelatin colonies: Circular, punctiform.

Gelatin stab: Slow rateriform liquefaction.

Agar colonies: Circular, punctiform, translucent.
Agar slant: Filiform, flat, smooth, soft, translucent, glistening growth with translueent sheen.

Broth: Slight growth.

Nitrites produced from nitrates in synthetic agar media.

Glucose, sucrose, mannitol, and less readily lactose and various organic acids are utilized as sources of carbon and energy when grown in synthetic media. To visible gas production, and probably no acid except carbonic acid.

Nitrogen may be ohtained from ammonium sulfate, asparagine, rystine, glycerol, aspartic acid, uric acid, tyrosin, potassium nitrate, urea and peptone.

Aerobic, facultative.

Optimum temperature $22^{\circ} \mathrm{C}$.

Source: Seventy cultures isolated from soil.

Habitat: Widely distributed in soil.

\section{Genus II. Listeria Pirie.*}

(Listerella Pirie, Publ. So. African Inst. for Med. Res., 3, 1927, 163; not Listerella Jahn, Ber. d. deutsch. Bot. Ges., 24, 1906, 538; not Listerella Cushman, Contr. Cushman Lab. Foram., Sharon, Mass., 9, 1933, 32; Pirie, Science, 91, 1940, 383.) Named for Joseph Lister, the English surgeon and bacteriologist.

Small rods, Gram-positive. Flagellation peritrichous. Aerobic. Catalase positive. Grow freely on ordinary media. Acid but no gas from glucose and a few additional carbohydrates. Pathogenic parasites. Infection characterized by a monorytosis. Parasitic on warm-blooded animals.

The type species is Listeria monocytogenes (Murray et al.) Pirie.

\section{Listeria monocytogenes (Murray} et al.) Pirie. (Bacterium monocytogenes Murray, Webb and Swann, Jour. Path. and Bact., 29, 1926, 407; Listerella hepatolytica Pirie, Publ. S. African Inst. for Med. Res., 3, 1927, 164; Listerella monocytogenes Pirie, ibid.; Listerella monocytogenes hominis Nyfeldt, Folia Haematologica, 47, 1932; Corynebacterium parvulum Schultz, Terry, Brice and Gebhardt, Proe. Soe. Exp. Biol. Med., 31, 1934, 1021; Pirie, Science, 91, 1940. 383; Bacillus monocytogenes Tobia, Areh. ital. med. colon., 23, 1912, 219; abst. in Cent. f. Bakt., I Abt., Ref., 144, 1943, 199.) Derived from the Greek, meaning generating monocytes.
Small rods : 0.4 to 0.5 by 0.5 to 2.0 microns, with rounded ends, slightly curved in some culture media. Occur singly, in $\mathrm{V}$-shaped or parallel pairs and in short chains. Motile, peritrichous (Paterson, Jour. Path. and Bact., 48, $1939,25)$ with four flagella at ordinary temperatures with tendency toward non-motility or single flagellum at $37^{\circ} \mathrm{C}$ (Griffin, Jour. Bact., 48, 1941, 114). Not acid-fast. Gram-positive.

Gelatin: No liquefaction. Growth is confined to the needle track.

In 0.25 per cent agar, 8.0 per cent gelatin, 1.0 per cent glucose semisolid meditim. growth along the stab in 24 hours at $37^{\circ} \mathrm{C}$, followed by irregular cloudy or

* Revised by Prof. E. G. D. Murray, MeGill Univ., Montreal, P. Q., Canada, September, 1938; further revision, January, 1945. 
granular extensions into the medium; growth does not spread through the entire medium. This is characteristic (Seastone, Jour. Exp. Med., 62, 1935, 203).

Sheep liver extract agar colonies: Circular, smooth, slightly flattened, transparent by transmitted and milk-white by reflected light. Viscid.

Sheep liver extract agar slant: Confluent, flat, transparent, viseid growth.

Peptone agar: Growth is thinner than on liver extract agar.

Blood agar: Improved growth with zone of hemolysis around colonies.

Peptone broth: Surface film with flocculent sediment.

Litmus milk: Slightly acid, decolorized. No coagulation.

Glycerol-potato: No apparent growth.

Inspissated ox serum: Grows as a very thin, transparent film.

Dorset's egg medium: Very thin film.

Indole not formed.

Hydrogen sulfide not formed.

Nitrites not produced from nitrates.

Acid but no gas from glucose, rhamnose and salicin promptly, more slowly from dextrin, sucrose, soluble starch and glycerol. Acid production may be variable and slow from maltose and lactose. No action on arabinose, galactose, xylose, mannitol, dulcitol, inulin and inositol.

All cultures give off a penetrating, rather unpleasant acid smell.

Aerobic, facultative.

Optimum temperature $37^{\circ} \mathrm{C}$. Thermal death point $58^{\circ}$ to $59^{\circ} \mathrm{C}$ in 10 minutes.

Animal inoculations: Injection of rabbits with cultures results in a very marked increase in monocytes circulating in the blood. This is the most striking character of the organism and is exhibited by strains derived from all sources. Infection is characterized by necrotic foci in various organs.

Serological characters: Agglutination and absorption of agglutinin reactions show a variation in degree with different strains but there is no definite indication that strains from different kinds of animal hosts are different species. Pater- son (Jour. Path. and Bact., 5\%, 1940, 127) concludes from his studies of the flagellar and somatic antigens of 54 cultures that four types may be recognized in this species. These do not bear any relation to the host species or to the geographical area from which they were isolated.

Possibly related to Erysipelothrix (Barber, Jour. Path. and Bact., 48, 1939, 11).

Habitat and source: Lesions in organs, blood, cerebrospinal fluid of rabbits, guinea pigs, sheep, cattle, foxes, hogs, fowls, gerbilles and man, in all of which natural disease occurs. Many cases have proved fatal. The cause of infectious mononucleosis in man (Nyfeldt, loc. cit.).

Appendix: The following binomials have also been proposed for species in this genus.

Bacterium hepatis Hülphers. (Sven. Vet.-Tidskrift, 2, 1911, 271.) From necrosis of the liver of a rabbit. Nyfeldt (Skand. Vet.-Tidskrift, 30, 1940, 284) regards this as a synonym of Listerella monocytogenes. However, failure to ferment lactose, rhamnose, sucrose and salicin with fermentation of xylose, and failure to infect guinea pigs and chickens indicate a possible difference between the two species.

Listerella hibiscus liquefaciens Nakahama. (Jour. Agr. Chem. Soc. Japan, 16, 1940, 345.) From retted kenaf (Hibiscus).

Listerella hominis, Listerella bovina, Listerella gallinarum, Listerella cunicula. and Listerella gerbilli Wramby. (Skand. Tet.-Tidskrift, 34, 1944, 280.) These names are given to indicate cultures of Listerella monocytogenes from man, cattle, chickens, rabbits and gerbilles, respectively.

Listerella ovis Gill. (Australian Vet. Jour., 13, 1937, 47.) Causes circling disease of sheep.

Burn (Jour. Bact., 30, 1935, 573) reports, but does not name, a new species in this genus. 


\section{Genus III. Erysipelothrix Rosenbach.*}

(Ztschr.f. Hyg., 63, 1909, 367.) From Greek erysipelas, a disease; and thrix, hair or thread.

Rod-shaped organisms with a tendency to the formation of long filaments. The filaments may also thicken and show characteristic granules. Non-motile. Grampositive. Microaerophilic. Catalase negative. Grow freely on ordinary media. Acid but no gas from glucose and a few additional carbohydrates. Parasitic on mammals.

The type species is Erysipelothrix rhusiopathiae (Migula) Winslow et al.

\section{Erysipelothrix}

rhusiopathiae

(Migula) Winslow et al. (Bacillus des Schweinerotlaufs, Loeffler, Arb. a. d. k. Gesundheitsamte, 1, 1886, 46; Bacillus thuillieri Trevisan, I generi e le specie delle Batteriacee, 1889, 13; Pasteurella thuillieri DeToni and Trevisan, in Saccardo, Sylloge Fungorum, 8, 1889, 995; Bacillus rhusiopathiae suis Kitt, Bakterienkunde u. path. Mikroscopie, 1893, 284; Bacterium erysipelatos suum (sic) Migula, in Engler and Prantl, Die natürl. Pflanzenfam., 1, 1a, 1895, 24; Bacterium rhusiopathiae suis Chester, Ann. Rept. Del. Col. Agr. Exp. Sta., 9, 1897, 98; Bacterium rhusiopethiae Migula, Syst. d. Bakt., 2, 1900, 43; Mycobacterium rhusiopathiae Chester, Man. Determ. Bact., 1901, 352; Erysipelothrix pori Rosenbach, Ztschr. f. Hyg., 63, 1909, 367; Winslow et al., Jour. Bact., 5, 1920, 198; Bacillus erysipelatos-suis Holland, Jour. Bact., 5, 1920, 218; Erysipelothrit erysipelatos-suis Holland, ibid. Bacillus ruboris suis Neveu-Lemaire, Précis Parasitol. Hum., 5th ed., 1921, 24; Nocardia thuillieri Vuillemin, Encyclopédie $\mathrm{My}$ cologique, Paris, 2, 1931, 125; Actinomyces thuillieri Nannizzi, in Pollacci, Tratt. Micopat. Umana, 4, 1934, 45.) From Greek rhusius, reddish; pathus, a disease; red disease.

Description taken in part from Karlson, Jour. Bact., 35, 1938, 205.

Slender rods: 0.2 to 0.3 by 0.5 to 1.5 microns, occurring singly and in chains. Non-motile. Gram-positive.

Gelatin colonies: Hazy, bluish-gray, racemose; situated a little below the surface, growing slowly.

Gelatin stab: Small, fimbriate colonies in the stab, at times definitely arborescent. No surface growth. No liquefaction.

Agar slant: Scant growth, translucent, moist, homogeneous.

Broth: Slight turbidity, with seant, grayish sediment.

Litmus milk: May become slightly acid.

Indole not formed.

Potato: Usually no growth.

Blood serum shows seant growth.

No gas from carbohydrates. Acid from glucose, galactose, fructose, lactose and more slowly from mannose and cellobiose. No acid from arabinose, xylose, rhamnose, maltose, melibiose, sucrose, trehalose, raffinose, melezitose, dextrin, stareh, inulin, amygdalin, salicin, glycerol, erythritol, adonitol, mannitol, sorbitol, dulcitol or inositol.

Esculin not hydrolyzed.

Hydrogen sulfide produced.

Voges-Proskauer test negative.

Methyl red test negative.

Methylene blue-reduction test negative.

Narrow green zone of hemolysis develops around deep colonies on blood agar ,

Catalase negative.

Out of 43 strains studied serologically (Watts, Jour. Path. and Bact., 50, 1940, $355), 38$ appeared to be of one antigenic group, and 5 of another.

* Revised by Prof. Robert S. Breed, New York State Experiment Station, August, 1938; further revision, January, 1945. 
Optimum pH 7.6.

Microaerophilic.

Optimum temperature $37^{\circ} \mathrm{C}$.

Source: From cases of swine erysipelas.

Habitat: The cause of swine erysipelas. Transmissible to gray and white mice, rabbits and pigeons. Has been transmitted to man by accidental inoculation.

\section{Erysipelothrix muriseptica (Flügge)}

Rosenbach. (Bacillus der Mäuseseptikämie, Koch, Mittheil. a. d. kaiserl. Gesundheitsamte, 1, 1881, 93; Bacillus insidiosus Trevisan, Car. di alc. nuov. gen. di Batter., 1885, 10; Bacillus murisepticus Flügge, Die Mikroorganismen, 2 Aufl., 1886, 250; Bacillus murinus Schroeter, in Cohn, Kryptogamen Flora v. Schlesien, 3, 1886, 162; Bacterium murisepticum Migula, in Engler and Prantl, Die natürl. Pflanzenfam., 1, la, 1895, 24; Mycobacterium murisepticum Chester, Manual Determ. Bact., 1901, 353; Rosenbach, Ztschr. f. Hyg., 63, 1909 , 367; Pasteurella muriseptica Bergey et al., Manual, 1st ed., 1923, 265; not Pasturella muriseptica Topley and Wilson, Princip. Bact. and Immun., 1, 1931, 482.) From Latin mus, muris, a mouse; Greek septicus, putrefying, septic.

Rods : 0.5 by 0.8 to 1.0 micron, oceurring singly. Non-motile. Gram-positive.

Gelatin colonies: Very small, whitish. dew-like, with indefinite margin.

Gelatin-stab: Filiform growth in stab, arborescent. No liquefaction.

Agar slant: Very slight, clear, dew-like streak.

Litmus milk: Lnchanged.

Potato: No growth.

Indole not formed.

Nitrites not produced from nitrates.

Microaerophilic.

Optimum temperature $37^{\circ} \mathrm{C}$

Source: From cases of mouse septicemia
Habitat: In fatal septicemia in white mice following injection of putrid meat infusion. Not infectious for field mice.

3. Erysipelothrix erysipeloidis (Lehmann and Neumann) Rosenbach. (Cladothrix des Erythema migrans, Rosenbach, Arch. klin. Chirurg., 36, 1887, 2 ; Oospora roscnbachi Sauvagais and $\mathrm{Ra}$ dais, 1892, aceording to Brumpt, Précis de Parasit., Paris, th ed., 1927, 1201; Oospora erysipeloidis Lehmann and Neumann, Bakt. Diag., 1 Aufl., 2, 1896, 392; Streptothrix rosenbachii Kruse in Flügge, Die Mikroorganismen, 3 Aufl., 2, 1896, 61; Actinomyces erysipeloidis Lachner-Sandoval, Ueber Strahlenpilze Strassburg, 1898, 64; Discomyces rosenbachi Gedoelst, Champ. Paras. Homme, 1902,177 ; Streptothrix erysipeloides Caminiti. Cent. f. Bakt., I Abt., Orig., 1907. 19s; Rosenbach, Ztschr. f. Hyg., 63. 1909, 367; Vocardia rosenbachi Castellani and Chalmers, Man. Trop. Med., 2nd ed., 1913, 815: Babesia erysipeloides Chalmers and Christopherson, Ann. Trop. Med. and Parasit.. 10, 1916, according to Nannizzi, in Pollacci, Tratt. Micopat. Lmana, 4, 1934. t5; Actinomyces rosenbachii Holland, Jour. Bact., 5, 1920. 216; Bacterium erysipeloidis Lehmann and Jeumann, Bakt. Diag., 7 Aufl., 2, 1927, 499.) From (ireek erysipelas, erysipelas; idus, shape, appearance.

Rosenbach (loc. cit.) made a comparative study of the three species in this genus and came to the conclusion that they were different, although closely allied to each other. However, Rickmann (Ztschr. f. Hyg., 64, 1909, 362) concluded that they were identical.

Source: Isolated by Rosenbach (Ver. handl. d. deutsch. Gesellsch. f. Chirurg., $2,1887,75$ ) in cases of human erysipeloid. 


\section{FAMILY IX. ACHROMOBACTERIACEAE BREED.}

(Jour. Bact., 50, 1945, 124.)

Rods, small to medium in size, cells usually uniform in shape. No branching on ordinary media, if at all. Gram-negative, rarely Gram-variable. Peritrichous or nonmotile. Growth on agar slants non-chromogenic to grayish-yellow, brownish-yellow or yellow to orange. The pigment does not diffuse through the agar. Characterized by lack of power or feeble powers of attacking carbohydrates. May form acid from hexoses but no gas. May or may not reduce nitrates. May or may not liquefy gelatin. Do not liquefy agar or attack cellulose, and are not phosphorescent. Litmus milk may become faintly acid but not sufficiently acid to curdle. Usually the reaction remains unchanged or becomes alkaline. Generally salt water, fresh water and soil forms and, less commonly, parasites. Some plant pathogens may belong here.

\section{Key to the genera of family Achromobacteriaceae.}

I. Non-chromogenic or at most little or no chromogenesis on agar or gelatin media.

A. Titmus milk turned alkaline. No acid from carbohydrates.

Genus I. Alcaligenes, p. $\$ 12$.

B. Litmus milk slightly acid (never curdled), unchanged or alkaline. Acid usually produced from hexose sugars.

Genus II. Achromobacter, p. 417.

II. Produces yellow to orange chromogenesis.

A. Litmus milk slightly acid (never curdled) unchanged or alkaline. Acid usually produced from hexose sugars.

Genus III. Flavobacterium, p. 427.

\section{Genus I. Alcaligenes Castellani and Chalmers.*}

(Manual Trop. Med., 3rd ed., 1919, 936.) From M.L., alkali and Latin genio, to produce.

Peritrichous to monotrichous, or non-motile rods. Gram-negative to Gramvariable. Do not produce acid or gas from carbohydrates. May or may not liquefy gelatin and solidified blood serum. Tum litmus milk alkaline and may or may not peptonize it. Do not form acetylmethylcarbinol. Chromogenesis when it occurs is grayish-yellow, brownish-yellow or yellow. Generally occur in the intestinal tract of vertebrates or in dairy products.

The type species is Alcaligenes faecalis Castellani and Chalmers.

* Revised by Prof. H. J. Conn, New York State Experiment Station, Geneva, New York, June, 1938; further revision by Prof. Robert S. Breed, New York State Experiment Station, Geneva, New York, June, 1945. 
Key to the species of genus Alcaligenes.

I. Gelatin not liquefied.

A. Motile.

1. Does not produce ropiness in milk. Found in the intestinal tract.

1. Alcaligenes faecalis.

2. Produces ropiness in milk.

2. Alcaligenes viscosus.

B. Non-motile.

1. Found in the intestinal tract.

3. Alcaligenes metalcaligenes.

II. Gelatin liquefied.

A. Motile.

1. Milk peptonized; blood serum liquefied.

4. Alcaligenes bookeri.

2. Milk not peptonized; blood serum not liquefied.

5. Alaligenes recti.

B. Ton-motile.

1. Milk peptonized, slimy.

6. Alcaligenes marshallii.

1. Alcaligenes faecalis Castellani and Chalmers. (Bacillus faecalis alcaligenes Petruschky, Cent. f. Bakt.. I Abt., 19, 1896, 187; Bacterium fecalis alcaligenes Chester, Ann. Rept. Del. Col. Agr'. Exp. Sta., 9, 1897, 73; Bacterium alcalig. enes Lehmann and Neumann, Bakt. Diag., 2 Aufl., 1899, 242; Bacillus alcaligenes Migula, Syst. d. Bakt., 2, 1900, 737; Castellani and Chalmers, Manual Trop. Med., 1919, 936; Bacillus fecalis-alcaligenes Holland, Jour. Bact., 5, 1920, 218; Bucterium fecalis-alcaligenes Holland, ibid.; Vibrio alcaligenes Lehmann and Neumann, Bakt. Diag., 7 Aufl., 2, 1927, 548: Bacterium faecale alcaligenes Mo nias, Jour. Int. Dis., 43, 1928, 330.) From Latin faex, dregs; M. L., fecal.

Rods: 0.5 by 1.0 to 2.0 microns, ocecurring singly and in pairs, and occasionally in long chains. Motile with peritrichous flagella. In some strains, the majority of the individual cells show only a single flagellum. This is apt to be in a lateral rather than in the polar position. Gramnegative.

Gelatin colonies: Circular, grayish, translucent.
Gelatin stab: Gray surface growth. No liquefaction.

Agar colonies: Transparent with opaque center, undulate margin.

Igar slant: White, glistening, opalesrent, undulate margin.

Broth: Turbill, with thin pellicle, and viscid sediment. (Gives off ammonia.

Litmus milk: Alkaline.

Potato: Seanty to abundant, yellowish to brownish growth.

Indole not formed.

Nitrite production from nitrates variable.

No acid or gas from arbohydrate media.

No characteristic odor.

Aerobic, facultative.

Optimum temperature $37^{\circ} \mathrm{C}$.

source: Feces, abscesses related to intestinal tract, occasionally blood stream.

Habitat: Intestinal canal. Generally considered non-pathogenic.

1a. Alcaligenes faecalis var. radicans Evans (Public Health Rpts., 46, 1931, 1676 ) is a gelatin liquefying strain. 
2. Alcaligenes viscosus (Weldin and Levine) Weldin. (Bacillus lactis viscosus Adametz, Cent. f. Bakt., 9, 1891, 698; Bacillus viscosus lactis Kruse, in Flügge, Die Mikroorganismen, 2, 1896, 359; Bacterium viscosus lactis Chester, Delaware Agr. Exp. Sta. 9th Ann. Rept., 1897, 89; Bacterium lactis viscosum Lehmann and Neumann, Bakt. Diag., 1 Aufl., 2, 1896, 198 (Eng. ed., 1901, 196); Bacterium subviscosum Migula, Syst. d. Bakt., 2, 1900, 326; Group I, varieties 1, 2, 3, 4 and 5 of Harrison, Rev. Gén. du Lait, 5, 1905, 100 ; Bacterium visco-coccoidium Buchanan and Hammer, Iowa Agr. Exp. Stat. Research Bull. 22, 1915, 260; Bacillus lactisviscosus Holland, Jour. Bact., 5, 1920, 218; Bacterium lactis-viscosus Holland, idem; Bacterium viscosum Weldin and Levine, Abst. Bact., 7, 1923, 16 (not Bacterium viscosum Migula, Syst. d. Bakt., 2, 1900, $647)$; Lactobacillus viscosus Bergey et al.. Manual, 1st ed., 1923, 244; Achromobacter viscosum Bergey et al., Manual, 2nd ed., 1925, 169; Weldin, Iowa State College Jour. Sci., 1, 1927, 186.) From Latin viscosus, viscous.

Description taken largely from Long and Hammer, Iowa state Coll. Jour. of Sci., 10, 1936, 262.

Rods : 0.6 to 1.0 by 0.8 to 2.6 microns, almost spherical cells frequently found, occurring singly, in pairs or short chains. Motile (Adametz, loc. cit.); non-motile (Long and Hammer, loc. cit.). Gramnegative, rarely Gram-positive. Capsules produced in milk cultures.

Gelatin colonies: Small, gray becoming yellowish.

Gelatin stab: White surface growth with villous growth in stab. No lique. faction.

Agar colonies: After 3 to 4 days, circu lar, 4 to $6 \mathrm{~mm}$ in diameter, white, viscid, shining, edge entire.

Agar slant: Abundant, white, spread ing, viscid, shining.

Broth: Turbid with thin pellicle and some sediment. Ropiness generally pro duced.
Litmus milk : Ropiness produced. Pellicle formed. Alkaline. No coagulation.

Potato: Moderately heavy, dirty-white, spreading, shining growth.

Indole not formed.

Nitrites ordinarily not produced or produced only in a trace from nitrates.

No $\mathrm{H}_{2} \mathrm{~S}$ produced.

slight, if any, acid production from carbohydrates.

Fat is hydrolyzed.

Methyl red reaction negative.

Voges-Proskauer reaction negative.

Temperature relations: Growth occurs at $10^{\circ}$ and at $20^{\circ} \mathrm{C}$. At $37^{\circ}$ and at $40^{\circ} \mathrm{C}$ grow th variable.

Aerobic,

Source: Originally isolated from water.

Habitat: Found in water and around dairy barns, dairy utensils. Produces ropiness in milk.

Long and Hammer (Iowa State Coll. Jour. Sci., 10, 1936, 264) have described a variety of this species (Alcaligenes viscosus var. dissimilis) which does not produce ropiness in milk.

3. Alcaligenes metalcaligenes Castellani and Chalmers. (Castellani and Chalmers, Man. Trop. Med., 1919, 936; Bacterium metalcaligones Weldin and Levine, Abst. Bact., 7, 1923, 13; Achromobacter metalcaligenes Bergey et al., Manual, 2nd ed., 1925, 169.) From Greek meta, in common with; M. L., resembling alcaligenes.

Rods : 0.6 by 1.5 microns, with rounded ends, occurring singly and in pairs. Nonmotile. Gram-negative.

Gelatin stab: No liquefaction.

Agar colonies : Circular, raised, smooth, amorphous, entire, gray.

Agar slant: Gray, scanty, filiform, contoured, viscid.

Broth: Membranous pellicle with heavy sediment.

Litmus milk: Alkaline.

Potato: Scanty, glistening, smooth, sometimes faint pink.

indole not formed. 
Nitrite production from nitrates variable.

Stareh not hydrolyzed.

Blood serum not liquefied.

No action on carbohydrates.

Aerobic, facultative.

Optimum temperature $22^{\circ} \mathrm{C}$.

Habitat: Intestinal canal.

4. Alcaligenes bookeri (Ford) Bergey et al. (Bacillus A of Booker, Trans. Ninth Internat. Med. Congress, 3, 1887, 598; Bacillus bookeri Ford, Studies from the Royal Victoria Hospital, Montreal, 1, 1903, 31 ; Bergey et al., Manual, 1st ed., 1923, 236; Bacterium bookeri Levine and Soppeland, Eng. Exp. Sta., Iowa State College, Bul. 77, 1926, 55.) Named for the bacteriologist who first isolated this species.

Rods: 0.5 by 1.5 to 2.0 microns, occurring singly. Motile with peritrichous flagella. Gram-negative.

Gelatin colonies: Circular, brown, variable in size.

Gelatin stab: Slow, saccate liqueficetion, becoming stratiform.

Agar colonies: Thin, transparent, with opaque center and indistinct margin.

Agar slant: Abundant, yellowish to yellowish-brown.

Broth: Turbid, with viscid sediment No pellicle.

Litmus milk: Alkaline. Soft curd Litmus reduced. Peptonization.

Potato: Luxuriant, yellowish-white, moist. Medium is darkened.

Indole not formed.

Nitrites not produced from nitrates.

No acid or gas from carbohydrate media.

Blood serum: Yellowish-brown growth. Gradual liquefaction.

No characteristic odor.

Aerobic, facultative.

Optimum temperature $37^{\circ} \mathrm{C}$.

Source: From alvine discharges of children suffering with cholera infantum.

Habitat: Intestinal canal.
5. Alcaligenes recti (Ford) Bergey et al. (Bacterium recti Ford, Studies from the Royal Victoria Hospital, Montreal, 1, 1903, 31 ; Bergey et al., Manual, 1st ed., 1923, 236.) From Latin rectus, rectum.

Rods : 0.5 by 1.5 to 2.0 microns, occurring singly, in pairs and in chains. Motile with peritrichous flagella. Gramnegative.

Gelatin colonies: Variable in size and shape, circular to oval, brown.

Gelatin stab: Rapid, saccate liquefaction.

Agar colonies: Large, grayish-white, with opaque center. Slightly spreading.

Agar slant: Grayish-white, echinulate

Broth: Turbid. No pellicle.

Litmus milk: Alkaline. No peptonization.

Potato: Luxuriant, moist, brownishred.

Indole not formed.

Nitrites produced from nitrates.

Noacid or gas from carbohydrate media.

Blood serum: Abundant white growth.

No liquefaction.

No characteristic odor.

Aerobic, facultative.

Optimum temperature $37^{\circ} \mathrm{C}$.

Source: Found but once from coecum and rectum (Ford).

Habitat: Intestinal canal.

6. Alcaligenes marshallii Bergey et al. (Bacillus B of Marshall, Cent. f. Bakt., II Abt., 11, 1903, 739; Bacterium lactis marshalli Conn, Esten and Stocking, Ann. Rept. Storrs Agr. Exp. Station, 1906, 141; Bergey et al., Manual, 1st ed., 1923, 237.) Named for Prof. C. E. Marshall, the American bacteriologist who first isolated this species.

Rods: 0.3 by 1.2 microns, occurring singly. Non-motile. Gram-negative.

Gelatin colonies: Gray, granular, irregular, glistening.

Gelatin stab: Slow, infundibuliform liquefaction.

Agar slant: Filiform, gray to creamywhite, raised, becoming lemon-yellow. 
Broth: Turbid, with gray ring and viscid sediment.

Litmus milk: Alkaline, slimy, peptonized, strong odor.

Potato: Luxuriant, lemon-yellow, smooth.

Indole not formed.

Nitrites not produced from nitrates.

To acid or gas from carbohydrates.

Aerobic, facultative.

Optimum temperature $30^{\circ} \mathrm{C}$.

Habitat: Milk.

Appendix: The following species have also sometimes been regarded as belonging in the genus Alcaligenes, or possess characters that indicate that they belong in this genus.

Achromobacter alcaliaromaticum (Berlin) Bergey et al. (Bacterium alcaliaromaticum Berlin, Rev. de Microbiol. et Epidemiol., 6, 1927; Bergey et al., Manual, 3rd ed., 1930, 212.) From feces. See Manual, 5th ed., 1939, 509 for a description of this species. This species is much like Alcaligenes faecalis.

Achromobacter cystinovorum Barber and Burrows. (Biochem. Jour., 30, 1936, 599.) From soil. See Manual, 5th ed., 1939,516 for a description of this species. This species is much like Alcaligenes marshallii.

Achromobacter lipidis (Anderson) Allison, Anderson and Cole. (Bacterium lipidis Anderson, Internat. Assoc. Milk Dealers, Proc, 30th Ann. Convention, Labora ory Section, October, 1937, 19; Allison, Anderson and Cole, Jour. Bact., 36, 1938, 571.) From rancid cream. See Manual, 5th ed., 1934, 521 for a description of this species. This species is much like Alcaligenes metalcaligenes.

Alcaligenes albus Bergey et al. (Bacitrium lactis album Conn, Esten, and Stocking, Ann. Rept., Storrs Agr. Exp. Station, 1906, 143; Bergey et al., Manual, 1st ed., 1923, 237.) From udder of cow. Gram-positive. See Manual, 5th ed., 1939,100 for a description of this species.

Alcaligenes alcalinofoetidus Hauduroy et al. (Bacillus alcalinofoetidus Cas- tellani, Jour. Trop. Med., 1930, 134; Hauduroy, Ehringer, Urbain, Guillot and Magrou, Dictionnaire des bactéries pathogènes. Paris, 1937, 29.) From tonsils of persons having an offensive breath.

Alcaligenes ammoniagenes (Cooke and Keith) Bergey et al. (Bacterium ammoniagenes Cooke and Keith, Jour. Bact., 13, 1927, 315; Bergey et al., Manual, 3rd ed., 1930, 367.) From feces of infants. Gram-positive. See Manual, 5th ed., 1939, 99 for a description of this species.

Alcaligenes denieri Corbet. (Organism No. 6, Denier and Vernet, Le Caoutchouc, 17, 1920, 10193; Quart. Jour. Rubber Research Inst., Malaya, 2, 1930, 152.) From the latex of Hevea brasiliensis (para rubber tree). Gram-positive. See Manual, 5th ed., 1939, 99, for a description of this species.

Alcaligenes faecalis var. mariense Hauduroy et al. (Bacillus mariense Klimenko quoted from Besson, Technique Microbiologique, p. 904; Hauduroy et al., Dict. Path. Bact., Paris, 1937, 31.) I hydrogen sulfide producing variety.

Alcaligenes lenis De Assis. (Boletim do Inst. Vital Brasil, Niteroi, No. 14, 1930, 1.) From human blood stream.

Alcaligenes stevensae Brown. (Amer. Museum Novit., No. 251, 1927, 6.) From crushed egg masses of the moth (Malacosoma americana). Said to be related to Alcaligenes bronchisepticus.

Bacillus coeci Ford. (Ford, Studies from Royal Victoria Hosp., Montreal, 1, No. 5, 1903, 45.) Found in stomach and rectum of a single human subject. Much like Alcaligenes bookeri.

Bacillus pylori Ford. (Ford, studies from Royal Victoria Hosp., Montreal 1, No. 5, 1903, 44.) Found in the human stomach. Liquefied gelatin and peptonized casein but did not liquefy hlond serum.

Flavobacterium fecale Bergey et al. Bacillus fecale aromaticum Stutzer, Cent.f. Bakt., I Abt., Orig., 91, 1923, 87; Bergey et al., Manual, 3rd ed., 1930, 150.) From feces. Resembles Alcaligenes marshallii. See Manual, 5th ed., 1939, 545 for a description of this species. 


\section{Genus II. Achromobacter Bergey et al.*}

(Bergey et al., Manual, 1st ed., 1923, 132; Achromobacterium Richards, Proc. Soc. Agr. Bact. (British), 15th Ann. Conf., 1944, 14.) From Greek achroma, without color and bactrum, a staft or rod.

Ton-pigment-forming (at most no pigment formed on agar or gelatin) rods. Motile with peritrichous flagella or non-motile. Gram-negative to Gram-variable. Litmus milk faintly acid to unchanged or alkaline. Occur in salt to fresh water and in soil.

The type species is Achromobacter liquefaciens (Eisenherg) Bergey et al.

Key to the species of genus Achromobacter.

I. Motile. Flagella peritrichous.

A. Gelatin liquefied.

1. Litmus milk unchanged.

a. Nitrites not produced from nitrates.

1. Achromobacter liquefaciens.

aa. Nitrites are produced from nitrates.

2. Achromobacter thalassius.

3. Achromobacter iophagum.

2. Litmus milk acid.

a. Nitrites are produced from nitrates.

4. Achromobacter delicatulum.

B. Gelatin not liquefied.

1. Litmus milk unchanged.

a. Nitrites are produced from nitrates.

5. Achromobacter aquamarinus.

6. Achromobacter cycloclastes.

2. Litnus milk slightly acid.

a. Nitrites not produced from nitrates.

7. Achromobacter superficiale.

II. Non-motile。

A. Gelatin liquefied.

1. Litmus milk unehanged.

a. Nitrites slowly produced from nitrates.

8. Achromobacter stenohalis.

aa. Nitrites not produced from nitrates.

9. Achromobacter builyri.

2. Litmus milk alkaline

a. Vitrites are produced from nitrates.

10. Achrumbacter stationis.

B. Gelatin not liquefied.

1. Litmus milk unchanged.

a. Action on nitrates not recorded.

11. Achromobacter eurydice.

2. Litmus milk acid, reduced in 5 days.

a. Nitrites are produced from nitrates.

12. Achromobacter delmarvae.

* Partially rearranged before his death by Prof. D. H. Bergey, Philadelphia, Pennsylvania, September, 1937; further revision by Prof. Robert S. Breed, New York State Experiment Station, Geneva, New York, August, 1945. 
1. Achromobacter liquefaciens (Eisenberg) Bergey et al. (Bacillus liquefaciens Eisenberg, Bakt. Diag., 3 Aufl., 1891, 112; not Bacillus liquejaciens Doyen, Jour. d. connaiss. médic., 1889, 108; not Bacillus liquefaciens Lucet, Ann. Inst. Past., 7, 1893, 327; not Bacillus liquefaciens Migula, Syst. d. Bakt., 2, 1900, 723 ; Bacillus sternbergii Migula, Syst. d. Bakt., 2, 1900, 726; Bergey et al., Manual, 1st ed., 1923, 135.) From Latin, liquefying.

Description emended by Bergey et al . (loc. cit.). This is reported to be a common water organism by Lustig (Diag. d. Bakt. des Wassers, 1893, 86), by Frankland and Frankland (Microorganisms in Water, 1894, 461) and by Horrocks (Bact. Exam. of Water, 1901, 54).

Short, rather thick rods, with rounded ends, occurring singly. Motile, possessing peritrichous flagella. Gram-negative.

Gelatin colonies: Circular, gray, entire, slimy. Liquefaction. In time a putrid odor.

Gelatin stab: Napiform liquefaction.

Agar slant: Dirty-white, spreading growth.

Broth: Turbid.

Litmus milk: Unchanged.

Potato: Light yellow streak.

Indole not formed.

Nitrites not produced from nitrates.

Aerobic, facultative.

Optimum temperature $20^{\circ}$ to $25^{\circ} \mathrm{C}$.

Habitat: Water.

\section{Achromobacter thalassius $\mathrm{Z}_{\mathrm{oBell}}$} and Upham. (Bull. Scripps Inst. of Oceanography, Univ. Calif., 5, 1944, 279.) From Greek thalassius, marine, of the sea.

Rods: 0.6 to 0.7 by 0.8 to 2.3 microns, with some variation in shape, occurring singly, in pairs and short chains and many cells lying side by side. Motile by means of peritrichous flagella. Gram-negative but cell walls tend to retain stain.

All media except the fresh-water broth, litmus milk, and potato were prepared with sea water.

Gelatin colonies: $1 \mathrm{~mm}$, circular, white.

Gelatin stab: Napiform liquefaction. Filiform growth along line of stab.

Agar colonies: Punctiform, rough, translucent, raised.

Agar slant: Moderate, glistening, beaded, watery, butyrous growth with no pigment.

Sea-water broth: No pellicle, slight turbidity, scanty powdery sediment.

Fresh-water broth: Fair growth.

Litmus milk: No visible change. Casein not digested.

Potato: No visible growth.

Indole not formed.

Nitrites are produced from nitrates.

Does not ferment glucose, lactose, maltose, sucrose, xylose, mannitol, glycerol, or salicin.

Starch not hydrolyzed.

Hydrogen sulfide not formed.

Ammonia produced from peptone but not from urea.

Fats not hydrolyzed.

Aerobic, facultative.

Optimum temperature $20^{\circ}$ to $25^{\circ} \mathrm{C}$.

Source: Marine bottom deposits.

3. Achromobacter iophagum (Gray and Thornton) Bergey et al. (Bacterium iophagum Gray and Thornton, Cent. f. Bakt., II Abt., 73, 1928, 89; Bergey et al., Manual, 3rd ed., 1930, 204.) From Greek ius, a poison and phagein, to eat or devour.

Rods : 0.8 to 1.0 by 1.0 to 5.0 microns. Motile by means of peritrichous flagella. Gram-negative.

Gelatin colonies: Quickly liquefied.

Gelatin stab: Liquefied.

Agar colonies: Circular or amoeboid, whitish, flat, raised, smooth, translucent, entire.

Agar slant: Filiform, white to buff, flat, undulate.

Broth: Turbid.

Litmus milk: Unchanged. 
Nitrites produced from nitrates.

Starch hydrolyzed.

Acid from glucose and sucrose. Occasionally from maltose and glycerol.

Attacks phenol and naphthalene.

Aerobic, facultative.

Optimum temperature $30^{\circ}$ to $35^{\circ} \mathrm{C}$.

Source: Fifteen cultures from soil.

Habitat : Soil

4. Achromobacter delicatulum (Jordan) Bergey et al. (Bacillus delicatulus Jordan, Report Mass. State Bd. of Health, 1890, 837; Bacterium delicatulus Chester, Ann. Rept. Del. Col. Agr. Exp. Sta., 9, 1897, 82; Bergey et al., Manual, 1st ed., 1923, 137.) From Latin delicatus, soft, delicate; M. L. delicatulus, somewhat delicate.

Characters added to Jordan's description by Bergey (loc. cit.) from his private notes are indicated. Steinhaus (Jour. Bact., 42, 1941, 771) apparently found the same organism and has added other characters.

Rods: 1.0 by 2.0 microns, occurring singly (Jordan). Motile, possessing peritrichous flagella. Gram-negative (Bergey).

Gelatin colonies: Whitish, homogeneous, with radiate margin.

Gelatin stab: Infundibuliform liquefaction.

Agar slant: Whitish, glistening.

Broth: Turbid, with gray pellicle and sediment.

Litmus milk: Acid. Slow reduction and peptonization (Steinhaus).

Potato: Thin, gray streak.

Acid from glucose, sucrose, maltose and lactose (slow) (Steinhaus).

No hydrolysis of starch (Steinhaus).

No $\mathrm{H}_{2} \mathrm{~S}$ produced (Steinhaus).

Indole not formed (Bergey).

Nitrites produced from nitrates.

Aerobic, facultative.

Optimum temperature $30^{\circ}$ to $35^{\circ} \mathrm{C}$.

Source: From the effluent of a septic tank (Jordan). From water (Bergey).
From the alimentary tract of an adult Colorado potato beetle (Leptinotarsa decemlineata Say) (Steinhaus).

Habitat: Presumably widely distributed in nature.

5. Achromobacter aquamarinus ZoBell and Upham. (Bull. Seripps Inst. of Oceanography, Univ. Calif., 5, 1944, 264.) From Latin aqua, water, and marinus, sea.

Rods: 0.8 by 1.2 to 2.0 microns, with rounded ends, occurring singly. Motile by means of a few peritrichous flagella. Gram-negative.

All media except the fresh-water broth, litmus milk, and potato were prepared with sea water.

Gelatin colonies: $2 \mathrm{~mm}$, convex, circular, entire, whitish.

Gelatin stab: Poor growth, no liquefaction, no pigment.

Agar colonies: $2 \mathrm{~mm}$, convex, smooth, circular.

Agar slant: Moderate, beaded, glistening, butyrous growth with no pigment.

Sea-water broth: Surface ring, moderate turbidity, heavy viscous sediment.

Fresh-water broth: Poor growth.

Litmus milk: No visible change. Casein not digested.

Potato: No visible growth.

Indole not formed.

Nitrites rapidly produced from nitrates.

Produces acid but no gas from glucose and maltose. Does not ferment lactose, sucrose, mannitol, glycerol, xylose, or salicin.

Starch not hydrolyzed.

Hydrogen sulfide not formed.

Ammonia produced from peptone but not from urea.

Fats are hydrolyzed.

Aerobic, facultative.

Optimum temperature $20^{\circ}$ to $25^{\circ} \mathrm{C}$.

Source: Found in sea water and on submerged slides.

Habitat: Sea water. 
6. Achromobacter cycloclastes (Gray and Thornton) Bergey et al. (Bacterium cycloclastes Gray and Thornton, Cent. f. Bakt., II Abt., 73, 1928, 89; Bergey et al., Manual, 3rd ed., 1930, 212.) From Greek cyclus, ring and clastus, breaking in pieces.

Rods : 1.0 to 1.5 by 1.5 to 8.0 microns. Motile with 1 to 12 peritrichous flagella. Gram-negative.

Gelatin colonies: Circular, white, raised, smooth, glistening, entire.

Gelatin stab: No liquefaction. Nail head growth.

Agar colonies: Circular to amoeboid, white, flat to convex, smooth, glistening, translucent with opaque center, entire.

Agar slant: Filiform, pale buff, raised, smooth, glistening, undulate.

Broth: Turbid.

Nitrites produced from nitrates.

Starch not hydrolyzed.

Litmus milk unchanged.

No acid from carbohydrate media .

Attacks phenol and naphthalene.

Aerobic, facultative.

Optimum temperature $30^{\circ}$ to $35^{\circ} \mathrm{C}$

Source: Three cultures from soil.

Habitat: Soil.

7. Achromobacter superficiale (Jordan) Bergey et al. (Bacillus superficialis Jordan, Report Mass. State $\mathrm{Bd}$. of Health, 1890, 833; Bacterium superficialis Chester, Amn. Rept. Del. Col. Agr. Exp. Sta., 9; 1897, 94; Bergey et al. Manual, 1st ed., 1923, 144.) From Latin superficialis, lying on the surface.

Characters added to Jordan's descrip tion by Bergey (loc. cit.) from his private notes are indicated.

Rods: 1.0 by 2.2 microns, occurring singly (Jordan). Motile, possessing peritrichous flagella. Gram-negative (Bergey).

Gelatin colonies: small, circular, gray, translucent.

Gelatin stab: Seanty surface growth. Slow liquefaction.

Agar slant: Limited, gray, filiform.

Broth: Slightly turhicl.
Litmus milk: No change. Later becoming slightly acid.

Potato: No growth (Jordan). Limited growth (Bergey). Abundant (Steinhaus).

Indole not formed (Bergey).

Nitrites not produced from nitrates.

Aerobic, facultative.

Optimum temperature $25^{\circ}$ to $30^{\circ} \mathrm{C}$.

Source: Sewage. Gibbons (Contrib. to Canadian Biol. and Fish., 8, No. 22, 1934, 279 ) reports this species as occurring in the slime and feces of the cod (Gadus callarias) and dogfish (Squalus acanthias). An organism apparently identical with. this organism has been found by Steinhaus (Jour. Bact., 42, 1944, 771) in the intestines of beetle larvie (Irographus jasciata De(r.).

Habitat: Presumably widely distributed in nature.

\section{Achromobacter stenohalis ZoBell}

and Tpham. (Bull. Seripps Inst. of Oceanography, Univ. Calif., 5, 1944, 257.) From Greek stenus, narrow or close, and halimus, salty ; adapted to a slight change of salinity only.

Rods: 0.8 to 0.9 by 0.8 to 1.6 microns, oceurring singly, in pairs and short chains. Non-motile. Capsulated. Gram-negutive

All media except the fresh-water broth, litmus milk, and potato were prepared with sea water.

Gelatin rolonies: $1 \mathrm{~mm}$, whitish, circuJar, convex, entire. No pigment.

Gelatin stah: Very slow crateriform liquefartion. Napiform in 50 days.

Igar colonies: Small, circular, opalescent, lobate edge, convex with slightly raised margin, smooth.

Agar slant: Monlerate, beaded, glistening, opalessent, beared growth with no pignent.

Sea-water broth: Moderate turbidity, viseid sediment, no pellicle or ring.

Fresh-water broth: No visible growth

Litmus milk: No visible changr Cascin not digested.

Potato: No visible growth. 
Indole not produced.

Nitrites slowly produced from nitrates.

No acid or gas from glucose, lactose, maltose, sucrose, mannitol, glycerol, xylose, or salicin.

Starch not hydrolyzed.

Hydrogen sulfide not produced.

Ammonia produced from peptone but not from urea.

Fats are not hydrolyzed.

Aerobic, facultative (poor anaerobic growth).

Optimum temperature $20^{\circ}$ to $25^{\circ} \mathrm{C}$.

Source: Sea water, marine mud, and marine phytoplankton.

Habitat: Sea water.

9. Achromobacter butyri Bergey et al. Micrococcus butyri-aromafaciens Kieith, The Technology Quarterly, 10, 1897,247 ; Bacillus butyri aromafaciens Grimm, Cent. f. Bakt., II Abt., 8, 1902, 589 ; Bergey et al., Manual, 1st ed., 1923, 148; Bacterium butyriaromafaciens Oneliansky, Jour. Bact., 8, 1923, 400.) From Latin butyrum, butter.

Rods : 0.5 to 1.0 micron, nearly spherical, occurring singly and in pairs. Nonmotile. Gram-negative.

Gelatin colonies: White, circular, smooth, glistening.

Gelatin stab: White surface growth, liquefaction with white sediment.

Agar slant: Abundant, white, glistening.

Broth: Turbid, with ring and sediment.

Litmus milk: Reaction unchanged. Aromatic odor.

Potato: Slow and limited, white growth.

Nitrites not produced from nitrates. Aerobic, facultative.

Optimum temperature $25^{\circ} \mathrm{C}$.

Habitat: Nilk.

10. Achromobacter stationis ZoBell and Upham. (Bull. Seripps Inst. of Oceanography, Univ. Calif., 5, 1944, 273.) From Latin statio, anchorage.
Ovoid rods : 0.4 by 0.5 to 0.6 microns, occurring singly or in chains of two to three. Non-motile. Gram-positive but easily destained.

All media except the fresh-water broth, litmus milk, and potato were prepared with sea water.

Gelatin colonies : 0.5 to $1 \mathrm{~mm}$, circular, convex, grayish-white.

Gelatin stab: Very slow napiform liquefaction.

Agar colonies: 1 to $2 \mathrm{~mm}$, convex, lobate edge, smooth, colorless.

Agar slant: Moderate, glistening, filiform, butyrous growth with no pigment.

Sea-water broth: Heavy pellicle, no turbidity, granular growth along walls, scanty sediment.

Fresh-water broth: Good growth.

Litmus milk: Becomes alkaline. Casein not digested.

Potato: No visible growth.

Indole not formed.

Nitrites rapilly produced from nitrates.

Produces acid but no gas from glucose. Does not ferment lactose, maltose, sticrose, mannitol, glycerol, xylose, or salicin.

Starch not hydrolyzed.

Hydrogen sulfide not formed.

Ammonia produced from peptone but not from urea.

Fats not hydrolyzed.

Aerobic, facultative.

Optimum temperature $20^{\circ}$ to $25^{\circ} \mathrm{C}$.

Source: Found in film of marine fouling organisms.

Habitat: Sea water.

11. Achromobacter eurydice (White) Bergey et al. (Bacterium eurydice White, U. S. Dept. of Agr., Bur. of Entomol., Cire. 157, 1912, 3 and U. S. Dept. of Agr. Bull. 810, 1920, 15; Bergey et al., Manual, 2nd ed., 1925, 170.) From Greek Eurydice, the wife of Orpheus.

Rods: Small, slender, with slightly rounded ends, occurring singly and in pairs. Ton-motile. Gram-negative. 
Gelatin stab: A bluish-gray growth occurs along the line of inoculation. No liquefaction.

Glucose agar colonies: Bluish-gray, circular, smooth, glistening, entire.

Broth: Uniform turbidity with viscid sediment.

Litmus milk: Unchanged.

Acid from glucose but little or no action on other carbohydrates.

Potato: Slight, grayish growth.

Aerobic, facultative.

Innocuous when fed to bees. Not pathogenic when inoculated subcutaneously in rabbits.

Source: Occurs as a secondary invader in European foulbrood of bees.

Habitat: Unknown.

12. Achromobacter delmarvae Smart. (Smart, Jour. Bact., 23, 1932, 41 and Jour. Agr. Research, 51, 1935, 363.) From Delmarva, coined from Del., Mar. and $\mathrm{Va}$, the regions in which the species was found.

Short rods: Average size 0.75 by 1.5 microns, with rounded ends, occurring singly, in pairs and in short chains. Nonmotile. Gram-negative.

Gelatin colonies: Similar to agar colonies.

Gelatin stab: Scanty growth. No liquefaction.

Beef-infusion agar colonies: Small, circular, raised, edges smooth, glistening, translucent, bluish-white, anorphous, margin entire.

Agar stab: Abundant growth. Surface growth round, smooth, glistening, bluish-white, raised. Filiform growth the whole length of stab, but growth best at top.

Agar slant: Abundant filiform growth, raised, glistening, smooth, translucent, bluish-white, no odor; old cultures slightly viscid. Medium unchanged.

Nutrient broth: Turbid. Delicate white pelliele. Sediment abundant, white, slightly stringy. No odor. Color of medium unchanged.
Sterile milk: Slow growth. No peptonization. Coagulation in 12 to It days. Milk turns chocolate brown beginning at top.

Litmus milk: Acid with reduction of litmus in 5 days. Coagulation with return of pink color in 12 to 14 days. Browning of medium.

Potato: Abundant growth, grayishwhite, glistening, smooth, raised. Medium changed from white to smoke-gray.

Indole not formed.

Nitrites produced from nitrates in 7 days at $26^{\circ} \mathrm{C}$.

No $\mathrm{H}_{2} \mathrm{~S}$ produced.

Ammonia not formed.

Diastatic action weak.

Acid but no gas from glucose, lactose, glycerol and mannitol. Alkaline reaction and no gas from sucrose.

Optimum pH 7.0.

Temperature relations: Optimum $26^{\circ} \mathrm{C}$. Good growth up to $31^{\circ} \mathrm{C}$. Very slight growth at $37^{\circ}$ and at $-8^{\circ} \mathrm{C}$.

Facultative anaerobe.

Source: Isolated from fresh strawberries from Delaware, Maryland and Virginia.

Habitat: Unknown.

Appendix: Many of the following species were described before Gram and flagella stains had been perfected. Hence it is impossible to identify them definitely as belonging to Achromobacter. Comparative study is needed in other eases before the remaining species can be placed in their proper place in the genus.

Achromobacter acidum (Chester) Bergey et al. (Species No. 56 of Conn, Storrs Agr. Exper. Sta., 7th Ann. Rept. for 1894, 1895, 83; Bacterium acidum Chester, Man. Determ. Bact., 1901, 146; Bergey et al., Manual, 1st ed., 1923, 151.) From milk. See Manual, 4th ed., 1934, 246 for a description of this organism.

Achromobacter agile (Ampola and Garino) Bergey et al. (Bacillus denitrificans apilis Ampola and Garino, Cent. 
f. Bakt., II Abt.. 2, 1596, 673; Bacterium denitrificans agilis Chester, Ann. Rept. Del. Col. Agr. Exp. Sta., 9, 1897, 76; Bacterium agile $\mathrm{H}$. Jensen, Cent. $\mathrm{f}$. Bakt., II Abt., 4, 1898, 408; Bacillus denitrificans Migula, Syst. d. Bakt., 2, 1900, 796; not Bacillus denitrificans Chester, Man. Determ. Bact., 1901, 274; Bacillus agilis Chester, Man. Determ. Bact., 1901, 226; not Bacillus agilis Tschistowitsch, Berl. klin. Wchnschr., 1892, 512; Bergey et al., Manual, 1st ed., 1923, 138.) From cow manure. See Manual, 4th ed., 1934, 219 for a description of this organism.

Achromobacter album (Eisenberg) Bergey et al. (Bacillus albus Eisenberg. Bakt. Diag., 3 Aufl., 1891, 171; Bacterium albus Chester, Ann. Rept. Del. Col. Agr. Exp. Sta., 9, 1897, 76; Bergev et al., Manual, 1st ed., 1923, 141.) From water. Cribbons (Contrib. to Canadian Biol. and Fish., 8, No. 22, 1934, 279) reports this species from the slime on cod (Gadus callarias). See Manual, th ed., 1934, 222 for a description of this organism.

Achromobacter amylororum (Rubentschick) Bergey et al. (Ĺrobacterium amylovorum Rubentschick. Cent. f. Bakt., II Abt., 64, 1925, 168; ibid., $66,1926,161$; Bergey et al., Manual, 3rd ed., 1930, 225.) From sewage slime. See Manual, 5 th ed., 1939,514 for a deseription of this organism.

Achromobacter anaerobium Shinwell. (Jour. Inst. Brewing, 43, 1937, 507.) From spoiled beer.

Achromobacter aromafaciens (Chester) Bergey et al. (Species No. 41 of Conn, Storrs Agr. Exper. Sta., 7th Ann. Rept. for 1894, 1895, 57; Bacterium connii Migula, Syst. d. Bakt.. 2, 1900, 440 ; not Bacterium connii Chester, Man. Determ. Bact., 1901, 1t6; Bacterium aromafaciens Chester, loc. cit., 115; Bergey et al., Manual, 1st ed., 1923. 151.) From milk sent from Uruguay to Chicago World's Fair. See Manual, 5 th ed., 1939, 519 for a description of this organism.
Achromblacter arcticum Rusakowa and Butkewitsch. (Microbiology (Russian), 10, 1941, 137; abst. in Cent. f. Bakt., II Abt., 105, 1942, 140.) From sea water (Barents Sea).

Achromobacter candicans (Frankland and Frankland) Bergey et al. (Bacillus candicans $\mathrm{G}$. and P. Frankland, Ztschr. f. Hyg., 6, 1889, 397; Bacterium candicans Chester, Ann. Rept. Del. Col. Agr. Exp. Sta., 9, 1897. 130; Bergey et al., Manual, 1sted., 1923, 149.) From soil. See Manual, 5 th ed., 1939,520 for a deseription of this organism.

Achromobacter centropunctatum (Jensen) Bergey et al. (Bacterium centropunctatus H. Jensen. Cent. f. Bakt., II Abt.. 4. 1898, 410 ; Bacillus centropunctatus Chester, Man. Determ. Bact., 1901, 225; Bergey et al., Manual, 1st ed., 1923, 139.) From cow manure. See Manual, tth ed., 1934, 220 for a deseription of this organism.

Achromobacter coccoideum (Chester) Bergey et al. (Species No. 16 of Conn, Storrs Agr. Exper. Sta., 6th Ann. Rept. for 1893, 1894, 51 ; Bacterium coccoidcum. Chester, Man. Determ. Bact., 1901, 147; Bergey et al., Manual, 1st ed., 1923, 152.) From ripening cream. See Manual, 5th ed., 1939, 520 for a description of this organism.

Achromobacter connii (Chester) Bergey et al. (Culture No. 55, Conn, Storrs Agr. Exp. Sta., Tth Annual Rept. for 1894, 1895, 83; Bacterium connii Chester, Man. Determ. Bact., 1901, 146; Bergey et al., Manual, 1sted., 1923, 149.) From milk. See Manual, th ed., 1934, 243 for a description of this organism.

Achromobacter dendriticum (Lustig) Bergey et al. (Bacillus dendriticus Lustig. Diagnostica dei hatteri delle acque, Torino, 1890 and Diagnostik der Bakterien des Wassers, 1893, 99; Bacterium dendriticus Chester, Ann. Rept. Del. Col. Agr. Exp. Sta., 9, 1897, 103 ; Bergey et al., Manual, 2nd ed., 1925, 156.) From water. See Manual, 5th ed., 1939,504 for a description of this organism. 
Achromobacter epsteinii Peshkov. (Peshkov, Jour. of Biology (Russian), 6 , 1937, 1003.) From water of a carp pond near Moscow.

Achromobacter fermentationis (Chester) Bergey et al. (Bacterium fermentationis Chester, Del. Agr. Exp. Sta. Rept. 1899, 53; Bergey et al., Manual, 1st ed., 1923, 152.) From soil. See Manual, 4th ed., 1934, 247 for a description of this organism. In Chester, Man. Determ. Bact., 1901, 231 this is listed as a synonym of Bacillus foetidus-liquefaciens Tavel, Ueber Aetiol. der Strumitis, Basel, 1892.

Achromobacter filefaciens (Jensen) Bergey et al. (Bacterium filefaciens H. Jensen, Cent. f. Bakt., II Abt., 4, 1898, 401; Bergey et al., Manual, 1st ed., 1923, 153.) From dust. See Manual, 4th ed., 1934, 247 for a description of this organism.

Achromobactor formosum (Ravenel) Bergey et al. (Bacillus formosus Ravenel, Memoirs Nat. Acad. Sei., 8, 1896, 12 ; not Bacillus formosus Bredemann and Heigener, Cent. f. Balt., II Abt., 93, 1935, 101; Bacterium formosus Chester, Ann. Rept. Del. Col. Agr. Exp. Sta., 9, 1897, 91; Bergey et al., Manual, 1st ed., 1923, 136.) From soil. Gibbons (Contrib. to Canadian Biol, and Fish., 8, No. $24,1934,308$ ) reports this species from fillets of haddock (Melanogrammus aeglefunus). See Manual, 5th ed., 1939, 505 for a description of this organism.

Achromobacter galophilum Bergey et al. (Culture No. 27, Baranik-Pikowsky, Cent. f. Bakt., II Abt., 70, 1927, 373; Bergey et al., Manual, 3rd ed., 1930, 223.) From sea water. See Manual, 5 th ed., 1939, 514 for a description of this organism.

Achromobacter gasoformans (Eisenberg) Bergey et al. (Gasbildner Bacillus, Tils, Zeitschr. f. Hyg., 9, 1890, 315; Bacillus gasoformans Eisenberg, Bakt. Diagnostik, 1891, 107; Bacterium yasoformans Chester, Ann. Rept. Del. Col. Agr. Exp. Sta., 9, 1897, 93; Bergey et al., Manual, 1st ed., 1923, 137.) From water. See Manual, 5th ed., 1939, 503 for a description of this organism. Gas bubbles observed in plain gelatin stab.

Achromobacter geminum (Chester) Bergey et al. (Bacillus geminus minor Ravenel, Memoirs Nat. Acad. Sci., 8, 1896, 28; Bacterium yeminus minor Chester, Ann. Rept. Del. Col. Agr. Exp. Sta., 9, 1897, 72; Bacillus geminus Chester, Man. Determ. Bact., 1901, 216; Bergey et al., Manual, 1st ed., 1923, 142.) From soil. See Manual, 5 th ed., 1939, 508 for a description of this organism.

Achromobacter gutlatum (Zimmermann) Bergey et al. (Bacillus guttatus Zimmermann, Bakt. unserer Trink- u. Nutzwässer, Chemnitz, 1, 1890, 56; Bacterium guttatus Chester, Ann. Rept. Del. Col. Agr. Exp. Sta., 9, 1897, 94; Bergey et al., Manual, 1st ed., 1923, 140.) From water. See Manual, 5th ed., 1939, 50s for a description of this organism.

Achromobacter halophilum Bergey etal. (Culture No. 36, Baranik-Pikowsky, Cent. f. Bakt., II Abt., 70, 1927, 373; Bergey et al., Manual, 3rd ed., 1930, 220.) From sea water. Sce Manual, 5 th ed., 1939, 513 for a description of this organism.

Achromobacter harllebii (Jensen) Bergey et al. (Bacterium hartlebii $\mathrm{H}$. Jensen, Cent. f. Bakt., II Abt., 4. 1898, 449; Bacillus hartlebii Chester, Man. Determ. Bact., 1901, 226; Bergey et al., Manual, 1st ed., 1923, 139.) From soil. See Manual, th ed., 1934, 219 for a description of this organism.

Achromobacter hyalinum (Jordan) Bergey et al. (Bacillus hyalinus Jordan, Rèport, Mass. State Bd. of Health, 1890, 835; Bacterium hyalinus Chester, Ann. Rept. Del. Col. Agr. Exp. Sta., 9, 1897, 95; Bergey et al., Manual, 1st ed., 1923, 135.) From sand in a septic tank. See Manual, 4th ed., 1934, 216 for a description of this organism. Also reported by Hateher (Jour. Elisha Mitchell Sei. Soc., 
$5 \tilde{5}, 1939,332)$ from the feces of a coekroach (Periplaneta americana). Litmus milk acid and coagulated. Gram-negative.

Achromobacter inunctum (Pohl) Bergey et al. (Bacillus inunctus Pohl, Cent. f. Bakt., 11, 1892, 143; Bacterium inunctus Chester, Ann. Rept. Del. Col. Agr. Exp. Sta., 9, 1897, 94; Bergey et al., Manual, 1st ed., 1923, 111.) From water. See Manual, 4th ed., 1934, 221 for a description of this organism.

Achromobacter lacticum bergey et al. (Kramer, Die Bakteriologie der Landwirtschaft, 2, 1892, 24; Bergey et al. Manual, 1st ed., 1923, 152.) From slimy milk. See Manual, 5th ed., 1939, 519 for a description of this organism. This appears to refer to Lneffler's slimy milk bacillus, more correctly known as $\mathrm{Bac}$ terium pituitosum Migula.

Achromobacter larvae (Stutzer and Wsorow) Bergey et al. (Enterobacillus larvae Stutzer and Wsorow; Cent. f. Bakt., II Abt., $71,1927,119$; Bergey et al., Manual, 3rd ed., 1930, 22\%.) From intestinal tract of normal and diseased caterpillars of winterwheat cutworm (Euxor segetum). See Manual, 5th ed. $1939,5+1$ for a description of this organ ism.

Achromobacter liquidum. (Frankland and Frankland) Bergey et al. (Bacillus liquidus G. and P. Frankland, Ztschr. f. Hyg., 6, 1889, 382; Bacterium liquidum Chester, Ann. Rept. Del. Col. Agr. Exp. Sta., 9, 1897, 137; Pseudomonas liquida Chester, Man. Determ. Bact., 1901, 311; Bergey et al., Manual, Ist ed., 1923, 145. From water. See Manual, 5th ed., 1939. 511 for a deseription of this organism. Achromobacter litorale (Russell) Bergey et al. (Bacillus litoralis Russell, Ztschr.f. Hyg., 11, 1891, 199; Bacterium litoralis Chester, Ann. Rept. Del. Col. Igr. Exp. Sta., 9, 1897, 94; Pseudomonas litoralis Migula, Syst. d. Bakt., 2, 1900, 8.9; Bergey et al., Manual, 1st ed., 1923, 138.) See Manual, 5th ed., 1939, 503 for a description of this organism. From mud bottom, Gulf of Xaples.
Achromobacter litorale var. 2, Bois and lioy. Naturaliste Canadien, 71,1915 , 259.) From intestine of the codfish (Gadus callarias L.).

Achromobacter middletownii (Chester) Bergey et al. (Species Yo. 53 of Conn, Storrs Agr. Exper. Sta., Tth Ann. Rept. for $1894,1895,82$; Bacterium middletownii Chester, Man. Determ. Bact., 1901, 147; Bergey et al., Manual, 1st ed., 1923, 151.) From milk. See Manual, 4th ed., 1934, 245 for a description of this organism.

Achromobacter mucidus Alford and McCleskey. (Proc. Louisiana Acad. Sci., $\gamma, 1943,25$.$) From crab meat having$ musty odor.

Achromobacter nijibetsui Takeda. (Cent. f. Bakt., II Abt., 94, 1936, 48.) From fish hatchery water. Not pathogenic to salmon eggs.

Achromobacter nitrororum (Jensen) Bergey et al. (Bacterium nitrozorum H. Jensen, Cent. f. Bakt., II Abt., ' 1898, 450; Bergey et al., MIanual, 1st ed., 1923, 15t.) From horse manure. see Manual, 4th ed., 1934, 248 for is description of this organism.

Achromobacter perolens Turner. (Australian Jour. Exp. Biol. and Med. Sci., 4, 1927, 57.) From musty eggs.

Achromobacter pestifer (Frankland and Frankland) Bergey et al. (Bacillus pestifer G. and P. Frankland, Philosoph. Trans. Roy. Soc., London, B, 178, 1888, 27; Bacterium pestifer Chester, Ann. Rept. Del. Col. Agr. Exp. Sta., 9, 1897, 96; Bergey et al., Manual, 1st ed., 1923, 140.) From dust. See Manual, 5th ed., 1939,507 for a description of this organism.

Achromobacler pitiowskyi Bergey et al. (Culture Jo. 25, Baranik-Pikowsky, Cent. f. Bakt., II Abt., 70, 1927, 373; Bergey et al., IIanual, 3rd ed., 1930, 222.) From sea water. See Manual, jth ed., 1939, 514 for a description of this organism.

Achromobacter pinnatum (Ravenel) Bergey et al. (Bacillus pinnatus 
Ravenel, Memoirs Nat. Acad. Sci., 8, 1896, 32; Bacterium pinnatus Chester, Ann. Rept. Del. Col. Agr. Exp. Sta., 9, 1897, 72; Bergey et al., Manual, 1st ed., 1923, 142). From soil. See Manual, th ed., 1934, 223 for a description of this organism.

Achromobacter ravenelii Bergey et al. (Bacillus geminus major Ravenel, Memoirs Nat. Acad. Sci., 8, 1896, 27; Bacillus raveneli Chester, Man. Determ. Bact, 1901, 217; Bergey et al., Manual, 1st ed., 1923, 143.) From soil. Gibbons (Contrib. to Canadian Biol. and Fish., 8 , No. 22, 1934, 279) reports this species from the slime on cod (Gadus callarias). See Nanual, 4th ed., 1934, 224 for a description of this organism.

Achromobacter refractans

(Wright) Bergey et al. (Bacillus refractans Wright, Mem. Nat. Acad. Sci., 7, 1894, 442; Bacterium refractans Chester, Ann. Rept. Del. Col. Agr. Exp. Sta., 9, 1897, 82; Bergey et al., Manual, 1st ed., 1923, 150.) From water. See Manual, 4th ed., 1934, 244 for a description of this organism.

Achromobacter reticulare (Jordan) Bergey et al. (Bacillus reticularis Jordan, Rept. Mass. State Bd. of Health, 1890, 834; Bergey et al., Manual, 1st ed., 1923, 144.) From the effluent of a septic tank. See Manual, 5th ed., 1939, 510 for a description of this organism.

Achromobacter rodonatum (Ravenel) Bergey et al. (Bacillus rodonatus Ravenel, Memoirs Nat. Acad. Sci., $8,1896,40$; Bacterium rodonatus Chester, Ann. Rept. Del. Col. Agr. Exp. Sta., 9, 1897, 83; Bergey et al., Manual, 1st ed., 1923, 150.) From soil. See Manual, 4th ed., 1934, 244 for a description of this organism.

Achromobacter rugosum (Chester) Bergey et al. (Species No. 27, Conn, Storrs Agr. Exp. Sta., 1893, 54; Bacillus rugosus Chester, Man. Determ. Bact. 1901 ,220; not Bacterium rugosum Henrici, Arb. Bakt. Inst. Tech. Hochschule Karlsruhe, 1, 1894, 43; not Bacillus rugosus Wright, Memoirs Nat. Arad.
Sci., 7, 1895, 438; Bacterium geminus major Chester, Ann. Rept. Del. Col. Agr. Exp. Sta., 9, 1897, 73; Bergey et al., Manual, 1st ed., 1923, 143.) From soil. See Manual, 4th ed., 1934, 224 for a description of this organism.

Achromobacter sewerinii Bergey et al. (Kultur No. 3, Sewerin, Cent. f. Bakt., II Abt., 1, 1895, 162; Vibrio denitrificans Sewerin, Cent. f. Bakt., II Abt., 3, 1897 , 517 ; Bergey et al., Manual, 1st ed., 1923, 140.) From horse manure.

Achromobacter solitarium (Ravenel) Bergey et al. (Bacillus solitarius Ravenel, Memoirs Nat. Acad. Sci., 8, 1896, 29; Bacterium solitarius Chester, Ann. Rept. Del. Col. Agr. Exp. Sta., 9, 1897, 71; Bergey et al., Manual, 1st ed., 1923, 143.) From soil. Gibbons (Contrib. to Canadian Biol. and Fish., 8, No. 22, 1934, 279) reports this species from the slime on cod (Gadus callarias). See Manual, 5 th ed., 1939 , 509 for a deseription of this organism.

Achromobacter stutzeri (Lehmann and Neumann) Bergey et al., (Bacillus denitrificans II, Burri and Stutzer, Cent. f. Bakt., II Abt., 1, 1895, 392; Bacterium slutzeri Lehmann and Neumann, Bakt. Diag., 1 Auf1., 2, 1896, 237 ; Bacillus nitrogenes Migula, Syst.d. Bakt., 2, 1900, 793; Bacillus stutzeri Chester, Man. Determ. Bact., 1901, 225; Bergey et al., Manual, 3rd ed., 1930, 207.) From horse manure. See Manual, 4th ed., 1934, 221 for a description of this organism.

Achromobacter tiogense (Wright) Bergey et al. (Bacillus tiogensis Wright, Memoirs Nat. Acad. Sci., 7, 1894, 441; Bacterium tiogensis Chester, Ann. Rept. Del. Col. Agr. Exp. Sta., 9, 1897, 82; Bergey et al., Manual, 1st ed., 1923, 150.) From water. See Manual, 4th ed., 1934, 244 for a description of this organism. Achrombbacter ubiquitum (Jordan) Bergey ctal. (Bacillus ubiquitus Jordan, Rept. Mass. State Bd. of Health, 1890, 830; Bacterium ubiquitus Chester, Ann. Rept. Del. Col. Agr. Exp. Sta., 9, 1897, 144; Bergey et al., Manual, 1st ed., 1923, 
153.) From sewage, water and dust. See Manual, 5th ed., 1939, 517 for a deseription of this organism.

Achromobacter venenosum (Vaughan) Bergey et al. (Bacillus venenosus Vaughan, Amer. Jour. Med. Sci., 104, 1892, 191; Bergey et al., Manual, 1st ed., 1923, 141.) From water. Gibbons (Contrib. to Canadian Biol. and Fish., 8 , No. 22, 1934, 279) reports this species from the slime on cod (Gadus callarias) and the feces of dogfish (Squalus acan- thias). See Manual, th ed., 1934, 222 for a description of this organism.

Achromobacter visco-symbioticum (Buchanan and Hammer) Bergey et al. (Bacillus visco-symbioticum Buchanan and Hammer, Iowa Agr. Exp. Sta. Res. Bull. 22, 1915, 261; Escherichia symbiot ica Bergey et al., Manual, 1st ed., 1923, 202 ; Bergey et al., 3rd ed., 1930, 209.) From ropy milk. See Manual, 4th ed., 1934,223 for a description of this organism.

\section{Genus III. Flavobacterium Bergey et al.*}

(Bergey et al., Manual, 1st ed., 1923, 97; Flavobacter Stewart, Jour. Mar. Biol. Assoc. Un. Kringdom, 13, 1932, 41.) From Latin flavus, yellow and bacterium, a small rod.

Rods of medium size forming a yellow to orange pigment on culture media. Motile with peritrichous flagella or non-motile. Generally Gram-negative. Characterized by feeble powers of attacking carbohydrates, occasionally forming acid from hexoses but no gas. Occur in water and soil.

The type species is Flavobacterium aquatile (Frankland and Frankland) Bergey et al.

\section{Key to the species of genus Flavobacterium.}

I. Non-motile, and slow or no liquefaction of gelatin.

A. Litmus milk unchanged.

1. Nitrites not produced from nitrates.

1. Flavobacterium aquatile.

II. Motile with peritrichous flagella.

A. Gelatin liquefied.

1. Litmus milk unchanged.

a. Nitrites produced from nitrates.

2. Flavobacterium diffusum.

3. Flavobacterium okeanokoites.

4. Flavobacterium rigense.

aa. Nitrites not produced from nitrates.

b. From fresh water.

5. Flavobacterium devorans.

bb. From sea water.

6. Flavobacterium marinotypicum.

7. Flavobacterium marinovirosum.

8. Flavobacterium halohydrum.

9. Flavobacterium neptunium.

* Partially rearranged before his death by Prof. D. H. Bergey, Philadelphia, Pennsylvania, Sept., 1937; further revision by Prof. Robert S. Breed, New York State Experiment Station, Geneva, New York, June, 1945. 
2. Litmus milk alkaline.

a. Nitrites produced from nitrates.

10. Flawobacterium suaveolens.

11. Flavobacterium rhenanus.

aa. Nitrites not produced from nitrates.

12. Flavobacterium marinum.

13. Flavobacterium harrisonii.

B. Gelatin not liquefied.

1. Litmus milk unchanged.

a. Nitrites not produced from nitrates.

14. Flavobacterium invisible.

2. Litmus milk acid.

a. Nitrites not produced from nitrates.

15. Flavobacterium lactis.

III. Non-motile.

A. Gelatin liquefied.

1. Litmus milk unchanged.

2. Litmus milk reduced.

16. Flawobacterium sewanense.

a. Nitrites not produced from nitrates.

17. Flavobacterium arborescons.

3* Litmus milk alkaline.

a. Nitrites proluced from nitrates.

18. Flavobacterium lutescens.

19. Flamobacterium fucatum.

4. Litmus milk peptonized.

a. Nitrites not produced from nitrates.

20. Flavobacterium esteroaromaticum.

5. Litmus milk acid.

a. Nitrites produeed from nitrates.

21. Flavobacterium balustinum.

22. Flavobacterium dormitator.

6. Action on litmus milk not recorded. Rust-colored on blood agar.

B. Cielatin not liquefied.

23. Flavobacterium ferugineum.

1. Titmus milk unchanged.

a. Nitrites produced from nitrates.

24. Flavobacterium proteus.

aa. Nitrites not produced from nitrates.

25. Flavblacterium breve.

26. Flavobacterium solare.

C. Action on gelatin not recorded.

1. Litmus milk unchanged.

a. Nitrites produced from nitrates.

27. Flavobacterium flavotenue.

1. Flavobacterium aquatile (Frank- Ztschr.f. Hyg., 6, 1889, 381; Bacterium land and Frankland) Borgey et al. aruatilis (thester, Ann. Rept. Del. Col. (Bacillus aquatilis G. and P. Frankland, Agr. Lxp. Sta., 9, 1897, 96; Bergey et 
al., Manual, 1st ed., 1923, 100.) From Latin aquatilis, aquatic.

Description taken from Frankland and Frankland and from studies by Dr. E. Windle Taylor, Metropolitan Water Board, London, on freshly isolated cultures.

Rods : 0.5 by 2.5 microns, with rounded ends, occurring singly, in pairs and in chains. Oscillatory movement only ; long threads often remaining motionless (Franklands). Gram-negative (Taylor).

Gelatin colonies : Center yellow-brown, with radiate arrangement of bundles of threads. Colorless margin. Very slow liquefaction (none in 6 weeks, Taylor).

Gelatin stab: Yellow surface growth. Slow liquefaction.

Agar slant: Yellow, smooth, glistening limited growth.

Broth: Turbid with whitish sediment. No pellicle.

Litmus milk: Unchanged (Taylor).

Potato: Limited, yellow streak to no growth.

Indole not formed (Taylor).

Nitrites not produced from nitrates.

Aerobic, facultative.

Optimum temperature $25^{\circ} \mathrm{C}$.

Distinctive characters: Resembles Flavobacterium arborescens microscopically; easily distinguished from this organism by its much slower and limited growth on ordinary gelatin and agar media, the marked difference in the appearance of colonies and the inability of Flavobacterium aquatile to produce more than a limited growth on potato.

Source: Isolated from the water of deep wells in the chalk region of Kent, England where it occurred as a practically pure culture. Found abundantly and reisolated by Taylor, 1941 from the same sources (personal communication).

Habitat: Water.

Note: The peritrichous, nitrate reducing and ammonia producing organism identified by Bergey (loc. cit.) in 1923, as Flavobacterium aquatile appears to have been something resembling Flavobacterium diffusum.
2. Flavobacterium diffusum (Frankland and Frankland) Bergey et al. (Bacillus diffusus G. and P. Frankland, Ztschr. f. Hyg., 6, 1889, 396; Bacterium diffusus Chester, Ann. Rept. Del. Col. Agr. Exp. Sta., 9, 1897, 97; Bergey et al., Manual, 1st ed., 1923, 100.) From Latin diffusus, spreading out, diffuse.

Description completed from Harrison (Canadian Jour. Res., 1, 1929, 233) as indicated.

Rods: 0.5 by 1.5 microns, occurring singly and in ehains. Motile, possessing peritrichous flagella. Gram-negative (Harrison).

Gelatin colonies: Thin, bluish-green, spreading, later faint yellow.

Gelatin stab: Thin, glistening, yellowish-green surface growth. Slow crateriform liquefaction.

Agar slant: Thin, light yellow, glistening.

Broth: Turbid, with greenish-yellow sediment.

Litmus milk: Unchanged (Harrison).

Potato: Thin, smooth, greenish-yellow, glistening growth.

Indole not formed (Harrison).

Nitrites produced from nitrates (Harrison).

Slight acidity from glucose. No acid from sucrose and lactose (Harrison).

Aerobic, facultative.

Optimum temperature $25^{\circ}$ to $30^{\circ} \mathrm{C}$.

Source: Originally found in soil. Found also by Tataroff (Die Dorpater Wasserbakterien, Dorpat, 1891, 58) in fresh water and by Harrison (loc, cit.) from skin of halibut from both the Atlantic and Pacific shores of Canada.

Habitat: Soil, fresh and sea waters.

\section{Flavobacterium okeanokoites Zo-} Bell and Upham. (Bull. Seripps Inst. of Oceanography, Univ. Calif., 5, 1944, 270.) From Greek Oceanus, the ocean god, the ocean and coites, bed.

Rods : 0.8 to 0.9 by 1.2 to 1.6 microns, with rounded ends, many coccoid, occurring singly and in long chains. 
Motile by means of peritrichous flagella. Gram-negative.

All media except the fresh-water broth, litmus milk, and potato were prepared with sea water.

Gelatin colonies: Small, circular, convex, entire, rust or orange colored, digest gelatin.

Gelatin stab: Slow napiform liquefaction, yellow growth.

Agar colonies: $2 \mathrm{~mm}$, circular, entire, smooth, convex.

Agar slant: Moderate, filiform, glistening, butyrous growth with yellow pigment.

Sea-water broth: No pellicle, moderate turbidity, moderate viscid sediment.

Fresh-water broth: Good growth.

Litmus milk: No visible change. Casein is digested.

Potato: No visible growth.

Indole not formed.

Nitrites slowly produced from nitrates.

Does not produce acid or gas from glucose, lactose, maltose, sucrose, glycerol, mannitol, xylose, or salicin.

Stareh not hydrolyzed.

Hydrogen sulfide is formed.

Ammonia produced from peptone but not from urea.

Fats not hydrolyzed.

Aerobic, facultative.

Optimum temperature $20^{\circ}$ to $25^{\circ} \mathrm{C}$.

Source: Marine mud.

Habitat: Sea water.

4. Flavobacterium rigense Bergey et al. (Bacillus brunneus rigensis Bazarewski, Cent. f. Bakt., II Abt., 15, 1905, 1; Bergey et al., Manual, 1st ed., 1923, 100.) From Riga, the name of the eity where the species was isolated.

Rods : 0.75 by 1.7 to 2.5 microns, occurring singly. Motile, possessing peritrichous flagella. Gram-negative.

Gelatin colonies: Circular, entire to undulate, grayish-white, homogeneous.

Gelatin stab: Smooth, yellowish surface growth. Infundibuliform liquefaction. Brownish-yellow sediment.
Agar slant: Narrow, whitish streak, becoming yellowish-brown, spreading. Pigment is water and alcohol soluble. Insoluble in ether.

Broth: Turbid with pellicle and brownish sediment. Cells capsulated.

Litmus milk: Unchanged.

Potato: Yellow, spreading growth. The growth turns brownish.

Hydrogen sulfide not formed.

Indole not formed.

Nitrites produced from nitrates.

Aerobic, facultative.

Optimum temperature $30^{\circ} \mathrm{C}$. Brownish colors develop best at lower temperatures. Orange-yellow colors develop best at $37^{\circ} \mathrm{C}$.

Habitat: Soil.

5. Flavobacterium devorans (Zimmermann) Bergey et al. (Bacillus devorans Zimmermann, Bakt. unserer Trink- $u$. Nutzwässer, Chemnitz, 1, 1890, 48; Bacterium devorans Chester, Ann. Rept. Del. Col. Agr. Exp. Sta., 9, 1897, 96; Bergey et al., Manual, 1st ed., 1923, 102.) From Latin devorans, devouring.

Characters added to Zimmermann's description by Bergey (loc. cit.) from his private notes are indicated. Steinhaus (Jour. Bact., 42, 1941, 771) apparently found the same organism.

Rods: 0.7 by 0.9 to 1.2 microns, oceurring singly, in pairs and chains. Motile (Zimmermann), possessing peritrichous flagella (Bergey). Gram-negative (Zimmermann).

Gelatin colonies: Circular, white, granular to filamentous, becoming yellowishgray.

Gelatin stab: Slow infundibuliform liquefaction.

Agar slant: Thin, gray, spreading.

Broth : Turbid.

Litmus milk: Unchanged.

Potato: No growth (Zimmermann). Yellowish-gray streak (Bergey).

Indole not formed.

Nitrites not produced from nitrates (Bergey). 
Aerobic, facultative.

Optimum temperature $25^{\circ}$ to $30^{\circ} \mathrm{C}$.

Source: From water at Chemnitz (Zimmermann). From water (Bergey). From alimentary tract of the nine-spotted lady beetle (Coccinella novemnotata Habst.) (Steinhaus).

Habitat: Water.

\section{Flavobacterium marinotypicum $\mathrm{Zo}_{\mathrm{O}}$}

Bell and Upham. (Bull. Seripps Inst. of Oceanography, Univ. Calif., 5, 1944, 268.) From Latin marinus, of the sea and typicus, typical.

Rods : 0.5 to 0.7 by $1 . t$ to 2.0 microns, occurring almost entirely as single cells. Motile by means of four or more peritrichous flagella. Gram-negative.

All media except the fresh-water broth, litmus milk, and potato were prepared with sea water.

Gelatin colonies: Very minute, yellow; with slow liquefaction.

Gelatin stab: Crateriform liquefaction becoming stratiform. Filiform along line of stab.

Agar colonies: Minute, circular, entire, convex, yellow.

Agar slant: Scanty, filiform, butyrous, shiny growth with yellow pigment.

Sea-water broth: Scanty, yellowish pellicle, heavy turbidity, slight viscid sediment.

Fresh-water broth: Good growth.

Litmus milk: Decolorized, neutral, greenish pellicle, slow peptonization.

Potato: Abundant, shiny, greenish-yellow growth. Potato darkened.

Indole not formed.

Nitrites not produced from nitrates.

Produces acid but no gas from glucose and glycerol. Does not ferment lactose, sucrose, mannitol, xylose, or salicin.

Starch not hydrolyzed.

Hydrogen sulfide is formed.

Ammonia produced from peptone but not from urea.

Fats not hydrolyzed.

Aerobic, facultative
Optimum temperature $20^{\circ}$ to $25^{\circ} \mathrm{C}$.

Source: Sea water and marine mud.

Habitat: Sea water.

7. Flavobacterium marinovirosum ZoBell and Upham. (Bull. Seripps. Inst. of Oceanography, Univ. Calif., 5, 1944, 271.) From Latin marinus, of the sea, and virosus, covered with slimy liquid or ooze.

Rods : 0.7 to 0.8 by 0.8 to 2.8 microns, with rounded ends, occurring singly and in long chains. Motile by means of peritrichous flagella. Gram-negative.

All media except the fresh-water broth, litmus milk, and potato were prepared with sea water.

Gelatin colonies: Small, circular, raised, rust-colored. Slowly digest gelatin.

Gelatin stab: Crateriform liquefaction becoming stratiform. Light orange pigment.

Agar colonies: 1 to $2 \mathrm{~mm}$, circular, convex, entire, smooth.

Agar slant: Moderate, filiform, glistening, mucoid growth with grayish-yellow pigment.

Sea-water broth: Heavy turbidity, no pellicle, abundant viscid sediment.

Fresh-water broth: Good growth.

Litmus milk: No visible change. Casein is digested.

Potato: No visible growth.

Indole not formed.

Nitrites not produced from nitrates.

Does not ferment glycerol, glucose, lactose, maltose, sucrose, mannitol, xylose, or salicin.

Starch not hydrolyzed.

Hydrogen sulfide is formed.

Ammonia produced from peptone but not from urea.

Fats not hydrolyzed.

Aerobic, facultative.

Optimum temperature $20^{\circ}$ to $25^{\circ} \mathrm{C}$.

Source: Sea water and marine mud.

Habitat: Sea water.

8. Flavobacterium halohydrium ZoBell and Upham. (Bull. Seripps Inst. of 
Oceanography, Univ. Calif., 5, 1944, 278.) From Greek hals, salt and hydror, water. Short rods : 0.6 by 0.8 to 1.0 microns, occurring singly. Motile by means of many peritrichous flagella. Gram-negative.

All media except the fresh-water broth, litmus milk, and potato were prepared with sea water.

Gelatin colonies: Small, circular, orange.

Gelatin stab: Napiform liquefaction becoming crateriform. Beaded along line of stab.

Agar colonies : $2 \mathrm{~mm}$, pulvinate, circu lar, entire, smooth.

Agar slant: Moderate, glistening, echinulate, butyrous growth with yellow pigment.

Sea-water broth: Yellow surface ring, heavy turbidity, moderate viscid sediment.

Fresh-water broth: No visible growth.

Litmus milk: No visible change. Casein not digested.

Very poorly tolerant of increases or decreases in salinity.

Potato: No visible growth.

Indole not formed.

Nitrites not produced from nitrates.

Produces acid but no gas from glucose, lactose, maltose, sucrose, and salicin. Does not ferment glycerol, mannitol, or xylose.

Starch is hydrolyzed.

Hydrogen sulfide not formed.

Ammonia produced from peptone but not from urea.

Fats not hydrolyzed.

Aerobic, facultative.

Optimum temperature $20^{\circ}$ to $25^{\circ} \mathrm{C}$.

Source: Sea water and marine muri.

Habitat: Sea water.

9. Flavobacterium neptunium ZoBell and Upham. (Bull. Scripps Inst, of Oceanography, Univ. Calif., 5, 1944, 278.) From Latin Neptunius, god of the sea.

Rods: 0.5 to 0.6 by 1.6 to 4.5 microns, many hent rods, occurring singly and in short chains. Motile by means of long, peritrichous flagella. Gram-negative.

All media except the fresh-water broth, litmus milk, and potato were prepared with sea water.

Gelatin colonies: Small, circular, darker centers, sink in gelatin, faintly yellow.

Gelatin stab: Slow napiform liquefaction. Filiform growth along line of stab.

Agar colonies: $2 \mathrm{~mm}$, circular, smooth, entire, convex, dark centers with buff pigment.

Agar slant: Luxuriant, echinulate, glistening, slightly mucoid growth with buff to yellow pigment. Agar discolored brown.

Sea-water broth: Heavy pellicle, scanty turbidity, scanty sediment.

Fresh-water broth: No visible growth.

Litmus milk: No visible change. Casein not digested.

Potato: No visible growth.

Indole not formed.

Nitrites not produced from nitrates.

Produces acid but no gas from glucose, lactose, maltose, and salicin. Does not ferment glycerol, mannitol, xylose, or sucrose.

Starch is hydrolyzed.

Hydrogen sulfide not formed.

Ammonia produced from peptone but not from urea.

Fats not hydrolyzed.

Aerobic, facultative.

Optimum temperature $20^{\circ}$ to $25^{\circ} \mathrm{C}$.

Source: Marine bottom deposits.

Hahitat: Sea water.

10. Flavobacterium suaveolens Soppeland. (Jour. Agr. Res., 28, 1924, 275.) From Latin suaveolens, of a sweet odor.

Rods : 0.6 to 0.5 by 1.0 to 1.2 microns, with rounded ends, oceurring singly and in pairs. Motile, with peritrichous flagella. Gram-negative on plain agar. Gram-positive in young culture on milk powder agar.

Gelatin stab: Rapid stratiform liquefaction. Medium hecomes brown. 
Agar colonies: Small, circular, smooth yellow, amorphous, undulate margin.

Agar slant: Moderate, flat, glistening, opaque, butyrous, yellow, with aromatic odor.

Broth: Turbid with seanty sediment Aromatic odor, becoming cheesy .

Litmus milk: Peptonized. Alkaline.

Potato: Abundant, yellow, glistening, becoming brown.

Indole formed.

Nitrites are produced from nitrates.

Hydrogen sulfide formed.

Slight acid but no gas from glucose, sucrose and glycerol. No acid from lactose.

Stareh hydrolyzed.

Blood serum is liquefied.

Aerobic, facultative.

Optimum temperature $25^{\circ} \mathrm{C}$.

Source: Dairy wastes.

Habitat: Unknown.

\section{Flavobacterium rhenanus (Migula)}

Bergey et al. (Rhine water bacillus of Burri, Frankland and Frankland, Microorganisms in Water, 1894, 483; Bacillus rhenanus Migula, Syst. d. Bakt., 2, 1900, 713 ; Bacillus rheni Chester, Manual Determ. Bact., 1901, 251; Bergey et al., Manual, 1st ed., 1923, 103.) Named for the Rhine River.

Characters added to Burri's description by Bergey (loc. cit.) from his private notes are indicated. Steinhaus (Jour. Bact., 42, 1941, 771) apparently found the same organism and has added other characters.

Rods: 0.7 by 2.5 to 3.5 microns, with rounded ends, occurring singly and in chains (Burri). Motile, possessing peritrichous flagella (Bergey). Gram-negative (Bergey).

Gelatin colonies: Convex, colorless, transparent, becoming yellowish.

Gelatin stab: Infundibuliform liquefaction.

Agar colonies: Small, smooth, convex, entire.

Glycerol agar slant: Thin, shining, honey-colored. Growth dry and tough.
Broth: Turbid, with orange-colored pellicle and sediment.

Litmus milk: Soft coagulum, becoming slightly alkaline with yellow ring.

Potato: Moist, glistening, thin, flat, orange to rust-colored.

Indole not formed (Bergey).

Nitrites produced from nitrates (Bergey).

Acid from glucose, maltose, and sucrose but not lactose (Steinhaus).

No hydrolysis of starch (Steinhaus).

No $\mathrm{H}_{2} \mathrm{~S}$ produced (Steinhaus).

Aerobic facultative.

Optimum temperature $30^{\circ} \mathrm{C}$.

Source: From Rhine River water (Burri). From water (Bergey). From eggs in ovary of a walking stick (Diapheromera femorata Say) (Steinhaus).

Habitat: Presumably widely distributed in nature.

12. Flavobacterium marinum Harrison. (Canadian Jour. of Research, 1, 1929, 234.) From Latin marinus, pertaining to the sea.

Rods: 0.8 by 1.2 to 1.3 microns, with rounded ends. Occur singly and in pairs. Motile with 4 to 5 peritrichous flagella. Encapsulated. Gram-variable. Show blue granules in Gram-negative rods.

Gelatin colonies: Circular, iridescent, whitish margin with pale yellow center. Liquefaction.

Gelatin stab: Saccate to stratiform liquefaction.

Agar colonies: Circular, pale yellow, smooth, convex, granular, reticulate edge.

Agar slant: Amber-yellow, slightly raised, spreading, smooth, glistening, transparent.

Ammonium phosphate agar: Scant growth.

Broth: Turbid, sediment.

Litmus milk: Alkaline. Digestion without coagulation. Clear serum.

Potato: Abundant, amber-yellow, becoming dirty yellow, spreading, glistening. 
Indole not formed.

Nitrites not produced from nitrates. Trace of ammonia formed.

Faint acidity from glucose. No action on lactose or sucrose.

Loeffler's blood serum not liquefied. Faint yellow spreading growth.

No $\mathrm{H}_{2} \mathrm{~S}$ formed.

Aerobic, facultative.

Optimum temperature $20^{\circ}$ to $25^{\circ} \mathrm{C}$.

Source: Isolated from living halibut obtained at 30 to 50 fathoms, Pacific Ocean. Gibbons (Contrib. to Canadian Biol. and Fish., 8, ㄱo. 22, 1934, 279) reports this species as occurring in the slime and feces of cod (Gadus callarias), halibut (IIippoglossus hippoglossus) and skate (Raja erinacea)

Habitat: Skin and feces of fishes.

13. Flavobacterium harrisonii Bergey et al. (Variety No. 6, Harrison, Rev. gén. du Lait, 5, 1905, 129; Bacillus lactis harrisonii Conn, Esten and Stocking, Ann. Rept. Storrs Agr. Exp. Sta., 1906, 169 ; Bergey et al., Manual, 1st ed., 1923, 104.) Named for Prof. F. C. Harrison, the Canadian bacteriologist who first isolated this species.

Rods : 0.25 to 0.75 by 0.3 to 3.5 microns, occurring singly and occasionally in short chains. Motile, possessing peritrichous flagella. Cram-negative.

Gelatin colonies: Small, gray, glistening, lobular, citron-yellow, slimy.

Gelatin stab: Villous growth in stab. Slow crateriform to napiform liquefaction.

Agar slant: Luxuriant, viscous, spreading, becoming dirty, to brownish citronyellow.

Broth: Turbid, with viseid ring and gelatinous sediment, sweetish odor, alkaline.

Litmus milk: Colorless to gray and slimy, becoming yellow, alkaline.

Potato: Luxuriant, yellow, spreading, slimy.

Indole not formed.

Gluccse, lactose, maltose and sucrose broth turn alkaline with a disagreeable odor. Reaction of glycerol broth remains neutral.

Aerobic, facultative.

Optimum temperature $25^{\circ} \mathrm{C}$.

Source: Slimy milk.

Habitat: Unknown.

\section{Flavobacterium invisibile (Vaughan)}

Bergey et al. (Bacillus invisibilis Vaughan, American Jour. Med. Sci., 104, 1892, 191 ; Bacterium invisibilis Chester, Ann. Rept. Del. Col. Agr. Exp. Sta., 9, 1897, 77; Bergey et al., Manual, 1st ed., 1923, 109.) From Latin invisibilis, not visibile.

Rods : 0.6 to 0.7 by 1.2 to 2.0 microns, occurring singly. Motile, possessing peritrichous flagella. Gram-negative.

Gelatin colonies: Pale yellow, burrlike, with irregular margin.

Gelatin stab: Scanty grow th on surface. Good growth in stab. No liquefaction.

Agar colonies: White, convex, smooth, serrate.

Igar slant: Limited, thick, white streak.

Broth: Turbid.

Litmus milk: Unchanged.

Potato: No growth.

Indole not formed.

Nitrites not produced from nitrates.

Aerobic, facultative.

Optimum temperature $35^{\circ} \mathrm{C}$.

Habitat: Water.

15. Flavobacterium lactis Bergey et al. (Bacillus uromaticus lactis Grimm, Cent. f. Bakt., Il Abt., 8, 1902, 584; Bacillus aromaticus (irimm, ibid., 589; not Bacillus aromaticus Pammel, Bull. 21, Iowa Agr. Exp. Sta., 1893, 792; Bergey et al., Manual, 1st ed., 1923, 108.) From Latin lac, milk.

Rods : 0.7 to 1.0 by 3.5 to 4.0 microns, vccurring singly, in pairs and in chains. Motile, possessing peritrichous flagella. Gram-negative.

Gelatin colonies: Circular, light yellow, slimy. Concentrically ringed, undulate.

Gelatin stab: Slimy surface growth. No liquefaction. 
Agar slant: Slimy, yellowish, smooth, moist.

Broth: Turbid, with abundant sediment.

Litmus milk: Slightly acid.

Potato: Thick, slimy, brownish, with yellowish margin.

Indole not formed.

Nitrites not produced from nitrates.

Cultures have pleasant odor.

Aerobic, facultative.

Optimum temperature $25^{\circ} \mathrm{C}$.

Source: Isolated from milk.

Habitat: Unknown.

16. Flavobacterium sewanense (Kalantarian and Petrossian) Bergey et al. (Bacterium sewanense Fialantarian and Petrossian, Cent. f. Bakt., II Abt., 85, 1932, 431; Bergey et al., Manual, 4th ed., 1934, 160.) From M. L., Sevan, a lake in Armenia .

Straight or curved rods : 1.0 to $2.0 \mathrm{by}$ 4.0 to 5.0 microns on Molisch's agar; on meat extract agar and potato agar they are short or even coccoid. Ends rounded, occurring singly or in pairs. Non-motile. Gram reaction not given. Presumably negative.

Gelatin stab: Slow liquefaction.

Agar colonies : Circular, raised, glistening, dirty white. Deep colonies yellow and lens-shaped.

Agar slant: Abundant, dirty yellow, glistening, raised.

Broth: Turbid with characteristic grow th forms. Pellicle formed in old cultures.

Milk: Unchanged.

Potato: Yellow, raised, glistening, with darkening of the medium.

No visible gas produced from carbohydrates.

Crystals of calcium carbonate form in old cultures on $\mathrm{CaCl}_{2}$ and Molisch's agar.

Aerobic, facultative.

Optimum temperature $20^{\circ} \mathrm{C}$.

Source: Isolated from pellicle formed on surface of fish infusions in Lake Sevan and tap waters containing 1 per cent $\mathrm{CaCl}_{2}$.
Habitat: Sea water. Thought to produce deposits of $\mathrm{CaCO}_{3}$ in Lake Sevan, S. S. R. Armenia.

17. Flavobacterium arborescens (Frankland and Frankland) Bergey et al. (Bacillus arborescens Frankland and Frankland, Ztschr. f. Hyg., 6, 1889, 379; also see Tils, Ztschr. f. Hyg., 9, 1890, 312; Zimmermann, Bakt. unserer Trink-u. Nutzwässer, 2, 1894, 20; and Wright, Mem. Nat. Acad. Sei., 7, 1894, 446, var. a and b; Bacterium arborescens Chester, Ann. Rept. Del. Col. Agr. Exp. Sta., 9, 1897, 106; Migula, Syst. d. Bakt., 2, 1900, 468 uses Bacillus arborescens in the text by mistake as Bacterium is used for other species in the genus and Bacterium arborescens is used in the index, p. 1058; not Bacillus arborescens Chester, Man. Determ. Bact., 1901, 249; Erythrobacillus arborescens Holland, Jour. Bact., 5, 1920, 217; Bergey et al., Manual, 1st ed., 1923, 113.) From Latin arborescens, becoming a tree or tree-like.

Rods: 0.5 by 2.5 microns, occurring singly and in chains. Non-motile (Franklands). Gram-negative (Zimmermann).

Gelatin colonies: Radiate branching filaments. Center yellowish, border translucent.

Gelatin stab: Liquefied with yellow deposit.

Agar slant: Dirty orange growth.

Broth: Turbid, with orange sediment. No pellicle.

Litmus milk: Slow coagulation; litmus reduced. Reaction unchanged (Wright).

Potato: Deep orange, luxuriant growth.

Nitrites not produced from nitrates.

Aerobic, facultative.

Optimum temperature $30^{\circ} \mathrm{C}$.

May belong to Corynebacterium (Lehmann and Neumann, Bakt. Diag., 7 Aufl., 2, 1927, 709).

Source: From river and lake water.

Habitat: Water.

17a. Bacillus arborescens Chester. (Bacillus arborescens non-liquefaciens Ravenel, Mem. Nat. Acad. Sci., 8, 1896, 
39; Bacterium arborescens non-liquefaciens Chester, Ann. Rept. Del. Col. Agr. Exp. Sta., 9, 1897, 103; not Bacterium arborescens non-liquefaciens von Rigler, Hyg. Rund., 12, 1902, 479; Chester, Man. Determ. Bact., 1901, 249.) Regarded by author as a non-liquefying strain of Bacillus arborescens Frankland and Frankland. Not a yellow chromogen. From soil.

18. Flavobacterium lutescens (Mig. ula) Bergey et al. (Der gelhe Bacillus, Lustig, Diagnostik der Bakterien des Wassers, 1893, 78; Bacterium lutescens Migula, Syst. d. Bakt., 2, 1900, 476; Bergey et al., Manual, 1st ed., 1923, 114.) From Latin lutum, yellow; lutescens, beroming yellowish.

Rods: 0.5 by 0.95 micron, occurring singly and in pairs. Non-motile. Gramnegative.

Gelatin colonies: Circular, yellow, lobate.

Gelatin stab: Slow liquefaction.

Agar slant: Pale yellow, heroming golden yellow.

Broth: Turbid.

Litmus milk: Alkaline.

Potato: Isuxuriant, golden-ycllow growth.

Indole not formed.

Nitrites produced from nitrates.

Aerobic, facultative

Optimum temperature $30^{\circ}$ to $35^{\circ} \mathrm{C}$.

Source: From water. Gibbons (Contrib. to Canadian Biol, and Fish., 8, No. $22,1934,279)$ reports this species as occurring in the slime of the cod (Gadus callarias).

Habitat: Fresh and salt water.

19. Flavobacterium fucatum Harrison. (Canadian Jour of Research, 1, 1929, 232.) From Latin fucatus, painted, colored.

Rods : 0.8 to 1.0 by 2.5 to 3.5 microns, slightly bent, with rounded ends. Granular with diphtheroid forms at $37^{\circ} \mathrm{C}$. Nonmotile. Gram-negative.
Gelatin colonies: Circular, yellow, entire, paler at edges.

Gelatin stab: Crateriform liquefaction.

Agar colonies: Circular, buff-yellow, smooth, shiny, convex to pulvinate, granular, entire.

Agar slant : Moderate, light buff-yellow, spreading, shiny, smooth.

Ammonium phosphate agar: Good growth in 6 days.

Broth : Turbid, becoming clear, pellicle and yellow sediment.

Litmus milk: Alkaline. Peptonized. Clear serum. Yellow sediment.

Potato: Abundant, pale buff-yellow, smooth, spreading, becoming orange-yellow,

Indole not formed.

Nitrites produced from nitrates.

Traces of ammonia formed.

No acid from glucose, lactose or sucrose

Loeffler's blood serum not liquefied. Light buff-yellow growth becoming ochraceus salmon.

No $\mathrm{H}_{2 \mathrm{~S}}$ formed.

Aerobic, facultative.

Optimum temperature $20^{\circ}$ to $25^{\circ} \mathrm{C}$.

Source: Repeatedly isolated from living halibut obtained at 30 to 50 fathoms, Pacifie Ocean. Also isolated by Gibbons (Contrib. to Canadian Biol. and Fish., 8, No. 22, 1934, 279) from cod (Gadus callarias) and dogfish (Squalus acanthias).

Habitat : Skin of tishes.

\section{Flavobacterium esteroaromaticum} (Omelianski) Bergey et al. (Bacterium esteroaromaticum Onelianski, Jour. Bact., 8, 1923, 407; Bergey et al., Manual, 3rd ed., 1930, 149.) From M. L., ester and Greek aromaticus, aromatic.

Rords: 0.5 by 1.0 to 3.0 microns. Nonmotile. Gram reaction not recorded.

Gelatin stab: Crateriform liquefaction with odor of musk melons.

Agar colonies: Circular, yellow-brown, with fimbriate margin and a fruity aroma. 
Broth: Turbid, slight sediment. Faint fruity aroma.

Litmus milk: Peptonized. Cheesy odor.

Potato: Abundant growth. Disagreeable odor.

Loeffler's blood serum: Liquefied.

Indole not formed.

Nitrites not produced from nitrates.

Ammonia formed.

Hydrogen sulfide formed.

Fat hydrolyzed.

Methylene blue reduced.

No acid from carbohydrates.

Aerobic, facultative.

Optimum temperature $30^{\circ} \mathrm{C}$.

Source: Aceidental contaminant in rahbit brain containing rabies virus.

Habitat: Presumably widely distributed.

\section{Flavobacterium balustinum Harri-}

son. (Canadian Jour. Research, 1, 1929. 234.)

Rods : 0.6 by 2.0 to 4.0 microns, forming short chains. Non-motile. Ciram-negative.

Gelatin colonies: Circular, bright yellow center, entire.

Gelatin stab: Liquefied.

Agar colonies: Punctiform, cadminmyellow, convex, shiny, transparent.

Agar slant: Egg yolk-yellow, semi transparent streak, smooth, shiny, be coming brownish-yellow.

Ammonium phosphate agar: Slight yellow growth.

Broth: Turbid, with yellow sediment.

Litmus milk: Slightly acid with vellow sadiment.

Potato: Scant, yellow growth.

Indole not formed.

Nitrites (trace) produced from nitrates. Ammonia not formed.

Faint acidity from glucose. No action on lactose or sucrose.

Loeffler's blood serum not liquefied. Egg yolk-like growth.

No $\mathrm{H}_{2} \mathrm{~S}$ formed.

Aerobic, facultative.
Optimum temperature $20^{\circ}$ to $25^{\circ} \mathrm{C}$.

Source: Isolated from living halibut obtained at 30 to 50 fathoms, Pacific Ocean.

Habitat: Skin of fishes.

\section{Flavobacterium dormitator}

(Wright) Bergey et al. (Bacillus dormitator Wright, Memoirs Nat. Acad. Sci., 7, 1895, 442; Bacterium dormitator Chester, Ann. Rept. Del. Agr. Exp. Sta., 9, 1897, 109 ; Bergey et al., Manual, 1st ed., 1923, 115.) From Latin dormitator, one who sleeps.

Description completed from Harrison (Canadian Jour. Res., 1, 1929, 233) whose cultures differed in some particulars from Wright's.

Rods with conical ends, occurring singly, in pairs and in chains. Non-motile. (iram-negative (Harrison).

Gelatin colonies: Small, yellow, slightly granular, liquef ying.

Gelatin stab: Infundibuliform liquefaction, yellow sediment.

Agar slant: Yellow, glistening, translucent.

Ammonium phosphate agar : Slight yellow growth.

Broth: Turbid, with slight pellicle and yellow sediment.

Litmus milk: Slightly acid; litmus reduced. Harrison reports no reduction.

Potato: Slight, transparent, yellow growth.

Indole not formed (Harrison).

Nitrite (trace) produced from nitrates (Harrison).

Acid from glucose, sucrose, glycerol and mannitol. No acid from lactose, raffinose, and inulin (Harrison).

Aerobic, facultative.

Optimum temperature $30^{\circ} \mathrm{C}$.

Source: Originally isolated from fresh water at Philadelphia. Later isolated by Harrison (loc. cit.) from skin of halibut taken in Pacific ocean off Canada. Gibbons Contrib. to Canadian Biol. and Fish., 8, No. 22, 1934, 279) reports this 
species as occurring in the slime of a haddock (Melanogrammus aeglefinus).

Habitat: Fresh and salt water.

23. Flavobacterium ferrugineum Sickles and Shaw. (Jour. Bact., 28, 1934, 421.) From Latin ferrugineus, resembling rust.

Small, slender rods: Less than 0.5 by 0.7 to 1.0 micron, occurring singly and in pairs. Non-motile. Gram-negative.

Gelatin : Liquefaction in one week at $37^{\circ} \mathrm{C}$; at room temperature liquefaction slower, napiform; yellow sediment along line of puncture.

Blood agar colonies: Dull, rust-colored, $1 \mathrm{~mm}$ in diameter, round, entire, umbilicate, rather dry.

Agar colonies: Similar to blood agar colonies but yellowish-gray in color.

Blood agar slants: Moderate growth, rust-colored, rather dry.

Agar slants: Growth very slight, thin, yellowish-gray.

Beef-infusion broth: No growth.

Beef extract broth: Moderate even turbidity. Adding type-specific carbohydrate results in a heavier growth with yellow sediment.

Potato : Moderate grow th, bright orange in color. Potato darkened.

Very active hydrolysis of starch.

Acid but no gas from glucose, lactose, sucrose, maltose, dextrin and inulin ; very slight action on mannitol; no action on salicin.

Limits of growth: Optimum $\mathrm{pH} 7.0$ to 7.5. Minimum 6.5. Maximum 9.0.

Temperature relations: Optimum $35^{\circ}$ to $37^{\circ} \mathrm{C}$. Minimum $22^{\circ} \mathrm{C}$. Maximum $39^{\circ} \mathrm{C}$. Thermal death point $52^{\circ} \mathrm{C}$ for 10 minutes. Enzyme produced by strain against pneumococcus carbohydrate withstands $56^{\circ} \mathrm{C}$ for 10 minutes.

Facultative aerobe.

Distinctive character: Decomposes the non-type-specific carbohydrate obtained from a degraded type I pneumococcus.

Source: Several. strains isolated from swamps and other uncultivated soils.

Habitat: Soil.
24. Flavobacterium proteus Shimwell and Grimes. (Bacterium Y, Shimwell and Grimes, Jour. Inst. Brewing, 42, N.S. 23, 1936, 119; Shimwell and Grimes, ibid., 348.) From Latin Proteus, a sea god who often changed in form.

Rods: 0.8 to 1.2 by 1.5 to 4.0 microns, occurring singly or in chains, and having rounded ends. Highly pleomorphic. Thickened filaments and spindle-shaped swellings common. Probably non-motile. Gram-negative.

Wort-gelatin plate: Surface colonies irregular, up to $1 \mathrm{~mm}$ in diameter, grayish-white or yellowish, flat or slightly raised, margin entire to lobate or crenate. Deep colonies circular, small, yellowish.

Wort-gelatin streak : Scanty, filiform or beaded, slightly raised, at first almost transparent, later more opaque and whitish-buff.

Wort-gelatin stab: Scanty, filiform or beaded, almost colorless. No liquefaction.

Wort-agar plate: Colonies small, pale, buff-colored, resembling bread-crumbs in shape.

Wort-agar streak : Similar to wort-gelatin streak. Sometimes a slight metallic sheen on old cultures.

Broth: Turbid in 24 hours at $30^{\circ} \mathrm{C}$, with a slight surface scum.

Litmus milk: Unchanged.

Potato: A slight, barely visible growth consisting of a narrow filiform dirty yellow line.

Indole not produced.

Nitrites are produced from nitrates.

Acetymethylearbinol not produced.

Starch not hydrolyzed.

Small amount of acid and gas from glucose and maltose. Trace of acid but no gas from sucrose. No acid or gas from lactose.

Acid, gas and ethyl alcohol produced in small quantity from wort together with a pronounced parsnip-like odor.

Optimum pH 5.0. No growth at $\mathrm{pH}$ 4.0 .

Temperature relations : Optimum $32^{\circ} \mathrm{C}$. 
Good growth at $18^{\circ} \mathrm{C}$. Thermal death point $54^{\circ} \mathrm{C}$ for five minutes.

Aerobic, facultative.

Distinctive character: Extreme pleomorphism in media of neutral or slightly alkaline reaction.

Source: Isolated from brewers' yeast.

Habitat: The common short rod bacterium of brewers' yeast.

25. Flavobacterium breve (Frankland and Frankland) Bergey et al. (Iiurzer Canalbacillus, Mori, Ztschr. f. Hyg., 4, 1888, 53; Bacillus brevis G. and P. Frankland, Microorganisms in Water, 1894, 429; not Bacillus brevis Migula, Syst. d. Bakt., 2, 1900, 583; Bacterium breve Chester, Man. Determ. Bact., 1901, 172; Bergey et al., Manual, 1st ed., 1923, 116.) From Latin brevis, short.

Rods: 0.8 to 1.0 by 2.5 microns, showing polar staining. Non-motile. Gramnegative.

Gelatin colonies: Minute, pale yellow, compact growth in 2 to 3 weeks.

Gelatin stab: Thin, yellowish growth on surface. Beaded growth in stab. No liquefaction.

Agar slant: Yellowish growth in 2 to 3 days.

Broth: Turbid with white sediment.

Blood serum: Growth of light gray color in 2 to 3 days.

Litmus milk: Unchanged.

Potato: No growth.

Aerobic, facultative.

Optimum temperature $35^{\circ} \mathrm{C}$.

Habitat: Water.

26. Flavobacterium solare (Lehmann and Neumann) Bergey et al. (Bacterium solare Lehmann and Neumann, Bakt. Diag., 1 Aufl., 2, 1896, 258; Bergey et al., Manual, 1st ed., 1923, 116.) From Latin solaris, solar.

Rods: 0.3 to 0.4 by 1.0 micron, occurring singly, in pairs and in chains. Nonmotile. Gram-negative.

Gelatin colonies: Circular, yellow, glistening, translucent. Projecting rays.
Gelatin stab: Yellow, arborescent growth in stab. No liquefaction.

Agar slant: Pale yellow, raised, arborescent.

Broth: Clear.

Litmus milk: Unchanged.

Potato: Soft, yellowish-brown streak.

Indole not formed.

Nitrites not produced from nitrates.

Aerobic, facultative.

Optimum temperature $30^{\circ} \mathrm{C}$.

Distinctive character: Resembles

Flavobacterium arborescens in type of growth.

Source: Würzburg tap water. Gibbons (Contrib. to Canadian Biol. and Fish., 8, No. 22, 1934, 279) reports this species as occurring in the slime of a skate (Raja erinacea) and of a hake (Urophycis tenuis).

Habitat: Fresh and salt water.

\section{Flavobacterium flavotenue}

Schrire. (Trans. Royal Soc. South Africa, 17, 1928, 45.) From Latin flavus, tawny yellow and tenuis, slender. Probably intended to mean, yellow and slender.

Small rods: Non-motile. Gram-negative.

Agar colonies: Small, circular, Iemon yellow, raised, entire.

Agar slant: Filiform, lemon yellow. Broth: Turbid.

Litmus milk: Unchanged.

Potato: Moist, yellow streak.

Indole not formed.

Nitrites are produced from nitrates. Acid from glucose, galactose and xylose.

Pathogenic to white mice and guiner pigs.

Aerobic, facultative.

Optimum temperature $20^{\circ} \mathrm{C}$.

Source: Isolated from a mold-like growth in a frog (Xenopus laevis).

Habitat: Unknown.

Appendix: Some of the following species were described before Gram and flagella stains had been perfected. 
Hence it is impossible to identify them definitely as belonging in Flavobacterium. Comparative study is needed in some cases before other species listed here can be placed in their proper place in the genus.

Flavobacterium acidificum Steinhaus. (Jour. Bact., 42, 1941, 772.) From the intestine of the grasshopper (Conocephalus fasciatus De G.), the Colorado potato beetle (Leptinotarsa decemlineata Say), several unidentified lady beetle larvae, and the white cabbage butterfly (Pieris rapae $L$.).

Flavobacterium antenniforme (Ravenel) Bergey et al. (Bacillus antenniformis Ravenel, Memoirs Nat. Acad. Sci., 8, 1896, 25; Bacterium antenniformis Chester, Ann. Rept. Del. Col. Agr. Exp. Sta., 9, 1897, 91; Bergey et al., Manual, 1st ed., 1923, 104.) From soil. See Manual, 5th ed., 1939. 531 for a description of this organism.

Flavobacterium aurantiacum (Frankland and Frankland) Bergey et al. (Bacillus aurantiacus (r. and P. Frankland, Zeitschr. f. Hyg., 6, 1889, 390; Bacterium aurantiacus (hester. Ann. Rept. Del. Col. Agr. Exp. Sta., 9, 1897, 109 ; Bergey et al., Manual, 1st ed., 1923, 107; Chromobacterium aurantiacum Topley and Wilson, Prine. Bact. and Immun., 1, 1931, 405.) From water. See Manual, 5 th ed., 1939, 533 for a description of this organism.

Flavobacterium aurantinum (Hammer) Bergey et al. (Bacillus aurantinus Hammer, Research Bull. No. 20, Iowa Exp. Sta., 1915, 149; Bergey et al., Manual, 1st ed., 1923, 107.) From milk. See Manual, 5th ed., 1939, 541 for a description of this organism.

Flanobacterium aurescens (Ravenel) Bergey et al. (Bacillus aurescens Ravenel, Memoirs Nat. Acad. Sci., 8, 1896, 8 ; not Bacillus aurescens Frankland and Frankland, Philo. Trans. Roy. Soc. London, B, 1878, 271; Bacterium aurescens Chester, Ann. Rept. Del. Col. Agr. Exp. Sta.. 9. 1897. 105; Bergey et al.. Mannitl, 1st erl., 1923, 1.).) Firon soil.
Gibbons (Contrib. Canadian Biol. and Fish., 8, No. 24, 1934, 307) found this species in fillets of haddock (Melanogrammus aeglefinus). See Manual, 4th ed., 1934, 142 for a description of this organism.

Flavobacterium brunneum (Copeland) Bergey et al. (Bacillus brunneus Copeland, Rept. Filtration Commission, Pittsburgh, 1899, 348; Bergey et al., Manual, 1st ed., 1923, 112.) From water. See Manual, 5th ed., 1939, 541 for a description of this organism. This may be Bacillus brunneus Schroeter, but not Bacillus brunneus Eisenberg. The latter forms spores.

Flavobacterium buccalis (Chester) Bergey et al. (Bacillus g, Vignal, Arch. d. phys. norm. et path., Sér. 3, 8, 1886, 365; Bacillus buccalis minutus Sternberg, Manual of Bact., 1893, 643; Bacterium buccalis minutus ('hester, Ann. Rept. Del. Col. Igr. Expt. Sta., 9, 1897, 108; Bacterium vignali Migula, Syst. d. Bakt., 2, 1900, 443; Bacterium bucallis (sic) Chester, Man. Determ. Bact., 1901. 167; not Bacterium buccale Migula, Syst. d. Bakt., 2, 1900, 445; Bacillus vignalis Nepveux, Thèse Fac. Pharm., Paris and Nancy, 1920, 112; Bergey et al., Manual, 1st ed., 1923, 113.) From saliva. See Manual, 5th ed., 1939, 541 for a description of this organism.

Flavobacterium butyri Bergey et al. (Bacillus aromaticus butyri Severin, Cent.f. Bakt., II Abt., 11, 1903, 264; Bergey et al., Manual, 1st ed., 1923, 106.) From sour cream. Produces an agreeable odor. See Manual, 5th ed., 1939, 534 for a description of this organism.

Flavobacterium chlorum Steinhaus. (Jour. Bact., 42, 1941, 772.) From the intestine of the nine-spotted lady beetle (Coccinella novemnotata Habst.).

Flavobacterium denitrificans (Lehmann and Neumann) Bergey et al. (Bacillus denitrificans I, Burri and Stutzer, Cent. f. Bakt., II Abt., 1, 1895, 360; Bacterium denitrificans I, Chester, Ann. Rept. Del. Col. Agr. Exp. Sta., 9, 1897, 77; Bacterium denitrificans Lehmann and Neu- 
mann, Bakt. Diag., 2 Aufl., 2, 1899, 273; Pseudomonas stutzeri Migula, Syst.d. Bakt., 2, 1900, 929; Bacillus denitrificans Chester, Man. Determ. Bact., 1901, 224; Bergey et al., Manual, 1st ed., 1923. 109; Chromobacterium denitrificans Topley and Wilson, Prine. Bact. and Immun., 1, 1931,405 .) From horse manure. See Manual, 5th ed., 1939, 534 for a description of this organism.

Flavobacterium desidiosum (Wright) Bergey et al. (Bacillus decidiosus (sic) Wright, Memoirs Nat. Acad. Sci, , 7, 1895, 443; not Bacillus desiduosus McBeth, Soil Sci., 1, 1916, 450; Bacterium desidiosus and Bactcrium decidiosus ('hester, Ann. Rept. Del. Col. Agr. Exp. Sta., 9, 1897, 107 and 133; Flarobacterium deciduosum Bergey et al.. Manual, 1st ed., 1923, 114.) From water. Gibbons (Contrib. Canadian Biol. and Fish.. 8, No. 24. 1934, 308) found this species in fillets of haddock (Melanogrammus aeglefinus). See Manual, 5th ed., 1939, $54 t$ for a description of this organism.

Flabobacterium flarescens (Poh1) Bergey et al. (Bacillus flavescens Pohl, Cent.f. Bakt., 11, 1s32. 144; Bergey et al., Manual, 1st ed.. 1923, 107.) From water. See Manual, 5th ed.. 1939, 535 for a description of this organism.

Flavobacterium flacum (Fuhmann) Bergey et al. (Bacillus flavus Fuhrmann, Cent. f. Bakt., II Abt., 19, 1907, 117; Manual, 1st ed., 1923, 101.) From beer. See Manual, 4th ed., 193t, $1+1$. for a description of this organism.

Flavobacterium gelatinum Sanborn. (Jour. Bact., 19, 1930, 376.) From sea water.

Flavobacterium halmephilum ElazariVolcani. (Studies on the mirroflora of the Dead Sea, Thesis, Hebrew Univ. Jerusalem, 1940, VIII and 85.) From the Dead Sea. A yellow halophilic speries.

Flavobacterium halophilum. Bergey et al. (Culture No. 30 of Baranik-Pikowsky, Cent. f. Bakt., II Abt., 70, 1927, 373; Bergey et al., Manual, 3rd ed., 1930, 147.) From sea water. See Manual,
5 th ed., 1939, 540 for a description of this organism.

Flavobacterium lacunatum (Wright) Bergey et al. (Bacillus lacunatus Wright, Memoirs Nat. Acad. Sci., 7, 1895, 435; Bacterium lacunatus Chester, Ann. Rept. Del. Col. Agr. Exp. Sta., 9, 1897, 110; Bergey et al., Manual, 1st ed., 1923, 117.) From water. See Manual, 5 th ed, 1939,552 for a description of this organism.

Flaiobacterium matzoonii (C'hester) Bergey et al. (species No. 46 of Conn, Storrs Agr. Exper. Sta., 7th Ann. Rept. for 1894, 1895, 80 ; Bacillus matazooni (sic) Chester, Man. Determ. Bact., 1901, 236; Bergey et al., Manual, 1st ed., 1923, 107.) From matzoon, a fermented milk from Armenia. See Manual, 5th ed., 1939, 536 for a deseription of this organism.

Flavobacterium ocale (Wright) Bergey et al. (Bacillus vialis Wright, Memoirs Nat. Acad. Sci.. 7, 1895, 435; Bacterium walis Chester, Ann. Rept. Del. Col. Agr. Exp. Nta., 9, 1897, 111; not Bacterium wale Migula, Syst. d. Bakt., 2, $1900,45 s$; Bergey et al., Manual, 1st ed., 1923. 117.) From water. See Manual, 5 th ed., 193!1, 551 for a description of this ()rganism.

Flavobacterium plicatum (Zimmermann) Bergey et al. (Bacillus plicatus Zimmermann, Bakt. unserer Trink- $u$. Nutzwässer, Chemnitz, 1, 1890, 54; not Bacillus plicatus Frankland and Frankland, Phil. Trans. Roy. Soe. Iondon, 178, B, 1587, 273; Bergey et al,, Manual, 1st ed., 1923, 105.) From water. Gramnegative. Non-motile. See Manual, 5th ed., 1939, 532 for a description of this organism. See p. 654 .

Flazobacterium pruneaeum Sanborn. (Jour. Bact., 19, 1930, 376.) From sea water.

Flavobacterium radiatum (Zimmermann) Bergey et al. (Bacillus radiatus Zimmermann, Bakt. unserer Trinku. Nutzwässer, Chemnitz, 1, 1890,58 ; Bacillus radiatus aquatilis Frankland and Frankland, Microorg. in Water, 
London, 1894, 458; Bergey et al., Manual, 1st ed., 1923, 104.) From water. See Manual, 5th ed., 1939, 531 for a description of this organism. Gram-variable. Slight motility of shorter rods.

Flavobacterium schirokikhii (H. Jensen) Bergey et al. (Salpeter zerstörenden Bacillus, Schirokikh, Cent. f. Bakt., II Abt., 2, 1896, 205; Bacterium schirokikhi H. Jensen, ibid., 4, 1898, 409; Bacillus denitrificans Chester, Man. Determ. Bact., 1901, 274; Bergey et al., Manual, 1st ed., 1923, 100). From horse manure. See Manual, 5th ed., 1939, 527 for a description of this organism.

Flavobacterium stolanatum (Adametz and Wichmann) Bergey et al. (Bacillus stolonatus Adametz and Wichmann, Mitt. Oest. Versuchsstat. f. Brauerei u. Mälz., Wien, Heft 1, 1888, 884; Bacterium stolonatus Chester, Ann. Rept. Del. Col. Agr. Exp. Sta., 9, 1897, 76; Bergey et al., Manual, 1st ed., 1923, 106.) See Manual, 5th ed., 1939, 535 for a description of this organism. From water.

Flavobacterium tremelloides

Bergey etal. (Bacillus tremelloides Tils, Ztschr. f. Hyg., 9, 1890, 292; Bacterium tremelloides Chester, Ann. Rept. Del. Col. Agr. Exp. Sta., 9, 1897, 105; Bergey et al., Manual, 1st ed., 1923, 105.) From river water at Freiburg. Forms a yellow, slimy milk. See Manual, 5th ed., 1939, 532 for a description of this organism.
Flavobacterium (Halobacterium) marismortui Elazari-Volcani. (Studies on the Microflora of the Dead Sea, Thesis, Hebrew Univ., Jerusalem, 1940, V and 48.) From the Dead Sea. This species and Flavobacterium (Halobacterium) halobium and Flavobacterium (Halobacterium) trapanicum are placed in a new subgenus of Flavobacterium named Halobacterium. All produce red pigment. The flagellation of these species was not determined. They may be polar flagellate, see Pseudomonas salinaria and $P$. cutirubra.

Flavobacterium (Halobacterium) halobium (Petter) Elazari-Volcani. (Microbe du rouge de morue, Le Dantec, Compt. rend. Soc. Biol., Paris, 58, 1902, 136; Bacillus halobius ruber Klebahn, Mitteil. a. d. Inst. f. allg. Bot. Hamburg, 4, 1919, 47; Bacterium halobium Petter, Over rood en andere bacterien van gesouten visch, Diss., Utrecht, 1932; Elazari-Volcani, Studies on the Microflora of the Dead Sea, Thesis, Hebrew Univ., Jerusalem, 1940, V and 59.) From reddened salted codfish.

Flavobacterium (Halobacterium) trapanicum (Petter) Elazari-Volcani. (Bacterium trapanicum Petter, Over rood en andere bacterien van gezouten visch, Diss., Utrecht, 1932; Elazari-Volcani, Studies on the Microflora of the Dead Sea, Thesis, Hebrew Univ. Jerusalem, 1940, V and 59.) From the Dead Sea. 


\section{FAMILY X. ENTEROBACTERIACEAE RAHN.}

(Cent. f. Bakt., II Abt., 96, 1937, 280.)

Gram-negative straight rods. Motile with peritrichous flagella, or non-motile. Grow well on artificial media. All species attack glucose forming acid, or acid and visible gas $\left(\mathrm{H}_{2}\right.$ present). Characteristically nitrites are produced from nitrates (exceptions in Erwinia only). Antigenic composition is best described as a mosaic which results in serological interrelationships among the several genera, even extending to other families. Many animal parasites, and some plant parasites causing blights and soft rots. Frequently occur as saprophytes causing decomposition of plant materials containing carbohydrates.

Note: Early attempts to develop a satisfactory basis for the recognition of species among the coliform-dysentery-typhoid group of bacteria are reviewed by Winslow, Kligler and Rothberg (Jour. Bact., 4, 1919, 429). These were largely based on differences in motility, production of indole, ability to liquefy gelatin, and, more particularly, differences in the ability to ferment carbohydrates, especially such compounds as glucose, lactose, sucrose, dulcitol and salicin. The more recent attempts to express differences in species of coliform bacteria by means of the IMViC reaction are reviewed by Parr (Amer. Jour. Public Health, 26, 1936, 39; Bact. Rev., 3, 1939, 1), this cryptic symbol indicating the indole test, methyl red acid determination, acetylmethylcarbinol production (Voges-Proskauer reaction) and the utilization of salts of citric acid. Stuart, Griffin and Baker (Jour. Bact., 36, 1938, 391) and Griffin and Stuart (Jour. Bact., 40, 1940,83) have applied these tests plus cellobiose fermentation to a study of a long series of cultures.

Capsulated types of coliform bacteria are still placed in this edition of the MaNtal in a separate genus, Klebsiella, although there is some question about the separation of these from the species in Escherichia and Aerobacter.

Meanwhile, the Kauffmann and White Antigenic Schema has been successfully applied to the recognition of serological groups and types among salmonellas and related organisms. The groupings recognized are outlined in the Salmonella Subcommittee Reports submitted to the 2nd and 3rd Congresses of Microbiology (Jour. Hyg., 34, 1934, 333 and Proc. 3rd Internat. Cong. for Microbiology, 1940, 832). The successful use of antigenic structure in this field has stimulated a study of the use of $\mathrm{H}$ and $\mathrm{O}$ antigens as a means of classifying the coliform group (Stuart, Baker, Zimmerman, Brown and Stone, Jour. Bact., 40, 1940,101) but this method of classifying the species of coliform bacteria has not proved particularly helpful as yet.

During this same period there has been an increasing appreciation of the closeness of the relationship between certain common chromogenic bacteria (Serratia) and the coliform bacteria (Breed and Breed, Cent. f. Bakt., II Abt., 71, 1927, 435). Moreover, the close relationship between bacteria producing soft rots of living vegetable and other plant tissue (now included in Erwinia) and the coliform bacteria has become more evident in recent studies (Waldee, Iowa State Coll. Jour. Sci., $19,1945,435)$. Many intermediate types are found in rotting vegetable materials, these rotting types having the ability to attack protopectin (Burkey, Iowa State Coll. Jour Sci., 3, 1928, 57) but not to cause soft rots of living plant tissue.

Borman, Stuart and Wheeler (Jour. Bact., 48, 1944, 351) have proposed a rearrangement of the species in the family Enterobacteriaceae which combines many forms that have previously been regarded as separate species, or even as belonging in separate genera. Only the future can determine which of all of these views best expresses the relationships of the bacteria belonging in the Family Enterobacteriaceae.-The Editors. 


\section{Key to the tribes of family Enterobacteriaceae.}

I. Ferment lactose with the formation of acid and visible gas within 24 hours at $37^{\circ} \mathrm{C}$ or within 48 hours at $25^{\circ}$ to $30^{\circ} \mathrm{C}$. Some transitional forms produce acid and gas from lactose slowly.

\section{Tribe 1. Eschericheae, p. 444.}

II. Plant parasites. Ferment lactose with formation of acid, or acid and visible gas. Usually attack middle lamellar substance in plant tissues, causing soft rots.

Tribe II. Erwineae, p. 463.

III. Ordinarily chromogenic producing a pink, red or orange-red pigment. Occasionally non-pigmented. Ferment glucose and lactose with formation of acid, or acid and visible gas.

Tribe III. Serrateae, p. 479.

IV. Lactose not fermented within 30 days either at $37^{\circ} \mathrm{C}$ or at $25^{\circ}$ to $30^{\circ} \mathrm{C}$. Urea decomposed within 48 hours.

Tribe IV. Proteae, p. 486.

V. Lactose rarely fermented within 30 days either at $37^{\circ} \mathrm{C}$ or at $25^{\circ}$ to $30^{\circ} \mathrm{C}$. Urea not decomposed within 48 hours.

$$
\text { Tribe V. Salmonelleae, p. } 492 .
$$

TRIBE I. ESCHERICHEAE BERFEY, BREED AND MURRAY.

(Preprint, Manual, 5th ed., October, 1938, vi.)

Ferment glucose and lactose with the formation of acid and visible gas within 24 hours at $37^{\circ} \mathrm{C}$, or within 48 hours at $25^{\circ}$ to $30^{\circ} \mathrm{C}$. Some forms produce acid and gas from lactose slowly (occasionally not at all). Do not liquefy gelatin except slowly in Aerobacter cloacac.

\section{Key to the genera of tribe Eschericheae.*}

I. Acetylmethylearbinol not produced. Methyl red test positive. Salts of citric acid may or may not be used as a sole source of carbon.

Genus I. Escherichia, p. 444.

II. Acetylmethylcarbinol produced. Methyl red test negative. Salts of citric acid used as sole source of carbon.

Genus II. Aerobacter, p. 453 .

III. Acetylmethylcarbinol may or may not be produced. Methyl red test variable. Salts of citric acid may or may not be used as sole source of carbon. Gas not as abundant as in previous genera. Capsulated forms from respiratory, intestinal and genito-urinary regions.

Genus III. Klebsiella, p. 457.

\section{Genus I. Escherichia C'astellani and Chalmerst.}

(Castellani and C'halmers. Manual Trop. Med., 3rd ed., 1919, 941; Colibacterium Orla-Jensen, Jour. Bact., 6, 1921, 272; Colobactrum (in part) Borman, Stuart and

* Levine (Jour. Bact., 1, 1916, 153) was the first to show the inverse correlation between the methyl red and Voges-Proskauer tests and used these characters for the primary separation of the Escherichia coli section and the Aerobacter aerogenes section (Amer. Jour. Public Health, 7, 1917, 784).

† Completely revised by Prof. M. W. Yale, New York State Experiment Station, Geneva, New York, Nov., 1938; further revision, July, 1943. 
Wheeler, Jour. Bact., 48, 1914, 357.) Named for Theodor Escherich, who first isolated the type species.

Short rods fermenting glucose and lactose with acid and gas production. Acetylmethylcarbinol is not produced. Methyl red test positive. Carbon dioxide and hydrogen produced in approximately equal volumes from glucose. Generally not able to utilize uric acid as a sole source of nitrogen. Found in feces and is occasionally pathogenic to man (colitis, cystitis, ete.). It is, however, also widely distributed in nature.

The type species is Escherichia coli (Migula) Castellani and Chalmers.

\section{Key to the species of genus Escherichia.}

I. Citric acid and salts of citric acid not utilized as sole source of carbon.

A. Hydrogen sulfide not produced.

1. Escherichia coli.

II. Citric acid and salts of citric acid utilized as sole source of carbon.

A. Hydrogen sulfide produced.

B. Hydrogen sulfide not produced.

2. Escherichia freundii.

\section{Escherichia intermedium.}

1. Escherichia coli (Migula) Castellani and Chalmers. (Bacterium coli commune Escherich, Die Darmbakterien des Neugeborenen und Säuglings, $1855 ; B a-$ cillus escherichii Trevisan, I generi e le specie delle Batteriacee, 1889, 15; Bacillus coli communis Sternberg, MLanual of Bacteriology, 1873, 439; Bacillus coli Migula, in Engler and Prantl, Natürlichen Pflanzenfam., 1, 1a, 1895, 27; Bacterium coli Lehmann and Neumann, Bakt. Diag., 1 Aufl., 2, 1896, 224; Bacillus coli verus Durham, Jour. Exp. Med., 5, 1900, 371; Bacillus coli communis verus Durham, ibid., 353; Aerobacter coli Beijerinck, Cent. f. Bakt., II Abt., 6 , 1900, 193; Castellani and Chalmers, Man. Trop. Med., 3rd ed., 1919, 941; Bacillus coli-communis Winslow, Kligler and Rothberg, Jour. Bact., 4, 1919, 483; Bacterium coli-communis Holland, Jour. Bact., 5, 1920, 217; Colobactrum coli Borman, Stuart and Wheeler, Jour. Bact., 48, 1944, 358.) From Latin colon, the large intestine.

Note: Weldin (Iowa State Jour. Sci., $1,1927,121$ ) considers the following identical with the above: Bacillus caricida Flügge, Die Mikroorganismen, 1886, 268 or more probably Brieger, Berlin. klin. Wochnschr., 1854, No. 14; Bacillus C, Booker, Trans. Ninth Internat. Med. Congress, 3, 1887, 598; Bacillus schafferi von Freudenreich, Landw. Jahrb. d. Schweiz, 4, 1890, 17; Bacterium cavicida Chester, Ann. Rept. Del. Col. Agr. Exp. Sta., 9, 1897, 130; Bacterium schafferi Chester, ibid., 7t; Bacillus mustelae septicus Matzuschita, Bakt. Diag., 1902; Bacillus communis Jackson, Jour. Inf. Dis., 8, 1911, 241; not Bacillus communis Migula, Syst. d. Bakt., 2, 1900, 725; Escherichia cavicida Castellani and Chalmers, Manual of Trop. Med., 3rd ed., 1919, 942; Escherichia schaefferi Bergey et al., Manual, 1st ed., 1923, 196.

Oesterle (Cent. f. Bakt., I Abt., Orig., $134,1935,115)$ has described a yellow strain Bacterium coli flavum, Parr (Proc. Soc. Exp. Biol. and Med., 35, 1937, 563) a golden-brown strain Bacterium aurescens (not Bacterium aurescens Migula, Syst. d. Bakt., 2, 1900, 466), and Tittsler (Jour. Bact., 33, 1937, 450) reddish-orange strains which are regarded as pigmented variants of Escherichia coli.

Rods : Usually 0.5 by 1.0 to 3.0 microns, varying from almost coccoid forms to long rods, occurring singly, in pairs and short chains. Motile or non-motile. Motile 
strains have peritrichous flagella. Not usually capsulated. Non-spore-forming. Gram-negative.

Gelatin colonies: Opaque, moist, grayish-white, entire.

Gelatin stab: Grayish-white, spreading, undulate. No liquefaction.

Agar colonies: Usually white, sometimes yellowish-white, rarely yellow, yellow-brown, golden-brown, reddishorange or red; entire to undulate, moist, homogeneous. Atypical forms occur frequently .

Agar slant: Usually white, sometimes yellowish-white, rarely yellow, yellowbrown, golden-brown, reddish-orange or red growth; moist, glistening, spreading.

Broth: Turbid, with heavy grayish sediment. No pellicle.

Litmus milk: Rapid acid formation with development of gas, usually coagulation, curd may or may not be broken up, no peptonization of the curd. Litmus may or may not be reduced.

Potato: Abundant, grayish to yellowish-brown, spreading.

Indole usually formed.

Nitrites produced from nitrates.

Blood agar plates: Different strains vary widely in their action, some being hemolytic (Buchgraber and Hilkó, Cent. f. Bakt., I Abt., Orig., 183, 1935, 449).

Heat resistance: Usually destroyed in 30 minutes at $60^{\circ} \mathrm{C}$, but certain heatresistant strains may withstand this exposure (Ayers and Johnson, Jour. Agr. Res., 3, 1914, 401; Stark and Patterson, Jour. Dairy Sci., 19, 1936, 495).

Antigenic structure: An antigenically heterogeneous species.

Methyl red test positive (Clark and Lubs, Jour. Inf. Dis., 17, 1915, 160); Voges-Proskauer test negative (Durham, Jour. Exp. Med., 5, 1901, 373); inverse correlation between methyl red and Voges-Proskauer tests (Levine, Jour. Bact., 1, 1916, 153).

Citric acid and salts of citric acid not utilized as sole source of carbon (Koser, Jour. Bact., 8, 1923, 493).

Uric acid not utilized as sole source of nitrogen (Koser, Jour. Inf. Dis., 23, 1918, $377)$; uracil utilized as sole source of nitrogen (Mitchell and Levine, Jour. Bact., 35, 1938, 19).

Gas ratio: Approximately equal volumes of carbon dioxide and hydrogen, ratio 1:1, produced from glucose (Harden and Walpole, Proc. Roy. Soc., Ser. B, 77, 1905, 399; Rogers, Clark and Davis, Jour. Inf. Dis., 14, 1914, 411).

Catalase produced.

No $\mathrm{H}_{2} \mathrm{~S}$ produced in peptone iron agar (Levine, Epstein and Vaughn, Amer. Jour. Public Health, 24, 1934, 505; Tittsler and Sandholzer, Amer. Jour. Public Health, 27, 1937, 1240). More sensitive indicators give positive tests for $\mathrm{H}_{2} \mathrm{~S}$ (Hunter and Weiss, Jour. Bact., 35, 1938, 20 ).

Trimethyleneglycol not produced from glycerol by anaerobic fermentation (Braak, Onderzoekingen over Vergisting van Glycerine, Thesis, Delft, 1928, 166; Werkman and Gillen, Jour. Bact., 23, 1932, 167).

Acid and gas from glucose, fructose, galactose, lactose, maltose, arabinose, xylose, rhamnose and mannitol. Sucrose, raffinose, salicin, esculin, dulcitol and glycerol may or may not be fermented. Variable fermentation of sucrose and salicin (Sherman and Wing, Jour. Bact., 33, 1937, 315; Tregoning and Poe, Jour. Bact., 34, 1937, 473). Inulin, pectin and adonitol rarely fermented. Dextrin, starch, glycogen and inositol not fermented. Cellobiose (Jones and Wise, Jour. Bact., 11, 1926, 359) and $\alpha$-methylglucoside (Koser and Saunders, Jour. Bact., 24, 1932, 267) not fermented. Certain strains produce variants which ferment lactose slowly or not at all (Rennebaum, Jour. Bact., 30, 1935, 625). Some strains of slow-lactose-fermenters appear to be intermediate between the coliform and paratyphoid groups (Sandiford, Jour. Path. and Bact., 41, 1935, 77). See Twort (Proc. Royal Soc. London, 79, 1907, 329) for utilization of unusual glucosides; Dozois et al. (Jour. Bact., 30, 1935, 189 and 32, 1936, 499) 
for utilization of certain sugar alcohols and their anhydrides; Poe and Klemme (Jour. Biol. Chem., 109, 1935, 43) for utilization of rare sugars. See Winslow, Kligler and Rothberg (Jour. Bact., 4, $1919,429)$ for review of literature relative to classification.

Fecal odor produced.

Aerobic, facultative.

Growth requirements: Good growth on ordinary laboratory media. Optimum grow th temperature $30^{\circ}$ to $37^{\circ} \mathrm{C}$. Growth takes place at $10^{\circ} \mathrm{C}$ and at $45^{\circ} \mathrm{C}$. Gas produced from glucose at $45^{\circ}$ to $46^{\circ} \mathrm{C}$. Eijkmann test positive (Eijkmann, Cent. f. Bakt., I Abt., Orig., 37, 1904, 74; Perry and Hajna, Jour. Bact., 26, 1933, 419).

Source: From feces of infants.

Habitat: Normal inhabitant of the intestine of man and all vertebrates. Widely distributed in nature. Frequently causes infections of the genitourinary tract. Invades the circulation in agonal stages of diseases.

1a. Escherichia coli var. acidilactici (Topley and Wilson) Yale.

(Milchsaurebacterium, Hueppe, Mit. d. kais. Gesund., 2, 188t, 340; Bacillus acidi lactici Zopf, Die Spaltpilze, 1885, 87; not Bacterium acidi lactici Zopf, Die Spaltpilze, 1884, 60; Bacillus acidi lactici I and II Grotenfelt, Fortschr.d. Med., 7, 1889, 121; possibly also Bacterium acidi lactici $I$ and II Grotenfelt, ibid., 123 ; Bacterium acidi lactici Migula, in Engler and Prantl, Natürlichen Pflanzenfamilien, 1, 1a, 1895, 25; not $\mathrm{Bac}$ terium acidi lactici Kruse, in Flügge, Die Mikroorganismen, 3 Aufl., 2, 1896, 357 ; not Bacterium B, Peters, Bot. Zeitung, 47, 1889, 422); possibly Bacterium grotenfeldtii Migula, Syst. d. Bakt., 2, 1900,408 , a synonym of Bacterium acidi lactici I Grotenfelt; Bacillus acidilactici Jackson, Jour. Inf. Dis., 8, 1911, 241 ; possibly Bacillus lacticus Macé, Traité pratique de bact., 1913, 452; not Bacillus lacticus Kruse, in Flügge, Die Mikroorganismen, 2, 1896, 356; Bacterium duodenale Ford, Studies from Victoria Hospital, Montreal, 1, 1903, 17 (according to Perkins, Jour. Inf. Dis., 37, 1925, 247); Encapsulatus acidi lactici Castellani and Chalmers, Manual of Trop. Med., 1919, 934; Bacillus lactici-acidi Holland, Jour. Bact., 5, 1920, 218; Bacterium acidilactici Holland, ibid.; (Encapsulata) Bacillus duodenale Perkins, Jour. Inf. Dis., 37, 1925, 247; Escherichia acidilactici Bergey et al., Manual, 1st ed., 1923, 199; Bacterium coli var. acidi lactici Topley and Wilson, Princip. Bact. and Immun., 1, 1931, 446; Yale, in Manual, 5th ed., 1939, 393.)

Identification: Includes strains of Escherichia coli which do not attack either sucrose or salicin. It is generally thought that Hueppe's cultures were contaminated with a spore-former.

Source: From milk.

1b. Escherichia coli var. neapolitana (Topley and Wilson) Yale. (Neapeler Bacterien, Emmerich, Deut. med. Wchnschr., 10, 1884, 299; Bacillus neapolitanus Flügge, Die Mikroorganismen, 1886, 270; Bacterium neapolitanus Chester, Ann. Rept. Del. Col. Agr. Exp. Sta., 9, 1897, 138; Escherichia neapolitana Castellani and Chalmers, Man. Trop. Med., 3rd ed., 1919, 942; Bacterium coli var. neapolitanum Topley and Wilson, Princip. Bact. and Immun., 1, 1931, 446; Yale, in Manual, 5th ed., 1939, 393.)

Identification: Includes strains of Escherichia coli which ferment sucrose and salicin.

Source: From cholera patients or cadavers, originally thought to be the cause of cholera.

1c. Escherichia coli var. communior (Topley and Wilson) Yale. (Bacillus coli communior Durham, Jour. Exp. Med. 5, 1900, 353; Bacillus communior Ford Studies from Victoria Hosp., Montreal 1, 1903, 17; Bacterium communior Jackson, Jour. Inf. Dis., 8, 1911, 241; Bacillus coli-communior Holland, Jour. Bact., 5, 1920, 217; Bacterium coli-communior 
Holland, idem; Escherichia communior Bergey et al., Manual, 1st ed., 1923, 200; Bacterium coli var. communior Topley and Wilson, Princip. Bact. and Immun., 1, 1931, 446; Yale, in Manual, 5th ed., 1939, 393.)

Yale (Cornell Vet., 23, 1933, 306) regards Bacterium astheniae Dawson (15th Ann. Rpt., Bur. Anim. Ind., U.S.D.A., 1898, 329; Bacillus astheniae Winslow, Kligler and Rothberg, Jour. Bact., 4, 1919, 487; Escherichia astheniae Bergey et al., Manual, 1st ed., 1923, 205) as a synonym of Escherichia communior.

Identification: Includes strains of Escherichia coli which ferment sucrose but not salicin. Levine (Iowa Eng. Exp. Sta. Bul. 62, 1921, 38) recognizes a strain which ferments salicin.

2. Escherichia freundii (Braak) Yále. (Bacterium freundii Braak, Onderzoekingen over vergisting van glycerine. Thesis, Delft, 1928, 140; Citrobacter freundii Werkman and Gillen, Jour. Bact., 23, 1932, 176; Yale, in Manual, 5th ed., 1939, 394; Colobactrum frendii Borman, Stuart and Wheeler, Jour. Bact., 48, 1944, 358.) Named for A. Freund, who first observed that trimethyleneglycol was a product of fermentation (1881).

Minkewitsch (Ztschr. f. Hyg., 111, 1930,180 ) proposed the name Bacterium coli citrovorum for the intermediates but this name is not acceptable since it is a trinomial.

Werkman and Gillen (Jour. Bact., 23, $1932,177)$ emended the deseription of Bacterium freundii, and created the genus Citrobacter. The following species renamed by Werkman and Gillen are regarded as identical with Escherichia freundii: Citrobacter album, Citrobacter decolorans, Citrobacter diversum and Citrobacter anindolicum.

Tittsler and Sandholzer (Jour. Bact., 29, 1935, 349) and Carpenter and Fulton (Amer. Jour. Pub. Health, 27, 1937, 822) suggest that the intermediates which give a positive methyl red and a negative Voges-Proskauer test be allocated to the genus Escherichia. Other strains are apparently more nearly related to the genus Aerobacter than to the genus Escherichia since they produce acetylmethylcarbinol. Barritt (Jour. Path. and Bact., 42, 1936, 441 ; 44, 1937, 679) has shown that some of the intermediates form traces of acetylmethylcarbinol which can be detected by the $\alpha$-naphthol test, but not by the standard VogesProskauer test as described in the Manual of Methods for the Pure Culture Study of Bacteria (Soc. Amer. Bact., 1937, 17).

Rods: Short rods with rounded ends, occurring singly, in pairs and short chains. Motile or non-motile. Gramnegative.

Gelatin stab: Liquefaction by 4 out of 15 cultures (Werkman and Gillen, Jour. Bact., 23, 1932, 177). No liquefaction by any strains (Tittsler and Sandholzer, Jour. Bact., 29, 1935, 353; Carpenter and Fulton, Amer. Jour. Pub. Health, 27, 1937, 822).

Agar slant: Smooth, gray, shining, filiform and butyrous growth.

Litmus milk: Acid in 2 days; coagulation may or may not take place; no peptonization.

Potato: Abundant, yellowish-white growth.

Indole may or may not be formed (Werkman and Gillen, loc. cit.; Tittsler and Sandholzer, loc. cit.).

Nitrites produced from nitrates.

Methyl red test positive. VogesProskauer test negative (Koser, Jour. Bact., 9, 1924, 59). Some strains give a positive methyl red and a positive VogesProskauer test (Parr, Jour. Bact., 36, 1938, 1).

Citric acid utilized as sole source of carbon; uric acid not utilized as the sole source of nitrogen (Koser, loc. cit.; Werkman and Gillen, loc, cit., 167).

Catalase produced.

Hydrogen sulfide produced in proteose peptone, ferric citrate agar (Levine, 
Epstein and Vaughn, Amer. Jour. Pub. Health, 24, 1934, 505; Tittsler and Sandholzer, Amer. Jour. Pub. Health, 27, 1937,1240 ).

Trimethyleneglycol produced from glycerol by anaerobic fermentation (Braak, loc. cit., 146; Werkman and Gillen, loc. cit., 167).

Acid and gas from glucose, fructose, galactose, arabinose, xylose, raffinose, lactose, maltose, mannose, rhamnose, trehalose, glycerol, mannitol and sorbitol. Sucrose, salicin, dulcitol, adonitol and inositol may or may not be fermented. Cellobiose usually fermented while $\alpha$ methyl-glucoside may or may not be fermented (Tittsler and Sandholzer, loc. cit.; Carpenter and Fulton, loc, cit.). No acid or gas from amygdalin, dextrin, erythritol, glycogen, inulin or melezitose.

Aerobic, facultative.

Growth requirements: Good grow th on ordinary laboratory media. Optimum growth temperature $30^{\circ}$ to $37^{\circ} \mathrm{C}$. Gas not produced in Eijkman test when carried out at $45^{\circ}$ to $46^{\circ} \mathrm{C}$ (Levine, Epstein and Vaughn, loc. cit.). No gas at $44^{\circ} \mathrm{C}$ (Wilson, Med. Res. Council, London. Special Rept. Ser. 206, 1935, 165).

Habitat: Normally found in soil and water and to a varying degree in the intestinal canal of man and animals. Widely distributed in nature.

3. Escherichia intermedium (Werkman and Gillen) Vaughn and Levine. (Citrobacter intermedium Werkman and Gillen, Jour. Bact., 23, 1932, 178; Vaughn and Levine, Jour. Bact., 44, 1942, 498.)

Citrobacter glycologenes Werkman and Gillen (loc. cit.) is also regarded as a synonym of Escherichia intermedium. Vaughn and Levine (loc. cit.) give a new deseription of Escherichia intermedium based on a study of 27 cultures.

Rods: Short rods with rounded ends. Occurring singly, in pairs and short chains in young nutrient agar or broth cultures. Actively motile with peritrichous flagella or non-motile. Gram-negative
Gelatin stab: No liquefaction after 60 days at $20^{\circ} \mathrm{C}$.

Agar slant: Smooth to wrinkled surface, grayish-white, abundant, raised and butyrous growth.

Nutrient broth: Turbid with slight ring at surface.

Litmus milk : Acid, sometimes coagulation and reduction, no proteolysis.

Potato: Growth abundant, white to ivory color.

Levine's eosine-methylene blue agar: Well-isolated colonies vary from 1 to 4 $\mathrm{mm}$ in size. No confluence of neighboring colonies. Colonies are slightly to moderately raised with surfaces varying from flat to convex and usually smooth and glistening but sometimes dull, rough and granular.

By transmitted light two types of colonies have been observed: (1) Colonies having almost the same appearance throughout but with a distinetly lighter center, the color being similar to the medium. (2) Colonies having a dark brownish central area which diffuses out to a lighter margin.

By reflected light three types of colonies have been observed: (1) Dark, button-like, concentrically ringed colonies possessing a strong, greenishmetallic sheen so characteristic for Escherichia coli. (2) Colonies with dark, purplish, wine-colored centers surrounded by a light pink zone. Some colonies are concentrically ringed.

Pink colonies with no suggestion of sheen but sometimes concentrically ringed.

Indole may or may not be formed.

Nitrites produced from nitrates.

Fermentation of glucose: The end products characteristic for the genus Escherichia are formed. Carbon dioxide and hydrogen gases are formed in approximately equimolar proportions (gas ratio 1:1) besides significant quantities of ethyl alcohol, and acetic, lactic and succinic acids with only traces of formic acid. Acetylmethylcarbinol and 2-3 
butylene glycol have not been found (Voges-Proskauer test negative).

Salts of citric acid are utilized as a sole source of carbon.

Catalase produced.

Hydrogen sulfide not detected in proteose peptone ferric-citrate agar.

Acid or acid and gas produced from xylose, arabinose, rhamnose, glucose, fructose, mannose, galactose, lactose, maltose, trehalose and mannitol. No acid or gas from melezitose, amygdalin and erythritol. Sucrose, raffinose, cellobiose, $\alpha$-methyl-glucoside, adonitol, dulcitol, glycerol, inositol, sorbitol, starch, aesculin, salicin and sodium malonate may or may not be fermented.

Aerobic, facultative.

Temperature requirements: Growth at $10^{\circ} \mathrm{C}$ and at $45^{\circ}$ to $46^{\circ} \mathrm{C}$. Optimum growth temperature $30^{\circ}$ to $37^{\circ} \mathrm{C}$. Gas not produced in Eijkman tests, although some cultures show grow th at $45^{\circ}$ to $46^{\circ} \mathrm{C}$.

Salt tolerance: Most cultures ferment glucose in the presence of sodium chloride in a concentration of 6.0 to 7.0 per cent. A few cultures tolerate 8.0 per cent sodium chloride.

$\mathrm{pH}$ range: Optimum about $\mathrm{pH}$ 7.0. Growth occurs at $\mathrm{pH} 5.0$ to $\mathrm{pH} 8.0$.

Habitat: Normally found to a varying degree in soil, water and in the intestinal canal of man and animals. Widely distributed in nature.

Appendix: The following described species have been placed in Escherichia or may belong here:

Bacillus alcalescens Ford. (Ford, Studies from the Royal Victoria Hosp., Montreal, 1, (5), 1903, 37 ; also see Jour. Med. Res., 6, 1901, 211; not Bacillus alkalescens Andrews, Lancet, 194, 1918, 560 ; Escherichia alcalescens Bergey et al., Manual, 1st ed., 1923, 202.) From feces.

Bacillus asiaticus Castellani. (Castellani, Cent.f. Bakt., I Abt., Orig., 65, 1912, 262; not Bacillus asiaticus Sakharoff, Ann. Inst. Past., 8, 1893, 550; Salmonella asiaticus Castellani and Chal- mers, Manual of Trop. Med., 3rd ed., 1919, 940; Proteus asiaticus Bergey et al., Manual, 1st ed., 1923, 211; Bacterium asiaticum Weldin and Levine, Abst. Bact., 1, 1923, 13.) From feces. Ferments lactose slowly or not at all.

Bacillus asiaticus mobilis Castellani. (Valérie 21, Boycott, Jour. Hyg., 6, 1906, 33 ; Castellani, Ann. di Med., Nav. e Colon., 11, 1916, 453; Salmonella asiaticus mobitis Castellani and Chalmers, Manual of Trop. Med., 3rd ed., 1919, 940; Bacterium valeriei Weldin and Levine, Abst. Bact., 7, 1923, 13; Proteus valeriei Bergey et al., Manual, 1st ed., 1923, 211.) From feces. A motile variety which Alves (Jour. Path. and Bact., 44, 1937, 485) found to be identical with Bacillus asiaticus.

Bacillus chylogenes Ford. (Ford, Studies from the Royal Victoria Hosp., Montreal, 1, (5), 1903, 62; also see Jour. Med. Res., 6, 1901, 219.) From feces.

Bacillus coli immobilis Kruse. (Kruse, in Flügge, Die Mikroorganismen, 3 Aufl, 2, 1896, 339; Bacterium coli immobilis Chester, Del. Agr. Exp. Sta., 9th Ann. Rept., 1897, 128; Enteroides entericus Castellani, Jour. Hyg., 7, 1907, 1; Bacillus schafferi MacConkey, Jour. Hyg., 9, 1909, 86 ; not Bacillus schafferi von Freudenreich, Landwirtschl. Jahrb. den Schweiz, 4, 1890, 17; Bacillus entericus Castellani and Chalmers, Manual of Trop. Med., 1st ed., 1910, 990; not Bacillus entericus Ford, Studies from Royal Victoria Hosp., Montreal, 1, (5), 1903, 40; Escherichia schaefferi Bergey et al., Manual, 1st ed., 1923, 196; Bacterium coli var. immobilis Winslow et al., Jour. Bact., 4, 1919, 486; Bacterium schafferi Weldin and Levine, Abst. Bact., 7, 1923, 13; not Bacterium schafferi Chester, Ann. Rept. Del. Col. Agr. Exp. Sta., 9, 1897, 74; Escherichia enterica Weldin, Iowa State Coll. Jour. Sci., 1, 1927, 134.) From feces. These were all described as non-motile variants of Escherischia coli (see Weldin, loc. cit.).

Bacillus coli mutabilis Neisser. 
(Neisser, Cent. f. Bakt., I Abt., Ref. (Supp.), 38, 1906, 98; Bacterium coli mutabile Massini, Arch. f. Hyg., 61, 1907, 250; Escherichia coli mutabilis Castellani and Chalmers, Man. Trop. Med., 3rd ed., 1919, 943; Escherichia coli-mutabile Deere et al., Jour. Bact., 31, 1936, 625.) From feces. An unstable variant closely related to Escherichia coli characterized by irregular lactose fermentation. When cultured on lactose indicator agar, it appears not to ferment lactose. After some days, lactose-fermenting papillae appear growing on or out of the original colonies. Subcultures from these secondary colonies give typical lactose fermentation but subculture from the primary colony, avoiding contact with the papillae, gives delayed fermentation of lactose and when again plated will produce non-fermenting colonies on which fermenting papillae later appear.

Bacillus gastricus Ford. (Ford, Studies from the Royal Victoria Hosp., Montreal, 1, (5), 1903, 58; also see Jour. Med. Res., 6, 1901, 213; Escherichia gastrica Bergey et al., Manual, 1st ed., 1923, 203.) From feces.

Bacillus gruenthali Morgan. (Das gruenthaler Bacterium, Fischer, Ztschr.f. Hyg., 39, 1902, 447; Morgan, Brit. Med. Jour., 1, 1905, 1257; Bacillus acidi lactici var. gruenthali Levine, Jour. Bact., 3, 1918, 270; Bacterium acidilactici var. gruenthali Winslow, Kligler and Rothberg, Jour. Bact., 4, 1919, 486; Escherichia gruenthali Castellani and Chalmers, Manual of Trop. Med., 1919, 942; Bacierium gruenthali Weldin and Levine, Abst. Bact., 3, 1923, 13.) From feces.

Bacillus iliacus Ford. (Ford, Studies from the Royal Victoria Hosp., Montreal, 1, (5), 1903, 61 ; also see Jour. Med. Res., 6, 1901, 213; Escherichia iliacus Bergey et al., Manual, 1st ed., 1923, 203; Proteus iliacus Bergey et al., Manual, 4th ed., 1934, 363.) From feces.

Bacillus infrequens Ford. (Ford, Studies from the Royal Victoria Hosp.,
Montreal, 1, (5), 1903, 42; also see Jour. Med. Res., 6, 1901, 219.) From feces. Bacillus jejunales Ford. (Ford, Studies from the Royal Victoria Hosp., Montreal, 1, (5), 1903, 66; also see Jour. Med. Res., 6, 1901, 219.) From feces.

Bacillus leporis Migula. (Bacillus leporis lethalis Sternberg, Manual of Bacteriology, 1893, 453; Bacterium leporis lethalis Chester, Ann. Rept. Del. Col. Agr. Exp. Sta., 9, 1897, 97; Migula, Syst. Bakt., 2, 1900, 651; Eberthella leporis Bergey et al., Manual, 1st ed., 1923, 229; Escherichia leporis Bergey et al., Manual, 2nd ed., 1925, 221.) From feces.

Bacillus para-gruenthali Castellani. (Castellani, 1914, quoted from Castellani and Chalmers, Ann. Past. Inst., 34 , 1920, 614; Escherichia paragruenthali Castellani and Chalmers, Manual of Trop. Med., 3rd ed., 1919, 942; Bacterium coli var. paragruenthali Weldin and Levine, Abst. Bact., 3, 1923, 13.) From feces. Weldin and Levine (Iowa State Coll. Jour. Sci., 1, 1926, 132) regard this species as identical with Bacillus gruenthali Morgan.

Bacillus plebeius Ford. (Ford, Studies from the Royal Victoria Hosp., Montreal, 1, (5), 1903, 41 ; also see Jour. Med. Res., 6, 1901, 213; Escherichia plebeia Bergey et al., Manual, 1st ed., 1923, 203.) From feces.

Bacillus subalcalescens Ford. (Ford, Studies from the Royal Victoria Hosp., Montreal, 1, (5), 1903, 37 ; also see Jour. Med. Res., 6, 1901, 217.) From feces.

Bacillus subgastricus Ford. (Ford, Studies from the Royal Victoria Hosp., Montreal, 1, (5), 1903, 58; also see Jour. Med. Res., 6, 1901, 219.) From feces.

Bacillus vekanda Castellani. (Castellani, Jour. Trop. Med. and Hyg., 20, 1917, 181; Enteroides vekanda Castellani and Chalmers, Manual of Trop. Med., 3rd ed., 1919, 941; Bacterium vekanda Weldin and Levine, Abst. Bact., 7, 1923, 13; Escherichia vekanda Bergey et al., Manual, 1st ed., 1923, 197.) From feces. Bacillus vesiculiformans Henrici. 
(Henrici, Arb. Bakt. Inst. Hochsch. Karlsruhe, 1, 1894, 25; Escherichia vesiculiformans Bergey et al., Manual, 2nd ed., 1925, 222.) From cheese.

Bacterium chymogenes Ford. (Ford, Studies from the Royal Victoria Hosp., Montreal, 1, (5), 1903, 63; also see Jour. Med. Res., 6, 1901, 219.) From feces.

Bacterium coli alcaligenes Chiari and Löffler. (Cent. f. Bakt., I Abt., Orig., 96, 1925, 95.) From feces.

Bacterium coli anindolicum Lembke. (Lembke, Arch. f. Hyg., 26, 1896, 299 ; Bacillus anindolicum Chester, Man. Determ. Bact., 1901, 207; Escherichia anindolica Bergey et al., Manual, 3rd ed., 1930, 325.) From feces.

Bacterium coli imperfectum Roelcke. (Cent. f. Bakt., I Abt., Orig., 145, 1939, 109.) From feces. Lactose not fermented.

Bacterium formicum Omelianski. (Omelianski, Cent. f. Bakt., II Abt., 11, 1904, 184; Achromobacter formicum Bergey et al., Manual, 1st ed., 1923, 144; Escherichia formica Bergey et al., Manual, 2nd ed., 1925, 220.) From soil.

Bacterium galactophilum Ford. (Ford, Studies from the Royal Victoria Hosp.. Montreal, 1, (5), 1903, 39; also see Jour. Med. Res., 6, 1901, 217; Escherichia galactophila Bergey et al., Manual, 1st ed., 1923, 202.) From feces.

Bacterium succinicum. Sakaguchi and Tada. (Cent.f. Bakt., II Abt., 101, 1940, 341.) From cheese.

Bacterium uromulabile Koch. (Cent. f. Bakt., I Abt., Orig., 193, 1935, 209.) From genito-urinary infections. A nonlactose-fermenting variety that developed the ability to ferment lactose slowly.

Bacterium vesiculosum Henrici. (Arb. bakt. Inst. Karlsruhe, 1, Heft 1, 1894, 37; Bacillus vesiculosus MacConkey, Jour. Hyg., 9, 1909, 86; Escherichia vesiculosa Castellani and Chalmers, Man. Trop. Med., 3rd ed., 1919, 942.) From cheese,

Escherichia alba schrire. (Trans.
Royal Soc. So. Africa, 17, 1928, 43.) From feces.

Escherichia brasitiensis Mello. (Sao Paulo Medico, Anno 10, 2, 1937, 11.) From feces.

Escherichia colofoetida (Castellani) Hauduroy et al. (Bacillus colofoetidus Castellani, Jour. Trop. Med. and Hyg., 1930, 134; Hauduroy et al., Dict. d. Bact. Path., 1937, 226.) From feces.

Escherichia coloides (Castellani) Castellani and Chalmers. (Bacillus coloides var. $A$ and Bacillus coloides var. $B$, Castellani; Castellani and Chalmers, Manual of Trop. Med., 1919, 942 and 946.) From feces.

Escherichia colotropicalis (Castellani) Castellani and Chalmers. (Bacillus colotropicalis Castellani, 1907 ; see Castellani and Chalmers, Manual of Trop. Med., 3rd ed., 1919, 942 and 946.) From feces.

Escherichia ellingeri (Metalnikov and (horine) Bergey et al. (Coccobacillus ellingeri Metalnikov and Chorine, Ann. Inst. Past., 42, 1928, 1635; Bergey et al., Manual, 3rd ed., 1930, 330.) Causes fatal infection in insects as Pyrausta nubilalis Hübn. (European corn borer) and Galleria mellonella L. (bee moth). See Manual, 5th ed., 1939, 606 for a description of this species.

Escherichia khartoumensis (Chalmers and Macdonald) Hauduroy et al. ( $\mathrm{Ba}$ cillus khartoumensis Chalmers and Macdonald, 1915; see Castellani and Chalmers, Manual of Trop. Med., 3rd ed., 1919, 948; Enteroides khartoumensis Castellani and Chalmers, ibid., 941 ; Hauduroy et al., Dict. d. Bact. Path., 1939, 230.) From feces.

Escherichia metacoli (Castellani) Castellani and Chalmers. (Bacillus metacoli Castellani, 1915; see Castellani and Chalmers, Manual of Trop. Med., 3rd ed., 1919, 942 and 948.) From feces.

Escherichia metacoloides (Castellani) Castellaniand Chalmers. (Bacillus metacoloides Castellani; see Castellani and Chalmers, Manual of Trop. Med., 3rd ed., 1919, 942 and 950.) From feces. 
Escherichia paradoxa (Toumanoff) Hauduroy et al. (Colibacillus paradoxus Toumanoff, Bull. Soc. Centr. de Méd. Vétér., 80, 1927, 367; Hauduroy et al., Dict. d. Bact. Path., 1937, 231.) From feces.

Escherichia paraenterica (Castellani) Hauduroy et al. (Bacillus paraentericus Castellani, Manual of Trop. Med., 1st ed., 1910, 991; Enteroides paraenterica Castellani and Chalmers, ibid., 3rd ed.. 1919, 941; Hauduroy et al., Diet. d. Bact. Path., 1937, 231.) From feces.

Escherichia pauloensis Mello. (Ass. Paulista de Medicina, 11, 1937, 73.) From feces.

Escherichia pseudocoli (Castellani) Castellani and Chalmers. (Bacillus pseudo-coli Castellani, Manual of Trop. Med., 1st ed., 1910, 990; Castellani and Chalmers, Manual Trop. Med., 3rd ed., 1919, 942.) From feces.

Escherichia pseudo-coliformis (Castellani) Hauduroy et al. (Bacillus pseudocoliformis Castellani, 1917; see Castellani and Chalmers, Manual of Trop. Med., 3rd ed., 1919, 952; Hauduroy et al., Dict. d. Bact. Path., 1937, 233.) From feces.

Escherichia pseudocoloides (Castellani)
Castellani and Chalmers. (Bacillus pseudocoloides Castellani, 1916; see Castellani and Chalmers, Manual of Trop. Med., 3rd ed., 1919, 954; ibid., 942; Bacterium pseudo-coloides Weldin and Levine, Abst. Bact., $\gamma, 1923,13$.$) From$ feces.

Escherichia pseudocoscoroba Castellani and Chalmers. (Bacillus coscoroba MacConkey, Jour. Hyg., 6, 1906, 570; not Bacillus coscoroba Trétrop, Ann. Inst. Past., 14, 1900, 224; Bacterium coscorobae Bergey and Deehan, Jour. Med. Res., 19, 1908, 182; Castellani and Chalmers, Man. Trop. Ned., 3rd ed., 1919, 942; Bacillus communior var. coscoroba Winslow, Kligler and Rothberg, Jour. Bact., 4, 1919, 486; Escherichia coscoroba Weldin, Iowa State Coll. Jour. Sci., 1, 1926,139 .) From feces and sewage. This organism described by MacConkey is quite different from the organism described by Trétrop (see Pasteurella appendix).

Escherichia pseudodysenteriae Bergey et al. (Bacterium pseudodysenteriae Kruse, Deutsche Med. Wehnschr., 27, 1901, 386; Bergey et al., Manual, 1st ed., 1923. 198.) From feces of normal persons and of dysentery patients.

\section{Genus II. Aerobacter Beijerinck.*}

(Beijerinck, Cent. f. Bakt., II Abt., 6, 1900, 193; Aerogenesbacterium Orla-Jensen, Jour. Bact., 6, 1921, 272; Colobactrum (in part) Borman, Stuart and Wheeler, Jour. Bact., 48, 1944, 357.) From Latin, air or gas, and rod.

Short rods, fermenting glucose and lactose with acid and gas production. Methyl red test negative; Voges-Proskauer test positive. Form two or more times as much carbon dioxide as hydrogen from glucose; trimethyleneglycol not produced from glycerol by anaerobic fermentation; citric acid and salts of citric acid utilized as sole source of carbon. Grow readily on ordinary media. Facultative anaerobes. Widely distributed in nature.

The type species is Aerobacter aerogenes (Kruse) Beijerinck.

Note: Kligler (Jour. Inf. Dis., 15, 1914, 18 $\bar{\gamma}$ ) found the fermentation of glycerol to be inversely correlated with gelatin liquefaction and considered the former the more reliable due to occasional loss of gelatin liquefying ability. This was confirmed by Levine (Amer. Jour. Pub. Health, 7, 1917, 781) who reports that the two characters do not correlate perfectly. Griffin and Stuart (Jour. Bact., 40, 1940, 93ff.) find a similar correlation of characters but feel that because these characters do not correlate perfectly, it would be better to rombine the two species into a single species.

* Completely revised by Prof. M. W. Yale, New York State Experiment Station, Geneva, New York, Nov., 1938; further revision, July, 1943. 


\section{Key to the species of genus Aerobacter.}

I. Glycerol fermented with acid and gas.

A. Gelatin not liquefied (rarely liquefied).

\section{Aerobacter aerogenes.}

II. Glycerol fermented with no visible gas.

A. Gelatin liquefied.

\section{Aerobacter cloacae.}

1. Aerobacter aerogenes (Kruse) Beijerinck. (Bacterium lactis aerogenes Escherich, Fortschr. d. Med., 3, 1885, 515; Bacterium lactis Baginsky, Ztschr. f. phys. Chem., 12, 1888, 437; not Bacterium lactis Lister, Quart. Jour. Micro. Sci., 13, 1873, 380; Bacterium aceticum Baginsky, ibid.; Bacillus lactantium Trevisan, I generi e le specie delle Batteriacee, 1889, 15; Bacillus lactis aerogenes Sternberg, Manual of Bacteriology, 1893, 447; Bacillus aerogenes Kruse, in Flügge, Die Mikroorganismen, 2, 1896, 340; not Bacillus aerogenes Miller, Deutsche med. Wchnschr., 12, 1886, 119; Bacterium aerogenes Chester, Del. Agr. Exp. Sta., 9th Ann. Rept., 1897, 53; not Bacterium aerogenes Miller, loc. cit.; Beijerinck, Arch. néerl. d. sci. exact. et nat., 4, 1900, 1 ; Encapsulatus lactis-aerogenes Castellani and Chalmers, Manual of Trop. Med., 1919, 934; (Encapsulata) Bacillus aerogenes Perkins, Jour. Inf. Dis., 37, 1925, 254; Colobactrum aerogenes Borman, Stuart and Wheeler, Jour. Bact., 48 , 1944, 358.) From Latin, gas-producing.

Rods : 0.5 to 0.8 by 1.0 to 2.0 microns, occurring singly. Frequently capsulated. (A variety showing a transverse arrangement of the capsule has been named Aerobacter transcapsulatus by Thompson, Jour. Bact., 28, 1934, 41.) Usually non-motile. Gram-negative.

Gelatin colonies: Thick, porcelainwhite, opaque, moist, smooth, entire.

Gelatin stab: Thick, spreading, white, opaque surface growth. No liquefaction.

Agar colonies: Thick, white, raised, moist, smooth, entire. More convex than colonies of Escherichia coli and of ten mucoid.
Agar slant: Abundant, thick, white, moist, glistening, spreading growth.

Broth : Turbid, with pellicle and abundant sediment.

Litmus milk: Acid with coagulation. No peptonization.

Potato: Thick, yellowish-white to yellowish-brown, spreading with nodular outgrowths over the surface.

Indole may or may not be formed (Ford, Studies from the Royal Victoria Hospital, Montreal, 1, 1901-1903, 16; Bardsley, Jour. Hyg. (Eng.), 34, 1934, 38 ; Wilson, Med. Res. Council, London, Spec. Rept. Ser. 206, 1935, 161).

Nitrites produced from nitrates.

Methyl red test negative (Clark and Lubs, Jour. Inf. Dis., 17, 1915, 160); Voges-Proskauer test positive (Durham, Jour. Exp. Med., 5, 1901, 373); inverse correlation between methyl red and Voges-Proskauer tests (Levine, Jour. Bact., 1, 1916, 153).

Citric acid and salts of citric acid utilized as sole source of carbon (Koser, Jour. Bact., 8, 1923, 493).

Uric acid utilized as sole source of nitrogen (Koser, Jour. Inf. Dis., 23, 1918, 377).

Gas ratio: Two or more volumes of carbon dioxide to one of hydrogen formed from glucose (Harden and Walpole, Proc. Roy. Soc. Series B, 77, 1905, 399; Rogers, Clark and Davis, Jour. Inf. Dis., 14, 1914, 411).

Catalase produced.

Hydrogen sulfide not produced in peptone iron agar (Levine, Epstein and Vaughn, Amer. Jour. Pub. Health, 24, 1934, 505; Tittsler and Sandholzer, Amer. Jour. Pub. Health, 27, 1937, 1240). More sensitive indicators give positive 
tests for hydrogen sulfide (Hunter and Weiss, Jour. Bact., 35, 1938, 20).

Trimethyleneglycol not produced from glycerol by anaerobic fermentation (Braak, Onderzoekingen over Vergisting van Glycerine, Thesis, Delft, 1928, 212; Werkman and Gillen, Jour. Bact., 23, 1932, 167).

Sodium hippurate hydrolyzed (Hajna and Damon, Amer. Jour. Hyg., 19, 1934, 545).

Acid and gas from glucose, galactose, lactose, fructose, arabinose, maltose, raffinose, cellobiose, salicin, esculin, starch, dextrin, glycerol, mannitol, sorbitol and inositol, $\alpha$-methyl-glucoside usually fermented (Koser and Saunders, Jour. Bact., 24, 1932, 267). Sucrose, inulin, dulcitol and adonitol may or may not be fermented. Protopectin not fermented. Variable fermentation of sucrose and mannitol (Sherman and Wing, Jour. Bact., 33, 1937, 315).

Aerobic, facultative.

Growth requirements: Good growth on ordinary laboratory media. Optimum growth temperature about $30^{\circ} \mathrm{C}$. Grows better at temperatures below $30^{\circ} \mathrm{C}$ than does Escherichia coli. Usually destroyed in 30 minutes at $60^{\circ} \mathrm{C}$, but certain heatresistant strains may withstand this exposure (Ayers and Johnson, Jour. Agr. Res., 3, 1914, 401; Stark and Patterson, Jour. Dairy Sci., 19, 1936, 495). Gas not produced in Eijkmann test when carried out at $45^{\circ}$ to $46^{\circ} \mathrm{C}$ (Eijkmann, Cent. f. Bakt., I Abt., Orig., 37, 1904, 74 ; Levine, Epstein and Vaughn, Amer. Jour. Pub. Health, 24, 1934, 505).

Habitat: Normally found on grains and plants and to a varying degree in the intestinal canal of man and animals. Widely distributed in nature.

2. Aerobacter cloacae (Jordan) Bergey et al. (Bacillus cloacae Jordan, Rept. Mass. State Bd. of Health, Part II, 1890, 836 ; Bacterium cloacae Lehmann and Neumann, Bakt. Diag., 1 Aufl., 2, 1896, 239 ; Bacillus lactis cloacae Conn, Esten and
Stocking, Storrs Agr. Exp. Sta., Conn., 18th Ann. Rept. for 1906, 180; Cloaca cloacae Castellani and Chalmers, Man. Trop. Med., 3rd ed., 1919, 938; Bergey et al., Manual, 1st ed., 1923, 207.) From Latin cloaca, sewer.

The following are also regarded as identical with Aerobacter cloacae: Aerobacter liquefaciens Grimes and Hennerty, Sci. Proc. Royal Dublin Society, (N. S.) 20, 1931, 93; not Aerobacter liquefaciens Beijerinck, Cent. f. Bakt., II Abt., 6, 1900, 199 (monotrichous); Bacillus levans Wolffin, Arch.f. Hyg., 21, 1894, 279 and Lehmann, Cent. f. Bakt., 15, 1894, 350 (Bacterium levans Lehmann and Neumann, Bakt. Diag., 1 Aufl., 2, 1896, 235; Cloaca levans Castellani and Chalmers, Man. Trop. Med., 3rd ed., 1919, 938; Aerobacter levans Bergey et al., Manual, 1st ed., 1923, 208).

Rods : 0.5 to 1.0 by 1.0 to 2.0 microns, occurring singly. Usually motile possessing peritrichous flagella. Not capsulated. Gram-negative.

Gelatin colonies : Thin, circular, bluish, translucent.

Gelatin stab: Slow liquefaction. Liquefying power sometimes lost (Kligler, Jour. Inf. Dis., 15, 1914, 199).

Agar colonies: Circular, thick, opaque with white center, entire.

Agar slant: Porcelain-white, smooth, glistening, spreading growth.

Broth: Turbid, with thin pellicle.

Litmus milk: Acid; coagulation, gas, slow peptonization.

Potato: Growth yellowish, moist, glistening.

Indole not formed (Levine, Epstein and Vaughn, loc. cit.; Wilson, Med. Res. Council, London, Spec. Rept. Ser. 206, 1935, 161).

Nitrites produced from nitrates.

Methyl red test negative; Voges-Proskauer test positive.

Citric acid and salts of citric acid utilized as sole source of carbon (Koser, Jour. Bact., 8, 1923, 493).

Uric acid utilized as sole source of 
nitrogen (Koser, Jour. Inf. Dis., 23, 1918, 377).

Gas ratio: Glucose fermented with at least two volumes of carbon dioxide to one of hydrogen (Rogers, Clark and Davis, Jour. Inf. Dis., 14, 1914, 411).

Catalase produced.

Hydrogen sulfide not produced in peptone iron agar (Levine, Epstein and Vaughn, Amer. Jour. Pub. Health, 24, 1934, 505).

Sodium hippurate not hydrolyzed (Hajna and Damon, Amer. Jour. Hyg., 19, 1934, 545).

Acid and gas from glucose, fructose, galactose, arabinose, xylose, lactose, maltose, raffinose, dextrin, salicin, trehalose, mannitol, sorbitol, cellobiose and $\alpha$-methyl-glucoside. Sucrose usually fermented. Inulin, esculin, starch, dulcitol, rhamnose and protopectin not attacked. Glycerol fermented with no visible gas (Kligler, loc. cit., 187; Levine, Amer. Jour. Pub. Health, $\gamma, 1917,781)$. Starch rarely fermented (Levine, ibid.). See Winslow, Kligler and Rothberg, Jour. Bact., 4, 1919, 429 for review of literature.

Fecal odor producerl.

Aerobic, facultative.

Growth requirements: Good growth on ordinary laboratory media. Optimum growth temperature $30^{\circ}$ to $37^{\circ} \mathrm{C}$. Cas not produced in Eijkmann test when carried out at $45^{\circ}$ to $46^{\circ} \mathrm{C}$ (Levine, Epstein and Vaughn, loc. cit.).

Habitat: Found in human and animal feces, sewage, soil and water.

Appendix: The following described species have been placed in Aerobacter or may belong here:

Actinobacter polymorphus Duclaux. (Duclaux, Ann. Inst. Nat. Agron., 5, 1882, 110; Bacillus actinobacter Migula, Syst. d. Bakt., 2, 1900, 689.) Causes swelling of cheese. Possibly this was Aerobacter cloacae.

Aerobacter chinense Bergey et al. (Bacillus capsulatus chinensis Hamilton, Cent. f. Bakt., II Abt., 4, 1898, 230;
Bacterium chinense Migula, Syst. d. Bakt., 2, 1900, 357; Bergey et al., Manual, 1st ed., 1923, 207.) From India ink. Acrobacter decolorans Burkey. (Iowa State Coll. Jour. Sci., 3, 1928, 77.) From rotted potato and hay infusions.

Aerobacter diversum Burkey. (Iowa State Coll. Jour. Sci., 3, 1928, 77.) From soil.

Aerobacter faeni Burkey. (Iowa State (oll. Jour. Sci., 3, 1928, 77.) From hay infusions.

Aerobacter hibernicum Grimes and Hennerty. (Sci. Proc. Royal Dublin Society, (N.S.) 20, 1931, 92.) From butter.

Aerobacter leporis Botta. (Giorn. Batteriol. e Immunol., 23, 1939, 217.) From liver abscess in a rabbit.

Aerobacter melezitovorum Burkey. (Iowa State Coll. Jour. Sci., 3, 1928, 77.) From soil.

Aerobacter pectinovorum Burkey. (Iowa State Coll. Jour. Sci., 3, 1928, 77.) From creek water.

Aerobacter oxytocum (Trevisan) Bergey et al. (Bacillus oxytocus perniciosus Flügge, Die Mikroorganismen, 1886, 268; Bacillus oxytocus Trevisan, I generi e le specie delle Batteriacee, 1889, 17; Bacterium oxylocus perniciosus Chester, Ann. Rept. Del. Col. Agr. Exp. Sta., 9, 1897, 139; Bacterium oxytocum Migula, Syst. d. Bakt., 2, 1900, 394; Escherichia oxytocus Castellani and Chalmers, Manual of Trop. Med., 3rd ed., 1919, 942; Bergey et al., Manual, 1st ed., 1923, 206.) From old milk.

Aerobacter paraoxytocum Mello. (Jorn. Dos Clinicos, No. 15, 1937.) From a dental abscess.

Bacillus aceris Edson and Carpenter. (Edson and Carpenter, Vermont Agr. Exp. Sta. Bull. 167, 1912, 475; Achromobacter aceris Bergey et al., Manual, 4th ed., 1934, 218.) From slimy maple sap. See Manual, 5th ed., 1939, 506 for a description of this organism. Identified by Fabian (Ind. and Eng. Chem., 27, 1935, 349) as Aerobacter aerogenes. 
Bacillus aromaticus Pammel. (Pammel, Bull. No. 21, Iowa Agr. Exper. Sta., 1893, 792; Pammel and Pammel, Cent. f. Bakt., II Abt., 2, 1896, 633; Bacterium aromaticus Chester, Ann. Rept. Del. Col. Agr. Exp. Sta., 9, 1897, 100; Flavobacterium aromaticum Bergey et al., Manual, 1sted., 1923, 105.) From cabbage. Used as a starter for cheese making. Acid and gas from glucose and sucrose. See Manual, 5th ed., 1939, 533 for a description of this organism.

Bacillus guillebeau $a, b$ and $c$, von Freudenreich. (Ann. de Micrographie, 2, 1890, 353.) From mastitis milk. Culture a may well have been Aerobacter aerogenes, $b$ appears to have been $A$. cloacae while $c$ was a mucoid variant (see Sternberg, Man. of Bact., 1893, 725).

Bacillus subcloacae Ford. (Studies from the Royal Victoria Hosp., Montreal, 1, (5), 1903, 60 ; also see Ford, Jour. Med. Res., 6, 1901, 213.) From feces.

Bacterium liquefaciens Ford. (Studies from the Royal Victoria Hosp., Montreal, 1, (5), 1903, 59; also see Ford, Jour. Med. Res., 6, 1901, 215.) From feces. While Ford regards this species as identical with Bacillus liquefaciens Eisenberg, neither is adequately described and they differ in important characters. The same holds true for Bacillus liquefaciens
Fuller and Johnson, Jour. Exp. Med., 4, 1899,627 .

Bacterium margaritaceum Migula. (Perlschnurbacillus, Maschek, Bakteriol. Untersuch. d. Leitmeritz. Trinkwässer, Leitmeritz, 1887 ; Migula, Syst. d. Bakt., 2, 1900, 422 and 1059.) From water. Possibly identical with Aerobacter aerogenes.

Bacterium subliquefaciens Ford. (Studies from the Royal Victoria Hosp., Montreal, 1, (5), 1903, 59; also see Ford, Jour. Med. Res., 6, 1901, 219.) From feces.

Bacterium zeae Comes. (Bacterial Disease of Corn, Burrill, Ill. Agr. Exp. Sta. Bull. 6, 1889, 164; Comes, Crittogamia Agraria, 1, 1891, 500; Bacillus secalis Ludwig, Lehrbuch der niederen Kryptogamen, 15:2, 95; Bacillus zeae Russell. Bacteria in their relation to vegetable tissue, Thesis, Johns Hopkins (niv, Baltimore, 1892, 36.) From corn blight. Moore (Agric. Sci., 8, 1894, 368 ) identified a culture received from Burrill as Bacillus cloacae Jordan.

Burkey (Iowa State College Jour. Sci., 3, 1928, 7\%) c'escribed five species (Aerobacter indologenes, Lerobacter motorium. Aerobacter mitificans, Acrobacter salicinovorum and Aerobacter pseudoproteus) which are regarded as varieties of Aerobacter cloacae.

\section{Genus III. Klebsiella Trevisan.*}

(Trevisan, Atti della accad. Fisio-Medico-Statistica in Milano, Ser. 4, 3, 1885, 105; Calymmalobacterium Aragão and Vianno, Mem. Inst. Oswaldo Cruz, 4, 1912, 222 ; Encapsulatus Castellani and Chalmers, Man. Trop. Med., 3rd ed., 1919, 934.) Named for Edwin Klebs (1834-1913), early German bacteriologist.

Short rods, somewhat plump with rounded ends, mostly occurring singly. Encapsulated in the mucoid phase. Non-motile. Gram-negative. Fermentation reactions are highly variable but usually a number of carbohydrates are fermented. Nitrites are produced from nitrates. Aerobic, growing well on ordinary culture media. Encountered frequently in the respiratory, intestinal and genito-urinary tracts of man, but may be isolated from a variety of animals and materials.

The type species is Klebsiella pneumoniae (Schroeter) Trevisan.

* Rearranged by Prof. M. W. Yale, New York State Experiment Station, Geneva, New York, Nov., 1938; further revision by Dr. O. B. Chapman, Syracuse Medical College, Syracuse, New York, December, 1945. 
1. Klebsiella pneumoniae (Schroeter) Trevisan. (Pneumoniecoccus, Friedlaender, Arch. f. Path. Anat., 87, 1882, 319; Bacterium pneumonie crouposae Zopf, Die Spaltpilze, 3 Aufl., 1885, 66; Klebsiella crouposa Trevisan, Atti della Accad. Fisio-Medico-Statistica in Milano, Ser. 4, 3, 1885, 105; Hyalococcus pneumoniae Schroeter, in Cohn, Kryptogamen Flora von Schlesien, 3(1), 1886 , 152; Bacillus pneumoniae Flügge, Die Mikroorganismen, 2 Aufl., 1886, 204; Trevisan, Rend. d. R. Istit. Lombardo, Ser. 2, 20, 1887, 94; Kilebsiella friedlanderi Trevisan, I generi e le specie delle Batteriacee, 1889, 26; Bacillus mucosus capsulatus Paulsen, Mittheil. f. d. Verein Schleswig-Holsteiner Aerzte, 2, 1893, No. 7; Bacterium pneumoniae Lehmann and Neumann, Bakt. Diag., 1 Aufl., 2, 1896, 200; Bacterium pneumonicum Migula, Syst. d. Bakt., 2, 1900, 350; Bacillus friedlanderi Macé, Traité Pratique de Bact., 4th ed., 1901, 771; Encapsulatus pneumoniae Castellani and Chalmers, Man. Trop. Med., 3rd ed., 1919, 934; Coccobacillus friedlanderi NeveuLemaire, Précis Parasitol. Hum., 5th ed., 1921, 20; Proteus pneumoniae Weldin, Iowa State Coll. Jour. Sci., 1, 1926, 149; Bacterium friedlander Weldin, idem; Bacillus mucosus-capsulatus Mason and Beattie, Arch. of Internal Med., 42, 1928, 331.) From Greek, of pneumonia.

Rods: 0.3 to 0.5 by 5.0 microns, with rounded ends, often four to five times as long as broad, occurring singly and in pairs. Encapsulated. Non-motile. Gram-negative.

Gelatin colonies: Dirty-white, smooth, opaque, entire, slightly raised.

Gelatin stab: Dirty-white surface growth. Filiform growth in stab. No liquefaction. Gas bubbles.

Agar colonies: White, shiny, convex, smooth, glistening, entire.

Agar slant: Slimy, white, somewhat translucent, raised growth.

Broth: Turbid, with thick ring or film. Litmus milk: Variable.
Potato: Yellowish, slimy, raised growth. Cias is formed.

Nitrites produced from nitrates.

Indole variable, usually not formed.

Fermentation of carbohydrates highly variable. Acid and gas may be formed from glucose, lactose, sucrose, fructose, galactose, maltose, mannitol and inositol.

Methyl red test variable.

Acetylmethylcarbinol production variable.

Blood agar: Usually no hemolysis.

Utilization of citrate as a sole source of carbon variable.

Aerobic, facultative.

Optimum temperature $37^{\circ} \mathrm{C}$.

Common name : Friedländer's pneumobacillus.

Source: Originally isolated from sputum in pneumonia.

Habitat: Associated with infections of the respiratory, intestinal and genitourinary tracts of man. Encountered in infections of animals and may be isolated from a wide variety of sources.

Note: The difficulty experienced in distinguishing members of this genus from those of Escherichia and Aerobacter is recognized. The members of these three genera exist in at least three growth phases, mucoid (capsulated), smooth and rough.

Working with the mucoid phase of Klebsiella, Julianelle (Jour. Exp. Med., $44,1926,113,683,735 ; 52,1930,539)$ described three serological types, A, B and $\mathrm{C}$ on the basis of capsular specific polysaccharides. There is evidence that other types exist. The presence of a generic specific somatic antigen pattern has not been definitely accepted.

Appendix: The following organisms may be placed in Klebsiella. The evidence for differentiating them into dis. tinct species is so meagre that for the present it may be better to consider them as varieties of Klebsiella pneumoniae.

Klebsiella adanti Hauduroy et al. 
(Dict. d. Bact. Path., 1937, 260.) From a case of pyelocystitis.

Klebsiella capsulata (Sternberg) Bergey et al. (Kapselbacillus, Pfeiffer, Ztschr. f. Hyg., 6, 1889, 145; Bacillus capsulatus Sternberg, Manual of Bact., 1893, 431 ; Bacterium capsulatum Migula, Syst. d. Bakt., 2, 1900, 349; Encapsulatus pfeifferi Bergey et al., Manual, 1st ed., 1923, 239; Bergey et al., Manual, 2nd ed., 1925, 265.) From purulent exudate from stomach of a guinea pig.

Kilebsiella crassa Trevisan. (Bacillus sputigenus crassus Kreibohm, Inaug. Diss., Göttingen, 1889; abst. in Cent. f. Bakt., 7, 1890, 313; Bacillus crassus sputigenus Flügge, Die Mikroorganismen, 2 Aufl., 1886, 260; Trevisan, I generi e le specie delle Batteriacee, 1889, 25; Bacterium sputigenes crassus Chester, Ann. Rept. Del. Col. Agr. Exp. Sta., 9, 1897, 88; Bacterium crassum Chester, Man. Determ. Bact., 1901, 151.) From sputum.

Klebsiella cuniculi Hauduroy et al. (Bacillus capsulatus pyaemiae cuniculi Koppinayi, Ztschr. f. Tiermed., 11, 1907, 429; Hauduroy et al., Dict. d. Bact. Path., 1937, 262.) From pleuropericarditis in a rabbit.

Klebsiella genitalium (Dimock and Edwards) Hauduroy et al. (Encapsulatus genitalium Dimock and Edwards, Jour. Amer. Veter. Assoc., 70, 1927, 469; Hauduroy et al., Dict. d. Bact. Path., 1937,264 .) From infections in the genito-urinary organs of mares.

Klebsiella granulomatis (Aragão and Vianna) Bergey et al. (Calymmatobacterium granulomatis Aragão and Vianna, Mem. do Inst. Oswaldo Cruz, Rio de Janeiro, 4, 1912, 211; Encapsulatus inguinalis Bergey et al., Manual, 1st ed., 1923, 238; Bergey et al., Manual, 2nd ed., 1925, 264.) From granuloma inguinale.
Klebsiella ozaenae (Abel) Bergey et al. (Bacillus mucosus ozaenae Abel, Cent. f. Bakt., 13, 1893, 167; Bacillus ozaenae Abel, ibid., 172; not Bacillus ozaenae Migula, Syst. d. Bakt., 2, 1900, 645,662 ; ? Bacillus capsulatus mucosus Fasching, Sitzgsber. Wien. Akad., III Abt., 100, 1891 (Bacterium capsulatus mucosus Chester, Ann. Rept. Del. Col. Agr. Exp. Sta., 9, 1897, 130; Bacterium faschingii Migula, Syst. d. Bakt., 2, 1900, 355 ; Bacillus capsulatus-mucosus Holland, Jour. Bact., 5, 1920, 217; Bacterium mucosum capsulatum Holland, ibid., 221; Bacterium mucosum-capsulatum Holland, ibid., 217); Bacterium ozaenae Lehmann and Neumann, Bakt. Diag., 1 Aufl., 2, 1896, 204; Bacterium mucosus ozaena Chester, Ann. Rept. Del. Col. Agr. Exp. Sta., 9, 1897, 138; Encapsulata ozenae Bergey et al., Manual, 1st ed., 1923, 240; Bergey et al., Manual, 2nd ed., 1925, 266.) From cases of ozena.

Klebsiella paralytica Cahn, Wallace and Thomas. (Wallace, Lyell, Thomas, Alvin and Cahn, Proc. Soc. Exp. Biol., 29, 1932, 1908; Cahn, Wallace and Thomas, Science, 76, 1932, 385; Wallace, Cahn and Thomas, Jour. Inf. Dis., 53, 1933, 386; Klebsiella alcis Hauduroy et al., Dict. d. Bact. Path., 1937, 260.) From intestine of tick (Dermacentor albipictus) and thought to be the cause of tick paralysis of moose.

Klebsiella rhinoscleromatis Trevisan. (Rhinoscleromabacillus, v. Frisch, Wien. med. Wchnschr., 1882; Cornil, Progris Medical, 1883; Trevisan, Rend. d. R. Istit. Lombardo, Ser. 2, 20, 1887, 95; Bacterium rhinoscleromatis Migula, Syst. d. Bakt., 2, 1900, 352; Bacterium nasalis Chester, Man. Determ. Bact., 1901, 134; Bacillus rhinoscleromatis Winslow, Kligler and Rothberg, Jour. Bact., 4, 1919, 491.) From cases of rhinoscleroma. 
*Appendix I. Tribe Eschericheae: Borman, Wheeler and Stuart (Jour. Bact., 48, 1944, 361) place coliform-like bacteria that are slow lactose-fermenters in a separate genus Paracolobactrum as follows:

\section{Genus A. Paracolobactrum Borman, Stuart and Wheeler.}

(Paracolibacille, Widal and Nobecourt, Semaine Méd., 1\%, 1897, 285; Borman, Stuart and Wheeler, Jour. Bact., 48, 194t,361.)

Short rods characterized by consistently delayed fermentation of lactose (occasionally negative). Glucose is fermented with formation of visible gas. Certain forms attack carbohydrates eharacteristically at $20^{\circ}$ to $30^{\circ} \mathrm{C}$ but not at $37^{\circ} \mathrm{C}$. Antigenic relationships to other genera in the family are common, even with respect to major antigens.

The type species is Paracolobactrum aerogenoides Borman, Stuart and Wheeler.

\section{Key to the species of genus Paracolobactrum.}

I. Acetylmethylearbinol produced.

II. Acetylmethylcarbinol not produced.

\section{Paracolobactrum aerogenoides.}

A. Citric acid utilized as a sole source of carbon.

2. Paracolobactrum intermedium.

B. Citric acid not utilized as a sole source of carbon.

3. Paracolobactrum coliforme.

1. Paracolobactrum aerogenoides Borman, Stuart and Wheeler. (Para-aerogenes, Stuart, Wheeler, Rustigian and Zimmerman, Jour. Bact., 45, 1943, 117; Borman, Stuart and Wheeler, Jour. Bact., 48, 1944, 361.) Latinized, resembling aerogenes.

Characters as for Aerobacter aerogenes and Aerobacter cloacae except for consistently delayed fermentation of lactose.

Source: From human gastroenteritis.

Habitat: Surface water, soils, grains, as well as the intestinal tract of animals. including man.

2. Paracolobactrum intermedium Borman, Stuart and Wheeler. (Parafreundii, Stuart et al., Jour. Bact., 45, 1943, 117; Borman, Stuart and Wheeler, Jour. Bact., 48, 1944, 361.) From Latin intermedius, intermediate.

Characters as for Escherichia freundii and Escherichia intermedium except for consistently delayed fermentation of lartose.

Source: From human gastroenteritis. Habitat: Surface water, soil, grains, as well as the intestinal tract of animals, including man.
3. Paracolobactrum coliforme Borman' Stuart and Wheeler. (Para-coli, Stuart et al., Jour. Bact., 45, 1943, 117; Borman, Stuart and Wheeler, Jour. Bact., 48, 1944, 361.) Latinized, resembling coli. Characters as for Escherichia coli except for consistently delayed fermentation of lactose.

Source: From human gastroenteritis.

Habitat: Surface water, soil, grains, as well as intestinal tract of animals, including man.

Note: The following also belong here: Bacterium paracoli Stutzer and Wsorow. (Non-lactose-fermenting Bacterium coli, Gilbert and Lion, Semaine Méd., 13, 1893, 130; Stutzer and Wsorow, Cent.f. Bakt., II Abt., 71, 1927, 115.) From intestines of healthy larvae of a moth (Euxoa segetum).

Salmonella para-colon (Day) Hauduroy et al. (Bacillus para-colon Day; see Castellani, Cent. f. Bakt., I Abt., Orig., 65, 1912, 264; also Castellani and Chalmers, Man. Trop. Med., 3rd ed., 1919,950; Hauduroy et al., Dict. d. Bact. Path., 1937, 461.)

* Prepared by Dr. E. K. Borman, Bureau of Laboratories, State Department of Health, Hartford, Connecticut, July, 1945. 
*Appendix II. Tribe Eschericheae: Gram-negative, peritrichous to non-motile rods similar to organisms placed in Paracolobactrum, Serratia and Salmonella have recently been described as causing diseases of reptiles, birds and mammals. They may be grouped here although they have been placed in several different genera.

1. Bacterium sauromali Conti and Crowley. (Jour. Bact., 36, 1938, 269.) From a generic name of lizards, Sauromalus.

Short rods: 0.2 to 0.5 by 1.0 to 2.0 microns, with rounded ends, occurring in groups. Motile with 4 to 6 peritrichous flagella. Cram-negative.

Gelatin: Infundibuliform liquefaction complete in 3 days at $37^{\circ} \mathrm{C}$. Black sediment. Medium browned.

Agar slant: Growth abundant, spreading, convex, faint yellowish-green, glistening, smooth, translucent, butyrous. Decided odor. Medium greened.

Nutrient broth: After 1 day at $37^{\circ} \mathrm{C}$. moderate turbidity. Ring. Decided odor. Scanty floceulent sediment.

Milk: Alkaline and complete peptonization in ten days.

Indole not formed.

Potato: Growth yellowish-green to olive.

Blood medium: Complete alpha hemolysis in 48 hours.

Peptone medium: Slight fluorescent greenish-yellow pigmentation.

Nitrites produced from nitrates.

Ammonia is produced.

Acid and gas from glucose, sucrose, maltose, galactose, fructose, salicin and mannitol. Acid but not gas from glycerol. No acid from lactose, arabinose, xylose, dextrin, inulin, dulcitol or starch.

Hydrogen sulfide produced.

Catalase positive

Methyl red test positive.

Pathogenic for animals.

Temperature relations: Optimum $37^{\circ} \mathrm{C}$. Minimum $20^{\circ} \mathrm{C}$. Maximum $45^{\circ} \mathrm{C}$.

Aerobic.

Source: From a tumor-like growth on the chuckawalla (Sauromalus varius).

Habitat: Causes tumors in lizards.
2. Serratia anolium Duran-Reynals and Clausen. (Jour. Bact., 33, 1937, 369.) From a generic name of lizards, Anolis.

Rods : 0.2 to 0.4 by 1.0 to 2.0 microns, occurring singly, in pairs, in clusters and palisades. Pleomorphic, other forms being 4 to 5 microns in length, curved, occasionally club-like, or 10 to 15 microns long and surrounded by a capsular material, or occasionally small and coccus-like. Motile (Duran-Reynals and Clausen) with 1 to 4 peritrichous flagella (Breed). Non-acid-fast. Gram-negative.

Gelatin stab: Rapid growth. Liquefaction infundibuliform. After 6 to 10 days a thick soft pellicle and blackish sediment is formed.

Agar colonies: After 24 hours at $37^{\circ} \mathrm{C}$, isolated colonies are low, convex, margin entire or slightly undulate. Colonies translucent, butyrous, glistening, smooth, 1.0 to $2.5 \mathrm{~mm}$ in diameter. While some colonies retain their smooth character. others become iarger, striated and wrinkled. showing opaque, radiated folds with irregularly crenated edges and a rougher texture. Penetrating acid smell produced.

Agar slant: After 24 hours at $37^{\circ} \mathrm{C}$, abundani, confluent, raised, whitish, butyrous, glistening, with entire or undulate edges.

Broth: Moderate growth with uniform turbidity. A pellicle is formed which disintegrates forming a ring on the walls of the tube. Sediment. Faint fluorescent yellowish coloration.

No visible gas in glucose broth (Breed).

Peptone water: After 6 to 10 days marked turbidity, medium darkened, blackish sediment formed.

Litmus milk: Coagulation and digestion. Partial discoloration of the litmus.

* Prepared by Prof. Robert S. Breed, New York State Experiment Station, Geneva, New York, June, 1946. 
Potato: Growth abundant, butyrous, glistening, raised, pinkish.

Indole not formed.

Blood is hemolyzed.

Loeffler's serum: Abundant, glistening growth. Liquefaction.

No $\mathrm{H}_{2} \mathrm{~S}$ produced.

Ammonia is produced.

Although Duran-Reynals and Clausen report nitrites not produced from nitrates, a retest of their cultures by Breed has shown that nitrites are actively produced from nitrates.

Acid from glucose, fructose, sucrose, mannitol, maltose, galactose and salicin. Dextrin, lactose, inulin, dulcitol, xylose and arabinose slightly attacked or not at all.

Pigment production: Water-soluble pigment produced. Pink coloration best shown on glycerol potato. Reddish coloration best shown in peptone water with 2 per cent glucose, the yellow coloration in glucose broth and the black coloration in the sediment of liquefied gelatin and peptone water. Some non-pigmented strains.

Temperature relations: Grows well at $20^{\circ} \mathrm{C}$. Growth more abundant at $37^{\circ} \mathrm{C}$. Practically no growth at $10^{\circ} \mathrm{C}$. Thermal death point $60^{\circ} \mathrm{C}$ for 20 minutes.

Aerobic.

Pathogenicity: Pathogenic for amphibians, reptiles and to some extent fish. Lesions are produced in the iguanid lizards (Anolis equestris and Anolis carolinensis), the gekkonid lizards (Tarentola mauritanica and Hemidactylus brookii), the garter snake (Thamnophis butleri) and the brown snake (Storeria dekayi), the musk turtle (Sternothaerus odoratus), the toad (Bufo americanus), the frog (Rana pipiens) and the catfish (Ameiuras melas). When the inoculated animal is kept at $37^{\circ} \mathrm{C}$, the disease becomes general and usually is fatal. Non-pathogenic for warm-blooded animals (Clausen and Duran-Reynals, Amer. Jour. Path., 13, 1937, 441).

Source: From tumor-like lesions in Cuban lizards (Anolis equestris). Also isolated from iguanid lizards (Basiliscus vittatus) from Mexico by Clausen and Duran-Reynals (loc. cit.).

Habitat: The cause of a natural, nonfatal, contagious disease of lizards.

3. Salmonella sp. (Type Arizona). (Salmonella sp., Dar es salaam Type var. from Arizona, Caldwell and Ryerson, Jour. Inf. Dis., 65, 1939, 245; Salmonella arizona Kauffmann, Acta Path. et Microbiol. Scand., 17, 1940, or 19, 1942; Arizona culture, Edwards, Cherry and Bruner, Jour. Inf. Dis., 73, 1943, 236; Salmonella arizona Group, Edwards, Jour. Bact., 49, 1945, 513.)

Ferments lactose and liquefies gelatin. Antigenic structure: XXXIII: $Z_{4}, Z_{23}$, $\mathrm{z}_{26}$ : - .

Source: Isolated by Caldwell and Ryerson (loc. cit.) from horned lizards, Gila monsters and chuckawallas. Also pathogenic for guinea pigs and rabbits. Found in snakes by Hinshaw and MeNeill (Cornell Vet., 34, 1944, 248). Also reported by Edwards (loc. cit.) from infants.

Habitat: Apparently widely distributed in lizards, snakes, and warmblooded animals. 
TRIBE II. ERWINEAE WINSLOW ET AL.

(Jour. Bact., 5, 1920, 209.)

Motile rods which normally require organic nitrogen compounds for growth. Produce acid with or without visible gas from a variety of sugars. In some species, the number of carbon compounds attacked is limited and lactose may not be fermented. May or may not liquefy gelatin. May or may not produce nitrites from nitrates. Invade the tissues of living plants and produce dry necrosis, galls, wilts and soft rots. In the latter case, a protopectinase destroys the middle lamellar substance.

There is a single genus.

\section{Genus I. Erwinia Winslow et al.*}

(Jour. Bact., 2, 1917, 560.) Named for Erwin F. Smith, pioneer American plant pathologist.

Characters as for the tribe.

The type species is Erwinia amylovora (Burrill) Winslow et al.

Key to the species of genus Erwinia.

I. **Pathogens which cause dry necrosis, galls or wilts in plants but not a soft rot (Erwinia sensu stricto).

A. Gas not produced in sugar media.

1. Gelatin liquefied.

a. Starch not hydrolyzed.

b. Nitrites not produced from nitrates.

1. Erwinia amylovora.

bb. Nitrites produced from nitrates.

2. Erwinia milletiae.

aa. Starch hydrolyzed.

b. Nitrites produced from nitrates.

3. Erwinia vitivora.

aaa. Action on starch not reported.

b. Nitrites produced from nitrates.

4. Erwinia cassavae.

2. Gelatin not liquefied.

a. Starch not hydrolyzed.

b. Nitrites produced from nitrates.

5. Erwinia salicis.

bb. Nitrites not produced from nitrates.

6. Erwinia tracheiphila.

* Completely revised by Prof. F. D. Chester, New York, N. Y., December, 1938; further revision by Prof. Walter H. Burkholder, Cornell University, Ithaca, New York, May, 1945.

** The genus Erwinia as defined here is heterogeneous in nature and is composed of at least two distinct groups. The first group constitutes Erwinia proper and does not produce visible gas from sugars. Waldee (Iowa State Coll. Jour. Sci., 19, 1945, 435 ) in a paper that appeared as this manuscript was ready for the press has suggested that the species in this first group be placed in a separate family Erwiniaceae. 
II. †Pathogens which normally cause a soft rot in plants (largely belong in the genus Pectobacterium Waldee).

A. Gas produced in sugar media.

1. Gelatin liquefied.

a. Nitrites produced from nitrates.

b. Hydrogen sulfide produced.

7. Erwinia betivora.

8. Erwinia carnegieana.

bb. Hydrogen sulfide not produced.

9. Erwinia atroseptica.

10. Eruinia carotovora.

aa. Nitrites not produced from nitrates.

11. Erwinia erivanensis.

12. Eruinia flavida.

2. Gelatin not liquefied.

a. Starch hydrolyzed.

aa. Starch not hydrolyzed.

13. Erwinia dissolvens.

14. Erwinia nimipressuralis.

B. Gas not produced in sugar media.

1. Gelatin liquefied.

a. Nitrites produced from nitrates.

b. Stareh hydrolyzed.

15. Erwinia ananas.

16. Erwinia cytolytica.

bb. Starch not hydrolyzed.

c. Acid from lactose.

17. Erwinia aroideae.

18. Erwinia mangiferae.

ce. No acid from lactose.

19. Erwinia citrimaculans.

2. Gelatin not liquefied.

20. Erwinia rhapontici.

3. Very slow gelatin liquefaction.

a. Nitrites not produced from nitrates.

21. Eruinia lathyri.

C. Gas production not reported.

1. Gelatin liquefied.

a. Nitrites produced from nitrates.

22. Erwinia lilii.

† The second group of species usually causes soft rots, but includes a few not very typical species. Waldee (loc. cit.) has proposed that the species that cause typical soft rot be placed in a new genus, Pectobacterium, with Pectobacterium carotovorum as the type species. The new genus is retained in the family Enterobacteriaceae. Waldee would place the atypical species in other genera, Erwinia dissolvens for example being placed in the genus Aerobacter. As further comparative studies are needed before such changes can be made with confidence, the older arrangement is allowed to stand in this edition of the MANLAL. 
1. Erwinia amylovora (Burrill) Winslow et al. (Micrococcus amylovorus Burrill, Illinois Indust. Univ., 11th Rept., 1882, 142; American Naturalist, 17, 1883, 319; Bacillus amylovorus Trevisan, I generi e le specie delle Batteriacee, 1889, 19; Bacterium amylovorus Chester, Del. Col. Agr. Exp. Sta., 9th Ann. Rept., 1897, 127; Bacterium amylovorum Chester, Manual Determ. Bact., 1901, 176; Winslow et al., Jour. Bact., 5, 1920, 209.) From Latin, starch devouring.

Description mainly from Ark, Phytopath., 27, 1937, 1.

Rods : 0.7 to 1.0 by 0.9 to 1.5 microns, occurring singly, in pairs and sometimes in short chains. Motile with peritrichous flagella. Gram-negative.

Gelatin colonies: Circular, whitish, amorphous, entire.

Gelatin stab: Slow crateriform liquefaction confined to the upper layer.

Agar colonies: Circular, grayish-white, moist, glistening, irregular margins.

Broth: Turbid, with a thin granular pellicle.

Potato: Growth white, moist, glistening. Medium not softened. No odor. No pigment.

Litmus milk: Coagulated after 3 to 4 days to a pasty condition, with a separation of whey. At first acid, becoming alkaline. Litmus reduced. There is a gradual digestion of the casein.

Blood serum: Growth similar to that on agar. No liquefaction.

Dunham's solution: Rapid growth, but clouding not dense.

Indole not produced.

Nitrites not produced from nitrates.

Most of the strains gave a positive test for ammonia in broth, a few showed only a slight positive test.

Acetylmethylcarbinol produced.

Growth in synthetic media with $\left(\mathrm{NH}_{4}\right)_{2} \mathrm{HPO}_{4}$ as a source of nitrogen and containing different carbohydrates.

Acid without gas from glucose, sucrose, arabinose, mannose, fructose, maltose, cellobiose, raffinose, salicin and amyg- dalin. Xylose, rhamnose, dulcitol and starch not fermented. Acid production from lactose and galactose variable. Utilizes salts of citric, malic, and hippuric acid. Action on salts of lactic and succinic acids variable. Salts of benzoic, maleic, malonic, oxalic, tartaric and valeric acid are not utilized.

Asparagine fermented with production of alkali. Glycine, valine, isoleucine, glutamic acid, cystine, tyrosine, tryptophane and urea not fermented.

Minimum temperature between $3^{\circ}$ and $\mathrm{S}^{\circ} \mathrm{C}$. Maximum below $37^{\circ} \mathrm{C}$.

Optimum pH 6.8. Minimum $\mathrm{pH} 4.0$ to 4.4. Maximum $\mathrm{pH} 8.8$.

Source: From the blossoms, leaves and twigs of the pear and apple.

Habitat: Attacks a large number of species in several tribes of the family Rosaceae (Elliott, Manual Bact. Plant Pathogens, 1930, 19).

2. Erwinia milletiae (Kawkami and Yoshida) Magrou. (Bacillus milletiae Kawkami and Yoshida, Bot. Mag., Tokyo, 34, 1920, 110; Magrou, in Hauduroy et al., Dict. d. Bact. Path., 1937, 213.) From Milletia, a genus named for A.J. Millett.

Rods : 0.4 to 0.6 by 0.9 to 2.5 microns. Motile with peritrichous flagella. Capsules. Gram-negative.

Gelatin: Liquefaction begins after 8 days.

Agar colonies: Circular, flat, smooth, shiny, opaque, waxy yellow. Margins entire.

Broth: Turbid. Heavy precipitate.

Milk: No coagulation. Clears with alkaline reaction.

Conjac: No liquefaction.

Nitrites produced from nitrates.

Acid but no gas from galactose, fructose, lactose, maltose, sucrose and mannitol. No acid from glycerol.

Starch not hydrolyzed.

Growth in 0.2 per cent but not in 0.3 per cent of the following acids in sucrose 
peptone broth: Acetic, citric, oxalic and tartaric.

Aerobic.

Grows well at $32^{\circ} \mathrm{C}$. Thermal death point, $53^{\circ} \mathrm{C}$ for $10 \mathrm{~min}$.

Source: From galls on the Japanese wisteria in various localities in Japan.

Habitat: Causes galls on the Japanese wisteria, Milletia floribunda.

3. Erwinia vitivora (Baccarini) du Plessis. (Bacillus vitivorus Baccarini, Bull. della Soc. Bot. Ital., 1894, 235; du Plessis, Dept. Agr. and Forestry Union of S. Africa, Science Bul. 214, 1940, 58.) From Latin, devouring the vine.

Note: Macchiati (Bol. dolla Soc. Bot., 1897,156 ) uses the name Bacillus baccarinii for Bacillus vitivorus. The description Macchiati gives is not of Erwinia vitivora but is evidently that of a saprophyte occurring with the pathogen. He condueted no inoculation experiments. Migula (System der Bakterien, 2, 1900, 778) gives Bacillus vitivorus Bace. (Malpighia, 6, 1892, 229) which is an incorrect citation and Bacillus baccarinii Maceh. 1897, as synonyms of Bacillus gummis Comes 18st. It is impossible to determine what this latter species is. Du Plessis (loc. cit.) does not believe Bacillus gummis is the same as Erwinia vitivora.

Rods: $0.74(0.44$ to 1.10$)$ by 1.46 (0.95 to 2.19$)$ microns. Cells sometimes dumbbell-shaped. Motile with peritrichous flagella. Gram-negative. Capsules present.

Gelatin: Liquefaction.

Agar colonies : First punctiform, irregularly circular or lenticular, ultimately circular, raised to pulvinate, glistening, spreading, light to orange-yellow. Agar becomes brown.

Broth: Turbid in 24 hrs. Whitish to lemon yellow pellicle.

Milk: Litmus reduced. Thread-like to spongy curd formed. Yellow whey about curd. Yellow growth on top of plain milk. Medium acid.
Uschinsky's solution: Slowly becomes turbid. Pellicle. Sediment whitishyellow.

Nitrites produced from nitrates.

Hydrogen sulfide produced.

Acid produced from glucose, fructose, xylose, lactose, sucrose, mannitol and salicin. No acid from raffinose or inulin. Starch hydrolyzed.

Facultative anaerobe.

Temperature relations : Optimum $25^{\circ} \mathrm{C}$. Maximum $35^{\circ}$ to $40^{\circ} \mathrm{C}$. Minimum $5^{\circ}$ to $10^{\circ} \mathrm{C}$.

Optimum pH 6.0. Minimum 4.2.

Source: Du Plessis used 5 isolates from various localities in South Africa.

Iabitat: Causes a disease of grape vines in South Africa, Italy and France.

4. Erwinia cassavae (Handsford) comb. nov. (Bacterium cassavae Handsford, Ann. Rept. Dept. Agric. Uganda for 1937, II, 1938, 48.) From cassava, the host plant.

Rods: Motile with a few peritrichous flagella. No apsules. Gram-negative. Gelatin is slowly liquefied.

Agar colonies: Smooth, lens-shaped, edges entire, trunslucent and of uniform structure. Yellow.

Broth: Turbid with a ring. A yellow precipitate in old cultures.

Milk becomes alkaline. Not cleared.

Nitrates are rapidly reduced to nitrites.

Methyl red test negative. Acetylmethylcarbinol produced (Dowson, Cent. f. Bakt., Il Abt., 100, 1939, 183).

Acid but no gas from glucose, sucrose, maltose and glycerol, but not from lactose.

Facultative anaerobe.

Source: From necrotic lesions on cassava leaves in Uganda.

Habitat: Pathogenic on cassava, Manihot $s p$.

5. Erwinia salicis (Day) Chester. (Bacterium salicis Day, Oxford For. Mem., 3, 1924, 14; Phytomonas salicis 
Magrou, in Hauduroy et al., Dict. d. Bact. Path., 1937, 408; Chester, in Bergey et al., Manual, 5th ed., 1939, 406.) From Latin salix, willow; M. L. generic name, Salix.

Description from Dowson, Ann. Appl. Biol., 24, 1937, 542.

Rods : 0.5 to 0.7 by 0.8 to 2.2 microns, occurring singly or in pairs, rarely in chains, with rounded ends. Motile with 5 to 7 long peritrichous flagella. Gramnegative.

Gelatin stab: Beaded growth. No liquefaction.

Infusion agar: Colonies appear slowly, circular, with slightly uneven margins, pale brown by transmitted light, pale gray by reflected.

Infusion agar slants: Growth thin, nearly transparent.

Broth: Moderate, uniform turbidity No pellicle.

Litmus milk: No change.

Potato: Bright yellow, later fading to pale brown, spreading, abundant, glistening, slimy growth.

Indole not formed.

Nitrites produced from nitrates (Dowson).

Hydrogen sulfide not produced.

Ammonia not produced.

Acetylmethylcarbinol

produced

Methyl red test negative (Dowson, Cent

f. Bakt., II Abt., 100, 1939, 183).

Acid, but no gas, from glucose, galactose, mannose, xylose, maltose, sucrose, raffinose, glycerol, mannitol and salicin. No growth in arabinose, fructose, rhamnose, inulin or dextrin.

No growth in Cohn's solution.

Stareh not hydrolyzed.

Temperature relations: Optimum $29^{\circ}$ to $30^{\circ} \mathrm{C}$. Minimum $5^{\circ}$ to $10^{\circ} \mathrm{C}$. Maximum $33^{\circ}$ to $37^{\circ} \mathrm{C}$. Thermal death point $50^{\circ}$ to $52^{\circ} \mathrm{C}$.

Aerobic, facultative anaerobic.

Source: From the cricket-bat willow (Salix caerulea) and from the white willow (Salix alba).

Habitat: Causes a water-mark disease of willow in England.
6. Erwinia tracheiphila (Erw. Smith) Holland. (Bacillus tracheiphilus Erw. Smith, Cent. f. Bakt., II Abt., 1, 1895, 364; Bacterium tracheiphilus Chester, Ann. Rept. Del. Col. Agr. Exp. Sta., $9,1897,72$; Smith, see Bacteria in Relation to Plant Diseases, 2, 1911, 286; Holland, Jour. Bact., 5, 1920, 215.) From Greek, trachea-loving, i.e., live in fibrovascular bundles.

Rods : 0.5 to 0.7 by 1.2 to 2.5 microns, with rounded ends, occurring singly and in pairs, more rarely in fours. Motile with peritrichous flagella. Capsulated. Gram-negative.

Gelatin colonies: Small, circular, grayish-white, smooth, glistening. Show internal striae by transmitted light.

Gelatin stab: Surface growth thin, spreading, grayish-white. Slight filiform growth in depth. No liquefaction.

Agar colonies: Small, circular, grayishwhite, smooth, glistening.

Agar slant: Growth gray, smooth, filiform, moist, glistening.

Broth: Slight turbidity. No ring or pellicle.

Potato: Growth white or color of substratum, smooth, moist, glistening. No action on the starch. Does not soften the middle lamella of potato cells.

Litmus milk: Not coagulated. Reaction unchanged. Litmus not reduced. Not peptonized.

Egg albumen: Not digested.

Blood serum: No liquefaction.

Cohn's solution: No growth.

Uschinsky's solution: Weak growth.

Fermi's solution: Weak growth.

Indole not formed in Dunham's solution.

Nitrites not produced from nitrates. Ammonia production moderate.

Cannot utilize asparagine, ammonium lactate or tartarate as sources of nitrogen.

Acid without gas from glucose, sucrose and fructose; growth in closed arm. Acid from glycerol. No growth in closed arm with lactose, maltose, dextrin, glycerol or mannitol. No acid from lactose

Starch not hydrolyzed. 
Hydrogen sulfide production feeble. Growth in broth with 1.0 per cent $\mathrm{NaCl}$ retarded; inhibited with 2.0 per cent.

Very sensitive to acid (phenolphthalein).

Temperature relations: Optimum $25^{\circ}$ to $30^{\circ} \mathrm{C}$. Minimum about $8^{\circ} \mathrm{C}$. Maximum $34^{\circ}$ to $35^{\circ} \mathrm{C}$. Thermal death point $43^{\circ} \mathrm{C}$ for one hour.

Aerobe and facultative anaerobe.

Source: From various curcurbits.

Habitat: Causes the wilt of cucumber, also affects cantaloupes, muskmelons, pumpkins and squashes.

6a. Bacillus tracheiphilus var. cucumis E. F. Smith. (An Introduction to Bact. Dis. of Plants, 1920, 135.) Smith states that squash is immune to this variety of Erwinia tracheiphila.

7. Erwinia betivora (Takimoto) Magrou. (Bacillus betivorus Takimoto, Ann. Phyt. Soc. Japan, 2, 1931, 356; Magrou, in Hauduroy et al., Dict. d. Bact. Path., Paris, 1937, 200). From Latin, devouring the beet.

Rod: Short rods, sometimes filaments. Motile with 2 to 6 peritrichous flagella. Gram-negative.

Gelatin : Liquefaction.

Agar colonies: Circular or amoeboid, homogenous, thin, edges smooth and entire.

Broth: Turbid with pellicle.

Milk: Acid, coagulated.

Nitrites produced from nitrates.

Indole produced.

Hydrogen sulfide produced.

Gas from glucose and sucrose.

Facultative anaerobic.

Optimum temperature $35^{\circ} \mathrm{C}$. Minimum $12^{\circ} \mathrm{C}$. Maximum $45^{\circ} \mathrm{C}$. Thermal death point $50^{\circ} \mathrm{C}$ for $10 \mathrm{~min}$.

Source: From rot of sugar beets in Korea.

Habitat: Pathogenic on roots of beets. Artificial inoculation of carrots, radishes, potato tubers and tomato fruits gave positive results.
8. Erwinia carnegieana Lightle, Standring and Brown. (Phytopath., 32, 1942, 310.) From the genus Carnegiea.

Rods: 1.12 to 1.79 by 1.56 to 2.90 microns. Motile with peritrichous flagella. Capsules. Gram-positive (Lightle et al.). Gram-negative; old cultures show Gram-positive granules in cells (Burkholder).

Gelatin: Slow liquefaction.

Agar colonies: Round, slightly raised, smooth, gray-white, wet-shining, margins entire.

Broth: Abundant growth.

Uschinsky's solution: Turbid, slight ring and sediment.

Milk: Litmus pink to reduced. No curdling.

Nitrites are produced from nitrates.

Hydrogen sulfide is formed (Burkholder).

Acid and gas from glucose, galactose, fructose, maltose, sucrose, raffinose, mannitol and salicin. Acid and gas from lactose and xylose and alkali from sodium tartrate (Burkholder).

Starch not hydrolyzed (Burkholder). No odor.

Aerobic.

Thermal death point $59^{\circ} \mathrm{C}$.

Source : From rotting tissue of the giant cactus (Carnegiea gigantia).

Habitat: Pathogenic on the giant cactus, but not on carrots.

9. Erwinia atroseptica (van Hall) Jennison. (Bacillus atrosepticus van Hall, Inaug. Diss., Amsterdam, 1902, 134 ; Jennison, Ann. Missouri Bot. Gard., 10, 1923, 43.) From Latin ater, black and septicus, putrefying.

Synonyms: Morse (Jour. Agr. Res., 8, $1917,79)$ lists the following synonyms: Bacillus solanisaprus Harrison, Cent.f. Bakt., II Abt., 17, 1906, 34 (Erwinia solanisapra Holland, Jour. Bact., 5, 1920, 222) and Bacillus melanogenes Pethybridge and Murphy, Roy. Irish Acad. Proc., 29, B, No. 1, 1911, 31.

Paine (Jour. Agr. Sci., 8, pt. 4, 1917, 
492) agrees and points out that Bacillus phytophthorus Appel is very similar to Bacillus melanogenes Pethybridge and Murphy.

Jennison (Ann. Missouri Bot. Gard., 10, 1923, 1) concurs and adds Bacillus phytophthorus Appel, Ber. d. Deut. Bot. Gesell., 20, 1902, 128 (nomen nudum) and K. Biol. Anst. f. Land. u. Forst. Arb., 3, 1903, 361. (This last reference contains Appel's description which is antedated by van Hall's description of the black leg pathogen.)

Stapp (Arb. d. Biol. Reichs. f. Landu. Forst., 16, 1928, 702) besides the above species adds Bacillus carotovorus Jones but uses the name Bacillus phytophthorus and states that the species contains 5 serological groups.

Description from Jennison (loc. cit.).

Rods : 0.6 by 1.5 microns. Motile with a few peritrichous flagella. No capsules. Gram-negative.

Gelatin liquefied.

Agar colonies: Small, round to somewhat irregular and whitish. Surface smooth with a glistening luster.

Broth: Turbid after a few days. Ring and sometimes a light pellicle.

Ammonia production feeble to moderate (Jennison). Ammonia production absent (Morse, loc. cit.).

Milk coagulated and acid. A slow peptonization. Litmus reduced.

Indole not formed.

Hydrogen sulfide not produced.

Nitrites are produced from nitrates.

Acid and gas from glucose, galactose, sucrose, lactose, maltose and mannitol. No acid and gas from dextrin and glycerol. Volume of gas is small.

Starch not hydrolyzed.

Cohn's solution: No growth.

Uschinsky's solution: Good growth.

Facultative anerobe (Morse, loc, cit.).

Optimum temperature $26^{\circ} \mathrm{C}$. Maximum $33^{\circ} \mathrm{C}$. Minimum below $5^{\circ} \mathrm{C}$ (Morse).

Slight growth with 3 per cent salt. None with 4 per cent salt.
Source: From stems of potatoes affected with black-leg.

Habitat: Causes a black rot on stem and tuber of potatoes and other vegetables.

Note: Smith (Science, 31, 1910, 748) regarded Erwinia solanisapra and Erwinia phytophthora as very closely related. Brooks, Nain and Rhodes (Jour. Path. and Bact., 28, 1925, 203) held that Erwinia phytophthora, Erwinia solanisapra and Erwinia carotovora are distinct serologically, although identical in cultural characteristics. Berridge (Ann. Appl. Biol., 13, 1926, 12) claimed from serological tests that Erwinia phytophthora and Erwinia solanisapra are different yet closely related organisms. Lacey (Ann. Appl. Biol., 13, 1926, 1) from cultural and serological tests considered Erwinia phytophthora, Erwinia solanisapra and Erwinia carotovora distinct species. Stapp (Arb. a. d. Biol. Reichanstalt f. Landw. u. Forstwirtsch., 16, 1928, 643) from serological tests places Erwinia phytophthora in one serological group and Erwinia carotovora in another. Leach (Phytopath., 20, 1930, 743) found that Erwinia phytophthora and Erwinia carotovora were indistinguishable in cultural and physiological characteristics, the most consistent difference being the inky black coloration of the tissues infected with the former.

Stapp (in Sorauer, Handb. d. Pflanzenk., 5 Aufl., 2, 1928, 229) states that it is generally believed that the disease caused by Bacillus solanincola Delacroix (Compt. rend. Acad. Sci., Paris, 133, 1901, 417 and 1030) is the same as stem rot of potato (blackleg).

10. Erwinia carotovora (Jones) Holland. (Bacillus carotovorus Jones, Cent. f. Bakt., II Abt., 7, 1901, 12; Holland, Jour. Bact., 5, 1920, 222; Bacterium carotovorum Lehmann and Neumann, Bakt. Diag., 7 Aufl., 2, 1927, 446; Pectobac- 
terium carotovorum Waldee, Iowa State Coll. Jour. Sci., 19, 1945, 469.) From Latin, carrot destroying.

Synonyms: Leach (Minnesota Agr. Exp. Sta. Tech. Bull. 76, 1931, 18) lists the following as synonyms:

Bacillus atrosepticus van Hall. (Van Hall, Inaug. Diss., Univ. Amsterdam, 1902, 134; Erwinia atroseptica Jennison, Ann. Missouri Bot. Gard., 10, 1923, 43.)

Bacillus phytophthorus Appel. (Ber. d. deut. Bot. Cres., 20, 1902, 128; Erwinia phytophthora Holland, Jour. Bact., 5, 1920, 222; Bacterium phytophthorum Burgwitz, Phytopath. Bacteria, Leningrad, 1935, 141; Pectobacterium phytophthorum Waldee, Iowa State Coll. Jour. Sci., 19, 1945, 471).

Bacillus solánisaprus Harrison. (Harrison, Cent. f. Bakt., II Abt., 17, 1907 , 34; Erwinia solanisapra Holland, Jour. Bact., 5, 1920, 222.)

Bacillus melanogenes Pethybridge and Murphy. (Roy. Irish Acad., 29, B, No. 1, 1911, 31.)

Bacillus oleraceac Harrison. (Harrison, Science, 16, 1902, 152; Erminin oleraceae Holland, Jour. Bact., 5, 1920, 222.)

Bacillus omnivorus van Hall. (Inaug. Diss., Univ. Amsterdam, 1902, 176.)

Bacillus apivorus Wormald. (Jour. Sci., 6, 1914, 203.)

Elrod (Bot. Giaz., 103, 1941, 270) holds that Erwinia aroideae is a synonym of Erwinia carolovora.

The following also have been considered as possible synonyms of Eruinia carotovora:

Bacillus cepivorus Delacroix. (Delacroix, Ann. Inst. Nat. Agron., Sér 2, 5, 1905, 368; Bacterium cepivorum Stapp, in Sorauer, Handb. d. Pflanzenkrankheiten, 5 Aufl., 2, 1928, 49; A planobacter cepivorus Elliott, Man. Bact. Plant Path. 1930, 4; Phytomonas cepivora Magrou, in Hauduroy et al., Dict. d. Bact. Path., 1937, 344.) Causes a rot of onion bulbs.

Bacillus cypripedii Hori. (Hori, Cent. f. Bakt., II Abt., 31, 1911, 85; Erwinia cypripedii Bergey et al., Manual, 1st ed., 1923, 171.)
Bacillus dahliae Hcr: and Bokur (Hori and Bokura, Imp. Agr. Expt. Sta. Nishigahara, 38, 1911, 69 ; Erwinia dahliae Magrou, in Hauduroy et al., Dict. d. Bact. Path., 1937, 205.)

Pseudomonas destructans Potter. (Potter, Proc. Univ. Durham Philos. Soc., 1899, 165 and Proc. Roy. Soc., 67, 1900, 449; Bacterium destructans Nakata, Nakajima and Takimoto, Tech. Rept. Korea Ind. Farm, 1922; Phytomonas destructans Bergey et al., Manual, 3rd ed., 1930, 264.) See Paine (Ann. Appl. Biol., 5, 1918, 64) for a discussion of this species.

Bacillus hyacinthi septicus Heinz. (Heinz, Cent. f. Bakt., 5, 1889, 539; Bacillus hyacinthi-septicus Lehmann and Neumann, Bakt. Diag., '2 Aufl., 2, 1899, 449; Bacterium hyacinthi septicus Chester, Ann. Rept. Del. Col. Agr. Exp. Sta., 9, 1897, 127; Bacillus hyacinthi Migula, Syst. d. Bakt., 2, 1900, 874; not Bacillus hyacinthi Trevisan, I generi e le specie delle Batteriacee, 1899, 19; Eruinia hyacinthi septica Magrou, in Hauduroy et al., Dict. d. Bact. Path., $1937,208$.

Rods: Usually 0.7 to 0.8 by 1.5 to 5.0 microns, oceasionally in chains. Stain rather slowly with aniline colors, well with Löffler's methylene blue. No capsules observed. Actively motile with peritrichous flagella. Gram-negative.

Gelatin stab: Rapid surface liquefaction, slower in depth (Jones). Some strains very slow liquefiers.

Agar colonies: After 2 days, circular, convex, smooth, grayish-white, moist, glistening. Margins sharp, entire.

Agar slant: Growth thin, grayishwhite, moist, glistening, butyrous. Medium not discolored.

Broth: Turbid, with pellicle and white flocculent sediment. Slow alkaline production.

Litmus milk: After 4 days, coagulated, acid, with separation of whey. Cheesy odor. Litmus reduced. Slightly peptonized. 
Potato: Growth thick, creamy-white; medium softened.

Dunham's solution: Feeble persistent turbidity.

Blood serum: Growth much as on agar. Not liquefied.

Uschinsky's solution: Strong turbidity.

Indole production none.

Nitrites produced from nitrates.

Diastase negative.

No $\mathrm{H}_{2} \mathrm{~S}$ produced or only a trace.

No ammonia produced.

Methyl red positive, Voges-Proskauer negative (Dowson, Cent. f. Bakt., II Abt., 100, 1939, 183).

Acid and gas from glucose, lactose, sucrose, fructose, raffinose, mannitol, arabinose, xylose, salicin and rhamnose. Acid without gas from glycerol and ethyl alcohol. Butyl alcohol, inulin and starch not fermented.

Facultative anerobe.

Temperature relations: Optimum 25 to $30^{\circ} \mathrm{C}$. Minimum $4{ }^{\circ} \mathrm{C}$. Maximum $38^{\circ}$ to $39^{\circ} \mathrm{C}$. Thermal death point $41^{\circ}$ to $51^{\circ} \mathrm{C}$

Pathogenesis: Causes a rapid soft rot of roots, rhizomes, fruits and the ffeshy stems of a variety of plants.

Source: From rotted carrots.

Habitat: Causes a soft rot in carrot, cabbage, celery, cucumber, egg-plant. iris, muskmelon, hyacinth, onion, parsnip, pepper, potato, radish, tomato, turnip, and other plants.

11. Erwinia erivanensis (Kalantarian) Bergey et al. (Bacterium erivanense Kalantarian, Cent. f. Bakt., II Abt., 65, 1925, 298; Bacillus erivanensis Stapp, in Sorauer, Handb. d. Pflanzenkr., 5 Aufl., 2, 1928, 202; Bergey et al., Manual. 3rd ed., 1930, 239.) Derived from Erivan, a city in Armenia.

Whether this organism is to be considered a chromogenic strain or a distinet species is impossible to determine; therefore, it occupies its present position tentatively. It cannot be separated from Erwinia carotovora on the basis of chromogenesis since the latter occasionally shows a tendency to the formation of a faint yellowish pigment.

Rods : 0.5 to 0.7 by 1.25 to 2.5 microns. Motile with peritrichous flagella. Gramnegative.

Gelatin colonies: After 3 days at $20^{\circ} \mathrm{C}$, circular, 1 to $1.5 \mathrm{~mm}$ in diameter, yellowish-white, convex, entire. Microscopically gray with opaque borders and darker patches.

Gelatin stab : Surface growth somewhat umbonate. In 10 to 12 days a slow liquefaction. Intense yellow growth.

Agar colonies: Grayish-white, fatty lustre, turning yellow after several days.

Agar slant: Growth grayish-white, fatty lustre, becoming yellow.

Broth: Strong more or less floceulent turbidity. No surface growth. Little sediment.

Potato: Growth somewhat raised, becoming yellowish.

Milk: Coagulated in 14 days, becoming alkaline, slowly clearing.

Indole is formed.

Nitrites not produced from nitrates.

Acid and gas from glucose, sucrose and mannitol. To gas from lactose and glycerol.

Optimum temperature $20^{\circ} \mathrm{C}$.

Source: From cotton plants.

Habitat: Causes a root-rot of cotton (Gossypium sp.).

12. Erwinia flavida (Fawcett) Magrou. (Bacillus flavidus Fawcett, Rev. Indust. y Agrico. de Tucuman, 13, 1922, 5; Rev. App. Mycology, 2, 1923, 338; not Bacillus flavidus Morse, Jour. Inf. Dis., 11, 1912, 284; Magrou, in Hauduroy et al., Dict. d. Bact. Path., 1937, 207.) From Latin flavus, yellow.

Morphology: Notile with peritrichous flagella. Gram-negative.

Gelatin: Yellow growth. Liquefaction.

Milk: Coagulated.

Potato: Yellow growth.

Indole is formed. 
Nitrites not produced from nitrates.

Acid and gas from glucose, lactose and sucrose.

Diastase not formed.

Source: From sugar cane.

Habitat : Causes a soft rot of sugar cane (Saccharum officinarum).

Note: If this decay is due to a simple organism as stated above, it is probable that it should be considered merely a chromogenic strain of Erwinia carotovora.

13. Erwinia dissolvens (Rosen) comb. nov. (Pseudomonas dissolvens Rosen, Phytopath., 12, 1922, 497; Phytomonas dissolvens Rosen, Phytopath., 16, 1926, 264; Bacterium dissolvens Rosen, ibid.; Aplanobacter dissolvens Rosen, ibid.; Aerobacter dissolvens Waldee, Iowa State Coll. Jour. Sci., 19, 1945, 473.) From Latin, dissolving.

Rods : 0.5 to 0.9 by 0.7 to 1.2 microns. Pairs, rarely in chains. Capsules present. First described as motile with a single flagellum; later as non-motile. Gram-negative.

Gelatin: Not liquefied.

Agar colonies: Round, margins entire, white, opaque, glistening, butyrous, emitting a strong odor of decaying vegetables.

Broth: Turbid with heavy surface growth consisting of ring, and floccules or compact slimy masses and streamers. Abundant sediment.

Uschinsky's solution: Good growth, but not viscid.

Litmus milk: Acid, coagulated.

Indole produced.

Nitrites produced from nitrates.

Hydrogen sulfide not produced.

Acid and gas from glucose, galactose, mannitol, sucrose, maltose, lactose and glycerol.

Starch hydrolyzed.

Optimum temperature $30^{\circ} \mathrm{C}$.

Good growth in 3 per cent salt. lie. tarded at 4 per cent.

Source: From rotting corn stalks.

Habitat: Pathogenic in corn plants.
14. Erwinia nimipressuralis Carter. (Illinois Nat. Hist. Survey Bull. 23, 1945, 423.) From Latin nimis, too much and pressuralis, pertaining to pressure.

Rods: Mostly 0.34 to 0.68 by 0.68 to 1.35 microns. Motile with as many as 6 peritrichous flagella. Capsules not observed. Gram-negative.

Gelatin: Not liquefied.

Potato glucose agar: Colonies eircular, smooth, whitish-cream, entire, flat to slightly raised and usually opaque. Gas produced when medium is stabbed.

Broth: Abundant with thin pellicle or floceulent surface growth. Sediment scant and viscid. Gas produced in nutrient broth plus glucose was 47 per cent $\mathrm{CO}_{2}$ and 2.4 per cent hydrogen. $\mathrm{CO}_{2}$ varied with age of culture, more being produced in young cultures.

Milk: Acid, coagulated. Litmus and bromocresol purple are reduced. Not peptonized.

Nitrites produced from nitrates.

Hydrogen sulfide produced.

Indole not produced.

Acid and gas produced from arabinose, rhamnose, xylose, glucose, fructose, galactose, mannose, lactose, maltose, trehalose, melibiose, cellobiose, mannitol, sorbitol and salicin; no acid or gas from inulin, dextrin or filterpaper; variable results from sucrose, raffinose, melezitose, dulcitol, glycerol and elm sawdust. Pectin is not fermented.

Starch not hydrolyzed.

Methyl red test positive. Acetylmethylcarbinol produced.

Facultative anaerobe.

Optimum temperature $24^{\circ}$ to $30^{\circ} \mathrm{C}$. Maximum $37^{\circ} \mathrm{C}$. Minimum $5^{\circ} \mathrm{C}$ or lower. Thermal death point $45^{\circ}$ to $55^{\circ} \mathrm{C}$.

Optimum pH for growth 6.8 to 7.5 . Maximum 10.0+. Minimum 4.6.

source: Five cultures from 5 different trees affected with wet wood.

IIabitat: Pathogenic in trunk wood of elms, Ulmus americana, U. pumila, $U$. julva and $U$. procera. 
15. Erwinia ananas Serrano. (Philippine Jour. Sci., 36, 1928, 271; Bacillus ananas Serrano, ibid.; Bacterium ananas Burgwitz, Phytopathogenic Bacteria, Leningrad, 1935, 44.) Named for the genus, Ananas.

Note: Not to be confused with Pseudomonas (Phytomonas) ananas Serrano, Philippine Jour. Sci., 36, 1928, 271.

Short rods: 0.6 by 0.9 micron, with rounded ends, occurring singly, in pairs and in short chains. Encapsulated. Motile with peritrichous flagella. Gramnegative.

Gelatin stab: Stratiform liquefaction, with a deep chrome-yellow sediment.

Potato glucose agar: After 24 hours, circular, $3 \mathrm{~mm}$ in diameter, convex, dense, homogeneous, entire, moist, strawyellow, mottled, becoming primuline yellow. Plates have a molasses odor. Show two types of colonies, rough and smooth. Rough colonies have crenate margins.

Potato glucose agar slant: Growth straw-yellow, raised, becoming primuline yellow, moist, glistening.

Broth: Turbid, with a straw-colored pellicle and ring.

Glucose broth: Growth sulfur yellow.

Litmus milk: Coagulated, faintly acid, becoming alkaline.

Potato: Copious grow th, moist, glistening, spreading, becoming primuline yellow.

Indole not formed.

Blood serum: Moderate growth, slightly raised, mustard yellow to primuline yellow. No liquefaction after 3 months.

Cohn's solution: No growth.

Phenol negative.

Diastase produced.

Nitrites produced from nitrates.

Slight amount of ammonia produced.

Slight amount of $\mathrm{H}_{2} \mathrm{~S}$ produced.

Small amount of alcohol and aldehyde produced.

No gas from carbohydrates. Acid from glucose, lactose, sucrose, mannitol, raffinose, glycerol, salicin, dextrin, maltose, fructose and mannose. No acid from arabinose, xylose, amygdalin, rhamnose, inositol, inulin, dulcitol, adonitol, asparagine or starch.

Source: From the pineapple (Ananas sativus) and sugar-cane (Saccharum officinarum).

Habitat: Causes a brown rot of the fruitlets of pineapple.

16. Erwinia cytolytica Chester. (Phytopath., 28, 1938, 431.) From Latin, cell dissolving.

Rods : 0.6 to 0.7 by 2.5 to 3.5 microns. Singly or in pairs. Gram-negative. Motile with peritrichous flagella.

Gelatin: Slow liquefaction.

Agar colonies : 2 to $3 \mathrm{~mm}$ in diameter, round, convex, moist, glistening, grayishwhite, watery, translucent. Light brownish-yellow by transmitted light.

Broth: Turbid.

Milk: Coagulated in 5 to 7 days. Slightly acid. Not digested.

Nitrites produced from nitrates.

Indole not formed.

Hydrogen sulfide not formed.

Acetylmethylcarbinol: A slight reaction.

Acid without gas from glucose, lactose, sucrose, raffinose, mannitol, salicin and isodulcitol. No acid from fructose, arabinose, xylose, glycerol and inulin.

Starch hydrolyzed.

Pectin dissolved.

Asparagine, peptone, and ammonia used as nitrogen sources in synthetic medium plus glucose. Potassium nitrate not used.

Optimum temperature $28^{\circ}$ to $30^{\circ} \mathrm{C}$. Growth at $37^{\circ} \mathrm{C}$. Slow growth at $20^{\circ} \mathrm{C}$ and no growth at $8^{\circ}$ to $10^{\circ} \mathrm{C}$.

Good growth at $\mathrm{pH} 6.8$ to 7.3 . Feeble growth at 5.0. No growth at 4.4.

Aerobic and facultative anaerobic.

Source: Several isolates from diseased dahlias in New York Botanical Garden.

Habitat: Causes a rot of the tuber and stems of dahlias. 
17. Erwinia aroideae (Townsend) Holland. (Bacillus aroideae Townsend, U. S. Dept. Agr., Bur. Plant Ind. Bull. 60, 1904, 40; Holland, Jour. Bact., 5, 1920, 222 ; Bacterium aroideae Stapp, in Sorauer, Handb. d. Pflanzenkr., 5 Aufl., 2, 1928, 41; Pectobacterium aroideae Waldee, Iowa State Coll. Jour. Sci., 19, 1945, 472.) From Greek, pertaining to the family Araceae.

Probable synonyms: Erwinia croci (Mizusawa) Magrou. (Bacillus croci Mizusawa, Kanag. Agr. Exp. Sta. Bull. 51, 1921, 1; Ann. Phytopath. Soc. Japan, 1, 1923, 1; Magrou, in Hauduroy et al., Dict. d. Bact. Path., 1937, 204.) Attacks Crocus sativus, also onion.

Eruinia melonis (Giddings) Holland. (Bacillus melonis Giddings, Vermont Agr. Exp. Sta. Bull. 148, 1910, 413; Holland, Jour. Bact., 5, 1920, 222 ; Pectobacterium melonis Waldee, Iowa State Coll. Jour. Sei., 19, 1945, 473.) E. F. Smith (An Introduction to Bact. Dis. of Plants, 1920, 240) considered Erwinia melonis and Erwinia aroideae identical. Causes a soft rot of muskmelon.

Erwinia papaveris (Ayyar) Magrou. (Bacillus papaveris Ayyar, Mem. Dept. Agr. India, Bact. Ser. 2, 1927, 29; Magrou, in Hauduroy et al., Dict. d. Bact. Path., 1937,214 .) The cause of a soft rot of the garden poppy.

Rods: 0.5 by 2 to 3 microns, with rounded ends, occurring singly, in pairs and in fours, also in chains under certain conditions. Motile with peritrichous flagella. No capsules. Gram-negative.

Gelatin stab: Narrow infundibuliform liquefaction.

Agar colonies: Circular to amoeboid, white, glistening. Borders sharp. Granular structure.

Agar slant: Growth white to grayishwhite, moist, glistening. Medium not discolored.

Broth: Turbid.

Potato: Growth whitish, with tinge of yellow. Medium grayed.

Litmus milk: Coagulated, acid, with separation of whey, not peptonized. Litmus reduced.

Indole not formed.

Nitrites produced from nitrates.

Acetylmethylcarbinol produced.

Methyl red negative (Dowson, Cent. f. Bakt., II Abt., 100, 1939, 183).

Acid without gas from glucose, lactose, sucrose, maltose, mannitol, glycerol, fructose, raffinose, arabinose and xylose. Growth in closed arm.

Diastase slight.

Hydrogen sulfide produced.

Uschinsky's solution: Good growth.

No growth in nitrogen. Growth feeble in $\mathrm{H}_{2}$ and $\mathrm{CO}_{2}$.

Temperature relations: Optimum $35^{\circ} \mathrm{C}$. Minimum $6{ }^{\circ} \mathrm{C}$. Maximum $41^{\circ} \mathrm{C}$. Thermal death point $50^{\circ} \mathrm{C}$ for 10 minutes.

Facultative anaerobe.

Differential characters: See Erwinia carotovora. Massey (Phytopath., 14, 1924, 460) considered Erwinia aroideae and Emwinia carotovora distinet species, though closely related. Link and Taliaferro (Bot. Gazette, 85, 1928, 198) found them distinct serologically. Dowson (Ann. Appl. Biol., 28, 1941, 102) differentiated them on their action on maltose and xylose.

Source: From rotted calla lily.

Habitat: Causes a soft rot of calla. Affects raw potato, egg-plant, cauliHower, radish, cucumber, cabbage, parsnip, turnip, salsify, tomato (ripe and green).

18. Erwinia mangiferae (Doidge) Bergey et al. (Bacillus mangiferae Doidge, Ann. Appl. Biol., 2, 1915, 1; Bergey et al., Manual, 1st ed., 1923, 173.) Named for the genus, Mangifera.

Rods: 0.6 by 1.5 microns, occurring singly and in chains, with rounded ends. Encapsulated. Motile with peritrichous flagella. Gram-negative.

Gelatin stab: Medium liquefied in 10 to 17 days. Growth yellow.

Agar colonies: Glistening, yellowish, undulate borders. 
Agar slant: Growth yellow, glistening.

Broth: Turbid, with yellow ring.

Litmus milk: Slow coagulation at $37^{\circ} \mathrm{C}$. Slight acidity. Casein slowly dissolved. Litmus reduced.

Potato: Growth spreading, glistening, yellowish. Medium not discolored.

Indole formed in peptone solution. Phenol negative.

Nitrites produced from nitrates.

No $\mathrm{H}_{2} \mathrm{~S}$ produced.

No ammonia in broth.

Feeble acid production without gas from glucose, lactose, sucrose, fructose and glycerol. No growth in closed arm with lactose and glycerol; more or less growth in closed arm with glucose, sucrose, fructose, maltose, raffinose and mannitol.

Diastase not formed.

Produces an enzyme capable of dissolving the middle lamella but without action on cellulose.

Cohn's solution: Slight turbidity.

Uschinsky's solution: No growth.

Fermis' solution with starch jelly: No growth.

Pigment insoluble in water, alcohol, ether, chloroform or dilute acids.

Temperature relations : Optimum $30^{\circ} \mathrm{C}$. Minimum $5^{\circ}$ to $6^{\circ} \mathrm{C}$. Maximum $45^{\circ} \mathrm{C}$. Thermal death point $60^{\circ} \mathrm{C}$.

Source: From the mango in Africa.

Habitat: Causes a disease of the mango (Mangifera indica).

\section{Erwinia citrimaculans (Doidge)} Magrou. (Bacillus citrimaculans Doidge, Ann. Appl. Biol., 3, 1917, 53; Bacterium citrimaculans Burgmitz, Phytopath. Bacteria, Leningrad, 1935, 154; Magrou, in Hauduroy et al., Dict. d. Bact. Path., 1937, 203.) From the genus Citrus and Latin maculans, spotting.

Rods : 0.45 to 0.7 by 0.8 to 3.2 microns. Motile with peritrichous flagella. Conspicuous capsule present. Gram-positive. Dowson thinks this species Gramnegative (Cent. f. Bakt.. II Abt., 100, 1939,1841 .
Gelatin: Liquefied.

Agar colonies: Subcircular, yellow, with dense grumose centers.

Broth: Turbid, with pellicle and sediment.

Milk: Coagulated, with precipitation of casein and extrusion of whey. Not peptonized. Litmus gradually reduced.

Blood serum : Not liquefied.

Indole is formed.

Nitrites produced from nitrates with evolution of gas.

Ammonia produced in broth.

Acid without visible gas from glucose, sucrose, fructose, galactose, maltose and mannitol. No acid from lactose, glycerol, dextrin or starch.

Diastase not produced.

Cohn's solution: No growth.

Uschinsky's solution: Growth present.

No growth in broth over chloroform.

Methylene blue and neutral red reduced.

Pigment insoluble in water, alcohol, ether, chloroform, carbon bisulfide, dilute acid or alkalis.

A turbid growth is produced in 10 per cent salt.

Temperature relations: Optimum $35^{\circ} \mathrm{C}$. Naximum $43^{\circ} \mathrm{C}$. Thermal death point $62^{\circ} \mathrm{C}$ for 10 minutes.

Facultative anaerobe.

Source: From diseased lemons and oranges.

Habitat: Causes a spot disease of citrus. In nature attacks lemons, oranges, naartjes and has also been successfully inoculated into limes, shaddock, grapefruit and citron. Seville oranges are resistant.

20. Erwinia rhapontici (Millard) comb. nov. (Phytomonas rhapontica Millard, Univ. Leeds and Yorkshire Council for Agr. Ed. Bul. 134, 1924, 111; Bacterium rhaponticum Millard, ibid.; A planobacter rhaponticum Elliott, Man. Bact. Plant Path., 1930, 12.) From Greek Rha pontic, rhubarh of Pontus, a province of Asia Minor; M. L. Rheum rhaponticum. 
Description from Metcalfe, Ann. of Appl. Biol., 27, 1940, 502, where he suggests it belongs in Erwinia.

Rods : 0.5 to 0.8 by 1.2 to 1.5 microns. Motile with 3 to 7 peritrichous flagella. Gram-negative.

Gelatin stab: Beaded growth. No liquefaction.

Infusion agar: Colonies circular, convex, smooth, glistening, translucent, with margins entire, 2 to $3 \mathrm{~mm}$ in diameter in 48 hours at $25^{\circ} \mathrm{C}$.

Rhubarb agar: Colonies slightly larger, often with a yellowish tinge.

Tryptophane broth: Turbid with fragile pellicle, a slight rim and slight flocculent deposit.

Milk: Acid in 3 to 4 days with or without slight eurd separation. No clotting.

Indole not produced.

Nitrites formed from nitrates.

Acetylmethylcarbinol produced.

No hydrogen sulfide produced.

Cohn's solution: Moderate growth.

Acid but no gas from arabinose, xylose, glucose, galactose, fructose, mannose, lactose, maltose, sucrose, mannitol, glycerol and salicin.

Growth in citrate solution.

Starch not hydrolyzed.

Chromogenesis: Water-soluble pinkish pigment in various media.

Growth from $0^{\circ} \mathrm{C}$ to $37^{\circ} \mathrm{C}$ and possibly higher.

Distinctive characters: Differs from Erwinia aroideae in that it does not liquify gelatin nor clot milk and is chromogenic. It also has a limited host range.

Source: From rotting rhubarb crowns. Metcalfe used 6 isolates from various sources in describing the pathogen.

Habitat: Causes a crown-rot of rhubarb.

21. Erwinia lathyri (Manns and Taubenhaus) Holland. (Bacillus lathyri Manns and Taubenhaus, Gardener's Chronicle, 53, 1913, 215; Manns, Delaware Agr. Exp. Sta., Bul. 108, 1915, 23 ; Holland, Jour. Bact., 5, 1920, 218; Bac- terium lathyri Burgwitz, Phytopath. Bacteria, Leningrad, 1935, 76.) From the genus Lathyrus.

Rods: After 24 hours at $25^{\circ}$ to $28^{\circ} \mathrm{C}$, 0.6 to 0.85 by 0.75 to 1.5 microns, with rounded ends. No capsules. Motile with peritrichous flagella. Gram-negative.

Gelatin colonies: After 8 days, circular, slightly convex, edges smooth. Liquefaction too slow to show on plate.

Gelatin stab: Growth best at surface. Line of stab filiform. Liquefaction slow, fairly well begun in four weeks, complete in three months.

Agar colonies: After 24 hours, yellow, stellate to amoeboid, smooth, glistening, slightly raised, entire. Centers granular, yellow.

Agar slant: Growth filiform, slightly convex, smooth, glistening, opaque, butyrous, light to deep yellow. Odor absent.

Broth: Strong turbidity in 24 hours, little or no pellicle. Sediment scant.

Litmus milk: Slow increase of acidity, not always sufficient to cause coagulation. Digestion of casein slow and variable.

Potato: Growth rapid, filiform, slightly convex, smooth, glistening, butyrous to slightly viscid. Light to deep yellow. Medium not discolored.

Indole is formed.

Cohn's solution: No growth.

Uschinsky's solution: Rapid growth, sometimes a pellicle. Fluid viscid.

Asparagine solution: Good growth.

Nitrites are not produced from nitrates.

Ammonia produced in broth and asparagine solution.

No gas from carbohydrates. Acid from glucose, lactose, sucrose, mannitol and glycerol. No growth in closed arm.

Diastase not formed or extremely weak.

Growth in broth over chloroform absent.

Growth inhibited by 4 per cent $\mathrm{NaCl}$.

Temperature relations: Optimum $28^{\circ}$ 
to $30^{\circ} \mathrm{C}$. Thermal death point $46^{\circ}$ to $48^{\circ} \mathrm{C}$ for 10 minutes.

Aerobic.

Source: From sweet peas.

Habitat: Stated to be pathogenic for sweet pea (Lathyrus odoratus) and other legumes. Considered by many to be a saprophyte.

22. Erwinia lilii (Uyeda) Magrou. (Bacillus litii Uyeda, by Bokura, Ann. Phytopath. Soc. Japan, 1 (2), 1919, 36; Magrou, in Hauduroy et al., Dict. d. Bact. Path., 1937, 210.) From Latin lilium, a name taken from the Greek but derived from the Celtic word li meaning white; M. L., generic name, Lilium.

Translated by Marion Okimoto.

Rods: 0.6 to 0.7 by 0.8 to 1.0 micron. No capsules. Motile with 6 to 8 peritrichous flagella. Gram-positive(?).

Gelatin: Liquefaction.

Gelatin plate: Colonies after 2 days, round and smooth with grayish surface.

Broth: Alkaline, ammonia produced. Milk: Curd formation.

Indole produced.

Nitrites produced from nitrates.

Hydrogen sulfide produced.

Sugar medium changes to a brown color. Conjac not utilized.

Aerobic, facultative.

Optimum temperature $32^{\circ}$ to $34^{\circ} \mathrm{C}$. Filled in $3 \mathrm{~min}$. at $50^{\circ} \mathrm{C}$. Resists $-20^{\circ} \mathrm{C}$ for $30 \mathrm{~min}$.

Source: From brown spots on lily bulbs in Japan.

Habitat: Causes a disease of lily bulbs and leaves.

Appendix: The following additional species are found in the literature. Many are incompletely described.

Bacillus brassicaevorus Delacroix. (Compt. rend. Acad. Sci., Paris, 140, 1905, 1356.) Presumably causes a rot of cabbage.

Bacillus farnetianus Pavarino. (Atti R. Accad. Naz. Lincei Rend. Cl. Sci. Fis., Mat. e Nat., 20, 1911, 233.)

Bacillus putrefaciens putridus Dela- croix. (Ann. Inst. Nat. Agron., 5, 1906, 154.) Pathogenic for tobacco.

Bacillus solaniperda Migula. (Syst. d. Bakt., 2, 1900, 573; Bacillus krameri Chester, Man. Determ. Bact., 1901, 282.) Causes a soft rot of potato.

Bacillus spieckermanni Jaczewski. (Elliott, Bacterial Plant Diseases, 1935, 67.) Name applied to a species described by Spieckermann (Landw. Jahrb., $31,1902,155$ ) but left unnamed.

Bacillus tabacivorus Delacroix. (Ann. Inst. Nat. Agron., 5, 1906, 266.) Said to cause collar rot of tobacco.

Bacillus tabificans Delacroix. (Compt. rend. Acad. Sci., Paris, 137, 1903, 871.) Said to cause spotting of tobacco leaves. Bacterium loehnisi Kalantarian. (Kalantarian, Cent. f. Bakt., II Abt., 65, 1925, 301; Phytomonas loehnisii Bergey et al., Manual, 3rd ed., 1930, 276.) From diseased cotton plants. Peritrichous.

Bacterium lycopersici Burgwitz. (Ztschr. f. Pflanzenkr., 34, 1924, 304.) From a blossom end rot of tomato.

Erwinia alliariae (Omori) Magrou. (Bacillus alliariae Omori, Official Gaz. of Japan, 11, 1896, No. 3758; Magrou, in Hauduroy et al., Dict. d. Bact. Path., 1937,195 .) Causes a root rot of horseradish.

Erwinia araliavora (Uyeda) Magrou. (Bacillus araliavorus Uyeda, Bull. Imp. Agr. Exp. Sta. Tokyo, 35, 1909, 61; Magrou, in Hauduroy et al., Dict. d. Bact. Path., 1937, 197.) Causes a root rot of ginseng.

Erwinia asteracearum (Pavarino) Magrou. (Bacillus asteracearum Paravino, Atti R. Accad. Naz. Lincei Rend. Cl. Sci. Fis., Mat. e Nat., Ser. 5, 21, 1912, 544; Magrou, in Hauduroy et al., Dict. d. Bact. Path., 1937, 199.) Pathogenic for the aster (Aster chinensis).

Erwinia bussei (Migula) Magrou. (Bacillus B, Busse, Ztschr. f. Pflanzenkr., 7, 1897, 74 ; Bacillus bussei Migula, Syst. d. Bakt., 2, 1900, 779; Bacillus betae Lehmann and Neumann, Bakt. Diag., 4 Aufl., 2, 1907, 599; not Bacillus betae Migula, Syst. d. Bakt., 2, 1900, 779; Magrou, in 
Hauduroy et al., Dict. d. Bact. Path., 1937, 200.) Pathogenic for the sugar beet.

Erwinia cacticida (Johnston and Hitchcock) Magrou. (B. cacticidus Johnston and Hitcheock, Trans. and Proc. Roy. Soc. South Australia, 47, 1923, 162 ; Magrou, in Hauduroy et al., Diet. d. Bact. Path., 1937, 201.) Causes a rot of cactus.

Erwinia edgeworthiae (Hori and Bokura) Magrou. (Bacillus edgeworthiae Hori and Bokura, Ideta Arata, Supplement to Handbook of the Plant Diseases of Japan, 1, 1925, 32; Magrou, in Hauduroy et al., Dict. d. Bact. Path., 1937, 206.) Pathogenic on Edgeworthia chrysantha, an oriental shrub.

Erwinia ixiae (Severini) Magrou. (Bacillus ixiae Severini, Annali di Botanica, Rome, 11, 1913, 413; Magrou, in Hauduroy et al., Dict. d. Bact. Path., 1937, 208.) Pathogenic on gladiolus and other bulbs.

Erwinia nelliae (Welles) Magrou. ( $B($ cillus nelliae Welles, Philippine Jour. Sci., 20, 1922, 279; Magrou, in Hauduroy et al., Dict. d. Bact. Path., 1937, 213.)

Erwinia papayae (Rant) Magrou. (Bacillus papayae Rant, Cent. f. Bakt., II Abt., 84, 1931, 483; Magrou, in Hatuduroy et al., Dict. d. Bact. Path., 1937, 214.) Pathogenic on papaya.

Erwinia sacchari Roldan. (Philippine Agric., 20, 1931, 256; Bacillus saccharum Roldan, idem; not Bacillus sacchari
Janse, Mededeel. uit's Lands. Plantentuin, 9, 1891, 1.)

Erwinia scabiegena (von Faber) Magrou. (Bacterium scabiegenum von $\mathbf{F a}$ ber, Arb. Kais. Biol. Anst. f. Land. u. Forstw., 5, 1907, 347; Bacillus scabiegenus Stapp, in Sorauer, Handb. d. Pflanzenkr., 5 Aufl., 2, 1928, 103; Magrou, in Hauduroy et al., Diet. d. Bact. Path., 1937, 217.) Pathogenic for the beet (Beta vulgaris).

Erwinia serbinowi (Potebnia) Magrou. (Bacterium beticola Serbinow, Zhurnal Bolezni Rastenii, 7, 1913, 237; not Bacterium beticola Smith, Brown and Townsend, Bur. Plant Ind., U. S. Dept. Agr., Bul. 213, 1911, 194; Bacterium serbinowi Potebnia, Kharkov Prov. Agr. Exp. Sta., 1, 1915, 1; Bacillus beticola Stapp, in Sorauer, Handb. d. Pflanzenkr., 5 Aufl., 2, 1928, 93; Bacillus serbinowi Elliott, Man. of Bact. Plant Pathogens, 1930, 75; Magrou, in Hauduroy et al., Diet. d. Bact. Path., 1937, 217.) Pathogenic for the sugar beet.

Erwinia uvae (Kruse) Magrou. (Bacillo della bacterosidei grappoli della vite, Cugini and Macchiati, Le Stazione sperimentali ital., 20, 1891, 579; Bacillus wvae Kruse, in Flügge, Die Mikroorganismen, 3 Aufl., 2, 1896, 329; Bacterium wae Chester, Ann. Rept. Del. Agr. Exp. Sta., 9, 1897, 53 and 127; Magrou, in Hauduroy et al., Dict. d. Bact. Path., 1937, 220.) Pathogenic for the grape.

Erwinia ritarera du Plessis syn. Clostridium baccarinii Bergey et al., Manual, 1st exl., 1923, 32S. 
TRIBE III. SERRATEAE BERGEY, BREED AND MURRAY.

(Preprint, Manual, 5th ed., October, 1938, vi.)

Small, aerobic rods, usually producing a bright red or pink pigment on agar and gelatin. There is a single genus.

\section{Genus I. Serratia Bizio emend. Breed and Breed.*}

(Bizio, Biblioteca italiana o sia Giornale de lettera, scienze e arti, 30, 1823, 288; Zaogalactina Sette, Sull'arrossimento straordinario di alcune sostanze alimentose osservato nella provincia di Padova l'anno 1819. Venezia, 1824, 51 ; Coccobacterium Schmidt and Weis, Die Bakterien, 1902, 10; Erythrobacillus Fortineau, Compt. rend. Soc. Biol., Paris, 58, 1905, 104; Dicrobactrum Enderlein, Sitzber. Gesell. Naturf. Freunde, Berlin, 1917, 309; Breed and Breed, Cent. f. Bakt., II Abt., 71, 1927, 435.) Named for Serafino Serrati, the Italian physicist who invented a steam boat at Florence before 1787 .

Small, aerobic, rapidly liquefying, nitrate reducing, Gram-negative, peritrichous rods which produce characteristic red pigments. White to rose-red strains that lack brilliant colors are common. Coagulate and digest milk. Liquefy blood serum. Typical species produce $\mathrm{CO}_{2}$ and frequently $\mathrm{H}_{2}$ from glucose and other sugars; also acetic, formic, succinic and lactic acids, acetylmethylcarbinol and 2,3 butylene glycol. Saprophytic on decaying plant or even animal materials.

The type species is Serratia marcescens Bizio.

\section{Key to the species of genus Serratia.}

I. Pigment not especially water-soluble, readily soluble in alcohol.

A. No visible gas from glucose.

1. Inconspicuous pellicle, if any, on plain gelatin.

1. Serratia marcescens.

2. Brilliant orange-red pellicle on plain gelatin.

2. Serratia indica.

B. Produce enough $\mathrm{H}_{2}$ with the $\mathrm{CO}_{2}$ from glucose to show gas in fermentation tubes.

1. Acetylmethylcarbinol produced.

2. Acetylmethylcarbinol not produced.

\section{Serratia plymuthicum.}

4. Serratia kilensis.

II. Pigment soluble in water and alcohol.

5. Serratia piscatorum.

1. Serratia marcescens Bizio. (Po- Sette, Memoria storico-naturale sull' lenta porporina, Biblioteca italiana, 30 , arrossimento straordinario di alcune 1823, 288.) From Latin, dissolving into a fluid or viscous matter.

Synonyms: Zoagalactina imetrofa 1838, see Trevisan, Rend. R. Inst. Lomb.

* Revised by Prof. Robert S. Breed, New York State Experiment Station, Geneva, New York, Nov., 1937; further revision by Prof. Robert S. Breed, Nov., 1945. 
di Sci.e Let., Ser. 2, 20, 1887, 797; Monas prodigiosa Ehrenberg, Bericht ü. d. z. Bekanntmachung geeigneten Verhandlungen d. Kgl. preuss. Acad. d. Wissenschaften, 1849, 354; Palmella prodigiosa Montague, Bul. Soc. nat. et cent. d. agric. Paris, Sér. 2, 7, 1853, 527; Micraloa prodigiosa Zanardini, 1863, see Trevisan, loc. cit., 1887, 799; Bacteridium prodigiosum Schroeter, in Cohn, Beiträge $z$. Biol. d. Pflanzen, 1, Heft 2, 1872, 109 ; Micrococcus prodigiosus Cohn, ibid., 127; Bacillus prodigiosus Flügge, Die Mikroorganismen, 1886, 284; Bacillus imetrophus Trevisan, loc. cit., 797; Bacillus marcescens De Toni and Trevisan, in Saccardo, Sylloge Fungorum, 8, 1889, 976 ; Bacterium prodigiosum Lehmann and Neumann, Bakt. Diag., 1 Aufl., 2, 1896, 259 ; Liquidobacterium prodigiosum OrlaJensen, Cent. f. Bakt., II Abt., 22, 1909, 339 ; Erythrobacillus prodigiosus Winslow et al., Jour. Bact., 5, 1920, 209; Dicrobactrum prodigiosum Enderlein, Bakterien Cyclogenie, 1925, 279; Salmonella marcescens and Salmonclla prodigiosa Pribram, Jour. Bact., 18, 1929, 384; Chromobacterium prodigiosum Topley and Wilson, Principles of Bacteriology, 1, 1931, 402.

Description largely taken from Breed and Breed, Jour. Bact., 9, 1924, 545.

Short rods, sometimes almost spherical : 0.5 by 0.5 to 1.0 micron, occurring singly and occasionally in chains of 5 or 6 elements. Motile, with four peritrichous flagella. Eight to ten flagella on cells grown at $20^{\circ}$ to $25^{\circ} \mathrm{C}$ (De Rossi, Rivista d'Igiene, 14, 1903, 000). Gramnegative.

Gelatin colonies: Thin, slightly granular, gray becoming red, circular, with slightly undulate margin. Liquefy the medium rather quickly.

Gelatin stab: Infundibuliform liquefaction. Sediment in liquefied medium usually red on top, white in the depth.

Agar colonies: Circular, thin, granular, white becoming red. $\mathrm{R}$ and $\mathrm{S}$ colonies with mucoid variants (Reed, Jour. Bact., 34, 1937, 255).

Agar slant: White, smooth, moist layer, taking on an orange-red to fuchsin color in three or four days, sometimes with metallic luster.

Broth: Turbid, may form a red ring at surface or slight pellicle, and gray sediment.

Litmus milk: Acid reaction with soft coagulum. A red surface growth develops. Little or no digestion takes place.

Potato: At first a white line appears, which rapidly turns red. The growth is luxuriant and frequently shows a metallic luster.

Produces acetic, formic, succinic and levolactic acid, ethyl alcohol, acetylmethylcarbinol, 2,3 butylene glycol, $\mathrm{CO}_{2}$ and a trace of $\mathrm{H}_{2}$ from glucose (Pederson and Breed, Jour. Bact., 16, 1928, 183).

Grows poorly or not at all in distilled water containing urea, potassium chloride and glucose.

Indole not produced.

Nitrites produced from nitrates.

Formation of $\mathrm{H}_{2} \mathrm{~S}$ : Produced from cysteine, cystine or organic sulfur compounds containing either of these molecules. Produced from sulfur but not from sulfites, sulfates or thiosulfates (Tarr, Biochem. Jour., 27, 1933, 1869; 28, 1934, 192).

Acetylmethylcarbinol is produced (Breed).

Pigment soluble in alcohol, ether, chloroform, benzol and carbon bisulfide (Schneider, Arb. Bakt. Hochsch. Karlsruhe, 1, 1894, 210). Pigment may diffuse through the agar, i.e., shows solubility in water where strains are very deeply pigmented (Breed). Pigment not formed at $35^{\circ} \mathrm{C}$.

Sodium formate broth (Stark and England, Jour. Bact., 29, 1935, 26) : Cultures do not produce visible gas (Breed).

Odor of trimethylamine is produced. Aerobic, facultative. 
Optimum temperature $25^{\circ}$ to $30^{\circ} \mathrm{C}$. No growth at $37^{\circ} \mathrm{C}$.

Source: Described by Bizio (loc. cit.) and Sette (loc. cit.) from growth on corn meal mush (polenta).

Habitat: Water, soil, milk, foods, silk morms and other insects.

2. Serratia indica (Eisenberg) Bergey et al. (Bacillus indicus Eisenberg, Bakt. Diag., 1 Aufl., 1886, 1; Bacillus indicus ruber Flügge, Die Mikroorganismen, 2 Aufl., 1886, 285; Micrococcus indicus Koch, Berichte ueber die Reise zur Erforschung der Cholera, 1887; Bacillus ruber indicus Kruse, in Flügge, Die Mikroorganismen, 3 Aufl., 2, 1896, 302; Bacterium ruber indicus Chester, Ann. Rept. Del. Col. Agr. Exp. Sta., 9, 1897, 112; Erythrobacillus indicus Holland, Jour. Bact., 5, 1920, 218; Bergey et al., Manual, 1st ed., 1923, 88; Breed and Breed, Jour. of Bact., 11, 1926, 76; Chromobacterium indicum Topley and Wilson, Princ. Bact. and Immun., 1, 1931, 402.) From Latin indicus, of India .

Small rods: 0.5 by 1.0 to 1.5 microns. Motile with four peritrichous flagella. Gram-negative.

Gelatin colonies: Resemble those of Serratia marcescens.

Gelatin stab: Liquefied rather quickly. Brilliant orange-red pellicle on plain gelatin.

Agar colonies: Pink, with slightly serrate margin, spreading, with green irridescence.

Agar slant: Luxuriant, dirty-white layer. Pigment produced best in alkaline media.

Broth: Turbid, with white sediment.

Litmus milk: Acid and coagulated. Digestion complete in 10 days.

Potato: Luxuriant growth with or without pigment formation.

Produces same products (except $\mathrm{H}_{2}$ ) from glucose as does Serratia marcescens
(Pederson and Breed, Jour. Bact., 16, 1928, 183).

Indole not produced.

Nitrites produced from nitrates.

Growth with pigment production in distilled water containing urea, potassium chloride and glucose.

Blood serum liquefied.

Odor of trimethylamine.

Sodium formate broth: Cultures do not produce visible gas (Breed).

Pathogenic for laboratory animals.

Acetylmethylcarbinol is produced (Breed).

Aerobic, facultative.

Optimum temperature $25^{\circ}$ to $35^{\circ} \mathrm{C}$. No growth at $37^{\circ} \mathrm{C}$.

Cultures of this organism lose their ability to produce the orange-red pellicle on gelatin and then become practically indistinguishable from cultures of Serratia marcescens. This would indicate that this so-called species is a rough strain of the former species (Breed). See Reed (Jour. Bact., 34, 1937, 255) for a discussion of dissociation phenomena in this genus.

Source: Isolated from alimentary tract of a Java ape in India; also from milk can from Ithaca, N. Y.

Habitat: Presumably widely distributed.

Apparently the following non-gelatin liquefying strain belongs with this species. Subcultures that are claimed to be derived from the original now liquefy gelatin.

2a. Serratia miquelii Bergey et al. (Named Bacillus ruber by Miquel and described in a letter to Hefferan, Cent. f. Bakt., II Abt., 11, 1903, 402; Erythrobacillus ruber Holland, Jour. Bact., 5, 1920, 223 ; Bergey et al., Manual, 1st ed., 1923, 95.)

Isolated from water by Miquel.

3. Serratia plymuthicum (Lehmann and Neumann) Bergey et al. (Roter 
Bacillus aus Plymouth, Fischer, Zeitschr. f. Hyg., 2, 1887, 74; Bacterium plymuthicum Lehmann and Neumann, Bakt. Diag., 1 Aufl., 2, 1896, 264; Bacillus plymouthensis Migula, Syst. d. Bakt., 2, 1900, 849; Erythrobacillus plymouthensis Holland, Jour. Bact., 5, 1920, 220 ; Bergey et al., Manual, 1st ed., 1923, 88.) Latinized from Plymouth, England.

Distinct rods : 0.6 by 1.5 to 2.0 microns with rounded ends, occurring singly and in short chains. Motile with peritrichous flagella. Gram-negative.

Gelatin colonies: Like Serratia marcescens. Original culture mucoid.

Gelatin stab: Crateriform liquefaction. Liquefaction as in Serratia marcescens.

Agar colonies: Like mucoid varieties of Serratia marcescens.

Agar slant: Sometimes show metallic luster. Pigment as in Serratia marcescens.

Broth: Like Serratia marcescens.

Litmus milk: Acid and coagulated.

Potato: Growth violet pink, with or without metallic luster.

Gas from glucose, lactose and sucrose, 70 to 80 per cent of it $\mathrm{CO}_{2}$. Remainder is $\mathrm{H}_{2}$. Gas is also produced in asparagine solutions.

Strong fecal odor produced.

Blood serum liquefied.

Acetylmethylcarbinol is produced (Breed).

Sodium formate broth: Cultures produce abundant gas (Breed).

Pigment soluble in alcohol, ether and sometimes water.

Aerobic, facultative.

Optimum temperature $30^{\circ} \mathrm{C}$.

Source: From water supply of Plymouth, England.

Habitat: Water and various foods.

\section{Serratia kilensis (Lehmann and} Neumann) Bergey et al. (Bacterium h, Breunig, Inaug. Diss., Kiel, 1888; Bacillus ruber balticus Kruse, in Flügge, Die Mikroorganismen, 3 Aufl., 2, 1896, 303; Bacterium kiliense Lehmann and
Neumann, Bakt. Diag., 1 Aufl., 2, 1896, 263; Bacterium ruber balticus Chester, Ann. Rept. Del. Col. Agr. Exp. Sta., 9, 1897, 142; Bacillus kiliensis Migula, Syst.d. Bakt., 2, 1900, 847; Erythrobacillus kiliensis Holland, Jour. Bact., 5, 1920 , 218; Bergey et al., Manual, 1st ed., 1923, 90 ; Chromobacterium kielense Topley and Wilson, Princip. Bact. and Immun., 1, 1931, 400.) From Kiel, a city on the Baltic Sea.

Description taken from Kruse (loc. cit.) and Bergey et al. (loc. cit.).

Slender rods : 0.7 to 0.8 by 2.5 to 5.0 microns, occurring singly. Motile with four peritrichous flagella. Gram-negative.

Deep gelatin colonies: Bright yellow. Gelatin liquefied slowly, usually becoming rose-red.

Glucose gelatin stab: Rapid liquefaction. Occasional gas bubbles (Breed).

Agar colonies: Small, red becoming magenta, smooth.

Agar slant: Bright red becoming darker in old cultures.

Agar stab: Turbid strongly pigmented water of condensation.

Broth: Turbid. Usually reddened.

Litmus milk: Acid; at $20^{\circ} \mathrm{C}$, coagulated slowly and pigment produced; at $35^{\circ} \mathrm{C}$, coagulated rapidly and no pigment produced.

Potato: Slight red growth, becoming luxuriant and darker.

Indole not formed.

Nitrites and free nitrogen produced from nitrates.

Blood serum liquefied.

Acid and gas from carbohydrates (Lehmann and Neumann, loc. cit.). Gas from glucose, lactose and sucrose, 20 to 30 per cent of it $\mathrm{CO}_{2}$ (Bergey). Inactive lactic acid produced and not more than a trace of acetylmethylcarbinol or 2,3 butylene glycol (Pederson and Breed, Jour. Bact., 16, 1928, 183).

Sodium formate broth: Gas produced (Breed). 
Acetylmethylcarbinol not produced by the Král culture (Breed).

Pigment formed at $37^{\circ} \mathrm{C}$. Pigment especially soluble in alcohol.

Optimum temperature $30^{\circ} \mathrm{C}$.

Aerobic.

Distinctive characters: It is not certain whether Breunig's original culture was a heavily pigmented strain of Serratia marcescens, or whether it was of the type described above. Cultures of both types have been widely distributed as the Kiel bacillus. Descriptions drawn up by Kruse (loc. cit.) and Lehmann and Jeumann (loc. cit.) in 1896 state that this bacterium produces visible gas, while Migula in 1900 gives a description which fits Serratia marcescens. Moreover, cultures obtained under this name from various laboratories in Europe and America are sometimes of one type and sometimes of the other. As the Král culture distributed as Bacillus ruber balticus is widely known and has now been shown to differ from Serratia marcescens in that it is a distinct rod in ordinary media, forms visible gas from carbohydrates and even more abundant gas from sodium formate media, the name Serratia kilensis is used here for the Král culture. Serratia kilensis is a distinct red like Serratia plymuthicum, but fails to produce acetylmethylcarbinol. This use of the name Serratia kilensis given here also accords with the description drawn up by Bergey for the first edition of the Manual based on the study of a culture which he obtained many years previously from Europe (Breed).

Source: From water at Kiel, Germany.

Habitat: Presumably widely distributed.

\section{Serratia piscatorum (Lehmann and} Neumann) Breed. (Microbe rouge de la sardine, Du Bois Saint-Sévrin, Ann. Inst. Past., 8, 1894, 155; Bacterium piscatorum Lehmann and Neumann, Bakt. Diag., 1 Aufl., 2, 1896, 263; Bacillus ruber sardinae Kruse, in Flügge, Die Mikroorganis- men, 3 Aufl., 2, 1896, 302; Bacterium ruber sardinae Chester, Ann. Rept. Del. Col. Agr. Exp. Sta., 9, 1897, 112; Bacillus sardinae Migula, Syst. d. Bakt., 2, 1900, 852; Bacillus piscatorus Chester, Man. Determ. Bact., 1901, 257.) From Latin piscatorum, of fishermen.

Short rods: 0.5 by 0.6 micron, occurring in pairs, sometimes in fours or (in broth) in long filaments. Actively motile. Gram-negative.

Gelatin colonies: Small, yellowish-gray becoming pink, very slimy. Carminered pellicle. Liquefaction.

Gelatin stab: Rapid liquefaction. Grayish pellicle which becomes red after 24 hours and later precipitates. Slimy.

Agar colonies: Dull, white to pinkish growth.

Broth: Rapid turbidity. Thick, slimy, white pellicle which later turns red. Purplish sediment. Liquid becomes pink and syrupy. In old cultures the broth is brown.

Potato: At $37^{\circ}$ to $39^{\circ} \mathrm{C}$, red pigment visible after 8 hours. At room temperatures growth is first white, slimy, later red.

Strong odor of trimethylamine.

Distinctive characters: Pigment soluble in alcohol, more soluble in water. Good pigment production at $37^{\circ} \mathrm{C}$. Sliminess.

Source: Isolated in 1893 from a box of oil-packed sardines at a canning-factory in France. Also found in the red pus from fishermen and sardine-factoryworkers suffering from felons. In these lesions, this organism is associated with an anaerobe, but by itself it is not pathogenic

Habitat: Presumably widely distributed.

Appendix: Serratia marcescens has frequently been described under other names, particularly where brilliantly pigmented cultures have been found. Some of these and other related species are listed below. It is known that white 
strains of these organisms occur in nature but these strains when found have probably been placed in non-chromogenic genera of the family Enterobacteriaceae.

Bacillus ruber Frank. (Frank, in Cohn, Beitr. z. Biol. d. Pflanz., 1, Heft 3, 1875, 181; not Bacillus ruber Zimmermann, Bakt. unserer Trink- u. Nutzwässer, Chemnitz, 1, 1890, 24; not Bacillus ruber Miquel, see Cent. f. Bakt., II Abt., 11, 1903, 402; Bacterium ruber Chester, Ann. Rept. Del. Col. Agr. Exp. Sta., 9, 1897, 113.) Grew in a warm place on rice cooked in chicken broth.

Bacillus subkiliensis Petrow. (Arb. bakt. Inst. Karlsruhe, 2, Heft 3, 1902, 273.) Dust contamination from air. Reported to resemble Bacillus kiliensis.

Bacterium aurescens Parr. (Proc. Soc. Exp. Biol. and Med., 35, 1937, 563). A reddish-brown organism. This and the reddish-orange organism described by Tittsler (Jour. Bact., 33, 1937, 450), which are regarded as pigmented variants of Escherichia coli, resemble the organisms in Serratia closely but do not liquefy gelatin. From water.

Serratia amylorubra (Hefferan) Bergey et al. (Bacillus amyloruber Hefferan, Cent. f. Bakt., II Abt., 11, 1903, 313; Erythrobacillus amyloruber Holland, Jour. Bact., 5, 1920, 217; Bergey et al., Manual, 1st ed., 1923, 90.) From Mississippi River water and buttermilk.

Serratia esseyana Combe. (Thèse, École de Méd. Univ. Besançon, 1934, 1.) From well water at Essey. A study of an authentic culture shows this to be Serratia marcescens (Breed).

Serratia fuchsina (Boekhout and De Vries) Bergey et al. (Bacillus fuchsinus Boekhout and DeVries, Cent. f. Bakt., II Abt., 4, 1898, 497; Erythrobacillus fuchsinus Holland, Jour. Bact., 5, 1920, 218; Bergey et al., Manual, 1st ed., 1923, 91.) Bacillus fuchsinus Migula. (Der rote Bacillus, Lustig, Diag. d. Bakterien d. Wassers, 1893, 72; Migula, Syst. d. Bakt., 2, 1900, 853.) Although these two organisms were named independently from different cultures, they were undoubtedly identical. The original cultures of these species appear to have been heavy pigmented strains of Serratia marcescens showing a metallic luster. No authentic cultures are available. From water.

Serratia gutturis Jan. (Bull. Soc. Sci. de Bretagne, 16, 1939, 34.) From sputum. Claimed to be different from Serratia marcescens on the ground that it will grow on an asparagine medium and that it reduces molybdates actively.

Serratia marinorubra Zobell and Upham. (Bull. Scripps Inst. Oceanography, LaJolla, 5, 1944, 255.) From sea water. Grew only on sea water media when first isolated but later a culture studied by Breed (1944) became adapted to growth on ordinary media and then showed the characteristics of Serratia marcescens.

Serratia miniacea (Zimmermann) Bergey et al. (Bacillus miniaceus Zimmermann, Die Bakterien unserer Trink- und Nutzwässer, Chemnitz, 1, 1890, 46; Erythrobacillus miniaceus Holland, Jeur. Bact., 5, 1920, 219; Bergey et al., Manual, 1st ed., 1923, 90.) Probably a heavily pigmented strain of Serratia marcescens or Serratia plymuthicum showing metallic luster. From water.

Serratia pyoseptica (Fortineau) Bergey et al. (Erythrobacillus pyosepticus Fortineau, Thesis, Faculty of Medicine, Paris, 1904; abstract in Bull. Inst. Pasteur, 3, 1905, 13; Bergey et al., Manual, 1st ed., 1923, 89.) No constant differences have been detected between Serratia marcescens and authentic cultures of Serratia pyoseptica. From the shirt of a hospital patient. Pathogenic for guinea pigs and birds. Forms a soluble toxin.

Serratia rubidaea Stapp. (Bacterium rubidaeum Stapp, Cent. f. Bakt., II Abt., 102, 1940, 251; ibid., 259.) From surface of plants and in composts. Characters much like those of Serratia marcescens. 
Serratia rutilescens (Hefferan) Bergey et al. (Bacillus rutilescens Hefferan, Cent. f. Bakt., II Abt., 11, 1903, 313; Erythrobacillus rutilescens Holland, Jour. Bact., 5, 1920, 220; Bergey et al., Manual, 1st ed., 1923, 91.) The characters given do not distinguish this species from strains of Serratia marcescens that have nearly lost their power of pigment production except that it is reported to grow rapidly at $37^{\circ} \mathrm{C}$. No authentic cultures appear to be available. From Mississippi River water.

Serratia rutilis (Hefferan) Bergey et al. (Bacillus rutilis Hefferan, Cent. f. Bakt., II Abt., 11, 1903, 313; Erythro- bacillus rutilis Holland, Jour. Bact., 5, 1920, 220 ; Bergey et al., 1st ed., 1923, 94.) The original of this species appears to have been a heavily pigmented strain of Serratia marcescens or of Serratia plymuthicum. No characters are given that distinguish it from these species and no cultures appear to be available. From Illinois River water.

Serratia stercoraria Jan. (Bull. Soc. Sci. de Bretagne, 16, 1939, 34.) From feces. Claimed to be different from Serratia marcescens because it attacks lactose, maltose and mannitol and reduces molybdates even more actively than Serratia gutturis. 


\section{TRIBE IV. PROTEAE CASTELLANI AND CHALMERS.}

(Manual of Trop. Med., 3rd ed., 1919, 932.)

Ferments glucose but not lactose with formation of acid and usually visible gas. There is a single genus.

\section{Genus I. Proteus Hauser.*}

(Hauser, Sitzber. d. phys.-med. Sozietät zu Erlangen, 1885, 156; Liquidobacterium Jensen, Cent. f. Bakt., II Abt., 22, 1909, 337; Spirilina Hueppe, Wiesbaden, 1886 , 146 ; Eisenbergia Enderlein, Sitzber. Ges. Naturf. Freunde, Berlin, 1917, 315.) From Latin, having a changeable form.

Straight rods. Gram-negative. Generally actively motile at $25^{\circ} \mathrm{C}$, motility may be weak or absent at $37^{\circ} \mathrm{C}$, peritrichous, occasionally very numerous flagella. Generally produce amoeboid colonies, swarming phenomenon, on moist medium. Marked pleomorphism characteristic only of very young, actively swarming cultures. Ferment glucose and usually sucrose but not lactose. Three species in fermentable carbohydrates produce small gas volumes even after prolonged incubation and an occasional culture does not produce gas. One species usually produces acid only. Urea decomposed and trimethylamine oxide reduced by all species.

The type species is Proteus vulgaris Hauser.

\section{Key to the species of genus Proteus.}

I. No action on mannitol.

A. Acid and gas from sucrose.

1. Acid and gas from maltose.

a. Indole formed.

1. Proteus vulgaris.

B. Acid and gas from sucrose (delayed).

1. No action on maltose.

a. Indole not formed.

2. Proleus mirabilis.

C. No action on sucrose (ordinarily).

1. No action on maltose.

a. Indole formed.

3. Proteus morganii.

II. Acid, occasionally a bubble of gas, from mannitol.

A. Acid from sucrose (delayed).

1. No action on maltose.

a. Indole formed.

4. Proteus rettgeri.

1. Proteus vulgaris Hauser. (Hauser, Sitzungsber. d. phys.-mediz. Sozietät zu Erlangen, 1885, 156; Bacillus proteus
Trevisan, I generi e le specie delle Batteriacee, 1889, 17; Bacterium vulgare Lehmann and Neumann, Bakt. Diag.,

* Originally revised by Prof. M. W. Yale, New York State Experiment Station, Geneva, New York, Nov, 1938; revised by Prof. C. A. Stuart and Dr. Robert Rustigian, Brown University, Providence, Rhode Island, May, 1943. 
1 Aufl., 2, 1896, 243; Bacillus proteus rulgaris Kruse, in Flügge, Die Mikroorganismen, 2, 1896, 272; Bacterium (Proteus) vulgaris Chester, Ann. Rept. Del. Col. Agr. Exp. Sta., 9, 1897, 101 Bacillus rulgaris Migula, Syst. d. Bakt., 2, 1900, 707; Bacterium proteus anindologenes van Loghem, Ann. Inst. Past. 32, 1918, 295; Bacillus proteus-vulgaris Holland, Jour. Bact., 5, 1920, 220.) From Latin, common.

Hauser described Proteus vulgaris as a rapid gelat in liquefier and Proteus mirabilis as a slow liquefier. Wenner and Rettger (Jour. Bact., 4, 1919, 332) found the property of liquefying gelatin too variable to serve as a basis for separation of species. They suggested that this differentiating character be set aside and the two species differentiated on the basis of maltose fermentation, the species fermenting the sugar receiving the name Proteus vulgaris and the species failing to attack it, Proteus mirabilis. This suggestion was accepted by Bergey et al., Manual, 1st ed., 1923 and Weldin, Iowa Jour. Sci., 1, 1927, 147; and their work was confirmed by Rustigian and Stuart (Jour. Bact., 45, 1913, 198) and by Thornton (Jour. Bact., 48, 1944, 123). Also see Moltke (Contributions to the Characterization and Systematic Classification of Bac. proteus vulgaris (Hauser), Levin and Munksgaard, Copenhagen, 1927, 156 ).

Rods: 0.5 to 1.0 by 1.0 to 3.0 microns, occurring singly, in pairs and frequently in long chains. Actively motile, with peritrichous flagella. Gram-negative

Gelatin colonies: Irregular, spreading, rapidly liquefying.

Gelatin stab: Rapid, stratiform liquefaction.

Agar colonies: Opaque, gray, spreading.

Agar slant: Thin, bluish-gray, spreading over entire surface.

Broth: Marked turbidity, usually with a thin pellicle.

Litmus milk: Slightly acid, becoming markedly alkaline. Quich peptonization.

Potato: Abundant, ereamy to yellowish-gray growth, becoming brown.

Indole formed.

Nitrites produced from nitrates.

Acetylmethylcarbinol not formed.

Acid and gas from glucose, fructose, galactose, maltose and sucrose. No acid or gas from dextrin, lactose or mannitol. See Moltke (loc. cit.) for other fermentation characters. Ratio $\mathrm{H}_{2}$ to $\mathrm{CO}_{2}$ is 1:1 (Speck and Stark, Jour. Bact., 44, 1912, 687).

Putrefactive odor produced.

Sodium citrate usually utilized as sole source of carbon.

Formation of $\mathrm{H}_{2} \mathrm{~s}$ : Produced from cysteine, cystine or organic sulfur compounds containing either of these molecules. Produced from sulfur and thiosulfates (Tarr, Biochem. Jour., 27, 1933, 1869; 28, 1934, 192). Lead acetate turned brown.

Aerobic, facultative.

Optimum temperature $37^{\circ} \mathrm{C}$.

Distinctive characters: X-Strains of Weil and Felix. Lehmann-NeumannBreed, Determinative Bact., Eng. Trans., 7 th ed., 2, 1931, 493: "The discovery of proteus strains which may be agglutinated by typhus serum is of very great importance. These are the so-called $\mathrm{X}$-strains from typhus patients found by Weil and Felix. They first cultivated strains $\mathrm{X}_{\text {and }} \mathrm{X}_{2}$ from the urine of typhus patients and later the famous $\mathrm{X}_{19}$. The two former were agglutinated weakly, the latter strongly (up to $1: 50,000$ ). The diagnosis of typhus by agglutination with strain $\mathrm{X}_{19}$ proved to be excellent and the reaction tock place in the serum of almost 100 per cent of those suffering from the disease... The typhus strains of proteus have recently been divided into the two types of Felix and Weil, the $H$ forms and the $\mathrm{O}$ forms. The former grows as a thin opaque film, the latter lacks this character and grows as non-spreading slimy colonies; frequently without dis- 
tinct flagella...." (For further description of $\mathrm{H}$ and $\mathrm{O}$ forms see Moltke, loc. cit.)

The $\mathrm{X}_{2}$ and $\mathrm{X}_{19}$ strains mostly ferment maltose.

Source: From putrid meat, infusions and abscesses.

Habitat: Putrefying materials.

2. Proteus mirabilis Hauser. (Hauser, Sitzungsber. d. phys.-mediz. Sozietät zu Erlangen, 1885, 156; Bacillus mirabilis Trevisan, I generi e le specie delle Batteriacee, 1889, 17 ; Bacillus proteus mirabilis Kruse, in Flügge, Die Mikroorganismen, 3 Aufl., 2, 1896, 276; Bacterium mirabilis Chester, Del. Coll. Agr. Exp. Sta., 9th Ann. Rept., 1897, 101; Bacillus pseudoramosus Migula, Syst. d. Bakt., 2, 1900, 817; not Bacillus pseudoramosus Distaso, Cent. f. Bakt., I Abt., Orig., 62, 1912, 441; Bacillus proteus-mirabilis Holland, Jour. Bact., 5, 1920, 220.) From Latin mirabilis, wonderful.

Short rods: 0.5 to 0.6 by 1.0 to 3.0 microns, occurring singly, in pairs and frequently in long chains. Motile, possessing peritrichous flagella. Gram-negative.

Gelatin colonies: Irregular, spreading.

Gelatin stab: Slow, stratiform liquefaction.

Agar colonies: Gray, irregular, spreading.

Agar slant: Thin, bluish-gray, spreading over surface.

Broth: Turbid, with thin gray pellicle and sediment.

Litmus milk: Slightly acid, becoming alkaline, peptonized.

Potato: Dirty-gray, spreading growth. Indole not formed.

Acetylmethylcarbinol frequently produced weakly.

Nitrites produced from nitrates.

Acid and gas from glucose, fructose and galactose. Acid and gas usually produced slowly from sucrose. No acid or gas from lactose, maltose, dextrin or mannitol.
The XK strains are mostly maltose negative.

Putrefactive odor produced.

Hydrogen sulfide is produced.

Sodium citrate usually utilized as a sole source of carbon.

Aerobic, facultative.

Optimum temperature $37^{\circ} \mathrm{C}$.

Source: From putrid meat, infusions and abscesses. Also reported as a cause of gastroenteritis (Cherry and Barnes, Amer. Jour. Pub. Health, 36, 1946, 484).

Habitat: Putrefying materials.

3. Proteus morganii (Winslow et al.) Rauss. (Organism No. 1, Morgan, Brit. Med. Jour., 1, 1906, 908; Bacillus morgani Winslow, Kligler and Rothberg, Jour. Bact., 4, 1919, 481; Bacterium morgani Holland, Jour. Bact., 5, 1920, 215; Bacterium metacoli or Escherichia morgani Thjøtta, Jour. Inf. Dis., 43, 1928, 349; Salmonella morgani Castellani and Chalmers, Man. Trop. Med., 1919, 939 ; Rauss, Jour. Path. and Bact., 42, 1936, 183; Morganella morganii Fulton, Jour. Bact., $46,1943,81$; regarded by Fulton as the type species of the genus Morganella.) Named for Morgan, who first isolated this organism.

Common name: Morgan's bacillus, type 1.

Rods : 0.4 to 0.6 by 1.0 to 2.0 microns, occurring singly. Motile with peritrichous flagella. See Rauss, loc. cit., for discussion of flagellation and relation to the swarming characteristic. Gramnegative.

Gelatin colonies: Bluish-gray, homogeneous, smooth, entire.

Gelatin stab: No liquefaction.

Agar colonies: Grayish or bluish-white, circular, entire.

Agar slant: Grayish-white, smooth, glistening growth.

Broth: Turbid.

Litmus milk: Neutral, or becoming alkaline.

Potato: Dirty-white, limited growth. 
Indole is formed.

Nitrites are produced from nitrates. Acetylmethylcarbinol not formed.

Acid and a small amount of gas from glucose, fructose, galactose and mannose. Rarely from xylose. Does not attack lactose, sucrose, maltose, arabinose, raffinose, dextrin, salicin, mannitol, dulcitol, sorbitol, adonitol or inositol.

Hydrogen sulfide not produced.

Sodium citrate not utilized as sole source of carbon.

Aerobic, facultative.

Optimum temperature $37^{\circ} \mathrm{C}$.

Source: Isolated from the feces of infants with summer diarrhea.

Habitat: In intestinal canal in normal or diarrheal stools.

\section{Proteus rettgeri (Hadley et al.)} Pustigian and Stuart. (Bacterium rettgeri Hadley, Elkins and Caldwell, Rhode Island Agr. Exp. Sta. Bull. 174, 1918, 169; Bacillus rettgeri St. John-Brooks and Rhodes, Jour. Path. and Bact., 26, 1923, 434; Eberthella rettgeri Bergey et al., Manual, 1st ed., 1923, 232; Shigella rettgeri Weldin, Iowa State College Jour. Sci., 1, 1927, 181; Atypical enteric organisms of the Shigella group, Cope and Kilander, Amer. Jour. Pub. Health, 32, 1942, 352; Proteus entericus Rustigian and Stuart, Jour. Bact., 45, 1943, 198; Rustigian and Stuart, Proc. Soc. Exp. Biol. and Med., 53, 1943, 241.) Named for L. F. Rettger, the American bacteriologist, who isolated this species in 1904.

Rods : 0.5 to 0.8 micron long, occurring singly, in pairs and oceasionally in chains. Usually non-motile at $37^{\circ} \mathrm{C}$, but actively motile variants possessing peritrichous flagella can be obtained at $25^{\circ} \mathrm{C}$. Gramnegative.

Gelatin colonies : Small, grayish, translucent, entire.

Gelatin stab: No liquefaction.

Agar colonies: Small, grayish, translucent, entire; under suitable conditions some strains show marked spreading.
Agar slant: Filiform to echinulate, grayish, thin, moist, translucent.

Broth: Turbid with flocculent to viscid sediment.

Litmus milk: Alkaline in eight days, becoming translucent.

Potato: Luxuriant, grayish growth.

Acid and occasionally slight gas from glucose, fructose, galactose and mannitol. Salicin may or may not be fermented. Slow and sometimes weak acid in sucrose. Lactose and maltose not fermented.

Indole is formed.

Nitrites are produced from nitrates. Acetylmethylcarbinol not formed.

Hydrogen sulfide not produced.

Sodium citrate utilized as sole source of carbon.

Aerobic, facultative.

Optimum temperature $37^{\circ} \mathrm{C}$.

Source: Originally isolated from cholera-like epidemic among chickens; recently isolated from sporadic and epidemic gastroenteritis patients.

Habitat: Fowl typhoid and some cholera-like diseases of birds.

Appendix: Acceptance of gelatin liquefaction and fermentation of glucose and sucrose but not lactose as the cardinal characteristics of Proteus without reference to urease production and small gas volumes has resulted in some cultures of Paracolobactrum (Borman et al., Jour. Bact., 48, 1944, 361) being described as Proteus (Rustigian and Stuart, Jour. Bact., 49, 1945, 419). Included in the appendix are species of Proteus whose taxonomic position is not clear. Where descriptions permit, the probable taxonomic position of the organism is indicated. For purposes of reference, organisms are also included which do not now merit species rank in the genus Proteus and organisms which will now be found in another genus.

Bacillus agglomerans Beijerinck. (Botan. Zeitung, 46, 1888, 740 or 749. ) From nodules on the roots of red clover. Colonies like those of Proteus. 
Bacillus murisepticus pleomorphus Karlinski. (Karlinski, Cent. f. Bakt.. 5, 1889, 193; Proteus of Karlinski, Sternberg, Man. of Bact., 1893, 460.) From a urine discharge and from abscesses in the uterus. Sternberg regards this species as probably identical with Proteus vulgaris Hauser.

Flarobacterium meningitidis Hauduroy et al. (Bacillus luteus liquefaciens Hau duroy, Duhamel, Ehringer and Mondin, Compt. rend. Soc. Biol., Paris, 110, 1932, 362 ; Hauduroy et al.. Dicl. d. Bact. Path., 1937, 236.) Related to this species but differing in that it ferments lactose is the following: Bacterium coli var. luteoliquefuciens Lehmann and Levy, in Lehmann and Neumann, Bakt. Diag., 4 Aufl., 2, 1907, 34 (Bacillus coli var. luteoliquefaciens Hauduroy, Duhamel, Ehringer and Mondin, loc. cit., 1932, 363).

Proteus alveicola Serbinow. (Jour. Microbiol., Petrograd, 2, 1915, 19.) From an infectious diarrhoea of honey bees (Apis mellifera).

Proteus americanus Pacheco. (Scien. cia Medica, 6, 1928.) From the blood of patients with liver abscesses. Assis (Brasil Medico, No. 42-45, 1934, 35), St. John-Brooks and Rhodes (3rd Internat. Cong. for Microbiology, Rept. of Proc., 1939, 167), Rustigian and Stuart (Jour. Bact., 45, 1943, 198) and Thornton (Jour. Bact., 48, 1944, 123) agree that Proteus americanus is Proteus mirabilis. See Manual, 5th ed., 1939, 434 for a description of this species.

Proteus ammoniae Magath. (Magath, Jour. Inf. Dis., 43, 1928, 181 ; Salmonella ammoniae Hager and Magath, Jour. Amer. Med. Assn., 85, 1925, 1352.) From urine in cystitis. St. John-Brooks and Rhodes (3rd Internat. Congr. for Microbiology, Rept. of Proc., 1939, 167), Levine (Jour. Bact., 43, 1942, 33), Rustigian and Stuart (Jour. Bact., 45, 1943, 198) and Thornton (Jour. Bact., 48, 1944, 123) agree that Proleus ammoniae is Proteus mirabilis. See Manual, 5th ed., 1939,
434 for a description of this species. See Fulton, Jour. Bact., 51, 1946, 685 for the view that Proteus ammoniae is a valid species.

Proteus bombycis Bergey et al. (A Gram-negative bacillus, Glaser, Jour. Bact., 9, 1924, 344; Bacterium bombycivorum Lehmann and Neumann, Bakt. Diag., 7 Aufl., 2, 1927, 445; Aerobacter bombycis Bergey et al., Manual, 3rd ed., 1930, 334; Bergey et al., Manual, 4th ed., 1934, 365.) From diseased silk worms (Bombyx mori). Proteus bombycis appears to be a strain of Paracolobactrum aerogenoides Borman et al. See Manual, 5 th ed., 1939, 436 for a description of this species.

Proteus diftuens (Castellani) Castellani and Chalmers. (Bacillus diffluens Castellani, 1915; Castellani and Chalmers, Man. Trop. Med., 3rd ed., 1919, 943.) From gastroenteritis patients. This may be a biochemical variant of Proteus mirabilis.

Proteus henricensis Shaw. (Sci., 65, 1927, 477.) From putrefying materials. Said to be related to Proteus diffuens.

Proteus infantum (Weldin and Levine) Weldin. (Dean, Med. Jour. Australia, 1, 1920, 27; Bacterium infantum Weldin and Levine, Abst. Bact., 7, 1923, 13; Weldin, Iowa State Coll. Jour. Sci., 1, 1926,148 .) From urine and feces of an infant.

Proteus insecticolens Steinhaus. (Jour. Bact., 42, 1941, 763.) From the stomach of the milkweed bug (Oncopeltus fasciatus). This appears to be a strain of Paiacolobactrum intermedium Borman et al.

Proteus melanovogenes Miles and Halnan. (Jour. Hyg., 37, 1937, 79.) From eggs showing black rot. This does not appear to be a member of the genus Proteus.

Proteus metadifluens (Castellani) Castellani and Chalmers. (Bacillus metadiffuens Castellani, 1915; Castellani and Chalmers, Manual Trop. Med., 1919. 943.) From gastroenteritis patients, 
This does not appear to be a member of the genus Proteus.

Proteus nadsonii Lobik. (Diseases of Plants, St. Petersburg, 9, 1915, 67.) From decomposed potatoes and tomatoes. This does not appear to be a member of the genus Proteus.

Proteus noctuarum (White) Bergey et al. (Bacillus noctuarum White, Jour. Agr. Res., 26, 1923, 488; Escherichia noctuarii Bergey et al., Manual, 3rd ed., 1930, 327; Bergey et al., Manual, 4th ed., 1934, 363.) A cause of cutworm (Fam. Noctuidae) septicemia. Culturally identical with but serologically different from Proteus sphingidis.

Proteus odorans Pribram. (Bacterium. aquatile odorans von Rigler, Hyg. Rund., 12, 1902, 479; Pribram, Klassifikation der Schizomyceten, Leipzig and Wien, 1933, 73.) From bottled mineral waters. Aromatic odor in milk.

Proteus paraamericanus Magalhães and Aragão. (Brasil Medico, 47, 1933, 815.) From urine. Assis (Brasil Medico, No. 42-45, 1934, 35) states that this is Proteus mirabilis.

Proteus paradiffuens (Castellani) Castellani and Chalmers. (Bacillus paradiffuens Castellani; Castellani and Chalmers, Manual Trop. Med., 3rd ed., 1919, 943.) This appears to be identical with Proteus mirabilis.

Proteus paramorganii Castellani and Chalmers. (Man. Trop. Med., 3rd ed., 1919, 943.) This is an $\mathrm{H}$ form of Proteus morganii.

Proteus photuris Brown. (Amer. Museum Nov., No. 251, 1927, 9.) From luminous organ of the firefly (Photuris pennsylvanicus). This does not appear to be a member of the genus Proteus.

Proteus piscicidus versicolor Babes and Riegler. (Babes and Riegler, Cent. f. Bakt., I Abt., Orig., 33, 1902-03, 449; Bacillus piscicidus versicolor Nepveux, Thèse, Fac. Pharm., Paris, 1920, 114.) From diseased carp (Cyprinus carpio). Resembles Proteus vulgaris.

Proteus pseudovaleriei Assis. (Jour.
Hyg., 27, 1927, 108.) Rustigian and Stuart (Proc. Soc. Exper. Biol. and Med., $53,1943,241)$ state that this is a paracolon organism, presumably Paracolobactrum coliforme Borman et al. See Manual, 5th ed., 1939, 435 for a description of this species.

Proteus recticolens Steinhaus. (Jour. Bact., 42, 1941, 763.) From pylorus and rectum of the milkweed bug (Oncopeltus fasciatus). This appears to be a strain of Paracolobactrum intermedium Borman et al.

Proteus sphingidis (White) Bergey et al. (Bacillus sphingidis White, Jour. Agr. Res., 26, 1923, 49; Escherichia sphingidis Bergey et al., Manual, 3rd ed., 1930, 327; Bergey et al., Manual, 4th ed., 1934, 366.) A cause of hornworm septicemia (Protoparce sexta Johan. and $P$. quinquemaculata Haw.). See Manual, 5th ed., 1939, 605 for a description of this species. White (loc. cit.) regards this species as possibly identical with Coccobacillus acridiorum d'Herelle.

Proteus sulfureus Holschewnikoff. (Holschewnikoff, Fortschr. d. Med., 7, 1889, 201 and Ann. de Microgr., 1, 18881889, 257; Bacillus lindenborni Trevisan, I generi e le specie delle Batteriacee, 1889, 17; Bacillus sulfureus Migula, Syst. d. Bakt., 2, 1900, 698; not Bacillus sulfureus Trevisan, I generi e le specie delle Batteriacee, 1889, 17.) From water. Similar to or perhaps identical with Proleus vulgaris Hauser. Produces $\mathrm{H}_{2} \mathrm{~S}$.

Proteus $s p$. Steinhaus. (Jour. Bact., 42, 1941, 764.) This organism appears to be a strain of Paracolobactrum intermedium Borman et al.

Proteus sp. Warren and Lamb. (Jour. Med. Res., 44, 1924, 375.) From feces and blood of patient with a fatal infection. This organism does not appear to be a member of the genus Proteus.

Urobacillus liquefaciens septicus Krogius. (Compt. rend. Soc. Biol, Paris, 2, 1890, 65.) Regarded by Lehmann and Neumann (Bakt. Diag., 1 Aufl., 2, 1896, 243) as a synonym of Proteus vulgaris. 
TRIBE V. SALMONELLEAE BERGEY, BREED AND MURRAY.

(Preprint, Manual, 5th ed., October, 1938, vi.)

Rods that are either motile with peritrichous flagella or non-motile. Attack numerous carbohydrates with the formation of acid, or acid and gas. Lactose, sucrose and salicin are not ordinarily attacked. Do not produce acetylmethylcarbinol. Gelatin not liquefied (exceptions have been noted, but are rare). Urea not hydrolyzed. Milk not peptonized. No spreading grow th on ordinary 2 to 3 per cent agar. Live in the bodies of warm-blooded animals, including man, occasionally in reptiles, and frequently in food eaten by these animals.

Key to the genera of tribe Salmonelleae.

I. Ferments glucose with the formation of acid and, with few exceptions, gas.

Genus I. Salmonella, p. 492.

II. Ferments glucose with the formation of acid but. with rare exceptions, no gas.

Genus II. Shigella, p. 535.

\section{Genus I. Salmonella Lignieres.*}

(Rec. de méd. vét., Sér. 8, 7, 1900, 389.)

Usually motile, but non-motile forms occur. Produce acid and gas from glucose, maltose, mannitol and sorbitol (except that in Salmonella typhosa and S. gallinarum no gas is produced). Lactose, sucrose and salicin not attacked. Do not clot milk, form indole or liquefy gelatin. Reduce trimethylamine oxide to trimethylamine. $\dagger$ All of the known species are pathogenic for warm-blooded animals, including man, causing food infections and enteric fevers. A few are found in reptiles. Some or all may also live in decomposing foods.

Although fermentation of lactose, sucrose and salicin, formation of indole, gelatin liquefaction and failure to produce gas have been deseribed for organisms serologically belonging to Salmonella, the practical recognition of this genus and studies of its constituent species suggest that these be looked upon as exceptions which do not invalidate the biochemical definition of the genus. Serological definition of the limits of the genus is fraught with many practical and theoretical difficulties. Indeed, there is increasing evidence of antigenic affinities of varying degree between Escherichia, Salmonella and Shigella. This is well reviewed by Bornstein (Jour. Immunol., 46, 1943, 439). Within the limits of the genus Salmonella, serological rela-

* Completely revised by Prof. Frederick Smith, McGill Univ., Montreal, P. Q., Canada, December, 1938; further revision, 1946. Manuscript read by Dr. F. Kauffmann, State Serum Institute, Copenhagen, Denmark and by Dr. Philip Edwards and Dr. D. W. Bruner, Agri. Exper. Sta., Lexington, Kentucky, May, 1946. These specialists have also assisted in completing references and in compiling records of the distribution of types.

$\dagger$ Wood and Baird, Jour. Fish. Res. Board Canada, 6, 1943, 194. 
tionships are the chief means of identifying new strains. There is general dissatisfaction with the granting of species rank to each one of the rapidly mounting number of types. The purposes of the greater number of bacteriologists, however, will be best served for the present by listing the known types.

There is a wide difference between the viewpoint of those who think of the serological types recognized in this genus as species, e.g., Schütze et al. (Jour. Hyg., 34, 1934, 333) and the more recently expressed viewpoint of Borman, Stuart and Wheeler (Jour. Bact., 48, 1944, 351). The latter authors recognize only three species in the genus, Salmonella choleraesuis, S. typhosa and S. kauffmannii. In the second report by Schütze et al. (Proc. 3rd Internat. Cong. Microbiol., New York, 1940, 832) the socalled species listed in the first report by this Sub-committee are designated as types.

Kauffmann, who recognizes nearly 150 serotypes in the group, nevertheless notes in a recent paper (Acta Path. et Microbiol. Scand., 22, 1945, 144) that five types are of special interest in the field of human medicine, Salmonella paratyphi $A$, Salmonella paratyphi B, Salmonella paratyphi $C$, Salmonella typhi and Salmonella sendai; and that six types are of special interest in the field of veterinary medicine, Salmonella typhimurium, Salmonella abortusequi, Salmonella abortusovis, Salmonella choleraesuis, Salmonella enteritidis and Salmonella gallinarum-pullorum.

The 150 or more serotypes are, in a way, comparable to the 50 or more serotypes of Diplococcus pneumoniae that are recognized on the basis of agglutination with immune serums. The serological methods used have proved to be of fundamental value as they provide useful diagnostic procedures by means of which unknown cultures can be accurately and quickly identified.

As the morphology, staining properties and physiology of the bacteria belonging to the various types are practically identical, only the antigenic structure, source and habitat (so far as the latter is known) have been recorded for the majority of the types listed. Even though there is much duplication, descriptions similar to those used elsewhere in the MANUAL are given for the eleven types that are of greatest interest. Special mention has also been made of unusual characters such as failure to produce gas from glucose, lactose fermentation, indole production and gelatin liquefaction.

The nomenclature used for this group presents a special problem. It developed from labelings used for cultures. These were designated by the name of a patient, e.g., Thompson; by the name of the hospital where the patient was placed, e.g., Virchow, Bispebjerg; or more frequently by the name of the village, locality or city where the outbreak occurred or was studied, e.g., Borbeck, Altendorf, Tel Aviv. The names of states and larger areas have also been used, e.g., Kentucky, Italia, etc. Recently several types have been named in honor of well-known bacteriologists, e.g., Berta, Gaminara, Arechavaleta. As this useful laboratory labeling is not in the form ordinarily used by taxonomists, various suggestions have been made regarding the development of a binomial nomenclature comparable to that more generally used. None of these suggestions has been generally accepted as yet. For example, Haupt (Ergebnisse d. Hyg., 13, 1932, 673) and others who have thought of the serotypes as species have added Latin endings to the place and other proper names that have been used, e.g., Salmonella readingensis, S. rostockensis. Schütze et al. (Jour. Hyg., 34, $1934,333)$ accepted the view that the place and other names should be used in binomials without adding Latin endings. Kauffmann (Ztschr. f. Hyg., 120, 1938, 193), on the other hand, has suggested that letters and numbers, e.g., Salmonella B2, or even (Acta Path. et Microbiol. Scand., 22, 1945, 147) the antigenic formula be used with the generic name, e.g., Salmonella IV, V, XII . . b b $\leftrightarrow 1,2 \ldots$ instead of Salmonella paratyphi $B$. 
The nomenclature used in the present edition of the MANUAL is slightly modified from that used in the fifth edition. The form adopted is in accordance with the view that the recognition of similar antigenic structures really identifies serotypes rather than species. In a way, serotypes are varieties in a taxonomic sense, though like horticultural varieties in higher plants, they do not exactly correspond with varieties as usually defined by taxonomists. Where cultural differences rather than antigenic structure have been used to subdivide species, these subdivisions are designated as varieties.

As it is not clear as yet how many and what species will eventually be recognized, the form Salmonella sp. has been used as before to indicate that the serotypes belong to species in the genus Salmonella which are not yet definitely defined. Geographic and other proper names are used to designate types as these have been used extensively in the literature. They have an historic significance and are not as easily confused as are letters and numbers. No Latin endings have been used for these place names as this might indicate that the serotype names are accepted as species names.

The genus Eberthella has been combined with the genus Salmonella as recommended by Schütze et al. (loc. cit.). With the exception of the typhoid organism, other species previously listed in Eberthella appear not to exist in type culture collections. As cultures are not available for study, these species are merely listed in an appendix to the genus Salmonella.

The type species is Salmonella choleraesuis (Smith) Weldin.

The table on pages 495 to 500 is used in place of the usual key. 


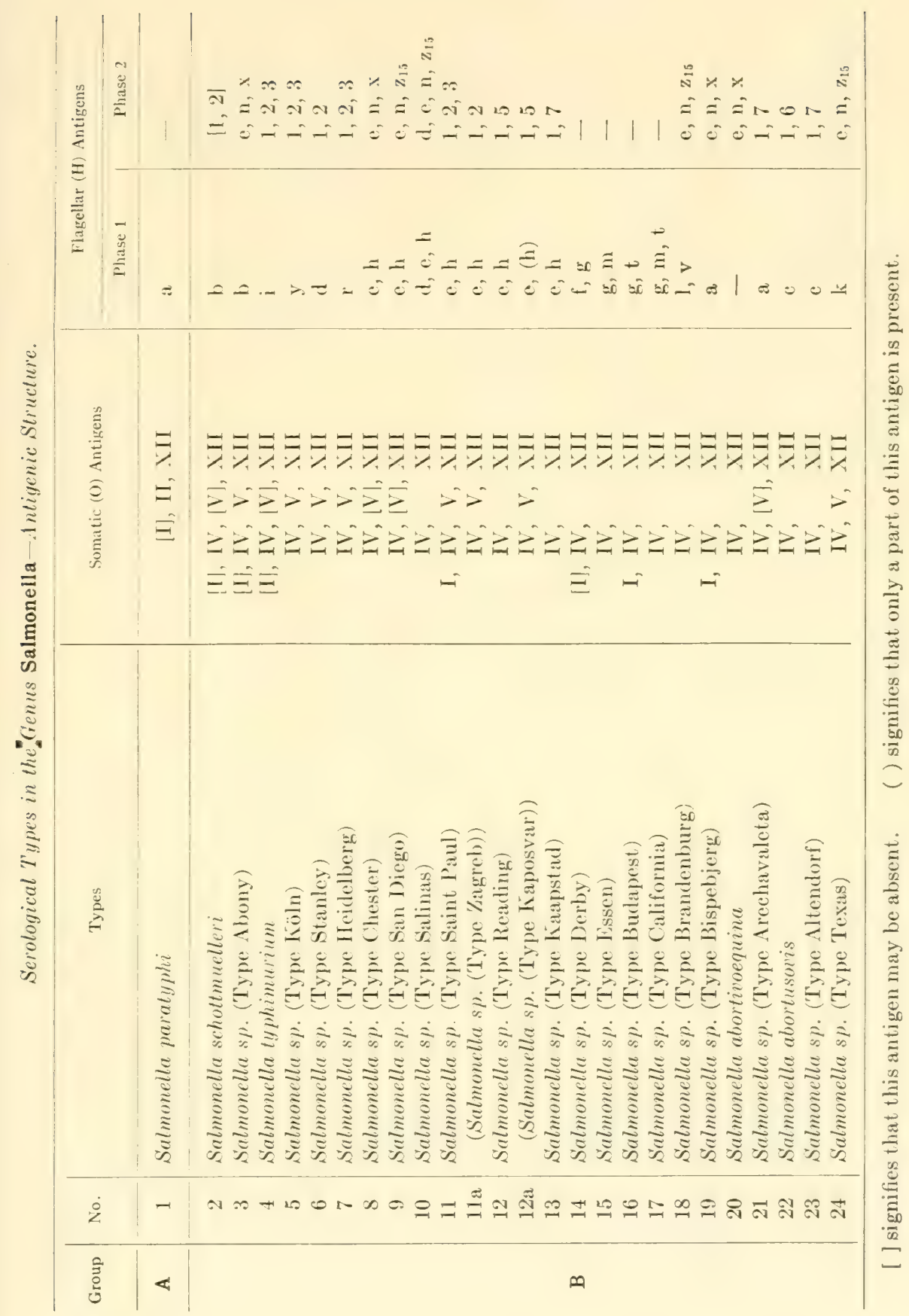




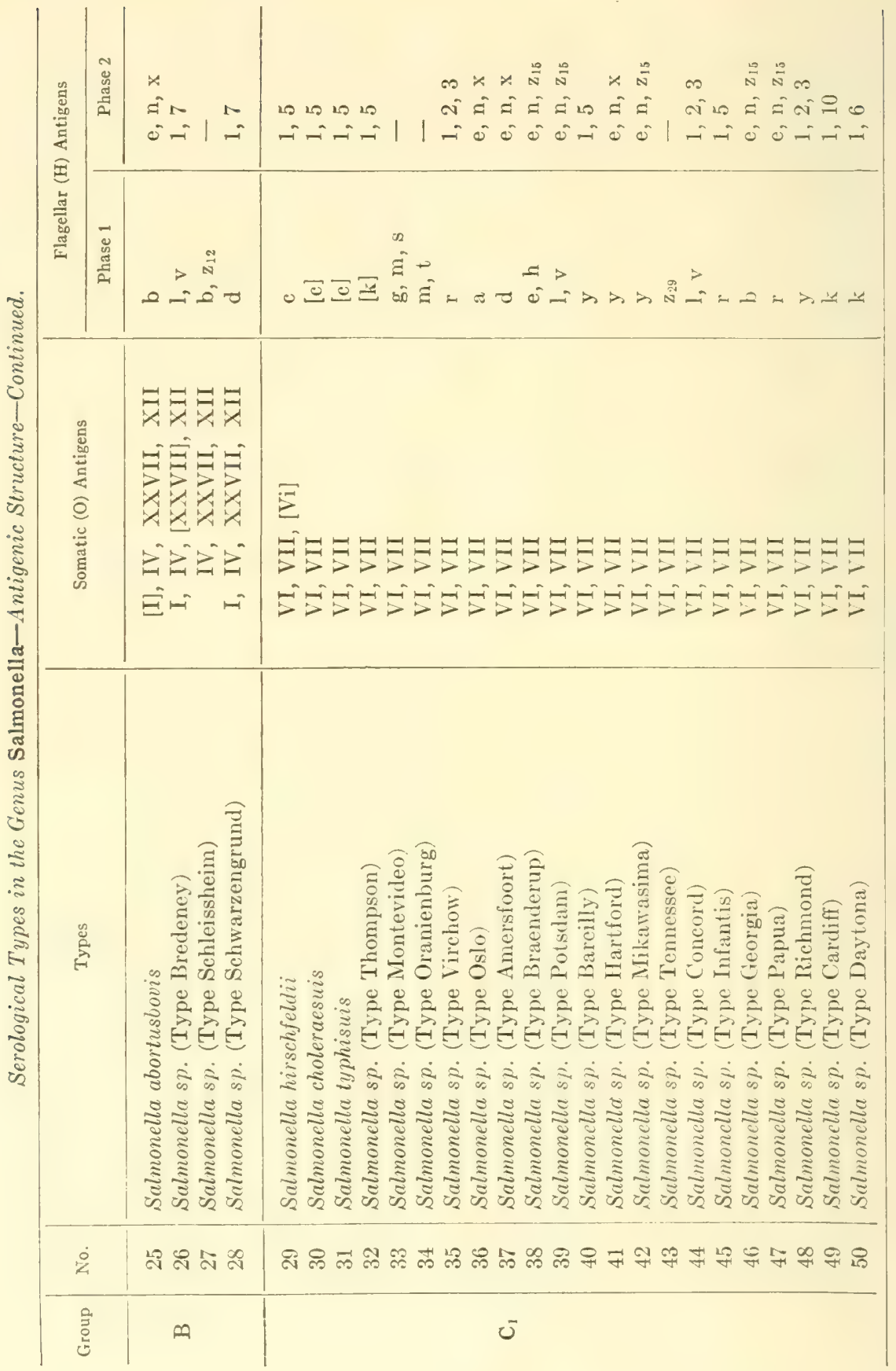




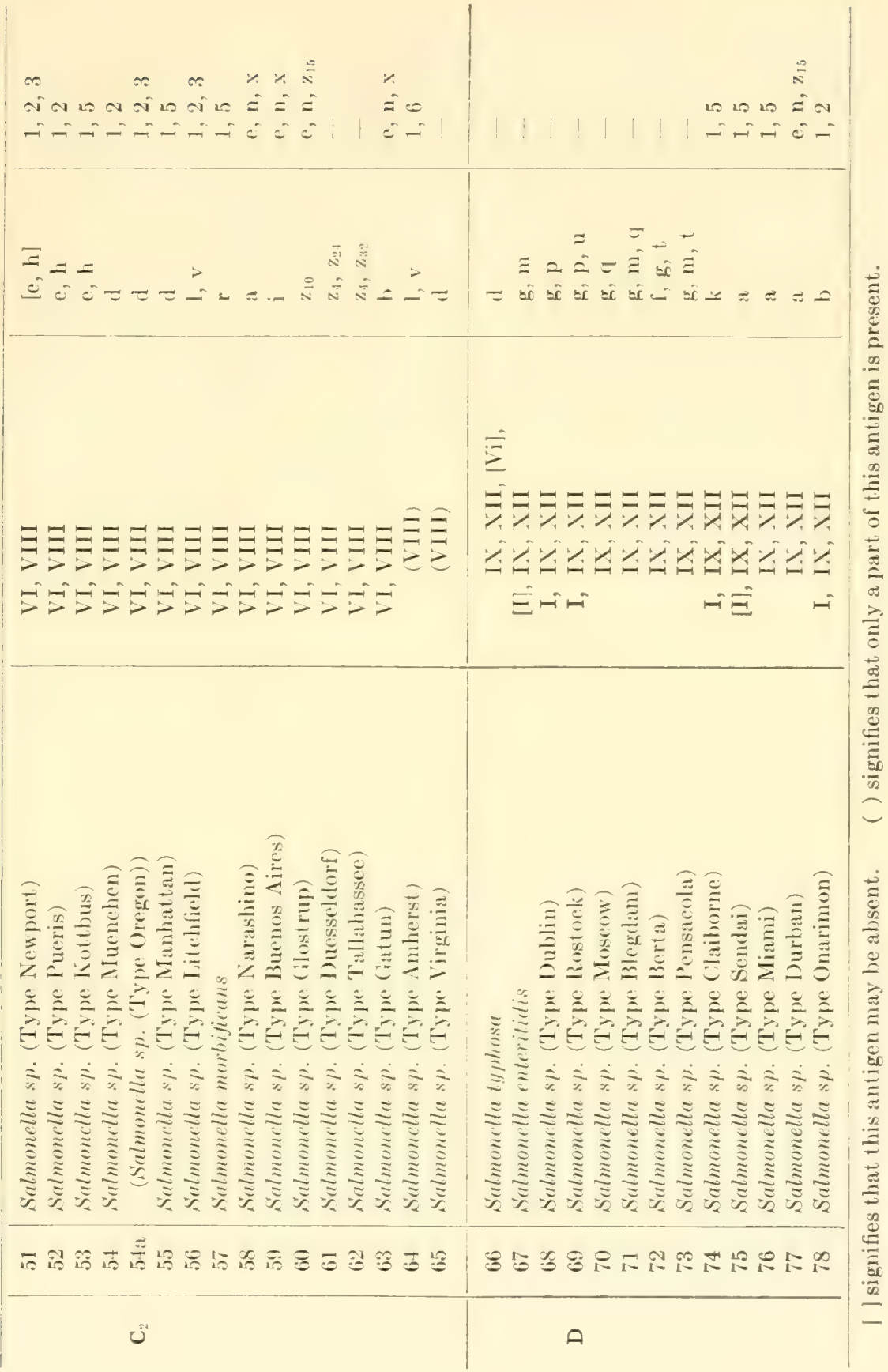




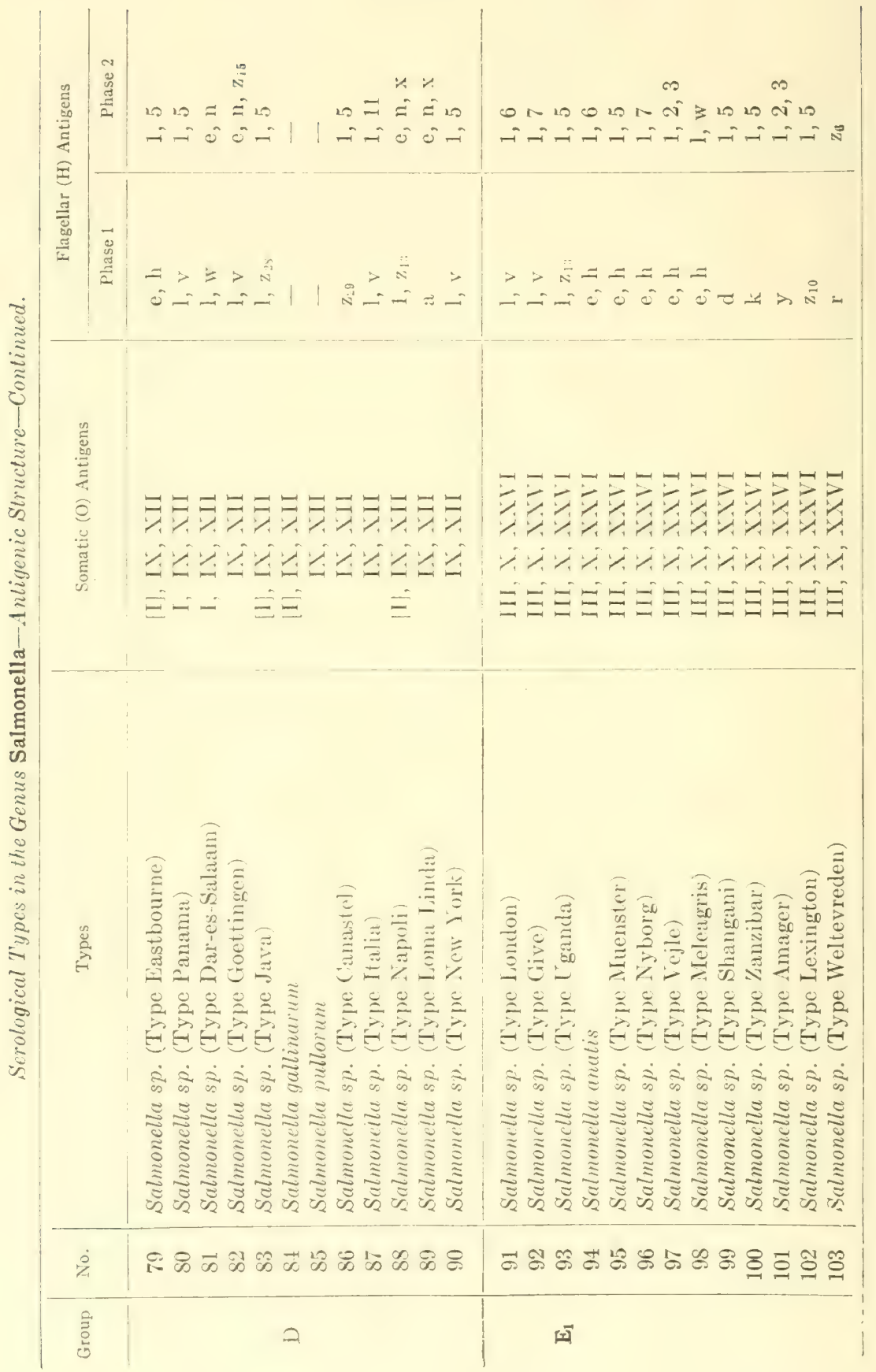




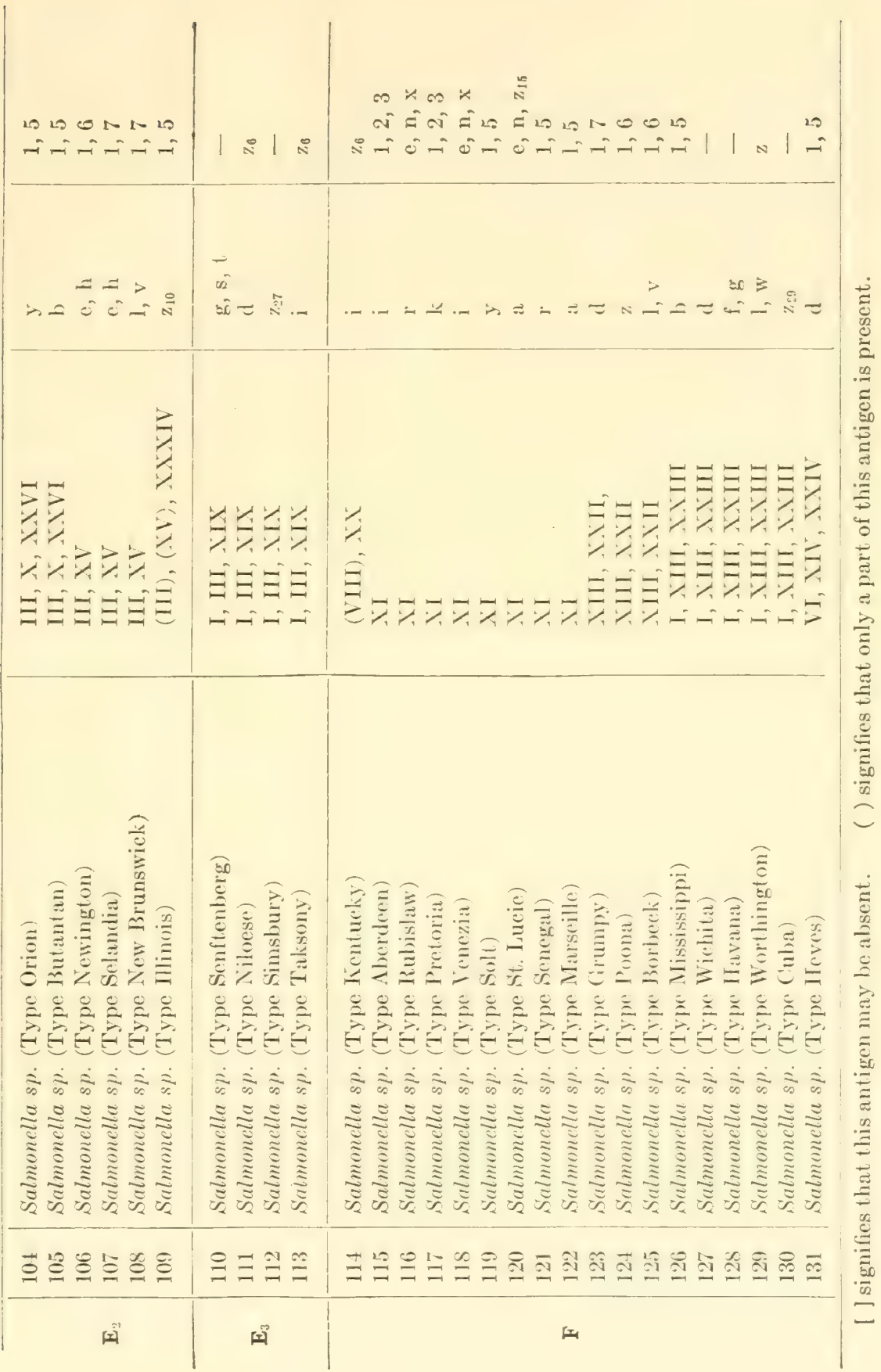




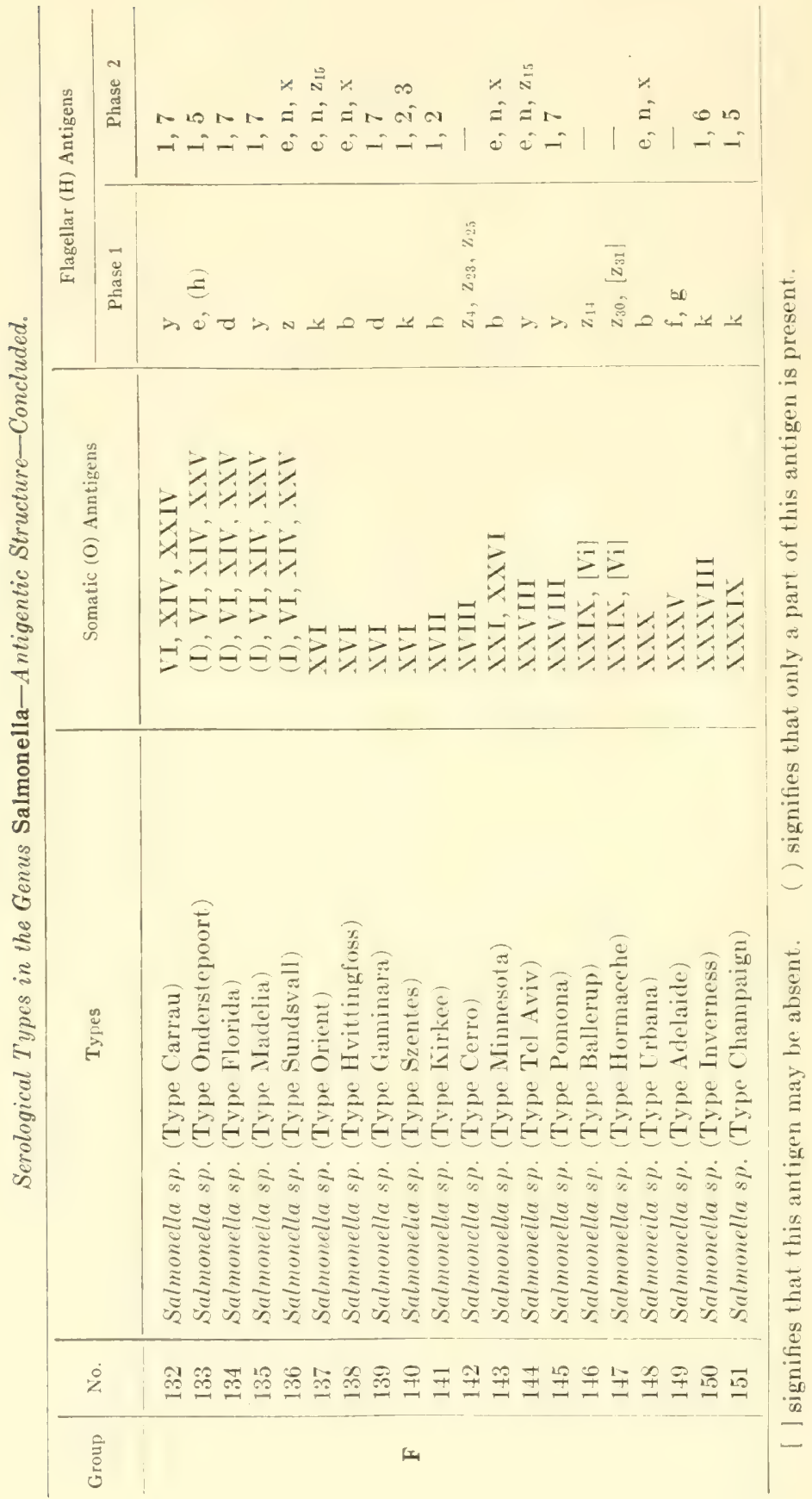


1. Salmonella paratyphi (Kayser) Castellani and Chalmers. (Bacterium paratyphi Typus A, Brion and Kayser, Münch. med. Wehnschr., 49, 1902, 611; Bacterium paratyphi Kayser, Cent. f. Bakt., I Abt., Orig., 31, 1902, 426; $\mathrm{Ba}$ cillus paratyphosus A Boycott, Jour. Hyg., 6, 1906, 33; Bacillus paratyphi Winslow and Kligler, Jour. Bact., 1, 1916, 81; Bacillus paratyphosus Winslow, Kligler and Rothberg, Jour. Bact., 4 , 1919, 474; Salmonella paratyphi and Salmonella paratyphi A Castellani and Chalmers, Man. Trop. Med., 3rd ed., 1919, 938 and 939; Bacterium paratyphosum A Holland, Jour. Bact., 5, 1920, 219.) From Latin para, like and typhus. typhoid.

Rods: 0.6 by 3.0 to 4.0 microns, oceurring singly. Motile with peritrichous flagella. Gram-negative.

Gelatin colonies: Bluish-gray, homogeneous, smooth, glistening, entire to slightly undulate.

Gelatin stab: Fair surface growth. No liquefaction.

Agar colonies: Grayish, homogeneous. smooth, glistening, entire to slightly undulate.

Agar slant: Filiform, grayish, smooth. glistening growth.

Broth: Turbid, with slight grayish sediment.

Litmus milk: Slightly acid.

Potato: Limited, dirty-white streak.

Indole not formed.

Nitrites produced from nitrates.

Acid and gas from glucose, fructose, galactose, mannose, arabinose, maltose, trehalose, dextrin, glycerol, mannitol, dulcitol, rhamnose and sorbitol. No acid or gas from lactose, suerose, raffinose, xylose, salicin, inulin, adonitol or inositol.

Reduces trimethylamine oxide (Wood and Baird, Jour. Fish. Res. Bd. Canada, $6,1943,198)$.

No hydrogen sulfide formed.

Aerobic, facultative.

Optimum temperature $37^{\circ} \mathrm{C}$.
Antigenic structure: [I], II, XII : a:(Type Durazzo lacks I).

Source: Isolated from enterie fever in man. Not known to be a natural pathogen of animals.

Habitat: A natural pathogen of man causing enteric fever.

2. Salmonella schottmuelleri (Winslow et al.) Bergey et al. (Bacilli paraty. phique, Achard and Bensaude, Soc. Méd. des Hôp. de Paris, 13, 1896, 679; Bacillus paratyphi alcaligenes Schottmüller, Deutsche med. Wchnschr., 32, 1900, 511; Bacterium paratyphi Typus B, Brion and Kayser, Münch. med. Wchnschr., 49, 1902, 611; Bacillus paratyphosus B Boycott, Jour. Hyg., 6, 1906 , 33; Bacterium paratyphosum B Le Blaye and Guggenheim. Manuel Pratique de Diag. Bact., 1914; Bacillus schottmülleri Winslow, Kligler and Rothberg, Jour. Bact., 4, 1919, 479; Salmonella paratyphi $B$ Castellani and Chalmers, Man. Trop. Ied., 3rd ed., 1919, 939; Bacterium schottmülleri Holland, Jour. Bact., 5, 1920. 222; included in Group IV of HechtJohansen, Copenhagen, 1923; Bergey et al., Manual, 1st ed., 1923, 213.) Named for Prof. Schottmüller who isolated this organism in 1899.

Ruds: 0.6 to 0.7 by 2.0 to 3.0 mierons, occurring singly and in pairs. Motile with peritrichous flagella. Gram-negative.

Gelatin stab: No liquefaction.

Agar colonies: Small, circular, bluishgray, transparent, homogeneous, entire to undulate.

Broth: Turbid with thin gray pellicle and sediment. Fecal odor.

Litmus milk: Slightly acid, becoming alkaline.

Potato: Grayish-white, viscous growth.

Indole not formed.

Nitrites produced from nitrates.

Acid and gas from glucose, fructose, galactose, mannose, arabinose, xylose, maltose, dextrin, trehalose, glycerol, 
mannitol, dulcitol, sorbitol, rhamnose and inositol. No acid or gas from lac. tose, sucrose, inulin, salicin or adonitol and usually not from raffinose.

Reduces trimethylamine oxide (Wood and Baird, loc. cit.).

Hydrogen sulfide produced.

Optimum temperature $37^{\circ} \mathrm{C}$.

Aerobic, facultative.

Antigenic structure: [I], IV, [V], XII: $\mathrm{b}:[1,2] \ldots$ Some strains lack antigen $\mathrm{V}$ and some have I.

Source: Isolated from cases of enteric fever in man. Not a natural pathogen of animals.

Habitat: A natural pathogen of man causing enteric fever. Also found rarely in cattle, sheep, swine, lower primates and chickens.

3. Salmonella sp. (Type Abony). (Salmonella abony Kauffmann, Acta Path. et Microbiol. Scand., 17, 1940, 1.)

Antigenic structure: [I], IV, V, XII: $\mathrm{b}: \mathrm{e}, \mathrm{n}, \mathrm{x}$. . .

Source: Isolated by Kauffmann from a mixed culture of Salmonella abortus bovis sent to him by Dr. K. Rauss, Budapest. Later three additional cultures were received from Dr. Rauss. Original culture from the feces of a normal person.

Habitat: All cultures thus far recognized have been from human sources.

4. Salmonella typhimurium (Loeffler) Castellani and Chalmers. (Bacillus typhi murium Loeffler, Cent. f. Bakt., 11, 1892, 192; Bacterium typhi murium Chester, Ann. Rept. Del. Col. Agr. Exp. Sta., 9, 1897, 70; Bacillus murium Migula, Syst. d. Bakt., 2, 1900, 761; Castellani and Chalmers, Man. Trop. Med., 3rd ed., 1919, 939; Bacillus typhi-murium Holland, Jour. Bact., 5, 1920, 221; Bacterium typhi-murium Holland, idem; Bacillus enteritidis B, Typ. murium Januschke, Ztschr. f. Infektionskr. d. Haustiere, $27,1924,182$.

The following are regarded as synonyms of this organism: Salmonella psittacosis Castellani and Chalmers (Man. Trop. Med., 3rd ed., 1919, 939; Bacillus psittacosis Nocard, Conseil d. Hyg. Publique et Salubrité du Dept. du Seine, Séance, March 24, 1893; Bacterium psittacosis Le Blaye and Guggenheim, Manuel Pratique de Diagnostic Bactériologique, 1914); Salmonella aertrycke Castellani and Chalmers (Man. Trop. Med., 3rd ed., 1919, 939; Bacillus acrtrycle De Nobele, Ann. Soc. Méd. Gand., 72, 1898, 281; Bacillus paraaertrycke Castellani, Ann. di Med. Nav. e Colon., 11, 1914, 453; Bacterium aertrycke Weldin and Levine, Abst. Bact., 7, 1923, 13); Kaensche's Bacillus and Basenau's Bacillus, Kaensche, Ztschr. f. Hyg., 22, 1896, 53; Bacillus pestiscaviae Wherry (Jour. Inf. Dis., 5, 1908, 519; Bacillus cholera-caviae Wherry, Pub. Health Repts., November, 1908; Pasturella pestis-caviae Holland, Jour. Bact., 5, 1920, 219); Bacillus paratyphosus $B$, Mutton type, Schütze, Lancet, 1 , 1920, 93; Group VII of Hecht-Johansen, Copenhagen, 1923; Salmonella aertrycke Ibrahim and Schütze, Brit. Jour. Exp. Path., 9, 1928, 353; Bacterium enteritidis Breslau and Salmonella breslau of German literature; Mouse-typhoid of many authors. Some strains are confused with Salmonella anatis because of their origin in ducklings, e.g., see Salmonella anatum var. aertrycke Olsen and Goetchins, Cornell Vet., 27, 1937, 354.

Hauduroy et al. (Dict. d. Bact. Path., Paris, 1937, 449) regard the following as synonyms of Salmonella aertrycke: $B a$ cillus breslaviensis Kruse, in Flügge, Die Mikroorganismen, 3 Aufl., 2, 1896, 377; Bacterium breslaviensis Chester, Ann. Rept. Del. Col. Agr. Exp. Sta., 9, 1897, 69; B. enteritidis breslaviense Berge, Deut. tierärztl. Wehnschr., 1926, 473; Salmonclla meleagridis Rettger, Plastridge and Cameron, Jour. Inf. Dis., 53, 1933, 279; Salmonella aertrycke var. meleagridis Cameron and Rettger, Jour. Bact., 27, 1934, 86.

See Edwards and Bruner, Kentucky 
Agr. Exp. Sta. Bull. 400, 1940, 43-70, for a discussion of this species.

Rods: 0.5 by 1.0 to 1.5 microns, occurring singly. Motile with peritrichous flagella. Gram-negative.

Gelatin colonies: Small, circular, grayish, granular, becoming yellowish-brown.

Gelatin stab: Flat surface growth. No liquefaction.

Agar colonies: Small, circular, grayish, entire to undulate.

Agar slant: Filiform, grayish, moist, entire growth.

Broth: Turbid.

Litmus milk: Slightly acid, becoming alkaline.

Potato: Grayish-white streak.

Indole not formed.

Nitrites produced from nitrates.

Acid and gas from glucose, fructose, galactose, arabinose, maltose, dextrin, mannitol, sorbitol and inositol. Acid from glycerol. No action on lactose, sucrose, raffinose, inulin, salicin or adonitol.

Reduces trimethylamine oxide (Wood and Baird, loc. cit.).

Hydrogen sulfide produced.

Optimum temperature $37^{\circ} \mathrm{C}$.

Aerobic, facultative.

Antigenic structure: [I], IV, [T], XII: $\mathrm{i}: 1,2,3 \ldots$

Source: Isolated during a mouse typhoid epidemic in the Hygienic Institute of Greifswald, Germany.

Habitat: Causes food-poisoning in man. A natural pathogen for all warmblooded animals. This type occurs more frequently than any other type not confined to a specific host. Also found in snakes by Hinshaw and McNeil (Amer. Jour. Vet. Res., 6, 1945, 264).

4a. Salmonella typhimurium (Type Binns). (Bacillus paratyphesus B Binns type, Schütze, Lancet, 1, 1920, 93; Group VI of Hecht-Johansen, Copenhagen, 1923; Typus-Binns, Kauffmann, Zb1. f. d. ges. Hyg., 25, 1931, 273; Salmonella typhimurium var. Copenhagen, Kauffmann,
Ztschr. f. Hyg., 116, 1934, 368; Salmonella typhi-murium var. Binns, Schütze et al., Jour. Hyg., 34, 1934, 339; Salmonella aertrycke var. Storrs, Edwards, Jour. Bact., 30, 1935, 471.)

Morphology and cultural characters indistinguishable from those of Salmonella typhimurium, except some strains ferment maltose late or are anaerogenic.

Antigenic structure: [I], IV, XII: i : 1, 2, 3... (Edwards, Jour. Hyg., 36, 1936, 348). Many colonies may be examined before the specific phase flagellar antigen is demonstrated. Differs from Salmonella typhimurium in lacking antigen $\mathrm{V}$.

Source: Isolated by Dr. McNee from a case of food poisoning in man, France, 1919.

Habitat: Natural host the pigeon, and may infect other animals, including man.

5. Salmonella sp. (Type Köln). (Salmonella köln Sievers, Cent. f. Bakt., I Abt., Orig., 150, 1913, 52; Salmonella coeln Kauffmann, Acta Path. et Microbiol. Scand., Suppl. 54, 1944, 33.)

Antigenic structure: IV, $\mathrm{V}, \mathrm{XII}: \mathrm{y}$ : $1,2,3 \ldots$

Source: A single culture isolated from a human case of enteritis.

Habitat: Not reported from other sources as yet.

6. Salmonella sp. (Type Stanley). (Bacillus paratyphosus B, Stanley type, Schüize, Lancet, 1, 1920, 93; Salmonella stanleyi Haupt, Ergebnisse d. Hyg., 13, 1932, 673; Salmonella Stanley type, White, Med. Res. Council, Spec. Rept. Ser. 103, 1926, 19; Salmonella stanley Warren and Scott, Jour. Hyg., 29, 1929, 415; Typus Stanley, Kauffmann, Ztschr. Hyg., 111, 1930, 210.)

Antigenic structure: IV, V, XII: d: $1,2 \ldots$

Source: Isolated from cases of human food poisoning in Stanley, England by Hutchens (1917). 
Habitat: Not known as a natural pathogen of animals.

7. Salmonella sp. (Type Heidelberg). (Bacterium enteritidis, Typus Heidelberg, Habs, Cent. f. Bakt., I Abt., Orig., 130, 1933, 367; Salmonella heidelberg Schutze et al., Jour. Hyg., 34, 1934, 340.)

Antigenic structure: IV, V, XII: r: $1,2,3 \ldots$

Source: Isolated from cases of human food poisoning in Heidelberg, Germany

Habitat: Not known as a natural pathogen of animals.

8. Salmonella sp. (Type Chester) (Salmonella chester Kauffmann and Tesdal, Ztschr. f. Hyg., 120, 1937, 168.)

Antigenic structure: IV, [V], XII: e, $h: e, n, x \ldots$

Source: Isolated by W. H. Grace, Chester, England, from gastroenteritis in man. Typed by Kauffmann and Tesdal (loc. cit.).

Habitat: Has usually been found in human feces.

9. Salmonella sp. (Type San Diego). (Salmonella san diego Kauffmann, Acta Path. et Microbiol. Seand., 17, 1940, 429.)

Antigenic structure: IV, [V], XII : e, h: e, $\mathbf{n}, \mathrm{z}_{15} \ldots$

Source: Originally isolated from cultures sent to Dr. Kauffmann by Dr. K. F. Meyer who obtained them from an outbreak of food poisoning near San Diego, California. Also reported from I)enmark, Uruguay and Kentucky.

Habitat: Usually has been isolated from human feces, but has been found in birds and other animals.

10. Salmonella sp. (Type Salinas). (Salmonella salinatis Edwards and Bruner, Jour. Bact., 44, 1942, 289.)

Antigenic structure: IV, XII : d, e, h: $\mathrm{d}, \mathrm{e}, \mathrm{n}, \mathrm{z}_{15} \ldots$

By cultivation in semi-solid agar containing agglutinating serum for Sal- monella typhosa, an organism having the antigenic formula for Salmonella $s p$. (Type San Diego) was isolated.

Source: From rat feces collected by Dr. Henry Welch near Salinas, California.

Habitat: Also found in normal human carriers.

11. Salmonella sp. (Type Saint Paul). (Salmonella saint paul Edwards and Bruner, Jour. Inf. Dis., 66, 1940, 220.)

Antigenic structure: I, IV, V, XII: e, $\mathrm{h}: 1,2,3 \ldots$

Source: A single culture isolated from the liver of a turkey poult by Dr. B. S. Pomeroy, St. Paul, Minnesota. Two cases in man.

Habitat: Also reported from hogs.

11a. Salmonella sp. (Type Zagreb). (Salmonella zagreb Kauffmann, Acta Path. et Microbiol. Scand., 17, 1940, 351.)

Antigenic structure: IV, V, XII : e, h: $1,2 \ldots$ This is a minor type of No. 11.

Source: Culture received by Dr. Kauffmann under the label $S$, reading from Dr. N. Cernozubov of Zagreb, Jugoslavia.

Habitat: Not reported from other sources as yet.

12. Salmonella sp. (Type Reading). (Bacillus paratyphosus B, Reading type, Schütze, Iancet, 1, 1920, 93; Salmonella reading Schutze, Brit. Jour. Exp. Path., 11, 1930, 34; Typus Reading, Kauffmann, Zentb1. f. d. ges. Hyg., 25, 1931, 273; Salmonella readingensis Haupt, Ergebnisse d. Hyg., 13, 1932, 673.)

Antigenic structure: IV, XII: e, h: $1,5 \ldots$

Source: Isolated from the Reading, England water supply by Dr. H. Schütze. Also found in hogs (Edwards and Bruner, Jour. Inf. Dis., 72, 1943, 64).

Habitat: A cause of gastroenteritis in man. 
12a. Salmonella sp. (Type Kaposvar). (Salmonella kaposvar Rauss, Cent. f. Bakt., I Abt., Orig., 147, 1941, 253; also see Kauffmann, Die Bakteriologie der Salmonella-Gruppe, Kopenbagen, 1941, 212.)

Antigenic structure: IV, V, XII: e, (h) $1,5 \ldots$ This is a minor type of No. 12 .

Source: From the feces of three members of a family suffering from gastroenteritis.

Habitat: Not reported from other sources as yet.

13. Salmonella sp. (Type Kaapstad). (Salmonella reading var. kaapstad Henning, Rhodes and Gordon-Johnstone, Onderstepoort Jour. Vet. Sci. Animal Ind., 16, 1941, 103; Salmonella kaapstad Kauffmann, Acta Path. et Nierobiol. Scand., 19, 1942, 523.)

Antigenic structure: IV, XII: e, h: $1,7 \ldots$

Source: From a child with meningitis.

Habitat: Not known from other sources as yet.

14. Salmonella sp. (Type Derby). ( $B a$ cillus enteritidis Peckham, Jour. Hyg., 22, 1923, 69; Derby type, Savage and White, Med. Res. Council Spec. Rept. Ser. 91, 1925, 19; Salmonella derby Warren and Scott, Jour. Hyg., 29, 1929, 415; Salmonella derbyensis Haupt, Ergebnisse d. Hyg., 13, 1932, 673.)

Antigenic structure: $[\mathrm{I}], \mathrm{IT}, \mathrm{XII}$ : f, $\mathrm{g}$ :- -

Source: Isolated from tank water at Derby, England.

Habitat: Widely distributed. Found in human feces, lymph glands of hogs, chickens, ete.

15. Salmonella sp. (Type Essen). (Salmonella essen 173 Hohn and Herrmann, Cent. f. Bakt., I Abt., Orig., 135, 1936, 505.)

Antigenic structure: IV, XII:g, m:-
Source: Isolated from the feces of an infant, Essen, Germany.

Habitat: Known only from human sources.

16. Salmonella sp. (Type Budapest). (Salmonella budapest Rauss, Ztschr. f. Immunitätsf., 95, 1929, 489.)

Antigenic structure: I, IV, XII: g, $t:-$.

Source: Originally isolated in Budapest from 3 normal persons and from 3 persons with enteric fever.

Habitat: Known only from human sources.

17. Salmonella sp. (Type California). (Salmonella california Edwards, Bruner and Hinshaw, Jour. Inf. Dis., 66, 1940, 127; Hinshaw, Hilgardia, 13, 1941, 583.)

Antigenic structure: IV, XII, g, m, $\mathrm{t}:$ : .

Sotrce: Six cultures isolated from infected turkey poults from California. The seventh culture was isolated from a turkey in a second outbreak of the infection. Reported by Pomeroy and Fenstermacher (Jour. Amer. Vet. Med. Assoc., 94, 1936, 90). Also found in hogs and man (Edwards and Bruner, Jour. Inf. Dis., 72, 1943, 64).

Habitat: Also reported from chickens and ducks. Widely distributed.

18. Salmonella sp. (Type Brandenburg). (Typus-Brandenburg, Kauffmann and Mitsui, Ztschr. f. Hyg., 111, 1930, 710; Kauffmann, Zentbl. f. d. ges. Hyg., 25, 1931, 273; Salmonella brandenburgensis Haupt, Ergebnisse d. Hyg., 13, 1932, 673; Salmonella brandenburg Schütze et al., Jour. Hyg., 34, 1934, 540.)

Antigenic structure: IV, XII: $1, \mathrm{v}$ : e, n, $z_{15}$. . . See Kauffmann, Ztschr. f. Hyg., 118, 1936, 540.

Source: Isolated from a case of gastroenteritis at the Virchow Hospital of Berlin. 
Habitat: Known only from human sources.

19. Salmonella sp. (Type Bispebjerg). (Salmonella bispebjerg Typus, Kauffmann, Ztschr. f. Hyg., 118, 1936, 540.)

Antigenic structure: I, IV, XII: a: $e, n, x . .$.

Source: Isolated from a case of gastroenteritis at the Bispebjerg Hospital in Copenhagen.

Habitat: Not reported from other sources as yet.

20. Salmonella abortivoequina (Good and Corbett) Bergey et al. (Bacillus abortivus equinus Good and Corbett, Jour. Inf. Dis., 13, 1913, 53; Bacillus abortus equi Meyer and Boerner, Jour. Med. Res., 29, 1913, 330; Bacillus abortivo-equinus Good and Corbett, Jour. Inf. Dis., 18, 1916, 586; Bacillus abortus equinus Weiss and Rice, Jour. Med. Res., 35, 1907, 403; Bacillus abortivus Winslow, Kligler and Rothberg, Jour. Bact., 4, 1919, 477; Bacillus abortus-equi Holland, Jour. Bact., 5, 1929, 216; Bacterium abortum-equi Holland, ibid.; Bergey et al., Manual, 1st ed., 1923, 217; Bacillus enteritidis B, Typ. equinus Januschke, Ztschr. f. Infektionskr. d. Haustiere, 29, 1924, 182; Salmonella abortus-equi Bergey et al., Manual, 2nd ed., 1925, 236.) From Latin, aborting and equine.

Antigenic structure: IV, XII: - : e, $\mathrm{n}, \mathrm{x}, \ldots$

Reduces trimethylamine oxide (Wood and Baird, loc. cit.).

Source: Isolated from afterbirth of mares that had aborted.

Habitat: A natural pathogen of mares, causing abortion. Infectious for guinea pigs, rabbits, goats, cows, producing abortion.

21. Salmonella sp. (Type Arechavaleta). (Salmonella arechavaleta Hormaeche and Peluffo, quoted from Hormaeche et al., Jour. Bact., 47, 1944, 323.)
Named in honor of Prof. Arechavaleta of Uruguay.

Antigenic structure: IV, [V], XII: a : $1,7 \ldots$

Source: From a human case of gastroenteritis. Also found by Dr. P. R. Edwards among cultures sent to him from the Canal Zone for identification.

Habitat: Known only from human sources.

22. Salmonella abortusovis (Lovell) Schütze et al. (Bacillus paratyphi abortus ovis Schermer and Ehrlich, Cent. f. Bakt., I Abt., Ref., 73, 1922, 252; Bacillus enteritidis $C$, Typ ovis Januschke, Ztsch. f. Infektionskr. d. Haustiere, 27, 1924, 182; Bacterium abortus ovis Lovell, Jour. Path. and Bact., 34, 1931, 13; Typus-Abortus ovis, Kauffmann, Zentlbl. f. d. ges. Hyg., 25, 1931, 273; Schütze et al., Jour. Hyg., 34, 1934, 340.)

Antigenic structure: IV, XII: c: 1, $6 . .$.

Reduces trimethylamine oxide (Wood and Baird, loc. cit.).

Source: Isolated from cases of abortion in sheep.

Habitat: Not known to infect any other animal.

23. Salmonella sp. (Type Altendorf). (Salmonella altendorf Hohn, Cent. f. Bakt., I Abt., Orig., 146, 1940, 218.)

Antigenic structure: IV, XII: c: 1, 7. . .

Source: Isolated from a case of acute gastroenteritis from Altendorf, Germany .

Habitat: Not reported from other sources as yet.

24. Salmonella sp. (Type Texas). (Salmonella texas Watt, De Capito and Moran, U. S. Public Health Repts., 62, 1947, 808.)

Antigenic structure: IV, V, XII: k: e, $\mathrm{n}, \mathrm{z}_{15} \ldots$.

Source: Isolated by Dr. James Watt from the feces of a boy convalescing from diarrhoea. 
Habitat: Not reported from other sources as yet.

25. Salmonella abortusbovis Kauffmann. (Kauffmann, Ztschr. f. Hyg., $120,1937,194$.

Antigenic structure: [I], IV, XXVII, XII : b: e, n, x. . .

Liquefies gelatin (Kauffmann, Ztschr. f. Hyg., 117, 1936, 778).

Source: Isolated and incompletely typed by H. Bernard, Ztschr. f. Hyg., $117,1935,352$.

Habitat: Normally found in cattle, causing abortion. Occasionally occurs in man.

26. Salmonella sp. (Type Bredeney). (Salmonella bredeney Kauffmann, Ztschr.

f. Hyg., 119, 1937, 356.)

Antigenic structure: I, IV, [XXVII], III : 1, v: $1,7 \ldots$.

Source: Found by Hohn and Herrmann in Bredeney, Germany. Typed by Kauffmann (loc. cit.). From cases of human gastroenteritis and an abscess of lower jaw.

Habitat: Isolated from human sources. Also found in normal hogs and chickens.

27. Salmonella sp. (Type Schleissheim). (Salmonella schleissheim Kauffmann and Tesdal, Ztschr. f. Hyg., 120, $1937,171$.

Antigenic structure: IV, XXVII, XII: $\mathrm{b}, \mathrm{z}_{12}:$ -

Liquefies gelatin (Kauffmann and Tesdal, loc. cit.).

Source: Isolated by Hopfengärtner (Münchener tierärz. Wchnschr., 1, 1929, 185) in Schleissheim. From cattle. Typed by Kauffmann and Tesdal (loc. cit.). Also found by Tillmanns in the liver of a horse (Ztschr. f. Fleisch. u. Milch Hyg., 50, 1940, 109). Caused an outbreak of gastroenteritis in 30 persons (Kauffmann, Acta Path. et Microbiol. Scand., 17, 1940, 1).

Habitat: Apparently widely distributed.

28. Salmonella sp. (Type Schwarzen- grund). (Salmonella schwarzengrund Kauffmann, Acta Path. et Microbiol. Scand., Suppl. 44, 1944, 34.)

Antigenic structure: I, IV, XXVII, XII: d: 1,7 .

Source: A single culture isolated by Dr. J. Hohn from a human case of enteritis that occurred in Schwarzengrund, near Breslau, Germany.

Habitat: Not reported from other sources as yet.

29. Salmonella hirschfeldii Weldin. (Bacillus paratyphosus $\beta_{5}$ Weil, Wien. klin. Wchnschr., 30, 1917, 1061; Bacillus erzinjan Neukirch, Ztschr. f. Hyg., 85, 1918, 103; Paratyphoid C bacillus, Hirschfeld, Lancet, 1, 1919, 296; "ParaC"', Mackie and Bowen, Jour. Roy. Army Med. Corps, 33, 1919, 154; Bacillus paratyphosus $C$ Andrewes and Neave, Brit. Jour. Exp. Path., 2, 1921, 157; Paratyphus $\mathrm{N}_{1}$, Iwaschenzoff, Arch. f. Schiffs- u. Trop. Hyg., 30, 1926, 1; Weldin, Iowa Sta. Coll. Jour. Sci., 1, 1927, 161; Bacterium hirschfeldii Weldin, ibid., 161; Typus-Orient, Kauffmann, Zbl. f. d. ges. Hyg., 25, 1931, 273; Salmonella paratyphi $C$ Castellani and Chalmers, Man. Trop. Med., 3rd ed., 1919, 939; Salmonella paratyphosus C Castellani and Chalmers, ibid., 952.) Named for Hirsehfeld who worked with this organism.

Rods : 0.3 to 0.5 by 1.0 to 2.5 mierons, occurring singly. Motile with peritrichous flagella. Gram-negative.

Gelatin colonies: Grayish, smooth, flat, glistening, margin irregular.

Gelatin stab: Flat, grayish surface growth. No liquefaction.

Agar colonies: Grayish, moist, smooth, translucent.

Broth: Turbid.

Litmus milk: Slightly acid, becoming alkaline.

Indole not formed.

Nitrites produced from nitrates.

Acid and gas from glucose, fructose, maltose, arabinose, xylose, dextrin, trehalose, mannitol, dulcitol and sorbitol. No action on lactose, sucrose, 
salicin, adonitol or inositol. Rarely may fail to form gas from sugars (Nabih, Jour. Hyg., 41, 1941, 39).

Reduces trimethylamine oxide (Wood and Baird, loc. cit.).

Hydrogen sulfide produced.

Optimum temperature $37^{\circ} \mathrm{C}$.

Aerobic, facultative.

Antigenic structure: VI, VII, [Vi]: c: $1,5 \ldots$

Source: Isolated from cases of enteric fever in man.

Habitat: A natural pathogen of man causing enteric fever.

\section{Salmonella choleraesuis (Smith)} Weldin. (Probably not the Bacillus of swine plague, Klein, Report of the Medical Officer of the Local Gov. Bd., England, 1877-78, Supplement, p. 168; Bacterium of swine plague, Salmon, U. S. Dept. Agr. Bur. An. Ind. Ann. Rep., 1885, 212; Bacterium of hog cholera, Salmon, ibid., 1886, 20; Bakterium der Schweinepest, Selander, Cent. f. Bakt., 3, 1888, 361; Pasteurella salmoni Trevisan, I generi e le specie delle Batteriacee, 1889, 21; Bacterium cholerae suis Th. Smith, U. S. Dept. Agr. Bur. An. Ind., Bull. 6, 1894, 9; Swine-feverbacillus, Klein, Cent. f. Bakt., I Abt., 18, 1895 , 105; Bacillus suipestifer Kruse, in Flügge, Die Mikroorganismen, 3 Aufl. 2, 1896, 401; Bacterium cholerae suum Lehmann and Neumann, Bakt. Diag. 1 Aufl., 2, 1896, 233; Bacterium suipestifer Chester, Ann. Rept. Del. Col. Agr. Exp. Sta., 9, 1897, 70; Bacillus cholerae suum Migula, Syst. d. Bakt., 2, 1900, 759; Le microbe du hog-cholera, Lignières, Bull. Soc. Cent. Méd. Vet., see Rec. de méd. vét., Paris, Sér. 8, \%, 1900 , 389; Bacillus salmoni. Chester, Manual Determ. Bact., 1901, 210; Bacterium intestinale suis Le Blaye and Guggenheim, Manuel Pratique de Diagnostic Bacteriologique, 1914; Bacillus suis Krumwiede, Kohn and Valentine, Jour. Med. Res., 38, 1918, 89; Bacterium (Salmonella) cholera suis Buchanan, Jour. Bact., 3, 1918, 53; Salmonella suipestifer Castellani and Chalmers, Man. Trop. Med., 3rd ed., 1919, 939; Bacillus cholerae-suis Winslow, Kligler and Rothberg, Jour. Bact., 4, 1919, 476; Bacterium cholerae-suis Holland, Jour. Bact., 5, 1920, 217; Bacillus paratyphosus $B$ (Arkansas type), Schütze, Lancet, 2, 1920, 93; included in Group I suipestifer, Andrewes and Neave, Brit. Jour. Exp. Path., 2, 1921, 157; Weldin, Iowa Sta. Coll. Jour. Sci., 1, 1927, 155; Typus suipestifer Amerika, Kauffmann, Zbl. f. d. ges. Hyg., 25, 1931, 273; the American Salmonella suipestifer of many authors.) From Latin, hog cholera.

Salmonella choleraesuis (Smith) Weldin is the type species of the genus Salmonella.

Rods : 0.6 to 0.7 by 2.0 to 3.0 microns, occurring singly. Motile with four to five peritrichous flagella. Gram-negative.

Gelatin colonies: Grayish, smooth, flat, glistening; margin irregular.

Gelatin stab: Flat, grayish surface growth. No liquefaction.

Agar colonies: Gravish, moist, smooth, translucent.

Agar slant: Grayish, moist, smooth, translucent growth.

Broth: Turbid, with thin pellicle and grayish-white sediment.

Litmus milk: Slightly acid, becoming alkaline, opalescent, translucent to yellowish-gray.

Potato: Grayish-white streak becoming brownish.

Indole not formed.

Nitrites produced from nitrates.

Acid and gas from glucose, fructose, galactose, mannose, xylose, maltose, glycerol, mannitol, dulcitol, rhamnose, sorbitol and dextrin. Arabinose, inositol, lactose, sucrose, salicin, inulin, raffinose and trehalose not attacked.

Reduces trimethylamine oxide (Wood and Baird, loc. cit.).

Hydrogen sulfide not produced.

Optimum temperature $37^{\circ} \mathrm{C}$.

Aerobic, facultative.

Antigenic structure: VI, VII: c: 1 , 
5... Serologically identieal with Sal. monella typhisuis, and cross-agglutinates to a varying degree with a number of other serotypes.

Habitat and source: Natural host the pig as an important secondary invader in the virus disease, hog cholera. Does not occur as a natural pathogen in other animals, although lethal for mice and rabbits on subcutaneous injection. Occasionally gives rise to acute gastroenteritis and enteric fever in man.

30a. Salmonella choleraesuis var. Kunzendorf Schütze et al.

The synonyms up to and including Weldin, 1927 for Salmonella choleraesuis apply equally well to the var. Kunzendorf, for these were not separated with certainty until 1926 (White, Med. Res. Council, London, Spee. Rep. Ser. 103, 27). Re-examined serologically a number of previously described strains agree with this variety. (Paratyphus $\mathrm{C} \mathrm{Ba-}$ cillus, Heimann, Cent. f. Bakt., I Abt., Orig., 66, 1912, 211; Paratyphosus C, Weil and Saxl, Wien. klin. Wehnschr., 30, 1917, 519; Typus-suipestifer Kunzendorf, Pfeiler, Ztschr. f. Infektskr. d. Haust., 20, 1920, 218; Bacillus paratyphosus B, G. type, Schütze, Lancet, 1. 1920, 93; Bacillus paratyphosus C, Dudgeon and Urquart, Lancet, 2, 1920, 15; included in Group II suipestifer, Andrewes and Neave, Brit. Jour. Exp. Path., 2, 1921, 157 and Group V of Hecht Johansen, Copenhagen, 1923; Salmonella suipestifer (European variety) Schütze. Brit. Jour. Exp. Path., 11, 1930 34; Typus-suipestifer Kunzendorf, Kauffmann, Zbl. f. d. gen. Hyg., 25, 1931, 273; Salmonella choleraesuis var. kuniendorf Schütze et al., Jour. Hyg., 34, 1934. 341; the European Salmonella suipestifer of many authors.)

Indistinguishable from Salmonella choleraesuis in morphology and cultural characters, except that the Kunzendorf variety forms hydrogen sulfide.

Antigenic structure: VI. VII : [c] : 1,5 . Differs from Salmonella choleraesuis in lacking the specific flagellar phase; serologically identical with Salmonella typhisuis var. voldagsen.

Source: From pigs with swine fever and once from a monkey in captivity.

Habitat: Causes acute gastro enteritis and enteric fever in man. Also found in cattle, sheep, carnivora and chickens.

31. Salmonella typhisuis (Glässer) Schütze et al. (Bacillus typhisuis Glässer, Deutsche tierärztl. Wchnschr., 17, 1909, 513; included in the Ferkeltyphus bacilli of German literature, Dammann and Stedefeder, Arch. f. wiss. u. prakt. Tierheilk., 36, 1910, 432; Bacillus glässer Neukirch, Ztschr. f. Hyg., 85, 1918, 103; Bacterium typhi-suis Holland, Jour. Bact., 5, 1920, 221; included in Group I suipestifer, Andrewes and Neave, Brit. Jour. Exp. Path., 2, 1921, 157; Typus-Glässer, Kauffmann, Zbl. f. d. ges. Hyg., 25, 1931, 273; Schütze et al., Jour. Hyg., 34, 1934, 342.) From Greek, typhus and Latin, pig.

Rods : 0.6 to 0.7 by 2.0 to 3.0 microns, occurring singly. Motile with four to five peritrichous flagella. Gram-negative.

Gelatin colonies: Grayish, smooth, flat, glistening, edge entire. No liquefaction.

Agar colonies: Grayish, moist, smooth, translucent.

Broth: Turbid.

Litmus milk: Slightly acid or neutral. Indole not formed.

Nitrites produced from nitrates.

Forms gas slowly and sparsely from all substances. Frowth poor on all ordinary media.

Acid from arabinose, xylose and trehalose. Delayed or variable fermentation from dextrin, maltose, rhamnose, dulcitol, sorbitol. Mannitol not fermented or very slowly. Inositol not fermented.

No $\mathrm{H}_{2} \mathrm{~S}$ produced.

Optimum temperature $37^{\circ} \mathrm{C}$.

Aerobic, facultative.

Antigenic structure: Identical with Salmonella choleraesuis, from which the 
organism differs in respect to arabinose and trehalose. Antigenic structure VI, VII : c: $1,5 . \ldots$

Habitat: Infects only the pig.

31a. Salmonella typhisuis var. voldagsen Schütze et al. (Included in Ferkeltyphus bacilli, Dammann and Stedefeder, Arch. f. wiss. u. prakt. Tierheilk., 36, 1910, 432; Bacillus voldagsen Neukirch, Ztschr. f. Hyg., 85, 1918, 103; included in Group II suipestifer, Andrewes and Neave, Brit. Jour. Exp. Path., 2, 1921, 157; Typus-voldagsen, Kauffmann, Zabl. f. d. ges. Hyg., 25, 1931, 273; Salmonella typhisuis var. voldagsen, Schütze et al., Jour. Hyg., 34, 1934, 342.)

Morphology and cultural characters identical with those of Salmonella typhisuis.

Antigenic structure: VI, VII: [c]: 1, 5 . . . Identical with that of Salmonella choleraesuis var. Kunzendorf from which species the organism differs culturally.

Habitat: Infects only the pig.

32. Salmonella sp. (Type Thompson). (Thompson type of Salmonelia, Scott, Jour. Hyg., 25, 1925, 398; Typus-Thompson-Berlin, Kauffmann, Zbl. f. d. ges. IIyg., 25, 1931, 273; Salmonella thompson. Schütze et al., Jour. Iyg., 34, 1934, 343.) Named after the family involved in the outbreak.

Antigenic structure, VI, VII: k: 1,5 . .

Source: Isolated from food poisoning in man. Also found in chickens and turkeys (Edwards and Bruner, Jour. Inf. Dis., 72, 1943, 64).

Habitat: Widely distributed in warmblooded animals.

32a. Salmonella sp. (Type Berlin) (Type Thompson). (Typus-Berlin, Kauffmann, Cent. f. Bakt., I Abt., Ref., 94, 1929, 282; Typus C Berlin, Boecker and Kauffmann, Cent. f. Bakt., I Abt., Orig., 116, 1930, 458; Typus-ThompsonBerlin, Kauffmann, Zbl. f. d. ges. Hyg.,
25, 1931, 273; Salmonella thompson var. berlin Schütze et al., Jour. Hyg., 34, 1934, 343.)

Antigenic structure: VI, VII: [k]: 1, 5 . . .

Source: Isolated from food poisoning in man. Not known to be a natural pathogen of animals.

Habitat: A natural pathogen of man causing food poisoning.

33. Salmonella sp. (Type Montevideo). (Salmonella montevideo Hormaeche and Peluffo, Arch. Urug. de Med., Cirug. y Espec., 9, 1936, 673.)

Antigenic structure: VI, VII: $\mathrm{g}, \mathrm{m}$, s: - .

Source: Originally isolated from human sources in Montevideo from an ape that died of an enterocolitis, and mesenteric glands of healthy hogs; also reported from chickens and powdered eggs (Schneider, Food Research, 11, $1946,313)$.

Habitat: Apparently widely distributed.

34. Salmonella sp. (Type Oranienburg). (Typus-Oranienburg, Kauffmann, Ztschr. f. Hyg., 111, 1930, 223; Salmonella oranionburgensis Haupt, Ergebnisse der Hyg., 13, 1932, 673; Salmonella oranienburg Schütze et al., Jour. Hyg., 34, 1934, 343.)

Antigenic structure: VI, VII: $\mathrm{m}, \mathrm{t}:-$. Source: From the feces of a child in a children's home near Oranienburg. Later isolated from gastroenteritis in man. Also from quail, chickens and powdered eggs (Schneider, loc. cit.).

Habitat: Reported from human suurees, from hogs and from birds.

35. Salmonella sp. (Type Virchow). (Typus-Virchow, Kauffmann, Ztschr. f. Hyg., 111, 1930, 221; Salmonella virchowii Haupt, Ergebnisse der Hyg., 13, 1932, 673; Salmonella virchow Schütze et al., Jour. Hyg., 34, 1934, 343.)

Antigenic structure: VI, VII: $r: 1$, $2,3 \ldots$ 
Source: Isolated from food poisoning in a man at the Rudolf Virchow Hospital in Berlin.

Habitat: A natural pathogen of man causing food poisoning.

36. Salmonella sp. (Type Oslo). (Salmonella oslo Tesdal, Ztschr. f. Hyg., 119, 1937, 451.)

Antigenic structure: VI, VII: a: e, $\mathrm{n}, \mathrm{x}, \ldots$

Source: Isolated in Oslo, Norway from cases of gastroenteritis in man.

Habitat: Not reported from other sources as yet.

37. Salmonella sp. (Type Amersfoort). (Salmonella amersfoort Henning, Jour. Hyg., 37, 1937, 561.)

Antigenic structure: VI, VII: d: e, $\mathrm{n}, \mathrm{x}, \ldots$

Source: Originally isolated from chickens from Amersfoort, Transvaal. Later found in a human mixed infection with Salmonella typhi murium.

Habitat: Not reported from other sources as yet.

38. Salmonella sp. (Type Braenderup). (Salmonella braenderup Kauffmann and Henningsen, Ztschr. f. Hyg., 120, 1937, 640.)

Antigenic structure: VI, VII: e, h: e, $n, z_{15} \ldots$.

Source: Isolated from a case of human gastroenteritis in Braenderup, Denmark. Also from a cat in the same home that had died from a diarrhoea. Reported later from So. Africa (see Kauffmann, Die Bakteriologie der SalmonellaGruppe, Kopenhagen, 1941, 237).

Habitat: Apparently widely distributed.

39. Salmonella sp. (Type Potsdam). (Typus-Potsdam, Kauffmann and Mitsui, Ztschr. f. Hyg., 111, 1930, 740; Salmonella potsdamensis Haupt, Ergebnisse der Hyg., 13, 1932, 673; Salmonella potsdam Schütze et al., Jour. Hyg., 34, 1934, 343.)

Antigenic structure: VI, VII: l, v: e, n. $z_{15}$. . .

Source: Isolated from food poisoning in man at Potsdam, Germany.

Habitat: A natural pathogen of man causing food poisoning.

40. Salmonella sp. (Type Bareilly). (Salmonella, Type Bareilly, Bridges and Scott, Jour. Roy. Army Med. Corps, 56, 1931, 241; Salmonella bareilly Schütze et al., Jour. Hyg., 34, 1934, 343.)

Antigenic structure: VI, VII: y: 1 , 5. . .

Source: Isolated in 1928 from cases of mild enteric fever that occurred in Bareilly, India. Also reported from chickens (Kauffmann, Die Bakteriologie der Salmonella-Gruppe, Kopenhagen, 1942, 235).

Habitat: A natural pathogen of man causing gastroenteritis and enteric fever. Widely distributed in fowls.

41. Salmonella sp. (Type Hartford). (Salmonella hartford Edwards and Bruner, Jour. Inf. Dis., 69, 1941, 223.)

Antigenic structure: VI, VII: y: c, $n, x . \ldots$

Source: One culture isolated from the stool of a man with persistent diarrhoea by Dr. E. K. Borman, Hartford, Conn.

Habitat: Not reported from other sources as yet.

42. Salmonella sp. (Type Mikawasima*). (Salmonella bareilly var. mikawasima Hatta, Japan Jour. Exper. Med., 16, 1938, 201; Salmonella mikawasima Hormaeche, quoted from Schütze et al., Proc. 3rd Internat. Cong. Microbiol., 1940, 337; also see Kauffmann, Acta Path. et Microbiol. Scand., 16, 1939, 347 and $i b i d ., 17,1940,429$.

Antigenic structures: VI, VII: y: e, $n, z_{15} \ldots$

* Correct spelling according to Prof. Kojima. 
Source: Isolated from a rat by Prof. Fojima and Prof. Hatta, 1937.

Habitat: Not reported from other sources as yet.

43. Salmonella sp. (Type Tennessee). (Salmonella tennessee Bruner and Edwards, Proc. Soc. Exp. Biol. and Med., $50,1942,174$.

Antigenic structure: VI, VII: $\mathrm{z}_{29}$ :-

Source: Culture isolated from feces of normal carrier by Dr. W. C. Williams, State Dept. of Health, Nashville, Tennessee.

Habitat: Also reported from turkeys and powdered eggs.

44. Salmonella sp. (Type Concord). (Salmonella var. concord Edwards and Hughes, Jour. Bact., 47, 1944, 574.)

Antigenic structure: VI, VII: 1, v: $1,2,3 \ldots$

Source: Two cultures isolated by Dr. J. R. Beach and one by Dr. C. U. Duckworth from fatal infections in chicks (U. S. A.) and one by Dr. Joan Taylor from the stool of a person affected with gast roenteritis (England).

Habitat: Also reported from turkeys.

45. Salmonella sp. (Type Infantis). (Salmonella infantis Wheeler and Borman, Jour. Bact., 46, 1943, 481.)

Antigenic structure: VI, VII: r: 1, 5. . . .

Source: Isolated at Hartford, Connecticut from the blood of an infant Subsequently also from stools.

Habitat: Not reported from other sources as yet.

46. Salmonella sp. (Type Georgia) (Salmonella georgia Morris, Brim and Sellers, Amer. Jour. Pub. Health, 34, 1944, 1279; Seligmann, Saphra and Wassermann, Amer. Jour. Hyg., 40, 1944 , 227.)

Antigenic structure: VI, VII: $b$ : e, $n, z_{15} \ldots$

Source: Isolated by Miss Jane Morris from the feces of a 16-year-old boy during routine examination of food handlers, State Dept of Health, Atlanta, Georgia.

Habitat: Not reported from other sources as yet.

47. Salmonella sp. (Type Papua). (Salmonella papuana Wilcox, Edwards and Coates, Jour. Bact., 49, 1945, 514.)

Antigenic structure: VI, VII: $r$ : e, $n, z_{15} \ldots$

Source: Isolated by Lt. Goldwasser from human feces from Port Moresby in Papua, New Guinea.

Habitat: Not reported from other sources as yet.

48. Salmonella sp. (Type Richmond). (Salmonella richmond Moran and Edwards, Proc. Soc. Exp. Biol. and Med., 62, 1946, 294.)

Antigenic structure: VI, VII: y: 1, 2, 3. . .

Source: Isolated by Mr. Forest Spindle in Rirhmond, Virginia from the feces of a child affected with gastroenteritis.

Habitat: Isolated as yet from human sources only.

49. Salmonella sp. (Type Cardiff). (Salmonella cardiff Taylor, Edward and Fidwrds, Brit. Med. Jour., 1945, i, 368.)

Antigenic structure: VI, VII: k: 1, $10 . .$.

Source: Isolated from human case of gastroenteritis from Cardiff, Wales.

Habitat: Isolated as yet from human sourres only

50. Salmonella sp. (Type Daytona). (Salmoneila daytona Moran and Edwards, Proe. Soe. Fxp. Biol. and Med., $62,1946,294$.)

Antigenic structure: VI, VII: k: 1, 6. ...

Source: Isolated by Mrs. Mildred Galton from human feces from Daytona, Florida.

Habitat: Not known from other sources as yet. 
51. Salmonella sp. (Type Tewport). (Paratyphus $\beta_{2}$, Weil and Saxl, Wien. klin. Wchnschr., 30, 1917, 519; Bacillus paratyphosus B, Newport type, Schütze, Lancet, 1, 1920, 93; Paratyphus Newport Bacillus, Kiauffmann, Cent. f. Bakt., I Abt., Ref., 94, 1929, 282; Salmonella newport Schütze, Brit. Jour. Exp. Path., 11, 1930, 34; Salmonella newportensis Haupt, Ergehnisse der Hyg., 13, 1932, 673.)

Antigenic structure: VI, VIII: e, h: $1,2,3 \ldots$.

Source: Isolated from food poisoning in man, Newport, England.

Habitat: Widely distributed in man, eattle, hogs. chickens, ete. Also in snakes (Hinshaw and MeNeil. Amer. Jour. Vet. Res., 6, 1945. 264).

5la. Salmonello sp. (Type Puerto Rico) Kauffmann. (Jordan, Amer. Jour. Trop. Dis,, 14, 1934, 27; Kauffmann, Cent. f. Bakt., I Abt., Orig.. 132, 1934, 162; Schütze et al., Jour. Hyg., 34, 1934, 344.)

Antigenic structure: VI. VIII: [e, h]: $1,2,3 \ldots$

This is regarded as a non-specific variant of Salmonella sp. (Type Newport) by Schütze et al. (Proc. 3rd Internat. Cong. Microbiol., New York, 1940,833 ).

52. Salmonella sp. (Type Pueris). (Salmonella pueris Wheeler and Borman, Jour. Bact., 46, 1943, 481.)

Antigenic structure: VI, VIII: e, h: $1,2 \ldots$.

Source: Isolated at Hartford. ('onnecticut from anal swabbings of a 14 year-old boy during an attack of gastroenteritis complicating measles.

Habitat: Not reported from other sources as yet.

53. Salmonella sp. (Type Kottbus). (Salmonella newport var. kottbus Kaufi- mann, C'ent. f. Bakt., I Abt., Orig., 132, 1934, 162; Salmonella kottbus Schütze et al., Proc. 3rd Internat. Nicrobiol. Cong., New York, 1940, 834.)

Antigenic structure: VI, VIII: e, h: $1,5 \ldots$

Source: From an acute case of gastroenteritis in Kottbus. Denmark.

Habitat: Not reported from other sources as yet.

54. Salmonella sp. (Type Muenchen). (Typus München, Mandelbaum, Cent. f. Bakt., I Abt., Ref., 105, 1932, 377; Salmonella muenchen Schütze et al., Jour. Hyg., 34, 193t, 34t.)

Antigenic structure: VI, VIII: d: $1,2 \ldots$.

Source: Isolated from a fatal case of enteric fever.

Habitat: Widely distributed. Reported from man, rabbits, hogs, camels and chickens (Kauffmann, Die Bakteriologie der Salmonella Gruppe, 1941, 244).

54a. Salmonella $s p$. (Type Oregon). (Salmonella oregon Edwards and Bruner, Amer. Jour. Hyg., 34, 1941, 21.)

Antigenic structure: VI, VIII: d: 1, 2. 3 .

Source: six cultures, one isolated from a turkey by Dr. E. M. Dickinson and five from the mesenteric glands of apparently normal hogs by Dr. H. L. Rubin. This is a minor type of No. 54 .

Habitat: Also reported from reptiles, rhickens and man. Also powdered eggs.

55. Salmonella sp. (Type Manhattan). (Salmonella manhattan Edwards and Bruner, Amer. Jour. Hyg., 34, 1941, 21.) Antigenic structure: VI, VIII: d:, $1,5 \ldots$

Source: Two cultures, one isolated from a chicken by Dr. L. D. Bushnell, Nankattan, Kansas, and the other from a turkey by Dr. W. R. Hinshaw. Also 
from reptiles, hogs and human sources (Edwards and Bruner, Jour. Inf. Dis., 72, 1942, 64).

Habitat: Apparently widely distributed.

56. Salmonella sp. (Type Litchfield). (Salmonella litchfield Edwards and Bruner, Jour. Inf. Dis., 66, 1940, 220.)

Antigenic structure: VI, VIII: 1, v: $1,2,3 \ldots$.

Source: Isolated from the liver of a young turkey poult from Litchfield, Minnesota by Dr. B. S. Pomeroy. Also isolated from a case of food poisoning in man by Miss Georgia Cooper.

Habitat: Not reported from any other source, as yet.

57. Salmonella morbificans (Migula) Haupt. (Bacillus bovis morbificans Basenau, Arch. f. Hyg., 20, 1894, 257; Bacillus morbificans bovis Kruse, in Flügge, Die Mikroorganismen, 3 Aufl., 2, 1896, 380; Bacterium morbificans bovis Chester, Ann. Rept. Del. Col. Agr. Exp. Sta., 9, 1897, 70; Bacillus morbificans Migula, Syst. d. Bakt., 2, 1900, 747; Flavobacterium morbificans Bergey et al., Manual, 3rd ed., 1930, 147; Haupt, Ergebnisse der Hyg., 13, 1930, 673; Salmonella bovis-morbificans Schütze et al., Jour. Hyg., 34, 1934, 344.)

Antigenic structure: VI, VIII: $\mathrm{r}$ : $1,5 . \ldots$

Source: Originally isolated from a septicemia in a cow.

Habitat: Also found in rabbits and in gastroenteritis in man.

58. Salmonella sp. (Type Narashino). (Salmonella narashino Nakaguro and Yamashita, quoted from Kauffmann, Die Bakteriologie der SalmonellaGruppe, Kopenhagen, 1941, 246.)

Antigenic structure: VI, VIII: a: e, $\mathrm{n}, \mathrm{x} . \ldots$

Source: From the blood and feces of a person suffering from enteric fever. Found in Japan.

Habitat: Not reported from other sources as yet.

59. Salmonella sp. (Type Buenos Aires). (Salmonella bonariensis Monteverde, $\mathrm{Na}$ ture, 149, 1942, 472.)

Antigenic structure: VI, VIII: i : e, n, x...

Source: Isolated by Dr. Monteverde, Buenos Aires from a mesenteric gland of a normal hog.

Habitat: Also reported from normal human carriers and from cases of gastroenteritis.

60. Salmonella sp. (Type Glostrup). (Salmonella glostrup Kauffmann and Henningsen, Acta Path. et Microb. Scand., 16, 1939, 99.)

Antigenic structure: VI, VIII: $z_{10}$ : e, $n, z_{15} \ldots$

Source: Isolated from cases of gastroenteritis in a family in Denmark. Also affected their dog. Later isolated in Jugoslavia and in Palestine.

Habitat: Evidently widely distributed.

61. Salmonella sp. (Type Duesseldorf). (Salmonella duesseldorf Hohn, Cent. f. Bakt., I Abt., Orig., 146, 1940, 218.)

Antigenic structure: VI, VIII: $\mathrm{z}_{4}, \mathrm{z}_{24}$ :

Source: Isolated from two patients, one of whom died. Found in Duesseldorf, Germany.

Habitat: Not reported from other sources as yet.

62. Salmonella sp. (Type Tallahassee). (Salmonella tallahassee Moran and Edwards, Proc. Soc. Exp. Biol. and Med., 62, 1946, 294.)

Antigenic structure: VI, VIII: $z_{4}$, $\mathrm{z}_{32}:$ -

Source: Isolated by Mrs. Mildred Gal. 
ton from feces of gastroenteritis patients and from normal human carriers, Tallahassee, Florida.

Habitat: Not known from other sources.

63. Salmonella sp. (Type Gatun). (Salmonella gatuni Wilcox and Coates, Jour. Bact., 51, 1946, 561.)

Antigenic structure: VI, VIII: b: e, $\mathrm{n}, \mathrm{x}$. . .

Source: Isolated from human feces from Gatun, Canal Zone.

Habitat: Not known from other sources as yet.

64. Salmonella sp. (Type Amherst). (Salmonella amherstiana Edwards and Bruner, Jour. Immunol., 44, 1942, 319.)

Antigenic structure: (VIII): 1, v: 1, $6 . .$.

Source: Isolated by Dr. H. Van Roekel from one of a group of poults affected with a fatal disease.

Habitat: Not reported from other sources as yet.

65. Salmonella sp. (Type Virginia). (Salmonella virginia Saphra and Seligmann, Proc. Soc. Exper. Biol. and Med., $58,1945,50$.

Antigenic structure: (VIII): d: - .

Source: Isolated by F. Spindle, Richmond, Virginia from the feces of an adult person suffering from a diarrhoea.

Habitat: Not known from other sources as yet.

66. Salmonella typhosa (Zopf) White. (Bacillus des Abdominal-Typhus, Eberth, Arch. f. path. Anat., 81, 1880 and 83, 1881; Typhus bacillen, Gaffky, Mitteil, a. d. kaiserl. Gesundheitsamte, 2, 1881, 372; Bacillus typhosus Zopf, Die Spaltpilze, 3 Aufl., 1885, 126; not $B a$ cillus typhosus Klebs, Handbuch d. path. Anat., 1880; Bacillus typhi Schroeter, in Cohn, Kryptogamen Flora v. Schlesien,
3, 1886, 165; Bacillus typhi abdominalis Flügge, Die Mikroorganismen, 2 Aufl., 1886, 198; Vibrio typhosus Trevisan, I generi e le specie delle Batteriacee, 1889, 23; Bacterium typhi Chester, Ann. Rept. Del. Col. Agr. Exp. Sta., 9, 1897, 73; Bacterium typhosum Twort, Proc. Royal Soc., London, 79, B, 1907, 329; Acystia typhi Enderlein, Sitzber. Gesell. Naturf. Freunde, Berlin, 1917, 517; Bacterium (Eberthella) typhi Buchanan, Jour. Bact., 3, 1918, 53; Eberthus typhosus Castellani and Chalmers, Man. Trop. Med., 3rd ed., 1919, 936; Eberthella typhi Bergey et al., Manual, 1st ed., 1923, 223; Eberthella typhosa Weldin, Iowa State College Jour. Sci., 1, 1927, 170; Salmonella typhi Warren and Scott, Jour. Hyg., 29, 1930, 416; White, Jour. Hyg., 29, 1930, 443.) Named from the disease, typhoid fever.

The species name typhosa should be used for the typhoid organism when it is placed in any genus other than $B a$ cillus in spite of the earlier use of this species name by Klebs for a different organism. There are two reasons for this: (a) This appears to be the proper course to follow under International Rules of Nomenclature (See Art. 54, p. 54) and (b) there is less chance for confusion regarding the nature of this organism among English-speaking persons who may carelessly interpret typhi as the name of a typhus rather than a typhoid bacillus.

Rods : 0.6 to 0.7 by 2.0 to 3.0 microns, occurring singly, in pairs, occasionally short chains. Motile with peritrichous flagella. Gram-negative.

Gelatin colonies: Grayish, transparent to opaque, with leaf-like surface markings.

Gelatin stab: Thin, white, opalescent growth. No liquefaction.

Agar colonies: Grayish, transparent to opaque.

Agar slant: Whitish-gray, glistening, echinulate, entire to undulate growth 
Broth: Turbid, moderate sediment and delicate pellicle in old cultures.

Litmus milk: Slight, transient acidity, followed by a return to neutral or to slight alkalinity

Potato: Delicate, moist, slightly spreading, barely visible growth.

Acid but no gas from glucose, fructose, galactose, xylose, maltose, raffinose, dextrin, glycerol, mannitol and sorbitol. No action on lactose, sucrose, inulin, rhamnose, inositol, salicin and usually arabinose and dulcitol.

Reduces trimethylamine oxide (Wood and Baird, loc. cit.).

Indole not formed.

No characteristic odor.

Nitrites produced from nitrates

Hydrogen sulfide produced

Aerobic, facultative

Optimum temperature $37^{\circ} \mathrm{C}$

Antigenic structure: IX, III, [Vi]: d:- The somatic antigens are related to those of Salmonella enteritidis and at number of other species of Salmonella. $V$ and $W$ forms are present (Felix and Pitt, Jour. Path. and Bact., 38, 1934, 409; Craigie and Brandon, Jour. Path. and Bact., 43, 1936, 233 and 239). Craigie and Yen (Canadian Public Health Journal, 29, 1938, 448 and 484) by the action of selected $\mathrm{Vi}$ phages recognize eleven distinct stable types of Salmonella typhosa which have been found to be of epidemiological importance.

Source: From the human intestine.

Habitat: The cause of typhoid fever. Pathogenic for laboratory animals on parenteral injection. Isolated once from a chicken by Henning, Onderstepoort, So. Africa.

Note: This species has previously been regarded as the type species of the genus Eberthella Buchanan (Acystia Enderlein, Sitzber. Gesell. Naturf. Freunde, Berlin, 1917, 317; Buchanan, Jour. Bact., 3, 1918, 53; Eberthus Castellani and Chalmers, Man. Trop. Med. 3rd ed., 1919, 934; Lankoides Castellani and Chalmers, ibid., 938; Wesenbergus Castellani and Chalmers, ibid., 940.)

67. Salmonella enteritidis (Gaertner) Castellani and Chalmers. (Bacillus enteritidis Gaertner, Correspond. d. Allgemein. Artzl. Verein Thuringen, 17, 1888, 573; Klebsiella enteritidis De Toni and Trevisan, in Saccardo, Sylloge Fungorum, 8, 1859, 923; Bacterium enteritidis Chester, Ann. Rept. Del. Col. Agr. Exp. Sta., 9, 1897, 68; Bacillus gaertner Morgan, Brit. Med. Jour., 1, 1905, 1257 ; Castellani and Chalmers, Manual Trop. Med., 3rd ed., 1919, 930.) Named for the disease, enteritis.

Rods: 0.6 to 0.7 by 2.0 to 3.0 microns, occurring singly, in pairs and occasionally in short chains. Motile with peritrichous flagella. Gram-negative.

Gelatin colonies: Circular, gray, translucent, granular, entire.

Gelatin stab: Abundant surface growth. No liquefaction.

Agar colonies: Circular, gray, translucent, moist, smooth, entire. Deskowitz and Buchbinder (Jour. Bact., 29, $1935,294)$ describe a variant that produces a soluble vellow pigment where certain peptone is present in the agar. Antigenic structure not determined.

Agar slant: Grayish-white, opalescent, smooth, moist, undulate growth.

Broth: Turbid, with thin pellicle and grayish-white sediment.

Litmus milk: Slightly acid, becoming alkaline, opaleseent, translurent to yelJow ish-gray.

Potato: Abundant, moist, yellowishbrown to brown growth

Indole not formed.

Nitrites produced from nitrates.

Acid and gas from glucose, fructose, galactose, mannose, arabinose, xylose, maltose, trehalose, dextrin, glycerol, mannitol, dulcitol and sorbitol. No acid or gas from lactose, sucrose, inulin, salicin, raffinose, adonitol and inositol. 
Reduces trimethylamine oxide (Wood and Baird, loc. cit.).

Hydrogen sulfide produced.

No characteristic odor.

Aerobic, facultative.

Optimum temperature $37^{\circ} \mathrm{C}$.

Antigenic structure: $[\mathrm{I}], \mathrm{IX}, \mathrm{XII}$ : $\mathrm{g}, \mathrm{m}:-\cdots$.

Source: First isolated from feces in an epidemic of meat poisoning at Frankenhausen, Germany.

Habitat: Widely distributed, occurring in man. Also in domestic and wild animals, particularly rodents.

67a. Salmonella enteritidis var. Danysz. (Bahr, Deutsche tierärztl. Wehnschr., 1928, 786 and 1930, 145; Typus-Gärtner Ratin, Kauffmann, Zbl. f. d. ges. Hyg., 25, 1931, 273; Sulmonella enteritidis var. danysz, Schütze et al., Jour. Hyg., 34, 1934, 345; Śalmonella danysii Gay et al., Agents of Disease and Host Resistance, 1935, 650.)

Differs from Salmonella enteritidis only in its negative action on glyecrol in Stern's medium.

Source: Isolated by Danysz in 1900.

Habitat: A natural pathogen of rodents and man.

67b. Salmonella enteritidis var. Chaco. (Savino and Menendez, Rev. Inst. Bact., $6,1934,347$; Kauffmann, Ztschr. f. Hyg. 117, 1935, 401.)

Differs from Salmonella enteritidis in its action on dulcitol when tested by the method of Bitter, Weigmann and Habs (Münch. med. Wchnschr., 73, 1926, 940.)

Habitat and source: Isolated from cases of fever during the Chaco war, South Amerim.

67e. Salmonella enteritidis var. Essen. (Hohn and Herrmann, Cent. f. Bakt., I Abt., Orig., 193, 1935, 183; ibid., 194, 1935, 277; Kauffmann, Ztschr. f. Hyg., $117,1935,401$.)

Differs from Salmonella cnteritidis when tested by the method of Bitter, Weigmann and Habs (Münch. med.
Wchnschr., 73, 1926, 940), giving a negative reaction with arabinose and dulcitol.

Habitat and source: Isolated from human gastroenteritis, ducks and duck pggs.

Note: Jansen (Cent. f. Bakt., I Abt., Orig., 135, 1935, 421) states that the organism named by him Salmonella enteritidis var. Wulheim is in reality Salmonella enteritidis var. Essen.

67d. Salmonella enteritidis var. Jena. (Fournier, Rev. Immunolog., Paris, 6, $1940-41,26+$.)

Source: Isolated from purulent pleural fluid.

Habitat: Not reported from other sources as yet.

68. Salmonella sp. (Type Dublin). (Bacillus enteritidis Pesch, Cent. f. Bakt., I Abt., Orig., 98, 1926, 22 ; Dublin Type, White, Med. Res. Counc., Syst. of Bact., 4, 1929, 86 and White, Jour. Hyg., 29, 1930, 443; Salmonella dublin Warren and Scott, Jour. Hyg., 29, 1930, 415; Typus-Dublin-Kiel, Kauffmann, Zzbl. f. d. ges. Hyg., 25, 1931, 273; Salmonella enteritidis var. dublin Schütze et al., Jour. Hyg., 34, 1934, 345.)

Antigenic structure: I, IX, XII: g, p: - .

Source: From meningitis in children (Pesch, loc. cit.). Also isolated by Dr. J. W. Bigger in Dublin, Eire from a fatal fever following a kidney operation. Typed by Dr. Bruce White (loc. cit.).

Habitat: Found in man. A natural pathogen of eattle. Widely distributed in cattle and foxes.

Two special fermentative types belong here: (1) Salmonella dublin $2=$ Salmonella dublin var. accra Kiauffmann, (2) Salmonella dublin $3=$ Salmonella dublin var. koln Kauffmann (Die Bakteriologie der Salmonella-Gruppe, Kopenhagen, 1941, 252).

69. Salmonella sp. (Type Trostock). (Gärtner-Poppe Typus, Bahr, Dtsch. Tierärzt. Wehnschr., 1930, 145; Typus 
Gärtner-Rostock, Kauffmann, Ztschr. f. Hyg., 111, 1930, 221; Salmonella enteritidis var. rostock Schütze et al., 34, 1934, 345; Salmonella rostockensis Haupt, Ergebnisse der Hyg., 13, 1932, 673.)

Antigenic structure: I, IX, XII: g, $\mathrm{p}, \mathrm{u}:-$.

Source: Originally isolated from cattle by Dr. Poppe in Rostock, Germany.

Habitat: Not known to infect man.

70. Salmonella sp. (Type Moscow). (Paratypus $\mathrm{C}_{1}$, Weigmann, Cent. f. Bakt., I Abt., Orig., 97, 1925, Beiheft, 299; Salmonella Type Moscow, Hicks, Jour. Hyg., 29, 1929, 446; Salmonella moscow Warren and Scott, Jour. Hyg., 29, 1929, 446; Typus Gärtner-Moskow, Kauffmann, Ztschr. f. Hyg., 111, 1930, 229; Salmonella moscowaensis Haupt, Ergebnisse der Hyg., 13, 1932, 673; Salmonella enteritidis var. moscow, Schütze et al., 34, 1934, 345).

Antigenic structure: IX, XII: g, q:-

Source: From patients with enteric fever. Isolated in Moseow, Russia.

Ilabitat: Infects man, horses, cattle.

71. Salmonella sp. (Type Blegdam). (Salmonella blegdam Kauffmann, Ztschr. f. Hyg., 117, 1935, 431.)

Antigenic structure: IX, XII: g, m, q:-.

- Source: Isolated in 1929, at State Serum Institute, Copenhagen from the blood of a pneumonia patient. Also found in the blood of a patient by Dr. Fournier, in Shanghai, China (Kauffmann, Die Bakteriologie der SalmonellaGruppe, Kopenhagen, 1941, 264).

Habitat: Not reported from other sources as yet.

72. Salmonella sp. (Type Berta). (Salmonella berta Hormaeche, Peluffo and Salsamendi, Arch. Urug. de Med., Cirug. y Espec., 12, 1938, 277.) Named in honor of Prof. Arnoldo Berta, Uruguay.

Antigenic structure: IX, XII: f, g, $\mathrm{t}:-$.
Source: Isolated from the mesenteric glands of normal hogs.

Habitat: Causes gastroenteritis in man. Also found in chickens.

73. Salmonella sp. (Type Pensacola). (Salmonella pensacola Moran and Edwards, Proc. Soc. Exper. Biol. and Med., $59,1945,52$.

Antigenic structure: IX, XII: g, m, $\mathrm{t}:-$.

Source: From a severe case of gastroenteritis in man.

Habitat: Not reported from other sources as yet.

74. Salmonella sp. (Type Claiborne). (Salmonella claibornei Wilcox and Lennox, Jour. Immunol., 49, 1944, 71.)

Antigenic structure: I, IX, XII: k: $1,5 . \ldots$.

Source: Culture isolated from human feces at Camp Claiborne, Louisiana.

Habitat: Not known from other sourees as yet.

75. Salmonella sp. (Type Sendai). (K type, Shimojo, quoted from Kauffmann, Die Bakteriologie der SalmonellaGruppe, Kopenhagen, 1941, 265; Atypical Paratyphosus A, Aoki and Sakai, Cent. f. Bakt., I Abt., Orig., 95, 1925, 152; Sendai type, White, Med. Res. Council, Spec. Rept. Ser. No. 103, 1926 , 118; Salmonella sendaiensis Haupt, Ergebnisse der Hyg., 13, 1932, 673; Salmonella sendai Schütze et al., Jour. Hyg., 34, 1934, 345; Eberthella sp. (Sendai Type) F. Smith, in Manual, 5th ed., 1939, 464.)

Antigenic structure: [I], IX, XII: a : $1,5 \ldots$.

Source: Isolated in 1922 by K. Shimojo in Japan from a case of paratyphoid. Later isolated by Aoki and Sakoi from feces, urine and blood of typhoid patients.

Habitat: A natural pathogen of man causing enteric fever. 
76. Salmonella sp. (Type Miami). (Salmonella miami Edwards and Moran, Jour. Bact., 50, 1945, 259.)

Antigenic structure: IX, XII: a: 1, 5 . . .

Differ culturally and biochemically from organisms of Sendai Type (Edwards and Moran, Jour. Bact., 50, 1945, 257)。

Source: Twenty-four cultures isolated by Mrs. Mildred Galton in Florida. Fourteen cultures were from cases of acute gastroenteritis, one from a patient with chronic diarrhoea, 4 from food handlers, 4 from chimpanzees thought to be affected with bacillary dysentery and one from pickles which caused an outbreak of food poisoning. One culture was from Borman, Wheeler, West and Mickle (Amer. Jour. Pub. Health, $33,1943,127$ ) and was isolated from a case of gastroenteritis in Connecticut. Another culture was from Seligmann, Saphra and Wassermann (Amer. Jour. Hyg., 38, 1913, 225) and was isolated from a case of enteric fever.

Habitat: Apparently widely distributed as a natural pathogen of man and apes.

77. Salmonella sp. (Type Durban). (Salmonclla durban Henning, Rhodes and Gordon-Johnstone, Onderstepoort Jour. Vet. Sci. An. Ind., 16, 1941, 103; also see Kauffmann, Acta Path. et Microbiol. Scand., 19, 1942, 523.)

Antigenic structure: IX, XII: $a$ : e, $\mathrm{n}, \mathrm{z}_{15} \ldots$.

Source: Isolated by Dr. J. GordonJohnstone in Durban, So. Africa from feces of a woman affected with gastroenteritis.

Habitat: Not reported from other sources as yet.

78. Salmonella sp. (Type Onarimon). (Salmonella onarimon Kisida, Kitasato Arch. of Exper. Med., 17, 1940, 1.)

Antigenic formula: I, IX, XII: b: $1,2 \ldots$.

Source: From the feces of a paraty- phoid B carrier. Later found in other cases of enteric fever resembling typhoid.

Habitat: Cause of a typhoid-like disease in man.

79. Salmonella sp. (Type Eastbourne). (Salmonella eastbourne Leslie and Shera, Jour. Path. and Bact., 34, 1931, 533.)

Antigenic structure: [I], IX, XII: e, $\mathrm{h}: 1,5 . \ldots$

May or may not produce indole (Kauffmann, Die Bakteriologie der SalmonellaGruppe, 1941, 12.)

Source: From human enteric fever at Eastbourne, England.

Habitat: A natural pathogen for man. Also found in turkeys.

80. Salmonella sp. (Type Panama). (Jordan, Amer. Jour. Trop. Med., 14, 1934, 27; Salmonella panama Kauffmann, Cent. f. Bakt., I Abt., Orig., 132, 1934, 160.)

Antigenic structure: I, IX, XII : l, v: $1,5 \ldots$.

Source: From human food poisoning at Fort Amador, in Panama, Canal Zone. Also isolated in New York City, Germany and Uruguay. Also in reptiles, hogs and chickens (Edwards and Bruner, Jour. Inf. Dis., 72, 1943, 64).

Habitat: Apparently widely dis1 ributed.

81. Salmonella sp. (Type Dar es Salaam). (Brown, Duncan and Henry, Lancet, 1, 1926, 117; Dar-es-Salaam Typus, Schütze, Arch. f. Hyg., 100, 1928, 192; Salmonella daressalaamensis Haupt, Ergebnisse der Hyg., 13, 1932, 673; Salmonella dar-es-salaam Schütze et al., Jour. Hyg., 34, 1934, 346.)

Antigenic structure: I, IX, XII : 1, w: e, n...

Liquefies gelatin (Jordan, Jour. Inf. Dis., 58, 1936, 126).

Source: Isolated by Butler in 1922 from a case of pyrexia at Dar es Salaam, East Africa. Cultures have also been reported from Zanzibar. 
Habitat: Known thus far from human sources only.

82. Salmonella sp. (Type Goettingen). (Salmonella goctlingen Hohn, Cent. f. Bakt., I Abt., Orig., 146, 1940, 218.)

Antigenic structure: IX, XII: l, v: $\mathrm{e}, \mathrm{n}, \mathrm{z}_{15} \ldots$. .

The complete formula was developed by Kauffmann (Acta Path, et Mierobiol. Scand., 17, 1940, 429.)

Source: Not given. Presumably from a human source.

Habitat: Not reported.

83. Salmonella sp. (Type Java). (Salmonella javiana Alley and Pijoan, Yale Jour. Biol. and Med., 15, 1942, 229; Edwards and Bruner, Jour. Immunol., 44, 1942, 319.)

Antigenic structure: [I], IX, XII: 1, $z_{\simeq 8}: 1,5 \ldots$

Source: From Eijkman Institute in Java. Isolated from feces of a child. Subsequently two cultures labeled N112 and N140, isolated in Panama from human carriers, were received from Col. Chas. G. Sinclair.

Habitat: Reported as yet from human sources only.

84. Salmonella gallinarum (Klein) Bergey et al. (Bacillus gallinarum Klein. Cent. f. Bakt., 5, 1889, 689; Pheasant bacillus, Klein, Jour. Path. \& Bact.. 2, 1893, 214; Bacillus phasiani septicus Kruse, in Flügge, Die Mikroorganismen, 3 Aufl., 2, 1896, 410; Bacterium sanguinarium Moore, 12th and 13th Ann. Rpt. for 1895-96, U. S. Dept. Agr. Bur. An. Ind., 1897, 188; see Moore, U. S. Dept. Agr. Bur. An. Ind., Bull. 8, 1895, 63; Bacillus phasiani Migula, Syst. der Bakt., 2, 1900, 769; Bacterium phasiani scpticus Chester, Ann. Rept. Del. Col. Agr. Exp. Sta., 9, 1597, 74; Bacterium gallinarum Chester, ibid., 80; Bacterium pyogenes sanguinarium Berry and Ernst, Jour. Med. Res., 10 (N. S. 5), 1903-04, 402; Bacillus pseudo-cholerae gallinarum Trincas, Giorn. della R. Soc. Ital.
d'Igiene, 1908, 385; Bacillus typhi gallinarum alcalifaciens and Bacillus typhi gallinarum Pfeiler and Rehse, Mitt. K. Inst. f. Landw. Bromberg, 5, 1913, 306; Eberthella sanguinaria Bergey et al., Manual, 1st ed., 1923, 231; Bergey et al., Manual, 2nd ed., 1925, 236; Shigella gallinarum Weldin, Iowa State Coll. Jour. Sei., 1, 1927, 179.) From Latin, of rhickens

Bacterium jeffersonii Hadley, Elkins and Caldwell, Rhode Island Agr. Exp. Sta. Bull. 174, 1918, 169 (Eberthella jeffersonii Bergey et al., Manual, 1st ed., 1923, 230; Shigella jeffersonii Bergey et al., Manual, 4th ed., 1934, 394). Shigella jeffersonii is identical serologically with Salmonclla gallinarum (St. John-Brooks and Rhodes, Jour. Path. and Bact., 26, $1923,433)$.

Rods: 0.4 to 0.6 by 0.8 to 1.6 microns, with rounded ends, occurring singly or (in blood) in short chains. Non-motile. Gram-negative.

Gelatin colonies: Small, grayish-white, finely grinular, circular, entire.

Gelatin stab: Slight, grayish-white surface growth with slight grayish, filiform growth in stab. No liquefaction.

Agar colonies: Moist, grayish, circular, entire.

Agar slant: Thin, gray streak, with irregular margin, moist, glistening.

Broth: Turbid with heavy, flocculent sediment.

Litmus milk: Reaction unchanged, becoming translucent. No coagulation.

Potato: Slight grayish growth.

Indole not formed.

Nitrites produced from nitrates.

Acid but no gas from glucose, fructose, galactose, mannose, xylose, arabinose, maltose, dextrin, mannitol, dulcitol and isodulcitol. Lactose, sucrose, glycerol, salicin and sorbitol are not attacked.

Reduces trimethylamine oxide (Wood and Baird, loc. cit.).

Hydrogen sulfide is sometimes formed. Aerobic, facultative.

Optimum temperature $37^{\circ} \mathrm{C}$.

Antigenic structure: [I], IX, XII:- : -. Identical with Salmonella pullorum, 
and related to Salmonella typhosa. [I] antigen noted by Kauffmann (Acta Path. et Microbiol. Scand., Suppl. 54, 1944, 36).

Source and habitat: The causative agent of fowl typhoid (clearly to be distinguished from fowl cholera), and identical with Noore's infectious leukemia of fowls. Infectious for rabbits and all poultry, canaries and certain wild birds (quail, grouse, pheasant) by feeding or by injection. Found once in a normal human carrier.

\section{Salmonella pullorum (Rettger)} Bergey et al. (Bacterium pullorum Rettger, Jour. Med. Res., 21 (X.S. 16), 1909, 117; also see Rettger, X. Y. Med. Jour., 71, 1900, 803; ibid., 73, 1901, 267; Rettger and Harvey, Jour. Med. Res.. 18 (N.S. 13), 1908, 277; Bacillus pullorum Smith and Ten Broeck, Jour. Ned. Res., 31 (N. S. 26), 1915, 547; Bergey et al., Manual, 1st ed., 1923, 218; Typus pullorum, Kauffmann, Zentbl. f. d. ges. Hyg., 25, 1931, 273.) From Latin, of chickens.

Rods: 0.3 to 0.5 by 1.0 to 2.5 microns, occurring singly. Ton-motile. Gramnegative.

Gelatin colonies: Grayish-white, moist, lobate, with grape-leaf surface.

Gelatin stab: Slight, grayish surface growth. No liquefaction.

Agar colonies: Grayish-white, smooth, glistening, entire to undulate.

Agar slant: Develops as discrete, translucent colonies.

Broth: Turbid.

Litmus milk: Acid, becoming alkaline. No coagulation.

Potato: Slow development, grayish.

Indole not formed.

Nitrites produced from nitrates.

Acid and gas from glucose, fructose, galactose, mannose, arabinose, xylose, mannitol and rhamnose. Does not attack lactose, sucrose, maltose, dextrin, salicin, raffinose, sorbitol, adonitol, dulcitol or inositol. Gas may be slight or absent (cf. Salmonella gallinarum).
Xylose may be fermented late (see Weldin, Iowa State Coll. Jour. Sci., 1, 1927, 165). Maltose fermenting strains may occur (Hinshaw, Browne and Taylor, Jour. Inf. Dis., 73, 1943, 197).

Reduces trimethylamine oxide (Wood and Baird, loc. cit.).

Hydrogen sulfide is formed.

Aerobic, facultative.

Optimum temperature $37^{\circ} \mathrm{C}$

Antigenic structure, IX, XII: The complete antigenic formula of S. pullorum is $\mathrm{IX}_{1} \mathrm{XII}_{1}, \mathrm{XII}_{2}, \mathrm{XII}_{3}$, while that of $S$. gallinarum seems to be $\mathbf{I X}$, $\mathrm{XII}_{1}, \mathrm{XII}_{3}$. Antigen $\mathrm{XII}_{2}$ is variable in S. pullorum (Edwards and Bruner, Cornell Vet., $36,1946,318$ ) and $\mathrm{XII}_{2}++$ and $\mathrm{NII}_{2}+$ forms occur. The $\mathrm{XII}_{2}++$ forms are synonymous with the $\mathrm{X}$ strains of Younie (Can. Jour. Comp. Med., 5, 1941,164 ).

Source: Isolated from chickens and other birds, as well as calves, hogs, rabbits and man. Occasionally produces food poisoning or gastroenteritis in man (Mitchell, Garlock and BrohKahn, Jour. Inf. Dis., 79, 1946, 57).

Habitat: The cause of white diarrhoea in young chicks. Infeets the ovaries and eggs of adult birds.

85a. Salmonella gallinarum var. Duisburg. (Müller, Münch. med. Wchnschr., 80, 1933, 1771; Kauffmann, Cent. f. Bakt., I Abt., Orig., 132, 1934, 337.)

Antigenically identical with Salmonella gallinarum and Salmonella pullorum. Differs from Salmonella gallinarum in its slow fermentation of maltose, failure to ferment d-tartrate and in not forming $\mathrm{H}_{2} \mathrm{~S}$.

Source and habitat: Isolated from ncute gastroenteritis in man.

86. Salmonella sp. (Type Canastel). (Salmonella canastel Randall and Bruner, Jour. Bact., 49, 1945, 511.) Source of name not given.

Liquefies gelatin.

Antigenic structure: IX, XII: $\mathrm{z}_{29}$ : $1,5 \ldots$ 
Source: Isolated in North Africa from American soldiers acting as food handlers.

Habitat: Not reported from other sources as yet.

87. Salmonella sp. (Type Italia). (Salmonella italiana Bruner and Edwards, Proc. Soc. Exp. Biol. and Med., $58,1945,289$.

Antigenic structure: IX, XII: l, v: $1,11 \ldots$.

Source: Two cultures, one isolated from a case of bloody diarrhoea and the other from a case of gastroenteritis in man. Found in Italy by Lt. Col. Robert Hebble and by Capt. Ira C. Evans.

Habitat: Not reported from other sources as yet.

S8. Salmonella sp. (Type Tapoli). (Salmonella napoli Bruner and Edwards, Proc. Soc. Exper. Biol. and Med., 58, 1945, 289.)

Antigenic structure: [I], IX, XII: 1, $z_{13}: e, n, x . .$.

Source: Ten cultures isolated from normal feces and from cases of gastroenteritis in Naples, Italy. The first culture was isolated by Capt. W. H. Ewing.

Habitat: Not reported from other sources as yet.

89. Salmonella sp. (Type Loma Linda). (Salmonella loma linda Edwards, Proc. Soc. Exper. Biol. and Med., 57, 1944, 104.)

Antigenie structure: IX, XII: a: e, $\mathrm{n}, \mathrm{x} . \ldots$

Source: Single culture isolated by Dr. T. F. Judefind, Loma Linda, California from the spinal fluid of a baby that died of meningitis.

Habitat: Not reported from other sources as yet.

90. Salmonella sp. (Type New York). (Salmonella new york Kaufimann, Acta Path. et Microbiol. Scand., Suppl. 5t, 194.4, 35.)
Antigenic structure: IX, XII: $1, v$ : $1,5 \ldots$

Source: Found by Dr. F. Schiff, New York in a study of a culture received under the label S. panama Strain No. 431. Regarded at the present time as a strain of Salmonella jariana by Dr. Kauffman (personal communication, March, 1947).

Habitat: Not reported from other sources as yet.

91. Salmonella sp. (Type London). (Salmonclla Type L, White, Med. Res. Council Spec. Rept. Ser. 103, 1926, 37; Salmonella londonensis Haupt, Ergebnisse der Hyg., 13, 1932, 675; Salmonella london Schütze et al., Jour. Hyg., 34, 1934, 346.)

Antigenic structure: III, X, XXVI: $\mathrm{l}, \mathrm{v}: 1,6 \ldots$

Source: Isolated in London from the feces of a gastroenteritis patient from IReading, England.

Habitat: Found in human infections, in hogs and in chickens.

92. Salmonella sp. (Type Give). (Salmonella give Kauffmann, Ztschr. f. Hyg., $120,1937,177$.)

Antigenic structure: III, X, XXVI: $1, v ; 1,7$.

Source: lrom feces of a patient with pernicious anemia. Also found in the L.S. A. and Germany. Oceurs in fowls and hogs (Edwards and Bruner, Jour. Inf. Dis., 72, 1943, 61).

Habitat: Apparently widely distributed.

93. Salmonella sp. (Type Uganda). (Salmonella uganda Kaufimann, Acta Path. et Microbiol. Scand., 17, 1940, 189.)

Antigenic structure: III, I, XXVI: $1, \mathrm{z}_{13}: 1,5$. .

Source: Isolated in Uganda by Dr. II. G. Wiltshire from a human spleen on autopsy. Typed by Dr. F. Kauffmann.

Habitat: Not reported from other sources as yet. 
94. Salmonella anatis (Rettger and Scoville) Bergey et al. (Bacterium anatis Rettger and Scoville, Abst. Bact., 3, 1910, S; not Bacterium anatis Migula, Syst. d. Bakt., 2, 1900, 364; Bacterium anatum Rettger and Scoville, Jour. Inf. Dis., 26, 1920, 217; Escherichia anata Bergey et al., MIanual, 1st ed., 1923, 198; Bergey et al., Manual, 2nd ed., 1925, 238; Salmonella anatum Bergey et al., Manual, 3rd ed., 1930, 344.) From Latin, of the duck.

With the transfer of this organism to the genus Salmonella, the original species name anatis again becomes available in spite of the earlier use of this species name by Migula for Cornil and Toupet's Bacillus der Enten-cholera (Compt. rend. Acad. Sci., Paris, 106, 1888, 1737). The latter organism is stated by Rettger and Scoville $(1920$, loc. cit., 220) to be indistinguishable from Pasteurella aviseptica.

Morphology and cultural characters like those of Salmonella enteritidis.

Kauffmann (Ztschr. f. Hyg., 119, 1937, 352) deseribes a lactose-splitting variant of this species.

Antigenic structure: III, X, XXYI: e, $\mathrm{h}: 1,6 \ldots$

Reduces trimethylamine oxide (Wood and Baird (loc. cit.).

Source: Isolated from an epizootic of keel in ducklings. Also found in intestinal infections in chickens and man. Frequently occurs in association with Salmonella typhimurium.

Habitat: Widely distributed in man and domestic animals.

95. Salmonella sp. (Type Muenster). (S'almonella anatum var. muenster, Kauffman $n$ and Silberstein, Cent. f. Bakt., I Abt., Orig., 132, 1934, 431; Salmonella muenster Kauffmann, Ztschr. f. Hyg., $140,1937,177$.

Antigenic structure: III, X, XXVI: e, $h: 1,5$.

Source: Isolated by Dr. Besserer in Muenster from food poisoning. Also isolated in Uruguay from human sources.
Habitat: Not known from any but human sources as yet.

96. Salmonella sp. (Type Nyborg). (Salmonella anatum var. nyborg, Kiristensen and Bojlén, Cent. f. Bakt., I Abt., Orig., 136, 1936, 294; Salmonella nyborg Kauffmann, Ztschr. f. Hyg., 120 . 1937, 189.)

Antigenic structure: III, X, XXTI: e, $\mathrm{h}: 1,7 \ldots$

Source: From a case of acute enteritis in a young girl in Nyborg, Denmark.

Habitat: Fnown only from human sources as yet.

97. Salmonella sp. (Type Tejle). (Salmonella vejle Harhoff, quoted from Kauffmann, Die Bakteriologie der SalmonellaGruppe, Kopenhagen, 1941, 274.)

Antigenic structure: III, X, XXVI:e, h: $1,2,3 \ldots$.

Source: Isolated by E. Møller, Copenhagen, from a case of acute gastroenteritis.

Habitat: Not reported from other sources as yet.

98. Salmonella sp. (Type Meleagris). (Salmonella meleagridis Bruner and Edwards, Amer. Jour. Hyg., 34, 1941, 82; not Salmonella meleagridis Rettger, Plastridge and Cameron, Jour. Inf. Dis., 53, 1933, 279.)

Antigenic structure: III, X, XXVI: e, $\mathrm{h}: \mathrm{l}, \mathrm{w} . .$.

Source: Original cultures isolated by Dr. B. S. Pomeroy, Lniv. of Minnesota, from two distinct outbreaks of infection in turkey poults. Stated to be the same as Salmonella bantam from Batavia, Java (Kauffmann, Acta Path. et Microbiol. Scand., 19, 1942, 529).

Habitat: In addition to the two strains isolated in Minnesota (Bruner and Edwards, Kentucky Agr. Exp. Sta., Bull. 434,1942 ), the same type was recognized among cultures received from Massachusetts, Michigan, Pennsylvania, Maryland, South America and Japan. Also isolated from German soldiers in 
Norway by Tesdal (Kauffmann, Die Bakteriologie der Salmonella-Gruppe, 1941, 295) and from snakes by Hinshaw and MeNeil (Amer. Jour. Tet. Res., 6, $1945,264)$.

99. Salmonella sp. (Type Shangani). (Salmonella shanguni Kauffmann, Acta Path. et Microbiol. Scand., 16, 1939, 347.)

Antigenic structure: III, X, XXVI: d: $1,5 \ldots$

Source: Isolated in Zanzibar by Dr. J. D. Robertson from a woman with enteric fever.

Habitat: Known only from human sources as yet.

100. Salmonella sp. (Type Zanzibar). (Salmonella zanzibar Kauffmann, Acta Path. et Microbiol. Scand., 16, 1939, 347.)

Antigenic structure: III, X, XXVI: $\mathrm{k}: 1,5 . \ldots$

Source: Isolated in Zanzibar by Dr. J. D. Robertson from a typhoid carrier.

Habitat: Also found in ehickens (Edwards).

101. Salmonella sp. (Type Amager). (Salmonella amager Kauffmann, Acta Path. et Microbiol. Sicand., 16, 1939,347.)

Antigenic structure: III, X, XXVI: y: $1,2,3 \ldots$.

Source: Isolated in Copenhagen from the feces of a person suffering from gastroenteritis.

Habitat: Known only from human sources as yet.

102. Salmonella sp. (Type Lexington). (Salmonella lexington Rubin, Jour. Bact.. 40, 1940, 463; Edwards, Bruner and Rubin, Proc. Soc. Exper. Biol. and Med., 44, 1940, 395.)

Antigenic structure: III, I, XXYI: $z_{10}: 1,5$.

According to Kauffmann (Die Bak teriologie der Falmonella-Gruppe, 1941, 276), Dr. Erber of Java has found a Salmonella type with the same antigenic structure and has given it the name Salmonella batavia.
Source: Isolated from mesenteric lymph glands of apparently normal hogs by I)r. H. L. Rubin, Univ. of Kentucky, Lexington, Ky.

Habitat: Also reported from turkeys.

103. Salmonella sp. (Type Weltevreden). (Salmonella weltevreden Mertens, quoted from Kauffmann, Acta Path. et Microbiol. Scand., 19, 1942, 529.)

Antigenic structure: III, X, XXVI: $r: z_{6} \ldots$

Source: Isolated by Dr. W. K. Mertens, Batavia, Java, according to Kauffmann (loc. cit.).

Habitat: Not reenrded in available literature.

10.1. Salmonella sp. (Type Orion). (Salmonella type, var orion and Salmonclla orion Barnes, Cherry and Myers, Jour. Bact. 50, 1915, 578.) From a seaman on the S. S. Orion.

Antigenic structure: III, X, XXVI: $y: 1,5 \ldots$

Source: From rectal swab specimen from a normal food handler.

Habitat: Not reported from other sources as yet.

105. Salmonella sp. (Type Butantan). (Salmonella butantan Peluffo, Arch. Urug. de Med., Cirug. y Espec., 18, 1944, 000.)

Antigenic structure: III, X, XXVI: b: $1,5 \ldots$.

Source: Isolated by Dr. C. A. Peluffo from a case of diarrhoea in a child.

Habitat: Tot reported from other sources as yet.

106. Salmonella sp. (Type Newington). (Anatum C 1 No.3071, N.C.T.C., London, Kauffmann and Silberstein, Cent. f. Bakt., I Abt., Orig., 132, 1934, 434; Salmonella newington Edwards, Jour. Hyg., 37, 1937, 38t.)

Antigenic structure: III, XV: e, h: $1,6 \ldots$

Source: Isolated from ducks from Newington, Connecticut by Dr. L. F. 
Rettger. Also found in hogs, silver foxes and man. Kauffmann (Ztschr. f. Hyg., 120, 1937, 177) has described a related type (Salmonella $\mathrm{tim}$ ) from a case of enteritis in Tim, Denmark.

Habitat: Widely distributed.

107. Salmonella sp. (Type Selandia). (Salmonella selandia Kauffmann, Ztschr. f. Hyg., 120, 1937, 189.)

Antigenic structure: III, XV: e, h: $1,7, \ldots$

Source: Isolated from the feces of a sailor on the S. S. Selandia after a voyage to Asia and Australia. Was patient in Bispebjerg Hospital with pleuropneumonia at the time.

Habitat: Known only from human sources as yet.

108. Salmonella sp. (Type New Brunswick). (Salmonella new brunswick Edwards, Jour. Hyg., 37, 1937, 381; also see Kauffmann, Ztschr. f. Hyg., 120, 1937 , 189.)

Antigenic structure: III, XV: 1, v : $1,7 \ldots$

Source: Isolated by Dr. F. R. Beaudette, New Brunswick, New Jersey from a chicken. Also isolated from gastroenteritis in man.

Habitat: Apparently widely distributed.

109. Salmonella sp. (Type Illinois). (Salmonella illinois Edwards and Bruner, Proc. Soc. Exper. Biol. and Med., 48 , 1941, 240.)

Antigenic structure: (III), (XV), XXXIV: $z_{10}: 1,5 \ldots$

Source: Isolated from hogs in Illinois by Dr. Robert Graham, from Hungarian partridges in Michigan by Miss Virginia Stoney and from turkeys in Minnesota by Dr. B. S. Pomeroy.

Habitat: Also reported from hogs and man (Edwards).

110. Salmonella sp. (Type Senftenberg). (Typus Senftenberg, Kauffmann, Ztschr. f. Hyg., 111, 1930, 221;
Salmonella senftenberg Schütze et al., Jour. Hyg., 34, 1934, 339; Śalmonella senftenbergensis Haupt, Ergebnisse der Hyg., 13, 1932, 673.)

Antigenic structure: I, III, XIX: g, s, t: - .

Source: From a case of acute gastroenteritis in a boy in Senftenberg, Denmark. Cultures have frequently been found from persons and also from young turkeys.

Habitat: Apparently widely distributed.

111. Salmonella sp. (Type Niloese). (Salmonella niloese Kauffmann, Acta path. et Microbiol. Scand., 16, 1939, 347$.

Antigenic structure: I, III, XIX: d: $z_{6} \ldots$

Source: Isolated in Copenhagen from a case of acute gastroenteritis in Niloese, Denmark. Later found frequently in gastroenteritis in Denmark.

Habitat: Known only from human sources as yet.

112. Salmonella sp. (Type Simsbury). (Salmonella simsbury Bruner and Edwards, Proc. Soe. Exper. Biol. and Med., $50,1942,174$.

Antigenic structure: I, III, XIX: $z_{27}$ :

Source: Original culture isolated by Dr. E. K. Borman, State Dept. Health Lab., Hartford, Conn., from a normal human carrier from Simsbury, Conn. Edwards states (1946) that this may be a variant of Salmonella sp. (Type Senftenberg).

Habitat: Also found in turkeys (Bruner and Edwards, Kentucky Agr. Exp. Sta., Bull. 434, 1942, 9).

113. Salmonella sp. (Type Taksony). (Salmonella taksony Rauss, Zutsehr. f. Immunit ätsforsch., 103, 1943, 220.)

Antigenic structure: I, III, XIX: i : $z_{6} \ldots$

Source: Isolated from a healthy carrier (Hungary). 
Habitat: Not reported from other sources as yet.

114. Salmonella sp. (Type Kentucky). (Salmonella kentucky Edwards, Jour. Hyg., 38, 1938, 306.)

Antigenic structure: (VIII), XX: i: $z_{6} \ldots$

Source: Isolated from the intestinal tract of a chick affected with coccidiosis and ulcerative enteritis. Found at Lexington, Kentucky.

Habitat: Also reported from many species of fowls, from hogs and from man (Edwards).

115. Salmonella sp. (Type Aberdeen). (Salmonella aberdeen J. Smith, Jour. Hyg., 34, 1934, 357.)

Antigenic structure: $\mathrm{XI}: \mathrm{i}: 1,2,3 \ldots$.

Source: Isolated in Aberdeen, Scotland, from the stool of a child suffering from acute enteritis. Also isolated by Timmerman in Utrecht from Ovomaltine, and by Fdwards in Fentucky from birds. See Kauffmann, Die Bakteriologie der Salmonella-Gruppe, Kopenhagen, 1941, 279.

Habitat: Apparently widely distributed.

116. Salmonella sp. (Type Rubislaw). (Salmonella rubislaw Smith and Kauffmann, Jour. Hyg., 40, 1940, 122.)

Antigenic structure: IX: $r: e, n, x$. . .

Source: Isolated in Aberdeen, Scotland from the feces of a child suffering from enteritis. Also found by Tesdal in Oslo, Norway. Reported by Hinshaw and McNeil from snakes (Amer. Jour. Vet. Res., 6, 1945, 264).

Habitat: Apparently widely distributed.

117. Salmonella sp. (Type Pretoria). (Salmonella pretoria Henning, Rhodes and Gordon-Johnstone, Onderstepoort Jour. Vet. Sci. An. Ind., 16, 1941, 103.)

Antigenic structure: XI: k: 1, 2, 3. . .
Source: Isolated by Dr. M. W. Henning in Pretoria, South Africa from an infection in garbage-fed hogs.

Habitat: Not reported from other sources as yet.

118. Salmonella sp. (Type Venezia). (Salmonella veneziana Bruner and Joyce, Jour. Bact., 50, 1945, 371.)

Antigenic structure: XI : i : e, n, x. . .

Source: Culture received from Capt. J. K. Hill. Isolated from an apparently normal Italian civilian food handler in Venice, Italy.

Habitat: Not known from other sources as yet.

119. Salmonella sp. (Type Solt)? (Salmonella solt Rauss, Ztschr. f. Immunitätsforsch., 103, 1943, 220.)

Antigenic structure: $\mathrm{XI}: \mathrm{y}: \mathbf{1}, 5 \ldots$

Source: Isolated from a healthy carrier (Hungary).

Habitat: Not reported from other sources as yet.

120. Salmonella sp. (Type St, Lucie). (Salmonella luciana Moran, Edwards and Bruner, Proc. Soc. Exp. Biol. and Med., 64, 1947, 89.) From St. Lucie, Florida.

Antigenic structure: $\mathrm{XI}$ : a: e, $\mathrm{n}$, $z_{15} \ldots$

Source: Single culture isolated by Mrs. Mildred Galton from feces of a normal human carrier.

Habitat: Not known from other sources as yet.

121. Salmonella sp. (Type Senegal). (Salmonella senegal Hinshaw and MeNeil, Jour. Bact., 52, 1946, 349.)

Antigenic structure: XI: r: 1, 5...

Source: Isolated by Dr. W. L. Hinshaw from a green mamba snake.

Habitat: Not known from other sources as yet.

122. Salmonella sp. (Type Marseille). (Salmonella marseille Moran, Edwards and Bruner, Proc. Soc. Exp. Biol. and Med., 64, 1947, 89.) 
Antigenic structure: XI: a: $1,5 . \ldots$

Source: Isolated in Marseilles, France by Capt. Wm. Sutton from feces.

Habitat: Not known from other sources as yet.

123. Salmonella sp. (Type Grumpy). (Salmonella grumpensis Hormaeche and Peluffo, quoted from Hormaeche et al., Jour. Bact., 47, 1941, 323.) Named for a person called grumpy.

Antigenic structure: IIII, XXIII, XXXVI: d: $1,7 \ldots$ as given by Kauffmann (Acta Path. et Microbiol. Scand., Suppl. 54, 1944, 37).

Source: Isolated in Lruguay from a guinea pig. Also studied by Kiauffmann (loc. cit.).

Habitat: Not reported from other sources as yet.

124. Salmonella sp. (Type Poona). (Salmonella poona Bridges and Scott, Jour. Roy. Army Med. Corps, 55, 1935, 221.)

Antigenic structure: XIII, XXII: z: $1,6 \ldots$

Source: Isolated by Dr. L. Dunbar in Poona from the stool of a child suffering from enteritis.

Habitat: Also reported from hogs (Edwards).

125. Salmonella sp. (Type Borbeck). (Salmonella borbeck Hohn and Herrimann, Cent. f. Bakt., I Abt., Orig., 145, 1940, 219.)

Antigenic structure: XIII, XXII: 1, $\mathrm{v}: 1,6 \ldots$

Source: Isolated from the feces of a child with typhoid. Found in the Borbeck section of Essen, Germany.

Habitat: Not reported from other sources as yet.

126. Salmonella sp. (Type Mississippi). (Salmonella mississippi Edwards, Cherry and Bruner, Proc. Soc. Exp. Biol. and Med., 54, 1943, 263.)

Antigenic structure: I, XIII, XXIII, $\mathrm{b}: 1,5, \ldots$
Source: Isolated by the State Dept. of Health of Mississippi from the stool of a normal food handler.

Habitat: Also reported from hogs (Edwards).

127. Salmonella sp. (Type Wichita). (Salmonella wichita Schiff and Strauss, Jour. Inf. Dis., 65, 1939, 125.)

Antigenic structure: I, XIII, XXIII: d: - .

Source: Isolated by Miss B. McKinlay in an epidemic of enteritis affecting babies, Wichita, Kansas. Also in fowls, turkeys and hogs (Edwards and Bruner, Jour. Inf. Dis., 72, 1942, 64).

Habitat: Apparently widely distributed.

128. Salmonella sp. (Type Havana). (Salmonella havana Schiff and Saphra, Jour. Inf. Dis., 68, 1941, 125.)

Antigenic structure: I, XIII, XXIII: f, g: -

Source: Isolated during an outbreak of 21 cases of meningitis in children in a maternity hospital in Havana, Cuba.

Habitat: Not reported from other sources as yet.

129. Salmonella sp. (Type Worthington). (Salmonella worthington Edwards and Bruner, Jour. Hyg., 38, 1938, 716.)

Antigenic structure: I, XIII, XXIII: $1, \mathrm{w}: \mathrm{z} \ldots$

Source: Isolated by Dr. B. S. Pomeroy from a turkey poult from Worthington, Minnesota. Also found in a hen. Later additional cultures were found in other birds, in rodents, cattle, hogs and man. (Edwards and Bruner, Jour. Inf. Dis., 72, 1943, 64).

Habitat: Apparently wideily distributed.

130. Salmonella sp. (Type Cuba). (Salmonella cubana Seligmann, Wasserman and Saphra, Jour. Bact., 51, 1946, 123.)

Antigenic structure; I, XIII, XXIII: $\mathrm{Z}_{29}:-$. 
Source: Isolated in Havana, Cuba by Dr. Arturo Curbelo from diseased baby chicks.

Habitat: Not reported from other sources as yet.

131. Salmonella sp. (Type Heves). (Salmonella heves Rauss, Ztschr. f. Immunitätsforsch., 103, 1943, 220.)

Antigenic structure: VI, XIV, IXIV: $\mathrm{d}: 1,5 \ldots$.

Source: Isolated from a healthy carrier (Hungary).

Habitat: Not reported from wther sources as yet.

132. Salmonella sp. (Type Carrau). (Salmonella carrau Hormaeche, Peluffo and Salsamendi, Arch. Lrug. de Med. Cirug. y Espec., 12, 1938, 377; Hormaeche, Peluffo and Perevra. Jour. Bact., 47, 1944, 323.)

Antigenic structure: VI, XIV, XXIV: $\mathrm{y}: 1,7 \ldots$

Source: Isolated in Lruguay from mesenteric glands of normal hogs.

Habitat: Also reported from feces and blood in man, once from flies and one culture from human blond from Mexico.

133. Salmonella sp. (Type Onderstepoort). (Salmonella onderstepoont Henning, Jour. Hyg., 36, 1936, 525.)

Antigenic structure: (I), VI, XIV, XXV: e, (h): 1, 5. .

Source: Isolated in So. Africa by 1)r.

J. H. Mason from sheep in Onderstepoort. Also isolated from man by $\mathrm{I}$ r. Hormaeche (Uruguay) and from turkeys (Edwards, Kentucky).

Habitat: Appitrently widely distributed in warm-blooded animals.

134. Salmonella sp. (Type Florida). (Salmonella florida Cherry, Edwards and Bruner, Proe. Soe. Exp. Biol. and Med.. 52, 1943, 125; Galton and Quan, Amer. Jour. Hyg., 38, 1943, 173.)

Antigenic structure: (I), II, XIV, XXV: d: $1,7 \ldots$
Source: Isolated by Mrs. Mildred Galton from feces of a patient with a febrile disease and diarrhoea.

Habitat: Also reported from reptiles (Edwards).

135. Salmonella sp. (Type Madelia). (Salmonella madelia Cherry, Edwards and Bruner, Proc. Soc. Exp. Biol. and Ned., 52, 1943, 125.)

Antigenic structure: (I), VI, XIV, $\mathrm{XXV}: \mathrm{y}: 1, \mathrm{i}$

Source: A single culture isolated by Dr. B. S. Pomeroy from the liver of a poult that died of septicemia. Found in Madelia, Minnesota.

Habitat: Also reported from man (Edwards).

136. Salmonella sp. (Type Sundsvall). (Salmonella sundsvall Olin and Alin, Acta Path. et Mierobiol. Scand., 20, $1943,607$.

Antigenic structure: (I), VI, XIV, XXV: $z: e, n, x \ldots$

Source: Isolated from a person suffer ing from gastroenteritis.

Habitat: Not reported from wher sources as yet.

137. Salmonella sp. (Type Orient). (Salmonella orientalis Carlquist and Conte, Bull. I'. '. Army Med. Dept., 6, $1946,343$.

Antigenir structure: XVI: k: e, n, $\mathrm{z}_{15} \ldots$.

Source: Isolated from U. S. Army personnel who had been prisoners of the Japanese Army in the Orient.

Habitat: Not known from other sources as yet.

138. Salmonella sp. (Type Hvittingfoss). (Salmonella hvittingfoss Tesdal, Ztschr. f. Hyg., 118, 1936, 533.)

Antigenic structure: XVI: b: e, $\mathbf{n}$. x. . .

Source: Isolated during a food poisoning outbreak in Hvittingfoss, a small town in Norway. Caused by eating 
pultoste, a kind of soft cheese. Cultures secured from the cheese, from the persons who were poisoned, from sewage and from a foal.

Habitat: Evidently rather nidely distributed.

139. Salmonella sp. (Type Gaminara). (Salmonella gaminara Hormaeche, Peluffo and Salsamendi, Arch. Urug. de Med., Cirug. y Espec., 12, 1938, 377; ibid., 14, 1939, 217.) Named in honor of Prof. Gaminara of Uruguay.

Antigenic structure: XVI: $d: 1,7 \ldots$.

source: Isolated from the feces of a child suffering from enteritis.

Habitat: Not known from other sources as yet.

140. Salmonella sp. (Type Szentes). (Salmonella szentes Rauss, Ztschr. f. Immunitätsforsch., 103, 1943, 220.)

Antigenic structure: XVI: k: 1, 2, 3. . .

Source: Isolated by Dr. K. Rauss from a healthy carrier (Hungary).

Habitat: Not reported from other sources as yet.

141. Salmonella sp. (Type Kirkee). (Salmonella kirkee Bridges and Dunbar, Jour. Roy. Army Med. Corps, 67, 1936, 289.)

Antigenic structure: XVII : b: $1,2 \ldots$.

Source: Isolated in Kirkee, India from the feces of a child suffering from acute enteritis. The source of the infection was thought to be a dog.

Habitat: Not reported from other sources as yet.

142. Salmonella sp. (Type Cerro). (Bacterium cerro Hormaeche, Peluffo and Salsamendi, Arch. Urug. Med., Cirug. y Espec., 12, 1938, 37т; Salmonella cerro Hormaeche, Peluffo and Aleppo, ibid., 19, 1941, 125.)

Antigenic structure: XVIII: $z_{4}, z_{23}$, $\mathrm{z}_{25}$ : -

Source: Isolated from the mesenteric glands of normal hogs from Cerro, Uruguay.
Habitat: Also isolated by the authors in 13 cases of infantile infections. Found also in chickens (Edwards).

143. Salmonella sp. (Type Minnesota). (Salmonella minnesota Edwards and Bruner, Jour. Hyg., 38, 1938, 716.)

Antigenic structure: XXI, XXVI: $\mathrm{b}: \mathrm{e}, \mathrm{n}, \mathrm{x} . \ldots$

Source: Isolated in Minnesota by Dr. B. S. Pomeroy from a young turkey.

Habitat: Also reported from eattle and man.

141. Salmonella sp. (Type Tel Aviv). (Salmonella tel-aviv Kauffmann, Acta Path. et Microbiol. Seand., 17, 1940, 1.)

Antigenic structure: XXYIII: y: e, $\mathrm{n}, \mathrm{z}_{15} \ldots$.

Source: Isolated in Tel Aviv, Palestine by Dr. G. B. Simmins during an epizootic affecting young chickens during which 50 per cent died.

Habitat: Not known from other sources as yet.

145. Salmonella sp. (Type Pomona). (Salmonella pomona Edwards, Proc. Soc. Exp. Biol. and Med., 58, 1945, 291.)

Antigenic structure: XXYIII: y: 1, 7....

Source: Single culture isolated from the intestine of a poult in 1941 by Dr. W. R. Hinshaw.

Habitat: Also reported from man (Edwards).

146. Salmonella sp. (Type Ballerup). (Salmonella ballerup Kauffmann and Møller, Jour. Hyg., 40, 1940, 246.)

Antigenic structure: XXIX, [Vi]: $\mathrm{Z}_{14}:$ -

source: From the feces of a woman from the town of Ballerup, Denmark. A cause of gastroenteritis.

Habitat: No knomn from other sources as yet.

147. Salmonella sp. (Type Hormaeche). (Salmonella hormaechei Monteverde, Nature, 154, 1944,676.) Named in honor of Dr. Hormaeche of Uruguay. 
Antigenic structure: $\mathrm{NXIX},\left[\mathrm{Vi}^{*}\right.$ : $z_{30},\left[z_{i 1}\right]:-\cdots$.

Source: From the ovary of a hen whose blood gave a positive reaction with the S. pullorum antigen. Found in Buenos Aires by Dr. Monteverde.

Habitat: Also reported from hogs and man (Edwards)

*Reported by Dr. P. R. Edwards (personal communication).

148. Salmonella sp. (Type Lrbana). (Salmonella urbana Edwards and Bruner, Jour. Inf. Dis., 69, 1941, 223.)

Antigenic structure: XXX: b: e, n, $x$. .

Source: One culture was received from Dr. Robert Graham, Lrbana, Illinois and was isolated from the contents of the colon of a hog affected with hemorrhagic enteritis. The second culture was isolated from the intestinal tract of a chicken by IJr. W. L. Mallmann, East Lansing, Michigan.

Habitat: Also reported from man (Edwards)

149. Salmonella sp. (Type Adelaide). (Salmonella adelaide Cleland. Med. Jour. Australia, 31, 1944, 59.)

Antigenic structure: XXXY: f, g: -

Source: Isolated in Adelaide, Australia by Miss Nancy Atkinson from two fatal cases resembling typhoid fever.

Habitat: Not reported from other sources as yet.

150. Salmonella sp. (Type Inverness). (Salmonella inverness Edwards and Hughes, Proc. Soc. Exp. Biol. and Med., $56,1944,33$.)

Antigenic structure: XXITIII k: $1,6 \ldots$

Source: Isolated by Mrs. Nildred Galton and Mr. MI. S. Quin of the Florida State Department of Health, from the stool of a normal food handler. Inverness, Florida

Habitat: Not reported from other sources as yet.

151. Salmonella sp. (Type ('hampaign). (Salmonella champaign Ed- wards, Proc. Soc. Exp. Biol. and Med., $58,1945,291$.)

Antigenic structure: XXXIX: $k$ : $1,5 \ldots$

Source: Single culture isolated from the liver of an adult hen by Dr. Robert Graham, Champaign, Illinois.

Habitat: Not reported from other sources as yet.

Appendix I: The following species and varieties are largely taken from Hauduroy, Ehringer, Urbain, Guillot and Magrou, Dictionnaire des Bactéries Pathogènes, Paris, 1937, 446-472. The relationships of many of these are not clear.

Bacillus canariensis Migula. (Bacillus der Kanarienvögelseptikämie, Ricek, Deutsche Ztschr. f. Thiermed., 15, 1889, 69; Migula, Syst. d. Bakt., 2, 1900, 770; Bacillus avisepticus Chester, Man. Determ. Bact., 1901, 220; not Ba. cillus avisepticus Kitt, in Folle and Wassermann, Handb. d. path. Mikroorg., 1 Aufl., 2, 1903, 544.) Associated with intestinal catarrh and liver changes in canaries. Hadley, Elkins and Caldwell (Rhode Island Agr. Exp. Sta., Bull. 174, $1918,178)$ regard this as probably $\mathrm{Ba}$ cillus gallinarum Klein.

Bacillus fricdebergensis Kruse. (Bacillus der Friedeberger Fleischvergiftung, Gaffky and Paak, Mitt. a. d. kaiserl. Gesundheitsamte, 6, 1890, 159; Kruse, in Flügge, Die Mikroorganismen, 3 Aufl., 2, 1896, 378; Bacterium friedebergensis Chester, Ann. Rept. Del. Col. Agr. Exp. Sta., 9, 1897, 73.) From sausage in meat poisoning.

Salmonella abortus canis Gard. (Ztschr. f. Hyg., 121, 1938, 139.) From the feces of four persons with paratyphoid apparently spread from an infected dog. Kitufimann regards this as identical with Salmonella schottmuelleri.

Salmonella annamensis Hauduroy et al. (Un bacille du groupe des Salmonella, Normet, Urbain and Chaillot, Compt. rend. Soc. Biol., Paris, 101, 1929, 752; Hauduroy et al., Dict. d. Bact. Path., 
1937, 450.) Isolated during an epidemic of dysentery at Hué (Annam) in 1925.

Salmonella archibaldii Castellani and Chalmers. (Man. Trop. Med., 3rd ed., 1919, 940.)

Salmonella carolina (Castellani) Castellani and Chalmers. (Bacillus carolinus Castellani, Ann. di Med. Nav. e Colon., 1, 1918; Castellani and Chalmers, Man. Trop. Med., 3rd ed., 1919, 940.)

Salmonella coagulans (Castellani) Hauduroy et al. (Bacillus coagulans Castellani, 1916; Balkanella coagulans C'astellani, 1916; see Castellani and Chalmers, Man. Trop. Med., 3rd ed., 1919, 935; Hauduroy et al., Dict. d. Bact. Path., 1937, 453.)

Salmonella columbensis (Castellani) Castellani and Chalmers. (Bacterium columbense Castellani, Proc. Mecting Ceylon Branch British Assoc., 1905, quoted from Castellani, Cent. f. Bakt., I Abt., Orig., 74, 1914, 197; Bacillus columbensis Castellani, Jour. Trop. Med. and Hyg., 20, 1917, 181; Castellani and Chalmers, Ann. Inst. Past., 34, 1920, 609; Morganclla columbensis Fulton, Jour. Bact., 46, 19+3, 81.) The cause of columbensis fever. Isolated from feces, urine and blood.

Salmonella enteritidis var. v, Hauduroy et al. (Bacille para-Gärtner $V$, Rochaix and Couture, Revue de Microbiologie appliquée, 2, 1936; Hauduroy et al., Dict. d. Bact. Path., 1937, 45-) Found associated with Salmonella enteritidis in meat pies and in the feces of individuals with food poisoning.

Salmonella enteritidis-yellow, a variety of Salmonella enteritidis Deskowitz and Buchbinder (Jour. Bact., 29, 1935, 293). Cultures differ from typical Salmonella enteritidis in producing a yellow, watersoluble pigment. From the feces of a rat with enteric infection.

Salmonella foctida Bergey et al. (Coccobacill's foetidus ozenae Perez, Ann. Inst. Past., 13, 1899, 937; Coccobacillus (foetidus) ozaenae Ward, Jour. Bact., 2, 1917, 619; Bergey et al., Manual, 1st ed., 1923, 220; Bacterium foetida Weldin and
Levine, Abst. Bact., 7, 1923, 13; Escherichia foetida Bergey et al., Manual, 2nd ed., 1925, 222.) From chronic rhinitis, ozena. See Manual, 4th ed., 1934, 380 for a description of this species.

Salmonella holsatiensis Roelcke. (Also Salmonella Typ Holstein, Roelcke, Cent. f. Bakt., I Abt., Orig., 137, 1936, 464.) According to Kauffmann (Ztschr. f. Hyg.. 119, 1937, 352) the O-antigens of this rapid fermenter of salicin and weak indole-former are identical with those of Salmonella poona. The H-antigens have not been compared as yet.

Salmonella icteroides (Sanarelli) Bergey et al. (Bacillo icteroide, Sanarelli, Il Policlinico, \&, 1897, 412 ; Bacillus icteroides Sanarelli, British Med. Jour., July 3, 1897, 7 ; Bacterium icteroides Lehmann and Teumann, Bakt. Diag., 2 Aufl., 2, 1899, 241; Bergey et al., Ianual, 1st ed., 1923, 218.) From yellow fever cadavers. See Manual, 5th ed., 1939, 604 for a description of this species.

Salmonella iwo-jima Lindberg and Bayliss. (Jour. Inf. Dis., 79, 1946, 92.) Isolated from a soldier on IwoJima during a routine examination of food handlers. Belongs to Group C Antigenic structure: VI, VIII: i: $1,5 \ldots$ Described too recently to be included in the main body of the text.

Salmonella liceagi Leon. (Rev. Inst. Salubridad y Enferm. Trop., 3, 19.42, 273.) From feces. This probably belongs in the coliform group.

Salmonella macfadyeanii (Weldin and Levine) Weldin. (Bacterium macfadyeanii Weldin and Levine, Abst. Bact. 7, 1923, 13; Weldin, Iowa State Jour. Sci., 1, 1927, 168.) Associated with hog cholera.

Salmonella mexicana Varela and Olarte. (Rev. Inst. Salubridad y Enferm. Trop., 4, 1943, 313.) From feces.

Salmonella monshaui Carlquist and Coates. (Jour. Bact., 53, 1947, 249.) Isolated from stump of a soldier who suffered traumatic amputation of a leg in the fighting around Monshau, Ger- 
many. Belongs to Group F. Antigenic structure: XXYV: m. t.:-Described too recently to be included in the main body of the text.

Salmonella nocardi Pacheco. (Compt. rend. Soc. Biol., Paris, 106, 1931, 372 and 1018.) Pathogenic for parrots and pigeons.

Salmonella oahu Lindberg and Bayliss. (Jour. Inf. Dis., 79, 1946, 92.) Isolated from a case of gastroenteritis in a soldier hospitalized on Oahu. Belongs to Group B. Antigenic structure: IV, I, XII: $1, \mathrm{v}: 1,2,3 \ldots$ Described too recently to be included in the main body of the text.

Salmonella ostrei (Besson and Ehringer) Hauduroy et al. (Bacillus ostrei Besson and Ehringer, Compt. rend. Soc. Biol., Paris, 87, 1922, 1017; Hauduroy et al., Dict. d. Bact. Path., 1937, 460.) Isolated from oysters. Not pathogenis for laboratory animals.

Salmonella para-asiatica (Castellani) Hauduroy et al. (Bacillus paraasiaticus Castellani, 1916; see Castellani and Chalmers, Man. Trop. Med., 3rd ed., 1919, 950; Hauduroy et al., Dict. d. Bact. Path., 1937, 461.)

Salmonella para-coagulans (Castellani) IIauduroy et al. (Bacillus para-coagulans Castellani, 1914; see Castellani and Chalmers, Man. Trop. Med., 3rd ed., 1919, 950; Hauduroy et al., Dict. d. Bact. Path., 1937, 461.)

Salmonella pauloensis Gomes. (Rev. Inst. Adolfo Lutz, 2, 1942, 231.) May be the same as Salmonella columbensis.

Salmonella pseudo-asiatica (Castellani) Castellani and Chalmers. (Bacillus pseudo-asiaticus Castellani, Cent. f. Bakt., I Abt., Orig., 65, 1912, 266; Castcllani and Chalmers, Man. Trop. Med., 3rd ed., 1919, 940.)

Salmonella pseudo-asiatica var. mobilis Hauduroy et al. (Bacillus pseudoasiaticus mobilis Castellani, see Castellimi and Chalmers, Man. Trop. Med., 3rd ed., 1919, 952; Hauduroy et al., Dict. d. Bact. Path., 1937, 463.)

Salmonella (?) pseudo-carolina (Cas- tellani) Hauduroy et al. (Bacillus pseudocarolinus Castellani, 1917; see Castellani and Chalmers, Man. Trop. Med., 3rd ed., 1919, 952; Hauduroy et al., Dict. d. Bact. Path., 1937, 463.)

Salmonella (?) pseudo-columbensis (Castellani) Hauduroy et al. (Bacillus pseudo-columbensis Castellani, see Castellani and Chalmers, Man. Trop. Med., 3rd ed., 1919, 954; Hauduroy et al., Dict. d. Bact. Path., 1937, 464.)

Salmonella pseudo-morganii (Castellani) Hauduroy et al. (Bacillus pseudomorgani Castellani, see Castellani and Chalmers, Man. Trop. Med., 3rd ed., 1919, 951; Hauduroy et al., Dict. d. Bact. Path., 1937, 464.)

Salmonella ranicida Hauduroy et al. (Bacille pathogène isolé des grenouilles, Gheorghiu and Balmus, Compt. rend. Soc. Biol., Paris, 108, 1931, 1002; Hauduroy et al., Dict. d. Bact. Path., 1937, 466.) Pathogenic for frogs.

Salmonella saipan Lindberg and Bayliss. (Jour. Inf. Dis., 79, 1946, 92.) Isolated from a case of gastroenteritis in a soldier hospitalized on Saipan. Belongs to Group E. Antigenic structure: III, X, XXVI: $z_{6} \ldots$ Described too recently to be included in the main borly of the text.

Salmonclla schotmülleri var. alvei Hauduroy et al. (Bacillus paratyphi alvei Bahr, Skand. Veterin. Tidsk., 9, 1919; Hauduroy et al., Diet. d. Bact. Path., 1937, 469.) Pathogenic for bees and wasps.

Salmonella veboda (Castellani) Castellani and Chalmers. (Bacillus veboda Castellani, Jour. Trop. Med. and Hyg., 20, 1917, 181; Castellani and Chalmers, Man. Trop. Med., 3rd ed., 1919, 939; Bacterium veboda Weldin and Levine, Abst. Bact., 7, 1923, 13.)

Salmonella watareka (Castellani) Bergey et al. (Bacillus waiareka Castellani, Rept. Advisory Committee for Trop. Dis. Research Fund for 1912, London, 1913; Bacterium watareka Weldin and Levine, Abst. Bact., \%, 1923, 13; Bergey et al., Manual, 1st ed., 1923, 219.) 
Salmonella werahensis (Castellani) Hauduroy et al. (Bacillus werahensis Castellani, see Castellani and Chalmers, Man. Trop. Med., 3rd ed., 1919, 956; Hauduroy et al., Dict. d. Bact. Path., 1937,471 .)

Salmonella wesenbergoides (Castellani) Hauduroy et al. (Bacillus vesenbergoides Castellani, 1916; see Castellani and Chalmers, Man. Trop. Med., 3rd ed., 1919, 935; Hauduroy et al., Dict. d. Bact. Path., 1937, 471.)

Salmonella willegoda (Castellani) Castellani and Chalmers. (Bacillus willegoda Castellani; Castellani and Chalmers, Man. Trop. Med., 3rd ed., 1919, 939.)

Salmonclla woliniae (Castellani) Castellani and Chalmers. (Bacillus woliniae Castellani, Jour. Trop. Med. and Hyg., 20, 1917, 181; Castellani and Chalmers, Man. Trop. Med., 3rd ed., 1919, 939; Bacterium woliniae Weldin and Levine, Abst. Bact., 7, 1923, 13.)

Appendix II: The following species have been thought to belong to the genus Eberthella, i.e., do not produce gas from glucose. Descriptions of nearly all of the species listed in the genus Eberthella will be found in the Manual, 5th ed., 1939, 464-169.

Bacillus subentericus Ford. (Studies from the Royal Victoria Hosp., Montreal, 1, 1903, 40; also see Jour. Med. Res., 1, 1901, 218.) From feces.

Bacterium typhi flavum Dresel and Stickl. (Deutsche med. Wchnschr., 54, 1928, 517.) From feces of persons with typhoid fever. Cruickshank (Jour. Hyg., 35, 1935, 354) reports that a variety of yellow chromogenic saprophytes have been identified as belonging to this species, none of which could be regarded as yellow variants of Salmonella typhosa (Zopf) White. They apparently belong in the genus Flavobacterium Bergey et al.

Eberthella alcalifaciens de Salles Gomes. (Rivista do Inst. Adolfo Lutz,
4, 1944, 191.) From catarrhal feces of an infant.

Eberthella belfastiensis (Weldin and Levine) Bergey et al. (Bacterium coli anaerogenes Lembke, Arch. f. Hyg., 26, 1896, 299; Bacterium lembkei Migula, Syst. d. Bakt., 2, 1900, 417; Bacterium anaerogenes Chester, Man. Determ. Bact., 1901, 135; Bacillus belfastiensis II, Wilson, Jour. Hyg., 8, 1908, 5ł3; Bacillus anaerogenes Holland, Jour. Bact., 5, 1920, 217; Bacterium belfastiensis Weldin and Levine, Abst. Bact., 7, 1923, 13; Bergey et al., Manual, 1st ed., 1923, 226; Bacillus coli anaerogenes Kerrin, Jour. Hyg., 28, 1928, 4; Escherichia anaerogenes Bergey et al., Manual, 3rd ed., 1930, 321; Castellanus colianaerogenes Castellani, Cent. f. Balt., I Abt., Orig., 125, 1932, 42.) From feces.

Eberthella bentotensis (Castellani and Chalmers) Bergey et al. (Bacillus bentotensis Castellani, Cent. f. Bakt., I Abt., Orig., 65, 1912, 262; Bacterium bentotensis Weldin and Levine, Abst. Bact., 7, 1923, 15; Bergey et al., Manual, 1st ed., 1923, 227; Castellanus bentotensis Castellani, Cent.f. Bakt., I Abt., Orig., 125, 1932, 42.) From the intestinal canal.

Eberthella chylogena (Ford) Bergey et al. (Bacillus chylogenes Ford, Studies from the Royal Victoria Hospital, Montreal, 1, No. 5, 1903, 62; Bergey et al., Manual, 1st ed., 1923, 224.) From the intestinal canal.

Eberthella dubia (Chester) Bergey et al. (Meiner Bakterie, Bleisch, Ztschr. f. Hyg., 13, 1893, 31; Bacillus dubius Liruse, in Flügge, Die Mikroorganismen, 3 Aufl., 2, 1896, 323; Bucillus bleischii Lruse, ibid., 704; Bacterium dubius Chester, Ann. Rept. Del. Col. Agr. Exp. Sta., 9, 1897, 93; Bergey et al., Manual, 1st ed., 1923, 225.) From the intestinal canal.

Eberthella enterica (Ford) Bergey et al. (Bacillus entericus Ford, Studies from the Royal Victoria Hospital, Montreal, 1, No. 5, 1903, 40; also see Jour. Med. Research, 1, 1901, 211; not Bacillus 
entericus Castellani, 1907 (Enteroides entericus Castellani and Chalmers, Man. Trop. Med., 3rd ed., 1919, 941); Bergey et al., Manual, 1st ed., 1923, 223.) From the intestinal canal.

Eberthella insecticola Steinhaus. (Jour. Bact., 42, 1941, 762 and 769.) From the intestinal tracts of grasshoppers, milkweed bugs and stinkbugs.

Eberthella kandiensis (Castellani) Bergey et al. (Bacillus kandiensis Castellani, Cent. f. Bakt., I Abt., Orig., 65, 1912, 262; Eberthus kandiensis Castellani and Chalmers, Man. Trop. Med., 3rd ed., 1919, 936; Bacterium kandiensis Weldin and Levine, Abst. Bact., 7, 1923, 13; Bergey et al., Manual, 1st ed., 1923, 225.) From feces.

Eberthella lewisii Weldin. (Organism B3., Lewis, Local Gov. Board Rept. Med. Suppl. London, 1910-11, Appen. B, No. 2, 1911, 314; Bacterium lewisii Weldin and Levine, Bact. Abst., $7,1923,13$; Weldin, Iowa State Col. Jour. Sci., 1, 1926, 172.) From feces of a normal child.

Eberthella oedematiens Assis. (Boletin do Inst. Vital, Brazil, 5, 1928,) From the intestinal canal.

Eberthella oxyphila (Ford) Bergey et al. (Bacterium oxyphilum Ford, Studies from the Royal Vietoria Hospital, Montreal, 1, No. 5. 1903, 49; Bergey et al., Manual, 1st ed., 1923, 224.) From the intestinal canal.

Eberthella pauloensis Mello. (Jornal dos Clinicos, Rio de Janeiro, No. 18-30, Sept., 1937, 7 pp.) From feces of a dysentery patient.

Eberthella priztnitzi (Custellani and Chalmers) Hauduroy et al. (Bacillus priztnitzi Castellani, Jour. Trop. Med. and Hyg., 20, 1917, 182; Eberthus prizlnitzi Castellani and Chalmers, Man. Trop. Med., 3rd ed., 1919, 936; Bacterium priztnitzi Weldin and Levine, Abst. Bact., 7, 1923, 13; Hauduroy et al., Dict. d. Bact. Path., 1937, 186.) From cases of paraenteric fever.

Eberthella proteosimilis Wassilien.
(Cent. f. Bakt., I Abt., Orig., 151, 1944, 423.) Colonies show motility on agar. From feces of a dysentery patient.

Eberthella pyogenes (Migula) Bergey et al. (Bacillus pyogenes foetidus Passet, Fortschr. der Med., 1885; Bacillus foetidus Trevisan, I generi e le specie delle Batteriacee, 1889, 16; Bacterium pyogenes foetidus Chester, Ann. Rept. Del. Col. Agr. Exp. Sta., 9, 1897, 141; Bacterium pyogenes Migula, Syst. der Bakt., 2, 1900, 381; Lankoides pyogenes Castellani and Chalmers, Man. Trop. Med., 3rd ed., 1919, 938; Bacillus pyogenes-foetidus Holland, Jour. Bact., 5, 1920 , 220; Bergey et al., Manual, 1st ed., 1923, 226; Castellanus pyogenes Castellani, Cent. f. Bakt., I Abt., Orig., 125, $1932,42$.$) From a rectal abscess.$

Eberthella talaiensis (Castellani) Bergey et al. (Bacillus talavensis Castellani, Cent. f. Bakt., I Abt., Orig., 65, 1912, 262; Eberthus talavensis Castellani and Chalmers, Man. Trop. Med., 3rd ed., 1919, 936; Bacterium talavensis Weldin and Levine, Abst. Bact., 7, 1923, 13; Bergey et al., Manual, 1st ed., 1923, 225.) From the intestinal canal.

Ebcrthella larda Assis. (Boletin do Inst. Vital, Brazil, 5, 1928.) From the intestinal canal.

Eberthella wesenbergi (Castellani and ('halmers) Hauduroy et al. (Bacillus wesenberg Castellani, 1913; Wesenbergus wesenbergi Castellani and Chalmers, Man. Trop. Med., 3rd ed., 1919, 940; Hauduroy et al.. Dict. d. Bact. Path., $1937,191$.

Eberthella wilsonii Weldin. (Bacillus belfastiensis 1-, Wilson, Jour. Hyg., 8, 1908, 543; Weldin, Iowa State Col. Jour. Sci., 1, 1926, 17t.) From feces.

Eberthella renopa Schrire. (Trans. Poyal Soe. So. Africa, $17,1928,43$. From wound infection in frogs.

Wesenbergus fermentosus Castellani and Chalmers. (Man. Trop. Med., 3rd ed., 1919,940.) From blood. Isolated by Arehibald in the Anglo-Egyptian Sudan. 


\section{Genus II. Shigella Castellani and Chalmers.*}

(Castellani and Chalmers, Man. Trop. Med., 3rd ed., 1919, 936; subgenera, Flexnerella and Shigella, Castellani and Chalmers, ibid., 938; Castellanus Carruti, Jour. Trop. Med. and Hyg., July 15, 1930; Proshigella Borman, Stuart and Wheeler, Jour. Bact., 48, 1944, 363.) Named for Prof. I. Shiga, the Japanese bacteriologist who discovered the dysentery bacillus in 1898 .

Ton-motile rods, although cultures of some of the less well-known species have been reported as motile. Produce acid but no gas from carbohydrates except with some types of Shigella paradysenteriae. Do not liquefy gelatin. Some species produce acid from lactose and form indole. Some species reduce trimethylamine oxide to trimethylamine, others do not. $\dagger$ Some species will grow at $45.5^{\circ} \mathrm{C}$ (Eijkman test). \pm Pathogenic (causing dysenteries) or non-pathogenic species, all living in the bodies of warm-blooded animals. Carried by polluted water supplies and by flies.

The type species is Shigella dysenteriae (Shiga) Castellani and Chalmers.

\section{Key to the species of genus Shigella.*k}

I. No acid from mannitol.

1. To acid from lactose. Milk not coagulated.

1. Indole not produced.

a. Acid but no gas from glucose.

1. Shigella dysenteriae.

aa. Acid and a small amount of gas from glucose.

4a. See Shigella paradysenteriae

(Type Newcastle).

2. Indole produced.

2. Shigella ambigua.

B. Acid formed slowly from lactose.

1. Indole not produced.

3. Shigella gintottensis.

II. Acid from mannitol (one type produces a small amount of gas).

1. To acid from lactose.

1. No acid from rhamnose, xylose or dulcitol.

2. Acid from rhamnose, xylose and dulcitol.

4. Shigella paradysenteriae.

5. Shigella alkalescens.

3. Acid from xylose but not from dulcitol.

6. Shigella pfaffii.

B. Acid formed slowly from lactose.

1. Indole not produced.

a. Acid from rhamnose. Tone from xylose.

7. Shigella sonnei.

aa. No acid from rhamnose. Acid from xylose.

8. Shigella equirulis.

* Completely revised by Dr. Frederick Smith, McGill University, Montreal, P. Q.: Canada, December, 1938; further revision, April, 1946.

$\dagger$ Wood, Baird and Keeping, Jour. Bact., 46, 1943, 106.

‡ Stuart and Rustigian, Jour. Bact., 46, 1943, 105.

** See Weil, Jour. Immunology, 55, 1947, 363-405. 
2. Indole produced.

a. Acid from dulcitol.

aa. No acid from dulcitol.

III. Action on mannitol unknown.

A. No acid from lactose.

1. Indole is produced.

1. Shigella dysenteriae (Shiga) Castellani and Chalmers. (Bacillus of Japanese dysentery, Shiga, Cent. f. Bakt., I Abt., 23, 1898, 599; Bacillus dysenteriae Shiga, Cent. f. Bakt., I tht., 2', 1898, 817; Bacillus japanicus Migula, Syst.d. Bakt., 2, 1900, 755; Bacillus shigae Chester, Man. Determ. Bact., 1901, 228; Bacillus dysentericus Ruffer and Willmore, Brit. Med. Jour., 2, 1909, 862; Bacterium dysenteriae Lehmann and Neumann, Bakt. Diag.,5 Aufl.,.2, 1912,348; not Bacterium dysenteriae Chester, Man. Determ. Bact., 1901, 145; Castellani and Chalmers, Man. Trop. Med., 3rd ed., 1919, 935; Bacterium shigae Holland, Jour. Bact., 5, 1920, 220; Ebcrthella dysenteriae Bergey et al., Manual, and ed., 1925, 250.) Latinized, of dysentery.

Rods: 0.4 to 0.6 by 1.0 to 3.0 microns, occurring singly. Non-motile. Gramnegative.

Gelatin colonies: Small, grayish, smooth, homogeneous, entire to slightly undulate.

Gelatin stab: Grayish surface growth. No liquefaction.

Agar slant: Grayish, filiform to echinulate, smooth, entire to undulate growth.

Broth: Slightly turbid, with grayish sediment.

Litmus milk: Slightly acid, then alkaline.

Potato: Delicate, grayish to slightly brownish streak.

Indole not produced.

Nitrites produced from nitrates.

Acid but no gas from glucose, fructose, raffinose, glycerol and adonitol. Joes not attack arabinose, xylose, maltose,
9. Shigella ceylonensis.

10. Shigella madampensis.

\section{Shigella septicemiae.}

lactose, sucrose, salicin, mannitol, dulcitol or rhamnose.

Does not reduce trimethylamine oxide (Woor et al., Jour. Bact., 46, 1943, 106).

Aerobic, facultative.

Optimum temperature $37^{\circ} \mathrm{C}$. Does not grow at $45.5^{\circ} \mathrm{C}$ (Eijkman's reaction, Stuart et al., Jour. Bact., $46,1943,105$ ).

Serologically homogeneous and different from the other species of Shigella. Forms a potent exotoxin.

Source: From widespread epidemics of dysentery in Japan.

Habitat: A cause of dysentery in man and monkeys.

2. Shigella ambigua (Andrewes) Weldin. (Bazillus Schmitz, Schmitz, Ztschr. f. Hyg., 84, 1917, 449; Bacillus ambiguus Andrewes, The Lancet, 194, 1918, 560; Bacillus dysenteriae "Schmitz", Murray, Jour. Roy. Army Med. Corps, 31, 1918, 257; Bacterium ambiguum Levine, Abst. Bact., 4, 1920, 15; not Bacterium ambiguum Chester, Del. Col. Agr. Exp. Sta. Ann. Rept., 11, 1900, 59; Eberthella ambigun Bergey et al., Manual, 1st ed., 1923, 229; Bacillus paradysenteriae $X$, Stutzer, Cent. f. Bakt., I Abt., Orig., 90, 1923, 12; Bacterium schmilzii Weldin and Levine, Abst. 13act., 7, 1923, 13; Weldin, Iowa State College Jour. Sci., 1, 1927, 177; Shigella schmilzii Hauduroy et al., Dict. d. Bact. Path., 1937, 496.) From Latin, uncertain.

Morphology and colony characters indistinguishable from those of Shigella dysenteriae.

Acid from glucose and rhamnose. 
Does not attack xylose, maltose, lactose, sucrose, dextrin, glycerol, mannitol or dulcitol.

Indole is produced.

Does not reduce trimethylamine oxide (Wood et al., Jour. Bact., 46, 1943, 106). Aerobic, facultative.

Optimum temperature $37^{\circ} \mathrm{C}$. Does not grow at $45.5^{\circ} \mathrm{C}$ (Stuart et al., Jour. Bact., 46, 1943, 105).

Serologically homogeneous and different from the other species of Shigelia. Does not form an exotoxin.

Source: Found in feces in a dysentery epidemic in a prison in Germany.

Habitat: A cause of human dysentery.

3. Shigella gintottensis (Castellani) Hauduroy et al. (Bacillus gintoltensis Castellani, 1910; see Castellani and Chalmers, Man. Trop. Med., 3rd ed., 1919, 948; Lankoides gintottensis Castellani and Chalmers, ibid., 938; Castellanus gintottensis Castellani, 1930; Castellani, Jour. Trop. Med. and Hyg., 36, 1933, 109; Hauduroy et al., Dict. d. Bact. Path., 1937, 488.)

Rods: Non-motile. Gram-negative.

Morphology and cultural characters indistinguishable from those of Shigella. dysenteriae.

Litmus milk: Acid and coagulation; decolorized.

Indole not formed.

Acid, but no gas, from lactose, glucose, arabinose and galactose. No acid from sucrose, dulcitol, mannitol, maltose, dextrin, raffinose, adonitol, inulin, sorbitol, levulose, inositol, salicin and glycerol.

Antigenic structure not known.

Source: From feces in cases of dysentery.

Habitat: A cause of human dysentery.

4. Shigella paradysenteriae (Collins) Weldin. (Bacillus dysenteriae Flexner, Phil. Med. Jour., 6, 1900, 414; Bacillus dysenteriae Hiss and Russell, Medical News, 82, 1903, 289; Bacillus dysenteriae Strong, Jour. Amer. Med. Assoc., 35,
1906, 498; Bacillus paradysenteriae Collins, Jour. Inf. Dis., 2, 1905, 620; includes weakly toxic strains of dysentery bacilli, Groups I and II, Sonne, Cent. f. Bakt., I Abt., Orig., 75, 1915, 408; Shigella flexneri Castellani and Chalmers, Man. Trop. Med., 3rd ed., 1919, 937; Shigella dysenteriae (Hiss and Russell, and Strong types) Castellani and Chalmers, ibid., 937; not Shigella paradysenteriae Castellani and Chalmers, ibid., 937; Bacillus flexneri Levine, Jour. Inf. Dis., 27, 1920, 31; Bacterium flexneri Levine, Abst. Bact., 4, 1920, 15; Bacterium dysenteriae (Flexner type) and Bacterium paradysenteriae Holland, Jour. Bact., 5, 1920, 215; Eberthella flexneri Weldin and Levine, Abst. Bact., 7, 1923, 13; Eberthella paradysenteriae Bergey et al., Manual, 1st ed., 1923, 230; Weldin, Iowa State College Jour. Sci., 1, 1927, 178.) Latinized, like dysentery.

Rods : 0.5 by 1.0 to 1.5 microns. Nonmotile. Gram-negative.

Morphologically these organisms are like Shigella dysenteriae.

Culturally these organisms differ from Shigella dysenteriae in that they ferment mannitol. No acid is produced from lactose, rhamnose, xylose or dulcitol.

Does not reduce trimethylamine oxide (Wood et al., Jour. Bact., 46, 1943, 106).

Does not form a potent exotoxin.

Aerobic, facultative.

Optimum temperature $37^{\circ} \mathrm{C}$. Does not grow at $45.5^{\circ} \mathrm{C}$ (Stuart et al., Jour. Bact., 46, 1943, 105).

Intigenically the organisms of this species are not homogeneous.

Boyd (Trans. Roy. Soc. Trop. Med. and Hyg., 33, 1940, 553) has shown that the mannitol-fermenting Shigella include many organisms previously unknown or unclassified because they did not agree with the classical types of Andrewes and Inman (Med. Res. Council, Special Rept. Ser. No.42, London, 1919). With these, on grounds of antigenic structure, will be included the gas-forming Manchester bacillus of Downie, Wade and Young (Jour. Hyg., 33, 1933, 196) and 
both mannitol-fermenting and non-fermenting Newcastle bacilli (Clayton and Warren, Jour. Hyg., 28, 1929, 355 and 29, 1929, 191).

The following tables are taken from Boyd (loc. cit.).

TABLE 1.-Classification of Shiyella paradysenteriae.

\begin{tabular}{c|c}
\hline New Name & Old Name \\
\cline { 1 - 2 } Bacillus dysenteriae Flexner I & $\begin{array}{cc}\text { Andrewes } \\
\text { Inman } \\
\text { (Flexner) }\end{array}$
\end{tabular}

Bacillus dysenteriae Flexner II

Bacillus dysenteriae Flexner III

Bacillus dysenteriae Flexner IV Bacillus dysenteriat Flexner V Bacullus dysenteriae Flexner VI

Bacillus dysenteriae Boyd I Bacillus dysenteriae Boyd II Bacillus dysenteriae Boyd III

Andrewes and Inman IV (Strong)

Andrewes and Inman $\mathrm{Z}$

Type 103

Type P 119

8S-Newcastle-

Manchester group

Type 170

Type $\mathrm{P} 288$

Type D1

The six Flexner types possess a common group antigen and separate typespecific antigens. The three Boyd types are distinct antigenically from each other and from the Flexner types.

Two new Flexner types (Type $953=$ provisional Type VII and Type 1296/\% = provisional Type VIII) have been described by Francis (Jour. Path. and Bact., 58, 1946, 320) as this section goes to press. Also see Boyd (ibid., 297).

TABLE 2.-Subclassification of Bacillus dysenteriae Flexner VI (including the Newcastle bacillus).

\begin{tabular}{|c|c|c|c|c|c|c|}
\hline & 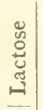 & 苞 & $\frac{\bar{\Xi}}{\stackrel{\Xi}{\Xi}}$ & Dulcitol & 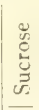 & $\frac{\stackrel{0}{\circ}}{\Xi}$ \\
\hline $\begin{array}{l}\text { Type } 88 \text { ( } 33 \text { per } \\
\text { cent of strains) }\end{array}$ & - & A & A & - & - & - \\
\hline $\begin{array}{l}\text { Type } 88 \text { ( } 66 \text { per } \\
\text { cent of strains) }\end{array}$ & - & A & A & (late) A & - & - \\
\hline $\begin{array}{l}\text { Manchester ba- } \\
\text { cillus......... }\end{array}$ & - & $\mathrm{AG}$ & $A G$ & (late) AG & & - \\
\hline $\begin{array}{r}\text { Newcastle bacil- } \\
\text { lus........... }\end{array}$ & - & $A G$ & - & (late) $\mathrm{AG}$ & & - \\
\hline
\end{tabular}

Source: From feces in rases of dysentery.

Habitat: A cause of dysentery in man. A cause of summer diarrhoea in children.

Note: The term Bacillus paradysenteriae is used by Kruse (Münch. med. Wchmschr., 1917, 1309) for the Escherichia coli-like motile and gas-forming Gram-negative rods that have been found to cause dysentery-like diseases. Kruse (Deut. med. Wchnschr., 27, 1901, 388) uses the term pseudodysentery for the group that includes the Flexner, Strong, and Hiss and Russell types. See Lehmann and Neumann, Bakt. Diag., 7 Aufl., 2, 1927, 456. Gardner (Med. Res. Council, System of Bacteriology, 4, 1929, 170) states that "Kruse's terms $B$. dysenteriac for Shiga, and $B a$ cillus pseudodysenteriae for the FlexnerSonne-Schmitz groups have, however, never taken root outside the Germanspeaking world".

4a. Shigella paradysenteriae (Type Newcastle). (Clayton and Warren, Jour. Hyg., 28, 1929, 355 and 29, 1929, 191; Bacillus dysenteriae Flexner VI in part, Boyd, Trans. Roy. Soc. Trop. Med. and Hyg., 33, 1940, 553.)

Rods: Non-motile. Gram-negative.

In peptone water solution, lactose, mannitol, and sucrose not fermented. Glucose, maltose and dulcitol fermented.

Peculiarities of the organism are: (1) Occasionally a slight bubble of gas is produced from glucose and dulcitol, (2) when the substrate is dissolved in beef extract broth, glucose, dulcitol and maltose are always fermented to gas and acid.

Does not reduce trimethylamine oxide (Wood et al., Jour. Bact., 46, 1943, 106).

Optimum temperature $37^{\circ} \mathrm{C}$. Does not grow at $45.5^{\circ} \mathrm{C}$ (Stuart et al., Jour. Bact., 46, 1943, 105).

Aerobic, facultative.

Serologically related to the mannitolfermenting strains of Shigella paradysenteriae. 
Source: Isolated in 1925 from a case of diarrhoea in Newcastle-on-Tyne, England.

Habitat: A cause of human dysentery.

4b. Shigella paradysenteriae (Type Manchester). (Downie, Wade and Young, Jour. Hyg., 33, 1933, 196; Bacillus dysenteriae Flexner VI in part, Boyd, Trans. Roy. Soc. Trop. Med. and Hyg., 33, 1940, 553.)

Characters as for Type Newcastle except that acid and gas are produced from mannitol. Does not produce gas from maltose.

Serologically related to the uon-mannitol-fermenting strains of Shigella paradysenteriae.

Source: Five st rains were isolated from cases of dysentery at Denton near Manchester, England. One strain came from a case of dysentery in Nigeria.

Hahitat: A cause of human dysentery.

\section{Shigella alkalescens (Andrewes)} Weldin. (Bacillus alkalescens Andrewes, The Lancet, London, 194, 1918, 560; Bacterium alkalescens Levine, Jour. Inf. Dis., 27, 1920, 31; Eberthella alkalescens Bergey et al., Manual, 1st ed., 1923, 231; Weldin, Iowa State College Jour. Sci., 1, 1927, 179; Proshigella alkalescens Borman, Stuart and Wheeler, Jour. Bact., 48, 1944, 363.) From the chemical term, alkaline.

Rods: 0.5 by 1.0 to 1.5 microns, occurring singly and in pairs. Non-motile. Gram-negative.

Gelatin stab: No liquefaction.

Agar slant: Abundant, transparent, of ten iridescent growth.

Broth: Turbid.

Litmus milk: Acid, then alkaline.

Potato: Moderate, grayish growth.

Indole is formed.

Acid but no gas from glucose, xylose, rhamnose, maltose, mannitol and dulcitol. Sucrose is fermented by some strains. Does not attack lactose, dextrin or salicin.

Reduces trimethylamine oxide to tri- methylamine (Wood et al., Jour. Bact., $46,1943,106)$. In contrast to all other species of the genus, will also produce trimethylamine from choline (Wood and Keeping, Jour. Bact., 47, 1944, 309).

Aerobic, facultative.

Optimum temperature $37^{\circ} \mathrm{C}$. Grows at $45.5^{\circ} \mathrm{C}$ (Eijkman's reaction, Stuart et al., Jour. Bact., 46, 1943, 105).

Not pathogenic. Not agglutinated by Shiga immune serum.

Source: From feces in cases of dysentery.

Habitat: Intestinal canal.

6. Shigella pfaffii (Hadley et al.) Weldin. (Bacillus der kanarienvögelseuche, Pfaff, Cent. f. Bakt., I Abt., Orig., 38, 1905, 276; Baclerium pfafi Hadley, EIkins and Caldwell, Rhode Island Agr. Exp. Sta. Bull. 17t, 1918, 169; Bacillus praffi Hadley, Elkins and Caldwell, ibid., 204; Eberthella pfaffi Bergey et al., Manual, 1st ed., 1923, 232; Weldin, Iowa State College Jour. Sci., 1, 1927, 180.) Named for Dr. Franz Pfaff of Prague who isolated this species.

Description largely from Hadley et al. (loc. cit., 180).

Rods: 0.5 by 1.0 to 2.0 microns, occurring singly. Non-motile. Gram-negative.

Gelatin colonies: Small, grayish, translucent.

Gelatin stab: No liquefaction.

Agar colonies: Small, yellowish-gray, homogeneous, translucent, entire. No odor.

Agar slant: Slight, yellowish-gray, translucent streak.

Broth: Turbid, with flocculent sediment (Pfaff, loc. cit., 250).

Litmus milk: Unchanged.

Potato: Moderate, whitish streak.

Acid but no gas from glucose, fructose, arabinose, xylose, maltose, dextrin, salicin and mannitol. Does not attack lactose, sucrose, raffinose, inulin, adonitol or dulcitol.

Indole not formed.

No hydrogen sulfide produced. 
Nitrites not produced from nitrates.

Pathogenic for canaries, sparrows, pigeons, white mice, guinea pigs and rabbits. Not pathogenic for chickens (Pfaff, loc. cit., 280).

Aerobic, facultative.

Optimum temperature $37^{\circ} \mathrm{C}$.

Source: First encountered in an epidemic of septicemia in canaries. Caused a necrotic enteritis.

Habitat: Not known from other sources.

7. Shigella sonnei (Levine) Weldin. (Duval's bacillus, Duval, Jour. Amer. Med. Assn., 43, 1904, 381; Pseudodysentery bacillus E, Kruse, Deutsche med. Wchnschr., 23, 1907, 292, 338; Bacillus ceylonensis A, Castellani, Jour. Hyg., 7, 1907, 1; Group III of Sonne, Sonne, Cent. f. Bakt., I Abt., Orig., 75, 1915, 408; Bacillus dispar (in part) Andrewes, Lancet, 1, 1918, 560 (see Shigella ceylonensis and Shigella madampensis); Bacillus of Sonne, Thjøtta, Jour. Bact., 4, 1919, 355; Bacterium sonnei Levine, Jour. Inf. Dis., 27, 1920, 31; Bacillus dysenteriae Sonne, Smith, Jour. Hyg., 23, 1924, 94; Weldin. Iowa Sta. Coll. Jour. Sei., 1, 1927, 182; Castellanus krusecastellani Cerruti, Jour. Trop. Med. and Hyg., 33, 1930, 207; Shigella paradysenteriae var. sonnei Bergey et al., Minual, 4th ed., 1934, 393; Proshigella sonnci Borman, Stuart and Wheeler, Jour. Bact., 48, 1944, 363.) Named for Dr. Carl Sonne, who worked with this organism.

Rods: Non-motile. Gram-iegative.

Gelatin: No liquefaction.

Agar colonies: Cultures dissociable into two types: (1) Glistening surface, $2 \mathrm{~mm}$ in diameter in 24 hours, soft, grayish, edge entire; (2) Granular surface, 3 to $4 \mathrm{~mm}$ in diameter in $2 t$ hours, soft, grayish, edge tending to spread unevenly, surface developing, after some days, papillae (daughter colonies) which are lactose-fermenting. Some colonies of type 1 change to type 2 on continued incubation. The colony types do not breed true.

Broth: Many authors stress the flocculent growth, associated with spontaneous agglutination in saline solution. These appear to be dependent on growth conditions and time of incubation.

Litmus milk: Acid and with about 50 per cent of strains coagulation. Coagulation tends to occur later than the fermentation of lactose in peptone water.

Indole is not produced.

Acid, but no gas, from lactose (about 2 per cent of strains are lactose-negative after 2 months incubation), glucose, fructose, maltose, galactose, rhamnose, mannitol, arabinose, raffinose and sucrose. No acid from dulcitol, inulin, inositol, adonitol, xylose (xylose is oceasionally fermented) and salicin.

Fermentation of substances other than the monosaccharides may require days or weeks.

Reduces trimethylamine oxide to trimethylamine (Wood et al., Jour. Bact., $46,1943,106$ ).

Serologically Shigella sonnei is divisible into two types, which do not correspond with the colony types described above. Most freshly isolated strains absorb agglutinins completely from all Shigella sonnei antisera, while most stock strains absorb only partially from other than antisera of the second serological type. There exist minor serological relationships between Shigella sonnei and Shigella paradysenteriae, Shigella alkalescens and Shigella madampensis.

Optimum temperature $37^{\circ} \mathrm{C}$. Grows at $45.5^{\circ} \mathrm{C}$ (Stuart et al., Jour. Bact., $46^{\circ}$, 1943, 105).

Source: From feces in cases of dysentery.

Habitat: A cause of mild dysentery in man; summer diarrhoea in children.

8. Shigella equirulis (de Blieck and van Heclsbergen) Edwards. (Bacillus nephritidis equi Meyer, Transvaal Dept. Agr. Rept. Gov. Bar., 1908-1909, 122 ; Bacterium viscosum equi Magnusson, 
Svensk. Veterinartijdskr., 1917, 81 ; also see Jour. Comp. Path. and Therap., 32, 1919,143 ; Bacillus equuli van Straaten, Verslay van den Werkzaamheden der Rijksseruminrichting voor 1916-1917, Rotterdam, 1918, 75; Bacterium pyosepticus equi de Blieck and van Heelsbergen, Tydschr. v. Diergeneesk., 46, 1919, 492; Bacillus equirulis de Blieck and van Heelshergen, ibid., 496; Bacterium pyosepticum riscosum Meissner, Deut. tierärtzl. Wehnschr., 29, 1921, 185; Bacterium pyosepticum (viscosum) equi Lütje, Deut. tierärztl. Wehnschr., 29, 1921, 463; Bacterium pyosepticum (viscosum) Meissner and Berge, Deut. tierärztl. Wchnschr., 30, 1922, 473; Bacterium pyosepticum Meissner, Deut tierärtzl. Wehnschr. 31, 1923, 348; Bacterium pyosepticum equi Landien, Inaug. Diss., Hanover, 1923; Bucillus pyosepticus Clarenberg, Ztschr. f. Infektskr. u. Hyg. d. Haust., , 27 , 1924, 193; Bacterium equi Weldin and Levine, Abst. Bact.. 7, 1923, 13; Eberthella vis$\cos a$ inviler. Jour. Amer. Vet. Med. Assoe., 66, 1925, 481; Shigella equi Weldin, Iowa sta. Col. Jour. Sci., 1, 1927, 121; Shigella viscosa Bergey et al., Manual, 3rd ed., 1930, 363; Edwards, Kentucky Agr. Exp. Sta. Res. Bul. 320, 1931.)

While awaiting further information, the binomial introduced by Edwards is used for this species although Haupt of Leipzig points out in a personal communication to Edwards (1934) that van Straaten's original name was Bacillus equuti. The binomial Bacillus equirulis is stated to have appeared first in the article by de Blieck and van Heelshergen, loc, cit.

Description from Edwards (loc. cit.).

Rods: 0.3 to 0.4 by 0.4 to 0.8 micron, occurring singly, in chains and filaments. Young cultures ( 8 to $10 \mathrm{hrs}$.) frequently show long filaments and streptococcuslike chains as well as large, yeast-like bodies with projections. Rough mucoid colonies consist of short, oval rods. Smooth colonies contain long filaments and streptococcus-like chains, Rough colonies are always mucoid. Non-mucoid colonies are always smooth. Capsules described but uncertain. Nonmotile. Gram-negative.

Gelatin colonies: Grayish-white, circular, translucent.

Gelatin stab: Nail-head, moderate growth along line of stab. No liquefaction.

Agar colonies: 3 to $5 \mathrm{~mm}$ at 48 hours. Semi-solid, tough, adherent, circular, grayish-white, smooth, moist, glistening. Rough variants and dwarf colonies

Agar slant: Grayish-white, viscid growth, covering the surface. Viable $\delta$ to 10 days.

Broth: Masses form on side of tube. At times a thin grayish pellicle. Grayish, tough, ropy sediment. Eventually diffuse turbidity which is highly viscous. Viability 2 to 4 reelis.

Litmus milk: Slowly acidified; slimy, viscid. Sometimes coagulation and reduction.

Potato: To visible growth.

Indole not formed.

Nitrites produced from nitrates.

Voges-Proskauer test negative.

Acid but no gas from glucose, fructose xylose, lactose, galactose, maltose, sucrose, mannitol and raffinose. Dextrin usually fermented. To action in rhamnose, dulcitol, sorbitol or inositol. Usually no action in salicin, adonitol and arabinose.

Does not reduce trimethylamine oxide (Wood et al., Jour. Bact., 46, 1943, 106).

Does not grow at $45.5^{\circ} \mathrm{C}$ (Stuart et al. Jour. Bact., 46, 1943, 105).

Optimum temperature $37^{\circ} \mathrm{C}$.

Aerobic, facultative

Not pathogenic for small experimental animals. Produces abscesses and stiffening of the joints when injected subcutaneously in horses.

Serologically heterogeneous. Nothing is known of its antigenic relations to other members of the genus. Haupt writes in a personal communication that comparative serological studies indicate 
that this species should be placed in the genus Actinobacillus.

Distinctive characters: Differentiation from Shigella sonnei is made on cultural and morphological grounds and immediate fermentation of lactose.

Source: Isolated from cases of jointill in foals.

Habitat: Causes joint-ill in foals.

\section{Shigella ceylonensis (Castellani)} Weldin. (Bacillus ceylonensis B, Castellani, Jour. Hyg., 7, 1907, 1; Bacillus dispar (in part) Andrewes, Lancet, 1, 1918, 560 (see Shigella madampensis and Shigella sonnei. Andrewes included in Bacillus dispar all lactose-fermenting members of the dysentery group); Lankoides ceylonensis $B$, Castellani and Chalmers, Man. Trop. Med., 3rd ed., 1919, 938; Eberthella dispar Bergey et al., Manual, 1st ed., 1923, 232 (sce Shigella madampensis); Weldin, Iowa Sta. Coll. Jour. Sci., 1, 1927, 182; Castallanus castellanii Cerruti, Jour. Trop. Med. and Hyg., 33, 1930, 207.) Latinized, pertaining to Ceylon.

Rods: Non-motile. Gram-negative.

Morphology and colony characters indistinguishable from those of shigella dysenteriae.

Gelatin not liquefied.

Litmus milk: Acid with roagulation. Indole is formed.

Acid, but no gas, from lactose, glucose, fructose, sucrose, mannitol, duleitol, maltose, xylose, arabinose, rhamnose, sorbitol, raffinose, dextrin and glycerol. Inulin, inositol, adomitol and salicin not fermented (saliein differentiates Shigella ceylonensis from Bacterium coli anaerogenes Lembke, Arch. f. Hyg., 26, 1896, 299).

Substances other than the monosaccharides are characteristically fermented slowly.

Reduces trimethylamine oxide to trimethylamine (Wood et al., Jour. Bact., $46,1943,106)$.

Pathogenic for guinea pigs and rabbits.

Serologieally the organism is stated by Castellani to be homogeneous and completely different from Shigella madampensis and Shigella sonnei. The relations to other members of the dysentery group have not been stated.

Optimum temperature $37^{\circ} \mathrm{C}$. Grows at $45.5^{\circ} \mathrm{C}$ (Stuart et al., Jour. Bact., 46 , 1943,105 ).

Source: Isolated from the stools and intestines of persons suffering from dysentery.

Habitat: A cause of dysentery in man.

10. Shigella madampensis (Castellani) Weldin. (Bacillus madampensis Castellani, Cent. f. Bakt., I Abt., Orig., 65, 1912, 262; Bacillus dispar (in part) Andrewes, Lancet, 1, 1918, 560 (see Shigella ccylonensis and Shigella sonnei); Lankoides madampensis Castellani and Chalmers, Man. Trop. Med., 3rd ed., 1919, 938; Bacterium dispar Levine, Abst. Bact., 4, 1920, 15; Eberthella dispar Bergey et al., Manual, 1st ed., 1923, 232 (see Shigella ceylonensis); Weldin, Iowa Sta. Coll. Jour. Sci., 1, 1927, 181a; Shigella dispar Bergey et at., Manual, 3rd ed., 1930, 364; Proshigella dispar Borman, Stuart and Wheeler, Jour. Bact., 48, 1944, 363.)

Neter (Bact. Rev., 6, 1942, 26) combines Shigella ceylonensis and S. madampensis into a single species which he names Shigella castellanii.

Strains currently existing in various Type Collections as Bacillus dispar have biochemicat properties indistinguishable from those described for Shigella madampensis (Glymn and Starkey, Jour. Bact., $37,1939,315)$.

Rods: Non-motile. Gram-negative.

Morphology and colony charactersindistinguishable from those of Shigella dysenteriae.

Gelatin not liquefied.

Indole is formed.

Litmus milk: Acid with coagulation.

Acid, but no gas, from lactose, maltose, sucrose, aribinose, xylose, glycerol, mannitol, rhamnose, glucose, fructose, galactoseand dextrin. Dulcitol, salicin, inulin, inositol and adonitol not fermented. 
Substances other than monosaccharides are characteristically fermented slowly.

Reduces trimethylamine oxide to trimethylamine (Wood et al., Jour. Bact, $46,1943,106)$.

Serologically the organism is stated by Castellani to be homogeneous and completely different from Shigella ceylonensis and Shigella sonnei. According to Andrewes (loc. cit.), Bacillus dispar is serologically distinct from Shigella alkalescens and Shigella paradysenteriae. Fifteen strains (Glynn and Starkey, loc. cit.) from various sources, labelled Bacillus dispar and conforming to the above description, proved to be serologically heterogeneous.

Optimum temperature $37^{\circ} \mathrm{C}$. Grows at $45.5^{\circ} \mathrm{C}$ (Stuart et al., Jour. Bact., 46, 1943,105 ).

Source: Isolated from human stools and intestines.

Habitat: Considered by Castellani to be a cause of colitis and cystitis.

11. Shigella septicaemiae (Bergey et al.) Bergey et al. (Bacillus septicaemiae anserum exsudativae Riemer, Cent. f. Bakt., I Abt., Orig., 37, 1904, 648; Eberthella septicaemiae Bergey et al., Manual, 2nd ed., 1925, 250; Bergey et al., Manual, 3rd ed., 1930, 358.) Latinized, of septicemia.

Small rods: 0.5 by 1.5 to 2.0 microns, occurring singly, in pairs and in threads. Motile. Gram-negative.

Gelatin colonies: Small, white, circular.

Gelatin stab: Slight, infundibuliform liquefaction, becoming complete in several weeks.

Agar colonies: Circular, transparent, smooth, homogeneous, entire.

Agar slant: Soft, grayish-white streak, slightly viscid, becoming transparent.

Does not grow on Endo agar.

Broth: Slight, uniform turbidity, with slight pellicle formation.

Litmus milk: Unchanged.

Potato: No growth.

Blood serum: Yellowish-white streak, the medium becoming brownish and slowly liquefied.
Indole is formed after several days. slight acid and no gas from glucose. To acid from lactose.

Hydrogen sulfide is formed.

Not pathogenic for white mice, guinea pigs, chickens or pigeons. Mildly pathogenic for ducks.

Aerobic.

Optimum temperature $37^{\circ} \mathrm{C}$.

Source: Isolated from blood, exudates and all of the internal organs of geese.

Habitat: Cause of a fatal septicemia in young geese.

Appendix: The following species are also found in the literature. Many are incompletely described.

Bacillus coli dysentericum Ciechanowski and Nowak. (Cent. f. Bakt., I Abt., Orig., 23, 1898, 445.) From a case of dysentery.

Bacillus dysenteriae Migula. (Bacillus of Japanese dysentery, Ogata, Cent. f. Bakt., 11, 1892, 264; Bacillus dysenteriae liquefaciens Kruse, in Flügge, Die Mikroorganisnen, 3 Aufl., 2, 1896, 281; Bacterium dysenteriae liquefaciens Chester, Ann. Rept. Del. Col. Agr. Exp. Sta., 9, 1897, 102; Migula, Syst. d. Bakt., 2, 1900,641 ; not Bacillus dysenteriae Shiga, Cent. f. Bakt., I Abt., 24, 1898, 817; not Bacillus dysenteriae Hiss and Russell, Medical News, 82, 1903, 289; not Bacillus dysenteriae Strong, Jour. Amer. Med. Assoc., 35, 1906, 498; not Bacillus dysenteriae Sonne, Smith, Jour. Hyg., 23, 1924, 94.) From a case of Japanese dysentery. Motile. Gram-positive.

Bacillus dysentericus Trevisan. (Bacillus der Dysenterie, Klebs, Cent. f. Bakt., 2, 1857, 248; Trevisan, I generi e le specie delle Batteriacee, 1889, 14; not Bacillus dysentericus Ruffer and Willmore, Brit. Med. Jour., 2, 1909, 862.) From feces.

Bacterium pseudodysentericum Küuse. (Kruse, Deutsche med. Wehnsehr., 27, 1901, 370, 386; Escherichia pseudodysenteriac Bergey et al., Manual, 1st ed., 1923, 198.) From feces. Motile.

Bacterium wakefield Berger. (Jour. 
Hyg., 44, 1945, 116-119.) From feces. A non-mannitol-fermenting organism of the Flexner group. Wheeler and Stuart (Jour. Bact., 51, 1946, 324) regard this as an anaerogenic paracolon.

Shigella albofaciens (Castellani) Hauduroy et al. (Bacillus albofaciens Castellani, Meetings of the Ceylon Branch of the British Medical Association, 1905; Hauduroy et al., Dict. d. Bact. Path., 1937, 482.)

Shigella arabinotardā, types $\mathbf{A}$ and $\mathbf{B}$, Christensen and Gowen. (Jour. Bact., 47, 1944, 171-176.) From cases of dysentery in U. S. Army in Tunisia. A lactose-negative, mannitol-negative Shigella.

Shigella bienstockii (Schroeter) Bergey et al. (Bacillus III, Bienstock, Ztschr. f. klin. Med., 8, 1884; Bacillus coprogenes parvus Flügge, Die Mikroorganismen, 2 Aufl, 1886, 269; Bacillus bienstockii Schroeter, Kryptogamen Flora von Schlesien, 3, 1, 1886, 163; Bacillus parvus Trevisan, I generi e le specie delle Batteriacee, 1889, 15; not Bacillus parvus Neide, Cent. f. Bakt., II Abt., 12, 190t, 341; Bactorium coprogenes paruus Chester, Ann. Rept. Del. Col. Agr. Exp. Sta., 9, 1897, 85; Bacterium bienstockii Chester, Man. Determ. Bact., 1901, 144; Eberthella bienstockii Bergey et al., Manual, 1st ed., 1923, 227; Bergey et al., Manual, 3rd ed., 1930, 360.) From feces. Shigella douglasi (Castellani and Chalmers) Hauduroy et al. (Bacillus douglasi Castellani and Chalmers, Man. Trop. Med., 3rd ed., 1919, 946; Hauduroy et al., Dict. d. Bact. Path., 1937, 484.)

Shigella etousae Heller and Wilson. (Jour. Path. and Bact., 58, 1946, 98.) From dysentery outbreak in an army camp in England.

Shigella faecaloides (Castellani) Hauduroy et al. (Bacillus faecaloides Castellani, 1915; see Castellani and Chalmers, Man. Trop. Med., 3rd ed., 1919, 946; Hauduroy et al., Diet. d. Bact. Path., 1937, 488 .

Shigella giumai (Castellani) Hauduroy et al. (Bacillus giumai Castellani, Cent. f. Bakt., I Abt., Orig., 65, 1912,
264; Wesenbergus giumai Castellani and Chalmers, Man. Trop. Med., 3rd ed., 1919, 940; Bacterium giumai Weldin and Levine, Abst. Bact., 7, 1923, 15; Salmonella giumai Bergey et al., Manual, 1st ed., 1923, 220; Hauduroy et al., Dict. d. Bact. Path., 1937, 488.)

Shigella lunavensis (Castellani) Hauduroy et al. (Bacillus lunavensis Castellani, Cent. f. Bakt., I Abt., Orig., 65, 1912, 264; Bacterium lunavensis Weldin and Levine, Abst. Bact., 7, 1923, 16; Haúduroy et al., Dict. d. Bact. Path., 1937, 489.) From feces.

Shigella metadysenterica var. $A, B, C$, and $D$ (Castellani) Hauduroy et al. (Bacillus metadysentericus var. $A, B, C$, and $D$, Castellani, 1904; see Castellani and Chalmers, Man. Trop. Med., 3rd ed., 1919, 946; Dysenteroides metadysentericus var. $A, B, C$, and $D$, Castellani and Chalmers, Ann. Inst. Past., 34, 1920, 607; Castellanus metadysentericus Castellani, 1930; Hauduroy et al., Dict. d. Bact. Path., 1937, 489.) From cases of dysentery.

Shigela negombensis (Castellani) Hauduroy et al. (Bacillus negombensis Castellani, Cent. f. Bakt., I Abt., Orig., $65,1912,262$; Hauduroy et al., Diet. d. Bact. Path., 1937, 490.)

Shigella oxygenes (Ford) Bergey et al. (Bacterium axygenes Ford, Studies from the Royal Victoria Hospital, Montreal, 1, No. 5, 1903, 47; Eberthella oxygenes Bergey et al., Manual, 1st ed., 1923, 228; Bergey et al., Manual, 3rd ed., 1930, 360.) From feces.

Shigella piscatora Bois and Roy. (Naturaliste Canadien, 71, 1945, 259.) From the intestine of a codfish (Gadus callarias $L$.).

Shigella tangallensis (Castellani) Hauduroy et al. (Bacillus tangallensis Castellani, Cent. f. Bakt., I Abt., Orig., 65, 1912, 266; Hauduroy et al., Dict. d. Bact. Path., 1937, 497.) From feces.

Shigella tarda (Castellani) Hauduroy et al. (Bacillus tardus Castellani, 1917; see Castellani and Chalmers, Man. Trop. Med., 3rd ed., 1919, 954; Hauduroy et al., Dict. d. Bact. Path., 1937, 497.) 


\section{FAMILY XI. PARVOBACTERIACEAE RAHN.*}

(Cent. f. Bakt., II Abt., 96, 1937, 281.)

Small, motile or non-motile rods. Gram-negative. Some will grow on ordinary media, but the majority either require or grow better on media containing body fluids or growth-promoting substances. Some invade living tissues. Usually do not liquefy gelatin. No visible gas formed in the fermention of carbohydrates. Infection in some cases may take place by penetration of organisms through mucous menabranes or skin. Parasitic to pathogenic on warm-biooded animals, including man.

\section{Key to the iribes of family Parvobacteriaceae.}

I. Usualiy grow on ordinary media.

A. Aerobic to facultative anaerobic.

1. Show bipolar staining. Majority ferment carbohydrates.

Tribe I. Pasteurelleae, p. 545.

2. Do not show bipolar staining. Tone ferment carbohydrates.

B. Anaerobic.

Tribe II. Brucelleae, p. วँ60.

Tribe III. Bacteroideae, p. 5ै6t.

II. On first isolation dependent on some factor or factors contained in blood or plant tissues. Aerobic to anaerobic.

Tribe IV. Hemophileae, p. 584.

TRIBE I. PASTEURELLEAE CASTELTANI AND CHALMERS.

(Man. Trop. Med., 3rd ed., 1919, 943.)

Small, motile or non-motile, ellipsoidal to elongated rods showing bipolar staining.

Key to the genera of tribe Pasteurelleae.

I. Milk not cuagulated.

1. Causes hemorrhagic septicemia, pseudotuberculosis, tularemia or plague.

Genus I. Pasteurella, p. 546.

II. Milk coagulated slowly and sometimes digested.

A. Causes glanders or glanders-like infections.

III. Milk unchanged to slightly acid.

Genus II. Malleomyces, p. 554 .

A. Associated with actinomycosis in cattle and in man.

Genus III. Actinobacillus, p. 556 .

* Revised by Prof. E. G. D. Murray, MeGill University, Montreal, Canada with the collaboration of Prof. Karl F. Meyer, Hooper Foundation, San Franciseo, California; Prof. W. A. Hagan, Cornell University, Ithaca, New York; Dr. Alice C. Evans and Dr. Margaret Pittman, National Institute of Health, Washington, D. C.; Prof. I. F. Huddleson, Michigan State College, East Lansing, Michigan; and others, December. 1938. 


\section{Genus 1. Pasteurella T'revisan.*}

(Octopsis 'Trevisan, Atti della Accad. Fisio-Medico-Statistica, Milano, Ser. 4, 3, 1885, 102; Trevisan, Rendiconti Reale Instituto Lombardo di Scienze e Lettere, 1887, 94; Coccobacillus Gamaleïa, Cent. f. Bakt., 4, 1888, 167; Eucystia Enderlein, Sitzber. Gesell. Naturf. Freunde, Berlin, 1917, 317.) Named for Louis Pasteur, the French scientist.

Small, Gram-negative, ellipsoidal to elongated rods showing bipolar staining by special methods; aerobic, facultative; may require low oxidation-reduction potential on primary isolation; majority ferment carbohydrates but produce only a small amount of acid; no or slight lactose fermentation; no gas production; gelatin not liquefied; milk not coagulated; parasitic on man, other mammals and birds.

The type species is Pasteurella multocida (Lehmann and Neumann) Rosenbusch and Merchant.

\section{Key to the species of genus Pasteurella.}

I. Growth on ordinary media. Growth in milk.

A. Non-motile and non-flagellated at $18^{\circ}$ to $26^{\circ} \mathrm{C}$. No change or slight acid in milk without coagulation.

1. Indole and $\mathrm{H}_{2} \mathrm{~S}$ produced. No growth in bile. Sorbitol fermented. No hemolysis on blood agar.

\section{Pasteurella multocida.}

2. Indole not formed. Hemolysis produced on blood agar.

2. Pasteurella hemolytica.

3. Neither indole nor $\mathrm{H}_{2} \mathrm{~S}$ produced. Growth in bile. Sorbitol not fermented. No hemolysis.

3. Pasteurella pestis.

B. Motile and flagellated at $18^{\circ}$ to $26^{\circ} \mathrm{C}$. Milk alkaline. Hydrogen sulfide produced. Indole not formed.

4. Pasteurella pseudotuberculosis.

II. No growth on plain agar or in liquid medium without special enrichment. No growth in milk.

\section{Pasteurella tularensis.}

1. Pasteurella multocida (Lehmann and Neumann) Rosenbusch and Merchant. (Virus der Wildseuche, Hueppe, Berlin. klin. Wochnschr., 23, 1886, 797; Bactérie ovoide, Lignières, Recueil de Méd. Vétér., 75, 1898, 836 (Bull. Soc. Centr. Méd. Vétér., N. S. 60, 1898, 836); Bacillus septicaemiae haemorrhagicae Sternberg, Man. of Bact., 1893, 408; Bacterium septicaemiae hemorrhagicae Lehmann and Neumann, Bakt. Diag., 2 Aufl., 2, 1899, 194; Bacterium multoci- dum Lehmann and Neumann, ibid., 196; Bacillus plurisepticus and Bacterium avicidum Kitt, in Kolle and Wassermann, Handb. d. path. Mikroorg., 1 Aufl., 2, 1903, 562; Bacillus pleurisepticus Jordan, General Bact., 1st ed., 1908, 289; Bacillus bipolaris septicus Hutyra, in Folle and Wassermann, Handb. d. path. Mikroorg., 2 Aufl., 6, 1913, 67; Bacillus bipolaris plurisepticus Hutyra, ibid.; Pasteurella septica Topley and Wilson, Princip. Bact. and Immun., 1st ed., 1, 1931, 488;

* Rearranged by Mrs. Eleanore Heist Clise, New York State Experiment Station, Geneva, New York, in accordance with the suggestions of Mr. Philip C. Harvey and Dr. Mark Welsh, Pearl River, New York, November, 1945. 
Pasteurella pluriseptica Gay et al., Agents of Disease and Host Resistance, 1935, 730; Rosenbusch and Merchant, Jour. Bact., 37, 1939, 85.) From Latin, killing many.

The following are regarded as identical with the above but are arranged here eccording to source:

Pasteurella bollingeri Trevisan. (Microparasiten bei eine neue Wild- und Rinderseuche, Bollinger, Über eine neue Wild- und Rinderseuche unsw., München, 1878; Bacterium bipolare multocidum Kitt, Sitz. Gesell. Morphol. u. Physiol., München, 1, 1885, 24; Trevisan, I generi e le specie delle Batteriacee, 1889, 21; Bacillus bovisepticus Kruse, in Flügge, Die Mikroorganismen, 3 Aufl., 2, 1896, 421; Bacterium bovisepticus Chester, Ann. Rept. Del. Coi. Agr. Exp. Sta., 9, 1897, 81; Bacterium multocidum Lehmann and Neumann, Bakt. Diag., 2 Auf., 2, 1899, 196; La Pasteurella bovine, Lignières, Recueil de Méd. Vétér., $77,1900,537$; Bacillus bipolaris borisepticus Hutyra, in Kolle and Wassermann, Handb. d. path. Mikroorg., 2 Aufl., 6, 1913, 67 ; Pasteurella boviseptica Holland, Jour. Bact., 5, 1920, 224; Pasteurella bovium Hutyra, in Kolle, Kraus und Uhlenhuth, Handb. d. path. Mikroorg., 3 Aufl., 6, 1927-1929, 487; Pasteurella ferarum Hauduroy et al., Dict. d. Bact. Path., 1937, 316.) From domestic cattle and deer.

Pasteurella avicida (Gamaleïa) Trevisan. (Microbe du cholera des poules, Pasteur, Compt. rend. Acad. Sci., Paris, $90,1880,239,952$ and 1030; Granules of fowl cholera, Salmon and Th. Smith, U. S. Dept. Agr. Ann. Rept., 1880, 438; Micrococcus cholerae gallinarum Zopf, Die Spaltpilze, 3 Aufl., 1885, 57; Octopsis cholerae gallinarum Trevisan, Atti della Accad. Fisio-Medico-Statistica, Milano, Ser. 4, 3, 1885, 102; Bacillus cholerae gallinarum Flügge, Die Mikroorganismen, 2 Aufl., 1886, 253; Bacterium cholerae gallinarum Schroeter, Firyptogamen Flora von Schlesien, 3, 1, 1886, 155; Hühnercholerabacterien, Kitt, Cent. f.
Bakt., 1, 1887, 305; Pasteurella cholerae gallinarum Trevisan, Rendiconti Reale Instituto Lombardo di Scienze e Lettere, 1887, 94; Coccobacillus avicidus Gamaleïa, Cent.f. Bakt., 4, 1888, 167; Trevisan, I generi e le specie delle Batteriacee, 1889,21 ; Bacterium avicidum Kitt, according to Chester, Man. Determ. Bact., 1901, 135; Bacterium cholerae Chester, idem; Bacillus avisepticus and Bacterium avisepticum Kitt, in Kolle and Wassermann, Handb. d. path. Mikroorg., 1 Aufl., 2, 1903, 544; not Bacillus avisepticus Chester, loc. cit., 220; Pasteurella avium Kitt, loc. cit., 562; Pasteurella gallinae Besson, Practical Bacteriology, London and New York, 1913, 447; Pasteurella cholerae-gallinarum Winslow et al., Jour. Bact., 2, 1917, 561; Bacillus cholerac-gallinarum Holland, Jour. Bact., 5, 1920, 217; Pasteurella aviseptica Holland, ibid., 224.) From fowls.

Pasteurella cuniculicida (Flügge) Trevisan. (Septicämiebacterien, Gaffky, Mit. kaiserl. Gesundheitsamte, 1, 1881, 98; Bacillus cuniculicida Flügge, Die Mikroorganismen, 2 Aufl., 1886, 251; Trevisan, I generi e le specie delle Batteriacee, 1889, 21 ; Bacterium septichaemiae Schroeter, Kryptogamen Flora von Schlesien, 3, 1, 1889, 155; Bacterium cuniculicida Chester, Ann. Rept. Del. Col. Agr. Exp. Sta., 9, 1897, 80 ; not Bacterium cuniculicida Chester, Man. Determ. Bact., 1901, 140; Bacillus cuniculisepticus Kitt, in Kolle and Wassermann, Handb. d. path. Mikroorg., 1 Aufl., 2, 1903, 562; Bacterium lepisepticum. Ferry and Hoskins, Jour. Lab. and Clin. Med., 5, 1920 , 311; Bacillus bipolaris septicus and Bacillus lepisepticus Ford, Textb. of Bact., 1927, 591; Pasteurella lepiseptica Holland, Jour. Bact., 5, 1920, 221 ; Pasteurclla cuniculi Schütze, Med. Res. Council, Syst. of Bact., London, 4, 1929, 469; Bacterium leporisepticum Hauduroy et al., Dict. d. Bact. Path., 1937, 314.) From rabbits.

Pasteurella suilla Trevisan. (Rothlaufstabchen, Loeffler, Arb. kaiserl. Ge- 
sundheitsamte, 1, 1886, 51; Rothlaufbacillen, Schütz, ibid., 74; Bacillus of swine plague, Salmon, Rept. U. S. Dept. Agr., Bur. An. Ind., 1886, 87; Bacillus parvus ovatus Flügge, Die Mikroorganismen, 2 Aufl., 1886, 273; Trevisan, Reale Instituto Lombardo d. Sci. e Let. Rend., Ser. 2, 20, 1887, 94; Bacterium suicida Migula, in Engler and Prantl, Natürl. Pflanzenfam., 1, 1a, 1895, 27; Bacillus suisepticus Kruse, in Flügge, Die Mikroorganismen, 3 Aufl., 2, 1896, 419; Bacterium suisepticus Chester, Ann. Rept. De1. Col. Agr. Exp. Sta., 9, 1897, 80; La Pasteurella porcine, Lignières, Recueil de Méd. Vétér., 77, 1900, 391; Pasteurella suiseptica Holland, Jour. Bact., 5, 1920 , 220; Pasteurella suum Hutyra, in Kolle, Kraus and Uhlenhuth, Handb. d. path. Mikroorg., '3 Aufl., 6, 1927-1929, 487.) From swine.

Bacterium bovicida Migula. (Microbo del barbone dei bufali, Oreste Armanni, Atti. d. R. Istit. d'incoragg. alle scienze natur. ecenom. e technol., 1.887; Letta nella tornata Accad., Sept. 16, 1886; Cent. f. Bakt., 2, 1887, 50; Atti della Commissione per le malattie degli animali, 121, 1887; Migula, Syst. d. Bakt., 2, 1900, 366; Pasteurella bubalseptica Kelser, Man. Vet. Bact., 1st ed., 1927, 195 ; Bacillus bubalsepticus Kelser, ibid.; Bacillus bipolaris bubalisepticus Hauduroy et al., Dict. d. Bact. Path., 1937, 312.) From buffaloes.

Pasteurella vituliseptica (Kitt) Ford. (Bacillus vitulisepticus Kitt, in Kolle and Wassermann, Handb. d. path. Mikroorg., 1 Aufl., 2, 1903, 562; Bacterium vitulisepticum Lehmann and Neumann, Bakt. Diag., 5 Aufl., 2, 1912, 282; Ford, Textb, of Bact., 1927, 597.) From calves.

Pasteurella muricida Meyer and Batchelder. Meyer and Batchelder, Jour. Inf. Dis., 39, 1926, 386; Pasteurella muriseptica Topley and Wilson, Princip. Bact. and Immun., 1st ed., 1, 1931, 482; not Pasteurella muriseptica Bergey et al., 1st ed., 1923, 265 (Bacillus murisepticus Flügge, Die Mikroorganismen, 2 Aufl.,
1886, 250; Erysipelothrix muriseptica Bergey et al., Manual, 2nd ed., 1925, 380).) From wild rats.

Bacillus bipolaris der malignen Meerschweinchen-Phlegmasie of Heymann and Kyriasides, Ztschr. f. Hyg., 114, 1932, 119 (Klebsiella caviae Hauduroy et al., Dict. d. Bact. Path., 1937, 261) is stated by the original authors to be closely related to this organism.

Plasaj and Pribram (Cent. f. Bakt., I Abt., Orig., 87, 1921, 1) also present a classification of the hemorrhagic septicenia bacteria.

Description from Schütze (Med. Res. Council, Syst. of Bact., London, 4, 1929, 451) who prepared it from studies of 230 strains described by 17 authors during the years 1908-1926.

Short ellipsoidal rods: 0.3 to 1.25 microns in length, occurring singly, in pairs, rarely in chains. Show bipolar staining. Non-motile. Gram-negative.

Gelatin: No liquefaction.

Agar: Fine translucent growth. Characteristic odor.

Broth: Uniform turbidity. Characteristic odor.

Milk: No change in reaction. No coagulation.

Potato: No visible growth.

Indole is formed.

Nitrites are produced from nitrates. Hydrogen sulfide is produced.

No hemolysis on blood agar.

Acid but no gas from glucose, mannitol (usually), sucrose, fructose, sorbitol, galactose, mannose, xylose (usually) and trehalose (usually). No acid from lactose, dulcitol, arabinose (usually), amygdalin, maltose (usually), raffinose, rhamnose, adonitol, dextrin, inulin, glyc:erol, salicin (usually) or erythritol.

Optimum temperature $37^{\circ} \mathrm{C}$. Killed at temperatures above $45^{\circ} \mathrm{C}$.

Aerobe to facultative anaerobe.

Three serological types have been found on the basis of agglutination tests (Little and Lyon, Amer. Jour. Vet. Res., 4, 1943, 110). 
Virulent for laboratory animals, especially mice and rabbits.

Distinctive characters: Grows on ordinary media. Bile salts inhibit growth.

Source: From numerous domestic animals and fowls, including cat, dog, cattle, horse, goat, sheep, pig, rabbit, chicken, and from reindeer, buffalo, rat, etc.

Habitat: The cause of hemorrhagic septicemia in birds and mammals.

2. Pasteurella hemolytica Newsom and Cross. (Jour. Amer. Vet. Med. Assoc., 80 (N.S. 33), 1932, 715.) From M. L., hemolytic.

Bipolar staining.

Blood agar: Hemolysis.

Indole not formed.

Acid from dextrin, fructose, galactose, glucose, glycerol (usually), inositol, lactose (usually), maltose, mannitol, raffinose, sorbitol, sucrose and xylose. No acid from arabinose, dulcitol, inulin, mannose, rhamnose or salicin.

No cross-agglutination between Pasteurclla multocida and this species.

Avirulent for rabbits.

Source: Twenty strains isolated from pneumonia in sheep and cattle.

Habitat: Occurs in pneumonia of sheep and cattle.

3. Pasteurella pestis (Lehmann and Jeumann) Holland. (Bacille de la peste, Tersin, Ann. Inst. Past., 8, 1894, 666; Pest Bacillus, Aoyama, Ztschr. f. Hyg., 21, 1895, 165; Bacterium pestis Lehmann and Neumann, Bakt. Diag., 1 Aufl., 2, 1896, 194; Bacillus pestis bubonicae Kruse, in Flügge, Dic Mikroorganismen, 3 Aufl., 2, 1896, 429; Bacterium pestis bubonicae Chester, Ann. Rept. Del. Col. Agr. Exp. Sta., 9, 1897, 81; Bacillus pestis Migula, Syst. d. Bakt., 2, 1900, 749; Eucystia pestis Enderlein, Sitzber. Gesell. Naturf. Freunde, Berlin, 1917, 317 ; Holland, Jour. Bact., 5, 1920, 219 ; Coccobacillus yersini Neveu-Lemaire, Précis Parasitol. Hum., 5th ed., 1921, 20.) From Latin pestis, plague.

Rods: 1.0 by 2.0 microns, occurring singly. Non-motile. Polar staining. Characteristic bladder, safety-pin and ring involution forms. Gram-negative.

Gelatin colonies: Flat, gray, with granular margin.

Gelatin stab: Flat surface growth. Arborescent growth in stab. No liquefaction.

Agar colonies: Grayish-white, translucent, irridescent, undulate.

Agar slant: Growth grayish, viscid, thin, moist, translucent. Growth slow, favored by the addition of blood or sodium sulfite.

Broth: Turbid or clear with flocculi in the fluid. Old cultures show a pellicle with streamers into the fluid (stalactites). Becomes alkaline more slowly than Pasteurella pseudotuberculosis. See Bessonowa and Lenskaja, Cent. f. Bakt., I Abt., Orig., 119, 1930, 430.

Litmus milk: Slightly acid or unchanged. No coagulation.

Potato: Scanty, grayish growth.

Indole not formed.

Lactose and rhamnose not attacked. Variable action on glycerol.

Nitrites are produced from nitrates. Temperature relations: Optimum $25^{\circ}$ to $30^{\circ} \mathrm{C}$. Minimum $0^{\circ} \mathrm{C}$. Maximum $43^{\circ}$ to $45^{\circ} \mathrm{C}$.

Aerobic, facultative.

Source: Buboes, blood, pleural effusion, spleen and liver of infected rodents and man. Sputum in pneumonic plague. Infected fleas.

Habitat: The causative organism of plague in man, rats, ground squirrels and other rodents. Infectious for mice, guinea pigs and rabbits. Transmitted from rat to rat and from rat to man by the infected rat flea.

Note: Pasteurella pestis and Pasteurella pseudotuberculosis are not definitely distinguishable by serological methods (Schütze, Med. Res. Council, Syst. of Bact., London, 4, 1929, 478, and $\mathrm{Wu}$ Lien-teh, in Chun, Pollitzer and $\mathrm{Wu}$, "Plague," National Quarantine Service, Shanghai, 1936). Malachite-green broth slowly decolorized by Pasteurella pestis 
and quickly by $P$. pseudotuberculosis; same for methylene blue, Janus green and thionin. No growth on Bessonowa media (pH 5.9). See Yersinia, p. 703.

\section{Pasteurella}

pseudotuberculosis

(Eisenberg) Topley and Wilson. (Bacillus der pseudotuberculose, Pfeiffer, Ueber die bacilläre Pseudotuberculose bei Nagetieren, Leipzig, 1889; Bacillus pseudotuberculosis Eisenberg, Bakt. Diag., 3 Aufl., 1891, 294; Streptobacillus pseudotuberculosis rodentium Preisz, Ann. Inst. Past., 8, 1894, 231; Bacterium pseudotuberculosis rodentium Lehmann and Neumann, Bakt. Diag., 1 Aufl., 2, 1896, 362 ; Bacillus pseudotuberculosis rodentium Lehmann and Neumann, ibid., 429; Bacterium pseudotuberculosis Migula, Syst. d. Bakt., 2, 1900, 37t; Corynebacterium rodentium Bergey et al., Manual, 1st ed., 1923, 386; Corynebacterium pseudotuberculosis Bergey et al., Manual, 2nd ed., 1925, 394; Topley and Wilson, Princip. Bact. and Immun., 1st ed., 2, 1931, 825; Corynebacterium pseudotuberculosis rodentium Iielser, Man. Vet. Bact., 2nd ed., 1933, 319; Malleomyces pseudotuberculosis rodentium Pribram, Klassifikation d. Schizomyceten, 1933, 93.) Latinized, false tuberculosis.

Bacillus tuberculosis zoogloeicae Malassez and Vignal (Ann. de Physiol., 1883, $370)$ is considered identical with this species by Hauduroy et al. (Dict. d. Bact. Path., 1937, 162).

The original tabular description by Eisenberg, Bakt. Diag., 3 Aufl., 1891, 294 is very incomplete. Description taken from Topley and Wilson, Princip. Bact. and Immun., 2nd ed., 1936, 607 and Bessonowa, Lenskaja and Molodtzowa, Office Internat. d'Hyg. Publ., 29, 1937, 2106.

Small rods: Variable in size and shape. Ellipsoidal or coccoid forms 0.8 by 0.8 to 2.0 microns, with rounded ends, oceurring singly. Rod-shaped forms 0.6 by 1.5 to 5.0 microns, with rounded ends, occurring singly, in groups or in short chains. Occasionally long curved fila- ments. Motile (Weitzenberg, Cent. f. Bakt., I Abt., Orig., 133, 1935, 343). Non-acid-fast. Gram-negative.

Gelatin stab : After 7 days at $22^{\circ} \mathrm{C}$, good filiform growth extending to bottom of tube. No liquefaction.

Agar colonies: After 24 hours at $37^{\circ} \mathrm{C}$, circular, 0.5 to $1.0 \mathrm{~mm}$ in diameter, umbonate, granular, translucent, grayish-yellow, butyrous; edge entire; dull, finely granular or beaten-copper surface; differentiated into a raised, more opaque center and a flat, clearer periphery with racial striation.

Igar slant: After 48 hours at $37^{\circ} \mathrm{C}$, growth moderate, confluent, raised, grayish-yellow, translucent, with glistening, wavy or beaten-copper surface and an irregularly lobate edge.

Blood agar plate: Good growth. No hemolysis.

Broth: After 24 hours at $37^{\circ} \mathrm{C}$, moderate growth with moderate turbidity which later clears. Viscous sediment. Incomplete surface and ring growth. Becomes alkaline more rapidly than Pasteurella pestis.

Potato: After 7 days at $22^{\circ} \mathrm{C}$, a thin yellowish membrane which later turns brown.

Indole not formed.

Litmus milk: Usually slightly alkaline.

Nitrites produced from nitrates.

Ammonia is produced.

Acid but no gas from glucose, maltose, mannitol, salicin, arabinose, xylose, rhamnose and glycerol. Sometimes acid from sucrose.

Hydrogen sulfide produced.

Catalase positive.

Methyl red positive.

Methylene blue is reduced.

Voges-Proskauer test negative.

Temperature relations: Optimum $30^{\circ} \mathrm{C}$. Minimum $5^{\circ} \mathrm{C}$. Maximum $43^{\circ} \mathrm{C}$. Thermal death point $60^{\circ} \mathrm{C}$ for ten minutes.

Pathogenicity: The cause of spontaneous disease in rabbits, rats and guinea pigs. Infectious for mice, rats, dogs, rats and horses.

Aerobic, facultative. 
Source: From a guinea pig inoculated with material from a horse suspected of having glanders.

Habitat: Lesions in natural disease in animals. Causes pseudotubereulosis in rodents, especially guinea pigs.

5. Pasteurella tularensis (McCoy and Chapin) Bergey et al. (Bacterium tularense McCoy and Chapin, Jour. Inf. Dis., 10, 1912, 61; McCoy and Chapin, Public Health Bull. 53, U. S. Treas. Dept., Public Health Service, 1912, 17; Bacillus tularense Vail, The Ophthalmic Record, 23, 1914, 487; also Francis, U. S. Hygienic Lab. Bull. 130, 1922; Bergey et al., Manual, 1st ed., 1923, 267; Brucella tularensis Topley and Wilson. Princip. Bact. and Immun., 1st ed., 1, 1931, 509; Coccobacterium tularense GalliValerio, Schweiz. med. Worhnschr., 68, 1938, 1206.) From Tulare, the county in California in which the disease was first observed.

Description taken from McCoy and Chapin (loc. cit.) and Francis (loc. cit.). Further revision by Francis, 1947 .

Equal numbers of cocei and rods; 0.2 by 0.2 to 0.7 mieron, oecurring singly. Bipolar staining may occur. Capsules rare or absent. Extremely pleomorphic (Hesselbrock and Foshay, J. Bact. 49, 1945, 209) Non-motile. Gram-negative.

No growth on plain agar or in liquid media without special enrichment. (Tamura and Gibby, J. Bact. 45, 1943, 361) Filterable through Berkefeld filters.

Growth occurs on coagulated egg-yolk (MeCoy and Chapin, loc. cit.), on bloodglucose-cystine agar (Francis, loc. cit.), on blood agar, glucose-blood agar and glucose serum agar. The addition of fresh sterile rabbit spleen to the surface of the last three media favors the growth of the organism.

Forms minute viscous colonies after 2 to 5 days which may attain a diameter of $4 \mathrm{~mm}$ if well separated. Growth readily emulsifiable.

Growth on blood media is gray. May cause green discoloration of the blood.
Rough, smooth and nucoid variants have not been reported.

Slight acid without gas may be produced from glucose, glycerol, maltose, mannose, fructose and dextrin.

Growth soluble in sodium ricinoleate.

Hydrogen sulfide produced in a cystine medium.

Aerobic. No growth anaerobically.

Optimum temperature $37^{\circ} \mathrm{C}$. Thermal death point $56^{\circ} \mathrm{C}$ for ten minutes. Survives best at low temperatures, even $-70^{\circ} \mathrm{C}$.

Pathogenicity: Penetrates unbroken skin to cause infection. Buboes and areas of necrosis produced in human and animal tissue. Infectious for man and most rodents, including rabbits, guinea pigs, rats, mice, squirrels, ground hogs, muskrats, beavers, water rats and lemmings.

Source: Originally isclated from California ground squirrels and later from more than 30 other forms of wild life in the United States and elsewhere. Found in lesions in man and animals with natural or experimental infections. Especially the liver, blood, lymph nodes, and spleen of animals.

Habitat: The cause of tularemia in man and transmitted from wild animals to man by blood-sucking inseets, by contact with infected animals, or by drinking water. Disease known in North Americr, Japan, Russia, Norway, Sweden, Austria, Turkey, Czechoslovakia and Central Germany. See Burroughs, Holdenreid, Longanecker and Meyer, Jour. Inf. Dis., $76,1945,115$ for a complete list of known vertebrate hosts.

Appendix: The following organisms may be identical with some of those listed above or related to them:

Bacillus coscoroba Trétrop. (Trétrop, Ann. Inst. Past., 14, 1900, 224; not Bacillus coscoroba MacConkey, Jour. Hyg., 6, 1906, 397.) The cause of swan cholera in the Antwerp Zoological Garden. Trétrop's description is that of a Pasteurella as is pointed out by Castellani 
and Chalmers (Man. Trop. Med., 3rd ed., 1919, 941). The organism described by Trétrop clearly was not the same as that in the culture sent by Binot of the Pasteur Institute to MacConkey and described by him (loc. cit.) as a member of the coliform group. Because of MacConkey's studies, the Binot culture has been accepted as determining the nature of Bacillus coscoroba in many subsequent studies of the coliform group, e.g., Bergey and Deehan, Jour. Med. Res., 19, 1908, 182; Levine, Amer. Jour. Pub. Health, 7, 1917, 785; Winslow, Kligler and Rothberg, Jour. Bact., 4, 1919, 485; Bergey et al., Manual, 1st ed., 1923, 204; etc.

Bacillus cuniculicida Migula. (Bacillus der Kaninchenseptikämie, Eberth and Mandry, Arch. f. path. Anat., 121, 1890; Bacillus cuniculicida mobilis Kruse, in Flügge, Die Mikroorganismen, 3 Aufl., 2, 1896, 406; Bacterium cuniculicide mobilis Chester, Ann. Rept. Del. Col. Agr. Exp. Sta., 9, 1897, 132; Migula, Syst. d. Bakt., 2, 1900, 757; not Bacillus cuniculicida Flügge, Dic Mikroorganismen, 2 Aufl., 1886, 251.) From peritoneal exudate of a rabbit.

Bacillus mustelaecida Trevisan.

(Bacillus der Frettchenseuche, Eberth and Schimmelbusch, Fortschr. d. Med., 6, 1888, 295; also see Arch. f. path. Anat, 115, 1889, 282; Trevisan, I generi e le specie delle Batteriacee, 1889, 13; Pasteurella mustelaecida DeToni and Trevisan, in Saccardo, Sylloge Fungorum, 8, 1889, 996; Bacillus mustelae septicus Kruse, in Flügge, Die Mikroorganismen, 3 Aufl., 2, 1896, 405; Bacterium mustelae septicus Chester, Ann. Rept. Del. Col. Agr. Exp. Sta., 9, 1897, 138; Bacillus muslelae Migula, Syst. d. Bakt., 2, 1900 , $\mathrm{v}$ and 756.) From a disease of ferrets.

Bacterium anatis Migula. (Bactéries du choléra des canards, Cornil and Toupet, Compt. rend. Acad. Sci. Paris, 106, 1888, 1747; Bacillus cholerae anatum Kruse, in Flügge, Die Mikroorganismen, 3 Aufl., 2, 1896, 417; Migula, Syst. d. Bakt., 2, 1900, 364.) Regarded as the cause of duck cholera and very similar to, if not identical with, Pasteurella avicida. See Rettger and Scoville, Jour. Inf. Dis., 26, 1920, 220. From the blood and other organs of infected ducks.

Bacterium cuniculi Migula. (Bacillus der Brustseuche des Kaninchens, Beck, Ztschr. f. Hyg., 15, 1893, 363; Bacillus cuniculi pneumonicus Kruse, in Flügge, Die Mikroorganismen, 3 Aufl., 2, 1896, 418; Bacterium cuniculi pneumonicus Chester, Ann. Rept. Del. Col. Agr. Exp. Sta., 9, 1897, 84; Migula, Syst. d. Bakt., 2, 1900, 370; Bacterium beckii Chester, Man. Determ. Bact., 1901, 142.) Associated with a lung plague of rabbits.

Bacterium haemorrhagicum (Kruse) Lehmann and Neumann. (Kolb, Arb. kaiserl. Gesundheitsamte, \%, 1892, 60; Bacillus haemorrhagicus Kruse, in Flügge, Die Mikroorganismen, 3 Aufl., 2, 1896, 424; Lehmann and Neumann, Bakt. Diag., 1 Aufl., 2, 1896, 194.) From the mucous membranes of fever patients.

Bacterium palumbarium Migula. (La bactérie de la maladie des palombes, Leclainche, Ann. Inst. Past., 8, 1894, 493; Bacillus cholerae columbarum Kiruse, in Flügge, Die Mikroorganismen, 3 Aufl., 2, 1896, 417; Bacterium cholerac columbarum Chester, Ann. Rept. Del. Col. Agr. Exp. Sta., 9, 1897, 84; Migula, Syst. d. Bakt., 2, 1900, 368; Bacterium columbarum Chester, Man. Determ. Bact., 1901, 141.) Associated with an epidemic in wild pigeons.

Bacterium phasianicida Klein (Klein, Cent. f. Bakt., I Abt., Orig., 31, 1902, 76; Bacterium phasianidarum mobile Enders, Berl. tierarztl. Wchnschr., No. 23, 1902; abst. in Cent. f. Bakt., 1 Abt., Ref., 34, 1904, 384.) From an epidemic in pheasants (England). Hadley, Elkins and Caldwell (Rhode Island Agr. Exp. Sta., Bull. 17t, 1918, 28) state that this species (which they call $B$. phasianicida) belongs in the group of paratyphoids (Salmonella).

Bacterium purpurum Chester. (Bacillus of purpura-haemorrhagica, Babes, Septiche Proz. Kindesalters, Leipzig: 1889; Bacillus haemorrhagicus septicus 
Tiruse, in Flügge, Die Mikroorganismen, 3 Aufl., 2, 1896, 424; Bacterium haemor rhagicus septicus Chester, Ann. Rept. Del. Col. Agr. Exp. Sta., 9, 1897, 85; Chester, Man. Determ. Bact., 1901, 143.) From a case of septicemia in man.

Bacterium tizzonii Migula. (Bacillus der haemorrhagischen Infektion, Tizzoni and Giovannini, in Zeigler, Beiträge, 7 , 1889, 300; Bacillus haemorrhagicus velenosus Kruse, in Flügge, Die Mikroorganismen, 3 Aufl., 2, 1896, 425; Bacterium haemorrhagicus velenosus Chester, Ann. Rept. Del. Col. Agr. Exp. Sta., 9, 1897, S5j; Migula, Syst. d. Bakt., 2, 1900, 386; Bacterium velenosum Chester, Man. Determ. Bact., 1901, 144.) From the blood of a child having a hemorrhagic infection.

Baclerium vassalei Migula. (Tizzoni and Giovannini, in Zeigler, Beiträge, 7 , 1859; Bacillus haemorrhagicus nephritidis Kruse, in Flügge, Die Mikroorganismen, 3 Aufl., 2, 1896, 424; Bacterium haemorrhagicus nephritidis Chester, Ann. Rept. Del. Col. Agr. Exp. Sta., 9, 1897, 85; Migula, Syst. d. Bakt., 2, 1900, 357; Bacterium nephritidis Chester, MIan. Determ. Bact., 1901, 145.) Isolated by Vassale from a case of hemorrhagic nephritis.

Pasteurella bouffardi Commes. (Commes, 1919; quoted from NeveuLemaire, Précis de Parasitol. Hum., 5th ed., 1921, 21.) The cause of a human pasteurellosis observed by Bouffard at Bamako in 1909.

Pasteurella caniseptica Hauduroy et al. (Pasteurella du rhien, Lignières, Recueil de Méd. Vét., $7 \gamma, 1900,469$; Bacterium canicida Lehmann and Neumann, Bakt. Diag., 4 Aufl., 2, 1907, 277; Hauduroy et al., Dict. d. Bact. Path., 1937, 312.) From dogs.

Pasteurella capriseptica (Lanfranchi and Pacchioni) Hauduroy et al. (Bacillus pneumoniae caprae Nicolle and RefikBey, Ann. Inst. Past., 10, 1596, 321; Pasteurella du chevre, Lignières, Recueil de Méd. Vétér., $7 \%, 1900,536 ;$ Bacillus caprisepticus Lanfranchi and Pacchioni,
1926; Bacillus bipolaris caprisepticus Chefik Kolayi and Raif, 1935; Hauduroy et al., Dict. d. Bact. Path., 1937, 313.) From hemorrhagic septicemia in goats. Pasteurella caviae Hauduroy et al. (Gaté and Billa, Compt. rend. Soc. Biol., Paris, 99, 1928, 814; Hauduroy et al., Dict. d. Bact. Path., 1937, 313.) From at guinea pig with a tuberculosis-like disease.

Pasteurella cariseptica (Schwer) Hauduroy et al. Pasteurella du cobaye, Phisalix, Compt. rend. Soc. Biol., Paris, 1, 1898, 761; Bacterium cavisepticum Schwer, C'ent. f. Bakt., I Abt., Orig., 33, 1902, 47; Hauduroy et al., Dict. d. Bact. Path., 1937, 314.) From hemorrhagic septicemia in guinea pigs.

Pasteurella desmodilli Pirie. (Pub. So. African Inst. Med. Res., 4, 1929, 191.)

Pastenrella equiseptica Kielser. (Bacille de la septicémie hémorragique du cheval, Lignières, Bull. Soc. Centr. d. Méd. Vét., 15, 1897, 437 and 16, 1898, 849 ; La Pasteurella equine, Lignières, Recueil de Méd. Vét., 7\%, 1900, 524; Kéelser, Man. Tet. Bact., 1st ed., 1927, 191; Bacillus cquisepticus Iielser, ibid.; Bacillus pneumoniae equi Poels.) From horses.

Pasteurella felis (Migula) Hauduroy et al. (Bacillus salivarius septicus felis Fiocea, Ann. d. Instit. d'igiene d. Univ. di Roma, 2, 1892 and Cent. f. Bakt., 11, 1892, 406; Bacillus felis septicus Kruse, in Flügge, Die Mikroorganismen, 3 Auf1., 2, 1896, 123; Bacterium felis septicus Chester, Ann. Rept. Del. Col. Agr. Exp. Sta., 9, 1897, 81; Pasteurella du chat, Lignières, Recueil de Méd. Vét., 77, 1900, 493; Bacterinm felis Migula, Syst. 1. Bakt., 2, 1960, 375; Hauduroy et al., 1)ict. d. Bact. Path., 1937, 316.) From the sputum of a cat.

Pasteurella mastidis Miessner and Schoop) Hauduroy et al. (Stäbchenbakterium, Dammann and Freese, Deut. tierärztl. Wchnschr., 15, 1907, 165; Bipolar organism of the Pasteurella group, Leyshon, Vet. Jour., 85, 1929, 299; Bacterium mastitidis Miessner and Schoop, Deut. tierärztl. Wchnschr., 40, 
1932, 69; Pasteurella, Marsh, Jour. Amer. Vet. Med. Assoc., 81 (N.S. 34), 1932, 376; Bacterium ovinum Haupt, Cent. f. Bakt., I Abt., Orig., 123, 1932, 365 ; Hauduroy et al.. Dict. d. Bact. Path., 1937, 316.) The cause of infectious mastitis of ewes.

Pasteurella necrophora Hauduroy et al. (Bacille de la nécrose infectieuse des Canaris, Cornell, The Vet. Record, 84, 1928, 350 ; Hauduroy et al., Dict. d. Bact. Path., 1937, 318.) From domestic canaries.

Pasteurella oviseptica Hauduroy et al (Galtier, Jour. d. méd. vét. et d. zoot., 1889-1890, 58, 113 and 481; La. Pasteurella ovine, Lignières, Recueil de Méd. Vétér., $77,1900,529$; Bacillus bipolaris ovisepticus Hutyra, in Folle and Wassermann, Hand. d. path. Mikro- org., 2 Aufl., 6, 1913, 67; Hauduroy et al., Dict. d. Bact. Path., 1937, 319.) From sheep.

Pasteurella pericarditis Hauduroy et al. Bacterium cavarum pericarditis Roth, Acta Pathol. et Microb. Scand., 11, 1934, 335; Hauduroy et al., Dict. d. Bact. Path., 1937, 319.) From guinea pigs.

Pasteurella strasburgensis Hauduroy et al. (Coccobacille de Strasbourg, Debre, Compt. rend. Soc. Biol., Paris, 82, 1919, 224; Hauduroy et al., Dict. d. Bact. Path., 1937, 323.) From a case of purulent pleurisy.

Pfeifferella anatipestifer Hendrickson and Hilbert. (Hendrickson and Hilbert, The Cornell Veterinarian, 22, 1932, 239; Hemophilus anatipestifer Hauduroy et al., Dict. d. Bact. Path., 1937, 247.) From a septicemic disease of ducks.

\section{Genus II. Malleomyces Pribram.*}

(Cladascus Enderlein (in part), Sitzber. Gesell. Naturf. Freunde, Berlin, 1917, 316; Pfeifferella Buchanan, Jour. Bact., 3, 1918, 54; Pribram, Klassification der Schizomyceten, Leipzig, 1933, 11 and 93; Loeflerella Gay et al., Agents of Disease and Host Resistance, Indianapolis, 1935, 782.) From Latin malleus, glanders and myces, fungus.

Because Pfeifferella was proposed inadvertently (Buchanan, Gen. Syst. Bact., 1925, 420 ) and because of a general feeling that it is inappropriate, Malleomyces Pribram is used as the earliest suitable name for this genus. The indefinite deseription of an organism (Malleomyces equestris) by Hallier (Ztschr. f. Parasitenkunde, 1870, 119) as the cause of glanders has not previously caused confusion and need not do so in the future.

Short rods, with rounded ends, sometimes forming threads and showing a tendeney toward branching. Motile or non-motile. Gram-negative. Tendency to bipolar staining. Milk slowly coagulated. Gelatin may be liquefied. Specialized for parasitic life. Grow well on blood serum and other body fluid media.

The type species is Malleomyces mallei (Flügge) Pribram.

\section{Key to the species of genus Malleomyces.}

I. Carbohydrates not fermented. Honey-like colonies on potato. Glycerol agar colonies slimy or tenacious, translucent. Non-motile.

\section{Malleomyces mallei.}

II. Carbohydrates femented. Profuse, creamy growth on potato. Glycerol agar colonies iridescent, becoming corrugated. Motile.

2. Malleomyces psendomallei.

* Revised by Prof. Robert S. Breed, New York State Experiment Station, Geneva, New York, December, 1938; further revision, December, 1945. 
1. Malleomyces mallei (Zopf) Pribram. (Rotzpilz, Löffier and Schütz, Deutsche med. Wchnschr., No. 52, 1882; Bacillus mallei Zopf, Die Spaltpilze, 3 Aufl., 1885, 89; Rotzbacillus, Löffler, Arb. kaiserl. Gesundheitsamte, 1, 1886, 222 ; Bacterium mallei Migula, in Engler and Prantl, Die ratürl. Pflanzenfam., 1, 1a, 1895, 21; Corynebacterium mallei Lehmann and Neumann, Bakt. Diag., 2 Aufl., 2, 1899, 366; Mycobacterium mallei Chester, Man. Determ. Bact., 1901, 353 ; Cladascus mallei Enderlein, Sitzber. Gesell. Naturf. Freunde Berlin, 1917. 395; Pfeifferella mallei Buchanan, Jour. Bact., 3, 1918, 51; Sclerothrix mallei Vuillemin, Encyclopédie Mycolog., Paris, 2, Champignons Parasites, 1931, 135; Brucella mallei Pacheco, Revista da Sociedado paulista de Medicina veterinaria, 3, 1933, 1; Actinobacillus mallei Thompson, Jour. Bact., 26, 1933, 226; also Jour. Bact., 25, 1933, 4 ; Pribram, Klassification der Schizomyceten. Leipzig and Vienna, 1933, 93; Loefflerella mallei Gay et al., Agents of Disease and Host Resistance, Indianapolis, 1935, 782.) From Latin malleus, glanders, a disease of horses.

Bacillus ozenae Trevisan (Corr. Ser., $1884, n .222$ ) is identical with this species according to Trevisan (I generi e le specie delle Batteriacee, 1889, 13).

Description largely from Kelser, Man. Vet. Bact., 2nd ed., 1933, 325.

Slender rods : 0.5 to 1.0 by 2.0 to 5.0 microns, with rounded ends, usually occurring singly, in pairs and in groups, but may grow into filaments. Branching involution forms on glycerol agar. Show irregular staining. Bipolar staining common. Non-motile. Gram-negative

Gelatin: Poor growth. Csually no liquefaction. May be slowly liquefied (Jordan, General Bact., 11th ed., 1935. 491),

Agar colonies: Moist, grayish-white layer, translucent, ropy, with regular borders. Later become yellowish or yellowish-brown.
Agar slants: Glistening, moist, ropy, grayish-white growth.

Löfler's serum : Good growth. Moist, viscid, yellowish colonies develop after 36 to 45 hours.

Broth: Turbid, sometimes with thin pellicle. Slimy or ropy sediment.

Litmus milk: Coagulation usually occurs after a week with some acid production. Litmus may or may not be reduced.

Potato: After 36 to 48 hours, pale yellow, heney-drop-like colonies. Later becoming darker, reddish-yellow or chocolate color. The medium sometimes has a faint greenish tinge around the growth.

Indole not formed.

Nitrites not produced from nitrates.

Carbohydrates usually not fermented. Some strains produce small amounts of acid from glucose.

Optimum temperature $37^{\circ} \mathrm{C}$. No growth below $20^{\circ} \mathrm{C}$ or above $44^{\circ} \mathrm{C}$.

Aerobic, facultative anaerobic.

Common name: Glanders bacillus.

Distinctive characters: Culture media of slightly acid reaction best suited for growth; addition of glycerol favors growth; honey-like growth on potato.

Source: Isolated by Löfller and Schütz from the liver and spleen of a horse. Jesions in animals and man.

Habitat: The cause of glanders, affecting horses, man, sheep and goats. Transmissible to dogs, eats, rabbits and guinea pigs.

\section{Malleomyces pseudomallei (Whit-} more) Breed. (Bacillus pseudomallei Whitmore, Jour. Hyg., 13, 1913, 1; Bacillus whitmori Stanton and Fletcher, Trans. 4th Cong. Far East Assn. Trop. Med., 2, 1921, 196; also Jour. Hyg., 23, 1925, 347; Pfeifferella pseudomallei Ford, Textb. of Bact., 1927, 294; Flavobacterium pseudomallei Bergey et al., Manual, 3rd ed., 1930, 146; Sclerothrix whitmori Vuillemin, Encyclopédie Mycolog., 2, Champignons Parasites, 1931, 136; Actinobacillus pseudomallei Thompson, Jour. Bact., 26, 1933, 226 ; also Jour. Bact., 
25, 1933, 44; Loefllerella whitmori Gay et al., Agents of Disease and Host Resistance, Indianapolis, 1935, 791 ; Breed, in Manual, 5th ed., 1939, 300.) Latinized, false glanders.

Short rods: With rounded ends, occurring singly and in short chains, showing bipolar staining. Motile. Gram-negative.

Gelatin stab: Moderate, crateriform liquefaction.

Agar colonies : Circular, slightly raised, thick, opaque, cream-colored with irregular margin.

Glycerol agar slant: Wrinkled, thick, rugose, cream-colored growth.

Broth: Turbid with pellicle.

Litmus milk: Curdling with slowly developed acidity, pink sediment; may be digested.

Potato: Vigorous, cream-colored growth.

Indole not formed.

Acid from glucose, maltose, lactose, sucrose and mannitol.

Blood serum slowly liquefied.

Aerobic, facultative.

Optimum temperature $37^{\circ} \mathrm{C}$.

Source: Lesions and blood in rats, guinea pigs, rabbits and man. Once from a transient nasal discharge in a horse (Stanton, Fletcher and Symonds) and once from a splenic abscess in a cow (Nicholls).

Habitat: Glanders-like infection (melioidosis) in rats, guinea pigs, rabbits and in man in India, Federated Malay States and Indo-China.

Appendix: The following may belong in this genus:

Flavobacterium orchitidis Sherwood, Irwin and Marts. (Sherwood, Irwin (not Edwin) and Marts, Amer. Jour. Diseases of Children, 45, 1933, 446; Sherwood, Jour. Kansas Med. Soc., 34, 1933, 220.) From a case of meningitis. Sherwood (personal communication, 1945) now considers this organism identica! with Bacillus rhitmori (Malleomyces pseudomallei). See Manual, 5th (d., 1939, 535 for a deseription of this species.

Malleomyces agliaceus Pribram. (Bacillo opale agliaceo, Vincenzi, Giorn. d. R. Acead. d. Med. Torino, 1890, No. 6; Pribram, Klassification der Schizomyecten, Lcipzig, 1933, 93.) Cause of pseudotuberculosis in frogs.

\section{Genus III. Actinobacillus Brumpt.*}

(Précis de Parasitologie, Paris, 1st ed., 1910, 849.)

Medium-sized, aerobic, Gram-negative rods which frequently show much pleomorphism. Coccus-like forms frequent. Tendency to bipolar staining. Acid but no gas produced from carbohydrates. Grow best, especially when freshly isolated, under increased $\mathrm{CO}_{2}$ tension. Pathogenic for animals; some species attack man. The outstanding characteristic of the group is the tendency to form aggregates in tissues or culture which resemble the so-called sulfur granules of actinomycosis.

The type species is Actinobacillus lignieresi Brumpt.

1. Actinobacillus lignieresi Brumpt. (Actinobacilo, Lignières and Spitz, Boletin d. Agri. y Ganaderia, Buenos Aires, 11, 1902, 169; Actinobacillus, Lignières and Spitz, Cent. f. Bakt., I Abt., Orig., 35, 1903, 294; Brumpt, Précis de Parasitol., Paris, 1st ed., 1910, 849; Bacillus lignieri (sic) Macé, Traité de Bactériolo-

* Revised by Prof. W. A. Hagan, New York State Veterinary College, Ithaca, New York, December, 1938; further revision, December, 1945. 
gie, 6th ed., 2, 1913, 743; Nocardia lignieresi Chalmers and Christopherson, Ann. Trop. Med. and Parasit., 10, 1916, 242 ; Discomyces lignieresi Brumpt, Précis de Parasitol., Paris, 3rd ed., 1922, 993; Pasteurella lignieresi Vuillemin, Encyclopédie Mycologique, Paris, 2, Champignons Parasites, 1931, 136.) Named for Lignières, who first worked with this organism.

Bacterium purifaciens Christiansen (Maanedsskr. f. Drylaeger, 29, 1917, 449; Pasteurella purifaciens Hauduroy et al., Dict. d. Bact. Path., 1937, 322) is regarded as identical with this organism by Tunnicliff (Jour. Inf. Dis., 69, 1941, $52)$.

Rods: 0.4 by 1.0 to 15.0 microns. Cocco-bacillary forms frequent. Nonmotile. Gram-negative.

Gelatin: Growth sparse or fails. No liquefaction.

Agar: Primary cultures usually succeed best when the inoculum is introduced by stab. Serum agar is more favorable than plain. Surface colonies are small, bluish, translucent at first, later becoming opaque.

Broth: Serum favors growth. Freshly isolated strains usually grow in form of small granules which adhere to sides of tube, leaving broth fairly clear. Later most strains grow diffusely, often forming a fragile pellicle.

Litmus milk: Most strains cause no change. Sometimes slight acid. No coagulation.

Potato: Little or no growth.

Acid but no gas within 48 hours from glucose, fructose, galactose, maltose, sucrose and mannitol. Acid after longer incubation from lactose, raffinose and glycerol.

Indole is formed in small amounts.

Nitrites not produced from nitrates.

Aerobic. Is favored by increased $\mathrm{CO}_{2}$ tension. Will not grow anaerobically.

Optimum temperature $37^{\circ} \mathrm{C}$.

Pathogenic for cattle and swine. A few cases reported in man. Rabbits and guinea pigs slightly susceptible to inoculation.

Source and habitat: Usually isolated from the lesions of actinobacillosis of cattle. This condition is of ten clinically diagnosed as actinomycosis. Lesions found in soft tissues, usually lymph nodes, where granulomatous tumors are formed. Eventually these break down to form abscesses.

\section{Actinobacillus actinomycetemcomi-} tans Topley and Wilson. (Bacterium actinomycetem comitans Klinger, Cent. f. Bakt., I Abt., Orig., 1912, 198; Actinobacillus actinomycetem camitans Topley and Wilson, Princip. of Bact. and Immun., 1, 1931, 256; Topley and Wilson, ibid., 2nd ed., 1936, 279.) From actinomycete, and Latin comitans, accompanying.

Description taken from Topley and Wilson (loc. cit.), Colebrook (Brit. Jour. Exp. Path., 1, 1920, 197), and Bayne-Jones (Jour. Bact., 10, 1925, 572 ).

Coceo-bacilli: Rods 1.0 to 1.5 microns long, cocci 0.6 to 0.8 micron in diameter. Occurring in densely-packed masses. Non-motile. Gram-negative.

Gelatin: No liquefaction.

Agar colonies: Small, tough, adherent. Glucose agar: Growth thin, dry, granular, hard, slightly yellow, adherent.

Liquid gelatin or broth: At $37^{\circ} \mathrm{C}$, numerous isolated, translucent granules, 0.5 to $1.0 \mathrm{~mm}$ in diameter, form along sides of tube. In a few days they fuse into a grayish-white mass, forming ring around tube and pellicle over surface. Later granules become opaque, grayish-white.

Glucose broth: Turbid. Yellowish flakes.

Milk: No growth.

Potato: No growth.

Acid but no gas from glucose and lactose.

Not pathogenic for laboratory animals. No growth at $20^{\circ} \mathrm{C}$. 
Aerobic, facultative.

Distinctive character: Manner of growth in liquid gelatin.

Source: Found in lesions of actinomycosis.

Habitat: Presumably in actinomyeotic lesions.

3. Actinobacillus actinoides (Smith) Topley and Wilson. (Bacillus actinoides Th. Smith, Jour. Exp. Med., 28, 1918, 333 ; Actinomyces actinoides Bergey et al., Manual, 1st ed., 1923, 346; Topley and Wilson, Princip. of Bact. and Immun., 1st ed., 1, 1931, 256.) From Greek, ray like.

Slender rods in tissues. In cultures may be bacillary or coccoid in form. Grows only under increased $\mathrm{CO}_{2}$ tension (so-called microaerophilic). Does not grow on ordinary agar or broth, except occasionally when transferred from more favorable media. Most characteristic growth on coagulated blood serum.

Gelatin: No growth.

Agar colonies: Very minute, pale, straw color.
Agar slant: Best growth seen in water of condensation. Serial transfers on this medium generally fail.

Broth: No growth.

Litmus milk: No growth.

Potato: No growth.

Coagulated blood serum (cow): Growth appears first in the condensation water. Appear as granules, consisting of capsular material in which bacillary forms are embedded. Surface mulberry-like because of club-like extensions of capsular material. In stained preparations, the capsular material appears amorphous.

Optimum temperature $37^{\circ} \mathrm{C}$.

Microaerophilic.

Not pathogenic for laboratory animals, except possibly the white rat in which a spontaneous chronic pneumonia oceurs caused by an organism indistinguishable from this one. Experiments with rats by artificial inoculation have not been reported.

Source: From lungs of calves suffering from chronic pneumonia.

Habitat: Has not been recognized in nature except in pathological processes. 


\section{* APPENDIX TO TRIBE PASTEURELLEAE.}

While the authors who deseribe the following new genus with its single species do not indicate its general relationships, it would appear to be as closely related to the species placed in Parrobacteriaceae as to those in any other family. It is therefore placed in this appendix pending a clarification of the situation.

\section{Genus A. Donovania Anderson, De Monbreun and Goodpasture.}

(Jour. Exp. Med., 81, 1945, 25.) Named for C. Donovan who first described the type species.

Pleomorphic non-motile rods, exhibiting single or bipolar condensations of chromatin. Occur singly and in clusters. May be capsulated or non-capsulated. Gram-negative. Growth outside human body occurs only in the yolk, yolk sac or amniotic fluid of developing chick embryo or in a medium containing embryonic yolk. Pathogenic for man causing granulomatous lesions, particularly in the inguinal region.

The type species is Donovania granulomatis Anderson, De Monbreun and Goodpasture.

1. Donovania granulomatis Anderson et al. (Epithelial cell parasites, Donovan, Indian Med. Gaz., 40, 1905, 414; Donovan bodies, Dienst, Greenblatt and Sanderson, Jour. Inf. Dis., 62, 1938, 112; Donovan organism, Anderson, Science, 97, 1913, 560; Anderson, De Monbreun and Goodpasture, Jour. Exp. Med., 81, 1945 , 25.) From M. L. granuloma, of granuloma.

Pleomorphic rods 1 to 2 microns in length, with rounded ends, occurring singly and in clusters. Intracellular forms usually capsulated. Non-motile. Gram-negative.

No growth on ordinary culture media. Chick embryo: Grows readily in yolk, yolk sac and feebly in amniotic fluid of developing chick embryo.

Embryonic yolk medium: Growth oceurs.

Distinctive characters: Capsulated forms readily demonstrated by means of Wright's stain as blue bacillary bodies surrounded by well-defined dense pinkish capsules. Non-capsulated forms variable in morphology. Characteristic safety-pin forms may be demonstrated.

Not pathogenic for the common experimental animals.

Source: Granulomatous lesions of man.

Habitat: Human lesions. The cause of granuloma inguinale.

* Prepared by Dr. Orren D. Chapman, Syracuse Medical College, Syracuse, New York, Mareh, 1946.

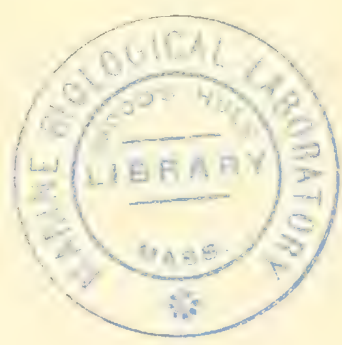


TRIBE II. BRUCELLEAE BERGEY, BREED AND MURRAY.

(Preprint, Manual, 5th ed., October, 1938, vi.)

Small, motile or non-motile rods or coccoids which grow on special media. There is a single genus Brucella.

\section{Genus I. Brucella Meyer and Shaw.*}

(Jour. Inf. Dis., 27, 1920, 173.) Named for Sir David Bruce, who first recognized the organism causing undulant fever.

Short rods with many coccoid cells, 0.5 by 0.5 to 2.0 inicrons ; non-motile; capsulated; Gram-negative; gelatin not liquefied; neither acid nor gas from carbohydrates; urea utilized; parasitic, invading all animal tissues, producing infection of the genital organs, the mammary gland, the respiratory and intestinal tracts; pathogenic for various species of domestic animals and man.

The type species is Brucella melitensis (Hughes) Meyer and shaw.

Key to the species of genus Brucella.

I. Non-motile.

A. Grow in special media containing basic fuchsin.

1. Grows in media containing thionin.

1. Brucella melitensis.

2. Does not grow in media containing thionin.

2. Brucella, abortus.

B. Does not grow in media containing basic fuchsin.

1. Grows in media containing thionin.

3. Brucella suis.

II. Motile.

4. Brucella bronchiseptica.

Differential characters of the three closely related species of genus Brucella.

\begin{tabular}{|c|c|c|c|c|c|c|c|}
\hline \multirow{2}{*}{ Species } & \multirow{2}{*}{$\begin{array}{l}\text { Infec- } \\
\text { tivity } \\
\text { for } \\
\text { guinea } \\
\text { pigs }\end{array}$} & \multirow{2}{*}{$\begin{array}{c}\mathrm{Re}- \\
\text { quires } \\
\mathrm{CO} 2 \\
\text { for iso- } \\
\text { lation }\end{array}$} & \multirow{2}{*}{$\begin{array}{c}\mathrm{H}_{2} \mathrm{~S} \\
\text { forma- } \\
\text { tion }\end{array}$} & \multirow{2}{*}{$\begin{array}{l}{ }^{*} \text { Glucose } \\
\text { utilized }\end{array}$} & \multirow{2}{*}{$\begin{array}{c}\text { Amino- } \\
\text { nitro- } \\
\text { gen } \\
\text { utilized }\end{array}$} & \multicolumn{2}{|c|}{$\begin{array}{l}\text { Growth in the } \\
\text { presence of }\end{array}$} \\
\hline & & & & & & $\begin{array}{l}\text { Thio- } \\
\text { nin }\end{array}$ & $\begin{array}{c}\text { Basic } \\
\text { fuchsin }\end{array}$ \\
\hline & & $\begin{array}{l}10 \text { per } \\
\text { cent }\end{array}$ & days & & & & \\
\hline Brucella melitensis. & ++ & 0 & \pm 1 & $+t+$ & + & $++t$ & $+t+$ \\
\hline Brucella abortus.... & ++ & ++ & 2 & + & ++ & 0 & $+t+$ \\
\hline Brucella suis..... & ++ & 0 & 4 & $+t+$ & + & $t+t$ & 0 \\
\hline
\end{tabular}

* All utilize glucose in shake cultures.

1. Brucella melitensis (Hughes) Meyer and Shaw. (Bruce, Practitioner, 39, 1887, 161; ibid., 40, 1888, 241; Rept. Army Med. Dept., London, 32, 1890,
Append. No. 4, 465; streptococcus Miletensis (sic) Hughes, The Mediterranean Naturalist, 2, February 1, 1892, 325; Micrococcus melitensis Bruce, Ann. Inst.

* Revised by Prof. I. F. Huddleson, Michigan State College, East Lansing, Michigan, December, 1942. 
Past., 7, April, 1893, 289; Hughes, La Riforma Med., 3, Aug. or Sept., 1893, 789 and Ann. Inst. Past., 7, Aug., 1893, 630; Bacterium melitense Saisawa, Ztschr. f. Hyg., 70, 1912, 1S1; Meyer and Shaw, Jour. Inf. Dis., 27, 1920, 173; Bacillus melitensis Holland, Jour. Bact., 5, 1920, 219; Alcaligenes melitensis Bergey et al., Manual, 1st ed., 1923, 235; Brucella melitensis var. melitensis Evans, U. S. Public Health Reports, 38, 1923, 1947.) From Latin, of Malta.

Short ellipsoidal rods : 0.3 to 0.4 micron in length, occurring singly and in pairs, rarely in short chains. Non-motile. Non-acid-fast. Gram-negative.

Gelatin colonies: Small, clear, entire.

Gelatin stab: Slow growth. No liquefaction.

Agar colonies: small, circular, convex, amorphous, smooth, glistening, entire, bluish-green, gravish if $\mathrm{R}$ type.

Agar slant: Growth slow, moist, honeylike, entire. After a week, the agar is turned brownish and crystals may appear.

Broth: After 10 days, moderate turbidity and grayish sediment. Reaction alkaline, $\mathrm{pH} 8.0$ or higher.

Litmus milk: Unchanged at 24 hours. Later becomes alkaline.

Potato: Scant growth, grayish beroming brownish.

Indole not formed.

Nitrates reduced, often with complete disappearance of nitrite (Zobell and Meyer, Jour. Inf. Dis., 51, 1932, 99). Because of the latter fact, reports in the literature are apparently contradictory.

Ammonia produced from urea.

Growth enhanced on beef liver or tryptose agar of $\mathrm{pH} 6.8$.

Neither acid nor gas from cartohydrate media.

Optimum reaction $\mathrm{pH} 7.4$.

Optimum temperature $37^{\circ} \mathrm{C}$. No growth at $6^{\circ}$ or at $45^{\circ} \mathrm{C}$. Killed at $59^{\circ} \mathrm{C}$.

Aerobic.

Distinctive characters: Requires no increased $\mathrm{CO}_{2}$ tension.
Source: Isolated by Bruce (1887, loc. cit.) from the spleen in fatal cases of Malta fever.

Habitat: Chief host the milch goat. The cause of undulant fever (brucellosis) in man and abortion in goats. May infect cows and hogs and be excreted in their milk. Infectious for all domestic animals.

2. Brucella abortus (Schmidt and Weis) Meyer and Shaw. (Bacillus of abortion, Bang, Ztschr. f. Thiermed., 1, 1897, 241; Bacterium abortus Schmidt and Weis, Bakterierne, 1901, 266; Bacterium abortivum Chester, Man. Determ. Bact., 1901, 121; Corynebacterium abortus endemici Preisz, Cent. f. Bakt., I Abt., Orig., 33, 1902, 194; Bacillus abortus Evans, Jour. Wash. Acad. Sci., 5, 1915, 122 ; Meyer and Shaw, Jour. Inf. Dis., 27, 1920, 173; Alcaligenes abortus Bergey et al., Manual, 1st ed., 1923, 234; Brucella melitensis var. abortus Evans, Public Health Reports, 38, 1923, 1947.) From Latin abortus, an untimely birth.

The morphological and cultural characters are similar to those of Brucella melitensis with the following exceptions: Requires 10 per cent $\mathrm{CO}_{2}$ for isolation, becomes aerobic after several transfers; the browning of the medium in agar slant culture is less marked; S cultures can be difierentiated from Brucella melitensis, but not from Brucella suis, by the agglutinin absorption test.

Source: From the genital organs and milk of infected cattle and from blood in human cases of undulant fever.

Habitat: Chief host the milch cow. The cause of infectious abortion in cattle. The same effects are produced in mares, sheep, rabbits and guinea pigs, and all domestic animals except hogs. Causes undulant fever (hrucellosis) in man.

3. Brucella suis Huddleson. (Organism resembling Bacillus abortus, Anonymous, U. S. D. A. Ann. Rept. Secy. Dept., Rept. of Chief Bur. Animal Ind., 
1914, 86 (30); authorship established by Traum in North Amer. Vet., 1, No. 2, 1920; described as Bacillus abortus by Good and Smith, Jour. Bact., 1, 1916, 415; Huddleson, Undulant Fever Symposium, Amer. Pub. Health Assoc., (Oct., 1928) 1929, 24; also Mich. Agr. Exp. Sta. Tech. Bull. 100, 1929, 12; Brucella melitensis var. suis Hardy, Jordan, Borts and Hardy, Public Healt h Reports, 45, 1930, 2433; Bacillus abortus suis Meyer, Amer. Jour. Pub. Health, 21, 1931, 503.) From Latin, of swine.

The morphological and cultural characters are similar to those of Brucella melitensis.

$\mathrm{S}$ cultures of Brucella suis can be differentiated from $\mathrm{S}$ cultures of Brucella melitensis, hut not from $S$ cultures of Brucella abortus, by the agglutinin absorption test.

Source: From urinogenital and many other organs of swine.

Habitat: Chief host the hog. Causes abortion in swine and undulant fever (brucellosis) in man. Also infectious for horses, dogs, cows, monkeys and laboratory animals.

The differentiation of the above species of Brucella by the bacteriostatic action of dyes depends upon the medium used. When tryptose agar (Difeo) is used, basie fuchsin and thionin should be used in a final dilution of $1: 100,000$.

There are several forms of the $\mathrm{R}$ and mucoid phases of Brucella spp. (Huddleson, Amer. Jour. Vet. Res., 7, 1946, 5). The true $\mathrm{R}$ type differs from the $\mathrm{S}$ type in its lack of pathogenicity, its antigenic properties, its susceptibility to agglutination by exposure of suspensions to heat and to basic dyes in concentration of $1: 2000$, and colonial appearance. The mucoid phases differ antigenically, morphologically and culturally. Colonies on agar are spherical or flat, regular in contour, grayish to mucoid in appearance. Suspensions are not agglutinated by heat or dyes, or always by special agglutinating serums. There is no change in their growth characteristics on media containing either basic fuchsin or thionin.

\section{Brucella bronchiseptica (Ferry)} Topley and Wilson. (Ferry, Amer. Vet. Rev., 37, 1910, 499; also see MeGowan, Jour. Path., 15, 1911, 372; Bacillus bronchicanis Ferry, Jour. Inf. Dis., 8, 1911, 402; Bacillus bronchisepticus Ferry, Amer. Vet. Rev., 41, 1912, 79; Bacterium bronchisepticus Evans, Jour. Inf. Dis., 18, 1916, 578; Bacterium bronchicanis Holland, Jour. Bact., 5, 1920, 221 ; Alcaligenes bronchisepticus Bergey et al., Manual, 1st ed., 1923, 234; Topley and Wilson, Princip. Bact. and Immun., 1st ed., 1, 1931, 508.) Latinized, disease of the bronchial tubes.

Evans (loc. cit., 593) regards Bacterium bronchisepticus as related to Bacterium abortus morphologically, culturally, biochemically and serologically.

Short slender rods: 0.4 to 0.5 by 2.0 microns, usually occurring singly, sometimes in pairs and chains. Motile with 4 to 6 peritrichous flagella (Topley and Wilson). Gram-negative.

Gelatin colonies: Similar to those on agar.

Gelatin stab: Slow filiform growth. No liquefaction.

Agar colonies: Small, opaque, white, slightly raised, porcellaneous, entire.

Agar slant: Growth moderate but more luxuriant than in Brucella melitensis, filiform, slightly raised, smooth, opalescent, lustrous, moist, entire.

Broth: Turbid, with thin, gray pellicle and ropy sediment. Musty odor develops.

Litmus milk: Alkaline. No coagulation.

Potato: Growth fairly abundant, brownish, glistening, moist, sticky. Medium is darkened.

Indole not formed.

Nitrites often produced from nitrates (Topley and Wilson). 
No acid or gas from glucose, sucrose, lactose, maltose or mannitol.

No $\mathrm{H}_{2} \mathrm{~S}$ produced (Topley and Wilson).

Catalase positive (Topley and Wilson).

Ammonia formed from urea and asparagine.

Optimum temperature $37^{\circ} \mathrm{C}$. Killed in twenty minutes at $55^{\circ} \mathrm{C}$.

Aerobic, facultative.

Source: From dogs affected with distemper.

Habitat: Causes acute, often fatal, pneumonia in dogs generally as a secondary invader in distemper. Also pathogenic for cats, rabbits, guinea pigs, ferrets, white rats and monkeys. Sometimes occurs in man.

Appendix: The following are recorded in the literature discussing this genus: Brucella evansi Pacheco (Revista da Sociedade Paulista de Med. Vet., 8,
$1933,9)$ is a name applied to a group of thirteen cultures referred to by Evans (Jour. Inf. Dis., 23, 1918, 35t) as abortuslike bacteria although she definitely indicates that these cultures do not agree with each other in their biochemical characteristics (loc. cit., Table 4, p. 361). The binomials Brucella paramelitensis, Brucella paraabortus and Brucella parasuis have been used for inagglutinable strains of these three species which are, according to Topley and Wilson (Princip. Bact. and Immun., 2nd ed., 1936, 632), now known to be merely rough variants, not deserving to be so named.

Micrococcus paramelitensis Negré and Raymond. (Compt. rend. Soc. Biol., Paris, 72, 1912, 791 and 1052.)

Micrococcus pseudomelitensis Sergent and Zammitt, 1908. Exact reference not known. 
TRIBE III. BACTEROIDEAE TRIB. NOV.

Motile or non-motile rods without endospores. May or may not require enriched culture media. Obligate anaerobes. Gram-negative.

Key to the genera of tribe Bacteroideae.

I. Cells with rounded ends.

Genus I. Bacteroides, p. 564 .

II. Cells with pointed ends.

Genus II. Fusobacterium, p. 581.

\section{Genus I. Bacteroides Castellani and Chalmers.*}

(Man. Trop. Med., 3rd ed., 1919, 959.)

Characters as for the tribe. From Greek, like a rod.

The type species is Bacteroides fragilis (Veillon and Zuber) Castellani and Chalmers.

Note: The descriptions have been taken largely from Weinberg et al. (Les Microbes Anaérobies, Paris, 1937, 658); Prévot (Ann. Inst. Past., 60, 1938, 285); Hauduroy, Ehringer, Urbain, Guillot and Magrou (Dict. Bact. Path., Paris, 1937, 51) ; and Eggerth and Gagnon (Jour. Bact., 25, 1933, 389). Because cultures of many of these organisms have not been subjected to critical study with identical tests and media, it is difficult to know how many should be considered as distinct species, and the present arrangement must be considered as tentative. The key, of necessity, has been drawn up from recorded characters which appeared useful for the purpose and these on further study may prove to be inarlequate.

\section{Key to the species of genus Bacteroides.}

I. Not requiring enriched media.

A. Gas formed from proteins.

1. Hydrogen sulfide not produred.

a. Non-motile.

1. Bacteroides fragilis.

aa. Motile.

2. Bacteroides serpens.

2. Hydrogen sulfide produced.

a. Indole not formed.

b. Very pleomorphic.

3. Bacteroides funduliformis.

bb. Not markedly pleomorphic.

4. Bacteroides siccus.

aa. Indole formed.

b. Gelatin liquefied.

5. Bacteroides coagulans.

* Completely revised by Dr. T. E. Roy, Bacteriologist to the Hospital for Sick ChilIren, Toronto, Ontario, Canada and Dr. C. D. Kelly, Assistant Professor of Bacteriology, McGill University, Montreal, P. Q., Canada, December, 1938; rearranged, Decem. ber, 1945 . 
bb. Gelatin not liquefied.

c. No acid from lactose and maltose.

6. Bacteroides varius.

cc. Acid from lactose and maltose.

d. Acid from sucrose. No acid from glycerol.

7. Bacteroides inaequalis.

dd. No acid from sucrose. Acid from glycerol.

8. Bacteroides insolitus.

B. No gas formed from proteins.

1. Indole not formed.

a. Hydrogen sulfide not formed.

b. No acid from lactose.

9. Bacteroides vescus.

bb. Acid from lactose.

c. No acid from salicin.

10. Bacteroides exiguus.

ce. Acid from salicin.

11. Bacteroides uncatus.

aa. Hydrogen sulfide formed.

b. No acid from salicin. Acid from arabinose.

c. Gelatin liquefied.

12. Bacteroides vulgatus.

cc. Gelatin not liquefied.

13. Bacteroides incommunis.

bb. Acid from salicin. No acid from arabinose.

14. Bacteroides distasonis.

bbb. No acid from salicin or arabinose.

c. Acid from sorbitol.

15. Bacteroides tumidus.

cc. No acid from sorbitol.

16. Bacteroides convexus.

2. Indole formed.

a. No acid from salicin or arabinose.

17. Bacteroides ovatus.

aa. Acid from salicin and arabinose.

b. No acid from mannitol.

c. No acid from rhamnose.

18. Bacteroides uniformis.

cc. Acid from rhamnose.

d. Not capsulated.

19. Bacteroides thetaiotaomicron.

dd. Capsulated.

20. Bacteroides variabilis.

bb. Acid from mannitol.

21. Bacteroides gulosus.

II. Requiring an enriched medium.

A. Producing a black pigment.

B. Not producing pigment.

22. Bacteroides melaninogenicus.

23. Bacteroides caviae. 
1. Bacteroides fragilis (Veillon and Zuber) Castellani and Chalmers. (Bacillus fragilis Veillon and Zuber, Arch. Méd. Exp. et Anat. Path., 10, 1898, 870; Castellani and Chalmers, Man. Trop. Med., 3rd ed., 1919, 959; Fusiformis fragilis Topley and Wilson, Princip. Bact. and Immun., 1st ed., 1, 1931, 302; Ristella fragilis Prévot, Ann. Inst. Past., 60, 1938, 290.) From Latin fragilis, fragile.

Rods with rounded ends, staining more deeply at the poles, occurring singly and in pairs. Non-motile. Gramnegative.

Gelatin : No liquefaction; small amount of gas.

Agar colonies: Small, gray, irregular.

Broth: Turbid.

Indole not formed.

Hydrogen sulfide not formed.

Litmus milk: No coagulation. Slight amount of gas.

Nitrites not produced from nitrates.

Acid from fructose, maltose, sucrose, galactose, glucose and arabinose. Some strains produce acid from lactose (Weinberg et al., Les Microbes Anaérobies, 1937,720 )

Anaerobic.

Optimum temperature $37^{\circ} \mathrm{C}$

Pathogenicity: Some strains produce subcutaneous abscesses in rabbits, guinea pigs or mice.

Source and habitat: From acute appendicitis, pulmonary gangrene, abscesses of the urinary tract, and septicaemias in man.

2. Bacteroides serpens (Veillon and Zuber) Hauduroy et al. (Bacillus serpens Veillon and Zuber, Arch. Méd. Exp. et Anat. Path., 10, 1898, 870; Bacillus radiiformis Rist and Guillemot, Arch. Méd. Exp. et Anat. Path., 1904; Hauduroy et al., Dict. d. Bact. Path., 1937, 74; Zuberella serpens Prévot, Ann. Inst. Past., 60, 1938, 293.) From Latin serpens, creeping.

Rods: Thick, with rounded ends, oc- curring singly, in pairs, or in short chains. Motile. Gram-negative.

Gelatin: Slow liquefaction, with gas.

Agar colonies: Punctiform.

Deep agar colonies: Small colonies in 48 hours, ray-like growth later. Gas produced.

Broth: Turbid, then floceulent grow th; some gas with foul odor.

Hydrogen sulfide not formed.

Iitmus milk: Acidified and coagulated in six days, with no digestion.

Acid from fructose, galactose, maltose and lactose.

Coagulated egg white and serum not liquefied.

Anaerobic.

Optimum temperature $37^{\circ} \mathrm{C}$.

lixperimental pathogenicity: Some strains produce abscesses in rabbits, guinea pigs and mice.

Source and habitat: Acute appendicitis, mastoiditis, pulmonary gangrene, hile tract of $\operatorname{dog}$, and sea water.

\section{Bacteroides funduliformis (Hallé)} Bergey et al. (Bacillus funduliformis Halle, Inaug. Diss., Paris, 1898; Bacillus thetoides Rist, Thèse de Paris, 1898; Bergey et al., Manual, 3rd ed., 1930, 373; Spherophorus funduliformis Prévot, Ann. Inst. Past., 60, 1938, 298.) From Latin funduliformis, sausage-shaped.

Rods: 1.5 to 3.0 microns long in pus, often spindle-shaped. Extremely pleomorphic in culture media, showing irregular filamentous and branching forms. Non-motile. Gram-negative.

Gelatin: Not liquefied.

Deep agar colonies: Lenticular, with some gas and foul odor.

Broth: Flocculent growth.

Glucose broth: Rapid growth with gas and foul odor.

Indole not formed; although sometimes found in old cultures.

Hydrogen sulfide is formed in small amounts.

Litmus milk: Acid and coagulation by some strains. 
Acid and gas from fructose, glucose and maltose. Some strains ferment mannitol, sucrose and lactose.

Anaerobic.

Optimum temperature $37^{\circ} \mathrm{C}$.

Experimental pathogenicity: Some strains are pathogenic for rabbits and guinea pigs but not for white rats and mice.

Source and habitat: Female genitalia, urinary infections, puerperal infections, acute appendicitis, otitis, pulmonary gangrene, liver abscesses, septicaemias and intestinal tract.

4. Bacteroides siccus Eggerth and Gagnon. (Eggerth and Gagnon, Jour. Bact., 25, 1933, 410; Spherophorus siccus Prévot, Ann. Inst. Past., 60, 1938, 299.) From Latin siccus, dry.

Short, thick rods: About 1.0 micron long. In glucose broth they are coccoid and often grow in short chains. Nonmotile. Gram-negative.

Gelatin: Not liquefied.

Blood agar colonies: Elevated, dry, difficult to emulsify, 1.0 to $1.5 \mathrm{~mm}$ in diameter.

Broth: Growth occurs as a powdery sediment with a clear supernatant fluid.

Indole not formed.

Hydrogen sulfide is formed.

Milk: Unchanged.

Nitrites not produced from nitrates.

Acid but no gas from fructose. No acid or gas from glucose, glycerol, mannitol, sorbitol, arabinose, saliein, trehalose, amygdalin, cellobiose, glycogen, rhamnose, xylose or lactose.

Non-pathogenic for white mice and rabbits.

Anaerobic.

Distinctive characters: Gas is formed in small amounts from peptone. Phenol red and brom cresol purple are decolorized in meat infusion broth.

Source: Two strains isolated from human teces.

Habitat: Probably intestinal canal of mammals.
5. Bacteroidés coagulans Eggerth and Gagnon. (Eggerth and Gagnon, Jour. Bact., 25, 1933, 409; Pasteurella coagulans Prévot, Ann. Inst. Past., 60, 1938, 292.) From Latin coagulans, coagulating.

Rods : 0.5 to 2.0 microns long. Bipolar staining. Non-motile. Gram-negative.

Gelatin: Liquefied in 8 to 12 days.

Blood agar colonies: Soft, transparent, $0.5 \mathrm{~mm}$ in diameter.

Broth: Diffuse growth.

Indole is formed.

Hydrogen sulfide is formed.

Milk: Coagulated in 8 days without acid production. The coagulum partly redissolves after 3 to 4 weeks.

Nitrites not produced from nitrates.

Non-pathogenic for white mice and rabbits.

Anaerobic.

Distinctive characters : No acid or gas from carbohydrates. A small amount of gas is formed from peptone. Phenol red and brom cresol purple are decolorized in a meat infusion broth.

Source: One strain isolated from human feces.

Habitat: Probably intestinal canal of mammals.

6. Bacteroides varius Eggerth and Gagnon. (Eggerth and Gagnon, Jour. Bact., 25, 1933, 409; Spherophorus varius Prévot, Ann. Inst. Past., 60, 1938, 299.) From Latin varius, diverse.

Rods : 1.0 to 3.0 microns long. Staining uneven. Non-motile. Gram-negative. Gelatin: Not liquefied in 45 days.

Blood agar colonies: Very flat cones, 2.0 to $3.0 \mathrm{~mm}$ in diameter.

Broth: Diffusely clouded.

Indole is formed.

Hydrogen sulfide produced.

Milk: Not acidified or coagulated.

Nitrites not produced from nitrates.

Acid and gas from fructose, galactose, glucose and mannose. No acid or gas from esculin, amygdalin, arabinose, cellobiose, dextrin, glycerol, glycogen, inulin, lactose, maltose, mannitol, melezitose, 
raffinose, rhamnose, salicin, sorbitol, starch, sucrose, trehalose or xylose.

Non-pathogenic for white mice and rabbits.

Anaerobic.

Distinctive characters: Gas is formed from peptone. Brom cresol purple and phenol red are decolorized in a meat infusion broth.

Source: Two strains isolated from human feces.

Habitat: Probably intestinal canal of mammals.

7. Bacteroides inaequalis Eggerth and Gagnon. (Eggerth and Gagnon, Jour. Bact., 25, 1933, 407; Spherophorus inaequalis Prévot, Ann. Inst. Past., 60, 1938, 298.) From Latin inaequalis, unequal.

Rods: Wide variation in size and form. Marked pleomorphism on blood agar. Non-motile. Gram-negative.

Gelatin: Not liquefied in 45 days.

Blood agar colonies: Pin-point in size. Broth: Diffusely clouded.

Indole is formed.

Hydrogen sulfide is produced.

Milk: Acidified but not coagulated.

Nitrites not produced from nitrates

Acid but no gas from esculin, anyg dalin, arabinose, fructose, galactose, glucose, lactose, maltose, mannose, raffinose, salicin, sucrose and xylose. No acid or gas from cellobiose, dextrin, glycerol, glycogen, inulin, mannitol, melezitose, rhamnose, sorbitol, starch and trehalose.

Non-pathogenic for white mice and rabbits.

\section{Anaerobic.}

Distinctive characters: Forms small amount ( 5 per cent in Smith tube) of gas from peptone water in the complete absence of carbohydrates. None of this gas is absorbed by alkali. Rapidly decolorizes brom cresol purple and phenol red in meat infusion broth; slowly or not at all in peptone water.

Source: One strain isolated from human feces.
Habitat: Probably intestinal canal of mammals.

8. Bacteroides insolitus Eggerth and Gagnon. (Eggerth and Gagnon, Jour. Bact., 25, 1933, 408; Ristella insolita Prévot, Ann. Inst. Past., 60, 1938, 291.) From Latin insolitus, uncommon.

Short thick rods: 1.0 to 2.0 microns long. Often slender, curved, 2.0 to 3.0 microns long. Non-motile. Gramnegative.

Gelatin: Not liquefied in 45 days.

Blood agar colonies: Minute, transparent.

Broth: Heavy, diffuse growth.

Indole is formed.

Hydrogen sulfide is formed.

Milk: Acidified and coagulated in 30 to 35 days.

Nitrites not produced from nitrates.

Acid but no gas from fructose, galactose, glucose, glycerol, lactose, maltose and mannose. No acid or gas from esculin, amygdalin, arabinose, cellobiose, dextrin, glycogen, inulin, mannitol, melezitose, raffinose, rhamnose, salicin, sorbitol, starch, sucrose, trehalose and xylose.

Non-pathogenic for white mice and rabbits.

Anaerobic.

Distinctive characters: Brom cresol purple and phenol red are rapidly decolorized in a meat infusion broth. A small amount of gas is formed from peptone.

Source: One strain isolated from human feces.

Habitat: Probably intestinal canal of mammals.

9. Bacteroides vescus Eggerth and Gagnon. (Eggerth and Gagnon, Jour. Bact., 25, 1933, 406; Fusiformis vescus Prévot, Ann. Inst. Past., 60, 1938, 300.) From Latin vescus, small or weak.

Slender, pointed rods: 1.0 to 2.0 microns long, sometimes slightly curved. 
Bipolar staining. Non-motile. Gramnegative.

Gelatin: Liquefied in 8 to 25 days.

Blood agar colonies: Very minute and transparent.

Broth: Diffusely elouded.

Indole not formed.

Hydrogen sulfide not produced.

Milk: Neither acidified nor coagulated.

Nitrites not produced from nitrates.

Peptone: No gas.

Acid but no gas from cellobiose (in 30 days), dextrin, glucose, maltose, mannose and rhamnose. No acid or gas from esculin, amygdalin, arabinose, galactose, mannitol, melezitose, raffinose, salicin, sorbitol, starch, sucrose, trehalose, xylose, glycerol, glycogen, inulin, lactose or fructose.

Non-pathogenic for white mice and rabbits.

Anaerobic.

Source: One strain isolated from human feces.

Habitat: Probably intestinal canal of mammals.

10. Bacteroides exiguus Eggerth and Gagnon. (Eggerth and Gagnon, Jour. Bact., 25, 1933, 407 ; Ristclla exigua Prévot, Ann. Inst. Past., 60, 1938, 292.) From Latin exiguus, small and narrow.

Very small slender rods: 0.5 to 1.0 micron long, occurring singly and in pairs. Non-motile. Gram-negative.

Gelatin: Liquefied in 16 to 20 days.

Blood agar colonies: These are of two types. One is pin-point in size, the other is large, gray, moist, 1.0 to $1.5 \mathrm{~mm}$ in diameter.

Broth: Diffusely clouded.

Indole not formed.

Hydrogen sulfide not formed.

Milk: Acidified and may or may not be coagulated in 35 to 40 days.

Nitrites not produced from nitrates.

Peptone: No gas.

Acid but no gas from fructose, galactose, glucose, lactose, maltose, mannose, sucrose and trehalose. One strain fer- ments raffinose. No acid or gas from esculin, amygdalin, arabinose, cellobiose, dextrin, glycerol, glycogen, inulin, mannitol, melezitose, rhamnose, salicin, sorbitol, starch or xylose.

Non-pathogenic for white mice and rabbits.

Anaerobic.

Source: Two strains isolated from human feces.

Habitat: Prebably intestinal canal of mammals.

11. Bacteroides uncatus Eggerth and Gagnon. (Eggerth and Gagnon, Jour. Bact., 25, 1933, 404; Ristella uncata Prévot, Ann. Inst. Past., 60, 1938, 291.) From Latin uncatus, hooked at the tip.

Rods: Extreme variations in size and form. The ordinary length is 5.0 to 8.0 microns. Curved and hooked forms common. Non-motile. Gram-negative.

Gelatin: Liquefied in 16 days.

Blood agar colonies: Very minute and transparent.

Broth: Turbid, growth is slow and light.

Indole not formed.

Hydrogen sulfide not formed.

Milk: Not acidified or coagulated.

Nitrites not produced from nitrates.

Peptone: No gas.

Acid but no gas after 8 to 30 days of incubation from dextrin, fructose, galactose, glucose, lactose, maltose, raffinose, rhamnose, salicin, starch and sucrose. No acid from esculin, amygdalin, arabinose, cellobiose, glycerol, glycogen, inulin, mannitol, mannose, melezitose, sorbitol, trehalose or xylose.

Non-pathogenic for white mice and rabbits.

Anaerobic.

Source: One strain isolated from human feces.

Habitat: Probably intestinal canal of mammals.

12. Bacteroides vulgatus Eggerth and Gagnon. (Eggerth and Gagnon, Jour. 
Bact., 25, 1933, 401; Pasteurella vulgata Prévot, Ann. Inst. Past., 60, 1938, 292.) From Latin vulgatus, common.

Oval rods : 0.7 to 2.5 microns long, usually occurring singly, sometimes in pairs. One strain formed filaments 10 microns long. Stain solidly, some strains show bipolar staining. Morphology very variable in glucose broth. Non-motile. Gram-negative.

Gelatin: Liquefied in 4 to 20 days by all but one strain.

Blood agar colonies: Soft, translucent, grayish, elevated, 1.5 to $2.0 \mathrm{~mm}$ in diameter. Half of the strains are hemolytic.

Broth: Heavy and diffuse growth.

Indole not formed.

Hydrogen sulfide is formed.

Milk: Acidified. Coagulated by some strains in 5 to 25 days.

Nitrites not produced from nitrates.

Acid and a small amount of gas from arabinose, dextrin, fructose, galactose, glucose, glycogen, inulin, lactose, maltose, mannose, raffinose, rhamnose, starch, sucrose and xylose. Seven strains fermented esculin. No acid or gas from amygdalin, cellobiose, glycerol, mannitol, melezitose, salicin, sorbitol, trehalose, dulcitol, erythritol or inositol.

Non-pathogenic for white mice and rabbits.

Anaerobic.

Distinctive characters: Does not form indole; does not produce gas from peptone. This is the commonest species found in the feces of adults. Differs from Bacteroides incommunis in that it does not ferment amygdalin and cellobiose, but does ferment glycogen and starch. Liquefies gelatin.

Source: Thirty-eight strains isolated from human feces.

Habitat: Probably intestinal canal of mammals.

13. Bacteroides incommunis Eggerth and Gagnon. (Eggerth and Gagnon, Jour. Bact., 25, 1933, 402; Ristella incommunis Prévot, Ann. Inst. Past., 60,
1938, 291.) From Latin incommunis, not common.

Rods : 0.5 to 1.5 by 1.0 to 3.0 microns, occurring singly. Stain solidly. Nonmotile. Gram-negative.

Gelatin: Not liquefied.

Blood agar colonies: Elevated, slightly yellowish, $1 \mathrm{~mm}$ in diameter. One strain formed soft colonies; the other was stringy when emulsified.

Broth: Growth is diffuse.

Indole not formed.

Hydrogen sulfide is formed.

Milk: Acidified but not coagulated; coagulates promptly on boiling.

Nitrites not produced from nitrates.

Peptone: No gas.

Acid and a small amount of gas from amygdalin, arabinose, cellobiose, dextrin, fructose, galactose, glucose, inulin, lactose, maltose, mannose, raffinose, rhamnose, sucrose and xylose. One strain fermented glycogen and starch. No action on esculin, glycerol, mannitol, melezitose, salicin, sorbitol or trehalose.

Non-pathogenic for white mice and rabbits.

Anaerobic.

Source: Two strains isolated from human feces.

Habitat: Probably intestinal canal of mammals.

14. Bacteroides distasonis Eggerth and Gagnon. (Eggerth and Gagnon, Jour. Bact., 25, 1933, 403; Ristella distasonis Prévot, Ann. Inst. Past., 60, 1938, 291.) Named for Distaso, Roumanian bacteriologist.

Rods : 0.5 to 0.8 by 1.5 to 2.5 microns, occurring singly. Staining solidly and having rounded ends. Some strains show a few bacilli 5.0 to 8.0 microns long. Non-motile. Gram-negative.

Gelatin: Not liquefied by 16 strains. The remaining 4 liquefied gelatin in 35 to 50 days.

Blood agar colonies: Soft, grayish, elevated colonies, 1.0 to $1.5 \mathrm{~mm}$ in diameter. Two strains markedly hemolytic. 
Broth: Growth is diffuse.

Indole not formed.

Hydrogen sulfide is produced.

Milk: Acidified. All but 4 strains coagulate milk.

Nitrites not produced from nitrates.

Peptone: No gas.

Acid but no gas from amygdalin, cellobiose, dextrin, fructose, galactose, glucose, inulin, lactose, maltose, mannose, melezitose, raffinose, rhamnose, salicin, sucrose, trehalose and xylose. Fifteen strains ferment esculin. Fifteen strains slowly ferment starch. No acid or gas from arabinose, glycogen, glycerol, mannitol or sorbitol.

Non-pathogenic for white mice and rabbits.

Anaerobic.

Distinctive characters: Usually fails to liquefy gelatin. Fails to ferment arabinose.

Source: Twenty strains isolated from human feces.

Habitat: Probably intestinal canal of mammals.

15. Bacteroides tumidus Eggerth and Gagnon. (Eggerth and Gagnon, Jour. Bact., 25, 1933, 405; Ristella tumida Prévot, Ann. Inst. Past., 60, 1938, 292.) From Latin tumidus, swollen.

Small, thick oval rods: 1.0 to 1.5 microns long and occurring singly. The staining is solid. On glucose broth many swollen forms with irregular staining from 1.0 to 4.0 by 1.5 to 10 microns. The bodies of these swollen forms are usually very pale, with only the ends staining. Non-motile. Gram-negative.

Gelatin: Liquefied in 12 to 20 days.

Blood agar colonies: Soft, grayish, elevated colonies, $1 \mathrm{~mm}$ in diameter.

Broth: Heavy, diffuse growth.

Indole not formed.

Hydrogen sulfide is produced.

Milk: Acidified but not coagulated.

Nitrites not produced from nitrates. Peptone: No gas.

Acid but no gas from dextrin, fruc- tose, galactose, glucose, glycogen, inulin, lactose, maltose, mannose, raffinose, sorbitol, starch and sucrose. No acid or gas from esculin, amygdalin, arabinose, cellobiose, glycerol, mannitol, melezitose, rhamnose, salicin, trehalose or xylose.

Non-pathogenic for white mice and rabbits.

Anaerobic.

Source: Four strains isolated from human feces.

Habitat: Probably intestinal canal of mammals.

16. Bacteroides convexus Eggerth and Gagnon. (Eggerth and Gagnon, Jour. Bact., 25, 1933, 406; Pasteurella convexa Prévot, Ann. Inst. Past., 60, 1938, 292.) From Latin convexus, convex.

Thick, oval rods: 0.8 to 1.5 microns long, occurring singly or in pairs. In glucose broth, the rods are usually 2.0 to 3.0 microns long. Non-motile. Gramnegative.

Gelatin: Liquefied in 20 to 30 days.

Blood agar colonies: Elevated, grayish, somewhat opaque colonies, 1.0 to 1.5 $\mathrm{mm}$ in diameter.

Broth: Heavy diffuse growth.

Indole not formed.

Hydrogen sulfide is produced.

Milk: Acidified and coagulated in 4 days.

Nitrites not produced from nitrates.

Peptone: No gas.

Acid and a small amount of gas from esculin, amygdalin, cellobiose, dextrin, fructose, galactose, glucose, glycogen, inulin, lactose, maltose, mannose, raffinose, starch, sucrose and xylose. No acid or gas from arabinose, glycerol, mannitol, melezitose, rhamnose, salicin, sorbitol or trehalose.

Non-pathogenic for white mice and rabbits.

Anaerobic.

Source: Five strains isolated from human feces.

Habitat: Probably intestinal canal of mammals. 
17. Bacteroides ovatus Eggerth and Gagnon. (Eggerth and Gagnon, Jour. Bact., 25, 1933, 405; Pasteurella ovata Prévot, Ann. Inst. Past., 60, 1938, 292.) From Latin ovatus, egg-shaped.

Small oval rods: 0.5 to 1.0 by 1.0 to 2.0 microns, occurring singly. Stains solidly. Yon-motile. Gram-negative.

Gelatin: Liquefied in 4 days.

Blood agar colonies: Soft, grayish, elevated colonies, 1.0 to $1.5 \mathrm{~mm}$ in diameter.

Broth: Diffuse, heavy growth.

Indole is formed.

Hydrogen sulfide is produced.

Milk: Acidified and coagulated in 4 days.

Nitrites not produced from nitrates.

Peptone: No gas.

Acid and a small amount of gas from esculin, amygdalin, cellobiose, dextrin, fructose, galactose, glucose, glycogen, inulin, lactose, maltose, mannose, raffinose, rhamnose, starch, sucrose and xylose. No acid or gas from arabinose, glycerol, mannitol, melezitose, salicin, sorbitol or trehalose.

Non-pathogenic for white mice and rabbits.

Anaerobic.

Source: One strain isolated from human feces.

Habitat: Probably intestinal canal of mammals.

18. Bacteroides uniformis Eggerth and Gagnon. (Eggerth and Gagnon, Jour. Bact., 25, 1933, 400; Ristella uniformis Prévot, Ann. Inst. Past., 60, 1938, 291.) From Latin uniformis, of a single form.

Small rods: 0.8 to 1.5 microns long, occurring singly, with rounded ends. Stain heavier at poles and around periphery. Non-motile. Gram-negative.

Gelatin: Liquefied by two strains in 15 to 40 days. Six strains did not liquefy.

Blood agar colonies: Transparent, soft, elevated, 0.5 to $0.75 \mathrm{~mm}$ in diameter.

Broth: Diffuse growth.

Indole formed.
Hydrogen sulfide produced slowly or not at all.

Milk: Acidified and coagulated in 8 to 12 days.

Nitrites not produced from nitrates.

Peptone: No gas.

Acid but no gas from esculin, amygdalin, arabinose, cellobiose, dextrin, fructose, galactose, glucose, glycogen, inulin, lactose, maltose, mannose, melezitose, raffinose, salicin, starch, sucrose, trehalose and xylose. No acid or gas from glycerol, mannitol, rhamnose, sorbitol, dulcitol, erythritol or inositol.

Non-pathogenic for white mice and rabhits.

Anaerobic.

Distinctive characters: Forms indole. Resembles Bacteroides vulgatus.

Source: Eight strains isolated from human feces.

Habitat: Probably intestinal canal of mammals.

19. Bacteroides thetaiotaomicron (Distaso) Castellani and Chalmers. (Bacillus thetaiotaomicron Distaso, Cent. f. Bakt., I Abt., Orig., 62, 1912, 444; Castellani and Chalmers, Man. Trop. Med., 3rd ed., 1919, 960; Spherocillus thetaiotaomicron Prévot, Ann. Inst. Past., $60,1938,300$.$) The combination theta,$ iota and omicron is used because the pleomorphic rods have the shape of these Greek letters.

Description taken from Distaso (loc. cit.). More complete description will be found in Eggerth and Gagnon (Jour. Bact., 25, 1933, 399).

Short, plump to oval rods. Stain solidly or only at poles. Sometimes with bar causing organism to resemble Greek letter theta. Motile (Distaso). Nonmotile (Eggerth and Gagnon). Gramnegative.

Gelatin: No liquefaction.

Glucose agar colonies: Large, transparent, entire. Sometimes form gas bubbles.

Broth: Turbid. 
Egg albumen broth: Albumen not attacked.

Indole is formed.

Hydrogen sulfide produced (Eggerth and Gagnon).

Litmus milk: Acid, coagulated. Curd shrinks with expulsion of turbid whey.

Nitrates not recorded (Distaso). Nitrites not produced from nitrates (Eggerth and Gagnon).

Peptone: No gas (Eggerth and Gagnon).

Acid and gas from esculin, amygdalin, arabinose, fructose, inulin, lactose, cellobiose, dextrin, galactose, glucose, glycogen, maltose, mannose, melezitose, raffinose, rhamnose, salicin, starch, sucrose, trehalose and xylose. Four strains fail to produce gas from any sugar. To acid or gas from glycerol, mannitol or sorbitol (Eggerth and Gagnon).

Anaerobic.

Distinctive characters: Resembles Bacteroides variabilis but is not capsulated, does not liquefy gelatin, usually forms gas from sugars, and ferments melezitose and trehalose. Differs from Bacteroides uniformis in morphology, forming gas from sugars and in fermenting rhamnose (Eggerth and Gagnon).

Source: Isolated frequently from human feces.

Habitat: Intestinal canal of mammals (common).

\section{Bacteroides variabilis (Distaso)} Castellani and Chalmers. Bacillus variabilis Distaso, Cent. f. Bakt., I Abt., Orig., 62, 1912, 441; Castellani and Chalmers, Man. Trep. Med., 3rd ed., 1919, 960; Capsularis rariabitis Prévot, Ann. Inst. Past., 60, 1938, 293.) From Latin variabilis, variable.

Short rods, with rounded ends, occurring singly. Some long flexuous rods. Capsulated. Non-motile. Gramnegative.

Gelatin: No growth on plain gelatin (Distaso); liquefaction (Eggerth and Gagnon, Jour. Bact., 25, 1933, 400).
Blood agar colonies : Smooth, glistening, elevated and very mucoid, about 1.0 $\mathrm{mm}$ in diameter.

Broth: Diffuse growth.

Indole is formed.

Hydrogen sulfide is formed.

Litmus milk: Unchanged (Distaso); acidified and some strains coagulating in 25 to 35 days (Eggerth and Gagnon).

Nitrites not produced from nitrates (Eggerth and Gagnon).

Peptone: No gas.

Acid and gas from glucose, lactose and sucrose (Distaso). Acid and no gas from esculin, amygdalin, arabinose. cellobiose, dextrin, fructose, galactose, glycogen, inulin, lactose, glucose, maltose, mannose, raffinose, rhamnose, salacin. starch, sucrose and xylose. No acid or gas from glycerol, mannitol, melezitose, sorbitol or trehalose (Eggerth and Gagnon).

Non-pathogenic for white mice and rabbits.

Anaerobic.

Optimum temperature $37^{\circ} \mathrm{C}$.

Distinctive characters: Capsulated. source: Isolated from human feces by Distaso, and by Eggerth and Gagnon (8 strains).

Habitat: Probably intestinal canal of mammals.

21. Bacteroides gulosus Eggerth and Gagnon. (Eggerth and Gagnon, Jour. Bact., 25, 1933, 398; Spherophorus gulosus Prévot, Ann. Inst. Past., 60, 1938, 298.) From Latin gulosus, gluttonous.

Small oval rods : 0.8 to 1.0 by 1.0 to 2.0 microns, staining deeper around periphery. Non-motile. Gram-negative.

Gelatin: Liquefied in 2 to 3 weeks.

Blood agar colonies: Soft, gray, entire, elevated, $2 \mathrm{~mm}$ in diameter.

Broth: Heavy and diffuse growth.

Indole formed.

Hydrogen sulfide is formed.

Milk: Acidified and coagulated in $t$ to 20 days.

Nitrites not produced from nitrates. 
Acid and a very small amount of gas from esculin, amygdalin, arabinose, cellobiose, dextrin, fructose, galactose, glycogen, inulin, lactose, glucose, maltose, mannitol, mannose, melezitose, raffinose, rhamnose, salicin, sorbitol, starch, sucrose, trehalose and xylose. Sorbitol and mannitol require 2 to 3 weeks for fermentation. Neither acid nor gas from glycerol, dulcitol, erythritol or inositol.

Peptone: No gas.

Non-pathogenic for white mice and rabbits.

\section{Anaerobic.}

Source: Seven strains isolated from human feces.

Habitat: Probably intestinal canal of mammals.

22. Bacteroides melaninogenicus (Oliver and Wherry) Roy and Kelly. (Bacterium melaninogenicum Oliver and Wherry, Jour. Inf. Dis., 28, 1921, 341; Hemophilus melaninogenicus Bergey et al., Manual, 3rd ed., 1930, 314; Ristella melaninogenica Prévot, Ann. Inst. Past., 60, 1939, 290; Roy and Kelly, in Manual, 5th ed., 1939, 569.) From Greek, blackproducing.

Description taken from Oliver and Wherry (loc. cit.) and Burdon (Jour. Inf. Dis., 42, 1928, 161).

Rods : 0.8 by 1.0 to 3.0 microns. Nonmotile. Gram-negative.

Serum gelatin stab: Dense flocculent growth at $37^{\circ} \mathrm{C}$. No liquefaction.

Blood agar slant: Confluent, black, dry layer. The blood is disintegrated in one to two weeks forming melanin. The medium becomes colorless.

Sodium phosphate broth: Turbid.

Litmus milk: Slow acidification but no coagulation.

Blood serum slant: Fairly luxuriant, white, moist layer.

Acid from fructose, glucose, lactose, maltose, sucrose and mannitol. No acid from galactose.

Non-pathogenic for rabbits, guinea pigs and white mice (Burdon). Anaerobic.

Optimum temperature $37^{\circ} \mathrm{C}$.

Distinctive characters: Growth very poor unless fresh body fluids are added to the medium. Grows more readily in mixed culture. When grown on a medium containing haemoglobin, a black pigment is produced (melanin).

Source: Oral cavity, external genitalia, infected surgical wound, urine and feces (Oliver and Wherry).

Habitat: Inhabits healthy mucous membranes of mammals, but may take a part in various pathological processes (Burdon).

23. Bacteroides caviae (Vinzent) Hauduroy et al. (Streptobacillus caviae Vinzent, Ann. Inst. Past., 42, 1928, 533; Hauduroy et al., Dict. d. Bact. Path., 1937, 53; Spherophorus caviae Prévot, Ann. Inst. Past., 60, 1938, 299.) From Cavia, a genus of rodents.

Rods: Small, sometimes curved. Usually 0.3 to 0.5 by 1.0 to 1.5 microns. Occurring singly and in chains. Pleomorphic in old cultures with long filamentous forms. Non-motile. Gramnegative.

Serum gelatin: No liquefaction.

Serum agar: Surface colonies, small, translucent, slightly raised, adherent to medium in 48 hours. Deep colonies, lenticular, $2 \mathrm{~mm}$ in size in 48 hours. Colonies difficult to break up. No gas.

Serum broth: Supernatant fluid clear, with small, stellate colonies, which tend to adhere to walls of the tube. No gas.

Indole not formed in serum peptone water.

Hydrogen sulfide not formed.

Milk: Unchanged.

Coagulated egg white and serum not liquefied.

No acid or gas from carbohydrates.

Pathogenic for guinea pigs, rabbits and mice.

Anaerobic.

Optimum temperature $37^{\circ} \mathrm{C}$. 
Distinctive characters: No growth unless serum is added to the medium.

Source : From epidemic benign cervical adenitis of guinea pigs.

Habitat: Infected guinea pigs so far as known.

Appendix I: Additional species which may belong here.

Bacteroides laevis (Distaso) Bergey et al. (Bacillus lacvis Distaso, Cent. f. Bakt., I Abt., Orig., 62, 1912, 114; Bergey et al., Manual, 1st ed., 1923, 259; not Bacillus laevis. Frankland and Frankland, Phil. Trans. Roy. Soc. London, 178, B, 1857, 278.) From feces.

Bacteroides liquefaciens (Distaso) Bergey et al. (Coccobacillus liquefaciens Distaso, Cent. f. Bakt., I Abt., Orig., 59, 1911, 102; Bergey et al., Manual, 1st ed., 1923, 262.) From feces.

Bacteroides rigidus (Distaso) Bergey et al. (Bacillus rigidus Distaso, Cent. f. Bakt., I Abt., Orig., 59, 1911, 103; Bergey et al., Manual, 1st ed., 1923, 263.)

Appendix II*: Prévot (Ann. Inst. Past., $60,1938,285$ and Man. de Class. et de Déterm. des Bact. Anaérobies, 1940, 38) has arranged some of the anaerobic, nonspore-forming, Gram-negative, largely parasitic rods in two families, Ristellaceac and Spherophoraceae, as follows:

\section{Family Ristellaceae Prévot.}

(Ann. Inst. Past., 60, 1938, 288.)

\section{Genus I. Ristella Prévot.}

(Loc. cit., 289.)

Straight or slightly bent, non-motile rods. Not eapsulated. Gram-negative. Anaerobic.

1. Ristella fragitis. See Bacteroides fragilis.
2. Ristella melaninogenica. See Bacteroides melaninogenicus.

3. Ristella haloseptica (Wyss) Prévot. (Bacterium halosepticum Wyss, Mitt. Grenz. Med. u. Chir., 13, 1904, 199; Prévot, loc. cit., 291.) From a fatal case of osteomyelitis in man. For a description of this species, see Manual, 5 th ed., 1939, 570,

4. Ristella putredinis (Weinberg et al.) Prévot. (Bacillus A, Heyde, Beitr. z. klin. Chirurg., 76, 1911, 1; Bacillus putredinis Weinberg et al., Les Microbes Anaérobies, 1937, 755; Prévot, loc. cit., 291.) Fifteen strains isolated from acute appendicitis. For a description of this species, see Manual, 5th ed., 1939, 571.

5. Ristella terebrans (Brocard and Pham) Prévot. (Bacillus terebrans Brocard and Pham, Compt. rend. Soc. Biol., Paris, 117, 1934, 997; Prévot, loc. cit., 291.) Two strains isolated from cases of gangrenous erysipelas, associated with a streptococcus. For a description of this species, see Manual, 5th ed., 1939, 571.

6. Ristella furcosa (Veillon and Zuber) Prévot. (Bacillus furcosus Veillon and Zuber, Arch. Méd. Exp. et Anat. Path., 10, 1898; Fusiformis furcosus Topley and Wilson, Princip. Bact. and Immun., 1st ed., 1, 1931, 302; Bacteroides furcosus Hauduroy et al., Dict. d. Bact. Path., 1937, 61; Prévot, loc. cit., 291.) From cases of appendicitis and from lung abscesses. For a description of this species, see Manual, 5th ed., 1939, 572 .

7. Ristella putida (Weinberg et al.) Prévot. (Bacillus gracilis putidus Tissier and Martelly, Ann. Inst. Past., 16, 1902, 865; Bacillus putidus Weinberg et al., Les Microbes Anaérobies, 1937, 790; not Bacillus putidus Kern, Arb. bakt. Inst. Narlsruhe, 1, Heft 4, 1896,

* Rearranged by Mrs. Eleanore Heist Clise, New York State Experiment Station, Geneva, New York, December, 1945. 
400 ; Prévot, loc. cit., 291.) From putrefying meat. For a description of this species, see Manual, 5th ed., 1939, 573.

\section{Ristella clostridiiformis (Ankersmit)} Prévot. (Bacterium clostridiiformis Ankersmit, Cent. f. Bakt., I Abt., Orig., 40, 1906, 115; Prévot, loc. cit., 291.) From the normal intestines of cattle. For a description of this species, see Manual, 5th ed., 1939, 574.

9. Ristella perfoetens (Weinberg et al.) Prévot. (Coccobacillus anaerobius perfoetens Tissier, Thèse Méd., Paris, 1900 ; Bacterium perfoetens Weinberg et al., Les Microbes Anaérobies, 1937, 790; Bacteroides perfoetens Hauduroy et al., Dict. d. Bact. Path., 1937, 67; Prévot, loc. cit., 291.) From the intestines of infants with diarrhoea. For a description of this species, see Manual, 5th ed., 1939, 575 .

10. Ristella thermophila $\beta$ (Weinberg et al.) Prévot. (Thermo $\beta$, Veillon, Ann. Inst. Past., 36, 1922, 430; Bacillus thermophilus $\beta$ Weinberg et al., Les Microbes Anaérobies, 1937, 800; Prévot, loc. cit., 291.) From manure. Nonpathogenic.

11. Ristella thermophila $\gamma$ (Weinberg et al.) Prévot. (Thermo $\gamma$, Veillon, Ann. Inst. Past., 36, 1922, 432; Bacillus thermophitus $\gamma$ Weinberg et al., Les Microbes Anaérobies, 1937, 800; Prévot, loc. cit., 291.) From manure. For a description of this species, see Manual, 5th ed., 1939, 575.

12. Ristella incommunis. See Bacteroides incommunis.

13. Ristella insolita. See Bacteroides insolitus.

14. Ristella halosmophila (Baumgartner) Prévot. (Bacteroides halosmophilus Baumgartner, Food Research, 2, 1937, 321 ; Prévot, Man. de Class. et de Déterm. des Bact. Anaérobies, 1940, 47.) From salted Mediterranean anchovies. Frequently found in the fish muscle and in the solar salt (the probable infecting agent) in which the fish is packed. For a description of this species, see Manual, 5 th ed., 1939, 584.

15. Ristella naviformis (Jungano) Prévot. (Bacillus naviformis Jungano, Compt. rend. Soc. Biol., Paris, 66, 1909, 122 ; Prévot, Ann. Inst. Past., 60, 1938, 291.) From the large intestine of the rat. For a deseription of this species, see Manual, 5th ed., 1939, 573.

16. Rislella lichenis-plani Prévot. (Bacillus of lichen planus, Jacob and Helmbold, Arch. Derm. Syph., 3, 1933 , 2s; Prévot, loc. cit., 291.) From the lesions of an inflammatory skin disease, lichen planus.

17. Ristella destillationis (Weinberg et al.) Prévot. (Bacterium, Tunnicliff, Jour. Inf. Dis., 13, 1913, 283; Bacterium destillationis Weinberg et al., Les Microbes Anaćrobies, 1937, 762; Prévot, loc. cit., 291.) From a case of chronic bronchitis.

18. Ristella uniformis. See Bacteroides uniformis.

19. Ristella distasonis. See Bacteroides distasonis.

20. Ristella uncata. See Bacteroides uncatus.

21. Ristella tumida. See Bacteroides tumidus.

22. Ristella exigua. See Bacteroides exiguus.

23. Ristella trichoides (Potez and Compagnon) Prévot. (Bacillus trichoides Potez and Compagnon, Compt. rend. Soc. Biol., Paris, 87, 1922, 339; Bac- 
teroides trichoides Hauduroy et al., Dict. d. Bact. Path., 1937, 78; Prévot, loc.cit.,292.) From a case of choleocystitis. For a description of this species, see Manual, 5th ed., 1939, 572.

24. Ristella glutinosa (Guillemot and Hallé) Prévot. (Bacillus glutinosus Guillemot and Hallé, Arch. Méd. Exp. et Anat. Path., 16, 1904, 599; Bacteroides glutinosus Hauduroy et al., Dict. d. Bact. Path., 1937, 61; Prévot, loc. cit. 292.) From purulent pleurisies.

25. Ristella capillosa (Tissier) Prévot. (Bacillus capillosus Tissier, Ann. Inst. Past., 22, 1908, 189; Prévot, loc. cit., 292.) From the intestines of infants. For a description of this species, see Manual, 5th ed., 1939, 573.

26. Ristella cylindroides (Rocchi) Prévot. (Bacterium cylindroides Rocchi, Lo stato actuale delle nostre cognizioni sui germi anaerobi Gamberine e Parmezziani, Bolegna, 1908; Prévot, loc. cit., 292.) From the human intestine. For a description of this species, see Manual, 5th ed., 1939, 574.

\section{Genus II. Pasteurella Trerisan.}

Four species. See Bacteroides vulgatus, Bacteroides oratus, Bacteroides convexus, and Bacteroides roagulans.

\section{Genus III. Dialister Bergey et al.}

Two species. See Dialister.

\section{Genus IV. Capsularis Prérot.}

(Loc. cit., 290.)

Characters as for the genus Ristella, but capsulated.

1. Capsularis zoogleiformans (Weinberg et al.) Prévot. (Bacillus mucosus anaerobius Prausnitz, Cent. f. Bakt., I Abt., Orig., 89, 1922, 126; Bacterium zongleiformans Weinberg et al., Les
Microbes Anaérobies, 1937, 725; Bacteroides praussnitzii Hauduroy et al., Dict. d. Bact. Path., 1937, 68; Prévot, loc. cit., 293.) From a purulent empyema in man. For a description of this species, see Manual, 5th ed., 1939, 576.

2. Capsulairs mucosus (Weinberg et al.) Prévot. (Coccobacterium mucosum anaerobicum Kilinger, Cent. f. Bakt., I Abt., Orig., 62, 1912, 186; Bacterium mucosum Weinberg et al., Les Microbes Anaérobies, 1937, 727; Bacteroides viscosus Hauduroy et al., Dict. d. Bact. Path., 1937, 81; Prévot, loc. cit., 293.) From a brain abscess following bronchiectasis in man. For a description of this species, see Manual, 5th ed., 1939, 575 .

3. Capsularis variabilis. See Bacteroides variabilis.

\section{Genus I. Zuberella Prevot.}

$$
\text { (Loc. cit., 290.) }
$$

Characters as for the genus Ristella, but motile with peritrichous flagella.

1. Zuberella serpens. See Bacteroides serpens.

2. Zuberella praeacuta (Tissier) Prévot. (Coccobacillus praeacutus Tissier, Ann. Inst. Past., 22, 1908, 189; Prévot, loc. cit., 293.) From the intestines of infants. For a deseription of this species, see Manual, 5th ed., 1939, 577.

3. Zuberella clostridiiformis mobilis Prévot. (Bacterium clostridiiformis Choukévitch, Ann. Inst. Past., 25, 1911, 345; Prévot, loc. cit., 293.) From the intestines of a horse. Choukévitch considered his organism the same as Ankersmit's Bacterium clostridiiformis, although the former was motile.

4. Zuberella aquatilis Prévot. (Spray and Laux, Amer. Water Works Assoc., 
22. 1930, 235; Prévot, loc. cil., 293.) From river water. For a description of this organism, see Manual, 5th ed., 1939, 577.

5. Zuberella variegata (Distaso) Prévot. (Bacillus variegatus Distaso, Cent. f. Bakt., I Abt., Orig., 62, 1912, 445; Bacteroides variegatus Castellani and Chalmers, Man. Trop. Med., 3rd ed., 1919, 960; Prévot, loc. cit., 293.) From the intestines. For a description of this species, see Manual, 5th ed., 1939, 578.

6. Zuberella rhinitis (Tunnicliff) Prévot. (Bacillus rhinitis Tunnicliff, Jour. Inf. Dis., 16, 1915, 493; Prévot, loc. cit., 293.) Thirty-two strains isolated from the nasopharynx in human beings suffering from pharyngitis, tonsilitis, bronchitis and rhinitis, as well as from the nasal mucosa of normal human beings, rabbits, guinea pigs and dogs. For a description of this species, see Manual, 5th ed., 1939, 576.

\section{Family Spherophoraceae Prévot.}

$$
\text { (Loc. cit., 289.) }
$$

\section{Genus I. Spherophorus Prérot.}

$$
\text { (Loc. cit., 297.) }
$$

Very pleomorphic rods. Metachromatic granules common in elongated forms. Non-motile. Non-spore-forming. Gram-negative.

1. Spherophorus necrophorus (Flügge) Prévot. (Bacillus der Kälberdiphtherie, Loeffler, Mitteil. kaiserl. Gesundheitsamte, 2, 1884, 493; Bacillus diphtheriae vitulorum Flügge, Die Mikroorganismen, 2 Aufl., 1886, 265; Bacillus necrophorus Flügge, ibid., 273; Bacillus diphtheriae-vitulorum Trevisan, I generi e le specie delle Batteriacee, 1889, 13; Bacillus filiformis Shütz; not Bacillus filiformis Tils, Ztschr. f. Hyg., 9, 1890, 294 ; not Bacillus filiformis Migula, Syst. d. Bakt., 2, 1900, 587; Nekrosebacillen, Bang, Maanedskrift f. Dyrleager, 2, 1890, 235 ; Streptolhrix cuniculi Schmorl, Deut. Ztschr. f. Tiermed., 17, 1891, 376; Actinomyces cuniculi Gasperini, Mittheil. 11 Internat. Med. Congr. Rome, see Cent. f. Bakt., 15, 1894, 684; not Actinomyces cuniculi Erikson, Med. Res. Council Spec. Rept. Ser. 203, 1935, 32; Oospora diphtheriae vitulorum Lehmann and Neumann, Bakt. Diag., 1 Aufl., 2, 1896, 393; Actinomyces necrophorus Lehmann and Neumann, Bakt. Diag., 2 Aufl., 2, 1899, 434; Streptothrix necrophora Kitt, Bakterienkunde, 1899 ; Corynebacterium necrophorum Lehmann and Neumann, Bakt. Diag., 4 Aufl., 2, 1907, 531; Bacillus nccroseos Salomonsen, quoted from Lehmann and Neumann, ibid., 532; Cladothrix cuniculi Macé, Traité de Bact., 6th ed., 2, 1913, 753; Bacterium necrophorum Lehmann and Neumann, Bakt. Diag., 7 Aufl., 2, 1927, 504; Fusiformis necrophorus Topley and Wilson, Princip. Bact. and Immun., 1st ed., 1, 1931, 299 ; Prévot, Ann. Inst. Past., 60,1938 , 298.) Because of the importance of this organism, a description is included here:

Rods: 0.5 to 1.5 microns wide, forming long filaments, up to $\mathrm{SO}$ to 100 microns long. Some authors report branching, others deny this. Short forms are reported by Schmorl to be motile. Gramnegative.

Gelatin stab: No liquefaction.

Agar colonies: Small, dirty-white, circular, opaque, with yellowish center under low power lens. Margin floccose.

Agar stab: Yellowish colonies along needle track. Gas bubbles produced.

Coagulated blood serum: Small, whitish colonies, becoming opaque, fimbriate.

Broth: Turbid, with gas. Cheese-like odor.

Indole is formed.

Litmus milk: Cheese-like odor. Acidified and generally coagulated.

Nitrites not produced from nitrates. Anaerobic. 
Optimum temperature $37^{\circ} \mathrm{C}$.

Produces a soluble exotoxin.

Source and habitat: Causes diphtheria in cattle with multiple sclerotic abscesses; gangrenous dermatitis in horses and mules; multiple necrotic foci in liver of cattle and hogs. One case of human infection reported. Transmissible to mice and rabbits.

2. Spherophorus funduliformis. See Bacteroides funduliformis.

3. Spherophorus necrogenes (Weinberg et al.) Prévot. (Bacillus, Kawamura, Jour. Jap. Soc. Vet. Sci., 5, 1926, 22 ; Bacillus necrogenes Weinberg et al., Les Microbes Anaérobies, 1937, 381; Prévot, loc. cit., 298.) From epidemic abscesses in hens.

\section{Spherophorus necroticus (Nati-} velle) Prévot. (Bacillus necroticus Nativelle, 1936, see Weinberg et al., Les Microbes Anaérobies, 1937, 693; Prévot, loc. cit., 298.) From a case of gangrenous appendicitis. For a description of this species, see Manual, 5th ed., 1939, 580.

5. Spherophorus peritonitis Prévot. (Bacillus, Ghon and Sachs, Cent. f. Bakt., I Abt., Orig., 38, 1905, 1 and 131; Prévot, loc. cit., 298.) From peritoneal exudate.

6. Spherophorus gulosus. See Bacteroides gulosus.

7. Spherophorus inaequalis. See Bacteroides inaequalis.

\section{Spherophorus varius. See Bac-} teroides varius.

9. Spherophorus siccus. See Bacteroides siccus.

10. Spherophorus mortiferus (Harris) Prévot. (Bacillus mortiferus Harris, Jour. Exp. Med., 6, 1901, 519; Prévot, loc. cit., 299.) From a liver abscess in man. For a description of this species, see Manual, 5th ed., 1939, 581.

\section{Spherophorus freundi (Hauduroy} et al.) Prévot. (Freund, Cent.f. Bakt., I Abt., Orig., 88, 1922, 9; Bacterium of Freund, Weinberg et al., Les Microbes Anaérobies, 1937, 706; Bacteroides freundii Hauduroy et al., Dict. d. Bact. Path., 1937, 57; Prévot, loc. cit., 299.) From a purulent meningitis following otitis in man. For a description of this species, see Manual, 5th ed., 1939, 581.

12. Spherophorus pyogenes (Hauduroy et al.) Prévot. (Buday, Cent. f. Bakt., I Abt., Orig., $7 \%, 1916,453$; Bacillus pyogenes anaerobius Bela-Johan, Cent. f. Bakt., I Abt., Orig., 87, 1922, 290 ; Bacteroides pyogenes Hauduroy et al., Dict. d. Bact. Path., 1937, 69; Prévot, loc. cit., 299.) From abscesses of the liver and lungs following septic mar wounds. Also from the blood stream following tonsillectomies. For a description of this species, see Manual, 5th ed., 1939, 582 .

13. Spherophorus gonidiaformans (Tunnicliff and Jackson) Prévot. ( $B a-$ cillus gonidiaformans Tunnicliff and Jackson, Jour. Inf. Dis., 36, 1925, 430; Actinomyces gonadiformis (sic) Bergey et al., Manual, 3rd ed., 1930, 469; Bacteroides gonidiaformans Hauduroy et al., Dict. d. Bact. Path., 1937, 62; Prévot, loc. cit., 299.) From a tonsil. For a description of this species, see Manual, 5th ed., 1939, 582.

14. Spherophorus floccosus (Weinberg et al.) Prévot. (Streptobacillus pyogenes floccosus Couromont and Cade, Arch. Med. Exp., 12, 1900, 393; Bacillus floccosus Weinberg et al., Les Microbes Anaérobies, 1937, 698; not Bacillus floccosus Kern, Arb. bakt. Inst. Karlsruhe, 1, Heft 4, 1896, 424; Bacteroides floccosus Hauduroy et al., Dict. d. Bact. 
Path., 1937, 55; Prévot, loc. cit., 299.) From blood in pyemia of man. For a description of this species, see Manual, 5th ed., 1939, 580 .

\section{Spherophorus influenzaeformis} (Russ) Prévot. (Bacillus influenzaeformis Russ, Cent. f. Bakt., I Abt., Orig., 39, 1905, 357; Bucteroides russii Hauduroy et al., Dict. d. Bact. Path., 1937, 73; Prévot, loc. cit., 299.) One strain isolated from a perianal abscess and two strains from purulent meningitis in man. For a description of this species, see Manual, 5th ed., 1939, 583.

16. Spherophorus cairae. See Bacteroides caviae.

\section{Genus II. Spherocillus Prérot.}

$$
\text { (Loc. cit., 297.) }
$$

Characters as for the genus Spherophorus, but motile with peritrichous flagella.

1. Spherocillus bullosus (Distaso) Prévot. (Bacillus bullosus Distaso, Cent. f. Bakt., I Abt., Orig., 62, 1912, 443; Bacteroides bullosus Castellani and Chalmers, Man. Trop. Med., Brd ed., 1919, 960; Prévot, loc. cit., 300.) From the intestinal canal. For a description of this species, see Manual, 5th ed., 1939, 583.

2. Spherocillus thetaiotaomicron. See Bacteroides thetaiotaomicron.

3. Spherocillus wirthi Prévot. (Bacillus, Wirth, Cent. f. Bakt., I Abt., Orig., 105, 1929, 201; Prévot, loc. cit., 300.) From a case of acute otitis.

Appendix III: The following additional species have been found in the literature.

Actinomyces pseudonecrophorus Harris and Brown. (Bull. Johns Hopkins Hosp.,
40, 1927, 203.) From the uterus in cases of puerperal infection. Probably should be classified near Spherophorus necrophorus. For a description of this species, see Manual, 5th ed,, 1939, 579.

Bacillus anaerobius gracilis Lewkowicz. (Arch. Méd. Exp., 13, 1901, 633.) From the mouths of infants.

Bacillus angulosus Garnier and Simon. (Presse Méd., 1909, 473.) From the blood of an infant with typhoid fever.

Bacillus annuliformans Massini. (Ztschr. f. gesam. Exp. Med., 1913, 81.) From a tuberculous cavity of man. Pathogenic.

Bacillus circularis major Heurlin. (Bakt. Unters. d. Keimgehaltes im Genitalkanale d. fiebernden Wöchnerinnen. Helsingfors, 1910, 168.) From the genital canal. Anaerobic. Gram-negative.

Bacillus limitans Heurlin. (Ibid., 165.) From the genital canal. Anaerobic. Gram-negative.

Bacillus nebulosus Hallé. (Hallé, Thèse de Paris, 1898; not Bacillus nebulosus Vincent, Ann. Inst. Past., 21, 1907, 69.) From the human vagina.

Bacillus stellatus Vincent. (Vincent, Ann. Inst. Past., 21, 1907, 62; not Bacillus slellatus Chester, Man. Determ. Bact., 1901, 274.) From water.

Bacillus symbiophiles Shottmuller. (Leitfarten f. d. klin. bakt. Kultur., Berlin, 1923.) From the blood in a case of puerperal fever.

Bacterium albarrani Jungano. (Compt. rend. Soe. Biol., Paris, 63, 1907, 302.) From a case of cystitis.

Bacterium minutissimum Le Blaye and Guggenheim. (Cocco-bacillus minutissimum gazogenes Jacobson, Ann. Inst. Past., 22, 1908, 300; Le Blaye and Guggenheim, Manuel Pratique de Diagnostic Bact, Viget Frères, 1914.) From intestines of infants.

Bricteroides splenomegaliae (Pinoy) Hauduroy et al. (Synbacterium splenomegaliae Pinoy, Compt. rend. Acad. Sci., Paris, 182, 1926, 1429; Hauduroy et al., 
Dict. d. Bact. Path., 1937, 75.) From cases of splenomegaly in Algeria. Pathogenic.

Pasteurella anaerobiontica Levinthal. (Cent. f. Bakt., I Abt., Orig., 106, 1928, 195.) From the naso-pharynx of man.
Streptobacillus gracilis Guillemot and Hallé. (Guillemot and Hallé, Arch. Méd. Exp. et Anat. Path., 16, 1904, 598; Bacteroides streptobacilliformis Hauduroy et al., Dict. d. Bact. Path., 1937, 76.) From putrid pleurisies.

\section{Genus $I I$. Fusobacterium Knorr.*}

(Knorr, Cent.f. Bakt., I Abt., Orig., 87, 1922, 536; Fusiformis Prévot and F usocillus Prévot, Ann. Inst. Past., 60, 1938, 300.)

Gram-negative, anaerobic rods, usually with tapering ends. Usually non-motile. Stain with more or less distinct granules.

The type species is Fusobacterium plauti-vincenti Knorr.

Key to the species of genus Fusobacterium.

I. Acid from maltose.

A. No gas produced.

B. Gas produced.

\section{Fusobacterium plauti-vincenti.}

2. Fusobacterium biacutum.

II. No acid from maltose.

A. Disagreeable odor produced on cultivation.

3. Fusobacterium nucleatum.

B. No odor produced.

\section{Fusobacterium polymorphum.}

1. Fusobacterium plauti-vincenti Knorr. (Knorr, Cent. f. Bakt., I Abt., Orig., 89, 1923, 16; Fusiformis plautivincenti and $F$ usiformis vincenti Hauduroy et al., Dict. d. Bact. Path., 1937, 240.) Named for H. C. Plaut and for H. Tincent who studied diseases of the respiratory tract.

The relationships between this organism and the following have not been clearly established: Fusiformis dentium Hoelling, Arch. f. Protistenkunde, 19, 1910, 240; Bacilius fusiformis Veillon and Zuber, Arch. de méd. expér., 10, 1898, 517 (Corynebaclerium fusiforme Lehmann and Neumann, Bakt. Diag., 4 Aufl., 2, 1907, 529); not Bacillus fusiformis Gottheil, Cent.f. Bakt., II Abt., 7, 1901, 724; Fusiformis fusiformis Topley and Wilson, Princip. of Bact. and Immun., 1st ed., 1, 1931, 300.

Weinberg, Nativelle and Prévot (Les Microbes Anaérobies, 1937, 804) and
Prévot (Ann. Inst. Past., 60, 1938, 285) make a distinction between Plaut's bacillus (Fusocillus plauti) and Vincent's bacillus (Fusiformis fusiformis), the former being actively motile and nonpathogenic and the latter non-motile and pathogenic.

Rods: 0.5 to 1.0 by 8 to 16 microns, occurring in pairs with blunt ends together and outer ends pointed, sometimes in short, curved chains or long spirillumlike threads. Granules present. Nonmotile. Gram-negative.

Serum agar shake culture: After 36 hours, colonies spherical, up to $0.5 \mathrm{~mm}$ in diameter, thin, yellowish-brown.

Serum agar plate: Matted growth. Medium around colonies becomes turbid from the precipitation of protein. No surface growth.

Serum broth: Milky turbidity.

Liver broth: No turbidity. Grayishwhite, flaky precipitate.

* Arranged by Prof. Robert S. Breed, New York State Experiment Station, Geneva, New York, December, 1938; rearranged, December, 1945. 
Indole not formed.

Acid from glucose, fructose, sucrose, maltose and sometimes from lactose. No acid from inulin or mannitol. (Hine and Berry, Jour. Bact., 34, 1937, 524.)

No $\mathrm{H}_{2} \mathrm{~S}$ produced.

No odor produced.

No gas formed.

Non-pathogenic for white mice (Hauduroy et al., loc. cit.).

Temperature relations: Optimum $35^{\circ}$ to $37^{\circ} \mathrm{C}$. Minimum $30^{\circ} \mathrm{C}$. (Hauduroy et al., loc. cit.)

Optimum pH 6.8 to 8.0 (Hauduroy et al., loc. cit.).

Anaerobe.

Source: Two strains isolated from deposit on teeth.

Habitat: Presumably the buccal cavity.

2. Fusobacterium biacutum Weinberg and Prévot. (Weinberg and Prévot, Compt. rend. Soc. Biol., Paris, 95, 1926, 519 ; Fusiformis biaculus Hauduroy et al., Dict. d. Bact. Path., 1937, 238.) From Isatin, double pointed.

Rods : 0.4 to 0.5 by 1.4 to 3.0 microns, with pointed ends, occurring singly, in pairs or sometimes in short chains. Nonmotile. Gram-negative.

Gelatin : No liquefaction.

Veillon's agar: Rapid growth. Colonies lens-shaped. Gas is produced which breaks up the medium.

Plain broth: Poor growth.

Glucose broth: Turbid. Medium rapidly acidified. Good growth.

Indole not formed.

Milk: Acid and coagulation in 2 to 8 days. Curd not digested.

Casein and coagulated egg-white not digested.

Neutral red reduced.

Acid from glucose, fructose, galactose, maltose and lactose.

Small amount of $\mathrm{H}_{2} \mathrm{~S}$ prociuced.

Does not require blood serum for growth.

Pathogenic for guinea pigs.

Killed in 60 minutes at $60^{\circ} \mathrm{C}$.
Anaerobic.

Source: Six strains isolated from a case of appendicitis.

Habitat: Unknown.

3. Fusobacterium nucleatum Knorr. (Knorr, Cent. f. Bakt., I Abt., Orig., 89, 1923, 17 ; Fusiformis nucleatus Bergey et al., Manual, 3rd ed., 1930, 514.) Latinized, nucleated.

Rods: 1.0 by 4.0 microns, spindleshaped, occurring singly. One or two granules present. Non-motile. Gramnegative.

Serum agar plate: Deep colonies lensshaped with offshoots.

Plain liver broth: No growth.

Liver broth with serum: After 1 to 3 days, flocculent deposit on the pieces of liver.

Indole not formed (Kinorr, loc. cit.). Indole formed (Hine and Berry, Jour. Bact., 34, 1937, 521).

Disagreeable odor produced on cultivation.

No gas produced.

Acid from glucose, usually from fructose, sometimes from sucrose and lactose. No acid from maltose, inulin or mannitol. (Hine and Berry, loc. cit.)

No $\mathrm{H}_{2} \mathrm{~S}$ formed.

Temperature relations: Optimum $35^{\circ}$ to $37^{\circ} \mathrm{C}$. Minimum $30^{\circ} \mathrm{C}$. (Hauduroy et al., Dict. d. Bact. Path., 1937, 239.) Survives $56^{\circ} \mathrm{C}$ for 15 minutes, but not $60^{\circ} \mathrm{C}$ for 10 minutes (Hine and Berry, loc. cit.).

Optimum pH 6.8 to 8.2 (Hauduroy et al., loc. cit.).

Anaerobe.

Source: One strain isolated from deposit on teeth in a healthy mouth.

Habitat: Presumably the buccal cavity.

4. Fusobacterium polymorphum Knorr. (Knorr, Cent.f. Bakt., I Abt., Orig., 89, 1923, 19; Fusiformis polymorphus Bergey et al., Manual, 3rd ed., 1930,515.) From Greek, assuming many forms.

Rods: 0.2 to 0.5 by 8 to 16 microns, 
occurring in pairs with the pointed ends adjoining. Often occurring in threads. Non-motile. Gram-negative.

Serum agar plates (alkaline): After 2 to 3 days, colonies $0.5 \mathrm{~mm}$ or larger, lensshaped with offshoots.

Tenacious sediment in liquid media.

Indole not formed (Knorr, loc. cit.). Indole formed (Hine and Berry, Jour. Bact., 34, 1937, 522).

No gas produced.

No odor produced.

Acid usually produced from glucose, fructose, and sucrose. No acid from lactose, maltose, inulin or mannitol. (Hine and Berry, loc. cit.)

$\mathrm{No}_{2} \mathrm{~S}$ produced.

Temperature relations: Optimum $35^{\circ}$ to $37^{\circ} \mathrm{C}$. Minimum $30^{\circ} \mathrm{C}$. (Hauduroy et al., Dict. d. Bact. Path., 1937, 242.) Survives $50^{\circ} \mathrm{C}$ for 15 minutes, $52^{\circ} \mathrm{C}$ for 10 minutes and $56^{\circ} \mathrm{C}$ for 5 minutes (Hine and Berry, loc. cit., 523).

Optimum pH 7.0 to 8.2 (Hauduroy et al., loc. cit.).

Anaerobe.

Source: One strain isolated from deposit on teeth in a case of gingivitis.

Habitat: Presumably the buccal cavity.

Appendix I: The following species are mentioned here because they appear to be related to the organisms in the genus: Fusobacterium:

Bacillus hastilis Seitz. (Ztschr. f. Hyg., 30, 1899, 47; Mycobacterium hastilis Chester, Man. Determ. Bact., 1901, 356.) A collective name for the organisms frequently found in stinking pus and in tonsillar pockets in both healthy and diseased mouths.

Fusocillus shmamini Prévot. (Ann. Inst. Past., 60, 1938, 300.) Feebly motile.

Fusiformis acnes, Fusiformis hodgkini and Fusiformis typhi-exanthematici (Plotz) of Holland (Jour. Bact., 5, 1920,
223) are names presumably intended for bacteria more properly placed in the genus Corynebacterium.

Fusiformis muris Hoelling. (Arch. f. Protistenkunde, 19, 1910, 239.) From the blind gut of a mouse. Stated by the author to be similar to Fusiformis termitidis Hoelling.

Fusiformis necrophorus Topley and Wilson. See Spherophorus necrophorus Prévot, page 578.

Fusiformis nodosus Beveridge. (Beveridge, Austral. Council Sci. and Indus. Res. Bul. 140, 1941, 56 pp.; Actinomyces nodosus Hagan, The Infectious Diseases of Domestic Animals. Ithaca, Nem York, 1943, 312.) Considered the primary cause of footrot of sheep. Also see Spirochacta penortha.

Appendix II: Because of the preferable form of the name and also because it is questionable whether the anaerobic fusiform bacteria of the mouth closely resemble the more or less aerobic bacteria found in termites, the genus name Fusobacterium Innor has been used for the mouth organisms. The termite organisms live in the intestinal tract bathed in digested wood and have the microscopic appearance of the cellulosedestroying C'ytophaga Winogradsky. These are shown by Stanier (Jour. Bact. $40,1910,619)$ to belong to Myxobacteriales.

The organisms placed in Fusiformis Hoelling are as follows:

Fusiformis hilli Duboscq and Grasse. (Arch. Zool. Expér. et Gén., 66, 1927, 454 and 486.) Found as an ectoparasite on flagellates Descovina spp. Which live in the rectum of termites (Calotermes (Glyptotermes) iridipennis).

Fusiformis termitidis Hoelling. (Arch. f. Protistenkunde, 19, 1910, 239.) From the intestinal tract of termites. 
TRIBE IV. HEMOPHILEAE WINSLOW ET AL.

(Jour. Bact., 5, 1920, 212.)

Minute parasitic forms growing on first isolation only in the presence of hemoglobin, ascitic fluid or other body fluids, or in the presence of certain growth accessory substances found in sterile, unheated plant tissue (potato). Motile or non-motile. Commonly found in the mucosa of respiratory tract or conjunctiva.

\section{Key to the genera of tribe Hemophileae.}

I. Aerobes to facultative anaerob's.

A. Non-motile.

1. Predominantly occurring singly.

2. Predominantly occurring as diplobacilli.

Genus I. Hemophilus, p. 581 .

Genus II. Moraxella, p. 590.

B. Motile, encapsulated.

II. Anaerobes.

A. Non-motile.

Genus III. Noguchia, p. 592.

Genus IV. Dialister, p. 594.

\section{Genus I. Hemophilus Winslow et al.*}

(Jour. Bact., 2, 1917, 561.) From Greek, loving blood.

Minute rod-shaped cells, sometimes thread-forming and pleomorphic. Non-motile. Gram-negative. Strict parasites growing best (or only) in the presence of hemoglobin and in general requiring blood serum, ascitic fluid, or certain growth accessory substances.

The type species is Hemophilus influenzae (Lehmann and Neumann) Winslow et al.

Key to the species of genus Hemophilus.

I. Affecting the respiratory tract.

1. Require both $\mathrm{V}$ and $\mathrm{X}$ growth factors for growth.

1. Hemophilus influenzae.

2. Hemophilus suis.

3. Hemophilus hemolyticus.

2. V growth factor sufficient for growth.

4. Hemophilus parainfluenzae.

5. Hemophilus pertussis.

II. Affecting the genital region.

3. $\mathrm{X}$ growth factor sufficient for growth.

6. Hemophilus ducreyi.

7. Hemophilus haemoglobinophilus.

* Revised by Dr. Margaret Pittman, National Institute of Health, Bethesda, Maryland, October, 1945. 
Where the relationship to growth accessory factors is known, the following table may serve as a key :

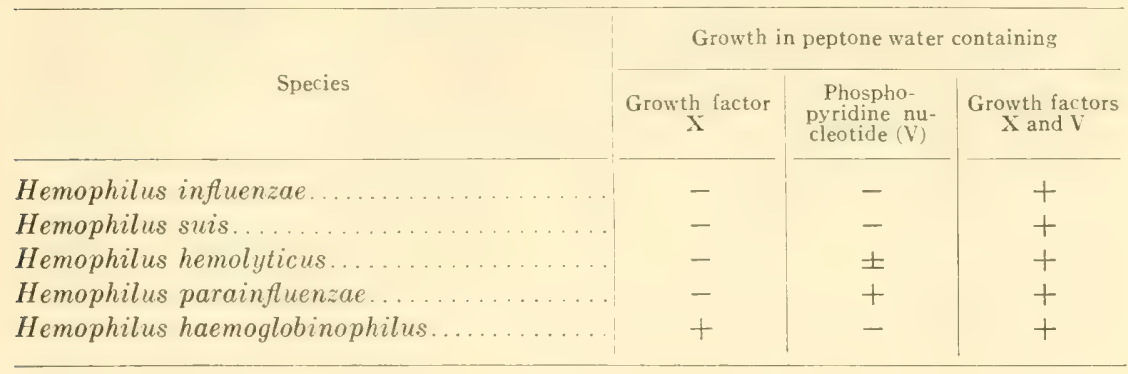

1. Hemophilus influenzae (Lehmann and Neumann) Winslow et al. (Korh, Wiener med. Wchnschr., 33, 1883, 1550; Weeks, New York Med. Record, 31, 1887, 571; Influenzabacillus, Pfeiffer, Deutsche med. Wchnschr., 1892, 28; Ztschr. f. Hyg., 13, 1893, 357; Bacterium influenzae Lehmann and Neumann, Bakt. Diag., 1 Aufl., 2, 1896, 187; Bacillus influenzae Kruse, in Flügge, Die Mikrnorganismen, 3 Aufl., 2, 1896, 434; Bacterium aegyptiacum Lehmann and Neumann, Bakt. Diag., 2 Aufl., 2, 1899 191; Hemophilus meningitidis cerebrospinalis septicemiae Cohen, Ann. Inst. Past., 23, 1909, 273; Winslow et al., Jour. Bact., 2, 1917, 561; Coccobrcillus pfeifferi Neveu-Lemaire, Précis Parasitol. Hum., 5th ed., 1921, 20; Hemophilus conjunctivitidis Bergey et al., Manual, 1st ed., 1923, 270.) From influenza, a disease of the respiratory tract.

Common name: The Koch-Weeks Bacillus.

Very small rods: 0.2 to 0.3 by 0.5 to 2.0 microns, occurring singly and in pairs, occasionally in short chains, and at times long thread forms are seen. Frequently show a marked tendency to bipolar staining. Some strains are encapsulated. Non-motile. Gram-negative.

Requires both the factors $\mathrm{X}$ and $\mathrm{V}$ for its growth.

Gelatin colonies: No growth.

Gelatin stab: No growth.
Blood agar colonies: Small, circular, transparent, homogeneous, entire. ratellitism with staphylococcus.

Blood agar slant: Thin, filiform, transparent growth.

Chocolate agar slant: Luxuriant growth.

Blood broth: Slightly turbid. No hemolysis.

Litmus milk, with blood: Some strains render it very slightly alkaline.

Sterilized potato slant: No growth.

Fresh unheated sterile potato added to broth favors development.

Indole is formed by some strains.

Nitrites are produced from nitrates.

Some strains attack none of the carbohydrates, while other strains attack various carbohydrates, provided a suitable medium is used. Mannitol and lactose never fermented.

Pathogenic.

Aerobic, facultative.

Optimum temperature $37^{\circ} \mathrm{C}$. Maximum $43^{\circ} \mathrm{C}$. Minimum $26^{\circ}$ to $27^{\circ} \mathrm{C}$. Killed at $55^{\circ} \mathrm{C}$ for thirty minutes.

Source: Isolated by Pfeiffer in cases of influenza. Found in conjunctiva, nasopharynx, sputum, sinuses, cerebrospinal fluid, blood, and pus from joints.

Habitat: Respiratory tract. A cause of acute respiratory infections, of acute conjunctivitis, and of purulent meningitis of children, rarely of adults. Re- 
garded by Pfeiffer and others to be the cause of influenza.

Note: Six types (a-f) of Hemophilus influenzae are recognized on the basis of precipitation of immune serum by capsular substance. Strains from cerebrospinal fluid are usually of type b. The majority of the strains from the respiratory tract are not type-specific.

2. Hemophilus suis Iauduroy et al. (Hcmophilus influenzae suis Lewis and Shope, Jour. Exp. Med., 54, 1931, 361 and 373 ; Bacteriumb influenzae suis Kole, Cent. f. Bakt., I Abt., Orig., 129, 1933, 161; Hauduroy et al., Dict. d. Bact. Path., 1937, 258.) From Latin, swine.

Resembles Hemophilus influenzae except it is relatively inert to growth, indole is not formed, nitrites are produced from nitrates, and maltose and sucrose are slightly fermented but not the carbohydrates fermented by Hemophilus influenzae.

Source: From cases of swine influenzal.

Habitat: With a filterable virus it causes swine influenz:

3. Hemophilus hemolyticus Bergey et al. (Bacillus X, Pritchett and Stillman, Jour. Exp. Med., 29, 1919, 259; Stillman and Bourn, Jour. Exp. Med., 32, 1920, 665; Bergey et al., Manual, 1st ed., 1923, 269.) From Greek, blood-dissolving.

Morphologically like Hemophilus infuenzae. Non-motile. Gram-negative.

Requires both the factors $\mathrm{X}$ and $\mathrm{V}$ for its growth. Valentine and Rivers (Jour. Exp. Med., 45, 1927, 993) isolated certain hemolytic strains which did not entirely agree with this

Blood agar colonies: Resemble Hemophilus influenzae but surrounded by at zone of hemolysis.

Blood agar slant: Thin, filiform, transparent growth.

Blood broth: Turbid, showing hemolysis.
Blood milk mixture: Slightly alkaline. Sterile unheated potato favors development.

Indole is formed by some strains.

Nitrites are produced from nitrates.

Some strains do not attack carbohydrates, other strains ferment various carbohydrates.

Aerobic, facultative.

Optimum temperature $37^{\circ} \mathrm{C}$.

Habitat: Found in upper respiratory tract of man. Non-pathogenic.

t. Hemophilus parainfluenzae Rivers. (Johns Hopkins Hosp. Bull., 33, 1922, 429.) From Latin, like, and the disease, influenza .

Morphologically like Hemophilus influenza. Non-motile. Gram-negative.

Requires the $V$ factor for its growth.

Blood agar colonies: Resemble Hemophilus influenzae. No hemolysis.

Blood agar slant: Thin filiform transplant.

Broth containing yeast extract: Floccular sediment.

Inclole is formed by some strains from cat.

Nitrites are produced from nitrates.

Some strains attack none of the carbohydrates; other strains ferment various carbohydrates.

Aerobic, facultative.

Optimum temperature $37^{\circ} \mathrm{C}$.

Habitat: Found in upper respiratory tract of man and cat. Usually nonpathogenic.

5. Hemophilus pertussis Holland. Microbe de coqueluche, Bordet and Gengou, Ann. Inst. Past., 20, 1906, 731; Holland, Jour. Bact., 5, 1920, 219; Bacillus pertussis Holland, idem; Baclerium tussis convulsivae Lehmann and Neumann, Bakt. Diag., 7 Aufl., 2, 1927, 317 (Bordet and Gengou's organism); not Bacterium tussis convulsivae Lehmann and Neumann, Bakt. Diag., 2 Aufl., 2, 1899, 192 (Czaplewski and 
Hensel's organism).) From Latin, whooping cough.

Short, oval rods: 0.2 to 0.3 by $1.0 \mathrm{mi}$ cron, occurring singly or sometimes in pairs and short chains. Show tendency to bipolar staining. Capsules may be demonstrated by special technic (Lawson). Non-motile. Gram-negative.

No growth on the usual liboratory media; adapted by repeated transfer with heavy inoculum. Adaptation accompanied by loss of original characteristics.

Bordet-Gengou medium or some modification containing at least 15 per cent blood is optimum for isolation and maintenance.

Colonies: Smooth, raised, entire. pearly, glistening. Surrounded by a zone of hemolysis.

Litmus milk: (After adaptation) brownish color. 1lkaline.

Indole not formed.

Nitrites not produced from nitrates.

No action in carbohydrate media .

Catalase positive.

Aerobic.

Optimum temperature $37^{\circ} \mathrm{C}$.

Serologically homogeneous when first isolated (Phase I of Leslie and Gardner). Dissociative changes, recognizable morphologically, culturally, antigenically, and by animal tests, take place when maintained on unfavorable media.

Source: From respiratory tract in cases of whooping cough, especially by the cough plate method.

Habitat: Etiologically associated with whooping cough.

Note: Bacillus para-pertussis Eldering and Kendrick. (Jour. Bact., 35, 1938, 561.) From cases of whoopingcough. Closely related antigenically to Hemophilus pertussis and Brucella bronchiseptica, but distinct from either.

\section{Hemophilus ducreyi (Neveu-} Lemaire) Bergey et al. (Ducrey, Cong. internat. de dermatol. et syph., Compt. rend., Paris, 1890, 229; Monatshít. f. prakt. Dermatol., 9, 1889, 387; Riforma med., 5, 1889, 98; Monatshft. f. prakt. Dermatol., 21, No. 2; Streptobacillus of soft chancre, Ducrey, Abstract in Cent. f. Bakt., I Abt., 18, 1895, 290; Bacillus ulceris cancrosi Kiruse, in Flügge, Die Mikroorganismen, 3 Aufl., 2, 1896, 456; Bacterium ulceris cancrosi Chester, Ann. Rept. Del. Col. Agr. Exp. Sta., 9, 1897, 67 ; Bacterium cancrosi Chester, Manual Determ. Bact., 1901, 120; Coccobacillus ducreyi Neveu-Lemaire, Précis Parasitol. Hum., 5th ed., 1921, 20; Bergey et al., Manual, 1st ed., 1923, 271.) Tamed for Ducrey who first isolated the organism.

Small rods : 0.5 by 1.5 to 2.0 microns, with rounded ends, occurring singly and in short chains. Non-motile. Gramnegative.

Requires the $\mathrm{X}$ factor for its growth.

Gelatin colonies: No growth.

Gelatin stab: No growth.

Blood agar colonies: Small, grayish, glistening, showing a slight zone of hemolysis around the colony in three or four days.

Best growth is obtained on clotted rabbit, sheep, or human blood heated to $55^{\circ} \mathrm{C}$ for 15 minutes, and in casein digest agar containing blood. Moisture is important for growth.

Aerobic, facultative.

Optimum temperature $37^{\circ} \mathrm{C}$.

Habitat: The cause of soft chancre (chaneroid).

\section{Hemophilus haemoglobinophilus}

(Lehmann and Neumann) Murray. (Bacillus haemoglobinophilus canis Friedberger, Cent. f. Bakt., I Abt., Orig., 33, 1903, 401; Bacterium haemoglobinophilus Lehmann and Neumann, Bakt. Diag., 4 Aufl., 2, 1907, 270; Hemophilus canis Rivers, Johns Hopkins Bull., 33, 1922, 149; Jour. Bact., 7, 1922, 579; Murray, in Manual, 5th ed., 1939, 309.) From Greek, hemoglobin-loving.

Small rods : 0.2 to 0.3 by 0.5 to 2.0 microns, occurring singly, in pairs and 
short chains. Non-motile. Gramnegative.

Requires the $\mathrm{X}$ factor for its growth.

Blood agar colonies: Small, clear, transparent, entire. Old colonies become opaque.

Blood broth: Turbid.

Blood milk mixture: Doubtful development.

Indole is formed.

Nitrites produced from nitrates.

Acid but no gas from glucose, fructose, galactose, mannitol, sucrose and xylose. No acid from maltose, lactose dextrin, arabinose or glycerol. (Rivers, loc cit.)

Optimum temperature $37^{\circ} \mathrm{C}$.

Aerobic, facultative.

Habitat: Occurs in large numbers in preputial secretions of dogs.

Appendix I:* The following species has been placed in the tribe Hemophileae by Van Rooyen (Jour. Path. and Bact., 43, 1936, 469). It has been pointed out by Buchanan (General Systematic Bacteriology, 1925, 490) that the genus name Streptobacillus is invalid.

Streptobacillus moniliformis Levaditi, Nicolau and Poincloux. (Compt. rend. Acad. Sci., Paris, 180, 1925, 1188.)

This organism is regarded as identical with Haverhillia multiformis Parker and Hudson (Amer. Jour. Path., 2, 1926, 357 ) by Van Rooyen (loc. cit.). Topley and Wilson (Princip. Bact. and Immun., 2nd ed., 1936, 270) regard it as identical with their Actinomyces muris (Streptothrix ratti Schottmüller), the cause of rat-bite fever. Asterococcus muris Heilman, Jour. Inf. Dis., 69, 1941, 32. See Actinomyces muris ratti in the Appendix to the genus Streptomyces. Jordan and Burrows (Textb. of Bact., 14th ed., 1946, 614 ) consider all these names synonymous. Dawson and Hobby (Proceedings, Third Internat. Congr. for Micro- biol., New York, 1940, Section I, 177) suggest that the pleuropneumonia-like cultures isolated from Streptobacillus moniliformis really represent variant phases in the growth of this organism.

Deseription from Levaditi et al. (loc. cit.) and Brown and Nunemaker (Bull. Johns Hopkins Hosp., 70, 1942, 201).

Streptubacilli: 2.0 to 3.0 microns in length, pleomorphic, with branching filaments up to 30 to 40 microns long, fragmented, bacillary and coccobacillary forms. Swollen and club-shaped cells are found. Morphology is best demonstrated by aniline dyes, e.g. Wayson's plague stain. Non-motile. Gram-negative.

Enriched media are required for good growth. Best liquid media are rabbit blood and broth containing serum or aseitic fluid. Best solid media are glyeerol extract of potato-infusion broth-egg yolk medium and nutrient agar containing serum.

Blood agar or ascitic serum agar: Colonies small, clear.

Blood plates: Growth slow. Numerous small whitish colonies appear on the third day.

Veillon's medium: Punctiform colonies, abundant in depth, less growth at surface. No gas.

Broth with ascitic fluid and globular extract: Good growth, forming clots which precipitate and are rather adherent to one another. Growth rapidly reduces the $\mathrm{pH}$ of the medium killing the bacteria in cultures $24 \mathrm{hrs}$. old.

Milk: Slow growth. No coagulation. Löffler's serum: Poor growth.

Virulent for rabbits and mice.

Good growth at $37^{\circ} \mathrm{C}$.

Facultative anaerobe. Grows better under anaerobic conditions in the presence of added $\mathrm{CO}_{2}$, than in the presence of air.

Source : Isolated from a case of a febrile,

* Appendixes I and II arranged by Prof. E. G. D. Murray, McGill Univ., Montreal, P. Q., Canada, March, 1946. 
septicemic disease, accompanied by arthritis, erythema and angina.

Habitat: The cause of an acute febrile disease sometimes ealled erythema multiforme.

Appendix II: The following species may be identical with some of those listed above or related to them:

Bacillus marianensis Leber and Prowazek. (Berlin. klin. Wochnschr., 1, 1911, 27.) Allied to the Koch-Weeks Bacillus. Associated with cases of pink eye.

Bacillus weeksi Yeveu-Lemaire. (Précis Parasitol. Hun., 5th ed., 1921, 24.) Deseribed by Weeks. The eause of trachoma or granular conjunctivitis in tropical countries. Transmitted by the domestic fly. Probably intended for the Week's bacillus (Weeks, New York Med. Record, 31, 1887, 571).

Bacterium tussis convulsirae Lehmann and Neumann. (Bacterium, Czaplewski and Hensel, Deut. med. Wochnschr., 23, 1897, 587; Lehmann and Neumann, Bakt. Diag., 2 Aufl., 2, 1899, 192; not Bacterium tussis convulsivae Lehmann and Neumann, ibid., 7 Aufl., 2, 1927, 317; Bacillus tussis convulsivae Lehmann and Neumann, ibid., 4 Aufl., 2, 1907, 269.) Considered the cause of whooping cough by the original isolators.

Hemophilus aphrophilus Khairat. (Jour. Path. and Bact., 50, 1940, 497.) From blood and from heart valve of a case of endocarditis.

Hemophilus cuniculi Hauduroy et al. (Hemophilus sp. Gibbons, Jour. Inf. Dis., 45, 1929, 288; Hauduroy et al.. Dict. d. Bact. Path., 1937, 249.) From skin abscesses of rabbits.

Hemophilus gallinarum Delaplane, Erwin and Stuart and Hemophilus gallinarum Eliot and Lewis. (Bacillus hemoglobinophilus coryzae gallinarum De Blieck, Tijdsch. v. Diergeneensk., 58. 1931, 310; also see Vet. Jour., 88, 1932, 9;
Delaplane, Erwin and Stuart, R. I. State Coll. Sta. Bull. 244, May, 1934; Eliot and Lewis, Jour. Amer. Vet. Med. Assoc., 84, 1934, 878.) From edematous fluid from the head of a chicken. The cause of an infectious rhinit is in chickens.

Hemophilus influenzae murium (Kairies and Schwartzer) Lwoff. (Bacterium influenzae murium Kairies and Schwartzer, Cent. f. Bakt., I Abt., Orig., 137, 1936, 351; Lwoff, Ann. Inst. Past., 62, 1939, 168.) From the lung of a mouse.

Hemophilus meningitidis (Martins) Hauduroy et al. (Coccobacillus meningitidis Martins, Compt. rend. Soc. Biol., Paris, 99, 1928, 955; Hauduroy et al., Dict. d. Bact. Path., 1937, 254.) Resembles Hemophilus influenzae except that it shows sluggish motility. From cerebro-spinal fluid.

Hemophilus muris Hauduroy et al. (Bacillus of an epizootic of stock mice, Mackie, Van Rooyne and Gilroy, Brit. Jour. Exp. Path., 14, 1933, 132; Hauduroy et al., Dict. d. Bact. Path., 1937, 255.) From heart blood, spleen and other organs of mice dying from an epizootic disease.

Hemophilus ouis Mitchell. (Jour. Amer. Vet. Assoc., 68, 1925, 8.) From bronchi of sheep.

Hemophilus pertussis Ford. (Bacillus pertussis cppendorf Jochmann and Krause, Ztschr. f. Hyg., 36, 1901, 193; Ford, Textb. of Bact., 1927, 615; not necessarily identical with Bordet and Gengou's organism, Hemophilus pertussis Holland, Jour. Bact., 5, 1920, 215.) From the respiratory tract and lungs in pertussis.

Hemophilus putoriorum Hauduroy et al. Bacterium infuenzae putoriorum multiforme Kairies, Ztschr. f. Hyg., 117, 1935, 12; Hauduroy et al., Dict. d. Bact. Path., 1937, 258.) From the respiratory tract of ferrets.

Lehmann and Neumann (Bakt. Diag., 6 Aufl., 2, 1920, and 7 Aufl., 2, 1927) list 
the following species as closely related to this group:

Bacillus catarrhalis Jundell. (Hygieae, 60, No. 6 and 7, p. 667.) From cases of acute bronchitis.

Bacillus trachomatis Lehmann and Neumann. (The Bacillus Müller, Luerssen, Cent. f. Bakt., I Abt., Orig., 39, 1905, 682.) From conjunctiva.

Bacterium czaplewskii Chester. (Bacillus bei Keuchhusten, Czaplewski, Cent. f. Bakt., 22, 1897, 641; Bacterium tussis convulsivae Lehmann and Neumann, Bact. Diag., 2 Aufl., 1899, 192; Chester, Man. Determ. Bact., 1901, 153.) From sputum in whooping cough. This is not now regarded as being etiologically associated with whooping cough. Bacterium exiguum Stäubli. (Münch. med. Wchnschr., No. 45, 1905.) From à case of septic endocarditis.

Bacterium microbutyricum Hellstein. From butter.

Bacterium minutissimus sputi (Luzzatto) Lehmann and Neumann. ( $B a-$ cillus minutissimus sputi Luzzatto, Cent. f. Bakt., I Abt., 27, 1900, 816.) From a case of pertussis.

Bacterium polymorphum convulsivum Melfi. (Cent. f. d. gesamte Hygiene, 7, 1924, 133.)

Bacterium septicaemiae canis Paranhos. (Cent. f. Bakt., I Abt., Orig., 50, 1909, 607.)

Streptobacillus urethrae Pfeiffer. (Cent. f. Bakt., I Abt., Ref., 36, 1905, 59.) From the normal urethra and from cases of chronic eystitis and urethritis.

\section{Genus II. Moraxella Lwoff.*}

(Diplobacillus McNab, Klinische Monatsbl. f. Augenheilk., 42, 1904, 64 ; not Diplobacillus Weichselbaum, Cent. f. Bakt., 2, 1887, 212; Lwoff, Ann. Inst. Past., 62, 1939, 168.) Named for Morax, who first isolated the type species.

Small, short, rod-shaped cells, usually occurring singly or in pairs. Non-motile. Parasitic. Aerobic. Gram-negative.

The type species is Moraxella lacunata (Eyre) Lwoff.

\section{Key to the species of genus Moraxella.}

I. No growth in gelatin.

II. Gelatin liquefied.

\section{Moraxella lacunata.}

A. Rapid liquefaction. No growth in milk.

2. Moraxella liquefaciens.

B. Very slow liquefaction. Cells capsulated. Growth in milk.

3. Moraxella bovis.

1. Moraxella lacunata (Eyre) Lwoff. (Diplobacille de la conjunctivite subaigue, Morax, Ann. Inst. Past., 10, 1896, 337 ; Diplobacillus of chronic conjunctivitis, Axenfeld, Cent. f. Bakt., I Abt., 21, 1897, 1; Bacterium conjunctivitis Chester, Ann. Rept. Del. Col. Agr. Exp. Sta., 9, 1897, 85; Bacillus lacunatus Eyre, Jour. Path. and Bact., 6, 1899, 1; not Bacillus lacunatus Wright, Memoirs Nat. Acad. Sci., 7, 1895, 435; Diplobacillus moraxaxenfeld McNab, Klinische Monatsbl. f. Augenheilk., 42, 1904, 64; Bacterium duplex Lehmann and Neumann, Bakt. Diag., 2 Aufl., 2, 1899, 193; Hemophilus lacunatus Holland, Jour. Bact., 5, 1920, 223; Bacillus duplex Hewlett, Med. Res. Council Syst. of Bact., 2, 1929, 417;

* Arranged by Prof. E. G. D. Murray, McGill University, Montreal, P. Q., Canada, September, 1945. 
Lwoff, Ann. Inst. Past., 62, 1939, 173; Hemophilus duplex Murray, in Manual, 5th ed., 1939, 308.) From Latin, pitted.

Audureau (Ann. Inst. Past., 64, 1940, 128) recognizes an atypical variety of this species. To distinguish between the two varieties, she designates these as Moraxella lacunata var. typica and Moraxella lacunata var. atypica.

Short rods: 0.4 to 0.5 by 2.0 microns, occurring singly and in pairs and short chains. Ends rounded or square in the chains. Non-motile. Gram-negative.

Gelatin colonies: No growth.

Gelatin stab: No growth.

Blood agar colonies: Small, circular, transparent, entire. Growth on subculture difficult. Certain strains are not surrounded by zones of hemolysis; others are (Oag, Jour. Path. and Bact., $54,1942,128)$.

Serum agar colonies: Delicate, grayish.

Löffler's blood serum : Slow but definite liquefaction (pitting) around the colonies.

Ascitic broth: Turbid with slight, grayish sediment.

Blood milk mixture: Doubtful development.

Litmus milk: Unchanged.

Potato: No growth.

Indole not formed.

Nitrites not produced from nitrates.

Various carbohydrates and mannitol are attacked.

Optimum temperature $37^{\circ} \mathrm{C}$.

Aerobic, facultative.

Source: From conjunctiva.

Habitat: The cause of subacute infectious conjunctivitis, or angular conjunctivitis.

2. Moraxella liquefaciens (McNab) comb. nov. (Diplobacille liquéfiant, Pettit, Annales d'oculistique, March, 1899, 166 and Thesis, Paris, 1900, 223; Diplobacillus liquefaciens McNab, Klinische Monatsbl. f. Augenheilk., 42, 1904, 64; Bacillus duplex liquefaciens Lwoff, Ann. Inst. Past., 62, 1939, 170; Moraxella duplex liquefaciens Lwoff, ibid., 171; Moraxella duplex Lwoff, ibid., 171; Moraxella duplex var. liquefaciens Audureau, Ann. Inst. Past., 64, 1940, 139.) From Latin, liquefying.

Diplobacilli : 1.0 to 1.5 by 2.0 microns, occurring singly and in pairs, and having rounded ends. Capsules not demonstrated. Non-motile. Stain uniformly with basic aniline dyes. Gram-negative.

Gelatin colonies: Round, 1.5 to 2.0 $\mathrm{mm}$ in diameter, yellowish-white.

Gelatin stab: Rapid liquefaction.

Blood agar: Ready growth in primary and subculture.

Ascitic agar colonies: Grayish, thick, round, viscous.

Peptone agar colonies: Same as above, but less abundant growth.

Coagulated serum: Liquefaction in 3 to 4 days; eventually complete.

Plain broth: Poor growth, if any. Slight uniform turbidity.

Ascitic broth: Abundant growth in 24 hours at $35^{\circ} \mathrm{C}$. Uniform turbidity. Later sediment and an opaque pellicle.

Milk: No growth. No coagulation.

Potato: Slight, yellowish-white, vis cous growth.

Optimum temperature between $20^{\circ}$ and $37^{\circ} \mathrm{C}$. Killed at $55^{\circ} \mathrm{C}$ for 15 minutes.

Aerobic.

Not pathogenic for laboratory animals.

Source: From cases of conjunctivitis associated with corneal ulceration in man.

Habitat: Conjunctivitis in man so far as known.

3. Moraxella bovis (Hauduroy et al.) comb. nov. (Diplobacillus, Allen, Jour. Amer. Vet. Med. Assn., 54, 1918, 307 ; Diplobacillus, Jones and Little, Jour. Exp. Med., 38, 1923, 139; Hemophilus bovis Hauduroy et al., Dict. d. Bact. Path., 1937, 247; Moraxella duplex des Bovidés, Lwoff, Ann. Inst. Past., 62, 1939, 174; Hemophilus ruminantium Reid and Anigstein, Texas Reports on 
Biol. and Med., 3, 1945, 187.) From Latin bovis, of the ox.

Short, plump rods: 0.5 by 1.5 to 2.0 microns, usually oecurring in pairs and short chains, with rounded ends. Capsulated. Non-motile. Gram-negative.

Gelatin: Slow growth at $22^{\circ} \mathrm{C}$. Very slow liquefaction.

Blood agar colonies: After 24 hours, round, translucent, grayish-white, surrounded by a narrow, clear zone of hemolysis. Deep colonies tiny with a clear hemolytic zone, usually $1.5 \mathrm{~mm}$ in diameter. After 48 hours, surface colonies somewhat flattened, 3.5 to $4 \mathrm{~mm}$ in diameter; deep colonies ellipsoidal and biconvex with hemolytic area of $2.5 \mathrm{to}$ $3 \mathrm{~mm}$ in diameter.

Blood agar slants: After 24 hours at $38^{\circ} \mathrm{C}$, heavy, viseid, grayish-white growth.

Coagulated serum liquefied.

Broth: Slow growth. Slight turbidity. Considerable sediment.

Litmus milk: Alkaline. Partial coagulation.

Indole not produced.

Potato: No growth.

No acid from glucose or other carbohydrates.

Not pathogenic for laboratory animals.

Killed at $58^{\circ}$ to $59^{\circ} \mathrm{C}$ in five minutes.

Aerobic.

Source : From cases of acute ophthalmia (pink eye) of cattle.
Habitat: In the exudate from cases of acute ophthalmia of cattle. The probable cause of bovine infectious keratitis (Baldwin, Amer. Jour. Vet. Res., 6, 1945,180 ).

Appendix: Other species placed in the genus Moraxella are as follows:

Moraxella josephi Lwoff. (Bacillus duplex josephi Scarlett, Annales d'Oculistique, 153, 1916, 100 and 485; Lwoff, Ann. Inst. Past., 62, 1939, 171; Moraxella duplex josephi Lwoff, ibid., 174; Bacillus josephi Audureau, Ann. Inst. Past., 64, 1940, 126.) Gram-positive. Pathogenic. From the conjunctiva of man.

Moraxella lwoffi Audureau. (Ann. Inst. Past., 64, 1940, 150.) Two varieties are recognized: var. bacteroides and var. brevis. From various types of conjunctivitis in natr.

Moraxella non liquefaciens Lwoff. (Bacterium duplex-nonliquefaciens Oliver and Wherry, Jour. Inf. Dis., 28, 1921,342 ; Bacillus duplex non-liquefaciens Hewlett, Med. Res. Council Syst. of Bact., 2, 1929, 418; Lwoff, Ann. Inst. Past., 62, 1939, 171; Moraxella duplex non liquefaciens Lwoff, ibid., 174; Bacillus duplex non liquefaciens Audureau, Ann. Inst. Past., 64, 1940, 126; Moraxella duplex var. non liquefaciens Audureau, ibid., 144.) From an ulcer of the cornea, and from bronchial sputum in man.

\section{Genus III. Noguchia Olitsky, Syverton and Tyler.*}

(Jour. Exp. Med., 60, 1934, 382.) Named for Noguchi, the bacteriologist who isolated the type species.

Small, slender, Gram-negative rods present in the conjunctiva of man and animals affected by a follicular type of disease; mucoid type of growth which on first isolation takes place with some difficulty in ordinary media; motile, flagellated, and encapsulated; aerobic and facultative anaerobic; optimum temperature for growth $28^{\circ}$ to $30^{\circ} \mathrm{C}$.

The type species is Noguchia granulosis (Noguchi) Olitsky, Syverton and Tyler.

\section{Key to the species of genus Noguchia.}

I. Acid from carbohydrates.

A. Acid from raffinose, maltose and salicin.

1. Noguchia granulosis.

* Arranged by Prof. C. D. Kelly, MeGill University, Montreal, October, 1938. 
B. No acid from raffinose, maltose and salicin.

\section{Noguchia simiae.}

II. No acid from carbohydrates.

\section{Noguchia granulosis (Noguchi)} Olitsky et al. (Bacterium granulosis Noguchi, Jour. Exp. Med., ł8, Supp. 2, 1928, 21; Olitsky, Syverton and Tyler, Jour. Exp. Med., 60, 1934, 382.) From Latin, granular.

Rods : 0.25 to 0.3 by 0.8 to 1.2 microns, motile by means of a single flagellum, usually polar. Pleomorphic. Gram-negative.

No growth on plain agar or broth.

Blood agar plate: Minute round colonies, shiny, somewhat raised, almost transparent or slightly grayish in 48 hours. Later the colonies increase in size, are grayish opalescent and somewhat sticky. Old colonies have a brownish or yellowish tint.

Semi-solid Leptospira medium: Grayish-white, diffuse growth, forming a delicate zone $1 \mathrm{~cm}$ deep.

Liquid Leptospira medium: Diffuse. slightly cloudy growth, with sticky grayish sediment at the bottom of the tube in old cultures.

Acid from glucose, fructose, mannose, sucrose, galactose, maltose, salicin, xylose, mannitol, dextrin, arabinose, amygdalin and lactose. Small amount of acid from raffinose, inulin, rhamnose and trehalose. No acid from dulcitol, sorbitol and inositol.

Non-pathogenic for rabbits, guinea pigs, rats and mice.

Optimum pH 7.8 .

Temperature relations: Optimum 15 to $30^{\circ} \mathrm{C}$. Grows at $37^{\circ} \mathrm{C}$.

Aerobe, facultative anaerobe.

Distinctive characters: Action on carbohydrates; agglutination reactions; motility at $15^{\circ}$, none at $37^{\circ} \mathrm{C}$.

Source: From trachoma of American Indians at Albuquerque, New Mexico.

Habitat: Regarded by Noguchi and others as a cause of trachoma in man.

\section{Noguchia cuniculi.}

Produces a granular conjunctivitis in monkeys and apes.

2. Noguchia simiae Olitsky et al. (Bacterium simiae Olitsky, Syverton and Tyler, Jour. Exp. Med., 57, 1933, 875; Olitsky et al., Jour. Exp. Med., $60,1934,382$.$) From Latin simia, ape.$

Slender rods: 0.2 to 0.3 by 0.8 to 1.2 microns, occurring singly; in pairs, in short chains or parallel arrangement of two or three, having pointed ends. Capsules are found. Actively motile by means of a single, rarely a double, flagellum, usually polar. Gram-negative.

Gelatin plates: Colonies more mucoid and raised than on agar.

Gelatin stab: Arachnoid growth along line of inoculation. No liquefaction.

Agar plates: Small, circular, grayish, translucent, smooth, convex, slightly raised colonies having a sticky or mucoid consistency .

Blood agar plates: More highly translucent and colorless in early growth than on plain agar, becoming grayish after two or three days.

Agar slants: Grayish-white to white, moist, mucoid, raised, glistening growth. Growth is more profuse when blood is added.

Leptospira medium: Homogeneous, dense growth in a $0.5 \mathrm{~cm}$ sharply defined layer, with a slight, nebulous, uniform opacity about $1 \mathrm{~cm}$ below. In three or four days the lower layer becomes more dense and in time extends to the bottom of the tube

Broth: Tniform turbidity, with a slight grayish sediment and no pellicle.

Litmus milk: Unchanged.

Potato: Light tan, spreading, abundant growth.

Indole not formed.

Nitrites not produced from nitrates. Acid but no gas from glucose, fructose, 
mannose, galactose, xylose, arabinose and rhamnose. Small amount of acid from dextrin. Some strains produce a small amount of acid from sucrose, lactose, inulin and mannitol. Raffinose, salicin, dulcitol, amygdalin, maltose, trehalose, sorbitol and inositol unchanged.

Serological reactions: Rabbit antiserum is specific for all strains and no cross agglutination with Noguchia granulosis.

Temperature relations: Optimum $28^{\circ}$ to $30^{\circ} \mathrm{C}$. Thermal death point $56^{\circ} \mathrm{C}$ for thirty minutes.

Aerobe, facultative anaerobe.

Distinctive characters: Action on carbohydrates; agglutination reactions.

Source: From inflammatory type (Type II) of spontaneous conjunctival folliculosis in Macacus rhesus monkeys.

Habitat: Causes conjunctival folliculosis in Macacus rhesus monkeys.

3. Noguchia cuniculi Olitsky, Syverton and Tyler. (Jour. Exp. Med., 60, 1934, 382.) From Latin cuniculus, rabbit.

Slender rods: 0.2 to 0.3 by 0.5 to 1.0 micron with pointed ends. Capsules are formed of much finer texture than those surrounding Noguchia gramulosis or Noguchia simiae. Actively motile with peritrichous flagella. Non-acidfast. Pleomorphic forms sometimes noted. Gram-negative.

Gelatin agar plates: Grayish, mucoid and confluent colonies.

Gelatin stab: Tenuous, arborescent, non-spreading growth. No liquefaction.

Agar plates: Small, spherical, translucent, slightly grayish, smooth, somewhat convex, moist and mucoid colonies with entire edges.

Blood agar plates: More profuse, more grayish and less translucent than on plain agar.
Agar slants: Slightly grayish, translucent, coalescent, glistening, mucoid, homogeneous and non-spreading growth. The water of syneresis appears uniformly cloudy or milky depending on amount of growth.

Leptospira medium: After 24 hours, a faint, nebulous surface growth followed by an ingrowing sac-like mass, with its base $5 \mathrm{~mm}$ across, lying at the center of the under surface and extending for 5 $\mathrm{mm}$ into the medium. The area spreads laterally until at about two or three days there is a uniform, opaque, whitish layer about $1 \mathrm{~cm}$ thick which progresses slowly until the bottom of the tube is reached in about seven days.

Broth: Uniform turbidity, without pellicle.

Litmus milk: Unchanged.

Potato: Faint, buff-colored (changing to brown after five days), non-spreading, sparse surface growth.

Indole not produced.

Nitrites not produced from nitrates.

No acid or gas from glucose, fructose, mannose, mannitol, sucrose, raffinose, inulin, galactose, maltose, salicin, xylose, dextrin, arabinose, amygdalin, lactose, dulcitol, rhamnose, trehalose, sorbitol or inositol.

Serological relations: Rabbit antiserum is specific for all strains, and no cross agglutination with Noguchia granulosis or Noguchia simiae.

Temperature relations: Optimum $28^{\circ}$ to $30^{\circ} \mathrm{C}$. Thermal death point $56^{\circ} \mathrm{C}$ for 15 to 30 minutes.

Aerobe, facultative anaerobe.

Distinctive characters: No action on carbohydrates; peritrichous flagella; agglutination.

Source: From spontaneous conjunctival folliculosis, Type II of rabbits.

Habitat: Causes conjunctival follicu. losis in rabbits.

\section{Genus IV. Dialister Bergey et al.*}

(Manual, 1st ed., 1923, 271.)

Minute rod-shaped cells, occurring singly, in pairs and short chains. Non-motile.

* Rearranged by Prof. D. H. Bergey, Philadelphia, Pennsylvania, 1933. 
Strict parasites. Growth occurs only under anaerobic conditions in media containing fresh, sterile tissue or ascitic fluid.

The type species is Dialister pneumosintes (Olitsky and Gates) Bergey et al.

1. Dialister pneumosintes (Olitsky and Gates) Bergey et al. (Bacterium pneumosintes Olitsky and Gates, Jour. Exp. Med., 33, 1921, 713; ibid., 35, 1922, 813; Bergey et al., Manual, 1st ed., 1923, 271 ; Bacillus pneumosintes Ford, Textb. of Bact., 1927, 634.) From Greek pneumon, lung and sintor, murderer or devastator.

Very short rods: 0.15 to 0.3 (in glucose broth 0.5 to 1.0 ) micron in length, occurring singly and occasionally in pairs, short chains or masses. The ends are rather pointed. Ton-motile. Gramnegative.

Blood agar colonies : Small, clear, circular, entire, translucent.

Growth occurs in media containing fresh sterile rabbit kidney and ascitic fluid. Under strict anaerobic conditions good growth on rabbit blood glucose agar plates.

Glucose broth in which Escherichia coli or Bacillus mesentericus (non-spore stage) has grown favors growth .

Acid but no gas from glucose. Neither acid nor gas from maltose, lactose, sucrose, inulin or mannitol.

Passes Berkefeld $V$ and $\mathrm{N}$ filters.

Optimum pH 7.t to 7.8. No growth at $\mathrm{pH} 7.0$ or $\mathrm{pH} 8.0$.

Optimum temperature $37^{\circ} \mathrm{C}$. Does not survive $56^{\circ} \mathrm{C}$ for half an hour.

Pathogenic for rabbits and guinea pigs.

Strict a naerobe.

Source: From filtered nasopharyngeal secretions from influenza patients in the early hours of the disease.

Habitat: Nasopharyngeal washings of man.
2. Dialister granuliformans (Pavlovic) Bergey et al. (Bacterium granuliformans Pavlovic, Cent. f. Bakt., I Abt., Orig., 112, 1929, 432 ; Bergey et al., Manual, 4th ed., 1934, 341.) From Latin, forming granules.

Small rods: Non-motile. Gram-negative.

Agar colonies: Very small, transparent. No gas.

Broth: Turbid.

Litmus milk: Unchanged.

Indole not formed.

Acid from glucose, sucrose and mannitol.

Passes through Chamberland $\mathrm{L}_{2}$ filters. Pathogenic for rabbits.

Optimum temperature $37^{\circ} \mathrm{C}$.

Anaerobic to microaerophilic.

Source: From respiratory tract in influenza.

Habitat: Mucous membrane of respiratory tract.

Appendix, Family Parvobacteriaceae:* De Bord (Iowa State Coll. Jour. Sci., $16,1942,471$ ) describes a new tribe, Himcae, which may belong in this family. The tribe includes three genera: Mima with the species. Mima polymorpha and the variety Mima polymorpha var. oxidans; Herellea with the single species Herellea raginicola; and Colloides with the single species Colloides anoxydana. The organisms are Gram-negative, pleomorphic, motile or non-motile rods, often showing bipolar staining, and were isolated from the normal vagina and from cases of vaginitis and conjunctivitis. Deacon (Jour. Bact., 49, 1945, 511) classifies nineteen cultures in these genera.

* Arranged by Dr. A. Parker Hitchens, University of Pennsylvania, Philadelphia, Pa., March, 1946. 


\section{HAMILY XII. BACTERIACEAE COHN.*}

(Arch. f. path. Anat. u. Physiol., 55, 1872, 237.)

liod-shaped cells without endospores. Motile or non-motile. Gram-positive and Gram-negative. Metabolism complex, amino acids being utilized, and generally carbohydrates.

This is a heterogeneous collection of species whose relationships to each other and to other groups are not clear.

Only a single genus is recognized at this time.

\section{Genus I. Bacterium Ehrenberg.}

(IV. Evertebrata, Berlin, 1828, 8.)

The original description of this genus follows:

Bacterium, Novum Genus, Familia Vibrionorum. Character Generis: Corpus polygastricum? anenterum? nudum, oblongum, fusiforme aut filiforme, rectum, monomorphum (contractione nunquam dilatatum), parum flexile (nec aperte undatum), transwerse in multas partes sponte dividuum.

This may be translated as follows:

Bacterium, new genus. Family of Vibriona. Charucter of the genus: Body with many stomachs? without an intestine? naked, oblong, spindle-shaped or filiform, straight, monomorphic (in contraction never dilated), not very pliant (and not definitely wavy), freely separated transversely into many parts.

The type species is Bacterium triloculare Ehrenberg.

The original deseription of this species follows:

B. triloculare nov. spec,; distincte triloculares. triarticulatum, subfusiformum, hyalinum.

Animalculum 1/300 lineae longtum, corpore tereti. Articuli s. septa interna divisionem instantem multiplicem transversam indicare videntur. Mobile sed pigrum animaleulum.

In Oasi Jovis Hammonis Siwae observatum, praeterea nullibi.

Bacterii Generis physiologia huiusque obscurra. Cibo colorato Ventriculos replere hae formae respuunt ideoque ad Polygastrica non misi dubitanter et interim collocantur.

This may be translated as follows:

B. triloculare new spec. Definitely with three compartments or three jointed, subfusiform, hyaline. Animalcules 1/300 of a line in length, with a smooth body. The joints or internal septa are observed to develoy preliminary to multiple transverse splitting. A notile but sluggish animalcule. Observer in the Oasis of Jupiter Ammon of Siwa, nowhere else.

The physiology of the genus Bucterium is thus far obscure. These forms refuse to fill their stomacbs with colored food and for this reason they are placed with hesitancy and only temporarily in the Polygastrica.

The original deseriptions are taken from Buchanan, General Systematic Bacteriology, 1925, 213, and the translations are also furnished by him. Buchanan in his book gives an excellent summary of the nomenclatural status of the term Bacterium on pages 213-230.

Since neither the genus nor the type species is characterized in a way to permit definite irtentification, the term Bacterium is used to cover species of non-sporeforming, rod-shaped bacteria whose position in the system of classification is not definitely established (Breed and Conn, Jour. Bact., 31, 1936, 517).

* Completely rearranged by Prof. Robert S. Breed and Mrs. Eleanore Heist Clise, New York State Experiment Station, Geneva, New York, May, 1946. 
Bacterium triloculare Ehrenberg. (Ehrenberg, IV. Evertebrata, Berlin, 1828, 8; Bacillus ehrenbergii Trevisan, I generi e le specie delle Batteriacee, 1899, 18; Bacterium chrenbergii De Toni and Trevisan, in Saccardo, Sylloge Fungorum, 8, 1889, 1022; Bacterium lineola Cohn, Beiträge z. Biol. d. Pflanzen, 1, Heft 2, 1872, 170.) Cohn also regards Vibrio lineola Miller, 1786 and Vibrio lineola (Bacillus lineola, Bactrium lineola) of other authors as synonyms of Bacterium triloculare as explained by Buchanan (loc. cit., 213 and 521). From Latin tri, three and loculus, cells or compartments.

I. Gram-positive.

Key to the remaining species of genus Bacterium.

A. Non-motile.

1. Nitrites produced from nitrates.

1. Bacterium erythrogenes.

2. Bacterium subrufum.

3. Bacterium linens.

4. Bacterium mycoides.

5. Bacterium mutabile.

6. Bacterium qualis.

2. Nitrites not produced from nitrates.

a. Grow on ordinary media.

7. Bacterium raccmosum.

8. Bacterium healii.

9. Bacterium insectiphilium.

10. Bacterium tegumenticola.

11. Bacterium minutaferula.

12. Bacterium fulvum.

aa. Grow only on sea water media on fresh isolation.

13. Bacterium marinopiscosus.

14. Bacterium sociovivum.

15. Bacterium immotum.

3. Action on nitrates unknown.

16. Bacterium ammoniagenes.

17. Bacterium minutissimum.

B. Motile in young cultures.

1. Nitrites not produced from nitrates.

18. Bacterium incertum.

19. Bacterium imperiale.

C. Motile. Proteus-like growth on media.

1. Nitrites not produced from nitrates.

20. Bacterium zopfii.

21. Bacterium zenkeri.

Appendixes I and II : These list 34 additional species of Gram-positive, motile or non-motile, non-spore-forming, rod-shaped bacteria. See p. 609 and 612 .

II. Gram-negative. Digest cellulose. Do not digest agar.

A. Non-motile. Gelatin liquefied. Chromogenic.

1. Milk acid. 
B. Non-motile. Gelatin liquefied. Non-chromogenic.

1. Milk acid.

a. Ammonia produced; indole not formed.

23. Bacterium udum.

C. Non-motile. Gelatin not liquefied. Ton-chromogenic.

1. Milk unchanged.

a. Ammonia not produced; indole not formed.

24. Bacterium lucrosum.

2. Milk acid.

a. Ammonia not produced; indole not formed.

25. Bacterium acidulum.

D. Motile. Gelatin liquefied. Chromogenic.

26. Bacterium castigatum.

1. Milk acid.

a. Ammonia produced; indole is formed.

27. Bacterium bibulum.

Appendices I to III: These list additional species of cellulose-digesting, Gram-negative, usually motile, rod-shaped bacteria. See p. 615 and 622 . Also similar species that utilize bacterial polysaceharides as a sole source of carbon. See p. 623.

III. Gram-negative. Digest agar.

A. Non-motile.

1. Nitrites not produced from nitrates.

a. Acid from glucose and other sugars.

28. Bacterium nenckii.

aa. Do not form acis from glucose.

29. Bacterium polysiphoniae.

30. Bacterium drobachense.

2. Action on nitrates unknown.

a. Do not form acid from glueose.

31. Bacterium delesseriae.

32. Bacterium boreale.

33. Bacterium ceramicola.

B. Motile but position of flagella not given. May be either peritrichous or polar.

1. Nitrites not produced from nitrates.

34. Bacterium rhodomelae.

2. Action on nitrites unknown.

35. Bacterium alginovorum.

36. Bacterium fucicola.

Appendices I and II : These describe 9 additional species and list others that digest agar. All are Gram-negative, motile or non-motile rod-shaped bacteria. See p. 627 .

IV. Gram-negative. Digest chitin.

A. Motile but position of flagella not given.

1. Non-chromogenic.

2. Yellow chromogenesis.

37. Bacterium chitinophilum.

38. Bacterium chitinochroma. 
Appendix I: One additional species is described. See p. 632.

V. Gram-negative. Phosphorescent bacteria.

A. Non-motile coccobacilli from sea water.

1. No liquefaction of gelatin.

39. Bacterium phosphoreum.

B. Motile rods from sea water. Position of flagella not given.

1. No growth in broth, and on coagulated blood serum or potato.

40. Bacterium phosphorescens indigenus.

C. Not stated whether motile or non-motile. From diseased insect larvae.

1. Yellow growth on potato.

41. Bacterium hemophosphoreum.

Appendix I: This includes a list of more than 40 additional so-called species of phosphorescent bacteria. See p. 634 .

VI. Gram-negative. Facultative autotrophic bacteria which secure energy from the oxidation of hydrogen and utilize carbon from $\mathrm{CO}_{2}$.

A. Non-motile.

1. Growth shows a red chromogenesis.

42. Bacterium erythrogloeum.

B. Motile with peritrichous flagella.

1. Yellow chromogenesis.

43. Bacterium lentulum.

2. Ivory-colored colonies.

44. Bacterium leucogloeum.

VII. Gram-negative. Plant pathogens.

A. Non-motile.

1. Gelatin not liquefied.

45. Bacterium stewartii.

B. Motile with a polar flagellum.

1. Gelatin not liquefied.

a. Colonies mustard yellow on agar.

46. Bacterium tardicrescens.

b. Colonies honey to Naples yellow on agar.

47. Bacterium albilineans.

Appendix I : This includes 19 additional species placed in Bacterium or Bacillus by their authors. All are reported to cause or to be associated with plant disease. See p. 639 .

VIII. Gram-negative. Miscellaneous species.

A. Produce a pink to red chromogenesis.

1. Motile.

a. Gelatin not liquefied.

48. Bacterium rubefaciens.

aa. Gelatin liquefied.

49. Bacterium rubidum.

2. Non-motile.

a. Gelatin not liquefied.

50. Bacterium latericcum. 
B. Do not produce pink or red chromogenesis.

1. Motile.

a. Produce clouding in alginic acid liquid medium.

b. From sea water.

\section{Bacterium alginicum.}

bb. From soil.

52. Bacterium terrestralginicum.

aa. Action on alginic acid unknown.

b. Causes a disease of swans.

53. Bacterium cygni.

2. Non-motile.

a. Causes red spot disease of carp.

54. Bacterium cyprinicida.

aa. Causes liberation of ammonia from a mixture of horse manure and urine.

55. Bacterium parvulum.

aa. Utilizes formates in a linuid medium with the formation of a reddish pellicle.

\section{Bacterium methylicum.}

Appendix I: Miscellaneous described species of non-spore-forming bacteria placed by their authors in the genus Bacillus. See p. 643.

Appendix II : Includes anaerobic bacteria that produce methane. See p. 645.

Appendix III : Miscellaneous species of non-spore-forming bacteria listed but not described. See p. 647 .

1. Bacterium erythrogenes Lehmann and Neumann. (Bacterium lactis erythrogenes Grotenfelt, Fortschr. d. Med., 7, 1889, 41; Bacillus lactis erythrogenes Stermberg, Mianual of Bact., 1893, 636; Lehmann and Neumann, Bakt. Diag., I Aufl., 2, 1896, 253; Bacillus erythrogenes Matzuschita, Bakt. Diagnostik, 1902, 220 ; Corynebaclerium erythrogenes Kisskalt and Berend, Cent. f. Bakt., I Abt., Orig., 81, 1918, 446; Erythrobacillus erythrogenes Holland, Jour. Bact., 5, 1920, 218; Erythrobacillus (lactis) erythrogenes Holland, ibid.; Serratia lactica Bergey et al., Minnual, 1st ed., 1923, 93; Chromobacterium lactis erythrogenes Topley and Wilson. Princip. Bact. and Immun., 1, 1931, 402.) from Greek, redproducing.

Microccccus lactis erythrogenes Conn, Fsten and Stocking, Ann. Rept. Storrs (Comn.) Agr. Exp. Sta., 18, 1906, 117 is stated to be allied to if not identical with the subove species

Reds: 0.3 to 0.5 by 1.0 to $1 . t$ micoms. in broth often up to 4.3 mierons long, occurring singly, and having rounded ends. Non-motile. Stain with the usual aniline dyes. (iram-positive (Lehmann and Neumann, loc. cit.).

Gelatin colonies: Small, circular, grayish, becoming yellow, sinking into the medium. Crateriform liquefaction. Yellow sediment. Medium becomes rose-colored.

Gelatin stab: Surface growth a whitish, later yellow, circular, thin layer. Weak growth in stab. Slow liquefaction at the surface, the liquid becoming red, with yellow sediment. The solid portion assumes a weak rose color.

Igar stab: Moist, fairly luxuriant. yellow growth, the medium assuming a rose to wine color.

Broth: Turbid, yellow. Pellicle (Fuller and Johnson, Jour. Fxp. Med., 4, 1899, 609)

Litmus milk: Arid. Slow coagulation, heving a clear fluid which beeomes bloodred in color. Reaction becomes alkaline. sterile milk: Casein slowly precipitated, later peptonized. Reaction neu- 
tral or alkaline A stratum of blood-red serum is seen above the precipitated casein and above this a yellowish-white layer of cream. An intensive sweet odor that becomes disagreeable.

Potato: Growth rapid, spreading. grayish, later yellow. On incubation a deep golden yellow color develops after 6 to 8 days. A darkening of the medium occurs around the culture, but soon disappears; later the whole potato becomes a weak yellowish-red.

Indole not formed (Fuller and Johnson, loc. cit.). Indole formed (Chester, Manual Determ. Bact., 1901, 174).

Blood serum: Liquefied (Fuller and Johnson, loc. cit.). Not liquefied (Hefferan, Cent. f. Bakt., II Abt., 11, 1903, 456).

Nitrites produced from nitrates.

No gas from carbohydrates.

Slight $\mathrm{H}_{2} \mathrm{~S}$ production (Matzuschita, loc. cit.).

Red pigment insoluble in water, alcohol, ether, chloroform, and benzol. Soluble (Hefferan, loc. cit., 529). Yellow pigment insoluble.

Distinctive character: Milk becomes blood-red in 12 to 20 days.

Non-pathogenic for mice (Fuller and Johnson, loc. cit.).

Optimum temperature $28^{\circ}$ to $35^{\circ} \mathrm{C}$

Aerobic (Fuller and Johnson, loc. cit.). Facultative anaerobe (Hefferan, loc. cit., 530).

Source: Isolated from red milk by Hueppe in Wiesbaden in 1886. Isolated from feces of a child by Baginsky (Cent. f. Bakt., 6, 1889, 137). Isolated from Ohio River water by Fuller and Johnson (loc. cit.). Isolated from Mississippi River water by Hefferan (loc. cit.). Tataroff isolated a rose fluorescent coccobacterium (Bacillus rosafluorescens Kruse, in Flügge, Die Mikroorganismen, 3 Aufl., 2, 1896, 305; Bacterium rosa Auorescens Chester, Ann. Rept. Del. Col. Agr. Exp. Sta., 9, 1897, 142) which Migula reports as identical, but which Hefferan considers atypical.
Habitat: Probably widely distributed in nature.

2. Bacterium subrufum Burri and Staub. (Burri and Staub, Landwirtsch. Jahrb. d. Schweiz, 40, 1926, 1006; Serratia subrufa Bergey et al., Manual, 3rd cd., 1930, 123.) From Latin sub, somewhat and rufus, red.

This organism is stated to be closely related to or possibly identical with Bacterium erythrogenes.

3. Bacterium linens Weigmann. (Organismus IX, Wolff, Milichwirt. Zent., 5, 1909, 145; Weigmann, in Wolff, Cent. f. Bakt., II Abt., 28, 1910, 422, and in Weigmann, Mykologie der Milch, 62, 1911, 220.) From Latin, daubing, smearing, or spreading over.

Also see Steinfatt, Milchwirt. Forsch., 9, 1930, 7; Kelly; Jour. Dairy Sci., 20, 1937, 239; Albert, Long and Hammer, Iowa Agr. Exp. Sta. Res. Bul. 328,1944 .

Rods: Average 0.62 by 2.5 microns when grown 1 to 2 days on tryptone glucose extract agar. Non-motile (Wolff). Gram-positive (Kielly, loc. cit.).

Gelatin colonies: At $18^{\circ} \mathrm{C}$ punctiform at first; after 12 days about $1 \mathrm{~mm}$ in diameter, compact, circular, shiny, brownish-yellow to red-brown. Liquefaction.

Gelatin stab: At $21^{\circ} \mathrm{C}$ crateriform liquefaction, becoming infundibuliform on extended incubation. Rate of liquefaction varies considerably with different cultures, some completing it in 15 days, others not completing it even on long incubation.

Agar colonies: On tryptone glucose extract agar at $21^{\circ} \mathrm{C}$ after 1 to 2 days, colonies convex, glistening, entire and cream-colored, becoming brown on extcnded incubation; diameters 2 to $5 \mathrm{~mm}$. On special cheese agar with incubation in oxygen, luxuriant growth, the color becoming bright orange to reddishbrown in 4 or 5 days. 
Agar stab: Heavy surface growth on tryptone glucose extract agar at $21^{\circ} \mathrm{C}$ with no growth along the line of inoculation.

Agar slant: On tryptone glucose extract agar at $21^{\circ} \mathrm{C}$ after 2 days growth abundant, glistening, filiform, non-viscid and cream-colored. After extended incubation the color usually is brown. On special cheese agar in an atmosphere of oxygen the growth is bright orange to reddish-brown in 4 or 5 days.

Broth: Turbidity and sediment.

Potato: At $21^{\circ} \mathrm{C}$ after 5 days, growth is scanty, smooth, glistening, and varies in color from grayish to brownish-orange.

Litmus milk: At $21^{\circ} \mathrm{C}$ the changes are very slow. After 6 or 7 days the reaction becomes alkaline and a yellow sediment appears. After approximately 10 days some digestion is evident, complete digestion generally requiring several weeks to over a month. A distinct ammoniacal odor, more or less objectionable, produced in old cultures. No coagulation. Ropiness often produced on extended incubation.

Indole not produced.

Nitrites produced from nitrates.

Methyl red and Voges Proskauer reactions negative.

Hydrogen sulfide produced in broth and on agar by some cultures but not by others.

Natural fats not hydrolyzed.

No acid or gas from arabinose, dextrin, glucose, dulcitol, galactose, inulin, lactose, fructose, maltose, mannitol, raffinose, rhamnose, salicin, sorbitol, sucrose or xylose.

Ethyl, propyl, butyl and amyl alcohols oxidized largely to corresponding acids; hexyl and heptyl alcohols attacked much less actively.

Catalase rapidly produced in or on various media.

Aerobic.

Growth temperatures: Growth at $8^{\circ}$ and $37^{\circ} \mathrm{C}$ but not at $45^{\circ} \mathrm{C}$, with the optimum at about $21^{\circ} \mathrm{C}$,
Heat resistance low, cultures being killed at $62.8^{\circ} \mathrm{C}$ in a few minutes.

Grow th in the $\mathrm{pH}$ range 6.0 to 9.8 ; no growth at $\mathrm{pH} 5.0$ or below.

Salt tolerant, cultures growing readily in a concentration of 15 per cent salt in broth or skim milk, with certain cultures apparently capable of growing somewhat in much higher concentrations.

Closely related to or identical with Bacterium erythrogenes Lehmann and Neumann.

Source: Originally isolated by Wolff from the surface flora of various soft cheeses.

Habitat: Widely distributed in and especially on the surface of dairy produets including blue, brick, camembert, limburger, oka and cheddar cheeses, butter, milk and eream. Also found in various feeds including grains, silage, green plants, hay and straw, and in water, soil, manure, and air.

\section{Bacterium mycoides (Grotenfelt)}

Migula. (Bacterium mycoides roseum Grotenfelt, Fortschr. d. Med., 7, 1889, 46; Bacillus mycoides roseus Sternberg, Manual of Bact., 1893, 640; Migula, Syst. d. Bakt., 2, 1900, 482; Bacillus mycoides-roseus Holland, Jour. Bact., 5, 1920, 219; Erythrobacillus mycoidesroseus Holland, ibid.; Serratia rosea Bergey et al., Manual, 1st ed., 1923, 96; Chromobacterium mycoides roseum Topley and Wilson, Princip. of Bact. and Immun., 1, 1931, 402.) From Greek mykes, fungus and eidos, form.

Rods: Non-motile. Gram-positive.

Gelatin colonies: Red, felt-like. Liquefaction.

Gelatin stab: Rapid liquefaction. Red pellicle. Red sediment.

Colonies composed of interlacing filaments (Crookshank, Textb. of Bact. and Inf. Dis., 1900, 524).

Agar stab: Red color produced if grown in dark; a white color in presence of light. 
Optimum temperature: Room temperature.

Pigment soluble in water.

Distinctive characters: Morphologically like the anthrax bacillus. Appearance in gelatin. Production of a brilliant rose color when grown in the dark; colonies grown in the light are white, but they assume the red color if developed further in the dark.

Source: Isolated from Wiesbaden soil by Scholl.

Habitat: Unknown.

Note: It has been claimed that this or a similar organism forms spores (Matzuschita, Bact. Diag., 1902, 168; Perlberger, Cent. f. Bakt., II Abt., 62, $1924,8)$. However cultures of Scholl's organism received from the Kràl collection by Hefferan (Cent. f. Bakt., II Abt., $11,1903,458$ ) and by Breed in 1926 (personal communication) did not form spores. These cultures produced nitrites from nitrates and failed to liquefy gelatin.

\section{Bacterium mutabile Steinhaus.} (Jour. Bact., 42, 1941, 775.) From Latin mutabilis, changeable.

Short rods: On agar, 0.7 to 0.9 by 1.0 to 2.0 microns. In fluid media, such as tryptophane broth, pleomorphic, bizarre forms frequently appearing slightly branched. Non-motile. Gram-positive.

Gelatin stab: Very slow liquefaction.

Agar colonies: Cream to yellow, circular, smooth, glistening, opaque.

Broth : Moderate turbidity, slight sediment.

Litmus milk: Alkaline, soft curd, slow peptonization.

Indole not produced.

Hydrogen sulfide not produced.

Nitrites produced from nitrates.

Starch not hydrolyzed.

Glucose, lactose, sucrose and maltose not fermented.

Aerobic.

Source: From the alimentary tract of the lyreman cicada, Tibicen linnei Smith and Grossbeck.

Habitat: Unknown.

6. Bacterium qualis Steinhaus. (Jour. Bact., 42, 1941, 774.) From Latin qualis, of what kind.

Short rods: Very short on solid media, frequently ellipsoidal in shape. In fluid media: 0.5 to 0.7 by 1.4 to 2.2 microns, occurring singly. Non-motile. Grampositive.

Gelatin stab: Liquefaction.

Agar colonies: Small (1 mm), white, glistening, transparent, circular, entire.

Agar slant: Filiform, smooth, glistening.

Broth: Almost clear; slight turbidity in serum and glucose broth.

Litmus milk: No change.

Indole not produced.

Hydrogen sulfide not produced.

Slight production of nitrites from nitrates.

Starch not hydrolyzed.

Acid from glucose, sucrose and maltose. Lactose not fermented.

Source: From the alimentary tract of the tarnished plant bug, Lygus pratensis $\mathrm{L}$.

Habitat: Unknown.

\section{Bacterium racemosum Kettnow .}

(Zettnow, Cent. f. Bakt., I Abt., Orig., 77, 1915, 209; Zettnowia racemosa Enderlein, Bakt. Cyclogenie, Berlin, 1925, 259; Flavobacterium racemosum Bergey et al., Manual, 1st ed., 1923, 115.) From Latin racemosus, branching.

Filaments: 0.5 to 0.8 by 10 to $12 \mathrm{mi}$ crons. Branching forms found. Nonmotile. Gram-positive.

Gelatin colonies: White, circular, soft, granular, brownish, entire.

Gelatin stab: White surface growth. Liquefaction napiform.

Agar slant: Light yellow, limited growth.

Broth: Turbid. 
Litmus milk: Coagulated, becoming alkaline.

Potato: Dirty-yellowish, linited streak.

Indole not formed.

Nitrites not produced from nitrates.

Aerobic, facultative.

Optimum temperature $20^{\circ} \mathrm{C}$

This species is selected as the type species for the genus Zettnowia Enderlein (loc. cit.).

Souree: Contamination on agar plate. Habitat: Lnknown.

\section{Bacterium healii Juchanan and} Hammer. (Buchanan and Hammer. Iowa Agr. Exp. Sta. Research Bull. 22. 1915, 249; Escherichia healii Bergey et al., Manual, 1st ed., 1923, 200; Achromobacter healii Bergey et al., Manual. 2nd ed., 1925, 157.)

Rods: 0.5 to 0.7 by 2.2 to 12.9 microns, occurring singly and in short chains. Non-motile. Cram-positive.

Gelatin stah: Stratiform liquefaction. Villous growth in stab.

Agar colonies: Large. White, rhizoid.

Agar slant: White, hard growth, with no tendency to stringiness.

Broth: Gray pellicle and sediment.

Litmus milk: Slightly acid, beeoming slimy, coagulated, peptonized.

Potato: Heavy, white, glistming growth

Indole not formed.

Nitrites not produced from nitrates.

Acid without gas from glucose, fruetose, maltose, sucrose salicin and starch. Fo acid from mannitol, lactose, raffinose or inulin.

Aerobic, facultative

Optimum temperature $220 \mathrm{C}$.

Source: Slimy milk.

Mabitat: Lnknown.

9. Bacterium insectiphilium Steinhaus. (Jour. Bitet.. 42, 1941, 777.) From M. I. insect, insect and Greek philos, loving.

Rods: 0.8 to 1.2 by 1.0 to 2.8 microns, occurring singly. At times appearing almost as cocci or coccobacilli. Nonmotile. Gram-positive.

Gelatin stab: Liquefaction.

Agar colonies: Light greenish-yellow, rircular, entire, raised, glistening, smooth, opaque.

Agar slant: Filiform, raised, smooth, glistening, opaque growth.

Broth: Moderate turhidity, slight viscid sediment.

Litmus milk: Alkaline, peptonization, and slow reduction.

Potato: Greenish-yellow, thick, moist growth.

Indole not produced.

Nitrites not produced from nitrates.

Hydrogen sulfide not produced.

Starch slightly hydrolyzed.

No action on the following arabohydrates: Glucose, lactose, sucrose, maltose, fructose, mannitol, galactose, arabinose, xylose, dextrin, salicin, rhamnose, raffinose, trehalose, sorbitol, inulin, dulcitol, glyeerol, adonitol, mannose.

Aerohic.

Source: From the body wall of the bagworm, Thyridopleryx ephemeraformis Hisw.

Habitat: Tonknown.

10. Bacterium tegumenticola Steinhaus. (Jour. Bact., 42, 1941, 775.) Fron Latin tegumentum, cover, skin and cola, dweller

Small rods: 0.5 to 0.8 by 1.0 to $1.5 \mathrm{mi}$ crons. Ifave a tendeney to be ellipsoidal on solid media. Non-motile. Grampositive

Gelutin stab: (iemerally no liquefaction. Virriable.

Igar colonies: Tiny (1 $\mathrm{mm}$ ), white, conver, glistening, circular, entire.

Agar slant: Filiform, glistening, grayish-white growth.

Broth: Slight turbidity; sediment.

Litmus milk: No change.

Indole not produced.

Hydrogen sulfide not produced.

Nitrites not produced from nitrates.

Starch not hydrolyzed.

Acid slowly produced from glucose 
and maltose. Acid from sucrose. Lactose not fermented.

Source: From the integument of the bed-bug, Cimex lectularius L.

Habitat: Lnnknown.

11. Bacterium minutaferula Steinhaus. (Jour. Bact., 42, 1941,778.) From Latin, small rod.

Very small rods : 0.4 to 0.9 by 0.7 to 1.0 micron, occurring singly. Non-motile. Gram-positive.

Gelatin stab: No liquefartion.

Igar colonies: Colorless to faint gray, circular, smooth, entire, glistening.

Agar slant: Very thin, transparent, glistening growth.

Broth: Slight turbidity and sediment.

Litmus milk: No change at first; slightly acid after one week.

Indole not produced.

Hydrogen sulfide not produced.

Nitrites not produced from nitrates.

Starch not hydrolyzed.

Acid from glucose after 4 days. Slight acid from sucrose. Lactose and maltose not fermented.

Aerobic.

Source: From triturated specimen of the mud-dauber wasp, Sceliphron cementarium Dru.

Habitat: Unknown.

12. Bacterium fulvum (Zimmermann) Chester. (Bacillus fulvus Zimmermann, Bakt. unserer Trink- u. Nutzwässer, Chemnitz, 1, 1890, 44; Chester, Ann. Rept. Del. Col. Agr. Exp. Sta., 9, 1897, 107; Flavobacterium fulvum Bergey et al., Manual, 1st ed., 1923, 115.) From Latin fulvus, dull yellow:

Rods: 0.8 by 0.9 to 1.3 microns, occurring singly, in pairs and in chains. Nonmotile. Gram-positive.

Gelatin colonies: Circular, convex, reddish-yellow.

Gelatin stab: Convex, reddish-yellow surface growth. Good growth in stab. Slow liquefaction.

Agar slant: Orange-red, glistening streak.
Broth: Turbid with yellow sediment.

Litmus lactose broth: Acid, or acid then alkaline (Dyar, Ann. N. Y. Acad. Sci., 8, 1895, 368).

Potato: Slowly spreading, yellowish, glistening growth.

Indole formed (Dyar, loc. cit.).

Nitrites not produced from nitrates (Bergey)

Aerobic, facultative.

Optimum temperature $30^{\circ} \mathrm{C}$.

Source: From Chemnitz and Döbeln tap water (Zimmermann). From dust and water (Dyar).

Habitat: Water.

13. Bacterium marinopiscosus $\% o B e l l$ and Lpham. (Bull. Scripps Inst. Oceanography, La Jolla, 5, 1944, 258.) From Latin marinus, pertaining to the sea, and piscosus, fish.

Rods: 1.2 to 1.6 by 2.0 to 4.7 microns, with rounded ends, show granular staining, occurring singly, in pairs and long rhains. Non-motile. Gram-positive, but many cells tend to decolorize leaving Gram-positive granules.

All differential media except the freshwater broth, litmus milk, and potato were prepared with sea water.

Gelatin colonies: Gray, circular, convex, $1 \mathrm{~mm}$. To pigment.

Gelatin stab: Liquefaction napiform, becoming crateriform to stratiform with age. Complete in 50 days.

Agar colonies : 2 to $4 \mathrm{~min}$, circular, conrex, entire, smooth, irregular edge.

Agar slant: Luxuriant, beaded, glistening, butyrous growth with no pigment.

Sea-water broth: No turbidity, abundant flocculent sediment, slight surface ring.

Fresh-water broth: Good growth.

Litmus milk: Decolorized, neutral, top peptonized.

Potato: Heavy, white, raised, mucoid, dull growth. Potato darkened.

Indole not formed.

Nitrites not produced from nitrates.

Acid but no gas from glucose and man- 
nitol. No acid from maltose, lactose, sucrose, glycerol, xylose or salicin.

Starch is hydrolyzed.

Hydrogen sulfide not formed.

Ammonia produced from peptone but not from urea.

Casein is digested.

Fats are not hydrolyzed.

Aerobic, facultative.

Optimum temperature $20^{\circ}$ to $25^{\circ} \mathrm{C}$.

Source: Found on the skin of marine fish.

Habitat: Not known from other sources.

14. Bacterium sociovivum $/ 20$ Bell and Upham. (Bull. Scripps Inst. Oceanography, La Jolla, 5, 1944, 269.) From Iatin socius, associate and vivum, to live.

Rods: 0.5 to 0.8 by 3.0 to 4.0 microns, with rounded ends, occurring singly, in pairs, and chains. Non-motile. Grampositive but tends to destain, leaving Gram-positive cell wall and granules.

All differential media except the freshwater broth, litmus milk and potato were prepared with sea water.

Gelatin colonies: Irregular, sunken, filamentous margin, grayish-white.

Gelatin stab: Crateriform liquefaction becoming stratiform.

Agar colonies: 2 to $t \mathrm{~mm}$, circular, convex, smooth, entire, darker center.

Agar slant: Luxuriant, beaded, glistening, butyrous growth with no pigment.

Sea-water broth: No pellicle, no turbidity, heavy floceulent sediment.

Fresh-water broth: Fair growth.

Litmus milk: Decolorized, neutral, completely peptonized in 20 days.

Potato: Abundant, dull, light creamcolored growth. Potato darkened.

Indole not formed.

Nitrites not produced from nitrates.

Acid but no gas from glucose, maltose, and mannitol. No acid from glycerol, lactose, sucrose, or salicin.

Starch is hydrolyzed.

Hydrogen sulfide not formerl.
Ammonia produced from peptone but not from urea.

Casein is digested.

Fats not hydrolyzed.

Aerobic, facultative.

Optimum temperature $20^{\circ}$ to $25^{\circ} \mathrm{C}$.

Source: Found associated with sedentary organisms in the sea.

Habitat: Commonly found on submerged surfaces and on sessile diatoms in sea water.

15. Bacterium immotum ZoBell and Upham. (Bull. Scripps Inst. Oceanography, 5, 1944, 271.) From Latin, meaning immobile or stationary.

Rods: 0.8 by 3.1 to 8.6 microns, with rounded ends, occurring singly, in pairs, and long chains. Non-motile. Grampositive but tend to destain leaving Gram-positive outline and granules.

All differential media except the freshwater broth, litmus milk, and potato were prepared with sea water.

Gelatin colonies: Small, circular, raised, gray, slowly digest gelatin.

Gelatin stab: Crateriform liquefaction becoming infundibuliform. Beaded growth along line of stab. No pigment.

Agar colonies: 1 to $2 \mathrm{~mm}$, circular, convex, smooth, lobate margin, darker centers.

Agar slant: Luxuriant, glistening, echinulate, mucoid growth with no pigment.

Sea-water broth: No pellicle, moderate turbidity, abundant, floceulent sediment.

Fresh-water broth: Scanty growth.

Litmus milk: Decolorized, neutral, partly peptonized in 20 days.

Potato: Luxuriant, mucoid, creamy growth which darkens potato.

Indole not formed.

Nitrites not produced from nitrates.

Acid but no gas from glucose, maltose, xylose, and mannitol. No acid from glycerol, lactose, sucrose, or salicin.

Starch is hydrolyzed.

Hydrogen sulfide not formed. 
Ammonia produced from peptone but not from urea.

Casein is digested.

Fats not hydrolyzed.

Aerobic, facultative.

Optimum temperature $20^{\circ}$ to $25^{\circ} \mathrm{C}$.

Source: Found associated with marine sedentary organisms.

Habitat: Not known from other sources.

16. Bacterium ammoniagenes Cooke and Keith. (Cooke and Keith, Jour. Bact., 13, 1927, 315; Alcaligenes ammoniagenes Bergey et al., Manual, 3rd ed., 1930, 367.) From M. L. ammonia and Latin genero, develop.

Rods with rounded ends, 0.8 by 1.4 to 1.7 microns, occurring singly. Nonmotile. Gram-positive.

Gelatin stab: No liquefaction.

Agar colonies: Circular, flat, smooth, entire, gray.

Agar slant: Growth moderate, smooth, flat, opaque, glistening, butyrous, amorphous.

Broth: Moderate turbidity, with flocculent sediment.

Litmus milk: Slightly alkaline.

Indole not formed.

No action on carbohydrates.

Blood serum not liquefied.

Urea is fermented forming ammonia.

Aerobic, facultative.

Optimum temperature $30^{\circ} \mathrm{C}$.

Source: From feces of infants.

Habitat: Presumably widely distributed in putrefying materials.

17. Bacterium minutissimum Migula. (Bacillus pyogenes minutissimus Kruse. in Flügge, Die Mikroorganismen, 3 Aufl., 2, 1896, 447; Bacterium pyogenes minutissimus Chester, Ann. Rept. Del. Col. Agr. Exp. Sta., 9, 1897, 89; Migula, Syst. d. Bakt., 2, 1900, 418; Eberthella minutissima Bergey et al., Manual, 1st ed., 1923, 22s; Shigella minutissima Bergey et al., Manual, 3rd ed., 1930, 359.) From Latin, smallest.
Description from Lruse (loc. cit.). Description not different from that of Bacillus tenuis sputigenes Pansini, according to Chester (Ann. Rept. Del. Col. Agr. Exp. Sta., 9, 1897, 89).

Rods: 0.5 by 1.0 micron, occurring singly and in pairs. Non-motile. Gram-positive.

Gelatin stab: No liquefaction. Yellowish growth spreading slightly on surface.

Growth on agar and blood serum is not characteristic.

Acid but no gas from glucose and lactose.

No characteristic odor.

Tot pathogenic for mice and rabbits.

Aerobic, facultative.

Optimum temperature $37^{\circ} \mathrm{C}$.

Source: Isolated from a facial abscess.

Habitat: Not known from other sources.

18. Bacterium incertum Steinhaus. (Jour. Bact., 42, 1941, 776.) From Latin incertus, uncertain.

Short rods: 0.5 to 0.8 by 1.0 to 1.5 microns, occurring singly and occasionally in pairs. Young cultures motile, after 48 hours generally non-motile. Gram-positive; after 48 hours many cells become Gram-negative.

Gelatin stab: No liquefaction.

Agar colonies: Tiny, grayish-white, smooth, almost transparent. Does not grow well on nutrient agar.

North's gelatin chocolate agar slant: Filiform, thin, transparent growth. Brown color of chocolate medium changes to yellowish-green.

Blood agar: Alpha hemolysis at first; after three day's beta hemolysis.

Broth: Almost clear; very slight growth.

Litmus milk: No change.

Indole not produced.

Hydrogen sulfide not produced.

Nitrites not produced from nitrates. Starch not hydrolyzed.

Acid but no gas from glucose, sucrose, 
fructose, mannose, and maltose. No fermentation of lactose, rhamnose, galactose, mannitol, dulcitol, inositol, or sorbitol.

Voges-Proskauer test: Negative.

Microaerophilic.

Source: From the ovaries of the lyreman cicada, Tibicen linnei Smith and Grossbeck.

Habitat: Unknown.

19. Bacterium imperiale Steinhaus. (Jour. Bact., 42, 1941, 777.) From Latin imperialis, referring to the imperial moth.

Small rods: 0.5 to 0.8 by 1.0 to 1.7 microns, occurring singly and in pairs. A few cells motile in young cultures. Gram-positive.

Gelatin stab: No liquefaction.

Agar colonies: Circular, entire, almost translucent, pinkish-orange to yellow pigment.

Agar slant: Filiform, glistening, opaque growth.

Broth: Slight to moderate turbidity; slight sediment.

Litmus milk: No change at first, later slightly acid.

Potato: Heavy, glistening, moist growth; reddish to yellowish-orange.

Indole not produced.

Hydrogen sulfide not produced.

Nitrites not produced from nitrates.

Starch not hydrolyzed.

Acid but no gas from glucose, sucrose, maltose, fructose, mannitol, galactose, arabinose, xylose, salicin, raffinose, trehalose, sorbitol, mannose, adonitol, esculin, and slight acid from lactose and dextrin. Inulin, dulcitol, glycerol, rhamnose, adonitol, and inositol not fermented.

Aerobic.

Source: From the alimentary tract of the imperial moth, Eacles imperialis Dru.

Habitat: Unknown.

20. Bacterium zopfii Kurth. (Kurth, Bericht. d. deutsch. Botan. Gesellschaft,
1, 1883, 97; Kurthic zopfii Trevisan, Atti della Accad. Fisio-Medico-Statistica in Milano, Ser. 4, 3, 1885, 92; Helikobacterium zopfii Escherich, Münch. med. Wchnschr., 33, 1886, 2, quoted from Enlows, U. S. Hygienic Lab. Bull. 121, 1920, 47; Bacterium (Proteus) zopfii Chester, Ann. Rept. Del. Col. Agr. Exp. Sta., 9, 1897, 103; Bacillus zopfii Migula, Syst. d. Bakt., 2, 1900, 815; Zopfius zopfii Wenner and Rettger, Jour. Bact., 4, 1919, 334.) Named for W. Zopf, German botanist.

This is the type species of the genus Kurthia Trevisan. (Trevisan, loc. cit.; Zopfius Wenner and Rettger, Jour. Bact., 4, 1919, 334.)

Rods: 0.8 by 3.5 microns, with rounded ends, occurring in long curved chains. Motile with peritrichous flagella. Gram-positive.

Gelatin colonies: Radiate, filamentous, gray.

Gelatin stab: Arborescent growth in stab. No liquefaction.

Agar colonies: Fimbriate.

Agar slant: Spreading, gray, fimbriate growth.

Broth: Slow, moderate growth.

Litmus milk: No change.

Potato: Moderate, gray growth; medium becoming dark.

No $\mathrm{H}_{2} \mathrm{~S}$ produced.

Indole not formed.

Nitrites not produced from nitrates. Aerobic, facultative.

Optimum temperature $25^{\circ}$ to $30^{\circ} \mathrm{C}$.

Habitat: Decomposing materials.

\section{Bacterium zenkeri (Hauser) Ches-} ter. (Proteus zenkeri Hauser, Ueber Fauinissbakterien, 1885; Bacillus zenkeri Trevisan, I generi e le specie delle Batteriacee, 1889, 17; Chester, Ann. Rept. Del. Col. Agr. Exp. Sta., 9, 1897, 103; Zopfus zenkeri Wenner and Rettger, Jour. Bact., 4, 1919, 334; Bacillus proteus-zenkeri Holland, Jour. Bact., 5, 1920, 220; Kurthia zenkeri Bergey et al., Manual, 2nd ed., 1925, 215.) Named for K. Zenker, German pathologist. 
Rods : 0.65 by 1.6 to 2.3 microns, occurring in pairs and in chains. Motile with peritrichous flagella. Gram-positive.

Gelatin colonies: Feathery, with filaments extending in all directions.

Gelatin stab: Surface growth like colonies. No arborescent growth in stab. No liquefaction.

Agar colonies: Thin, filamentous, spreading, grayish.

Agar slant: Thin, bluish-gray, filamentous growth

Broth: Slightly turbid, with gray sediment.

Litmus milk: No change.

Potato: Barely visible, yellowishgray, glistening growth.

Indole not formed.

Nitrites not produced from nitrates.

No $\mathrm{H}_{2} \mathrm{~S}$ formerl.

Aerobic, facultative.

Optimum temperature $30^{\circ} \mathrm{C}$.

Habitat: Decomposing materials.

Note: Wenner and Rettger, loc. cit., consider the last two species to be identical.

Appendix I : The following Gram-posi tive, motile species may belong with the above group. All have been placed at one time or another in the genus Achromobacter or in the genus Flarobacterium.

1. Achromobacter lipolyticum (Huss Bergey et al. (Bactridium lipolyticum Huss, Cent. f. Bakt., II Abt., 20, 1908, 474; Bergey et al., Manual, 2nd ed. 1925, 158.) From Greek, fat-dissolving.

Small, oval rods: 0.3 to 0.5 by 0.7 to $1 . t$ microns. Motile, possessing peritrichous flagella. Gram-positive.

Gelatin colonies: Circular, grayish to transparent with irregular margin.

Gelatin stab: Infundibuliform liquefaction.

Agar colonies: Growth circular, gray smooth, glistening, with entire margin.

Broth: Turbid with granular sediment.

Litmus milk: Coagulated, peptonized. becoming alkaline.
Potato: Moist, glistening, grayish growth.

Indole is formed.

Acid from glucose, sucrose, raffinose, xylose, mannitol and glycerol.

Fats are split in milk, giving rise to a rancid odor and a bitter taste.

Aerobic, facultative.

Optimum temperature $35^{\circ} \mathrm{C}$.

Source: From the udder of a cow giving abnormal milk.

Habitat: Milk.

2. Achromobacter stearophilum (Weinzirl) Bergey et al. (Bacillus stearophilus Weinzirl, Jour. Med. Res., 39, 1919, 404; Bergey et al., Manual, 1st ed., 1923, 145.)

Rods: 0.8 by 5.0 microns, occurring singly. Motile. Gram-positive.

Gelatin colonies: Scanty development.

Pumplin gelatin stab : Filiform growth in stab. No liquefaction.

Pumpkin agar colonies: Small, smooth, convex, gray, entire.

Pumpkin juice: Slightly turbid.

Pumpkin milk: Acid, coagulated.

Potato: Slight, smooth, gray, glistening, filiform growth

Indole not formed.

Nitrites not produced from nitrates.

No acid from carbohydrate media.

Starch from pumpkin hydrolyzed.

Aerobic, facultative.

Optimum temperature $20^{\circ} \mathrm{C}$.

Source: Canned pumpkin.

Habitat: Lnknown.

3. Achromobacter sulfureum Bergey et al. Bakt. 4, Rubentschick, Cent. f. Bakt., II Abt., 72, 1927, 123; Bergey et al., Manual. 3rd ed., 1930, 220.)

Rods: 0.7 to 0.8 by 1.7 to 2.2 microns, occurring singly and in pairs. Motile. Gram-positive.

Gelatin stab: Saccate liquefaction.

Agar colonies: Circular, grayish-white, flat, homogeneous.

Agar slant: Filiform, grayish-white, smooth, homogeneous growth. Metallic luster. 
Broth: Turbid.

Litmus milk: Peptonized.

Potato: Yellowish-brown layer.

Indole not formed.

Nitrites produced from nitrates with gas formation.

Hydrogen sulfide formed.

Ammonia formed.

Urea is attacked.

Methylene blue reduced.

Aerobic, facultative.

Optimum temperature $30^{\circ}$ to $33^{\circ} \mathrm{C}$. Can grow at $0^{\circ} \mathrm{C}$.

Source: Sewage filter beds.

Habitat: Putrefying materials.

Note: See Pseudomonas urease Bergey et al. for another motile, Gram-positive organism described by Rubentschick (Bakt. 3) from the same source.

\section{Achromobacter aerophilum (Rubent-} schick) Bergey et al. (Urobacterium aerophilum Rubentschick, Cent. f. Bakt., II Abt., 64, 1925, 168; Bergey et al., Manual, 3rd ed., 1930, 224.)

Rods : 0.75 to 0.85 by 2.0 to 4.5 microns, occurring singly, in pairs, and in chains. Motile. Gram-positive.

Urea gelatin colonies: Small, circular, dirty-gray, entire.

Urea gelatin stab: No liquefaction.

Urea agar colonies: Circular, grayish, smooth.

Urea agar slant: Dirty-gray, glistening to dry growth.

Urea broth: 'Turbid.

Urea milk: Unchanged.

Urea potato: Slight, grayish-white streak.

Indole not formed.

Nitrites produced from nitrates.

$\mathrm{H}_{2} \mathrm{~S}$ not formed.

Ammonia not formed.

Aerobic, facultative.

Source: Sewage slime.

Habitat: Putrefying materials.

5. Achromobacter citrophilum (Rubentschick) Bergey et al. (Urobacterium citrophilum Rubentschick, Cent.f. Bakt., II Abt., 64, 1925, 168; 66, 1926, 161; 67,
1926,$167 ; 68,1926,327$; Bergey et al., Manual, 3rd ed., 1930, 224.)

Rods : 0.75 to 0.85 by 2.5 to 6.0 microns, occurring singly and in pairs. Motile. Gram-positive.

Urea gelatin colonies: Small, grayishwhite, smooth, undulate.

Urea gelatin stab: No liquefaction.

Urea agar slant: Filiform, grayishwhite, thin, dry growth.

Urea broth: Turbid.

Urea milk: Unchanged.

Urea potato: Dirty-gray, thin streak.

Indole not formed.

Nitrites produced from nitrates.

Hydrogen sulfide not formed.

Ammonia not formed.

Can derive oxygen from sodium citrate.

Aerobic, facultative.

Optimum temperature $30^{\circ} \mathrm{C}$.

Source: Sewage slime.

Habitat: Putrefying materials.

6. Flavobacterium sulfureum Bergey et al. (Bacterium punctans sulfureum Zetnow, Cent. f. Bakt., I Abt., Orig., $7 \gamma, 1916,222$; Bergey et al., Manual, 1st ed., 1923, 103.)

Rods : 0.5 to 0.7 by 0.7 to 1.5 microns. Motile, possessing peritrichous flagella. Gram-positive.

Gelatin colonies: Very small, barely visible, becoming brownish-yellow, granular.

Gelatin stab: Spreading growth on the surface only. Later crateriform liquefaction.

Agar slant: Sulfur-yellow growth.

Broth: Turbid.

Litmus milk: Alkaline, peptonized, yellow.

Potato: Sulfur-yellow streak.

Indole not formed.

Nitrites not produced from nitrates.

Blood serum: Sulfur-yellow growth.

Partial liquefaction.

To acid from glucose.

Aerobic, facultative.

Optimum temperature $25^{\circ} \mathrm{C}$. 
Source: Air.

Habitat: Unknown.

7. Flavobacterium acetylicum Levine and Soppeland. (Bull. 77, Engineering Exp. Sta., Iowa State Agricultural College, 1926, 46.) From the chemical term acetyl.

Rods : 0.9 by 1.1 microns, with rounded ends, occurring singly and in pairs. Motile. Gram-positive.

Gelatin stab: Stratiform liquefaction.

Agar colonies: Irregular in form, yellow, smooth, flat, amorphous, entire.

Agar slant: Abundant, echinulate growth, flat, peach yellow, smooth and butyrous.

Broth: Ring growth on surface. Turbid with scant sediment.

Litmus milk: Slight acidity, with granular curd. Peptonization. Litmus reduced.

Potato: Moderate, orange growth.

Indole not formed.

Nitrites not produced from nitrates.

Starch hydrolyzed.

Blood serum liquefied.

Acid from glucose with formation of acetylmethylcarbinol.

Aerobic, facultative.

Optimum temperature $22^{\circ} \mathrm{C}$.

Source: From skimmed milk.

Habitat: Unknown.

\section{Flavobacterium fuscum (Zimmer-}

mann) Bergey et al. (Bacillus fuscus Zimmermann, Bakt. unserer Trink- und Nutzwässer, Chemnitz, 1, 1890, 70; not Bacillus fuscus Flügge, Die Mikroorganismen, 2 Aufl., 1886, 290; Bacterium fuscus Chester, Ann. Rept. Del. Col. Agr. Exp. Sta., 9, 1897, 111; Bergey et al., Manual, 1st ed., 1923, 113; Chromobacterium fuscum Topley and Wilson, Prine. Bact. and Immun., 1, 1931, 405.) From Latin fuscus, tawny.

Rods: 0.6 by 1.5 microns, occurring singly. Ton-motile. Gram-positive.

Gelatin colonies: Small, with brownish center and yellowish border.
Gelatin stab: Gray, filiform growth in stab. Slow crateriform liquefaction.

Agar colonies: Circular, pale yellow, smooth, slightly convex, entire.

Agar slant: Growth greenish-yellow, plumose, smooth, raised, undulate.

Broth: Turbid, with pellicle and sediment.

Litmus milk: Slightly acid, becoming alkaline, with yellow ring.

Potato: Thick, moist, chrome-yellow streak.

Indole not formed.

Nitrites produced from nitrates.

Aerobic, facultative.

Optimum temperature $30^{\circ} \mathrm{C}$.

Source: From Zwönitz River water.

Habitat: Water.

8a. Bacterium fuscum liquefaciens (Dyar) Chester. (Bacillus fuscus liquefaciens Dyar, Ann. N. Y. Acad. Sci., 8, 1895, 375; Chester, Ann. Rept. Del. Col. Agr. Exp. Sta., 9, 1897, 108.) Received from the Kràl collection labeled Bacillus fuscus; also from air. Differs from the above only in liquefying gelatin more slowly and completely.

9. Flavobacterium maris Harrison. (Canadian Jour. Research, 1, 1929, 232.) From Latin mare, sea.

Rods: 0.7 to 0.8 by 1.0 to 1.2 microns, occurring singly and in pairs. At $37^{\circ} \mathrm{C}$ coccoid. Non-motile. Encapsulated. Gram-positive

Gelatin colonies: Punctiform, redorange, granular, entire.

Gelatin stab: Red-orange surface growth, filiform growth in stab. No liquefaction.

Agar colonies: Circular, orange-yellow, smooth, glistening, convex.

Agar slant: Growth moderate, orangeyellow, becoming cadmium-orange to red-orange, spreading, glistening.

Broth: Clear with orange pellicle and sediment.

Litmus milk: At first faintly alkaline, becoming faintly acid with orange sediment.

Potato: Seant growth. 
Indole not formed.

Nitrites produced from nitrates.

Traces of ammonia formed.

Faint acidity from glucose. No action on lactose or sucrose.

Loeffler's blood serum not liquefied.

No $\mathrm{H}_{3} \mathrm{~S}$ formed.

Aerobic, facultative.

Optimum temperature $20^{\circ}$ to $25^{\circ} \mathrm{C}$.

Sonrce: Isolated from the skin of fishes. Steinhaus (Jour. Bact., 42, 1941, 771) found a similar organism in the intestine of a caterpillar.

Habitat: Unknown.

Appendix Ii: Among the following species reported as Gram-positive are many that appear to be similar to the species listed above, while others may belong to the so-called parasitic lactohacilli of the intestine or even to Corynebacterium. The majority have been placed by their authors in genera other than Bacterium.

Bacillus achrous Migula. (No. 6, Lembke, Arch. f. Hyg., 26, 1896, 301; Migula, Syst. d. Bakt., 2, 1900, 676.) From feces. Motile.

Bacillus asteriformis Migula. (Microbe astériforme, de Klecki, Ann. Inst. Past., 9, 1895, 735; Migula, Syst. d. Bakt., 2, 1900, 816.) From feces. Motile. Colonies resemble those of Bacillus mycoides but no spores are mentioned.

Bacillus casei limburgensis Orla-Jensen. (Orla-Jensen, Cent. f. Bakt., II Abt., 13, 1904, 702; Bacterium casei limburgensis Yale, N. Y. S. Agr. Exp. Sta., Tech. Bul. 268, 1943, 5.) From limburger cheese. Presumably the same as Bacterium linens Weigmann.

Bacillus choulevitchi Herter. (Microbe VIII, Choukévitch, Ann. Inst. Past., 25, 1911, 265; Merter, Just's Botan. Jahresber., 39, 2 Abt., Heft 4, 1915, 748.) From the large intestine of a horse.

Bacillus colorabilis Migula. (Bacillus der Gallenblase, Naunyn, Deutsche med. Wochnschr., 1891, 193; Bacillus cuniculicida havaniensis Steruberg, Man. of
Bact., 1893, 450; Bacillus coli colorabilis Kruse, in Flügge, Die Mikroorganismen, 3 Aufl., 2, 1896, «34; Bacterium coli colorabilis Chester, Ann. Rept. Del. Col. Agr. Exp. Sta., 9, 1897, 87; Bacterium cuniculicida havaniensis Chester, idem; Migula, Syst. d. Bakt., 2, 1900, 736.) From a diseased gall-bladder (Naunyn) and from the intestines and liver of yellow-fever cadavers (Sternberg).

Bacillus cupularis Migula. (No. 12, Lembke, Arch. f. Hyg., 26, 1896, 307; Migula, Syst. d. Bakt., 2, 1900, 677.) From feces. Motile.

Bacillus fumigatus Migula. (No, 14, I.embke, Arch. f. Hyg., 29, 1897, 320; Migula, Syst. d. Bakt., 2, 1900, 679.) From feces. Motile

Bacillus gazogenes Heurlin. (Heurlin, Bakt. Unters. d. Keimgehaltes im Genitalkanale der fiebernden Wöchnerinnen. Holsingfors, 1910,97.) From the vagina.

Bacillus glacialis Migula. (Giftproduzierender Bacillus, Vaughan and Perkins, Arch. f. Hyg., 27, 1896, 305; Migula, Syst. d. Bakt., 2, 1900, 807.) From ice cream and cheese. Motile.

Bacillus involutus Wälsch. (Cent. f. Bakt., I Abt., Orig., 38, 1905, 645.) From preputial secretion.

Bucillus laseri Migula. (Gasbildender aërober Bacillus, Laser, Cent., f. Bakt., 13, 1893, 217; Migula, Syst. d. Bakt., 2, 1900 , 763.) From the lungs and liver of a calf. Motile.

Bacillus membranifer Migula. (No.7, Lembke, Arch. f. Hyg., 26, 1896, 302; Migula, Syst. d. Bakt., 2, 1900, 675.) From feces. Motile.

Bacillus pseudotyphosus Migula. ('Typhusähnlicher Bacillus, Lustig, Diag. d. Bakt. d. Wassers, 1893, 18; Migula, Syst. d. Bakt., 2, 1900, 730; not Bacillus pseudotyphosus Kruse, in Flügge, Die Mikroorgmismen, 3 Aufl., 2, 1896, 383.) Motile. From several samples of water from Thales de Aosta, Italy.

Bacillus pulmonum Migula. (Proteus bei Lungengangrän des Menschen, Babes, Progrès médical roumain, April 6, 1889; Migula, Syst. d. Bakt., 2. 1900, 
755.) From gangrenous lung exudate and the spleen. Motile.

Bacillus radiatus Zimmermann. (Zimmermann, Bakt. unserer Trink- u. Nutzwässer, Chemnitz, 1, 1890, 58; not $\mathrm{Ba}$ cillus radiatus Lüderitz, Ztschr. f. Hyg., 5, 1889, 149; not Bacillus radiatus Chester, Man. Determ. Bact., 1901, 241; Bacillus aquatilis radiatus Kruse, in Flügge, Die Mikroorganismen, 3 Aufl., 2, 1896,315 ; quoted as $B$. radiatus aquatilis Zimmermann by Kruse, idem; Bacterium radiatus aquatilis Chester, Ann. Rept. Del. Col. Agr. Exp. Sta.. 9; 1897, 100; Bacterium aquatilis radiatus Chester, idem; Bacillus pseudoradiatus Migula, Syst. d. Bakt., 2, 1900, 830; Bacterium aquatilis Chester, Man. Determ. Bact., 1901, 160; not Bacterium aquatile. Migula, loc. cit., 469.) From water. Motile. In certain stages of development (presumably old cultures) Zimmermann states that this organism is Gramnegative.

Bacillus splendens Miguli. (Bacillus putidus splendens Bernabei, Bull. d. Soc. Lancisiana d. Ospedali di Roma, 13; see abst. in Cent. f. Bakt., 17, 1895. 469; Migula, Syst. d. Bakt., 2, 1900, 754.) From a case of fetid bronchitis. Notile.

Bacterium acidiformans (Sternberg) Chester. (Bacillus acidiformans Sternberg, Man. of Bact., 1893, 449 ; Chester, Ann. Rept. Del. Col. Agr. Exp. Sta., 9, 1897, 79.) From the liver of a yellow fever patient. Also reported from beeswax (White, L. S. Dept. Agr. Bur. Entomol., Bull. 14, 1906, 14).

Bacterium cactivorum Pasmetti and Buzzati-Traverso. (Nuovo Gior. Bot. Ital., X.S. 42, 1935, 117.) From a diseased cactus.

Bacterium dendrobii Pavarino. (Rev. di Pat. Veg., 5, 1912, 242.) From Dendrobium $s p$.

Bacterium (?) musae (Grumann) Elliott. (Pseudomonas musae Gaumann, Med. van het Inst. voor Plantz., No. 48, 1921, 58; Elliott, Manual Bact. Plant Path., 1930, 170; Phytomonas (?) musae Magrou, in Hauduroy et al., Dict. d.
Bact. Path., 1937, 385.) From diseased bananas.

Bacterium nicotinovorum Bucherer. (Cent. f. Bakt., II Abt., 105, 1942, 169.) From a mixture of soil, manure and mud.

Bacterium pseudotyphosum Migula. (Typhusähnlicher Bacillus, Lustig, Diagnostik der Bakterien des Wassers, 1893, 17; Migula, Syst. d. Bakt., 2, 1900, 428.) Non-motile. Isolated by Sartori from Rome tapwater.

Bacterium teutlium Metcalf. Metcalf, Cent. f. Bakt., II Abt., 13, 1904, 28; Aplanobacter teutlium E. F. Smith, Intro. to Bact. Dis, of Plants, 1920, 474; Phytomonas teutlia Bergey et al., Manual, 3rd ed., 1930, 280.) From a soft rot of sugar beets. See Manual, 5th ed., 1939,216 for a description of this species.

Flavobacterium dehydrogenans Armaudi. (Micrococcus dehydrogenans Arnaudi, Boll. Sez. ital. Soc. intern. Microbiol., 18, 1939, 000; Arnaudi, Cent.f. Bakt., II Abt., 105, 1942-43, 352.) From a pressed yeast cake.

Flavobacterium vitarumen Kinutsen. (Knutsen, in Bechdel, IIoneywell, Dutcher and Knutsen, Jour. Biol. ('hem., 80, 1928, 234.) From the rumen of a cow.

Kurthia bessoni Severi. (Quoted from Giorn. Batteriol. e Inmunol., 34, 1946, $10 \overline{\mathrm{T}}$.)

Kurthia variabilis Severi. (Giorn. Batteriol. e Immunol., 34, 1946, 107.) From feces.

22. Bacterium idoneum MeBeth. McBeth, Scil Science, 1, 1916, 460 ; Cellulomonas idonea Bergey et al., Manual, 1st ed., 1923, 165.) From Latin idoneus, capable.

Rods: 0.5 by 1.5 microns. Non-moile. Gram-negative.

Gelatin stab: Moderate, yellowish growth. Slight napiform liquefaction.

Agar colonies: Circular, convex, soft, becoming brittle, grayish, granular, entire.

Agar slant: Scant, yellowish-white growth, becoming distinctly yellow. 
Gelatin stab: Moderate, yellowish growth. Slight napiform liquefaction.

Agar colonies: Circular, convex, soft, becoming brittle, grayish, granular, entire.

Agar slant: Scant, yellowish-white growth, becoming distinctly yellow.

Ammonia cellulose agar: Enzymatic zone shows a diameter of 2 to $3 \mathrm{~mm}$ at the end of 30 days.

Peptone cellulose agar: Enzymatic zone shows a diameter of 1.5 to $2.0 \mathrm{~mm}$ at the end of 30 days.

Broth: Turbid.

Filter paper broth: Paper reduced to thin, limp sheet which falls apart on slight agitation at end of 15 days.

Litmus milk: Acid, not digested,

Potato: Abundant, moist, glistening, grayish-white growth, becoming distinctly yellow.

Indole not formed.

Nitrites produced from nitrates.

Ammonia not produced.

Acid from glucose, maltose, lactose, starch and glycerol.

Aerobic, facultative.

Optimum temperature $20^{\circ} \mathrm{C}$

Source: Soil from California.

Habitat: Soil.

23. Bacterium udum Kellerman et al. (Kellerman, McBeth, Scales and Smith, Cent. f. Bakt., II Abt., 39, 1913, 514; Cellulomonas uda Bergey et al., Manual, 1st ed., 1923, 166; Proteus cellulomonas var. Proteus udus Pribram, Klassifikation der Schizomyceten, Leipzig und Wien, 1933, 72.) From Latin udus growing in marshy ground.

Rods: 0.5 by 1.5 microns. Non-motile. Gram-negative.

Gelatin stab: Liquefaction.

Agar slant: Luxuriant, faintly yellowish growth.

Cellulose agar: Enzymatic zone 0.5 mm wide.

Broth: Turbid.

Litmus milk: Acid.

Potato: Good growth.

Indole not formed.

Nitrites produced from nitrates.
Ammonia is produced.

Acid from glucose, fructose, arabinose, xylose, maltose, lactose, sucrose, dextrin and starch.

Aerobic, facultative.

Optimum temperature $20^{\circ} \mathrm{C}$.

Source: Compost from Arlington, Va. Habitat: Soil.

24. Bacterium lucrosum MeBeth. (McBeth, Soil Science, 1, 1916, 461; Cellulomonas lucrosa Bergey et al., Manual, 1st ed., 1923, 167.) From Latin lucrosus, Iucrative.

Rods: 0.4 by 1.3 microns. Non-motile. Gram-negative.

Gelatin stab: No growth.

Agar colonies: Circular, convex, semitransparent, granular, entire.

Agar slant: Moderate, flat, grayishwhite growth, becoming somewhat iridescent.

Ammonia cellulose agar: On crowded plate, the colonies show an enzymatic zone of $1 \mathrm{~mm}$ or more.

Peptone cellulose agar: Enzymatic zone 2 to $3 \mathrm{~mm}$ wide in 25 days.

Broth: Turbid.

Filter paper broth: Paper is reduced to a grayish-white pulpy mass whose fibers separate on slight agitation.

Litmus milk: No change.

Potato: No growth.

Indole not formed.

Nitrites not produced from nitrates.

Ammonia not produced.

Acid from glucose, maltose, lactose, sucrose, starch and mannitol.

Aerobic, facultative.

Optimum temperature $20^{\circ} \mathrm{C}$.

Source: Soil from California.

Habitat: Soil.

25. Bacterium acidulum Kellerman et al. Kellerman, MeBeth, Scales and Smith, Cent. f. Bakt., II Abt., 39, 1913, 513; Cellulomonas acidula Bergey et al., Manual, 1sted., 1923, 167.) From Latin acidus, acid.

Rods: 0.3 by 1.0 micron. Non-motile. Gram-negative. 
Gelatin stab: No liquefaction.

Agar slant: Slight, grayish growth.

Cellulose agar: Enzymatic zone 0.5 to $1 \mathrm{~mm}$ in width.

Broth: Clear.

Litmus milk: Acid.

Potato: No growth.

Indole not formed.

Nitrites not produced from nitrates.

Ammonia not produced.

Acid from glucose, maltose, lactose and sucrose. None from glycerol or mannitol.

Starch not hydrolyzed.

Aerobic.

Optimum temperature $20^{\circ} \mathrm{C}$.

Source: Soil from Utah.

Habitat: Soil.

26. Bacterium castigatum McBeth. (McBeth, Soil Science, 1, 1916, 458; Cellulomonas castigata Bergey et al., Manual, 1st ed., 1923, 168.) From Latin castigatus, subdued.

Rods: 0.4 by 1.2 microns. Non-motile. Gram-negative.

Gelatin stab: Moderate surface growth. No liquefaction.

Agar colonies: Circular, slightly convex, brittle, grayish-white, granular, entire.

Agar slant: Abundant, glistening, grayish-white growth.

Ammonia cellulose agar: Enzymatic zone may attain a diameter of $2.5 \mathrm{~mm}$ in 30 days.

Peptone cellulose agar: Enzymatic zone may reach a diameter of $2 \mathrm{~mm}$ in 30 days.

Broth: Slightly turbid.

Filter paper broth: Paper completely disintegrated and reduced to a pulp-like mass in 15 days.
Litmus milk: Acid, not digested.

Indole not formed.

Nitrites not produced from nitrates.

Ammonia not produced.

Acid from glucose, maltose, lactose, sucrose, starch and glycerol.

Aerobic.

Optimum temperature $20^{\circ} \mathrm{C}$.

Source: Soil from California.

Habitat: Soil.

27. Bacterium bibulum (McBeth and Scales) Holland. (Bacillus bibulus McBeth and Scales, Bur of Plant Industry, L. S. Dept. of Agr., Bul. No. 266, 1913, 35; Holland, Jour. Bact., 5, 1920, 217 and 221; Cellulomonas bibula Bergey et al., Manual, 1st ed., 1923, 158.) From Latin bibulus, thirsty.

Rods: 0.4 by 1.3 microns. Motile. Gram-negative.

Gelatin stab: Crateriform liquefaction.

Cellulose agar colonies: Circular, convex, smooth, soft, grayish to faintly yellowish-white, finely granular. Enzymatic zone $0.3 \mathrm{~mm}$ in some cases.

Agar slant: Luxuriant, glistening, smooth, moist, raised growth.

Broth: Slightly turbid.

Litmus milk: Faintly acid.

Potato: Smooth, glistening, canary yellow growth.

Indole is formed.

Nitrites not produced from nitrates.

Ammonia is produced.

Acid from glucose, maltose, lactose, sucrose, starch, glycerol and mannitol.

Aerobic, facultative.

Optimum temperature $20^{\circ} \mathrm{C}$.

Source: From sewer slimes and cultivated soils.

Habitat: Soil.

Appendix I : The genus Cellulomonas as originally proposed was based on a single physiological property and included such diverse types of bacteria as (1) polar flagellate species, now placed in Pseudomonas, (2) Gram-variable, non-motile rods now placed in Corynebacterium, and (3) peritrichous, non-spore-forming, Gram-negative rods. Unfortunately the name is unsuitable for the third of these groups so that it has not been inserted in the outline used in this edition of the MANTAL. Descriptions of species previously placed in this genus are given below. 


\section{Genus A. Cellulomonas Bergey et al.}

(Manual, 1st ed., 1923, 154.)

Small rods, with rounded ends, non-spore-forming, motile with peritrichous flagella, occurring in soil and having the property of digesting cellulose. Growth on ordinary culture media often not vigorous. Gram-negative.

The type species is Cellulomonas biazotea (Kellerman) Bergey et al.

Key to the species of genus Cellulomonas.

I. Motile with peritrichous flagella.

A. Gelatin liquefied. Chromogenic.

1. Milk acid.

a. Ammonia not produced; indole not formed.

1. Cellulomonas biazotea.

2. Milk acid; digested.

a. Ammonia produced; indole not formed.

2. Cellulomonas aurogenes.

aa. Ammonia produced; indole formed.

3. Milk alkaline.

3. Cellulomonas galba.

a. Ammonia produced; indole not formed.

4. Cellulomonas folia.

4. Litmus milk unchanged.

¿. Ammonia produced; indole not formed.

5. Cellulomonas flara.

B. Gelatin liquefied. Non-chromogenic.

1. Milk acid.

a. Ammonia not produced; indole not formed.

6. Ccllulomonas cellasea.

an. Ammonia produced; indole not formed.

7. Cellulomonas ingis.

aar. Ammonia not produced; indole formed.

2. Milk acid; digested.

S. Cellulomonas concitata.

a. Ammonia produced; indole not formed.

9. Cellulomonas caesia.

C. Gelatin not liquefied. Chromogenic.

1. Milk acid.

a. Ammonia produced; indole formed.

2. Milk alkaline.

10. Cellulomonas gilva.

a. Ammonia not produced; indole not formed.

11. Cellulomonas ferrugineu.

D. Gelatin not liquefied. Non-chromogenic.

1. Milk acid; not digested.

a. Ammonia not produced; indole not formed.

12. Cellulomonas albida.

13. Cellulomonas alma.

aa. Ammonia not produced; indole formed.

14. Cellulomonas desidiosa.

aaa. Ammonia produced; indole not formed. 
15. Cellulomonas pusilla.

16. Cellulomonas gelida.

II. Motility not recorded.

A. Gelatin liquefied. Chromogenic.

1. Milk arid.

a. Ammonia not produced. Acid from glucose.

17. Cellulomonas flavigena.

a a. Ammonia produced. No acid from carbohydrates.

18. Cellulomonas rossica.

1. Cellulomonas biazotea (Kellerman et al.) Bergey et al. (Bacillus biazolous Kellerman, MrBeth, Scales and Smith, Cent. f. Bakt., II .1\}t., 39, 1913, 506; Bergey et al., Manual, Ist ed., 1923, 158; Protens cellulomonas var. Proteus biazoleus Pribram, Klassifikation der Schizonyerten, Leipzig und Wien, 193:3, 72.)

This is the type species of the genus Cellulomonas.

Rods: 0.5 by 0.8 micron. Notile with one to three peritrichous flagella. Gram-negative.

Gelatin stab: Liquefaction.

- Agar slant: Luxuriant yellow growth.

Cellulose agar: Enzymatic zone 0.25 $\mathrm{mm}$ or less in width.

Peptone cellulose agar: To enzymatic zone.

Broth: Turbid.

Litmus milk: Acid. No curdling or digestion.

Potato: Crows well.

Indole not formed.

Nitrites produced from nitrates.

Ammonia not produced.

Acid from glucose, maltose, lactose, sucrose, and glyeerol. No acid from mannitol.

Aerobir, fucultative.

Optimum temperature $20^{\circ} \mathrm{C}^{\mathrm{C}}$.

Source: Soil from Ltah.

Hebitat: Soil.

2. Cellulomonas aurogenes (Kollermu (t al.) Bergey et al. (Bacillus aurogenes Kellerman, McBeth, Seales and Smith. Cent. f. Bakt., II Abt., 39, 1913, 505; Bergey et al., Manual, lst nd., 1923,
157.) From Latin and Greek, gold-producing.

Rods: 0.4 by $1 . t$ mierons. Motile with one to three peritrichous flagella. Gram-negative.

Gelatin stab: Liquefaction.

Agar slant: Luxuriant yellow growth. Cellulose agar: Enzymatic zone 0.5 to $1.5 \mathrm{~mm}$ wide.

Broth: Turbid.

Litmus milk: Acid, digested.

Potato: Iuxuriant growth.

Indole not formed.

Nitrites produced from nitrutes.

Ammonia produced.

Aeid from glucose, maltose, lactose, sucrose, stareh and glycerol. No acid from mannitol.

Aerobic, facultative.

Optimum temperature $20^{\circ} \mathrm{C}$.

Source: From soil from Louisiana and Maine.

Habitat: s'oil.

3. Cellulomonas galba (Kellerman et al.) Bergey et al. (Bacillus galbus Kiellerman, McBeth, Scules and Smith, Cent. f. Bakt., II Abt., 39, 1913, 509; Bergey et al., Manual, 1st ed., 1923, 157.) From Latin galbus, yellow.

Rods: $0 . \pm$ by 1.0 micron. Intile with one to three peritrichous flagella. Gram-negative.

Gelatin stab: Iiquefisction.

Agar slant: Luxuriant yellow growth. Cellulose agar: Enzymatic zone $0.5 \mathrm{~mm}$ in width.

Broth: Turbid.

Litmus milk: Acid, digested.

Potato: No growth.

Indole is formed. 
Nitrites not produced from nitrates. Ammonia produced.

Acid from glucose, maltose, lactose, sucrose, starch and glycerol. No acid from mannitol.

Aerobic, facultative.

Optimum temperature $20^{\circ} \mathrm{C}$.

Source: Soil from Louisiana.

Habitat: Soil.

4. Cellulomonas folia Sanborn. (Jour. Bact., 12, 1926, 1 and 343.) From Latin folium, leaf.

Description from Sanborn (Jour. Bact., 18, 1929, 170) and also from his unpublished notes.

Rods: 0.8 to 1.0 by 1.0 to 1.5 microns, occurring singly and in short chains. Motile with four to six peritrichous flagella. Gram-negative.

Gelatin stab: Slow crateriform liquefaction, becoming stratiform.

Agar slant: Growth moderate, dirtywhite, echinulate, raised, glistening, opaque, butyrous.

Broth: Turbid with yellowish sediment.

Litmus milk: Alkaline.

Potato: Thick, moist, yellowish-brown growth.

Indole not formed.

Nitrites produced from nitrates.

Acid and gas slowly produced from glucose, sucrose, glycerol and mannitol after prolonged incubation. No acid or gas from lactose.

Starch hydrolyzed.

Ammonia produced.

No $\mathrm{H}_{2} \mathrm{~S}$ formed.

Aerobic, facultative.

Optimum temperature $25^{\circ}$ to $30^{\circ} \mathrm{C}$.

Resembles Cellulomonas rossica.

Source: From decomposing leaves.

Habitat: Occurring in soil and active in decomposing leaves in composts, having the property of digesting cellulose.

5. Cellulomonas flava Sack. (Cent. f. Bakt., II Abt., 62, 1924, 79.) From Latin flavus, yellow.

Rods: 0.2 by 1.5 microns. Motile. Gram-negative.
Gelatin colonies: Circular, citron yellow.

Gelatin stab: Very slow liquefaction. Agar colonies: Large, circular, citron yellow.

Agar slant: Abundant, citron yellow streak.

Broth: Turbid with pellicle and sediment.

Litmus milk: Unchanged.

Potato: Light brown streak.

Indole not formed.

Nitrites and ammonia produced from nitrates.

Hydrogen sulfide produced.

Cellulose hydrolyzed.

Aerobic, facultative.

Optimum temperature $20^{\circ} \mathrm{C}$.

Habitat: Soil.

6. Cellulomonas cellasea (Kiellerman et al.) Bergey et al. (Bacillus cellaseus Kellerman, McBeth, Scales and Smith, Cent. f. Bakt., II Abt., 39, 1913, 508; Bergey et al., Manual, 1st ed., 1923, 158.)

Rods: 0.5 by 1.2 microns. Motile with one to three peritrichous flagella. Gram-negative.

Gelatin stab: Liquefaction.

Agar slant: Limited grayish growth .

Cellulose agar: Enzymatic zone $0.5 \mathrm{~mm}$ or less.

Broth: Clear.

Litmus milk: Acid.

Potato: No growth.

Indole not formed.

Nitrites not produced from nitrates.

Ammonia not produced.

Acid from glucose, maltose, lactose, sucrose, starch, glycerol and mannitol.

Aerobic, facultative.

Optimum temperature $20^{\circ} \mathrm{C}$.

Soures: Soil from Ltah.

Habitat: Soil.

7. Cellulomonas iugis (MeBeth) Bergey et al. (Bacillus iugis MeBeth, Soil Seience, 1, 1916, 456; Bergey et al., Manual, 1st ed., 1923, 158.) From Latin, joined together.

Rods: 0.4 by 1.4 microns. Motile with 
one to three peritrichous flagella. Gram-negative.

Gelatin stab: Napiform liquefaction.

Agar colonies: Circular, convex, soft, grayish-white, granular, entire.

Agar slant: Scant, grayish-white, filiform growth.

Ammonia cellulose agar: After 20 days, all colonies show an enzymatic zone of $1 \mathrm{~mm}$ or more.

Peptone cellulose agar: Enzymatic zone continues to increase up to 30 days at which time it may reach $5 \mathrm{~mm}$ in width.

Broth: Turbid.

Filter paper broth: After 15 days, the paper shows many ragged holes but disintegrates readily.

Litmus milk: Acid, not digested.

Potato: Abundant, glistening, grayish-white growth.

Indole not formed.

Nitrites produced from nitrates.

Ammonia produced.

Acid from glucose, maltose, lactose, sucrose, starch, glycerol and mannitol.

Aerobic, facultative.

Optimun temperature $20^{\circ} \mathrm{C}$.

Source: Soil from Citlifornia.

Habitat: Soil.

\section{Cellulomonas concitata (McBeth)}

Bergey et al. (Bacillus concitatus MeBeth, Soil Science, 1, 1916, 448; Bergey et al., Manual, 1st ed., 1923, 159.) From Latin concitatus, rapid.

Rods: 0.5 by 1.2 microns. Motile with one to four peritrichous flagella. Gram-negative.

Gelatin stab: Napiform liquefaction.

Agar colonies: Irregularly circular, decidedly convex, soft, becoming viscid, grayish-white, sometimes slightly fluorescent, granular, entire.

Agar slant: Abundant, flat, moist, faint yellowish-white growth.

Ammonia celiulose agar: Surface colonies show an enzymatic zone of 1.0 to $1.5 \mathrm{~mm}$. Deep colonies no zone but colony somewhat clearer than surrounding medium.
Peptone cellulose agar: Enzymatic zone, surface colonies, 2 to $2.5 \mathrm{~mm}$; bottom colonies, $1 \mathrm{~mm}$ or less.

Broth: Turbid.

Filter paper broth: In 15 days, the paper is a disintegrated fibrous mass which retains its pure white color.

Litmus milk: Acid, not digested.

Potato: No growth.

Indole is formed.

Nitrites not produced from nitrates.

Ammonia not produced.

Acid from fructose, maltose, lactose, sucrose, starch and glycerol.

Aerobic, facultative.

Optimum temperature $20^{\circ} \mathrm{C}$.

Source: Soil from California.

Habitat: Soil.

9. Cellulomonas caesia (Kellerman et al.) Bergey et al. (Bacillus caesius Kellerman, McBeth, Scales and Smith, Cent. f. Bakt., II Abt., 39, 1913, 507; Cellulomonas caseia (sic) Bergey et al., Manual, 1st ed., 1923, 159; Cellulomonas casei (sic) Bergey et al., Manual, tth ed., 1934, 199.) From Latin caesius, bluishgray

Rods: 0.4 by 1.5 microns. Motile with one or two peritrichous flagella. Gramnegative.

Gelatin stab: Liquefaction.

Beef agar streak: Moderate, flat, thin growth, slightly bluish fluorescence.

Cellulose agar: Enzymatic zone, 0.5 to $1.0 \mathrm{~mm}$ in 15 days.

Broth: Turbid. Slight sediment in 5 days.

Litmus milk: Acid, digested.

Potato: No growth.

Indole not formed.

Nitrites produced from nitrates.

Ammonia produced.

Acid from glucose, maltose, lactose, sucrose, starch, glycerol and mannitol.

Aerobic, facultative.

Optimum temperature $20^{\circ} \mathrm{C}$.

Source: Soil from Louisiana, Wisconsin and New Hampshire.

Habitat: Soil. 
10. Cellulomonas gilva (MeBeth) Bergey et al. (Bacillus gilous MeBeth, Sorl Science, 1, 1916, 453; Bergey et ial.. Manual, 1st ed., 1923, 160.) From Latin gilvus, pale yellow.

Rods: 0.5 by 1.5 microns. Motile with one to five peritrichous flagella. Gram-negative.

Gelatin stab: Moderate, yellowishwhite surface growth. No liquefaction.

Agar colonies: Circular, convex, butyrous, canary-yellow, sometimes with brownish rings, granular, entire.

Agar slant: Filiform, yellowish-white growth.

Ammonia cellulose agar: Enzymir zone not more than $1 \mathrm{~mm}$. Entire colony semitransparent

Peptone cellulose agar: Enzymutis zone, 3 to $4 \mathrm{~mm}$ in 25 days.

Broth: Slightly turbid.

Filter paper broth: In 15 days, the paper is reduced to a thin, white filmy mass which disintegrates readily.

Litmus milk: Acid, not digested.

Potato: Abundant, canary-yollow growth.

Indole is formed.

Nitrites produced from nitrates.

Ammonia is produced.

Acid from glucose, maltose, lactose, sucrose, starch and glyeerol. No arid from mannitol.

Aerobie, facultative.

Optimum temperature $200^{\circ} \mathrm{C}$

Source: Soil from Californiat.

Habitat: Soil.

11. Cellulomonas ferruginea (Rullmann) Bergey et al. (Bacillus ferrugineus Rullmann, Cent. f. Bakt., I Abt. Orig., 24, 1898, 465; van Iterson, Cent. f. Bakt., II Abt., 11, 1904, 694; not Bacillus ferrugineus Rullmann, C'ent. f Bakt., I Abt., 24, 1898, 467; Bergey ot al., Manual, 1st ed., 1923, 150.) From Latin, rust-colored.

Rods : 0.5 to 0.8 by 1.5 to 2.0 mierons, occurring singly. Motile, possessing peritrichous flagella. Gram-negative.
Gelatin colonies: Brown, the pigment diffusing into the medium.

Gelatin stab: No liquefaction.

Agar slant: Rusty-brown streak.

Broth: Turbid.

Litmus milk: Dark-yellow ring; alkaline.

Potato: Rusty-brown streak.

Indole not formed.

Nitrites not produced from nitrates.

Ammonia not produced.

Aerobic, facultative.

Optimum temperature $25^{\circ} \mathrm{C}$.

Habitat: Water.

12. Cellulomonas albida (McBeth) Bergey et al. (Bacillus albidus MeBeth, Suil Science, 1, 1916, 445; Bergey et al., Manual, 1st ed., 1923, 160.) From Latin albidus, white.

Rods: 0.4 by 1.0 micron. Motile with one to three peritrichous flagella. Gram-negative.

Gelatinstab: Scant growth. Noliquefaction.

Agar colonies: Circular, convex, soft, grayish-white, granular, entire.

Agar slant: Scant, white streak.

Ammonia cellulose agar: After 30 days the colonies show an enzymatic zone of 1 to $2 \mathrm{~mm}$.

Broth: Clear.

Filter paper broth: In 15 days, the paper is reduced to a thin, filmy, grayishwhite mass which readily breaks up.

Litmus milk: Slightly acid, not digested.

Potato: No growth.

Indole not formed.

Nitrites not produced from nitrates.

Ammonia not produced.

Acid from glucose, maltose, lactose, sucrose, starch, glycerol and mannitol.

Aerobic, facultative.

Optimum temperature $20^{\circ} \mathrm{C}$.

Source: Soil from California.

Habitat: Soil.

13. Cellulomonas alma (McBeth) Bergey et al. (Bacillus almus McBeth, Soil Science, 1, 1916, 446; Bergey et al., 
Manual, 1st ed., 1923, 161.) From Latin almus, nourishing.

Rods : 0.5 by 1.2 microns. Motile with one to five peritrichous flagella. Gramnegative.

Gelatin stab: Scant growth. No liquefaction.

Agar colonies: Circular, convex, soft, becoming brittle, grayish-white, granular, entire.

Ammonia cellulose agar: Enzymatic zone 3 to $4 \mathrm{~mm}$ in 25 days.

Peptone cellulose agar: Enzymatic zone 2.5 to $3.5 \mathrm{~mm}$ in 30 days.

Agar slant: Scant, grayish-white growth, becoming yellowish-irhite.

Broth: Slightly turbid.

Filter paper broth: Paper reduced to a loose felt-like white mass in 15 days.

Litmus milk: Slightly acid, not digested.

Potato: No growth.

Indole not formed.

Nitrites not produced from nitrates.

Ammonia not produced.

Acid from glucose, maltose, lactose, sucrose, starch and glycerol. No acid from mannitol.

Aerobic, facultative.

Optimum temperature $20^{\circ} \mathrm{C}$.

Source: Soil from California.

Habitat: Soil.

\section{Cellulomonas desidiosa (McBeth)} Breed. (Bacillus desiduosus (sic) McBeth, Soil Science, 1, 1916, 450; Cellulomonas deciduosa (sic) Bergey et al., Manual, 1st ed., 1923, 162; Breed, in Manual, 5th ed., 1939, 495.) From Latin desidiosus, inactive.

Rods: $0 . \pm$ by 1.0 micron. Motile with one to three peritrichous flagella. Gram-negative.

Gelatin stab: Moderate growth. No liquefaction.

Agar colonies: Circular, slightly convex, soft, becoming somewhat viscid, grayish-white, granular, entire.

Agar slant: Seant, flat, grayish-white growth.
Ammonia cellulose agar: Enzymatic zone 3 to $3.5 \mathrm{~mm}$ in 25 days.

Peptone cellulose agar: Enzymatic zone 1 to $2 \mathrm{~mm}$ around surface colonies. Bottom colonies frequently show no enzymatic zone until after 20 days.

Broth: Slightly turbid.

Filter paper broth: Paper is divided into gray white mass which readily disintegrates.

Litmus milk: Acid, not digested.

Potato: No growth.

Indole is formed.

Nitrites produced from nitrates.

Ammonia not produced.

Acid from glucose, lactose, maltose and starch. No acid from mannitol, sucrose or glycerol.

Aerobic, facultative.

Optimum temperature $20^{\circ} \mathrm{C}$.

Source: Soil from California.

Habitat: Soil.

15. Cellulomonas pusilla (Kellerman et al.) Bergey et al. (Bacillus pusilus (sic) Kellerman, McBeth, Scales and Smith, Cent. f. Bakt., II Abt., 39, 1913, 513; Cellulomonas pusila (sic) Bergey et al., Manual, 1st ed., 1923, 161.) From Latin pusilla, very small.

Rods: 0.6 by 1.1 microns. Motile with one to three peritrichous flagella. Gram-negative.

Gelatin stab: No liquefaction.

Agar slant: Scant, grayish-white growth.

Cellulose agar: Enzymatic zone $1 \mathrm{~mm}$ or less in width.

Broth: Turbid.

Litmus milk: Acid.

Potato: No growth.

Indole not formed.

Nitrites produced from nitrates.

Ammonia is produced.

Acid from glucose, maltose, lactose, sucrose, starch and glycerol. No acid from mannitol.

Aerobic, facultative.

Optimum temperature $20^{\circ} \mathrm{C}$. 
Source : Soil from District of Columbia and South Carolina.

Habitat: Soil.

16. Cellulomonas gelida (Kellerman et al.) Bergey et al. (Bacillus gelidus Kellerman, McBeth, Scales and Smith, Cent. f. Bakt., II Abt., 39, 1913, 510; Bergey et al., Manual, 1st ed., 1923, 162.) From Latin gelidus, stiff.

Rods: 0.4 by 1.2 microns. Motile with one to three peritrichous flagella. Gram-negative.

Gelatin stab: No liquefaction.

Agar slant: Luxuriant, grayish-white growth.

Cellulose agar: Enzymatic zone 1.5 $\mathrm{mm}$ in width.

Broth: Turbid.

Litmus milk: Acid, peptonized.

Potato: Grows well.

Indole not formed.

Nitrites not produced from nitrates.

Ammonia is produced.

Acid from glucose, maltose, lactose, sucrose, starch and glycerol. No acid from mannitol.

Aerobic, facultative.

Optimum temperature $20^{\circ} \mathrm{C}$.

Source: Soil from Connecticut.

Habitat: Soil.

17. Cellulomonas flavigena (Kellerman and MeBeth) Bergey et al. (Bacillus flavigena Kellerman and McBeth, Cent. f. Bakt., II Abt., 34, 1912, 488; Bergey et al., Manual, 1st ed., 1923, 165.) From Latin, yellow-producing.

Rods: 0.4 by 1.0 micron. Motility not recorded. Gram-negative.

Gelatin stab: Liquefaction.

Agar slant: Luxuriant, yellow growth.

Cellulose agar: Enzymatic zone 0.75 to $1.5 \mathrm{~mm}$ in width.

Broth: Turbid.

Litmus milk: Acid.

Potato: Grows well.

Indole not formed.

Nitrites produced from nitrates.

Ammonia not produced.

Acid from glueose, fructose, arabinose, xylose, maltose, lactose, sucrose, dextrin, starch, inulin, salicin, glycerol and mannitol.

Aerobic, facultative.

Optimum temperature $20^{\circ} \mathrm{C}$.

Source: From contaminated culture. Habitat: Soil.

18. Cellulomonas rossica (Kellerman and McBeth) Bergey et al. (Bacillus rossicus Kellerman and MeBeth, Cent. f. Bakt., II Abt., 34, 1912, 492; Bergey et al., Manual, 1st ed., 1923, 157; Proteus cellulomonas var. Proteus rossicus Pribram, Klassifikation der Schizomyceten, Leipzig und Wien, 1933, 72.)

Rods: 0.3 by 1.2 microns. Motility not recorded. Gram-negative.

Gelatin stab: Rapid liquefaction.

Agar slant: Luxuriant, yellow growth. Cellulose agar: Enzymatic zone 0.5 to $1.0 \mathrm{~mm}$ in width.

Broth: Turbid.

Litmus milk: Alkaline.

Potato: Grows well.

Indole not formed.

Nitrites produced from nitrates.

Ammonia produced.

No acid from carbohydrate media.

Aerobic, facultative.

Optimum temperature $20^{\circ} \mathrm{C}$.

Souree: From contaminated culture. Habitat: Soil.

Appendix II : The following cellulosedigesting bacteria are not included above:

Achromobacter picrum Fuller and Norman. (Jour. Bact., 46, 1943, 276.) From soil.

Bacillus aurogenes var. albus Kellerman, MeBeth, Scales and Smith. (Cent. f. Bakt., II Abt., 39, 1913, 506.) From soil from New York State. Differs from Cellulomonas aurogenes in that it shows no chromogenesis.

Bacillus rossicus var. castaneus Kellerman et al. (loc. cit., 508; Proteus cellulomonas var. Proteus castaneus Pribram, Klassifitation der Schizomyceten, Leipzig und Wien, 1933, 72.) From soils 
from Maine, Connecticut and New Fork. Peritrichous. No liquefaction of gelatin. Chestnut color on potato.

Bacillus subalbus Kellerman et al. (loc. cit., 512). From soils from Georgia, Kentucky and New York.
Bacillus subalbus var. batatatis Kellerman et al. (loc. cit., 513). From soil from South Carolina. Differs from the above species in that it liquefies gelatin and forms a very scant yellowish growth on potato.

Appendix III : The following genus has been proposed for Gram-negative rods that utilize bacterial polysaccharides as a sole source of carbon.

\section{Genus 1. Saccharobacterium Sickles and Shaw.}

$$
\text { (Jour. Bact., 28, 1934, 430.) }
$$

Pleomorphic, non-motile, non-spore-forming rods. Gram-negative. Grow in mineral solutions containing bacterial polysaccharides as the sole source of carbon. Found in swamp and other uncultivated soils. Placed by the authors in the Family Mycobacteriaceae because of resemblances between these bacteria and those placed in Cytophaga, Cellfalcicula and Cellvibrio. As the latter genera are no longer placed in this family, Saccharobacterium is placed temporarily in this appendix to the genus Bacterium near bacteria that decompose cellulose and agar.

The type species is Saccharobacterium ovale Sickles and Shaw.

1. Saccharobacterium ovale Sickles and Shaw. (Jour. Bact., 28, 1934, 422.) From Latin ovum, egg, ellipse.

Extremely pleomorphic, Foung cells ellipsoidal, 1.5 by 2.0 microns, usually in pairs, contain granules which stain deeply with basic dyes. Older cultures contain cells which may be from 12 to 15 microns long. Non-motile. Gramnegative.

No growth on ordinary media such as beef-extract agar, blood agar, beef-extract agar slants, nutrient gelatin, potato slants, litmus milk, beef-infusion broth and beef-extract peptone broth.

Medium A plus pneumococcus II carbohydrate and 0.8 per cent agar: Very small, round, pink colonies, pinpoint in size after about 5 days. After 2 weeks $1 \mathrm{~mm}$ in diameter. Coherent.

\section{Litmus milk: No growth.}

Beef-extract peptone with 1 per cent sucrose: Moderate turbidity. Yellowish sediment.

Starch: Hydrolyzed in Medium A containing pneumococcus II carbohydrate. Growth in lactose and sucrose broths.
Growth in maltose, xylose and dextrin broths in some strains. No acid from inulin, mannitol, saliein and glucose broths.

Aerobic.

Minimum temperature $20^{\circ} \mathrm{C}$. Optimum $34^{\circ}$ to $35^{\circ} \mathrm{C}$. Maximum $37^{\circ} \mathrm{C}$. Thermal death point $54^{\circ} \mathrm{C}$ for 10 minutes.

Minimum pH 6.4. Optimum pH 7.0 to 7.4. Maximum $\mathrm{pH}$ 7.8.

Distinctive characters: The addition of 0.5 per cent sodium chloride to any favorable medium completely prevents growth of the organism (Medium A is that used by Dubos and Avery in 1931, $\left(\mathrm{NH}_{4}\right)_{2} \mathrm{SO}_{4}, 1 \mathrm{~g}, \mathrm{~K}_{2} \mathrm{HPO}_{4}, 2.0 \mathrm{~g}$, tap water $1000 \mathrm{ml}$ ). Decomposes the carbohydrate of pneumococcus type II.

Source: Swamps and other uncultivated soils.

Habitat: Soil.

2. Saccharobacterium acuminatum Sickles and Shaw. (Jour. Bact., 28, 1934, 425.) From Latin acuminare, to sharpen.

Extremely pleomorphic. Young organisms are pointed, often curved rods, 
0.5 by 2 microns, having a densely staining granule. The tapering pointed ends remain unstained. Older cells have rounded ends, are spherical, pearshaped or a long ellipsoid, stain weakly. Non-motile. Gram-negative.

No growth on ordinary media. See preceding species.

Medium S with pneumococcus I carbohydrate and 0.8 per cent agar: Very tiny, pale yellow colonies. Less than $0.5 \mathrm{~mm}$ in diameter.

Starch not hydrolyzed.

Growth in sucrose broth. No growth in glucose, lactose, maltose dextrin, inulin, mannitol and salicin broths.

Aerobic.

Minimum temperature $20^{\circ} \mathrm{C}$. Optimum $28^{\circ}$ to $32^{\circ} \mathrm{C}$. Maximum $34^{\circ} \mathrm{C}$. Thermal death point $48^{\circ} \mathrm{C}$ for 10 minutes.

Minimum pH 6.0. Optimum pH 6.6 to 7.2. Maximum $\mathrm{pH} 7.8$.

Distinctive characters: Decomposes the carbohydrate of pneumococcus Type I. The addition to any favorable medium of 0.7 per cent sodium chloride, of 0.3 per cent beef extract or of 0.5 per cent peptone completely inhibits grow th.

The composition of Medium $\mathrm{S}$ is as follows: $\mathrm{MgSO} \cdot 7 \mathrm{H}_{2} \mathrm{O}, 0.2 \mathrm{~g}, \mathrm{NH}_{4} \mathrm{H}_{2} \mathrm{PO}_{4}$, $1.5 \mathrm{~g}, \mathrm{CaCl}_{2}, 0.1 \mathrm{~g}, \mathrm{FeCl}_{3}$, tr, $\mathrm{KCl}, 0.1 \mathrm{~g}$, 10 ce $\mathrm{N} / 1 \mathrm{NaOH}$. Distilled water 1,000 $\mathrm{ml}, \mathrm{pH} 7.2$ to 7.4 . To this was added the specific pneumococcus carbohydrate as a source of carbon in concentrations varying from 0.002 to 0.01 per cent.

Source: From swamps and other uncultivated soils.

Habitat: Soil.

28. Bacterium nenckii Biernacki. (Biernacki, Cent. f. Bakt., II Abt., 29, 1911, 166; Achromobacter nenckii Bergey et al., Manual, 3rd ed., 1930, 227.) Named for Nenchi, a chemist at the Medical Institute in Warsaw.

Rods: 0.8 by 1.25 to 2.0 microns, with rounded ends, occurring singly and in pairs. Capsulated. Non-motile. Gram-negative.
Gelatin colonies: Circular, convex, yellowish-white, granular.

Glucose and sucrose gelatin: Colonies larger and slimy.

Gelatin stab: No liquefaction.

Agar colonies: Circular, grayish-white, glistening, concentric, finely granular.

Agar slant: The medium is liquefied.

Glucose and sucrose agar: Heavy slimy growth with gas. Faint fruity odor.

Broth: Slightly turbid with gray sediment and slight odor.

Litmus milk: Acid and gas formation.

Potato: Slight growth.

Glycerol potato: Heavy growth with the appearance and consistency of cream.

Indole not formed.

Nitrites not produced from nitrates.

Acid and gas from glucose, fructose, galactose, maltose, sucrose, raffinose and mannitol.

Fruity odor in cultures.

Facultative anaerobe.

Optimum temperature $37^{\circ} \mathrm{C}$.

Source: From Spanish dried grapes. Irabitat: Unknown.

29. Bacterium polysiphoniae Lundestad. (Lundestad, Cent. f. Bakt., II Abt., 75, 1928, 331; Flavobacterium polysiphoniae Bergey et al., Manual, 3rd ed., 1930, 152.) From Greek, many tubes.

Rods: 0.5 to 0.6 by 2.0 to 4.0 microns, with rounded ends, occurring singly. Non-motile. Gram-negative.

Fish-gelatin colonies: Circular, slightly glistening, bright yellow, transparent, with denser center.

Fish-gelatin stab: Slight yellowish growth on surface. Slow saccate liquefaction.

Seq-weed agar colonies: Circular, flat, with concentric rings, diffuse margin, light yellow. Agar is disintegrated.

Fish-agar slant: Yellow, flat growth, with undulate margin.

Broth: Turbid with flocculent pellicle and yellowish sediment.

Indole not formed. 
Nitrites not produced from nitrates. No action on carbohydrates.

Slight hydrolysis of starch.

Aerobic, facultative.

Optimum temperature $30^{\circ} \mathrm{C}$.

Source: Sea water of Norwegian Coast.

Habitat: Sea water.

30. Bacterium droebachense Lundestad. (Lundestad, Cent. f. Bakt., II Abt., 1928, 329; Flavobacterium droe bachense Bergey et al., Manual, 3rd ed.. 1930, 153; Pseudomonas droebachense Stanier, Jour. Bact., 42, 1941, 54t.) Latinized, from Dröbak, where this organism was isolated.

Rods : 0.5 to 0.6 by 2.0 to 2.6 microns, with rounded ends, occurring singly. Non-motile. Gram-negative.

Fish-gelatin colonies: Small, circular. compact, opaque, glistening, orange yellow.

Fish-gelatin stab: Liquefaction in fundibuliform becoming stratiform.

Sea-weed agar colonies: Small, circu lar, flat, opaque, glistening, orangeyellow. Agar is disintegrated.

Fish-agar slant: Flat, opaque, glistening, slimy, orange-yellow, entire growth.

Broth: Slight flocculent turbidity yellow.

Indole not formed.

Nitrites not produced from nitrates.

Starch hydrolyzed.

Slow growth on surface of glucose agat stab. No gas.

Aerobic, facultative.

Optimum temperature about $37^{\circ} \mathrm{C}$. Minimum temperature $5^{\circ}$ to $10^{\circ} \mathrm{C}$. Maximum $40^{\circ} \mathrm{C}$.

Stanier (loc. cit.) identified cultures isolated from sea water on the Pacific Coast as belonging to this species. Some liquefied gelatin while others did not. Nitrates were reduced. A yellow membraneous pellicle was formed on broth, and the temperature range is given as $5^{\circ}$ to $35^{\circ} \mathrm{C}$. Optimum $25^{\circ} \mathrm{C}$. He renamed the organism Pseudomonas droebachensis, but reported it nonmotile.

Source: From sea water at Dröbak on the Norwegian Coast.

Habitat: Sea water.

31. Bacterium delesseriae Lundestad. (Lundestad, Cent. f. Bakt., II Abt., $75,1928,332$; Flavobacterium delesseriae Bergey et al., Manual, 3rd ed., 1930, 153.)

Rods : 0.5 to 0.6 by 1.6 to 2.6 microns, with rounded ends, occurring singly. Non-motile. Gram-negative.

Fish-gelatin colonies: Circular, transparent, glistening, concentrically ringed, vellow.

Fish-gelatin stab: Crateriform liquefaction, with yellow sediment.

Sea-weed agar colonies: Circular, flat, concentrically ringed, light yellow. Agar is disintegrated.

Fish-agar slant: No growth.

Broth: Turbid with flocculent pellicle and sediment, light yellow.

Indole not reported.

Nitrites not reported.

No action on carbohydrates.

Slight hydrolysis of stareh.

Aerobic, facultative.

Optimum temperature $23^{\circ} \mathrm{C}$.

Source: Sea water of Norwegian Coast.

Habitat: Sea water.

32. Bacterium boreale Lundestad. (Lundestad, Cent. f. Bakt., II Abt., 75, 1928, 333; Flavobacterium boreale Bergey et al., Manual, 3rd ed., 1930, 154.) From Latin borealis, northern.

Rods: 0.5 to 0.6 by 1.6 to 2.6 microns, with rounded ends, occurring singly. Non-motile. Gram-negative.

Fish-gelatin colonies: Circular, opaque, glistening, concentrically ringed, yellow.

Fish-gelatin stab: Yellow, with crateriform liquefaction.

Sea-water agar colonies: Circular, flat, opaque, glistening, diffuse margin, light yellow. Agar is disintegrated. 
Fish-agar slant: Yellow, flat, glistening, opaque, entire growth.

Broth: Finely flocculent, yellow sediment.

Indole not reported.

Nitrites not reported.

No action on carbohydrates.

Slight hydrolysis of starch.

Aerobic, facultative.

Optimum temperature $23^{\circ} \mathrm{C}$

Source: Sea water of Norwegian Coast.

Habitat: Sea water.

33. Bacterium ceramicola Lundestad. (Lundestad, Cent. f. Bakt., II Abt., 75 , 192S, 332; Flavobacterium ceramicola Bergey et al., Manual, 3rd ed., 1930, 154.) From Greek, living in earthenware.

Rods : 0.5 to 0.6 by 1.4 to 2.4 microns, with rounded ends, occurring singly and Iying side-by-side. Jon-motile. Crramnegative.

Fish-gelatin colonies: Circular, glistening, transparent, yellow.

Fish-gelatin stab: Slight, yellow surface growth. Liquefaction crateriform.

Sea-water agar colonies: Circular, flat, transparent, glistening, diffuse margin, light yellow. Agar is disintegrated.

Fish-agar slant: Moderate, yellow, flat, entire, glistening, opaque growth.

Broth: Light yellow pellicle and sediment.

Indole not reported.

Nitrites not reported.

No action on carbohydrates.

Slight hydrolysis of starch.

Aerobie, facultative.

Optimum temperature $23^{\circ} \mathrm{C}$.

Source: Sea water of Norwegian Coast.

Habitat: Sea water.

34. Bacterium rhodomelae Lundestad. (Lundestad, Cent. f. Bakt., II Abt., 75, 1928, 331; Flavobacterium rhodomelae Bergey et al., Manual, 3rd ed., 1930, 146.)

Rods : 0.5 to 0.8 by 1.2 to 2.0 microns, with rounded ends, occurring singly, in pairs, and at times in short chains. Motile. Gram-negative.

Fish-gelatin colonies: Circular, slightly glistening, opaque, white.

Fish-gelatin stab: Rapid infundibuliform liquefaction.

Sea-weed agar colonies: Circular, flat, thin, transparent, glistening, entire. Agar is dissolved.

Glucose agar slant: Moderate growth, white, becoming orange-yellow, flat, undulate margin, opaque, glistening.

Broth: Turbid, with pellicle and grayish-ycllow, slimy sediment.

Indole not formed.

Nitrites not produced from nitrates.

No action on carbohydrates.

Very slight hydrolysis of stareh.

Aerobic, facultative.

Optimum temperature $20^{\circ}$ to $25^{\circ} \mathrm{C}$.

Source: Sea water of Norwegian Coast.

Habitat: Sea water.

35. Bacterium alginovorum Waksman, Carey and Allen. (Jour. Bact., 28, 1934, 215.) From M. L., alginic and Latin voro, devour.

Rods: 0.75 to 1.2 by 1.5 to 2.0 microns, with rounded to almost elliptical ends, especially when single, occurring frequently in pairs and even in chains. Actively motile. Capsule-forming. Gram-negative.

Alginic acid plate: Colony large, white in appearance with coarse granular center, entire margin. Clears up turbidity caused by the alginic acid on plate. No odor.

Alginic acid licuid medium: Heavy pellicle formation. Active production of an enzyme, alginase, which brings about the disappearance of alginic precipitate in sea water medium.

Salt water medium: A slimy pellicle of a highly tenacious nature is produced, the whole medium later turning to a soft jelly.

Sea water gelatin: Active and rapid liquefaction in two to six days, at $18^{\circ} \mathrm{C}$; 
highly turbid throughout the liquefied zone.

Agar liquefaction: Extensive softening of agar, no free liquid.

Sea water glucose broth: Abundant uniform turbidity, with surface pellicle; some strains give heavier turbidity and others heavier pellicle.

Litmus milk containing 3.5 per cent salt: No apparent growth.

Potato moistened with sea water: Moist, spreading growth, ivory-colored; heavy sediment in free liquid at the bottom.

Starch plate: Abundant, cream-colored, slimy growth; extensive diastase production.

Aerobic, microaerophilic.

Optimum temperature $20^{\circ} \mathrm{C}$.

Source: From sea water, sea bottom sediments and from the surface of algal growth in the sea.

Habitat: Very common in the ser.

36. Bacterium fucicola Waksman, Carey and Allen. (Jour. Bact., 28, 1934, 213.) From Latin fucus, seaweed and cola, dweller.

Short rods: 0.6 to $1.0 \mathrm{by} 1.0$ to 1.5 microns, with ends rounded to almost coccoid; slightly curved. Actively motile, with twirling motion. Gram-negative.

Alginic acid plate: Colonies finely granular, entire; at first whitish, turning brown in three to five days, and later almost black, producing a deep brown soluble pigment.

Alginic acid liquid medium: Limited growth on surface in the form of a pellicle. Frequently produces no growth at all.

Sea water gelatin: Active liquefaction; no growth in stab; thin, fluorescent growth throughout liquefied zone.

Agar liquefaction: Positive, although limited; only softening of agar.

Sea water glucose broth: Faint turbidity; no pellicle, no sediment.

Litmus milk containing salt: No apparent growth.

Potato moistened with sea water: No growth.

Starch plate: No growth.

Aerobic.

Optimum temperature $20^{\circ} \mathrm{C}$.

Source: From sea water near the surface of the sand bottom.

Habitat: Rare in sea water.

Appendix I: Additional agar-digesting bacteria placed in genera other than Bacterium.

I. Achromobacter.

A. Motile with peritrichous flagella.

1. Nitrites produced from nitrates.

1. Achromobacter pastinatar.

II. Agarbacterium.

A. Non-motile.

1. Nitrites produced from nitrates.

2. Agarbacterium bufo.

B. Motile, but position of flagella not recorded.

1. Nitrites produced from nitrates.

3. Agarbacterium reducans.

4. Agarbacterium viscosum.

2. Nitrites not produced from nitrates.

5. Agarbacterium mesentericus.

6. Agarbacterium aurantiacum.

7. Agarbacterium cyanoides.

3. Seven additional species that are numbered but not named. 


\section{Flavobacterium.}

A. Non-motile.

1. Nitrites produced from nitrates.

B. Peritrichous flagella.

8. Flavobacterium uliginosum.

1. Nitrites produced from nitrates.

9. Flavobacterium amocontactum.

1. Achromobacter pastinator Goresline. (Jour. Bact., 26, 1933, 442.) From Latin pastinator, one who digs a trench.

Short rods: 0.4 by 1.5 microns, occurring singly and in pairs. Motile with two to five peritrichous flagella. Gramnegative.

Plain gelatin stab: No growth.

Nutrient gelatin stab: Surface growth very scanty. No liquefaction.

Nutrient agar colonies: At first tiny, almost colorless, becoming yellowish and ring-like. Agar liquefied rapidly.

Nutrient agar slant: Growth good, flat, not thick. Agar liquefied along streak often to the depth of a quarter of an inch. Pocket formed at bottom of slant filled with a rather viseous, yellowish fluid.

Nutrient broth: Slight turbidity after 5 days. Subsurface but no surface growth. No sediment.

Litmus milk: Slightly aceid after 201 days. No curd. Only a trace of reduction at bottom of tube.

Potato: No growth

Indole not formed.

Nitrites produced from nitrates.

No $\mathrm{H}_{2} \mathrm{~S}$ produced.

Acid from arabinose, glucose, galac tose, lactose, fructose, maltose, mannose, melezitose, pectin, raffinose, rhamnose. salicin, sucrose, stareh and dextrin. No growth in duleitol, erythritol, manni tol, sorbitol, glycerol, xylose and inulin.

Stareh is hydrolyzed.

Limits of growth: pH 5.9 to 9.0 .

Temperature relations: Optimum $28^{\circ} \mathrm{C}$. Good growth at $25^{\circ} \mathrm{C}$. Moderate growth at $20^{\circ}$ and $37^{\circ} \mathrm{C}$. No growth at $10^{\circ}$ and $42^{\circ} \mathrm{C}$.

Facultative anierohe.

Distinctive characters: Digests agar rapidly; colonies sink through to the glass of the Petri dish. Fehling's solution reduced by the liquefied agar. Considerable change in viscosity of agar due to the digestion.

Source: From a trickling filter receiving ereamery wastes.

Habitat: Probably widely distributed in uature.

2. Agarbacterium bufo Angst. (Puget Sound Biol. Sta. Pub., 7, 1929, 49.)

Short rods with rounded ends, 0.6 by 0.5 mieron, oecurring singly and in pairs. Ton-motile. No capsules. Gram-negative.

Fish gelatin stab: Stratiform liquefaction, growth best at top.

Fish gelatin colonies: Circular, erateriform, granular.

Fish agar slant: Abundant, filiform, raised, glistening, opaque, yellow, membranous grow th.

Fish agar colonies: Circular, concentrically ringed, sunken, entire, granular, yellow to orange.

Digests agar; cellulose not attacked.

Potato: No growth.

Plain milk unchanged, surface growth yellow.

Does not produce $\mathrm{H}_{2 \mathrm{~S}}$ or indole.

Nitrites produced from nitrates.

Acid from mannitol. No acid from xylose, rhimnose, arabinose, glucose, sucrose or lactose.

Starch not hydrolyzed.

Aerohic.

Optimum temperature $25^{\circ}$ to $28^{\circ} \mathrm{C}$. Maximum under $36^{\circ} \mathrm{C}$.

Source: Isolated from Odonthalia kamtschatica.

Habitat: On marine algae. 
3. Agarbacterium reducans Angst. (Puget Sound Biol. Sta. Pub., 7, 1929, 49.)

Short rods with rounded ends, 0.6 by 0.8 micron, occurring singly and in pairs. Motile. No capsules. Gram-negative.

Fish gelatin colonies : Circular, sunken, entire, crateriform, granular.

Fish gelatin stab: Crateriform liquefaction, growth only near surface.

Fish agar slant: Abundant, filiform, flat, glistening, smooth, opaque, white, butyrous growth.

Fish agar colonies: Moderate, circular, smooth, flat, entire, granular, white to buff or colorless.

Digests agar; cellulose not attacked.

Fish broth: Turbid, no sediment, no surface growth.

Potato: No growth.

Nitrites produced from nitrates.

No $\mathrm{H}_{2} \mathrm{~s}$ or indole formed.

Plain milk unchanged.

Acid from sucrose, arabinose, rhamnose and mannitol. No acid from xylose or lactose.

Starch is hydrolyzed.

Aerobic.

Optimum temperature $25^{\circ}$ to $28^{\circ} \mathrm{C}$; thermosensitive.

Source: Isolated from Nercocystis luetkeana.

Habitat: On marine algae.

\section{Agarbacterium viscosum Angst.} (Puget Sound Biol. Sta. Pub., 7, 1929, 49.)

Short rods with rounded ends, 0.6 to 0.8 micron, occurring singly or in pairs. Motile. No capsules. Gram-negative.

Fish gelatin colonies : Circular, sunken, entire, crateriform, granular.

Fish gelatin stab: Stratiform liquefaction, grow th best at surface.

Fish agar slant: Abundant, raised, glistening, smooth, opaque, gray, vesicular, viscid growth.

Fish agar colonies: Circular, contoured, raised, lobate, granular, fluorescent green.

Digests agar; cellulose not attacked.
Fish broth: Flocculent pellicle, turbid, no sediment, fluorescent green.

Potato: Abundant, filiform, glistening, smooth, yellowish-brown, butyrous growth.

Nitrites produced from nitrates.

No $\mathrm{H}_{2} \mathrm{~S}$ or indole formed.

Plain milk unchanged; surface growth greenish.

No acid from rhamnose, sucrose, lactose, mannitol, xylose or arabinose.

Starch is hydrolyzed.

Aerobic.

Optimum temperature $20^{\circ}$ to $28^{\circ} \mathrm{C}$; thermosensitive.

Source: Isolated from Iridaea cordata. Habitat: On marine algae.

5. Agarbacterium mesentericus Angst. (Puget Sound Biol. Sta. Pub., 7, 1929, 49.)

Short rods with rounded ends, 0.6 by 0.8 micron, occurring singly or in pairs Motile. No eapsules. Grom-negative.

Fish gelatin stab: Infundibuliform liquefaction; growth best at top.

Cielatin rolonies: Circular, sunken, irregular, crateriform, granular.

Fish agar slant: 1bundant, filiform, raised, glistening, finely wrinkled when old or dry, opaque, buff, membranous growth.

Fish agar colonies: Circular, concentrically ringed, flat, entire, granular, white to buff.

Digests agar; cellulose not attacked.

Fish broth: Membranous pellicle, moderate clouding, no sediment.

Potato: Spreading, raised, glistening, wrinkled, buff to yellowish, membranous growtb.

Does not produce $\mathrm{H}_{2}$ or indole.

Nitrites not produced from nitrates.

Plain milk unchanged.

Acid from mannitol. No acid from xylose, rhamnose, arabinose, glucose or lactose.

Starch is hydrolyzed.

Aerobic.

Optimum temperature $20^{\circ}$ to $28^{\circ} \mathrm{C}$; thermosensitive. 
Source: Marine algae; isolated from Nercocystis luetkeana.

Habitat: On marine algae.

\section{Agarbacterium aurantiacum Angst.} (Puget Sound Biol. Sta. Pub., 7, 1929, 49.)

Short rods with rounded ends, 0.6 to 0.8 micron, occurring singly or in pairs. Motile. No capsules. Gram-negative.

Fish gelatin colonies: Circular, sunken, crateriform, entire.

Fish gelatin stab: Stratiform liquefaction, no growth along line of stab.

Fish agar slant: Abundant, filiform, flat, glistening, smooth, opaque, orange, butyrous growth.

Fish agar colonies: Circular, smooth, flat, erose, sunken, granular.

Digests agar; cellulose not attacked.

Fish broth: Membranous pellicle, turbid, no sediment.

Plain milk unchanged; surface growth orange.

Potato: Abundant, filiform, flat, dull, smooth, orange, butyrous growth.

Nitrites not produced from nitrates.

No $\mathrm{H}_{2} \mathrm{~S}$ or indole formed.

Acid from lactose and mannitol. No acid from xylose, rhamnose, arabinose, glueose or suerose.

Starch not hydrolyzed.

Aerobic.

Optimum temperature $20^{\circ}$ to $25^{\circ} \mathrm{C}$; thermosensitive.

Source: Isolated from Porphyia perforata.

Habitat: On marine algae.

\section{Agarbacterium cyanoides Angst.} (Puget Sound Biol. Sta. Pub., 7, 1929, 49.)

Short rods with rounded ends, $0.8 \mathrm{by}$ 1.4 microns, occurring singly or in pairs. Motile. No capsules. Gram-negative.

Fish gelatin colonies: Circular, sunken, entire, crateriform, granular.

Fish gelatin stab: Stratiform liquefaction, growth only at top.

Fish agar slant: Abundant, filiform, raised, glistening, smooth, opaque, gray, butyrous growth.
Fish agar colonies: Circular, smooth, flat, lobed, granular, greenish to yellowish.

Digests agar; cellulose not attacked.

Fish broth: Flocculent pellicle, turbid, no sediment, fluorescent green.

Potato: Abundant, filiform, raised, glistening, smooth, buff, butyrous growth.

Nitrites not produced from nitrates.

No H.S or indole formed.

Plain milk aridified, greenish surface growth.

Acid from sucrose. No acid from xylose, arabinose, glucose, lactose, mannitol or rhamnose.

Stareh is hydrolyzed.

Aerobic.

Optimum temperature $20^{\circ}$ to $28^{\circ} \mathrm{C}$; thermosensitive.

Source: Isolated from Iridaea cordata. Hahitat: On marine algae.

Note: Seven additional species are described with as much detail by Angst (loc. cit.) as are the six above species; but he refers to them only as Agarbucterium Nos. $5,6,7,8,9,13,14$, and 15 . All digest ngir.

8. Flavobacterium uliginosum ZoBell and Lpham. (Bull. Seripps Inst. of Oceanography, Univ. Calif., 5, 194., 263.) From Iatin uligo, ooze or moist mud.

Rods: 0.4 to 0.6 by 1.2 to 3.9 microns, some slightly curved, occurring mostly singly with some short chains. Nonmotile. Gram-negative.

All differential media except the freshwater broth, litmus milk, and potato were prepared with sea water.

Gelatin colonies: $1 \mathrm{~mm}$, orange, sunken.

Gelatin stab: Infundibuliform liquefaction. Yellow pigment. Gelatin discolored brown.

Agar colonies: Sunken, uneven, irregular, gumny colonies which liquefy agar. Produces orange to ycllow pigment and discolors agar brown.

Agar slant: Luxuriant, yellowish- 
orange, glistening, filiform, adherent growth which slowly liquefies agar.

Sea-water broth: Dense yellow pellicle, moderate turbidity, slightly viscid sediment.

Fresh-water broth: No visible growth.

Litmus milk: Completely decolorized, neutral.

Potato: సo visible growth.

Indole not formed.

Nitrates rapidly reduced to nitrites.

Produces acid but no gas from xylose, glucose, maltose, lactose, sucrose and salicin. Does not ferment glycerol or mannitol.

Starch not hydrolyzed.

Hydrogen sulfide not formed.

Ammonia produced from peptone but not from urea.

Casein digested.

Fats not hydrolyzed.

Agar liquefied rapidly. However, after prolonged laboratory cultivation this organism gradually loses its ability to digest agar.

Aerobic, obligate.

Optimum temperature $20^{\circ}$ to $25^{\circ} \mathrm{C}$.

Source: Marine bottom deposits.

9. Flavobacterium amocontactum $\mathrm{ZO}$ Bell and Allen. (Jour. Bact., 29, 1935, 246.) From Latin amo, to love and contactus, touching, contacting.

Slender rods: 0.4 to 0.7 by 1.6 to 2.3 microns, with rounded ends, occurring singly and in irregular clumps. Stain very lightly. Possess well-defined capsules. Actively motile by means of peritrichous flagella. Gram-negative.

Gelatin stab: Grood filiform growth with rapid saccate liquefaction.

Agar colonies: Circular, 2.0 to $4.0 \mathrm{~mm}$ in diameter, yellow.

Agar slant: Abundant, filiform, smooth, glistening, abundant, bright yellow growth having a butyrous consistency. Originally liquefied agar, but this property was lost following artificial cultivation.

Sea water broth: Good growth with ring at surface. Strong turbidity and abundant viscid sediment. No odor.
Nilk: No growth.

Potato: No growth.

Potato dialyzed in sea water: Slight yellow growth.

Indole not formed.

Nitrites produced from nitrates.

Ammonia liberated from peptone.

Hydrogen sulfide produced.

To acid from glucose, lactose, sucrose, xylose or mannitol.

Starch not attacked.

Optimum reaction $\mathrm{pH} 8.0$.

Optimum temperature $18^{\circ}$ to $21^{\circ} \mathrm{C}$.

Facultative aerobe.

Distinctive character: Adheres firmly to submerged glass slides; cannot be removed with running water.

Source: Many cultures isolated from glass slides submerged in sea water.

Habitat: Sea water.

Appendix II: Another species described recently is:

Bacillus exedens Wieringa. (Wieringa, Jour. Microbiol. and Serol., \%, 1911,121 ; Bacillus agar-exedens Wieringa, idem.) From stable manure, leafmold and soil. Liquefies agar.

37. Bacterium chitinophilum Hock. (Jour. Marine Res., 4, 1941, 103.) From M. L., chitin and Greek philos, loving.

Short rods: 0.35 to 0.65 by 0.95 to 1.5 microns. Motile. Gram-negative.

Sea water gelatin: Liquefaction; growth absent in stab but abundant in liquefied zone.

Sea water agar plate: Colonies circular, smooth, entire, raised, white.

Sea water liquid medium: Moderate growth, sometimes with formation of ring or pellicle. Scant granular sediment.

Decomposes natural chitinous material such as horseshoe crab shells and also purified chitin.

Four out of five strains produce nitrites from nitrates.

Acid from glucose and usually from sucrose, glycerol and mannitol. One of five cultures produced acid from lactose. Does not digest cellulose. 
Does not hydrolyze starch.

Does not produce hydrogen sulfide. Aerobic.

Optimum temperature $20^{\circ} \mathrm{C}$.

Source: From the shell of a decomposing horseshoe crab, Limulus polyphemus, and from the intestinal tracts of Venus mercenaria, Ovalipes ocellatus, Mustelus mustelus and Spheroides maculatus.

Habitat: Common in marine sand, mud and water.

38. Bacterium chitinochroma Hock. (Jour. Marine Res., 4, 1941, 105.)

Short rods: 0.45 to 0.75 by 0.90 to 1.4 microns. Motile. Gram-negative.

Sea water gelatin: Active liquefaction; no growth in stab, but thick bright yellow growth throughout the liquefied zone.

Basic agar plate: Colonies circular, smooth, entire, raised, varying in color from lemon to deep orange.

Basic liquid medium: Abundant growth with production of pellicle. Scant granular sediment, increasing with age of culture.

Decomposes natural chitinous material such as horseshoe crab shells and also purified chitin.

Does not produce nitrites from nitrates.

Acid from glucose and sucrose. but not lactose, glycerol and mannitol. Does not digest cellulose.

Hydrolyzes starch.

Does not produce hydrogen sulfide.

Aerobic.

Optimum temperature $20^{\circ} \mathrm{C}$.

Source: From the intestinal tract of the squid, Loligo pealeii. Common.

Habitat: Marine sand, mud and water.

Appendix I : The first species of chitinovorous bacteria that was deseribed and named was placed in the genus Bacillus because it was a motile rod.

1. Bacillus chitinovorus Benecke. (Bot. Zeitung, 63, 1905, 227.) From M. I. shitin, chitin; vorus, devouring.
Rods : 0.75 by 2.0 microns. Sometimes in pairs and chains. Motile with peritrichous flagella. Gram-negative.

Gelatin stab: Liquefaction.

Mineral agar containing chitin: Good growth if no sugar is added to produce acid. Non-chromogenic.

Peptone mincral agar containing chitin: Good growth if reaction is neutral to slightly alkaline.

Salt in concentrations up to $1 \frac{1}{2}$ per cent is favorable for growth. Maximum 4 per cent.

Peptone broth: Turbid with heavy, slimy, whitish to brownish pellicle.

Nitrites produced from nitrates.

Ammonia produced in peptone-chitin media.

Acid from glucose and sucrose.

Optimum temperature $20^{\circ} \mathrm{C}$.

Source: Isolated at Kiel from media rontaining decomposing crabshells and from media containing purified chitin; also from soil.

Habitat: Brackish water and soil.

Notes: Bacillus tumescens Zopf, $B a$ cillus cohaerens Gottheil, Bacillus proteus vulgaris Kruse, Bacillus coli communis Sternberg, Bacillus fuorescens liquefaciens Flügge, Bacillus megatherium De Bary, Vibrio aquatilis Günther and Spirillum rubrum von Esmarch did not attack chitin under the conditions tested by Benecke (loc, cit.).

Benton (Jour. Bact., 29, 1935, 449) describes but does not name 17 types of rhitinovorous bacteria isolated from water, mud and plankton of fresh water lakes, from decaying May fly nymph shells, intestinal contents of fish, frogs, bats, snipe, and crayfish. Also shore soil, composts, etc. Twelve types are reported to be monotrichous, two are peritrichous and three, position of flagella not stated. Of two Gram-positive types, one may have been a sporeformer and the other a Corynebacterium. Two types digested cellulose.

ZoBell and Rittenberg (Jour. Bact., $35,1938,275)$ isolated and studied but 
did not name 31 cultures of chitinoclastic bacteria from marine sources. Out of 16 cultures studied intensively, all were Gram-negative. All but 4 of the 31 cultures were motile. One culture was a coccus and two species were vibrios. None digested cellulose.

*39. Bacterium phosphoreum (Cohn) Molisch. (Micrococcus phosphoreus Cohn, see letter addressed to J. Penn. Verzameling van stukken betreffende het geneeskundig staatstoezicht in Nederland, 1878, 126; Bacterium phosphorescens Fischer, Cent. f. Bakt., 3, 1888, 107; Photobacterium phosphorescens Beijerinck, Arch. Néerl. d. Sci. Exactes, 23, 1889, 401; Streptococcus phosphoreus Trevisan, I generi e le specie delle Batteriacee, 1889, 31; Bacillus phosphoreus Macé, Traité de Bact., Paris, 4th ed., 1901, 995; Micrococcus phosphorescens Chester, Man. Detern. Bact., 1901, 181; Molisch, Die Leuchtende Pflanzen, 1912, 66; Photobacter phosphoreum Beijerinck, Folia Microbiologica, Delft, 4, 1916, 15; Photobacterium phosphoreum Ford, Textb. of Bact., 1927, 615.)

Description from Fischer (loc, cit.).

Coccobacilli: Occur frequently as zooglea. Non-motile. Stain lightly with aniline dyes.

Gelatin: No liquefaction.

Gelatin streak: Cray-white growth.

Broth: No growth.

Milk: No growth.

Potato: No growth.

Ferments carbohydrates.

Blue-green phosphorescence.

Minimum temperature $5^{\circ} \mathrm{C}$. Maximum $25^{\circ}$. Optimum for luminescence $10^{\circ} \mathrm{C}$.

Aerobic, facultative.

Source: Isolated from luminous fish.

Habitat: Found commonly on dead fish, meat, etc.

\section{Bacterium phosphorescens indige-} nus (Eisenberg) Chester. (Einheimischer Leuchtbacillus, Fischer, Cent, f. Bakt.; 3, 18ss, 107; Photobacterium fischeri Beijerinck, Arch. Néerl. d. Sci. Exactes, 23, 1889, 401; Bacillus fischeri Trevisan, I generi e le specie delle Batteriacee, 1889, 18; Bacillus phosphorescens indigenus Eisenberg, Bakt. Diag., 3 Aufl., 1891, 124; Vibrio fischeri Lehmann and Neumann, Bakt. Diag., 1 Aufl., 2, 1896, 342; Chester, Ann. Rept. Del. Col. Agr. Exp. Sta., 9, 1897, 121; Microspira fischeri Chester, Man. Determ. Bact., 1901, 333; Spirillum phosphorescens Holland, Jour. Bact., 5, 1920 , 225; Vibrio phosphorescens Holland, ibid., 226; Achromobacter fischeri Bergey et al., Manual, 3rd ed., 1930, 220.)

Description from Fischer (loc, cit.).

Short thick rods: 0.4 to 0.7 by 1.3 to 2.1 microns, with rounded ends, occurring singly and in pairs. Motile. Stain with the usual aniline dyes.

Johnson, Zworykin and Warren (Jour. Bact., 46, 1913, 167) made pictures with the electron microscope of a culture which they identify with this species. The organisms have a tuft of polar flagella, indicating that this species belongs in the genus Pseudomonas.

Gelatin stab: Liquefaction.

Gelatin colonies: Liquefaction. After one week, circular, $1 \mathrm{~mm}$ in diameter, entire.

Broth: No growth.

Milk: No growth.

Blood serum: No growth.

Potato: No growth.

Cooked fish: Abundant growth. Entire surface covered with a gray-white, slimy, phosphorescent mass.

Temperature relations: Minimum $5^{\circ}$ to $10^{\circ} \mathrm{C}$. Optimum $22^{\circ} \mathrm{C}$.

Aerobic.

Source: From sea water at Fiel and from herring.

* Dr. Frank H. Johnson, Dept. Bacteriology, Princeton Univ., Princeton, New Jersey, assisted in preparing the section on phosphorescent bacteria, May, 1946 


\section{Habitat: Live on dead fish and in sea water.}

41. Bacterium hemophosphoreum Pfeiffer and Stammer. (Pfeiffer and Stammer, Ztschr. f. Morph. u. Oköl. d. Tiere, 20, 1930, 136; Brucella (?) haemophosphoreum Pribram, Klassifikation der Schizomyceten, Leipzig und Wien, 1933, 67.)

Rods: 1.0 by 4.5 microns, the size varying with the medium. Seem to show bipolar staining.

Fish agar with 3 per cent sea salt: Good growth.

Litmus milk: Acid. Reduction.

Potato: Yellow growth, medium becoming orange.

Indole not formed.

Nitrites not produced from nitrates.

Acid from glucose, sucrose, lactose, maltose, galactose, mannitol and fructose.

\section{Phosphorescent.}

Pathogenic for other insects.

Source: Isolated from the blood of diseased larvae of the mealworm, Tenebrio molitor.

Habitat: From diseased insect larvae.

Appendix 1: The following phosphorescent species have been described in the literature. Many are incompletely described and they have been placed in various genera without adequate study.

Achromobacter argenteophosphorescens (Katz) Bergey et al. (Bacillus argenteophosphorescens I, Katz, Cent. f. Bakt., 9, 1891, 157; Bacterium argenteo-phosphorescens Chester, Ann. Rept. Del. Col. Agr. Exp. Sta., 9, 1897, 121; Bacillus argenteo-phosphorescens Migula, Syst. d. Bakt., 2, 1900, 869; Photobacillus I, Miquel and Cambier, Traité de Bact., Paris, 1902, 881; Bergey et al., Manual, 3rd ed., 1930, 221.) From sea water in Elizabeth Bay, Sydney, Australia. Silver-white luminescence. Probably at variety of Photobacterium fischeri Beijerinck, according to Katz.

Achromobacter cyaneophosphorescens
(Katz) Bergey et al. (Bacillus cyaneophosphorescens Katz, Cent. f. Bakt., 9, 1891, 158; Photobacterium cyaneum Ludwig, according to Kruse, in Flügge, Die Mikroorganismen, 3 Aufl., 2, 1896, 331; Photobacterium cyano-phosphorescens (sic) Ford, Textb. of Bact., 1927, 619; Vibrio cyaneo-phosphorescens Lehmann and Neumann, Bakt. Diag., 7 Aufl., 2, 1927, 543; Bergey et al., Manual, 3rd ed. $1930,221$.$) From sea water in Little$ Bay, near Sydney, Australia. Bluishgreen luminescence. Identical with or similar to Photobacterium indicum Beijerinck, according to Katz.

Achromobacter luminosum Bergey et al. (Bacillusargenteo-phorphorescens II, Katz, Cent. f. Bakt., 9, 1891, 157; Bacterium argenteo-phosphorescens Migula, Syst. d. Bakt.. 2, 1900, 435; Photobacillus II, Miquel and Cambier, Traité de Bact., Paris, 1902, 882; Bergey et al., Manual, 3rd ed., 1930, 226.) From fish obtained in the market. Greenish-silver luminescence.

Achromobacter phosphoreum (Migula) Bergey et al. (Bacillus argenteo-phosphorescens liquefaciens Katz, Cent. f. Bakt., 9, 1891, 157; Bacillus phosphoreus Migula, Syst. d. Bakt., 2, 1900, 867; Bergey et al., Manual, 3rd ed., 1930, 222.) From sea water along the coast near Sydney, Australia. Luminescence slight. Probably identical with Photobacterium luminosum B eijerinck:

Achromobacter phosphoricum (Migula) Bergey et al. (Bacillus argenteo-phosphorescens III, Katz, Cent. f. Bakt., 9, 1891, 157; Bacillus phosphoricus Migula, Syst.d. Bakt., 2, 1900, 870; Photobacillus III, Miquel and Cambier, Traité de Bact., Paris, 1902, 882; Bergey et al., Manual, 3rd ed., 1930, 223.) From cuttlefish (Sepia sp.) obtained in the fish market. Bluish-greenish-white luminescence.

Achromobacter smaragdinophosphorescens (Katz) Bergey et al. (Bacillus smaragdino-phorphorescens Katz, Cent. f. Bakt., 9, 1891, 159; Bacterium smaragdino phosphorescens Chester, Ann. Rept. 
Del. Col. Agr. Exp. Sta., 9, 1897, 124; Bacterium smaragdino-phosphorescens Migula, Syst. d. Bakt., 2, 1900, 435; Bacterium smargadinum (sic) Chester, Man. Determ. Bact., 1901, 181; Bergey et al., Manual, 3rd ed., 1930, 225.) From herring in a fish market in Sydney, Australia. Green luminescence. Probably identical with Photobacterium phosphorescens Beijerinck.

Bacillus fischeri Dyar. (Dyar, Ann. N. Y. Acad. Sci., 8, 1895, 370; Bacterium fischeri Chester, Man. Determ. Bact., 1901,165 .) Dyar added to the confusion in the nomenclature of phosphorescent organisms by giving this name to four cultures received by him from the Kràl collection labeled Photobacterium phosphorescens, Photobacterium balticum, Photobacterium fischeri and Photobacterizm pflügeri.

Bacterium chironomi Issatschenko. (Bulletin du Jardin Impérial botanique à St. Pétersbourg, 11, 1911, 37; Photobacterium chironomi Issatschenko, ibid., 43.) A phosphorescent bacterium from a genus of midges, Chironomus.

Bacterium giardi (Kruse) Billet. (Giard and Billet, Compt. rend. Soc. Biol., Paris, 1889, 533; Photobacterium pathogenicum Giard, quoted from Eijkmann, see abst. in Cent. f. Bakt., 12, 1892, 656; Photobacterium giardi Kruse, in Flügge, Die Mikroorganismen, 3 Aufl. 2, 1896, 333; Bacillus phosphorescens giardi Kruse, idem; Bacterium phosphorescens giardi Chester, Ann. Rept. Del. Col. Agr. Exp. Sta., 9, 1897, 125; Billet, Bull. Sei. France et Belgique, 21, 1598, 1tt; Bacterium phosphorescens. giardi Chester, Man. Determ. Bact. 1901, 182.) Pathogenic for marine crustaceans.

Bacterium hippanici Issatschenko (loc. cit., 47). From fresh water fish. Bacterium lucens (van Tieghem) Nüesch. (Micrococcus lucens van Tieghem; Nüesch, Karsten's Deutsche Flora, 1880; quoted from Ludwig, Cent. f. Bakt., 2, 1887, 375.) From luminous meat. Considered identical with Bacterium phosphoreum.

Bacterium luminosus (Beijerinck) Chester. (Photobacterium luminosum Beijerinck, Arch. Néerl. d. Sci. Exactes, 23, 1889, 401; Vibrio luminosus Beijerinck, Bot. Zeit., 1889, 763, according to Trevisan, I generi e le specie delle Batteriacee, 1889, 23; Bacillus luminosus DeToni and Trevisan, in Saccardo, Sylloge Fungorum, 8, 1889, 982; Chester, Ann. Rept. Del. Col. Agr. Exp. Sta., 9, 1897, 121; Microspira luminosa Migula, Syst. d. Bakt., 2, 1900, 1015; Photobacter luminosum Beijerinck, Folia Microbiologica, Delft, 4, 1916, 15.) From sea water.

Bacterium pelagia Dubois. (Dubois, Compt. rend. Acad. Sci., Paris, 10\%, 1888, 502 and 111, 1890, 363; Bacillus pelagia DeToni and Trevisan, in Saccardo, Sylloge Fungorum, 8, 1889, 959.) Isolated from the surface of Pelagiae noctilucae.

Bacterium pfluegeri Ludwig. (Ludwig, Ztschr. f. wissensch. Mikrosk., 1, 1881, 181; Micrococcus pflügeri Ludwig, Hedwigia, No. 3, 1884; Arthrobacterium pflügeri DeBary, 1887; Photobacterium pflïgeri Beijerinck, Cent. f. Bakt., 8, 1890, 617; Bacterium phosphorescens pfugeri Chester, Ann. Rept. Del. Col. Agr. Exp. Sta., 9, 1897, 125.) From fish and meat. Considered identical with Bacterium phosphoreum.

Bacterium pholas Dubois. (Compt. rend. Acad. Sci., Paris, 107, 1888, 502.) Isolated from Pholadis dactyli.

Bacterium phosphorescens Hermes. (Hermes, Sitzungsber. naturf. Freunde, April 19, 1887, quoted from Cent. f. Bakt., 2, 1887, 404; Bacillus hermesi Trevisan, I generi e le specie delle Batteriacee, 1889, 18.) From sea water. Macé (Traité de Bact., Paris, tth ed., $1901,994)$ says this may be the same as Micrococcus phosphoreus Cohn. Emerald-green luminescence.

Bacterium phosphorescens gelidus (Eisenberg) Chester. (Phosphorescirenden Mikroorganismen, Forster, Cent. f. 
Bakt., 2, 1887, 337; Bacillus phosphorescens gelidus Eisenberg, Bakt. Diag., 3 Aufl., 1891, 182; Chester, Ann. Rept. Del. Col. Agr. Exp. Sta., 9, 1897, 125.) From phosphorescent sea fish. Fischer (Cent.f. Bakt., 4, 1888, 89) states that this organism is the same as his Bacterium phosphorescens.

Coccobacillus acropoma Yasaki and Haneda. (Yasaki and Haneda, 1936; quoted from Harvey, Living Light, Princeton, 1940,33.) From a fish (Acropoma japonicum).

Coccobacillus coelorhynchus. (Studied by Hsu, Sei-i-kai Med. Jour., 56, 1937, 1; qucted from Harvey, Annual Rev. of Biochem., 10, 1941, 543.) From a deepsea fish (Coclorhynchus sp.).

Coccobracillus ikiensis. (Quoted from Harvey, Living Light, Princeton, 1940, 263.

Coccobacillus loligo Kishitani. (Kishitani, Proc. Imp. Acad. Tokyo, 4, 1928, 69; quoted from Harvey, Living Light, Princeton, 1940, 35.) From the squid (Loligo edulis).

Micrococcus cyanophos. (Studied by Claren, Ann. d. Chemie, 535, 1938, 122; quoted from Harvey, Living Light, Princeton, 1940, 184.)

Micrococcus physiculus.

(Quoted

from Harvey, Living Light, 1940, 3t.) The cause of luminesecnce of a fish (Physiculus japonicus).

Microspira phosphoreum Yasaki. (Yasaki, see Sei-i-kai-zasshi, 45, 1926; quoted from Harvey, Living Light, 1940, 239.) Caused luminescence of a freshwater shrimp in Japan.

Photobacter hollandiae Beijerinck. (Proc. Sect. Sci., Kion. Akad. v. Wetensch., Amsterdam, 3, 1900, 352.) Simi lar to Photobacterium luminosum.

Photobacter hollandicum Beijerinck. (Folia Microbiologica, Delft, 4, 1916, 15.)

Photobacter hollandicum parvum Beijerinck. (Folia Microbiologica, Delft, 4, 1916, 15.)

Photobacter splendidum. Beijerinck. (Beijerinck, Proc. Sect. Sci., Kon..Akad. v. Wetensch., Amsterdam, 3, 1900, 352;
Vibrio splendidus Lehmann and Neumann, Bakt. Diag., 7 Aufl., 2, 1927, 543; Photobacterium splendidum, quoted from Harvey, Living Light, Princeton, 1940, 204.) May be a variety of Photobacterium indicum.

Photobacter splendor maris Beijerinck. (Proc. Seet. Sei., Kon. Akad. v. Wetensch., Amsterdam, 3, 1900, 352.) May be a variety of Photobacterium indicum.

Photobucterium Beijerinck. (Beijerinck, Arch. Néerl. d. Sci. Exactes, 23, 1859, 401; Photobacter Beijerinck, Proc. Sect. Sci., Kon. Akad. v. Wetensch., Amsterdam, 3, 1900, 352; Photospirillum Miquel and Cambier, Traité de Bact., Paris, 1902, 888; Photomonas Orla-Jensen, Jour. Bact., 6, 1921, 271.) Photobacterium phosphorescens is the type species of this genus. See Bacterium phosphoreum. Several species are placed in this genus by Fischer.

Photobacterium annulare Fischer. (Fischer, Ergebnisse d. Plankton-Expedition d. Humboldt-Stiftung, 4, 1894, 41; Microspira annularis Migula, Syst. d. Bakt., 2, 1900, 1014.) From sea water.

Photobacterium balticum Beijerinck. (Einheimischer Leuchtbacillus, Fischer, Cent.f. Bakt., 3, 18s8, 105; Beijerinck, Akad. v. Wetenschappen, Afdeel. Natuurk., 2de Reeks, 7, 1890, 239; see abst. in Cent. f. Bakt., 8, 1890, 617; Vibrio balticus Lehmann and Neumann, Bakt. Diag., 1 Aufl., 2, 1896, 341.) From water of the Baltic Sea. The relationship of Photobacterium balticum to Bacterium phosphorescens indigenus is not clear. The former species is based on a culture sent by Fischer to Beijerinck laboled Einheimischer Leuchtbacillus which Beijerinck considered to be different from his Photobacterium fischeri.

Photobacterium caraibicum Fischer. (Fischer, loc. cit., 1894, 41; Microspira caraibica Migula, loc. cit., 1015.) From sea water.

Photobactorium coronatum Fischer. (Fischer, loc cit., 41; Microspira coronata 
Migula, loc. cit., 1013.) From seat water.

Photobacterium degenerans Fischer. (Fischer, loc. cit., 37; Microspira degenerans Migula, loc. cit.. 1015; Bacillus degenerans Beijerinck. Folia Microbiologiea, Delft, I, 1912, 1.) From sea water.

Photobacterium delgadense Fischer. (Fischer, loc. cit., 37; Miciospira delgadensis Migula, loc. cit., 1014. From sea water.

Photobacterium glutinosum Fischer. (Fischer, loc. cit., 41; Microspira glutinosa Migula, loc. cit., 1014.) From sea water.

Photobacterium hirsutum Fischer (loc. cit., 41). From marine fish.

Photobacterium. papillare Fischer. (Fischer, loc. cit., 11 ; Microspira papillaris Migula, loc. cit., 1016.) From sea water.

Photobacterium sepiae. (Quoted from Doudoroff, Jour. Bact., 44, 1942, 451 , who obtained a culture so labeled which had come from Prof. Hluyver's collection at Delft.)

Photobacterium tuberosum Fischer. (Fischer, loc cit., 37; Microspira tuberosa Migula, loc. cil., 1014; Photobacter tuberculatum Beijerinck, Folia Microbiologica, Delft, 4, 1916, 15.) From sea water.

Pseudomonas toyamensis. (Quoted from Harvey, Living Light, Princeton, 1940, 263.)

Sarcina noctiluca Heller. (Heller, Arch. f. Physiol, path. Chem, u. Mikx., N.F., 6, 1853-54, 44; see Harvey, Living Light, Princeton, 1940, 6.) From fish. Possibly the same as Bacterium phosphoreum Molisch.

42. Bacterium erythrogloeum Ruhland and Grohmann. (Cent. f. Bakt., II Abt., 61, 1924, 256.) From Greek erythros, red and glota, glue.

Rods : 0.5 by 2.0 microns. Non-motile. Gram-negative.

Gelatin plate: Red, droplet-like colonies.
Gelatin stab: No liquefaction.

Agar plate: Red, droplet-like colonies.

Agar slant: Raised, non-spreading, glistening, brick-red growth.

Potato: Abundant, brick-red, warty. Aerobic.

Facultative autotroph.

Oxidizes hydrogen in an inorganic medium under an atmosphere of $\mathrm{H}_{2}, \mathrm{O}_{2}$, and $\mathrm{CO}_{2}$. Produces a pellicle on the inorganic liquid medium.

Source: Calcareous soil.

Habitat: Prohably widely distributed in soil.

43. Bacterium lentulum Grohmann. (Cent. f. Bakt., II Abt., 61, 1924, 256.)

Rods: 0.5 by 1 to 2 microns. Motile by long thin peritrichous flagella. Gramnegative.

Gelatin plate: Colonies appear like milk droplets.

Gelatin stab: No liquefaction.

Agar plate: Tough, ochre yellow coloniess about $7 \mathrm{~mm}$ in diameter.

Agar streak: Parchment-like, folded, yellow streak about $1 \mathrm{~cm}$ broad.

Potato: Heavy, yellow growth.

Aerobic

Facultative autotroph.

Oxidizes hydrogen in an inorganic medium under an atmosphere of $\mathrm{H}_{2}, \mathrm{O}_{2}$, and $\mathrm{CO}_{2}$. Produces a heavy folded pellicle on the inorganic liquid medium.

Source: Soil poor in lime.

Habitat: Probably widely distributed in soil.

44. Bacterium leucogloeum Ruhland and Grohmann. (Cent, f. Bakt., II Abt.,61, 1924, 256.) From Gireek leukos, white and glota, glue.

Rods: 0.5 by 0.7 to 52 (?) microns. Motile by means of peritrichous flagella.

Gelatin stab: No liquefaction.

Agar streak: Wide, slimy, wet, ivorycolored growth.

Potato: Gray-brown slime.

Aerobic.

Facultative autotroph.

Oxidizes hydrogen in an inorganic medium under an atmosphere of $\mathrm{H}_{2}, \mathrm{O}_{2}$, 
and $\mathrm{CO}_{2}$. Produces a pellicle on the inorganic liquid medium.

Source: Calcareous soil.

Habitat: Probably widely distributed in soil.

*45. Bacterium stewartii Erw. Smith. (Sweet corn bacillus, Stewart, N. Y. Agr. Exp. Sta. Bul. 130, 1897, 423; Pseudomonas stewarti Smith, Proc. A. A. A. Sei., 47, 1898, 422; Smith, Bact. in Rel. to Plant Dis., 3, 1914, 89; Aplanobacter stewarti McCulloch, Phytopath., 8, 1918, 440; Bacillus stewarti Holland, Jour. Bact., 5, 1920, 220; Phytomonas stewartii Bergey et al., Manual, 1st ed., 1923, 192.) Named for F. C. Stewart, American plant pathologist.

Description from Smith, U. S. Dept. Agric., Div. Veg. Phys. and Path., Bul. 28, 1901 .

Rods: 0.4 to 0.7 by 0.9 to 2.0 microns. Capsules. Non-motile (McCulloch, loc. cit.). Gram-negative.

Gelatin: No liquefaction.

Nutrient agar colonies: Small, round, yellow colonies.

Broth: Growth feeble with whitish ring and yellow precipitate.

Milk: Yellow ring but no visible action on the milk. Slightly acid.

Nitrites not produced from nitrates. McNew (Phytopath., 28, 1938, 773) states that less virulent strains assimilate only organic nitrogen; those of intermediate virulence assimilate nitrogen from inorganic salts without reduction of nitrates to nitrites; virulent strains reduce nitrates to nitrites.

Hydrogen sulfide not formed.

Indole production slight or none

Reduction of methylene blue in Dunham's solution feeble or doubtful.

Acid but no gas from glucose, galactose, sucrose, mannitol and glycerol. No acid from maltose. Acid from fruetose, arabinose and xylose (McNew, loc. cit.).
Starch not hydrolyzed.

Optimum temperature $30^{\circ} \mathrm{C}$. Maximum $39^{\circ} \mathrm{C}$. Minimum $8^{\circ} \mathrm{C}$.

Optimum pH 6.0 to 8.0. Limits about $\mathrm{pH} 4.5$ to 8.5 .

$\$$ per cent salt restricts growth.

Strict aerobe.

Source: From wilted sweet corn.

Habitat: Pathogenic on corn, Zea mays. Sweet corn very susceptible and field corn slightly so.

46. Bacterium tardicrescens MeCulloch. (McCulloch, Phytopath., 27, 1937, 135; Phytomonas tardicrescens Burkholder, Phytopath., 27, 1937, 617.) From Latin, slow growing.

Rods : 0.6 to 0.8 by 1.58 microns. Motile with a polar flagellum. Gram-negative.

Gelatin: No liquefaction.

Beef-extract agar colonies: Circular, mustard yellow, edges entire, 1 to 1.5 $m m$ in diameter.

Broth: Light clouding.

Milk: Slightly alkaline. Clearing after 5 to 6 weeks.

Nitrites are produced from nitrates.

Indole not produced.

No $\mathrm{H}_{2} \mathrm{~S}$ produced or feebly so.

Acid but no gas from glucose, fructose, galactose, arabinose, xylose and rhamnose. Alkaline reaction from salts of citric, malic and succinic acid.

Stareh is not hydrolyzed.

Not lipolytic (Starr and Burkholder, Phytopath., 32, 1942, 603).

Optimum temperature $26^{\circ} \mathrm{C}$. Maximum $32^{\circ} \mathrm{C}$. Minimum $5{ }^{\circ} \mathrm{C}$ (McCulloch, Phytopath., 28, 1938, 648).

Optimum pH 6.5 to 7.5. Grow th slight at 5.8 and 8.0 ( MeCulloch, loc. cit.).

No growth with 3 per cent salt (McCulloch, loc. cit.).

Aerobic.

Distinctive character: Very slow grower.

* The section covering species of interest to plant pathologists has been prepared by Prof. Walter H. Burkholder, Cornell Univ., Ithaca, New York, May, 1946. 
Source: Isolated by McCulloch and by Burkholder from blighted iris leaves.

Habitat: Pathogenic on Iris $s p p$.

47. Bacterium albilineans Ashby. (Ashby, Trop. Agr., Trinidad, 6, 1929, 135; Phytomonas albilineans Magrou, in Hauduroy et al., Dict. d. Bact. Path., Paris, 1937, 326.) From Latin, producing white streaks.

Description taken from Martin, Carpenter and Weller, The Hawaiian Planters' Record, 36, 1932, 181.

Rods: 0.25 to 0.3 by 0.6 to 1.0 micron, occurring singly or in chains. Motile with a polar flagellum. Gram-negative.

Agar colonies: After 7 to 10 days, minute transparent drops, moist, shining. Honey yellow to Naples yellow.

Gelatin: No liquefaction.

Milk: Growth, but no visible change in the milk.

No growth with ammonium salts, nitrates, or asparagine as a source of nitrogen.

No growth in peptone water without carbohydrates. Invertase secreted.

Starch is not hydrolyzed.

Optimum temperature about $25^{\circ} \mathrm{C}$. Maximum $37^{\circ} \mathrm{C}$.

Distinctive characters: Differs from Xanthomonas vascularum which produces a large gummy type of colony, and which is a very active organism biochemically. The two pathogens also differ in the type of lesion they produce on sugar cane.

Source: Isolated by D. S. North (Colonial Sugar Ref. Co., Sidney, N.S. Wales, Agr. Rept., 8, 1926, 1) from white stripe and leaf scald of sugar cane in Australia.

Habitat: Vascular pathogen of sugar cane, Saccharum officinarum.

Appendix I: The following species have been described from diseased plant tissues but may not, in some cases at least, have been the cause of the disease.

Bacillus betae Migula. (Kramer, Oesterreich. landwirtsch. Centralb., 1891,
Heft 2 and 3; Migula, Syst. d. Bakt., 2, 1900, 779.) The cause of a disease of the sugar beet (Beta vulgaris).

Bac. caryophyllacearum Dufrenoy. (Compt. rend. Soc. Biol., Paris, 81, 1918, 920 ; probably there is an earlier reference to this species.) On Dianthus, Saponaria and Lychnis.

Bacillus coffeicola Steyaert. (Rev. Zoo. et Bot. Afr., 22, 1932, 137.) From nodules on coffee roots.

Bacillus lacerans Migula. (Bacillus $\alpha$, Busse, Ztschr. f. Pflanzenkr., 7, 18-, 72 ; Migula, Syst. d. Bakt., 2, 1900, 780.) From diseased sugar beets.

Bacillus maculicola Delacroix. (Delacroix, Compt. rend. Acad. Sci. Paris, 140, 1905, 680; Bacterium maculicola Stapp, in Sorauer, Handb. d. Pflanzenkrankheiten, 5 Aufl., 2, 1928, 276; Aplanobacter maculicola Elliott, Manual Bact. Plant Path., 1930, 8; Phytomonas nicotianae-tabaci Magrou, in Hauduroy et al., Dict. d. Bact. Path., 1937, 386.) From diseased spots on leaves of tobacco.

Bac. nucleophyllus Dufrenoy. (Compt. rend. Soc. Biol., Paris, 81, 1918, 920, nomen nudum.) On Rhododendron ferrugineum.

Bac. tritici Dufrenoy. (Compt. rend. Soc. Biol., Paris, 81, 1918, 920, nomen nudem; not Pseudomonas tritici Hutchinson, India Dept. of Agr., Bact. Ser., 1, 1917, 174.) On wheat.

Bacillus vitis Montemartini. (Rev. Patol. Veg., 6, 1913, 175.) Pathogenic on the grape (Vitis vinifera).

Bacterium apii Brizi. (Lav. e Relaz. d. Reg. Staz. di Patol. Veg., Roma, Gennio-Giugno, 15, 1896 and Atti R: Accad. Naz, Lincei, Rend. Cl. Sc. Fis., Math. e Nat., Ser. 5, 6, 1897, 233.) Motile. From rot of celery.

Bacterium betae Chester. (Bacterial parasite, Arthur and Golden, Indiana Agr. Exp. Sta., Bull. 39, 1892, 61; Chester, Ann. Rept. Del. Col. Agr. Exp. Sta., 9, 1897, 128; Bacillus arthuri Migula, Syst. d. Bakt., 2, 1900, 681.) Motile. From diseased sugar beet tubers.

Bacterium briosianum Pavarino. 
(Atti del. R. Accad. Naz. Lincei, Rend. Cl. Sci. Fis., Math. et Nat., 20, 1911, 161.) Motile. From lesions on the vanilla vine.

Bacterium castinicolum Cavara. (Rev. d. Pat. Veg., 7, 1914, 5.) Motile From chestnut canker.

Bacterium corylii Brzezinski. (Bull. Intern. Acad des Sci. Cracovie, Cl. Sci. Math. e Nat., 1903, 139.) Motile. From diseased filbert trees.

Bacterium dendrobii Pavarino. (Rev. di Pat. Veg., 5, 1912, 242.)

Bacterium dianthi Chester. (Parasitic bacteria, Arthur and Bolley, Purdue Univ. Agr. Exp. Str., Bull. 59, 1896, 21; Chester, Ann. Rept. Del. Col. Agr. Exp. Sta., 9, 1s97, 106; Bacillus dianthi Chester, Man. Determ. Bact., 1901, 253; Pseudomonas dianthi E. F. Smith, U. S Dept. Agr., Div. Veg. Phys. and Path., Bull. 28, 1901, 153.) Motile. From lesions on carnation leaves.

Bacterium fici Cavara. (Ist. Bot. del. R. Univ. di Catania, Atti Aead. Gioen., 18, Mem. 14, 1905, 1; Phylomonas (?) fici Magrou, in Hauduroy et al., Dict. d Bact. Path., 1937, 354.) Motile Causes a blight of figs.

Bacterium lycopersici var. vitiati Strzalkowska. (Strzalkowska, Acta Soc. Bot. Poloniae, Warsaw, 7, 1930, 611; Phytomonas vitiati Burkholder, in Manual, 5th ed., 1939, 216.) From rotting tomato

Bacterium mali Brzezinski. (Bull Intern. Acad. Sci. Cracovie, Cl. Sci. Math. e Nat., 1903, 100.) Motile From apple canker.

Bacterium montemartinii Pavirino. (Rev. di Pat. Veg., 5, 1911, 65.) Motile From wisteria canker.

Bacterium (?) oncidii Peglion. (Peglion, 1899, quoted from Hauduroy et al., Dict. Bact. Path., 1937, 388; Bacillus oncidii Stevens, 1913; Phytomonas (?) oncidii Hauduroy et al., idem.) From an orchid (Oncidium sp.).

Bacterium pini Chester. (Bacillus des tumeurs du Pin d'Alep, Vuillemin, Compt. rend. Acad. Sci., Paris, 107, 188s, 874 and 1181; Bacillus vuillemini Trevi- san, I generi e le specie delle Batteriacee, 1889, 19; Chester, Ann. Rept. Del. Col. Agr. Exp. Sta., 9, 1897, 127; Pseudomonas pini Petri, Ann. Ist. Supt. For. Naz. Firenze, 9, 1924, 187.) From galls on pine (Pinus halepensis).

Bacterium putredinis Davaine. (Davaine, Bactéries, in Dictionnaire Encyclopédique des Sci. Médicales, 1866; Bacillus putredinis Trevisan, Add. ad Gen., p. 36; see DeToni and Trevisan, in Saccardo, Sylloge Fungorum, 8, 1889, 1025; not Bacillus pulredinis Weinberg, Nativelle and Prévot, Les Microbes Anaérobies, Paris, 1937, 755.) Causes a soft rot of several plants.

Bacterium pyri Brzezinski. (Bull. Internat.d.l'Acad. des Sci.de Cracovie, (l. Sci. Math. e Nat., 1903, 130.) Motile. From pear canker.

Bacterium rubefaciens Burr. (Burr, Ann. App. Biol., 15, 1928, 570; Phytomonas rubefaciens Magrou, in Hauduroy et al., Dict. Bact. Path., 1937, 406; not Bacterium rubefaciens Chester, Ann. Rept. Del. Col. Agr. Exp. Sta., 9, 1897, 115.)

Bacterium suberfacions Burr. (Burr, Ann. App. Biol., 15, 1928, 570; Phytomonas suberfaciens Magrou, in Hauduroy et al., Dict. Bact. Path., 1937, 417.) Motile. From diseased potato tubers.

48. Bacterium rubefaciens (Zimmermann) Chester. (Bacillus rubefaciens Zimmermann, Die Bakterien unserer Trink- und Nutzwässer, Chemnitz, 1, 1890, 26; Chester, Ann. Rept. Del. Col. Agr. Exp. Sta., 9, 1897, 115; Erythrobacillus rubefaciens Holland, Jour. Bact., 5, 1920, 223; Serratia rubefaciens Bergey ct al., Manual, 1st ed., 1923, 92; Chromobacterium rubefaciens Topley and Wilson, Prine. Bact and Immun., 1, 1931, 402.) From Latin ruber, red and facio, to make.

Rods: 1.0 to 1.6 microns in length, occurring singly and in pairs. Actively motile. Gram-negative.

Gelatin colonies: Minute, white.

Gelatin stab: Surface growth yellowish, the medium taking on a red tinge. No liquefaction. 
Agar colonies: Small, white, with erose margin.

Agar slant: White, smooth, glistening, somewhat luxuriant, the medium taking on a wine red eolor.

Broth: Turbid with white pellicle, the medium slowly assuming a reddish tinge.

Litmus milk: Acid, with slow coagulation and reduction of the litmus. Beroming alkaline.

Potato: A heavy, white, creamy layer, which later becomes yellowish-brown.

Indole not produced.

Nitrites produced from nitrates.

Aerobic, facultative.

Optimum temperature $25^{\circ} \mathrm{C}$. To growth at $37^{\circ} \mathrm{C}$.

Habitat: Water.

49. Bacterium rubidum (Eisenberg) Chester. (Bacillus rubidus Eisenberg, Bakt. Diag., 3 Aufl., 1891, 88; Bacterium rubidus (sic) Chester, Ann. Rept. Del. Col. Agr. Exp. Sta., 9, 1897, 107 and 115; Serratia rubida Levine and Soppeland, Iowa State Coll. Engineering Exp. Sta. Bull. 77, 1926, 53.) From Latin rubidus red.

Description from Eisenberg (loc. cil.). Levine and Soppeland (loc. cit.) found an organism in buttermilk which they identified as Serratia rubida. Their description is more complete than that given by Eisenberg but differs from the original in several respects.

Rods: Medium size with rounded ends, often in long chains. Motile.

Gelatin colonies: Circular, finely granular, entire, with reddish center. Slow growth.

Gelatin stab: Liquefaction. Brownish-red sediment.

Agar colonies: Small, flat, smooth, amorphous, entire, hrownish-red. Slow growth.

Agar slant: Brownish-red streak. Spreading over surface.

Potato: Brownish-red growth.

Blood serum liquefied, red pigment

Aerobic, facultative

Does not grow well at $37^{\circ} \mathrm{C}$.

Source: Water.
50. Bacterium latericeum (Adametz) Lehmann and Neumann. (Bacillus latericeus Adametz, Die Bakterien der Trink- und Nutzwässer, Mitteil. der oestrr. Versuchsanst. f. Brauerei u. Mälzerei in Wien, 1888, 50; Bacillus erythraeus Trevisan, I generi e le specie delle Batteriacee, 1889, 19; Bacterium latericeum Lehmann and Neumann, Bakt. Diag., 1 Aufl., 2, 1896, 258; Serratia latericea Bergey et al., Manual, 1st ed., 1923, 94.) From Latin latericeus, brick.

Rods : 0.5 to 0.7 by 1.0 to 1.3 microns. Non-motile. Gram-negative.

Gelatin colonies: Small, white, granular, with slightly irregular margin.

Gelatin stab: A thin, dry, spreading, cream-pink surface growth. No liquefaction.

Agar colonies: Dry, glistening, whitish, with irregular margin.

Agar slant: Brick-red, smooth, glistening, butyrous.

Broth: Thick pellicle; fluid clear.

Litmus milk: Alkaline.

Potato: Brick-red streak.

No gas from carbohydrate media.

Indole not produced.

Nitrites produced from nitrates.

Aerobic facultative.

Optimum temperature $25^{\circ}$ to $30^{\circ} \mathrm{C}$.

Habitat: Water.

51. Bacterium alginicum Waksman, Carey and Aller. (Jour. Bact., 28, 1934, 213.)

Rods short to almost spherical, 0.6 to 1.0 mieron in diameter. Sluggishly motile. Capsule-forming. Gramnegative.

Alginic acid plate: White, finely granulated colonies, with entire margin. Does not clear up the turbidity in plate. Odor formed, resembling that of old potatoes.

Alginic acid liquid medium: Thin pellicle, weak alginase formation.

Sea water gelatin: 'Thin growth throughout gelatin stab, no liquefaction in 7 days at $18^{\circ} \mathrm{C}$.

Agar liquefaction: None.

Sea water glucose broth: Uniform but 
very limited turbidity; no pellicle; no sediment.

Litmus milk containing salt: No apparent growth.

Potato moistened with sea water: Moist, spreading growth, cream-colored; heavy sediment in free liquid at bottom.

Starch plate: Limited, pale blue growth; no diastase.

Aerobic.

Optimum temperature $20^{\circ} \mathrm{C}$.

Source: From sea water, and from the surface of algal growth.

Habitat: Common in sea water.

52. Bacterium terrestraiginicum Waksman et al. (Waksman, Carey and Allen, Jour. Bact., 28, 1934, 217.)

Long rods, with somewhat rounded ends, usually single, but also in pairs, and occasionally in chains of shorter rods. 1.0 to 1.5 by 1.5 to 2.5 microns. Motile. Granular. Gram-negative.

Alginic acid plate: Colonies small, whitish in appearance with a slight metallic sheen.

Alginic acid liquid medium: Medium at first clouded. Later, a pellicle is formed on the surface of the medium, which is soon broken up due to active gas formation. Reaction of medium becomes slightly alkaline.

Gelatin medium : Slow growth throughout stab, slow liquefaction at surface of medium at $18^{\circ} \mathrm{C}$.

Agar liquefaction: None.

Glucose broth: Abundant turbidity, some sediment, no pellicle, slightly fluorescent.

Litmus milk: Acid, milk coagulated, only limited digestion of coagulum.

Potato: Abundant, pinkish, compact, dry growth on surface of plug, the rest of plug becoming gray, with a tendency to darkening.

Starch plate: Limited growth along streak, no diastese.

Aerobic to facultative anaerobic.

Optimum temperature $30^{\circ} \mathrm{C}$.

Source: From New Jersey soil.

Habitat: Soil.
53. Bacterium cygni Migula. (Septikämiebacillus der Schwäne, Fiorentini, Cent. f. Bakt., 19, 1896, 935; Migula, Syst. d. Bakt., 2, 1900, 365; Bacillus cygneus Chester, Manual Determ. Bact., 1901, 221.) From Latin cygnus, swan.

Rods: Motile. Gram-negative.

This organism may have been the fowl cholera or septicemia organism (Pasteurella avicida Trevisan); but is more probably closely related to the organism which causes keel in ducklings (Salmonella anatis Rettger and Scoville).

Source: From a swan.

Habitat: The cause of an infectious disease of swans in the city park at Milan, Italy in 1895.

\section{Bacterium cyprinicida Plehn.} (Plehn, Cent. f. Bakt., I Abt., Orig., 35, 1903-04, 461; Klebsiella cyprinicida Bergey et al., Manual, 2nd ed., 1925, 266.) From Greek kyprmos, carp and Latin caedo, to kill.

Rods: 0.8 by 1.0 micron, occurring singly and in chains. Capsulated. Non-motile. Gram-negative.

Gelatin colonies: White, glistening, convex, with slight fluorescence around the colony in three or four days.

Gelatin stab: White, convex surface growth. No liquefaction.

Agar slant: White, glistening layer, becoming slimy.

Broth: Turbid, with thick gray pellicle and slimy sediment.

Litmus milk: Slightly alkaline. No coagulation.

Potato: Light yellowish layer, becoming dark brownish. The medium is dark violet-gray.

Indole not formed.

Nitrites not produced from nitrates.

No acid from carbohydrate media.

Aerobic, facultative.

Optimum temperature $10^{\circ}$ to $20^{\circ} \mathrm{C}$.

Habitat: The cause of a fatal disease in carp, showing as red spots on the ventral surface.

55. Bacterium parvulum Conn. (N. Y. 
Agr. Exp. Sta. Bul.494, 1922, 26.) From Latin, very small.

Very small rods: 0.1 to 0.2 by 0.3 to 0.5 micron. Non-motile. Gram-negative.

Gelatin plate: Punctiform colonies.

Agar plate: Punctiform colonies.

Grows poorly in liquid media.

Indole not formed.

Nitrites produced from nitrates.

No acid from glucose, lactose, sucrose, glycerol or ethyl alcohol in either liquid or solid media.

Starch not digested.

Optimum temperature $25^{\circ} \mathrm{C}$.

Strictly aerobic.

Distinctive character: Causes strong volatilization of ammonia from a mixture of horse feces and urine.

Source: From manure.

Habitat: Soil.

56. Bacterium methylicum (Loew) Migula. (Bacillus methylicus Loew, Cent. f. Bakt., 12, 1892, 465; Migula, Syst.d. Bakt., 2, 1900, 447.) From the chemica! term, methyl.

Short, thick rods: 1.0 by 2.0 to 2.5 microns. Gram stain not recorded.
Gelatin colonies: After 2 days, round to oval, yellowish, entire; later edges ciliate. Liquefaction.

Glucose gelatin stab: Liquefaction crateriform. Whitish-yellowish sediment. No liquefaction in depth.

Glucose gelatin stab: In depth, little or no growth, slowly liquefied near surface.

Agar stab: Surface growth spreading, grayish-white. No growth in depth.

Broth: No turbidity. On the surface and adherent to the walls, a white ring which precipitates on shaking.

Potato: Growth very slow, pure white, adherent.

Grows well in 0.5 per cent methyl alcohol, 0.05 per cent dicalcium phosphate, and 0.01 per cent magnesium sulfate, on which broth it forms a reddish pellicle.

Possesses the ability to decompose formaldehyde and formic acid salts with formation of a reddish pellicle.

Aerobe.

Source: A culture contamination from the air.

Habitat: Probably soil.

Appendix I: A few of the numerous Gram-negative, motile or non-motile, nonspore-forming rods that do not belong in the groups previously listed in this genus are described here. All have been placed in the genus Bacillus by those who have described them, although none form spores.

I. Produce a pink to red chromogenesis.

A. Motile.

B. Non-motile.

1. Bacillus lactorubefaciens.

1. Gelatin liquefied.

2. Bacillus rubricus.

3. Bacillus rufus.

2. Gelatin not liquefied.

a. Salmon pink on agar.

4. Bacillus mycoides corallinus.

aa. Vinous red on agar.

5. Bacillus bruntzii.

II. Produces a water-soluble orange to emerald green pigment.

A. Motile.

1. Gelatin liquefied.

6. Bacillus aurantiacus tingitanus 
1. Bacillus lactorubefaciens Gruber (Gruber, Cent. f. Bakt., II Abt., 8, 1902, 457; Serratia lactorubefaciens Bergey et al., Manuil, 1st ed., 1923, 92.) From Latin, to make milk red.

Small rods : 0.4 to 0.6 by 3.5 microns, occurring singly and in pairs. Motile with peritrichous flagella. Gram reaction not given.

Gelatin colonies: Grayish-white, smooth, glistening, spreading.

Gelatin stab: At times arboreseent; the medium tinged with red. No liquefaction.

Agar colonies: Circular, lobed, grayish, contoured.

Agar slant: White, spreading growth.

Broth: Turbid, with grayish pellicle and slimy sediment.

Limus milk: Becomes rose red, slimy, slightly acid, without coagulation.

Potato: White, spreading growth.

No gas from carbohydrate media.

Indole not produced.

Nitrites produed from nitrates.

Aerobie, farultative

Optimum temperature $25^{\circ} \mathrm{C}^{\circ}$

Habitat: Milk

2. Bacillus rubricus Hefferun. (Hef feran, Cent. f. Bakt., II Abt., 11, 1903 403; Erythrobacillus rubricus Holland Jour. Bact., 5, 1920, 220; Serratia rubrica Bergey et al., Manual, 1st ed., 1923, 313; Chromobacterium rubricum Topley and Wilson, Prine. Bact, and Immun., 1. 1931, 402.)

Rods: 0.7 to 0.9 by 1.0 to 4.0 microns, occurring singly. Non-motile. Gram reaction note given.

Gelatin colonies: Small, circular, ye] low-orance, deepening to red.

Gelatin stab: Slow liquefaction. Old cultures lose this property

Agar colonies: Circular, raised, entire

Agar slant: Moist, spreading, white to pink, gradually deepening in color.

Broth: Turbid, with viscid sediment. Litmus milk: Alkaline.

Potato: Slight growth, bright pink, turning coral red.

Indole not produced.

Nitrites not produced from nitrates.
No acid or gas from carbohydrate media.

Aerobic, facultative.

Optimum temperature $25^{\circ}$ to $30^{\circ} \mathrm{C}$. No growth at $37^{\circ} \mathrm{C}$.

Source: Isolated from Mississippi river water, also from buttermilk.

3. Bacillus rufus Hefferan. (Hefferan, Cent. f. Bakt., II Abt., 11, 1903, 313; Erythrobacillus rufus Holland, Jour. Bact., 5, 1920, 220; Serratia rufa Bergey et al., Manual, 1st ed., 1923. 95.) From Latin rufus, red.

Differs from Bacillus rubricus in showing more luxuriant grow th on potato and slower action in milk.

Source: From Mississippi River water.

4. Bacillus mycoides corallinus Hefferan. (Hefferan, Cent. f. Bakt., II Abt., 11, 1903, 459; Serratia corallina Bergey et al., Manual, 1st ed., 1923, 93.)

imall, slender rods: 1.2 to 2.0 microns in length, occurring singly and in pairs. Non-motile. Gram reaction not given.

Cielatin rolonies: Minute, becoming pink, smooth, raised.

Gelatin stab: Slow growth. Raised, smooth, glistening, pink surface growth. Fine, feethery grow th in stab. Noliquefaction.

Agar colonies: Minute, with filamentous margin.

Agar slant: Smooth, moist, salmon pink.

Broth: Turbid, with pink flakes on surface.

Litmus milk: Alkaline, with red surface.

Potato: Like agar slant

Indole not formed.

Nitrites produced from nitrates.

No gas from carbohydrate media.

Aerobic, facultative.

Optimum temperature $25^{\circ}$ to $30^{\circ} \mathrm{C}$.

Source: Isolated from Mississippi river water.

5. Bacillus bruntzii Nepveux. (Nepveux, Compt. rend. Soc. Biol., Paris, 72, 1920, 242; Thise, Far. de Pharm., Jouve et Cie, Paris, 1920, 136 pp.; Serratia bruntzii Bergey et al., Manual, 
3rd ed., 1930, 125.) Named for Prof. Bruntz of Paris.

Bacillus roseus fluorescens Marchal (Trav. Lab. Microbiol. Fac. Pharm. Naney, 1937, 90) is regarded by Lasseur (personal communication, 1938) as identical with Bacillus bruntzii Nepveux.

Rods : 0.3 to 0.5 by 1.25 to 1.5 microns, occurring singly and in pairs. Nonmotile. Gram-negative. The cells store volutin and glycogen as reserve materials.

Gelatin colonies: Circular, gray, smooth, contoured, glistening, undulate margin, becoming red.

Gelatin stab: No liquefaction.

Agar colonies: Circular, flat, smooth, contoured, radiate margin, vinous red.

Agar slant: Smooth, echinulate, butyrous, vinous red in color.

Broth: Turbid.

Litmus milk: Unchanged.

Indole not formed.

Nitrites produced from nitrates.

Acid from glucose, fructose, maltose, lactose, sucrose, mannitol, dulcitol and glycerol.

Aerobic, facultative.

Optimum temperature $20^{\circ}$ to $25^{\circ} \mathrm{C}$.

Habitat: Water.

\section{Bacillus aurantiacus tingitanus}

Remlinger and Bailly. (Compt. rend. Soc. Biol. Paris, 119, 1935, 246.)

Short rods: Lsually 2 to 3 microns, sometimes 5 to 6 microns long. Actively motile. Gram-negative.

Growth occurs on all the ordinary nutrient media. Fluorescent bright orange pigment.
Gelatin: Rapid liquefaction.

Milk: Slow coagulation.

Synthetic broth: Lasseur, DupaixLasseur and Marion (Trav. Lab. Microbiol. Fac. Pharm. Nancy, Fasc. 9, 1936, 34) recognize two rough types of this organism, one of which forms a smooth and the other a wrinkled pellicle. The smooth type gives a rough $(\mathrm{pH} 4.7)$ or a smooth ( $\mathrm{pH} 6.3$ ) pellicle according to the $\mathrm{pH}$ of the medium.

Indole not formed.

Artichoke media: Luxuriant growth. Emerald green pigment produced. On transferring the culture to potato, the bright orange pigment reappears.

Coagulated serum: No liquefaction.

Acid from sucrose, lactose, glucose, mannitol and maltose.

Non-pathogenic.

Optimum pH 6.6. No growth at $\mathrm{pH}$ 6.2 , but grows at pH 7.8 .

Optimum temperature $20^{\circ} \mathrm{C}$. Good growth from $15^{\circ}$ to $37^{\circ} \mathrm{C}$.

Aerobic.

Pigment: Orange or eapucine pigment which diffuses throughout the medium. Not affected by the presence or absence of light. Pigment production depends on the growth of the culture, not on the acidity of the medium. Insoluble in acetone, amyl alcohol and gasoline. Partially soluble in ether and ethyl alcohol which are colored yellow.

Distinctive character: A fluorescent pigment of an unusual shade (bright orange).

Source: From water at Tangiers. Habitat: Presumably water.

Appendix II:* The anaerobic genus Methanobacterium was proposed tentatively by Kluyver and Van Niel in 1936 with indication that they regarded Söhngen's methane bacterium as the type species of the genus. Later, Barker (1936) found organisms that he regarded as identical with those previously isolated by Söhngen and he proposed the name. Methanobacterium söhngenii for this species. A second species found at the same time was named Methanobacterium omelianskii and it was identified as the species previously described but not named by Omeliansky. At the time, he felt that these anaerobes should be included in the family Mycobacteriaceae (1936,

* The manuseript for this section has been reviewed by Dr. H. Albert Barker, University of California, Berkeley, California, February, 1945. 
p. 422). In 1940, he discovered that the second species produced spores. In a personal communication (March 20,1945) he suggests that further work is needed before the relationships of these organisms can be clarified.

\section{Genus A. Methanobacterium Kluyver and Van Niel.}

(Cent. f. Bakt., II Abt., 94, 1936, 399.)

Straight or slightly bent rods, sometimes united in bundles or long chains. Usually non-motile. Endospores sometimes formed. Anaerobic. Chemo-heterotrophic or chemo-autotrophic oxidizing various organic or inorganic compounds and reducing carbon dioxide to methane. Gram-variable, usually negative.

The type species is Methanobacterium soehngenii Barker.

1. Methanobacterium soehngenii Barker. (Methanebacterium, Söhngen, Dissertation, Delft, 1906; Barker, Arch.f. Mikrobiol., 7, 1936, 433.) Named for Prof. N. L. Söhngen who first studied this organism.

Rods: Straight to slightly bent, moderately long. Non-motile. Non-sporeforming. Gram-negative.

In liquid cultures cells are characteristically joined into long chains which of ten lie parallel to one another so as to form bundles.

Acetate and $\mathrm{n}$-butyrate but not propionate are fermented with the production of methane and carbon dioxide.

Ethyl and n-butyl alcohols not fermented.

Obligate anacrobe.

Source: Enrichment cultures containing acetate or butyrate as the only organic compound. Four strains were isolated from acetate enrichment cultures. The cultures were highly purified but not strictly pure.

Hahitat: Canal mud, sewage. Probably occurs widely in fresh water sediments where anacrobic conditions prevail.

\section{Methanobacterium omelianskii Bar-}

ker. (Bacille de la décomposition méthanique de l'alcohol ethylique, Omeliansky, Ann. Inst. Past.. 30, 1916, 80; Barker, Arch f. Mikrobiol., 7, 1936 , 436; also see Barker, Antonie van Leeuwenhoek, 6, 1940, 201 and Jour. Biol. Chem., 137, 1941, 153.) Named for Prof. W. Omeliansky who first observed the organism.
Rods: 0.6 to 0.7 by 1.5 to 10 microns, usual length 3 to 6 microns, unbranched, straight or slightly bent. Usually nonmotile, occasionally feeble motility is observed. Spores of low heat resistance formed, spherical, terminal, swelling the rods.

Primary alcohols, including ethyl, propyl, n-butyl and n-amyl alcohols, are oxidized to the corresponding fatty acids. Secondary alcohols, including isopropyl and sec-butyl, are oxidized to the corresponding ketones. Hydrogen is oxidized.

Fatty and hydroxy acids, glucose, polyalcohols and amino acids are not attacked.

Carbon dioxide is used and converted to methane. Grow th and alcohol oxidation are directly proportional to the carbon dioxide supply at low concentrations.

Nitrate, sulfate and oxygen cannot be used as oxidizing agents.

Utilizes ethyl alcohol best of all organic compounds.

Utilizes ammonia as a nitrogen source. Growing range: pH 6.5 to 8.1.

Optimum temperature $37^{\circ}$ to $40^{\circ} \mathrm{C}$. Maximum $46^{\circ}$ to $48^{\circ} \mathrm{C}$

Obligate anaerobe.

Source: Soil, fresh water and marine muds, rabbit feces, sewage. Pure cultures were isolated from fresh water and marine muds (Barker, loc. cit., 1910).

Habitat: Wherever organic matter is decomposing in an anaerobic, approximately neutral environment. 
Appendix III : Miscellaneous species of non-motile, or motile, non-spore-forming rod-shaped bacteria not previously listed or described.

Ascobacterium luteum Babes. (Babes, in Cornil and Babes, Les Bactéries, 3rd ed., 1, 1890, 155; also see Petri, Cent. f. Bakt., II Abt., 26, 1910, 359.) From water in Budapest (Babes) and the olive fly (Petri).

Bacillus $a, b, c, d, e, f, h$ and $i$, Vignal. (Arch. d. phys. norm. et path., Sér. 3, 8, 1886, 350-373; also see Flavobacterium buccalis Bergey et al. and Bacillus buccalis fortuitus Sternberg.) From saliva and the teeth.

Bacillus acido-aromaticus Van der Leck. (Cent. f. Bakt., II Abt., 17, 1907, 652.) From milk.

Bacillus acutangulus Migula. (No. 13, Lembke, Arch. f. Hyg., 29, 1897, 319; Migula, Syst. d. Bakt., 2, 1900, 680.) From feces.

Bacillus acutus Kern. (Arb. bakt. Inst. Farlsruhe, 1, Heft 4, 1896, 433.)

From the stomach of a bird.

Bacillus adametzii Migula. (Bacillus No. XIII, Adametz, Landwirtsch. Jahrb., 18, 1889, 246; Migula, Syst. d. Bakt., 2, 1900, 686; not Bacillus adametsi Trevisan, I generi e le specie delle Batteriacee, 1589, 19.) From cheese.

Bacillus aeris Chester. (Bacillus riolaceus sacchari Ager, X. Y. Med. Jour. 1894, 265; see Dyar, Ann. X. Y. Acad. Sci., 8, 1895, 369; Bacterium violaceous sacchari Chester, Ann. Rept. Del. Col. Agr. Exp. Sta., 9, 1897, 116; (hester, Man. Determ. Bact., 1901, 260.) From air. Produces a violaceus black pigment in old cultures in milk.

Bacillus aerobius Doyen. (Bacillus urinae aerobius Doyen, Jour. d. connaiss. médic., 1889, 107; Doyen, ibid.. 108.) From urine.

Bacillus aerogenes Miller. (Miller. Deutsche med. Wchnschr., 12, 1886, 119; see Miller, Dic Mikroorganismen der Mundhöhle, Leipzig, 1889, 262; not $B(t-$ cillus aerogenes Kruse, in Flügge, Die Mikroorganismen, 3 Aufl., 2, 1896, 340.) From the stomach.

Bacillus aërogenes sputigenus capsulatus Herla. (Arch. de Biol., 14, 1895, 403; abst. in Cent. f. Bakt., 25, 1899, 359.) From the blood of a mouse which had been inoculated with the sputum of a pneumonia patient.

Bacillus aeschynomenus Trevisan. (Bacille de l'air j, Babes, in Cornil and Babes, Les Bactéries, 2nd ed., 1886, 150; Trevisan, I generi e le specie delle Batteriacee, 1889, 20.) From air.

Bacillus aëthebius Trevisan. (Bacille de l'air $c$, Babes, in Cornil and Babes, Les Bactéries, 2nd ed., 1886, 149; Trevisan, I generi e le specie delle Batteriacee, 1889,20 .) From air.

Bacillus agilis Trevisan. (Bacillus der Vagus-Pneumonie, Schou, Fortschr. d. Medicin, 3, 1885, Yo. 15; Bacillus pneumonicus agilis Flügge, Die Mikroorganismen, 2 Aufl., 1886, 262; Trevisan. I generi e le specie delle Batteriacee, 1889, 14; not Bacillus agilis Tschistowitsch, Berl. klin. Wochnschr., 1892, 512; not Bacillus agilis Chester, Man. Determ. Bact., 1901, 226; not Bacillus agilis Mattes, Sitzungsber. d. Gesells. z. Beforderung d. gesam. Naturw. z. Marburg. 62, 1927, 406; not Bacillus agilis Hauduroy et al., Dict. d. Bact. Path. l'aris, 1937, 33; Bacterium pneumonicus agilis Chester, Ann. Rept. Del. Col $\therefore$ gr. Exp. Sta., 9, 1897, 140; Bacterium vagus pneumonie Chester, ibid., 144; $B a-$ cillus pneumonicus Migula, Syst. $d$. Bakt., 2, 1900, 752.) From the lungs of rabbits having vagus pneumonia.

Bacillus agnlis Mattes. (Sitzungsber. d. Gesells. z. Beforderung d. gesam. Naturw. z. Marburg, 62, 1927, 406. From the Mediterranean flour moth (Ephestia huchniella).

Bacillus agillimus DeToni and Trevisan. (Bacillus luteus putidus Maggiora, Giorn. d. Soc. ital, d'Igiene, 11, 1889, 344; DeToni and Trevisan, in Saccardo, Sylloge Fungorum, 8, 1889, 969.) From the skin. 
Bacillus agnorum Trevisan. (Bacterium subtile agnorum Rivolta, Giorn. di Anat. fisiol. degli animali, 1881, 31 and 1883, 78; Trevisan, I generi e le specie delle Batteriacee, 1889, 13.) From diseased lambs.

Bacillus alacer Eekstein. (Ztschr. f. Forst- u. Jagdwesen, 26, 1894, 13.) Found associated with the eggs of the nun moth (Lymantria monacha).

Bacillus alatus Grieg Smith. (Proc. Linn. Soc. New So. Wales, 30, 1905, 570.)

Bacillus albatus Kern. (Arb. bakt. Inst. Karlsruhe, 1, Heft 4 , 1896, 408.) From the stomarh and intestines of : bird.

Bacillus albus Pagliani, Maggiora and Fratini. (Weisser Bacillus, Eisenberg, Bakt. Diag., 1 Aufl., 18\$6, Table 7 ; Pagliani et al., Giorn. d. Soc. ital. d'Igiene, 9, 1857, 587; not Bacillus albus Trevisan, I generi e le specic delle Batteriacee, 1889, 14; not Bacillus albus Bergey et al., Manual, 3rd ed., 1930, 395; Bacterium albus Chester, Ann. Rept, Del. Col. Agr. Exp. Sta., 9, 1897, 76.) From water.

Bacillus albus anaerobiescens Vaughan. (Amer. Jour. Med. Sci., 104, 1892, 191.) From water.

Bacillus altus putidus Debary. (Quoted from Sternberg, Man. of Bact., 1893, 675.) From water.

Bacillus albus-putidus C'hester. (Maschek, see Adametz, Bakt. Nutz. u. Trinkwässer, 1888; Chester, Man. Determ. Bact., 1901, 237.) From water.

Bacillus albus putridus Vaughan. (Amer. Jour. Med. Sci., 104, 1S92, 186.) From water.

Bacillus alpha Dyar. (Ann. X. I. Acad. Sci., 8, 1895, 366.) From air.

Bacillus amabilis Dyar. (Dyar, Ann. N. Y. Acad. Sci., 8, 1895, 358; Bacterium amabilis Chester, Ann. Rept. Del. Col. Agr. Exp. Sta., 9, 1897, 110.) From air.

Bacillus amarillae Trevisan. (Bacille de la fiévre jaune, Babes, in Cornil and Babes, Les Bactéries, 2nd ed , 1886, 529;
Trevisan, I generi e le specie delle Batteriaces, 1889, 13.) From a case of yellow fever.

Bacillus amarus Migula. (Bacillus liquefaciens lactis amari v. Freudenreich, Landwirtsch. Jahrb. d. Schweiz, 8, 1894; Migula, Syst. d. Bakt., 2, 1900, 694; not Bacillus amarus Hammer, Iowa Agr. Exp. Sta. Res. Bull. 52, 1919, 198.) From bitter milk.

Bacillus amerimnus Trevisan. (Bacille de l'air b, Babes, in Cornil and Babes, Les Bactéries, 2nd ed., 1886, 149; Trevisan, I generi e le specic delle Batteriacee, 1\$89, 20.) From air.

Bacillus amygdaloides Weiss. (Arb. bakt. Inst. Karlsruhe, 2, Heft 3, 1902, 246.) From brine on salted pickles.

Bacillus anceps Trevisan. (Bacille du mucus intestinal normal $a$, Babes, in Cornil and Babes, Ies Bactéries, 2nd ed., 1586, 153; Trevisan, I generi e le specie delle Batteriacee, 1889, 15.) From normal intestinal mucous.

Bacillus anthraciformis Wilhelmy. (Arb. bakt. Inst. Karlsruhe, 3, 1903, 28.) From meat extract.

Bacillus anthracoides Trevisan. (Bacille de l'air li, Babes, in Cornil and Babes, Les Bactéries, 2nd ed., 1886, 151; Trevisan, I generi e le specie delle Batteriacee, 1889, 20; not Bacillus anthracoides Kruse, in Flügge, Die Mikroorgunismen, 3 Aufl., 2, 1896, 232.) From air.

Bacillus annulatus Zimmermann. (Bakt. unserer Trink- u. Nutzwässer, Chemnitz, 2, 1894, 30.) From water.

Bacillus anularius Henrici. (Arb. bakt. Inst. Karlsruhe, 1, Heft 1, 1894, 32.) From Emmenthal cheese.

Bacillus apicum Iruse. (Canestrini, Atti Soc. Ven. Trent. Sei. Nat., 12, 1892, 13t; Kruse, in Flügge, Die Mikroorganismen, 3 Aufl., 2, 1896, 233.) From infected bees and their larvae.

Bacillus apisepticus Burnside. (Jour. Econ. Ent., 21, 1928, 379.) Pathogenic for the honey bee (Apis mellifera).

Bacillus aquatilis Migula. (Bacillus 
aquatilis sulcatus $T^{T}$, Weichselbaum, Das österreichische Sanitïtswesen, 1889 , No. 14-23; Bacterium aquatilis sulcatus quartus Chester, Ann. liept. Del. Col. Agr. Exp. Sta., 9, 1897, 72; Migula, Syst. d. Bakt., 2, 1900, 733; not Bacillus aquatilis Frankland and Frankland, Ztschr. f. Hyg.. 6, 1889, 381; Bacillus aquatilissu!calus-quartus Chester, Man. Determ. Bact., 1901, 216.) From water.

Bacillus aquatilis Trevisan. (Bacille de l'eau $a$, Babes, in Cornil and Babes, Les Bactéries, 2nded., 1886, 167 ; Trevisan, I generi e le specie delle Batteriacee. 1889, 19; not Bacillus aquatilis Frankland and Frankland, Ztschr. f. Hyg., 6, 1889, 381.) From water.

Bacillus aquatilis communis Kruse. (Iruse, in Flügge, Die Mikroorganismen, 3 Aufl., 2, 1896. 315; Bacterium aquatilis communis Chester, Amn. Rept. Del. Col. Agr. Exp. Sta., 9, 1897, 91.) Found commonly in water. Listed by Kiruse as a non-chromogenic strain of Bacillus fuorescens liquefaciens (Pseudomonas fluorescens Migula).

Bacillus arborescens Jamieson and Edington. (Brit. Med. Jour., 1, 1857, 1265.) From the desquamation of searlet fever patients.

Bacillus arboreus Migula. (Bitumchenbacillus, Masehek, Bakt. Entersuch. d. Leitmeritzer Trinkwässer, Leitmeritz, 1887; Migula, Syst. d. Bakt., 2, 1900, 710.) From water.

Bacillus aromaticus Beijerinck. (Quoted from Van der Leck, Cent. f. Bakt., II Abt., 17, 1907, 490; not Bacillus aromaticus Pammel, Bull, 20, Iowa Agr. Exp. Sta., 1893, 792; not Bacillus aromaticus Grimm, Cent. f. Bakt., II Abt. 8, 1902, 589; not Bacillus aromaticus Van der Leck, loc. cit., 659.) From milk.

Bacillus aromaticus Van der Leck. (Van der Leck, Cent. f. Bakt., II Abt., 17, 1907, 659.) From soft cheeses.

Bacillus assimitis Trevisan. (Bacille de l'air $i$, Babes, in Cornil and Babes, Les Bactéries, 2nd ed., 1886, 150; Trevisan, I generi e le specie delle Batteriacee, 1899,20 .) From air.

Bucillus aurantius Trevisan. (Orangerother $W$ asserbacillus, Adametz and Wichmann, Mitth. Oest. Vers. Stat. f. Brauerei u. Mälz. in Wien, 1, 1888, 50; Trevisan, I generi e le specie delle Batteriacee, 1889, 19; not Bacillus aurantius Bergey et al., Manual, 3rd ed., 1930, 121.) From water.

Bacillus aureus Eckstein. (Ztschr. f. Forst-u. Jagdwesen, 26, 1894, 9; probably not Bacillus aureus Frankland and Frankland, Philos. Trans. Roy. Soc. London, 178, B, 1887, 272 and probably not Bacillus aureus Pansini, Arch. f. path. Anat. u. Physiol., 122, 1890, 436; not Bacillus aureus Adametz, quoted from Sternberg, Man. of Bact., 1893, 621.) Capable of infecting the larvae of various insects.

Bacillus azureus Zimmermann. (Bakt. unserer Trink- u. Nutzwässer, Chemnitz, 2, 1894, 24.) From water

Bacillus babesi Trevisan. (Bacille du nucus intestinal normal b, Babes, in Cornil and Babes, Les Bactéries, 2nd ed., 1856, 153; Trevisan, I generi e le speeie delle Batteriaces, 1889, 15.) From normal intestinal mucus.

Bacillus belfantii Migula. (Eine neue pathogene Bakteriumart im Tetanusmaterial, Belfanti and Pescarolo, Cent. f. Bakt., 4, 1888, 513; Bacillus accidentalis tetani Kruse, in Flügge, Die Miliroorganismen, 3 Aufl., 2, 1896, 433; Bacterium accidentalis tetani Chester, Ann. Rept. Del. Col. Agr. Exp. Sta., 9, 1897, 88; Migula, Syst. d. Bakt., 2, 1900, 767; not Bacillus belfanti Carbone and Venturelli, Boll. Ist. Sieroter., Milan, 4, 1925, 59; Bacillus accidentalis Chester, Man. Determ. Bact., 1901, 229.) From pus in a case of tetanus.

Bacillus benzoli Tausson. (Planta, $\gamma$, 1929 , 735.) From soil. Oxidizes benzene,

Bacillus beritericus Trevisan. (Caratt. di alc. nuov, gen. di Batt., 1885, 12.) From cases of beri-beri in Japan. 
Also see Ogata, abst. in Cent. f. Bakt., 3, 18S8, 75 .

Bacillus berolinensis Migula. CRoter Bacillus aus Wasser, Fraenkel, Cirundriss der Bakterienkunde, 3 Auft., 1890, 252; Bacillus ruber berolinensis Kruse, in Flügge, Die Mikroorganismen, 3 Auff., 2, 1896, 303; Bacterium ruber berolinensis Chester, Ann. Rept. Del. Col. Agr. Exp. Sta., 9, 1897, 113; Migula, Syst. d. Bakt., 2, 1900, 856; not Bacillus berolinensis Chester, Man. Determ. Bact., $1901,305$.$) From water. Rust-red to$ orange-yellow pigment on potato.

Bacillus bete Dyar. (Ann. N. Y. lear. Sci., 8, 1895, 366.) From air.

Bacillus beyerinckii DeToni and Trevisan. (Bacillus radicicolu var. liquefaciens Beijerinck, Bot. Zeitung, 1888, 750; DeToni and Trevisan, in Saccardo, Sylloge Fungorum, 8, 1889, 972; not $\mathrm{Ba}$ cillus beijerinckii Henneberg, Ztschr. f. Spiritusindustrie, 26, 1903, 22; sce Cent. f. Bakt., II Abt., 11, 1903, 159.) From soil and the roots of legumes.

Bacillus billingsi Chester. (Bacillus of corn-stalk disease of cattle, Billings, in Baumgarten, Jahresbericht, 1889, 184; (hester, . Ian. Determ. Bac1., 1901, 214.) Isolated by Billings from corn-stalk disease of cattle, and by Nocard from bronchopneumonia in oxen.

Bacillusbombycis (hatton. CChatton, ('ompt. rend. Acad. Sci., Paris, 156, 1913, 1708; not Bacillus bombucis Marechiati, Stazioni sperimentali Agrarie Italiane, 20, 1\$91, 121; Bacterium bombycis Paillot, L'infection chez les insectes, 1933, 131.) From diseased silkworms (Bumbyx mori).

Bacillus bookeri Dyar. (Dyar, Ann. N. Y. Acad. Sei., 8, 1895, 378; not Bacillus bookeri Ford, Studies from the lioval Victoria Hospital, Montreal, 1, 1903, 31.) Found by Dr. Prudken in at case of rystitis.

Bracillus brachythrix DeToni and Trevisan. (Bacillus $r$, Maggiora, Giorn. Soe ital. d'Igiene, 11, 1859, 348; DeToni and Trevisan, in Saceardo, Sylloge
Fungorum, 8, 1889, 967.) From the skin.

Bacillus brunncus (Schroeter) Schronter. (Bacteridium brunneum Schroeter, in Cohn, Beitr. z. Biol. d. Pflanz., 1, Heft 2, 1872, 126; Schroeter, in Cohn, Kryptog. Flora v. Schlesien, 3 (1), 1886, 158; not Bacillus brunneus Adametz and Wichmann, Die Bakt. der Nutz-und Trinkwässer, Wien, 1888; Bacillus fuscus Flügge, Die Mikroorganismen, 2 Aufl., 1886, 290; not Bacillus fuscus Zimmermann, Bakt. unserer Trink- u. Nutzwïsser, Chemnitz, 1, 1890, 70.) Bacterium brunneum Schroeter or Cohn is given as a synonym by Flügge (1886) and hy Trevisan (1889) but this appears to be an ineorrect spelling of Bacteridium brunneum Schroeter. Neither Schroeter nor Cohn used Bacterium brunneum in 1872 or later so far as can be determined by a careful study of their papers. From corn, wheat and potato infusions.

Bacillus buccalis fortuitus Sternberg. (Bacillus j, Vignal, Arch. Phys. norm, et path., Sér. 3, 8, 1886, 337; Sternberg, Man. of Bact., 1893, 685; Bacterium bucalis (sic) fortuitus Chester, Ann. Rept. Del. Col. Agr. Exp. Sta., 9, 1897, 91 and 130 ; Bacillus bucalis (sic) Chester, Man. Determ. Bact., 1901, 234; not Bacillus buccalis Trevisan, I generi e le specie delle Batteriacee, 1889, 15.) From the mouth.

Bacillus buccalis muciferens Miller. (Miller, Dental Cosmos, 39, 1891, 792 and 800 .) From the blood. I slimy capsulated bacillus.

Bacillus buccalis septicus Miller. (Miller, Dental Cosmos, 33, 1891, 792 and 802.) From the mouth and in pus of an abscess caused by a dental instrument.

Bacillus butyri Migula. (Bacillus butyri I, v. Klecki, ('ent.f. Bakt., 15, 1894, 357; Migula, Syst. 1. Bakt., 2, 1900, v and 811.) From rancid butter.

Bacillus caeci Ford. (Studies from the Royal Victoria Hosp., Montreal, 1, (5), 1903, 45; also see Jour. Med. Res., 
1, 1901, 217.) Fron the stomach and rectum.

Bacillus canalensis Castellani. (Proc. Soc. Exp. Biol. and Med., 25, 1928, 540.) From human feces.

Bacillus canus Migula. (Grauer Bacillus, Maschek, Lntersuch. d. Leitmerit zer Trinkwässer, Leitmeritz, 1887; Migula, Syst. d. Bakt., 2, 1900, 711.) From water.

Bacillus canus Eckstein. (Ztschr. f. Forst- u. Jagdwesen, 24, 1894, 15.) From larvae of the nun moth (Lymantria monacha.)

Bacillus carabiformis Raczyscli. (Diss. milit. merlic. Acad. Petropolitanae Ruteniae, 1885; abst. in Cent. f. Bakt., $6,1889,113$.) From the stomach of a dog.

Bacillus carnis Wilhelmy. (Wilhelmy, Arb. bakt. Inst. Karlsruhe, 3, 1903, 21; not Bacillus carnis Klein, Cent. f. Bakt., II Abt., 35, 1903, 459.) From meat extract.

Bacillus cascolyticus Lochmann. (Cent. f. Bakt., I Abt., Orig., 31, 1902, 385.) From the organs of guinea pigs which had been inoculated with tubercle bacilli.

Bacillus cathetus Trevisan. (Bacille de l'air $g$, Babes, in Cornil and Babes, Les Bactéries, 2nd ed., 1886, 150; Trevisan, I generi e le specie delle Bat teriacee, 1889, 20.) From air.

Bacillus caviae Trevisan. (Bacille du mucus intestinal normal du cobaye $e$, Babes, in Cornil and Babes, Les Bactéries, 2nd ed., 1886, 154; Trevisan, I generi e le specie delle Batteriacee, 1889, 15; Pasteurella caviae DeToni and Trevisan, in Saccardo, Sylloge Fungorum, 8 , 1889, 996; not Pasteurella caviac Hauduroy et al., Dict. d. Bact. Path., 1937, 313.) From the intestinal mucus of guinea pigs.

Bacillus centralis Zimmermann. (Bakt. unserer Trink. u. Nutzwässer, (hemnitz, 2, 1894, 10.) From water.

Bacillus charrini Trevisan. (Bacille de la pseudo-tuberculuse bacillaire du cobaye, Charrin and Roger, Compt. rend. Acad. Sci., Paris, 106, 1885, 868; Trevisan, I generi e le specie delle Batteriacee, 1889, 13.) From pulmonary tuberculosis of guinea pigs.

Bacillus chlorinus Migula. (Grüngelber Bacillus, Tataroff, Inaug. Diss., Dorpat, 1891, 50; Migula, Syst. d. Bakt., 2, 1900, 820 ; not Bacillus chlorinus Frankland and Frankland, Philos. Trans. Roy. Soc. London, 178, B, 1887, 274.) From water.

Bacillus chyluriae Trevisan. (Bacillus of chyluria, Wilson, Brit. Med. Jour., No. 1249, 188t, 1128; Trevisan, Atti Acad. Med.-Fis.-Stat. Milan., Ser. Y. 3, 1885, 99.) From chyluria.

Bacillus citreus (Inna and Tommasoli) Iruse. (Ascobacillus citreus Unna and Tommasoli, Monats. f prakt. Dermatol., 9, 1890, 60; Kruse, in Flügge, Die Mikroorganismen, 3 Aufl., 2, 1S96, 309; not Bacillus citreus Frankland and Frankland, Philos. Trans. Roy. Soc., London, $178,1887, \mathrm{~B}, 272$; Bacterium citreus Chester, Ann. Rept. Del. Col. Agr. Exp. Sta., 9, 1897, 104.) From the human skin in cases of eczema.

Bacullus citricus Kern. (Kern, Arb. bakt. Inst. Karlsiuhe, 1, Heft 4, 1896. 426; not Bacillus citricus Weiss, ilid., 2, Heft 2, 1902, 234.) From the intestines of birds.

Bacillus citmus Migula. (Citronge)ber Bacillus, Maschek, Bakt. Intersuch. d. Leitmeritzer Trinkwässer, 1Ss7; Migula, Syst.d. Bakt., 2, 1900, 832.) From water.

Bacillus cladogenes Trevisan. (Bactérie de l'air No. 3, Babes, in Cornil and Babes, Les Bactéries, 2nd ed., 1886, 140; Trevisan, I generi e le specie delle Batteriacee, 1889, 20.) From air.

Bacillus claviformis Doyen. (Bacillus urinae claviformis Doyen, Jour. d. connaiss. médic., 1889, 106; Doyen, ibid., 108; Bacillus doyeni DeToni and Trevi- 
san, in Saccardo, Sylloge Fungorum, 8, 1889, 949.) From urine.

Bacillus clconi Picard. (Bull. Soc. d'Étude et de Vulgarisation Zool. Agric., $12,1913,134$.$) A fluorescent cocco-$ bacillus. From diseased larvae of weevils (Temnorrhinus (Cleonus) mendicus).

Bacillus coccineus Catiano. (Catiano, in Cohn, Beitr. z. Biol. d. Pflanz., 7, 1896, 339; not Bucillus coccuneus Pansini, Arch. f. path. Anat., 122, 1890, 437; Bacillus subcoccineus Migula, Syst. d. Bakt., 2, 1900, 857.) From the vagina. Reddish pigment.

Bacillus coeruleo-viridis Trevisan. (Blaugrün fluorescirende Bacterium, Adametz, Mitth. Oest. Vers. Stat. f. Brauerei u. Mälz. in Wien, 1, 1888, 46; Trevisan, I generi e le specie delle Batteriacee, 1889, 20; Bacterium coeruleoviride De'Toni and Trevisan, in Saccardo, Sylloge Fungorum, 8, 1889, 1087.) From water.

Bacillus coeruleus Eckstein. (Eckstein, Ztschr. f. Forst-u. Jagdwesen, 26, 189.1, 14; not Bacillus coeruleus Smith, Med. News, 1887, 758; probably not Bacillus coeruleus Voges, Cent. f. Balit., 14, 1893, 301.) From larvae of the nun moth (Lymantria monacha).

Bacillus columbarum Chester. (Bacillus of pigeon cholera, Moore, U.S. D.A., Bur. Anim. Ind., Bull. 8, 1895; Chester, Man. Determ. Bact., 1901, 209.) From a disease of pigeons. Hadley et al. (Rhode Island Agr. Exp. Sta., Bull. 174, 1918, 178) regard this as probably a paracolon.

Bacillus constrictus Zimmermann. (Zimmermann, Bakt, unserer Trink- u. Nutzwässer, Chemnitz, 1, 1890, 42; Bacterium constrictus Chester, Ann. Rept. Del. Col. Agr. Exp. Sta., 9, 1897, 112.) From water.

Bacillus convolutus Wright. (Wright, Mem. Nat. Acad. Sci., 7, 1895, 461; Bacterium convolutus Chester, Ann. Rept. Del. Col. Agr. Exp. Sta., 9, 1897, 101.) From river water.

Bacillus coprogenes foetidus Sternberg.
(Darmbacillus, Schottelius, 1885; Sternberg, Man. of Bact., 1893, 468.) From the intestinal contents of pigs which had died of swine erysipelas.

Bacillus coronatus Keck. (Inaug. Diss., Dorpat, 1890, 43.) From water.

Bacillus corvi Kem. (Arb. bakt. Inst. Karlsruhe, 1, Heft 4, 1896, 394.) From the stomach and intestines of birds.

Bacillus courmontii Migula. (Courmont, Compt. rend. Soc. Biol., Paris, 1889; Bacillus pseudotuberculosis simitis Kruse, in Flügge, Die Mikroorganismen, 3 Aufl., 2, 1896, 454; Migula, Syst. d. Bakt., 2, 1900, 770.) From tubercles of cattle.

Bacillus crassus Lucet. (Bacillus crassus pyogenes bovis Iucet, Ann. Inst. Past., 7, 1897, 327; Bacillus crassus pyogenes Lueet, ibud., 327; Lucet, ibid., 328; Bacillus pyogenes crassus Kruse, in Flügge, Die Mikroorganismen, 3 Aufl., 2, 1896, 343; Bacterium pyogenes crassus Chester, Ann. Rept. Del. Col. Agr. Exp. Sta., 9, 1897, 141; Bacillus bovis Migula, Syst. d. Bakt., 2, 1900, 765.) From bovine abscesses. Regarded by Kruse as a synonym of Bacillus pneumoniae.

Bucillus crinitus Migula. (No. 15, Lemblie, Arch. f. Hyg., 29, 1897, 321; Migula, Syst. d. Bakt., 2, 1900, 678.) From feces.

Bacillus cubonianus Cuboni and Garbini. (Atti. dei Lincei, Ser. 4, 6, 1890, 26-27, quoted from Steinhaus, Bact. Assoc. Extracell. with Insects and Ticks, Minneapolis, 1942, 53; not Bacillus cubonianus Macchiati, Staz. Sperim. Agr. Ital., 23, 1892, 228.) From silkworms (Bombyx mori).

Bacillus cuenoti Mercier. (Bakterienähnlichen Gebilden, Blochmann, Ztschr. f. Biol., 24, 1857, 1; Compt. rend. Soc. Biol., Paris, 61, 1906, 682; also in Arch. f. Protistenkunde, 9, 1907, 346.) From the fat body of the cockroach (Periplaneta orientalis).

Bacillus cuniculi Migula. (Bacillus septicus cuniculi Lucet, Ann. Inst. Past., $6,1892,564$; Bacillus cuniculi septicus Kruse, in Flügge, Die Mikroorganismen, 
3 Aufl., 2, 1896, 406; Bacterium cuniculi septicus Chester, Ann. Rept. Del. Col. Agr. Exp. Sta., 9, 1897, 76; Migula, Syst. d. Bakt., 2, 1900, 758.) Associated with a s pontaneous epizootic of rabbits.

Bacillus cuniculicida immobitis Kruse. (Kruse, in Flügge, Die Mikroorganismen, 3 Aufl.. 2, 1896, 417; Bucterium cuniculicida immobilis Chester, Ann. Rept. Del. Col. Agr. Exp. Sta., 9, 1897. 84; Bacterium cuniculicida var. immobile Chester, Man. Determ. Bact., 1901, 140.) Cause of a disease of rabbits.

Bacillus cystiformis Clado. (Quoted from Sternberg, Man of Bact., 1893. 649). From urine in a rase of cystitis

Bacillus cystitidis Migula. (C'occohacillus aerogenes vesicae Schow. Cent. f. Bakt., 12, 1892, 749; Bacillus aerogenes vesicae Lehmann and Neumann, Bakt. Diag., 1 . Aufl., 2. 1896. 237; Bacterium aerogenes vesicae Chester, Ann. Rept. Del. Col. Agr. Exp. Sta., 9. 1897, 128; Migula, Syst. d. Bakt., 2, 1900, 771; Bacillus aerogenes (hester, Man. Determ. Bact., 1901, 227; not Bacillus aerogenes Miller, Deutsrhe med. Worhnschr.. 12. 1886, 119; not Bacillus aerogenes Kruse, in Flügge. Die Mikroorganismen, 3 Aufl., $2,1896.340 .1$ From urine in a ease of eystitis.

Bacillus dacryoidens Migula. (Bacillus oogenes hydrosulfurens $\eta$, Zörkendöfer. Arch. f. Hyg.. 16, 1s93, 389; Migula, Syst. d. Bakt.. 2, 1900, 791.1 From hens' eggs.

Bacillus decolor Erkstein. (Ztschr. f. Forst- u. Jagdwesen, 26, 1894, 15.) From the larvae of a butterfly (Tanesen utricae).

Bacillus decolorans major Dyar (Ann. X. Y. Acad. Sei., 8, 1895, 362.) From air.

Bacillus decolorans minor Dyar (Ann. N. Y. Arad. Sci., 8, 1895, 359.) From air.

Bacillus defessus Kern. (Arb, bakt Inst. Karlsruhe, 1, Heft 4, 1896, 397. From the stomach and intestines of birds.

Bacillus delta Dyar. (Dyar, Ann.
N. Y. Acad. Sci., 8, 1895, 368; Bacterium delta Chester, Ann. Rept. Del. Col. Agr. Exp. Sta., 9, 1897, 114.) From water.

Bacillus denitrificans Giltay and Aberson. (Giltay and Aberson, Arch. Néerl. Sei. exact et nat., 25, 1891, 341; quoted from Sternberg, Man. of Bact., 1893, 727 ; not Bacillus denitrificans Migula, Syst. d. Bakt.. 2, 1900, 796; not Bucillus denitrificans C'hester, Man. Determ. Bact., 1901, 274.) From soil and air.

Bacillus dentrofuorescens van Iterson. (Cent.f. Bakt., II Abt., 9, 1902, 772; 12, 1904, 111.) Fluorescent. From soil.

Bacillus dentalis viridans Miller. (Miller, Die Mikroorganismen der Mundhöhle, Leipzig, 1859, 218.) From carious teeth.

Bacillus dermoides Tataroff. (Inaug. Diss., Dorpat, 1891. 19.) From water.

Bacillus diaphanus Migula. (Halibacterium pellucidum Fischer, Die Bakterien des Meeres, 1894, 22; Migula, Syst. d. Bakt.. 2, 1900, 712.) From sea water.

Bacillus difjtuens Doyen. (Bacullus urmae difluens Doyen, Jour. d, connaiss médic.. 18s9, 107; Doyen, ibid., 10s; not Bacillus difiluens: Castellani, 1915, see Castellani and ('halmers, Man. Trop. Med., 3rd ed., 1919, 943.) From urine.

Bacillus digitatus Migula. (Bacillus No. 7, Pansini, Arch. f. path. Anat., 122, 1890, 443; Migula, Syst. d. Bakt., 2, 1900, 659.) From sputum.

Bacillus dissimilis Trevisan. (Bacil lus I, Leube, Arch. f. path. Anat., 100, 1885,556 ; Trevisan, I generi e le specie delle Battriacee, 1889, 16.) From urine.

Bacillus domesticus Dyar. (Dyar, Ann. Y. I. Acad. Sci., 8, 18!5, 358; Bacterium domestzcus Chester, Ann. Rept. Del. Col. Agr. Exp. Sta., 9, 1897,110 .) From air.

Bacillus droserae (Troili-Petersson) Buchanan and Hammer. (Bacterium droserae Troili-Petersson, Cent. f. Bakt., II Abt., 38, 1913, 1; Buchanan and Hammer, Iowa Sta. Coll. Agr. Exp. Sta., Res. Bull. 22, 1915, 256.) Isolated by placing leaves of a sundew (Droser $t$ intermedia) in milk and isolating the 
slimy milk organisms developing. Closely related to Bacterium lactorubefaciens Gruber, according to $\mathrm{Bu}$ chanan and Hammer.

Bacillus duclauxii (Miquel) DeToni and Trevisan. (Urobacillus duclauxii Miquel, Ann. d. Microgr., 2, 1889, 58; DeToni and Trevisan, in Saccardo, Sylloge Fungorum, 8, 1889, 963.) From sewage.

Bacillus eczemicus Trevisan. (I generi e le specie delle Batteriacee, 1889, 14.) From exudate in cases of eczema.

Bacillus egregius Zopf. (Quoted from Papenhausen, Arb. bakt. Inst. Karlsruhe, 3, 1903, 59.) A reddish-yellow non-spore-forming rod.

Bacillus elipsoideus Migula. (Bacillus saprogenes vini $\mathrm{v}$, Kramer, Die Bakterien in ihren Bezichungen zur Landwirtschaft, 2, 1892, 138; Migula, Syst. d. Bakt., 2, 1900, 681.) From wine.

Bacillus ellingtonii Chester. (Bacillus No. 21, Conn, Rept. Conn. (Storrs) Agr. Exp. Sta., 1893, 52; Chester, Man. Determ. Bact., 1901, 264.) From milk.

Bacillus eminans Weiss. (Arb. bakt. Inst. Farlsruhe, 2, Heft 3, 1902, 232.) From vegetable infusions.

Bacillus emulsinus Ferni and Montesano. (Cent. f. Bakt., 15, 1894, 722.) From air. Decomposes amygdalin.

Bacillus endocarditidis Migula. (Bacillus endocarditidis griseus Weichselbaum, Beitr. z. path. Anat., 4, 1889, 119; Bacterium endocarditidis griseus Chester, Ann. Rept. Del. Col. Agr. Exp. Sta., 9, 1897, 88; Migula, Syst. d. Bakt., 2, 1900, 750.) From a case of endocarditis.

Bacillus engelmanni Trevisan. (Bacterium chlorinum Engelmann, see Flügge, Die Mikroorganismen, 2 Aufl,, 1886, 289; not Bacterium chlorinum Migula, Syst. d. Bakt., 2, 1900, 471; Trevisan, I generi e le specie delle Batteriacee, 1889 , 18.) Soure not given.

Bacillus enteromyces Trevisan. (Bacille des selles $f$, Babes, in Cornil and Babes, Les Bactéries, 2nd ed., 1886, 154; Trevisan, I generi e le specie delle Batteriacee, 1889, 15.) From feces.
Bacillus entomotoxicon Duggar. (Bull. Illinois State Lab. Nat. Hist., 4, 1896, 340-379.) From the squash bug (Anasa tristis).

Bacillus epsilon Dyar. (Dyar, Ann. N. Y. Acad. Sci., 8, 1895, 369; Bacterium. epsilon Chester, Ann. Rept. Del. Col. Agr. Exp. Sta., 9, 1897, 114.) From air.

Bacillus equi Migula. (Bacillus equi intestinalis Dyar and Keith, Technol. Quarterly, 6, 1893, No. 3; abst. in Cent.f. Bakt., 16, 1894, 838; Bacterium equi intestinalis Chester, Ann. Rept. Del. Col. Agr. Exp. Sta., 9, 1897, 70; Migula, Syst. d. Bakt., 2, 1900, 874; Bacillus intestinalis Chester, Man. Determ. Bact., 1901, 213.) From the intestines of a horse.

Bacillus erubescens Migula. (Bacillus oogenes hydrosulfureus $\kappa$, Zörkendörfer, Arch. f. Hyg., 16, 1893, 391; Migula, Syst. d. Bakt., 2, 1900, 792; Bacillus rubescens Nepveux, Thèse, Fac. Pharm. Paris, 1920, 113.) From hens' eggs.

Bacillus erythrogenes rugatus Dyar. (Ann. N. Y. Acad. Sci., 8, 1895, 374.) A wrinkled variety of Bacillus lactis erythrogenes Hueppe.

Bacillus erythrosporus Miflet. (Miflet, in Cohn, Beitr. z. Biol. d. Pflanz., 3, Heft 1, 1879, 135; Bacillus (Streptobacter) erythrospores (sic) Schroeter, in Cohn, Krypt. Flora v. Schles., 3, 1, 1886, 158; Bacterium erythrosporus Chester, Ann. Rept. Del. Col. Agr. Exp. Sta., 9, 1897, 123.) From putrefying egg-white and meat infusion. According to Chester, the author mistook reddish granules for spores. Fluorescent.

Bacillus esterificans fluorescens Maasen. (Arb. a. d. k. Gesundsheitsamte, 15, 1899, 501-507.) From grains and from rotting vegetation in river water.

Bacterium esterificans stralauense Maasen. (Arb. a. d. k. Gesundheitsamte, 15, 1899, 504-507.) From Spree River water.

Bacillus eta Dyar. (Dyar, Ann. N. Y. Acad. Sci., 8, 1895, 374; Bacterium eta Chester, Ann. Rept. Del. Col. Agr. Fxp. Sta., 9, 1897, 107.) From air.

Bacillus ethareticus Frankland and 
Frankland. (Proc. Roy. Soc. London, 46, 1889.) Ferments mannitol, glycerol and glucose to ethyl alcohol and acetic acid with a trace of formic and succinic acids.

Bacillus ethacetosuccinicus Frankland and Frew. (Transactions of the Chemical Society, 1892, 275.) Ferments mannitol and dulcitol to ethyl alcohol, acetic acid, succinic acid, hydrogen and carbonic acid.

Bacillus exapatus Trevisan. (Bacillus der conjunctivalsack $f$, Fick, Ueber Mikroorg. in Conjunctivalsack, Wiesbaden, 1887; Trevisan, I generi e le specie delle Batteriacee, 1889, 15.) Found frequently in the human eye.

Bacillus exiguus Wright. (Wright, Mem. Nat. Acad. Sci., 7, 1895, 447; Bacterium exiguum Chester, Ann. Rept. Del. Col. Agr. Exp. Sta., 9, 1897, 114; not Bacterium exiguum Stäubli, Münchner med. Wochnschr., No. 45, 1905. From water.

Bacillus famiger Trevisan. (Bacillus bei Erysipel am Kaninchenohr, Flügge, Die Mikroorganismen, 2 Aufl., 1886, 283; Trevisan, I generi e le specie delle Batteriacee, 1889, 14.) From a case of erysipelas of the ear of a rabhit.

Bacillus felis (Rivolta) Trevisan. (Cocco-tacterium felis Rivolta, Giorn. di Anatomia, No. 1, 1888; Trevisan, 1 generi e le specie delle Batteriacee, $1889,14$.$) From an infection in a cat.$

Bacillus fermentationis Chester. ( $B a$ cillus foetidus liquefaciens Tavel, Leber d. Aetiol. d. Strumitis, Basel, 1892; Chester, Man. Determ. Bact., 1901, 233.) From strumit is.

Bacillus ferrugineus Rullmann. (Rullmann, Cent. f. Bakt., I Abt., 24, 1898, 467; not Bacillus ferrugineus Van Iterson, Cent . f. Bakt., II Abt., 11, 1903, 694.) From canal water.

Bacillus ferrugineus Dyar. (Dyar, Ann. N. Y. Acad. Sci., 8, 1895, 361; not Bacillus ferrugineus Rullmann, Cent. f. Bakt., I Abt., Orig., 24, 1898, 465; Bacterium furrugineus (sic) Chester, Ann. Rept. Del. Col. Agr. Exp. Sta., 9, 1897,
115; Bacterium ferrugineum Chester, Man. Determ. Bact., 1901, 177.) From air and from a fresh leaf of the pitcher plant (Sarracenia purpurea).

Bacillus fertilis DeToni and Trevisan. (Bacillus urinae fertilis Doyen, Jour. d. connaiss. médic., 1889, 107; DeToni and Trevisan, in Saceardo, Sylloge Fungorum, 8, 1889, 949.) From urine.

Bacillus figurans Vaughan. (Vaughan, Amer. Jour. Med. Sci., 104, 1892, 107; not Bacillus figurans Crookshank, Man. of Bact., 1st ed., 1886.) From water.

Bacillus finitimus ruber Dyar. (Ann. N. Y. Acad.Sci., 8, 1895, 361.) From air.

Bacillus flavidescens Migula. (Bacillus aquatilis sulcatus v, Weichselbaum, Das österreichische Sanitätswesen, 1889, No. 14-23; Bacillus aquatilis sulcatus hruse, in Flügge, Die Mikroorganismen, 3 Aufl., 2, 1896, 382; Migula, Syst. d. Bakt., 2, 1900, 734; Bacillus weichsellaumii Chester, Man. Determ. Bact., 1901, 218.) From water.

Bacillus flavoides Castellani. (Proc. Soc. Exp. Biol. and Med., 25, 1928, 539.) From the human shin.

Bacillus flavus Eckstein. (Ztschr. f. Forst- u. Jagdwesen, 26, 1891, 12; not Bacillus flaws Fuhrmann, Cent. f. Bakt., II Abt., 19, 1907, 117; not Bacillus flavus Bergey et al., Manual, 1st ed., 1923, 286.) From dead larvae of a butterfly (Tanessa polychlorus).

Bacillus flexuosus Wright. (Wright, Mem. Nat. Acad. Sci., $\tau, 1895,460$; Bacterium flexuosus Chester, Ann. Rept. Del. Col. Agr. Exp. Sta., 9, 1897, 100.) From river water.

Bacillus floccosus Kern. (Kern, Arb. bakt. Inst. Farlsruhe, 1, Heft 4,1896 , 424; not Bacillus floccosus Weinberg et al., Les Microbes Anaérobies, 1937, 698.) From the stomach and intestines of birds.

Bacillus fluidificans DeToni and Trevisan. (Bacillus fluidificans parvus Maggiora, Giorn. Soc. ital. d'Igiene, 11, 1889, 344; DeToni and Trevisan, in Saccardo, Sylloge Fungorum, 8, 1889, 969.) From the skin. 
Bacillus fluorescens Chester. (Bacillus fluorescens aureus Zimmermann, Bakt. unserer Trink-u. Nutzwässer, Chemnitz, 1, 1890, 14; Bacterium fluorescens aureus Chesier, Ann. Rept. Del. Col. Agr. Exp. Sta., 9, 1\$97, 109; Chester, Man. Determ. Bact., 1901, 255; not $\mathrm{Ba}$ cillus fuorescens Trevisan, I generi e le specie delle Batteriacee, 1889, 19; not Bacillus fluorescens Bergey et al., Man ual, 1st ed., 1923, 287.) From water.

Bacillus fllorescens Pagliani, Maggiora and Fratini. (Pagliani et al., Giorn.d. Soc. ital. d'Igiene, 9, 1887, 587; not $B a$ cillus fluorescens Bergey et al., Manual, 1st ed., 1923, 287; Bacillus paglianii Trevisan, I generi e le specie delle Batteriacee, 1589, 19; not Bacullus paglianii Carbone and Venturelli, Boll. Ist Sieroter., Milan, 4, 1925, 59.) From water and soil.

Bacillus fluorescens albus Zimmermann. (Bakt unserer Trink-u. Nutzwässer, (hemnitz, 1, 1890, 18.) From water.

Bacillus fuorescens bregensis Robine and Hauduroy. (Compt. rend. Soc. Biol. Paris, 98, 1928, 25.) From the water of hot sulfur springs. Fourment (Compt. rend. Soe. Biol. Paris. 98, 1928, 588 ) states that this organism is a variety of Bacillus fuorescens liquefaciens hiruse. but Robine and Hauduroy (Compt. rend. Soc. Piol. Paris, 99, 1928, 317) deny this.

Bacillus fluorescens liquefaciens minu tissimus Lnna and Tommasoli. (Quoted from Sternberg, Man. of Bact., 1893, 636.) From the surface of the body in eases of eczema seborrhoeicum.

Bacillus fluorescens longus Zimmermann. (Zimmermann, Bakt. unserer Trink- 11. Nutzwässer, Chemnitz, 1, 1590, 20; Bacterium fluorescens longus Chester, Ann. Rept. Del. Col. Agr. Exp. Sta., 9, 1897, 124.) From water.

Bactllus fluorescens tenuis Zimmer mann. (Zimmermann, Bakt. unserer Trink- u. Nutzwässer, Chemnitz, 1, 1890, 14; Bacterium fluorescens tenuis Chester, Ann. Rept. Del. Col. Agr.
Exp. Sta., 9, 1897, 124.) From water. Bacillus foetidus Eckstein. (Ztschr. f. Forst- u. Jagdwesen, 26, 1894, 12.) From dead larvae of a butterfly ( $V$ anessa urticae).

Bacillus foetidissimus Migula. (Bacillus pyogenes foetidus liquefaciens Lanz, Cent. f'. Bakt., 14, 1893, 269; Bacterium pyogenes foetidus liquefaciens Chester, Ann. Rept. Del. Col. Agr. Exp. Sta., 9, 1897,92 ; Migula, Syst. d. Bakt., 2, 1900, 643.) From a brain abscess.

Bacillus fortissimus Weiss. Arb. bakt. Inst. Karlsruhe, 2, Heft 3, 1902, 209.) From asparagus and brewer's grain infusions.

Bacillus fuliginosus Weiss. (Arb. bakt. Inst. Karlsruhe, 2, Heft 3, 1902, 224.) From vegetable infusions.

Bacillus fulvus Edington. (Edington, Brit. Med. Jour., June 11. 1887, 1262; not Bucillus fulnus Zimmermann. Bakt. unserer Trink- u. Nutzwässer, Chemnitz, 1, 1890, 44; not Bacillus fulvus Migula, Syst. d. Bakt.. 2, 1900, 84t.) Assoriated with rases of searlatina. Not pathogenic.

Bacillus fulvus Migula. (Bacillus tuberigenus 4, Gonnermann, Landwirtsch. Jahrb., 23, 1894, 656; Migula, Syst. d. Bakt., 2, 1900, 84t; not Bacillus fulvus Zimmermann, Bakt. unserer Trink-u. Nutzwiisser, Chemnitz, 1, 1890, 44.) From root nodules on lupine.

Bacillus fumeus Vigula. (No. 5 , Lembke, Arch. f. Hyg., 29, 1897, 313; Migula, Syst. d. Bakt., 2, 1900, 787.) From fecess.

Bacillus fumosus Migula. No. 4, Lembke. Arch. f. Hyg., 29, 1897, 312; Migula, Syst. d. Bakt., 2, 1900, 788.) From feces.

Bacillus fungosus Weiss. (Arb. bakt. Inst. Karlsruhe, 2, Heft 3. 1902, 255.) From fermenting beets.

Bacillus fuscans Miller. (Miller, Die Mikroorganismen der Mundhöhle, Leipzig, 1889, 70.) From the mouth.

Bacillus fuscescens Migula. (Bacillus fuscus limbatus Scheibenzuber, Allgem. Wiener med. Zeitung, 34, 1889, 171; 
Bacterium fuscus limbatus Chester. Ann. Rept. Del. Col. Agr. Exp. Sta., 9, 1897, 117; Migula, Syst. d. Bakt., 2, 1900, 836; Bacterium fuscescens Chester, Man. Determ. Bact., 1901, 179; Bacillus fuscus Chester, ibid., 261; not Bacillus fuscus Flügge, Dic Mikroorganismen, 2 Aufl, 1886, 290; not Bacillus fuscus Zimmermann, Bakt. unserer Trink-u. Nutzwässer, Chemnitz, 1, 1890, 70.) From rotten eggs.

Bacillus galtieri Trevisan. (Microbe pathogène chromo-aromatique du pore, Galtier. Compt rend. Acad. Sci. Paris, 106, 1888, 1368; Trevisan, I generi e le specie delle Batteriacee, 1889, 14; $B a$ cillus chromoaromaticus Eisenberg. Bakt . Diag., 3 Aufl., 1891, 360; Bacterium chromo-aromaticus Chester, Ann. Rept. Del. Col. Agr. Exp. Sta., 9, 1897, 119; Bacillus helvolus Chester, Man. Determ. Bact. 1901, 264; not Bacillus helvolus Zimmermann, Bakt. unserer Trink- u. Nutzwässer, Chemnitz, 1, 1890, 52.) From lesions in a case of broncho-pneumonit in hogs.

Bacillus gasoformans Pribram. (Bacterium aquatile gasoformans non liquefaciens von Rigler, Hyg. Rund., 12, 1902, 482; Bacterium gasoformans non liquefaciens von Rigler, ibid., 485 and Cent. f. Bakt., I Abt., Ref., 31, 1902, 682; Bacillus aquatilis gasoformans non liquefaciens Pribram, Klassification der Schizomyceten, Leipzig und Wien, 1933, 83; ibid., 83.) From bottled mineral waters. Similar to coliform bacteria except that it is a yellow chromogen.

Bacillus gaytoni Cheshire. (Bees and Bee Keeping, London, 2, Part 13, 1886, 543 and 569.) Found in black bees (.1 pis mellifera), i.e., black because of the loss of hairy covering.

Bacillus gelatinosus Migula. (Bac terium gelatinosum betae Glaser, Cent. f. Bakt., II Abt., 1, 1895, 879; Migula, Srst. d. Bakt., 2. 1900, 805.) From beet juice

Bacillus gelalogenes IBlack. (Trans. Ill. State Dental Sor.. 2?, 18S6, IST. From the mouth.
Bacillus geton Trevisan. (Barille de l'eau $b$, Babes, in Cornil and Babes, Ides Bactéries, 2nd ed.. 1886, 168; Trevisan, I generi e le specie delle Batteriaces. $1889,19$.$) From water.$

Bacillus gigas Goot. (Goot, Mrol. Proefstat. voor de Java Suikerindustrir. Pt. 5, . Fo. 10, 60 pp., quoted from Steinhaus, Bact. Assoc. Extracell, with Insects and Ticks, Minneapolis, 1942, 5s; not Bacillus gigas Zeissler and Rassefeld, Areh. f. wiss, u. prakt. Tierheilk. $59,1929,419$.) From larval and adult stages of a beetle (Adoretus compressus).

Bacillus (?) gingivae Migula. (Bacterium gingivae pyogenes Miller. Die Mikroorganismen der Mundhöhle. Leipzig. 1889, 217; Bacillus myogenes gingivae Kruse, in Flügge. Die Mikroorganismen, 3 Aufl. 2, 1896, 287; Migula, Syst. I. Bakt., 2, 1900, 642.) From the oral ('avity.

Bacillus glaucus Maschek. Maschek. Bakt. Intersuch. d. Leitmeritz. Trinkwässer, Jahresber. d. Oberrealschule zu Leitmeritz. 1887; quoted from Sternberg. Man. of Bact., 1893, 637; Bacterium ylaucus Chester, Ann. Rept. Del. Col. Agr. Exp. Sta., 9, 1897, 116.) From water.

Bacillus globosus Migula. (Bacterium 1, Peters, Botan. Zeit., 47, 1859; Migula, Syst. d. Bakt., 2, 1900, 798.) From fermenting dough.

Bacillus globulosus Weiss. (Arb, bakt. Inst. Karlsruhe, 2, Heft 3, 1902, 253. From sauerkraut and bean infusions.

Bacillus gonnermanni Migula. (Bacillus tuberigenus II, Gonnermann, Landwirtsch. Jahrb., 23, 1894, 656; Migula. Syst. d. Bakt., 2, 1900, 682.) From root nodules on a lupine.

Bacillus gortynae Paillot. (Compt. rend. Acad. Sci., Paris, 157, 1913, 611. From caterpillars of Gortyna ochracca. A coccobacillus.

Bacillus gracilescens Henrici. (Arb. bakt. Inst. Farlsruhe, 1, IIeft 1, 1894. 26.) From Swiss cheese

Bacillus gracilis Kern. Kiern, Arb bakt. Inst. Karlsruhe, 1, IIcft 4, 1896. 421; not Bacillus gracitis Timmermann. 
Bakt. unserer Trink- u. Nutzwässer, Chemnitz, 1, 1890, 50; Bacillus gracilior Migula, Syst. d. Bakt., 2, 1900, 664.) From the stomach and intestines of birds.

Bacillus gracilis aerobiens Vaughan. (Amer. Jour. Med. Sci., 104, 1892, 187.) From water.

Bacillus gracilis anaerobiescens Vaughan. (Amer. Jour. Med. Sci., 104, 1892, 187.) From water.

Bacillus gracilis cadareris Sternberg. (Sternberg, Man. of Bact., 1893, 733; Bacterium gracilis cadieris Chester, Ann. Rept. Del. Col. Agr. Exp. Sta., 9, 1897. 84.) From the human liver.

Bacillus grandis Trevisan. (Batolle de l'air $h$, Babes, in Cornil and Babes. Les Bactéries, 2nd ed., 1886, 150; Trevisan, I generi e le specie delle Batteriacee. 1889, 20.) From air.

Bacillus gramulatus Chester. (Bacillus aquatilis solidus Lustig. Diag. Bakt. d. Wassers, 1893; Bacterium aquatilis solidus Chester, Ann. Rept. Del. Col. Agr. Exp. Sta., 9, 1897, 76; Chester, Man. Determ. Bact., 1901, 223.) From water.

Bacillus gramulosus Losshi. (Lossti, Inaug. Diss., Dorpat, 1893, 25; not Bacillus gramulosus Russell, Zischr. f. Hyg., 11, 1892, 99; not Bacillus granulosus Gerstne, Arb. bakt. Inst. Karlsruhe, 1. Heft 2, 1894, 167; Bacillus subqranulosus Migula, Syst. d. Bakt., 2, 1900, \$20.) From sand on the Riga coast.

Bacillus grareolens Bordoni-Tffreduzzi. (Bordoni-Lffreduzzi, Fortschr. d. Med., 4, 1886, 157; not Bacillus graveolens Russell, Ztschr. f. Hyg., 11, 1892. 99 ; not Bacillus graveolens Gotheil, Cent. f. Bakt., II Abt., 7, 1901, 496; Bacterium graceolens Eisenberg, 13akt. Diag., 3 Aufl., 1891, 108.) From skin between the toes.

Bacillus graritzi Trevisan. (Bareillus der Acne Contagiosa des Pfrerdes, Dieckerhoff and Grawitz, Arch. f. path. Anat., 102, 1885, 148; Trevisan, I generi e le specie delle Batteriacec, Milan, 18s?, 13; Bacillus acues contagiosae Kruse, in Flügge, Die Mikroorganismen, 3 Aufl.,
2, 1896, 445; Bacterium acnes contagiosac Chester, Ann. Rept. Del. Col. Agr. Exp. Sta., 9, 1897, 89; Bacterium acnes Migula, Syst. d. Bakt., 2, 1900, 385; Bacterium grauitzii Chester, Man. Determ. Bact., 1901, 154.) From aene pustles in horses.

Bacillus griseus Migula. (Grauer Bacillus, Keck, Inaug. Diss., Dorpat, 1891, 51 ; Migula, Syst. d. Bakt., 2, 1900, 785.) From water.

Bacillus hatmatoides Wright. (Wright, Mem. Nat. Acad. Sci., 7, 1895, 448; Bacterium haemaloides Chester, Ann. Rept. Del. Col. Agr. Exp. Sta., 9, 1897, 115.) From river water.

Bacillus hajeki Trevisan. (Bacillus foetulus ozaenac Hajek, Münch. med. Wochnschr., 1887 and Berliner klin. Wochnschr., 1868, 662; Trevisan, I generi e le specie delle Batteriacee, 1889 , 16; Bacllus ozaenae Migula, Syst. d. Batkt., 2, 1960, 645; not Bacillus ozaenae Abel, Cent. f. Bakt., 13, 1893, 167; Bacterum foetudus ozaenae (hester, Ann. Rept. Del. ('ol. Agr. Exp. Sta., 9, 1897, 134.) From nasal mucus in human ozena.

Pacillus halobicus Horowitz-Wlassowa. (Ztsehr.f. Inters. d. Lebensm., 62, 1931, 597. From brines used in salting fish.

Bacillus halophilus Russell. (Russell, Ztschr. f. Hyg., 11, 1891, 200; Bacterium hulophilus ('hester, Ann. Rept. Del. ('ol. Agr. Exy). Sta., 9, 1897, 93 and 135.) From sea water and marine mud.

Bacillus haramiensis Migula. (Bacillus haraniensis liquefaciens Sternberg, Man, of Bart., 1893, 686; Bacterium hataniensis liquefaciens Chester, Ann. Rept. Del. Col. Agr. Exp. Sta., 9, 1897, 97; Migula, Syst. d. Bakt., 2, 1900, 725; not Bacillus havaniensis Sternberg, loc. cit., 71s: From the skin

Bacillus heleolus gramulatus Dyar. (Ann. X. Y. Acad. S'ci., 8, 1895, 374.) Apparently a variety of Bacillus helvolus Zimmermann.

Bacillus heminecrobrophilus Arloing. (Compt. rend. Acad. Sci., Paris, 107, 1888,1169 and $108,1889,458$.) From the 
lymph glands of an experimental guinea pig.

Bacillus hepaticus fortuitus Sternberg. (Sternberg, Man. of Bact., 1893, 649; Bacterium hepaticus fortuitus Chester, Ann. Rept. Del. Col. Agr. Exp. Sta., 9, 1897, 136.) From the liver of a yellowfever cadaver.

Bacillus herrmanni Migula. (Ein neuer Kapselbacillus, Herzfeld and Herrmann, Hyg. Rundschau, 5, 1895, 6t2; Migula, Syst. d. Bakt., 2, 1900, 647.) From a nasal secretion.

Bacillus hofmanni Migula. (Hofmann, Wochnsehr. f. Forstwirtsch., 1891 , No. 1-6 and No. 35-39; Migula, Syst. d. Bakt., 2, 1900, 7+2.) From the larvae of the nun moth (Lymantria monacha).

Bacillus hudsonii Dyar. (Ann. N. Y. Acad. Sci., 8, 1895, 369; Bacterium hudsonil Chester, Ann. Rept. Del. Col. Agr. Exp. Sta., 9, 1897, 106.) From air.

Bacillus humilis Trevisan. (Bactérie de l'air No. 1, Babes, in Cernil and Babes, Les Bactóries, 2nd ed., 1856, 140; Trevisan, I generi e le specie delle Batteriacee. 1889, 20.) From air.

Bacilues hydrocharis Trevisan. (Baeille de l'eau $c$, Babes, in Cornil and Babes, Les Bactŕries, 2nd ed., 1886, 168; Trevisan, I generi a le specie delle Batteriacee, 1889, 19.) From water.

Baci!lus hydrosulfureus Migulat. ( $B a$ cillus oogenes hydrosulfureus $\zeta$ ZörkenGörfer, Arch. f. Hyg., 16, 1843, 3s5; Migula, Syst. d. Bakt., 2, 1900, 695.) From hens' eggs.

Bacillus iclerogenes Kruse. (Guarnieri, Ace. med. Roma, $87 / 88$ and Vinrent, Semaine médicale, 1893, 29; Kruse. in Flügge, Die Mikroorganismen, 3 Aufl., 2, 1896, ?72; Bacterium icterogenes Chester, Ann. Rept. Del. Col. Agr. Exp. Sta., 9, 1897, 69.) From the liver and blood in cases of acute yellow atrophy.

Bacillus incanus Pohl. (Pohl, Cent. f. Bakt., 11, 1892, 142; Bacterium incanus Chester, Ann. Rept. Del. Col. Agr. Exp. Sta., 9, 1897, 99; Bacterium incannum (sic) ('hester, Man. Determ. Bact., 1901, 157.) From swamp water.
Bacillus indigogenus Alvarez. (Alvarez, Compt rend. Acad. Sei., Paris, 105, 1887, 286; Bacterium indigogenus Chester, Ann. Rept. Del. Col. Agr. Exp. Sta., 9, 1597,136 .) From an infusion of leaves of the indigo plant.

Bacillus innesi Trevisan. (Bacille de l'éléphantiasis des Arabes, Innes, Bull. Ist. Égypt. de 1886, Cairo, 1887; Trevisan, I generi e le specie delle Batteriacee, 1859 , 13.) From the blood in cases of elephantiasis in Egypt.

Bacillus inodorus Trevisan. (I generi e le specie delle Batteriacee, $1889,16$. From pus.

Bacillus intestinus motilis Sternberg. (Sternberg, Man. of Bact., 1893, 649; Bacterium intestinus motilis Chester, Ann. Rept. Del. Col. Agr. Exp. Sta., 9, $1897,74$.$) From intestines of yellow$ fever cadavers.

Bacillus inutilis Dyar. (Ann. N. Y. Acad. Sei., 8, 1895, 364.) From air.

Bacillus kappa Dyar. (Ann. N. Y. Acad. Sci., 8, 1895, 375.) From diseased larva of a moth (Scolioptery.x libatrix).

Bacillus lilebsii Trevisan. (Bacillus typhosus Klebs, Handb. d. path. Anat., 1850 and Arch. f. exper. Pathol. u. Pharmac., 13, 1881, Heft 5-6; Trevisan, Car. di alc. nuov. gen. di Battr., 1885, 10; Trevisan, I generi e le specie delle Batteriacee, 1889, 14; not Bacillus typhosus Zopf, Die Spaltpilze, 3 Aufl., 1885, 126.) From an intestinal necrosis.

Bacillus klectii Migula. (Bacillus butyri II, v. Klecki, Cent. f. Bakt., 15, 1894, 360; Migula, Syst. d. Bakt.. 2, 1900, 810.) From rancid butter.

Bacillus kleinii Trevisan. (Bacillus de la diarrhée choleriforme, Klein, Micro-organisms and Disease, 1885, 87; Trevisan, in DeToni and Trevisan, in Saccardo, Sylloge Fungorum, 8, 1889, 946; not Bacillus kleinii Migula, Syst. d. Bakt., 2, 1900, 766; not Bacillus kleinii Buchanan and Hammer, Iowa Agr. Exp. Sta. Res. Bull. 22, 1915, 276.) From the blood in fatal cases of choleraic diarrhoea.

Bacillus kleinii Migula. (Ein neuer 
Bacillus des malignen Oedens, Klein, C'ent. f. Bakt., 10, 1891, 186; Bacillus iscudo-oedematis maligni Sanfelice, 7.tschr. f. Hyg., 14, 1893, 353; Bacillus vedematis aerobicus Sternberg, Man. of Bact., 1893, 465; Bacillus oedematis aerobius Iruse, in Flügge, Die Mikroorganismen, 3 Aufl., 2, 1896, 214; Bacterium oedematis aerobius Chester, Ann. Rept. Del. Col. Agr. Exp. Sta., 9, 1897, 75; Migula, Syst. d. Bakt., 2, 1900, 766; not Bacillus kileinii Trevisan, in DeToni and Trevisan, in Saccardo, Sylloge Fungorum, 8, 1859, 946; not Bacillus likinii Buchanan and Hammer, Iowa Agr. Exp. Sta. Res. Bull. 22, 1915, 276; Bacillus aerobius Chester, Man. Determ. Bact., 1901, 221.) From a guinea pig inoculated with soil.

Bacillus kornii C'hester. (Barillus bei rinem Leberabseess, Fion, Cent. f. Bakt., 21, 1\$97, 438; Chester, Man. Determ. Bact., 1901, 252.) From a case of liver abscess.

Bacillus lactis Chester. (Bacillus b. Giuillebeau, Ann. Microg., 11, 1898-1899, 225; Chester, Man. Determ. Bact., 1901 238; not Bacillus lactis Neide, Cent. f Bakt., II tht.. 12, 1804, 337.) From milk.

Burillus lastofoetielus Migula. Bacil lus foctidus lactis Jensen, 22de Beretning frat den Kigl. Veterin og Iandbohöjskoles Laboratorium for landökonomiske Forsoeg. Copenhagen, 1891, 15; Migula, Syst. d. Bakt., 2, 1900, 7f(1.) From tainted milk and butter.

Bacillus lanceolatus Mattes. (Sitzungsber. d. Gesells. z. Beförderung d. gesam. Naturm. z. Marburg, 62, 1927, 381-417.) From benign foulbrool of bees (A pis mellifera)

Bacillus larvicida Dyar. (Ann. X. I Acad. Sei.. 8, 1895, 377; Bacterium larvicider Chester, Ann. Rept. Del. Col. Agr. Fxp. Sta.. 9. 1897, 103.) From the rxudate of a diseased linva of a moth (Clisiocampa fragilis).

Bacillus lassari Trevisan. (Bacillus des lichen ruber, Lassar, see Flïgge, Dic Mikroorganismen, 2 Auf., 1886, 239;
Trevisan, I generi e le specie delle Batteriacee, 1889, 14.) From lichen ruber, a skin disease.

Bacillus lentiformis Kern. (Arb. bakt. Inst. Karlsruhe, 1, Heft 4, 1896, 418.) From the stomach and intestines of birds.

Bacillus leptinotarsae White. (Proc. Ent. Soc. Wash., 30, 1928, 71; Jour. Agr. Res., 51, 1935, 223.) From diseased larvae of the Colorado potato-beetle (Leptinotarsa decemlineata).

Bacillus lesagci Trevisan. (Bacille de la diarrhée verte des enfants, Lesage, Bull. Acad. Méd., Paris, October, 1887; Trevisan, I generi e le specie delle Batteriacee, 1889, 14; Bacillus viridis Iiruse, in Flügge, Die Mikroorganismen, 3 Aufl, 2, 1896, 292; Bacterium viridis Chester, Ann. Rept. Del. Col. Agr. Exp. Sta., 9, 1897, 118.) Associated with green diarrhoea of children.

Bacillus limbatus Migula. (Bacillus limbatus butyri von Klecki, Cent. f. Bakt., 15, 1894, 359; Migula, Syst.d. Bakt., 2, 1900,62.) From rancid butter.

Bucillus limicola Russell. (Bot. Gaz.. 18, 1893, 383.) From sea water and marine mud at Woods Hole. Massachusetts.

Bacillus lineatus Eckstein. (Ztschr. f. Forst- u. Jagdwesen, 26, 1894, 17.) From larvae of the nun moth (Lymantria monacha).

Bacillus lineatus Migula. (Bakterie V, Weigmann and Zirn, Cent. f. Bakt., 15, 1894, 467; Migula, Syst. d. Bakt.. 2. 1900, 806; not Bacillus lineatus Eckstein, Ztschr. f. Forst-und Jagdwesen, 26, 1894, 17.) From soapy milk.

Bacillus liparis Paillot. (Compt. rend. Acad. Sci., Paris, 164, 1917, 527.) From larvae of the gypsy moth (Portheria (Lymantria) dispar).

Bacillus liquefaciens Doyen. (Bacillus urinae liquefaciens Doyen, Jour. d. connaiss. médie., 1889, 108; Doyen, idem; not Bacillus liquefaciens Eisenberg, Bakt. Diag., 3 Aufl., 1891, 112.) From urine.

Bacillus liquefaciens Migula. (Baril- 
lus sulcatus liquefaciens Kiruse, in Flügge. Die Mikroorganismen, 3 Aufl., 2, 1896. 318; Migula, Syst. d. Bakt., 2, 1900, 723: not Bacillus liquefaciens Eisenberg. Bakt. Diag.. 3 Aufl., 1891, 112.) From water.

Bacillus liquefaciens albus Vaughan (Amer. Jour. Med. Sci.. 10\%. 1892. 185. From water.

Bacillus liquefaciens communis Sternberg. (Sternberg, Man. of Bact., 1S93. 686; Bacterium liquefaciens communis Chester, Amn. Rept. Del. Col. Agr. Exp. Sta., 9, 1897, 137.) From the feees of yellow fever patients. Consislered by Chester (loc. cit., 91) to be synonymous with Bacillus aquatilis communis Kruse

Bacillus liquidus communis Stemberg Manual of Bart.. 1893. (isfo.) From feces.

Barillus litorosus Russell. (Bot. Craz. 18. 1893. H4.) From sea water and marine mud at Woods Hole. Massa chusetts.

Bacillus loxiacide Tartakowsky

Arch. 1. Veterinarwiss., 1sss; quoted from Chester. Man. Determ. Bact. 1901. 211.) Assoriated with an infectious disease of crossbills.

Bacillus lucidus Migula. No. s. Lembke, Arch. f. Hyg.. 26, 1\$96, 303: Migula, Srst, 1. Bakt., 2. 19k00, 67t. From feces.

Bacillus lupi Trevisan. (I generi e lo specie delle Batteriacee, 1ss!) 12 From lupus, a skin disease.

Bacillus lupini Migula. (Bacillus tu berigenus $\gamma$. Cionnermann. Landwirtsch. Jahrh., 23, 1894, 657: Migulat. Syst. d. Bakt., 2, 1900, 7913.) From root nodules on lupine.

Bacillus lustigi Trevisan. (Bacillo) inoffensivo del Mytilus elulis. Lustig. Arch. per le sci. med.. 12, 1887. 17: Trevisan, see DeToni and Trevisan, in Saccardo, Sylloge Fungorum, 8, 1889 , 95s; not Bacillus lustigii Carbone and Venturelli, Boll. Ist. Sieroter., Milan, 4. 1925, 59. 1 From the liver of a mussel (.Mytilus edulis).

Bacillus luteo-albus Beijerinck.
(Botan. Zeit., 46, 18s8, 749.) From ront nodules on legumes.

Bacillus lutetiensis Chester. (Bucillus violaceus luteliensis Fruse, in Flügge, Die Mikroorganismen, 3 Auf1., 2, 1896 , 311; Chester, Man. Determ. Bact.. 1001. 306.) From water.

Bucillus luteus Flügge. (Die Mikrnorganismen, 2 Aufl., 1886, 290; not Bacillus luteus von Dobrzynierki, Cent. f. Bakt., I Abt., 21, 1s97, \$35; not Bacillus luteus Garbowski, C'ent. f. Bakt., II 1bt., 19, 1907,641.) From air.

Bacillus lymantriae Picard and Blanc. (Picard and Blanc. Compt. rend. Acad. Sci., Paris, 15\%, 1913, 80; Bacillus lymantria a P'aillot, ibid., 168, 1919, 258; Bacil. lus (Bacterium) lymantriae Paillot, L'infection chez les insectes, 1933, 131; Coceobacillus lymantriae Steinhaus, Catalogue of Bacteria Issociated Extracellularly with Insects and Ticks, Minneapolis, 1942, 64 and 183.) From diseased larvae of the gypsy moth (Portheria (L, mmantria) disp(r).

Bacillus lymantriae $\beta$ Paillot. (Comp: rend. Acad. Sei., Paris, 168, 1919, 258. from diseased larvae of the gypsy moth (Portheria (Limmantria) dispar).

Bacillus lymantricola adiposus Paillot. (compt. rend. Acad. Sci.. Paris, 168. 1919, 25s; Bacterium lymantricola artiмжм. Paillut. L'infection chez les insectes, 1933, 135.) From eaterpillars of Portheria (Lymantria) dispar

Bacillus madidus Migula. (No. 5. Lembke, Arch. f. Hyg., 26, 1s:96, 300; Migula, Syst. d. Bakt., 2, 1900, \$12. From bread.

Bacillus maggiorae DeToni and Trevisan. (Bacillus B, Maggiora, Ciiorn. Sor. ital. d'Igiene, 11, 1889, 340; DeToni and Trevisan, in Saccardo, Sylloge Fungorum, 8, 1889, 968.) From the skin of the human foot and from air.

Bacillus major Doyen. (Bacillusurinat major Doyen, Jour. d. connaiss. médic., 1889, 107; Doven ibid., 108.) From urine.

Bacillus malariae Ǩlebs and TommasiCrudeli. (Arch. f. expar. Pathol., 2, 
1879.) From swamp soil. See Sternberg, Man. of Bact., 1893, 523.

Bacillus mammitidis Migula. (Bacillus a, Guillebeau, Ann. de Microg., 2, 1890, No. 8; Migula, Syst. d. Bakt., 2, 1900,810 .) From the milk of cows having mastitis.

Bacillus manganicus Beijerinck. (Folia Microbiol., Delft, 2, 1913, 130.) From soil. Motile. Is able to oxidize manganese carbonate.

Bacillus margarineus Migula. (Diplococcus capsulatus margarineus Jolles and Winkler, Ztschr. f. Hyg., 20, 1895, 103; Migula, Syst. d. Bakt.. 2, 1900, 694.) From margarine.

Bacillus maricola Migula. (Halibacterium polymorphum Fischer, Die Baktierien des Meeres, 1894, 36; Migula, Syst. d. Bakt.. 2, 1900, 709.) From sea water.

Bacillus marsilliensis Kruse. (Bacillus of Marseilles swine plague, Rietsch and Jobert, Compt. rend. Acad. Sei. Paris, 106, 1885, 1096; Kruse, in Flügge, Die Mikroorganismen, 3 Aufl., 2, 1896. 405; Bacterium marsiliensis C'hester Ann. Rept. Del. Col. Agr. Exp. Sta., $9,1897,67$.) Associated with a disease of swine.

Bacillus martinez Sternberg. (Sternberg, Man. of Bact., 1893, 651; Barillus martinezii Dyar, Ann. X. Y. Acad. Sci. 8, 1895, 364; Bacterium martinezii Chester, Ann. Rept. Del. Col. Agr. Exp. Sta., 9, 1897, 83.) From the liver of a yellow fever cadaver. Dyar isolated an organism from the air to which he applier Sternberg's name as the descriptions of the two species did not disagree.

Bacillus meleagridis Migula. (McFadyean, Jour. Comp. Path. and Therap., 6, 1893, 334; Migula, Syst. d. Bakt., 2, 1900, 770; Bacillus meleagris Chester, Man. Determ. Bact., 1901, 220.) The cause of epizootic pneumo-carditis in turkeys.

Bacillus melleus Schrocter. (In Cohn, Kryptog. Flora v. Sehlesien, 3, 1, 1886, 158.) From feces and other sources.

Bacillus melolonthae Chat ton. (Compt. rend. Acad. Sci., Paris, 156, 1913, 1708.) From diseased cockchafers (Melolontha melolontha).

Bacillus melolonthae liquefaciens $\alpha, \beta$ and $\gamma$ Paillot. (Compt, rend. Acad. Sci., Paris, 167, 1918, 1046; Annales des Épiphyties, 8, 1922, 108-110; B. melolonthae liquefaciens $\alpha, \beta$ and $\gamma$ Paillot, I'infection chez les insectes, 1933, 173, 196 and 189 respectively. According to the index the $B$. is used for Bacterium.) From diseased cockehafers (Melolontha melolontha).

Bacillus melolonthae non-liquefaciens $\alpha, \beta$ and $\gamma$ Paillot. (Compt. rend Acad. Sci., Paris, 163, 1916, 553; Annales des Epiphyties, 8, 1922, 111-113.) From diseased cockchafer (Welolontha melolontha).

Bacillus melolonthae non-liquefaciens $\delta$ Paillot. (Compt. rend Acad. Sci., Paris, 167, 1918, 1046; Annales des Épiphyties, 8, 1922, 113.) From diseased cockehafers (Melolontha melolontha).

Bacillus melolonthae non-liquefaciens $\epsilon$ Paillot. (Compt. rend. Acad. Sei., Paris, 169, 1919, 1122; Annales des Épiphyties, 8, 1922, 114.) From diseased rockchafers (Melolontha melolontha).

Bacillus membranaceus Kern. (Arb. bakt. Inst. Karlsruhe, 1, Heft 4, 1896, 407.) From the stomach and intestines of a bird.

Bacillus meningitidis Migula. (Bacillus aerogenes meningitidis Centanni, Arch. per le seienze mediche, 17, 1893, No. 1; Bacterium meningitidis aerogenes Chester, Ann. Rept. Del. Col. Agr. Exp. Sta., 9, 1897, 96; Migula, Syst.d. Bakt., 2, 1900, 642; Bacillus radiatus ('hester, Man. Determ. Bact., 1901, 241.) From two cases of meningitis.

Bacillus metabolicus DeToni and Trevisan. (Bacillus H, Maggiora, Giorn. Soc ital. d'Igiene, 11, 1889, 350: DeToni and Trevisan, in Saccardo, Sylloge Fungorum, 8, 1889, 968.) From the skin of the human foot.

Bacillus metaflavus Castellani. (Proc. Soc. Expt. Biol. and Med., 25, 1928, 539.) From the human skin. 
Bacillus minimus Eckstein. (Ztschr. f. Forst- u. Jagdwesen, 26, 1894, 16.) From caterpillars of the nun moth (Lymantria monacha).

Bacillus minutissimus Migula. (Basillus aureus minutissimus Kruse, in Flügge, Die Mikroorganismen, 3 Aufl. 2, 1896, 441; Migula, Syst. d. Bakt., 2, 1900, 833.) From air.

Bacillus mitidus Henrici. (Arb. bakt. Inst. Karlsruhe, 1, Heft 1, 1894, 29.) From Gouda cheese.

Bacillus mobilissimus Migula. (Bacillus oogenes hydrosulfureus $\delta$, Zörkendörfer, Arch. f. Hyg., 16, 1893, 390; Migula, Syst. d. Bakt., 2, 1900, 791.) From hens' eggs.

Bacillus mollis Doyen. (Bacillus urinae mollis Doyen, Jour. d. connaiss. médic., 1889, 107; Doyen, ibid., 108.) From urine.

Bacillus morulans Bouequet. (Phytopath., 7, 1917, 286.) From diseased sugar beets. Associated with curly top of sugar beet.

Bacillus mottei Trevisan. (Motte and Protopopoff, Wratsch., 1857, No. 21, 415; abst. in Cent. f. Bakt.. 2, 1887, 450; Trevisan, I generi e le specie delle Batteriacee, 1889, 13.) Associated with a rabies-like disease of rabbits and dogs.

Bacillus (?) multiformis Castellani. (Proc. Soc. Exp. Biol. and Med., 25, 1928 . 539.) From the human skin.

Basillus murimus Chester. (Bacillus of rat plague, Issatschenko, Cent. f. Bakt., 23, 1898, 873; Chester, Man. Determ. Bact., 1901, 224; not Bacillus murinus Schroeter, in Cohn, Kiryptoy. Flora v. Schlesien, 3, 1886, 162.) From the spleen and liver of rats attacked in St. Petersburg by a plague.

Bacillus mycogenes Edwarks. (Jour. Inf. Dis., 2, 1905, 431; Bacterium muco. genum Edwards, idem.) From exudate of wound infections. Belongs to the Bacillus mucosus capsulqulus group.

Bacillus mytili Trevisan. (Bacillo patogeno del Mytilus edulis, Lustig, Arch. per le Sci. med., 12, 1887, 17; Trevisan. see DeToni and Trevisan, in Saceardo,
Sylloge Fungorum, 8, 1889, 958.) From the liver of a mussel (Mytilus edulis).

Bacillus naphthatinicus liquefaciens Tausson. (Planta, 4, 1927, 214.) From oil-soaked soils at Baku, Russia. Oxidizes naphthalene.

Bacillus naphthalinicus non-liquefaciens Tausson. (Planta, 4, 1927, 214.) From oil-soaked soils at Baku, Russia. Oxidizes naphthalene.

Bacillus nebulosus Migula. (Bacillus luberigenus 3, Gonnermann, Landwirtsch. Jahrb., 23, 1894, 656; Migula, Syst. d. Bakt., 2, 1900, 84t; not Bacillus nebulosus Wright, Mem. Nat. Acad. Sci., 7, 1894, 465; not Bacillus nebulosus Hallé, Thèse de Paris, 1898; not Bacillus nebulosus Vincent, Ann. Inst. Past., 21, 1907, 69; not Bacillus nebulosus Goresline, Jour. Bact., 27, 1934, 52.) From root nodules on lupine.

Bacillus necans Trevisan. (Bacille consécutif au charbon, Babes, in Cornil and Babes, Les Bactéries, 2nd ed., 1886, 231; Trevisan, I generi e le specie delle Batteriacee, 1889 , 14.) From rabbits dead from anthrax.

Bacillus nephriticus Trevisan. (Barille de la néphrite bactérienne. Babes, in Cornil and Babes, Les Bactéries, 2nd ed., 1856, 373; Trevisan, I generi e le specie delle Batteriacee, 1889, 14.) From uine in rases of nephritis.

Bacillus neurotomae Paillot. (Compt. rend. Acad. Sci., Paris, 178, 1924, 247; probably identical with Bacterium neurotomae Paillot, L'infection chez les insectes, 1933, 146.) From diseased larvae of a sawfly (Neurotoma nemoralis L.)

Bacillus nitens Migula. (Bacillus oogenes hydrosulfureus $\iota$, Zörkendörfer, Arch. f. Hyg., 16, 1893, 390; Migula, Syst. d. Balit., 2, 1900, 793.) From hens' eggs.

Bacillus ochrolencus Migula. (Bacillus oogenes hydrosulfureus $\epsilon$, Zörkendörfer, Arch. f. Hyg., 16, 1893, 387; Migula, Syst. d. Bakt., 2, 1900, S44.) From hens' eggs.

Bacillus olloratus Weiss. (Weiss, Arb. bakt. Inst. Farlsruhe. 2, 1902, 213; not 
Bacillus odoratus Migula, Syst. d. Bakt. 2, 1900,686 ; Bactorium odoratum Omeliansky, Jour. Bart., 8, 1923, 394.) From fermented beets.

Bacillus odorificans Migula. (Weisser stinkender Bacillus, Maschek, Bakt. Untersuch. d. Leitmeritzer Trinkwässer, Leitmeritz, 1887; Migula, Syst , l. Bakt., 2, 1900, 711.) From water. Intense odor resembling that of liquid manure.

Bacillus odorificus Omeliansky. (Jour. Bact., 8, 1923, 393.) Probably intended for Bacillus odorificans Migula.

Bacillus odorus Henrici. (Arb. bakt. Inst. Karlsruhe, 1, Heft 1, 1894, 30.) From cream cheese.

Bacillus oonergasius Trevisan. (Bacille du mucus intestinal normal $c$, Babes, in Cornil and Babes, Les Bactéries, 2nd ed., 1886, 153; Trevisan, I generi e to specie delle Batteriacee, 1889 , 15.) From normal intestinal mucus.

Bacillus ostcomyeliticus Trevisan. (Bacille de l'ostéomyélite, Rodet; Trevisan, 1884; sce Trevisan, I generi e le specie delle Batteriacee, 1889, 16.) From a case of osteomyelitis.

Bucillus oxylacticus Dyar.

(Ann I. Y. Acad. Sci., 8, 1895, 369.) Culture received from hrit's laboratory labeled Bacillus oxylacticus; also from air.

Bacillus pallescens Migula. (Bacillus luteus pallescens Tosski, Inaug. Diss. Dorpat, 1893, 44; Migula, Syst. d. Bakt., P. 1900, s19.) From garden soil.

Bacillus pallidus schroeter. (Schroe. tor, in Cohn, Kryptog. Flora v. Schlesien, 3, 1, 1s\$6, 155; not Bacillus pallidus Bredemamn and Heigener, ('ent. f. I3akt., II Aht., 9.3, 1935, 98.) From arokerl potato.

Bacillus panifions laturent. (Bull. soce. 12. But. Bolge., 1885, 175.) From fermenting dough.

Basillus pummosus kern. (drt). Wakt. Inst. Karlsruhe, 1, Theft 4, 1896,409. From the stomiallis and intestines of birds.

Bacillus pansinii Migula. (Bacillus No. 12, Pansini, Arch. f. path. Anat.,
122, 1s90, 477; Migula, Syst. d. Bakt., 2. 1900, 660.) From sputum.

Bacillus parallelus Edson and Carpenter. (Vermont Agr. Exp. Sta. Bull. 167, 1912, 593.) From maple sap. Capsulated. At times feebly fluorescent.

Bacillus paullulus Trevisan. (Bacille de l'air d, Babes, in Cornil and Babes, Les Bactéries, 2ud ed., 1886, 149; Trevisun, I generi o le specie delle Batteriacee, 1889, 20.) From air.

Bacillus pectinophorae White and Voble. (Jour. Eeon. Entomol., 29, 1936, 123.) from discased pink bollworm larvae (Pectinophora gossypiella).

Bacillus pediculi Arkwright and Bacot. (Parasitol., 13, 1921, 26.) From the genital atpparatus of the louse (Pediculus hum(anus).

Barillus pellucillus Doyen. (Bacillus urinae pellucidus Doven, Jour. d. connaiss. médic.. 1s\$9, 107; Doyen, ibid, 108; not Bucillus pellucidus Kern, Arb. bakt. Inst. Karlsruhe, 1, Heft t, 1896 , 404.) From urine

Bacillus perlitratus Beijerinck. (Cent. f. Bakt., 1/, 1893, S31.) From a hean infusion.

Bacillus perroncili Trevisau. (Bacillo della pneumonite nodulare dei vitellini, Perroncito, Parassiti dell'uomo e degli animali utili, 1882, 52; Trevisan, I generi c le specie delle Batteriacee, 18s:) 13.) From pulmonary nodules in contagious pheumonia in calves.

Bacillus pelersii Migula. (Bactorium B, Peters, Botan. Zeit., 47, 1889; Bacterium acidi lactici Kruse, in Flügge, Dic Mikroorganismen, 3 Auf1., 2, 1896 , $35 \%$; not Bacterium acidi lactici \%opf, Dic Spaltpilze, 2 Aufl., 1884, 60; not Baclerium acidi lactici $I$ and $I I$ Cirotenfelt, Fortschr. 1. Med., r, 1889, 123; not Bacterium acidi lactici Migula, in Engler and Prantl, Dic naturl. Pflanzenfam., 1. 1a, 1895, 25; Migula, Syst. d. Bakt., $\therefore$ 1900, 79!). forom fermenting (lough. P'robably Bacterium levans Wolftin.

Bacillus phenanthenicus baliensis Tausson. (Planta, 5, 1928, 239.) From 
soil. Etilizes phenanthrene and other hydrocarbons.

Bacillus phenanthrenicus guricus Tausson. (Planta, 5, 1928, 239.) From soil. Utilizes phenanthrene and other hydrocarbons.

Bacillus phenologenes Berthelot. (Ann. Inst. Past., 32, 1918, 20.) From feces. Forms phenol.

Bacillus pieris agilis Paillot. (Compt. rend. Acad. Sci., Paris, 168, 1919, 477.) From diseased caterpillars of the eabbage butterfly (Pieris brassicae).

Bacillus pieris fuorescens Paillot. (Compt. rend. Acad. Sci., Paris, 168. 1919, 477; Ann. Epiphyt., 8, 1922, 124.) From diseased caterpillars of the cabbage butterfly (Pieris brassicae).

Bacillus pieris liquefaciens Paillot. (Compt. rend. Acad. Sci., Paris, 168, 1919, 477; Bacillus pieris liquefaciens $\alpha$ Paillot, Annales des Epiphyties, 8, 1922, 125. From diseased caterpillars of the cabbage butterfly (Pieris brassicae). If this author followed his usual custom, this is identical with his Bact. pieris liquefaciens in his book, L'infection chez les insectes, 1933, 135 .

Bacillus pieris liquefacions $\beta$ Paillot. (Annales des Epiphyties, 8, 1922, 126; name occurs as $B$. pieris liquefaciens $\beta$ Paillot, L'infection chez les insectes, 1933, 299. Aceording to the index $B$. stands for Bucterium.) From diseased caterpillars of the cabbage butterfly (Pieris brassicae).

Bacillus pieris non-liquejaciens $\alpha$ Paillot. (Compt. rend. Acad. Sci., Paris, 168, 1919,477 ; $B$. pieris non-liquefacius $\alpha$ Paillot, L'infection chez les insectes, 1933, $135 \mathrm{ff}$. According to the index $B$. stands for Bacterium.) From the eahbage butterfly (Pieris brassicae).

Bacillus pieris non-liquefaciens $\beta$ Paillot. (Compt. reud. Acarl. Sei., Paris, $168,1919,474 ; B$. pieris non-liquefaciens $\beta$ Paillot, L'infection chez les insectes. 1933,299 ; according to the index, the $B$. stands for Bacterium.) From diseased caterpillars of the cabbage butter Hy (Pieris brassicae).
Bacillus pleomorphus Migula. (Bacillus murisepticus pleomorphus Tiurliński, Cent. f. Bakt., 5, 1889, 193; Bacterium murisepticus pleomorphus ('hester, Ann. Rept. Del. Col. Agr. Exp. Sta., 9, 1897, 102; Migula, Syst. d. Bakt., 2, 1900, 649; Bacillus murisepticus Chester, Man. Determ. Bact., 1901, 247; not Bacillus murisepticus Flügge, Die Mikroorganismen, 2 Aufl., 1886, 250.) From pus.

Bacillus plicatus Frankland and Frankland. (Philos. Trans. Roy. Soc. London, 178, B, 1888, 273; not Bucillus plicatus Zimmermann, see Frankland and Frankland, Microorganisnis in Water, London, 189ł, 459.) From air.

Bacillus plumbeus Migula. (Grau verHüssigender Bacillus, Keck, Inaug. Diss.. Dorpat, 1890, 54; Migula, Syst. d. Bakt.. 2, 1900, 719.) From water.

Bacillus pneumo-enteritidis murium Schilling. (Arb. a. d. kaise1l. (iesundheitsamte, 18, Heft 1, 1900.) From at disease of rats.

Bacillus pncumosepticus kiruse. (Pucumonie bacillus, Klein, C'ent. f Bakt.. 5, 1889, 625; Kruse, in Flügge Die Mikroorganismen, 3 Aul1., 2, $1 \times 96$ 408; not Bacillus pncumosepticus Babes. Progris méd. roumain., 6, 1889; Bac terium preumoscpticus Chestrer, Anm. Rept. Del. Col. Agr. Exp. Sta., 9, 1897 , 76.) From rusty sputum. ('onsidered the cause of an epidemic of pnemmonit in Lngland.

Bacillus poelsii Chester. Mleessherer giftung te Rotterdam, Poels and Dhont; Tweede Rapport van de des hundigen; ('hester, Man. Determ. Bat ., 1901, 209.) From beef in meat poisoning.

Bacillus pomodoriferus ('astellani. (Pror. Soc. Exp. Biol. and Med., 25, 1928, 540.) From the urine in a rase of rystitis and from feces.

Bucillus poncei Glaser. (Ann. Entomol. Soc. Am., 11, 1918, 19.) Pathogenic for the insects, Mclanoplus femuirubrum and Encoptolopus sordidus.

Bacillus praequollens Maassen. (Arb. a. d. kitiserl. Gesundheitsamte, 15 . 
1899, 507.) From sweat of a cholera patient.

Bacillus primus fullesi Dyar. (Ann. N. Y. Acad. Sci., 8, 1895, 360; Bacterium primus fullesi Chester, Ann. Rept. Del. Col. Agr. Exp. Sta., 9. 1997, 72.) From a leaf of the pitcher plant (Sarracenia purmereu). Regarded by Dyar as identical with Bacillus No. 1, isolated by Fulles (Ztschr. f. Hyg., 10, 1891, 250) from forest soil.

Bacillus promissus hiem. (Arb. bakt Inst. Karlsruhe, 1, Ieft $4,1896,420$. From the intestines of a dove.

Bacillus proteidis Paillot. (Annales des Ejuphyties, 8, 1022, 130.) From diseased larvae of the cabbage buterfly (Pieris brassicae).

Bacillus protervus Trevisan. (Barillus der Conjunctivalsaeli d, Fick, Microorgan, in Conjunctivalsark, Wic,baden, 1857; Trevisan, I generi e le specie delle Batcriace, 1889, 17.) From the ron junctiva.

Bacillus pruddeni Dyar. (Ann. N. Y Acad. Sei., 8, 1805, 378.) Fround by Dr. Prudken in a case of erstitis

Bacillus pseudomirabitis Migula. (Bacillus mirabitis Tataroff, Inaug: Diss., Lorpat, 1891, 18; Migula, Syst. d bakt., 2, 1900, S18.) from water. Ac ewroling to Migula, Tataroff mistakenly believed that he had Zimmernaann's Bacillus mirabilis.

Bacillus psendotuberculosis Migula. (Du Cazal and Taillard, Ann. Inst. Pust., 5, 1891, 25e3; Buctlu. pseudotuber rulwix liquefaciens Liruse, in Flügge, Dic Miliroorgunismen. 3 Aufl. 2, 1896, 455; Migula, Syst. d. Bak! .. 2, 1900, 644. From nodules in the peritoneum.

Bacillus pseudmphowas Iiruso (L̈̈sener, Arb. a. d. kaisenl. Gesundheitsante, 11, 1805. 2; Krruse, in Flügge, Die Mikroorganismen, 3 Auf1., 2, 1896, 383; Bactorium pseudotyphosus Chester, Ann. Teppt. Del. Col. Agr. Mxp. Sta., 9, 1897. 73; not Bacterium pseudotyhposum Migulat, Syst. d. Bakt., 2, 1960, 428.) Isolin al by Lösoner from peritoneal fluid of a hog, from water, cle.; by Pansini from a liver abscess; by Babes from a variety of sources. Kruse used this as a general name for any typhoid-like organism.

Bacillus mulpae pyogenes Miller. (Miller, Die Mikroorganismen der Mundhöhle, Leipzig, 1859, 219.) From gangrenous pulp of a tooth.

Bacillus punctatus Shul'gina and Kalinicker. (Rep. Eur. Appl. Ent., Leningrat, 3, No. 1, 1927, 99-104, quoted from Steinhaus, Bact. Assoc. Extracell. with Insects and Ticks, Minneapolis, 1942, 79; not Bucillus punctatus Zimmermann, Bakt unserer Trink- u. Nutzwässer, Chemnitz, I lieihe, 1s90, 38.) From the locust (Iocusta mimatoria).

Bacillus puncticulatus Migula. (No. 16, Lembles, Arrh. f. Hyg., 29, 1897, 322; Migula, Syst. d. Bakt., 2, 1900, 678.) From feecos.

Bacillus putidus licrn. (Kern, Arb. bakt. Inst. Karlsruhe, 1, Heft 4, 1896, 400; not Bracillus putidus Chester, Man. Deform. Bact., 1901, 237; not bacillus pulidus Weinberg et al., Les Microbes Anacrolues, 1937, 790.) from the stomarh and intestines of birds.

Bucillus pylori Ford. (studies from the Royal Vietoria Ifrisp., Montreal, 1 (5), 1903, 44; also see Jour. Med. lies., 1, 1901, 217.) From the stomach.

Bacillus mogenes Lucet. (Bacillus pyogenes hovis Lucet, Ann. Inst. Past., 7, 1893, 372; Lucet, ibil., 328; not Bacillas progenes Gilage, Ztschr. f. Fleish. u. Milehhyg., 13, 1903, 166.) From bovine absecsses.

Bacillus myogenes var. liquefaciens Chester. (Bacillus pyogenes foetidus liquefaciens Lanz, Cent. f. Bakt., 14, 1893, 277; Bucterium pyogenes foctidus liquefaciens Chester, Ann. Rept. Del. Col. Agr. Exp. Sta., 9, 1897, 92; Chester, Man. Determ. Bact., 1901, 235.) From a brain abseess after otitis media.

Bacillus progenes soli Bolton. (Ann. Jour. Med. Sci., June, 1892; quoted from Stemberg, Min. of Bact., 1893, 728.) From garien earth.

Bacillus pyrameis $I$ and $I I$, Paillot. (Compt, rend. Acad. Sci., Paris, 157, 
1913, 611.) From tissues and blood of the caterpillar of Pyrameis (Vanessa) cardui. A coccobacillus.

Bacillus radians Trevisan. (Bactérie de l'air No. 2, Babes, in Cornil and Babes, Les Bactéries, 2nd ed., 1886, 140; Trevisan, I generi e le specie delle Batteriacee, 1889, 20.) From air.

Bacillus ramificans Migula. (Bacillus No. 9, Pansini, Arch. f. path. Anat., 122, 1890, 445; Migula, Syst. d. Bakt., 2, 1900, 661; Bacillus pansini Chester, Man. Determ. Bact., 1901, 246; not $\mathrm{Ba}$ cillus pansinii Migula, loc. cit., 660.) From sputum.

Bacillus recuperatus Wright. (Wright, Mem. Nat. Acad. Sci., 7, 1895, 439; Bacterium recuperatus Chester, Ann. Rept. Del. Col. Agr. Exp. Sta., 9, 1897, 74.) From Schuylkill River water.

Bacillus repazii Herter. (Bacillus moliniformis Repazi, Compt. rend. Soc. Biol., Paris, 68, 1910, 410; not Bacillus moniliformis Garnier, Arch. Méd. expér. et Anat. pathol., 19, 1908, 785; Herter, in Just's Botan. Jahresber., 39, 2 Abt., Heft 4, 1915, 750.) From a lung abscess.

Bacillus rhinitis atrophicans Paulsen. (Paulsen, quoted from Chester, Ann. Rept. Del. Col. Agr. Exp. Sta., 9, 1897, 79; Bacterium rhinitis atrophicans Chester, ibid., 141.) From nasal secretions.

Bacillus rigidus afis White. (Jour. Path. and Bact., 24, 1921, 70.) From intestine of bee.

Bacillus rogerii Migula. (Bacillus septicus putidus Roger, Revue de Méd., 1891, 10; Migula, Syst. d. Bakt., 2, 1900, 647; Bacterium septicus putidus Chester, Ann. Rept. Del. Col. Agr. Exp. Sta., 9, 1897, 92; Bacillus putidus Chester, Man. Determ. Bact., 1901, 237; not Bacillus putidus Kern, Arb. bakt. Inst. Karlsruhe, 1, Heft 4, 1896, 400; not Bacillus putidus Weinberg et al., Les Microbes Anaérobies, 1937, 790.) From the spinal fluid and liver in a case of cholera.

Bacillus rosaceus Migula. (Bacillus rosaceus margarinicus Jolles and Winkler, Ztschr.f. Hyg., 20, 1895, 105; Migula,
Syst. d. Bakt., 2, 1900, 859.) From margarine. Red pigment.

Bacillus rosaceus metaloides Dyar. (Bacterium rosaceus metaloides Dowdeswell, Ann. de Micrographie, 1, 1888-89, 310; Dyar, Ann. N. Y. Acad. Sci., 8, 1895 , 376; not Bacillus rosaceus metalloides Tataroff, Inaug. Diss., Dorpat, 1891, 65, see Hefferan, Cent. f. Bakt., II Abt., 8, 1902, 689; Bacterium rosaceum Lehmann and Neumann, Bakt. Diag., 2 Aufl., 2, 1899, 261.) Culture from Dr. Crookshank, London.

Bacillus roseus Migula. (Halibacterium roseum Fischer, Die Bakterien des Meeres, 1894, 22; Migula, Syst. d. Bakt., 2, 1900, 860; Bacillus roseus fischeri Nepveux, Thèse, Fac. Pharm. Paris, 1920, 115.) From sea water. Red pigment .

Bacillus rosenthalii Migula. (Rosenthal, Inaug. Diss., Berlin, 1893, 37; Migula, Syst. d. Bakt., 2, 1900, 656.) From the oral cavity.

Bacillus rubescens Edington. (Ann. Rept. Fish. Board for Scotland, 6, 1887, 204.) From reddened salted codfish.

Bacillus rubescens Jordan. (Jordan, Massachusetts State Board of Health, Boston, 1890, 835; Bacterium rubescens Chester, Ann. Rept. Del. Col. Agr. Exp. Sta., 9, 1897, 115.) From sewage.

Bacillus rubiginosus Catiano. (In Cohn, Beitr. z. Biol. d. Pflanzen, 7, 1896 , 538.) From the vagina. Red pigment.

Bacillus rubiformis Kern. (Arb. bakt. Inst. Karlsruhe, 1, Heft 4, 1896, 431.) From the stomach of a bird.

Bacillus rubrofuscus (Fischer) Migula. (Halibacterium rubrofuscum Fischer, Die Bakterien des Meeres, 1894, 36; Migula, Syst.d. Bakt., 2, 1900, 865.) From sea water.

Bacillus rubus (sic) Chester. (Der rother Bacillus, Lustig, Diag. d. Bakt. d. Wassers, 1893, 72; Bacillus ruber aquatilis Kruse, in Flügge, Die Mikroorganismen, 3 Aufl., 2, 1896, 303; Bacterium ruber aquatilis Chester, Ann. Rept. Del. Col. Agr. Exp. Sta., 9, 1897, 112; Chester, 
Man. Determ. Bact., 1901, 257; Bacillus lustigi Chester, ibid., 304.) From water.

Bacillus salmoneus Dyar. (Dyar, Ann. X. Y. Acad. Sci., 8, 1895, 361; Baclerium sulmoneus Chester, Ann. Rept. 1)el. (ol. Igr. Exp). Sta., 9, 1897, 116.) From air.

Bacillus sulutarius Metchnikoff. (Metehnikolf, Mialadies des hannetons du ble, Odessa (in Russian), (quoted from Paillot, I'inferion chez les insectes, Paris, 1933, 123.) From diseased larvae of a beetle (A nisoplia austriara).

Bacillus strnquineus Schroeter. (In (ohn, Kryptog. Flora v. Schlesien, 3, 1, 1ss6, 156.) From stagnant water.

Bacillus suponaceus Migula. (Bacillus lactis saponacei Weigman and Zirn, Cent. f. Balkt., 15, 1894, 464; Migula, Syst. 1. Bakt., 2, 1900. 692.) From soipy milk.

Bacillus saprogenes Chester. (Bacillus saprogenes rini rl liramer, Bakteriol. Landwirtseh., 1\$30, 139; ('hester, Man. Determ. Bact., 1901, 289.) From diseased wine

Bacillus stpmogfrnes Trevisin. (Bacilliss saprogenes 1, Rosenbach, Mikroorganismen bei den Wundinfectionskrankheiten des Menschen, Wiesbaden, 1sst; Trevisan, I generi e le specie delle Batteriaces, 18s9, 17; Bacterium saprofenes C'hester, Ann. Rept. Del. Col. Agr. Exp. Stit., 9, 1897, 142.) From feees.

Bacillus sarracenicolus Dyar. (Dyar, Ann. Y. Y. Acad. Sci., 8, 1895, 357; Bacterium sorracenicolus (sic) Chester, Ann. Rept. Del. Col. Agr. Exp. Sta., 9, 1897, s1.) From a fresh leaf of the pitcher plant (Saracenia purpurea).

Bacillus scallalinae Jamieson and Ed. ington. (Jamieson and Edington, Brit. Mrol. Jour., 1, 1sis, 1265; Bacillus sudaminis Trevisan. I generi e le specie dello 13attriacer, Ist9, 15.) From the skin of scarlet fover patients.

Bacillus scoticus Migula. (Bacillus der Grouse-disease, Klein, Cent. f. Bakt., $6,1889,36$ and 593 ; ibid., $\gamma, 1890$. 81; Migula, Syst. d. Bakt., 2, 1900, 76s;
Bacillus tetraonis Chester, Man. Determ. Bact., 1901, 221.) The cause of a disease of grouse (Lagopus scoticus) in lingland and Scotland.

Bacillus secundus Trevisan. (Bacillus II, Leube, Arch. f. path. Anat., 100, 1885, 000 ; Trevisan, I generi e le specie delle Batteriacee, 1889, 16.) From urine.

Bacillus secundus fullesi Dyar. (I)yar, Ann. X. Y. Acad. Sci., 8, 1895, 35.); Bacterium secundus fulessii (sic) ('hester, Ann. Rept. Del. Col. Agr. Exp. Sta., 9, 1897, 82; Bacterium secundus fullesi ('hester, ibid., 143.) From air. Dyar regarded his organism as identical with Bacillus No. 2 of Fulles (Ztschr. f. Iyg., 10, 1891, 250) which was from soil. Bacillus seplicacmiac lophyri Shiperovich. Shiperovich, Protect. Plants I'kraiur, 1925, +1-46; Abs, in Rev. Appl. Ent., A., 14, 1926, 209.) From larvae of sawflies (I)iprion sertifer).

Bucillus septicuemicus Trevisan. (Ba(cillus of septicemia of man, Klein, Mieroorganisms and Disease, 1885, 84; Trevisim, in DeToni and Trevisan, in Sac(ardo, sylloge fungorum, 8, 1889, 945.) from blood and infected lymph glands.

Bucillus scptieus hominis Mironoff. (.Miromoff, ('nt. f. Ciynäkol., 1892, 42; Bacterium septicus hominis Chester, Inn. Rept. J)el. ('ol. Agr. Exp. Sta., 9, $1497,143$.$) From a case of septic infec-$ fion of the uterus. Regarded by Ches10. (M:m. I)etem. Batet., 1901, 143) as a synonym of P'asteurella agrigena Trevisan

Bacillus septicus iesicae Clado. (Bull. de la Soc anatom. de Paris, 1887, 339.) From the urine of a person suffering from rystitis.

Bacillus sericess Vimmermann. (Bakt. unserer Trink- u. Nutzwässer, ('hemnitz, 2, 1894, 52.) From water.

Bacillus serratus Migula. (Bacillus No. 1t, Pansini, Irch. f, path. Anat., 122, 1890, 449; Migula, Syst. d. Bakt., 2, 1900, 658.) From sputum.

Bacillus sctosus Migula. (Bacillus No. XYIII, Adametz, Landwirtsch. Jahrb., 
18, 1889, 250; Migula, Syst. d. Bakt., 2, 1900, 812.) From cheese.

Bacillus sillerschmidii Chester. (Bacillus der Fleischvergiftung, Silberschmidt, Correspondenz-Blatt f. Schweizer Aerzte, 1896, No. 8; Chester, Mar. Determ. Bact., 1901, 212, From poisonous meat.

Bacillus simulans Trevisan. (Barille de l'air a, Bubes, in Cornil and Bubes, Les Bactéries, 2nd ed., 18\$6, 149; Trevisan, I generi e le specie delle Batteriaree, 1889, 20.) From air.

Bacillus singularis Losski. (Inaug. Diss., Dorpat, 1893, 45.) From garden soil.

Bacillus siticulosus Kern. (Arb. bakt Inst. Karlsruhe, 1, Heft 4, 1896, 423.1 From the stomachs and intestines of birds.

Bacillus sordidus Drar. (1) yar. Ann. X. Y. Acad Sci., 8, 1595, 379; Bacterium sordidus Chester, Ann. Rept. Del. Col. Agr. Exp. Sta., 9, 1897, 79.) Culture received by Dyar as Microrocms sordidus from Kràl's laboratory.

Bacillus sordidus Kern. (Arb. Bakit. Inst. Karlsruhe, 1. Heft 4 . 1596, 396.) From the stomach of a bird.

Bacillus spermophilinus Issatchenko. (Ein aus Zieselmäusen ausgeschiedener Bacillus, Mereschkowsky, Cent. f. Bakt. 17, 1895, 742; Issatchenko, Seripta Bo tanica Hort. Lniv. Imp. Petropolitanae, Fasc. $X Y, 1897$; quoted from Migula. Syst. d. Bakt., 2, 1900,618.) Apparently resembled Salmonella tuphimurium. From ground squirrels (Stpermophilus musicus).

Bacillus spirans Weiss. (Art). bakt Inst. Karlsruhe, 2, In oft 3. 1902, 222.) From bean infusions

Bacillus spumosus Zimmermann. (Bakt, unserer Trink-u. Nutzwïsser, Chemnitz, 2, 1894, 28.) From water.

Bacillus squamosus Pansini. (Arch. f. path. Inat., 122, 1890, 4.48.) From sputum.

Bacillus strassmanni Trevisan. (Bacillus albus cadaveris Strassmann and Strecker, Ztschr. f. Medicinalbeamte,
1888; Trevisan, I generi e le specie delle Batteriacee, 1889, 17; Bacterium albus cadaceris Chester, Ann. Rept. Del. Col. Agr. Exp. Sta., 9, 1897, 102; Bacillus cadateris Migula, Syst. d. Bakt., 2, 1900, 616.) From the blood of an infant.

Bucillus striatus Doyen. (Bacillus urinae striatus Doyen, Jour. d. connaiss. médic., 1889, 107; Doyen, ibid., 108.) From urine.

Bacillus striatus albus von Besser. (Ziegler's Beiträge, 4, 1889, 331.) Found in normal nasal mucus.

Bacillus striatus flames von Besser. ('Ziegler's Beiträge, 4, 1889, 331; Bacterium striatus flatus Chester, Ann. Rept. Del. Col. Agr. Exp. Stat., 9. 1897, 111.) From nasal mucus. Rare.

Bacillus strumitidis Migula. (Bacillus strumitis $\alpha$, Tavel, Ueber die Aetiologie der Strumitis, Basel, 1892, 81; Migula, Syst. 1. Balit., 2, 1900, 741.) From a ase of strumitis.

Bacillus strumitis Tavel. (Tavel, 1889; see Viquerat, Ann. de Mierographie, 2, 1889-1890, 228.) From acute catarrhal strumitis.

Bacillus subcoccoideus Migula. (Barillus aquatilis sulcatus $I I I$, Weichselhaum, Das österreichische Sanitätswesen, 1859, No. 14-23; Migula, Syst. (1. Bakt., 2, 1900, 732.) From water.

Bacillus subflavus Zimmermann. (Zimmermann, Bakt. unserer Trink. u. Nutzwässer, Chemnitz, 1, 1890, 62; Bacterium sulflavus Chester, Ann. Rept. Del. Col. Agr. Exp. Sta., 9, 1897, 109.) From water. According to Chester (Man. Detcrm. Bact., 1901, 254), Bacillus flacescens $\mathrm{P}^{\mathrm{O}} \mathrm{hl}$ is identical with this species.

Bacillus subgastricus White. (U. S. Dept. Agr. Bur. Ent., Tech. Bul. 14, $1906,23$.$) From intestinal contents of$ honey bee (Apis mellifera). While this does not appear to be the same as Bacillus gastricus Ford (see Steinhaus, Bacteria Associated Extracellularly with Insects and Ticks. Minneapolis, 1942. 85 ), it may have been described by some 
previous author as White does not indicate that he regards it as new.

Bacillus subochraceus Dyar. (Dyar, Ann. N. Y. Acad. Sei., 8, 1595, 358; Bacterium subochraceus Chester, Ann. Rept. Del. Col. Agr. Exp. Sta., 9, 1897. 110.) From air.

Bacillus subrubiginosus Migula. (Braunroter Barillus, Maschek, Bakt. Untersuch. d. Leitmeritzer Trinkwässer, Leitmeritz, 1887; Migula, Syst. d. Bakt. 2, 1900, 836.) From water.

Bacillus subsulcatus Migula. (Bacillus aquatilis sulcatus II, Weichselbaum, Das österreichische Sanitätswesen, 1889 , No. 14-23; Migula, Syst. d. Bakt., 2, 1900, 732.) From water.

Bacillus sulcalus (hester. (Bacillus sulcatus liquefaciens Kruse, in Flïgge, Die Mikroorganismen, 3 Aufl., 2, 1896 , 318; Bacterium sulcatus liquefaciens Chester, Ann. Rept. Del. Col. Agr. Exp. Sta., 9, 1897, 97; Chester, Man. Determ. Bact., 1901, 213; not Bacillus sulcatus Migula, Syst. d. Bakt., 2, 1900, 731.) From water.

Bacillus sulcatus Migula. (Bacillus aquatilis sulcatus I, Weichselbaum, Das österreichische Sanitätswesen, 1SS9, No. 14-23; Migula, Syst. d. Bakt., 2, 1900, 731.) From water.

Bacillus sulfhydrogenus Miquel. (Ann. de Micrographie, 1, 1888-1889, 369.) From sewage.

Bacillus tardissimus DeToni and Trevisan. (Bacillus fluidifueans tardissimus Maggiora, Giorn. Soc. ital. d'Igiene, 11, 1589, 347; DeToni and Trevisan, in Saccardo, Sylloge Fungorum, 8, 1889, 967.) From the skin of the human foot.

Bacillus tartricus Cirimbert and $\mathrm{Fi}$ quet. (Jour. Pharm. et de Chim., 6 Sér., 7, 1898, 97; Compt. rend. Soc. Biol., 49, 1897, 962.) Decomposes d-tartrates. Probably identical with Aerobacter cloacae (Vaughn et al., Jour. Bact., 52, $1946,324)$.

Bacillus telmatis Trevisan. (Bacillus saprogenes 2, Rosenbach, Mikroorganismen bei den Wundinfectionskrankheiten des Menschen, Wiesbarlen, 1884; Trevi- san, I generi e le specie delle Batteriacee, $1889,14$.$) From perspiration of feet.$

Bacillus tenuis Doyen. (Bacillus urinae tenuis Doyen, Jour. d. connaiss. médic., 1889, 107; Doyen, ibid., 108; not Bacillus tenuis Migula, Syst. d. Bakt., 2, 1900, 587; Bacillus tenuatus Trevisan, in DeToni and Trevisan, in Saccardo, Sylloge Fungorum, 8, 1889, 948.) From urine.

Bacillus tenuis apis White. (Jour. Path. and Bact., 24, 1921, 72.) From intestine of bee.

Bacillus terrigenus Frank. (Berichte deutsch. botan. Gesellsch., 4, 1886,000.) From soil.

Bacillus thermophilus Miquel. (Miqucl. Ann. de Microgr., 1, 1888-1889, 6; not Bacillus thermophilus Chester, Man. Determ. Bact., 1901, 265; not Bacillus thermophilus Bergey et al., Manual, 1st. od., 1923, 315.) From water, sewage, suil, ete.

Bacillus theta Dyar. (Ann. N. Y. Acad. Sci., 8, 1895, 375; Bacterium theta Chester, Ann. Rept. Del. Col. Agr. Exp. Sta., 9, 1897, 144.) From air.

Bacillus tingens Eckstein. (Ztschr.f. Forst-u. Jagdwesen, 26, 1894, 10.) From dead larvae of Orgyia pudibunda.

Bacillus toluolicum Tausson. (Planta, \%, 1929, 735.) From soil. Oxidizes toluene.

Bacillus torigenus Chester. (Bacillus of ice cream poisoning, Vaughn and Perkins, Arch. f. Hyg., 27, 1896, 308; Chester, Man. Determ. Bact., 1901, 208.) From poisonous ice eream.

Bacillus trambustii Kruse. (Trambusti and Cialeotti, Cent. f. Bakt., 11, 1892, 717; Kruse, in Flügge, Die Mikroorganismen, 3 Aufl., 2, 1896, 319; Bacterium trambusti Chester, Ann. Rept. Del. Col. Agr. Exp. Sta., 9, 1897, 97.) From water.

Bacillus tremaergasius Trevisan. (Bacille du mucus intestinal normal $d$, Babes, in Cornil and Babes, Les Bactéries, 2nd ed., 1886, 153; Trevisan, I generi e le specie delle Batteriacee, 1889. 15.) From normal intestinal mucus. 
Bacillus trimethylamin Beijerinck. (Bot. Zeitung, 46, 1888, 726.)

Bacillus truttae Mersch. (Quoted from Lehmann and Neumann, Bakt. Diag., 7 Aufl., 2, 1927, 481.) Closely related to Bacterium salmonicida Lehmann and Neumann.

Bacillus tumidus Chester. (Anaerobe Bacillus I, Sanfelice, Ztschr. f. Hyg., 14. 1893, 268; Chester, Man. Determ. Bact., 1901, 265.) From putrefying flesh. Strict anaerobe.

Bacillus uffeduzzii Trevisan. (Batterio della setticemia salivare nei conigli, Bordoni-Cffreduzzi and Di-Mattei. Arch. per le scienze med., 10, 1886; Trevisan, see DeToni and Trevisan, in Saccardo, Sylloge Fungorum, 8, 1889, 951.) From normal human saliva.

Bacillus ulna Cohn. (Beitr. z. Biol. d. Pflanz., 1, Heft 2, 18:2, 177.) From water, air, etc,

Bacillus umbilicatus Zimmermann. (Bakt. unserer Trink- u. Nutzwässer, Chemnitz, 2, 1894, 6.) From water.

Bacillus urinae Migula. (Ein Harnbacterium, Karplus, Arch. f path. Anat.. 131, 1893, 211; Migula, Syst.d. Bakt., 2, 1900, 739.) From urine.

Bacillus utpadeli Trevisan. (Bacillus aus Zwischendeckenfüllung, [tpadel, Arch. f. Hyg., 6, 1857, 359; Trevisan, I generi e le specie delle Batteriacee, 1889. 15.) Frcm the intestine.

Bacillus vacuolatus Dyar. (Dyar, Ann. X. Y. Acad. Sci., 8, 1895, 357; Bacterium racuolatus Chester, Ann. Rept. Del. Col. Agr. Exp. Sta., 9, 1897, 81.) Frcm a trap of the carnivorcus water plant, Cricularia vulgaris.

Bacillus regetus Fiern. (Arb. bakt. Inst. Karlsruhe, 1, Heft 4, 1896, 399.) Frcm the stomach and intestines of birds.

Bacillus velox Kern. (Arb. bakt. Inst. Karlsruhe, 1, Heft 4, 1896, 405.) From the stomach and intestines of birds.

Bacillus venenosus brevis Vaughan. (Arer. Jour. Med. Sci., 104, 1892, 192; Bacterium venenosus and Bacterium venenosus brevis Chester, Ann. Rept. Del.
Col. Agr. Exp. Sta., 9, 1897, 76.) From water.

Bacillus venenosus Chester. (Bacillus venenosus invisibitis Vaughan, Amer. Jour. Med. Sci., 104, 1892, 192; Bacterium venenosus imisibilis Chester, Ann. Rept. Del. Col. Agr. Exp. Sta., 9, 1897, 76; Chester, Man. Determ. Bact., 1901, 224; not Bacillus renenosus Vaughan, Amer. Jour. Med. Sci., 104, 1892, 191.) From water.

Bacillus renenosus liquefaciens Taughan. (Amer. Jour. Med. Sei., 104, 1892, 193.) From water.

Bacillus ventricosus Weiss. (Weiss, Arb. bakt. Inst. Karlsruhe, 2, Heft 3, 1902 , 233; not Bacillus ventricosus Bredemann and Heigener, Cent. f. Bakt., II Abt., 93, 1935, 102.) From vegetable infusions.

Bacillus ventriculi Raczynski. (Diss. milit. medic. Acad. Petropolitanae Ruteniae, 1888; abst. in Cent. f. Bakt., 6, 1889,113 .) From the stomach of a dog.

Bacillus vermiculosus Zimmermann. (Zimmermann, Bakt. unserer Trink-u. Nutzwässer, Chemnitz, 1, 1890, 40; Bacterium vermiculosus Chester, Ann. Rept. Del. Col. Agr. Exp. Sta., 9, 1897, 99.) From water

Bacillus versatilis DeToni and Trevisan. (Bacillus A, Maggiora, Giorn. Soc. ital. d'Igiene, 11, 1889, 339; De Toni and Trevisan, in Saccardo, Sylloge Fungorum, 8, 1889, 968.) From the skin of the human foot and from air.

Bacillus vesiculiferus Migula. (Bacillus strumitis $\beta$, Tavel, Leber die Aetiologie der Strumitis, 1892, 110; Migula, Syst. d. Bakt., 2, 1900, 741.) From a case of strumitis.

Bacillus vialis Hansgirg. (Oesterr. bot. Ztschr., 1858, 6.) From roadside soil from near Prague.

Bacillus viator Trevisan. (Bacille de l'air $e$, Babes, in Cornil and Babes, Les Bactéries, 2nd ed., 1886, 150; Trevisan, I generi e le specie delle Batteriacee, 1889, 20.) From air.

Bacillus villosus Migula. (Bacillus aquatilis villosus Tataroff, Inaug. Diss., 
Dorpat, 1891, 47; Migula, Syst. d. Bakt.. 2, 1900, 828; not Bacillus villosus Tieck, Inaug. Diss., Dorpat, 1890, 47.) From water.

Bacillus vinicola Migula. (Bacillus saprogenes vini $I I$, Kramer, Die Bakterien in ihren Beziehungen zur Landwirtschaft, 2, 1892, 136; Migula, Syst. d. Bakt., 2, 1900, 685.) From wine.

Bacillus viniperda Migula. (Bacillus saprogenes vini $I$, Kramer, Die Bakterien in ihren Beziehungen zur Landwirtschaft, 2, 1892, 135; Migula, Syst. d. Bakt., 2, 1900, 684.) From wine.

Bacillus virens van Tieghem. (Bull. Soc. bot. France, 27, 1880, 175.) From aquatic plants.

Bacillus viridans Zimmermann. (Bakt. unserer Trink. "1. Nutzwässer, Chemnitz, 2, 1894, 22.) From water.

Bacillus viridescens non-liquefaciens Ravenel. (Ravenel, Mem. Nat. Acad. Sci., 8, 1896, 14; Bactcrium viridescens non-liquefaciens Chester, Ann. Rept Del. Col. Agr. Exp. Sta., 9, 1897, 72 and 123.) From soil.

Bacillus vulpinus von Iterson. (Cent. f. Bakt., II Abt., 12, 1904, 111.) From fresh garden soil, ranal water.

Bacillus wardii Chester. (Gas-and taint-producing bacillus in cheese curd, Moore and Ward, Cornell Univ. Igr. Expt. Sta., Bull. 158, 1899, 221-227; Chester, Man. Determ. Bact.. 1901, 206. From tainted, gassy cheese curd and from milk drawn directly from the udder. Presumably this was a coliform orginism.

Bacillus weckeri Trevisan. (Bacillus der Jequirity-Ophthalmie, de Wecker, 1882; see Flügge, Die Mikroorganismen, 2 Aufl, 1886, 279; Trevisan, I generi o le specie delle Batteriacee, 1889, 17.) From infusions of jequirity seed ( $\mathrm{Abr}$. precatorii)

Bacillus wesenbergii Chester. (Bacillus der Fleischvergiftung, Wesenberg, Ztschr. f. Hyg., 28, 1898, 494; Chester, Man. Determ. Bact., 1901, 247; not Bacillus wesenberg Castellani.) From meat which caused a meat poisoning outbreak. Closely related to Proteus vulgaris Hauser.

Bacillus wichmanni Trevisan. (Goldgelber Wasserbacillus, Adametz and Wichmann, Mitth. Oest. Vers. Stat.f. Brauerei u. Mälz., 1, 1888, 49; Trevisan, I generi e le specie delle Batteriacee, 1889, 19; Bacillus chryseus Migula, Syst d. Bakt., 2, 1900, 833.) From water.

Bacilluszcta Dyar. (Dyar, Ann. N. Y. Acad. Sci., 8, 1895, 369; Bacterium zeta Chester, Ann. Rept. Del. Col. Agr. Exp. Sta., 9, 1897, 114.) From air.

Bacillus zonatus Migula. (Bacillus No. 15, Pansini, Arch. f. path. Anat., 122, 1890, 450; Migula, Syst. d. Bakt., 2, 1900, 658.) From sputum.

Bacillus zörkendörferi Migula. (Bacillus oogcnes hydrosulfureus $\gamma$, Zörkendörfer, Arch.f. Hyg., 16, 1893, 385; Migula, Syst. d. Bakt.,2, 1900,696.) From hens' eggs.

Bacillus zymoseus (Leube) Trevisan. (Coccobacillus zymogenes Leube, Arch. f. path. Anat., 1885; Trevisan, I generi e le specie delle Batteriacee, 1889, 16.) From fermenting infusions.

Bacterium acidi propionici Weigmann. (Weigmann, quoted from Pribram, Klassifikation der Schizomyceten, Leipzig und Wien, 1933, 76; Plocamobacterium acidi propionici Pribram, idem.)

Bacterium aeris Migula. (Bacillus aeris minutissimus Kruse, in Flügge, Die Mikroorganismen, 3 Aufl., 2, 1896, 441; Bacterium aeris minutissimus Chester, Ann. Rept. Del. Col. Agr. Exp. Sta., 9, 1897, 109; Migula, Syst. d. Bakt., 2, 1900, 445; Bacterium aeris-minutissimum Chester, Man. Determ. Bact., 1901, 168.) From air.

Bacterium aerogenes $I$ and $I I$ Miller. (Miller, Deutsche med. Wochnschr., 12, 1886, 119; see Miller, Die Mikroorganismen der Mundhöhle, Leipzig, 1889, 261 and 262; not Bacterium aerogenes Chester, Ann. Rept. Del. Col. Agr. Exp. Sta., 9, 1897, 53; Bacillus millerianus DeToni and Trevisan, in Saceardo, Sylloge Fungorum, 8, 1889, 952.) From the digestive tract of man. 
Bacterium agreste Löhnis. (Löhnis, Cent.f. Bakt., I Abt., Orig., 40, 1906, 177; Bacillus agrestis de Rossi, Microbiol. agraria e technica, Torino, 1927, 828; not Bacillus agrestis Werner, Cent. f. Bakt., II Abt., 8\%, 1933, 468.) From soil.

Bacterium agrigenum (Trevisan) Migula. (Bacillus septicus agrigenus Flügge, Die Mikroorganismen, 2 Aufl.. 1886, 257; Pasteurella agrigena Trevisan. I generi e le specie delle Batteriacee, 1889, 21; Bacterium septicus agrigenus Chester, Ann. Rept. Del. Col. Agr. Exp. Sta., 9, 1897, 85; Migula. Syst. d. Bakt., 2, 1900, 372; Bacterium septicum Chester, Man. Determ. Bact.. 1901, 143.) From soil.

Bacterium album Migula. (Weisser Bacillus, Tataroff. Inaug. Diss., Dorpat. 1891, 35; Migula, Syst. 1. Bakt., 2, 1900, 419.) From water.

Bacterium algeriense Migula. (Gayon and Dubourg, Ann. Inst. Past., 8, 1894. 108; Migula, Syst . d. Bakt., 2, 1900, 513. Isolated in Algiers from wine where it causes a mannitol fermentation.

Bacterium aliphaticum Tausz and Peter. (Cent.f. Bakt., II Abt.. 49, 1919. 505.) From garden soil.

Bacterium aliphaticum liqucfacions Tausz and Peter. (Cent. f. Bakt., II Abt., 49, 1919, 505.) From gatrden soil.

Bacterium allantoides (Klein) Chester. (Bacillus allantoides Klein, C'ent. f Bakt., 6, 1889, 383; Chester, Ann. Rept Del. Col. Agr. Exp. Sta., 9, 1897, 103. Isolated as a culture contamination.

Bacterium alutaceum Migula. (Cioldgelber chagrinierter Bacillus, Tataroff Inaug. Diss., Dorpat, 1891, 62; Migula. Syst. d. Bakt.. 2, 1900, 464.) From water.

Bacterium ambiguum Chester. (C'hester, Ann. Rept. Del. Col. Agr. Exp. Sta. 11, 1900, 59; not Bacterium ambiguus Chester, ibid.. 9, 1897, 71; not Bucterium ambiguum Levine. Abst. Bart.. 4, 1920. 15.) From soil.

Bacterium amforeti Issatchenko. ( $\mathrm{R}^{2}-$ cherches sur les microbes de l'Océan
Glacial Arctique (in Russian), Petrograd, 1914, 237.) From sea water.

Bacterium anaerobium Migula. (Fuchs, Inaug. Diss., Greifswald, 1890; Migula, Syst. d. Bakt., 2, 1900, 388.) Obligate anaerobe. Pyogenic. Possibly a spore-former.

Bacterium anguillarum (Canestrini) Migula. (Bacillus anguillarum Canestrini, Atti d. R. Instituto Veneto di Scienze, Ser. 7, 1892-93; Migula, Syst. d. Bakt., 2, 1900, 442.) From diseased cels in the valleys of Comacchio.

Bacterium angustum Migula. (Lembke, Arch. f. Hyg., 26, 1896, 305; Migula, Syst.d. Bakt., 2, 1900, 474.) From feces.

Bacterium aphthosum (Iiruse) Chester. (Bacillus der Mundseuche des Menschen. Siegel, Deutsch. med. Wochnsehr., 1891, No. 49, 1328; Bacillus aphthosus Kruse, in Flügge, Die Mikroorganismen, 3 Aufl., 2, 1896, 427; Chester, Ann. Rept. Del. Col. Agr. Exp. Sta., 9, 1897, 85.) From the liver and kidneys of cattle affected with foot and mouth disease.

Bacterium apis No. 1, No. 2 and No. 3. Metalnikov and Kostritsky. (Compt. rend. Soc. Biol., Paris, 114, 1933, 1291. From diseased bees (Apis mellifera.)

Bacterium aquatile auruntiacum von Rigler. (Hyg. Rund., 12, 1902, 480.) From bottled mineral waters.

Bacterium aquatile citreum von Rigler. (Hyg. Pund., 12, 1902, 481.) From bottled mineral waters.

Bacterium aquatile debile von Rigler. (Hyg. Rund., 12, 1902, 481.) From hottled mineral waters.

Bacterium aquatile flarum von Rigler. (Hyg. Rund.. 12, 1902, 480.) From bottled mineral waters.

Bacterium aquatile luteum von Pigler. (Hyg. Rund., 12, 1902, 480.) From bottled mineral waters.

Bacterium arborescens non liquefaciens von Rigler. (Hyg. Rund., 12, 1902, 479; not Bacterium arborescons non-liquefaciens Chester, Ann. Rept. Del. Col. Agr. Exp. Sta., 9, 1897, 103.) From bottled mineral waters.

Bacterium arcticum Issatchenko.

(Re- 
cherches sur les microbes de l'Océan Glacial Arctique (in Russian). Petrograd, 1914, 148.) From sea water.

Bacterium arthritidis Migula. (Schüller, Berliner klin. Wochnschr., 1893, No. 36; Bacillus arthritidis chronicae liruse, in Flügge, Die Mikroorganismen, 3 Aufl., 2, 1896, 287; Migula, Syst. d. Bakt., 2, 1900, 443.) From a case of chronic: arthritis.

Bacterium asporiferum

Migula. (Flügge, Ztschr. f. Hyg., 17, 1894, 290; Anaërobier No. II, Kruse, in Flügge, Die Mikroorganismen, 3 Aufl., 2, 1896, 251; Migula, Syst. d. Bakt., 2, 1900, 446.) From milk.

Bacterium aurantii (Viron) Migula. (Bacillus aurantii Viron, Compt. rend. Acad. Sci., Paris, 114, 1892, 179; Migula, Syst. d. Bakt., 2, 1900, 512.)

Bacterium aurantium-roseum. Honing. (Honing, Cent. f. Bakt., II Abt., 37, 1913, 373; Plocamobacterium aurantium Pribram, Klassifikation der Schizomyceten, Leipzig und Wien, 1933, 77.) From fermenting tobacco.

Bacterium aurescens (Frankland and Frankland) Migula. (Bacillus aurescens Frankland and Frankland, Philos. Trans. Royal Soc of London, 178, 1887, B, 271; not Bacillus aurescens Ravenel, Mem. Nat. Acad. Sci., 8, 1896, 8; Migula, Syst. d. Bakt., 2, 1900, 466.) From air.

Bacterium aureum (Frankland and Frankland) Migula. (Bacillus aureus Frankland and Frankland, Philos. Trans. Royal Soc of London, 178, 1887, B, 272; Migula, Syst. d. Bakt., 2, 1900, 480.) From air.

Bacterium aureum (Adametz) Chester. (Bacillus aureus Adametz, quoted from Sternberg, Man of Bact., 1893, 621; not Bucillus aureus Frankland and Frankland, Philos. Trans. Roy. Soc. London, $178,1887, \mathrm{~B}, 272$; not Bacillus aureus Pansini, Arch. f. path. Anat., 122, 1890 , 436; Bacillus aureo-flaves Kruse, in Flügge, Die Mikroorganismen, 3 Aufl., 2, 1896, 310; Bacterium aureo farus Chester, Ann. Rept. Del. Col. Agr. Exp. Sta., 9, 1897, 109; Chester, ibid., 129; not Bac- terium aureum Migula, Syst. d. Bakt., 2, 1900, 480; Bacillus flarus Chester, Man. Determ. Bact., 1901, 255; not Bacillus flaves Fuhrmann, Cent. f. Bakt., II Abt., 19, 1907, 117; not Bacillus flavus Bergey et al., Manual, 1st ed., 1923, 286.) From water (Adametz); from the skin in cases of eezema (Tommasoli, Monats. f. prakt. Dermatol., 9.)

Bacterium arium Chester. (Bacillus of roup in fowls, Moore, U. S. Dept. Agr. Bur. Animal Industry, Bull. 8, 1895; ('hester, Man. Determ. Bact., 1901, 138.) From exudate of fowls in roup or diphtheria.

Bacterium babcsii Migula. (Bacillus septicus acuminatus Babes, Bakteriologische Untersuchungen der septischen Prozesse des Kindesalters, Leipzig, 1889; see Eisenberg, Bakt. Diag., 3 Aufl., 1891, 327 ; Bacterium septicus acuminatus Chester, Ann. Rept. Del. Col. Agr. Exp. Sta., 9, 1897, 66; Migula, Syst. d. Bakt., 2, 1900, 507; Bacterium acuminatum (hester, Man. Determ. Bact., 1901, 119.) From blood and organs of a new-born infant with septicemia.

Bacterium balbianii Billet. (Billet, Compt. rend. Acad. Sci., Paris, 107, 1888, 423; also in Bull. Sci. de la France et de la Belgique, 21, 1890, 108; Bacillus balbianii Trevisan, I generi e le specie delle Batteriacee, 1889, 17.) From sea water.

Bacterium barentsianum Issatchenko. (Recherches sur les microbes de l'Océan Glacial Arctique (in Russian). Petrograd, 1914, 155.) From sea water.

Bacterium beijerincki Issatchenko. (Recherches sur les microbes de l'Océan Glacial Aretique (in Russian). Petrograd, 1914, 157.) From sea water.

Bacterium benzoli $a$ and $b$ Wagner. (Ztschr. f. Garungphysiol., 4, 1914, 289.) From soil. Utilize benzene and certain benzene derivatives.

Bacterium besseri Migula. (Besser, Cent. f. Bakt., 13, 1893, 590; Migula, Syst. d. Bakt., 2, 1900, 508.) From smallpox.

Bacterium betae viscosum Panek. (Bull. Acad. Sci. Cracovie, 1, 1905, 5.) 
From fermenting beets. Reported to liquefy agar-gelatin (Biernacki, Cent. f. Bakt., 29, 1911, 166). Stanier (Jour. Bact., 42, 1941, 548) thinks this was a heterofermentative Lactobacillus.

Bacterium bossonis Chester. (Bacillus über eine neue Infektionskrank. des Rindviehs, Bosso, Cent. f. Bakt., 22, 1897, 537 and 23, 1898, 318; Chester, Man . Determ. Bact., 1901, 153.) Associated with an infectious disease of cattle

Bacterium boutrouxii (Trevisan) DeToni and Trevisan. (Micrococcus capable d'acétifier l'alcohol, Boutroux, Ann. Inst. Past., 2, 1888, 209; Bacillus boutrouxii Trevisan, I generi e le specie delle Batteriacee, 1889, 16; DeToni and Trevisan, in Saccardo, Sylloge Fungorum, 8, 1889, 1021.) From alcoholic infusions.

Bacterium boris Migula. (Pneumobacillus liquefaciens bovis Arloing, Compt . rend. Acad. Sci., Paris, 99, 18-, 109 and 116; Bacillus pneumonicus liquefaciens Kruse, in Flügge, Die Mikroorganismen, 3 Aufl., 2, 1896, 285; Bacterium pneumonicus liquefaciens Chester, Ann. Rept. Del. Col. Agr. Exp. Sta., 9, 1897, 99; Migula, Syst. d. Bakt., 2, 1900, 442; Bacterium pneumonicum Chester, Man. Determ. Bact., 1901, 158.) From the exudate of lung plague in cattle. A Gram-positive coccus-like bacterium.

Bacterium brassicac Conrad. (Bacterium brassicae acidae Lehmann and Conrad, in Lehmann and Teumann, Bakt. Diag., 1 Aufl., 2, 1896, 232; Conrad, Arch. f. Hyg., 29, 1897, 82; not Bacterium brassicae Wehmer, Cent. f. Bakt., II Abt., 10, 1903, 628; not Bacterium brassicae Migula, Syst. d. Bakt., 2, 1900, 296 (Bacillus brassicae Pommer, Mitt. botan. Inst. Graz, 1, 1886, 95); Bacillus brassicae Migula, loc. cit., 737.) From sauerkraut.

Bacterium breitfussi Issatchenko. (Recherches sur les microbes de l'Océan Glacial Aretique (in Russian). Petrograd, 1914, 152.) From sea water.

Bacterium brevissimum Weiss. (Arb. bakt. Inst. Karlsruhe, 2, Heft 3, 1902, 227.) From vegetable infusions.

Bacterium brunneoflavum (Dyar) Chester. (Bacillus brunneoflavus Dyar, Ann. N. Y. Acad. Sci., 8, 1895, 362; Chester, Ann. Rept. Del. Col. Agr. Exp. Sta., 9, 1897,112 .) Culture received from Kràl's laboratory as Micrococcus brunneus.

Bacterium bullosum Migula. (Bacillus No. 18, Pansini, Arch.f. pathol. Anat. u. Physiol., 122, 1890, 451; Migula, Syst.d. Bakt., 2, 1900, 415.) From feces.

Bacterium cadaceris (Sternberg) Chester. (Bacillus cadaveris Sternberg, Man. of Bact., 1893, 492; Chester, Ann. Rept. Del. Col. Agr. Exp. Sta., 9, 1897, 126.) From liver and kidneys of yellow fever cadavers. Anaerobe

Bacterium canalis Migula. (Kapseltragender Kanalbacillus, Mori, Ztschr. f. Hyg., 4, 1888, 52; Bacillus canalis capsulatus Sternberg, Man. of Bact., 1893. 476; Bacterium canalis capsulatus Ches. ter, Ann. Rept. Del. Col. Agr. Exp. Sta., 9, 1897, 130; Migula, Syst. d. Bakt., \&, 1900, 351.) From sewage.

Bacterium canalis parrum (Sternberg) Chester. (Bacillus canalis parvus Sternberg, Man. of Bact., 1893, 476; Chester, Ann. Rept. Del. Col. Agr. Exp. Sta., y, $1897,130$.$) Obtained by Mori (1888)$ from sewage

Bacterium carneum (Liruse) Chester. (Fleischfarbiger Bacillus, Tils, Ztschr. f. Hyg., 9, 1890, 294; Bacillus carnicolor Frankland and Frankland, Microorganisms of Water, 1894, 477; Bacillus carneus Kruse, in Flügge, Die Mikroorganismen, 3 Aufl., 2, 1596, 304; Chester, Ann. Rept. Del. Col. Agr. Exp. Sta., 9, 1897, 113.) From water.

Bacterium carnosum Kern. (Arb. bakt. Inst. Karlsruhe, 1, Heft 4, 1896, 448.) From the intestines of birds.

Bacterium cartilagineum (Olsen-Sopp) Buchanan and Hammer. (Bacillus cartilagineus Olsen-Sopp, Cent. f. Bakt., II Abt., 33, 1912, 49; Buchanan and Hammer, Iowa Sta. Agr. Exp. Sta., Res. Bull. 22, 1915, 271.) From slimy or ropy sour milk called false taette. 
Baclerium caseicola Migula. (Bacillus No. XII, Adametz, Iandw. Jahrb., 18, 1859, 245; Migula, Syst. d. Bakt., 2, $1900,475$.$) \quad From cheese.$

Bacterium castellum Henrici. (Arh). bakt. Inst. Kiarlsruhe, 1, Heit 1, 1891, 38.) From cheese.

Bacterium catenula Dujardin. (Dujardin, Hist. natur. des zooph., 18+1; Bacillus catenula Trevisan, I generi e le specie delle Batteriacee, 1889, 18; not Bacillus catemula Migula, Syst. d. Bakt., 2, 1900, 588.) From rice paddies and swamps.

Bacterium caratum hern. (Arb. bakt Inst. Karlsruhe, 1, Heft 4,1896 , 419.) From the intestines of birds.

Bacterium cavernae Migula. (Bucillus cavernae mimutissimus hruse, in Flügge, I) Mie Mroorganismen, 3 Aufl., 2, 1896. 4t0; Pfeiffer und Beck, Deutsch. med. Wochnschr., 1892, No. 21; Migula, Syst. (1. Bakt., 2, 1900, 509.) From human tuberculosis.

Bacterium cariae forluitum (Sternberg) Chester. (Bacillus caviae fortuitus Sternberg, Ian. of Bact., 1893, 650; ('hester, Ann. Rept. Del. Col. Igr. Exp. Sta., 9, 1897, 74.) From the liver of a yellow fever endaver.

Bacterium caricida havaniensis (Sternberg) Chester. (Bacillus cavicida harawicnsis Sternberg, Man. of Bact.. 1893, 425; Chester, Ann. Ropt. Del. Col. Agr. Exp. Sta., 9, 1897, 74.) From the intestine of a yellow fever cadaver.

Bacterium centricum Migulà. (Huber, Armin, Arch. f. pathol. Ana1. u. Physiol. 134, 1893, 216; Migula, Syst. d. Bakt., 2, 1900, 390; not Bacterium concentricum, a typographical error, see Migula, ibid. page v.) From a case of rystitis.

Bacterium cerinum Henrici. (Arb. hakt. Inst. Karlsruhe, 1, Ieft 1, 1894. 50.) From cheese.

Bacterium chlorinum Engelmann. (Bot. Zeitung, 1882, 324.) Green pigment.

Bacterium chologenes (Kruse) Chester. (Colonbacillus, Stern, Deutsche med. Wochnschr., 1893, 613; Bacillus cholo- yenes Kruse, in Flügge, Die Mikroorganismen, 3 Aufl., 2, 1896, 374; Chester, Ann. Rept. Del. Col. Agr. Exp. Sta., 9, 1897, 69.) From a case of purulent meningit is .

Bacterium chryseum Migula. (BacilLis nova species II, Freund, Inaug. I)iss., Erlangen, 1S93, 37; Migula, Syst. 1. Bakt., 2, 1900, 477.) Chromogenic bacterium from the mouth cavity.

Bacterium chrysogloea Zopf. (Zopf, in Overbeck, Nova Acta d. kais. Leop.Carol. Akad. d. Naturf., 55, 1891, No. 7; Bucillus chrysogloia (sic) Zimmermann, Bakt. unserer Trink-u. Nutzwässer, 2, 1894, 12.) From water.

Bacterium citreum (Frankland and Frankland) Migula. (Bacillus citreus Frankland and Frankland, Philos. Trans. Royal Sor of London, $178,1887, \mathrm{~B}, 272$; Migula, Syst. d. Bakt., 2, 1900, 459.) lrom air.

Bacterium coli apium Serbinow. (Jour. Microbiol. Petrograd., 2, 1915, 19.) From honey hees (.1 pis mellifera). Bacterium coli similis (Sternberg) Chester. (Bacillus coli similis Sternherg, Man. of Bact., 1893, 650; Chester, Ann. Rept. Del. Col. Agr. Exp. Sta., 9, 1897, 132.) From a human liver.

Bacterium colloideum Migula. (Bacterium butyri colloideum Lafar, Arch. f. Hyg., 13, 1s91, 17; Migula, Syst. d. Bakt., 2, 1900, 409.) From butter.

Bacterium comes Bersteyn. (Arb. bakt. Inst. Karlsruhe, 3, 1903, 93.) From soil.

Bacterium compactum (Kruse) Migula. (Bacillus compactus Kruse, in Flügge, Die Mikroorganismen, 3 Aufl., 2, 1896, 353; Migula, Syst. d. Bakt., 2, 1900, 438.) From air.

Bacterium concentricum liern. (Arb. bakt. Inst. Karlsruhe, 1, Heft 4, 1896, 437.) From the intestines of birds.

Bacterium conjunctiritidis (Kruse) Migula. Foch, Berichte aus Aegypten an den preuss. Staatsminister des Innern; see Arb. at. d. kaiserl. (iesundheitsamte, 3, 1887; Kiartulis, Cent. f. Bakt., 1, 1887, 289; Bacillus acgyptius 
Trevisan, I generi e le specie delle Batteriacee, 1889, 13; Bacillus conjunctivitidis Kruse, in Flügge, Die Mikroorganismen, 3 Aufl., 2, 1896, 440; Baclerium conjunctivitis Chester. Ann. Rept. Del. Col. Igr. Exp. Sta., 9, 1897, 67; Migula, Syst.d. Bakt., 2, 1900, 509; not Bacterium conjunctiritidis Chester, Man. Determ. Bact., 1901, 120; Bacterium aegyptium (hester, ibid., 121.) Associated with conjunctival catarrh in Egypt .

Bactorium corticale (Haenlein) Migula. (Bacillus corticalis Haenlein, Deutsch. Gerberzeitung, 1894, No. 18-34; Migula, Syst. d. Bakt., 2, 1900, 449.) Found on pine bark; in acid dyeing-liquor.

Bacterium crenatum Weiss. (Ar). bakt. Inst. Karlsruhe, 2, Heft 3, 1902. 221.) From fermenting malt.

Bacterium cristalliferum Gicklhorn. (Cent. f. Bakt.. II (ht.. 50, 1920, 420.) A sulfur bacterium from soil. See Manual, 5th ed., 1939, 86 for a descrintion of this organism.

Bacterium cuticularis (Tils) Chester. (Bacillus cuticularis Tils, Ztschr. f. Hyy., 9, 1890, 293; Chester, Ann. Rept. Del. Col. Agr. Exp. Sta., 9, 1897, 105.) From water.

Bacterium debile Berstyn. (Arb. bakt Inst. Karlsruhe, 3, 1903, 96.) From soil. Bacterium delendae-muscae Roubaud and Descazeaux. (Compt. rend. Acad. Sci., Paris, 17\%, 1923, 716.) From fly larvae (Stomoxys calcitrans and Musca domestica).

Bacterium deliense Honing. (Cent. f. Bakt., II Abt., 37, 1913, 37\%.) From tobacco plants in Sumatra.

Bacterium diatrypeticum Migula. (Bacillus diatrypeticus casei Baumann, Landwirtsch. Versuchsstationen, 42, 1893, 181; Migula, Syst. d. Bakt., 2, 1900, 404.) From cheese.

Bacterium enchelys Ehrenberg. (Ehrenberg, Abhandl. d. Akad. d. Wissensch. zu Berlin, 1830, 61; Bacillus enchelys Trevisan, I generi e le specie delle Batteriacee, $1889,18$.$) From$ water.
Bacterium endocarditidis Migula. (Bacillus endocarditidis capsulatus Weichselbaum, Beitr. z. pathol. Anat. u. z. allgem. Pathol., 4, 1887, 197; Migula, Syst. d. Bakt., 2, 1900, 359.) Found in the aorta, the left ventricle, the splren and kidneys of cadavers.

Bacterium endometritidis (Kruse) Chester. (Bacillus endometritidis Kruse, in Flügge, Die Mikroorganismen, 3 Aufl., 2, 1896, 432; Chester, Ann. Rept. Del. Col. Agr. Exp. Sta., 9, 1897, 88.) From a liver abscess.

Bacterium endomelitis canis Meyer. (Meyer, quoted from Pribram, Klassifikation der Schizomyceten, Leipzig und Wien. 1933, 7 ; Plocamobacterium (ndometritis Pribram, idem.) From a case of endometritis in a dog.

Bacterium enterocoliticum Schleifstein and Coleman. (A motile, Cram-negative bacillus, Schleifstein and Coleman, X. I. State Jour. Med., 39, 1939, 1749; Ann. Rept. Div Lab. and Res., N. Y. State Dept. Health for 1943,56 .) From lesions about the face, from an ulcer in the intestine and from the intestinal contents. Resembles Bacillus lignieri and Pasteurella pseudotuberculosis.

Bacterium crythromyxa (Zopf) Migula. (Micrococcus (Staphylococcus) erythromyxa Zopf, Ber. d. deutschen bot. Gesellsch., 1891, 22; Rhodococcus erythromzxa Zopf, loc. cit.; Migula, Syst. 1. Bakt., 2, 1900, 457; Bacillus erythromyxa Matzuschita, Bakt. Diag., 1902, 389.) Frequently listed as a Micrococcus.

Bacterium exanthematicum (Kruse) Chester. (Bacille, Babes and Oprescu, Inn. Inst. Past., 5, 1891, 273; Bacillus cxanthematicus Kruse, in Flügge, Die Mikroorganismen, 3 Aufl., 2, 1896, 426; Chester, Ann. Rept. Del. Col. Agr. Exp. Sta., 9, 1897, 87.) From a case of kemorrhatric septicemia.

Bacterium fausseki Is satchenko. (Recherches sur les microbes de l'Océan Glacial Arctique (in Russian). Petrograd, 1914, 157.) From sea water.

Bacterium ferophilum (sic) Migula. 
(Die ferrophilen Bakterien, Marpmann, Cent. f. Bakt., II Abt., 4, 1898, 21; Migula, Syst. d. Bakt., 2, 1900, 455 and 1058.) Isolated during studies on black discoloration of cheese.

Bacterium finitimum Chester. (Bacillus finitimus ruber Dyar, Ann. N. Y. Acad. Sci., 8, 1895, 361; Bacterium finitimus ruber Chester, Ann. Rept. Del. Col. Agr. Exp. Sta., 9, 1897, 116; Chester, Man. Determ. Bact., 1901, 177.) From air.

Bakterium flaveum Wilhelmy. (Arb. bakt. Inst. Karlsruhe, 3, 1903, 15.) From meat extract.

Bacterium flazocoriacum (Eisenberg) Chester. (Schwefelgelher bacillus, Adametz and Wichmann, Die Bakterien der Trink- und Nutzwässer, Mitt. Oest. Versuchsstat. f. Brauerei u. Mälz., Wien, Heft 1, 1888, 49; Bacillus flavocoriaceus Eisenberg, Bakt. Diag., 3 Aufl., 1891, 141; Chester, Ann. Rept. Del. Col. Agr. Exp. Sta., 9, 1897, 112.) From water.

Bacterium flarofuscum Migula. (No. 9, Lembke, Arch.f. Hyg., 26, 1896, 304; Migula, Syst. d. Balit., 2, 1900, 479.) From meat.

Bacterium flavum Issatchenko. (Recherches sur les microbes de l'Océan Glacial Aretique (in Russian). Petrograd, 1914, 151.) From sea water.

Bacterium foliicola. (Miehe) de Jongh. (Bacillus folicola Miehe, see Jahrb. wiss. Bot., 53, 1914, 1; ibid., 58, 1919, 29; de Jongh, On the Symbiosis of Ardisia crispa. Thesis, Univ. Leiden, 1938, 33.) A bacterial symbiont isolated from germinating seeds and embryos.

Bacterium freundii Migula. (Bacillus nova species I, Freund, Inaug. Diss., Erlangen, 1893, 31; Migula, Syst. d. Bakt., 2, 1900, 472.) From the mouth ravity.

Bacterium fungoides (Tschistowitsch) Migula. Bacillus fungoides Tschistowitsch, Berl. klin. Wochnsehr., 1892, 513; Migula, Syst. d. Bakt., 2, 1900, 391.) From pus.

Bacterium fuscum (Flügge) Migula
(Bacillus fuscus Flügge, Die Mikroorganismen, 2 Aufl., 1886, 290; Migula, Syst. d. Bakt., 2, 1900, 463.) From water.

Bacterium gamma (Dyar) Chester. (Bacillus gamma Dyar, Ann. N. Y. Acad. Sci., 8, 1895, 367; Chester, Ann. Rept. Del. Col. Agr. Exp. Sta., 9, 1897, 106.) From air.

Bacterium yammari Vejdovsky. (Cent. f. Bakt., II Abt., 11, 1904, 484.) From sections of a fresh water crustacean (Gammarus zscholkiei). Cells exhibit nuclei, showing mitosis.

Bacterium gelechiae No. 1 and No. 2, Metalnikov and Metalnikov. (Compt. rend. Acad. Agr., France, 18, 1932, 204.) From dead and dying larvate of a moth (Gelechia gossypiella).

Bacterium gelechiae No. 5, Metalnikov and Meng. (Compt. rend. Soc. Biol., Paris, 113, 1933, 170.) From dead larvae of a moth (Gelechia gossypiella). Bacterium gemmiforme Migula. (Lembke, Areh. f. Hyg., 29, 1897, 313; Migula, Syst. d. Bakt., 2, 1900, 391.) From intestinal contents.

Bacterium gibbosum. Weiss. (Arb. bakt. Inst. Karlsruhe, 2, Heft 3, 1902, 230.) From fermenting beets and malt.

Bacterium gingivae pyogenes Miller. (Miller, Die MIikroorganismen der Mundhöhle, Leipzig, 18s8, 217; Bacillus gingivae pyogenes Sternberg, Manual of Bact., 1893, 471.) From an alveolar abscess.

Bacterium gingivitidis (Kruse) Migula . (Babes, Deutsch. med. Wochnsehr., 1893, 1035; Bacillus gingivitidis Kruse, in Flügge, Die Mikroorganismen, 3 Aufl., 2, 1896, 427; Migula, Syst. d. Bakt., 2, 1900, 393.) Isolated in an epidemic of seurvy in Jilssy.

Bacterium gliscrogenum Malerba and Sanna-Salaris. Malerba and SannaSalaris, Lavori eseguiti nell'Istituto fisiol. di Napoli, 2, 1888, 13 and 95; Bacillus gliscrogenus Trevisan, I generi e le specie delle Batteriacee, $1889,14$. From urine

Bacterium gonnermanni Migula. (Bacillus tuberigenus 6 Gonnermann, Land- 
wirtsch. Jahrb., 23, 1894, 657; Migula, Syst. d. Bakt., 2, 1900, 418.) From root ncdules of lupine.

Bacterium gracilescens Weiss. (Arb. bakt. Inst. Karlsruhe, 2, Heft 3, 1902, 259.) From fermenting asparagus and milt.

Bacterium gracillimum Weiss. (Arb. bakt. Inst. Karlsruhe, 2, Heft 3, 1902, 235.) From bean and asparagus infusions.

Bacterium granulatum Henrici. (Henrici, Arb. bakt. Inst. Farlsruhe, 1 , Heft 1, 1894, 33; not Bacterium granulatum Chester, Man. Determ. Bact., 1901, 189.) From cheese.

Bacterium granulosum Weiss. Weiss, Arb. bakt. Inst. Karlsruhe, 2, Heft 3, 1902, 212; not Bacterium granulosum Lehmann and Neumann, Bakt. Diag., 5 Aufl., 2, 1912, 306.) From vegetable infusions.

Bacterium gryllotalpae Metalnikov and Mong. (Compt. rend. Acad. Sci., Paris, $201,1935,367$.) From diseased larvae of the ericket (Gryllotalpa gryllotalpa).

Bacterium gummosum Ritsert. (Ritsert, Ber. d. pharmaz. Gesell., 1, 1891 , 389 ; abst. in Cent. f. Bakt., 11, 1892, 730; Bacillus gummosus Migula, Srst. d. Bakt., 2, 1900, 873.) I mixture of a spore-forming rod and a streptococeus. See Bacillus gummosus IIapp and Vicrococcus gummosus Happ.

Bacterium halans (Zimmermann) Migula. Bacillus halans Zimmermann, Die Bakterien unserer Trink-u. Nutzwässer, Chemnitz, 2, 1894, 54; Migula, Syst. d. Bakt., 2, 1900, 429.) From water.

Bacterium hebetisiccus Steinhaus. (Jour. Bact., 42, 1941, 762 and 773.) From the walking stick (Diapheromera femorata).

Bacterium herbicola a aureum Gieilinger. Mitteil. a. d. Gebiete d. Lebensmitteluntersuchungen u. Hyg., 12, 1921, 262.) From corn meal. This is a variety of Bacillus herbicola Burri and Düggeli.

Bacterium herbicola rubrum Düggeli. (Düggeli, Cent. f. Bakt., II Abt., 12,
1904, 605; Bacterium herbicola $\beta$ rubrum Lehmann and Jeumann, Bakt. Diag., 4 Aufl., 2, 1907, 356.) From germinating plants, roots and barley seeds.

Bacterium hexacarbovorum Störmer. (Jihresber. d. Vereinigg. f. angew. Botanik, 5, 1907, 116.) From soil. Utilizes benzene and certain benzene derivatives.

Bacterium hidium Goobin. (Russian Health Resort Service, 5, 1923, 3.) Attacks ethane and other hydrocarbons.

Bacterium hirudinicolicum Lehmensick. (Cent.f. Bakt., I Abt., Orig., 147, 1941, 317; see Biol. Abst., 18, 1944, No. 6761.) Symbiotic in the intestines of Hirudo offcinalis and $H$. medicinalis.

Bacterium hoshigaki var. glucuronicum $I I$ and $I I I$ Takahashi and Asai. (Cent. f. Bakt., II Abt., 87, 1933, 395 and 405.) From dried persimmons (hoshigaki).

Bacterium infecundum Chester. (Bacillus filiformis haraniensis Sternberg, Man. of Bact., 1893, 650; Bacterium filiformis haraniensis Chester, Ann. Rept. Del. Col. Agr. Exp. Sta., 9, 1897, 126; Chester, Man. Determ. Bact., 1901, 181.) From the liver of a rellow fever cadaver. Anaerobic.

Bacterium inocuum Chester. (Wilde, Wien. klin. Wochnschr., 1892, No. 1-2; Bacillus lactis inocuus Kruse, in Flügge, Die Mikroorganismen, 3 Aufl., 2, 1596, 352; Bacterium lactis inocuus Chester, Ann. Rept. Del. Col. Agr. Exp. Sta., 9, 1897, 82; Chester, Man. Determ. Bact., 1901, 138.) From milk.

Bacterium intrinsectum Steinhaus. (Jour. Bact., 42, 1941, 764 and 774.) From an unidentified leaf beetle.

Bacterium iogenum Baumgartner. (Deutsche Monatschr. f. Zahnheilk., 2r, 764; Bacterium jogenum Baumgartner, Ergeb. d. gesam. Zahnheilk., 1, 1911, 752 and $779 ; B$. iogenum Kligler, Jour. Allied Dent. Soc., 10, 1915, 152.) From the mouth. Regarded as identical with Jodococcus vaginatus Miller by Kiligler (loc. cit.).

Bacterium keratomalaciae Migula. (Bacillus septicus keratomalaciae Babes, 
Bakteriol. Untersuch. d. sept. Prozesse d. Kindesalters, Leipzig, 1889; Migula, Syst. d. Bakt., 2, 1900, 363.) From an infected cornea.

Bacterium knipowitchi Issatchenko. (Recherches sur les microbes de I'Océan Glacial Aretique (in Russian). Petrograd, 1914, 150.) From sea water. A phosphorescent bacterium found to be pathogenic for the mealworm (Tenebrio molitor) by Pfeiffer and Stammer (Ztschr. f. Morph. u. Ökol. d. Tiere, 20, 1930, 157).

Bacterium liralii (Dyar) Chester. (Bacillus kralii Dyar, Ann. 1. Y. Icad. Sci., 8, 1895, 376; Chester, Ann. Rept. Del. Col. Agr. Exp. Sta., 9, 1897, 93; not Bacterium kralii Chester, Man. Determ. Bact., 1901, 166.) Received as Bacillus butyricus from Kiràl's laboratory by Dyar. The 1900 Iiril Catalogue lists cultures of Bacillus butyricus Botkin and Bacillus butyricus Hueppe. Is Dyar found that the characters of his culture differed from those of Bacillus butyricus Hueppe, Dyar's culture was probably Bacillus butyricus Botkin.

Bacterium kralii Chester. (Bacillus fuscus liquefaciens Dyar, Ann. X. Y. Acad. Sci., 8, 1895, 375; Bacterium fuscus liquefaciens Chester, Ann. Rept. Del. Col. Agr. Exp. Sta., 9, 1897, 108; Chester, Man. Determ. Bact., 1901, 166.) Re ceived as Bacillus fuscus from Krall's laboratory by Dyar who also found it in air. The 1920 Iiral catalogue lists Bacil lus fuscus Flügge syn. Bacterium brun neum Schrötter (sic); braunrother Ba cillus Maschek.

Bacterium laerii Migula. (Bacillus viscosus No. 1, van Laer, Extrait des mémoires couronnés et autres mémoires, Acad. Royale de Belgique, 1889, 36; see Kramer, Bakteriol. in ihren Beziehungen zur Landwirtsch., 2, 1892, 119; Bacillus viscosus cerevisiae Kruse, in Flügge, Die Mikroorganismen, 3 Aufl., 2, 1896, 359; Bacterium viscosus cerevisiae Chester, Ann. Rept. Del. Col. Agr. Exp. Sta., 9, 1897, 78; Migula, Syst. d. Bakt., 2, 1900, 402; Bacterium viscosum Chester, Man.
Determ. Bact., 1901, 128.) From beer, yeast, air, bread. Causes a slimy fermentation.

Bacterium lacvolacticum Migula. (Bacillus acidi laevolactici Schardinger, Monatsh. f. Chemie, 11, 1890, 544; Migula, Syst. d. Bakt., 2, 1900, 406; Bacterium acidi laevolactici Lehmann and Neumann, Bakt. Diag., 4 Aufl., 2, 1907. 178.) From milk.

Bacterium laminariae Billet. (Compt. rend. Acad. Sci., Paris, 106, 1888, 293; Billetia laminariae Trevisan, I generi e le specie delle Batteriacee, 1889, 11; Kurthia laminariae De Toni and Trevisan, in Saceardo, Sylloge Fungorum, 8, $1889,931$.$) From rotting sea weed.$ The type species of the genus Billetia Trevisan.

Bacterium langkatense Honing. (Cent. f. Bakt., II Abt., 37, 1913, 381.) From tobaceo plants in Sumatra.

Bacterium largum (v. Kilecki) Migula. (Bacillus largus v. Kilecki, Ann. Inst. Past., 9, 1898, 728; Migula, Syst.d. Bakt.,2, 1900, 448.) From the intestines of dogs.

Bacterium lepierrei Chester. (Bacille fluorescent pathogène, Lepierre, Ann. Inst. P'ast., 9, 1895, 643; Chester, Man. Determ. Bact., 1901, 182.) From cistern water.

Bacterium lethalis (Babes) Chester. (Proteus lethalis Babes, Progrès Médical Roumain, 1889; Chester, Ann. Rept. Del. Col. Agr. Exp. Sta., 9, 1897, 104; Bacillus lethalis Chester, Man. Determ. Bact., 1901, 249.) From lung gangrene in man.

Bacterium leucaemiae Migula. (Lucet, Jahresher. ü. Fortschr. in d. Lehre v. d. path. Mikroorg., $7,1891,319$; Bacillus lencaemiae canis Kruse, in Flügge, Die Mikroorganismen, 3 Aufl., 2, 1896, 285; Bacterium leucaemine canis Chester, Ann. Rept. Del. Col. Agr. Exp. Sta., 9, 1897, 119; Migula, Syst. d. Bakt., 2, 1900, 442; Bacillus leucaemiac Chester, Man. Determ. Bact., 1901, 264.) From a dog with leukemia.

Bacterium limbatum Migula. (Bacterium limbatum acidi lactici Marpmann, 
Ergänzungshefte d. Centralh. f. allg. Gesurdheitspflege, 2, 122; Bacillus limbatus acidi lactici Sternberg, Nan. of Bact., 1s93, 645; Migula; Syst. d. Bakt., 2. 1900, 407.) From fresh milk.

Bacterium lincolı (Müller) ('olnn. (Tibrio lineola Müller, Vermium His 1cria, 1773, 39; Cohn, Beitr. z. Biol. d. 1'flunz., 1, Heft 2, 1872, 170; Bacillus lineola Trevisan, I generi e le spocie delle Batteriacee, 18s9, 18.) From stagnant water, infusions, ete.

Bacterium lini Migula. (Winogradsky, Compt rend. Acad. Sci., Paris, 121 , 1893, 742; Migula, Syst. d. Bakt., 2, 1900. 513.) From retting hemp.

Bacterium linkoi Issutchenko. (Rewherches sur les microbes de l'ocium Giacial Aretique (in Russian). Petrograd, 1914, 154.) From sea water.

Bacterium liquefacions communis (Sternberg) ('hester. (Bacillus liquefaciens communis Sternberg, Man, of Bact., 1893, 686; Chester, Ann. Iiept Del. Col. Agr. Exp. Sta., 9, 1897, 91 Bacillus communis Migula, Syst. d Bakt., ?, 1900, 725; not Bacillus communis Jackson, Jour. Inf. Dis., 8, 1911, 241. From the feres of yellow fever patients

Bucterium litoreum W:irming. (Warming, Danmarks Krster levende Bak terier, 1875, 39s; Bacillus litoreus Trovi san, I generi e le specie delle Batteriacee 1889, 18.) From sea water.

Bacterium loculosum Migula. (Fächerbacillus, Clauss, Inaug. Diss., Würzburg, 1859, 27; Migula, Syst. rl. Bakt., 2, 1900, 408.) From milk.

Bacterium luceli Migula. (Lucet, Ann. Inst. Past., 3, 1959, 401; Bacillus cuniculicida thermophilus hruse, in Flügge, Die Mikroorganismen, 3 Auft. 2, 1896, 418; Migula, Syst. d. Bakt., 2, 1900, 507; Bacterium cuniculicida thermophitus Chester, Anm. Rept. Del. Col Agr. Exp. Stat., 9, 1897, 83; Bactorium curiculicida Chester. Man. Determ. Bact., 1901, 140.) Associated with an cpizootic in rabbits and guinea pigs.

Bacterium luduigi Karlinski. (Hyg. liundschau, 5, 1895, 685.) From the water of the hot springs at Ilidze in Bosnia.

Bacterium luteolum Henrici. Henrici, Arb. bakt. Inst. Karlsruhe, 1, Heft 1, 1894, 51; Migula, Syst. d. Bakt., 2, 1900 , 455.) From cheese.

Bacterium lutescens Migula. (Der gelbe Bacillus, Lustig, Diag. d. Bakt. d. Wassers, 1893, 78; Migula, Syst. d. Bakt. 2, 1900, 476.) From water.

Bacterium luteum Adametz. (List, Inaug. Diss., Leipzig, 1885, 53; Adametz, Bakt. Nutz-u. Trinkwässer, Mitteil. d. österr. Versuchsstation für Brauerei und Mälzerei in Wien, 1888, 48.) From the stomach contents of sheep and from water.

Bacterium margarineum Migula. (Margarinbacillus $\alpha$, Jolles and Winkler, Ztschr. f. Hyg., 20, 1895, 102; Migula, Syst. d. Bakt., 2, 1900, 410.) From margarine.

Bacterium marinum Issatchenko. (Recherches sur les microbes de l'Océan Glacial Iretique (in Russian). Petroyrad, 1914, 238.) From sea water.

Bacterium maydis Maiocchi. (Maiocchi, Bollet. d. Acead. medic. d. Roma, October, 18s1; Bacillus maydis Trevisan, I generi e le specie delle Batteriacee, $1889,17$.$) From corn (maize) infusions.$

Bacterium medanense Honing. (Cent f. Bakt., II Abt., 37, 1913, 382.) From the peanut plant (Arachis hypogaea).

Bacterium melolonthae liquefaciens Paillot. (Compt. rend. Sor. Biol., Paris, $68,1916,1102$.) From the rockchafer (Melolontha melolontha). Aceording to the author's system of nomenclature, this is presumably a synonym of Bacillus melolonthac liquefaciens $\alpha$.

Bacterium meningitidis (Neumann and Schaffer) (hester. (Bacillus meningitidis purulentae Neumann and Schaeffer, Arch. f. path. Anat., 109, 1887, 477; Chester, Ann. Rept. Del. Col. Agr. Exp. Stit., 9, 1897, 71; Bacillus neumanni Miguli, Srst. d. Bakt., 2, 1900, 751; not Bacillus neumanni Herter, in Just, Botan. Jahresber., 2 Abt., 39, Heft 4 , 1915, 748; Bacillus meningitidis Chester, 
Man. Determ. Bact., 1901, 213.) From pus from an individual who died of purulent meningitis.

Bacterium merismopedioides Zopf. (Zopf, Die Spaltpilze, 1 Auff., 1883, 56; Bacillus synchyseus Trevisan, I generi e le specie delle Batteriacee, 1889, 18; Bacterium synchyseus DeToni and Trevisan, in Saccardo, Sylloge Fungorum, 8, 1889, 1022.) From canal water.

Bacterium microsporum Trevisan. (Trevisan, Rendic. d. Instit. Lombardo, Ser. 2, 13, 1879; Bacillus microtis Trevisan, I generi e le specie delle Batteriacee, 1889, 18; Bacterium microtis DeToni and Trevisan, in Saccardo, Sylloge Fungorum, 8, 1889, 1025.) From water and putrefying infusions.

Bacterium minutum (Zimmermann) Migula. (Bacillus minutus Zimmermann, Die Bakterien unserer Trink- und Nutzwässer, Chemnitz, 2, 1894, 56; Migula, Syst. d. Bakt., 2, 1900, 423.) From water.

Bacterium monachae von Tubeuf. (v. Tubeuf, Forstlich - naturwissensch. Ztschr., 1, 1892, 34; Bacillus monachae Migula, Syst. d. Bakt., 2, 1900, 7ł2.) From the larvae of a moth (Lymantria monacha).

Bacterium multipediculum (Flügge) Chester. (Bacillus multipediculus Flügge, Die Mikroorganismen, 2 Aufl., 1886, 323; Chester, Ann. Rept. Del. Col. Agr. Exp. Sta., 9, 1897, 104.) Isolated frequently as a contamination on potato media.

Bacterium muripestifer (Kruse) Chester. (Bacillus dor Mäuseseuche, Laser, Cent.f. Bakt., 11, 1892, 181; Bacillus muripestifer Kiruse, in Flügge, Die Mikroorganismen, 3 Aufl., 2, 1896, 432; Chester, Ann. Rept. Del. Col. Agr. Exp. Sta., 9, 1897, 87.) From the spleen of a field mouse. Associated with a plague of field mice.

Bacterium nacreaceum ('Limmermann) Migula. (Perlmutterglänzender Baeillus, Keck, Inaug. Diss., Dorpat, 1890, 40; Eberbach, Inaug. Diss., Dorpat. 1890; Bacillus nacreaceus Zimmermann,
Die Bakterien unserer Trink- und Nutzwässer, Chemnitz, 2, 1894, 34; Migula, Syst. d. Bakt., 2, 1900, 426.) From water.

Bacterium naphthalinicus Tausson. (Planta, 4, 1927, 214.) From oil-soaked soils at Baku, Russia. Oxidizes naphthalene.

Bacterium nicolaieri Migula. (Kapselbacillus, Nicolaier, Cent. f. Bakt., 16, 1894, 601; Migula, Syst. d. Bakt., 2, $1900,354$.$) Associated with purulent$ nephritis.

Bacterium nicotianum Bucherer. (Cent. f. Bakt., II Abt., 105, 1942-43, 446.) From fermenting tobaceo leaves.

Bacterium nicotinobacter Bucherer. (Cent. f. Bakt., II Abt., 105, 1942, 170.) From a mixture of soil, manure and rotting materials. Gram-variable.

Bacterium nicotinophagum Bucherer. (Cent. f. Bakt., II Abt., 105, 1942, 167.) From a mixture of soil, manure, and rotting materials. Also from fermenting tobacco leaves (ibid., 446).

Bacterium nitens liern. (Arb. bakt. Inst. Karlsruhe, 1, Heft 4, 1896, 459.) From the intestines of birds.

Bacterium nomae (Schimmelbusch) Migula. (Bacillus nomae Schimmelbusch, Deutsch. med. Wochnsehr., 1889, No. 26; Migula, Syst. d. Bakt., 2, 1900, 384.) Found in necrotic tissues.

Bacterium oblongum (Boutroux) DeToni and Trevisan. (Micrococcus oblongus Boutroux, Annales de l'École normale supérieure, Sér. 2, 5, 1881, 67; Bacillus oblongus Trevisan, I generi e le specie delle Batteriacee, 1889, 16; DeToni and Trevisan, in Saceardo, Sylloge Fungorum, 8, 1889, 1021; Bacterium gluconirum Miquel and Cambier, Traité de Bact., 1902, 605; not Bacterium gluconicum Hermann, Biochem. Zeit., 192, 1928, 198.) From vinegar. May be an acetobacter.

Bacterium ogalae Migula. (Ogata, Cent.f. Bakt., 9, 1891, 442; Migula, Syst. d. Bakt., 2, 1900, 389.) From dust.

Bacterium orchiticum (Kruse) Chester. (Bacillus zur Rotzdiagnose, Kutscher, 
Ztschr. f. Hyg., 21, 1895, 156; Bacillus orchiticus Kruse, in Flügge, Die Mikroorganismen, 3 Aufl., 2, 1896, 455; Chester, Ann. Rept. Del. Col. Agr. Exp. Sta., 9, 1897, 99.) From nasal secretions of a glandered horse.

Bacterium osteophilum Billet. (Contribution à l'étude de la morphologie et du développement des Bactériacées, Bull. Sei. de la France et de la Belgique, Paris, 21, 1890, 149.) From macerated human bones.

Bacterium ovale Migula. (Bacillus No. 17, Pansini, Arch. f. pathol. Anat. u. Physiol., 122, 1890, 451; Migula, Syst. d. Bakt., 2, 1900, 458; not Bacterium ovale Chester, Man. Determ. Bact., 1901, 171 (Bacillus ovalis Wright, Mem. Nat. Acad.Sci., 7, 1895, 435).) From feces.

Bacterium ovatum Migula. (Bacillus ovatus minutissimus Unna-Tommasoli, Monatsh. f. prakt. Dermatol., 9, 1889, 59; Migula, Syst. d. Bakt., 2, 1900, 417; Bacterium oratus minutissimus Chester, Ann. Rept. Del. Col. Agr. Exp. Sta., 9, 1897, 139; not Bacterium ovatum. Chester, Man. Determ. Bact., 1901, 177 (Bacillus ruber ovatus Bruyning, Arch. néerl. Sci. exact. et nat., Sér. II, 1898, 297).) From human skin with seborrheic eczema.

Bacterium pallens Henrici. (Arb. bakt. Inst. Karlsruhe, 1 , Heft 1, 1894, 36.) From cheese.

Bacterium pallescens Henrici. (Arb. bakt. Inst. Karlsruhe, 1 , Heft 1, 1894, 35.) From cheese.

Bacterium pallidum Henrici. (Arb. bakt. Inst. Karlsruhe, 1 , Heft 1, 1894, 34; Ulvina pallida Pribram, Klassifikation der Schizomyceten, Leipzig und Wien, 1933, 76.) From cheese.

Bacterium pallidior Chester. (Bacillus fuscus pallidior Dyar, Ann. N. Y Acad. Sci., 8, 1895, 361; Bacterium fuscus pallidior Chester, Ann. Rept. Del. Col. Agr. Exp. Sta., 9, 1897, 111; Chester, Man. Determ. Bact., 1901, 171.) Culture received by Dyar from Liràl's laboratory labeled Bacillus latericeus. Dyar renames this because the culture does not agree with Bacillus latericeus
Eisenberg. However, the 1900 Irràl catalogue indicates that this was Bacillus latericcus Adametz and Wichmann syn. ziegelrother Bacillus, Adametz; Bacterium lactericeum Lehmann and Neumann.

Bacterium papillare Issatchenko. (Recherches sur les microbes de l'Océan Glacial Aretique (in Russian). Petrograd, 1914, 149.) From sea water.

Bacterium paradoxus (Kruse) Chester. (Typhus ahnlicher Bacillus, Kruse and Pasquale, Ztschr. f. Hyg., 16, 1894, 19 ; Bacillus paradoxus Kruse, in Flügge, Die Mikroorganismen, 3 Aufl., 2, 1896, 373; Chester, Ann. Rept. Del. Col. Agr. Exp. Sta., 9, 1897, 71.) From the liver in a case of dysentery.

Bacterium paraviscosum Buchanan and Hammer. (Iowa Sta. Coll. Agr. Exp. Sta., Res. Bull. 22, 1915, 266.) Stated to be similar to Bacterium viscosum of various authors.

Bacterium patelliforme Honing. (Cent. f. Bakt., II Abt., 3\%, 1913, 378.) From tobacco plants in Sumatra.

Bacterium pateriforme Migula. (Bacillus albicans pateriformis Unna-Tommasoli, Monatsh. prakt. Dermatol., 9, 1889, 58; Migulat, Syst. d. Bakt., 2, 1900, 415.) Found on human skin with seborrheic eczema.

Bacterium petersii Migula. (Bacterium C, Peters, Botan. Zeitung, 47, 1889 ; Bacillus aceticus petersii Kruse, in Flügge, Die Mikroorganismen, 3 Aufl., 2, 1896, 355; Bacterium aceticus petersii Chester, Ann. Rept. Del. Col. Agr. Exp. Sta., 9, 1897, 77; Migula, Syst. d. Bakt., 2, 1900, 397; Bacterium aceticum Chester, Man. Determ. Bact,, 1901, 127; not Bacterium aceticum Baginsky, Ztschr. f. phys. Chem., 12, 1888, 437.) From fermenting dough.

Bacterium photometricum Engelmann. (Engelmann, Jour. Roy. Microscop. Soc., 1882, 656 and 1883, 256; Bacillus photometricus Trevisan, I generi e le specie delle Batteriacee, $1889,18$.$) Sapro-$ phytic.

Bacterium piluliformans (Müller-Thur- 
gau) Migula. (Bacillus piluliformans Müller-Thurgau, Jahresber, d. Versuchsstation zu Wädenswil 1892/3, 3, 1894, 92; Migula, Syst. d. Bakt., 2, 1900, 513.) From a disease of red wine.

Bacterium pituitosum Migula. ( $\mathrm{Ba}$ cillus lactis pituitosi Löeffler, Berliner klin. Wochnschr., 1887, 631; Bacterium lactis pituitosi Chester, Ann. Rept. Del. Col. Agr. Exp. Sta., 9, 1897, 86; Migula, Syst. d. Bakt., 2, 1900, 403; Bacterium lactis Chester, Man. Determ. Bact., 1901, 148; not Bacterium lactis Baginsky, Ztschr. f. phys. (hem., 12, 1888, 437.) From milk.

Bacterium pityocampae Dufrency. (Compt. rend. Soc. Biol., Paris, 71, 1919. 288.) From diseased caterpillars of the processionary moth ('nethocampa pityo)campa).

Bacterium pleuropneumoniae Migula. (Diplococeus der Brustseuche der Pferde, Schütz, Arch. f. pathol. Anat. u. Physiol., 107, 374; Migula, Syst. d. Bakt.. 2, 1900, 348.) Frequently isolated from horses with pneumonia.

Bacterium plicatum (Zimmermanu) Chester. (Bacillus plicatus Zimmermann, Dic Bakterien unserer Trink- und Nutzwässer, Chemnitz, 1, 1890, 54; ('hester, Ann. Rept. Del. Col. Agr. Exp. Sta. 9, 1897, 108; Bacterium plicatimu Migula, Syst. (l. Bakt., 2, 1900, v and 453 ). From water.

Bacterium pneumopecurium C'hester. (Bacillus of sporadic pneumonia of cattle, Smith, I. S. I)ept. Igr. Bur. Animal Husbandry, 1895, 136; Chester, Man. Determ. Bact, 1901, 137.) Similar to Pasteurella suilla.

Bacterium pneumosepticum (Babes) Migula. (Bacillus pnoumosepticus Babes, Progrès méd. roumain, 6, 1889 ; not Bacillus pneumosepticus Kruse, in Flügge, Dis Mikroorganismen, 3 Aufl., 2, 1896, 408; Migula, Syst. d. Bakt., 2, $1900,377.1$ From a case of septic pneumonia .

Bacterium polymorphum (Frankland and Frankland) Migula. (Bacillus polymorphus Frankland and Frankland,
Philos. Trans. Royal Soc. London, 178, 1887, B, 275; Migula, Syst. d. Bakt., 2, 1900, 420.) From air.

Bacterium porri Majocchi. (Majocchi, in Tommasi-Crudeli, Anatomia patologica, 1, 1852; Bacillus verrucae vulgaris Kuhnemann, Monatsh. f. prakt. Dermatol., 9, 1889; Bacillus porri Trevisan, I generi e le specie dello Batteriacee, $1889,13$.$) From warts.$

Bacterium prodeniae Metalnikov and Metalnikov, (Compt. rend, Acad. Agric., France, 18, 1932, 206.) From a blackened dead larva of a moth (Prodenia litura).

Baclerium profusum (Frankland and Frankland) Migula. (Bacillus profusus Frankland and Frankland, Philos. Trans. lioyal soe. London, $178,1887, \mathrm{~B}, 276$; Migula, Syst. d. Bakt.. 2, 1900, 421.) From air

Bacterium psendoaquatile Migula. (Bacillus aquatilis $\alpha$, Tataroff, Inaug. Diss., Dorpat, 1891, 44; Migula, Syst.d. Bakt., 2, 1900, 470.) From water.

Bacterium pseudoconjunctivitidis (Kruse) Chester. (Kartulis, Cent. f. Bakt., 1, 1857, 289; Bacillus pseudoconjunctiritidis Liruse, in Flügge, Die Mikroorganismen, 3 Aufl., 2, 1896, 441; Chester, Ann. Rept. Del. Col. Agr. Exp. Sta., 9, 1897, 108.) From ronjunctival seretions

Bacterium pseudofilicinum Migula. (Farlenbacillus, Maschek, Bakteriologische Lntersuchungen der Leitmeritzer Trinkwässer, Iceitmeritz, 1887; Migula, Syst. (l. Bakt., 2, 1900, 454.) From water.

Bacterium pseudoinfluenzae (Kruse) ('hester. (Pseudoinfluenzabacillus, Pfeiffer, Ztschr. f. Hyg., 13, 1893, 357; Barillus psendoinfluenzae hruse, in Flügge, Die Mikroorganismen, 3 Aufl., 2, 1\$96, 439; Chester, Ann. Rept. Del. (ol. Agr. Exp. Sta., 9, 1897, 66.) From water.

Bacterium pseudokeratomalaciae Migula. (Loch, Cent. f. Bakt., 10, 1891. 369; Migula, Syst. d. Bakt., 2, 1900, 359.) 
A capsulated bacterium from infected cornea of a child.

Bactcrium pseudomultipediculum Migula. Bacillus multipediculus flavus Zimmermann, Bakt. unser. Trink- u. Nutzwässer, Chemnitz, 2, 1894, 42; Migula, Syst.d. Bakt., 2, 1900, 332.) From sewage.

Bacterium pseudopneumonicum (Passet) Chester. (Bacillus pseudopneumonicus Passet, Untersuchungen über die Aetiologie der eiterigen Phlegmone des Menschen, 1885, 40; Chester, Ann. Rept. Del. Col. Agr. Exp. Sta., 9, 1897, 140; Brucella pseudopneumonicum Pribram, Klassifikation der Schizomyceten, Leipzig und Wien, 1933, 68.) From pus.

Bacterium punctum (Mueller) Ehrenberg. (Monas punctum Mueller, Infusoria, 1786,3 ; Bacillus punctum Trevisan, I generi e le specie delle Batteriacee, 1889, 18.) From swamps and stagnant water.

Bacterium putidum Chester. (Bacillus gracilis cadaceris Sternberg, Man. of Bact., 1893, 733; Chester, Man. Determ. Bact., 1901, 140.) From a liver.

Bacterium pyaemicum Migula. (Levy, Cent.f. klin. Med., 1890, No. 4; abst. in Cent. f. Bakt., 8, 1890, 86; Migula, Syst. d. Bakt., 2, 1900, 443.) From a rase of pyemia.

Bacterium pyocinnabareum (Kruse) Chester. (Ferchmin, Ceber rote Eiterung, Wratsch, 1892, No. 24 and 25; abst. in Cent. f. Bakt., 13, 1893, 103; Bacillus pyocinnabareus Kruse, in Flügge, Die Mikroorganismen, 3 Aufl., 2, 1896, 304; Chester, Ann. Rept. Del. Col. Agr. Exp. Sta., 9, 1897, 113.) From a case of red pus.

Bucterium pyogenes Chester. (Fuchs, Inaug. Diss., Greifswald, 1890; Bacillus pyogenes anaerobius Kruse, in Flügge, Die Mikroorganismen, 3 Autl., 2, 1896, 244; not Bacillus pyogenes anaerobius Béla-Johan, Cent. f. Balit., I Abt., Orig. 87, 1922, 290; Bacterium pyogenes anaerobius Chester, Ann. Rept. Del. Col. Agr. Exp. Sta., 9, 1897, 127; Chester, Man. Determ. Bact., 1901, 184; not
Bacterinm pyogenes Migula, Syst. d Bakt., 2, 1900, 381; not Baclerium pyogenes Ward, Jour. Bact., 2, 1917, 619.) From stinking pus from a rabbit.

Bacterium pyrauslae Nos. 1-7 Metalnikov and Chorine. (Internat. Corn Borer Invest., Sci. Repts., 1, 1928, 52.) From diseased corn borer larvae ( $P y$ rausta nubilalis $\mathrm{Hb}$.).

Bacterium radiatum Chester. (Del. College Agr. Expt. Sta. Ann. Rept., 11, 1900, 56.) From soil.

Bacterium ramificans Weiss. (Arb. bakt. Inst. FarIsruhe, 2, Heft 3, 1902, 229.) From bean infusions.

Bacterium rangiferinum Honing. (Honing, Cent. f. Bakt., II Abt.. 37, 1913, 379; Plocamobacterium rangiferinum Pribram, Klassifikation der Schizomyceten, Leipzig und Wien, 1933, 78.) From fermenting tobaceo.

Bacterium repens Miehe. In organism associated with Bacterium folicola de Jongh.

Bacterium retiformans Gichlhorn. (Cent. f. Bakt., II Ibt., 50, 1920, 421.) I sulfur bacterium from garden soil. See Manual, 5th ed., 1939, 86 for a description of this organism.

Bacterium rhizopodicum Migula. (Bacillus rhizopodicus margarineus Jolles and Winkler, Ztsehr. f. Hyg., 20, 1895, 105; Migula, Syst. d. Bakt., 2, 1900, 452.) From margarine.

Bacterium roseum Losski. (Losski, Inaug. Dise., Dorpat, 1893; quoted from Migula, Syst. d. Bakt., 2, 1900, 484; Bacillus roseus Nepveux, Thèse, Fac. Pharm., Paris, 1920, 115.) From sand.

Bacterium rubigenosum Kern. (Kern, Arb. bakt. Inst. Tiarlsruhe, 1, Heft, 4, 1896, 456; Bacillus rubigenosus Nepveux, Thèse, Fac. Pharm., Paris, 1920, 113; not Bacillus rubiginosus Catiano, in Cohn, Beitr. z. Biol. d. Pflanzen, r, 1896, 538.) From the intestines of birds.

Bacterium rubrum Schneider. (Bahterium rubrum Schneider, Arb. bakt. Inst. Karlsruhe, 1, Heft 2, 1894, 213; also see Migula, Syst. d. Bakt., 2, 1900, 488; Bacillus rubrum Nepveux, Thèse, 
Fac. Pharm., Paris, 1920, 115.) From swamp water. Difficult to distinguish from Bacterium erythromyxa.

Bacterium rubrum Metalnikov and Metalnikov. (Compt. rend. Acad. Agric., France, 18, 1932, 204; not Bacterium rubrum Schneider, Arb. bact. Inst. Karlsruhe, 1, Heft 2, 1894, 213.) From the cotton worm (Gelechia gossypiella).

Bacterium salivae Migula. (Bacillus salivae minutissimus Kruse, in Flügge, Die Mikroorganismen, 3 Aufl., 2, 1896 , 440; Bacterium salivae minutissimus Chester, Ann. Rept. Del. Col. Agr. Exp. Sta., 9, 1897, 86; Migula, Syst. d. Bakt., 2, 1900, 418.) From secretions of the mouth.

Bacterium salmonicida Lehmann and Neumann. (Bacillus der Forellenseuche, Fmmerich and Weibel, Arch. f. Hyg., 21, 1894, 1; Lehmann and Neumann, Bakt. Diag., 1 Aufl., 2, 1896, 240; Bacillus salmonicida Kruse, in Flügge, Die Mikroorganismen, 3 Aufl., 2, 1896, 322; Bacterium salmonica Chester, Ann Rept. Del. Col. Agr. Exp. Sta., 9, 1897. 99; see Mackie et al., Final Rept. of the Furunculosis Committee, H. M. Stationery Office, Edinburgh, 1935; and Duff, Jour. Bact., 34, 1937, 49.) Pathogenic for trout.

Bacterium sanguinis Migula. (Bacillus sanguinis typhi Sternberg, Man, of Bact., 1893, 732; Bacterium sanguinis typhi Chester, Ann. Rept. Del. Col. Agr. Exp. Sta., 9, 1897, 89; Migula, Syst.d. Bakt., 2, 1900, 506.) From the blood of typhus fever patients.

Bacterium schüfineri Honing. (Cent. f. Bakt., II Abt., 37, 1913, 370.) From tobaceo plants in Sumatra.

Bacterium septentrionale Issatehenko. (Recherches sur les microbes de 1 Océan Glacial Aretique (in Russian). Petrograd, 1914, 239.) From sea water

Bactorium (Proteus) septicus (Babes) Chester. (Proteus seplicus Babes, Septische Processe des Kindesalters, 1889; Bacillus protens septicus Kruse, in Flügge, Die Mikronrganismen, 3 Aufl., 2,
1896, 279; Chester, Ann. Rept. Del. Col. Agr. Exp. Sta., 9, 1897, 102; Bacillus septicus Chester, Man. Determ. Bact., 1901, 245; not Bacillus septicus Macé, Traité pratique de Bact., 1st ed., 1889, 455; not Bacillus septicus Migula, Syst. d. Bakt., 2, 1900, 646; not Bacillus septicus Crookshank, Textb. of Bact., 4th ed., 1900, 632.) From the intestine of a child having septicemia.

Bacterium setosum Henrici. (Arb. bakt. Inst. Karlsruhe, 1, Heft 1, 1894, 46.) From cheese.

Bacterium siccum Issatchenko. (Recherches sur les microbes de l'Océan Glacial Aretique (in Russian). Petrograd, 1914, 235.) From sea water.

Bacterium sieberti Migula. (Siebert, Inaug. Diss., Würzburg, 1894, 13; Migula, Syst. d. Bakt., 2, 1900, 456.) From hair follicles.

Bacterium soriferum Migula. (Severin, Cent.f. Bakt., II Abt., 1, 1895, 799; Migula, Syst. d. Bakt., 2, 1900, 438.) From manure.

Bacterium spiniferum. (Unna-Tommasoli) ('hester. (Bacillus spiniferus Unna-Tommasoli, Monatsh. f. prakt. Dermatol., 9, 1889, 58; Chester, Ann. Rept. Del. Col. Agr. Exp. Sta., 9, 1897, 110 and 143.) From human skin with seborrheic eczema.

Bacterium spinosum Weiss. (Arb. bakt. Inst. Karlsruhe, 2, Heft 3, 1902 , 219.) From fermenting beets.

Bacterium spirale Issatchenko. (Recherches sur les microbes de l'Océan Glacial Arctique (in Russian). Petrograd, 1914, 238.) From sea water.

Bacterium sputigenum Chester. (Bacillus aerogenes sputigenus capsulatus Herla, Archiv de Biol., 14, 1895, 403; Chester, Man. Determ. Bact., 1901, 133; not Bacterium sputigenum Migula, Syst. d. Bakt., 2, 1900, 378.) From the blood of at mouse which had been inoculated with the sputum of a pneumonia patient.

Bacterium sputigenum Migula, (Kreibohm, Inaug. Diss., Helmstedt, 1898, 29; Migula, Syst. d. Bakt., 2, 1900, 378.) From the mouth. 
Bacterium squamatum Weiss. (Arb. bakt. Inst. Karlsruhe, 2, Heft 3, 1902 , 242.) From vegetable infusions.

Bacterium squamosum Kern. (Arb. bakt. Inst. Karlsruhe, 1, Heft 4, 1897, 436.) From the stomachs and intestines of birds.

Bacterium stalactitigenes Honing. (Cent. f. Bakt., II Abt., 37, 1913, 375.)

From tobacco plants in Sumatra.

Bacterium sternbergii Migula. (Bacillus anaerobius liquefaciens Sternberg, Man. of Bact., 1893, 693; Migula, Syst. d. Bakt., 2, 1900, 444; Bacterium anaerobicum Chester, Man. Determ. Bact. 1901, 198; Bacillus sternbergii Winslow, Kiligler and Rothberg, Jour. Bact., 4 , 1919, 487.) From intestines of yellow fever cadavers.

Bacterium steroidiclasium. Arnaudi and Ercoli. (Boll. Sez. ital. Soc. intern. Microbiol., 20 (3), 1941, 000; also see Arnaudi, Cent. f. Bakt., II Abt., 105 , $1942-43,352$.$) Source not given in$ second paper. From bakers' yeast.

Bacterium streckeri (Trevisan) Migula (Bacillus citreus cadaveris Strassmann and Strecker, Ztschr. f. Medizinalbeamte, 1888, No. 3; Bacillus streckeri Trevisan, I generi e le specie delle Batteriacee, 1889, 17; Bacterium citreus cadareris Chester, Ann. Rept. Del. Col. Agr. Exp. Sta., 9, 1897, 108; Migula, Syst. d. Bakt., 2, 1900, 460; Bacterium citreum Chester, Man. Determ. Bact., 1901, 167.) From a cadaver.

Bacterium subcitricum Weiss. (Arb. bakt. Inst. Karlsruhe, 2, Heft 3, 1902, 257.) From vegetable infusions.

Bacterium subfuscum Kern. (Arb. bakt. Inst. Karlsruhe, 1, Heft 4, 1896, 461.) From the intestines of birds.

Bacterium subluteum Migula. (Bacillus luteus von Dobrzyniecki, Cent. f Bakt., I Abt., 21, 1897, 835; Migula, Syst. d. Bakt., 2, 1900, 456.) From the mouth.

Bacterium sulfureum Holschewnikoff. (Holschewnikoff, Fortschr. d. Med., 7, 1889, 204 and Ann. de Microgr., 1, 18881889, 261; Bacillus sulfureus Trevisan, I generi e le specie delle Batteriacee, 1889 , 17.) From sewage

Bacterium sumatranum Honing. (Cent. f. Bakt., II Abt., 37, 1913, 37t.) From tobaceo plants in Sumatra.

Bacterium surgeri (Dornic and Daire) Buchanan and Hammer. (Bacillus surgeri Dornic and Daire, Bull. mens. de l'Office de renseignements agricoles, 6 , 1907, 146; Buchanan and Hammer, Iowa Sta. Coll. Agr. Exp. Sta., Res Bull. 22, 1915, 254.) From serum produced in the manufacture of casein Causes slimy milk. Closely related to the Bacterium bulgaricum group, accord ing to Buchanan and Hammer.

Bacterium sycosiferum Migula. (Bacillus sycosiferus foetides Unna-Tommasoli, Monatsh.f. prakt. Dermatol., 8, 1889, 183; Migula, Syst. d. Bakt., 2, 1900, 385.) From the beard of a patient with bacillogenic sycosis

Bacterium syphitidis (Kruse) Migula (Syphilisbacillus, Lustgarten, Wiener med. Wochnschr., 1884 and Wiener med. Jahrbücher, 1885; Pacinia syphititica Trevisan, I generi e le specie delle Batteriacee, 1889, 23; Bacillus syphilidis Kruse, in Flügge, Die Mikroorganismen, 3 Aufl., 2, 1896, 514; Migula, Syst.d. Bakt., 2, 1900, 496.) From syphilis.

Bacterium tachytonum Fischer. (Fischer, Deutsche med. Wochnschr., 1894, No. 25-28; Bacillus tachytonus Migula, Syst. d. Bakt., 2, 1900, 655.) From feces in a case of cholera.

Bacterium tenue Migula. (Bacillus tenuis sputigenes Pansini, Arch. f. path. Anat., 122, 1890, 453; Bacillus sputigenus tenuis (sic) Kruse, in Flügge, Die Mikroorganismen, 3 Aufl., 2, 1896, 431 ; Bacterium sputigenes tenuis Chester, Ann. Rept. Del. Col. Agr. Exp. Sta., 9, 1897, s9; Migula, Syst. d. Bakt., 2, 1900, 457.) Associated with advanced phthisis and catarrhal pneumonia.

Bacterium termo (Mueller) Ehrenberg. (Monas termo Mueller, Infusoria, 1786; Ehrenberg, Abhandl. Akad. Berl., 1830; Palmella infusionum Ehrenberg, Infusionsthierchen, 1838, 526; Zoogloea 
termo Cohn, Xova Acta Leop. Carol., 24, 1853, 123; Bacillus termo Trevisan, I generi e le specie delle Batteriacee, 1889, 18.) From infusions.

Bacterium termo viar. subterianeum Hansgirg. (Hansgirg, Oest. Bot. Ztschr., 1888, 6; quoted from DeToni and Trevisan, in Saccardo, Sylloge Fungorum, 8, 1889, 1025.) From (lamp) walls in a cellar.

Bacterium thiogenes Lehmumn. (Thionsäurebakterien, Trautwein, Cent. f. Bakt., II Abt., 53, 1921, 513; ibid., 61, 1924, 1; Tehmann, in Lehmann and Nellmann, Bakt. Diag., 7th Aufl., 2, 1927, 516; Thiobacillus trautweinii Bergey. et al., Manual, 2ncl ed., 1925, 39.) From canal water, sewage and soil. Regarded by Trautwein (loc. cit., 1924, 5) as closely related to Bacterium denitrificans Loh. mann and Neumann. See Flarobar terium denitrificans Bergey et al. Ifetero trophic and therefore wrongly placed in Thiobacillus (Starkey, Jour. Bart., 28, 1934, 387; Jour. (ien. Physiol., 18, 1935. 325).

Bacterium tholoeidenm (iessner. (Gessner, Arch. I. Hyg., 9, 18s9, 129; Bacillus tholoeideus DeToni and Treviwin, in Saceardo, Sylloge Fungorum, 8 . 1889, 952.) From the human duolenum.

Bacterium tortuosum Zukal. (Zukial. Verh. d. zoolog. botan. Ciesellsch., Wien. 35, 1885; Bacillus torluosus Trevisan, I generi e le specie delle Batteriacee, 1889. 18; not Bacillus tortuosus Debono, Cent. f. Bakt., I Abt., Orig., 62, 1912, 233. From muddy water.

Bacterium tremulans Ehrenbery. (Ehrenberg, Abhandlungen d. Berliner Akad., 1830, 38; Tibrio Iremulans Fhrenherg, Die Infusionsthierchen, 1838, 79; Trevisan, Rend. Ist. Lomb., 1879, 145; Bacillus tremulans Trevisan, I generi e le specie delle Batteriacee, 1889, 1א.) From stagnant water, infusions, etc.

Bacterium trichorhexidis Migula (Bacillus multiformis trichorrhexidis Hodara. Monatsh. f. prak1. Dermatol., 19. 1894, 173; Migula, Syst. d. Bakt., 2,
1900,437 .) From healthy hair showing trichorrhexis.

Bacterium truncatum Chester. (Ba('illus No. XII, Adametz, Landwirtsch. Jahrh., 18, 1889; Chester, Man. Determ. Bact., 1901, 15r; not Bacterium truncatum Migula, Syst. d. Bakt., 2, 1900, 407; not Baclerium truncatum (hester, loc. cit.. 195.) From Emmenthat cheese.

Bacterium luberosum Liern. (Arb. bakt. Inst. Karlsruhe, 1 , Heft 4, 1896, 455; Bacillus tuberosus Nepveux, Thèse, Fac. Phatm., Paris, 1920, 113.) From the intestines of birds.

Bacterium turcosum. (Quoted from Franke and Rudloff, Biochem. Ztschr., $310,1942,207$.) Souree not given.

Bacterium uniforme Weiss. (Arb. bakt. Inst. Kitrlsruhe, 2, Heft 3, 1902, 250.) lirom fermenting malt.

Bacterium wreac Ieube and Graser. (Iente and Graser, Arch. f. pathol. Anat. u. Physiol., 100, 1885, 558; Bacillus ureae Dyar, Amn. X. Y. Acad. Sci., 8, 1895, 357; not Bacillus ureac Miquel, Bull. Sioc. Chim, d. Paris, 31, 1879, 391; Trobacillus lembei Micuel and Cambier, Traité de Bactériologie, Paris, 1902, 635; not Lrobacillus leubei Beijerinck, Cent. f. Bakt., II Abt., 7, 1901, 51; Plocamobacterium ureae Pribram, Klassifikation ler Schizomyceten. Leipzig und Wien, 1933, 78.) From urine. Leube makes no statement regarding spore formation. While Miquel's and Leube's organisms are sometimes regarded as having been identical, Miquel did not regard his Bacillus ureac as being identi"al with Leube's Bacterium ureae and gave them separate names Urobacillus duclaurii and Trobncillus leubei (Miquel and (ambier, loc. cit., 631 and 635). The latter name had however been previously used by Beijerinck (loc. cit.) for a different organism. Dyar credits the name Bacillus ureae to Jaksch (Ztschr. f. physiol. Chem., 5, 1881, 395) who, however, spoke only of a Harnstoffpilz and evidently had no pure cultures. Dyax's culture which came from Kràl is listed in the 1900 Kril catalogue as 
Bacillus ureae Leube. Also see Gibson (Jour. Bact., 24, 1935, 493). Löhnis (Handb. f. landwirtsch. Bakt., 1910, 459) thinks that this species belongs in the Proteus group.

Bacterium vaillardi Migula. (Kelsch and Vaillard, Ann. Inst. Past., 4, 1890, 276; Migulit, Syst. d. Bakt., 2, 1900, 437.) Found in swellings of the lymph system in leukemia.

Bacterium varicosum Migula. (Gombert, Recherches expér. microbes conjonctives, Paris, 18s9; Bacillus varicosus conjunctivae Sternberg, Man. of Bart., 1893, 474; Bacterium raricosus conjunctivae Chester, Ann. Rept. Del. Col. Agr. Exp. Sta., 9, 1897, 100; Migula, Syst. d. Bakt., 2, 1900, 444.) From the normal conjunctiva of man.

Bacterium variosum Weiss. (Arb. bakt. Inst. Karlsruhe, 2, Heft 3, 1902, 218.) From vegetable infusions.

Bacterium velatum Migula. (Bucillus tuberigenus 5, Gonnermann, Landwirtsch. Jahrb., 23, 1894, 657; Migula, Syst. d. Bakt., 2, 1900, 45t; Bacillus velatus Nepvenx, Thèse, Fae. Pharm., Paris, 1920, 113.) From lupine root nodules.

Bacterium vernicosum Zopf. (Zopf, Beitr. z. Physiol. u. Morphol, niederer Organismen, Heft 1, 1892, 63; Bacillus vernicosus Migula, Syst. d. Bakt.. 2, 1900. 781.) From cutton-seed meal.

Bacterium vesiculosum Henrici. (Arb. a. d. bakt. Inst. d. techn. Hochschule zu Karlsruhe, 1, Heft, 1, 1894, 37.) From cheese.

Bacterium villosum (Keck) Migulat (Bacillus millosus Keek, Inaug. Diss. Dorpat, 1890, 47; Migula, Srst. d. Bakt. 2, 1900, 429; Plocamobacterium rillosum Pribram, Klassifikation der fichizomyceten, Leipzig und Wien, 1933, 79.) From water.

Bacterium vinicola Migula. (Bacillus viscosus vini Kramer, Bakteriol. in ihren Beziehungen z. Landwirtsch., 2, 1892, 144; Migula, Syst. d. Bakt., 2, 1900, 510.) From wine.

Bacterium viniperda Migula. (Bacil- lus saprogenes vini IV, Framer, Bakteriol. in ihren Beziehungen z. Landwirtsch., 2, 1892, 135; Migula, Syst. d. Bakt., 2, 1900, 416.) From diseased wine.

Bacterium viride van Tieghem. (Bull. Soe bot. France, 27, 1880, 174.) Found on a fungus.

Bacterium riscidum Migula, (Bacillus viscosus margarineus Jolles and Winkler, Ztschr. f. Hyg., 20, 1895, 104; Migula, Syst. d. Bakt., 2, 1900, 450.) From matrgarine.

Bacterium viscosum Migula. (Bacillus viscosus sacchari Kramer, Sitzungsb. d. kais. Akad. d. Wiss., Wien, 1889: Bakteriol. in ihren Beziehungen $z$ Landwirtsch., 2, 1892, 156; Migula, Syst. d. Bakt., 2, 1900, 447.) Similar to Leuconostoc mesenteroides except that it liquefies gelatin.

Bacterium viscosum non-liquefaciens Stutzer and Wsorow. (Cent. f. Bakt., II Abt.. $71,1927,117$.) From pupae of moth (Euxor segetum). Resembles $\mathrm{Ba}$ cillus viscosus Frankland.

Bacterium vitulinum Chester. (Bacillus der Septikämie bei einem Seekalbe, Bosso, Cent. f. Bakt., 25, 1899, 52; (hester, Man. Determ. Bact., 1901, 143.) From a septicemia of the sea-calf (Phoca vitulina).

Bacterium vitulorum Migula. (Bacillus der weissen Ruhr der Kälber, Jensen. Monatsh. f. prakt. Tierheilk., 3, 1892, 92; Maanedskrift for Dyrlaeger, 4, 189293, 140; Bacillus dysenteriae vitulorum Kruse, in Flügge, Die Mikroorganismen, 3 Aut1., 2, 1896, 412; Bacterium dysenteriae rilulorum Chester, Ann. Rept. Del. Col. Agr. Exp. Sta., 9, 1897, 86; Migula, Syst. d. Bakt., 2, 1900, 394; Bacterium dysenteriae Chester, Man. Determ. Bact., $1901,145$.$) Associated with dysentery$ of ealves.

Bacterium winkleri Migula. Margarinbacillus $\beta$, Jolles and Winkler, Ztschr. f. Hyg., 20, 1895, 102; Migula, Syst. d. Bakt., 2, 1900, 485.) From margarine.

Bacterium wrightii ('hester. (Capsule Bacillus of Mallory and Wright, 
Ztschr. f. Hyg., 20, 1895, 220; Chester, Man. Determ. Bact., 1901, 133.) From a ease of bronchopneumonia.

Bacterium zinnioides Honing. (Cent. f. Bakt., II Abt., 37, 1913, 371.) From tobacco, peanut and other plants in Sumatra.

Bacterium zuernianum. (List) Chester. (Bacillus zucrnianus List, Inaug. Diss., Leipzig, 1885, 36; Chester, Ann. Rept. Del. Col. Agr. Exp. Sta., 9, 1897, 83.) From fresh manure and intestines of sheep; also found in water.

Coccobacillus acridiorum Picard and Blanc. (Coccobacille des sauterelles, d'Herelle, Compt. rend. Acad. Sci. Paris, 152, 1911, 1413; Picard and Blanc, ibid., $156,1913,1335$; Bacillus acridiorum Chatton, ibid., 156, 1913, 1708.) From a locust (Schistocerca americana Drury). Coccobacillus cajae Pieard and Blanc. (Compt. rend. Acad. Sci., Paris, 156, 1913, 1334; Bacillus cajus Marchol, Revue de Phytopath. Appl., 1, 1914, 11.) From diseased caterpillars of Arctia caja.

Coccobacillus gibsoni Chorine. (Internat. Corn Borer Invest., Sci. Repts., 2, 1929, 42; B. gibsoni Paillot, B. presumably indicates Baclerium, see index, p. 522, L'infection chez les insectes, 1933, 134; Bacillus gibsoni Steinhaus, Bacteria Associated Extracellularly with Insects, Minneapolis, 1942, 58.) From diseased corn borer larvae (Pyraustra nubilalis).

Coccobacillus insectorum Hollande and Vernier. (Compt. rend. Acad. Sci., Paris, 171, 1920, 207.) From diseased caterpillars of a moth (Malacosoma castrensis).

Coccobacillus insectorum var. malacosomae Hollande and Vernier. (Compt. rend. Acad. Sei., Paris, 171, 1920, 208.) From diseased caterpillars of a moth (Malacosoma castrensis).

Denitrobacterium thermophilum Ambroz. (Cent. f. Bakt., II Abt., 37, 1913, 3.) A thermophilie bacterium from soil. Diplobacillus melolonthae Paillot. (Compt. rend. Soc. Biol., Paris, 69, 1917, 5; Annales des Epiphyties, 8, 1922, 117.)
From larvae of cockchafers (Meloloniha melolonitha).

Diplobacillus pieris Paillot. (Annales des Épiphyties, 8, 1922, 129.) From diseased caterpillars of the cabbage butterfly (Pieris brassicae).

Helicobacterium aerogenes Miller. (Deutsche Med. Wchnschr., 12, 1886, 119; Bacillus helicoides DeToni and Trevisan, in Saccardo, Sylloge Fungorum, 8, 1889, 952.) From the stomach. This is the type species of the genus Helicobacterium.

Helicobacterium klebsii Miller. (Die Mikroorganismen der Mundhöhle, 2 Aufl., Leipzig, 1892, 370; quoted from Buchanan, Gen. Syst. Bact., Baltimore, 1925, 327.) From the mouth.

Microbucillus citreus baregensis Robine and Hauduroy. (Compt. rend. Soc. Biol., Paris, 98, 1928, 26.) From hot sulfur springs at Barèges. Fourment (Compt. rend. Soc. Biol., Paris, 98, 1928, 588) states that this species is Bacillus luteus Flügge, but Robine and Hauduroy (Compt. rend. Soc. Biol., Paris, 99, 1928, 317) deny this.

Micrococcobacillus necroticans Pascheff. (See Pascheff, Bericht. d. ophthalmol. Gesellsch., Heidelberg, 1916, 418 or Klin. Monatsbl. f. Augenheilk., $57,1916,517$ and $58,1917,97$; Coccobacillus xolymor phus necroticans, quoted from Bayer and v. Herrensehwand, Arch. f. Ophthalmol., 98, 1919, 358; Micrococcobacillus rolymorphus necroticans Pascheff, Arch. d'Ophthalmol., 38, 1921, 28; Pascheff, ibid., 97.) From the human eye. Reported as the causal organism of conjunctivitis.

Nitrosobacillus thermophilus Campbell. (Sci., 75, 1932, 23.) From soil. Oxidizes ammonia to nitrite.

Pacinia ferrarii Trevisan. (Bacillo dell' ulcera molle, Ferrari, 1885; Trevisan, I generi e le spccie delle Batteriacee, 1889, 23.)

Pacinia fickii Trevisan. (Bacillus e des Conjunctivalsackes, Fick, 1887; Trevisan, I generi e le sperie c'elle Batteriacee, $1889,23$. 
Pacinia micheli Trevisan. (Michel, Luftstäbchen des Conjunctivalsecretes, 1882; Trevisan, I generi e le specie delle Batteriacee, 1889, 23.) From the conjunctiva.

Plocamobacterium acidi lactici Pribram. (Lange Milchsaurestäbchen, Wolff, Cent. f. Bakt., II Abt., 20, 1908, 545; Pribram, Klassifikation der Schizomyceten, Leipzig und Wien, 1933, 76.) From milk.

Plocamobacterium epidermidis (Bizzozero) Pribram. (Leptothrix epidermidis Bizzozero, Arch. f. path. Anat., 98, 1896, 455; Pribram, loc. cit., 77.) From the skin.

Plocamobacterium proteolyticum (Wollman) Pribram. (Glycobacter proteolyticus Wollman, Ann. Inst. Past., 26, 1912, 617; Pribram, loc. cit., 118.)

Plocamobacterium rubrum Pribram, loc. cit., 78. Red cheese bacterium (Kiel).

Plocamobacterium tilsitense Pribram, loc. cit., 78. From Tilsit cheese (Kiel).

Proteus hominis Bordoni-Uffreduzzi. (Bacterium, Bordoni-Uffreduzzi and Di Mattei, Arch. per le scienze mediche, 10, 1886, No. 7; abst. in Cent. f. Bakt., 1 , 1887, 345; Bordoni-Uffreduzzi, Ztschr. f. Hyg., 3, 1888, 333; Proteus hominis capsulatus Bordoni-Uffreduzzi, ibid.; Proteus capsulatus septicus Banti, Lo Sperimentale, 88; Klebsiella bordonii Trevisan, I generi e le specie delle Batteriacee, 1889 , 25; Bacillus capsulatus septicus Kruse, in Flügge, Die Mikroorganismen, 3 Aufl., 2, 1896, 345; Baclerium hominis capsulatus Chester, Ann. Rept. Del. Col. Agr. Exp. Sta., 9, 1897, 136; Bacterium capsu- latus and Bacterium capsulatus septicus Chester, ibid., 130; Bacterium proteus Migula, Syst. d. Bakt., 2, 1900, 362; Bacterium bordonii Chester, Manual of Determ. Bact., 1901, 152.) From a case of ragpicker's disease which may have been anthrax or malignant edema.

Urobacillus beijerinckii Christensen. (Christensen, Cent. f. Bakt., II Abt., 27, 1910, 357; Bacillus beijerinckii De Rossi, Microbiologia Agraria e Technica, 1927, 646.) From humus. Utilizes urea.

Urobacillus jakschii Söhngen. (Söhngen, Cent.f. Bakt., II Abt., 23, 1909, 93; Bacillus jakschii De Rossi, Microbiologia Agraria e Technica, 1927, 646.) From garden earth. Utilizes urea.

Urobacillus miquelii Beijerinck. (Cent. f. Bakt., 7, 1901, 47.) From garden earth. Löhnis (Handb. f. landwirtsch. Bakt., 1910, 459) regards this as belonging to the genus Proteus.

Urobacillus schütenbergii $I$ and $I I$ Miquel. (Miquel, Ann. de Micrograph., $5,1893,321$ and 323 ; Bacillus schützenbergii Migula, Syst. d. Bakt., 2, 1900 , 727.) From sewage and river water. These may belong to Proteus (Löhnis, Handb. f. landwirtsch. Bakt., 1910, 459).

Urobacterium aerophilum Rubentschik. (Cent. f. Bakt., II Abt., 66, 1925, 175.) From salt water, Lake Liman near Odessa.

Urobacterium citrophilum Rubentschik. (Cent. f. Bakt., II Abt., 66, 1925, 172.) From black mud and salt water, Lake Liman near Odessa.

Viscobacterium lactis foetidum Laxa. (Cent.f. Bakt., II Abt., 95, 1936, 130.) From milk having a fetid odor. 


\section{APPENDIX TO SUBORDER EUBACTERINEAE}

Record of species and synonyms discovered too late to be entered in the main body of the text. Arranged alphabetically by genera.

Acetobacter aceti (Kïtzing) Beijerinck syn. Bacterium aceticus Chester, Ann. Rept. Del. Col. Agr. Exp. Sta., 9, 1897, 77.

Acctobacter acetosum Bergey et al. syn. Ulvina acetosa Pribram, Klassifikation der Schizomyeeten, Leipzig und Wien, 1933,75 .

Acetobacter ascendens Bergey et al. syn. Ulvina ascendens Pribram, Klassifikation der Archizomyeren, Leipzig und Wien, 1933, 75.

Acetobacter dirersum Humm. (Duke Univ. Marine Lab., North Carolina, Bull. 3, 1946, 63.) From sea water, Beaufort, North ('arolina and marine algae, Miani, Florida. Digests agar.

Acetobacter mobile Tǒsic and Walker. (Jour. of Brewing, 50, 1944, 296.) From bottled ale.

Acetobacter pesteurianum (Hansen) Beijerinck syn. Bacillus pastenrianum Flügge, Die Mikroorganismen, 2 Auft. 1886, 314; not Bacillus pastemriamus Lehmann and Xeumann, Bakt. Diag., $t$ Aufl. 2, 1907. 82; Thime pasteuriane Pribram, Klassifikation der Schizomyceten, Leipzig und Wien, 1933, 76.

Acetobacter potens Humm. (Duke Univ. Marine Lab., North Carolina, Bull. 3, 1946, 63.) From intertidal sand, Beaufort, North Carolina. Digests agar.

Acetobucter rancens Beijerinck syn Tluina ranens Pribram. Klassifikation der sehizomyerten. Leipzig und Wien, $1933,76$.

Acctobacter singulare Humm. (Duke Univ. Marine Lab., North Carolina, Bull. 3, 1946, 62.) From sea water. Beaufort, North Carolina. Digests agar. Acetobacter xylinum (Brown) Holland syn. Bacillus xylinus Trevisan, I generi e le specie delle Batteriacee, 1889, 16; Ulvina xylina Pribram, Klassifikation der Schizomyceten, Leipzig und Wien, $1933,76$.

Achromobacter caseinicum Gahl. (Jour. Bact., 16, 1928, 38.) From a solution of sodium caseinate. Polar flagellate. Possibly a strain of Pseudomonas fluorescens Migula that had lost the power of forming pigment.

Achromobacter nijibetsui Takeda. (Cent. f. Bakt., II Abt., 94, 1936, 48.) From diseased salmon eggs. Not found to be virulent. I polar-flagellated, Gram-negative, yellow chromogen, presumably belonging in the genus Janthomonus.

Actinolarillus actinomycetemcomitans Topley and Wilson syn. Bacillus actinomycetemcomitans Rosebury, Bact. Rev., 8, 1944, 205.

Acrobucter liquefaciens Beijerinck. (C'ont. f. Bakt., II Abt., b, 1900, 199; not Aerobueter liquefaciens Grimes and Hennerty, Aci. Proe. Roy. Dublin Soc., (N.S.) 20, 1931, 93.) From mud and water in swamus. Monotrichous, otherwise like Aerobacter cloacae. This may have been at speries of gas-forming Pseudemomas.

Aerobacter tartarivorum Nijdam. (Thesis, Ieiden, 1907.) Decomposes dtartrates. Probably identical with Aerobacter aerogence (Vaughn, Marsh, Stadtman and Cantino, Jour. Bact., 52, 1946, 324).

Alcaligenes marshallii Bergey et al. syn. Bacterium marshalli Buchanan and Hammer, Iowal sita. Coll. Agr. Exp. Sta., Ress. Bull. 22, 1915, 272.

Alealifenes viscosum Weldin syn. Plocamobacterium viscosum Pribram, Klassifikation der Schizomyceten, Leipzig und Wien, 1933,79 .

Ascococcus bucculis Miller. (Die Mikroorganismen der Mundhöhle, Leipzig, 1889, 65.) From the mouth. 
Bacillus annulatus Wright Syn. Bacterium annulatus Chester, Ann. Rept. Del. Col. Agr. Exp. Sta., 9, 1897, 105.

Bacillus cubonianus Macchiati syn. Bacterium cubonianus Chester, Ann. Rept. Del. Col. Agr. Exp. Sta., 9, 1897, 132 .

Bacillus duplicatus Wright. (Wright, Mem. Nat. Acad. Sci., \%, 1895, 457; Bacterium duplicatus Chester, Ann. Rept. Del. Col. Agr. Exp. Sta., 9, 1897, 90.) From Schuylkill River water. Monotrichous.

Bacillus fluorescens mutabilis Wright. (Wright, Mem. Nat. Acad. Sci., 7, 1895, 449; Bacterium fuorescens mutabilis Chester, Ann. Rept. Del. Col. Agr. Exp. Sta., 9, 1897, 120.) From Sehuylkill River water.

Bacillus fluorescens nivalis Eisenberg syn. Bacterium fluorescens nivalis Chester, Ann. Rept. Del. Col. Agr. Exp. Sta., $9,1897,120$.

Bacillus hayducti Henneberg syn. Plocamobacterium hayducki Pribram, Klassifikation der Schizomyreten, Leipzig und Wien, 1933, 77.

Bacillus influenzoides apis White. (Jour. Path, and Bact., 24, 1921, 71.) From intestine of bee. Monotrichous.

Bacillus mesentericus aureus Winkler syn. Bacillus winkleri Chester, Man. Determ. Bact., 1901, 256.

Bacillus pabuli acidi II Weiss syn. Plocamobacterium pabuli Pribram, Klassifikation der Schizomyceten, Leipzig und Wien, 1933, 78.

Bacillus iaginae Kruse syn. Bacterium raginae Chester, Ann. Rept. Del. Col. Agr. Exp. Sta., 9, 1897, 67.

Bacillus viridi-luteus Trevisan. (Grüngelber Bacillus, Eisenherg, Bakt. Diag., 1 Aufl., 1886, 10; Trevisan, I generi e le specie delle Batteriacee, 1889, 19.) From water. This probably was the same as Bucillus fluorescens Trevisan, ibid., 19 and Pseudomonas fluorescens Migula .
Bacillus wortmannii Henneberg syn. Plocamobacterium uortmanni Pribram, Klassifikation der Schizomyceten, Leipzig und Wien, 1933, 79.

Bacterium granulosum Lehmann and Neumann syn. Plocamobacterium granulosum Pribram, Klassifikation der Schizomyceten, Leipzig und Wien, 1933, 77 .

Bacterium lipolyticum Huss syn. Kurthia lipolyticum Pribram, Klassifikation der Schizomyceten, Leipzig und Wien, $1933,75$.

Bacterium orleanense Henneberg syn. Llrina orleanensis Pribram, Klassifikation der Achizomyceten, Leipzig und Wien, 1933, 75.

Bacterium xylinoides Henneberg syn. Tluina xylinoides Pribram, Klassifikation der Schizomyceten, Leipzig und Wien, 1933, 76.

Brucella byzantinea (Montsouris) Pribram. (Coceobacterium byzantineum Montsouris, quoted from Pribram, Klas sifikation der Schizomyceten, Leipzig und Wien, 1933, 67; Pribram, idem.)

Brucella cocciformis (Jaiser) Pribram. (Bacterium cocciforme Jaiser, quoted from Pribram, Klassifikation der Schizomyceten, 1933. 67; Coccobacterium thermophilum Negre, Compt. rend. Soc. Biol., Paris, 75, 1913, 814 and \$67; Pribram, idem.) From sputum.

Chlorobucterium lactis Guillebeau. (Landw. Jahrb. d. Schweiz., 4, 1890, 32.) From the udder of cows with mastitis. Produces a green pigment. Presumably identical with Pseudomonas aeruginosa Migula. The type species of the genus Chlorobacterium. Guillebeau.

Chromobacterium chocolalum linutsen. Quoted from Lasseur, Dupaix-Lasseur and Meleion, Travaux du Lab. de Microbiol., Fac. Pharm. de Nancy, Fasc. XIII, 1942-43-44, 1944, 164, 187, 293, and 313.) Isolated by M. H. Inutsen, State Coll., Pennsylvania. Source not known. Dull violet with brown tinge. Disso- 
ciates into a violet and an orange strain (Chromobacterium orangium Kinutsen, loc. cit., 294).

Chromobacterium iodinum Davis. (Davis, Cent.f. Bakt., II Abt., 100, 1939, 273; also see Clemo and MeIlwain, Jour. Chem. Soc., Pt. 1, 1938, 479; Pseudomonas iodinum Tobie and Pseudomonas clemo Tobie, Bull. Assoe. des Diplômés de Microbiol., Fac. Pharm. Naney, No. 18, 1939, 16.) From plate inoculated with milk. This non-motile organism does not have the characters of Chromobacterium sensu stricto so that this species is retained with Bactorium for the present.

Coccus cumulus minor Black. (Trans. Ill. State Dental Soc., 22, 1S86, 192.) From the mouth.

Corynebacterium hemolyticum MacLean, Licbow and Rosenberg. (Jour. Inf. Dis., 79, 1946, 69.) From infections among American soldiers and natives in the South and West Pacific. Similar in many ways to Corynebacterium pyogenes and $C$. ovis.

Corynebacterium piriforme Honing. (Cent. f. Bakt., II Abt., 37, 1913, 383.) From tobaceo plants in Sumatra.

Diplococcus aquatilis Vaughan. (Amer. Jour. Ned. Sci., 104, 1892, 184.) From water.

Diplococcus glycinophilus Cardon and Barker. (Jour. Bact., 52, 19.6, 629.) From marine mud.

Diplococcus luteus Adametz and Wichmann. (Adametz and Wichmann, Die Bakterien der Trink- und Nutzwässer, Mitt. Oest. Versuchsstat. f. Brauerei u. Mälzerei, Heft 1, 1888, 49; Planococcus luters Migula, Syst. Bakt., 2, 1900, 274.) From water.

Escherichia Castellani and Chalmers syn. Colibacter Pestana and Andrade, Ann. Paulistas de Med. e Cir., 39, 1940, 462.

Escherichia coli Castellani and Chalmers syn. Colibacter commune Pestana and Andrade, loc. cit.

Flavobacterium harrisonii Bergey et al. syn. Bacillus harrisonii Buchanan and Hammer, Iowa Sta. Coll. Agr. Exp. Sta., Res. Bull. 22, 1915, 257.

Flavobacterium tabidum Iimata. (Cent. f. Bakt., II Abt., 105, 1942, 120.) From spoiled semi-dried fish (Trachurus japonicus). Polar flagellate.

Fusiformis grandis Grassé. (C'ompt. rend. Soc. Biol., Paris, 94, 1926, 1014; Arch. Zool. Expér. et Cién., 65, 1926, 463.) From the surface of the body of a flagellate (Polymastix mclolonthae), in the intestine of larvae of beetles and tipulids, possibly also free in the intestine of the insects.

Fusiformis legeri Grassé. (Compt. rend. Soc. Biol., Paris, 94, 1926, 1014; Arch. Zool. Expér. et Gén., 65, 1926, 467.) From the surface of the body of a flagellate (Polymastix legeri) and in the intestine of diplopods.

Fusiformis lophomonadis Grassé. (Compt. rend. Soc. Biol., Paris, 94, 1926, 1015; Arch. Zool. Expér. et Gén., $65,1926,468$.) From the surface of the body of a flagellate (Lophomonas striata) and in the intestine of corkroaches.

Fusiformis melolonthae Grassé. (Compt. rend. Soc. Biol., Paris, 94, 1926, 1014; Arch. Zool. Expér. et Gén., $65,1926,465$.) From the surface of the body of a flagellate (Polymastix melolonthae) and in the intestine of larvae of beetles and tipulids.

Gluconoucetotacter cerinus Takahashi and Asai. (Cent. f. Bakt., II Abt., 93, 1936, 252.) From fruits.

Glueonoacetobacter liquefaciens Takahashi and Asai, loc. cit. From fruits.

Gluconoacetobacter roseus Takahashi and Asai. (Bacterium industrium var. hoshigaki Takahashi and Asai, Cent.f. Bakt., II Abt., 82, 1930, 400; Bacterium hoshigaki var. glucuronicum I Takahashi and Asai, ibid., 87, 1933, 385; Takahashi and Asai, ibid., 93, 1936, 252.) From dried persimmons (hoshigaki).

Gluconobacter liquefaciens Asai. (Jour. Agr. Chem. Soc. Japan, 10, 1934, 
621 and $11,1935,50$; see Cent. f. Bakt., II Abt., 93, 1936, 248.) From fruits.

Jodococcus magnus Miller. (Deutsche med. Wehnschr., 14, 1888, 612.) From the mouth. The type species of the genus Jodococcus (syn. Iodococcus) Miller.

Jodococcus parvus Miller (ibid., 612). From the mouth.

Lactobacillus bulgaricus Holland syn. Bacterium bulgaricum Buchanan and Hammer, Iowa Sta. Agr. Exp. Sta., Res. Bull. 22, 1915, 250.

Lactobacillus buchneri Bergey et al. syn. Ulvina buchneri Pribram, Ḱlassifikation der Schizomyceten, Leipzig und Wien, 1933, 75.

Lactobacillus delbrueckii Beijerinch syn. Ulvina delbruecki Pribram, loc. cit., 75; Plocamobacterium delbruechi Pribram, ibid., 77.

Lactobacillus helveticus Holland syn. Plocamobacterium casei Pribram, loc. cit., 77; Plocamobacterium helveticum Pribram, ibid., 78.

Lactobacillus pastorianus Bergey et al. syn. Plocamobacterium pastorianum Pribram, loc. cit., 78 .

Lactobacillus pentoaceticus Fred, $\mathrm{Pe}$ terson and Davenport syn. Plocamobacterium fentoaceticum Pribram, loc. cit., 78 .

Lactobacillus plantarum Holland syn. Ulvina cucumeris fermentati Pribram, loc. cit., 75 .

Lactobacillus tactte Olsen-Sopp. (Cent. f. Bakt., II Abt., 33, 1912, 14.) From ropy milk.

Leptotrichia Trevisan partial syn. Leucothrix Oersted, De regionibus marinis, 1844,44 .

Listeria monocytogenes Pirie syn. Brucella monocytogenes Pribram, Klassifikation der Schizomyceten, Leipzig und Wien, 1933, 68 .

Mammococcus gorini. (Quoted from L. Gorini, Enzymologia, 10, 1942, 102.) From the udder.

Micrococcus afermentans Castellani. (Proc. Soc. Exp. Biol. and Med., 25, 1928,
536 ; also see Jour. Trop. Med. and Hyg., 35,1932 , 372.) From an ulcerative lesion of the skin.

Micrococcus albus var. maltigenes $\mathrm{Du}$ mais and Albert. (Quebec Laitier, 5 (2), 1946, 19.) From Richelieu cheese. Regarded as an important ripening agent.

Micrococcus aquatilis Vaughan. (Vaughan, Amer. Jour. Med. Sci., 104, 1892, 190; not Micrococcus aquatilis Chester, Man. Determ. Bact., 1901, 88; not Micrococcus aquatilis Bolton, Ztschr. f. Hyg., 1, 1886, 94.) From water.

Micrococcus aquatilis albissimus von Rigler. (Hyg. Rund., 12, 1902, 4\$2.) From bottled mineral waters.

Micrococcus aquatilis albus Vaughan. (Vaughan, Amer. Jour. Med. Sci., 104, 1892,182 ; not Micrococcus aquatilis albus Toporoff, Cent. f. Bakt., 13, 1893, 487.) From water.

Micrococcus aquatilis magnus Vaughan. (Amer. Jour. Med. Sci., 104, 1892, 182.) From water.

Hicrococcus aquivivus ZoBell and $\mathrm{Cp}$ ham. (Bull. Scripps Inst. of Oceanography, Univ. Calif., 5, 1944, 275. From sea water.

Micrococcus cyaneus (Schroeter) Cohn syn. Bacterium cyaneus White, U. S. D.A., Bur. Entomol. Tech. Ser. Bull. $14,1906,16$.

Micrococcus enteroideus Castellani (Proe. Soc. Exp. Biol. and Med., 25, 1928, 536; also see Jour. Trop. Med. and Hyg., 35, 1932, 372.) From feces.

Micrococcus euryhalis ZoBell and $\mathrm{Cp}_{\mathrm{p}}$ ham. (Bull. Seripps Inst. of Oceanography, Univ. Calif., 5, 1944, 255.) From sea water.

Hicrococcus griseus Winter syn. Bacillus griseus Trevisan, I generi e le specie delle Batteriacee, 1889, 18.

Micrococcus himonoi Kimata. (Cent. f. Bakt., II Abt., 105, 1942, 116.) From spoiled semi-dried fishes (Scomber japonicus and Trachurus japonicus). Resembles Micrococcus caseolyticus and II. mucofaciens. 
Micrococcus infimus ZoBell and Upham. (Bull. Scripps Inst. of Oceanography, Lniv. Calif., 5, 1944, 262.) From marine bottom deposits.

Micrococcus laevulosinertis Castellani. (Proc. Soc. Exp. Biol. and Med., 25, 1928, 536; also see Jour. Trop. Med. and Hyg. $35,1932,372$.) From a ease of stomatitis.

Micrococeus maripuniceus ZoBell and Upham. (Bull. Seripps Inst of Oceanography, Univ. Calif., 5, 1944, 264.) Sessile form found on slides submerged in sea water.

Micrococcus metentericus Castellani. (Quoted from Jour. Trop. Med. and Hyg., 35, 1932, 372.) From case of ulcerative colitis.

Micrococeus moricolor Holmes and Wilson. (Jour. Bact., 49, 1945, 311.) From contaminated wounds. Produces a mulberry pigment on potato.

Micrococcus myceticus Castellani. (Arch. Dermat. and Syphil., 18, 1928, 857.) From cases of pseudomycosis.

Micrococcus nexifer Millor. (Miller, Die Mikroorganismen der Mundhöhle, Leipzig, 1889, 65.) From the mouth. Probably Streptococcus brevis according to Goadby (Mycology of the Mouth, London, 1903, 60).

Micrococcus putatus Ravenel. (Mem. Nat. Acad. Sci., 8, 1896, 21.) From soil.

Vicrococcus putneus Castellani. (Quoted from Jour. Trop. Med. and Hyg., 35, 1932, 372.) From a case of glossitis

Micrococeus rhodochrous Migula syn. Bacillus rhodochrous Dyar, Ann. N. Y. Acad. Sci., 8, 1895, 362; Bacterium rhodochrous Chester, Ann. Rept. Del. Col. Agr. Exp. Stit., 9, 1897, 116.) Dyar had the original Hicrococcus rhodochrous culture from Kràl and felt as have others who have examined this culture that it is not a true Micrococens.

Micrococeus sedentarius ZoBell and Lpham. (Bull. Scripps Inst. of Oceanongraphy, Iniv. Calif., 5, 1944, 260.)
Sessile form found on slides submerged in sea water.

Micrococcus sedimenteus ZoBell and Lpham. (Bull. Seripps Inst. of Oceanography, Lniv. Calif., 5, 1944, 265.) Sessile form found on slides submerged in sea water and in marine mud.

Micrococcus visicidus Castellani. (Proc. Soc. Exp. Biol. and Med., 25, 1928, 536; also see Jour. Trop. Med. and Hyg., 35, 1932, 372.) From an inflamed upper lip.

Microspira vacillans Gicklhorn. (Cent. f. Balit., II Abt., 50, 1920, 422.) From the pool in the Botanical Garden, Univ. Graz, Austria. Contains grains of sulfur.

Neisseria babesi Trevisan. (Bactérie de l'hémoglobinurie du boeuf, Babes, 1888; Trevisan, I generi e le specie delle Batteriacee, 1889, 32.)

Neisseria lulea (Adametz) Trevisan. (Diplucoccus luteus Adametz, 1887; Trevisan. I generie le specie delle Batteriacee, 1859, 32.)

Neisseriu micheli Trevisan. (Trachomeoccus, Michel, 1886; Trevisan, I generi e le specic delle Batteriacee, 1889, 32.)

Neisseria pharyngis syn. Nicrococcus pharyngis Cruikshank and Cruikshank, Med. Res. Council Syst. of Bact., 8; 1931,349 .

Pacinia decipiens Trevisan. (Spirillum aus der Luft, Babes, Ztschr. f. Hyg., $5,1888,183$; Trevisan, I generi e le specie delle Batteriacee, 1889,24 .) From the air.

Pacinia rabida Trevisan. (Spirillum bei Rabies, Babes, Ztschr. f. Hyg., 5, 1888, 181; Trevisan, I generi e le specie delle Batteriacee, 1889,23 .)

Pectobacterium delphinii Waldee. (Ark, Phytopath., 28, 1938, 281; Waldee, Iowa State Coll. Jour. Sci., 19, 1945, 471.) Causes larkspur bacterial blight.

Phylomonas asplenii Ark and Tompkins. (Phytopath., 36, 1946, 760.) Causes leaf blight of bird's nest fern.

Phytomonas maculifclium-gardeniae 
Ark. (Phytopath., 36, 1946, 867.) From gardenia (Gardenia jasminoides). A xanthomonad.

Phytomonas syringae populans Smith. (Jour. Agr. Res., 68, 1944, 269.) Considered the cause of blister spot, a disease of apple.

Phytomonas washingtoniae Pine. (Phytopath., 33, 1943, 1203.) From the Washington palm. Washingtonia filifera. A pseudomonad.

Pneumococcus flavescens Arloing. (Compt. rend. Acad. Sci., 109, 1859, 428 and 459.) From lesions of cattle having peripneumonia.

Pneumococcus gutta cerei Alloing, loc. cit. From lesions of cattle having peripneumonia.

Pneumococcus lichnoides Arloing, loc. cit. From lesions of cattle having peripneumonia.

Pseudomonas aestumarina ZoBell and Lpham. (Bull. Scripps Inst of Oceanogriphy, Univ. Calif., 5, 1944, 269.) A marine sedentary organism.

Pscudomonas allii (Griffiths) Migula syn. Bacillus allii Sternberg, Man. of Bact., 1893, 629.

Pseudomonas ambigua (Wright) (hester syn. Bacterium ambiguus Chester, Ann. Rept. Del. Col. Agr. Exp. Sta., 9. $1897,71$.

Pseudomonas atlantica Humm. (Duke Univ. Marine Lab., North Carolina, Bull. 3, 1946,58.) From seaweed (Gracilaria blodgettii) and beach sand. Digests agar.

Pseudomonas aurea Migula syn. Bacterium fluorescens aureus Chester, Ann. Rept. Del. Col. Agr. Exp. Sta., 9, 1897, 109.

Pseudomonas azotogena \%oBell and Upham. (Bull. Seripps Inst. of Oceanography, Univ. Calif., 5, 1944, 260.) From sea water and marine mud.

Pseudomonas beaufortensis Humm (loc. cit., 58). From seawater, bottom mud and on algae. Digests agar.

Pseudomonas berolinensis Migula. (Indigoblauer Bacillus, Claessen, Cent. f. Bakt., 7, 1890, 13; Bacillus berolinensis indicus Germano, Cent. f. Bakt., 12, 1892, 517; Bacillus indigoferus Zimmermann, Bakt. unserer Trink-u. Nutzwässer, Chemnitz, 2, 1894, 16, not indicated as being the same as Bacillus indigoferus Voges, Cent. f. Bakt., 14, 1893, 307; Bacillus indigonaceus Sihneider, Arb. bakt. Inst. Karlsruhe, 1, Heft 2, 1894, 225; Migula, in Engler and Prantl, Die natürl. Pflanzenfam., 1, 1:1, 1895 , 29; Bacterium indigonaceum Lehmann and Neumann, Bakt. Diag., 1 Auf., 2, 1896, 267; Bacterium berolinensis indicus Chester, Ann. Rept. Del. Col. Agr. Exp. Sta., 9, 1897, 118.) From Spree River water.

Pseudomonas butyri Migula syn. Bactorium butyri fluorescens Chester, Ann. Rept. Del. Col. Agr. Exp. Sta., 9, 1897, 120.

Pseudomonas centrifugans (Wright) Chester. (Bacillus centrifugans Wright, Mem. Nat. Acad. Sci., $7,1895,462 ;$ Bacterium centrifugans Chester, Ann. Rept. Del. Col. Agr. Exp. Sta., 9, 1897, 95; Chester, Man. Determ. Bact., 1901, 312.) From water.

Pseudomonas coadunata Chester syn. Bacterium coadunatus Chester, Ann. Rept. Del. Col. Agr. Exp. Sta., 9, 1897, 90 .

Pseudomonas coenobios ZoBell and Lpham. (Bull. Seripps Inst. of Oceanography, Univ. Calif., 5, 1944, 272.) From film of marine fouling organisms.

Pseudomonas cohaerea (Wright) Chester, not Bacillus cohaerens Gottheil, Cent. f. Bakt., II Abt., \%, 1901, 458; Bacterium cohaereus (sic) Chester, Ann. Rept. Del. Col. Agr. Exp. Sta., 9, 1897, 93.

Pseudomonus coli communis Conn, Esten and Stocking. (Storrs Agri. Exp. Sta., Conn., 15th Ann. Rept. for 1906, 186.) From cheddar cheese. Like Bacillus coli communis except that it has a single, long flagellum.

Pseudomonas convexa ('hester syn. Bacterium fluorescens convexus Chester, 
Ann. Rept. Del. Col. Agr. Exp. Sta., 9, 1897, 123.

Pseudomonas corallina Humm (loc. cit., 59). From marine algae of all common species at Beaufort, Nor. Car. Digests agar.

Pseudomonas delabens (Wright) Chester syn. Bacterium delabens Chester, Ann. Rept. Del. Col. Agr. Exp. Sta., 9, 1897, 97.

Pseudomonas eisenbergii Migula syn. Bacterium fluorescens non-liquefaciens Chester, Anm. Rept. Del. Col. Agr. Exp. Sta., 9, 1897, 124.

Pseudomonas elongata Humm (loc. cit., 60). From intertidal sand, Atlantic Beach, Nor. Car. Digests agar.

Pseudomonas enalia ZoBell and Lpham. (Bull. Scripps Inst. of Oceanography, Univ. Calif., 5, 1944, 254.) From sea water and marine mud.

Pseudomonas fairmountensis (Wright) Chester syn. Bacterium fairmountensis Chester, Ann. Rept. Dol. Col. Agr. Exp. Sta., 9, 1897, 90.

Pseudomonas felthami ZoBell and Cpham. (Bull. Scripps Inst. of Oceanography, Univ. Calif., 5, 1944, 267.) From marine mud,

Pseudomonas fimbriata (Wright) Chester syn. Bacterium fimbriatus Chester, Ann. Rept. Del. Col. Agr. Exp. Sta., 9, 1897, 95.

Pseudomonas floridana $1 \mathrm{fumm}$ (loc. cit., 60). From algae and beach sand at Miami, Fla., and Beaufort, Nor. Car. Digests agar.

Pseudomonas fluorescens Migula syn. Bacterium fluorescens liquefaciens Chester, Ann. Rept. Del. Col. Agr. Exp. Sta., 9, 1897, 120.

Pseudomonas foliacea Chester syn. Bacterium fluorescens foliaceus Chester, Ann. Rept. Del. Col. Agr. Exp. Sta., 9, 1897, 122 .

Pseudomonas geniculatus (Wright) Chester. Bacillus geniculatus Wright; not Bacillus geniculatus DeBary, Inaug. Diss., Strassburg, Leipzig, 1885; not Bacillus geniculatus Trevisan, I generi e le specie delle Batteriacee, 1889, 16; syn. Bacterium geniculatus Chester, Ann. Rept. Del. Col. Agr. Exp. Sta., 9, 1897, 95.

Pseudomonas humicola Bersteyn. (Arb. bakt. Inst. Karlsruhe, 3, 1903, 97.) From soil.

Pseudomonas hypothermis ZoBell and Upham. (Bull. Scripps Inst. of Oceanography, Univ. Calif., 5, 1944, 276.) From marine bottom deposits.

Pseudomonas incognita Chester syn. Bacterium fluorescens incognitus Chester, Ann. Rept. Del. Col. Agr. Exp. Sta., 9, 1897, 122 .

Pseudomonas indoloxidans Gray. (Proc. Roy. Soc. London, B, 102, 1928, 263.) From soil from Italian Tyrol.

Pseudomonas indigoferus (Voges) Migula. (Bacillusindigoferus Voges, Cent. f. Bakt., 14, 1893, 307; Bacterium indigoferus Chester, Ann. Rept. Del. Col. Agr. Exp. Sta., 9, 1897, 118; Migula, Syst.d. Bakt., 2, 1900, 950.) From Tiel tap water (Voges); from Delft ditch water, mud and garden soil (Elazari-Volcani. Arch.f. Mikrubiol., 10, 1939, 357). Some authors regard Voges' organism as identical with Claessen's indigo blue bacillus, see Pseudomonas berolinensis.

Pseudomonas indigoferus var. immobilis Elazari-Volcani. (Arch. f. Mikrobiol., 10, 1939, 350.) From ditch mud. See Lehmann and Neumann (Bakt. Diag., 1 Aufl., 2, 1896, 267) who also had a non-motile strain (Bacterium indigonaceum) from Kràl which they considered identical with Claessen's indigo blue bacillus.

Pseudomonas inertia Humm (loc. cit., 61). From intertidal sand, Atlantic Beach, Nor. Car. Digests agar.

Pseudomonas iris Migula syn. Bacillus fluorescens crassus Kruse, in Flügge, Die Mikroorganismen, 3 Aufl., 2, 1896, 294; Bacterium fluorescens crassus Chester, Ann. Rept. Del. Col. Agr. Exp. Sta., 9, 1897, 134; Bacterium iris Chester, idem, 137.) From sputum.

Pseudomonas jaegeri Migula syn. Bac- 
terium proteus fluorescens Chester, Ann. Rept. Del. Col. Agr. Exp. Sta., 9, 1897, 119; and Bacillus urinae Chester, Man. Determ. Bact., 1901, 263.

Pseudomonas javanica (Eijkmann) Migula syn. Bacterium javaniensis Chester, Ann. Rept. Del. Col. Agr. Exp. Sta., 9, 1897, 111.

Pseudomonas liquida Chester. (Bacillusliquidus Frankland and Frankland, Ztschr.f. Hyg., 6, 1889, 382; Chester, Man. Determ. Bact., 1901, 311; Achromobacter liquidum Bergey et al., Manual, 1sted., 1923, 145.) From water. Originally described merely as motile; Chester recognizes the species as polar flagellate and lists Bacillus liquefaciens communis Sternberg and Bacillus aquatilis communis Kruse as synonyms.

Pseudomonas longa Migula syn. Bacterium fluorescens longus Chester, Ann. Rept. Del. Col. Agr. Exp. Sta., 9, 1897, 124.

Pseudomonas macroselmis Migula syn. Bacillus fluorescens putidus Chester, Ann. Rept. Del. Col. Agr. Exp. Sta., 9, $1897,124$.

Pseudomonas marinopersica ZoBell and Upham. (Bull. Scripps Inst. of Oceanography, Univ. Calif., 5, 1944, 275.) From marine bottom deposits.

Pseudomonas melochlora (Winkler and Schrötter) Migula syn. Bacterium melochlorus Chester, Ann. Rept. Del. Col. Igr. Exp. Sta., 9, 1897, 120; see abst. in Cent.f. Bakt., 9, 1891, 700.

Pseudomonas membranula ZoBell and Upham. (Bull. Scripps Inst. of Oceanography, Univ. Calif., 5, 1944, 270.) Sessile form found on slide submerged in sea.

Pseudomonas minutissima Migula syn. Bacterium fluorescens minutissimus Chester, Ann. Rept. Del. Col. Agr. Exp. Sta., 9, 1897, 120.

Pseudomonas monadiformis (Kiruse) Chester syn. Bacterium monadiformis Chester, Ann. Rept. Del. Col. Agr. Exp. Sta., 9, 1897, 69; Bacterium coli mobilis Chester, ibid., 69.
Pseudomonas multistriata (Wright) Chester syn. Bacterium multistriatus Chester, Ann. Rept. Del. Col. Agr. Exp. Sta., 9, 1897, 90.

Pseudomonas nebulosa (Wright) Chester syn. Bacterium nebulosus Chester, Ann. Rept. Del. Col. Agr. Exp. Sta., 9, 1897, 93.

Pseudomonas neritica ZoBell and Upham. (Bull. Scripps Inst. of Oceanography, Univ. Calif., 5, 1944, 255.) From sea water and marine mud.

Pseudomonas nexibilis (Wright) Chester syn. Bacterium nexibilis Chester, Ann. Rept. Del. Col. Agr. Exp. Sta., 9, 1897,74 .

Pseudomonas obscura ZoBell and L $\mathrm{p}$ ham. (Bull. Seripps Inst. of Oceanography, Univ. Calif., 5, 1944, 274.) From marine bottom deposits.

Pseudomonas oceanica ZoBell and Upham. (Bull. Seripps Inst. of Oceanography, Univ. Calif., 5, 1944, 266.) From marine mud.

Pseudomonas ochracea (Zinmermann) Chester syn. Bacterium ochraceus Chester, Ann. Rept. Del. Col. Agr. Exp. Sta., $9,1897,104$.

Pseudomonas oralis Chestersyn. Bacterium fluorescens ovalis Chester, Ann. Rept. Del. Col. Agr. Lxp. Sta., 9, 1897, 123.

Pseudomonas pallescens Migula syn. Bacterium viridis pallescens Chester, Ann. Rept. Del. Col. Agr. Exp. Sta., 9, 1897,124 .

Pseudomonas perfectomarinus ZoBell and Upham. (Bull. Seripps Inst. of Oceanography, Univ. Calif., 5, 1944, 277.) From sea water and marine nud.

Pseudomonas periphyta ZoBell and Epham. (Bull. Seripps Inst. of Oceanography, Lniv. Calif., 5, 1944, 276.) Sessile form found in film of marine fouling organisms.

Pseudomonas phosphorescens (Fisher) Bergey et al. syn. Pasteurella phosphorescens Trevisan, I generi e le specie delle Batteriacee, 1889, 21; Bacillus phosphorescens indicus Eisenberg, Bakt. 
Diag., 3 Aufl., 1891, 123; Vibrio indicus Lehmann and Neumann, Bakt. Diag., 1 Aufl., 2, 1896, 341; Bacterium phosphorescens indicus Chester, Ann. Rept. Del. Col. Agr. Exp. Sta., 9, 1897, 120; Photobacter indicum Beijerinck, Proc. Sect. Sci, lion. Akad. v. Wetensch., Amsterdam, 3, 1900, 352; Microspira phosphorescens Chester, Man. Determ. Bact., 1901, 333; Bacillus indicus Beijerinck, Folia Mikrobiologica, Delft, I, 1912, 1. Beijerinck (loc. cit., 1900) discusses two variants of this species: Photobacter indicum var. obscurum and Photobacter indicum var. parrum. Later, Beijerinck (loc. cit., 1912), in discussing mutants of this species, proposes the species names Bacillus indicus parvus, Bacillus indicus semiobscurus and Bacillus indicus obscurus.

Pseudomonas piscoma Hanzatwa and Takeda. (Jozogaku Zasshi, Osaka, Japan (Jour. of Zymology), 9, 1931, 571; quoted from Takeda, Cent. f. Bakt., II Abt., 94, 1936, 46.) From diseased salmon eggs.

Pseudomonas pleomorpha ZoBell and Upham. (Bull. Scripps Inst. of Oceanography, Univ. Calif., 5, 1944, 275.) From marine bottom deposits.

Psendomonas pullulans (Wright) Chestersyn. Bacterium pullulans Chester, Ann. Rept. Del. Col. Agr. Fxp. Sta., 9, 1897, 105.

Pseudomonas puris Patrick and Werkman. (Proc. Iowa Acad. Sci., 37, 1930, 57.) From a typhoid-like infection of snakes.

Pseudomonas ribicola Bohn. (Jour. Agr. Res., 73, 1916, 285.) From the native currant, Ribes ameum.

Pseudomonas riboflacinus Foster. (Jour. Bact., 47, 1944, 30.) Oxidizes riboflavin to lumichrome. From riboflavin-rich soil.

Pseudomonas roscola Humm (loc cit., 62). From intertidal sand, Atlantic Beach, Nor. Car. Digests agar.

Pseudomonas rugosa. (Wright) Chester syn. Bacterium rugosus Chester. Ann.
Rept. Del. Col. Agr. Exp. Sta., 9, 1897, 122.

Pseudomonas schuyltilliensis Chester syn. Bacterium fluorescens schuylkilliensis Chester, Ann. Rept. Del. Col. Igr. Exp. Sta., 9, 1897, 119.

Pseudomonas scissa (Frankland and Frankland) Migula syn. Bacterium scissus Chester, Ann. Rept. Del. Col. Agr. Exp. Sta.. 9, 1897, 143.

Pseudomonas sessilis ZoBell and Upham. (Bull. Seripps Inst, of Oceanography, Univ. Calif., 5, 1944, 259.) Sessile form found on solid surfaces submerged in the sea.

$P$ seudomonas sinuosa (Wright) Chester syn. Bacterium simuosus Chester, Ann. Rept. Del. Col. Agr. Exp. Sta., 9, 1897, 69.

Pseudomonas smaragdina Migula syn. Bacterium smaragdino foetidus Chester, Ann. Rept. Del. Col. Agr. Exp. Sta., 9, 1897, 119; and Bacillus smaragdinus Chester, Man. Determ. Bact., 1901, 263.

Pseudomonas sterotropis $\mathrm{ZOB}$ Bell and Upham. (Bull. Seripps Inst of Oceanography, Univ. Calif., 5, 1944, 272.) From a film of marine fouling organisms.

Pseudomonas striata Chester syn. Bacterium striatus viridis Chester, Ann. Rept. Drl. Col. Agr. Exp. Sta., 9, 1897, 123.

Pseudumonas syncyanea Migula syn. Bacterium syncyanus (sic) Schroeter, Beitr. z. Biol. d. Pflanz., 1, Heft 2, 1872, 126 and Bactorium cyanogenum Zopf, Die Spaltpilze, 2 Aufl., 18St, 50; may be in 1 Aufl.

Pseudomonas synxantha (Ehrenberg) Holland. (Vibrio synxanthus Ehrenberg, Verhandl. d. Berl. Akad., 1840, 202; Vibrio xanthogenes Fuchs, Magazin f.d. ges. Tierheilk. I, 1841, 193; Bacterium. xanthinum Schroeter, in Cohn, Beiträge z. Biol. d. Pflanzen, 1, Heft 2, 1872, 120; Bacillus synxanthus Flügge, Die Mikroorganismen, 2 Aufl., 1886, 290; Flavobacterium synxanthum Bergey et al., Manual, 1st ed., 1923, 102; Holland, Jour. Bact., 5, 1920, 220.) Bergey et al. 
(Manual, 1st ed., 1923, 102) give $\mathrm{Bac}$ terium xanthogenes as a synonym. From milk and cream. Polar flagellate (Hammer, personal communication). See Hammer, Res. Bul. 20, Iowa Agr. Exp. Sta., 1915, for a description of this organism.

Pseudomonas tenuis Migula syn. Bacterium fluorescens tenuis Chester, Ann. Rept. Del. Col. Agr. Exp. Sta., 9, 1897, 124.

Pseudomonas turcosa Migula syn. Türkisfarbener Bacillus, Tataroff, Inaug. Diss., Dorpat, 1891, 52.

Pseudomonas vadosa ZoBell and $\mathrm{T}^{\top} \mathrm{p}$ ham. (Bull. Seripps Inst. of Oceanography, Univ. Calif., 5, 1944, 263.) From sea water and marine bottom deposits.

Pseudomonas vendrelli Tobie. (Pseudomonas vendralli (sic), mentioned by Farrell and Wolff, Jour. Ind. and Eng. Chem., 33, 19.1, 1186; L. S. Patent 2,227,716, Mar. 1942, issued to Lockwood et al.; Bacillus vendrelli Lasseur, Dupaix-Lasseur and Meleion, Travaux Lab. Microbiol. Fac. Pharm. Nancy, Fase. 13, 1942-43-44, 1914, 293.) Isolated by W. C. Tobie in 1935 from well near Ponce, Puerto Rico owned by Mr. Tendrell (Tohie, Jour. Bact., 52, 1946, 685). Presumably Pseudomonas aeruginosa.

Pseudomonas virescens (Frick) Migula syn. Bacterium virescens Chester, Ann. Rept. Del. Col. Agr. Exp. Sta., 9, 1897, 124.

Pseudomonas viscosa (Frankland and Frankland) Migula syn. Bacterium viscosus Chester, Ann. Rept. Del. Col. Agr. Exp. Sta., 9, 1897, 145; not Bacterium viscosum Weldin and Levine, Abst. Bact., 7, 1923, 16.

Pseudomonas xanthochrus ZoBell and Upham. (Bull. Scripps Inst. of Oceanography, Univ. Calif., 5, 1914, 279.) From marine bottom deposits.

Ramibacterium alactolyticum Prévot and Taffanel. (Ann. Inst. Past., 68. 1942, 259.) From an osteophlegmon of the maxillary bone.
Salmonella atherton Ferris, Hertzberg and Atkinson. Med. Jour. Australia, 32, 1945, 368.) From 29 cases of gastroenteritis in an army hospital.

Salmonella typhosa (Zopf) White syn. Bacillus typhicus Cabral and Da Rocha, I. Trabalhos do Cabinette de Microbiologia; abst. in Ann. de Micrographie, 2, 1889-1890, 295.

Sarcina pelagia ZoBell and Upham. (Bull. Seripps Inst. of Oceanography, Univ. Calif., 5, 1944, 279.) From sea water and marine bottom deposits.

Serratia fuchsina Bergey et al. syn. Proteus fuchsinus Pribram, Klassifikation der s'chizomvceten, Leipzig und Wien, 1933. 73.

Serratia indica (Eisenberg) Bergey et al. syn Bacillus indicus ruber Flügge, Die Mikroorganismen, 2 Aufl., 1886, 285; Bacterium indicum. Crookshank, Manual, 18s 7.240.

Spirillum paruum Esmarch. (Cent. 1. Bakt., I Abt., Orig., 32, 1902, 565; also see Zettnow, ibid., $78,1916,1$. From decaying organic matter.

Spirillum sputigenum Flügge. (Lewis, Lancet, Sept. 20, 1s5t; Flügge, Die Mikroorganismen, 2 Aufl., 1886, 387; $P a$ cinia lewisi Trevisan, I generi e le specie delle Batteriacee, 1889, 24.) From sputum.

Staphylococcus activus Prévot and Taffanel. (Ann. Inst. Past., 71, 1945, 102.) From puerperal septicemia. Anaerobic

Staphylococcus cilreus duodenalis Gessner. (Areh. f. Hyg., 9, 1889, 136.) From the human duodenum.

Staphylococcus magnus Black. (Trans. Ill. State Dental Soc., 22, 1886, 188.) From the mouth.

Staphylococcus medius Black. (Trans. Ill. State Dental Soc., 22, 1886, 190.) From the mouth.

Staphylococcus pyogenes bovis Lucet. (Ann. Inst. Past., 7, 1593, 327.) From borine abscesses.

Staphylococeus riscosus Croadby. 
(Mycology of the mouth, London, 1903, 172.) From the mouth.

Streptobacterium dextranicum Perquin. (Jour. Microbiol. and Serol., 6, 1940, 226.) Produces slime from sucrose solutions.

Streptococcus aquatilis Vaughan. (Amer. Jour. Med. Sci., 104, 1892, 184.) From water.

Streptococcus liquefaciens Frankland and Frankland. (Phil. Trans. Roy. Soc. London, 178, B, 1888, 264.) From air. After the section covering Streptococcus liquefaciens Sternberg emend. Orla-Jensen was in page proof, it was discovered that Frankland and Frankland had discovered and named a liquefying streptococcus earlier than Sternberg. The Franklands described this species as producing a yellow pigment.

Streptococcus pyogenes duodenalis Gessner. (Arch. f. Hyg., 9, 1889, 132.) From the human duodenum.

Streptococcus taette (Olsen-Sopp) Buchanan and Hammer. (Bacterium lactis longi Troili-Petersson, Ztschr. f. Hyg., 32, 1899, 361 and Milchzeitung, 28, 1899, 438; Streptobacillus taette Olsen-Sopp, Cent. f. Bakt., II Abt., 39, 1912, 9; Buchanan and Hammer, Iowa Sta. Coll. Agr. Exp. Sta., Res. Bull. 22, 1915, 277.) Probably the characteristic organism of Swedish ropy milk. Olsen-Sopp (loc. cit.) misquotes Troili-Petersson's name as Bacillus acidi lactis longus (see Troili-Petersson, Cent. f. Bakt., II Abt., $38,1913,1)$.

Thiospira agilissima (Gicklhorn) Bavendamm. (Spirillumagilissimum Gicklhorn, Cent. f. Bakt., II Abt., 50, 1920, 418; Bavendamm, Die farblosen und roten Schwefelbakterien, Pflanzenforschung, Heft 2, 1924, 116.) From the pond in the Annen Castle Park, Graz, Austria. Contains grains of sulfur.

Thiospira elongata Perfiljev. (Ber. d. Sapropel Kiomm. Petrograd, 1923, 56.) From mud containing $\mathrm{H}_{2} \mathrm{~S}$.

Thiospira propera Hama. (Jour. Sci.
Hiroshima Univ., Ser. B, Bot., 1, 1933, 157; abst. in Cent. f. Bakt., II Abt., 91, 1934, 200.)

Thiospira sulfurica Issatchenko. (Biological observations on the sulfur bacteria (Russian), about 1927, $16 \mathrm{pp}$.)

Vibrio adaptatus ZoBell and Upham. (Bull. Scripps Inst. of Oceanography, Univ. Calif., 5, 1944, 258.) From sea water and marine sediments.

Vibrio agarlyticus Cataldi. (Rev. d. Inst. Bact. (D.N.H.), Buenos Aires, 9, 1940, 375.) From activated sludge. Digests agar.

Vibrio albensis Lehmann and Neumann syn. Vibrio dunbari Holland, Jour. Bact., 5, 1920, 226; probably Vibrio phosphorescens Jermoljewa, Cent. f. Bakt., I Abt., Orig., 100, 1926, 170; not Vibrio phosphorescens Holland, loc. cit.

Vibrio algosus ZoBell and Upham. (Bull. Seripps Inst. of Oceanography, Univ. Calif., 5, 1944, 257.) Associated with marine kelp.

Vibrio amphibolus Trevisan. (Babes, Ztschr. f. Hyg., 5, 1888, 183; Trevisan, I generi e le specie delle Batteriacee, 1889, 23.) Anaerobe.

Vibrio avidus Humm. (Duke Univ. Marine Lab., North Carolina, Bull. 3, 1946,54 .) From intertidal sand, Beaufort, North Carolina. Digests agar.

Vibrio choleroides $\alpha$ and $\beta$ Bujwid syn. Bacterium choleroides Chester, Ann. Rept. Del. Col. Agr. Exp. Sta., 9, 1897, 131.

Vibrio costicolus Smith and Vibrio costicolus var. liquefaciens Smith. (Roy. Soc. Queensland, Proc. for 1937, 49, 1938, 29 and 32.) From tainted ribs of bacon and tank brines in bacon factories. Active growth in 4 to 15 per cent brines.

Tibrio euprima, Tibrio yasakii, Coccobacillus tolega and Coccobacillus sepiola Majima, Sci-i Kwai-Med. Jour. 50, 1931, 41-67; see Warren, Jour. Bact., 49, 1945, 548.) Phosphorescent bacteria.

Vibrio fortis Humm (loc. cit., 55). From seaweed (Gracilaria confervoides). Digests agar. 
Vibrio frequens Humm (loc. cit., 56). From marine algae (Cladophoropsis, Laurencia poitei, etc.) Digests agar.

Vibrio halonitrificans Smith. (Roy. Soc. Queensland, Proc. for 1937, 49, 1938, 29.) From tank brines in bacon factories. Active growth in $t$ to 10 per cent brines.

Vibrio haloplanktis ZoBell and Upham. (Bull. Scripps Inst. of Oceanography, Univ. Calif., 5, 1944, 261.) Sessile form found associated with marine phytoplankton.

Vibrio hyphalus ZoBell and Upham. (Bull. Scripps Inst. of Oceanography, Univ. Calif., 5, 1944, 277.) From marine bottom deposits.

Vibrio marinagilis ZoBell and Upham. (Bull. Scripps Inst. of Oceanography, Univ. Calif., 5, 194t, 264.) From sea water and marine mud.

Vibrio marinoflavus ZoBell and $\mathrm{Up}$ ham. (Bull. Seripps Inst. of Oceanography, Univ. Calif., 5, 1944, 258.) From sea water.

Vibrio marinofulvus ZoBell and Upham. (Bull. Scripps Inst. of Oceanography, Univ. Calif., 5, 1944, 262.) From sea water.

Vibrio marinopraesens ZoBell and Upham. (Bull. Scripps Inst. of Oceanography, Univ. Calif., 5, 1944, 256.) From sea water.

Vibrio marinovulgaris ZoBell and Upham. (Bull. Scripps Inst. of Oceanography, Univ. Calif., 5, 1944, 261.) From sea water.

Vibrio notus Humm (loc. cit., 56). From intertidal sand, Atlantic Beach, North Carolina. Digests agar.

Vibrio perimastrix Alarie. (Alarie, Thesis, MacDonald Coll. MeGill Univ., 1945; see Perlin and Michaelis, Sci., 103, 1946, 673.) Will decompose cellulose only in presence of $\mathrm{CO}_{2}$.

Vibrio phytoplanktis ZoBell and Upham. (Bull. Scripps Inst. of Oceanography, Univ. Calif., 5, 1944, 261.)
From sea water and marine phytoplankton.

Vibrio pieris Paillot. (Compt. rend. Soc. Biol., Paris, 94, 1926, 68.) From caterpillars of the cabbage butterfly (Pieris brassicae) which had been parasitized by larvae of $A$ panteles glomeratus.

Vibrio ponticus ZoBell and Upham. (Bull. Seripps Inst. of Oceanography, Univ. Calif., 5, 1944, 259.) From sea water.

Vibrio rumpel Lode. (Cent. f. Bakt., I Abt., Orig., 35, 1903, 526; see Ballner, Cent. f. Bakt., II Abt., 19, 1907, 572.) From water. Phosphorescent.

Vibrio stanieri Humm (loc. cit., 57). From seaweed (Acanthophora spicifera), Miami, Fla. Digests agar.

Vibrio turbidus Humm (loc. cit., 57). From seaweed (Gracilaria confervoides). Digests agar.

Vibrio viridans Miller. (Quoted from Miller, Microorganisms of Human Mouth, Phila., 1890, 85; see Miller, Die Mikroorganismen der Mundhöhle, Leipzig, 1889.) From the mouth.

Xanthomonas translucens var. phleipratensis Wallin and Reddy. (Phytopathology, 35, 1945, 939.) The cause of a bacterial streak disease on timothy grass (Phleum pratense).

Xanthemonas vignicole Burkholder. (Phytopath., 34, 1944, 431.) From cowpea, Vigna sinensis.

Yersinia van Loghem (Ann. Past. Inst., 72, 1946, 975), a genus proposed to include Pasteurella pestis and P. pseudotuberculosis.

Ein neuer für Thiere path. Mikroorg. aus dem Sputum eines Pneumoniekranken, Bunzel and Federn, Arch. f. Hyg., 19, 1893; 326; Bacillus dubius pneumoniae Kruse, in Flügge, Die Mikroorganismen, 3 Aufl., 2, 1896, 419; Bacterium sub. pneumonicum Migula, Syst.d. Bakt., 2, 1900, 376; Bacterium dubium Chester, Man. Determ. Bact., 1901, 142. From the sputum of a pneumonia patient. 


\section{FAMILY XIII. BACILLACEAE FISCHER. *}

(Jahrb. f. wiss. Bot., 27, 1895, 139.)

Rod-shaped cells, capable of producing spores, either with peritrichous flagella or non-motile; monotrichous flagellation has been reported but is doubtful. Endospores are eylindrical, ellipsoidal or spherical, and are located in the center of the cell, sub-terminally or terminally. Sporangia do not differ from the vegetative cells except when bulged by spores larger than the cell diameter. Such sporangia are spindleshaped when spores are central, or wedge- or drumstick-shaped when spores are terminal. Usually Gram-positive. Pigment formation is rare. Aerobic, microaerophilic or anaerobic. Gelatin is frequently liquefied. Sugars are generally fermented, sometimes with the formation of visible gas. Some species are thermophilic, i.e., will grow readily at $55^{\circ} \mathrm{C}$. Mostly saprophytes, commonly found in soil. A few are animal, especially insect, parasites or pathogens.

\section{Key to the genera of family Bacillaceae.}

I. Aerobic; catalase positive.

Genus I. Bacillus, p. 705.

II. Anaerobic or microaerophilic; catalase not known to be produced.

Genus II. Clostridium, p. 763.

INTRODUCTION TO THE GENUS BACILLUS,

In the fifth edition of the MANUAL, the late F. D. Chester stated: "It is difficult to offer a rational system of classification for the described forms of the genus Bacillus because of the incompleteness of the data". He prepared a splendid review of the literature but naturally could not supply the data that were missing. He stated further that "The majority of the so-called species in the genus have been imperfectly presented,... the net result being that there are comparatively few clearly and definitely described species among the nany herein recorded. The development of a better knowledge will be a work of the future". He then discussed the type of work that should be done. A reading of his statement is recommended to anyone contemplating naming a new species.

During the past few years, the writer with the assistance of Francis E. Clark and Ruth E. Gordon has made a study of the genus Bacillus along the lines indicated by Chester. Representative cultures have been obtained from various laboratories, institutions, and private collections. Special mention should be made of the private collection of Prof. J. R. Porter, now at the Iowa State University. It contained about 200 named species and was invaluable for the work. As a result of this study, it appears that many species have been differentiated by such simple characters as mucoid, folded, adherent or rhizoid growth, pigment production, the fermentation of a specific carbohydrate, etc. Others have been grouped because of some special physiological activity such as the decomposition of calcium n-butyrate, xylan, cellulose, etc. Chester rightly considered that these physiological groups had no taxonomic value.

Species have been characterized upon a broad basis in the present arrangement on the assumption that one species should not dissociate into another species. Since certain characters are more stable than others, these have been used to establish a

* Revised by Mr. Nathan R. Smith, U. S. Bureau of Plant Industry Station, Beltsville, Maryland (Bacillus), August, 1943, and Prof. R. S. Spray, School of Medicine, West Virginia University, Morgantown, West Virginia (Clostridium), May, 1942. 
species pattern. This has reduced the number of species of the mesophilic members of the genus from many poorly defined organisms to a few well characterized and delimited species. Intermediates oceur between related species and have been treated as such. The report on which this arrangement is based has recently been published by Smith, Gordon and Clark (C. S. Dept. Agr. Misc. Pub. To. 559, 1946, 112 pp.).

Some workers may think that the cut in the number of species has been too drastic and that certain organisms listed as varieties, morphotypes, or biotypes should be retained as species. This would not be consistent with the newer knowledge of bacteriology that has been developed during the past two decades. No doubt other species occur in nature that are not included herein. But before jumping to the conclusion that a culture is a new species, closely related organisms as well as the isolate should be studied along the lines given by Chester in the fifth edition of the MANUAL.

The production of indole and the formation of $\mathrm{H}_{2} \mathrm{~S}$ have been omitted from the descriptions because these characters have no taxonomic value. Certain other properties, such as colony form, character of the growth on slants, in litmus milk, etc., have a very limited value. They are included for the sake of completeness.

\section{Genus I. Bacillus Cohn.*}

(Beiträge z. Biol. d. Pflanzen, 1, Heft 2, 1872, 146 and 175.) From Latin bacillum, a small stick.

Synonyms: ? Bactiella Morren, Bull. d. Sei. natur et de Geol., No. 27, 1830, 203; Metallacter Perty, Zur Kenntniss kleinster Lebensformen, 1852, 180 ; ? Bacteridium Davaine, Dict. Encyclop. d. Sci. Méd., Ser. I, 8, 1868, 21; Pollendera Trevisan, 1884 (see DeToni and Trevisan, in Saccardo, Sylloge Fungorum, 8, 1889, 943); Zopfiella Trevisan, Atti della Accademia Fisio-Medico-Statistica in Milano, Ser. 4, 3, 1885, 93; Cornilia Trevisan, I generi e le specie delle Batteriacee, 1889, 21; Lrobacillus Miquel, Ann Microg., 1, 1859, 517; Bacterium Migula, Arb. bakt. Inst. Karlsruhe, 1, 1894, 237 (not Bacterinm Ehrenberg, Symbolae Physicae seu Icones et Descriptiones Animalium, ete., Berlin, 1828, 8); Bactrinum, Bactridium, Bactrillum, Clostrillium, Clostrinium and Paracloster Fischer, Jahresb. f. wissensch. Bot., 27, 1895, 139; Endobacterium Lehmann and Neumann, Bakt. Diag., 1 Aufl., 2, 1896, 103; Astasia Meyer, Flora, 84, 1897, 185; Saccharobacter Beijerinck, Cent. f. Bakt., II Abt., 6, 1900, 200 ; Fenobacter Beijerinck, ibid.; A planobacter E. F. Smith, Bact. in Relation to Plant Dis., 1, 1905, 171; Semiclostridium Maassen, Arb. a. d. k. Gesundheitsamte, Biol. Abt., 5, 1905, 5; M yxobacillus Gonnermann, Ztschr. f. Zuckerind. u. Landwirtsch., 36, 1907, 877; Plennobacterium Gonnermann, ibid.; Serratia Vuillemin, Ann. Mycolog., 11, 1913, 521 (not Serratia Bizio, Polenta porporina, Biblio. Ital., 30, 1823, 288); Schaudinnum, Theciobactrum, Zygostasis, Eisenbergia, Migulanum and Rhagadascia Enderlein, Sitzber. Gesell. Naturf. Freunde, Berlin, 1917,309; Cellulobacillus Simola, Ann. Ac. Se. Fenn., Ser. A, 34, No. 1 and 6, 1931 (abst in Cent. f. Bakt., II Abt., 86, 1932, 89); not Cellulobacillus Orla-Jensen, Cent. f. Bakt., II Abt., 22, 1909, 343; Zymobacillus Kiluyver and Van Niel, Cent. f. Bakt., II Abt., 94, 1936, 369.

Rod-shaped bacteria, sometimes in chains. Sporangia usually not different from the vegetative cells. Catalase present. Aerobic, sometimes showing rough colonies and

* Revised by Mr. Nathan R. Smith, U. S. Bur. Plant Industry Station, Beltsville, Maryland, August, 1943. 
forming a pellicle on broth. Usually oxidize carbohydrates or proteins more or less completely, often producing slight acidity, without pronounced accumulation of characteristic products. Soil is the most common habitat.

The internationally accepted (Jour. Bact., 33, 1937, 445) type species is Bacillus subtilis Cohn emend. Prazmowski.

\section{Key to the species of genus Bacillus.}

I. Mesophilic (good grow th at $30^{\circ} \mathrm{C}$ ), aerobic (sometimes also grow at low concentrations of oxygen).

A. Spores ellipsoidal to cylindrical, central to terminal, walls thin. Sporangia not distinctly bulged. Gram-positive.

1. Diameter of rods less than 0.9 micron. Cells from glucose or glycerol nutrient agar stain uniformly.

a. Growth at $\mathrm{pH} 6.0$. Acetylmethylcarbinol produced.

b. Gelatin hydrolyzed (Frazier method). Acid from xylose or arabinose with ammoniacal nitrogen.

c. Starch hydrolyzed. Nitrites produced from nitrates.

1. Bacillus subtilis.

d. Black pigment on carbohydrate media only.

1a. Bacillus subtilis var. aterimus.

dd. Black pigment on tyrosin media only.

1b. Bacillus subtilis var. niger.

cc. Starch not hydrolyzed. Nitrites not formed from nitrates.

2. Bacillus pumilus.

bb. Gelatin not hydrolyzed. No acid from xylose or arabinose.

3. Bacillus coagulans.

aa. No growth at pH 6.0. Acetylmethylearbinol not formed.

b. Casein digested. Urease not formed.

4. Bacillus firmus.

bb. Casein not digested. Urease produced.

5. Bacillus lentus.

2. Diameter of rods 0.9 micron or more. Cells from glucose or glycerol nutrient agar appear vacuolated if lightly stained.

a. Acid from xylose or arabinose with ammoniacal nitrogen. Acetylmethylcarbinol not produced.

6. Bacillus megatherium.

aa. No acid from xylose or arabinose. Acetylmethylcarbinol produced.

b. Saprophytic, sometimes pathogenic but not causing anthrax; usually motile.

c. Growth on agar not rhizoid.

7. Bacillus cereus.

ce. Rhizoid growth on agar; usually non-motile.

7a. Bacillus cereus var, mycoides.

bb. Pathogenic. Causative agent of anthrax; non-motile.

8. Bacillus anthracis.

B. Spores ellipsoidal, central to terminal, walls thick, remnants of sporangium often adhering. Sporangia distinctly bulged, spindle and racket forms. Gram-variable.

1. Acid and gas from carbohydrates.

a. Acetylmethylcarbinol produced. Crystalline dextrins not formed from starch. 
9. Bacillus polymyxa.

aa. Acetylmethylcarbinol not produced. Crystalline dextrins formed from starch.

10. Bacillus macerans.

2. No visible gas from carbohydrates.

a. Saprophytic. Growth on ordinary media.

b. $\mathrm{pH}$ of glucose, proteose-peptone broth cultures less than 8.0.

Citrates not used as source of carbon.

c. Starch hydrolyzed. Acid from sucrose with ammoniacal nitrogen.

d. Acid from xylose or arabinose with ammoniacal nitrogen. Acetylmethylcarbinol not formed.

11. Bacillus circulans.

dd. No acid from xylose or arabinose. Acetylmethylearbinol produced.

12. Bacillus alvei.

cc. Starch not hydrolyzed. No acid from sucrose.

13. Bacillus laterosporus.

bb. $\mathrm{pH}$ of glucose, proteose-peptone broth cultures 8.0 or higher.

Citrates used as source of carbon.

14. Bacillus brevis.

aa. Parasitic. No growth on ordinary media.

b. Cause of American foulbrood of honey bees.

15. Bacillus larvae.

bb. Cause of the milky disease of Japanese beetles (Popillia japonica Newm.).

Type A. 16. Bacillus popilliae.

Type B. 17. Bacillus lentimorbus.

C. Spores spherical, central to terminal. Sporangia definitely bulged, drumsticks and spindles. Carbohydrates not attacked. Gram-variable.

1. Growth on nutrient agar without urea or free ammonia.

a. Urease not formed.

18. Bacillus sphaericus.

aa. Urease produced.

18a. Bacillus sphaericus var. fusiformis.

2. No growth on nutrient agar without urea or free ammonia. Urease formed.

19. Bacillus pasteurii.

II. Thermophilic, optimum temperatures $55^{\circ} \mathrm{C}$ or above; slight if any growth at $37^{\circ} \mathrm{C}$. Aerobic.*

A. Spores ellipsoidal to cylindrical, central to terminal; sporangia not distinctly bulged.

1. Diameter of rods less than 0.8 micron.

a. Gas from carbohydrates.

20. Bacillus thermoamylolyticus.

aa. No gas from carbohydrates.

* The data on the species of this group are so meager that it is not possible to offer a rational system of classification. Many of the characters used for separating the various species are probably as variable in this group as they have been found to be in the mesophilic group. Lacking a knowledge of the limits of variability and lacking other pertinent data, the present arrangement is regarded as temporary only. 
b. Growth below $50^{\circ} \mathrm{C}$.

c. Nitrites from nitrates, of ten with liberation of nitrogen gas.

21. Bacillus kaustophilus.

21a. Bacillus pepo.

ce. No nitrites from nitrates.

bb. No growth below $50^{\circ} \mathrm{C}$.

22. Bacillus thermoindifferens.

23. Bacillus thermodiastaticus.

2. Diameter of rods greater than 0.8 micron.

a. Growth on nutrient agar.

b. Remnants of sporangium adherent.

24. Bacillus cylindricus.

bh. Remnants of sporangium not adherent.

25. Bacillus robustus.

25a. Bacillus losanitchii.

aa. No growth on nutrient agar.

26. Bacillus calidolactis.

B. Spores ellipsoidal to rylindrical, central to terminal; sporangia distinctly bulged.

1. Diameter of rods less than 0.9 micron.

a. Starch hydrolyzed.

b. Nitrites from nitrates, sometimes with liberation of nitrogen gas.

27. Bacillus michaelisii.

27a. Bacillus lobatus.

$27 \mathrm{~b}$. Bacillus thermononliquefaciens.

bb. No nitrites from nitrates.

c. Action on cellulose not recorded.

28. Bacillus thermotranslucens.

28a. Bacillus stearothermophilus.

28b. Bacillus aerothermophilus.

cc. Cellulose hydrolyzed.

29. Bacillus thermocellulolyticus.

aa. Starch not hydrolyzed.

b. Nitrites from nitrates, sometimes with gaseous nitrogen.

c. Milk unchanged.

30. Bacillus thermoalimentophilus.

ec. Milk acid, coagulated.

31. Bacillus thermoliquefaciens.

2. Diameter of rods greater than 0.9 micron.

a. Starch hydrolyzed.

b. No nitrites from nitrates.

32. Bacillus tostus.

C. Spores spherical, central to terminal; sporangium not distinctly bulged.

33. Bacillus viridulus.

1. Bacillus subtilis Cohn emend. Prazmowski. (Cohn, Beitr. z. Biol. d. Pflanzen, 1 , Heft 2, 1872, 174; Heft 3, 1875, 188; 2, Heft 2, 1876, 249; Prazmowski, Untersuchungen über die Entwicklungsge- schichte und Fermentwirkung einigen Bakterien-Arten. Inaug. Diss., Leipzig, 1880.) From Latin subtilis, thin, siender.

The identity of this species has been 
the subject of some controversy owing to the indefiniteness of the original descriptions, to the distribution of cultures under the name Bacillus subtilis that were incorrectly identified, to variations in the forms of growth that may be observed, and to confusion with Bacillus cereus. In cases where Bacillus subtilis is said to be "anthrax-like," or "similar to the anthrax bacillus," it should be remembered that these terms apply to Bacillus cereus and not to Bacillus subtilis. Conn (Jour. Inf. Dis., 46, 1930, 341) concluded that the so-called Marburg strain fitted the earliest recognizable description of this species which is that given by Prazmorski (loc. cit.), and his view was accepted after a study of cultures by the International Committee on Bacteriological Nomenclature (Jour. Bact., 33, 1937, 445).

During the past two decades much progress has been made in the study of variations in the stages of growth of bacteria, the rough, smooth, mucoid, etc., and in the variability in physiology as well. From the recent work of Smith, Gordon, and Clark (loc. cit.) it appears that many species have been characterized on such simple grounds as growth folded, mucoid, adherent, colored, rhizoid, ete., all of which are subject to variation, either induced or spontaneous. The present arrangement of this species is the result of their work combined with data supplied by the work of Conn and others.
Species probably identical with or variants of Bacillus subtilis:

Bacillus geniculatus de Bary, Beitrag zur Kenntnis der niederen Organismen im Mageninhalt, Inaug. Diss., Strassburg, Leipzig, 1885; Bacillus mesentericus fuscus Flügge, Die Mikroorganismen, 2 Aufl., 1886, 321 (Bacillus mesentericus Trevisan, I generi e le specie delle Batteriacee, 1889, 19; not Bacillus mesentericus as interpreted by Chester, Del. Agr. Exp. Station 15th Ann. Report, 1903, 86; not Bacillus mesentericus as given by Lawrence and Ford, Jour. Bact., 1, 1916, 295); * Bacillus mesentericus vulgatus Flügge, Die Mikroorganismen, 2 Aufl., 1886, 322 (Bacillus v'ulgatus Trevisan, I generi e le specie delle Batteriacee, 1889, 19); Bacillus liodermos Flügge, Die Mikroorganismen, 2 Iufl., 1886, 323 (Bacillus No. X, Flügge, Ztschr. f. Hyg., 17, 1894, 296; Bacillus lactis No. X, Kruse, in Flügge, Die Mikroorganismen, 3 Aufl., 2, 1896, 209; Bacillus intermedius Migula, Syst. d. Bakt., 2, 1900, 579; Bacillus cremoris Chester, Man. Determ. Bact., 1901, 274); Bacillus laevis Frankland and Frankland, Philos. Trans. Roy. Soc. London, 178, B, 1887, 278 (not Bacillus laevis Distaso, Cent. f. Bakt., I Abt., Orig., 62, 1912, 444); Tyrothrix tenuis Duclaux, Ann. Inst. Nat. Agron., 4, 1852, 23 (Bacillus tenuis Trevisan, I generi e le specie delle Batteriacee, 1889, 16); Kartoffelbacillus, Globig, Ztschr. f. Hyg., 3. 1888, 294 (Bacillus roseus Trevisan, loc. cit., 19; Bacillus mesentericus ruber Kruse, in Flügge, Die

*T. Gibson, University of Edinburgh (personal communication), has found that the European and supposedly the original st rains of Bacillus mesentericus hydrolyzestarch and reduce nitrates to nitrites, whereas the American strains are negative in both of these characters. Furthermore, the latter are usually smooth and when the rough stage exists, it does not resemble a mesentery from which the organism derived its name. This term, however, can still be applied to the European strains. Since the American strains are identical with Bacillus pumilus (Chester, Del. Agr. Exp. Station, 15th Ann. Report, 1903, 87; Lawrence and Ford, Jour. Bact., 1, 1916, 300), it has been recommended (Smith, Gordon, and Clark, loc. cit.) that they be designated as Bacillus pumilus to avoid ambiguity. Since the European Bacillus mesentericus is only a stage of growth of Bacillus subtilis, the former name should be dropped. 
Mikroorganismen, 2, 1896, 199; Bacillus globigii Migula, Syst. der Bakt., 2, 1900, 554; Bacillus vitalis Chester, Man. Determ. Bact., 1901, 286); Bacillus leptosporus Klein, Cent. f. Bakt., 6, 1889, 316; Bacillus No. 6, Pansini, Arch. f. path. Anat. u. Physiol., 122, 1890, 422 (Bacillus coccoideus Migula, Syst. der Bakt., 2, 1900, 558); Bacillus radians Migula, Syst. d. Bakt., 2, 1900, 580 (Bacillus No. IX, Flügge, Ztschr. f. Hyg., 17, 1894, 296; Bacillus lactis No. IX, Kruse, in Flügge, Die Mikroorganismen, 3 Aufl., 2, 1896, 209; Bacillus stellatus Chester, Man. Determ. Bact., 1901, 27t; not Bacillus stellatus Vincent, Ann. Inst. Past., 21, 1907, 69); Bacillus mesentericus panis viscosi II Vogel, Ztschr. f. Hyg., 26, 1897, 404 (Bacillus panis Migula,** Syst. der Bakt., 2, 1900, 576); Bacillus armoraciae Burchard, Arb. a. d. bakt. Inst. d. techn. Hochschule zu Karlsruhe, 2, 1898, 46; Bacillus idosus Burchard, ibid., 47; Bacillus subtilis a Gottheil, Cent. f. Bakt., II Abt., 7, 1901, 635; Bacillus natto Sawamura, Bull. Coll. Agr., Tokyo, 7, 1906, 108; Bacillus mesentericus var. flavus Laubach, Jour. Bact., 1, 1916, 497 (Bacillus flavus Bergey et al., Manual, lst ed., 1923, 286; not Bacillus flavus Fuhrmann, Cent. f. Bakt., II Abt., 19, 1907, 117); Bacillus truffauti Truffaut and Bezssonoff, Compt. rend. Acad. Sci., Paris, $175,1922,544$; Bacillus mesentericus hydrolyticus Hermann and Neuschul, Biochem. Ztschr., 281, 1935, 219.

The name Vibrio subtilis Ehrenberg (Infusionsthierchen als vollkommene Or- ganismen, Leipzig, 1838) seems to have given rise to the species name.

Spores : 0.6 to 0.9 by 1.0 to 1.5 microns, ellipsoidal to cylindrical, central or paracentral. Germination prevailingly equatorial.

Sporangia: Ovoid to cylindrical, only slightly bulged if at all.

Rods : 0.7 to 0.8 by 2.0 to 3.0 microns, single or in short chains, rounded ends, stain uniformly. Motile. Gram-positive. The following variations have been observed: Smaller or larger rods, filaments, encapsulated cells (the slimy bread organisms), few shadow forms, nonmotile and Gram-variable. Rods on glucose nutrient agar store small amount of fat.

Gelatin stab: Liquefaction.

Agar colonies: Usually rough, finely wrinkled, opaque, dull, adherent, slightly spreading, brownish tinge. Variations may be smooth, soft, thin, translucent, non-adherent, dendroid, coarsely wrinkled, creamy-white to yellowish to orange.

Agar slants: Growth abundant, flat, spreading, usually has a dull mat surface, finely wrinkled, adherent, becoming slightly brownish. Variations may be coarsely wrinkled or folded, non-adherent, smooth, thin, translucent, dendroid, creamy-white to yellow to orange. Some strains show a greenish fluorescence when grown at $45^{\circ} \mathrm{C}$ on nutrient agar.

Broth: Turbid becoming clear with formation of a tough, wrinkled pellicle.

Milk: Slowly peptonized, becoming alkaline.

** There has been confusion about the identity of the so-called slimy bread bacteria. Lehmann and Neumann (Bakt. Diag., 7 Aufl., 2, 1927, 616) stated that they were interrelated and also more or less closely related to Bacillus mesentericus and to Bacillus vulgatus. Laubach (Jour. Bact., 1, 1916, 501) isolated a strain of Bacillus panis that lost its capsules on artificial media, although it still remained slimy. From this and the work of Smith, Gordon and Clark (loc.cit.) it is apparent that the slimy bread organisms are mucoid variants of Bacillus subtilis, which may or may not be encapsulated, and motile or non-motile (see also Bacillus subtilis var. viscosus Chester, Del. Agr. Exp. Station, 15th Ann. Report, 1903, 84). 
Milk agar plate: Casein hydrolyzed.

Potato: Growth luxuriant, warty or wrinkled to coarsely folded, whitish to pink or yellow, becoming brownish with age.

Nitrites formed from nitrates.

Starch is hydrolyzed.

Acid with ammoniacal nitrogen from xylose, arabinose, glucose, fructose, galactose, mannose, maltose, sucrose, salicin, glycerol, and mannitol. Usually acid from dextrin. Variable reactions on rhamnose, raffinose, and inulin. Usually no action on lactose.

Acetylmethycarbinol produced.

Citrates utilized.

Optimum temperature $30^{\circ}$ to $37^{\circ} \mathrm{C}$. Will usually grow from $50^{\circ}$ to $56^{\circ} \mathrm{C}$.

Aerobic, facultative.

Source: Original cultures isolated by Cohn from an infusion of lentils (1872), from a boiled infusion of cheese and white beets (1875), and from boiled hay infusions (1876). Hence, frequently called the hay bacillus. The folded, non-adherent stage of growth (Bacillus vulgatus and the European strain of Bacillus mesentericus) is often called the potato bacillus. Manner of germination of spores established by Prazmowski (loc. cit.).

Habitat: Widely distributed in soil and in decomposing organic matter.

Note: Bacillus vulgatus has long been separated from Bacillus subtilis by the folded character and the non-adherence of its growth. Recently Lamanna (Jour. Bact., 44, 1942, 611) has attempted to separate this species from Bacillus subtilis by the splitting of the spore sheath along the transverse axis upon germination. Since the two species are otherwise morphologically and physiologically alike and since these characters are subject to much variation, there seems to be no valid reason for this separation. One can, if he desires, indicate the different stages of growth; for instance, Bacillus subtilis morphotype vulgatus (or mesentericus) for the folded growth, Bacillus subtilus morphotype panis for the slimy growth, and Bacillus subtilis morphotype globigii for those that produce a red or orange pigment. These terms would apply to the present condition of the culture and would have to be changed if the character of the growth changed.

1a. Bacillus subtilis var. aterrimus comb. nov. (Potato bacillus, Biel, Cent. f. Bakt., II Abt., 2, 1896, 137; Bacillus aterrimus Lehmann and Neumann, Bakt. Diag., 1 Aufl., 2, 1896, 303; Bacillus mesentericus niger Lunt, Cent. f. Bakt., II Abt., 2, 1896, 572; Bacillus niger Chester, Man. Determ. Bact., 1901, 306.) From Latin aterrimus, very black.

Synonyms: Bacillus nigrificans Fabian and Nienhuis, Mich. Agric. Exp. Station, Tech. Bull. 140, 1934, 24; Bacillus tyrosinogenes Rusconi, as referred to by Carbone et al., Instit. Sierot. Milan., 2, 1921-1922, 29; not Bacillus tyrosinogenes Hall and Finnerud, Proc. Soc. Expl. Biol. and Med., 19, 1921, 48 and Hall, Abstr. Bact., 6, 1922, 6.

In the early accounts the production of a blue-black to black pigment on potato was stressed. It was also said to resemble Bacillus subtilis and Bacillus vulgatus on gelatin plates. Recent work (Clark and Smith, Jour. Bact., 37, 1939, 280) has shown that pigmentation occurs only in the presence of a carbohydrate. In addition (Gordon and Smith, Jour Bact., 43, $1942,55)$, it was established that the abil ity to form the pigment could be lost through serial transfers and colony selection and that the resultant dissociants could not be differentiated from Bacillus subtilis.

Source: Isolated from rye bread in moist chamber used for growing some aspergilli (Biel).

Habitat: Widely distributed in soil.

1b. Bacillus subtilis var. niger comb. nov. (Bacillus lactis niger Gorini, Gior. d. Reale Soc. Ital. Ig., 16, 1894, 9; Bacil- 
lus niger Migula, Syst. der Bakt., 2, 1900, 636.) From Latin niger, black.

The black pigment characterizing this organism is formed only in media containing tyrosine (Clark and Smith, Jour. Bact., 37, 1939, 279). The ability to form the pigment may be lost through serial transfer and colony selection. It then cannot be separated from Bacillus subtilis (Gordon and Smith, loc. cit.).

Souree: First isolated from milk.

Habitat: Widely distributed in soil.

\section{Bacillus pumilus Gottheil. (Cent.}

f. Bakt., II Abt., \%, 1901, 681.) From Latin pumilus, dwarfish, little.

Synonyms: Bacillus mesentericus as interpreted by Chester, Del. Agric. Exp. Station, 15th Ann. Report, 1903, 87; Bacillus mesentericus as given by Lawrence and Ford, Jour. Bact., 1, 1916, 295 and $300 ;$ Bacillus mesentericus var. flavus Laubach, Jour. Bact., 1, 1916, 497 ; perhaps also Bacillus parme Veide, Cent. f. Bakt., II Abt., 12, 1904, 34t; Bacillus leptodermis Burchard, Arb. a. d. bakt. Inst. d. techn. Hochschule zu Karlsruhe, 2, 1898, 33 .

Spores : Ellipsoidal to cylindrical, thin walled, naked, central or paracentral, usually about 0.5 by 1.0 micron although some may approach the size of those of Bacillus subtilis.

Sporangia: Ellipsoidal to cylindrical, not bulged.

Rods : 0.6 to 0.7 by 2.0 to 3.0 microns, usually occurring singly or in pairs. Chains, filaments and shadow forms may be found in some strains. Cells grown on glucose nutrient agar have few small fat globules. Motile with peritrichous flagella. Gram-positive.

Gelatin stab: Slow liquefaction.

Agar colonies: Thin, flat, spreading, dendroid, snooth, translucent. The rough stage also occurs.

Agar slants: Growth moderate, smooth, soft, thin, glistening, non-adherent, spreading, usually whitish although it may be yellowish. The rough stage is trugh and finely wrinkled, sometimes resembling certain strains of Bacillus subtilis.

Broth: Uniform turbidity, with or without a ring or half-formed pellicle. The rough stage forms a pellicle.

Milk: Peptonized, sometimes coagulated.

Milk agar plate: Casein hydrolyzed.

Potato: Growth is smooth, thin, spreading, moist to slimy, yellowish, turning somewhat brown. The rough stage is dry and finely wrinkled.

Nitrites not produced from nitrates.

Starch not hydrolyzed.

Acid with ammoniacal nitrogen from arabinose, xylose, glucose, fructose, galactose, mannose, sucrose, salicin, glycerol and mannitol; usually also from maltose and raffinose. Reaction variable with dextrin. [sually no acid from rhamnose, lactose, and inulin.

Acetylmethylcarbinol produced.

Citrates utilized as sole source of (arbon.

Optimum temperature about $30^{\circ} \mathrm{C}$. Maximum temperature allowing growth usually about $50^{\circ} \mathrm{C}$.

Aerobic.

Source: Isolated from plants, cheese, dust, and as a contaminant of media.

Habitat: Widely distributed in nature.

3. Bacillus coagulans Hammer. (Iowa Agric. Exp. Station, Research Bull. 19, 1915, 129; Sarles and Hammer, Jour. Bact., 23, 1932, 301.) From Latin coagulans, rurdling, coagulating.

Synonyms: Bacillus thermoacidurans Berry, Jour. Bact., 25, 1933, 72; Bacillus dextrolacticus Andersen and Werkman, Iowa State Coll. Jour. of Sci., 14, 1940, 187.

Spores: Ellipsoidal to cylindrical, terminal or subterminal, thin walled, 0.6 to 0.9 by 1.0 to 1.5 microns. Sporulation better on acid proteose peptone agar (Stern, Hegarty, and Williams, Food Research, \%, 1942, 186). 
Sporangia: Only slightly swollen, if at all.

Rods: 0.5 to 0.9 by 2.5 to 3 mierons, singly or in short chains, resemble $B a$ cillus subtilis. Cells from glucose agar contain few small fat globules. Motile. Gram-positive.

Gelatin: No growth at $20^{\circ} \mathrm{C}$. No change in gelatin by Frazier method at $45^{\circ} \mathrm{C}$.

Agar colonies: small, entire, raised, not characteristic.

Agar slants: Growth scant to moderate, thin, flat. On acid proteose peptone agar growth is more abundant and microscopically the cells appear healthier.

Broth: Moderate uniform turbidity, followed by clearing. Glueose broth attains a $\mathrm{pH}$ of 4.0 to 4.4 .

Milk: Coagulated.

Milk agar plate: Weak hydrolysis of casein.

Potato: Growth scant to moderate, thin, spreading, white to cream-colored. May have a sour odor.

Nitrites usually not formed from nitrates.

Stareh is hydrolyzed.

Acid from glucose, galactose, fructose, lactose, maltose, sucrose, dextrin, and glycerol. Usually no acid from arabinose and sorbitol. No acid from xylose and mannitol. Organic nitrogen preferable to inorganic.

Acetylmethylcarbinol produced.

Citrates not used as sole source of carbon.

Optimum temperature about $45^{\circ} \mathrm{C}$ (Hammer, $55^{\circ} \mathrm{C}$ ). Maximum temperature allowing growth $54^{\circ} \mathrm{C}$ to $60^{\circ} \mathrm{C}$. Slow growth, if any, at $25^{\circ} \mathrm{C}$.

Aerobic, facultative.

Source: Isolated from evaporated milk (Hammer) and tomato juice (Berry).

Habitat: Canned goods; probably widely distributed in nature.

4. Bacillus firmus Werner. (Cent. f. Bakt., II Abt., 87, 1932, 470.) From Latin firmus, firm, strong.
Spores: Usually ellipsoidal, central to subterminal, 0.6 to 0.7 by 1.0 to 1.2 microns on Ca-n-butyrate agar (Werner); 0.7 to 0.9 by 1.0 to 1.4 microns on nutrient agar. Sporulation better on plain peptone agar than on nutrient agar.

Sporangia: Ellipsoidal to cylindrical, sometimes slightly bulged.

Rods : 0.6 to 0.9 by 1.5 to 4.0 microns, single or in short chains, few filaments. On glucose nutrient agar there are swollen, shadow, and other abnormal forms, few small fat globules. Motile with peritrichous flagella. Gram-positive.

Gelatin stab: Slow liquefaction. Gelatin plate shows wide zone of hydrolysis.

Agar colonies: Small, smooth, dense, entire, white to pink.

Agar slants: Growth moderate, smooth, opaque, not spreading, whitish. Pink variations may occur. Growth inhibited when glucose is added, because of the production of acid. No growth at $\mathrm{pH}$ 6.0 or below.

Broth: Scant uniform turbidity or a flocculent growth.

Milk agar plate: Weak to strong casein hydrolysis.

Potato: No growth.

Nitrites produced from nitrates.

Starch is hydrolyzed.

Acid from glucose. No acid from arabinose and xylose. Ammonium salts not used as sole source of nitrogen.

Acetylmethylcarbinol not produced.

Citrates usually not utilized.

Urease not produced.

Salt tolerance: Will grow in nutrient broth containing 4 to 7 per cent $\mathrm{NaCl}$.

Optimum temperature about $28^{\circ} \mathrm{C}$. Maximum temperature allowing growth $37^{\circ} \mathrm{C}$ to $45^{\circ} \mathrm{C}$.

Source: Seven strains isolated from soils in Central Europe and Egypt.

Habitat: Widely distributed in soil.

5. Bacillus lentus Gibson. (Cent. f. Bakt., II Abt., 92, 1935, 368.) From Latin lentus, slow. 
Spores: Ellipsoidal, central to subterminal, 0.7 to 0.8 by 1.0 to 1.3 microns.

Sporangia: Ellipsoidal to cylindrical, may be slightly swollen.

Rods : 0.6 to 0.7 by 2.0 to 3.0 microns, occurring singly or in pairs. Motile with peritrichous flagella. Gram-positive.

Gelatin stab: No liquefaction. No change in gelatin by Frazier method.

Agar colonies: Small, smooth, entire, glistening, white, opaque.

Agar slants: Growth only moderate, slow, thin, gray to white, opaque, not spreading. Nogrow th at pH 6.0 or below. Growth inhibited by glucose because of the change to acid reaction.

Broth : Faint uniform turbidity, grinular sediment.

Milk: Unchanged.

Milk agar plate: C'asein not hydrolyzed.

Potato: No growth.

Nitrites not produced from nitrates.

Starch is hydrolyzed.

Acid from arabinose, xylose, glucose, sucrose, and lactose. Inorganic nitrogen not utilized.

Acetylmethylearbinol not formed.

Citrates not used as sole source of carbon.

Urease produced. Urea decomposed at room temperature, feebly at $37^{\circ} \mathrm{C}$.

Salt tolerance: Will grow in nutrient broth containing 4 per cent $\mathrm{NaCl}$.

Optimum temperature about $25^{\circ} \mathrm{C}$. Maximum temperature allowing growth $37^{\circ} \mathrm{C}$.

Growth on most media is increased by the addition of urea.

Acrobic.

Source: Nine strains isolated from soils.

Habitat : Common in soils.

\section{Bacillus megatherium De Bary.} (Bacillus megaterium (sic) De Bary, Vergleichende Morph. und Biol. der Pilze, 1884, 499.) Generally assumed that the original spelling was a typographical error and that the later spelling megatherium comes from Greek roots meaning big animal (Breed, Science, $7_{0}$, 1929, 480). Ripple (Arch. Mikrobiol., $11,1940,470$ ) holds that the original spelling meaning big rod is the correct form.

Synonyms as given by Smith, Gordon, and Clark (loc. cit.): Bacillus capri Stapp, Cent. f. Bakt., II Abt., 51, 1920, 19 ; Bacillus carotarum Koch, Bot. Zeit., 18, 1888, 277 (Bacterium carotarum Migula, Syst. d. Bakt., 2, 1900, 293); Bacillus cobayae Stapp, Cent. f. Bakt., II Abt., 51, 1920, 10; Bacillus danicus Löhnis and Westermann, Cent. f. Bakt., II Abt., 22, 1908, 253; Bacillus graveolens Gottheil, Cent.f. Bakt., II Abt., 7, 1901, 496 and 535; Bacillus malabarensis Löhnis and Pillai, Cent. f. Bakt., II Abt., 19, 1907, 91; Bacillus musculi Stapp, Cent. f. Bakt., II Abt., 51, 1920, 39 ; Bacillus oxalaticus Migula, Arb. a. d. bakt. Inst. d. Tech. Hochschule z. Karlsruhe, 1, Heft, 1, 1894, 139; Bacillus petasites Gottheil, Cent. f. Bakt., II Abt., 7, 1901, 535 (Lawrence and Ford, Jour. Bact., 1, 1916, 273); Bacillus ruminatus Gottheil, ibid., 496; Bacillus silvalicus Neide, Cent. f. Bakt., II Abt., 12, 1904, 32; Bacillus tumescens Zopf, Die Spaltpilze, 1 Auf1., 1883, 66 (Zopfiella tumescens Trevisan, Car. d. alc. nuov. gen. di Batter., 1885, 4).

Other possible synonyms given by Neide (loc. cit., 11): Bacterium hirtum Henrici, Arb. bakt. Inst. Karlsruhe, 1, 1894, 44 (Pseudomonas hirtum Ellis, Cent. f. Bakt., II Abt., 11, 1903, 213; Bacillus hirtus Ellis, Ann. Bot., 20, 1906, 233); Bacillus brassicae Pommer, Mitt. botan. Inst. Graz, 1, 1886, 95 (Bacterium brassicae Migula, Syst. d. Bakt., 2, 1900, 296 ).

Although the name Bacillus tumescens Zopf (which is here regarded as a probable synonym) has priority over Bacillus megatherium, the latter name is preferred because of general usage. Neither of the original descriptions is sufficiently detailed to characterize adequately the 
species named, and Zopf (Die Spaltpilze, 3 Aufl., 1885, 82-83) regarded the two species as distinct. The modern work on which the present description of $B a$ cillus megatherium is based has been largely carried out with cultures identified as Bacillus megatherium, and the true nature of the species is really fixed by the informal emendations made in these more recent descriptions. The emended descriptions give this name a more certain meaning than is given Bacillus tumescens by the descriptions existent in the literature.

Spores: Ellipsoidal, sometimes nearly round, central to paracentral, 1.0 to 1.5 by 1.5 to 2.0 microns (larger dimensions have been reported).

Sporangia: Ellipsoidal to cylindrical, often in short chains; not swollen.

Rods : 1.2 to 1.5 by 2.0 to 4.0 microns, occurring singly and in short chains. Larger and smaller cells, irregular, twisted, and shadow forms are present in some strains, depending upon the substrate. Cells from glucose or glycerol nutrient agar usually store much fat and stain unevenly (vacuolated) with dilute stains. Motility with peritrichous flagella, usually slow, although some strains may show active motility. Gram-positive.

Gelatin stab : Slow liquefaction.

Agar colonies : Large, smooth, soft, convex, entire, opaque, creamy-white to yellow. The rough stage is usually concentrically ridged with a thin edge.

Agar slants: Growth abundant, soft, butyrous, creamy-white to yellow with pellucid dots. Browning with age; a few strains become black if the medium contains tyrosine.

Broth: Medium to heavy uniform turbidity.

Milk: Peptonized.

Milk agar plate: Casein hydrolyzed.

Potato: Growth abundant, smooth, soft to slimy, spreading, creamy-white, pale to lemon-yellow or pink. A few strains are orange-colored, some blacken the potato. The rough stage is wrinkled.

Nitrites usually not produced from nitrates.

Starch is hydrolyzed.

Acid with ammoniacal nitrogen from arabinose, glucose, fructose, sucrose, maltose, dextrin, inulin, salicin, glycerol and mannitol. Usually acid from xylose, galactose, mannose, and raffinose; variable from lactose. Generally no acid from rhamnose.

Acetylmethylcarbinol not formed.

Citrates used as sole source of carbon.

Uric acid hydrolysis: Variable.

Optimum temperature $28^{\circ} \mathrm{C}$ to $35^{\circ} \mathrm{C}$. Maximum temperature allowing growth usually between $40^{\circ} \mathrm{C}$ and $45^{\circ} \mathrm{C}$.

Source: Originally isolated from cooked cabbage.

Habitat: Widely distributed in soil, water, and decomposing materials.

Note: A description of Bacillus megatherium-Bacillus cereus intermediates follows the description of Bacillus cereus.

7. Bacillus cereus Frankland and Frankland. (Philosoph. Transact. Roy. Soc. London, 178, B, 1887, 279.) From Latin cereus, waxy.

Synonyms: Bacillus ellenbachensis alpha Stutzer and Hartleb, Cent. f. Bakt., II Abt., 4, 1898, 31; Bacillus ellenbachensis Gottheil, Cent. f. Bakt., II Abt., 7, 1901, 540; Bacterium petroselini Burchard, Arb. bakt. Inst. Karlsruhe, 2, 1898, 39 (Bacillus petrosclini Lehmann and Neumann, Bakt. Diag., 4 Aufl., 2, $1907,414)$.

The following are given as possible synonyms by Gottheil, Cent. f. Bakt., II Abt., 7, 1901, 540: Bacillus ramosus liquefaciens Flügge, Die Mikroorganismen, 2 Aufl., 1886, 342; Bacillus stoloniferus Pohl, Cent. f. Bakt., 11, 1892, 142 (Bacterium stoloniferus Chester, Ann. Rept. Del. Col. Agr. Exp. Sta., 9, 1897, 91; Achromobacter stoloniforum. Bergey et al., Manual, 1st ed., 1923, 136) ; Bacillus limosus Russell, Ztschr. f. Hyg., 11, 
1892, 196 (not Bacillus limosus Klein, Ber. d. deutseh. bot. Gesellsch., \%, 1889, 65; Bacillus limophilus Migula, Syst. d. Bakt., 2, 1900, 550); Bacillus brevis o Flügge, Ztschr. f. Hyg., 17, 1894, 294; Bacillus lutulentus Kern, Arb. bakt. Inst. Karlsruhe, 1, 1897, 402; Bacillus goniosporus Burchard, Arb. bakt. Inst. Karlsruhe, 2, 1898, 14; Bacterium turgescens Burchard, ibid., 18; Bacillus cursor Burchard, ibid., 25; Bacillus loxosus Burchard, ibid., 37.

The following are also listed as synonyms or biotypes of Bacillus cereus by Smith, Gordon and Clark: Bacillus sessilis Klein, Cent. f. Bakt., 6, 1889, 349 and 377 (Bacterium sessile Migula, Syst. d. Bakt., 2, 1900, 290); Bacillus albolactis Migula, ibid., 577 (Bacillus lactis albus Loeffler, Berlin. klin. Wehnsehr., 1887, 630); Bacillus lacticola Neide, Cent. f. Bakt., II Abt., 12, 1904, 168 (Bacillus No. V, Flügge, Ztschr. f. Hyg., 17, 1894 , 294; Bacillus lactis No. V, Kruse, in Flügge, Die Mikroorganismen, 3 Aufl., 2, 1896, 208; Bacterium lacticola Migula, Syst. d. Bakt., 2, 1900, 305; Bacillus excurrens Migula, ibid., 582; Bacillus cereus Chester, Man. Determ. Bact., 1901, 278; not Bacillus cereus Frankland and Frankland, Philos. Trans. Roy. Soc. London, 178, 13, 1857, 279); Bacillus lactis Neide, Cent. f. Bakt., II Abt., 12, 1904, 337 (Bacillus No. I, Flügge, Ztschr. f. Hyg., 17, 1894, 294; Bacillus flüggei Chester, Manual Determ. Bact., 1901, 281); Bacillus robur Neide, Cent. f. Bakt., II Abt., 12, 1904, 18; Bacillus thuringiensis Berliner, Zeit. f. angew. Entomol., 2, 1915, 29 (see also Mattes. Gesellschaft zur Beförd. der Gesam. Naturw., 62, 1927, 381; Bacterium thuringiensis Chorine, Internat. Corn Borer Invest., 2, 1929, 50); Bacillus cereus var. fluorescens Laubach, Jour. Bact., 1, 1916, 508 (Bacillus fluorescens Bergey et al., Manual, 1st ed., 1923, 298; not Bacillus fluorescens Trevisan, I generi e le specie delle Batteriacee, $1889,18)$; Bacillus sublilis Michigan strain, Conn., Jour. Inf. Dis., 46, 1930,
341; Bacillus undulatus den Dooren de Jong, Cent. f. Bakt., I Abt., Orig., 122, 1931, 277 (see also den Dooren de Jong, Arch. f. Mikrobiol., 4, 1933, 36); Bacillus siamensis Siribaed, Jour. Inf. Dis., 57, 1935, 143 (see also Bacillus cereus var. siamensis Clark, Jour. Bact., 33, 1937, 435); Bacillus metiens Charlton and Levine, Iowa Eng. Exp. Station, Bull. 132, 1937, 18; (see also Levine, Buchanan and Lease, Iowa State Coll. Jour. Sci., 1, 1927, 379); Bacillus tropicus Heaslip, Med. Jour. Australia, 28, 1941, 536.

Neide (loc. cit.) gave the following as possible synonyms of Bacillus lacticola: Bacillus butyricus Hueppe, Mitteil. a. d. kaiserl. Gesundheitsamte, 2, 1884, 309; not Bacillus butyricus Macé, Traité de Bact,, 1st ed., 1888 (Clostridium butyricum Prazmowski, Untersuchungen über die Entwickelungsgeschichte und Fermentwirkung einiger Bacterien-Arten. Inaug. Diss., Leipzig, 1880, 23); Bacillus aureus Pansini, Arch. f. pathol. Anat. u. Physiol., 122, 1890, 436 (not Bacillus aureus Frankland and Frankland, Philos. Trans. Roy. Soc. London, 178, B, 1887, 272); Bacillus lacteus Migula, Syst.d. Bakt., 2, 1900, 571 (No. 17, Lembke, Arch. f. Hyg., 29, 1897, 323); Bacillus goniosporus Burchard, loc cit.

Neide also gave the following as possible synonyms of Bacillus lactis: Bacillus lutulentus Kern, loc. cit.; Bacillus aggomeratus Migula, Syst. der Bakt., 2, 1900, 557; Bacillus amarificans Migula, ibid., 584; Bacillus cylindrosporus Burchard, Arb. bakt. Inst. Karlsruhe, 2 1898,31 .

Other possible synonyms of Bacillus cereus are: Bacillus anthracoides Hüppe and Wood, Ber. klin. Wehnschr., 16, 1889, 347 (Kruse, in Flügge, Die Mikroorganismen, 3 Aufl., 2, 1896, 232; Bacterium anthracoides Migula, Syst. der Bakt., 2, 1900, 281; not Bacterium anthracoides Trevisan, I generi e le specie delle Batteriacee, 1889, 20); Bacillus pseudanthracis Wahrlich, Bakteriol. Studien, Petersburg, 1890-91, 26 (not 
Bacillus pseudanthracis Kruse, in Flügge, Die Mikroorganismen, 3 Aufl., 2, 1896, 233 ; Bacterium pseudoanthracis Migula. loc. cit., 282); Bacterium flexile Burchard, Inaug. Diss., Karlsruhe, 1897 and Arb. bakt. Inst. Karlsruhe, 2, 1898, 16; Bacillus ellenbachi Sawamura, Tokyo Imp. Univ. Coll. Agr. Bull. 7, 1906, 105; Bacillus hoplosternus Paillot, Compt. rend. Acad. Sci., Paris, 163, 1916, 772; Bacillus fulminans Schrire and Greenfield, Trans. Roy. Soc. So. Africa, 17, $1929,309$.

Spores : Ellipsoidal, average size 1.0 by 1.5 microns (considerable variation has been noted by various writers), central or paracentral, usually freely formed in 24 hours. Germination prevailingly polar.

Sporangia: Ellipsoidal or cylindrical, only slightly swollen, if at all. In short to long chains.

Rods : 1.0 to 1.2 by 3.0 to 5.0 microns, occurring in long chains, ends square. Cells appear granular or foamy if lightly stained, especially if grown on glucose or glycerol nutrient agar; fat usually stored. Smooth strains are motile with many peritrichous flagella, rough strains weakly motile or non-motile. Gram-positive.

Gelatin stab: Rapid liquefaction.

Agar colonies: Large, flat, entire or irregular, whitish with characteristic appearance by transmitted light described by various observers as ground glass, moire silk, or galvanized iron. All stages occur from the thin, spreading. very rough and arboreseent, to the smooth dense form of colony.

Agar slant: Growth abundant, usually non-adherent, spreading, dense, whitish to slightly yellowish. Old slants show characteristic whip-like outgrowths. Some strains produce a yellowish-green fluorescence.

Broth: Heavy uniform turbidity, with or without a fragile pellicle.

Milk: Rapid peptonization, with or without slight eoagulation.

Blood serum: Partially liquefied. Hemolysis on blood agar.
Potato: Growth abundant, thick, soft, creamy-white to pinkish, spreading over the potato. Rough strains may be folded and more pigmented.

Nitrites usually produced from nitrates.

Starch is hydrolyzed.

Acid (with ammoniacal nitrogen) from glucose, fructose, maltose, dextrin, and glycerol. Acid usually from sucrose and salicin. Usually no acid from mannose and lactose. No acid from arabinose, rhamnose, xylose, raffinose, inulin, and mannitol.

Acetylmethylcarbinol produced.

Citrates usually utilized as sole source of carbon.

Optimum temperature about $30^{\circ} \mathrm{C}$. Maximum temperature allowing growth varies from $37^{\circ} \mathrm{C}$ to $48^{\circ} \mathrm{C}$, usually about $43^{\circ} \mathrm{C}$.

Aerobic.

Source: From soil, dust, milk, plants, and as contaminant of media.

Habitat: Widely distributed. Oceurs more often in soil than any other member of the genus. See Chester, Del. Agr. Exp. Station, 15th Ann. Report, 1903, 73; Lawrence and Ford, Jour. Bact., 1, 1916, 284; Conn, N. Y. Exp. Station, Tech. Bull. 58, 1917; Conn and Breed, Jour. Bact., 4. 1919, 273; Soriano, Thesis, Univ. Buenos Aires, 1935, 569.

\section{Bacillus megatherium-Bacillus} cereus intermediates.

According to Smith, Gordon, and Clark (loc. cit.) intermediate forms occur between Bacillus megatherium and Bacillus cereus which cannot be represented by a distinct species. These intermediates are characterized morphologically by the early appearance on agar of shadow or distorted forms, long filaments, and generally only a few spores. Fat globules are smaller and less numerous. Physiologically the group is erratic, showing a progression of characters from Bacillus megatherium on the one hand to Bacillus cereus on the other. Acetylmethylcarbi- 
nol and nitrites are not usually formed. Fermentation of the pentoses and mannitol, the ability to grow well on glucose nitrate agar, susceptibility to the bacteriophage active against Bacillus megatherium or Bacillus cereus and the general character of the growth determines whether the intermediate is more closely related to Bacillus megatherium or to Bacillus cereus.

Bacillus cohaerens Gottheil (Cent. f. Bakt., II Abt., 7, 1901, 458 and 689) may be taken as a representative of this intermediate group resembling Bacillus megatherium more closely than Bacillus cereus. Gottheil gave as possible synonyms: Bacillus vermicularis Frankland and Frankland, Ztschr. f. Hyg., 6, 1889, 384 (Bacterium vermiculare Migula, Syst. d. Bakt., 2, 1900, 302); Bacillus filiformis Tils, Ztschr. f. Hyg., 9, 1890, 293; Bacillus lactis albus Eisenberg, Bakt. Diag., 3 Aufl., 1891, 110; Bacillus virgatus Kern, Arb. bakt. Inst. Karlsruhe, 1, 1897, 416; Bacillus cylindrosporus Burchard, Arb. bakt. Inst. Karlsruhe, 2, 1898, 31; Bacillus bipolaris Burchard, ibid., 34 .

Other strains which apparently belong to this same group are : Bacterium pansinii Migula, Syst. d. Bakt., 2, 1900, 303 (Bacillus No. 3, Pansini, Arch. f. path. Anat., 122, 1890, 439; Bacterium granulatum Chester, Man. Determ. Bact., 1901, 189) ; Bacterium tomentosum Henrici, Arb. bakt. Inst. Karlsruhe, 1, 1897, 40; Bacillus teres Neide, Cent. f. Bakt., II Abt., 12, 1904, 161.

Representing those strains in this intermediate group more closely related to Bacillus cereus is Bacillus simplex Gottheil (loc. cit., 685). Gottheil gave the following as possible synonyms: Bacillus vacuolosis Sternberg, Manual of Bact., 1893, 717; Bacillus natans Kern, Arb. bakt. Inst. Karlsruhe, 1, 1897, 413; Bacillus loxosporus Burchard, Arb. bakt. Inst. Karlsruhe, 2, 1898, 49.

7a. Bacillus cereus var. mycoides (Flügge) comb. nov. (Bacillus mycoides
Flügge, Die Mikroorganismen, 2 Aufl., 1886, 324.) From Greek mykes, fungus ; eidos, form, shape, i.e., fungus-like.

Gottheil, Cent. f. Bakt., II Abt., 7, 1901, 589, gave the following as probable synonyms: Wurzelbacillus, Eisenberg, Bakt. Diag., 1st ed., 1886, 4; Bacillus figurans Crookshank, Manual, 1st ed., 1886 (Bacterium figurans Chester, Ann. Rept. Del. Col. Agr. Exp. Sta., 9, 1897, 134); Bacillus brassicae Pommer, Mitt. a. d. botan. Inst. zu Graf, 1, 1886, 95 (Bacterium brassicae Migula, Syst. d. Bakt., 2, 1900, 296); Bacterium casei Migula, Syst. d. Bakt., 2, 1900, 304 (Bacillus No. XVI, Adametz, Landw. Jahrb., 18, 1889, 248; Bacterium proteum Chester, Man. Determ. Bact., 1901, 195); Bacillus ramosus Frankland and Frankland, '/tschr. f. Hyg., 6, 1889, 388 (not Bacillus ramosus Veillon and Zuber, Arch. Méd. Exp. et Anat. Path., 10, 1898,542 ); Bacillus radicosus Zimmermann, Die Bakterien unserer Trink- u. Nutzwässer, etc., I Reihe, 1890, 30 (Bacterium radicosum Migula, Syst.d. Bakt., 2, 1900, 283); Bacillus implexus Zimmermann, ibid. (Bacterium implexum Migula, ibid., 298); Bacillus intricatus Migula, ibid., 546 (Cladothrix intricata Russell, Ztschr. f. Hyg., 11, 1892, 191).

Another possible synonym is Bacillus praussnitzii Trevisan (I generi e le specie delle Batteriacee, 1889, 20). Laubach, Jour. Bact., 1, 1916, 495, found that this differed from Bacillus mycoides only in the fermentation of lactose. This has been substantiated by later work.

Holzmüller (Cent. f. Bakt., II Abt., 23, 1909, 304) described four varieties of Bacillus mycoides which he designated by Greek letters and in addition named four new species which were apparently only variations of Bacillus mycoides: Bacillus effusus, Bacillus olfactorius, Bacillus nanus and Bacillus dendroides (not $B a$ cillus dendroides Thornton, Ann. Appl. Biol., 9, 1922, 247).

Bacillus cereus var. mycoides is identi- 
cal in all respects with Bacillus cereus except in the following characters :

Agar colonies: Grayish, thin, widely spreading by means of long twisted chains of cells, turning to the right or left.

Agar slants: Growth thin, rhizoid, grayish, widely spreading, adhering to or growing into the agar. Later, growth becomes thicker and softer.

The physiological similarity between Bacillus cereus and Bacillus mycoides has often been noted. Gordon (Jour. Bact., 39, 1940, 98) showed that the rhizoid character of the growth of Bacillus mycoides was readily lost by cultivation in flasks containing $100 \mathrm{ml}$ of broth and that the resulting dissociants could not be differentiated from Bacillus cereus. It is, therefore, a question whether $B a$ cillus mycoides should be given the dignity of a variety of Bacillus cereus or merely designated as a stage of growth (morphotype).

Source: Isolated from soil.

Habitat: Widely distributed in soil.

\section{Bacillus anthracis Cohn emend.} Koch. (Les infusories de la maladie charbonneuse, Davaine, Compt. rend. Acad. Sci., Paris, 69, 1864, 393; Cohn, Beiträge z. Biol.d. Pflanzen, 1, Heft 2, 1872, 177; Koch, ibid., 2, Heft 2, 1876 , 279 ; Bactéridie des charbon, Pasteur and Joubert, Compt. rend. Acad. Sci., Paris, 84, 1877, 900; Bacterium anthracis Zopf, Die Spaltpilze, 2 Aufl., 1884, 45; Bacillus (Streptobacter) anthracis Schroeter, Kryptogamen Flora v. Schlesien, 3, 1, 1886, 163; Pollendera anthracis Trevisan, 1884, see Trevisan, I generi e le specie delle Batteriacee, 1889, 13; Bacterium anthracis Migula, in Engler and Prantl, Die natürlichen Pflanzenfam., 1, 1a, 1895, 21; Aplanobacter anthracis Erw. Smith, Bacteria in Relation to Plant Diseases, 1905, 171; Bacillus (Bacteridium) anthracis Buchanan, Jour. Bact., 3, 1918, 37.) From Greek, gen. of anthrax, charcoal, a carbuncle, the disease anthrax.
According to Smith, Gordon, and Clark (loc. cit.) this species is a pathogenic variety of Bacillus cereus. They worked extensively with the latter but not with many strains of Bacillus anthracis. The only difference between the two seemed to be pathogenicity and motility, and some strains of Bacillus cereus are weakly pathogenic and some practically nonmotile. It would appear that Bacillus cereus is a so-called parent species from which two varieties (var. anthracis and var. mycoides) and several morpho- and biotypes have sprung.

Spores : Ellipsoidal, 0.8 to 1.0 by 1.3 to 1.5 microns, central or paracentral, often in chains. Germination polar.

Sporangia: Ellipsoidal to cylindrical, not swollen, in chains.

Rods : 1.0 to 1.3 by 3 to 10 microns with square or concave ends, occurring in long chains, resemble Bacillus cereus. Cells from glucose or glycerol nutrient agar appear granular (vacuolated) if stained lightly; many fat globules present. Nonmotile. Gram-positive.

Gelatin stab: Arborescent in depth, inverted pine tree. Liquefaction crateriform becoming stratiform.

Agar colonies : Large, irregular, dense, curled structure composed of parallel chains, similar to certain strains of Bacillus cereus.

Agar slant: Growth abundant, grayish, dense, spreading, with fimbriate borders.

Broth: Little or no turbidity, thick pellicle.

Milk: Coagulated, slightly acid, peptonized.

Potato: Growth abundant, spreading, white to creamy.

Nitrites formed from nitrates.

Starch is hydrolyzed.

Acid from glucose, fructose, sucrose, maltose, trehalose, and dextrin. Some strains produce late and slight acidity in glycerol and salicin. No definite fermentation occurs in arabinose, rhamnose, mannose, galactose, lactose, raffinose, inulin, mannitol, dulcitol, sorbitol, inosi- 
tol, and adonitol (Stein, Vet. Med., 38, 1943).

Acetylmethylcarbinol produced.

Optimum temperature about $35^{\circ} \mathrm{C}$. Maximum temperature allowing growth about $43^{\circ} \mathrm{C}$.

Aerobic, facultative.

Pathogenic for man, cattle, swine, sheep, rabbits, guinea pigs, mice, etc.

Source : From blood of infected animals .

Habitat. The cause of anthrax in man. cattle, sheep and swine.

\section{Bacillus polymyxa (Prazmowski)} Migula. (Clostridium polymyxa Prazmowski, Inaug. Diss., Leipzig, 1880, 37; Migula, Syst. der Bakt., 2, 1900; 638; Granulobacter polymyxa Beijerinek, K. Akad. Wetenschap., Amsterdam, Sec. 2, 1, 1903, No. 10; Granulobacter polymyxa var. mucosum and var. tenax Beijerinck and Van Delden, Cent. f. Bakt., II Abt., 9, 1902, 13; further description by Gruber, Cent. f. Bakt., II Abt., 14, 1905, 353; Aerobacillus polymyxa Donker, loc. cit., 138.) From Greek poly, many or much; myxa, slime or mucus.

This and the species immediately following (Bacillus macerans) are sometimes placed in the sub-genus Aerobacillus Donker emend. Kluyver and Van Neil (Donker, Inaug. Diss., Delft, 1926, 138; Kluyver and Van Neil, Cent. f. Bakt., II Abt., 94, 1936, 402; not Aerobacillus Pribram, Jour. Bact., 18, 1929, 374; not Aerobacillus Janke, Cent. f. Bakt., II Abt., 80, 1930, 481).

For a study of this group and a review of the literature see Porter, McCleskey and Levine, Jour. Bact., 33, 1937, 163. They give the following as synonyms of Bacillus polymyxa: Bacillus asterosporus Migula (Astasia asterospora Meyer, Flora, Erg. Bd., 84, 1897, 185; Migula, Syst. der Bakt., 2, 1900, 528; Aerobacillus asterosporus Donker, loc. cit., 141); Bacillus ovoaethylicus Pribram (Bacillus mycoides var. ovoaethylicus Wagner, Ztschr. f. Untersuch. d. Nahrungs-u. Genussmittel, 31, 1916, 234; Pribram, Klassifikation der Schizomyceten, Leip- zig und Wien, 1933, 86); Bacillus aerosporus Greer, Jour. Inf. Dis., 42, 1928, 508.

Gottheil (Cent. f. Bakt., II Abt., 7, 1901,727 ) regarded the following as synonyms: Bacillus thalassophilus Russell, Ztschr. f. Hyg., 11, 1892, 190; Bacillus subanaerobius Migula, Syst. der Bakt., 2, 1900,600 .

Bredemann (Cent. f. Bakt., II Abt., $23,1909,45)$ admitted that the organisms, Bacillus asterosporus alpha, Bacillus dilaboides, and Bacillus clostridioides, named by Haselhoff and himself in an earlier article (Landwirtsch. Jahrb., 35, $1906,420,426,432)$ were merely variants of Bacillus asterosporus.

The following is usually considered a variety or strain of Bacillus polymyxa differing from the latter mainly in the production of a violet pigment on potato and agar in the presence of peptone: Bacillus violarius acetonicus Bréaudat, Ann. Inst. Pasteur, 20, 1906, 874 (Aerobacillus violarius Donker, Inaug. Diss., Delft, 1926, 141).

Also a probable synonym of Bacillus polymyxa is Bacillus amaracrylus Viosenet (Bacille de l'amertume, Voisenet, Compt. rend. Acad. Sci., Paris, 153, 1911, 363; Voisenet, Ann. Inst. Pasteur, 32, 1918, 477; Aerobacillus amaracrylus Donker, loc. cit., 141). The chief character in which it differs from Bacillus polymyxa is its ability to dehydrate glycernl with the formation of acrolein.

Also a probable variant of Bacillus polymyxa is Bacillus pandora Corbet (Jour. Bact., 19, 1930, 321). The chief characters in which the latter differs from the former are the production of acid without gas from glucose and the lack of diastatic action.

Spores: Ellipsoidal, 1.0 to 1.5 by 1.5 to 2.5 microns, central to subterminal, wall usually thick and stainable. Freely formed.

Sporangia: Swollen, spindle-shaped (clostridia), sometimes clavate.

Rods : 0.6 to 1.0 by 2.5 to 6.0 microns, 
occurring singly or in short chains. Cells contain small fat globules then grown on glucose nutrient agar. Motile with peritrichous flagella. Gram-variable.

Gelatin stab: Slow liquefaction. $\mathrm{Hy}$ drolysis of gelatin always positive by Frazier technic.

Agar colonies: Thin, inconspicuous, lobed, spreading over entire plate. Rough forms are round, whitish, and sometimes tough.

Agar slant: Grow th scant to moderate, indistinct to whitish. On glucose agar, the growth is much heavier, raised, gummy, glistening; gas is formed.

Broth: Uniform to granular turbidity, flocculent to slimy sediment. Rough stage forms pellicle. Final $\mathrm{pH}$ of glucose broth cultures 5.2 to 6.8 .

Milk: Not coagulated, gas usually formed.

Milk agar plate: Casein hydrolyzed.

Potato: Growth moderate to abundant, whitish to light tan, potato decomposed with formation of gas. Growth of rough stains is denser and heaped up.

Nitrites are produced from nitrates.

Starch is hydrolyzed. Crystalline dextrins are not produced.

Acid and gas (with ammoniacal nitrogen) from arabinose, xylose, glucose, fructose, galactose, mannose, maltose, sucrose, lactose, trehalose, cellobiose, raffinose, melezitose, dextrin, inulin, salicin, glycerol, and mannitol. Gum is also usually formed. Erythritol, adonitol, dulcitol and inositol not fermented. With organic nitrogen no acid or gas from rhamnose or sorbitol (Porter, McCleskey, and Levine, loc. cit., also Tilden and Hudson, Jour. Bact., 43, 1942, 530). This, however, could not be confirmed by Smith, Gordon, and Clark (loc. cit.) who found that acid and gas were produced from both carbohydrates.

Hemicellulose and pectin are attacked (Ankersmit, Cent. f. Bakt., I Abt., Orig., 40, 1905, 100). In glucose broth, ethyl alcohol and butylene-glycol are produced also small amounts of acetone and butyl alcohol.

Acetylmethylcarbinol is produced.

Citrates usually not utilized as sole source of carbon.

Optimum temperature about $30^{\circ} \mathrm{C}$. To growth at $42^{\circ} \mathrm{C}$ to $45^{\circ} \mathrm{C}$; good growth at $20^{\circ} \mathrm{C}$, slow at $13^{\circ} \mathrm{C}$.

Not agglutinated by Bacillus macerans sera, results with homologous sera irregular (Porter, McCleskey, and Levine, loc. cit.).

Aerobic, facultative.

Source: First isolations were from grain, soil, and pasteurized milk.

Habitat: Widely distributed in water, soil, milk, feces, deeaying vegetables, ete.

In addition see: Chester, Del. Agr. Exp. Station, 15th Ann. Report, 1903, 65; Wund, Cent. f. Bakt., I Abt., Orig., 42, 1906, 193, 289, 385; Wahl, Cent. f. Bakt., II Abt., 16, 1906, 489; Ritter, Cent. f. Bakt., II Abt., 20, 1908, 21 ; Meyer, Cent. f. Bakt., I Abt., Orig., 49, 1909, 305; Bredemann, Cent. f. Bakt., II Abt., 23, 1909, 41; Virtanen and Kurstom, Biochem. Ztschr., 161, 1925, 9; Stapp and Zycha, Arch. f. Mikrobiol., 2, 1931, 493; Zycha, Arch.f. Mikrobiol., 3, 1932, 194; Patrick, Iowa State Coll. Jour. Sci., 7, 1933, 407 .

10. Bacillus macerans Schardinger. (Rottebazillus 1, Schardinger, Wiener klin. Wochenschr., 17, 1904, 207; Schardinger, Cent. f. Bakt., II Abt., 14, 1905, 772; Aerobacillus macerans Donker, Inaug. Diss., Delft, 1926, 139; Zymobacillus macerans Kluyver and Van Niel, Cent. f. Bakt., II Abt., 94, 1936, 402.) From Latin macerans, softening, macerating or retting.

Porter, MeCleskey, and Levine, Jour. Bact., 33, 1937, 163, regard the following as a synonym of Bacillus macerans: Bacillus acctoethylicum Northrup, Ashe, and Senior, Jour. Biol. Chem., 39, 1919, 1 (Aerobacillus acetoethylicus Donker, loc. cit.).

The following is probably a variant of 
Bacillus macerans: Aerobacillus schuylkilliensis Eisenberg, Jour. Amer. Water Works Assoc., 34, 1942, 365. It is said to differ from Bacillus macerans in that sorbitol is not fermented, hydrogen sulfide is produced and gelatin is liquefied.

Spores : Ellipsoidal, 1.0 to 1.5 by 1.5 to 2.5 microns, terminal to subterminal ; wall thick and stainable.

Sporangia: Swollen terminally, clavate.

Rods : 0.6 to 1.0 by 2.5 to 6.0 microns, oceurring singly or in pairs, cells are larger on sugar media than on sugar-free media, and contain a few small fat globules. Motile. Gram-variable.

Gelatin stab: Liquefaction variable (see optimum temperature). Gelatin is hydrolyzed as determined by the Frazier technic $\left(30^{\circ} \mathrm{C}\right)$.

Agar colonies: Small, thin, transparent to whitish, irregular, usually smooth.

Agar slant: Growth moderate, spreading, inconspicuous.

Broth: Turbid, slight sediment. In sugar broths some strains produce slime. Glucose broth cultures, pH 5.0 to 5.5 .

Milk: Acid and gas. No visible peptonization.

Milk agar plate: Casein not hydrolyzed in one week; later usually slight hydrolysis.

Potato: Growth indistinct, gas is formed and the potato is digested. Fruity odor sometimes produced.

Nitrites produced from nitrates.

Starch is hydrolyzed.

Acid and gas from arabinose, rhamnose, xylose, glucose, fructose, galactose, mannose, suerose, maltose, lactose, trehalose, cellobiose, raffinose, melezitose, dextrin, inulin, salicin, pectin, xylan, glycerol, mannitol, and sorbitol. Erythritol, adonitol, dulcitol, and inositol not fermented (Porter, McCleskey, and Levine, loc. cit.).

Produces acetone and ethyl but never butyl alcohol; ratio acetone to alcohol is $1: 2$.

Acetylmethylcarbinol not produced.
Citrates not utilized as sole source of carbon.

Optimum temperature about $37^{\circ} \mathrm{C}$. Good growth at $42^{\circ}$ to $45^{\circ} \mathrm{C}$ and sometimes slightly higher; poor growth, if any, at $20^{\circ} \mathrm{C}$.

Differentiated from Bacillus polymyxa by the production of erystalline dextrins from starch, lack of formation of acetylmethylcarbinol, and by growth at $42^{\circ} \mathrm{C}$ to $45^{\circ} \mathrm{C}$.

All strains agglutinated by homologous sera but not by Bacillus polymyxa serum.

Aerobie, facultative.

For additional literature, see Porter, MeCleskey and Levine, Jour. Bact., 33, $1937,180$.

Source: Originally isolated from vats in which flax was retting.

Habitat: Widely distributed in soil, water, decomposing starchy materials, retting flax, etc.

11. Bacillus circulans Jurdan. (Jordan, Exp. Inv., Mass. State Board Health, Part II, 1890, S31; Baclerium circulans Chester, Ann. Rept. Del. Col. Agr. Lxp. Sta., 9, 1897, 92; also see Ford. Jour. Bact., 1, 1916, 519.) From Latin circulans, making round or circular.

Smith, Gordon, and Clark (loc. cit.) consider Bacillus circulans as a complex (see also Gibson and Topping, Soc. Agric. Bact. (British), Abstr. Proc., 1938, 43) because of the variations in the character of the grow th and quantitative differences in physiology. All stages of growth may be found from the smooth actively motile strains that have motile colonies to the mucoid, non-spreading strains. The species is more saccharolytic than proteolytic, considerable variation being found in its action on gelatin and casein. The following are regarded as variants: Bacillus closteroides Gray and Thornton, Cent. f. Bakt., II Abt., 73, 1928, 93 ; Bewegungstypus sehwarmender Bakterien, RussMunger, Cent. f. Bakt., I Abt., Orig., 142, 1938, 175; Bacillus krzemienicwski 
Kleczkowska, Norman and Snieszko, Soil Sci., 49, 1940, 185 (a mucoid variant).

Also probable variants : Bacillus platus Soriano, Thesis, Univ. Buenos Aires, 1935, 572; Bacillus naviformis Soriano, ibid., 574 (not Bacillus naviformis Jungano, Comp. rend. Soc. Biol., Paris, 1, 1909, 122).

Spores: Ellipsoidal, 0.8 to 1.2 by 1.1 to 2.0 microns, terminal or subterminal. Spore wall thick and stainable. In some strains spores may be kidney-shaped and remnants of sporangium may adhere.

Sporangia : Swollen terminally, clavate.

Rods : 0.5 to 0.9 by 2.0 to 5.0 microns, sometimes curved, usually occurring singly. Cells contain small fat globules when grown on glucose agar. Motile, some strains exceedingly so. Gramvariable, usually negative

Gelatin stab: Slow cone-shaped liquefaction, liquefied portion evaporating (Jordan); no liquefaction (Ford). Gelatin hydrolyzed if tested by the Frazier method.

Agar colonies: Thin, transparent, spreading over entire surface of plate. Often nearly invisible. The colonies of the rougher or mucoid strains are small, entire, whitish, non-spreading.

Giant agar colonies: If the surfaces of agar plates are dried before inoculation with very motile strains, instead of spreading as a thin layer of individual cells, minute rotating colonies proceed out from the edge of the colony, sometimes becoming entirely disconnected from it. In moving out across the agar surface, non-motile cells are left behind. These may grow later. Eventually the whole plate is covered.

Agar slant: Growth thin, transparent, spreading, becoming denser. Mucoid strains are dense, non-spreading, entire, gummy and adherent.

Broth: Light to fair turbidity with floceulent to slimy sediment. Some strains do not grow perceptibly. In glurose broth cultures the final $\mathrm{pH}$ is usually 5.0 to 5.8 .
Milk: Usually acid, slowly coagulated.

Milk agar plate: Casein not hydrolyzed. Weak hydrolysis with some strains.

Potato: Growth is very variable, from none to good growth, from colorless to yellow ish, pink, or brownish.

Nitrites usually produced from nitrates.

Starch is hydrolyzed. Crystalline dextrins usually not formed.

Acid without gas (with ammoniacal nitrogen) from glucose, fructose, mannose, galactose, sucrose, maltose, raffinose, salicin, and dextrin. Usually acid from arabinose, xylose, lactose, glycerol, and mannitol. Reaction variable with rhamnose and inulin.

Acetylmethylcarbinol not produced.

Citrates usually not utilized.

Methylene blue reduced and then reoxidized.

Urease produced by some strains.

Optinum temperature about $30^{\circ} \mathrm{C}$. Maxinum temperature allowing growth, $40^{\circ} \mathrm{C}$ to $48^{\circ} \mathrm{C}$. A few strains will grow up to $52^{\circ} \mathrm{C}$.

This species is closely related to Bacillus macerans from which it is distinguished by the lack of gas formation from carbohydrates and the lack of crystalline dextrins from starch. It is also close to Bacillus alvei as indicated by the key.

Source: Found occasionally in tap water, Lawrence, Mass. (Jordan).

Habitat: Widely distributed in soil, water, and dust.

12. Bacillus alvei Cheshire and Cheyne. (Jour. Roy. Mic. Soc., Ser. II, 5, 1885, 592.) From Latin alveus, beehive.

Probably identical with the above: Bacillus paraalvei Burnside, Amer. Bee Jour., 72, 1932, 433; Burnside and Foster, Jour. Econ. Entomol., 28, 1935, 578.

Spores: Ellipsoidal, 0.7 to 1.0 by 1.5 to 2.5 microns, central to terminal. Free spores frequently lie in parallel arrangement like the rods. 
Sporangia: Swollen, spindle-shaped to clavate.

Rods : 0.5 to 0.8 by 2 to 5.0 microns. Cells frequently lie parallel, side by side, like cartridges in a clip. Usually noncapsulated and very motile. Few small fat globules in cells from glucose agar. Gram-variable (young cells Gram-positive, becoming Gram-negative).

Gelatin stab: Slow liquefaction.

Agar colonies: Thin, translucent, smooth, quickly spreading as a thin layer over entire plate. The growth thickens with age. Rough and mucoid strains do not spread.

Giant agar colonies: If the surfaces of agar plates are dried before inoculation with the motile strains, instead of spreading as a thin layer, minute bullet-shaped colonies proceed out from the edge of the colony and move across the sterile agar. Non-motile and sometimes motile cells are left behind along the path made by the motile colony (Smith and Clark, Jour. Bact., 35, 1938, 59). Eventually the whole plate is covered.

Agar slant: Growth thin, inconspicuous, spreading, becoming thicker. Rough and mucoid strains do not spread, growth is heaped, and sometimes gummy.

Broth: Uniform turbidity. Rough strains may form a pellicle. Glucose broth cultures have a pH of 5.0 to 6.0 .

Milk: Usually congulated, little or no acid, peptonized.

Milk agar plate: Casein hydrolyzed.

Potato : Growth scant to moderate, soft, spreading, usually creamy yellow.

Nitrites not produced from nitrates.

Starch is hydrolyzed.

Acid (with ammoniacal nitrogen) from glucose, fructose, galactose, sucrose, maltose, dextrin and glycerol. Reaction variable on mannose, lactose, raffinose, salicin, and mannitol. No acid from arabinose, rhamnose, xylose, and inulin.

Acetylmethylcarbinol is produced.

Citrates not utilized.

Optimum temperature about $30^{\circ} \mathrm{C}$.
Maximum temperature allowing growth $43^{\circ} \mathrm{C}$ to $45^{\circ} \mathrm{C}$.

Putrefactive odor on media rich in proteins (egg).

Aerobic.

Source: Isolated from diseased brood of bees.

Habitat: Associated with European foulbrood of honey bees; widely distributed in soil.

Note: The following must be considered in connection with Bacillus alvei:

Bacillus pluton White. (U. S. Dept. of Agric., Bur. Entomol., Circ. 157, 1912; Diplococcus pluton Bergey et al., Manual, 2nd ed., 1925, 45.)

See also Lochhead, Science, 67, 1928, 159 and Proc. IV Intern. Congr. Entomol., 2, 1929, 1005; Burnside, Jour. Econ. Entomol., 27, 1934, 656; Tarr, Ann. Appl. Biol., 24, 1935, 614; Burri, Beihefte z. schweiz. Beinenz., 1, Heft 1, 1941.

White considered Bacillus pluton to be the cause of European foulbrood though the evidence was indirect since the organism was not cultivated. Lochhead suggested that Bacillus pluton and Streptococcus apis are variants or stages in the life history of Bacillus alvei, a hypothesis supported by Burnside who included, in addition, Bacterium eurydice. According to Burri, rod forms identical with Bacterium eurydice give rise to Bacillus pluton which is not directly cultivable. Tarr considers European foulbrood to be caused by Bacillus pluton, distinct from Bacillus alvei, and considers it a strict parasite able to multiply only in the intestines of young larvae.

Source: Larvae of the honey bee infected with European foulbrood.

13. Bacillus laterosporus Laubach. (Jour. Bact., 1, 1916, 511.) From Latin latus, lateris, the side; Greek sporus, seed; M.L., spore.

Synonym: Bacillus orpheus White. (U. S. Dept. of Agric., Bur. Entomol., Cire. 157, 1912,3.) Although named by White, the organism was not described 
until 1917 (McCray, Jour. Agr. Research, $8,1917,410)$. Resembles Bacillus laterosporus (White, U. S. Dept. Agric. Bull. $810,1920,14)$. According to the rules of priority, the name to be used is Bacillus laterosporus.

Spores : Ellipsoidal, 1.0 to 1.3 by 1.2 to 1.5 microns, central to subterminal, formed close to one side, remnants of the sporangium adhering to the other side.

Sporangia: Swollen, spindle-shaped.

Rods : 0.5 to 0.8 by 2.0 to 5.0 microns, occurring singly and in pairs. Ends pointed or poorly rounded. Cells from glucose nutrient agar may have few small fat globules. Motile. Gram-variable.

Gelatin stab: Slow liquefaction.

Agar colonies : Thin, transparent, irregular, spreading. Colonies of rough strains are small, round, convex, nonspreading.

Agar slant: Growth moderate, flat, translucent to opaque, moist, sometimes with a silvery sheen.

Broth: Uniform to granular turbidity; usually no pellicle. Glucose broth cultures, $\mathrm{pH} 6.0$ to 6.4 .

Milk: Usually curdled, peptonized.

Milk agar plate: Occasionally weak hydrolysis of casein.

Potato: Growth thin, spreading, grayish to pinkish, turning light brown with age. Sometimes finely wrinkled.

Nitrites produced from nitrates.

Starch is not hydrolyzed.

Acid (with ammoniacal nitrogen) from glucose, fructose, maltose, glycerol, and mannitol. Reaction variable on galactose, mannose, and salicin. No acid from arabinose, rhamnose, xylose, sucrose, lactose, raffinose, inulin, and dextrin.

Acetylmethylcarbinol not produced.

Citrates not utilized as sole source of carbon.

Optimum temperature about $28^{\circ} \mathrm{C}$. Maximum temperature allowing growth $37^{\circ} \mathrm{C}$ to $45^{\circ} \mathrm{C}$.

Aerobic.

Source: Isolated from water.

Habitat: Widely distributed in soil, water and dust.
14. Bacillus brevis Migula emend. Ford. (Bacillus No. I, Flügge, Ztschr.f. Hyg., 17, 1894, 294; Bacillus lactis No. I, Kruse, in Flügge, Die Mikroorganismen, 3 Aufl., 2, 1896, 208; Migula, Syst. d. Bakt., 2, 1900, 583; not Bacillus brevis Frankland and Frankland, Microorganisms in Water, 1894, 429; Bacillus flüggei Chester, Man. Determ. Bact., 1901, 281; Ford, Jour. Bact., 1, 1916, 522.) From Latin brevis, short.

Synonym: Bacillus centrosporus Ford, Jour. Bact., 1, 1916, 524.

There is some doubt as to the identity of Migula's Bacillus brevis which originally was Flügge's Bacillus No. I. Neide (Cent. f. Bakt., II Abt., 12, 1904, 337) also renamed Flügge's organism. He called it Bacillus lactis and described it sufficiently that it may be recognized as a strain of Bacillus cereus. Ford believed that his isolations from milk, soil and dust conformed to Migula's description of Bacillus brevis. Ford's interpretation has been accepted. The species has apparently become well established in Europe (Gibson and Topping, Soc. Agric. Bact. (British), Abstr. Proc., 1938, 43) as well as in America.

Description from Ford and from Smith, Gordon, and Clark (loc. cit.).

Spores: Ellipsoidal, 1.0 to 1.3 by 1.5 to 2.0 microns, central to subterminal. Spore walls thick and stainable. An occasional strain shows the relationship of this species to Bacillus laterosporus in that some of the spores may be lateral and remnants of the sporangia may adhere to one side of the spore.

Sporangia: Definitely swollen, spindleshaped to clavate.

Rods : 0.4 to 0.8 by 1.5 to 5.0 microns, with pointed ends, occurring singly or in pairs. On glucose agar cells contain numerous small fat globules. Motile. Gram-variable.

Gelatin stab: Slow liquefaction.

Agar colonies: Thin, flat, translucent, smooth, quickly spreading over plate.

Agar slants: Growth smooth, moist, spreading, grayish white. 
Broth: Usually heavy uniform turbidity, sometimes with a fragile pellicle. Glucose broth cultures have a $\mathrm{pH}$ of 8.0 to 8.6 after 7 days.

Milk: Peptonized.

Milk agar plate: Casein hydrolyzed.

Potato: Growth scant to moderate, flat, spreading, soft, creamy-yellow to pink to brownish.

Nitrites usually formed from nitrates. Starch is not hydrolyzed.

Acid (with ammoniacal nitrogen) from glucose, fructose, maltose, and sucrose. Usually acid from galactose and glycerol. Reaction is variable on rhamnose and mannitol. No acid from arabinose, xylose, mannose, lactose, raffinose, inulin, dextrin and salicin.

With organic nitrogen, the acid formed from carbohydrates is masked by the alkalinity due to proteolysis.

Acetylmethylcarbinol is not produced.

Citrates usually utilized as a sole source of earbon.

Optinum temperature about $30^{\circ} \mathrm{C}$. Maximum temperature allowing growth varies from $43^{\circ} \mathrm{C}$ to $54^{\circ} \mathrm{C}$.

Produces antibiotic substances (tyrothricin, gramicidin; sec Dubos and Hotchkiss, Jour. Exp. Med., 73, 1941, 629).

\section{Aerobic.}

Source: From milk (Flügge); from milk, soil and dust (Ford).

Habitat: Widely distributed in soil, water, dust, milk, etc.

\section{Bacillus larvae White. (Bac-} terium X, Moore and White, New York State Dept. Agr., 11th Ann. Rept. Com. Agr. for 1903, 1904, 111; Bacillus X, Moore and White, ibid., Rept. for 1904, 1905, 106; White, Thesis, Cornell Univ. Ithaca, N. Y., 1905; White, U. S. Dept. Agr. Bur. Entomol., Tech. Ser. Bul. 14, 1906, 32 ; White, U. S. Dept. Agr. Bul. 809 , 1920, 13.) From Latin larva, a ghost; M. L., of a larva.

Synonyms: Bacillus brandenburgiensis Maassen, Mittl.a. d. kaiserl. biol. Anstalt f. Land- u. Forstw. in Dahlem, Heft 2,
1906, 28; ibid., Heft 7, 1908, 24 pp.; Arb, a. d. Anstalt f. Land- u. Forstw., 6, 1908. 61; Bacillus burri Cowan, British Beekeeper's Guide Book. 20th ed., London, $1911,171$.

Description from Lochhead, Sci. Agr., $9,1928,84$.

Spores: Ellipsoidal, central to subterminal.

Sporangia: Swollen, spindle form.

Rods : 0.5 to 0.8 by 2.5 to 5.0 microns, occurring singly and in chains. Motile. Gram-variable.

Gelatin stab: No growth. In carrotgelatin, slow liquefaction.

Yeast-carrot agar colonies: Small, whitish, somewhat transparent, smooth, slightly glistening.

Agar slant: No growth. With addition of carrot extract, noticeable growth along line of inoculation. More abundant growth if yeast extract is also added.

Yeast-earrot broth : Fungoid in appearance, floating masses which may be broken up by shaking to produce a uniform elouding.

Carrot-milk: Acid with curdling. No peptonization.

Potato: No growth.

Nitrites formed from nitrates (see Lochhead, Can. Jour. Res., C, 15, 1937, 79).

Stareh not hydrolyzed (carrot-starch agar).

Acid (in yeast extract-peptone broth) from xylose, glucose, fructose, galactose, salicin. Slight acidity by some strains from lactose and sucrose. No acid from mannitol or dulcitol.

Thiamin replaces the growth factor in vegetable or yeast extracts, ete. (Lochhead, Jour. Bact., 44, 1942, 185).

Optimum temperature about $37^{\circ} \mathrm{C}$. Maximum temperature about $45^{\circ} \mathrm{C}$.

Source: From diseased brood.

Habitat : Causal organism of American foulbrood of honey bees.

See in addition: White, Science, 49 , 1919, 362; Sturtevant, Jour. Agr. Res., 28, 1924, 129; Borchert, Die seuchenhaften Krankheiten der Honigbiene. Berlin, 
1926; Sturtevant, Jour. Agr. Res., 45, 1932, 257 ; Hitchcock, Jour. Econ. Entomol., 29, 1936, 895; Stoilowa, Cent. f. Bakt., II Abt., 99, 1938, 124; Tarr, Ann. Appl. Biol., 25, 1938, 633; Holst and Sturtevant, Jour. Bact., 40, 1940, 723.

16. Bacillus popilliae Dutky. (Jour. Agr. Research, 61, 1940, 59.) From the genus name of the Japanese beetle, Popillia japonica Newm.

Spores: Cylindrical, 0.9 by 1.8 microns, central. Free spores have not been observed.

Sporangia: Swollen, spindle-shaped. Contains a refractile body at the broader pole of the cell which is about half the size of the spore and reacts similarly to stains.

Rods: Unstained, 0.9 by 5.2 microns. Stained by crystal violet after fixing in Schaudinn's solution, 0.3 by 3.5 microns. Non-motile. Gram-positive.

Unheated egg yolk-beef infusion agar slants: Growth occurs as small discrete colonies.

Optimum temperature about $30^{\circ} \mathrm{C}$. Maximum temperature about $36^{\circ} \mathrm{C}$.

Aerobic, facultative.

Source: From infected larvae.

Habitat: Cause of type A milky disease of Japanese beetle, Popillia japonica. See Hawley and White, N. Y. Ent. Soc. Jour., 43, 1935, 405.

\section{Bacillus lentimorbus Dutky.}

(Jour. Agr. Res., 61, 1940, 65.) From Latin lentus, slow, lingering, and morbus, death.

Spores : Ellipsoidal, 0.9 by 1.8 microns, central.

Sporangia: Swollen, spindle-shaped. No refractile granule at pole.

Rods: Unstained, 1.0 by 5.0 microns. Stained by erystal violet after fixing in Schaudinn's solution, 0.5 by 4.0 microns. No growth on artificial media.

Optimum temperature about $25^{\circ} \mathrm{C}$. Maximum temperature about $30^{\circ} \mathrm{C}$.

Aerobic, facultative.

Source: Diseased larvae.
Habitat : Cause of type B milky disease of Japanese beetle, Popillia japonica.

18. Bacillus sphaericus Neide. (Cent. f. Bakt., II Abt., 12, 1904, 350.) From Latin sphaericus, spherical.

Neide (loc. cit.) gave the following as possible synonyms: Bacillus (Streptobacter) albuminus Schroeter, in Cohn, Kryptogamenflora von Schlesien, 3, 1, 1886,162 ; Bacillus putrificus coli Flügge, Die Mikroorganismen, 2 Aufl., 1886, 303; Bacillus gracilis Zimmermann, Die Bakterien unserer Trink- u. Nutzwässer, etc., 1, 1890, 50 (Bacterium gracile Chester, Man. Determ. Bact., 1901, 198); Bacillus butyricus Botkin, Ztschr. f. Hyg., 11, 1892, 421; Bacillus thalassophilus Russell, Ztschr. f. Hyg., 11, 1892, 190; Bacillus pseudotetanicus aerobius Kruse, in Flügge, Die Mikoorganismen, 3 Aufl., 2, 1896, 267 (Bacillus pseudotetanicus Migula, Syst. der Bakt., 2, 1900, 626; not Bacillus pseudotetanicus Kruse, idem; not Bacillus pseudotetanicus Chester, Man. Determ. Bact., 1901, 302; Bacillus pscudotetanicus var. aerobius Chester, ibid., 303); Plectridium paludosum Fischer, Jahr. f. Wiss. Bot,, 27, 1895, 147; Pseudotetanicusbacillus, Tavel, Cent. f. Bakt., I Abt., 23, 1898, 538 (Bacillus pseudotetani Migula, Syst. der Bakt., 2, 1900, 59s; Bacillus taveli Chester, loc. cit., 304).

Also apparently identical with Bacillus sphaericus: Bacillus subtetanicus Migula, Syst. der Bakt., 2, 1900, 629; Bacillus lactimorbus Jordan and Harris, Jour. Amer. Med. Assoc., 50, 1908, 1669 (see also Jour. Inf. Dis., 6, 1909, 465) ; Bacillus serositidis Lacorte, Memorias do Instit. Oswaldo Cruz, 26, 1932, 1.

There has been considerable confusion over the correct name to be applied to the non-pathogenic aerobic organisms resembling Clostridium tetani. Kruse (loc. cit.) isolated his cuture of Bacillus pseudotetanicus aerobius from a case of human tetanus. It was aerobic at ordinary temperatures but produced spores 
only at higher temperatures and under anaerobic conditions. Migula called this Bacillus pseudotetanicus. Ford (Jour. Bact., 1, 1916, 520) stated that this name had priority over Neide's Bacillus sphaericus which he thought was identical. On the other hand, Tavel (loc. cit.) isolated a pseudotetanus bacillus that was apparently anaerobic. Its spores were ellipsoidal and it formed more gas than the tetanus bacillus. Migula named this organism Bacillus pseudotetani. Subsequently both of Migula's names have been applied to the aerobic organism. Bacillus pseudoletaricus and Bacillus pseudotetani are nomina dubia and Bacillus sphaericus should therefore be used.

Spores : Spherical, 0.7 to 1.3 mierons in diameter, terminal to subterminal. Young spores in sporangia may be oval. Spore wall thick; remnants of sporangium may adhere.

Sporangia: Definitely swollen, clavate to spindle-shaped.

Rods : 0.6 to 1.0 by 1.5 to 7.0 microns, occurring singly or in short chains. On glucose agar cells contain few small fat globules. Motile. Gram-variable; often Gram-negative with Gram-positive granules.

Gelatin stab: Scant growth. No liquefaction. Gelatin hydrolyzed if tested by the Frazier technic.

Agar colonies : Small, thin, flat, translucent, often spreading over the plate.

Giant agar colonies: If the surface of the agar is fairly dry, many strains exhibit minute colonies that swarm out from the point of inoculation and cover the plate (cf. Bacillus alvei and Bacillus circulans).

Agar slants: Growth thin, smooth, spreading, translucent, becoming yellowish-brown. Growth occurs at pH 6.0.

Broth: Uniform turbidity. Glucose broth cultures have $\mathrm{pH}$ of 8.3 to 8.6 after 7 days.

Milk: No change.

Milk agar plate: Scant, if any, hydrolysis of casein.
Potato: Growth seant, thin, spreading, soft, gray, becoming yellowish-brown with age.

Nitrites not formed from nitrates.

Starch not hydrolyzed.

No acid from carbohydrates.

Acetylmethylcarbinol not produced.

Citrates not utilized.

Urease not formed.

Salt tolerant. Growth occurs in broth containing 4 per cent $\mathrm{NaCl}$.

Optimum temperature about $30^{\circ} \mathrm{C}$. Maximum temperature allowing growth $40^{\circ} \mathrm{C}$ to $45^{\circ} \mathrm{C}$.

Not pathogenic for guinca pigs.

Aerobic, facultative.

Source: From mud of a pond, rotting cypress wood, rotting oak wood, and from soil.

Habilat: Widely distributed in nature.

Bacillus rotans Roberts (Jour. Bact., 29, 1935, 229) differs from Bacillus sphaericus in that it will not grow as low as pH 6.0 nor in broth containing 4 per cent $\mathrm{NaCl}$. Originally characterized by motile colonies, this phenomenon has been noted with certain other members of the genus and with some strains of Bacillus sphaericus. Smith, Gordon, and Clark (loc. cit.) consider it a variety of Bacillus sphaericus.

Source: From intestine of a termite.

Habitat: Probably widespread in soil.

18a. Bacillus sphaericus var. fusiformis comb. nov. (Bacillus fusiformis Gottheil, Cent.f. Bakt., II Abt., 7, 1901, 724.) From Latin fusus, spindle; formis, shape.

This organism differs from Bacillus sphaericus only in that it produces urease.

Source: One strain isolated from Beta vulgaris lutea (beet). Also from milk, dust, soil and contaminated hirudin.

Habitat: Widely distributed in nature.

Bacillus loehnisii Gibson (Jour. Bact., $29,1935,495$ ) is very closely related to the above. It will not grow at $\mathrm{pH} 6.0$ or below, prefers media containing urea, and produces nitrites from nitrates. Gibson (loc. cit., 500) in discussing the 
organisms of this group stated "each species contains strains dissimilar in several features and each is connected to the others by transitional forms"'. Smith, Gordon, and Clark (loc. cit.) tentatively have placed it as a variety of Bacillus sphaericus.

Source: From soil.

Habitat: Widely distributed in soil.

19. Bacillus pasteurii (Miquel) Chester. (Urobacillus pasteurii Miquel, Ann. Micrographie, 1, 1889, 552; sive Bacillus ureae $\alpha$, ibid., 2, 1890, 13, 53, 122, 145, 367, 488; Chester, Ann. Rept. Del. Col. Agr. Exp. Sta., 10, 1898, 110.) Named for the French scientist. Iouis Pasteur (1822-1895).

Viehover, Cent. f. Bakt., II Abt., 39, 1913, 209, gave the following as possible synonyms: L'robacillus maddoxii Miquel, Ann. Micrographie, 3, 1891, 27.5 and 305 (Bacterium maddoxi Chester, Ann. Rept. Del. Col. Agr. Exp. Sta., 9, 1897, 98; Bacillus maddoxi Chester, ibid., 10, 1898, 110); Urobacillus leubei Beijerinck, Cent. f. Bakt., II Abt., 7, 1901, 51.

Synonyms according to Gibson, Jour. Bact., 29, 1935, 494 and 496: Bacillus probatus Viehover, Cent. f. Bakt., II Abt., 39, 1913, 209; Urobacillus psychrocartericus and Urobacillus hesmogenes Rubentschik, Cent. f. Bakt., II Abt., 66, 1925, 166 (Bacillus psychrocartericus and Bacillus hesmogenes Bergey et al., Manual, 3rd ed., 1930, 403, 404). Gibson also included the following as possibly identical with the above although they were incompletely described: Bacillus ureae II and III Burri, Herfeldt and Stutzer, Jour. Landw., 42, 1894, 329; Urobacillus duclauxii Miquel, Ann. Micrographie, 2, $1890,53,122$, and 145 ; Urobacillus maddoxii Miquel, ibid., 3, 1891, 275 and 305.

Description taken from Löhnis and Kuntze, Cent. f. Bakt., II Abt., 20, 1908, 684; Gibson, Jour. Bact., 28, 1934, 295 and 313; Smith, Gordon, and Clark (loc. cit.).

This species has been designated as the type species of the genus Urobacillus Miquel (Ann. de. Micrographie, 1, 1889,
517) by Enlows (U. S. Pub. Health Ser., Hyg. Lab. Bull. 121, 1920, 96).

Spores: Spherical, 1.0 to 1.2 microns, terminal to subterminal.

Sporangia: Prevailingly clavate. Not in chains.

Rods : 0.7 to 0.8 by 1.5 to 2.0 microns (1.0 to 1.5 by 4.0 to 5.0 microns, Löhnis and Kuntze), occurring singly or in pairs. Motile. Gram-variable.

Urea gelatin stab: Slow liquefaction.

Urea agar colonies: Small, entire, not characteristic.

Urea agar slope: Growth thin, very little spreading, colorless or white to yellowish. Will not grow at $\mathrm{pH} 6.0$ or less.

Urea broth: Moderate to heavy uniform turbidity. Will grow with 4 per cent $\mathrm{NaCl}$ added.

Nitrites produced from nitrates in urea nitrate nutrient broth.

Starch not hydrolyzed.

Carbohydrates not attacked.

Acetylmethylcarbinol not formed.

Urease produced.

Optimum temperature about $30^{\circ} \mathrm{C}$, minimum $6^{\circ} \mathrm{C}$. Maximum temperature allowing growth $40^{\circ} \mathrm{C}$ in water bath. Optimum temperature for urease activity $50^{\circ} \mathrm{C}$.

\section{Aerobic.}

The distinguishing character of this species is that growth oceurs only in peptone media containing urea or free ammonia under neutral or alkaline conditions.

Source: From decomposing urine.

Habitat: Widely distributed in soil, dust, manure, and sewage.

20. Bacillus thermoamylolyticus Coolhaas. (Cent. f. Bakt., II Abt., 75, 1928, 344.) From Greek thermos, hot, amylon, starch, and lytikos, able to loose; hence, dissolving. Probably intended to mean thermophilic and starch digesting.

Spores: Slightly elongated, ellipsoids 0.6 by 1.5 microns, central.

Sporangia: Cylindrical, not swollen, not in chains. 
Rods: 0.6 by 5 to 8 microus. Motile. Gram-positive.

Gelatin stab : No liquefaction.

Agar colonies: At $60^{\circ} \mathrm{C}$ of two types, large and small, circular, translucent, granular, slimy.

Broth: Very weak growth, no surface growth, no sediment.

Milk: Not coagulated, slowly peptonized.

Potato: Slight growth.

Nitrites produced from nitrates.

Starch actively hydrolyzed.

Acid and gas from glucose, fructose, galactose, maltose, dextrin, starch and glycerol. Arabinose, xylose and mannitol not fermented

Thermophilic, optimum temperature $50^{\circ}$ to $55^{\circ} \mathrm{C}$.

Aerobic.

Source: From sewage.

Habitat: Probably in decaying matter.

21. Bacillus kaustophilus Prickett. (N. Y. Agr. Exp. Sta. Teeh. Bul. 147, 1928, 38.) From Greek kaustós, burnt, red-hot; philos, loving; heat-loving.

Spores: Ellipsoidal, 0.5 by 0.6 to 0.8 micron, terminal to suhterminal. No free spores obscrved.

Sporangia: Only slightly swollen if at all.

Rods: On yeast extract-nutrient agar at $56^{\circ} \mathrm{C}, 0.7$ by 2.0 to 4.5 microns, with rounded ends. Actively motile. Grampositive.

Gelatin stab: No growth at $20^{\circ} \mathrm{C}$. Liquefied at $56^{\circ} \mathrm{C}$.

Agar colonies: At $56^{\circ} \mathrm{C}$, circular, convex, smooth. Borders entire to irregular. Show curled structure, strands of chains. Brown by transmitted light.

Agar slant: Growth abundant, raised, glistening, contoured, bluish-green to bluish-white by transmitted light. After three weeks at $37^{\circ} \mathrm{C}$, growth has a distinct reddish-brown color, butyrous, viscid.

Broth: Slightly turbid, no sediment. No surface growth, alkaline.

Litmus milk: Rennet coagulum, peptonization feeble, litmus reduced.
Potato: Amount of growth variable, brownish, spreading, glistening, slimy. Some strains do not grow.

Nitrites produced from nitrates, often with the production of nitrogen.

Starch is hydrolyzed.

Acid from glucose and salicin. Rhamnose, maltose, sucrose, raffinose, mannitol, sorbitol and inulin not fermented.

Acetylmethylearbinol not produced.

Thermophilic, optimum temperature $60^{\circ} \mathrm{C}$ to $65^{\circ} \mathrm{C}$. Growth at $73^{\circ} \mathrm{C}$ to $75^{\circ} \mathrm{C}$ but none at $80^{\circ} \mathrm{C}$ on agar slants.

Aerobic, facultative.

Good growth occurs in synthetic media containing potassium nitrate, sodium ammonium phosphate, aspartic acid, and sodium asparaginate, respectively, as only sources of nitrogen with glucose as source of carbon.

Source: Forty-eight cultures isolated from pastequrized milk at a single milk plant (Buffalo, X. Y.).

Habitat: Probably originally from soil and dust.

Thermobacillus digestans Feirer (Soil Sci., 23, 1927, 50) seems to be closely related to the preceding. It is more strongly proteolytic, digesting milk completely in 7 days.

Source: Four strains isolated from soil.

21a. Bacillus pepo shaw. (Jour. Inf. Dis., 43, 1928, 473.) From Cucurbita pepo, the pumpkin.

From the brief original description, this organism seems to vary from Bacillus kaustophilus only in its distinctive viscid or slimy character.

Source: Two cultures isolated from swelled cans of pumpkin.

Habitat: Probably found in soil and dust.

22. Bacillus thermoindifferens Weinzirl. (Jour. Med. Research, 39, 1919, 402.) From Greek thermos, hot and Latin indifferens, indifferent. Indifferent to or tolerant of heat .

Spores: Ellipsoidal, 0.5 by 0.8 micron, terminal. 
Sporangia: Cylindrical, not swollen.

Rods : 0.7 by 2.0 to 4.5 microns, occurring singly and in short chains, with rounded ends. Motile. Gram-positive.

Gelatin stab: Growth filiform. Slow infundibuliform liquefaction.

Agar colonies: Circular, convex, smooth, entire, amorphous.

Agar slant: Growth flat, spreading, glistening, translucent, butyrous, contoured.

Broth: Turbid, abundant sediment. No surface growth.

Litmus milk: Alkaline. Litmus reduced.

Potato: No growth.

Indole not formed.

Nitrites not produced from nitrates.

Starch is hydrolyzed.

Acid from glucose. No acid from lactose, sucrose or mannitol.

Thermophilic, optimum temperature $55^{\circ} \mathrm{C}$. Grows at $20^{\circ}$ to $37^{\circ} \mathrm{C}$.

Aerobic.

Source: Isolated from canned pumpkin.

Habitat: Probably found in soil and dust.

Thermobacillus reductans Feirer (Soil Sci., 23, 1927, 51) is said to resemble Bacillus thermoindifferens except that nitrites are formed from nitrates and the minimum temperature is $40^{\circ} \mathrm{C}$.

Source: Two strains isolated from soil.

Thermobacillus catenatus Feirer (Soil Sci., 23, 1927, 53) may be related to this group. The description is very incomplete. Its distinctive feature is the production of indole.

Source: Two strains isolated from soil.

23. Bacillus thermodiastaticus Bergey et al. (Type 1, Berger, Jour. Bact., 4, 1919, 304; Bergey et al., Manual, 1st ed., 1923, 310.) From Greek thermos, hot and diastalikos, separative; M. L., enzymatic, diastatic; hence diastatic at high temperatures.

Spores: Of less diameter than that of the rods, ellipsoidal, central.

Sporangia: Cylindrical.

Rods: 0.5 to 0.7 by 2 to 3 microns, occurring in chains, with square ends. Motile with peritrichous flagella. Grampositive.

Gelatin stab: Liquefaction.

Agar colonies: Grayish, spreading, with lobate to fimbriate borders.

Agar slant: Growth thin, limited, bluish-gray.

Broth: Turbid.

Litmus milk: Not coagulated, peptonized.

Potato: Growth slight, grayish.

Titrites produced from nitrates.

Starch is hydrolyzed.

Thermophilic, optimum temperature $65^{\circ} \mathrm{C}$. No growth at $50^{\circ} \mathrm{C}$. Growth at $75^{\circ} \mathrm{C}$.

Aerobic.

Source: Isolated from dust and contaminated milk.

Habitat: Probably widely distributed in soil and dust.

Thermobacillus diastasius Feirer (Soil Sci., 23, 1927, 49) differs from Bacillus thermodiastaticus only in that nitrites are not formed from nitrates (Feirer).

Source: Two strains isolated from soil.

24. Bacillus cylindricus Blau. (Cent. f. Bakt., II Abt., 15, 1905, 119.) From Greek kylindrikos, cylindrical.

Spores : Somewhat elongated, 0.7 to 1.1 by 1.8 to 2.5 microns, terminal. Remnants of sporangium adherent. Germination equatorial and oblique.

Sporangia: Cylindrical or only slightly swollen at end, not in chains.

Rods: On glucose agar at $60^{\circ} \mathrm{C}, 0.8$ to 1.1 by 5.0 to 7.5 microns, occurring singly and in pairs. Motile with peritrichous flagella. Cells store glycogen. Gram-positive.

Gelatin stab: No liquefaction.

Glucose agar colonies: Grayish-white, entire to lobed to dentate. By transmitted light yellowish-brown centers with brownish-yellow borders. Finely fibrous structure.

Glucose agar slant: Growth thin, dull, grayish-white.

Litmus milk: Unchanged. 
Potato: No growth.

Nitrites not produced from nitrates.

Starch not hydrolyzed.

Thermophilic, optimum temperature $60^{\circ} \mathrm{C}$ to $70^{\circ} \mathrm{C}$.

Source: Isolated from moist field soil in Germany .

Habitat: Probably found in dust and soil.

Bacillus calidus Blau (Cent. f. Bakt., II Abt., 15, 1905, 134) differs so little from the preceding species that it cannot be considered as distinct.

Source: Isolated from field soil in Germany (Blau). Dust, ground feeds, ete., about dairies and various dairy products (Prickett, N. Y. Agr. Exp. Sta. Tech. Bul.147, 1928, 45).

25. Bacillus robustus Blau. (Cent. f. Bakt., II Abt., 15, 1905, 126.) From Latin robustus, oaken, hard, firm.

Spores: IIllipsoidal, 1.0 by 1.6 to 2.2 microns, polar to medial. Remnants of sporangium not adherent. Germination prevailingly equatorial.

Sporangia: Ellipsoidal to eylindrical, not in chains.

Rods: 1.0 to 1.2 by 3 to 4 microns, occurring singly and in short chains. Motile. Gram-positive.

Glucose agar colonies: Circular, graywhite. By transmitted light brownishyellow. Borders distinct, serrate to lobed, finely granular.

Glucose agar slant: Growth yellowishwhite, translucent, becoming grayishwhite, spreading, dull.

Potato: Growth yellowish-white, moist, glistening, smooth.

Nitrites not produced from nitrates.

Starch not hydrolyzed.

Thermophilic, optimum temperature $55^{\circ} \mathrm{C}$ to $60^{\circ} \mathrm{C}$. Grows at $65^{\circ} \mathrm{C}$.

Aerobic.

Source: Isolated from field soil near a forest in Germany.

Habitat: Probably found in soil and dust.

Thermobacillus restatus Feirer (Soil Sci., 23, 1927, 51) is said to correspond in some respects with Bacillus robustus. Feirer states that it is not possible to definitely establish the identity because Blau failed to record the action of $\mathrm{Ba}$ cillus robustus on nitrates and several other media and did not note the production of $\mathrm{H}_{2} \mathrm{~S}$.

Source: Three strains isolated from soil.

25a. Bacillus losanitchii Bergey et al. (Bacillus thermophilus losanitchii Georgevitch, Cent. f. Bakt., II Abt., 27, 1910, 164; Bergey et al., Manual, 1st ed., 1923, 313.) Named for Losanitch, near Vranje.

As far as can be determined from the meager description, this organism does not differ from Bacillus robustus except perhaps as to the maximum temperature allowing growth. Growth limits are $45^{\circ} \mathrm{C}$ to $78^{\circ} \mathrm{C}$.

Source: Isolated from water of hot sulfur spring. Temperature of water $83^{\circ} \mathrm{C}$

Habitat: Probably in natural hot waters.

Note: Georgevitch (Arch. f. Hyg., 72, 1910,201 ) has described a thermophilic aerobic spore-forming sulfur bacillus from a hot sulfur spring at Vranje (Servia) under the name Bacillus thermophilus vranjensis. This does not grow on ordinary media unless sulfur compounds are added. It has a tuft of flagella at either end. Spores are ellipsoidal, terminal, distend the rod, and show polar germination.

Georgevitch (Cent. f. Bakt., II Abt., $27,1910,150)$ describes a second thermophilic, motile, capsulated, ellipsoidal. spored rod from a chalybeate hot spring near Vranje under the name Bacillus thermophilus jiroini.

26. Bacillus calidolactis Hussong and Hammer. (Jour. Bact., 15, 1928, 186.) From Latin calidus, warm, hot and lac, lactis, milk.

Gorini states (R. Ist. Lombardo Sci. e Lett. Rend., 76, 1942, 3) that Bacillus 
calidolactis is the same organism as Bacillus lactis termophilus (sic) Gorini (Giorn. d. R. Soc. Ital. d'Igiene, 16, 1894, 16). From the descriptions this appears to be probable.

Spores: Ellipsoidal, of slightly greater diameter than the rods, terminal.

Sporangia: Slightly swollen, clavate.

Rods : 0.7 to 1.4 by 2.6 to 5.0 microns, occurring singly, in pairs and short chains. Ton-motile. Gram-positive, some cells becoming Gram-negative with age.

Gelatin stab: No liquefaction.

No growth on plain nutrient agar.

Glucose agar colonies: Thin, white, opaque, filamentous.

Glucose agar slant: Growth abundant, echinate, dull, white.

Glucose agar stab: Growth abundant, beaded, gray.

Glucose broth: Turbid.

Litmus milk: Acid, coagulation. Litmus reduced.

Potato: No growth.

Nitrites produced from nitrates by some strains.

Acid from glucose, lactose, fructose, galactose and maltose. No acid from inulin, sucrose or glycerol.

Thermophilic, optimum temperature $55^{\circ} \mathrm{C}$ to $65^{\circ} \mathrm{C}$. No growth at $37^{\circ} \mathrm{C}$.

Aerobic, facultative.

Source: Isolated from normal pasteurized skim milk (Hussong and Hammer). Milk and milk powder (Prickett, $\mathrm{X}$. $\mathrm{X}$. Agr. Exp. Sta. Tech. Bul. 147, 1928, 47).

Habitat: Probably in dairy products.

27. Bacillus michaelisii Prickett. (Bacillus thermophilus aquatilis liquefaciens Michaelis, Arch. f. Hyg., 36, 1899, 285; Prickett, N. Y. Agr. Exp. Sta. Tech. Bul. 147, 1928, 45.) Named for Georg Michaelis of Berlin who first isolated the species.

Spores: Of greater diameter than the rods, terminal.

Sporangia: Swollen, clavate.

Rods: 0.6 to 0.8 by 2 to 4 microns. Motile. Gram-positive.
Gelatin stab: Liquefaction.

Agar colonies : Circular, raised, smooth, glistening.

Agar slant: Growth moderate, smooth, glistening.

Broth: Slight turbidity.

Milk: Not coagulated, alkaline.

Potato: Growth moist, glistening, yellowish, becoming brownish.

Nitrites with gas produced from nitrates.

Starch is hydrolyzed.

Acid from glucose and sucrose. No acid from rhamnose, maltose, lactose, glycerol, mannitol or inulin.

Thermophilic, optimum temperature $50^{\circ} \mathrm{C}$ to $60^{\circ} \mathrm{C}$.

Aerobie, facultative.

Source: Isolated from fountain waters (Michaelis). From fodder, dust, dairy utensils (Prickett).

Habitat: Probably found in soil and dust.

27ia. Bacillus lobatus Bergey et al. (Type 3, Bergey, Jour. Bact., 4, 1919, 304; Bergey et al., Manual, 1st ed., 1923, 311.) From Greek lobatos, having the form of a lobe.

Judging from the meager description, there is no essential difference between this organism and the preceding.

Source: Isolated from dust, soil, and horse manure.

Habitat: Probably widely distributed in soil and decaying matter.

Bacillus nondiastaticus Bergey et al. (Type 2, Bergey, Jour. Bact., 4, 1919, 304; Bergey et al., Manual, 1st ed., 1923, 310.) From Greek, no diastase.

The description of this organism is practically identical with Bacillus lobatus, the only difference noted being that this species hydrolyzes starch while Bacillus nondiastaticus does not.

Source: Isolated from dust and soil (Bergey). Ground grains, raw and pasteurized milk (Prickett, N. Y. Agr. Exp. Sta. Tech. Bul. 147, 1928, 47).

Thermobacillus vulgaris Feirer (Soil Sci., 23, 1927, 50) liquefies gelatin, does 
not reduce nitrates to nitrites nor alter litmus milk. According to Feirer it is otherwise similar to Bacillus nondiastaticus.

Source: Two strains isolated from soil.

27b. Bacillus thermononliquefaciens Bergey et al. (Type 4, Bergey, Jour. Bact., 4, 1919, 304; Bergey et al., Manual, 1st ed., 1923, 312.) From Greek thermos, hot; and Latin non, not and liquefaciens, making liquid. Probably intended to mean thermophilic and non-gelatinliquefying.

Aside from the non-liquefaction of gelatin, there seems to be no difference in the description of this organism and the two immediately preceding.

Source: Isolated from dust, soil, and horse manure.

Habitat: Probably found in soil and decaying matter.

28. Bacillus thermotranslucens Bergey et al. (Type 5, var. b, Bergey, Jour. Bact., 4, 1919, 304; Bergey et al., Manual, 1st ed., 1923, 312.) From Greek thermos. hot and Latin translucens, translucent. Probably intended to nean thermophilic and translucent.

Spores: Of larger diameter than the rods, terminal.

Sporangia: Terminally swollen, clavate, not in chains.

Rods : 0.3 to 0.4 by 1.0 to 1.5 microns, occurring singly. Motile with peritrichous flagella. Gram-positive.

Gelatin stab: No liquefaction.

Agar colonies: Thin, transparent, spreading widely.

Agar slant: Growth thin, spreading, veil-like.

Broth: Turbid.

Litmus milk: Not coagulated, slightly acid.

Potato: No growth.

Nitrites not produced from nitrates.

Starch slightly hydrolyzed.

Thermophilir, optimum temperature $60^{\circ} \mathrm{C}$. Slight grow th at $37^{\circ} \mathrm{C}$. No Nrowth at $70^{\circ} \mathrm{C}$.
Aerobic.

Source: Isolated from guinea pig feces, dust and from cheese.

Habitat: Probably found in soil and decaying matter.

Thermobacillus linearius Feirer (Soil Sci., 23, 1927, 53) is said to be similar in some respects to the preceding. Feirer states that formation of acid from several sugars is the distinctive feature of this species, a character not mentioned by Bergey.

Source: Five strains isolated from soil.

2Sa. Bacillus stcarothermophilus Donk. (Jour. Bact., 5, 1920, 373.) From Greek stérir, tallow and thermophilos, heatloving. Intended meaning obscure.

From the descriptions, the vegetative rods of this organism seem to be slightly larger than the preceding, otherwise no difference is noted.

Source: Isolated from samples of spoiled canned corn and string beans.

Habitat: Probably found in soil and dust.

28b. Bacillus aerothermophilus Weinzirl. (Jour. Med. Research, 39, 1919, 40.3.) From Greek aeros, air and thermophilus, heat-loving. Probably intended to mean aerobic and thermophilic.

There is nothing in the original account of this organism which is at variance with that of the preceding.

Source: Isolated from canned string beans (Weinzirl). From milk, water, hay, dust, beef extract, and agar (Pricket.t, N. Y. Agr. Exp. Station Tech. Bull. $147,1928,46$ ).

Habitat: Probably found in soil and dust.

Thermobacillus alcalinus Feirer (Soil Sci., 23, 1927, 52) is said to differ from the preceding in that it does not change litmus milk.

Source: Four strains isolated from soil.

Thermobacillus nuber Feirer (Soil Sci., $23,1927,52$ ) apparently is closely related to this group. Its distinguishing character is the production of a pink pigment 
in meat, brain, and blood serum, no color on other media.

Source: Isolated from soil.

\section{Bacillus thermocellulolyticus Cool-} haas. (Cent. f. Bakt., II Abt., 76, 1928, 43.) From Greek thormos, hot; and Latin cellula, a small room; M. L., cellulose and Greek lytikos, dissolving. Probably intended to mean thermophilic and cellulose-digesting.

Spores: Ellipsoidal, 0.8 by 1.5 microns, terminal.

Sporangia : Terminally swollen, clavate.

Rods : 0.3 by 3.5 to 6 microns, occurring singly and in pairs. No reserve material. Non-motile. Gram-positive.

Gelatin stab: No liquefaction.

Glucose agar colonies: Small, glistening, translucent.

Cellulose agar colonies: Circular, borders undulate to lobate.

Broth: Slight growth, no surface growth or sediment.

Milk: No growth.

Nitrites not produced from nitrates.

Starch is hydrolyzed.

No acid from carbohydrates.

Cellulose hydrolyzed.

Thermophilic, optimum temperature $50^{\circ} \mathrm{C}$ to $55^{\circ} \mathrm{C}$. Maximum $60^{\circ} \mathrm{C}$ to $65^{\circ} \mathrm{C}$. Minimum $35^{\circ} \mathrm{C}$ to $37^{\circ} \mathrm{C}$.

Aerobic, facultative.

Source: Isolated from sewage.

Habitat: Probably found in decaying matter.

\section{Bacillus thermoalimentophilus}

Weinzirl. (Jour. Med. Research, 39, 1919, 404.) From Greek thermos, hot; Latin alimentum, food; and Greek philos, loving. Loving hot food.

Spores: Ellipsoidal, 0.8 by 1.0 micron, terminal.

Sporangia: Swollen, clavate, not in chains.

Rods: 0.6 by 3.0 microns, occurring singly, with rounded ends. Motile, flagella not stated. Gram-positive.

Gelatin stab : No growth at $20^{\circ} \mathrm{C}$.
Agar colonies: Circular, raised, smooth, amorphous, entire.

Agar slant: Growth spreading to effuse, smooth, glistening, butyrous.

Broth: Turbid, surface ring.

Litmus milk: Unchanged.

Potato: No growth.

Nitrites with gas produced from nitrates.

Starch not hydrolyzed.

Neither acid nor gas from glucose, lactose, sucrose or mannitol.

Thermophilic, optimum temperature $55^{\circ} \mathrm{C}$. No growth at $20^{\circ} \mathrm{C}$. Growth at $37^{\circ} \mathrm{C}$.

\section{Aerobic.}

Source: Isolated from canned blueberries (Weinzirl). Pasteurized milk and filter cloth (Prickett, N. Y. Agr. Exp. Sta. Tech. Bul. 147, 1928, 46).

Habitat: Probably found in soil and dust.

Thermobacillus violaceus Feirer (Soil Sci., 23, 1927, 52) corresponds in some respeets with the preceding. Feirer also states that his cultures did not reduce nitrates to nitrites and produced acid on glucose and sucrose.

Source: Four strains isolated from soil.

31. Bacillus thermoliquefaciens Bergey et al. (Type 5, var. a, Bergey, Jour. Bact., 4, 1919, 304; Bergey et al., Manual, 1st ed., 1923, 313.) From Greek thermos, hot, and Latin liquefaciens, liquefying. Probably intended to mean thermophilic and gelatin-liquefying.

Spores: Ellipsoidal, polar, of greater diameter than the rods.

Sporangia: Terminally swollen, clavate.

Rods: 0.2 to 0.4 by 2 to 3 microns, occurring singly, with rounded ends. Motile with peritrichous flagella. Grampositive.

Gelatin stab: Liquefaction.

Agar colonies: Moderately dense, lobate.

Agar slant: Growth dense, grayish, lobate to fimbriate margins.

Litmus milk: Coagulated, acid. Litmus reduced. 
Potato: No growth.

Nitrites and ammonia produced from nitrates.

Starch not hydrolyzed.

Thermophilic, optimum temperature $60^{\circ} \mathrm{C}$. Slight growth at $37^{\circ} \mathrm{C}$. No growth at $70^{\circ} \mathrm{C}$.

Aerobic.

Source: Isolated from dust, guinea pig feces and horse manure (Bergey). Water and milk (Prickett, N. Y. Agr. Exp. Sta. Tech. Bul. 147, 1928, 47).

Habitat: Probably originally from soil nd dust.

32. Bacillus tostus Blau. (Cent. f. Bakt., II Abt., 15, 1905, 130.) From Latin tostus, parched, dried.

Spores: Ellipsoidal, 0.8 to 1.6 by 1.5 to 2.2 microns. Germination prevailingly polar.

Sporangia : Terminally swollen, clavate, not in chains.

Rods : 1.2 by 4.5 to 5.0 microns, occurring in pairs and in short chains. Cells store glycogen. Motile with peritrichous flagella.

Agar colonies: Small, circular, dense. By transmitted light bright yellow to yellowish-brown. Borders sharp, entire to lobate. Older colonies porcelain-like.

Agar slant: Growth thin, grayish-white, spreading, smooth, glistening.

Potato: No growth.

Nitrites not produced from nitrates.

Starch is hydrolyzed.

Ammonia is produced.

Thermophilic, optimum temperature $60^{\circ} \mathrm{C}$ to $70^{\circ} \mathrm{C}$.

Aerobic.

Source: Two cultures isolated from soil in Germany.

Habitat: Probably found in soil and dust.

33. Bacillus viridulus (Migula) Bergey et al. (Bacillus thermophilus II Rabinowitsch, Ztschr. f. Hyg., 20, 1895, 154; Bacterium viridulum Migula, Syst. der Bakt., Q, 1900, 343; Bacterium thermophilum II Chester, Manual, 1901, 186 ; Bacillus thermophilus Bergey et al., Manual, 1st ed., 1923, 315; Bergey et al., Manual, 4th ed., 1934, 464; not Bacillus thermophilus Miquel, Ann. d. Microg., 1, 1888, 6; not Bacillus thermophilus Chester, Man. Determ. Bact., 1901, 265.) From Latin, dim. adj. viridis, green, somewhat green.

Spores: Spherical, central.

Sporangia: Cylindrical, not swollen.

Rods: Rather large, slightly bent, occurring singly and in pairs. Nonmotile. Gram-positive.

Agar colonies: Irregular, spreading, granular, greenish.

Broth: Alkaline.

Potato: Grow th grayish-yellow ; margin undulate.

Nitrites not produced from nitrates.

Starch is hydrolyzed.

Thermophilic, grows at $60^{\circ} \mathrm{C}$ to $70^{\circ} \mathrm{C}$. Optimum temperature $62^{\circ} \mathrm{C}$. Grows at $33^{\circ} \mathrm{C}$.

Aerobic, facultative.

Source: Isolated from soil, snow, feces, corn grains.

Habitat: Probably found in soil and dust.

Appendix: The following additional aerobic spore-forming bacteria are found in the literature. Because of insufficient data it has not been possible to classify them. Some of these may be synonyms of well-known species, some may be varieties, whereas others may actually be separate species.

Aromabacillus weigmanni Omeliansky. (Isolated by Weigmann, 1890; Omeliansky, Jour. Bact., 8, 1923, 398.) From milk.

Bacillus abysseus ZoBell and Upham. (Bull. Scripps Inst. of Oceanography, Univ. Calif., 5, 1944, 273.) Subterminal spores. From marine mud.

Bacillus acidifaciens Patrick and Werkman. (Iowa State Coll. Jour. Sci., 7, 1933, 413.) One of a group characterized by the fermentation of xylan. A single culture isolated from decayed maple wood.

Bacillus acidificans presamigenes casei 
Gorini. (Rend. R. Accad. dei Lincei, 8, 1928, 598.) From manure, fodder and milk. Regarded by Gorini (personal communication, 1925) as identical with Bacillus circulans Jordan.

Bacillus acido-proteolyticus casei Gorini. (Le Lait, 9, 1912, 98.) From Parmesan and Emmenthal cheese. Regarded by Gorini (personal communication, 1925) as equivalent to one of the species of Tyrothrix of Duclaux.

Bacillus adametzi Trevisan. (Brauner Wasserbacillus, Adametz and Wichmann, Mittheil. d. oesterr. Versuchsstat. f. Brauerei u. Mälzerei, Wien, Heft 1, 1888, 51 ; Trevisan, I generi e le specie delle Batteriacee, 1889, 19; not Bacillus adametzii Migula, Syst. d. Bakt., 2, 1900, 686; Bacillus brunneus Eisenberg, Bakt. Diag., 3 Aufl., 1891, 142; Bacterium brunneum Migula, ibid., 331; not Bacterium brunneneum Schroeter, in Cohn, Beitr. z. Biol. d. Pflanz, 1, Heft 2, 1882, 125.) From water.

Bacillus adhaerens Laubach. (Jour. Bact., 1, 1916, 503.) One culture isolated from dust.

Bacillus aegyptiacus Werner. (Cent. f. Bakt., II Abt., 87, 1933, 459.) Good growth on $\mathrm{Ca}$ n-butyrate agar. One culture isolated from Egyptian soil.

Bacillus aerifaciens Steinhaus. (Jour. Bact., 42, 1941, 782.) Author states that it probably belongs to the Aerobacillus group. From triturated specimens of the white cabbage butterfly (Pieris rapae).

Bacillus aerobius von WahI. (Cent. f. Bakt., II Abt., 16, 1906, 496.) Reported to resemble Bacillus mesentericus fuscus. From canned peas.

Bacillus aerophilus Flügge. (Flügge, Die Mikroorganismen, 2 Aufl., 1886, 321; Bacterium aerophilum Chester, Man. Determ. Bact., 1901, 191.) From dust.

Bacillus afanassieff Trevisan. (Bacillus tussis convulsivae Afanassief, St. Petersburg. med. Wochnschr., 1887, No. $38-42$; not Bacillus tussis convulsivae Lehmann and Neumann, Bakt. Diag., 4 Aufl., 2, 1907, 269; Trevisan, I generi e le specie delle Batteriacee, 1889,$13 ; \mathrm{Ba}$ - cillus pertussis Migula, Syst. d. Bakt., 2, 1900, 754.) From mucus and pus.

Bacillus agilis Tschistowitsch. (Tschistowitsch, Berl. klin. Wochnschr., 1892, 512; not Bacillus agilis Trevisan, I generi e le specie delle Batteriacee, 1889, 14; not Bacillus agilis Chester, Man. Determ. Bact., 1901, 226; not Bacillus agilis Mattes, Sitzungsber. d. Gesellsch. z. Beforderung d. gesam. Naturw. z. Marburg, 62, 1927, 406; not Bacillus agilis Hauduroy et al., Dict. d. Bact. Path., Paris, 1937, 33.) From pus.

Bacillus agilis Hauduroy et al. ( $\mathrm{Ba}$ cillus agilis larvae Toumanoff, Bull. Soc. Cent. de Méd. Vétér., 80, 1927, 367; Hauduroy, Ehringer, Urbain, Guillot and Magrou, Dictionnaire de Bactéries Pathogènes, Paris, 1937, 33.) Found in foulbrood of bees.

Bacillus agrestis Werner. (Cent. f. Bakt., II Abt., 87, 1933, 468; not Bacillus agrestis de Rossi, Microbiol. agraria e technica, Torino, 1927, 828.) One of a group of species described as being able to grow on a $\mathrm{Ca}$-butyrate agar. Three cultures were isolated from German and Italian soils.

Bacillus agri Laubach and Rice. (Jour. Bact., 1, 1916, 516.) Isolated twice from soil in Baltimore.

Bacillus agrophilus Stührk. (Cent.f. Bakt., II Abt., 93, 1935, 189.) Only moderate growth on Ca n-butyrate agar. One culture isolated from soil from Cuba.

Bacillus agrotidis typhoides Pospelov. (Rept. Bur. Appl. Ent., Russian, 3, $1927,8$.$) Found in diseased larvae of$ the moth, Euxoa (Agrotis) segctum.

Bacillus (Streptobacter) albuminis Schroeter. (Bacillus aus Faeces V, Bienstock, Ztschr. f. klin. Med., 8, Heft 1, 1884, 1; Schroeter, in Cohn, Kryptog. Flora v. Schlesien, 3, 1, 1886, 162; Bacillus putrificus coli Flügge, Die Mikroorganismen, 2 Aufl., 1886, 303; Bacillus diaphthirus Trevisan, I generi e le specie delle Batteriacee, 1889, 15.) From feces.

Bacillus albus (Sack) Bergey et al. (Cellulomonas albus Sack, Cent. f. Bakt., II Abt., 62, 1924, 79; Bergey et al., Man- 
ual, 3rd ed., 1930, 398; not Bacillus ulbus Trevisan, I generi e le specie delle Batteriacee, 1889, 14; not Bacillus albus Copeland, Report Filtration Comm., Pittsburgh, 1899, 314.) Cellulose is hydrolyzed. From soil in Germany.

Bacillus alcalophilus Vedder. (Ned. Tijdschr. v. Hyg. Microbiol. en Serolog., 1, 1934, 141.) Grows only in and on highly alkaline culture media. Sixteen strains isolated from the feces of healthy animals.

Bacillus alopecuri Nogtev. (Botanicheskii Zhurnal, U.S.S.R., 23, 1938, 149.) Causes nodule formation on fox grass (Alopecurus pratensis).

Bacillus alpha Dyar. (Ann. N. Y. Acad. Sci., 8, 1895, 366.) From the air.

Bacillus alpinus Werner. (Cent. f. Bakt., II Abt., 87, 1933, 465.) Good growth on calcium salts of formic, acetic, propionic and butyric acids. One culture isolated from soil from Austria.

Bacillus alveolaris Kisenjoposky. (Review of pests of Volhymia, Volhymia Ent. Bur., Zemstvo of Volhymia, Zitomir, 1916, 24 pp.) From diseased bees (Apis mellifera).

Bacillus amarus Hammer. (Iowa Agr. Exp. Station Res. Bull. 52, 1919, 198.) From evaporated milk.

Bacillus aminovorans den Dooren de Jong. (Cent. f. Bakt., II Abt., 71, 1927, 215.) From soil.

Bacillus amyloaerobius Crimi. (Abst. in Cent. f. Bakt., II Abt., 61, 1924, 63.) From potato rot.

Bacillus amylolyticus Kellerman and MeBeth. (Cent. f. Bakt., II Abt., 34, 1912, 490.) Decomposes cellulose. One culture isolated from manure.

Bacillus annuliformis Migula. (Fadenähnlicher Bacillus, Masehek, Bakt. Untersuch. d. Leitmeritzer Trinkwässer, Leitmeritz, 1887; Migula, Syst. d. Bakt, 2, 1900, 783.) From water.

Bacillus anthracis similis McFarland. (Cent. f. Bakt., I Abt., 24, 1895, 556.) From dust.

Bacillus apicum Canestrini. (Atti Soc. Ven. Trent. Sci. Nat., 91 ; according to Kruse, in Flügge, Die Mikroorganismen, 3 Aufl., 2, 1896, 233.) From diseased bees and their larvae.

Bacillus aporrhoeus Fuller and Norman. (Jour. Bact., 46, 1943, 277.) From soil. Decomposes cellulose.

Bacillus arachnoideus Migula. (Bacillus No. III, Flügge, Ztschr. f. Hyg., 17, 1894, 294; Bacillus lactis No. III, Kiruse, in Flügge, Die Mikroorganismen, 3 Aufl., 2, 1896, 208; Migula, Syst. d. Bakt., 2, 1900, 583; Bacterium lacteum Migula, ibid., 321.) From milk.

Bacillus arenarius Stührk. (Cent. f. Bakt., II Abt., 93, 1935, 187.) Good growth on $\mathrm{Ca}$-butyrate agar. One strain isolated from Cuban soil.

Bacillus aridus Migula. (Bacillus No. 8, Pansini, Arch. f. path. Anat., 122, 1590, 44t; Migula, Syst. d. Bakt., 2, 1900, 559.) From sputum.

Bacillus arlongii (sic) DeToni and Trevisan. (Bacillus de la septicèmie gangrèneuse, Arloing and Chauveau, see Crookshank, Man. of Bact., 3rd ed., 1890, 305; DeToni and Trevisan, in Saccardo, Sylloge Fungorum, 8, 1889, 950.) From wounds in gangrenous septicaemia.

Bacillus asiaticus Sakharoff. (Sakharoff, Ann. Inst. Past., 8, 1893, 550; not Bacillus asiaticus Castellani, Cent.f. Bakt., I Abt., Orig., 65, 1912, 262.) From feces in a case of cholera.

Bacillus asteris Verona. (Riv. Pat. Veg., 25, 1935, 15.) Pathogenic for China aster (Aster chinensis).

Bacillus asthenogenes Bernard. (Ann. Inst. Past., 35, 1921, 459.) Grows aerobically as well as anaerobically. Under anaerobic conditions it is said to play a role in gastric derangement and infection commonly confused with beriberi. Author reports that it is very similar to Bacillus megatherium.

Bacillus aterrimus tschitensis Kilimenko. (Cent. f. Bakt., II Abt., 20, 1908,1 .) Reported to be like the black potato bacillus except that it forms a black pigment on gelatin and the potato is brown instead of black. From air.

Bacillus aurantius (Sack) Bergey et al. 
(Cellulomonas aurantius Sack, Cent. f. Bakt., II Abt., 62, 1924, 78; Bergey et al., Manual, 3rd ed., 1930, 421; not Bacillus aurantius Trevisan, I generi e le specie delle Batteriacee, 1889, 19.) From soil.

Bacillus badius Batchelor. (Jour. Bact., 4, 1919, 25.) From the intestinal tract of children.

Bacillus balcanus Bartels. (Cent. f. Bakt., II Abt., 103, 1940, 25.) Growth on media containing $\mathrm{m} / 50$ phenol. Eight strains isolated from soil.

Bacillus barbitistes Statelov. (Mitt. bulg. ent. Gesells., 7, 1932, 56-61.) From diseased tettigonids (Isophya (Barbitistes) amplipennis).

Bacillus batatae Otani. (Trans. Tottori Soc. Agric. Sci., Japan, 6, 1939, 222.) From rotten sweet potatoes (Ipomaea batatas).

Bacillus bellus Heigener. (Cent. f. Bakt., II Abt., 93, 1935, 96.) Probably a strain of Bacillus brevis. One culture isolated from garden soil of Germany.

Bacillus bernensis Lehmann and Neumann. (Aromabacillus, Burri, Cent. f. Bakt., II Abt., 3, 1897, 609; Lehmann and Neumann, Bakt. Diag., 2 Aufl., 2, 1899,$304 ;$ Bacillus odoratus Migula, Syst . d. Bakt., 2, 1900, 686; Bacterium odoratum Omeliansky, Jour. Bact., 8, 1923, 394.) From Emmenthal cheese. Reported as producing the aroma of this cheese.

Bacillus beta Dyar. (Ann. I. I. Acad. Sci., 8, 1895, 366.) From dust.

Bacillus betainovorans Heigener. (Cent. f. Bakt., II Abt., 93, 1935, 94.) Good growth on betaine and valine agar. One culture isolated from soil from Mantua.

Bacillus betanigrificans Cameron, Esty and Williams. (Food Research, 1, 1936, 75.) From blackened canned beets where juice contains an abnormally high amount of iron.

Bacillus biacutum Soriano. (Revista del Inst. Bacteriol., Univ. Buenos Aires, $6,1935,564$.) From soil.

Bacillus bombycis Macchiati. (Des vibrions, Pasteur, Études sur la maladie des vers à soie, La Flacherie, Chapitre
II, Paris, 1870; Macchiati, Stazioni sperimentali Agrarie Italiane, 20, 1891, 121 ; not Bacillus bombycis Chatton, Compt. rend. Acad. Sci., Paris, 156, 1913, 1708; Bacillus megaterium bombycis Sawamura, Tokyo Imp. Coll. Agric. Bull., 6, 1905, 375.) Pasteur originally isolated this large bacillus from the intestine of silkworms (Bombyx mori) suffering from wilt disease. Regarded by Sawamura as a variety of Bacillus megatherium.

Bacillus bombycis non-liquefaciens Paillot. (Ann. Epiphyt., 8, 1922, 131; L'infection chez les insectes, 1933, 288.) Larvae of the gypsy moth (Lymantria (ispar) are immune to this bacillus.

Bacillus bombycoides Paillot. (Compt. rend. Acad. Agr. France, 28, 1942, 158.) Causes lesions because of a bacterial toxin. From infected silkworms.

Bacillus bombysepticus Hartman. (Lingnan Sei. Jour., 10, 1931, 280.) Causes a disease of the silkworm (Bombyx mori).

Bacillus borborokoites ZoBell and Upham. (Bull. Scripps Inst. of Oceanography, Univ. Calif., 5, 1944, 274.) Central spores. From marine bottom deposits.

Bacillus borstelensis Stührk. (Cent. f. Bakt., II Abt., 93, 1935, 179.) Grows well on Ca n-butyrate agar. Resembles Bacillus rufescens of the same group except that it does not show the typical brown coloration of media. Тжо strains isolated from soils in Germany.

Bacillus bredemannii Chester. ( $B a$ cillus adhaerens Stührk, Cent. f. Bakt., II Abt., 93, 1935, 183; not Bacillus adhaerens Laubach, Jour. Bact., 1, 1916, 503; Chester, in Manual, 5th ed., 1939, 675.) Weak growth on Cit n-butyrate agar. One strain isolated from Cuban soil.

Bacillus bronchitidis Migula. (Bacillus der putriden Bronchitis, Lumnitzer, Cent. f. Bakt., 3, 1888, 621; Bacillus bronchitidis putridae Lumnitzer, Wien. med. Presse, 1888, 666 ; Migula, Syst. d. Bakt., 2, 1900, 641; Bacterium lumnitzeri Chester, Man. Determ. Bact., 1901, 120.) 
From sputum in cases of putrid bronchitis.

Bacillus bruneus Migula. (Maschek, Bakt. Untersuch. d. Leitmeritzer Trinkwasser, 1887; Migula, Syst. d. Bakt., 2, 1900, 835; not Bacillus brunneus Eisenberg, Bakt. Diag., 3 Aufl., 1891, 142.) From water.

Bacillus brunneus Eisenberg. (Brauner Wasserbacillus, Adametz and Wichmann, Die Bakt. d. Nutz- u. Trinkwässer, Mitth. Oesterreich. Versuchssta. f. Brauerei u. Mälzerei, Wien, Heft 1, 1888, 51; Eisenberg, Bakt. Diag., 3 Aufl., 1891, 142; not Bacillus brunneus Schroeter, in Cohn, Kryptog. Flora v. Schlesien, 3, 1, 1886, 158; Bacterium brunneum Migula, Syst. d. Bakt., 2, 1900, 331.) From water. Non-motile.

Bacillus butlerovii Serbinow. (Věst. Russ. obšč pčelovod. (Messager de la Soc. russe d'Apiculture), No. 3 and No. 11, 1912; see Rev. Appl. Entomol., Ser. A, 1, 1913, 94 and 4t1.) From black brood of bees.

Bacillus bütschlii Schaudinn. (Arch. f. Protistenkunde, 1, 1902, 306.) Characterized by its large size (3.0 to 6.0 by 24.0 to 80.0 microns) and granular protoplasm. From the intestine of a cockroach (Blatta (Periplaneta) orientalis).

Bacillus butyricus Hueppe. (Hueppe, Mitteil. kaiserl. Gesundheitsamte, 2, 1884, 309; not Bacillus butyricus Macé, Traité de Bact., 1st ed., 1888; not Bacillus butyricus Botkin, Ztschr. f. Hyg., 11, 1892, 121; Clostridium hueppei Trevisan, I generi e le specie delle Batteriacee, 1889, 22; Bacillus pseudobutyricus Kruse, in Flügge, Die Mikroorganismen, 3 Aufl., 2, 1896, 207; Bacillus hueppei Chester, Man. Determ. Bact., 1901, 276.)

Bacillus calfactor Miehe. (Arb. der deutsch. Landw. Gesel., Berlin, Heft 3, 1905, 76; Die Selbsterhitzung des Heues, Jena, 1907, 49.) Thought to be the most important thermogenic microorganism in the fermentation of hay. From heating hay.

\section{Bacillus canaliculatus Wilhelmy.}

(Arb. bakt. Inst. Karlsruhe, 3, 1903, 20.) From meat extract.

Bacillus canceris Migula. (Syst. d. Bakt., 2, 1900, 625.) From a case of stomach cancer.

Bacillus caniperda Migula. (Ovalbacillus der Hundestaupe, Galli-Valerio, Cent. f. Bakt., I Abt., 19, 1896, 694; Migula, Syst. d. Bakt., 2, 1900, 76t; Bacterium canis Chester, Man. Determ. Bact., 1901, 198.) From nasal mucus and urine of dogs.

Bacillus capillaceus Wright. (Mem. Nat. Acad. Sci., 7, 1895, 456.) From water.

Bacillus capsici Pavarino and Turconi. (Atti Istit. Bot. R. Univ. Pavia, 15, 1918, 207.) Causes leaf wilt of pepper (Capsicum annuum). May be identical with disease caused by Pseudomonas vesicatoria (Stapp, in Sorauer, Handbuch der Pflanzen-Krankheiten, 2, 5 Aufl., 1928, 262).

Bacillus carniphilus Wilhelmy. (Arb. bakt. Inst. Karlsruhe, 3, 1903, 19.) From meat extract.

Bacillus carnosus Zimmermann. (Tils, Bakt. Untersuch. d. Freiburg. Leitungswässer, Leipzig, 1890, No. 57; Zimmermann, Bakt. unserer Trink-u. Nutzwässer, Chemnitz, II Reihe, 1894, 4.) From water.

Bacillus catenulatus Bartels. (Cent.f. Bakt., II Abt., 103, 1940, 27.) Growth on media containing $\mathrm{M} / 100$ phenol. Four strains isolated from soil.

Bacillus cepae Bassalik and EdelszteinKosowa. (Acta Soc. Bot. Polon., 10, 1933, 519.) From diseased onions ( $A l$ lium cepa).

Bacillus cerealium Gentner. (Cent. f. Bakt., II Abt., 50, 1920, 428; Pseudomonas cerealia Stapp, in Sorauer, Handbuch der Pflanzen-Ǩrankheiten, 2, 1928, 22; Bacterium cerealinum Elliott, Manual Bacterial Plant Pathogens, 1930, 111.) Pathogenic for barley (Hordeum vulgare), rye (Secale cereale) and wheat (Triticum sp.).

Bacillus cinctus Ravenel. (Mem. Nat. Acad. Sci., 8, 1896, 30.) From soil. 
Bacillus cirroflagellosus ZoBell and Upham. (Bull. Scripps Inst of Oceanography, Univ. Calif., 5, 1944, 266.) Central spores. Found in marine mud.

Bacillus cladoi Trevisan. (Bacille pedunculé, Clado, Bull. Soc. Anat. Paris, 1887, 339; Trevisan, I generi e le specie delle Batteriacee, 1889, 14; Bacillus pendunculatus (sic) Eisenberg, Bakt. Diag., 3 Aufl., 1891, 340; Bacillus septicus vesicae Sternberg, Man. of Bact., 1893, 475.) From urine in a case of cystitis.

Bacillus closteroides Gray and Thornton. (Cent. f. Bakt., II Abt., 73, 1928, 93.) Decomposes phenol. Probably identical with or a variety of Bacillus circulans. Sixteen strains isolated from Rothamsted soils.

Bacillus coccineus Pansini. (Pansini, Arch. f. path. Anat., 122, 1890, 437; not Bacillus coccineus Catiano, in Cohn, Beitr. z. Biol. d. Pflanzen, 7, 1896, 339.) From sputum. Red pigment.

Bacillus colorans Libermann. (Jour. of Microbiol., Ukraine, 5, 1938, 73; abst. in Cent. f. Bakt., II Abt., 101, 1940, 81.) From fruit conserves containing 10 to 20 per cent sugar.

Bacillus comesii Rossi. (Ann. d. Scuola d. Agricult. in Portici, 1903; Arch. di Farmacologia sperim., 3, 1904, fase. 10.) Similar to Bacillus mesentericus. Said to have the ability to dissolve plant particles.

Bacillus concoctans Patrick and Werkman. (Iowa State Coll. Jour. Sci., \%, 1933, 415.) Ferments xylan. One culture isolated from soil.

Bacillus conjunctivitidis subtiliformis Michalski. (Cent. f. Bakt., I Abt., Orig., 36, 1904, 212.) From more than 50 cases of acute conjunctivitis. Similar to Bacillus subtilis.

Bacillus consolidus Stührk. (Cent.f. Bakt., II Abt., 93, 1935, 191.) Good growth on $\mathrm{Ca}$-butyrate agar. One strain isolated from Cuban soil.

Bacillus contextus Migula. (Bacillus $D$, Peters, Botan. Zeitung, 47, 1889; Migula, Syst. d. Bakt., 2, 1900, 522.) From leaven.
Bacillus corrugatus Migula. (Bacillus mesentericus vulgatus Flügge, Die Mikroorganismen, 2 Aufl., 1886, 322; Bacillus No. II, Flügge, Ztschr. f. Hyg., 17, 1894, 294 ; Bacillus lactis No. II, Kruse, in Flügge, Die Mikroorganismen, 3 Aufl., 2, 1896, 208; Migula, Syst. d. Bakt., 2, 1900, 583.) From milk.

Bacillus corruscans Schroeter. (In Cohn, Krryptog. Flora v. Schlesien, 3, 1, 1886, 158.) From cooked potato.

Bacillus costatus Lloyd. (Jour. Bact., 21, 1931, 94.) From sea water off Scotland. Nitrates and nitrites reduced to nitrogen.

Bacillus crinatus Chester. (Bacillus No. 5, Pansini, Arch. f. path. Anat., 122, 1890, 441; Chester, Man. Determ. Bact., 1901, 281.) From sputum.

Bacillus crinitus Wright. (Wright, Mem. Nat. Acad. Sci., 7, 1895, 453; Bacterium crinatum (sic) Chester, Man. Determ. Bact., 1901, 192.) From river water.

Bacillus crystalloides Dyar. (Dyar, Ann. N. Y. Acad. Sci., 8, 1895, 371; Bacterium crystaloides (sic) Chester, Man. Determ. Bact., 1901, 191.) From the air.

Bacillus cubensis Stührk. (Cent. f. Bakt., II Abt., 93, 1935, 192.) Good growth on $\mathrm{Ca}$ n-butyrate agar. Two cultures isolated from soils from Cuba.

Bacillus cystiformis Trevisan. (Bacille cystiforme, Clado, Bull. Soc. Anat. Paris, 1887, 339; Trevisan, I generi e le specie delle Batteriacee, 1889, 14.) From urine in a case of cystitis.

Bacillus cytaseus McBeth and Scales. (U. S. Dept. Agr., Bur. Plant Industry, Bull. 266, 1913, 39; Bacterium cytaseum Holland, Jour. Bact., 5, 1920, 218.) Requires cellulose for best growth. From decomposing materials and soil.

Bacillus cylaseus var. zonalis Fellerman et al. (Cent. f. Bakt., II Abt., 39, 1913,511.) From soil from Utah. While no spores were observed, this organism was like Bacillus cytaseus except that colonies on cellulose agar showed con- 
centric opaque or semi-opaque and transparent zones.

Bacillus danteci Kruse. (Bacille rouge de Terre-Neuve, Le Dantec, Ann. Inst. Past., 5, 1891, 662 ; Kruse, in Flügge, Die Mikroorganismen, 3 Aufl., 2, 1896, 270.) From reddened salt cod fish.

Bacillus daucarum von Wahl. (Cent. f. Bakt., II Abt., 16, 1906, 494.) Ipparently a strain of Bacillus subtilis. From canned carrots.

Bacillus demmei Trevisan. (Bacillus der Erythema nodosum, Demme, Fortschr. d. Med., 6, 1888, 257; Trevisan, I generi e le specie delle Batteriacee, 1889 , 14 ; Bacillus erythematis Kruse, in Flügge, Die Mikroorganismen, 3 Aufl., 2, 1896, 426; Bacillus erythematis maligni Kruse, ibid., 479; Bacterium erythematis Migula, Syst. d. Bakt., 2, 1900, 346.) From ery thema nodosum (skin)

Bacillus dendroides Holzmüller. (Cent. f. Bakt., II Abt., 23, 1909, 331.) From frog feces. Closely related to Bacillus mycoides.

Bacillus dendroides Thornton. (Thornton, Ann. Appl. Biol., 9, 1922, 247 ; not Bacillus dendroides Holzmüller, loc. cit.) Common in Rothamsted soil. Said to belong to the Bacillus subtilis group.

Bacillus dentatus Heigener. (Cent.f. Bakt., II Abt., 93, 1935, 106.) Good growth on valine agar. Two cultures isolated from soil of Jugoslavia and North Carolina.

Bacillus destruens von Wahl. (Cent. f. Bakt., II Abt., 16, 1906, 502.) From boiled asparagus.

Bacillus detrudens Wright. (Mem. Nat. Acad. Sci., 7, 1895, 452.) From mater.

Bacillus diastaticus Boyarska. (Izvestia Acad. Sci., L. S. S. R., Biol ser. 1941.) Thermophilic.

Bacillus disciformans Zimmermann. (Zimmermann, Bakt. unserer Trink. u. Nutzwässer, Chemnitz, II Reihe, 1894, 48; Bacterium disciformans Lehmann and Neumann, Bakt. Diag., 1 Aufl., 2, 1896, 238.) From waste water. Apparently not identical with Bacillus disci- formis Gräfenhan, although the name suggests possible relationship.

Bacillus disciformis Gräfenhan. (Inaug. Diss., Halle, 1891, 1.) From water. From the description, this organism may be Bacillus subtilis.

Bacillus distortus (Duclaux) Trevisan. (Tyrothrix distortus Duclaux, Ann. Inst. Nat. Agron., 4, 1852, 23; Trevisan, I generi e le specie delle Batteriacee, 1889, 16.) From milk.

Bacillus dobelli Dubosca and Grassé. (Arch. Zool. Expér. et Gén., 66, 1927, 457; Bacillus (Flexilis) dobelli Duboseq and Grassé, ibid., 487.) Similar to $\mathrm{Ba}$ cillus flexilis Dobell. Found in rectum of a termite (Calotermes (Glyptotermes) iridipennis). These authors suggest that Bacillus flexitis Dobell, Bacillus buischlii schatudinn and Bacillus dobelli be grouped under the name Flexilis.

Bacillus duclauxii (Miquel) Chester. (Bacillus ureae Miquel, Bull. Soc. Chim. d. Paris, 31, 1879, 391; Urobacillus duclauxii sive Bacillus ureae $\beta$ Miquel, Ann. d. Microg., 2, 1889-1890, 53, 122 and 145; Chester, Ann. Rept. Del. Col. Agr. Exp. Sta., 10, 1898, 123.) From water and soil.

Bacillus dysodes Zopf. (Die Spaltpilze, 3 Aufl., 1885, 90.) From fermenting dough.

Bacillus elegans Heigener. (Cent. f. Bakt., II Abt., 93, 1935, 103.) Four cultures isolated from soil, one from Jugoslavia and three from Germany.

Bacillus emulsionis Beijerinck. (Folia Microbiol., 1, 1912, 377; see Perquin, Jour. Microbiol. and Serol., 6, 1940, 226.) Produces slime in sucrose solutions.

Bacillus encephaloides Trevisan. (Bacille de l'air $f$, Babes, in Cornil and Babes, Les Bactéries, 1886, 150; Trevisan, I generi e le specie delle Batteriacee, 1889, 20.) From the air.

Bacillus enterothrix Collin. (Arch. Zool. Expér. et Gén., 51, 1913, Notes and Revue, No.3.) Found in the rectum of toad tadpoles (Alytes sp.).

Bacillus epidermidis (Bizzozero) Bordoni-Lffreduzzi. (Leptothrix epidermi- 
dis Bizzozero, Arch. f. path. Anat., 98 1884, 441; Bordoni-Ufireduzzi, Fortschr. d. Med., 4, 1856, 156; Carcinombacillus, Scheurlen, Deutsche med. Wochnschr. 1887, 1083; Bacillus mesentericus rubiginosus Senger, Cent. f. Bakt., 3, 1888, 603 ; Bacillus bizzozerianus Trevisan, I generi e le specie delle Batteriacee, 1889, 14; Bacillus scheurleni Dyar, Ann. I. I. Acad. Sci., 8, 1895, 367.) From the human mouth and skin. Macé (Traité pratique de Bact., th ed., 1901, 1071, says that this organism is the nrdinary potato barillus, i.e., Bacillus vulgatus.

Bacillus epiphytus ZoBell and Cpham. (Bull. Seripps Inst. of Oceanography, Univ. Calif., 5, 194t, 266.) Central spores. Found associated with marine phytoplankton.

Bacillus erodens Ravenel. (Mem. Nat. Acad. Sci., 8, 1896, 35.) From soil.

Bacillus esterificans Maassen. (Arb. a. d. Kaiserl. Gesundheitsamte, 15, 1899 , 504; Plectridium esterificans Huss, Cent f. Bakt., II Abt., 19, 1907, 52.) Found in a solution of decomposing litmus; also isolated from butter.

Bacillus evanidus Grohmann. (Morphologisch-biologische Beiträge zur Kenntnis der Wasserstoffbakterien, Inaug. Diss., Univ. Leipzig, 1923; Cent. f. Bakt., II Abt., 61, 1924, 207; Ruhland and Grohmann, Jahrb. Wissensch. Botanik, 63, 1924, 321.) Oxidizes hydrogen in the presence of oxygen to form water. Presumably widely distributed in soil.

Bacillus exiguus Saito. (Jour. Coll. Sci., Imp. Univ., Tokyo, 23, Art. 15, $1908,44$.$) Isolated 3$ times from garden air.

Bacillus exilis Bariels. (Cent. f Bakt., II Abt., 103, 1940, 29.) Growth in media containing $\mathrm{m} / \mathrm{100}$ phenol. Eight strains isolated from soil.

Bacillus fastidiosus den Dooren de Jong. (Cent. f. Bakt., II Abt., 79, 1929, 344.) Six strains isolated from unheated garden soil.

Bacillus ferrigenus Bargaglio-Petrucci. (Nuovo Giornale botanico italiano, 1913, 1914, 1915; quoted from De Rossi,
Nicrobiol. Agraria e Technica, 1927 , 904.) A facultative thermophile, growing up to $65^{\circ}$ to $70^{\circ} \mathrm{C}$.

Bacillus festinus McBeth. (Soil Sei., 1, 1916, 451.) Filter paper decomposed to a grayish-white felt-like mass. From soil in California.

Bacillus filamentosus Kilein. (Klein, see Migula, Syst. d. Bakt., 2, 1900, 285; Bacterium filamentosum Burchard, Arb. bakt. Inst. Karlsruhe, 2, Heft 1, 1902, 22.)

Bacillus filaris Migula. (Bacillus No. XI, Flügge, Ztschr. f. Hyg., 17, 1894, 296; Bacillus lactis No. XI, Kruse, in Flügge, Die Mikroorganismen, 3 Aufl., 2, 1896, 209; Migula, Syst. d. Bakt., 2, 1900, 579 ; Bacillus aromaticus Chester, Man. Determ. Bact., 1901, 276; not Bacillus aromaticus Pammell, Bull. No. 21, Iowa Agr. Exp. Sta., 1893, 792; not Bacillus aromaticus Grimm, Cent. f. Bakt., II Abt., 8, 1902, 584.) From milk.

Bacillus filicolonicus ZoBell and Upham. Bull. Scripps Inst. of Oceanography, Lniv. (alif., 5, 1944, 270.) Subterminal spores. From sea water and marine mud.

Bacillus filiformis (Duclaux) Trevisan. (Tyrothrix filiformis Duclaux, Ann. Inst. Nat. Agron., 4, 1882, 23; Trevisan, I generi e le specie delle Batteriacee, 1889 , 16 ; not Bacillus filiformis Tils, Ztschr. f. Hyg., 9, 1890, 317; not Bacillus filiformis Migula, Syst. d. Bakt., 2, 1900, 587.) From cheese.

Bacillus fissuratus Ravenel. (Mem. Nat. Acad. Sci., 8, 1896, 38.) From soil.

Bacillus fitzianus Zopf. (Fitz, Ber. d. deutsch. chem. Gesellsch., 6, 1873, 48; ibid., 9, 1876, 1348; ibid., 10, 1877, 276; Glycerinaethylbacterie, Buchner, in Xägeli, Untersuch. ü. niedere Pilze, 1882, 220 ; Zopf, Die Spaltpilze, 1 Aufl., 1883, 52; Bacterium fitzianum Zopf, Die Spaltpilze, 2 Aufl., 188t, 49.) From boiled hay infusions. Forms butyric acid.

Bacillus fagellifer Migula. (Bacillus No. VI, Flügge, Ztschr. f. Hyg., 17, 1894, 294; Bacillus lactis No. VI, Kruse, in Flügge, Die Mikroorganismen, 3 Aufl., 2, 1896, 209; Migula, Syst. d. Bakt., 
2, 1900, 581 ; Bacillus rudis Chester, Man. Determ. Bact., 1901, 279.) From milk. Bacillus flavescens Weiss. (Weiss, Arb. bakt. Inst. Karlsruhe, 2, Heft 3, 1902, 258; not Bacillus flavescens Pohl, Cent. f. Bakt., 11, 1892, 144.) From brewer's grains. Uncommon.

Bacillus flavidus Stührk. (Cent. f. Bakt., II Abt., 93, 1935, 185; not Bacillus flavidus Fawcett, Rev. Indust. y Agrico. de Tucuman, 13, 1922, 5; not Bacillus flavidus Morse, Jour. Inf. Dis., 11, 1912, 284.) Good growth on Ca n-butyrate agar. One culture isolated from soil from Egypt.

Bacillus flavidus alvei Klamann. (Bienenwirtschaftl. Cent., Hanover, 1890, No. 2.) Associated with foulbrood of bees. Bacillus flavoviridis Migula. (Maschek, Bakt. Untersuch. d. Leitmeritzer Trinkwässer, Leitmeritz, 1887; Migula, Syst. d. Bakt., 2, 1900, 821.) From water.

Bacillus flexilis Dobell. (Quart. Jour. Microse. Sci., 52, 1908, 121; Arch. f. Protistenk., 26, 1912, 117.) Reported as being similar to Bacillus bütschlii sichaudinn although only half its size. From the large intestine of frogs (Tiana temporaria) and toads (Bufo vulgaris).

Bacillus flexus Batchelor. (Jour. Bact., 4, 1919, 23.) Resembles Bacillus megatherium. From intestinal contents of a child.

Bacillus fluorescens undulatus Ravenel. (Mem. Nat. Acad. Sci., 8, 1896, 20.) From soil.

Bacillus foliaceus Migula. (Bacillus mesentericus fuscus Flügge, Die Mikroorganismen, 2 Aufl., 1886, 321; Bacillus No. IV, Flügge, Ztschr. f. Hyg., 17, 1894, 294 ; Bacillus lactis No. IV, Kruse, in Flügge, Die Mikroorganismen, 3 Aufl., 2, 1896, 208; Migula, Syst. d. Bakt., 2, 1900, 582.) From milk, air and soil.

Bacillus formosus Heigener. (Cent.f. Bakt., II Abt., 93, 1935, 101; not Bacillus formosus Ravenel, Mem. Nat. Acad. Sci., 8, 1896, 12.) One culture isolated from soil from Montenegro.
Bacillus foutini Chester. (Bacillus D, Foutin, Cent. f. Bakt., 7, 1890, 373; Chester, Man. Determ. Bact., 1901, 285.) From hail.

Bacillus frankei (sic) DeToni and Trevisan. (Sarkombacillen, Francke, Münch. med. Wochnschr., 1888, No. 4; abst. in Cent. f. Bakt., 3, 1888, 601; DeToni and Trevisan, in Saccardo, Sylloge Fungorum, 8, 1889, 967.) From cases of sarcoma.

Bacillus freudenreichii (Miquel) Chester. (Urobacillus freudenreichii sive $B a$ cillus ureae $\gamma$ Miquel, Ann. d. Micrographie, 2, 1890, 367 and 488; Chester, Ann. Rept. Del. Col. Agr. Exp. Sta., 10, 1898, 110.) Löhnis (Cent. f. Bakt., II Abt., 14, 1905, 719) considered this a variety of Bacillus pumilus. Gibson (Jour. Bact., 29, 1935, 493) believed it belonged to the Bacillus pasteurii group although no authentic cultures were available. From soil.

Bacillus frutodestruens Madhok and Ud-Din. (Indian Jour. Agr. Sci., 13, 1943, 129.) Causes a rot of tomato fruit.

Bacillus funicularis Kluyver and Van Niel. (Planta, Arch. f. wissensch. Botanik, 2, 1926, 507.) Exhibits contact irritability. From soil.

Bacillus furus Goadhy. (Dental Cosmos, 42, 1900, 322.) From the mouth. Associated with dental caries.

Bacillus (Streptobacter) fusisporus Schroeter. (In Cohn, Kirypt. Flora v. Schlesien, 3, 1, 1886, 161.) From waste water in a sugar factory.

Bacillus fusus Batchelor. (Jour. Bact., 4, 1919, 27.) Said to resemble Bacillus centrosporus, i.e., Bacillus brevis. From feces.

Bacillus gangliformis Ravenel. (Ravenel, Mem. Nat. Acad. Sci., 8, 1896, 34; Bacterium gangliforme Chester, Man. Determ. Bact., 1901, 193.) From soil.

Bacillus gangraenae Chester. (Bacillus gangraenae pulpae Arkövy, Cent. f. Bakt., 23, 1897, 921; Chester, Man. Determ. Bact., 1901, 275; not Bacillus gangraenae Tilanus, Nederl. Tijdschr. v. 
Geneeskunde, 21, 1885, 110.) Associated with gangrene of tooth pulp and caries of teeth.

Bacillus gasoformans Pammel. (Pammel, Cent. f. Bakt., II Abt., 2, 1896, 642; not Bacillus gasoformans Eisenberg, Bakt. Diag., 3 Aufl., 1891, 107; Bacillus pammelii Chester, Man. Determ. Bact, 1901, 270.) From cheese.

Bacillus gelatinosus Migula. (Bacterium gelatinosum betae Glaser, Cent. f. Bakt., II Abt., 1, 1895, 879 ; Migula, Syst. d. Bakt., 2, 1900, 805.) Produces slime in sucrose solutions. Probably a variety of Bacillus vulgatus according to Sacchetti (Cent. f. Bakt., II Abt., 95, 1936, 115).

Bacillus geniculatus (Duclaux) Trevisan. (Tyrothrix geniculatus Duclaux, Ann. Inst. Nat. Agron., 4, 1882, 23; Trevisan, I generi e le specie delle Batteriacee, 1889 , 16; not Bacillus geniculatus De Bary, Inaug. Diss., Strassburg, Leipzig, 1885; not Bacillus geniculatus Wright, Mem. Nat. Acad. Sci., 7, 1894, 459; Bacillus gonatodes DeToni and Trevisan, in Saccardo, Sylloge Fungorum, 8, 1889, 964; Bacterium geniculatum Migula, Syst. d. Bakt., 2, 1900, 322.) From milk.

Bacillus gigas (Koch) Trevisan. (Streptobacteria gigas-pericardii Billroth, Die Vegetat ionsformen von Coccobacteria septica, Berlin, 1874, 60; Streptobacteria gigas Koch, in Cohn, Beitr. z. Biol. d. Pflanz., 2, Heft 3, 1877, 429; Metallacter gigas Trevisan, Batter. ital., 1879, 25; Trevisan, Atti. d. Accad. Fis.-Med.Stat., Milan, Ser. 4, 3, 1886, 96; not Bacillis gigas van der Goot, Med. Proefstation voor de Java Suikerindust., pt. 5, No. 10, 1915, 28t; not Bacillus gigas Zeissler and Rassefeld, Arch. f. wiss. u. prakt. Tierheilk., 59, 1929, 419.) From pericardial exudate.

Bacillus ginglymus Ravenel. (Mem. Nat. Acad. Sci., 8, 1896, 37.) From soil. Bacillus glaciformis Wilhelmy. (Arb. bakt. Inst. Karlsruhe, 3, 1903, 29.) From meat extract.
Bacillus globifer Bartels. (Cent. f. Bakt., II Abt., 103, 1940, 26.) Growth on media containing $\mathrm{is} / 100$ phenol. Author considers it similar to Bacillus alvei. Five strains isolated from soil.

Bacillus glutinis Patrick and Werkman. (Iowa State Coll, Jour. Sci., 7, 1933, 414.) Ferments xylan. Two strains isolated from decayed apple mood.

Bacillus glycinophitus Rippel. (Rippel, Arch. f. Mikrobiol., 8, 1937, 42; also see Rippel and Nabel, ibid., 10, 1938, 359.) From intestines of cattle. Fresh cultures on agar form protein from glycine and glucose.

Bacillus gossypina Stedman. (Alabama Agr. Exp. Sta. Bul. 55, 1894, 6; Earle, Alabama Agr. Exp. Sta. Bul. 107, 1899, 311.) Reported as cause of boll rot on cotton (Gossypium sp.)

Bacillus gramularis Stührk. (Cent. f. Bakt., II Abt., 93, 1935, 180.) Very good growth on Ca n-butyrate agar. One culture isolated from garden soil in Germany.

Bacillus granulosus Russell. (Russell, Ztschr.f. Hyg., 11, 1892, 194; Bacterium granulosum Chester, Man. Determ. Bact., 1901, 194.) From mud from the Gulf of Naples.

Bacillus grossus Migula. (Bacterienart No. 13, Lembke, Arch. f. Hyg., 26, 1896, 308; Migula, Syst. d. Bakt., 2, 1900, 570.) From the intestines of infants.

Bacillus gryllotalpae Metalnikov and Meng. (Compt. rend. Acad. Sci., Paris, 201, 1935, 367.) From diseased larvae of the cricket (Gryllotalpa gryllotalpa).

Bacillus guano Stapp. (Cent.f. Bakt., II Abt., 51, 1920, 29.) From soil previously fertilized with guano.

Bacillus gummosus Happ. (Bakt. und Chem. Untersuch. über die schleimige Gahrung. Univ. Basel, 1893, 34; abst. in Cent. f. Bakt., 14, 1893, 175.) From digitalis infusions. Presumably a mucoid form of a common spore-former. See Bacterium gummosum Ritsert.

Bacillus harai Hori and Miyake. (Rpt. Imp. Agr. Exp. Sta. Nishigahara, 38, 
1911, 69.) Pathogenic for willow (Salix sp.).

Bacillus hessii (Guillebeau) Kruse. (Bacterium hessii Guillebeau, Landw. Jahrb. d. Schweiz, 5, 1891, 138; Kruse, in Flügge, Die Mikroorganismen, 3 Aufl., 2, 1896,210 .) There is some question whether the original culture was a sporeformer or whether it was mixed with one of the common slimy milk organisms. From slimy milk.

Bacillus hirudinis Schweizer. (Arch. f. Mikrobiol., $7,1936,235$.) From the digestive slime of leeches (Hirudo medicinalis and Hirudo officinalis).

Bacillus hollandicus Stapp). (Cent. f. Bakt., II Abt., 51, 1920, 47.) From soil from Delft

Bacillus hoplosternus Paillot. (Compt. rend. Acad. Sei., Paris, 163, 1916, 774; Ann. Inst. Past., 33, 1919, 403.) Isolated once from the body fluid of a June bug. Pathogenic for several species of insects.

Bacillus imminutus MeBeth. (Soil Sci., 1, 1916, 455.) Growth only in the presence of cellulose. From ten different soils of California.

Bacillus immobilis Steinhaus. (Jour. Bact., 42, 1941, 783.) The author states that it probably belongs to the Bacillus arthaerens group. From rectum of larvae of the sphinx moth (Ceratomia calalpae).

Bacillus imomarinus ZoBell and Upham. (Bull. Scripps Inst. of Oceanography, Univ. Calif., 5, 1944, 265.) Subterminal spores. From marine bottom deposits in shoal waters.

Bacillus indifferens Soriano. (Thesis, Univ. Buenos Aires, 1935, 565.) One strain isolated from soil.

Bacillus infantilis Kicndall. (Jour. Biol. Chem., 5, 1909, 419 and 439.) From the intestine in cases of infantilism. Saprophytic.

Bacillus intermittens Wilhelmy. (Arb. bakt. Inst. Karlsruhe, 3, 1903, 23.) From meat extract.

Bacillus intrapallans Forbes. (Bull. Illinois State Lab. Natur. Hist., Art. IV, 1896, 283, 288 and 297.)
Bacillus jubatus Bartels. (Cent. f. Bakt., II Abt., 103, 1940, 24.) Very good growth on media containing $\mathrm{M} / 50$ phenol. Nine strains isolated from soil.

Bacillus kaleidoscopicus Wilhelmy. (Arb. bakt. Inst. Karlsruhe, 3, 1903, 31.) From meat extract.

Bacillus kefir Kuntze. (Cent. f. Bakt., II Abt., 24, 1909, 116.) From kefir, a Caucasian milk beverage.

Bacillus kermesinus Migula. (Karminroter Bacillus, Tataroff, Inaug. Diss., Dorpat, 1891, 67 ; Migula, Syst. d. Bakt., 2, 1900, 858.) From water.

Bacillus kildini Issatehenko. (Recherches sur les Microbes de l'Océan Glacial Arctique. Petrograd, 1914.) From sea water.

Bacillus koubassoffii Chester. (Bacillus der krebsartigen Neubildungen, Loubassoff, Vortrag. Moskauer Militürärztlichen Verein, 1888, No. 22; abst. in Cent. f. Bakt., 7, 1890, 317; Chester, Man. Determ. Bact., 1901, 282.) From eancerous growths of the human stomach.

Bacillus lacca Kiern. (Arb. bakt. Inst. Karlsruhe, 1, Heft 4, 1896, 411.) From the stomachs and intestines of birds.

Bacillus lactis-albus Chester. (Bacillus lactis albus Sternberg, Man. of Bact., 1893, 680; Chester, Man. Determ. Bact., 1901, 277.) From milk.

Bacillus lactucae Voglino. (Ann. R. Accad. Agr. Torino, 46, 1903, 25.) Pathogenic for lettuce (Lactuca sativa).

Bacillus lasiocampa Brown. (Amer. Museum Novitates, No. 251, 1927, 7.) Said to belong to Bacillus subtilis group. From ovaries and egg tubes of tent caterpillar moth.

Bacillus latvianus Kalnins. (Latvijas Univ. Raksti, Serÿa I, No. 11, 1930, 265.) Cellulose attacked at $34^{\circ} \mathrm{C}$ but not at $37^{\circ} \mathrm{C}$. Probably from soil.

Bacillus lautus Batchelor. (Jour. Bact., \&, 1919, 30.) One culture from feces.

Bacillus legrosii Hauduroy et al. (Legros, Thèse Méd. Paris, 1902 ; Hauduroy et al., Dict. d. Bact. Path., 1937, 43.) 
Facultative anaerobe producing gaseous gangrene. From a gaseous suppuration.

Bacillus leguminiperdus von Oven. (Cent. f. Bakt., II Abt., 16, 1906, 67; Bacterium leguminiperdum Stevens, The Fungi which Cause Plant Disease, 1913 , 28.) Pathogenic for lupine (Lupinus $s p$.), kidney bean (Phaseolus vulgaris), pea (Pisum sativum), tomato (Lycopersicum esculentum).

Bacillus lehmanni Herter. (Microbe 5A, Choukévitch, Ann. Inst. Past., 25. 1911, 350; Herter, Just's Botan. Jahresber., 39, 2 Abt., Heft 4, 1915, 748.) From the large intestine of the horse.

Bacillus lekitosis Perlman. (N. Y. State Dept. Agr. and Markets, Ann. Rept., 1931, 115.) Egg lecthin hydrolyzed completely by powerful extracellular enzyme. From contaminated sample of whole mixed eggs.

Bacillus lesagei Trevisan. (Bacille de la diarrhée verte des enfants, Lesage, Bull. Acad. Méd. Paris, Oct. 25, 1887; Trevisan, I generi e le specie delle Batteriacee, 1889, 14.) From the intestine of infants.

Bacillus levaniformans Greig Smith (Proc. Linn. Soc. New South Wales, 26, 1901, 589, 67t and 684; Cent. f. Bakt., II Abt., 8, 1902, 596.) Produces slime in sucrose solutions. Probably a variety of Bacillus vulgatus according to Sacchetti (Cent. f. Bakt., II Abt., 95, 1936, 115).

Bacillus licheniformis (Weigmann) Chester. (Bacterie a, Weigmann, Cent. f. Bakt., II Abt., 2, 1896, 155; Clostridium licheniforme Weigmann, loc. cit., 4, 1898, 822 ; Chester, Man. Determ. Bact., 1901, 287 ; see also Gibson, Soc. Agric. Bact. (British); Abstr. Proc., 1927, Paper No. 10; Gibson and Topping, itid., 1938, 43; Gibson, ibid., 1943, 13.) Gibson plares this with Bacillus subtilis although it was originally deseribed as heing Gramnegative and forming clostridial sporangia. Spore germination polar.

Bacillus lichenoides Grohmann. (Morphologisch-biologische Beiträge zur Kenntnis der Wasserstoffbakterien, In- aug. Diss., Univ. Leipzig, 1923; Cent. f. Bakt., II Abt ., 61, 1924, 267; Ruhland and Grohmann, Jahrb. Wissensch. Botanik, $63,1924,321$.) Oxidizes hydrogen in the presence of oxygen to form water. Presumably widely distributed in soil.

Bacillus lignivorans Patrick and Werkman. (Iowa State Coll. Jour. Sci., 7, 1933, 410.) Ferments xylan. One culture isolated from decayed maple wood.

Bacillus lignorum Patrick and Werkman. (Iowa State Coll. Jour. Sci., 7, 1933, 410.) Ferments xylan. One culture isolated from decayed apple wood.

Bacillus limnophilus Stührk. (Cent. f. Bakt., II Abt., 93, 1935, 190.) Good growth on Ca n-butyrate agar. One culture isolated from soil of Germany.

Bacillus lingardi Trevisan. (Bacillus de la stomatite ulcereuse du veau, Lingard and Batt; Trevisan, I generi e le specie delle Batteriacee, 1889, 14.) From ulcerations on the tongue and mucous membrane of the mouth of calves.

Bacillus lividus Zimmermann. (Bakt. unserer Trink-u. Nutzwässer, Chemnitz, II Reihe, 1894, 18; not Bacillus lividus Voges, Cent. f. Bakt., 14, 1893, 303.) From water.

Bacillus longior Saito. (Jour. Coll. Sci., Imp. Univ., Tokyo, 23, Art. 15, 1908, 57.) Isolated once from garden air.

Bacillus longus Migula. (Bacillus No. VII, Flügge, Ztschr. f. Hyg., 17, 1894, 296; Bacillus luctis No. VII, Kruse, in Flügge, Die Mikroorganismen, 3 Aufl., 2, 1896, 209; Migula, Syst. d. Bakt., 2, 1900 , 581; not Bacillus longus Chester, Man. Determ. Bact., 1901, 303; Bacillus plicatus Chester, ibid., 275; not Bacillus plicatus Frankland and Frankland, Philos. Trans. Royal Soc. London, 178, B, 1887, 273; not Bacillus plicatus Zimmermann, Bakt. unserer Trink-u. Nutzwässer, Chemnitz, I Reihe, 1890, 54; not Bacillus plicatus Deetjen, Inaug. Diss., Würzburg, 1890; not Bacillus plicatus Copeland, Rept. Filtration Commission, Pittsburgh, 1899, 348.) From milk. 
Bacillus loxosporus Burchard. (Arb. bakt. Inst. Karlsruhe, 2, Heft 1, 1902, 49.) From the air. Synonym of Bacillus simplex according to Gottheil, Cent. f. Bakt., II Abt., 7, 1901, 633.

Bacillus luteus Garbowski. (Bacillus luteus sporogenes Smith and Baker, Cent. f. Bakt., II Abt., 4, 1898, 788; Garbowski, Cent. f. Bakt., II Abt., 19, 1907, 641.) From two samples of beet sugar.

Bacillus lutzae Brown. (Amer. Museum Novitates, No. 251, 1927, 8.) Pathogenic for certain flies. Dying individuals of the green blow fly (Lucilia sericata) yielded pure cultures.

Bacillus maculatus Stührk. (Cent.f. Bakt., II Abt., 93, 1935, 184.) Good growth on Ca n-butyrate agar. Two cultures isolated from soils from Cuba and Germany.

Bacillus maïdis Paltauf and Heider. (Paltauf and Heider, Wiener med. Jahrb., 3, 1888, 383; Paltauf, Med. Jahrb., No. S, 1889.) From an infusion of maize; also from feces in cases of pellagra. This species was originally described by Cuboni, probably in the Rendic. $R$. Acead. dei Lincei, 1, 1886 . It was later shown to be a spore-former of the Bacillus mesentericus group. It was quite different from the organism isolated by Tataroff (Pseudomonas maidis Migula) and identified by him as Bacillus maidis.

Bacillus malakofaciens von Wahl. (Cent. f. Bakt., II Abt., 16, 1906, 499.) Reported to he similar in morphology and physiology to Bacillus asterosporus. From preserved asparagus and from green beans.

Bacillus maritimus Russell. (Russell, Bot. Gazette, 18, 1893, 445; Bacterium maritimum Chester, Man. Determ. Bact., 1901, 189.) From sea-mud.

Bacillus mazun Weigmann, Gruber and Huss. (Cent. f. Bakt., II Abt., 19, 1907, 72.) From the Armenian milk product, mazun.

Bacillus mediosporus Migula. lus No. VIII, Flügge, Ztschr. f. Hyg., 17, 1894, 296; Bacillus lactis No. VIII, Kruse, in Flügge, Die Mikroorganismen, 3 Aufl., 2, 1896, 209; Migula, Syst.d. Bakt., 2, 1900, 580; Bacillus magnus Chester, Man. Determ. Bact., 1901, 276.) From milk.

Bacillus medio-tumescens Saito. (Jour. Coll. Sci. Imp. Univ., Tokyo, 23, Art. 15, 1908, 45.) Isolated twice from garden air.

Bacillus megatherium Ravenel. (Ravenel, Mem. Nat. Acad. Sci., 8, 1896, 11; not Bacillus megatherium De Bary, Vergleich. Morph. u. Biol. d. Pilze, 1884, 499; Bacillus megatherium var. ravenelii ('hester, Man. Determ. Bact., 1901, 271.) From soil.

Bacillus melanosporus Schroeter. (Eine Bacillus Art, Eidam, Beitr. z. Biol. d. Pflanz., 1, 3, 1875, 216; Schroeter, in Cohn, Kryptog. Flora v. Schlesien, 3, 1, 1886, 159.) From cooked potato.

Bacillus melonis Patrick and Werkman. (Iowa State Coll. Jour. Sei., 7, 1933, 411 ; not Bacillus melonis Giddings, Vermont Agr. Exp. Station Bull. 148, 1910, 413.) Ferments xylan. One culture isolated from decayed watermelon.

Bacillus mesentericus fuscus consistens Dyar. (Amn. N. Y. Acad. Sci., 8, 1895, 373.) Found as a contamination in a milk rulture.

Bacillus mesentericus juscus granulatus Dyar. (Ann. X. Y. Acad. Sci., 8, 1895, 3i3.) Found abundantly in a jar of sterilized milk.

Bacillus mesentericus roseus Zimmermann. (Bakt. unserer Trink-u. Nutzwässer, Chemnitz, 2, 1894, 26.) From water. Zimmermann received this culture from Fral under the above name. Krìl (Sanmlung v. Mikroorganismen, Prague, 1900, 7) lists it as a synonym of Bacillus mesentericus ruber Globig.

Bacillus mesentericus vulgatus mucosus Ivanovics. (Cent. f. Bakt., I Abt., Orig., 142, 1938, 52.) Author believed it to be identical or near to Bacillus vulgatus 
but produces much slime. From drainage water.

Bacillus mesenterioides Deetjen. (Inaug. Diss., Würzburg, 1890.) From sausage.

Bacillus micans Wilhelmy. (Arb. bakt. Inst. Karlsruhe, 3, 1903, 30.) From meat extract.

Bacillus milii (sic) Howard. (Gleanings in Bee Culture, 28, 1900, 124.) From black brood of the honey bee (Apis mellifera).

Bacillus mitochrondrialis Alexeieff. (Arch. f. Protist., 49, 1924, 399.) From horse manure.

Bacillus modestus Schieblich. (Cent. f. Bakt., I Abt., Orig., 124, 1932, 269.) Prefers carbohydrate media and $37^{\circ} \mathrm{C}$. From grass and meadow plants.

Bacillus monachae (von Tubeuf) Eckstein. (Bacillus B, Hofmann, Die Schlaffsucht (Flacherie) der Nonne (Liparis monacha), 1891, Frankfort am Main; Bacterium monachae von Tubeuf, Forstl. naturwissensch. Ztschr., 1, 1892, 34, 277; Eckstein, Ztschr. f. Forst-u. Jagdwesen, 26, 1894, 6.) From diseased caterpillars of the nun moth (Lymantria monacha).

Bacillus montanus Werner. (Cent.f. Bakt., II Abt., 87, 1933, 458.) Good growth on Ca n-butyrate agar. One culture isolated from soil of Germany.

Bacillus moulei DéToni and Trevisan. (Bacille des viandes à odeur de beurre rance des halles de Paris, Nocard and Moulé, Recueil de médecine vétér., Sér. $7,6,18-, 67$; DeToni and Trevisan, in Sacardo, Sylloge Fungorum, 8, 1889, 971.) From spoiled meat.

Bacillus mucilaginosus Migula. (Happ, Inaug. Diss., Berlin, 1893, 28; Migula, Syst. d. Bakt., 2, 1900, 696.) From a slimy fermentation.

Bacillus mucosus Zimmerman. (Bakt. unserer Trink- u. Nutzwässer, Chemnitz, II Reihe, 1894, 8; Bacterium mucosum Migula, Syst. d. Bakt., 2, 1900, 315.) From water.

Bacillus mucronatus Saito. (Jour. Coll. Sei., Imp. Univ., Tokyo, 23, Art. 15,
1908, 58.) Isolated twice from garden air.

Bacillus multipediculus flavus Zimmermann. (Bakt. unserer Trink-u. Nutzwässer, Chemnitz, II Reihe, 1894, 42.) From water.

Bacillus muralis Tomaschek. (Botan. Zeit., 46, 1887, 665.)

Bacillus mutabilis Soriano. (Estudio sistematico de algunas bacterias esporuladas aerobias, Thesis, Univ. Buenos Aires, 1935, 570.) Four strains isolated from fecal matter and Argentine soils.

Bacillus myxodens Burchard. (Arb. bakt. Inst. Karlsruhe, 2, Heft 1, 1898, 41.) From beer yeast.

Bacillus nebulosus Goresline. (Goresline, Jour. Bact., 27, 1934, 72; not Bacillus nebulosus Wright, Mem. Nat. Acad. Sci., 7, 1894, 465; not Bacillus nebulosus Halle, Thèse de Paris, 1898; not Bacillus nebulosus Vincent, Ann. Inst. Past., 21, 1907, 69; not Bacillus nebulosus gazogenes Jacobson, Ann. Inst. Past., 22, 1908, 300.) From a trickling filter receiving creamery wastes.

Bacillus neumanni Herter. (Microbe 5B, Choukévitch, Ann. Inst. Past., 25, 1911, 350; Herter, Just's Botan. Jahresber., 39, 2 Abt., Heft 4, 1915, 748.) From the large intestine of the horse.

Bacillus nigrescens Bartels. (Cent. f. Bakt., II Abt., 103, 1940, 22.) Good growth on media containing $\mathrm{M} / 25$ phenol. Three strains isolated from soil.

Bacillus nigricans Kern. (Arb. bakt. Inst. Karlsruhe, 1, Heft 4, 1896, 428.) From the stomach of a bird.

Bacillus nitidis Heigener. (Cent. f. Bakt., II Abt., 93, 1935, 99.) One culture isolated from soil from Washington, D. C.

Bacillus nitri Ambrož. (Cent. f. Bakt., I Abt., Orig., 51, 1909, 193.) Used for cytological studies because of its large size. Found on gelatin plates poured for the isolation of yeasts.

Bacillus nitroxus Beijerinck. (Cent. f. Bakt., II Abt., 25, 1909, 45.) In the absence of air forms $\mathrm{N}$ gas, $\mathrm{CO}_{2}$, and $\mathrm{N}_{2} \mathrm{O}$ in nitrate broth. Under aerobic condi- 
tions only a weak reduction. From garden soil.

Bacillus nobilis Adametz. (See Freudenreich, Cent. f. Bakt., II Abt., 7, 1901, 857 ; ibid., 8, 1902, 674.) This organism was sold under the name Tyrogen; it was said to play a part in the ripening of hard cheese. This was doubted by Freudenreich who identified it as one of the Tyrothrix group. Original deseription apparently in Osterreichen MokereiZeitung, Nov. 15, 1900; Dec. 1 and 15, 1900; Milchzeitung, No. 4\$, 1900.

Bacillus novus (Huss) Bergey et al. (Plectridium novum Huss, Cent. f. Bakt., II Abt., 19, 1907, 256; Bergey et al., Manual, 1st ed., 1923, 304.) From sterilized milk.

Bacillus oblongus Eckstein. (Ztschr. f. Forst- u. Jagdwesen, 26, 1894, 16.) From the larvae of a moth (Hyponomeuta evonymella).

Bacillus oehensis Bartels. (Cent. f. Bakt., II Abt., 103, 1940, 28.) Growthon media containing $\mathrm{M} / 100$ phenol. One culture isolated from soil.

Bacillus oleae Schiff-Giorgini. (Cent. f. Bakt., II Abt., 15, 1905, 203.) Thought to be the cause of tubercles on the olive tree from which it was isolated.

Bacillus omelianskii Serbinoft. (Zhurnal Bolezni Rastenii, Leningrad, 9, 1915, 105.) Causes a rot of sorghum.

Bucillus ontarioni (Chorine) Steinhaus. (Bacterium ontarioni Chorine, Internat. Corn Borer Invest., Sci. Rpts., 2, 1929, 44; also Ann. Inst. Past., 43, 1929, 1658; $B$. ontarioni Paillot, $B$. presumably indicates Bacterium, see index, p. 522, L'infection chez les insectes, 1933, 134; Steinhaus, Bacteria Associated Extracellularly with Insects, Minneapolis, 1942, 72.) From diseased larvae of the corn borer (Pyraustra nubilalis $\mathrm{Hb}$.).

Bacillus oogenes Migula. (Bacillus oogenes hydrosulfureus $\alpha$, Zörkendörfer, Arch. f. Hyg., 16, 1893, 385 ; Migula, Syst. d. Bakt., 2, 1900, 573.) From hens' eggs. Bacillus opacus Weiss. (Arb. bakt. Inst. Karlsruhe, 2, Ieft 3, 1902, 214.)
From bean infusions and fermenting cabbage.

Bacillus orae Werner. (Cent. f. Bakt., II Abt., 8\%, 1933, 464.) Weak growth on agar containing calcium salts of formic, acetic, and butyric acids. One culture isolated from European soil.

Bacillus oxylacticus Dyar. (Dyar, Ann. 1. Y. Acad. Sci., 8, 1895, 369; Bacterium oxylacticus Chester, Ann. Rept. Del. Col. Agr. Exp. Sta., 9, 1897, 107.) From air and a culture from Kràl's Iaboratory labeled Bacillus oxylacticus. The latter is given in the Kràl 1900 catalogue as Bacillus oxalaticus Zopf and undoubtedly was the organism received by Migula from Zopf and studied by him (Migula, Arb. tech. Hochschule Karlsruhe, 1, Heft 1, 1894, 139 and Migula, Syst. d. Bakt., 2, 1900, 538). This is now regarded as having been Bacillus megatherium De Bary.

Bacillus pabuli Schieblich. (Cent. f. Bakt., II Abt., 58, 1923, 204.) Commonly isolated from green and fermenting fodder.

Bacillus pallidus Heigener. (Cent. f. Bakt., II Abt., 93, 1935, 98.) One strain isolated from soil from New York State.

Bacillus palustris Sickles and Shaw. (Jour. Bact., 28, 1934, 418; Rhodobacillus palustris Sickles and Shaw, Jour. Bact., 38, 1939, 241.) Decomposes the specific carbohydrate of pneumococcus type III. From swamp and other uncultivated soils.

Bacillus palustris var. gelaticus Sickles and Shaw (loc. cit., 119). A variety that decomposes agar slightly. Found only once.

Bacillus paucicutis Burchard. (Arb. bakt. Inst. Karlsruhe, 2, Heft 1, 1902, 27.) From rye bread.

Bacillus pectocutis Burchard. (Arb. bakt. Inst. Karlsruhe, 2, Heft 1, 1902, 24.) From the air.

Bacillus pelagicus Russell. (Bot. Gaz., 18, 1893, 383.) From sea water and marine mud from Woods Hole, Massachusetts.

Bacillus pellucidus Soriano. (Revista 
del Instit. Bacteriol., Buenos Aires, 6, 1935, 567.) Author says colonies resemble Bacillus simplex. Habitat probably in soil.

Bacillus peptogenes (Buchanan and Hammer) Bergey et al. (Bacterium peptogenes Buchanan and Hammer, Iowa Agr. Exp. Sta. Res. Bull. 22, 1915, 273; Bergey et al., Manual, 1st ed., 1923, 293.) From a tube of litmus milk after autoclaving.

Bacillus peptonans Chester. (Bacillus lactis peptonans Sterling, Cent. f. Bakt., II Abt., 1, 1895, 473; Chester, Man. Determ Bact., 1901, 271.) From milk. Very similar to Bacillus mesentericus vulgatus Flügge.

Bacillus peptonificans Lubenau. (Cent. f. Bakt., I Abt., Orig., 40, 1906, 435.) Similar to Bacillus subtilis. Believed to be the cause of an epidemic of gastroenteritis.

Bacillus perlucidulus Saito. (Jour. Coll. Sci., Imp. Univ., Tokyo, 23, Art. 15, 1908, 43.) Isolated 3 times from garden air.

Bacillus petiolatus Saito. (Jour. Coll. Sci., Imp. Univ., Tokyo, 23, Art. 15, 1908, 48.) Isolated twice from garden air.

Bacillus phaseoli von Wahi. (Cent.f. Bakt., II Abt., 16, 1906, 500.) From canned beans.

Bacillus phenolphilos Bartels. (Cent. f. Bakt., II Abt., 103, 1940, 21.) Good growth on media containing $\mathrm{w} / 50$ phenol. One culture isolated from soil.

Bacillus picrogenes Patrick and Werkman. (Iowa State Coll. Jour. Sci., 7, 1933, 410.) Ferments xylan. One culture isolated from decayed watermelon.

Bacillus piliformis Tyzzer. (Jour. Med. Research, 37, 1917, 307.) All attempts to cultivate the organism failed except when mixed with a streptotoccus. Considered aerobic by Ford (Textbook of Bact., 1927, 712). Causes fatal disease of Japanese waltzing mice.

Bacillus piscicidus Migula. (Bacillus piscicidus agilis Siebert, Gazeta lekarska, 1895, No. 13-17; abst. in Cent. f. Bakt.,
17, 1895, 888; Bacterium piscicidus agilis Chester, Ann. Rept. Del. Col. Agr. Exp. Sta., 9, 1897, 140; Migula, Syst. d. Bakt., 2, 1900, 652; Bacillus piscicidus nobilis (sic) Babes and Riegler, Cent. f. Bakt., II Abt., Orig., 33, 1902-03, 440.) Cause of a fish epidemic in St. Petersburg.

Bacillus pisi von Wahl. (Cent. f. Bakt., II Abt., 16, 1906, 502.) From young peas.

Bacillus platychoma Gray and Thornton. (Cent. f. Bakt., II Abt., 73, 1928, 93.) Phenol is attacked. Three strains isolated from soil.

Bacillus plexiformis Goadby. (Dental Cosmos, 42, 1900,322.) From the mouth.

Bacillus plicatus Deetjen. (Deetjen, Inaug. Diss., Würzburg, 1890; not Bacillus plicatus Frankland and Frankland, Philos. Trans. Royal Soc. London, 178, B, 1887, 273; not Bacillus plicatus Zimmermann, Bakt. unserer Trink- u. Nutzwässer, Chemnitz, I Reihe, 1890,54 ; not Bacillus plicatus Copeland, Rept. Filtration Commission, Pittsburgh, 1899, 348; not Bacillus plicatus Chester, Man. Determ. Bact., 1901, 275.) From sausage.

Bacillus pollacii Pavarino. (Atti R. Accad. Naz. Lincei Rend. Cl. Sei. Fis. Math. e Nat., 20, 1911, 233.) Reported to cause depressed spots on leaves of Odontoglossum citrosmum.

Bacillus populi Brisi. (Atti Cong. Nat. Ital. Pom. della Soc. Ital. di Sci. Nat. Milano, 1907, 376.) Reported as cause of galls on branches of poplar trees (Populus sp.).

Bacillus pseudanthracis Kruse. (Milzbrandähnlicher Bacillus, Burri, Hyg. Rundschau, 4, 1894, 339; abst. in Cent. f. Bakt., 16, 1894, 374; Kruse, in Flügge, Die Mikroorganismen, 3 Aufl., 2, 1896, 233 ; not Bacillus pseudanthracis Wahrlich, Bakteriol. Studien, Petersburg, 1890-91, 26; Bacillus pseudo-anthracis Chester, Man. Determ. Bact., 1901, 280.) From South American bran.

Bacillus pseudococcus Migula. (Bacil- 
lus No. 11, Pansini, Arch. f. path. Anat., 122, 1890, 446; Migula, Syst. d. Bakt., 2, 1900, 557.) From sputum.

Bacillus pseudodiphthericum magnus Ødegaard. (Acta Path. et Microbiol. Scand., 21, 1944, 451; see Endicott, Biol. Abst., 20, 1946, 12926.) From the nose of a child suspected of having diphtheria. Resembles Corynebacterium diphtheriae in young cultures. Non-pathogenic.

Bacillus pseudofusiformis Saito. (Jour. Coll. Sci., Imp. Univ., Tokyo, 23, Art. 15, 1908, 47.) Isolated once from garden air.

Bacillus pseudosubtilis Migula. (Bacillus subtilis similis Sternberg, Manual of Bact., 1893, 679; Migula, Syst. d. Bakt., 2, 1900, 618.) From the liver of a yellow fever cadaver.

Bacillus punctiformis Chester. (Bacillus No. 23, Conn, Storrs Agr. Exp. Sta. Rept., 1893, 53; Chester, Man. Determ. Bact., 1901, 284.) From milk.

Bacillus pycnoticus Grohmann. (Cent. f. Bakt., II Abt., 61, 1924, 261; Ruhland and Grohmann, Jahrb. wissensch. Botanik, 63, 1924, 321; Hydrogenomonas pycnotica Bergey et al., Manual, 3rd ed., $1930,34$.$) Oxidizes hydrogen in the$ presence of oxygen to form water. Presumably widely distributed in soil.

Bacillus quercifolius Deetjen. (Dectjen, Inaug. Diss., Würzburg, 1890 ; Bacterium quercifolium Migula, Syst. d. Bakt., 2, 1900, 309.) From sausage.

Bacillus rarerepertus Schieblich. (Cent. f. Bakt., I Abt., Orig., 124, 1932, 269.) From beet leaves.

Bacillus rarus Werner. (Cent. f. Bakt., II Abt., 87, 1933, 456.) Good growth on $\mathrm{Ca}$-butyrate agar. One culture isolated from forest soil of Germany .

Bacillus repens Gibson. (Cent. f. Bakt., II Abt., 92, 1935, 370.) Decomposes urea. Eight strains isolated from soil.

Bacillus reptans Ghosh. (Compt. rend. Soc. Biol., Paris, 86, 1922, 914.) From a case of appendicitis.
Bacillus retaneus Grohmann. (Morphologisch- biologische Beiträge zur Kenntnis der Wasserstoffbakterien, Inaug. Diss., Univ. Leipzig, 1923; Cent. f. Bakt., II Abt., 61, 1924, 267 ; Ruhland and Grohmann, Jahrb. wissensch. Botanik, $63,1924,321$.) Oxidizes hydrogen in the presence of oxygen to form water. Presumably widely distributed in soil.

Bacillus retiformis Migula. (Netzbacillus, Maschek, Bakt. Untersuch. d. Leitmeritzer Trinkwässer, Leitmeritz, 1887 ; Migula, Syst. d. Bakt., 2, 1900, 712.) From water.

Bacillus robustus Weiss. (Weiss, Arb. bakt. Inst. Karlswuhe, 2, Heft 3, 1902, 247; not Bacillus robustus Blau, Cent. f. Bakt., II Abt., 15, 1905, 134.) From fermenting beets.

Bacillus ruber Zimmermann. (Limmermann, Die Bakt. unserer Trink-u. Nutzwässer, Chemnitz, 1, 1890, 24; not Bacillus ruber Cohn, Beitr. z. Biol. d. Pflanz., 1, Heft 3, 1875, 181; Bacillus pseudoruber Migula, Syst. d. Bakt., 2, 1900, 850; Erythrobacillus ruber Holland, Jour. Bact., 5, 1920, 223, line 15; Serratia rubra Bergey et al., Manual, 1st ed., 1923, 92; Chromobacterium ruber Topley and Wilson, Princip. of Bact. and Immun., 1, 1931, 402.) From Chemnitz tap water. Spherical spores. See Manual, 5 th ed., 1939, 607 for a description of this species.

Bacillus rufescens Stührk. (Cent. f. Bakt., II Abt., 93, 1935, 178.) Characterized by good growth on Ca n-butyrate agar. One culture isolated from garden soil of Germany.

Bacillus rufulus Saito. (Jour. Coll. Sci., Imp. Univ., Tokyo, 23, Art. 15, 1908, 59.) Isolated 3 times from garden air.

Bacillus rugosus Henrici. (Henrici, Arb. bakt. Inst. Karlsruhe, 1, Heft 1, 1894, 28; not Bacillus rugosus Wright, Mem. Nat. Acad. Sci., 7, 1895, 438; not Bacillus rugosus Chester, Man. Determ. Bact., 1901, 220.) From Swiss cheese.

Bacillus rugulosus Stührk. (Cent.f. Bakt., II Abt., 93, 1935, 181.) One of a 
group of species described as growing well on $\mathrm{Ca} n$-butyrate agar. Three strains isolated from soils of Germany, Cuba, and Italy.

Bacillus sacchari Janse. (Mededeel. uit's Lands. Plantentuin, 9, 1891, 1.) Reported to cause sereh, a disease affecting sugar cane (Saccharum officinarum). Went (Arch. voor de Java Suikerindustrie, 1895, 589) regards this as probably Bacillus subtilis.

Bacillus saccharolyticus Nepomnjatschjaja and Libermann. (Jour. f. Mikrobiol., Ukraine, 5, 1938, 57 ; abst. in Cent. f. Bakt., II Abt., 101, 1940, 81.) From plum preserves. A gas-producing rod.

Bacillus saccobranchi Dobell. (Ouart Jour. Micro. Sci., 56, 1911, 411.) From heart blood of a fish (Saccobranchus fossilis).

Bacillus santiagensis Stührk. (Cent. f. Bakt., II Abt., 93, 1935, 188.) Good growth on $\mathrm{Ca}$ n-butyrate agar. One culture isolated from Cuban soil.

Bacillus saprogenes Migula. (Bacillus saprogenes vini III, Kramer, Bakteriologie in ihren Beziehungen zur Landwirtschaft, 2, 1892, 137; Migula, Syst. d. Bakt., 2, 1900, 572; not Bacillus saprogenes $I, I I$ and $I I I$, Herfeldt, Cent. f. Bakt., II Abt., 1, 1895, 77; not Bacillus saprogenes Salus, Areh. f. Hyg., 51, 1904 , 115.) From wine.

Bacillus saprogenes Chester. (Bacillus saprogenes vini VI, Kramer, Bakteriol. Landwirtsch., 2, 1892, 139; Chester, Man. Determ. Bact., 1901, 289; not Bacillus saprogenes Trevisan, I generi e le specie delle Batteriacee, 1889, 17.) From wine.

Bacillus scaber (Duclaux) Trevisan. (Tyrothrix scaber Duclaux, Ann. Inst. Nat. Agron., \&, 1882, 23; Trevisan. I generi e le specie delle Batteriacee, 1889 , 16.) From milk.

Bacillus schottelii Trevisan. (Darmbacillus, Lydtin and Schottelius, Der Rotlauf der Schweine, Weisbaden, 1885, 214; Bacillus coprogenes foetidus Flügge, Die Mikroorganismen, 2 Aufl., 1886, 305;
Trevisan, I generi e le specie delle Batteriacee, 1889, 17; Bacterium coprogenes Migula, Syst. d. Bakt., 2, 1900, 327; Bacterium schottelii Chester, Man. Determ. Bact., 1901, 197.) From the intestinal contents of swine.

Bacillus segetalis Werner. (Cent. f. Bakt., II Abt., 87, 1933, 467.) Characterized by growth on Ca $\mathrm{n}$-butyrate agar. One strain isolated from soil in Germany.

Bacillus septico-aerobius Hauduroy et al. (Bacille septique aérobie, Legros, Thèse Méd., Paris, 1902 ; Hauduroy et al., Dict.d. Bact. Path., 1937, 46.) Aerobic, facultative. From a case of acute gaseous gangrene.

Bacillus septicus insectorum Krassilstschik. (Memoires de la Soc. Zool. de France, 6, 1893, 250.) From cockchafer larvae (Melolontha melolontha).

Bacillus serrulatus Stührk. (Cent. f. Bakt., II Abt., 93, 1935, 193.) Only moderate growth on Ca n-butyrate agar. One culture isolated from Cuban soil.

Bacillus sesami Malkoff. (Cent. f. Bakt., II Abt., 16, 1906, 665.) Pathogenic on sesame (Sesamum).

Bacillus siccus Chester. (Bacillus No. 25, Conn, Storrs Agr. Exp. Sta. Rept., 1893, 63; Chester, Man. Determ. Bact., 1901, 284.) From milk.

Bacillus similis Schroeter. (Bacillus II, Bienstock, Ztschr. f. klin. Med., 8, 1884, Heft 1 and 2; Schroeter, in Cohn, Kryptog.-Flora v. Schlesien, 3, 1, 1886, 160 ; Bacillus coprocinus Trevisan, I generi e le specie delle Batteriacee, Milan, 1889, 15; Bacillus faecalis No. II, Kruse, in Flügge, Die Mikroorganismen, 3 Aufl., 2, 1896, 215; Bacterium simile Chester, Man. Determ. Bact., 1901, 197.) From feces.

Bacillus similis Eckstein. (Arch. f. Forst-u. Jagdwesen, 26, 1894, 11.) From infected larvae of the nun moth (Lymantria monacha), ete.

Bacillus similityphosus Migula. (Typhusähnlicher Bacillus, Maschek, Bakt. Untersuch. d. Leitmeritz. Trinkwässer, Leitmeritz, 1887; Migula, Syst. d. Bakt., 2, 1900, 730.) From water. 
Bacillus sinapivagus Kossowicz. (Cent. f. Bakt., II Abt., 23, 1909, 241.) From pickles.

Bacillus sombrosus Kern. (Arb. bakt. Inst. Karlsruhe, 1, Heft 4, 1896, 429.) From the stomach of a bird.

Bacillus sorghi Burrill. (The Microscope, 7, 1887, 321; Proc. Amer. Soc. Microscopists, 1888, 193; Bacterium sorghi Chester, Delaware Agr. Exp. Sta. Ann. Rept., 9, 1897, 127; Elliott and Smith, Jour. Agr. Res., 38, 1928, 1.) Reported to cause a disease of sorghum (Holcus sorghum).

Bacillus sotto Paillot. (Sotto-Bacillus, Ischivata, quoted from Aoki and Chigasaki, Mitteil. d. Med. Fakul. d. k. Univ. z. Tokyo, 13, 1915, 419 and 14, 1915, $59 ;$ Bacterium sotto Metalnikov and Chorine, Internat. Corn Borer Invest., Sci. Repts., 1, 1928, 56; Paillot, ibid., 1, 1928, 77-106.) From silkworms (Bombyx mori). Sotto is Japanese for "plötzlich hinfallen".

Bacillus spatiosus Saito. (Jour. Coll. Sci., Imp. Univ., Tokyo, 23, Art. 15, 1908, 56.) Isolated once from garden air.

Bacillus spermatozoides Eckstein. (Ztschr. f. Forst- u. Jagdwesen, 26, 1894, 13.) From dead moths (Hyponomeuta evonymella).

Bacillus sphaerosporus Beijerinck. (Cent. f. Bakt., II Abt., 25, 1909, 45.) This organism has round terminal spores and produces nitrous oxide from nitrates. From garden soil.

Bacillus sphaerosporus calco-aceticus Beijerinck (loc. cit., 46). A variety of the above having spherical to ellipsoidal spores.

Bacillus spinosporus Soriano. (Thesis, Univ. Buenos Aires, 1935, 562.) Spores spinate like some strains of Bacillus polymyxa. No gas formed. One strain isolated from soil.

Bacillus spiralis Migula. (Syst. d. Bakt., 2, 1900,624.) From water.

Bacillus spïrogyra Dobell. (Quart. Jour. Micro. Sci., 53, 1909, 579 and 56, $1911,434$.$) From large intestine of frog$ (Rana temporaria) and toad (Bufo vulgaris)
Bacillus spongiosus Aderhold and Ruhland. (Cent. f. Bakt., II Abt., 15, 1905, 376.) Found in the gum masses discharged by cherry trees.

Bacillus sporonema.Schaudinn. (Arch. f. Protistenkunde, 2, 1903, 421.) From sea water.

Bacillus spurius Grohmann. (Morphologisch-biologische Beiträge zur Kenntnis der Wasserstoffbakterien, Inaug. Diss., Univ. Leipzig, 1923; Cent. f. Bakt., II Abt., 61, 1924, 267; Ruhland and Grohmann, Jahrb. wissensch. Botanik, 63, 1924, 321.) Oxidizes hydrogen in the presence of oxygen to form water. Presumably widely distributed in soil.

Bacillus sputi Chester. (Bacillus No. 6, Pansini, Arch. f. path. Anat., 122, 1890, 442 ; Chester, Man. Determ. Bact., 1901, 280.) From sputum.

Bacillus squamiformis Saito. (Jour. Coll Sci., Imp. Univ., Tokyo, 23, Art. 15, 1908, 54.) Isolated 9 times from garden soil.

Bacillus stellaris Saito. (Jour. Coll. Sci., Imp. Univ., Tokyo, 23, Art. 15, 1908, 52.) Isolated 6 times from garden air.

Bacillus stellatus Zimmermann. (Zimmermann, Bakt. unserer Trink- u. Nutzwässer, II Reihe, 1894, 14; not Bacillus stellatus Chester, Man. Determ. Bact., 1901, 274; not Bacillus stellatus Vincent, Ann. Inst. Past., 21, 1907, 62.) From water.

Bacillus streptoformis Migula. (Salpeter zerstörenden Bacillus, Schirokikh, Cent. f. Bakt., II Abt., 2, 1896, 204; Migula, Syst. d. Bakt., 2, 1900, 682; Bacillus schirokikhi Chester, Man. Determ. Bact., 1901, 252; Bacillus denitrificans Chester, ibid., 274.) From horse feces. Utilizes potassium nitrate.

Bacillus suaveolens Sclavo and Gosio. (Quoted from Omeliansky, Jour. Bact., 8, 1923, 398.) No source given.

Bacillus subcuticularis Migula. (Bacillus cuticularis albus Tataroff, Inaug. Diss., Dorpat, 1891, 24; Migula, Syst. d. Bakt., 2, 1900, 623; Bacillus cuticularis Chester, Man. Determ. Bact. 1〔01, 285.) From water. 
Bacillus sublanatus Wright. (Mem. Nat. Acad. Sci., 7, 1895, 455.) From water.

Bacillus sublustris Schieblich. (Cent. f. Bakt., II Abt., 58, 1923, 206.) Commonly isolated from green and fermenting fodders.

Bacillus submarinus ZoBell and Upham. (Bull. Scripps Inst. of Oceanography, Univ. Calif., 5, 1944, 267.) Central ovate spores. From marine bottom deposits.

Bacillus (Streptobacter) subtiliformis Schroeter. (Bacillus I, Bienstock, Ztschr. f. klin. Med., 8, Heft 1 and 2, 1884; Schroeter, in Cohn, Kryptog.Flora v. Schlesien, 3, 1, 1886, 160; Bacillus mesenthericus (sic) Trevisan, I generi e le specie delle Batteriacee, Milan, 1859, 15; not Bacillus mesentericus Trevisan, ibid., 19; Bacillus subtilis simulans $I$, Eisenberg, Bakt. Diag., 3 Aufl., 1891, 189; Bacillus faecalis No. I, Kruse, in Flügge, Die Mikroorganismen, 3 Aufl, 2, 1896, 215; Bacterium subtile Migula, Syst. d. Bakt., 2, 1900, 292; Bacterium subtiliforme Chester, Man. Determ. Bact., 1901, 197.) From feces.

Bacillus succinicus Fitz. (Quoted from DeToni and Trevisan, in Saccardo, Sylloge Fungorum, 8, 1889, 966.) From infusions.

Bacillus suffuscus Grohmann. (Morphologisch-biologische Beiträge zur Kenntnis der Wasserstoffbakterien, Inaug. Diss., Univ. Leipzig, 1923; Cent. f. Bakt., II Abt., 61, 1924, 267; Ruhland and Grohmann, Jahrb. wissenseh. Botanik, 63, 1924, 321.) Oxidizes hydrogen in the presence of oxygen to form water. Presumably widely distributed in soil.

Bacillus supraresistans Stührk. (Cent. f. Bakt., II Abt., 93, 1935, 185.) Very good growth on Ca n-butyrate agar. One culture isolated from soil in Germany.

Bacillus surgeri Dornic and Daire. (Bull. mensuel de l'Office de renseignements agricoles, 6, 1907; abst. in Rev. Gén. du Lait., 6, 1907, 164.) Spores not observed but author stated that they were probably present because this species could withstand $85^{\circ} \mathrm{C}$ for 5 minutes. From whey.

Bacillus tabaci III, Koning. (Tijdschr. voor toegepaste scheikunde en hygiene. Deel 1, 1897. See Behrens, Mykologie der Tabakfabrikation, in Lafar, Handbuch der techn. Mykologie, 5, 1905, 11.) Thermophilic. Probably from soil.

Bacillus tardivus Stührk. (Cent. f. Bakt., II Abt., 93, 1935, 177.) Very slight growth on $\mathrm{Ca}$ n-butyrate agar. One culture isolated from garden soil of Germany.

Bacillus technicus Morikawa and Prescott. (Jour. Bact., 13, 1927, 58; also see Morikawa, Bull. Agr. Chem. Soc. Japan, 3, 1927, 28.) Produces butyl and isopropyl alcohols. Source not given.

Bacillus tenax Eckstein. (Ztschr. f. Forst- u. Jagdwesen, 26, 1894, 14.) From larvae of the nun moth (Lymantria monacha).

Bacillus tenuis non-liquefaciens Choukévitch. (Ann. Inst. Past., 25, 1911, 352.) From large intestine of horse.

Bacillus terminalis Migula. (Bacillus No. XII, Flügge, Ztschr. f. Hyg., 17, 1894, 296 ; Bacillus lactis No. XII, Kruse, in Flügge, Die Mikroorganismen, 3 Aufl., 2, 1896, 269; Migula, Syst. d. Bakt., 2, 1900, 578; Bacillus lacteus Chester, Man. Determ. Bact., 1901, 291.) From milk. A duplicate of Bacterium sempervivum Migula.

Bacillus terminalis var. thermophilus Prickett. (N. Y. Agr. Exp. Sta. Tech. Bull. 147, 1928, 44.) Produces a brown water soluble pigment on agar; optimum temperature $55^{\circ} \mathrm{C}$ to $65^{\circ} \mathrm{C}$. Fourteen strains from raw and pasteurized milk, milk powder, and skin of a cow.

Bacillus terrestris Werner. (Cent. f. Bakt., II Abt., 87, 1933, 461.) Weak growth on $\mathrm{Ca}$-butyrate agar. Two strains isolated from soils of Germany.

Bacillus tetanoides Saito. (Jour. Coll. Sci., Imp. Univ., Tokyo, 23, Art. 15, 1908, 49.) Isolated once from garden air.

Bacillus thalassokoites ZoBell and Upham. (Buil. Scripps Inst. of Oceanography, Univ. Calif., 5, 1944, 268.) Central spores. From marine bottom deposits. 
Bacillus theae Hori and Bokura. (Jour. Plant Protection, Tokyo, 2, 1915, 1.) Pathogenic for tea (Thea sinensis).

Bacillus thermoabundans Beaver. (Dissertation, Ohio State University, Columbus, 1932,31.) Thermophilic, subterminal spores. Growth at $55^{\circ} \mathrm{C}$, less growth at $37^{\circ} \mathrm{C}$. From malted milk powder.

Bacillus thermoacetigenitus Beaver, loc. cit., 25. Thermophilic, central spores. No growth at $37^{\circ} \mathrm{C}$. From vinegar.

Bacillus thermoacidificans Renco. (Ann. Microhiol., 2, 1942, 000.) From Grana cheese whey. This is stated by Gorini (R. Ist. Lombardo Sci. e. Lett.,

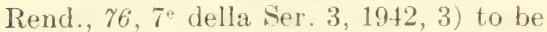
the same as Bacillus lactis termophilus Gorini.

Bacillus thermoactivus Beaver, loc. cit., 27. Thermophilic, central spores. No growth at $37^{\circ} \mathrm{C}$. From home-canned beets.

Bacillus thermoannulatus Beaver, loc. cit., 17. Thermophilic, subterminal spores. No growth at $37^{\circ} \mathrm{C}$. From commercially canned tomatoes.

Bacillus thermoaquatitis Beaver, loc. cit., 18. Thermophilic, subterminal spores. No growth at $37^{\circ} \mathrm{C}$. From a warm spring at Springfield, Ohio.

Bacillus thermoarborescens Beaver, loc. cit., 30. Thermophilic, sub-terminal to central spores. Growth at $55^{\circ} \mathrm{C}$, less grow th at $37^{\circ} \mathrm{C}$. From candy.

Bacillus thermobutyrosus Beaver, loc. cit., 15. Thermophilic, subterminal spores. No growth at $37^{\circ} \mathrm{C}$. From commercially canned tomatoes.

Bacillus thermocompactus Beaver, loc. ict., 20. Thermophilic, subterminal spores. No growth at $37^{\circ} \mathrm{C}$. From red grapes stored in sawdust.

Bacillus thermodactylogenitus Beaver, loc. cit., 28. Thermophilic, central to subterminal spores. Growth at $37^{\circ} \mathrm{C}$ and $55^{\circ} \mathrm{C}$. From commercially packed dates.

Bacillus thermocffervescens Beaver, loc. cit., 23. Thermophilic, central spores. No growth at $37^{\circ} \mathrm{C}$. From commercially canned peas.

Bacillus thermofaecalis Beaver, loc. cit., 30. Thermophilic, subterminal spores. Growth at $55^{\circ} \mathrm{C}$. From feces of robin.

Bacillus thermofibrincolus Itano and Arakawa. (Ber. d. Ohara Inst. f. landwirsch. Forsehungen, Japan, 4, 1929, 265.) Thermophilic; decomposes cellulose. From soil containing decomposed leaves.

Bacillus thermofiliformis Beaver, loc. cit., 22. Thermophilic, subterminal spores. No growth at $37^{\circ} \mathrm{C}$. From commercially canned peas.

Bacillus thermograni Beaver, loc. cit., 16. Thermophilic, subterminal spores. No growth at $37^{\circ} \mathrm{C}$. From commercially canned corn.

Bacillus thermolongus Beaver, loc. cit., 19. Thermophilic, subterminal spores. No growth at $37^{\circ} \mathrm{C}$. From commercially ranned tomatoes.

Bacillus thermolubricans Beaver, loc. cit., 26. Thermophilic, central spores. No grow th at $37^{\circ} \mathrm{C}$. From lubricating oil.

Bacillus thermononodorus Beaver, loc. cit., 26. Thermophilic, central spores. No growth at $37^{\circ} \mathrm{C}$. From tap water.

Bacillus thermonubilosus Beaver, loc. cit., 19. Thermophilic, subterminal spores. No growth at $37^{\circ} \mathrm{C}$. From soil, Yellow Springs, Ohio.

Bacillus thermoodoratus Beaver, loc. cit., 29. Thermophilic, central spores. Growth at $55^{\circ}$, less grow that $37^{\circ} \mathrm{C}$. From spoiled gelatin.

Bacillus thermopellitus Beaver, loc. cit., 22. Thermophilic, central spores. No growth at $37^{\circ} \mathrm{C}$. From old sour milk. Bacillus thermophilus Miquel. (Miquel, Ann. d. Microgr., 1, 1888, 4; $B a-$ cillus thermophilus miquelii Kruse, in Flügge, Die Mikroorganismen, 3 Aufl., 2, 1896, 269; Bacterium termophilum (sic) Migula, Syst. d. Bakt., 2, 1900, 342; Bacterium miquelii Chester, Man. Determ. Bact., 1901, 186.) From the 
intestine, water and soil. Thermophilic. No growth below $40^{\circ} \mathrm{C}$.

Bacillus thermophilus sojae Rokusho and Fukutome. (Jour. Agr. Chem. Soc., Japan, 13, 1937, 1235.) From spontaneously heating soy-bean cake.

Bacillus thermosuavis Beaver, loc. cit., 24. Thermophilic, central spores. No growth at $37^{\circ} \mathrm{C}$. From commercially canned mincemeat.

Bacillus thermotenax Beaver, loc. cit., 28. Thermophilic, subterminal spores. Growth at $37^{\circ} \mathrm{C}$ and $55^{\circ} \mathrm{C}$. From ground horseradish.

Bacillus thermourinalis Beaver, loc. cit., 16. Thermophilic, subterminal spores. No growth at $37^{\circ} \mathrm{C}$. From human urine.

Bacillus thermoviscidus Beaver, loc. cit., 21. Thermophilic, subterminal spores. No growth at $37^{\circ} \mathrm{C}$. From fresh pig ovary.

Bacillus thoracis Howard. (Gleanings in Bee Culture, 28, 1900, 124.) From black brood of the honey bee (Apis mellifera).

Bacillus tracheitis sive graphitosis Krassilstschik. Memoires de la Soc. Zool. de France, 6, 1893, 250.) From diseased larvae of the cockchafer (Melolontha melolontha).

Bacillus tricomii Trevisan. (Bacillo della gangraena senilis, Tricomi, Riv. internaz. di Med. e Chir., 3, 1886, 73; Trevisan, I generi e le specie delle Batteriacee, 1889, 13; Bacterium tricomii Migula, Syst. d. Bakt., 2, 1900, 310.) From a case of senile gangrene.

Bacillus trifolii Voglino. (Ann. R. Accad. Agr. Torino, 39, 1896, 85.) Pathogenic for clover (Trifolium pratense, $T$. repens, $T$. resupinatum).

Bacillus tritus Batchelor. (Jour. Bact., 4, 1919, 29.) One culture from feces.

Bacillus tuberis von Wabl. (Cent. f. Bakt., II Abt., 16, 1906, 503.) From cooked truffles (Tuber oestivum).

Bacillus tuberosus Weiss. (Arb bakt.
Inst. Karlsruhe, 2, Heft 3, 1902, 248.) From fermenting beets.

Bacillus tubifex Dale. (Annals of Bot., 26, 1912, 133.) Reported to cause a leaf disease of potato (Solanum tuberosum) and tomato (Lycopersicum esculentum).

Bacillus turgidus (Duclaux) Trevisan. (Tyrothrix turgidus Duclaux, Ann. Inst. Nat. Agron., 4, 1882, 23; Trevisan, I generi e le specie delle Batteriacee, 1889 , 16.) From milk.

Bacillus tympani-cuniculi Morcos. (Jour. Bact., 23, 1932, 454.) Causes tympanitis in young rabbits.

Bacillus ubicuitarius Soriano. (Estudio sistematico de algunas bacterias esporuladas aerobias, Thesis, Univ. Buenos Aires, 1935, 569.) Four cultures isolated from soil.

Bacillus ulna Cohn. (Cohn, Beitr.z. Biol. d. Pflanz., 1, Heft 2, 1872, 177; also see Prazmowski, Untersuch. ü. d. Entwickelungsges. u. Fermentwirk. einiger Bakterienarten, Leipzig, 1880, 20.) Found once in an infusion of cooked egg-white.

Bacillus undulatus den Dooren de Jong. (Bull. Assoc. Diplômés de Microbiol. Nancy, No. 26-27, 1946, 12.) From soil.

Bacillus uvaeformis Kern. (Arb. bakt. Inst. Karlsruhe, 1, Heft 4, 1896, 415.) From the stomachs and intestines of birds.

Bacillus vaculatus Ravenel. (Mem. Nat. Acad. Sci., 8, 1896, 31.) From soil. Bacillus validus Heigener. (Cent. f. Bakt., II Abt., 93, 1935, 97.) Four cultures isolated from soil from Germany, Cuba, and Egypt.

Bacillus valinovorans Heigener. (Cent. f. Bakt., II Abt., 93, 1935, 104.) Good grow th on valine agar. Five strains isolated from soils from Egypt, Germany, Italy, and Palestine.

Bacillus varians Saito. (Jour. Coll. Sci,, Imp. Univ., Tokyo, 23, Art. 15, 1908, 50.) Isolated 11 times from garden air.

Bacillus ventricosus Heigener. (Cent. 
f. Bakt., II Abt., 93, 1935, 102; not Bacillus ventricosus Weiss, Arb. bakt. Inst. Karlsruhe, 2, 1898, 233.) One culture isolated from soil from Italy.

Bacillus ventriculus Kioch. (Botan. Zeitung, 46, 1888, 341.) From slices of carrot exposed to the air. Formed two spores in a spindle-shaped sporangium.

Bacillus vernicosus Zimmermann. (Bakt. unserer Trink- u. Nutzwässer, Chemnitz, II Reihe, 1894, 46; not Bacillus vernicosus Migula, Syst. d. Bakt., 2, 1900, 781.) From waste water.

Bacillus verticillatus Ravenel. (Ravenel, Mem. Nat. Acad. Sei., 8, 1896, 13; Bacterium verticillatum Chester, Man. Determ. Bact., 1901, 192.) From soil.

Bacillus vesicae Migula. (Bacillus septicus vesicae Clado, quoted from Eisenberg, Bakt. Diag., 3 Aufl., 1891, 341 ; Migula, Syst. d. Bakt., 2, 1900, 620.) From urine in a case of cystitis. Probably is Bacillus cladoi Trevisan.

Bacillus villosus Zimmermann. (Bakt. unserer Trink- u. Nutzwässer, Chemnitz, II Reihe, 1894, 38; not Bacillus villosus Keck, Inaug. Diss., Dorpat, 1890, 47.) From water.

Bacillus violaceus Eisenberg. (Bakt. Diag., 2 Aufl., 1888, 8; not Bacillus violaceus Schroeter, Kryptogamen-Flora von Schlesien, 3, 1886, 157; not Bacillus violaceus Frankland and Frankland, Ztschr. f. Hyg., 6, 1888, 394.) Said to produce central spores. From water. Bacillus viridi-glaucescens Sack. (Cent. f. Bakt., II Abt., 65, 1925, 113.) From several kinds of soil.

Bacillus viridiluteus Pagliani et al. (Grüngelber, nicht verflüssiger Bacillus, Eisenberg, Bakt. Diag., 1 Aufl., 1886, Tab. 6; Pagliani, Maggiora and Fratini, Soc. ital. d'igiene, 1887,586 , see Trevisan, I generi e le specie delle Batteriacee, 1889, 19). From water.

Bacillus viscosus bruxellensis van Laer. (Cent. f. Bakt., II Abt., 23, 1909, 159.) From beer wort.

Bacillus viticola Burgwitz. (Bacillus vitis Merjanian and Kovaleva, Prog. Agric. et Vitic., 95, 1930, 594 and 96, 1931,
17; not Bacillus vitis Montemartini, Rev. Patol. Veg., 6, 1913, 175; Burgwitz, Phytopath. Bacteria, Leningrad, 1935, 37.) Pathogenic for the grape vine.

Bacillus vitreus Migula. (No. 11, Lembke, Arch. f. Hyg., 26, 1896, 306; Migula, Syst. d. Bakt., 2, 1900, 569.) From the intestines of infants.

Bacillus vogelii Migula. (Roter Kartoffelbacillus, Vogel, Ztschr. f. Hyg., 26, 1897, 404; Migula, Syst. d. Bakt., 2, 1900,556 ; Bacillus viscosus Chester, Man. Determ. Bact., 1901, 286; not Bacillus viscosus Frankland and Frankland, Ztschr. f. Hyg., 6, 1889, 391.) From stringy bread.

Bacillus watzmannii Werner. (Cent.f. Bakt., II Abt., 87, 1933, 462.) Weak growth on $\mathrm{Ca}$ n-butyrate agar. One culture isolated from soil of Germany.

Bacillus weigmanni Migula. (Bakterie II, Weigmann and Zirn, Cent. f. Bakt., 15, 1894, 465; Migula, Syst. d. Bakt., 2, 1900, 693.) From soapy milk.

Bacillus xylanicus Patrick and Werkman. (Iowa State Coll. Jour. Sci., 7, 1933, 415.) Ferments xylan. One culture isolated from decayed apple wood.

Bacillus xylophagus Patrick and Werkman. (Iowa State Coll. Jour. Sci., 7, 1933, 414.) Ferments xylan. One culture isolated from decayed apple wood.

Bacillus zirnii Migula. (Bakterie III, Weigmann and Zirn, Cent. f. Bakt., 15, 1894, 466 ; Migula, Syst. d. Bakt., 2, 1900, 693.) From soapy milk.

Bacterium adametzii Migula. (Bacillus No. XIV, Adametz, Landwirtsch. Jahrb., 18, 1889, 246; Migula, Syst. d. Bakt., 2, 1900, 338; Bacterium rugosum Chester, Man. Determ. Bact., 1901, 194.) From cheese.

Bacterium aloes Passalacqua. (Rev. Pat. Veg., 19, 1929, 110.) From diseased aloes.

Bacterium angulans Burchard. (Inaug. Diss., 1897 ; Arb. bakt. Inst. Karlsruhe, 2, Heft 1, 1902, 43.) From water.

Bacterium aqueum Migula. (Bacillus thermophilus VIII, Rabinowitsch, Ztschr. f. Hyg., 20, 1895, 160; Migula, 
Syst. d. Bakt., 2, 1900, 345; Bacterium thermophilum VIII, Chester, Man. Determ. Bact., 1901, 186.) From feces and corn.

Bacterium articulatum Kern. (Arb. bakt. Inst. Karlsruhe, 1, Heft 4, 1897, 445.) From the stomach and intestines of birds.

Bacterium asparagi von Wahl. (Von Wahl, Cent. f. Bakt., II Abt., 16, 1906 , 498; Bacillus asparagi Lehmann and Neumann, Bakt. Diag., 4 Aufl., 2, 1907, 436.) From boiled asparagus.

Bacterium brachysporum Burchard. (Inaug. Diss., 1897; Arb. bakt. Inst. Farlsruhe, 2, Heft 1, 1902, 20.) From bakery bread.

Bacterium canadensis Chorine. (Internat. Corn Borer Invest., Sci. Rpts., 2, 1929, 39; also Ann. Inst. Past., 43, 1929, 1658; Bac. canadensis Chorine and Metalnikov, Ann. Inst. Past., 43, 1929, 1392; also Paillot, L'infection chez les insectes, 1933, $13 \pm$ where Bac. equals Baclerium, see index p. 522; Bacillus canadensis Steinhaus, Bacteria Associated Extracellularly with Insects, Minneapolis, 1942,50 .) In its general characters said to resemble Bacillus meqatherium and other bacteria isolated from insects (Bacillus thuringiensis, Bacillus hoplosternus, etc.). Pathogenic for larvae of Pyrausta nubilalis, Galleria mellonella, and Ephestia kuhniella. From diseased larvae of the corn borer.

Bacterium cattleyae Pavarino. (Atti R. Accad. Naz. Lincei Rend. Cl. Sci. Fis., Mat. e. Nat., 20, 1911, 233.) From diseased orchids.

Bacterium cazaubon Metalnikov. Compt. rend. Soc. Biol., Paris, 105, 1930, 536; two varieties, Bacterium cazaubon I and II, are recognized by Metalnikov, Ermolaev and Schobaltzyn, Internat. Corn Borer Invest., Sci. Repts., 3, 1930, 30 and Ann. Inst. Past., 46, 1931, 469.) From diseased corn borer larvae (Pyrausta nubilalis Hb.).

Bacterium christiei Chorine. (Internat. Corn Borer Invest., Sci. Rpts., 2, 1929, 46; also Anu. Inst. Past., 43, 1929, 1666.) According to the author, this closely resembles Bacterium ontarioni. Several strains isolated from diseased corn borers.

Bacterium colomatii Chester. (Colomatii, Breslauer arztliche Ztschr., 1883, No. 4 ; Chester, Man. Determ. Bact., 1901, 186.) From xerotic masses in conjunctivitis.

Bacterium deliense Swellengrebel (Archiv f. Protist., 31, 1913, 277.) Observed in stained smears from the spleen of diseased cattle but not isolated. Two spores may form in a single cell if division is delayed.

Bacterium ephestiae No. 1 and No. 2 Metalnikov and Chorine. (Ann. Inst. Past., 43, 1929, 1394.) Not pathogenic for corn borer although the size of the larvae was reduced. Later, Ellinger and Chorine (Internat. Corn Borer Investigations, Sci. Rpts., 3, 1930, 37) identified these as strains of Bacillus thuringiensis. From diseased larvae of Ephestia kuehniella.

Bacterium filiforme Henrici. (Henrici, Arb. bakt. Inst. Karlsruhe, 1 , Heft 1, 1894, 41; not Bacterium filiforme Migula, Syst. d. Bakt., 2, 1900, 296; Bacterium subfiliforme Migula, ibid., 297.) From Swiss cheese.

Bacterium galleriae No. 1, Chorine. (Bâtonnet mince, Metalnikov, Compt. rend. Acad. Sci., Paris, 175, 1922, 69; Chorine, Ann. Inst. Past., 41, 1927, 1115.) From diseased larvae of the bee moth (Galleria mellonella).

Bacterium galleriae Chorine. (Plus grand bâtonnet, Metalnikov, Compt. rend. Acad. Sci., Paris, 175, 1922, 70; Chorine, Compt. rend. Soc. Biol., Paris, 95, 1926, 200; Bacterium galleriae No.2, Chorine, Ann. Past. Inst., $41,1927,1117$. From diseased larvae of the bee moth (Galleria mellonella). Resembles Bacillus megatherium. Pathogenic for the corn borer (Internat. Corn Borer Invest., Sci. Repts., 1, 1927, 46).

Bacterium galleriae No. 3, Chorine. (Ann. Inst. Past., 41, 1927, 1118.) From diseased larvae of the bee moth (Galleriae mellonella). Resembles Bacillus subtilis and Bacillus mesentericus, 
Bacterium giganteum Kern. (Arb. bakt. Inst. Karlsruhe, 1, Heft 4, 1896 , 453.) From the stomach and intestines of birds.

Bacterium glaucescens Migula. ( $\mathrm{Ba}$ cillus thermophilus VI, Rabinowitsch, Ztsehr. f. Hyg., 20, 1895, 158; Migula. Syst. d. Bakt., 2, 1900, 344; Bacterium thermophilum VI, Chester, Man. Determ. Bact., 1901, 185.) From feces.

Bacterium glutinosum Kern. (Arb. bakt. Inst. Karlsruhe, 1, Heft 4, 1896. 441.) From the stomach of a dove

Bacterium ilidzense Migula. (Bacillus ilidzensis capsulatus Karlinski, $\mathrm{Hy}_{-}$ gienische Rundschau, 5, 1895, 688; Migula, Syst. d. Bakt., 2, 1900, 340.) From the water of a hot spring. Thermophilic.

Bacterium implectans Burchard. (Inaug. Diss., 1897 ; Arb. bakt. Inst. Karlsruhe, 2, Heft 1, 1898, 29.) From drinking water.

Bacterium insulosum Wilhelmy. (Arb. bakt. Inst. Karlsruhe, 3, 1903, 16.) From meat extract.

Bacterium insulum IVeiss. (Arb. bakt Inst. Karlsruhe, 2, Heft 3, 1902, 252.) From fermenting malt

Bacterium intactum Migula. (Bacillus thermophilus $V$, Rabinowitsch, Ztschr. f. Hyg., 20, 1895, 158; Migula, Syst. d. Bakt., 2, 1900, 344; Bacterium thermophilum V, Chester, Man. Determ. Bact., 1901, 185.) From feces and corn.

Bacterium iris Migula. (Irisierender Bacillus, Tataroff, Inaug. Diss., Dorpat, 1891, 57; Migula, Syst. d. Bakt., 2, 1900. 313; not Bacterium iris Chester, Anm. Rept. Del. Col. Agr. Exp. Sta., 9, 1897, 125.) From water

Bacterium italicum No. 1 and No. 2, Metalnikov, Ermolaev and Skobaltzyn. (Ann. Inst. Past., 46, 1931, 470; No. 2 is also described in Internat. Corn Borer Invest. Sci. Repts., 3, 1930, 30.) From larvae of the corn borer (Pyrausta nubilalis)

Bacterium longum Kern. (Kern, Arb. bakt. Inst. Karlsruhe, 1, Heft 4, 1894, 391 ; Bacterium squamosum longum Kern, ibid., 458; Bacillus squamosus longus Chorine, Ann. Inst. Past., 41, 1927, 1114.) From the intestines of a dove (Columba oenas).

Bacterium lunula Dobell. (Guart. Jour. Micro. Sici., 53, 1909, 579.) From rectum of the toad (Bufo vulgaris). Resembles Bacterium hinuclealum Swellengrebel.

Bacterium lydiae Migula. (Bacillus thermophilus I, Rabinowitsch, Ztschr.f. Hyg., 20, 1895, 156; Migula, Syst. d. Bakt., 2, 1900, 343; Bacterium thermophilum I, Chester, Man. Determ. Bact., 1901, 185.) Widely distributed in soil, snow, feces, corn.

Bacterium mansfieldii Chester. (Bacillus No. 18, Conn, Storrs Agr. Expt. Sta., 1893, 51; Chester, Man. Determ. Bact., 1901, 197.) From milk.

Bacterium markusfeldii Chester. (Bacillus der trichorrhexis nodosa, Markusfeld, Cent. f. Bakt., I Abt., 21, 1897, 230 ; Chester, Man. Determ. Bact., 1901, 192.) Associated with the disease, trichorrhexis nodosa.

Bacterium mesentericum Migula. (Bacillus mesentericus panis viscosi $I$, Vogel, Ztschr. f. Hyg., 26, 1897, 404; Migula, Syst.d. Bakt., 2, 1900, 314; Bacterium panis Chester, Man. Determ. Bact. 1901, 196.) From stringy bread dough.

Bacterium mesenteroides Migula. (Bacillus No. XVII, Adametz, Landw. Jahrb., 18, 1889, 249; Migula, Syst.d. Bakt., 2, 1900, 312; Bacterium viscosum Chester, Man. Determ. Bact., 1901, 194.) From cheese.

Bacterium modestum Bersteyn. (Arb. bakt. Inst. Karlsruhe, 3, 1903, 95.) From soil.

Bacterium monstrosum Henrici. (Arb. bakt. Inst. Karlsruhe, 1, Heft 1, 1894 , 47.) From Swiss cheese.

Bacterium nephritidis Migula. (Bacillus nephritidis interstitialis Letzerich, Ztschr. f. klin. Med., 13, 188-, 33; Migula, Syst. d. Bakt., 2, 1900, 310; not Bacterium nephritidis Chester, Man. Determ. Bact., 1901, 145.) From urine in cases of nephritis.

Bacterium ochraceum Migula. (Bacillus viscosus ochraceus Freund, Martin, 
Inaug. Diss., Erlangen, 1893, 37; Migula, Syst. d. Bakt., 2, 1900, 333.) From the oral cavity.

Bacterium olivae Montemartini. (Atti Inst. Bot. Pavia Univ., 2 ser., 14, 1914, 154.) From diseased olive branches.

Bacterium paludosum MeBeth. (Soil Sci., 1, 1916, 463.) Filter paper reduced to a white pulp-mass. From two soils in California.

Bacterium perittomaticum Burchard. (Arb. bakt. Inst. Karlsruhe, 2, 1898, 11.) Similar to or identical with Bacillus ruminatus (Gottheil, Cent. f. Bakt., II Abt., 7, 1901, 485). From soil.

Bacterium pituitans Burchard. (Inaug. Diss., 1897; Arb. bakt. Inst. Karlsrube, 2, Heft 1, 1898, 8.) From a brown concretion in a cooked egg.

Bacterium plicativum Weiss. (Weiss, Arb. bakt. Inst. Karlsruhe, 2, Heft 3, 1902, 223; not Bacterium plicativum Migula, Syst. d. Bakt., 2, 1900, v and 453.) From fermenting beets and malt.

Bacterium plicatum Henrici. (Henrici, Arb. bakt. Inst. Karlsruhe, 1 , Heft 1 , 1894, 49 ; not Bacterium plicalum Chester, Man. Determ. Bact., 1901, 166.) From brick cheese.

Bacterium pseudaceti Migula. (Ba cillus No. XV, Adametz, Landw. Jahrb., 18, 1889, 247; Migula, Syst. d. Bakt., 2, 1900, 320; Bacterium turgidum Chester, Man. Determ. Bact., 1901, 195.) From cheese. Characteristic involution forms very similar to those of Bacillus aceti Hansen.

Bacterium pseudomycoides Migula. (Migula, Syst. d. Bakt., 2, 1900, 486 ; Bacillus pseudomycoides roseus Nepveux, Thèse, Fac. Pharm., Nancy, 1920, 112.) From soil.

Bacterium pseudovermiculosum Saito. (Jour. Coll. Sci., Imp. Univ., Tokyo, 23, Art. 15, 1908, 62.) Isolated twice from garden air.

Bacterium pyrenei No. 1, No. 2 and No 3, Metalnikov, Ermolaev and Skobaltzyn. (Internat. Corn Borer Invest., 3, 1930, 28 and Ann. Inst. Past., 46, 1931, 467, 468 and 469 respectively; presumably the same as Bacillus pirenei Pospelov, Lenin Acad. Agr. Sci. (U.S.S.R.), Ann. Rept. 1936, 318-321.) No. 1 from diseased larvae of the corn borer (Pyrausta nubilalis) that had become black after death; No. 2 from larvae that had become brown; and No. 3 from larvae that had become pinkish-brown.

Bacterium radiatum Kern. (Kern, Arb. bakt. Inst. Karlsruhe, 1, Heft 4, 1896, 438; Bacterium barbatum Migula, Syst. d. Bakt., 2, 1900, 317.) From the stomach of a finch.

Bacterium rusticum Kern. (Arb. bakt. Inst. Karlsruhe, 1, Heft 4, 1896, 440.) From the stomach of a sparrow.

Bacterium sempervivum Migula. (No. XII, Flügge, Ztschr. f. Hyg., 17, 1894, 296 ; Bacillus lactis No. XII, Kruse, in Flügge, Die Mikroorganismen, 3 Aufl., 2, 1896, 269; Migula, Syst. d. Bakt., 2, 1900, 321.) From milk.

Bacterium serratum Kern. (Arb. bakt. Inst. Karlsruhe, 1, Heft 4, 1896, 451.) From the intestine of a dove.

Bacterium sewerini Migula. (Sewerin, Cent. f. Bakt., II Abt., 3, 1897, 709; Migula, Syst. d. Bakt., 2, 1900, 330.) From manure.

Bacterium spissum Kern. (Arb. bakt. Inst. Karlsruhe, 1, Heft 4, 1896, 446.) From the intestine of a bird.

Bacterium sputicola Migula. (Bacillus No. 4, Pansini, Arch. f. path. Anat., 122, 1890, 440; Migula, Syst. d. Bakt., 2, 1900, 306; Bacterium sputi Chester, Man. Determ. Bact., 1901, 190.) From sputum.

Bacterium streptococciforme Migula. (Bacillus thermophilus III, Rabinowitsch, Ztschr. f. Hyg., 20, 1895, 156; Migula, Syst. d. Bakt., 2, 1900, 343; Bacterium thermophilum III, Chester, Man. Determ. Bact., 1901, 185.) From soil, feces, corn.

Bacterium subdenticulatum Migula. (Bacillus thermophilus VII, Rabinowitsch, Ztschr. f. Hyg., 20, 1895, 158; Migula, Syst. d. Bakt., 2, 1900, 345; Bacterium thermophilum VII, Chester, Man. Determ. Bact., 1901, 185.) From feces. 
Bacterium subrubeum Kern. (Arb. bakt. Inst. Karlsruhe, 1, Heft 4, 1896, 450; Bacillus subrubeus Nepveux, Thèse, Fac. Pharm., Naney, 1920, 115.) From the intestines of birds.

Bacterium subsquamosum Migula. (Bacterium squamosum longum Kern, Arb. bakt. Inst. Karlsruhe, 1, Heft 4, 1896, 458; Migula, Syst. d. Bakt., 2, 1900, 335.) From the intestines of a dove.

Bacterium subthermophilum Migula. (Bacillus thermophilus IV, Rabinowitsch, Ztschr. f. Hyg., 20, 1895, 157 ; Migula, Syst. d. Bakt., 2, 1900, 344; Bacterium thermophilum IV, Chester, Man. Determ. Bact., 1901, 186.) From soil and feces.

Bacterium subtilis var. galleriae Chorine. (Ann. Inst. Past., 41, 1927, 1120.) From diseased larvae of the bee moth (Galleria mellonella).

Bacterium tenax Kern. (Arb. bakt. Inst. Karlsruhe, 1, Heft 4, 1896, 443.) From the stomachs of birds.

Bacterium terrae (Ucke) Chester. (Streptobacillus terrae Ucke, Cent. f. Bakt., I Abt., 23, 1898, 1001; Chester, Man. Determ. Bact., 1901, 199.) From soil.

Bacterium truncatum Chester. (Bacillus No. 51, Conn, Storrs Agr. Exp. Sta., 1894, 81; Chester, Man. Determ. Bact., 1901, 195; not Bacterium truncatum Migula, Syst. d. Bakt., 2, 1900, 407 ; not Bacterium truncatum Chester, ibid., 157.) From milk.

Bacterium verrucosum Kern. (Arb. bakt. Inst. Karlsruhe, 1, Heft 4, 1896, 434.) From the stomachs and intestines of birds.

Bacterium virgula (Duclaux) Migula. (Tyrothrix virgula Duclaux, Ann. Inst. Nat. Agron., 4, 1882, 23; Migula, Syst. d. Bakt., 2, 1900, 323.) From cheese.

Bacterium viride van Tieghem. (Van Tieghem, Bull. Soc. Bot. France, 1880, 174; Bacillus viridis Trevisan, I generi e le specie delle Batteriacee, 1889, 18.)

Cellulobacillus mucosus Simola. (Ann. Ac. Sc. Fenn., Ser. A, 34, 1931 ; abst. in
Cent. f. Bakt., II Abt., 86, 1932, 89.) Thermophilic; cellulose decomposed quickly at $55^{\circ}$ to $60^{\circ} \mathrm{C}$, more slowly at $37^{\circ} \mathrm{C}$.

Cellulobacillus myxogenes Simola (loc. cit.). Not slimy as above.

Clostridium gelatinosum Laxa. (Eine thermophilen Bacillus, Laxa, Cent. f. Bakt., II Abt., 4, 1898, 362 ; Laxa, ibid., $6,1900,286 ; 8,1902,154$; Bacterium sacchariphilum Migula, Syst. d. Bakt., 2, 1900, 341; Bacterium laxae Chester, Man. Determ. Bact., 1901, 187.) From sugar factory wastes. Produces slime in sucrose solutions. Probably a variety of Bacillus vulgatus according to Sacchetti (Cent. f. Bakt., II Abt., 95, 1936, 115).

Denitrobacterium thermophilum Ambroz. (Cent. f. Bakt., II Abt., 37, 1913, 3.) From soil.

Lactobacillus sporogenes HorowitzWlassowa and Nowotelnow. (Cent. f. Bakt., II Abt., 87, 1933, 331.) Resembles Lactobacillus delbrueckii but forms ellipsoidal, terminal spores.

Metabacterium polyspora Chatton and Perard. (Compt. rend. Soc. Biol., Paris, $65,1913,1232$.) The type species of the genus Metabacterium, characterized by forming one to eight spores in a single cell. From the caecum of guinea pigs. See Buchanan (Jour. Bact., 3, 1918, 39).

Myxobacillus betae Gonnermann. (Oesterreich-Ungarische Ztschr. f. Zuckerind. u. Landwirtsch., 36, 1907, 877; see Cent. f. Bakt., II Abt., 21, 1908, 258.) Produces slime in sucrose solutions. Appears to be closely related to Bacillus subtilis.

Nitrosobacillus thermophilus Campbell. (Science, 75, 1932, 23.) A thermophilic aerobic rod with swollen clavate sporangia; forms nitrites from ammonium salts. From surface layers of soil from North Carolina and Florida.

Semiclostridium commune, S. citreum, $S$. flavum and S. rubrum Maassen. (Arbt. a. d. biol. Abt. f. Land- u. Forstwirtsch. am kaiserl. Ges. Amt., 5, 1907, 1.) Produce slime in sucrose solutions. 


\section{Genus II. Clostridium Prazmowski.*}

(Prazmowski, Untersuchungen über die Entwickelungsgeschichte und Fermentwirkung einiger Bacterien-Arten, Inaug. Diss., Leipzig, 1880, 23; Vibrio Müller, Vermium terrestrium et fluviatilum, 1, 1773, 39; Bacterium Ehrenberg, Evertebrata, Berlin, 1828, (8? ***; Metallacter Perty, Zur Kenntniss kleinster Lebensformen, 1852, (180?); Amylobacter Trecul, Compt. rend. Acad. Sci., Paris, 61, 1865, 435; Bacillus Cohn, Beitr. z. Biol. d. Pflanzen, 1, Heft 2, 1872, 175; Tyrothrix Duclaux, Ann. Inst. Nat. Agron., 4, 1882, (79?); Pacinia Trevisan, Atti della Accad. Fis.-Med.-Statist., Milano, Ser.4, 3, 1885, (83?); Cornilia Trevisan, I generi e le specie delle Batteriacee, Milano, 1889, 21 ; Granulobacter Beijerinck, Verhandl. d. k. Akad. v. Wetensch., Amsterdam, Tweedie Sect., Deel I, 1893, 4; Bactridium, Paraplectrum, Diplectridium and Plectridium Fischer, Jahrb. f. Wissensch. Botan., 27, 1895, 139; Granulobacillus Schattenfroh and Grassberger, Cent. f. Bakt., II Abt., 5, 1899, 702; Streptobacillus Rist and Khoury, Ann. Inst. Past., 16, 1902, 70; Botulobacillus, Butyribacillus, Cellulobacillus, Putribacillus and Pectobacillus Orla-Jensen, Cent. f. Bakt., II Abt., 22, 1909, 342-343; Pectinobacter Makrinov, Arch. Sci. Biol. (Russ.), 18, 1915, 442; Bacteroides Castellani and Chalmers, Man. of Trop. Med., 3rd ed., 1919, 959; Butyriclostridium and Putriclostridium Orla-Jensen, Jour. Bact., 6, 1921, 263; Rivoltillus and Metchnikovillus Heller, Jour. Bact., 6, 1921, 550; Omelianskillus, Macintoshillus, Douglasillus, Henrillus, Flemingillus, Vallorillus, Multifermentans, Hiblerillus, Welchillus, Stoddardillus, Arloingillus, Meyerillus, Novillus, Seguinillus, Reglillus, Robertsonillus, Nicollaierillus, Martellillus, Recordillus, Tissierillus, Putrificus, Ermengemillus and Weinbergillus Heller, Jour. Bact., 7, 1922, 5-9; Peptoclostridium Donker, Inaug. Diss., Delft, 1926, 23; Botulinus, Chauvoea and Welchia Pribram, Jour. Bact., 18, 1929, 374; Anaerobacillus, Verrucosus and Euclostridium Janke, Cent.f. Bakt., II Abt., 80, 1930, 490 ; Butylobacter Bakonyi, U. S. Letters Pat., 1,818,782, 1931 ; Caduceus, Endosporus, Inflabilis, Palmula and Terminosporus Prévot, Ann. Inst. Past., 61, 1938, 76-86; Acuformis (syn. Palmula) Prévot, Man.d. Class., etc., 1940, 152.) From Greek, clostridium, a little spindle.

Rods, frequently enlarged at sporulation, producing clostridial or plectridial forms. The cells possess no catalase. Anaerobic or microaerophilic. Biochemically very active. Many species ferment carbohydrates producing various acids (frequently including butyric) and gas $\left(\mathrm{CO}_{2}, \mathrm{H}_{2}\right.$ and sometimes $\left.\mathrm{CH}_{4}\right)$. Others cause rapid putrefaction of proteins producing offensive odors. Commonly found in soil and in human or animal feces. Some species, while growing saprophytically on decomposing vegetable matter or on dead tissue within an animal host, form various toxic and lytic substances and are thereby pathogenic.

The type species is Clostridium butyricum Prazmowski.

\section{Key to the species of genus Clostridium.}

I. Strictly anaerobic.

A. Not typically fermenters of cellulose.

1. Do not characteristically produce distinctive pigments.

a. Spores central, excentric, to subterminal.

b. Spores oval.

* Revised by Prof. R. S. Spray, School of Medicine, West Virginia University, Morgantown, West Virginia, November, 1938; further revision May, 1942.

** In a few instances the original records were inaccessible. In such cases the page is indicated as (8?). In all other cases the page indicates what is believed to be the earliest record of the designation cited. 
c. Rods distinetly swollen at sporulation.

d. Motile.

e. Gelatin, or glucose gelatin, not liquefied.

f. Glucose fermented.

g. Coagulated albumin not liquefied.

h. Stormy fermentation, or at least active coagulation of milk. Also see hhh.

i. Glycerol not fermented.

j. Mannitol fermented.

k. Starch, lactose and sucrose fermented.

1. Clostridium butyricum.

kk. Starch not fermented. Lactose and sucrose fermented.

1a. Clostridium beijerinckii.

jj. Mannitol not fermented.

k. Starch and lactose not fermented.

1b. Clostridium pasteurianum.

ii. Glycerol fermented.

j. Mannitol not fermented.

k. Starch, lactose, sucrose and salicin fermented.

1c. Clostridium multifermentans.

hh. Milk slowly coagulated; not stormily. Also see hhh.

i. Glycerol and mannitol not fermented.

2. Clostridium fallax.

ii. Glycerol not recorded.

j. Acid, but no gas, from lactose and sucrose.

3. Clostridium fissum.

hhh. Milk not coagulated.

i. Glycerol not fermented.

4. Clostridium difficile.

gg. Coagulated albumin not recorded.

h. Milk acidified, but not coagulated.

5. Clostridium viscifaciens.

ee. Gelatin, or glucose gelatin, liquefied.

f. Glucose fermented.

g. Coagulated albumin not liquefied.

h. Milk slowly coagulated. Clot not digested.

i. Glycerol and mannitol not fermented.

j. Lactose fermented.

k. Sucrose not fermented. Salicin fermented.

6. Clostridium septicum.

kk. Sucrose fermented. Salicin not fermented.

7. Clostridium feseri.

ii. Glycerol fermented.

8. Clostridium hemolyticum.

hh. Milk acidified but not coagulated.

i. Glycerol fermented.

j. Mannitol not fermented.

k. Starch fermented. Lactose, sucrose and salicin 
not fermented. Exotoxin formed; toxic on injection but not on feeding.

9. Clostridium novyi.

kk. Starch not recorded.

1. Lactose, sucrose and salicin not fermented.

m. Adonitol fermented.

10. Clostridium botulinum.

mm. Adonitol not fermented.

10a. Clostridium botulinum. Type C

gg. Coagulated albumin slowly to rapidly liquefied.

h. Stormy fermentation, or at least active coagulation of milk. Clot not digested.

11. Clostridium acetobutylicum.

hh. Milk slowly and softly coagulated; not stormily. Clot slowly to rapidly digested.

i. Glycerol and mannitol not fermented. Also see iii.

j. Starch not recorded.

$\mathrm{k}$. Lactose fermented.

12. Clostridium aerofoetidum.

kk. Lactose not fermented.

13. Clostridium sporogenes.

13a. Clostridium sporogenes var. A. P. Marie.

13b. Clostridium sporogenes var. equine.

13c. Clostridium tyrosinogenes.

13d. Clostridium flabelliferum.

13e. Clostridium parasporogenes.

ii. Glycerol fermented. Also see iii.

j. Mannitol not fermented.

14. Clostridium parabotulinum

Types $\mathrm{A}$ and $\mathrm{B}$.

iii. Glycerol not recorded.

j. Mannitol and starch not recorded.

k. Lactose and sucrose weakly fermented.

1. Gas formed from earbohydrates.

15. Clostridium saccharolyticum.

11. Gas not formed from carbohydrates.

16. Clostridium regulare.

ff. Glucose not fermented. (Carbohydrates not fermented.)

g. Coagulated albumin not digested. Lab-coagulation of milk; increasing alkalinity. Clot digested.

17. Clostridium hastiforme.

gr. Coagulated albumin not recorded. Slow, mildly acid coagulation of milk. Clot digested.

18. Clostridium subterminale.

dd. Non-motile.

e. Gelatin, or glucose gelatin, not liquefied.

19. Clostridium malenominatum.

ce. Rods not swollen at sporulation. 
d. Motile.

e. Gelatin, or glucose gelatin, liquefied.

f. Glucose fermented.

g. Coagulated albumin liquefied. Milk slowly coagulated. Clot slowly digested.

20. Clostridium bifermentans.

gg. Coagulated albumin not recorded.

h. Milk slowly coagulated; slimy.

i. Gas formed from glucose.

21. Clostridium mucosum.

ii. Acid but no gas from glucose.

22. Clostridium pruchii.

ee. Iron-gelatin (Spray), no growth.

23. Clostridium cylindrosporum.

dd. Non-motile.

e. Gelatin, or glucose gelatin, liquefied.

f. Glucose fermented.

g. Coagulated albumin not liquefied.

h. Milk stormily fermented. Clot not digested.

i. Glycerol fermentation variable.

j. Mannitol not fermented. Starch, lactose and sucrose fermented. Salicin rarely fermented. Types identified by specific toxin-antitoxin neutralization.

24. Clostridium perfringens

Types A, B, C and D.

bb. Spores spherical.

c. Rods distinctly swollen at sporulation.

d. Motile.

e. Gelatin, or glucose gelatin, not liquefied.

f. Glucose fermented.

g. Coagulated albumin not liquefied.

h. Milk acidified; slowly and softly coagulated; not stormily. Clot not digested.

25. Clostridium sphenoides.

hh. Milk acidified but not coagulated.

26. Clostridium innominatum.

cc. Rods not swollen at sporulation.

d. Non-motile.

e. Gelatin, or glucose gelatin, not liquefied.

f. Glucose fermented.

g. Coagulated albumin not liquefied.

h. Milk acidified but not coagulated.

27. Clostridium filiforme.

aa. Spores terminal.

b. Spores distinctly oval to ellipsoid.

c. Rods distinctly swollen at sporulation.

d. Motile.

e. Trelatin, or glucose gelatin, not liquefied. Also see eee.

f. Glucose fermented. 
g. Coagulated albumin not liquefied.

h. Milk slowly coagulated. Clot not digested.

i. Glycerol not fermented.

j. Mannitol fermented.

28. Clostridium sartagoformum.

jj. Mannitol not fermented.

29. Clostridium paraputrificum.

ff. Glucose not fermented.

g. Coagulated albumin not liquefied. Milk unchanged.

30. Clostridium cochlearium.

gg. Coagulated albumin not recorded.

h. Milk, or iron-milk (Spray), no growth.

i. Carbohydrates not fermented.

j. Ethyl alcohol fermented chiefly to caproic acid.

31. Clostridium kluyverii.

jj. Ethyl alcohol not fermented to caproic acid.

32. Clostridium acidiurici.

ee. Gelatin, or glucose gelatin, liquefied. Also see eee.

f. Glucose fermented.

g. Coagulated albumin liquefied.

h. Milk often, but not always, coagulated. Clot, if formed, not digested.

33. Clostridium capitovale.

hh. Milk acidified but not coagulated. Slow peptonization.

i. Glycerol and mannitol not recorded.

j. Starch not fermented.

34. Clostridium parabifermentans.

ji. Starch not recorded. Lactose weakly fermented.

35. Clostridium ovalare.

eee. Gelatin, or glucose gelatin, not recorded. Glucose fermented with acid but no gas.

36. Clostridium zoogleicum.

bb. Spores spherical, or nearly so.

c. Rods distinctly swollen at sporulation.

d. Motile.

e. Gelatin, or glucose gelatin, not liquefied. Also see eee.

f. Glucose fermented.

g. Coagulated albumin not liquefied.

h. Milk slowly coagulated, not stormily. Clot not digested. Also see hhh.

37. Clostridium thermosaccharolyticum.

hh. Milk not coagulated; unchanged. Also see hhh.

38. Clostridium caloritolerans.

hhh. Milk slowly alkalinized; casein slowly separated.

39. Clostridium tetanoides.

ee. Gelatin, or glucose gelatin, liquefied. Also see eee.

f. Glucose not fermented. 
g. Coagulated albumin slowly liquefied.

h. Milk may show soft lab-coagulation. Clot not definitely digested.

40. Clostridium tetani.

hh. Milk shows slow, soft lab-coagulation. Clot slowly digested.

41. Clostridium lentoputrescens.

ff. Glucose weakly fermented.

g. Coagulated albumin slowly liquefied.

h. Milk variably coagulated. Clot, if formed, variably digested.

42. Clostridium filamentosum.

eee. Gelutin records at variance.

f. Glucose fermented.

g. Coagulated albumin not liquefied.

h. Milk not coagulated; unchanged.

dd. Non-motile.

43. Clostridium tetanomorphum.

e. Gelatin, or glucose gelatin, not liquefied.

f. Glucose fermented.

g. Coagulated albumin not recorded.

44. Clostridium alcaligenes.

ee. Gelatin, or glucose gelatin, liquefied.

f. Glucose fermented.

g. Coagulated albumin not liquefied.

45. Clostridium angulosum.

gg. Coagulated albumin liquefied.

46. Clostridium putrefaciens.

2. Characteristically produce pigments of varied colors.

a. Spores central, excentric, to subterminal.

b. Spores oval.

c. Rods distinctly swollen at sporulation.

d. Motile.

e. Gelatin, or glucose gelatin, not liquefied.

f. Black pigment formed around colonies in deep agar.

47. Clostridium nigrificans.

ff. Violet pigment formed in potato mash.

g. Indole is formed.

48. Clostridium belfantii.

gg. Indole is not formed.

48a. Clostridium maggiorai.

fff. Green pigment formed on potato slant.

$\mathrm{g}$. Indole is formed.

48b. Clostridium derossii.

48c. Clostridium ottolenghii.

48d. Clostridium paglianii.

$\mathrm{gg}$. Indole is not formed.

48e. Clostridium lustigii.

48f. Clostridium sclavoi. 
fff. Red pigment formed in potato mash.

g. Indole not recorded.

49. Clostridium venturelli.

ee. Gelatin, or glucose gelatin, liquefied.

f. Red to orange-red pigment formed, especially in starchy media.

g. Indole is not formed.

h. Stormy fermentation of milk. Clot slowly softened.

50. Clostridium roseum.

hh. Slow, spongy coagulation of milk. Clot slowly digested.

51. Clostridium chromogenes.

ff. Yellow-orange pigment formed in various media.

g. Indole is not formed.

h. Milk actively coagulated, not stormily. Clot is not digested.

52. Clostridium felsineum.

aa. Spores terminal.

b. Spores oval.

c. Rods distinctly swollen at sporulation.

d. Non-motile.

e. Gelatin, or glucose getamin, no liquefied.

f. Deep red pigment formed on potato slants.

53. Clostridium carbonei.

Typical fermenters of cellulose.

1. Do not characteristically produce distinctive pigments.

a. Spores terminal.

b. Spores distinctly oval to ellipsoid.

c. Rods distinctly swollen at sporulation.

d. Motile.

e. Gelatin, or glucose gelatin, liquefied. Ferments a variety of carbohydrates, other than cellulose, after prolonged cultivation.

54. Clostridium spumarum.

pe. Gelatin, or glucose gelatin, not recorded. Carbohydrates, other than cellulose, not fermented.

55. Clostridium werneri.

bb. Spores spherical, or nearly so.

c. Rods distinctly swollen at sporulation.

d. Non-motile.

56. Clostridium cellulosolvens.

Characteristic pigments produced in certain media.

a. Spores terminal.

b. Spores distinctly oval to ellipsoid. Rods distinctly swollen at sporulation.

57. Clostridium dissolvens.

bb. Spores spherical, or nearly so. Rods distinetly swollen at sporulation,

58. Clostridium omelianskii. 
II. Microaerophilic. Grow customarily as anaerobes, but are able to produce scan sometimes atypical, growth on aerobic agar slants.

A. Not typically fermenters of cellulose.

1. Do not characteristically produce distinctive pigments.

a. Spores central, excentric, to subterminal. Spores oval. Rods dis tinctly swollen at sporulation.

59. Clostridium carnis.

ee. Gelatin, or glucose gelatin, liquefied.

f. Carbohydrates not fermented.

60. Clostridium histolyticum.

aa. Spores terminal. Spores distinctly oval to ellipsoid. Rods distinctly swollen at sporulation.

\section{Clostridium tertium.}

1. Clostridium butyricum Prazmowski. (Untersuch. ü. d. Entwickelungsgeschischte ü. Fermentwirk. einiger BacterienArten, Inaug. Diss., Leipzig, 1880, 23; Bacillus butyricus Flügge, Die Mikroorg., 2 Aufl., 1886, 296.) From M. L., acidum butyricum, butyric acid.

Described from the original incomplete records of Prazmowski, as amplified by the studies of Adamson, Jour. Path. and Bact., 22, 1919, 371, and of Hall, Jour. Inf. Dis., 30, 1922, 467.

Rods : 0.7 by 5.0 to 7.0 microns, straight or slightly curved, with rounded ends, occurring singly, in pairs, in short chains and occasional long filaments. Motile. Spores oval, excentric to subterminal, swelling rods to clostridial forms. Grampositive, becoming Gram-negative.

Granulose positive in clostridial stage (blue color with iodine).

Gelatin and glucose gelatin: Not liquefied.

Plain agar slant (anaerobic): Little or no growth.

Glucose agar surface colonies (anaerobic): Circular or slightly irregular, slightly raised, moist, creamy-white.

Deep glucose agar colonies: Biconvex, dense, yellowish-white, entire. Agar fragmented early by abundant gas.

Blood agar not hemolyzed.

Plain broth : Little or no growth.

Glucose broth: Abundant, diffuse turbidity; much gas.

Litmus milk: Acid and early coagulation. Litmus is reduced. Stormy fer- mentation; clot fragmented but not digested.

Indole not formed.

Nitrites not produced from nitrates.

Fixes atmospheric nitrogen.

Acid and gas from xylose, glucose, lactose, sucrose, starch, salicin, esculin and mannitol. Amygdalin, pectin, cellulose, glycerol and Ca-lactate not fermented.

Fermentation products include butyl, ethyl and iso-propyl alcohols, acetone, organic acids, $\mathrm{H}_{2}$ and $\mathrm{CO}_{2}$.

Coagulated albumin not liquefied.

Blood serum not liquefied.

Brain medium not blackened or digested.

Non-pathogenic for guinea pig and rabbit.

Grows well from $30^{\circ} \mathrm{C}$ to $37^{\circ} \mathrm{C}$.

Anaerobic.

Source: Originally isolated from cheese. Commonly encountered in naturally soured milk, in naturally fermented starchy plant substances and in soil.

Habitat: Probably rather widely dispersed in soils rich in humus.

Note: Many butyric acid-producing anaerobes are recorded in the literature. The questionable purity and the incomplete descriptions, particularly of the older species, make it difficult to determine the degree of relationship of these species to Clostridium butyricum Prazmowski. The following list cites the outstanding historic or recently described species. 
Ferment butyrique, Pasteur, Compt. rend. Acad. Sci., Paris, 52, 1861, 345 (Vibrion butyrique, Pasteur, ibid., 1261); Bacillus amylabacter van Tieghem, Bull. de la Soc. Botan. de France, 24, 1877, 128 (Metallacter amylobacter Trevisan, Reale Ist. Lombardo d. Sci. e Lett., Rendiconti, 1879, 147; Clostridium amylobacter Holland, Jour. Bact., 5, 1920, 217); Bacterium navicula Reinke and Berthold, Untersuch. a. d. Bot. Lab. d. Univ. Göttingen, 1, 1879, 21 (Amylobacter navicula Wehmer, Cent. f. Bakt., II Abt., 4, 1898, 696; Bacillus naricula Chester, Ann. Rept. Del. Col. Agr. Exp. Sta., 10, 1898, 128; Clostridium naviculum Prévot, Ann. Inst. Past., 61, 1938, 80); Bacillus butylicus Fitz, Ber. d. Deuts. Chem. Gesellsch., 15, 1882, 867 (Bacterium fitz Buchner, Ztschr. f. Physiol. Chem., 9, 1885, 384); Butylbacillus, Buchner, ibid., 391; Clostridium butyricum (Bacillus amylobacter) I, II, III, Gruber, Cent. f. Bakt., 1, 1887, 370-371; Bacillus butylicus Migula, Syst. d. Bakt., 2, 1900, 598 (Clostridium butyricum I, Gruber, Cent. f. Bakt., 1, 1887, 370); Bacillus gruberi Migula, loc. cit., 599 (Clostridium butyricum II, Gruber, loc. cit., 371); Bacillus subanaerobius Migula, loc. cit., 600 (Clostridium butyricum III, Gruber, loc. cit., 371); Bacille amylozyme, also bacille amylocyme, Perdrix, Ann. Inst. Past., 5, 1891, 290 (Bacillus amylozyma Migula, Syst. d. Bakt., 2, 1900, 626; Bacillus amylozymicus Peterson, Scott and Thompson, Biochem. Ztschr., 219, 1930, 1; Clostridium amylozyme Prévot, Ann. Inst. Past., 61, 1938, 79; Clostridium var. amylozyme Prévot, Man. d. Class., ete., 1940, 109); Bacillus orthobutylicus Grimbert, Inn. Inst. Past., 7, 1893, 353; Granulobacter butylicum Beijerinck, Verhandl. d. K. Akad. v. Wetensch., Amsterdam, Tweedie Sectie, Deel I, 1893, 3 (Clostridium butylicum Donker, Thesis, Delft, 1926, 149; Amylobacter butylicum van Beynum and Pette, Cent. f. Bakt., II Abt., 93,
1935,200 ; this species is probably identical with Clostridium butyricum I Gruber, Cent. f. Bakt., I Abt., 1, 1887, 370); Granulobacter saccharobutyricum Beijerinck, loc. cit., 3, also in Arch. Néerland. d. Sci. Exactes et Nat., 29, 1896, 1 (commonly identified with Bacillus butylicus Fitz, Ber. d. Deuts. Chem. Gesellsch., 15, 1882, 867; Bacillus humosus Migula, Syst. d. Bakt., 2, 1900, 600 ; Clostridium saccharobutyricum Donker, Thesis, Delft, 1926, 147; Amylobacter saccharobutyricus van Beynum and Pette, Cent. f. Bakt., II Abt., 93, 1935, 200); Bacillus saccharobutyricus von Klecki, Cent. f. Bakt., II Abt., 2, 1896, 169; Bactridium butyricum Chudiakow, Zur Lehre von der Anaerobiose (Russ.), Teil I, 1896, (?), cited by Rothert, Cent. f. Bakt., II Abt., 4, 1898, 390 ; Granulobacillus saccharobutyricus mobilis non-liquefaciens Schattenfroh and Grassberger, Cent. f. Bakt., II Abt., 5, 1899, 702 (bewegliche Buttersäurebacillus, Grassberger and Schattenfroh, Arch. f. Hyg., 42, 1902, 219; Bacillus saccharobutyricus mobilis Hopffe, Ztschr. Infekrnkh. d. Haust., 14, 1913, 396; Bacillus amylobacter mobilis Gratz and Vas, Cent. f. Bakt., II Abt., 41, 1914, 509); Plectridium pectinovorum Störmer, Mitteil. d. Deuts. Landwirts. Gesellsch., 18, 1903, 195 (Microbe du rouissage, Winogradsky, Compt. rend. Acad. Sci., Paris, 121, 1895, 74t; Granulobacter pectinovorum Beijerinck and van Delden, Arch. Néerland. d. Sci. Exactes et Nat., Ser. II, 9, 1904, 423; Clostridium pectinovorum Donker, Thesis, Delft, 1926, 145); Bacillus holobutyricus Perdrix, Compt. rend. Soc. Biol., Paris, 57, 1904, 481; Bacillus amylobacter Bredemann, Cent. f. Bakt. II Abt., 23, 1909, 385 (Clostridium amylobacter Prévot, Ann. Inst. Past., 61, 1938, 79); Amylobacter nonliquefaciens Ruschmann and Bavendamm, Cent. f. Bakt., II Abt., 64, 1925, 359; Clostridium intermedium Donker, Thesis, Delft, 1926, 147 (Strain No. 3, Donker, ibid., 39); Clostridium butyricum iodophilum 
Svartz, Jour. Inf. Dis., 47, 1930, 138 (Clostridium iodophilum Prévot, Ann. Inst. Past., 61, 1938, 80); Granulobacter saccharobutyricus immobile nonliquefaciens McCoy, Fred, Peterson and Hastings, Jour. Inf. Dis., 46, 1930, 121 ; Bacillus amylobacter $S$ and $W$, Wertheim, U. S. Letters Pat., 1,917,676, 1933; Clostridium tyrobutyricum van Beynum and Pette, Cent. f. Bakt., II Abt., 93, 1935, 208; Clostridium polyfermenticum, Clostridium saccharopetum, Clostridium saccharophilicum and Clostridium succharopostulatum Partansky and Henry. Jour. Bact., 30, 1935, 564.

1a. Clostridium beijerinckii Donker. (Donker, Thesis, Delft, 1926, 145.) Named for M. W. Beijerinck, the Dutch bacteriologist.

Has the general characters of Clostridium butyricum.

Distinctive character: Non-fermentation of starch.

Acid and gas from glucose, lactose, sucrose, inulin, galactose, fructose and mannitol. Glycerol and starch not fermented.

Source : From soil and fermenting plant tissues.

Habitat: Apparently widely distributed in agricultural soils.

1b. Clostridium pasteurianum Winogradsky. (Winogradsky, Arch. Sci. Biol. (Russ.), 3, 1895, 330; Clostridium pastorianum Winogradsky, Cent. f. Bakt., II Abt., 9, 1902, 43; Bacillus pasteurianus Lehmann and Neumann, Bakt. Diag., 4th Auf., 2, 1907,82; Bacillus pastorianus Lehmann and Neumann, ibid., 462; not Bacillus pastorianus Macé, Traité Prat. d. Bact., 4th ed., 1901, 957; Bacillus winogradskyi Weinberg et al., Les Microbes Anaér., 1937, 645.) Named for Louis Pasteur, the French scientist.

Probably related species: Bodily, Univ. Colorado Studies, 26, 1938, 30, records 5 new species isolated from 10 strains re- ceived labeled $C$. pasteurianum. These have been designated as Bacillus dulcitofermentans, Bacillus rhamnoticus, Bacillus inulofugus, Bacillus nonpentosus and Bacillus azoticus.

Has the general characters of Clostridium butyricum.

Distinctive characters: Prolonged retention of the spore within a peculiar brush-like spore-capsule, and the nonfermentation of starch. Assimilates free atmospheric nitrogen.

Distinguished from Clostridium beijerinckii by the non-fermentation of lactose and mannitol, and from Clostridium butyricum by the non-fermentation of starch.

Acid and gas from glucose, sucrose, inulin, galactose, fructose and dextrin. Glycerol, starch, lactose and mannitol not fermented.

Source: Originally isolated from soil.

Habitat: Not determined, but apparently of restricted and local distribution in soil.

1c. Clostridium multifermentans Bergey et al. (Bacillus multifermentans tenalbus Stoddard, Lancet, 1, 1912, 12; Multifermentans tenalbus Heller, Jour. Bact., 7, 1922, 6; Bergey et al., Manual, 1st ed., 1923, 324.) From Latin, multus, many, and fermentans, fermenting.

Has the general characters of Clos. tridium butyricum, and is probably only a variety.

Distinctive character: Blood agar colonies show a zone of hemolysis in 24 hours.

Nitrites are produced from nitrates.

Distinguished from Clostridium butyricum by the above characters and by the fermentation of glycerol and nonfermentation of mannitol.

Distinguished from Clostridium beijerinckii by the fermentation of starch and of glycerol.

Distinguished from Clostridium pasteurianum by fermentation of starch and of lactose. 
Acid and gas from glucose, fructose, galactose, maltose, lactose, sucrose, raffinose, starch, salicin, inulin and glycerol. Mannitol and dulcitol not fermented.

Source: Originally isolated from human gaseous gangrene.

Habitat: Found in soil and milk. Widely distributed in nature.

2. Clostridium fallax (Weinberg and Seguin) Bergey et al. (Bacille $\mathrm{A}$. Weinberg and Seguin, Compt. rend. Soc. Biol., Paris, 78, 1915, 277; Bacillus fallax Weinberg and Seguin, ibid., 686; not Bacillus fallax Ornstein, Ztschr. f. Hyg., 91, 1920, 159; Vallorillus fallax Heller, Jour. Bact., 7, 1922, 6; Bergey et al., Manual, 1st ed., 1923, 325.) From Latin, fallax, deceptive.

Rods : 0.6 by 1.2 to 5.0 microns, occurring singly or rarely in pairs. Motile with peritrichous flagella. Eneapsulated in body fluids. Spores rarely observed, oval, excentric to subterminal, swelling rods. Gram-positive.

Gelatin not liquefied.

Glucose agar surface colonies (anaerobic): Circular, flat, with transparent, crenated margin.

Glucose agar deep colonies : Lenticular, bean-shaped, irregular, smooth.

Agar slant (anaerobic): Grayish film.

Broth: Poor growth; slight diffuse turbidity.

Glucose broth: Abundant turbidity and gas. Clearing by sedimentation.

Indole not formed (Duffett, Jour. Bact., 29, 1935, 576).

Litmus milk: Acid, slowly coagulated. Litmus reduced. Clot channeled by gas, but not digested.

Acid and gas from glucose, galactose, fructose, maltose, lactose, sucrose, inulin, salicin and starch. Glycerol and mannitol not fermented. Records vary in regard to action on lactose, inulin and salicin.

Coagulated albumin not liquefied.

Blood serum not liquefied.

Brain medium not blackened or digested.
Meat medium reddened; not blackened or digested.

Pathogenicity for guinea pig variable, and commonly lost in cultivation. Forms a weak exotoxin.

Optinum temperature not recorded; grows well at $37^{\circ} \mathrm{C}$.

Anaerobic.

Source: From war wounds, appendicitis, and once from black-leg of sheep.

Habitat: Not determined, other than these sources.

3. Clostridium fissum (Debono) Bergey et al. (Bacillus fissus Debono, Cent. f. Bakt., I Abt., Orig., 62, 1912, 232; Bergey et al., Manual, 1st ed., 1923, 332.) From Latin, fissum, separated.

Rods: Variable in size, rounded or square ends, occurring singly, in pairs and in chains and filaments. Motile. Spores small, oval, subterminal, slightly swelling rods. Gram-positive.

Gelatin: Not liquefied.

Deep gelatin colonies at $22^{\circ} \mathrm{C}$ : Small, brownish, globular, opaque and entire.

Deep glucose agar colonies: Small, white, globular. Gas is formed. No pigment formed.

Broth: Uniformly turbid.

Milk: Acid, coagulated after 3 days.

Indole not formed.

Acid and gas from glucose. Acid only in lactose and sucrose.

Coagulated albumin not liquefied.

Grows at $22^{\circ} \mathrm{C}$ and $37^{\circ} \mathrm{C}$.

Anaerobic.

Distinctive character: All cultures smell strongly of butyric acid.

Source: From human feces.

Habitat: Not determined, other than this source.

4. Clostridium difficile (Hall and O'Toole) Prévot. (Bacillus difficilis Hall and O'Toole, Amer. Jour. Dis. Child., 49, 1935, 390; Clostridium difficilis Prévot, Ann. Inst. Past. 61, 1938, 84.) From Latin, difficilis, difficult.

Rods :Heavy-bodied. Actively motile. 
Spores elongate, subterminal swelling rods. Gram-positive.

Gelatin : Not liquefied.

Blood agar surface colonies (anaerobic): Irregular, flat and non-hemolytic.

Deep agar colonies: Minute, flat, opaque disks, becoming lobate.

Milk: Poor growth. Gas formed in traces, but milk unchanged.

Acid and gas from glucose, fructose, mannitol, salicin and xylose. Traces of gas, but no acid, from galactose, maltose, sucrose, lactose, raffinose, inulin and glycerol.

Coagulated albumin not liquefied.

Blood serum not liquefied.

Brain medium with iron is moderately blackened. Digestion not recorded.

Pathogenic for guinea pig and rabbit. Subcutaneous inoculation induces marked edema. Death may occur in from 1 to 9 days.

Toxicity: Glucose broth culture filtrates kill guinea pig and rabbit in 24 to 36 hours.

Grows well at $37^{\circ} \mathrm{C}$.

Anaerobic.

Source : From feces of new-born infants.

Habitat: Not determined, other than this source.

5. Clostridium viscifaciens Sherman and Erb. (U. S. Pat., 2,017,572, 1935.) From Latin, viscus, birdlime, glue; $f a$ ciens, making.

Rods: Vegetative cells 3 to 10 microns long; average about 6 microns. Motile. Spores oval, 1 by 2 microns, central to subterminal, sometimes swelling rods to club-like and spindle-shaped cells. Gram-negative.

Granulose reaction positive.

Gelatin: Not liquefied.

Plain agar slant (anaerobic): No growth.
Plain agar stab : No growth.

Liquid media: Tendency toward flocculent growth.

Milk: Acidified but not coagulated. Casein not digested.

Corn mash : Not fermented or digested.

Indole not formed.

Nitrites produced from nitrates.

Ammonia produced from peptone.

Acid, gas and alcohols produced from glucose and maltose.

Acid and gas from sucrose, lactose, dextrin, starch, glycerol, mannitol and salicin.

Calcium lactate: Not fermented.

Fermentation products include butyl alcohol (66 parts), iso-propyl alcohol (31 parts), and small amounts of acetone (3 parts).

Limiting reaction for grow th: About $\mathrm{pH}$ 4.0 to about $\mathrm{pH} 8.0$.

Optimum temperature $32^{\circ} \mathrm{C}$ to $36^{\circ} \mathrm{C}$. Grows from $15^{\circ} \mathrm{C}$ to $42.5^{\circ} \mathrm{C}$.

Anaerobic.

Distinctive character: In fermentable sugar broths it produces a copious flocculum.

Source: From soil and from grains and other plant materials in contact with soil.

Habitat: Apparently widely dispersed in agricultural soils.

6. Clostridium septicum (Macé) Ford.* (Vibrion septique, Pasteur and Joubert, Compt. rend. Acad. Sci., Paris, 85, 1877, 113, and Bull. Acad. Med., $2^{\circ}$ Ser., 6, 1877, 794; Vibrio pasteurii Trevisan, Reale Ist. Lombardo d. Sci. e. Lett., Rendiconti, Ser. 2, 12, 1879, 147; Bacillus septicus Macé, Traité Prat. d. Bact., 1st ed., 1888, 455; not Bacillus septicus Migula, Syst. d. Bakt., 2, 1900, 646 (Unnamed aerobic bacillus, Babes, Sept. Proc. d. Kindesalters, Leipzig, 1889, 32; Bacillus septicus ulceris gangraenosi

* Note: In an editorial, Jour. Amer. Vet. Med. Assoc., 62, 1922-23, 565, the name Clostridium septicum is ascribed to Winslow et al., Jour. Bact., 5, 1920, 191. Search fails to confirm the reference. Casual mention is not regarded as sufficient to establish priority. Hence, Ford is regarded as the author of this binomial. 
Sternberg, Man. Bact., 1893, 472); not Bacillus septicus Klein, Micro-organisms and Disease, 1884, 78; Cornitia pasteuri 'Trevisan, I generi e le specie delle Batteriacee, 1889, 22; Bacillus septicus gangrenae Arloing, Leçons sur la tuberculose et certaines septicémies, Paris, 1892, 451; Vibriogene septique, Rosenthal, Compt. rend. Soc. Biol., Paris, 64, 1908, 398; Vibrio septique LeBlaye and Guggenheim, Man. Prat. d. Diag. Bact., 1914, 438; Rivoltillus vibrion Heller, Jour. Bact., 7, 1922 , 6; Bacillus parasarkophysematos Miessner, Cent. f. Bakt., I Abt., Orig., 89 (Bhft.), 1922, 126, and Deuts. Tierarztl. Wchnschr., 30, 1922, 416 (Bacillus parasarcophysematos Zeissler, Cent. f. Bakt., I Abt., Orig., 89 (Bhft.), 1922, 119); Vibrio septicus Rottgardt, Deuts. Tierarztl. Wehnschr., 34, 1926, 553; Ford, Textbook of Bact., 1927, 726; Clostridium septicus Scott, Cornell Vet., 18, 1928, 259; Clostridium septique Topley and Wilson, Princ of Bact. and Immunol., 1st ed., 2, 1929, 1161.) From Greek, septicus, putrefactive, septic.

Probable synonym: Bacillus of Ghon and Sachs, Cent. f. Bakt., I Abt., Orig., 34, 1903, 289.

Identical or closely related species: Clostridium balaenae Prévot, Ann. Inst. Past., 61, 1938, 81 (Walfischseptikämie Bacillus, Nielsen, Cent. f. Bakt., 7, 1890, 269; Bacille de la septicémie de la baleine, Christiansen, Compt. rend. Soc. Biol., Paris, 83, 1920, 324; Wal fischbazillus, Christiansen, Cent. f. Bakt., I Abt., Orig., 84, 1920, 127); Bacillus gastromycosis ovis Kitt, Bakt. u. Path. Mikros., 2 Aufl., 1893, 239 (Bradsotbacillus, Nielsen, Monats. Prakt. Tierhlk., 8, 1897, 59); Bacillus tumefaciens Wilson, Lancet, 196, 1919, 657 (Clostridium tumefaciens Prévot, Ann. Inst. Past., 61, 1938, 81); not Bacillus tumefaciens Israilsky, Cent. f. Bakt., II Abt., 67, 1926, 236; Bacillus seu Clostridium sarcophysematos bovis Kitt, Bakterienkunde u. Path. Mikros., 2 Aufl., 1893, 232 (Bacillus sarcophysematos Kitt, ibid., Index, X; not Bacillus sarcophysematos Zeissler, Cent. f. Bakt., I
Abt., Orig., 89 (Bhft.), 1922, 119.) (See Clostridium feseri.)

Confused in the older literature with Koch's bacillus of malignant edema, Mitt. a. d. kais. Gesundhts., 1, 1881, 54 ( $B a$ cillus oedematis maligni Zopf, Die Spaltpilze, 3 Aufl., 1885, 88; Clostridium oedematis malignis Fischer, Jahrb. f. Wissen. Bot., 27, 1895, (146?); Bacillus oedematis Schroeter, in Cohn's Kryptogamen-Flora v. Schlesien, 3, 1, 1886, 163; Clostridium edematis Holland, Jour. Bact., 5, 1920, 218; Clostridium oedematis-maligni Bergey et al., Manual, 1st ed., 1923, 325).

It is commonly believed at present that Koch's bacillus of malignant edema was a culture of Clostridium septicum contaminated with Clostridium sporogenes or some closely related organism.

Described from Weinberg and Seguin, La Gang. Gaz., Paris, 1918, 79, and from Hall, Jour. Inf. Dis., 30, 1922, 486.

Rods : 0.6 to 0.8 by 3.0 to 8.0 microns, rounded ends, occurring singly, in pairs and in short chains in cultures; long chains and filaments commonly predominate in body exudates. Motile, with peritrichous flagella. Spores oval, excentric to subterminal, swelling rods. Gram-positive.

Gelatin: Liquefied, with gas bubbles.

Agar surface colonies (anaerobic): Small, transparent, of variable shape.

Blood agar surface colonies (anaerobic): Delicate, flat, leaf-like, irregular. Hemolytic.

Deep agar colonies: Variable; usually finely filamentous, cottony, spherical.

Broth: Slight, diffuse turbidity, with clearing.

Litmus milk: Litmus reduced; slow coagulation and moderate gas. Clot not digested.

Indole not formed.

Nitrites produced from nitrates.

Acid and gas from glucose, fructose, galactose, maltose, lactose and salicin. Sucrose, inulin, mannitol and glyeərol not fermented (Hall, loc. cit., 489).

Coagulated albumin not liquefied.

Blood serum not liquefied. 
Brain medium not blackened or digested.

Meat medium reddened; not blackened or digested.

Pathogenic for guinea pig, rabbit, mouse and pigeon. Forms an exotoxin for which an antitoxin is prepared.

Optimum temperature about $37^{\circ} \mathrm{C}$.

Anaerobic.

Source: Originally isolated from animals inoculated with soil; later from malignant edema of animals, and from human war wounds and from appendicit is

Habitat: Animal intestine, and in manured soils.

7. Clostridium feseri Trevisan. (Beweglichen Bakterien, Feser, Ztschr. f. Prakt. Vet.-Wissensch., 4, 1876, 19; Trevisan, Atti Accad. Fis.-Med.-Stat. di Milano, 3, 1885, 116; Bacterium chauvoei Arloing, Cornevin and Thomas, Le charbon symptomatique du boeuf, Paris, 2nd ed., 1887, 82; Bacillus chauvoei De Toni and Trevisan, in Saccardo, Sylloge Fungorum, 8, 1889, 1004; Bacillus chauvaei Trevisan, I generi e le specie delle Batteriacee, 1889, 22; Bacillus feseri Kitt, Bacterienkunde, ete., 2 Aufl., 1893, 233; Bacillus anthracis symptomatici Liruse, in Flügge, Die Mikroorg., 3 Aufl., 2, 1896, 245; Bacillus carbonis Migula, in Engler and Prantl, Die natür. Pflanzenfam., 1, 1a, 1895, 26; Butyribacillus chauvoei Orla-Jensen, Cent. f. Bakt., II Abt., 2E, 1909, 342; Bacillus gangraenae emphysematosae Hutyra and Marek, Spez. Path. u. Ther. d. Haust., 3 Aufl., 1, 1910, 39; Bacillus chauvei Holland, Jour. Bact., 5, 1920, 217; Clostridium chauvei Holland, ibid., 217; Bacillus anthracis-symptomatici Holland, ibid., 217; Clostridium anthracis-symptomatici Holland, ibid., 217; Bacillus sarkophysematos Miessner, Cent. f. Bakt., I Abt., Orig., 89 (Bhft.), 1922, 123 (Bacillus sarcophysematos Zeissler, ibid., 119; not Bacillus sarcophysematos Kitt, Bakterienkunde, etc., 2 Aufl., 1893, Index, X); Bacillus symptomaticus Matouschek, Cent. f. Bakt., II Abt., 58, 1923, 472; Clostridium chauvoei Scott, Jour. Inf. Dis., 38, 1926, 262; Clostridium chauvaei Scott, Cornell Vet., 18, 1928, 259.) Named for Feser, an early German bacteriologist.

Possible synonyms: Bacterio ocello cuneato, Rivolta, Giorn. di Anat., Fisiol. e Patol. d. Animali, Piso, 14, 1882, 33; Bacillum cuneatum, Rivolta, ibid., 67; Bacillum ocello-cuneat um, Rivolta, ibid., 67; Bacterium cuneatum Rivolta, ibid., 77; Bacterium ocello cuneatum Rivolta, ibid., 78; Bacillus sarcophysematosi Peppler, Cent. f. Bakt., I Abt., 29, 1901, 354.

Rods : 1.0 by 3.0 to 8.0 microns, occurring singly, in pairs and in short chains. Usually show a dark chromatic point near each end. Motile with peritrichous flagella. Spores oval, excentric to subterminal, swelling rods. Gram-positive.

Gelatin: Liquefied. Gas bubbles.

Agar surface colonies (anaerobic): Simall, grayish, semi-opaque, filamentous.

Agar slant (anaerobic): Grayish, spreading growth.

Broth: Turbid, slightly peptolytic.

Litmus milk: Acid; slowly coagulated. Gas may be formed. Clot not digested.

Indole not formed (early studies record only a trace).

Acid and gas from glucose, fructose, galactose, maltose, lactose and sucrose. Inulin, salicin, mannitol, glycerol and dextrin not fermented (Hall, Jour. Inf. Dis., 30, 1922, 486).

Coagulated albumin not liquefied.

Blood serum not liquefied.

Brain medium not blackened or digested.

Egg-meat modium: Small gas bubbles in 8 hours. Meat becomes pinkish and the liquid slightly turbid. No blackening or digestion.

Pathogenic for guinea pig, mouse and rabbit. Forms an exotoxin.

Optimum temperature $37^{\circ} \mathrm{C}$. Can grow at $50^{\circ} \mathrm{C}$.

Anaerobic

Source: The cause of black leg, black quarter or symptomatic anthrax in cattle and other animals.

Habitat: Probably soil; especially where heavily manured. 
8. Clostridium hemolyticum (Hall) Hauduroy etal. (Clostridium hemolyticus bovis Vawter and Records, Jour. Amer. Vet. Med. Assoc., 68 (N.S. 21), 1925-26, 512 ; Bacillus hemolyticus Hall, Jour. Inf. Dis., 45, 1929, 156; Hauduroy et al., Dict. d. Bact. Path., 1937, 125.) From Greek, haemo, blood; lyticus, dissolving.

Related species: Clostridium hemolyticum var. sordelli Hauduroy et al., loc. cit., 126 (Bacillus sp. (?), Sordelli, Prado and Ferrari, Compt. rend. Soc. Biol., Paris, 106, 1931, 142; Unnamed anaerobe of Matte, Inst. Biol. Soc. Nac. Agric., Chile, 2A, 1921, (31?) (cited from Vawter and Records, loc. cit., 172).

Rods : 1.0 to 1.3 by 3.0 to 5.6 microns, with rounded ends, occurring singly, in pairs and in short ehains. Motile with long peritrichous flagella. Spores oval to elongate, subterminal, swelling rods. Gram-positive.

Gelatin: Liquefied.

Blood agar surface colonies (anaerobic): Light, diffuse growth. Blood hemolyzed.

Deep agar colonies: At first lenticular, becoming densely woolly masses with short peripheral filaments. Little or no gas formed.

Broth plus liver: Luxuriant diffuse turbidity, followed by agglutinative clearing. Moderate gas formed.

Milk: Acid and slow coagulation. Clot not digested.

Acid and gas from glucose, fructose, galactose and glycerol. Lactose, maltose, sucrose, raffinose, arabinose, xylose, inulin, salicin, mannitol and dulcitol not fermented. Subsequent studies show that pure galactose is not fermented (Records and Vawter, Nevada Agr. Exp. Sta., Bull. 173, 1945, 48 pp.).

Indole is formed.

Methyl red and Voges-Proskauer tests are negative.

Nitrites are not produced from nitrates.

Hydrogen sulfide is produced. The four characteristics given above are from Records and Vawter (loc. cit., 30).
Coagulated albumin not liquefied.

Blood serum not liquefied.

Brain medium not blackened or digested.

Meat medium reddened, not blackened. No digestion.

Pathogenic and toxic for guinea pig and rabbit. Effect due to an unstable hemolytic toxin.

Grows well at $37^{\circ} \mathrm{C}$.

Anaerobic.

Source : From blood and tissues of cattle dying of icterohemoglobinuria.

Habitat: Not determined. Thus far isolated only from animals.

9. Clostridium novyi (Migula) Bergey et al. (Bacillus oedematis maligni No. II, Novy, Ztschr. f. Hyg., 17, 1894, 212; Bacillus oedematis thermophilus Kruse, in Flügge, Die Mikruorg., 3 Aufl., 2, 1896, 242 ; Bacillus novyi Migula, Syst. d. Bakt., 2, 1900, 872; Bacterium oedematis ther. mophilus Chester, Ann. Rept. Del. Col. Agr. Exp. Sta., 9, 1897, 126; Bacillus thermophilus Chester, Man. Determ. Bact. 1901, 265; Bacillus oedematiens Weinberg and Seguin, Compt. rend. Soc. Biol., Paris, 78, 1915, 507 (Bacille B. Weinberg and Seguin, ibid., 177); Novillus maligni Heller, Jour. Bact., 7, 1922, 7; Clostridium oedematiens Bergey et al., Manual, 1st ed., 1923, 326; Bergey et al., idem; Clostridium thermophilum Pribram, Jour. Bact., 22, 1931, 430; Clostridium novyi Type A, Scott, Turner and Vawter, Proc., 12th Internat. Vet. Congr., 2, 1934, 175.) Named for F. G. Novy, the American bacteriologist who first isolated this organism.

Related or possibly identical species : Neuen pathogenen anaeroben Bacillus, Kerry, Osterr. Ztsehr. f. Wiss. Veterinärk., 5, 1894, 228; Bacterium nivosum LeBlaye and Guggenheim, Man. Prat. d. Diag. Bact., 1914, 344 (Bacille neigeux, Jungano, Compt. rend. Soc. Biol., Paris, 62, 1907, 677; Bacillo nevoso, Jungano, Il Tommasi, 2, 1907, (731?); Gasödembazil lus, Aschoff, Deuts. med. Wchnschr., 42. 1916, 512; Bacillus bellonensis Sacquépée, 
Compt. rend. Soc. Biol., Paris, 80, 1917, 850 (Bacille de l'oedème gazeux malin, Sacquépée, ibid., 78, 1915, 316; Clostridium bellonensis Prévot, Ann. Inst. Past., 61, 1938, 81); Bacillus gigas Zeissler and Rassfeld, Arch. Wiss. u. Prakt. Tierhlk., 59, 1929, 419 (Clostridium novyi Type B, Scott et al., loc. cit., 175; Clostridium gigas Prévot, Ann. Inst. Past., 61, 1938, 82); not Bacillus gigas Trevisan, Atti. d. Acead. Fis.-Med.-Stat., Milano, Ser. 4, 3, 1885, 96; Clostridium novyi Type C, Scott et al., loc. cit., 175 (nonpathogenic bacillus of osteomyelitis of buffalo, Kraneveld, Nederl. Ind. Bl. Diergeneesk., 42, 1930, 561; Clostridium bubalorum Prévot, Ann. Inst. Past., 61, 1938, 82; Bacillus osteomyelitis bubalorum Prévot, Man. d. Class., etc., 1940, 123).

Rods : 0.8 to 0.9 by 2.5 to 5.0 microns, occurring singly and in pairs, not in chains. Motile with peritrichous flagella. Spores large, oval, subterminal, swelling rods. Gram-positive.

Gelatin : Liquefied and blackened.

Agar surface colonies (anaerobic): Small, white, with darker center, filamentous.

Agar slant (anaerobic): Grayish, spreading growth.

Deep agar colonies: Compact, opaque, becoming filamentous with age.

Broth: Turbid, with flocculent sediment.

Litmus milk: Acid, not coagulated. Litmus reduced.

Acid and gas from glucose, fructose, maltose, xylose, starch and glycerol. Lactose, sucrose, mannitol, dulcitol, inulin and salicin not fermented (Hall, Jour. Int. Dis., 30, 1922, 491).

Coagulated albumin not liquefied.

Blood serum not liquefied.

Brain medium not blarkened of digested.

Pathogenic for guinea pig, rabbit, mouse, rat and pigeon. Forms an exotoxin, toxic on injection but not on feeding growth.

Optimum temperature $35^{\circ} \mathrm{C}$ to $38^{\circ} \mathrm{C}$.

Anaerobic.
Source: From a guinea pig inoculated with peptonized casein; later from gaseous gangrene.

Habitat: Probably occurs in manured soils.

10. Clostridium botulinum (Van Ermengem) Holland. (Bacillus botulinus Van Ermengem, Cent. f. Bakt., I Abt., 19, 1896, 443, and Ztschr. f. Hyg., 26, 1897, 48; Holland, Jour. Bact., 5, 1920 , 217; Ermengemillus botulinus Heller, Jour. Bact., 7, 1922, 8.) From Latin, botulus, sausage; M.L., botulinus, relating to sausage.

Clostridium botulinum comprises a number of toxic species, conveniently divided by Bengtson, U. S. Public Health Serv., Hyg. Lab. Bull. 136, 1924, 33, and by Meyer and Gunnison, Jour. Inf. Dis., $45,1929,96$ and 108, and by Gunnison and Meyer, Jour. Inf. Dis., 45, 1929, 130, into a non-ovolytic (Clostridium botulinum) and an ovolytic (Clostridium parabotulinum) group. Authorities are not yet in agreement on fermentations and on variant sub-types, and the present groupings are only tentative, and subject to revision. Meyer and Gunnison cite some 15 sub-types on the basis of toxicity, agglutination and fermentation.

The original Van Ermengem strain is not available, and his description is inadequate for classification purposes. Description follows Bengtson (loc. cit.) who used Lister Institute Strain No. 94 (Brit. Med. Res. Counc., Spec. Rept. Ser. No. 12, 1917, 29; ibid., Spec. Rept. Ser. No. 39, 1919, 26) as a type culture.

Rods : 0.5 to 0.8 by 3.0 to 8.0 microns, with rounded ends, occurring singly, in pairs and in short to occasional long chains. Motile with peritrichous flagella. Spores oval, central, subterminal, to terminal at maturation, slightly swelling rods. Gram-positive.

Gelatin: Liquefied.

Deep liver agar colonies: Fluffy with dense center.

Liver agar surface colonies (anaerobic) : No perceptible growth. 
Broth: Scant or no growth.

Liver broth: Luxuriant turbidity, with considerable gas.

Milk: Slowly increasing acidity. No coagulation. No gas.

Acid and gas from glucose, fructose, maltose, dextrin, glycerol, adonitol and inositol. Galactose, sucrose, lactose, raffinose, inulin, dulcitol, mannitol, xylose, arabinose, rhamnose and salicin not fermented (Bengtson, loc. cit., 22-25).

Coagulated albumin not liquefied.

Blood serum not liquefied.

Brain medium not blackened or digested.

Meat medium not blackened or digested.

Pathogenic for animals. Forms a powerful exotoxin which is neurotoxic both on injection and feeding. Toxin is neutralized by Clostridium parabotulinum Type B antitoxin.

Optimum temperature $20^{\circ}$ to $30^{\circ} \mathrm{C}$ (Van Ermengem, Ztschr. f. Hyg., 26, 1897,42 ); $30^{\circ} \mathrm{C}$ (Van Ermengem, Arch. d. Pharmacodyn., 3, 1897, 213 and 499; see Williams and Reed, Jour. Inf. Dis., 71, 1942, 227). Starin (Jour. Inf. Dis., $38,1926,103)$, growth usually earlier at $37^{\circ} \mathrm{C}$. Toxin production probably best around $28^{\circ} \mathrm{C}$.

Anaerobic.

Source: Unknown. Culture received through Reddish from Robertson as Bacillus botulinus No. 94, Strain A, Institute of Infectious Diseases at Berlin. Similar strains have been isolated from canned foods.

Habitat: Probably occurs in soil.

10a. Clostridium botulinum Type C. (Toxin producing anaerobe, Bengtson, $\mathrm{U}$. S. Pub. Health Repts., 37, 1922, 164 and 2252 ; Bacillus botulinus Type C, Bengtson, ibid., 38, and U. S. Pub. Health Serv., Hyg. Lab. Bull. 136, 1924, 7; Clostridium luciliae Bergey et al., Manual, 1st ed., 1923, 336.) From Latin, botulus, sausage.

Probably identical variety : Clostridium parabotulinum equi Theiler and Robin- son, Rev. Gén. de Med. Vet., 36, 1927, 199 (Clostridium botulinum Type E, Topley and Wilson, Principles of Bact. and Immunol., 2nd ed., 1936, 688; Bacillus (Clostridium) botulinum D, Weinberg and Ginsbourg, Données Recentes sur les Microbes Anaér., Paris, 1927, 107, but shown to be a Type C by Robinson, Union S. Africa, 16th Ann. Rept., Dir. Vet. Serv. and Animal Indus., 1930, 126; not Clostridium botulinum Type D, Meyer and Gunnison, vide infra). From a rat carcass presumably responsible for botul ism in mules in South Africa.

Related varieties: Bacillus parabotulinus Seddon, Jour. Comp. Path. and Therap., 35, 1922, 155 and 275 (Clostridium parabotulinum Ford, Text-Book of Bact., 1927, 743, although this name was used earlier in the "group" sense by Bengtson, U. S. Pub. Health Serv., Hyg. Lab. Bull. 136, 1924, 32). First isolated from bones considered the source of "bulbar paralysis" of cattle in Australia.

Clostridium parabotulinus bovis Theiler et al., Union S. Africa, Dept. Agric., 11th and 12th Repts. of the Dir. Vet. Educ. and Res., Part II, 1927, 1202 (Clostridium botulinum Type D, Meyer and Gunnison, Proc. Soc. Expt. Biol. and Med., 26, 1928-29, 88, also Jour. Inf. Dis., 45, 1929, 106; not Clostridium botulinum Type D, Weinberg and Ginsbourg, vide supra). From "lamziekte" of cattle in South Africa.

Clostridium botulinum Type E, Gunnison, Cummings and Meyer, Proc. Soc. Expt. Biol. and Med., 35, 1936, 278. An organism received by them from the Russian Ukraine; source of isolation not stated.

Clostridium botulinum Type C may be regarded as a variety of Clostridium botulinum, as it has morphologic and cultural characters very similar to those of the Van Ermengem strain. Only divergent or additional characters are recorded here.

Rods : 0.5 to 0.8 by 3.0 to 6.0 microns, commonly slightly curved.

Agar stab: Slight growth. No gas. 
Deep liver agar colonies: Lenticular, becoming loosely fluffy. Gas is formed. Deep glucose agar colonies: Fluffy, without central nucleus. Gas is not formed.

Agar surface growth (anaerobic): Very seant, thin.

Broth: Seant growth.

Milk: Slowly increasing acidity. No coagulation. No digestion.

Acid and gas from glucose, fructose, galactose, maltose, glycerol and inositol. Dextrin is weakly fermented. Sucrose, lactose, raffinose, inulin, adonitol, dulcitol, mannitol, xylose, arabinose, rhamnose and salicin not fermented.

Pathogenic for animals. Forms a powerful exotoxin which is neurotoxic both on injection and feeding. Toxin is neutralized by homologous (Type $\mathrm{C} \alpha$ ) antitoxin, but not by Bacillus parabotulinus Seddon (Type C $\beta$ ) antitoxin, although Seddon-toxin is neutralized by Type C $\alpha$ antitoxin (Pfenninger, Jour. Inf. Dis., 35, 1924, 347).

Grows well at $37^{\circ} \mathrm{C}$.

Anaerobic .

Source: Larvae of blue-bottle fly (Lucilia caesar). Produces limberneck in chickens.

Habitat: Not determined, other thin this source.

11. Clostridium acetobutylicum Mc Coy, Fred, Peterson and Hastings. (McCoy et al., Jour. Inf. Dis., 39, 1926, 483; ibid., 46, 1930, 11s; Clostridium aceto-butylicum Legg, U. S. Pat., 1,668,814, 1928; Clostridium acetonobutylicum Prévot, Ann. Inst. Past., 61, 1938, 80; Clostridium acetobutyricum Prévot, Man. d. Class., ete., 1940, 110.) From Latin, acetum, vinegar and butylicus, butylic, relating to butyl alcohol.

Synonyms: Bacillus granulobacter pectinovorum Speakman, Jour. Biol. Chem., 41, 1920, 319; Clostridium acetonigenum Donker, Inaug. Diss., Delft., 1926, 144.

Rods: Vegetative cells 0.6 to 0.72 by 2.6 to 4.7 microns; clostridia 1.3 to 1.6 by 4.7 to 5.5 microns. Straight, with rounded ends, occurring singly and in pairs, not in chains. No capsules. Motile with peritrichous flagella. Spores oval, excentric to subterminal, swelling rods to clostridia. Gram-positive, becoming Gram-negative.

Granulose reaction positive in clostridial stage.

Glucose gelatin: Liquefied.

Glucose agar surface colonies (anaerobic): Compact, raised, fairly regular.

Deep glucose agar colonies: Compact, typically lenticular and smooth. Agar fragmented early by abundant gas.

Blood agar not hemolyzed.

Pigmentation : None; colonies creamywhite, opaque.

Plain broth: No growth.

Glucose broth: Abundant, uniform turbidity, with much gas.

Litmus milk: Acid and active, often stormy, coagulation. Litmus reduced. Clot fragmented by gas, but not visibly digested. Proteolysis demonstrable, however, on milk agar.

Potato: Growth ereamy-yellow. Potato digested to a yellow slime.

Corn mash: Much gas with butylie odor.

Indole not formed.

Acetylmethylcarbinol formed from many carbohydrates.

Nitrites not produced from nitrates.

Nitrites reduced to ammonia.

Acid and gas from arabinose, xylose, rhamnose, glucose, galactose, mannose, fructose, sucrose, maltose, lactose, raffinose, melezitose, starch, dextrin, inulin, glycogen, $d$-mannitol, $\alpha$-methyl glucoside and salicin. Esculin, amygdalin and trehalose are weakly fermented. Melobiose, dulcitol, $d$-arabitol, perseitol, lactositol, sorbitol, erythritol, adonitol, inositol, quercitol, glycerol, pectin and cellulose are not fermented.

Fermentation products include acetone, butyl and ethyl alcohols, butyrie and acetic acids, $\mathrm{H}_{2}$ and $\mathrm{CO}_{2}$.

Coagulated albumin cubes: Softened and browned by slow digestion. 
Hydrogen sulfide produced from thiosulfate or sulfite; generally negative from proteinaceous sources.

Blood serum not liquefied.

Brain medium not blackened or digested.

Non-pathogenic for guinea pig and rabbit.

Optimum temperature probably about $37^{\circ} \mathrm{C}$. Grows from $20^{\circ} \mathrm{C}$ to $47^{\circ} \mathrm{C}$.

\section{Anaerobic.}

Source: From corn, molasses, potato and garden soil.

Habitat: Widely, but apparently sparsely, dispersed in agricultural soils.

NоTE: A number of acetone and butyl alcohol-fermenting anaerobes have been described. Present knowledge, however, does not permit any expression of the degree of possible relationship. Only a few well-described species are cited. Bacillus butylicus B. F., Ricard, U. S. Pat., 1,385,888, 1921; Bacillus butylaceti cum Freiberg, U. S. Pat., 1,537,597, 1925; Clostridium butyricum (Prazmowski-Pike-Smyth) Pike and Smyth, L. S. Pat., 1,655,435, 1928; Butylobacter betae, Butylobacter sinense, Butylobacter solani and Butylobacter zeae Bakonyi, British Pat., 328,723, 1930, and U. S. Pat., 1,818,782, 1931; Bacillus saccharobutyricus liquefaciens MeCoy et al., Jour. Inf. Dis., 46, 1930, 121 (Bacillus saccharobutyricum liquefaciens Legg and Stiles, I. S. Pat., $1,27,813,1933)$; Clostridium saccharobutylicum-gamma Izsak and Funk, L. s. Pat., 1,908,361, 1933 (Clostridium saccharobutylicum gamma and Clostridium. saccharobutyricum gamma Izsak and Funk, I. S. Pat., 2,016,112, 1935); Clostridium saccharo-acetobutylicum-alpha McCoy, British Pat., 415,311, 1934; Clostridium propyl-bulylicum Muller and Legg, British Pat ., $415,312,1934$ (C'lostridium propyl butylicum Legg and Stiles, U. s. Pat., 2,063,448, 1936); Clostridium saccharobutyl-acetonicum Loughlin, British Pat., 409,730, 1934, and I. S. Pat., 1,996,428, 1935, and 1,992,921, 1935; Clostridium saccharo-acetobutylicum Stiles and Legg, British Pat., 437,121, 1935 (Clostri- dium saccharo-acetobutylicum Legg, U. S. Pat., 2,063,449, 1936); Clostridium saccharo-acetobutylicum-beta Arzberger, U. S. Pat., 2,050,219, 1936; Clostridium saccharo-acetobutylicum-gamma Arzberger, ibid.; Clostridium inverto-acetobutylicum Legg and Stiles, British Pat., 437,120, 1935, and Legg, U. S. Pat., 2,063,449, 1936; Clostridium (Bacillus) tetrylium Owen, Mobley and Arroyo, Cent. f. Bakt., II Abt., 95, 1936, 131; Clostridium saccharobutyl-isopropyl-acetonicum Loughlin, U. S. Pat., 2,085,666, 1937 (Clostridium saccharo-butyl-isopropyl-acetonicum Loughlin, ibid., and U. S. Pat., $2,096,377,1937$ ).

12. Clostridium aerofoetidum (Weinberg and Seguin) Bergey et al. (Bacille D, Weinberg, Compt. rend. Soc. Biol., Paris, 79, 1916, 117; Bacillus aerofoetidus Weinberg and Seguin, ibid., 1028; Bacillus aero-foetidus McIntosh, Med. Res. Counc., Spec. Rept. Ser. No. 39, 1919, 42; Seguinillus aerofoetidus Heller, Jour. Bact., 7, 1922, 7; Bergey et al., Manual, 1st ed., 1923, 327.) From Latin, aer, air and foetidus, fetid.

Rods : 0.4 to 0.6 by 3.0 to 5.0 microns, occurring singly, in pairs and in short chains. Motile with peritrichous flagella. Spores rare, oval, subterminal, slightly swelling rods. Gram-positive.

Gelatin : Rapidly liquefied.

Agar surface colonies (anaerobic): Circular, transparent, with faintly bluish tint, fimbriate.

Deep agar colonies : Lenticular, becoming indented and lobate.

Blood agar not hemolyzed.

Glucose broth : Turbid; with sediment.

Litmus milk: Acid; slowly coagulated; followed by slow peptonization. Gas is formed.

Acid and gas from glucose, fructose, galactose, mannose, maltose, lactose, xylose, amygdalin, salicin, esculin and glycogen. Sucrose, inulin, glycerol and mannitol not fermented.

Coagulated albumin slowly liquefied.

Blood serum is liquefied. 
Brain medium blackened and digested.

Meat medium reddened, then blackened and slowly digested.

Slightly pathogenic for guinea pig.

Optimum temperature $30^{\circ} \mathrm{C}$ to $35^{\circ} \mathrm{C}$.

Anaerobic.

Source: From gaseous gangrene and from feces.

Habitat: Not determined other than these sources. Probably occurs in soil.

13. Clostridium sporogenes (Metchnikoff) Bergey et al. (Bacillus sporogenes var. A, Metchnikoff, Ann. Inst. Past., 22, 1908, 944; Bergey et al., Manual, 1st ed., 1923, 329; not Clostridium sporogenes Holland, Jour. Bact., 5, 1920, 220 (Bacillus enteritidis sporogenes Klein, Cent. f. Bakt., I Abt., 18, 1895, 737; Bacillus sporogenes Migula, Syst. d. Bakt., 2, 1900,560 ; Bacillus (enteritidis) sporogenes and Bacillus enteritidis Kilein, Local Govt. Bd., Ann. Rept. Med. Off., London, 33, 1903-04, 442 and 443.) From Greek, sporus, seed; M.L., spore; genes, producing.

Two varieties, $A$ and $B$, were described. Bacillus sporogenes var. A, Metchnikoff, loc. cit., 944 (Metchnikovillus sporogenes Heller, Jour. Bact., \%, 1922, 9; Clostridium sporogenes var. A, Prévot, Ann. Inst. Past., 61, 1938, 83) is regarded as the typical form and is described here. Var. B, see Clostridium bifermentans.

Synonyms or probably related species: Oedembacillen, Koch, Mitt. a. d. kaiserl. Gesundheitsamte, 1, 1881, 54; Bacillus oedematis maligni Zopf, Die Spaltpilze, 3 Aufl., 1885, 88 (not Bacillus oedematis maligni Liborius, Ztschr.f. Hyg., 1, 1886 , 158; Bacillus oedematis Migula, Syst.d. Bakt., 2, 1900, 604); Bacillus oedematis Chester, Man. Determ. Bact., 1901, 292; Clostridium oedematis maligni Bergey et al., Manual 1st ed., 1923, 325 (see Species No. 6, Clostridium septicum Ford, p. 774): Paraplectrum foetidum Weigmann, Cent. f. Bakt., II Abt., 4, 1898, 827 (Bacterie $\beta$, Weigmann, Cent. f. Bakt., II Abt., 2, 1896, 155; Bacillus weigmanni Chester,
Man. Determ. Bact., 1901, 300; Plectridium foetidum Weigmann, Mykologie der Milch, Leipzig, 1911, 70; Bacillus anaerobius foetidus LeBlaye and Guggenheim, Man. Prat. d. Diag. Bact., 1914, 329 ; Endosporus foetidus Prévot, Ann. Inst. Past., 61, 1938, 75); Bacillus saprogenes carnis Salus, Arch. f. Hyg., 51, 1904, 114 (Bacillus saprogenes Salus, ibid., 115; not Bacillus saprogenes $I, I I$, III, Herfeldt, Cent. f. Bakt., II Abt., 1, 1895, 77; Bacillus carnis saprogenes Salus, Arch. f. Hyg., 51, 1904, 124; Plectridium saprogenes Prévot, Ann. Inst. Past., 61, 1938, 87); Bacillus sporogenes coagulans Debono, Cent. f. Bakt., I Abt., Orig., 62, 1912, 229 (Clostridium coagulans Bergey et al., Manual, 1st ed., 1923, 335); Reading Bacillus, Donaldson and Joyce, Lancet, 2, 1917, 448; Bacillus pulrificus rerrucosus Zeissler, Ztschr. f. Infkrnkh. u. Hyg. Haust., 21, 1920-21, 13 (Bacillus verrucosus Lehmann and Süssmann, in Lehmann and Neumann, Bakt. Diag., 7 Aufl., 2, 1927, 662 ).

Rods : 0.6 to 0.8 by 3.0 to 7.0 microns, with rounded ends, occurring singly, in pairs, or less frequently in short to long chains and filaments. Motile with peritrichous flagella. Spores oval, excentric to subterminal, swelling rods. Grampositive.

Gelatin: Liquefied and blackened.

Agar surface colonies (anaerobic): Small, irregular, transparent, becoming opaque, yellowish-white, fimbriate.

Deep agar colonies: Woolly balls with dense, nodular center.

Agar slant (anaerobic): Grayish, opaque, spreading.

Blood agar is hemolyzed.

Broth: Turbid. Gas is formed. Putrid odor.

Litmus milk: Softly coagulated. Litmus reduced. Slow peptonization, leaving a dark, amber-colored liquid.

Indole formed (trace). Not formed (Hall, Jour. Inf. Dis., 30, 1922, 482).

Nitrites not produced from nitrates.

Acid and gas from glucose, fructose, 
galactose and maltose. Lactose, sucrose, salicin, glycerol, mannitol and inulin not fermented. (Records vary on many sugars.)

Coagulated albumin liquefied.

Blood serum liquefied to a dark, putrid liquid.

Brain medium blackened and digested. Foul odor.

Meat medium reddened, then blackened and digested with foul odor. Gas is produced. Tyrosin erystals not obvious.

Non-pathogenic to guinea pig and rabbit, other than a slight, temporary local tumefaction. Filtrate non-toxic on injection and feeding.

Optimum temperature $37^{\circ} \mathrm{C}$. Can grow at $50^{\circ} \mathrm{C}$.

Anaerobic.

Source: From intestinal contents, gaseous gangrene, and from soil.

Habitat: Common in soil, especially where heavily manured.

The following species are commonly regarded as variants of the typical Clostridium sporogenes.

13a. Clostridium sporogenes var. A. P. Marie Prévot, Ann. Inst. Past., 61, 1938, 83 (Bacille anaérobie, Marie, Compt. rend. Soc. Biol., Paris, 93, 1925, 21).

Resembles the typical Clostridium sporogenes except in the sharp but not putrid odor of its cultures.

Pathogenicity: Large abscesses are induced on subcutaneous injection into guinea pigs.

From spontaneous putrefaction of macerated pork.

13b. Clostridium sporogenes var. equine Prévot, Ann. Inst. Past., 61, 1938, 83 (Unnamed anaerobe No. IV, Choukévitch, Ann. Inst. Past., 25, 1911, 259).

Sporulation is delayed and restricted. Spores are long and almost rectilinear. Litmus milk is coagulated, then the clot is digested after 3 to 4 weeks.

Coagulated albumin is slowly dissolved.
Not pathogenic for guinea pig or mouse. From large intestine of horse.

13c. Clostridium tyrosinogenes (Hall) Bergey et al. (Culture No. 106, Hall and Finnerud, Proc. Soc. Expt. Biol. and Med., 19, 1921-22, 48; Bacillus tyrosinogenes Hall, Abst. Bact., 6, 1922, 7; not Bacillus tyrosinogenes Rusconi, as cited by Carbone, Ramazotti, Mazzucchi and Monti, Boll. Ist. Sieroter., Milan, 2, 1921, (29?), see Clark and Smith, Jour. Bact., 37, 1939, 278; Bergey et al., Manual, 1st ed., 1923, 329; Clostridium sporogenes var. tyrosinogenes Prévot, Ann. Inst. Past., 61, 1938, 83.) From Greek, tyrus, cheese; M. L., tyrosinum, tyrosine; genes, producing.

Ferments monosaccharides but not higher carbohydrates (Hall, Jour. Inf. Dis., 30, 1922, 482).

Traces of gas, but no acid, from glycerol, sorbitol, mannose, xylose, lactose, sucrose, arabinose, galactose, salicin, inulin, dextrin and starch (F. E. Clark, personal communication).

Distinctive character: Forms large amounts of tyrosin which precipitate in cultures in protein media.

Source: Originally isolated from a culture erroneously labeled Bacillus tetani. Later isolated from an amputated arm.

Habitat: Not determined. Only two isolations on record.

13d. Clostridium flabelliferum Sturges and Reddish. (Fish-tailed putrefactive anaerobe, Reddish and Sturges, Abst. Bact., 8, 1924, 5; Sturges and Reddish, Jour. Bact., 11, 1926, 37; Clostridium sporogenes var. caudapiscis Prévot, Ann. Inst. Past., 61, 1938, 83.) From Latin, flabellum, a little fan; fer, bearing.

Glucose agar surface colonies (anaerobic): Coarse, raised, with long peripheral intertwining outgrowths.

Deep plain agar colonies: Irregular, becoming woolly.

Sucrose is fermented (in contrast with Clostridium sporogenes).

Distinctive character: Spores are long 
retained within the sporangium, of which the distal end frays out to fibrils, giving the characteristic fish-tail appearance. Otherwise closely resembles Clostridium sporogenes.

Source: From soured hams and from salt.

Habitat: Not determined, other than these sources.

13e. Clostridium parasporogenes (Bulloch et al.) Bergey et al. (Bacillus Type XII, MeIntosh and Fildes, Med. Res. Counc., Spec. Rept. Ser. No. 12, 1917, 36; Bacillus parasporogenes Bulloch et al., Med. Res. Counc., Spec. Rept. Ser. No. 39, 1919, 39; Bergey et al., Manual, 1st ed., 1923, 327; Clostridium sporogenes var. parasporogenes Prévot, Ann. Inst. Past., $61,1938,83$.)

Deep agar colonies: Lenticular to slightly irregular. Not woolly.

Pathogenic for young guinea pigs. Filtrate non-toxic on injection or on feeding.

Optimum temperature $30^{\circ} \mathrm{C}$ to $35^{\circ} \mathrm{C}$.

Distinctive character : Resembles Clos tridium sporogenes, but does not form woolly colonies in deep agar, and is agglutinatively distinct. Probably merely a variety.

Source: From gaseous gangrene.

Habitat: Not determined. Probably occurs in soil.

14. Clostridium parabotulinum Bengtson. (U. S. Pub. Health Serv., Hyg. Lab. Bull. 136, 1924, 32; Types A and B, Burke, Jour. Bact., 4, 1919, 556; Clostridium botulinum Types A and B, Bergey et al., Manual, 1st ed., 1923, 328.) From Latin, para, like; M.L., botulinum, a species name.

Note: This group comprises the putrefactive (ovolytic) species, including strains commonly referred to as Memphis and Canton (Type A), and Nevin (Type B). Growth of these types is more easily obtained than with the Clostridium botulinum strains, and the reactions are more obvious.
Gunnison and Meyer (Jour. Inf. Dis., $45,1929,130)$ propose an intermediate group between Clostridium botulinum and Clostridium parabotulinum, which they call Clostridium metabotulinum. Such a group would provisionally include certain European Type B strains, the Australian Type C, certain American Type C strains, and the South African Type D.

Rods : 0.5 to 0.8 by 3.0 by 8.0 microns, with rounded ends, occurring singly, in pairs, and in short chains. Motile with peritrichous flagella. Spores oval, subterminal, distinctly swelling rods. Gram-positive.

Gelatin: Liquefied.

Deep liver agar colonies: Type $\mathbf{A}$ tend to be restricted to compact disks, with sharp outline and small, opaque nucleus at periphery. Type B tend rather to form loose, woolly colonies (indicative (only).

Liver agar surface growth (anaerobic): Profuse, moist.

Broth: Fairly abundant diffuse turbidity. Many strains spontaneously agglutinate.

Liver broth: Luxuriant turbidity. Profuse gas.

Milk: Slight acidity ; slow curdling precipitation, with subsequent digestion and darkening.

Fermentation records are variable: Acid and gas from glucose, fructose, maltose, dextrin, glycerol and salicin. Galactose, sucrose, lactose, rhamnose, raffinose, inulin, adonitol, dulcitol, mannitol, xylose, arabinose and inositol not fermented (Bengtson, loc. cit., 22-25).

Coagulated albumin liquefied: Action of Type B usually more marked than that of Type A.

Blood serum liquefied.

Brain medium blackened and digested, with putrefactive odor.

Meat medium blackened and digested. Putrefactive odor. Tyrosine crystals not observed.

Pathogenic for animals. Forms a powerful exotoxin which is neurotoxic both on injection and feeding, and which 
is neutralized only by the homologous type antitoxin.

Optimum temperature : Records at variance. Grows best at 35 to $37^{\circ} \mathrm{C}$. Toxin production best at about $28^{\circ} \mathrm{C}$.

\section{Anaerobic.}

Distinctive character: Types are identified chiefly by protection tests with known-type antitoxin, and to less extent by agglutination.

Source: Chiefly from spoiled, non-acid canned goods, from soil and from silage.

Habitat: Found rather widely dispersed in soil.

\section{Clostridium saccharolyticum Ber-} gey et al. (Bacillus sporogenes saccharolyticus Distaso, Cent. f. Bakt., I Abt., Orig., 59, 1911, 100; Bergey et al., Manual, 1st ed., 1923, 334.) From Greek, saccharum, sugar; lyticus, dissolving, digesting.

Rods: Short, rounded ends, occurring singly, in pairs and in short chains. Motile. Spores large, oval, excentric to subterminal, swelling rods. Gram-positive.

Gelatin : Liquefied.

Deep glucose agar colonies: Gray, biconvex, lenticular, granular, entire. Gas is formed.

Broth: Turbid.

Milk: Soft coagulation; casein precipitated, then peptonized, leaving a clear, yellow-amber supernatant fluid.

Indole is formed.

Acid and gas feebly formed from glucose. Lactose and sucrose feebly, or doubtfully, fermented.

Coagulated albumin slowly liquefied.

Grows well at $37^{\circ} \mathrm{C}$.

Anaerobic.

Distinctive character: All cultures give a mixed butyric and fecal odor.

Source: From feces of a chimpanzee.

Habitat: Not determined, other than this source.

16. Clostridium regulare Bergey et al. (Bacillus sporogenes regularis Distaso, Cent.f. Bakt., I Abt., Orig., 59, 1911, 100 ;
Bergey et al., Manual, 1st ed., 1923, 334.) From Latin, straight.

Long rods: With rounded ends, occurring singly and in pairs. Motile. Spores oval, small, subterminal, slightly swelling rods. Gram-positive.

Gelatin: Liquefied.

Deep agar colonies: Small, opaque, irregular.

Milk: Acid; slowly coagulated, then elot slowly digested.

Indole formed in small quantity.

Slight acidity from glucose, lactose and sucrose. Gas is not formed. Odor of scatol and valerianic acid.

Coagulated albumin slowly liquefied.

Grows at $37^{\circ} \mathrm{C}$.

Anaerobic.

Source: From human feces.

Habitat: Not determined, other than this source.

\section{Clostridium hastiforme MacLen-} nan. (A4, Cunningham, Cent. f. Bakt., II Abt., 82, 1930-31, 487, and B4a, Cunningham, ibid., 83, 1931, 11; MacLennan, Jour. Path. and Bact., 49, 1939, 543.) From Latin, resembling a spear.

Rods : Slender, 0.3 to 0.6 by 2.0 to 6.0 microns, with rounded ends, occurring singly, in pairs, and rarely in short chains. Filaments not observed. Motile, with delicate peritrichous flagella; motility persists even after sporulation. Spores ellipsoidal, subterminal, swelling rods. Polar-cap of protoplasm remains long attached to free spores. Gram-positive.

Gelatin: Rapidly liquefied. Blackening not recorded.

Plain agar surface colonies (anaerobic): Minute translucent dots, becoming irregularly round, granular, grayish-white, with opaque center and delicate translucent border.

Blood agar surface colonies (anaerobic): As above, but larger and more opaque. Old colonies show grayish pigmentation. No hemolysis.

Deep plain agar colonies : Small, irregularly round with coarsely filamentous 
horder. A little gas is occasionally formed.

Broth: Transient uniform turbidity, quickly settling as a heavy, white, floceulent deposit. Culture assumes a cheesy odor.

Milk: Abundant growth, with labcoagulation in 2 to 3 days. No increase in acidity; becoming slightly alkaline. Clot completely digested in 10 to 14 days, leaving a white, semi-translucent fluid of cheesy odor.

Indole not formed.

Ammonia not formed.

Hydrogen sulfide not formed.

Glucose not fermented.

Carbohydrates not fermented.

Egg medium: No digestion or other visible change,

Coagulated albumin not digested or blackened.

Blood serum not digested or blackened.

Meat medium not digested or blackened, even in presence of metallic iron. Meat particles slightly reddened.

Brain medium not digested or blackened.

Grows well between $22^{\circ} \mathrm{C}$ and $37^{\circ} \mathrm{C}$.

Anaerobic.

Non-pathogenic to guinea pigs on subcutaneous inoculation (Cunningham, Cent.f. Bakt., II Abt., 83, 1931, 12).

Source: Originally isolated by Cunning ham as a dissociant from a culture of Bacillus saccharobutyricus von Klecki. Later isolated by MacLennan; 1 strain from a culture of Clostridiun sporogenes, and 2 strains from street dust.

Habitat: Not determined, other than these sources.

18. Clostridium subterminale (Hall and Whitehead) comb. nov. (Bacillus subterminalis Hall and Whitehead, Jour. Inf. Dis., 41, 1927, 66.) Named from the characteristic position of the spores.

Rods: Occurring singly, in pairs and rarely in short chains. Motile. Spores oval, subterminal, swelling rods. Grampositive
Gelatin: Slowly liquefied, with slight turbidity and black sediment.

Blood agar surface colonies (anaerobic): Delicate. At first mildly, later actively, hemolytic.

Deep agar colonies: Opaque, compact, biconvex or lobate dises.

Agar slant (anaerobic): No surface growth.

Glucose broth: Turbidity, but no acid or gas formed.

Indole not formed.

Milk: Slowly coagulated (2 to 3 days), with mild acidity and gas. Slow but complete digestion of casein ( 8 to 18 days).

Glucose, fructose, galactose, maltose and lactose not fermented.

Brain medium: Slight turbidity in supernatant fluid. Slight gas formation and slow digestion.

Iron brain medium : Blackened in 2 to 3 days.

Tyrosin erystals not observable.

Non-pathogenic to guinea pigs on subcutaneous injection.

Grows well at $37^{\circ} \mathrm{C}$.

Obligately anaerobic.

Source: From an African arrowhead.

Habitat: Not determined, other than this source.

\section{Clostridium malenominatum}

(Weinberg et al.) comb. nov. (Pseudo-coli anaérobie, Jungano, Compt. rend. Soc. Biol., Paris, 05, 1908-09, 457; Bacillus pseudo-coli anaérobie Jungano and Distaso, Les Anaérobies, 1910, 162; Bacillus pseudocoli anaerobius LeBlaye and Guggenheim, Man. Prat. d. Diag. Bact., 1914, 345; Bacillus malenominatus Weinberg et al ., Les Microbes Anaérobies, 1937, 763; Paraplectrum malenominatum Prévot, Ann. Inst. Past., 61, 1938, 75.) From Latin, meaning badly named.

Rods: Short, cocco-bacillary, becoming elongated to short filaments in old cultures-especially in sugar broth. Ends rounded. Distinct bipolar staining tendency. Non-motile. Capsulated, especially in body fluids. Spores oval, 
subterminal, slightly swelling rods. Gram-negative.

Gelatin: No growth.

Deep agar colonies: Small, round, very regular, almost transparent. Gas not formed.

Plain broth: Uniform turbidity, settling after 48 hours, forming a fine, powdery sediment.

Indole not produced.

Milk: Growth with no coagulation.

Glucose and sucrose not fermented.

Coagulated albumin: Not attacked.

Meat medium: Abundant growth. No record of changes. Capsules are demonstrable in this medium.

Very pathcgenie for guinea pigs, which die of septicemia in 24 hours after intraperitoneal inoculation. Less pathogenic for rabbit, which dies after one week.

Toxin not demonstrable in cultures.

Grows at $22^{\circ} \mathrm{C}$ to $37^{\circ} \mathrm{C}$.

Obligately anaerobic.

Source: From feces of a diarrheal infant.

Habitat: Not determined, other than this single isolation.

20. Clostridium bifermentans (Weinberg and Seguin) Bergey et al. (Bacillus bifermentans sporogenes Tissier and Martelly, Ann. Inst. Past., 16, 1902, 894; Bacillus bifermantans Weinberg and Seguin, La Gangrène Gazeuse, Paris, 1918, 128; Martellillus bifermentans Heller, Jour. Bact., $7,1922,8$; Bergey et al., Manual, 1sted.. 1923,323.) From Latin. bis, twice, and fermentum, a ferment.

Closely related if not identical species Bacillus centrosporogenes Hall, Jour. Inf. Dis., 30, 1922, 464 (Clostridium centrosporogenes Bergey et al., Manual, 1st ed., 1923, 322); Bacillus oedematis sporogenes Sordelli, Compt. rend. Soc. Biol., Paris, 89, 1923, 55 (Anaérobie agent de gangrène gazeuse, Sordelli, ibid., 87, 1922, 838; Bacillus sordelli Hall and Scott, Jour. Inf. Dis., 41, 1927, 329; Bacillus sporogenes oedematis Piening, Thesis, Hanover, 1931, (?), cited from McCoy and Mcclung, The Anaer. Bact., etc., 2, 1939, 492; Clostridium sordelli Prévot, Ann.
Inst. Past., 61, 1938, 83); Clostridium oedematoides Meleney, Humphreys and Carp, Proc. Soc. Expt. Biol. and Med., 24, 1926-27, 67\%.

Varying degrees of virulence and toxicity occur in the above group. The more toxic and virulent strains are commonly referred to as Bacillus sordelli, although otherwise an apparently homogeneously organized group.

Probable synonyms: Clostridium foetidum Liborius, Ztschr. f. Hyg., 1, 1886, 160 (Cornilia foetida Trevisan, I generi e le specie delle Batteriacee, 1889, 22; Bacillus foetidus Chester, Ann. Rept. Del. Col. Agr. Exp. Sta., 10, 1898, 128; not Bacillus foetidus Trevisan, loc. cit., 16); Bacillus liquefaciens magnus Lüderitz, Ztschr. f. Hyg., 5, 1889, 146 (Cornilia magna Trevisan, loc. cit., 22; Bacillus magnus Herfeldt, Cent. f. Bakt., II Abt., 1, 1895, 78; Bacillus magnus liquefaciens LeBlaye and Guggenheim, Man. Prat. d. Diag. Bact., Paris, 1914, 327; Bacillus foetidus clostridiformis LeBlaye and Guggenheim, idem, 327); Clostridium. foetidum carnis Salus, Arch. f. Hyg., 51, 1904, 121 (Clostridium carnis foetidum and Clostridium foetidum Salus, ibid., 121 and 121: Clostridium carnofoetidus Mc Crurden, Jour. Biol. Chem., 8, 191011. 109; Clostridium carnofoetidum Prévot, Ann. Inst. Past.. 61, 1938, 84); Bacillus sporogenes var. $B$, Metchnikoff, Ann. Inst. Past., 22, 1908, 941 (Clostridium sporogenes var. $B$, Prévot, Ann. Inst. Past., 61, 1938, 83); Bacillus sporogenes foetidus Choukévitch, Ann. Inst. Past., 25, 1911, 257 (Bacillus foetidus Choukévitch, ibid., 258); Bacillus putrificus tenuis Zeissler, Ztschr. f. Infkrnkh. u. Hyg. Haust., 21, 1920-21, 13; Bacillus nonfermentans Hall and Whitehead, Jour. Inf. Dis., 41, 1927, 65.

Rods : 0.8 to 1.0 by 5.0 to 6.0 microns, occurring singly, in pairs, and in short chains. Sporesoval, central to excentric, not distinctly swelling rods. Motile in very young cultures only (less than 24 hours old). Gram-positive.

Gelatin: Liquefied and blackened. 
Agar surface colonies (anaerobic): Circular, crenated to amoeboid.

Blood agar surface colonies (anaerobic): Small, transparent, hemolytic, becoming opaque, yellowish, spreading.

Broth: Turbidity and gas. Thick mucoid sediment.

Litmus milk: Slowly coagulated. Slowly peptonized, with little gas.

Indole is formed.

Nitrites not produced from nitrates.

Hydrogen sulfide is produced.

Acid and gas from glucose, fructose, mannose and maltose. Lactose, sucrose and inulin not fermented. Records suggest variability in glycerol and salicin.

Coagulated albumin rapidly liquefied and blackened.

Blood serum liquefied and blackened.

Brain medium digested and blackened.

Egg-meat medium digested and blackened. Tyrosin crystals in 8 to 10 days.

Pathogenicity: Variable with the strain; some kill rabbits in 24 hours; others produce only slight edema, while some show no effect.

Toxicity : Likewise variable, from acute to none.

Optimum temperature from $30^{\circ} \mathrm{C}$ to $37^{\circ} \mathrm{C}$. Can grow at $50^{\circ} \mathrm{C}$.

Anaerobic.

Source: Originally from putrid meat; subsequently from gaseous gangrene.

Habitat: Occurs commonly in feces, soil and sewage. Widely distributed in nature.

21. Clostridium mucosum (Klein) Bergey et al. (Bacillus mucosus Klein, Cent. f. Bakt., I Abt., 29, 1901, 991; not Bacillus mucosus Zimmermann, Die Bakt. unserer Trink- u. Nutzwässer, Chemnitz, 2, 1894, 8; Bacterium mucosum Migula, Syst. d. Bakt., 2, 1900, 315; Bacillus kleinii Buchanan and Hammer, Iowa Agric. Exp. Sta. Res. Bull. 22, 1915, 276 ; not Bacillus kleinii Migula, Syst. d. Bakt., 2, 1900, 766; not Bacillus kleinii Trevisan, in litt. eited from De Toni and Trevisan, in Saccardo, Sylloge Fungorum, 8, 1889, 946; Clostridium kleinit
Bergey et al., Manual, 1st ed., 1923, 321; Bergey et al., Manual, th ed., 1934, 472; not Clostridium mucosum Simola, cited from Prévot, Man. d. Class., etc., 1940, 112; Endosporus mucosus Prévot, Ann. Inst. Past., 61, 1938, 75.) From Latin, slimy.

Rods : 1.3 by 2.0 to 5.0 microns, occurring singly, in pairs and in chains. Motile. Spores oval, central, not swelling rods. Gram-negative (Klein, loc. cit.). Young cultures Gram-positive (Buchanan and Hammer, loc. cit.).

No growth in media without carbohydrates.

Glucose gelatin: Slowly liquefied.

Glucose gelatin surface colonies (anaerobic): Small, gray.

Glucose gelatin stab: Villous growth. Slow liquefaction.

Glucose agar slant (anaerobic): Thin, veil-like layer. Slimy condensation water.

Glucose broth: Turbid. Gas bubbles.

Litmus milk: Acid; slowly coagulated, slimy. Gas formed. Odor of butyric acid.

Potato: No growth.

Indole not formed.

Nitrites not produced from nitrates.

Acid and gas from glucose.

Blood serum : No growth.

Non-pathogenic.

Grows at $37^{\circ} \mathrm{C}$.

Anaerobic.

Source: Blood sausage (Blutwurst).

Habitat: Not determined, other than this source.

22. Clostridium pruchii (Buchanan and Hammer) Bergey et al. (Bacillus lactis pruchii Conn, Esten and Stocking, 18th Ann. Rept. Storrs Agric. Exp. Sta., 1906, 179; Bacillus pruchii Buchanan and Hammer, Iowa Agric. Exp. Sta. Res. Bull. No. 22, 1915, 276; Bergey et al., Manual, 1st ed., 1923, 322.) Named for M. J. Prucha, American bacteriologist.

Rods: Variable in size, with clubshaped ends. Motile, with peritrichous 
flagella. Spores central, oval, not swelling rods. Gram-positive.

Gelatin: Rapid, stratiform liquefaction. Reddish-yellow sediment.

Agar surface colonies (anaerobic): Round, flat, white, smooth, opaque.

Agar slant (anaerobic): Luxuriant, white, viscid.

Broth: Turbid, with flocculent pellicle and gray viscous sediment.

Litmus milk: Acid; slowly coagulated, becoming slimy yellow.

Potato: Thin, brownish, spreading.

Indole not formed.

Nitrites not produced from nitrates.

Acid but no gas from glucose.

Coagulated albumin not recorded.

Blood serum not liquefied.

Non-pathogenic.

Optimum temperature $30^{\circ} \mathrm{C}$. Grows $\pi$ ell between $20^{\circ} \mathrm{C}$ and $37^{\circ} \mathrm{C}$.

Anaerobic.

Source : From slimy milk.

Habitat: Not determined, other than this source.

23. Clostridium cylindrosporum Barker and Beck. (Jour. Biol. Chem., 141, 1941, 3.) Named from the characteristic spore morphology .

Rods : 1.0 by 4.0 to 7.0 microns, straight. Motile with peritrichous flagella. Spores elongate to cylindrical, 1.0 to 1.1 by 1.7 to 3.0 microns, central, subterminal to terminal, with little or no swelling of rods. Gram-negative.

Iron-gelatin (Spray): No growth.

Deep plain agar: No growth.

Deep uric acid agar colonies: Whitish, compact, lobate, 1 to $2 \mathrm{~mm}$ in diameter, with irregular edges, surrounded by a zone of precipitated ammonium ureate which gradually disappears.
Plain broth: No growth.

Glucose broth : No growth.

Iron-milk (Spray) : No growth.

Indole not recorded (probably negative).

Nitrites not recorded (probably negative).

Glucose not fermented.

Carbohydrates not fermented.

Cellulose not fermented.

Coagulated albumin not liquefied.

Blood serum not liquefied.

Brain medium not digested or blackened.

Pathogenicity not recorded (probably non-pathogenic).

Optimum temperature about $35^{\circ} \mathrm{C}$.

Optimum reaction about $\mathrm{pH} 7.5$; lower limit for growth $\mathrm{pH} 6.5$.

Anaerobic.

Distinctive characters: Requires uric acid, or certain other purines, as a primary source of carbon and energy. The purines are converted into ammonia, $\mathrm{CO}_{2}$, acetic acid and a little glycine. This organism is physiologically similar to Clostridium acidi-urici, but may be readily distinguished from the latter by its morphology.

Source: A single strain isolated from soil.

Habitat: Probably soil, although only this single isolation is recorded.

24. Clostridium perfringens (Veillon and Zuber) Holland.* Clostridium perfringens Type A, Wilsdon. (Bacillus aerogenes capsulatus Welch and Nuttall, Johns Hopkins Hosp. Bull. 3, 1892, 81 (Bacillus capsulatus aerogenes Lehmann and Neumann, Bakt. Diag., 2 Aufl., 2, 1899, 327); Bacillus phlegmones emphysematosae Fraenkel, U'eber Gasphleg-

* Because of use of the species name perfringens by the Permanent Standards Commission of the Health Organization of the League of Nations (Report of the Permanent Commission on Biological Standardization, London, June 23, 1931), the use of this name has been continued although it is preceded by a valid binomial (Bacillus emphysematosus Kruse). 
monen, Leipzig, 1893, 47; Bacillus emphysematosus Kruse, in Flügge, Die Mikroorg., 3 Aufl., 2, 1896, 242; Bacterium aerogenes capsulatus Chester, Ann. Rept. Del. Col. Agr. Exp. Sta., 9, 1897, 125; Bacterium emphysematosus Chester, ibid.. 126; Bacillus emphysematis vaginae Lindenthal, Wien. klin. Wchnschr., 10, 1897, 42; Bacillus perfringens Veillon and Zuber, Arch. Méd. Expt. et Anat. Path., 10, 1898, 539; Bacillus capsulatus anaerobius and $B a$ cillus capsulatus aerogenes Binaghi, Cent. f. Bakt., II Abt., 4, 1898, 920; Granulobacillus saccharobutyricus immobilis liquefaciens Schattenfroh and Grass berger, Cent. f. Bakt., II Abt.. 5, 1899, 702 (Granulobacillus immobilis Schattenfroh and Firassberger, Arch. f. Hyg., 37, 1900, 68; Bacillus amylobacter immobilis Gratz and Vas, Cent. f. Bakt., II Abt., 41, 1914, 509); Bacterium welchii Migula, ibid., 392; Bacillus welchii Lehmann and Neumann, Bakt. Diag., 4 Aufl., 2, 1907, 457; Bacillus butyricus asporogenes im mobilis Rocchi, Cent. f. Bakt., I Abt., Orig., 60, 1911, 580; probably Bacillus multiformis Distaso, ('ent. f. Bakt., I Abt., Orig., 59, 1911, 101 (Bacleroides multiformis Bergey et al., Manual, 1st. ed., 1923, 263; Cillobacterium multiforme Prévot, Ann. Inst. Past., 60, 1938, 297; not Bacillus multiformis van Senus. Inaug. Diss., Leiden, 1890, (?), quoted from Herfeldt, Cent. f. Bakt., II Abt., 1, 1895, 117); Bacillus aerogenes-capsu latus Holland, Jour. Bact., 5, 1920, 217 Clostridium aerogenes-capsulatum. Hol land, ibid., 217; Bacillus phlegmonesemphysematosae Holland, ibid., 219; Clostridium phlegmones-emphysematosae Holland, ibid., 219; Clostridium phleg mones emphysematosae Holland, ibid. 222; Clostridium welchii Holland, ibid., 221; Clostridium perfringens Holland, ibid., 219; Welchillus acrogenes Heller, Jour. Bact., 7, 1922, 6; Butyribacillus immobilis-liquefaciens Heller, ibid., 18; Bacillus welchii Type A Wilsdon, Univ Cambridge, Inst. Animal Path., 2nd Rept. of Dir., 1931, 72; Clostridium saccharobulyricum liquefaciens van Beynum and Pette, Cent. f. Bakt., II Abt., 93, 1935-36, 205; Welchia perfringens Prévot, Ann. Inst. Past., 61, 1938, 78.) Latinized, very fringed.

Related varieties: Clostridium egens Bergey et al., Manual, 1st ed., 1923, 324 (Bacillus egens Stoddard, Jour. Exp. Med., 29, 1919, 187; Stoddardillus egens Heller, Jour. Bact., 7, 1922, 6; Clostridium perfringens var. egens Hauduroy et al., Dict. d. Bact. Path., 1937, 124; Welchia perfringens var. egens Prévot, Ann. Inst. Past., 61, 1938, 78).

Clostridium perfringens Type B, Wilsdon. (Bacillus of lamb dysentery, Dalling, Jour. Path. and Bact., 28, 1925, 536, and ibid., 29, 1926, 316; L. D. Bacillus, Dalling, Handbook Ann. Congr. Nat. Vet. Med. Assoc., Gt. Britain and Ireland, 1928, 56; Brcillus welchii Type B, Wilsdon, Univ. Cambridge, Inst. Animal Path., 2nd Rept. of Dir., 1931, 73; Clostridium welchii (Type agni) Glenny et al., Jour. Path. and Bact., 97, 1933, 53; Bacillus agni. Weinberg et al., Les Mic. Anzér., 1937, 233; Welchia agni Prévot, Ann. Inst. Past., 61, 1938, 78.)

Clostridium perfringens Type C, Wilsdon. (Bacillus paludis McEwen, Jour. Compar. Path. and Ther., 43, 1930, 1; Bacillus welchii Type C, Wilsdon, Univ. Cambridge, Inst. Animal Path., 2nd Rept of Dir., 1931, 73; Welchia agni var. paludis Prévot, Ann. Inst. Past., 61, 1938, 78; Welchia paludis Prévot, Man. d. ('lass., etc., 1940, 217.

Clostridium perfringens Type D, Wilsdon. (Bacillus welchii Type D, Wilsdon, Tniv. Cambridge, Inst. Animal Path., 2nd Rept. of Dir., 1931, 74; Bacillus ovitoxicus Bennetts, Austral. Inst. Sci. and Indus., Bull. No. 57, 1932, 5, and Vet. Jour., 88, 1932, 250; Welchia agni var. oritoxicus Prévot, Ann. Inst. Past., 61, 1938, 78; Clostridium ovitoxicus Spray, in Manual, 5th ed., 1939, 773; Welchia ovitoxicus Prévot, Man. d. Class., ete., 1940, 217.)

Probably related (or possibly identical) varieties: Bacille du rhumatisme, 
Achalme, Compt. rend. Soc. Biol., Paris, 43, 1891, 651, and Ann. Inst. Past., 11, 1897, 848 (Bacille and bacterium d'Achalme, Thiroloix, Compt. rend. Soc. Biol., Paris, 49, 1897, 268; Bacillus achalmei Neveu-Lemaire, Précis Parasitol. Hum., 5th ed., 1921, 24); Bacillus emphysematis maligni Wicklein, Arch. f. Path. Anat. u. Physiol., 125, 1891, 91; Bacillus cadaveris Sternberg, Researches relating to the etiology and prevention of yellow fever, Washington, 1891, 212 (Bacterium cadaveris Migula, Syst. d. Bakt., 2, 1900, 510; not Bacillus cadaveris Klein, Cent. f. Bakt., I Abt., 25, 1899 , 279 ; not Clostridium cadaveris Sternberg. loc. cit., 213; not Bacillus cadarcris Migula, loc. cit., 646); Bacillus cadaceris butyricus Buday, Cent. f. Bakt., I Abt. 24, 1898, 374 (Bacillus budayi and Bacterium cadaveris butyricum LeBlaye and Guggenheim, Man. Prat. de Diag. Bact., 1914, 378; Eubacterium cadaveris Prévot, Ann. Inst. Past., 60, 1938, 295).

Bacillus zoodysenteriae Weinberg et al.. Les Mic. Anaér., 1937, 256 (Bacillus zoodysenteriae hungaricus Detre, Cent. f. Bakt., I Abt., Orig., 104, 1927, 251; Welchia perfringens var. zoodysenteriae Prévot, Ann. Inst. Past., 61, 1938, 78).

Clostridium perfringens var. anaerogenes Hauduroy et al., Dict. d. Bact. Path., 1937, 122 (Unnamed species of Grootten, Compt. rend. Soc. Biol., Paris, 100, 1929, 499).

Rods: Short, thick, 1.0 to 1.5 by 4.0 to 8.0 microns, occurring singly and in pairs, less frequently in short chains. Nonmotile. Sporesoval, central to excentric, not swelling rods. Encapsulated. Grampositive.

Gelatin: Liquefied and blackened.

Agar surface colonies (anaerobic): Circular, moist, slightly raised, opaque center, entire.

Broth: Turbid; peptolytic. Clearing with viscid sediment.

Litmus milk: Acid, coagulated. Clot torn with profuse gas formation, but not digested.
Potato: Thin, grayish-white streak; gas in subtended liquid.

Indole not formed.

Nitrites produced from nitrates.

Acid and gas from glucose, fructose, galactose, mannose, maltose, lactose, sucrose, xylose, trehalose, raffinose, starch, glycogen and inositol. Mannitol not fermented. Salicin rarely fermented. Action on inulin and glycerol variable.

Coagulated albumin not liquefied.

Blood serum not liquefied.

Brain medium not blackened or digested.

Egg-meat: Profuse gas production in 8 hours. The meat is reddened and the liquid becomes turbid. No digestion.

Pathogenic for guinea pig, pigeon and mouse. Produces an exotoxin for which an antitoxin can be prepared.

Optimum temperature $35^{\circ} \mathrm{C}$ to $37^{\circ} \mathrm{C}$. Can grow at $50^{\circ} \mathrm{C}$.

Anaerobic.

Distinctive characters: Stormy fermentation of milk, combined with nonmotility.

Source: Gaseous gangrene, feces, milk and soil.

Habitat: Widely distributed in feces, sewage and soil.

25. Clostridium sphenoides (Bulloch et al.) Bergey etal. (Bacillus sphenoides Bulloch, Bulloch, Douglas, Henry, MeIntosh, O'Brien, Robertson and Wolf, Med. Res. Coune., Spec. Rept. Ser. No. 39, 1919, 43; Douglasillus sphenoides Heller, Jour. Bact., 7, 1922, 5; Bergey et al., Manual, 1st ed., 1923, 331; Plectridium sphenoides Prévot, Ann. Inst. Past. 61, 1938, 88.) From Greek, wedgeshaped.

Described from Bulloch et al., loc. cit., as amplified by Hall, Jour. Inf. Dis. 30, 1922, 502 .

Rods: Small, fusiform in vegetative state, occurring singly, in pairs and occasionally in short chains. Sporulating cells cuneate. Motile. Spores spherical, subterminal, becoming terminal on 
maturation, swelling rods. Gram-positive only in young cultures.

Gelatin: Not liquefied.

Agar surface colonies (anaerobic): Circular, or slightly irregular, entire.

Blood agar surface colonies (anaerobic): Minute dew-drops, becoming whitish, opaque. Blood is hemolyzed.

Deep agar colonies: Minute, opaque, smooth disks.

Broth: Turbid.

Litmus milk: Acid; slowly and softly coagulated. Clot not digested.

Indole not formed (indole formed by Tholby strain, Stanley and Spray, Jour. Bact., 41, 1941, 256).

Nitrites produced from nitrates.

Acid and gas from glucose, galactose, maltose, lactose and saliein. Inulin, glyecrol and dulcitol not fermented. Strains are apparently variable on mannitol, sucrose, dextrin and starch.

Coagulated albumin not liquefied.

Blood serum not liquefied.

Brain medium not blackened or digested.

Non-pathogenic for guinea pig and rabbit.

Optimum temperature not determined. Grows well at $30^{\circ} \mathrm{C}$ to $37^{\circ} \mathrm{C}$.

Anaerobic.

Source: From gangrenous war wounds.

Habitat: Not determined, other than this source.

26. Clostridium innominatum Prévot. (Bacillus E, Adamson, Jour. Path. and Bact., 22, 1918-19, 391; Prévot, Ann. Inst. Past., 61, 1938, 85.) From Latin, remaining unnamed.

Rods: Very small, thick, tapering at one or both ends, occurring singly, paired, in chains and filaments. Involution forms abundant on glucose agar. Motile. Spores small, spherical, subterminal, swelling rods. Gram-positive, quickly becoming Gram-negative.

Gelatin: Not liquefied.

Glucose agar surface colonies (anaerobic) : Two forms are produced : 1) Circu- lar, entire edge, opaque; 2) Diffuse, spreading, irregular and translucent.

Plain agar surface colonies (anaerobic): Small, circular, entire edge, whitishtranslucent, becoming opaque-yellowish with age.

Plain broth: Moderate turbidity, clearing by sedimentation in 3 to 4 days.

Glucose broth: More abundant turbidity and slight gas production.

Milk: Slowly acidified but not clotted. No further change.

Glucose, maltose, lactose and mannitol fermented with acid and gas.

Sucrose not fermented.

Coagulated albumin: Not digested or blackened.

Meat medium: Not digested or blackened.

Blood serum: Not digested or blackened.

Brain medium: Not digested or blackened.

Non-pathogenic (Prévot, loc. cit.).

Grows well at $37^{\circ} \mathrm{C}$.

Anaerobic.

Source: From septic and gangrenous war wounds.

Habitat: Not determined, other than this source.

27. Clostridium filiforme Bergey et al. (Bacillus regularis filiformis Debono, Cent. f. Bakt., I Abt., Orig., 62, 1912, 234; Bergey et al., Manual, 1st ed., 1923, 331.) From Latin, thread-like.

Rods : 0.5 to 0.8 by 3.0 to 5.0 microns, slender, occurring singly, in pairs, in chains and filaments. Non-motile. Spores very small, spherical, subterminal, or occasionally terminal, not swelling rods. Gram-positive.

Gelatin : Not liquefied.

Deep gelatin colonies: Small, gray, filamentous.

Deep agar colonies: Irregular, gray, translucent, filamentous.

Broth: Uniform turbidity.

Litmus milk: Acid, but no further change. 
Potato: Gray, filamentous; substance not digested.

Acid and gas from glucose and lactose. Acid only from sucrose and dulcitol. Starch not fermented.

Coagulated albumin not liquefied.

Grows in gelatin at $22^{\circ} \mathrm{C}$.

Anaerobic.

Source: From human feces.

Habitat: Not determined, other than this source.

28. Clostridium sartagoformum Partansky and Henry. (Jour. Bact., 30, 1935, 570.) From Latin, shaped like a frying-pan.

Rods : 0.3 to 0.5 by 3.5 to 6.0 microns. Slender, curved, with rounded ends, occurring singly. Motile. Spores oval, terminal, swelling rods. Gram-positive.

Gelatin: Not liquefied.

Agar surface colonies (anaerobic): Convex, discrete, circular, transparent to white and opaque. Surface moist and smooth.

Blood agar not hemolyzed.

Deep agar colonies : Regular, lent icular, smooth.

Broth: Clear; no growth.

Glucose broth: Turbid, with gas bubbles.

Litmus milk: Acid; slowly coagulated, with some gas formation. Clot not digested.

Potato: Very scant growth. No gas in surrounding liquid.

Indole not formed.

Nitrites not produced from nitrates.

Acid and gas from xylose, glucose, fructose, galactose, sucrose, lactose, maltose, raffinose, inulin, salicin, mannitol, acetate and butyrate. Starch, ethanol, glycerol and dulcitol not fermented.

Coagulated albumin not liquefied.

Blood serum not liquefied. Scant growth.

Brain medium not blackened or digested. Some gas is formed.

Optimum temperature $37^{\circ} \mathrm{C}$.

Anaerobic.

Distinctive character : Ferments sulfite waste liquor in 40 per cent concent ration, forming butyric and acetic acids, $\mathrm{H}_{2}$ and $\mathrm{CO}_{2}$.

Source: From garden soil and from stream and lake mud.

Habitat: Not recorded; obviously soil. Distribution undetermined.

29. Clostridium paraputrificum (Bienstock) Snyder. (Art V, Bienstock, Fortschr. d. Med., 1, 1883, 612; Bacillus diapthirus Trevisan, I generi e le specie delle Batteriacee, 1889, 15; Bacillus paraputrificus Bienstock, Ann. Inst. Past., 20, 1906, 413, and Strassburger Med. Zeit., 3, 1906, 111; Bacillus paraputrificus coli Henry, Brit. Med. Jour., 1, 1917, 763; Tissierillus paraputrificus Heller, Jour. Bact., 7, 1922, 27; Snyder, Jour. Bact., 32, 1936, 401; Plectridium paraputrificum Prévot, Man. d. Class., ete., 1940, 160.) From Latin, also putrefying.

Probable synonyms: Köpfchenbakterien, Escherich, Fortschr. d. Med., 8, 1885, 515; Bacillus No. 3, Rodella, Ztschr. f. Hyg., 39, 1902, 209 (Plectridium fluxum Prévot, Ann. Inst. Past., 61, 1938, 87); Art XI, Hibler, Cent. f. Bakt., I Abt., 25, 1899, 516 (Art IX, Hibler, Untersuch. ü. d. Path. Anaer., 1908, 3 and 407; Plectridium nonum Prévot, Ann. Inst. Past., 61, 1938, 88); Anaerobe b, Dalyell, Jour. Path. and Bact., 19, 1914-15, 281; Bacillus innutritus Klcinschmidt, Monatsehr. f. Kinderheilkunde, 62, 1931, 18 (Palmula innutrita Prévot, Ann. Inst. Past., 61, 1938, 89; Acuformis innutritus Prévot, Man. d. Class., etc., 1940, 165).

Described from Hall and Snyder, Jour. Bact., 28, 1934, 181.

Rods : 0.3 to 0.5 by 2.0 to 6.0 microns. Straight or slightly curved, single, in pairs, or in short chains. Ends rounded. Motile with peritrichous flagella. Spores oval, terminal, swelling rods. Grampositive.

Gelatin : Not liquefied. Gas is formed. Blood agar surface colonies (anaerobic): Delicate, irregular, round-topped dewdrops. Non-hemolytic. 
Deep agar colonies: Small, irregular, opaque, dense, cottony masses. Gas is formed.

Broth: Diffuse turbidity.

Milk: Usually coagulated in from 6 to 10 days. Abundant gas, but no peptonization.

Indole is not formed.

Acid and gas from glucose, fructose, galactose, maltose, lactose, sucrose, raffinose, dextrin, soluble starch, amygdalin and salicin. Xylose, inulin, mannitol and glycerol not fermented.

Coagulated albumin not liquefied.

Blood serum not liquefied or discolored.

Brain medium not blackened or digested. Non-proteolytic.

Non-pathogenic for guinea pig and rabbit.

Grows well at $37^{\circ} \mathrm{C}$.

Anaerobic.

Source: Feces, gaseous gangrene, and postmortem fluid and tissue cultures.

Habitat: Undetermined, other than these sources. Evidently occurs commonly in intestinal canal of human beings.

30. Clostridium cochlearium (Bulloch et al.) Bergey et al. (Bacillus Type IIIc, McIntosh, Med. Res. Counc., Spec. Rept. Ser. No. 12, 1917, 20; Bacillus cochlearius Bulloch, Bulloch, Douglas, Henry, McIn tosh, O'Brien, Robertson and Wolf, Med. Res. Counc., Spec. Rept. Ser. No. 39, 1919, 40; Flemingillus cochlearius Heller, Jour Bact., 7, 1922, 5; Bergey et al., Manual 1st ed., 1923, 333; Plectridium cochlearium Prévot, Ann. Inst. Past., 61, 1938 88; Plectridium incertum Prévot, idem.) From Latin, spoon-shaped.

Rods: Slender, straight, occurring chiefly singly, or infrequently in pairs and in short chains. Motile with peritrichous flagella. Spores oval, terminal, swelling rods. Weakly Gram-positive.

Gelatin: Not liquefied.

Agar surface colonies (anaerobic): Circular, clear, entire, or with crenated edge.

Deep agar colonies : Lenticular, entire. Broth: Turbid.
Litmus milk: Unchanged.

Glucose not fermented.

C'arbohydrates not fermented.

Congulated albumin not liquefied.

Blood serum not liquefied.

Brain medium not blackened or digested.

Meat medium: Slightly reddened. Not blackened or digested. Little gas of nonputrefactive odor.

Non-pathogenic.

Optimum temperature $30^{\circ} \mathrm{C}$ to $35^{\circ} \mathrm{C}$.

Anaerobic.

Source: From human war wounds and septic infections.

Habitat: Not determined, other than these sources. Probably oceurs in soil.

31. Clostridium kluyveri Barker and Taha. (Jour. Bact., 43, 1942, 347.) Named for A.J. Kluyver, in whose laboratory the organism was discovered.

Rods : 0.9 to 1.1 by 3.0 to 11.0 microns. Straight to slightly curved; usually single, but also paired and occasionally in long chains. Motile with peritrichous flagella. Spores oval, terminal, swelling rods. Generally Gram-negative; some strains weakly Gram-positive when young.

Iron-gelatin (Spray): No growth.

Surface agar colonies (anaerobic): Growth slow and restricted by residual traces of oxygen. Rough and smooth colonies are produced.

Deep agar colonies (yeast autolysate and $\mathrm{C}_{2} \mathrm{H}_{5} \mathrm{OH}$ ): Small colonies ( 1 to 3 $\mathrm{mm}$ ) after 2 to 3 days; two types are formed: a) fluffy spheres with dense nuclear center and filamentous periphery ; b) compact, lenticular colonies. Little gas is formed.

Plain broth: No growth.

Glucose broth : No growth.

Milk or iron-milk (Spray): No grow th.

Indole not recorded (probably negative).

Nitrites not recorded (probably negative).

Glucose not fermented.

Carbohydrates not fermented. 
Cellulose not fermented.

Coagulated albumin not liquefied.

Blood serum not liquefied.

Brain medium not digested or blackened.

Probably non-pathogenic.

Optimum temperature about $34^{\circ} \mathrm{C}$. Grows between $19^{\circ} \mathrm{C}$ and $37^{\circ} \mathrm{C}$.

Optimum reaction about $\mathrm{pH} \quad 6.8$. Range for growth $\mathrm{pH} 6.0$ to 7.5 .

Anaerobic.

Distinctive characters: Large size of cells, and slow growth, accompanied by non-putrefactive odor of caproic acid and of higher alcohols. Growth is exceptionally favored by synergistic association with Methanobacterium omelianskii. In pure culture a high concentration of yeast autolysate is required. Caproic acid is formed from ethyl alcohol.

Source: From black mud of fresh water and marine origin.

Habitat: Not determined, other than these sources. Evidently widely dispersed in nature.

\section{Clostridium acidiurici (Liebert)} Barker. (Bacillus acidi urici Liebert, Koninkl. Akad. v. Wetensch., Proc. Sect. Sci., Amsterdam, 12, 1909, 55; Barker, Jour. Bact., 36, 1938, 323.) Named from its characteristic ability to ferment uric acid.

Rods : 0.5 to 0.7 by 2.5 to 4.0 microns; straight. Motile with peritrichous flagella. Spores oval, terminal, swelling rods. Most strains Gram-negative. A few strains weakly Gram-positive, quickly becoming Gram-negative.

Iron-gelatin (Spray): No growth.

Deep plain agar : No growth.

Deep uric acid agar colonies: Whitish, compact, lobate, 1 to $2 \mathrm{~mm}$ in diameter, with irregular edge; surrounded by a temporary zone of precipitated ammonium ureate which gradually disappears.

Surface uric acid agar colonies (anaerobic): Variable with strain and with moisture of medium. Colonies 1 to 2 $\mathrm{mm}$ in diameter, opaque, white, raised, round, smooth edge, with concentric surface markings, and of rubbery consistency. Other colonies may be very thin, soft, transparent, with fimbriate projections, spreading to cover almost the entire plate. Intermediate colony types also observed.

Plain broth: No growth.

Glucose broth: No growth.

Iron-milk (Spray): No growth.

Indole not recorded (probably negative).

Nitrites not recorded (probably negative).

Glucose not fermented.

Carbohydrates not fermented.

Cellulose not fermented.

Coagulated albumin not liquefied.

Blood serum not liquefied.

Brain medium not digested or blackened.

Probably non-pathogenic.

Optimum temperature about $35^{\circ} \mathrm{C}$.

Optimum reaction about $\mathrm{pH} 7.5$; lower limit for growth about $\mathrm{pH} 6.5$.

Anaerobic.

Distinctive characters: Requires uric acid, or certain other purines, as a primary source of carbon and energy. The purines are converted mainly into ammonia, $\mathrm{CO}_{2}$ and acetic acid. During growth the medium tends to become alkaline ( $\mathrm{pH} 8.0$ to 8.5 ); there is no visible evolution of gas.

Source: From soils of diverse origin.

Habitat: Evidently widely dispersed in soils. Present in fecal material of yellow-shafted flicker (Colaptes auratus).

33. Clostridium capitovale (Snyder and Hall) Snyder. (Bacillus capitovalis Snyder and Hall, Cent. f. Bakt., I Abt., Orig., 135, 1935, 290; Clostridium capitovalis Snyder, Jour. Bact., 32, 1936, 401; Plectridium capitovalis Prévot, Ann. Inst. Past., 61, 1938, 87.) From Latin, oval-headed.

Rods : 0.5 to 0.8 by 2.0 to 2.5 microns. Slender, commonly curved, with rounded ends, occurring singly, in pairs, and rarely in short ehains. Motile with long peri- 
trichous flagella. Spores oval, terminal, swelling rods. Gram-positive.

Gelatin : Liquefied.

Blood agar surface colonies (anaerobic): Tiny, transparent, round or irregular dew-drops, becoming opaque. Non-hemolytic.

Deep agar colonies: Small, opaque, lenticular to heart-shaped.

Tryptone broth: Turbid. Gas is formed.

Milk: Often, but not invariably, clotted. Acid is formed. Clot, when formed, is not digested.

Indole not formed.

Nitrites not produced from nitrates.

Acid and gas from glucose, fructose and galactose. Maltose, lactose, sucrose, raffinose, xylose, inulin, dextrin, starch, cellulose, amygdalin, salicin, mannitol and glycerol not fermented.

Coagulated albumin liquefied.

Blood serum slowly softened and partially liquefied. Not blackened. Mildly proteolytic.

Brain medium is blackened; slightly softened, but not conspicuously liquefied.

Pathogenicity: Guinea pig may show slight subcutaneous edema; usually no effect. Non-pathogenic for rabbit.

Grows at $37^{\circ} \mathrm{C}$.

Anaerobic.

Source: Human feces, gaseous gangrene and septicemia.

Habitat: Not determined, other than these sources.

\section{Clostridium parabifermentans} comb. nov. (Bacillus parabifermentans sporogenes de Gasperi, Compt. rend. Soc. Biol., Paris, 67, 1909, 492.) From Greek, para, near, and Latin, bis, twice, and fermentum, a ferment.

Rods : 0.5 to 0.7 by 4.0 to 5.0 microns, occurring singly, in pairs and in chains of 3 to 5 cells. Motile. Spores oval, terminal, swelling rods. Gram-positive.

Glucose gelatin: Rapid growth with liquefaction.

Deep glucose agar colonies : Lenticular, regular, opaque, whitish. Agar dis- rupted by considerable gas of putrefactive odor.

Glucose broth: Abundant growth with uniform turbidity and with viscous sediment.

Milk: Acidified but not coagulated. Casein slowly precipitated with slow, but complete, digestion.

Indole formed in trace.

Glucose, lactose and sucrose fermented to acids. (Gas not recorded.) Starch is not fermented.

Coagulated albumin actively liquefied.

Non-pathogenic for mouse.

Grows between $22^{\circ} \mathrm{C}$ and $37^{\circ} \mathrm{C}$.

Anaerobic.

Source: From putrefying game (pheasant and guinea-fowl).

Habitat: Undetermined, other than this source.

35. Clostridium ovalare Bergey et al. (Bacillus putrificus ovalaris Debono, Cent. f. Bakt., I Abt., Orig., 62, 1912, 231 ; Bergey et al., Manual, 1st ed., 1923, 336; Plectridium ovalaris Prévot, Ann. Inst. Past., 61, 1938, 88.) From Latin, oval.

Rods : 0.3 to 0.4 by 6.0 to 8.0 microns, straight or curving, ends rounded, occurring singly, in pairs and in short chains. Motile. Spores oval, terminal, swelling rods. Gram-positive.

Gelatin : Rapidly liquefied.

Deep glucose agar colonies: Small, globular, entire, becoming brownish. Scant gas is formed.

Broth: Turbid.

Litmus milk : Acid, peptonized without coagulation .

Indole not formed.

Acid and scant gas from glucose and lactose. Acid only from sucrose. Dulcitol not fermented.

Coagulated albumin rendered transparent, then slowly peptonized, with a putrefactive odor.

Grows at $22^{\circ} \mathrm{C}$ and at $37^{\circ} \mathrm{C}$.

Anaerobic.

Source: Originally from putrid meat, later from feces. 
Habitat: Not determined, other than these sources.

36. Clostridium zoogleicum Bergey et al. (Bacillus sporogenes zoogleicus Distaso, Cent. f. Bakt., I Abt., Orig., 59, 1911, 99; Bergey et al., Manual, 1st ed., 1923, 335.) From Greek, zoogleal.

Rods : Fairly long, occurring singly, in pairs and in short chains. Motile. Spores large, oval, terminal, swelling rods. Gram-positive.

Gelatin: Growth and liquefaction not recorded.

Deep agar colonies: Small, gray, slightly opaque, becoming heart-shaped. Gas is not formed.

Broth: Turbid, then clearing with zoogleal sediment.

Litmus milk: Slowly coagulated, then digested. Litmus reduced.

Indole is formed in trace.

Acid but no gas from glucose. Lactose and sucrose not fermented.

Coagulated albumin liquefied, leaving a clear fluid and zoogleal sediment.

Grows at $37^{\circ} \mathrm{C}$.

Anaerobic.

Source: From human feces.

Habitat: Undetermined, other than this source.

37. Clostridium thermosaccharolyticum MicClung. (Jour. Bact., 29, 1935, 200; Terminosporus thermosaccharolyticus Prévot, Ann. Inst. Past, 61, 1938, 86.) From Greek, heat, and sugar-digesting.

Rods : 0.4 to 0.7 by 3.5 to 7.5 microns, slender, granulated, occurring singly and in pairs, not in chains. Motile with peritrichous flagella. Spores spherical, terminal, swelling rods. Gram-negative.

Gelatin : Not liquefied.

Pea-infusion agar surface colonies (anaerobic): Granular, grayish-white, raised center, with feathery edges.

Deep glucose-tryptone agar colonies: Small, lenticular, smooth.

Liver-infusion broth over liver meat: Turbidity and gas.

Litmus milk: Litmus reduced. Acid and slow but firm coagulation; coagulum split with gas. Clot not digested.

Indole not formed.

Nitrites not produced from nitrates.

Cellulose not fermented.

Acid and gas from arabinose, fructose, galactose, glucose, mannose, xylose, cellobiose, lactose, maltose, sucrose, trehalose, dextrin, glycogen, corn-starch, amygdalin, esculin, $\alpha$-methyl glucoside and salicin. Raffinose weakly fermented. Rhamnose, inulin, pectin, erythritol, inositol, mannitol, glycerol, quereitol and Ca-lactate not fermented.

Coagulated albumin not liquefied.

Blood serum not liquefied.

Brain medium not blackened or digested.

Meat-medium not blackened or digested.

Non-pathogenic on feeding to white rat, or by injection into rabbit.

Optimum temperature $55^{\circ} \mathrm{C}$ to $62^{\circ} \mathrm{C}$. Thermophilic.

Anaerobic.

Source: From hard-swell of canned goods, and from soil.

Habitat: Not determined, other than these sources.

38. Clostridium caloritolerans Meyer and Lang. (Jour. Inf. Dis., 39, 1926, 321 ; Plectridium caloritolerans Prévot, Ann. Inst. Past., 61, 1938, 87.) From Latin, heat-enduring.

Rods : 0.5 to 0.8 by 8.0 to 10.0 microns, with rounded ends, occurring singly, in pairs, in chains and in curved filaments. Motile with peritrichous flagella. Spores spherical or pear-shaped, terminal, swelling rods. Gram-positive.

Gelatin : Not liquefied.

Glucose blood agar surface colonies (anaerobic): Small, flat, grayish, rhizoidal. Non-hemolytic.

Deep liver agar colonies: Small, flat, transparent disks with large polar tufts. Some colonies become fluffy.

Broth: Slight turbidity.

Glucose broth: Abundant turbidity; 
with clearing by sedimentation. Gas is formed.

Brom cresol purple milk: No change.

Indole not formed.

Acid and gas from glucose, galactose and maltose. Fructose feebly fermented. Lactose, sucrose, raffinose, inulin, salicin, mannitol, inositol and glyeerol not fermented.

Coagulated albumin not liquefied.

Blood serum not liquefied.

Brain medium not blackened or digested.

Beef-heart mash medium: Reddened; not blackened or digested.

Non-pathogenic for mouse, guinea pig and rabbit.

Optimum temperature not determined. Grows at $37^{\circ} \mathrm{C}$.

Anaerobic.

Source: From an old culture of Clos tridium parabotulinum Type A.

Habitat: Not determined, other than this single isolation.

39. Clostridium tetanoides (Adamson) Hauduroy et al. (Unnamed anaerobe, Adamson and Cutler, Lancet, 1, 1917, 688 ; Bacillus tetanoides (A) Adamson, Jour. Path. and Bact., 22, 1918-19, 382; Hauduroy et al., Dict. d. Bact. Path., 1937 140; Palmula macrospora Prévot, Ann Inst. Past., 61, 1938, 88; Acuformis macrosporus Prévot, Man. ¿. Class., etc. 1940, 166.) From Latin, tetanus-like.

Rods : 1.0 to 2.0 by 4.0 to 12.0 microns (averaging 1.0 to 1.5 by 6.0 to $7.0 \mathrm{mi}$ crons), with rounded to slightly tapered ends, oecurring singly, in pairs and in chains of 3 to 5 cells, but not in filaments. Motile only in young cultures. Spores large, spherical, terminal, swelling rods. Gram-positive in young cultures, soon becoming Gram-negative.

Gelatin : Not liquefied.

Glucose agar surface colonies (anaerobic): Circular, regular, opaque, hluishgray, moist, shining, thick, raised. Surface flat, becoming comical in center with age. On moist medium showing radiating, dendritic branching. Growth becomes tenacious-mucoid.

Plain agar surface colonies (anaerobic): Confluent, becoming an opaque film. Isolated colonies circular to slightly irregular. Dendritic branching and mucoid tendency less evident than on glucose agar.

Glucose agar stab: Thick growth along stab, starting $0.5 \mathrm{~cm}$ below surface. No gas or splitting of medium.

Neutral-red glucose agar: Reduced to orange by transmitted, and greenishfluorescent by reflected light.

Plain broth: Early slight turbidity, with clearing and mucoid sedimentation.

Glucose broth: Abundant turbidity and profuse mucoid sediment.

Milk: Slight and slowly increasing alkalinity, with slow separation of casein. No further change.

Indole: Trace formed in broth.

Glucose and maltose fermented with acid but nogas. Lactose, sucrose, mannitol, starch and cellulose not fermented.

Coagulated albumin: Not digested or blackened.

Meat medium: Not digested or blackened.

Blood serum: Not digested or blackened.

Brain medium : Not digested or blackened.

Non-pathogenic for guinea pig and rabbit.

Optimum temperature not recorded. Grows well at $37^{\circ} \mathrm{C}$.

Anaerobic.

Source: From war wounds, from postmortem blood culture, and from garden soil.

Habitat: Not determined, other than these sources.

40. Clostridium tetani (Flügge) Holland. (Tetanusbacillen and Tetanuserreger, Nicolaier, Deuts. Med. Wchnschr., 10, 1884, 843; Bacillus tetani Flügge, Dif Mikroorg., 2 Aufl.. 1886, 27t; Pacinic nicolaieri Trevisan, I generi e le speci, delle Batteriacee, 1889, 23; Plectridium. 
letani Fischer, Jahrb. f. Wissensch. Botanik, 27, 1895, (147?); Holland, Jour. Bact., 5, 1920, 220; Nicollaierillus tetani Heller, Jour. Bact., 7, 1922, 7.) From tetanus, lockjaw.

Rods : 0.4 to 0.6 by 4.0 to 8.0 microns, rounded ends, occurring singly, in pairs, and often in long chains and filaments. Motile with peritrichous flagella. Spores spherical, terminal, swelling rods. Gram-positive.

Gelatin: Slowly liquefied and blackened.

Serum agar surface colonies (anaerobie): Small, transparent, villous to fimbriate margin.

Blood agar is hemolyzed.

Deep agar colonies: Fluffy, cottony spheres, usually without visible central nucleus.

Broth: Slightly turbid. Gas is formed. Some strains clear quickly by sedimentation.

Litmus milk: Slow precipitation of casein, or soft clotting. Clot slowly softened, but not definitely digested. Little gas is formed.

Indole is formed.

Vitrites not produced from nitrates.

Glucose not fermented.

Carbohydrates not fermented.

Coagulated albumin slowly liquefied.

Blood serum slowly softened, with feeble digestion.

Brain medium blackened and slowly digested. Not actively proteolytic.

Pathogenic and toxic. Forms a potent exotoxin for which an antitoxin is prepared. Toxin intensely toxic on injection but not on feeding.

Optimum temperature $37^{\circ} \mathrm{C}$

Anaerobic.

Source: Originally isolated from animals inoculated with garden soil extract. Frequently isolated from wounds in human tetanus.

Habitat: Common in soils, and in human and horse intestine and feces.

41. Clostridium lentoputrescens Hartsell and Rettger. (Bacillus der Eiweiss- fäulniss, Bienstock, Fortschr. d. Med., 1, 1883, 614 (Art IT, Bienstock, ibid., 612; Eiweissbacillus, Bienstock, Ztschr. f. klin. Med., 8, 1884, 38); Bacillus albuminis Schroeter, in Cohn's KryptogamenFlora von Schlesien, 3, 1. 1886, 162; Bacillus putrificus coli Flügge, Die Mikroorg., 2 Aufl., 1886, 303; Pacinia putrifica Trevisan, I generi e le specie delle Batteriacee, 1889, 23; Bacillus putrificus Bienstock, Ann. Inst. Past. 13, 1899, 861; Bacillus butyricus putrefaciens Rodella, Ann. Inst. Past., 19 1905, 804; Putribacillus vulgaris OrlaJensen, Cent. f. Bakt., II Abt., 22, 1909, 343; Clostridium putrificum Holland, Jour. Bact., 5, 1920, 220; Putrificus bienstocki Heller, Jour. Bact., 7, 1922, 8; Bacillus putrificus (coli) Lehmann and Neumann, Bakt. Diag., 7 Aufl., 2, 1927. 661 ; Hartsell and Rettger, Jour. Bact., 27, 1934, 39 and 497; Plectridium putrificum and Plectridium putrificum var. lentoputrescens Prévot, Ann. Inst. Past., 61, 1938, 88.) From Latin, slowly made putrid.

Probable synonyms: Bacillus radiatus Lüderitz, Ztschr. f. Hyg., 5, 1889, 149 (Cornilia radiata Trevisan, loc. cit., 22; Bacillus radiatus anaerobius Hopffe, Ztschr. f. Infkrnkh. u. Hyg. Haust., 14, 1913, 392); Barillus cadaveris sporogenes (anaerobicus) Klein, Cent. f. Bakt., I Abt., 25, 1899, 279 (Bacillus cadaveris Klein, idem; not Bacillus cadaveris Sternberg, Researches relating to the etiology and prevention of yellow fever, Washington, 1891, 212; not Bacillus cadaveris Migula, Syst. d. Bakt., 2, 1900, 646 ; Bacillus cadaveris sporogenes Klein, loc. cit., 281; Plectridium cadaveris Prévot, Ann. Inst. Past., 61, 1938, 88); Art XIV, von Hibler, Untersuch. u. d. Path. Anaer., 1908, 3 and 413; Bacillus tetanoides $(B)$ Adamson, Jour. Bact. and Path., 22, 1918-19, 388.

Hartsell and Rettger, loc. cit., conclude that their organism differs very materially either from Clostridium cochlear. ium or from Bacillus putrificus, as described by Cunningham, Jour. Bact., 
24, 1932, 61, and, as it cannot be definitely related to any other anaerobic species (including the Eiweissbacillus, Bienstock, loc. cit., Bacillus putrificus coli Flügge, loc. cit., Bacillus putrificus Bienstock, loc. cit., ete.), they propose the name of Clostridium lentoputrescens for this species.

Rods : 0.4 to 0.6 by 7.0 to 9.0 microns, with rounded ends, occurring singly, in pairs and in chains. Motile with peritrichous flagella. Spores spherical, terminal, swelling rods. Weakly Grampositive, becoming Gram-negative.

Gelatin : Liquefied.

Agar surface colonies (anaerobic): Small, circular, flat, edge crenated to filamentous spreading. Develop a ground-glass appearance.

Deep agar colonies: Fluffy spheres with fibrils radiating from a central nucleus.

Blood agar is hemolyzed.

Litmus milk: Slow, soft coagulation or flocculent precipitation. Casein is slowly digested.

Indole is formed (Hall, Jour. Inf. Dis., $30,1922,141$ ). Not formed (Hartsell and Rettger, loc. cit., 509).

Nitrites not produced from nitrates.

Hydrogen sulfide formed in egg-meat medium.

Carbohydrates not fermented. Glucose slightly attacked without distinct acid (Hartsell and Rettger, loc. cit., 508).

Coagulated albumin slowly liquefied and blackened.

Blood serum is liquefied. Gas is formed.

Brain medium slowly blackened and digested.

Egg-meat medium; Slightly turbid liquid. Meat reddened in 7 to 10 days, then digested with a foul odor.

Non-pathogenic for white mouse, guinea pig and rabbit. Filtrate non-toxic on injection or feeding.

Grows well at $37^{\circ} \mathrm{C}$.

Anaerobic.

Source : From putrefying meat.

Habitat: Intestinal canal of human. Widely dispersed in soil.
42. Clostridium filamentosum Bergey et al. (Bacillus putrificus filamentosus Distaso, Cent. f. Bakt., I Abt., Orig., 59, 1911, 98; Bergey et al., Manual, 1st ed., 1923, 333; Palmula filamentosa Prévot, Ann. Inst. Past., 61, 1938, 88; Acuformis filamentosus Prévot, Man. d. Class., etc., 1940, 165.) From Latin, filamentous.

Rods: Slender, occurring singly, in pairs and in chains. Motile. Spores spherical, or nearly so, terminal, swelling rods. Gram-positive.

Gelatin : Liquefied.

Deep glucose agar colonies: Delicate, cottony flocculi. Only a trace of gas formed.

Broth: Turbid.

Litmus milk : May or may not coagulate and digest slowly (variable).

Indole formed in scarcely detectable trace. Odor of scatol.

Glucose is feebly fermented, with little gas. Lactose and sucrose not fermented.

Coagulated albumin: Rendered transparent, then slowly liquefied.

Grows well at $37^{\circ} \mathrm{C}$.

Anaerobic.

Source: From human feces.

Habitat: Not determined, other than this source.

43. Clostridium tetanomorphum (Bulloch et al.) Bergey et al. (Bacillus pseudo-tetanus, Type No. IX,-Tetanuslike Bacillus (Pseudotetanus Bacillus), MeIntosh and Fildes, Med. Res. Coune., Spec. Rept. Ser. No. 12, 1917, 11 and 32; Bacillus tetanomorphus Bulloch et al., Med. Res. Counc., Spee. Rept. Ser. No. 39, 1919, 41; Macintoshillus tetanomorphus Heller, Jour. Bact., 7, 1922, 5; Bergey et al., Manual, 1st ed., 1923, 330; Plectridium tetanomorphum Prévot, Ann. Inst. Past., 61, 1938, 87.) From Greek, shaped like the tetanus organism.

Synonyms or possibly related species: Bacillus pseudotetani Migula, Syst. d. Bakt., 2, 1900, 598 (Tetanusähnlicher Bacillus and Pseudotetanusbacillus, Tavel and Lanz, Mitteil. a. klin. Med. Inst. d. Schweiz, 1, 1893, 162; Bacillus taveli 
Chester, Man. Determ. Bact., 1901, 304; Plectridium pseudotetanicum Prévot, Ann. Inst. Past., 61, 1938, 87; Plectridium pseudo-tetanicum Prévot, Man. d. Class., etc., 1940, 158); possibly identical with Bacillus fragilis Veillon and Zuber, Arch. d. Méd. Expt. et d'Anat. Path., 10, 1898, 536 and Bacillus ramosus Veillon and Zuber, ibid., 537.

Rods: Slender, with rounded ends, occurring singly and in pairs, not in chains. Motile with peritrichous flagella. Spores spherical, or nearly so, terminal, swelling rods. Gram-positive.

Gelatin: Not liquefied. Gelatin is liquefied (Hall, Jour. Inf. Dis., 30, 1922, 501 ).

Agar surface colonies (anaerobic): Small, flat, irregularly circular, translucent, crenated.

Déep agar colonies: Small, opaque, irregular; not woolly or branched.

Agar slant (anaerobic): Grayish, translucent growth.

Broth: Turbid.

Litmus milk: Unchanged; or occasional slight reduction of litmus.

Acid and gas from glucose and maltose. Fructose, galactose, lactose, sucrose, salicin, inulin, mannitol and glycerol not fermented.

Coagulated albumin not liquefied.

Blood serum not liquefied.

Brain medium not blackened or digested.

Egg-meat medium : Slight gas formation in 48 hours. White crystals are deposited.

Non-pathogenic for guinea pig and rabbit.

Grows at $30^{\circ} \mathrm{C}$ and $37^{\circ} \mathrm{C}$.

Anaerobic.

Source: From war wounds and from soil.

Habitat: Not determined other than these sources. Probably rather common in soil.

44. Clostridium alcaligenes Bergey et al. (Bacillus anaërobicus alcaligenes Debono, Cent. f. Bakt., I Abt., Orig., 62,
1912, 232; Bergey et al., Manual, 1st ed., 1923, 331; Palmula alcaligenes Prévot, Ann. Inst. Past., 61, 1938, 89; Acuformis alcaligenes Prévot, Man. d. Class., etc., 1940, 165.) From French alcali, alkali and Latin suffix, producing.

Rods: Long, slender, occurring singly, in pairs and in short chains. Non-motile. Spores spherical, terminal, swelling rods. Gram-positive.

Gelatin: Not liquefied.

Deep glucose agar colonies: Lenticular to irregular, or spherical, white, granular, entire.

Broth: Uniform turbidity. Fecal odor. Milk: Alkaline; casein slowly precipitated, with yellowish supernatant fluid.

Indole is formed in abundance.

Acid and gas from glucose and lactose. Sucrose and dulcitol not fermented. Cultures have odor of valerianic acid.

Grows at $22^{\circ} \mathrm{C}$ and at $37^{\circ} \mathrm{C}$.

Anaerobic.

Source: From human feces.

Habitat: Not determined, other than this source.

45. Clostridium angulosum (Distaso) Hauduroy et al. (Bacillus angulosus Distaso, Cent. f. Bakt., I Abt., Orig., 62, 1912, 439; not Bacillus angulosus Garnier and Simon, Bull. et Mem. Soc. Méd. Hôp. Paris, 24, 1907, 1034; Bacteroides angulosus Bergey et al., Manual, 1st ed., 1923, 260; Hauduroy et al., Dict. des Bact. Path., 1937, 91.) From Latin angulosus, having angles, hooked.

Rods: Short, thick, with rounded ends, occurring singly and in pairs. Long rods sometimes bent to form an obtuse angle. Encapsulated. Non-motile. Spores very small, spherical, terminal, slightly swelling rods. Gram stain not recorded.

Plain gelatin: No growth at $20^{\circ} \mathrm{C}$ or at $37^{\circ} \mathrm{C}$.

Glucose gelatin: Grows well at $37^{\circ} \mathrm{C}$. Growth cloudy at first, then clears and liquefies, with whitish, powdery precipitate.

Glucose agar deep colonies: Large, 
angular, opaque, yellowish. Gas bubbles are formed.

Broth: Turbid.

Litmus milk: Acid and congulated in 14 days.

Indole is formed.

Acid and gas from glucose, lactose and sucrose. Butyric acid is formed.

Coagulated albumin not liquefied. Odor of skatol.

Optimum temperature $37^{\circ} \mathrm{C}$.

Anaerobic.

Distinctive character: Resembles the Bacille neigeux, Jungano, Compt. rend. Soc. Biol., Paris, 62, 1907, 677, in form, but not in other respects.

Source: From human feces.

Habitat: Not determined, other than this source.

46. Clostridium putrefaciens (McBryde) Sturges and Drake. (Bacillus putrefaciens McBryde, U. S. D. A., Bur. An. Ind., Bull. 132, 1911, 6; Sturges and Drake, Jour. Bact., 14, 1927, 175; Palmula putrefaciens Prévot, Ann. Inst. Past., 61, 1938, 89; Acuformis putrefaciens Prévot, Man. d. Class., etc., 1940, 165.) From Latin, putrefying.

Deseription from McBryde (loc, cit.) and amplified from Sturges and Drake (loc. cit.)

Rods : 0.5 to 0.7 by 3.0 to 15.0 microns, rounded ends, occurring singly, in pairs, and in chains and filaments. Non-motile. Spores spherical, terminal, swelling rods. Gram-positive.

Gelatin : Liquefied.

Agar surface colonies (anaerobic): Small, filamentous.

Agar slant (anaerobic): Scanty, white, beaded, glistening growth.

Broth: Moderate turbidity. Heavy, floceulent sediment.

Litmus milk: Rennet coagulation, peptonized. Litmus reduced.

Indole not formed.

Nitrites not produced from nitrates.

Slight production of hydrogen sulfide. Acid and gas from glucose. Lactose, sucrose, maltose and starch not fermented.

Coagulated albumin liquefied.

Blood serum liquefied.

Brain medium blackened and slowly digested.

Minced pork medium: Slight disintegration; sour, putrefactive odor.

Non-pathogenic.

Optimum temperature $20^{\circ} \mathrm{C}$ to $25^{\circ} \mathrm{C}$. Slow growth at $\mathrm{O}^{\circ} \mathrm{C}$ and no visible growth at $37^{\circ} \mathrm{C}$.

Anaerobic.

Source: From musele tissue of hogs at slaughter.

Habitat: Not determined, other than this source.

47. Clostridium nigrificans Werkman and Weaver. (Iowa State Coll. Jour. Sci., 2, 1927-28, 63; Werkman, Iowa State Coll. Research Bull. 117, 1929, 165.) From Latin niger, black and faciens, making.

Rods : 0.3 to 0.5 by 3.0 to 6.0 microns, with rounded ends. Motile. Spores oval, subterminal, slightly swelling rods. Gram-positive.

Gelatin : Not liquefied.

Deep agar colonies: Show blackening of medium around colonies. Black increased by adding 0.1 per cent ferric chloride to medium.

Milk: Not recorded.

Indole not formed.

Nitrites not produced from nitrates.

Glucose not fermented.

Carbohydrates not fermented.

Coagulated albumin not liquefied.

Blood serum not liquefied.

Brain medium blackened but not digested.

Hydrogen sulfide produced from cystine.

Non-pathogenic to man, guinea pig, mouse, rat and rabbit.

Optimum temperature $55^{\circ} \mathrm{C}$. Thermophilic, growing at $65^{\circ} \mathrm{C}$ to $70^{\circ} \mathrm{C}$.

Anaerobic.

Distinctive character: Black colonies in agar media. 
Source: From canned corn showing sulfur stinker spoilage; also occasionally from soil and manure.

Habitat: Presumably soil, although detected with great difficulty.

48. Clostridium belfantii (Carbone and Venturelli) Spray. (Bacillus belfantii Carbone and Venturelli, Boll. Ist. Sieroter., Milan, 4, 1925, 59; not Bacillus belfantii Migula, Syst. d. Bakt., 2, 1900, 767; Spray, in Manual, 5th ed., 1939, 759; Endosporus belfantii Prévot, Ann. Inst. Past., 61, 1938, 75.) Named for Belfanti, an Italian bacteriologist.

Rods : 0.4 to 0.6 by 1.5 to 7.0 microns, thick and straight, occurring singly, in pairs and in short chains. Motile. Spores large, oval, central to subterminal, swelling rods. Usually Gram-negative, occasional cells Gram-positive

Granulose reactior negative.

Gelatin : Not liquefied.

Plain agar surface colonies (anaerobic) : Large, round, opaque, with filamentous edge,

Deep agar colonies: Arborescent along the stab. Gas is formed.

Plain broth: Diffuse turbidity, clearing by precipitation. No pigmentation. Gas is formed.

Potato mash: Forms a foam becoming violet in 24 to 48 hours, persisting 3 to 6 days, but disappearing on exposure to air.

Potato slant: Grayish pellicle, becoming violet in 24 to 48 hours. Gas of alcoholic odor is produced. No acetone.

Glycerinated potato: Thin, grayish pellicle, not becoming violet.

Milk: Coagulated in 24 to 48 hours. Clot broken by gas.

Milk agar: Abundant growth. Gas of butyric odor is liberated.

Indole is formed.

Hydrogen sulfide not formed.

Acid and gas from glucose, fructose, maltose, sucrose, lactose and mannitol. Starch and inulin weakly fermented.

Coagulated albumin not liquefied.

Blood serum not liquefied.

Grows well at $37^{\circ} \mathrm{C}$
Anaerobic .

Specifically agglutinated only by homol ogous antiserum.

Source: From retting beds and from air.

Habitat: Not determined, other than these sources.

Note : Six other strains of similar pigmenting, sporulating anaerobes are described by the authors. These have the general characters of Clostridium belfantii, but differ in certain particulars, such as color of pigment, fermentation and specific agglutination. Present information does not permit accurate systematic differentiation.

48a. Clostridium maggiorai (Carbone and Venturelli) Spray. (Bacillus maggiorai Carbone and Venturelli, loc. cit., 59; Spray, in Manual, 5th ed., 1939, 759; Endosporus maggiorai Prévot, loc. cit., 76.) Named for Maggiora, an Italian bacteriologist.

Characters in general those of the group, but does not produce indole.

Violet pigmentation persisting only 24 hours.

No alcoholic odor from cultures.

Specifically agglutinated only by homol ogous antiserum.

From mud from bed of stream in Italy.

48b. Clostridium derossii (Carbone and Venturelli) Spray. (Bacillus de rossii Carbone and Venturelli, loc. cit., 59; Spray, in Manual, 5th ed., 1939, 760; Endosporus rossii Prévot, loc. cit., 76.) Named for G. de Rossi, an Italian bacteriologist.

Characters in general those of the group. Greenish pigmentation on potato slant, changing to violet or orange.

Indole is formed.

Spedifically agglutinated only by homologous antiserum.

From soil in Italy.

48c. Clostridium ottolenghii (Carbone and Venturelli) Spray. (Bacillus ottolenghii Carbone and Venturelli, loc. cit., 59; Spray, in Manual, 5th ed., 1939, 760; 
Endosporus ottolenghii Prévot, loc. cit., 76.) Named for Ottolenghi, an Italian bacteriologist.

Characters in general those of the group. Potato slant digested to a grayishbrown mash. Greenish pigment changing to reddish. Gas of disagreeable odor is formed.

Indole is formed.

Specifically agglutinated only by homologous antiserum.

From mud from bed of a stream in Italy.

48d. Clostridium paglianii (Carboneand Venturelli) Spray. (Bacillus paglianii Carbone and Venturelli, loc. cit., 60 ; not Bacillus paglianii Trevisan, I generi e le specie delle Batteriacee, 1889, 19; Spray, in Manual, 5th ed., 1939, 760; Endosporus paglianii Prévot, loc. cit., 76.) Named for Pagliani, an Italian bacteriologist.

Characters in general those of the group. One or two subterminal spores are said to be formed.

Greenish pigmentation on potato, browning with age.

Indole is formed.

Specifically agglutinated only by homologous antiserum.

From soil in Italy.

48e. Clostridium lustigii (Carbone and Venturelli) Spray. (Bacillus lustigii Carbone and Venturelli, loc. cit., 59; not. Bacillus lustigii Trevisan, in litt., quoted from DeToni and Trevisan, in Saccardo, Sylloge Fungorum, 8, 1889, 958; not Bacillus lustigi Chester, Man. Determ. Bact., 1901, 304; Spray, in Manual, 5th ed., 1939, 760; Endosporus lustigii Prévot, loc. cit., 76.) Named for Lustig, an Italian bacteriologist.

Characters in general those of the group. Green pigmentation on potato slant.

Indole is not formed.

Specifically agglutinated only by homologous antiserum.

From mud and soil in Italy.

48f. Clostridium sclavoi (Carbone and Venturelli) comb. now. (Bacillus sclavoi
Carbone and Venturelli, loc. cit., 60; Endosporus sclavoei Prévot, loc. cit., 76.) Named for Sclavo, an Italian bacteriologist.

Characters in general those of the group. Greenish to brown pigmentation on potato slant.

Indole is not formed.

Specifically agglutinated only by homol ogous antiserum.

From retting flax in Italy.

49. Clostridium venturelli (de Tomasi) Spray. (Bacillus venturelli de Tomasi, Boll. Ist. Sieroter. Milanese, 4, 1925, 203; Endosporus venturelli Prévot, Ann. Inst. Past., 61, 1938, 76; Spray, in Manual, 5th ed., 1939, 769.) Named for Venturelli, an Italian bacteriologist.

Rods: Pleomorphic, fusiform to straight or slightly curved, with rounded ends. Size variable with medium, 0.5 to 0.8 by 2.5 to 8.0 , and up to 20.0 microns. Occurring singly, in pairs, in chains, or frequently in parallel groupings. Motile. Capsulated. Spores oval, central to excentric, swelling rods. Gram-negative.

Granulose reaction positive; showing violet granules with iodine.

Gelatin: No growth; no liquefaction.

Glucose agar surface colonies (anaerobic) : Round, becoming rose-colored.

Plain agar slant (anaerobic): No growth.

Maltose agar stab: Colonies lenticular, yellowish, turning to rose. Odor of acetone.

Plain broth: No growth.

Potato slant (anaerobic): Becomes mucilaginous. Bubbles of gas of amylic odor.

Potato mash: Very abundant growth; rose color, with red spots.

Milk with $\mathrm{CaCO}_{3}$ : Coagulated; becoming yellow, then pale rose. Amylic odor.

Acid and gas from glucose, maltose, sucrose, fructose, lactose, inositol, dextrin and starch. Arabinose, glycerol, mannitol and inulin not fermented (cited from Weinberg et al., Les Mic. Anaér., $1937,800)$. 
Fermentation products include especially acetone and amyl alcohol, and -maller amounts of propyl, butyl and isobutyl alcohols, and acetic acid.

Coagulated albumin not digested.

Blood serum not liquefied; forms a small amount of yellowish liquid.

Optimum temperature $18^{\circ} \mathrm{C}$ to $20^{\circ} \mathrm{C}$. Inhibition of growth and pigmentation above $25^{\circ} \mathrm{C}$.

\section{Anaerobic.}

Distinctive character: Forms a rosecolored pigment which is soluble in alcohol, but not in water, ether or chloroform.

Source: From potato.

Habitat: Not determined, other than this source.

50. Clostridium roseum McCoy and McClung. (Arch. f. Mikrobiol., 6, 1935, 237.) From Latin roseum, pink.

Rods : 0.7 to 0.9 by 3.2 to 4.3 microns, sccurring singly, in pairs and in short chains. Motile with peritrichous flagella. Spores oval, subterminal, swelling rods to clostridia. Gram-positive, becoming Gram-negative.

Granulose reaction positive in clostridial stage.

Glucose gelatin : Liquefied.

Plain agar slant (anaerobic): Surface growth scant, scarcely perceptible.

Glucose agar surface colonies (anaerobic): Raised, smooth, edges slightly irregular. Pink to orange pigment.

Deep glucose agar colonies: Compact, lenticular, pink to red-orange.

Blood agar not hemolyzed.

Pigmentation (anaerobic): Colonies red-orange, becoming purplish-black on aeration.

Plain broth: No growth.

Glucose broth: Abundant, uniform turbidity, with much gas.

Litmus milk: Stormy coagulation. Litmus reduced, but obseured by pink pigment. Clot slowly softened. Proteolysis demonstrable on milk agar.

Potato: Rapid digestion to a clear yellow fluid and bluish sediment. Much gas with butylic odor.
Corn mash: Resembling reaction of Clostridium acetobutylicum, but with flesh-orange pigment, becoming slowly purple at surface on ageing.

Hydrogen sulfide formed from thiosulfate and sulfite.

Nitrates reduced to ammonia.

Nitrites reduced to ammonia.

Indole not formed.

Acid and gas from xylose, arabinose, glucose, mannose, fructose, galactose, lactose, maltose, sucrose, raffinose, starch, dextrin, glycogen, inulin, pectin and salicin. Esculin and amygdalin weakly fermented. Mannitol, erythritol, glycerol, $\alpha$-methyl glucoside, Ca-lactate and cellulose not fermented.

Coagulated albumin cubes: Softened and yellowed by slow digestion.

Blood serum not liquefied.

Brain medium not blackened or digested.

Non-pathogenic for guinea pig and rabbit.

Optimum temperature about $37^{\circ} \mathrm{C}$. Grow th occurs from $8^{\circ} \mathrm{C}$ to $62^{\circ} \mathrm{C}$.

Anaerobic.

Source : From German maize.

Habitat: Probably occurs in soil.

51. Clostridium chromogenes Prévot. (Chromogenic anaerobe, Ghon and Mucha, Cent.f. Bakt., I Abt., Orig., 42, 1906, 406; Bacillus anaerobius chromogenes LeBlaye and Guggenheim, Man. Prat. d. Diag. Bact., 1914, 321; Prévot, Ann. Inst. Past., 61, 1938, 85.) From Latin, color-producing.

Rods: Moderate size, coccoid to elongate, ends rounded to slightly pointed; straight to slightly curved. Occurring singly, paired, in short chains and in long, curved to coiled filaments. Capsulate, especially in serum media. Motile, with many peritrichous flagella. Spores abundant, oval, central, subterminal, to apparently terminal at maturation, swelling rods to clubs and clostridia. Grampositive.

Granulose negative with iodine solution. 
Gelatin: Liquefied in 48 hours. Diffuse turbidity, clearing with abundant, whitish-gray sediment, which later becomes red to violet-red. Upper $(1 \mathrm{~cm})$ layer shows diffuse, red pigment.

Deep plain agar (without peptone): Growth sparse. Pigment not formed in absence of peptone.

Blood agar surface colonies (anaerobic): Grayish, moist, shining, flat; edges lobate with finely dendritic-tufted edges. Blood agar is hemolyzed.

Glucose agar surface colonies (anaerobic): As on blood agar. Growth slightly less profuse.

Glucose agar deep colonies: Grayishwhite, multi-lobate, with dense center and dendritic, tufted edges. Growth begins about $1 \mathrm{~cm}$ below surface. Gas abundantly formed. Diffuse, red pigment appears in superficial layers after 4 to 5 days.

Glucose meat-infusion broth: Abundant, diffuse turbidity with much gas. Gradual, profuse sedimentation, but with prolonged turbidity.

Peptone water: Grow th variable; sometimes fails. At best, moderate turbidity and sediment. No gas.

Synthetic fluid media (Uschinsky, etc.): No growth (unless peptone is added). Growth is proportionate to added peptone.

Potato slant (anaerobic): Growth deli cate, shining, grayish-yellow. Fecal odor.

Milk: Spongy coagulation after 3 to 4 days. Abundant gas. Turbid, yellowish whey is expressed. Casein clot gradually digested in 4 to 5 weeks. Fecal odor.

Indole is not formed.

Hydrogen sulfide is abundantly formed.

Coagulated albumin (hydrocoel- and ascitic-fluid): Digested and blackened, with moderate gas of fecal odor. When covered with agar, the agar plug shoms diffuse, red pigmentation

Pathogenicity: Weakly pathogenic for white mice and guinea pigs. Produces hemorrhagic, serous peritonitis after in- traperitoneal inoculation. Death due apparently to a weak toxin. Virulence increased by animal passage.

Grows well at $21^{\circ} \mathrm{C}$ and at $37^{\circ} \mathrm{C}$.

Anaerobic.

Distinctive character: Red pigmentation which is increased on addition of chlorine-, or of bromine-water. Although produced by an anaerobe, pigment appears only in aerated zone and depends on peptone content of medium.

Source: From pus of a human perinephritic abscess.

Habitat: Not determined, other than this single source.

52. Clostridium felsineum (Carbone and Tombolato) Bergey et al. (Bacillus felsineus Carbone and Tombolato, Le Staz. Sper. Agrar., Ital., 50, 1917, 563; Rusehmann and Bavendamm, Cent. f. Bakt., II Abt., 64, 1925, 340; Van der Lek, Thesis, Delft, 1930, (143?); Clostridium felsinus Bergey et al., Manual, 3rd ed., 1930,453 .) Named for Felsinea, the ancient name of Bologna, Italy.

Described from Ruschmann and Bavendamm (loc. cit.), from the Kluyver strain used by Van der Lek (loc. cit.), and from McCoy and MeClung, Areh. f. Mikrobiol., 6, 1935, 230.

Rods : 0.3 to 0.4 by 3.0 to 5.0 microns, occurring singly, in pairs and in short chains. Motile with peritrichous flagella. Spores oval, subterminal, swelling rods to clostridia. Gram-positive, becoming Gram-negative.

Granulose positive in the clostridial stage.

Glucose gelatin : Liquefied.

Plain agar slant (anaerobic): Surface growth seant, scarcely perceptible.

Glucose agar surface colonies (anaerobic): Raised, smooth, slightly irregular, yellow-orange.

Deep glucose agar colonies: Compact, lenticular, opaque, yellow.

Blood agar not hemolyzed.

Pigmentation (anaerobic): Yelloworange, ageing to brownish. Not changing on aeration. 
Plain broth: No growth.

Glucose broth: Abundant, uniform turbidity, with much gas. Yellow slimy sediment.

Litmus milk: Acid and coagulation. Litmus reduced. Clot torn and yellowed. No visible digestion.

Potato: Digested to a yellow slime. Much gas with butylic odor.

Corn mash: Resembling reaction of Clostridium acetobutylicum, but with flesh to orange pigment.

Indole not formed.

Nitrates reduced to ammonia.

Nitrites reduced to ammonia.

Acid and gas from arabinose, xylose, glucose, mannose, fructose, galactose, lactose, mal tose, sucrose, raffinose, starch, dextrin, inulin, glycogen, pectin and salicin. Mannitol, erythritol, glycerol, Ca-lactate and cellulose not fermented.

Fermentation products include butyl and ethyl alcohol, acetone, organic acids (probably butyric and acetic), $\mathrm{H}_{2}$ and $\mathrm{CO}_{2}$.

Coagulated albumin cubes: Softened and yellowed by slow digestion.

Blood serum not liquefied.

Brain medium not blackened or digested.

Non-pathogenic for guinea pig and rabbit.

Grows at $37^{\circ} \mathrm{C}$.

Anaerobic.

Source: From retting flax.

Habitat: Not determined. Found in soil in Italy. Argentina and in the Cnited States.

53. Clostridium carbonei Arnaudi. (Soc. Intern. Microbiol., Boll. Sez. Ital., 8, 1936, 251, and Boll. Ist. Sieroter, Milano, 16, 1937, 650; Inflabilis carbonei Prévot, Man. d. Class., etc., 1940, 96.) Named for Carbone, an Italian bacteriologist .

Rods : 0.8 to 1.0 by 3.5 to 4.5 microns, with ends slightly tapered. Non-motile. Spores oval, terminal, 0.8 to 1.0 by 1.0 to 1.75 microns, swelling rods. Grampositive.
Granulose reaction strongly positive with iodine solution.

Gelatin: To growth.

Glucose and lactose gelatin: No growth.

Plain agar surface colonies (anaerobic): Flat, shining, colorless, with irregular edges.

Malt agar surface colonies (anaerobic): Creamy to slightly reddish colonies with irregular edges.

Roux-potato slant (anaerobic) : Punctiform, raised, opaque, deep red colonies, becoming almost violet.

Plain agar stab: Only traces of growth along stab.

Glucose and maltose agar stab: No growth.

Plain broth: Very slight, colorless, diffuse turbidity.

Glucose broth: Very slight turbidity.

Maltose broth: Intense turbidity, with profuse, reddish-yellow sediment.

Tarozzi broth: slight, diffuse turbidity. Indole not formed.

Hydrogen sulfide not formed.

Milk: Soft coagulation, with slight, fine reddish floceulence. Whey turbid and colorless. Reaction acid. Clot not digested.

Digest-milk (optimum medium) : Very abundant turbidity, with bright red flocculent sediment, diffusing uniformly on shaking.

Coagulated egg-yolk broth: Slight turbidity; no digestion.

Coagulated egg-albumin broth: Slight turbidity; no digestion.

Coagulated serum (Loeffler, anaerobic): Poor growth; flat, red surface colonies. No digestion.

Brain medium : Not recorded.

Cellulose not attacked. Hemp is not retted.

Ferments weakly: Glucose, maltose, sucrose, galactose, fructose and raffinose. Slow and partial fermentation of lactose (only in acidified medium). Starch slightly fermented. Fermentation products include $\mathrm{H}_{2}, \mathrm{CO}_{2}, \mathrm{CH}_{4}$, butyric acid and traces of ethyl alcohol. 
Non-pathogenic for sheep, rabbit, guinea pig or white mouse.

Optimum reaction $\mathrm{pH} 7.0$ to 7.2 ; minimum $\mathrm{pH} 6.0$; maximum $\mathrm{pH} 8.0$.

Optimum temperature $37^{\circ} \mathrm{C}$. Grows slowly at $25^{\circ} \mathrm{C}$ to $30^{\circ} \mathrm{C}$; growth ceases at $40^{\circ} \mathrm{C}$.

Anaerobic.

Distinctive character : Production of a brilliant red pigment, soluble in amyl alcohol, petrol-ether, xylol and aniline oil. Partly soluble in ether, chloroform and acetone. Pigment very stable in light.

Source: From macerated raw potato infusion.

Habitat: Not recorded, other than from this single source.

54. Clostridium spumarum (Prévot and Pochon) comb. nov. (Plectridium spumarum Prévot and Pochon, Compt. rend. Soc. Biol., Paris, 130, 1939, 966.) From Latin, foam or froth.

Rods: 0.5 by 4.0 microns, motile. Spores are oval and terminal, swelling rods. Gram-positive.

Gelatin : Liquefied in 15 days.

Deep agar: Forms small cottony colonies and a few gas bubbles.

Peptone water: Turbidity and slight sediment.

Milk: Coagulated in 5 days, but clot is not digested.

Indole is produced.

Hydrogen sulfide is formed (medium not stated).

Sugars not attacked immediately after isolation.

After 1 month cultivation, ferments slowly glucose, fructose, galactose, maltose, arabinose, xylose, sucrose, mannitol and starch. Inulin is not fermented.

Cellulose (in synthetic medium) is fermented chiefly to acetic and butyric acids, together with inflammable gas and traces of ethyl alcohol.

Coagulated albumin not attacked.

Brain medium not blackened.

Optimum temperature around $37^{\circ} \mathrm{C}$. Not thermopbilic.
Anaerobic.

Distinctive characters: Does not produce pigment, and ferments a variety of carbohydrates.

Source: From the scum of sugar refining vats.

Habitat: Not determined, other than from this source.

55. Clostridium werneri Bergey et al. (Bacillus cellulosam fermentans Werner, Cent. f. Bakt., II Abt., 67, 1926, 297; Bergey et al., Manual, 3rd ed., 1930, 452; Bacterium cellulosam Jepson, Bull. Entomol. Res., 28, 1937, 163; Terminosporus cellulosarn fermentans Prévot, Ann. Inst. Past., 61, 1938, 86; Terminosporus cellulosam-fermentans Prévot, Man. d. Class., etc., 1940, 148.) Named for Erich Werner, the German bacteriologist who first isolated this organism.

Related species: Probably closely related to Clostridium omelianskii.

Rods : 0.5 to 0.7 by 1.5 to 7.0 microns, occurring singly and in pairs, but not in chains. Motile with peritrichous flagella. Spores oval, terminal, swelling rods. Gram-negative.

Cellulose agar slant (anaerobic): Growth only in contact with cellulose. Growth grayish black; agar is darkened. Gas is formed.

Agar slant (anaerobic): No growth.

Broth: Nogrowth.

Broth with filter paper: Poor growth; cellulose weakly attacked.

Omelianski solution with filter paper: Abundant growth; cellulose digested with formation of $\mathrm{H}_{2}$ and $\mathrm{CO}_{2}$.

Hydrogen sulfide is formed in the Omelianski medium, presumably from the $\left(\mathrm{NH}_{4}\right)_{2} \mathrm{SO}_{4}$ and $\mathrm{MgSO}_{4}$.

Glucose not fermented.

Carbohydrates, other than cellulose, not fermented.

Non-pathogenic for mice.

Optimum temperature $33^{\circ} \mathrm{C}$ to $37^{\circ} \mathrm{C}$.

Anaerobic.

Source: From larvae of rose leaf beetle (Potosia cuprea). 
Habitat: Occurs in soil and in feces of herbivorus animals.

56. Clostridium cellulosolvens Cowles and Rettger. (Jour. Bact., 21, 1931, 167; Caduceus cellulosolvens Prévot, Ann. Inst. Past., 61, 1938, 86.) From chemical term, cellulose, and Latin, dissolving.

Rods : 0.5 by 2.0 to 6.0 microns, commonly curving, occurring singly and in pairs, not in chains. Non-motile. Spores spherical, terminal, swelling rods. Gram stain uncertain; usually Gram-negative.

Does not grow in routine media, except where cellulose or certain few carbohy drates are added.

Surface colonies on dextrin-cysteine meat infusion agar (anaerobic): Tiny, round, transparent dew-drops; finely granular, with smooth edge.

Acid and gas from cellulose, dextrin, arabinose, xylose and soluble starch. Glucose, fructose, mannose, lactose, maltose, sucrose, melezitose, raffinose, inulin, salicin, amygdalin, adonitol, dulcitol, erythritol, glycerol, inositol, mannitol, sorbitol and gum arabic not fermented.

Cellulose decomposed to $\mathrm{H}_{2}, \mathrm{CO}_{2}$ and organic acids.

Grows at $37^{\circ} \mathrm{C}$.

Anaerobic.

Source: From horse feces.

Habitat: Not determined, other than this source. Probably widely dispersed in manured soil.

\section{Clostridium dissolvens Bergey et} al. (Bacillus cellulosae dissolvens Khouvine, Ann. Inst. Past., 37, 1923, 711; Bergey et al., Manual, 2nd ed., 1925, 344; Caduceus cellulosae dissolvens Prévot, Ann. Inst. Past., 61, 1938, 86.) From Latin, dissolving.

Rods : Slender, ranging from 2.5 to 12.5 microns in length, occurring singly, occasionally in pairs, but not in chains. Non-motile. Spores oval, terminal, swelling rods. Gram-negative.

Cellulose is digested by the formation of an endocellulase which acts only when the bacteria are attached to the cellulose.
Saccharides are formed from cellulose, also $\mathrm{CO}_{2}, \mathrm{H}_{2}$, ethyl alcohol, acetic, lactic and butyric acids.

A yellow pigment is formed in presence of cellulose.

Glucose not fermented.

Carbohydrates, other than cellulose, not fermented.

Non-pathogenic for guinea pig.

Optimum temperature : Grows between $35^{\circ} \mathrm{C}$ and $51^{\circ} \mathrm{C}$, without a definite optimum.

Anaerobic.

Distinctive character: Grows only in media containing cellulose, in the presence of which it produces a yellow pigment .

Source: From human feces.

Habitat: Intestinal canal of man.

58. Clostridium omelianskii (Henneberg emend. Clausen) comb. nov. (Cellulose fermenting microbe, Omelianski, Compt. rend. Acad. Sci., Paris, 121, 1895, 653 (Bacillus fermentationis cellulosae Omelianski, Arch. Sci. Biol. (Russ.), 7, 1899, 419; Bacterium cellulosis Migula, Syst. d. Bakt., 2, 1900, 513); Bacille hydrogénique, Omelianski, Arch. Sci. Biol. (Russ.) , 9, 1902-03, 263 (Wasserstoffbacillus, Omelianski, Cent. f. Bakt., II Abt., 8, 1902, 262; Bacillus fossicularum Lehmann and Neumann, Bakt. Diag., 1 Auf1., 2, 1907, 466; Bacillus hydrogenii Jungano and Distaso, Les Anaérobies, 1910, 1tī; Bacillus omelianshii Henneberg, Cent. f. Bakt., II Abt., 55, 1922, 276, emend. Clausen, Cent. f. Bakt.. II Abt., 84, 1931, 40 and 54; Omelianskillus hydrogenicus Heller, Jour. Bact., 7, 1922, 5; C'aduceus cellulosae hydrogenicus Prévot, Ann. Inst. Past., 61, 1938, 86); Bacille formenique, Omelianski, Arch. sci. Biol. (Russ.), 9, 1902-03, 263 Methanbacillus, Omelianski, Cent. f. Bakt., II Abt., 11, 1903-04, 370; Bacillus methanigenes Lehmann and Neumann, Bakt. Diag., 4 Aufl., 2, 1907, 466; Cellobacillus methanigenes Orla-Jensen, Cent. f. Bakt., II Abt., 22, 1909, 343; Bacillus methanii Jungano and Distaso, Les 
Anaérobies, 1910, 146; Caduceus cellulosae hydrogenicus var. cellulosae methanicus Prévot, Ann. Inst. Past., 61, 1938, 86; ('aduceus cellulosae methanicus Prévot, Man. d. Class., ete., 1940, 150).) Named for Omelianski, the Russian bacteriologist who first described this type.

This species was apparently first isolated and studied in pure culture by Clausen (loc. cit.). From his studies he concludes that Omelianski's Wasserstoffand Methanbacillus are but a single species, and that the gaseous fermentation products $\left(\mathrm{H}_{2}, \mathrm{CO}_{2}\right.$ and $\left.\mathrm{CH}_{4}\right)$ were affected by the symbiotic forms always present in Omelianski's cultures.

His evidence is quite convincing, and the organism is presented here from his deseription.

Rods: Length varying with the medium, 0.5 to 0.7 by 5.0 to 15.0 microns, straight to slightly curved. Occurring chiefly singly, occasionally in pairs, frequently parallel in groups, never in chains or filaments. Young cells motile, but motility disappearing with sporulation. Flagella not demonstrable. Spores spherical, terminal, swelling rods. Spores 1.0 to 1.5 microns in diameter, varying with medium. Gram-positive, becoming Gram-labile on sporulation.

Young vegetative cells colored wine-red with iodine solution.

Gelatin (plus asparagine) : Liquefied in 6 to 10 days. Medium remains perfectly clear.

Asparagine agar deep colonies : Grayishwhite, delicate, cottony, with fine radial outgrow ths.

Asparagine agar surface colonies (anaerobic) : Poor growth, delicate, translucent, filmy, searcely discernible.

Cellulose-liver broth : Solution remains visibly clear and does not darken with age. Occasional large gas bubbles arise.

Indole not formed.

Ammonia not formed.

Nitrates not reduced to nitrites.

Traces of $\mathrm{H}_{2} \mathrm{~S}$ produced in inorganic solutions.

Milk: Soft coagulation in 24 hours.
Anorphous clot shrinks and settles, forming a yellowish-red to orange sediment, with turbid supernatant whey.

Brain medium : Not digested or blackened; no visible evidence of growth.

None of the following carbohydrates attacked: Maltose, mannitol, lactose, glucose, sucrose, galactose, fructose, starch, salicin, glycerol and inulin.

Cellulose apparently the primary Csource, but is only weakly attacked by pure cultures.

Yellow pigment not recorded in presence of cellulose (see Clostridium dissolvens).

Non-pathogenie for mice; other animals not recorded.

Optimum reaction $\mathrm{pH} 7.0$ to 7.4 ; grows between pH 6.0 and 8.4 .

Optimum temperature $37^{\circ} \mathrm{C}$ to $42^{\circ} \mathrm{C}$.

Anaerobic: Growing at 25 to $30 \mathrm{~mm}$ mercury pressure.

Distinctive characters: Ability to liquefy gelatin (plus asparagine); to coagulate milk with orange sediment, and to grow in media containing asparagine without requiring presence of cellulose. Spores resist heating at $100^{\circ} \mathrm{C}$ for 90 minutes.

Source: From human, cow and horse excreta, from cow's stomach contents, from cheese and from soil.

Habitat: Intestinal canal of animals, and presumably thence widely disseminated in soil.

59. Clostridium carnis (Klein) Spray. (Art V, von Hibler, Cent. f. Bakt., I Abt., 25, 1899, 515; Bacillus carnis Klein, Cent. f. Bakt., I Abt., Orig., 35, 1904, 459, also Trans. Path. Soc, London, 55, 1904, 74; Art. VI, von Hibler, Untersuch. ü. d. Path. Anaer., 1908, 3 and t06; Hiblerillus sextus IIcller, Jour. Bact., $7,1922,6$; Bacillus lactiparcus Lehmann and Süssmann, in Lehmann and Teumann, Bakt. Diag., 7 Aufl., 2, 1927, 647; Plectridium carnis Prévot, Ann. Inst. Past., 61, 1938. 87; Spray, in Bergey et al., IIanual, 5th ed., 1939, 750; Clostridium 
sextum Prévot, Man. d. Class., etc., 1940, 136.) From Latin, of flesh.

Description from Hall and Duffett, Jour. Bact., 29, 1935, 269.

Rods : 0.5 to 0.7 by 1.5 to 4.5 microns, occurring singly, in pairs, rarely in chains of 3 to 4 cells. Motile with peritrichous flagella. Sporesoval to elongate, subterminal, slightly swelling rods. Gram-positive.

Gelatin : Not liquefied or blackened.

Agar surface colonies (aerobic): Minute, transparent dew-drops, becoming flat and lobate.

Blood agar surface colonies (aerobic): Similar to plain agar. Slight hemolysis.

Deep agar colonies : Lenticular, becoming nodular to arborescent.

Milk: Abundant gas, but no coagulation or other change.

Indole not formed.

Acid and gas from glucose, galactose, fructose, maltose, lactose, sucrose, amygdalin, salicin and dextrin. Trehalose, raffinose, xylose, arabinose, starch, inulin, mannitol, dulcitol, sorbitol, glycerol and inositol not fermented.

Coagulated albumin not liquefied.

Blood serum not liquefied.

Brain medium not blackened or digested.

Pathogenic for guinea pig, white rat and rabbit. Forms an exotoxin of moderate intensity, producing edema, necrosis and death on sufficient dosage.

Grows well at both $37^{\circ} \mathrm{C}$ and at room temperature.

Anaerobic and microaerophilic; growing delicately on aerobic agar slants.

Source: Originally isolated from a rabbit inoculated with garden soil (von Hibler); from contaminated beef infusion (Klein).

Habitat : Probably oceurs in soil.

60. Clostridium histolyticum (Weinberg and Seguin) Bergey et al. (Bacillus histolyticus Weinberg and Seguin, Compt. rend. Acad. Sci., Paris, 163, 1916, 449; Weinbergillus histolyticus Heller, Jour. Bact., 7, 1922, 9; Bergey et al., Manual, 1st ed., 1923, 328.) From Greek, tissuedissolving.

Rods : 0.5 to 0.7 by 3.0 to 5.0 microns, occurring singly and in pairs. Motile with peritrichous flagella. Spores oval, subterminal, swelling rods. Grampositive.

Gelatin: Complete liquefaction in 24 hours

Blood agar surface colonies (aerobic): Minute, round dew-drops. Blood is hemolyzed.

Deep agar colonies: Variable; from lenticular, lobate, to fluffy, according to the agar concentration.

Agar slant (acrobic) : Grows aerobically in barely perceptible film, or in tiny, smooth, discrete colonies.

Broth: Turbid, with slight precipitate. Indole not formed.

Nitrites not produced from nitrates.

Litmus milk: Softly coagulated, then slowly digested. Little gas is formed.

Carbohydrates are not fermented.

Coagulated albumin slowly liquefied.

Blood serum slowly liquefied with darkened, putrid fluid.

Brain medium blackened and digested with putrefactive odor.

Egg-meat medium : Little gas is formed. Meat first reddened, then darkened in 3 days. Digestion apparent in about 24 hours. Nauseous odor. Tyrosin crystals are abundant after about a week.

Pathogenic for small laboratory animals. Produces a cytolytic exotoxin which causes extensive local necrosis and sloughing on injection. Not toxic on feeding.

Grows well at $37^{\circ} \mathrm{C}$.

Anaerobic and microaerophilie. Grows feebly on aerobic agar slant.

Source: Originally isolated from war wounds, where it induces active necrosis of tissue.

Habitat: Not determined, other than source stated. Found occasionally in feces and soil. Apparently widely, but sparsely, dispersed in soil. 
61. Clostridium tertium (Henry) Bergey et al. (Bacillus tertius Henry, Jour. Path. and Bact., 21, 1916, 347; Henrillus tertius Heller, Jour. Bact., 7, 1922, 5; Bergey et al., Manual, 1st ed., 1923, 332; Plectridium tertium Prévot, Ann. Inst. Past., 61, 1938, 87.) From Latin tertius, third.

Probable synonyms: Bacillus gazogenes parvus Choukévitch, Ann. Inst. Past., 25, 1911, 271 (Bacillus gazogenes Choukévitch, ibid., 268; Plectridium gazogenes Prévot, Ann. Inst. Past., 61, 1938, 87); Bacillus Y, Fleming, Lancet, 2, 1915, 376; Bacillus aero-tertius Bulloch et al., Med. Res. Coune., Spec. Rept. Ser. 39, 1919,4 ; Bacillus spermoide Ninni, Pathologica, 21, 1920, 385 (Clostridium spermoides Bergey et al., Manual, 1st ed., 1923, 336; Palmula spermoides Prévot, Ann. Inst. Past., 61, 1938, 88; Acuformis spermoides Prévot, Man.d. Class., ete., $1940,164)$.

Rods : 0.4 to 0.6 by 3.0 to 6.0 microns, occurring singly and in pairs, not in chains. Motile. Spores oval, terminal, swelling rods. Gram-positive.

Gelatin: Not liquefied.

Agar surface colonies (aerobic) : Circular with opalescent, crenated margin.

Deep agar colonies: Small, lentieular. regular, smooth.

Agar slant (aerobic): Grayish, filmy, opalescent growth.

Blood agar is hemolyzed.

Broth : Turbid, with sediment.

Litmus milk: Acid, coagulated, with some gas formation. Clot is not digested.

Indole not formed.

Nitrites produced from nitrates.

Acid and gas from glucose, fructose, galactose, mannose, lactose, maltose, sucrose, arabinose, xylose, trehalose, melezitose, soluble starch, esculin, mannitol, inositol and salicin. Inulin and glycerol not fermented.

Coagulated albumin not liquefied.

Blood serum not liquefied.

Brain medium not blackened or ligested.

Meat medium reddened; acid and gas formed. Meat not blackened or digested. Non-putrefactive.

Non-pathogenic for guinea pig and rabbit.

Optimum temperature $30^{\circ} \mathrm{C}$ to $35^{\circ} \mathrm{C}$. Can grow at $50^{\circ} \mathrm{C}$.

Anaerobic and microaerophilic. Grows feebly on aerobic agar slant.

Source: From gangrenous wounds and from feces.

Habitat: Widely distributed in soil, feces and sewage.

Appendix I: The following species of anaerobes are listed, chiefly for their historic interest, from descriptions too incomplete to permit present definite classification. Many of these original cultures are now lost, and the descriptions are often too brief even to permit comparison with recognized species. Several were described from cultures of doubtful purity, and even the anaerobic status of some is open to question. The synonymy cited is not to be regarded as definitely established in all instances.

Many strains have been described by number or by common names only. These are not included here, but many may be found listed in Les Microbes Anaérobies, Weinberg, Nativelle and Prévot, Paris, 1937, and in The Anacrobic Bacteria and their Activities in Nature and Disease, McCoy and McClung, Univ. ('alif. Press, 1939.

Species are listed alphabetically under the first valid binomial, regardless of their original designation.

Acuformis caninus Prévot. (Unnamed species of Wolbach and Saiki, Jour. Med. Res., 21, 1909, 267; Palmula canina Prévot, Ann. Inst. Past., 61, 1938, 88; Prévot, Man. d. Class., ete., 1940, 164.) From liver of healthy dog.

Acuformis dubitatus Prévot. (Species No. 1 of Rodella, Cent. f. Bakt., II Abt., 17, 1907, 374; Palmula dubitata Prévot, Ann. Inst. Past., 61, 1938, 89; Prévot, Man. d. Class., ete., 1940, 165.) From normal and from spoiled milk. 
Acuformis immobilis Prévot. (Bacillus putrificus immobilis Distaso, Ann. Inst. Past., 23, 1909, 955; Palmula immobilis Prévot, Ann. Inst. Past., 61, 1938, 89; Prévot, Man. d. Class., etc., 1940, 165.) From intestine of the flying fox (Pteropus).

A mylobacter butylicus Duclaux. (Ann. Inst. Past., 9, 1895, 813.) From fermenting macerated potato inoculated with soil.

A mylobacter ethylicus Duclaux. (Ann. Inst. Past., 9, 1895, 814.) Probably only a facultative anaerobe. From fermenting macerated potato inoculated with soil.

Bacillus acidi acetici Thöni. (Thesis, Bern, 1906, (?); quoted from McCoy and McClung, The Anaer. Bact. ete., 2, 1939, 413.) Source not known.

Bacillus aerogenes gangrenosae Weinberg and Ginsbourg. (Bacillo aerogene gangrenosa, Nacciarone, Riforma Med., 33, 1917, 778; Weinberg and Ginsbourg, Bull. Inst. Past., 23, 1925, 825.) From human gaseous gangrene.

Bacillus amyloclasticus Renshaw and Fairbrother. (Bacillus amyloclasticus intestinalis Renshaw and Fairbrother, Brit. Med. Jour., 1, 1922, 675; ibid., 818.) Stated by authors to be a facultative anaerobe. From intestine of diabetic persons.

Bacillus anaerobic No. VIII Chester. (Anaerobe No. VIII, Sanfelice, Ztschr. f. Hyg., 14, 1893, 375; Pseudo-Rauschbrandbacillus, Kruse, in Flügge, Die Mikroorg., 3 Aufl., 2, 1896, 250; Chester, Man. Det. Bact., 1901, 296.) From putrefying meat infusions, soil, and from tissues of guinea pigs after inoculation with soil.

Bacillus butyricus Botkin. (Botkin, Ztschr. f. Hyg., 11, 1892, 421; Bacillus No. I, Flügge, Ztschr. f. Hyg., 1 7,1894 , 290; Bacillus botkini Migula, Syst. d. Bakt., 2, 1900, 594; Bacillus parabutyricus LeBlaye and Guggenheim, Man. Prat. de Diag. Bact., 1914, 324; not Bacillus parabutyricus Gratz and Vas, Cent. f. Bakt., II Abt., 41, 1914, 510; Clostridium butyricum Holland, Jour. Bact., 5, 1920, 217 and 222.) From milk.
Bacillus butyricus dimorphus Rocchi. (Cent. f. Bakt., I Abt., Orig., 60, 1911, 580.) A collective name for a group of butyric anaerobes considered denaturable and mutually interconvertible.

Bacillus cadaveris grandis Sternberg. (Researches relating to the etiology and prevention of yellow fever, Washington, 1891, 213.) From liver of a yellow fever cadaver.

Bacillus cannabinus Makrinow and Tchijowa. (Arch. Sci. Biol. (Russ.), 29, 1929, 52; also Cent. f. Bakt., II Abt., 80 , 1930,59 .) Stated by the authors to be a facultative anaerobe. From soil and from retting of kenaf (Hibiscus cannabinus).

Bacillus cincinnatus Gerstner. (Gerstner, Thesis, Basel, 1894, 17; Bacillus cincinnati Jungano and Distaso, Les Anaérobies, 1910, 88.) From soil and sewage.

Bacillus clavatus Migula. (Anaerobe No. III, Flügge, Ztschr. f. Hyg., 17, 1894, 289; Migula, Syst. d. Bakt., 2, 1900, 597; Bacillus cuneatus Chester, Man. Determ. Bact., 1901, 299.) From boiled cow's milk.

Bacillus colicogenes Tissier. (Ann. Inst. Past., 26, 1912, 523.) From stool of an infant with diarrhea and colic.

Bacillus coprogenes Hopffe. (Bacillus coprogenes foetidus Herfeldt, Cent. f. Bakt., II Abt., 1, 1895, 77; not Bacillus coprogenes foetidus Flügge, Die Mikroorg., 2 Aufl., 1886, 305; Hopffe, Ztschr. Infkrnkh. u. Hyg. Haust., 14, 1913, 404.) From manure and from horse intestine.

Bacillus cresologenes Rhein. (Rhein, Compt. rend. Soc. Biol., Paris, 87, 1922, 576; Clostridium cresologenes Prévot, Ann. Inst. Past., 61, 1938, 85.) From brain medium inoculated with soil.

Bacillus de baryanus Klein. (Ber. d. Deuts. Bot. Gesellsch., 7 (Bhft.), 1889, 60.) Migula (Syst. d. Bakt., 2, 1900, $640)$ says this is probably anaerobic. Observed in swamp water, but not cultivated on artificial media.

Bacillus diffrangens Gerstner. (Thesis, Basel, 1894, 19.) From soil and sewage. 
Bacillus dimorphobutyricus Lehmann and Neumann. (Dimorpher Buttersäurebacillus, Grassberger and Schattenfroh, Arch. f. Hyg., 60, 1907, 59; Lehmann and Neumann, Bakt. Diag., 4 Aufl., 2, 1907, 441.) From milk.

Bacillus elegans Romanoviteh. (Compt. rend. Soc. Biol., Paris, 71, 1911, 169.) From normal human intestine and from diseased appendix.

Bacillus fibrosus Gerstner. (Thesis, Basel, 1894, 26.) From soil and sewage.

Bacillus flüggei Migula. (Anaerobe No. IV, Flügge, Ztschr. f. Hyg., 17, 1894, 290 ; Migula, Syst. d. Bakt., 2, 1900, 597.) From boiled cow's milk.

Bacillus fossicularum Lehmann and Neumann. (Wasserstoffbacillus, Omelianski, Cent.f. Bakt., II Abt., 8, 1902, 262; Lehmann and Neumann, Bakt. Diag., 4 Aufl., 2, 1907, 466, may have been named in the 3 Aufl.) From mud from canals. Forms hydrogen and $\mathrm{CO}_{2}$ from anaerobic cellulose fermentations.

Bacillus funicularis Gerstner. (Thesis, Basel, 1894, 24.) From soil and sewage.

Bacillus gangraenae Tilanus. (Nederl. Tijdschr. v. Geneeskunde, 21, 1885, 110.) From a gangrenous human leg.

Bacillus gracilis ethylicus Achalme and Rosenthal. (Compt. rend. Soc. Biol., Paris, 58, 1906. 1025.) From stomach contents in a case of gastritis.

Bacillus granulosus Gerstner. (Gerstner, Thesis, Basel, 1894, 21; Bacillus granulatus Migula, Syst. d. Bakt., 2, 1900, 613.) From soil and sewage.

Bacillus haumani Soriano. (Unnamed plectridium, Sordelli and Soriano, Compt. rend. Soc. Biol., Paris, 99, 1928, 1517; Plectridio amarillo, Soriano, Tomo commemorativo del XXV aniversario de la fundación de la Facultad de Agronomia y Veterinaria, Buenos Aires, 1929, (?); Soriano, Rev. Inst. Bact., Buenos Aires, 5, 1930, 743; Plectridium amarillum Stampa, Ann. Brass. et Distill., 29, 193031 , (253, 271 and 302?); Bacillus haumanni Arnaudi, Boll. Ist. Sieroter., Milano, 16, 1937, 650; Clostridium haumanni
Prévot, Ann. Inst. Past., 61, 1938, 81.) From flax-retting beds.

Bacillus ichthyismi Konstansoff. (Vestnik Obshtch. Hig. Sudeb. i Prakt. Med., Petrograd, 51, 1915, (766?); quoted from Weinberg et al., Les Microbes Anaérobies, 1937, 341.) From fish responsible for a condition simulating botulism.

Bacillus indolicus Gratz and Vas. (Gratz and Vas, Cent. f. Bakt., II Abt., 41, 1914, 511; Inflabilis indolicus Prévot, Ann. Inst. Past., 61, 1938, 77.) From cheese.

Bacillus infantilism Hill and Whitcomb. (Amer. Jour. Pub. Health, 3, 1913, 926.) From human intestine.

Bacillus inflatus Koch. (Koch, Botan. Zeit., 46, 1888, 328; Clostridium inflatum Trevisan, I generi e le specie delle Batteriacee, 1889, 22; not Bacillus inflatus Distaso, Ann. Inst. Past., 23, 1909, 955.) Anaerobic status insecure; aerobic according to Kruse, in Flügge, Die Mikroorg., 3 Aufl., 2, 1896, 259. Observed in swamp waters, but not isolated. Formed two spores in a spindle-shaped sporangium.

Bacillus irregularis Choukévitch. (Choukévitch, Ann. Inst. Past., 25, 1911, 348; Clostridium irregularis Prévot, Ann. Inst. Past., 61, 1938, 85.) From large intestine of horse.

Bacillus kedrowskii Migula. (Bacillus No. 2, Kedrowski, Ztschr. f. Hyg., 16, 1894, 451; Bacillus acidi butyrici Kruse, in Flügge, Die Mikroorg., 3 Aufl., 2, 1896, 256 ; not Bacillus acidi butyrici $I$ Weigmann, Cent. f. Bakt., II Abt., 4, 1898, 830 (see Bacillus pseudonavicula); Migula, Syst. d. Bakt., 2, 1900, 589.) From cheese and rancid butter.

Bacillus lactopropylbutyricus Tissier. (Apparently identical with Bacillus lactopropylbutyricus non liquefaciens Tissier and Gasching, Ann. Inst. Past., 17, 1903, 546; Tissier, Ann. Inst. Past., 19, 1905, 282; Clostridium lactopropylbutylicum Prévot, Ann. Inst. Past., 61, 1938, 85; Bacillus lactopropylbutylicus non 
liquefaciens Prévot, Man. d. Class., etc., 1940, 141.) From milk.

Bacillus lichenoides Piening. (Cent. f. Bakt., I Abt., Orig., 124, 1932, 217; not Bacillus lichenoides Grohmann, Cent. f. Bakt., II Abt., 61, 1924, 267.) Cited only by name, without deseription. From dried sheep intestines used for preparation of catgut sutures.

Bacillus limosus Klein. (Ber. d. Deutsch. Bot. Gesellsch., 7 (Bhft.), 1889, 60.) Migula (Syst.d. Bakt., 2, 1900, 640) says this is probably anaerobic. Observed in swamp water, but not cultivated on artificial media.

Bacillus longus Chester. (Bacillus muscoides colorabilis Ucke, Cent. f. Bakt., I Abt., 29, 1898, 1000; Chester, Man. Determ. Bact., 1901, 303.) From garden soil.

Bacillus lubinskii Kruse. (Tetanusähnlicher Bacillus, Lubinski, Cent. f. Bakt., 16, 1894, 771; Kruse, in Flügge, Die Mikroorg., 3 Aufl., 2, 1896, 267.) From a fetid human abdominal abscess in peritonitis

Bacillus lyticus Costa and Troisier. (Compt. rend. Soc. Biol., Paris, 78, 1915, 433.) From gangrenous war wounds. Stated to be intermediate between Clostridium perfringens and Clostridium septicum.

Bacillus macrosporus Klein. (Ber.d. Deutsch. Bot. Gesellsch., 7 (Bhft.), 1889, 60.) Migula (Syst. d. Bakt., 2, 1900, 640) says this is probably anaerobic. Observed in swamp water, but not cultivated on artificial media.

Bacillus makrono-filiformis Becker. (Ztschr. f. Infkrnkh. d. Haust., 23, 1922, 20.) From a guinea pig cadaver.

Bacillus maymonei Carbone. (Boll. Sez. Ital., Soc. Intern. Microbiol., 1, 1929,74 .) A retting organism of doubtful purity and anaerobic status. Cultivated from kenaf (Hibiscus cannabinus).

Bacillus megalosporus Choukévitch. (Choukévitch, Ann. Inst. Past., 25, 1911, 351; Hiberillus megalosporus Heller, Jour. Bart., 7, 1922, 17; Inflabitis megalo- sporus Prévot, Ann. Inst. Past., 61, 1938, 77.) From large intestine of horse.

Bacillus multiformis Distaso. (Distaso, Cent. f. Bakt., I Abt., Orig., 59, 1911, 101; not Bacillus multiformis van Senus, Dissert., Leiden, 1890, (?) ; Bacteroides multiformis Bergey, Manual, Ist ed., 1923, 263; Cillobacterium multiforme Prévot, Ann. Inst. Past., 60, 1938, 297.) Stated by Distaso (loc. cit.) not to produce spores, but to belong probably to the Welch Group. From feces of dog.

Bacillus multiformis van Senus. (van Senus, Dissert., Leiden, 1890 and Koch's Jahrsber., 1, 1890, 138.) From mud and from decomposing vegetation.

Bacillus muscoides Liborius. (Liborius, Ztschr. f. Hyg., 1, 1886, 163; Cornilia muscoides Trevisan, I generi e le specie delle Batteriacee, 1889,22.) From mouse inoculated with soil, from cheese, and from bovine feces.

Bacillus muscoides non colorabilis Ucke. (Cent. f. Bakt., I Abt., 23, 1898, 1000.) From hay infusion.

Bacillus nanus Romanovitch. (Compt. rend. Soc. Biol., Paris, 71, 1911, 239.) From human intestine.

Bacillus nebulosus Vincent. (Ann. Inst. Past., 21, 1907, 69; not Bacillus nebulosus Wright, Mem. Nat. Acad. Sci., 7, 1894, 465; not Bacillus nebulosus Hallé, Thèse de Paris, 1898, 33; not Bacillus nebulosus Migula, Syst. d. Bakt., 2, 1900, 844; not Bacillus nebulosus Goresline, Jour. Bact., 27, 1934, 52.) From well and river water.

Bacillus oedematis Migula. (Bacillus cedematis maligni Liborius, Ztschr. f. Hyg., 1, 1886, 158; not Bacillus oedematis maligni Zopf, Die Spaltpilze, 3 Aufl., 1885, 88; Migula, Syst. d. Bakt., 2, 1900, 604; not Bacillus oedematis Chester, Man. Determ. Bact., 1901, 292.)

Bacillus otitidis sporogenes putrificus von Hibler. (Cent. f. Bakt., I Abt., Orig., 68, 1913, 282.) From a human brain abscess.

Bacillus otricolare Weinberg and Ginsbourg. (Bacillo otricolare, Nacciarone, Riforma Med., 33, 1917, 778; Weinberg 
and Ginsbourg, Bull. Inst. Past., 23, 1925, 825; Endosporus otricolare Prévot, Ann. Inst. Past., 61, 1938, 75.) From human gaseous gangrene.

Bacillus pappulus de Gasperi. (de Gasperi, Cent. f. Bakt., I Abt., Orig., 58, 1911, 1; Paraplectrum pappulum Prévot, Ann. Inst. Past., 61, 1938, 75.) From rancid sausages.

Bacillus parabutyricus Gratz and Vas. (Gratz and Vas, Cent. f. Bakt., II Abt., 41, 1914, 510; not Bacillus parabutyricus LeBlaye and Guggenheim, Man. Prat. de Diag. Bact., 1914, 324.) From Liptauer cheese.

Bacillus penicillatus Gerstner. (Inaug. Diss., Basel, 1894, 27.) From soil and sewage.

Bacillus peroniella Klein. (Ber. d. Deutsch. Bot. Gesellsch., 7 (Bhft.), 1889, 60.) Migula (Syst. d. Bakt., 2, 1900, $640)$ says this is probably anaerobic. Observed in swamp water, but not cultivated on artificial media.

Bacillus polypiformis Liborius. (Liborius, Ztschr. f. Hyg., 1, 1886, 162; Cornilia polypiformie Trevisan, I generi e le specie delle Batteriacee, 1889, 22; Anaerobe No. II, Sanfelice, Ztschr. f. Hyg., 14, 1893, 369; Bacillus cephaloideus Migula, Syst. d. Bakt., 2, 1900, 631.) From mouse inoculated with soil.

Bacillus postumus Heim. (Heim, Lehrbuch d. Bakt., 3 Aufl., 1906, (?) (p. 259 in 6 Aufl.); quoted from Würcker, Sitzungsber. d. Physik.-Med. Soz. in Erlangen, 41, 1909, 230; also Heim, Cent. f. Bakt., I Abt., Orig., 55, 1910, 341.) From various spontaneously putrefying infusions.

Bacillus pseudomagnus Migula. (Anaerobe No. VI, Sanfelice, Ztschr. f. Hyg., 14, 1893, 373; Migula, Syst. d. Bakt., 2, 1900, 608; Bacillus caris Chester, Man. Determ. Bact., 1901, 293.) From putrefying meat infusions, soil and animal excreta.

Bacillus pseudonavicula Migula. (Species No. 1, Kedrowski, Ztschr. f. Hyg., 16, 1894, 445; Bacillus acidi butyrici I Weigmann, Cent. f. Bakt., II Abt., 4,
1898, 830; not Bacillus acidi butyrici Kruse, in Flügge, Die Mikroorg., 3 Aufl., 2, 1896, 256; Migula, Syst.d. Bakt., 2, 1900, 590.) From cheese and rancid butter.

Bacillus pseudooedematis Kruse. (Pseudo - Oedembacillen, Liborius, Ztschr. f. Hyg., 1, 1886, 163; Bacillus pseudo-oedematis maligni Gerstner, Inaug. Diss., Basel, 1894, 35; not Bacillus pseudooedematis maligni Sanfelice, Ann. dell'Ist. d'Ig. di Roma, n.s. 1, 1891, 370; Anaerobe No. VII, Sanfelice, Ztschr. f. Hyg., 14, 1893, 374; Kruse, in Flügge, Die Mikroorg., 3 Aufl., 2, 1896, 239; Bacillus pseudoedematis Chester, Man. Determ. Bact., 1901, 293; presumably identical with Bacillus pseudo-septicus Perrone, Ann. Inst. Past., 19, 1905, 371, and, according to Perrone, with Proteus hominis capsulatus Bordoni-Uffreduzzi, Ztschr.f. Hyg., 3, 1888, 333.) From mouse inoculated with soil, from putrefying meat infusions and from animal excreta.

Bacillus pseudosolidus Migula. (Anaerobe No. III, Sanfelice, Ztschr. f. Hyg., 14, 1893, 371; Migula, Syst. d. Bakt., 2, 1900, 630; Bacillus tardus Chester, Man. Determ. Bact., 1901, 294; not Bacillus tardus Choukévitch, Ann. Inst. Past., 25, 1911, 350.) From putrefying meat infusions, soil, and from animal excreta.

Bacillus pseudotetani Migula. (Pseudotetanusbacillus, Tavel and Lanz, Mitt. a. klin. med. Inst. d. Schweiz, 1, 1893, 162; Migula, Syst. d. Bakt., 2, 1900, 598; Bacillus taveli Chester, Man. Determ. Bact., 1901, 304.) From cases of intestinal abscesses.

Bacillus pseudotetanicus Fruse. (Anaerobe IX, Sanfelice, 'Ztschr. f. Hyg., 14, 1893, 375; Kruse, in Flügge, Die Mikroorg., 3 Aufl., 2, 1896, 267; not Bacillus pseudotetanicus Migula, Syst. d. Bakt., 2, 1900, 626; not Bacillus pseudotetanicus Chester, Man. Determ. Bact., 1901, 302; Bacillus subtetanicus Migula, loc. cit., 629.) From putrefying meat infusions and from soil. 
Bacillus putrificus coagulans Distaso. (Cent. f. Bakt., I Abt., Orig., 59, 1911, 97.) From human and animal intestine. Bacillus putrificus var. non liquefaciens Putzu. (Policlin., Sez. Chir., 23, 1916, 225.) From human gas gangrene.

Bacillus putrificans immobitis Distaso. (Ann. Inst. Past., 23, 1909, 962.) From the feces of the flying fox (Pteropus).

Bacillus pyogenes foetidus Herfeldt. (Cent. f. Bakt., II Abt., 1, 1895, 77.) From manure and from horse intestine.

Bacillus ieniformis Gerstner. (Gerstner, Inaug. Diss., Basel, 189., 22; Bacterium reniforme Migula, Syst. d. Bakt., 2, 1900,329.) From soil and sewage.

Bacillus rubellus Okada. (Okada, Cent. f. Bakt., 11, 1892, 4; Clostridium rubellum Prévot, Ann. Inst. Past., 61, 1938, 85.) From dust and dirt.

Bacillus saccharofermentans de Gas peri. (Compt. rend. Soc. Biol., Paris, 67, 1909, 494.) From putrefying carcasses of game birds.

Bacillus saccharogenes Romanovitch. (Compt. rend. Soc. Biol., Paris, 71, 1911, 168.) From human intestine, both normal and during appendicitis.

Bacillus saprogenes $I, I I$ and $I I I$, Herfeldt. (Herfeldt, Cent. f. Bakt., II Abt., 1, 1895, 77; not Bacillus saprogenes Salus, Arch. f. Hyg., 51, 1904, 115.) From manure and from horse intestine.

Bacillus saprogenes intestinalis Romanovitch. (Compt. rend. Soc. Biol., Paris, 71, 1911, 237.) From human intestine.

Bacillus saprophyticus Maes. (Surg. Clin. North Amer., 10, 1930, 792.) Only casual mention of this organism as one of the common gas bacilli. Othemise unidentified.

Bacillus saprotoxicus sordelli, Soriano, Ferrariand Torino. (Sordelli et al., Rev. d. Inst. Bact., Buenos Aires, 6, 1934, 432; Clostridium saprotoxicum Prévot, Ann. Inst. Past., 61, 1938, 83.) From human gaseous gangrene.

Bacillus sarcoemphysematodes hominis Conradi and Bieling. (Münch. med.
Wehnschr., 63, 1916, 133.) From human lesions.

Bacillus satellitis Loris-Melikov. (Unnamed species of Loris-Melikov, Compt. rend. Soc. Biol., Paris, 70, 1911, 865; Loris-Melikov, Ann. Inst. Past., 27, 1913, 545; Bacillus satelitis (sic) LorisMelikov, Compt. rend. Acatd. Sci., Paris, 156, 1913. 346; Inflahilis satellitis Prévot, Ann. Inst. Past., 61, 1938, 77.) From human typhoid feces.

Bacillus scatologenes Weinberg and Ginsbourg. (Skatol-liberating Clostridium, Fellers, Abst. Bact., 7, 1923, 351 ; Bacillus skatol, Fellers and Clough, Jour. Bact., 10, 1925, 105; Weinberg and Ginsbourg. Les Microbes Anaérobies, Paris, 1927, 5t.) From spoiled canned maearoni and salmon.

Bacillus septicus Klein. (Klein, Micro-Organisms and Disease, London, 1884, 78; not Bacillus septicus Macé, Traité Prat. d. Bact., 1st ed., 1888, 455; not Bacillus septicus Migula, Syst. d. Bakt., 2, 1900, 646.) Probably synonymous with Clostridium perfringens Type A. From earth, putrid blood and other albuminous fluids, and occasionally in blood-vessels of man and animals after death.

Bacillus solidus Lüderitz. (Lüderitz, Ztschr.f. Hyg., 5, 1889, 152; not Bacillus solidus Chester, Ann. Rept. Del. Col. Igr. Exp. Sta., 10, 1sts, 129; Cornilia solida Trerisan, I generi e le specie delle Batteriacee, 1589, 22.) From mouse and guine: pig inoculated with soil.

Bacillus solmsii Klein. (Klein, Ber. d. Deutsch. Bot. Gesellsch., $\gamma$ (Bhft.), 1889, 60; Diplectridium solmsii Fischer, Jahrb. f. Wiss. Bot., 27, 1895, 148). Migula (Syst. d. Bakt., 2, 1900, 640) says this is probably anaerobic. Observed in swamp water, but not cultivated on artificial media.

Bacillus spinosus Lüderitz. (Lüderitz, Ztschr. f. Hyg., 5, 1889, 152; Cornilia spinosa Trevisan, I generi e le specie delle Batteriacee, 1889,22.) From mouse and guinea pig inoculated with soil. 
Bacillus sporogenes Migula. (Bacillus enteritidis sporogenes Klein, Cent. f. Bakt., I Abt., 18, 1895, 737; Migula, Syst. d. Bakt., 2, 1900, 560; Bacillus (enteritidis) sporogenes and Bacillus enteritidis Kilein, Loc. Govt. Bd., Ann. Rept. Med. Off., London, 33, 1903-04, 442 and 443; Bacillus sporogenes capsulatus Rettger, Jour. Biol. Chem., 2, 1906-07, 84; Bacillus enteritidis-sporogenes Holland, Jour. Bact., 5, 1920, 218; Clostridium enteritidis-sporogenes Holland, ibid., 218; Clostridium enteritidis sporogenes Holland, ibid., 222; Clostridium sporogenes Holland, ibid., 220.) Probably a culture of Clostridium perfringens contaminated with Clostridium bifermentans or with Clostridium sporogenes. From epidemic diarrheal feces, and from milk presumably causing the epidemic.

Bacillus sporogenes non liquefaciens Jungano. (Jungano, Compt. rend. Soc. Biol., Paris, 65, 1908-09, 716; Bacillus sporogenes liquefaciens Jungano, ibid., 718; Bacillus sporogenes non liquefaciens anaerobius LeBlaye and Guggenheim, Man. Prat. d. Diag. Bact., 1914, 395.) From the intestine of the flying fox (Pteropus).

Bacillus sporogenes foetidus Choukévitch. (Ann. Inst. Past., 25, 1911, 257.) From the large intestine of a horse.

Bacillus sporogenes parvus Choukévitch. (Ann. Inst. Past., 27, 1913, 251.) From intestine of cattle.

Bacillus stellatus Vincent. (Vincent, Ann. Inst. Past., 21, 1907, 69; Bacillus stellatus anaerobius LeBlaye and Guggenheim, Man. Prat. d. Diag. Bact., 1914, 368; Bacterium stellatum LeBlaye and Guggenheim, idem.) Anaerobic status uncertain, but Vincent compares it with Bacillus polypiformis Liborius, and with Anaerobe No. II, Sanfelice. From water.

Bacillus subfoetidus Migula. (Anaerobe V, Sanfelice, Ztschr. f. Hyg., 14, 1893, 372; Migula, Syst. d. Bakt., 2, 1900, 609; Bacillus anaerobic No. $V$ Chester, Man. Determ. Bact., 1901, 296; Bacillus pseudotetanicus Chester, ibid., 302.) From putrefying meat infusions, soil, and from animal excreta.
Bacillus tachysporus Wesbrook. (Jour. Path. and Bact., 4, 1896-97, 8.) From infection in human tetanus.

Bacillus tenuis glycolyticus Distaso. (Ann. Inst. Past., 23, 1909, 955.) From intestine of the flying fox (Pteropus).

Bacillus tenuis spatuliformis Distaso. (Distaso, Cent. f. Bakt., I Abt., Orig., 59, 1911, 101; Bacteroides tenuis Bergey et al., Manual, 1st ed., 1923, 263; Cillobacterium spatuliforme Prévot, Ann. Inst. Past., 60, 1938, 297; Bacillus spatuliformis Prévot, Man. d. Class., etc., 1940, 79.) Spores not observed by Distaso, but placed by him in the perfringens-group. From feces of dog.

Bacillus teras Knorr. (Kinorr, Cent. f. Bakt., I Abt., Orig., 82, 1918-19, 225; Inflabilis leras Prévot, Ann. Inst. Past., 61, 1938, 77.) From soil and from fluid aspirated in hematopneumothorax.

Bacillus thalassophilus Russell. (Russcll, Ztschr. f. Hyg., 11, 1892, 189.) Variably recorded as a strict or facultative anaerobe (see Bacillus polymyxa and Bacillus sphaericus). From sea water and mud from depth of sea.

Bacillus thermofibrincolus Itano and Arakawa. (Bull. Agric. Chem. Soc., Japan, 5, 1929, 33.) Source not recorded.

Bacillus tympani-cuniculi Morcos. (Jour. Bact., 23, 1932, 454.) From viscera, muscles and blood of rabbits dying of infectious tympanitis.

Bacillus ukilii Weinberg, Prévot, Davesne and Renard. (Unnamed species of Ckil, Compt. rend. Soc. Biol., Paris, 87, 1922, 1009; Weinberg et al., Ann. Inst. P'ast., 42, 1928, 1199; Bacillus oedematogenes Frei, Ergeb. d. allgem. Path. Mensch. u. Tiere, 31, 1936, 52; Clostridium ukilii. Hauduroy et al., Dict. d. Bact. Path., 1937, 142; Clostridium oedematis-benigni Prévot, Ann. Inst. Past., 61, 1938, 82; Clostridium oedematis benigni Prévot, Man. d. Class., etc., 1940, 120.) From diseased human appendix.

Bacillus ventriculosus Koch. (Koch, Botan. Zeit., 46, 1888, 311; Clostridium ventriculosus Trevisan, I generi e le specie delle Batteriacee, 1889, 22.) Prob- 
ably not anaerobic. Observed in decaying vegetation and in swampy waters.

Bacterium coprophilum Migula. (Anaerobe No.2, Sewerin, Cent. f. Bakt., II Abt., 3, 1897, 708; Migula, Syst. d. Bakt., 2, 1900, 323; Bacillus coprophilus Weinberg, Nativelle and Prévot, Les Microbes Anaér., 1937, 643. ) From horse manure.

Bacterium lini Migula. (Unnamed species of Winogradsky, Compt. rend. Acad. Sci., Paris, 121, 1895, 744; Migula, Syst.d. Bakt., 2, 1900,513. From retting flax.

Bacterium pseudoclostridium Migula. (Clostridium foctidum lactis von Freudenreich, Cent. f. Bakt., II Abt., 1, 189.5, 856 ; Migula, Syst. d. Bakt., 2, 1900, 511.) From cheese.

Bacterium sternbergii Migula. ( $B a-$ cillus anaerobicus liquefaciens Sternberg, Researches relating to the etiology and prevention of yellow fever, Washington, 1891, 214; Bacillus anaerobius liquefaciens Kruse, in Flügge, Die Mikroorg., 3 Aufl., 2, 1896, 241; Migula, Syst. d. Bakt., 2, 1900, 444; Bacterium anaerobicum Chester, Man. Determ. Bact., 1901, 198.) From intestine of a yellow fever cadaver.

Bactridium butyricum Chudiakow. (Chudiakow, Zur Lehre von der Anaerobiose, Teil I, Moskau, 1896, (?); quoted from Rothert, Cent. f. Bakt., II Abt., 4, 1898, 390.) Stated by Rothert to be a pathogenic, obligate anaerobe, but source of culture is not specified.

Caduceus thermophilus $\alpha$ Prévot. (Anaerobie thermophile $\alpha$ (Thermo $\alpha$ ), Veillon, Ann. Inst. Past., 36, 1922, 428; Prévot, Ann. Inst. Past., 61, 1938, 86; Bacillus thermophilus $\alpha$ Prévot, Man. d. Class., etc., 1940, 150; Caduceus thermophilus Prévot, ibid., 149; Caduceus thermophilus alfa Prévot, ibid., 150.) From horse manure.

Clostridium aceticum Wieringa. (Jour. Microbiol. and Serol., 6, 1940, 257.) From soil. Oxidizes $\mathrm{H}_{2}$, using $\mathrm{CO}_{2}$ as the hydrogen acceptor, forming acetic acid, thus using $\mathrm{H}_{2}$ as sole source of growth energy and $\mathrm{CO}_{2}$ as sole carbon source for cell growth.

Clostridium albo-lacteum Killian and Fehér. (Ann. Inst. Past., 55, 1935, 620.) From Sahara Desert soil.

Clostridium alboluteum Killian and Fehér. (Ann. Inst. Past., 55, 1935, 595.) From Sahara Desert soil.

Clostridium album liquefaciens Killian and Fehér. (Ann. Inst. Past., 55, 1935, 595.) From Sahara Desert soil.

Clostridium album minor Killian and Fehér. (Ann. Inst. Past., 55, 1935, 620.) Presumably identical with Clostridium minor Killian and Fehér, ibid., 597. From Sahara Desert soil.

Clostridium album non liquefaciens Killian and Fehér. (Ann. Inst. Past., 55, 1935, 599.) Presumably identical with Clostridium non liquefaciens Killian and Fehér, ibid., 597. From Sahara Desert soil.

Clostridium americanum Pringsheim. (Eine Alkohole bildende Bakterienform, Pringsheim, Cent. f. Bakt., II Abt., 15, 1906, 300; Bacillus pringsheim Pringsheim, ibid., 311; Pringsheim, Cent. f. Bakt., II Abt., 16, 1906, 796; Clostridium butyricum var. americanum Prévot, Man. d. Class., ete., 1940, 109.) Anaerobic status uncertain. From spontaneously fermenting potato.

Clostridium aurantibutyricum Hellinger. (Commemorative Vol. to Dr. Ch. Weizmann's 70th Birthday, Nov., 1944, 46.) From retted Hibiscus from So. Africa.

Clostridium balaenae Prévot. (Walfischseptikämie Bacillus, Nielsen, Cent. f. Bakt., 7, 1890, 269; Bacille de la septicémie des baleines, Christiansen, Compt. rend. Soc. Biol., Paris, 83, 1920, 324; Walfischseptikämiebazillus, Christiansen, Cent. f. Bakt., I Abt., Orig., 84, 1920, 127; Prévot, Ann. Inst. Past., 61, 1938, 81.) From flesh of whales dying of septicemia. Later isolated from same material by Christiansen.

Clostridium canadiense Dernby and Blane. (Jour. Bact., 6, 1921, 420.) From a human case of gangrene.

Clostridium caproicum Prévot. 
cillus anaerobicus der Capronsäuregruppe, Rodella, Cent. f. Bakt., II Abt., 16, 1906, 58; Prévot, Ann. Inst. Past., 61, 1938, 84; Bacillus anaerobicus caproicus Prévot, Man. d. Class., etc., 1940, 140.) From cheese.

Clostridium cellobioparus Hungate. (Jour. Bact., 48, 1944, 499.) From rumen of cattle.

Clostridium cellulosae Horowitz-Wlassowa and Novotelnow. (Cent. f. Bakt., II Abt., 91, 1935, 477.) Cited only by name. Source not stated.

Clostridium corallinum Prévot and Raynaud. (Ann. Inst. Past., \%0, 1944, 182.) From serous fluid obtained post mortem from a mouse inoculated with an emulsion of street dust.

Clostridium cuniculi Galli. (Galli, Boll. Ist. Sieroter., Milan, S, 1923-24, 337; Clostridium gallii Prévot, Ann. Inst. Past., 61, 1938, 83.) From necrotic visceral lesion in a rabbit.

Clostridium disporum Vuillemin. (Compt. rend. Acad. Sci., Paris, 136, 1903, 1583.) Probably not anaerobic. Encountered in cultures of Blastomyces; said to form two spores.

Clostridium foetidum fecale Romanovitch. (Compt. rend. Soc. Biol., Paris, 71, 1911, 238.) From normal human intestine.

Clostridium ghoni Prévot. (Unnamed species of Ghon and Mucha, Cent. f. Bakt., I Abt., Orig., 39, 1905, 497; Prévot, Ann. Inst. Past., 61, 1938, 83.) From post-operative human peritonitis.

Clostridium giganteum Benecke and Keutner. (Ber. d. Deutsch. Botan. Gesellsch.. 21, 1903, 340.) From sea water.

Clostrifium haemolysans Hauduroy et al. Bacillus anaerobius haemolysans Markoff, Cent. f. Bakt., I Abt., Orig., 77, 1915-16. 421; H:auduroy et al., Dict. d. Bart. Path., 1937, 105; Plectridium hemolysans Prévot, Ann. Inst. Past., 61, 1958, 57.) From putrid human buecal tissues.

Clostridium hueppei Trevisan. (Buttersäurebarillen, Hueppe, Nitteil. a. d. kais. Gesundhts., 2, 1881, 353; Bacillus butyricus Flügge, Die Mikroorg., 2 Aufl., 1886, 300; Trevisan, I generi e le specie delle Batteriacee, 1889, 22; Bacillus pseudo-butyricus Krruse, in Flügge, Die Mikroorg., 3 Aufl., 2, 1896, 207; Bacillus hueppei Chester, Man. Determ. Bact., 1901, 276.) From milk.

Clostridium hyalinum Killian and Fehér. (Ann. Inst. Past., 55, 1935, 595.) From Sahara Desert soil.

Clostridium kluyverii Barker and Taha. (Jour. Bact., 43, 1942, 347.) From alcohol-containing enrichment cultures of Mcthanobacterium omclianskii inoculated with black mud from fresh water and marine sources.

Clostridium liborii Trevisan. (Liborius buttersiurebildender Bacillus, Flügge, Die Mikroorg., 2 Aufl., 1886, 299; Trevisan, I generi e le specie delle Batteriacee, 1889,22.) Presumably the same as Clostridium foctidum Liborius, Ztschr. f. Hyg., 1, 1886, 160. From mice inoculated with garden soil.

Clostridium medium Henneberg. (Cent. f. Bakt., II Abt., 55, 1922, 248.) From human and animal feces.

Clostridium mitelmani Prévot. (Ann. Inst. Past., 61, 1938, 84.) Stated by Prévot to have been isolated by Mitelman from diarrheal human intestine.

Clostridium mucosus Simola. (Simola, Ann. Acad. Seient. Fennicoe, Helsinki, Ser. A, 34, 1931, (115?); Clostridium mucosum Prévot, Man. d. Class., ete., 1940, 112; not Clostridium mucosum Bergey et al., Manual, th ed., 1934, 472; quoted from Prévot, Ann. Inst. Past., $61,1938,80$ who records it as a facultative anaerobe.) Source of isolation unknown.

Clostridium myxogenes Simola. (Simolit, Ann. Acad. Scient. Fennicoe, Helsinki, Ser. A, 34, 1931, (115?); quoted from Prévot, Ann. Inst. Past., 61, 1938, 80 who records it as a facultative anaerobe.) Source of isolation unknown.

Clostridium necrosans Prévot. ( $\mathrm{Ba}$ cillus aerogenes necrosans Schupfer, Policlin., Sez. Med., 12, 1905, 261 ; Prévot, Ann. Inst. Past., 61, 1938, 84.) Isolated 
from a gaseous, necrotic thoracic abscess in a woman.

Clostridium nothnageli Henneberg. (Cent. f. Bakt., II Abt., 55, 1921-22, 245.) Cultivated, but not isolated in pure culture, from human and animal feces.

Clostridium partum Prévot. (Unnamed anaerobe of Levens, Cent. f. Bakt., I Abt. Orig., 88, 1922, 479; Prévot, Ann. Inst. Past., 61, 1938, 85.) From a cow in post-partum rausch-brand.

Clostridium propionicum Cardon and Barker. (Jour. Bact., 52, 1916, 629.) From marine mud.

Clostridium proteolyticum Choukévitch. (Ann. Inst. Past., 27, 1913, 253.) Said to be a facultative anaerobe. From intestine of cattle.

Clostridium pygmaeum Henneberg. (Cent.f. Bakt., II Abt., 55, 1921-22, 250.) From human and animal feces.

Clostridium sarcoemphysematodes Prévot. (Bacillus sarcemphysematodes hominis Conradi and Bieling, Münch. med. Wochnschr., 63, 1916, 134; Clostridium sarcemphysematodes (sic) Prévot, Ann. Inst. Past., 61, 1938, 81; Prévot, Man. d. Class., etc., 1940, 120.) From human gaseous gangrene.

Clostridium sardiniensis Prévot. (Ann. Inst. Past., 61, 1938, 81.) Referred to Altara by Prévot. Cited by name only from Prévot.

Clostridium secundum Hauduroy et al. (Unnamed species of Ghon and Sachs, Cent. f. Bakt., I Abt., Orig., 48, 1909, 399; Hauduroy et al., Dict. d. Bact. Path., 1937, 130.) From human emphysematous liver.

Clostridium solidum Sanfelice. (Sanfelice, Ztschr. f. Hyg., 14, 1893, 372; Bacillus solidum Chester. Ann. Rept. Del. Col. Agr. Exp. Sta., 10, 189s, 129; Bacillus sanfelicei Migula, Syst. d. Bakt., 2, 1900, 630; not Bacillus solidus Lüderitz, Ztschr. f. Hyg., 5, 1859, 152.) From putrefying meat infusions, soil and from animal excreta.

Clostridium sphaeroides Killian and Fehér. (Ann. Inst. Past., 55, 1935, 598.) From Sahara Desert soil.
Clostridium strasburgense Hauduroy et al. (Unnamed species of Vaucher, Boez, Lanzenberg and Kehlstadt, Bull. et Mem. Soc. Méd. Hôp. Paris, 49, 1925, 1641; Hauduroy et al., Dict. d. Bact. Path., 1937, 135; Inflabilis sanguicole Prévot, Ann. Inst. Past., 61, 1938, 77.) Isolated by blood culture in human puerperal septicemia.

Clostridium tenue Hauduroy et al. (Bacillus anaerobicus tenuis Distaso, Cent. f. Bakt., I Abt., Orig., 62, 1912, 439; Bacillus anaerobius tenuis LeBlaye and Guggenheim, Man. Prat. d. Diag. Bact., 1914, 337; Hauduroy et al., Dict. d. Bact. Path., 1937, 136.) From normal human intestine.

Clostridium thermoacidophilus Damon and Feirer. (Damon and Feirer, Jour. Bact., 10, 1925, 41; Palmula thermoacidophila Prévot, Ann. Inst. Past., 61, 1938, 89; Acuformis thermoacidophilus Prévot, Man. d. Class., etc., 1940, 165.) Isolated anaerobically, but not strictly anaerobic. From horse manure.

Clostridium thermoaerogenes Damon and Feirer. (Damon and Feirer, Jour. Bact., 10, 1925, 40; Caduceus thermoaerogenes Prévot, Ann. Inst. Past., 61, 1938. 86.) From horse manure.

Clostridium thermocellum Viljoen, Fred and Peterson. (Viljoen et al., Jour. Agric. Sci. (London), 16, 1926, 7; Terminosporus thermocellus Prévot, Ann. Inst. Past., 61, 1938, 86.) From horse manure.

Clostridium thermochainus Damon and Feirer. (Jour. Bact., 10, 1925, 42.) From horse manure.

Clostridium thermophilum Jemel'jantschik and Borissowa. (Microbiology (Russian), 10, 1941, 236-241; Bacillus thermophilus anaerobicus, idem; abst. in Cent. f. Bakt., II Abt., 105, 1942, 148; not Clostridium thermophilum Pribram, Jour. Bact., 22, 1931, 430.) From fish conserves.

Clostridium thermoputificum Damon and Feirer. (Damon and Feirer, Jour. Bact., 10, 1925, 39; Palmula thermopu- 
trifica Prévot, Ann. Inst. Past., 61, 1938, 89; Acuformis thermoputrificus Prévot, Man. d. Class., ete., 1940, 165.) From horse manure.

Clostridium toxinogenes Prévot. (Unnamed anaerohe of Kojima, Ztschr. f. Hyg., 99, 1923, 86; Prévot, Ann. Inst. Past., 61, 1938, 82.) From muscle of a cow dying of symptomatic anthrax.

Clostridium ureolyticum Prévot. (Ann. Inst. Past., 61, 1938, 85; presumably Erde A, Geilinger, Cent. f. Bakt., II Abt., 47, 1917, 252.) From manured soil. Clostridium valerianicum Prévot. (Fäulnisanaerobien der Baldriansäure, Rodella, Cent.f. Bakt., II Abt., 16, 1906, 62; Prévot, Ann. Inst. Past., 61, 1938, 84.) From cheese.

Clostridium viscosum Chudiakow. (Zur Lehre von der Anaerobiose, Teil I, 1896 (Russ.); quoted from Rothert, Cent. f. Bakt., II Abt., 4, 1898, 390.) A facultative anaerobe.

Clostridium xanthogenum DeGraaff. (Nederl. Tijd. Hyg., Microbiol. en Serol., 4, 1930, 219.) From cultured buttermilk undergoing atypical fermentation.

Clostridium zuntzii Henneberg. (Cent. f. Bakt., II Abt., 55, 1922, 249.) Cultivated, but not isolated in pure culture, from human and animal feces.

Cornilia parva Trevisan. (Bacillus liquefaciens parus Lüderitz, Ztschr. f. Hyg., 5, 1889, 148; Trevisan, I generie le specie delle Batteriacee, 1889, 22; Bacterium parvum Migula, Syst. d. Bakt., 2, 1900,324 .) From animals inoculated with garden soil.

Endosporus otricolare Prévot. (Bacillo otricolare, Nacciarone, Riforma Med., Napoli, 33, 1917, 778; Prévot, Ann. Inst. Past., 61, 1938, 75.) From gangrenous war wounds.

Granulobacillus sporogenes Andre. (Granulobacillus sp., Sommer, Deutsch. Monatschr. f. Zahnheilk., 33, 1915, 328; Andre, Ztschr. f. Hyg., 114, 1933, 412.) From infected, necrotic pulp of human teeth.

Granulobacter lactobutyricum Bei- jerinck. (Ferment de lactate de chaux, Pasteur, Compt. rend. Acad. Sci., Paris, $56,1863,416$; Beijerinck, Verhandl. d. k. Akademie v. Wetensch., Amsterdam, Tweedie Sectie, Deel I, 1893, 8; Bacillus lactobutyricus Migula, Syst. d. Bakt., 2, 1900, 601; Amylobacter lactobutyricus van Beynum and Pette, Cent. f. Bakt., II Abt., 93, 1935, 200.) From fermenting grain mash and from soil.

Granulobacter pectinovorum Beijerinck and van Delden. (Plectridium pectinovorum Störmer, Mitteil. d. deutseh. Landwirts. Gesellsch., 18, 1903, 195; Beijerinck and van Delden, Arch. Néerl. d. Sci. Exactes et Nat., Ser. II, 9, 1904, 423; Bacillus pectinovorus LeBlaye and Guggenheim, Man. Prat. d. Diag. Bact., 1914, 324; Bacillus pectinororum Henneberg, Cent. f. Bakt., II Abt., 55, 1922, 279; Clostridium pectinovorum Donker, Thesis, Delft, 1926, 145.) From retting plant tissues.

Granulobacter reptans Beijerinck and van Delden. (Cent. f. Bakt., II Abt., 9, 1902, 13.) Probably aerobic or microaerophilic. From garden and other soils.

Granulobacter sphaericum Beijerinck. (Cent. f. Bakt., II Abt., 7, 1901, 568.) Probably aerobic or microaerophilic. From garden and other soils.

Gramulobacter urocephalum Beijerinck and van Delden. (Arch. Néerl. d. Sci. Exactes et Nat., Ser. II, 9, 1904, 423.) From retting plant tissues.

Hiblerillus rectus Heller. (Streptobacillus anaerobicus rectus Choukévitch, Ann. Inst. Past., 25, 1911, 350; Bacillus anaerobius rectus LeBlaye and Guggenheim, Man. Prat. d. Diag. Bact., 1914, 337; Heller, Jour. Bact., 7, 1922, 17; Inflabilis rectus Prévot, Ann. Inst. Past., 61, 1938, 77.) From large intestine of the horse.

Hiblerillus septimus Heller. (Art VII, von Hibler, Untersuch. ü. d. Path. Anaer., 1908, 3 and 406; Heller, Jour. Bact., 7, 1922, 17; Clostridium septimum Prévot, Ann. Inst. Past., 61, 1938, 84.) From spleen of a guinea pig inoculated with soil. 
Inflabilis barati Prévot. (Bacille de Barat, Tissier, Compt. rend. Soc. Biol., Paris, 81, 1918, 426; Prévot, Ann. Inst. Past., 61, 1938, 77.) From beer wort.

Inflabilis magnus Prévot. (Streptobacillus anaerobicus magnus Choukévitch, Ann. Inst. Past., 25, 1911, 251; Bacillus anaerobius magnus LeBlaye and Guggenheim, Man. Prat. d. Diag. Bact., 1914, 337; Prévot, Ann. Inst. Past., 61, 1938, 77.) From large intestine of the horse.

Inflabilis plagarum Prévot. (Bacillus S, Adamson, Jour. Path. and Bact., 22, 1918-19, 373; Prévot, Ann. Inst. Past., 61, 1938, 77.) From war wounds.

Inflabilis pseudo-perfringens Prévot. (Presumably Bacillus L, Adamison, Jour. Path. and Bact., 22, 1918-19, 372; Prévot, Ann. Inst. Past., 61, 1938, 77.) From war wounds.

Inflabilis setiensis Prévot and Raynaud. (Ann. Inst. Past., 70, 1944, 50.) From oysters.

Marlellillus proteolyticus Heller. (Orgauism II, Hempl, Jour. Hyg., 17, 1918, 16; Heller, Jour. Bact., 7, 1922, 26.) From musele in human gaseous gangrene.

1 yerillus sadowa Heller. (Jour. Bact., 7, 1922, 7; also Barney and Heller, Arch. Surg., 4, 1922, 477.) From a gangrenous human arm.

Pectinobacter amylophilum Makronov. (Arch. Sci. Biol. (Russ.), 18, 1915, 141.) Stated by author to be anaerobic, but description does not make this evident. From soil.

Plectridium cellulolyticum Pochon. (Compt. rend. Soc. Biol., Paris, 113, 1933, 1325.) Isolated anaerobically, but not strictly anaerobic. From stomach of ruminants.

Plectridium pectinovorum liquefaciens sjolander and MeCoy. (Cent. f. Bakt., II Abt., 97, 1937, 315 and 322; probably identical with Amylobacter liquefaciens Ruschmann and Bavendamm, Cent. f. Bakt., II Abt., 64, 1937, 359.) From spontaneously retting plant tissues.

Plectridium snieszkoi Prévot. (Lnnamed anaerobic thermophilis cellulose- fermenting species, Snieszko, Cent. f. Bakt., II Abt., 88, 1933, 403; Prévot, Man. d. Class., ete., 1940, 154.) From soil and manure.

Recordillus fragilis Heller. (Jour. Bact., 7, 1922, 8 and 27.) From a liver infaret in a cow.

Reglillus progrediens Heller. (Jour. Bact., 7, 1922, 7; also Barney and Heller, Arch. Surg., 4, 1922, 477.) From muscle of a gangrenous human arm.

Robertsonillus primus Heller. (Organism I, Hempl, Jour. Hyg., 17, 1918, 13 ; Heller, Jour. Bact., 7, 1922, 7.) From a gangrenous war wound of human maxilla.

Streptobacillus terrae Ucke. (Cent. f. Bakt., I Abt., 23, 1898, 1000.) Fromgarden soil.

Terminosporus raabi Prévot. (Lnnamed anaerobe of Raab, Jour. Amer. Water Works Assoc., 10, 1923, 1051; Prévot, Ann. Inst. Past., 61, 1938, 86). From Minneapolis city water.

Terminosporus thermocellulolyticus Pochon. (Ann. Inst. Past., 68, 1942, 354,383 and 467.) Strict anaerobe. Optimum growth at $60^{\circ}$ to $66^{\circ} \mathrm{C}$.

Tyrothrix catenula Duclaux. (Duclaux, Ann. Inst. Nat. Agron., 4, 1882, 23; Cornilia catenula Trevisan, I generi e le specie delle Batteriacee, 1889, 22 ; Bacillus catenula Chester, Ann. Rept. Del. Col. Agr. Exp. Sta., 10, 1898, 123.) Commonly regarded as anaerobic, but not by Migula (Syst. d. Bakt., 2, 1900, 588). From cheese.

Tyrothrix claviformis Duclaux. (Duclaux, Ann. Inst. Nat. Agron., 4, 1882, 23; Pacinia claiformis Trevisan, in litt., quoted from DeToni and Trevisan, in Saccardo, Sylloge Fungorum, 8, 1889, 1017; Bacterium claviforme Migula, Syst. d. Bakt., 2, 1900, 322; Bacillus claviformis Vincent, Ann. Inst. Past., 21, 1907, 70.) From cheese.

Tyrothrix urocephalum Duclaux. (Duclaux, Ann. Inst. Nat. Agron., 4, 1882 , 23; Bacterium urocephalum Chester, Ann. Rept. Del. Col. Agr. Exp. Sta., 10, 189S, 123; Bacillus urocephalum Migula, Syst. d. Bakt., 2, 1900, 588.) 
Appendix II. The following organisms are listed in the text as probable synonyms or possibly related species. They are cited here again in order to record the source of the original isolation. For convenience, they are listed alphabetically under the names of the species to which such presumed relationship is ascribed.

\section{Clostridium butyricum Prazmow-} ski.

Amylobacter non liquefaciens Ruschmann and Bavendamm. From retting plant tissues.

Bacille amylozyme, Perdrix. From city water of Paris, and from the Seine River water.

Bacillus amylobacter is and $W$ Wertheim. From soil and tissues of field plants.

Bacillus butylicus Fitz. From glycerol solutions undergoing butylic fermentation after inoculation with fresh cow feces.

Bacillus holobutyricus Perdrix. From putrefying milk.

Bacillus orthobutylicus Grimbert From soil, grains and from legumes.

Bacillus saccharobutyricus von Klecki. From cheese.

Bacterium navicula Reinke and Berthold. Observed and described from decomposing plant tissues. Not isolated in pure culture.

Bactridium butyricum Chudiakow Cited by Rothert, and source not stated by abstractor.

Butylbacillus, Buchner. From glycerinated hay infusion.

Clostridium butyricum (Bacillus amylobacter) I, II, III, Gruber. From sugar solutions undergoing butyric fermentation. Source of inoculum not stated. (III is probably not strictly anaerobic.)

Clostridium butyricum iodophilum Svartz. From human feces.

Clostridium intermedium Donker. From retting flax.

Clostridium polyfermenticum Partansky and Henry. From mud of streams and lakes and from soil.
Clostridium saccharobutyricum Donker. From various farinaceous materials and from soil.

Clostridium saccharopetum. Partansky and Henry. From mud of streams and lakes and from soil.

Clostridium saccharophilicum Partansky and Henry. From mud of streams and lakes and from soil.

Clostridium saccharopostulatum Partansky and Henry. From mud of streams and lakes and from soil.

Clostridium tyrobutyricum van Beynum and Pette. From soil, water, milk, cheese and various farinaceous materials. Widely dispersed in nature.

Ferment butyrique (Vibrion butyrique) Pasteur. Cultivated and presumably isolated from sugar solutions undergoing butyric fermentation after inoculation with yeast. Purity of cultures seriously questioned.

Granulobacillus saccharobutyricus mobilis non-liquefaciens schattenfroh and Grassberger. From milk and from soil.

Gianulobacter butylicum. Beijerinck. From fermenting grain mash and from soil.

Granulobacter succharobutyricum Beijerinck. From fermenting grain mash and from soil.

Gianulobacter saccharobutyricus immobile nonliquefaciens McCoy et al. Source of isolation not recorded.

Plectridium pectinonorum Störmer. From retting flax and hemp. Probably not strictly anaerobic.

1b. Clostridium pasteurianium Winogradsky .

Bacillus azoticus, Bacillus dulcitofermentans, Bacillus inulofugus, Bacillus nompentosus and Bacillus rhamnoticus Bodily. Source of isolation not specified, other than from cultures received from various sources labeled $C$. pasteurianum.

6. Clostridium septicum Ford.

Bacillus tumefaciens Wilson. From human gaseous gangrene. 
Bradsotbacillus, Nielsen. From tissues and organs of sheep dring of braxy.

Walfischseptikïmie Bacillus, Nielsen. From whales evidently dead of sept icemia resulting from harpoon wounds.

9. Clostridium novyi Bergey et al.

Bacille neigeux, Jungano. From a human case of fetid cystitis, from abscess of kidney, and from various perineal infections.

Bacillus bellonensis Sacquépée. From human gaseous gangrene.

Bacillus gigas Zeissler and Rassfeld. From tissues of a sheep dying of a braxylike disease.

Bacillus oedematiens Weinberg and Seguin. From human gaseous gangrene.

Clostridium bubalorum Prévot. Isolated, but not named, by Kraneveld from cases of osteomyelitis of the East Indian buffalo.

Gasödembazillus, Aschoff. From human gaseous edema resulting from war wounds.

\section{Clostridium ace tobutylicum $\mathrm{McCoy}$} et al.

Bacillus butylaceticum Freiberg. From grains, soil and natural waters. Widely distributed in nature.

Bacillusbutylicus B.F., Ricard. From drains from slaughter houses.

Bacillus saccharobutyricus liquefaciens McCoy et al. Source of isolation unknown; records only from the collection of the Dept. Agric. Bact. of the Univ. of Wis. Received from a commercial laboratory.

Butylobacter betae Bakonyi. From beets (Beta vulgaris) contaminated with soil.

Butylobacter sinense Bakonyi. From Jaffa oranges.

Butylobacter solani Bakonyi. From German potatoes.

Butylobacter zeae Bakonyi. From Hungarian maize.

Clostridium butyricum (PrazmowskiPike-Smyth) Pike and Smyth. From spontaneously fermenting corn meal mash.
Clostridium inverto-acetobutylicum Legg and Stiles. From soil and from plant materials in contact with soil.

Clostridium propyl-butylicum Muller and Legg. From soil and from plant materials in contact with soil.

Clostridium saccharo-acetobutylicum Stiles and Legg. From soil and from plant materials in contact with soil.

Clostridium saccharo-acetobutylicumalpha MeCoy. From soil.

Clostridium saccharo-acetobutylicumbeta Arzberger. From soil, rotten wood, grain, cornstalks and river mud.

Clostridium saccharo-acetobutylicumyamma Arzberger. From soil, rotten nood, grain, corn stalks and river mud.

Clostridium saccharobutyl-acetonicum Loughlin. From potato; found in soil and on plant materials grown in or near soil.

Clostridium saccharobutylicum-gamma Izsak and Funk. From rice.

Clostridium saccharobutyl-isopropylacetonicum Loughlin. From potatoes, grains and other plant materials grown in or above soil.

Clostridium (Bacillus) tetrylium Owen, Mobley and Arroyo. From soil and from roots of sugar cane.

\section{Clostridium sporogenes Bergey} et al.

Bacillus putrificus verrucosus Zeissler. From animals suffering from a liauschbrandlike infection; later from gangrenous war wounds.

Bacillus saprogenes carnis salus. From human feces by enrichment in meat mash medium.

Bacillus sporogenes coagulans Debono. From normal human intestine.

Paraplectrum foetidum Weigmann. From cheese and milk.

Reading Bacillus, Donaldson and Joyce. From gangrenous human war wounds.

\section{Clostridium bifermentans Bergey} et al.

Bacillus centrosporogenes Hall. From a sterility test of tuberculin, from canned spinach and from garden soil. 
Bacillus liquefaciens magnus Lüderitz From mice and guinea pigs inoculated with garden soil.

Bacillus nonfermentans Hall and Whitehead. From poisoned African arrowheads.

Bacillus oedematis sporogenes Sordelli. From human gaseous gangrene.

Bacillus putrificus tenuis Zeissler. From malignant edema of various animals and from human gaseous gangrene.

Bacillus sporogenes foetidus Choukévitch. From large intestine of horse.

Clostridium foetidum Liborius. From mice inoculated with garden soil.

Clostridium foetidum carnis Salus. From human feces by enrichment in meat mash medium.

Clostridium oedematoides Meleney, Humphreys and Carp. From a case of human post-operative gaseous gangrene.

24. Clostridium perfringens Holland.

Bacille du rhumatisme, Achalme. Isolated by blood culture from human cases of acute articular rheumatism.

Bacillus amylobacter immobilis Gratz and Vas. From Liptauer cheese.

Bacillus cadaveris Sternberg. From liver and kidney of a yellow fever cadaver.

Bacillus cadaveris butyricus Buday. From organs of human cadavers undergoing postmortem emphysema.

Bacillus egens Stoddard. From muscle in a fatal case of human gaseous gangrene.

Bacillus emphysematis maligni Wicklein. From human gaseous gangrene.

Bacillus emphysematis vaginae Lindenthal. From human kolpohyperplasia cystica or vaginitis emphysematosa.

Bacillus multiformis Distaso. From feces of dog.

Bacillus ovitoxicus Bennetts. From blood, tissues and organs of sheep in Australia dying of entero-toxemia.

Bacillus paludis McEwen. From intestines and viscera of sheep in the Romney Marsh in England suffering from a disease called struck.

Bacillus perfringens Veillon and Zuber. Originally isolated from pus in human appendicitis; later from a variety of sources.

Bacillus phlegmones emphysematosae Fraenkel. From human gaseous phlegmons; later from a variety of related conditions of human beings and animals.

Bacillus zoodysentriae hungaricus Detre. Isolated in Hungary from intestines of diarrheal pigs and lambs.

Clostridium perfringens var. anaerogenes Hauduroy et al. An unnamed culture isolated by Grooten by blood culture from a fatal human septicemia.

Granulobacillus saccharobutyricus immobilis liquefaciens Schattenfroh and Grassberger. Originally isolated from market milk; later from cheese, soil, water, human and animal feces, and from various grain meals.

29. Clostridium paraputrificum Snyder. Bacillus innutritus Kleinschmidt. From stools of newborn infants.

Plectridium fluxum Prévot. From feces of nursing newborn infants.

Plectridium nonum Prévot. From emphysematous muscle of an amputated human arm.

41. Clostridium lentoputrescens Hart: sell and Rettger.

Bacillus cadaveris sporogenes (anaerobicus) Klein. From normal intestines of man and animals.

Bacillus radiatus Lüderitz. From mice and guinea pigs inoculated with garden soil.

Bacillus tetanoides (B) Adamson. From human septic and gangrenous war wounds.

43. Clostridium tetanomorphum Bergey et al.

Bacillus fragilis Veillon and Zuber. From human cases of purulent appendicitis.

Bacillus ramosus Veillon and Zuber. From human cases of purulent appendicitis and from pulmonary gangrene.

45. Clostridium angulosum Hauduroy et al. 
Bacillus angulosus Garnier and Simon. Isolated from gangrenous human war From blood of a child suffering from typhoid fever.

wounds. Stated to be aerobic.

Bacillus gazogenes parvus Choukévitch.

61. Clostridium tertium Bergey et al. From large intestine of horse.

Bacillus aero-tertius Bulloch et al. Bacillus spermoide Ninni. From soil. 


\section{Suborder II. Caulobacterineae Breed, Murray, and Hitchens.*}

(Caulobacteriales Henrici and Johnson, Jour. Bact., 29, 1935, 3; ibid., 30, 1935, 83; Breed, Murray and Hitchens, Bact. Rev., 8, 1944, 255.)

Non-filamentous, attached bacteria growing characteristically upon stalks, sometimes sessile. The stalked cells are asymmetrical in that gum, ferric hydroxide or other material is secreted from one side or one end of the cell to form the stalk. Multiply by transverse fission. In some species the stalks are very short or absent. In the latter case the cells may be attached directly to the substrate in a zoogloeic mass. Cells occur singly, in pairs or short chains, never in filaments; not ensheathed. Nonspore-forming. Typically aquatic in habitat.

\section{Key to the families of suborder Caulobacteriineae.}

I. Long axis of cell transverse to long axis of stalk; stalks dichotomously branched. A. Stalks lobose, composed of gum, forming zoogloea-like colonies.

B. Stalks are bands of ferric hydroxide. Family I. Nevskiaceae, p. 830.

\section{Family II. Gallionellaceae, p. 830 .}

II. Long axis of cell coincides with axis of stalk.

A. Reproducing by transverse fission, stalks unbranched.

Family III. Caulobacteriaceae, p. 832 .

III. Sessile, capsulated colonies of cocci and short rods attached to water plants.

A. Deposit of ferric hydroxide about a zoogloeic mass.

Family IV. Siderocapsaceae, p. 833.

As a result of discussions that have taken place since the fifth edition of the MANUAL was issued, certain readjustments have been made in the arrangement of the stalked bacteria. The organisms in all of the typical species are simple rigid bacteria which are like ordinary bacteria except that they develop a stalk. For tbis reason the group has been made a suborder of the order Eubacteriales.

Stanier and Van Niel (Jour. Bact., 42, 1941, 454) emphasize the fact that the family Pasteuriaceae includes species which reproduce by methods (longitudinal fission, budding) different from those found in other groups of bacteria, and Henrici and Johnson (loc. cit., 81) state that it is at least doubtful whether these species are phylogenetically related to the other stalked bacteria. While waiting for pure culture studies and a clarification of these relationships, this family has been placed in an appendix to the suborder Caulobacteriineae.

The family Siderocapsaceae has been included in the suborder as the absence of a stalk in attached forms is a natural modification. As stated by Cholodny (Die Eisenbakterien, Jena, 1926, 34-58), these bacteria are similar in morphology and physiology to those found in the family Gallionellaceae. This is an arrangement that retains all of the simple non-filamentous types of iron bacteria in one general group.

The stalked bacteria studied by Henrici and Johnson (loc.cil.) were of fresh water origin. Bacteria of this type are found, however, equally if not more abundantly in marine habitats where they play their part in starting the fouling of underwater surfaces. ZoBell and Upham (Bull. Scripps Inst. Oceanography, La Jolla, Cali-

* Completely revised by Prof. A. T. Henrici, University of Minnesota, Minneapolis, Minnesota, December, 1938; further revision by Prof. Robert S. Breed, New York State Experiment Station, Geneva, New York, July, 1946. 
fornia, 5, 1944, 253) summarize this situation as follows: "Many of the bacteria found in sea water are sessile or periphytic, growing preferentially or exclusively attached to solid surfaces. The sessile habit of marine bacteria is most pronounced when they are growing in very dilute nutrient solutions such as sea water to which nothing has been added. . . Most sessile bacteria appear to attach themselves tenaciously to solid surfaces by exuding a mucilaginous holdfast. I few have stalks. Some of the sessile bacteria grow on the walls of the eulture receptacle without clouding the medium itself".

The emphasis placed on the presence of a stalk by Henrici and Johnson (loc. cit.) seems artificial. In fact it may be questioned whether mere attachment by a holdfast or otherwise is a character of fundamental importance from the taxonomic standpoint. Henrici and Johnson's arrangement of these poorly known bacteria, however, has certain practical advantages and it has therefore been retained in this edition of the MANUAL with such modifications as seem to be clearly indicated by the progress that has been made since their outline was published.

The submerged slide technique as employed by Henrici (Jour. Bact., 25, 1933, 277) and by ZoBell and Allen (Proc. Soc. Exper. Biol. and Med., 30, 1933, 1409) has proved to be most useful for studying bacteria that live attached to a substrate. 


\section{FAMILY I. NEVSKIACEAE HENRICI AND JOHNSON.}

(Jour. Bact., 29, 1935, 4; ibid., 30, 1935, 83.)

Stalked bacteria, the long axis of the rod-shaped cells being set at right angles to the axis of the stalk. Stalks lobose, dichotomously branched, composed of gum. Multiplication of cells by transverse binary fission. Growing in zoogloea-like masses in water or in sugar vats.

There is a single genus Nevskia.

\section{Genus I. Nevskia Famintzin.}

(Bul. Acad. Imp. Sci., St. Pétersb., 34, N.S. 2, 1892, 484.) From the Neva River at St. Petersburg.

Description as for the family.

The type species is Nevskia ramosa Famintzin.

1. Nevskia ramosa Famintzill. (Bul. Acad. Imp. Sci., St. Pétersb., Ser. IV, 34, N.S. 2, 1892, 484.) From Latin ramosus, branched.

Globular, bush-like or plate-like colonies of gummy consistency floating upon the surface of water. Colonies composed of gummy material arranged in dichotomously branched stalks arising from a common base, with the bacterial cells contained in the gum, a single cell at the tip of each stalk. At times cells are set free from the stallis to start new colonies.

Rod-shaped cells set with their long axis at right angles to the axis of the broad lobe-like stalk. Cells 2 by 6 to 12 microns, containing a number of highly refractile globules of fat or sulfur.

Multiplication by binary fission. Not cultivated on artificial media.

Source: From the aquarium in the Botanical Garden, St. Petersburg. Similar but smaller organisms found by Henrici and Johnson (Jour. Bact., 30, 1935, 63) in a jar of water from the lily pond of the Lniversity of Minnesota greenhouse in Minneapolis.

Habitat: Water.

2. Nevskia pediculata (Kioeh and Hosaeus) Henrici and Johnson. (Bacterium pediculatum Koch and Hosaeus, Cont. f. Bakt., 16, 1894, 225; Henrici and Johnson, Jour. Bact., 30, 1935, 83.) From Latin pediculus, diminutive of pes, foot.

Composed of twisted, short, thick, sausage-like strands, often branched. These strands are stalks, composed of gum.

The cells occur at the tips of the stalks and are smaller than those of Nevskia ramosa and are without internal globules. Not cultivated.

This organism is very similar to, and may be identical with, the cultivated species described and named Betabacterium vermiforme by Mayer (Das TibiTionsortium. Thesis, Utrecht, 1938). See p. 362 .

Source: Found growing in the syrup of a sugar refinery as zoogloeae.

\section{FAMILY II. GALLIONELLACEAE HENRICI AND JOHNSON.}

(Jour. Bact., 29, 1935, 4; ibid., 30, 1935, 83.)

Stalked bacteria, the long axis of the rod-shaped cells being set at right angles to the axis of the stalks. Stalks are slender, twisted bands, dichotomously branched, composed of ferric hydroxide, completely dissolving in dilute hydrochloric acid. Multiplication of cells by transverse binary fission. Grow in iron-bearing waters.

There is a single genus Gallionella. 


\section{Genus I. Gallionella Ehrenberg.}

(Ehrenberg, Die Infusionsthierchen, 1838, 166; not Gaillonella Bory de St. Vincent, Dict. Classique d'Hist. Nat., 4, 1823, 393; Glocotila Kützing, Phycologia Generalis, 1813, 245; Didymohelix Griffith, Ann. Mag. Nat. Hist., Ser. 2, 12, 1853, 438; Spirophyllum Ellis, Cent. f. Bakt., II Abt., 19, 1907, 502; Nodofolium Ellis, Proc. Roy. Soc. Edinburgh, 28, Part 5, 1908, 339.) From an incorrect spelling of the algal genus name, Gaillonella.

Description as for the family.

The type species is Gallionella ferruginca Ehrenberg.

\section{Key to the species of genus Gallionella.}

I. Cells kidney-shaped; stalks branched.

A. Stalks slender, spirally twisted.

1. Cells small, stalks very slender.

2. Cells larger, stalks broader.

1. Gallionella ferruginea.

\section{Gallionella major.}

B. Stalks thick, not definitely in spirals.

3. Gallionella minor.

II. Cells oval or round; stalks unbranched.

4. Gallionella corneola.

1. Gallionella ferruginea Ehrenberg. (Gaillonella ferruginea Ehrenberg, Vorl. Mittheil. ü. d. wirkl. Vorkommenn fossiler Infusionen u. ihre grosse Verbreitung, Ann. Phys., Ser. 2, 8, 1836, 217; Ehrenberg, Die Infusionsthierchen, 1838, 166; Melosira ochracea Ralfs, Ann. and Mag. Nat. His., Ser. 1, 12, 1813, 351 (quoted from Buchanan, General Systematic Bacteriology, 1925, 363); Glocotila ferruginea Kützing, Species Algarum, 1819, 363; Didymohelix ferruginea Griffith, Ann. Mag. Nat. Hist., Ser. 2, 12, 1853, 438; Gloeosphaera ferminia Rabenhorst, Alg. Mitteleur., no. 387; Hedwigia, 8, no. 9, 1851, 43; Melosira minutula Breb., Alg. Fal., 5, 42 (quoted from DeToni and Trevisan, see below); Spirulina ferruginea Kirchner, Algen, Kryptogamenflora v. Schlesien, 2, 1, 1878, 250; Spirschaete ferruginea Hansgirg, Oestr. botan. Ztschr., no. 7-8, 1888, 5; Spiritlu:n ferrugineum DeToni and Trevisan, in Saccardo, Sylloge Fungorum, 8, 1889, 1007; Chlamydothrix ferruginea Migula, Syst. d. Bakt., 2, 1900, 1031; Spirophyllum ferrugineum Ellis, Cent. f. Bakt.,
II Abt., 19, 1907, 502; Spirophyllum tenue, Nodofolium ferrugineum, Spirosome ferruginoum and Spirosoma solenoide Ellis, Proc. Roy. Soc. Edinburgh, 28, Part 5, 1908, 311; also see Cent. f. Bakt., II Abt., 26, 1910, 321; Gallionella tacniata Enderlein, Bakterien-Cyclogenie, Berlin and Leipzig, 1925, 252.) From Latin ferruginus, iron rust.

Kidney-shaped bacteria, the cells 0.5 by 1.2 microns, which secrete colloidal ferric hydroxide from the concave portion of the cell, forming band-like stalks. 1 rotatory motion of the cells gives rise to a spiral twisting of the stalks.

In the older studies, the stalks were described as the organism, the minute cells at the tip having been dislodged or at least overlooked. The cells lie at the tip of the stalk, and multiply by transverse binary fission. This gives rise to a dichotomous branching of the stalks. Stalks become very long and slender, with smooth edges.

Not cultivated in artificial media.

Habitat: Cool springs and brooks which carry reduced iron in solution. 
2. Gallionella major ('hololny. (Trav. Station. hiolog. du Dnitpre. Arad. des Sci. de l'Tlkraine, Classe Sci. Phys. et Math., 3, Livre 4, 1927.) From Latin major, larger.

Very similar to Gallionella ferruginea, but the cells are distinctly larger (1 by 3 microns), and, some cells failing to divide, reach a length of 7 microns or more. These form stalks of double the normal width.

The cells contain one or more vacuoles, apparently filled with an iron compound.

Source: Found in a spring in the Caucasus.

Habitat: Iron-hearing water.

3. Gallionelia minor Cholodny. (Ber. d. deutsch. Bot. Ges., 42, 1921, 35.) From Latin minor, smaller.

Cells as in Galliunclla ferruginea, hut stalks are shorter, thicker, encrusted with nodules of iron and not definitely band-like or twisted.

Habitat: Iron-bearing water.

4. Gallionella corneola Dorit. (I)ie Eisenorganismen, Pflanzenforschung, Heft 16, 1934, 25.) From Latin corneolus, a little horn.
Cells spherical or ellipsoidal, or lenticular in cross section, 0.5 by 2.5 to 3.0 microns.

Stalks short, unbranched, not spirally twisted, completely dissolving in dilute hydrochloric acid. Stalks slender at the base, expanded at the tip, slightly curved, 15 to 30 microns long, attached to the substrate by a holdfast, 3 to 8 stalks arising from a single holdfast.

Halitat: Iron-bearing water.

Appendix: Additional species of Gallionella have been described as follows:

Gallionella glomerata described by Naumann (Kungl. Svenska Vetenskapsakad. Handl., I, 62, 1921, Part 4, 45) is not a valid species according to Cholodny (Die Eisenbakterien, Jena, 1926, 40). From the Aneboda region, Sweden. Gallionella reticulosa Butkevich. (Ber. d. Wiss. Meeresinst. Moseow, 3, 1928, 58 and 80.) From the White Sea.

Gallionella sideropous described by Naumann (Kungl. Svenska Vetenskapsakad. Handl., I, 62, 1921, Part 4, 33) is not a valid species, according to Cholixhy (Die Eisenbakterien, Jena, 1926, 39). From the Ineboda region, Sweden. Giallionflla tortuosa Butkevich. (Ber. 4. wiss. Merresinst. Moscow, 3, 1925, 57 and 79.) From the Petschora Sea.

\section{FAMILY III. CAULUBACTERIACEAE HEXRIOI AND JOHNSON.}

(Jour. Bact., 29, 1935, 4; ibid., 30, 1935, 83.).

Stalked bacteria, the long axis of the elongated cells coinciding with the long axis of the stalks. Stalks are slender, flagellum-like, often attached to the substrate by a button-like holdfast, unbranched. Multiplication of cells by transverse binary fission. The outermost cell of a pair may form a stalk before cell division is complete. Periphytic, growing upon submerged surfaces.

There is a single genus Caulobacter.

\section{Genus I. Caulobacter Henrici and Johnson.}

(Jour. Bact., 29, 1935, 4; ibid., 30, 1935, 83.) From Latin caulis, stalk and bacter, a small rod.

Description as for the family.

The type species is Caulobacter vibrioides Henrici and Johnson.

1. Caulobacter vibrioides Henrici and Johnson. (Jour. Bact., 30, 1935, 84.) From Latin, like a vibrio.
Cells elongated, curved, vibrio-like, with rounded ends, 0.7 to 1.2 by 2.0 to 2.5 mierons. 
Growing upon firm substrates in water. Iot cultivated on artificial media.

Habitat: Water.

Appendix: Henrici and Johnson (Jour. Bact., 30, 1935, 62) list the following as possibly belonging in this genus:

Bacillus flagellatus Omeliansky. (Jour. Microbiol. (Russian), 1, 1914, 24.) Probably the same as the organism described by Jones (Henrici and Johnson, loc. cit., 62).
Polar flagellate organism, Jones. (Cent. f. Bakt., II Abt., 14, 1905, 459.) From water and sewage.

Vibriothrix tonsillaris Tunnicliff and Jackson. (Tunnicliff and Jackson, Jour. Inf. Dis., 46, 1930, 12; see Henrici and Johnson, loc. cit., 62.) From tonsil crypts. See p. 219 for a different viewpuint regarding this species.

Six additional types are figured but not named by Henrici and Johnson (loc. cit., 84).

\section{FAMILY IV. SIDEROCAPSACEAE PRIBRAM.}

(Tribe Siderocapseae Buchanan, Jour. Bact., 2, 1915, 615; Pribram, Jour. Bact., 18, 1929, 377.)

Cells spherical or ovoid. Motile stages, if any, unknown. Not yet cultivated on artificial media. Thick capsules enclosing the cells become encrusted with ferric hydroxide. Attached to the surface of leaves and other parts of water plants.

\section{Key to the genera of family Siderocapsaceae.}

I. Cocci, occurring singly and in groups, and embedded in small irregular gelatinous masses.

Genus I. Siderocapsa, p. 833.

II. Coccobacteria, occurring in chains, and embedded in large gelatinous masses.

Genus II. Sideromonas, p. 834 .

\section{Genus I. Siderocapsa Molisch.}

(Ann. Jard. Bot. Buitenzorg, 2 Sér., Supp. 3, 1909, 29; also Die Eisenbakterien, Jena, 1910, 11.) From Greek sideros, iron and Latin capsa, box.

One to many spherical to ovoid small cells embedded in a mass of capsular material surrounded by ferric hydroxide. Best recognized by staining with Schiff's reagent. Motility unknown. Grow attached to the surface of water plants.

The type species is Siderocapsa treubii Inlisch.

1. Siderocapsa treubii Molisch. Ann. Jard. Bot. Buitenzorg, 2 Sér., Supp. 3, 1909, 29: also Die Eisenbakterien, Jena. 1910. 11.) Named for Prof. Treub, the director of the tropical garden at Buitenzorg, Java.

Coeci: 0.4 to 0.6 micron in diameter embedded in zoogloeal masses surrounded by ferric hydroxide.

Deposit ferric hydroxide on the surfaces of water plants.

Source: Found attarhed to the roots, root hairs and leaves of water plants
Elodea. Numphaea, Sugittaria, Salvinia, etc.).

Habitat: Widely distributed, on fresh water plants.

2. Siderocapsa major Molisch. (Ann. Jart. Bot. Buitenzorg, 2 Sér., Supp. 3, 1909, 29; also Die Eisenbakterien, Jena, $1910,13$.$) From Latin major, larger.$

Cells colorless, coceus-like short rods, 0.7 by 1.8 microns. A colony consists of 2 to 100 or more cells.

Similar to Siderocapsa treubii, except 
that the cells are larger and the gelatinous capsule is less sharply defined.

Source: Isolated from Spirogyra $s p$. Habitat: Epiphytic on fresh water plants.

Appendix: Two additional species have been placed in the genus Siderocapsa by later investigators:
Siderocapsa coronata Redinger. (Arch. f. Hydrobiol., 22, 1931, 410.) From lake water. A free floating form. Siderocapsa monoica Naumann. (Kungl. Svenska Vetenskapsakad. Handl., I, 62, 1921, Part 4, 49; quoted from Cholodny, Die Eisenbakterien, Jena, 1926, 59.) Found on Polamogeton natans in Sweden. Cells occur singly.

\section{Genus II. Sideromonas Cholodny.}

(Ber. d. deutsch. Bot. Ges., 40, 1922, 326; also Die Eisenbakterien, Jena, 1926, 55.) From Greek sideros, iron and monas, a unit.

Small cocci or coccobacteria which grow in chains in gelatinous masses containing ferric hydroxide attached to thread algae, generally of the genus Conferva.

The type species is Sideromonas confervarum Cholodny.

\section{Sideromonas confervarum Cho-} lodny. (Ber. d. deutsch. Bot. Ges., 40, 1922, 326; also Die Eisenbakterien, Jena, 1926, 55; Siderocystis confervarum Naumann, quoted from Dorff, Die Eisenorganismen, Pflanzenforschung, Heft 16, 1934, 13.) From Iatin, of the genus Conferva.

Coccobacteria: 0.5 to 0.6 by 0.8 to 0.9 micron, occurring in chains embedded in gelatinous masses, 10 to 100 mierons in diameter. Chains become visible when the gelatinous mass is treated with formalin followed by dilute $\mathrm{HCl}$, washed in water, and stained with gentian violet or carbol fuchsin. No motility observed.

Form deposits of ferric hydroxide in the gelatinous mass surrounding the bacteria.

Source: Found on the surface of thread algae in water containing iron salts.

Habitat: Widely distributed on fresh water green algae.

Appendix: Additional species of simple, sessile, non-filamentous bacteria which cause deposits of ferric hydroxide have been described. The majority are rodshaped bacteria and resemble Sideromonas. The list follows:

Ferribacterium calceum Brussoff.
(Brussoff, Cent. f. Bakt., II Abt., 48, 1918, 208; Sidcrobacter calceum Naumann, Kungl. Svenskia Vetenskapsakad. Handl., I, 62, Part 4, 1921, 53 and 63; Bacillus calceus De Rossi, Microbiol. Agraria e Technica, Torino, 1927, 903.) From slime in drainage ditches at Aachen.

Ferribacterium duplex Brussoff. (Brussoff, Cent. f. Bakt., II Abt., 45, 1916, 547; Sideroderma duplex Naumann, Kungl. Svenskil Vetenskapsakad. Handl., I, 62, Part t, 1921, 53 and 63; Bacterium duplex De Rossi, Microbiol. Agraria e Technica, Torino, 1927, 903.) A non-motile, diplobacterium from water samples from Breslau (Schwentniger and Pirschamer).

Naumanniclla minor Dorff. (Die Eisenorganismen, Pflanzenforschung, Heft 16, 1934, 21.) From iron-bearing spring water at Worms (Rhein).

Naumanniella neustonica Dorff. (Die Eisenorganismen, Pflanzenforschung, Heft 16, 1934, 2J.) From Neuston on Tuefelsee near Freienwalde. Dorff (loc. cit.) indicates this species as the type for a new grenus Naumanniella.

Siderobacter duplex Naumann. (Kungl. Svenski Vetenskapsakad. Handl., I, 62, Part 4, 1921, 55.) From Aneboda region, Sweden. 
Siderobacter lineare Naumann (loc. cit., 55). From Aneboda region, Sweden. The type species of the genus Siderobacter.

Siderococcus communis Dorff. (Die Eiscnorganismen, Pflanzenforschung, Heft 16, 1934, 11.) Widely distributed in Germany, Finland, Russia, Sweden, Czechoslovakia and the U. S. A.

Siderococcus limoniticus Dorff (loc. cit.). From a swamp iron ore deposit. This is the type species of the genus Siderococcus Dorff (loc. cit.).

Siderocystis duplex Naumann. (Kungl. Svenska Vetenskapsakad. Handl., I, 62, Part 4, 1921, 43.) From Aneboda region, Sweden.

Siderocystis minor Naumann (loc. cit., 43). From Aneboda region, Sweden.

Siderocystis vulgaris Naumann (loc. cit., 42). From Aneboda region, Sweden. The type species of the genus Siderocystis.

Sideroderma limneticum Naumann.
(Kungl. Svenska Vetenskapsakad. Handl., I, 62, Part 4, 1921, 32; Ochrobium tectum Perfiliev, Verh. d. Internat. Vereinigung $\mathrm{f}$. theoret. u. angew. Limnologie, 1925, Stuttgart, 1927; quoted from Dorff, Die Eisenorganismen, Pflanzenforschung, Heft 16, 1934, 18.) From Aneboda region, Sweden. The type species of the genus Sideroderma. Perfiliev regards this species as type for a new genus, Ochrobium.

Sideroderma rectangulare Naumann (loc. cit., 54). From Aneboda region, Sweden.

Sidcroderma tenue Naumann (loc. cit., 54). From Aneboda region, Sweden.

Siderothece major Naumann. (Kung!. Svenska Vetenskapsakad. Handl., I, 62, Part 4, 1921, 17.) From Aneboda region, Sweden. The type species of the genus Sidterothece.

Siderothece minor Naumann (loc. cit., 17). From Aneboda region, Sweden. 


\section{APPEXDIX TO SLBORDER CACIOBACTERINEAE}

The fumily Pastsuriacear include l among the stalket bacteria by Henrici and Johnson (boc. cit.) has been placed in this appendix as the organisms belonging to the genera Pusteuria and Blusteculis reprolues by methods of fission or budding, or both, that are different from the methods of reproduction found in other bacteria. Further information regarding the organisms in these genera is greatly needed

FAMIIY A. PASTELRTACEAE LALRENT EMEND. HENRICI AND JOHNSON.

(Laurent, Compt rend. Acad. Sci., Paris, 3, 1S90, 75t; Henrici and Johnson, Jour. Bact., 30, 1935, 84.)

Stalked bacteria with spherical or pear-shaped cells; if cells are elongated, long axis of cell coincides with axis of stalk. Stalks may be very short or absent, but when present are usually very fine and at times arranged in whorls attached to a common holdfast. Cells multiply by longitudinal fission or by budding, or by both. Mostly periphytic, one species is parasitic.

\section{Key to the genera of family Pasteuriaceae.}

I. Stalks lacking, cells sessile.

Genus I. Pasteuria, p. 836 .

II. Stalks long and slender, often in whorls.

Genus II. Blastocaulis, p. 836 .

\section{Genus I. Pasteuria Metchnikoff.}

(Ann. Inst. Past., 2, 1888, 166.) Tamed for Louis Pasteur, the French chemist and bacteriologist.

Pear-shaped cells attached to each other or to a firm substrate by holdfasts secreted at the narrow end, multiplying by longitudinal fission and by budding of spherical or ovoid cells at the free end.

The type species is Pasteuria ramosa Metchnikoff.

\section{Pasteuria ramosa Metehnikutì.} (Ann. Inst. Past., 2, 1888, 465.) From Latin ramosus, branchel.

Cells grow attached to each other in cauliflower-like masses, multiplying ly. longitudinal fission and by intracellular spores (?) which are extruded as bud-like bodies.

Habitat: Parasitic in the body cavities of Daphria pulex and Daphnia magna.

\section{Genus II. Blastocaulis Henrici and Johnson.}

(Jour. Bact., 30, 1935, 8t.) From Greek blastos, bud, germ and Latin caulis, stalk.

Pear-shaped or globular cells attached to a firm substrate by long slender stalks with a holdfast at the base. Stalks may occur singly or may arise in clusters from a common holdfast. Growing on firm substrates in fresh water. Not cultivated on artificial media.

The type species is Blastocaulis sphaerica Henrici and Johnson.

1. Blastocaulis sphaerica Henrici and Johnsou. (Jour. Bact., 30, 1935, S4.) From Latin sphaera, sphere.

('ells spherical, multiplying character. istically by bulding, often staining deeply at the free pole an 1 faintly at the attacherl pole, 1 to 2 microns in diameter. Habitat: Water 
Appendix: Henrici and Johnson (loc. cit., 84) figure but do not name four additional types of these organisms which they regard as additional species belonging to this genus.

Stanier and Van Niel (Jour. Bact., 42, $1941,454)$ regard the following as belonging to this group:

Hyphomicrobium vulgare Stutzer and Hartleb. (Saltpeterpilz, Stutzer and Hartleb, Cent. f. Bakt., II Abt., 3, 1897, 621; Stutzer and Hartleb, Untersuchungen über die bei der Bildung von Saltpeter beobachteten Mikroorganismen, I Abt. Mittheil. landwirtsch. Inst. Univ. Breslau, 1898, abst. in Cent. f. Bakt., II Abt., 5, 1899, 678.)
From tap water and soil. The position of this organism in relation to other Schizomyceles is very uncertain. It is regarded by Boltjes (Arch.f. Mikrobiol., $\tau, 1936,188$ as an organism which may he transitional between Schizomycetes and Phycomycetes. The cells possess structures which appear to be polar flagella; but with dark field illumination show an attached thread of ultramicroscopic size. Reproduction by cell division was not observed. Possibly this may be by budding from the attached thread. Associated with Vitrobacter $s p p$. This is the type species of the genus Hyphomicrobium Stutzer and Hartleb. 


\section{Suborder III. Rhodobacterineae Breed, Murray and Hitchens.*}

(Family Rhodobacteriacene Migula, Syst. d. Bakt., 2, 1900, 1042; Breed, Murray and Hitehens, Bact. Rev., 8, 1944, 257.)

Cells spherical, rod-, vibrio-, or spiral-shaped. Size of individual cells from less than 1 to over 20 microns. Motility, when exhibited, due to the presence of polar flagella. Gram-negative so far as known. No endospores formed. Red, purple, brown or green bacteria which contain bacteriochlorophyll or other chlorophyll-like green pigments, and usually also possess one or more carotenoid pigments. Capable of carrying out a photosynthetic metabolism which differs from that of green plants in that it does not proceed with the evolution of oxygen, and depends upon the presence of extraneous oxidizable compounds which are dehydrogenated with the simultaneous reduction of carbon dioxide. As oxidizable substrates a variety of simple substances can be used, such as sulfide, or other reduced sulfur compounds, molecular hydrogen, alcohols, fatty acids, hydroxy- and keto-acids, etc. All can be grown in strictly anaerobic cultures when illuminated. Those members which can grow in the presence of air can also be cultured in the dark under aerobic conditions. Color depends markedly on environmental conditions; small individuals appear colorless unless observed in masses. May contain sulfur globules. Described species have largely been found in fresh water habitats. Some species occur in marine habitats.

\section{Key to the families of suborder Rhodobacteriineae.}

I. Purple bacteria whose pigment system consists of bacteriochlorophyll and various carotenoids capable of carrying out a photosynthetic metabolism.

A. Contain sulfur globules in the presence of hydrogen sulfide. The sulfur purple bacteria.

Family I. Thiorhodaceae, p. 841.

B. Do not contain sulfur globules even in the presence of hydrogen sulfide. All require organic grow th factors. The non-sulfur purple and brown bacteria.

Family II. Athiorhodaceae, p. 861.

II. Green sulfur bacteria containing a pigment system which has the characteristics of a chlorophyllous compound although it differs from the chlorophyll of green plants and from the bacteriochlorophyll of the purple bacteria.

Family III. Chlorobacteriaceae, p. 869.

The organisms previously included in the order Thiobacteriales Buchanan do not constitute a taxonomic entity; they represent rather a physiological-ecological community. In this sense, however, a special treatment of this group as a unit has decided advantages from a determinative point of view.

When first proposed as a systematic assemblage, the order Thiobacteria Migula (Syst. (1. Bakt., 2, 1900, 1039) was intended to include the morphologically conspicuous organisms which, in their natural habitat, contain globules of sulfur as cell inclusions. Since Winogradsky (Beitr. z. Morph. u. Physiol. d. Bact., I, Schwefelbacterien, 1888) had elucidated the function of hydrogen sulfide and of sulfur in their metabolism, the characteristic inclusions appeared linked with a hitherto unrecognized type of physiology, viz. the oxidation of an inorganic substance instead of the decomposition of organic materials. From this oxidation the sulfur bacteria derive their energy for maintenance and growth.

* Completely revised by Prof. C. B. van Niel, Hopkins Marine Station, Pacific Grove, California, January, 1944. 
Two groups of sulfur bacteria could be distinguished, one consisting of colorless, the other of red or purple organisms. The members of both groups presented an unusual morphology apart from the sulfur droplets; in all cases the individual cells were considerably larger than those of the common bacteria, while many species grew as distinctive colonial aggregates. Migula separated these sulfur bacteria into two families, Beggiatoaceae and Rhodobacteriaceae. Even at that time, however, some difficulties existed as to just what organisms should properly be considered as sulfur bacteria. Miyoshi (Jour. Coll. Sci., Imp. Univ., Tokyo, 10, 1897, 143) had discovered a bacterium which forms stringers, incrusted with sulfur, in sulfur springs, but which does not store sulfur globules in its cells. Although physiologically this organism appeared to comply with Winogradsky's concept of a sulfur bacterium, the absence of the typical cell inclusions made Miyoshi decide it could not be considered as such. The problem was aggravated when Nathansohn, Beijerinck, and Jacobsen published their studies on small, colorless, Pseudomonas-like bacteria capable of oxidizing hydrogen sulfide, sulfur, and thiosulfate, and evidently dependent upon this oxidation process for their development. Morphologically these organisms have little in common with the Beggiatoaceae; they were designated by Beijerinck as species of Thiobacillus and have since been rightly considered as members of the order Eubacteriales (see p. 78). Nevertheless, these organisms are physiologically in no way different from the Beggiatoaceae, so that if physiology only is considered, a good case could be made out for their incorporation in the Thiobacteriales.

Furthermore, Molisch (Die Purpurbakterien, Jena, 1907, 95 pp.) described in some detail a number of bacterial species which, in view of their characteristic pigment system, appeared closely related to the Rhodobacteriaceae, but which develop only in organic media and are, therefore, not sulfur bacteria in the sense of Winogradsky or Migula. In stressing the importance of pigmentation Nolisch combined the red sulfur bacteria and the newly discovered purple bacteria into an order Rhodobacteria with the two families Thiorhodaceae and Athiorhodaceae. It is this grouping that has been followed in the present edition of the MANCAL.

Only a very small number of typical sulfur bacteria have been studied in pure cultures. As a result the descriptions of genera and species rest mainly on observations made with collections from natural sources or crude cultures. Most investigators have implicitly accepted differences in cell size or in colonial appearance as a sufficient justification for establishing independent species. Evidently this procedure presupposes a considerable degree of constancy of such characteristics in the organisms in question. It is true that Winogradsky's investigations have provided a reasonable basis for this belief, but later studies with pure cultures of certain purple bacteria have established beyond a doubt that environmental conditions, such as composition of the medium and temperature, may exert a profound influence on the general morphology of these organisms. By this, it is not intended to infer that the previously proposed genera and species of sulfur bacteria should be abandoned. But it does follow that a cautious evaluation of the distinguishing features is necessary. In the absence of carefully conducted investigations on morphological constancy and variability of most of the previously recognized species of sulfur bacteria with pure cultures grown under a variety of external conditions, the best approach appears to be a tentative arrangement of these organisms based upon those characteristics which are readily ascertainable. Experience with this group over the past twenty years has shown that, while Winogradsky's fundamental work must remain the foundation of present taxonomic efforts, it is advisable to simplify the much more elaborate classification developed by Buchanan which was followed in previous editions of this Mantal. 
Certain genera of sulfur purple bacteria, created by Winogradsky, will very probably be consolidated when detailed information concerning the morphology of the organisms is available. Lntil such time it seems, however, best to retain most of them, even though the distinguishing characteristics are not always very clear. For the benefit of those who are familiar with previous methods of classification it will be indicated where deviations have been adopted.

The non-sulfur purple bacteria (Athiorhodaceae Molisch; Rhodobacterioideae Buchanan) have been subjected to a comparative morphological and physiological study comprising more than 150 strains, among which all previously proposed genera and species are represented (Van Niel, Bact. Rev., 8, 1944, 1-118). It has been found that the characteristics upon which Molisch based the seven genera of this group are inadequate, and a new classification with only two distinguishable genera has been proposed. This system will be followed here.

Nadson (Bull. Jard. Imper. Bot., St. Petersburg, 12, 1912, 64) described a new type of small, green bacteria, not containing sulfur globules in the presence of hydrogen sulfide, but excreting elementary sulfur. These appear incapable of oxidizing sulfur compounds other than sulfides. They are photosynthetic and are capable of growing in anaerobic culture when illuminated. The green pigment differs from the green plant chlorophylls and from the bacteriochlorophyll of the purple bacteria, but has the characteristics of a chlorophyllous compound. These are grouped in the family Chlorobacteriaceae. 


\section{FAMIIY I. THIORHODACEAE MOLISCH.}

(Molisch, Die Purpurbakterien, Jena, 1907, 27; Subfamily Chromativideae Buchanan, Jour. Bact., 3, 1918, 464; Rhodo-Thiobacteria Bavendamm, Die farblosen und roten Schwefelbacterien, Pflanzenforschung. Heft 2, 1924, 102; Rhodothiobacteria Bavendamm, Ergeb. Biol., 13, 1936, 49.)

Unicellular organisms, often developing as cell aggregates or tamilies of variable size and shape. Single cells have the form of spheres, ovoids, short rods, vibrios, spirals, long rods or, occasionally, chains. They occur in nature in environments containing sulfides and require light for their development; infra-red irradiation of a wave-length extending to about 900 millimicrons is effective. They produce a pigment system composed of green bacteriochlorophyll, and yellow and red carotenoids. As a result they appear as pale purple, brownish to deep red cell masses. Single cells, unless they are of considerable size, usually appear to be unpigmented. These are anaerobic or microaerophilic organisms, with a photosynthetic metabolism in which carbon dioxide is reduced with the aid of special hydrogen donors without the liberation of molecular oxygen. Where these organisms are found in nature, hydrogen sulfide acts as a hydrogen donor, and sulfur. the first intermediate oxidation product, accumulates as sulfur droplets in the cells. Probably all members of the group can utilize a number of organic substances other than hydrogen sulfide as hydrogen donors for photosynthesis. Thus they are potentially mixotrophic.

Characterization of the genera in this group has since Winogradsky's studies (Beiträge zur Morphologie und Physiologie der Schwefelbacterien, Leipzig, 1888) been based upon the mode of development of the cell aggregates. Pure culturc studies (Bavendamm, Die farblosen und roten Bakterien, I. Schwefelbakterien, Pflanzenforschung, Heft 2, 1924, 74 pp.; van Niel, Arch. f. Mikrobiol., 3, 1931, 1-112; Manten, Antonie van Leeuwenhoek, 8,1942, $164 \mathrm{pp}$.) have since shown that not only the sequence of events in the formation of the aggregates but also the appearance and form of the latter even including the size and shape of the component cells are influenced to a considerable extent by environmental conditions. This obviously casts doubt upon the usefulness of the previously used diagnostic criteria for genera and species. On the other hand, the scope of pure culture studies has not yet attained sufficient breadth to warrant the use of a different approach. As a provisional measure, Winogradsky's genera are therefore maintained. Even the larger taxonomic units must be regarded as being of tentative value only.

\section{Key to the genera of the family Thiorhodaceae.}

I. Cells usually combined into aggregates.

A. Cells grouped as regular sarcina packets.

Genus I. Thiosarcina, p. 842.

B. Cells not in sarcina packets.

1. Aggregates in the form of a flat sheet.

a. Cells in regular arrangement, with tetrads as the common structural unit.

Genus II. Thiopedia, p. \$43.

aa. Cells in irregular aggregates.

Genus III. Thiocapsa, p. 844. 
2. Aggregates in the form of three-dimensional masses.

a. Cells distinctly rod-shaped, and arranged in a net-like structure. Genus IV. Thiodictyon, p. 815.

aa. Cells not so arranged.

b. Cells in a common capsule, individuals rather scattered and loosely grouped.

Genus V. Thiothece, p. 846.

bł. Cells in rather dense clumps.

c. Iggregates embedded in conspicuous common slime capsule.

1. Aggregates small, compact, often several of them enclosed together in a common capsule.

Genus VI. Thiocystis, p. 846 .

did. Aggregates large and solid, later break up into small chusters.

Genus VII. Lamprocystis, p. 847.

ce. ('ommon capsule lacking or very transient.

1. Aggregates as a whole exhibit amoeboid movements.

Genus VIII. Imocbobacter, p. 848.

d.. Agregates devoid of amoeboid movements.

Genus IX. Thiopolycoccus, p. 850 .

II. Cells usually oceurring singly.

A. Cells clearly spiral-shaped.

B. Cells not spiral-shaped.

Genus 1. Thiospirillum, 1. 850 .

1. Cells irregular, often swollen, distorted, or composed of long, crooked and bent rods to filaments.

Genus XI. Rhabdomonas, p. 853 .

2. Cells regular, spherical to short rods or bean-shaped.

a. Cells spherical, as a rule non-motile, and each one surrounded by a rather wide capsule.

Genus XII. Rhodothece, p. 855 .

aa. Cells cllipsoidil, ovoid, short rods or vibrios, actively motile.

Cienus XIII. Chromatium, p. 856 .

\section{Genus 1 . Thiosarcina Winogradsky.}

(Winogradsky, Zur Morphologie und Physiologie der Bacterien, I. Schwefelbacterien, Leipzig, 1888, 104; Rhodosurcina Orla-Jensen, Cent. f. Bakt., II Abt., 22, 1909, 344; Rhodothiosarcine Ellis, Sulphur Bacteria, London and New York, 1932, 163.) From Greek theion, sulfur and Latin sarcina, bundle, packet.

Individual cells spherical, forming regular cubical packets of sarcina-shape, resulting from consecutive division in three perpendicular planes. Packets commonly containing s to bt cells. Notility infrequent. Non-spore-forming. Contain bacteriochlorophyll and carotcnoid pigments, hence, pigmented purplish to red. Capable of carrying out a photosynthetic metabolism in the presence of hydrogen sulfide, cells then store sulfur globules. Anaerobic.

The type species is Thiosarcina rosea (Schroeter) Winogradsky.

1. Thiosarcina rosea (Schroeter) Win ogradsky. CSarrime rosed Sehroeter, liryptog. Flore von thhlesion, 3, 1, 1s\$6, 15t; Sarcina sulphurala Winogradsky, Bot. Ztg., 45, 1857, 576; Winogradsky, Zur Morphologie und Physiologio. 
der Schwefelbacterien, Leipzig, 1888 , 104; Rhodothiosarcina rosea Ellis, Sul phur Bacteria, London and New lork, 1932, 163.) From Latin roseus, rosecolored.

Cells spherical, 2 to 3 microns in diameter, occurring in packets containing 8 to 64 cells. Infrequent motility. Color ranging from purplish-rose to nearly black.
Anaerobic

Habitat: Mud and stagnant bodies of water containing hydrogen sulfide and exposed to light; sulfur springs.

Distribution: Probably ubiquitous. One of the less frequent among the purple sulfur bacteria.

Illustration: Issatchenko, Recherches sur les microbes de l'océan glacial aretique, Petrograd, 1914, Plate II, fig. 5.

\section{Genus II. Thiopedia Winogradsky.}

(Zur Morphologie und Physiologie der Bacterien, I. Schwefelbacterien, Leipzig, 1888, 85.) From Greek theion sulfur and pedion, plane.

Individual cells spherical to short rod-shaped, the latter shortly before cell division. Arranged in flat sheets with typical tetrads as the structural units. These arise from divisions of the cells in two perpendicular directions. Cell aggregates of various sizes, ranging from single tetrads to large sheets composed of thousands of cells. Motility infrequent. Non-spore-forming. Contain bacteriochlorophyll and carotenoid pigments. Capable of photosynthesis in the presence of hydrogen sulfide, and then storing sulfur globules. Anaerobic.

The type species is Thiopedia rosea Winogradsky.

\section{Thiopedia rosea Winogradsky} (Erythroconis littoralis Oerstedt, Nit turhist. Tidskrift, 3, 1810-18t1, 555; Merismopedia littoralis Rabenhorst, Flora Europaea Algarum, Leipzig, 2, 1865, 57; Winogradsky, Zur Morphologie und Physiologie der Schwefelbacterien, Leipzig, 1888, 85; Pediococcus roseus Trevisan, I generi e le specie delle Batteriacee, Milan, 1889, 28; Lampropedia rosea De'Toni and Trevisan, in Saccardo, Sylloge Fungorum, 8, 1889, 1049; Planococcus roseus Migula, in Engler and Prantl, Die natürlichen Pflanzenfamilien, 1, 1a, 1895, 19.) From Latin roseus, rose-colored.

Size: 1 to 2 microns, often appearing as slightly elongated cocei regularly arranged in platelets. Color, pale red to nearly black, depending upon the amount of sulfur stored. Red color visible only with large cell masses, not in individuals.

Aceorling to Winogradsky, the cells are often embedded in a common slime capsule; the extensive studies of Utermöhl (Archiv f. Hydrobiol., Suppl. Vol.
$5,1925,251-276)$ make the regular occurrence of such eapsules extremely doubtful. On the other hand, Itermöhl emphasizes as quite characteristic the (ommon presence of a relatively large pseudoracuole, or aerosome, in the cells of this species encountered in plankton samples. Winogradsky does not mention this; nevertheless, it appears to be a regular and valuable distinguishing feature.

Anaerobic.

Habitat: Mud and stagnant bodies of fresh, brackish and salt water containing hydrogen sulfide and exposed to light; sulfur springs.

Distribution: L'biquitous. C'ommon, frequently giving rise to very extensive mass developments.

Illustrations: Warming, Videnskab. Meddel. naturhist. Forening, Kjöbenhavn, 1876, Plate VIII, fig. 2; Winogradsky, loc. cil., Plate III, fig. 18; Pringsheim, Naturwissensch., $20,1932,481$, the last one a truly excellent photomicrograph. 
Appendix: The following genus was formerly placed near Thiopedia. Winogradsky, Migula, E. F. Smith and others disregard this genus. A record is included here because of its historic interest.

Genus A. Lampropedia Schroeter.

(Schroeter, in Cohn, Kryptog. Flora v. Schlesien, 3, 1, 18\$6, 151.) From Greek lampros, bright and pedion, plane.

Trevisan (I generi e le specie delle Batteriacee, 1889, 28) and DeToni and Trevisan (in Saccardo, Sylloge Fungorum, 8, 1889, 1048) list as synonyms: Erythroconis Oersted, Naturhistorisk Tidsskrift, 2, 15t0, 55̃̃ in part, Pediococcus Lindner, Inaug. Diss., Berlin, 1857, 97; Thiopedia Winogradsky, Schwefelbacterien, Leipzig, 1888, 85.

Cells united into tetrads, forming flat, tubular masses, contain sulfur globules and bacteriochlorophyll and yellow and red carotenoids.

The type species is Lampropedia hyolina (Ehrenberg) Schroeter.

1. Lampiopedia hyalina (Ehrenberg Schroeter. Gonium hyalinum Ehren berg, Abhandl. d. Berl. Akat., 1830; Merismoperlia hyalina Kützing, Species Algarum, 1819; Saciua hyulina Winter, in Rabenhorst, Kryptogamen-Flora, 2 Aufl., 1, 1884, 51; Schroeter, in Cohn. Kryptogram. Flora v. Schlesien, 3, 1. 1886, 151; Pedincoccus hyalinus Trevisan, I generi e le specie delle Batteriacee, 1889, 28; Mieroroceus hyalimus Migula, Syst. d. Bakt., 2, 1900, 195.) From swamp water and decomposing materials from sugar refineries.

2. Lampropediu reitenbachii (C"aspary) DeToni and Trevisan. (Merismopedium. reitenbachii Caspary, Schriften d. phy sik. ökon. Gesellsch. zu Königsterg, 15, 1874,104 ; Sarcina reitenbachii Winter, in Rabenhorst, Kryptogamen-Flora, 2 Aufl., 1, 1854, 50; Pediocoerse reichenbachii (sic) Trevisan, I generi a le specic delle Batteriaces. 1889, 28; DeToni and
Trevisan, in Siceardo, Sylloge Fungorum, 8, 1889, 1048.) Found on rotting water-plints.

3. Lampropertia violacea (Breb.) DeToni and Trevisan. (Agmencllum violaceum Brob., quoted from DeToni and Trevisan, in Sitcardo, Sylloge Fungorum, 8, 1ss9, 1049; Merismopedia violacea liützing, Species Algarum, 1849, 472; Pediococrus vinlaceus Trevisan, I generi a le sperie delle Batteriacee, 1889, 28; DeToni and Trevisan, loc. cit., 1048.) From stagnant water. Common.

4. Lampropedia achracea (Mettenheimer) DeToni and Trevisan. (Merismopedia ochracea Mettenheimer, Abhandil. d. senkemberg. naturforsch. Gesellsch. in Frankf., 2, 1856-58, 41; DeToni and Trevisan, in Saccardo, Sylloge Fungorum, 8, 18s9, 1049.) From the yellowish slime from a well at Frankfurt

\section{Genus III. Thiocapsa Winogradsky.}

(Schwefelbacterien, Leipzig, 1885, 84.) From (treek theion, sulfur and Latin capsa, container, capsule.

Cells spherical, occurring in families of irregularly arranged individuals held together in a common slime capsule. The aggregates are spread out flat on the substrate. Motility not observed. As the colony grows, the capsule bursts, and the cells are spread apart. General morphology and development thus appears similar to that in the genus A phanocapsa among the blue-green algae. Contain bacteriochlorophyll and carotenoid pigments; capable of photosynthesis in the presence of hydrogen sulfide. Lnder such conditions sulfur is stored in the form of globules in 
the cells. This genus is so much like Thiothece that it is doubtful whether a distinction can be maintained.

The type species is Thiocapsa roseopersicina Winogradsky.

\section{Key to the species of genus Thiocapsa.}

I. Individual cells about 3 microns in diameter.

1. Thiocapsa roseopersicina.

II. Individual cells about 1.5 microns in diameter.

2. Thiocapsa floridana.

1. Thiocapsa roseopersicina Winogradsky. (Schwefelbacterien. Leipzig, 1885. 84.) From Latin roseus, rose-colored and persicum, pearh; MIL., peachrolored.

Cells: Spherical, 2.5 to 3 microns in diameter, occurring in families of irregularly arranged individuals held together in a common slime capsule. Motility not observed. Esually a distinct rosered. Stored sulfur droplets may attain a considerable size.

Habitat: Mul and stagnant bodies of water containing hydrogen sulfile and exposed to light; sulfur springs.

Illustration: Winograrksy, loc. cit. Plate IV, fig. 15 .

2. Thiocapsa floridana Uphof. (Uphof,
Arch. f. Hydrobiol., 18, 1927, 84; Thiocapsa minima Issatchenko, Borodin Juhilee Volume, p. 6, 1929?.) From the locality, Florida, where the organism was first found.

Cells: Spherical. About 1.5 microns in diameter. In groups of irregular colonies, each surrounded by a common capsule, several colonies being stuck together. Motility not observed.

Source: Palm Springs, Florida and Lake Sakskoje, near Eupatoria, Crimea.

Habitat: Mud and stagnant water containing hydrogen sulfide and exposed to light; sulfur springs. Probably ubiquitous.

Illustration: Uphof, loc. cit., 83, fig. VI.

\section{Genus $I V$. Thiodictyon Winogradsky.}

(Winogradsky, Schwefelbacterien, Leipzig, 1888, 80; Rhododictyon Orla-Jensen, Cent. f. Bakt., II Abt., 22, 1909, 334.) From Greek theion, sulfur and dictyon, net.

Cells rod-shaped, frequently with pointed ends, somewhat resembling spindles. Form aggregates in which the cells become arranged end to end in a net-like structure, somewhat reminiscent of the shape of the green alga $H$ ydrodictyon. The shape is not constant; cells may also form more compact masses. Sometimes groups of cells separate from the main aggregate by active movements. Common gelatinous capsule not observed. Contain bacteriochlorophyll and carotenoid pigments; cells usually very faintly colored. Capable of photosynthesis in the presence of hydrogen sulfide; the cells then store sulfur as small globules.

The type species is Thiodictyon elegans Winogradsky.

1. Thiodictyon elegans Winogradsky. Winogradsky, Schwefelbacterien, Leipzig, 1888, 80; Thiodictyon minus Issatchenko, Recherches sur les microbes de. l'océan glacial arctique, Petrograd, 1914, 251.) From Latin elegans, tasteful, elegant.

Rods: 1.5 to 1.7 by 2.5 to 5 mierons; or longer just prior to cell division. Usually contain a large pseudovacuole (aerosome), leaving a rather thin protoplasmic sheath along the cell wall.

Sulfur droplets: Generally quite small, and deposited exclusively in the thin protoplasmic layer. 
Issatchenko (Études microbiologiques des Lacs de Boue, Leningrad, 1927, 113114) recognizes a forma minus and a forma magna, differentiated mainly by the size of the individual rods.
Habitat: Mud and stagnant water, containing hydrogen sulfide, and exposed to light; sulfur springs.

Illustrations: Winogradsky, loc. cit., Plate III, fig. 13-17.

\section{Genus V. Thiothece Winogradsky.}

(Winogradsky, Schwefelbacterien, Leipzig, 1888, 82; Thiosphaera Miyoshi, Jour. Coll. Sci., Imp. Univ. Tokyo, Japan, 10, 1897, 170.) From Greek thcion, sulfur and theke, container.

Purple sulfur bacteria which, in their growth characteristics, resemble the bluegreen alga Aphanothece. Cells spherical to relatively long cylindrical-ellipsoidal, embedded in a gelatinous capsule of considerable dimensions. Following cell division the daughter cells continue to secrete mucus which causes the individual bacteria to remain clearly separated by an appreciable distance; the common capsule thus appears only loosely filled. The cells may become actively motile and separate themselves from the colony. Such swarmers closely resemble the cells of certain species of Chromatium. Contain bacteriochlorophyll and carotenoid pigments. Capable of photosynthesis in the presence of hydrogen sulfide, producing elementary sulfur as an intermediate oxidation product which is stored as sulfur globules inside the cells.

The type species is Thiothece gelatinosa Winogradsky.

1. Thiothece gelatinosa Winogradsky. (Winogradsky, Schwefelbacterien, Leipzig, 1888, 82; Thiosphaera gelatinosa Miyoshi, Jour. Coll. Sci., Imp. Univ. Tokyo, Japan, 10, 1897, 170; Lamprocystis gelatinosa Migula, Syst. d. Bakt., 2, 1900, 1044; Chromatium sphneroides Hama, Jour. Sci. Hiroshima Univ., Ser. B, Div. 2, Bot., 1, 1933, 158.) From Latin gelatio, freezing, indicating solidification or clumping.

Cells: 4 to 6 by 4 to 7 microns, spherical to cylindrical. Color of individual cells, faint, often grayish-violet, or even dirty yellowish. Sulfur globules usually deposited in outermost layers of protoplasm, and generally small.

Habitat: Mud and stagnant water containing hydrogen sulfide and exposed to light; sulfur springs.

Illustrations: Winogradsky, loc. cit., Pl. III, fig. 9-12; Miyoshi, loc. cit., Pl. XIV, fig. 25.

\section{Genus VI. Thiocystis Winogradsky.}

(Schwefelbacterien, Leipzig, 1888, 60.) From Greek theion, sulfur and kystis, sac, bladder.

Purple sulfur bacteria which form compact colonies, many of which may be loosely embedded in a common gelatinous capsule. Individual cells spherical to ovoid, often diplococcus-shaped. Colonies may emerge as more or less large units from out of the common capsule and break up afterwards, sometimes into single swarmers; or the aggregates may split up inside the original capsule, and release small motile units or single swarmers. In pure cultures frequently developing as single cells and diplococci. Produce bacteriochlorophyll and carotenoid pigments, coloring the cell masses purplish to red. Capable of photosynthesis in the presence of hydrogen sulfide, whereby elementary sulfur is formed as an intermediate oxidation product which is deposited as droplets inside the cells. 
The type species is Thiocystis violacea Winogradsky.

\section{Key to the species of genus Thiocystis.}

I. Individual cells more than 2 microns in width.

1. Thiocystis violacea.

II. Individual cells about 1 micron or less in width.

2. Thiocystis rufa.

1. Thiocystis violacea Winogradsky. (Winogradsky, Schwefelbacterien, Leipzig, 1888, 65; Planosarcina violacea Migula, in Engler and Prantl, Die natürl. Pflanzenfamilien, 1, 1a, 1895, 20.) From Latin violaceus, violet-colored.

Cells: About 2.5 to 5.5 microns in diameter, spherical to ovoid. Swarmers actively motile by means of polar flagella.

Colonies: Small, inside a common capsule, containing not over 30 cells. Several such colonies form loosely arranged aggregates, most characteristically composed of about 10 to 20 colonies in a single capsule. The result is a nearly spherical zoogloea. In small colonies, the cells appear as rather distinct tetrads; in larger colonies, the cells become somewhat compressed and the tetrad-like arrangement may be lost.

In pure cultures, the species often fails to produce the characteristic capsules; the organisms then occur as actively motile single cells or diplococei, with little or no slime formation. No pseudo. capsules are formed.

Habitat: Mud and stagnant water containing hydrogen sulfide and exposed to light; sulfur springs.

Illustrations: Zopf, Zur Morphologie der Spaltpflanzen, Leipzig, 1882, Pl. V, fig. 12; Winogradsky, loc. cit., Pl. II, Fig. 1-7.

2. Thiocystis rufa Winogradsky. (Schwefelbacterien, Leipzig, 1888, 65.) From Latin rufus, red.

Cells: Less than 1 micron in diameter. Color red, usually darker than in the type species. When the cells are stuffed with sulfur globules, the aggregates appear almost black.

The common gelatinous capsule usually contains a far greater number of closely packed individual colonies than is the case in Thiocystis violacea.

Habitat: Mud and stagnant water containing hydrogen sulfide and exposed to light; sulfur springs.

Illustration: Winogradsky, loc. cit., Pl. II, fig. S.

\section{Genus VII. Lamprocystis Schroeter.}

(In part, Clathrocystis Cohn, Beitr. Biol. Pfl., 1, Heft 3, 1875, 156; in part, Cohnia Winter, in Rabenhorst, Kryptogamen-Flora, 2 Aufl., 1884, 48; Schroeter, Die Pilze Schlesiens, in Cohn, Kryptogamen-Flora von Schlesien, 3, 1, 1886, 151; Cenomesia? de Toni and Trevisan, in Saccardo, Sylloge Fungorum, 8, 1889, 1039; Lankasteron Ellis, Sulphur Bacteria, London and New York, 1932, 135.) From Greek lampros, bright, shining, and kystis, sac or bladder.

Purple sulfur bacteria which form more or less large aggregates of cells enclosed in a common gelatinous capsule. Individual cells spherical to ovoid. Small aggregates closely resemble those of Thiocystis, even to the extent of the tetrad-like arrangement of cells in the small colonies. Behavior of the large aggregates during development appears to be different; the small individual cell groups or colonies do not emerge from the slime capsule until the initially relatively compact cell mass becomes broken up into smaller clusters, these eventually forming a somewhat net- 
like structure. This behavior has been ascribed to a change in the mode of cell division which at first appears to take place in three perpendicular planes, and later presumably changes to a division in only two directions. Cells when free are motile by means of polar flagella. In pure culture also this type rarely, if ever, produces large aggregates with the development here mentioned as characteristic for the genus (Bavendamm, Die farblosen und roten Schwefelbakterien, Pflanzenforschung, Heft 2, 1924, 76). This, along with the other similarities, makes it doubtful whether future studies will result in the retention of the genera Lamprocystis and Thiocystis side by side. Produce bacteriochlorophyll and carotenoid pigments, coloring the cell masses purplish-pink to red. Capable of photosynthesis in the presence of hydrogen sulfide, storing elementary sulfur as globules inside the cells.

The type species is Lamprocystis roseopersicina (Kützing) Schroeter.

1. Lamprocystis roseopersicina (Küłzing) Schroeter. (Hicrolou rosea Kützing, Linnaea, 8, 1833, 371; Cryptococcus roseus Kützing, Phycologia generalis, Leipzig, 1843,149 ; Pritococcus roseopersicinus Iiützing, Species $\mathrm{Algarum}$, Leipzig, 1849, 196; Palmollu persicino Cohn, Leonhard's Jarhb. f. Mineralog. 1864, 606; Pleurnescus roseo-persicinus Rabenhorst, Flora Lur. Algarum, Leipzig, 3, 1868, 28; Bacterium rubescens Lankester, Quart. Rev. Micro. Sci. 13, 1573, 408; not Bacterium rubescens Chester, Ann. Rept. Del. Col. Agr. Exp. Sta., 9, 1897, 115; Clathrocystis roseopersicina Cohn, Beitr. Biol. Pfl., 1, Heft $3,1875,157$; Cohnia roseo-persicina Winter, in Rabenhorst, Kryptogamen Flora 2 Aufl., 1, 1884, 48; Schroeter, in Cohn, Kryptogamen-Flora von Schlesien, 3, 1, 1886, 151; Planosarcina roseo-persicin Migula, in Engler and Prantl, Die na türlichen Pflanzenfam., 1, 1a, 1895, 20; Lankasteron rubescens Ellis, Sulphur Bacteria, London and New York, 1932,
135.) From Latin roseus, rose-colored and persicum, peach; M.L., peach-colored.

In all probability, Thioderma rubrum Miyoshi (Jour. Coll. Sci., Imp. Univ. Tokyo, Japan, 10, 1897, 170) is identical with this species.

Cells: Spherical to ovoid, 2 to $2.5 \mathrm{mi}$ rrons in diameter, up to 5 microns long hefore rell division. Motile. Polar flagellate.

Winogralsky (loc, cit.) reports that the rells frequently contain pseudovacuoles.

Habitat: Mud and stagnant water containing hydrogen sulfide and exposed to light; sulfur springs.

Illustrations: Warming, Videnskab. Meddel. naturhistor. Foren., Kjöbenhavn, 1876 , Pl. VIlI, fig. $3 \mathrm{~g}$; Zopf, $Z$. Morphol. d. Spaltpflanzen, Leipzig, 1882, Pl. V, fig. 8, 13; Winogradsky, Schwefelbacterien, Leipzig, 1885, Pl. II, fig. 9-15; Bavendamm, Die farblosen und roten Schwefelbakterien, Jena, 192t, Pl. II, fig. 3.

\section{Genus VIII. Amoebobacter Winogradsky.}

(Winogradsky, Schwefelbacterien, Leipzig, 1888, 71; Amoebomonas Orla-Jensen, Cent. f. Bakt., II Abt., 22, 1909, 334.) From amoeba, one of the protozoa characterized by a constantly changing shape, and Greek baktron, rod.

Purple sulfur bacteria, usually occurring in aggregates composed of many individuals without a characteristic common capsule. Slime formation can, nevertheless, be observed with very small colonies. With growth of the individual cells, the capsule bursts and the cell mass slowly moves out while the bacteria remain united. The colonies change their shape during growth and in response to environmental influences; the individual cells appear motile and cause the movements of the entire 
colony. Winogradsky ascribes the coherence of the cell masses to the existence of interconnecting protoplasmic filaments between cells, but these have never been observed, and their occurrence is extremely doubtful. It is much more probable that the bacteria are held together by mucus, though not so much of the latter is produced as to form a clearly discernible capsule.

Produce bacteriochlorophyll and carotenoid pigments. Capable of photosynthesis in the presence of hydrogen sulfide, and then store sulfur as droplets inside the cells.

The type species is Amoebobacter roseus Winogradsky.

Since the characterization of the genera Amocbobacter, Lamprocystis, Thiocystis, Thiocapsa and Thiothece is based upon the arrangement of individual bacteria in a common capsule, which, from Winogradsky's descriptions of Amoebobacter and from pure culture studies with Thiocystis and Lamprocystis, has been shown to vary considerably, depending upon developmental stages and environmental conditions, it is quite possible that future investigations will show the desirability of restricting the number of genera.

\section{Key to the species of genus Amoebobacter.}

I. Cells spherical to ovoid, about 2.5 to 3.5 microns in diameter and up to 6 microns in length prior to cell division.

1. Amoebobacter roseus.

II. Cells distinctly rod-shaped, about 1.5 to 2 microns in width by 2 to 4 microns in length.

2. Amoebobacter bacillosus.

III. Cells spherical, quite small, about 0.5 to 1 micron in diameter.

3. Amoebobacter granula.

1. Amoebobacter roseus Winogradsky. (Schwefelbacterien, Leipzig, 1888, 77.) From Latin roseus, rose.

Cells spherical to ovoid, 2.5 to 3.5 microns in width and up to 6 microns in length. Notile. Often contain pseudovacuoles. Cell-aggregates often form transitory hollow spheres or sacks, with the bacteria occupying the periphery as a shallow layer. These are reminiscent of stages in the development of Lamprocystis.

Habitat: Mud and stagnant water containing hydrogen sulfide and exposed to light; sulfur springs.

Illustrations: Winogradsky, loc. cit., PI. III, fig. 1-6.

\section{Amoebobacter bacillosus Winograd-} sky. (Winogradsky, Schwefelbacterien, Ieipzig, 1888, 78; Thioderma roseum Miyoshi, Jour. Coll. Sei., Imp. Univ.
Tokyo, Japan, 10, 1897, 158.) From Latin bacillus, resembling a rod.

Cells rod-shaped, about 1.5 to $2 \mathrm{mi}$ crons by 2 to 4 microns. Cells contain pseudovacuoles (aerosomes). Sulfur globules deposited exclusively in peripheral protoplasmic layer, usually quite small.

Habitat: Mud and stagnant water, containing hydrogen sulfide and exposed to light; sulfur springs.

Illustrations: Zopf, Z. Norphol. 1. Spaltpfl., Leipzig, 1882, Pl. V', fig. 26-27; Winogradsky, loc. cit., Pl. III, fig. 7 .

Miyoshi's incomplete description of Thioderma roseum (loc. cit.), type species of genus Thioderma, is sufficient to make practically certain that it is identical with Amoebobacter bacillosus. The description of Thiodictyon clggans Winogradsky (loc cit.) suggests that it cannot the distinguished from this speeies. 
3. Amoebobacter granula Winogradsky. (Schwefelbacterien, Leipzig, 1888, 78.) From Latin granulus, a granule.

Cells: Spherical, small, about 0.5 to 1 micron in diameter. Faint pigmentation; the sulfur inclusions give the cell masses a black appearance. Aggregates are apt to consist of closely-knit masses which are difficult to separate.

When sulfur is stored, a single droplet usually fills most of the cell. Because of the high refractive index of this globule, it becomes difficult if not impossible to make accurate observations of the cell shape.

Habitat: Mud and stagnant water containing hydrogen sulfide and exposed to light; sulfur springs.

Illustration: Winogradsky, loc. cit., Pl. III, fig. 8.

\section{Genus IX. Thiopolycoccus Winogradsky.}

(Winogradsky, Schwefelbacterien, Leipzig, 1888, 79; Rhodopolycoccus OrlaJensen, Cent. f. Bakt., II Abt., 22, 1909, 334.) From Greek theion, sulfur; polys, many; and kokkos, granule or small cell.

Purple sulfur bacteria which form dense aggregates of rather solid construction and irregular shape. The colonies appear, in contrast with Amoebobacter, non-motile and do not tend to form hollow zoogloeal structures by which they are differentiated from Lamprocystis. Cell masses held together by mucus which does not, however, appear as a regular capsule. Large clumps may fissure with the formation of irregular shreds and lobes which continue to break up into smaller groups of cells. Individual bacteria spherical, motility not observed. Contain bacteriochlorophyll and carotenoid pigments, so that the aggregates, in accord with the dense packing with individual cells, appear distinctly red. Capable of photosynthesis in the presence of hydrogen sulfide, when the cells store elementary sulfur as droplets inside the cells.

The type species is Thiopolycocess ruber Winogradsky.

1. Thiopolycoccus ruber Winogradsky (Winogradsky, Schwefelbacterien, Leipzig, 1888, 79; Micrococcus ruber Migulit, in Engler and Prantl, Die natürlichen Pflanzenfamilien, 1, 1a, 1895, 18.) From Latin ruber, red.

Cells: Spherical, about 1.2 microns in diameter. No motility observed.
Habitat: Mud and stagnant water containing hydrogen sulfide and exposed to light; sulfur springs.

Illustrations: Winogradsky, loc. cit., Pl. IV, fig. 16-18; Issatchenko, Recherches sur les microbes de l'océan glacial arctique, Petrograd, 1914, Pl. II, fig. 7 .

\section{Genus $X$. Thiospirillum Winogradsky.}

(Ophidomonas Ehrenberg, I)ie Infusionstierchen, Leipzig, 1838, 43; Winogradsky, Schwefelbacterien, Leipzig, 1888, 10t; Thiorhodospirillum Fuhrmann, Vorlesungen ü. techn. Mykologie, Jena, 1913, 325; Rhodothiospirillum Bavendamm, Schwefelbakterien, Jena, 1924, 115.) From Greek theion, sulfur, and diminutive of spira, screw.

Purple sulfur bacteria, occurring singly, as spirally wound cells, motile by means of polar flagella. Contain bacteriochlorophyll and carotenoid pigments, coloring the cells brownish-to purplish-red. Capable of photosynthesis in the presence of hydrogen sulfide, during which they produce and store, as an intermediate oxidation product, elementary sulfur in the form of droplets inside the cells.

The differentiation of species in this group has been based exclusively on observa- 
tions with material from natural collections and from laboratory mass cultures. The criteria used are the size and shape of the spirals, and the color of the organisms. Not a single representative has so far been obtained and studied in pure culture, so that no information is available concerning the constancy or variability of these characteristics. It is, however, likely that such properties may be greatly influenced by environmental factors. Hence, the following key and descriptions of species are apt to be modified when more extensive studies have been made. The published descriptions of some species make it seem probable that they should not even be incorporated in Thiospirillum.

The type species is Thiospirillum jenense (Ehrenberg) Winogradsky.

\section{Key to the species of genus Thiospirillum.}

I. Width of cells 2.5 microns or more.

1. Color of cells, especially in masses, yellowish-brown to orange-brown.

1. Thiospirillum jenense.

2. Color of cells deep red or violet.

a. Cells long, typical spirals; clearly red.

2. Thiospirillum sanguineum.

aa. Cells short, slightly curved, vibrio-shaped; color purple to violet-red.

3. Thiospirillum violaceum.

II. Width of cells less than 2.5 microns.

1. Width of cells 1.5 to 2.5 microns.

4. Thiospirillum rosenbergii.

2. Width of cells about 1 micron.

5. Thiospirillum rufum.

1. Thiospirillum jenense Ehrenberg Winogradsky. (Ophidomonas jenensis Ehrenberg, Die Infusionstierchen, Leipzig, 1838, 44; Spirillum jenense Trevisan, Batter. ital., 1879, 26; Winogradsky, Schwefelbacterien, Leipzig. 1888, 104; Rhodothiospirillum jenense Ellis, Sulphur Bacteria, London and New York, 1932, 161; Thiospirillum crassum Hama, Jour. Sci. Hiroshima Univ., Ser. B, Div. 2, Bot., 1, 1933, 157.) Named for the city of Jena, Germany, where Ehrenberg discovered this organism.

Cells: Cylindrical, sometimes pointed at ends, 2.5 to 4 mierons long, coiled as spirals. Generally 30 to 40 microns in length but may be as long as 100 microns. Shape of individual coils varies, complete turns measuring about 15 to 40 microns in length, and from $\frac{1}{2}$ to $1 / 10$ of the width in height. Polar flagellate. Tufted at both ends. Olive-brown, sepia-brown and reddish-brown
This coloring appears to be the only recognizable difference from Thiospirillum sanguinoum. Thiospirillum crassum Hama (loc cil.) reported to be 3.7 to 4 by 12 to 40 microns and yellowishbrown in color, thus becomes indistinguishable from Thios pirillum jenense; the 80 mierons long Thiospirillum jenense forma maxima Szafer (Bull. Acad. Sci. Cracovie, Sér. B, 1910, 162) does not at present justify recognition as a special taxonomic entity.

It is even doubtful whether the observed color difference between Thiospirillum jenense and Thiospirillum sanguineum constitutes a valid criterion for their maintenance as two distinet species (Buder, Jahrb. wiss. Bot., 56, 1915, 53t; Bavendamm, Die farblosen und roten Schwefelbakterien, Pflanzenforschung, Heft 2, 1924, 131).

Habitat: Mud and stagnant water containing hydrogen sulfide and exposed to light; more rarely in sulfur springs. 
Illustrations: Zettnow, Ztschr. f. Hyg., 24, 1897, Pl. II, fig. 49-52; Buder, lor cit., fig. 1; Szafer, loc. cit., Pl. IV, fig. 4; Hama, loc. cit., Pl. 18, fig. 1, 8a; Pl. 19, fig. 1.

2. Thiospirillum sanguineum (Ehrenberg) Winogradsky. (Ophidomonas sanguinea Ehrenberg, Verhandl. Akad. Wiss. Berlin, 1840, 201; Spirillum sanguineum. Cohn, Beitr. Biol. Pf., 1, Heft 3, 1875, 169; Winogradsky, Schwefelbacterien, Leipzig, 1888, 104.) From Latin sanguineus, blood-colored, red.

Cells: Cylindrical, sometimes attenuated at ends, spirally coiled; 2.5 to 4.0 microns in width, commonly about 40 microns long with a range of from 10 to 100 microns. Size and shape of coils variable, complete turns measuring from 15 to 40 microns in length and from $\frac{1}{2}$ to $1 / 10$ of the length in width. Polar flagellate, usually tufted at both ends. Individual cells rose-red with a grayish hue, groups of cells deep red. Sulfur droplets numerous under appropriate conditions.

Habitat: Mud and stagnant water containing hydrogen sulfide and exposed to light; rarely in sulfur springs.

Illustrations: Cohn, loc. cit., Pl. VI, fig. 15; Warming, Vidensk. Meddel, naturhist. Foren., Kjöbenhavn, 1876, Pl. VII, fig. 8; Buder, Jahrb. wiss. Bot., $56,1915,534$, fig. 2 .

3. Thiospirillum violaceum (Warming) Winogradsky. (Spirillum violaceum Warming, Vidensk. Meddel. naturhist. Foren., Kjöbenhavn, 1876, 395; Winogradsky, Schwefelbacterien, Leipzig, 1888, 104.) From Latin violaceus, violetcolored.

Cells: Short and fat, 3 to 4 by 8 to 10 microns, ends smoothly rounded. Slightly curver, bean- or vibrio-shaped. Only rarely are they twisted suggesting a spirillum. Polarly flagellated.

The shape of cell seems to fit the genus Chromatium rather than Thiospirillum and Warming (loce cil.) emphasizes the resemblance to Chromatium okenii.

Color bluish-violet; this color may be related to a scarcity of sulfur droplets in the cells.

Habitat: Mud and stagnant water.

Illustration: Warming, loc. cit., Pl. VII, fig. 3 .

\section{Thiospirillum rosenbergii (Warm-} ing) Winogradsky. (Spirillum rosenbergii Warming, Vidensk. Meddel, naturhist. Foren., Kjöbenhavn, 1876, 346; Winogradsky, Schwefelbacterien, Leipzig, 1888, 104.) Named for the Danish algologist, Rosenberg.

Cells: 1.5 to 2.5 by 4 to 12 microns; coiled, with turns of about 6 to $7.5 \mathrm{mi}$ crons in length and variable width up to 3 or 4 microns. Color very dark, due to numerous sulfur globules. Color of protoplasm not recorded.

Habitat: Mud and stagnant water containing hydrogen sulfide and exposed to light.

Distribution: Probably ubiquitous, but less frequently recorded as the organism is not as spectacular as the large Thiospirillum jonense and Thiospirillum sanguineum.

Illustration: Warming, loc. cit., Pl. I, fig. 12 .

5. Thiospirillum rufum (Perty) Migula. (Spirillum rufum Perty, Bern, 1852, 179; Migula, Syst. d. Bakt. 2, 1900, 1050.) From Latin rufus, red, reddish.

General characteristics presumably those of the genus, although it does not appear either from Perty's description, or from those of Migula (loc. cit.), Bavendamm (Die farblosen und roten Schwefelbakterien Jena, 1924, 132) and HuberPestalozzi (Die Binnengewässer, 16, Heft 1, Das Phytoplankton des Süsswassers, Stuttgart, 1938, 304) that the cells ever contain sulfur globules. Only the red color is emphasized. Consequently, it is quite possible that this 
organism belongs in the genus Rhordospirillum .

Cells: 1.0 by 8 to 18 microns; coiled to oceupy $1 \frac{1}{2}$ to $t$ turns, the lat ter commonly 4 microns wide by 4 microns long. These dimensions agree with those of Rhodospirillum rubrum (Esmarch) Molisch and the identity of the two organisms is probable.

Habitat: Found in red slime spots on the side of a well. Mud and stagnant bodies of water.

Illustration: Migula, Syst. d. Bakt., 1, 1897, Pl. III, fig. 7.

Appendix: Three species have been placed in the genus Thiospirillum without convincing evidence that they conform to the generic diagnosis.

Thiospirillum agilis Kolkwitz. (Kolkwitz, Kryptogamenflora d. Mark Brandenburg, 5, Pilze, 1909, 162; Thiospira agilis Bavendamm, Die farblosen und roten Schwefelbakterien, Pflanzenforschung, Heft 2, 1924, 116.) This is not known to have been a purple bacterium and hence may represent a member of the genus Thiospira.

Thiospirillum agilis var. polonica Strzeszewski. (Bull. Acad. Sci., Cracovie, Sér. B, 1913,322.) This also may belong in the genus Thiospira.

Thiospirillum pistiense Czurda. (Cent. f. Bakt., II Abt., 92, 1935, 409.) Not described as pigmented and does not contain sulfur globules. Reported to be a probable agent in the production of hydrogen sulfide from sulfates or sulfur. It may therefore be the spirillar form of Vibrio desulfuricans Beijerinck or, being thermophilie, of Vibrio thermodesulfuricans Elion.

Thiospirillum winogradstii Omeliansky. (Cent. f. Bakt., II Abt., 14, 1905, 764.) This is colorless and is included in Thiospira.

\section{Genus XI. Rhabdomonas Cohn:}

(Cohn, Beitr. Biol. Pfl., 1, Heft 3, 1875, 167; Mantegazzaea Trevisan, R. Inst. Lombardo de Sci. e Lett., IV, Ser. 2, 12, 1879, 137; Rhabdochromatium Winogradsky, Schwefelbacterien, Leipzig, 1888, 100; in part, Rhodocapsa Molisch, Die Purpurbakterien, Jena, 1907, 17.) From Greek rhabdos, a rod, and monas, a unit (cell).

Purple sulfur bacteria, as a rule occurring singly, in the form of rather irregular, long rods to filaments, exhibiting more or less pronounced swellings, or club and spindle shapes. Filamentous structures sometimes with constrictions giving the filament the appearance of a string of beads. These may be surrounded by a relatively inconspicuous slime capsule which can be rendered visible by India ink. The less distorted cell types are frequently motile, flagella polar. Produce bacteriochlorophyll and carotenoid pigments, coloring the cells pinkish- to purplish-red. Capable of photosynthesis in the presence of hydrogen sulfide and then storing sulfur globules as an intermediate oxidation product inside the cells.

The status of this genus is doubtful. Winogradsky (loc. cit.) recognized the similarity of its members to species of Chromatium and the occurrence of many intermediate forms which make a sharp distinction between the two genera impossible He preferred the designation of Rhabdochromatium as a sub-genus. Warming (Videnskab. Meddel. naturhist. Foren., Kjöbenhavn, 1876, 320 ff.), Nadson (Bull. Jard. Impér. Bot. St. Pétersb., 3, 1903, 116), van Niel (Arch. f. Mikrobiol., 3, 1931, 61), and Ellis (Sulphur Bacteria, London and New York, 1932, 151) considered the species of Rhabdochromatium as abnormal growth forms (involution forms) of corresponding species of Chromatium, while Lauterborn (Verhandl, naturhistor.-medizin. Vereins, Heidelberg, N.F., 13, 1915, 424), Buder (Jahrb. wiss. Bot., 58, 1919, 534) and Baven- 
damm (Die farblosen und roten Schwefelbakterien, Pflanzenforschung, Heft 2, 1924, 129) favor generic rank.

The type species is Rhabdomonas roseus Cohn.

\section{Key to the species of genus Rhabdomonas.}

I. Cells not containing calcium carbonate inclusions in addition to sulfur globules.

a. Cells more than 3 microns in width.

aa. Cells less than 3 microns in width.

1. Rhabdomonas rosea.

2. Rhabdomonas gracilis.

II. Cells containing calcium carbonate inclusions in addition to sulfur globules.

3. Rhabdomonas linsbaueri.

1. Rhabdomonas rosea Cohn. (Cohn, Beitr. Biol. Pfl., 1, Heft 3, 1875, 167; Beggiatoa roseo-persicina ' $/ \mathrm{opf}, \mathrm{K}$. Morphol. d. Spaltpflanzen, Leipzig, 1882, 30; Rhabdochromatium roseum Winogradsky, Schwefelbacterien, Leipzig, 1888, 100; Rhabdochromatium fusiforme Winogradsky, ibid., 102; Pseudomonas rosea Migula, in Engler and Prantl, Die natürlichen Pflanzenfam., 1, 1a, 1895, 30.) From Latin roseus, rose-colored.

Cells: Uneven in width and length, often swollen to spindle-shaped, sometimes tending towards filamentous growth. The greatest width of a spindle-shaped or fusiform cell may be close to 10 microns; in the more filamentous structures it is usually around 5 microns. The length varies between 10 and 30 microns for single cells; filamentous forms, frequently showing bulges and constrictions suggestive of compound structures in which cell division has been incomplete, may attain considerably greater lengths, up to 100 microns. The ends of spindle-shaped cells often taper to very fine points or attenuated fibers; also filaments are generally thinner toward the extremities. Single individuals and short filaments are motile by means of polar flagella, long filaments rarely motile. The ends of a filament may become pinched off and swim away.

Color rose-red; cells are usually filled with sulfur globules.

There is no good reason for maintain- ing Rhabdomonas fusiformis (Rhabdochromatium fusiforme Winogradsky) as a separate species; the variations in size and shape bring this form well within the range of Rhabdomonas rosea. Present indications strongly suggest that the latter species should be regarded as a peculiar developmental form of Chromatium okenii.

Habitat: Mud and stagnant water containing hydrogen sulfide and exposed to light; sulfur springs.

Illustrations: Cohn, loc, cit., Pl.VI, fig. 14; Warming, Vidensk. Meddel. naturhistor. Foren., Kjöbenhavn, 1876, Pl. VII, fig. 1c-e; Kopf, loc. cit., Pl. V, fig. 2b; Winogradsky, loc. cit., Pl. IV, fig. 9-11, 13-14.

\section{Rhabdomonas gracilis (Warming)} Migula. (Wonas gracilis Warming, Vidensk. Medrlel. naturhist. Foren., Kjöbenhavn, 1876, 331; Rhabdochromatium minus Winogradsky, Schwefelbacterien, Leipzig, 1888, 102; Rhabdochromatium gracile Migula, Syst. d. Bakt., 2, 1900, 1049; Rhodocapsa suspensa Moliseh, Die Purpurbakterien, Jena, 1907, 17; Rhabdomonas minor Bergey et al., Manual, 3rd ed., 1930, 532.) From Latin gracilis, slender.

Cells: Much smaller than those of Rhabdomonas rosea, and with less tendency to form fusiform cells. Usually filamentous, more or less cylindrical, often with constrictions, but found up 
to 60 microns in length. Shorter filaments motile. Polar flagellate. Slime formation may occur under special conditions. Rose-red. Sulfur globules. Probably an abnormal growth form of Chromatium virosum.

Habitat: Mud and stagnant water containing hydrogen sulfide and exposed to light; sulfur springs.

Illustrations: Warming, loc. cit., PI. VII, fig. 5; Winogradsky, loc. cit., Pl. IV, fig. 12; Molisch, loc cit., PI. II, fig. 11-12.

\section{Rhabdomonas linsbaueri (Gickl-} horn) comb. not. (Rhubdochromatium linsbaueri Girklhorn, Ber. d. deut bot. Ges., 39, 1921, 312.) Named for the botanist, $\mathrm{K}$. Linsbauer.

Cells: Resemble Rhabdomonas rosea, irregular, rod-shaped, 3 to 5 microns wide, up to 39 microns in length.

The characteristic feature of the species, and the chief means of differentiation, is the occurrence of ealcium carbonate inclusions in addition to the sulfur globules in the cells. Whether this is strictly an environmentally conditioned characteristic, due to the photosynthetic development of the bacteria in a medium rich in calcium ions, so that calcium carbonate is precipitated as the alkalinity increases, has not yet been established, but seems possible. In that case the identity of this species with Rhabdmonas rosea would become evident.

Source: From a pond near Graz, Austria.

Habitat: Fresh water.

\section{Genus XII. Rhodothece Molisch.}

(Die Purpurbakterien, Jena, 1907, 19.) From Greek rhodon, rose and theke, container, capsule.

Purple sulfur bacteria, occurring singly, not aggregated in families. Cells spherical, each surrounded by a rather wide capsule which is, however, rarely visible without special staining. Motility not observed. Contain bacteriochlorophyll and carotenoid pigments, coloring the cells reddish. Capable of photosynthesis in the presence of hydrogen sulfide; the cells then store sulfur globules, arising as an intermediate oxidation product of the sulfide.

In view of the experiences of Bavendamm and others that a number of representatives of the purple sulfur bacteria, characterized by typical colonial aggregates when found in nature, may develop as single cells in pure culture, it is quite conceivable that the genus Rhodothece is synonymous with some other genus, e.g., Lamprocystis, and that the two genera represent different growth forms induced by environmental conditions.

The type species is Rhodothece pendens Molisch.

1. Rhodothece pendens Molisch. (Die Purpurbakterien, Jena, 1907, 19.) From Latin pendeo, to be suspended.

Cells: Spherical, frequently occurring as diplococei, occasionally as very short chains or clumps of 3 to 5 individuals. 1.8 to 2.5 microns in diameter. Produce rather abundant slime. Cells embedded in individual capsules which are rarely visible without staining (India ink). Characteristic is the regular occurrence of pseudovacuoles (aerosomes) which are supposed to keep the cells suspended in liquid media. Refractive phenomena due to the pseudovacuoles and to the sulfur globules distort the cell shape under ordinary illumination so that bacteria appear as polygons rather than round cells. Isually 2 aerosomes and 2 sulfur globules per cell. Color not observable in individual bacteria. Cell groups are rose-red. Motility not observed.

Habitat: Mud and stagnant water con- 
taining hydrogen sulfide and exposed to light. Not reported from sulfur springs.
Illustrations: Moliseh, Die Purpurbakterien, Jena, 1907, Pl. II, fig. 13-14.

\section{Genus XIII. Chromatium Perty.}

(Perty, Zur Kenntniss kleinster Lebensformen, Bern, 1852, 174; Rhodomonas OrlaJensen, Cent. f. Bakt., II Abt., 22, 1909, 334.) From Greek chroma, color.

Cells occur singly, more or less ovoid, bean- or vibrio-shaped, or short rods. The last-mentioned are often thick-cylindrical with rounded ends. Motile by means of polar flagella. Contain bacteriochlorophyll and carotenoid pigments, coloring the cells various shades of red. Capable of photosynthesis in the presence of hydrogen sulfide and storing elementary sulfur as an incomplete oxidation product in the form of globules inside the cells.

At present, the genus contains 11 deseribed species and one variety. In addition, two more purple sulfur bacteria, Pseudomonas molischii Bersa (Planta, 2, 1926, 375) and Thiospirillum coccineum Hama (Jour. Sci. Hiroshima Univ., Ser. B, Div. 2, Bot., 1, 1933, 158), have been incorporated here as species of Chromatium because the descriptions and illustrations furnished by the original authors leave no doubt as to their taxonomic affiliations.

Differentiation of species has, in the past, been based almost entirely upon size and shape of individual cells, often with complete disregard for the variability of these criteria. The unsatisfactory and arbitrary nature of such a classification has occasionally been pointed out, and with much justification. Winogradsky (Schwefelbacterien, Leipzig, 1888, 98) mentions the many transitional stages that can be observed between Chromatium okenii and Chromatium weissei; Strzeszewski (Bullet. Acad. Sci., Cracovie, Sér. B, 1913, 321) holds that it is impossible to distinguish, on the basis of sizes or otherwise, between Chromatium weissei and Chromatium minus. Such contentions, derived from observations on material from natural collections or crude cultures, have been greatly strengthened by studies with pure cultures of species of Chromatium. Thus van Niel (Arch. f. Mikrobiol., 3, 1931, 59) reported variations in width from 1 to 4 microns, and in length from 2 to 10 microns or even up to 50 microns; Manten (Antonie van Leeuwenhoek, 8, 1942, $164 \mathrm{ff}$.) found size differences of 1 to 14 microns with a pure culture of an organism that he identified as Chromatium okenii. Often the differences in size of a pure culture can be related to special environmental conditions. On account of such results a designation of species on the basis of size relations alone is manifestly unsatisfactory. Moreover, the available data do not suggest that differences in shape, color or arrangement of sulfur globules can be used more effectively. Lack of adequate experimental results with a sufficiently large number and variety of pure cultures prevents a more rational classification at present.

The previously proposed species have been listed below with their respective characteristics and arranged as far as possible in the order of decreasing width.

Two Chromatium species have been deseribed as containing inclusions of calcium carbonate in addition to sulfur globules. Is in the case of Rhabdomonas linsbaueri, it is not known whether this feature may be a direct consequence of the calcium ion content and $\mathrm{pH}$ of the environment, and thus fail to have taxonomic significance.

The type species is Chromatium okenii Perty.

1. Chromatium gobii Issatchenko. (Rerherehes sur les microbes de l'oréan glarial aretique, Petrograd, 191 to 253.) Named for Prof. X. Cinhi. 
Cells : 10 microns by 20 to 25 microns. Source: From sea water of Arctic Ocean.

Habitat: Presumably ubiquitous in the colder portions of the Ocean at least.

Illustration: Issatchenko, loc. cit., Pl. II, fig. 12 .

2. Chromatium warmingii (Cohn) Migula. (Monas warmingii Cohn, Beitr. Biol. Pf., 1, Heft 3, 1875, 167; Migula, Syst. d. Bakt., 2, 1900, 1048.) Named for the Danish botanist, Eugene Warming.

Cells: 8 by 15 to 20 microns, also smaller (Cohn).

Illustration: Cohn, loc. cit., PI. VI, fig. 11.

2b. Chromatium warmingiiforma minus Bavendamm. (Die farblosen und roten Schwefelbakterien, Pflanzenforschung, Heft 2, 1924, 127.) Named for the Danish botanist, Eugene Warming.

Cells: \pm by 6 to 10 microns.

Illustrations: Bavendamm, loc. cit., 91, fig. 7, and Pl. II, fig. 12, a-b.

3. Chromatium linsbaueri Gicklhorn. (Ber. d. deut. botan. Ges., 39, 1921, 312.) Named for the Austrian botanist, K. Linsbauer.

Cells: 6 by up to 15 microns (Gicklhorn); 6 to 8 mierons in width (Ellis. Sulphur Bacteria, London and Tew Tork, 1932, 147). Special characteristic is the occurrence of calcium carbonate inclusions. Otherwise resembles Chromatium okenii.

Source: From a pool in the Stiftingtal, near Graz, Austria.

Habitat: Fresh water.

Illustrations: Gicklhorn, loc. cit., 31t, fig. 1; Ellis, loc. cit., 148, fig. 31.

\section{Chromatium okenii (Ehrenberg)} Perty. (Monas okenii Ehrenberg, Infusionsthierchen, Leipzig, 1838; Perty, Zur Kenntniss kleinster Lebensformen,
Bern, 1852, 174; Bacillus okenii Trevisan, I generi e le specie delle Batteriacee, 1889, 18; Bacterium okenii DeToni and Trevisan, in Saccardo, Sylloge Fungorum, 8, 1889, 1027; Pseudomonas okenii Migula, in Engler and Prantl, Die nat ürlichen Pflanzenfamilien, 1, 1a, 1895, 30.) Named for the German naturalist, $L$. Oken. This is the type species of genus Chromatium.

Cells: 5.6 to 6.3 by 7.5 to 15 microns (Cohn); minimum width 4.5 microns (Issatchenko, Borodin Jubilee Vol., 1929?, 8); with many transitions to Chromatium weissei (Winogradsky, Schwefelbacterien, Leipzig, 1888, 92). Also: 3.5 by 8 to 12 microns and varying in size from 1 to 15 microns (Manten, Antonie van Leeuwenhoek, 8, 1942, 16t).

Illustrations: Cohn, Beitr. Biol. Pfl., 1, Heft 3, 1875, Pl. VI, fig. 12; Winogradsky, loc. cit., Pl. IV, fig. 3-4; Issatchenko, Recherches sur les microbes de l'océan glacial aretique, Petrograd, 1914, Pl. II, fig. 9.

5. Chromatium weissei Perty. (Perty, Zur Kenntniss kleinster Lebensformen, Bern, 1852, 174; Bacillus weissii Trevisan, I generi e le specie delle Batteriacee, 1889, 18; Bacterium weissii DeToni and Trevisan, in Saccardo, Sylloge Fungorum, 8, 1889, 1027.) Named for the zoologist, J. F. Weisse, consequently the more common spellings, Chromatium weissii or $C$. weisii are in error.

Cells: 4.2 by 5.7 to 11.5 microns (Perty); also 3 to 4 by 7 to 9 microns (Issatchenko, Borodin Jubilee Volume, 1929?, 8); transitions to Chromatium okenii (Winogradsky, Schwefelbacterien, Leipzig, 1888, 92); transitions to Chromatium minus (Strzeszewski, Bull. Acad. Sci., Cracovie, Sér. B. 1913, 321).

Illustrations: Winogradsky, loc. cit., Pl. IV, fig. 1-2; Miyoshi, Jour. Coll. Sci., Imp. Univ. Tokyo, Japan, 10, 1897, Pl. XIV, fig. 15. 


\section{Chromatium cuculliferum Gickl-} horn. (Cent. f. Bakt., II Abt., 50, 1920, 419.) From Latin cucullus, eap or hood and fero, to bear.

Cells: 4 by 6 to 8 microns (Gicklhorn); according to Bavendamm (Sehwefelbakterien, Jena, 1924, 127) identical with Chromatium warmingii forma minus. Gicklhorn elaims this organism to be colorless, which appears very doubtful.

Source: From the pond in the Annen Castle Park, Graz, Austria.

Habitat: Fresh water ponds.

Illustration: Gicklhorn, loc. cit., fig. 2 .

7. Chromatium minus Winogradsky. (Winogradsky, Schwefelbacterien, Leipzig, 1888, 99; Bacillus minor Trevisan, I generi e le specie delle Batteriacee, 1889, 18; Bacterium minus DeToni and Trevisan, in Saccardo, Sylloge Fungorum, 8, 1889, 1027.) From Latin mimus, small.

Cells : 3 by 3.5 to 7 microns (Winogradsky); also 1.7 to 3 microns in width and up to 8.5 mierons in length (Issat chenko, Borodin Jubilee Volume, 1929?, 9); all transitions to Chromatium weissei from which it cannot be distinguished (Strzeszewski, Bull. Acul. Sci., ('racovie, Sér. B, 1913, 321).

Illustrations: Winogradsky, loc. cit., Pl. IV, fig. 5; Miyoshi, Jour. Coll. Sei., Imp. Univ., Tokyo, Japan, 10, 1897, Pl. XIV, fig. 16; Issatehenko, Recherches sur les microbes de l'océan glacial arctique, Petrograd, 1914, Pl. II, fig. 10-11.

\section{Chromatium vinosum (Ehrenberg)} Winogradsky. (Monas vinosa Ehrenberg, Die Infusionsticrehen, Leipzig, 1838, 11; Winogradsky, Schwefelbacterien, Leipzig, 18s8, 99; Bacillus vinosus Trevisan, I generi e le specie delle Batteriacee, 1889, 18; Bacterium vinosum DeToni and Trevisan, in Saceardo, Sylloge Fungorum, 8, 1889, 1027.) From Latin vinosus, pertaining to wine, wine. colored.
Cells: 2 by 2.5 to 5 microns; also 1.4 to 3 by 1.5 to 5 microns (Jimbo, Botan. Magaz. Tokyo, 51, 1937, 872); 1.7 to 2 by 2 to 9 microns (Issatchenko, Borodin Jubilee Volume, 1929?, 9); or 1 to $1.3 \mathrm{mi}$ crons by 2.5 to 3 microns (Schrammeck, Beitr. Biol. d. Pflanzen, 22, 1935, 317). Jimbo considers Thioderma roseum Miyoshi to be identical with Chromatium vinosum.

Illustrations: Winogradsky, loc. cit., PI. IV, 6-7; Miyoshi, Jour. Coll. Sci., Imp. Univ. Tokyo, Japan, 10, 1897, Pl. XIV, fig. 17; Nadson, Bull. Jard. Imp. Botan., St. Pétersbourg, 12, 1912, Pl. III, fig. 1-2.

9. Chromatium violaceum Perty. (Zur Kenntniss kleinster Lebensformen, Bern, 1852, 174.) From Latin violaceus, violet-colored.

Cells: About 2 by 2 to 3 microns. According to Cohn (Beitr. Biol. Pfl., 1, Heft $3,1875,166$ ) probably identical with Chromatium vinosum. Apparently includes various sizes.

10. Chromatium molischii (Bersa) comb.nov. (Pseudomonas molischii Bersa, Planta, 2, 1926, 375.) Named for the Austrian botanist, H. Molisch.

Cells: About 2 by 2.5 to 8 microns. Supposedly contains calcium carbonate ats inclusions.

Illustration: Bersa, loc. cit., 376, fig. 3 .

11. Chromatium gracile Strzeszewski. (Bull. Acad. Sci., Cracovie, Sér. B, 1913, 321.) From Latin gracilis, slender.

('ells: 1 to 1.3 by 2 to 6 microns; also to 1.5 micron in width (Issatchenko, Ftudes microbiologiques des Lacs de B oue, Leningrad, 1927, 114).

Illustration: Strzeszewski, loc. cit., P1. XXXIX, fig. 1-2; Tokuda, Botan. Magaz., Tokyo, 50, 1936, 339, fig. 1-23.

12. Chromatium minutissimum Winogradsky. (Winogradsky, Schwefelbac- 
terien, Leipzig, 1888, 100; Bacillus minutissimus Trevisan, I generi e le specie delle Batteriacee, 1889, 18; Bacterium minutissimum DeToni and Trevisan, in Saccardo, Sylloge Fungorum, 8, 1889, 1028.) From Latin minutus and diminutive, very minute.

Cells: About 1 to 1.2 micron by $2 \mathrm{mi}$ crons. Also from 0.5 to 0.7 micron by 0.6 to 1 micron (Issatchenko, Recherches sur les microbes de l'océan glacial aretique, Petrograd, 1914, 253); and 1 to 3 microns by 2 to 5 mierons (Issatchenko, Borodin Jubilee Volume, 1929?, 9).

Illustrations: Winogradsky, loc cit. Pl. IV', fig. S; Miyoshi, Jour. Coll. Sci.,
Imp. Univ., Tokyo, Japan, 10, 1897, Pl. XIV, fig. 18.

Appendix: The measurements for Thiospirillum coccineum Hama (Jour. Sei. Hiroshima Univ., Ser. B, Div. 2, Bot., 1, $1933,158)$ which, according to description and figures (ibid., Pl. 18, fig. 2; Pl. 19, fig. 2), is an unquestionable species of Chromatium, are given as 2 by 4 to 15 microns. It thus closely resembles the bacteria of the Chromatium minus, C. vinosum, C. violaceum, and C. molischii group.

Chromatium sphaeroides Hama, loc. cit. Thiospirillum violaceum (Warming) Winogradsky is probably also a member of this assemblage.

\section{APPENDIX TO FAMILY THIORHODACAE.}

Three genera of sulfur purple bacteria have been proposed whose place and nature are at present very doubtful. They follow here:

a. Thiosphaerion Miyoshi, with the single species Thiosphaerion violaceum Miyoshi (Jour. Coll. Sei., Imp. Univ., Tokyo, Japan, 10, 1879, 170). Occurs in round colonies in which numerous bacteria are held together by mucus, though not in a clearly discernible common capsule. Individual cells ovoid, about 1.5 to 2 by 2.5 microns; motile. Resembles Lamprocystis roseopersicina in many respects. Reported once from Yumoto Hot Springs, near Nikko, Japan.

Illustrations: Miyoshi, loc. cit., Pl. XIV, fig. 24 a-b.

b. Pelochromatium Lauterborn, with the single species Pelochromatium roseum Lauterborn (Verhandl, naturhist. medizin. Vereins, Heidelberg, N.F. 13, 1915, 424). Forms small colonies in which the bacteria are regularly arranged in about 5 rows, from 2 to 4 cells high, around a colorless central body. The entire colony actively motile and behaves like a single unit. Individual cells bean-or vibrio-shaped, about $1 \mathrm{mi}$ cron or less by 2 microns; the harrel shaped colony measures 2.5 to + by + to 8 microns. The structure may represent a complex of a colorless central bacterium surrounded by purple bacteria, analogous to Chlorochromatium aggregatum Lauterborn. Whether such structures have generic or even specific taxonomic significance remains to be determined. The lack of information concerning the occurrence of sulfur globules in the cells makes it doubtful whether the organisms are sulfur purple bacteria at all. Found twice by Lauterborn in mud samples.

Illustrations: Lauterborn, loc. cit., $\mathrm{Pl}$. III, fig. 28, a-c.

Utermöhl suggested the name Lauterborniola minima Utermöhl (Biol. Zentralbl., 43, 1924, 605) for the small brownish bacteria which form the covering of the central body of Pelochromatium roseum; according to this author the central body is a larger bacterium, 1.5 by 7 microns which he named Endosoma palleum.

c. Thioporphyra Ellis, with the single species Thioporphyia rolutans Ellis (Jour. Roy. Technic. Coll. Glasgow, 
1926, 165). The account of this pleomorphic organism, which is claimed to multiply by fission, budding, and probably spore formation, is wholly unconvincing. The shape and size of some of the cells make it appear likely that Ellis observed mixtures of various kinds of purple sulfur bacteria.

Illustrations: Ellis, loc. cit., 166, fig. 1-14; 171, Micro. I; 172, Micro. II; Sulphur Bacteria, London and New York, $1932 ; 153$, fig. 33 ; 154, fig. $34 ; 156$, fig. $35 ; 158$, fig. 36 .

Finally, there exist some, as yet unnamed, red to purple bacteria which contain bacteriochlorophyll and carotenoid pigments, are capable of photosynthesis in the presence of hydrogen sulfide, but excrete elementary sulfur as an intermediate oxidation product instead of storing sulfur globules inside their cells (van Niel, Arch. f. Mikrobiol., 3, 1931, 63 ). They are small motile rods, vibrios or spirilla, about 0.5 by 1 to 2 microns. They may also occur as spherical cells of about 1 micron in diameter. They can readily be grown in organic media, under anaerobic conditions, in illuminated cultures and may be included either with the sulfur purple bacteria or with the non-sulfur purple bacteria, among which Rhodopseudomonas palustris is equally capable of photosynthesis in the presence of reduced inorganice sul fur compounds. 


\section{FAMILY II. ATHIORHODACEAE MOLISCH.*}

(Molisch, Die Purpurbakterien, Jena, 1907, 28; Rhodobacterioideae Buchanan, Jour. Bact., 3, 1918, 471; Athiorhodobacteria Bavendamm, Ergeb. Biol., 13, 1936, 49.)

Unicellular bacteria, of relatively small size, occurring as spheres, short rods, vibrios, long rods and spirals. Motility is due to the presence of polar flagella. Gram-negative. They produce a pigment system composed of bacteriochlorophyll and one or more carotenoids, coloring the cells yellowish-brown, olive brown, dark brown or various shades of red. Color usually not observable with single cells but only with cell masses. Generally microaerophilic, although many representatives may grow at full atmospheric oxygen tension. Capable of development under strictly anaerobic conditions, but only in illuminated cultures by virtue of a photosynthetic metabolism. The latter is dependent upon the presence of extraneous hydrogen donors, such as alcohols, fatty acids, hydroxy-and keto-acids, and does not proceed with the evolution of molecular oxygen. Those members which can grow in the presence of air can also be cultivated in darkness, but only under aerobic conditions.

\section{Key to the genera of family A thiorhodaceae.}

I. Cells rod-shaped or spherical, not spiral-shaped.

II. Cells spiral-shaped.

Genus I. Rhodopseudomonas, p. 861.

Genus II. Rhodospirillum, p. 866 .

Genus I. Rhodopseudomonas Kluyver and van Niel emend. van Niel.

(Includes Rhodobacillus Molisch, Die Purpurbakterien, Jena, 1907, 14; Rhodobacterium Molisch, ibid., 16; Rhodococcus Molisch, ibid., 20; Rhodovibrio Molisch, ibid., 21 ; Rhodocystis Molisch, ibid., 22; Rhodonostoc Molisch, ibid., 23; Rhodosphaera Buchanan, Jour. Bact., 3, 1918, 472; Rhodorrhagus Bergey et al., Manual, 3rd ed., 1930, 535; Rhodomonas Kluyver and van Niel, Cent. f. Bakt., II Abt., 94, 1936, 397; not Rhodomonas Orla-Jensen, Cent. f. Bakt.. II Abt., 22, 1909, 331; Kluyver and van Niel, in Czurda and Maresch, Arch. f. Mikrobiol., 8, 1937, 119; van Niel, Bact. Rev., 8, 1944, 86.) From Greek rhodon, red and pseudomonas, false unit.

Spherical and rod-shaped bacteria, motile by means of polar flagella. Gramnegative. Contain bacteriochlorophyll which enables them to carry out a photosynthetic metabolism. The latter is dependent upon the presence of extraneous oxidizable substances and proceeds without the evolution of molecular oxygen. Though some members can oxidize inorganic substrates, none appears to be strictly autotrophic, due to the need for special organic growth factors. Produce accessory pigments causing the cultures, especially when kept in light, to appear in various shades of brownish-yellow to deep red.

The genus includes the members of Molisch's genera Rhodobucterium, Rhodobacillus, Rhodovibrio, Rhodocystis, Rhodonostoc and Rhodococcus, as well as the genera Rhodosphaera Buchanan, Rhodorrhagus Bergey et al. and Rhodomonas Kluyver and van Niel.

The type species is Rhodopseudomonas palustris (Molisch) van Niel.

* Completely revised by Prof. C. B. van Niel, Hopkins Marine Station, Pacific Grove, California, January, 1944. 
Keys to the species of genus Rhodopseudomonas.

I. Based upon morphological characters.

1. Cells clearly rod-shaped in all media.

a. Cells short, somewhat curved, to long branched rods, size of young and short cells 0.6 to 0.8 by 1.2 to 2 microns; in older cultures up to $10 \mathrm{mi}$ crons long; do not form slime; liquid cultures, when young, or after shaking, evenly turbid. Color red to dark brown-red.

1. Rhodopseudomonas palustris.

aa. Cells slender rods, 0.5 by 1.2 microns usually clumped together in extensive slime masses. Cultures pale brown to peach-colored.

2. Rhodopseudomonas gelatinosa.

2. Cells more or less spherical in media at $\mathrm{pH}$ below 7 .

a. In media at $\mathrm{pH}$ about 7 elearly rod-shaped, 1 by 1 to 2.5 microns. Chains of cells frequent, and in characteristic zigzag arrangement.

3. Rhodopseudomonas capsulatus.

a. In media at $\mathrm{pH}$ above 7 cells still predominantly spherical, 0.7 to $4 \mathrm{mi}$ crons in diameter. Mostly single, little tendency to chain formation.

4. Rhodopseudomonas spheroides.

II. Based chiefly on physiological properties.

1. Gelatin liquefied.

2. Gelatin not liquefied.

2. Rhodopseudomonas gelatinosa.

a. Does not produce mucus in media at $\mathrm{pH}$ above 8 . Color the same under aerobic and anacrobic conditions of growth.

1. Rhodopseudomonas palustris.

aa. Produce mucus in media at pH above 8 . Color brown in anaerobic, red in aerobic culture.

b. Develops readily in media with 0.2 per cent propionate as the chief oxidation substrate. Mucus production marked at $\mathrm{pH}$ above 8 , but very limited between 7 and 8 .

3. Rhodopseudomonas capsulatus.

bb. Does not develop in media with 0.2 per cent propionate as the main oxidation substrate. Slime formation extensive at $\mathrm{pH}$ above 7 .

4. Rhodopscudomonas spheroides.

III. Based principally upon biochemical characters.

1. Thiosulfate used as main oxidation substrate

1. Rhodopseudomonas palustris.

2. Thiosulfate not used.

a. Propionate $(0.2$ per cent) used.

3. Rhodopseudomonas capsulatus.

aa. Propionate not used.

b. Mannitol and sorbitol (0.2 per cent) used.

4. Rhodopseudomonas spheroides.

bb. Mannitol and sorbitol not used.

2. Rhodopseudomonas gelatinosa. 
1. Rhodopseudomonas palustris (MI)lisch) van Niel. (Rhodobacillus palustris Molisch, Rhodobacterium capsulatum Molisch and Rhodovibrio parvus Molisch, Die Purpurbakterien, Jena, 1907, 14, 18 and 21; Rhodomonas palustris Kluyver and van Niel, Cent.f. Bakt., II Abt., 94 , 1936, 397; Rhodopscudomonas No. 9 and No. 16, Czurda and Maresch, Arch. f. Mikrobiol., 8, 1937, 120; van Niel, Bact. Rev., 8, 1944, 89.) From Latin paluster, boggy, marshy.

Cells: Usually distinctly rod-shaped, though in young cultures very short. lightly curved rods may often predominate. Size variable, even for the same strain, and strongly influenced by age of culture and composition of medium. Rather consistently short cells in young cultures in yeast extract, especially when incubated anaerobically in the light, or in anaerobic cultures with substrates which permit only a slow and scanty development, such as malonate. Dimensions in such cultures 0.6 to 0.8 by 1.2 to 2 microns. More often, especially in older cultures, rells are much longer, up to 10 mierons. Highly characteristic is the pronounced tendency to the formation of irregularly shaped, bent and crooked long rods, occasionally swollen at one or both extremities, and frequently suggesting branching. Such cells usually form rlusters reminiscent of Corynebacterium and Mycobacterium cultures.

Cells in young cultures actively motile by means of polar flagella; irregular and long cells as a rule non-motile. Gramnegative.

Growth in liquid media never mucoid; sediment in older cultures homogeneous and smooth, readily redispersible.

Color: Varies considerably, depending upon the medium, and especially in anaerobic illuminated cultures. Where development is slight (as in malonate, thiosulfate, and, usually, glycerol media), the color is a light pink; in fatty acid-containing media more nearly dark reddish-brown. Color due to bacterio- chlorophyll and a number of different carotenoid pigments; most strains produce in addition a water-soluble, noncarotenoid, bluish-red pigment which diffuses into the culture medium.

In yeast extract cultures growth is possible over the range $\mathrm{pH} 6$ to 8.5. With certain substrates, especially fatty acids, the combined effect of low $\mathrm{pH}$ and a substrate concentration of 0.1 to 0.2 per cent may prevent growth. No characteristic odors save that old cultures may develop a distinct ionone-like fragrance. Gelatin is not liquefied; leucine is generally utilized as a substrate.

Most strains are able to grow on the surface of agar plates or slants; a few, especially when first isolated, appear more sensitive to oxygen and develop only in stabs in which the upper region may remain free of growth. Generally such strains can be adapted to grow at full atmospheric oxygen tension.

Most fatty acids and hydroxy acids are adequate oxidation substrates. All cultures can grow at the expense of thiosulfate and produce rapid and profuse growth in glutarate and ethanol media. No development in media containing as the chief oxidation substrate 0.2 per cent sorbitol, glucose or mannose, even though these substances are not inhibitory. Molecular hydrogen can be oxidized

All cultures can develop anaerobically in illuminated cultures by photosynthesis.

Temperature optimum generally rather high, good development being possible up to $37^{\circ} \mathrm{C}$. However, certain strains exhibit a lower temperature optimum.

Distinguishing characteristies: Morphological resemblance to species of Mycobacterium in old cultures; ability to grow with thiosulfate as the chief oxidizable substrate, and failure to develop in media which contain carbohydrates or sugar alcohols in a concentration of 0.2 per cent as the main oxidizable compounds. 
Habitat: Regularly found in mud and stagnant bodies of water.

Illustrations: Molisch, loc. cit., Plate I, fig. 1, 2; Plate II, fig. 10; van Niel, loc. cit., fig. 1-3, p. 18, and fig. 18-26, p. 90.

\section{Rhodopseudomonas gelatinosa (Mo-} lisch) van Niel. (Rhodocystis gelatinosa Molisch, Die Purpurbakterien, Jena, 1907, 22; van Niel, Bact. Rev., 8, 1944, 98.) From Latin gelatio, freezing, indicating solidification, or in this case, clumping.

Cells: In young cultures, short and small rods, approximately 0.5 by 1 to 2 microns. In old cultures much longer, up to 15 microns, and then irregularly curved rods, often swollen and gnarled in places up to 1 micron in width. In this stage the cells bear some resemblance to those found in old cultures of Rhodopseudomonas palustris, but the characteristic Mycobacterium-like clusters of the latter are absent. Single cells infrequent due to a copious mucus production in all media which causes the cells to clump together. While young cells are actively motile by means of polar flagella, motility is often difficult to ascertain as a result of the pronounced tendency to conglomerate; the individuals in the clumps appear to be non-motile. Gram-negative. Gelatin is liquefied; of the single amino acids alanine, asparagine, aspartic and glutamic acids appear generally satisfactory substrates.

Color: Quite distinctive in most anaerobic cultures as a pale, delicate, pinkish shade, rather peach-colored. Only in the presence of rather high concentrations of yeast extract (when a much heavier growth is obtained than with low concentrations supplemented with 0.2 per cent of various single oxidation substrates) do the slimy cell masses appear a dirty, faded brown. Color is due to bacteriochlorophyll and carotenoid pigments. Occasionally a water-soluble, non-carotenoid, bluish-red pigment is produced which diffuses into the culture medium.

In yeast extract, growth occurs over a $\mathrm{pH}$ range extending from at least 6.0 to 8.5 .

Cultures produce a characteristic acrid odor.

More sensitive to fatty acids than other species of Rhodopseudomonas; with 0.2 per cent propionate no growth occurs. The best single oxidizable substrates appear to be ethanol, glucose, fructose and mannose, as well as a variety of amino acids. Citrate also permits good growth; not, on the other hand, glycerol, mannitol, sorbitol or tartrate in the usual concentration of 0.2 per cent.

Thiosulfate is not oxidized; behavior towards molecular hydrogen unknown.

More pronouncedly microaerophilic than the other Rhodopseudomonas species; most cultures cannot develop on aerobically incubated slants or agar plates.

Capable of strictly anaerobic development in illuminated cultures by virtue of a photosynthetic metabolism.

Temperature relations so far unknown.

Distinguishing properties: The small size of the individual cells, and the pronounced clumping which causes the cultures to be exceptionally stringy; the unusual color of the cell masses; the ability to liquefy gelatin, to utilize citrate and a number of amino acids. Correlated with these is the failure to grow in media with 0.2 per cent propionate, tartrate and glycerol.

Habitat: Regularly present in stagnant bodies of water and in mud.

Illustrations: Molisch, loc. cit., Plate I, fig. 8; van Niel, loc. cit., fig. 55-60, p. 99 ; fig. 61-66, p. 100 .

3. Rhodopseudomonas capsulatus (Molisch) van Niel. (Rhodonostoc capsulatum Molisch, Die Purpurbakterien, Jena, 1907, 23; van Niel, Bact. Rev., 8, 1944, 92.) From Latin capsula, container (sheath). 
Cells: Depending upon the $\mathrm{pH}$ of the medium, cells nearly spherical, or as distinct rods, often devoid of motility. Motility due to polar flagella. The spherical cells are found in media with a $\mathrm{pH}$ below 7 ; they are usually arranged in chains resembling streptococei. Rodshaped cells are characteristic for media with $\mathrm{pH}$ above 7 ; the higher the $\mathrm{pH}$, the longer the rods. Individual cells slightly less than 1 micron wide, al though attenuated rods (about 0.5 micron in width) are frequent at $\mathrm{pH}$ above 8 , and slightly swollen cells (to 1.2 microns) are found in media containing sugars. Length varies from 1 to 6 microns; most common dimensious in approximately neutral media 2 to 2.5 microns. At $\mathrm{pH}$ above 8 abnormal growth in the form of irregular filaments. Outstandingly characteristic is the zigzag arrangement of the cells in chains.

Cultures in media of $\mathrm{pH} 8$ or above are distinctly mucoid. Gram-negative.

Color: Anaerobic cultures develop with a brown color, the shade ranging from a light yellowish-brown to a deep mahogany brown. When grown in the presence of oxygen, the cultures are dark red. Even the pigmentation of the brown-colored organisms from an anacrobic culture can be changed into a distinct red by shaking a suspension with air for some hours; light enhances the rate of this color change. Color due to bacteriochlorophyll and carotenoid pigments. To diffusible water-soluble pigment is produced.

Growth possible over a $\mathrm{pH}$ range from at least 6 to 8.5 , morphology becoming abnormal in the alkaline media.

Most cultures are odorless, although occasionally a faint peach-like odor can be detected.

Growth is not inhibited by the presence of oxygen, although the pigmentation is thereby affected.

Fatty acids and most substituted acids are satisfactory substrates. Rapid and abundant growth with propionate at a concentration of 0.2 per cent. At this same concentration glutaric acid leads, at best, to very meager cultures, while tartrate, citrate and gluconate fail to induce growth, as do also ethanol, glycerol, mannitol and sorbitol. In media with 0.2 per cent glucose or fructose good growth is obtained. No growth with mannose. Thiosulfate is not, but molecular hydrogen can be, oxidized by this species.

Gelatin is not liquefied; of the amino acids alanine and glutamic acid are satisfactory substrates, while leucine is not utilized.

Distinguishing properties: Cell shape and arrangement in chains; brown color of anaerobic, red pigmentation of aerobic cultures; ability to grow in media with 0.2 per cent propionate, glucose, fructose, alanine and glutamic acid; failure to develop with leucine, as well as with ethanol, glycerol, mannitol and sorbitol in the above-mentioned concentration.

All cultures can develop anaerobically in illuminated cultures by a photosynthetic metabolism.

Temperature optimum distinctly lower than for Rhodopseudomonas palustris, and, as a rule, around $25^{\circ} \mathrm{C}$.

Habitat: Regularly found in stagnant bodies of water and in mud.

Illustrations: Molisch, loc. cit., Plate II, fig. 9; van Niel, loc. cit., fig. 4-6, p. 19; fig. 27-32, p. 92; and fig. 33-38, p. 93.

\section{Rhodopseudomonas spheroides van} Niel. (Rhodococcus capsulatus Molisch, Die Purpurbakterien, Jena, 1907, 20; Rhcdococcus minor Molisch, ibid., 21; Rhodosphaera capsulata Buchanan, Jour. Bact., 3, 1918, 472; Rhodosphaera minor Bergey et al., Manual, 1st ed., 1923, 405; Rhodorrhagus minor Bergey et al, Manual, 3rd ed., 1930, 535; Rhodorhagus capsulatus Bergey et al., Manual, 3rd ed., 1930, 535; van Niel, Bact. Rev., 8, 1944, 95.) From Latin sphaera, a round body and Greek eidos, form of. 
Cells: Ciencrally single, nearly spherical, diameter without slime capsule variable, depending upon medium, ranging from 0.7 to 4 microns. In young cultures actively motile by means of polar flagella; motility soon ceases in media which are or become alkaline. Copious slime production in media at $\mathrm{pH}$ above 7 . In strongly alkaline cultures abnormal cell-shapes occur in the form of irregular, swollen and distorted rods, often having the appearance of spore-bearing cells, simulated by the production of fat bodies. In sugar-containing media egg-shaped cells, measuring as a rule 2.0 to 2.5 by 2.5 to 3.5 microns, are frequently found. Gramnegative.

Color: Anaerobic cultures develop with brown color, ranging in shade from a light, dirty greenish-brown to a dark brown. Cultures grown in the presence of oxygen are distinctly red. As in the case of Rhodopseudomonas capsulatus, the brown color of an anaerobic culture ean be ehanged to red by shaking with air, light stimulating the color change. Color due to bacteriochlorophyll and carotenoid pigments. The large majority of cultures of this species produces in addition a water-soluble, non-carotenoid, bluish-red pigment which diffuses into the culture medium.

Gelatin is not liquefied, and growth with single amino acids appears somewhat erratic. No definite correlations have been observed.

Development is possible over a wide $\mathrm{pH}$ range, extending from at least 6.0 to 8.5 .

All cultures exhibit an unpleasant putrid odor.

Requires for optimal development higher concentrations of yeast extract as a supply of growth factors than either Rhodopseudomonas palustris or Rhodopseudomonas capsulatus and is more sensitive to low fatty acid concentrations. With 0.2 per cent propionate in a neutral medium, no growth occurs; caproic and pelargonic acids are toxic in concentrations below 0.1 per cent. On the other hand, tartrate and gluconate can serve as oxidation substrates, as can also ethanol, glycerol, mannitol, sorbitol, glucose, fructose and mannose in 0.2 per cent concentrations.

In sugar-eontaining media, acid is produced; the $\mathrm{pH}$ may drop to below 4.0 before development ceases. Acid production from glucose occurs both in presence and absence of air, and in illuminated as well as in non-illuminated cultures. In cultures exposed to light, the acid usually disappears later on.

Thiosulfate is not oxidized; hydrogen oxidation has not been observed.

Oxygen does not prevent growth; colonies develop on the surface of agar plates exposed to air, with a red pigmentation. Capable of strictly anaerobic development in illuminated cultures by photosynthesis.

Temperature optimum below $30^{\circ} \mathrm{C}$.

Distinguishing properties: Spherical cell-shape in most media; brown color of anacrobic and red pigmentation of ierobic cultures; growth with 0.2 per cent tartrate, gluconate, ethanol, glycerol, mannitul, sorbitol, glucose, fructose and mannose; failure to grow with 0.2 per cent propionate.

Habitat: Regularly found in stagnant bodies of water and in mud.

Illustrations: Molisch, loc. cil., Plate II, fig. 15; van Niel, loc. cil., fig. 7-8, p. 19 ; fig. $39-45$, p. 96 ; fig. $46-54$, p. 97 .

\section{Genus II. Rhodospirillum Molisch emend. van Nicl.}

(Molisch, Die Purpurbakterien, Jena, 1907, 24; van Niel, Bact. Rev., 8, 1944, 86; the genus now includes the genus Phacospirillum Kluyver and van Niel, Cent. f. Bakt., If Abt., 94, 1936, 396.) From Greek rhodon, red and M.L. spirillum, spirillum.

Spiral-shaped bacteria, motile by means of polar flagella. Gram-negative. 
Contain bacteriochlorophyll and are potentially photosynthetic in the presence of extraneous oxidizable substances. Molecular oxygen is not produced. Unable to grow in strictly mineral media, even when possessed of the ability to utilize hydrogen as oxidizable substrate, due to the need for organic nutrilites. Produce accessory pigments causing the cultures, especially when grown in the light, to appear in various shades of red to brown.

The type species is Rhodospirillum rubrum (Esmarch) Molisch.

\section{Key to the species of genus Rhodospirillum.}

I. Cultures red; cells well over 0.5 micron, usually about 1 to 1.2 microns in width. 1. Rhodospirillum rubrum.

II. Cultures brown to orange; cells 0.5 micron or less in width.

2. Rhodospirillum fulvum.

1. Rhodospirillum rubrum (Esmarch) Molisch. (Spirillum rubrum Esmarch, Cent. f. Bakt., 1, 1887, 225; Moliseh, Die Purpurbakterien, Jena, 1907, 25; Rhodospirillum photometricum Molisch, ibid., 24; Rhodospirillum giganteum Molisch, ibid., 2t; Rhodospirillum longum Hama, Jour. Sci. Hiroshima Univ., Ser. B, Div. 2, 1, 1933, 135; Rhodospirillum gracile Hama, ibid., 159.) From Latin ruber, red.

Cells: Characteristically spiralshaped, but size of elements variable within wide limits, depending upon environmental conditions during growth Width of cells from 0.5 to 1.5 microns; length from 2 to 50 microns, and over; even in a single culture such differences may be found. Also the shape and size of the spiral coil varies much; it usually ranges between 1 to $t$ microns in width, and from 1.5 to 7 microns in length. In alanine media the majority of the cells occurs in the form of half-circles to complete rings; malate media tend to produce much flattened spirals.

In old cultures involution forms appear, straightened spirals and irregularly swollen cells, the latter common in media with higher fatty acids. Such cells stain irregularly, contain fatty in clusions, and are occasionally branched.

Mueus is not produced. In ealeium deficient media the growth is flocculent, as if agglutinated. With an adecguate calcium supply the growth in liquid media is homogeneous, suspended, and consists of single cells.

Young cultures show active motility, due to polar flagella. Ciram-negative.

Gelatin is not liquefied; the amino acids alanine, asparagine, aspartic and glutamic acids are satisfactory oxidizable compounds.

Color: Ordinarily deep and dark red, without any brownish tinge. In ethanol media lighter, and a characteristic pink. Pigment production markedly influenced by oxygen and light. Slants incubated in darkness present a pale grayish surface growth with a faint reddish hue, while often showing deep-red cell masses in the region between glass wall and agar surface where development proceeds at low oxygen tension. The color is due to bacteriochlorophyll and carotenoid pigments. Among the latter spirilloxan thin is quantitatively predominant. Water-soluble, diffusible pigments are not produced.

Development possible over a $\mathrm{pH}$ range of at least 6 to 8.5 , although, as in other cases, the combination of an acid reaction and the presence of fatty acids may prevent growth.

Cultures produce a distinctive odor, reminiscent of slightly putrid yeast.

In general, grow well with fatty acids as the chief oxidizable substrate; however, are prevented from growing by 0.2 per cent propionate in a neutral medium. Most substituted acids are equally satisfactory, with the exception 
of tartrate, gluconate and citrate. In a concentration of 0.2 per cent, ethanol is a suitable substrate, whereas the carbohydrates and their corresponding polyalcohols are not utilized.

Thiosulfate is not oxidized; molecular hydrogen can be used by some strains.

Rather microaerophilic; many strains upon initial isolation incapable of growth at atmospheric oxygen tension. Subsequent adaptation can be induced. But even such adapted cultures exhibit negative chemotaxis to air.

Capable of strictly anaerobic development in illuminated cultures on the basis of a photosynthetic metabolism.

Temperature optimum generally between $30^{\circ}$ and $37^{\circ} \mathrm{C}$.

Distinguishing properties: The most important characteristics of the species are the spiral shape, combined with the ability to produce a red pigment with a definite absorption maximum at 550 millimicrons in the intact cells. Diagnostically useful are the good growth in media with 0.2 per cent ethanol, alanine, asparagine, aspartate or glutamate, and the inadequacy of similar concentrations of carbohydrates and thiosulfate as substrates.

Habitat: Regularly present in stagnant bodies of water and in mud.

Illustrations: Molisch, loc. cit., Plate 1, fig. 5-7; van Niel, Bact. Rev., 8, 1944, fig. $9-10$, p. 19 ; fig. $11-16$, p. 24 ; fig. $67-$ 75, p. 103; fig. 76-84, p. 104; fig. 85-90, p. 106; fig. 91-96, p. 107.

2. Rhodospirillum fulvum van Niel. (Bact. Rev., 8, 1944, 108.) From Latin fulvum, yellowish, tawny.

Characteristic for the species is the very small size of the individual cells. These are not over 0.5 micron wide, and generally not longer than 2.5 microns. The most common shape consists of a complete turn of about 1 by 1.5 microns. In media with fatty acids as a substrate the spirals appear somewhat steeper than in fumarate, succinate or malate cultures. Swollen individuals resembling vibrios are encountered in cultures which do not appear quite healthy. Formation of mucus or clumping has not been observed.

Gelatin is not liquefied; aspartate has been the only amino acid capable of inducing growth. Thiosulfate is not oxidized.

Color: Quite distinct from that of Rhodospirillum rubrum; colonies and stab cultures are a reddish-brown, while liquid cultures often appear brownishorange. The color is due to bacteriochlorophyll and carotenoid pigments; among the latter spirilloxanthin, as evidenced by the absence of an absorption maximum at 550 millimicrons, is not represented as a major constituent. Does not produce water-soluble, diffusible pigments.

Capable of strictly anaerobic development in illuminated cultures, due to photosynthetic metabolisn.

Fatty acids and the four-earbon dicarboxylic acids are uniformly good substrates; glutarate is not used. Ethanol and glucose, in a concentration of 0.2 per cent, have yielded satisfactory cultures; other carbohydrates, as well as the corresponding polyalcohols, have given negative results.

Little information available concerning $\mathrm{pH}$ and temperature relations. 13ehaves generally as a strict anaerobe; adaptation to microaerophilie conditions has not been achieved. Negative aerotaxis very pronounced.

Distinguishing properties: The small size and the color of the cultures serve as adequate criteria for its differentiation from Rhodospirillum rubrum. The strictly anaerobic nature and the failure to grow with glutarate and various amino acids except aspartate can probably be used as supplementary specific properties.

Habitat: Bodies of stagnant water and mud.

Illustrations: Vin Niel, loc. cit., fig. $9 \overline{7}-102$, p. 109. 


\section{FAMILY III. CHLOROBACTERIACEAE GEITLER AND PASCHER.*}

(Cyanochloridinae-Chlorobacteriaceae Geitler and Pascher, Die Süsswasserflora Deutschlands, Österreichs und der Schweiz, Jena, 12, 1925, 451; Chlorothiobacteria Bavendamm, Ergeb. Biol., 13, 1936, 49.)

Green bacteria, usually of small size, occurring singly or in cell masses of various shapes and sizes, developing in environments containing rather high concentrations of hydrogen sulfide and exposed to light. As a rule not containing sulfur globules but frequently depositing elementary sulfur outside the cells. Contain green pigments of a chlorophyllous nature, though not identical with the common green plant chlorophylls nor with bacteriochlorophyll. Capable of photosynthesis in the presence of hydrogen sulfide; do not liberate oxygen.

A number of genera have been proposed, characterized by special colonial growth forms, others on the basis of a supposed symbiotic habitus, where the green bacteria grow in more or less characteristic aggregates together with other micro-organisms. In view of the variations in size and shape exhibited by the only member of this group which has so far been obtained and studied in pure culture (van Niel, Arch. f. Mikrobiol., 3, 1931, 65ff.) the validity of many of these genera is doubtful. The following keys and deseriptions, therefore, bear a strictly provisional character. Here, as in the case of the sulfur purple bacteria, significant advances can only be expected from pure culture studies under controlled environmental conditions.

\section{Key to the genera of family Chlorobacteriaceae.}

I. Free-living bacteria not intimately associated with other microbes.

a. Bacteria not united into well defined colonies.

Genus I. Chlorobium, p. 869.

aa. Bacteria united into characteristic aggregates.

b. Bacteria without intracellular sulfur globules.

Genus II. Pelodictyon, p. 870.

bb. Bacteria with intracellular sulfur globules.

Genus III. Clathrochloris, p. 872 .

II. Green bacteria found as symbiotic aggregates with other organisms.

a. Aggregates composed of green bacteria and protozoa.

Genus IV. Chlorobacterium, p. 872.

aa. Aggregates composed of two different types of bacteria.

b. Aggregates small, barrel-shaped, actively motile, and consisting of a central, polarly flagellated, rod-shaped bacterium with a covering of green sulfur bacteria.

Genus V. Chlorochromatium, p. 873.

bb. Aggregates large, cylindrical, non-motile, and composed of a central filamentous bacterium with a more or less extensive covering of green sulfur bacteria .

Genus VI. Cylindrogloea, p. 873.

\section{Genus I. Chlorobium Nadson.}

(Nadson, Bull. Jard. Impér. Botan., St. Pétersb., 12, 1912, 6t (Russian), \$3 (German); Chloronostoc Pascher, Die Süsswasserflora Deutschlands, Österreichs und der Schweiz, Jena, 12, 1925, 456; Tetrachloris Pascher, ibid., 455; Sorochloris? Pascher,

* Completely revised by Prof. C. B. van Niel, Hopkins Marine Station, Pacific Grove, California, January, 1944. 
ibid., 455; Chloropseudomonas? Czurda and Maresch, Arch. f. Mikrobiol., 8, 1937, 123; in part, Pelogloea Lauterborn, Verhandl. naturhistor.-medizin. Vereins, Heidelberg, N.F. 13, 1915, 430.) From Greek chloros, green and bios, life.

Green sulfur bacteria, occurring singly or in chains, individual cells of various sizes and shapes, from spherical to relatively long rod-shaped, the latter sometimes coiled into tight spirals; often united in chains, and generally embedded in a slime capsule. Non-motile. Contain a chlorophyllous pigment different from the common green plant chlorophylls and from bacterio-chlorophyll. Capable of photosynthesis in the presence of hydrogen sulfide, during which they produce elementary sulfur which is excreted outside the cells. Do not form spores.

The type species is Chlorobium limicola Nadson.

\section{Chlorobium limicola Nadson.} (Nadson, Bull. Jard. Impér. Botan., St. Pétersb., 12, 1912, 61 (Russian), \$3 (German); Chloronostoc abbreviatum Pascher, Süsswasserflora Deutschlands, Österreichs und der Schweiz, Jena, 12, 1925, 456; Tetrachloris inconstans Pascher, ibid., 456; Sorochloris aggregata? Pascher, ibid., 455; in part Pelogloea chlorina Lauterborn, Verhandl. naturhistor.-medizin. Vereins, Heidelberg, N.F. 13, 1915, 430.) From Latin, mud-dweller.

Cells: Various shapes and sizes, markedly dependent upon environmental conditions. In young and heal thy state predominantly spherical to ovoid, about 0.5 to 1 micron in diameter, frequently united in chains resembling streptococci. Often cells become elongated and appear as rods, generally about 0.7 micron by 1 to 2.5 microns; also these may remain united in chains. Regularly produce mucus, causing the formation of cellconglomerates of different size and shape, but not, as a rule, of characteristic appearance.
Color yellowish-green. Non-motile. Abnormal cell forms (involution forms) rather common. These may be larger spherical cells, up to 5 to 6 microns in diameter, the larger ones generally vacuolated, or long rods, occasionally club-shaped but more often coiled. In rare cases the latter may be Ioosely wound. More frequently they are tightly-coiled serews, with cells of about 0.5 micron in diameter by as much as $\mathbf{1 5}$ microns in length. The spherieal involution forms are normally encountered in acid, the coiled ones in alkaline enviromments.

Strictly anaerotic and apparently dependent upon hydrogen sulfide and light. Development in organic media has not been obtained.

Habitat: Mud and stagnant water containing rather high concentrations of hydrogen sulfide and exposed to light; more rarely in sulfur springs.

Illustrations: Nudson, loc. cit., Pl. III, fig. 3-12; van Niel, Areh. f. Mikrobiol., 3, 1931, 66, fig. 8 .

\section{Genus II. Pelodictyon Laulerborn.}

(Lauterborn, Allgem. botan. Ztschr., 19, 1913, 98; Verhandl naturhistor.-medizin. Vereins, Heidelberg, N.F. 13, 1915, 431; Schmidlea loc. cit., Lauterborn, Allgem. botan. Zeitschr., 19, 1913, 97; in part, Pelogloea Lauterborn, Verhandl. naturhist. medizin Vereins, Heidelberg, N.F. 13, 1915, 430; Pediochloris Geitler, Die Süsswasserflora Deutschlands, Österreichs und der Schweiz, Jena, 12, 1925, 457.) From Greek pelos, mud and dictyon, net.

Green sulfur bacteria, individual cells ovoid to distinctly rod-shaped, producing rather extensive mucoid capsules, and generally united into large colonies of characteristic shapes. Non-motile. Contain chlorophyllous pigments different from 
the common green plant chlorophylls and from bacteriochlorophyll. Capable of photosynthesis in the presence of hydrogen sulfide, but do not store sulfur globules inside the cells.

The type species is Pelodictyon clathratiforme (Szafer) Lauterborn.

\section{Key to the species of genus Pelodictyon.}

I. Cells united in colonies in a net-like fashion.

\section{Pelodictyon clathratiforme.}

II. Cells arranged in tightly packed colonies without net-like structure.

a. Colonies composed of irregularly arranged cell-masses, extending in three dimensions.

2. Pelodictyon aggregatum.

aa. Colonies consisting of parallel strands and extending in two dimensions.

3. Pelodictyon parallelum.

1. Pelodictyon clathratiforme (Szafer) Lauterborn. (Aphanothece clathratiforme Szafer, Bull. Acad. Sci., Cracovic, Sér. B. 3, 1910, 162; Lauterborn, Allgem. botan. Ztschr., 19, 1913, 98; Lauterborn, Verhandl. naturhist.-medizin. Vereins, Heidelberg, N.F. 13, 1915, 430; Pelodictyon clathratiforme Geitler, Die Süsswasserflora Deutschlands, Österreichs und der Schweiz, Jena, 12, 1925, 458; Pclodictyon lauterbornii Geitler, ibid., 458.) From Greek clathros, trellis and formis, shape.

Cells: Generally rod-shaped, ranging from slightly elongated ovoids to distinct rods, often vacuolated, about 0.5 to 1.5 micron by 2 to 4 microns, producing rather wide slime capsules, and characteristically united into threedimensional colonies which present a net-like appearance, with mazes of about 10 to 50 microns.

Color yellowish-green. Non-motile.

Abnormal cell forms (involution forms) not uncommon, consisting of elongated and curved, forked, or clubshaped and swollen rods, occasionally suggesting rudimentary branching at the extremities. Such cells may be found as elements in chains for the greater part composed of normal individuals.

Habitat: Mud and stagnant water containing rather high concentrations of hydrogen sulfide and exposed to light; sulfur springs.

Illustrations: Szafer, loc. cit., PI. VI, fig. 5; Perfiliev, Jour. Microbiol. (Russian), 1, 1914, Pl. II, fig. 1, 5-12; Lauterborn, loc. cit., 1915, Pl. III, fig. 33.

2. Pelodictyon aggregatum Perfiliev. A phanothece lutecla Schmidle, Beihefte Botan. Cent., 10, 1901, 179; Schmidlea luteole Lauterborn, Allgem. botan. Ztschr., 19, 1913, 97; Latuterborn, Verhandl. naturhistor.-medizin. Vereins, Heidelberg, N.F., 13, 1915, t29; Pelogloea bacillifera Lauterborn, ibid., 430; Perfiliev, Jour. Microbiol. (Russian), 1, 1914, 197.) From Latin aggregatus, leading together, grouping.

Cells: Csually rod-shaped, about 1 to 1.5 microns by 2 to 4 microns, often vacuolated, producing mucus capsules, and united into irregularly shaped, three-dimensional colonies in which the cells are more or less tightly packed, without orderly arrangement. Colonies may attain a size of up to $1 \mathrm{~mm}$; frequently they are not fully compact, but contain less dense areas, or appear perforated, thus forming transition stages to Pelodictyon clathratiforme.

Color yellowish-green. Non-motile.

Abnormal cell forms (involution forms) usually in the shape of elongated and curved, forked or club-shaped and swollen rods, occasionally suggesting branching at extremities.

Habitat: Mud and stagnant water, containing rather high concentrations of 
hydrogen sulfide, and exposed to light; sulfur springs.

Illustrations: Perfiliev, loc. cit., Pl. II, fig. 2; Lauterborn, loc. cit., Pl. III, fig. $29-31$.

3. Pelodictyon parallelum Perfiliev. (Aphanothece parallela Szafer, Bull. Acad. Sci., Cracovie, Sér. B, 3, 1910, 163; Perfiliev, Jour. Microbiol. (Russian), 1, 1914, 198; Pediochloris parallela Geitler, Die Süsswasserflora Deutschlands, Österreichs und der Schweiz, Jena, 12, 1925, 457.) From Latin parallelus, beside one another.

Cells: Rather small, spherical to ovoid, or even rod-shaped; about 0.5 to 1 micron by 1 to 3 microns, occurring in chains, and forming flat, plate-like, two-dimensional aggregates in which the chains are arranged as parallel strands.

Color yellowish-green. Non-motile.

Abnormal cell forms not specifically mentioned, but likely to occur, and to resemble those of other species.

This species may well be a special growth-form of Chlorobium limicola.

Habitat: Mud and stagnant water containing rather high concentrations of hydrogen sulfide and exposed to light; sulfur springs.

Illustrations: Szafer, loc. cit., Pl. VI, fig. 7; Perfiliev, loc. cit., Pl. II, fig. 2.

\section{Genus III. Clathrochloris Geiller.}

(Die Süsswasserflora Deutschlands, Österreichs und der Schweiz, Jena, 12, 1925, 457.) From Greek clathros, trellis and chloros, green.

Green sulfur bacteria of small size, generally spherical, and arranged in chains which are united into loose, trellis-shaped aggregates, somewhat similar to those of Pelodictyon clathratiforme and Pelodictyon aggregatum. Cells usually contain sulfur globules. Color yellowish-green. Non-motile.

The type species is Clathrochloris sulphurica (Szafer) Geitler.

\section{Clathrochloris sulphurica (Szafer)} Geitler. (Aphanothece sulphurica Szafer, Bull. Acad. Sci., Cracovie, Sér. B, 3, 1910, 162; Geitler, Die Süsswasserflora Deutschlands, Österreichs und der Schweiz, Jena, 12, 1925, 457.) From Latin, containing sulfur.

Cells: Spherical, about 0.5 to $0.7 \mathrm{mi}$ cron in diameter, usually containing sulfur globules. Color yellowish-green. Non-motile.

The reported occurrence of sulfur globules in the cells of this very small species is surprising; it is the only one among the green sulfur bacteria in which these inclusions have been encountered. The published descriptions are even more fragmentary than those of other members of the group.

Source: Reported only from sulfur springs in Lubién Wiclki, near Lwow, Poland.

Habitat: Mud and stagnant water containing rather high concentrations of hydrogen sulfide and exposed to light; sulfur springs.

Illustration: Szafer, loc. cit., Pl. VI, fig. 6 .

\section{Genus IV. Chlorobacterium Lauterborn.}

(Lauterborn, Verhandl. naturhist.-medizin. Vereins, Heidelberg, N.F., 13, 1915, 429; not Chlorobacterium Guillebeau, Landw. Landw. Jahrb. d. Schweiz, 4, 1890, 32; Chroostipes Pascher, Die Süsswasserflora Deutschlands, Österreichs und der Schweiz, Jena, 12, 1925, 116.) From Greek chloros, green and Latin bacterium, a small rod.

Green sulfur bacteria(?) which grow symbiotically as an outside covering on cells of protozoa, such as amoeba and flagellates. Cells rod-shaped, often slightly curved, greenish. Non-motile.

The type species is Chlorobacterium symbioticum Lauterborn. 
1. Chlorobacterium symbioticum Lauterborn. (Lauterborn, Verhandl. naturhist.-medizin. Vereins, Heidelberg, N.F., 13, 1915, 429; Chroostipes linearis Pascher, Die Süsswasserflora Deutschlands, Österreichs und der Schweiz, Jena, 12, 1925, 116.) From Greek, living symbiotically.

Cells: Rod-shaped, about 0.5 by 2 to 5 microns, often slightly curved. Nonmotile.

Occur as a peripheral covering of certain protozoa with which they may form a symbiotic unit.
It is not certain that this is a green sulfur bacterium; the description of localities where it was found fail to mention the presence of hydrogen sulfide in the environment which should be a prerequisite for a nember of this group.

Source: Reported from a number of pools in Germany.

Habitat: Stagnant water.

Illustrations: Lauterborn, loc cit, Pl. III, fig. 34-36; Pascher, loc. cit., fig. 149 .

\section{Genus r. Chlorochromatium Lauterborn.}

(Lauterborn, Allgem. botan. Ztschr., 19, 1906, 196; Chloronium Buder, Ber. d. deut. bot. Ges., 31, 1914, Generalversammlungsheft, 80.) From (ireek chloros, green and chroma, color.

Green sulfur bacteria, ovoid to rod-shaped with rounded ends, occurring as barrelshaped aggregates, consisting of a rather large colorless bacterium with a polar flagellum as the center, surrounded by the green bacteria, arranged in 4 to 6 rows, ordinarily from 2 to 4 cells high. The entire conglomerate behaves like a unit, is motile, and multiplies by the more or less simultaneous fission of its components.

The green constituents contain a chlorophyllous pigment which is not identical with the common green plant chlorophylls or with bacteriochlorophyll. Capable of photosynthesis in the presence of hydrogen sulfide, but do not store sulfur globules in the cells.

The type species is Chlorochromatium aggregatum Lauterhorn.

1. Chlorochromatium aggregatum Lauterborn. (Lauterborn, Allgem. botan. 'Ztschr., 19, 1906, 196; Chloronium mirabile Buder, Ber. deut. botan. Ges., 31, 1914, Generalversammlungsheft, s0.) From Latin aggregatus, grouped.

Cells of the green component 0.5 to 1.0 by 1.0 to 2.5 microns, mostly from is to 16 individuals surrounding the central bacterium. Size of the total harrelshaped unit variable, generally 2.5 to 5 by 7 to 12 microns. Occasionally a group of the complex colonies may remain attached in a chain.

Anaerobic.

Habitat: Mud and stagnant water con- taining rather high concentrations of hydrogen sulfide and exposed to light.

There is at present no good reason for distinguishing 2 varieties (forma typica and forma minor) or even species, on the basis of size differences of the colony, as Cieitler proposed (Die Süsswasserflora Deutschlinds, Österreichs und der schweiz, Jena, 12, 1925, 460). The reported and personally observed sizes of such units show that the extreme limits are linked by a complete series of transitions.

Illustrations: Buder, loc. cit., Pl. XXIV, fig. 1-5; Perfiliev, Jour. Microhiol. (Russian), 1, 1914, 213, fig. 1-5.

\section{Genus VI. Cylindrogloea Perfiliev.}

(Jour. Microbiol. (Russian), 1, 1914, 223.) From Latin cylindrus, cylinder and Greek gloios, a glutinous substance. 
Green sulfur bacteria, consisting of small ovoid to rod-shaped cells, growing in association with a filamentous, colorless, central bacterium, thus forming colonies of a cylindrical shape. Non-motile. The green component contains a chlorophyllous pigment different from the common chlorophylls of green plants and from bacteriochlorophyll. Capable of photosynthesis in the presence of hydrogen sulfide, without depositing sulfur globules in the cells.

The type species is Cylindrogloea bacterifera Perfiliev.

1. Cylindrogloea bacterifera Perfiliev. (Jour. Microbiol. (Russiun), 1, 1914,223. From Latin bacter, rod and fero, to bear.

Individual green components ovoid to rod-shaped, about 0.5 to 1 by 2 to $4 \mathrm{mi}$ crons, very similar to those of the complex Chlorobacterium symbioticum and Chlorochromatium aggregalnm with which they may well be identical. The central filamentous bacterium is embedded in a slime capsule of considerable dimensions. This, in turn, is surrounded by a layer of green bacteria, usually one cell thick. The green organisms ma form a very dense outer covering, or they may be more sparsely distributen! over the mueus eapsule. The entire unit is again surrounded by a sizeable slime zone. Igeregates measure about 7 to 8 microns in width, and up to 50 microns in length; they are non-motile. Both components appear to be non-spore-forming.

Habitat: Mud and stagnant water containing rather high concentrations of hydrogen sulfide and exposed to light.
Illustration: Perfiliev, lec. cit., 213, fig. 6-11.

Perfiliev rightly emphasizes, as Buder had done for Chloronium mirabile, the provisional nature of thus using a gencric designation for an apparently stable romplex romposed of two different organisms. It remains possible that the last three genera of symbiotic entities represent fortuitous combinations whose occurrence is anditioned by environmental factors. If so, the generic terminology would be devoid of any taxonomic significance, and the green bacteria should bo relegated to more appropriate genera. Indications suggestire of this state of affairs can be found in the literature: for sxample in I'termöhl's observation (Arehiv f. Hydrobiol., Suppl. 5. 1925, 279, that the complex Chlorochromatium aggregatum may, especially in the presence of oxygen, disintegrate, whereupon the green constituents appear as small Pelodictyon aggregatum (Schmillea lutenla) colonies.' 


\section{ORDER II. ACTINOMYCETALES BUCHANAN.}

(Jour. Bact., 2, 1917, 162.)

Organisms forming elongated cells which have a definite tendency to branch. These hyphae do not exceed 1.5 mierons and are mostly about 1 micron or less in diameter. In the $\boldsymbol{M}$ ycobacteriaceae the mycelium is rudimentary or absent; no spores are formed; the cells are acid-fast. The Actinomycetaceae and Streptomycetaceae usually produce a characteristic branching mycelium and multiply by means of special spores, oidiospores or conidia. Special spores are formed by fragmentation of the plasma within straight or spiral-shaped spore-bearing hyphae; the oidiospores are formed by segmentation, or by transverse division of hyphae, similar to the formation of oidia among the true fungi; the conidia are produced singly, at the end of simple or branching conidiophores. They grow readily on artificial media and form well-developed colonies. The surface of the colony, especially in the Actinomycetaceae and Streptomycrtaceae, may become covered with an aerial mycelium. Some form colorless or white colonies, whereas others form a variety of pigments. Some species are partially acid-fast. In relation to temperature, most are mesophilic, while some are thermophilic. Certain forms are capable of growing at low oxygen tension. The Order as a whole is composed of saprophytic species, but also includes species that are parasitic and sometimes pathogenic on both animals and plants.

Key to the families of order Actinomycetales.

I. Mycelium rudimentary or absent, no spores formed. Acid-fast.

\section{Family I. Mycobacteriaceae, p si5.}

II. True mycelium produced.

A. Vegetative mycelium divides by segmentation into bacillary or coccoid elements. Some species partially acid-fast.

Family II. Actinomycetaceae, p. 892.

B. Vegetative mycelium normally remains undivided.

Family III. Streptomycetaceae, p. 929.

Among the recent systems of classification of this order it is sufficient to mention the following: Baldacei (Mycopath., 2, 1939, 84) divided the order Actinomycetales into two families: (a) Mycobacteriaceae Chester with two subfamilies, Leptotrichioideae Baldacci and Proactinomycoideae Baldacci, each with five genera, and (b) Actinomycetaceae Buchanan, with two genera, Micromonospora and Actinomyces. Krassilnikov (Ray fungi and related organisms, Izd. Akad. Nauk, Moskow, 1938) divided the order into (a) Actrnomycetaceae, with four genera, Actinomyces, Proactinomyces, Mycobacterium and Mycococcus, and (b) Micromonosporaceae, with one genus, Micromonospora. Waksman (Jour. Bact., 39, 1940, 549) divided the order into four families: Mycobacteriaceae, Proactinomycetaceae, Actinomycetaceae and Micromonosporaceae.

\section{FAMILY I. MYCOBACTERIACEAE CHESTER.*}

(Chester, Man. Determ. Bact., 1901, 349 ; Proactinomycetaceae Lehmann and Haag, in Lehmann and Neumann, Bakt. Diag., 7 Aufl., 2, 1927, 674.)

* Completely revised by Prof. G. B. Reed, Queens University, Kingston, Ontario, Canada, December, 1938; minor revisions, December, 1914; with a complete revision of $M$ yeobacterium leprae and $M$. lepracmurium by Dr. John H. Hanks, Leonard Wood Memorial, American Leprosy Foundation, New York, X. Y. 
Slender filaments, straight or slightly curved rods, frequently irregular in form with only slight and occasional branching. Often stain unevenly, i.e., show variations in staining reaction within the cell (beading). No conidia. Non-motile. Aerobic. Gram-positive. Acid-fast. $\dagger$ Pathogenic species grow slowly (several weeks); those from soil, water and vegetation more rapidly (several days).

There is a single genus Mycobacterium Lehmann and Neumann.

\section{Genus I. Mycobacterium Lchmann and Neumann.}

(Coccothrix Lutz, Zur Morphologie des Mikroorganismus der Lepra. Dermatologische Studien, Heft 1, 1886, 22; Schlerothrix Metschnikoff, Arch. f. path. Anat. u. Physiol., 113, 1888, 70; Lehmann and Neumann, Bakt. Diag., 1 Aufl., 2, 1896, 108; Mycomonas Orla-Jensen, Cent. f. Bakt., II Abt., 22, 1909, 329; Eumyces Battaglia, Soc. Internaz. Microbiol. Boll. Sez. Ital., 10, 1938, 166.) From Greek myces, fungus and bacterium, a little rod.

Characters as for the family.

The type species is Mycobacterium tuberculosis (Schroeter) Lehmann and Neumann.

\section{Key to the species of yenus Mycobacterium.}

I. Parasites in warm-blooded animals; grow slowly on all media.

A. Grow slowly on glycerol agar in atmospheric air; experimentally infect guinea pigs and fowls.

1. Experimentally produces generalized tuberculosis in guinea pigs but not in rabbits and fowls. Growth enhanced by the addition of glycerol to most media. Generally pale yellow to orange pigmentation on serum media.

1a. Mycobacterium tuberculosis var. hominis.

2. Experimentally produces generalized tuberculosis in guinea pigs and rabbits but not in fowls. Growth not enhanced by addition of glycerol to media. Never pigmented.

1b. Mycobacterium tuberculosis var. bovis.

3. Experimentally produces generalized tuberculosis in fowls and rabbits but not in guinea pigs.

2. Mycobacterium arium.

B. Grows in primary culture on glycerol agar only when extracts of, or heatkilled acid-fast bacilli are added. Experimentally fails to infect guinea pigs or fowls.

3. Mycobacterium paratuberculosis.

C. Have not been grown on culture media thus far devised. Experimentally fail to infect guinea pigs or fowls.

1. Has not experimentally been transmitted to any animal species.

4. Mycobacterium leprae.

2. Occurs in wild rats, and can be experimentally transmitted to rats and some strains of mice.

5. Mycobacterium lepracmurium.

$\dagger$ Most acid-fast bacteria treated with carbol-auramin and decolorized with $\mathrm{NaCl}$ $\mathrm{HCl}$-alcohol show fluorescence under the microseope when they are radiated by long wavelength ultraviolet light (Haitinger, Fluorescenz-mikroskopie, Leipzig, 1938, 108 pp.; Ellinger, Biol. Revs., 15, 1940, 323-350 ; Richards, Jour. Bact., 44, 1942, 721).

For a discussion of the influence of environment on acid-fastness, see Salle and Moser, Internat. Jour. Leprosy, 5, 1937, 163. 
II. Saprophytes or parasites on cold-blooded animals; grow rapidly on most media.

A. Fail to survive $60^{\circ} \mathrm{C}$ for 1 hour.

1. Fail to grow at $47^{\circ} \mathrm{C}$.

a. Unable to utilize sorbitol.

6. Mycobacterium piscium.

7. Mycobacterium marinum.

8. Mycobacterium ranae.

9. Mycobacterium thamnopheos.

aa. Utilize sorbitol.

10. Mycobacterium friedmannii.

11. Mycobacterium spp.

2. Grows at $47^{\circ} \mathrm{C}$.

12. Mycobacterium lacticola.

B. Survives $60^{\circ} \mathrm{C}$ for 1 hour; grows at $47^{\circ} \mathrm{C}$.

13. Mycobacterium phlei.

\section{Mycobacterium tuberculosis} (Schroeter) Lehmann and Neumann. (Tuberkelbacillen, Koch, Mitteil. a.d. kaiserlich. Gesundheitsamte, 2, 1884, 6; Bacillus tuberculosis Schroeter, in Cohn, Kryptogamen Flora v. Schlesien, 3, 1886, 164; Bacillus tuberculosis Flügge, Die Mikroorganismen, 2 Aufl., 1886, 208; Coccothrix tuberculosis Lutz, Dermatol. Studien, 1, 1886, 22; Sclerothrix kochii Metchnikoff, Arch. f. path. Anat. u. Physiol., 113, 1888, 70; Bacterium tuberculosis Migula, in Engler and Prantl, Die natürlichen Pflanzenfamilien, I Abt., 1a, 1895, 23; Lehmann and Neumann, Bakt. Diag., 1 Aufl., 2, 1896, 363; Bacillus kochii, quoted from Lehmann and Neumann, idem; Discomyces tuberculosus (sic), quoted from Neveu-Lemaire, Précis de Parasitol. Humaine, 5th ed. 1921, 25; Sclerothrix tuberculosis Vuillemin, Encyclopédie Mycologique, Paris, 2, 1931, 134; Eumyces tuberculosis Battaglia, Soc. Internaz. Microbiol. Boll. Sez. Ital., 10, 1938, 166.) From M. L. tuberculosis, tuberculosis.

Two varieties of this species are commonly recognized, the human and the bovine.

1a. Mycobacterium tuberculosis var. hominis Lehmann and Neumann. (Human tubercle bacilli, Th. Smith, Trans. Assoc. Am. Phys., 11, 1896, 75; Mycobacterium tuberculosis typus humanus Lehmann and Neumann, Bakt. Diag.,
4 Aufl., 2, 1907, 550.) From Latin hominis, of man.

Common name: Human tubercle bacillus.

Description from Koch (loc. cit.) and Topley and Wilson (Princip. of Bact. and Immun., London, 2nd ed., 1936, 315).

Rods, ranging in size from 0.3 to 0.6 by 0.5 to 4.0 microns, straight or slightly curved, occurring singly and in occasional threads. Sometimes swollen, clavate or even branched. Stain uniformly or irregularly, showing banded or beaded forms. Acid-fast and acid-alcohol-fast. Gram-positive. Growth in all media is slow, requiring several weeks for development.

This bacterium contains mycolic acid (Stodola, Lesuk, and Anderson, Jour. Biol. Chem., 126, 1938, 505-513). The acid-fast mycolic acid combines more firmly with carbol-auramin than with carbol-fuchsin and this apparently accounts for the increased sensitivity of fluorescence microscopy for this bacterium (Richards, Science, 93, 1941, 190; Richards, Kline, and Leach, Amer. Rev. Tuberc., 44, 1941, 255-266).

Nutrient agar: No growth.

Glycerol agar colonies: Raised, thick, cream-colored, with a nodular or wrinkled surface and irregular thin margin.

Glycerol agar slant: After 4 weeks, raised, thick, confluent, cream-colored growth.

Nutrient broth: No growth. 
Glycerol broth: After 8 weeks, thick, white or cream-colored, wrinkled pellicle extending up the sides of the flask, no turbidity; granular or scaly deposit.

Dorset's egg slants: After 4 weeks, rather sparse, discrete or confluent, slightly raised, grayish-yellow growth with finely granular surface.

Glycerol egg slants: After 4 weeks, luxuriant, raised, confluent, gray to yellow growth, with granular surface, generally with nodular heaped-up areas.

Coagulated beef serum: After 4 weeks, thin, effuse, confluent, gray to yellow growth, with a very fine granular surface.

Glycerol beef serum: After 4 weeks, luxuriant, thick, raised, confluent, yellow: to orange-yellow growth, with coarsely granular surface, generally with irregularly heaped-up areas.

Litmus milk: Growth, but no change in the milk.

Glycerol potato: After 4 weeks, luxuriant, raised, confluent, cream-colored growth with a nodular or warty surface.

Carbohydrates: Glucose, fructose, arabinose and galactose are utilized; sucrose and lactose not utilized (Merrill, Jour. Bact., 20, 1930, 235, based on the examination of one strain)

Optinum temperature $37^{\circ} \mathrm{C}$

Optimum pH 7.4 to 8.0 (Ishimori, Ztschr. f. Hyg., 102, 192t, 329); pH 6.0 to 6.5 (Dernby and Näslund, Biochem. Zeit., 132, 1922, 392).

Pathogenicity: Produces tuberculosis in man, monkey, dog and parrot. Experimentally, it is highly pathogenic for guinea pigs but not for rabbits, cats, goats, oxen or domestic fowls

Intermediate mammalian types: Griffith (Lancet, 1, 1916-17, 721; Jour. Path. and Bact., 21, 1924, 54) has found aberrant types particularly in skin lesions of both man and ox, which are in certain characteristics intermediate between the human and the bovine varieties. He finds no evidence, however, that the one variety may change into the other.

Variation: Variation in colony structure of the two mammalian varieties, comparable with that in other species, has been described by several authors, as Petroff et al. (Jour. Exp. Med., 60, 1934, 515), Birkhaug (Ann. Inst. Past., 57, 1933, 428), Kahn et al. (Jour. Bact., 25, 1933, 157), Uhlenhuth and Sieffert (Zeit. Immun., 59, 1930, 187), Reed and Rice (Canad. Jour. Res., 5, 1931, 111), Smithburn (Jour. Exp. Med., 63, 1936, 95) and Shaffer (Jour. Path. and Bact., 40, 1935, 107). Several of these authors have found associated variation in cell structure and in virulence though Boquet (Compt. rend. Soc. Biol. Paris, 103, 1930, 290), Birkhaug (Ann. Inst. Past., 49, 1932,630 ), and others, have failed to find differences in virulence. Reed and Rice (Jour. Immunol., 23, 1932, 385) found the $\mathrm{S}$ form to contain an antigenic substance lacking in the $\mathrm{R}$ form.

Antigenic structure: By agglutination, absorption of agglutinins and complement fixation a distinction may be made between the mammalian varieties and Mycobacterium avium, but it has been impossible to distinguish, by these means, between the two mammalian varieties (Tullock et al., Tubercle, 6, Oct.-Dec., 1924, 18, 57 and 105; Wilson, Jour. Path. and Bact., 28, 1925, 69; Griffith, Tubercle, 6, May, 1925, 417; Rice and Reed, Jour. Immunol., 23, 1932, 385; Kauffman, Ztschr. f. Hyg., 114, 1932, 121). Tuberculins prepared from the human and the bovine varieties are ordinarily indistinguishable in their action but Lewis and Seibert (Jour. Immunol., 20, 1931, 201) detected a difference by cross anaphylactic reactions.

Distinctive characters: Tubercle bacilli pathogenic for guinea pigs and rabbits, not for fowls. Mycobacterium tuberculosis var. hominis produces generalized tuberculosis in guinea pigs but not in rabbits. Mycobaclerium tuberculosis var. bovis produces generalized disease in both guinea pigs and rabbits. Growth of the human variety is enhanced by the addition of glycerol to most media. The growth of the bovine variety is not enhanced by the addition 
of glycerol. The human variety generally develops yellow to red pigment on serum media, while the bovine variety never produces pigment. Antigenically the two varieties are not distinguishable.

Source: From tuberculous lesions in man.

Habitat: The cause of tuberculosis in man. Transmissible to rabbits and guinea pigs.

1b. Mycobacterium tuberculosis var. bovis Lehmann and Neumann. (Bovine tubercle bacilli, Th. Smith, Trans. Assoc. Am. Phys., 11, 1896, 75; 13, 1895, 417; Jour. Exp. Med., 3, 1898, 451; Hycobacterium tuberculosis typus bovinus Lehmann and Neumann, Bakt. Diag., 4 Aufl., 2, 1907, 550.) From Latin bovis, of the ox.

Common name: Bovine tubercle bacillus.

Description from Th. Smith (loc. cit.) and Topley and Wilson (Princip. of Bact. and Immun., 2nd ed., 1936, 315).

Rods which are shorter and plumper than the human type. Range in size from 1.0 to 1.5 microns. Very short forms are frequently intermixed with somewhat larger forms. Stain regularly or irregularly. Acid-fast and acid-alcohol-fast. Gram-positive. Less easily cultivated than the human variety.

Nutrient agar: No growth.

Glycerol agar colonies : Small, irregular, with granular surface, no pigment.

Glycerol agar slant: After 4 weeks, thin, granular or effuse, confluent growth.

Nutrient broth: No growth.

Glycerol broth: After 8 weeks, thin grayish-white film, slightly nodular, no turbidity. Slight granular deposit.

Dorset's egg slants: After 4 weeks, similar to var. hominis but generally poorer growth and no pigmentation.

Glycerol egg slants: After 4 weeks, similar to Dorset's egg slants.

Coagulated beef serum: After 4 weeks, thin, effuse, confluent, white to gray growth with very fine granular surface.
Generally less luxuriant than in the human variety.

Glycerol beef serum: After 4 weeks, similar to plain beef serum.

Glycerol potato: After 4 weeks, thin, effuse, grayish growth.

Litmus milk: Growth, but no change in the milk.

Optimum temperature $37^{\circ} \mathrm{C}$.

Optimum pH 5.8 to 6.9 (Ishimori, Ztschr. f. Hyg., 102, 1924, 329); 6.0 to 6.5 (Dernby and Näslund, Biochem. Zeit., 132, 1922, 392).

Pathogenicity: Produces tuberculosis in ox, man, monkey, goat, sheep, pig, cat, parrot, cockatoo and possibly some birds of prey. Experimentally, it is highly pathogenic for rabbit and guinea pig, slightly pathogenic for dog, horse, rat and mouse; not pathogenic for fowls.

Variation: See Mycobacterium tuberculosis var. hominis.

Antigenic structure: See Mycobacterium tuberculosis var. hominis.

Distinctive characters: See Mycobacterium tuberculosis var. hominis.

Source: From tubercles in cattle.

Habitat: The cause of tuberculosis in cattle. Transmissible to man and domestic animals. More highly pathogenic for animals than the human type.

2. Mycobacterium avium Chester. (Tuberculose des oiseaux, Strauss and Gamaléia, Arch. Méd. exp. et Anat. path., 1891; Bacillus der Hühnertuberculose, Maffucci, Ztschr. f. Hygiene, 11, 1892, 449; Bacillus tuberculosis gallinarum Sternberg, Man. of Bact., 1893, 392; Mycobacterium tuberculosis avium Lehmann and Neumann, Bakt. Diag., 1 Aufl., 1, 1896, 370; Bacillus tuberculosis avium Kruse, in Flügge, Die Mikroorganismen, 3 Aufl., 2, 1596, 506; Mycobacterium avium Chester, Manual Determ. Bact., 1901, 357; Mycobacterium tuberculosis typus gallinaceus Lehmann and Neumann, Bakt. Diag., 4 Aufl., 2, 1907, 553.) From Latin avis, bird.

Common name: Avian tubercle bacillus. 
Description from Strauss and Gamaléia (loc. cit.) and Topley and Wilson (Princip. of Bact. and Immun., 2nd ed., 1936, 315).

Rods resembling those of the bovine type of tubercle organism.

Nutrient agar: After 4 weeks, slight growth, effuse, translucent with fine granular surface.

Glycerol agar colonies: After 3 to 4 weeks, raised, regular, hemispherical, creamy or white colonies.

Nutrient broth: After 4 weeks, very slight viscous to granular bottom growth, no pellicle, no turbidity.

Glycerol broth: After 4 weeks, diffuse, turbid growth with a viscous to granular deposit.

Dorset's egg slants : After 4 weeks, confluent, slightly raised growth, with smooth regular surface.

Glycerol egg slants: After 4 weeks, luxuriant, raised, confluent, creamy to yellow growth with perfectly smooth surface.

Coagulated beef serum: After 4 weeks, thin, effuse, grayish-yellow growth with smooth surface.

Glycerol beef serum: After 4 weeks, luxuriant, raised, confluent, yellow to orange-yellow or occasionally pale pink growth, with a smooth glistening surface.

Glycerol potato: After 4 weeks, luxuriant, raised, confluent, with smooth to nodular surface.

Litmus milk: Growth, but no change in the milk.

Carbohydrates : Fructose, arabinose and sucrose are utilized, glucose is slightly utilized, galactose and lactose are not utilized (Merrill, Jour. Bact., 20, 1930, 235 , based on the examination of one strain).

Optimum temperature $40^{\circ} \mathrm{C}$; range $30^{\circ}$ to $44^{\circ} \mathrm{C}$ (Bynoe, Thesis, MeGill University, Montreal, 1931).

Optimum pH 6.8 to 7.3 (Bynoe, loc. cit.).

Pathogenicity: Produces tuberculosis in domestic fowls and other birds. In pigs it produces localized and sometimes disseminated disease. Experimentally in the rabbit, guinea pig, rat and mouse it may proliferate without producing macroscopic tubercles-tuberculosis of the Yersin type. Man, ox, goat, cat, horse, dog and monkey are not infected.

Variation: Winn and Petroff (Jour. Exp. Med., 57, 1933, 239), Kahn and Schwartzkopf (Jour. Bact., 25, 1933, 157), Birkhaug (Ann. Inst. Pasteur, 54, 1935, 19), Reed and Rice (Canad. Jour. Res., 5, 1931, 111) and others, have shown variation to follow the course described for many species. Winn and Petroff have separated four colonial types: smooth, flat smooth, rough, deep yellow smooth. These also differ in chemical and physical properties. The smooth form exhibited the greatest degree of virulence, the flat smooth a lower virulence, while the chromogenic smooth and the rough were relatively benign. Some authors have failed to demonstrate this difference in virulence. The above description applies primarily to the smooth form.

Antigenic structure: By agglutination, absorption of agglutinins and complement fixation $M$ ycobacterium avium may be distinguished from other members of the genus (Tullock et al., Tubercle, 6, 1924, 18, 57 and 105; Wilson, Jour. Path. and Bact., 28, 1925, 69; Mudd, Proc. Soc. Exp. Biol. and Med., 23, 1925, 569, and others). Furth (Jour. Immunol., 12, 1926, 273) and Shaffer (Jour. Path. and Bact., 40, 1935, 107) on this basis divided Mycobacterium avium into 1 or 2 subgroups.

Distinctive characters: Tubercle bacilli pathogenic for fowls, not for guinea pigs or rabbits. Culturally distinguished from the mammalian types by the absence of pellicle formation in fluid media and the habit of growth on most solid media. Antigenically distinguished from other species.

Source: From tubercles in fowls, widely distributed as the causal agent of tuberculosis in birds and less frequently in pigs. 
Habitat: The cause of tuberculosis in chickens. Transmissible to pigeon, other birds, mouse, rabbit and pig.

\section{Mycobacterium paratuberculosis}

Bergey et al. (Darmtuberculose bacillen, Johne and Frothingham, Deutsch. Ztschr. Tiermed., 21, 1895, 138; Pseudotuberkulose bacillen, Bang, Berl. tierärztl. Wchnschr., 1906, 759; Bacillus of Johne's Disease, M'Fadyean, Jour. Comp. Path., 20, 1907, 48; Twort, Proc. Roy. Soc., B, 83, 1910, 156; Bergey et al., Manual, 1st ed., 1923, 37t.) From M. L. paratuberculosis, of the disease paratuberculosis.

Common name: Johne's bacillus.

The organism from a similar disease in sheep is probably identical though more difficult to cultivate Dunkin and Balfour-Jones. Jour. Comp. Path., 48, 1935, 236).

Description from M'Fadyean (loc. cit.) and Twort and Ingram (A Monograph on Johne's Disease, London, 1913 ).

Plump rods, 1.0 to 2.0 microns in length, staining uniformly, but occasionally the longer forms show alternately stained and unstained segments. Yon-motile. Acid-fast .

The organism is difficult to cultivate and, in primary cultures, has only been grown in media containing dead tubercle bacilli or other dead acid-fast bacteria (Boquet, Ann. Inst. Pasteur, 37, 1928, 495). In a few instances cultures have been acclimatized to a synthetic medium free from added dead bacteria (Dunkin. Jour. Comp. Path. and Therap., 46, 1933, 159; Watson, Canad. Pub. Health Jour.. $26,1935,268$ ).

Colonies on glycerol agar containing heat-killed Mycobacterium phlei: After \pm to 6 weeks, just distinguishable, dullwhite, raised, circular colonies.

Colonies on Dorset's glycerol egg containing heat-killed Mycobacterium phlei: After 4 to 6 weeks, minute, dull-white. raised, circular, with a thin, slightly. irregular margin. Older colonies become more raised, radially striated or irregularly folded and dull yellowish-white.

Dorset's glycerol egg containing sheep's brain and heat-killed Iycobacterium phlei: Growth slightly more luxuriant.

Glycerol broth containing heat-killed Mycobacterium phlei: Thin surface pellicle which later becomes thickened and folded.

Dorset's synthetic fluid containing heat-killed Mycobacterium phlei: As on glycerol broth with. Mycobacterium phlei.

Pathogenicity: Produces Johne's disease, chronic diarrhea, in cattle and sheep. Experimentally it produces a similar disease in bovine animals, sheep and goats. Guinea pigs, rabbits, rats and mice are not infected. Very large doses in laboratory animals produce slight nodular lesions comparable with those produced by Mycobacterium phlei.

Antigenic structure: Johnin, prepared as tuberculin, gives positive reactions in cattle with Johne's disease. According to M'Fadyean et al. (Jour. ('omp. Path. and Therap., 29, 1916, 62) tuberculous animals may also give a reaction. Plumb (Den Kong. Vet. Landbohøjskole Arssk., 1925, 63) has shown that a reaction may be produced in animals sensitized to avian tuberculin and that avian tuberculin causes a reaction in some animals infected with Johne's bacillus.

Distinctive characters: A small acidfast bacillus producing characteristic lesions in cattle and growing only in the presence of dead acid-fast bacilli.

Source: From the intestinal mucous membrane of cattle suffering from chronic diarrhea. Apparently an obligate parasite.

Habitat: The cause of Johne's disease, a chronic diarrhea in cattle. The bacteria are found in the intestinal mucosa. Not pathogenic for guinea pigs or rabbits.

4. Mycobacterium leprae (ArmauerHansen) Lehmann and Neumann. (Bacillus leprae Armauer-Hansen, Norsk. Mag. I aegevidensk., 9, 1S7t, 1; Arch. f. path. Anat. u. Physiol., 79, 1879, 
32; Nord. Med. Ark., 12, 1880, 1; Quart. Jour. Micro. Sci., 20, 1880, 92; Coccothrix leprae Lutz, Dermatol. Stud. 1, 1886, quoted from DeToni and Trevisan, in Saccardo, Sylloge Fungorum, 8, 1889, 944; Lehmann and Neumann, Bakt. Diag., 1 Aufl., 2, 1896, 372; Discomyces leprae Neveu-Lemaire, Précis Parasit. Hum., 5th ed., 1921, 27; Sclerothrix leprae Vuillemin, Encyclopédie Mycologique, Paris, 1, 1921, 135; Mycobacterium leprae hominus Lowe, Internat. Jour. Leprosy, 5, 1937, 312.) From Greek lepra, leprosy

Common name: Leprosy bacillus.

Armauer-Hansen (loc. cit.) was the first to observe the bacilli in the tissues of lepers. The disease is now known as Ilansen's disease. The bacilli occur in enormous numbers in lepromatous (nodular) cases of the disease and sparsely in the neural form. The present bacteriological means of identification depend on: (a) acid-fast staining, and (b) failure of the organism to grow in bacteriological media or in laboratory animals. Heated suspensions of the bacilli (obtained from nodules) produce a positive lepromin reaction in 75 to 97 per cent of normal persons and of neural cases of leprosy, but usually produce no reaction in lepromatous individuals (Mitsuda: See Hayashi, Int. Jour. Leprosy, 1, 1933, 31-38). The failure of lepromatous persons to respond to injected leprosy bacilli constitutes : fundamental criterion for testing the validity of microorganisms such as other acid-fast or diphtheroid cultures which can at times be recovered from leprous tissues by inoculation of bacteriological media.

Many organisms have been isolated from leprous tissues, some of which are acid-fast and have been styled $M y c o-$ bacterium leprae. The strains which have been adequately studied have proven to fall into the saprophytic groups (see No. 11, Mycobacterium spp.) Hanks (Int. Jour. Leprosy, 9, 1941, 275298) found that acid-fast cultures of this type, as well as the diphtheroids which also have repeatedly been isolated from leprosy, were recoverable only from lesions located proximally with respect to open ulcers in the skin.

Description of organisms seen in leprosy tissue from Armauer-Hansen (loc. cit.) and Topley and Wilson (Princip. Bact. and Immun., 2nd ed., 1936, 316).

Rods : 0.3 to 0.5 by 1 to 8 microns, with parallel sides and rounded ends, staining evenly or at times beaded. When numerous, as from lepromatous cases, they are generally arranged in clumps, rounded masses or in groups of bacilli side by side. Strongly acid-fast. Grampositive.

Pathogenicity: The communicability of leprosy from man to man is accepted (Rogers and Muir, Leprosy, 2nd ed., Balt imore, 1940, 260 pp.). Experimental transmission to humans or to animals has not been successful.

Source: Human leprous lesions. In the lepromatous form of the disease bacilli are so abundant as to produce stuffed-cell granulomas; in the tuberculoid and ncutral lesions they are rare.

Habitat: Obligate parasite in man. Confined largely to the skin (especially to convex and exposed surfaces) and to peripheral nerves. The microorganisms probably do not grow in the internal organs.

5. Mycobacteriun lepraemurium Marchoux and Sorel. (Bacillus der Rattenlepra, Stefansky, Cent. f. Bakt., I Abt., Orig., 33, 1903, 4.41; Mycobacterium leprae murium Marchoux and Sorel, Ann. Inst. Past., 26, 1912, 700; Bucillus leprae murium Muir and Henderson, Indian Jour. Med. Res., 15, 1927, 15.)

Mycobacterium pulviforme Marchoux (Ann. Derm., 1921, No. 21 and Ann. Inst. Past., 37, 1923, 348) from leprosy. like lesions in a man from Hayti is thought by the author to be identical with Mycobacterium lepraemurium.

Common name: Rat leprosy bacillus. 
Rods: 3.0 to 5.0 microns in length with slightly rounded ends. When stained, often show irregular appearance. Strongly acid-fast. Gram-positive.

Like the human leprosy bacillus, this organism has not been cultivated in vitro; but can be passed experimentally through rats and some strains of mice.

Distinctive features: The heat-killed bacilli produce lepromin reactions in lepratomous humans. The bacilli from lesions are not bound together in clumps, rounded masses and palisades as in human lesions. For further details see review by Lowe (Internat. Jour. Leprosy, 5, 1937, 310 and 463)

Source: An endemic disease of rats in various parts of the world, having been found in Odessa, Berlin, London, New South Wales, Hawaii, San Francisco and elsewhere.

Habitat: The natural disease occurs chiefly in the skin and lymph nodes, causing induration, alopecia (loss of hair) and eventually ulceration.

\section{Mycobacterium piscium Bergey et} al. (Bacillus tuberculosis piscium Dubard, Bull. acad. de méd., 3 sér., 38, 1897, 580; Bataillon, Dubard and Terre, Compt. rend. Soc. Biol., 4, sér. 10, 1897, 446; Bergey et al., Manual, 1st ed., 1923, 375.) From Latin piscis, fish.

Description from Bataillon et al. (loc. cit.) and Aronson (Jour. Inf. Dis., 39, 1926, 319).

Slender rods, occurring singly and in threads, occasionally showing branching. Acid-fast. Non-motile. Gram-positive.

Agar colonies: Small, circular, white, moist, with lobate margin and fine granular surface.

Agar slant: Scant, white, moist, creamlike.

Glycerol agar colonies: Thin, flat, smooth, glistening, yellow.

Dorset's egg medium: Flat, smooth, moist, greenish.

Broth: Thin pellicle, with floceulent sediment.
Litmus milk: Thickened. No coagulation. Slightly alkaline.

Potato: White, warty, butyrous colonies.

Carbohydrates: Utilizes glucose and fructose but not sucrose, lactose, arabinose or galactose (Merrill, Jour. Bact., 20, 1930,235 , based on examination of one strain).

Antigenic structure: By agglutination and complement fixation (Mudd, Proc. Exp. Biol. and Med., 23, 1925, 569; and Furth, Jour. Immunol., 12, 1926, 286) Mycobacterium piscium has been distinguished from Mycobacterium friedmannii, Mycobacterium ranae and probably Mycobacterium marinum. From the limited number of cultures examined it is not evident whether this is due to species or strain specificity.

Pathogenicity: Experimentally produces tubercles in carp, frog and lizard, but not pathogenic for rabbit, guinea pig or birds (Dubard, Rev, de la Tuberc., 6, 1898, 13). Not pathogenic for salt water fish except eels (Betegh, Cent. f. Bakt., I Abt., Orig., 53, 1910, 374; 54, 1910, 211).

Distinctive characters: Mycobacterium piscium, Mycobacterium marinum, Mycobacterium ranae, Mycobacterium thamnopheos and Mycobacterium friedmannii constitute a closely related group--possibly one species. They differ from other members of the genus in their pathogenicity for cold-blooded animals, their failure to survive $60^{\circ} \mathrm{C}$ for an hour, their failure to grow at $47^{\circ} \mathrm{C}$ and their inability to utilize sorbitol.

Mycobacterium marinum is distinguished by its diffuse growth in broth, acid production in milk and deep yellow to orange pigmentation on most media. The other species grow in broth as a pellicle and render milk alkaline. Mycobacterium piscium, Mycobacterium ranae, Mycobacterium thamnopheos and Mycobacterium friedmannii may be distinguished from each other by their habit of growth on solid media. But relatively few cultures have been studied 
and the reports in certain important respects are conflicting, especially concerning pigmentation and utilization of carbohydrates. Aronson, Mudd and Furth found them to differ antigenically, but too few cultures were used to distinguish between species and strain specificity.

Source: From tubercles in earp.

Habitat: The cause of nodule and tumor-like formations in carp (Ciprinus carpio). Infectious for carp, frogs, lizards. Not infectious for guinea pigs and pigeons.

7. Mycobacterium marinum Aronson. (Jour. Inf. Dis., 39, 1926, 315.) From Latin marinus, marine.

Description from Aronson (loc. cit.).

In lesions, short, thick, uniformly staining organisms are seen frequently occurring in clumps, while long, thin, beaded or barred rods are scattered more discretely. In cultures the organisms have the same appearance. Non-motile. Acid-fast, and acid-alcohol-fast. Grampositive.

Agar slant (slightly acid): In five to seven days, moist, glistening, elevated colonies, becoming lemon-yellow.

Gelatin: Not liquefied.

Agar colonies: In 5 to 7 days, smooth, moist, slimy, lemon-yellow, later orangecolored.

Glycerol agar colonies: In 14 to 18 days, grayish-white, moist, elevated with irregular margins. Old growths lemonyellow and still later orange-colored.

Dorset's and Petroff's egg media : Similar to growth on glycerol agar but more luxuriant.

Broth and glycerol broth: Growth is diffuse, no pellicle formed.

Litmus milk: Acidified and coagulated.

Indole not formed.

Nitrites not produced from nitrates.

Carbohydrates: Utilizes arabinose and fructose, fails to utilize sorbitol and galactose (Gordon, Jour. Bact., 34, 1937, 617 ).

Aerobic, facultative.
Optimum temperature $18^{\circ}$ to $20^{\circ} \mathrm{C}$. Fails to survive $60^{\circ} \mathrm{C}$ for 1 hour, fails to grow at $47^{\circ} \mathrm{C}$ (Gordon, Jour. Bact., 34, 1937, 617).

Pathogenicity : Experimentally infects salt water fish, goldfish, frogs, mice and pigeons, but not rabbits or guinea pigs.

Antigenic structure: By agglutination and complement fixation distinguished from Mycobacterium ranae, Mycobacterium friedmannii, and probably Mycobacterium piscium (Mudd, Proc. Soc. Exp. Biol. and Med., 23, 1925, 569; Furth, Jour. Immunol., 12, 1926, 286). See Mycobacterium piscium.

Distinctive characters: See Mycobacterium piscium.

Source: From areas of focal necrosis of the liver of sergeant majors (Abudefduf mauritii), croakers (Micropogon undulatus) and sea bass (Centropristes striatus).

Habitat: Causes spontaneous tuberculosis in salt water fish.

\section{Mycobacterium ranae (Küster)} Bergey et al. (Küster, Münch. med. Wchnschr., 52, 1905, 57; Bergey et al., Manual, 1st ed., 1923, 374.) From Latin rana, frog.

Description from Küster (loc cit.), Bynoe (Thesis, Mefill University, Montreal, 1931) and Aronson (Jour. Inf. Dis., 44, 1920, 222).

Slender rods, 0.3 to 0.5 by 2 to $8 \mathrm{mi}$ crons, smaller in old cultures. Uniformly acid-fast in cultures 2 weeks old or older. In younger cultures the staining is irregular, many organisms are not acid-fast. Non-motile. Gram-positive.

Gelatin stab: No liquefaction.

Agar colonies: Irregular, raised colonies, 1 to $3 \mathrm{~mm}$ in diameter with moist glistening surface, later becoming coarsely granular.

Agar slant: Thick, spreading, glistening. In old cultures dry and scaly. Putrid odor. Grayish-white.

Glycerol agar colonies: Similar to gelatin colonies but slightly creamy and becoming dry and wrinkled in old cultures. 
Dorset's egg medium: Spreading, raised, glistening, later wrinkled.

Loeffler's medium: Similar to Dorset's egg medium, white to buff-colored.

Litmus milk: Becomes alkaline.

Glycerol broth: Grayish flaky pellicle which breaks up early and settles.

Broth: Slightly turbid, with slight sediment.

Potato: Scanty, grayish growth, raised with a warty surface.

Indole not formed.

Nitrites are produced from nitrates.

Carbohydrates: Glucose, fructose and arabinose are utilized; sucrose, lactose and galactose not utilized (Merrill, Jour. Bact., 20, 1930, 235). Fructose, mannitol and trehalose are utilized; sorbitol, arabinose and galactose are not utilized (Gordon, Jour. Bact., 34, 1937, 617).

No $\mathrm{H}_{2} \mathrm{~S}$ formed.

Optimum temperature $28^{\circ} \mathrm{C}$ (Küster), $37^{\circ} \mathrm{C}$ (Bynoe).

Optimum pH 6.6 to 7.3 , range 4.0 to 10.0 .

Antigenic structure: By agglutination and complement fixation Mycobacterium ranae may be distinguished from $\mathbf{M}$ ycobacterium piscium and Mycobacterium friedmannii (Mudd, Proc. Soc. Exp. Biol. and Med., 28, 1925, 569; Furth, Jour. Imnunol., 12, 1926, 286). See Mycobacterium piscium.

Pathogenicity: Experimentally causes tubereulosis in frogs, lizards, turtles; not pathogenic for rabbits, guinea pigs, rats or mice.

Distinctive characters: See Mycobucterium piscium.

Source: From the liver of a frog.

Habitat: In a group of 215 cultures belonging to the genus, isolated from soils, Gordon (Jour. Bact., 34, 1937, 617) found 65 to sufficiently resemble $I$ ycobacterium ranae to indicate at least a very close relationship. If they prove to be identieal, the species is widely distributed.

9. Mycobacterium thamnopheos Aronson. (Jour. Inf. Dis., 44, 1929, 222.) From Thamnophis, a genus of snakes.
Tuberculbacillen bei Schlangen, Sibley, Arch. f. pathol. Anat. u. Physiol., $116,1889,104$ (Mycobacterium tropidonatum (sic) Bergey et al., Manual, 1st ed., 1923,376 ) is probably identical, but the descriptions are too meager to be conclusive. Acid-fast bacilli described by Gibbes and Shurley (Amer. Jour. Med. Sci., $100,1890,145$ ) as the cause of tuberculosis in boas and pythons; by Shattock (Trans. Path. Soc., London, $53,1902,430)$ and by von Hanseneann (Cent. f. Bakt., I Abt., Orig., 34, 1903, 212) as causing tuberculosis in a Python molurus, are possibly identical, but the descriptions do not permit us to draw any conclusions. According to Aronson, similar organisms isolated from pathological lesions in boa constrictors and Caluber catonifer differ antigenically from Mycobacterzum thamnopheos.

Description taken from Aronson (loc. cit.) and Bynoe (Thesis, McGill University, Montreal, 1931).

Slender rods : 0.5 by 4 to 7 microns, frequently slightly curved, beaded and barred forms frequently occur. Nonmotile. Acid-fast in cultures of 4 days or older, in younger cultures some organisms are not acid-fast. Not alcoholfast. Gram-positive.

Gelatin stab: Growth occurs along the line of inoculation. No liquefaction.

Agar colonies : 0.5 to $1 \mathrm{~mm}$ in diameter, irregular, raised, moist and glistening.

Glycerol agar: Spreading, raised, dry, pale pink to buff growth.

Glycerol broth: A thin pellicle appears in 5 to 6 days, gradually becomes thicker and falls as a sediment.

Dorset's egg medium: Raised, moist, pinkish growth after 10 days, later becoming salmon-colored.

Loeffler's serum : Small, raised, convex, dry growth.

Litmus milk: Alkaline.

Glycerol potato: Raised, hemispherical, dry and granular growth.

Indole not produced.

Nitrates: Not reduced by 2 strains, 
reduced by 1 strain (Aronson); slightly reduced (Gordon); not reduced (Bynoe).

Carbohydrates: Utilizes fructose, mannitol and trehalose; fails to utilize arabinose, sucrose, galactose and sorbitol (Gordon, Jour. Bact., 34, 1937, 617).

Temperature relations : Fails to survive $60^{\circ} \mathrm{C}$ for 1 hour, fails to grow at $47^{\circ} \mathrm{C}$ (Gordon); good growth at $25^{\circ} \mathrm{C}$, no growth at $37^{\circ} \mathrm{C}$ (Aronson); optimum for growth $25^{\circ} \mathrm{C}$, range $10^{\circ}$ to $35^{\circ} \mathrm{C}$ (Bynoe).

Range of $\mathrm{pH}: 6.6$ to 7.8 (Aronson); optimum 7.3 to 8.0 , range 5.0 to 11.0 (Bynoe).

Pathogenicity: Experimentally produces generalized tuberculosis in snakes, frogs, lizards and fish but not pathogenic for guinea pigs, rabbits or fowls.

Antigenic structure: By agglutination and absorption of agglutinins Mycobacterium thamnopheos may be distinguished from Mycobacterium marinum, Mycobacterium friedmannii and Mycobacterium ranae. See Mycobacterium piscium.

Variation: According to Bynoe and Wyckoff (Amer. Rev. Tub., 29, 1934, 389) $\mathrm{S}$ and $\mathrm{R}$ forms may be distinguished by colony structure and individual cell arrangement.

Distinctive characters: See Mycobacterium piscium.

Source: From the lungs and livers of garter snakes (Thamnophis sirtalis).

Habitat: Present as a parasite in the garter snake and possibly other coldblooded vertebrates.

\section{Mycobacterium friedmannii Hol-} land. (Schildkröten tuberkelbacillus, Friedmann, Cent. f. Bakt., I Abt., Orig., 34, 1903, 647; Bacillus friedmanii (sic) Holland, Jour. Bact., 5, 1920, 218; Mycobacterium friedmanii Holland, ibid.; Mycobacterium chelonei Bergey et al., Manual, 1st ed., 1923, 376.) Named for Dr. Friedmann, who isolated this organism.

Common name: Turtle bacillus.

Description from Friedmann (loc. cit.) and Aronson (Jour. Inf. Dis., 44, 1929, 222).
Slender rods: 0.2 to 0.4 by 0.5 to 5 microns. Beaded forms are common. Acid-alcohol-fast in young cultures but in cultures two weeks old generally there are many non-acid-fast rods. Non-motile. Gram-positive.

Gelatin stab: White surface growth, scanty growth along the line of stab. No liquefaction.

Agar colonies : 1 to $3 \mathrm{~mm}$ in diameter, irregularly round, raised, moist, glistening, white.

Glycerol agar slants: Thick, spreading growth, at first moist, later granular, yellowish-white (Friedmann); olive-gray (Bynoe); white (Aronson).

Glycerol broth: Thick wrinkled pellicle after two to three days growth, later some membranous sediment. Gravishyellow (Friedmann); grayish-white (Bynoe).

Dorset's egg medium: Spreading, raised, slightly moist, pale buff.

Loeffler's serum: Scant growth, raised, dry, crumb-like.

Litmus milk: Slightly alkaline after 10 days growth.

Glycerol potato: Thick, wrinkled, gray after 2 days growth.

Indole not formed.

Carbohydrates: Glucose, fructose and arabinose utilized, sucrose slightly utilized, galactose and lactose not utilized (Merrill, Jour. Bact., 20, 1930, 235). Arabinose not utilized (Gordon, Jour. Bact., 34, 1937, 617).

Optimum temperature $25^{\circ}$ to $30^{\circ} \mathrm{C}$.

Pathogenicity: Experimentally produces tubercles in most species of coldblooded animals, possibly in guinea pigs but not in other warm-blooded animals.

Variation: According to Gildemeister (Cent. f. Bakt., I Abt., Orig., 86, 1921, 513) S and P types may be distinguished on glycerol agar. The S grows as smooth, moist, glistening, convex colonies; the $\mathrm{R}$ as flat, dry, spreading colonies. Wykoff (Amer. Rev. Tub., 29, 1934, 289) has shown a difference in the form of cell division and corresponding cell arrangement of the two types. 
Distinctive characters: See Mycobacterium piscium.

Source: From the lungs of turtles in the Berlin aquarium.

Habitat: A parasite in turtles and possibly sparingly distributed in soils. Gordon (Jour. Bact., 34, 1937, 617) found 65 out of 215 soil cultures of members of the genus to closely resemble this species.

11. Mycobacterium spp. (A miscellaneous group many of which have been incorrectly identified as Mycobacterium leprae Lehmann and Neumann.)

Clegg (Phil. Jour. Sci., 4, 1909, 77 and 403), Duval (Jour. Exp. Med., 12, 1910, 649), Duval and Wellman (Jour. Inf. Dis., 11, 1912, 116), Currie, Brinckerhoff and Hollmann (Pub. Health Rep., 25, 1910, 1173 ) and others have described as $\mathrm{Myco-}$ bacterium leprae a group of organisms isolated from leprosy lesions. Much evidence, summarized by McKinley (Medicine, 13, 1931, 377), points to the conclusion that these organisms are not pathogenic and not the causal agent of leprosy. They cannot therefore be included under Mycobacterium leprae as defined above.

Thomson (Amer. Rev. Tub., 26, 1932, 162), Gordon (Jour. Bact., 34, 1937, 617), and Gordon and Hagan (Jour. Bact., 36, 1938 , 39) recently separated the saprophytic members of the genus Mycolacterium into three main groups and several subgroups. Species names as here defined have been added to the key as follows :

Group I. Fail to survive $60^{\circ} \mathrm{C}$ for 1 hour. Grow at $47^{\circ} \mathrm{C}$.

a. Utilizes arabinose. IIycobacterium lacticola.

b. Unable to utilize arabinose.

Mycobacterium sp.

Group II. Fail to survive $60^{\circ} \mathrm{C}$ for 1 hour. Do not grow at $47^{\circ} \mathrm{C}$. a. Unable to utilize sorbitol.

1. Unable to utilize arabinose.

Mycobacterium ranae.

Mycobacterium thamnopheos.

Mycobacterium sp.

2. Utilize arabinose.

Mycobacterium mar. inum.

Mycobacterium sp.

b. Utilizes sorbitol.

Mycobacterium spp.

c. Unable to utilize most carbohydrates.

Mycobacterium friedmannii.

Mycobacterium sp.

Group III. Survive $60^{\circ} \mathrm{C}$ for 1 hour. Grow at $47^{\circ} \mathrm{C}$.

a. Utilizes arabinose.

Mycobacterium phlei.

b. Unable to utilize arabinose.

Mycobacterium $s p$.

In this study Gordon and Hagan included many recently isolated soil forms, named saprophytic species, pathogens for cold-blooded animals and 19 cultures, from various collections, which bore the name Mycobacterium leprae. Of these so-called Mycobacterium leprae, six belong to Group I which corresponds with Mycobacterium lacticola and includes many soil forms, two belong to Group IIa which includes Mycobacterium ranae, Mycobacterium thamnopheos and a number of undefined soil forms, while eleven belong to Group IIb. The lat ter group includes a number of soil cultures but no other defined species.

In the several groups to which so-called Mycobacterium leprae strains belong, some appear to be indistinguishable from soil forms, others are distinguished by habit of growth, utilization of carbohydrates or by pigmentation.

12. Mycobacterium lacticola Lehmann and Neumann. (Bakt. Diag., 2 Aufl., 2, 
1899, 409.) From Latin lac, lactis, milk and colo, to dwell; hence, a milk dweller.

From the fact that Lehmann and Neumann (loc. cit., 411) refer to the binomial Bacillus friburgensis Korn, it is evident that the species name friburgensis (see Appendix) published the same year (1899) has priority over the species name lacticola. However, since it has never been used with the broad meaning given Mycobacterium lacticola by Lehmann and Neumann in the original description, it is not substituted for the more commonly used Mycobacterium lacticola in this edition of the Manual.

Deseription from Lehmann and Neumann (loc. cil) and Jensen (Proe. Linnean Soc. of New ho. Wales, 59, 1934, 19$)$.

Slender rods : 0.5 to 0.7 by 2 to 8 microns in young cultures, in older cultures the rods are shorter and frequently coccoid in shape. (urved and irregular forms oceur oceasionally. Branched forms if they oreur, are very rare. Staining is generally uniform but slight beading oceurs occasionally. Strongly acid-fast except organisms from glueose-containing nedia which are sometimes only faintly acid-fast. (iram-positive.

Gelatin colonies: Similar to those on agar.

Gelatin stab: Filiform growth in stab. No liquefaction.

Agar colonies : Convex, glistening, with entire margins, at first smooth but after 10 to 14 days growth folded or wrinkled. Opaque, at first white, after 2 or 3 days growth becomes yellow

Glucose agar: Similar to agar but more rapid growth and less intensely pigmented.

Glycerol agar slants: spreading, moist, wrinkled, pale cream-colored to yellow.

Nutrient broth: Diffuse growth, later with yellowish pellicle.

Litmus milk: Small white granules of growth at the surface, later a dry yellowish pellicle. After some weeks' growth the milk becomes alkaline and clear. No coagulation
Dorset's egg medium: As on glycerol agar.

Coagulated serum: As on glycerol agar.

Potato: Spreading, raised, wrinkled growth, pale vellow to orange.

Long's medium lacking glycerol: No growth. Long's medium with 5 per cent glycerol: Acid formed. (Thomson, Amer. Rev. Tub., 26, 1932, 162.)

Indole not formed.

Nitrates: Reduced, doubtful (Jensen).

Carbohydrates: Glucose, fructose, arabinose and galactose are utilized; lactose is not utilized; sucrose is not utilized by 3 strains, utilized by 1 strain (Mycobacterium friburgensis) (Merrill, Jour. Bact., $20,1930,235)$. Sorbitol, arabinose, galactose, trehalose, mannitol and fructose are utilized; sucrose is not utilized (Gordon, Jour. Bact., 34, 1937, 617).

Optimum temperature $37^{\circ} \mathrm{C}$, maximum temperature for growth $52^{\circ} \mathrm{C}$, minimum $15^{\circ}$ to $18^{\circ} \mathrm{C}$. Fails to survive $60^{\circ} \mathrm{C}$. for 1 hour, grows at $47^{\circ} \mathrm{C}$ (Gordon, Jour. Bact., 34, 1937, 617; Gordon and Hagan, Jour. Bact., 36, 1938, 39).

Optimum pH 6.8 to 7.2 . Limits for growth 4.5 to 10.0 .

Distinctive characters: Saprophytic acid-fast organism. Grows rapidly on most media, develops a yellow or orange pigmentation after 3 to 4 days growth. Fails to grow on Long's medium lacking glycerol and produces acid when glycerol is present. Fails to survive $60^{\circ} \mathrm{C}$ for an hour, grows at temperatures as high as $47^{\circ} \mathrm{C}$

Variation: Lehmann and Neumann (Bakt. Diag., 2 Aufl., 2, 1899, 408) and Haag (Cent.f. Bakt., II Abt., $\boldsymbol{\gamma}_{1}, \mathbf{1 9 2 7}, 1$ ) describe three forms: a flat smooth form, a moist, slimy, smooth form and a dry, friable perrugose form. The two former correspond with $\mathbf{S}$ and the latter with $\mathbf{R}$ types described by Bynoe as characteristic of Mycobacterium stercoris, Mycobacterium berolinensis, Mycobacterium butyricum and Mycobaclerium graminis which in turn correspond with $\mathrm{S}$ and $\mathbf{R}$ types of other members of the genus. Schwabacher (Spec. Rep. Ser. Med. Res. 
Coun., London, No. 182, 1933) finds a difference in the arrangement of the individual cells of the $S$ and $R$ types.

Source: From butter, plant dust, cow manure.

Habitat: Gordon (Jour. Bact., 34, 1937, 617 ) found 1 culture isolated from nasal exudate, 1 from bovine lymph gland and 94 isolated from soil, out of a group of 215 soil cultures belonging to the genus, to be either identical with or very elosely related to this species. If these strains are valid members of the species, $\boldsymbol{M}_{y} \mathrm{co}-$ bncterium lacticola is widely distributed in soil, dust, dairy products, etc.

13. Mycobacterium phlei Lehmann and Neumann. (Timotheebacillus or Grasbacillus I, Moeller, Therapeutischen Monatsheften, 12, 1898, 607; Moeller. Deut. med. Wehnsehr., 24, 1898, 376; Lehmann and Neumann, Bakt. Diag., 2 Aufl., 2, 1899, 411; Mycobacterium moelleri Chester, Manual Determ. Bact. 1901, 35s; Sclerothrix phlei Vuillemin, Encyclopédie Mycologique, Paris, 2, 1931, 160.) From MI. L. Phleum, a genus of grasses.

Description from Moeller (loc. cit.) and Jensen (Proc. Linnean Soc. New So. Wales, 59, 1934, 32).

Slender rods : 0.2 to 0.5 by 1 to 4 microns, sometimes club-shaped, frequently beaded, rarely branched. Strongly acidfast and acid-alcohol-fast in cultures older than 2 to 3 days, in younger cultures there are generally many non-acid-fast cells. Non-motile. Gram-positive.

Gelatin colonies: Small, 0.5 to $1 \mathrm{~mm}$ in diameter; irregular, raised, moist and glistening, finely granular, orange.

Gelatin stab : Filiform, opaque, orange. No liquefaction.

Agar colonies: Similar to gelatin colonies, yellow to orange.

Agar slant: Spreading, raised, dry with roughened granular surface, yellow to orange.

Broth: Turbid, with yellow pellicle.

Dorset's egg medium: Spreading, raised, dry, orange.
Loeffler's serum: Similar to Dorset's egg medium, creany to yellow.

Glycerol broth: Thin transparent pellicle, later becoming thickened, rough, wrinkled and yellow to pink, still later a flaky sediment.

Litmus milk: Yellow floceuli on surface, slowly becomes alkaline. To coagulation.

Potato: Thick, dry, yellow, adherent growth.

Long's medium lacking glycerol : Abundant growth. Long's medium with 5 per cent glycerol: No acid formed (Thomson, Amer. Rev. Tub., 26, 1932, 162)

Nitrites are produced from nitrates.

Indole not formed.

Carbohydrates : Glucose, fructose, arabinose, trehalose, mannitol and galactose are utilized; sucrose and lactose are not utilized (Merrill, Jour. Bact., 20, 1930, 235 ; Gordon, Jour. Bact., 34, 1937, 617).

Temperature relations: Survives $60^{\circ} \mathrm{C}$ for 1 hour, grows at $47^{\circ} \mathrm{C}$ (Thomson, Amer. Jour. Tub., 26, 1932, 162); optimum for growth $37^{\circ} \mathrm{C}$, range $20^{\circ}$ to $58^{\circ} \mathrm{C}$ (Bynoe).

Optimum pH 6.8 to 7.3 ; range 5.5 to 10.0 .

Pathogenicity: The injection of large numbers of organisms into guinea pigs results in a local abscess of a few weeks' duration, occasionally small abscesses develop in the regional lymph glands or the visceral organs. According to Mayer (Cent.f. Bakt., I Abt., 26, 1899, 331) and others, the injection of the organisms along with butter or other fat increases the pathological reaction.

Variation: Haag (Cent. f. Bakt., II 1bt., 71, 1927, 1) and Bynoe (Thesis, MeGill University, Montreal, 1931) find two or three colony types: an R form which fits into the description of the species given above and an s type which grows as a perfectly smooth, raised, moist, glistening colony with an entire margin. Cooper (Jour. Inf. Dis., 54, 1934, 236) distinguished pigmented and non-pigmented types. 
Distinctiva characters: Saprophyti acid-fast organism, grows rapidly on most media. Shows yellow pigmentation as soon as growth is visible. Grows well on Long's medium lacking glycerol and fails to produce acid when glycerol is present. Survives $60^{\circ} \mathrm{C}$ for 1 hour and grows at $47^{\circ} \mathrm{C}$.

Source: Originally isolated from hay and grass. Frequently found in soil, dust and other sources. Out of 215 cultures of the genus recovered from soils by Gordon (Jour. Bact., 34, 1937, 617) Mycobacterium phlei was isolated on 22 occasions. The same author reports 3 cultures of a closely related if not identical organism recovered from bovine lymph glands, 1 recovered from bovine skin and 1 recovered from a hen's spleen.

Habitat: Widely distributed in soils, dust, hay, etc.

Appendix I: The following saprophytic species have been placed in this genus. Their relationships are not clear. Some are related to or possibly identical with Mycobacterium lacticola.

Mycobacterium album Söhngen. (Cent. f. Bakt., II Abt., 37, 1913, 599.) From garden earth.

Mycobacterium bekkerii Bekker. (Antonie van Leeuwenhoek, 9, 1943, 81 ; abst. in Cent. f. Bakt., I Abt., Orig., 149, 1944, 500.) From urine.

Mycobacterium berolinense Bergey et al . (Tuberkelähnlichen Bacillen, Rabinowitsch, Ztschr. f. Hyg., 26, 1897, 90; Mycobacterium lacticola $\beta$ perrugosum Lehmann and Neumann, Bakt. Diag., 2 Aufl., 2, 1899, 410; Mycobacterium lacticola perrugosum Haag, Cent. f. Bakt., II Abt., 71, 1927, 3; Bergey et al., Manual, 1st ed., 1923, 377.) From butter.

Mycobacterium butyri Chester. (Man. Determ. Bact., 1901, 357.) This name includes both the Tuberkelähnlichen Bacillen of Rabinowitsch and the Butter Bacillus of Petri. From butter.

Mycobacterium butyricum Bergey et al. (Butter Bacillus, Petri, Arb. kaiserl.
cGesundheitsamte, 14, 1898, 1; Bergey et al., Manual, 1st ed., 1923, 377.) From butter.

Mycobacterium cholesterolicum Tak. (Antonie van Leeuwenhoek, 8, 1942, 39.) From garden soil.

Mycobacterium friburgensis (Korn) Chester. (Bacillus friburgensis Korn, Cent. f. Bakt., I Abt., 25, 1899, 532; Mycobacterium lacticola $\gamma$ friburgensis Lehmann and Neumann, Bakt. Diag., 2 Aufl., 2, 1899, 411; Chester, Man. Determ. Bact., 1901, 359.) From butter.

Mycobacterium graminis Chester. (Grasbacillus II, Moeller, Cent f. Bakt., I Abt., 25, 1899, 369; Mycobacterium lacticola $\alpha$ planum Lehmann and Neumann, Bakt. Diag., 2 Aufl., 2, 1899, 408; Chester, Man. Determ. Bact., 1901, 358; Mycobacterium lacticola planum Haag, Cent. f. Bakt., II Abt., $\gamma_{1}, 1927,3$.) From hay dust.

Mycobacterium hyalinum Söhngen. (Cent. f. Bakt., II Abt., 37, 1913, 599.) From garden earth.

Mycobacterium luteum Söhngen (loc. cit.). From garden earth.

Mycobacterium muris Simmons. (Jour. Inf. Dis., 41, 1927, 13.) From the feces of gray mice.

Mycobacterium phlei perrugosum Haag. (Cent. f. Bakt., II Abt., 71, 1927, 6.) From soils and manure.

Mycobacterium phlei planum Haag (loc. cit.). From soils.

Mycobacterium ranicola $I$ and $I I$ Haag (loc. cit.). From frogs.

Mycobacterium rubrum Söhngen (loc. cit.). From garden earth.

Iycobacterium smegmatis (Trevisan) Chester. (Smegma bacillus, Alvarez and Tavel, Arch. Phys. norm. et path., $6,1885,303$; Bacillus smegmatis Trevisan, I generi e le specie delle Batteriacee, 1889, 14; Bacterium smeymatis Migula, Syst. d. Bakt., 2, 1900, 497; Chester, Man. Determ. Bact., 1901, 357.) From smegma. Weber (Arb. kaiserl. Gesundheitsamte, 19, 1902, 251) finds Mycobacterium smegmatis acid-but not alcoholfast in contrast to the mammalian 
tubercle bacilli which are both acid-and alcohol-fast. Later observers (Bynoe, Thesis, McGill University, Montreal, 1931) have not found this a valid distinction.

Mycobacterium smegmatis var. muris Galli-Valerio. (Cent. f. Bakt., I Abt., Orig., 75, 1915, 49.) From the preputial glands of the black rat (Mus rattus).

Mycobacterium stercoris Bergey et al. (Mist Bacillus, Moeller, Berlin, thierärztl. Wochnschr., 1898, 100; Mycobacterium stercusis (sic) Bergey et al., Manual, 1st ed., 1923, 378; Bergey et al., Manual, 4th ed., 1934, 542.) From manure.

Mycobacterium testudinis Friedmann and Piorkowski. (See Haag, Cent. f. Bakt., II Abt., 71, 1927, 5; apparently the same as Mycobacterium testudo, loc. cit., 10.) This is probably Mycobacterium friedmannii. From turtles.

Appendix II: Krassilnikov (Milirobiol., 7, 1935, 335; and Ray Fungi and
Related Organisms, Izd. Acad. Nauk. Moskow, 1938, 121-130) describes a genus Mycococcus distinct from Hansgirg's (Österr. Bot. Ztschr., 38, 1888, 266) family Mycococcaceae (which is related to the fungi) and distinct from Mycococcus Bokor (Arch. f. Mikrobiol., 1, 1930, 1).

Mycococcus Krassilnikov includes species that produce coccus-like cells, genetically related to the species included in Mycobacterium; reproduction is by fission or budding in different directions, often forming short, irregular chains with side branches; in old cultures, the vegetative cells change into resting cells, the latter germinating in a manner similar to the spores of actinomycetes. Seven species are listed, with incomplete descriptions. Mycococcus ruber, M. capsulatus, $M$. luteus, $M$. citreus and $M$. albus are described in Krassilnikov's origina] paper. One of these (Mycococcus luteus) is dropped in his later monograph while descriptions of two new species are added (Mycococcus tetragenus and $M$. mucosus). 


\title{
FAMILY II. ACTINOMYCETACEAE BLCHANAY*
}

\author{
(Jour. Bact., 3, 1918, 403.)
}

Mycelium is non-septate during the early stages of growth but later may become septate and break up into short segments, rod-shaped or spherical in shape, or the mycelium may remain non-septate and produce spores on aerial hyphae. The organisms in culture media are either colorless or produce various pigments. Some species are partially acid-fast. This family is distinguished from the previous one by the formation of a true mycelium. As compared with the next family, it is characterized by the manner of spore formation.

\section{Key to the genera of family Actinomycetaceae.}

I. Obligate aerobic. The colonies are bacteria-like in nature, smooth, rough or folded, of a soft to a dough-like consistency, sometimes compact and leathery in young stages. Most forms do not produce any aerial mycelium; a few produce a limited mycelium, the branches of which also break up into oidiospores ur segmentation spores. Some species are partially acid-fast.

Genus I. Nocardia, p. \$12.

II. Anaerobic or microaerophilic, parasitic; non-acid-fast, non-proteolytic and nondiastatio

Genus II. Actinomyces, p. 925.

\section{Genus 1. Nocardia Trevisan.}

(Trevisan, I generi e le specie delle Batteriacee, 1889, 9; Actinomyces Gasperini, Cent. f. Bakt., 15, 1894, 6st and Atti dell' XI congresso med. internaz. Roma, 6 , 1895, 82; not Actinomyces Harz, Jahresher. d. Münch. Thierarzneischule for 1877 1878, 1879, 125; Actinobacterium Hats, C'ent. f. Bakt., I Abt., Orig., 40, 1906, 180; Sampietro, Ann. d'Igiene, Roma, 18, 1908, 331; Actinococcus Beijerinck, Folio Microbiol., Delft, 3, 1914, 196; not Actinococcus Kützing, species Algarum, 1849; Brevistreptothrix Lignières, Ann. Parasit. hum. et comp., 2, 1924, 1; Asteroides Puntoni and Leonardi, Boll. e Atti d. R. Acead. Med., 61, 1935, 90; Proactinomyces Jensen, Proc. Linn. Soc. New to. Wales, $56,1931,345$.) Named for Prof. E. Nocard who first described the type species.

Slender filaments or rods, frequently swollen and occasionally branched, forming a mycelium which after reaching a certain size assumes the appearance of bacteriumlike growths. Shorter rods and coccoid forms are found in older cultures. Conidia not formed. Stain readily, occasionally showing a slight degree of acid-fastness. Non-motile. No endospores. Aerobic. Gram-positive. The colonies are similar in gross appearance to those of the genus Mycobacterium. Paraffin, phenol and m-cresol are frequently utilized as a source of energy.

In their early stages of growth on culture media (liquid or solid), the structure of nocardias is similar to that of actinomyces in that they form a typical mycelium hyphae branch abundantly, the branching being true. The diameters of the hyphae vary hetween 0.5 and 1 micron, usually 0.7 to 0.8 micron, according to the species. The mycelium is not septate. However, the further development of nocardias differs sharply from that of actinomyces: the filaments soon form transverse walls and the whole mycelium breaks up into regularly cylindrical short cells, then into coccoid

* Completely revised by Prof. S. A. Waksman, New Jersey Experiment Station, New Brunswick, New Jersey and I'mf. A. T. Henrici, Tniversity of Minnesota, Minneapolis, Minnesota, May, 1943. 
cells. On fresh culture media, the coccoid cells germinate into mycelia. The whole cycle in the development of nocardias eontinues for 2 to 7 days. Most frequently the coccoid cells are formed on the third to fifth day, but in certain species (e.g., Nocardia rubra) they can be found on the second day.

Numerous chlamydospores may be found in older cultures of nocardias. They are formed in the same way as the chlamydospores in true fungi: the plasma inside the filaments of the mycelium condenses into elongated portions. In older cultures of nocardias many coccoid cells are changed into resistant cells. The latter are larger than the vegetative coccoid cells; the plasma of these cells is thicker than the plasma of vegetative cells; on fresh media they germinate like the spores of actinomyces; they form 2 to 3 germ tubes. Besides the cells mentioned, numerous involution forms can often be found in older cultures of nocardias; the cells are thin, regularly cylindrical or coccoid, are often transformed into a series of spherical or elliptical ampules and a club-like form (2 to 3 microns and more).

The multiplication of nocardias proceeds by fission and budding; occasionally they form special spores. Budding occurs often. The buds are formed on the lateral surface of the cells; when they have reached a certain size, they fall off and develop into rod-shaped cells or filaments. The spores are formed by the breaking up of the cell plasm into separate portions usually forming 3 to 5 spores; every portion becomes rounded, covered with a membrane and is transformed into a spore; the membrane of the mother cell dissolves and disappears. The spores germinate in the same way as those of actinomyces. They form germ tubes which develop into a mycelium.

The colonies of nocardias have a paste-like or mealy consistency and can easily be taken up with a platinum loop; they spread on glass and occasionally render the broth turbid. The surface colonies are smooth, folded or wrinkled. Typical nocardias never form an aerial mycelium, but there are cultures whose colonies are covered with a thin coating of short aerial hyphae which break up into cylindrical oidiospores.

Yany species of nocardias form pigments; their colonies are of a blue, violet, red, yellow or green color; more often the cultures are colorless. The color of the culture serves as a stable character.

Krassilnikov (Ray fungi and related organisms, Izd. Acad. Nauk, I S.S.R., Moscow, 1938) divides the genus into two groups: 1. Well developed aerial mycelium; substrate mycelium seldom produces cross-walls; the threads break up into long, threadlike rods; branches of the aerial mycelium produce segmentation spores and oidiospores; the latter are cylindrical with sharp ends; no spirals or fruiting branches. This group is the same as group B of Jensen (loc. cit.). 2. Typical forms; mycelium develops only at early stages of growth, then breaks up into rod-shaped and coccoid bodies; smooth and rough colonies, dough-like consistency; never form an aerial mycelium; similar to bacterial colonies; aerial mycelium may form around colonies. This genus can also be divided, on the basis of acid-fastness, into two groups: Group 1. Partially acid-fast organisms, which are non-proteolytic, non-diastatic and utilize paraffin; usually yellow, pink, or orange-red in color. Group 2. Non-acid-fast organisms, which are diastatic, largely proteolytic and do not utilize paraffin; yellow, orange to black in color.

The type species is Nocardia farcinica Trevisan.

\section{Key to the species of genus Nocardia.}

I. Partially acid-fast* organisms with strongly refractive cells; non-proteolytic and generally non-diastatic; constantly capable of utilizing paraffin.

* Acid-fastness is not marked in cultures, is apparent in infected tissues, pro. nounced in sputum or other exudates. 
A. Initial mycelium well developed, richly branching, dividing into rods and generally into cocei.

1. Vegetative mycelium soft, without macroscopically visible aerial mycelium.

a. Vegetative mycelium yellow, orange or red.

b. Pathogenic.

c. Vegetative mycelium white, buff, or pale yellow.

1. Nocardia farcinica.

cc. Vegetative mycelium yellow to red.

2. Nocardia asteroides.

bb. Not pathogenic.

3. Nocardia polychromogenes.

aa. Vegetative mycelium white to pink.

b. Gelatin not liquefied.

c. Growth on nutrient agar opaque, cream-colored; coccoid forms in broth.

4. Nocardia opaca.

cc. Growth on nutrient agar watery, no coccoid forms in broth.

5. Nocardia erythropolis.

ecc. Growth on nutrient agar pink.

d. White aerial mycelium on milk.

6. Nocardia leishmanii. .

dd. Pink pellicle on milk.

7. Nocardia caprae.

ddd. Yellow pellicle on milk.

bb. Gelatin liquefied.

8. Nocardia pretoriana.

9. Nocardia pulmonalis.

2. Vegetative mycelium hard, yellow, with white aerial mycelium; hyphae divide into chains of acid-fast cocci.

10. Nocardia paraffinae.

B. Initial mycelium very short, rapidly dividing into rods and cocci.

1. Slowly growing organisms; cells 0.5 to 0.7 micron in diameter.

11. Nocardia minima.

2. Rapidly growing organisms; cells 1.0 to 1.2 microns in diameter.

a. Growth pink.

b. Cystites (swollen cells) not formed.

c. No indigotin from indole.

12. Nocardia corallina.

cc. Indigotin from indole.

13. Nocardia globerula.

bb. Cystites formed.

14. Nocardia salmonicolor.

aa. Growth coral red.

15. Nocardia rubropertincta.

aaa. Growth dark red.

16. Nocardia rubra.

aaa. Growth white.

b. No aerial mycelium.

17. Nocardia coeliaca.

bb. Aerial mycelium.

18. Nocardia transvalensis. 
II. Non-acid-fast organisms with weakly refractive cells; no distinct formation of cocci. Constantly diastatic.

A. Not proteolytic.

1. Growth on agar pale cream.

2. Growth on agar yellow.

19. Nocardia mesenterica.

20. Nocardia flava.

3. Growth on agar green.

21. Nocardia viridis.

4. Growth on agar yellow-green.

22. Nocardia citrea.

5. Growth on agar pink to crimson.

23. Nocardia madurae.

6. Growth consistency soft, sparse aerial mycelium.

24. Nocardia lutea.

7. Growth consistency medium, good aerial mycelium.

25. Nocardia blackwellii.

8. Good action on milk. Growth consistency firm, liberal, aerial mycelium.

26. Nocardia cuniculi.

9. Deep brown pigment on protein media.

27. Nocardia rangoonensis.

10. Light brown pigment on protein media.

28. Nocardia caviae.

B. Proteolytic.

1. Growth on nutrient agar with rapid formation of unbranched diphtheroid-like rods; no typical cystites; broth turbid.

29. Nocardia actinomorpha.

2. Growth on nutrient agar with extensive mycelia; simple unbranched rods not formed; cystites present. Broth clear.

30. Nocardia flavescens.

3. Colonies orange-yellow to orange-red, which may change to black.

31. Nocardia maculata.

4. Light brown pigment on protein media.

32. Nocardia rhodnii.

5. Green to greenish-brown pigment on protein media.

33. Nocardia gardneri.

1. Nocardia farcinica Trevisan. (Bacille du farcin, Nocard, Ann. Inst. Past., 2, 1888, 293; Trevisan, I generi e le specie delle Batteriacee, Milan, 1889, 9 ; Streptotrix farcinica Rossi Doria, Ann. d. Ist. d'Igi. Sper. Univ. di Roma, 1, 1891, 424; Actinomyces farcinicus Gasperini, Ann. Ist. d'Igiene, Roma, 2, 1892, 222; Oospora farcinica Sauvageau and Radais, Ann. Inst. Past., 6, 1892, 248; Actinomyces bovis farcinicus Gasperini, Cent. f. Bakt., 15, 1894, 684; Bacillus farcinicus Gas- perini, ibid.; Cladothrix farcinica Macé, Traité de Bactériologie, 3rd ed., 1894, 1047; Streptothrix farcini bovis Kitt, Bakterienkunde und pathologische Mikroskopie, Vienna, 3 Auf., 1899, 511; Bacterium nocardi Migula, Syst. d. Bakt., 2, 1900, 345; Streptothrix nocardii Foulerton, Jour. Compt. Path. and Therap., 14, 1901, 51; Discomyces farcinicus Gedoelst, Les champignons parasites de l'homme et des animaux domestiques, Brussels, 1902, 167; Ac- 
tinomyces noeardii Buchanan, Veterinary Bacteriology, Philadelphia, 1911, 37s; Nocardia abida Clialmers and Christopherson, Ann. Trop. Med. and Parasit. 10, 1916, 2\%i, according to Dodge, Medical Mycology, St. Louis, 1935, 746.) From M. L. farcinicus, of farey.

Filaments 0.25 microm in thickness, branched. Markedly acid-fast.

Gelatin colonies: Small, circular, transparent, glistening.

Gelatin stab: No liquefaction.

Agar colonies: Yellowish-white, irregular, refractive, filamentous.

Agar slant: Grayish to yellowish-white growth, surface roughened.

Broth: Clear, with granular sediment, often with gray pellicle.

Litmus milk: Unchanged.

Potato: Abundant, dull, crumpled, whitish-yellow growth.

Nitrites not produced from nitrates.

No soluble pigment formed.

Proteolytic action absent.

Starch not hydrolyzed.

Aerobic, facultative.

Optimum temperature $37^{\circ} \mathrm{C}$.

Source: From cases of bovine farcy.

Habitat: Associated with a disease in cattle, resembling chronic tuberculosis Transmissible to guinea pigs, cattle and sheep, but not to rabbits, dogs, horses or monkeys.

\section{Nocardia asteroides (Eppinger)}

Blanchard. (Cladothrix asteroides Eppinger, Beitr. z. path. Anat., 9, 1891, 287 ; Streptotrix (sic) eppingerii Rossi Doria, Ann. Inst. d'Ig. sper. d. Univ. Roma, 1, 1891, 423; Streptotrix (sic) asteroides Gasperini, Ann. Inst. d'Ig. sper. d. Univ. Roma, 2, 1892, 183; Oospora asteroides Sauvageau and Radais, Anm. Inst. Past., $6,1892,252$; Actinomyces asteroides Gasperini, Cent. f. Bakt., 15, 1894, 684; Blanchard, in Bouchard, Traité Path. Gén., 2, 1s95, 811; Discomyces asteroides Gedoelst, Champ. Paras. Homme et Anim., 1902, 173, Actinomyces eppingeri Namyslowski, Cent. f. Bakt., I Abt.
Orig., 62, 1912, 566; Asteroides asteroides Puntoni and Leonardi, Boll. e Atti 1. R. Acrad. Med. di Roma, 61, 1935, 92; Mycobacterium asteroides, quoted from Puntoni and Leonardi, idcm; Proactinomyces asteroides Baldacei, Soe. Internat. di Mierob., Boll. d. Sez. Ital., 9, 1937, 141.) From Greek aster, star and cides, shape.

Probable synonyms : Streptotrix aurantiaca Rossi Doria, loc. cit., 417 (Actinomyces aurantiacus Gasperini, loc. cit., 1892, 222 ; Oospora aurantiaca Lehmann and Neumann, Bakt. Diag., 1 Aufl., 2, 1896, 388; Cladothrix aurantiaca Macé, Traité Pratique de Bact., 4th ed., 1901, 1026; Nocardia aurantiaca Chalmers and Christopherson, Ann. Trop. Med. and Parasit., 10, 1916, 268) and Streptothrix freeri Musgrave and Clegg, Philippine Jour. Sci., Med. Ściences, 2, 1907, 477 (Discomyces freeri Brumpt, Précis de Parasitol., Paris, 1st ed., 1910, 858; Nocardia freeri Pinoy, Bull. Inst. Past., 11, 1913, 936; Oospora freeri Sartory, Champ. Paras. Homme et Anim., 1923, 785; Actinomyces freeri Bergey et al., Mamual, 1st ed., 1923, 346). According to Chalmers and Christopherson (loc. cil.) another synonym of this organism is Streptothrix hominis Sabrazès and Rivière, Le remaine Médecine, 1895, no. 44 .

Straight, fine mycelium, 0.2 micron in thickness, which breaks up into small, cocoid conidia. Acid-fast.

Gelatin stab: Yellowish surface growth. No growth in stab. No liquefaction.

Synthetic agar : Thin, spreading, orange growth. No aerial mycelium.

Starch agar: Restricted, scant, orange growth.

Plain agar: Much folded, light yellow growth, becoming deep yellow to yellowish-red.

Glucose broth: Thin, yellowish pellicle.

Litmus milk: Orange-colored ring. No coagulation. No peptonization.

Potato: Growth much wrinkled, whitish becoming yellow to almost brick-red. 
Nitrites produced from nitrates.

No soluble pigment formed.

Proteolytic action doubtful.

Starch not hydrolyzed.

Transmissible to rabbits and guinea pigs but not to mice.

Aerobic.

Optimum temperature $37^{\circ} \mathrm{C}$.

Source : From a cerebral abscess in man.

Habitat: Also found in conditions resembling pulmonary tuberculosis.

A number of strains of acid-fast actinomycetes isolated from human lesions have deviated in certain particulars from the description of Nocardia asteroides, but not sufficiently to warrant separation as species. The following varieties are described by Baldacci (Mycopathologia $1,1938,68)$ :

Nocardia asteroides var. crateriformis (Baldacci) comb. nov. (Proactinomyces asteroides var. crateriformis Baldacci, loc. cit.) Less tendency to fragmentation of mycelium. Complete lack of aerial mycelium. Growing as discrete colonies, disk-or erater-shaped.

Vocardia asteroides var. decolor. (Baldacci) comb. nov. (Proactinomyces asteroides var. decolor Baldacci, loc. cit.) Greater tendency to produce white aerial mycelium; vegetative mycelium colorless

Nocardia asteroides var. gypsoides (Baldacci) comb. nov. (Actinomyces gypsoides Henrici and Gardner, Jour. Inf. Dis., 28, 1921, 248; Discomyces gypsoides Brumpt, Précis de Parasitol., Paris. 3rd ed., 1922, 980; Oospora gypsoides Sartory, Champ. Paras. Homme et Anim., 1923, 802; Proactinomyces asteroides var. gypsoides Baldacci, loc. cit.) White aerial mycelium; darkening of peptone media.

\section{Nocardia polychromogenes (Vallée)} comb. nov. (Streptothrix polychromogenes Vallée, Ann. Inst. Past., 1\%, 1903, 288; Streptothrix pluricromogena Caminiti. Cent. f. Bakt., I Abt., Orig., 44, 1907, 198; Actinomyces polychromogenes Lieske,
Morphol. u. Biol. d. Strahlenpilze, Leipzig, 1921, 32; Proactinomyces polychromogenes Jensen, Proc. Linnean Soc. New So. Wales, 56, 1931, 79 and 363; Oospora polychromogenes Sartory, quoted from Nannizzi, in Pollacci, Tratt. Micopat. Umana, 4, 1934, 51; Actinomyces plurichromogenus Dodge, Medical Mycology, St. Louis, 1935, 737.) From Greek, producing many colors.

Description from Jensen (loc. cit.).

Long wavy filaments: 0.4 to 0.5 by 70 to 100 microns, extensively branched but without septa. Older cultures consist entirely of rods 4 to 10 microns, frequently in V, Y, or smaller forms. Still older cultures consist of shorter rods and coccoid forms. Gram-positive, frequently showing bands and granules.

Gelatin stab: Thin yellowish growth along the stab with thin radiating filaments. Surface growth flat, wrinkled, red. No liquefaction.

Nutrient agar: Scant, orange-red growth.

Glucose agar: After 3 to 4 days raised, flat, glistening, rose-colored growth. After 1 to 3 weeks becoming folded and coral-red.

Glucose broth: After 3 to 4 days turbid; after 2 to 3 weeks an orange flaky sediment. No surface growth.

Milk: Growth starts as small orangecolored surface granules. After 1 to 2 weeks a thick, soft, orange-colored sediment forms.

Optimum lemperature $22^{\circ}$ to $25^{\circ} \mathrm{C}$.

Distinctive characters: Differs from locardia corallina in the formation of very long filaments and in filiform "growth in gelatin stabs.

Source: From the blood of a horse; from soil in France and Australia.

Habitat: Soil.

4. Nocardia opaca (den Dooren de Jong) comb. nov. (Mycobacterium opacum den Dooren de Jong, Cent. f. Bakt., II Abt., 71, 1927, 216; Mycobacterium crystallophagum Gray and Thornton, Cent. 
f. Bakt., II Abt., 73, 1928, 86; Actinomyces crystallophagus Bergey et al., Manual, 3rd ed., 1930, 473; Proactincmyces opacus Jensen, Proc. Linnean Soc. New So. Wales, 57, 1932, 369; Proactinomyces crystallophagus Reed, in Manual, 5th ed., 1939, 834.) From Latin opacus, shady, darkened.

Description from Gray and Thornton (loc. cit.), Bynoe (Thesis, MeGill University, Montreal, 1931), and Jensen (loc. cit.).

Long, curved, irregular and branching filaments or rods : 0.8 to 1.0 by 2 to 16 microns, or occasionally longer. Few chains or clumps are formed. In older cultures shorter rods or cocci are generally formed. Readily stained. Not acid-fast. Gram-positive.

Gelatin colonies : Round, convex, whitish, smooth, shining, edges slightly arborescent. Deep colonies: Burrs, with slightly irregular processes.

Gelatin stab : Convex, whitish, smooth, resinous, filiform, erose.

Broth: Turbid with broken white scum, or clear with granular suspension.

Dorset's egg medium: Spreading, smooth, moist, salmon-colored growth.

Loeffler's medium: Scanty growth, smooth, moist, light buff-colored.

Glycerol potato: Dry, rough, crumpled, pink to buff-colored growth.

Litmus milk: Grayish pellicle; slightly alkaline.

Nitrites are produced from nitrates.

No acid from sucrose, lactose, maltose or glucose.

Phenol and naphthalene are utilized as sources of energy.

Optimum pH 6.8 to 7.3 .

Optimum temperature $30^{\circ} \mathrm{C}$.

Distinctive characters: Differs from Nocardia corallina and Nocardia polychromogenes in that the cells are much longer than those of the former and much shorter than those of the latter. Grows in smooth convex surface colonies and burr-like deep colonies.
Source: Twenty-four strains isolated from soils in Great Britain.

Habitat: Probably sparingly distributed in soils.

\section{Nocardia erythropolis (Gray and} Thornton) comb. nov. (Mycobacterium erythropolis Gray and Thornton, Cent. f. Bakt., II Abt., 73, 1928, 87; Actinomyces erylhropolis Bergey et al., Manual, 3rd ed., 1930, 472; Proactinomyces erythropolis Jensen, Proc. Linnean Soc. of New So. Wales, 5\%, 1932, 371.) From Greek erythrus, red and polis, city.

Description from Gray and Thornton (loc. cit.) and from Bynoe (Thesis, McGill University, Montreal, 1931).

Long uneven-sided rods and filaments, curved and branching up to 11 microns long by 0.8 micron. Coccoid forms not formed. Stains readily. Not acid-fast. Gram-positive.

Gelatin colonies: After 12 days, round, flat, white, shining; edge entire. Deep colonies: Round, smooth.

Gelatin stab: After 8 to 14 days, convex, white, smooth, shining, radiate from center, borders cleft. Line of puncture filiform, erose.

Agar colonies: Round, 2 to $3 \mathrm{~mm}$ in diameter, convex, watery-white; edge entire. Deep colonies: Lens-shaped.

Agar slant: Filiform, flat, watery growth; edge undulate.

Broth: Growth slight, turbid.

Dorset's egg medium: After 2 weeks, raised, moist, finely granular, irregular margin, flesh-colored.

Loeffler's medium: After 7 days growth as on Dorset's egg medium, but pink.

Glycerol potato: After 7 days, flat, dry, rough, orange-colored.

Litmus milk: Pale pink pellicle.

Nitrites not produced from nitrates.

No acid from glucose, lactose, sucrose or glycerol.

Phenol is utilized.

Optimum pH 6.8 to 8.0 .

Optimum temperature $25^{\circ} \mathrm{C}$. 
Distinctive characters: Differs from Nocardia coeliaca and Nocardia actinomorpha in the filiform growth and absence of liquefaction of gelatin. Long rods and filaments.

Source: Six strains isolated from soils in Great Britain.

Habitat: Presumably soil.

6. Nocardia leishmanii Chalmers and Christopherson. (A new acid-fast streptothrix, Birt and Leishman, Jour. Hyg., 2, 1902, 120; Chalmers and Christopherson, Ann. Trop. Med. and Parasit., 10, 1916, 255; Discomyces leishmani Brumpt, Précis de Parasitol., Paris, 3rd ed., 1922, 984; Actinomyces leishmani Sartory and Bailly, Mycoses pulmonaires, 1923, 253.) Named for Leishman, one of the original isolators.

Description from Erikson (loc. cit., p. 27).

Initial cells frequently swollen, large and irregular, aggregated in short chains and then branching out into regular narrow filaments; at margin of colony on synthetic glycerol agar may be seen comparatively long thick segments with accompanying fringe of normal hyphae; later entire colonies asteroid in appearance, very fine and close angular branching, with aerial hyphae situated singly; aerial mycelium generally abundant with irregularly cylindrical conidia. Slightly acid-fast. The latter property must have been attenuated during artificial cultivation, for the organism is reported as markedly acid-fast by the original isolators.

Gelatin: Small pink colonies in depths of stab. No liquefaction.

Glucose agar: Rounded elevated colonies with paler frosting of aerial mycelium ; growth becoming piled up, aerial myce. lium sparse.

Glycerol agar: Small round pink colonies, tending to be umbilicated and piled up, stiff white aerial spikes.

Coon's agar: Small round colorless colonies, stiff white aerial spikes; later a pink tinge.

Potato agar: Minute colorless round colonies, small raised patches of white aerial mycelium.

Dorset's egg medium: Colorless confluent growth studded with little wartlike projections bearing stiff aerial spikes; growth becomes pinkish with a white aerial mycelium; later, growth drab gray, medium discolored.

Serum agar: Minute round colorless colonies with pinkish tinge in confluent raised patch.

Inspissated serum: Small round pale pink colonies, umbilicated and raised up.

Broth: Liberal growth, white flocculent colonies; later pink surface colonies.

Synthetic sucrose solution: Colorless flocculent sediment, thin colorless pellicle.

Milk: Surface growth, white aerial mycelium, solid coagulum; later partly peptonized with pink aerial mycelium.

Litmus milk: Pink surface growth, aerial mycelium, milky opaque after 40 days.

Carrot plug: Small irregularly round raised colonies, colorless, covered with stiff aerial spikes; later buff-colored, convoluted and ribbed growth with small patches of white aerial mycelium; aerial mycelium pink in two months.

Source: From fatal case of lung disease and pericarditis in man.

Habitat: Human infections so far as known.

\section{Nocardia caprae (Silberschmidt)} comb. nov. (Streptothrix caprae Silberschmidt, Ann. Inst. Past., 13, 1899, 841; Cladothrix caprae Macé, Traité Pratique de Bact., 4th ed., 1901, 1094; Discomyces caprae Gedoelst, Champ. Paras. Homme et Anim., 1902, 174; Oospora caprae Sartory, Champ. Paras. Homme et Anim., 1923, 813; Actinomyces caprae Ford, Textb. of Bact., 1927, 205.) From Latin capra, goat. 
Description from Erikson (Med. Res. Council Spee. Rept. Ser. 203, 1935, 26 ).

Initial cells only slightly enlarged; early development of aterial hyphae, while substratum threads are still short; frequent slipping of branches; aerial mycelium abundant on all media with tendency to form coherent spikes; mycelium not very polymorphous, but occasional thicker segments appear. Slightly acid-fast.

Gelatin: Extensive dull growth with small raised patches of pink aerial mycelium; later ribbon-like, depressed. $N_{0}$ liquefaction.

Glucose agar: Irregular bright pink growth tending to be heaped up; later abundant masses frosted over with thin white aerial mycelium.

Glycerol agar: Abundant growth, small round pink colonies, partly covered with white aerial mycelium.

Potato agar: Extensive thin growth, pink in raised patches, covered by white aerial mycelium; later aerial mycelium also becomes pink

Starch agar: Minute colorless colonies covered by white aerial mycelium.

Blood agar: Minute round colorless colonies aggregated in broad pink zones, paler aerial mycelium. No hemolysis.

Dorset's egg medium: Few colorless colonies, some pink, white aerial mycelium; later, growth becoming dull pink. irregular, with scant white aerial mycelium.

Ca-agar: Minute colorless colonies, white aerial mycelium; later a pinkish tinge.

Serum agar: Small round pink colonies frosted over with thin white aerial mycelium.

Inspissated serum: No growth.

Broth: Superficial pellicle composed of pink colonies with white aerial mycelium; moderate floceulent sediment.

Glucose broth: Small sediment of fine flocculi; later pellicle composed of small pink colonies; superficial skin entire and salmon-colored in 16 days.
Synthetic glycerol solution: Round pink disc-like colonies on surface and tenuous white wispy growth in suspension and sediment; after 20 days, surface colonies bearing white aerial mycelium extending $2 \mathrm{~cm}$ up tube.

Synthetic sucrose solution: Minute white colonies in suspension and sediment in 3 days; thin dust-like pellicle in 10 days; some surface colonies with white aerial mycelium in 17 days.

Milk : Red surface skin; solid coagulum.

Litmus milk: Red surface growth, no change in liquid; after 4 weeks, liquid decolorized, opaque.

Potato plug: Abundant growth, small colonies, mostly confluent, entirely covered with pale pink aerial mycelium; growth becomes membranous, considerably buckled; later superficial colonies with pink aerial mycelium on liquid at base of tube, bottom growth of round white colonies.

Starch not hydrolyzed.

Source: From lesions in goats.

8. Nocardia pretoriana Pijper and Pullinger. (Pijper and Pullinger, Jour. Trop. Med. Hyg., 30, 1927, 153; Actinomyces pretorianus Nannizzi, in Pollacei, Tratt. Micopat. Umana, 4, 1934, 38.) Named for Pretoria in South Africa.

Description from Erikson. (Med. Res. Council Spee. Rept. Ser. 203, 1935, 30 ).

Minute flat colonies are formed con sisting of angularly branched filaments, and bearing a few short straight aerial hyphae; later the growth becomes spreading and extensive, the slipping of the branches is well marked and the aterial hyphae are divided into cylindrical ronidia. Slightly acid-fast.

Gelatin: A few colorless flakes. No liquefaction.

Glucose agar: Pale buff umbilicated and piled up colonies.

Glycerol agar: Piled up pink mass, very scant white aerial mycelium at margin. 
Ca-agar: Yellowish wrinkled coherent growth with white aerial mycelium on apices and at margin.

Coon's agar: Colorless mostly submerged growth, scant white aerial mycelium.

Dorset's egg medium: A few round colorless colonies in 3 days; after 3 weeks, irregular raised pink mass, warted appearance, moderate degree of liquefaction.

Serum agar: Raised, convoluted, slightly pinkish growth.

Inspissated serum: No growth.

Broth: Moderate quantity of flakes and dust-like surface growth.

Synthetic sucrose solution: A few colorless flakes on the surface, lesser bottom growth.

Milk: Yellowish surface growth; solid coagulum in one month; later, partly digested, pale pink growth up the wall of the tube.

Litmus milk: Colorless surface growth, liquid blue; becoming hydrolyzed and decolorized.

Potato plug: Small raised pale pink colonies with white aerial mycelium; after 2 months, plug and liquid discolored, growth dull buff, dry and convoluted at base, round and zonate at top of slant, white aerial mycelium, surface and bottom growth on liquid.

Source: From a case of mycetoma of the chest wall in a South African native.

Habitat: Human infections so far as known.

\section{Nocardia pulmonalis (Burnett)} comb. nov. (Actinomyces pulmonalis Burnett, Ann. Rept. I. Y. State Vet. Coll., 1909-1910, 167.) From Latin pulmo, lung.

Gram-positive mycelium breaking up readily into oval-shaped conidia. Acidfast, especially in early stages of growth.

Gelatin: Small, whitish, spherical colonies; edges of colony becoming chalky white; limited liquefaction.
Agar: Moist, raised growth in the form of small, spherical colonies.

Glucose agar: Dull, whitish, convoluted growth.

Broth: Delicate, translucent film on surface, becoming corrugated with some whitish, spherical colonies in medium.

Milk: Colonies on the surface of the medium; milk is coagulated in a few days, later digested.

Potato: Luxuriant growth in the form of small, translucent, round colonies; becoming colored lemon-yellow; later, growth becomes convoluted or folded with chalky white aerial mycelium, color of plug brownish.

Non-pathogenic for rabbits and guinea pigs.

Aerobic.

Source: From the lungs of a cow.

Habitat: Bovine infections so far as known.

10. Nocardia paraffinae (Jensen) comb. nov. (Proactinomyces paraffinae Jensen, Proc. Linn. Soc. New So. Wales, 56, 1931, 362.) From M. L. paraffina, paraffine.

In agar media, the organism initially forms an extensive mycelium of long, richly-branching hyphae, 0.4 to $0.5 \mathrm{mi}$ eron thick. After 5 to 6 days, at room temperature, numerous end branches swell to about double thickness, become more refractive, exhibit fine incisions along their external contours, and divide into oval, spore-like elements, 0.8 to 1.0 by 1.2 to 1.5 microns. This process of division starts at the tips of the swollen branches and proceeds basipetally until most of the hyphae appear divided. Primary septa have not been seen in the hyphae. A similar process of division takes place in liquid media, where also the filaments often fall into fragments of variable length. The spore-like elements, but not the undivided filaments, are markedly acid-fast. The aerial mycelium consists of rather short, straight, not very much branched hyphae, 0.4 to 
0.6 micron thick, which never show any differentiation into spores.

Gelatin: No liquefaction.

Sucrose agar: Very scant growth. Thin colorless veil, sometimes with a trace of white aerial mycelium.

Glucose agar: Fair growth. Vegetative mycelium flat, growing into medium; pale ochre-yellow to orange, with raised outgrowths on the surface. Growth of a crumbly consistency. Scant, white, aerial mycelium.

Nutrient agar: Slow but good growth. Vegetative mycelium superficial, somewhat raised, ochre-yellow, hard, but with a loose, smeary surface. Aerial mycelium scant, small white tufts. No pigment.

Potato: Fair growth. Vegetative mycelium granulated, first pale-yellow, later deep ochre-yellow to orange. Scant, white, aerial mycelium. No pigment.

Liquid media (milk, broth, synthetic solutions): Small, round granules of various yellow to orange colors, firm, but can be crushed into a homogeneous smear. In old broth cultures a thick, hard, orange to brownish surface pellicle is formed.

Sucrose not inverted.

Starch not hydrolyzed.

Cellulose is not decomposed.

Nitrates are not reduced to nitrites.

Milk is not coagulated or digested.

Final reaction in glucose $\mathrm{NH}_{4} \mathrm{Cl}$ solution, $\mathrm{pH} 4.6$ to 4.4 .

All strains show a marked power of utilizing paraffin wax as a source of energy.

Source: Isolated from soil.

Habitat: Soil.

11. Nocardia minima (Jensen) comb. nov. (Proactinomyces minimus Jensen, Proc. Linnean Soc. New So. Wales, 56, 1931, 365.) From Latin minimus, very small.

Filaments and rods : 0.4 to 0.6 by 2 to 10 microns. In older cultures mostly short rods, frequently $\mathrm{V}, \mathrm{Y}$, swollen forms, or cocci. Irregularly stained with ordinary dyes, generally show bars and bands. Generally a few cells from cultures are acid-fast, most are not acidfast. Gram-positive.

Gelatin stab: Filiform, granulated, cream-colored growth. No liquefaction.

Agar : Slow growth, raised, folded, with finely myeloid margins. At first colorless, after 6 to 8 weeks flesh pink or coral pink.

Potato: Growth slow, after 6 to 8 weeks abundant, spreading, much raised, finely wrinkled, coral pink.

Paraffin is utilized.

Optimum temperature $22^{\circ}$ to $25^{\circ} \mathrm{C}$.

Distinctive characters: Closely resembles Nocardia corallina but differs in the much slower growth and the smaller size of the cells.

Source: From soil in Australia.

Habitat: Soil.

12. Nocardia corallina (Bergey et al.) comb. nov. (Bacillus mycoides corallinus Hefferan, Cent. f. Bakt., II Abt., 11, 1904, 459; Serratia corallina Bergey et al., Manual, 1st ed., 1923, 93; Streptothrix corallinus Reader, Jour. Path. and Bact., 29, 1926, 1; Mycobacterium agreste Gray and Thornton, Cent. f. Bakt., II Abt., 73, 1928, 84; Actinomyces agrestis Bergey et al., Manual, 3rd ed., 1930, 472; Proactinomyces agrestis Jensen, Proc. Linnean Soc. New So. Wales, 56, 1931, 345; Proactinomyces corallinus Jensen, ibid., 57, 1932, 364.) From Latin corallinus, coral red.

Description from Gray and Thornton (loc. cit.), Jensen (loc. cit.) and Bynoe (Thesis, McGill University, Montreal, 1931).

Branching rods, generally curved, 1 to 1.5 by 3 to 10 microns. In older cultures generally shorter rods and cocci. Nonmotile. Not acid-fast. Gram-positive.

Gelatin colonies: Round, convex, smooth, pink, shining, edge filamentous. Deep colonies: Burrs. 
Gelatin stab: Nailhead; line of stab arborescent. No liquefaction.

Agar colonies: Round, convex, or umbonate, smooth, pink, shining or matte; border lighter, edge filamentous or with arborescent projections. Deep colonies: Burrs, or lens-shaped, with arborescent projections. In their very early stages colonies consist of branching filamentous rods. As the colony grows, the cells in the interior break up into short rods and cocci which eventually form the mass of the colony. Cells on the outside remain filamentous, giving the colony a burrlike appearance, and often forming long arborescent processes.

Agar slant: Filiform, convex, smooth, pink, shining or matte; arborescent or with projections from undulate border.

Litmus milk: Alkaline. Reddish pellicle.

Glycerol potato: Filiform, raised, dry, wrinkled, yellowish-brown to coral red.

Broth: Usually turbid. Pink scum.

Dorset's egg medium: Filiform, raised, dry, wrinkled, orange.

Loeffler's medium: Similar to growth on Dorset's egg medium, but pink.

Nitrites produced from nitrates.

Acid from glycerol and glucose with some strains. No acid or gas from sucrose, maltose or lactose.

Phenol and m-cresol are utilized. Some strains utilize naphthalene. (Gray and Thornton.) Some strains utilize phenol or m-cresol (Jensen).

Optimum $\mathrm{pH} 6.8$ to 8.0 .

Optimum temperature $22^{\circ}$ to $25^{\circ} \mathrm{C}$.

Distinctive characters: Soil organism forming $M$ ycobacterium-like colonies after 2 to 4 days on simple media. Pale pink chromogenesis. Nailhead growth in gelatin stab. Branching rods and short filaments.

Source: Seventy-four strains isolated from soils in Great Britain and Australia.

Habitat: Soil.

13. Nocardia globerula (Gray) comb. nov. (Mycobacterium globerulum Gray,
Proc. Roy. Soc. London, B, 102, 1928, 265; Proactinomyces globerulus Reed, in Manual, 5th ed., 1939, 838.) From Latin globus, a sphere.

Description from Gray (loc. cit.) and from Bynoe (Thesis, MeGill University, Montreal, 1931).

Curved rods and filaments: 1 by 2 to 9 microns, with many coccoid cells, especially in old cultures. Rods and filaments are frequently irregularly swollen. Not acid-fast. Capsules may be present. Gram-positive.

Gelatin: After 19 days surface colonies irregularly round, 1 to $2 \mathrm{~mm}$ in diameter, convex, light buff, smooth, shining; edge entire. Deep colonies: Round, with entire edge.

Gelatin stab: After 8 days nailhead, irregularly round, convex, pinkish-white, smooth, shining; line of stab erose.

Agar: After 4 days surface colonies irregularly round, 3 to $5 \mathrm{~mm}$ in diameter, convex, white, smooth, shining; edge undulate, erose. After 7 days, more convex and of a watery appearance. Deep colonies: After 4 days, lens-shaped.

Agar slant: After 3 days, filiform, flat, watery; edge irregular.

Nutrient and peptone broth: Turbid with viscous suspension.

Indole not formed.

Litmus milk: Alkaline.

Glycerol potato: After 24 hours, filiform, moist, smooth, pale pink.

Dorset's egg medium: After 2 weeks, spreading, raised, moist, orange-colored.

Loeffler's medium: Growth las on Dorset's egg medium, but salmon-colored.

Nitrites not produced from nitrates.

No acid from glucose, lactose, maltose, sucrose or glycerol.

Phenol is utilized.

Indole agar: Blue crystals of indigotin formed.

Optimum temperature $25^{\circ}$ to $28^{\circ} \mathrm{C}$.

Optimum pH 6.8 to 7.6 .

Distinctive characters: This organism resembles most closely Nocardia corallina. It is distinguished by pro- 
ducing a more watery type of surface growth, more nearly entire deep colonies and more particularly by the production of indigotin from indol.

Source: From soil in Great Britain.

Habitat: Presumably soil.

\section{Nocardia salmonicolor (den Dooren} de Jong) comb. not. (.Mycobacterium salmonicolor den Dooren de Jong, Cent. 1. Bakt., II Abt., 71, 1927, 216; Flacobacterium sulmonicolor Bergey et al., Manual, 3rd ed., 1930, 157; Proactinomyces sulmonicolor Jensen, Proc. Linnean Soc. New so. Wales, 57, 1932, 368.) From Latin salmo, salmon and color, color.

Closely related to Nocardia corallina.

On glucose-asparagine-agar after 18 to $24 \mathrm{hrs}$., long branching rods are formed, 1.0 to 1.3 microns in thickness, with small refractive granules of aerial mycelium, sometimes stretching into quite long filaments; after 2 to 3 days small definite mycelia are present, and after 5 to 6 days these have largely divided into short rods and cocei; the colonies have the same burr-like appearance as those of Nocardia corallina. Many cells at the edge of the colonies show, after 3 to 4 days, elub- or pear-shaped swellings, up to 2.5 to 3.0 microns in width; after 5 to 6 days, many of these swollen cells are seen to germinate with the formation of two more slender sprouts. (Orskov, Investigations into the Morphology of the ray fungi. Copenhagen, 1923, 82, gives an almost identical picture of Streptothrix rubra; it is questionable, indeed, whether these two organisms are not really identical.)

Gelatin: At $20^{\circ}$ to $22^{\circ} \mathrm{C}$, scant arborescent growth in stab; small wrinkled orange surface colony. No liquefaction.

Glucose-asparagine-agar: Good growth, restricted, rather flat, edges lobate, surface warty, glistening, first pale orange, later ochre-yellow; consistency crumbly. After 5 to 6 weeks the growth is paler with many small round raised yellow secondary colonies.

Glucose-nutrient agar: Excellent growth, spreading, flat, dense, edges lobate, surface frolded, glistening, yellow, gradually changing to deep orange-red.

Nutrient broth: Fair growth; thin pellicle and granular sediment, at first cream-colored. later red; broth clear at first, slightly turbid after 3 weeks.

Milk: Good growth; pellicle of small cream-colored granules after 2 days, later a thick orange sediment. Not coagulated, but appears slightly cleared after 5 weeks, the reaction becoming alkaline.

Potato. Good growth, raised, warty, crumbly, glistening, at first buff, changing to orange and finally to almost bloodred.

Indole not formied.

Nitrites produced from nitrates.

Nitrate, ammonium salts, asparagine and peptone are utilized almost equally well with glucose as source of carbon, although the growth is most rapid with peptone.

Sucrose not inverted, although readily utilized with sodium nitrate as a source of nitrogen.

Paraffin readily utilized as a source of earbon.

Phenol not utilized.

To acid from glucose or glycerol.

Starch not hydrolyzed.

No growth in oxygen-free atmosphere.

Source: Isolated from soil by means of an ethylamine enriched medium, at $37^{\circ} \mathrm{C}$.

Iabitat: Probably soil.

15. Nocardia rubropertincta (Hefferan) comb. nov. (Butterbacillus, Grassherger, Münch. med. Wochnschr., 46 , 1899, 343; Bacillus rubropertinctus Hefferan, Cent.f. Bakt., II Abt., 11, 1903, 460 ; Serratia rubropertinctus Bergey et al., Manual, 1st ed., 1923, 96; Mycobaclerium rubropertinctum Ford, Textb. of Bact., 1927, 255; Proactinomyces rubropertinctus Reed, in Manual, 5th ed.. 
1939, 835.) From Latin, colored very red.

Büttner (Arch. Hyg., 97, 1926, 17) regards Mycobacterium eos as probably identical with Mycobacterium rubrum Söhngen (Cent. f. Bakt., II Abt., 3\%, 1913, 599), Grassberger's organism (loc. cit.), Hormann and Morgenrot's organism (Hyg. Rundsch., 7, 1898, 229), and Weber's organism (Arb.a. d. k. Gesundheitsamte, Berlin, 19, 1903).

To this list Lehmann and Neumann (Bakt. Diag., 7 Aufl., 2, 1927, 764) also add the organism of Ascher (Ztschr. f. Hyg., 32, 1899, 329) and the butter bacillus of Aujeszky (Cent. f. Bakt., I Abt., Orig., 31, 1902, 132).

Jensen (Proc. Linnean Soc. New So. Wales, 49, 1934, 32) regards the following organisms as probably identical: $B a c-$ terium rubrum Migula (Syst. d. Bakt., 2, 1900, 488) a preliminary deseription of which is given by Schneider (Arb. bakt . Inst. Karlsruhe, 1, Heft 2, 1894, 213); probably this is also the organism referred to by Haag (Cent. f. Bakt., II Abt., 71, 1927, 35) as Bacterium rubrum; and Mycobacterium rubrum Söhngen (loc. cit.).

Description taken from Grassberger (loc. cit.), Hefferan (loc. cit.) and Jensen (loc. cit.).

Small rods: 0.3 to 0.9 by 1.5 to 3.0 microns. Cells in 18 to 24 hour agar culture in beautiful angular arrangement, after 2 to 3 days nearly coccoid, 0.6 by 0.8 micron. Tendency for branching on gly cerol agar after 2 to 3 days, but branching does not occur commonly though granules of aerial mycelium are sometimes seen (Jensen). Not acid-fast (Grassberger). Acid-fast (Hefferan). Variable (Jensen). Non-motile. Grampositive.

Gelatin colonies : Irregular with crenate margin and folded surface. Coral red.

Gelatin stab: Surface growth like the colonies. Growth in stab at first thin, then granular to arborescent with chromogenesis. No liquefaction.
Agar colonies: Small, granular, becoming pink to red depending on composition of agar.

Agar slant: Dry, lustreless (R) to glistening (S), pink to vermillion red.

Broth: Faint uniform turbidity with salmon-pink pellicle (in scales) which is renewed on surface as it settles to form a red sediment (Hefferan, Jensen).

Litmus milk: Thick, fragile, dull coral red surface scales and sediment. Unchanged (Hefferan) to alkaline and somewhat viseid after 3 to 4 weeks (Jensen).

Potato: Slow but excellent intensive red growth becoming dull orange (Jensen).

Nitrites not produced from nitrates; nitrates, ammonia and asparagine are almost as good sources of nitrogen as peptone (Jensen).

Benzine, petroleum, paraffin oil and paraffin are utilized as sources of energy (Söhngen). To action on manganese dioxide (Söhngen, Cent. f. Bakt., II Abt., 40, 1914, 55t).

Optimum pH 6.8 to 7.2. Growth stops at $\mathrm{pH} 4.9$.

Temperature relations: Grows well between $20^{\circ}$ and $37^{\circ} \mathrm{C}$ (Jensen).

Aerobic to facultative anaerobe.

Distinctive characters: Mycobacterium-like colonies with coral to vermillion red chromogenesis on asparagine agar, potato, zelatin and other media. Short rods, seldom forms filaments. Generally not acid-fast.

Source: Six cultures isolated from butter (Grassberger). Several cultures isolated from soil in Holland (Söhngen) and Australia (Jensen). Two cultures as contaminants in tuberculin flasks (Hagan, Breed).

Habitat: Probably widely distributed in soil.

16. Nocardia rubra (Ḱrassilnikov) comb. nov. (Proactinomyces ruber Krassilnikov, Bull. Acad. Sci. U. S. S. R., No. 1, 1938, 139.) From Latin ruber, red. 
Mycelium produced at first but soon breaks up into rods and cocei. The latter multiply by fission, cross-wall formation and budding. Cells are Gram-positive, weakly acid-fast.

Gelatin : No liquefaction.

Colonies smooth or folded and rough, shiny or dull, dark red color. Pigment belongs to the carotinoids, does not diffuse into the mediun:

Milk: No coagulation or peptonization.

Sucrose not inverted.

Starch not hydrolyzed.

No growth on cellulose.

Readily assimilates fats and paraffin, and, to a less extent, wax.

Various strains of this organism may vary considerably from type.

Habitat: Soil.

17. Nocardia coeliaca (Gray and Thornton) comb. nov. (Mycobacterium cocliacum Gray and Thornton, Cent. f. Bakt., II Abt., 73, 1928, 88; Flavobacterium coeliacum Bergey et al., Manual, 3rd ed., 1930, 156; Proactinomyces coeliacus Reed, in Manual, 5th ed., 1939, S36.)

Description from Gray and Thornton (loc. cit.) and from Jensen (Proc. Linnean Soc. New So. Wales, 56, 1931, 201).

Short, curved, uneven-sided rods : 0.8 by 5 microns with occasional filaments up to 10 to 12 microns long, frequently beaded, occasionally swollen or branched. Coccoid forms 0.8 to 1.2 microns in diameter are common, especially in older cultures. Stain readily. Not acid-fast. or occasionally slightly acid-fast. Grampositive.

Gelatin colonies : After 12 days, irregular, raised, white, rugose, dull, edge entire. Deep colonies: Irregular, smooth or slightly broken.

Gelatin stab: Convoluted, buff-white to yellowish, dull; below surface the growth forms many irregular hollow lobes, giving a glistening appearance, to a depth of 3 to $4 \mathrm{~mm}$.

Agar colonies: After 11 days, less than
$1 \mathrm{~mm}$ in diameter, round or irregular, raised, white, resinous, edge irregular, burred. Deep colonies: Irregularly round or oval, edge slightly broken.

Agar slant: Filiform, convex, white, rugose, resinous, edge undulate.

Broth: Turbid.

Litmus milk: Slightly alkaline after 5 to 7 days.

Glycerol potato: After 2 days, dry, crumpled, orange, becoming brown after about 10 days.

Dorset's egg medium: Raised, smooth, moist, verrucose, buff-colored.

Loeffler's medium: After 10 days, slight growth, dry, granular, pale buffcolored.

Nitrites are not produced from nitrates.

No acid from glucose, lactose, sucrose or glycerol.

Phenol is utilized.

Optimum $\mathrm{pH} 7.6$ to 8.0 .

Optimum temperature $22^{\circ}$ to $25^{\circ} \mathrm{C}$.

Distinctive characters: Differs from the previously described members of the genus in the absence of chromogenesis. Forms hollow lobes in deep gelatin cultures. Cells are rods, seldom filaments.

Source: From soil in Great Britain and Australia.

Habitat : Presumably soil.

15. Nocardia transvalensis Pijper and Pullinger. (Pijper and Pullinger, Jour. Trop. Med. Hyg., 30, 1927, 153; Actinomyces transvalensis Nannizzi, in Pollacei, Tratt. Micopat. Umana, \&, 1934, 46.) Named for the Transvaal, a state of South Africa.

Description from Erikson (Med. Res. Council Spec. Rept. Ser, 203, 1935, 28).

Initial mycelium unicellular, but with the central branch frequently broader and showing dense granular refractile contents, small colonies quickly covered with aerial myeelium, the straight aerial hyphae in some cases becoming clustered into irregular spikes, colorless drops are exuded and a pink coloration produced in 
the densest part of the growth on synthetic glycerol agar. Angular branching with division of the substratum filaments can be seen, the aerial hyphae also being irregularly segmented. Acid-fast.

Gelatin: Poor growth, a few irregular colorless flakes. No liquefaction.

Agar: No growth.

Glucose agar: Raised, granular, pink colonies with white aerial mycelium.

Glycerol agar: Small pink coiled masses with thin white aerial mycelium.

Potato agar: No growth.

Coon's agar: Colorless growth with liberal white aerial mycelium.

Dorset's egg medium : Small irregularly raised and coiled dull pink mass.

Serum agar: Very poor growth.

Inspissated serum: Scant colorless flaky growth; later a minute tuft of pale pink aerial mycelium.

Broth: Moderate flaky growth.

Synthetic sucrose solution: Poor growth, a few flakes on surface, a few at bottom.

Potato plug: Dry, raised, convoluted, pink growth with white aerial mycelium in one month; dull, pink, brittle surface colonies, with paler aerial mycelium floating coherently on liquid at base in 2 months.

Milk: No change.

Starch not hydrolyzed.

Source : From a case of mycetoma of the foot in South Africa.

Habitat: Human infections so far as known.

19. Nocardia mesenterica (Orla-Jensen) comb. nov. (Microbacterium mesentericum Orla-Jensen, The Lactic Acid Bacteria, 1919, 181; Proactinomyces mesentericus Jensen, Proc. Linnean Soc. New So. Wales, 57, 1932, 373.) From Greek mesenterium, mesentery.

Extensive mycelium composed of richly branching hyphae of a somewhat variable thickness, 0.4 to 0.8 micron; no aerial hyphae are seen. With increasing age the hyphae divide into fragments of varying size and shape, partly diphtheroid rods, but no real cocci. There is, particularly in richer media, a tendency to form large, swollen, fusiform to almost spherical cells, up to 3.5 microns in diameter. These may stain intensely with carbol fuchsin; when transferred to fresh media, they germinate and produce a new mycelium.

Gelatin: Good growth; finely arborescent, cream-colored growth in the stab; raised, folded, pale-yellow, surface colony. No liquefaction.

Glucose-asparagine-agar: Fair growth, narrow, raised, granular, very pale yellow, glistening; condensation water clear, with small granules. At $30^{\circ} \mathrm{C}$ only scant growth consisting of small irregular white granules, growing deeply down into the agar.

Glucose-nutrient-agar: Good growth, restricted, with undulate edges, surface with high transverse folds, creamcolored; the consistency is firm and cartilaginous after 2 days, later looser and more brittle. Growth at $28^{\circ}$ to $30^{\circ} \mathrm{C}$ rather scant; smooth, soft, glistening, cream-colored smear.

Sabouraud's agar: Excellent growth, spreading, at first flat and smooth, pale straw-yellow, perfectly hard and cartilaginous, later raised and strongly folded, of a loose, curd-like consistency, bright lemon-yellow. Growth at $28^{\circ}$ to $30^{\circ} \mathrm{C}$ only fair, restricted, folded, creamcolored, soon becoming s oft and smeary .

Potato: Seant growth; restricted, soft, cream-colored smear.

Broth: Good growth; voluminous, flaky, whitish sediment; broth clear.

Milk: At $28^{\circ}$ to $30^{\circ} \mathrm{C}$, small creamcolored granules along the tube; the milk undergoes no visible changes within 4 weeks. No proteolytic action.

Indole not formed.

Sucrose is inverted.

Starch is hydrolyzed.

Cellulose is not decomposed.

Nitrates are reduced to nitrites.

No growth in oxygen-free atmosphere. 
Nitrogen is utilized as sodium nitrate, ammonium phosphate and asparagine, although these are inferior to peptone as sources of nitrogen.

Soure: Fermented beets.

\section{Nocardia flava (Krassilnikov)} comb. nov. (Proactinomyces flavus Kirassilnikov, Bull. Acad. Sci. U. S. S. R., No. 1, 1938, 139.) From Latin flarus, yellow.

Cells at first filamentous, 0.7 to 0.8 micron in diameter; after 2 to 3 days broken into long rods and then into cocci 0.7 micron in diameter. No spores, some strains form chlamydospores. Cell multiplication by fission, cross-wall formation, rarely by budding. Cells Gram-positive; not acid-fast.

Gelatin: No liquefaction.

Synthetic agar colonies: Bright yellow or gold color.

Meat peptone media: Dirty yellow pigmentation.

Agar colonies: Pigment bright yellow or gold color on synthetic media, dirty yellow on meat peptone media. P'igment not soluble in medium. Surface of colony somewhat shiny or rough and folded, of a dough-like consistency

Milk: No peptonization or coagulation. Sucrose weakly inverted.

Stareh is hydrolyzed.

Does not grow on paraffin and wax but makes weak growth on fat.

Habitat: Soil, not common.

\section{Nocardia viridis (Krassilnikov)} comb. nov. (Proactinomyces viridis Krassilnikov, Bull. Acad. Sci. U. S. S. R., No. 1, 1938, 139). From Latin viridis, green.

Mycelial cells often branching, 0.7 to 0.8 micron in diameter with cross-wall; after 5 to 7 days the cells break up into rods 5 to 15 microns long. Cocci not observed. Cells multiply by fission, seldom by budding. Spores not formed. Cells Gram-positive, not acid-fast.

Gelatin: No liqueftection.
Colonies colored dark green. Pigment not soluble in medium, in water or in organic solvents. Surface of colony somewhat shiny. On potato, rough, much folded, broken up into small colonies.

Milk: No peptonization or coagulation. Sucrose readily inverted.

Stareh weakly hydrolyzed.

Grows well on fats and paraffin and less on wax.

\section{Habitat: Soil.}

\section{Nocardia citrea (Krassilnikov)} comb. nov. (Proactinomyces citrcus Krassilnikov, Bull. Acad. Sci. U. S. S. R., No. 1, 1938, 139.) From M. L. citreus, lemon yellow.

Mycelium in young cultures consists of very fine threards 0.3 to 0.5 micron in diameter. After several days the cells break up into short rods 0.5 by 1.5 to 5 mierons and into rocei 0.3 to 0.5 micron in diameter. Multiplies by fission and bud formation; spores not formed. Cells not acirl-fast.

Gelatin: Liquefaction.

Colonies: Vellow-green, usually lough and folded.

Milk: Coagulation and peptonization. Sucrose is inverted.

Starch is hydrolyzed.

Weak growth on fat. No growth on paraffin or wax.

Habitat: Soil and water.

23. Nocardia madurae (Vincent) Blanchard. (Streptothrix madurae Vincent, Ann. Inst. Past., 8, 1894, 129; Blanchard, Bouchard's Traité de Path. Gén., 2, 1896, 868; Oospora madurae Lchmann and Neumann, Bakt. Diag., 1 Aufl., 2, 1896, 388; Actinomyces madurae Lachner-Sandoval, Ueber Strahlenpilze, 1898, 64; Cladothrix madurae Macé, Traité Pratique de Bact., 4th ed., 1901, 1090; Discomyces madurae Gedoelst, Champ. Paras. Ilomme et Animaux, Paris. 1902. 169.) Nimed for the disease 
Madura foot, with which this organism is associated.

Oospora indica Kanthack (Kanthack, Jour. Path. and Bact., 1, 1893, 140; Nocardia indica Chalmers and Christopherson, Ann. Trop. Med. and Parasit., 10, 1916, 231 ; Discomyces indicus NeveuLemaire, Précis de Parasitol. Hum. 5th ed., 1921, 42; Actinomyces indicus Brumpt, Précis de Parasitol., Paris. th ed., 1927, 1196) is regarded by some authors as identical with Nocardia madurae Blanchard. If this is established, then the correct name of the organism is Nocardia indica (Kanthack) ('halmers and Christopherson.

The species described under the name Actinomyces madurae in previous editions of Bergey's Manual is definitely not the true causative agent of the disease and is probably a contaminant carried as a culture of this species.

Morphology in tissues, growth in form of granules consisting of radiating actinomycosis. In cultures, initial branched mycelium fragmenting into rod-shaped and coccoid bodies. No aerial mycelium or spores. Not acid fast.

Gelatin: Growth scant, whitish; no liquefaction.

Gelatin colonies: Round, glistening, at first white, then buff to rose or crimson. Pigment production is irregular and unpredictable. Occasionally red soluble pigment is produced. Growth eventu. ally wrinkled. No aerial mycelium.

Potato: Wrinkled friable growth; buffcolored, sometimes red.

Broth: Growth as a floceular sediment.

Milk: No rhange, or slight slow peptonization.

Diastatic (?) action.

Not pathogenic for the usual laboratory animals; pathogenic for monkeys (Musgrave and Clegg, Philippine Jour. Sci. Ser. B., Med. Sci., 3, 1908, 470).

Habitat: Cause of some cases of Madura foot.
24. Nocardia lutea Christopherson and Archibald. (Christopherson and Archibald, Lancet, 2, 1918, 847; Actinomyces luteus Brumpt, Précis de Parasitol., Paris, tth ed., 1927, 1206.) From Latin luteus, yellow.

Description from Erikson (Med. Res. Council Spec. Rept. Ser. 203, 1935, 30).

Initial elements swollen and segmented, giving rise to irregular spreading polymorphous colonies composed of cells of all shapes and sizes with markedly granular contents. Later more monomorphous, the filaments being arranged in angular apposition. Sometimes (e.g., on synthetic glycerol agar) the segments are so granular as to appear banded. On potato agar, small filamentous colonies are formed with irregular angular branching and bear a few isolated short straight derial hyphae.

Gelatin: Pale pink wrinkled growth on wall of tube and colorless punctiform and stellate colonies in medium; no liquefaction.

Agar : Abundant, coherent, moist, pink membranous growth with round discrete colonies at margin ; after 3 weeks colorless fringed margin, round confluent portion.

Glucose agar: Scant reddish smeary growth.

Glycerol agar: Yellowish-pink, wrinkled membrane.

Potato agar: Coherent pink moist growth, centrally embedded with small round discrete colonies at margin.

Dorset's egg medium: Poor growth, dull pink, spreading.

Serum agar: Conflueut granular pink membrane.

Broth: Pink flakes and surface growth.

Inspissated serum: Raised convoluted pink mass; becoming orange, much wrinkled, scalloped margin.

Synthetic sucrose solution: Red granules and abundant minute colorless colonies at bottom; in 2 weeks a colorless dust-like surface pellicle.

Glucose broth: Abundant, pinkish 
flaky surface growth, breaking up easily and sinking to bottom.

Litmus milk: Orange-red surface and bottom growth, liquid blue.

Potato plug: Carrot-red, moist, thick, granular growth in bands, partly raised and with discrete round colonies; sparse colorless very thin aerial mycelium at top of slant in 2 months.

Source: From actinomycosis of the lachrymal gland.

\section{Nocardia blackwellii (Erikson)} comb. nov. (Actinomyces blackwellii Erikson, Med. Res. Counc. Spec. Rept. Ser. 203, 1935, 37.)

Description from Erikson (loc. cit., p. 32).

Initial elements short, rod-like, growing out into longer forms sparsely branching; small radiating colonies are produced with short straight aerial mycelium, frequently large round or ovoid cells are interposed in the irregularly segmented chains of cells, being sometimes isolated in company with 2 or 3 short filaments and sometimes terminal.

Gelatin: Few colorless minute colonies along line of inoculation; after 30 days abundant colorless colonies to $10 \mathrm{~mm}$ below surface, larger pink-yellow surface colonies with white aerial mycelium; no liquefaction.

Agar: Confluent wrinkled growth with small, round, pinkish, discrete colonies at margin.

Glucose agar: Abundant, pale pink growth, small conical colonies, piled up, convoluted.

Glycerol agar: Extensive, granular, irregular, thin, pinkish growth; after 40 days, a few discrete colonies with depressed margins, center piled up, pink.

Serum agar: Smooth, cream, umbilicated colonies, with submerged growth extending into medium in scallops 5 to 8 $\mathrm{mm}$ deep; a pale pink mass in 2 weeks.

Potato agar: Small, round, colorless colonies covered with white aerial mycelium; after 2 weeks colonies dull pink, submerged margins, few aerial spikes, moderate aerial mycelium at top of slant.

Broth: Flakes, later innumerable minute colonies, some adhering to wall just above liquid level.

Synthetic sucrose solution: Delicate, round, white colonies; later abundant minute colonies in suspension, thick cream pellicle on surface and pink grains in sediment.

Milk: Heavy convoluted bright yellow surface pellicle, no coagulation.

Litmus milk: Yellow surface growth, milky sediment, liquid unchanged.

Carrot plug: Small, round, smooth, cream-colored elevated colonies in 10 days; sparse stiff colorless aerial spikes in 16 days; abundantly piled up, convoluted, ochreous growth in 25 days.

Source: From hock joint of foal.

26. Nocardia cuniculi Snijders. (Snijders, Geneesk. Tijdsch. Med. Ind., $64,1924,47$ and 75 ; Actinomyces cuniculi Erikson, Med. Res. Council Spec. Rept. Ser. 203, 1935, 32; not Actinomyces cuniculi Gasperini, Cent. f. Bakt., 15, 1894, 684; Actinomyces sumatrae Erikson, loc. cit., 32.) From Latin cuniculus, rabbit.

Description taken from Erikson (loc. cit., p. 31).

Large swollen cells give rise to ramify ing filaments or to small chains of short thick segments which branch out into more regular hyphae; sometimes the irregular elements are beset with spiny processes before giving rise to typical long branching filaments; later the picture becomes more monomorphous and short straight aerial hyphae are borne, which presently exhibit irregular segmentation.

Gelatin: Few flakes. No liquefaction.

Agar: Small, round, elevated, creamcolored colonies, umbilicated and radially wrinkled.

Glucose agar: Minute, colorless colon. 
ies; becoming dull pink, partly confluent and piled up, few stiff pink aerial spikes.

Glycerol agar: Small round elevated cream-colored colonies, margins depressed; becoming smooth, discrete, yellowish.

Dorset's egg medium: Scant pinkish smeary growth.

Serum agar: Small, raised, creamcolored colonies, becoming confluent and piled up.

Inspissated serum: Thick, colorless, ribbed membrane; no liquefaction.

Broth: Small and larger cream-colored, scale-like surface colonies, abundant, flocculent bottom growth.

Synthetic sucrose solution; Thin surface pellicle, small colorless flakes, minute particles at bottom, scant growth.

Milk: Heavy yellow growth attached to walls; solid coagulum in 1 month.

Litmus milk: Yellow surface growth, liquid unchanged.

Potato plug: Coral-pink, dry, granular growth, covered to a considerable extent with white aerial mycelium, piled up in center, discrete colonies at margin, pink surface pellicle on liquid and colorless colonies at base.

Source: Infected rabbits.

\section{Nocardia rangoonensis (Erikson)} comb. nov. (Actinomyces rangoon Erikson, Med. Res. Council Spec. Rept. Ser. 203, 1935, 37.)

Description from Erikson (loc. cit., p. 33).

Swollen round initial cells, giving rise to branching hyphae which segment and present slipping and angular arrangement; few short straight aerial hyphae, which later develop into a profusely branching long waving aerial mycelium. Non-acid-fast.

Gelatin: Abundant minute colonies in depths and larger cream-colored ones on surface with white aerial mycelium; brown pigment surrounding growth. No liquefaction.

Agar colonies: Round, lobate, umbili- cated, raised up, cream-colored to pale pink; later, medium discolored dark brown, colonies colorless.

Glucose agar: Convoluted, coherent, cream-colored growth, medium discolored. After 23 days, wrinkled, biscuit-colored growth, colorless margin, border white aerial mycelium, medium dark brown.

Glycerol agar: Dull, mealy, pink, mrinkled growth, scant white aerial mycelium at top, medium slightly discolored.

Coon's agar: Minute colorless colonies in streaks.

Potato agar: Small, round, lemoncolored colonies, partly confluent, with white aerial mycelium; later medium discolored light brown; submerged growth greenish.

Dorset's egg medium : Extensive colorless growth, pale pink aerial mycelium in center; later covered with a powdery pinkish-white aerial mycelium.

Serum agar colonies: Irregular, small, elevated, cream-colored, frequently umbilicated.

Inspissated serum : Poor growth, small piled up pink mass.

Broth: Abundant colorless growth, flocculent mass at bottom and pellicle at surface, medium slightly discolored.

Synthetic sucrose solution: Small white colonies with pinkish tinge on surface, lesser bottom growth.

Milk: Coagulation, yellow surface ring, becoming partly peptonized, liquid discolored dark brown, brownish growth up side of tube.

Litmus milk: Colorless growth, liquid partly decolorized; coagulation; later partly digested.

Carrot plug: Small round colorless colonies, velvety white aerial mycelium; in 2 months, piled up pink granular mass with warted prominences, marginal zone white aerial mycelium and thin all-over central aerial mycelium.

Source: Human pulmonary case of streptothricosis. 
Habitat: Human infections so far as known.

\section{Nocardia caviae Snijders.} (Snidjers, Geneesk. Tijdschr. Ned. Ind., 64, 1924, 47 and 75; Actinomyces caviae Erikson, Med. Res. Council Spec. Rept. Ser. 203, 1935, 37.) From the generic name of the guinea pig.

Description from Erikson (loc. cit., p. 32 ).

Initial segmentation, producing elements of approximately even thickness arranged in angular apposition, and later long profusely ramifying threads with strongly refractile protoplasm. Aerial mycelium straight and branching, the aerial hyphae with occasional coiled tips divided into cylindrical conidia.

Gelatin: A few colorless flakes. No liquefaction.

Glucose agar: Piled up, convoluted, cream-colored to pale pink growth, white aerial mycelium.

Glycerol agar: Scanty growth.

Coon's agar: Colorless scant growth, partly submerged, white aerial mycelium.

Potato agar : Colorless spreading growth with dense white aerial mycelium.

Dorset's egg medium: Heavily corrugated pale pink growth with submerged margin, dense white aerial mycclium in center; after 3 weeks, colorless transpired drops.

Serum agar: Pale pink wrinkled growth, partly submerged; after 4 weeks, piled up with scant white aerial mycelium, medium discolored reddish-brown.

Inspissated serum: Pale pink raised growth, coiled, white aerial mycelium.

Broth : Cream-colored wrinkled surface pellicle extending up wall and breaking easily, moderate bottom growth, flaky, medium discolored.

Synthetic sucrose solution: Round white colonies in suspension and attached to one side of tube, pink surface colonies with white aerial mycelium.

Milk: Colorless surface growth, white aerial mycelium; coagulation.
Litmus milk: Liquid blue, surface growth; after 1 month, white aerial mycelium, colorless sediment, liquid still blue.

Potato plug: Small colorless colonies, white powdery aerial myeelium; later abundant raised pale pink confluent growth, discolored plug; after 2 months, raised buckled pink colonies with white aerial mycelium floating on liquid at base

Source: Infected guinea pigs, Sumatra.

29. Nocardia actinomorpha (Gray and Thornton) comb. nov. (Mycobacterium. actinomorphum Gray and Thornton, Cent. f. Bakt.. II Abt., 73, 1928, 88; Actinomyces actinomorphus Bergey et al., Manual, 3rd ed., 1930, 471; Proactinomyces actinomorphus Jensen, Proc. Linnean Soc. New So. Wales, 56, 1931, 363.) From Greek actis, ray and Latin morpha, shape, form.

Description from Gray and Thornton (loc. cit.), Jensen (loc. cit.), and Bynoe (Thesis, McGill University, Montreal, 1931 ).

Long branching filaments and rods : 0.5 to 0.8 by up to 10 microns. In older cultures rods 2 to 3 microns long generally predominate. On some media extentively branching hyphae occur. Readily stained. Not acid-fast. Gram-positive.

Gelatin colonies: After 12 days, round, saucer-like, white, raised rim, edges burred. Liquefaction. Deep colonies: Burrs.

Gelatin stab: After 8 to 1.4 days, saecate liquefaction, 5 to $8 \mathrm{~mm}$.

Agar colonies: After 11 days, round, 1 $\mathrm{mm}$ in diameter, convex, white, granular or resinous; long arborescent processes from the edge. Deep colonies: Arborescent burrs; processes about equal to diameter of colony.

Agar slant: Filiform, raised to convex, white, rugose, dull; edge undulate, with strong tufted projections below surface.

Broth: Turbid, or clear with white scum. 
Dorset's egg medium: After 2 weeks, raised, dry, smooth, salmon-buff growth.

Loeffler's medium: After 2 days, smooth, moist, warty, salmon-colored growth.

Litmus milk: Alkaline after 5 to 7 days .

Glycerol potato: After 2 days, dry, wrinkled, pink to orange growth.

Nitrites are produced from nitrates.

No acid from glucose, lactose, sucrose or glycerol.

Phenol and naphthalene are utilized.

Optimum temperature $25^{\circ}$ to $30^{\circ} \mathrm{C}$.

Optimum pH 7.8 to 8.5 .

Distinctive characters: Differs from Nocardia coeliaca in saccate liquefaction of gelatin. Long rods and filaments.

Source: A few strains have been isolated from soil in Great Britain and Australia.

Habitat: Presumably soil.

\section{Nocardia flavescens}

(Jensen) comb. nov. (Proactinomyces flavescens Jensen, Proc. Linnean Soc. New So. Wales, 56, 1931, 361.) From Latin flavescens, becoming golden yellow.

On media where a firm growth is produced, the vegetative mycelium appears as long, branched, non-septate hyphae, 0.4 to 0.6 micron thick. In other media, as on nutrient agar and potato, septa are formed and the mycelium appears in preparations as fragments of very variable size, partly resembling highly branched mycobacteria. In several cases-for instance, on nutrient agar at $28^{\circ}$ to $30^{\circ} \mathrm{C}$, in 5 to 6 weeks old eultures in glucose broth, and in glucose $\mathrm{NH}_{4} \mathrm{Cl}$ solution-short elements assume swollen, fusiform to lemon-shaped forms. The aerial mycelium consists of fairly long hyphae of the same thickness as the vegetative hyphae, not very much branched, without spirals, of en clinging together in wisps. A differentiation into spores is never visible by direct microscopic examination. Neither is this the case in stained preparations; here the aerial hyphae break up into fragments of quite variable length, from 1.2 to 1.5 up to 10 to 12 microns, showing an irregular, granulated staining.

Gelatin: Slow liquefaction.

Sucrose agar: Good growth. Vegetative mycelium superficially spreading, much raised and wrinkled, cracking, white to cream-colored, of a dry, but loose and crumbly, consistency. Aerial mycelium scant, thin, white. Faint yellow soluble pigment after 2 to 3 weeks.

Glucose agar: Good growth. Vegetative mycelium superficial, wrinkled, honey-yellow, of a hard and cartilaginous consistency. Aerial mycelium thin, smooth, white. Yellow soluble pigment.

Nutrient agar: Good growth. Vegetative mycelium raised and much wrinkled, first dirty cream-colored, later dark yellowish-gray, of a soft, moist, curd-like consistency. No aerial mycelium. No pigment.

Potato: Good to excellent growth. Vegetative mycelium much raised and wrinkled, first cream-colored, later yellowish-brown, soft and smeary. No aerial mycelium, no pigment.

Glucose broth: Rather scant growth. Granulated, yellowish sediment; no surface growth. Broth clear. No pigment. To acidity.

Sucrose is inverted.

Starch is hydrolyzed.

Cellulose is not decomposed.

Nitrates are reduced slightly or not at all with various sources of energy.

Milk: Coagulated and slowly redissolved with acid reaction.

Final reaction in glucose- $\mathrm{NH}_{4} \mathrm{Cl}$ solution, pH 3.9 to 3.6 .

No growth under anaerobic conditions. Habitat: Soil.

31. Nocardia maculata (Millard and Burr) comb. nor. (Actinomyces maculatus Millard and Burr, Ann. Appl. Biol., 13, 1926, 580; Proactinomyces maculatus Umbreit, Jour. Bact., 38, 1939, 84.) From Latin maculatus, spotted.

Description taken from Umbreit 
Filamentous organisms possessing a tough shiny colony which is cartilaginous, rarely producing an aerial mycelium, though in certain strains, it may occur frequently. Retains the mycelium form for long periods. Not acid-fast.

Gelatin : Liquefaction.

In the young colony an orange-yellow to orange-red intercellular pigment is produced on all media, which may or may not change to black as the culture ages.

Milk: \o digestion.

Starch is hydrolyzed.

Does not utilize paraffin.

Habitat: Soil.

32. Nocardia rhodnii (Erikson) comb. nov. (Actinomyces rhodnii Erikson, Med. Res. Council spec. Rept. Ser. 203, 1935, 37.) Named for the insect genus, Rhodnius.

Description from Erikson (loc. cil., p. 29).

In early stages, the minute colonies are composed of hyphal segments arranged in angular apposition; the aerial mycelium being short and straight. Later the growth becomes extensive and spreading, made up partly of long, genuinely branching filaments and partly of short segments exhibiting slipping branching, each giving rise to aerial hyphae. After 2 weeks the angular branching is very marked, delicate spreading herring-bone patterns being formed.

Gelatin: Rapid liquefaction; pale pink colonies in superficial pellicle and sediment.

Coon's agar: Colorless pinpoint colonies.

Czapek's agar : Minute, colorless, round colonies.

Glucose agar: Abundant, coral pink, convoluted, piled up growth.

Glycerol agar: Extensive growth, dull pink colonies round and umbilicated, becoming piled up and deeper coral; later partly submerged.
Dorset's egg medium: Salmon-pink, granular membrane; later piled up.

Serum agar: Extensive, reddish, confluent mass, granular, tending to be piled up; the medium around the growth shows reddish coloration in 2 weeks.

Inspissated serum: Smooth, round, pale pink colonies, centrally depressed and irregularly coiled larger mass; no liquefaction.

Broth : Salmon-pink flakes in sediment and colonies on surface; after 2 weeks abundant growth, discoloration of me. dium.

Glucose broth: Thin, pink, superficial pellicle, easily breaking up, and small flakes in sediment; after 2 weeks abundant growth extending up tube.

Synthetic sucrose solution: Colorless to pink colonies in superficial pellicle, and minute round white colonies coherent in loosely branching mass in sediment.

Milk: Bright orange growth; medium unchanged.

Potato agar: Abundant, pink growth, piled up, scant stiff white aerial mycelium at top of slant.

source: From reduvid bug, Rhodnius prolixus.

33. Nocardia gardneri (Waksman) comb. nov. (Actinomycete, Gardner and Chain, Brit. Jour. Exp. Path., 23, 1942, 123 ; Proactinomyces gardneri Waksman, in Waksman, Horning, Welsch and Woodruff, Soil Sci., 54, 1942, 289.) Named for Prof. Gardner who first isolated this organism.

Gram-positive, branching mycelium.

Gelatin: Cream-colored surface ring. Rapid liquefaction. Green to greenishbrown soluble pigment gradually diffuses through the liquefied portion.

Nutrient agar: Cream-colored, elevated, lichenoid growth, soft, not leathery; no aerial mycelium; very faint brownish pigment.

Glucose agar: Brownish, lichenoid 
growth, with wide, cream-colored edge; white to grayish aerial mycelium gradually covering surface. Reverse of growth yellowish; no soluble pigment.

Glucose-asparagine agar: Aerial mycelium develops slowly.

Tryptone broth: Growth occurs as small pellets at the base of the flask; later, a thin surface pellicle appears, which consists of a branching mycelium. Black pigment slowly produced.

Litmus milk: Tnchanged.

Potato: Barnacle-like, brownish, spreading growth; no aerial mycelium. Medium brownish around growth.

Indole not formed.

No acid from glucose, lactose, maltose, mannitol, sucrose and dulcitol.

Good growth at $25^{\circ} \mathrm{C}$. Slow yrowth at $37^{\circ} \mathrm{C}$.

Distinctive character: Produces an antibiotic substance (proactinomycin) upon synthetic and organic media which is primarily active against various Grampositive bacteria.

Source: Isolated as an air contaminant at Oxford, England.

*Appendix I: The following species probably belong to this genus. Many are incompletely described. Some of the species listed here may belong in the genus Streptomyces.

Actinomyces albus acidus Nenkirch. Neukirch, Inaug. Diss, Strassburg, 1902, 50; Actinomyces albus var. acidus Nannizzi, in Pollacei, Tratt. Micopat. Umana, 4, 1934, 9.) From a case of keratitis.

Actinomyces avadi Dodge. (Streptothrix madurae Koch and Stutzger, Ztschr. f. Hyg., 69, 1911, 17; not Streptothrix madurae Vincent, Ann. Inst. Past., 8, 1894, 129; Dodge, Medical Mycology,
St. Louis, 1935, 729.) From a Madura foot in Egypt, case of Dr. Avad.

Actinomyces bolognesii-chiurcoi (Vuillemin) Dodge. (Malbrachea bolognesiichiurcoi Vuillemin, in Bolognesi and Chiureo, Archivi di Biol., 1, 1925; Dodge, Medical Mycology, St. Louis, 1935, 766.) From ulcers on the thorax.

Actinomyces cameli (Mason) Sartory and Bailly. (Streptothrix cameli Mason, Jour. Trop. Med. and Therap., 32, 1919, 34; Oospora cameli Sartory, Champ. Paras. Homme et Anim., 1923, 822; Sartory and Bailly, Mycoses Pulmonaires, 1923, 253.) From pseudotuberculosis lesions in a camel.

Actinomyces canis (Rabe) Gasperini. (Discomyces pleuriticus Vachetta, Studi e ricordi clin. Milano, 1882; Pleuromyces canis familiaris Rivolta, Giornali d. Anat. Fisiol. e Patol., 16, 1884, 4; Cladothrix canis Rabe, Berlin. tierärztl. Wochnschr., 1885, 65; Gasperini, Ann. Ist. d'Ig. sper. Univ. Roma, 2, 1892, 222; Streptothrix canis and Actinomyces pleuriticus canis familiaris, quoted from Gasperini, Cent. f. Bakt., 15, 1894, 684; Leptothrix pleuriticus Piana and Galli-Tallerio, 1896, quoted from Nannizzi, in Pollacei, Tratt. Micopat. Lmana, 4, 1934, 37; Nocardia canis Chalmers and Christopherson, Ann. Trop. Med. and Parasit., 10, 1916, 255; Oospora canis Sartory, Champ. Paras. Homme et Anim., 1923, 821.) Rabe isolated this organism in two cases of phlegmon and a case of peritonitis in dogs.

Actinomyces citrocremeus Nannizzi. (Mycobacterium diphtheriae acium Trincas, 1907, quoted from Nannizzi, in Pollacci, Tratt. Micopat. Umana, 4, 1934,50; Nannizzi, idem.) From a disease in birds.

Actinomyces dassonvillei BrocqRousseu. (Brocq-Rousseu, Thèse Sci.

* The appendix was originally prepured by Prof. S. A. Waksman and Prof. A. T. Henrici, May, 1943; it has been developed further by Mrs. Eleanore Ineist Clise, Geneva, Lew Tork, August, 1945 
Nat. Paris, 1907; Nocardia dassonvillei Liégard and Landrieu, Ann. d'Occulistique, $46,1911,418 ;$ Discomyces dassonvillei Brumpt, Précis de Parasitologie, Paris, 2nd ed., 1913, 976.) Reported from a cervical abscess (Brumpt), from a case of conjunctivitis (Liégard and Landrieu), and from grain (Pinoy, Bull. Inst. Past., 11, 1913, 923).

Actinomyces dermatonomus Oxer. (Bull. Austral. Jour. Exp. Biol. Med. Sci., 6, 1929, 301, quoted from Dodge, Medical Mycology, St. Louis, 1935, 719; Oxer, 1930, quoted from Nannizzi, in Pollacci, Tratt. Micopat. Umana, 4, 1934, 51.) From lesions on sheep in Australia.

Actinomyces donnae Dodge. (Strcptothrix sp. Donna, Ann. Ig. Sperim., 14, 1904, 449; Dodge, Medical Mycology, St. Louis, 1935, 745.) From sputum in a pulmonary infection.

Actinomyces dori (Beurmann and Gougerot) Brumpt. (Sporotrichum, Dor, Presse Méd., 14, 1906, 234; Sporotrichum dori Beurmann and Gougerot, Ann. Derm. Syphiligr. IV, 7, 1906, 996; Discomyces dori Beurmann and Gougerot, Les Nouvelles Mycoses, 1909, 59; Rhinocladium dori Neveu-Lemaire, Précis Parasitol., 1921, 84; Oospora dori Sartory, Champ. Paras. Homme et Anim., 1923, 770; Brumpt, Précis de Parasitol., 4th ed., 1927, 1206; Nocardia dori Vuillemin, Encyclopédie Mycologique, Paris, 2, 1931,284 .) Found in subcutaneous abscesses resembling sporotrichosis.

Actinomyces hominis Berestneff. (Berestneff, Inaug. Diss., Moskow, 1897; not Actinomyces hominis Waksman, Soil Science, 8, 1919, 129; Nocardirt hominis Chalmers and Christopherson, Ann. Trop. Med. and Parasit., 10, 1916; Discomyces hominis Brumpt, Précis de Parasitul., Paris, 3rd ed., 1922, 984.) From a case of pseudoactinomycosis.

Actinomyces japonicus Caminiti. Streplothrix sp. Aoyama and Miyamoto, Mitteil. Med. Fak. K. Jap. Univ. Tokio, 4, 1901, 231; abst. in Cent. f. Bakt., I
Abt., Orig., 29, 1901, 262; Caminiti, Cent. f. Bakt., I Abt., Orig., 4t', 1907, 198; Streptothrix japonica Petruschky, in Folle and Wasserman, Handb. d. path. Mikroorg., 2 Aufl., 5, 1913, 295; Discomyces japonicus Brumpt, Précis de Parasitol., Paris, 3rd ed., 1922, 984.) From a case of actinomycosis of the lungs.

Actinomyces keralolytica Acton and McGuire. (Indian Med. Gaz., 65, 1930, 61 and 66, 1931, 65; Proactinomyces keratolyticus, author unknown.) Produces cracked heels among the ryots of India .

Actinomyces lepromatis de SouzaAraujo. (Compt. rend. Soc. Biol., 100, 1929, 937.) From a leproma, Brazil. Actinomyces levyi Dodge. (Actinomyces sp. Levy; Oospora sp. Sartory, Champ. Paras. Homme et Anim., 1923, \$27; Dodge, Medical Mycology, St. Louis, 1935, 730.) From pus.

Actinomyces micetomae Greco. (Streptothrix. micetomae argentinae $\beta$, Greco, in Durante, Segunda Observación de Pié de Madura o Micetoma en la República Argentina, Thesis, Buenos Aires, 1911; Greco, Origine des Tumeurs, 1916, 759 ; Oospora micetomae Sartory, Champ. Paras. Homme et Anim., 1923, 783.) From a case of mycetoma pedis.

Actinomyces minimus (LeCalve and Nalherbe) Dodge. (Oospora forme de Microsporum (Audouini var. equinum), Bodin, Arch. de Parasitol., 2, 1899, 606; Trichophyton minimum LeCalve and Malherbe, Arch. de Parasitol.; Microsporum minimum Castellani and Chalmers, Man. Trop. Med., 3rd ed., 1919, 993; Dodge, Medical Mycology, St. Louis, 1935, 728.) From ringworm of horse and dog.

Actinomyces mucosus Basu. (Indian Med. Gaz., 78, 1943, 577.) From bronchial actinomyeosis.

Actinomyces neddeni Namyslowski. (Streptothrix sp. zur Nedden, İlin. Monatsbl. f. Augenheilk., 45, 1907, 152; Namyslowski, Cent. f. Bakt., I 
Abt., Orig., 62, 1912, 564.) From a case of keratitis.

Actinomyces nodosus (Beveridge) Hagan. (Fusiformis nodosus Beveridge, Austral. Council Sci. and Indus. Res. Bul. 140, 1941, 56 pp.; Hagan, The Infectious Diseases of Domestic Animals. Ithaca, New York, 1943, 312.) Considered the primary cause of foot-rot of sheep. Also see Spirochaeta penortha.

Actinomyces phenotolerans Werkman. (In Gammel, Arch. Derm. Syphilol., 29, 1934, 286.) From granuloma in man.

Actinomyces puntonii López Ortega. (López Ortega, Annali d'Igiene, Rome, 44, 1934, 867; Asteroides puntonii Puntoni and Leonardi, Boll. e Atti d. R. Accad. Med. di Roma, 61, 1935, 94.) From a pulmonary abscess.

Actinomyces purpureus Cavara. (Orloff, Vestnik Ofth., 29, 1912, 653; Cavara, Micosi Oce., 1928, 89; not Actinomyces purpureus Killian and Fehér, Ann. Inst. Past., 55, 1935, 620.) From a case of keratitis in Russia.

Actinomyces ribeyro Dodge. (Hongo aitrosporado Ribeyro, Ann. Fac. Med. Lima, 3, 1919, 1; Dodge, Medical Mycology, St. Louis, 1935, 735.) From a generalized infection on the arms, legs and chest of a patient in Peru.

Actinomyces rodellae Dodge. (Streptothrix sp. Rodella, Cent. f. Bakt., I Abt., Orig., 84, 1920, 450; Dodge, Medical Mycology, St. Louis, 1935, 734.) From abscesses of the tooth and jaw.

Actinomyces ruber (Kruse) Sanfelice. (In Cladcthrix cromogeno, Ruiz Casabó, Cronica medico-quirurgica de la Habana, 20, No. 13, 1894, 340; see Cent. f. Bakt., I Abt., 17, 1895, 466; Streptothrix rubra Kruse, in Flügge, Die Mikroorganismen, 3 Aufl., 2, 1896, 63; Cladothrix rubra Macé, Traité Pratique de Bact., th ed., 1901, 1097; not Actinomyces ruber Krainsky, Cent. f. Bakt., II Abt., 41, 1014, 662; Sanfelice, Cent. f. Bakt., I Abt., Orig., 36, 1904, 355; Nocardia rubra Chalmers and Christopherson, Ann. Trop. Med. and Parasit., 10, 1916, 265;
Discomyces ruber Brumpt, Préeis de Parasitol., Paris, 3rd ed., 1922, 981.) From sputum. Some authors consider the following synonymous with this organism: Streptothrix mineacea (Actinomyces mineaceus Lachner-Sandoval, Ueber Strahlenpilze, 1898, 66).

Actinomyces rubidaureus LachnerSandoval. (Cladothrix mordoré, Thiry, Areh. Physiol. Norm. et Path., 9, 1897, 283 ; Lachner-Sandoval, Ueber Strahlenpilze, Inaug. Diss., Strassburg, 1898, 66; Actinomyces mordoré, Thiry, Thèse, Nancy, 1900, 82; Nocardia mordoré, Chalmers and Christopherson, Ann. Trop. Med. and Parasit., 10, 1916, 265; Nocardia thiryei de Mello and Pais, Arq. Hig. Pat. Exot., 6, 1918, 193; Dis. comyces thiryi Brumpt, Précis de Para. sitol., Paris, 3rd ed., 1922, 981; Oospora mordoré, Sartory, Champ. Paras. Homme et Anim., 1923, 824; Actinomyces thiryi Sartory and Bailly, Mycoses Pulmonaires, 1923, 252.) From a case showing anginous exudate with edema.

Actrnomyces salvati Langeron. (Langeron, Bull. Soc. Path. Exot., 15, 1927, 526; Fontoynont and Salvat, ibid., 596.) From generalized nodular lesions in the Madagascar rat.

Actinomyces sartoryi Dodge. (Oospora pulmonalis var. acido-resistant, Sartory, Arch. Med. Pharm. Milit., ro, 1916, 605; Dodge, Medical Mycology, St. Louis, 1935, 756.) From a patient. showing symptoms of pulmonary tuberculosis.

Actinomyces septicus Mac Teal and Blevins. (Jour. Bact., $\{$ $9,1945,605$.) From human endocarditis.

Actinomyces serratus Dodge. (Actinomyces asteroides var. serratus Sartory, Meyer and Meyer, Ann. Inst. Past., 44, 1930, 298; also see Compt. rend. Acad. Sci. Paris, 188, 1929, 745; Dodge, Medical Mycology, St. Louis, 1935, 745.) From a case of actinomycosis of bones with yellow grains.

Actinomyces sommeri Greco. (Streptothrix madurae Greco, Primer Caso de 
Pié de Madura o Micetoma en la República Argentina, Thesis, Buenos Aires, 1904; Streptothrix micetomas argentinae $\alpha$, Greco, in Durante, Segunda Observación de Pié de Madura o Micetoma en la República Argentina, Thesis, Buenos Aires, 1911; Greco, Origine des Tumeurs, 1916, 726; Nocardia micetomae-argentinae Durant, 1911, quoted from Brumpt, Précis de Parasitol., Paris, 3rd ed., 1922, 985; Oospora sommeri Sartory, Champ. Paras. Homme et Anim., 1923, 783.) From a case of mycetoma pedis in Argentina.

Actinomyces tossicus Dodge. (Actinomyces albus var. tossica Rossi, Ann. Ig. Sperim., 9, 1905, 693; Oospora alba var. toxique, Sartory, Champ. Paras. Homme et Anim., 1923, 829; Dodge, Medical Mycology, St. Louis, 1935, 719.) From tumors in the abdominal cavities of domestic fowl

Actinomyces urethridis Brumpt. (Précis de Parasitol., Paris, th ed., 1927, 1206.) Isolated by Roček in 1920 from cases of prostatitis.

Actinomyces variabilis Cohn. (Cent. f. Bakt., I Abt., Orig., 70, 1913, 301.) From pus in the bladder in a case of cystitis and from the prostate.

Asleroides pseudocarneus Puntoni and Leonardi. (Boll. e Atti d. R. Accad. Med. di Roma, 61, 1935, 93.)

Bacillus berestnewi Lepeschkin. (Cent. f. Bakt., II Abt., 12, 1904, 641 and 13, 1904, 13.) From sputum of a pneumonia patient.

Bacillus (Micrococcus?) havaniensis Sternberg. (Sternberg, Manual of Bact., 1893, 718; Bactorium havaniensis Chester, Ann. Rept. Del. Col. Agr. Exp. Sta., 9, 1897, 116; Serratia havaniensis Bergey et al., Manual, 1st ed., 1923, 95.) From human intestinal canal.

Cladothrix matruchoti Mendel. (Mendel, Compt. rend. Soc. Biol., 82, 1910, 583; Nocardia matruchoti Pettit, 1921, quoted from Nannizzi, in Pollacci, Tratt. Micopat. Umana, 4, 1934, 51; Oospora matruchoti, quoted from Nan- nizzi, idem; Actinomyces matruchoti Nannizzi, idem.) From the roots of a decaying tooth with tumefaction. Species dubia.

\section{Cohnistrepothrix misri Carpano.} (Riv. di Parassitologia, n. 2, p. 107; quoted from Boll. d. Sez. Ital. d. Soc. Internat. d. Microbiol., 10, 1938, 62.) From human dermatosis in Egypt.

Discomyces berardinisi Brumpt. Streptothrix sp. de Berardinis, Ann. Ottalmol. Lavori Clin. Oculist. Napoli, 33, 1904, 914; Actinomyces de Berardinis, Namyslowski, Cent. f. Bakt., I Abt., Orig., 62, 1912, 566; Brumpt, Précis de Parasitol., Paris, 3rd ed., 1922, 977; Actinomyces bernardinisi Brumpt, ibid., tth ed., 1927, 11s9; Nocardia berardinisi Vuillemin, Encyclopédie Mycologique, Paris, 2, 1931, 126.) From a case of keratitis.

Discomyces brasiliensis Lindenberg. (Lindenberg, Rev. med. de S. Paolo, 1909, No. 18, and Arch. de Parasitol., 13, 1909, 265; Nocardia brasiliensis Pinoy, Bull. Inst. Past., Paris, 11, 1913, 936; Streptothrix brasiliensis Greco, Origine des Tumeurs, 1916, 724; Oospora brasitiensis Sartory, Champ. Paras. Homme et Anim., 1923, 786; Actinomyces brasiliensis Gomes, Ann. Palistas Med. Cirurg., 14, 1923, 150.) From a case of mycetoma of the leg. According to Pinoy and others, idential with Nocardia asteroides.

Discomycescongolensis Baerts. (Baerts, Bull. Méd. Katanga, 2, 1925, 67; Actinomyces congolensis Brumpt, Précis de Parasitol., Paris, 4th ed., 1927, 1206.) From lesions in a case of actinomycosis from the Belgian Congo.

Discomyces dispar (Vidal) Brumpt. (Microsporon dispar Vidal, 1882, Microsporon anemoeon Vidal, 1882 and Sporotrichum dispar, quoted from Brumpt, Précis de Parasitol., Paris, 3rd ed., 1922, 095 and from Nannizzi, in Pollacci, Tratt. Micopat. Umana, 4, 1934, 25; Brumpt, idem; Actinomyces dispar Brumpt, ibid., th ed., 1927, 1206.) 
From a case of pityriasis. Species dubia.

Discomyces mexicanus Boyd and Crutchfield. (Boyd and Crutchfield, Amer. Jour. Trop. Med., 1, 1921, 268; Actinomyces mexicanus Brumpt, Précis de Parasitol., Paris, th ed., 1927, 1192; Nocardia mexicana Ota, Jap. Jour. Derm. Urol., 28, 1928.) From a nycetoma of the foot.

Discomyces minutissimus (Burcharit) Brumpt. (Microsporon minutissimum Burchardt, in Uhle and Wagn. Pat. Gen., 1859; Trichothecium sp. J. Neumann, 1868; Microsporon gracile Balzer, Ann. Derm. Syphiligr., II, 4, 18s3, 681; Sporotrichum minutissimum Saccardo, Sylloge Fungorum, 4, 1886, 100; Microsporoides minutissimus Neveu-Lemaire, Précis Parasitol., 1906; Brumpt, Précis de Parasitol., 1st ed., 1910, 863; Oospora minutissima Ridet, Oospora et Oosporoses, 1911, 68; Nocardia minutissima Verdun, Précis parasitol., 1912; Actinomyces minutissimus Brumpt, ibid., 4th ed., 1927, 1199.) Reported as the etiological agent of erythrasma.

Mycobacterium alluvialum Bergey et al. (Kersten, Cent. f. Bakt., I Abt., Orig., 51, 1909, 494; Bergey et a!., Manual, 1st ed., 1923, 379.) From soil.

Mycobacterium convolutum Gray and Thornton. (Gray and Thornton, Cent. f. Bakt., II Abt., 73, 1928, 87; Actinomyces convolutus Bergey et al., Manual, 3rd ed., 1930, 473.) From soil. Resembles Nocardia opaca.

Nocardia arborescens (Edington) Trevisan. (Bacillus arborescens Edington, Brit. Med. Jour., June 11, 1887, 1262; Trevisan, I generi e le specie delle Batteriacee, 1889, 9; Actinomyces arborescens Gasperini, Cent. f. Bakt., 15, 1894, 684.) From human skin in cases of searlatina.

Nocardia bahiensis da Silva. (Da Silva, Brasil Med., 33, 1919, 81 and Mem. Inst. Butantan, 1, 1918-1919, 187; Discomyces bahiensis Neveu-Lemaire, Précis de Parasitol. Hum., 5th ed., 1021, 44;
Oospora bahiensis Sartory, Champ. Paras. Homme et Inim., 1923, 784; Actinomyces bahiensis Brumpt, Précis de Parasitol., th ed., 192 $7,1195$.$) \quad From$ an actinomycotic mycetoma in Brazil.

Nocardia berestneffi Chalmers and Christopherson. (Streptothrix cas I, II, Berestneff, Inaug. Diss., Moscow, 1897; Chalmers and Christopherson, Ann. Trop. Med. and Parasitol., 10, 1916, 263; Discomyces berestneffi Brumpt, Précis de Parasitol., Paris, 3rd ed., 1922, 992; Actinomyces berestneff Sartory and Bailly, Mycoses pulmonaires, 1923, 256.) From a case of pulmonary pseudotuberculosis.

Vocardia bicolor (Trolldenier) de Mello and Fernandes. (Actinomyces bicolor Trolldenier, Ztschr. f. Tiermedizin, $\gamma$, 1903, S1; de Mello and Fernandes, Mem. Asiatic Soe. Bengal, 7, 1919, 106.) Found in cerebromeningitis, bronchitis and lymphadenitis in a dog.

Nocardia convoluta Chalmers and Christopherson. (Chalmers and Christopherson, Ann. Trop. Med. and Parasit., 10, 1916, 25i; Discomyces conrolutus Neveu-Lemaire, Précis Parasitol. Hum., 5 th ed., 1921, 44; Oospora comoluta Sartory, Champ. Paras. Homme et Anim., 1923, 769; Actinomyces convolutus Brumpt, Précis de Parasitol., Paris, 4th ed., 1927, 1195.) From a yellow grain mycetoma of the foot in the Sudan.

Nocardia cyindracea de Fiorté. (De Fiorté, Ann. Trop. Med. Parasit., 11, 1918, 205; Discomyces cylindraceus Neveu-Lemaire, Précis Parasitol. Hum., 5th ed., 1921, 4.; Oospora cylindracea Sartory, Champ. Paras. Homme et Anim., 1923, 74 ; Actinomyces cylindraceus Brumpt, Précis de Parasitologie, Paris, 4th ed., 192 $\bar{\gamma}$, 1206.) From an infection of the outer ear, resembling mycetoma.

Nocardia enteritidis (Pottien) Castellani and Chalmers. (Streptothrix enteritidis Pottien, 1902, according to Sanfelice, Cent. f. Bakt., I Abt., Orig., 36, 
1904, 357; Bacillus enteritidis Pottien, 1902, according to Nannizzi, in Pollacci, Tratt. Micopat. Umana, 4, 1934, 25; not Bacillus cnteritidis Gaertner, Correspond. d. Allgemein. Artz. Vereins Thuringen, 17, 1888, 573; Castellani and Chalmers, Man. Trop. Med., 2nd ed., 1913, 819; Oospora enteritidis, quoted from Nannizzi, loc. cit.; Discomyces enteritidis Brumpt, Précis de Parasitol., Paris, 3rd ed., 1022, 980; Actinomyces enteritidis Brumpt, ibid., 4th ed., 1927, 1191.) From a case of enteritis.

Nocardia equi Chalmers and Christopherson. (Streptothrix sp. Dean, Trans. Path. Soc. London, 51, 1900, 26; ('halmer's and Christopherson, Ann. Trop. Med. Parasit., 10, 1916, 263; Discomcyes equi Brumpt, Précis de Parasitologie, 3rd ed., 1922, 092; Actinomyces equi Sartory and Bailly, Mycoses Pulmonaires, 1923, 256.) From an abscess on the jaw of a horse.

Nocardia erythrea (Foulerton) Chalmers and Christopherson. (Streptothrix erythrea Foulerton, 1910; Chalmers and Christopherson, Ann. Trop. Med. and Parasit., 10, 1916, 268.)

Nocardia foulertoni Chalmers and Christopherson. (Streptothrix hominis Foulerton and Jones, Trans. Path. Soc. London, 53, 1902, 56; Streptothrix hominis $I$ Foulerton, in Allbutt and Rolleston, Syst. of Medicine, 2, 1906, 302; Nocardia hominis Castellani and Chalmers, Man. Trop. Med., 2nd ed., 1913, 819; Oospora hominis Ridet, 1911, according to Castellani and Chalmers, idem; Chalmers and Christopherson, Ann. Trop. Med. and Parasit., 10, 1916, 263; Discomyces foulertoni Brumpt, Préeis de Parasitol., Paris, 3rd ed., 1922, 993; Actinomyces foulertoni Brumpt, ibid., 4th ed., 1927, 1204.) From abscesses of the chest and from sputum.

Nocardia genesii Fróes. (Fróes, Do mycetoma pedis no Brasil 50, 1930; Actinomyces genesii Dodge, Medical Mycology, St. Louis, 1935, 761.) From a case of Genesio Salles from Bahia.

Nocardia jollyi Vuillemin. (Vuillemin, in Jolly, Rev. Med. de l'Fst, 48
1920, 42; Discomyces jollyi Brumpt, Précis de Parasitol., Paris, 3rd ed., 1922, 84; Oospora jollyi Sartory, Champ. Paras. Homme et Anim., 1923, 770; Actinomyces jollyi Brumpt, ibid., 4th ed., 1927, 1196.) From a case clinically resembling bubonic plague.

Nocardia lasserei Verdun. (Nocardia $s p$. Lasserre, Thèse Toulouse, 1904; Verdun, Précis Parasitol., 1912; Discomyces lasserrei Neveu-Lemaire, Précis Parasitol. Hum., 5th ed., 1921, 43; Oospora lasserei Sartory, Champ. Paras. Homme et Anim., 1923, 798; Actinomyces lasserei Brumpt, Précis de Parasitol., th ed., 1927, 1206.) From an ulcerative lesion on the pharynx and upper lip.

Nocardia lingualis (Eisenberg) Chalmers and Christopherson. (ZungenbelagVibrio, Weibel, Cent. f. Bakt., 4, 1888, 227 ; Tibrio lingualis Eisenberg, Bakt. Diag., 3 Aufl., 1891, 212; Spirillum linguae Sternberg, Man. of Bact., 1893, 697; Spirosoma lingualis Migula, in Engler and Prantl, Die natürl. Pflanzenfam., 1, la, 1895, 31; Streptothrix lingualis Bajardi, Cent. f. Bakt., I Abt., Orig., 35, 1904, 129; Chalmers and Christopherson, Ann. Trop. Med. and Parasit., 10, 1916, 265; not Nocardia lingualis Castellani and Chalmers, Man. Trop. Med., 2nd ed., 1913, 819; Discomyces lingualis Brumpt, Précis de Parasitol., Paris, 3rd ed., 1922, 980; Actinomyces lingualis Sartory and Bailly, Mycoses Pulmonaires, 1923, 252.) From a deposit on the tongue. Jensen (Proc. Linn. Soc. New So. Wales, 59, 1934, 43) regards this species as closely related to Corynebacterium filamentosum. Also see paye 205.

Nocardia londinensis (sic) Chalmers and Christopherson. (Streptothrix. hominis II, Foulerton, Lancet, 1, 1906, 970; Chalmers and Christopherson, Ann. Trop. Med. and Parasit., 10, 1916, 256; Discomyces londinensis Brumpt, Précis de Parasitol., Paris, 3rd ed., 1922, 993; Actinomyces londincusis Brumpt, ibid., 
4th ed., 1927, 1204.) From cases of human actinomycosis.

Nocardia macrodipodidarum Fox. (Fox, Disease in Captive Wild Mammals and Birds, 1923, 570; Actinomyces macrodipodidarum Dodge, Medical Mycology, St. Louis, 1935, 747.) Found in lumpy jaw with septicemia and gasteroenteritis of kangaroos.

Nocardia nicollei Delanoë. (Delanoë, Arch. Inst. Past. Tunis, 17, 1928, 257; Actinomyces nicollei Nannizzi, in Pollacci, Tratt. Micopat. Umana, 4, 1934, 36.) From a mycetoma of the thigh.

Vocardia nigra (Castellani) Castellani and Chalmers. (Streptothrix nigra Castellani, 1913, according to Castellani and Chalmers, Man. Trop. Med., 3rd ed., 1919, 1062; presumably not Streptothrix nigra Rossi Doria, Ann. Ist. Ig. sper. Univ. Roma, 1, 1891, 399; Castellani and Chalmers, idem.) From black granules in a case of tonsillar nocardiomycosis. Usually forms black colonies on agar.

Nocardia panginensis de Mello and Fernandes. (De Mello and Fernandes, Mem. Asiatic Soc. Bengal, 7, 1919, 130; Actinomyces panginensis Dodge, Medical Mycology, St. Louis, 1935, 718.) From a dermatosis.

Nocardia pijperi Castellani and Chalmers. (Nocardia sp. Pijper, Folia Microbiol., 5, 1917, 50 and Med. Jour. So. Africa, 12, 1917, 141; Castellani and Chalmers, Man. Trop. Med., 3rd ed., 1919, 1060; Discomyces pijperi NeveuLemaire, Précis Parasitol. Hum., 5th ed., 1921, 44; Actinomyces pijperi Sartory and Bailly, Mycoses pulmonaires, 1923, 256.) From sputum in a case of chronic bronchitis.

Nocardia pinoyi de Mello and Fernandes. (De Mello and Fernandes, Mem. Asiatic Soc. Bengal, 7, 1919, 130; Actinomyces pinoyi Dodge, Medical Mycology, St. Louis, 1935, 723.) From a case of ery thrasma.

Nocardia ponceti Verdun. (Verdun, Précis Parasitol., 1912; Discomyces pon- ceti Neveu-Lemaire, Précis Parasitol. Hum., 5th ed., 1921, 43; Oospora ponceti Sartory, Champ. Paras. Homme et Anim., 1923, 778; Actinomyces ponceti Brumpt, Précis de Parasit., Paris, 4th ed., 1927, 1205.) Isolated by Moorhof, Dor and Poncet from a muscular pseudoactinomycosis. This species may be synonymous with Nocardia krausei (Chester).

Nocardia putridogenes (Vezspremi) de Mello and Pais. (Cladothrix putridogenes Vezspremi, Cent. f. Bakt., I Abt., 44, 1907, 408 and 515; de Mello and Pais, Arq. Hig. Pat. Exot., 6, 1918, 187; Oospora putridogenes Sartory, Champ. Paras. Homme et Anim., 1923, 823; Actinomyces putridogenes Nannizzi, in Pollacci, Tratt. Micopat. Umana, 4, 1934, 41.) From greenish pus from gingival ulcers and abscess of the jaw.

Nocardia repens (Eklund) Vuillemin. (Epidermophyton sp. Lang, 1879; Lepocolla repens Eklund, 1883; Achorion repens Guéguen, 1904; Epidermophyton repens; all quoted from Nannizzi, in Pollacci, Tratt. Micopat. Umana, 4, 1934; Vuillemin, Encyclopédie Mycologique, Paris, 2, 1931, 124; Actinomyces repens Nannizzi, idem.) From the skin in cases of psoriasis.

Nocardia rivierei Verdun. (Streptothrix sp. Rivière, Cong. Franç. Méd. Bordeaux 1895, 2, 1896, 1003; Actinomyces Sabrazes et Rivieri, Berestnev, Inaug. Diss., Moscow, 1897; Verdun, Précis Parasitol., 1912; Discomyces rivierei Neveu-Lemaire, Précis Parasitol. Hum., 5th ed., 1921, 43; Oospora rivierei Sartory, Champ. Paras. Homme et Anim., 1923, 792; Actinomyces rivierei Brumpt, Précis de Parasitol., 4th ed., 1927, 1201.) From sputum and pus in a bronchopleuropulmonary infection followed by multiple abscesses.

Nocardia sanfelicei Redaelli. (Streptothrix acido-resistente, Sanfelice, Boll. Ist. Sieroterapico Milanese, 2, 1922, 327 ; Redaelli, Ist. Sieroterapico Milanese, $7,1928,75$ and 121; Actinomyces 
sanfelicei Nannizzi, in Pollacei, Tratt. Micopat. Umana, 4, 1934, 51.) From fatal lesions in a rat.

Nocardia splenica Gibson. (Gibson, 1930, quoted from Nannizzi, in Pollacei, Tratt. Micopat. Umana, 4, 1934, 50; Actinomyces splenica Nannizzi, idem.) From a case of splenomegalia.

Nocardia tenuis Castellani. (Castellani, Brit. Jour. Derm. Syphilis, 23, 1911, 341; Discomyces tenuis Castellani, Proc. Roy. Soc. Med., 6, Derm., 1912, 23; Cohnistreptothrix tenuis Chalmers and Christopherson, Ann. Trop. Med. and Parasit., 10, 1916, 273; Actinomyces tenuis Dodge, Medical Mycology, St. Louis, 1935, 715.) From cases of trichomycosis flava.

Nocardia valvulae Chalmers and Christopherson. (Streptothrix valvulae destruens bovis Luginger, Monats. f. prakt. Tierheilk., 15, 1904, 289; Chalmers and Christopherson, Ann. Trop. Med. and Parasit., 10, 1916, 263; Oospora valvulae destruens bovis Sartory, Champ. Paras. Homme et Anim., 1923, 788; Actinomyces valuularis Ford, Textb. of Bact., 1927, 211; Actinomyces valuulae Nannizzi, in Pollacei, Tratt. Micopat Umana, 4, 1934, 51.) From endocarditis in cattle.

Oospora anaerobies Nartory. (Actinomyces sp. Butterfield, Jour. Inf. Dis., 2, 1905, 421; Sartory, Champ. Parasit. Homme et Anim., 1923, $830 ;$ Actinomyces anaerobies Plaut, quoted from Dodge Medical Mycology, St. Louis, 1935, 717.) From pus from human lung.

Oosporabronchialis Sartory and Levasseur. (Sartory and Levasseur, 1914, quoted from Neveu-Lemaire, Précis Parasitol. Hum., 5th ed., 1921, 13; Actinomyees bronchialis Sartory, Bull. Sei. Pharm., 23, 1916, 12; Discomyces bronchialis Neveu-Lemaire, idem.) From sputum in a case of pulmonary oosporosis.

Oospora buccalis Roger, Bory and Sartory. (Roger, Bory and Sartory, Bull. Mem. Soc. Méd. Hôp. Paris, 27, 1909 ,
319 and Compt. rend. Soc. Biol., 66, 1909, 301; Discomyces buccalis Brumpt, Précis de Parasitol., Paris, 1st ed., 1910, 861; Nocardia buccalis Castellani and Chalmers, Man. Trop. Med., 2nd ed., 1913, 819; Actinomyces buccalis Sartory and Bailly, Mycoses pulmonaires, 1923, 256.) From a case of creamy stomatitis with tonsillar abscess.

Oospora catarhalis Sartory and Bailly. (Sartory and Bailly, Thèse Univ. Strasbourg Fac. Pharm., 4, 1921, 57; Discomyces catarrhalis Brumpt, Précis de Parasitol., Paris, 3rd ed., 1922, 984; Actinomyces catarrhalis Brumpt, ibid., th ed., 1927, 1195.) From sputum in a case of pulmonary oosporosis.

Oospora hominis Ridet. (Streptothrix hominis IV Foulerton, 1906; Streptothrix hominis III Foulerton, 1910; Ridet, 1911, according to Nannizzi, in Pollacei, Tratt. Nicopat. Umana, 4, 1934, 9; Nocardia appendicis Chalmers and Christopherson, Ann. Trop. Med. and Parasit., 10, 1916, 256; Discomyces appendicis Brumpt, Précis de Parasitol., 3rd ed., 1922, 977; Actinomyces appendicis Brumpt, ibid., th ed., 1927, 1189.) From a case of appendicitis and an iliac abscess.

Oospora lingualis Guéguen. (Guéguen, Compt. rend. Soc. Biol., 64, 1908, 852; Discomyces lingualis Brumpt, Précis de Partisitol., Paris, 1st ed., 1910, 865 and 2nd ed., 1913, 976; not Discomyces lingualis Brumpt, ibid., 3rd ed., 1922, 980; Nocurdia lingualis Castellani and Chalmers, Man. Trop. Med., 2nd ed., 1913, $\$ 19$; not Nocardia lingualis Chalmers and Christopherson, Ann. Trop. Med. and Parasitol., 10, 1916, 265; Discomyces guegueni Brumpt, ibid., 3rd ed., 1922, $880 ;$ Actinomyces guegueni Brumpt, ibid., 4th ed., 1927, 1191, Nocardia guegueni Ota, Jap. Jour. Derm. Urol., 28, 1928.) From cases of lingua nigra.

Oospora pulmonalis Roger, Sartory and Bory. (Roger, Sartory and Bory, Compt. rend. Soc. Biol., 66, 1909, 150; Discomyces pulmonalis Brumpt, Précis 
de Parasitologie, 1st ed., 1910, 860; Nocardia pulmonalis Castellani and Chalmers, Man. Trop. Med., 2nd ed., 1913, 817; Actinomyces pulmonalis Sartory and Bailly, Mycoses Pulmonaires, 1923, 256.) From the sputum of a patient with pulmonary mycosis.

Oospora pulmonalis var. chromogena Sartory. (Sartory, 1913; Actinomyces pulmonalis var. chromogenus Nannizzi, in Pollacci, Tratt. Micopat. Umana, 4, 1934, 39.) From sputum of a patient suspected of having pulmonary tubereulosis.

Proactinomyces albus Krassilnikov. (Bull. Acad. Sci., U. S. S. R., No. 1, 1938, 139.) Cells at first produce mycelium with frequent branching varying in diameter from 0.6 to 1.0 micron. Breaks up after 2 or 3 days into rods and sometimes later into cocci. Multiply by fission, cross-wall formation and budding. Does not form spores; cells are Gram-positive and are not acid-fast. Colorless growth. Colonies vary in the different strains, somewhat rough, folded, shiny or dull, of a dough-like consistency. Tirassilnikov listed several strains of this organism, including Proactinomyces oligocarbophilus.

Proactinomyees uquosus Turfitt. (Jour. Bact.. 47, 1944, 490.) From soil. Decomposes cholestero!.

Proactinomyces cyaneus (Beijerinck) Krassilnikov. (Actinococcus cyaneus Beijerinck, Folia microbiol., Delft, 2, 1914, 196; Krassilnikov, loc. cit.) Blue pigment produced on synthetic media. Cells are rod-shaped. 0.7 to 0.8 by 3 to 7 microns. Branching cell material on potato multiplies by means of bud formation, by fission, and cross-wall formation; no true spores formed.

Proactinomyces cyaneus-antibiolicus Gause. (Jour. Bact., 51, 1946, 649.) From soil. Produces litmocidin, a new antibiotic.

Proactinomyces moormani Franklin (Ann. Intern. Med., 13, 1940, 12155.)
From the pus of multiple molar abscesses in a dental patient.

Proactinomyces paraguayensis Almeida. (Mycopath., 2, 1940, 201.) From a thoracic mycetoma with heavy, dark grains affecting a Canadian patient living in the Paraguayan Chaco. Sabouraud's glucose agar: Pseudomembranous colony with raised, dark center surrounded by a white band, progressively increasing in size, and then by a light chocolate area.

Proactinomyces restrictus: Turfitt. (Jour. Bact., 4\%, 1944, 491.) From soil. Decomposes cholesterol.

Proactinomyces sp. Helzer. Found in sputum of tuberculous patient. Pathogenic for guinea pigs and rabbits.

Streptothrix buccalis Goadby. (Mycology of the Mouth, London, 1903, 200.) From the mouth in cases of pyorrhoea. Chalmers and Christopherson (Ann. Trop. Med. and Parasit., 10, 1916, 234) regard this as a synonym of Nocardia liquefaciens.

Streptothrix flava Chester. (Actinomyces sp. Bruns, Cent. f. Bakt., 26, 1899, 11; Chester, Man. Determ. Bact., 1901, 362; Nocardia bruni Chalmers and Christopherson, Ann. Trop. Med. and Parasit., 10, 1916, 256; Streptothrix hominis Bruns, according to Chalmers and Christopherson, idem; Discomyces bruni Brumpt, Précis de Parasitol., Paris, 3rd ed., 1922, 992; Actinomyces bruni Brumpt, ibid., 4th ed., 1927, 1204; Actinomyces flavus Dodge, Medical Mycology, St. Louis, 1935, 752.) From pus from a case of actinomycosis of the abdominal wall.

Streptothrix fusca Karwacki. (Farwacki, Compt. rend. Soc. Biol., 66, 1911, 180; not Streptothrix fusca Corda, Prachtflora europäischer Schimmelbildungen, Leipzig and Dresden, 1839, 27 ; Nocardia fusca Castellani and Chalmers, Man. Trop. Med., 2nd ed., 1913, 818; Discomyces fuscus Brumpt, Précis de Parasitol., Paris, 3rd ed., 1922, 993; Oospora fusca Sartory, Champ. Paras. 
Homme et Anim., 1923, 809; Actinomyces fuscus Sartory and Bailly, Mycoses pulmonaires, 1923, 256; not Actinomyces fusca Söhngen and Fol, Cent. f. Bakt., II Abt., 40, 1914, 87.) From the sputum of a tuberculosis patient.

Streptothrix luteola Foulerton and Jones. (Foulerton and Jones, Trans. Path. Soc. London, 53, 1902, 75; also see Foulerton, Lancet, 1, 1905, 1200 and 1, 1906, 970; Nocardia luteola Castellani and Chalmers, Man. Trop. Med., 2nd ed., 1913, 815; Discomyces luteolus Brumpt, Précis de Parasitol., Paris, 3rd ed., 1922, 981; Oospora luteola Sartory, Champ. Paras. Homme et Anim., 1923, 812; Actinomyces luteolus Ford, Textb. of Bact., 1927, 213.) From a lung infection, from a case of conjunctivitis, and from a dental abscess.

Streptothrix madurae Solari. (Solari, Semana Méd., 24, 1917, 573; not Streptothrix madurae Vincent, Ann. Inst. Past., 8, 1894, 129; not Streptothrix madurae
Koch and Stutzger, Ztschr. f. Hyg., $69,1911,17$.$) From Madura foot. See$ Actinomyces avadi.

Streptothrix muris-ratti Schottmüller. (Schottmüller, Derm. Wochnschr., 58, 1914, 77; Nocardia muris de Mello and Pais, Arq. Hig. Pat. Exot., 6, 1918, 183; Actinomyces muris-ratti Nannizzi, in Pollacci, Tratt. Micopat. Umana, 4, 1934, 51.) From sodoku or rat-bite fever.

Streptothrix tarozzii Miescher. (Actinomyces albus Tarozzi, Archivio Sci. Med., 39, 1909, 553; Miescher, Arch. Derm. Syphilis, 124, 1917, 297; Actinomyces tarozzii Dodge, Medical Mycology, St. Louis, 1935, 735.) From a case of Madura foot.

Streptothrix verrucosa (Adler) Miescher. (Actinomyces verrucosus Adler, 1904, see Nannizzi, in Pollacei, Tratt. Micopat. Umana, 4, 1934, 46; Miescher, Arch. Derm. Syphilis, 124, 1917, 314.) From mycetoma pedis. 


\section{Genus II. Actinomyces Harz.}

(Harz, in Bollinger, Centbl. f. med. Wissensch., 15, 1877, 485; Harz, Jahresber. d. Münch. Thierarzneischule for 1877-78, 1879, 125; not Actinomyce Meyen, Linnaea, 2, 1827, 442; Discomyces Rivolta, Clinica Veter., Milano, 1, 1878, 208; Actinocladothrix Afanasiev, St. Petersb. med. Wehnschr., 4, 1887, 323; Micromyces Gruber, Cent. f. Bakt., 10, 1891, 648; not Micromyces Dangeard, Le Botaniste, 1, 1888, 55; Actinobacterium Haas, Cent. f. Bakt., I Abt., Orig., 40, 1906, 180; Carteria and Carterii Musgrave, Clegg and Polk, Philippine Jour. Sci., Ser. B, Med. Sci., 3, 1908, 470; Cohnistreptothrx Pinoy (in part), 1911, see Pinoy, Bull. Inst. Past., 11, 1913, 929; Anaeromyces Castellani, Douglas and Thomson, Jour. Trop. Med. Hyg., 24, 1921, 149 ; Brevistreptothrix Lignières, Ann. Parasit. Hum. Comp., 2, 1924, 1.)

True mycelium produced. The vegetative mycelium fragments into elements of irregular size and may exhibit angular branching. No conidia produced. Tot acidfast. Anaerobic to microaerophilic. Pathogenic for man and animals.

The type species is Actinomyces bovis Harz.

\section{Key to the species of genus Actinomyces.}

I. Colonies soft, smooth, uniform, not adherent to medium. To aerial hyphae.

1. Actinomyces bovis.

II. Colonies tougher in texture and warted in appearance, adherent to medium. Scanty aerial growth of hyphae.

2. Actinomyces israeli.

1. Actinomyces bovis Harz. (Harz, in Bollinger, Cent. f. med. Wissensch., 15, 1877, 485; Jahrb. d. Münch. Thierarzeneischule, 1877,781 ; Discomyces bovis Rivolta, La clinica Veterinaria, 1, 1878, 169 or 208; Bacterium actinocladothrix Afanasiev, St. Petersburger Med. Wochnschr., 13, 1888, 84; Nocardia actinomyces Trevisan, I generi e le specie delle Batteriacee, Milan, 1889, 9 ; Streptotrix actinomyces Rossi Doria, Ann.d. Ist. d'Igi. Sper., Univ. di Roma, 1, 1891, 425; Cladothrix bovis Macé, Traité de Bact., 2nd ed., 1891, 666; Oospora bovis Sauvageau and Radais, Ann. Inst. Past., Paris, 6, 1892, 271; Actinomyces bovis sulphureus Gasperini, Cent. f. Bakt., 15, 1894, 684; Nocardia bovis Blanchard, in Bouchard, Traité de Path. Générale, 2, 1896, 857; Actinomyces sulphureus Gasperini, Atti Soc. Tosc. Scienz. Nat., P. V., 11, 1896; Cladothrix actinomyces Macé, Traité de Bact., 3rd ed., 1897, 1038; Streptothrix actinomycotica Foulerton, Lancet, 2, 1899, 780; Streptothrix bovis communis Foulerton, Jour. Comp. Path. and Therap., 14, 1901, 50; Strepto- thrix bovis Chester, Man. Determ. Bact., 1901, 361; Streptothrix sulphurea Caminiti, Cent. f. Bakt., I Abt., Orig., 44, 1907, 197; Sphaerotilus bor'is Engler, Syllabus der Pflanzenfam., 5 Aufl., 1907,5 ; Nocardia sulphurea Vuillemin, Encyclopédie Mycologique, Paris, 2, 1931, 129; Proactinomyces boris Henrici, Biology of Bact., 2nd ed., 1939, 409.) From Latin bovis, of the ox.

Synonyms previous to 1919 as given by Breed and Conn, Jour. Bact., 4, 1919, 596.

Probable synonym: Brevistreptothrix spıtzi Lignières, Annales de Parasit., 2, 1924, 2 (Streptothrix spitzi Lignières and Spitz, Cent. f. Bakt., I Abt., Orig., 35, 1904, 453; Actinobacterium isreali var. spitzi Sampietro, Ann. Igiene, 18, 1908, 391; Discomyces spitzi Brumpt, Précis de Parasitol., Paris, 1st ed., 1910, 847; Actinomyces spitzi Lieske, Morphol. u. Biol. d. Strahlenpilze, Leipzig, 1921, 32 ; Oospora spitsi Sartory, Champ. Paras. Homme et Anim., 1923, 775). Found in mycosis of the upper jaw of oxen in Argentina. 
Description from Erikson, Med. Res. Council, London, Special Report Ser. 240, 1940, $63 \mathrm{pp}$.

No aerial hyphae. Radiate, sulfurcolored granules occur in the pus found in cases of actinomycosis. Large club shaped hyphae are seen in morbid tissues. Gram-positive. Non-motile. Not acidfast.

Mycelium: Undergoes fragmentation very rapidly, extensive branching is rare. Hyphae less than 1 micron in diameter.

Colonies: Smoother and softer in consistency, and more uniform than in the following species. The colonies are not adherent to the medium and growth is scantier.

Semi-solid media: Excellent growth, especially with paraffin seal.

Gelatin: Occasionally scant, flaky growth. No liquefaction.

Liquid media: Occasional turbidity with a light floceulent growth.

Acid from glueose, sucrose and maltose. No acid from salicin and mannitol.

Pigments: No soluble pigments produced on protein media. No insoluble pigments produced by growth.

Egg or serum media: No proteolytic action.

Litmus milk: Becomes acid but usually no coagulation, no peptonization. Sometimes no growth.

No hemolysis in blood broth or blood agar.

Serology: To cross agglutination between five bovine strains and human strains of Actinomyces israeli. No cross reactions with representative aerobic strains.

Optimum temperature $37^{\circ} \mathrm{C}$.

Anaerobic to microaerophilic. Bovine strains are more oxygen-tolerant on egg or serum media than strains of human origin belonging to the following species.

As pointed out by Lignières and Spitz (Bull. Soc. cent. Méd. vet., 20, 1902, 457 and 546) and ot hers, distinction should be made between the infections produced by Actinomyces bovis and those produced by the Gram-negative Actinobacillus now known as Actinobacillus lignieresi. These infections frequently occur in mixed form and are also frequently complicated by the presence of pyogenic cocci (Magnussen, Acta path. Microbiol. Scand., 5, 1928, 170; and others ).

Source: Originally found in lumpy jaw of cattle.

Habitat: Frequently found in and about mouth of cattle and probably other animals. Lesions may also be produced in the liver, udder or other organs of cattle and hogs. Possibly also in human mouth (Naeslund, Acta path. Microbiol. Scand., 2, 1925, 110).

This and the following species are sometimes regarded as being identical (see Emmons, Public Health Repts., U.S.P.H.S., 53, 1935, 1967; Rosebury, Bact. Rev., 8, 1944, 190; and others).

\section{Actinomyces israeli (Kiruse)}

Lachner-Sandoval. (Strahlenpilz, Wolff and Israel, Arch. f. path. Anat., 126, 1891, 11; Streptothrix israeli Kruse, in Flügge, Die Mikroorganismen, 3 Aufl., 2, 1896, 56 ; Actinomyces israeli LachnerSandoval, Inaug. Diss., Strassburg, 1898, 64; Discomyces israeli Gedoelst, Les champignons parasites de l'homme et des animaux domestiques, 1902, 163; Actinobacterium israeli Sampietro, Ann. l'Ig. sperim., 18, 1908, 331; Cohnistreptothrix israeli Pinoy, Bull. Inst. Pasteur, Paris, 11, 1913, 931; Nocardia israeli Castellani and Chalmers, Man. Trop. Med., 2nd ed., 1913, 814; Anaeromyces bronchitica Castellani, Douglas and Thomson, Jour. Trop. Med. Hyg., 24, 1921, 149; Cohnistreptothrix bronchitica Verdun and Mandoul, Précis Parasitol., 1924, 754; Brevistreptothrix israeli Lignières, Amnales de Parasit., 2, 1924, 2; Proactinomyces israeli Negroni, Compt. rend. Soc. Biol., Paris, 117, 1934, 1239; Corynebacterium israeli Haupt and Zeki, Cent. f. Bakt., I Abt., Orig., 130, 1933, $95 ;$ Oospora isracli, cuoted from Nannizzi, 
Tratt. Micopat. Lmana, 4, 1934, 53 ; Actinomyces wolf-israel Lentze, Cent. $f$. Bakt., I Abt., Orig., 141, 1938, 21.) Named for Prof. Israel, one of the original isolators of this organism.

Synonyms previous to 1919 essentially as given by Breed and Conn, Jour. Bact., 4, 1919, 597.

Description from Erikson, Med. Res. Council, London, Special Rept. Ser. 240, 1940, 63 pp.

Erect aerial hyphae produced in an atmosphere of reduced oxygen tension. These hyphae are occasionally septate but no definite spores are formed. One micron or more in diameter. Large club-shaped forms are seen in morbid tissues. Gram-positive. Non-motile. Not acid-fast.

Substrate mycelium: Initially unicellular and the branches may extend into the medium in long filaments or may, more or less quickly, exhibit fragmentation and characteristic angular branching. The latter resembles the similar phenomenon found in Corynebacterium.

Colonies: These exhibit a considerable degree of polymorphism but no stable variants have been established. Tougher in texture than those of Actinomyces bovis. Old colonies warted in appearance. Adherent to the medium.

Gelatin: Occasionally scant, flaky growth. To liquefaction.

Liquid media: Esually clear.

Acid from sugars: According to Slack (Jour. Bact., 43, 1941, 193-209) acid from glucose, maltose, mannitol, sucrose and lactose; according to Negroni and Bonfiglioli (Physics, 15, 1939, 159) acid from glucose, galactose, lactose, fructose, maltose, raffinose, sucrose and xylose.

Pigments: No soluble pigments on protein media. No insoluble pigments produced by growth.

Egg or serum media: No proteolytic action.

Litmus milk : Becomes acid but usually does not clot. No peptonization. Frequently no growth.

No hemolysis.

Serology: No cross agglutination between 12 human strains and bovine strains of Actinomyces. No cross reactions with representative aerobic strains.

Optimum temperature $37^{\circ} \mathrm{C}$.

Anaerobic to microaerophilic.

Source: From 2 cases of human actinomycosis : (1) A retromaxillary tumor, (2) actinomycosis of lung and breast (Wolff and Isreal).

Habitat : From human sources (mouth, tonsillar crypts, etc.). Also reported from various domestic animals such as dogs (Baudet, Ann. Parasit., 12, 1934, 296) and cats (Edington, Vet. Record., 14, 1934, 311).

Appendix: The following names have been applied to anaerobic or semi-anaerobic species, with descriptions which do not permit clear separation from the above or from each other.

Actinobacterium meyeri Prévot. (Anacrobe Streptothrix-Art, Meyer, Cent. f. Bakt., I Abt., Orig., 60, 1911, 75; Prévot, Ann. Inst. Past., 60, 1938, 303.) From fetid pus.

Actinomyces discofoliatus Grüter. (Grüter, Ztschr. f. Augenheilk., 13, 1933, 477; redescribed by Negroni, Mycopathologia, 1, 1938, 81.) From lachrymal concretion and human infection.

Actinomyces lanfranchii Sani. (Sani, 1916, quoted from Dodge, Medical Mycology, St. Louis, 1935, 731; Nocardia lanfranchii de Mello and Pais, Arq. Hig. Pat. Exot., 6, 1918, 178.) From glandular and ganglionar actinomycosis of the ox. Regarded as a variety of Actinomycrs bovis.

Actinomyces thjoettae Dodge. (Cohnistreptothrix $s p$. or Streptothrix $s p$. Thjøtta and Gundersen, Jour. Bact., 10, 1925, 1; Dodge, Medical Mycology, 1935, 713.) From the blood in a case of acute rheumatism. 
Cohnistreptothrix neschezadimenki (sic) Chalmers and Christopherson. (Eine Streptothrix, Neschezadimenko, Cent. f. Bakt., I Abt., Orig., 46, 1908, 573; Chalmers and Christopherson, Ann. Trop. Med., 10, 1916, 273; Actinomyces neschczadimenki Dodge, Medical Mycology, 1935, 712; Actinobacterium abscessus Prévot, Ann. Inst. Past., 60, 1938, 303.) From a human infection.

Discomyces carougeaui Gougerot. (Gougerot, Compt. rend. Soc. Biol., Paris, 67, 1909, 580; Nocardia carougeaui Castellani and Chalmers, Man. Trop. Med., 2nd ed., 1913, 817; Cohnistreptothrix carogeanui (sic) Chalmers and Christopherson, Ann. Trop. Med., 10, 1916, 273; Streptothrix carougeaui Greco, Origine des Tumeurs, 1916, 724; Actinomyces carougeaui Brumpt, Précis de Parasitol., 4th ed., 1927, 1206.) From a human infection.

Discomyces thibiergei Ravaut and Pinoy. (Ravaut and Pinoy, Ann. Derm. et Syph., 10, 1909, 417; Nocardia thibiergei Castellani and Chalmers, Man.
Trop. Med., 2nd ed., 1913, 817; Cohnistreptothrix thibiergei Pinoy, Bull. Inst. Past., 11, 1913, 938; Oospora thibiergei Sartory, Champignons Parasites de l'Homme et des Animaux, Fasc. 11, 1923, 792; Actinomyces thibierger Greco, Origine des Tumeurs, 1916, 723.) From a human infection.

Streplothrix cuniculi Schmorl. (Schmorl, Deutsch. Ztschr. f. Thiermed., 17, 1891, 375; Actinomyces cuniculi Gasperini, Ann. Ist. Ig. sper. Univ. Roma, 2, 1892, 222; Cladothrix cuniculi Macé, Traité de Bact., 6th ed., 2, 1913, 753; Cohnistreptothrix cuniculi Chalmers and Christopherson, Ann. Trop. Med., 10, 1916, 273; Nocardia cuniculi Froilani de Mello and Fernandes, Mem. Asiatic Soc. Bengal , 7, 1919, 107; Oospora cuniculi Sartory, Champignons parasites de l'Homme et des Animaux, Fasc. 11, 1923, 824.) From infections in rabbits. Generally regarded as probably identical with Spherophorus necrophorus (Flügge) Prévot. See page 678 . 


\section{FAMILY III. STREPTOMYCETACEAE WAKSMAN AND HENRICI.*}

(Jour. Bact., 46, 1943, 339.)

Vegetative mycelium not fragmenting into bacillary or coccoid forms. Conidia borne on sporophores. Primarily soil forms, sometimes thermophilic in rotting manure. A few species are parasitic.

\section{Key to the genera of family Streptomycetaceae.}

I. Conidia produced in aerial hyphae in chains.

Genus I. Streptomyces, p. 929.

II. Conidia produced terminally and singly on short conidiophores.

Genus II. Micromonospora, p. 978.

\section{Genus I. Streptomyces Waksman and Herici.}

(Streptothrix Cohn, Beitr. zur Biol. der Pflanzen, I, Heft 3, 1875, 186; not Streptothrix Corda, Prachtflora Europaescher Schimmelbildung, 1839; Nocardia Wright, Jour. Med. Research, 13, 1905, 349; Nocardia Winslow et al., Jour. Bact., 2, 1917, 554; not Nocardia Trevisan, I generi e le specie delle Batteriacee, 1889, 9; Cohnistreptothrix (Group I) Orskov, Investigations in the Morphology of the Ray Fungi. Copenhagen, 1923, 147; not Cohnistreptothrix Pinoy, in Liégard and Landrieu, Bull. Soc. Opht., 24, 1911, 253 and Bull. Inst. Past., 11, 1913, 929; Aerothrix Wollenweber (in part), Ber. deut. Bot. Gesel., 39, 1921, 26; Euactinomyces Langeron (in part), Nouv. Traité de Méd., 4, 1922; Waksman and Henrici, Jour. Bact., 46, $1943,339$.

Organisms growing in the form of a much-branched mycelium with a typical aerial mycelium. Conidiospores formed in chains. Aerobic. Saprophytic soil forms, less commonly parasitic on plants or animals.

This genus can be divided, on the basis of the structure of sporulating hyphae, into five groups:

Group 1. Straight sporulating hyphae, monopodial branching, never producing regular spirals.

Group 2. Spore-bearing hyphae arranged in clusters.

Group 3. Spiral formation in aerial mycelium; long, open spirals.

Group 4. Spiral formation in aerial mycelium; short, compact spirals.

Group 5. Spore-bearing hyphae arranged on mycelium in whorls or tufts.

The type species is Streptomyces albus (Rossi Doria emend. Krainsky) Waksman and Henrici.

\section{Key to the species of genus Streptomyces.}

I. Saprophytes; psychrophilic to mesophilic.

A. Soluble pigment on organic media faint brown, golden-yellow, or blue; pigment may also be absent entirely.

1. Pigment absent, or faint brown pigment formed at first and later lost; aerial mycelium abundant, white.

1. Streptomyces albus.

2. Pigment blue or red, when present. The red (insoluble) phase occurs when the reaction is distinctly acid, the blue (soluble) phase when it is alkaline.

2. Streptomyces coelicolor.

* Revised by Prof. S. A. Waksman, New Jersey Experiment Station, New Brunswick, New Jersey and Prof. A. T. Henrici, University of Minnesota, Minneapolis, Minnesota, May, 1943. 
3. Pigncut at first green becoming brown; aerial mycelium usually absent.

3. Streptomyces verne.

4. Pigment yellowish-green; growth on synthetic agar penetrating into medium, pink.

4. Streptomyces californicus.

5. Pigment golden-yellow; growth on synthetic agar yellow, with yellow soluble pigment.

5. Streptomyces flaveolus.

6. Pigment brown (only on certain protein media, as gelatin, glucose broth).

a. Grown on synthetic agar red to pink. Scant, white aerial mycelium.

6. Streptomyces boliliae.

aa. Growth on synthetic agar colorless; aerial mycelium thin, rose-colored.

7. Streptomyces roseochromogenus.

aaa. Growth on synthetic agar mouse-gray; powdery aerial mycelium.

8. Streptomyces griseolus.

aaaa. Growth on synthetic agar white turning yellowish, aerial mycelium white.

9. Streptomyces erythreus.

B. Soluble yellow pigment on Ca-malate agar.

1. Proteolytic action strong in milk and gelatin.

a. Yellow pigment formed.

b. Cellulose decomposed; starch is hydrolyzed.

10. Streptomyces cellulosae.

bb. Cellulose not decomposed.

11. Streptomyces parous.

2. Proteolytic action weak.

12. Streptomyces malenconi.

C. Soluble brown pigment formed on synthetic agar.

1. Yellowish-green pigment on potato.

13. Streptomyces diastaticus.

2. Red-brown pigment on potato plug.

14. Streptomyces fimicarius.

D. Greenish-yellow soluble pigment formed; sulfur-yellow pigment on potato.

15. Streptomyces flacovirens.

E. Soluble brown pigment formed in all media containing organic substances.

1. Pigment deep brown (chromogenic type).

a. Pigment faint brown on organic media, becoming greenishbrown to black; reddish aerial mycelium on glucose agar.

16. Streptomyces olivochromogenus.

aa. Aerial mycelium yellowish with gray margin; weak diastatic action.

17. Streptomyces diastatochromogenes.

aaa. Acrial mycelium yellowish; diastatic action weak.

18. Streptomyces flavochromogenes.

aaa. Aerial mycelium gray; sporophores in clusters; strongly antibiotic.

19. Streptomyces antibioticus. 
2. Growth and aerial mycelium green on synthetic agar.

20. Streptomyces viridochromogenes.

3. Deep brown to black pigment on synthetic agar.

a. Orange-red on potato; no aerial mycelium on synthetic agar; growing feebly.

21. Streptomyces purpeochromogenus.

aa. Brown to black on potato; abundant cottony aerial mycelium on synthetic agar.

b. Brown ring on milk culture; coagulated; peptonized.

22. Streptomyces phaeochromogenus.

bbb. Black ring on milk; no coagulation; peptonization doubtful.

23. Streptomyces aureus.

$c^{1}$. Pied to rose-red pigment on glucose, maltose, and starch agar.

24. Streptomyces erythrochromogenes.

$c^{2}$. Lavender-colored aerial mycelium.

25. Streptomyces lavendulae.

$c^{3}$. Growth on potato gray with black center.

26. Streptomyces reticuli.

$\mathrm{c}^{4}$. Gromth on potato cream-colored, becoming pink to dark red.

27. Streptomyces rubrireticuli.

$c^{5}$. Growth on potato greenish-olive.

d. Aerial mycelium straw-colored.

28. Streptomyces flavus.

dd. Aerial mycelium chrome-orange.

29. Streptomyces ruber.

F. No soluble pigment formed on gelatin or other media.

1. Proteolytic action strong in milk and gelatin.

$a^{1}$. Yellowish-green growth on starch with pinkish aerial mycelium.

30. Streptomyces citreus.

$\mathrm{a}^{2}$. Golden-yellow growth, later becoming orange to red-brown, on synthetic media.

31. Streptomyces fulvissimus.

$\mathrm{a}^{3}$. Cream-colored growth on starch media.

32. Streptomyces gougeroti.

$\mathrm{a}^{4}$. Bluish-black color on synthetic media, with white aerial mycelium.

33. Streptomyces violaceoniger.

$a^{5}$. Yellowish pigment on potato.

b. Aerial mycelium thick, powdery, water-green; starch is hydrolyzed.

34. Streptomyces griseus.

bb. Aerial mycelium white; starch meakly hydrolyzed.

35. Streptomyces griseoflavus.

$a^{6}$. Greenish-black pigment on potato; aerial mycelium white.

36. Streptomyces albidoflavus.

$a^{7}$. Reddish-brown pigment on potato; aerial mycelium white; starch is not hydrolyzed.

37. Streptomyces poolensis. 
$a^{8}$. Gray to sulfur-yellow pigment on potato.

b. Aerial mycelium mouse-gray to light drab; starch is hydrolyzed.

38. Streptomyces olivaceus.

bb. Aerial mycelium yellowish-white.

39. Streptomyces lieskci.

bbb. No aerial mycelium; starch is hydrolyzed.

40. Streptomyces microflavus.

$a^{9}$. No soluble pigment on potato.

41. Streptomyces cacaoi.

2. Proteolytic action weak.

a. Soluble pigment formed on synthetic agar.

b. Pigment blue or blue-black.

42. Streptomyces novaecaesareae.

bb. Pigment brown to black.

43. Streptomyces exfoliatus.

44. Streptomyces gelaticus.

aa. No soluble pigment on synthetic agar.

$\mathrm{b}^{1}$. Growth turning black; diastatic action strong.

c. Growth on synthetic agar scant with abundant spirals in aerial mycelium.

45. Streptomyces rutgersensis.

cc. No spirals on synthetic agar; characteristic greencolored growth on protein-glycerol medium.

46. Streptomyces lipmanii.

ccc. No spirals on synthetic agar; growth dark, almost black.

47. Streptomyces halstedii.

$\mathrm{b}^{2}$. Moist aerial mycelium, many spirals.

48. Streptomyces hygroscopicus.

$\mathrm{b}^{3}$. Growth orange-colored.

49. Streptomyces fradiae.

$\mathrm{b}^{4}$. Growth yellowish.

50. Streptomyces alboflavus.

$b^{5}$. Growth rose to red; aerial mycelium white.

51. Streptomyces albosporeus.

$b^{6}$. Growth cream-colored; aerial mycelium flaky.

52. Streptomyces flocculus.

$b^{7}$. Growth red; aerial mycelium black.

c. Complete decomposition of cellulose; weakly diastatic.

53. Streptomyces melanosporeus.

cc. Incomplete decomposition of cellulose; strongly diastatic.

54. Streptomyces melanocyclus.

3. No proteolytic action or very little.

a. Acid-resistant strains.

55. Streptomyces acidophilus.

aa. Non-acid-resistant.

56. Streptomyces rubescens. 
II. Saprophytes; thermophilic.

A. Starch hydrolyzed. Yellowish growth on potato.

57. Streptomyces thermophilus.

B. Starch not hydrolyzed. Abundant, dark-colored growth on potato.

58. Streptomyces thermofuscus.

III. Plant parasites.

A. Tyrosinase reaction positive; aerial mycelium gray-white.

59. Streptomyces scabies.

B. Tyrosinase reaction negative; attacks sweet potatoes.

60. Streptomyces ipomoea.

IV. Isolated from animal tissues. In the animal body, hyphae often show clavate enlargements at the ends.

A. Limited proteolytic action in gelatin, milk, coagulated egg-albumin or fibrin.

1. Color of vegetative growth golden-brown.

2. Color of vegetative growth pink.

61. Streptomyces fordii.

a. Sparse white aerial mycelium.

62. Streptomyces africanus.

aa. Formation on mycelium of bodies similar to Thermoactinomyces.

63. Streptomyces gallicus.

aaa. Yellowish-pink growth on potato plug; scant white aerial mycelium.

64. Streptomyces pelletieri.

3. Color of vegetative growth white.

65. Streptomyces listeri.

4. Vegetative growth cream-colored, scant white aerial mycelium.

66. Streptomyces upcottii.

5. Growth very limited on various media, except on potato plug; no liquefaction of gelatin.

67. Streptomyces hortonensis.

B. Strong proteolytic action in gelatin and milk.

1. No pigment produced.

$a^{1}$. No growth on potato plug.

68. Streptomyces gibsonii.

$a^{2}$. Moist, membranous growth on potato plug; diastase formed.

69. Streptomyces beddardii.

70. Streptomyces kimberi.

$a^{3}$. Extensive growth on potato media; white powdery aerial mycelium.

$a^{4}$ Abundant growth on potato plug, becoming black; white-gray aerial mycelium; plug discolored.

71. Streptomyces somaliensis.

$a^{5}$. Pink-colored growth on some media.

72. Streptomyces panjae.

$a^{6}$. Profuse white aerial mycelium on most media, spiral formation.

73. Streptomyces willmorei.

1. Streptomyces albus (Rossi Doria emend. Krainsky) Waksman and Henrici.
(Streptotrix alba Rossi Doria, Ann. d'Ist. d'Ig. sper. di. Univ. di Roma, 1, 1891 , 
399; Cladothrix alba Macé, Traité Pratique Bact., 3rd ed., 1897; Actinomyces albus Krainsky, Cent. f. Bakt., II Abt., $41,1914,662 ;$ Nocardia alba Chalmers and Christopherson, Ann. Trop. Med. and Parasit., 10, 1916, 270; Waksman and Henrici, Jour. Bact., 46, 1943, 339.) From Latin albus, white.

Additional synonyms as given by Baldacci (Mycopathologia, 2, 1940, 156): Cladothrix dichotoma Macé, not Cohn, 1886 ; Streptothrix foersteri Gasperini, not Cohn, 1890; Streptothrix No. 2 and 3, Almquist, 1890; Actinomyces saprophyticus Gasperini, 1892; Oospora doriae Sauvageau and Radais, 1892; Cladothrix liquefaciens Hesse, 1892 (according to Duché); Cladothrix invulnerabilis Acosta and Grande Rossi, 1893; Actinomyces chromogenus Gasperini, 1894 (Streptotrix nigra Rossi Doria, 1891); Streptothrix gedanensis I Scheele and Petruschky, 1897; Streptothrix graminearum Berestneff, 1898; Actinomyces thermophilis (Berestneff) Miehe, not Gilbert, 1898; Cladothrix odorifera Rullmann, 1898; Actinomyces chromogenes Gasperini $\beta$ alba Lehmann and Neumann, 1899; Oospora sp. Bodin, 1899 (according to Duché); Oospora alpha Price-Jones, 1900 (according to Chalmers and Christopherson); Streptothrix leucea Foulerton, 1902 (according to Chalmers and Christopherson); Streptothrix candida Petruschky, 1903; Streptothrix lathridii Petruschliy, 1903; Streptothrix dassonvillei BrocqRousseau, 1907 (according to Duché); Streptothrix pyogenes Caminiti, 1907 (according to Chalmers and Christopherson); Streptothrix sanninii Ciferri, 1922; Actinomyces almquisti Duché, 1934; Actinomyces gougeroti Duché, 1934. Doubtful synonyms: Oospora metchnikowi Sauvageau and Radais, 1892; Oospora guignardi Sauvageau and Radais, 1892; Actinomyces albus Waksman and Curtis, 1919; Actinomyces thermodiastaticus Bergey, (1919) 1925. Varieties: Actinomyces albus var. acidus Neukirch, 1902 (according to Nannizzi); Actino- myces albus var. ochroleucus Neukirch, 1902 (according to Wollenweber); $A c$ tinomyces albus var. toxica Rossi, 1905; Actinomyces albus var. cretaceus (Krüger) Wollenweber, 1920; Actinomyces albus var. $\alpha$ Ciferri, 1927.

More complete information regarding these species will be found in the text or in the Appendix to Genus Streptomyces.

The description of this species by Rossi Doria is incomplete. The characters given below are taken from Krainsky (loc. cit.) with some supplementary information from later authors. Other descriptions which may vary from this in certain details are given by Waksman and Curtis (Soil Sci., 1, 1916, 117), Bergey et al. (Manual, 1st ed., 1923, 367), Duché (Les actinomyces du groupe albus, Paris, 1934, 257) and Baldacei (loc. cit.).

Vegetative hyphae: Branched, 1 micron in diameter.

Aerial mycelium: Abundant, white. Hyphae 1.3 to 1.7 microns in diameter with ellipsoidal spores (1 micron long) in coiled chains on lateral branches of the aerial hyphae.

Gelatin: Liquefaction. Colonies gray, no soluble pigment.

Ca-malate agar: Colonies of medium size, the center only is covered with a white aerial mycelium.

Starch agar: Aerial mycelium white but covers the whole surface.

Glucose agar: Gray aerial mycelium becoming brownish.

Peptone and bouillon agar: No aerial mycelium but a chalky white deposit forms on old colonies.

Odor: Earthy or musty.

Broth: Flaky growth on bottom with surface pellicle in old cultures.

Potato: Colonies and aerial mycelium white.

Carrots and other vegetables: Excellent growth (Duché).

No growth on cellulose.

No hydrolysis of starch.

Actively proteolytic.

Nitrites produced from nitrates. 
Milk: Peptonized after coagulation. Reaction becomes alkaline (Duché).

Aerobic.

Source: From air and soil (Rossi Doria); from garden soil (Kriainsky).

Habitat: Dust, soil, grains and straw. Widely distributed.

2. Streptomyces coelicolor (ReinerMüller) comb. nou. (Streptothrix coelicolor Reiner-Müller, Cent. f. Bakt., I Abt, Orig., 46, 1908, 197; Nocardia coelicolor Chalmers and Christopherson, Ann. Trop. Med. and Parasit., 10, 1916, 271; Actinomyces coelicalor Lieshe, Morphol. u. Biol. d. Strahlenpilze, Leipzig, 1921, 28.) From Latin caelum, sky and color, color.

Regarded by the authors of this section as the same as Actinomyces violaceus Waksman and Curtis, Soil Science, 1, 1916, 110 (Actinomyces violaceus-ruber Waksman and Curtis, ibid., 127; Actinomyces waksmanii Bergey et al., Manual, 3rd ed., 1930, 4S9) and Actinomyces tricolor Wollenweber, Arbeiten d. Forschungsinstitut für Kartoffelbau, 1920, 13. It is, however, pointed out by J. E. Conn (Jour. Bact., 46, 1943, 133) that certain differences between the descriptions of Waksman and Curtis, and that of Müller may correspond to actual chemica] differences in the pigments produced; and that the organism of Waksman and Curtis may be a separate species.

Description by Müller except as noted.

Morphology of Streptomyces coelicalor has not been fully described. According to Waksman and Curtis who deseribed Actinomyces violaceus-ruber, this is as follows: Straight filaments with open, dextrorse spirals, breaking up into conidia. Conidia oval or rod-shaped, 0.7 to 1.0 by 0.8 to 1.5 microns.

Gelatin: Good growth. No pigment formation. Liquefaction fairly rapid, beginning in 4 to 7 days.

Plain agar: Good growth. Pigment lacking or faint blue (Conn).
Czapek ayar (according to Waksman and Curtis concerning Actinomyces riola(eus-ruber): Thin, spreading, colorless at first, becoming red, then blue. Aerial mycelium thin, white, powdery, becoming mouse-gray.

Asparagine agar (synthetic): With glycerol as source of carbon, good growth, violet to deep blue, with pigment diffusing through medium; final $\mathrm{H}$-ion concentration about $\mathrm{pH} 7.0$ to 8.0. With glucose as source of carbon, poorer growth, red, no diffusion of pigment; final $\mathrm{H}$-ion concentration about $\mathrm{pH} 6.0$ to 5.0 (Conn).

Broth: Good growth. Cretaceous layer around edge.

Milk: No change at $25^{\circ} \mathrm{C}$ (Conn). At $37^{\circ} \mathrm{C}$, coagulation. Peptonization beginning in 3 to 5 days.

Potato: Strong pigment production, sometimes greenish-blue or violet, but usually sky-blue, diffusing through medium and coloring water at base of tube.

Nitrites produced from nitrates.

Blood agar: Hemolysis showing on 4 th day,

Müller reports no acid from carbohydrates on organic media. Conn, however, finds acid from glucose and lactose, and sometimes from sucrose and mannitol when grown on synthetic media.

Pigment: The most striking characteristic of this organism is a litmus-like pigment usually produced on potato or synthetic media, which is deep blue and water-soluble at alkaline reactions (beyond $\mathrm{pH} 8.0$ ), violet around neutrality, and red (insoluble in water) at about $\mathrm{pH}$ 6.0. Conn points out that the primary pigment has a spectrophotometric curve almost identical with that of azolitmin; but that there are undoubtedly other pigments produced, especially in the case of the strains believed to be typical of Actinomyces violaceus-ruber (as previously pointed out by Waksman and Curtis).

Good growth at room temperature and at $37^{\circ} \mathrm{C}$. 
Aerobic.

Distinctive character : Litmus-like pigment.

Source: Dust contamination on a potato slant.

Habitat: Soil and plant surfaces. Very abundant.

Note: Because of the numerous colors and shades shown by the pigment according to final $\mathrm{H}$-ion concentration and other less understood factors, this species may have been described under various names. On the other hand, it is entirely possible, as pointed out by Conn (loc. cit.), that careful study of the pigments may show that more than one species is actually involved.

3. Streptomyces verne (Waksman and Curtis) comb. nov. (Actinomyces verne Waksman and Curtis, Soil Science, 1, 1916, 120.) Derivation uncertain.

Filaments with close branching of the hyphae. No conidia demonstrated.

Gelatin stab: Small, cream-colored colonies. Rapid liquefaction.

Synthetic agar: Abundant, spreading, wrinkled, elevated, glossy, yellowish growth, becoming brownish, lichenoid margin.

Starch agar: Scant, brownish, restricted growth.

Glucose agar: Abundant, much folded growth, center raised, gray with purplish tinge, entire.

Plain agar: Small, grayish colonies with depressed center, becoming wrinkled.

Glucose broth : Slightly flaky sediment.

Litmus milk: Pinkish-brown ring; coagulated; peptonized, with alkaline reaction.

Potato: Cream-colored growth, becoming gray, wrinkled.

Nitrites produced from nitrates.

Soluble brown pigment formed. Soluble green pigment produced when freshly isolated.

Starch is hydrolyzed.

Aerobic.

Optimum temperature $37^{\circ} \mathrm{C}$.
Source: Isolated once from upland California soil.

Habitat: Soil.

4. Streptomyces californicus (Waksman and Curtis) comb. nov. (Actinomyces californicus Waksman and Curtis, Soil Science, 1, 1916, 122.) Named for the State of California.

Filaments with long, narrow, open spirals. Spherical to oval conidia from straight and spiral hyphae.

Gelatin stab: Gray, moist, abundant surface growth. Liquefaction in 30 days.

Synthetic agar: Spreading, vinaceouscolored growth. Aerial mycelium powdery, thin, light neutral gray.

Starch agar: Growth spreading, pink center with colorless to gray margin.

Glucose agar : Restricted, much folded, cream-colored growth, with sulfur-yellow tinge.

Plain agar: Thin, restricted, yellowish to cream-colored growth.

Glucose broth: Solid cream-colored mass on surface, with pink tinge.

Litmus milk: Faint, brownish surface growth; coagulated; peptonized in 40 days.

Potato: Glossy, yellow to red growth, turning red-brown.

Nitrites produced from nitrates.

No soluble pigment formed.

Starch is hydrolyzed.

Aerobic.

Optimum temperature $37^{\circ} \mathrm{C}$.

Source: Isolated once from California sandy loam.

Habitat: Soil.

\section{Streptomyces flaveolus (Waksman)} comb.nov. (Actinomyces 168, Waksman, Soil Science, 8, 1919, 134; Actinomyces flaveolus Waksman, in Manual, 1st ed., 1923, 368; Actinomyces heimi Duché, Actinomyces du groupe albus. Encyclopédie Mycologique, Paris, 6, 1934, 359.) From Latin flavus, yellow and eolus, diminutive ending; hence, somewhat yellow. 
Numerous closed and open spirals on all media. Conidia oval to elliptical.

Gelatin stab: Liquefied; abundant, yellowish, spreading pellicle.

Synthetic agar: Growth light sulfuryellow turning to cadmium-yellow, penetrating deep into medium. Aerial mycelium as white to ash-gray patches.

Starch agar: White, spreading growth.

Glucose agar: Restricted growth, surface folded, raised.

Plain agar: White, glistening, wrinkled growth.

Glucose broth: Thin, yellow pellicle.

Litmus milk: Sulfur-yellow ring; coagulated; peptonized, with faintly alkaline reaction.

Potato: Abundant, wrinkled, creamcolored growth.

Nitrites produced from nitrates.

Soluble empire-yellow pigment formed.

Starch is hydrolyzed.

Aerobic.

Optimum temperature $25^{\circ} \mathrm{C}$.

Habitat: Soil.

6. Str eptomyces bobiliae (Waksmanand Curtis) comb. nov. (Actinomyces bobili Waksman and Curtis, Soil Science, 1, 1916, 121.) From the name of a person.

Mycelium with branching hyphae. Few close spirals of a dextrorse type.

Gelatin stab: Dense, cream-colored to brownish surface growth. Rapid liquefaction.

Synthetic agar: Abundant, glossy, wrinkled, elevated, coral-red growth becoming deep red. Scant, white aerial mycelium.

Starch agar: Restricted, finely wrinkled, coral-red growth with hyaline margin .

Plain agar: Restricted, glossy, gray growth, becoming brownish.

Glucose broth : Round colonies in fluid. Flaky sediment.

Litmus milk: Dark brown ring. No coagulation. Peptonized.

Potato: Thin, yellowish growth, becoming red, dry and wrinkled.
Nitrites produced from nitrates.

Soluble brown pigment formed.

Starch is hydrolyzed.

Aerobic.

Optimum temperature $37^{\circ} \mathrm{C}$.

Source: Isolated once from adobe and garden soils.

Habitat: Soil.

\section{Streptomyces roseochromogenus}

(Jensen) comb.nov. (Actinomyces roseus Krainsky, Cent. f. Bakt., II Abt., 41, 1914, 662; Waksman and Curtis, Soil Science, 1, 1916, 125; Waksman, Soil Science, 8, 1919, 148; not Actinomyces roseus Namyslowski, Cent. f. Bakt., I Abt., Orig., 62, 1912, 567; Nocardia rosea Chalmers and Christopherson, Ann. Trop. Med. and Parasit., 10, 1916, 270; Actinomyces roseochromogenus Jensen, Proc. Linnean Soc. New So. Wales, 56, 1931, 359.) From Latin, producing rose color.

Filaments with numerous open and closed spirals. Conidia 1.0 to 1.2 by 1.5 to 3.0 microns .

Gelatin stab : Liquefaction, with small, cream-colored colonies in bottom of liquid.

Synthetic agar: Thin, spreading, colorless growth. Aerial mycelium thin, pale, brownish.

Starch agar: Colorless, spreading growth.

Glucose agar: Growth extensive, spreading, colorless, entire.

Plain agar: White growth, becoming yellowish.

Glucose broth: Cream-colored ring, with flaky sediment.

Litmus milk: Brownish ring. No coagulation. Peptonized in 10 to 15 days, becoming strongly alkaline.

Potato: Much wrinkled, brownish growth.

Nitrites produced from nitrates.

Purple pigment on egg media; brown on gelatin.

Starch is hydrolyzed.

Aerobic. 
Optimum temperature $37^{\circ} \mathrm{C}$. Habitat: Soil.

8. Streptomyces griseolus (Waksman) comb. nov. (Actinomyces 96, Waksman, Soil Science, 8, 1919, 121; Aclinomyces griseolus Waksman, in Manual, 1st ed., 1923, 369.) From Latin griseus, gray and eolus, diminutive ending; hence, somewhat gray.

Branching mycelium; no spirals observed. Conidia spherical or ovalshaped.

Gelatin stab : Liquefied with yellowish, flaky pellicle and sediment.

Synthetic agar: Colorless, thin, spreading growth, chiefly in the medium; surface growth limited almost entirely to the aerial mycelium. Aerial mycelium at first gray, later becoming pallid, neutralgray.

Starch agar: Grayish-brown growth, with dark ring.

Glucose agar: Spreading growth, both on the surface and into the medium; center raised, cream-colored, turning dark.

Plain agar: Brownish growth, with smooth surface.

Glucose broth: Thick, brown ring.

Litmus milk: Abundant growth, pink pellicle; coagulated; peptonized, becoming alkaline.

Potato: Cream-colored growth, becoming black, spreading.

Nitrites produced from nitrates.

Faint brownish soluble pigment formed.

Starch is hydrolyzed.

Aerobic.

Optimum temperature $25^{\circ} \mathrm{C}$.

Habitat: Soil.

9. Streptomyces erythreus (Waksman) comb.nov. (Actinomyees 161, Waksman, Soil Science, 8, 1919, 112; Actinomyces erythreus Waksman, in Manual, 1st ed., 1923, 370; Actinomyces krainskii Duché, Encyclopédie Mycologique, Paris, G, 1934, 306.) From Greek erythrus, red.
Mycelium fine, branching; numerous open spirals formed as side branches of the main hyphae.

Gelatin stab: Abundant, dense, gray growth with pinkish tinge, chiefly on surface of liquefied medium.

Synthetic agar: Spreading growth with irregular margin, developing deep into the medium; color at first white, later turning yellowish, agar around growth has a white, milky surface. Aerial mycelium, thick, solid, white.

Starch agar: Cream-colored, circular colonies, with faint greenish tinge.

Glucose agar: Abundant, spreading, cream-colored growth, later turning brown chiefly on surface; center raised, lobate margin.

Plain agar: Cream-colored growth.

Glueose broth: Abundant, creamcolored surface growth,

Litmus milk: Yellowish surface zone; coagulated; peptonized, becoming alkaline.

Potato: Wrinkled, cream-colored growth, becoming yellowish.

Nitrites produced from nitrates.

Soluble purple pigment formed.

Starch is hydrolyzed.

Aerobic.

Optimum temperature $25^{\circ} \mathrm{C}$.

Similar to Streptomyces erythrochromogenes (Species No. 24) except that no brown soluble pigment is formed.

Source: From California and Hawaiian soils.

Habitat: Soil.

10. Streptomyces cellulosae (Krainsky) comb. nov. (Actinomyces cellulosae Krainsky, Cent. f. Bakt., II Abt., 41, 1914, 662., From M. L. cellulosa, cellulose.

Conidia almost spherical, 1.3 microns in diameter, of ten arranged in chains.

Gelatin colonies: Circular, yellowish.

Gelatin stab: Liquefied.

Plain agar: White aerial mycelium. Ca-malate agar: Yellowish colonies; 
gray aerial mycelium. Soluble yellow pigment formed.

Glucose agar: Abundant growth, gray aerial mycelium. Soluble yellow pigment.

Starch agar: Same as on glucose agar.

Glucose broth: Coarse, flaky growth. Yellow pigment.

Litmus milk: Peptonized.

Potato: Light gray growth; gray aerial mycelium.

Nitrates show slight reduction.

Strong diastatic action. Esculin is hydrolyzed.

Cellulose is decomposed.

Aerobic.

Optimum temperature $30^{\circ}$ to $35^{\circ} \mathrm{C}$.

Habitat: Soil.

11. Streptomyces parvus (Krainsky) comb. nov. (Actinomyces parvus Iirainsky, Cent. f. Bakt., II Abt., 41, 1914, 662; Nocardia parva Chalmers and Christopherson, Ann. Trop. Med. and Parasit., 10, 1916, 268.) From Latin parvus, small.

Conidia more or less oval, 0.9 to 1.3 by 1.2 to 1.8 microns.

Gelatin: Colonies yellow. Slow liquefaction.

Ca-malate agar: Small, yellow colonies with light yellow aerial mycelium.

Glucose agar: Same as on Ca-malate agar.

Starch agar: Same as on Ca-malate agar.

Glucose broth: Hemispherical colonies in bottom of tube.

Litmus milk: Peptonized.

Nitrate slightly reduced.

Moderate diastatic action.

Cellulose not decomposed.

Aerobic.

Optimum temperature.

Source: Garden soil.

Habitat: Soil.

12. Streptomyces malenconii (Duché) comb. nov. (Actinomyces malenconi Duché, Encyclopédie Mycologique, Paris,
6, 1934, 353.) Named for Mr. Malençon from whom the original culture was obtained.

Gelatin: Poor growth; liquefaction.

Asparagine glucose agar: Rapid opaque growth, later becoming covered with white aerial mycelium; amber-colored pigment, dissolved in medium.

Peptone agar: Cream-colored lobous growth, covered with whitish aerial mycelium.

Asparagine glucose solution: Long, much branching filaments, 0.5 to 0.7 micron; somewhat heavier aerial mycelium with a few irregular conidia; some flaky growth on bottom of tube; surface growth is cream-colored with rare white aerial mycelium; licuid becomes slightly yellow.

Peptone solution: Whitish growth with yellowish soluble pigment.

Milk: Surface growth with whitish aerial mycelium; slow peptonization, liquid becoming brownish-colored.

Potato: Rapid growth with thin white mycelium; no soluble pigment.

Coagulated serum: Radiating creamcolored growth covered with white aerial mycelium; slow liquefaction.

No pigment on tyrosine medium.

Source: Culture obtained from Mr. Malençon, an inspector in Morocco.

\section{Streptomyces diastaticus (Krain-} sky) comb. nov. (Actinomyces diastalzcus Krainsky, Cent. f. Bakt., II Bakt., 41, 1914, 662; Waksman and Curtis, Soil Science, 1, 1916, 116.) From M. L. diastaticus, diastatic.

Actinomyces roseodiastaticus Duché, Encyclopédie Mycologique, Paris, 6, 1934, 329 is said to differ from both Krainsky's, and Waksman and Curtis'strains.

Filaments may show fine, long, narrow spirals. Conidia oval, 1.0 to 1.2 by 1.1 to 1.5 microns.

Gelatin stab: Liquefied with small, cream-colored flakes in liquid.

Synthetic agar: Thin, gray, spreading 
growth. Aerial mycelium white, becoming drab gray.

Starch agar: Thin, colorless, spreading growth. Aerial mycelium gray.

Glucose agar: Yellowish, spreading growth. No aerial mycelium.

Plain agar: Cream-colored growth. Thin aerial mycelium.

Glucose broth: Gray ring with grayish colonies in bottom of tube.

Litmus milk: Brownish ring; coagulated; peptonized in 25 to 30 days, becoming faintly alkaline.

Potato: Abundant, wrinkled, creamcolored growth with greenish tinge.

Nitrites produced from nitrates.

Brown to dark brown soluble pigment formed.

Starch is hydrolyzed.

Aerobic.

Optimum temperature $37^{\circ} \mathrm{C}$.

Habitat: Soil.

\section{Streptomyces fimicarius (Duché)} comb. nov. (Actinomyces fimicarius Duché, Encyclopédie Mycologique, Paris, 6, 1934, 346.) From Latin fimus, dung and carus, loving.

Gelatin: Punctiform colonies with whitish aerial mycelium; reddish soluble pigment. Liquefaction.

Asparagine agar: Cream-colored growth with whitish aerial mycelium; reverse side, cream-colored to slight ochre.

Czapek's agar: Yellowish masses of growth with yellowish-white aerial mycelium; reverse side orange-colored; faint yellowish soluble pigment.

Peptone agar: Cream-colored growth with white aerial mycelium; reverse side, yellowish.

Asparagine solution: Vegetative filaments 0.5 to 0.6 micron long; branching aerial mycelium 0.8 to 1.0 micron, forming numerous conidia ; flaky grow th produced on bottom; surface growth becomes covered with a white aerial mycelium; reverse side, brownish-red.

Czapek's solution: Cream-colored punctiform growth with yellowish aerial mycelium; no soluble pigment.

Peptone solution: Whitish growth that flakes throughout liquid; yellowish pigment.

Tyrosine medium: White growth with yellowish reverse; yellowish soluble pigment.

Milk: Colorless growth becoming covered with whitish aerial mycelium; slow peptonization of milk which becomes rosecolored, finally changing to brownish-red.

Potato: Cream-colored to yellowish growth with whitish aerial mycelium; reddish-brown pigmentation of plug.

Coagulated serum: Cream-colored growth with whitish aerial mycelium; rapid liquefaction of serum.

Distinctive eharacters: Abundant growth upon neutral and acid media; whitish acrial mycelium; marked odor; soluble brownish-red pigment. This species seems to form the transition type between the Actinomyces albus group and the Actinomyces chromogenus group.

Iabitat : Found abundantly in manure.

\section{Streptomyces flavovirens (Waks-} man) comb. nov. (Actinomyees 128, Waksman, Soil Science, 8, 1919, 117; Actinomyces flavovirens Waksman, in Manual, 1st ed., 1923, 352; Actinomyces albatiridis Duché, Encyclopédie Mycologique, Paris, 6 , 1934, 317.) From Latin flavus, yellow and virens, becoming green.

Large masses of minute tufts; the hyphae coarse, straight, short, relatively unbranched, beaded; open spirals may be produced in certain substances. Conidia spherical, oval to rod-shaped, 0.75 to 1.0 by 1.0 to 1.5 microns.

Gelatin stab: Yellowish-green surface pellicle, consisting of a mass of small colonies, on the liquefied medium.

Synthetic agar: Growth spreading deep into the substratum, yellowish with greenish tinge. Aerial mycelium, gray, powdery.

Starch agar: Greenish-yellow, spreading growth, developing deep into the medium. 
Glucose agar: Restricted growth, developing only to a very small extent into the medium, yellow, turning black, edge entire.

Plain agar: Yellowish growth; the reverse dark in center with yellowish zone and outer white zone.

Glucose broth: Thick, sulfur-yellow pellicle or ring.

Litmus milk: Cream-colored to brownish ring; coagulated; peptonized, becoming faintly alkaline.

Potato: Sulfur-yellow, wrinkled growth.

Only a trace of nitrite is formed from nitrates.

Greenish-yellow soluble pigment formed.

Starch is hydrolyzed.

Aerobic.

Optimum temperature $25^{\circ} \mathrm{C}$.

Habitat: Soil.

\section{Streptomyces olivochromogenus} (Bergey et al.) comb. nov. (Actinomyces chromogenus 205, Waksman, Soil Science, 8, 1919, 106; Actinomyces olivochromogcnus Bergey et al., Manual, 2nd ed., 1925, 368.) From Greek, producing an olive color.

Filaments with numerous close spirals. Conidia oval or elliptical.

Gelatin stab : Cream-colored, spreading surface growth. Rapid liquefaction.

Synthetic agar: White, spreading growth. Aerial mycelium ash gray with brownish tinge.

Starch agar: Transparent, spreading growth.

Glucose agar: Abundant, natal brown to almost black growth, entire margin.

Plain agar: Wrinkled, brown growth, becoming gray-green.

Glucose broth: Thin, brown growth, flaky sediment.

Litmus milk: Dark brown ring; coagulated; peptonized, becoming alkaline.

Potato : Small, wrinkled, black colonies.

Faint traces of nitrites formed from nitrates.
Soluble brown pigment formed.

Starch is hydrolyzed.

Aerobic.

Optimum temperature $37^{\circ} \mathrm{C}$.

Habitat: Soil.

\section{Streptomyces diastatochromo-}

genes (Krainsky) comb. nov. (Actinomyces diastatochromogenes Krainsky, Cent. f. Bakt., II Abt., 41, 1914, 662.) From Greek, probably intended to mean producing both diastase and color.

Conidia spherical or oval, about 1.2 microns.

Gelatin colonies : Light gray-colored.

Gelatin stab: Liquefied.

Plain agar: Medium-sized colonies, with white to gray aerial mycelium.

Ca-malate agar : Medium-sized colonies, colorless, with gray aerial mycelium.

Glucose agar: Same as on Ca-malate agar.

Starch agar: Same as on Ca-malate agar.

Glucose broth: Flaky colonies in depth at first, later also over surface.

Potato: Light gray colonies; gray aerial mycelium; medium colored black.

Soluble brown pigment formed in gelatin.

Weakly diastatic.

No growth on cellulose.

Tyrosinase formed.

Aerobic.

Optimum temperature $35^{\circ} \mathrm{C}$.

Habitat : Soil.

18. Streptomyces flavochromogenes (Krainsky) comb. nov. (Actinomyces flavochromogenes. Krainsky, Cent. f. Bakt., II Abt., 41, 1914, 662.) From Latin flavus, yellow and Greek, producing color.

Conidia oval, 1.7 microns.

Gelatin colonies : Yellowish colonies.

Gelatin stab: Slight liquefaction.

Plain agar: Aerial mycelium formed late, at first white, later gray. Gray soluble pigment formed.

Ca-malate agar: Colonies yellow with white aerial mycelium forming late. 
Glucose agar: Brown soluble pigment formed.

Starch agar: Yellow colonies, with white aerial mycelium.

Glucose broth : Fine flakes, with small spherical colonies adherent to glass. Medium colored brown.

Potato: Yellow colonies, with white aerial mycelium.

Nitrites produced from nitrates.

Weakly diastatic. Esculin acted upon.

Slow growth on cellulose.

Tyrosinase formed.

Aerobic.

Optimum temperature $35^{\circ} \mathrm{C}$.

Habitat: Soil.

19. Streptomyces antibioticus (Waksman and Woodruff) comb. nov. (Actinomyces antibioticus Waksman and Woodruff, Jour. Bact., 42, 1941, 232 and 246.) From Greek, antibiotic.

Spore-bearing hyphae produced in the form of straight aerial hyphae. The conidiophores are arranged in clusters; no spirals formed. The conidia are nearly spherical to somewhat elliptical.

Gelatin : Dark brown growth on surface, with patches of gray aerial mycellium. Dark pigment produced, which gradually diffuses into the unliquefied part of the gelatin. Liquefaction at first very slow, later becoming rapid.

Czapek's agar: Thin, whitish growth. Thin, gray aerial mycelium.

Peptone media: Production of dark pigment at early stage of growth is very characteristic. Growth brownish, thin, with a yellowish-gray to yellowish-green aerial mycelium.

Potato plug: Folded, brown-colored growth, with a thin black ring on plug, fading into a bluish tinge. No aerial mycelium.

Carrot plug: Cream-colored to faint brownish growth. No aerial mycelium. No pigment.

Litmus milk: Thick, brownish ring on surface of milk. Mouse-gray aerial mycelium with greenish tinge; growth be- comes brown, especially in drier portions adhering to glass. No reaction change, no coagulation of milk, no clearing; whitish sediment at bottom of tube. Old cultures: Heavy growth ring on surface of milk, heavy precipitation on bottom; liquid brownish to black in upper portion.

Odor: Very characteristic soil odor.

Antagonistic properties: Has a marked antagonistic effect on Gram-positive and Gram-negative bacteria, much more on the former than on the latter, as well as on actinomycetes. It is also active against fungi, which vary in degree of sensitivity. Produces a specific bacteriostatic and bactericidal substance known as actinomycin (Waksman and Woodruff, Jour. Bact., 40, 1940, 581).

Source: Isolated from soil on Escherichia coli-washed-agar plate, using living cells of $E$. coli as the only source of available nutrients.

Habitat: Soil.

\section{Streptomyces viridochromogenes} (Krainsky) comb. nov. (Actinomyces viridochromogenes Krainsky, Cent. f. Bakt., II Abt., 41, 1914, 662; Waksman and ('urtis, Suil seienee, 1, 1916, 114.) From Latin. green and Greek, producing color.

Filaments with numerous open spirals, 3 to 5 microns in diameter, occurring as side branches and terminal conidia, short ovals or spheres, 1.25 to 1.5 mierons.

Gelatin stab: Cream-colored surface growth, becoming greenish. Slow liquefaction.

Synthetic agar: Spreading growth, cream-colored with dark center, becoming dark green; reverse yellowish to light cadmium. Aerial mycelium abundant, spreading, white, becoming light green.

Starch agar: Circular, spreading, yellowish colonies.

Glucose agar: Abundant, spreading, wrinkled, gray growth, becoming black.

Plain agar: Abundant, restricted, gray growth, with greenish tinge. 
Glucose broth: Dense, solid ring, brownish, becoming dark green.

Litmus milk: Dark brown surface growth; coagulated; peptonized, with faintly alkaline reaction.

Potato: Abundant, gray-brown growth.

Nitrites produced from nitrates.

Soluble brown pigment formed.

Starch is hydrolyzed.

Aerobic.

Optimum temperature $37^{\circ} \mathrm{C}$.

Habitat: Soil.

21. Streptomyces purpeochromogenus (Waksman and Curtis) comb. nov. (Actinomyces purpeochromogenus Waksman and Curtis, Soil Science, 1, 1916, 113.) From Latin, purple and Greek, producing color.

Branching mycelium and hyphae with few imperfect spirals. Conidia spherical, 0.75 to 1.0 micron in diameter.

Gelatin stab: Slow, brownish surface growth. Slow liquefaction.

Synthetic agar: Slow, restricted, smooth, gray growth, becoming brown with purplish tinge; center raised. Margin yellow.

Starchagar : Small, dark brown colonies.

Glucose agar: Abundant, restricted, gray growth, becoming brown to dark brown.

Plain agar: Gray to brownish growth, becoming dark brown, almost black.

Glucose broth: Slight, flaky sediment.

Litmus milk: Dark-brown ring; coagulated; slowly peptonized, with faintly alkaline reaction.

Potato: Restricted, orange to orangered growth.

Nitrites not produced from nitrates.

Soluble dark brown pigment formed.

Starch shows slight hydrolysis.

Aerobic.

Optimum temperature $25^{\circ} \mathrm{C}$.

Source: Isolated once from California adobe soil.

Habitat: Soil.
22. Streptomyces phaeochromogenus (Conn) comb. nov. (Actinomyces pheochromogenus (sic) Conn, N. Y. State Agr. Exp. Sta. Tech. Bull. No. 60, 1917, 16.) From Greek, producing a brown color.

Branching filaments and hyphae, spirals narrow, open, elongated, sinistrorse.

Gelatin stab: Abundant, spreading, cream-colored surface growth, becoming brown. Slow liquefaction.

Synthetic agar: Colorless growth, becoming brown to almost black. Aerial mycelium abundant, white with brownish shade.

Starch agar: Spreading, brownish growth, becoming brown.

Glucose agar: Restricted, much folded, brown growth

Plain agar: Thin, cream-colored growth, becoming gray.

Glucose broth: Dense, wrinkled pellicle.

Litmus milk: Dark, almost black ring; coagulated, with slow peptonization, faintly alkaline reaction.

Potato: Brown to almost black growth.

Nitrites produced from nitrates.

Soluble brown pigment formed.

Starch is hydrolyzed.

Aerobic.

Optimum temperature $25^{\circ} \mathrm{C}$.

Source: Isolated from soil.

Habitat: Soil.

23. Streptomyces aureus (Waksman and Curtis) comb. nov. (Actinomyces aureus Waksman and Curtis, Soil Science, 1, 1916, 124; not Actinomyces aurea Ford, Textb. of Bact., 1927, 220.) From Latin aureus, golden.

Mycelium shows numerous spirals. Conidia spherical to oval, 0.6 to 1.0 by 0.8 to 1.4 microns.

Gelatin stab: Fair, cream-colored surface growth, becoming brown, spreading. Liquefied.

Synthetic agar: Thin, spreading, color- 
less growth. Aerial my celium thin, gray, powdery, becoming cinnamon drab.

Starch agar : Thin, transparent, spreading growth.

Glucose agar: Spreading, light orange growth, raised center, hyaline margin.

Plain agar: Restricted, gray growth.

Glucose broth: Thin, brownish ring; flaky sediment.

Litmus milk: Black ring. No coagulation. Peptonization doubtful.

Potato: Abundant, wrinkled, brown growth, becoming black.

Nitrites produced from nitrates.

Soluble brown pigment formed.

Starch is hydrolyzed.

Aerobic.

Optimum temperature $25^{\circ} \mathrm{C}$.

Source: Isolated many times from a variety of soils.

Habitat: Soil.

\section{Streptomyces erythrochromogenes}

(Krainsky) comb. nov. (Actinomyces erythrochromogenes Krainsky, Cent. f. Bakt., II Abt., 41, 1914, 662; Waksman and Curtis, Soil Science, 1, 1916, 112.) From Greek, producing a red color.

Conidia oval, about 2.0 microns long.

Gelatin colonies: Slow growth.

Gelatin stab: Liquefied. A soluble brown pigment formed.

Plain agar: Brown soluble pigment. White aerial mycelium.

Ca-malate agar: Colonies circular, with grayish-white margined aerial mycelium.

Glucose agar: Red pigment formed.

Starch agar: A soluble rose pigment on old cultures.

Glucose broth: Abundant growth. Floating colonies, later a pellicle is formed. Brown soluble pigment.

Potato: Gray aerial mycelium. Medium colored black.

Nitrates show slight reduction.

Weakly diastatic.

No proteolytic enzyme formed.

No growth in cellulose.

Aerobic.

Optimum temperature $30^{\circ} \mathrm{C}$.
Source: Soil and roots of Alnus (alder). Habitat: Soil.

25. Streptomyces lavendulae (Waksman and Curtis) comb. nov. (Aclinomyces lavendulae Waksman and Curtis, Soil Science, 1, 1916, 126.) From M. L., lavender.

Hyphae coarse, branching. Spirals close, 5 to 8 microns in diameter. Conidia oval, 1.0 to 1.2 by 1.6 to 2.0 microns.

Gelatin stab : Creamy to brownish surface growth. Liquefied.

Synthetic agar: Thin, spreading, colorless growth. Aerial mycelium cottony, white, becoming vinous-lavender.

Starch agar: Restricted, glistening, transparent growth.

Plain agar: Gray, wrinkled growth.

Glucose broth: Abundant, flaky sediment.

Litmus milk: Cream-colored ring. No coagulation; peptonized, with strong alkaline reaction.

Potato: Thin, wrinkled, cream-colored to yellowish growth.

Nitrites produced from nitrates.

Soluble brown pigment formed.

Starch is hydrolyzed.

Aerobic.

Optimum temperature $37^{\circ} \mathrm{C}$.

Certain strains of this organism produce antibiotics. One such antibiotic, designated as streptothricin, is active both in vitro and in vivo against various Gram-positive and Gram-negative bacteria, fungi and actinomycetes (Waksman and Woodruff, Proc. Soe. Exp). Biol. Med., 49, 1942, 207; Waksman, Jour. Bact., 46, 1943, 299)

Source: Isolated once from orchard soil. Habitat: Soil.

26. Streptomyces reticuli (Waksman and Curtis) comb. nov. (Actinomyces reticuli Waksman and Curtis, Soil Science, 1, 1916, 118.) From Latin reticulum, a small net.

Mycelium in whorls; spirals formed on 
glucose agar are sinistrorse. Conidia spherical, 1.0 to 1.4 microns in diameter.

Gelatin stab: Liquefied with small, brown flakes.

Synthetic agar: Colorless growth, with yellowish tinge, becoming brownish, spreading. Aerial mycelium thin, white, cottony.

Starch agar: Brownish-gray growth.

Glucose agar: Restricted, brownish growth, center raised.

Plain agar: Gray, wrinkled growth, becoming brownish.

Glucose broth: Sedinent consisting of large colonies.

Litmus milk: Reaction unchanged; coagulated; peptonized.

Potato: Gray growth, with black center.

Nitrites produced from nitrates.

Dark brown pigment formed.

Starch is hydrolyzed.

Aerobic.

Optimum temperature $25^{\circ} \mathrm{C}$.

Source : From upland and adobe soils in California.

Habitat : Soil

27. Streptomyces rubrireticuli nom. nov. (Actinomyces reticulus-ruber Waksman, Soil Science, 8, 1919, 146; Actinomyces reticulus Bergey et al., Manual, 2nd edi., 1925, 373.) From Latin ruber, red and reticulum, a small net.

Branching filaments with both primary and secondary whorl formation. Spirals formed on glucose agar. Conidia ovalshaped.

Gelatin stab: Surface growth yellowish-red to dragon-pink. Liquefied.

Synthetic agar: Abundant, spreading growth, usually pink. Aerial mycelium thin, rose to pink.

Starch agar: White growth with red tinge.

Glucose agar: Abundant, spreading, rose-red, entire growth.

Plain agar: Red growth, with yellowish margin, becoming red.

Glucose broth: Thin, flaky sediment.
Litmus milk: Abundant, red pellicle; coagulated; peptonized. Reaction unchanged.

Potato: Cream-colored growth, later pink to dark red.

Nitrites produced from nitrates.

Soluble dark brown pigment formed.

Starch is hydrolyzed.

Aerobic.

Certain strains of this organism produce an antibiotic.

Source: Isolated from New Jersey orehard and California upland soils.

Optimum temperature $37^{\circ} \mathrm{C}$.

Habitat : Soil.

28. Streptomyces flavus (Krainsky) comb. nov. (Actinomyces flavus Krainsky, Cent. f. Bakt., II Abt., 41, 1914, 662 ; Waksman and Curtis, Soil Science, 1, 1916, 118; not Actinomyces flavus Sanfelice, Cent. f. Bakt., I Abt., Orig., 36, 1905, 359.) From Latin flavus, yellow.

Coarse filaments with branching hyphae. Conidia formed by budding and breaking up of hyphae into oval forms.

Gelatin stab: Small, yellowish masses on surface of liquefied medium.

Synthetic agar: Circular, yellow or sulfur-yellow colonies. Aerial mycelium straw-yellow.

Starch agar: Spreading, cream-colored growth, with pink tinge.

Glucose agar: Restricted, raised, folded, sulfur-yellow growth, center shading to brown.

Plain agar: Gray, spreading, folded growth.

Glucose broth: Small, white colonies in bottom of tube.

Litmus milk: Coagulated; peptonized, becoming distinetly alkaline.

Potato: Elevated, much wrinkled, greenish-olive growth.

Traces of nitrite formed.

Soluble brown pigment formed.

Starch is hydrolyzed.

Aerobic. 
Optimum temperature $25^{\circ} \mathrm{C}$.

Habitat: Soil.

29. Streptomyces ruber (Krainsky) comb. nov. (Actinomyces ruber Krainsky, Cent. f. Bakt., II Abt., 41, 1914, 662 ; Waksman, Soil Science, 8, 1919, 149; not Actinomyces ruber Sanfelice, Cent. f. Bakt., I Abt., Orig., 36, 1904, 355; Nocardia krainstii Chalmers and Christopherson, Ann. Trop. Med. and Parasit., 10, 1916, 268.) From Latin ruber, red.

Straight, branching mycelium, radiating. A few spirals may be formed.

Gelatin stab : Liquefaction, with yellow flakes.

Synthetic agar: Abundant, spreading, red growth. Aerial mycelium abundant, cottony, chrome-orange.

Starch agar: Abundant, spreading, red growth.

Glucose agar: Restricted, abundant, entire, coral-red growth.

Plain agar: Restricted, elevated, wrinkled, olive-green growth.

Glucose broth: Red ring, with spongy colonies on the surface.

Litmus milk: Dark ring with red tinge; coagulated; peptonized, with alkaline reaction.

Potato: Elevated, wrinkled, greenish growth.

Nitrites produced from nitrates.

Soluble brown pigment formed.

Starch is hydrolyzed.

Aerobic.

Optimum temperature $37^{\circ} \mathrm{C}$.

Habitat: Soil.

30. Streptomyces citreus (hrainsky) comb. nov. (Actinomyces citreus Krainsky, Cent. f. Bakt., II Abt., 41, 1914, 662; Waksman and Curtis, Soil Science, 1, 1916, 99; not Actinomyces citreus Gasperini; Cent. f. Bakt., 15, 1894, 684.) From M. L. citreus, lemon-yellow.

Filaments with long, narrow open spirals. Conidia spherical to oval, 1.2 to 1.5 by 1.2 to 1.8 microns .
Gelatin stab : Yellowish, restricted surface growth. Liquefaction in 35 days.

Synthetic agar: Abundant, spreading, raised, wrinkled, citron-yellow growth. Aerial mycelium covering surface ; citronyellow.

Starch agar: Abundant, yellowishgreen growth.

Glucose agar: Extensive, glossy, oliveyellow, entire growth; center elevated.

Plain agar: Restricted, cream-colored growth.

Glucose broth : Thin, wide, yellow ring; flaky sediment.

Litmus milk: Cream-colored surface growth; coagulated; peptonized, becoming alkaline.

Potato: Yellowish growth, aerial mycelium white.

Trace of nitrite production from nitrate.

The pigment formed is not soluble.

Starch hydrolyzed.

Aerobic.

Optimum temperature $37^{\circ} \mathrm{C}$.

Habitat: Soil.

31. Streptomyces fulvissimus (Jensen) comb. nov. (Actinomyces fulvissimus Jensen, Soil Science, 30, 1930,66.) From Latin fulvissimus, very yellow.

Vegetative mycelium without any special characteristics; aerial mycelium of short, straight, of ten trifurcated hyphae, 1.0 to 1.2 microns broad; no spiral formation; branches of hyphae break up into conidia, 1.0 to 1.2 by 1.2 to 1.5 microns.

Gelatin: Vegetative mycelium narrow, smooth, yellowish-brown to red-brown; no aerial mycelium; no pigment; gelatin completely liquefied in 10 to 12 days.

Nutrient agar: Good growth; vegetative mycelium raised, finely wrinkled, deep red-brown; no aerial mycelium; brownishyellow pigment.

Czapek's agar: Good growth (one strain very scant), vegetative mycelium flat, narrow, first light golden, later deep orange to red-brown; aerial mycelium scant, sometimes almost absent, first 
white, later light grayish-brown; pigment very characteristic, bright golden to orange.

Glycerol agar: Good growth; vegetative mycelium narrow, raised, smooth, golden to dark bronze; aerial mycelium scant, in patches, white to light cinnamon-brown; pigment intensely golden to orange.

Starch-casein agar: Good growth; vegetative mycelium spreading, folded, yellowish-brown; aerial mycelium abundant, smooth, lead-gray; pigment dull yellow to orange.

Potato: Good growth; vegetative mycelium raised, much wrinkled, rustbrown; aerial mycelium absent or traces of white; pigment gray to faint lemonyellow.

Loeffler's blood s̀erum: Vegetative mycelium red-brown; no aerial nycelium; yellowish pigment; no liquefaction.

Distinctive characters: The characteristic golden pigment is formed in nearly all media in which the organism groms, but becomes most typical and attains its greatest brightness in synthetic agar media; it has indicator properties, turning red in strongly acid solutions. The species is easily recognized on agar plates by its bronze-colored colonies, surrounded by haloes of bright yellow pigment.

Source: Very common in Danish soils. Habitat: Soil.

\section{Streptomyces gougeroti (Duché)} comb. nov. (Actinomyces gougeroti Duché, Encyclopédie Mycologique, Paris, 6, 1934, 272.) Named for Prof. Gougerot, from whom the culture was obtained.

Gelatin: Cream-colored colonies developing slowly with faint aerial mycelium; no pigment; liquefaction.

Plain agar: Cream-colored grow th forming concentric ring with age, with brownish reverse; faint yellowish soluble pigment.

Synthetic agar: Slow growth as punctiform colonies ; cream-colored with smooth edge; no aerial mycelium; no soluble pigment.

Peptone broth: Cream-colored ring on surface of medium with flakes throughout the medium; no soluble pigment.

Synthetic solution: Submerged mycelium in the form of flakes, later forming a surface pellicle; filaments of aerial mycelium 1 micron in diameter, with $\mathrm{nu}$ merous conidia ; cream-colored growth; no soluble pigment.

Tyrosine medium: Good growth with white aerial mycelium; no soluble pigment.

Litmus milk: Growth in the form of colonies which remain separated from one another; also flakes in the bottom of the tube with bluish tinge on reverse of growth; milk turns blue in 10 to 12 days.

Coagulated serum: Cream-colored growth covered with white aerial mycelium; rapid liquefaction of serum.

Potato: Slow grow th of a greenish tinge; aerial mycelium; no black pigment.

Distinctive character: Intermediate between Streptomyces albus with its abundant aerial mycelium and Actinomyces almquisli with its very scanty aerial mycelium.

Source: Culture obtained from the collection of Prof. Gougerot.

33. Streptomyces violaceoniger (Waksman and Curtis) comb. nov. (Actinomyces violaceus-niger Waksman and Curtis, Soil Science, 1. 1916, 111.) From Latin violaceus, violet and niger, black.

Gelatin: Gray growth, with no production of aerial mycelium. Gelatin around colony rapidly liquefied, but without any change in color.

Czapek's agar: Colony at first dark gray, turning almost black, 2 to $4 \mathrm{~mm}$ in diameter. Surface glossy, much folded with a very thin gray margin. A white to gray aerial mycelium is produced after the colony has well developed. A bluishblack pigment is produced at a later stage of its growth. The pigment slowly dis solves in the medium, turning almost 
black. Odor fairly strong. Microscopically two types of mycelium are found: the thin, branching filaments of the substratum, and the thick filaments of the aerial mycelium. The aerial mycelium fragments not very rapidly, producing a few conidia, spherical and oval, 1.2 to 1.5 by 1.2 to 2.3 microns. These often occur in chains.

Czapek's solution: Colonies large, 2 to $3 \mathrm{~mm}$ in diameter, appearing at the bottom and surface of the solution, but none throughout the medium. Colonies bluish in color, with a regular margin. Me. dium not colored.

Potato plug: Growth at first very slight, but after 45 hours develops into a yellowish-gray continuous thick smear which later turns brown, with a white aerial mycelium covering the growth. Medium not colored.

Source: Isolated once from the upland California soil.

\section{Habitat: Soil.}

34. Streptomyces griseus (Krainsky) comb. nou. (Actinomyces griseus Krainsky, Cent. f. Bakt., II Abt., 41, 1914, 662.) From M. L. griseus, gray.

Branching filaments; a few spirals have been observed. Conidia rod-shaped to short cylindrical, 0.8 by 0.8 to 1.7 mierons . Aerial mycelium greenish-gray.

Gelatin stab : Greenish-yellow or crcamcolored surface growth with brownish tinge. Rapid liquefaction.

Synthetic agar: Thin, colorless, spreading growth, becoming olive buff. Aerial mycelium thick, powdery, water-green.

Starch agar: Thin, spreading, transparent growth.

Glucose agar: Growth elevated in center, radiate, cream-colored to orange, erose margin.

Plain agar: Abundant, cream-colored, almost transparent growth.

Glucose broth: Abundant, yellowish pellicle with greenish tinge, much folded.

Litmus milk: Cream-colored ring; co- agulated with rapid peptonization, becoming alkaline.

Potato: Yellowish, wrinkled growth.

Nitrites produced from nitrates.

The pigment formed is not soluble.

Starch is hydrolyzed.

Aerobic.

Optimum temperature $37^{\circ} \mathrm{C}$.

Different strains of this organism proluce different antibiotics. One of these, streptomycin, was isolated in erystalline form. It is active against a large number of bacteria and actinomycetes, but not against fungi and viruses. It is not very toxic to animals, and has found extensive application in the treatment of various diseases, mostly caused by Gram-negative bacteria and certain forms of tuberculosis.

Source: Garden soil. .

Habitat: Soil.

35. Streptomyces griseoflavus (Kirainsky) comb. nov. (Actinomyces griseoflarus Krainsky, Cent. f. Bakt., II Abt., 41, 1914, 662.) From M. L. griseus, gray and Latin flavus, yellow.

Conidia oval, 1.2 microns.

Gelatin colonies : Yellowish. Concentric rings.

Gelatin stab : Rapidly liquefied.

Plain agar: Colonies yellowish, with white aerial mycelium.

Ca-malate agar : Large colonies covered with yellow to greenish-gray aerial mycelium.

Glucose agar: White aerial mycelium is slowly formed.

Starch agar: White aerial mycelium.

Glucose broth: Flaky growth.

Litmus milk: Peptonized.

Potato: Yellowish growth, aerial mycelium gray .

Nitrites produced from nitrates.

Weakly diastatic. Acts on esculin.

Grows well on cellulose.

Aerobic.

Optimum temperature $35^{\circ} \mathrm{C}$.

Habitat: Soil. 
36. Streptomyces albidoflavus (Rossi Doria) comb. nov. (Streptotrix (sic) albido-flava Rossi Doria, Ann. d. Ist. d'Ig. sper. d. Univ. di Roma, 1, 1891, 407; Actinomyces albido-flavus Gasperini, ibid., 2, 1892, 222; Streptothrix albido Chester, Man. Determ. Bact., 1901, 365; Cladothrix albido-flava Macé, Traité Pratique de Bact., 4th ed., 1901, 1097; Nocardia albida Chalmers and Christopherson, Ann. Trop. Med. and Parasit., 10, 1916, 271.) From Latin albidus, white and flavus, yellow.

Description from Duché, Encyclopédie Mycologique, Paris, 6, 1934, 294.

Gelatin: Punctiform colonies with white aerial mycelium on surface of liquid; no soluble pigment; rapid liquefaction.

Synthetic asparagine agar: Growth becomes rapidly covered with white aerial mycelium, later becoming whitishyellow; brown on reverse side; yellowish soluble pigment.

Peptone agar: Cream-colored growth covered with fine white aerial mycelium; yellow soluble pigment.

Tyrosine agar: Fine growth with orange-yellow on reverse side; medium becomes colored yellowish to yellowishrose.

Synthetic asparagine solution: Long branching filaments, 0.6 micron in diameter. Thicker aerial mycelium producing irregular spores; flaky growth dropping to bottom of tube. Surface growth becomes covered with yellowishwhite aerial mycelium; brownish on reverse side; soluble pigment yellowish.

Peptone solution: Rapid, much folded growth, partly covered with white mycelium on surface of medium; soluble yellow-ochre pigment.

Milk: Rapid growth becoming covered with whitish aerial my celium; never fully covering the surface; no coagulation; peptonization begins slowly and is completed in 13 days, liquid becoming colored yellowish-orange.
Coagulated serum: Crean-colored growth of surface becoming covered with white aerial mycelium; rapid liquefaction of serum.

Starch medium: Cream-colored growth rapidly colored with yellow aerial mycelium; after 20 days growth becomes much folded; greenish on reverse side; slightly amber color in medium.

This strain is closely related to Streptomyces albus. Develops poorly on Czapek's medium without asparagine.

Source: From dust.

37. Streptomyces poolensis (Taubenhaus) comb. nov. (Actinomyces poolensis Taubenhaus, Jour. Agr. Res., 13, 1918, 446.) Named for Prof. R. F. Poole, plant pathologist.

Description from Waksman, Soil Sci., 8, 1919, 140.

Fine, branching mycelium; spirals usually not seen. Conidia oval to elliptical.

Gelatin stab: Liquefied, with small, brownish flakes in fluid.

Synthetic agar: Thin, colorless, spreading growth. Aerial mycelium white to gray.

Starch agar: Restricted, cream-colored growth.

Glucose agar: Growth abundant, light brown, glossy, raised center, entire.

Plain agar: Yellowish, translucent growth.

Glucose broth : Thin, brownish ring.

Litmus milk: Brownish ring; coagulated; peptonized, with strongly alkaline reaction.

Potato: Thin, reddish-brown; medium becoming purplish.

Nitrites produced from nitrates.

Faint trace of soluble brown pigment.

Starch not hydrolyzed.

Aerobic.

Optimum temperature $37^{\circ} \mathrm{C}$.

Source: Associated with disease of sweet potato. 
38. Streptomyces olivaceus (Waksman) comb. nov. (Actinomyces 206, Waksman, Soil Science, 7, 1919, 117; Actinomyces olivaceus Waksman, in Manual, 1st ed., 1923, 354.) From Latin, olive-colored.

Small clumps, with straight and branching hyphae. No spirals on most media. Conidia spherical and oval, 0.9 to 1.1 by 0.9 to 2.0 microns.

Gelatin stab: Liquefied with creamcolored, flaky, yellow sediment.

Synthetic agar: Growth abundant, spreading, developing deep into medium, yellow to olive-ochre, reverse yellow to almost black. Aerial mycelium mousegray to light drab.

Starch agar: Thin, yellowish-green, spreading growth.

Glucose agar: Growth abundant, restricted, entire, center raised.

Plain agar: White, glistening growth.

Glucose broth: Sulfur-yellow ring.

Litmus milk: Faint, pinkish growth; coagulated; peptonized, becoming alkaline.

Potato: Growth abundant, much wrinkled, elevated, gray, turning sulfuryellow on edge.

Nitrites produced from nitrates.

The pigment formed is not soluble.

Starch is hydrolyzed.

Aerobic.

Optimum temperature $25^{\circ} \mathrm{C}$.

Habitat: Soil.

39. Streptomyces lieskei (Duché) comb. nov. (Actinomyces lieskei Duché, Encyclopédie Mycologique, Paris, 6, 1934, 289.) Named for Prof. Lieske of Leipzig.

Gelatin: Cream-colored growth becoming covered with white aerial mycelium; no soluble pigment. Rapid liquefaction.

Plain agar: Cream-colored growth becoming covered with white aerial mycelium; yellowish soluble pigment.

Synthetic agar: Cream-colored growth with delayed white aerial mycelium growing from the edge toward the center; mycelium later yellowish. Reverse of growth yellowish to green. Dirty yellow to yellow-green soluble pigment.

Synthetic solution: Long branching filaments 0.7 micron in diameter. Yellowish-white aerial mycelium does not readily produce spores; flakes drop to the bottom of the tube.

Peptone solution: Cream-colored colonies on surface with flakes in the liquid dropping to the bottom of the tube. Liquid becomes yellowish in color.

Tyrosine medium: Rapid growth on surface with whitish-yellow aerial mycelium; yellowish to orange-yellow soluble pigment.

Milk: Cream-colored growth; colorless on reverse side; no aerial mycelium. Peptonization without coagulation. After 20 days the whole milk becomes a clear yellowish liquid.

Coagulated serum: Clear-colored growth. Rapid liquefaction.

Culture related to Streptomyces alboflams and Streptomyces albidoflaws.

40. Streptomyces microflavus (Krainsky) comb. nov. (Actinomyces microflavus Krainsky, Cent. f. Bakt.; II Abt., 41, 1914, 662; Micromonospora microflava Duché, Encyclopédie Mycologique, Paris, 6, 1934, 29.) From Greek micrus, small, and Latin flavus, yellow.

Conidia large, spherical to rod-shaped, of ten in pairs or chains, 2.0 by 2.0 to 5.0 microns.

Gelatin colonies: Small, yellow.

Gelatin stab: Liquefied.

Plain agar: Yellow colonies, with roseyellow aerial mycelium in 3 to 4 weeks.

Ca-malate agar: Minute yellow colonies. No aerial mycelium.

Glucose agar : A rose-yellow aerial mycelium develops in about 12 days.

Starch agar: Same as on glucose agar.

Glucose broth : Small spherical colonies in depth.

Litmus milk: Peptonized.

Potato: Yellow growth. No aerial mycelium.

Nitrites produced from nitrates. 
Strongly diastatic.

Scant growth on cellulose.

Starch is hydrolyzed.

Aerobic.

Optimum temperature $25^{\circ} \mathrm{C}$.

Habitat: Soil.

41. Streptomyces cacaoi (Waksman) comb. nov. (Actinomyces cacaoi Waksman, in Bunting, Ann. Appl. Biol., 19, 1932, 515.) Named for the chocolate tree (Theobroma cacao).

Long aerial mycelium with considerable spiral formation; the spirals are long and open, not compact.

Gelatin: Flocculent growth. Jo aerial mycelium. Rapid liquefaction. No pigment production.

Nutrient agar: Brown-colored growth covered with tiny patches of ivorycolored aerial mycelium.

Glucose agar: Thin yellowish growth, later turning reddish-brown; no soluble pigment; light gray to mouse-gray mycelium, with white edge. Typical odor of streptomyces.

Czapek's agar: Same as on glucose agar

Potato: Abundant brownish growth with white to mouse-gray aerial mycelium.

Biochemical characteristics: Strong proteolytic enzymes acting on casein and gelatin; strong diastatic action, no sugar or dextrin left in 1 per cent starch solution after a few days. Limited reduction of nitrate.

Source: Three strains isolated from cacao beans in Nigeria. There were slight differences among the three strains; the above description is of Strain $I$.

42. Streptomyces novaecaesareae nom. nov. (Actinomyces violaceus-caeseri Walisman and Curtis, Soil Science, 1, 1916, 111.) From Nova Caesarea, Latin name for the State of New Jersey.

Filaments with both straight and spiral aerial hyphae;spirals dextrorse. Conidia oval to elongate.

Gelatin stab: Small, cream-colored surface colonies with slow liquefaction.
Synthetic agar: Growth gray, becoming bluish, glossy, much wrinkled. Aerial mycelium appears late; white.

Starch agar: Restricted, circular, blueish-violet colonies.

Glucose agar: Restricted, gray growth, becoming red.

Plain agar: Thin, cream-colored growth.

Glucose broth: Fine, colorless, flaky sediment.

Litmus milk: Gray ring; coagulated; slow peptonization, becoming faintly alkaline.

Potato: Growth cream-colored, wrinkled, turning yellowish.

Nitrites produced from nitrates.

Soluble purple pigment formed.

Starch is hydrolyzed.

Aerobic.

Optimum temperature $37^{\circ} \mathrm{C}$.

Source: Isolated once from upland California soil.

Habitat: Soil.

43. Streptomyces exfoliatus (Waksman and Curtis) comb. nov. (Actinomyces exfolialus Waksman and Curtis, Soil Science, 1, 1916, 116.) From Latin, stripped of foliage.

Slightly wavy filaments with tendency to form spirals. Conidia oval, 1.0 to 1.5 by 1.2 to 1.8 microns.

Gelatin stab: Cream-colored surface growth. Liquefied.

Synthetic agar: Growth colorless, becoming brown, smooth, glossy. Aerial mycelium in white patches over surface.

Starch agar: Restricted, gray growth, becoming brown.

Plain agar: Grows only in depth of medium.

Glucose broth: Small, grayish colonies in depth.

Litmus milk: Cream-colored ring, soft coagulum in 12 days; slow peptonization, becoming strongly alkaline.

Potato: Growth somewhat wrinkled, gray, becoming brown.

Nitrites produced from nitrates.

Brown, soluble pigment formed. 
Starch is hydrolyzed.

Aerobic.

Optimum temperature $37^{\circ} \mathrm{C}$.

Source: Isolated several times from adobe and upland soils in California.

Habitat: Soil.

\section{Streptomyces gelaticus (Waks-} man) comb. nov. (Actinomyces 104, Waksman, Soil Science, 8, 1919, 165; Actinomyces gelaticus Waksman, in Manual, 1st ed., 1923, 356.) From M. L. gelaticus, gelatinous.

Branching mycelium with open spirals.

Gelatin stab: Liquefied with creamcolored flaky sediment.

Synthetic agar: Growth colorless, spreading, chiefly deep into the medium. Aerial mycelium thin, white, turning grayish.

Starch agar: Thin, spreading, creamcolored growth.

Glucose agar: Abundant, spreading, white growth.

Plain agar: Wrinkled, cream-colored growth only on the surface.

Glucose broth: Thin, cream-colored pellicle; slight flaky sediment.

Litmus milk : Pinkish ring; coagulated; peptonized with distinctly alkaline reaction.

Potato: Growth abundant, much wrinkled, greenish, becoming black with yellowish margin.

Nitrates show slight reduction to nitrites.

Soluble brown pigment formed.

Starch is hydrolyzed.

Aerobic.

Optimum temperature $25^{\circ} \mathrm{C}$

Habitat: Soil.

45. Streptomyces rutgersensis (Waksman and Curtis) comb. nov. (Actinomyces rutgersensis Waksman and Curtis, Soil Seience, 1, 1916, 123.) Named for Rutgers University, New Brunswick, New Jersey.

Branching filaments with abundant open and closed spirals; hyphae fine, long, branching. Conidia spherical and oval,
1.0 to 1.2 microns, with tendency to bipolar staining.

Gelatin stab: Cream-colored, spreading surface growth. Liquefied.

Synthetic agar: Growth thin, colorless, spreading, becoming brownish to almost black. Aerial mycelium thin, white, becoming dull-gray .

Starch agar: Gray, spreading growth.

Glucose agar : Abundant, brown mycelium, becoming black with cream-colored margin.

Plain agar: Thin, wrinkled, creamcolored growth.

Litmus milk: Cream-colored ring; coagulated; slow peptonization, becoming alkaline.

Potato: Abundant, white-gray, much folded growth.

Nitrites produced from nitrates.

The pigment formed is not soluble.

Stareh is hydrolyzed.

Aerobic.

Optimum temperature $37^{\circ} \mathrm{C}$.

Source: Isolated many times from a variety of soils.

Habitat: Common in soil.

46. Streptomyces lipmanii (Waksman and Curtis) comb. nov. (Actinomyces lipmanii Waksman and Curtis, Soil Science, 1, 1916, 123.) Named for Prof. J. G. Lipman, New Jersey Agricultural Experiment Station.

Straight, branching mycelium and hyphae. Conidia oval, 0.8 to 1.1 by 1.0 to 1.5 microns.

Gelatin stab: Liquefied with creamcolored, flaky sediment.

Synthetic agar: Growth abundant, raised, colorless, becoming light brown and wrinkled. Aerial mycelium white, turning gray .

Starch agar: Transparent growth, becoming dark with age.

Glucose agar: Light yellow, irregular, spreading growth

Plain agar: Yellow, glossy, radiately wrinkled growth.

Glucose broth: White ring, with abundant, colorless flaky sediment. 
Litmus milk: Cream-colored ring; coagulated; peptonization with alkaline reaction.

Potato: Abundant, cream-colored, wrinkled growth.

Nitrites produced from nitrates.

The pigment formed is not soluble.

Starch is hydrolyzed.

Aerobic.

Optimum temperature $25^{\circ} \mathrm{C}$.

Source: Isolated many times from a variety of soils.

Habitat: Common in soil.

47. Streptomyces halstedii (Waksman and Curtis) comb. nov. (Actinomyces halstedii Waksman and Curtis, Soil Science, 1, 1916, 124.) Named for a person.

Branching mycelium and hyphae with close spirals. Conidia oval or rodshaped, 1.0 to 1.2 by 1.2 to 1.8 mierons.

Gelatin stab: Liquefied, with small, cream-colored masses in bottom of tube.

Synthetic agar: Growth abundant, heavy, spreading, raised, light, becoming dark, almost black. Aerial mycelium white, turning dull-gray.

Starch agar: Abundant, brownish, glossy growth.

Glucose agar : Growth spreading, colorless, wrinkled, center elevated, edge lichenoid, becoming brown.

Plain agar: Restricted, wrinkled, cream-colored growth.

Glucose broth : Small, colorless colonies in bottom of tube.

Litmus milk: Cream-colored ring; coagulated; peptonized, becoming alkaline.

Potato: Growth abundant, moist, wrinkled, cream-colored with green tinge.

Nitrites produced from nitrates.

The pigment formed is not soluble.

Starch is hydrolyzed.

Optimum temperature $37^{\circ} \mathrm{C}$.

Aerobic.

Source: Isolated many times from the deeper soil layers.

Habitat: Common in subsoil.

48. Streptomyces hygroscopicus (Jen- sen) comb. nov. (Actinomyces hygroscopicus Jensen, Proc. Linn. Soc. New So. Wales, 56, 1931, 257.) From Greek, hygroscopic.

Hyphae of vegetative mycelium 0.6 to 0.8 micron in diameter. Aerial hyphae long, tangled, branched, 0.8 to 1.0 micron in diameter; spirals numerous, sinistrorse, narrow, usually short, only 1 or 2 turns, closed, typically situated as dense clusters on the main stems of the aerial hyphae. Conidia oval, 0.8 to 1.0 by 1.0 to 1.2 microns.

Gelatin: Slow liquefaction. No pigment produced.

Nutrient agar: Good growth. Vegetative mycelium raised, wrinkled, glossy, cream-colored; later yellowish-gray with yellowish-brown reverse. Occasionally a scant white aerial mycelium.

Sucrose agar: Good to abundant growth. Vegetative mycelium heavy, superficially spreading, folded, glossy surface, white to cream-colored, later sulfur-yellow to yellowish-gray, with golden to light orange reverse. Soluble pigment of the same color. Aerial mycelium scant, thin, white or absent.

Glucose agar: Good growth. Vegetative mycelium superficially spreading, surface granulated, cream-colored to straw-yellow, later dull chrome-yellow to brownish-orange. Aerial mycelium thin, smooth, dusty, white to pale yellowishgray, after 1 or 2 weeks more or less abundantly interspersed with small, moist, dark violet-gray to brownish patches which gradually spread over the whole surface. Light yellow soluble pigment.

Potato: Fair growth. Vegetative mycelium raised, wrinkled, cream-colored, later yellowish-gray to dull brownish. Aerial mycelium absent or trace of white.

Milk: Completely digested in 3 to 4 weeks at $30^{\circ} \mathrm{C}$ without any previous coagulation. The reaction becomes faintly acid ( $\mathrm{pH} 6.0$ or less).

Nitrates not reduced with sucrose as source of energy.

Sucrose is inverted. 
Starch is hydrolyzed.

Cellulose is decomposed readily by some strains.

Distinctive character: In this species, the aerial mycelium (which in other actinomycetes is strikingly hydrophobic) on certain media (glucose or glycerol asparagine agar) becomes moistened and exhibits dark, glistening patches. These patches, when touched with a needle, prove to be a moist, smeary mass of spores. This characteristic feature is not equally distinct in all strains.

Source: Seven strains isolated from soils.

Habitat: Soil.

49. Streptomyces fradiae (Waksman and Curtis) comb. nov. (Actinomyces fradii Waksman and Curtis, Soil Science, 1, 1916, 125.) From the name of a person.

Straight, branching filaments and hyphae. No spirals. Conidia rod-or ovalshaped, 0.5 by 0.7 to 1.25 microns.

Gelatin stab : Cream-colored to brownish, dense growth on liquid medium.

Synthetic agar: Smooth, spreading, colorless growth. Aerial mycelium thick, cottony mass covering surface, sea-shell pink.

Starch agar: Spreading, colorless growth.

Glucose agar: Growth restricted, glossy, buff-colored, lichenoid margin.

Plain agar : Growth yellowish, becoming orange-yellow, restricted.

Glucose broth: Dense, narrow, orangecolored ring; abundant, flaky, colorless sediment.

Litmus milk: Faint, cream-colored ring; coagulated; peptonized, becoming alkaline.

Potato: Restricted, orange-colored growth.

Nitrites not produced from nitrates. The pigment formed is not soluble.

Starch is hydrolyzed.

Aerobic.

Optimum temperature $25^{\circ} \mathrm{C}$.
Source: Isolated once from adobe soil in California.

Habitat: Soil.

50. Streptomyces alboflavus (Waksman and Curtis) comb. nov. (Actinomyces alboflavus Waksman and Curtis, Soil Science, 1, 1916, 120.) From Latin albus, white and flavus, yellow.

Straight, branching mycelium, with very little tendency to form spirals. Very few oval-shaped conidia formed.

Gelatin stab: Abundant, colorless surface growth. Liquefaction occurs in 35 days.

Synthetic agar: Growth glossy, colorless, spreading, becoming yellowish. Aerial mycelium white, powdery, with yellow tinge.

Starch agar: Thin, yellowish, spreading growth.

Glucose agar: Growth restricted, much-folded, creamy with sulfur-yellow surface.

Plain agar: Restricted, cream-colored growth.

Glucose broth: White, cylindrical colonies on surface, later flaky mass in bottom of tube.

Litmus milk : Pinkish ring. No coagulation. Peptonized, becoming alkaline.

Potato: Moist, cream-colored, wrinkled growth.

Nitrites produced from nitrates.

The pigment formed is not soluble.

Starch is hydrolyzed.

Aerobic.

Optimum temperature $37^{\circ} \mathrm{C}$.

Source: Isolated once from orchard soil. Habitat: Soil.

51. Streptomyces albosporeus (Krainsky) comb. nov. (Actinomyces albosporeus Krainsky, Cent. f. Bakt., II Abt., 41, 1914, 649; Nocardia albosporea Chalmers and Christopherson, Ann. Trop. Med. and Parasit., 10, 1916, 268; Waksman and Curtis, Soil Science, 1, 1916, 99.) From Latin albus, white and Greek spora, spore. 
Straight, branching filaments with straight, branching hyphae, and occasional spirals. Conidia spherical or oval, 0.8 to 1.2 by 1.0 to 1.8 microns.

Gelatin stab: Growth yellow, changing to red, with hyaline margin. Liquefaction in 35 days.

Synthetic agar: Growth spreading, colorless with pink center, becoming brownish. Aerial mycelium white at first, later covering the surface.

Starch agar: Growth thin, spreading, transparent, with red tinge.

Glucose agar: Growth spreading, red, wrinkled, radiate, entire.

Plain agar: Minute, cream-colored colonies.

Glucose broth: Pinkish ring.

Litmus milk: Scant, pink ring. No coagulation. No peptonization.

Potato: Growth thin, spreading, wrinkled, gray, becoming brown with greenish tinge.

Nitrites produced from nitrates.

The pigment formed is not soluble.

Starch is hydrolyzed.

Aerobic.

Optimum temperature $37^{\circ} \mathrm{C}$.

Habitat: Soil.

52. Streptomyces flocculus (Duché) comb. nov. (Actinomyces flocculus Duché, Encyclopédie Mycologique, Paris, 6, 1934, 300.) From Latin, somewhat woolly, referring to the appearance of the aerial mycelium.

Gelatin: Very limited growth. Slow liquefaction.

Asparagine glucose agar: Weak growth; limited cream-colored colonies hardly raised above the surface of the medium; occasionally abundant grow th is produced with white aerial mycelium and colorless on reverse side.

Czapek's agar: Cream-colored growth, later covered with white aerial mycelium; no soluble pigment.

Peptone agar: Cream-colored growth, later covered with white aerial mycelium; no soluble pigment.
Asparagine glucose solution: Branching immersed filaments, 0.8 micron in diameter; aerial mycelium 1.0 by 1.2 microns with numerous conidia; flakes settle to the bottom of the tube.

Peptone solution: Pointed colonies; cream-colored on surface of medium.

Tyrosine medium: Whitish growth without any pigment.

Milk: Rose-colored growth; slow peptonization.

Potato: Punctiform growth covered with white aerial mycelium; faint yellowish pigment.

Coagulated serum: Cream-colored growth; fine white aerial mycelium; slow liquefaction of serum.

Source: Culture obtained from $\mathrm{Mr}$. Malençon, an inspector in Morocco.

53. Streptomyces melanosporeus (Krainsky) comb. nov. (Actinomyces melanosporeus Krainsky, Cent. f. Bakt., II Abt., 41, 1914, 662; Nocardia melanosporea Chalmers and Christopherson, Ann. Trop. Med. and Parasit., 10, 1916, 26s.) From Greek melas, black and spora, spore.

Conidia almost spherical, 1.2 microns in diameter.

Gelatin colony: Small, reddish colonies.

Gelatin stab: Liquefied.

Ca-malate agar: Colonies red, with black aerial mycelium.

Glucose agar: Same as on Ca-malate agar.

Starch agar: Same as on Ca-malate agar.

Glucose broth: Flaky, orange-red colonies adherent to glass.

Litmus milk: Peptonized.

Potato: Red colonies with black aerial mycelium.

Nitrites produced from nitrates.

Weakly diastatic.

Grows well on cellulose. Cellulose is decomposed.

Aerobic. 
Optimum temperature $25^{\circ} \mathrm{C}$.

Habitat: Soil.

\section{Streptomyces melanocyclus (Mer-} ker) comb. nov. (Hicrocuccus melanocyclus Merker, Cent. f. Bakt., II Abt.. 31, 1911, 589; Aclinomyces melanocyclus Krainsky, Cont. f. Bakt., II Abt., 41, 1914, 662; Nocardia melanocycla Chalmers and Christcpherson, Ann. Trop. Med. and Parasit., 10, 1916. 268.) From Greek melas, black and cyclus, eircle.

Conidia almost spherical, 0.9 micron in diameter.

Gelatin colonies: Growth poor.

Gelatin stab: Rapid liquefaction.

Ca-malate agar: Colonies small, flat, orange-red. Aerial mycelium black, occurring along the edges.

Glucose broth: Same as on Ca-malate agar

Starch agar: Same as on Ca-malate agar.

Glucose broth: Colorless, spherical colonies.

Litmus milk: Peptonized.

Nitrites produced from nitrates.

Good diastatic action.

Cellulose is decomposed.

Aerobic.

Optimum temperature $25^{\circ} \mathrm{C}$.

Habitat: Soil.

55. Streptomyces acidophilus (Jensen) comb. nov. (Actinomyces acidophilus Jensen, Soil Sci., 25, 1928, 226.) From Greek, acid-loving.

Vegetative mycelium profusely branched, hyphae 0.6 to 0.8 micron in diameter with homogeneous protoplasm and no visible septa. Aerial mycelium with hyphae 1.0 to 1.2 microns in diameter, somewhat branched, forming either very few or very numerous sinistrorse spirals. Oval conidia 1.0 to 1.2 by 1.2 to 1.5 microns

Gelatin: After 10 days growth very scant, thin, colorless, semi-transparent. Slow liquefaction.

Nutrient agar: No growth.

Gluense agar: Good growth at $25^{\circ} \mathrm{C}$. Substratum mycelium raised, somewhat wrinkled, colorless in young cultures. Aerial mycelium thin, white at first, later gray or yellowish-brown.

Starch agar: Good growth at $25^{\circ} \mathrm{C}$. Substratum mycelium flat, smooth, colorless. Aerial mycelium abundant, smooth, white.

Czapek's agar: No growth.

Plain broth: No growth.

Milk: No growth.

Potato: Growth good, raised, folded. No discoloration.

Nitrites not produced from nitrates except a trace in two strains.

Diastatic.

Weakly proteolytic.

Inversion of sucrose: Negative.

Distinctive character: The ability to live in acid media only.

Source: Four strains isolated from three acid humus soils.

Habitat: Acid humus soils.

56. Streptomyces rubescens (Jarach) comb. nov. (Streptothrix rubescens Jarach, Boll. Sez. Ital. Soc. Intern. Microb., 3, 1931, 43.) From Latin rubescens, becoming red.

Gelatin: No liquefaction; limited nonpigmented growth.

Glucose agar: Large number of small round colonies raised in the center and growing together, as well as deep into the medium; of a whitish opalescent color.

Czapek's agar: Poor growth, becoming pigmented salmon-red, edge entire.

Milk agar medium : Rose-coral-colored, thin growth with edge entire.

Broth: Minute flakes, the liquid later becoming reddish-colored.

Milk: No coagulation and no digestion; slight red coloration of milk.

Potato plug: Reddish growth, not extensive; opalescent surface.

Source: From soil.

57. Streptomyces thermophilus (Gilbert) comb. now. (Actinomyces thermophilus Gilhert, Ztschr. f. Hyg., 47, 1904, 383 ; not Actinomyces thermophilus Berestnew, Inaug. Diss., Moskow, 1897; 
Nocardia thermophila Chalmers and Christopherson, Ann. Trop. Med. and Parasit., 10, 1916, 271.) From Greek thermus, heat and philus, loving.

Description from Waksman, Cmbreit and Gordon, Soil Sci., 47, 1939, 49.

Hyphae straight, conidia formed.

Gelatin: Liquefaction.

Czapek's agar: At $28^{\circ} \mathrm{C}$, deep colorless growth, thin white aerial mycelium; no soluble pigment.

Starch agar: Yellowish growth with white-gray, powdery aerial mycelium.

Milk: Proteolysis.

Potato plug: Yellowish growth with no aerial mycelium, the plug usually being colored brown.

Starch is hydrolyzed.

No pigment produced on nutrient agar or gelatin.

Temperature relations : Optimum $50^{\circ} \mathrm{C}$. Good growth at $28^{\circ} \mathrm{C}$. Usually no growth at $60^{\circ} \mathrm{C}$. Some strains are incapable of growing at $28^{\circ} \mathrm{C}$, whereas others seem to grow well even at $65^{\circ} \mathrm{C}$.

Aerobic.

Habitat: Soil, hay, composts.

58. Streptomyces thermofuscus (Waksman, Umbreit and Gordon) comb. nor. (Actinomyces thermofuscus Waksman, Umbreit and Gordon, Soil Sci., 47, 1939, 49.) From Greek thermus, heat and Latin fuscus, dark. Presumably derived to mean heat-Ioving and dark in color.

Hyphae spiral-shaped; conidia produced.

Gelatin: Liquefaction. At $50^{\circ} \mathrm{C}$, a grayish ring is produced and soluble pigment is formed. At $28^{\circ} \mathrm{C}$, growth with no soluble pigment.

Czapek's agar: Poor growth at $28^{\circ} \mathrm{C}$, deep-gray, with but little aerial mycelium. At $50^{\circ} \mathrm{C}$, growth dark to violet, with gray to lavender aerial mycelium and soluble brown pigment.

Milk: Proteolysis.

Potato: Abundant, dark-colored growth, no aerial mycelium, or few white patches, dark soluble pigment.
Starch is hydrolyzed.

Temperature relations: Good growth at $50^{\circ}$ and $60^{\circ} \mathrm{C}$. Will grow at $65^{\circ} \mathrm{C}$. Faint growth at $28^{\circ} \mathrm{C}$.

Aerobic.

Distinctive characters: This species is distinguished from Streptomyces thermophilus by the brown-colored aerial mycelium on synthetic media, spiral-shaped hyphae, and ability to grow readily at $65^{\circ} \mathrm{C}$.

Habitat: Soils and composts.

59. Streptomyces scabies (Thaxter) comb. nov. (Oospora scabies Thaxter, Ann. Rept. Conn. Agr. Exp. Sta., 1891, 153; Actinomyces scabies Gussow, Science, N. S. 39, 1914, 431.) From Latin scabies, scab.

Wavy or slightly curved mycelium, with long branched aerial hyphae, showing a few spirals. Conidia more or less cylindrical, 0.8 to 1.0 by 1.2 to $1.5 \mathrm{mi}$ crons.

Gelatin stab: Cream-colored surface growth, becoming brown. Slow liquefaction.

Synthetic agar: Abundant, creamcolored, wrinkled, raised growth. Aerial mycelium white, scarce.

Starch agar: Thin, transparent, spreading growth.

Glucose agar: Restricted, folded, cream-colored, entire growth.

Plain agar: Circular, entire colonies, smooth, becoming raised, lichenoid, wrinkled, white to straw-colored, opalescent to opaque.

Glucose broth: Ring in form of small colonies, settling to the bottom.

Litmus milk: Brown ring with greenish tinge; coagulated; peptonized with alkaline reaction.

Potato: Gray, opalescent growth, becoming black, wrinkled.

Nitrites produced from nitrates.

Brown soluble pigment formed.

Starch is hydrolyzed.

Optimum temperature $37^{\circ} \mathrm{C}$.

Aerobic.

The potato scab organism, like other 
acid-fast organisms, can be selectively impregnated with carbol-auromin and when exposed to ultraviolet radiation fluoresces bright yellow. This technic confirms Lutman's conclusion that the hyphae are intercellular and grow within the middle lamellae (Richards, Stain Tech., 18, 1943, 91-94).

Source: Isolated from potato scab lesions.

Habitat: Cause of potato scab; found in soil.

60. Streptomyces ipomoea (Person and Martin) comb. nov. (Actinomyces ipomoea Person and Martin, Phytopath., 30, 1940, 313.) From M. L. Ipomoea, a generic name.

Conidia on glucose-casein agar: Oval to elliptical, 0.9 to 1.3 by 1.3 to 1.8 microns.

Gelatin : After 25 days at $20^{\circ} \mathrm{C}$, scanty growth, no aerial mycelium; no soluble pigment; liquefaction.

Synthetic agar: Abundant growth, mostly on surface of medium, moderately wrinkled, olive-yellow.

Nutrient agar: Moderate growth in the form of small, shiny, crinkled colonies both on the surface and imbedded in the medium, silver-colored.

Starch agar: Growth moderate, smooth, deep in medium, ivory-colored. Aerial mycelium white with patches of bluishgreen. No soluble pigment. Complete hydrolysis after 12 days.

Milk: Growth in form of ring; hydrolysis, without visible coagulation.

Potato: Growth moderate, light brown, shiny, wrinkled. No aerial mycelium. No soluble pigment.

Nitrites are produced from nitrates.

Starch is hydrolyzed.

No growth on cellulose.

Source: From diseased sweet-potato (Ipomoea $s p$.) tubers and small rootlets from several localities in Louisiana.

\section{Streptomyces fordii (Erikson)} comb. nov. (Actinomyces fordii Erikson, Med. Res. Counc. Spec. Rept. Ser. 203, 1935, 15 and 36.) Presumably named for the surgeon who first secured the culture.

Mycelium: Filaments of medium length, no spirals or markedly wavy branches. Short, straight, sparse aerial mycelium. Small oval conidia on potato agar and starch agar.

Gelatin: No visible growth, slight softening in 20 days; half-liquefied after 40 days.

Agar: Small, creamy-golden, ringshaped colonies, and heaped-up patches, becoming golden-brown in color and convoluted.

Glycerol agar: Extensive, goldenbrown, convoluted, thin layer.

Serum agar : Golden-brown ring-shaped and coiled smooth colonies; no liquefaction.

Ca-agar: Yellow, scale-like closely adherent colonies; scattered white aerial mycelium.

Blood agar: Innumerable small yellowish ring-shaped colonies; no hemolysis.

Broth: Few flakes at first; later abundant coherent puffball growth.

Synthetic sucrose solution: Moderate sediment of minute round white colonies.

Synthetic glycerol solution: Light white fluffy colonies, minute and in clusters.

Inspissated serum: Innumerable colorless piupoint colonies; scant white aerial mycelium; after 15 days colonies large, hollow on reverse side; margin depressed; no liquefaction.

Dorset's egg medium: Minute, creamcolored, elevated colonies, becoming golden-brown, raised, convoluted.

Milk: Coagulated; brownish surface ring.

Litmus milk: No change in reaction.

Potato plug: Yellowish growth in thin line, terminal portion tending to be piled up, scant white acrial mycelium at top of slant; after 12 days, growth abundant, golden-brown, confluent, partly honeycombed, partly piled up.

Starch not hydrolyzed.

Tyrosine agar: Reaction negative.

Source: Human spleen in a case of acholuric jaundice. 
62. Streptomyces africanus (Pijper and Pullinger) comb. nov. (Nocardia africana Pijper and Pullinger, Jour. Trop. Med. and Hyg., 30, 1927, 153; Actinomyces africanus Nannizzi, in Pollaci, Tratt. Micopat. Umana, 4, 1934, 8.) From Latin Africanus, relating to Africa.

Description from Erikson, Med. Res Counc. Spec. Rept. Ser. 203, 1935, 18.

Unicellular branching mycelium forming small dense pink colonies with short straight sparse white aerial mycelium.

Gelatin: Irregular pink flakes; no liquefaction.

Agar: A few flat pink discoid colonies.

Glucose agar: Minute red discrete round colonies and piled up paler pink mass with thin white aerial mycelium.

Glycerol agar: After 2 weeks, small heaped-up colorless masses with pink tinge around the colorless colonies, margin depressed; after 3 weeks, abundant, piled up, pale pink growth.

Ca-agar: After 1 week, small, round, colorless colonies with red centers, margins submerged; after 2 weeks, growth bright cherry-red, confluent, with colorless margin.

Dorset's egg medium: Small colorless blister colonies, partly confluent; becoming wrinkled, depressed into medium; slight liquefaction.

Serum agar : Irregularly round, raised, wrinkled, colorless colonies; becoming dry, pink and flaky; later piled up, brownish, friable.

Inspissated serum: After one week, smooth, round, colorless colonies with submerged margin, in confluent patches pink and pitted into medium; after 2 weeks, medium broken up, slight liquefaction; after 3 weeks, liquid dried up, colonies umbilicated, raised, dry and friable.

Broth: Small pink colonies embedded in coherent flocculent mass.

Synthetic sucrose solution: Small pink granules in sediment after 1 week; colonies of medium size, coherent, after 3 weeks.

Potato agar: Bright red growth, small round colonies with colorless submerged margins, and piled up patches with stiff sparse white aerial mycelium.

Litmus milk: Bright red surface growth, liquid unchanged after one month; liquid opaque reddish-purple after 2 months; hydrolyzed, clear winered after 3 months.

Source: From a case of mycetoma of a foot in South Africa.

63. Streptomyces gallicus (Erikson) comb. nov. (Actinomyces gallicus Erikson, Med. Res. Counc. Spec. Rept. Ser. 203, 1935, 36.) From Latin gallicus, of the Gauls (French).

Description from Erikson (loc. cit., p. 24).

Mycelium shows lateral highly refractive bodies which appear almost identical with the singly situated spores found in Micromonospora chalceae.

Gelatin: Scant irregular pink growth; liquefaction very slow, only slight degree in 20 days.

Agar: A few transparent minute pink colonies; growth becomes partly confluent.

Glucose agar: No growth.

Glycerol agar: No growth.

Czapek's agar: No growth.

Coon's agar: Minute colorless to pinkish colonies.

Ca-agar: Glossy pink pinhead colonies.

Potato agar: Pale pink, moist, granular growth.

Serum agar: Pinpoint colonies, pink, shining.

Blood agar: Abundant growth, minute, discrete, round, pink colonies, some aggregated in confluent narrow bands. No hemolysis.

Dorset's egg medium: Minute colonies, becoming confluent, tangerine-colored.

Inspissated serum: Abundant, pink, membranous growth, becoming reddishbrown; later discrete colonies at margin, clear on reverse side. No liquefaction.

Broth : Pinkish flakes.

Synthetic sucrose solution: A few fine white flocculi. 
Synthetic glycerol solution: A few small round white colonies.

Milk: Coagulated; peptonized; yellowish-pink surface ring.

Litmus milk: No coagulation or peptonization; no change in color.

Potato plug: Very slow growth, a few minute translucent pink colonies after 16 days; after 21 days, considerable increase in number of colonies, still small and discrete. After 2 months, colonies 1 to $2 \mathrm{~mm}$ in diameter, bright coral, tending to be umbilicated and heaped up.

Tyrosine agar: Reaction negative.

Source: From blood culture in a case of Banti's disease.

64. Streptomyces pelletieri (Laveran) comb. nov. (Micrococcus pelletieri Laveran, Compt. rend. Soe. Biol., Paris, 61, 1906, 340; Oospora pelletieri Thiroux and Pelletier, Bull. Soc. path. exot., 5, 1912, 585; Nocardia pelletieri Thiroux, see Pinoy, Bull. Inst. Past., 11, 1913, 935; Discomyces pelletieri Brumpt, Précis de Parasitol., Paris, 2nd ed., 1913, 970; Actinomyces pelletieri Brumpt, ibid., 4 th ed., 1927, 1204.) Named for M. Pelletier who first isolated this species.

Description from Erikson, Med. Res. Council Spec. Rept. Ser. 203, 1935, 21.

Thiroux and Pelletier (Bull. Soc. path. exot., 5, 1912, 585) considered that their cultures resembled Nocarilia madurae, but they grew the organism only on Sabouraud's gelatin, on which it appeared in a constantly red, easily detachable form. Nocardia indica was regarded as identical by Pinoy, although in the original description by Laveran the organism was called Micrococcus pellefieri, owing to the fact that no mycelium was seen, merely coccoid bodies. Nocardia genesii Froes (Bull. Inst. Past., $29,1931,1158$ ) is described as closely allied, the distinction being founded upon the fact that the red grains were smaller in size and much more numerous, but no cultural details are given.

Mycelium composed of slender straight and not very long filaments, forming small dense pink colonies with a few short straight isolated aerial branches.

Gelatin: Slight liquefaction; few pink flakes; later almost completely liquefied.

Agar: Minute colorless colonies and piled up pale pink masses.

Glucose agar: Poor growth, a few minute pink colonies.

Glycerolagar : Poor growth, a few moist pink colonies.

Ca-agar : Colorless small colonies; after 1 week, confluent skin, pink, buckled; medium discolored later.

Coon's agar: Poor growth, creamcolored with pink center, mostly submerged.

Potato agar: Colorless blister colonies; after 3 weeks, colonies larger, showing concentric zones, submerged margins and occasional zone or tuft of white aerial mycelium, pinkish coloration.

Dorset's egg medium: Abundant, wrinkled, pink skin with small discrete colonies at margin in six days; later surface rough, mealy; considerable liquefaction in 17 days.

Serum agar: Moist cream-colored growth tending to be heaped up, discrete colonies at margin; becoming umbilicated.

Inspissated serum: Round, moist, colorless colonies.

Blood agar: At first a few pinhead, cream-colored colonies, no hemolysis; later colonies dense, button-shaped, with narrow fringed margin.

Broth: Small, minute, pink, clustered colonies.

Synthetic sucrose solution: Small, pink colonies in sediment; later minute colonies adhering to side of tube.

Milk: Soft curd; half-digested; peptonization complete in 20 days.

Litmus milk: Pink surface growth, semi-solid, no color change; after 20 days, coagulum cleared, liquid purple.

Potato plug: After one month growth sparse; yellowish-pink, irregularly piled up, portions with scant white aerial mycelium; after 6 months abundant highly piled up small rounded pink 
masses, scant white aerial mycelium persistent.

Source : From a case of crimson-grained mycetoma in Nigeria (E. C. Smith, Trans. R. Soe. Trop. Med. Hyg., 22, 192S, 157).

Habitat: Human infections so far as known.

\section{Streptomyces listeri (Erikson)} comb. nov. (Actinomyces listeri Erikson, Med. Res. Council Spec. Rept. Ser. 203, 1935, 36.) Named for Dr. Joseph Lister, the father of antiseptic surgery.

Description from Erikson (loc. cit., p. 23 ).

Long slender filaments, many loosely wavy, forming a dense spreading mycelium which rapidly grows into a membrane on most media. Aerial mycelium very slow and inconstant in appearance, short and straight, conidia oval.

Gelatin: Slight liquefaction; round white surface colonies; after 45 days, confluent skin, almost completely liquefied.

Agar: Smooth, round, moist, creamcolored, margin depressed, center elevated, closely adherent; becoming umbilicated, with a myceloid margin.

Glucose agar: Cream-colored, glistening, pinpoint colonies; later aggregated in convoluted skin.

Glycerul agar: Abundant, moist, cream-colored growth, colonies elevated, piled up; powdery white aerial mycelium . After 20 days, skin deeply buckler; colorless with exuded drops.

Ca-agar: Poor growth, a slight biscuitcolored membrane.

Potato agar: After one week, extensive growth, colorless submerged colonies, warted surface; dirty pink coloration after 2 weeks; scant white aerial mycelium after 4 months.

Dorset's egg medium: No growth.

Blood agar: Small, round, creamcolored colonies, smooth translucent surface; no hemolysis.

Serum agar: Small, irregular, moist, cream-colored colonies, tending to be heaped up; later somewhat transparent. Inspissated serum: Abundant growth, colorless shiny colonies, centrally elevated, becoming confluent.

Broth: Small, round, white colonies in sediment.

Glucose broth: Small, white, nodular colonies; later abundant floceuli.

Synthetic sucrose solution: Delicate white colonies in suspension and in sediment.

Litmus milk: Coagulation. No change in reaction.

Potato plug: Abundant, dull, brownish, wrinkled skin with white aerial mycelium; large, stellate, fluffy, white colonies in liquid at base.

Source: From human material. Strain from Lister Collection.

Habitat: From human infections so far as known.

66. Streptomyces upcottii (Erikson) comb. nov. (A new pathogenic form of Streptothrix, Gibson, Jour. Bact. and Path., 23, 1920, 357 ; Actinomyces upcottii Erikson, Med. Res. Council Spec. Rept. Ser. 203, 1935, 36.) Named for Dr. Harold Upcott, the surgeron who first secured the culture.

Description from Erikson (loc. cit., p. 22).

Filaments characteristically long, straight, much interwoven and ramified; typical unicellular mycelium, usually forming medium to large heavy cartilaginous colonies. Gibson states that the threads vary in thickness and show septa, but this has not been confirmed. A very slight transient aerial mycelium appeared on one agar slope, but this has not been repeated on any slide microculture on any medium. Slightly acidfast.

Gelatin: Abundant flocculent growth along streak, round eream-colored colonies on surface. Partly liquefied in 14 days; complete liquefaction in 2 months.

Agar: Smooth, shining, round, creamcolored colonies, margin submerged, 
scant white aerial mycelium in one week; colonies large (up to $10 \mathrm{~mm}$ in diameter), centers elevated, greenish tinge, very sparse aerial mycelium in two weeks; the aerial mycelium disappears and large radial grooves appear in most colonies in 3 weeks.

Glucose agar: Smooth, round, creamcolored colonies, margin depressed, centers elevated, hollow on reverse side; later a coherent membranous growth, piled up, yellowish.

Glycerol agar: Small, round, creamcolored, glistening colonies, heavy texture, margins submerged; later, colonies umbilicated, tending to be piled up; after 6 weeks, growth very much convoluted and raised, broad submerged margin, slightly reddish medium.

Coon's agar: Small, radiating, white colonies, growth mostly submerged.

Ca-agar: Small, colorless membranous growth with undulating margin; later, centrally depressed into medium.

Potato agar: Poor growth, small, colorless blister colonies, medium slightly discolored.

Dorset's egg medium: Round, flat, colorless, scale-like colonies, some marked by concentric rings and slightly hollowed in center; growth becomes yellow-brown.

Serum agar: Large colonies ( 3 to $4 \mathrm{~mm}$ in diameter), colorless, granular, centrally elevated, depressed at margin, resembling limpets.

Blood agar: Large drab heavily textured colonies; no aerial mycelium; no hemolysis.

Broth: Large coherent mass composed of fluffy colonies.

Synthetic sucrose solution: Fair growth, minute white colonies.

Carrot plug: Colorless, spreading, moist, wrinkled growth in six weeks; later a dull greenish-brown, moist, very much wrinkled and depressed skin.

Source: From the spleen in a case of acholuric jaundice.

Habitat: From human infections so far as known.
67. Streptomyces hortonensis (Erikson) comb. nov. (Actinomyces horton Erikson, Med. Res. Council Spec. Rept. Ser. 203, 1935, 36.) Named for the Horton War Hospital at Epsom, England from which the culture was obtained.

Description from Erikson (loc. cit., p. 22).

Typical germination into very slow growing unicellular mycelium composed of long slender straight branching filaments. Very sparse straight aerial mycelium produced only once on potato. Non-acid-fast.

Gelatin: Round cream-colored colonies on surface and a few $\mathrm{mm}$ below. No liquefaction.

Agar: Very slow growth, a few smooth cream-colored colled colonies in 19 days; after 2 months, liberal, irregular, convoluted growth.

Glucose agar: Coiled and heaped up cream-colored translucent masses; after 2 months, growth rounded, elevated, ridged outwards from hollow center.

Glycerol agar: Coiled, colorless, lustrous patches, isolated colony with central depression.

Serum agar: Poor growth, small amorphous cream-colored mass.

Inspissated serum: Intricately coiled cream-colored growth. No liquefaction.

Broth : Flakes.

Synthetic sucrose solution: Poor growth, a few flakes.

Synthetic glycerol solution: Delicate white floceulj at base.

Litmus milk: Green surface growth, liquid hydrolyzed, partly clear purple; later decolorized, brown.

Potato agar: Colorless blister colonies in one week; dull green heaped and coiled mass after 3 weeks; medium becomes slightly discolored.

Potato plug: After 3 weeks, abundant, colorless, umbilicated, round colonies, some coiled in raised masses; later, liberal olive-green growth, piled up, dense, velvety gray-green aerial mycelium at top of slant, small round fluffy white colonies in liquid at base. 
Source: From pus containing typical actinomycotic granules from parotid abscess.

Habitat: From human infections so far as known.

68. Streptomyces gibsonii (Erikson) comb. nov. (Actinomyces gibsonii Erikson, Med. Res. Council Spec. Rept. Ser. 203, 1935, 36.) Named for Prof. Gibson of Oxford.

Description from Erikson (loc. cit., p. 15).

Young growing mycelium branches profusely at short intervals; later grows out into long frequently wavy filaments; twisted hyphae also seen on water agar. Power of producing aerial mycelium apparently lost.

Gelatin: Dull white flakes sinking as medium liquefies; liquefaction complete in 12 days.

Agar: Small, cream-colored, depressed, partly confluent colonies, becoming an extensive wrinkled cream-colored skin.

Glucose agar: Cream-colored wrinkled membranous growth.

Potato agar: Wrinkled glistening membranous growth.

Serum agar : Small moist cream-colored colonies growing into medium.

Dorset's egg medium: Small, round, smooth, colorless colonies with conically elevated centers.

Inspissated serum : Innumerable colorless pinpoint colonies with scant white aerial mycelium at top; after 8 days, a coherent wrinkled skin with brownishred discoloration at reverse, medium becoming transparent; completely liquefied, pigmented brown in 15 days.

Blood agar: Yellowish confluent bands, irregularly wrinkled, with small discrete colonies, clear hemolytic zone.

Broth : Sediment of floceuli, some round and fan-shaped colonies.

Synthetic sucrose solution: Very delicate white flocculi.

Potato plug: No growth.

Starch not hydrolyzed.

Milk: Coagulated; partly peptonized.
Tyrosine agar: Negative reaction.

Source: From the spleen in a case of acholuric jaundice. Injected into a monkey, and reisolated.

Habitat: From human infections so far as known.

69. Streptomyces beddardii (Erikson) comb. nov. (Actinomyces beddardii Erikson, Med. Res. Council Spec. Rept. Ser. 203, 1935, 36.) Presumably named for the surgeon who first secured the culture.

Description from Erikson (loc. cit.; p. 13).

Rapidly growing, dense, spreading mycelium composed of very long slender filaments, many wavy or closely coiled, particularly on glucose agar; spirals less marked or lacking on poorer nutritive media like synthetic glycerol agar or water agar. Aerial mycelium sparse, short, straight on synthetic glycerol agar, much slower and more plentiful on glucose agar; later shows long, very fine spirals breaking up into small oval conidia; aerial hyphae straighter and more branched with shorter conidiophores on starch agar. Non-acid-fast.

Gelatin: Dull white flakes sinking to bottom as medium liquefies; liquefaction complete in 8 days.

Agar: Colorless, coherent, wrinkled, membranous growth with submerged margin; after 3 months, medium discolored, scant white aerial mycelium at top.

Glucose agar: Wrinkled membranous growth; after 2 months, scant white aerial mycelium.

Glycerol agar: Small, cream-colored, discrete colonies becoming confluent, under surface much buckled.

Potato agar : Moist, cream-colored skin, convoluted, closely adherent.

Ca-agar: Extensive, moist, creamcolored, wrinkled, membranous growth.

Coon's agar: Scant, cream-colored, membranous growth.

Starch agar: Spreading, colorless growth, considerable white aerial mycelium. 
Blood agar: Hemolysis. Growth in uniformly striated colorless bands, occasional round colonies at margin.

Dorset's egg medium: Extensive, very wrinkled, membranous growth, surface bright yellow. After 2 months, considerable liquefaction.

Serum agar: Wrinkled, glistening, cream-colored, membranous growth.

Inspissated serum: Colorless smeary growth, reverse becoming transparent; starting to liquefy at base; completely liquefied and brown in 12 days.

Broth: Suspended and sedimented colorless flocculi, some small round colonies.

Synthetic sucrose solution: Abundant white colonies in coherent mass near bottom of tube; large shell-shaped masses.

Synthetic glycer 1 solution: At first, at few round white colonies in suspension; later, large branched feathery mass at bottom.

Milk: Coagulated; later peptonized.

Litmus milk: Medium deep blue, becoming hydrolyzed to clear purple

Potato plug: Colorless moist membranous growth with scant white aerial mycelium at top of plug.

Stareh is hydrolyzed.

Tyrosine agar: Reaction negative.

Source: Human spleen in a case of spleenic anemia.

Habitat: From human infections so far as known.

70. Streptomyces kimberi (Erikson) comb. nor. (Actinomyces kimberi Erikson, Med. Res. Council Spec. Rept. Ser. 203, 1935, 36.) Presumably named for the surgeon who first secured the culture.

Description from Erikson (loc. cit., p. 14).

Mycelium of long straight profusely branching filaments forming circumscribed colonies on all media with abundant production of short straight and branched aerial mycelium; small round conidia. Non-acid-fast.

Gelatin: Liquefied. Smooth shining colonies becoming powdery white with aerial mycelium, floating on liquefied medium. No pigmentation.

Agar: Smooth round moist creamcolored colonies, $1 \mathrm{~mm}$ in diameter; after 17 days, white powdery aerial mycelium.

Glucose agar: Discrete cream-colored colonies becoming confluent, white aerial mycelium.

Glycerul agar: Moist cream-colored colonies becoming confluent, white aerial mycelium.

Potato agar: Extensive growth covered by white powdery aerial mycelium; large colorless exuded droplets.

Wort agar: Heavy brownish lichenoid colony; after 30 days, a white aerial mycelium.

Ca-agar: Dull crean-colored scaly growth, covered by chalky white aerial mycelium.

Coon's agar: Extensive growth, white aerial mycelium in annular arrangement.

Czapek's agar: Small colonies covered with white aerial nycelium.

Blood agar : Many large colonies, creamcolored, tough, smooth, glistening, with margin depressed; no hemolysis .

Serum agar: Moist, cream-colored honeycombed skin, scant white aerial myeelium.

Dorset's egg medium : Closely adherent seale-like colonies, centrally elevated, with white aerial mycelium.

Inspissated serum: Rapid spreading grow th, discrete round colonies at margin, completely rovered with white aerial mycelium, colorless transpired drops; slight softening at base.

Broth: small round colonies in sediment in 2 days; supernatant colonies with white acrial mycelium and large hollow flakes in sediment in 15 days; oceasional reddish-brown coloration.

Synthetic sucrose solution: Round white colonies at bottom; later small stellate colonies in suspension and a few supernatant with white aerial mycelium.

Synthetic glycerol solution: Round white colonies at bottom; later coherent mulberry-like mass composed of fluffy 
round portions; after 15 days, irregular wispy flocculi and large coherent mass.

Milk: Coagulation; no peptonization; initial pinkish-brown ring descends until medium is dark brown throughout (2 months).

Litmus milk: Blue coloration, hydrolyzed to clear purple in 2 months.

Starch not hydrolyzed.

Tyrosine agar: Reaction negative.

Source: Blood culture of a woman with acholuric jaundice.

Habitat: From human infections so far as known.

71. Streptomyces somaliensis (Brumpt) comb. nov. (Indiclla somaliensis Brumpt, Arch. Parasit., Paris, 10, 1906, 489; Discomyces somalicnsis Brumpt, Précis de Parasitologie, Paris, 2nd ed., 1913, 967; Indiellopsis somalicnsis Brumpt, ibirl.; Nocardia somaliensis Chalmers and Christopherson, Ann. Trop. Med. and Parasit., 10, 1916, 239; Streptothrix somaliensis Miescher, Arch. Derm. Syphilis, 124, 1917, 297; Actinomyces somaliensis St. John-Brooks, Med. Res. Council Syst. of Bact., London, 8, 1931, 75.) Tamed for the country of origin, French Somaliland.

Description from Erikson (Med. Res. Council Spec. Rept. Ser. 203, 1935, 17 ).

Simple branching unicellular mycelium with long straight filaments, forming circumseribed colony erowned with short straight aerial mycelium.

Gelatin: Cream-colored colonies, medium pitted; complete liquefaction in 10 days; hard black mass at bottom.

Agar: Abundant yellowish granular growth with small discrete colonies at margin; later growth colorless, colonies umbilicated.

Glucose agar: Poor growth, moist cream-colored elevated patch.

Glycerol agar: Abundant growth, minute round to large convoluted and piled up masses, colorless to dark gray and black.

Ca-agar : Round cream-colored colonies, depressed, umbilicated, piled up, thin white aerial mycelium; colonies become pale brown.

Potato agar: Small round colorless colonies, zonate margin depressed, confluent portion dark greenish-black.

Blood agar: Small dark brown colonies, round and umbilicated, piled up confluent bands, reverse red-black; hemolysis.

Dorset's egg medium : Extensive colorless growth, partly discrete; becoming opaque, cream-colored, very wrinkled; later rough, yellow, mealy, portion liquid.

Serum agar: Spreading yellow-brown skin, intricately convoluted.

Inspissated serum: Cream-colored coiled colonies, medium pitted, transparent and slightly liquid.

Broth: A few round white colonies at surface, numerous fluffy masses in sediment; later large irregular mass breaking into wisps.

Synthetic sucrose solution: Minute round white fluffy colonies in sediment; after 17 days, scant wispy growth.

Milk: Soft semi-liquid coagulum which undergoes digestion; heavy wrinkled surface pellicle, completely liquefied in 12 days.

Litmus milk: Soft coagulum, partly digested, blue surface ring; clear liquid in 12 days.

Potato plug : Abundant grow th, colonies round and oval, partly piled up in rosettes, frosted with whitish-gray aerial mycelium, plug discolored; after 16 days, aerial mycelium transient, growth nearly black.

Although Streptomyces somaliensis has been known for a long time, there has been until recently no detailed descriptions of the organism beyond the fact that it possesses a distinctly hard sheath around the grain which is insoluble in potash and eau de javelle. The rare occurrence of septa and occasional intercalary chlamydospores is reported by Brumpt (Arch. Parasit., 10, 1905, 562), but has not been confirmed by Erikson (loc. cit.). Chalmers and Christopherson (Ann. Trop. Med. Parasit., 10, 1916. 223) 
merely mentioned the growth on potato as yellowish-white and lichenoid without describing any aerial mycelium. Balfour in 1911 reported a case but gave no data, and Fülleborn limited his description to the grain (Arch. Schiffs. Trop. Hyg., 15, 1911, 131). This species was first placed in Indiella, a genus of fungi, by Brumpt (1906, loc. cit.). Later Brumpt (1913, loc. cit.) proposed a new genus or subgenus, Indiellopsis, containing the single species Indiellopsis somaliensis.

Source: Yellow-grained mycetoma, Khartoum (Balfour, 4th Rept. Wellcome Trop. Res. Lab., A. Med., London, 1911, 365).

Habitat: This condition has been observed by Baufford in French Somaliland, by Balfour (loc. cit.) in the Anglo-Egyptian Sudan, by Fülleborn (loc. cit.) in German So. West Africa and by Chalmers and Christopherson (loc. cit.) in the Sudan.

\section{Streptomyces panjae (Erikson)} comb. nov. (Actinomyces panja Erikson, Med. Res. Council Spec. Rept. Ser. 203, 1935, 36.) Named for Dr. Panja who first secured the culture.

Deseription from Erikson (loc. cit., p. 16).

Unicelluks mycelium with slender branching filaments; very small round colonies; no aerial mycelium visible on any medium, but occasional isolated aerial branches. Non-acid-fast.

Gelatin: Complete liquefaction in 4 days.

Agar: Colorless irregularly piled up convoluted growth; after 1 month, easily detachable, brownish.

Glucose agar: Small colorless coiled mass in 1 week; heaped up green growth in 2 weeks.

Glycerol agar: Poor growth, scant colorless pateh.

Ca-agar: Colorless to pink spreading growth with minute discrete colonies at margin; after 2 weeks, bright red mass, buckled and shining, colorless submerged margin.

Coon's agar: Small submerged colorless growth.

Potato agar : Small elevated convoluted colorless masses with purple tinge in center.

Dorset's egg medium: Small round tough colorless colonies, margin well embedded; after 3 weeks, colonies elevated, warted, darkened, medium discolored and broken; slight degree of liquefaction, medium dark brown.

Serum agar : Colorless, glistening, piled up, convoluted mass.

Inspissated serum: Small round blister colonies and irregularly convoluted patches deeply sunk in pitted medium; after 2 weeks, medium transparent, slight degree of liquefaction.

Broth: Flakes and minute colorless colonies.

Glucose broth: Poor growth, scant flakes, pinkish.

Synthetic sucrose solution: Pinkish flocculi; after 3 weeks, moderate growth, minute colorless colonies.

Milk: Coagulation; pale green surface growth; mostly digested in 2 weeks.

Litmus milk: Soft coagulum, color unchanged; after 2 months, mostly digested, residue coagulum light purple.

Source : From an ulcer of the abdominal wall, Calcutta.

73. Streptomyces willmorei (Erikson) comb. nov. (Actinomyces willmorei Erikson, Med. Res. Council Spec. Rept. Ser. 203, 1935, 36.) Named for Dr. Willmore who isolated the culture.

Description from Frikson (loc. cit., p. 19)

Germination usual, but growing unicellular inycelium frequently branches at very short intervals, presenting peculiar clubbed and budding forms with occasional separate round swollen cells which may represent the cystites of other writers. The filaments are characteristically long, homogeneous, and much interwoven. Aerial mycelium is profuse 
in most media, with a marked tendency to produce loose spirals (water and synthetic glycerol agar) with chains of ellipsoidal conidia. Thick aerial clusters may also be formed.

Gelatin: Minute colorless colonies; liquefaction.

Agar : Heavy folded colorless lichenoid growth, rounded elevations covered with white aerial mycelium; later, submerged margin, round confluent' growth, aerial mycelium marked in concentric zones.

Glucose agar: Colorless wrinkled confluent growth with smooth entire margin, large discrete colonies like flat rosettes; after 4 months, scant white aerial mycelium.

Glycerol agar: Round smooth creamcolored colonies, heavy texture, margin submerged, stiff sparse aerial spikes; after 3 weeks, colonies large (up to $10 \mathrm{~mm}$ in diameter).

Ca-agar: Spreading colorless growth, pitting medium, submerged undulating margin; very scant white aerial mycelium.

Coon's agar: Opaque white growth extending irregularly (up to $3 \mathrm{~mm}$ ) into medium, margin smooth and submerged, center raised, greenish tinge covered with white aerial mycelium; after 3 weeks, margin green, central mass covered by gray aerial mycelium.

Potato agar: Fair growth, partly submerged, covered with grayish-white aerial mycelium; medium becomes discolored.

Blood agar: Heavily textured small drab colonies, aerial mycelium microscopical; no hemolysis.

Dorset's egg medium: Large, round, colorless, scale-like colonies, radially wrinkled; growth brownish, medium discolored in 2 weeks.

Serum agar: Smooth colorless discoid colonies; marked umbilication after 2 weeks.
Broth : Large fluffy white hemispherical colonies, loosely coherent.

Synthetic sucrose solution: A few large round white colonies with smooth partly zonate margins, lightly coherent in sediment; later smaller colonies in suspension attached to side of tube.

Milk: Coagulation; one-third peptonized.

Carrot plug: Colorless raised colonies with powdery white aerial mycelium; after 1 month, very much piled up, aerial mycelium gray; after 2 months, superabundant growth around back of plug, confluent, greatly buckled, all-over gray aerial mycelium.

Source: Streptothricosis of liver (Willmore, Trans. Roy Soc. Trop. Med. Hyg., 17, 1924, 344).

Habitat: From human infections so far as known.

*Appendix: The following names have been used for species of Streptomyces. Many of them are regarded as new by their authors merely because they were isolated from a new type of lesion, or from some animal other than man. Others are inadequately described species from air, soil or water. Relationships to other better described species are usually very obscure. Some of the species listed here may belong in the uppendix to the genus Nocardia.

Actinomyces aerugineus Wollenweber. (Arb. d. Forschungsinst. f. Kartoffelbau, 1920, 16.) From deep scab on potato.

Actinomyces albidofuscus Neukirch. (Actinomyces albido fuscus Berestnew, Inaug. Diss., Moskow, 1897; see Cent. f. Bakt., I Abt., 24, 1898, 707; Neukirch, Inaug. Diss., Strassburg, 1902, 3.) From grain.

Actinomyces albidus Duché. (Encyclopédie Mycologique, Paris, 6, 1934, 266.)

Actinomyces alboatrus Waksman and

* This appendix was originally prepared by Prof. S. A. Waksman and Prof. A. T. Henrici, May, 1943; it has been developed further by Mrs. Eleanore Heist Clise, Geneva, New York, August, 1945. 
Curtis. (Soil Science, 1, 1916, 117.) From adobe soil.

Actinomyces alboviridis Duché. (Encyclopédie Mycologique, Paris, 6, 1934, 317.)

Actinomyces albus (Rossi Doria) Gas. perini. (Streptotrix (sic) alba Rossi Doria, Ann. d. Ist. d'Ig. sper. d. Univ. di Roma, 1, 1891, 421; Streptothrix Nos. 2 and 3, Almquist, Ztschr. f. Hyg., 8, 1890, 189; Oospora doriae Sauvageau and Radais, Ann. Inst. Past., 6, 1892, 251; Actinomyces bovis albus Gasperini, Atti Soc. Tose. Scienz. Nat., P. Y., 9, 1894; Gasperini, Cent. f. Bakt., 15, 1894, 685.) A general name applied to the most common streptomyces in air and water.

Actinomyces albus asporogenes Berestnew. (Inaug. Diss., Moskow, 1897; see Cent. f. Bakt., I Abt., 24, 1898, 708.)

Actinomyces albus var. ochraleucus Wollenweber. (Arb. d. Forschungsinst. für Kartoffelbau, 1920, 16.)

Actinomyces albus - vulgaris Ciani. (Quoted from Baldacci, Boll. Sez. Ital. Soc. Internaz. di Microbiol., 9, 1937, 140.)

\section{Actinomyces almquisti Duché.}

(Duché, Encyclopédie Mycologique, Paris, 6, 1934, 278.) From eulture labeled Actinomyces albus (Krainsky) Waksman and Curtis (Soil Sci., 1, 1916 , 117 ; said to resemble Streptothrix No. 1 , Almquist, Ztschr. f. Hyg., 8, 1890, 189). Actinomyces alni Peklo. (Cent. f. Bakt., II Abt., 27, 1910, 451.) From swellings of the roots of Alnus glutinosa. Actinomyces annulatus Wollenweber. (Arb. Forschungsinst. für Kartoffelbau, $1920,16$.$) From dark-colored potato$ stem.

Actinomyces (Streptothrix) annulatus Beijerinck. (Folia Microbiologica, 1. $1912,4$.

Actinomyces aurea (du Bois Saint Sévérin) Ford. (Streptothrix aurea du Bois Saint Sévérin, Arch. de méd. nav., 1895, 252; Nocardic aurea Castellani and Chalmers, Man. Trop. Med., 2nd ed., 1913, 818; Oospora aurea Sartory, Champ. Paras. Homme et Anim., 1923, 818; Ford,
Textb. of Bact., 1927, 220; not Actinomyces aureus Waksman and Curtis, Soil Science, $1,1916,124$.) Possibly synonymous with Actinomyces aureus LachnerSandoval, Die Strahlenpilze, 1893, according to Lieske, Morphol. u. Biol. d. Strahlenpilze, Leipzig, 1921, 26. Found in conjunctivitis.

Actinomyces bellisari Dodge. (Streptothrix alba Bellisari, Ann. Ig. Sperim., 14, 1904, 467; Oospora alba Sartory, Champ. Paras. Homme et Anim., 1923, S19; Dodge, Medical Mycology, St. Louis, 1935, 744.) Isolated in a warehouse in Naples from the dust of cereal coming from California.

Actinomyces bovis var. nigerianus Erikson. (Med. Res. Council Spec. Rept. Ser. 203, 1935. 20 and 36.) From streptothricosis of the skin of cattle in Nigeria.

Actinomyces candidus (Petruschky) Bergey et al. (Streptothrix candida (Gedanensis II) Petruschky, Verhandl. d. Kongr. f. innere Med., 1898; see Petruschky, in Kolle and Wassermann, Handb. d. path. Mikroorg., 2 Aufl., 5, 1913, 285 and 294; Nocardia candida Castellani and Chalmers, Man. Trop. Med., 2nd ed., 1913, 818; Discomyces candidus Brumpt, Précis de Parasitol., Paris, 3rd ed., 1922, 980; Bergey et al., Manual, 1st ed., 1923, 347.) From human lung.

Actinomyces carneus (Rossi Doria) Gasperini. (Streptotrix carnea Rossi Doria, Ann. Ist. d'Ig. sper. Univ. Roma, 1, 1891, 415; Gasperini, ibid., 2, 1892, 222 ; Oospora carnea Lehmann and Neumann, Bakt. Diag., 1 Aufl., 2, 1896, 388; Cladothrix carnea Macé, Traité Pratique de Bact., 4th ed., 1901, 1096; Discomyces carneus Brumpt, Précis de Parasitol., 2nd ed., 1913, 976; Nocardia carnea Castellani and Chalmers, Man. Trop. Med., 2nd ed., 1913, 818.) From air.

Actinomyces carnosus Millard and Burr. (Ann. Appl. Biol., 13, 1926, 601.) From scab on potato.

Actinomyces casei Bernstein and Morton. (Jour. Bact., 27, 1934, 625.) Thermophilic. From pasteurized cheese. 
Actinomyces cati (Rivolta) Gasperini. (Discomyces cati Rivolta, 1878; Gasperini, Cent. f. Bakt., 15, 1894, 684.) Cause of a disease in a cat.

Actinomyces cerebriformis Namyslowski. (Namyslowski, Cent. f. Bakt., I Abt., Orig., 62, 1912, 564: Streptothrix cerebriformis Chalmers and Christopherson, Ann. Trop. Med. and Parasit., 10, 1916, 273; Nocardia cerebriformis Vuillemin, Encyclopédie Mycologique, Paris, 2, 1931, 126.) From an infection of the cornea of the human eye.

Actinomyces cereus. (Quoted from Lieske, Morphol. u. Biol. d. Strahlenpilze, Leipzig, 1921, 33.)

Actinomyces chromogenus (Gasperini) Gasperini. (Streptotrix nigra Rossi Doria, Ann. d. Ist. d'Ig. sper. d. Univ. di Roma, 1, 1891, 419; Streptotrix cromogena (sic) Gasperini, according to Rossi Doria, idem; Gasperini, ibid., 2, 1892, 222; Oospora chromogenes Lehmann and Neumann, Bakt. Diag., 1 Aufl., 2, 1896, 389; Cladothrix chromogenes Macé, Traité Pratique de Bact., 4th ed., 1901, 1075; Actonomyces niger Brumpt, Précis de Parasitol., Paris, 4th ed., 1927, 1206.) A general name for streptomyces from air producing a dark chromogenesis on protein media.

Actinomyces cinereonigeraromaticus Neukirch. (Actinomyces cinereus niger aromaticus Berestnew, Inaug. Diss., Moskow, 1897 ; see Cent. f. Bakt., I Abt., 24, 1898, 707; Neukirch, Inaug. Diss., Strassburg, 1902, 3; Nocardia cinereonigra Chalmers and Christopherson, Ann. Trop. Med. and Parasit., 10, 1916, 271; Streptothrix cinereonigra aromatica, attributed to Berestnew by Chalmers and Christopherson, idem; Actinomyces cinereo-niger, quoted from Lieske, Morphol. u. Biol. d. Strahlenpilze, Leipzig, 1921, 33.) From grain.

Actinomyces citreus Gasperini. (Gasperini, Cent. f. Bakt., 15, 1894, 684; Streptothrix citrea Kruse, in Flügge, Die Mikroorganismen, 3 Aufl., 2, 1896, 63; not Actinomyces citreus Lirainsky, Cent. f. Bakt., II Abt., 41, 1914, 662.)
Actinomyces clavifer Millard and Burr. (Ann. Appl. Biol., 13, 1926, 601.) From scab on potato and from soil.

Actinomyces coroniformis Millard and Burr. (Ann. Appl. Biol., 13, 1926, 601.) From peat soil.

Actinomyces craterifer Millard and Burr. (Ann. Appl. Biol., 13, 1926, 601.) From scal, on potato.

Actinomyces cloacae Brussoff. (Cent.f. Bakt., II Abt., 49, 1919, 97.) Frommud. Actinomyces cretaceus (Irüger) Wollenweber. (Oospcra cretacea Krüger, Berichte der Versuchsstat. f. Zuckerrohrs, Kergok-Legal, 1890; Wollenweber, Arbeiten d. Forschungsinstitut für Kartoffelbau, 1920, 16.) From potatoscab.

Actinomyces dicksonii Erikson. (Med. Res. Council Spec. Rept. Ser. 203, 1935, 17.)

Actinomyces elastica söhngen and Fol. (Cent. f. Bakt., II Abt., 40, 1914, 92.) From garden earth.

Aclinomyces ferrugincus Naumann. (Kungl. Svenska Vetenskapsakad. Handl., I, 6., Part 4, 1921, 45.) From Aneboda refion of Sweden. Deposits ferric hydroxide about the mycelial threads.

Actinomyces filiformis (Boas) Nannizzi. (Bacillus filiformis Boas, 1897; not Bacillus filiformis Tils, Ztschr. f. Hyg., 9, 1890, 294; not Bacillus filiformis Migula, Syst. d. Bakt., 2, 1900, 387; Nocardia filiformis Vuillemin, Encyclopédie Mycologique, Paris, 2, 1931, 132; Nannizzi, in Pollacei, Tratt. Micopat. Umana, 4, 1934, 26.) From the human stomach.

Actinomyces fimbriatus Millard and Burr. (Ann. Appl. Biol., 13, 1926, 601.) From scab on potato.

Actinomyces flavogriseus Duché. (Encyclopédie Mycologique, Paris, 6, 1934, 341.) From volcanic soils (Martinique).

Actinomyces flavus Sanfelice. (Sanfelice, Cent. f. Bakt., I Abt., Orig., 36, 1904, 359; Streptothrix flava Sanfelice, ibid.; not Streptalhrix flara Chester, Manual Determ. Bact., 1901, 362; not Actinomyces flavus Krainsky, Cent. f. 
Bakt., II Abt., 4l, 1914, 662.) From air.

Actinomyces flacus Millard and Burr. (Millard and Burr, Ann. Appl. Biol., 13, 1926, 601; not Actinomyces flavus Sanfelice, Cent. ؟. Bakt., I Abt., Orig., $36,1904,359$; not Actinomyces flaur Krainsky, ('ent. f. Bakt., II Abt., 41, 1914, 662; not Actinomyces flarus Dodge, Medical Mycology, St. Louis, 1235, 752.) From seab on potato.

Actinomyces foersteri (Cohn) Gassperini. (Streptothrix foersteri Cohn, Beitr. z. Biel. d. Pflanz., 1, Heft 3, 1855, 186; Cladothrix foersteri Winter, Die Pilze, in Rabenhorst's KiryptogamenFlora, I Abt., 1, 1884, 60; Nocardia foersteri Trevisan, I generi e le specie delle Batteriacee, 1889, 9; Oospora foerster $i$ Sauvageau and Radais, Amn. Inst. Past., 6, 1892, 252; Gasperini, Cent. f. Bakt., 15, 1894, 68t; Discomyces foersteri Gedoelst, Les champignons parasites de l'homme et des animaux domestiques. Brussels, 1902, 176; Cohnistreptothrix. foersteri Pinoy, Bull. Inst. Pasteur, Paris, 11, 1913, 937.) The first streptomyces to be deseribed. Probably not identifiable. From an inflamed tear duct. Chalmers and ('hristopherson Ann. Trop. Mel. and Parasit., 10, 1916, 273) include Leptothrix oculorum Sorokin, 1881 as a synonym of this species.

Actinomyces fuscu Söhngen and Fol. (Cent. f. Bakt., II Abt., 40, 1914, s7.) From garden earth.

Actinomyces gabritschewsii Neukirch. (Actinomyces of Gabritschewsky, Berestnew, Inaug. Diss., Moskow, 1597; see Cent. f. Bakt., I Abt., 24, 1898, 708; Neukirch, Inaug. Diss.. Strassburg, 1902, 3.) From water.

Actinomyces gedanensis (Löhlein) Bergey et al. (Strcptothrix gerlanensis I, Scheele and Petruschky, Verhandl. d. Kongr. f. innere Med., 1S\$7, 500; Streptothrix gedanensis Löhlein, Ztsehr. f. Hyg., 63, 1909, 1!; Nocardia gedanensis Chalmers and Christopherson, Ann. Trop. Med. and Parasit., 10, 1916, 255; Discomyces gedanensis Brumpt, Précis de
Parasitol., Paris, 3rd ed., 1922, 984; Bergey et al., Manual, 1st ed., 1923, 347.) From sputum of patient with chronic lung disease.

Actinomyces gibsoni Dodge. (Streptothrix sp. Gibson, Jour. Path. Bact., 23, 1920, 357; Oospora sp. Sartory, Champ. Paras. Homme et Anim., 1923, 776; Dodge, Medical Mycology, St. Louis, 1935, 722.) See page 961.

Actinomyces gracilis Millard and Burr. (Ann. Appl. Biol., 13, 1926, 601.) From scab on potato.

Actinomyces graminearum Berestnew. (Berestnew, Inaug. Diss., Moskow, 1897; see Cent. I. Bakt., I Abt., 24, 1898, 707; Nocardia graminarium (sic) Chalmers and Christopherson, Ann. Trop. Med. and Parasit., 10, 1916, 265; Streplothrix graminarium Chalmers and Christopherson, idem.) From grain.

Actinomyces graminis Topley and Wilson. (Aktinomyces, Bostroem, Beitr. path. Anat. u. Path., 9, 1891, 1; Topley and Wilson, Princip. Bact. and Immun., 1st ed., 1, 1931, 250; Actinomyees bostroemi Baldacei, Boll. Sez. Ital. Soc. Internat. Microbiol., 9, 1937, 141.) From bovine actinomycosis.

Actinomyces gruberi Terni. (Terni, Cent.f. Bakt., 16, 1894, 362; Nocardia gruberi Blanchard, in Bouchard, Traité Path. Gén., 2, 1896, 855; Streptothrix grueberi (sic) Sanfelice, Cent. f. Bakt., I Abt., Orig., 36, 1904, 356; Oospora gruberi, quoted from Nannizzi, Tratt. Micopat. (mana, 4, 1934, 51.) From soil. Produces several pigments on culture media.

Actinomyces guignardi (Sauvageau and Radais) Ford. (Oospora guignardi Sauvageau and Radais, Ann. Inst. Past., 6, 1892, 255; Ford, Textb. of Bact., 1927, 220.) From dust. Gasperini (loc. cit.) regards this as a possible synonym of Actinomyces chromogenus.

Actinomyces halotrichis ZoBell and Upham. (Bull. Scripps Inst. Oceanography Univ. California, 5, 1944, 273.) From mariue mud and kelp.

Actinomyces heimi Duché. (Eneyclo- 
pédie Mycologique, Paris, 6, 1934, 359.)

Actinomyces hoffmanni (Gruber) Gasperini. (Micromyces hofmanni (sic) Gruber, Münch. med. Wochnschr., 1891; also Areh. f. Hyg., 16, 1893, 35; Oospora hoffmanni Sauvageau and Radais, Ann. Inst. Past., 6, 1892, 252; Gasperini, Cent. f. Bakt., 15, 1894, 684; Streptothrix hofmanni Kruse, in Flügge, Die Mikroorganismen, 3 Aufl., 2, 1896, 62; Cladothrix hoffmanni Macé, Traité Pratique de Bact., 4th ed., 1901, 1081.) Pathogenic. See page 976 .

Actinomyces holmesi (Gedoelst) Nannizzi. (Discomyces holmesi Gedoelst, Champ. Paras. Homme et Anim., 1902; Nannizzi, Tratt. Micopat. Umana, 4, 1934, 49.)

1ctinomyces hominis Waksman. (Soil Science, 8, 1919, 129.) Culture received from K. Meyer from Foulerton who isolated it in 1911 from an abscess of the palm. Waksman (loc. cit.) and Baldacei (Mycopathologia, 2, 1940, 160) regard this as identical with Bostroem's organism (see Actinomyces graminis above) and Baldacei has renamed it Actinomyces innominatus.

Actinomyces incanescens Wollenweber. (Arb. Forschungsinst. für Kartoffelbau, 1920, 16.) From the soil of potato fields near Berlin.

Actinomyces intermedius (Krüger) Wollenweber. (Oospora intermedia Krüger, Berichte der Versuchsstat. f. Zuckerrohrs, Kergok-Legal, 1890 ; Wollenweber, Arb. d. Forschungsinst. für Kartoffelbau, 1920, 16.) From the soil of potato fields near Berlin.

Actinomyces interproximalis (Fennel) Ford. (Streptothrix interproximalis Fennel, Jour. Inf. Dis., 22, 1918, 567; Ford, Textb. of Bart., 192-, 195.) From the mouth.

Actinomyces invulnerabilis (Acosta and Grande Rossi) Lachner-Sandoval. (Cladothrix invulnerabilis Acosta and Grande Rossi, Cronica medicoquirurgica de la Habana, No. 3, 1893; see Cent. f. Bakt., 14, 1893, 14; Streptothrix invulnerabilis
Kruse, in Flügge, Die Mikroorganismen, 3 Aufl., 2, 1896, 64; Lachner-Sandoval, Ueber Strahlenpilze, Strassburg, 1898; Nocardia invulnerabilis Chalmers and Christopherson, Ann. Trop. Med. and Parasit., 10, 1916, 271.) From river water.

Actinomyces krausei (Chester) Ford. (Streptothrix aus Eiter, Krause, Münch. med. Wchnschr., 46, 1899, 749 and Cent. f. Bakt., I . Ibt., 26, 1899, 209; also see Petruschky, in Kolle and Wassermann, 2 Aufl., 5, 1913, 267; Streptothrix krausei Chester, Manual Determ. Bact., 1901, 364; Nocardia krausci Chalmers and Christopherson, Ann. Trop. Med. and Parasit., 10, 1916, 263; Discomyces krausei Brumpt, Précis de Parasitol., Paris, 3rd ed., 1922, 993; Ford, Textb. of Bact., 1927, 208.) From actinomycotic pus.

Actinomyces lacertac Terni. (Terni, L'Officiale Sanitario, 1896, 160; Streptothrix lacertae Foulerton, in Allbutt and Rolleston, Syst. of Med., 2, 1912, 309; Oospora lacertae Sartory, Champ. Paras. Homme et Anim., 1923, quoted from Nannizzi, Tratt. Micopat. Umana, 4, 1934, 51.) From grayish nodules in the liver of Italian lizards (Lacerta viridis and $L$. agilis).

Actinomyces lathridii (Petruschky) Ford. (Streptothrix lathridii Petruschky, Verhandl. d. Kong. f. innere Med., 1S98; Ford, loc. cit., 205. From the beetle, Lathridius rugicollis.

Actinomyces loidensis Millard and Burr. (Ann. Appl. Biol., 13, 1926, 601.) From seab on potato

Actinomyces luteo-roseus Sanfelice. (Actinomyces bovis luteoroseus Gasperini, Cent.f. Bakt., 15, 1894, 68t; Sanfelice, Cent. f. Bakt., I Abt., Orig., 36, 190t, 355.) Isolated from actinomycotic lesion in cattle.

Actinomyces marginatus Millard and Burr. (Ann. Appl. Biol., 13, 1926, 601.) From seab on potato.

Actinomyces marinolimosus ZoBell and 
Upham. (Bull. Scripps Inst. Oceanog raphy Univ. California, 5, 1944, 256.) From marine mud.

Actinomyces melanoroseus Roisin. (Wisti Nauk Doslid. Kat. biol. Odessa, 1, 1929,60.)

Actincmyces metchnikovi (Sauvageau and Radais) Ford. (Oospora metchnikowi Sauvageau and Radais, Ann. Inst. Past., 6, 1892, 242; Ford, loc. cit., 220.) From water. Gasperini (loc. cit.) regards this organism as a possible synonym of Actinomyces chromogenus.

Actinomyces muris ratti Lieske. (Streptothrix ratti Schottmüller, Dermat. Wochnschr., 58, 1914, Supplement, 77; Streptothrix muris-ratti Dick and Tunnicliff, Jour. Inf. Dis., 23, 1918, 186; Lieske, Morphol. u. Biol. d. Strahlenpilze, Leipzig, 1921, 31; regarded as identical with Streptobacillus moniliformis Levaditi, Nicolau and Poincloux, Compt. rend. Acad. Sei. Paris, 180, 1925, 1188 by Topley and Wilson, Princip. of Bact. and Immun., 2nd ed., 1936, 274. The latter organism is regarded as identical with Haverhillia multiformis Parker and Hudson, Amer. Jour. Path., 2, 1926, 357 by Van Rooyen, Jour. Path. and Bact., 43, 1936, 469; Actinomyces muris Topley and Wilson, loc. cit.) From a case of rat-bite fever.

Actinomyces musculorum Hertwig. (Actinomyces musculorum suis Duncker, Ztschr.f. Microskopie u. Fleischbeschau, 3, 1884. No. 3; Hertwig, Arch. f. wissensch. u. prakt. Thierheilk., 12, 1886, 365; Oospora musculorum suis Lehmann and Neumann, Bakt. Diag., 1 Aufl. $2,1896,383$.) Seen in calcareous deposits in the muscles of swine.

Actinomyces myricae Peklo. (Cent. f. Bakt., II Abt., 27, 1910, 451.) From the roots of Myrica.

Actinomyces from Neddeni, Namyslowski. (Cent. f. Bakt., I Abt., Orig., 62, 1912, 564.) From the human eyelid.

Actinomyces nigricans Killiau and Fehér. (Ann. Inst. Past., 55, 1935, 620.) From desert soil.

Actinomyces nigrificans (Krüger) Wol- lenweber. (Oospora nigrificans Krüger, Berichte der Versuchsstat. f. Zuckerrohrs, Kergok-Legal, 1890 ; Wollenweber, Arb. Forschungsinst. für Kartoffelbau, 1920, 16.) From potato scab.

Actinomyces nitrogenes Sartory, Sartory, Meyer and Walter. (Bull. Acad. Méd., Paris, 116, 1936, 186; also Ann. Inst. Past., 58, 1937, 684.) From sputum.

Actinomyces nivea. (Ineorrectly attributed to Krainsky, 1914 by Chalmers and Christopherson, Ann. Trop. Med. and Parasit., 10, 1916, 270.)

Actinumyces nondiastaticus Bergey et al. (Var. b, Bergey, Jour. Bact., 4, 1919, 304; Bergey et al., Manual, 1st ed., 1923, 371.) from air.

Actinomyces ochraceus Neukirch. (Ueber Actinomyceten, Strassburg, 1902, 4.) From soil.

Actinomyces ochroleucus Neukrich. (Ueber Actinomyceten, Strassburg, 1902, 4.) From soil.

Actinomyces odorifera lioelz. (Inaug. Diss., Kiel, 1934; Le Lait, 16, 1936, 154.) Actinomyces oligocarbophilus Lantzsch. (Lantzsch, Cent. f. Bakt., II Abt., 57, 1922, 309; Proactinomyces oligocarbophilus Krassilnikov, Bull. Acad. Sci., U. S. S. R., No. 1, 1938, 139.) Lantzsch regards this organism as identical with Bacillus oligocarbophilus Beijerinck and Van Delden, Cent. f. Bakt., II Abt., 10, 1903, 33 (Carboxydomonas oligocarbophila Orla-Jensen, Cent. f. Bakt., II Abt., 22, 1909, 311). Secures growth energy by oxidizing $\mathrm{CO}$ to $\mathrm{CO}_{2}$. From soil. See Manual, 5th ed., 1939, 81 for a description of the bacillary stage of this organism. Carboxyodomonas oligocarbophila Orla-Jensen is the type species of the genus Carboxydomonas Orla-Jensen (loc. cit.).

Actinomyces orangico-niger. (Quoted from Lieske, Morphol. u. Biol. d. Strahlenpilze, Leipzig, 1921, 33.)

Actinomyces orangicus. (Quoted from Lieske, Morphol. u. Biol. d. Strahlenpilze, Leipzig, 1921, 33.)

Actinomyces pelogenes Sawjalow 
(Cent. f. Bakt., II Abt., 39, 1913, 440.) From mud containing hydrogen sulfide.

Actinomyces pluricolor Terni. (Streptothrix pluricolor Fuchs; Terni, quoted from Gasperini, Cent. f. Bakt., 1j, 1894 , 684; Nocardia pluricolor Chalmers and Christopherson, Ann. Trop. Med. and Parasit., 10, 1916, 268.)

Actinomyces pluricolor diffundens Berestnew. (Inaug. Diss., Moskow, 1897; see Cent. f. Bakt., I Abt., 24, 1898, 708.) From air.

Actinomyces praecox Millard and Burr. (Ann. Appl. Biol., 13, 1926, 601.) From scab on potato.

Actinomyces pratecundus Millard and Burr. (Ann. Appl. Biol., 13, 1926, 601.) From seab on potato and from soil.

Actinomyces protea (Schürmayer) Ford. Oospora proteus and Streptothrix proteus Schürmayer, Cent. f. Bakt., I Abt., 27, 1900, 58; Ford, loc. cit., 208.) From an abscess of the foot

Actinomyces pseudotuberculosae (Flexner) Brumpt. (Streptothrix pseudotuberculosa Flexner, Jour. Exp. Med., 3, 1898, 438; Brumpt, Précis de Parasit., Paris, th ed., 1927, 1206.)

Actinomyces pseudotuberculosus Lehmann and Neumann. (Actinomyces atypica pseudotuberkulosa Hamm and Keller, Cent. f. Bakt., I Abt., Ref., 42, 1909, 729; Lehmann and Neumann, Bakt. Diag., 5 Aufl., 2, 1912, 621; Nocardia pseudotuberculosis de Mello and Fernandes, Mem. Asiatic Soe, Bengal, 7, 1919, 110.)

Actinomyces purpureus Killian and Fehér. (Ann. Inst. Past., 55, 1935, 620.) From desert soil.

Actinomyces putorii (Dick and Tunnieliff) Ford. (Streptothrix putorii Diek and Tunnicliff, Jour. Inf. Dis., 23, 1918, 183; Ford, Textb. of Bact., 1927, 216.) From the blood of a patient bitten by a reasel.

Actinomyces pyogenes Lieske. (Eine neue Streptothrixspecies, Caminiti, Cent. f. Bakt., I Abt., Orig., 44, 1907, 193; Streptothrix pyogenes Chalmers and Christopherson, Ann. Trop. Med. and
Parasit., 10, 1916, 270; Lieske, Morphol. u. Biol. d. Strahlenpilze, Leipzig, 1921, 32.) From air.

Actinomyces radiatus Namyslowshi. (Namyslowski, Cent. f. Bakt., I Abt., Orig., 62, 1912, 564; Streptothrix radiatus Chalmers and Christopherson, Ann. Trop. Med. and Parasit., 10, 1916, 273; Nocardia radiata Vuillemin, Encyclopédie Mycologique, Paris, 2, 1931, 126.) From an infection of the cornea of the human eye.

Actinomyces rosaceus. (Quoted from Lieske, Morphol. u. Biol. d. Strahlenpilze, Leipzig, 1921, 33.)

Actinomyces roseodiastaticus Duché. (Encyclopédie Mycologique, Paris, 6, 1934, 329.)

Actinomyces roseus Namyslowski. (Actinomyces $s p$. Lowensteins Klin. Monatsbl. f. Augenheilk., 48, 1910, 185; Namyslowski, Cent. f. Bakt., I Abt., Orig., 62, 1912, 567; not Actinomyces roseus Trainsky, Cent. f. Bakt., II Abt., ł1, 1914, 662; Discomyces roseus Brumpt, Préeis de Parasitol., Paris, 3rd ed., 1922, 981.)

Actinomyces saharae Kiillian and Fehér. (Ann. Inst. Past., 55, 1935, 621.) From desert soil.

Actinomyces salmonicolor Millard and Burr. (Ann. Appl. Biol., 13, 1926, 601.) From sour soil.

Actinomyces sampsonii Millard and Burr. (Ann. Appl. Biol., 13, 1926, 601.) From seab on potato.

Actinomyces sanguinis Basu. (Ind. Jour. Med. Res., 25, 1937, 325.) From the blood of a patient with bronchial pneumonia.

Actinomyces sanninii Ciferri. (Quoted from Baldacei, Boll. Sez. Ital. Soc. Internaz. di Microbiol., 9, 1937, 140.)

Actinomyces setonii Millard and Burr. (Ann. Appl. Biol., 13, 1926, 601.) From scab on potato.

Actinomyces spiralis Millard and Burr. (Ann. Appl. Biol., 13, 1926, 601.) From decaying grass.

Actinomyces taraxeri cepapi (Schottmüller) Ford. (Streptothrix taraxeri 
cepapi Schottmüller, Dermat. Wchnschr., 58, 1914, Supplement, 77; Ford, loc. cit., 196.) From a case resembling rat-bite fever following the bite of a South African squirrel (Taraxerus cepapi).

Actinomyces tenuis Millard and Burr. (Ann. Appl. Biol., 13, 1926, 601.) From seab on potato.

Actinomyccs thermodiastaticus Bergey et al. (Var. a, Bergey, Jour. Bact., 4, 1919, 301; Bergey et al., Manual, 1st ed., 1923, 370.) From stomach contents of a rabbit.

Actinomyces thermotolerans Stadler. (Arch.f. Hyg., 33, 1899, 40.) From milk and butter.

Actinomyces variabilis Cohn. (Cent. f. Bakt., I Abt., Orig., 70, 1913, 301.) From pus in the bladder in a case of eystitis, and from the prostate.

Actinomyces verucosus Nadson. Nadson, Die Mikroorganismen als geologische Faktoren I. Potersburg, 1903; quoted from Dorff, Dic Eisenorganismen, Pflanzenforschung, Jena, Heft 16, 1934, 43; not Actinomyces verrucosus Adler, 1904, see Nannizzi, in Pollacci, Tratt. Micopat. Umana, 4, 1934, 46.) From sea mud. Deposits ferric hydroxide about the mycelial threads.

Actinomyces violaceus (Rossi Doria) Gasperini. (Streptotrix violacea Rossi Doria, Ann. d. Ist. d'Ig. sper. d. Univ. di Roma, 1, 1891, 411; Oospora violacea Sauvageau and Radais, Ann. Inst. Past., 6, 1892, 252 ; Gasperini, Cent. f. Bakt., 15, 1894, 684; Cladothrix violacea Macé, Traité Pratique de Bact., 4th ed., 1901, 1075; Nocardia violacea Chalmers and Christopherson, Ann. Trop. Med. and Parasit., 10, 1916, 270; Discomyces violaceus Brumpt, Précis de Parasitol., Paris, 3rd ed., 1922, 995.) From air and water.

Actinomyces viridis (Lombardo-Pellegrino) Sanfelice. (Streptothrix viridis Lombardo-Pellegrino, Riforma Med., 19, 1903, 1065; also see Cent. f. Bakt., I Abt., Ref., 35, 1904, 761; Sanfelice, Cent. f. Bakt., I Abt., Orig., 36, 1904, 355.) From soil.

Actinomyces viridis Millard and Burr.
(Millard and Burr, Ann. Appl. Biol., 13, 1926, 601; not Actinomyces viridis Sanfelice, Cent. f. Bakt., I Abt., Orig., 36, 1904, 355; not Actinomyces viridis Duché, Encyclopédie Mycologique, Paris, 6, 1934, 311.) From scab on potato.

Actinomyces xanthostromus Wollenweber. (Arb. Forschungsinst. f. Kartoffelbau, 1920, 16.)

Actinomyces wedmorensis Millard and Burr. (Amn. Appl. Biol., 13, 1926, 601.) From peat soil.

Asteroides lieskeyi Puntoni and Leonardi. (Boll. e Atti d. R. Accad. Med. di Roma, 61, 1935, 94.) A renaming of Actinomyces lieskey, a culture whose source was unknown. This may possibly be the same as Actinomyces lieskei Duché (see Streptomyces lieskei).

Cladothrix odorifera Rullmann. (Rullmann, Inang. Diss., Munich, 1895; see Cent. f. Bakt., I Aht., 17, 1895, 884 and Cent. f. Bakt., II Abt., 2, 1896, 116; Oospora odorifera Jehmann and Neumann, Bakt. Diag., 1 Aufl., 2, 1896, 392 ; Actinomyces odorifer Lachner-Sandoval, Ueber Strahlenpilze, 1898, 65; Streptothrix odorifera Foulerton and Jones, Trans. Path. Soc. London, 53, 1902, 112; Nocardia odorifera Castellani and Chalmers, Man. Trop. Med., 2nd ed., 1913, 818.) From sputum in a case of chronic bronchitis.

Cladothrix placoides Kiligler. (Leptothrix placoides alba. Dobrzyniecki, Cent. f. Bakt., I Abt., 21, 1897, 225; Kligler, Jour. Allied Dental Soc., 10, 1915, 141, 282 and 445; Leptotrchia placnides Bergey et al., Manual, 3rd ed., 1930, 458.) From a tooth canal. For a description of this species see Manual, 5th ed., 1939, 829 . The description indicates that this organism belongs to Nocardia or Streptomyces.

Coccobacillus pseudo - actinomycosis polymorphus Berestneff. (Berestneff, 1898, quoted from Chalmers and Christopherson, Ann. Trop. Med. and Parasit., 10, 1916, 273.)

Cohnistreptothrix americana Chalmers and Christopherson. (Streptothrix $s p$. 
Bloomfield and Bayne-Jones, Johns Hopkins Hosp. Bull. 26, 1915, 230; Chalmers and Christopherson, Ann. Trop. Med. and Parasit., 10, 1916, 273; Actinomyces americanus Dodge, Medical Mycology, St. Louis, 1935, 716.) From a liver abscess.

Cohnistreptothrix misri Carpano. (Riv. di Parasit., No. 2, 1937, 107.) From human dermatosis in Egypt.

Cohnistreptothrix silberschmidtii Chalmers and Christopherson. (Streptothrix, Silberschmidt, Cent. f. Bakt., I Abt., 27, 1900, 486; Chalmers and Christopherson, Ann. Trop. Med. and Parasit., 10, 1916, 273; Nocardia silberschmidti Froilano de Mello and Fernandes, Mem. Asiatic Soc. Bengal, 7, 1919, 111; Actinomyces silberschmidti Dodge, Medical Mycology, St. Louis, 1935, 711.) From cases of dacryocystitis.

Discomyces decussalus Langeron and Chevallier. (Langeron and Chevallier, Compt. rend. Soc. Biol., 72, 1912, 1030; Nocardia decussata Castellani and Chalmers, Man. Trop. Med., 2nd ed., 1913, 817; Oospora decussata Sartory, Champ. Paras. Homme et Anim., 1923, 825; Actinomyces decussatus Brumpt, Précis de Parasitol., 4th ed., 1927, 1206.) From dry, scaly lesions. Not considered pathogenic.

Nocardia chalmersi de Mello and Fernandes. (De Mello and Fernandes, Mem. Asiatic Soc. Bengal, 7, 1919, 130; Actinomyces chalmersi Dodge, Medical Mycology, St. Louis, 1935, 734.) From the saliva of a horse.

Nocardia christophersoni de Mello and Fernandes. (De Mello and Fernandes, Mem. Asiatic Soc. Bengal, 7, 1919, 130; Actinomyces christophersoni Dodge, Medical Mycology, St. Louis, 1935, 723.)

From the air.

Nocardia citrea Chalmers and Christopherson. (Ann. Trop. Med. and Parasit., 10, 1916, 270.) A blanket name proposed to include Actinomyces griseoflavus Krainsky, Actinomyces flavus Krainsky, Streptothrix flava Sanfelice and Streptothrix flava Bruns.
Nocardia cruoris Macfie and Ingram. (Macfie and Ingram, Ann. Trop. Med. and Parasit., 15, 1921, 283; Discomyces cruoris Brumpt, Précis de Parasitol. Paris, 3rd ed., 1922, 984; Oospora cruoris Sartory, Champ. Paras. Homme et Anim., 1923, 809; Actinomyces cruoris Brumpt, ibid., 4th ed., 1927, 1195.) From blood.

Nocardia dichotoma (Macé) Chalmers and Christopherson. (Cladothrix dichotoma Macé, Compt. rend. Acad. Sci. Paris, 6, 1888, 1622; not Cladothrix dicho toma Cohn, Beitr. z. Biol. d. Pflanzen 1, Heft 3, 1875, 185; Chalmers and Christopherson, Ann. Trop. Med. and Parasit., 10, 1916, 270.)

Nocardia ferruginea Trevisan. (Bakterium bei Chorea St. Viti, Naunyn, Mittheil. aus der Med. Klinik zu Königsberg, 1888, 292; Trevisan, I generi e le specie delle Batteriacee, 1889, 9; Actinomyces ferrugineus Gasperini, Cent. f. Bakt., 15, 1894, 684.) From pia mater in a case of St. Vitis' dance.

Nocardia garteni (Brumpt) Castellani and Chalmers. (Cladothrix liquefaciens No. 2, Garten, Deutsche Ztschr. f. Chirurg., 41, 1895, 432; Discomyces garleni Brumpt, Précis de Parasitol., Paris, 1st ed., 1910, 860; Castellani and Chalmers, Man. Trop. Med., 2nd ed., 1913, 818; Oospora garteni Sartory, Champ. Paras. Homme et Anim., 1923, 778; Actinomyces garteni Brumpt, loc. cit., 4 th ed., 1927, 1191; Actinomyces liquejaciens Ford, Textb. of Bact., 1927, 202.) From cases of human actinomycosis.

Nocardia goensis de Mello and Fernandes. (De Mello and Fernandes, Mem. Asiatic Soc. Bengal, 7, 1919, 130; Actinomyces goensis Dodge, Medical Mycology, St. Louis, 1935, 723.) From lesions of vitiligo. Saprophytic.

Nocardia liguire Urizer.

(Urizer, 1904; Actinomyces liguire Nannizzi, in Pollacci, Tratt. Micopat. Umana, 4, 1934, 49.)

Nocardia liquefaciens (Hesse) Castel- 
lani and Chalmers. (Cladothrix liquefaciens Hesse, Deutsche Ztschr. f. Chirurg., 41, 1895, 432; Discomyces liquefaciens Brumpt, Précis de Parasit., Paris, 1st ed., 1910, 860; Castellani and Chalmers, Man. Trop. Med., 2nd ed., 1913, 818; Streptothrix liquefaciens Chalmers and Christopherson, Ann. Trop. Med. and Parasit., 10, 1916, 233, 265; Oospora liquefaciens Sartory, Champ. Paras. Homme et Anim., 1923, 778; Actinomyces liquefaciens Brumpt, loc. cit., 4th ed., 1927, 1192.) From an inguinal abscess.

Nocardia microparva Chalmers and Christopherson. (Ann. Trop. Med. and Parasit., 10, 1916, 268.) Listed as synonymous with Actinomyces microparva Krainsky, 1914 which may be intended for Actinomyces microflavus Lirainsky, Cent. f. Bakt., II Abt., 41, 1914, 662.

Nocardia orangica (Berestneff) Chalmers and Christopherson. (Streptothrix orangica Berestneff, Inaug. Diss., Moscow, 1897, quoted from Chalmers and Christopherson, Ann. Trop. Med. and Parasit., 10, 1916, 271; Chalmers and Christopherson, idem.)

Nocardia rogersi de Mello. (Nocardia (Cohnistreptothrix) rogersi de Mello, A Med. Contemp., 1919; Discomyces rogersi Neveu-Lemaire, Précis Parasitol. Hum., 5th ed., 1921, 44; Actinomyces rogersii Brumpt, Précis de Parasitol., 4th ed., 1927, 1206.) From sputum.

Nocardia nubea Chalmers and Christopherson. (Ann. Trop. Med. and Parasit., 10, 1916, 271.) Nomen nudum. According to Dodge (Medical Mycology, St. Louis, 1935, 765), this is a synonym of Oospora rubra Wilbert, Recueil Hyg. Méd. Vét. Militaire, 1908.

Nocardia saprophytica Chalmers and Christopherson. (Streptothrix leucea saprophytica Foulerton, 1902, quoted from Chalmers and Christopherson, Ann. Trop. Med. and Parasit., 10, 1916, 270; Chalmers and Christopherson, idem.)

Nocardia urinaria Pijper. (Pijper,
1918, quoted from Castellani and Chalmers, Man. Trop. Med., 3rd ed., 1919, 1057; Actinomyces urinarius Nannizzi, in Pollacei, Tratt. Micopat. Umana, 4, 1934, 50.)

Oospora hoffmanni (Gruber) Sauvageau and Radais. (Mikromyces hofmanni (sic) Gruber, Trans. Int. Congr. Hyg. Derm., VI, 2, 1891-1892, 65 and Arch. f. Hyg., 16, 1893, 35; Sauvageau and Radais, Anm. Inst. Past., 6, 1892, 251; Actinomyces hoffmanni Gasperini, Cent. f. Bakt., 15, 1894, 684; Streptothrix hofmanni Kruse, in Flügge, Die Mikro. organismen, 3 Aufl., 2, 1896, 62; Cladothrix hoffmanni Macé, Traité Pratique de Bact., 4th ed., 1901, 1081; Nocardin hoffmanni Chalmers and Christopherson, Ann. Trop. Med. and Parasit., 10, 1916, 268.) From a sample of vaccine.

Oospora spumalis Sartory. (Sartory, in Sartory and Bailly, Mycoses pulmonaires, 1923, 318; Actinomyces spumalis Dodge, Medical Mycology, St. Louis, 1935, 751.) From human sputum.

Streptothrix aaser Johan-Olsen. (Inaug. Diss., Christiania, 1893, 91; quoted from Neukirch, Ueber Actinomyceten, Strassburg, 1902, 69.)

Streptothrix alpha Price-Jones. (PriceJones, 1900; quoted from Chalmers and Christopherson, Ann. Trop. Med. and Parasit., 10, 1916, 270.) Considered synonymous with Streptothrix alba (Rossi Doria).

Streptothrix aquatilis Johan-Olsen. (Inaug. Diss., 1893, 93; quoted from Johan-Olsen, Cent. f. Bakt., II Abt., 3, 1897, 279.)

Streptothrix beta Price-Jones. (PriceJones, 1900; quoted from Chalmers and Christopherson, Ann. Trop. Med. and Parasit., 10, 1916, 270; Nocardia beta Chalmers and (hristopherson, idem.)

Streptothrix chondri Johan-Olsen. (Inaug. Diss., 1893, 95; quoted from Johan-Olsen, Cent. f. Bakt., II Abt., 3, 1897, 278.)

Streptothrix enteritidis Pottien. (Quoted from Sanfelice, Cent. f. Bakt., I Abt., Orig., 36, 1904, 355.) 
Streptothrix foersteri Gasperini. (Gasperini, Annales de Micrographie, 2, 1890, 462; not Streptothrix foersteri Cohn, Beitr. z. Biol. d. Pflanzen, 1, Heft 3, 1875, 196; Actinomyces saprophyticus Gasperini, Ann. d. Ist. d'Ig. sper. d. Univ. Roma, 2, 1592, 226; Actinomyces saprophyticus var. cromogenus Gasperini, ibid., 229.) From air.

Streptothrix gelatinosus Johan-Olsen. (Cent. f. Bakt., II Abt., 3, 1897, 279.)

Streptothrix humifica Johan - Olsen. (Cent.f. Bakt., II Abt., 3, 1897, 278.)

Streptothrix lemani Johan-Olsen. (Inaug. Diss., 1893, 96; quoted from Johan-Olsen, Cent. f. Bakt., II Abt., $3,1897,279$.)

Streptothrix necrophora Wilhelm. (Monats.f. prakt. Tierheilk., 14, 1902, 193.) See page 578.

Streptothrix leucea Foulerton. (In All. butt and Rolleston, Syst. of Med., 2, 1912, 310.)

Streptothrix melanotica Price-Jones. (On the General Characteristics and Pathogenic Action of the Genus Streptothrix, 1901; also see Foulerton, in Allbutt and Rolleston, Syst. of Med., 2, 1912, 304.)

Streptothrix oidicformis Johan-Olsen.
(Inaug. Diss., 1893, 96; quoted from Neukirch, Ueber Actinomyceten, Strassburg, 1902, 69.)

Streptothrix spirilloides Johan-Olsen. (Inaug. Diss., 1893, 96; quoted from Neukirch, Ueber Actinomyceten, Strassburg, 1902, 69.)

Streptothrix tartari Sanfelice. (Cent.

f. Bakt., I Abt., Orig., 36, 1904, 355.)

Streptothrix wallemia Johan-OIsen. (Inaug. Diss., 1893, 96; quoted from Neukirch, Ueber Actinomyceten, Strassburg, 1902, 69.)

Streptothrix zopfi Casagrandi. (Quoted from Caminiti, Cent. f. Bakt., I Abt., Orig., 44, 1907, 198.)

Drechsler (Botan. Gazette, 67, 1919, 65 and 147) described eighteen morphological types of Actinomyces (Streptomyces). The relationships of these types to species previously described in the literature are not explained except in four instances. Actinomyces III is regarded as Actinomyces lavendulae Waksman and Curtis; Actinomyces $\mathrm{X}$ is regarded as Streptothrix alba Rossi Doria (possibly Actinomyces griseus Krainsky); Actinomyces XII is regarded as Actinomyces aureus Waksman and Curtis; and Actinomyces XVII is Actinomyces scabies Güssow. 


\section{Genus II. Micromonospora Ørskov.}

(Orskov, Investigations into the morphology of the ray fungi. Copenhagen, 1923, 147; includes Thermoactinomyces Tsilinsky, Ann. Inst. Past., 13, 1899, 501; ibid., 17, 1903, 206.)

Well developed, fine, non-septate mycelium, 0.3 to 0.6 micron in diameter. Grow well into the substrate. Not forming at any time a true aerial mycelium. Multiply by means of conidia, produced singly at end of special conidiophores, on surface of substrate mycelium. Conidiophores short and either simple, branched or produced in clusters. Strongly proteolytic and diastatic. Many are thermophilic and can grow at $65^{\circ} \mathrm{C}$. Csually saprophytes. These organisms occur mostly in hot composted manure, dust, soil and in lake bottoms.

The type species is Micromonospora chalcea (Foulerton) Orskov.

\section{Key to the species of genus Micromonospora (Orstiov Group III).}

I. Vigorously growing organisms, typically with copious spore formation on glucoseasparagine-agar.

A. Vegetative mycelium pale pink to deep orange, no typical soluble pigment.

1. Micromonospora chalcea.

B. Vegetative mycelium orange changing to brownish-black, brown soluble pigment.

2. Micromonospora fusca.

II. Slowly and feebly growing organisms, with scant spore formation on glucoseasparagine-agar, no soluble pigment.

A. Vegetative mycelium pale pink to pale orange.

3. Micromonospora paria.

B. Vegetative mycelium yellow to orange-red.

4. Micromonospora globosa.

C. Vegetative mycelium blue.

5. Micromonospora vulgaris.

Note: This genus could be subdivided on the basis of the relations of the organisms to temperature, since it includes a number of thermophilic forms which grow readily at $55^{\circ}$ to $65^{\circ} \mathrm{C}$, mesophilic forms having their optimum temperature at $30^{\circ} \mathrm{C}$, and organisms growing at low temperatures in lakes. Each of these can be divided into 3 groups, based on the structure of the spore-bearing hyphae. Among the thermophilic forms, only representatives of the first group have so far been isolated in pure culture although the existence of the other two groups has definitely been demonstrated in microscopic preparations. These are:

Group 1. Simple spore-bearing hyphae.

Group 2. Branching spore-bearing hyphae.

Group 3. Spore-bearing hyphae in elusters.

1. Micromonospora chalcea (Foulerton) Orskov. (Streptothrix chalcea Foulerton, Lancet, 1, 1905, 1200; Nocardia chalcea Chalmers and Christopherson, Ann. Trop. Med. and Parasit., 10, 1916, 268; Orskov, Thesis, Copenhagen, 1923, 156; Actinomyces chalcea Ford, Texth, of
Bact., 1927, 221.) From Greek chalceus, bronze.

Description from Jensen, Proc. Linn. Soc. New So. Wales, 57, 1932, 173.

Formation of a unicellular mycelium which forms distally placed, singly situated spores. No aerial hyphae. Nosur- 
face growth in liquid medium. The organism resists desiccation for at least $\delta$ months. Comparison between the power of resistance of the mycelium and the spores, respectively, will no doubt present great difficulty, because it is almost impossible to ensure that the two constituents are actually detached. Otherwise, the nycelium is but slightly capable of germinating, which may be ascertained by inoculating a water agar plate liberally with a mixture of mycelial threads and spores. While practically all the spores germinate, the mycelial threads were never found to form new colonies.

Vegetative mycelium on glucose-asparagine-agar: Heavy, compact, raised, pale pink to deep orange, not spreading much into the medium. Spore-layer well developed, moist and glistening, brownish-black to greenish-black, this color sometimes spreading through the whole mass of growth.

Gelatin is liquefied.

Grows in liquid media as small firm orange granules or flakes.

Milk is digested with a faintly acid reaction, mostly after a previous coagulation.

Many strains invert sucrose.

Some strains produce nitrites from nitrates.

Starch is hydrolyzed.

Most strains decompose cellulose.

Proteolytic action seems stronger in this than in the other species of this genus.

Optimum temperature for growth $30^{\circ}$ to $35^{\circ} \mathrm{C}$. Thermal death point of $\mathrm{my}$ celium, $70^{\circ} \mathrm{C}$ in 2 to 5 minutes. Spores resist $80^{\circ} \mathrm{C}$ for 1 to 5 minutes.

Habitat: Soil, lake mud and other substrates. In addition to the above references, see Erikson (Jour. Bact., 41. 1911, 299) and Lmbreit and MeCoy (A Symposium on Hydrobiology, Cniv. of Wisconsin Press, 1941, 106-114).

2. Micromonospora fusca Jensen. (Proc. Linn. Soc. New So. Wales, 57, 1932, 178.) From Latin fuscus, dark.
Vegetative mycelium on glucose-asparagine-agar heavy, compact, orange, rapidly changing to deep brown and nearly black; spore-layer moist, glistening, grayish - to brownish-black. Deep brown soluble pigment.

Gelatin is liquefied.

Grows in liquid media as small brown granules and flakes.

Milk is slowly digested; no coagulation.

Sucrose is inverted.

Reduction of nitrates, positive or negative.

Cellulose is attacked to a slight extent. Starch is hydrolyzed.

Habitat: Soil.

\section{Micromonospora parva Jensen.} (Proc. Linn. Soc. New So. Wales, 57, 1932, 177.) From Latin parvus, small.

Scant growth on glucose-asparagineagar; vegetative mycelium thin, spreading widely into the agar, almost colorless to pale pink or orange. Sporulation scant, giving rise to thin grayish, moist crusts on the surface

Gelatin is liquefied.

Milk is left unchanged; or coagulated, slowly redissolved with faintly acid reaction.

Sucrose not inverted.

Nitrates not reduced.

Cellulose not decomposed.

Starch is hydrolyzed.

Habitat : Soil.

4. Micromonospora globosa Lirassilnikov. (Ray Fungi and Related Organisms. Izd. Acad. Nauk, Moskow, 1938, 13t; Microbiology, U. S. S. R., 8, 1939, 179.) From Latin globosus, spherical.

$A$ fine $(0.5$ to 0.8 micron in diameter) monopodially branching mycelium. This mycelium breaks soon into separate pieces of varying length and irregular outline. Conidia are formed at the ends of short branches, one on each. Individual branches with conidia resemble grape vines. The conidia are spherical 1.0 to 1.3 microns; they arise by the 
swelling of the branch tips. The swellings become round, acquire the shape of spheres, which, as the formation of the conidia proceeds, are divided from the branch by a transverse septum.

Gelatin is liquefied.

Colonies: Rugose, at first very compact, later acquire a pasty consistency, and their bond with the medium becomes not so fast. The color of the cultures varies from light yellow to orange-red. During fruit-bearing the colonies are covered with a brownish-black tarnish of conidia.

In meat-peptone broth, ammonia is produced.

Milk: Coagulation; peptonization.

Nitrites are produced from nitrates.

Sucrose is inverted.

Cellulose not decomposed.

Starch is hydrolyzed.

Habitat: Soil.

5. Micromonospora vulgaris (Tsilinsky) Waksman, Umbreit and Gordon. (Thermophile Cladothrix, Kedzior, Arch. Hyg., 27, 1896, 328; Thermoactinomyces vulgaris Tsilinsky, Ann. Inst. Past., 13, 1899, 501; Actinomyces monosporus Schütze, Arch. f. Hyg., 67, 1908, 50 (Nocardia monospora Chalmers and Christopherson, Ann. Trop. Med. and Parasit., 10, 1916, 271); Actinomyces glaucus Lehmann and Schütze, in Lehmann and Neumann, Bakt. Diag., 5 Aufl., 2, 1912, 641 (Nocardia glauca Chalmers and Christopherson, loc. cit.); Micromonospora coerulea Jensen, Proc. Linn. Soc. New So. Wales, 57, 1932, 177; Waksman, Umbreit and Gordon, Soil Sci., 47, 1939, 51.) From Latin vulgaris, common.

Morphologically the development of this organism is entirely comparable to that of the mesophilic form described by Jensen. The young mycelium shows slightly more branching than that produced by species of Streptomyces. Spores are borne at the end of short branches from which they are easily broken. The aerial mycelium, though present, is usually rudimentary, rarely exhibiting the tangled network of strands typical of species of Streptomyces. Thermophilic strains of Micromonospora vulgaris differ thus from the mesophilic forms, which show no trace of aerial mycelium. Fragmentation has not been seen in slide cultures of the organism thus far isolated, but it was found to occur in smear preparation.

According to Jensen, the mesophilic strains grow slowly on glucose-asparagine-agar; vegetative mycelium dense, dark greenish-blue, with a hard and glossy surface. Sporulation very scant. The surface sometimes shows a thin white veil resembling aerial mycelium, but without aerial spores.

Gelatin: Liquefaction.

Good growth on beef-peptone agar, potato, milk, beef-peptone broth, etc. Grows in liquid media as fairly large, firm, round, white to pink granules (Jensen). Usually a white, powdery, thin aerial mycelium is produced which is hardly raised above the surface. No soluble pigment is formed.

Czapek's agar: Growth white, powdery, slightly raised.

Broth: A tough white pellicle and in many instances a considerable number of ball-like colonies at the bottom of the tube. No turbidity.

Milk: Coagulated and digested.

Nitrites not produced from nitrates.

Sucrose not inverted.

Cellulose not decomposed.

Starch is hydrolyzed.

Optimum temperature of thermophilic forms $57^{\circ} \mathrm{C}$. Growth range $48^{\circ}$ to $68^{\circ} \mathrm{C}$.

Habitat: Straw, soil, high temperature composts.

Appendix: The following anaerobic species has been described:

Micromonospora propionici Hungate. (Alst. in Jour. Bart., 48, 1944, 380 and 499; Jour. Bact., 51, 1946, 51.) From the alimentary tract of the woorleating termite (Amilermes minimus). Ferments glucose or cellulose to form acetic and propionic acids and $\mathrm{CO}_{2}$. Obligate anaerobe. 


\title{
ORDER III. CHLAMYDOBACTERIALES BUCHANAN.
}

\author{
(Jour. Bact., 2, 1917, 162.)
}

Filamentous, colorless, alga-like bacteria. May or may not be ensheathed. They may be unbranched or may show false branching. False branching arises from a lateral displacement of the cells of the filament within the sheath which gives rise to a new filament, so that the sheath is branched while the filaments are separate. The sheath may be composed entirely of iron hydroxide, or of an organic matrix impregnated with iron, or may be entirely organic. The filaments themselves may show motility by a gliding movement like that found in the blue-green algae (Oscillatoriaceae). Conidia and motile flagellate swarm cells may be developed, but never endospores. Fresh water and marine forms.

\section{Liey to the families of order Chlamydobacteriales.*}

I. Alga-like filaments which do not contain sulfur globules. False branching may occur.

A. Usually free floating filaments. Motile swarm cells may he formed.

Family I. Chlamydobacteriaceae, p. 981.

B. Attached filaments which show a differentiation of base and tip. Non-motile conidia formed in the swollen tips of the filaments.

Family II. Crenothrichaceae, p. 987.

II. Alga-like, unbranching filaments which may contain sulfur globules when growing in the presence of sulfides. Filaments may be motile by a creeping or sliding movement along a solid substrate.

Family III. Beggiatoaceae, p. 988.

\section{FAMILY I. CHLAMYDOBACTERIACEAE MIGLIA.**}

(Arb. Bakt. Inst. Hochschule, Fiarlsruhe, 1, 1894, 237.)

Filamentous bacteria which frequently show false branching. Sheaths may or may not be impregnated with ferric hydroxide. Cells divide only transversely. Swarm cells, it developed, are usually motile by means of flagellat Usually found in fresh water.

\section{Key to the genera of family Chlamydobacteriaceae.}

I. Showing typical false branching.

1. Sheaths entirely organic, not impregnated with ferric hydroxide.

Genus I. Sphaerotilus, p. 982.

B. Sheaths impregnated with ferric hydroxide.

Genus II. Clonothrix, p. 983.

II. Unbranched or rarely showing false branching.

A. Sheaths or holdfasts impregnated with ferric hydroxide.

Genus III. Leptothrix, p. 983.

* In Appendix I, p. 996, will be found a group of non-filamentous, non-sheathforming, colorless sulfur bacteria, as the family Achromatiaceae. Their true relationships are as yet obscure, and they have been attached as an Appendix to the Chlamydobacteriales largely on account of the similarity of their metabolism to that of the Beggiatoaceae.

** Completely revised by Prof. A. T. Henrici, Lniversity of Minnesota, Minneapolis, Minnesota, December, 1938; further revision by Prof. Robert S. Breed, New York State Experiment Station, Geneva, New York, July, 1946. 


\section{Genus I. Sphaerotilus Kützing.}

(Kützing, Linnaea, 8, 1833, 385; Cladothrix Cohn, Beitr. z. Biol. d. Pflanz., 1, Heft $3,1875,185$.) From Greek sphaera, sphere.

Attached, colorless threads, showing false branching, though this may be rare in some species. Filaments consist of rod-shaped or ellipsoidal cells, surrounded by a firm sheath. Multiplication occurs both by non-motile conidiat and by motile swarm cells, the latter with lophotrichous flagella.

The type species is Sphaerotilus natans Kützing.

\section{Sphaerotilus natans Kützing.} (Kützing, Linnaea, 8, 1833, 385; not Sphacrotilus natans Sack, Cent. f. Bakt., II Abt., 65, 1925, 116; Cladothrix natans Migula, in Engler and Prantl, Die natürl. Pflanzenfam. 1, 1a, 1895, 46.) From Latin natans, swimming.

Cells cylindrical, surrounded by a sheath which is slimy in character, 2 to 3 microns in diameter. False branching rare.

Multiplication necurs through the formation of conidia within the sheath of the vegetative cells, from which they swarm out at one end, swim about for a time, then attach themselves to objects and develop into delicate filaments.

Gelatin rapidly liculuefied, requires organic nitrogen, does not grow in the ordinary peptone solution, grows best with low concentrations of meat extract (Zikes, Cent. f. Bakt., II Abt., 4.9, 1915, 529)

The culture cultivated and described as Sphaerotilus natans by Sack (Cent. f. Bakt., II Abt., 65, 1925, 116) was identified as Bacillus mycondes by Haag (ibid., $69,1926,4)$.

Source: Originally found in polluted waters. May become a real nuisance in sewage purification plants of the activated sludge type (Lackey and Wattie, L. S. T'ub. Health Ser., Pub. Health Repts., 55, 1940, 975) and in streams polluted with sulfite liquor from pulp and paper mills (Lackey, Mimeographed Rept. U. S. Pub. Health Ser., 1941).

Habitat: Stagnant and running water, especially sewage polluted streams.

2. Sphaerotilus dichotomus (Cohn) Migula. (Cladothrix dichotoma Cohn,
Beitr. z. Biol. d. Pflanz., 1, Heft 3, 1875, 185; Migula, Syst. d. Bakt., 2, 1900, 1033; Sphaerotilus natans var. cladothrix Butcher, Trans. Brit. Myc. Soc., 17, 1932, 112.) From Greek dichotomos, cut in two parts, forked.

The identity of this species as distinet from Sphaerotilus nalans has been questioned. Cohn's description applied to filaments 0.3 micron in diameter, while all later authors have applied the name to a much larger organism (2 to 4 microns in diameter).

Zikes (Cent. f. Bakt., II Abt., 43, 1915, $529)$ gives the following differential characters: Cells smaller than Sphaerotilus natans, 1.5 to 2.5 microns; false branching constant; grows best in high concentrations of meat extract; will grow in ordinary peptone solutions; can utilize inorganic nitrogen; liquefies gelatin slowly.

Source: Found by Cohn in water containing Myconostoc.

Habitat: Comparatively unpolluted fresh water capable of sustaining algae.

3. Sphaerotilus fluitans (Migula) Schikora. (Streptothrix fluitans Migula, in Engler and Prantl, Dic natürl. Pflanzenfam., 1, 1a, 1895, 39; Schikora, Ztschr. f. Fischerei, 7, 1899, 1-28; Chlamydothrix fluitans Migula, Syst. d. Bakt., 2, 1900, 1033; Leptothrix fuitans Chester, Man. Determ. Bact., 1901, 370.) From Latin fluitans, flowing, floating.

Very thin attached filaments surrounded by a soft sheath, from which almost spherical conidia issue, usually attaching themselves to the exterior of the sheath, where they multiply. 
Habitat: Swamp water or sewage polluted waters.

Appendix: Additional species have been deseribed as belonging in this genus. Those described by Ravenel have generally been overlooked although he was one of the earliest workers to culture these organisms. The list follows:

Cladothrix fungiformis Ravenel. (Mem. Nat. Acad. Sci., 8, 1896, 19.) From deep virgin soil.

Cladothrix intestinalis Rarenel (Ioc. cit., 18). From virgin soil.

Cladothrix non-liquefacions Ravenel (loc. cit,, 16). From deep made soil. Cladothrix profundus Ravenel (loc. cit., 17). From deep made soil.

Cladothrix ramosa Gasperini. (Atti d. Soc. toscana d'Ig., 2, 1912, 000.$)$ From water.

Cladothrix retzcularis Jaumann. (Küungl. Svenska Tetenskapsakad. Handl., I, 62, Part 4, 1921, 41; Sphaerotilus reticularis Cataldi, Thesis, Iniv. Buenos Aires, 1939, 55.) From Aneboda region, Sweden.

Sphaerotilus roseus Zopf. Beiträge z. Physiol. u. Morph. nieder. Organismen, 1892, 32.) From water.

\section{Genus II. Clonothrix Ruze.}

(Jour. d. Bot., 10, 1896, 325.) From Greek klon, a twig and thrix, hair.

Attached filaments shoming false branching as in Sphacrotilus. Sheaths organic, encrusted with iron or manganese, broader at the base and tapering toward the tip. Cells colorless, cylindrical. Reproduction by spherical conidia formed in chains by transverse fission of cells; conidia formation acropetal, limited to short branches of the younger portion of the filaments.

The type species is Clonothrix fusca Roze.

1. Clonothrix fusca Roze. (Roze, Jour. d. Bot., 10, 1896, 325; Clonothrix fusca Schorler, Cent. f. Bakt., II Abt., 12, 1904, 689; Crenothrix fusca Dorff, Die Eisenorganismen, Pflanzenforsehung, Heft 16, 193t, 41.) From Iatin fuscus, brown.

Cells cylindrieal with rounded ends, 2 by 10 microns, becoming larger toward the base and smaller toward the tips of the filaments.

Sheaths 7 microns at the base to $2 \mathrm{mi}$ crons at the tips.

Conidia about 2 microns in diameter.

This organism was described by Roze as a blue-green alga, but subsequent observers have failed to find pigment. It was deseribed independently by Schorler who gave it the same name. Cholodny considered it identical with Crenothrix polyspora but liolk (Amer.
Jour. Bot., 25, 1938, 11) has clearly differentiated these species.

Habitat: Waterworks and pipes.

Appendix: Apparently the following species resemble Clonothrix fusca:

Clonothrix tenuis Kolkwitz. (Kolkwitz, Schizomycetes in Kryptogamenflora der Mark Brandenburg, 5, 1915, 14t; Crenothrix tenuis Dorff, Die Eisenorganismen, l'flanzenforschung, Heft 16, 1934, 42.) From the settling basin of a sewage plant near Berlin. Dorff thinks this may have been a growth form of Crenothrix fusca Dorff.

Mycothrix abundans Nilumann. (Kungl. Svenska Vetenskapsakad. Hand1. I, 62, 1921, Part 4, 4t.) From the Aneboda region, Sweden. The type species of the genus Mycothrix.

Hycothrix clonotricoides Naumann (loc. cit., 54). From the Aneboda region, Sreden.

\section{Genus III. Leptothrix Kützing.}

(I ützing, Phyoologia Generalis, 1843, 198; not Leptotrichia Trevisan, Reale Ist . Lombardo di Sci. e Lettere, Ser. 2, 12, 1879, 138; Detoniella DeToni and Trevisan, in 
Saccardo, Sylloge Fungorum, 6, 1889, 929; Chlamydothrix Migula, Syst. d. Bakt., 2, 1900, 1030; Conidiothrix Benecke, Bau u. Leben d. Bakt., 1912, 489; Megalothrix Schwers, Cent. f. Bakt., II Abt., 33, 1912, 273; Syncrotis Enderlein, Sitzber. Gesell. Naturf. Freunde, Berlin, 1917, 312.) From Greek leptos, small and thrix, hair.

Filaments of cylindrical colorless cells, with a sheath at first thin and colorless, later thicker, yellow or brown, encrusted with ferric hydroxide. The oxide may be dissolved by dilute acid, whereupon the inner cells show up well. Multiplication is by division and abstraction of cells and by motile cylindrical swarmers. False branching may occur.

The type species is Leptothrix schracca liüzing.

Key to the species of genus Leptothrix.

I. Filanents not spirally twisted.

1. Free swimming, not attached.

1. Sheath homogeneous, cylindrical.

1. Leptothrix ochracea.

2. Sheath composed of a bundle of fine parallel filaments.

2. Leptothrix trichogenes.

B. Attached to at substrate by a holdfast.

1. Arising singly, each filament from its own holdfast.

a. Filaments show false branching.

3. Leptothrix discophora.

aa. Filaments unbranched.

1. Leptothrix sideropous.

2. Numerous filaments arising from a common holdfast.

a. Filaments large, uniform in diameter.

5. Leptothrix lopholea.

aa. Filaments smaller, tapering toward the tip.

6. Leptothrix échinata.

II. Filaments spirally twisted.

A. Epiphytic, growing twisted around filamentous algae.

B. Not epiphytic.

7. Leptothrix epiphylica.

8. Leptothrix pseudoracuolata.

1. Leptothrix ochracea Tíüzing. (Kützing, Phycologia Generalis, 1843, 198; Lyngbya ochracea Thuret, Ann. Sei. Nat. Bot., VT, 1, 1875, 279; Beggiatod. ochracea (iasperini, Inn. d'Igiene Sper. 2, 1912, 000; ('hlumydothix ochracea Migula, Syst. d. Bakt., 2, 1900, 1031.) From Iatin ochra, yellow.

Long filaments, free-floating, never attached to a substrate, never branch. ing. Filaments 1 micron in thickness, composed of rod-like colorless cells, surrounded in young filaments by a delicate sheath which later heermes yellow to brown in color.
Sheath homogeneous, completely dissolving in dilute hydrochloric acid.

When the sheath hecomes very thick, the filaments creep out of the sheath and secrete a new one, so that many cmpty sheaths ate found. Polat Hagellate. motile, swarn-cells have been observed.

Habitat: Iron-bearing waters.

2. Leptothrix trichogenes Cholodny. (choledny, cent. f. Bakt., II Abt., 61, 192. 292; Toxolhrix ferruginea Molisch, Die Eisenbakterien in Japan, Scc. Report Tohoku J. Univ., 4 Ser. Biol., 1, 1925. 
13.) From Greek thrix, hair and geno, producing.

Long, slender, articulated filaments, free-floating, never branched. Filaments 0.5 micron in thickness, composed of rod-like colorless cells.

Filaments surrounded by a fine sheath . This sheath ruptures longitudinally and rolls up as a fine hair-like body at one side of the filament. This process continually repeated leads to the development of a thick sheath composed of numerous hair-like bodies arranged in parallel bundles, which are easily separated from the filament. The sheath is completely dissolved in dilute hydrochloric acid.

Mode of reproduction is unknown.

Habitat: Iron-bearing waters.

3. Leptothrix disccphora (Schwers) Dorff. (Megalothrix discophora Schwers, Cent. f. Bakt., II Abt., 33, 1912, 273; Leptothrix crassa Cholodny, Cent. f. Bakt., II Abt., 61, 1924, 292; Chlamydothrix discophora Naumann, Ber. d. Deutsch. Bot. Ges., 46, 1928, 141; Dorff, Die Eisenorganismen, Pflanzenforschung, Heft 16, 1934, 31.) From Latin liscus, disk and Greek phorous, to bear.

Long, slender, articulated filaments composed of elements of varying length showing false branching (Cholodny, loc. cit., 297). Usually attached to a submerged substrate but may be free-floating.

Filaments surrounded by a heavy sheath, thick (10 to 15 microns) at the base, tapering toward the free tip, heavily impregnated with ferric hydroxide.

Reproduction by motile swarm cells liberated from the tip, and also by the emergence of the filament from the sheath, with subsequent breaking up into individual non-motile cells (conidia).

Habitat: Water.

\section{Leptothrix sideropous (Molisch)} Cholodny. (Chlamydothrix sideropous
Molisch, Die Eisenbakterien, 1910, 14; Gallionella sideropous Naumann, Kungl. Svenska Vetenskapsakad., 62, 1921, 33; Cholodny, Die Eisenbakterien, Pflanzenforschung, Heft 4, 1926, 25.) From Greek sideros, iron.

Short, unbranched filaments composed of rod-shaped cells of varying length, 0.6 micron in diameter.

Sheath very thin, colorless, giving an iron reaction only at the base of the filament. Attached by a broad holdfast. which gives a marked iron reaction.

Habitat: Found in water, growing on submerged surfaces.

5. Leptothrix lopholea Dorff. (Die Eisenorganismen, Pflanzenforschung, Heft 16, 1934, 33.) From Greek lophos, crest, tuft.

Short, slender unbranched filaments, uniform in diameter, attached to a substrate, 5 to 13 filaments arising from a common holdfast. Filaments 20 to 33 microns long, cells 0.5 by 1.0 to 1.3 mic rons.

Sheaths composed of ferric hydroxide dissolve completely in dilute hydrochloric acid.

Filaments creep out of the sheath as in Leptothrix ochracea.

Habitat: Water.

6. Leptothrix echinata Beger. (Cent. f. Bakt., II Abt., 92, 1935, 401.) From Latin echinatus, bristled.

Similar to the preceding species, but occurring in larger colonies, 20 to 50 filaments arising from a common holdfast. Filaments are shorter $(9$ to 10 microns).

Sheath is thicker at the base and tapers toward the free tip of the filaments, which are slightly spiral. The sheath contains an organic matrix visible after treatment in dilute hydrochloric acid.

Habitat: Found in water, especially in manganese-bearing waters.

7. Leptothrix epiphytica (Migula) Chester. (Streptothrix epiphytica Migula, 
in Engler and Prantl, Die natürl. Pflanzenfam., 1, 1a, 1895, 38; Lyngbya cpiphytica Hieronymus, in Kirchner, ibid., 67; Chester, Manual Determ. Bact., 1901, 370; Leptothrix volubilis Cholodny, Cent.f. Bakt., II Abt., 61, 1924, 292; Chlamydothrix epiphytica Naumann, Ber.d. Deutsch. Bot. Gies., 46, 1928, 141.) From M.L.epiphyticus, epiphytic.

Long eylindrical unbranched filaments growing spirally around filaments of Tolypothrix, Oedogonium, etc. Cells rod-shaped, 1 by 2 nierons.

Sheaths cylindrical, encrusted with iron.

Cells may leave the sheaths as in Leptothix ochraceu.

Habitat: Water.

8. Leptothrix pseudovacuolata (Perfiliev) Dorff. (Spirothrix pseudovacuolata Perfiliev, Verh. d. Int. Verein. f. theor. 11. angew. Limnologie, 1925, Stuttgart, 1927; Dorff, Die Eisenorganismen, Pflanzenforschung, Heft 16, 1934, 36.) From Greek, having false vareuoles.

Filaments 85 to 250 microns in length, unbranched, spirally wound, oceasionally straight. Strongly encrusted with ferric hydroxide. Spirals 20 to $24 \mathrm{mi}$ crons from erest to crest.

Cells rounded at the ends, thin-walled, granular, 1.7 to 2.8 by 3.5 to 30 microns.

Apparently heterotrophic.

Habitat: Found in bot tom muds of deep lakes with very low oxygen eontent.

Appendix: The following simple, filamentous organisms have also been placed in the genus Leptothrix or appear to belong here :

Chlamydothrix thermalis Molisch. (Die Eisenbakterien in Japan. Sc. Report Tohoku J. Univ., 4 Ser. Biol., 1, 1923, 135; Leptothrix thermalis Dorff, Die
Eisenorganismen, Pflanzenforschung, Heft 16, 1934, 38.) From hot springs in Japan.

Leptothrix hyalina (Migula) Bergey et al. Streptothrix hyalina Migula, in Engler and Prantl, Die natürl. Pflanzenfam., 1, 1a, 1895, 38; Chlamydothrix hyalina Migula, Syst. d. Bakt., 2, 1900, 1003; Bergey ot al., ManuaI, 1st ed., 1923, 391.) From swamp water.

Leptothrix major Dorff. (Die Eisenorganismen, Pflanzenforschung, Heft 16, 1934, 35.). From Spree River water near Berlin.

Leptothrix winogradskii Cataldi. (Thesis, Univ. Buenos Aires, 1939, 64.) From water.

Lieskcella bifuda Perfiliev. (Perfiliev. Verh. d. internat. Vereinigung f theoret. u. angew. I imnologie, 1925, Stuttgart, 1927; quoted from Dorff, Die Eisenorganismen, Pflanzenforschung, Heft 16, 1934, 27; also designated Liesheella bifilaris by Perfiliev.) From iron bearing water. Shows gliding movements similar to blue-green algae. The type species of the genus Lieskeella Perfiliev.

Sideromyees glomerata Naumann. (Quoted from Dorff, Die Eisenorganismen, Pflanzenforschungen, Heft 16, 1934, 27.) From swamps in the Aneboda region of Sweden. This is the type species of the genus Sideromyces syn. Mycogallionella Naumamn.

Sphaerothrix latens Perfiliev. (Perfiliev, Verh. d. internat. Vereinigung $f$. theoret. u. angew. Limnologie, 1925, Stuttgart, 1927; quoted from Dorff, Die Eisenorganismen, Pflanzenforschung, Heft 16, 1934, 29.) From a peat bog in a small pond near Leningrad. This is the type species of the genus Sphaerothrix Perfiliev. Grows in disks showing a concentrie structure. 


\section{FAMILY II. CRENOTHRICHACEAE HANSGIRG. *}

\section{(Österr. Bot. Ztschr., 36, 1888, 228.)}

Filaments not branched, attached to a firm substrate, showing differentiation of base and tip. Sheaths plainly visible, thin and colorless at the tip, thick and encrusted with iron at the base. Cells cylindrical to spherical, dividing in three planes to produce the spherical non-motile conidia.

\section{Genus I. Crenothrix Cohn.}

(Cohn, Beitr. z. Biol. d. Pflanz., 1, Heft 1, 1870, 108; Phragmidiothrix Engler, Verh. Bot. Ver. Brandenb., 24, 1882, 19.) From Greek crenos, spring and thrix, hair.

Characters as for the family.

The type species is Crenothrix polyspora Cohn.

1. Crenothrix polyspora Cohn. (Beitr. z. Biol. d. Pflanz., 1, Heft 1, 1870, 10s; Hypheothrix kuehniana Rabenhorst, Flora europ. algarum, Sect. II, 88; Leptothrix kuehniana Rabenhorst, Algen Sachsens, No. 284; Crenothrix kuehniana Zopf, Zur Morphologie der Spaltpilzen, 1882, 36; Crenothrix manganifera Jackson, Hyg. Rund., 14, 1904, 19.) From Greek, many spores.

Long, articulated filaments, unbranched, enclosed in a sheath which becomes expanded toward the tip. The sheath is composed of organic matter encrusted with iron. Filaments, including the sheath, measure 2 to $9 \mathrm{mi}$ crons in diameter.

Vegetative cells vary markedly in length from long eylindrical to short ovoid forms.

Conidia, spherical, 1 to 2 microns in diameter, are liberated from the expanderl tips of the sheaths. They are non-motile.

Cultivation: Has not been grown on artificial media in pure culture.

Conidia may germinate upon the ex- terior of the sheath from which they have been liberated, giving rise to new filaments attached to the surface of the older one, presenting a simulation of false brunching.

Cholodny believed Clonothrix fusca to be identical with Crenothmx polyspora. However, Clonothrix fusca shows genuine false branching and produces conidia by fission in only one plane, so that the filaments taper toward the tir instead of expanding (see Folk, Amer. Jour. Bot., 25, 1938, 11) for a clear cut differentiation of these two species.

Source: This organism is wide-spread in water pipes, drain pipes and springs where the water contains iron. It frequently fills pipes under such circumstances and causes a real nuisance. Found by Cohn in samples of water from springs in the neighborhood of Breslau, Germany .

Habitat: In stagnant and running waters containing organic matter and iron salts, growing as thick brownish or greenish masses.

* Completely revised by Prof. A. T. Henrici, Lniversity of Minnesota, Minneapolis, Minnesota, December, 1938; further revision by Prof. Robert S. Breed, New York State Experiment Station, Geneva, New York, July, 1946. 


\section{FAMILY III. BEGGIATOACEAE MIGULA.*}

(Arb. Bakt. Inst. Karlsruhe, 1, 1894, 238: in part, Leuco-Thiobacteria Bavendamm, Die farblosen and roten Schwefelbakterien, Pflanzenforschung, Heft 2, 1924, 102.)

Filamentous organisms, composed of chains of cells. Individual cells generally not visible without staining. Structure very similar to that of Oscillatoriaceae, but devoid of chlorophyll and phycocyanin. When growing in the presence of hydrogen sulfide, the filaments contain sulfur globules. Special reproductive structures unknown.

In proposing the family Beggiatoaceae for the two genera of this subgroup known in 1894, Migula remarked that "it would be best to combine them with the Oscillatoriaceae and classify them among the Schizophyta" (Arb. Bakt. Inst. Karlsruhe, 1, 1894, 238). The same authority has stated: "Also in view of their internal structure the species of Beggiatoa are so similar to those in the genus Oscillaria that they can hardly be separated generically" (in Engler and Prantl, Die nat ürl. Pflanzenfam., $1,1 \mathrm{a}, 1895,41)$.

Since then, the close relationship hetween the filamentous, colorless sulfur bacteria and the blue-green algae of the family Oscillatoriaceae has become increasingly clear. A particularly important line of evidence is supplied by the discovery of sulfur bacteria paralleling each of the major genera of the Oscillatoriaceae. The family Beggiatoaceae Migula is retained for these filamentous sulfur bacteria. Taxonomically they could readily be classified as colorless members of the class Schizophyceae.

\section{Ley to the genera of family Beggiatoaceae.}

I. Filaments non-motile. Grow attached by means of holdfast at base.

Genus I. Thiothrix, p. 988.

II. Filaments motile, like Oscrllaloria, by creeping or sliding movements along a solid substrate. Not attached.

A. Occurring singly, not embedded in a common slime-sheath.

1. Filaments straight or bent, but not permanently coiled.

Genus II. Beggiatoa, p. 990 .

2. Filaments coiled or spirally wound.

Cienus III. Thiospirillopsis, p. 993.

B. Occurring in bundles, cmbedded in a common slime-sheath.

Genus IV. Throploca, p. 993.

Genus 1 . Thiothrix Winogradsky.

(Beitr. z. Morph. u. Physiol. d. Bakt., I, Schwefelbacterien, Leipsig, 1888, 39.) From Greek theion, sulfur, and thrix, hair.

Filaments non-motile, segmented, with a delicate sheath, and differentiated into base and tip. Grow attached at base to solid objects by means of gelatinous holdfast. Reproduction by transverse fission of the segments, and by rod-shaped socalled conidia, probably arising by the apical segments becoming free. Temporarily, the conidia show creeping motility, settle on solid objects, and grow out into new filaments.

The type species is Thiothrix nivea (Rabenhorst) Winogradsky.

The following key to the species of the genus Thiothrix is based upon the diameter

* Completely revised by Prof. C. B. Van Niel, Hopkins Marine Station, Pacific Grove, California, January, 1944. 
of the filaments and their habitat, the only criteria used by previous authors for the differentiation of the seven published species. The validity of these distinguishing characteristies is, however, doubtful because their constancy has not been sufficiently established; so far the morphology of the Thiothrix spreies has not been studied in pure cultures.

\section{Key to the species of genus Thiothrix.}

I. Found in fresh water environments.
1. Diameter of filaments about $2(1.4$ to 3.0$)$ microns.

1. Thiothrix nivea.

B. Diameter of filaments about 1 mieron.

\section{Thiothrix tenuis.}

C. Diameter of filaments less than 0.5 micron.

3. Thiothrix tenuissima.

II. Found in marine environments.

1. Diameter of filaments averages about 20 microns (actual range 15 to $30 \mathrm{mi}$ crons).

t. Thiothrix voukii.

B. Diameter of filaments about $t(4.4$ to 6.6$)$ microns. Segments about 25 microns long.

5. Thiothrix longiarticulala.

C. Diameter of filaments about 3 (1.8 to 5) microns. Segments about 1 micron long.

6. Thiothrix annulata.

D. Diameter of filaments about $1(0.8$ to 1.3$)$ micron.

7. Thiothrix marina.

1. Thiothrix nivea (Rabenhorst) Winogradsky. (Beggiatoa nivea Rabenhorst, Flora europaea algarum, 2, 1865, 9ł; Leptotrichia nivea De Toni and Trevisan, in Saccardo, Sylloge Fungorum, 8, 1889, 934; Symphyothrix nivea Wartman and Schenk, Schweiz. Kryptogamenflora; Winogradsky, Beitr. z. Morph. u. Physiol. d. Bact., I, Schwefelbacterien, 1888, 39.) From Latin niveus, snowy.

Filaments with a thin sheath, diameter 2.0 to 3.0 microns at base, 1.7 mierons in the middle, 1.4 to 1.5 microns at tip. As long as the filaments contain sulfur globules, segmentation is invisible; length of segments 4 to 15 microns, the longer ones usually near a pex, the shorter ones near base.

Motile segments (so-called conidia) mostly single, 8 to 15 microns long, sometimes in short filaments of 2 to 4 cells and up to 40 microns long. These segments may settle and develop near the base of the mother filament or on a filament itself, forming verticillate structures. These have been described as Thiothrix nivea var. verticillata Miyoshi (Jour. Coll. Sci., Imp. Univ. Tokyo, 10, 1897,156 ).

Habitat: Fresh water cuvironments where hydrogen sulfide is present (sulfur springs, stagnant pools, on submerged decaying vegetation, etc.).

2. Thiothrix tenuis Winogradsky (Beggiatoa alba var. uniserialis Engler, Üb. die Pilz-Vegetation des weissen oder todten Grundes in der Kieler Bucht, 1883, 4; Winogradsky, Beitr. z. Morph. u. Physiol. d. Bact., I, Schwefelbacterien, 1888, 40.) From Latin tenuis, slender.

Filaments about 1.0 micron in diameter, of nearly uniform thickness. Often in dense, felted masses. Segments 4 to 5 microns long.

Habitat: Fresh water environments 
where hydrogen sulfide occurs. According to Bavendamm (Die farblosen $u$. roten Schwefelbakt., Pflanzenforschung, Heft $2,1924,107$ ) also found in sea water.

3. Thiothrix tenuissima Winogralsky. (Beitr. z. Morph. u. Physiol. d. Bact., I, Schwefelbacterien, 1888, 40; Thiothrix minutissima L phof, Arch. f. Hydrobiol, 18, 1927,77.) FromLatin tenuis, diminu tive, very slender.

Filaments less than 0.5 micron in diameter, usually in dense masses.

Habitat: Fresh water environments where hydrogen sulfide oceurs.

4. Thiothrix voukii Klas. (Arch. f. Protistenk., 88, 1936, 123.) Named for Vouk, a Pussiun scientist.

Filaments 15 to 30 , most frequently 17 microns in diameter, of rather uniform thickness. Segments visible without. special treatment. Segments generally somewhat longer than wide, rarely square, oceasionally barrel-shaped. Length of segments 15 10 30, nostly 19 to 23 mierons. Motile segments not yet observed.

Apart from the lack of motility. this species closely resembles the notile Beggiatoa mirabilis.

Source: Found in effluent of sulfur springs at seashore near Split, Jugoslavia. So far reported only onee.

Habitat: Marine enviromments eontaining hydrogen sulfide.

\section{Thiothrix longiarticulata Klas.} (Arch. f. Protistenk., 88, 1936, 126. From Latin longus, long :nel articulatus jointed.

Filaments 3.3 to 6.6 , most frequently 4.2 microns in diameter, of unifom thickness. Orcur in dense, folted masses. Segments long, measuring 19 to 33 , mostly 26 microns in length. Sulfur droplets usually absent in the proximity of cross-walls. Motile segments not yet reported.

Source: Found in effluent of sulfur springs at seashore near Split, Jugoslavia. So far reported only once.

Habitat: Marine environments containing hydrogen sulfide.

6. Thiothrix annulata Molisch. (Cent. f. Bakt., II Abt., 33, 1912, 58.) From Latin annulatus, ringed.

Filaments 3 to 4 , oceasionally up to 5 microns in diameter, thinner at base (2 microns) and at tip (1.8 microns). Segments only about 1 micron in length. Often found with narrow bands which are free of sulfur, thus giving a ringed appearance to the filaments. Old filaments may shom spacial thickening and distortion, but this is not characteristic for the species.

Hahitat : Marine enviromments containing hydrogen sulfide; frequently on dearing algar.

7. Thiothrix marina Molisch. (Cent. f. Bakt., II Abt., 83, 1912, 58.) From Latin marims, pertaining to the sea. Filaments about 1 (0.8 to 1.3$)$ micron in diancter, of rather uniform thickness. Lsually in felted masses.

Resembles Thiothrix tenuis. Since the latter has been reported from marine environments (Bavendamm, Dir farblosen u. roten Schwefelbakterien, Pflanzenforschung, Ileft 2, 1924, 107), Thiothrix marina may not be an independent species, but identical with Thisthrix tenuis.

Hah,itat: Mariue (?) environmentsconbiining hydrogen sulfide; frequently on deeaying algae.

\section{Genus II. Beggiatoa Trevisan.}

(Prospetto della Flora Euganea, 1842, 56.) Named for the Vicenza physician, F. S. Beggiato.

Filamentous, motile, segmented organisms, oceurring singly or in white to creamy folted masses in which the separate filaments retain their individuality. Not at- 
tached. Existence of a sheath not definitely established. Movenents of the filaments dependent upon a solid substratum over which they slide in the same manner as species of Oscillatoria. Sliding movements often accompanied by rotation of filaments around long axis. Réproduction by transverse fission of segments; the filaments may also break up into smaller units, each continuing a separate existence. The latter mode of multiplication corresponds to that by means of the so-called motile conidia or segments in Thiothrix.

The type species is Beggiatoa alba (Vaucher) Trevisan.

In this genus, also, the species so far described are differentiated on the basis of dimensions. The range of sizes for separate species appears, in most cases, quite arbitrary, especially in view of the existence of practically all intermediate stages. Since the smaller forms have heen found both in fresh water and marine environments (Bavendamm, Die farblosen $u$. roten Schwefelbakterien, Pflanzenforschung, Heft 2, 1924, 104), the previously described Beggiatoa marina has been omitted as a separate species. Pure culture studies may establish more staisfactory methods of differentiation and a better understanding of speciation.

\section{Key to the species of genus Beggiatoa.}

I. Diameter of filaments greater than 25 microns.

\section{Beggiatoa gigantea.}

II. Diameter of filaments less than 25 microns.

A. Diameter of filaments greater than 15 microns.

2. Beggiatoa mirabilis.

B. Diameter of filaments less than 15 microns.

1. Diameter of filaments greater than 5 microns.

3. Beggiatoa arachnoidea.

2. Diameter of filaments less than 5 microns.

a. Diameter of filaments greater than 2.5 microns.

4. Beggiatoa alba.

aa. Diameter of filaments less than 2.5 microns.

b. Diameter of filaments greater than 1 micron.

5. Beggiatoa leplomitiformis.

bb. Diameter of filaments less than 1 micron.

6. Beggiatoa minima.

1. Beggiatoa gigantea Kilas. (Arch. f. Mikrobiol., 8, 1937, 318; includes the arge forms of Beggiatoa mirabilis Colnn, Hedwigia, 4, 1865, 81.) From Greek gigas, giant.

Filaments 26.4 to 55 , average 35 to 40 microns in diameter. Klas, in his diagnosis, gives 26.4 to 42.9 microns as dimensions. This would exclude the largest forms of Beggiatoa mirabilis described by Hinze (Ber. d. deut. bot. Ges., 19, 1901, 369). Since the proposal of a separate species for such organisms appears at present unjustified, the maximum diameter has here been inereased. Fila- ments clearly segmented; length of segments 5 to 13 , average 8.5 microns. Teminal cells rounded or tapering.

When the filaments are in healthy condition they are of uniform width; bulging of the sides indicates unfavorable conditions.

Habitat: Ipparently restricted to marine environments containing hydrogen sulfide. Frequent on decaying marine algae

2. Beggiatoa mirabilis Cohn emend. Klas. (Cohn, Hedwigia, 4, 1865, 81; 
Kilas, Arch. f. Mikrobiol., 8, 1937, 318.) From Latin mirabilis, wonderful.

Filaments 15 to 21.5 , average $17 \mathrm{mi}$ crons in diameter. The so-defined species does not overlap with Beggiatoa gigantea according to Klas (loc. cit.). Segmentation usually observable without special treatment; segments 5 to 13 , average 8.5 microns long. Terminal cèlls rounded or tapering, sometimes bent.

When the filaments are in healthy condition they are of uniform width; an unfavorable environment induces bulging of the sides.

Habitat: Apparently restricted to marine environments containing hydrogen sulfide. Common on decaying marine algae.

Uphof (Arch. f. Hydrobiol., 18, 1927, 83) has ereated a species, Beggiatoa maxima, which on account of its diameter (10 to 20 microns) falls partly within the range of Beggiatoa mirabilis, partly within Beggiatoa arachnoidea. Sinec it was found in a fresh water environment, the habitat of Beggiatoa mirabitis may not be restricted to marine media.

\section{Beggiatoa arachnoidea (Agardh)} Rabenhorst. (Oscillatoria arachnoidea Agardh, Regensburger Flora, 1827, 634; Rabenhorst, Flora europaea algarum, 1865, 94; Beggiatoa pellucida Cohn, Hedwigia, 4, 1865, 82; ? Oscillatoria beggiatoides Arzichowsky, Bull. Jard. Imp. Bot., St. Pétersb., 2, 1902, 38, 47; includes the larger members of Beggiatoa major Winogradsky, Beitr. z. Morph. u. Physiol. d. Bact., I, Schwefelbacterien, 1888, 25; and the smaller ones of Beggiatoa maxima Uphof, Arch. f. Hydrobiol., 18, 1927, 80.) From Greek, resembling a cobweb.

Filaments 5 to 14 microns in diameter. Segmentation generally observable only after special staining or removal of sulfur globules; segments 5 to 7 microns in length. Terminal cells rounded, often tapering. Filaments of uniform width.
Habitat: Both fresh water and marine environments containing hydrogen sulfide.

4. Beggiatoa alba (Vaucher) Trevisan. (Oscillatoria alba Vaucher, Histoire des Conferves d'eau douce, 1803, 198; Beg-d gialoa punctata Trevisan, Prospetto della Flora Euganea, 1842, 56; Beggiatoa alba var. marina Cohn, Hedwigia, 4 , 1865, 83; Beggiatoa marina Molisch, Cent. f. Bakt., II Abt., 33, 1912, 58; in part, Beggiatoa major Winogradsky, Beitr. z. Morph. u. Physiol. d. Bact., I, Schwefelbacterien, 1888, 25.) From Latin albus, white.

This is the type species of the genus. Filaments 2.5 to 5 , most commonly 3 microns in diameter, of even width. Segmentation difficult to detect in filaments containing many sulfur globules; segments 3 to 9 microns long, shortly after division practically square. Terminal cells rounded.

Habitat: Both fresh water and marine environments eontaining hydrogen sulfide.

Distribution: Lbiquitous, and probably the most common of the filimentous sulfur bacteria.

\section{Beggiatoa leptomitiformis (Mene-} ghini) Trevisan. (Oscillatoria leptomitiformis Meneghini, Delle Alghe viventi nelle terme Euganee, 1844, 122; Trevisan, Prospet to della Flora Euganea, 1842, 56; Beggiatoa media Winogradsky, Beitr. z. Morph, u. Physiol. d. Bact., I, Schwefel. bacterien, 18ss, 25.) From Greek leptos, small and mitos, thread and Latin forma, shape.

Filaments 1 to 2.5 microns in diameter, of uniform width. Segmentation only observable after removal of sulfur globules; segments 4 to 8 microns in length. Terminal cells usually rounded.

Habitat: Fresh water and marine environments containing hydrogen sulfide. 
6. Beggiatoa minima Winogradsky. (Winogradsky, Beitr. z. Morph. u. Physiol. d. Bacterien, I. Schwefelbacterien, 1888, 25; Beggiatoa minor Uphof, Arch. f. Hydrobiol., 18, 1927, 79; not Beggiatoa minima Warming, Om Nogle ved.Danmarks Kyster levende Bakterier, 1876,52 , which from the description is not a Beggiatoa.) From Latin minimus, least.

Filaments less than 1 micron in diameter, of uniform width. Normally appears unsegmented; length of segments about 1 micron.

Habitat: Fresh water and marine environments containing hydrogen sulfide.

\section{Genus III. Thiospirillopsis Uphof.}

(Arch. f. Hydrobiol., 18, 1927, 81.) From Greek theion, sulfur M.L.spirillum, spirillum and Greek opsis, appearance.

Filamentous, colorless sulfur bacteria, segmented, and spirally wound. Exhibit creeping motility, combined with rotation, so that the filaments move forward with a corkscrew-like motion. The tips produce oscillating movements. Resembles Spirulina among the Oscillatoriaceae.

The type species is Thiospirillopsis floridana Uphof.

1. Thiospirillopsis floridana Uphof. (Arch. f. Hydrobiol., 18, 1927, 83.) Named from Florida, the place where it was first found.

Iilaments 2 to 3 microns in diameter. Segmentation difficult to observe without special precautions; segments about 3 to 5 microns long. The spiral windings are regular.

Source: Found in the sulfur spring water at Weliwa Springs and Palm Springs, Florida. A very similar organism has been observed at Pacific Grove, California, in a marine aquarium where hydrogen sulfide had been generated by sulfate reduction. The genus Thiospirillopsis may, therefore, be more wide-spread than is generally believed.

Habitat: Probably widely distributed in water containing sulfur.

\section{Genus $I V$. Thioploca Lauterborn.}

(Ber. d. deut. botan. Ges., 25, 1907, 238.) Name derived from Greek theion, sulfur, and ploka, braid.

Filaments of Beggiatoa-like appearance, but occurring in parailel or braided bundles, enclosed by a common wide slime-sheath. The latter is frequently incrusted on the outside with detritus. Within the sheath the individual filaments are motile in the manner of Beggiatoa; the filaments are segmented, the terminal segments often tapering.

Resembles closely the genera Hydrocoleus and Microcoleus among the Oscillatoriaceae.

It is doubtful whether the members of the genus Thioploca are true colorless sulfur bacteria; most investigators of these forms have reported a greenish-blue coloration of the filaments. Only the regular occurrence of sulfur droplets in filaments taken from their natural habitat stamps the organisms as sulfur bacteria. In view of the close relationship of the Beggiatoaceae to the blue-green Oscillatoriaceae, this is, however, a minor issue.

Four species have been deseribed to date. Three correspond, with respect to the individual filaments, to Béggiatoa arachnoidea, Beggiatoa alba, and Beggiatoa leptomitiformis respectively; the fourth appears to be a combination of the first and third 
species of Beggiatoa in a common shealh. This oceurrence of two distinct species of Beggiatoa in a common sheath makes the genus a doubtful taxonomic entity.

The type species is Thioploca schmidlei Lauterborn.

\section{Key to the species of genus Thioploca.}

I. Filaments in a common sheath of fairly uniform diameter.

A. Diameter of individual filaments 5 to 9 microns.

1. Thioploca schmidlei.

B. Diameter of individual filaments 2 to 5 mierons.

2. Thioploca ingrica.

C. Diameter of individual filaments 1 to 2 microns.

3. Thioploca minima.

II. Filaments in common sheath of greatly different diameter.

4. Thioploca mixta.

1. Thioploca schmidlei Lauterborn. (Ber. d. deut. bot. Ges., 25, 1907, 238.) Named for Mr. Schmidle.

Individual filaments in a common sheath 5 to 9 microns in diameter, clearly segmented. Segments 5 to 8 microns in length. Mucilaginous sheath 50 to 160 microns in diameter. Number of filaments embedded in one sheath variable.

Source: Various Iocalities in Central Europe.

Habitat: So far reported only in fresh water mud, containing hydrogen sulfide and calcium carbonate.

2. Thioploca ingrica Wislouch. (Ber. d. deut bot. Ges., 30, 1912, 470.) From Ingria, an ancient district of Leningrad.

Individual filaments in common sheath 2 to 4.5 microns in diameter, clearly segmented. Segments 1.5 to 8 microns in length. Mucilaginous sheath up to 80 microns in diameter. Number of filaments in one sheath variable.

Source: Various localities in Central Europe.

Habitat: Found in fresh water and marine mud containing hydrogen sulfide.

\section{Thioploca minima Tíoppe. (Arch.} f. Hydrobiol., 14, 1923, 630.) From Latin minimus, least.

Individual filaments in a common sheath 0.8 to 1.5 microns in diameter, segmentation generally observable only after removal of sulfur droplets. Segments 1 to 2 microns long. Mucilaginous sheath up to 30 microns in diameter. Number of filaments in one sheath variable.

Sourec: Various localities in Central Europe.

Habitat: Fresh water and marine mud containing hydrogen sulfide.

4. Thioploca mixta Koppe. (Arch. f. Hydrobiol., 14, 1923, 630.) From Latin mixtus, mixed.

Individual filaments in a common sheath of two clearly different sizes, comprizing both filaments of 6 to $8 \mathrm{mi}$ crons, and filaments of about 1 micron in diameter. The former are clearly segmented, with segments of 5 to $8 \mathrm{mi}$ crons in length. In the latter segmentation is visible after removal of sulfur droplets; segments 1 to 2 microns long. Mucilaginous sheath usually about 50 microns thick. Number of filaments in one sheath variable.

Source: Reported so fir only from Lake Constanza.

Habitat: Fresh water mud containing hydrogen sulfide.

Appendix: In addition to the above genera and species, a number of insufficicntly characterized, filamentous sulfur bacteria which may be related to the 
Beggiatoaceae have been described under various names as follows:

\section{Conidiothrix sulphurea Petersen.} (Dansk Botan. Arkiv., 1, 1921, 1.)

Filamentous, nonmotile organisms, of uniform width, between 0.5 and $1 \mathrm{mi}$ cron in diameter, covered on the outside with sulfur. Segmentation not reported. The outstanding characteristic of the genus Conidiothrix is the supposed multiplication of the filaments by means of conidia which arise by budding on the filament. Apart from this reported occurrence of a budding process, the description is similar to that of Leptothrix sulphurea and of Thiothrix temuis and Thiothrix tenuissima. Since consecutive observations on growing organisms are lacking, it seems adivisable to consider Conidiothrix sulphurea as probably identical with Thiothrix tenuis or Thiothrix tenuissima.

Leptothrix sulphurea Miyoshi. (Jour. Coll. Sci., Imp. Eniv. Tokyo, Japan, 10, 1897,154 .)

Filamentous, non-motile organisms, of uniform width, not exceeding $0.7 \mathrm{mi}$ cron in diameter. The filaments are covered on the outsicle with a powdery deposit of elementary sulfur. Segmentation observable only after special staining; length of segments not published.

Found by Miyoshi in sulfur springs in Japan. Although not reported as containing sulfur globules inside the filaInents, the description would closely fit Thiothrix tenuis or Thiothrix tenuissima Winogradsky. The latter have been observed in masses covered on the outside with elementary sulfur. Therefore, it seems likely that Leptothrix sulphurea is a synonym for Thiothrix tenuis or Thiothrix tenuissima.

Thionema vaginalum Kolkwitz. (Ber. d. deut bot. (ies., 56, 1938, 11.)
The type species of the genus Thionema.

Described as a filamentous, colorless sulfur bacterium, non-motile, attached in the manner of Thiothrix. Filaments 1.5 to 2 microns in diameter, segmented. Segments 2 to 5 microns long. Reproduction, as in the case of Thiothrix, by means of detached segments.

While this part of the description fits that of Thiothrix nivea, the new generic name was proposed on the basis of the occurrence of a distinct sheath, frequently impregnated with iron compounds. Since Winogradsky mentions the occurrence of a sheath also in Thiothrix nivea, it seems desirable to consider Thionema raginatum, at least for the time being, as a probable synonym of Thiothrix nivea.

Source: Found on waterplants in the Teltow-Canal near Berlin, the water containing hydrogen sulfide and iron salts.

Thiosiphon adriaticum Kilas. (Sitzungsber. Akad. d. Wissensch. Wien, Mathem.-naturw. K]., I, 145, 1936, 209.)

Described as a filamentous sulfur bacterium, non-motile, but without segmentation, hence tubular and unicellular. Multiplication by means of conidia arising from restriction of the apical part of the cell. Length of fiJament about 1 to $1.5 \mathrm{~mm}$, widt th 17 to 53 microns, usually tapering towards apex. Conidia 13 to 30 microns by 30 to 50 microns.

The description is at variance with the appearance of the organism in the published photomicrograph in so far as the size of the conidia is concerned. From the photomicrograph this appears to be about 30 by 200 microns. The entire appearance is strongly reminiscent of that of Beggiatoa mirabilis (Beggiatoa gigantea) in certain cultures. The short conidia, described in the text, strikingly resemble species of Achromatium. Consecutive observations on growing cultures of Thiosiphon do not appear to 
have been made. Since (a) the internal structure of the large Beggiatoaceae is easily damaged, (b) the segmentation in living individuals is diffieult to observe when the filaments are filled with sulfur, (c) the presence of Achromatium in the locality from which Thiosiphon was col lected is almost certain, and (d) the de velopmental cycle is merely a reconst rue tion of simultaneously observed elements, considerable doubt as to the validity of the genus appears justified.

* Appendix I: The group of large, unicellular, colorless sulfur bacteria is placed here as a single family, Achromatiaceae Massart as in previous editions of the MANUAL. It includes organisms which are similar in physiology to the Beggiatoaceae.

Massart (Ree. Inst. Bot. Univ. Bruxelles, 5, 1902, 251) proposed the family Achromatiaceae for the bacteria described by Schewiakof' (Ưber einen neuen bacterienähnlichen Organismus des Süsswassers, Habilitationsschrift, Heidelberg, 1893) as Achromatium oxaliferum. The family diagnosis was modified by Nadson (Jour. Microbiol., St. Pétersb., 1, 1914, 72) and by Nadson and Wislouch (Bull. Princip. Jard. But. Républ. Russe, 22, 1923, 33) to include the genera Thiophysa and Thiosphaerella.

In this form, the family represents a homogeneous group of organisms, all chir acterized by a pronounced similarity in cell-shape, strueture, method of reproduction and motility. They exhibit very slow, jerky and rotating movements, but are de. void of flagella or other visible organs of locomotion. They closely resemble the blue-green algae of the genus Synechococcus, even in size.

By including the genus Thiospira in the family Achromatiaceae, Buchman (Jour. Bact., 3, 1918, 462) modified the diagnosis to read:

Unicellular, large, motile (by means of flagella?). Cells containing granules of sulf ur (or in one form possibly oxalate) but no bacteriopurpurin.

Thus was proposed a family in which the spiral sulfur bacteria, indubitably related to species of Spirillum among the Eubacteriales, were linked with the taxomomically obseure species included in Achromatium and Thiophysa. Four genera, Achromatium, Thiophysa, Thiospira and Hillhousia were recognized.

Bavendamm (Die farblosen und roten Schwefelbakterien, Pflanzenforschung, Heft 2, 1924, 109), following the same trend, also combined all non-filamentous forms of the colorless sulfur bacteria into a family Achromaliaceac, with the diagnosis: Cells free, motile. As he realized that Hillhousia should be regarded as a synonym of Achromatium and added the genus Thiovulum Hinze (Ber. d. deut. bot. Ges., 31, 1913, 195), four genera we re again included in the funily. Thiosphaerella was adrled as an appendix to Thiophysa.

Thiovulum is morphologically similar to Achromatium, Thiophysa, and Thiosphaerella with respect to cell size and structure, but differs conspicuously in being actively and rapidly motile. The manner of locomotion suggests the presence of polarly inserted flagella. However; these have never been demonstrated convincingly .

While it is conceivable that a relationship exists between Thiovulum and the organisms of the Achromatium type, the combination of the representatives into one family should be regarded as tentative and open to question. There certainly is no justification at present for including the sulfur spirilla in this family. These are placed in this edition of the MANUAL in Spirilleae among the Eubacteriales.

* Completely revised by Prof. C. B. Van Niel, Hopkins Marine Station, Pacific Grove, California, January, 1944. 


\section{FAMILY A. ACHROMATIACEAE MASSART.}

(Rec. Inst. Bot. Univ. Bruxelles, 5, 1902, 251.)

Cells large, spherical to ovoid in shape, sometimes rod-shaped, may contain globules of sulfur and/or calcium carbonate crystals. Do not possess photosynthetic pigments. Fresh water and marine forms.

A satisfactory differentiation of the genera Achromatium, Thiophysa, and Thiosphaerella is at present well-nigh impossible. They have here been combined into a single genus, Achromatium.

Achromatium mobile Lauterborn (Verhandl. Natur-histor.-Mediz. Vereins Heidelberg, N.F., 13, 1915, 413) is fundamentally different from the other members of the genus. It possesses a clearly visible polar flagellum, suggesting its close affinity with the Pseudomonadaceae among the Eubacteriales. Whether it is a true sulfur bacterium has not been established with certainty; this appears very doubtful in the case of the two similar forms deseribed as Pseudomonas bipunctata and Pseudomonas hyalina by Gicklhorn (Cent. f. Bakt., II Abt., 50, 1920, 425, 426). Utermöhl and Koppe (Verhandl. Intern. Ver. f. theoret. u. angew. Limnologie, 1913, 86 and Arehiv f. Hydrobiol., Suppl. Bd. 5, 1925, 234) have proposed the generic name Macromonas for this group. This has been adopted here.

All of the above mentioned organisms have so far been studicd exclusively as found in their natural habitats. Pure culture studies are greatly needed. These may show that the peculiar calcium carbonate inclusions (not calcium oxalate as thought by Schewiakoff, nor calcium thiosulfate as believed by Hannevart) in Achromatium oxaliferum and in Macromonas bipunctata occur only under special environmental conditions.

\section{Key to the genera of family Achromatiaceae.}

I. Large, ovoid to spherical organisms, normally containing sulfur globules when found in the presence of hydrogen sulfide.

A. Non-motile, or slowly, jerkily sliding across the substrate.

Genus I. Achromatium, p. 997.

B. Actively motile, independent of the substrate.

Genus II. Thiovulum, p. 999.

II. Rod-shaped and curved organisms, motile by means of polar flagella.

1. Bean-shaped to short rod-shaped organisms which may contain small sulfur globules, but are chiefly characterized by large, round spherules of calcium carbonate as cell inclusions. The polar flagellum is often visible in the larger forms without special staining.

Genus III. Macromonas, p. 1000.

\section{Genus I. Achromatium Schewiatioff.}

(Schewiakoff, Üh. einen neuen bacterienähnlichen Organismus des Süsswassers, Habilitationsschr., Heidelberg, 1893; Modderula Frenzel, Biol. Centralbl., 17, 1897, 801; Hillhousia West and Griffiths, Proe. Roy. Soc., B, 81, 1909, 389). From Greek $a$, without and chroma, color.

Thiophysa Hinze (Ber. d. deut. bot. (ies., 21, 1903, 309) and Thiosphaerella Nadson (Jour. Microbiol., St. Pétersb., 1, 1914, 72) are also included in the genus as defined here

Cnicellular organisms with large cells, shortly cylindrical with hemispherical extremities, also ellipsoidal to spherical. Cells divide by a constriction in the 
middle. Movements, if any, are of a slow, rolling, jerky type and are dependent upon the presence of a substrate. No special organs of locomotion a re known. In their natural habitat, the cells contain sulfur droplets and sometimes additional inclusions, such as large spherules of calcium earbonate.

The type species is Achromatium oxaliferum Schewiakoff.

It is not easy as yet to determine whether several species should be recognized in this genus. There appears to be some justification for differentiating between the forms which contain the characteristic and conspicuous calcium carbonate inclusions and forms in which these large spherules are lacking. The former have been reported mostly from fresh or brackish water environments, while the characteristic habitat of the latter seems to be marine. It is, of course, probable that the internal deposition of calcium carbonate depends upon the composition of the environment, so that the distinction may prove arbitrary and non-specific.

Achromatium cells of widely different sizes have been described. Schewiakoff (Üb. einen neuen bacterienähnlichen Organismus des Süsswassers, Habilitations schrift, Heidelberg, 1893) mentions a variation of 15 to 43 microns in length, and 9 to 22 microns in width for Achromatium oxaliferum. Larger cells have been observed by Warming (Videnskab. Meddel, naturhistor. Foren., Kjöbenhavn, 1875, No. 20-28, 360; size to S5 microns), and by Virieux (Ann. Sci. Natur., Sér. 9, 18, 1913, 265; size to 95 microns in length).

Nadson (Bull. Jard. Imp. Botan., St. P'étersb., 13, 1913, 106; Jour. Microb., St. Pétersb., 1, 1914, 52) proposed the name Achromatium gigas for the larger organisms; also West and Griffiths (Ann. Bot., 97, 1913, 83) created two species, Hillhousia mirabilis, with sizes of 42 to 86 microns long by 20 to 33 microns wide, and Hillhousia palustris, measuring on the average 14 by 25 microns, for the same group of sulfur bacteria.

However, Bersa (Sitzungsber. Akad. Wiss., Winn, Mathem.naturw. Kl., I, 129, 1920,233 ) observed so many intermediate sizes that he recognized only a single species. Nadson and Wislouch (Bull. Princ. Jard. Botan., Républ. Russe, 22, 1923, Suppl. 1, 33) arrived at the same conclusion, and this view is acepted here.

The marine Achromatium types which do not contain calcium carbonate crystals, also have been segregater into species on the basis of their size. Here again, there does not seem to be any valid reason for mantaining several speries as there is a continuous series of intermediate forms.

Thus, the organisms previously deseribed as Achromatium oxaliferum, Achromatium gigas, Hillhousia mirabilis and Hillhousia palustris are provisionally treated here as one species, while the marine counterpart, Thiophysa volutans, is combined with Thiophysa macrophysa and Thosphacrella amylifera, all three being regarded as Achromatium rolutans.

\section{Key to the species of genus Achromatium.}

I. Organisms characteristically containing calcium carbonate crystals in the form of highly refractile, large spherules. Oecur mestly in fresh water and brackish murls.

1. Achromatium oxaliferum.

II. Organisms naturally oceurring without such calcium carbonate inclusions. Found in marine mud.

2. Achromatium volutans. 
1. Achromatium oxaliferum Schewi:koff. (Schewiakoff, Üb, einen neuen bacterienähnlichen Organismus des Süsswassers, Habilitationsschrift, Heidelberg, 1893; Modderula hartwigi Frenzel, Biol. Cent ralbl., 17, 1897, 801; Hillhousia mirabilis West and Griffiths, Proc. Roy. Soc., B, 81, 1909, 389; Hillhousia palustris West and Griffiths, Ann. Bot., 27, 1913, 83; Achromatium gigas Nadson, Bull. Jard. Imp. Bot., St. Pétersb., 13, 1913, 106). From Latin oxalis, intended to refer to the supposed presence of oxalate crystals and foro, to bear.

Enicellular organisms, varying in shape from spherical or ovoid to shortly cylindrical with hemispherical extremities. Division by eonstriction in the middle. Cells vary in size from spheres of about 7 microns or even less in diameter to giant forms 100 microns long by 35 microns wide. The extremes are connected by a continuous series of intermediate sizes.

Organisms may show motility of a jerky and rotating kind, always very slow, and dependent upon a substrate. Typical organs of locomotion absent.

Normally contain small sulfur globules, accompanied by much larger calcium carbonate crystals, the latter in the form of large highly refractile spherules. [nder favorable environmental conditions these may disappear before the sulfur globules. Cells with calcium carbonate inclusions have a very high specific gravity. They are, therefore, found only in the bottom of pools, streams, ete., usually in the mud.

Strictly microaerophilic, and apparently require hydrogen sulfide.

Habitat: Fresh water and brackish mud containing hydrogen sulfide and caleium salts. According to Tadson and Wisloueh (Bull, princip. Jard. bot., Républ. Russe, 22, 1923, Suppl. 1, 33) also in marine mud.

\section{Achromatium volutans (Hinze) comb.} nov. (Thiophysa volutans Hinze, Ber.d. deut. bot. Ges., 21, 1903, 309; Thiophysa macrophysa Nidison, Bull. Jard. Imp. Bot., St. Pétersb., 19, 1913, 106 and Jour. Microb., St. Pétersb., 1, 1914, 54; Thiosphaerella amylifera Nadson, Bull. Jard. Imp. Bot., St. P'étersb., 13, 1913, 106 and Jour. Microb., St. Pétersh., 1, 1914, 54.) From Latin volutans, rolling.

Enicellular organisms, spherical to ovoid in shape, dividing by constriction in the middle. Size variable, ranging from spheres about 5 microns in diameter to ovoids up to 40 microns in length.

Cells may show motility of a jerky and rotating hind, always very slow, and dependent upon a substrate. Typieal organs of locomotion absent.

Normally contain sulfur globules, but lack large internal calcium carbonate deposits.

Microaerophilie, apparently reguiring hydrogen sulfide.

Habitat: Marine mud containing hydrogen sulfide; decaying seaweeds.

\section{Genus II. Thiovulum Hinze.}

(Ber. d. deut. bot. Ges., 31, 1913, 195.) From Greek theion, sulfur and Latin orum, egg.

Lnicellular organisms, round to ovoid. Cytoplasm often concent rated at one end of the cell, the remaining space being occupied by a large vacuole. Multiplication by constriction which, in late stages, merges into fission. Actively motile; movements accompanied by rapid rotation. Flagellation not definitely demonstrated, but type of locomotion suggests polar flagellation. Normally contain sulfur globules in the cytoplasm, hence, these are frequently concentrated at one end of the cell

The type species is Thiovulum majus Hinze. 
As in the case of Achromatium, it is difficult to establish distinct species. Those that have been described differ only in size, and the differences appear to be far from constant. For Thiovulum (Monas) mülleri (Warming) Lauterborn (Verhandl. Naturhist.-medizin. Vereins, Heidelberg, N. F., 13, 1915, 414) the diameter is stated by Warming (Videnskab. Meddel. naturhistor. Foren., Kjöbenhavn, 1875, No. 20-28, 363), Hinze (Ber. d. deut. bot. Ges., 31, 1913, 191) and Lauterborn (loc. cit., 415) respectively to be 5.6 to 15,13 to 15 and 5 to 13 microns. The ovoid cells of Thiorulum majus are noted as being 11 to 18 microns long and 9 to 17 microns wide, while Thiovulum minus comprises the smaller forms from 9.6 to 11 mierons long by 7.2 to 9 microns wide. In view of the regular occurrence of all intermediate sizes it seems best to recognize only a single species at present.

1. Thiovulum majus Hinze. (Hinze, Ber. d. deut. bot. Ges., 31, 1913, 195; including Thiovulum minus Hinze, idem.; Monas mulleri Warming, Videnskab. Meddel. naturhistor. Foren., Kjöbenhavn, 1875, No. 20-28, 363; Achromatium mulleri Migula, Syst. d. Bakt. 2, 1900, 1038; Thiovulum mulleri Lauterborn, Verhandl. Naturhist.-medizin. Vereins, Heidelberg, N.F., 13, 1915, 414.) From Latin major, large.

Lnicellular organisms, spherical to ovoid. Cytoplasm often concentrated at one end of the cell, the remainder heing occupied by a vacuole. Multiplication by constriction which, in late stages, merges into fission. Size of cells, 5 to 20 microns in diameter.
The most characteristic feature is its motility; it is the only one of the spherical to ovoid, colorless sulfur bacteria capable of rapid movement. Flagellation has not been definitively demonstrated, but the type of locomotion suggests the presence of polar flagella.

Normally contains sulfur droplets in rytoplasm, frequently encentrated at one end of cell.

Microaerophilic; apparently requires hydrogen sulfide.

Habitat: In sulfide-containing water, usually accumulating near the surface. Often in cultures of decaying algae. Both in fresh water and marine environments.

\section{Genus III. Macromonas Utermöhl and Koppe.}

(Verhandl. Intern. Ver. f. Theoret. u. angew. Limnologie, 1923, 86.) From Latin macro, large and monas, a unit or cell.

Colorless, eylindrical to bean-shaped bacteria, actively motile by means of a single polar flagellum. Multiplication by constriction (fission). Chiefly characterized by the occurrence of caleium carbonate inclusions in the form of large spherules. In their natural habitat they may also contain small sulfur globules.

The type species is Macromonas mobilis (Iauterborn) Utermöhl and Koppe.

Two species have primarily been distinguished on the basis of cell size. Whether this is sufficiently constant to serve as a specific character has not been definitely established. From studies still limited in seope and extent on the organisms in their natural habitat, it appears at present that the two species should be maintained, at least provisionally. It is possible, however, that further observations, especially with cultures under different environmental conditions, will show the occurrence of intermediate types and of a greater range of variation in size of pure cultures than what has previously been reported. 


\section{Ke'! to the species of genus Macromonas.}

I. Cells measuring 12 microns or more in length and 8 microns or more in width.

1. Macromonas mobilis.

II. Cells measuring less than 12 microns in length and 5 microns or less in width.

2. Macromonas bipunctata.

1. Macromonas mobilis (Lauterborn) Utermöhl and Koppe. (Achromatium) mobie Lauterborn, Verhandl. Naturhist.-Medizin. Vereins, Heidelberg, X. F. 13, 1915, 413; Microspira racillans Cickhorn, Cent, f. Bakt., II Abt., 50, 1920 . 422; Ltermöhl and Koppe, Verhandl. Intern. Ver. f. theoret. u. angew. Limnologie, 1923, 86 and [termöhl and Koppe, Arch. f. Hydrobiol, Suppl. Bd. 5. 1925,234 .)

Colorless sulfur bacteria, always occurring singly, slightly curved, elongated ellipsoids or eylinders with broad hemispherical ends. Itength varies from 12 to 30 mierons, widt h from 8 to 14 microns; most common size 20 by 9 microns. Multiplication by constriction in the middle.

Cells actively motile by means of at single polar flagellum, distinctly visible without special staining. It is 20 to 40 microns long, and, with respect to the direction of motion, always posteriorly placed. Rate of movement somewhat sluggish, about 800 mierons per minute. probably on account of high specific gravity of cells.

Tormally contain small sulfur droplets and, in addition, large, roughly spherical inclusions of calcium carbonate. Two to four such erystal masses almost fill a single rell. Under unfavorable conditions the calcium carbonate crystals may disappear before the sulfur globules.

Microaerophilic; apparently require hydrogen sulfide.

Habitat: Fresh water cnvironment containing sulfide and calcium ions; in shallow basins and streams in the upper layers of the mud.

2. Macromonas bipunctata (Gicklhorn)
Ctermiöhl and Koppe. (Pseudomonas bipunctata Gicklhorn, Cent. f. Bikt., II Abt., 50, 1920, 425; Ctermöhl and Koppe, Areh. f. Hyclrobiol., Suppl. Bd. 5, 1925, 235.)

Cells colorless, wecurring singly, eylindrical with hemispherieal ends; after cell division often temporarily pearshaped. Length 5 to 12 mierons, width 3 to 5 mierons. Multiplication by eonstriction in the middle.

Aetively motile by means of a single polar flagellum, about 10 to 15 microns long. and always posteriorly placed with respert to the direction of movement. Flagellum delicate, not visible without staining. Rate of morement sluggish, abeut 600 microns per minute. Probably this slow motion is on aceount of the high specifie gravity of the eells.

Normally eontain calcium carbonate crystals as inclusions. These are in the form of large spherules, one or two of which nearly fill the individual cells. Sulfur globules have not been demonstrated with eertainty as yet.

Microaerophilic, but it is uncertain whether hydrogen sulficle is required.

Source: From stems, leaves, etc. of fresh water plants in ponds near Graz, Austria.

Habitat: Fresh water enviromments containing caleium ions; but it has been found in sulfide-containing as well as in sulfide-free water. In shallow basins and streams in upper layers of the mud.

Note: Another species in this genus is Macromonas hyalina (Gicklhorn) Utermöhl and Fioppe. (Pseudomonas hyalina Gicklhorn, Cent. f. Bakt., II Abt., 50, 1920, 426; Etermöhl and Koppe, Arch.f. Hydrobiol., Suppl., 5, 1925, 235.) Similiar to Macromonas bipunctata. 


\title{
APPENDIX TO ORDER CHLAMYDOBACTERIALES
}

A recently recognized order of filamentous bacteria includes organisms similar in many ways to those included in Chlamydobacteriales.

\section{ORDER CARYOPHANALES PESHKOFF.*}

\author{
(Jour. Gen. Biol., (Russian), 1, 1940, 611, 616.)
}

Filamentous or bacillary bacteria of variable size characterized either by the presence of a central body or a ring-like nucleus which frequently takes the form of a disk. These bodies are clearly visible in the living cells. The nuclear elements give a clearcut Feulgen reaction. The filaments may be enclosed in a sheath. Colorless. The individuals consist of cylindrical cells enclosed in a continuous sheath or they are tube-like coenocytic organisms containing varying numbers of ring or disk-like nuclei separated from each other by alternating protoplasmic segments. These may disintegrate into mononucleate coccoid cells. Gonidia sometimes formed. Found in water and in the intestines of arthropods and vertebrates.

\section{FAMIL I. PONTOTHRICACLAE PLSHAOFF. Jour. Gen. Biol. (Russian), 1, 1940, 611, 616.)}

Long, unbranched filaments which consist of separate cells in a continuous sheath. Multiplication by rell division, homogonia and unicellular gonidia. Resemble the blue green algae but they are non-motile and photosynthetic pigments are lacking. Free living forms.

\section{Genus I. Pontothrix Nadson and Krassitnikon.}

(Comp. rend. Acad. Sei. de U.R.S.S., A, No. 1, 1932, 243-247.)

Characters as for the family.

The type species is Pontothrix longissima (Molish) Nadson and Krassilnikow.

1. Pontothrix longissima (Molish) Nadson and Kirassilnikow. (Chlamydothrix longissima Molish, Cent. f. Bakt., II Abt., 33, 1912, 60; Nadson and Krassilnikow, loc. cit., 243.) Cells in the fila- ments, 1.5 to 2.0 by 1.0 to 5.0 mierons. Filaments $0.5 \mathrm{~cm}$ in length. Cells show a central chromatin body. Found on Zostera marina in the Bay at Sebastopol on the Black Sea.

\section{FAMILI II. ARTHROAITACEAE PESHKOFF.}

(Jour. Gen. Biol. (Russian), 1, 1940, 611, 616.)

Filaments probably divided into cells although septa (protoplasmic?) disappear during sporulation. Disk-like nuclei alternate with thin protoplasmic segments (septa). Spores form in the distal ends of filaments. Non-motile. The filaments are attached by a spherical body in groups to the intestinal wall of insects, crustaceans and tadpoles.

\section{Genus: $I$. Arthromitus Lecily.}

\section{(Proc. Acad. Nat. Sei., Philadelphia, 4, 1849, 227.)}

Characters as for the family. Although the description is worded somewhat differently, there does not seem to be any essential difference between this and the following genus.

The type species is Arthrominus cristatus Leidy.

* Arrangerl by Prof. Michael A. Peshkoff, Institute of Cytology, Acart. of Sci., Moscow, I.S.S.R., April, 1947. 
1. Arthromitus cristatus Leidy. (Proc. Acad. Nat. Sci., Phila., 4, 1819, 227 and Jour. Acad. Nat. Sei. Phila., 8, 1881, 413.) From the intestine of the milliped (Julus marginatus) and the termite (Reticulitermes flavipes). Filaments delicate, straight or inflected, growing in tufts usually of moderate density, from minute, attached, yellowish rounded or oval bodies. Articuli short, cylindric, uniform, length 2.75 microns, width 0.6 micron, no trace of interior structure. Length of filament 67 to 543 microns, breadth 0.6 micron.

2. Arthromitus intestinalis (Valentin) Peshhoff. (Hygrocrocis intestinalis Talentin, Report. f. Anat. u. Phys., 1, 1836 , 000 ; Peshhoff, Jour. Gen. Biol. (Russian), 1, 1940,597.) From the intestine of the cockroach (Blatla oricnlalis). C'hatton and Perard (Compt. rend. Soc. Biol., Paris, 74, 1913, 1160) conclude that this species and Arthromitus cristatus Leidy are of the same genus although they accept the name Hygrococis as having priority. However, the latter is invalid as a bacterial genus because it was given earlier as the name of a genus of algae. See Buchanan, General Systematic Bacteriology, 1925, 183.

3. Arthromitus nitidus Leidy. (Smithsonian Contributions to Knowledge, $5,1852,35$.) From the intestine of the milliped (Julus marginatus).

4. Arthromitus batrachorum Collin. (Areh. Zøol. Expér. et Gén., 51, 1913, 63.) From the alimentary tract of toad tadpoles (Bufo calamita).

\section{Genus 1I. Coleomitus Duboscq and Grassé.}

(Coleonema Duboseq and Grassé, Arrh. Zool. Expér. et Gén., 68, 1929, Notes et Revue, 14; not Coleonema Bartl. and Wendl., 192t, fam. Rutaceae; Duboscq and Grassé, Arch. Zool. Expér. et Gén., 70, 1930, X. et R., 28.)

Long filaments, divided by partitions. Bacillary elements in basal region. Ovoid or ellipsoidal spores in other parts of filament originating by transformation from these bacillary elements through sporoblasts.

The type species is Coleomitus pruvoti Duboseq and Grassé.

1. Coleomitus pruvoti (Duboseq and Grassé) Duboseq and Grassé. (Coleonema pruioti Duboseq and Grassé, Arch. Zool. Expér. et Gén., 68, 1929, Notes et Revue, 14; Arch. Zool. Expér. et Gén., 70, 1930, N. et R., 28.) In the intestine of a termite (Kalotermes $s p$.) from the Loyalty Islands.
Filaments with hyaline sheath, length variable up to 320 microns, breadth 1.3 microns. Bacillary elements 3 to 4 microns long, also elements up to $6 \mathrm{mi}$ crons with a chromatic granule or disc in the middle of the body. Spores ellipsoidal 0.8 to 0.9 by 1.7 to 2.0 microns, all containing an excentrically placed granule of volutin.

\section{FAMILI III. OSCILLOSPIRACEAE PESHKOFF.}

\section{(Jour. Gen. Biol. (Russian), 1, 1910, 611, 616.)}

Bacillary and filamentous forms. Filaments are most probably partitioned to form narrow cells each containing a central chromatin body (disk-like nucleus). These give a clear Feulgen reaction, and are embedded in hyaline protoplasm.
Spores are formed by a fusion of 2-3 protoplasts of neighboring cells. Actively motile. The character of the motion suggests the presence of peritrichous flagella. Parasitic in the intestinal tract of vertebrates. 


\section{Genus I. Oscillospira C'hatton and Perard.}

(Comp. rend. Soc. Biol., Paris, 65, 1913, 1159.)

Characters as for the family.

The type species is Oscillospira guilliermondii Chatton and Perard.

1. Oscillospira guilliermondii Chatton and Perard. (Chatton and Perard, idem.) From the intestine of a guinea pig. Spores 2.0 by 4.0 to 5.9 microns (Krassilnikow, Microbiol. Jour. (Ru;$\operatorname{sian}), 6,1928,249)$.

\section{FAMILY IV. CARYOPHANACEAE PESHKOFF \\ (Jour. Gen. Biol. (Russian), 1, 1940, 611, 616.)}

Large filamentous and bacillary forms. Individuals not divided into cells; they are virtually coenocytic tubular organ isms containing alternating ring, horseshoe or disk-like nuclei and protoplasmic segments. Such nuclei are most comparable with single chromosomes reproducing themselves by means of a true endomitosis (Peshkoff, Nature, 154, 1946, 137). No spores formed. When motile, possess peritrichous flagella. They are found on the nuucous membranes of the mouth eavities of man and various animals, and in the alimentary tracts of ruminants.

\section{Genus I. Caryophanon Prshoft}

$$
\text { (Inc. cit.) }
$$

Characters as for the family.

The type species is Caryophanon lalum Prohkoff.

\section{Caryophanon latum Peshkoff.} (Compt. rend (Doklady) Acad. Sei., U.R.S.S. Nouvelle série. 25, 1939, 244; Jour. Gen. Biol. (Russian), 1, 1940, 527; Microbiology (Russian), 15, 1946, 189.)

Slightly curved rods 3.1 by 15 to 20 microns. Grow on cow manure-extract agar $\mathrm{pH} 7.8$ to 8.0 . Also grow on yeast extract agar at same $\mathrm{pH}$. Aerobic. Isolated from 20 to 30 per cent of samples of fresh cow manure. Non-pathogenic. Isolated at least 20 times in Moscow (U.S.S.R.) and its vicinity by Peshkoff. In 1945 isolated and successfully cultivated in England by Robinow and Pringsheim. Apparently ubiquitous, connected with ruminants. Colonies round, 1 to $2 \mathrm{~mm}$ in diameter with slightly undulate margins. Subject to distinct S-R variation. $R$ forms tend to grow in long motile filaments and are much thinner than the plump $S$ individunls. May occur in the form of mononucteate corcoids (especially on yeast-extract agar) and polynucleate bacilli. When grown from old cultures may develop irregular giant forms.

2. Caryophanon tenue Peshkoff. (Comp. Rend. (Doklady) Acad. Sci. I. R. S. S., Nouvelle Sér., 25, 1939, 244 ; Jour. ('́nn. Biol. (Russian), 1, 1940, 597.)

Similar to the above species, but more slender. Diameter 1.5 microns. Grow's on cow manure extract agar and yeastextract agar at $p H$ 7.8 to 8.0. From fresh cow manure.

3. Caryophanon muelleri comb. nov. (Simonsiella muelleri Simons, Cent. f. Bakt., I Abt., Orig., 88, 1922, 50.) Nonmotile. Found on mucous membrane of oral cavity of healthy humans, 3.0 to 4.5 by 10.0 microns.

4. Caryophanon crasse comb. nov. (Simonsiella crassa Simons, loc. cit., 509.) Non-motile. Found on mucous membrane of domestic animals.

5. Caryophanon filiformis comb. nov. (Simonsiclla filiformis Simons, loc. cit., 509.) Long filaments from mucous mem. brane of oral cavity of domestic animals. 


\title{
ORDER IV. MYXOBACTERIALES JAHN.*
}

\author{
(Frryptogamenflora der Mark Brandenburg, V, Pilze 1, Lief. 2, 1911, 201.)
}

Synonymy: Myxobacteriaeae Thaxter, Bot. Gaz., 17, 1892, 389; Myxobactrales Clements, The genera of fungi. Minneapolis, 1909, 8; Synbacteriés Pinoy, Compt. rend. Acad. Sci., Paris, 157, 1913, 77 ; Myxobacterieae Heller, Jour. Bact., 6, 1921, 521 ; Polyangidae Jahn, Beiträge zur botanischen Protistologie, I, Die Polyangiden, Geb. Borntraeger, Leipzig, 1924; Myxobacteriae Stanier and van Niel, Jour. Bact., 42, $1941,437$.

The name Myxobacteriaceae, although having the form of a family designation, mas proposed by Thaxter (loc. cit.) in an article bearing the title "On the Myxobacteriaceae, a new order of Schizomycetes." Apparently the first ordinal name was that given by Clements (loc. cit.), but does not follow the spelling fixed by the precedent of Thaxter. The revised spelling was given by Jahn as Myxobacteriales. Pinoy (loc. cit.) suggested Synbacteries. The name Myxobacterieae was proposed by Heller (loc.cit.) as a class designation, Bacteria being regarded as the designation of a phylum. Polyangidae is likewise a class designation, Jahn (1924, loc. cit.) concluding this group should be coordinate in rank with the Schizomycetes. Buchanan (Jour. Bact., 3, 1918, 541) proposed the name Myxobacteriales, not knowing of the previous use of the term. He has therefore at times been incorrectly designated as the author of the name.

It may be argued that a more appropriate ordinal designation might be Polyangiales, inasmuch as the generic name Myxobacter proposed by Thaxter was soon found to be a synonym of Polyangium Link. However, there would seem to be justification of the retention of a name based upon an "ancient generic name" in Rule 21 of the Brussels Code.

The group is herein regarded as an order, though Jahn, and Stanier and van Niel agree in regarding it as a class.

Common or trivial names. The slime bacteria, myxobacteria or polyangids.

Brief characterization of the order. The relatively long, slender, flexible, nonflagellate vegetative cells produce a thin, spreading colony (pseudoplasmodium, swarm). The cells are often arranged in groups of 2 or 3 to a dozen or more, their long axes parallel. The group moves as a unit, by means of a crawling or creeping motion, away from the center of the colony. The moving cells pave the substrate with a thin layer of slime on which they rest.

During sporulation (which occurs in all forms except members of the genus Cytophaga) the cells are much shortened, in some cases becoming spherical or coccoid, thick-walled and highly refractile. Fruiting bodies are formed by the species of all families except the Cytophagaceae and the genus Sporocytophaga of the family Myxococcaceae. The fruiting bodies may consist of aggregations of cysts in which the spores (resting cells) are inclosed, or of masses of mucilaginous slime surrounding large numbers of shortened, rod-shaped, or coccoid spores. Fruiting bodies may be sessile or stalked. They are usually pigmented a bright shade of orange, yellow, red or brown, though colorless fruiting bodies, as well as black, have been described.

* The section covering the order Myxobacteriales was first developed in its present. form by Professor R. E. Buchanan, Iowa State College, Ames, Iowa, for the fourth edition of the Manual issued in 1934. It was revised by Professor Buchanan for the fifth edition in 1939. The present review has been carried out by Dr. J. M. Beebe and Professor R. E. Buchanan who had had material assistance from Dr. R. Y. Stanier, April, 1943. 
Members of the genus Sporocytophaga are not known to produce fruiting bodies as such, but often dense agglomerations of shortened rods or cocci have been noted; these may be interpreted as primitive forms of fruiting bodies.

Physiologically most species show great similarity, preferring substrates rich in cellulosic or other complex carbohydrate materials.

Most of the known species are saprophytic or coprophilic and may be found on dung, in soil, on rotten wood, straw, leaves, etc. They frequently appear to live in close association with various true bacteria and are probably parasitic on them. Many have becn cultivated on dung. One species is aquatic and parasitic on an alga, Cladophora sp. (Geitler, Arch. f. Prostistenol., 50, 1924, 67). One is parasitic (?) on lichens and some are halophilic marine forms (Stanier, Jour. Bact., 40, 1940, 623). Another species is reported as pathogenic for fish (Ordal and Rucker, Proc. Exper. Biol. and Med., 56, 1944, 15).

Culture media. The myxobacteria are frequently cultured by transferring to dung. For certain species sterilized dung has been reported as less favorable than the unsterilized. Dung decoction agar has often been employed. Among the early investigators, Quehl (Cent.f. Bakt.., II, Abt., 16, 1906, 9) secured slow growth of some species on malt extract-gelatin at $18^{\circ}$ to $20^{\circ} \mathrm{C}$ with digestion of the gelatin. Potato-nutrient agar was reported better than dung agar, while no grow th occurred on sterilized potato alone. Peptone was considered necessary; glucose had little effect. Pinoy (Comp. rend. Acad. Sci., Paris, 157, 1913, 77) claimed that satisfactory development of Chondromyces crocatus depended upon the presence of a species of Micrococcus in the medium. Kofler (Sitzber. d. k. Akad. Wiss., Wien, Math. Nat. Klasse, 122 Abt., $1913,845)$ successfully used a sucrose-peptone agar to which was added potassium and magnesium salts.

Recent evidence indicates that the carbon requirements of these organisms are met satisfactorily by the more complex earbohydrates, and frequently by their produets of hydrolysis. Mishustin (Microbiology, Moscow, 7, 1938, 427), Imšenecki and Solntzeva (Microbiology, Moscow, 6, 1937, 3), Krzemicniewski and Kírzemieniew ska (Acta Soc. Bot. Po1., 5, 1927, 102) and others have reported good growth of several species of myxobacteria on cellulose.

Beebe (Jour. Bact., 40, 1940, 155) claimed several species to be facultative parasites on various true bacteria. Good growth was obtained on suspensions of killed bacterial cells in 1.5 per cent agar. Sniesko, Hitchner and McAllister (Jour. Bact., 41, 1941, 26 ) showed the destruction of living bacterial colonies by colonies of myxobacteria.

Temperature range. Most species cultivated in the laboratory show a minimum between $17^{\circ}$ and $20^{\circ} \mathrm{C}$ though some species grow at $10^{\circ} \mathrm{C}$. Maximum growth usually occurs at about $35^{\circ} \mathrm{C}$ and the maximum growth temperature is about $40^{\circ} \mathrm{C}$. More normal fruiting bodies are produced at lower temperatures.

The Krzemieniewskis (Acta Soe. Bot. Pol., 5, 1927, 102) report that the fruiting bodies of Melittangium boletus, Myxococcus virescens, Chondrococcus coralloides, Archangium gephyra and Archangium primigenium var. assurgens first develop, followed by Polyangium fuscum and $P$. fuscum var. velatum. At $30^{\circ} \mathrm{C}$ they appear in about 5 to 7 days, at $17^{\circ}$ to $20^{\circ} \mathrm{C}$ in 8 to 12 days, and at $11^{\circ}$ to $14^{\circ} \mathrm{C}$ in 24 to 30 days. Each $10^{\circ} \mathrm{C}$ rise in temperature approximately halves the time. Other species are slower in developing.

The vegetative rods. The vegetative cells are loing, flexuous rods, of ten 30 times as long as broad. Thaxter noted rods up to 15 microns in length though these appear abnormally long. In general the cells are cylindrical, more rarely tapered or pointed at the ends. Jahn (1924, loc. cit.) described spindle-shaped cclls. Thaxter (Bot. Gaz., 37, 1904, 405) believed that a highly elastic wall was present; other authors have 
failed to prove it by plasmolytic agents. Jahn states that tinctorial and chemical methods failed to definitely show the presence of a membrane, but that the elasticity of the cells show this clearly. The cells are flexible, not rigid as are ordinary bacteria. Beebe (Jour. Bact., 41, 1941,214) reported the presence of a cell membrane in Myxococcus xanthus, often made visible with proper staining procedures. The cells frequently show one or more refractive granules. Thaxter also noted nucleus-like granules in the spores of Myxococcus, while Bauer (Arch. f. Protistenk., 5, 1905, 92) reported that during germination of the spores of Myxococcus a refractile granule is found at each end of the cell. Badian (Acta Soc. Bot. Pol., 7, 1930, 55) stated that the cell of Myxococcus virescens lacks a true nucleus, but that there is present a basophilic structure probably nuclear in nature. It is dumb-bell-shaped and divides longitudinally in mitosis. In spore formation an autogamy occurs followed by what appears to be a reduction division. All chromatin material was Gram-negative except during reduction; it may be stained by hematoxylin. Beebe noted a condensed mass of nuclear material in the vegetative cells of Myxococcus xanthus that divided by constriction prior to each cell fission. Nuclear division is considered to be nonrandom amitosis. Cell division is by means of constriction at a point near the center and is always complete. The nucleus is stained by gentian violet and by iron-hematoxylin and gives a faintly positive Feulgen reaction. What appears to be an autogamous fusion of chromosomes takes place during sporulation, followed by a nuclear division during germination of the spores. The spores germinate by a process analagous to budding. Vahle (Cent. f. Bakt., II Abt., 25, 1909, 178) found fat globules and occasional small volutin granules in 3 to 4 day old cultures. Glycogen was not found.

In masses the vegetative rods may be somewhat reddish in color. Thaxter suggested the possibility that the color might be bacteriopurpurin. Treated with concentrated sulfuric acid the pigment gives a blue reaction, hence Jahn $(1924$, loc. cit. $)$ concludes it to be carotin.

Motility of the cells. Baur (loc. cit.) states that cells have a power of forward movement at a rate of about 10 microns per minute. No flagella are present. The cells do not "swim." They may bend and are unlike most true bacteria in this respect, though Dobell (Quart. Jour. of Microscop. Science, 56, 1911, 395 and Arch. f. Protistenkunde, $26,1912,117$ ) describes such flexibility for the giant bacteria (see Bacillus flexilis). This is characteristic also of Beggialoa, Oscillatoria and Spirochaeta.

The cells en masse move in a "front," advancing and leaving behind a slime. The cells in general tend to lie on rather than in the slime. The exact mechanism of motion has proved puzzling. Jahn believes the motion to be related to that of forms like Oscillatoria, and to be due to excretion of slime from the cell, probably an asymmetrical excretion which pushes the cell along.

The colony. This has been variously termed a swarm, pseudoplasmodium, plasmodium and reproductive communalism. It bears a faintly superficial resemblance to the plasmodium of certain of the slime molds (Myxomycetes) but differs in that the true plasmodium is composed of the fused bodies of large numbers of amoeboid cells. The myxobacterial colony is an aggregation of individual rod-shaped, bacterial cells that are not amoeboid. The slime produced by the cells is not protoplasmic, and the colony is not motile but increases in size as the cells move away from the center. Larger numbers of cells are to be found at the margins than on the central portions of the colony; in consequence, fruiting bodies tend to be found in concentric rings on the colony. The cells lie on the surface of the slime which they secrete, not in it. 
Thaxter proposed the term "pseudoplasmodium" as a satisfactory descriptive name for the vegetative colony, while Jahn preferred the use of "swarm stage." Inasmuch as the term "colony" in relation to bacterial growth implies large numbers of vegetative cells developing as a unit without regard for size, shape or structure, it is equally suitable. Stanier (Bact. Rev., 6, 1942, 183) speaks of the condition as "reproductive communalism."

Pigmentation of the fruiting bodies is commonly employed in the differentiation of species. Species that produce colorless cysts and some with black pigment have been reported; in general the fruiting bodies are brightly colored in shades of yellow, red, orange or brown. The color seems to originate in the slime or cyst walls rather than in the encysted cells; its nature is not well understood. The Krzemieniewskis (Bull. Acad. Polon. Sci. Lettres, Classe Sci. Math. Nat., Sér. B, Sci. Nat., I, 1937, 11) noted that the orange-red fruiting bodies of Sorangium compositum became graybrown in strong alkali; the pigment was highly soluble in acetic acid and alcohol and easily soluble in ether and chloroform. It was insoluble in benzol, carbon di-sulfide and petroleum ether. They suggested that it was a carotin derivative rather than true carotin.

Beebe $(1941$, loc. cit.) found that the pigments of Polyangium fuscum, Podangium erectum, Myxococcus virescens, Chondrococcus blasticus and Myxococcus xanthus gave typical carotin reactions in concentrated sulfuric acid, but were insoluble in chloroform, ether, acetone and methyl and ethyl alcohol. An atypical carotin reaction resulted with hydrochloric and nitric acids. He concluded the pigments to be related to the carotins.

The fruiting bodies. After growth as a vegetative colony the pseudoplasmodium usually forms fruiting bodies which may in the different species be of many shapes and sizes. Differentiation of species, genera and families is based almost entirely upon the character of fruiting body developed. In some cases a stalk is produced, in some not.

In some forms the stalk is delicate and white, consisting of little-changed slime, in other cases it may be stiff and colored. The rods evidently are carried up by the slime which they secrete. In some forms the stalk is simple and short, in others relatively long and branched.

The rods ordinarily associate in more or less definite clumps to form cysts. These cysts may be sessile or stalked. Usually the rods shorten and thicken materially before the cyst ripens. In some forms they shorten so much as to become short ovoid or cylindrical, functioning as spores. They are not endospores such as are found in the genus Bacillus.

The cysts may or may not possess a definite membrane produced from slime. Usually the cysts are bright colored, frequently red, orange or yellow. The spores within the cysts when dried retain their vitality for considerable periods of time. Jahn records germination of Polyangium fuscum after $5 \frac{3}{4}$ years, of Myxococcus fulvus after 8 years.

Methods of isolation. One technic of isolation used by the Krzemieniewskis (1927, loc. cit.) was to sieve the fresh soil, place it on blotting paper in petri dishes, and add sterilized rabbit dung. The soil was saturated with water to 70 to 100 per cent, and the plates incubated at $26^{\circ}$ to $30^{\circ} \mathrm{C}$. After 5 to 10 days fruiting bodies began to appear on the dung. Numerous species were isolated by this method.

Mishustin (loc. cit.) employed silica gel plates on the surface of which sterilized filter paper had been placed. Small lumps of soil were placed on the filter paper and the plates incubated for several days at various temperatures. Vegetative myxo- 
bacterial colonies developed around the inocula and were purified by transfer to fresh cellulose plates.

Beebe reported a modified Krzemieniewski technic to be satisfactory. Fruiting bodies that had developed on sterilized rabbit dung were transferred to bacterial suspension agar plates. Associated hacteria failed to grow well, but myxobacteria developed rapidly.

The species of the genera Cytophaga and Sporocytophaga require special technics (Stanier, Bact. Rev., 6, 1942, 143). The soil forms which decompose cellulose may be enriched with a medium consisting of cellulose (usually in the form of filter paper) and a neutral or slightly alkaline mineral base containing either ammonium or nitrate salts as nitrogen source. For certain species chitin may be substituted for cellulose. Pure cultures may be secured by use of soft agar (1 per cent or less) with finely divided cellulose or with cellulose dextrins (Fuller and Norman, Jour. Bact., 46, 1943, 281).

Cultivation of organisms. Pure cultures of many species have been grown upon various media and substrates. Sterilized dung, dung decoction agar, nutrient agar, potato and potato agar, sterilized lichens, etc. have all been used. Little study has been made of the food requirements. Recent evidence indicates the utilization of some of the more complex carbohydrates. Imšnecki and Solntzeva (Microbiology, Moscow, 6, 1937, 3) reported the growth of certain species on cellulose with partial decomposition of that compound. Mishustin (Microbiology, Moscow, 7, 1938, 427) isolated five species of cellulose-decomposing myxobacteria, cultivating them on a mineral salt-silica gel medium to which filter paper had been added as a source of carbon. Krzemieniewska (Acta. Soc. Bot. Polon., 7, 1930, 507) grew species of the Cytophagaceae on cellophane, while stapp and Bortels (Cent. f. Bakt., II Abt., 90, 1934,28 ) record the growth of other members of the same family on media containing such carbon sources as mannitol, glucose, sucrose, dextrin, cellotriose and cellobiose. Inorganic nitrogen sources compared favorably with organic, in some cases appearing to be preferable. Stanier (Jour. Bact., 40, 1940, 623) observed peptone and yeast extract to be the only suitable nitrogen sources for the Cytophagaceae, inorganic salts and amino acids failing in this respect. Agar and cellulose were decomposed, while chitin and starch were not utilized. Johnson (Jour. Bact., 24, 1932, 335) and Benton (Jour. Bact., 29, 1935, 449) both reported chitinovorous myxobacteria. Beebe (Iowa State Coll. Jour. Sci., 15, 1941, 319 and 17, 1943, 227) claimed growth of species of Polyangium, Podangium, Chondrococcus and Myxococcus on 1.5 per cent agar with no other nutrients added. Peptone appeared to aid development, while the addition of beef extract had no favorable effect. Moderate growth occurred on a mineral saltagar medium without the addition of carbon or nitrogen sources. Growth was stimulated by the addition of various complex carbohydrates including cellulose and starch, the latter being hydrolyzed; complete inhibition resulted with pentoses and hexoses. Best grow th was reported on a medium composed of dried bacterial cells suspended in 1.5 per cent agar. The suspended cells were lysed by the myxobacteria.

The Krzemieniewskis (Acta Soc. Bot. Pol., 5, 1927, 102) showed that the optimum hydrogen inn concentrations for growth of different species were found between $\mathrm{pH}$ 3.6 and 8.0. Beebe $(1941$, loc. cit.) reported no growth of any species below pH 6.0 , while moderate development was noted up to $\mathrm{pH} 9.0$.

Habitat and distribution. Many species have been described from dung. The work of the Krzemieniewskis (Acta Soc. Bot. Pol., 5, 1927, 102), Mishustin (Microbiology, Moscow, $7,1938,427$ ), Imšenecki and Solntzeva (loc. cit.) and others seems to indicate that they oceur commonly in soils, particularly soils under cultivation or high in organic materials.

Different species appear to be characteristic of various 
types of soils. Polyangium cellulosum var. ferrugineum Mishustin and Polyangium cellulosum var. fuscum Mishustin (loc. cit.) were observed to be common in the black soils of Eastern European Russia, while a similar variety of the same species was reported only from podzol soils. Species of the families Polyangiaceae, Sorangiaceae and to a lesser degree Archangiaceae appear to predominate in Russian and European soils, while the soils of Central and Western United States seem to be more suitable for the growth of the Myxococcaceae. Soils of mountainous regions are said to contain fewer numbers of myxobacteria than those of lowland areas.

The distribution of myxobacteria in the soil seems to show a relationship to the hydrogen ion concentration. Some species are found only in neutral or alkaline soils ( $\mathrm{pH} 7.0$ to 8.0 ), others only in acid soils ( $\mathrm{pH} 3.6$ to 6.4 ). Some species show a wide tolerance ( $\mathrm{pH} 3.6$ to 8.0 ).

Relationships of the Myxobacteria. The resemblance of the pseudoplasmodium of the myxobacteria to the plasmodium of the slime molds is as noted above probably to be regarded as without significance, as is also the superficial resemblances of the fruiting bodies of the two groups. Jahn $(1924$, loc. cit.) dismisses the relationship to the Thiobacteriales suggested by Thaxter as improbable. Thaxter believed the pos session of the red color might show presence of bacteriopurpurin; but Jahn found a carotin reaction which argues against this idea. Jabn insists upon a close relationship to the blue-green algae, particularly because of the mobility of the cells and the creeping motion. He does not believe all Schizophytae that do not belong to the Cyanophyceae (blue-green algae) should be grouped as bacteria. He believes the myxobacteria to be more closely related to the blue-green algae than to the true bacteria, and creates the class Polyangidae to be coordinate with the class Schizomycetes. In this he ignores the equal evidence of close relationship of the sulfur bacteria to the Cyanophyceae. His argument would lead to the recognition of all the orders of bacteria recognized in this MANUAL as classes. The wisdom of this is not apparent. The Myxobacteriales may be regarded as a well-differentiated order of the Schizomycetes showing some resemblance to the true bacteria on the one hand and the $M y x o-$ phyceae (Cyanophyceae) and Thiobacteriales on the other.

Families of the Myxobacteriales. The division of the order Myxobacteriales into families has been based, in all classifications proposed, upon morphology. The final demonstration by Stanier (Jour. Bact., 40, 1940, 636) of the close relationship between species of the genus Cytophaga and the myxobacteria led him to propose the recognition of a new family, Cytophagaceae.

The principal character differentiating this family from the four previously recognized is the absence of differentiated fruiting bodies. The resting cells are rod-shaped in the genus (Cytophaga). In another genus recognized by Stanier (Sporocytophaga) the resting cells are spherical. This brings the taxonomist face to face with the problem of deciding whether the presence of fruiting bodies or the spherical shape of the spores should be the primary basis of differentiation. The formation of spherical spores is believed to be of sufficient significance to require the inclusion of all organisms producing such in the family $M$ yxococcaceae. Sporocytophaga, although it produces no fruiting body, is therefore placed in this family, while those forms which produce neither spherical spores nor fruiting bodies (genus Cytophaga) are placed in the new family Cytophagaceae.

Krzemieniewska's (Acta Soc. Bot. Pol., 7, 1930, 507 and Arch. Microbiol., 4, 1933, 394) conclusion that two distinct cell shapes appear in the myxobacteria (short, thick rods with ends almost truncate, and long, slender rods almost spindle-shaped in some cases with pointed tips) is supported by Stanier (Bact. Rev., 6, 1942, 143) as also the 
conclusion that the family Archangiaceae should be abandoned (Krzemieniewski and Krzemieniewska, Bull. Acad. Pol. Sci. Lettres, Classe Sci. Math. Nat., Sér. B., Sci. Nat., I, 1937, 11-31) and the genera and species redistributed. The validity of the argument is accepted, but the family is retained until a satisfactory revision can be effected. This should be based on a careful comparative study of the species.

\section{Key to the Families of Order Myxobacteriales.}

I. Neither definite fruiting bodies (cysts) nor spores (microcysts) produced. Family I. Cytophagaceae, p. 1012.

II. Spores (resting cells, microcysts) produced.

A. Resting cells (spores, microcysts) elongate, not spherical or ellipsoidal. Fruiting bodies (cysts) produced.

1. Fruiting bodies (cysts) not of definite shape; cells heap up to produce mesenteric masses or finger-like (columnar) bodies.

Family II. Archangiaceae, p. 1017.

2. Fruiting bodies (cysts) of definite shape.

a. Cysts usually angular. Vegetative cells usually thick and short, with blunt, rounded ends.

Family III. Sorangiaceae, p. 1021.

aa. Cysts usually rounded. Vegetative cells long and thin, sometimes spindle-shaped with pointed ends.

Family IV. Polyangiaceae, p. 1025.

R. Resting cells (spores, microcysts) spherical or ellipsoidal. Fruiting bodies produced except in genus Sporocytophaga.

Family V. Myxococcaceae, p. 1040. 


\section{FAMILY I. CYTOPHAGACEAE STANIER.}

(Jour. Bact., 40, 1940, 630.)

Flexible, sometimes pointed rods, showing creeping motility. No fruiting bodies or spores (microcysts) formed. There is a single genus Cytophaga.

\section{Genus I. Cytophaga Winogradsky.}

(Ann. Inst. Pasteur, 43, 1929, 578.)

Diagnosis: As for family. From Greek kytos, hollow place or cell; and phagein, to eat, devour.

The type species is Cytophaga hutchinsonii Winogradsky.

Key to the species of genus Cytophaga.

I. From soil.

A. Do not utilize starch.

1. Produce yellow pigment on cellulose.

1. Cytophaga hutchinsonii.

2. Cytophaga lutea.

2. Produces orange pigment on cellulose.

3. Cytophaga anrantiaca.

3. Produces pink pigment on cellulose.

4. Cytophaga rubra.

4. Produces olive-green pigment on cellulose.

B. Utilize starch.

5. Cytophaga tenuissima.

1. Produces yellow to orange pigment on starch.

6. Cytophaga deprimata.

2. Produces cream to pale yellow pigment on starch.

7. Cytophaga albogilva.

II. From sea water.

A. Dark pigment on cellulose.

B. No pigment on cellulose.

8. Cytophaga krzemienieuskae.

9. Cytophaga diffluens.

C. Liquefies agar.

10. Ciytophaga sensitiva.

1. Cytophaga hutchinsonii Winogradsky. (Winogradsky, Ann. Inst. Pasteur, 43, 1929, 578; Cytophaga strain 8, Jensen, Proc. Limn. Soc. N. A. Wales, 65, 1940, 547; not Cylophaga hutchinsoni Inšenecki and Solntzeva, Bull. Acad. Sici. C.S.S.R., Ser. Biol., No. 6, 1936, 1129.)

Etymology : Named for H. B. Hutehinson.

Rods: Highly flexible, occurring singly, 0.3 to 0.4 microns wide at the center and tapering to both ends. Length 3.0 to
6.0 microns, according to Krzemieniewska (Arch. Mikrobiol.. 4, 1933, 396); 1.8 to t.0 microns, according to. Jensen (loc. cit.). May be straight, bent, I-shaped or Sshaped. Stain poorly with ordinary aniline dyes. With Giemsa's or Winogradsky's stain young cells are colored uniformly except for the tips, which remain almost colorless; in older cells there is a concentration of chromatin material at the center. Old cultures show large coccoid cells which are not rearlily seen. Gram-negative. 
Growth on cellulose, cellobiose, cellulose dextrins and glucose. On mineral salts-silica gel plates covered with filter paper, bright yellow glistening mucilaginous patches are produced after a few days. The filter paper in these regions is gradually completely dissolved and the patches become translucent.

Ammonia, nitrate, asparagin, aspartic acid and peptone can serve as sources of nitrogen, according to Jensen (loc. cit.).

Strictly aerobic.

Optimum temperature $28^{\circ}$ to $30^{\circ} \mathrm{C}$.

Source: Isolated from soil.

Habitat: Soil. Decomposes plant residues.

2. Cytophaga lutea Winogradsky. (Ann. Inst. Pasteur, 43, 1939, 599.)

Etymology: Latin luteus, yellow.

Dimensions of the cells approximately those of $C$ ytophaga aurantiaca (see below) but rather larger and thinner and without marked central swelling. Gramnegative.

Produces a brilliant yellow pigment similar to that of Cytophaga hutchinsonii.

This species differs only in size from Cytophaga hutchinsoni, and is probably a variety of it.

Source: Isolated from soil.

Habitat: Soil. Decomposes plant residues.

\section{Cytophaga aurantiaca Wino-} gradsky. (Ann. Inst. Pasteur, 43, 1929, 597; probably Mycococcus cytophagus Bokor, Arch. Microbiol., 1, 1930, 34.)

Etymology : Modern Latin aurantiacus, orange-colored.

Cells 1.0 micron wide at the center by 6 to 8 microns long. Except for size, very similar to those of Cytophaga hutchinsonii. Gram-negative.

Produces orange mucilaginous patches on filter paper-silica gel plates. Fibrolysis is very rapid and intense.

Source: Isolated from soil.

Habitat: Soil. Decomposes plant residues.
4. Cytophaga rubra Winogradsky. (Ann. Inst. Pasteur, 43, 1929, 598.)

Etymology: Latin ruber, red.

Pointed rods, straight or sometimes slightly bent, occasionally hooked at one end. Length approximately 3 microns. Gram-negative.

Produces diffuse, rapidly-spreading, pink to brick-red patches on filter papersilica gel plates. Fibrolysis is much slower and less extensive than that caused by Cytophaga hutchinsonii.

Source: Isolated from soil.

Habitat: Soil. Decomposes plant residues.

5. Cytophaga tenuissima Winogradsky . (Ann. Inst. Pasteur, 43, 1929, 599; incorrectly spelled Cytophaga ternissima in Bergey et al., Manual, 4 th ed., 1934, 559.)

Etymology: Latin tenuissimus, most tenuous, very slender.

Dimensions of cells not given, but described as being extremely slender. Gram-negative.

Produces mucilaginous, greenish to olive patches on filter paper-silica gel plates.

Source: Isolated from soil.

Habitat: Soil. Decomposes plant residues.

6. Cytophaga deprimata Fuller and Norman. (Jour. Bact., 45, 1943, 566.)

Etymology : Latin deprimo, to depress or sink down.

Rods: Long and flexuous with pointed ends, 0.3 to 0.5 by 5.5 to 10 microns, arranged singly. Creeping motility on solid surfaces. Gram-negative.

Growth on starch agar is at first smoky to faint yellow becoming bright yellow later. Colonies are irregular and concave in elevation. The edge spreads indistinguishably into the surrounding medium and shallow depressions develop around the colony. Small colonies give the plate a characteristic pitted appearance.

Growth on cellulose dextrin agar is 
milky white. Colonies are depressed in medium.

Gelatin is liquefied in 4 days.

Glucose, lactose, maltose, sucrose, pectin, starch, cellulose dextrin and hemicellulose are utilized. Very scant growth on cellulose may be found on first isolation.

Yeast extract, ammonium nitrate and peptone are suitable nitrogen sources.

Indole not formed.

Nitrites not produced from nitrates.

No visible change in litmus milk.

Highly aerobic.

Optimum temperature $25^{\circ}$ to $30^{\circ} \mathrm{C}$.

Source: Isolated from soil.

Habitat: Soil. Decomposes organic matter.

7. Cytophaga albogilva Fuller and Norman. (Jour. Bact., 45, 1943, 566.)

Etymology: Latin albus, white, and gilvus, pale yellow.

Long flexuous rods with pointed ends, 0.3 to 0.5 by 4.5 to 7.5 microns, arranged singly. Creeping motility on solid surfaces. Gram-negative.

Growth on starch agar is cream to pale yellow. Colonies are small, concave, and irregulary round. Edge is entire and irregular.

Growth on cellulose dextrin agar is restricted. Colonies are pin-point, milky white in color, round and concave.

Gelatin is liquefied in 7 days.

Glucose, galactose, lactose, maltose, sucrose, gum arabic, pectin, starch, cellulose dextrin and hemicellulose are utilized. Very scant growth on cellulose may be found on first isolation.

Ammonia, nitrate and peptone are suitable nitrogen sources.

Indole not formed.

Nitrites not produced from nitrates.

No visible change in litmus milk.

Highly aerobic.

Optimum temperature $22^{\circ}$ to $30^{\circ} \mathrm{C}$.

Source: Isolated from soil.

Habitat: Soil. Decomposes organic matter.
8. Cytophaga krzemieniewskae Stanier. (Incorrectly spelled Cytophaga krzemieniewskii in Stanier, Jour. Bact., 40, 1940, 623; Jour. Bact., 42, 1941, 532.)

Etymology: Named for H. Krzemieniewska.

Long, flexible rods, usually of even width with blunt ends, occasionally somewhat pointed and spindle-shaped, 0.5 to 1.5 by 5 to 20 microns. Starshaped aggregates occur in liquid media. Creeping motility on solid surfaces, non-motile in liquids.

Growth on a sea water-peptone agar plate begins as a smooth, thin, pale pink, rapidly spreading swarm. After a few days, the older portions of the swarm assume a warty appearance due to the accumulation of cells in drop-like masses, resembling immature fruiting bodies but always containing normal vegetative cells. A diffusible brown to black pigment which masks the pink color of the swarm is produced after about a week. Agar is rapidly decomposed, and ultimately liquefaction becomes almost complete.

Sea water-gelatin stab: Liquefaction.

Growth in liquid media is turbid and silky with a pink sediment; the medium turns dark brown or black after 1 or 2 weeks.

Xylose, glucose, galactose, lactose, maltose, cellobiose, cellulose, alginic acid, agar and stareh are utilized, but not arabinose, sucrose and chitin.

Yeast extract and peptone are the only suitable nitrogen sources known.

Weakly catalase positive.

Indole not formed.

Nitrites produced from nitrates.

Hydrogen sulfide not produced.

Salt concentration range: $\mathbf{1 . 5}$ to 5.0 per cent.

Strictly aerobic.

Optimum temperature $22^{\circ}$ to $25^{\circ} \mathrm{C}$.

Source: Isolated from sea water.

Habitat: Sea water. Probably on decaying marine vegetation. 
9. Cytophaga diffluens Stanier. (Jour. Bact., 40, 1940, 623; Jour. Bact., 42, 1941, 546.)

Etymology: Latin diffuens, spreading, flowing away.

Pointed, sometimes spindle-shaped, flexible rods, 0.5 to 1.5 by 4 to 10 microns. In old cultures involution forms consisting of long, twisted, thin threads are found. Star-shaped aggregates of cells occur in liquid media. Creeping motility on solid surfaces, non-motile in liquids.

Growth on a sea water-peptone agar plate begins as a thin, pink, rapidly spreading swarm which often covers the entire surface in a few days. The swarm gradually increases in thickness and develops an irregular, beaten-copper surface due to the liquefaction of the underlying agar. After 4 to 5 days the color becomes orange. Liquefaction of the agar is ultimately almost complete.

Sea water-gelatin stab: Papid liquefaction.

Growth in liquid media is turbid, often with suspended floccules and a heavy pellicle.

Xylose, glucose, galactose, lactose, maltose, cellobiose, cellulose, agar and alginic acid are utilized, but not arabinose, sucrose, chitin or starch.

Yeast extract and peptone are the only suitable nitrogen sources known.

Weakly catalase positive.

Indole not formed.

Nitrites produced from nitrates.

Hydrogen sulfide not produced.

Salt concentration range: 1.5 to 5.0 per cent.

Slightly aerobic.

Optimum temperature $22^{\circ}$ to $25^{\circ} \mathrm{C}$.

Source: Isolated from sea water.

Habitat: Sea water. Probably on decaying marine vegetation.

\section{Cytophaga sensitiva Humm.} (Duke Univ. Marine Lab., North Carolina, Bull, 3, 1946, 64.) Etyomology: Latin sensus, to perceive.

Cells long, slender, flexous rods.
Apparently not flagellated, 0.8 to 1.0 by 7.0 to 20 microns. Cell ends not tapered or only slightly so. Gramnegative. Cells exhibit creeping motility on agar with ability to reverse direction of movement without turning. Bending movements occur in liquid media.

Colonies light orange, thin and shining. Irregular margin. Outer part composed of a single layer of cells, spreading rapidly, the center somewhat thicker and more or less opaque, sunken in the agar. Agar liquefied. Single colony may nearly cover the surface of the agar in the Petri dish within one week; center of colony sinks to the bottom of the dish and may develop vertical sides. Usually the colony begins to die after a week or ten days from the center outward, as shown by loss of pigment. Apparently no water-soluble pigment is produced. Colony $18 \mathrm{~mm}$ in diameter and gelase field $25 \mathrm{~mm}$ in diameter after three days on agar containing 0.8 per cent potassium nitrate and 0.8 per cent peptone (iodine stain).

Gelatin: No growth.

Milk: To growht.

Nitrate apparently not produced from nitrate (agar medium).

Optimum nitrate concentration of medium appeared to be 0.5 per cent. Fair growth on sea water plus agar only, and on agar containing 1.0 per cent. potassium nitrate. Slight growth on 2.0 per cent nitrate agar.

Optimum peptone concentration appeared to be about 0.1 per cent; growth inhibited by concentrations of peptone exceeding $0 . t$ per cent.

No growth on agar media containing any one of the following substances in a concentration of 0.2 per cent glucose, starch, ammonium sulfate. The basal medium, however, supported excellent growth.

Repeated efforts were made to obtain a pure culture by streaking plates and by pouring plates. These were finally suc- 
cessful by the use of an agar medium that contained 0.1 per cent peptone, 0.05 per cent beef extract, 0.05 per cent glucose, and traces of yeast extract and ferric phosphate. Good growth on broth of this composition was also obtained. Apparently the yeast extract supplied necessary growth substances.

fource: Isolated september 19, 1945 from a mixed rulture with l'srudomomes corallina, by streaking a piece of Dictyolu dichotoma on agar containing 0.2 per cent potassium nitrate.

IIabitat: From seaweed. Beaufort, North Carolina.
Appendix: Stapp and Bortels (Cent.f. Bakt., II Abt., 90, 1934, 28) described four new obligate cellulose-decomposing species: Cytophaga siluestris, Cylophaga unularis, Cytophaga flavicula and Cytophaga crocea. The differences between them are small and, while it is impossible to make positive identifications on the basis of present knowledge, they seem to be very similar to Cytophaga hutchinsonii. In the absence of comparative pure culture studies on the obligate rellulose-decomposing members of the genus, the proper delimination of speeies is not possible. Their inclusion in keys must await additional information. 


\section{FAMILY II. ARCHANGIACEAE J.AHN.}

(Beiträge zur botan. Protistologie, I. Die Polyangiden. Geb. Borntraeger, Leipzig, 1924.)

In the organisms belonging to this family the swarm (pseudoplasmodium) produces irregular swollen or twisted fruiting bodies, or develops columnar or finger-like growths, usually without a definitely differentiated membrane.

\section{Key to the genera of family Archangiaceae.}

I. Fruiting body depressed, usually irregularly delimited, the interior usually consisting of swollen or intestine-like twisted or inter-twined masses, whose windings may be constricted or may jut out (project) as free ends.

Genus I. Archangium, p. 1017.

II. Fruiting body consists of single (separate) columnar or finger-like structures arising from the substrate.

Genus II. Stelangium, p. 1020.

\section{Genus I. Archangium Jahn.}

(Jahn, Beiträge zur botan. Protistologie, I. Die Polyangiden. Geb. Borntraeger, Leipzig, 1924, 67; Ophiocystia Enderlein, Bermerkungen zur Systematik der Chondromyciden, Berlin, 1924, 6 pp.)

Etymology: Greek arche, primitive, and angion, vessel (according to Jahn, this genus is the most primitive).

The mass of shortened rods cmbedded in slime forms a pad-shaped or more rounded, superficially swollen or tuberous fruiting body, even with horny divisions. The fruiting body has no membrane. In the interior can be seen a mass resembling coiled intestines. The windings of this coil may be uniform, or irregularly jointed, free or stuck together; the ends may be extended and horny. Instead of a membrane there may be loosely enveloping slime.

The type species is Archangium gephyra Jahn.

\section{Key to the species of genus Archangium.}

I. No slimy capsules.

A. Fruiting body usually wound, irregularly constricted, sometimes swollen and vesicular, appressed.

1. Fruiting body red.

a. The shortened rods 2.5 to 3 microns.

aa. The shortened rods 4 to 6 microns.

1. Archangium gephyra.

2. Archangium primigenium.

2. Fruiting body yellow.

3. Archangium flavum.

B. Tube usually uniformly thick, loosely wound, often branched.

4. Archangium serpens.

II. Fruiting body consisting of a reddish coiled tube, embedded in yellow slime.

5. Archangium thaxteri.

1. Archangium gephyra Jahn. (Chondromyces serpens Quehl, Cent.f. Bakt., II Abt., 16, 1906, 16; Jahn, Beiträge zur botanischen Protistologie. I. Die Polyangiden, Geb. Borntraeger, Leipzig, $1924,67$. 
Etymology: Greek gephyra, a bridge. So named because a transition form between the Archangiaceae and the Myxococcaceae.

Swarm stage (pseudoplasmodium): Grows easily in manure decoction, forming a pseudoplasmodium and ring of fruiting bodies. The vegetative rods are about 10 microns long, 0.5 micron in diameter.

Fruiting bodies: Up to $1 \mathrm{~mm}$ in diameter, of irregular form and with swollen or padded surface. Average sized fruiting bodies are a reddish flesh color by reflected light; smaller fruiting bodies, a light rose. On a dark background large fruiting bodies when fresh appear bluish violet. By transmitted light the fruiting bodies appear yellowish to light red. Upon addition of alcohol or when heated in glycerine, they lose the color quickly and appear gray or colorless.

The inner structures are for the most part a mesenteric mass of tubes 40 to 60 microns wide, without any membrane, and without any enclosing slime. The convolutions are often pressed together. On the inside of these tubes there appears definitely a septation by straight or slightly arched cross walls which, however, do not always cut entirely through the spore masses from one side of the tube to the other. Upon pressure, the fruiting body breaks up into a number of small fragments about 15 to 30 microns in diameter. Within these fragments the shortened rods lie parallel and in bundles.

The rods in the fruiting bodies are so shortened that they resemble the spores of the Myxococcaceae. The spores are 2.5 to 2.8 microns long and about 1.4 microns wide. Often they are somewhat bent so that they appear to be beanshaped. In the smooth, transparent tips of fruiting bodies they stand closely parallel to each other, so that in transmitted light one sees only their cross section and is at first led to believe that he is dealing with one of the Myxococcaceae.

Source and habitat: Found frequently in the region of Berlin on the dung of deer, rabbits, and hare, once also on old decaying lichens. Easily overlooked on account of its usual bluish color. According to Krzemieniewski (1927) the most common of myxobacteria in the soils of Poland. Isolated on rabbit dung.

Illustrations: Quehl (loc. cit.) Pl. 1, Fig. 7. Jahn (1924, loc. cit.) PI. 1, Fig. 5. Krzemieniewski, Acta Soc. Bot. Poloniae, 4, 1926, Pl. III, Figs. 25-26.

\section{Archangium primigenium (Quehl)}

Jahn. (Polyangium primigenium Quehl, Cent. f. Bakt., II Abt., 16, 1906, 16; Jahn, Beiträge zur botanischen Protistologie. I. Die Polyangiden, Geb. Borntraeger, Leipzig, 1924.)

Etymology : Latin, primigenius, primitive, referring to the simple and primitive character of the fruiting body.

Swarm stage (pseudoplasmodium): In manure decoction cysts germinate readily. Vegetative rods 4 to 8 microns in length.

Fruiting bodies: Up to $1 \mathrm{~mm}$ in diameter, sometimes larger, with irregularly padded swollen surface; when fresh a lively red color which is quite prominent especially against a dark background; when dried, dark red. In transmitted light flesh red to yellowish red. In alcohol and upon heating it is quickly bleached.

In transmitted light one sees that the fruiting body is made up of numerous intestine-like convolutions closely appressed, not however, always definitely delimited. These tubes usually have a diameter of from 70 to 90 microns, of ten constricted and attenuated. No membrane is present. The rods in the fruiting bodies are about 4 microns long and 0.8 micron wide. Upon pressure on the fruiting bodies, the rods remain together in small fragments of various sizes.

2a. Archangium primigenium var. 
assurgens Jahn. (Jahn, Beiträge zur botanischen Protistologie. I. Die Polyangiden, Geb. Borntraeger, Leipzig, 5, 1924, 69; Archangium assurgens Krzemieniewski, Acta Soc. Bot. Poloniae, 1927, 95.)

Etymology : Latin assurgens, rising up.

Size and color of the fruiting body as in the species, likewise the inner structure, size and arrangement of the rods. However, the tubules which together constitute the fruiting bodies are more or less free at their ends and stand up from the substrate. Their diameter is somerhat less (about 45 microns), they are often convoluted so that they many times appear to be constricted (like pearls).

Pronounced races of the species and of the variety are so different in habits that they may be regarded as distinct species. Jahn believes the presence of intermediate strains makes a separation difficult.

Source and habitat: According to $\mathrm{Jahn}$, Archangium primigenium is not particularly common. It is usually found on rabbit dung, sometimes on roe dung. The variety assurgens is relatively rare (found three times on rabbit dung) Kofler (1930) on rabbit dung, Vienna. Very rare in Polish soils according to Krrzemieniewski (1927).

Illustrations: Quehl, Cent. f. Bakt., II Abt., 16, 1906, 16, Pl. 1, Fig. 5; Jahn, Kryptogamenflora d. Mark Brandenburg, V, Pilze I, Lief. 2, 1911, 201, Pl. 1, Fig. 5; Jahn (1924, loc. cit.) Pl. 1, Fig. 4, also Fig. G, page 37; Krzemieniewski (1926, loc. cit.) Pl. II, Fig. 23; (1927, loc. cit.) $\mathrm{Pl}$. IV, Fig. 3. var. assurgens, Pl. IV, Fig. 1 and 2.

3. Archangium flavum (Kofler) Jahn. (Polyangium flavum Kiofler, Sitzber. d. Kais. Akad. Wiss. Wien. Math.-Nat. Klasse, 122 Abt., 1913, 861; Jahn, Beiträge zur botanischen Protistologie. I. Die Polyangiden, Geb. Borntraeger, Leipzig, 1924, 71.)
Etymology: Latin flavus, golden or reddish-yellow.

Swarm stage (pseudoplasmodium) : Not described.

Fruiting bodies: About $0.5 \mathrm{~mm}$ in diameter, yellow, spherical or oval, with humped or padded surface. The mass of cells quite homogeneous, upon pressure under cover glass single sections tend to adhere. No membrane, though the rods are so tightly linked that when cautiously placed under a cover glass, the form of the fruiting body is retained. Rods 2 to 4 microns.

Source and habitat: Kofler (1924) on hare dung found in Danube meadows. Reported as frequent in Polish soils by Krzemieniewski $(1926,1927)$.

Illustrations: Krzemieniewski, Acta Soc. Bot. Poloniae, 4, 1926, Pl. II, Fig. 24. (1927), Pl. IV, Fig. 4, 5 and 6.

\section{Archangium serpens (Thaxter)} Jahn. (Chondromyces serpens Thaxter, Bot. Gaz., 17, 1892, 403; Jahn, Beiträge zur botanischen Protistologie. I. Die Polyangiden, Geb. Borntraeger, Leipzig, 1924, 72.)

Etymology: Latin serpens, creeping.

Swarm stage (pseudoplasmodium): Rods cylindrical, 0.6 by 5 to 7 microns. Cultures on agar develop convoluted form.

Fruiting body: About $1 \mathrm{~mm}$ in diameter, recumbent, consisting of numerous loosely intertwined cysts, confluent in an anastomosing coil, flesh-colored, when dry dark red, 50 microns in diameter, bent, occasionally somewhat broadened or constricted, branched.

Source and habitat: Thaxter, Bot. Gaz., 17, 1892, 389. On decaying lichens. Cambridge, Mass.

Illustrations: Thaxter (loc. cit.), $\mathrm{Pl}$. 24, Fig. 24.

5. Archangium thaxteri Jahn. (Beiträge zur botanischen Protistologie. I. 
Die Polyangiden, Geb. Borntraeger, Leipzig, 1924, 71.)

Etymology: Named for Dr. Roland Thaxter.

Swarm stage (pseudoplasmodium): Vegetative stages not observed. Either no germination or prompt cessation of growth on lung extract. May be transferred on dung.

Fruiting body: Usually 0.25 to 0.5 $\mathrm{mm}$, occasionally $0.75 \mathrm{~mm}$ in diameter. Irregularly rounded, superficially sulfur yellow. Upon pressure numerous reddish convoluted tubules are observed embedded in a yellow slime. The average diameter of the tubules is about. 50 microns. No membrane surrounds the tubes. They contain the shortened rods.
The fruiting body is bleached by alcohol or heat, becoming yellowish. Enveloping slime is variable. In well developed specimens the slime forms a stalk, giving the whole the appearance of a morel. In small specimens the rods are embedded in the slime. The fruiting bodies stand loosely separated on surface of dung, never in large groups. Shortened rods (spores) 0.5 micron by 3 microns, very slender.

source and habitat: According to Jahn rare, on rabbit dung. Races with well developed stalks even less common.

Illustrations: Jahn (loc. cit.), Pl. 1, Fig. 1 and 2. Krzemieniewski, Acta Soc. Bot. Poloniae, 4, 1926, Pl. II, Fig. 27.

\section{Genus II. Stelangium Jahn.}

(Kryptogamenflora der Mark Brandenburg, V, Pilze I, Lief. 2, 1911, 205.)

Etymology: Greek stele, pillar or column and angion, vessel.

Diagnosis: Fruiting bodies are columnar or finger-like, sometimes forked, without definite stalk, standing upright on the substrate.

The type species is Stelangium muscorum (Thaxter) Jahn.

\section{Stelangium muscorum (Thaxter)} Jahn. (Chondromyces muscorum Thaxter, Bot. Gaz., 37, 1904, 411; Jahn, Kryptogamenflora der Mark Brandenburg, V, Pilze I, Lief. 2, 1911, 205.)

Etymology: Latin muscus, moss.

Swarm stage (pseudoplasmodium) : Not described.

Fruiting body: Bright yellow-orange,
90 to 300 microns long, 10 to 50 microns wide, without differentiated stalk, simple or rarely furcate, upright, elongate, compact or slender, narrowed at tip. Rods (spores) 1 to 1.3 by 4 to 6 microns.

source and habitat: According to Thaxter (loc. cit.) on liverworts on living beech trunks in Indiana.

Illustrations: Thaxter (loc. cit.) Pl. 27, Figs. 16-18. 


\section{FAMILY III. SORANGIACEAE JAHN.}

(Beiträge zur botan. Protistologie. I. Die Polyangiden. Geb. Borntraeger, Leipzig, $1924,73$.

Diagnosis: The shortened rods of the fruiting body lie in angular, usually relatively small eysts of definite polygonal shape. Often many of these cysts are surrounded by a common membrane. The primary eyst may be differentiated from the angular or secondary cysts. Jo stalked forms are known.

\section{Genus I. Sorangium Jahn.}

(Jahn, Beiträge z. botan. Protistologie. I. Die Polyangiden. Geb. Borntraeger, Leipzig, 1924, 73; Cystoecemia Enderlein, Bemerkungen z. Systematik d. Chondromyciden, Berlin, 1924, 73.)

Etymology: Greek soros, heap and angion, vessel.

Diagnosis: As for the family. The cysts are united into rounded fruiting bodies. Eight species have been allocated to this genus.

The type species is Sorangium schroeteri Jahn.

\section{Key to the species of Genus Sorangium.}

I. Fruiting bodies not black when ripe.

A. Primary cysts absent; fruiting body shows only angular, spherical or oval small cysts.

1. Cysts angular.

a. Fruiting body very small (50 to 80 microns), often irregularly cerebriform; the angular cysts often completely separated from each other, and about 13 microns in diameter.

1. Sorangium schroeteri.

aa. Fruiting body composed of many small cysts.

b. Cysts orange-red in color; over 5.0 microns in diameter.

2. Sorangium sorediatum.

bb. Rusty brown color; cysts less than 3.5 microns in diameter.

3. Sorangium cellulosum.

2. Cysts spherical or oval.

\section{Sorangium spumosum.}

B. Both primary and secondary eysts present.

1. Primary cysts small and numerous, about 20 microns, with definite mem. brane and few angular secondary cysts.

5. Sorangium septatum.

2. Primary cysts large, with delicate, often indefinite, membrane.

6. Sorangium compositum.

11. Fruiting bodies black or brownish-black when ripe.

A. Primary cysts generally not formed.

B. Primary cysts generally formed.

7. Sorangium nigrum.

8. Sorangium nigrescens.

1. Sorangium schroeteri Jahn. (Jahn, Beiträge zur botanischen Protistologie.
I. Die Polyangiden. Geb. Borntraeger, Leipzig, 1924, 73; regarded as a synonym 
of Sorangium compositum by Krzemieniewski, Acta Soe. Bot. Poloniae, 5, 1927, 96.)

Etymology : Named for Julius Schroeter (1837-1894).

Swarm stage (pseudoplasmodium) : Not described.

Fruiting bodies: Very small, circular, swollen, often kidney-shaped with brainlike convolutions, usually 60 microns (occasionally 120 microns) in diameter, bright orange-red. Surrounded by a delicate slime membrane about 0.7 micron thick, apparent only with high magnifications. Divided secondarily into angular cysts, by sutures extending inward which divide the mass regularly into well delimited portions, many angled, usually about 12 microns in diameter, and in other places into areas less well delimited and about 14 microns in diameter. Resembles gelatin which has dried in a sheet and cracked into regular areas. Rods in cysts 5 microns long. Cysts sometimes occur together in large numbers, covering an area to $0.5 \mathrm{~mm}$.

Source and habitat: Found by Jahn (loc. cit.) five times on rabbit dung in environs of Berlin.

Illustrations: Jahn (1924, loc. cit.), Pl. 2, Fig. 22.

\section{Sorangium sorediatum (Thaxter)} Jahn. (Polyangium sorediatum Thaxter, Bot. Gaz., 37, 1904, 414; Jahn, Beiträge zur botanischen Protistologie. I. Die Polyangiden, Geb. Borntraeger, Leipzig, 1924, 73.)

Etymology: From Greek, soros, heap, probably through the botanical term soredium, one type of reproductive body in the lichen, and sorediate, with surface patches like soredia.

Swarm stage (pseudoplasmodium): Rods 0.8 by 3 to 5 microns. Attempts to cultivate have failed.

Fruiting body: Orange-red, irregularly lobed, consisting of a compact mass of small angular cysts. Average size of eysts 6 to 7 microns, smallest 3 microns, with thick and sharply defined edges. Rods 0.8 by 3 to 5 microns. The Krzemieniewskis (1927, loc. cit., 96) have described a variety, Sorangium sorediatum var. macrocystum, consisting of cysts 6 to 14 by 7 to 16 microns, about twice as large as in the type.

Source and habitat: Reported once by Thaxter (loc. cit.) on rabbit dung from South Carolina. Krzemieniewski (1927, loc. cit.) common in Polish soils.

Illustrations: Thaxter (loc, cit.) $\mathrm{Pl}$. 27, Figs. 22-24. Quehl, Cent. f. Bakt., II Abt., 16, 1906, 9, Pl. 1, Fig. 2. Jahn, Kryptogamen-flora d. Mark Brandenburg, V, Pilze I, Lief. 2, 1911, 202, Fig. 1. Krzemieniewski, Acta Soc. Bot. Pol., 4, 1926, Pl. IV, Figs. 39-41. (1927, loc. cit.) Pl. V, Fig. 17, var. macrocystum Fig. 18.

3. Sorangium cellulosum Imšenecki and Solntzeva. (Microbiology, Moscow, 6, 1937, 7.)

Etymology: Modern Latin cellulosum, cellulose.

Fruiting body: Mature fruiting body rusty brown, 400 to 500 microns in diameter, sessile on layer of partially dried slime. No outer wall or limiting membrane. Composed of numerous cysts, irregular in shape, 1.6 to 3.2 microns in diameter, each containing less than ten shortened rods. No discernable cyst wall or membrane.

Spores: 0.3 by 1.5 to 2.0 microns (no other data).

Vegetative cells: Flexible, rod-shaped cells with rounded ends, occurring singly; no flagella but motile by means of a crawling motion; 0.4 to 0.6 by 2.2 to 4.5 microns.

Vegetative colony : No data.

Physiology: Good growth on starch, cellulose. Decompose up to 24 per cent cellulose in ten days, but does not form fruiting bodies. Very poor growth on arabinose with formation of many involution forms including very much elongated 
cells. Fail to grow on nutrient agar, washed agar, potato, carrot, milk.

Source: Isolated from soil.

Habitat: Soil. Decomposes organic matter.

4. Sorangium spumosum Krzemieniewski and Krzemienewska. (Acta Soc. Bot. Poloniae, 5, 1927, 97.)

Etymology : Latin spumosus, frothy or foamy.

Swarm stage (pseudoplasmodium): Rods 0.7 to 0.9 by 2.6 to 5.2 microns.

Fruiting bodies: Consist of numerous cysts, spherical or oval, not surrounded by a common membrane, but united into bodies embedded in slime. Often in double or single rows. Cyst walls colorless, or slightly brownish, transparent, so that the characteristic arrangement of the rods may be seen within. Cysts 8 to 26 by 7 to 20 microns.

Source and habitat: Krzemienierski (1927, loc. cit.) from Polish soil, isolated on rabbit dung.

Illustrations: Krzemieniewski (1927, loc. cit.) Pl. V, Fig. 19.

5. Sorangium septatum (Thaxter) Jahn. (Polyangium septatum Thaxter, Bot. Gaz., 37, 1904, 412; Jahn, Beiträge zur botanischen Protistologie. I. Die Polyangiden, Geb. Borntraeger, Leipzig, 1924, 75.)

Etymology: Latin saeptatus, fenced, i.e., divided by walls.

Swarm stage (pseudoplasmodium): Rods 0.8 to 1 by 3 to 5 microns.

Fruiting bodies: Yellowish-orange. When dried, dark orange-red, 50 microns to more than 100 microns in diameter, cysts rounded or ovoid, angular or eylindrical, inner portion of the envelope divided into a variable number of secondary cysts. Cysts 18 to 22 by 12 to 22 microns in diameter. Secondary cysts 10 to 12 microns. The Krzemieniewskis $(1927$, loc. cit., 96) recognize a variety, Sorangium septatum var. micro- cystum, which has secondary cysts with dimensions 4 to 10 by 3 to 8 microns.

Source and habitat: Collected twice (Thaxter, Bot. Gaz., 37, 1904, 412) on horse dung in Cambridge, Mass. Reported by Krzemieniewski (Acta Soc. Bot. Poloniae, 5, 1927) as common in Polish soil.

Illustrations: Thaxter (loc. cit.) PI. 27, Figs. 25-28. Jahn, Kryptogamen-flora d. Mark Brandenburg, V, Pilze I. Lief 2, 1911, 202, Fig. 2. Krzemieniewski, Acta Soc. Bot. Pol., 4, 1926, Pl.27, Figs. 27-38; ibid., 1927, Pl. V, Fig. 15, var. microcystum, Fig. 16.

6. Sorangium compositum (Thaxter) Jahn. (Polyangium compositum Thaxter, Bot. Gaz., 37, 1904, 413; Jahn, Beiträge zur botanische Protistologie. I. Die Polyangiden, Geb. Borntraeger, Leipzig, 1924, 74; Polyangium sorediatum Quehl, Cent. f. Bakt., II Abt., 16, 1906, 17; not Polyangium sorediatum Thaxter, ibid.)

Etymology: Latin compositus, compound.

Swarm stage (pseudoplasmodium) : Not described.

Fruiting bodies: Dull yellowish-orange changing to dark red on drying. Rounded, small, 0.5 to $1 \mathrm{~mm}$, usually as a whole or even in larger clumps surrounded by a delicate and evanescent membrane. In large fruiting bodies the eysts are bound together in balls 70 to 90 microns in diameter by a delicate membrane. The balls readily fall apart. Secondary cysts are angular, 7 by 11 mierons, surrounded by a delicate orange-red membrane, about 0.4 micron in thickness. Length of rods in the cysts 5 microns.

Source and habitat: Thaxter (loc. cit.) rabbit dung, South Carolina. Jahn (1904, loc. cit.) found it four times on rabbit dung near Berlin, and twice on hare dung in Oberharg. Common in soils of Poland according to Krzemieniewski (1927, los. cit.). 
Illustrations: Thaxter (loc. cit.) Pl. 27, Figs. 29-30, Jahn (1924, loc. cit.) Pl. I, Fig. 6. Krzenieniewski, Acta Soc. Bot. Pol., 4, 1926, Pl. III, Figs. 32-36; ibid., 1927, 5, Pl. IV, Figs. 7, 8, 9, 10, 11, 12 ; PI. V, Figs. 13, 14; Pl. VI, Fig. 36.

7. Sorangium nigrum Kirzemieniewski. (Bull. Int. l'Acad. Pol. Sci. et Lettres, Classe Sci. Math. et Nat., Sér. B, 15, 1937.)

Etymology : Latin niger, black.

Fruiting body : Primary cysts generally not formed; when observed, appeared as smoke-colored slime envelope surrounding clumps of a few cysts. Secondary cysts usually arranged in rows within cellulose fibers, the material of the fiber forming a common sheath. Each individual cyst inclosed by a cyst wall, clearly differentiated from the tubularshaped cellulose fibers. Cysts measure 9 to 16 by 9 to 23 microns; average 10 by 18 microns. Cyst wall moderately thick, colorless, transparent, becoming light brown with age, and finally black.

Spores: No data.

Vegetative cells: 1.1 to 1.3 by 2.5 to 5.5 microns.

Vegetative colony: Young colonies dead black in color. On filter paper a bright orange margin is noted, the vegetative cells of which cover the cellulose fibers. On cotton eloth the margin is bright dirty-yellow, tinged with pink. Under low power magnification, renter of the colony appears similar to matted fungal hyphae, due to chararteristic: compact accumulation of cysts and cellulose fibers.

Physiology: Cellulose fibers become swollen by the action of this organism, and become gray-brown with a violet tinge. Fibers lose the properties of cellulose and give no characteristic reactions.

Source: Isolated from soil.
Habitat: Soil. Decomposes cellulose fibers.

Illustrations: Krzemieniewski (loc. cit.) Plate IV, Figs. 22-26.

8. Sorangium nigrescens Krzemieniewski. (Bull. Int. l'Acad. Pol. Sci. et Lettres, Classe Sci.. Math. et Nat., Sér. B, 15, 1937.)

Etymology : Latin nigrescens, becoming dark or black.

Fruiting body: Primary cysts vary in size up to 200 microns in diameter, irregular in shape and inclosed in a colorless slime envelope. Formed by an aceumulation of secondary cysts. Secondary cysts at first colorless, transparent, later becoming brownish with a limiting membrane; the young cysts appear dirty-yellow, the older ones grayishbrown to black. Color originates not only from the brownish cyst wall but from the gray mass of encysted cells. Secondary eysts measure 5 to 12 by 6 to 15 microns; average 6 by 10 microns. On filter paper not only well-formed primary rysts are formed, but also free secondary cysts are noted embedded in the slime of the colony.

Spores: No data.

Vegetative cells : 1.2 to 1.4 by 2.5 to 6.4 microns. Younger cells somewhat shorter.

Vegetative colony: Mass of dark fruiting bodies develops at center of colony on filter paper; margin grayish-yellow. Cellulose fibers covered with vegetative rells on outside, and contain many cells within.

Physiology : Destroys cellulose. Cultivated six years with cellulose as carbon source.

Source: Isolated from sandy soil in pine woods in Ciemianka (?).

Habitat: Snil. Deromposes cellulose fibers.

Illustrations: Iirzemieniewski (loc. cit.) Plate III, Figs. 17-21 


\section{FAMILY IV. POLYANGIACEAE JAHN} 1924.)

(Beiträge zur botan. Protistologie. I. Die Polyangiden. Geb. Borntraeger, Leipzig,

Diagnosis: In the fruiting bodies the more or less shortened rods lie in rounded cysts of definite form. The well-defined wall is composed of hardened slime, and is yellow, red or brownish. The cysts may be united by a definitely visible slime membrane, the remnant of the vegetative slime, or they may be tightly appressed and cemented by the scarcely visible remnants of the slime, or they may develop singly or in numbers on astalk. In the more highly developed forms the stalk branches and carries the cysts at the tips of the branches.

\section{Key to the genera of family Polyangiaceae.}

I. Cysts rounded, not stalked, usually many (one in Polyangium simplex) lying loosely in a slime membrane or closely appressed.

II. Cysts not as in I.

Genus I. Polyangium, p. 1025.

A. Cysts pointed at the apex, often completely concrescent, and united to large disks or spheres.

B. Cysts free, single or many on a stalk.

Genus II. Synangium, p. 1032.

1. Cysts forming a disk, flattened dorsoventrally, like the cap of a Boletus, on a white stalk.

2. Cysts not forming a disk.

Genus III. Melittangium, p. 1033.

a. Cysts rounded or elongate, single on stalks.

Genus IV. Podangium, p. 1034.

aa. Cysts rounded or elongate or pointed, numerous on the ends of stalks which may be branched.

Genus V. Chondromyces, p. 1036.

\section{Genus I. Polyangium Link.}

(Link, Mag. d. Ges. Naturforsch. Freunde zu Berlin, 3, 1809, 42; Cystobacter Schroeter, in Cohn, Kryptogamenflora v. Schlesien, 3, 1, 1886, 170; Myxobacter Thaxter, Bot. Gaz., 17, 1892, 394.)

Etymology : Greek poly, many and angion, vessels, referring to the numerous cysts.

Diagnosis: Cysts rounded or coiled, surrounded by a well-developed membrane, either free or embedded in a second slimy layer.

The type species is Polyangium vitellinum Link.

\section{Key to the species of genus Polyangium.}

I. Not parasitic on water plants (algae).

A. Sorus not white or grayish in color.

1. Cysts rounded to spherical.

a. Ripe cysts yellow, reddish-yellow, orange or light red; not brown.

b. Cysts several or numerous and small.

c. Not closely appressed.

d. Slime envelope transparent white or colorless.

e. Usually 10 to 15 cysts. Rods in cysts, 3 microns long. Cysts 75 to 200 microns.

1. Polyangium vitellinum. 
ee. Cysts numerous. Rods 1.3 to 2.0 microns long. Cysts 20 to 80 microns.

2. Polyangium minus.

dd. Slime envelope bright yellow.

3. Polyangium luteum.

cc. Closely appressed; often polygonal due to pressure.

d. Bright yellow.

\section{Polyangium morula.}

dd. Orange.

5. Polyangium cellulosum.

bb. Cysts single, large.

c. Large, 250 to 400 microns; reddish-yellow.

6. Polyangium simplex.

ce. Smaller, 30 to 60 by 50 to 130 microns; orange to light red.

7. Polyangium ochraceum.

aa. Ripe cysts reddish-brown to dark brown.

b. Cysts lying free, covered by a more or less definite slime envelope.

c. About 60 microns in diameter; slime envelope delicate and colorless.

8. Polyangium fuscum.

cc. About 35 microns in diameter; slime envelope yellow.

9. Polyangium aureum.

bb. Cysts rounded, in stellate arrangements on a slimy substrate.

10. Polyangium stellatum.

2. Cysts elongate, coiled.

a. Cysts brownish-red.

11. Polyangium ferrugineum.

aa. Cysts bright orange-yellow.

12. Polyangium indivisum.

B. Sorus white or gray in color.

1. Hyaline slime envelope white, foamy in appearance; cysts average 28 by 34 microns.

13. Polyangium spumosum.

2. Sorus flat, crust-like, smoke-gray in color due to slime envelope; cysts average 36 by 44 microns.

14. Polyangium fumosum.

II. Aquatic, parasitic on Cladophora.

\section{Polyangium parasiticum.}

1. Polyangium vitellinum Link. microns, occasionally 200 microns in (Link, Mag. d Ges. Naturforschender diameter, almost always surrounded Freunde zu Berlin, 3, 1809, 42; Myxo- by a white slimy envelope, about 10 to bacter aureus Thaxter, Bot. Gaz., 17, 15 cysts in a mass. Rods in the cysts 1892, 403.)

Etymology: Modern Latin vitcllus, like an egg yolk.

Swarm stage (pseudoplasmodium): When rising to form cysts, milky white. Rods large, cylindrical, rounded at either end, 0.7 to 0.9 by 4 to 7 microns.

Fruiting body: Cysts golden yellow, usually relatively spherical, 75 to 150 about 3 microns in length.

Source and habitat: Thaxter (loc. cit.) on very wet wood and bark in swamps. Maine, Belmont. Jahn (1924, loc. cit.) states it is not common; on old wood, lying in moist ditches, also on old poplar bark which was kept moist in a dish, also found twice on rabbit dung.

Illustrations: Thaxter (loc. cit.) Pl. 
25, Figs. 34-36. Zukal, Ber. d. deutsch. Bot. Ges., 15, 1897, 542, Pl. 27, Figs. 6-10. Jahn, Kryptogamenflora d. Mark Brandenburg, V, Pilze I, Lief. 2, 1911, 199, Fig. 3. Jahn, Beiträge zur botanischen Protistologie. I. Die Polyangiden. Geb. Borntraeger, Leipzig, 1924, 77, and PI. II, Fig. 13.

2. Polyangium minus Krzemieniewski. (Acta Soc. Bot. Poloniae, 4, 1926, 33.)

Etymology : Latin minor, less or small.

Swarm stage (pseudoplasmodium): Vegetative rods 0.4 to 0.6 by 3 to 7 microns.

Fruiting bodies: Cyst masses commonly cover the substrate to an area of $0.5 \mathrm{sq} . \mathrm{mm}$. Cysts are spherical or oval, small, 20 to 80 by 20 to 50 microns, light rose in color, becoming brownish, embedded in a transparent coloriess slime. Cyst membrane. light colored, relatively thick, 0.5 to 1.0 micron, transparent, revealing the contents. Rods in cyst 0.8 to 1.0 by 1.3 to 2.0 microns.

Source and habitat: On rabbit dung sterilized and placed on soil (Poland). Rather rare. Relatively slow in appearance, only after many days.

Illustrations: Krzemieniewski (loc. cit.) Pl. IV, Fig. 47-48; Pl. V, Fig. 49.

3. Polyangium luteum Krzemieniewski. (Acta Soc. Bot. Poloniae, 5, 1927, 98.)

Etymology: Latin luteus, saffron- or golden-yellow.

Swarm stage (pseudoplasmodium) : Not described.

Fruiting bodies: Golden yellow, consisting of a few cysts surrounded by a common bright yellow very thick slime wall. The cysts have colorless thin walls. Rods 0.7 to 0.8 by 3.8 to 5.8 microns.

Source and habitat: Isolated from soil on rabbit dung by Krzemieniewski (1927).
Illustration : Krzemieniewski (loc. cit.) PI. V, Fig. 22, 23.

4. Polyangium morula Jahn. (Kiryptogamenflora der Mark Brandenburg., I, Pilze I, 1911, 202.)

Etymology : Modern Latin from Greek mōra, mulberry. A diminutive referring to shape of cysts.

Swarm stage (pseudoplasmodium) : Not described.

Fruiting bodies: Cysts bright yellow, closely packed into a mulberry-shaped sorus; cysts with thick membrane (3 microns), often made polygonal by pressure, 20 to 35 microns, bound together by slime. The whole sorus is 100 to 200 microns broad. Rods in cysts about 3 microns in length. Jahn states he has not studied fresh cysts. In the older cysts the rods are difficult to observe.

Source and habitat: Observed once only by Jahn (loc. cit.) on rabbit dung.

Illustration: Jahn (1924, loc. cit.) Pl. 2, Fig. 21.

5. Polyangium cellulosum Imšenecki and Solntzeva.* (On aerobic cellulosedecomposing bacteria. Akademiia Nauk, Leningrad, Isvestiia, 1936, 1115; English summary, 1168.)

Etymology: Modern Latin cellulosum, cellulose.

Fruiting body: Rods at center of the colony non-motile, forming large orange aggregates. Shorter than those at margin : 0.7 to 0.9 by 3.4 to 5.6 microns. Later a concentration of cells occurs. Rods come closer together, form rounded or oval aggregates from which cysts become delimited. Cysts orange in color, 8 to 24 microns, average 20 to 25 microns. In addition to bacterial cells droplets of fat, 1.5 to 3.5 microns, are sometimes seen within the cyst. When treated with $\mathrm{H}_{2} \mathrm{SO}_{4}$, cysts are easily broken up under the cover glass. Fruiting bodies are composed of clumps of cysts. Fruiting bodies oval or pear-

* Translated from the original by E. V. Prostov, Iowa State College Library, Ames, Iowa. 
shaped, 40 to 55 by 110 to 160 microns, reddish-brown. Covered with a slime membrane (flakes of dried slime). Each composed of 12 to 40 cysts which become polygonal from pressure. No cystophore, except those formed from slimy threads which have a stratified structure. Cysts sometimes arranged in chains.

Spores : 0.7 to 0.8 by 2.2 to 3.5 microms.

Vegetative cells: Thick, bent rods, with rounded ends, 0.8 to 1.2 by 3.5 to 8.5 microns. Motile, no flagella. Young rods have 1 chromatin granule, older have 2. Found in cellulose fibers at the margin of the colony. Fibers solidly stuffed near the margin. At the periphery individual cells may be seen.

Vegetative colony: Cysts germinate on filter paper producing vegetative colonies. Colonies large, orange, moist, increasing in size. The older colonies have orange margins while the center is dark brown, corresponding to the color of the fruiting bodies. Often show several concentric rings.

Physiology : Rods cover cellulose fibers, partially or completely destroying them. Paper becomes transparent.

Optimum tenperature $18^{\circ}$ to $22^{\circ} \mathrm{C}$. At $30^{\circ}$ growth very slow

Grows only on wet cellulose; not in ordinary media. No growth in a hanging drop of broth.

\section{Aerobic.}

Source and habitat: Soil.

Illustrations : Imšenecki and Solntzev? (loc. cit.) Table II, 2; figures 1 to 5 .

5a. Polyangium cellulosun var. ferrugineum Mishustin. (Microbiology, Mos cow, 7, 1938, 427.)

Etymology: Latin ferrugineus, of the color of iron-rust.

Fruiting body: Composed of numerous cysts having definite wall. Mass of rods has a yellowish tinge, and the cysts are colored reddish-yellow. Color probably confined to the cyst walls. Cysts round or egg-shaped, or may be angular due to pressure. Each cyst contains numerous shortened rods. Cysts usually 12 to 40 microns in diameter. Numerous cysts grouped in to fruiting bodies having bright red or drabbish red color when ripe. Form of fruiting body variable: most commonly rounded, ellipsoidal or biscuit shaped, sometimes sausage-shaped. Cysts confined by an orange-colored slime membrane or envelope. To cystophore present. Fruiting bodies not easily broken up. Vary in size from 80 to 240 microns.

Spores: No data.

Vegetative cells: Long, flexible, nonflagellate cells, motile by crawling, 0.8 to 1.2 by 3.0 to 5.0 microns. Become shortened and highly refractile during fruiting body formation.

Vegetative colony: On silica gel with cellulose at first pale pink. After six days fruiting bodies of red color appear, together with free cysts and many nonencysted shortened rods. Fruiting bodies numerous at center of colony, and later form in concentric rings around center. Margin of colony composed of vegetative cells; periphery pink. Mature colonies 2 to $5 \mathrm{~cm}$ in diameter, bright red, becoming drabbish red; pigmentation appears to be confined to limited areas. Surface dull, moist. Margin not definite.

Physiology: Cellulose at center of colony completely destroyed; not entirely hroken down under remainder of colony.

The author considers this a color variant of Polyangium cellulosum Imsenecki and Solntzeva.

Source: Isolated from the black soils of Eastern European Russia.

Habitat: Digests organic matter in soil.

5b. Polyangium cellulosum var. fuscum Mishustin. (Microbiology, Moseow, 7, $1938,427$.

Etymology: Latin fuscus, dark, swarthy, dusky, tawny.

Fruiting body : Composed of individual cysts, each with separate cyst wall, and held together by a common slime membrane or envelope. Shortened rodshaped spores inclosed within the cyst 
walls. Cysts forming outside the large masses usually rounded; those within often polygonal or angular. Cysts 5 to 24 microns long, oval or egg-shaped. Eneysted cells give cysts granular appearance. Ripe eysts brown to light brown in color; immature, yellow to pink. Fruiting bodies pinkish-yellow when young, becoming brown when ripened. Considerable variation in form : round, oval or sausage-shaped, and from 50 to 80 microns up to several hundred microns. Outer slime envelope often indistinct; no dried slime noticeable between the cysts.

Spores: No data.

Vegetative cells: Identical with those of Polyangium cellulosum var. ferrugineum.

Vegetative colony: A faint yellow cast on cellulose-silica gel after 2 to 3 days. Becomes yellow-orange to yellow-pink after 6 to 8 days, while center is brownishgray. Margin pinkish to yellow-pink. Surface dull, moist. As fruiting bodies ripen, colony becomes darker, finally dark brown. Reaches diameter of 2 to $5 \mathrm{~cm}$. Fruiting bodies often arranged in form of pigmented, closely set, concentric rings. Margin of colony not clearly defined. Esually regularly rounded or oval. Cellulose completely destroyed only at center of colony.

Source: Common in black soils of Sumy Experiment Station. Found only once in podzol soils.

Habitat: Digests organic matter in soil.

5c. Polyangium cellulosum var. fulvum Mishustin. (Microbiology, Moscow, \%, 1938, 427.)

Etymology: Latin fulus, reddishyellow, gold-colored.

Fruiting body: Rose or pink in color, composed of numerous eysts. Young cysts yellow to yellow-orange, becoming pink, rose or red, or pinkish-yellow. Cysts same shape as others of the species; 6 to 24 microns in diameter, average 10 to 12 microns; contain many short rods. Fruiting bodies vary in shape, often elongated,flagella (?)-shaped (columnar?), up to 20 to 25 by 350 to 450 microns. Also globular, mace-shaped, ete. Lisually 25 to 40 by 50 to 80 microns. Cysts inclosed by outer common envelope or slime membrane. Easily broken up mechanically.

Spores: No data.

Vegetative cells : 0.8 to 1.2 by 3.5 to 6.0 microns.

Vegetative colony: On cellulose-silica gel form a hardly visible white (colorless ?) colony at 2 days. After 6 days becomes pink in color. Fruiting bodies first form near center. After 9 to 10 days central area reddish-pink while periphery has yellowish cast. Mature colony 2.5 to $7.5 \mathrm{~cm}$ in diameter, pinkorange color, fairly regularly round or oval in shape. Pigmented concentric rings of fruiting bodies.

Physiology: Cellulose entirely destroyed at center of colony and often at other points.

Source: Podzol soils of Timiriazev Agricultural Academy. Seldom in black soils of Sumy Experiment Station.

Habitat: Digests organic matter in soils.

5d. Polyangium cellulosum var. luteum Mishustin. (Microbiology Moscow, 7, 1938, 427.)

Etymology: Latin luteus, saffron-yellow, orange-yellow.

Fruiting body: Poorly organized agglomerations of colorless to yellow cysts inclosing sporulated cells. Cysts regularly egg-shaped to oval, 8 to 20 microns in diameter; predominantly 6 to 10 microns. Matured cysts loosely connected into rounded or elongate masses 40 to 80 by 100 to 150 microns. Ripe fruiting bodies easily pulled apart.

Spores: No data.

Vegetative cells: Similar to others of the species.

Vegetative colony: On cellulose colonies regularly rounded or oval, surface has moist appearance. Yellowish cast 2nd or 3rd day, becoming deeper yellow. 
Ochre yellow formations resembling fruiting bodies by 5 to 6 days. Many free eysts at center of colony. Later colony becomes pale dirty-yellow, while periphery remains bright yellow. Sometimes one or two brightly pigmented rings consisting of agglomerations of fruiting bodies are found in older colonies. Mature colonies 1.5 to $3.0 \mathrm{~cm}$ in diameter.

Physiology: Filter paper completely destroyed at center of colony. Developed better below pH 7 (around pH 6) than others of the species.

Source: Isolated from soils of the Timiriazev Agricultural Academy. Common in podzol soils.

Habitat: Digests organic matter in soil.

6. Polyangium simplex Thaxter. (Myxobacter simplex Thaxter, Bot. Gaz., 18, 1893, 29; Thaxter, Bot. Gaz., 37, 1904,414 .)

Etymology : Latin simplex, simple, i.e., not compound.

Swarm stage (pseudoplasmodium): Rods, large, eylindrical, rounded at either end, 0.7 to 0.9 by 4 to 7 microns.

Fruiting bodies: Cysts single, very large, 250 to 400 microns, bright reddish yellow, irregularly rounded. Rods flesh colored in mass. Upon pressure adhering together in sheaves.

Source and habitat: Found by Thaxter (loc. cit.) in U. S. A. on very wet wood and bark in swamps.

7. Polyangium ochraceum Krzemieniewski. (Acta Soc. Bot. Poloniae, 4, 1926,34 .)

Etymology: Modern Latin from Greek ochra, yellow ochre, hence ochraceous.

Swarm stage (pseudoplasmodium) : Not described.

Fruiting bodies: The orange to light red fruiting body in form of a single spherical or oval cyst 60 to 80 by 50 to 130 microns, each with a thick yellow-brown membrane. The cyst content often (particularly in the oval eysts) is constricted by the membrane which pene- trates deeply. From the side the cyst appears to be divided. Rods in eysts 0.5 by 4 to 8 microns.

Source and habitat: From sterilized rabbit dung on soil (Poland).

Illustrations: Krrzemieniewski (loc. cit.) PI. V, Fig. 50, 51.

\section{Polyangium fuscum (Schroeter)} Thaxter. (Cystobacter fuscus Schroeter, in Cohn, Kryptogamenflora v. Schlesien, 3, 1, 1886, 170; Thaxter, Bot. Gaz., 37, 1904, 414.)

Etymology: Latin fuscus, fuscus, brown.

Swarm stage (pseudoplasmodium): I lods slender, elongate, 0.6 by 5 to 12 microns. Grows readily on agar, also on dung agar. Baur states rods are 15 to 20 microns in length and move about 2 to 3 microns per minute in hanging drop, on agar 5 to 10 microns per minute.

Fruiting bodies: Cysts flesh-colored when young, chestnut brown when ripe, spherical, about 60 microns (Thaxter, 50 to 150 by 50 to 70 microns) in diameter, with definite membrane, lying in considerable numbers in large sori, usually 30 to 40 sometimes up to 100 . The slime envelope is much more delicate and evanescent than in $P$. vitellinum. Occasionally a form is found with cysts measuring 100 microns: under these often lie kidney shaped cysts even 150 microns in length; apparently, a variety. Rods in cysts about 0.8 to 1.5 by 3 to 3.5 microns. Cysts (Baur) on dung decoction break in 10-12 hours, and rods pour out, apparently passively at first.

P. fuscum var. velatum Krzenieniewski differs from the type in that the membrane is thin, separated from cysts, folded.

Source and habitat: Thaxter (Bot. Gaz., 23, 1897), on rabbit dung from southern California. Fofler (Sitzber.d. Kais. Akad. Wiss. Wien. Math.-Nat. Klasse., 122 Abt., 1913, 845) on rabbit dung, Vienna. Jahn (Die Polyangiden, Geb. Borntraeg., 1924), common on dung, 
also occurs on decaying lichens and on poplar bark kept moist. Quite common in Polish soils according to Krzemieniewski (1927, loc. cit.).

Illustrations: Thaxter (1897, loc. cit.), Pl. 31, Figs. 37-39. Baur, Arch. Protistenkunde, 5, 1905, Pl. 4, Figs. 14, 15, and 17. Quehl, Cent. f. Bakt., II Abt., 16, 1906, Pl. 1, Figs. 8 and 16 . Jahn, Beiträge zur botanischen Protistologie. I. Die Polyangiden, Geb. Borntraeger. Leipzig, 1924, Pl. 2, Fig. 12. Also Fig. 1, p. 9. Krzemieniewski, Acta Soc. Bot. Poloniae, 4, 1926, 34, Pl. IV, Figs. 42-43, also var. velatum Plate IV, Figs. 44-46.

9. Polyangium aureum Krzemieniewski . (Acta Soc. Bot. Pol., 7, 1930, 255.)

Etymology : Latin, aureus, golden.

Separated from Polyangium morula on basis of pigmentation.

Fruiting body: Cysts reddish-brown, variable in number, embedded in yellow slime to form a sorus with a common slime envelope. Cysts nearly spherical or slightly elongate, averaging 32 by 37 microns. Cyst wall orange-yellow, about 3.0 microns thick. Older cysts contain shortened rods, a granular mass, and a colorless or yellowish oleaginous liquid.

Spores: Rod-shaped.

Vegetative cells: Straight rods, of uniform diameter, with rounded ends, 0.7 to 0.9 by 2.8 to 5.3 microns.

\section{Habitat : Soil.}

Illustrations: Krzemieniewski (loc. cit.) Plate XVII, Figs. 14-17.

10. Polyangium stellatum Fofler. (Sitzber. d. kais. Akad. Wiss. Wien, Math.-Nat. Klasse, 122 Abt., 1913, 19.)

Etymology: Latin stellatus, stellate. Swarm stage (pseudoplasmodium) : Not described.

Fruiting bodies: Cysts elongate, 80 to 120 microns broad, 160 to 200 microns long, flesh-colored when young, brownishred when old, star-shaped with 2 to 9 rays fixed by the narrowed base upon a kind of hypothallus.
Source and habitat: Found by Kofler (1913, loc. cit.) on hare dung at Vienna.

Illustrations: Kofler (1913, loc. cit.) Pl.?, Fig. 6.

11. Polyangium ferrugineum Krzemieniewslii. (Acta Soc. Bot. Poloniae, 5, 1927, 97.)

Etymology: Latin ferrugineus, dark red, like iron rust.

Swarm stage (pseudoplasmodium) : Not described.

Fruiting bodies: Irregular, branched and occasionally constricted coils. Branches of same diameter as the main tube. Cyst wall is brown-red. In the interior no differentiation is visible. Rods in cysts are relatively short and thick, 0.8 to 1.1 microns by 2 to $2.5 \mathrm{mi}$ crons, not definitely arranged. Close to Archangium gephyra, but with cyst walls.

Source and habitat: Krzemieniewski (loc. cit.) from soil in Poland and on rabbit dung.

Illustrations : Krzemieniewski (loc. cit.) Pl. V, Fig. 21.

12. Polyangium indivisum Krzemieniewski. (Acta Soc. Bot. Poloniae, 5, 1927, 97.)

Etymology: Latin indivisus, undivided.

Swarm stage (pseudoplasmodium) : Not described.

Fruiting bodies: Similar to Polyangium ferrugineum, but much smaller and bright orange-yellow. Enclosed in a similarly colored slime membrane. Interior of coils undifferentiated. Cyst rods 0.8 to 1.0 by 3 to 6 microns, straight, and rounded on ends. Arranged perpendicularly to the wall, giving a netted appearance resembling Melitiangium.

Source and habitat: From soils in Poland, Krzemieniewski (1927, loc. cit.).

13. Polyangium spumosum Krrzemieniewski. (Acta Soc. Bot. Pol., 7, 1930, 254.)

Etymology: Latin spumosus, foaming, full of foam. 
Fruiting body : Colorless sori embedded in hyaline slime forming a common envelope around the cysts. Surface white, foamy in appearance; cysts in irregularly rounded accumulations, 100 to $150 \mathrm{mic}$ rons in diameter. Cysts usually spherical, sometimes elongate; 18 to 38 by 20 to 50 microns; average 28 by 34 microns. Cyst membrane colorless. Cysts contain bundles of shortened cells, a granular colorless mass, and a clear oleaginous fluid.

Spores: Shortened rods.

Vegetative cells: Straight rods, uniformly thick, with rounded ends; 0.6 to 0.8 by 3.9 to 6.8 microns.

Habitat: Soil.

Illustrations: Krrzemieniewski (loc. cit.) Plates XVI-XVII, Figs. 10-13.

\section{Polyangium fumosum Krzemien-} iewski. (Acta Soc. Bol. Pol., \%, 1930, 253.)

Etymology: Latin fumosus, smoky.

Fruiting body: A flat, crust-like layer of 2 to 20 (or more) cysts arranged to form a sorus. Sori rounded, up to 90 microns in diameter, or irregularly shaped; often elongate up to 400 microns long. Smokygray color due to surrounding slime walls. Outer profile of sheath (or cortex) irregular. Cyst wall 2.4 to 3.5 mierons thick; cysts often nearly spherical, 13 to 48 microns in diameter, though frequently elongate. Average 36 by 44 microns. Colorless, single, inclosed in a transparent membrane.

Spores: No data.

Vegetative cells: Long, straight, cylindrical with rounded ends; 0.7 to 0.9 by 2.7 to 5.7 microns. Encysted cells similar.

Habitat : Soil.

Illustrations: Kírzemieniewski (loc. cit.) Plate XVI, Figs. 6-9.

15. Polyangium parasiticum Geitler. (Arch.f. Protistenkunde, 50, 1924, 67.)

Etymology: Latin parasiticus, parasitie.

Swarm stage (pseudoplasmodium): In water, on surface of the alga Cladophora. Pseudoplasmodia small. Rods long, cylindric, rounded at end and 0.7 by 4 to 7 microns. At first saprophytic, later entering and destroying the Cladophora rell.

Fruiting bodies: Sometimes single, usually 2 to 8 microscopically small, united in irregular masses, spherical or somewhat elongated. From 15 to $\mathbf{5 0}$ microns, usually 25 to 40 microns, with hyaline slime. When mature, red-brown in color, with firm wall.

source and habitat: Found on Cladophora (fracta?) in pool at Vienna (Geitler, 1924).

Illustrations: Geitler (1924, loc. cit.) Figs. 1-10.

\section{Genus II. Synangium Jahn.}

(Jahn, Beiträge zur botan. Protistologie. I. Die Polyangiden, Geb. Borntraeger, Leipzig, 1924, 79; A pelmocoena Enderlein, Bakterien-Cyclogenie, Berlin, 1924, 243.)

Etymology : Greek syn, together and angion, vessel, referring to the clustering of the cysts.

Diagnosis: Cysts provided with an apical point, united more or less completely to rosette-shaped, hemispherical or spherical fruiting bodies.

The type species is Synangium sessile (Thaxter) Jahn.

\section{Key to the species of genus Synangium.}

I. Cysts irregular, pointed, united as a rosette on a slimy base, without a stalk.

$$
\text { 1. Synangium sessile. }
$$

II. The fused cysts on a simple or branched stalk.

A. Cyst group spherical, with the points of the cysts covered as with hair, reddish.

2. Synangium lanuginosum. 
B. Cyst group an oblate spheroid, yellow. Points of cysts less numerous.

3. Synangium thaxteri.

1. Synangium sessile (Thaxter) Jahn . (Chondromyces sessilis Thaxter, Bot. Gaz., 37, 1904, 111; Jahn, Beiträge zur botanischen Protistologie. I. Die Polyangiden, Geb. Borntraeger, Leipzig, 1924, 79.)

Etymology: Latin sessilis, sessile, not stalked.

Swarm stage (pseudoplasmodium) : Not described.

Fruiting body: Cysts form on the base a clump or rosette without trace of stalk. Diameter of rosettes 100 to 250 microns. Individually the cysts are quite variable in form, irregularly spindle-shaped, usually short-pointed, wrinkled surface toward the tip. At the base they fuse or unite to irregular masses. Cysts 18 to 55 by 25 to 75 microns, average 40 by 50 microns.

Source and habitat: Thaxter (loc. cit.) found this on decaying wood in Florida.

Illustration: Thaxter (loc. cit.) Pl. 27, Figs. 14-15.

\section{Synangium lanuginosum (Kofler)} Jahn. (Chondromyces lanuginosus Kofler, Sitzber. d. Kais. Akad. Wiss. Wien. Math.-Nat. Ǩlasse, 122 Abt., 1913, 861; Jahn, Beiträge zur botanischen Protistologie. I. Polyangiden, Geb. Borntraeger, Leipzig, 1924, 79.)

Etymology: Latin lanuginosus, woolly.

Swarm stage (pseudoplasmodium) : Not described.

Fruiting body : Cyst eluster, consisting of united cysts, spherical or oval, 80 to 200 microns in diameter, when dry, dark flesh-colored, covered with hairs 15 to 50 microns long, originating from the individual cysts and giving the cyst cluster the appearance of a hairy ball. Skin of the eysts not definite. Rods within the cysts 3 to 6 microns. The cyst clusters are terminal on more or less forked stalks, about $1 \mathrm{~mm}$ high.

Source and habitat: Kofler (loc. cit.) found this on rabbit dung at Vienna.

Illustrations: Kofler (loc. cit.) Pl. 1, Figs. 1-3.

3. Synangium thaxteri (Faull) Jahn. (Chondromyces thaxteri Faull, Bot. Gaz., $62,1916,226$; Jahn, Beitrïge zur botanischen Protistologie, I. Die Polyangiden. Geb. Borntraeger, Leipzig, 1924, 79.) Regarded as a synonym of Synangium lanuginosum by Krzemieniewski, Acta Soc. Bot. Poloniae, 4, 1926, 39.

Etymology: Named for Dr. Roland Thaxter, American botanist.

Swarm stage (pseudoplasmodium): Cultured for 2 years on dung, best in mixed cultures. Piods 0.5 by 3 to 6 microns.

Fruiting body : Fruit cluster flattened, spherical, yellow to flesh color or reddish-orange, with a stalk which varys in length, about 140 mierons in diameter. The bristles corresponding to the single eysts are 15 to 30 microns long, at the base 10 to 12 microns wide. Sometimes cyst single, usually 3 to 4 , orcasionally 20 to 30 . Rods 0.5 by 3 to 6 microns. Stalk maximum length $0.75 \mathrm{~mm}$, usually 350 microns, single or branched. Broad based, narrowing to apex and yellow in color. In germination rods move from basal sear of membrane, leaving the empty sack behind.

Source and habitat: On deer dung in Ontario, Canada (Faull).

Illustrations: Faull (loc. cit.) PI. 5 and 6. Jahn (loc. cit.) Fig. X, p. 80.

\section{Genus III. Melittangium Jahn.}

(Jahn, Beiträge zur botan. Protistologie. I. Die Polyangiden, Geb. Borntraeger, Leipzig, 1924, 78.)

Etymology: Greek melitta, bee and angion, vessel, because of the honey-comb pattern of the membrane. 
Diagnosis: Cysts brownish orange-red, on short white stalk, like a mushroom. Has appearance of a white-stalked Boletus. The rods inside stand at right angles to the membrane. Upon germination the covering membrane is left colorless and with an appearance of honey-comb.

The type species is Melitlangium boletus Jahn.

1. Melittangium boletus Jahn. (Beiträge zur botanischen Protistologie. I. Die Polyangiden, Geb. Borntraeger, Leipzig, 1924, 78.)

Etymology: Latin boletus, a kind of mushroom.

Swarm stage (pseudoplasmodium): No descriptiou.

Fruiting bodies: Cyst stalked, mushroom-like, white when immature, then yellowish-flesh colored, finally yellowishbrown to nut brown, when dried more reddish-brown. Larger diameter of cyst about 100 microns, height 40 to 50 microns, length of white stalk about 40 microns, length of rods in the eyst 3 to 4 microns by 0.5 microns. Sometimes the cyst is smaller and spherical (50 to 60 microns diameter), sometimes there is fusion of neighboring cysts, occasionally the stalk is abortive.

Source and habitat: Jahn (loc. cit.) found this not uncommon on rabbit and deer dung in the vicinity of Berlin, also on deer dung from Denmark. Krzemieniewski (1927, loc. cit.) reported it as common in Polish soils.

Illustrations: Jahn (loc. cit.), Pl. 2, Fig. 17 and 18. Also Fig B, p. 11, C-F, p. 23, O-Q, p. 43, T-U,p. 55. Krremieniewski, Acta Soc. Bot. Poloniae, 4, 1926, 1, Pl. V, Fig. 55-56.

\section{Genus IV. Podangium Jahn.}

(Cystobacter Schroeter, in Cohn, Kryptogamenflora v. Sehlesien, 3, 1, 1886, 170; Jahn, Beitrüge zur botan. Protistologie. I. Die Polyangiden. Geb. Borntraeger, Leipzig, 1924, 80; Monocystia Enderlein, Bakterien-Cyclogenie, Berlin, 1924, 243.) Etymology: Greck pus, podis, foot and angion, vessel.

Diagnosis: Cysts chestmut-brown or red-brown, single on a more or less definite white stalk.

The type species is Podangium erectum (Schroeter) Jahn.

\section{Key to the species of genus Podangium.}

I. Stalk scarcely definite, cysts short, appressed, if clongate then passing over from the white stem into the club-shaped cyst. Ripe eysts chestnut-brown.

1. Podangium erectum.

II. Stalk well dillerentiated.

A. Cysts spherical, often irregular, confluent, the white stalk short.

\section{Podangium lichenicolum.}

13. Cysts lengthened ellipsuidal, red-brown, definitely differentiated from the white, slender stalks.

\section{Podangium gracilipes.}

\section{Podangium erectum (Schroeter)} Jahn. (Cystobacter erectus Schroeter, in Cohn, Krytogamenflora v. Schlesien, 3, 1, 1886, 170; Chondromyces erectus Thaxter, Bot. Gaz, 23, 1897, 407; Jahn, Beiträge zur botanischen Protistologie. I. Die Polyangiden, Geb. Borntraeger, Leipzig, 1924, S0.)

Etymology: Latin erectus, erect, upright. 
Swarm stage (pseudoplasmodium): Kofler states rods are 2 to 5 microns in length.

Fruiting bodies: Cysts usually short, almost spherical, compact, rounded above, orange-red changing to chestnut-brown, single on a white to yellow hypothallus constituted from the slime remaining behind. A definite "foot" of whitish slime is seldom observed. Fifty to hundreds together. Usually about 80 microns high and 40 to 50 microns broad above, smaller below, often spherical cysts 60 microns in diameter. Rods in cysts 0.6 by 4 microns .

Jahn believes the European form to be distinct from that deseribed by Thaxter. Thaxter's form produces cystophores 60 to 300 microns long which wither at maturity so that cysts appear sessile.

Source and habitat: Thaxter (loc.cit.), horse dung in laboratory cultures, Massachusetts. Kofler (Sitzber. d. Kais. Akad. Wiss. Wien. Math.-Nat. Klasse, 122 Abt., 1913), mouse dung. Jahn (1924) common on manure of different kinds, also on bark covered with lichens. Krzemieniewski (Acta Soc. Bot. Pol., 5, 1927, 102) reported this species from Polish soil, but rare.

Illustrations: Thaxter (loc. cit.) Pl. 31, Figs. 16-19. QuehI, Cent. f. Bakt., II Abt., 16, 1906, Pl. 1, Figs. 4. Jahn (loc. cit.) Pl. I, Figs. 7, 8, and 9. Iirzemieniewski, Acta Soc. Bot. Poloniae, 4, 1926, 1, Pl. V, Figs. 52-53.

\section{Podangium lichenicolum (Thaxter)} Jahn. (Chondromyces lichenicolus Thaxter, Bot. Gaz., 1\%, 1892, 402; Jahn, Beiträge zur botanischen Protistologie. I. Die Polyangiden, Geb. Borntraeger, Leipzig, 1924, 81.)

Etymology: Greek lichen, tree moss, lichen. Latin -colus, dwelling.

Swarm stage: Reddish, rods cylindrical, tapering slightly, 0.6 by 5 to 7 microns. Germinate readily after drying for 18 months when sown on moist lichens.

Fruiting bodies: Cysts single, rounded or irregularly lobed, often confluent. Cystophore short, squarish, often lacking or misshapen. Cysts 28 to 35 microns, stem 7 to 8 by 10 microns.

Source and habitat: Thaxter (1892), parasitic upon living lichens, which it destroys, New Haven, Conn. Thaxter $(1904$, loc. cit.), lichens, Indiana, on algae, seen on wet boards, in mill race, Massachusetts.

Illustrations: Thaxter (1892, loc. cit.) Pl. 23, Figs. 20 to 23. Quehl, Cent. f. Bakt., II Abt., 16, 1906, 9, Pl. 1, Fig. 6.

\section{Podangium gracilipes (Thaxter)} Jahn. (Chondromyces gracilipes Thaxter, Bot. Gaz., 23, 1897, 406; Jahn, Beitrïge zur botanischen Protistologie. I. Die Polyangiden, Geb. Borntraeger, Leipzig, 1924, 82.)

Etymology: Latin gracilipes, slender footed.

Swarm stage: Rods 5 to 7 microns.

Fruiting bodies: Cysts bright orangered, or red, 25 by 35 microns, elongate, rounded, on a white pointed stalk, rigid and persistent on substratum, rods also in stalk. Shortened rods in cyst 3 to 5 microns. Cysts sometimes pear-shaped, caducous.

Source and habitat: Thaxter (loc.cit.), from rabbit dung, Massachusets. Kofler (1913, loc. cit.), dung, Vienna. Jahn (loc. cit.) relatively common. Twice on rabbit dung near Berlin, once on goat dung in Norway. Krzemieniewski (192i loc. cit.) reported this species from Polish soil, but rare.

Illustrations: Thaxter (loc. cit.) Pl. 31, Figs. 20-24. Quehl, Cent. f. Bakt., II Abt., 16, 1906, Pl. 1, Fig. 12. Jahn (loc. cit.) Pl. II, Figs. 19, 20. Krzemieniewski (1926, loc. cit.), Pl. V, Fig. 54. 


\section{Genus V. Chondromyces Berkeley and Curtis.}

(See Berkeley, Introduction to Cryptogamic Botany, London, 185i, 313; Stigmatella Berkeley and Curtis, ibid. 1857, 313 (figure but no deseription); Berkeley (deseription), Notes on North American Fungi, Crevillea, 3, 1874, 97; see Berkeley and Curtis, in Saccardo, Sylloge Fungorum, 4, 1S86, 679, Polycephalum? Kalchbrenner and Cooke, Grevillea, 9, 1880, 22; Myrobotyrs Zukal, Ber. d. deutsch. Bot. Gesellseh., 14. 1896, 316; Cystodesmia Enderlein, Bakterien-Cyclogenie, Berlin, 1924, 243.)

Synonymy: A species was tigured and named in 1857 by Berkeley as Chondromyces crocatus Berkeley and Curtis, but not described. The generic name was finally deseribed in 187t. Probably the date of the name should be the date of its description, although it is possible that an adequate labeled illustrat in should be interpreted as valid publication.

Etymology : Greek chondros, grain and myces, (fungus).

Diagnosis: Cysts compactly gmuped at the end of a colored stalk (cystophore). Cystophore simple or branched.

The type species is Chondromyces crocatus Berkeley and Curtis.

Key to the species of genus Chondromyces.

I. Cysts not in chains.

A. Cysts sessile when ripe.

1. Cysts not pointed.

a. Cysts rounded.

b. Yellow.

1. Chondromyces crocatus.

bb. Bright orange-red.

2. Chondromyces aurantiacus.

aa. Cysts cylindrical.

3. Chondromyces cylindricus.

2. Cysts pointed.

4. Chondromyces apiculatus.

B. Cysts borne on stalk or stipe when ripe.

1. Cysts orange-colored and truncate or rounded at distal end.

a. Cystophore usually simple.

5. Chondromyces pediculatus.

aa. Cystophore usually branched.

6. Chondromyees medius.

2. Cysts copper-red when ripe; pear-shaped.

7. Chondromyces minor.

II. Cysts in chains at end of a compact stalk.

8. Chondromyces catenulatus.

1. Chondromyces crocatus Berkeley and Curtis. (Berkeley, Introduction to Cryptogamic Botany, London, 1857, 313 ; Berkeley, Notes on North American Fungi, Grevillea, 3, 1874,64; Myxobotyrs variabilis Zukal, Ber. d. deutsch. bot. Ges., 15, 1896, 340, according to hrzemieniewski, Acta Soc. Bot. Poloniae, 4, 1926, 38.)
Etymology: Latin crocatus, saffron yellow.

Swarm stage (pseudoplasmodium): Pale orange-red. Rods cylindrical or tapering slightly, straight or slightly curved, 0.6 to 0.7 by 2.5 to 6 microns. Cultivated on nutrient agar and sterilized horse dung. Cysts placed in moist chamber germinate in one or two days. 
The contents are first contracted within the cyst walls, showing the individual rods. The cyst wall is then absorbed or disappears at the base, and the rods escape in a regular stream until only the empty cyst is left.

Fruiting bodies: Cysts nearly conical, rounded at tip, average 12 by 28 microns ( 6 to 20 by 15 to 45 microns), straw yellow, in spherical heads of variable numbers ( 70 to 90 microns) at tips of branches. Cystophore orange-colored, slender, striated, often twisted or irregularly bent, simple or branched as many as 5 times. Ahout 600 microns high, rarely $1 \mathrm{~mm}$.

Source and habitat: Thaxter (1892, loc. cit.), melon rind from south Carolina and old straw from Ceylon and Cambridge, Mass. Zukal (loc. cit.), Vienna. Quehl, dung from Java and on deer dung, near Berlin. Thaxter (1904, loc cit.), New Haven, Conn., Tabor, Iowa, Florida, Laubach, Java.

Illustrations: Berkeley, Introduction to Cryptogamic Botany, London, 1857. 313. Thaxter, Bot. Gaz., 17, 1892, 389. Pl. 22 and 23, Figs. 1-11. Quehl, Cent. f. Bakt.. II tht., 16, 1906, 9, Pl. 1, Fig. 10. Jahn, Kryptogamenfora der Mark Brandenburg, I, Pilze I, Lief 2, 1911, 199, Fig. 6. Jahn, Beiträge zur botanischen Protistologie, I. Die Polyangiden, Geb. Borntraeger, Leipzig, 1924, Pl. 2, Figs. 14,15 , and 16 .

2. Chondromyces aurantiacus (Berkeley and Curtis) Thaxter. (Stigmatella aurantiaca. Berkeley and Curtis (no description). Introduction to (ryptogamic Botany. London, 1857. 313; Berkeley (description), Notes on North Ameri can Fungi. Grevillea, 3, 1sit, 97; Stilbum rhytidosporum Berkeley and Browne. 1873, 96, see Saccardo, Sylloge Fungorum, 4. 1886, 571; Polycephalum aurantiacum Kalchbrenner and Cooke. Australian Fungi, Grevillea, 9, 1880, 23; Myxobotrys rariabilis Zukal, Ber. deutsch. Bot. Ges., 14, 1896, 340; Chon- dromyces aurantiacus Jahn, Kryptogamenflora der Mark Brandenburg, V, Pilze I, 1911, 206.)

Etymology : Modern Latin aurantiacus, orange-colored.

Swarm stage (pseudoplasmodium): Flesh-colored, distinctly reddish. Rods large, tapering somewhat, normally straight, rounded at either extremity, 0.6 to 1 by 7 to 15 microns, average 0.5 by 7 microns (?). Easily cultivated on nutrient agar, but on this rarely produces well formed cystophores, though cultivable on its ordinary substrate without difficulty.

Fruiting bodies: Cysts oval, elliptical or spherical, average 30 by 50 microns, at first stalked then sessile, united in small numbers at one end of cystophores, bright orange-red, chestnut-brown when kept moist for a considerable period or flesh-colored. Cystophore colorless, often yellowish at the tip, usually simple, rarely forked, 200 to 400 microns high.

The Krzemieniewskis (Acta Soc. Bot. Poloniae, 5, 1927, 96) have described a Chondromyces aurantiacus var. frutescens in which the fruiting body consists of a greenish, later yellowish mass of rods which develops into a thick cystophore with numerous terminal cysts. The cysts are oval or spherical, sometimes with cross-striations, first orange-colored, later brown, about 40 to 120 by 30 to 90 mierons. The eyst rods are 0.9 to 1.0 by 2.3 to 3.4 microns.

source and habitat: Berkeley (1857, loc. cit.), on lichen. Berkeley and Brown (1873, loc. cit.), on rotten wood from Cevlon. Thaxter (1892, loc, cit.) in North America not uncommon on old wood and fungi. Zukal (loc. cit.), Vienna. Thaxter, Bot. Gaz., 23, 1897, 395, on antelope dung from Africa. Thaxter, Bot.Gaz., 37, 1904, 405, Florida, Philippines. Quehl, Cent. f. Bakt., II tbt., 16, 1906, 9, dung from Java. Krzemieniewski, Acta Soc. Bot. Poloniae, 4, 1926, 1, rare in Polish soils.

Illustrations: Berkeley and Brown 
(1873, loc. cit.) Pl. 4, Fig. 16. Kalchbrenner and Cooke (loc. cit.). Thaxter (1892, loc. cit.) Pl. 23 and 24, Figs. 12-19 and 25-28. Zukal (loc. cit.) Pl. 20. Quehl (loc. cit.) PI. 1, Fig. 10. Jahn, Beiträge zur botanischen Protistologie, I. Die Polyangiden, Geb. Borntraeger, Leipzig, 1924, Fig. V, p. 57, Fig. W, p. 59. Krzemieniewski (1926, loc. cit.), Pl. V, Figs. 57-60; (1927) var. frutescens Pl. VI, Figs. 27-35.

3. Chondromyces cylindricus Krzemieniewski. (Acta Soc. Bot. Pol., 7, 1930, 260.)

Etymology: Greek kylindrikos, cylindrical.

This organism was at first thought to be a variety of Chondromyces aurantiacus. It is separated from it on the basis of size, shape and pigmentation of the cysts.

Fruiting body: Cystophore composed of bundles of cells, develops from a thick, greenish-yellow mass of rods; unbranched or with short branches, colorless to pale orange-yellow; up to 200 microns high. Numerous eysts develop from cystophore and branches; at first borne on slender stipe 20 microns long, later becoming sessile on cystophore. Young cysts orange-yellow, later becoming deeper orange, and finally bright orange-brown when ripe. Shape variable: oval, irregularly rounded; predominantly cylindrical with rounded ends, 16 to 49 by 30 to 90 microns; average 29 by 56 microns.

Spores: Shortened rods 0.8 to 1.1 by 1.8 to 3.3 microns.

Vegetative cells: Long rods, tapered at ends, 0.5 to 0.6 by 6.7 to 11.0 microns . Habitat: Soils.

Illustrations: Krzemieniewski (loc. cit.) Plate XVII, Fig. 18.

4. Chondromyces apiculatus Thaxter. (Bot. Gaz., 23, 1897, 405.)

Etymology: Modern Latin from Latin apex, a point: with a small point.

Swarm stage (pseudoplasmodium): Rods 1 by 3 to 20 microns. Does not grow as well on nutrient agar as Chondromyces crocatus and produces cysts and cystophores rarely. Cultivated on dung. Kofler states rods are 3 to 5 microns in length.

Fruiting bodies: Cysts of variable form, cylindrical to broadly turnip-shaped, usually with basal and apical appendages, the latter longer and pointed, bright orange, 28 by 35 microns. Cysts united in a single spherical terminal head, about 200 microns in diameter. Cystophore rigid, stiff, seldom branched, to $1 \mathrm{~mm}$ high, colorless, longitudinally striate. Cysts germinate at both base and apex.

Source and habitat: Thaxter (1897, loc. cit.), on antelope dung from Africa. Thaxter (1904, loc. cit.), deer dung, Philippines, Florida. Baur (1906, loc. cit.), on rabbit dung near Berlin. Kofler (1913, loc. cit.), on rabbit dung near Vienna.

Illustrations: Thaxter (1897, loc, cit.) Pl. 30, Figs. 1 to 15. Quehl (1906, loc. cit.), Pl. 1, Figs. 13 to 14. Jahn (1911, loc. cit.) p. 199, Fig. 5.

\section{Chondromyces pediculatus Thaxter.} (Bot. Gaz., 37, 1904, 410.)

Etymology: From Latin pediculus, a small foot; small footed (stalked).

Swarm stage (pseudoplasmodium): Rods 0.6 to 0.7 by 2 to 4 microns.

Fruiting bodies: Cysts rounded to bellshaped, truncate at distal end, orangeyellow, when dry orange-red, 35 to 50 microns. Sessile on stalks 40 to 60 microns in length, which are arranged as an umbel on the tip of the eystophore. Cystophore 300 to 700 microns in length, solitary, simple, usually rather slender and somewhat wrinkled.

Source and habitat: Thaxter (loc. cit.), on goose dung in South Carolina.

Illustrations: Thaxter (loc. cit.) Pl. 26, Figs. 7 to 13 .

6. Chondromyces medius Krzemieniewski. (Acta Soc. Bot. Pol., 7, 1930, 263.) 
Etymology: Latin medius, medial, moderate.

Fruiting body: Glistening, orangecolored cysts attached to cystophore in clusters by means of filamentous stipes about 40 microns long. Deciduous. Mass of rod-shaped cells from which cystophore develops colorless to pink. Cystophore composed of bundles of cells, often branched; appear similar to those of Chondromyces aurantiacus var. frutescens. Cysts variable in shape; predominant are those rounded or flattened at the apex and tapered toward the base, 24 to 78 by 26 to 93 microns. Average 51 by 55 microns.

Spores: No data.

Habitat: Soil.

Illustrations: Krzemieniewski (loc. cit.) Plate XVII, Figs. 20-22.

7. Chondromyces minor Krzemieniewski. (Acta Soc. Bot. Pol., 7, 1930, 265.)

Etymology: Latin minor, less, little, small.

Fruiting body: Cell masses from which cystophore develops, reddish-violet in color. Cystophore white, simple or branched, up to 120 microns high, 17 to 89 microns thick. Cysts borne in clumps of 2 to 20 at apex of cystophore and branches on delicate colorless stipes. Cysts rose-red becoming copper-red when dry; pear-shaped, tapering toward base and broad at the apex; 20 to 47 by 20 to
65 microns; average 28 by 38 microns. Deciduous. Stipes 3 to 6 by 10 to 25 microns.

Spores : 0.6 to 0.8 by 2.9 to 4.3 microns. Vegetative cells: 0.6 by 3.8 to 7.2 microns.

Habitat: Soil.

Illustrations: Krzemieniewski (loc. cit.) Plate XVII, Figs. 23-24.

8. Chondromyces catenulatus Thaxter. (Bot. Gaz., 37, 1904, 410.)

Etymology : Modern Latin from catena, a chain, = occurring in chains.

Swarm stage (pseudoplasmodium): Cultivated only on original substrate. Rods 1 to 1.3 by 4 to 6 microns.

Fruiting bodies: Cysts light yelloworange, 20 to 50 by 18 microns in rosarylike chains, which may be branched once or twice, sessile on a short compact stalk, cysts separated by shriveled isthmuses. Chains to 300 microns. Cystophore simple 180 to 360 microns, cleft above, and passing over into the chains, rather broad at base and spreading somewhat on substratum. The divisions of the cystophore are pointed, short and slightly swollen.

Source and habitat: Thaxter (loc. cit.), on decaying poplar wood, New Hampshire.

Illustrations: Thaster (loc. cit.) Pl. 26, Figs. 1 to 5 . 


\section{FAMILY V. MYXOCOCCACEAE JAHN.}

(Beiträge zur botan. Protistologie. I. Die Polyangiden, Geb. Borntraeger, Leipzig, 1924, 84.)

Diagnosis: The rods become shortened when fruiting occurs (resting cells are formed), and develop into spherical or ellipsoidal spores or microcysts. Upon germination the vegetative cell develops from the spore by a process analogous to budding, pinching off at the point of emergence, leaving the spore wall entirely empty. In three of the genera, definite fruiting bodies are produced. In Sporocytophaga, the spores (microcysts) are produced from the vegetative cells without development of fruiting bodies.

\section{Key to the genera of family Myxococcaceae.}

I. Definite fruiting bodies formed.

A. Fruiting body not containing or made up of eysts.

1. Fruiting bodies deliquescent.

Genus 1. Myxococcus, p. 1040.

2. Fruiting bodies firm, not deliquescent.

Genus II. Chondrococcus, p. 1044.

B. Fruiting body made up of cysts.

Genus III. Angiococcus, p. 1047.

II. No definite fruiting bodies formed.

Genus IV. S'porocytophaga, p. 1048.

\section{Genus 1. Myxococcus Thaxter.}

(Bot. Gaz., 17, 1892, 403.)

Etymology: Greek myxa, mucus and kokkos, berry; slime sphere.

Diagnosis: Spherical spores in conical or spherical or occasionally ovoid upright fruiting bodies, united by a loose more or less mobile slime.

The type species is Myxococcus fulvus (Cohn emend. Schroeter) Jahn.

\section{Key to the species of genus Myxococcus.}

I. Stalk lacking or indicated only by a constriction.

A. Spores average less than 1.4 microns in diameter.

1. Fruiting body red or brownish-fiesh color.

2. Fruiting body light blood-red.

1. Myxococcus fulvus.

2. Myxococcus cruentus.

B. Spores average 2.0 microns in diameter.

1. Fruiting body yellow to greenish-yellow.

3. Myxococcus virescens.

2. Fruiting body yellow-orange to orange.

4. Myxococcus xanthus.

II. Well developed stalk supporting spherical spore mass above.

A. Spores spherical.

B. Spores oval.

5. Myxососcus stipitatus.

6. Myxococcus ovalisporus. 
1. Myxococcus fulvus (Cohn emend. Schroeter) Jahn. (Micrococcus fulvus Cohn?, Beiträge z. Biologie d. Pflanzen, 1, Heft 3, 1875, 181; Jahn (1924) states that the description of Cohn is too inadequate to determine whether be was dealing with a true species of the genus Myxococcus. Cohn described the organism from horse dung, as producing conical, rust-red droplets $\frac{1}{2} \mathrm{~mm}$ in diam. eter, the cells bound together by an intercellular slime, cells large, 1.5 microns in diameter; Micrococcus fulvus Schroeter, Schizomycetes, in Cohn, Kryptogamenflora v. Schlesien, 3, 1, 1886, 144. Observed on horse dung and rabbit dung at various localities. Jahn insists that this organism must be the same as Myxococcus rubescens Thaxter. Myxococcus rubescens Thaxter, Bot. Gaz., 17, 1892, 403; Myxococcus ruber Baur, Arch. f. Protistenkunde, 5, 1905, 95; Myxococcus pyriformis A. L. Smith, Jour. Bot., 39, 1901, 71; Myxococcus javanensis de Kruyff, Cent.f. Bakt, II Abt., 21, 1908, 386; Rhodococcus fulvus Winslow and Winslow, Systematic Relationships of the Coccaceae, 1908, 262; Myxococcus fulvus Jahn, Beiträge zur botanischen Protistologie. I. Die Polyangiden, Geh. Borntraeger, Leipzig, 1924, 84.)

Etymology: Latin fulvus, reddishyellow.

Swarm stage (pseudoplasmodium): Thaxter states that the rod masses are reddish, rods slender, irregularly curved, 0.4 by 3 to 7 microns. Bauer followed spore germination in hanging drop. Spores 0.8 to 1.3 microns, without structure, in five hours swollen to 1 to 1.5 microns, and no longer as refractive. The membrane is not burst; the cell becomes egg-shaped, then elongate and cylindric. He regards his Myxococcus ruber as distinct from Thaxter's Myxococcus rubescens in part because of differences in spore germination. The cells become motile after doubling or trebling in length. It is a creeping motion in contact with the substrate; the cells do not "swim." Rate of motion 5 to 10 microns per ninute. Rods eventually are 0.5 to 0.7 microns by 4 to 10 microns. Cell division by transverse fission. Spore formation is through shortening and rounding of the cells, the converse of germination. In hanging drop the cells tend to congregate after three days and to transform into spores. Rods sporulate in 3 to 4 hours. The rods continue to congregate, and the spore mass increases, held together by viscous matrix. Vegetative cells are light flesh color.

Gelatin is quickly liquefied, completely in 1 to 2 days, but no fruiting bodies are formed.

Kofler secured good growth on Hasting's milk agar, and determined digestion of casein.

Baur could not secure good growth on any agar medium of known composition. With peptone, sugars, ete., some growth but not normal when peptone present. He carried one strain $3 \frac{1}{2}$ months on peptone sugar agar. Good growth on dung agar. Addition of peptone to dung agar not significant in effect, the addition of glucose altered the form of the fruiting bodies.

De Kruyff secured best results with a dung extract agar to which was added ammonium nitrate and potassium phos. phate.

Fruiting bodies: Spherical or elongate pear-shape, constricted below, often with definite slimy stalk, flesh red to brownish-red, when dry rust-red to brown, about 300 microns in diameter. Spores 1 to 1.2 microns. Jahn $(1924$, Toc. cit.) notes two varieties.

var. albus. (Latin albus, white) Constantly white, even when transferred. Fruiting bodies somewhat smaller than the type.

var. miniatus. (Latin miniatus, painted with red lead or cinnabar.) Color cinnabar-red, fruiting bodies somewhat larger.

The form described by de Kruyff had spores 1.6 microns in diameter. 
Source and habitat: Thaxter (1892, loc. cit.), on various decaying substances, lichens, paper, dung, etc. Smith (loc. cit.), on rabbit dung from Wales. Baur (loc. cit.), on cow and dog dung. De Kruyff (loc. cit.), on stable manure in Java. Jahn (1924, loc. cit.), very common, on almost all specimens of dung, also on bark, decaying wood, and lichens. Krzemieniewski (1927, loc. cit.) very common in Polish soil. Kiofler (loc. cit.), dung of rabbit, horse, goat, mouse, roe, deer, on stem of clematis and decaying leaves and in bird nest.

Illustrations: Cohn (loc. cit.) PI. 6, Fig. 18. Smith (loc. cit.) Fig. 1. Baur (loc.cit.) Figs. 1, 2, 3, and Pl. 4, Figs. 1-13, 16. Jahn (1924, loc. cil.) Figs. L-M, p. 43, Fig. R, p. 47. Krzemieniewski, Acta Soc. Bot. Poloniae, 4, 1926, Pl. I, Figs. 7-8. Kofler, Sitzber d. Fais. Akad. Wiss. Wien. Math.-Nat Klasse, 122 Aht., 1913, 845, P1. 2, Figs. 10 and 12 .

Cultures: Baur (loc. cit.) states that he deposited a pure culture in the Zentralstelle für Pilzkulturen.

\section{Myxococcus cruentus Thaxter.} (Thaxter, Bot. Gaz., 23, 1897, 395; Chondrococcus crucntus Krzenieniewski, Acta Soc. Bot. Poloniae, 5, 1927, 79.)

Etymology: Latin cruentus, blood-red.

Swarm stage (pseudoplasmodium): Rods 0.8 by 3 to 8 microns. Was not. cultivated.

Fruiting body: Cysts regularly spherical, 90 to 125 microns, blood-red. Slime forms on the surface a more or less definite membrane, in which the spores lie. Spores oval or irregularly oblong about 0.9 to 1 by 1.2 to 1.4 microns. Cysts are densely aggregated.

Source and habitat: Thaxter (loc. cit.), on cow dung, Temessee. Irzemieniewski (1927, loc. cit.) rare in Polish soils.

Illustrations: Thaxter (loc. cil.) Pl. 31, Figs. 28-29.
3. Myxococcus virescens Thaxter. (Bot. Gaz., 17, 1892, 404.)

Etymology : Latin virescens, becoming green.

Swarm stage (pseudoplasmodium): Rod masses greenish-yellow. Rods slender, irregularly curved, 0.4 by 3 to 7 microns. When cultivated in potato agar tends to lose its green color and becomes yellowish. Badian (1930) reports the presence of a dumb-bell-shaped nuclear structure which splits longitudinally in cell division, and shows autogamy preceding and a reduction division during spore formation.

Fruiting body: Spherical or conical, usually less rounded than other species of the genus, yellowish, occasionally greenish, in culture on artificial media, easily becoming white, 150 to 500 microns. The slime deliquesces in continued moisture. Spores large, about 2 microns.

Source and habitat: Thaxter (loc. cit.), on hen's and dog's dung, New England. Jahn $(1924$, loc. cit.), not very abundant on dung of rabbit, horse, stag and black rock. Krzemieniewski (1927, loc. cit.), common in soil in Poland. Badian (loc. cit.), Poland.

Illustrations: Krzemieniewski, Acta Soe. But. Poloniae, 4, 1926, Pl. I, Fig. 9. Badian, Acta Soc. Bot. Poloniae, 7, 1930, $55, \mathrm{Pl} .1,8$ figures.

4. Myxococcus xanthus Beebe. (Jour. Bact., 42, 1941, 193.)

Etymology: Greek xanthos, orange, golden.

Fruiting body : Spherical to subspherical, usually sessile but occasionally constricted at the base giving the appearance of a short stalk or foot. Mature fruiting body up to 300 to 400 microns in diameter, of ten slightly flat tened on top or one side. Color varies from light yellowish-orange when young to bright orange when mature; color constant, never tending toward greenish-yellow. No outer eyst wall or 
limiting membrane discernible, the spores being imbedded in the slime holding the mass together. Usually single, though two or three fruiting bodies may become joined to form an irregular mass; each is attached to the substrate, however, and never bud one from another.

Spores: Spherical, with thick outer wall or membrane. Highly refractile. Stain very easily with any of the ordinary bacterial or nuclear dyes. 2.0 microns in diameter, seldom larger.

Vegetative cells : Large, flexible, single, Gram-negative rods with rounded ends. No flagella, but move on surface of solid or semi-solid substrate with a crawling or creeping motion. Vary in size from 0.5 to 1.0 by 4 to 10 microns; average 0.75 by 5 microns. Nore or less distinct cell wall often evident.

Vegetative colony: Characteristics vary with the substrate.

On plain 1.5 per cent agar (no nutrients added): Very thin and transparent, often hardly visible except by transmitted light. Little or no pigmentation. Surface covered with fine, more or less regularly spaced ridges causing a dull macroscopic appearance without gloss or sheen. Margin very thin and quite regular.

On rabbit dung decoction agar: Col ony thicker, the surface being broken by veins or ridges radiating from the center. Thick central area of ten smooth and glossy while margin much the same as that on plain agar. Teins or ridges extend outward from center in loose spiral, always in clock-wise direction. Pigmentation, yellow to pale orange, confined to thicker central portion, extends part way along veins to margin.

On nutrient agar: Growth poor. Colony thick, at first heavily veined, the veins later merging to form an irregular glossy surface. Colony remains small, pigmentation usually fairly heavy; mar gin thick, irregular to lobate.

Physiology: Grows well on mineral salt-agar to which has been added dulcitol, inulin, cellulose, reprecipitated cellulose or starch; hydrolyzes starch; does not destroy cellulose to any appreciable extent. Best growth on suspension of killed bacterial cells in agar; suspended cells in growth area lysed. Development completely inhibited by arabinose, largely by maltose and mannose.

Source: Isolated from dried cow dung, Ames, Iowa.

Habitat: Decomposed bacterial cells in dung.

Illustrations: Beebe (loc. cit.) Figs. $1-28$.

5. Myxococcus stipitatus Thaxter. (Bot. Gaz., 23, 1897, 395.)

Etymology: From Latin stipes, stalk; stalked.

Swarm stage (pseudoplasmodium): Rods 0.5 to 0.7 by 2 to 7 microns or longer. Grows well on nutrient agar, but does not fruit readily.

Fruiting body: Spore mass nearly spherical, 175 microns in diameter, deliquescent, sessile on a well developed compact stalk, white to yellowish and flesh color. Spores 0.8 to 1.2 by 1.0 to 1.15 microns. Stalk 100 to 200 microns long, 30 to 50 microns wide.

Source and habitat: Thaxter (loc. cit.), repeatedly on dung in laboratory cultures at Cambridge, Mass., Maine, Tennessee. Krrzemieniewski (1927, loc. cit.) rommon in Polish soils.

Illustrations: Thaxter (loc. cit.) Pl. 31, Figs. 30 to 33 . Krzemieniewski, Acta Soc. Bot. Poloniae, 4, 1926, PI. II, Figs. 13-14.

6. Myxococcus ovalisporus Krzemieniewski. (ActaSoc. Bot., Pol., 4, 1926, 15.)

Etymology : Modern Latin ovalis, oval, Greek sporos, seed. Oval spored.

Swarm stage (psendoplasmodium): Not described.

Fruiting bodies: Produces almost spherical, characteristically shortened, ovoid 
spore masses of light milky yellow color. These are often raised on a poorly developed stalk. This stalk always shows some bacterial cells remaining, and in this and color is differeniated from $M$. stipitatus. From the base of the stalk or directly from the substrate one or more small fruiting bodies develop. Spores are oval, sometimes irregularly spherical, 1.3 to 1.9 by 1.0 to 1.4 microns. In culture retains its differences from Myxococcus stipitatus. The latter sporulates best at room temperature, but
Myxococcus ovalisporus in an incubator (presumably at $37^{\circ} \mathrm{C}$ ).

Source and habitat: Develops on rabbit dung (sterilized) on soil in Poland (Krzemieniewski).

Appendix: Rippel and Flehmig (Arch. f. Mikrobiol., 4, 1933, 229) describe a new type of aerobic cellulose destroying bacteria under the new genus name of Itersonia. This genus includes a single species, Itersonia ferruginea. This organism shows similarity to those included in Myxococcus.

\section{Genus II. Chondrococcus Jahn.}

(Jahn, Beiträge zur botan. Protistologie. I. Die Polyangiden, Geb. Borntraeger, Leipzig, 1924; Dactylocoena Enderlein, Bakterien-Cyclogenie, Berlin, 1924, 243.)

Synonymy: A segregate from Myxococcus Thaxter.

Etymology: Greek chondros, grain and kokkos, ball (coccus).

Diagnosis: Spores embedded in a viscous slime which hardens. Fruiting bodies divided by joints or constrictions, often branched, usually relatively small.

Seven species are included, of which the first described by Thaxter and best described, Chondrococcus coralloides (Thaxter) Jahn, may be designated as the type. The first species listed by Jahn is regarded as doubtful and should not be regarded as the type for there is no evidence that Jahn ever saw the species.

\section{Key to the species of genus Chondrococcus.}

I. Not parasitic on fish.

A. Erect, simple or somewhat branched fruiting bodies.

1. Secondary fruiting bodies not produced.

a. Fruiting bodies constricted or jointed.

1. Chondrococcus coralloides.

aa. Fruiting body simple, columnar, club-or cushion-shaped.

b. Fruiting body thick below, lesser above.

2. Chondrococcus cirhosis.

bb. Not as in b.

c. Spores 1.6 to 2.0 microns in diameter.

d. Fruiting body cushion-shaped.

3. Chondrococcus megalosporus.

dd. Fruiting body branched.

4. Chondrococcus macrosporus.

cc. Fruiting body smaller below, above club-shaped. Spores 1.0 to 1.2 microns in diameter.

1a. Chondrococcus coralloides var. clavatus.

2. Secondary fruiting bodies arise as bud-, finger-or coral-like growths from primary fruiting body.

5. Chondrococcus blasticus.

B. Recumbent, simple swelling or cyst heap constituting the fruiting body. 
1. Cysts 60 to 170 microns, without definite envelope, in swollen brain-like arrangement.

6. Chondrococcus cerebriformis.

2. Cysts 30 to 35 microns, numerous, and embedded in a thick slime envelope. 1b. Chondrococcus coralloides var. polycystus.

II. Parasitic on fish.

\section{Chondrococcus columnaris.}

1. Chondrococcus coralloides (Thaxter) Jahn. (Myxococcus coralloides Thaxter, Bot. Gaz., 17, 1892, 404; Myxococcus digitatus Quehl, Cent. f. Bakt., II Abt., $16,1906,18$ (pro parte); Myxococcus clavatus Quehl, ibid.; Myxococcus polycystus Kofler, Sitzberg. d. kais. Wiss., Wien. Mat.-Nat. Klasse, 122 Abt., 1913,865 (pro parte); Myxococcus exiguus Kofler, ibid., 867 (pro parte); Chondrococcus polycystus Krzemieniewski, Act. Soc. Bot. Poloniae, 4, 1926, 46.)

Etymology: Greek korallion, coral, eidos, like.

Swarm stage (pseudoplasmodium): Rod masses pale pinkish, thin, rods slender, curved 4 to 7 by 0.4 microns. Readily cultivated on lichens and on potato agar.

Fruiting bodies: Very variable in shape, usually with rounded coral-like processes, recumbent or upright, sometimes with finger-like outgrowths or rounded constrictions, usually small, about 50 microns in diameter, protuberances 20 to 30 microns wide, light rose to flesh color. Spores 1 to 1.2 microns. Jahn concludes that the species segregated by Quehl and by Kofler are of varietal rank only. Krzemieniewski (1926) regards Chondrococcus polycystus (Kofler) Krzemieniewski as a distinct species.

Source and habitat: Thaxter (1892), uncommon in America, on lichens. Very common in Europe. Jahn (1924), relatively common. On dung of rabbit, hare, horses, deer, old bark and old lichens. Goat dung from Lapland and Italy. Kofler (1913), dung of field mice, horses, hares, goats, roe and deer. Krzemieniewski (1927), common in Polish soil.
Illustrations: Thaxter (1892, loc. cit.) Pl. 24, Figs. 29-33. Quehl (1906, loc. cit.) Pl. 1, Figs. 1 and 9. Fofler (1913, loc. cit.) Pl. 1, Fig. 4, Pl. 2, Fig. 9. Tirzemieniewski (1926, loc. cit.) Pl. II, Figs. 15-18. Jahn (1924, loc. cit.) Fig. Y, p. 87 .

1a. Chondrococcus corralloides var. clavatus varies from Chondrococcus coralloides in having fruiting bodies simple or branched rather than constricted or jointed.

1b. Chondrococcus corralloides var. polycystus varies from Chondrococcus coralloides, in having its fruiting bodies simple swellings or "cyst heaps" rather than branched, and in being recumbent rather than erect.

2. Chondrococcus cirrhosus (Thaxter) Jahn. (Myxococcus cirrhosus Thaxter, Bot. Gaz., 23, 1897, 409; Jahn, Beiträge zur botanischen Protistologie. I. Die Polyangiden, Geb. Borntraeger, Leipzig, 1924, 200.)

Etymology: Modern Latin from Greek cirrhos, tawny.

Swarm stage (pseudoplasmodium): Rods 0.8 by 2 to 5 microns.

Fruiting bodies: Elongate, upright, thickened below, slender above, extended to a rounded point, 50 to 100 mierons long, 20 microns in diameter at base, light red to flesh-colored. Spores about 1 micron.

Source and habitat: Thaxter (loc.cit.), once only on grouse dung, Mass.

Illustrations: Thaxter (loc. cit.) $\mathrm{Pl}$. 31, Figs. 25-27.

3. Chondrococcus megalosporus Jahn. (Beiträge zur botanischen Protistologie. 
I. Die Polyangiden, Geb. Borntraeger, Leipzig, 1924, 86.)

Etymology: Greek megalos, large; sporos, seed, spore; large spored.

Swarm stage (pseudoplasmodium) : Not described.

Fruiting bodies: About 80 to $160 \mathrm{mi}$ crons wide, rounded, cushion-shaped, dark flesh color. Spores 2 microns.

Source and habitat: Jahn (loc. cit.), on stag dung near Berlin.

Illustrations: Jahn (loc. cit.) Fig. I, i to $\mathrm{k}, \mathrm{p} .87$.

4. Chondrococcus macrosporus Kirzemieniewski. (Acta Soc. Bot. Poloniae, 4, 1926). According to Kirzemieniewski, not to be confused with Zukal's species, Myxococcus macrosporus (Ber. d. deutsch Bot. Gesellsch., 15, 1897, 542.)

Etymology : Greek makros, long, large; sporos, seed, spore; large-spored.

Swarm stage (pseudoplasmodium) : Not described.

Fruiting bodies: Much like Chondrococcus coralloides, differing in color and in size of spores. Spores 1.6 to 2.0 microns. Fruiting body yellow or light brown color, with long branches.

Source and habitat: Irzemieniewski (loc. cit.), found it first on leaves, later isolated from soil on rabbit dung.

Illustrations: Krzemieniewski (loc. cit.) Pl. II, Fig. 19.

5. Chondrococcus blasticus Beebe. (Iowa State Col. Jour. Sci., 15, 1941, 310.)

Etymology: Greek blastikos, budding.

Fruiting body: Primary: Spherical to subspherical, usually sessile but oceasionally with a short stalk or foot; pale pink to bright salmon pink; 300 to 600 microns in diameter. No outer wall or limiting membrane evident. Develops on sterilized rabbit dung in from 3 to 6 days at room temperature. Secondary: Arising as bud-like growth from the primary fruiting body. Develops into irregularly shaped, finger-, coral-or budlike protuberance. Seldom branched; occasionally stalked but usually sessile on primary fruiting body until latter is utilized in formation of several secondary fruiting bodies. Deep pink to salmon pink in color. Variable in size and shape; 50 to 150 by 75 to 225 microns. No outer wall or limiting membrane evident.

Spores: Spherical, thick-walled, highly refractile; 1.2 to 1.4 microns in diameter. Held together in the fruiting body by the mass of slime.

Vegetative cells : Long, slender, flexible rods, straight or curved to bent, ends rounded to slightly tapered, Gramnegative. 0.5 to 0.6 by 3.0 to 5.0 microns. Usually found in groups of 2 to 12 lying parallel on the surface of the slimy colony, the group moving as a unit. Motile by a crawling or creeping motion, no flagella.

Vegetative colony: Thin, colorless, transparent at margin; surface broken by many small ridges or veins. Center smooth, slightly thicker, often showing pale pink color. Fruiting bodies first form at or near center, later distributed irregularly on other parts of colony. Margin enmposed of active vegetative ("ells.

Physiology: Good growth on mineral salt agar to which has been added such complex carbohydrates as dulcitol, inulin, cellulose, reprecipitated cellulose or stareh; starch hydrolyzed, cellulose not destroyed appreciably. Can utilize agar as both ( and $\mathrm{N}$ sources. Best growth on suspensions of killed bacterial cells in agar. Growth inhibited partially or entirely by arabinose, mannose and maltose.

Source: Goat dung and soil, Ames, Iowa.

Habitat: Soil. Decomposes organic matter, especially bacterial cells in dung.

Illustrations: Beebe (loc. cit.) Pl. II, Figs. 5-6, pl. IV, Fig. 18.

\section{Chondrococcus cerebriformis} (hofler) Jahn. (Myxococcus cerebriformis Kofler, Sitzber. d. kais. Akad. Wiss., Wien. Math.-Nat. Klasse, 122 
Abt., 1913, 866; Jahn, Beiträge zur botanischen Protistologie. I. Die Polyangiden, Geb. Borntraeger, Leipzig, 1924, 86.)

Etymology: Latin cerebrum, brain; formis, shape.

Swarm stage (pseudoplasmodium): Rods 4 to 12 microns.

Fruiting bodies: About $1 \mathrm{~mm}$ long, clumped masses with swollen upper surface, brain-like, violet rose, often leadgray. Cysts 100 to 170 microns, without slime envelope. Spores 1.1 to $1.6 \mathrm{mi}$ erons. Jahn (loc. cit.) suggests that this may be Archangium gephyra.

Souree and habitat: Kofler (loc.cit.), on hare dung in the vicinity of Vienna.

Illustrations: Kofler (loc. cit.) Pl. 2, Figs. 7 and 8 .

7. Chondrococcus columnaris (Davis) Ordaland Rucker. (Bacillus columnaris Davis, Bull. U. S. Bur. Fisheries, 38, 1923, 261; Ordal and Rucker, Proc. Sioc. Exper. Biol. and Med., 56, 1944, 18; also see Fish and Rucker, Trans. Amer. Fish. Soc., 73, 1944 in press; Cytophaga columnaris Garnjobst, Jour. Bact., 49, 1945, 113.)

Etymology: From Latin columnaris, rising in the form of a pillar.

Vegetative cells: Flexible, weakly refractive, Gram-negative rods, 0.5 to 0.7 by 4 to 8 microns. Creeping motion observed on solid media, and flexing movements in liquids.

Spores (microcysts) : 0.7 to $1.2 \mathrm{microns}$, spherical to ellipsoidal, occurring on both liquid and solid media.

Physiology: Growth best on 0.5 to 0.9 per cent agar with 0.25 to 0.50 per cent Bactotryptone at $\mathrm{pH}$ 7.3. Colonies on tryptone agar yellow, flat and irregular. Edge uneven with swarming apparent. Gelatin liquefied rapidly. No indole. Noreduction of nitrates. Starch, cellulose and agar not attacked. Sugars not fermented, but glucose oxidized.

Fruiting bodies on agar not deliquescent, and surrounded by a firm membrane. A peculiar type of fruiting body formed in liquid media. Where organisms are in contact with infected tissues or with scales, produce columnar, sometimes branched, fruiting bodies in which typical spores (microcysts) develop in 7 to 10 days.

Source and habitat: First deseribed as cause of hacterial disease of warm water fishes (Davis, loc. cit.) and later in fingerlings of the cold water blue hlack salmon (Oncorhynchus nerka). Transmissible to salmonid fishes.

\section{Genus III. Angiococcus Jahn.}

(Beiträge zur Protistologie. I. Die Polyangiden, Geb. Borntraeger, Leipzig, 1924, 89.)

A segregate from Myxococcus Thaxter.

Diagnosis: Fruiting body consisting of numerous round (disk-shaped) cysts, cyst wall thin, spores within.

Etymology: Greek angion, vessel and kokkos, coccus (ball).

The type species is Angiococcus disciformis (Thaxter) Jahn.

\section{Key to the species of genus Angiococcus.}

A. Cysts yellow to dark orange-yellow; disk-shaped; 35 microns in diameter.

1. Angiococcus disciformis.

B. Cysts colorless to yellow; round; up to 15 microns in diameter.

2. Angiococcus cellulosum.

1. Angiococcus disciformis (Thaxter) Jahn. (Myxococcus disciformis Thaxter, Bot., Gaz., 37, 1904, 412; Jahn, Beiträge zur botanischen Protistologie. I. Die Polyangiden, Geb. Borntraeger, Leipzig, 1924, 89.) 
Etymology: Greek diskos, a quoit, discus; Latin formis, shape.

Swarm stage (pseudoplasmodium): Rods 0.5 to 0.6 by 2 to 3 microns.

Fruiting bodies: Cysts disk-shaped, crowded, sessile, attached by a more or less ragged scar-like insertion, or in masses. Cysts yellowish when young, when old dark orange-yellow, about 35 by 10 microns. Cyst wall distinct, thin, becoming very slightly wrinkled. Spores irregularly spherical, embedded in visrous slime, difficult to see in the ripe cyst.

Source and habitat: Thaxter (loc. cit.), dung of muskrat and deer, Massachuset ts and New Hampshire. Krzemieniewski (1927, loc. cit.), rare in Polish soils.

Illustrations: Thaxter (loc. cit.) Pl. 27, Figs. 19-21. Krzemieniewski Acta Soc. Bot. Poloniae, 4, 1926, PI. II, Figs. 21-22.

\section{Angiococcus cellulosum Mishustin.} (Microbiology, Moseow, 7, 1938, 427.)

Etymology: Modern Latin cellulosum, cellulose.

Fruiting body : Regularly rounded (less frequently extended or angular), 20 to 150 microns in diameter; yellow or pink in color, to drabbish when old. Encysted cells surrounded by a colorless cyst wall or envelope. Usually 1 to 3 short stalks or cystophores up to 10 microns high. Within outer wall are numerous cysts containing resting cells (spores). Cysts have regularly rounded form; unpigmented to yellow; 5 to 15 microns in diameter, average 6 microns. Number of cysts in fruiting body increases with age.

Spores: Cocci (term used is shortened rods) combined into globular aggregations easily broken up. Size not given.

Vegetative cells : 0.4 to 0.5 by 1.5 to 2.0 microns. Cell contents pigmented gray, and of indefinite outline (?).

Vegetative colony : Fairly rapid growth on cellulose with silica gel. Colony has a yellowish cast. Reaches diameter of 1.5 to $2.0 \mathrm{~cm}$ after 6 days with center yellowish-pink and margin tinged light pink. Surface moist. Fruiting bodies more numerous at center, but distributed over entire area. Fruiting bodies do not noticeably protrude above the surface of the colony.

Physiology : Cellulose attacked but not completely destroyed. Lower fibers remain intact, but on treatment with hot soda solution they fall spart.

Habitat: Soils.

\section{Genus IV. Sporocytophaga Stanier.}

(Jour. Bact., 40, 1940, 629.)

Diagnosis: Spherical or ellipsoidal microcysts formed loosely in masses of slime among the vegetative cells. Fruiting bodies absent.

Etymology: Greek sporos seed, spore; kytos hollow place, cell and phagein to eat. The type species is Sporocytophagu myxococcoides (Krzemienierska) Stanier.

\section{Ley to the species of genus Sporocytophaga.}

I. Microcysts spherical.

A. Does not utilize starch.

1. Sporocijtophaga myxococcoides.

B. Utilizes starch

II. Microcysts ellipsoidal.

2. Sporocytophaga congregala.

3. Sporocylophaga ellipsospora. 
1. Sporocytophaga myxococcoides (Krzemieniewska Stanier. (Spirochaeta cytophaga Hutchinson and Clayton, Jour. Agr. Sci., 9, 1919, 150; Cytophaga myxococcoides Krzemieniewska, Arch. Mikrobiol., 4, 1933, 400; Cytophaga globulosa Stapp and Bortels, Cent. f. Balit., II Abt., 90, 1934, 47; Cytophaga hutchinsonii Imšenecki and Solntzeva, Bull. Acad. Sci. U.S.S.R., Ser. Biol. No. 6, 1936, 1129; not Cytophaga hutchinsoni Winogradshy, Ann. Inst. Pasteur, 43, 1929, 578; Stanier, Jour. Bact., 40, 1940, 630.)

Etymology : Modern Latin from generic name Myxococcus, and eidos, like.

Vegetative morphology: Flexible, singly occurring rods, 0.3 to 0.4 micron wide at the center, tapering to both ends. Length 3 to 8 microns according to Krzemieniewska (loc. cit.), 2.5 to 5 microns according to Jensen (Proc. Linn. Soc. N. So. Wales, $65,1940,547)$. May be straight, bent, U-shaped or S-shaped. Show creeping motility (Stapp and Bortels, loc. cit.). Stain poorly with ordinary aniline dyes; with Giemsa's stain, the young cells are colored uniformly except for the tips. As the rods shorten and swell to form microcysts, the chromatin becomes concentrated and moves toward the center of the cell, generally in the form of two parallel bands (Krzemieniewska, Acta Soc. Bot. Pol., 7, 1930, 514).

Microcysts: Spherical, 1.3 to 1.6 microns in diameter, covered with a sheath of mucus. According to Kirzemieniewska (1930, loc. cit.), germination is by emergence of the shortened rod from the sheath, followed by elongation; according to Stapp and Bortels (loc. cit.) and Imšenecki and Solntzeva (loc. cit.), by a simple elongation of the entire microcyst.

Growth is strictly confined to cellulose. On mineral salts-silica gel plates covered with filter paper, yellow, glistening, slightly mucilaginous patches are pro- duced after a few days. The color gradually assumes a light brownish tinge on aging. The filter paper in these regions is eventually completely dissolved and the patches become translucent.

Ammonia, nitrate, asparagin, aspartic acid and peptone can serve as sources of nitrogen (Jensen, loc. cit.).

Strictly aerobic.

Optimum temperature 28 to $30^{\circ} \mathrm{C}$.

Source: Isolated from soil.

Habitat: Soil. Decomposes cellulose.

2. Sporocytophaga congregata Fuller and Norman. (Jour. Bact., 45, 1943, 567.)

Etymology: Latin congrēgo, to assemble.

Vegetative cells are long, flexuous rods with pointed ends, 0.5 to 0.7 by 5.5 to 8.0 microns. Creeping motility on solid surfaces.

Spores (microcysts): Spherical, 0.7 to 1.1 microns in diameter. Usually occur in localized regions within the colony.

Growth on starch agar is smoky, later turning yellow. Colonies are irregularly round, slightly concave. Edge is smooth and entire at first, later becoming irregular. Marginal and internal swarming may be prominent. The vegetative cells gather into groups and in these regions a large number of spherical spores are found.

Growth on cellulose dextrin agar is pale; colonies are small and concave. Hollowing of the agar is limited to the area of colony growth.

Glucose, galactose, lactose, maltose, sucrose, arabinose, calcium gluconate, starch, cellulose dextrin, pectin, and hemicellulose are utilized. Filter paper is not attacked.

Ammonium, nitrate, and peptone are suitable nitrogen sources.

Indole not formed.

Nitrites not produced from nitrates. 
Litmus milk: Growth but no digestion or curd formation.

Highly aerobic.

Optimum temperature $25^{\circ}$ to $30^{\circ} \mathrm{C}$.

Source: Isolated from soil.

Habitat: Soil. Decomposes organic: matter.

3. Sporocytophaga ellipsospora (Insenecki and Solntzeva) Stanier. (Cytophaga cllipsospora Imšenecki and Solntzeva, Bull. Acad. Sci. I.S.S.R., Ser. Biol., No. 6, 1936, 1137; Stanier, Bact. Rev., $6,1942,153$ and 190 .)

Etymology: Greek ellipsis, an ellipse, and sporos, seed.

Vegetative morphology: Flexible, singly occurring rods, 0.4 micron wide at the center and tapering to both ends. Length 7.5 microns. May be straight, bent, U-shaped, or S-shaped. Show creeping motility.

Microcysts: Oval or somewhat elon. gated, 0.9 to 1.2 by 1.6 to 1.8 microns Amost always situated in closely-packed aggregates, isolated individual micro. rysts rare. Germinate by elongation.

Grow th is strictly confined to cellulose. On mineral salts-siliea gel plates covered with filter paper, orange, glistening, mucilaginous patehes are produced. Ultimately the filter paper is completely dissolved and the patches become translucent.

Ammonia, nitrate and peptone can serve as sources of nitrogen.

Strictly aerobic.

Optimum temperature $28^{\circ}$ to $30^{\circ} \mathrm{C}$.

Source: Isolated from soil.

Habitat: Soil. Decomposes cellulose 


\section{ORDER V. SPIROCHAETALES BUCHANAN.*}

(Jour. Bact., 3, 1918, 5ł2.)

Slender, flexuous cell body in the form of a spiral with at least one complete turn, 6 to 500 microns in length. Some forms may show an axial filament, a lateral crista or ridge, or transverse striations; otherwise no significant protoplasmic pattern. Smaller forms may have a lower refractive index than bacteria, and so living organisms can be seen only with dark field illumination. Some forms take aniline dyes with difficulty. Giemsa's stain is uniformly successful. Multiplication by transverse fission. To sexual cycle known. Granules formed by some species in vector hosts. All forms are motile. No organs of locomotion**; motility serpentine or by spinning on the long axis without polarity. Free-living, saprophytic and parasitic.

\section{Key to the families of order Spirochaetales.}

I. Spirals 30 to 500 microns in length, having definite protoplasmic structures.

Family I. Spirochaetaceae, p. 1051.

II. Spirals 4 to 16 microns in length, having no obvious protoplasmic structure.

Family II. Treponemataceae, p. 1058.

\section{FAMIIY I. SPIROCHAETACEAE SWELLENGREBEL.}

(Ann. Inst. Past., 21, 1907, 581.)

Coarse spiral organisms, 30 to 500 microns in length, having definite protoplasmic structures. Found in stagnant, fresh or salt water and in the intestinal tract of bivalve molluses (Lamellibranchiata).

\section{Key to the genera of family Spirochaetaceae.}

I. No obvious periplast membrane and no cross-striations.

Genus I. Spirochaeta, p. 1051.

II. Periplast membrane present. Cross-striations prominent in stained specimens.

A. Free-living in marine ooze.

B. Parasitic on lamellibranch molluses. Crista prominent.

Genus III. Cristispira, p. 1055.

\section{Genus 1. Spirochaeta Ehrenberg.}

(Ehrenberg, Abhandl. Berl. Akad., 1833, 313; Spirochoeta Dujardin, Hist. nat. Jes Zoophytes, 18t1, 209; Spirochaete Cohn, Beitr. z. Biol. d. Pflanz., 1, Heft 1, 1872,

* Originally prepared by Prof. D. H. Bergey, Philadelphia, Pa., October, 1922. Revised by Prof. E. G. D. Murray, MeGill Lniversity, Montreal, P. Q.. Canada, December, 1938. Further revision by Dr. G. H. Robinson, Wm. H. Singer Memorial Research Laboratory of the Allegheny General Hospital, Pittsburgh, Pa., September, 1943. Appendices prepared by Mrs. Eleanore Heist Clise, Geneva, X. Y., September, 1943.

** Recent photographs taken with the electron microscope indicate the presence of structures resembling flagella (Mudd, Polevitzky and Anderson, Jour. Bact., 46, 1943, 15). Whether these can be considered as organs of locomotion awaits decision. At present it seems best to confine deseriptions of structure to features seen by use of the oil immersion lens. 
180; Ehrenbergia Gieszezykiewicz, Bull. Acad. Polonaise d. Sci. et Lettres, Cl. Sci. Math. et Nat., Sér. B, 1939, 24.)

Non-parasitic, with flexible, undulating body and with or without flagelliform tapering ends. Protoplast wound spirally around a well-defined axial filament. No obvious periplast membrane and no cross-striations. Motility by a creeping motion. Primary spiral permanent. Free-living in fresh or sea water slime, especially in the presence of $\mathrm{H}_{2} \mathrm{~S}$. Common in sewage and foul waters.

The type species is Spirochaeta plicatilis Ehrenberg.

\section{Key to the species of genus Spirochaeta.}

I. Large spirals with rounded ends.

II. Smaller spirals with pointed ends.

1. Spirochaeta plicatilis.

2. Spirochaeta marina.

3. Spirochaeta curystrepta.

4. Spirochacta stenostrepta.

5. Spirochaeta daxensis.

1. Spirochaeta plicatilis Ehrenberg. (Ehrenberg, Die Infusionstierchen, 1838, 83; Spirillum plicatile Dujardin, Infus., 1, 1841, 225; Spirulina plicatilis Cohn, Nova Acta Acaul. Caes. Leop. Carol., 24, I, 1853, 125; Spirochacte plicatilis Cohn, Beitr. z. Biol. d. Pflanz., 1, Heft 2, 1872, 180; Spirillum portae Mantegazza, Giorn. Ist. Lomb., 3, p. 48i, according to De Toni and Trevisan, in Saccardo, Sylloge Fungorum, 8, 1889, 1006; Spirochaeta plicatilis plicatilis Zuelzer, Arch. f. Protistenk., 24, 1912, 17; Ehrenbergia plicatilis Gieszczykiewicz, Bull. Acad. Polomaise d. Sci. et Lettres, Cl. Sei. Math. et Nat., Sér. B, 1939, 24.) From Latin, folded.

Cylindrical : 0.5 to 0.75 by 100 to 500 microns, with blunt ends.

Spiral amplitude : 2.0 microns, regular. Spiral depth: 1.5 microns, regular. Waves, several, large, inconstant, irregular.

Axial filament distinct in stained specimens, consisting of chitin or cutin-like substance. Flexible, elastic.

Division transverse.

Stains violet by Giemsa's stain and gray by iron-hemotoxylin.

Cytoplasmic spirals stain with eosin, rubin, ete. Contain volutingranules.

Trypsin digestion: Axial filament resistant
Bile salts (10 per cent) : Bècomes shadowy, pale, but is not dissolved.

Saponin (10 per cent): Lives $30 \mathrm{~min}$ utes. Later becomes shadowy, but is not ‘lissolved.

Grows best under low oxygen tension.

Optimum temperature $20^{\circ}$ to $25^{\circ} \mathrm{C}$.

Habitat: Free-living in fresh or salt water.

2. Spirochaeta marina Zuelzer. (Spirochacta plicatilis marina Zuelzer, Arch. f. Protistenk., 24, 1912, 17; Zuelzer, ibid., 51.) From Latin, of the sea.

Probably a subspecies or variant of Spirochaeta plicatilis.

Cylindrical, 0.5 by 100 to 200 microns with blunt ends.

Axial filament present. Flexible, clastic.

Division transverse.

Contains smaller and more irregularly distributed volutin granules than Spirochaeta plicatilis. Cytoplasmic spirals stain.

Grows best at low oxygen tension.

Optimum temperature $20^{\circ} \mathrm{C}$.

Habitat: Sea water.

3. Spirochaeta eurystrepta Zuelzer. (Spirochaeta plicatilis eurystrepta Zuelzer, Arch. f. Protistenk., 24, 1912, 17; 
Zuelzer, ibid., 51.) From Greek, broadly twisted.

Probably a subspecies or variant of Spirochacta plicatilis.

Cylindrical: 0.5 micron in thickness and up to 300 mierons in length.

Spiral amplitude: More shallow than spirals of Spirochaeta plicatilis with blunt. ends.

Axial filament present. Flexible, slastic.

Division transverse.

Fewer volutin granules than in Spirochacta plicatilis. Cytoplasmic spirals stain.

Optimum temperature $20^{\circ} \mathrm{C}$.

Habitat: Swamp water and in grossly polluted water containing $\mathrm{H}_{2} \mathrm{~S}$.

4. Spirochaeta stenostrepta Zuelzer. (Zuelzer, Arch. f. Protistenkunde, 24, 1912, 16 ; Treponema stenostrepta Enderlein, Bakterien-Cyclogenie, 1925, 253.) From Greek, narrowly twisted.

Cylindrical : 0.25 micron in thickness and 20 to 60 , occasionally up to 200 , microns in length, with pointed ends.

Spiral amplitude very narrow with steep windings.

Axial filament present. Flexible, elastic.

Division transverse.

Fewer volutin granules than in Spirochaeta plicatilis.

Cytoplasmic spirals stain.

Optimum temperature $20^{\circ} \mathrm{C}$.

Habitat: Water containing $\mathrm{H}_{2} \mathrm{~s}$.

\section{Spirochaeta daxensis Cantacuzène} (Compt. rend. Soc. Biol., Paris, 68, 1910, 75.) Named for Dax, a watering place in France.

Large spirals: 0.5 to 2.5 by 30 to 100 microns, possessing a longitudinal chromatin filament, and tapering at the ends.

They are flattened and exhibit a double series of curls, smaller waves being superimposed on larger undulations.

Optimum temperature $44^{\circ}$ to $52^{\circ} \mathrm{C}$.
Source: Found in water of hot spring of Dax $\left(52^{\circ}\right.$ to $\left.56^{\circ} \mathrm{C}\right)$.

Habitat: Hot springs.

Appendix: The following species may belong in this genus. Descriptions are usually inadequate.

Spirochaeta agilis Adehmann. (Cent. f. Bakt., I Abt., Orig., 88, 1922, 413.) From mud from the harbor at Kiel. Original culture had a weak odor of hydrogen sulfide.

Spirochaeta aurantia Vinzent. (Compt. rend. Soc. Biol., Paris, 95, 1926. 1472.) From drain water. Forms small yellowish-orange colonies on agar after 5 to $S$ days. Shows involut ion forms. This species definitely does not belong in the genus Spirochaeta, although it is placed here for the present. Its description suggests that it may belong among the vibrios.

Spirochacta flexibilis Nägler. (Cent. f. Bakt.. I Abt., Orig., 50, 1909, 445.) From the mud of a lake.

Spirochaeta fulgurans Dobell. (Arch. f. Protistenk., 26, 1912, 117.) From water of the river Granta at Cambridge. May be a synonym of Spirochaeta stenostrepta.

Spirochaeta gigantea Warming. (Warming, Videnskabl. Neddel. Naturh. Foren., Copenhagen, 1875, 70; Spirillum giganteum Trevisan, I generi e le specie delle Batteriacee, 1859, 24; not Spirillum giganteum Migula, Syst. d. Bakt., 2, 1900, 1025.) Large, 3 microns in diameter. Inadequately described and possibly not a spirochaete.

Spirachaeta graminea Zuelzer. (Zuelzer, 1925, in Prowazek, Handb. d. path. Protoz., 3, 1931, 1670.) From an infusion of herbs. Size 0.25 by 100 microns. From rivers and marshes where it digests cellulose.

Spirochaeta graminea marina Zuelzer. (Cent. f. Balit., I Abt., Orig., 96, 1925. 426.) From sea water.

Spirochaeta icterogenes marina Zuelzer. 
(Cent. f. Bakt., I Abt., Orig., 96, 1925, 426.) From sea water.

Spirochaeta minima Dobell. (Dobell, Arch. f. Protistenk., 26, 1912, 117; not Spirochaeta minima Pettit, Contribution ¿ l'Etude des Spirochétidés, Vanves, II, 1928, 187 (Treponema minimum Beaurepaire-Aragĩo and Vianna, Mem. Inst. Oswaldo Cruz, 5, 1913, 211).) One of the smallest known Spirochaeta, 0.5 by 2.0 to 2.5 microns. From water of the river Granta at Cambridge. Sinilar to Spirochaeta fulyurans.

Spirochacta vivax (Dohell) Zuelzer. (Treponema vivax Dobell, Areh. f. Protistenk., 26, 1912, 117; Zuelzer, 1925, in Prowazek, Handh. d. path. Protoz., 3,
1931, 1669.) From fresh water of the river Granta at Cambridge.

Spirochaete kochii Trevisan. (Spirochate des Wollsteiner See, Koch, in Cohn, Beitr. z. Biol. d. Pflanzen, 2, Heft 3, 1877, 420; Trevisan, Batter. Ital., 1879, 26; Spirillum kochii Trevisan, I generi e le specie delle Batteriacee, 1889, 24.) From water.

Spirochacte schroeteri Cohn. (Jahresber. d. Schles. Gesellch. f. 1883, 198; quoted from Schroeter, in Cohn, Kryptog. Flora v. Schlesien, 3, 1, 1889, 168; Spirillum schroeteri Cohn, quoted from DeToni and Trevisan, in Saccardo, Sylloge Fungorum, 8, 1889, 1007.) Similar to spirochaete cohnii. From cellar walls.

\section{Genus II. Saprospira Gross.}

\section{(Mittheil. Zool. Stat. zu Neapel, 20, 1911, 190.)}

Spiral protoplasm without evident axial filament. Spirals rather shallow. Transverse markings or septa (?) seen in unstained and stained specimens. Periplast membrane distinct. Notility active and rotating. Free-living in marine ooze.

The type species is Saprospira grandis Ciross.

1. Saprospira grandis Gross. Mitteil. Zool. Station zu Neapel, 20, 1911, 190.) From Latin, great.

Cylindrical, 1.2 by so microns in length, with obtuse ends.

Spiral amplitude is 24 microns.

Waves large, inconstant, shallow, irregular, 3 to 5 in number, sometimes almost straight.

Axial filament absent.

Cross-striations present

Membrane distinct.

Division transverse.

Flexible, elastic.

Crista absent.

Terminal spiral filament alssent.

Highly motile end portion absent.

Trypsin digestion.

Source: Found in intestinal tract of the oyster.

Habitat: Free-living in foraminiferoms sand.

2. Saprospira puncta Dimitroff.
(Jour. Bact., 12, 1926, 146.) From Latin, pitted.

Large spirals: 1.0 by 86 microns with pointed ends.

The spiral amplitude is 4 to 8 microns. The average number of turns is 3 .

Axial filament absent.

Cross-striations present

Membrane distinct.

Division transverse.

couree: Found in oysters.

3. Saprospira lepta Dimitroff. (Jour. Bact., 12, 1926, 144.) From Latin, small.

Large spirals : 0.5 by 70.0 mierons, with pointed ends.

The spiral amplitude ranges from 5 to 13 microns.

The spiral width varies from 1.6 to 4.8 microns.

The average number of turns is 6 .

Axial filament absent .

Coss-striations present. 
Membrane distinct.

Division transverse.

Source: Found in oysters in Baltimore, Narvland.

Appendix: The following species have been placed in this genus.
Saprospira flexuosa Dobell. (Arch. f. Protistenk., 26, 1912, 117.) Isolated once from water of the river Granta at Cambridge

Saprospira nana Gross. (Mittheil. Zool. Sta. zu Neapel, 20, 1911, 188.) From foraminiferous sand.

\section{Genus III. Cristispira Gross.}

(Mittheil. Zool. Stat. zu Neapel, 20, 1910, 41.)

Flexuous cell bodies in coarse spirals, 28 to 120 microns in length. Characterized by a crista or thin membrane of varying prominence on one side of the body extending the entire length of the organism. Cross-striations. Actively motile. Found in the intestinal tract of molluscs.

The type species is Cristispira balbianii (Certes) Gross.

1. Cristispira balbianii (Certes) Gross. (Trypanosoma balbianii Certes, Bull. Soc. Zool. de France, 7 , 1882, 347; Spirochaeta balbianii Swellengrebel, Ann. Inst. Past., 21, 1907, 562; Spirochacte balbianii Borrel and Cernovodeanu, Compt. rend. Soc. Biol., Paris, 62, 1907, 1102; Gross, Cent. f. Bakt., I Abt., Orig., 65, 1912, 90.)

Cylindrical: 1.0 to 3.0 by 40 to 120 microns, with obtuse ends.

Spiral amplitude is 8 microns. Spiral depth is 1.6 microns. Waves 2 to 5 , sometimes more, large, irregular, shallow.

Axial filament absent.

Cross-striations present.

Membrane distinct.

Flexible, elastic.

Crista present, a ridge-like membrane making one to two complete turns.

Terminal spiral filament absent. Highly motile end portion absent.

Stains: Cell membrane behaves like chitin or cutin substance. Stains violet by Giemsa's solution, and light gray by iron-hemotoxylin.

Trypsin digestion: Membrane resistant, crista and striations disappear.

Bile salt (10 per cent): Crista quickly dissolves.

Saponin (10 per cent): Crista becomes fibrillar, then indistinct.
Source: From the crystalline style of oysters.

Habitat: Parasitic in alimentary tract of shell-fish.

2. Cristispira anodontae (Keysselitz) Gross. (Spirochacta anodontae Keysselitz, Arb.a.d. kaiserl. Gesundheitsamte, 23, 1906, 566; Gross, Cent. f. Bakt., I Abt., Orig., 65, 1912, 900.) From M.L., mussels.

0.8 to 1.2 by 44 to $8 S$ microns with sharply pointed ends; flattened and possessing an undulating membrane. The periplast is fibrillar in appearance and there is a dark granule at each end of the undulating membrane. The chromatin material is distributed in the form of globules or elongated bands.

Large spirals: The average width of the spiral is 2 microns. The average wave length is 8 microns.

The number of complete turns ranges from 5 to 11.

Habitat: Found in the crystalline style of fresh water mussels, Anodonta cygnea. and 1. mutabilis, also in intestinal tract of oysters.

3. Cristispira pinnae (Gonder) Bergey et al. (Spirochaete pinnae Gonder, Cent. f. Bakt., I Abt., Orig., 47, 1908, 491 ; Spirochaeta pinnae Schellack, Arb. a. d. 
kaiserl. Gesundheitsamte, 30, 1909, 379; Bergey et al., Manual, 1st ed., 1923, 423.) From Latin, of a mussel.

Spirals : 0.5 to 3.0 by 10 to 60 microns, round in section with blunt ends, the one being slightly more pointed than the other.

They have a ridge or comb running along one side but no terminal filaments.

Cross-striations distinct.

The chromatin granules are grouped in fours.

An undulating membrane can be demonstrated.

Source: Found in the intestinal canal of the scallop (Pecten jacobaeus).

Habitat: From the crystalline style of molluses.

Appendix: Additional species which appear to belong in this genus are:

Cristispira acuminata (Schellack) Ford. (Spirochaeta acuminata Schellack, Arb. kais. Gesundheitsamte, 30, 1909, 379; not Spirochaeta acuminata Castellani, Brit. Med. Jour., 2, 1905, 1330; Ford, Textb. of Bact., 1927, 939.) From the crystalline style of a molluse, Tapes lacta.

Cristispira cardii-papillosi (Schellack) Ford. (Spirochaeta cardii-papillosi Schellack, Arb. kais. Gesundheitsamte, 30, 1909, 379; Ford, Textb. of Bact., 1927, 939.) From the crystalline style of a molluse, Cardium papillosum.

Cristispira chamae (Schellack) Noguchi. (Spirochacta chamae Schellack, Arb. kais. Gesundheitsamte, 30, 1909, 379; Noguchi, Jour. Exp. Med., 27, 1918, 583.) From the crystalline styles of nolluses, Chama spp.

Cristispira gastrochaenae (Schellack) Ford. (Spirochaeta gastrochaenae Schellack, Arb. kais. Gesundheitsamte, 30, 1909, 379; Ford, Textb. of Bact., 1927, 940.) From a shellfish, Gastrochaena dubia. Constant length 29 microns.

Cristispira helgolandica Collier. (Cent. f. Bakt., I Abt., Orig., 86, 1921, 132.) Found three times in the body fluid of an echinoderm, Asterias rubens, in the North sea. Average length 68 microns. Named for the place where the investigation was made (Helgoland).

Cristispira interrogationis Gross. (Mittheil. Zool. Station zu Neapel, 20, 1910, 41.) From the intestinal canal of the scallop, Pecten jacobaeus.

Cristispira limae (Schellack) Ford. (Spirochaeta limae Schellack, Arb. kais. Gesundheitsamte, 30, 1909, 379; Ford, Textb. of Bact., 1927, 939.) From the crystalline styles of molluses, Lima spp. Similar to Cristispira balbianii.

Cristispira mactrae (Prowazek) Ford. (Spirochaeta mactrae Prowazek, Arch. f. Schiffs- u. Tropenhyg., 14, 1910, 297; Ford, Textb. of Bact., 1927, 940.) From the digestive tract of a shellfish, Mactra sulcataria.

Cristispira mina Dimitroff. (Jour. Bact., 12, 1926, 159.) Found in oysters. Cristispira modiolae (Schellack) Noguchi. (Spirochaeta modiolae Schellack, Arb. kais. Gesundheitsamte, 30, 1909, 379; Noguchi, Jour. Exp. Med., 27, 1918, 583.) Found in mussels and oysters.

Cristispira ostrcae (Schellack) Noguchi. (Spirochaeta ostreae Schellack, Arb. kais. Gesundheitsamte, 30, 1909, 379; Noguchi, Jour. Exp. Med., 27, 1918, 583.) From the erystalline style of the oyster, Ostrea edulis. Identical with Cristispira anodonlae Gross.

Cristispira pachelabrae de Mello. (Compt. rend. Soc. Biol., Paris, 84, 1921, 241.) From the digestive tract of a shellfish, Pachelabra moestra.

Cristispira parvula Dobell. (Arch. f. Protistenk., 26, 1912, 117.) From the crystalline style of a mollusc, Venus (Meretrix) castra, in Ceylon. The smallest Cristispira known- 0.4 to 0.5 by 20 to 45 microns.

Cristispira pectinis Gross. (Mittheil. Zool. Sta. zu Neapel, 20, 1910,41.) From the digestive tract of a scallop, Pecten jacobaeus. Identical with Cristispira balbianii Gross.

Cristispira polydorae Mesnil and Caul- 
lery. (Compt. rend. Soe. Biol., Paris, 79, 1916, 1118; Cristispirella polydorae Hollande, Compt. rend. Acad. Sci. Paris, 172, 1921, 1696). From a marine annelid, Polydora flava.

Cristispira pusilla (Schellack) Ford. (Spirochaeta pusilla Schellack, Arb. kais. Gesundheitsamte, 30, 1909, 379; Ford, Textb. of Bact., 1927, 940.) From the digestive tract of a mussel, Anodonto mutabilis.

Cristispira saxicavae (Schellack) Ford. (Spirochaeta saxicavae Schellack, Arb. kais. Gesundheitsamte, 30, 1909, 379; Ford, Textb. of Bact., 1927, 940.) From the crystalline style of a molluse, Saxicava arctica.

Cristispira spiculifera (Schellack) Dimitroff. (Spirochaeta spiculifera Schellack, Arb. kais. Gesundheitsamte, 30, 1909, 379; Dimitroff, Jour. Bact., 12, $1926,157$.$) Found in mussels.$

Cristispira tapetos (Schellack) Gross. (Spirochaeta tapetos Schellack, Arb. kais. Gesundheitsa ste, 30, 1909, 379; Gross, Cent. f. Bakt., I Abt., Orig., 65, 1912, 84.) From the crystalline style of a mollusc, Tapes decussata.

Cristispira tenua Dimitroff. (Jour. Bact., 12, 1926, 160.) Found in oysters. Cristispira veneris Dobell. (Quart. Jour. Mierose. Sei., London, 54, 19101911, 507 and ibid., 56, 1911, Part 3.) From a clam, Venus (Meretrix) castra, in Ceylon. Identical with Cristispira balbianii Gross

Cristispirella caviae Hollande. (Compt. rend. Acad. Sci., Paris, 172, 1921, 1693.) From the intestine of a guinea pig. Probably a protozoan. Evidently the same as Heliconema (see appendix to Borrelia). Both Cristispirilla polydorae and Cristispirella caviae have characteristics at variance with accepted ideas of spirochaetes.

Spirochaeta solenis Fantham. (Ann. Trop. Med. and Parasitol., 5, 1911, 479.) A parasite of a molluse, Solen ensis. 


\section{FAMILY II. TREPONEMATACEAE SCHAUDINN.}

(Deutsche med. Wochnschr., 31, 1905, 1728.)

Coarse or slender spirals, 4 to 16 microns in length; longer forms due to incomplete or delayed division. Protoplasm with no obvious structural features. Some may show terminal filaments. Spirals regular or irregular, flexible or comparatively rigid. Some visible only with dark field illumination. Parasitic on vertebrates with few exceptions. Some pathogenic. Many can be cultivated.

\section{Key to the genera of family Treponemataceae.}

I. Stains easily with ordinary aniline dyes.

Genus I. Borrelia, p. 1058.

II. Stain with difficulty except with Giemsa's stain and silver impregnation.

A. Strict anaerobes.

B. Aerobes.

Genus II. Treponema, p. 1071.

Genus III. Leptospira, p. 1076.

\section{Genus I. Borrelia* Swellengrebel.}

(Swellengrebel, Amn. Inst. Past., 21, 1907, 582; Spiroschaudinnia Sambon, in Manson, Tropical Diseases, August, 1907, 833 ; Cacospira Enderlein, Sitzber. Ges. Naturf. Freunde, Berlin, 1917, 309; Entomospira Enderlein, ibid.; Spironema Bergey et al., Manual, 1st ed., 1923, 424; not Spironema Vuillemin, Compt. rend. Acad. Sci. Paris, 140, 1905, 1567; Sprochaeta Gieszezykiewiez, Bull. Acad. Polonaise d. Sei. et Lettres, C1. Sci. Math. et Nat., Sér. B, 1939, 24.)

Length 8 to 16 microns. Coarse, shallow, irregular, with a few obtuse angled spirals. Generally taper terminally into fine filaments. Stain easily with ordinary aniline dyes. Refractive index approximately the same as that of true bacteria. Parasitic upon many forms of animal life. Some are pathogenie for man, other mammals and birds. Generally hematophytes are found on mucous membranes. Some are transmitted by the bites of arthropods.

The type species is Borrelia anserina (Sakharoff) Bergey et al.

1. Borrelia anserina (Sakharoff) Bergey et al. (Spirorhaeta anserina Sakharoff, Ann. Inst. Past., 5, 1891, 564; Spirillum anserum (sic) Sternberg, Man. of Bact., 1893, 499; Spirillum anserinum Macé, Traité Pratique de Bact., 4th ed., 1901, 1060; Spirochacte anserina Macé, ibid.; Spiroschaudinnia anserina Castellani and Chalmers, Man. Trop. Med., 2nd ed., 1913, 403; Spironema anserina Noguchi, Jour. Exp. Med., 27, 1918, 584; Bergey et al., Manual, 2nd ed., 1925, 435; Treponema anserina Noguchi, in Jordan and Falk, Newer Kinowledge Bact. and Immun., 1928, 456.) From Latin, pertaining to geese.
Synonyms: Spirochaeta marchouxi Nuttall, Epidemiol. Soc., London, 24, 1904, 12 (Spirille de la poule, Marchoux and Salimbeni, Ann. Inst. Past., 17, 1903, 569; Spirochacta gallinarum Stephens and Christopher, Practical Study of Malaria and Other Blood Parasites, Liverpool, 1905; Borrclia gallinarum Swellengrebel, Ann. Inst. Past., 21, 1907, 623; Spirochaete gallinarum Lehmann and Neumann, Bakt. Diag., 4 Aufl., 2, 1907, 623 ; Spironema gallinarum Gross, Cent. f. Bakt., I Abt., Orig., 65, 1912, 92; Spiroschaudinnia marchouxi Castellani and Chalmers, Man. Trop. Med., 2nd ed., 1913, 403; Spironema marchouxi Ford,

* Further revision of the genus by Prof. E. G. D. Murray, MeGill Univ., Montreal, P. Q., Canada, April, 1947. Reviewed by Dr. Gordon E. Davis, Rocky Mountain Laboratory, U.S.P.H.S, Hamilton, Montana. 
Textb. of Bact., 1927, 955; Spirochaeta gallinae Ford, idem; Treponema gallinarum Noguchi, in Jordan and Falk, Newer Knowledge Bact. and Immun., 1928, 461 ; Treponema marchouxi Gay et al., Agents of Disease and Host Resistance, 1935, 1077). The cause of septicaemia in chickens.

Spirochaeta granulosa penetrans Balfour, Jour. Trop. Med. and Hyg., 10, 1907, 153 (Spiroschaudinnia granulosa Balfour, Jour. Trop. Veter., Calcutta, 5, 1910, 309 ; Spironema granulosa Ford, Textb. of Bact., 1927, 957). From spirochetosis of fowls in Sudan.

Spirochaeta nicollei Brumpt, Bull. Soc. Path. Exot., 2, 1909, 285 and/or Précis de Parasitol., Paris, 1st ed., 1910 (GalliValerio, Cent. f. Bakt., I Abt., Orig., $50,1909,189$ and $61,1912,529$; Spironema nicollei Ford, Textb. of Bact., 1927, 958; Treponema nicollei Gay et al., Agents of disease and Host Resistance, 1935, 1077). From spirochetosis of geese in Tunisia.

Spirochaeta neveuxi Brumpt, Bull. Soc. Path. Exot., 2, 1909, 285 (Spiroschaudinnia neveuxii Castellani and Chalmers, Man. Trop. Med., 2nd ed., 1913, 404; Spironema neveuxi Ford, Textb. of Bact., 1927, 958; Treponema neveuxi Gay et al., Agents of Disease and Host Resistance, 1935,1077 ). The cause of fowl spirochetosis in Senegal.

Spirochacta gallinarum var. hereditaria Neumann and Mayer, in Lehmann, Med. Atlanten, 11, 1914, 276. A North African strain of fowl spirochetosis.

Borrelia pullorum Redowitz, Amer. Jour. Med. Technol., 2, 1936, 91. From diseased chickens.

Spirochata anatis Parrot, Bull. Soc. Path. Exot., 13, 1920, 647. Pathogenic for domestic ducks in Algeria.

Morphology : 0.25 to 0.3 by 8 to 20 microns, averaging about 1 spiral per micron.

Actively motile, with lashing movements.

Stains readily with aniline dyes and Giemsa's stain.
Cultivation: Can be cultivated in Noguchi's ascitic fluid-rabbit kidney medium.

Immunology: Antigenically distinct from species found in mammals.

Arthropod vectors: Transmitted by the bites of ticks (Argas persicus, A. miniatus, A. reflexus and Ornithodoros moubata.)

Pathogenic for birds but not for mammals.

Source: From blood of infected geese, ducks, fowls and vector ticks.

Habitat: The cause of spirochetosis of fowls.

2. Borrelia recurrentis (Lehert) Bergey et al. (Obermeier, Berlin. klin. Wochschr., 1873, 152; Protomycetum recurrentis Lebert, Ziemssen's Handbuch, 2, 1874, 267 ; Spirochaete obermeieri Cohn, Beitr. z. Biol. d. Pflanzen, 1, Heft 3, 1875, 196; Spirillum abermeieri Zopf, Die Spaltpilze, 3 Aufl., 1S\$5, 71; Spirochaeta obermeieri Migula, in Engler and Prantl, Die natürl. Pfanzenfam., 1, 1a, 1895, 35; Spirochate recurrentis Lehmann and Neumann, Bakt. Diag., 4 Aufl., 2, 1907, 621 ; Spirochacta recurrentis Castellani and Chalmers, Man. Trop. Med., 1st ed., 1910, 305; Spironema recurrentis Gross, Cent. f. Bakt., I Abt., Orig., 65, 1912, 85; Spiroschaudinnia recurrentis Castellani and Chalmers, Man. Trop. Med., 2nd ed., 1913, 398; Spironema obermeieri Park and Williams, Pathogenic Microorganisms, 6th ed., 1917, 513; Cacospira recurrentis Enderlein, Sitzungsber. d. Gesellsch. naturf. Freunde, 1917, 313; Treponema recurrentis Brumpt, Nouveau Traité de Médecine, Paris, 4, 1922, 508; Treponema obermeieri Brumpt, ibid.; Cacospira obermeieri Enderlein, Bakterien-Cyclogenie. 1925, 254; Bergey et al., Manual, 2nd ed., 1925, 433; Spirillum recurrentis Ford, Textb. of Bact., 1927, 948; Spiroschaudinnia obermeieri Ford, ibid.) From Latin, recurring.

Cylindrical or slightly flattened, 0.35 to 0.5 by 8 to 16 microns, with pointed ends. 
Spiral amplitude 1.5 microns.

Spirals large, wavy, inconstant, about 5 in number.

Terminal finely spiral filaments present.

Highly motile end portion absent.

Motility : By active cork-screw motion without polarity. Lashing movements common in drawn blood.

Stains with common aniline dyes. Gram-negative. Violet with Giemsa's stain.

Bile salts (10 per cent): Disintegration complete.

Saponin (10 per cent): Immobilized in 30 minutes, then broken up in a few hours. In some a skeletal structure remains.

Cultivation: Can be cultured in ascitic or hydrocoel fluid to which a piece of sterile rabbit kidney is added. Optimum reaction $\mathrm{pH} 7.2$ to 7.4 .

Immunology: Serum does not agglutinate Borrelia duttoni.

Accidental and experimental transmission by conjunctival sac and skin abrasions.

Disease in experimental animals (small rodents after monkey passage) mild.

Arthropod vector: Louse (Pediculus humanus) which exhibits normal transmission from the 16 th to the 28 th day. Found in the bed-bug (Cimex lectularius) and ticks, but not transmitted by them. No evidence of hereditary transmission in the louse.

Habitat: The cause of European relapsing fever. Transmissible to man, monkeys, mice and rats.

3. Borrelia duttonii (Breinl) Bergey et al. (Dutton and Todd, Brit. Med. Jour., 2, 1905, 1259; Spirochaeta duttoni Breinl, Lancet, 2, 1906, 1690; Spirillum duttoni Novy and Knapp, Jour. Inf. Dis., 3, 1906, 296; see Dutton and Todd, Jour. Trop. Med., 10, 1907, 385; Spirochaete duttoni Lehmann and Neumann, Bakt. Diag., 4 Aufl., 2, 1907, 623; Spironema duttoni Gross, Cent. f. Bakt., I Abt., Orig., 65, 1912, 94; Spirochaeta Micro- spironema duttoni Duboscq and Lebailly, Compt. rend. Acad. Sci., 154, 1912, 662; Spiroschaudinnia duttoni Castellani and Chalmers, Man. Trop. Med., 2nd ed., 1913, 399; Treponema duttoni Brumpt, Nouveau Traité de Médecine, Paris, 4, 1922, 497; Cacospira duttoni Enderlein, Bakterien-Cyclogenie, 1925, 254; Bergey et al., Manual, 2nd ed., 1925, 434.) Named for Dutton, who discovered this organism.

Morphology : Similar to Borrelia recurrentis.

Cultivation: Growth occurs under anaerobic conditions in serum water, hydrocoel or ascitic fluid to which a piece of sterile rabbit kidney is added.

Immunology: This organism is antigenically distinct from other causes of relapsing fever.

Pathogenic for mice and rats. Disease in small rodents and many other experimental animals very severe.

Arthropod vector: This species is transmitted to man through the bite of the tick (Ornithodoros moubata) by fecal contamination of the bite. In the tick the organism goes through some granulation or fragmentation phenomenon, the nature of which is not understood. Hereditary transmission to at least the third generation of the tick. Not transmitted by the louse.

Habitat: The cause of Central and South African relapsing fever.

4. Borrelia kochii (Novy) Bergey et al. (Spirochaeta kochi Novy, Proc. Path. Soc. Philadel., N. S. 10, 1907, 1; Spirochaeta rossi Nuttall, Jour. Roy. Inst. Pub. Health, London, 16, 1908, 385; Spiroschaudinnia rossii Castellani and Chalmers, Man. Trop. Med., 2nd ed., 1913, 400; Spironema kochii Noguchi, Jour. Exp. Med., 27, 1918, 584; Treponema kochi Brumpt, Nouveau Traité de Médecine, Paris, 4, 1922, 497; Treponema rossi Brumpt, ibid.; Bergey et al., Manual, 2nd ed., 1925, 434; Borrelia rossi Steinhaus, Insect Microbiology, 1946, 452.) 
Named for Koch, who first observed spirochetes in East African relapsing fever.

Morphology : Similar to that of Borrelia recurrentis .

Cultivation: Same as for Borrelia recurrentis.

Immunology: Antigenically distinct from both Borrelia recurrentis and $B$. duttonii.

Pathogenic for mice and rats.

Arthropod vector: No record.

Habitat: The cause of African relapsing fever.

5. Borrelia novyi (Schellack) Bergey et al. (Spirochaete from relapsing fever, Norris, Pappenheimer and Flournoy, Jour. Inf. Dis., 3, 1906, 266; Spirochaeta novyi Schellack, Arb. kaiserl. Gesundheitsamte, 27, 1907, 199 and 364; Spironema novyi Gross, Archiv f. Protistenk., 24, 1912, 115; Spiroschaudinnia novyi Castellani and Chalmers, Man. Trop. Med., 2nd ed., 1913, 400; Treponema novyi Brumpt, Nouveau Traité de Médecine, Paris, 4, 1922, 508; Cacospira novyi Enderlein, Bakterien-Cyclogenie, 1925, 254; Bergey et al., Manual, 2nd ed., 1925, 434.) Named for Novy, the American bacteriologist.

Morphology : Similar to that of Borrelin recurrentis.

Cultivation: Same as for Borrelia recurrentis.

Immunology: Antigenically distinct from other relapsing fever organisms.

Pathogenic for monkeys, white rats and white mice.

Arthropod vector: Unknown.

Habitat: Recovered from a patient in Bellevue Hospital, New lork. Origin of infection unknown.

6. Borrelia berbera (Sergent and Foley) Bergey et al. (Spirochaeta berbera Sergent and Foley, Ann. Inst. Past., 24, 1910, 337; Spiroschaudinnia berbera Castellani and Chalmers, Man. Trop. Med., 2nd ed., 1913, 402; Spironema berbera
Noguchi, Jour. Exp. Med., 27, 1918, 584; Spirochaeta berbera Kolle and Hetsch, Exper. Bakt. u. Infekt., 6 Aufl, 1, 1922, 811; Treponema berberum Brumpt, Nouveau Traité de Médecine, Paris, 4, 1922, 496; Bergey et al., Manual, 2nd ed., 1925, 435.) Named for the Berbers, a tribe of Northern Africa.

Morphology: More tenuous than other relapsing fever organisms, 0.2 to 0.3 by 12 to 24 microns.

Cultivation: No record of its cultivation.

Immunology: Antigenically distinct from Borrelia recurrentis.

Arthropod vector: Possibly carried by the louse (Pediculus vestimenti).

Source: Found in cases of relapsing fever in Algiers, Tunis and Tripoli.

Habitat: Cause of relapsing fever in North Africa. Is virulent for monkeys. Produces non-fatal infections in rats and mice.

7. Borrelia carteri (Mackie) Bergey et al. (Spirochacta carteri Mackie, Ann. Trop. Med. and Parasitol., 1, 1907, 157 and Indian Med. Gazette, 44, 1908, 370; Spirillum carteri Mackie, Lancet, 2, 1907, S32, according to Ford, Textb. of Bact., 1927, 950; Spiroschaudinnia carteri Castellani and Chalmers, Man. Trop. Med., 2nd ed., 1913, 401; Spironema carteri Noguchi, Jour. Exp. Med., 27, 1918, 584; Treponema carteri Brumpt, Nouveau Traité de Médecine, Paris, 4, 1922, 497; Bergey et al., Manual, 2nd ed., 1925, 435.) Named for Carter, who in 1879 described this organism in the blood of patients with Indian relapsing fever.

Morphology : Similar to Borrelia berbera.

\section{Cultivation: Not recorded.}

Immunology : Probably a distinct species. A succession of distinct serological types oceurs with the relapses in a single infection (Cumningham et al., Far Eastern Association of Tropical Medicine, Tokyo, 1925; Indian Journal of Medical 
Research, 22, 1934-1935, 105 and 595; ibid., 2/4, 1937, 571 and 581).

Arthropod vector: Carricel by either Pediculus vestimenti or Cimex rotundatus or by both.

Habitat: The cause of Indian relapsing fever. Transmissible to monkeys, rabbits, rats and mice.

\section{Borrelia theileri (Laveran) Bergey} et al. (Spirochacla theilcri Iuaveran, Compt. rend. Acad. Sci., Paris, 136, 1903, 939; Spiroschaudinnia theileri Castellani and Chalmers, Man. Trop. Med., 2nd ed., 1913, 404; Spironema theileri Noguchi, Jour. Exp. Med., 27, 1918, 5st; Bergey et al., Manual, 2nd ed., 1925, 435 ; Spirillum theileri and Spirochaete theileri Pettit, Contribution ì l'Etude des Spirochétidés, Vanves, II, 1928; Treponemu. theileri Noguchi, in Jordan and Falk, Newer Knowledge Bact. and Inmun., 1928, 461.) Named for Theiler, who discovered this organism in 1902 in Transvaal, South Africa.

Morphology : 0.25 to 0.3 micron by 20 to 30 microns with pointed ends.

Cultivation: No record.

Immunology: Is distinct from the species infecting man.

Arthropod vector: Transmitted by the tick (Rhipicephalus decoloratus).

Source: Blood of cattle.

Habitat: Blood of cattle and other mammals in South Africa.

\section{Borrelia glossinae (Novy and} Knapp) Bergey et al. (Spirillum glossinae Novy and Knapp, Jour. Inf. Dis., 3, 1906, 385; Spirochaeta glossinae Castellani and Chalmers, Man. Trop. Med., 1st ed., 1910, 310; Spiroschaudinnia glossinae Castellani and Chalmers, ibid., 3rd ed., 1919, 454; Spironema glossinae Bergey et al., Manual, 1st ed., 1923, 425 ; Bergey et al., Manual, 2nd ed., 1925, 435; Entomospira glossinae Euderlein, Bakterien-Cyclogenie, 1925, 254; Treponoma glossinae Ford, Textb. of Bact., 1927.
988.) Named for the genus of insects, Glossina.

Morphology: 0.2 by 8.0 microns, occurring singly, sometimes in pairs. Generally 4 spirals. Shorter, narrower and has more turns than has Borrelia recurrentis.

Habitat: Found in the stomach contents of the tse-tse fly (Glossina palpalis)

10. Borrelia buccale (Steinberg) Brumpt. (Spirochaeta buccalis Steinberg, 1862, accorling to Hoffmann and Prowazek, Cent. f. Bakt., I Aht., Orig., 41, 1906, \$19; Spirochacte cohnii Winter, Die Pilze, 1879, 61; (?) Microspira buccalis Lewis, The Lancet, 18st, quoted from Sehroeter, in Cohn, Kryptog. Flora v. Schlesien, 3, 1, 1889, 169; Spirochaetc buccalis, quoted from Schroeter, ibid., 16s; Spirillum cohnii Trevisan, I generi e le specie delle Batteriacee, 1889, 2t; Spirillum buccale Macé, Traité Pratique de Bact., th ed., 1901, 1062; Spirochaeta inaequalis Gerber, ('ent. f. Bakt., I Abt., Orig., 56, 1910, 508; Spirochaeta undulata Gerber, idem; Treponema buccale Dobell, Areh. f. Protistenk., 26, 1912, 117; Spironema buccale Gross, Cent. f. Bakt.. I Abt., Orig. 65, 1912, 84; Spiroschandinnia buccalis Castellani and Chalmers, Man. Trop. Med., 3rd ed., 1919, 450 ; Brumpt, Nouveau Traité de Médecine, Paris, 4, 1922, 495; Treponema inaequale Brumpt, ibirl.; Treponema undulatum Brumpt, ibid., 514.) From Latin buccalis, buecal.

Morphology : 0.4 to 0.9 by 7 to $20 \mathrm{mi}$ crons. The largest of the mouth spirochetes.

Motility: Active, serpentine, rotating and Hexuous.

Staining: Stains with aniline dyes and is violet with Giemsa's stain.

Cultivation: IIas not been obtained in pure culture and probably does not grow in any medium tried to date.

Habitat: In normal mouths and invades formed lesions of the respiratory mucous membrane 
11. Borrelia vincentii (Blanchard) Bergey et al. (Spivuchacta rincenti Blanchard, Arch. f. Protistenk., 10, 1906, 129; Spirochacta schaudinni Prowazek, Arb. kaiserl. Gesundheitsante, 22, 1907, 23; Spirochacte plaut-vincenti Lehmann and Neumann, Bakt. Diag., 5 Aufl., 2, 1912, 579; Spiroschaudinnia vincenti Castellani and Chalmers, Man. Trop. Med., 2nd ed., 1913, 402; Spiroschaulinnia schaudinni Castellani and Chalmers, idem; Spironema vincenti Park and Williams, Pathogenic Microorganisms, 6 th ed., 1917, 506; Treponema vincen! $i$ Brumpt, Nouveau Traité de Médecine, Paris, 4, 1922, 514; Treponema schaudinni Brumpt, idem; Bergey et al., Manual, 2nd ed., 1925, 435.) Named for Vincent, the French bacteriologist.

Norphology : 0.3 by $\&$ to 12 microns, 3 10 \& irregular shallow spirals. Stains easily with the common aniline dyes and is Gram-negative.

Motility : Has a rapid progressive and viluratory motion.

Cultivation: Can be cultivated under anaerobic conditions. Cultures may show long forms with only a writhing motion.

Not pathogenic for laboratory animals.

Habitat: Found on normal respiratory mucous nembrane and is associated with a fusiform bacillus (Fusobacterium. plauti-vincenti) in Vincent's angina.

12. Borrelia refringens (Schaudinn and Hoffmann) Bergey et al. (Spirochaeta refringens Schaudinn and Hoffmann, Arbeiten kaiserl. Gesundheitsamte, 22, 1905, 528; Spirochaeta refringens Hoffmann and Prowazek, Cent. f. Bakt., I Abt., Orig., 41, 1906, 742; Spironema refringens Gross, Arch. f. Protistenk., 24, 1912, 115; Spiroschaudinnia refringens Castellani and Chalmers, Man. Trop. Med., 3rd ed., 1919, 459; Treponema refringens Castellani and Chalmers, ibid., 461; Bergey et al., Manual, 2nd ed., 1925, 436.) From Latin, refractive.

Morphology : 0.5 to 0.75 by 6 to $20 \mathrm{mi}$ crons. Spirals are coarse and shallow.
Spirals are generally smoothly rounded and regular, tapering towards the end into a fine projection. Stains easily by common dyes. In stained specimens the spirals appear irregular.

Motility: Artive serpentine and rotating motion with marked flexion.

Cultivation: Lncertain.

Pathogenicity : None.

Source: Found with Treponema pallidum in some cases of syphilis as originally described by Schaudinn.

Habitat: Genital mueous membranes and necrotic lesions of the genitalia of man.

13. Borrelia hyos (King and Drake) Bergey et al. (Hog cholera virus, King and Baeslack, Jour. Inf. Dis., 12, 1913, 39; Spirochaetu suis King, Baeslack and Hoffmann, Jour. Inf. Dis., 12, 1913, 235; not Spirochacta suis Bosanquet, Spirochetes, Saunders, 1911 ; Spirochaeta hyos King and Drake, Jour. Inf. Dis., 16, 1915, 54; Spironema hyos Bergey et al., Manual, 1st ed., 1923, 426; Bergey et al., Manual, 2nd ed., 1925, 436; Spironema suis Ford, Textb. of Bart., 1927, 959.) From Greek, hog.

Morphology : 1 mieron by 5 to 7 mierons. Distinctly shorter and thicker than other members of the genus.

Motility: Aetive spimning motion, spirals fixed.

Cultivation: (irows under anaerobic conditions in the presence of tissue.

Habitat: Found in the blood, intestinal ulcers and other lesions of hogs suffering from hog cholera.

14. Borrelia hermsi (Davis) Steinhaus. (Spirochaeta hermsi Davis, Amer. Assoc. Adv. Sci., Pub. No. 18, 1942, 46; Steinhaus, Insect Microbiology, 1946, 453.)

Investigations by Davis (loc. cit.) indicate that each species of Ornithodoros that is a relapsing fever vector carries a spirochete that is tick-host specific and that this host-specific relationship offers a more accurate approach to the differentiation of relapsing fever spirochetes 
than any of the several criteria previously used.

This was shown to be the case for Borrelia hermsi and Borrelia parkeri. For this reason no attempt is made to describe the morphology and other characters of the relapsing fever spirochetes of North and South America.

Borrelia hermsi is transmitted by Ornithodoros hermsi.

A cause of relapsing fever in the Western part of the U.S. A.

15. Borrelia parkeri (Davis) Steinhaus. (Spirochaeta parkeri Davis, loc. cit.; Steinhaus, loc. cit.)

Transmitted by Ornithnodoros parkeri.

A cause of relapsing fever in the Western part of the U.S. $\Lambda$.

16. Borrelia turicatae (Brumpt) Steinhaus. (Spirochaela turicalae Brumpt, Comp. rend. Soe. Biol., Paris, 119, 1933, 1369; Steinhaus, loc. cit.)

Transmitted by Ornilhodoros turicata.

A cause of relapsing fever in Mexicn, Texas and nearby areas.

17. Borrelia venezuelensis Brumpt. (Treponema venezuelensis Brumpt, Nouveau Traité de Médecine, Paris, 4, 1922, 492; Brumpt, ibid., 495; Spirochaela venezuelensis Pettit, Contributions à l'Êtude des Spirochétidés, Vanves, 2,1928, 295.)

Transmitted by Ornithodoros rudis (O. venezuelensis).

A cause of South American relapsing fever.

Brumpt (Précis de Parasitologie, 3rd ed., Paris, 1936) regards this species as identical with Borrelia neotropicalis (Bates, Dunn and St. John) Steinhaus. (Treponema neotropicalis Bates, Dunn and St. John, Amer. Jour. Trop. Med., 1, 1921, 183; Spirochaeta neotropicalis St. John and Bates, Amer. Jour. Trop. Med., 2, 1922, 251; Steinhaus, loc. cil.) Transmitted by Ornithodoros venezuelensis. A cause of relapsing fever in Panama.
Appendix: Many of the species included in this appendix are so inadequately described that it is not certain that they belong in this group.

Borrelia phagedenis (Noguchi) Bergey et al. (Spirochaeta phagedenis Noguchi, Jour. Exp. Med., 16, 1912, 261; Spiroschaudinnia phagedenis Castellani and Chalmers, Man. Trop. Med., 2nd ed., 1913, 403; Treponema phagedenis Brumpt, Nouveau Traité de Médecine, Paris, 4, 1922, 511; Spironema phagedenis Bergey et al., Manual, 1st ed., 1923, 426; Bergey et al., Manual, 2nd ed., 1925, 435.) From phagedenous ulcer.

Heliconema pyrphoron Scholer. (Cent. f. Bakt., I Abt., Orig., 138, 1937, 342.) From human blood. Pathogenic. Heliconema vincenti Sanarelli. (Ann. Inst. Past., 41, 1927, 701.) From the intestine of a guinea pig. Shows stages between spirochetes and fusiform bacilli. (See Hindle, Med. Res. Council Syst. of Bact., 8, 1931, 130.)

Microspiromema merlangi Duboseq and Lebailly. (Compt. rend. Acad. Sci. Paris, 154, 1912, 662.) From the whiting, Merlangus merlangus. May be a synonym of spirochaeta gadi.

Spirillum gondii Nicolle. (Nicolle. Compt. rend. Soc. Biol., Paris, 63, 1907, 213; Spirochaeta gondi Zuelzer, 1925, in Prowazek, Handb. d. path. Protoz., 3, 1931, 1680.) Found in the blood of a rodent, Ctcnodactylus gondi. Not pathogenic. Associated with a piroplasma. Probably not a spirochete.

Spirillum latapici Laveran. (Laveran, Bull. Soc. Path. Exot., 1, 1908, 148; Spirochaeta latapiei Zuelzer, 1925, in Prowazek, Handb. d. path. Protoz., 3, 1931, 1683; Spironema latapici (sic) Ford, Textb. of Bact., 1927, 964.) From the blood of a shark.

Spirillum pitheci Thiroux and Dufougeré. (Thiroux and Dufourgeré, Compt. rend. Acad. Sci. Paris, 150, 1910, 132; Spirochaeta pitheci Zuelzer, 1925, in Prowazek, Handb. d. path. Protoz., 3, 1931, 1676; Spironema pitheci Ford, 
Textb. of Bact., 1927, 961.) From the b'ood of an African monkey, Cercopithecits palas. Pathogenic for monkeys, rats and field mice. Closely related to Borrelia duttonii.

Spirochaeta aboriginalis Cleland. (Cleland, Jour. Trop. Med., 12, 1909, 143; Spiroschaudinnia aboriginalis Castellani and Chalmers, Man. Trop. Med., 2nd ed., 1913, 402; Treponema aboriginalis Brumpt, Nouveau Traité de Médecine, Paris, 4, 1922, 496.) Found in cases of granuloma inguinale in West Australia. Probably saprophytic.

Spirochaeta acuminata Castellani (Castellani, Brit. Med. Jour., 2, 1905, 1330 ; Spirochaeta tenuis acuminata Castellani, idem and/or Arch. f. Schiffs-u. Tropenhyg., 12, 1908, 311 ; Spiroschaudinnia acuminata Castellani and Chalmers, Man. Trop. Med., 3rd ed., 1919, 449; T'reponema acuminatum Brumpt, Nouveau Traité de Médecine, Paris, 4, 1922, 496.) From ulcerated lesions of yaws.

Spirochaeta acuta Kritchewshi and Séguin. (Rev. de Stomatol., 22, 1920 613.) From the oral cavity.

Spirochacta aeglefini Henry. (Jour. Path. and Bact., 18, 1913, 222.) From haddock.

Spirochaeta aegyptica Gonder. (Gonder, in Prowazek, Handb. d. path. Protoz., 6, 1914, 671; Spironema aegyptica Noguchi, Jour. Exp. Med., 27, 191S, 584; Treponema egypticum Brumpt, Nouveau Traité de Médecine, Paris, 4, 1922, 500; Borrelia aegypticum Steinhaus, Insect Microbiology, 1946, 452.) Observed in cases of relapsing fever in Sudan. Prolably a synonym of Borrelia recurrentis.

Spirochaeta ambigua Séguin and Virızent. (Séguin and Vinzent, Compt. rend. Soc. Biol., Paris, 121, 1936, 408; Treponema ambigua Prérot, Man. Class. et Determ. d. Bactéries Anaérobies, Paris, 1910, 209.) From the oral cavity and the lungs. Pathogenir. Strict anae. robe.

Spirochaeta amphibiae Yakimoff and Miller. (Bull. Soc. Path. Exot., 18, 1925,
306.) From the intestines of frogs, Rana temporaria.

Spirochacta argentinensis Iiuhn and Steiner. (Kuhn and Steiner, Med. Klin., 13, 1917, 1007; Spirochaeta polysclerolica Arzt and Iierl, aceording to Pettit, Contribution à l'Etude des Spirochétidés, Vanves, II, 1928, 134; Treponema (?) aryentinensis Noguchi, in Jordan and Falk, Newer Kinowledge Bact. and Immun., 1928, 478.) From the livers of guinea pigs and rabbits inoculated with blood from patients having multiple sclerosis. Pathogenic for man, monkeys, dogs, rabbits and guinea pigs. Named for the Latin name of the town of Strasbourg (Argentoratum).

Spirochacta balanitidis Hoffmann and Prowazek. (Hoffmann and Prowazek, Cent. f. Balit., I Abt., Orig., 41, 1906, 741; Spiroschautinnia balanitidis Castellani and Chalmers, Min. Trop. Med., 2nd ed., 1913, 404; Spironoma balanitidis Park and Williams, Pathogenic Microorganisms, 6th ed., 1917, 505; T'reponema balanitidis Brumpt, Nouveau Traité de Nédecine, Paris, 4, 1922, 496.) From a case of balanitis.

Spirochacta bovis-caffris Nuttall. (Nuttall, Parasitology, 3, 1910, 10s; Spironema bovis-caffris Ford, Textb. of Bact., 1927, 960.) From the blood of a buffalo.

Spirochaeta bronchialis Castellani. (Castellani, Ceylon Medical Reports, 1907; Spiroschaudinnia bronchialis Castellani and Chalmers, Man. Trop. Med., 2nd ed., 1913, 402; Treponema bronchiale Brumpt, Nouveau Traité de Médecine, Paris, 4, 1922, 496.) Found in cases of bronchitis in Ceylon. A mixture of several species of mouth spirochates is apparently deseribed under this designation.

Spirochaeta bucco-pharyngei Macfie. Macfic, Ann. Trop. Med. and Parasitol., 10, 1916, 329 ; Treponema bucco-pharyngei Brumpt, Nouveau Traité de Médecine, Paris, 4, 1922, 497.) From the throat of a native of the Gold Coast. May be identical with Spirochaeta dentium or $S$. buccalis. 
Spirochaeta bufonis Dobell. (Dobell, Quart. Jour. Microse. Sci., 52, 1908, 121; Spiroschaudinnia bufonis Castellani and Chalmers, Man. Trop. Med., 3rd ed., 1919, 454; Spironema bufonis Ford, Textb. of Bact., 1927, 986; Treponema bufonis Ford, ibid.) From the intestines of a toad, Bufo vulgaris.

Spirochaeta caesirae retortiformis Hellmann. (Arch. f. Protistenk., 29, 1913, 22.) From the urinary sac of a tunicate, Caesira retortiformis.

Spirochaeta caesirae septentrionalis Hellmann. (Arch. f. Protistenk., 29, 1913,22 .) From the urinary sac of a tunicate, Caesira seplentrionalis.

Spirochacta canina Bosselut. Soc. Path. Exot., 18, 1925, 702.) From the blood of a dog.

Spirochaela canis Macfie. (Ann. Trop. Med. and Parasitol., 10, 1916, 305.) From dog feces.

Spirochaeta cobayae Knowles and Basu (Knowles and Basu, Indian Jour. Med. Res., 22, 1935, 449; Treponema cobayae Topley and Wilson, Princip. Bact. and Immun., 2nd ed., 1936, 725; Borrelia cobayae Steinhaus, Insect Mierobiology, 1946 , 454.) From the blood of guinea pigs. Blood parasite belonging to the relapsing fever group. Pathogenic for guinea pigs, rabbits and white rats.

Spirochaeta comandoni Séguin and Vinzent. (Séguin and Vinzent, Compt. rend. Soc. Biol., Paris, 191, 1936, 408; Treponema comandoni Prévot, Man. Class. et Determ. d. Bactéries Anaérobies, Paris, 1940, 208.) From the oral cavity. Rather common. Non-pathogenic. Strict anaerobe.

Spirochaeta crocidurae Leger. (Leger, Bull. Soc. Path. Exot., 10, 1917, 280; Treponema crocidurae MeFarland, Pathogenic Bacteria and Protozoa, 2nd ed.. 1933, 136.) From a shrew-mouse, Crocidura stampflii, in Senegal. Transmitted by Ornithodoros erraticus.

Spirochacta ctenocephali Patton. (Patton, Ann. Trop. Med. and Parasitol., 6, 1912, 357; Treponema ctenocephali Ford, Texth. of Bact., 1927, 989.) Para- sitic in the digestive tract of the larvae of the Indian cat-flea, Ctenocephalus felis.

Spirochacta cubensis Hoffman. (Sanidad y Beneficienca Boletin oficial, Havana, 28, 1923, 76.) From the feces of IIyla septentrionalis.

S'pirochacta culicis Jaffé. (Jaffé, Arch. f. Protistenk., 9, 1907, 100; Spironema culicis Gross, Cent. f. Bakt., I Abt., Orig., 65, 1912, 87; Entomospira culicis Enderlein, Sitzungsber. Ges. Naturf. Freunde, Berlin, 1917, 313; Spiroschaudinnia culicis Castellani and Chalmers, Man. Trop. Med., 3rd ed., 1919, 454; S'pirillum culicis Pringault, Compt. rend. Soc. Biol., Paris, 84, 1921, 209; Treponema culicis Ford, Textb. of Bact., 1927, 989.) Found in the intestines and Malpighian tubules of mosquito larvae, Culex sp.

Spirochaeta didelphis Vianna, de Figueiredo and Cruz. (Brasil-Medico, 26, 1912 , 912.) From the blood of an opossum, Didelphis aurita.

Spirochaeta equi (Novy and Knapp) Castellani and Chalmers. (Spirillum equi Novy and Knapp, Jour. Inf. Dis', 3, 9106, 294; Castellani and Chalmers, Man. Trop. Med., 1st ed., 1910, 309; Spiroschaudinnia equi Castellani and Chalmers, Man. Trop. Med., 2nd ed., 1913, 404; Spironema equi Noguchi, Jour. Exp. Med., 27, 1918, 584; Treponema equi Noguchi, in Jordan and Falk, Newer Knowledge Bact and Immun., 1928, 461.) From the blood of a horse. May be identical with Borrelia theileri.

Spirochaeta equina. (Dodd ?, Jour. Comp. Pathol and Therap., 19, 1906, 318; quoted from Pettit, Contribution à l'Etude des spirochétidés, Vanves, II, 1928, 111.)

Spirochaela eurygyrata Werner. (Werner, Cent. f. Bakt., I Abt., Orig., 52, 1909, 241; Spironema curgyratum (sic) Noguehi, Jour. Exp. Med., 27, 191S, 584; Spiroschaudinnia eurygyrata Castellani and Chalmers, Man. Trop. Med., 3rd ed., 1919, 451 ; Spirillum eurygyrata Castellani and Chalmers, ibid.; Borrelia eur!y- 
gyrata Brumpt, Nouveau Traité de Médecine, Paris, 4, 1922, 495; Treponema eurygyratum Brumpt, ibid., 50.) From the intestinal contents of man.

Spirochacta exanthematotyphi Futaki. (Futaki, Brit. Med. Jour., Oct. 13, 1917; Treponema exanthemalotyphi Savini, Compt. rend. Soe. Biol., Paris, 88 , 1923, 958.) Found in the kidneys and urine of cases of exanthematous typhus. Non-pathogenic.

Spirochaeta febris Chester. (Afanassiew, Cent.f. Bakt., I Abt., 25, 1899, 405; Chester, Man. Determ. Bact., 1901, 347.) From a case of recurrent fever.

Spirochaeta gadi Neumann. (Neumann, Ztschr. f. Hyg., 64, 1909, 79; Mierospironema gadi Duboseq and Lebailly, Compt. rend. Acad. Sci. Paris. 154, 1912, 662; Treponema gadi Dubosea and Lebailly, Arch zool. expér. et génér., 10, 1912, 331; Spironema gadi Ford, Textb. of Bact., 1927, 964.) From the blood of a sea fish, Gadus minutus.

Spirochacta gallica Couvy and Dujarric de la Rivière. (Couvy and Dujarric de la Rivière, Compt. rend. Soc. Biol., Paris, 81, 1918, 22; Treponema yallicum Brumpt, Nouveau Traité de Médecine, Paris, 4, 1922, 500.) From the blood of trench fever patients.

Spirochacta gangraenae carcinomatosae Hoffmann. (Berl. klin. Wochnschr., 42, 1905, 880.) From malignant tumors.

Spirochaeta gangraenosa nosocomialis Róna. (Róna, Verhandl. d. deutseh. dermat. Gesellsch., 9, 1907, t71; Treponema gangracnosa nosocomialis Noguchi, Jour. Exp. Med., 16, 1912, 261.) From ulcers of the genital region.

Spirochaeta hacmophilus Trosier and Sifferlen. (Ann. Inst. Past., 58, 1937. 233.) From a child with intestinal trouble and continuous fever.

Spirochacta hispanica de Buen. (De Buen, Ann. Parasitol., 4, 1926, 185; Treponema hispanicum Noguchi, in Jordan and Falk, Newer Knowledge Bact. and Immun., 1928, 481; Spirochacta marocanum Nicolle and Anderson, Compt. rend. Acad. Sci. Paris, 187, 1928, 747 ;
Spirochaeta hispanicum. var. marucanum Nicolle, Anderson and Colas-Belcour, Arch. Inst. Past. Tunis, 18, 1929, 343; Treponema hispanicum var. marocanum Gay et al., Agents of Disease and Host Resistance, 1935, 1074; Borrelia hispanicum Steinhaus, Insect Microbiology, 1946, 453.) The cause of Spanish and Noroccan relapsing fever. Transmitted by Ornithodoros marocanus. Not agglutinated by serum of Borrelia recurrentis. Pathogenic for man and laboratory animals.

Spirochacta intestinalis Macfie and Carter. (Marfie and Carter, Ann. Trop. Med. and Parasitol., 11, 1917, 79; Treponema intestinale Brumpt, Nouveau Traité de Médecine, Paris, 4, 1922, 505.) From human feces.

Spirochacta jonesii Dutton, Todd and Tobey. (Dutton, Todd and Tobey, Jour. Med. Res., 15 (N.S. 10), 1906, 491 ; Spironema jonesii Ford, Textb. of Bact., 1927, 964.) From the blood of an African mudfish, Clarias angolensis.

Spirochacta lagopodis Fantham. (Fantham, Proc. Zool. Society, London, 1910, 692; Spironema lagopodis Noguchi, according to Pettit, loc. cit.) From the blood of the grouse, Lagopus scoticus.

Spirochaeta leucotermitis Hollande. (Arch. zool. expér. et gén., 61, 1922, 23.) From an insect, Leucotermes lucifugus.

Spirochaeta lovati Fantham. (Proc. Zool. Society, London, 1910.) From the intestinal contents of the grouse, Lagopus scoticus.

Spirochaeta lowenthali Besson. (Besson, p. 736, according to Ford, Textb. of Bact., 192 $\bar{\jmath}$, 1001.) From malignant tumors.

Spirochaeta lutrae Prowazek. (Prowazek, Arb. kaiserl. Gesundheitsamte, $26,1907,31$; Spiroschaudinnia lutrae Castellani and Chalmers, Man. Trop. Med., 2nd ed., 1913, 404; Spironema lutrae Ford, Textb. of Bact., 1927, 961.) From the blood of an otter, Lutra sp.

Spirochaeta lymphaticus Proescher and White. (Proescher and White, Jour. Amer. Med. Assoc., 49, 1907, 1988; 
Treponoma lymphaticum Brumpt, Nouveau Traité de Médecine, Paris, 4, 1922, 506.) From cases of lymphatic leukemia. Pathogenic for guinea pigs, rats and monkeys.

Spirochaeta macaci Castellani and Chalmers. (Castellani and Chalmers, 1908, see Castellani and Chalmers, Man. Trop. Merl., 1st ed., 1910, 310; Spiroschaudinnia macaci Castellani and Chalmers, Man. Trop. Med., 2nd ed., 1913. 403; Spironema macaci Ford, Textb. of Bact., 1927,960.) From monkeys in Ceylon. Closely related to or possibly idlentical with Borrelia carteri.

Spirochaeta marmotae Carpano. (Ann. d'Igien. sper., 23, 1913, 215.) From is rodent, Arctomys marmola.

Spirochacta melanogenes canis Lukeš. (Deutsch. Tierärztl. Wochnschr., 1923, 137; see Ann. Inst. Past., 38, 1921, 523.) From the intestines of a dog. The rause of a dog plague.

Spirochacla melophagi Porter. (Quart. Jour. Microse. Sci., London, 55, 1910, 189.) From the intestine, ovaries, and pupa of Melophagus ovinus.

Spirochaeta metritis de Andrade. (Compt. rend. Soc. Biol., Paris, 86, 1922, 1048.) From a uterine excretion.

Spirochacta microgyrata gaylordi Calkins. (Calkins, Jour. Inf. Dis., 4, 1907. 173 ; Spirochacta microgyrata var. gaylerdi Calkins, ibid., 171; Spironema microgyrata var. ganlordi Ford, Textb. of Bact. 1927,1001 .) From breast tumors of mice. Spirochacta naganophila Savini. (Compt. rend. Soc. Biol., Paris, 88, 1923, 956.) From the blood of mice. No description given.

Spirochacta noelleri Zuelzer. (1925, in Prowazek, Handb. (l, path. Protoz. 3, 1931, 1684.) From the intestine of the larvae of Simulium noclleri.

Spirochaeta noguchii Strong. (Str.)ng, United Fruit Co. Med. Dept. 14th Ann. Rept., 1925; Treponema noquehii Noguchi, in Jordan and Falk, Newer Lnowledge Bact. and Immun., 1928, 483.) From a type of skin uleer in south America.
Spirochaeta normandi Nicolle, Anderson and Colas-Belcour. (Compt. rend. Acad. Sci. Paris, 185, 1927, 334.) From Ornithodoros normandi, the natural agent of transmission. Pathogenic for white mice.

Spirochacta normandi var. carthaginensis. (Quoted from Hindle, Med. Res. Council Syst. of Bact., 8, 1931, 169.) From ticks in Tunis.

Spirochate nosocomialis Hofimann and Gonder. (Hoffmann and Gonder, 1914, according to Brumpt, Nouveau Traité de Médecine, Paris, 4, 1922, 508; Treponema nosocomiale Brumpt, ibid.) Probably a synonym of Borrelia vincentii.

Spirochacla obtusa Castellani. (Castollani, Brit. Med. Jour., 2, 1905, 1330; Spirochacta lenuis obtusa Castellani, 1908, according to Ford, Textb. of Bact., 1927, 973; Spiroschaudinnia obtusa Castellani, Arch. f. Schiffs- u. Tropenhyg., 12, 1908. 311 (see Castellani and Chalmers, Man.. Trop. Med., 3rd ed., 1919 449); Treponema oblusum Brumpt, Nouveau Traité de Médecine, Paris, 4, 1922, 508; Treponema tenue cbtusum Brumpt, ibid.) From ulcerated lesions of yaws.

Spirochacla ovis (Novy and Knapp) Bergey et al. (Spirillum ovis Novy and Inapp, Jour. Inf. Dis., 3, 1906, 294; Spirochacta ovina Blanchard, Semaine Médicale, 20, 1906, 1; Spiroschaudinnia ovina Castellani and Chalmers, Man. Trop. Med., 2nd ed., 1913, 404; Spironema ouis Ford, Textb. of Bact., 1927, 960 ; Treponema ovis Noguchi, in Jordan and Falk, Newer Kinowledge Bact. and Inmun., 1928, 461; Treponoma ovinum Noguchi, ibil., 480; Bergey et al., Manual, 5th ed., 1939, 960.) From the blood of sheep. May be identical with Borrelia theileri.

Spirochaeta pelamidis Neumann. (Neumann, Ztschr. f. Hyg., 64, 1909, 80; Spironema pelamidis Ford, Textb. of Bact., 1927, 96t.) From the blood of a fish, Pelamys sarda. Resembles Spirochaeta gadi.

Spirochueta perforans Cavalié and Mandoul. (Compt. rend. Soc. Biol., Paris, 
85, 1921, 1068.) From cases of pyorrhea alveolaris. Associated with fusiform bacilli. Probably synonymous with Borrelia vincentii.

Spirochaeta periplanetae Laveran and Franchini. (Bull. Soc. Path. Exot., 13, 1920, 332.) From the digestive tract of the cockroach, Periplaneta orientalis.

Spirochaeta persica Dschunkowsky. (Dschunkowsky, Deutsch. med. Wochnschr., 39, 1913, 419; Treponema persicum Brumpt, Nouveau Traité de Médecine, Paris, 4, 1922, 509; Borrelia persica Steinhaus, Insect Microbiology, 1946, 452.) From a case of relapsing fever (Mianeh fever) in Persia. Transmitted by Ornithodoros tholozani and $O$. latiorensis. Serum not agglutinated by Borrelia recurrentis. Disease in man fairly severe and in gerbilles and monkeys very mild.

Spirochaeta pollachii Henry. (Spirochaeta gadi pollachii Henry, Jour. Path. and Bact., 14, 1910, 463; Henry, ibid., 17, 1912, 160; Treponema fallax Duboseq and Lebailly, Arch. Zool. Expér. et Gén., 10, 1912, 331; Spirochaeta fallax Zuelzer, 1925, in Prowazek, Handb. d. path. Protoz., 3, 1931, 1671; Spironema pollachii Ford, Textb. of Bact., 1927, 965.) From the blood of the pollack, Gadus pollachius.

Spirochaeta pseudobuccalis Zuelzer. (Cent. f. Bakt., I Abt., Orig., 85, 1921, *154.)

Spirochaeta pseudorecurrentis Zuelzer. (Cent. f. Bakt., I Abt., Orig., 85, 1921, *154.)

Spirochaeta pyorrhoeica Kolle. (Med. Klin., 13, 1917, 59.) Commonly found in pus from pyorrhea alveolaris.

Spirochaeta raillieti Mathis and Leger. (Compt. rend. Soc. Biol., Paris, 70, 1911, 212.) From the blood of a rabbit. Not pathogenic.

Spirochaeta ranarum Yakimoff and Miller. (Bull. Soc. Path. Exot., 18, 1925, 306.) From the intestines of frogs, Rana temporaria.

Spirochaeta recta Gerber. (Gerber, Cent.f. Bakt., I Abt., Orig., $56,1910,513$;
Treponema rectum Brumpt, Nouveau Traité de Médecine, Paris, 4, 1922, 511.) May be a synonym of Borrelia vincentii.

Spirochacta regaudi Ball and Roquet. (Ball and Roquet, 1911, according to Pettit, Contribution à l'Etude des Spirochétidés, Vanves, II, 1928; Spirella regaudi Ball and Roquet, 1911, according to Brumpt, Nouveau Traité de Médecine, Paris, 4, 1922, 517; also see Edkins, Parasitology, 15, 1923, 296.) From the stomachs of cats and dogs. Possibly belongs among the spirilla (Noguchi); is in the same group as Cristispirella and Heliconema.

Spirochaeta sinensis Pons. (Compt. rend. Soc. Biol.', Paris, 89, 1923, 1028.) From the blood of $a$ fever patient in China. Pathogenic for rabbits and monkeys.

Spirochaeta sogdianum Nicolle and Anderson. (Nicolle and Anderson, Compt. rend. Acad. Sci. Paris, 187, 1928, 746; Borrelia sogdianum Steinhaus, Insect Microbiology, 1946, 453.) Not pathogenic for guinea pigs or fowls. Probably synonymous with Borrelia recurrentis.

Spirochaeta sporogenes psoriasis Rasek. (Individual publications, Christiania, 1920-1921, 4.)

Spirochaeta sporogona rheumatismi Rasck. (Individual publications, Christiania, 1920-1921, 4.) From the blood in cases of acute arthritis.

Spirochaeta staphylina Ghidini and Archetti. (Riv. Biol. Coloniale, 2, 1939, 131.) From the intestine of a termite, Reticulitermes lucifugus, Italy.

Spirochaeta stenogyrata Werner. (Werner, Cent. f. Bakt., I Abt., Orig., 52, 1909, 241; Treponema stenogyratum Brumpt, Nouveau Traité de Médecine, Paris, 4, 1922, 514.) From human feces.

Spirochaeta suilla Dodd. (Jour. Compt. Path. and Therap., 19, 1906, 216.) From cutaneous lesions of pigs. Pathogenic for pigs.

Spirochaeta temporariae Yakimoff and Miller. (Bull. Soc. Path. Exot., 18, 
1925, 306.) From the intestines of frogs, Rana temporaria.

Spirochaeta tenuis Gerber. (Gerber, Cent. f. Bakt., I Abt., Orig., 56, 1910, 508; Treponema tenue Brumpt, Nouveau Traité de Médecine, Paris, 4, 1922, 514.) May be identical with Spirochaeta dentium or with Borrelia vincentii.

Spirochaeta termitis (Leidy) Dobell. (Vibrio termitis Leidy, Jour. Acad. Nat. Sei., Phila., 2nd Ser., 8, 18\$1, 441 ; Spirochaeta minei Prowazek, Arch. f. Schiffsu. Tropenhyg., 14, 1910, 297; Dobell, Spolia ceylanica, 3, 1910, 78; Treponema minei Dobell, ibid.; ? Spirochaele grassii Döflein, Prob. der Protistenk., 2, 1911, 17; Spirochaeta grassii Döflein, Die Natur der Spirochäten, Jena, 1911; Treponema termitis Dohell, Arch. f. Protistenk., 26, 1912, 117; Entomospira grassii Enderlein, Sitzungsber. Ges. Naturf. Freunde, Berlin, 1917, 313; Cristispira termitis Hollande, Arch. Zool. Expér. et Gén., 61, 1923, X. and R., 25; Treponema grassi Ford, Textb. of Bact., 1927, 988.) From the intestines of Termes lucifugus and Calotermes spp.

Spirochaeta tropidonoti Dobell. (Dobell, Spolia ceylanica, $7,1911,65$; Spironema tropidonoti Ford, Textb. of Bact., 1927, 962.) Isolated once from the blood of a snake, Tropidonotus stolatus, in Ceylon.

Spirochacta urethrae Marfie. Macfie, Ann. Trop. Med. and Parasitol., 10, 1916. 305 ; Spiroschaudinnia urethrae Castellani and Chalmers, Man. Trop. Med., 3rd ed., 1919, 451; Treponema urethrae Brumpt, Nouveau Traité de Médecine. Paris, 4. 1922, 514.) From the urine of Gold Coast natives. Causes acute arthritis.

Spirochacta usbekistanica Pickoul. (Russ. Jour. Trop. Mrel., 6, 1928, 612.) From cases of relapsing fever in Bokhara. Spirochaeta vespertilionis (Novy and Kinapp) Castellani and Chalmers. (Spirillum vespertilionis Vovy and Knapl, Jour. Inf. Dis., 3, 1906, 294; Castellani and Chalmers, Man. Trop. Med., 1st ed., 1910,309 ; Spiroschaudinnia vespertilionis Castellani and Chalmers, Man. Trop.
Med., 3rd ed., 1919, 454; Spironema vespertilionis Ford, Textb. of Bact., 1927, 961.) From the blood of a bat, Vespertilio kuhlii.

Spirochaeta vincenti var. bronchialis Delamare. (Compt. rend. Soc. Biol., Paris, 90, 1924, 611.)

Spirochacta zlatogorovi Yakimoff. (Bull. Soc. Path. Exot., 14, 1921, 532.) From feces.

Spirochacte exanthematica Lewascheff. (Cent. f. Bakt., I Abt., 18, 1895, 133.) From the blood in cases of typhus fever.

Spirochate forans Reiter. (Reiter, Deutsch. med. Wochnsehr., No. 50, 1916, 10; see Cent. f. Bakt., I Abt., Orig., 79, 1917, 176; Treporema forans Brumpt, Nouveau Traité de Médecine, Paris, 4, 1922,500 ; Spirochacta forans Pettit, Contribution à l'Étude des Spirochétidés, Vanves, II, 1928, 164.) From the blood in a case of articular rheumatism. Not pathogenic for guinea pigs or mice.

Spirochaete gracilis Veszpremi. (Veszpremi, Cent. f. Bakt., I Abt., Orig., 44, 1907,332 ; not Sipirochacta gracilis Ievaditi and Stanesco, Compt. rend. Soe . Biol., Paris, 67, 1909, 188 (Treponema levaditii Brumpt. Nouveau Traité de Médecine, Paris, 4, 1922, 501; Treponema gracile Brumpt, idem); Treponema gracile Ford, Texth. of Bact.. 1927, 978.) From a gangrenous phlegmon of the mouth. Found in association with fusiform bacilli and therefore may be identical with Borrelia vincentii or Spirochaeta dentium or Treponema macrodentium.

Spirochate reparis. (Quoted from Lehmann and Neumann, Bakt. Diag., 6 Aufl., 2, 1920, 809.) From the oral cavity. Spironema caviae Ford. (Spirochaete (?), Macfie, Ann. Trop. Med. and Parasitol., 8, 1914, 4t7; Ford, Textb. of Bact., 1927, 961.) From the blood of a guinea pig at Lagos.

Spironema vesperuginis (Gonder) Ford. (Spirochacta vesperuginis Gonder, Arb. kais. Gesundheitsamte, 27, 1908, 406; Ford, Textb. of Bact., 1927, 961.) From the blood of a bat, Tesperugo huhlii.

Spiroschaudinnia caviae sangiorgi. 
(Sangiorgi, Pathologica Rivista, 5, 1913, 128; Spirochaeta caviae Hindle, Med. Res. Council Syst. of Bact., 8, 1931, 174.) From the blood of a guinea pig.

Spiroschaudinnia mitis Castellani and Chalmers. (Castellani and Chalmers, Man. Trop. Med., 3rd ed., 1919, 451; Treponema mite Brumpt, Nouveau Traité de Médecine, Paris, 4, 1922, 506.) From urine in mild cases of camp jaundice. Probably not pathogenic.

Treponema lincola (Donné) Brumpt. (Vibrio lineola Donné, Recherches Microse. Nature d. Mucus des Organs Genitourinaires, Paris, 1837 ; Brumpt, Nouveau Traité de Médecine, Paris, 4, 1922, 505.) From secretions of the genitalia.

\section{Genus II. Treponema Schaudinn.}

(Spironema Vuillemin, Compt. rend. Acad. Sci. Paris, 140, 1905, 1567 ; not Spironema Bergey et al., Manual, 1st ed., 1923, 424; Schaudinn, Deutsche med. Wochnschr., 31, 1905, 172s; Microspironema Stiles and Pfender, Amer. Med., 10, 1905, 936.)

Length 3 to 18 microns. Longer forms due to incomplete division. Protoplasm in acute, regular or irregular spirals. Terminal filament may be present. Some species stain only with Giemsa's stain. Weakly refractive by dark field illumination in living preparations. Cultivated under strictly anaerobic conditions. Pathogenic and parasitic for man and animals. Generally produce local lesions in tissues.

The type species is Treponema pallidum (Schaudinn and Hoffmann) Schaudinn.

1. Treponema pallidum (Schaudinn and Hoffmann) Schaudinn. (Spirochaete pallida Schaudinn and Hoffmann, Arb. kaiserl. Gesundheitsamte, 22, 1905, 528; Schaudinn, Deutsche med. Wochnschr., 31, 1905, 1728; Spironema pallidum Vuillemin, Compt. rend. Acad. Sci. Paris, 140 , 1905, 1567; Microspironema pallidum Stiles and Pfender, Amer. Med., 10, 1905, 936; Trypanosoma luis Krrystalowicz and Siedlecki, 1905, see abst. in Bull. Inst. Past., 4, 1906, 204; Spirochaeta pallida Hoffmann and Prowazek, Cent. f. Bakt., I Abt., Orig., 41, 1906, 741.) From Latin, pale.

Morphology: Very fine protoplasmic spirals 0.25 to 0.3 by 6 to 14 microns.

Spiral amplitude : 1.0 micron, regular, fixed.

Spiral depth: 0.5 to 1.0 micron.

Terminal spiral filament present.

Weakly refractive in living state by dark field illumination. Miz appear as a series of bright dots or string of radiant beads with poor dark field illumination.

Staining: Stain with difficulty except with Giemsa's stain by which they appear pink or rose. Appear black with silver impregnation methods.
Motility: Sluggish, drifting motion, stiffly flexible, rarely rotating.

Trypsin digestion: Resistant for many days

Bile salts (10 per (ent): Disintegration complete.

Saponin (10 per cent): Broken up in time.

Cultivation: With difficulty under strict anaerobiosis in ascitic fluid with addition of fresh rabbit kidney.

Habitat: The cause of syphilis in man. Can be transmitted experimentally to anthropoid apes and rabbits.

2. Treponema pertenue Castellani. (Castellani, Jour. Trop. Med., 8, 1905, 253; Spirochaeta pertenuis Castellani, Jour. Ceylon Branch Brit. Med. Assoc., June, 1905 ; Spirochaeta pallidula Castellani, Brit. Jour. Med., 2, Nov., 1905, 1330; Spirochaete pertenuis Lehmann and Neumann, Bakt. Diag., 5 Aufl., 2, 1912, 677; Spironema pertenue Gross, Archiv f. Protistenk., 24, 1912,115; Treponema pallidulum Brumpt, Nouveau Traité de Médecine, Paris, 4, 1922, 508.) From Latin, very fine. 
Morphologically indistinguishable from Treponema pallidum.

Cultivable under anaerobic conditions in the same medium used for Treponema pallidum.

Habitat: The cause of yaws-tropica frambesia. Patients with the disease give a positive Wassermann test. Prboably transmitted by contact.

3. Treponema microdentium Noguchi. (Jour. Exp. Med., 15, 1912, 81.) From Greek mikros, small and Latin, teeth.

The organism is less than 0.25 micron in thickness in the middle and tapers toward each extremity, which is pointed. The length varies with age but may reach 8 microns and show an average of 14 curves. Sometimes a long, thin flagella-like projection is observed at each extremity .

Growth occurs under anaerobic conditions in serum water medium containing fresh tissue. The serum is slightly coagulated and gives off a strong, fetid odor.

Habitat: Normal oral cavity.

\section{Treponema mucosum Noguchi} (Jour. Exp. Med., 16, 1912, 194; Spirochacta mucosa Pettit, Contribution i l'Êtude des Spirochétidés, Vanves, II, 1928, 190.) From Latin, mucous.

Spirals : 0.25 to 0.3 by 8 to 12 microns. The number of curves varies from 6 to 8 . Both extremities are sharply pointed and of ten possess a minute curved projection, $S$ to 10 microns long.

Cultivable under anaerobic conditions, forming mucin.

The cultures give off a strong, putric! odor.

Takes the red in Giemsa's stain.

Strict anaerobe

Source: From pus in a case of pyorrhoea.

Habitat: Found in pyorrhea alveolaris. It possesses pyogenic properties.

5. Treponema calligyrum Noguchi (Noguchi, Jour. Exp. Med., 17, 1913, 96; Spirochaeta calligyra Zuelzer, 1925, in Prowazek, Hand. d. path. Protoz., 3, 1931,
1673.) From M. L., with beautiful circles.

Morphology : 0.35 to 0.4 by 6 to $14 \mathrm{mi}$ crons, average 9 to 12 microns. Spirals are regular and deep but more rounded than those of Treponema pallidum. The organism is of uniform width until near the extremities which end in sharp points with delicate projections.

Motility: Active, chiefly rotating.

Stains reddish-violet with Giemsa's stain.

Cultivation: Grows under anaerobic conditions.

Not pathogenic for monkeys or rabbits.

Source: From smegma.

Habitat : Lesions and membranes of the pudenda.

\section{Treponema genitalis Noguchi}

(Treponema minutum Noguchi, Jour. Exp. Med., 27, 1918, 671 ; not Treponema minutum Dobell, Arch. f. Protistenk., 26, 1912, 151; not Treponema minutum Castellani, 1916; Noguchi, Laboratory Diagnosis of Syphilis, New York, 1923, 260; Spirochaeta minutum Zuelzer, 1925, in Prowazek, Handb. d. path. Protoz., 3, 1931, 1673; Spirochaeta genitalis Séguin and Vinzent, Ann. Inst. Past., 61, 1938, 255.) From Latin, genital.

Morphology: 0.25 to 0.3 by 3 to $14 \mathrm{mi}$ crons. Spirals round, regular and shallow. Smaller than Treponema pallidum and spirals are closer together.

Motility: Active.

Culture: Grows anaerobically and requires fresh tissue.

Non-pathogenic.

Habitat: Found on male and female genitalia.

7. Treponema carateum Brumpt. (Treponema de un caso de pinta, Saenz, Grau Triana and Alfonso, Arch. de Med. Int., Havana, 4, 1938, 3 ; Brumpt, Compt. Rend. Soc. Biol., Paris, 130, 1939, 942; Treponema herrejoni León y Blanco, Rev. de Med. Trop. y Parasitol., Habana, 6, 1940, 5; Treponema pictor Pardo-Cas. tello, Rev. de Med. Trop. y Parasitol 
Habana, 6, 1940, 117; Treponema pintae Curbelo, Elementos de Bacteriología Médica, 1941, 34.) From carate, spotted sickness.

Deseription taken from León y Blaneo (loc. cit.).

Cylindrical: 0.25 to 0.30 by 7.8 to 36.8 microns, average length 17.8 microns. With sharp-pointed ends.

Spiral amplitude : 1 mieron, regular.

Spiral depth: 0.8 to 1.0 micron.

Number of waves, 6 to 27 , according to length. Ten to twelve (Brumpt, lor. cit.).

Actively motile. At times undulating or creeping movements are shown.

Staining reactions: Readily takes silver impregnations, Giemsa's stain, carbolfuchsin and gentian violet.

Saponin (10 per cent) : Disintegrates in six hours at room temperature. Same result with sodium taurocholate (10 per (ent) and with bile.

Distilled water: Produces swelling.

Loses motility on heating for 15 minutes at $50^{\circ} \mathrm{C}$ or for 3 hours at $41^{\circ} \mathrm{C}$.

Wassermann, Kahn and Meinicke rear. tions positive.

Has not yet been cultivated artificially. Experimental transmission unsuecessful so far.

Source: From the border of cutaneous lesions of persons having pinta (spotted sickness).

Habitat: The cause of pinta (or carate). Common in Mexico and Colombia. Also found in other northern countries of South America, in Central America and the West Indies. Rare in Cuba. Possibly found in other tropical regions of the world.

\section{Treponema cuniculi Joguchi.} Spirochaetr paraluis cuniculi Jakobsthal, Dermatol. Wehnschr., 71, 1920, 569; Noguchi, Jour. Amer. Med. Assoc., $7 \gamma$, 1921, 2052; also see Noguchi, Jour. Exp. Med., 35, 1922, 395; Treponema pallidum var. cuniculi Klarenbeek, Cent. f. Bakt.. I Abt., Orig.. 87, 1921, 203; Spirochaeta cuniculi Seraditi, Marie and Isaien,
Compt. rend. Soc. Biol., Paris, 85, 1921 , 51; Spirochaeta pallida var. cuniculi Zuelzer, 1925, in Prowazek, Handb. d. path. Protoz., 3, 1931, 1765; Spirochaeta paraluis Pettit, Contribution à l'Etude des Spirochétidés, Vanves, II, 1928, 91 ; Spirochacta paraluis-cuniculi Hindle, Med. Res. Council Syst of Bact., 8, 1931, 187.) From Latin, rabbit.

Deseription from Noguchi (loc. cit.).

Closely resembles Treponema pallidum, but longer.

Width 0.25 micron; length 10 to $16 \mathrm{mi}$ crons; long specimens up to 30 mirrons frequent.

Spirals 8 to 12 in number, regular, deep.

Spiral amplitude 1 to 1.2 microns.

Spiral depth 0.6 to 1.0 micron.

Delicate terminal filament at one, sometimes both, ends.

Often forms entangled masses of long threads; occurs sometimes in a stellate arrangement.

Staining properties same as for Treponema pallidum. Both readily stained by ordinary basic analine dyes when fixed in a buffered formaldehyde solution.

Wassermann reaction negative.

Pathogenesis: Disease transmissible to healthy rabbits, producing papular lesions in the genitoperineal region. Not pathogenif for monkeys, mice or guinea pigs.

Source: From lesions in the genitoperineal region of five rabbits.

Habitat: The cause of rabhit spirochetosis.

Appendix: Many of the species in this appendix are so inadequately deseribed that it is not rertain that they belong in this group.

Microspironema legeri Duboseq and Lebailly. (Duboseq and Lebailly, Compt. rend. Acad. Sci. Paris, 154, 1912, 662; Treponema legeri Zuelzer, 1925, in Prowazek, Handb. d. path. Protoz., 3, 1931, 1683.) From a fish, Box boops.

Spirochaeta microgyrata Loewenthal. (Loewenthal, Berl. klin. Worhnschr., 43, 1906, 283; Spironema microgyrata Nogu- 
chi, Jour. Exp. Med., 27, 1918, 584; Spiroschaudinnia microgyrata Castellani and Chalmers, Man. Trop. Med., 3rd ed., 1919, 454; Treponema microgyratum Brumpt, Nouveau Traité de Médecine, 4, 1922, 506.) From cancerous ulcers of man, dogs, and mice. Regarded by Hoffmann and Prowazek (Cent. f. Bakt., I Abt., Orig., 41, 1906, 819) as identical with Spirochaeta dentium.

Spirochaeta parotitidis Lehmann. (In Lehmann and Neumann, Bakt. Diag., 7 Aufl., 2, 1927, 580.) Pathogenie, producing a disease similar to mumps in experimental animals (cats) and causing parotitis and orchitis in apes.

Spirochaeta penortha Beveridge. (Austral. Jour. Expt. Biol. and Med. Sci., 14, $1936,307$.$) This organism is also called$ Treponema podovis according to Shahan, in Keeping Livestock Healthy, U. S. D. A. Yearbook of Agriculture, Part 6, 1942, 830.) Present as an accessory factor in foot-rot of sheep. Also see Actinomyces nodosus.

Spirochaeta phlebotomi Pringault. (Compt. rend. Soc. Biol., Paris, 84, 1921, 209.) From the sand fly, Phlebotomus perniciosus.

Spirochaeta pseudopallida Mulzer. (Mulzer, Berl. klin. Wchnschr., 42, 1905, 1144; Spiroschaudinnia pseudopallida Castellani and Chalmers, Man. Trop. Med., 3rd ed., 1919, 449; Treponema pseudopallidum Brumpt, Nouveau Traité de Médecine, Paris, 4, 1922, 511.) Found in ulcerating carcinomata.

Spirochaeta skoliodonta Hoffmann. (Hoffmann, Cent. f. Bakt., I Abt., Orig., 86, 1920, 137 ; Spirochacta acuta Kritchevsky and Séguin, 1920, according to Séguin and Vinzent, Ann. Inst. Past., 67, 1941, 62; Treponema skoliodontum Noguchi, in Jordan and Falk, Newer Knnowledge Bact. and Immun., 1928, 481.) From the peritoneal exudate of a guinea pig. One of the smallest known spirochetes. From the oral cavity.

Spirochaeta subtilis Castellani. (Castellani, 1907; Spiroschaudinnia subtilis Castellani and Chalmers, Man. Trop.
Med., 3rd ed., 1919, 450; Treponema subtile Brumpt, Nouveau Traité de Médecine, Paris, 4, 1922, 514; Spironema subtilis Pettit, Contribution à l'Êtude des Spirochétidés, Vanves, II, 1928.) From the oral mucosa and from intestinal contents. May be a synonym of Spirochaeta dentium.

Spirochaeta urethralis Castellani. (Castellani, 1915; Treponema urethrale Castellani and Chalmers, Man. Trop. Med., 3rd ed., 1919, 1944.) From a purulent urethral discharge.

Spirochaeta vaccinae Bonhoff. (Bonhoff, Berl. klin. Wochnschr., 42, 1905, 1142 ; Treponema vaccinae Brumpt, Nouveau Traité de Médecine, Paris, 4, 1922, 515.) From vaceinia.

Spirochacta vaginalis Macfie. (Macfie, Ann. Trop. Med. and Parasitol., 10, 1916, 315; Treponema vaginalis Brumpt, Nouveau 'Traité de Médecine, Paris, 4, 1922, 514.) From a case of vaginitis in a Gold Coast native.

Spirochaete hartmanni Gonder. (Gonder, Cent. f. Bakt., I Abt., Orig., 47, 1908, 491 ; Spironema hartmanni Gross, Cent. f. Bakt., I Abt., Orig., 65, 1912, 88; Spirochaeta hartmanni Noguchi, Jour. Exp. Med., 34, 1921, 297; Treponema hartmanni Ford, Textb. of Bact., 1927, 990.) From the digestive tract of molluses, Pinna spp.

Spirochaete polyspira Wolff. (Wolff, Cent. f. Bakt., II Abt., 18, 1907, 448; Treponema polyspirum Wolff, ibid.; Spirochaeta polyspira Pettit, Contribution à l'Étude des Spirochétidés, Vanves, II, 1928, 14.) From rotten potatoes.

Treponema carpanoi Yakimoff and Rastjapin. (Arch. f. Protistenk., 71, 1930, 543.) From stomatitis of horses.

Treponema cotti Duboseq and Lebailly. (Duboseq and Lebailly, Arch. Zool. Expér. et Gén., 10, 1912, 331; Microspironema cotti Duboseq and Lebailly, Compt. rend. Acad. Sci., Paris, 154, 1912, 662.) From the marine bullhead, Cottus bubalis. Treponema dentium (Miller) Dobell. (Spirochaete im Zahnschleim, Cohn, Beitr. z. Biol. d. Pflanzen, 1, Heft 2, 1872, 
180; Spirochaete denticola Flügge, Die Mikroorganismen, 2 Aufl., 1886, 390; Spirochaete dentium Miller, Microorganisms of the Human Mouth, Philadelphia, 1890, 80; Spirillum dentium Sternberg, Manual of Bact., 1893, 694; Spirochaeta dentium Migula, in Engler and Prantl, Die natürl. Pflanzenfam., 1, 1a, 1895, 35; Spirochaeta denticola Arndt, according to Hoffmann and Prowazek, Cent. f. Bakt., I Abt., Orig., 41, 1906, 819; Dobell, Arch. f. Protistenk., 26, 1912, 117; Spironema dentium Gross, Cent. f. Balit., I Abt., Orig., 65, 1912, 88; Spirochaeta dentinum McFarland, Pathogenic Bacteria and Protozoa, 7th ed., 1912, 546; Treponema microdentium Noguchi, Jour. Exp. Med., 15, 1912, 81; Spirochaeta orthodonta Hoffmann, Deutsch. med. Wochnschr., 46, 1920, 257; Spirochaeta microdentium Heim, Lehr. d. Bakt., 6 and 7 Aufl., 1922, 477; Treponema denticola Brumpt, Nouveau Traité de Médecine, Paris, 4, 1922, 497; Treponema orthodontum Noguchi, in Jordan and Falk, Newer Knowledge Bact. and Immun., 1928, 481; Treponema dentium-stenogyratum Pettit, Contribution à l'Étude des Spirochétidés, Vanves, II, 1928, 240.) The smallest of the mouth spirochaetes. Non-pathogenic. This term probably includes several morphologically similar species which have not as yet been sufficiently characterized.

Treponema drosophilae Chatton. (Compt. rend. Soc. Biol., 73, 1912, 212.) From Drosophila confusa. Six to thirty microns in length, tapers at both ends, four spirals, movement helicodal.

Treponema gallicolum Lebailly. (Compt. rend. Soc. Biol., Paris, 75, 1913, 389.) From the caecum of the hen, Gallus $s p$.

Treponema hilli Duboseq and Grassé. (Compt. rend. Soc. Biol., Paris, 94, 1926, 34; Arch. Zool. Expér. et Gén., 66, 1927, 484.) From the surface of the body of a flagellate, Devescovina hilli, and in the intestine of a termite, Glyptotermes iridipennis. A very small organism.

Treponema intermedium Dobell. (Mittelformen, Lühe, Handb. d. Tropen- krankh., 3, 1906; see Hoffmann and Prowazek, Cent. f. Bakt., I Abt., Orig., 41, 1906, 819; Dobell, Arch. f. Protistenk., 26, 1912, 117; Treponema macrodentium Noguchi, Jour. Exp. Med., 15, 1912, 81; Spirochaeta media oris Hoffmann, Deutsch. med. Wochnschr., 46, 1920, 257; Treponema medium. Brumpt, Nouveau Traité de Médecine, Paris, 4, 1922, 505; Spirochacta intermedia Pettit, Contribution à l'Êtude des Spirochétidés, Vanves, II, 1928, 146; Spirochaeta macrodentium Pettit, ibid., 182; Spirochaeta media and Spironema media Pettit, ibid., 240.) The middle-sized spirochete of the mouth.

Treponema lari Lebailly. (Compt. rend. Soc. Biol., Paris, $75,1913,389$. Found in the caecum of birds, also in the guinea-pig. Named for one of the birds, Larus ridibundus.

Treponema minutum Dobell. (Treponema sp. Dobell, Quart. Jour. Microse. Sci., 52, 1908, 121; Dobell, Arch. f. Protistenk., 26, 1912, 151; not Treponema minutum Castellani, 1916; not Treponema minutum Noguchi, Jour. Exp. Med., 27, 1918, 671; Spirochaeta minutum Zuelzer, 1925, in Prowazek, Handb. d. path. Protoz., 3, 1931, 1682.) From the large intestines of toads, Bufo vulgaris.

Treponema parvun Dobell. (Dobell, Arch. f. Protistenk., 26, 1912, 117; Spirochaeta parvum Zuelzer, 1925, in Prowazek, Handb. d. path. Protoz., 3, 1931, 1685.) From the intestine of the cockroach, Stylopyga (Blatta, Periplaneta) orientalis. Very small organism.

Treponema pavonis Duboseq and Lebailly. (Arch. Zool. Expér. et Gén., 10, 1912, 331.) From the intestine of the blenny, Blennius pavo.

Treponema perexile Duboseq and Lebailly. (Duboseq and Lebailly, Arch. Zool. Expér. et Gén., 10, 1912, 331 ; Spirochaeta perexilis Hindle, Med. Res. Council Syst. of Bact., 8, 1931, 180.) From the blood of a marine fish, Lepadogaster bimaculatus.

Treponema podovis Ludovic and Blaizot. (Compt. rend. Acad. Sci. Paris, 18\%, 
1928, 911.) Pathogenic. Cause of a disease in sheep.

Treponema querquedulae Lebailly. (Compt. rend. Soc. Biol., Paris, 75, 1913, 389.) From caeca of birds. Named for the teal, Querquedula querquedula.

Treponema rhinopharyngeum Brumpt. (Treponema minutum Castellani, 1916; Spiroschaudinnia minuta Castellani and Chalmers, Man. Trop. Med., 3rd ed., 1919, 1881; Brumpt, Nouveau Traité de Médecine, Paris, 4, 1922, 514.) From man in cases of rhinopharyngitis. Probably a synonym of Spirochaeta gracilis.

Treponema rigidum Zinsser and Hopkins. (Jour. Bact., 1, 1916, 489.) From the tissues in five different strains of rabbit syphilis. Probably a synonym of Treponema cuniculi.

Treponema spermiformis Duboseq and Grassé. (Arch. Zool. Expér et Gén., 66, 1927,453 .) From the rectum of a termite, Glyptotermes iridipennis.

Treponema squatarolae Lebailly. (Compt. rend. Soc. Biol., Paris, 75, 1913. 389.) From the raecum of a bird, squatarola squatarola.
Treponema stylopygae Dobell. (Dobell, Arch. f. Protistenk., 26, 1912, 117; Spirochaeta stylopygae Zuelzer, 1925, in Prowazek, Handb. d. path. Protoz., 3, 1931, 1685.) From the intestines of the cockroach, Stylopyga orientalis.

Treponema tricalle Cohn. (Cohn, 1872, quoted from Castellani and Chalmers, Man. Trop. Med., 2nd ed., 1913, 414.)

Treponema triglae Duboseq and Lebailly. (Arch. Zool. Expér et Gén., 10, 19[2,331.) From the rectum of a fish, Trigla lucerna.

Treponema tropiduri Neiva, Marques da Cunha and Travassus. (Mem. do Inst. Oswaldo (ruz, 6, 1914, 180.) From the blood of a South American lizard, Tropidurus torquatus.

The following species are listed in the index of Castellani and Chalmers, Manual of Tropical Medicine, 2nd ed., 1913, 17181719 , but are not mentioned in the text (pp. 136-141): Treponema bovidae, $T$. camelidae, $T$. canidae, T. felidae, T. hippopotami, $T$. reptilia, $T$. rhinoceri, $T$. selachii, T. suidae, T. ungulata and $T$. ursidae.

Genus III. Leptospira Noguchi

(Jour. Exp. Med., 25, 1917, 753.

Finely coiled organisms 6 to 20 microns in length. Spirals 0.3 micron in depth and 0.4 to 0.5 micron in amplitude. In liquid medium one or both ends are bent into a semicircular hook each involving $\frac{1}{10}$ to $\frac{1}{8}$ of the organism. Spinning movements in liquid and vermiform in semisolid agar, forward or backward. Seen in living preparations only with dark field. Stain with difficulty except with Giemsa's stain and silver impregnation. Require oxygen for growth.

The type species is Leptospira icterohaemorrhagiae (Inada and Ido) Noguchi.

\section{Leptospira icterohaemorrhagiae}

Inada and Ido) Niguchi. (Spirochueta icterohaemorrhagiae Inada and Ido, Tokyo Ijishinski, 1915; Inada, Ido, Hoki, Kaneko and Ito, Jour. Exp. Med., 23, 1916, 377 ; Spirochaeta icterogenes Uhlenhuth and Fromme, Med. Klin., 11, 1915, 1202 ; Spirochaeta nodosa Huebner and Reiter, Deutseh. med. Wochnschr., 41, 1915, 1275 ; Noguchi, Jour. Exp. Med., 25, 1917,
755; Spiroschaudinniu icterohaemorrhagiae Castellani and Chalmers, Man. Trop. Med., 3rd ed., 1919, 447; Treponema icterogenes Gonder and Gross, Arch. f. Protistenk., 39, 1919, 62; Spirochaete ictero-haemorrhagica (sic) Lehmann and Neumann, Bakt. Diag., 6 Aufl., 2, 1920, 810; Treponema ictero-hemorragiae (sic) Brumpt, Nouveau Traité de Médecine, Paris, 4, 1922, 501; Treponema nodosum 
Brumpt, ibid., 508; Leptospira icterogenes Ford, Textb. of Bact., 1927, 994; Leptospira nodosa Ford, ibid., 993.) From Greek icterus, jaundice and hemorrhagiae, bleeding.

Morphology : 0.25 to 0.3 by 6 to $9 \mathrm{mi}$ crons and oceasionally 20 to 25 microns.

Spiral amplitude: 0.4 to 0.5 micron, regular, rigid.

Spiral depth: 0.3 micron, regular.

Waves: One or more gentle waves throughout entire length. When in liquid media, one or both ends may be semicircularly hooked, while in semisolid media the organism appears serpentine, waved or bent. Very active flexibility.

Terminal filament and flagella absent.

Body stains reddish by Giemsa's stain.

Bile salts (10 per cent): Easily dissolved.

Saponin (10 per cent): (ompletely resistant.

Cultured easily in medium containing 10 per cent rabbit serum, 0.2 per cent agar, slight amount of hemoglobin in salt or Ringer's solution. Does not grow in surface colonies.

Temperature range: $25^{\circ}$ to $37^{\circ} \mathrm{C}$. Remains alive longer at $25^{\circ} \mathrm{C}$.

Pathogenir for guinea pigs and deermice.

Habitat: The cause of infectious jaundice in man (Weil's disease). Found in the kidneys, urine and blood of wild rats No insect vector known. Found freeliving in water and slime (in mines).

2. Leptospira hebdomadis (Ido et al.) Noguchi. (Spirochaeta nanukayami Ido, Hoki, Ito and Wani, Nippon Gakkai Zasshi, 5. 1917, No. 5; Spirochaeta hebdomadis Ido, Ito and Wani, Jour. Exp. Med., 28, 191s, 435̃; Spiroschaudinnia hebdomadis Castellani and Chalmers, Man. Trop. Med., 3rd ed., 1919, 448; Noguchi, Jour. Exp. Med., 30, 1919, 17; Treponema hebdomadis Brumpt, Jouveau Traité de Médecine, Paris, 4, 1922, 501.) From Latin, seven days.

Morphologically indistinguishable from
Leptospira icteroheamorrhagiae but can be distinguished serologically.

In man causes less jaundice than Leptospira icterohaemorrhagiae and is never fatal.

Identical with Type B, Leptospira autumnalis.

Slightly pathogenic for young guinea pigs.

Is carried by the field vole (Microtus montibelli).

Habitat: Cause of seven-day fever or gikiyami in Japan.

3. Leptospira biflexa (Wolbach and Binger) Noguchi. (Spirochaela biflexa Wolbach and Binger, Jour. Med. Res., 30, 1914, 23; Noguchi, Jour. Exp. Med., 27, 1918. 585; Spirochaeta pseudo-icterogenes (aquatilis) Thlenhuth and Zuelzer, Cent. f. Bakt., I Aht., Orig., 85. 1921,*141; Spirochaeta pseudoicterogenes $\mathrm{C} h \mathrm{hlenhuth}$ and Zuelzer, Klin. Wochnschr., 1, 1922, 2124; Spirochaeta psendo-icterohemorrhagiae Vinzent, Compt. rend. Soc. Biol., Paris, 95. 1926, 1472; Leptospira pseudoicterogenes Noguchi, in Jordan and Falk, Newer Knowledge Bart and Immun., 1928, 461.) From Latin, doubly bent.

Size : 0.2 to 0.25 by 5 to 7 microns with tapering ends. Spiral amplitude 0.2 to 0.25 micron. Will pass through an L5 candle filter.

Waves : 22 to 30 in number.

Stains: Best results with Giemsa's stain.

Culture: Can grow in distilled water plus 0.1 per cent potassium nitrate. Rabbit serum in distilled water is best medium.

Optimum temperature $20^{\circ} \mathrm{C}$.

Antigenically distinct from Leptospira icterohaemorrhagiae.

Not pathogenic.

Source: From tap water, ponds and pools in Berlin.

Habitat: Fresh water.

4. Leptospira canicola Okell et al. (Okell, Dalling and Pugh, Vet. Jour., 81, 1925, 3.) From Latin, dog-dweller. 
Morphologically indistinguishable from Leptospira icterohaemorrhagiae.

Cultivation: Same as Leptospira icterohaemorrhagiae.

Immunology : Some cross-reaction with Leptospira icterohaemorrhagiae, but specific in higher dilutions of immune serum.

Source: From blood of dogs.

Habitat: A natural parasite of dogs. Causes a chronic disease of old dogs characterized by uremia, not jaundice. Fatal in 80 per cent of those infected. No intermediate host known. Probably transmitted by direct contact; possibly by healthy carriers.

Appendix: The species listed below are inadequately described and may be identical with those described in full.

Leptospira aqueductum (sic) Ford. (Spirochaeta pseudoicterogenes aquaeductuum Uhlenhuth and Zuelzer, Cent. f. Bakt., I Abt., Orig., 85, 1921, ${ }^{*} 150$; Ford, Textb. of Bact., 1927, 998.) From fresh water of aqueducts. Probably a synonym of Leptospira biflexa.

Leptospira asthenoalgiae Carbo-Noboa. (Bull. Inst. Past., 22, 1924, 898.) From blood, urine and organs of persons having dengue.

Leptospira autumnalis Topley and Wilson. (Akiyami Type A, Koshina, Shiwozawa and Kitayama, Japan. Med. Wld., 4, 1924, 268; see also Jour. Exp. Med., 42, 1925, 873; Topley and Wilson, Princip. Bact. and Immun., 1st ed., 2, 1931, 1202; Spirochaeta autumnalis $A$, quoted from Hindle, Med. Res. Council Syst. of Bact., 8, 1931, 312 ; Spirochaeta autumnalis Hindle, ibid.) The cause of akiyami or harvest sickness in Japan. May be identical with Leptospira icterohaemorrhagiae.

Leptospira bataviae. (1925, quoted from Gispen and Schüffner, Cent. f. Bakt., I Abt., Orig., 144, 1939, 427.) From a case of fever in the Dutch East Indies. Probably a synonym of Leptospira hebdomadis.

Leptospira biliohemoglobinuriae (Blanchard and Lefrou) Noguchi. (Spirochaeta bilio-hemoglobinuriae Blanchard and Lefrou, Compt. rend. Acad. Sci., Paris, 175, 1922, 602; Noguchi, in Jordan and Falk, Newer Knowledge Bact. and Immun., 1928, 490.) From cases of blackwater fever.

Leptospira bonariensis Savino and Rennella. (Rev. Inst. Bact. "Dr. Carlos G. Malbram”, 12, 1944, 182.) From gray rats.

Leptospira bovis Noguchi. (New York State Med. Jour., 22, 1922, 426.) From the gastric mucosa of the $\mathrm{ox}$.

Leptospira couvyi Gomes de Faria. (Compt. rend. Soc. Biol., Paris, 90, 1924, 55 ; Spirochaeta couvyi Hindle, Med. Res. Council Syst. of Bact., 8, 1931, 317.) From the blood of persons having dengue.

Leptospira dentale Perrín. (Rev. Mex. de Biol., 2, 1922, 171.) Found in the pus of bucco-maxillary gangrene.

Leptospira grippo-typhosa Topley and Wilson. (Topley and Wilson, Princip. Bact. and Immun., 2nd ed., 1936, 728; Spirochaeta dmitrovi Rimpau, Schlossberger and Kathe, Cent. f. Bakt., I Abt., Orig., 141, 1938, 320.) The cause of swamp fever in Europe. Probably synonymous with Leptospira hebdomadis. Also see Baschenin, Cent. f. Bakt., I Abt., Orig., 113, 1929, 438 and 450; Dinger and Verschaffelt, Ann. Inst. Past., 45, 1930, 396.

Leptospira haemoglobinuriae Schüffner. (Geneesk. Tijdschr. Ned. Indië, 58, 1918, 352 ; Spirochaeta haemoglobinuriae Hindle, Med. Res. Council Syst. of Bact., 8, 1931, 314.) From the blood of a Javanese patient suffering from an attack of blackwater fever.

Leptospira icterohemoglobinuriae Schüffner. (Schüffner, Geneesk. Tijdschr. v. Ned. Indië, 58, 1918, 352, according to Pettit, Contribution à l'Étude des Spirochétidés, Vanves, II, 1928; Spirochaeta icterohemoglobinuriae Schüffner, Mededeel. Burgerl. Geneesk. Dienst in Nederl. Indië, 58, 1918, 7 (according to Blanchard and Lefrou, Compt. rend. Acad. Sci., Paris, 175, 1922, 602); Treponema icterohemoglobinuriae Brumpt, 
Nouveau Traité de Médecine, Paris, 4, 1922, 501.) From the blood in a case of blackwater fever.

Leptospira interrogans (Stimson) Noguchi. Spirochaeta interrogans Stimson, U. S. Public Health Rept., Part I, 22, 1907, 541; Leptospira icteroides Noguchi, Jour. Exp. Med., 29, 1919, 581; Treponema interrogans Brumpt, Nouveau Traité de Médecine, Paris, 4, 1922, 505; Treponema icteroides Brumpt, ibid.; Spirochaeta icteroides Lehmann and Neumann, Bakt. Diag., 7 Aufl., 2, 1927, 576; Noguchi, in Jordan and Falk, Newer Knowledge Bact. and Immun., 1928, 454.) Noguchi (1928) regards this species as identical with Leptospira icterohaemorrhagiae.

Leptospira pettiti (Fiessinger) Hindle. (Spirochaete pettiti Fiessinger, Ann. de Méd., 5, 1918, 156; Treponema pettiti Brumpt, Nouveau Traité de Médecine, Paris, 4, 1922, 510; Hindle, Med. Res. Council Syst of Bact., 8, 1931, 316 ; Spirochaeta pettiti Hindle, idem; not Spirochaeta pettiti Row, Jour. Trop. Med. and Hyg., 1922, 36t.) From urine. Morphologically indistinguishable from Leptospira icterohaemorrhagiae.

Leptospira pyrogenes Vervoort. (Vervoort, Geneesk. Tijdschr. v. Ned. Indië, 63, 1923, 800; Spirochaeta febrilis Vervoort, Rep. Far East. Assoc. Trop. Med., London, 1923, 683; Spirochaeta pyrogenes Hindle, Med. Res. Council Syst of Bact., 8, 1931, 314.) From the blood of persons suffering from dengue-like fevers in Sumatra. Pathogenic.

Leptospira saxkoebing Petersen. (Acta Path. et Microbiol. Scand., 21, 1944, 165.) A new serological type.

Leptospira salina Ford. Spirochaeta pseudoicterogenes salina Uhlenhuth and
Zuelzer, Cent. f. Bakt., I Abt., Orig., $85,1921,{ }^{*} 150$; Ford, Textb. of Bact., 1927, 998.) From salt water.

Leptospira trimerodonta (Hoffmann) Noguchi. (Spirochaeta trimerodonta Hoffmann, Deutsch. med. Wochnschr., 46, 1920, 257; Leptospira dentium Hoffmann, ibid., 625; Leptospira buccalis Fontana, according to Pettit, Contribution à l'Étude des Spirochétidés, Vanves, II, 1928, 232; Noguchi, in Jordan and Falk, Newer Knowledge Bact. and Immun., 1928, 461.) From the oral cavity. May be synonymous with Leptospira icterohaemorrhagiae.

Spirochaeta anthropopitheci Wilbert and Delorme. (Ann. Inst. Past., 41, 1927, 1147.) Pathogenic for chimpanzees in French Guinea. Probably identical with Leptospira icterohaemorrhagiae.

Spirochaeta elusa Wolbach and Binger. (Wolbach and Binger, Jour. Med. Res., 30, 1914, 9; Treponema elusum Bergey et al., Manual, 1st ed., 1923, 428.) From pond water. Not pathogenic. For a description of this species, see Bergey et al., Manual, 5th ed., 1939, 957.

Spirochaeta ictero-uraemia canis Klarenbeek. (Tijdschr. Diergeneesk., 55, 1928, 227.) From the kidneys of dogs. Pathogenic for guinea pigs. May be synonymous with Leptospira icterohaemorrhagiae or $L$. canicola.

Spirochaeta pseudohebdomadis Zuelzer. (1925, in Prowazek, Handb. d. path. Protoz., 3, 1931, 1671.) Probably identical with Leptospira hebdomadis.

\section{Spirochaeta trimeres Hoffmann.}

(Deutsch. med. Wochnschr., 46, 1920, 257.) From the oral cavity. May be synonymous with Leptospira trimerodonta. 

SUPPLEMENT NO. 1.

\section{ORDER RICKETTSIALES}

April, 1947. 



\section{ORDER RICKETTSIALES GIESZCZYKIEWICZ.}

(Bull. Intern. Acad. Polon. Sci., Classe Math. Nat., B (1), 1939, 9-30.)

Small, rod-shaped, coccoid, spherical and irregularly-shaped microorganisms which stain lightly with aniline dyes. Gram-negative. Usually not filterable. Cultivated outside the body, if at all, only in living tissue, embryonated eggs or rarely in media containing body fluids. Parasitic organisms intimately associated with tissue cells and erythrocytes, chiefly in vertebrates and of ten in arthropods which act as vectors. The intracellular parasites of Protozoa may also belong here. May cause diseases in man or animals, or both.

\section{Key to the families of order Rickettsiales.}

I. Intracellular parasites, or parasites intimately associated with tissue cells. Do not occur in erythrocytes. Frequently cause diseases of vertebrates transmitted by arthropod vectors.

Family I. Rickettsiaceae, p. 1083.

II. Facultative intracellular or extracellular parasites found characteristically in or on the ery throcy tes of vertebrates. May be transmitted by arthropod vectors.

Family II. Bartonellaceae, p. 1100.

III. Intracellular parasites found in vertebrate tissues and not transmitted by arthropod vectors.

Family III. Chlamydozoaceae,p.1114.

\section{*FAMILY I. RICKETTSIACEAE PINKERTON.}

(Pinkerton, Parasitology, 28, 1936, 186; Rickettsiales Buchanan and Buchanan, Bacteriology, 4th ed., New York, 1938, 49.)

Small, of ten pleomorphic, rod-shaped, ovoid, coccoid and coccus-shaped bacteriumlike organisms, intimately associated with arthropod tissues, usually in an intracellular position. Stain lightly with aniline dyes. Gram-negative. Have not been cultivated to date in cell-free media. May be parasitic to man and other animals causing diseases (typhus and related ills) that are transmitted by arthropod vectors (lice, fleas, ticks, mites and probably other ectoparasites).

\section{Key to the genera of family Rickettsiaceae. $\dagger$}

I. Cells rod-shaped, ellipsoidal and coccoid.

A. Non-filterable.

B. Filterable.

Genus I. Rickettsia, p. 1084 .

Genus II. Coxiella, p. 1092.

II. Cells spherical, occasionally elongated.

Genus III. Cowdria, p. 1094.

* Prepared by Dr. Ida A. Bengtson (retired), National Institute of Health, Bethesda, Maryland, November, 1946. Through the courtesy of Dr. Edward A. Steinhaus much use was made of material from his book, Insect Microbiology, Ithaca, $1946,763 \mathrm{pp}$. before it was generally available.

† Includes only those rickettsiae which have been rather completely studied. For additional rickettsiae, see appendix. 


\section{Genus I. Rickettsia da Rocha-Lima.}

(Berl. klin. Wehnschr., 53, 1916, 566-569.) Named for Howard Taylor Ricketts who lost his life studying typhus fever.

Small, often pleomorphic, rod-shaped to coccoid organisms occurring intracytoplasmically in lice, fleas, ticks and mites, or sometimes intranuclearly. Stain lightly with aniline dyes. Gram-negative. Non-filterable. Have not been cultivated in cell-free media. Parasites of man and animals which are the etiological agents of epidemic typhus, murine or endemic typhus, Rocky Mountain spotted fever, tsutsugamushi disease, rickettsialpox and other diseases.

For reasons that are diseussed elsewhere (Bengtson, Jour. Bact., 53, 1947, 325) the genus Dermocentroxenus has been united with the genus Rickettsia.

The type species is Rickettsia prowazekii da Rocha-Lima.*

Key to the species of genus Rickettsia.

\section{Louse-borne.}

II. Flea-borne.

III. Tick-borne.

IV. Mite-borne.

\section{Rickettsia prowazekii.}

\section{Rickettsia typhi.}

3. Rickettsia rickettsii.

4. Richeltsia conorii.

\section{Richettsia tsutsugamushi.}

6. Rickettsia akari.

1. Rickettsia prowazekii da RochaLima. (da Rocha-Lima, Berl. klin. Wchnschr., 53, 1916, 567; Riclettsia exanthematotyphi Kodama, Kitasato Arch. Exper. Med., 9, 1932, 360; Richett sia prowazeki var. prowazeki Pinkerton, Parasitology, 28, 1936, 186; Richettsia prowazeki sub-species prowazeki Philip, Amer.Jour. Hyg., 37, 1943, 307.) Named for S. von Prowazek who lost his life studying typhus fever.

Minute coccoid, ellipsoidal and ovoid forms to short rods, sometimes long rods and occasionally filamentous forms, often in pairs and occasionally in chains. In infected lice the minute coccoid and paired coccoid forms predominate over the short and long rods and the filamentous forms which are up to 40 microns in length. Single elements 0.25 by 0.4 to 0.3 by 0.45 micron. Pairs range from 0.25 by 0.7 to 0.3 by 1.1 microns. In yolk sacs the organisms vary in size from minute coccoid forms in heavily infected tissue to rod forms resembling small bacteria in lightly infected tissue. Within the same smear of infected mammalian cells and in chick embryo tissue the organisms are quite uniform in size and morphology. Occur intracytoplasmically in vascular endothelial cells and in serosal cells. Non-motile.

* The Editors of the MANual follow Recommendation XL of the International Botanical Code (see p. 59) in regard to the endings used for specific names. This calls for the use of the $i i$ ending for epithets taken from the name of a man ending in a consonant (except names ending in er). Some students of the Rickettsiaceae follow the International Rules of Zoological Xomenclature which use $i i$ only in case the name used was employed and declined in Latin. Zoologists use the single $i$ for modern patronymics based on all other names of men. 
The organisms are colored purplish with the Giemsa stain, the two individuals of a pair being connected by a zone of faintly blue stained material. They are colored blue with Castañeda stain (Jour. Inf. Dis., 47, 1930, 416) and bright red against a blue background with Machiavello stain (Rev. Chilena de Hig. y Med. Prev., 1, 1937, 101). Gramnegative.

Cultivation: In plasma tissue cultures of mammalian cells, in the louse intestine, in modified Maitland media with and without agar, on chorio-allantoic membrane and yolk sac of chick embryo, the latter being currently the medium of choice.

Optimum temperature $32^{\circ} \mathrm{C}$ in plasma tissue culture, $35^{\circ} \mathrm{C}$ in chick embryo cells.

Immunology : Immunity prolonged but may not be complete in man. Indistinguishable from endemic (murine) typhus in cross immunity tests in guinea pigs, but distinguishable from Rocky Mountain spotted fever and other rickett sial diseases in such tests. Neutralizing antibodies are found in the serum of recovered guinea pigs and convalescent humans up to 2 to 3 weeks after defervescence. Iilled vaccines produced from infected lice and from infected yolk sacs afford a high degree of protection against the disease. Hyperimmune antisera for therapeutic use have been produced in rabbits by injection with infected yolk sac suspensions and in horses and donkeys with infected mouse lung suspensions.

Serology: Strains from various parts of the world are closely related as determined by complement fixation; are distinguishable from other rickettsiae by agglutination, complement fixation and precipitin tests; have a common antigenic factor (alkali stable polysaccharide) with Proteus OX19; and have a soluble antigen in yolk culture.

Lethal effect: Heavily infected yolk sac cultures injected intravenously or intraperitoneally are fatal to white mice in a few hours.

Resistance to chemical and physical agents: Readily inactivated by heat and chemical agents. A temperature of $50^{\circ} \mathrm{C}$ kills the organism in 15 to 30 minutes, and 0.5 per cent phenol and 0.1 per cent formalin kill the organism.

Pathogenicity: Pathogenic for man, apes, monkeys, guinea pigs, cotton rats, gerbilles, the louse (Pediculus humanus). Inapparent infections occur in white mice, white rats and rabbits. A characteristic febrile reaction with no mortality and without testicular swelling occurs in the guinea pig. Passage in guinea pigs is accomplished by transfer of blood or brain from infected animals. Causes a febrile disease with exanthema and high mortality in man.

Source: Seen in the blood of typhus patients and in smears of epithelial cells of the intestinal tract of lice fed on typhus patients.

Habitat: The body louse (Pediculus humanus var. corporis), head louse (Pediculus humanus var. capitis) and Pedicinus longiceps. The etiological agent of epidemic typhus (European typhus, classical typhus, typhus exanthematicus).

2. ${ }^{*}$ Rickettsia typhi (Wolbach and Todd) Philip. (Dermacentroxenus

* Some may regard the binomial Ricliettsia typhi as invalid because of its previous use by do Amaral and Monteiro for the organism causing eastern Rocky Mountain spotted fever. However, because the binomial Dermacentroxenus typhi Wolbach and Todd clearly has priority and because the binomial proposed by do Amaral and Monteiro has never come into general use, Rickettsia typhi Philip has been accepted for use in the MANYAL. If Philip's binomial had been rejected, then it would have been necessary to accept Rickettsia manchuriae Kodama et al. as this appears to have priority over the more generally used Rickettsia mooseri Monteiro.-Editors. 
typhi Wolbach and Todd (not Tood), Ann. Inst. Past., 34, 1920, 158; minute intracellular bodies, Mooser, Jour. Inf. Dis., 43, 1928, 261 ; Rickettsia manchuriae Kodama, Takahashi and Kono, Saikingaku-Zasshi (Jap.), No. 426, 427, Aug. and Sept., 1931; see Kodama, Kono and Takahashi bibliography, Kitasato Arch. Exper. Med., 9, 1932, 95; Rickettsia mooseri Monteiro, Mem. Inst. Butantan, 6, 1931, 97 (pub. July, 1932), see do Amaral and Monteiro, bibliography, ibid., 7, 1932, 367; Rickettsia exanthematofebri Kodama, Kitasato Arch. Exp. Med., 9, 1932, 360; Rickettsia muricola Monteiro and Fonseca, Brazil Med., 46, 1932, 1032; Rickettsia murina and Rickettsia fletcheri Megaw, Trans. Roy. Soc. Trop. Med. Hyg., 29, 1935, 105; Rickettsia prowazeki var. mooseri Pinkerton, Parasitology, 28, 1936, 185; Rickettsia prowazeki sub-species typhi Philip, Amer. Jour. Hyg., 37, 1943, 304; Rickettsia typhi Philip, idem; not Rickettsia typhi do Amaral and Monteiro, Rev. Sud. Amér. de Méd. et Chirug., 4, 1933, 806.) From M. L. typhus, typhus.

Resembles Rickettsia prowazekii in morphological and staining properties. Non-motile. Gram-negative.

Cultivation: May be cultivated in plasma tissue culture of mammalian cells, in modified Maitland media with and without agar, in fleas, in the peritoneal cavity of $\mathrm{X}$-rayed rats, in the lungs of white mice and in white rats following intranasal inoculation, in the lungs of rabbits following intratracheal inoculation, in the chorio-allantoic membrane and the yolk sac of the chick embryo.

Optimum temperature $35^{\circ} \mathrm{C}$ in chick embryo cells.

Immunology : Prolonged immunity in man and animals following infection. Complete cross immunity between epidemic and endemic typhus in guinea pigs recovered from infections with Rickettsia prowazekii and Rickettsia typhi. No cross immunity between en- demic typhus and Rocky Mountain spotted fever, Q fever or tsutsugamushi disease in guinea pigs.

Serology: Distinguishable from the rickettsiae of spotted fever, $\mathrm{Q}$ fever and tsutsugamushi disease by complement fixation, agglutination and precipitin tests, less readily from $R$. prowazekii by these tests. Has common antigenic factor with Proteus OX19, and soluble antigen in yolk-sac cultures.

Lethal effect: Heavily infected yolk sac cultures injected intravenously or intraperitoneally fatal to white mice in a few hours.

Pathogenicity: Pathogenic for man, apes, monkeys, rabbits, guinea pigs, white rats, eastern cotton rat, white mice, gerbilles. Other susceptible animals include the woodchuck, house mouse, meadow mouse, white-footed mouse, old-field mouse, cotton mouse, golden mouse, wild rat (Rattus norvegicus), wood rat, rice rat, flying squirrel, gray squirrel, fox squirrel, gophers, cotton-tail rabbit, swamp rabbit, chipmunk, skunk, opossum and cat. A characteristic febrile reaction occurs in the guinea pig with testicular swelling without ulceration, after intraperitoneal inoculation. Passage in guinea pigs is accomplished by transfer of testicular washings or blood from infected animals. Cause of a febrile disease with exanthema in man, with low mortality.

Source: Seen by Wolbach and Todd (loc. cit.) in the endothelial cells of the capillaries, arterioles and veins in sections of skin from cases of Mexican typhus (tabardillo). Also described by Mooser (loc. cit.) in sections and smears of the proliferated tunica vaginalis of guinea pigs reacting to the virus of Mexican typhus.

Habitat: Infected rat fleas (Xenopsylla cheopis, Xenopsylla astia), infected chicken fleas (Echidnophaga gallinacea) found on wild rats, and the rat louse (Polyplax spinulosus). Wild rats and field mice act as the reservoir of infection. The etiological agent of en- 
demic (murine) typhus which is transmitted to man by the rat flea.

\section{Rickettsia rickettsii (Wolbach)}

Brumpt. (Dermacentroxenus rickettsi Wolbach, Jour. Med. Res., 41, 1919-20, 87; Rickettsia rickettsi Brumpt, Précis de Parasitologie, 3rd ed., 1922, 757; Rickettsia brasiliensis Monteiro, Mem. Inst. Butantan, 6, 1931, 3; *Rickettsia typhi do Amaral and Monteiro, Rev. Sud. Amér. de Méd. et Chirurg., 4, 1933, 806; Dermacentroxenus rickettsi var. brasiliensis Pinkerton, Parasitology, 28, 1936, 186.) Rickettsia dermacentroxenus, a corruption of Dermacentroxenus rickettsi, though widely used, has no genuine taxonomic standing. Named for Howard Taylor Ricketts, who first transmitted the disease from human cases to monkeys and guinea pigs with the production of characteristic symptoms and lesions and fatal effect.

Minute paired organisms surrounded by a narrow clear zone or halo and often lanceolate, resembling in appearance a minute pair of pneumococci. Approximately 0.2 to 0.3 micron by 1 micron. Non-motile.

In smears of mammalian tissues there occur in addition to the lanceolate forms, slender rod-shaped forms stained blue with the Giemsa stain, sometimes exhibiting polar granules, stained purplish or reddish. There are also minute pale blue-staining rounded forms. In the tick there are three forms: (1) Pale blue bacillary forms curved and club-shaped, (2) smaller bluish rods with deeply staining chromatoid granules and (3) more deeply staining, purplish, lanceolate forms. A very minute form may appear in tightly packed masses in the nuclei of the cells. Occurs in the cytoplasm and nucleus in all types of tissue in the tick and in the vascular endothelium, in the serosal cells of the peritoneal cavity, in the smooth muscle cells of arteriolar walls and in the macrophages of mammals.

In yolk sac cultures and in the Maitland media cultures, bacillary forms of ten occur in pairs. In single smears from infected yolk sacs, the rickettsiae are rather uniform in size and morphology and are definitely larger than Rickettsia prowazekii and Rickettsia typhi. They also grow more sparsely. Stain blue with the Castañeda stain and bright red against a blue background of tissue with the Machiavello stain.

Cultivation: May be cultivated in plasma tissue culture of mammalian cells, in Maitland media with and without agar, on the chorio-allantoic membrane and in the yolk sac of the chick embryo, and in ticks.

Optimum temperature $32^{\circ} \mathrm{C}$ in plasma tissue culture, $35^{\circ} \mathrm{C}$ in chick embryo cells.

Immunology: Prolonged immunity in man and animals after recovery from infection. Killed vaccines produced from infected ticks and from infected yolk sacs afford considerable protection against the disease. Therapeutic antisera have been produced by the injection of rabbits with tick virus and with infected yolk sac. No cross immunity between spotted fever in guinea pigs recovered from infections with Rickettsia rickettsii and typhus in guinea pigs recovered from infections with Rickettsia prowazekii and Rickettsia typhi. Cross immunity between spotted fever in guinea pigs recovered from infections with Rickettsia rickettsii and boutonneuse fever in guinea pigs recovered from infections with Rickettsia conorii, but spotted fever vaccine does not protect against boutonneuse fever of the Mediterranean area or against infections with the South African strains of Rickettsia conorii.

* Erroneously applied by do Amaral and Monteiro to the so-called eastern type of Rocky Mountain spotted fever.-Editors. 
Serology: Distinguishable from Rickettsia prouazekii and Rickettsia typhi by complement fixation and agglutination with specific antigens. Distinguishable from Rickettsia conorii by complement fixation, though some degree of cross fixation indicates antigenic relationship. Has common antigenic factor with Proteus OX19 but not distinguishable from Rickettsia prowazekii and Rickettsia typhi by Weil-Felix test.

Resistance to chemical and physical agents: Readily inactivated by heat and chemical agents. Destroyed by a temperature of $50^{\circ} \mathrm{C}$ in 10 minutes, and by 0.5 per cent phenol and 0.1 per cent formalin. Destroyed by desiccation in about 10 hours.

Pathogenicity: Pathogenic for man, monkeys and guinea pigs. Rabbits and white rats are moderately susceptible. Animals susceptible in varying degrees include species of ground squirrels, tree squirrels, chipmunks, cotton-tail rabbits, jack rabbits, snowshoe rabbits, marmots, wood rats, weasels, meadow mice and deer mice. In Brazil the opossum, rabbit, dog and cavy have been found naturally infected and the Brazilian plains dog, capybara, coati and certain bats are also susceptible. Sheep are mildly susceptible.

A febrile reaction occurs in guinea pigs with typical scrotal lesions, involving petechial hemorrhages in the skin, which may become necrotic. Virulent strains kill 80 to 90 per cent of the animals, milder strains kill 20 to 25 per cent. Passage in guinea pigs is accomplished by transfer of blood from infected animals. A febrile reaction accompanied by exanthema occurs in man. Mortality is high in some localities, low in others.

Source: Seen by Ricketts (Jour. Amer. Med. Assoc., 52, 1909, 379) in the blood of guinea pigs and monkeys experimentally infected with Rocky Mountain spotted fever and in the salivary glands, alimentary sac and ovaries of infected female Dermacentor ticks and in their ova.

Habitat: Infected wood tick (Dermacentor andersoni) and the dog tick (Dermacentor variabilis), also the rabbit tick (Haemaphysalis leporis-palustris), Amblyomma brasitiensis, Amblyomma cajennense, Amblyomma striatum, Amblyomma americanum and Ixodes dentatus. A number of ticks belonging to the genera Amblyomma, Dermacentor, Rhipicephalus, Ornithodoros and Haemaphysalis have been experimentally infected. The virus is transmissible through the ova of female ticks. The etiological agent of Rocky Mountain spotted fever, Saõ Paulo exanthematic typhus of Brazil, Tobia fever of Colombia and spotted fever of Minas Geraes which are all transmitted to man by the bite of infected ticks.

\section{Rickettsia conorii Brumpt.} (Brumpt, Compt. rend. Soc. Biol., Paris, 110, 1932, 1199; Rickettsia megawi var. pijperi do Amaral and Monteiro, Mem. Inst. Butantan, 7, 1932, 361; Rickettsia blanci Caminopétros, $1^{\mathrm{rr}}$ Cong. Internat. Hyg. Mediterr., Rapports et Compt. rend., 2, 1932, 202; Dermacentroxenus rickettsi var. pijperi Mason and Alexander, Onderst. Jour. Vet. Sci. and An. Indust., 13, 1939, 74; Dermacentroxenus rickettsi var. conori Mason and Alexander, ibid.; Dermocentroxenus conori Steinhaus, Insect Microbiology, 1946, 339.) Named for A. Conor who with A. Bruch published in 1910 the first cliniral description of boutonneuse fever.

Resembles Rickettsia rickettsii. In the tick, diplococcoid and diplobacillary forms predominate, though when the rickettsiae occur in compact masses they are smaller and more coccoid. In tissue cultures the organisms are lanceolate, diplococcoid, and diplobacillary, oecurring in the nuclei as well as in the cytoplasm of the cells. Size 0.3 to 0.4 by 1 to 1.75 microns. Non-motile.

Stain purplish with the Giemsa stain, 
blue with the Castañeda stain and bright red with a blue background with the Machiavello stain. Gram-negative.

Cultivation: May be cultivated in plasma tissue culture of manmalian cells, in modified Maitland media, and in the yolk sacs of chick embryos.

Immunology: The disease is related immunologically to Rocky Mountain spotted fever with which it cross immunizes, but the spotted fever vaccine does not protect against the Mediterranean and South African strains of boutonneuse fever.

Serology: Distinguishable from Rickettsia rickettsii by complement fixation. Has a common antigenic factor with Proteus OX19 and OX2.

Pathogenicity : Pathogenic for man and guinea pigs. It is also pathogenic in varying degrees for dogs, horses, spermophiles, monkeys, rabbits, gerbilles and white mice.

Boutonneuse fever is a much less virulent infection for the guinea pig than Rocky Mountain spotted fever. A temperature reaction occurs, accompanied by scrotal swelling but there is no sloughing. There is practically no mortality. Passage in guinea pigs is accomplished by transfer of blood from an infected animal.

In man, localized primary sores (taches noires) and an inflammatory reaction in the regional lymph nodes occur at the site of the tick bite. A febrile reaction with exanthema occurs and mortality is low.

Source: Seen by Caminopétros (Compt. rend. Soc. Biol., Paris, 110, 1932, 344 ) in smears from the tunica vaginalis of guinea pigs inoculated with infected dog ticks (Rhipicephalus sanguineus).

Habitat: The brown dog tick (Rhipicephalus sanguineus) and also the ticks, Amblyomma hebraeum, Haemaphysalis leachi, Rhipicephalus appendiculatus and Boopphilus decoloratus. Transmissible through the ova of adult female ticks. The probable animal reservoir is the dog. The etiological agent of boutonneuse fever in man, also known as eruptive, Mediterranean or Marseilles fever and probably Kenya typhus and South African tick bite fever, though the identity of the latter with boutonneuse fever has been questioned.

5. Rickettsia tsutsugamushi (Hayashi) Ogata. (Theileria tsutsugamushi

- Some may question the use of this binomial on the ground that Hayashi thought that this species was possibly or probably protozoan in nature when he proposed the name Theileria tsutsugamushi (loc. cit.) in 1920. However he questions whether Theileria is the correct generic name in this paper and accepts the viewpoint that this organism is a rickettsia in a paper published in 1924 entitled, On Rickettsia, Trans. Jap. Path. Soc., 14, 1924, 198-201. He does not use the binomial Rickettsia tsutsugamush in this paper as indicated by some of his friends in latter papers (Ogata, loc. cit., Kawamura, loc. cit.) and apparently first uses it himself in a paper entitled, On Tsutsugamushi Disease, Jap. Path. Soc., 22, 1932, 686.

Hayashi was not the first to rerognize the probable rickettsial nature of the organism of the tsutsugamushi disease (see Blake et al., Amer. Jour. Hyg., 41, 1945, 257-262) and some even question whether any of the bodies that he found in human lymphocytes from lymph nodes, in mononuclear endothelial phagocytes of the spleen and lymph nodes, and in tissues taken from the region of the mite bite in patients suffering from tsutsugamushi fever were the same as organisms described as Rickettsia orientalis by Nagayo et al. (loc. cit.).

This position is not supported, however, by Nagayo and his associates who admit that their organisms are identical with some of the organisms deseribed by Hayashi. Mitamura (Trans. Jap. Path. Soc.. 21. 1931, 463) sums this un as follows: "Wir stellen 
Hayashi, Jour. Parasit., 7, 1920, 63; *Rickettsia orientalis Nagayo, Tamiya, Mitamura and Sato, Jikken Igaku Zasshi, 14, May 20,1930,8 pp.; †Rickettsia tsutsugamushi Ogata, Cent. f. Bakt., I Abt., 122, 1931, 249; Rickeltsia akamushi Kawamura and Imagawa, ibid., 122, 1931, 258; Rickettsia orientalis var. schiffneri do Amaral and Monteiro, Mem. Inst. Butantan, 7, 1932, 360; Rickettsia megawi do Amaral and Monteiro, idem; Rickettsia megawi var. fletcheri do Amaral and Monteiro, ibid., 361; Rickettsia tsutsugamushi-orientalis Kawamura, Nisshin Igaku, 23, 1934, 000; Rickettsia pseudotyphi Vervoort, see Donatien and Lestoquard, Acta Conv. Tertii Trop. atque malariae morbis, pars I, 1938, 564; Rickettsia sumatranus (sic) Kouwenaar and Wolff, Proc. 6th Pacific Sci. Cong. (1939), 5, 1942, 636; Dermacen- troxenus orientalis Moshkovsky, Uspekhi Souremennoi Biologii (Russian) (Advances in modern biology), 19, 1945, 13.) From two Japanese ideographs transliterated tsutsuga, something small and dangerous, and mushi, a creature now known to be a mite. If the $i$ ending is accepted as forming a Latin genitive, the modern meaning of the species name, tsutsugamushi, would be 'of a dangerous mite'.

Small pleomorphic bacterium-like microorganisms, usually thicker than Rickettsia prowazekii, Rickettsia typhi, Rickettsia rickettsii and Coxiella burnetii and less sharply defined. Ellipsoidal or rodshaped, of tei appearing as a diplococcus or as a short bacillus with bipolar staining resembling the plague bacillus. Diffusely distributed in the cytoplasm of the cell. Size 0.3 to 0.5 by 0.8 to 2

nicht in Abrede dass Herr Hayashi bei einem kleinen Teil der von ihn beschriebenen Förperchen unsere Rickettsia orientalis vor sich gehalot hat". Hayashi vigorously defends his own observations in the same discussion and the following year after making comparative studies of strains of Rickettsia crientalis and his own Rickettsia tsutsugamushi reaches the following conclusion (loc. cit.) "Riclettsia tsutsugamushi and Rickettsia orientalis refer to one and the same species of microorganisms and there seems to be no way in which one can be recognized as differing from the other." Under these conditions the only valid name appears to be Rickettsia tsutsugamushi.Editors.

* These authors publish practically the same preliminary paper in three other places as follows: Compt. rend. Soc. Biol., Paris, 104, June 14, 1930, 637-641; Jap. Jour. Exper. Med., 8, Aug. 20, 1930, 309-318 and Trans. Jap. Path. Soc., 20, 1930, 556-566. The complete report on this work did not appear until the following year: Jap. Jour. Exper. Med., 9, March 20, 1931, 87-150.-Eilitors.

† This binomial apparently first appears in the literature in a review article by Kawamura (Handbuch der path. Microorganismen, Kolle and Wassermann, 3 Aufl., $8,1930,1398$ ) where it is used incidentally and is attributed to Hayashi, 1923 . The fact that Hayashi did not use Ricketlsia tsutsugamushi before 1931 is confirmed by Mitamura (Trans. Jap. Path. Soc., 21, 1931, 463) who states in a footnote: Kawamura und Ogata geben an, dass Hayashi 1923 für den Erreger den Namen Rickettsia tsutsugamushi vorgeschlagen hat. Eine solche Angabe Hayashi, is nicht nur uns, sondern auch dem Autor, wie er uns persönlich erzählt, unhekannt." Ogata apparently first used Ricliettsia tsutsugamushi in the title of a paper that he presented in 1930 to the 8th Cong. Far East Assoc. Trop. Med. which, however, appeared in the Transactions of the Congress, 2, June, 1932, 167-171. Meanwhile, the same paper with an added discussion of the nomenclature appeared in the Cent. f. Bakt., I Abt., Orig., 122, Oct. 1, 1931, 249-253 and it is this paper that is usually regarded as establishing the use of Richettsia tsutsugamushi for this species.-Editors. 
microns. Non-motile. Colored purplish with the Giemsa stain, and red against a blue background with the Machiavello stain. Stains well with azur III and methylene blue. Gram-negative.

Cultivation: In plasma tissue culture of mammalian cells; on the chorio-allantoic membrane and in the yolk sac of the chick embryo; in rabbit testes and in the endothelial cells overlying Descemet's membrane of the rabbit eye.

Immunology: Immunity conferred by infection appears less complete than in typhus and Rocky Mountain spotted fever. Strains from several different areas have been found to cross immunize in guinea pigs, but the true relationship of the disease occurring in different localities remains to be determined. Reciprocal cross-immunity between mite strains and human strains has been demonstrated in rabbits, hamsters and mice.

Serology: Antigens from different strains vary in sensitivity when tested hv complement fixation with immune sera. There are probably a number of different types on the basis of complement fixation with immune sera. Has a mmmon antigenic factor with Proteus nX-K.

Resistance to chemical and physical g.gents: Readily inactivated by heat and chemical agents. Destroyed by a temperature of $50^{\circ} \mathrm{C}$ for 10 minutes, and by 0.1 per cent formalin and 0.5 per cent phenol.

Pathogenicity: Pathogenic for man, monkeys, gibbons, guinea pigs, hamsters, rats, voles, mice, gerbilles, rabbits (by intraocular injection) and chick embryo. There is wide variation in the virulence of different strains for laboratory animals, infection being established with great difficulty with some, while others may cause a high mortality .

A febrile reaction occurs in guinea pigs. Passage in guinea pigs and mice is accomplished by inoculation of infected spleen or blood from an infected animal, passage in rabbits by intraocular inoculation of blood, lymph node or organ emulsions of infected animals. Ascites, enlarged spleen often with a fibrinous deposit are characteristic.

In man an eschar with adenopathy develops at the site of the mite bite. In scrub typhus the eschar is not present. A febrile reaction with exanthema occurs and mortality is variable.

In rabbits infection of Descemet's membrane follows intraocular injection of infected material.

Source: Seen by Hayashi in smears and sections of the lesion (eschar) at the site of the mite bite and in smears and sections of the adjacent lymph nodes from cases of the disease; also seen by Nagayo et al. (loc. cit.) in the endothelial cells overlying Descemet's membrane in rabbits inoculated intraocularly with infectious material.

Habitat: The mites (Trombicula atamushi, Trombicula deliensis syn. T. walchi, Trombicula fletcheri and probably several others). Infective through the ova of the adult female. Only the larvae feed on rodents or man. Reservoir hosts are probably certain wild rodents, including house and field rats, mice and voles and probably some birds. The etiological agent of tsutsugamushi disease and scrub typhus (for numerous other designations of the disease see Farner and Katsampes, U. S. Naval Med. Bull., 43, 1944, 800).

Note : Rickettsia nipponica Sellards. (Sellards, Amer. Jour. Trop. Med., 3, 1923, 545; Rickettsoides nipponica da Rocha-Lima, in Kolle and Wasserman, Handb. d. path. Mikroorganismen, 3 Aufl., 8, 1930, 1350.) This problematical organism was thought by its author to be the cause of tsutsugamushi disease. Because it was cultivatible by the methods used by Sellards, it is not now regarded as identical with Ricliettsia tsutsugamushi Ogata. Rickeltsoides nipponica is the type species (monotypy) of the 
genus Rickettsoides da Rocha-Lima (loe. cit.).

6. Rickettsia akari Huebner, Jellison and Pomerintz. (Pub. Health Rept., 61, 1946, 1682.) From Acarus, a genus of mites.

Minute diplobacilli, oceurring intracellularly and extracellularly, and bipolarly stained rods. Resemble typical rickettsiae morphologically. Ton-motile.

Stain well by Machiavello's method, the organisms appearing bright red against a blue background. Stain poorly with methylene blue. Gram-negative. Occur intracytoplasmically and have been seen intranuclearly in yolk sac cells.

Cultivation: In the yolk sar of the chick embryo. No growth on artificial eulture media.

Immunology : Guinea pigs recovered from rickettsialpox are immune to infection with strains isolated from inferted mites.

Serology: Antigens prepared from infected yolk sacs are highly specific except for cross reactions with Rorky Mountain spotted fever antigens. Sera from convalescent patients fixed complement with the homologous antigen and usually with Rocky Mountain spotted fever antigens though at a lower titer. Does not have a common antigenic factor with Proteus strains except that low titers were obtained in a few recovered cases in agglutination tests with Proteus OX19.

Pathogenicity: Pathogenic for man with focal initial erythematous lesion and adenopathy, followed by fever and appearance of macular rash. No mortality. Experimental infections have been produced in white mice and guinea pigs by the inoculation of infected blood (irregularly), and of infected liver and spleen suspensions, infected brain, infected lymph nodes, tunica washings of infected animals and by infected yolk sacs. Symptoms in mice include inactivity, arcelerated respiration, ruffled fur, with occasional deaths; in guinea pigs, fever and marked scrotal reactions. Infeeted embryos are killed in 4 to 7 days. It has not been found pathogenic for monkeys, distinguishing it from Rickeltsia conorii. It is also probably more pathogenic for white mice than Riclictlsia conorii.

Source: Blood of a human case of rickettsialpox in New York City.

Habitat: Blood of human cases and an ectoparasite of rodents, the mite (Alloderman!lssus sanguineus Hirst). The etiological agent of human rickettsialpox.

\section{Genus II. Coxiella Bengtson, gen. nem.}

(Subgenus Coxiella Philip, Amer. Jour. Hyg., 37, 1943, 306; generic status recommended by Steinhaus, Insect Microbiology, 1946, 263.) Named for Herald R. Cox who first lescribed the organism in guinea pigs inoculated with infected ticks collected in Montana.

Small, pleomorphic, rod-shaped and coccoid organisms, occurring intracellularly in the cytoplasm and extracellularly in infected ticks. Stain lightly with aniline dyes. Gram-negative. They are filterable. Have not been cultivated in cell-free media. Parasites of man and animals which include the etiological agent of Q fever.

The type species is Coxiella buinetii (Derrick) Bengtson.

1. Coxiella burnetii (Derrick) Bengtson comb. nov. (Rickettsia burneti Derrick, Med. Jour. Australia, 1, 1939, 14; Rickellsia diaporica Cox, Pub. Health Rep., 54, 1939, 1826; Rickettsia burneti var. americana, Anon., Brit. Med. Jour. 2, 1911,588; Rickettsia (Coxiella) burneti Philip, Amer. Jour. Hyg., 37, 1943, 306.) Named for F. M. Burnet who discovered the organism in Australia. 
Small bacterium-like, pleomorphic organisms varying in size from coccoid forms to well marked rods. Occur as cytoplasmic micro-colonies with diffuse or compact distribution of the organisms through the cytoplasm. Also seen extracellularly, where they appear as small lanceolate rods, diplobacilli and oceasionally segmented filamentous forms. Chains of 3 to 6 elements often seen. Quite uniform in size and morphology in infected yolk sacs and in mouse spleen with exceedingly minute forms in heavily infected material. Snall lanceolate rods, 0.25 by 0.4 to 0.5 micron, bipolar forms 0.25 by 1.0 micron, diplobacilli 0.25 by 1.5 microns. Non-motile.

With Giemsa's stain they appear reddish-purple, with Machiavello's stain bright red against a blue background. Gram-negative.

Cultivation: May be cultivated in plasma tissue cultures, in modified Maitland media and in the yolk sac of chick embryos.

Immunology: There is complete crosis immunity between Australian and American strains of $\mathrm{Q}$ fever in guinea pigs. Strains from other parts of the world also cross immunize.

Serology: American and Australian strains are identical by agglutination and agglutinin absorption. Strains from various countries are serologically related as shown by complement fixation. Q fever is distinguishable from other rickettsial diseases by complement fixation tests. No common antigenic factor with any Proteus strain has been demonstrated.

Filterability: The infectious agent of Q fever readily passes Berkefeld $\mathrm{X}$ filters which are impermeable to ordinary bacteria and $W$ filters which are impermeable to typhus and spotted fever rickettsiae.

Resistance to chemical and physical agents: Comparatively resistant to heat, drying and chemical agents. Survives at least 109 days in cell-free media with- out loss of titer, resistant to $60^{\circ} \mathrm{C}$ for 1 hour and to 0.5 per cent formalin and 1 per cent phenol when tested in fertile eggs.

Pathogenicity: Pathogenic for man, guinea pig and the white mouse. The monkey, dog, white rat and rabbit are mildly susceptible. Certain bush animals in Australia, particularly the bandicoot, are susceptible and these animals have been found naturally infected. Other rodents and marsupials are mildly susceptible. Calves have been experimentally infected and cows have been found recovered from naturally acquired infections.

A febrile reaction occurs in guinea pigs but mortality is low except with heavily infected yolk sac which causes a high mortality. On subcutaneous or intradermal inoculation a marked inflammatory thickening of the skin occurs at the site of inoculation. On autopsy the spleen is enlarged from 2 to 12 times by weight and is engorged with blood. Transfer in guinea pigs and mice is accomplished by transfer of infected liver and spleen. A febrile reaction often accompanied by pneumonitis occurs in man, but mortality is low.

Source: First seen in smears from mice inoculated intraperitoneally with infectious material by Burnet and Freeman (Med. Jour. Australia, 2, 1937 (2), 281).

Habitat: The wood tick (Dermacentor andersoni) and the ticks, Dermacentor occidentalis, Amblyomma americanum, Haemaphysalis leporis-palustris, Ixodes dentatus and Haemaphysalis humerosa. Several other species of ticks have been shown to transmit experimentally the virus of $\mathrm{Q}$ fever. It has been found to survive in the ova of the female ticks (Dermacentor andersoni and Haemaphysalis humerosa). The bandicoot (Isodon macrurus) is probably the natural reservoir of the disease in Australia. The etiological agent of $\mathrm{Q}$ (Queensland) fever in man. 


\section{Genus III. Cowdria Bengtson, gen. nov.}

Named for E. V. Cowdry who first described the organism in heartwater of three ruminants, sheep, goats and cattle.

Small pleomorphic, spherical or ellipsoidal, occasionally rod-shaped organisms, occurring intracellularly in ticks. Gram-negative. Have not been cultivated in cell-free media. Parasites which are the etiological agent of heartwater of cattle, sheep and goats.

The type species is Cowdria ruminantium (Cowdry) Bengtson.

\section{Cowdria ruminantium (Cowdry)} Bengtson, comb. nov. (Rickettsia ruminantium Cowdry, Jour. Exp. Med., 42, 1925, 231; Rickettsia (Cowdria) ruminantium Moshkovsky, Uspekhi Souremennoi Biologii (Russian) (Advances in Modern Biology), 19, 1945, 18.) From M. L. Ruminantia, the cud-chewing mammals.

Differ morphologically from typical rickettsiae, showing usually spherical and ellipsoidal forms; occasionally bacillary forms. Irregular pleomorphic forms occur. Grow in the cytoplasm of cells, sometimes in densely packed masses. Size of cocei from 0.2 to 0.5 micron in diameter in the endothelial cells of animals, 0.2 to 0.3 micron in diameter in ticks. Bacillary forms 0.2 to 0.3 by 0.4 to 0.5 micron and pairs 0.2 by 0.8 micron in ticks. Non-motile.

Stain blue with the Giemsa stain and can also be stained by methylene blue and other basic aniline dyes. Gramnegative.

Cultivation not reported.

Immunology: Immunity incomplete after recovery from the infection. The organisms are found in the tissues long after recovery. There is some evidence of a variety of strains.

Pathogenicity: Pathogenic for goats, sheep and cattle. Transmissible to goats by inoculation of infected blood intrajugularly. The most characteristic lesion is the hydropericardium of infected animals. The only small animal shown to be susceptible is the ferret.

Source: Seen in the endothelial cells of renal glomeruli and in the endothelial cells of the cerebral cortex of animals suf- fering from heartwater and in the tick, Amblyomma hebraeum.

Habitat: The bont tick (Amblyomma hebraeum) and also Amblyomma variegatum. When the tick is infected in the larval state, it can transmit the infection to the nymphal and adult stages, but the disease is not transmissible through the ova of the adult female tick. The etiological agent of heartwater in sheep, goats and cattle in South Africa.

Appendix I: Further studies of the organism of trench fever are required before the relationship of Rickettsia quintana to the other more firmly established species of rickettsiae can be determined. Therefore, it is placed in this appendix.

1. Rickeltsia quintana Schminke. (Schminke, Münch. med. Wchnschr., 64, July 17, 1917, 961; Riclettsia wolhynica Jungmanm and Kuczyuski, Ztschr. klin. Med., 85, 1918, 261; Fossilis quintana suggested as a possible subspecies "if necessary" by Megaw, Trop. Dis. Bull., 40, 1913, 828.)

Probable synonym : Rickettsia pediculi Munk and da Rocha-Lima, Münch. med. Wchnschr., 64, 1917, 1423.

Coccoid or ellipsoirlal organisms, often occurring in pairs, more plump and staining more deeply with the Giemsa stain than Rickctlsia prowazekii. Da Rocha-Lima gives their size as 0.2 to 0.4 micron by 0.3 to 0.5 micron. In lice appear as short rods, frequently in pairs and often bipolarly stained. Non-motile.

Stain reddish-violet with the Giemsa stain. Gram-negative. Occur extra- 
cellularly in the region of the epithelial lining of the gut of the louse.

Cultivation: Has not been cultivated in tissue culture or any cell-free medium, though Rickettsia pediculi, considered by some identical with Rickettsia quintana, has been cultivated on human and horse blood agar.

Pathogenicity: Pathogenic for man, causing recurrent fever. No strain has been definitely established in laboratory animals.

Immunology : Partial immunity is produced after an attack of the disease. The disease is characterized by relapses which may occur as long as two years after the initial attack.

Distinctive characteristics: The organism resists a temperature of $60^{\circ} \mathrm{C}$ moist heat ior 30 minutes or a dry heat at $80^{\circ} \mathrm{C}$ for 20 minutes. It resists desiccation in sunlight for 4 months. It is filterable under certain conditions but not when in plasma or serum. It is present in filtrates of infected vaccine sediments and excrements of infected lice.

Source: Seen in lice fed on trench fever patients by Töpfer (Münch. med. Wchnschr., 61, 1916, 1495).

Habitat: The epithelial lining of the gut of the body louse (Pediculus humanus var. corporis) where they occur extracellularly, and Pediculus capitis. The virus is not transmissible through the ova. May be the etiological agent of trench fever (Wolhynian fever, shin bone fever, five-day fever).

Appendix II : Additional named species are included in Chapter $\mathrm{V}$, Riclettsiae, in Steinhaus, Insect Microbiology. Ithaca, 1946, 304-328. Some differ morphologically and tinctorially from typical rickettsiae, some are not associated with an arthropod vector, some have been incompletely studied and described, some have been cultivated in cell-free media. Pending the completion of further studies involving possible cultivation in fertile eggs, the determination of biological properties, and adequate comparative immunological and serological studies, no attempt is made to classify these organisms. The descriptions are condensed from those given by Steinhaus :

Ehrlichia (Rickettsia) kurlovi Moshkovsky. (Compt. rend. Soc. Biol., Paris, 126, 1937, 379; Ehrlichia kurlovi Moshkovsky, Uspekhi Souremennoi Biologii (Russian) (Advances in Modern Biology), 19, 1945, 12.) Found in the monocytes of guinea pigs. Described by Kurloff in 1889 as inclusions in the mononuclear cells of guinea pigs and other animals. These becane known as Kurloff bodies. However, the parasitism of these bodies is questionable.

Rickettsia avium Carpano. (Riv. Pat. Comp., Jan.-Feb., 1936, 1.) Minute bodies in the leucocytes and tissue cells of a bullfinch (Pyrrhula europea) brought to Egypt from Germany. Donatien and Lestoquard (Arch. Inst. Pasteur Algerie, 15, 1937, 142) suggested that this organism might have been that of psittacosis.

Rickettsia bovis Donatien and Lestoquard. (Donatien and Lestoquard, Bull. Soc. Path. Exot., 29, 1936, 1057; Ehrlichia bovis Moshkovsky, Uspekhi Souremennoi Biologii (Russian) (Advances in Modern Biology), 19, 1945, 18.) Concerned in a disease of cattle which is transmitted by an unidentified tick of the genus Hyalomma. The organism occurs in circular or round-angled polygonal masses which consist of a large number of tightly pressed, minute spherical granulations. These masses are situated in the cytoplasm of various monocytes. The organism causes a relatively light febrile disease in cattle, and an inapparent infection in sheep and fever in monkeys.

Rickettsia canis Donatien and Lestoquard. (Donatien and Lestoquard, Bull. Soc. Path. Exot., 28, 1935, 418; Ehrlichia (Rickettsia) canis Moshkovsky, Compt. 
rend. Soc. Biol., Paris, 126, 1937, 382; Ehrlichia canis Moshkovsky, Uspekhi Souremennoi Biologii (Russian) (Advances in Modern Biology), 19, 19.45, 18.) Moshkovsky selects this species as the type species of the subgenus Ehrlichia Moshkovsky (loc. cit.). Found in dogs used for experimental purposes in Algeria. Appears to be transmitted naturally by the dog tick (Rhipicephalus sanguineus). All active stages of the tick transmit the organism and it passes intraovarially from the female to the larvae of the next generation. The organisms are generally spherical in shape and can be seen in the circulating monocytes. The infection causes a serious and often fatal illness in dogs. Small laboratory animals are not susceptible to the disease.

Rickettsia conjunctivae, Rickettsia conjunctivae bovis and Rickettsia conjunctivae gaili, see Family III, Chlamydozoaceae.

Rickettsia ctenocephali Sikora. (Arch. Schiffs- u. Tropenhyg., 22, 1918, 442.) Found in cat fleas (presumably Ctenocephalides felis) on the surface of the organs in the body cavity and in the coelomic fluid. Two forms were found which might be two species, one resembling Rickettsia pediculi and the other Rickettsia melophagi. Hertig and Wolbach (Jour. Med. Res., 44, 1924, 329) found Rickettsia clenocephali to vary in size and shape from minute cocci to rather large, swollen, curved rods, staining reddish with the Giemsa stain.

Ricketlsia culicis Brumpt. (Ann. Parasitol. Hum. et Comp., 16, 1938, 153.) Found in the stomach epithelium of mosquitoes (Culex fatigans) 12 days after they had been fed on a patient carrying Microfilaria bancrofli. Thought to be pathogenic for the mosquito and possibly for man. Occurs in the form of small granules and more often as small bipolar rods. Stains with haemalum, erythrosine-orange and toluidine blue. Gramnegative.
Richettsia dermacentrophila Steinhaus. (Pub. Health Repts., 57, 1942, 1375.) Found in all stages of the wood tick (Dermacentor andersoni). In the epithelial cells of the intestinal diverticula and other tissues of the tick, usually extracellularly but sometimes intracellularly. Not seen in the nuclei of cells. Gramnegative and staining red with the Machiavello stain, and bluish-purple with the Giemsa stain. Stains less deeply with ordinary bacterial stains than most bacteria. Resembles Rickettsia rickettsii morphologically but is slightly larger. Not pathogenic for laboratory animals or for some of the natural hosts of Dermacentor andersoni.

Rickettsia hirundinis Cowdry. (Jour. Exp. Med., 37, 1923, 431.) An organism observed by Arkwright, Atkin and Bacot (Parasitology, 13, 1921, 27) in the tissues of Cimex hirundinis which is probably the same organism to which Cowdry referred as Rickettsia hirundinis. Considered by Steinhaus as a nomen nudum.

Rickeltsia kairo da Rocha-Lima. (Cairo rickettsia, Arkwright and Bacot, Brit. Jour. Exper. Path., 4, 1923, 70; da Rocha-Lima, in Kolle and Wasserman, Handb. d. path. Mikroorg., 3 Aufl., 8, 1930, 1361.) Resembles Rickettsia rochalimae and Rickettsia prowazekii.

Ricketlsia lectularia Arkwright, Atkin and Bacot. (Parasitology, 13, 1921, 27.) Found in the gut of the bedbug (Cimex lectularius) as filamentous and rodshaped organisms. It seems probable that all bedbugs harbor the organism and it is also present in the developing ova. The location is intracellular. Very pleomorphic, ranging from small coccoid forms to thread-like forms. The small coccoid and diplococcoid forms stain deep purple with the Giemsa stain, while bacillary, lanceolate and thread forms stain more red than purple with the Giemsa stain. Not infective for small laboratory animals or for man.

Rickettsia linognathi Hindle. (Parasitology, 13, 1921, 152.) Found in the 
alimentary tract of the goat louse (Linognathus stenopsis). Resembles Rickettsia trichodectae morphologically and occurs only extracellularly in the lumen of the gut.

Rickettsia melophagi Nöller. (Arch. Schiffs- u. Tropenhyg., 21, 1917, 53.) Found upon and in the cuticular layer covering the epithelium of the midintestine of the sheep tick (Melophagus ovinus). Occurs characteristically in pairs of fairly uniform size, coccoid and sometimes rod-shaped. Gram-negative but stains fairly well with carbol-fuchsin and gentian violet. Stains deep purple with Giemsa's method and bright red with Machiavello's method. Has been cultivated on non-living culture media, a glucose-blood-bouillon agar medium. The ability of Rickettsia melophagi to infect sheep has been the subject of contradictory claims. Small laboratory animals seem not to be susceptible.

Rickettsia ovina Lestoquard and Donatien. (Lestoquard and Donatien, Bull. Soc. Path. Exot., 29, 1936, 108; Ehrlichia ovina Moshkovsky, Usphekhi Souremennoi Biologii (Russian) (Advances in Modern Biology), 19, 1945, 18.) Found in the blood of diseased sheep from Turkey and Algeria. The organisms occur as minute coccoid granules, grouped in masses and present only in the monocytes and never in endothelial cells. They stain uniformly dark red with the Giemsa stain but did not stain with the Castañeda technic. Infected ticks (Rhipicephalus bursa) are thought to be the vectors.

Rickettsia pisces Mohamed. (Ministry Agr., Egypt., Tech. Sci. Serv. Bull. 214, 1939,6 pp.) In the monocytes and plasma of the blood of a fish (Tetraodon fahaka) showing necrotic ulcers on its head and both sides of the body. The heart, liver and intestines showed lesions. The organisms were minute coccoid forms varying from 0.2 to 0.4 micron in diameter and frequently occurring in pairs.
Riclettsia rocha-limae Weigl. (Przglad. Epidemj., 1, 1921, 375.) Occurs in lice (Pediculus humanus) but is ap. parently non-pathogenic either to lice or to vertebrates. Larger and more pleomorphic than Rickettsia prowazekii. In smears or sections of the gut of lice, Rickettsia rocha-limae occurs in agglomerated masses, grouped like staphylococci. They occur both extracellularly and intracellularly and stain more deeply than Rickettsia prowazelii. Weigl claims to have cultivated this species on artificial culture media under anaerobic conditions. Not pathogenic for laboratory animals or man.

Rickettsia suis Donatien and Gayot. (Bull. Soc. Path. Exot., 35, 1942, 324.) Causes a disease in swine, the pathology of which resembles heartwater of ruminants. See Genus III, Coudria, Family Richettsiaceat

Rickettsia trichodectae Hindle. (Parasitology, 13, 1921, 152.) In the species of biting lice (Trichodectas pilosus) which may be found on horses. This insect does not suck blood. The organisms oceur extracellularly in the alimentary tract of the louse. The average size is 0.3 to 0.5 by 0.5 to 0.9 micron and occasionally longer forms occur.

Rickettsia weigli Mosing. (Arch. Inst. Pasteur, Tunis, 25, 1936, 373.) Concerned in an epidemic disease which broke out in 1934 among employees of the Institute of Biology in Lwow who were engaged in feeding supposedly uninfected lice on their persons. Mosing and others have suggested the possibility that this rickettsia may be an extreme mutant of Rickettsia pediculi. small coccoid to rod-shaped organisms staining well with the Giemsa stain, usually slightly longer than Rickettsia prowazekii. In the louse (Pediculus humanus), the rickettsiae occur extracellularly in the intestinal lumen forming a layer covering the surface of the epithelial lining. Not pathogenic for the louse as is Rickettsia prowazekii and Rickettsia 
rocha-limae. It causes a febrile illness in man in which relanses occurred 3 to 5 times as in trench fever. Rickettsiu weigli was agglutinated by convalescent sera but not by sera from typhus patients. Convalescent sera gave no positive Weil-Felix reaction.

Wolbachia pipientis Hertig. (Rickettsia of Culex pipiens, Hertig and Wolbach, Jour. Med. Res., 44, 1924, 329; Hertig. Parasitology, 28, 1936, 453.) This is the type species of the genus Wolbachice Hertig (loc. cit). Found in the ovaries or testes of the mosquito, and present in all stages of the mosquito's development. The outstanding morphological characteristic of the organism is great pleomorphism. Minute coccoids and short rods may be considered typical, but the usual microscopic field consists of various shapes and sizes. Some forms show bipolar staining with the Giemsa stain. The organism is a harmless parasite of the mosquito. Laboratory animals are apparently not susceptible.

The following unnamed rickettsiae isolated from animals or seen in animals are included in Steinhaus' list of rickettsiae (Insect Microbiology. Ithaca, 1946, 344):

A rickettsia was isolated by Parker, Kohls, Cox and Davis (Pub. Health Rept., 5/, 1939, 1482) from a tick (Amblyomma maculatum). It is pathogenic for guinea pigs and the disease is referred to as the maculatum disease. There is complete cross immunity in guinea pigs between this infection and Rocky Mountain spotted fever and boutonneuse fever, but it dillers from these diseases in some particulars.

A rickettsia-like organism was isolaterl from the reduviid bng (Triatoma rubrofasciata) by Webb (Parasitology, 32, $1940,355)$. It was pathogenic for some laboratory animals and was maintained in guinea pigs for 5 passages. The rickettsiae were transmissible to the next generation through the egg of the reduviid bug.

$\Lambda$ spotted fever type of rickettsia was isolated by Anigstein and Bader (Texas Repts. Biol. Med., 1, 1943, 105) from the dog tick (Rhipicephalus sanguineus) taken from normal dogs. It was pathogenic for rabbits and guinea pigs.

A rickettsia was isolated by Anigstein and Bader (Texas Repts. Biol. Med., 1, 1943, 298, 389) from ticks (Amblyomma americanum) collected in Texas. They believed it to be the cause of bullis fever.

Rickettsiae were observed by Enigh (Berl. u. Münch. Tierärztl. Wchnschr., $1942,25)$ in the leucocytes of a bison calf. No arthropod was associated with this rickettsia.

A rickettsia-like agent pathogenic for guinea pigs was reported by Tatlock (Proc. Soc. Exp. Biol. and Med., 5\%, $1944,95)$. The animals had been injected with blood from a patient with "pretibial" fever. No arthropod vector was indicated.

Three species of rickettsia-like organisms isolated from the wood-tick (Dermacentor andersoni) are described by Noguchi (Jour. Exper. Med., 43, $1926,518-521)$. These were named $B a$ cillus vickeltsiformis, Bacillus pseudoxerosis and Bacillus equidistans. All could be cultivated on cell-free media and none was pathogenic for laboratory animals.

Appendix III: Unnamed rickettsialike organisms seen in the tissues of insects.

Hertig and Wolbach (Jour. Med. Res., 44, 1921, 329) list sixteen species of arachnids and twenty-three species of insects which are hosts to rickettsiae or rickettsia-like organisms.

Wolbach (Jour. Amer. Med. Assoc., 84, $1925,723)$ reports hosts of non-pathogenic rickettsiae which include fourteen species of arachnids (ticks, mites and spiders) and twenty-two species of insects distributed in nine orders, including 
numerous non-blood-sucking insects as well as lice and ticks.

Cowdry (Arch. Path. and Lab. Med., 2, 1926, 59) lists seven species of arachnids and twenty-four species of insects which are hosts to non-pathogenic rickettsiae.

Buchner, P. (Tier und Pflanze in Symbiose. Gebrüder Borntraeger, Berlin, 1930, 900 pp.) Through the text, and particularly on pages $300-664$, the rickettsia-like and bacterium-like microorganisms occurring intracellularly in insects and other small animals are discussed, principally from the viewpoint of the biologist.

Paillot, A. (L'infection chez les Insects, Paris, 1933, 535 pp.). Concerned principally with bacterial infections of insects, but also includes information in intracellular symbiotes and rickettsialike and bacterium-like microorganisms. Steinhaus, Edward A. (Insect microbiology. Ithaca, 1946, 188-255.) Intracellular bacterium-like and rickettsialike symbiotes are discussed. 


\section{FAMILY II. BARTONELLACEAE GIESZCZYKIEWICZ.*}

(Bull. Intern. Acad. Polon. Sci., Classe Sci. Math. Nat., B (I), 1939, 9-30.)** Small, of ten pleomorphic, rod-shaped, coccoid, ring-shaped, filamentous and beaded micro-organisms, staining lightly with aniline dyes, but well with Giemsa's stain. Gram-negative. Parasites of the erythrocytes in man and other vertebrates. Known to be transmitted by arthropod vectors in some cases. The causative organisms of bartonellosis in man, haemobartonellosis, grahamellosis and eperythrozoönosis in the lower animals. Differ from the protozoa that also parasitize erythroeytes in that the entire parasite stains with no differentiation into rytoplasm and nucleus.

\section{Key to the genera of family Bartonellaceae.}

1. Parasites of the erythrocytes and of fixed tissue in man.

\section{Genus I. Bartonella, p. 1100.}

2. Parasites of the erythrocytes of lower mammals, increased in susceptible animals by splenectomy. Eradicated by arsenicals.

Genus II. Maemobartonella, p. 1102.

3. Parasites of the erythrocytes of lower mammals. Not inereased in susceptible animals by splenectomy. Not eradicated by arsenicals.

Genus III. Grahamella, p. 1109.

4. Blood parasites, found on the erythrocytes and in the plasma of lower mammals. Appear as rings, coccoids and short rods. Splenectomy activates latent infections.

Genus IV. Eperythrozoon, p. 1111.

\section{Genus I. Bartonella Strong, Tyzzer and Sellards.}

(Bartonia Strong, Tyzzer, Brues, Sellards and Gastiaburú, Jour. Amer. Med. Assoc., 61, 1913, 1715; not Bartonia Muhlenberg, in Willdenow, Neue Schrift Ges. Nat. Fr., Berlin, 3, 1801, 444; not Bartonia Sims, Bot. Mag., 1804; not Bartonia Crossman, Essais de Paleoconchologie Comparée, the Livr., Paris, 1901 ; Strong, Tyzzer and Sellards, Jour. Amer. Med. Assoc., 64, 1915, 808; emend. Tyzzer and Weinman, Amer. Jour. Hyg., 30 B, 1939, 143.) Named for A. L. Barton who described these organisms in 1909.

Parasites of the erythrocytes which also multiply in fixed tissue cells. On the red blood cells in stained films, they appear as rounded or oval forms or as slender, straight, curved or bent rods occurring either singly or in groups. Characteristically in chains of several segmenting organisms, sometimes swollen at one or both ends and

* Prepared by Dr. Ida A. Bengtson (retired), National Institute of Health, Bethesda, Maryland and Dr. David Weinman, Parasitologist to the 1937 Harvard Expedition to Peru, Boston, Mass., April, 1947.

** Partial syn. Anaplasmidae has been proposed as a family name to unite the four genera Anaplasma, Grahamella, Bartonella and Eperythrozoon by Neitz, Alexander and du Toit (Onderst. Jour. Vet. Sci. and An. Ind., 3, 1934, 268). Since the name is derived from Anaplasma, the nature of which is not fully understood and since these authors consider the 4 genera as belonging to the protozoan order Haemosporidia, it seems advisable not to consider this nomenclature for the present. The genus Anaplasma (parasites of the red blood cells of cattle) created by Theiler (Transvaal Govt. Vet. Bact. Rept. 1908-9, 7-64, 1910) consists of two species A naplasma marginale and A maplasma centrale. Recent workers are inclined to consider them to be bacterial in nature as they do not show a differentiation into eytoplasm and nucleus. 
frequently beaded (Strong et al., loc. cit., 1913), without a distinct differentiation of nucleus and cytoplasm. In the tissues they are situated within the cytoplasm of endothelial cells as isolated elements and grouped in rounded masses. These parasites occur spontaneously in man and in arthropod vectors, are endowed with independent motility, reproduce by binary fission, and may be cultivated by unlimited serial transfers on cell-free media. One species has been recognized. It is known to be established only on the South American continent and perhaps in Central America. Human bartonellosis may be manifested clinically by one of the two syndromes constituting Carrión's disease (Oroya fever or verruga peruana) or as an asymptomatic infection (definition by Strong, Tyzzer and Sellards emend. Tyzzer and Weinman (in Weinman, Trans. Amer. Philosoph. Soc., N.S., 33, pt. 3, 1944, 246 ).

The type species is Bartonella bacilliformis (Strong et al.) Strong et al.

\section{Bartonella bacilliformis (Strong,} Tyzzer, Brues, Sellards and Gastiaburú) Strong, Tyzzer and Sellards. (Bartonia bacilliformis Strong et al., Jour. Amer. Med. Assoc., 61, 1913, 1715; Bartonella bacilliformis Strong, Tyzzer and Sellards, Jour. Amer. Med. Assoe., 64, 1915, 808; emend. Tyzzer and Weinman, Amer. Jour. Hyg. 30(B), 1939, 143; also see Weinman, Trans. Amer. Philosoph. Soc. N.S., 33, pt. 3, 1944, 246. Partial or com plete synonyms: Bartonella cocoide (sic) Hercelles, Ann. de Fac. de Med., Lima. 9, 1926, 231; Bartonclla peruciana Escomel, Bull. Soc path. Exot.. 22, 1929, 35t; Eperthyrozoom noguchii Lwoff and Vaucel, Compt. rend. Soc. Biol., Paris, 103, 1930, 975.) From Latin bacillus, rod and forma, shape.

Small, pleomorphic organisms, show ing greatest morphological range in the blood of man, appearing as red-violet rods or coccoids situated on the red cells. when stained with Giemsa's stain. Bacilliform bodies are the most typical, measuring 0.25 to 0.5 by 1 to 3 microns Often curved and may show polar enlargement and granules at one or both ends. Rounded organisms measure about 0.75 micron in diameter and a ringlike variety is sometimes abundant. On semi-solid media a mixture of rods and granules appear. The organisms may oceur singly or in large and small, irregular dense collections, measuring up to 25 microns or more in length. Puncti- form, spindle-shaped and ellipsoial forms of the organism occur, varying in size from 0.2 to 0.5 by 0.3 to 3 microns.

Gram-negative and non-acid-fast. Stain poorly or not at all with the usual aniline dye stains, but satisfactorily with Romanorsky and Giemsa stains.

Motile in the blood and in cultures. One to four unipolar flagella.

Cultivation: Growth in semi-solid agar with fresh rabbit serum and rabbit hemoglobin and in semi-solid agar with blood of man, horse or rabbit with or without the addition of fresh tissue and certain carbohydrates, in other culture media containing blood, serum or plasma, Huntoon's hormone agar at 20 per cent, semi-solid gelatin media, blood-glucosecystine agar, chorio-allantoic fluid and yolk sac of chick embryo.

Gelatin not liquefied.

No acid or gas $n$ glucose, sucrose, galactose, maltose, fructose, xylose, lactose, mannose, mannitol, dulcitol, arabinose, raffinose, rhamnose, dextrin, inulin, salicin and amygdalin.

No action on lead acetate.

Aerobic obligate.

Optimum temperature $28^{\circ} \mathrm{C}$.

Immunology: Natural immunity to infection has not been demonstrated in susceptible species. Acquired immunity apparent both during and after the disease. Bartonellae from different sources appear to provoke similar responses. Bartonellae from Oroya fever protect 
against infection with organisms obtained from verruga cases.

Serology: Immune sera fix complement and agglutination of suspensions of Bartonella by sera from recovered cases has been reported.

Pathogenicity: Three forms of the disease occur in man; the anemic (Oroya fever), the eruptive (verruga peruana) and mixed types of both of the other forms. Experimental Oroya fever has not been successfully produced in ani- mals, except rarely in an atypical form in monkeys. Experimental verruga peruana has been produced in man, in a number of species of monkeys and occasionally in dogs.

Source: Blood and endothelial cells of lymph glands, spleen and liver of human cases of Oroya fever.

Habitat: Blood and endothelial cells of infected man, probably also in sand flies (Phlebotomus verrucarum and Phlebotomus noguchii).

\section{Genus II. Haemobartonella Tyzzer and Weinman.}

(Amer. Jour. Hyg., 30(B), 1939, 141.) From Greek haemos, blood and the generic name Bartonella.

Includes parasites of the red blood cells in which there is no demonstrable multiplication in the tissues and which do not produce cutaneous eruptions. They are typically rod- or coccoid-shaped, showing no differentiation into nucleus and cytoplasm, occurring naturally as parasites of vertebrates, and are transmitted by arthropods. They are distributed over the surface of the erythrocytes, and possibly sometimes within them. They stain well with Romanowsky type stains and poorly with other aniline dyes. Gram-negative. Not cultivated indefinitely in cell-free material. Rarely produce disease in animals without splenectomy, are markedly influenced by arsenotherapy, and are almost all of world-wide distribution. The experimental host range is restricted, infectivity of a rodent species for other rodents being common, but for primates unknown.

The type species is Haemobartonella muris (Mayer) Tyzzer and Weinman.

\section{Key to the species of genus Haemobartonella.}

I. The etiological agent of haemobartonellosis of the white rat.

1. Haemobartonella muris.

II. The etiological agent of haemobartonellosis of the dog.

2. Haemobartonella canis.

III. The etiological agent of haemobartonellosis of the vole.

3. Haemobartonella microtii.

IV. The etiological agent of haemobartonellosis of the guinea pig.

4. Haemobartonella tyzzeri.

V. The etiological agent of haemobartonellosis of cattle.

5. Haemobartonella bovis.

VI. The etiological agent of haemobartonellosis of the buffalo.

6. Haemobartonella sturmanii.

VII. The etiological agent of haemobartonellosis of the deer mouse.

7. Haemobartonella peromyscii.

VIII. The etiological agent of haemobartonellosis of the gray-backed deer mouse.

7a. Haemobartonella peromyscii var. maniculati.

IX. The etiological agent of haemobartonellosis of the short-tailed shrew.

8. Haemobartonella blarinae.

$\mathrm{X}$. The etiological agent of haemobartonellosis of the gray squirrel.

9. Haemobartonella sciurii. 
1. Haemobartonella muris (Mayer) Tyzzer and Weinman. (Bartonella muris Mayer, Arch. f. Schiffs.- u. Tropenhyg., 25, 1921, 151; Bartonella muris ratti Regendanz and Kikuth, Compt. rend. Soc. Biol., Paris, 98, 1928, 1578; Tyzzer and Weinman, Amer. Jour. Hyg., 30 (B), 1939, 143.) From Latin mus, muris, mouse.

Slender rods with rounded ends, frequently showing granules or swellings at one or both extremities, and dumbbell, coccoid or diplococcoid forms. May occur individually, in pairs, or in short chains of 3 or 4 elements, and, when abundant, in parallel grouping. The rods measure 0.1 by 0.7 to 1.3 microns and as much as half the length of a red cell. The coccoids have a diameter of 0.1 to 0.2 micron.

They have been found on and in the erythrocytes and in the plasma. Preferred stains are those of the Romanowsky type. With Giemsa's stain various investigators report an intense red coloration, a bluish tinge with distinct pink shading, blue with purple granules. With Wright's stain, the organisms stain bluish, with reddish granules at the ends. With Schilling's methylene blue-eosin stain the organisms stain a bright red color with the ery throcyte staining blue. They stain faintly with Manson's stain, pyronin-methyl green and fuchsin. Gram-negative.

There is lack of agreement concerning visibility in the fresh state and motility. Various authors report Brownian movement, slow and sinuous motion in the red cell or rapid motion.

Cultivation: Cultivated with difficulty and divergent results have been reported. Growth on various media reported (blood agar, agar with 2 per cent defibrinated rat blood, horse blood agar, N. N. X.. Blutrösplatte of Wethmar, hormone agar with blood of rabbit, horse or man, ascitic fluid agar, chocolate agar, semi-solid rabbit serum agar, semi-solid rabbit blood agar, Noguchi-Wenyon medium, defibrinated rat blood, glucose broth,
Tarozzi broth, peptone water) but usually growth was scant or could not be continued by transfer to the same medium or the organism isolated was noninfectious or the possibility of latent infections in the animal was not excluded. Best results are apparently obtained with semi-solid rabbit serum agar and semisolid rabbit blood agar.

No conclusive results have been reported in tissue culture. The organism has been cultivated on the chorio-allantoic membrane of the chick embryo.

Filterability: Non-filterable with Seitz or Berkefeld $\mathrm{X}$ filters.

Immunology: No authentic case of true natural immunity in rats has been established. Acquired immunity occurs in (1) the latently-infected rat, (2) the infected rat after splenectomy and recovery from the disease, the period of resistance corresponding to the duration of latency, (3) the non-splenectomized non-carrier rat following infection, (4) animals other than the rat following infection.

Serology : No precipitins, thrombocytobarin, isoagglutinins, or cold hemolysins have been reported in the serum of anemic rats. Complement deviation and agglutination have been reported with sera from rabbits, rats and guinea pigs injected with cultures. Rabbits immunized with cultures have given positive Weil-Felix reactions with Proteus OX19 and OXK and rat sera recovered from haemobartonellosis have given a positive Weil-Felix reaction and positive agglutination in low dilution with Rickettsia prowazekii.

Pathogenicity: Infected blood, liver suspension, defibrinated laked blood, washed red cells, plasma and hemoglobinuric urine may produce infection by the subcutaneous, intravenous, intraperitoneal or intracardiac routes. Slight, transient or no haemobartonellosis occurs in adult non-splenectomized haemobartonella-free albino rats, adult non-splenectomized albino rats of carrier stock, adult splenectomized rats pre- 
viously infected, until 15 weeks to 8 months after infection. Typical haemobartonellosis occurs in adult splenectomized haemobartonella-free albino rats and in young non-splenectomized haemobartonella-free albino rats weighing 20 to 30 grams at 3 weeks. Variable results have been obtained by different investigators with wild mice, guinea pigs. rabbits, hamsters, pigeons and monkeys (Macacus rhesus and Macacus sp.). It is known to be infectious for wild rats, albino mice, rabbits and for two Palestinian rodents (Sphallax (Spalax correct designation) typhlops and Meriones tristrami). Negative results have been reported in dogs, kittens, eats, sheep and various birds. Causes a definite and characteristic anemia without cutaneous eruption.

Arsenical therapy: True sterilization of latent or recognized infection with organic arsenical compounds.

Source: Blood of infected albino rats. Habitat: Ectoparasites such as the rat louse (Polyplax (Haematopinus) spinulosus), the flea (Xenopsylla cheopis) and possibly the bedbug (Cimex lectularius). Also found in the erythroeytes of susceptible animals. World wide in distribution.

2. Haemobartonella canis (Kikuth) Tyzzer and Weinman. (Bartonella canis Kikuth, Klin. Wchnschr., 1928, 1729; Tyzzer and Weinman, Amer. Jour. Hyg., 30(B), 1939, 151.) From Latin canis, dog.

One of the most pleomorphic of the haemobartonellae, occurring as thin rods, straight or slightly curved, dumbbellshaped organisms, dots, coccoids, or rings. Chains of rods, coccoids or rings occur. These consist of only one type of these forms or a mixture of types. The chains may be straight, curved, branched or annular. Variable in size. Round forms vary from 0.2 or 0.5 micron to the limit of visibility. Single rods are 0.2 by 1 to 5 microns, while the composite forms vary from 1 to 4 microns. Situation is epi-erythrocytic.

Giemsa's fluid stains the organism red-violet, usually intensely. Methylene blue used as a vital stain colors the organism distinctly. Gram-negative and non-acid-fast.

Considered non-motile by most investigators.

Cultivation: Cultivation has not been demonstrated in semi-solid rabbit serumagar medium nor in media containing serum of splenectomized dogs, N.N.N., Noguchi's medium for leptospira, blood broth, Chatton's medium covered with vaseline for Trichomastix.

Filterability: Results equivocal.

Immunology: The outstanding phenomena resemble those found in the rat infected with Haemobartonella muris.

Pathogenicity: Splenectomy is essential to infection accompanied by anemia in the dog. Negative results in splenectomized haemobartonella-free guinea pig, rat, rabbit, and mukey (Cercopithecus sabaeus). No infection or anemia in unoperated mice, white rats, young rabbits, young dogs and young guinea pigs. The splenectomized cat has been found to carry the infection by serial passage.

Arsenical therapy: Complete sterilization obtained by neoarsphenamine.

Source: Erythrocytes of infected splenectomized dogs.

Habitat: Found in dog fleas (Ctenocephalus) and erythocytes of infected animals. Distribution wide-spread, the infection occurring spontaneously in Europe, India, North and South Africa, North and South America.

3. Haemobartonella microtii Tyzzer and Weinman. (Tyzzer and Weinman, Amer. Jour. Hyg. 30 (B), 1939, 143; also see Weinman, Trans. Amer. Philosoph. Soc., N. S., 33, 1944, 312; questionable synonym Bartonella arvicolae Yakimoff, Arch. Inst. Past. de Tunis, 17, 1928, 350; Haemobartonella arricolae Weinman, loc. 
cit., 290.) From the genus of voles, Microlus.

In infected animal, morphology resembles that of Haemobartonella canis, the organisms occurring as rods, coccoids, filaments, club forms, ring forms and granular masses. In addition to these forms there occur in Giemsa-stained blood films ovoids, diamond- or flameshaped small forms as well as coarse segmented or unsegmented filaments up to 5 microns in length. Filaments may contain one or more rings, or may be composed in part or entirely of diamondshaped, coccoid or ovoid elements, sometimes in parallel rows. Rods of ten show intense bipolar staining. Coccoid forms, usually scattered, may occur as aggregates or clumps on the red cell, apparently embedded in a faint biue matrix.

A pale blue veil-like substance may cover nearly half of one surface of the red cells and show at its border typical red-violet stained rods or filaments in the Giemsa-stained specimens. A bowshaped arrangement of elements is characteristic. Organisms lie on the surface of the red cells. In cultures organisms are more uniform in morphology resembling Bartonella bacilliformis. Individual organisms are fine rods, 0.3 by 1.0 to 2 microns, sometimes occurring in chains and often in clumps. Small round forms occur, measuring 0.5 micron in diameter, and occasionally round disk-like structures.

Cultivation: Growth in Noguchi's semi-solid serum agar 2 weeks after inoculation with eitrated or heparinized blood and incubated at $23^{\circ} \mathrm{C}$ shows as white rounded masses, measuring up to about $1 \mathrm{~mm}$ in the upper $15 \mathrm{~mm}$ of the tube. In tissue culture the organism grows in small, rounded compact masses within the cytoplasm of infected cells. Indefinite maintenance of the strains isolated on artificial media has not been possible.

Pathogenicity: Splenectomized white mice and splenectomized laboratory reared voles are readily susceptible to infection. No marked anemia or any mortality in heavily infected animals. Splenectomized dogs, white rats and deer mice are not susceptible.

Source and habitat: Erythrocytes of the vole (Microtus pennsylvanicus pennsylvanicus) following splenectomy. The natural mode of transmission has not been determined though ticks or mites are suspected.

\section{Haemobartonella tyzzeri (Wein-} man and Pinkerton) Weinman: (Bartonella tyzzeri Weinman and Pinkerton, Ann. Trop. Med., 32, 1938, 217; Weinman, Trans. Amer. Philosoph. Soc., 33, 1944, 314.) Named for Prof. Tyzzer who studied haemobartonellae.

Single or composite rods from about 0.25 micron by 1.4 to 4.0 microns. Occasional granular swellings and enlarged poles. Short rods also occur averaging 0.2 to 0.3 by 0.8 micron and also round forms with diameters of 0.2 to 0.3 micron. Distributed irregularly in the red cells. Stain intensely red-violet with Giemsa's or May-Grünwald-Giemsa's solutions. Gram-negative.

Cultivation: Initial cultures on Noguchi's semi-solid serum agar obtained irregularly. When incubated at $28^{\circ} \mathrm{C}$, colonies appear as isolated white spheres about $1 \mathrm{~mm}$ in diameter in the upper 8 $\mathrm{mm}$ border of the medium. The clumps are composed of rods and granules, with larger round structures or disks occurring occasionally. Also cultivated on the Zinsser, Wei and Fitzpatrick modification of the Maitland medium. Prolonged maintenance on semi-solid media has not been obtained.

Pathogenicity: Splenectomized haemobartonella-free guinea pigs may be infected by blood or cultures injected subcutaneously or intraperitoneally. Splenectomized Haemobartonella murisfree rats are insusceptible when inoculated with infected guinea-pig blood. Lacacus rhesus monkeys are also in- 
susceptible to inoculations of infected blood, tissue and cultures. Infection of the guinea pig is subclinical in its manifestations, probably due to the small number of parasites in the blood. No definite anemia accompanies infection.

Source and habitat: Erythrocytes of the Peruvian guinea pig (Cavia porcellus). Has also been encountered in Colombia but not in other parts of the world. Observed in latently infected animals only after splenectomy. The natural mode of transmission is unknown. though the flea may be a possible vector.

\section{Haemobartonella bovis (Donatien} and Lestoquard) Weinmin. (Donatien and Lestoquard, Bull. Soc. Path. Exot., 27, 1934, 652; Bartonella sergenti Adler and Ellenbogen., Jour. Comp. Path. and Therap., 47, 1934, 221; (?) Bartonella bovis Rodriguez, Rev. del Inst. Llorente, 13, 1935, 5; abst. in Bull. Inst. Past., 34 , 1936, 1033; Weinman, Trans. Amer. Philosoph. Soc., X. S., 33, 1944, 308; Haemobartonella sergenti Weinman, loc. cit., 290.) From Latin bos, bovis, ox.

Resembles Haemobartonella muris and H. canis. Occurs as rods, coccobacilli and cocei, singly, in pairs or short chains or groups of 10 or more elements. The rods measure 1.2 to 2 microns in length and are very slender. The coccobacilli occur singly or in pairs measuring 0.3 by 0.6 to 0.8 micron and the diameters of the cocci are about 0.3 micron. The parasite may occupy a central or marginal position on the red cell; the number on a cell varying from 1 to 20 . Tot more than 20 per cent of the cells are parasitized.

Using the Romanowsky stain, the organisms stain similarly to the chromatin of Piroplasma spp.

Source and habitat: In the blood of bulls in Algeria and in a non-splenectomized calf in Palestine.

6. Haemobartonella sturmanii Grinberg. (Grinberg, Ann. Trop. Med., 33,
1939, 33; Weinman, Trans. Amer. Philosoph. Soc., N. S., 33, 1944, 313.)

Similar to Haemobartonella bovis and $H$. canis in morphology and staining properties. Occurs as rods, cocco-bacillary and coccoid forms, varying in length from 0.5 to 1.5 microns. The number of parasites per infected cell varies from 1 to 15 and they occur individually, scattered irregularly in clumps or sometimes in chains stretching across the cell. At the height of the infection more than 90 per cent of the cells are infected.

Pathogenicity: Causes a temperature rise in buffaloes and slight anemia after direct blood inoculation. Splenectomized rabbits, hamsters and splenectomized calves inoculated with blood from infected buffaloes remained free of the parasite.

Source and habitat: In the blood of huffaloes in Palestine.

\section{Haemobartonella peromyscii Tyzzer.} (Proc. Amer. Philos. Soc., 85, 1942, 377.) Named for the genus of deer mice, Peromyscus.

Occurs as delicate filamentous forms (which may be branched) on the red blood colls. These filaments may become beaded and give rise to a number of roccoids and rods from which ring forms may develop.

Stains by Ciemsa's method, but staining process must be intense in order to demonstrate the organism.

Pathogenicity: Infection transmissible to splenectomized white rats, white mice and voles, producing a more or less severe illness with anemia.

Habitat: In the blood of the deer mouse (Peromyscus lencopus novaboracensis).

7a. Haemobartonella peromyscii var. maniculati Tyzzer. (Proc. Amer. Philos. Soc., 85, 1942, 381.) Named for the species of mouse from which it was isolated.

Occurs as rods and filamentous 
branched forms. Coarser filaments appear to rise from rounded granules. Delicate rods are preponderant, and minute coccoids appear occasionally. When transferred to the common deer mouse, coarser forms appear, including filaments and large coccoids, sometimes in chains.

Pathogenicity: Pathogenic for graybacked deer mice and the common deer mouse, but non-infective for splenectomized white mice.

Habitat: Blood of the gray-backed deer mouse (Peromyscus maniculatus gracilis).

\section{Haemobartonella blarinae Tyzzer}

(Proc. Amer. Philos. Soc., 85, 1942, 382.) Named for the genus of shrews, Blarina.

Extremely pleomorphic with delicate rods and coccus-like forms, often occurring in chains which also contain larger elements which have a deeply stained, bead-like granule. In the early stages of infection they may occur as thick bands or filaments stretching over the red cells usually with a bead or granule.

The bands take a bluish tint with Geimsa's stain, while the more delieate form stains a slaty violet. The head is distinctly reddish. In the fully developed infection, rods and filaments pre dominate over rounded forms. The organisms may be scattered on the surface of the red cells or may form a dense cap which is intensely stained. Rudimentary mycelia may be found radiating from a central portion and reddish stained material with ill-defined contours may occur at the ends of the mycelial branches.

Pathogenicity: Pathogenic for the short-tailed shrew but not for deer mice or white mice. Causes anemia in the shrew.

Habitat: In the blood of the short tailed shrew (Blarina brevicauda).

9. Haemobartonella sciurii Tyzzer. (Proc. Amer. Philos. Soc., 55, 1942, 385.)
Named for the genus of gray squirrels, Sciurus.

Very pleomorphic. Occurs as minute rods and filaments which are continuous or segmented. The rods and filaments vary in thickness, some are very uneven and some very coarse. Beaded chains may develop from the thickened forms.

The bead-like elements stain a dull reddish at the periphery with Giemsa's stain while the remainder is very faintly stained in contrast to the intensely staining basophilic rods and filaments. Some of the rounded forms have the appearance of large, thick rings. Beads and rings may arise from slender deeply staining rods, simulating very closely spores within bacilli, though no germination of filaments from them has been observed.

Pathogenicity: Slightly pathogenic for the gray squirrel, non-pathogenie for normal white mice.

Habitat: Blood of the gray squirrel (Sciurus carolinensis leucotis).

Appendix: Here are included (1) Haemobartonella of undetermined specific rank, (2) Haemobartonella-like structures in non-splenectomized mammals and in cold-blooded animals, (3) Invalid species (see Weinman, Trans. Amer. Philosoph. Soc.. X. S., 33, 1944, 315).

1. Hameobartonellae of undetermined specifie rank. Microorganisms are grouped according to host of origin and are considered to be haemobartonellae from the description of the original author; but the information furnished is not sufficient for further classification.

Haemobartonellae similar to Haemobartonella muris in wild rats: Mus decumanus, Wus norvegicus, Ratlus rattus frugivorus, Mus rattus griseiventer, Mus rattus rattus, Mus sylvaticus. In various rats; technical names not given.

Haemohartonellae similar to Haemobartonella muris in albino mice. Schilling (Klin. Wehnschr., 1929, 55) separated the haemobartonella of the mouse from 
that of the rat and named it Bartonella muris musculi var. albinoi (Haemobartonella muris musculi var. albinoi Weinman, loc. cit., 290).

Haemobartonellae similar to Haemobartonella muris in other mammals: Haemobartonella glis glis (Kikuth) Weinman (Bartonella glis glis Kikuth, Cent. f. Bakt., I Abt., 123, 1931, 356; Weinman, loc. cit., 317) in dormice (Glis glis).

Haemobartonella opossum (Regendanz and Kikuth) Weinman (Bartonella opossum Regendanz and Kikuth, Arch. f. Schiffs- u. Tropenhyg., 32, 1928, 587; Weinman, loc. cit., 290) in the marsupial rat (Metachirus opossum) and in the opossum (Didelphys didelphys).

Haemobartonella spp. in Lophuromys ansorgei, in Lophuromys laticeps, in Oenomys bacchante editus, in Praeomys jacksoni, in Arvicanthus striatus, in deer mouse (Peromyscus leucopus novaboracensis), in Chinese hamsters (Cricetulus griseus, Cricetulus griseus fumatus), in A podemus agrarius and Phodopus praedilectus), and in squirrels (Sciurusvulgaris). Mixed infections, including haemobartonellae are found in jerboa, the gerbille. and various rodents (see Weinman, loc. cit., 317-319).

2. Haemobartonella-like structures in non-splenectomized mammals and coldblooded animals.

Various bodies whose proper classification in the genus Hacmobartonella has not been established (Weinman, loc.cit., 319)

In non-splenectomized mammals:

Bartonella melloi Yakimoff and Rastegaieff, Bull. Soc. Path. Exot., 24, 1931, 471 (Haemobartonella melloi Weinman, loc. cit., 290) in the ant eater (Manis pentadactyla).

Bartonella pseudocebi Pessõa and Prado, Rev. biol. e hyg., 1, 1927, 116 (Haemobartonella pseudocebi Weinman, loc. cit., 290) in the monkey (Pseudocebus apella).

Bartonella rocha-limai Faria and Pinto, Compt. rend. Soc. Biol., Paris, 95, 1926,
1500 (Haemobartonella rocha-limai Weinman, loc. cit., 290) in the bat (Hemiderma brevicauda).

Bartonella sp. in the rat (Ratlus rufes. cens) and Bartonella $s p$. in the dormouse (Myoxus glis).

In cold-blooded animals:

Bartonella pavlovskii Epstein, All Union. Inst. Exper. Med., Moscow, 1935, 398, see Ray and Idnani, Indian Jour. Vet. Sci. and Animal Husb. 10, 1910, 259, (Haemobartonella pavlovskii, Weinman loc. cit., 290) in the lamprey (Petromyzon marinus).

Bartonella nicollei Yakimoff, Arch. Inst. Pasteur Tunis, 17, 1928, 350 (Haemobartonella nicollei Weinman, loc. cit., 290) in the brochet (Esox lucius).

Bartonella ranarum da Cunha and Munez, Compt. rend. Soc. Biol., Paris, 97, 1927, 1091 (Haemobartonella ranarum Weinman, loc. cit., 290) in the frog (Leptodactylus ocellatus). This is probably identical with Bartonella batrachorum Zavattari, Boll. d. Soc. ital. biol. sper., 6, 1931, 121 (Haemobartonella batrachorum Weinman, loc. cit., 290) from the same species.

Bartonella sp. in the gecko (Platydactylus mauritanicus), Bartonella $s p$. in the lizard (Lacertilia sp.), Bartonella $s p$. in the lizard (Tropidurus peruvianus), Bartonella $s p$. in the tench (Tinca tinca) and Bartonella $s p$. in the tortoise (Testudo graeca).

\section{Invalid species:}

Bartonella caviae Campanacci, Ateneo parmense, 1, 1929, 99 (Haemobartonella caviae Weinman, loc. cit., 290) from the guinea pig

Bartonella ukrainica Rybinsky, Rev. Microbiol. epidem. et parasitol., 8, 1929, 296 (Haemobartonella ukrainica Weinman, loc. cit., 290) from the guinea pig.

Weinman (loc. cit., 314) states that the parasitism of these structures was not proven and no illustrations are furnished by the authors. 


\section{Genus III. Grahamella Brumpt.}

(Brumpt, Bull. Soc. Path. Exot., 4, 1911, 514; Grahamia Tartakowsky, Trav. IX Cong. Int. Med. Vet., 4, 1910, 242; not Grahamia Theobald, Colonial Office, Misc. Pub. No. 237, 1909.) Named for G. S. Graham-Smith who discovered the parasite in the blood of voles.

Parasites occurring within the erythrocytes of the lower mammals which morphologically bear a resemblance to Bartonella, but which are less pleomorphic, more plump, and more suggestive of the true bacteria. They stain more deeply than bartonellae with Giemsa's stain, stain lightly with aniline dyes and with methylene blue. They are Gram-negative, non-acid-fast and non-motile. Splenectomy has no effect on the source of infection except in rats. They are non-pathogenic and not affected by arsenicals. Several species have been cultivated on cell-free media.* The etiological agent of grahamellosis of rodents and some other vertebrates.

The type species is Grahamella talpae Brumpt.

1. Grahamella talpae Brumpt. (Bull. Soc. Path. Exot., 4, 1911, 514.) Named for the genus of moles, Talpa.

Long or short rods of irregular contour lying within the red blood cells, many with a marked curve, of ten near one of the extremities. One or both ends of the longer forms enlarged, giving a wedgeor club-shaped appearance. Some of the medium-sized forms definitely dumbbellshaped, small forms nearly round.

With Giemsa's stain, the protoplasm of the organism stains light blue, with darker areas at the enlarged ends. Dark staining areas of longer forms give the organism a banded appearance. Length varies from 0.1 to 1 micron. Parasites occasionally free in the plasma, but usually in groups. Most of the infected corpuscles contain between 6 and 20 parasites (Graham-Smith, Jour. Hyg., 5, 1905, 453).

Pathogenicity: Pathogenic for moles.

Appendix: In addition to Grahamella talpae Brumpt, descriptions of the following species occur in the literature. The list may not be complete and the validity of these species may be questioned in some cases.

Grahamella acodoni Carini. (Ann. Parasit., 2, 1924, 253.) From Acodon serrensis, Brazil.

Grahamella alactagae Tartakowsky. (Katalogue der Exponaten der Landwirthschaftlichen Ausstellung (Russisch), St. Petersburg, 1913.) From Alactaga saliens and Alactaga aconitus in Transcaucasia and steppes of Astrakhan (Alactaga misspelled Alactoga). Quoted from Yakimoff, Arch. f. Protistenk., 66, 1929, 303.

Grahamella arvalis Tartakowsky. (Katalogue der Exponaten der Landwirthschaftlichen Ausstellung (Russisch) St. Petersburg, 1913.) From Microtus arvalis in Transcaucasia. Quoted from Yakimoff, Arch. f. Protistenk., 66, 1929, 304.

Grahamella balfourii Brumpt. (Grahamella sp. Balfour, Rept., Wellcome Tropical Research Laboratory, 2, 1906, 97; Grahamella balfouri Brumpt, Bull. Soc. Path. Exot., Paris, 4, 1911, 517.) From the desert rat (Jaculus jaculus) in the Sudan.

* Tyzzer (Proc. Amer. Philos. Soc., 85, 1942, 375) finds that grahamellae isolated in culture show a close relationship to Streptobacillus moniliformis (Actinomyces muris) and proposes the inclusion of the genus Grahamella in the family Actinomycetaceae. The latter relationship appears to be very doubtful. 
Grahamella blarinae Tyzzer. (Proc. Amer. Philos. Soc., 85, 1942, 370.) From the short-tailed shrew (Blarina brevicauda) in Massachusetts.

Grahamella bovis Marzinowsky. (Med. Obosrenie, 1917, No. 1-2.) From the ox (Bos taurus) in Russia. Quoted from Yakimoff, Arch. f. Protistenk., 66, 1929, 304.

Grahamella brumptii Ribeyro and del Aquilla. (Ann. Fac. Med., Lima, 1, 1918, 14-20.) From Desmodus rufus in Peru.

Grahamella canis lupus Kamalow. (Cent. f. Bakt., I Abt., Orig., 128, 1933, 197.) From the wolf, Tiflis.

Grahamella couchi Neitz. (Onderst. Jour. Vet. Sci. and An. Ind., 10, 1938, 29.) From the multimammate mouse (Mastomys coucha) in South Africa.

Grahamella criceti domestici Parzwanidze. (Das Material zum Hämoparasitismus der Tiere bei (ns. Tiflis, 1925.) From Cricelus domesticus in Transcaurasia. Quoted from Yakimoff, Arch. f. Protistenk., 66, 1929, 304.

Grahamella cricetuli Patton and Hindle. (Proc. Roy. Soc., London, $\mathrm{B}(100)$, 1926, 387.) From Cricetulus griseus in China.

Grahamella dschunkowstii Tartakow sky. Grahamiasp., Dschunkowsky and Luhs, Trav. IX. Cong. Internat. Med. Vet., 1909, 4, 1910, 242; Grahamia dschunlowski Tartakowsky, 1910.) From the bat (Tespertilio noctula) in Transeaucasia. Ref. to Tartakowsky quoted from Yakimoff, Arch. f. Protistenk., 66, 1929, 304.

Grahamella dudtschenkoi Iakimoff. Grahamella sp., Dudtschenko, Cent. f. Bakt., I Abt., Orig., 74, 1914, 241; Grahamella dudtschenkoi Yakimoff, Arch. f. Protistenk., 66, 1929, 304.) From the hamster (Cricetulus sp.) in Transbaikal.

Grahamella ehrlichii lakimoft. (Grahamella ehrlichi Yakimoff, Arch. f. Protistenk., 66, 1929, 305.) From the perch (Perca fluviatilis) in Russia.
Grahamella francai Brumpt. (Grahamella sp. Franca, Arch. Inst. Bacter. Camara, Pestana, 3, 1911, 277; Grahamella francai Brumpt, Précis de Parasitologie, 2éme éd., 1913, 102.) From the jumping rat (Eliomys quercinus) in Portugal.

Grahamella gallinarum Carpano. (Ann Parasit. hum. et comp., 13, 1935, 238.) From leghorn chickens in Egypt.

Grahamella gerbilli Sassuchin. (Grahamia gerbilli Sassuchin, Arch. f. Protistenk., 74, 1931, 526.) From Gerbillus tamaricinus in southeast Russia.

Grahamella hegneri Sassuchin. (Grahamia hegneri Sassuchin, Arch. f. Protistenk., $75,1931,152$.$) From Citellus$ pygmaeus in Russia.

Grahamella joyeuxii Brumpt. (Grahamella sp., Joyeux, Bull. Soc. Path. Exot., Paris, 6, 1913, 614; Grahamella joyeuxi Brumpt, Précis de Parasitologie, 2éme éd., 1913, 102.) From Golunda fallax and Mus raltus in French Guinea.

Grahamella merionis Adler. (Trans. Roy. Soc. Trop. Ned., 24, 1930, 78.) From Meriones tristrami in Palestine.

Grahamella microli Lavier. (Bull. Soc. Path. Exot., Paris, 14, 1921, 573.) From Vicrotus arvalis in France.

Grahamella microti pennsylvanici Tyzzer. (Proc. Amer. Philos. Soc., 85, 1942, 366.) From the common vole (Microtus pennsylranicus ponnsylranicus) in Massachusetts.

Grahamella muris C'arini. (Bull. Soc. Path. Exot., Paris, 8, 1915, 104.) From the house rat (Mus decumanus) in Brazil.

Grahamella muris musculi iberica Parzwanidze. (Das Material zum Hämoparasitismus der Tiere bei uns. Tiflis, 1925.) From Mus musculus in Transcaucasia. Quoted from lakimoff, Arch. f. Protistenk., 66, 1929, 304.

Grahamella musculi Benoit-Bazille. (Bull. Soc. Path. Exot., Paris, 13, 1920, 408.) From Hus musculus var. albinos in France.

Grahamella ninae kohl-yakomovi Takimoff. (Bull. Soc. Path. Exot., Paris, 10, 
1917, 99.) From the hamster (Cricetus phoca) in Transcaucasia.

Grahamella peramysci Tyzzer. (Proc. Amer. Philos. Soc., 85, 1942, 363.) From the deer mouse ( $P$.leucopus novaboracensis) in Massachusetts.

Grahamella peromysci var. maniculati Tyzzer. (Proc. Amer. Philos. Soc., 85, 1942, 365.) From the gray-backed deer mouse (Peromyscus maniculatus) in Massachusetts.

Grahamella phyllotidis Tyzzer. (Proe. Amer. Philos. Soc., 85, 1942, 371.) From the Peruvian mouse (Phyllotis darwini linatus).
Grahamella pipistrelli Markow. (Grahamia pipistrelli Markow, Russian Jour. Trop. Med., 1926, No. 5, 52.) From the bat (Pipistrellus nathusii) in Russia.

Grahamella rhesi Leger. (Bull. Soc. Path. Exot., Paris, 15, 1922, 680.) From the monkey (Macacus rhesus) in Annam.

Grahamella sanii Cerruti. (Arch. Ital. Sci. Med. Col., 11, 1930, 522.) From Testudo graeca in Sardinia.

Grahamella talassochelys Cerruti. (Arch. Ital. Sci. Med. Col., 12, 1931, 321.) From Tallasochelys carelta in Sardinia. (Misspelled for Thalassochelys.)

\section{Genus IV. Eperythrozoon Schilling.*}

(Schilling. Kilin. Wehnschr., 1928, 1854; Gyromorpha Dinger, Nederl. tijdschr. geneesk., 72, 1928, 5903.) From Greek meaning animal on red blood cell.

Microscopic blood parasites found in the plasma and on the erythrocytes. They stain well with Romanowsky type dyes, and then appear as rings, coccoids or short rods, 1 to 2 microns in greatest dimension, staining bluish or pinkish violet. They show no differentiation of nucleus and cytoplasm. The organisms are not known to retain the violet in Gram's method or to be acid-alcohol-fast. Splenectomy activates latent infection. Not cultivated in cell-free media. Arthropod transmission has been established for one species (Weinman, Trans. Amer. Philosoph. Soc., N.S. 33 , pt. $3,1944,321$ ).

The type species is Eperythrozoon coccoides Schilling.

\section{Key to the species of genus Eperythrozoon.}

I. Etiological agent of eperythrozoönosis of white mice.

1. Eperythrozoon coccoides.

II. Etiological agent of eperythrozoünosis of sheep.

2. Eperythrozoon ovis.

III. Etiological agent of eperythrozoünosis of cattle.

3. Eperythrozoon wenyonii.

IV. Etiological agent of eperythrozoünosis of gray-backed deer mice.

4. Eperythrozoon varians.

$V$. Etiological agent of eperythrozoönosis of voles and dwarf mice.

5. Eperythrozoon dispar.

* This genus has been considered as belonging to the Protozoa by Neitz, Alexander and Du Toit (Onderst. J. Vet. Sei., 3, 1934, 268) and to the bacteria by Mesnil (Bull. Soc. Path. exot., 22, 1929, 531 and by Tyzzer (in Weinman, Trans. Amer. Philosoph. Soc., N.S., 33, pt. 3, 1944, 244). The evidence at hand favors the inclusion of this group among those organisms which are not protozoan in nature but which are closely related to bacteria. 
1. Eperythrozoon coccoides Schilling. (Sehilling, Klin. Wehnschr., 1928, 1854; Gyromorpha musculi Dinger, Nederl. tijdschr. geneesk., 72, 1928, 5905.) From Greek, coccus-shaped.

In stained blood films these oryanisms appear as rings, coccoids and rods, the majority as rings of regular outline with clear centers. They are in the plasma and on the red cells. Measure 0.5 to 1.4 microns in greatest dimension.

Stain pale red or reddish-blue with the Giemsa or the May-Grunwald-Giemsa technics. Gram-negative.

Suggested methods of multiplication by binary fission, budding, development of small coccoidal to annular forms.

Cultivation: Negative results.

Immunology: Immunological state in animals that of the premunition type. Latent infection in mice which is made manifest by splenectomy.

Pathogenicity: Pathogenic for white mice, rabbits, white rats, wild mice, usually in young animals or in splenertomized adults.

Source: Blood of splenectomized white mice.

Habitat: Blood of infected animals, mouse louse (Polyplax serrata) and probably other arthropods.

2. Eperythrozoon ovis Neitz, Alexander and Du Toit. (Neitz et al., Address, Biological Society, Pretoria, Mar. 15, 1934; from Neitz, Onderst. Jour. Vet. Sci. and An. Ind., 9, 1937, 9.) From Latin ovis, sheep.

Delicate rings approximately 0.5 to 1.0 mieron in diameter though occasionally larger. In addition there are triangles with rounded angles, ovoid, comma, rod, dumbbell and tennis racket forms. Found supra-cellularly on the erythrocytes but often free. Colored pale purple to pinkish-purple with Giemsa's stain. Suggested mode of multiplication by budding.

Cultivation: Negative results.
Immunology: Immunological state in sheep appears to be that of the premunition type.

Pathogenicity: Sheep, antelopes and probably goats and splenectomized calves are susceptible. Dogs, rabbits and guinea pigs are refractory. The distinctive feature of Eperythrozoon ovis is its ability to provoke illness in normal animals without resorting to splenectomy.

Source: Blood of infected South African sheep.

Habitat: Blood of infected animals. No ectoparasites found on sheep naturally infected, but an arthropod is suspected.

3. Eperythrozoon wenyonii Adler and Ellenbogen. (Adler and Ellenbogen, Jour. Comp. Path. and Therap., 47, 1934 (Sept. 3), 220; see Bartonella wenyoni in appendix.) Named for Dr. C. M. Wenyon, a student of these organisms.

Morphologically similar to Eperythrozoon coccoides. Coccoid and often vesicular, staining pale red with Giemsa's stain and varying from 0.2 to 1.5 microns in diameter. Multiplication seems to be by budding and fission, and by filamentous grow ths from the ring forms, suggesting resemblance to Hyphomycetes. Up to 50 or 60 parasites are found on one cell. These are arranged in irregular chains or in tightly packed groups.

Cultivation not reported.

Immunology: The organism creates a state of premunition and latent infection is made manifest by splenectomy.

Pathogenicity: Cattle are susceptible, but sheep are not infected either before or after splenectomy.

Source: Blood of infected cattle.

Habitat: Blood of infected cattle, arthropod transmission not proven.

4. Eperythrozoon varians Tyzzer. (Proc. Amer. Philos. Soc., 85, 1942, 387.) From Iatin varians, varying. 
Occur in rings, coccoids of varying size, some very minute, bacillary forms.

Many of the bacilliform elements show an unstained lens-like swelling, indicating the formation of a ring within the substance of the rod. At the height of the infection most of the organisms are found in the plasma. Whenever an organism comes in contact with a red cell, it stains intensely.

Pathogenesis: Pathogenic for the graybacked deer mouse (causing anemia) and for the splenectomized common deer mouse. Not pathogenic for splenectomized white mice.

Habitat: Blood of the gray-backed deer mouse (Peromyscus maniculatus gracilis).

5. Eperythrozoon dispar Bruynoghe and Vassiliadis. (Ann. de Parasitol., 7, 1929, 353.)

Resembles Eperythrozoon coccoides in staining, distribution on the erythrocytes and also in appearance except that circular disks with solid staining centers may greatly outnumber the ring forms. Found on the red blood cells and in the plasma. Size range that of Eperythrozoon coccoides, also some larger ring forms.

Cultivation: Not successful.

Immunology: Infection is followed by premunition and latent infection is made manifest by splenectomy. Splenectomized rabbits premunized against $E$. coccoides do not react to inoculation with $E$. dispar; if the latter is injected first, they do not react to $E$. coccoides.

Infectivity: Infective for the European vole (Arvicola[Microlus] arvalis), the American vole (Microtus pennsylvanicus pennsylvanicus), the dwarf mouse (Mus minutus), the rabbit, and Mus acomys.
Not infective for albino rats or albino mice.

Source: Blood of infected animals.

Appendix: 1) Species incompletely studied, Eperythrozoon spp. and Eperythrozoon-like structures (Weinman, Trans. Amer. Philosoph. Soc., N. S. 33, pt. 3, 1944, 320).

Eperythrozoon noguchii Lwoff and Vaucel. (Bull. Soc. path. exot., 26, 1933, 397.) Probably not a valid species.

Eperythrozoon perekropovi Yakimoff. (Arch. f. Protistenk., 73, 1931, 271.) Classification in genus Eperythrozoon questionable.

Bartonella wenyoni Nieschulz. (Ztschr. f. Infektionskr., 53, 1938, 178.) Probably identical with Eperythrozoon wenyoni. If valid, Haemobartonella wenyoni.

Possible human infection (Schüffner, Nederl. tijdschr. v. geneesk., 73, 1939, 3778 ).

2) Animals infected with parasites which are definitely eperythrozoön-like but of uncertain specificity or which are eperythrozoön-like in some features but which can not be definitely classified generically :

Jerboa sp. Kikuth. (Cent. f. Bakt., I Abt., Orig., 123, 1931, 356.)

Arvicola arvalis Zuelzer. (Zuelzer, Cent. f. Bakt., I Abt., Orig., 102, 1927, 449 ; Kikuth, Ergebn. Hyg. Bakt., Immunitätsforsch. u. Exper. therap., 13, 1932, 559.)

Rattus rattus Schwetz. (Ann. Soc. belge de med. trop., 14, 1934, 277.)

Sciurus vulgaris Nauck. (Arch. f. Schiffs- u. Trop.-Hyg., 31, 1927, 322.)

Leptodactylus pentadactylus Carini. (Compt. rend. Soc. Biol., Paris, 103, 1930, 1312.) 


\section{FAMILY III. CHLAMYDOZOACEAE MOSHKOYSKY.*}

(Uspekhi Souremennoi Biologii (Russian) (Advances in Modern Biology), 19, $1945,12$.

Small, pleomorphic, often coccoid microorganisms usually with characteristic development cycle. Stain with aniline dyes. Gram-negative. Behave as obligate intracytoplasmic parasites. Have not been cultivated in cell-free media. Criteria adequate for classification lacking for more recently isolated members. The attribution of Crenus III, Colesiota, either to Rickettsiaceae or to Chlamydozoaceae is still in doubt.

\section{Key to the genera of family Chlamydozoaceae.}

I. Cells coccoid and with life cycle.

A. Non-cultivatable in chicken embryonic tissues.

Genus I. Chlamydozoon, p. 1114.

B. Cultivatable in chicken embryonic tissues.

Genus II. Miyagawanella, p. 1115.

II. Cells pleomorphic.

Genus III. Colesiota, p. 1119.

Genus 1. Chlamydozoon Halberstaedter and von Prowazek.

(Arb. a. d. kaiserl. Gesundheitsamte, 26, 1907, 44.) From Greek chlamydos, cloak and zoon, animal.

Coccoid spherical cells with developmental cycle. Gram-negative. Intracytoplasmic habitat. Non-cultivatable in chicken embryonic tissues. Susceptible to sulfonamide and penicillin action.

The type species is Chlamydozoon trachomatis Foley and Parrot.

1. Chlamydozoon trachomatis Foley and Parrot. (Rickeltsia trachomae Bus:icea, Arch. Ophthalm., 52, 1935, 567; Foley and Parrot, Arch. Inst. Past. d'Algérie, 15, 1937, 339; Ricletlsia trachomalis Foley and Parrot, idem.) Tamed for the disease, trachoma.

Coccoid bodies: Small microorganisms 200 to 350 millimicrons in diameter form the elementary bodies. Initial bodies up to 800 millimicrons in diameter and plaques up to 10 microns also found. All larger forms encapsulated with substanee derived either from the agent or from the cytoplasm of the parasitized cells. Elementary body is the basic unit. Paired forms or clusters occur. Gramnegative. Stains poorly with aniline dyes; blue or reddish-blue with the Ciemsa stain and red or blue, depending on the metabolic state, with the Macchiavello stain. Matrix of plaques gives a strong reaction for glyeogen. Nonmotile.

Cultivation: Has never been cultivated.

Immunological aspects: Has one or more antigens in common with or closely resembling one or more present in Miyagawanella spp. Produces, in low conrentrations, antibodies which fix complement with antigen from Miyagawanella lymphogranulomatis.

Pathogenicity: Pathogenic for man, apes and monkeys where it affects only

* Prepared by Dr. Geoffrey Rake, The Squibh Institute for Medical Research, New Brunswick, New Jersey, September, 1946. 
the cornea and conjunctiva causing highly destructive lesions.

Chemotherapy: Susceptible to sulfonamides and penicillin.

Source: Found in scrapings of cornea or conjunctiva in cases of trachoma.

Habitat: The etiological agent of trachoma in man.

2. Chlamydozoon oculogenitale Moshkovsky. (Moshkovsky, Uspekhi Souremennoi Biologii, 19, 1945, 12.) From Latin oculus, eye and genitalis, genital.

Morphology and staining reactions: As for Chlamydozoon trachomatis.

Cultivation: Has never been cultivated.
Immunological aspects: As for $C$. trachomatis.

Pathogenicity: Pathogenic for man, baboons and monkeys. Causes an acute conjunctivitis and, in man, an inflammation of the lower genito-urinary eract.

Chemotherapy: Susceptible to sulfonamides and penicillin.

Source: Found in conjunctival exudates, and in exudates from infected urethra or cervix. Also present in contaminated pools of water.

Habitat: The etiological agent of swimming pool conjunctivitis, neonatal conjunctivitis or inclusion conjunctivitis.

\section{Genus II. Miyagawanella Brumpt.}

(Ann. de Parasit., 16, 1938, 153.) Named for Prof. Miyagawa, the Japanese bacteriologist, who first (1935) grew the type species in the chick embryo.

Coccoid to spherical cells with a developmental cycle. Gram-negative. Intracytoplasmic habitat. Cultivatable in chicken embryonic tissues. Some species are susceptible to sulfonamide or penicillin action.

The type species is Miyagawanella lymphogranulomatis Brumpt.

\section{Key to the species of genus Miyagawanella.}

I. The etiological agent of lymphogranuloma venereum, lymphogranuloma inguinale, climatic bubo, and esthiomène in man.

\section{Miyagawanella lymphogranulomatis.}

II. The etiological agent of psittacosis or parrot fever.

\section{Miyagawanella psittacii.}

III. The etiological agent of ornithosis (Meyer).

3. Miyagawanella ornithosis.

IV. The etiological agent of one type of viral pneumonia.

4. Miyagawanella pneumoniae.

V. The etiological agent of mouse pneumonitis (Gönnert).

5. Miyagawanella bronchopneumoniae.

VI. The etiological agent of feline pneumonitis (Baker).

6. Miyagawanella felis.

VII. The etiological agent of Louisiana pneumonia.

7. Miyagawanella louisianae.

VIII. 'The etiological agent called the Illinois virus, the cause of one type of viral pneumonia.

\section{Miyagawanella illinii.}


1. Miyagawanella lymphogranulomatis Brumpt.(Brumpt, Ann. de Parasit., 16, 1938, 153; Ehrlichia lymphogranulomalosis Mauro, (Reference not found.) Named for the disease, lymphogranuloma.

Coccoid bodies: Small microorganisms 200 to 350 millimicrons in diameter form the elementary bodies. Initial bodies up to 1 micron and plaques up to 10 microns also found. All larger forms encapsulated with a substance derived either from the agent or from the cytoplasm of parasitized cells. Elementary body is the basic unit. Paired forms or clusters occur. Gram-negative. Stain with aniline dyes, purple with the Giemsa stain and red or blue, depending on metabolic state, with the Macchiavello stain. Matrix of the plaque does not give the reaction for glycogen. Non-motile.

Filterability: Passes through Chamberland $L_{2}$ and $L_{3}$, Berkefeld $V$ and $N$ and sometimes through Seitz EK filters.

Cultivation: In plasma tissue cultures of mammalian cells, in mammalian cells on agar, in the chorio-allantoic membrane or particularly in the yolk sac of the chicken embryo but has not been cultivated in the allantoic sac. Optimum temperature $37^{\circ} \mathrm{C}$ in tissue cultures, $35^{\circ} \mathrm{C}$ in the chicken embryo.

Immunological aspects: Has one or more antigens in common with or closely resembling one or more present in the chlamydozoa and other miyagawanellae. Antisera against any of these two genera react with antigens from Miyagawanella lymphogranulomatis or the other miyagawanellae thus far tested. One common antigen has been isolated as a soluble fraction distinct from the bodies of the agent. Distinguished sharply from the other miyagawanellae by antitoxic neutralization of toxic factor or by neutralization of infections in mice with chicken antisera. Evidence exists that these two serological reactions are with distinct specific antigens. Immunity in man or animals is probably poor in the absence of continuing apparent or inapparent infection.

Toxic factor: Infected yolk sac or yolk injected intravenously or intratraperitoneally is rapidly fatal to mice. Produces characteristic lesions on the skin of normal guinea pigs.

Pathogenicity: Pathogenic for man, apes, monkeys, guinea pigs, cotton rats, hamsters, mice, chicken embryos. Inapparent infections may occur with the agent harbored in the organs. Causes local genital lesions, septicemia, lymphadenitis, meningitis, ophthalmitis and rarely pneumonitis in man.

Tissue tropisms: In laboratory rodents this species is infective by the intranasal (pneumonitis), the intracerebral (meningitis) and the intradermal routes.

Chemotherapy: Susceptible to relatively high concentrations of penicillin, to the sulfonamides and to some antimony compounds.

Source: Most commonly the genital secretions of infected individual or the draining lymph nodes. Also occasionally in blood, spinal fluid and ocular secretions.

Habitat: The etiological agent of lymphogranuloma venereum, lymphogranuloma inguinale, climatic bubo, esthiomène and some forms of anorectal inflammation.

\section{Miyagawanella psittacii (Lillie)} Moshkovsky. (Rickettsia psittaci Lillie, Publ. Health Repts., 45, 1930, 773; Microbacterium multiforme psittacosis Levinthal,* 1st Cong. internat. de Microbiol., 1, 1930, 523; Moshkovsky, Uspekhi Souremennoi Biologii (Russian) (Advances in Modern Biology), 19, 1945, 12; Ehrlichia psittaci Moshkovsky, ibid., 19.) From Psittaci, an order of birds.

* This is the type species of the genus Microbacterium Levinthal which is invalid because of the earlier Microbacterium Orla-Jensen, 1919, see p. 370. 
Coccoid bodies: As for Miyagawanella lymphogranulomatis.

Filterability: Partly filterable through Berkefeld N, Chamberland L and Q or Seitz EK filters.

Cultivation: As for Miyagawanella lymphogranulomatis but grows readily in allantoic sac without adaptation.

Immunological aspects: As for $M$. lymphogranulomatis but no soluble fraction yet demonstrated.

Toxic factor: Infected yolk sac or yolk injected intravenously or intraperitoneally is rapidly fatal to mice.

Pathogenicity: Pathogenic for birds (particularly psittacine and finch species), man, monkeys, guinea pigs, pocket gophers, hamsters, white rats, kangaroo rats, mice, rabbits and chicken embryos. Inapparent infections may occur with the agent harbored in the organs. Causes a highly fatal pneumonitis with septicemia in man.

Tissue tropisms: Causes a septicemia. In man this species shows predilection for the respiratory tract. In laboratory rodents, it is infective by the intranasal, the intraperitoneal (peritonitis and septicemia), the intracerebral and the intravenous routes.

Chemotherapy: Susceptible to relatively high concentrations of penicillin. Some strains are susceptible to sulfonamides.

Source: Found in the organs and nasal secretions of infected birds and, from the latter, spreads to the plumage by preening and other methods. Plentiful in droppings or dust from infected cages. Relatively resistant under such conditions.

Habitat: The etiological agent of psittacosis or parrot fever. Also of some cases of atypical pneumonia.

3. Miyagawanella ornithosis Rake, spec. nov. From Greek ornithos, bird.

Coccoid bodies: As for Miyagawanella lymphogranulomatis.

Cultivation: As for Miyagawanella psittaci.
Immunological aspects: Has one or more antigens in common with, or closely resembling, one or more present in chlamydozoa and other miyagawanellae as shown by a cross reaction in complement fixation tests. Sharply distinguished from other miyagawanellae by toxinantitoxin neutralization or by neutralization of infection in mice with chicken antisera. The latter test however suggests that the agent of meningopneumonitis (Francis and Magill, Jour. Exp. Med., 68, 1938, 147) is this species rather than something distinct. Immunity in man or animals is probably poor except in the presence of continuing apparent or inapparent infections. Cross reactions suggest that Miyagawanella ornithosis may be more closely related to Miyagawanella lymphogranulomatis than is $M$. psittaci.

Toxic factor: As for Miyagawanella psittaci.

Pathogenicity: Pathogenic for birds (especially non-psittacine species), man, ferrets, guinea pigs, hamsters, white rats, kangaron rats, mice, rabbits and chicken embryos. Inapparent infections may occur. Causes a moderately severe pneumonitis with septicemia in man.

Tissue tropisms: Causes a septicemia. In birds and man shows a predilection for the lungs. In laboratory rodents, this species is infective by the intranasal, intracerebral, intravenous and with relatively large inocula of most strains) intraperitoneal routes.

Chemotherapy: Susceptible to relatively large doses of penicillin. Not susceptible to sulfonamides.

Source: Found in organs and nasal secretions of finches, pheasants (including domestic chickens), domesticated doves, fulmar petrels and other birds. Spreads from the secretions to plumage and droppings.

Habitat: The etiological agent of ornithosis (Meyer) and meningopneumonitis (Francis and Magill). 
4. Miyagawanella pneumoniae Rake, spec. nov. Named for the disease, pneumonia.

Coccoid bodies: As for Miyagawanella lymphogranulomatis but slightly smaller, circa 200 millimicrons in diameter.

Cultivation: As for Miyagawanella psittaci.

Immunological aspects: As for Miyagawanella psittaci. Distinet from Miyagawanella ormithosis by the neutralization test with chicken antisera.

Pathogenicity: Pathogenic for birds, man, cotton rats, hamsters, white rats, kangaroo rats, mice and chicken embryos. Causes a fatal pneumonitis in man.

Tissue tropisms: As for Miyagawanella ornithosis.

Chemotherapy: As for Miyagawanclla ornithosis.

Source: Occurs in lungs of infected humans. Possibly originally of avian origin.

Habitat: The etiological agent of one type of viral pneumonia. The type strain is the so-called strain S-F (Eaton, Beck and Pearson, Journ. Exp. Med., 73, 1941, 641 ).

5. Miyagawanella bronchopneumoniae Moshkovsky. (Moshkovsky, Yspekhi Souremennoi Biologii, 19, 1945, 19; Ehrlichia bronchopneumoniae Moshkovsky, idem.) Named for the disease, bronchopneumonia.

Coccoid bodies: As for Miyagawanella pneumoniae.

Cultivation: As for Miyagawanella lymphogranulomatis. Does not grow in the allantoic cavity of the chick.

Immunological aspects: As for Miyagawanella lymphogranulomatis but no soluble antigen has been demonstrated.

Toxic factor: Heavily infected yolk sacs and yolk injected intravenously are very rapidly fatal to mice.

Pathogenicity: Pathogenie for mice, hamsters and ferrets. Produces a mod erately severe pneumonitis.

Tissue tropisms: Shows a predilection for the lungs. In mice, it is also infective by the intravenous route.

Chemotherapy: Susceptible to sulfonamides and to relatively large doses of penicillin.

Source: Found in lungs of certain stocks of the laboratory mouse.

Habitat: The agent of mouse pneumonitis. Bronchopneumonie virus (Gönnert, Cent. f. Bakt., I Abt., Orig., 147, $1941,151)$.

6. Miyagawanella felis Rake, spec. nov. From Latin felis, cat.

Coceoid bodies: As for Miyagawanella iymphogranulomatis.

Cultivation: As for Miyagawanella psitlaci.

Immunological aspects: As for Miyagawanclla psittaci but nothing known about inapparent infections in the natural host, the domestic eat.

Toxic factor: Infected yolk sac or other membranes and yolk or other fluids, injected intravenously into mice or chicken embryos or intraperitoneally into mice are rapidly fatal.

Pathogenicity: Pathogenic for cats, hamsters, mice and chicken embryos. Causes a fatal pneumonitis with acute conjunctivitis in eats.

Tissue tropisms: Prediliction for lungs and conjunctivae. In laboratory rodents, this species is infective by the intranasal, intraperitoneal, intracerebral and intravenous routes.

Chemotherapy: As for Miyagawanella ornithosis.

Source: Lungs of infected cats.

Habitat: The etiological agent of one form of cat nasal catarrh, influenza or distemper (Baker, Science, 96, 1942, 475) and feline pneumonitis.

7. Miyagawanella louisianae Rake, spec. nor. Named for the State of Louisiana.

Coccoid hodies: Is for Miyagawanella psitlaci. 
Filterability : Filters thruugh Berkefeld $\mathrm{N}$ and Mandler 6,7 and 9 filters.

Cultivation: In the yolk sac of the chicken embryo.

Immunological aspects: Indistinguishable from other miyagawanellae by complement fixation tests with yolk sac antigens. Partly distinguished from $M_{i}$ yagawanella psittaci and $M$. ornithosis by active immunization in mice and guinea pigs.

Pathogenicity: Pathogenic for man, guinea pigs, cotton rats, mice and chicken embryos. Slightly pathogenic for white rats, golden hamsters and deer mice. Macacus rhesus monkeys, rabbits, muskrats and nutria are unaffected. Causes a highly fatal pneumonitis and septicemia in man.

Tissue tropisms: Causes a septicemia. In man this species shows predilection for the respiratory tract. In laboratory rodents it is infective by the intranasal, intraperitoneal, intracerebral, intramuscular and subcutaneous routes.

Chemotherapy: As for Miyagawanella ornithosis.

Source: Sputum and organs of infected persons.
Habitat: The etiolugical agent of Louisiana pneumonia (Olson and Larson, U. S. Pub. Health Repts., 59, 1944, 1373), so-called Borg strain.

\section{Miyagawanella illinii Rake, spec.} not. Named for the State of Illinois.

Coccoid bodies: As for Miyagawanella lymphogranulomatis.

Filterability: Passes through Berkefeld $\mathrm{N}$ or $\mathrm{IV}$ filters.

Cultivation: In the yolk sac of chicken embryo.

Immunological aspects: Distinguished from other miyagawanellae by neutralization tests in mice with chicken antisera and partly from Miyagawanella psittaci, M. ornithosis and $M$. pneumonia by active immunization in mice.

Pathogenicity : Pathogenic for man and white mice. Causes a highly fatal pneumonitis in man.

Tissue tropisms: Infective in mice by the intranasal, intraperitoneal, intracerebral and subeutaneous routes.

Source: Lungs of infected persons.

Habitat: The etiological agent called the Illinois virus (Zichis and Shaughnessy, Science, 102, 1945, 301).

\section{Genus III. Colesiota Rake, gen. nov.}

(Rickettsia Coles, 17th Rept. Direct. Vet. Serv. and An. Ind. Un. South Africa, 1931, 175.) Named for Prof. Coles who first studied these organisms.

Pleomorphic cells which may be coccoid, triangular, rod-shaped or in the form of rings. Gram-negative. Intracytoplasmic habitat.

The type species is Colesiota conjunctivae (Coles) Rake.

\section{Colesiota conjunctivae (Coles)} Rake, comb. nov. (Riclictsia conjunctirae Coles, 17th Rept. Direct. Tet. Serv. and An. Ind. Ln. South Africa, 1931, 17:; Chlamydozoon conjunclivac Moshkovsky, Uspekhi Souremennoi Biologii, 19, 1945, 19.) From M. L. conjunctiva, the conjunctiva.

Pleomorphic bodies: Average diameter 600 to 950 millimicrons. May be solid and coccoid, rod-shaped, or triangular, or in form of open rings or horse-shoes.
No chains. Masses frequent. No capsule. Stains with ordinary aniline dyes but less intensely than bacteria. Gramnegative. Non-motile.

Cultivation: Has never been cultivated.

Immunological aspects: Unknown.

Pathogenicity: Pathogenic for sheep, cattle and goats. Causes acute conjunctivitis and keratitis.

Tissue tropisms: Affects only the conjunctiva and cornea. 
Habitat: Found in scrapings of cornea or conjunctiva or in discharges from affected eyes. Etiological agent of infectious or specific ophthalmia in sheep, cattle and goats.

\section{Colesiota conjunctivae-gallii (Coles)} Rake, comb. nov. (Riclieltsia conjunctivae-galli Coles, Onders. Jour. Vet. Sci. and Indust., 14, 1940, 469.) From conjunctiva and Latin gallus, hen.

Pleomorphic bodies: Similar to Colesiota conjunctivae. Stain purplish-red or blue with the Giemsa stain.

Cultivation: Has never been cultivated.

Immunological aspects: Unknown.

Pathogenicity: Pathogenic for the domestic fowl. Causes an acute conjunctivitis and keratitis.
Tissue tropisms: As for Colesiota conjunctivae.

Source: As for Colesiota conjunctivae. Habitat: The etiological agent of one form of ocular roup in fowls.

Appendix: The following are similar to or identical with the above:

Rickettsia conjunctivae-bovis (Coles, South Afr. Vet. Med. Assoc., 7, 1936, 1) cannot be distinguished from Colesiota conjunctivae by any described characteristics.

Rickettsia lestoquardi Donatien and Gayot. (Bull. Soc. Path. Exot., 35, 1942, 325.) Found in benign conjunctivitis in swine similar to that which occurs in ruminants. 
* Appendix to Order Rickettsiales: The following are described species of intracytoplasmic and intranuclear parasites of Protozoa whose relationships to similar parasites of arthropods and vertebrates are not yet clear. All of the protozoon intracellular parasites are of larger size than typical members of Rickettsiales and some have been placed in genera (Cladothrix, Micrococcus) where the typical species do not live intracellularly.

\section{Genus A. Caryococcus Dangeard.}

(Compt. rend. Acad. Sci., Paris, 194, 1902, 1365.)

Genus established for a bacterial parasite of the nucleus of Euglena; organisms rounded.

The type species is Caryococcus hypertrophicus Dangeard.

\section{Caryococcus hypertrophicus Dan-} geard. (Compt. rend. Acad. Sci., Paris, 134, 1902, 1365.) Parasitic in the nucleus of a flagellate (Euglena deses).

Occurs in the nucleus as an agglomeration of close-set, rounded corpuscles. The nucleus increases considerably in volume, the chromatin is reduced to thin layers against the membrane, the interior of the nucleus is divided into irregular compartments by chromatic trabeculae.

2. Caryococcus cretus Kirby. (Univ. Calif. Publ. Zool., 49, 1944, 240.) Parasitic in the nucleus of a flagellate (Trichonympha corbula) from the intestine of a termite (Procryptotermes sp.), Madagascar.

Spherules 1 to 1.5 microns or more in diameter, in preparations appearing clear with usually a chromatic, sharply defined, crescentic structure peripherally or interiorly situated, sometimes with two such bodies or several chromatic granules; parasitic in nucleus; parasitized nucleus enlarged only moderately or not at all, chromatin altered but not greatly diminished in amount.

\section{Caryococcus dilatator Kirby.} (Univ. Calif. Publ. Zool., 49, 1944, 238.) Parasitic in the nucleus of flagellates (Trichonympha chattoni and other species of Trichonympha) from the intestine of termites (Glyptotermes iridipennis), Australia, and other species.

Spherules 0.5 micron or less in diameter, internally differentiated with stainable granule or stainable region peripherally situated; parasitic in nucleus and nucleolus; nuc peus becomes greatly enlarged and the chromatin mostly or entirely disappears.

\section{Caryococcus invadens Kirby.} (Univ. Calif. Publ. Zool., 49, 1944, 238.) Parasitic in the nucleus of a flagellate (Trichonympha peplophora) from the intestine of a termite (Neotermes howa), Madagascar.

Spherules 1 to 1.5 microns in diameter, sometimes arranged in pairs, often internally differentiated with stainable central or peripheral granules or stained areas; parasitic in the nucleolus or endosome and nucleus; parasitized nucleolus becoming greatly enlarged and crossed by trabeculae, eventually consumed; nucleus becoming moderately enlarged, but chromatin not disappearing.

5. Caryococcus nucleophagus Kirby. (Univ. Calif. Publ. Zool., 49, 1944, 236.) Parasitic in the nucleus of a flagellate (Trichonympha corbula) from the intestinc of termites (Procryptotermes $s p$.), Madagascar, and three species of Kalotermes (s. 1.) from Madagascar.

* Prepared by Prof. Harold Kirby, Jr., University of California, Berkeley, California, October, 1946. 
Spherules with a diameter of about 0.5 micron, sometimes arranged in pairs, sometimes with a thicker, crescentic, stainable area of the periphery on one side; parasitic within the nucleus, ex- terior or interior to the chromatin mass, which may be diminished in amount, but does not disappear, nor is the parasitized nucleus appreciably enlarged.

\section{Genus B. Drepanospira Petschenko.}

(Arch. f. Protistenk., 22, 1911, 282.)

Cell incurved in two spiral turns that are not abrupt, one of the ends pointed, the other a little rounded, no flagella, movement helicoid by means of all the body, no cell division, endospores formed, regular spherical colonies formed by individuals at certain stages of development.

The type species is Drepanospira milleri Petschenko.

1. Drepanospira mülleri Petschenko. (Müllerina paramecii Petschenko, Cent. f. Bakt., I Abt., Orig., 56, 1910, 90; Petschenko, Arch. f. Protistenk., 22, 1911, 252; see also Kirby, in Calkins and Summers, Protozoa in Biological Research, 1941, 1036.) Parasitic in the cytoplasm of Paramecium caudatum.
Developing from a group of curved rods in the cytoplasm to a large, ellipsoidal mass almost filling the body. $\mathrm{Nu}$ clear portion occupying part of the cell.

The author regards this genus as belonging in the family Spirillaceae betwcen Spirosoma and Microspira.

\section{Genus C. Holospora Hafjine.}

(Ann. Inst. Past., 4, 1890, 151.)

Genus established for bacterial parasites of the ciliate, Paramecium aurelia (= Paramecium caudatum?).

The type species is Holospora undulata Haffkine.

\section{Holospora undulata Haffkine.}

(Ann. Inst. Past., Paris, 4, 1890, 151.) In the micronucleus of the ciliate Paramecium aurelia $(=P$. caudatum?).

Gradually tapered at ends; $1 \frac{1}{2}, 2$ and $2 \frac{1}{2}$ spiral turns; develops from a small, fusiform body which grows and divides transversely; brings about a great enlargement of the micronucleus, which becomes filled with the spirals (see Drepanospira mülleri Petschenko).

\section{Holospora elegans Haffline. (Haff-} kine, Ann. Inst. Past., Paris, 4, 1890, 154 ; see also Kirby, in Calkins and Summers, Protozoa in Biological Researeh, New York, 1941, 1035.) In the micronucleus of the ciliate, Paramecirm aurelia ( $=P$, caudatum?).
Vegetative stage fusiform; elongated, elliptical, nucleus-like body in some; divides equatorially, budding at one end; transformation into spore entails enlargement, clear space separating membrane at sides, spore pointed at ends.

3. Holospora obtusa Haffkin. (Haffkine, Ann. Inst. Past., Paris, 4, 1890, 153 ; also see Fiveiskaja, Arch. f. Protistenk., 65, 1929, 276.) In the macronucleus of the ciliate Paramecium aurelia $(=P$. caudatum?).

Spores not spiralled and both ends are rounded. Reproduction by fission, also by formation of a bud at one of the extremities of the fusiform cell. Bodies with rounded ends 12 to 30 microns long; also spindle-shaped bodies with pointed 
ends, 0.5 by 3 to 6 microns (Fiveiskaja, loc. cit.).

The following species have been placed in genera belonging in the orders Chlamydobacteriales and Eubacteriales respectively :

Cladothrix pelomyxae Veley. (Veley, Jour. Linn. Soc., Zool., 29, 1905, 375; see also Leiner, Arch. f. Protistenk., 4\%, 1924, 282; Kirby, in Calkins and Summers, Protozoa in Biological Research, New York, 1941, 1025; Hollande, Bull. Biol. France Belg., $79,1945,49$.$) In the$ cytoplasm of the rhizopod, Pelomyxa palustris and probably also other species of Pelomyxa.

Rods, 1.5 to 22 microns or more in length, divided into several to many sections by transverse partitions, generally aggregated in proximity to the nuclei, which may be thickly invested by close-set bacteria applied to the surface.

Micrococcus batrochorum (sic) Takimoff. (Arch. f. Protistenk., \%2, 1930, 137.) In the cytoplasm of the flagellate, Trichomonas batrachorum from the tree toad (Hyla arborea). Also seen free in preparations of the intestinal contents of Hyla.

Round, 1 to 1.5 microns in diameter, grouped generally in aggregates of irregular form, but also occur individually .

Note: Further deseriptions of bacterial and other parasites of Protozoa with bibliography will be found in Calkins and Summers, Protozoa in Biological Research, New lork, 1941, 1009-1113 and in Kirby, Lniv. of Calif. Pub. in Zoology, $53,1946,193-207$. 

SUPPLEMENT NO. 2

\section{ORDER VIRALES \\ THE FILTERABLE VIRUSES}





\section{FILTERABLE VIRUSES*}

The so-called filterable viruses, today generally called merely viruses, are still of unknown affiliations so far as relationships to established groups of microorganisms are concerned. They are treated here as members of an order, consisting of 13 families, 32 genera and 248 species.

Among viruses as we know them, there are three constituent groups that have come to be recognized, and to some extent named and classified, through the largely separate efforts of bacteriologists, animal pathologists, and plant pathologists. Taxonomic overlapping of the three groups, viruses affecting bacteria, viruses having only animal hosts, and viruses invading higher plants, can hardly be justified as yet by available evidence. Nevertheless it has been shown that a single virus may multiply both in a plant host and in an insect vector. This seems to dispose of the thought that adaptation to a plant or animal enviromment would necessarily preclude utilization of other sources of the materials needed for multiplication.

For the present it seems feasible to continue with the custom, tacitly accepted in the past, of classifying bacteriophages separately as one sub-group, riruses causing diseases in seed plants as a second sub-group, and those causing diseases in animals as a third sub-group. It should be recognized that this may prove to be only a temporary arrangement, necessary because we have no eridence to warrant taxonomic overlapping of the three groups and useful while we await critical investigations and possible development of a substitute plan capable of displaying natural relationships to better advantage. Eventually evidence may become arailable to show that some bacteriophages can infect higher plants or animals and can increase in the new enriromment, or that viruses known to attack animals or plants can similarly enlarge their host ranges. Or, there may be discoveries of common physical properties that would aid in formulating an interlocking classification, for which at present we lack any substantial basis.

It is of especial significance now that the three fields be unified at least by a parallel derelopment of nomenclature. Toward this end the present section of this supplement is directed.

* Supplement No. 2 has been prepared by Francis O. Holmes, The Rockefeller Institute for Medical Research, Princeton, N. J., September, 1944. In this section, authorities for the names of plant hosts are in general as given by Gray's New Manual of Botany, 7th edition, and Bailey's Manual of Cultivated Plants, 1938; in each of these standard works will be found a list of abbreviations customarily used in botany in citing authorities for binomials. 


\section{ORDER VIRALES Breed, Murray and Hitchens.}

(Jour. Bact., 47, 1944, 421.)

Viruses. Etiological agents of disease, typically of small size and capable of passing filters that retain bacteria, increasing only in the presence of living cells, giving rise to new strains by mutation, not arising de novo. A considerable number of viruses have not been proved filterable; it is nevertheless customary to include these viruses with those known to be filterable, because of similarities in other attributes and in the diseases induced. Some not known to be filterable are inoculable only by special techniques, as by grafting or by use of insect vectors, and suitable methods for testing their filterability have not been developed; moreover, it is not certain that so simple a criterion as size measured in terms of filterability will prove to be an adequate indicator of the limits of the natural group. Cause diseases of bacteria, plants and animals.

Key to the suborders of order Virales.

I. Infecting bacteria.

Suborder I. Phagineae, p. 1128.

II. Infecting higher plants.

Suborder II. Phytophagineae p. 1145.

III. Infecting animals (insects, mammals).

Suborder III. Zoophagineae, p. 1225.

Suborder I. Phagineae subordo novus.

Viruses pathogenic in bacteria; bacteriophages. Containing at present only one family, the Phagaceae.

FAMILY I. PHAGACEAE HOLMES.

(Handb. Phytopath. Viruses, ${ }^{*} 1939,1$. )

Characters those of the suborder. There is a single genus.

\section{Genus I. Phagus Holmes.}

(Loc. cit., 1.)

Characters those of the family. Generic name from Greek phagein, to eat.

The type species is Phagus minimus Holmes.

Note: Bacteriophagum d'Herelle (Compt. rend. Soc. Biol., Paris, 81, 1918, 1161) a genus name applied in connection with early studies of bacteriophages, had as its type species Bacteriophagum intestinale d'Herelle, a bacteriophage that is not now identifiable or, more probably, a mixture of such unidentifiable bacteriophages, for filtrates containing it were said to be capable of killing outright a culture of bacteria (ibid., 1160). The genus name Bacteriophagum is, therefore, regarded as a nomen dubium, if not also a nomen confusum; subsequently it was abandoned by its author, for reasons that are not clear, in favor of the genus name Protobios d'Herelle 1924 (Immunity in natural infectious disease; page 343 of authorized English edition by George H. Smith, Baltimore, Williams \& Wilkins Co., 1924, 399 pp). Protobios protobios

* Holmes, F. O., Handbook of Phytopathogenic Viruses, Burgess Publishing Company, Minneapolis, Minn., 1939, 221 pp. 
d'Herelle (loc.cit., 345), presumably the type species of this genus, was not an ordinary virus but was said to be non-parasitic (i.e., free-living) in nature, was capable of reducing sulphur, and is not now identifiable. The genus name Protobios and the corresponding binomial Protobios bacteriophagus d'Herelle are therefore regarded also as nomina dubia and are not used here. Bacteriophagus Thornberry (Phytopath., 31, 1941, 23) appears to represent a variant spelling of d'Herelle's earlier genus name; it was not accompanied by any indication of what recognizable single bacteriophage served as type and thus does not modify the standing of Bacteriophagum.

\section{Key to the species of genus Phagus.}

I. Dysentery-coli bacteriophages.

A. Producing large plaques, 8 to $12 \mathrm{~mm}$ in diameter.

1. Particle size small, 8 to 12 millimicrons.

1. Phagus minimus.

2. Particle size 15 to 20 millimicrons.

\section{Phagus minor.}

B. Producing moderately large plaques, 2 to $6 \mathrm{~mm}$ in diameter, with distinct halo.

1. Particle size 20 to 30 millimicrons.

3. Phagus parvus.

4. Phagus primarius.

5. Phagus secundarius.

6. Phagus dysenteriae.

C. Plaques medium size, 1 to $3 \mathrm{~mm}$ in diameter, with distinct halo.

1. Particle size 25 to 40 millimicrons.

7. Phagus medius.

8. Phagus astrictus.

D. Plaques small, 0.5 to $1.5 \mathrm{~mm}$ in diameter, with soft edge or narrow halo.

1. Particle size 30 to 45 millimicrons.

9. Phagus major.

10. Phagus coli.

11. Phagus artus.

E. Plaques very small, 0.1 to $1.2 \mathrm{~mm}$ in diameter, with sharp edges.

1. Particle size 50 to 75 millimicrons,

12. Phagus maximus.

II. Bacteriophages attacking Agrobacterium tumefaciens Conn, Pseudomonas solanacearum Smith, Xanthomonas citri Dowson, Xanthomonas pruni Dowson, Erwinia carotovora Holland, Erwinia aroideae Holland, Bacterium stewarti E. F. Smith. 
A. Specific for bacterial hosts named above.
13. Phagus tumoris.
14. Phagus solanacearum.
15. Phagus citri.
16. Phagus pruni.
17. Phagus deformans.
18. Phagus contumax.
19. Phagus maidis.

III. Bacteriophages attacking Salmonella enteritidis Castellani and Chalmers.

20. Phagus enteritidis.

21. Phagus commutabilis.

22. Phagus tertius.

23. Phagus dubius.

IV. Bacteriophage attacking Salmonella typhosa.

24. Phagus indicens.

V. Bacteriophages attacking Bacillus megalherium DeBary, Bacillus mycoides Flügge, and Rhizobium leguminosarum Frank.

A. Thermal inactivation at $75^{\circ} \mathrm{C}$ in 10 minutes in vitro.

1. Host may be freed from bacteriophage by heating at $80^{\circ} \mathrm{C}$ for 10 minutes.

25. Phagus testabitis.

2. Host retains virus even when heated at $90^{\circ} \mathrm{C}$ for 10 minutes.

26. Phagus indomitus.

B. Thermal inactivation at $60^{\circ} \mathrm{C}$ in 30 minutes.

27. Phagus subvertens.

VI. Bacteriophages attacking streptococci.

28. Phagus ineptus.

29. Phagus streptococci.

30. Phagus maculans.

31. Phagus lacerans.

32. Phagus tolerans.

33. Phagus michiganensis.

VII. Bacteriophages at tacking staphylococci.

34. Phagus fragilis.

35. Phagus intermedius.

36. Phagus caducus.

37. Phagus alpha.

38. Phagus beta. 
39. Phagus durabilis.

40. Phagus liber.

VIII. Bacteriophages attacking vibrios.

\section{Phagus cholerae. \\ 42. Phagus celer. \\ 43. Phagus effrenus. \\ 44. Phagus lentus.}

IX. Bacteriophages attacking Corynebacterium diphtheriae Lehmann and Neumann.

45. Phagus diphtheriae.

46. Phagus futilis.

1. Phagus minimus Holmes. (Handb. Phytopath. Viruses, 1939, 141.) From Latin minimus, least, in reference to size. Common name: Bacteriophage S13.

Hosts: Escherichia coli Castellani and Chalmers; Shigella dysenteriae Castellani and Chalmers.

Induced disease : On plate cultures that are uniformly covered with confluent colonies of host organisms, this bacteriophage produces large cleared plaques, 8 to $12 \mathrm{~mm}$ in diameter, with wide shelving edges.

Serological relationships: No crossneutralization reactions with bacteriophages C13, C36, D5, D20, C18, D3, S8, C21, C16, and D6.

Immunological relationships: Member of Resistance Group I.

Other properties: Particle size 8 to 12 millimicrons. Not affected by 26.3 per cent urea solution. Little or no inactivation by $1: 25,000$ methylene blue in $2 \mathrm{~mm}$ layer $20 \mathrm{~cm}$ from 100 candle-power light for 30 minutes. Lysis completely inhibited by 0.25 per cent solution of sodium citrate.

Literature: Burnet and McKie, Jour. Path. and Bact., 36, 1933, 299-306, 307318; 37, 1933, 179-184; Burnet et al., Austral. Jour. Exp. Biol. and Med. Sci., 15, 1937, 227-368.

2. Phagus minor H. (loc. cit., 141). From Latin minor, lesser.
Common names: Bacteriophage $\mathrm{C} 13$, C8, and D44.

Hosts: Escherichia coli Castellani and Chalmers; Shigella dysenteriae Castellani and Chalmers.

Induced disease: Large plaques, 8 to $12 \mathrm{~mm}$ in diameter, with wide shelving edges.

Serological relationships: Cross reactions with bacteriophages C8 and D44 but not with bacteriophages S13, C36, D5, D20, D13, C18, D3, S8, C21, C16, D6.

Immunological relationships: Member of Resistance Group I.

Other properties: Particle size, 15 to 20 millimicrons. Completely inactivated by $1: 25,000$ methylene blue in $2 \mathrm{~mm}$ layer $20 \mathrm{~cm}$ from 100 candle-power light for 30 minutes. Specific soluble substance formed in lysed cultures blocks phageantiphage reaction.

Literature: Burnet, Jour. Path. and Bact., 36, 1933, 307-318; Brit. Jour. Exp. Path., 14, 1933, 100-108.

3. Phagus parvus H. (loc. cit., 142). From Latin parvus, small.

Common names: Bacteriophage C36, S18, C38, M, and C37 of Burnet.

Hosts: Escherichia coli Castellani and Chalmers; Shigella dysenteriae Castellani and Chalmers.

Induced disease: Moderately large plaques, 2 to $6 \mathrm{~mm}$ in diameter, with distinct halo. 
Serological relationships: Induces formation of antibody capable of neutralizing bacteriophages $\mathrm{S} 18$, C38, M, and C37, but not bacteriophages S13, C13, D5, D20, D13, C18, D3, S8, C21, C16, or D6, which represent distinet serological groups.

Immunological relationships: Member of Resistance Group I.

Other properties : Particle size, 20 to 30 millimicrons. Completely inactivated by $1: 25,000$ methylene blue in $2 \mathrm{~mm}$ layer $20 \mathrm{~cm}$ from 100 candle-power light for 30 minutes.

Literature: Burnet, Jour. Path. and Bact., 36, 1933, 307-318.

4. Phagus primarius H. (loc. cit., 143). From Latin primarius, chief or first.

Common names: Bacteriophage D5, C51, C50, and D48.

Hosts: Escherichia coli Castellani and Chalmers; Shigella dysenteriae Castellani and Chalmers.

Induced disease: Moderately large plaques, 2 to $6 \mathrm{~mm}$ in diameter, with distinct halos.

Serological relationships: Cross-neutralization reactions with bacteriophages C51, C50, and D48, but not with S13, C13, C36, D20, D13, C18, D3, S8, C21, C16, D6.

Immunological relationships: Member of Resistance Group I.

Other properties: Particle size, 20 to 30 millimicrons. Completely inactivated by $1: 25,000$ methylene blue in $2 \mathrm{~mm}$ layer $20 \mathrm{~cm}$ from 100 candle-power light for 30 minutes.

Literature: Burnet, Jour. Path. and Bact., 36, 1933, 307-318.

5. Phagus secundarius H. (loc. cit., 143). From Latin secundarius, inferior or second.

Common names: Bacteriophage D20 and $\mathrm{G}$.

Hosts : Escherichia coli Castellani and Chalmers; Shigella dysenteriae Castellani and Chalmers.

Induced disease: Moderately large plaques, 2 to $6 \mathrm{~mm}$ in diameter, with distinet halo.

Serological relationships: No crossneutralization reactions with bacteriophages S13, C13, C36, D5, D13, C18, D3, $\mathrm{S} 8, \mathrm{C} 21, \mathrm{C} 16$, or D6.

Immunological relationships: Member of Resistance Group II.

Other properties: Nearly all inactivated by $1: 25,000$ methylene blue in $2 \mathrm{~mm}$ layer $20 \mathrm{~cm}$ from 100 candle-power light for 30 minutes. Particle size, 20 to 30 millimicrons.

Literature: Burnet, Jour. Path. and Bact., 36, 1933, 307-318.

6. Phagus dysenteriae H. (loc. cit., 144). From Latindysenteria, dysentery.

Common names: Bacteriophage D13, specific dysentery phage.

Host: Shigella dysenteriae Castellani and Chalmers.

Insusceptible species: Escherichia coli Castellani and Chalmers.

Induced disease: Moderately large plaques, 2 to $6 \mathrm{~mm}$ in diameter, with distinet halo.

Serological relationships: Antiserum to this strain is not known to be effective against any other strain of bacteriophage ; in particular, no cross reactions with bacteriophages S13, C13, C36, D5, D20, C18, D3, S8, C21, C16, or D6.

Immunological relationships: Member of Specific Dysentery Resistance Group.

Other properties: Particle size, 20 to 30 millimicrons. Completely inactivated by $1: 25,000$ methylene blue in $2 \mathrm{~mm}$ layer $20 \mathrm{~cm}$ from 100 candle-power light for 30 minutes.

Literature: Burnet, Jour. Path. and Bact., 36, 1933, 307-318.

7. Phagus medius H. (loc. cit., 144), From Latin medius, moderate, in reference to particle size.

Common name: Bacteriophage C18, C35, C26, C47, or C34.

Hosts: Escherichia coli Castellani and Chalmers; Shigella dysenteriae Castellani and Chalmers. 
Induced disease : Medium size plaques, 1 to $3 \mathrm{~mm}$ in diameter, with distinct halo.

Serological relationships: Cross reactions with bacteriophages C35, C26, C47, and C34, but not with S13, C13, C36, D5, D20, D13, D3, S8, C21, C16, or D6.

Immunological relationships: Member of Resistance Group II.

Other properties: Particle size, 25 to 40 millimicrons.

Literature: Burnet, Jour. Path. and Bact., 36, 1933, 307-318.

8. Phagus astrictus H. (loc. cit., 145). From Latin astrictus, limited, in reference to inability to lyse Escherichia coli Castellani and Chalmers.

Common vames: Bacteriophage D3; "smooth" dysentery phage.

Host: Shigella dysenteriae Castellani and Chalmers.

Insusceptible species: Escherichia coli Castellani and Chalmers.

Induced disease: Medium size plaques, 1 to $3 \mathrm{~mm}$ in diameter, with distinct halo.

Serological relationships: No crossneutralization reactions with bacteriophages S13, C13, C36, D5, D20, D13, C18, $\mathrm{S} 8, \mathrm{C} 21, \mathrm{C} 16$, or D6.

Immunological relationships: Member of Smooth Dysentery Resistance Group.

Other properties : Particle size, 25 to 40 millimicrons. Nearly all inactivated by $1: 25,000$ methylene blue in $2 \mathrm{~mm}$ layer 20 $\mathrm{cm}$ from 100 candle-power light for 30 minutes.

Literature: Burnet, Jour. Path. and Bact., 36, 1933, 307-318.

9. Phagus major H. (loc, cit., 146). From Latin major, greater, in reference to particle size.

Common name: Bacteriophage S8, L, $\mathrm{S} 28, \mathrm{C} 33$, or $\mathrm{S} 41$.

Hosts : Escherichia coli Castellani and Chalmers; Shigella dysenteriae Castellani and Chalmers.

Induced disease: Small plaques, 0.5 to $1.5 \mathrm{~mm}$ in diameter, with soft edge or narrow halo.
Serological relationships: No crossneutralization reactions with bacteriophages S13, C13, C36, D5, D20, D13, C18, D3, C21, C16, or D6.

Immunological relationships: Member of Resistance Group I.

Other properties: Particle size, 30 to 45 millimicrons.

Literature: Burnet, Jour. Path. and Bact., 36, 1933, 307-318; Brit. Jour. Exp. Path., 14, 1933, 100-108; Gough and Burnet, ibid., 38, 1934, 301-311.

10. Phagus coli H. (loc. cit., 146). From Latin colon, the colon.

Common names: Bacteriophage C21 or C5; specific coli phage.

Host: Escherichia coli Castellani and Chalmers.

Insusceptible species: Shigella dysenteriae Castellani and Chalmers.

Induced disease: Small plaques, 0.5 to $1.5 \mathrm{~mm}$ in diameter, with soft edge or very narrow halo.

Serological relationships: No crossneutralization with bacteriophages S13, C13, C36, D5, D20, D13, C18, D3, S8, $\mathrm{C} 16$, or D6.

Immunological relationships: Member of Specific Escherichia coli Resistance Group.

Other properties: Particle size, 30 to 45 millimicrons. Completely inactivated by $1: 25$, 000 methylene blue in $2 \mathrm{~mm}$ layer $20 \mathrm{~cm}$ from 100 candle-power light for 30 minutes.

Literature: Burnet, Jour. Path. and Bact., 36, 1933, 307-318.

11. Phagus artus H. (loc. cit., 148). From Latin artus, narrow, in reference to limited host range.

Common names: Bacteriophage D6, D33; smooth dysentery phage.

Host: Shigella dysenteriae Castellani and Chalmers, smooth strains.

Induced disease: Small plaques, 0.5 to $1.5 \mathrm{~mm}$ in diameter, with soft edge or very narrow halo.

Serological relationships: Not neu- 
tralized by sera specific for bacteriophages $\mathrm{S} 13, \mathrm{C} 13, \mathrm{C} 36, \mathrm{D} 5, \mathrm{D} 20, \mathrm{D} 13, \mathrm{C} 18$, D3, S8, C21, or C16.

Immunological relationships: Member of Smooth Dysentery Resistance Group.

Other properties: Particle size, 30 to 45 millimicrons.

Literature: Burnet, Jour. Path. and Bact., 36, 1933, 307-318.

12. Phagus maximus $H$. (loc. cit., 147). From Latin maximus, greatest, in reference to particle size.

Common names: Bacteriophage C16, C4, C15, C20, C32, C46, D4, D12, D29, D53, H, J, K, and W. L. L.

Hosts : Escherichia coli Castellani and Chalmers; Shigella dysenteriae Castellani and Chalmers.

Induced disease: Small plaques, 0.1 to $1.2 \mathrm{~mm}$ in diameter, with sharp edges.

Serological relationships: No crossneutralization reaction with bacteriophages S13, C13, C36, D5, D20, D13, C18, D3, S8, C21, D6, or staphylococeus bacteriophage Au2. Agglutinated and inactivated by homologous, though not by other, antisera. For agglutination an original titer of $2 \times 10^{\circ}$ or higher is required; the reaction is visible to the unaided eye after 24 hours at $50^{\circ} \mathrm{C}$ and succeeds even after inactivation by heat (70 to $85^{\circ} \mathrm{C}$ for 30 minutes), formaldehyde, or a photodynamic dye (proflavine).

Immunological relationships: Member of Resistance Group II.

Thermal inactivation: At or below $70^{\circ}$ to $85^{\circ} \mathrm{C}$ for 30 minutes.

Other properties: Particle size estimated by filtration as 50 to 75 millimicrons, by centrifuging as 79 to 90 millimicrons, from photographs as 50 to 60 millimicrons. Rapidly inactivated by 26.3 per cent urea solution. Little or no inactivation by $1: 25,000$ methylene blue in $2 \mathrm{~mm}$ layer $20 \mathrm{~cm}$ from 100 candlepower light for 30 minutes. Lysis not inhibited by 1.5 per cent or weaker solutions of sodium citrate. Thermolabile specific soluble substance formed in lysed cultures blocks phage-antiphage reaction.

Literature: Burnet, Brit. Jour. Exp. Path., 14, 1933, 93-100, 100-108, 302-308; Jour. Path. and Bact., 36, 1933, 307-318; 37, 1933, 179-184; Burnet and Lush, ibid., 40, 1935, 455-469; Burnet and MeKie, ibid., 36, 1933, 299-306.

13. Phagus tumoris H. (loc. cit., 150). From Latin tumor, a swelling, in reference to association of this bacteriophage with bacterial tumors.

Common name: Agrobacterium tumefaciens bacteriophage.

Host: Agrobacternum tumefaciens Conn, most strains.

Insusceptible species: Some strains of Agrobacterium tumefaciens, Bacterium stewarti E. F. Smith, Erwinia atroseptica Bergey et al., E. carotovora Holland, Pseudomonas tabaci Stapp, Xanthomonas beticola Burkholder, X. campestris Dowson, $X$. citri Dowson, X. phaseoli Dowson X. pruni Dowson and $X$. vesicatoria Dowson.

Geographical distribution: United States, Russia.

Induced disease: Plaques 2 to $6 \mathrm{~mm}$ in diameter in $t$ to 6 hours, edges of plaques spotted, moth-eaten in appearance until 40 hours after seeding; enlargement then stops and the edges of the plaques become smooth, double-ringed. Infection of plants by Agrobacterium tumefaciens is progressively inhibited by increasing amounts of bacteriophage in inoculum.

Thermal inactivation: At $95^{\circ} \mathrm{C}$ in 10 minutes (another report says $70^{\circ} \mathrm{C}$, time not recorded).

Other properties: Resists dilution to $1: 10^{11}$; storage at $5^{\circ} \mathrm{C}$ for over 25 months; prompt, though not gradual, drying; 1 per cent hydrogen peroxide for 72 hours; 95 per cent ethyl alcohol for 1 hour; 70 per cent ethyl alcohol for 6 hours; $2 \frac{1}{2}$ per cent phenol for 1 hour; $1: 3000$ nitric acid for 1 hour; $\mathrm{N} / 64$ sodium hydroxide for 1 hour.

Literature: Israilsky, Cent. f. Bakt., 
II Abt., 67, 1926, 236-242; $71,1927,302-$ 311 ; 79, 1929, 354-370; Kent, Phytopath., 27, 1937, 871-902; Muncie and Patel, Phytopath., 20, 1930, 289-305.

14. Phagus solanacearum H. (loc. cit., 148). From name of host.

Common name: Pseudomonas solanacearum bacteriophage.

Host: Pseudomonas solanacearum Smith.

Geographical distribution: Formosa (Taiwan).

Induced disease: Medium size plaques on plate cultures of Pscudomonas solanacearum.

Serological relationships: When injected into rabbits, this bacteriophage stimulates the production of a specific precipitating antibody not giving cross reactions with anti-bacterial antibodies. Antiphagic serum inactivated at $90^{\circ} \mathrm{C}$ in 10 minutes.

Thermal inactivation: At $63^{\circ} \mathrm{C}$ in 10 minutes $\left(61^{\circ} \mathrm{C}\right.$ in 30 minutes; $66^{\circ} \mathrm{C}$ in about 1 minute).

Other properties: Optimum temperature for increase, $34^{\circ} \mathrm{C}$.

Literature: Matsumoto and Okabe, Jour. Plant Prot., 22, 1935, 15-20; Jour. Soc. Trop. Agr., 7, 1935, 130-139; 9, 1937 , 205-213.

15. Phagus citri H. (loc. cit., 149). From name of host.

Common name: Xanthomonas citri bacteriophage.

Host: Xanthomonas citri Dowson, the citrus canker organism.

Geographical distribution: Formosa. (Taiwan).

Induced disease: Lysis. This bacteriophage has been isolated from soil under diseased trees, and once from infected leaves. It may play a role in the destruction of the citrus canker organism in the soil.

Other properties: Optimum temperature for increase, $30^{\circ} \mathrm{C}$.

Literature: Matsumoto and Okabe,
Agriculture and Horticulture, 12, 1937, 2055-2059.

16. Phagus pruni H. (loc. cit., 151). From name of host.

Common name: Xanthomonas pruni bacteriophage.

Host: Xanthomonas pruni Dowson.

Geographical distribution: United States (from soil beneath infected peach trees).

Induced disease: Lysis in broth cultures; plaques on agar cultures, but characteristics of plaques not described.

Other properties: Estimated diameter 11 millimicrons in broth. Resists dilution to $1: 10^{5}$ or more.

Literature: Anderson, Phytopath., 18, 1928, 144; Thornberry, ibid., 25, 1935, 938-946.

17. Phagus deformans $H$. (loc. cit., 151). From Latin deformare, to disfigure, in reference to malformation of infected host cells.

Common name: Erwinia carotovora bacteriophage.

Host : Erwinia carotovora Holland.

Insusceptible species: Agrobacterium tumefaciens Conn, except in some early tests with possibly mixed bacteriophages; Erwinia amylovora Winslow et al., $E$. melonis Holland, Salmonella pullorum Bergery et al., S. gallinarum Bergey et al., Shigella dysenteriae Castellani and Chalmers, Tanthomonas pruni Dowson.

Geographical distribution: United States (Michigan).

Induced disease: In Erwinia carotovora, cells reduced in motility, agglutinated, malformed, some elongated, others swollen, bulged at one end, bulged in middle, or enlarged and spherical.

Other properties: Resists dilution to $1: 10^{3}$, and storage in sterile medium at room temperature for $5 \frac{1}{2}$ months.

Literature: Coons and Kotila, Phytopath., 15, 1925, 357-370; Mallmann and Hemstreet, Jour. Agr. Res., 28, 1924, 599-602. 
18. Phagus contumax spec. nov. From Latin contumax, refractory, in reference to ability of this bacteriophage to withstand heating sufficient to destroy accompanying host cells.

Common name: Erwinia aroideae bacteriophage.

Host : Erwinia aroideae Holland.

Insusceptible species: Agrobacterium tumefaciens Conn, Bacterium formosanum Okabe, Erwinia carotovora Holland, Pseudomonas andropogoni Stapp, $P$. solanacearum Smith, $P$. tomato Burkholder, Xanthomonas campestris Dowson, $X$. citri Dowson, $X$. malvacearum Dow. son, $X$. nakatae Dowson, X. phaseoli Dowson, $X$. ricinicola Dowson.

Geographical distribution: Formosa (Taiwan).

Induced disease: Very small plaques, 0.1 to $1.0 \mathrm{~mm}$ (mostly less than $0.5 \mathrm{~mm}$ ) in diameter.

Thermal inactivation: Resists heating at $60^{\circ} \mathrm{C}$ for 30 minutes without appreciable loss of titer, but host organism is killed by this treatment.

Other properties: Optimum temperature for inerease, about $25^{\circ} \mathrm{C}$. This bacteriophage may be prepared by heating centrifuged cultures at $60^{\circ} \mathrm{C}$ for $30 \mathrm{~min}$ utes as efficiently as by filtration to remove bacteria.

Literature: Matsumoto, Trans. Nat. Hist. Soc. Formosa, 29, 1939, 317-338; 30, 1940, 89-98; 31, 1941, 145-154; Matsumoto and Sawada, ibid., 28, 1938, 247-256.

19. Phagus maidis $H$. (loc. cit., 152). From New Latin mais, corn (maize), host of Bacterium stewarti.

Common name: Bacterium stewarti bacteriophage; Phytomonas stewarti bacteriophage; Aplanobacter stewarti bacteriophage.

Host: Bacterium stewarti E. F. Smith (= Pseudomonas stewarti E. F. Smith, Phytomonas stewarti Bergey et al. and Aplanobacter stewarti McCulloch).

Geographical distribution: United States.
Induced disease: In Bacterium stewarti, variation or loss of yellow color, change of viscosity of growth, reduction or loss of virulence. Infection of corn plants by seed-borne Bacterium stewarti is much reduced by treating seeds with this bacteriophage before they are planted.

Thermal inactivation: Above $65^{\circ} \mathrm{C}$ in 30 minutes.

Other properties: Infective in dilutions to $10^{-7}$. Soon lost from cultures maintained at $\mathrm{pH} 3.85$ to 4.00 , or on Ivanoff's medium, which contains oxidizing compounds.

Literature: Thomas, Phytopath., 25, 1935, 371-372; Science, 88, 1938, 56-57; Phytopath., 30, 1940, 602-611.

20. Phagus enteritidis H. (loc. cit., 153). From name of host.

Common names: Salmonella enteritidis bacteriophage 1, 12, or 33; Group A bacteriophages.

Hosts : Salmonella enteritidis Castellani and Chalmers, S. gallinarum Bergey et al., Shigella dysenteriae Castellani and Chalmers.

Induced disease: Plaques of medium size, usually with surrounding translueent halo.

Immunological relationships: Member of Resistance Group $A$; host individuals that have acquired resistance to this bacteriophage are resistant to lines 12 and 33 , but susceptible to Salmonella enteritidis bacteriophages 8,20 , and 11 , as well as to other strains of Resistance Groups B, C, and D.

Literature: Burnet, Jour. Path. and Bact., 32, 1929, 15-42.

21. Phagus commutabilis H. (loc. cit., 153). From Latin commutabilis, variable, in reference to differences within Resistance Group B, typified by this bacteriophage.

Common names: Salmonella enteritidis bacteriophage $8,18,28,31,34,38$; Group $\mathrm{B}$ bacteriophages.

Hosts : Salmonella enteritidis Castellani 
and Chalmers, Shigella dysenteriae Castellani and Chalmers, Shigella gallinarum Weldin, Salmonella typhosa White.

Induced disease: Small plaques with sharp edges, or moderately large plaques with characteristic halo.

Immunological relationships: Nember of Resistance Group B; host individuals that have acquired resistance to this bacteriophage are resistant to lines 18,28 , 31,34 , and 38 , but susceptible to Salmonella enteritidis bacteriophages 1, 20, and 11 , as well as to other strains of Resistance Groups A, C, and D.

Literature: Burnet, Jour. Path. and Bact., 32, 1929, 15-42.

22. Phagus tertius H. (loc. cit., 154). From Latin tertius, third, in reference to the third Resistance Group of Salmonella enteritidis bacteriophages, Group C, typified by this bacteriophage.

Common names: Salmonella enteritidis bacteriophage $20,25,32,35$; Group C bacteriophages.

Hosts : Salmonella enteritidis Castellani and Chalmers, S. gallinarum Bergey et al., Shigella dysenteriae Castellani and Chalmers.

Induced disease: Plaques of small size, with sharp edges.

Immunological relationships: Member of Resistance Group C. Host individuals that have acquired resistance to this bacteriophage are resistant to lines 25,35 , and 32 , but susceptible to Salmonella enteritidis bacteriophages of Resistance Groups A, B, and D.

Literature: Burnet, Jour. Path. and Bact., 32, 1929, 15-12.

23. Phagus dubius H. (loc. cit., 155). From Latin dubius, doubtful, in reference to uncertainty of distinction between Resistance Groups C and D.

Common names: Salmonella enteritidis bacteriophage 11, 13; Group D bacteriophages.

Hosts : Salmonella enteritidis Castellani and Chalmers, Shigella dysenteriae Cas- tellani and Chalmers, Shigella gallinarum Weldin.

Induced disease: Very large plaques, up to $8 \mathrm{~mm}$ in diameter on 1.2 per cent agar.

Immunological relationships: Member of Resistance Group D. Host individuals that have acquired resistance to this bacteriophage are resistant to line 13 , but susceptible to Salmonella enteritidis bacteriophages of Resistance Groups A, B, and $\mathrm{C}$.

Literature: Burnet, Jour. Path. and Bact., 32, 1929, 15-42.

24. Phagus indicens spec. nov. From Latin indicere, to disclose or indicate, in reference to diagnostic use of this bacteriophage in identifying $\mathrm{V}$ forms of the typhoid bacillus.

Common name: Phage Q151.

Host: Salmonella typhosa White (= Bacillus typhosus Zopf).

Insusceptible species: W forms of the typhoid organism and various Salmonella species.

Geographical distribution : Canada.

Induced disease : In Salmonella typhosa, small plaque formation (lysis) and complete inhibition of growth in cultures of the $\mathrm{V}$ form (bearing $\mathrm{Vi}$ antigen; resisting $O$ agglutination) and no lysis or restraining effect on growth of the $W$ form (lacking $\mathrm{Vi}$ antigen; agglutinated by $\mathrm{O}$ antiserum). In the presence of the virus, mixed cultures are quickly transformed since only $W$ variants can increase. Pure $V$ cultures can be identified by the test for their complete inhibition; this inhibition is regularly followed by secondary growth representing the pure $\mathrm{W}$ form of the host, a readily formed variant.

Filterability: Passes Seitz EK filter.

Other properties: Filtrates active in dilutions to $10^{-9}$ or $10^{-11}$.

Literature: Craigie, Jour. Bact., 31, 1936, 56 (Abst.); Craigie and Brandon, Jour. Path. and Bact., 43, 1936, 233-248, 249-260. 
25. Phagus testabilis H. (loc. cit., 155). From Latin testabilis, able to bear witness, in reference to evidence that this bacteriophage has given, by virtue of its easy destruction when heated in spores, against the hypothesis of frequent spontaneous origin of bacteriophage from the bacterial host.

Common name: Bacillus megatherium. bacteriophage.

Host: Bacillus megatherium De Bary.

Geographical distribution: United States.

Induced disease : Plaques $0.5 \mathrm{~mm}$ or less in diameter, with surrounding translucent zone.

Thermal inactivation: In vitro, at $75^{\circ}$ $\mathrm{C}$ in 10 minutes. Spores from infected cultures, after being heated for $10 \mathrm{~min}$ utes at $80^{\circ} \mathrm{C}$, regularly give rise to subcultures that do not show the presence of this bacteriophage spontaneously during subsequent growth but that are susceptible to lysis if the bacteriophage is again introduced.

Literature: Adant, Compt. rend. Soc. Biol., Paris, 99, 1928, 1246; Cowles, Jour. Bact., 20, 1930, 15-23.

26. Phagus indomitus H. (loc. cit., 156). From Latin indomitus, unrestrained, in reference to the ability of this bacteriophage to increase after heat treatment of infected spores.

Common name: Bacillus mycoides bacteriophage.

Host: Bacillus mycoides Flügge, some strains.

Insusceptible species: Bacillus cereus Frankland and Frankland, B. subtilis Cohn emend. Prazmowski, B. megatherium De Bary, B. anthracis Cohn emend. Koch. Some strains of B. mycoides.

Geographical distribution. United States.

Induced disease: Large plaques, with some secondary growth of host organism.

Thermal inactivation: In vitro, at $75^{\circ} \mathrm{C}$ in 10 minutes. Spores from infected cultures, heated at $90^{\circ} \mathrm{C}$ for 10 minutes give no bacteriophage on grinding, but lytic cultures when grown.

Literature: Lewis and Worley, Jour. Bact., 32, 1936, 195-198.

27. Phagus subvertens H. (loc. cit., 156). From Latin subvertere, to subvert, in reference to suspected action of this bacteriophage in causing running-out of alfalfa fields through destruction of nodule organisms.

Common name: Rhizobium leguminosarum bacteriophage.

Host: Rhizobium leguminosarum Frank. It has been shown that this bacteriophage is unable to increase in clover roots without the nodule-forming organism, $R$. leguminosarum, and that the bacteriophage plays no obviously essential role in nodule formation.

Induced disease: Very small plaques, with edges not sharply defined.

Thermal inactivation: At $60^{\circ} \mathrm{C}$ in 30 minutes.

Other properties: Not inactivated by drying for 2 months.

Literature: Gerretsen et al., Cent. f. Balit., II Abt., 60, 1923, 311-316; Grijns, ibid., 71, 1927, 248-251; Hitchner, Jour. Bact., 19, 1930, 191-201 ; Vandecaveye and Katznelson, Jour. Bact., 31, 1936, 465-477.

28. Phagus ineptus H. (loc. cit., 157). From Latin ineptus, unsuitable, in reference to inability of this bacteriophage to adapt itself to lysis of strain RW of its host.

Common name : Streptococcus bacteriophage R.

Host : Streptococcus cremoris Orla-Jensen, strain $\mathbf{R}$.

Insusceptible species: Streptococcus cremoris, strain RW.

Geographical distribution: New Zealand.

Induced disease: Plaques 0.25 to 0.6 $\mathrm{mm}$ in diameter.

Serological relationships: Antisera specific for streptococcus bacteriophage $\mathrm{RW}$ 
and its strain $\mathrm{RW} 1$ are ineffective in neutralizing this bacteriophage.

Immunological relationships: Cultures of host-strain R, after exposure to this bacteriophage, furnish subcultures only partly resistant to this bacteriophage and completely susceptible to streptococcus bacteriophage RW and its substrain RW1.

Literature: Whitehead and Hunter, Jour. Path. and Bact., 44, 1937, 337-347.

29. Phagus streptococci H. (loc. cit., 158). From generic name of host.

Common name : Streptococcus bacteriophage RW.

Host: Streptococcus cremoris Orla-Jensen, strain RW.

Geographical distribution: New Zealand.

Induced disease: Plaques 0.25 to 0.6 $\mathrm{mm}$ in diameter.

Thermal inactivation: At $70^{\circ}$ to $75^{\circ} \mathrm{C}$, time not recorded, probably 30 minutes (pH 6.0).

Literature: Whitehead and Hunter, Jour. Path. and Bact., 44, 1937, 337-347.

Strains : One variant has been described and distinguished from the type variety, typicus H. (loc. cit., 158) :

29a. Phagus streptococci var. virilis $\mathrm{H}$. (loc. cit., 158). From Latin virilis, vigorous. Common name: Strain RW1 of streptococcus bacteriophage RW. Differing from the type variety in being able to increase at the expense of strain RW1 of Streptococcus cremoris (Whitehead and Hunter, Jour. Path. and Bact., 44, 1937, 337-347).

30. Phagus maculans spec. nov. From Latin maculare, to speckle, in reference to tiny plaques produced by this bacteriophage.

Common name: Streptococeus bacteriophage A.

Hosts : Streptococcus 646, 751, 775.

Geographical distribution: United States (Massachusetts).

Induced disease: Plaques exceedingly minute, scarcely visible to the unaided eye.

Serological relationships: Specific antisera neutralize but there is no cross reaction with respect to streptococcus bacteriophage $\mathrm{B}, \mathrm{C}$, or $\mathrm{D}$.

Thermal inactivation: At $60^{\circ} \mathrm{C}$ in 1 hour.

Other properties: Withstands storage at about $5^{\circ} \mathrm{C}$ for at least 145 days with but little loss of virulence.

Literature: Evans, Science, 80, 1934, 40-41; U.S.P.H.S., Public Health Reports, 49, 1934, 1386-1401.

31. Phagus lacerans spec. nov. From Latin lacerare, to tear, in reference to ragged edges of plaques produced by this bacteriophage.

Common name: Streptococcus bacteriophage $\mathbf{B}$.

Hosts: Streptococcus 563,639 : Streptococcus mucosus Howard and Perkins.

Insusceptible species: Streptococcus erysipelatos Rosenbach.

Geographical distribution: United States (Wisconsin).

Induced disease : Medium size plaques, the largest about $3 \mathrm{~mm}$ in diameter, edges ragged, centers clean.

Serological relationships: Specific neutralization, but no cross reactions with streptococcus bacteriophages A, C, and D.

Thermal inactivation: At $60^{\circ} \mathrm{C}$ in 1 hour.

Other properties: Withstands storage at about $5^{\circ} \mathrm{C}$ for at least 261 days.

Literature: Clark and Clark, Jour. Bact., 11, 1926, 89; Proc. Soc. Exp. Biol. and Med., 24, 1927, 635-639; Colvin, Jour. Inf. Dis., 51, 1932, 17-29; Evans, U.S.P.H.S., Public Health Reports, 49, 1934, 1386-1401; Jour. Bact., 39, 1940, 597-604; Shwartzman, Jour. Exp. Med., $46,1927,497-509$.

32. Phagus tolerans spec. nov. From Latin tolerans, tolerating, in reference to the unusual ability of this streptococcus 
bacteriophage to remain viable under certain adverse conditions.

Common name : Streptococcus bacteriophage C.

Hosts : Streptococcus 646, 594, 756, 806.

Geographical distribution: Uniterl States (Ohio, Massachusetts, Connecticut).

Induced disease: Small plaques, the largest about $1.0 \mathrm{~mm}$ in diameter.

Serological relationships: Specific neutralization, but no cross reactions with streptococeus bacteriophages A, B, and $\mathrm{D}$.

Thermal inactivation: At $63^{\circ}$ to $65^{\circ} \mathrm{C}$ in 1 hour.

Other properties: Withstands storage in $1: 200$ phenol at about $5^{\circ} \mathrm{C}$ for at least 261 days; equally resistant to storage in $1: 10,000$ sodium ethyl mercurithiosalicylate (merthiolate), or to storage without preservatives.

Literature: Evans, U. S. Pub. Health Ser., Public Health Reports, 49, 1934, 1386-1401.

33. Phagus michiganensis spec. nov. From name of state. Michigan, where this bacteriophage was first isolated.

Common name : Streptococe us bacteriophage D.

Host : Streptococeus 693.

Geographical distribution: Lnited States (Michigan).

Induced disease: Small plaques, about $0.75 \mathrm{~mm}$ in diameter, edges clear-cut, centers clean.

Serological relationships: Specific neutralization, but no cross neutralization with streptococeus bacteriophages A, B, and $\mathrm{C}$.

Thermal inactivation: At $60^{\circ}$ to $63^{\circ} \mathrm{C}$ in 1 hour.

Other properties: Withstands storage at about $5^{\circ} \mathrm{C}$ for at least 261 days.

Literature: Evans, ¿. s. Pub. Health Ser., Public Health Reports, 49, 1934, 1386-1401.

34. Phagus fragilis, H. (loc. cit., 159). From Latin fragilis, fragile, in reference to easy destruction of this bacteriophage by light and by concentrated urea solutions.

Common names: Staphylococcus bacteriophage Au2, Au3, Au4, or D, perhaps bacteriophage $\mathrm{H}$ of Gratia.

Hosts: Staphylococcus aureus Rosenbach and Staphylococcus albus Rosenbach.

Geographical distribution: United States.

Induced disease: Small plaques, 0.2 to $1.0 \mathrm{~mm}$ in diameter, with sharp edges.

Serological relationships: Cross-neutralization reactions with staphylococcus bacteriophages Au1, Au3, Aut, and D, but not with staphylococcus bacteriophages Au21, Au12, A, B, C, or bacteriophage C'16.

Thermal inactivation: At about $57^{\circ} \mathrm{C}$ in 30 minutes.

Other properties: Particle diameter 50 to 75 millimicrons. Readily inactivated photodynamically. Completely inactivated by 27 per cent urea solution in 1 hour at $37^{\circ}(\therefore$. Lysis not inhibited even by 1.5 per cent sodium citrate in agar medium.

Literature: Burnet and Lush, Jour. Path. and Bict ., 40, 1935, 455-469; Burnet and MeKie, Austral. Jour. Exp. Biol. and Med. Sci., 6, 1929, 21-31; Fisk, Jour. Inf. Dis., $71,1942,153-160$.

35. Phagus intermedius H. (loc. cit, 160). From Latin intermedius, intermediate, in reference to position of this bacteriophage between staphylococcus bacteriophages that multiply readily in broth cultures of host organisms and those that do not.

Common name: Staphylococcus bacteriophage Au21.

Host: Staphylococcus aureus Rosenbach.

Geographical distribution: Australia.

Induced disease: Small plaques, 0.1 to $0.3 \mathrm{~mm}$ in diameter, with sharp edges.

Serological relationships: Specific neutralization reaction but no cross-neutralization reaction with staphylococcus bacteriophages Au2 or Aul2. 
Other properties: Not readily inactivated photodynamically; completely inactivated by 27 per cent urea solution in 1 hour at $37^{\circ} \mathrm{C}$; lysis inhibited by 1 per cent sodium citrate in agar medium but not by 0.5 per cent or lower concentrations.

Literature: Burnet and Lush, Jour. Path. and Bact., 40, 1935, 455-469.

36. Phagus caducus H. (loc. cit., 160). From Latin caducus, perishable, in reference to the easy destruction of this bacteriophage by concentrated urea solutions.

Common name: Staphylococcus bacteriophage Au12.

Host: Staphylococcus aureus Rosenbach.

Geographical distribution: Australia.

Induced disease: Small plaques, 0.2 to $0.5 \mathrm{~mm}$ in diameter, with sharp edges.

Serological relationships: Cross-neutralization reactions with staphylococeus bacteriophages Aul1 and Au13, but not with staphylococcus bacteriophages $\mathbf{A} 2$, Au21, A, and C. Antiserum to staphylococcus bacteriophage $B$ gives no neutralization of Au12, though the reciprocal reaction occurs to $1: 200$ dilution.

Other properties: Not readily inactivated photodynamically; completely inactivated by 27 per cent urea solution in 1 hour at $37^{\circ} \mathrm{C}$; lysis inhibited by as little as 0.25 per cent sodium citrate in agar.

Literature: Burnet and Lush, Jour. Path. and Bact., 40, 1935, 455-469.

37. Phagus alpha H. (loc. cit., 161). From Greek equivalent of common name.

Common name: Staphylococcus bacteriophage A.

Host : Staphylococcus albus Rosenbach.

Geographical distribution: Australia.

Induced disease: Plaques of medium size, 1.5 to $2.5 \mathrm{~mm}$ in diameter, with hazy periphery.

Serological relationships: Specific neutralization reaction, but no cross-neutralization reactions with staphylococeus bacteriophages $\mathrm{Au} 2, \mathrm{~B}$, or $\mathrm{C}$.
Immunological relationships: Colonies of Staphylococcus albus appearing after lysis with this bacteriophage are resistant to staphylococcus bacteriophages $\mathrm{B}, \mathrm{C}$, and $\mathrm{D}$.

Thermal inactivation: At $68^{\circ}$ to $70^{\circ} \mathrm{C}$ in 30 minutes.

Other properties: Not readily inactivated photodynamically; not completely inactivated by 27 per cent urea solution in 1 hour at $37^{\circ} \mathrm{C}$; lysis not inhibited even by 1.5 per cent sodium citrate in agar.

Literature: Burnet and Lush, Jour. Path. and Bact., 40, 1935, 455-469; Burnet and McKie, Austral. Jour. Exp. Biol. and Med. Sci., 6, 1929, 21-31.

38. Phagus beta H. (loc. cit., 162). From Greek equivalent of common name.

Common name: Staphylococcus bacteriophage $B$.

Host : Staphylococcus albus Rosenbach. Geographical distribution: Australia.

Induced disease: Plaques of medium size, 0.7 to $1.5 \mathrm{~mm}$ in diameter, with sharp edges.

Serological relationships: Specific neutralization reaction, but no cross-neutralization reaction with respect to staphylococcus bacteriophages Au2, Au12, $A$, or C , except that ant iserum made with Au12 neutralizes this bacteriophage in low dilutions (See Phagus caducus).

Immunological relationships: Colonies appearing after lysis of Staphylococcus albus with this bacteriophage furnish organisms susceptible to staphylococeus bacteriophages $\mathrm{A}$ and $\mathrm{D}$.

Thermal inactivation: At $63^{\circ}$ to $65^{\circ} \mathrm{C}$ in 10 minutes.

Other properties: Readily inactivated photodynamically; completely inactivated by 27 per cent urea solution in 1 hour at $37^{\circ} \mathrm{C}$; lysis not inhibited even by 1.5 per cent sodium eitrate in agar medium.

Literature: Burnet and Lush, Jour. Path. and Bact., 40, 1935, 455-469; Burnet 
and McKie, Austral. Jour. Exp. Biol. and Med. Sci., 6, 1929, 21-31.

39. Phagus durabilis H. (loc. cit., 162). From Latin durabilis, lasting, in reference to the stability of this bacteriophage in concentrated urea solution and other unfavorable media.

Common name: Staphylococcus bacteriophage C.

Host: Staphylococcus albus Rosenbach.

Geographical distribution: Australia.

Induced disease: Plaques 2.0 to $3.0 \mathrm{~mm}$ in diameter. Vitreous change in peripheral zone.

Serological relationships: Cross-neutralization reaction with staphylococcus bacteriophage $\mathrm{C}^{\prime}$, and less strongly with B, but not with Au2 or A.

Immunological relationships: Colonies of Staphylococcus albus appearing after lysis with this bacteriophage furnish organisms resistant to it but susceptible to staphylococcus bacteriophages A, B, and $\mathrm{D}$.

Thermal inactivation : At $61^{\circ}$ to $63^{\circ} \mathrm{C}$ in 30 minutes.

Other properties: Not readily inactivated photodynamically; not completely inactivated by 27 per cent urea solution in 1 hour at $37^{\circ} \mathrm{C}$; lysis not inhibited even by 1.5 per cent sodium citrate in agar medium.

Literature: Burnet and Lush, Jour. Path. and Bact., 40, 1935, 455-469; Burnet and MeKie, Austral. Jour. Exp. Biol. and Med. Sci., 6, 1929, 21-31 ; Rakieten et al., Jour. Bact., 32, 1936, 505-518.

40. Phagus liber H. (loc. cit., 163). From Latin liber, independent, in reference to demonstrated independence of this virus, its bacterial host, and its dipterous superhost, in respect to origin.

Common name: Staphylococcus muscae bacteriophage.

Host: Staphylococcus muscae Glaser.

Geographical distribution: United States.

Induced disease: Lysis in broth cul- tures; plaques in agar cultures, but characteristics of plaques not recorded.

Thermal inactivation: At a little above $50^{\circ} \mathrm{C}$ in 5 minutes.

Other properties: A characteristic nucleoprotein has been isolated from lysed staphylococci. Sedimentation constant, $650 \times 10^{-13} \mathrm{~cm}$ dyne $^{-1}$ sec..$^{-1}$, corresponding to a molecular weight of about $300,000,000$. Denatured at acidities beyond pH 5.0. Digested by chymotrypsin, not by trypsin. Apparent density, about 1.20. Diffusion coefficient, varying with dilution.

Literature: Glaser, Amer. Jour. Hygiene, 27, 1938, 311-315; Northrop, Jour. Gen. Physiol., 21, 1938, 335-366; Shope, Jour. Exp. Med., 45, 1927, 1037-1044; Wyckoff, Jour. Gen. Physiol., 21, 1938, 367-373.

41. Phagus cholerae H. (loc. cit., 164). From former name of host.

Common name : Vibrio comma bacteriophage.

Host: Vibrio comma Winslow et al. (formerly $V$. cholerae Neisser); Indian strains usually carry this bacteriophage, but Chinese and Japanese strains lack it, are susceptible, and upon inoculation become lysogenic.

Geographical distribution: India.

Induced disease: In both $\mathrm{R}$ and $\mathrm{S}$ forms of Vibrio comma, no plaques on ordinary agar plates, but vibrios become lysogenic. Egg-white in 1:25 dilution enhances activity enough to allow visible lysis, occasional plaques, or stippling at the site of inoculation.

Immunological relationships: Vibrio comma organisms that have been infected with this bacteriophage and are resistant to its further action are still susceptible to cholera bacteriophages $\mathrm{A}, \mathrm{C}$, and D.

Literature: White, Jour. Path. and Bact., 44, 1937, 276-278.

42. Phagus celer H. (loc. cit., 164). From Latin celer, quick, in reference to relatively quick action of this bacteriophage. 
Common name: Cholera bacteriophage A.

Host: Vibrio comma Winslow et al, smooth types, except non-agglutinable vibrios.

Geographical distribution: India.

Induced disease: Lysis in 2 hours, followed by abundant secondary growth. Only smooth elements of the culture are attacked.

Serological relationships : Antigenically distinct from cholera bacteriophage C .

Immunological relationships: Secondary growth resistant to this virus, but susceptible to cholera bacteriophages C and D.

Other properties: Selectively inactivated by specific polysaccharide of smooth strains, not by a lipoid emulsion that is effective against cholera bacteriophage $\mathrm{C}$. Active in dilution of $1: 10^{9}$ or $1: 10^{10}$. Multiplication rate, $\mathrm{n} \times 10^{5}$ in 2 hours.

Iiterature: Asheshov et al., Indian Jour. Med. Res., 20, 1933, 1127-1157; White, Jour. Path. and Bact., 43, 1936, 591-593.

43. Phagus effrenus H. (loc. cit., 165). From Latin effrenus, unbridled, in reference to the ability of this bacteriophage to attack all tested strains of the cholera organism.

Common name: Cholera bacteriophage C.

Host : Vibrio comma Winslow et al., all strains.

Geographical distribution: India.

Induced disease: Sometimes death without lysis. When lysis occurs, it is rarely complete and is followed by secondary resistant growth.

Serological relationships: Antigenically distinct from cholera bacteriophage $\mathrm{A}$.

Immunological relationships: Secondary growth resistant to this bacteriophage, but susceptible to cholera bacteriophages $\mathrm{A}$ and $\mathrm{D}$.

Other properties: Selectively inactivated by lipoid from smooth strain of host, but not by specific polysaccharide.
Active in dilution of $1: 10^{8}$ or $1: 10^{10}$. Multiplication rate, $\mathrm{n} \times 10^{1}$ in 2 hours.

Literature: Asheshov et al., Indian Jour. Med. Res., 20, 1933, 1127-1157; White, Jour. Path. and Bact., 43, 1936, 591-593.

44. Phagus lentus H. (loc. cit., 166). From Latin lentus, slow, in reference to the relatively slow and incomplete lysis induced by this bacteriophage.

Common name: Cholera bacteriophage $\mathrm{D}$.

Host: Vibrio comma Winslow et al.

Geographical distribution: India.

Induced disease: Incomplete lysis in about 5 hours, followed, in rough cultures, by slow development of resistant secondary growth.

Immunological relationships: Secondary growth resistant to this bacteriophage, but susceptible to cholera bacteriophages $\mathrm{A}$ and $\mathrm{C}$.

Other properties: Not inactivated by specific polysaccharide effective against cholera bacteriophage $A$, nor by lipoid effective against cholera bacteriophage C. Multiplication rate, $\mathrm{n} \times 10^{2}$ in 2 hours.

Literature: Asheshov et al., Indian Jour. Med. Res., 20, 1933, 1127-1157; White, Jour. Path. and Bact., 43, 1936, 591-593.

45. Phagus diphtheriae H. (loc. cit., 167). From name of host.

Common name: Corynebacterium diphtheriae bacteriophage.

Host: Corynebacterium diphtheriae Lehmann and Neumann, many strains, especially 122 of 127 Australian type II gravis isolates; type I gravis isolates are lysogenic (carriers); all intermediate isolates are susceptible.

Insusceptible species : Corynebacterium diphtheriae, all tested mitis isolates, except 2 lysogenic. A strain of $C$. diphtheriae from Swan Hill, 200 miles north of Melbourne, was found to be resistant to this bacteriophage and to the small- 
plaque diphtheria bacteriophage, $P$. futilis.

Geographical distribution: Australia.

Induced disease: In Coryncbacterium diphtheriae on agar, plaques 1.0 to $1.5 \mathrm{~mm}$ in diameter, with shelving edge. A few resistant bacterial colonies often appear in the central clear area.

Literature: Kieogh et al., Jour. Path. and Bact., 46, 1938, 565-570; Smith and Jordan, Jour. Bact., 21, 1931, 75-88; Stone and Hobby, Jour. Bact., 27, 1934, 403-417.

46. Phagus futilis H. (loc. cit., 168). From Latin futilis, vain, in reference to regular appearance of resistant organisms in plaques on agar cultures lysed by this bacteriophage.

Common name: Small-plaque diphtheria bacteriophage.

Host: Corynebacterium diphtheriae Lehmann and Neumann, gravis type I isolates and all but 5 gravis type II isolates.

Insusceptible species: All tested intermediate and mitis strains of $C$. diphtheriae.

Geographical distribution: Australia.

Induced disease: In Corynebacterium diphtheriae on agar, pin-point plaques or confluent plaques, with confluent growth of secondary, resistant organisms.

Literature: Keogh et al., Jour. Path. and Bact., 46, 1938, 565-570. 


\section{Suborder II. Phytophagineae subordo novus.}

Viruses infecting higher plants; vectors typically homopterous or hemipterous insects (leafhoppers, aphids, white flies, true bugs) or thysanopterous insects (thrips). From Greek phagein, to eat, and phyton, a plant.

Key to the families of suborder Phytophagineae.

1. Inducing yellows-type diseases; vectors typically cicadellid or fulgorid leafhoppers.

Family I. Chlorogenaceae, p. 1145.

2. Inducing mosaic diseases; vectors typically aphids.

Family II. Marmoraceae, p.1163.

3. Inducing ringspot diseases; vectors unknown.

Family III. Annulaceae, p. 1212.

4. Inducing leaf-curl diseases; vectors typically white flies.

Family IV. Rugaceae, p. 1218.

5. Inducing leaf-savoying diseases; vectors, true bugs.

Family V. Savoiaceae, p. 1221.

6. Inducing spotted wilt; vectors, thrips.

Family VI. Lethaceae, p. 1223.

\section{FAMILI I. CHIOROGENACEAE HOLMES EMEND.}

(Handb. Phytopath. Tiruses, 1939, 1.)

Viruses of the Yellows-Disease Group; pathogenic in flowering plants, causing diseases in which effects on chlorophyll are usually diffuse or stripe-like, no typical spotting or spotty mottling being involved. Vectors, so far as known, leafhoppers (CICADELLIDAE and FULGORID.AE).

\section{Key to the genera of family Chlorogenaceae.}

I. True Yellows Group. Tiruses inducing diseases usually characterized by stimulation of normally dormant and adventitious buds to produce numerous slender shoots with long internodes and by chlorosis without spotting; invaded parts abnormally erect in habit. Vectors cicadellid leafhoppers so far as known.

Genus I. Chlorogenus, p. $11+h$.

II. Peach X-Disease Group. Viruses inducing diseases characterized by rosetting of foliage and sometimes death of host.

Genus II. Carpophthora, p. 1151.

III. Phloem-Necrosis Group. Viruses inducing diseases characterized by progressive degeneration of the host plant or by wilting and sudden death; sometimes by root discoloration. Vectors cicadellid leafhoppers so far as known.

Genus III. Morsus, p. 1153.

IV. Yellow-Dwarf Group. Viruses inducing diseases characterized by chlorotic effects somewhat resembling true mottling but often more diffuse. Vertors cicadellid (agallian) leafhoppers.

Genus IV. Aureogenus, p. 1154.

I. Fiji-Disease Group. Viruses inducing diseases characterized by marked vascular proliferation. The vector of one is known to be a leafhopper of the subfamily Delphacinae, family FULGORIDAE.

Genus V. Galla, p. 1157. 
VI. Stripe-Disease Group. Viruses inducing diseases characterized by chlorotic striping; hosts grasses. Vectors, cicadellid and fulgorid leafhoppers.

Genus VI. Fractilinea, p. 1159.

\section{Genus I. Chlorogenus Holmes.}

(Loc. cit., 1.)

Viruses of the Typical Yellows Group, inducing diseases usually characterized by stimulation of normally dormant and adventitious buds to produce numerous slender shoots with long internodes, by chlorosis without spotting, or by both growth of numerous slender shoots and chlorosis. Invaded parts abnormally erect in habit. Affected flowers often virescent. Hosts, dicotyledonous plants. Vectors, so far as known, exclusively cicadellid leafhoppers. Generic name from Greek chloros, light green or yellow, and suffix, gen, signifying producing, from Greek genos, descent.

The type species is Chlorogenus callistephi Holmes.

\section{Key to the species of genus Chlorogenus.}

I. Natural hosts many, in various families of plants.

1. Chlorogenus callistephi.

2. Chlorogenus australiensis.

II. Known natural hosts relatively few.

A. Natural hosts rosaceous.

B. Natural hosts solanaceous.

C. Natural host sandal.

D. Natural host cranberry.

E. Natural host locust.

F. Natural host alfalfa.

G. Natural host hop.

3. Chlorogenus persicae.

4. Chlorogenus solani.

5. Chlorogenus santali.

6. Chlorogenus vaccinii.

7. Chlorogenus robiniae.

8. Chlorogenus medicaginis.

9. Chlorogenus humuli.

1. Chlorogenus callistephi Holmes. (Handb. Phytopath. Viruses, 1939, 2.) From New Latin Callistephus, generic name of the China aster.

Common names: Aster-yellows virus, lettuce white-heart virus, Erigeron-yellows virus.

Hosts: Callistephus chinensis Nees, the China aster, is the host that has been studied most. 170 or more species in 38 different families of dicotyledonous plants have been shown susceptible. Lettuce, endive, carrot, buckwheat, parsnip, and New Zealand spinach are among the hosts of economic importance.
Insusceptible species: All tested species of the family Leguminosae and some species of all other tested families have appeared naturally immune.

Geographical distribution: U. S., Canàda, Bermuda, Japan, and Hungary. In California the celery-yellows strain of this virus replaces the type.

Induced disease: In most host species the characteristics of disease are clearing of veins, followed by chlorosis of newly formed tissues, stimulation of normally dormant buds to growth, malformation, virescence of flowers, sterility, and upright growth habit. Stimulation of nor- 
mally dormant buds to adventitious growth and abnormally erect habit are the most constant features. Chlorosis is absent or inconspicuous in some hosts.

Transmission: By leafhopper, Macrosteles divisus (Uhl.) (= Cicadula sexnotata (Fall.), C. divisa (Uhl.)) (CICA$D E L L I D A E)$. Incubation period, about 2 weeks. Some strains of this virus are transmitted also by the leafhoppers Thamnotettix montanus Van D. and $T$. geminatus Van D. (CICADELLIDAE). By grafting. By dodder. Not through seeds of diseased plants. Not by mechanical inoculation of plants, but virus has been passed from insect to insect mechanically in Macrosteles divisus; juice from viruliferous insects contains little virus just after inoculation, but the effective concentration increases at least 100 -fold between the 2 nd and 12 th day of a 17-day incubation period; it seems greatest before the insects begin to infect the aster plants on which they are maintained.

Thermal inactivation: In juice from viruliferous insects, at about $40^{\circ} \mathrm{C}$ in 10 minutes; at $25^{\circ} \mathrm{C}$ in 2 to 3 hours. In plant tissues, at $38^{\circ}$ to $42^{\circ} \mathrm{C}$, in 2 to 3 weeks; cured plants fully susceptible to reinfection. In insect vector, $M$. divisus, at $31^{\circ} \mathrm{C}$ in 12 days.

Other properties: Virus in juices derived from insects is more stable at $0^{\circ} \mathrm{C}$ than at $25^{\circ} \mathrm{C}$ or when frozen; at $0^{\circ} \mathrm{C}$ it withstands storage 24 , not 48 , hours in 0.85 per cent $\mathrm{NaCl}$ solution at $\mathrm{pH} 7.0$ but most of the virus is inactivated in this time; it withstands dilution $1: 1000$ in neutral 0.85 per cent $\mathrm{NaCl}$ solution; for brief (less than 5-minute) exposures, it remains viable over the range from $\mathrm{pH} 5$ to 9 .

Literature: Black, Phytopath., 31, 1941, 120-135; 33, 1943, 2 (Abst.) ; Johnson, ibid., 31, 1941, 649-656; Kunkel, Am. Jour. Bot., 13, 1926, 646-705; Contrib. Boyce Thompson Inst., 3, 1931, 85-123; 4, 1932, 405-414; Am. Jour. Bot., 24, 1937, 316-327 ; 28, 1941, 761-769; Linn, Cornell Agr. Exp. Sta. (Ithaca), Bull.
742, 1940; Ogilvie, Bermuda Dept. Agr., Agr. Bull. 6, 1927, 7-8; Severin, Hilgardia, 3, 1929, 543-583; Phytopath., 20, 1930, 920-921; Hilgardia, 7, 1932, 163-179; 8, 1934, 305-325, 339-361 ; Phytopath., 30, 1940, 1049-1051 ; Hilgardia, 14, 1942, 411440; Severin and Haasis, Hilgardia, 8, 1934, 329-335.

Strains : Two variant strains, one found in nature, the other derived experimentally, have been given varietal names to distinguish them from the type variety, vulgaris $\mathrm{H}$. (loc. cit., 2):

1a. Chlorogenus callistephi var. californicus H. (loc. cit., 3). From California, name of state in which this strain was first recognized. Common name: Celery-yellows strain of aster-yellows virus. Differing from the type variety by ability to infect celery (Apium graveolens L.UM BELLIFERAE) and zinnia (Zinnia elegans Jacq.-COMPOSITAE) (Kunkel, Contrib. Boyce Thompson Inst., 4, 1932, 405-414; Severin, Hilgardia, 3, 1929, $543-583 ; 8,1934,305-325)$.

1b. Chlorogenus callistephi var. attenuatus H. (loc. cit., 4). From Latin attenuatus, weakened. Common name: Heat-attenuated strain of aster-yellows virus. Differing from the type variety by inducing less severe chlorosis and less uprightness of new growth in affected aster plants (Kunkel, Am. Jour. Bot., 24, 1937, 316-327).

2. Chlorogenus australiensis comb. nov. From Australia, name of continent. Synonym: Galla australiensis H. (loc. cit., 107).

Common names : Tomato big-bud virus ; virescence virus; perhaps also stowboor virus, tobacco stolbur or montar virus, eggplant little-leaf virus.

Hosts : SOLA NACEAE-Datura stramonium L., Jimson weed; Lycopersicon esculentum Mill., tomato; Nicotiana tabacum L., tobacco; Solanum melongena L., eggplant; S. nigrum L., black nightshade. Recently a long list of species in this and 
other families have been reported as susceptible to virescence virus, presumed to be an isolate of tomato big-bud virus. (Hill, Jour. Counc. Sci. Ind. Res., 16, $1943,85-92$ ).

Geographical distribution: Australia, especially New South Wales; viruses causing somewhat similar diseases have been reported also from the Crimea and the northwestern United States.

Induced disease: In tomato, flowers erect, virescent, calyx bladder-like, pollen sterile; floral proliferation. Growth of axillary shoots stimulated. New leaves progressively smaller. Yuungest leaves yellowish-green in color, especially at their margins; usually purplish underneath. Hypertrophy of inner phloem. No intracellular inclusions. Fruit reddens imperfectly and becomes tough and woody. Roots appear normal. In Solanum nigrum, axillary shoots numerous, leaves small, internal phloem adventitious. In tobaceo, plants dwarfed; leaves recurved, distorted, twisted, thickened, brittle, yellowish green, hanging down close to stem; small leaves on shoots from axillary buds; proliferation and virescence of flowering parts; chlorotic clearing of veins as early effect of disease; upper surface of foliage appears glazed; some necrosis of veins, in old leaves, near tips and margins or on midrib; viable seed rarely produced; calyx bladder-like, floral axis may form short branches bearing small leaves; disease sometimes called bunchy top.

Transmission: By leafhopper, Thamnotettix argentata Evans (C'ICADELLI$D A E)$. Experimentally by budding and other methods of grafting. Not by inoculation of expressed juice

Literature: Cobb, Agr. Gaz. New South Wales, 13, 1902, 410-414; Dana, Phytopath., 30, 1940, 866-869; Hill, Jour. Austral. Inst. Agr. Sci., 6, 1940, 199-200; Jour. Council Sci. Ind. Res., 10, 1937, 309-312; 16, 1943, 85-92; Michailowa, Phytopath., 25, 1935, 539-558; Rischkov et al., Ztschr. Pflanzenkr., 43, 1933, 496-
498; Samuel et al., Phytopath., 23, 1933, 641-653.

3. Chlorogenus persicae H. (loc. cit., 5). From New Latin Persica, former generic name of peach.

Common names: Peach-yellows virus, little-peach virus.

Hosts: ROSACEAE-Prunus persica (L.) Batsch, peach; $P$. salicina Lindl., Japanese plum; and all other tested species of the genus Prunus.

Geographical distribution: Eastern United States and Canada, south to North Carolina. First occurred near Philadelphia in this country. Origin perhaps oriental; introduction in oriental plums suspected. Not in Europe.

Induced disease: In peach, clearing of veins, production of thin erect shoots bearing snall chlorotic leaves, followed by death in a year or two. In early stages of the disease there is premature ripening of fruit. In Japanese plum, systemic infection but no obvious symptoms.

Transmission: By the leathopper, $M a$ cropsis trimaculala (Fitch) (CICADEL$I I D A E)$. By budding; virus spreads down stem from point of bud insertion faster than up. Not by inoculation of expressed juice, despite numerous attempts. Not by pollen of diseased trees.

Immunological relationships: Presence of peach-yellows virus immunizes tree against little-peach virus, formerly considered an independent entity.

Thermal inactivation: In peach tissues, at $34^{\circ}$ to $35^{\circ} \mathrm{C}$ in 4 to 5 days; at $44^{\circ} \mathrm{C}$ in 30 minutes; at $47^{\circ} \mathrm{C}$ in 10 minutes; at $50^{\circ}$ $\mathrm{C}$ in 3 to 4 minutes; at $56^{\circ} \mathrm{C}$ in 15 seconds.

Other properties: Trees and bud sticks may be treated safely with heat sufficient to kill the virus. Cured trees are susceptible to reinfection.

Literature : Blake, N. J. Agr. Exp. Sta., Bull. 226, 1910; Kunkel, Contrib. Boyce Thompson Inst., 5, 1933, 19-28; Phytopath., $26,1936,201-219,809-830 ; 28,1938$, 491-497; Manns, Trans. Peninsula Hort. Soc., 23, 1933, 17-19; Manns and Manns, 
ibid., 24, 1934, 72-76; McCubbin, Pennsylvania Dept. Agr., Gen. Bull. 382, 1924.

Strains: Numerous strains of peachyellows virus probably exist in nature. One of these has been given a varietal name, distinguishing it from the type variety, vulgaris $\mathrm{H}$. (loc. cit., 5):

3a. Chlorogenus persicae var. micropersica H. (loc. cit., 6). From Greek micros, small, and New Latin Persica, former generic name of peach. Common name: Little-peach strain of peatch-yellows virus. Differing from the type variety by tend. ency to cause a mild type of disease, characterized by distortion of young leaves, production of many short branches on main trunk, later yellowing of mature leaves, twiggy growth, shoots slightly less erect than in typical peach yellows. (Kunkel, Phytopath., 26, 1936, 201-219; $26,1936,809-830$; 28, 1938, 491-497; Manns, Trans. Peninsula Hort. Soc., 23, 1933, 17-19;24, 1934, 72-76.)

4. Chlorogenus solani H. (loc. cit., 7). From New Latin Solanum, generic designation of potato. Synonym: Chlorophthora solani MeKinney, Jour. Washington Acad. Sci., 34, 1944, 151 .

Common names: Potato witches'-broom virus, potato wilding or semi-wilding virus.

Hosts: SOLANACEAE-Solanum tuberosum L., potato. Experimentally, also SOLANACEAE-Lycopersicon esculentum Mill., tomato; Nicotiana tabacum L., tobacco; $N$. glutinosa L.; $N$. rustica L. APOCYNACEAE-Vinca rosea L., periwinkle. CHENOPODIACEAEBeta vulgaris L., sugar beet.

Geographical distribution: United States (Montana, Washington), Russia.

Induced disease: In potato, increasingly pronounced flavescence in new leaflets on one or more stems, production of new dwarfed leaflets with marginal flavescence on stems with unusually long internodes and enlarged nodes, growth of spindling axillary and basal branches, profuse blooming and fruiting, lack of dormancy in tuber buds, formation of many small underground tubers as well as some aerial tubers; plants grown from diseased tubers form thread-like stems and small simple leaves; infected plants survive several seasons, with progressive degeneration. In tomato, experimentally, extreme leaf dwarfing, marginal Havescence of leaves and abnormally numerous axillary branches; stems become hollow and die; plants do not survive long after infection. In tobacco, experimentally, slender axillary branches with dwarfed leaves, flowers on spindling pedicels, numerous, small; later leaves flavescent or marginally flavescent.

Transmission: By tuber-core grafts with prepatent period of 29 to 114 days. By stem grafts. By dodder, Cuscuta campestris Yuncker (CONVOLVULA$(E A E)$. Not by inoculation of expressed juice. Not by Macrosteles divisus (Uhl.) (CICADELLIDAE). No insect vector is known. Not through seeds of diseased tomatoes.

Thermal inactivation: at $42^{\circ} \mathrm{C}$ in 13 days, in tissues of I inca rosea; at $36^{\circ} \mathrm{C}$ in 6 days in small potato tubers.

Literature: Hungerford and Dana, Phytopath., 14, 1924, 372-383; Kunkel, in Virus Diseases, Cornell Univ. Press, Ithaca, N. Y., 1943, 63-82; Proc. Am. Philosoph. Soc., 86, 1943, 470-475; McLarty, Scient. Agr., 6, 1926, 395; Whipple, Montana Agr. Exp. Sta., Bull. 130, 1919; Young, Science, 66, 1927, 304-306; Am. Jour. Bot., 16, 1929, 277-279; Young and Morris, Jour. Agr. Res., 36, 1928, \$35-854.

5. Chlorogenus santali H. (loc. cit., 8). From New Latin Santalum, generic designation of sandal.

Common names: Sandal spike-disease virus, sandal spike-rosette virus.

Hosts: SANTALACEAE-Santalum album L., sandal. Spike-like diseases have been found also in RHAMNEAEZizyphus oenoplia Mill., SAPINDA$C E A E$-Dodonaea riscosa .Jacq., IER- 
BENACEAE-Stachytarpheta indica Vahl, and $A P O C Y N A C E A E-V i n c a$ rosea L.

\section{Geographical distribution: South} India.

Induced disease : In sandal, abnormally profuse blooming at first, suppression of blooming later; reduction in leaf size and internode length; death ensues in the third year or earlier. In all but the youngest leaves of affected branches, vacuolate intracellular bodies with definite peripheral membrane, 4 to 9 microns in maximum diameter, are found.

Transmission: By twig grafts, inserted buds, and patch grafts, with success decreasing in the order named. Prepatent period 3 to 4 months. Best results in May and June; poorest in October. Perhaps through seeds, but not through pollen of diseased plants. Insect transmission claimed, but species not identified. Reported transmission by Moonia albimaculata (CICADELLIDAE) requires further confirmation. Not by inoculation of expressed juice. Not by root grafts.

Literature: Coleman, Mysore Dept. Agr., Mycol. Ser., Bull. 3, 1917; Indian Forester, 49, 1923, 6-9; Dover, ibid., 60, 1934, 505-506; Narasimhan, Phytopath., 23, 1933, 191-202 ; Rangaswami and Sreenvasaya, Current Science, 4, 1935, 17-19; Sreenvasaya, Nature, 126, 1930, 957; Venkata Rao and Gopala Iyengar, Mysore Sandal Spike Invest. Comm., Bull. 4, 1934, 1-12; Indian Forester, 60, 1934, 689-701.

6. Chlorogenus vaccinii $H$. (loc. cit., 10). From New Latin Vaccinium, generic designation of cranberry.

Common names: Cranberry false-blossom virus, Wisconsin false-blossom virus.

Hosts : ERICACEAE-Vaccinium macrocarpon Ait., cranberry; $V$. oxycoccus L. Experimentally, also $A P O C Y N A$ CEAE-Vinca rosea I., periwinkle. COMPOSITAE-Calendula officinalis L., calendula. SOLANACEAE-Lycopersicon esculentum Mill., tomato; Nico- tiana glutinosa L.; $N$. tabacum L., tobacco; Solanum tuberosum L., potato.

Geographical distribution: Eastern United States and Canada. It is believed that the virus does not spread in bogs with alkaline ( $\mathrm{pH} 7.4$ to 8.8) flooding water in Wisconsin though it spreads rapidly in the more productive bogs with nearly neutral ( $\mathrm{pH} 6.0$ to 7.0 ) flooding water.

Induced disease : In cranberry, flowers erect, instead of pendent as in healthy plants; calyx lobes enlarged, petals short, streaked with red and green, stamens and pistils abnormal. Flowers may be replaced by leaves or short branches. Dormancy of axillary buds is broken, producing numerous erect shoots, forming a witches' broom. Diseased fruits small, irregular in shape, erect.

Transmission: By leafhopper, Ophiola striatula (Fall.) (=Euscelis striatulus (Fall.)) (CICADELLIDAE). Not by inoculation of expressed juice. By dodder, Cuscuta campestris Yuncker (CONVOLVULACEAE).

Thermal inactivation: At $40^{\circ} \mathrm{C}$ in 2 weeks in tissues of periwinkle.

Literature : Dobroscky, Contrib. Boyce Thompson Inst., 3, 1931, 59-83; Fracker, Phytopath., 10, 1920, 173-175; Kunkel, Science, 95, 1942, 252; Torreya, 43, 1943, 87-95; Shear, U. S. Dept. Agr., Bull. 444, 1916; Stevens, Phytopath., 15, 1925, 8591; 34, 1944, 140-142; U. S. Dept. Agr., Circular 147, 1931; Stevens and Sawyer, Phytopath., 16, 1926, 223-227; Wilcox and Beckwith, Jour. Agr. Res., 47, 1933, 583-590.

7. Chlorogenus robiniae H. (loc, cit., 13). From New Latin Robinia, generic designation of locust. Synonym: Polycladus robiniae McKinney, Jour. Washington Acad. Sci., 34, 1944, 151.

Common names: Locust witches'broom virus; locust brooming-disease virus.

Hosts : LEGUMINOSAE-Robinia pseudoacacia L., black locust.

Geographical distribution: United 
States (southern Pennsylvania to northeastern Georgia, west to southwestern Ohio and Tennessee).

Induced disease : In black locust, clearing of veins, followed by progressive reduction in size of newly formed leaves; growth of spindly shoots to form witches' brooms. Roots more brittle, shorter, and darker than normal, with excessive branching of rootlets, giving the appearance of root brooms.

Transmission: By grafting and budding. Not by inoculation of expressed juice. No insect vector is known.

Literature : Grant et al., Jour. Forestry, 40, 1942, 253-260; Hartley and Haasis, Phytopath., 19, 1929, 163-166; Jackson and Hartley, Phytopath., 23,1933, 83-9 0; Waters, Plant World, 1, 1898, 83-84.

8. Chlorogenus medicaginis $\mathrm{H}$. (loc. cit., 14). From New Latin Medicago, generic designation of alfalfa (lucerne).

Common names: Alfalfa witches'broom virus, lucerne witches'-broom virus, spindle-shoot virus, mistletoe virus, Kurrajong virus, bunchy-top virus.

Hosts: LEGUMINOSAE-Medicago sativa L., alfalfa (lucerne).

Geographical distribution: Australia, especially New South Wales; perhaps United States.

Induced disease: In alfalfa, plant dwarfed; leaves small, rounded, chlorotic at edge, puckered, distorted; stems short, spindly, numerous. Flowers usually not formed, but sometimes pale and small, sometimes replaced by leafy structures. Seed rarely produced.

Transmission: By grafting. Not by inoculation of expressed juice. No insect vector is known.

Literature : Edwards, Jour. Australian Inst. Agr. Sci., 1, 1935, 31-32; New South Wales Dept. Agr., Science Bull. 52, 1936; Agr. Gaz. New South Wales, 47, 1936, 424-426; Richards, U. S. Dept. Agr., Plant Disease Reporter, Supplement, 71 , 1929, 309-310.

9. Chlorogenus humuli $H$. (loc. cit., 15). From New Latin Humulus, generic designation of the hop.

Common names: Hop-nettlehead virus, silly-hill disease virus, virus of infectious sterility of the hop.

Hosts : MORACEAE-Humulus lupulus L., European hop.

Geographical distribution: England, Czechoslovakia, Germany, Poland.

Induced disease : In hop, stems numerous, spindly, short, plants weak. Leaves curled upward at margin; cone production greatly reduced.

Transmission: By grafting. Not by inoculation of expressed juice. Not through soil. No insect vector is known.

Literature: Blattný and Vukolov, Rec. Inst. Rech. Agron. Rép. Tehécosl., 137, 1935, 3-18; Goodwin and Salmon, Jour. Inst. Brew., 33, 1936, 209-210; Salmon, ibid., 32, 1935, 235-237 ; 39, 1936, 181-186; Salmon and Ware, Jour. South-Eastern Agr. College, Wye, Kent. 37, 1936, 21-25.

\section{Genus II. Carpophthora McKinney emend.}

(Jour. Washington Acad. Sci., 34, 1944, 152.)

Peach X-Disease Group; viruses inducing diseases characterized in general by rosetting of foliage and sometimes death of host. Generic name from Greek, meaning fruit and ruin or destruction.

The type species is Carpophthora lacerans MeKinney.

\section{Key to the species of genus Carpophthora.}

I. Inducing chlorosis, reddening, and tattering of foliage, with rosette formation late in the disease in some hosts. 
II. Indueing rosette formation characteristically, but not tattering of affected foliage.

\section{Carpophthora rosettae.}

1. Carpophthora lacerans (Holmes) McKinney. (Marmor lacerans Holmes, Handb. Phytopath. Viruses, 1939, 82; McKinney, Jour. Washington Acad. Sci., 34, 1944, 152.) From Latin lacerare, to lacerate, in reference to characteristic foliage injury.

Common name: Peach $\mathrm{X}$-disease virus; virus of peach yellow-red virosis.

Hosts: ROSACEAE-Prunus persica (L.) Batsch, peach; $P$. virginiana $\mathrm{I}_{\text {., }}$ chokecherry

Geographical distribution: United States, Canada.

Induced disease: In peach, foliage normal each spring but yellowish areas appear in June at base of leaves; affected trees appear lighter green than neighboring healthy trees; discolored spots occur at random on the leaf blade, becoming red and yellow with remainder of leaf becoming chlorotic; the discolored areas usually fall out, leaving the foliage tattered; subsequently, affected leaves drop except at tips of branches; young trees may die, older ones survive indefinitely. Fruit either shrivels and falls or ripens prematurely, with bitter flavor and no viable seed. In chokecherry, conspicuous premature reddening of foliage, development of fruits with dead embryos in the pits; in the second and third seasons after infection, duller colors of foliage, rosettes of small leaves on terminals; death follows the advanced stage of disease.

Transmission: By budding. Not by inoculation of expressed juice. No insect vector has been reported.

Literature: Berkeley, Div. of Botany and Plant Path., Science Service, Dominion Dept. Agr., Ottawa, Canada, Publication 678, 1941 ; Boyd, C. S. Dept. Agr., Plant Dis. Rep., 22, 1938, 334; Hildebrand, Contrib. Boyce Thompson Inst., 11, 1941, 485-496; Hildebrand and Palmiter, U.S. Dept. Agr., Plant Dis. Rep.,
22, 1938, 394-396; Hildebrand et al., Handbook of virus diseases of stone fruits in North America, Michigan Agr. Exp. Sta., Mise. Publ., 1942, 21-24; Stoddard, Connecticut Agr. Exp. Sta., Circ. 122, 1938, 5t-60; Proc. Connecticut Pomol. Soc., 48, 1938, 29-32.

\section{Carpophthora rosettae (Holmes)} comb. nov. (Chlorogenus rosettae H., nomen nudum, Phytopath. 29, 1939, 434; Nanus rosettae H., Handb. Phytopath. Viruses, 1939, 125.) From rosette, common name of induced disease, from French, diminutive of rose, a rose.

Common name: Peach-rosette virus.

Hosts: ROSACEAE-Prunus persica (I.) Batsch, peach; P. communis Fritsch, atmond; $P$. domestica L., plum. Experimentally, also- $\triangle P O C Y N A C E A E-$ Tiner rosea I., periwinkle. ROSA. CEAE-I' americana Marsh., wild plum; $P$. armeniaca $\mathrm{L}$., apricot; $P$. cerasus $\mathrm{L}$., cherry; $P$. pumila L., sand cherry. SOLANACEAE-Lycopersicon esculentum Mill., tomato; Nicotiana glutinosa L.

Geographical distribution: United States (Georgia, Alabama, South Carolina, Tennessee, West Virginia, Missouri, Oklahoma).

Induced disease: In peach, sudden wilting and death, or growth of abnormally short stems bearing dwarfed leaves with clearing and thickening of veins, followed by death in a few months.

Transmission: By budding. By dodder, Cuscuta campestris Yuncker. Not by inoculation of expressed juice. Not through soil. No insect vector is known.

Immunological relationships: No protection is afforded by previous infection of peach trees with Chlorogenus persicae, peach-yellows virus.

Thermal inactivation: At $50^{\circ} \mathrm{C}$ in 10 minutes (in tissues of peach). Rosetted trees are abnormally susceptible to heat 
injury and heat treatments cure peachrosette disease only in recently infected trees.

Literature: Kunkel, Phytopath., 26, 1936, 201-219, 809-830; in Virus Diseases,
Cornell Univ. Press, Ithaca, X. Y., 1943, 63-82; McClintock, Jour. Agr. Res., 24, 1923, 307-316 ; Phytopath., 21, 1931, 373386 ; Smith, U. S. Dept. Agr., Div. Yeg. Path., Bull. 1, 1891.

\section{Genus III. Morsus gen. nov.}

Alfalfa-Dwarf Group; viruses inducing diseases characterized in general by sudden wilting and death or by gradual decline of vigor with foliage of darker green color than normal. Vectors, like those of the typical yellows subgroup, cicadellid leafhoppers so far as known. Generic name from Latin morsus, sting or vexation.

The type species is Morsus suffodiens spec. nov.

\section{Key to the species of genus Morsus.}

I. Affecting alfalfa and grape.

II. Affecting tobacco.

III. Affecting elm.

1. Morsus suffodiens spec. nov. From Latin suffodere, to sap or undermine, in reference to process leading to sudden collapse of long infected, but sometimes not obviously injured, grape vines as well as to progressive decline in size of infected alfalfa plants, the foliage of which may remain green to the last.

Common names: Alfalfa-dwarf virus, lucerne dwarf-disease virus, virus of Pierce's disease of the grape, virus of Anaheim disease.

Hosts: LEGUMINOSAE-Medicago sativa L., alfalfa (lucerne). VITA$C E A E$ - Vitis vinifera L., grape.

Geographical distribution: United States.

Induced disease: In alfalfa, leaves small but green, plant progressively smaller, wood of roots discolored yellow, transpiration decreased; wilting may occur; starch of root diminished; plant eventually succumbs, thinning stand prematurely. In grape, dark green color of leaves retained along veins, not between them, or no abnormality in appearance of foliage; wilting and sudden death of plant in summer of second year. In late summer of first year, there may be

\section{Morsus suffodiens.}

\section{Morsus reprimens.}

3. Morsus ulmi.

dying leaf margins and dying back of cane tips.

Transmission: By budding and root grafting. By leafhoppers, Draeculacephala minerva Ball, Carneocephala fulgida Nott., C. triguttata Nott., Helochara delta Oman, Neokolla circellata (Baker), $N$. gothica (Sign.), N. confluens (Uhler), N. heiroglyphica (Say), and Cuerna occidentalis Oman and Beamer (CICA$D E L L I D A E)$; these vectors all belong to the subfamily Amblycephalinae; all tested species of this, but none of any other, subfamily have proved capable of transmitting this virus. Not by inoculation of expressed juice. Not through soil.

Literature: Frazier, Phytopath., 34, 1944, 1000-1001; Hewitt, Phytopath., 29, 1939, 10; 31, 1941, 862; Blue Anchor, 18, 1941, 16-21, 36; Hewitt et al., Phytopath., 32, 1942, 8; Houston et al., Phytopath., 32, 1942, 10; Milbrath, Calif. Dept. Agr., 20th Ann. Rept., Bull. 28, 1940, 571; Pierce, U. S. Dept. of Agr., Div. of Veg. Path., Bull. 2, 1892, 1-222; Weimer, Phytopath., 21, 1931, 71-75; 27, 1937, 697-702 ; Jour. Agr. Res., 47, 1933, 351368 ; 53, 1936, 333-347 ; 55, 1937, 87-104. 
2. Morsus reprimens spec, nov. From Latin reprimere, to restrain, check, or curb, in reference to the inhibiting effect on growth of the host plant, tobacco.

Common name: Tobacco yellow-dwarf virus.

Hosts : SOLANACEAE-Nicotiana tabacum L., tobacco; N. rustica L., Indian tobaceo; $N$. trigonophylla Dun. Experimentally, also $N$. glauca Grah. (symptomless) and $N$. glutinosa $\mathrm{L}$.

Geographical distribution: Australia (Victoria, New South Wales, South Australia, and southern Queensland).

Induced disease : In tobacco, internodes of stem shortened, leaves small; downward bending of tips and rolling under of margins of young apical leaves; young leaves darker than normal at first, bunched, later appear ribbed; leaves become yellow-green, pale first between veins; old leaves rugose, thickened, later savoyed. Root system small, roots slightly brown externally and in the region of the phloem. Affected plants may survive the winter and show diseased new growth in the spring.

Transmission: By grafting and budding. By nymphs and adults of the leaf- hopper. Thamnotettix argentata (Evans) (CICA DELLIDAE).

Literature: Dickson, Australia, Council Sci. Indust. Res., Pamphlet 14, 1929, 22; Hill, Australia, Journal of the Council Sci. Indust. Res., 10, 1937, 228-230; 14, 1941, 181-186; 15, 1942, 13-25.

3. Morsus ulmi spec. nov. From Latin ulmus, elm.

Common name: Elm phloem-necrosis virus.

Host : URTICACEAE-Ulmus americana L., American elm.

Geographical distribution: United States (Ohio, Indiana, Illinois, Missouri, Tennessee, Kentucky, and West Virginia).

Induced disease: In elm, gradual decline over a period of 12 to 18 months before death or sudden wilt, drying of leaves, and death within 3 to 4 weeks. All ages susceptible, from seedling to large tree.

Transmission: By patch grafting. Not by inoculation of expressed juice.

Literature: Leach and Valleau, U. S. Dept. Agr., Plant Dis. Rept., 23, 1939, 300-301; Swingle, Phytopath., 30, 1940, 23.

\section{Genus IV. Aureogenus Black.}

(Proc. Am. Philos. Soc., 88, 1944, 141.)

Viruses of the Yellow-Dwarf Group, inducing diseases characterized by yellowing without typical mosaic-type mottling. Vectors agallian leafhoppers (CICADELLI$D A E)$. Generic name from Latin aureus, yellow or golden, and genus, group.

The type species is A ureogenus vastans (Holmes) Black.

\section{Key to the species of genus Aureogenus.}

I. Mechanically transmissible in some hosts by rubbing methods of inoculation. Not producing enlarged veins or club-leaf in clover.

1. Aureogenus vastans.

II. Not known to be transmissible by rubbing methods of inoculation.

A. Producing enlarged veins in clover.

2. Aureogenus magnivena.

B. Producing club-leaf in cluver.

\section{Aureogenus clavifolium.}


1. Aureogenus vastans (Holmes) Black. (Marmor vastans Holmes, Handb. Phytopath. Viruses, 1939, 94; Black, Proc. Am. Philos. Soc., 88, 1944, 141.) From Latin vastare, to devastate. Common name: Potato yellow-dwarf virus.

Hosts: SOLANACEAE-Solanum tuberosum L., potato. COMPOSITAEChrysanthemum leucanthemum L., var. pinnatifidum Lecoq and Lamotte, daisy; Rudbeckia hirta L., black-eyed Susan. CRUCIFERAE-Barbarea vulgaris $\mathrm{R}$. $\mathrm{Br}$., common winter cress. LEGU MINOSAE-Trifolium pratense L., red clover. Experimentally to numerous species in these and other families.

Geographical distribution: Northeastern United States and southeastern Canada.

Induced disease: In potato, yellowing of leaves, necrosis of stem, dwarfing of plant; the stem, if split, shows rusty specks especially at nodes and apex; the apex dies early; tubers are few, small, close to the stem, often cracked, with flesh discolored by scattered brown specks; seed tubers tend to remain unrotted in the ground, becoming hard and glassy; some of them do not germinate in warm soil, others produce shoots that die before reaching the surface, giving poor stands. In Chrysanthemum leucanthemum var. pinnatifidum, at first, clearing of veins; later, young leaves distorted, thick, stiff, small; petioles short, leaves erect, forming a rosette at the crown of the plant; with passing of the early phases of the disease, foliage tends to appear nearly normal, but remains darker green and more erect than that of healthy plants; virus is recoverable both during and after the period of obvious disease and infected plants may constitute an important reservoir. In Trifolium incarnatum L., crimson clover, experimentally, clearing of veins and yellowing of younger leaves (in summer the yellowing is usually replaced in part by an inter- veinal reddish-brown color on both leaf surfaces extending from the margins inwards); dwarfing of entire plant; death or a chronic disease characterized by milder manifestations without, however, vein enlargement or cupping of leaves. In Nicotiana rustica L., experimentally, yellowish primary lesions followed by clearing of veins and systemic chlorosis; the primary lesions facilitate quantitative estimation of concentrations of this virus.

Transmission: By inoculation of expressed juice, in the presence of finely powdered carborundum, to Nicotiana rustica; mechanical transmission very difficult in other hosts tested. By grafting. By clover leafhopper, Aceratagallia sanguinolenta (Provancher) ; experimentally, by other closely related leafhoppers, Aceratagallia lyrata (Baker), A. obscura Oman, and A. curvata Oman; not (for the type variety of the virus) by Agallia constricta Van Duzee; very rarely by Agallia quadripunctata (Provancher) and Agalliopsis novella (Say) (CICADELLI$D A E)$. The vector Aceratagallia sanguinolenta remains infective as an overwintering adult; incubation period not less than 6 days, commonly much longer; virus does not pass to progeny of viruliferous leafhoppers through eggs or sperm; this leafhopper varies genetically in ability to transmit.

Immunological relationships: No protection is afforded against necrotic effects of a testing strain of this virus (var. lethale Black) by prior inoculation of Nicotiana rustica with isolates of Marmor medicaginis, $M$. cucumeris, $M$. upsilon, Annulus tabaci, A. orae, or A. dubius, but the varieties vulgare Black and agalliae Black protect; these specifically protecting strains give no similar protection against formation of necrotic lesions by subsequently applied isolates of Marmor tabaci, M. lethale, Annulus tabaci, or A. orae.

Thermal inactivation : At 50 to $52^{\circ} \mathrm{C}$ in 10 minutes. 
Filterability: Passes Berkefeld W filter.

Other properties: Tirus viable at 23 to $27^{\circ} \mathrm{C}$ less than 13 hours after extraction of juice from diseased plant; not infective after drying in leaf tissues.

Literature: Barrus and Chupp, Phytopath., 12, 1922, 123-132; Black, Am. Potato Jour., 11, 1934, 148-152; Cornell Univ. Agr. Exp. Sta., Mem. 209, 1937, 1-23; Phytopath., 28, 1938, 863-874; Am. Jour. Bot., 27, 1940, 386-392; Am. Potato Jour., 18, 1941, 231-233; Phytopath., 33, 1943, 363-371; Geneties, 28, 1943, 200209; Proc. Am. Philos. Soc., 88, 1944, 132-14t; Hansing, Cornell Univ. Agr. Exp. Sta., Bull. 792, 1943; Price and Black, Am. Jour. Bot., 28, 1941, 594-595; Taylor, Am. Potalo Jour., 15, 1938, 37-40; Walker and Larson, Jour. Agr. Res., 59, 1939, 259-280; Watkins, Jour. Econ. Ent ., 32, 1939, 561-564; Comell Lniv. Agr. Exp. Sta., Bull. 758, 1941, 1-21; Younkin, Am. Potato Jour., 19, 1942, 6-11.

Strains: Beside the type variety, Aureogenus rastans var. vulyare Black (Am. Jour. Bot., 27, 1940, 391), on which the species is based, two distinctive strains have been given varietal names:

1a. Anreogenus vastans var. agalliae Black. (Am. Potato Jour., 18, 1941, 233.) From New Latin Agallia, generic name of vector of this strain. Common name: New Jersey strain of potato yellow-lwarf virus. Differing from the type especially in its distinctive vector, the leafhopper, Agallia constricta Van Duzee, which is incapable of transmitting the type strain, and in not being transmitted by $A$ ceratagallia sanguinolenta (Provancher), common vector of the type variety. Experimentally, transmitted also by Agallia quadripunctata (Provancher); perhaps rarely by Agalliopsis novella (Say); Differing but little from the type in effects on potato (var. Green Mountain) and Nicotiana rustica but more definitely in effects on crimson clover, in affected plants of which a rusty-brown necrosis along the veins, not induced by the type strain, is always present in some degree.

1b. Aureogenus vastans var. lethale Black. (Am. Jour. Bot., 27, 1940, 391.) From Latin lethalis, causing death. Common name: Strain B5 of potato yellow-dwarf virus. Differing from the type variety especially in a tendency to induce in Nicotiana rustica, experimentally, brown primary lesions with necrotic gray centers, systemic yellowing, extensive necrosis of veins, collapse of large areas of leaf, and sometimes death of the host; not known to occur in nature as a separate strain, but readily isolated as a variant from strains collected in nature.

\section{Aureogenus magnivena Black.} (Proc. Am. Philos. Soc., 88, 1944, 144.) From Latin magnus, large, and vena, vein.

Common name: Clover big-vein virus.

Host : Experimentally, LEGUMINOSAE-Trifolium incarnatum L., crimson clover.

Insusceptible species: $\quad$ SOLANA$C E A E-N i c o t i a n a$ rustica $\mathrm{L}$., Indian tobacen; Solanum tuberosum L., potato.

Geographical distribution: United States (presumably, Washington, D. C.).

Induced disense: In crimson clover, experimentally, unevenly thickened veins which are depressed below the upper surface of the leaf; these enlarged veins, best observed from below, sometimes bear enations that arise from their lower surfaces, leaves often curl upward and inward marginally; in summer, yellowing of leaves progresses from margins inward, the yellow color being later replaced in part by red or purple red; petioles undulating; plants dwarfed; internodes shortened; no clearing of veins; no rusty-brown necrosis.

Transmission: Not by inoculation of expressed juice. By leafhoppers, AgalLiopsis novella (Say), Agallia constricta Van Duzee, A. quadripunctata (Provancher); not by Aceratagallia sanguinolenta (Provancher) (CICADELLIDAE). 
3. Aureogenus clavifolium Black. (Proc. Am. Philos. Soe., 88, 194t, 141.) From Latin clava, club, and folium, leaf.

Common name: ('lover club-leaf virus. Host : Experimentally, LEGL $H I N O$ $S A E$ - Trifolium incarnatum L., crimson clover.

Insusceptible species: SOLA.YACEAE-Nicotiana rustica L., Indian tobacco; Solanum tuberosum L., potato.

Geographical distribution: Lnited States (Princeton, N. J.).

Induced disease: In crimson clover, experimentally, youngest leaves lighter green than normal, slow to unfold; leaf margins yellowed or colored red or purple red; affected leaves narrow, smooth or savoyed; plant dwarfed, new shoots from leaf axils slightly stimulated; new growth of spindly stems and small leaves; no rusty-brown necrosis of veins, no obvious enlargement of veins, and no obvious clearing of veins at the onset of disease.

Transmission: Not by inoculation of expressed juice. By leafhopper, Agalliopsis novella (Say) (CICADELLI. $D A E$ ) ; not by leafhoppers, Aceratagallia sanguinolenta (Provancher), Agallia constricta Van Duzee, nor A. quadripunciata (Provancher) (CICADELLIDAE).

\section{Genus V. Galla Holmes.}

$$
\text { (Loc. cit., 106) }
$$

Viruses of the Fiji-Disease Group, inducing diseases characterized by vascular proliferation. Generic name from Latin galla, a gall nut

The type species is Galla fijiensis Holmes.

\section{Key to the species of genus Galla.}

I. Infecting sugar cane.

1. Inducing formation of conspicuous galls.

1. Galla fijiensis.

B. Jot inducing formation of conspicuous galls.

2. Galla queenslandiensis.

II. Infecting anemone.

III. Infecting peach.

IV. Infecting corn.

1. Galla fijiensis Holmes. Handb. Phytopath. Viruses, 1939, 106.) From name of Fiji Islands.

Common name: Fiji-disease virus.

Host : GRA.IINEAE-Saccharum officinarum L., sugar cane.

Geographical distribution: Fiji Islands, New South Wales, Java, Philippine Islands, New Guinea and New Caledonia.

Induced disease: In sugar cane, galls on vascular bundles, formed by proliferation of phloem and nearby cells. Affected cells show characteristic spherical or oval inclusion bodies. Developing leaves shortened, crumpled, abnormally

\section{Galla anemones.}

\section{Galla verrucae.}

\section{Galla zeae.}

dark green. Infected stools of cane become bushy. Roots small, bunchy.

Transmission: By leafhoppers, Perkinsiella saccharicida Kirk. (in Queensland) and $P$. vastatrix Breddin (in Philippine Islands) ( $F L L G O R I D A E$, subfamily Delphacinae). Not by grafting. Not by inoculation of expressed juice. Not through eggs of $P$. vastatrix. Cuttings taken from affected canes produce some healthy and some diseased plants, because virus does not become uniformly distributed throughout the host tissues.

Literature: Kunkel, Bull. Exp. Sta., Hawaiian Sugar Planters' Assoc., Bot. 
Ser., 3, 1924, 99-107; Lyon, ibid., 3, 1921, 1-43; Hawaiian Planters' Rec., 12, 1915, 200; Mungomery and Bell, Queensland, Bur. Sugar Exp. Sta., Div. Path., Bull. 4, 1933; Ocfemia, Am. Jour. Bot., 21, 1934, 113-120; Ocfemia and Celino, Phytopath., 29, 1939, 512-517; Reinking, Phytopath., 11, 1921, 334-337.

2. Galla queenslandiensis $H$. (loc cit., 109). From Queensland, where the induced disease was first studied.

Common name: Sugar-cane dwarf-disease virus.

Host : GRAMINEAE-Saccharum offcinarum L., sugar cane.

Geographical distribution: Queensland.

Induced disease: In sugar cane, young leaves marked with scattered chlorotic streaks, leaves stiff and erect, spindle twisted, abnormally short and pale. As leaves mature, streaks disappear, leaves become darker than normal green. In recently infected plants, vascular bundles are enlarged, irregular in shape, fused and characterized by abnormal proliferation of thin-walled lignified cells.

Literature: Bell, Queensland, Bur. Sugar Exp. Sta., Div. Path., Bull. 3, 1932.

3. Galla anemones H. (loc. cit., 108). From Latin anemone, anemone or windflower.

Common name: Anemone-alloiophylly virus.

Hosts : RANUNCULACEAE-Anemone nemorosa $L$., vernal windflower; $A$. ranunculoides L.; A. trifolia L.

Geographical distribution: Germany.

Induced disease : Leaves thickened and distorted, petioles thickened. Flowers distorted or not formed. Vaseular bundles larger and more numerous than in healthy plants. Palisade cells short, chloroplasts smaller and fewer than normal.

Transmission: By needle puncture into rhizomes immersed in filtered juice of diseased plant. By contamination of soil with fragments of diseased leaves or rhizomes.
Literature: Klebahn, Bericht. d. Deutsch. Bot. Gesellsch., 15, 1897, 527536 ; Ztschr. wissensch. Biol., Abt. E, Planta, 1, 1926, 419-440; 6, 1928, 40-95; Phytopath. Ztschr., 4, 1932, 1-36; 9, $1936,357-370$.

4. Galla verrucae Blodgett. (Phytopath., 39, 1943, 30.) From Latin verruca, wart. Originally spelled verruca, apparently by a typographical error, which was corrected twice on the following page, once in a statement that the genitive verrucae had been given as specific epithet.

Common name: Peach-wart virus.

Host : ROSACEAE-Prunus persica (L.) Batsch, peach.

Geographical distribution: United States (Idaho, Washington, Oregon).

Induced disease: In peach, no characteristic effect on foliage. Fruits blistered, welted, later with warty outgrowths conspicuously raised. Affected tissues light tan to red, rough, cracked and russeted, or smooth. Gumming usual, often severe. Warty tissue superficial; underlying tissues coarse, filled with gum pockets, but not abnormal in flavor. Warty tissue may be hard and bony, but more often it is merely tougher than normal.

Transmission: By budding and inarching.

Literature: Blodgett, Phytopath., 31, 1941, 859-860 (Abst.) ; 33, 1943, 21-32.

j. Galla zeae Mchinney. (Jour. Washington Acad. Sci., 34, 1944, 328.) From Latin zea, a kind of grain.

Common name: Wallaby-ear disease virus.

Host: GRAMINEAE-Zea mays L., corn (maize).

Geographical distribution: South(astern Queensland, Australia.

Induced disease: In corn (maize), small swellings on secondary veins on undersides of young leaves, spreading to base and tip of leaf along veins; plant dwarfed, becoming abnormally deep green and deficient in development of pollen; silk, cobs, and grain retarded in growth. 
Transmission: By leafhopper, Cicadula bimaculata Evans (CICADELLIDAE).
Literature: Schindler, Jour. Austral. Inst. Agr. Sci., 8, 1912, 35-37.

\section{Genus VI. Fractilinea McKinney.}

(Jour. Washington Acad. Sci., 34, 1944, 148.)

Viruses of the Stripe-Disease Group; hosts grasses; insect vectors, cicadellid and fulgorid leafhoppers. Generic name from Latin, meaning interrupted and line.

The type species is Fractilinea maidis (Holmes) McKinney.

\section{Key to the species of genus Fractilinea.}

I. Vectors, cicadellid leafhoppers.

II. Vectors, fulgorid leafhoppers.

1. Fractilinea maidis.

2. Fractilinea oryzae.

3. Fractilinea tritici.

4. Fractilinea quarta.

\section{Fractilinea zeae.}

6. Fractilinea avenae.

1. Fractilinea maidis (Holmes) McKinney. (Marmor maidis Holmes, Handb. Phytopath. Viruses, 1939, 56; Fractilinea maidis McKinney, Jour. Washington Acad. Sci., 34, 1944, 149.) From New Latin mays, corn (i.e. maize). Common name: Maize-streak virus.

Hosts : GRA.MINEAE-Zea mays L., corn (maize); Digitaria horizontalis Willd., Eleusine indica Gaert.; Saccharum officinarum L., sugar cane.

Geographical distribution: Africa.

Induced disease : In corn, pale spots at base of young leaf, followed by chlorotic spotting and streaking of subsequently formed leaves. Virus moves rapidly (up to $40 \mathrm{~cm}$ in 2 hours at $30^{\circ} \mathrm{C}$ ) after introduction into host plant by insect. More virus in chlorotic spots than in green areas of affected leaves.

Transmission: By leafhoppers, Cicadulina (= Balclutha) mbila (Naude), $C$. zeae China, and $C$. storeyi China (CICADELLIDAE). In C. mbila ability to transmit this virus is controlled by a sex-linked dominant gene; active male $(\mathrm{AX})(\mathrm{Y})$, inactive male $(\mathrm{aX})(\mathrm{Y})$, inactive female $(\mathrm{aX})(\mathrm{aX})$, active female $(\mathrm{AX})(\mathrm{AX})$ or $(\mathrm{AX})(\mathrm{aX})$. Inactive individuals ingest virus when feeding, but can become infective only if the intestine is wounded purposely or accidentally. If inoculated artificially by introducing virus into blood, both active and inactive insects become infective. Incubation period, 6 to 12 hours at $30^{\circ} \mathrm{C}$. Young not infected through the egg. Infective leafhopper cannot transmit virus unless feeding puncture exceeds a minimum period, about 5 minutes in duration. This virus has not been transmitted to its plant hosts by inoculation of expressed juices.

Filterability: At $\mathrm{pH}$ 6, passes Chamberland $\mathrm{L}_{1}$ and $\mathrm{L}_{3}$, Berkefeld $\mathrm{V}$ and $\mathrm{N}$, filters; retained by Seitz E K filter disc.

Literature: Storey, Ann. Appl. Biol., $12,1925,422-439 ; 15,1928,1-25 ; 19,1932$, 1-5; Proc. Roy. Soc., B, 112, 1932, 46-60; $113,1933,463-485$; 125, 1938, 455-477; Ann. Appl. Biol., 21, 1934, 588-589; 24, 1937, 87-94; East Afr. Agr. Jour., 1, 1936, 471-475; Storey and MeClean, Ann. Appl. Biol., 17, 1930, 691-719.

Strains: Two strains that differ radically from the type, var. typicum $\mathrm{H}$. (loc. cit., 56), have been given varietal names, as follows:

1a. Fractilinea maidis var. sacchari $\mathbf{H}$. (loc. cit., 57). From New Latin Saccharum, generic name of sugar cane. Common name: Cane-streak strain of 
maize-streak virus. Differing from the type strain in being specialized for attacking sugar cane, in which the type (from maize) tends to be localized or finally lost with resultant spontaneous recovery of the temporary host. The cane-streak strain usually spreads readily in the cane plant; leaves become much marked with broken, narrow, pale, longitudinal stripes and spots; stems remain unaffected. One variety of sugar cane, P.O.J. 213, is resistant and, if infected, tends to recover. (McClean, Intern. Soc. Sugar Cane Techn., Bull. 27, 1932; Proc. So. Afr. Sugar Techn. Assoc., 1936, 1-11; Storey, Rept. Imp. Bot. Conf., London, 1924, 132-144; Union So. Afr. Dept. Agr., Sci. Bull. 39, 1935; Ann. Appl. Biol., 17, 1930, 691-719.

1b. Fractilinea maidis var. mitis $H$. (loc. cit., 58). From Latin mitis, mild. Common name: Mottle strain of maize streak virus. Differing from the typical strain by the mildness of the disease induced in corn (maize), transitory chlorotic mottling of newly developed leaves, followed by fading of mottling and production of apparently normal leaves. Young leaves, while mottled, are less rigid than normal and may not remain as nearly erect as healthy leaves. (Storey, Ann. Appl. Biol., 24, 1937, 87-94.)

\section{Fractilinea oryzae (Holmes) comb.} nov. (Marmor oryzae Holmes, loc. cit., 64.) From Latin oryza, rice.

Common name: Rice dwarf-disease virus.

Hosts: GRAMINEAE-Oryza sativa L., rice. Experimentally, also Alopecurus fulvus L.; Avena sativa L., oat; Echinochloa crusgalli Beauv. var. edulis Honda; Panicum miliaceum L.; Poa pratensis L.; Secale cereale L., rye; Triticum vulgare Vill., wheat.

Insusceptible species: GRAMINEAE -Zea mays L., corn (maize); Hordeum vulgare L., barley ; Setaria italica Beauv., foxtail millet; Andropogon sorghum Brot. (= Holcus sorghum L.), sorghum.

Geographical distribution : Japan, Philippine Islands .

Induced disease: In rice, yellowish green spots along veins of young leaf, followed by chlorotic spotting and streaking of subsequently formed leaves. Growth stunted, internodes and roots abnormally short, forming a dwarf plant. Vacuolate intracellular bodies, 3 to 10 by 2.5 to 8.5 microns in size, close to nuclei of cells in affected tissues.

Transmission: By leafhoppers, Nephotettix apicalis var. cincticeps ${ }^{\circ}$ Uhler, $N$. bipunctatus Fabr., and Deltocephalus dorsalis Motsch. (CICADELLIDAE). Virus transmitted through some of the eggs but through none of the sperm of infected individuals of $N$. apicalis. Transfer from individuals thus infected through the egg to their progeny likewise demonstrated, even to the 7 th generation. This is the only confirmed instance of transmission of a phytopathogenic virus through the eggs of an insect vector and is considered as evidence that the virus multiplies within the body of its vector as well as in its plant hosts. Incubation period in insect usually 30 to 45 days after first feeding on an infected plant, sometimes as short as 10 or as long as 73 days; nymphs from viruliferous eggs do not become infective until 7 to 38 (average 19) days after emergence. Transmission by inoculation of expressed juice has not been demonstrated. No transmission through seeds from diseased rice plants. No soil transmission.

Ijiterature: Agati et al., Philippine Jour. Agr., 12, 1941, 197-210; Fukushi, Trans. Sapporo Nat. Hist. Soc., 12, 1931, 35-41; Proc. Imp. Acad., Tokyo, 9, 1933, 457-460; Jour. Fac. Agr. Hokkaido Imp. Univ., 3\%, 1934, 41-16t; Trans. Sapporo Nat. Hist. Soc., 13, 1934, 162-166; Proc. Imp. Acad., Tokyo, 11, 1935, 301-303; 13, 1937, 328-331; 15, 1939, 142-145; Jour. Fac. Agr. Hokkaido Imp. Univ., 45, 1940, 83-154; Katsura, Phytopath., 
26, 1936, 887-895; Takata, Jour. Japan Agr. Soc., 171, 1895, 1-4; 172, 1896, 13-32 (Takata's papers, in Japanese, constitute the first published record of transmission by an insect of a virus causing disease in a plant, the leafhopper Deltocephalus dorsalis Motsch. transmitting dwarf-disease virus to rice; see Fukushi, 1937, cited above).

3. Fractilinea tritici McKinney. Jour. Washington Acad Sci., 34, 1944, 327. From Latin triticum, wheat.

Common name: Winter-wheat mosaic virus.

Hosts : GRA.YI.VEAE-Triticum aestivum L., wheat; Secale cereale L., rye; Avena byzantina; A. fatua L., wild oat; A. sativa L., oat; Hordeum vulgare I., barley.

Geographical distribution: Union of Soviet Socialist Republics.

Induced disease: In winter wheat and rye, chlorotic mottling; profuse branching. In winter wheat, phloem necrosis; chloroplasts few, small; vacuolate inclusions in cells; nuclei enlarged and with extra nucleoli; no protein crystals of the pupation-disease type in affected cells. In spring wheat, barley, and oats, chlorotic mottling without profuse branching; no proliferation of flowers, but grain is rarely formed, most infected plants dying before this stage of growth.

Transmission: By leafhopper, Deltocephalus striatus L. (CICADELLIDAE), with incubation period of 15 to 18 days. Not by inoculation of expressed juice. Not through soil.

Literature: Zazhurilo and Sitnikova, Compt. rend. Acad. Sci. U. R. S. S., 25, 1939, 798-801; 26, 1940, 474-478; 29, 1940, 429-432; Proc. Lenin Acad. Agr. Sci., L'. R. S. S., 6, 1941, 27-29. [Rev. Appl. Myc., $19,1940,268 ; 20,1941,157,396 ; 22,1943$, $59]$.

4. Fractilinea quarta (Holmes) comb. nov. (Marmor quartum Holmes, loc. cit., 65.) From Latin quartus, fourth.
Common name: Sugar-cane chloroticstreak virus or fourth-disease virus.

Host : GRA.UI VEAE-Saccharum officinarum L., sugar cane.

Geographical distribution: Java, Queensland, Hawaii, Puerto Rico, Colombia, United States (Louisiana).

Induced disease: In sugar cane, reduction of growth rate; wilting at midday; long, narrow, longitudinal streaks, of creamy or white color, in the leaves. Streaks 1/16 to $3 / 16$ inches wide, generally less than 1 foot long, fragmenting.

Transmission: By leafhopper, Draeculacephala portola Ball (CICADELLI$D A E)$. Not demonstrated by inoculation of expressed juice.

Thermal inactivation: In cuttings, at $52^{\circ} \mathrm{C}$ in less than 20 minutes.

Literature: Abbott, Phytopath., 28, 1938, S55-857; Sugar Bull., 16, 1938, 3-4; Abbott and Ingram, Phytopath., 32, 1942, 99-100; Bell, Queensland Agr. Jour., 40, 1933, 460-164; Martin, Ha maiian Planters' Rec., 34, 1930, 375-378; Hawaiian Sugar Planters' Assoc. Proc., 53, 1934, 24-35.

5. Fractilinea zeae (Holmes) comb. nov. (Marmor zeae Holmes, loc. cit., 59.) From New Latin Zea, generic name for corn (maize), from Latin zea, a kind of grain.

Common name: Maize-stripe virus.

Host: GRAMI VEAE-Zea mays L., corn (maize).

Insusceptible species: GRAMINEAE -Saccharum officinarum L., sugar cane. Geographical distribution: Hawaii, Tanganyika, Mauritius, Trinidad, Cuba. Not in Lnited States.

Induced disease: In corn (maize), at first few, elongated, chlorotic lesions near base of young leaf, later enlarging and fusing to form continuous stripes. Subsequently formed leaves banded and striped variously. Vacuolate intracellular inclusions in cells of affected areas.

Transmission: By leafhopper, Peregrinus maidis (Ashm.) (FULGORI$D A E)$; the incubation period in this in- 
sect is usually between 11 and 29 days, although shorter periods have been demonstrated in a few cases. Virus may persist in the insect host until death, but may become exhausted earlier. Not by aphid, Aphis maidis Fitch (APHIDI$D A E)$. Not by inoculation of expressed juice.

Literature: Briton-Jones, Trop. Agr., 10, 1933, 119-122; Carter, Ann. Ent. Soc. Am., 34, 1941, 551-556; Kunkel, Bull. Hawaiian Sugar Planters' Assoc., Bot. Ser., 3, 1921, 44-57 ; 1924, 108-114; Hawaiian Planters' Rec., 26, 1922, 58-64; Phytopath., 17, 1927, 41 (Abst.); Stahl, Trop. Pl. Res. Found., Bull. 7, 1927; Storey, Rept. of Plant Pathologist, Amani Agr. Res. Station, 4th Ann. Rept., 1931-32, pp. 8-13.

\section{Fractilinea avenae McKinney.} (Jour. Washington Acad. Sei., 34, 1944, 327.) From Latin avena, oats.

Common name: Pupation-disease virus.

Hosts: GRAMINEAE-Avcna sativa L., oat; Triticum aestivum I., wheat;
Echinochloa crusgalli Beauv.; Setaria viridis; rarely, Agropyron repens (L.) Beauv. and Bromus inermis Leyss. Experimentally, also Hordeum vulgare L., barley; Panicum miliaceum L., millet; Oryza sativa L., rice; Secale cereale L., rye; Zea mays L., corn (maize).

Geographical distribution: West Siberia.

Induced disease: In oat, chlorotic mottling, profuse development of shoots, proliferation of flowers with change to leaf-like structures. Protein crystals in affected cells have been regarded as accumulated virus.

Transmission: By leafhopper, Delphax striatella Fallan (FULGORIDAE), especially first and second instar nymphs; fifth instar nearly immune to infection. Incubation period, 6 days or more. Virus overwinters in insect as well as in plants. Not transmitted from an infected leafhopper to its progeny. Not through soil. Not through seeds from infected plants.

Literature : Sukhov et al., Compt. rend. Acad. Sci., U. R. S. S., 20, 1938, 745-748; $26,1940,479-482,483-486$. 


\section{FAMILY II. MARMORACEAE HOLMES EMEND.}

(Handb. Phytopath. Viruses, 1939, 16.)

Viruses of the Mosaic Group, inducing diseases usually characterized by persistent chlorotic or necrotic spotting, and often by mottling. The family is here extended to include several small groups of viruses, formerly assigned independent family rank, but sharing a tendency to aphid transmission, so far as known, and inducing diseases characterized by abnormal growth habit, thickening and rolling of leaves, or dwarfing, traits not incompatible with the characters of the present group. Should any one of these small groups become the center of a large assemblage of new viruses in the future, separate familial status for it might again be advantageous. In the combined grouping here used, specific vectors, so far as known, are aphids ( $A P H I D I D A E)$.

Key to the genera of family Marmoraceae.

I. Viruses of the Typical Mosaic-Disease Group.

Genus I. Marmor, p. 1163.

II. Viruses of the Spindle-Tuber Group.

Genus II. Acrogenus, p. 1202.

III. Viruses of the Leaf-Roll Group.

Genus III. Corium, p. 1203.

IV. Viruses of the Dwarf-Disease Group.

Genus IV. Nanus, p. 1206.

V. Viruses of the Rough-Bark Group.

Genus V. Rimocortius, p. 1208.

VI. Viruses of the Symptomless Group.

Genus VI. Adelonosus, p. 1211.

\section{Genus I. Marmor Holmes.}

(Loc. cit., 16)

Viruses inducing typical mosaic diseases in various plants. Generic name from Latin marmor, a mottled substance.

The type species is Marmor tabaci Holmes.

Key to the groups within genus Marmor.

A. Relatively resistant to heat inactivation, usually requiring more than 10 minutes at 85 to $90^{\circ} \mathrm{C}$ for complete inactivation.

1. Tobacco-Mosaic Virus Group.

B. Relatively susceptible to heat inactivation, requiring less than 10 minutes at 85 to $90^{\circ} \mathrm{C}$ for complete inactivation.

a. Replacing potato-veinbanding virus in mixed infections.

2. Tobacco-Etch Virus Group.

aa. Not replacing potato-veinbanding virus in mixed infections.

3. Cucumber-Mosaic Virus Group.

C. Many additional species cannot yet be grouped into definite subdivisions of the genus; they constitute a residual or

\section{Miscellaneous Mosaic-Virus Group.}

Key to the species of the Tobacco-Mosaic Virus Group.

Viruses relatively resistant to heat inactivation, requiring in most cases more than 10 minutes at 85 to $90^{\circ} \mathrm{C}$ for complete inactivation. Insect vectors as yet unknown under natural conditions. 
I. Found in nature principally in solanaceous plants; Cucurbitaceae insusreptible. Chlorotic mottling in some hosts, necrotic lesions in others as result of experimental infection. Suspensions show anisotropy of flow.

1. Marmor tabaci.

2. Marmor constans.

II. Found in nature only in cucurbitaceous plants; Solanaceae insusceptible. Only mottling as result of experimental infection. Suspensions show marked anisotropy of flow.

\section{Marmor astrictum.}

III. Found only in leguminous plants. Chlorotic lesions in some varieties of the common snap-bean plant, necrotic lesions in others, as a result of experimental infection.

\section{Marmor laesiofaciens.}

IV. Found in greenhouses confined to roots and lower parts of plants. Only necrotic lesions as result of experimental infection. Suspensions do not show anisotropy of flow.

\section{Marmor lethale.}

V. Found in tomato and experimentally transmissible to a number of species of plants in this and other families. Resembling the preceding in a number of physical characteristics, including failure to show anisotropy of flow.

5a. Marmor dodecahedron.

1. Marmor tabaci Holmes. (Holmes, Handb. Phytopath. Viruses, 1939, 17; Musivum tabaci Valleau, Phytopath., 30, 1940, 822; Phytovirus nicomosaicum Thornberry, Phytopath., 31, 1941, 23.) From New Latin Tabacum, early generic name for tobacco.

Common names: 'Tobaceo-mosair virus. tomato-mosaic virus.

Hosts: SOLANACEAE-Nicotiana tabacum L., tobacco; Lycopersicon esculentum Mill., tomato; and Capsicum frutescens L., garden pepper, among crop plants; nearly all, if not all, solanaceous plants can be infected, although in some the virus remains localized at or near the site of inoculation. PLANTAGINA $C E A E-\mathrm{A}$ strain of this virus has been found in nature infecting Plantago lanceolata L., ribgrass, $P$. major $\mathrm{L}$. and $P$. rugelii Dene., common broad-leaved plantains. Experimental hosts are widely distributed through many related families of plants.

Geographical distribution: World-wide.

Induced disease: In most varieties of tobacco, yellowish-green primary lesions, followed by clearing of veins, distortion and greenish-yellow mottling of newly formed leaves. In Ambalema tobacco, no symptoms, virus being restricted to inoculated leaves or those nearby. Strains of tobacco showing necrotic effects have been produced recently. In tomato, no obvious primary lesions, systemic disease characterized by greenishyellow mottling of foliage, moderate distortion of leaf shape, and a reduction of fruit yield not exceeding 50 per cent. If some strain of potato-mottle virus (Marmor dubium) is also present, a more severe disease is induced; this is known as double-virus streak, and is characterized by systemic necrosis. In most varieties of garden pepper, yellowish primary lesions followed by systemic chlorotic mottling. In the Tabasco pepper and its recent derivatives, recovery by abscission of inoculated leaf, after localization of virus in necrotic primary lesions. Vacuolate intracellular inclusions are found in ehlorotic tissues of all hosts that show distinet chlorotic mottling.

Transmission: By slight abrasive contacts. By inoeulation of expressed juice. To some extent by the aphids, Myzus pseudosolani Theob., M. circumflexus (Buckt.), Macrosiphum solanifolii Ashm., and Myzus persicae (Sulz.) (APHIDI$D A E)$. By grafting. Through soil. 
Through dodder, Cuscuta campestris Yuncker (CONVOLVULACEAE), without infecting this plant vector. Not through pollen from diseased plants. Not through seeds from diseased tobacco; seed transmission has been reported in the case of recently ripened seeds from diseased tomato.

Serological relationships: Precipitin test gives cross reactions between all known strains, except those characterized by failure to spread systemically in tobacco. No cross reactions with other viruses except weakly with cucurbitmosaic virus (Marmor astrictum). Type and other strains of tobacco-mosaic virus give cross reactions in complement-fixation and neutralization tests.

Immunological relationships: Plant protection tests, particularly in Nicotiana sylvestris Spegaz. and Comes, have demonstrated that tissues invaded by any strain of this virus are immune to subsequent infection by the tomato aucubamosaic strain of tobacco-mosaic virus, indicating a group relationship not shared by other viruses, such as cucumbermosaic virus or tobacco-ringspot virus.

Thermal inactivation: It 88 to $93^{\circ} \mathrm{C}$ in 10 minutes; at 86 to $92^{\circ} \mathrm{C}$ in 30 minutes. Filterability: Tobacen-mosaic virus was the first virus shown to be filterable. by Iwanowski in 1892 ; its filterability was confirmed and interpreted by Beijerinck in 1898 .

Other properties: The ultimate particles of tobacco-mosaic virus have been shown to be rod-shaped and isotropic, sometimes associated in pairs, end to end. Lnder proper conditions, thread-like paracrystals are formed. Specific gravity has been determined as about 1.37 , refractive index as about 1.6. Isoelectric point between pH 3.2 and 3.5. Suspensions in media of lower refractive indices show anisotropy of flow. Sedimentation constants, at $20^{\circ} \mathrm{C}, 187 \times 10^{-13} \mathrm{~cm}$ per sec. per dyne at infinite dilution for unaggregated virus and $216 \times 10^{-13} \mathrm{~cm}$ per sec. per dyne for associated particles. The computed average lengt h of the virus unit is about 272 millimicrons; diameter, 13.8 millimicrons. Electron micrographs show that characteristic particles are rodlike, between 10 and 20 millimierons in width, variable in length, but in some preparations averaging 270 millimicrons in length for single units, 405 to 540 millimicrons in length for associated pairs; X-ray measurements in air-dry gel show width $15.20 \pm 0.05$ millimierons. Solutions st ronger than about 1.3 per cent separate into layers, the lower spontaneously doubly refracting and more concentrated than the upper. At concentrations of electrolytes somewhat less than are required to precipitate the virus as fibres or needle-shaped paracrystals, the solutions form clear gels that become fluid on shaking or diluting (at $\mathrm{pH} 7$ and $30^{\circ}$ C). The virus is destroyed by high-frequency sound radiation, by pressures betreen 6000 and 8000 kilograms per square centimeter, and by hydrogen-ion concentrations above $\mathrm{pH} 11$ or below $\mathrm{pH} 1$. It is relatively stable between $\mathrm{pH} 2$ and $\mathrm{pH}$ 8. It is rapidly broken down in $6 \mathrm{MI}$ urea solutions, in the presence of salts, to low-molecular-ireight components devoid of activity. Analysis of purified virus : carbon 47.7 per cent, hydrogen 7.35 per eent, nitrogen 15.9 per cent, sulfur 0.24 per cent, phosphorus 0.60 per cent, lipoid 0.0 per cent, carbohydrate 1.6 to 2.0 per cent. A revised estimate of the sulfur content is 0.20 per cent, probably all in cysteine; no methionine has been detected in the typical variety of this virus. The percentagas of the following substances in the virus are: tyrosine 3.9 , tryptophane 4.5 , proline 4.6 , arginine 9.0 , phenylalanine 6.0 , serine 6.4 , threonine 5.3 , cysteine 0.68 , alanine 2.4 , aspartic acid 2.6 , glutamic acid 5.3 . leucine 6.1 , valine 3.9 , nucleic acid 5.8, and amide nitrogen 1.9, collectively accounting for about 68 per cent of the total weight. Virus formation ceases in infected host tissues immersed in 0.0002 molar sodium cyanide solution, beginning again after removal of cyanide. 
Literature: The literature dealing with tobacco-mosaic virus is too voluminous to permit citation of more than a few representative publications. Allard, U. S. Dept. Agr., Bull. 40, 1914; Bawden and Pirie, Proc. Roy. Soc. London, Ser. B., 123, 1937, 274-320; Beale, Jour. Exp. Med., 54, 1931, 463-473; Beijerinck, Verhandel. Konink. Akad. Wetenschappen te Amsterdam, II, 6, 1898, 3-22; Grant, Phytopath., 24, 1934, 311-336; Hoggan, Jour. Agr. Res., 49, 1934, 1135-1142; Iwanowski, Bull. Acad. Imp. Sci. St. Petersburg, Ser. 4, 3, 1892, 67-70; Jensen, Phytopath., 23, 1933, 964-974; Johnson, Science, 64, 1926, 210; Kausche et al., Naturwiss., 27, 1939, 292-299; Knight, Jour. Biol. Chem., 147, 1943, 663-666; Kunkel, Phytopath., 24, 1934, 437-466; Lauffer, Jour. Am. Chem. Soc., 66, 1944, 1188-1194; Price, Phytopath., 23, 1933, 749-769; Stanley, Phytopath., 26, 1936, 305-320; Takahashi and Rawlins, Proc. Soc. Exp. Biol. and Med., 30, 1932, 155157; Valleau and Johnson, Kentucky Agr. Exp. Sta., Bull. 376, 1937; Vinson, Science, $66,1927,357-358$; Woods, Science, 91, 1940, 295-296.

Strains: A great number of variant strains have been isolated both experimentally and from plants infected in nature. These usually share with the type variety most of the fundamental properties, particle size, especially width, stability at relatively high temperatures, longevity in storage, some common antigens. The following bave been distinguished from the type, var. vulgare $\mathrm{H}$. (loc. cit., 17), by varietal names :

1. Marmor tabaci var. aucuba $\mathrm{H}$. (loc. cit., 20). A group of isolates producing necrotic local lesions in inoculated leaves of Nicotiana sylvestris Spegaz. and Comes; useful in identifying many other strains of this virus which on prior application protect the tissues of this plant from the necrotic effects of aucuba-type strains (Smith, Ann. Appl. Biol., 18, 1931, 471-493; Kunkel, Phytopath., 24, 1934, 437-466). 1b. Marmor tabaci var. deformans $H$. (loc. cit., 22). Producing exceptionally severe malformation of tomato foliage. (Ainsworth, Ann. Appl. Biol., 24, 1937, $545-556)$.

1c. Marmor tabaci var. canadense $\mathrm{H}$. (loc. cit., 23). Producing a necrotic type of streak disease in tomatoes (Jarrett, Ann. Appl. Biol., 17, 1930, 248-259).

1d. Marmor tabaci var. lethale $\mathrm{H}$. (loc. cit., 24). Producing spreading necrotic lesions in tobacco and tomato under experimental conditions (Jensen, Phytopath., 27, 1937, 69-84 ; Norval, Phytopath. 28, 1938, 675-692).

1e. Marmor tabaci var. plantaginis $\mathrm{H}$. (Phytopath., 31, 1941, 1097). Specially adapted in nature for systemic spread in species of Plantago. This variety contains histidine $(0.55$ per cent $)$ and methionine ( 2 per cent) not found in the type of the species.

1f. Marmor tabaci var. obscurum H. (Handb. Phytopath. Viruses, 1939, 25). Systemic in tobacco without producing obvious disease under experimental conditions (Holmes, Phytopath., 24, 1934, 845-873; 26, 1936, 896-904; Jensen, Phytopath., 27, 1937, 69-84).

1g. Marmor tabaci var. immobile $\mathrm{H}$. (loc. cit., 26). Produces chlorotic primary lesions in experimentally infected tobacco, but rarely becomes systemic. (Jensen, Phytopath., 23, 1933, 964-977 ; 27, 1937, 69-84).

1h. Marmor tabaci var. artum $\mathrm{H}$. (loc. cit., 27). Necrotic lesions experimentally induced in Nicotiana glutinosa L. (SOLA NACEAE) are much smaller than those of the type variety (Jensen, Phytopath., 27, 1937, 69-84).

1i. Marmor tabaci var. siccans Doolittle and Beecher. (Phytopath., 32, 1942, 991). Causing necrosis and shriveling of tomato foliage. 


\section{Marmor constans MeKinney.}

(Jour. Washington Acad. Sci., 34, 1944, 326.) From Latin constans, fixed.

Common name: Tobacco mild darkgreen mosaic virus.

Hosts: $\quad S O L A N A C E A E-N i c o t i a n a$ glauca R. Grah., tree tobacco.

Insusceptible species: SOLANA$C E A E$-Lycopersicon esculentum Mill., tomato. CUCURBITACEAE-

Cucumis sativus L., cucumber.

Geographical distribution: Islands of Grand Canary and Teneriffe.

Induced disease: In Nicotiana glauca, systemic chlorotic mottling.

Transmission: By inoculation of expressed juice. No insect vector is known.

Thermal inactivation: At about $86^{\circ} \mathrm{C}$ in 10 minutes.

Literature: McKinney, Jour. Agr. Res., 39, 1929, 557-578; Am. Jour. Bot., 28, 1941, 770-778; Peterson and McKinney, Phytopath., 28, 1938, 329342 ; Thornberry and McKinney, ibid., 29, 1939, 250-260.

\section{Marmor astrictum Holmes.}

(Holmes, Handb. Phytopath. Viruses, 1939, 27; Musivum astrictum Valleau, Phytopath., 30, 1940, 823.) From Latin astrictus, limited, in reference to host range.

Common names: Cucurbit-mosaic virus, English cucumber-mosaic virus.

Hosts : CUCURBITACEAE-Cucumis sativus $\mathrm{L}$., cucumber; $C$. anguria $\mathrm{L}$., gherkin; C. melo L., melon; Citrullus vulgaris Schrad., watermelon; only cucurbitaceous plants have appeared to be susceptible thus far.

Insusceptible species: All tested solanaceous species. CUCURBITACEAE-Bryonia dioica L.; Cucurbita pepo L., vegetable marrow. LEGU$M I N O S A E$-Phaseolus vulgaris L, var. Golden Cluster.

Geographical distribution: England.

Induced disease : In cucumber, clearing of veins and crumpling in young leaves, followed by a green-mosaic mottling, with blistering and distortion of newly formed leaves. Plant stunted. Fruit unmarked or slightly mottled. Diseased plants less obviously affected during winter months.

Transmission: By inoculation of expressed juice. No insect vector is known.

Serological relationships: Weak cross precipitin reactions and full cross-neu. tralization reactions with tobacco-mosaic virus (Marmor tabaci). Two common antigens postulated. Preparations of virus that have been inactivated by treatment with nitrous acid or $\mathrm{X}$-rays are still antigenic.

Thermal inactivation : At 80 to $90^{\circ} \mathrm{C}$ in 10 minutes.

Filterability: Passes Pasteur-Chamberland filters $\mathrm{L}_{1}$ to $\mathrm{L}_{7}$, and membranes of 150 millimicrons average pore diameter.

Other properties: Virus, infectious in dilution of $10^{-10}$, is present to the extent of 0.2 to 0.3 gram per liter of juice from diseased plants. Preparations show sheen and anisotropy of flow, indicating rod-shaped particles. Solutions stronger than 2.5 per cent separate into 2 layers at room temperature, the lower being the more concentrated and birefringent. Precipitates with ammonium sulfate show needle-shaped paracrystals. Sedimentation constants $\mathrm{S}_{20}{ }^{\circ}=173 \times 10^{-13} \mathrm{~cm}$ sec..$^{-1}$ dyne $^{-1}$ and about $200 \times 10^{-13} \mathrm{~cm}$. sec..$^{-1}$ dyne $e^{-1}$. Virus withstands drying without inactivation but with partial loss of ability to show anisotropy of flow and with reduction of serological activity to about half. Tryptophane content 1.4 per cent, phenylalanine 10.2 per cent, the first lower and the second bigher than in tobacco-mosaic virus.

Literature: Ainsworth, Ann. Appl. Biol., 22, 1935, 55-67; Bawden and Pirie, Nature, 199, 1937, 546-547; Brit. Jour. Exp. Path., 18, 1937, 275-291; Knight, Arch. Virusf., 2, 1942, 260-267; Knight and Stanley, Jour. Biol. Chem., 141, 1941, 29-38 ; 141, 1941, 39-49; Price, Am. Jour. Bot., 27, 1940, 530-541; Price and Wyckoff, Nature, 141, 1938, 685. 
Strains: A distinctive strain has been distinguished from the type, var. chlorogenus $\mathrm{H}$. (loc. cit., 27), by the varietal name:

3a. Marmor astrictum var. aucuba H. (loc. cit., 29). Differing from the type of this species by inducing a yellow-mottling, rather than a green-mottling, mosaic in cucumber (Ainsworth, Ann. Appl. Biol., 22, 1935, 55-67).

4. Marmor laesiofaciens Zaumeyer and Harter. (Jour. Agr. Res., 67, 1943, 305.) From Latin laesio, substantive from laedere, to injure, and participle from facere, to make.

Common name: Bean-mosaic virus 4 ; southern bean mosaic virus 1 .

Hosts: LEGUMINOSAE-Phaseolus vulgaris $\mathrm{L}$., bean. Experimentally, also Phaseolus lunatus L., sieva bean; Soja max Piper var. Virginia, Virginia soy bean.

Insusceptible species: All tested species in families other than the $L E G U$ MINOSAE.

Geographical distribution: United States (Louisiana).

Induced disease: In bean, systemic chlorotic mottling in some varieties, localized necrosis in others; in a few varieties, systemic necrosis. In mottling-type varieties, chlorotic mottling of foliage; pods marked by dark yreen blotches or shiny areas, slightly malformed, short, frequently curled at end. In necrotic-type varieties with localized response, bearing a dominant gene lacking in mottling-type varieties, reddish necrotic lesions at the site of inoculation; no evidence of systemic spread of virus. In varieties showing systemic necrosis, pinpoint or slightly larger necrotic primary lesions with veinal neerosis of inoculated leaf ; systemic veinal necrosis, distortion and curling of affected leaves, drooping at the pulvini; stem and petiole streak; eventual death of plant.

Transmission: By inoculation of expressed juice. Through seeds from infected plants.
Serological relationships: Not demonstrated.

Immunological relationships: Previous infection with bean-mosaic virus, Marmor phaseoli, does not protect against infection with this virus.

Thermal inactivation: At 90 to $95^{\circ} \mathrm{C}$, time not stated, probably 10 minutes.

Other properties: Withstands dilution of $1: 500,000$ and aging 32 weeks at $18^{\circ} \mathrm{C}$.

Literature : Zaumeyer and Harter, Phytopath., 32, 1942, 438-439; 33, 1943, 16; 34, 1944, 510-512; Jour. Agr. Res., 67, 1943, 295-300, 305-328.

Strains: A strain differing from the type has been given the varietal name:

4a. Marmor laesiofaciens var. minus Zaumeyer and Harter. (Jour. Agr. Res., 67, 1943, 305.) From Latin minor, lesser. Differing from the type by inducing formation of slightly less diffuse and spreading lesions in necrotic-type bean leaves; also by inducing milder early symptoms and more severe late symptoms in mottling-type beans. Passes through seeds from infected plants to infect seedlings grown from them. Found in additional localities in the United States (California, Colorado, Idaho, Maryland).

5. Marmor lethale H. (loc. cit., 86). From Latin lethalis, causing death.

Common name: Tobacco-necrosis virus.

Hosts: SOLANACEAE-Nicotiana tabacum I., tobaceo; N. glutinosa L.; $N$. langsdorffii Weinm; Lycopersicon esculentum Mill., tomato; Solanum nigrum I. COMPOSITAE-Aster. GERANIICEAE-Pelargonium hortorum Bailey. LEGUMINOSAE-Phaseolus vulgaris $I_{\text {.., }}$ bean. Confined to roots of these natural hosts except in the cases of Nicotiana tabacum and $N$. glutinosa in which lower leaves are sometimes invaded; necrotic lesions along midrib and larger veins in these. No obvious manifestations of disease in infected roots. Experimentally to plants in many families with production of localized necrotic lesions only. 
Geographical distribution: England, Scotland, Australia. This virus has been found only in greenhouses.

Induced disease: In tobacco, necrosis of midrib and larger veins of first-developed pair of leaves, between November and February. Virus also in roots of many healthy-looking plants throughout the year. Upon artificial inoculation of foliage, numerous small brown necrotic local lesions are produced. Yield of virus from infected plant $0.02 \mathrm{mg}$ per cc of expressed juice, on the average.

Transmission: By contamination of soil with virus. No insect vector is known. Experimentally, by inoculation of expressed juice.

Serological reactions: Precipitates with homologous antiserum. No cross reaction with tomato bushy-stunt or tobaccomosaic viruses.

Immunological relationships: Protection tests show lack of relationship to tobacco-mosaic virus, tobacco-ringspot virus, tomato-ringspot virus, cucumbermosaic virus, and the severe-etch strain of tobacco-etch virus.

Thermal inactivation: At 90 to $92^{\circ} \mathrm{C}$ in 10 minutes.

Filterability: Average particle diameter 20 to 30 millimicrons as determined by filtration through Gradocol membranes; other reports give diameter as 13 to 20 millimicrons by filtration (14 to 19 millimicrons by radiation experiments, about 20 millimicrons from electron micrographs).

Other properties: Infectious after storage for months in dried leaves and after storage for half a year in absolute ethyl alcohol at room temperature. Specific gravity 1.3. Nore soluble in ammonium sulfate solutions at $0^{\circ} \mathrm{C}$ than at room temperature. Composition: Carbon 44.8 to 45.3 per cent, nitrogen 15.5 to 16.5 per cent, hydrogen 6.5 to 7.0 per cent, phosphorus 1.4 to 1.7 per cent, sulfur 1.1 to 2.0 per cent, carbohydrate 7.0 to 9.0 per cent; ash 5.8 to 7.0 per cent ( 3 to 5 per cent after prolonged dialysis at $\mathrm{pH} 3$ ). Nucleic acid of the ribose type has been isolated. To anisotropy of flow in solution but crystals are birefringent, showing sharp extinctions parallel to, and at right angles to, the plane of the crystal when examined edge-on in a polarizing microscope. Sedimentation constant, $\mathrm{S}_{20}{ }^{\circ}=112 \times 10^{-13}$; in other preparations a crystalline component with sedimentation constant $130 \times 10^{-13}$ and an amorphous component with sedimentation constant $58 \times 10^{-13}$ have been reported, as well as small amounts of a substance with sedimentation constant $220 \times 10^{-13}$.

Strains: Isolates of tobacco-necrosis virus serologically distinct but not otherwise different from each other appear to imply the existence of several strains of this virus, or of a closely related group of viruses, in England.

Literature: Bawden, Brit. Jour. Exp. Path., 22, 1911, 59-70; Bawden et al., ibid., 23, 1942, 314-328; Cohen, Proc. Soc. Exp. Biol. and Med., 48, 1941, 163-167; Lea, Nature, 146, 1940, 137-138; Pirie et al., Parasitol., 30, 1938, 543-551; Price, Am. Jour. Bot., 25, 1938, 603-612; Am. Jour. Bot., 27, 1940, 530-541; Arch. Virusf., 1, 1910, 373-386; Price and Wyckoff, Phytopath., 29, 1939, 83-94; Smith, Parasitol., 29, 1937, 70-85; 29, 1937, 86-95; Smith and Bald, Parasitol., 27, 1935, 231-245; Smith and MacClement, Parasitol., 32, 1940, 320-332.

5a. Marmor dodecahedron H. (loc. cit., 30). From Greek dōdekahedron, dodecahedron.

Common name: Tomato bushy-stunt virus.

Hosts : SOLANACEAE-Lycopersicon esculentum Mill., tomato. Experimentally, also SOLANACEAE-Datura stramonium L.; Nicotiana glutinosa L.; $N^{*}$. langsdorffii Weinm.; $N$. tabacum L., tobacco; Solanum nigrum L. LEGUMINOSAE-Phaseolus vulgaris L., bean; Vigna sinensis (L.) Endl., cowpea. COIIPOSITAE-Zinnia clegans Jacq., zinnia.

Geographical distribution: British Isles. 
Induced disease: In tomato, some primary lesions neerotic, ring-like or spot-like, others masked, or disclosed only by chlorophyll retention in yellowing leaves. In young plants, systemic necrotic lesions may cause death; in older plants, growth ceases, young leaves become pale yellow; growing points may die, inducing growth of axillary buds to produce a bushy top; older leaves become yellowed and show some purple coloration. In White Burley tobacco, local necrosis only, lesions small, red at first. then white. In cowpea, reddish necrotic primary lesions only

Transmission: By inoculation of expressed juice. Through dodder, Cuscuta campestris Yuncker (CONTOLVULA$C E A E)$. Not through seeds of diseased plants. No insect vector is known.

Serological relationships: A specific antiserum, prepared by a single intravenous injection of rabbits with $2 \mathrm{mg}$ of purified virus, gives granular, compact precipitates, serving for quantitative estimation of this virus, antiserum being used at dilutions of $1: 200$ or $1: \$ 00$, virus at dilutions to $10^{-6}$.

Immunological relationships: 11 ill infect plants previously invaded by tobacco-mosaic virus, tomato spotted-wilt virus, tohacco-ringspot virus, and Bergerac-ringspot virus.

Filterability: Passes membranes down to 40 millimicrons average pore diameter.

Other properties: Virus crystallizes from solutions of ammonium sulfate as isotropic, rhombic dodecahedra, which shrink and swell reversibly on drying and rewetting; shrinkage reduces size to 80 per cent of the wet dimensions. In the presence of heparin, non-birefringent prisms, rather than dodecahedra, appear. $S_{20}{ }^{\circ}=132 \times 10^{-13} \mathrm{~cm}$. sec. ${ }^{-1}$ dyne $^{-1}$. Particle approximately spherical, 27.4 millimicrons in diameter by $\mathrm{X}$-ray measurements (average diameter by filtration data, 14 to 20 millimicrons). Solutions do not show anisotropy of flow. Inactivated by drying. Molecular weight $8,800,000$. Density 1.353. Molecular weight may be as high as $24,000,000$ in solution, but the density is then lower, 1.286. Analysis : carbon 47 to 50 per cent, nitrogen 15.8 to 16.4 per cent, phosphorus 1.3 to 1.5 per cent, ash 1.7 to 5 per cent, hydrogen 7.2 to 8.2 per cent, sulfur 0.4 to 0.8 per cent, carbohydrate 5 to 6 per cent.

Literature: Ainsworth, Jour. Ministry Agr., 43, 1936, 266-269; Bawden and Pirie, Nature, 141, 1938, 513; Brit. Jour. Exp. Path., 19, 1938, 251-263; Bernal and Fankuchen, Jour. Gen. Physiol., 25, 1941, 111-165; Bernal et al., Nature, 142, 1938, 1075; Cohen, Jour. Biol. Chem. 144, 1942, 353-362; Proc. Soc. Exp. Biol. and Med., 51, 1942, 104-105; Lauffer, Jour. Phys. Chem., 44, 1940, 1137-1146; Lauffer and Stanley, Jour. Biol. Chem., 135, 1940, 463-472; Neurath and Cooper, Jour. Biol. ('hem., 135, 1910, 455-462; Smith, Nature, 135, 1935, 908; Ann. Appl. Biol., 22, 1935, 731-711; Jour. Roy. Hort. Soc., 60, 1935, 448-451; Smith and MacClement, Parasitol., 33, 1941, 320-330; Stanley, Jour. Biol. Chem., 135, 1940, 437-454.

\section{Key to the species of the Tobacco-Etch Virus Group.}

Viruses relatively susceptible to heat inactivation (inactivated at 52 to $58^{\circ} \mathrm{C}$ in 10 minutes). A small, closely allied group, tending to replace or to be replaced by each other, if present in mixture in tobaceo.

I. Not replaced, if in mixture, by other viruses of this group; dominant member of the group in tobacco.

\section{Marmor erodens.}

II. Replaced by No. 6, not by No. s, if in mixture with it in tobacco.

7. Marmor hyoscyami.

III. Replaced by No. 6 or 7 if in mixture with cither in tobacco.

8. Marmor upsilon. 
6. Marmor erodens Holmes. (Holmes, Handb. Phytopath. Viruses, 1939, 40; Foliopellis erodens Valleau, Phytopath., $30,1940,825$.) From Latin erodere, to erode or gnaw away

Common name: Tobacco-etch virus.

Hosts: SOLAYACEAE-Capsicum frutescens L., pepper; Datura stramonium L., Jimson weed; Lycopersicon esculentum Mill., tomato; Nicotiana tabacum L., tobacco; Petunia sp., petunia; Physalis heterophylla Nees.

Geographical distribution: United States.

Induced disease: In tobaceo, systemic mild-mottling chlorosis, with traces of necrotic etching; int ranuclear crystalline inclusions and intracytoplasmic granular and amorphous inclusions that tend to crystallize, forming needle-shaped birefringent bodies, 2 to 10 microns in length.

Transmission : Experimentally, by . Iy zus persicae (Sulz.), M. circumflexus (Buckt.), A phis rhamni Boyer, A. fabate (Scop.), and Macrosiphum gei (Koch) ( $A P H I D I D A E)$; by inoculation of expressed juice.

Serological relationships: Precipitin reactions with homologous antisera, but no cross-reactions with tobaceo-mosaic virus, tobacco-ringspot virus, potatomottle virus, potato aucuba-mosaic virus, potato mild-mosaic virus, hyoscyamusmosaic virus, potato-veinbanding virus, or pea-mosaic virus.

Immunological relationships: Protects tobacco against subsequent infection by potato-veinbanding virus and hyoscyimus-mosaic virus. In mixed infections, it suppresses and replaces these two viruses.

Thermal inactivation : At 53 to $55^{\circ} \mathrm{C}$ in 10 minutes.

Filterability: Passes Pasteur-Chamberland $L_{1}$, not $L_{3}$, filter candle.

Other properties: Sedimentation constant $S_{20}{ }^{\circ}=170 \times 10^{-13} \mathrm{~cm} . \mathrm{sec}^{-1} \mathrm{dyne}^{-1}$. Concentrated preparations show anisotropy of flow, indicating elongated particle shape.

Literature: Bawden and Kassanis, Ann.
Appl. Biol., 28, 1941, 107-118; Fernow, Cornell Agr. Exp. Sta. (Ithaca), Mem. 96, 1925; Holmes, Phytopath., 32, 1942, 1058-1067; Johnson, Kentucky Agr. Exp. Sta., Res. Bull. 306, 1930.

Strains: A distinctive severe-symptom strain, isolated from plants infected in nature and studied intensively, has been distinguished from the type, var. vulgare H. (loc. cit., 40 ), by the varietal name:

6a. Marmor erodens var. severum H. (loc.cit., 41). Differing from the type by a tendency to induce more pronounced necrotic etching and a greater stunting effect in infected tobacco.

7. Marmor hyoscyami spec. nov. From New Latin Hyoscyamus, genus name of plant from which this virus was first isolated.

Common names: Hyoscyamis-mosaic virus, $\mathrm{Hy}_{y}$. III virus, Hyoscyamus-IIIdisease virus.

Hosts : SOLA VACEAE-Hyoscyamus niger L., henbane. Experimentally, also Nicotiana tabacum L., tobacco.

Insusceptible species : $C U C L R B I T A$ $C E A E$-Cucumis sativus L., cucumber.

Geographical distribution: England.

Induced disease : In henbane, chlorotic clearing of veins followed by yellow-mot tling mosaic.

Transmission: By inoculation of $\mathrm{ex}-$ pressed juice to dilutions of $10^{-4}$. By aphids, Myzus persicae (Sulz.), M. circumflexus (Buckt.), and Macrosiphum solanifolii Ashm. (= II. gei Koch) (APHIDID AE).

Serological relationships: Several isolates of this virus give mutual crossprecipitin reactions but no precipitation occurs when antiserum prepared with this virus is mixed with cucumber-mosaic virus, tobacco-etch virus, or potato-veinbanding virus.

Immunological relationships: No immunity with respect to this virus is induced in tobaceo by previous infection with cucumber-mosaic virus. Potatoveinbanding virus is unable to multiply in the presence of this virus and is replaced by it. Tobacco-etch virus pro- 
tects against this virus and replaces it in mixed infections.

Thermal inactivation: At $58^{\circ} \mathrm{C}$ in 10 minutes.

Filterability : Passes Chamberland $\mathrm{L}_{1}$, but not $\mathrm{L}_{3}$, filter candles.

Other properties: Concentrated solutions show anisotropy of flow. Field of virus, 1 to $3 \mathrm{mg}$ per liter of juice expressed from diseased tobacco plants.

Literature: Bawden and Kassanis, Ann. Appl. Biol., 28, 1941, 107-118; Hamilton, ibid., 19, 1932, 550-567; Sheffield, ibid., 25, 1938, 781-789; Watson and Roberts, Proc. Iroy. Soc. London, Ser. B, 127, 1939, 543-576.

8. Marmor upsilon comb. nov. (Marmor cucumeris var. upsiton Holmes, loc. cit., 33; Murialba venataenia Valleau, Phytopath., 30, 1940, 824.) From Greek name of the letter $Y$, sometimes used to denote this virus.

Common names: Potato-veinbanding virus, potato virus $\mathrm{Y}$.

Hosts: SOLANACEAE-Solanum tuberosum L., potato; Nicotiana tabacum I., tobacco. Experimentally, also $L y$ cium barbarum L.

Geographical distribution: England, France, United States, Brazil. Rare in Scotland and part of Ireland.

Induced diserase: In some potato varieties, leaf drop and necrotic stem-streak; in others, no signs of disease; in still others, chlorotie mottling with or without necrosis. In combination with strains of the potato-mottle virus (Marmor dubium), this virus causes rugose mosaic, a common and destructive double-virus disease.
Transmission: By inoculation of expressed juice. By aphid, Myzus persicae (Sulz.); experimentally, also by Aphis rhamni Boyer (synonym for Aphis abbreviata Patch) (APHIDIDAE).

Serological relationships : Precipitin reactions with homologous antisera. No cross reactions with tobacco-mosaic virus, tobacco-etch virus, hyoscyamus-mosaic virus, potato-mottle virus, potato mildmosaic virus, potato aucuba-mosaic virus, tobacco-ringspot virus, or common peamosaic virus. Reported cross reaction with cucumber-mosaic virus needs confirmation.

Immunological relationships: A mild strain protects against subsequent infection with the typical virus. This virus is suppressed and replaced by hyoscyamus-mosaic virus and by tobacco-etch virus in mixed infections.

Thermal inactivation: At $52^{\circ} \mathrm{C}$ in 10 minutes.

Filterability: Passes with difficulty through Gradocol membrane of 42 millimicron average pore diameter.

Other properties: Inactivated by drying.

Literature: Demis, Nature, 142, 1938, 154; Johnson, Phytopath., 25, 1935, 650-952; Jones and Vincent, Jour. Agr. Res., 55, 1937, 69-79; Kassanis, Ann. Appl. Biol., 29, 1942, 95; Koch, Phytopath., 23, 1933, 319-342; Kramer and Silberschmidt, Arquivos Inst. Biol., São Paulo, Brazil, 11, 1940, 165-188; Salaman, Nature, 139, 1937, 924; Smith, Proc. Roy. Soc., Ser. B, 109, 1931, 251-267; Smith and Dennis, Ann. Appl. Biol., 27, 1940, 65-70.

Key to the species of the Cucumber-Mosaic Virus Group.

Viruses relatively susceptible to heat inactivation, requiring less than 10 minutes at 85 to $90^{\circ} \mathrm{C}$ for complete inactivation. Not replacing potato-veinbanding virus in mixed infections.

I. Infecting both dicotyledonous and monocotyledonous plants.

9. Marmor cucumeris.

II. Infecting dicotyledonous, but not monocotyledonous, plants.

10. Marmor solani.

11. Marmor aucuba.

12. Marmor umbelliferarum. 
13. Marmor cruciferarum.

14. Marmor brassicae.

15. Marmor betae.

16. Marmor lactucae.

17. Marmor dahliae.

18. Marmor phaseoli.

19. Marmor leguminosarum.

20. Marmor pisi.

21. Marmor medicaginis.

III. Infecting monocotyledonous, but not dicotyledonous, plants.

22. Marmor tulipae.

23. Marmor mite.

24. Marmor iridis.

25. Marmor sacchari.

26. Marmor cepae.

27. Marmor scillearum.

9. Marmor cucumeris Holmes (Holmes, Handb. Phytopath. Viruses, 1939, 31; Murialba cucumeris Valleau, Phytopath., 30, 1940, 823.) From Latin cucumis, cucumber.

Common name: Cucumber-mosaic virus.

Hosts : Very wide range of hosts among dicotyledonous and monocotyledonous plants; cucumber, celery, spinach, tobacco, and pepper are sometimes seriously affected. Overwintering hosts are : $S O L$ ANACEAE-Physalis subglabrata Mackenzie and Bush, $P$. heterophylla Nees. ASCLEPIADACEAE-Asclepias syriaca L. PHYTOLACCACEAE-Phytolacca decandra L. LABIATAE-Nepeta cataria L. Probably there are also other susceptible perennials.

Geographical distribution: Probably almost world-wide.

Induced disease: In cucumber, Cucumis sativus $\mathbf{L}$., yellowish-green systemic mottling. Leaves small, distorted, curled; plants dwarfed, internodes shortened. Few fruits set. Fruits mottled, misshapen, giving the disease the name "white pickle." In black cowpea, Vigna sinensis (L.) Endl., small reddish necrotic local lesions only. No intracellular bodies are found in plants infected with cucumber-mosaic virus.

Transmission: By inoculation of expressed juice. By aphids, Myzus persicae (Sulz.), M. pseudosolani Theob., $M$. circumflexus (Buckt.), Macrosiphum solanifolii Ashm., and Aphis gossypii Glov. (APHIDIDAE). Through seeds of diseased plants in Echinocystis lobata (Michx.) Torr. and Gray, wild cucumber, in Cucumis melo L., muskmelon, and in Cucurbita pepo L., vegetable marrow. By several species of dodder, Cuscuta californica Choisy, C. campestris Yuncker, and C. subinclusa Dur. and Hilg. (CONVOLVULACEAE).

Immunological relationships: Infection with the type and other chlorotic-mottling strains protects zinnia against subsequent infection by an indicator strain of this virus (var. judicis).

Thermal inactivation: At 70 to $80^{\circ} \mathrm{C}$ in 10 minutes.

Filterability: Passes Berkefeld $W$ and $\mathrm{N}$ filters and collodion membranes of 45 millimicron average pore diameter.

Other properties: Inactivated by drying or 3 to 4 days' storage in juice at room temperature.

Literature: Ainsworth, Ann. Appl. Biol., 25, 1938, 867-869; Chamberlain, New Zealand Jour. Science and Technology, 21, 1939, 73A-90A ; Celino, Philippine Agr., 29, 1940, 379-414; Doolittle, Phytopath., 6, 1916, 145-147; U. S. Dept. Agr., Bull. 879, 1920; Doolittle and Walker, Jour. Agr. Res., 31, 1925, 1-58; Gilbert, Phytopath., 6, 1916, 143-144; Hoggan, Jour. Agr. Res., 47, 1933, 689704 ; Jagger, Phytopath., 6, 1916, 148-151 ; 
8, 1918, 32-33; Fendrick, Phytopath., 24, 1934, 820-823; Mahoney, Proc. Am. Soc. Hort. Sci., 332, 1935, 477-480; Price, Phytopath., 25, 1935, 776-789; 29, 1939, 903-905; Am. Jour. Bot., 27, 1940, 530 541; Storey, Ann. Appl. Biol., 26, 1939, 298-308.

Strains: Various host plants seem to have induced specialization of cucumbermosaic virus in strains particularly adapted to existence in their tissues. Several of these and certain laboratoryderived strains useful in technical procedures have been distinguished from the type, var. vulgare $\mathrm{H}$. (loc. cit., 31), by varietal names, as follows:

9a. Marmor cucumeris var. commelinue H. (loc. cit., 35). From New Latin Commelina, generic name of weed serving as a natural reservoir of this strain. Common name: Southern celery-mosaic strain of cucumber-mosaic virus. Differing from the type in severity of disease induced in celery and some other plants. Transmitted by Aphis gossypii Glov., A. maidis Fitch, and Pentalonia nigronervosa Coq. (APHIDIJ)AE). (Price, Phytopath., 25, 1935, 947-954; Wellmutr, ibid., 24, 1934, 695-725, 1032-1037; 25, $1935,289-308,377-404$.)

9b. Marmor cucumeris var. phaseoli H. (loc, cit., 36). From New Latin Phaseolus, generic designation of lima bean. Common name: Lima-bean strain of cucumber-mosaic virus. Differing from type of species in ability to cause a chlorotic mottling disease in lina bean in nature. (Harter, Phytopath., 26, 1936, 94; Jour. Agr. Res., 56, 1938, 895-906: McClintock, Phytopath., 7, 1917, 60.)

9c. Marmor cucumeris var. lilii H. (loc. cit., 37). From Iatin lilium, lily. Common name: Lily-mosaic strain of cucumber-mosaic virus. Differing from the type variety by ability to persist in nature in lilies, producing masked infection or chlorotic mottling unless in mixture with lily-symptomless virus (Adelonosus litii), when a more severe disease involv- ing necrosis is induced. (Brierley, Phytopath., 29, 1939, 3; 30, 1940, 250-257; Brierley and Doolittle, ibid., 30, 1940, 171-174; Ogilvie and Guterman, ibid., 19, 1929, 311-315; Price, ibid., 27, 1937, $561-569$.)

9d. Marmor cucumeris var. judicis $\mathrm{H}$. (loc. cit., 38). From Latin judex, judge. Common name: Indicator strain of $\mathrm{cu}$ cumber-mosaic virus. Differing from the type variety in inducing the formation of necrotic local lesions in zinnia (Zinnia elegans Jacq., COMPOSITAE). Previous infection of zinnia by other strains of cucumber-mosaic virus inhibits the formation of these necrotic local lesions, identifying the strains as related to cach other and to the indicator strain. (Price, Phytopath., 24, 1934, $743-761 ; 25,1935,776-789$.

9e. Murmor cucumeris var. vignae $\mathbf{H}$. (loc. cit., 39). From New Latin Vigna, generic name of cowpea. Common name: cowpea-mottling strain of cucumbermosaic virus. Differing from the type variety in producing systemic chlorotic mottling, rather than reddish-brown nerotic local lesions, in Black cowpea. Not known in nature but derived experimentally from a mild-mottling strain of cucumber-mosaic virus during serial passage in cowpea. (Price, Phytopath., $24,1934,743-761 ; 25,1935,776-759$.

10. Marmor solani H. (loc. cit., 47). From New Latin Solanum, generic name of potato.

Common names: Polato mild-mosaic virus, potato virus $A$.

Hosts: SOLA VACEAE-Solanum tuberosum L., potato. Experimentally, also Nicotiana tabacum L., tobaceo; Solanum nigrum L. var. nodiflorum; and Datura stramonium L., Jimson weed.

Geographical distribution: United States, Fingland, Holland.

Induced diseise: In potato, very mild chlorotic mottling or masked symptoms in some varieties (as Irish Chieftain), systemic necrosis in others (for example, 
British Queen). Immunity to aphid infection with this virus is found in the varieties Katahdin and Earlaine. A combination disease, characterized by pronounced yellow-mosaic patterns, is caused by this virus in the variety Irish Chieftain if the potato-veinbanding virus (Marmor upsilon) is also present. In tobacco, experimentally, faint veinbanding mosaic.

Transmission: To potato, by rubbing methods of inoculation of expressed juice, using carborundum powder; to tobacco, by rubbing without carborundum. By aphids, Aphis abbreviata Patch and Myzus persicae (Sulz.) (APHIDIDAE).

Serological relationships: No crossprecipitin reactions with potato aucubamosaic virus, potato-veinbanding virus, tobacco-mosaic virus, tobacco-etch virus, tobacco-ringspot virus, or pea-mosaic virus.

Immunological relationships: A feeble strain of this virus has been found to protect fully against the typical strain in the Netherlands.

Thermal inactivation: At $50^{\circ} \mathrm{C}$ in 10 minutes.

Literature: Bawden, Ann. Appl. Biol., 23, 1936, 487-497; Chester, Phytopath., 25, 1935, 686-701 ; Dykstra, Phytopath., 29, 1939, 40-67 ; Hansen, Tidsskr. Planteavl, 42, 1937, 631-681; Murphy and Loughnane, Sci. Proc. Roy. Dublin Soc., 21, 1936, 419-430; Murphy and McKay, ibid., 20, 1932, 227-247; Oortwijn Botjes, Tijdsch. Plantenziekten, 45, 1939, 2529 ; Schultz et al., Phytopath., 27, 1937. 190-197; 30, 1940, 944-951.

11. Marmor aucuba H. (loc. cit, 49). From New Latin Aucuba, a genus of plants having mottled foliage

Common name: Potato aucuba-mosaic virus.

Hosts : SOLAVACEAE-Solanum tuberosum L., potato. Experimentally, also Atropa belladonna L. (symptomless); Capsicum frutescens L., pepper; Datura stramonium L., Jimson weed (symptomless); Hyoscyamus niger L., henbane (symptomless); Lycopersicon esculentum Mill., tomato; Petunia hybrida Vilm., petunia (symptomless); Nicotiana tabacum L., tobaceo (symptomless); Solanum dulcamara L., bittersweet; S. nigrum I. var. nodiflorum.

Geographical distribution: United States, Great Britain, Europe.

Induced disease: In potato, yellow spots on lower leaves of some varieties; in the variety Irish Chieftain, brilliant yellow mottle over whole plant, perhaps because of simultaneous presence of potato mild-mosaic virus in this variety. Necrosis of the cortex and of the pith in tubers in many varieties.

Transmission: By inoculation of expressed juice. Probably by aphid, $M y$ zus persicae (Sulz.) (APHIDIDAE).

Serological relationships: No precipitin cross-reactions with potato mild-mosaic virus, potato-veinbanding virus, tobaceomosaic virus, tobacco-eteh virus, tobaceoringspot virus, or pea-mosaic virus. Precipitin cross-reactions with the Canada-streak strain of potato aucubamosaic virus.

Thermal inactivation: At 65 to $68^{\circ}$ C in 10 minutes.

Filterability: Passes Pasteur-Chamberland $L_{1}$ filter, but not $L_{3}$ or $L_{5}$.

Literature: Chester, Phytopath., 25, 1935, 686-701 ; 27, 1937, 903-912; Clinch, Sci. Proc. Roy. Dublin Soc., 22, 1941, 435-445; Clinch et al, , ibid., 21, 1936, 431448; Dykstra, Phytopath., 29, 1939, 917933.

Strains: One strain differing from the type has been given a varietal name:

11a. Marmor aucuba var. canadense Black and Price. (Phytopath. 30, 1940, 144.) From common name of strain.

Common name: Canada-streak strain of potato aucuba-mosaic virus. Differing from the type variety by tendency to produce necrosis in stems, veins, petioles, and leaves and also, about 2 months after harvest, in pith of tuber, especially at stem end. (Chester, Phytopath., 27, 
1937, 903-912; Dykstra, Phytopath., 29, $1939,917-933$.

12. Marmor umbelliferarum H. (loc. cit., 67). From New I atin Umbelliferae, family name of plants among which celery is classified.

Common name: Celery-mosaic virus, western celery-mosaic virus.

Hosts: UMBELLIFERAE-Apium graveolens L., celery and celeriac; Daucus carota L., carrot. Experimentally, also Ancthum graveolens L., dill; Anthriscus cerefolium (L.) Hoffn., salad chervil; Carum carvi L., caraway; Coriandrum sativum L., coriander; Petroselinum hortense Hoffm., parsley.

Insusceptible species: Cucumis sativus I., cucumber, and all other tested species not of the family Umbelliferae.

Geographical distribution: Lnited States (California).

Induced disease: In celery, at first, clearing of veins in young leaves; later, foliage yellowed, plint stunted, young petioles shortencd, older petioles horizontal, giving plant a flat appearance. Foliage mottled green and yellow; leaflets narrow, twisted or cupped; older leaves with some necrosis; petioles with white streaks or spots. In celeriac, clearing of veins, followed by systemic chlorotic mottling. In carrot, chlorotic spotting of young leaves, followed by systemic chlorotie mottling.

Transmission: By inoculation of $\mathrm{ex}$ pressed juice, in dilutions to $1: 4000$. No specifie insect vector is known, but 11 species of aphids capable of breeding on celery transmit the virus, though they do not long retain the power of transmission after leaving diseased plants. These vectors are Aphis apigrancelens Essig, A. apii Theob., 1. ferruginea-striata Essig, A. gossypii Glov., A. middletonii Thomas, A. rumicis Linn., Cacariella capreae (Fabr.), Myzus circumflexus (Buckit.), M. convolvuli (Kalt.), M. persicae (Sulz.), Rhopalosiphum molliferum (Hottes) (APHIDIDAE). Some aphids not able to breed on celery also transmit this virus.

Thermal inactivation: At 55 to $60^{\circ} \mathrm{C}$ in 10 minutes.

Filterability: Passes all grades of Chamberland filters.

Other properties: Virus active after storage at $-18^{\circ} \mathrm{C}$ for 18 months.

Literature: Severin and Freitag, Hilgardia, 11, 193S, 493-558.

13. Marmor cruciferarum H. (loc. cil., 69). From New Iatin Cruciferae, family name of plants among which cauliflower is classified.

Common name: Cauliflower-mosaic virus.

Hosts: ('RUCIFERAE-Brassica oleracea I., cauliflower, kale, Brussels sprouts, cabbage, and broccoli; B. campestris I., wild yellow mustard; Matthiola incana R. Br., annual stock. Experimentally, also Brassica adpressa Boiss; $B$. alba Rabenh., white mustard; $B$. arvensis (L.) Kłze, charlock; $B$. juncea Coss., leaf mustard (one strain not susceptible); B. napus L., rape; B. petsai Bailey, pe-tsai; B. nigra Kioch, black mustard; B. rapa L., turnip; Capsella bursa-pastoris Medic., shepherd's purse ; Iberis amara L., rocket candytuft ; Lepidium sativum L., garden cress; Lunariu annua L., honesty; Raphanus ruphanistrum L., white charlock; $R$. sativus L., radish.

Insusceptible species: CHENOPODI$A C E A E-$ Spinacia oleracea L. COMPOSITAE-Lactuca sativa L. CRUCIFERAE-Alyssum saxatile L.; $A$. maritimum Lam.; Arabis albida Stev.; Athysanus pusillus Greene; Brassica juncea Coss. (Japanese strain; another strain susceptible); Cheiranthus cheiri L.; Erysimum perofstianum Fisch. and Mey.; Hesperis matronalis L.; Malcomia maritima R. Br.; Roripa nasturtium Rusby; Stanleya pinnata (Pursh.) Britt.; Thysanocarpus radians Benth. LEGUII VUSAE- Vicia jaba I. SOLANA('EAE-Capsicum frutescens L.; Lycoper- 
sicon esculentum Mill.; L. pimpinellifolium Mill.; Nicotiana glutinosa L.; $N$. langsdorffi Weinm.; N. tabacum L. vars. Turkish and White Burley. TROPAEOLACEAE-Tropaeolum majus L. UMBELLIFERAE-A pium graveolens $\mathrm{L}$.

Geographical distribution: United States, England.

Induced disease: In cauliflower, clearing of veins, followed by mild chlorotic mottling, veins usually banded with dark green, necrotic flecks later in chlorotic areas. Midrib curved, leaves distorted. Plant stunted; termiual head or curd dwarfed. Solanaceous plants appear to be immune, a point of distinction between this virus and turnip-mosaic virus, $M a r$ mor brassicae.

Transmission: By inoculation of expressed juice, using carborundum powder. By many aphid species, Brevicoryne brassicae (Linn.), cabbage aphid; Rhopalosiphum pseudobrassicae Davis, false cabbage aphid; Myzus persicae (Sulz.), peach aphid; Aphis graveolens Essig, celery leaf aphid; A. apigraveolens Essig, celery aphid; $\boldsymbol{A}$. middletonii Thomas, erigeron root aphid; A. gossypii Glov., cotton aphid; Cavariella capreae (Fabr.), yellow willow aphid; Myzus circumflexus (Buckt.), lily aphid; Rhopalosiphum melliferum (Hottes), honeysuckle aphid. ( $A P H I D I D A E)$. No seed transmission.

Thermal inactivation: At $75^{\circ} \mathrm{C}$ in 10 minutes.

Literature: Caldwell and Prentice, Ann. Appl. Biol., 29, 1942, 366-373, 37t379 ; Rawlins and Tompkins, Phytopath., 24, 1934, 1147 (Abst.); Tompkins, Jour. Agr. Res., 55, 1937, 33-46.

14. Marmor brassicae H. (H., loc. cit., 70; Marmor matthiolae H., loc. cit., 71.) From New Latin, Brassica, generic name of turnip.

Ccmmon name: Turnip-mosaic virus.

Hosts : CRUCIFERAE-Brassica rapa I., turnip; B. napobrassica Mill., swede or rutabaga; $B$. napus $\mathrm{L}$., rape; $B$. nigra (L.) Koch, black mustard; B. oler. acea L., cabbage; Armoracia rusticana Gaertn., horse-radish; Cheiranthus cheiri L., wallflower; Matthiola incana R. Br., stock; Sinapis alba L., white mustard. Experimentally, also CRUCIFERAEBerteroa incana (L.) DC.; Brassica alba Rabenh., white mustard; $B$. arvensis (L.) Ktze.; B. chinensis L., Chinese cabbage; $B$. juncea (L.) Coss.; Capsella bursa-pastoris (L.) Medic.; Cardamine heterophylla (Forst. f.) O. E. Schultz; Cheiranthus allionii Hort.; Coronopus didymus Smith; Hesperis matronalis L.; Lepidium ruderale L.; $L$. sativum L., $L$. virginicum $\mathrm{L}$.; Nasturtium officinale $\mathrm{R}$. Br.; Neslia paniculata (L.) Desv.; Radicula palustris (L.) Moench.; Raphanus sativus L.; Sisymbrium altissimum L.; S. officinale (L.) Scop.; Thlaspi arvense I. CHENOPODIACEAE-Beta vulyaris L.; Spinacia oleracea L., spinach. COMPOSIT AE-Calendula officinalis $\mathrm{L}$. Zinnia elegans Jacq. RANUNCULACEAE-Delphinium ajacis L. SOLANA$C E A E$-Lycopersicon pimpinellifolium Mill.; Nicotiana bigelovii S. Wats.; $N$. glutinosa L.; $N$. langsdorffi Weinm.; $N$. repanda Willd.; $N$. rustica L.; $N$. sylvestris Speg. and Comes; N. tabacum L., tobacco; Petunia hybrida Vilm.

Geographical distribution: United States, England, New Zealand.

Induced disease: In turnip, systemic chlorotic mottling; plants stunted, leaves distorted. In tobacco, experimentally, characteristic necrotic primary lesions only.

Transmission: By inoculation of expressed juice. By cabbage aphid, Brevicoryne brassicae (Linn.), and by the peach aphid, Myzus persicae (Sulz.) ( $A P H I D I D A E)$.

Thermal inactivation: At $54^{\circ} \mathrm{C}$ in 10 minutes.

Strains: A considerable number of strains of this virus appear to occur in nature, but those that have been studied often have been considered as distinct. viruses and not compared with each other 
under identical circumstances. More work is needed to show existing alliances.

Literature : Chamberlain, New Zealand Jour. Agr., 53, 1936, 321-330; New Zealand Jour. Science and Technology, 21, 1939, 212A-223A; Clayton, Jour. Agr. Res., 40, 1930, 263-270; Gardner and Kendrick, ibid., 22, 1921, 123-12t ; Hoggan and Johnson, Phytopath., 25, 1935, 640-644; Larson and Walker, Jour. Agr. Res., 59, 1939, 367-392 ; 62, 1941, 475-491; Schultz, Jour. Agr. Res., 22, 1921, 173-178; Smith, Ann. Appl. Biol., 22, 1935, 239-212; Tompkins, Jour. Agr. Res., 57, 1938, 589-602; 58, 1939, 63-77; Tompkins et al., ibid., 57, 1938, 929-943.

15. Marmor betae H. (loc. cit., 72). From Latin beta, beet.

Common name: Sugar-beet mosaic virus.

Hosts: CHENOPOI)IACEAE-Beta vulgaris L., beet; Spinacia oleracea I.., spinach

Geographical distribution: France, Denmark, Germany, Sweden, Lnited States, England.

Induced disease: In beet, discrete yellowish secondary lesions or clearing of veins on young leaves, followed by chlorotic mottling of newly formed leaves Darkening of vascular tissue. Leaves bend back near tips, which sometimes die. Intracellular bodies formed. In spinach, 6 to 21 days after infection, chlorotic flecks on young leaves. Plant stunted, outer leaves killed, dying from their tips back. Center of plant survives for a time, but firally dies.

Transmission: By inoculation of $\mathrm{ex}$ pressed juice, in dilutions to $10^{-3}$. By aphids, Myzus persicae (Sulz.), Aphis rumicis Linn., and perhaps Macrosiphum solanifolii Ashm. (=.M. gei Koch) $(A P H I D I D A E)$. No seed transmission.

Thermal inactivation: It 55 to $60^{\circ} \mathrm{C}$ in 10 minutes.

Other properties: Inactivated by standing in expressed juice for 24 to 45 hours at about $70^{\circ} \mathrm{F}$.
Literature : Böning, Forsch. Geb. Pflanzenkr. u. Immun. Pflanzenreich, 3, 1927, 81-128; Cent. f. Bakt., II Abt., 71, 1927, 490-497; Gratia and Manil, Compt. rend. Soc. Biol., Paris, 118, 1935, 379-381; Hoggan, Phytopath., 23, 1933, 446-474; Jones, Washington Agr. Exp. Sta. Bull. 250, 1931; Lind, Tidsskr. Planteavl, 22, 1915, 44-457; Robbins, Phytopath., 11, 1921, 349-365; Schmidt, Ber. Deutsch. Bot. Ges., 45, 1927, 598-601.

16. Marmor lactucae H. (loc. cit., 81). From Iatin lactuca, lettuce.

Common name: Lettuce-mosaic virus.

Hosts: COMPOSITAE-Lactuca sativa L., lettuce; Senecio vulgaris L., groundsel. Experimentally, also $\mathrm{COM}$ POSITAE-Sonchus asper Hoffm., prickly sow-thistle. LEGUMINOSAE -Lathyrus odoratus I., sweet pea; Pisum sativum L., pea.

Insusceptible species: COMPOSITAE --Sonchus oleraceus I., S. arvensis L., Taraxacum officinale Web., Carduus arwhsis Curt. CRLCIFERAE-Brassica oleracea L. CUCURBITACEAECucumis sativus I. SOLANACEAELycopersicon esculentum Mill., Nicotiana tabacum L., I. glutinosa L., Datura stramonium $\mathrm{L}$.

Geographical distribution: United States, England, Germany, Bermuda.

Induced disease: In lettuce varieties, clearing of veins followed by systemic chlorotic mot ting, dwarfing and defective hearting; sometimes by scorching of leaf edges, vein necrosis or necrotic flecking between veins.

Transmission: $\mathrm{By}$ inoculation of expressed juice, in dilutions to $1: 100$ if mixed with a little 0.5 per cent sodium sulphite solution and a trace of powdered carborundum. By aphids, Myzus persicae (Sulz.) and Macrosiphum gei Koch (APHIDIDAE). Through seeds from diseased plants. It is believed that seedhorne virus is the most important source of primary inoeulum in the spring.

Thermal inactivation: At 55 to $60^{\circ} \mathrm{C}$ in 10 minutes. 
Filterability : Fails to pass $\mathrm{L}_{1}$ PasteurChamberland filter.

Literature: Ainsworth and Ogilvie, Ann. Appl. Biol., 26, 1939, 279-297; Jagger, Jour. Agr. Res., 20, 1921, 737-740; Newhall, Phytopath., 13, 1923, 10t-106.

17. Marmor dahliae H. (loc. cit., 85 ). From New Latin Dahlia, generic name of host plant.

Common name: Dahlia-mosaic virus.

Hosts: COMPOSITAE-Dahlia pinnata Cav., dahlia. Experimentally, also D. imperialis Roezl.; D. maxonii Safford.

Geographical distribution: Lnited States, Holland, Germany, England.

Induced disease : In intolerant varieties of dahlia, chlorotic mottling of foliage, leaf distortion, dwarfing of all stems and of roots, occasionally necrotic streaking of midveins. In tolerant varieties, inconspicuous chlorotic mottling or masked symptoms.

Transmission: By aphid, Myzus persicae (Sulz.) ( $A P H I D I D A E)$. By graft ing. Not by inoculation of expressed juice. Not through soil. Not through seeds from diseased plants.

Literature: Brierley, Am. Dahlia Soc. Bull., Ser. 9, No. 65, 1933; Contrib. Boyce Thompson Inst., 5, 1933, 235-288; Goldstein, Bull. Torrey Bot. Club, 54, 1927 , 28.5-293.

18. Marmor phaseoli H. (loc. cit., 87). From New Latin Phaseolus, generic name of bean.

Common name: Bean-mosaic virus.

Hosts: LEGL II NOSAE-Phaseolus vulgaris L., bean. Experimentally, alsu Phaseolus acutifolius Gray var. latifolius Freem.; P. aureus Roxb.; P. calcaratus Roxb.; P. lunatus L.; Lespedeza striata (Thumb.) Hook. and Arn.; Vicia faba L.; $V$. sativa $\mathrm{L}$., spring vetch.

Insusceptible species: LEGL'MINOSAE-Pisum sativum I., garden pea; Lathyrus odoratus L., sweet pea.

Geographical dist ribution: World-wide, wherever beans are grown.

Induced disease: In bean, first leaves to be affected are crinkled, stiff, chlorotic; later leaves show chlorotic mottling; leaf margins often rolled down. Optimum temperature for expression of disease, 20 to $28^{\circ} \mathrm{C}$, partial masking at 28 to $32^{\circ} \mathrm{C}$, complete masking at 12 to $18^{\circ} \mathrm{C}$.

Transmission: By inoculation of expressed juice in dilutions to $1: 1000$, using carborundum or other abrasive powder. By aphids, Aphis rumicis Linn., Macrosiphum (= Illinoia) solanifolii Ashm., M. pisi Kalt., Aphis gossypii Glov., A. medicaginis Koch, A. spiraecola, Brevicoryne brassicae (Linn.), Hyalopterus atriplicis Linn., Macrosiphum ambrosiae Thos., Rhopalosiphum pseudobrassicae Davis, and Myzus persicae (Sulz.) ( $A P H I D I D A E)$. In beans, there is seed transmission to 30 to 50 per cent of plants grown from infected parents; pollen from infected plants is said to transmit virus.

Thermal inactivation: At 56 to $58^{\circ} \mathrm{C}$ in 10 minutes.

Literature: Fajardo, Phytopath., 20, 1930, 469-494, 883-888; Murphy, ibid., 30, 1940, 779-784; Murphy and Pierce, ibid., 28, 1938, 270-273; Parker, Jour. Agr. Res., 52, 1936, 895-915; Pierce, Phytopath., 24, 1934, 87-115; Jour. Agr. Res., $49,1934,183-188 ; 51,1935,1017-1039$; Reddick, II Congr. Intern. Path. Comp., 1931, 363-366; Reddick and Stewart, Phytopath., 8, 1918, 530-534; Richards and Burkholder, Phytopath., 33, 1943, 1215-1216; Wade and Andrus, Jour. Agr. Res., 63, 1941, 389-393; Wade and Zaumeyer, L. S. Dept. Agr., Circ. 500, 1938; Walker and Jolivette, Phytopath., 33, 1943, 778-785; Zaumeyer and Liearns, ibid., 26, 1936, 614-629; Zaumeyer and Wade, Jour. Agr. Res., 51, 1935, 715-749.

19. Marmor leguminosarum H. (loc. cit., 89). From New Latin Leguminosae, family name of pea.

Common name: Pea-mosaic virus.

Hosts: LEGU IIINOSAE-Lathyrus odoratus L., sweet pea; Pisum sativum L., pea ; Trifolium pratense L., red clover ; Vicia faba L., broad bean. Experiment- 
ally, also Cicer arietinum L.; Desmodium canadense (I.) DC.; Lathyrus sativus L. grass pea ; Lupinus albus L., white lupine; $L$. angustifolius, blue lupine; $L$. densiflorus Benth.; L. hartwegii Lindl.; L. nanus Dougl.; Medicago arabica Huds., spotted bur clover; $M$. hispida Gaertn. toothed bur clover; Melilotus alba Desr. white sweet clover; 11 . indica All., annual yellow sweet clover; $M$. officinalis (L.) Lam., yellow sweet clover; Phaseolus acutifolius Gray, tepary bean; $I^{\prime}$. vulgaris L., bean; Trifolium agrarium L. ; T. carolinianum Michx.; T. dubium Sibth.; $T$. glomeratum I., cluster clover; T. hy-

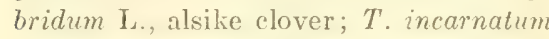
L., crimson clover; T. procumbens I.; $T$. reflexum L.; T. suaveolens, Persian clover ; Vicia sativa I., common vetch.

Insusceptible species: All tested species in families other than the Leguminosae.

Geographical distribution: United States, British Isles, Europe, New Zealand.

Induced disease: In pea, clearing of veins in young leaves, followed by chlorosis of newly formed leaves, stunting of plant, and systemic chlorotic mottling. In sweet pea, systemic chlorosis and chlorotic mottling, flower colors broken. In lupine, necrotic streak on one side of stem, stunting of plant and bending of growing point to injured side. Plant soon wilts and dies. In Vicia jaba, mottled leaves contain characteristic isometric crystals in host-cell nuclei (especially within nucleoli) as well as in cell eytoplasm.

Transmission: By inoculation of expressed juice, with ease. By aphids, Macrosiphum pisi lialt., .H. solanifolit Ashm. (= H. gei Koch), and Aphis rumicis Linn. (APHIDIDAE). Tot transmitted through seed.

Serological relationships: Specific precipitin reactions differentiate this virus from tobacco-mosaic virus, tobacco-etch virus, potato-mottle virus, potato mildmosaic virus, potato aucuba-mosaic virus, and tobacco-ringspot virus.
Thermal inactivation: At $60^{\circ} \mathrm{C}$ in 10 minutes.

Literature: Chester, Phytopath., 25, 1935, 686-701 ; Doolittle and Jones, ibid., 15, 1925, 763-772; Johnson and Jones, Jour. Agr. Res., 54, 1937, 629-638; MeWhorter, Phytopath., 31, 1941, 760-761; Murphy and Pierce, ibid., 27, 1937, 710-721; Osborn, ibid., 27, 1937, 589-603; Pierce, Jour. Agr. Res., 51, 1935, 1017-1039; Spierenburg, Tijdschr. Plantenz., 42, 1936, 71-76; Zaumeyer and Wade, Jour. Agr. Res., 53, 1936, 161-185.

20. Marmor pisi H. (loc. cit., 90). From Latin pisum, pea.

Common name: Pea enation-mosaic virus.

Hosts: LEGUMINOSAE-Pisum sativum L., pea; Vicia faba L., broad bean. Experimentally, also Lathyrus odoratus L., sweet pea; Soja max (L.) Piper, soy bean; Trifolium incarnatum L., crimson clover.

Insusceptible species: $L E G U M I N O$. SAE-Arachis hypogaea I., peanut; Medicago sativa L., alfalfa; Melilotus alba Desr., white sweet clover; $M$. officinalis (L.) Lam., yellow sweet clover; Phaseolus aureus Roxb., mung bean; $P$. vulgaris I., bean; Trifolium hybridum L., alsike clover; $T$. pratense L., red clover; $T$. repens L., white Dutch elover. SOLA.ACEAE-Lycopersicon esculentum Mill., tomato; Solanum tuberosum L., potato.

Geographical distribution: United states, perhaps Ciermany.

Induced disease: In peas, systemic chlorotic mottling; in some varieties, as Alderman, occasional necrotic spots and numerous enations on lower surfaces of leaves. Pods distorted. In broad bean, systemic chlorotic spotting and striping of leaves. In sweet pea and soy bean, experimentally, systemic chlorotic mottling.

Transmission: By inoculation of expressed juice, using carborundum; more readily from aphid-inoculated plants than from mechanically-inoculated plants. 
Infective in dilutions to $10^{-3}$. By aphids, Macrosiphum pisi Kalt, and M. solanifolii Ashm. (= II. gei Koch) (APHI$D I D A E$ ), with incubation periods of about 12 hours before the insects can infect. Not through sceds from diseased plants.

Thermal inactivation: it $66^{\circ} \mathrm{C}$ in 10 minutes.

Literature : Böning, Forsch. Geb. Pflanzenkr. u. Immun. Pflanzenreich, 4, 1927, 43-111; Johnson and Jones, Jour. Agr Res., 54, 1937, 629-638; Loring et al., Proc. Soc. Exp. Biol. and Med., 38, 1938 239-241; Osborn, Phytopath., 25, 1935. 160-177; 28, 1938, 749-75⿺ 923-934; Pieree. Jour. Agr. Res., 51, 1935, 1017-1039 Snyder, Phytopath., 24, 1934, 78-80; Stubbs, ibid., 27, 1937, 242-266.

21. Marmor medicaginis $\mathrm{H}$. (loc cit., 91). From New Latin Medicago, generic name of alfalfa (lucerne).

Common name: Alfalfa-mosaic virus.

Hosts: LEGLWINOSAE-Medicago sativa L., alfalfa (lucerne). SOLANA CEAE-Solanum tuberosum Is., potato. Experimentally, also transmissible to many species of dicotyledonous plants (summarized by Price, Am. Jour. Bot., 27, 1940, 530-541) including $C U C U R B I$ $T A C E A E$-Cucumis sativus $\mathrm{L}$., cucumber. COMPOSITAE-Zinnia elegans Jacq., zinnia. LEGUM I NOSAEPhaseolus vulgaris L., bean; Trifolium incarnatum L., crimson clover. SOLANACEAE-Capsicum frutescens L., pepper; Lycopersicon esculentum IIIll., tomato; Nicotiana tabacum L., tobacco.

Geographical distribution: United States.

Induced disease: In alfalfa, systemic chlorotic mottling, tending to be masked at times. In bean, (most varieties) small necrotic primary lesions, reddish brown at periphery. No secondary lesions. Some bean varieties show no lesions after inoculation; one of these, Refugee Rogue, possesses two dominant genes either of which will confer this type , resistance. In tobacco, white necrotic flecks, small rings and ares on inoculated leaves; later, systemic mottling, followed by production of necrotic oak-leaf patterns; virus content may be low in plants long diseased, especially in summer.

Transmission: By inoculation of expressed juice. By aphids, IIacrosiphum pisi Kalt. (for typical strain) and $M$. solanifolii Ashm. (for potato-calico strain) ( $A P H T D I D A E$ ). Tot through seeds from diseased plants.

Immunological relationships: Resistance to superinfection with the type of this virus is conferred by earlier infection with potato-calico virus (now considered a related strain but earlier regarded as distinct), but not by earlier infection with potato-mottle virus, cucumbermosaic virus, or the Canada-streak strain of potato aucuba-mosaic virus.

Thermal inactivation: At 65 to $70^{\circ} \mathrm{C}$ in 10 minutes.

Other properties: Sedimentation constant, $73.9 \pm 5.2 \times 10^{-13} \mathrm{~cm}$. per sec. in a unit centrifugal field. Specific volume 0.673 . Particles spherical or nearly so. Diameter 16.5 millimicrons; weight $2.1 \times 10^{6}$ times hydrogen unit. Isoelectric point about $\mathrm{pH}$ 4.6. Inactivated and, more slowly, hydrolyzed by trypsin.

Literature: Black and Price, Phytomth. . 30, 1940, 414-4t7; Lauffer and Ross, Jour. Am. Chem. Soc., 62, 1940, 3296-3300, Pierce, Phytopath., 24, 1934, 87-115; Price, Am. Jour. Bot., 27, 1940, 530-541; Ross, Phytopath., 31, 1941, 394-410, 410420; Wade and Zaumeyer, Jour. Am. Soc. Agron., 32, 1940, 127-134; Zaumeyer, Jour. Agr. Res., 56, 1938, 7\$7-772.

Strains: At least one strain of alfalfamosaic virus was formerly considered as an independent virus, causing a disease known as calico in potato. It has now been given varietal rank and distinguished from the type, var. typicum Black and Price (Phytopath., 30, 1940, 446) by the following name:

21a. Marmor medicaginis var. solani Black and Price (Phytopath., 30, 1940, 
446). From New Latin Solanum, generic name of potato.

Common name: Potato-calico strain of alfalfa-mosaic virus. Differing from the type by inducing a more severe disease in potato, in which it is commonly found in nature. (Price and Black, Phytopath., 30, 1940, 444-447 ; Dykstra, ibid., 29, 1939, 917-933; Porter, Potato Assoc. Amer. Proc., 18, 1931, 65-69; Hilgardia, 6, 1931, 277-294; 9, 1935, 383-394.)

22. Marmor tulipae H. (loc. cit., 52), From New Latin Tulipa, generic name of tulip.

Common name: Tulip color-adding virus.

Hosts: LILIACEAE-T'ulipa gesneriana L., garden tulip; $T$. eichleri Regel; T. greigi Regel.

Insusceptible species: $A M A R Y L L I$ $D A C E A E-N$ arcissus sp., narcissus. IRIDACEAE-Iris germanica L., iris. LILIACEAE-Allium cepa L., onion. SOLANACEAE-Nicotiana tabacum $\mathrm{L}$., tobacco.

Geographical distribution: Wherever hybrid tulips are grown.

Induced disease: In tulip, no obvious effect on leaves but dark striping of flower by pigment intensification. Little interference with growth of plant. No intracellular bodies.

Transmission: By hypodermic injections of expressed juice in dilutions to $10^{-5}$. By aphids, Myzus persicae (Sulz.), Macrosiphum solanifolii Ashm. (= M. gei Koch, Illinoia solanifolii Ashm.), Aphis (= Anuraphis) tulipae B. de Fonsc. (on bulbs), and perhaps Macrosiphum pelargonii lialt. (APHIDIDAE). Not through seeds from diseased plants.

Thermal inactivation: At 65 to $70^{\circ} \mathrm{C}$ in 10 minutes.

Literature: Hughes, Ann. Appl. Biol., $18,1931,16-29 ; 21,1934,112-119$; McWhorter, Phytopath., 22, 1932, 998 (Abst.); 25, 1935, 898 (Abst.); Ann. Appl. Biol., 25, 1938, 254-270.
23. Marmor mite H. (loc. cit., 53). From Latin mitis, mild.

Common name: Lily latent-mosaic virus.

Hosts : LILIACEAE-Lilium amabile; $L$. auratum Lindl.; $L$. canadense L.; $L$. candidum L.; L. cernuum; L. chalcedonicum L.; L. croceum Chaix.; L. davmottiae; $L$. elegans Thunb.; $L$. formosanum Stapf.; L. giganteum; L. henryi Baker; $L$. leucanthum; L. longiflorum Thunb.; $L$. myriophyllum; L. pumilum; L. regale Wils.; L. sargentiae Wils.; L. speciosum Thunb.; L. superbum L.; L. testaceum Lindl.; L. tigrinum Ker; $L$. umbellatum Hort.; L. wallacei; Tulipa gesneriana L., garden tulip; $T$. clusiana Vent.; T. linifolia Regel.

Insusceptible species : $L I L I A C E A E-$ Allium cepa L., onion; Lilium hansoni Leichtl. IRIDACEAE-Iris germanica L., iris. SOLANACEAE-Nicotiana tabacum L., tobaceo.

Geographical distribution: Wherever lilies and tulips are cultivated.

Induced disease : In Easter lily, masked symptoms or systemic chlorotic mottling, in either case without necrotic flecking. In tulip, systemic chlorotic mottling in foliage and flower "breaking" (color removal, except in a few varieties in which color intensification occurs instead). Intracellular bodies characterize invaded tissues.

Transmission: By inoculation of expressed juice (rubbing surface of leaves), in both lily and tulip. By plugging and grafting of dormant bulbs of tulip. By aphids, Myzus persicae (Sulz.), Macrosiphum solanifolii Ashm. (= M. gei Foch), and Aphis (= Anuraphis) tulipae B. de Fonse. (APHIDIDAE). Not through seeds from mosaic Lilium longiflorum.

Thermal inactivation: At 65 to $70^{\circ} \mathrm{C}$ in 10 minutes.

Literature: Atanasoff, Bull. Soc. Bot. Bulgarie, 2, 1928, 51-60; Brierley, Phytopath., 29, 1939, 3 (Abst.) ; 30, 1940, 250- 
257 ; 31, 1941, 838-843 ; Brierley and Doolittle, ibid., 30, 1940, 171-174; Cayley, Ann. Appl. Biol., 15, 1928, 529-539;19, 1932, 153-172; Guterman, Hort. Soc. N. Y., Yearbk., 1930, 51-102; Hall, Gard. Chron., 93, 1933, 330-331 ; Hughes, Ann. Appl. Biol,. 21, 1934, 112-119; McKay and Warner, Nat. Hort. Mag., 12, 1933, 179-216; McWhorter, Phytopath., 25, 1935, 898 (Abst.); Science, 86, 1937, 179; Ann. Appl. Biol., 25, 1938, 254-270; Science, 88, 1938, 411; Ogilvie and Guterman, Phytopath., 19, 1929, 311-315.

24. Marmor iridis H. (loc. cit., 55). From New Latin Iris, generic name of iris.

Common rame: Iris-mosaic virus.

Hosts : IRIDACEAE-Iris filifolia Boiss., I. tingitana Boiss. and Reut., and I. xiphium L., bulbous irises; Iris ricardi Hort.; I. unguicularis Poir.; bearded iris, variety William Mohr.

Insusceptible species: SOLANA $C E A E$-Lycopersicon esculentum Mill., tomato; Nicotiana tabacum L., tobacco; Petunia hybrida Vilm., petunia. LILIA CEAE-Tulipa gesneriana L., tulip.

Geographical distribution: United States (Washington, Oregon, California), Holland, Bulgaria, France, England.

Induced disease: In bulbous irises, dwarfing of plant, chlorotic mottling of foliage, breaking of flowers. Rate of increase in planting stock decreased. Flower breaks usually darker than normal color of flower. Vacuolate intracellular bodies in some affected tissues.

Transmission: By injection of freshly extracted juice of diseased plants into internodal tissue. By aphids, Macrosiphum (= Illinoia) solanifolii Ashm. and Myzus persicae (Sulz.) (APHIDI$D A E)$.

Literature: Brierley and McWhorter, Jour. Agr. Res., 53, 1936, 621-635.

25. Marmor sacchari H. (loc. cit., 60). From New Latin Saccharum, generic name of sugar cane, from Latin saccharum, sugar.

Common name: Sugar-cane mosaic virus.

Hosts : GRAMINEAE-Saccharum officinarum L., sugar cane; Holcus sorghum L., sorghum; H. sudanensis Bailey, Sudan grass; Brachiaria platyphylla Nash; Chaetochloa magna Seribn.; C.verticillata Scribn.; Paspalum boscianum Fluegge; Syntherisma sanguinale Dulac. Experimentally, also Zea mays L., corn (maize); Chaetochloa lutescens Stuntz; Echinochloa crusgalli Beauv. ; Miscanthus sinensis Anderss., eulalia; Panicum dichotomiflorum Michx.; Pennisetum glaucum R. Br., pearl millet; Saccharum narenga Wall.

Insusceptible species: All tested species other than Gramineae.

Geographical distribution: Originally in Far East; now in nearly all countries where sugar cane is grown; believed still to be absent from Mauritius.

Induced disease: In sugar cane, systemic mottling chlorosis, light areas of pattern elongated, but crossing veins. Occasionally, stem cankers. Regularly, discoloration and necrosis in mature inner stalk tissues. Vacuolate intracellular bodies occur in diseased tissues. Canes sometimes recover, spontaneously losing the virus and becoming susceptible to reinfection.

Transmission: By inoculation of expressed juice (puncture through inocuIum into young leaf). By aphids, A phis maidis Fitch, Carolinaia cyperi Ainslie, Hysteroneura setariae (Thomas), and Toxoptera graminum Rond.; not by Sipha flava Forbes ( $A P H I D I D A E)$. Not by Draeculacephala mollipes (Say) (CICADELLIDAE).

Serological relationships: Specific neutralizing and precipitating antibodies have been demonstrated.

Thermal inactivation: At 53 to $54^{\circ} \mathrm{C}$ in 10 minutes in leaf tissues. 
Other properties: Active after storage 27 days at $-6^{\circ} \mathrm{C}$.

Literature: Brandes, Jour. Agr. Res., $19,1920,131-138,517-522$; 24, 1923, 247262; Desai, Current Science, 3, 1935, 18; Forbes and Mills, Phytopath., 39, 1943, 713-718; Ingram and Summers, Jour. Agr. Res., 52, 1936, 879-888; Kunkel, Bull. Exp. Sta. Hawaiian Sugar Planters' Assoc., Bot. Ser., 3, 1924, 115-167; Matz, Jour. Agr. Res., 46, 1933, 821-839; Rafay, Indian Jour. Agr. Science 5, 1935, 663670; Seín, Jour. Dept. Agr. Porto Rico, 14, 1930, 49-68; Stoneberg, U. S. Dept. Agr., Tech. Bull. 10, 1927; Tate and Vandenberg, Jour. Agr. Res., 59, 1939, 73-79.

26. Marmor cepae H. (loc. cit., 66). From Latin cepa, onion.

Common name: Onion yellow-dwarf virus.

Host : LILIACEAE-Allium cepa L., onion (the variety viviparum Metz. is symptomless when infected and may serve as an unrecognized reservoir of virus).

Geographical distribution: United States, Germany, Czecho-Slovakia, Russia, New Zealand.

Induced disease : In onion (most varieties), yellow streaks at base of developing leaf, followed by yellowing, crinkling, and flattening of newly formed leaves; leaves prostrate, flower stalks bent, twisted, stunted; plants reduced in size, bulbs small, yield of seeds reduced. A few varieties of onion are relatively tolerant, and the tree-onion, var. viviparum is symptomless after infection.

Transmission: By inoculation of expressed juice. By 48 of 51 tested species of aphid, principally A phis rumicis Linn., A. maidis Fitch, and Rhopalosiphum prunifoliae Fitch ( $A P H I D I D A E)$. Not through seeds from diseased plants. Not by contaminated soil.

Thermal inactivation: It 75 to $80^{\circ} \mathrm{C}$ in 10 minutes.

Other properties: Virus withstands dilution to $10^{-4}$, storage at $29^{\circ} \mathrm{C}$ for about
100 hours and storage at $-14^{\circ} \mathrm{C}$ for more than time tested ( 6 hours), but is inactivated by drying in leaf tissues.

Literature: Andreyeff, Rev. Appl. Mycol., 17, 1938, 575-576; Blattny, Ochrana Rostlin, 10, 1930, 130-138; Bremer, Phytopath. Ztschr., 10, 1937, 79-105; Brierley and Smith, Phytopath., 34, 1944, 506-507; Chamberlain and Baylis, New Zealand Jour. Science and Technology, 21, 1939, 229A-236A; Drake et al., Iowa State Coll. Jour. Science, 6, 1932, 347355; Jour. Econ. Ent., 26, 1933, 841-846; Henderson, Phytopath., 20, 1930, 115 (Abst.); Iowa State Coll., Research Bull. 188, 1935, 211-255; Melhus et al., Phytopath., 19, 1929, 73-77; Porter, U. S. Dept. Agr., Plant Dis. Rept., 12, 1928, 93; Tate, Iowa State Coll. Jour. Science, 14, 1940, 267-294.

27. Marmor scillearum Smith and Brierley (Phytopath., 34, 1944, 503.) From New Latin Scilleae, name of tribe in which hosts are classed.

Common name: Ornithogalum-mosaic virus.

Hosts: LILIACEAE (of the tribe Scilleae -Ornithogalum thyrsoides Jacq. ; probably also Galtonia candicans Decne.; Hyacinthus orientalis I., hyacinth; $L a$ chenalia sp.

Insusceptible species: LILIACEAE (of the tribe Scilleae)-Muscari botryoides Mill. ; Scilla peruviana L. ; Camassia leichtlinii (Baker) S. Wats.; Hyacinthus azureus (Fenzl.) Baker. AMARYLLI$D A C E A E-P$ ancratium maritimum; Zephyranthus sp. IRIDACEAE-Tritonia crocata (L.) Ker. LILIACEAEAgapanthus africanus; Allium cepa, onion; A. cernuum Roth.; A. fistulosum L. ; A . porrum L. ; Gloriosa rothschildiana O'Brien; Lilium formosanum Stapf.; and $L$. longiflorum. SOLANACEAE-Nicotiana tabacum $\mathrm{L}$.

Geographical distribution: United States (Oregon; probably also Alabama and presumed to be widespread in plants of the squill tribe, Scilleae, of the family LILI ACEAE). 
Induced disease: In Ornithogalum thyrsoides, young leaves finely mottled with light and dark green, and becoming more conspicuously mottled with gray or yellow as the leaves mature; flower stalks sometimes boldly marlied with light and dark green blotches. In periantl segments, thin longit udinal streaks.
Transmission: By inoculation of expressed juice in the presence of fine carborundum powder, with difficulty. By aphids, Aphis gossypii Glov., Macrosiphum lilii Monell, M. solanifolii Ashm., and Myzus persicae (Sulz.); less efficiently by Myzus circumflexus (Buckt.) (APHIDIDAE).

\section{Key to the species of the Miscellaneous Mosaic-Virus Group.}

Many of the following viruses, although described in some detail in the literature, stand in need of reinvestigation to determine additional properties and possible relationships to preceding groups.

I. Affecting species of $M A L V A C E A E$.

28. Marmor abutilon.

II. Affecting species of $C E L A S T R A C E A E$.

29. Marmor euonymi.

III. Affecting species of $O L E A C E A E$.

30. Marmor ligustri.

IV. Affecting species of $L E G C M I N O S A E$ (and no. 39, other families also).

31. Marmor laburni.

32. Marmor arachidis.

33. Marmor trifolii.

34. Marmor pachyrhizi.

35. Marmor vignae.

36. Marmor repens.

37. Marmor fastidiens.

38. Marmor iners.

39. Marmor efficiens.

V. Affecting species of GRAMINEAE.

40. Marmor tritici.

41. Marmor graminis.

VI. Affecting species of MUSACEAE.

42. Narmor abaca.

VII. Affecting species of PASSIFLORACEAE.

VIII. Affecting species of ROSACEAE.

43. II armor passiflorae.

44. Marmor flaccumfaciens.

45. Marmor rosae.

46. Marmor veneniferum.

47. Narmor mali.

48. Narmor fragariae.

49. Marmor marginans.

50. Narmor rubi.

51. Marmor persicae.

52. Marmor astri.

53. Marmor rubiginosum.

5t. Marmor cerasi.

55. Marmor lineopictum.

56. Marmor pallidolimbatus.

57. Marmor nerviclarens. 
IX. Affecting species of VITACEAE.

X. Affecting species of SANTALACEAE.

58. Marmor viticola.

59. Marmor santali.

XI. Affecting species of CONVOLVULACEAE and, experimentally, of other families.

60. Marmor secretum.

XII. Affecting species of GERANIACEAE.

61. Marmor pelargonii.

XIII. Affecting species of SOLANACEAE and in most cases also of other families.

62. Marmor angliae.

63. Marmor aevi.

64. Marmor raphani.

XIV. Affecting species of PRIMULACEAE.

65. Marmor primulae.

XV. Affecting species of MORACEAE.

66. Marmor caricae.

XVI. Affecting species of $R U T A C E A E$.

67. Marmor italicum.

28. Marmor abutilon H. (loc. cit., 50). From New Latin Abutilon, generic name of a host.

Common name : Abutilon-mosaic virus.

Hosts : $M A L V A C E A E-A b u t i l o n$ striatum Dicks. var. thompsonii Veitch. Experimentally, also Abutilon arboreum Sweet; A. avicennae Gaertn.; A. esculentum St. Hil.; A. indicum Sweet; $A$. insigne Planch.; A. megapotamicum St. Hil. and Naud.; A. regnellii Miq.; A. sellowianum Regel; A.venosum Lem.; A.vitifolium Presl.; Althaea ficifolia Cav.; $A$. officinalis L.; A. rosea Cav.; Anoda hastata Cav.; Kitaibelia vitifolia Willd.; Malva borealis; $M$. crispa; M. mauritiana Mill.; M. sylvestris L.; M. verticillata L.; Malvastrum capense Gareke; Modiola decumbens G. Don.; Sida mollis Herb.; S. napaea Cav.; Sidalcea candida A. Gray.

Insusceptible species: $M A L Y A C E A E$ -Althaea taurinensis; Sidalcea purpurea; Sphaeralcea umbellata G. Don.

Geographical distribution: Germany, France, England, United States; originally obtained from a single variegated seedling found among green plants of Abutilon striatum imported from the West Indies in 1868 by Veitch and Sons; subsequently the infected plant was propagated vegetatively as an ornamental variety.

Induced disease: In Abutilon, systemic chlorotic mottling. Recovery occurs if there is persistent removal of affected leaves, suggesting that the virus does not increase in stems. After recovery, plants are susceptible to reinfection.

Transmission: By grafting, except patch-bark-grafting, which is ineffective. Occasionally through seeds from diseased planis. Not by inoculation of expressed juice. No insect vector is known.

Varieties: Distinctive strains have been noted, but not separately named; one isolate originally occurring in Abutilon darwini var. tesselatum, seems to belong here; it differs from the type principally in severity of induced disease and in ability to infect Lacatera arborea.

Literature: Baur, Ber. d. Deutsch. Bot. Gesellsch., 22, 1904, 453-460; 24, 1906, 416-428; 25, 1907, 410-413; K. Preuss. Akad. Wiss., Sitzungsber., 1906, 11-19; Davis, Ann. Missouri Bot. Gard., 16, 1929, 145-226; Hertzsch, Ztschr. f. Bot., 20, 1927, 65-85; Keur, Phytopath., 23, 1933, 20 (Abst.); 24, 1934, 12-13 (Abst.); Bull. Torrey Bot. Club, 61, 1934, 53-70; Lindemuth, Gartenflora, 51, 1902, 323326. 
29. Marmor euonymi H. (loc. cit., 51). From New Latin Euonymus, generic name of host.

Common name: Euonymus-mosaic virus.

Hosts : CELASTRACEAE-Euonymus japonica L. f. (sometimes written Evonymus japonicus). Probably also $E$. radicans Sieb.

Geographical distribution: Germany.

Induced disease: In Euonymus japonica, persistent yellowing along veins.

Transmission : By grafting.

Literature: Baur, Ber. d. Deutsch. Bot. Gesellsch., 26a, 1908, 711-713; Rischkow, Biol. Zentralbl., 4\%, 1927, 752-764.

30. Marmor ligustri $\mathrm{H}$. (loc. cit., 52). From New Latin Ligustrum, generic name of host, from Latin ligustrum, ancient name of privet plant.

Common name: Ligustrum-mosaic virus.

Host : OLEACEAE-Ligustrum vulgare $\mathrm{L}$., common privet.

Geographical distribution: Germany.

Induced disease: Systemic chlorotic spotting.

Transmission: By grafting. Not through seeds from diseased plants.

Literature: Baur, Ber. d. Deutsch. Bot. Gesellsch., 25, 1907, 410-413.

31. Marmor laburni H. (loc. cit., 51). From generic name of a host plant, Laburnum vulgare.

Common name: Laburnum-mosaic virus.

Hosts : LEGU II INOSAE-Laburnum vulgare Griseb. (= L. anagyroides Medic.), bean tree. Experimentally, also Cytisus hirsutus L.

Insusceptible species: $L E G U M I N O$ $S A E$-Laburnum alpinum Griseb.; $C y$ tisus purpureus.

Geographical distribution: Germany.

Induced disease: Systemic chlorotic variegation.

Transmission: By bark grafts or by budding. Not through seeds from diseased plants of Laburnum vulgare.

Literature: Baur, Ber. d. Deutsch. Bot. Gesellsch., 25, 1907, 410-413.

32. Marmor arachidis $\mathrm{H}$. (loc. cit., 67). From New Latin Arachis, generic name of peanut.

Common name: Peanut-rosette virus

Host : $L E G U M I N O S A E-$ Arachis $h y$ pogaea L., peanut.

Geographical distribution: Union of South Africa, Madagascar, Tanganyika Territory, Cganda, Senegal, Gambia, Sierra Leone, Java.

Induced disease: In peanut, yellowing of young leaves, at first with green veins; reduction in leaf size, petiole length, and internode length, producing rosette; curling and distortion of later-formed, wholly chlorotic or chlorotically mottled leaflets. Seed formation inhibited. No abnormal proliferation of tissues.

Transmission: By grafting. By both winged and wingless individuals of the aphid, Aphis laburni Kalt. $(=A$. leguminosae Theob.) (APHIDIDAE). Not by 13 tested species of leafhoppers. Not by inoculation of expressed juice. Not through seed from diseased plants. Not through soil.

Literature : Hayes, Trop. Agr., 9, 1932, 211-217; McClintock, Science, 45, 1917, 47-48; Soyer, Publ. Inst. Nat. Étud. Agron. Congo Belge, Sér. Sci., 21, 1939, 23 pages (Rev. Appl. Mycol., 19, 1940, 386 , Abst.); Storey and Bottomley, Ann. Appl. Biol., 15, 1928, 26-45; Zimmerman, Der Pflanzer, 3, 1907, 129-133; 9, 1913, 59-63.

33. Marmor trifolii H. (loc. cil., 93) From New Latin Trifolium, generic name of red clover, from Latin trifolium, clover.

Common name: Red-clover vein-mosaic virus.

Hosts: LEGCMINOSAE-Trifolium pratense $\mathrm{L}$., red clover; Lathyrus odoratus L., sweet pea; Vicia faba L., broad bean. Experimentally, also Trifolium hybridum L., alsike clover ; T. incarnatum L., crim- 
son clover; $T$. repens $I_{\text {., }}$, white clover; Melilotus alba Desr., white sweet clover; Pisum sativum L., pea.

Insusceptible species: $L E G U M I N O$ SAE--Phaseolus vulgaris $\mathrm{I}$., bean; $P$. aureus Roxb., mung bean; Medicago satira I., alfalfa. SOL $A N A C E A E-L y c o-$ persicon csculentum Mill., tomato; Nicotiana tabacum I., tobaceo; N. glutinosa I.; N. langsdorffi Weinm.; N. rustica I.; N. sylvestris Spegaz. and Comes; Solanum tuberosum L., potato.

Geographical distribution: Enited States.

Induced disease: In red clover, yellow color along veins, but no mottling. Sometimes small yellow spots in interveinal areas. Little or no stunting. In Vicia faba, experimentally, necrotic splotches or rings sometimes at site of inoculation. Clearing of veins followed by appearance of whitish bands along the veins. Stalks discolored, purplish. Diseased plants are stunted and often die back to a point near the base of the stalk, inducing new growth from buds on the stem.

Transmission: By inoculation of expressed juice, using carborundum. $\mathrm{By}$ aphid, Macrosiphum pisi Tialt. (APHI$D I D A E$ ), without incubation period and without long retention. Not by aphids, Macrosiphum solanifolii $\Lambda$ shm. (= M. gei Koch) or Aphis rumicis Linn. ( $A P H I D I D A E)$.

Thermal inactivation: At $60^{\circ} \mathrm{C}$ in 10 minutes.

Literature: Osborn, Phytopath., 27, 1937, 1051-1058; Zaumeyer, Jour. Agr. Res., 56, 1938; 747-772; Zaumeyer and Wade, Phytopath., 27, 1937, 1009-1013.

34. Marmor pachyrhizi spec. nov. From New Latin Pachyrhizus, generic name of sincamas.

Common name : Sinc:amas-mosaic virus. Host: . LEGUMINOSAE-Pachyrhizus erosus (L.) Urb., sineamas (yam bean).

Insusceptible species: $L E G U M I N O$. SAE-Phaseolus vulgaris $\mathrm{L}$., bean.
Geographical distribution: Philippine Islands.

Induced disease : In sincamas, chlorotic mottling of foliage; in plants infected when young, dwarfing.

Transmission: By inoculation of $\mathrm{ex}$ pressed juice, in the presence of sand as abrasive. Through about 25 percent of the seeds from infected plants. Not through soil, int erlacing of roots, or casual contacts of leaves and stems. No insect vector is known.

Literature: Fajardo and Marañon, Philippine Jour. Science, 48, 1932, 129142.

35. Marmor vignae spec. nov. From $\mathrm{New}$ Latin Vigna, generic name of cowpea, from family name of an Italian botanist, Domenico Vigna.

Common name: Cowper-mosaic virus.

Hosts: LEGUMINOSAE-Vigna sinensis (L.) Endl., cowpea. Experimentally, also Phascolus lunatus L., lima bean.

Geographical distribution: United States (Arkansas, Oklahoma, Louisiana, Indiana, Georgia, Iora, Mississippi, Kansas, New Jersey).

Induced disease : In cowpea, clearing of veins followed hy chlorotic mottling, slight convex cupping of leaflets, shortcned internodes, abortion of flowers, twisting of petioles, delayed maturity. Malformation of leaves, stunting of plants, and reduction of yield more pronounced in some varicties of compea than in others.

Transmission: By inoculation of expressed juice, especially in the presence of fine carborundum powder. By aphids, Macrosiphum solanifolii Ashm., M. pisi Kalt., Aphis gossypii Glov. (APHIDI$D A E$ ) ; not by various beetles nor by the bean leafhopper, Empoasca fabae LeB. (CICADELLIDAE). Through 5 per cent of seeds from infected cowpea plants.

Thermal inactivation: At 72 to $75^{\circ} \mathrm{C}$ in 10 minutes.

Other properties: Infectious in dilutions as high as 1:1000 and after 2 days 
storage in expressed juice at room temperature, 20 to $25^{\circ} \mathrm{C}$.

Literature: Elliott, Phytopath., 11, 1921, 146-148; Gardner, Indiana Acad. Science Proc., 36, 1927, 231-247; 37, 1928, 417; McLean, Phytopath., 31, 1941, 420430; Smith, Science, 60, 1924, 268.

36. Marmor repens Johnson. (Phytopath., 32, 1942, 114.) From Latin repens, unlooked for, in reference to unexpected discovery of this virus as a constituent of a complex formerly regarded as a single virus, so-called "white-clover mosaic virus".

Common name: Pea-wilt virus.

Hosts : LEGUMINOSAE-Trifolium repens L., white clover. Experimentally, also Lathyrus odoratus L., Lens esculenta Moench.; Lupinus albus L.; Medicago lupulina L.; Melilotus alba Desr.; Phaseolus aureus Roxb., mung bean; $P$. vulgaris L., bean; Pisum sativum L., pea; Trifolium hybridum L.; T. incarnatum L.; $T$. pratense L.; Ticia faba L.; V. sativa L.; Vigna sinensis (L.) Endl., cowpea.

Insusceptible species : $C A R Y O P H Y L$ LACEAE-Stellaria media (L.) Cyrill. CHENOPODIACEAE-Beta vulgaris L.; Spinacia oleracea L. COMPOSI$T A E-C a l l i s t e p h u s$ chinensis Nees; Lactuca sativa L.; Taraxacum officinale Weber; Zinnia elegans Jacq. CRUCIFERAE-Barbarea vulgaris R. Br.; Brassica oleracea L.; Raphanus sativus L. CUCURBITACEAE-Cucumis sativus L. GRAMTNEAE-Zea mays I. LEGUMINOSAE-Glycine max Merr.; Lupinus hirsutus L.; Medicago sativa L. LILIACEAE-Lilium formosanum Stapf. PLANTAGINACEAE-Plantago lanceolata L.; P. major L. POLY GONACEAE-Rumex acetosella L. SCROPHULARIACEAE - Antirrhinum majus L. SOLANACEAE-Datura stramonium L.; Lycopersicon esculentum Mill.; Nicotiana glutinosa L.; $N$. rustica L.; N. sylvestris Spegaz. and Comes; $N$. tabacum L.; Solanum nigrum $\mathrm{L}$.
Geographical distribution: United States (Washington).

Induced disease: In white clover, systemic chlorotic mottling. In pea, experimentally, originally infected leaves wilt and die, remaining attached to the stem by their shriveled petioles; a few adjacent lower leaves may also wilt and die; in most varieties the top foliage remains green, but in two varieties, Alaska and Canada White, it mottles faintly; stems show faint grayish discoloration; plants are retarded in growth and dwarfed. If pea-mottle virus, Marmor efficiens Johnson, is also present, a severe streak disease occurs. Intracellular inclusions absent. In mung bean, experimentally, necrotic zonate local lesions. In cowpea, experimentally, brown necrotic local lesions in inoculated primary leaves, diffuse areas of bleaching in uninoculated trifoliate leaves. In bean, experimentally, mild chlorotic mottling except in three varieties that appear insusceptible (varieties Ideal Market, Iientucky Wonder, and Navy Robust).

Transmission: By inoculation of expressed juice. Not by dodder, Cuscuta campestris Yunck. (CONVOLVULACEAE). Not by pea aphid, Macrosiphum pisi Kalt. (APHIDIDAE). No insect vector is known.

Thermal inactivation: At 58 to $60^{\circ} \mathrm{C}$ in 10 minutes.

Filterability : Passes Berkefeld W filter candle.

Other properties : Infectious in dilution of $1: 100,000$. Not inactivated by storage in juice of infected plants at about $25^{\circ} \mathrm{C}$ for one month or by similar storage in dried tissues of infected pea plants.

Literature: Johnson, Phytopath., 32, 1942, 103-116; Pierce, Jour. Agr. Res., 51, 1935, 1017-1039.

37. Marmor fastidiens spec. nov. From Latin fastidiens, disdaining, in reference to slight irregularities in the reported host ranges of constituent strains and failure of this virus to infect certain varieties of the pea although it 
may utilize many other varieties of this species as host.

Common name: Alsike-clover mosaic virus.

Hosts: LEGUMINOSAE-Trifolium hybridum L., alsike clover; Pisum sativum L., pea (except the varieties Horal, Perfection, and Surprise). Experimentally, also Crotalaria striata DC:; C. retusa $\mathrm{L}$.; and $C$. spectabilis Roth the two last-named species are reported to be insusceptible to the type strain of the virus, but susceptible to one or more of the other tested strains); Lupinus albus L.; L. angustifolius L.; Medicago sative L.; Melilotus alba Desr.; Phaseolus vulgaris L., bean ; Trifolium incarnatum L. ; T. pratense L.; Vicia faba $\mathrm{L}$.

Insusceptible species: $S O L A N A$ CEAE-Datura stramonium L.; Nicotiana glauca Graham; N. glutinosa L.; N. tabacum L.; Petunia hybrida Vilm. LEGUMINOSAE-Phascolus aureus Roxb., mung bean; P. lunatus L., sieva bean; Soja max (L.) Piper, soybean; Trifolium repens L., white clover; Ticia sativa $\mathrm{L}$,, spring vetch.

Induced disease: In pea and bean, experimentally, systemic chlorotic mottling; some isolates kill inoculated leaves and even cause death of infected plants.

Transmission: By inoculation with expressed juice, at dilutions to $1: 6000$ or 1: 8000 . No insect vector is known.

Thermal inactivation: At 60 to $65^{\circ} \mathrm{C}$ in 10 minutes; one strain at lower temperature, 54 to $58^{\circ} \mathrm{C}$.

Strains: Several strains have been distinguished by the severity of their effects on host plants. These may be characterized as follows: var. fastidiens, var. nov., type variety, the first of the strains to be described (originally known as alsike clover mosaic virus 1 ), induces mild disease in pea, does not infect red clover; var. mite, var. nov., lescribed as nea mosaic virus 4 , indures mild symptoms on pea, infects red clover; var. reprimens, var. nov., described as pea mosaic virus 5 , stunts peas severely; var. denudans, var. nov., described as alsike clover mosaic virus 2, defoliates pea plants. Varietal names from New Latin fastidiens, epithet of the species, and from Lat in milis, mild; reprimere, to restrain; and denudare, to denude; all three in reference to induced symptoms.

Literature: Wade and Zaumeyer, Phytopath., 28, 1938, 505-511; Zaumeyer, Jour. Agr. Res., 60, 1940, 433-452.

38. Marmor iners spec. nov. From Lit in iners, sluggish or inert, in reference to failure of the virus to spread systemically in certain of its hosts.

('ommon name: Pea-streak virus.

Hosts: LEGL II IOSAE-Pisum sutimm L., pea. Experimentally, also Galega officinalis L., goat's rue; Glycine soja Sieb. and Zuce., soya bean; Lathyrus odoratus L., sweet pea; Lotus hispidus Desf.; Lupinus angustifolius L., blue lupin; L. luteus L., yellow lupin; $L$. mulabilis Sweet; Phaseolus vulgaris L., bean; Trifolium arvense L., haresfoot trefoil; T. cernum Brot., nodding clover; $T$. fragiferum I., strawberry clover; $T$. glomeratum L., cluster clover; T. hybridum $\mathrm{L}$., alsike clover; $T$. pratense $\mathbf{L}$., red clover; T. repens $\mathrm{L}$., white clover; Vicia villosa Roth., hairy vetch. $C U C U R$ BITACEAE-C'ncumis melo L., rock melon; C. satims L., cucumber; Cucurbita pepo L., marrow.

Insusceptible species: $C H E N O P O D I$ ACEAE-Spinacia oleracea L., spinach; Beta vulgaris L., beet. COMPOSITAE - Calendula officinalis L., calendula; Lactuca sative $\mathrm{I}$., lettuce; Zinnia elegans Jacq., zinnia. CRUCIFERAE-Brassica napus L., swede; B. oleracea L., cabhage; $B$. rapa L., turnip; Malthiola incana R. Br., stock; Raphanus sativus I., radish; Sisymbrium officinale (L.) Scop., hedge mustard. LEGUMINOSAEArachis hypogaea L., peanut; Lathyrus latifolius I.., perennial sweet pea; $L$. pubescens Hook. and Arn., Argentine sweet pea; Lotus corniculatus L. ; Lupinus arborens sims, tree lupin; Medicago arabica Huds.; M. sativa L., lucerne (alfalfa); Phaseolus multiflorus Willd., run- 
ner bean; Trifolium striatum L., striated clover; T. subterraneum L., subterranean clover; Vicia faba L., broad bean. PLANTAGINACEAE - Plantago lanceolata L., plantain. SCROPHU LARIACEAE-Antirrhinum majus L. SOLANACEAE-Cyphomandra belacea Sendt., tree tomato; Datura stramonium I., Jimson weed; Nicotiana glauca $\mathrm{R}$. (irah.; N. rustica L., Turkestan tobacco; 1. tabacum L., tobacco; Physalis peruviana I., Cape gooseberry; Solanum nigrum L., black nightshade. TROPAE OLACEAE-Tropaeolum majus L., nasturtiun. CWBELLIFERAE-Apium graveolens L., celery.

Geographical distribution: New Zealand.

Induced disease: In the pea, stunting, wilting of young leaves, purple or purplebrown spotting on young leaves, dark streak on stem. Near tip, stem may die. Stem becomes brittle, tip bent to one side. Pods may remain flat and turn dark purple or purple-brown, or if already formed may show purple or purple-brown markings. Older leaves turn yellow, then brown and shrivelled. Infected plants usually die within two or three weeks. In inoculated plants small brown primary lesions, rapidly increasing in size especially along veins, eventually involve the whole leaf; petiole and stem streak follows. Among garden peas, the varieties Pride of the Market, Little Marvel, Wm. Massey and Autocrat are little affected; among field peas, the varieties Unica and White Irory are equally resistant. In cucumber, experimentally, numerous brown, necrotic local lesions, each with light colored center and surrounding light-yellow halo. In bean, experimentally, local and systemic' necrosis, stem streak, death of plant.

Transmission: $\mathrm{By}$ inoculation of expressed juice, best with an abrasive powder such as fine sand. Not by Myzus persicae (Sulz.), Macrosiphum solani (APHIDIDAE), nor Thrips tabaci Lind. (THRIPIDAE). No insect vector is known.
Thermal inactivation: At 78 to $80^{\circ} \mathrm{C}$ in 10 minutes.

Filterability : Passes Mandler filters of preliminary, regular, and fine grades.

Other properties: Dilution end point $1: 10^{5}$. Not inactivated at room temperature in 41 days.

Literature: Chamberlain, New Zealand Jour. Science and Technology, 20 , $1939,365 \mathrm{~A}-381 \mathrm{~A}$.

39. Marmor efficiens Johnson. (Phytopath., 32, 1942, 114.) From Latin efficiens, effective, in reference to ability of this virus to cause mottling in all tested varieties of pea in contrast with inability of pea-wilt virus, a second constituent of the complex earlier known as "whiteclover mosaic virus," to produce such chlorotic symptoms in tested varieties other than Alaska and Canada White.

Common name: Pea-mottle virus.

Hosts: LEGL MINOSAE-Trifolium repens L., white clover; Pisum sativum L., pea. Experimentally, also $C A R Y O$ PHYLLACEAE-Stellaria media (L.) Cyrill. CHENOPODIACEAE-SPinacia oleracea L., spinach. CUCURBITACEAE-Cucumis sativus L. LEGLUINOSAE-Lathyrus odoratus L.; Lens esculenta Moench.; Lupinus albus L.; L. hirsutus L.; Medicago lupulina L. ; I. sativa L., alfalfa (lucerne); Melilotus alba Desr.; Phaseolus aureus Roxb.; P. vulgaris L., bean; Trifolium hybridum L.; T. incarnatum L.; $T$. pratense L.; Vicia faba L.; V. sativa L. SCROPHL'LARIAC'EAE-Antirrhinum majus L.

Insusceptible species: ( $H E$ VOPODIAC'EAE-Beta v'ulgaris L., sugar beet. COMPOSITAE-Callistephus chinensis Nees; Lactuca sativa L.; Taraxacum officinale Weber; Zinnia elegans Jacq. CRICIFERAE-Barbarea vulgaris R. $\mathrm{Br}$.; Brassica oleracea L.; Raphanus sativus L. GRAMINEAE-Zea mays L. LEGL'MINOSAE-Glycine max Merr.; Vigna sinensis (L.) Endl. LILIACEAE-Lilium formosanum Stapf. PLANTAGINACEAE - Plantago 
lanceolata L.; $P$. major L. POLYGON ACEAE-Rumex acetosella L. SOLAN. ACEAE-Datura stramonium L.; Lycopersicon esculentum Mill.; Nicotiana glutinosa L. ; N. rustica L.; N. sylvestris Spegaz. and Comes; N. tabacum L.; Solanum nigrum $\mathrm{L}$.

Geographical distribution: Enited States (Washington).

Induced disease: Experimentally, in pea, developing leaves late in opening; clearing of veins, chlorotic spotting, stunting, chlorotic mottling; stipules mottled; stems, pods, and seeds appear normal. If pea-wilt virus (Marmor repens Johnson) is also present, a severe streak disease occurs. Intracellular inclusions absent. In bean, light yellow spots and clearing of veins. In spinach, severe chlorotic mottling, dwarfing. In alfalfa, streaks of yellowing along veins, chlorotic mottling.

Transmission: By inoculation of expressed juice. By dodder, Cuscuta campestris Yunck. (CONVOLVULACEAE). Not by pea aphid, Macrosiphum pisi Kalt. (APHIDIDAE). No insect veetor is known.

Thermal inactivation: At 60 to $62^{\circ} \mathrm{C}$ in 10 minutes.

Filterability : Passes Berkefeld W filter candle.

Other properties: Infectious in dilution of $1: 10,000$ and after storage in expressed juice or dried tissues for one month at about $25^{\circ} \mathrm{C}$.

Literature: Johnson, Phytopath., 32, 1942, 103-116; Johnson and Jones, Jour. Agr. Res., 54, 1937, 629-638; Pierce, ibid., 51, 1935, 1017-1039; Zaumeyer and Wade, ibid., 51, 1935, 715-719.

40. Marmor tritici H. (loc. cit., 61). From Latin triticum, wheat.

Common names: Wheat-mosaic virus, wheat-rosette virus.

Hosts: GRA.MINEAE-Triticum aestivum L., wheat; Secale cereale L., rye. Experimentally, also all tested species of the tribe Hordeae; Triticum compactum Host; T. turgidum L.; T. durum Desf.;
T. dicoccum Schrank; T. spelta L.; T. polonicum L.; T. monococcum L., Hordeum vulgare L., barley.

Insusceptible species : GRAMINEAE - Bromus inermis Leyss., awnless bromegrass (of the tribe Festuceae).

Geographical distribution: United States, Japan.

Induced disease: In wheat, systemic chlorotic mottling, with dwarfing in some varieties; vacuolate, rounded intracellular bodies in diseased cells, usually close to nucleus. Some selections of Harvest Queen wheat are resistant.

Transmission: Through soil; remains infectious in soil 6 or more years. By inoculation of expres:sed juice (needle punctures in stem). Not through seeds or stubble of diseased plants. No insect vector is known.

Thermal inactivation: Contaminated soil becomes incapable of infecting wheat plants if heated for 10 minutes at $60^{\circ} \mathrm{C}$ though not if heated for the same length of time at $50^{\circ} \mathrm{C}$.

Literature: Johnson, Science, 95, 1942, 610; MeKinney, Jour. Agr. Res., 23, 1923, 771-800; U. S. Dept. Agr., Bull. 1361, 1925; U. S. Dept. Agr., Circ. 412, 1937 ; Jour. Agx. Res., 40, 1930, 547-556; MeKinney et al., ibid., 26, 1923, 605-603; Wada and Hukano, Agr. and Hort., 9, 1934, 1778-1790 (Rev. Appl. Mycol., 14, 1935, 61S, Abst.); Jour. Imp. Agr. Exp. Sta., 3, 1937, 93-12S (Rev. Appl. Mycol., 16, 1937, 665, Abst.); Webb, Jour. Agr. Res., 35, 1927, 587-614; 36, 1928, 53-75.

41. Marmor graminis McKinney. (Jour. Washington Acad. Sci., 34, 1944, 325.) From Latin gramen, grass.

Common name: Brome-grass mosaic virus.

Hosts : GRAMINEAE-Bromus inermis Leyss., awnless brome-grass. Experimentally, also Triticum aestivum L., wheat; Avena sativa L., oat.

Geographical distribution: United States (Kansas).

Induced disease: In awnless bromegr:ss, systemic chlorotic mottling of the 
type commonly called yellow mosaic berause of the distinctly yellow color of the chlorotic areas in affected leaves.

Transmission: $\mathrm{By}$ inoculation of expressed juice or of aqueous suspensions of dried diseased tissues; not inactivated by drying in diseased tissues for at least 51 days. No insect vector is known.

Literature: McKinney et al., Phytopath., 32, 1942, 331 .

42. Marmor abaca H. (loc. cit., 63). From rommon name of host plant.

Common name: Abacá bunchy-top virus.

Host : MCSACEAE-Musa. textilis Née, abacá (Manila hemp plant).

Insusceptible species : MLSACEAEMusa sapientum I. vars. cinerea (Blaneo) Teodoro, compressa (Blanco) Teodoro, lacatan (Blanco) Teodoro, and suaveolens (Blanco) Teodoro; M. cavendishii Lamb.

Geographical distribution: Philippine Islands.

Induced disease: In abacá (MIanila hemp plant), chlorotic lines and spots along veins of young leaves, followed by growth of distorted leaves, successively shorter, narrower, stiffer, and mor: curled along their margins. The green areas of mottled leaves, petioles, and leaf sheaths are darker than normal. Newly formed diseased leaves unfurl early, but are short, producing the bunchy top that is referred to in the common name of the disease.

Transmission: By the aphid, Pentalonia nigroneriosa Coc. (APIIIDI$D A E$ ), vector also of the apparently distinct banana bunchy-top virus of Australia. The incubation period of abacá bunchy-top virus in this aphid is bet reen 24 and 48 hours in length. The progeny of viruliferous aphids do not receive the virus directly, but must feed on diseased plants before they can infect healthy abacá. Transmission by inoculation of expressed juice has not been demonstrated. No soil transmission.

Literature: Oefemin, Am. Jour. Bot.,
17, 1930, 1-18; Philippine Agriculturist, 22, $1934,567-581$.

43. Marmor passiflorae H. (loc. cit., 77). From New Lat in Passiflora, generic name of passion fruit.

Common name: Passion-fruit woodiness virus.

Hosts: PASSIFLORACEAE-Passiflora edutis Sims, passion fruit; $P$. coerulea L. Experimentally, also $P$. alba Link and Otto.

Insusceptible species: $S O L A N A$ CEAE-Datura stramonium L.; Lycopersicon esculentum Mill., tomato; Nicotiana glutinosa L.; N. tabacum L., tobacco.

Geographical distribution: Australia (New South Wales, Queensland, Victoria), Kienya.

Induced disease: In passion fruit, growth checked; leaves puckered, slightly chlorotic or obscurely mottled, curled, twisted, deformed. Clearing of veins has been observed. C'olor of stems darker green than normal in some places. Fruits short or deformed, discolored, surface sometimes roughened by cracks; so hard as not to be cut through readily. Pericarp or rind of fruit abnormally thick. Pulp deficient, color deepened. At temperatures below $80^{\circ} \mathrm{F}$, some abscission of young chlorotic leaves; above $85^{\circ} \mathrm{F}$, masking of the disease in most plants.

Transmission: By inserting cotton in stem wound after soaking it in expressed juice of diseased plant. By aphids, $M y$ zus persicae (Sulz.), Macrosiphum solanifolii Ashm., and two dark-colored species of the genus Aphis ( $A P H I D I D A E$ ).

Literature: Cobb, Agr. Gaz. New South Wales, 12, 1901, 407-418; Noble, Jour. and Proc. Roy. Soc. New South Wales, 62, 1928, 79-98; Noble and Noble, ibid., 72, 1939, 293-317; Simmonds, Queensland Agr. Jour., 45, 1936, 322-330.

44. Marmor flaccumfaciens $H$. (loc. cit., 73). From Lat in flaccus, flabby, and facere, to make.

Common names: Rose-wilt virus, rose dieback virus. 
Hosts: ROSACEAE-Rosa hybrids roses.

Geographical distribution: Australia, especially Victoria; New Zealand; possibly Italy.

Induced disease : In rose, leaflets crowd ed, brittle, recurved. Defoliation progresses from tip to base of plant. Tips of branches discolor and die back an inch or two. Stem darkens at base. Buds remain green and begin development, but growth is soon checked by necrosis at tips. Plant may recover temporarily, but not permanently.

Transmission: By inoculation of expressed juice (needle-puncture and scratch methods). No insect vector is known.

Filterability : Passes Seitz filter (Seit $z$ EK Schichten type, size 6).

Literature: Gigante, Boll. Siaz. Pat. Veg. Roma, n. s. 16, 1936, 76-94; Grieve, Austral, Jour. Exp. Biol, and Med. Science, 8, 1931, 107-121; Jour. Dept. Agr. Victoria, 1932 and 1933, pages 30-32.

45. Marmor rosae H. (loc. cit., 74). From Latin rosa, rose.

Common name: Rose-mosaic virus.

Hosts: ROSACEAE-Rosa rugosa Thunb.; R. chinensis Jacq. var. manetti Dipp.; $R$. multiflora Thunb.; $R$. odorata Sweet, tea rose; $R$. gymnocarpa; Rubus parviflorus Nutt.

Geographical distribution: United States, England, Bulgaria, Brazil.

Induced disease : In Rosa rugosa and $R$. chinensis var. manetti, systemic chlorotic: mottling.

Transmission: By budding and other forms of graftage. Not by inoculation of expressed juice. No insect vector is known.

Literature: Baker and Thomas, Phyto path., 32, 1942, 321-326; Brierley. Phytopath., 25, 1935, 8 (Abst.); Brierley and Smith, Am. Nurseryman, 72, 1!40, 5-8; Jour. Agr. Res., 61, 1940, 625-660; Lramer, Revista de Agricultura, 15, 1940, 301-311; O Biologico, 6, 1940, 365-368; McWhorter, U. S. Dept. Agr., Plant Dis. Rep., 15,
1931, 1-3; Milbrath, West. Florist, 13, 1930, 29-30; Nelson, Phytopath., 20, 1930, 130 (Abst.); Newton, Rep. Domin. Bot., 1930, Div. Bot., Canad. Dept. Agr., 1931, 23; Thomas and Massey, Hilgardia, 12 , 1939, 645-663; Vibert, Jour. Soc. Imp. et ('ent. Hort., 9, 1863, 14t-145; White, Phytopath., 22, 1932, 53-69; 24, 1934, 1124-1125.

46. Marmor veneniferum H. (loc. cit., 75). From Lat in venenifer, poisonous, in reference to occasional killing of tissues near inserted bud in graft transmission.

Common name: Rose-streak virus.

Hosts: ROSACEAE-Rosa multiflora Thunb.; R. vdorata Sweet; Rosa hybrids.

Geographical distribution: Eastern United States.

Induced disease : In various rose species and hybrids, brownish or reddish ring and veinbanding patterns on leaves, and ring patterns on stems. Sometimes necrotic areas near inserted bud, causing girdling of stem and wilting of foliage.

Transmission: By grafting. Not by inoculation of expressed juice. No insect vector is known.

Literature: Brierley, Phytopath., 25, 1935, 7-8 (Abst.); Brierley and Smith, Jour. Agr. Res., 61, 1940, 625-660.

47. Marmor mali H. (loc. cit., 75). From Latin malus, apple tree.

Common name: Apple-mosaic virus.

Hosts: ROSACEAE-Pyrus malus L., apple. Experimentally, also Cotoneaster harroviana; Eriobotrya japonica Lindl., loquat; Photinia arbutifolia Lindl., toyon; Rosa sp., rose; Sorbus pallescens.

Insusceptible species: ROSACEAEAmelanchier alnifolia Nutt.; Crataegus douglasii Lindl.; Pyrus communis L., pear.

Geographical distribution: United States, Australia, Bulgaria, British Isles.

Indured disease: In apple, clearing of veins and systemic chlorotic spotting. The chlorotic areas sometimes become necrotic during months of intense sunlight.

Transmission: By grafting. No insect 
vector is known. Transmission by inoculation of expressed juice has not been demonstrated.

Thermal inactivation: Not demonstrated. Virus in stem tissues withstands at least $50^{\circ}$ C' for as much as 60 minutes without being inactivated.

Literature: Blodgett, Phytopath., 28, 1938, 937-938; Bradford and Joley, Jour., Agr. Res., 46, 1933, 901-908; Christofi, Phytopath. Zeitschr., 7, 1934, 521-536; 8, 1935, 285-296; Thomas, Hilgardia, 10, $1937,581-58 s$.

48. Marmor fragariae H. (loc. cit., 7s). From New Latin Fragaria, generic name of straberry, from Latin fraga, strawberries.

Common name: Strawberry-crinkle virus.

Hosts: $\quad R O S A C E A E-F r a g a r i a ~ h y-$ brids, cultivated strawberries. Experimentally, also Fragaria vesca L., woodland strawberry.

Geographical distribution: Inited States, England.

Induced disease: In cultivated strawberry, crinkling and chlorosis of leaves. At first, minute chlorotic flecks appear in young leaves. These flecks enlarge, and small necrotic spots may appear in their centers. Vein-clearing appears frequently. Affected foliage lighter and less uniformly green than normal. The variety Royal Sovereign may appear normal through carrying this virus.

Transmission: By aphid, Myzus fragaefolii Ckll. (= Capitophorus fragariae Theob.) (APHIDIDAE). By grafting. Not by inoculation of expressed juice.

Literature: Harris, Ann. Rept. East Malling Res. Sta. for 1936, 1937, 201-211, 212-221; ibid., for 1937, 1938, 201-202; Harris and Hildebrand, Canad. Jour. Res., C , 15, 1937, 252-280; Ogilvie et al., Ann. Rept. Long Ashton Res. Sta. for 1933, 1934, 96-97; Vaughan, Phytopath., 23, 1933, 738-740; Zeller, Oregon Agr. Exp. Sta., Sta. Bull. 319, 1933; Zeller and Vaughan, Phytopath., 22, 1932, 709-713.
49. Marmor marginans H. (loc. cit., 79). From Latin marginare, to provide with a margin.

Common name: strawberry yellowedge virus.

Hosts: ROSACEAE-Fragaria hybrids, strawberries; Fragaria californica C. and S.; F. chiloensis Duch. (symptomless). Experimentally, also Fragaria vesca L.; $F$. virginiana Duch. (some clones appear to be immune to infection by runner inarching).

Geographical distribution: United States, England, France, New Zealand.

Induced disease: In strawberry, plant appears flat with outer zone of leaves more or less normal, central leaves dwarfed, vellow-edged, deficient in red pigmentation. The variety Premier may earry this virus without showing any obvious manifestation of disease.

Transmission: By aphid, Myzus fragaefolii Ckll. (APIIIDIDAE). By grafting. Not by inoculation of expressed juice. Not through seeds from diseased plants.

Literature : Chamberlain, New Zealand Jour. Agr., 49, 1934, 226-231 ; Harris, Jour. Pom. and Hort. Science, 11, 1933, 56-76; Harris and Hildebrand, Canad. Jour. Res., C , 15, 1937, 252-280; Hildebrand, ibid., C, 19, 1941, 225-233; Plakidas, Phytopath., 16, 1926, 423- $\$ 26$; Jour. Agr. Res., 35, 1927, 1057-1090.

50. Marmor rubi H. (Holmes, loc. cit., s0; Poecile rubi McKinney, Jour. Washington Acad. Science, 34, 1944, 148.) From Lat in rubus, bramble bush.

Common name: Red-raspberry mosaic virus.

Hosts: ROSACEAE-Rubus idaeus L., red raspberry; $R$. occidentalis L., black raspberry.

Geographical distribution: United States.

Induced disease : In red raspberry, systemic chlorotic mottling, masked at high temperatures of summer. Foliage development delayed in spring. In some varieties, leaf petioles and cane tips die, 
canes remain short and become rosetted.

Transmission: By aphids, principally Amphorophora rubi Kalt., but also $A$. rubicola Oestl. and A. sensoriata Mason (APHIDIDAE). Not by inoculation of expressed juice.

Literature: Bennett, Michignu Agr. Exp. Sta., Techn. Bull. 80, 1927; 125, 1932; Cooley, New York Agr. Exp. Sta. (Geneva), Bull. 675, 1936; Harris, Jour. Pom. and Hort. Science, 11, 1933, 237-255; 17, 1940, 318-343; Rankin, New York Agr. Exp. Sta., Geneva, Bull. 543, 1927 ; New York Agr. Exp. Sta., Geneva, Tech. Bull. 175, 1931.

51. Marmor persicae H. (Holmes, loc. cit., 81; Flavimacula persicae MeKinney, Jour. Washington Acad. Science, 34, 1914, 149.) From New Latin Persica, former generic name of peach.

Common name: Peach-mosaic virus.

Hosts: ROSACEAE-Prunus persica (L.) Batsch, peach and nectarine, all tested varieties. Experimentally, also $P$. armeniaca L., apricot; $P$. communis Fritsch, almond; $P$. domestica L., plum and prune.

Insusceptible species: Attempts to infect sweet and sour cherries have thus far failed.

Geographical distribution: United States (Colorado, California, Utah, Oklahoma, Texas, New Mexico, Arizona).

Induced disease : In peach, short internodes in spring growth, sometimes breaking in flower pattern, chlorotic mottling and distortion of foliage carly in season, masking of leaf symptoms or excision of affected areas of leaf lamina in midsummer; fruit small, irregular in shape, unsalable. Some peach varieties are less damaged than others, but all are thought to be equally susceptible to infection, and equally important as reservoirs of virus when infected. In almond, experimentally, symptomless infections; symptoms appear in some apricot and plum varieties when experimentally infected, not in others.

Transmission: By budding and other methods of grafting. Not by inoculation of expressed juice. Not through soil. No insect vector is known. Not through pollen or seed from diseased plants.

Thermal inactivation: Not demonstrated; virus not inactivated by temperatures effective in inactivating peachyellows virus.

Literature : Bodine, Colorado Agr. Exp. Sta., Bull. 421, 1936; Bodine and Durrell, Phytopath., 31, 1941, 322-333; Cation, ibid., 2t, 1934, 1380-1381; Christoff, Phytopath. Ztschr., 11, 1938, 360-422; Cochran, California Cultivator, 87, 1940, 164-165; Cochran and Hutchins, Phytopath., 28, 1938, 890-892; Hutchins, Science, 76, 1932, 123; Hutehins et al., U. S. Dept. Agr., Circ. 427, 1937, 48 pp.; Kunkel, Am. Jour. Bot., 23, 1936, 683686; Phytopath., 28, 1938, 491-497; Thomas and Rawlins, Hilgardia, 12, 1939, 623-644; Valleau, Kentucky Agr. Exp. Sta., Bull. 327, 1932, 89-103.

52. Marmor astri H. (loc. cit., 83). From Latin astrum, star.

Common name: Peach asteroid-spot virus.

Host : ROSACEAE-Prunus persica (L.) Batsch, peach.

Geographical distribution: California. Induced disease: In peach, discrete chlorotic lesions spreading along veins, forming star-like spots; developing leaves normal in appearance, becoming affected as they mature. Some chlorophyll retained in lesions as leaves turn yellow. Affected leaves shed early.

Transmission: By grafting. Not by inoculation of expressed juice. No insect vector is known.

Literature : Cochran and Smith, Phytopith., 28, 1938, 278-281.

53. Marmor rubiginosum Reeves. (Phytopath., 30, 1940, 789.) From Latin rubiginosus, rusty.

Common name: Cherry rusty-mottle virus.

Host : ROSACEAE-Prunus avium L., sweet cherry. 
Geographical distribution: United States (Washington).

Induced disease: In sweet cherry, chlorotic mottling 4 to 5 weeks after full bloom, first on small basal leaves, later on all leaves. The older affected leaves develop autumnal colors and abseiss, 30 to 70 per cent of the foliage being lost. The remaining foliage appears somewhat wilted, shows increased mottling, chlorotic spots, and areas becoming yellowish brown, appearing rusty. Blossoms normal. Fruits smaller than normal, in sipid, not misshapen. Growth rate of tree reduced slightly.

Transmission: By grafting. Not by inoculation of expressed juice. No insect vector is known.

Literature: Reeves, Phytopath., 30, 1940, 789 (Abst.); Jour. Agr. Res., 62, 1941, 555-572 (see 566-567).

54. Marmor cerasi Zeller and Evans. (Phytopath., 31, 1941,467.) From Latin cerasus, cherry tree; originally spelled cerasae, by error.

Common name: Cherry mottle-leaf virus.

Hosts: ROSACEAE-Prunus avium L., sweet cherry; P. cmarginata (Dougl.) Walp., wild cherry. Experimentally, also $P$. cerasus $\mathrm{L}$. (tolerant) and $P$. mahaleb L. (tolerant).

Geographical distribution: United States (Washington, Oregon, Idaho, California) and Canada (British Columbia).

Induced disease: In sweet cherry, chlorotic mottling; leaves puckered, wrinkled, distorted, not perforated. Blossoms not affected. Fruit small, hard, insipid, uneven or delayed in ripening. Crop reduced. Branches shortened, tree eventually stunted.

Transmission: By budding. No insect vector is known. Not by the black cherry aphid, Myzus cerasi (F.) ( $A P H I$ $D I D A E)$. Not by inoculation of expressed juice.

Thermal inactivation: Not demonstrated; not at $46^{\circ} \mathrm{C}$ in 60 minutes nor at $49^{\circ} \mathrm{C}$ in 10 minutes in bud sticks.
Literature : Reeves, Washington State Hort. Assoe. Proc., 31, 1935, 85-89; Jour. Agr. Res., 62, 1941, 555-572; Zeller, Oregon State Hort. Soc. Report, 26, 1934, 92-95; Phytopath., 31, 1941, 463-467.

\section{Marmor lineopictum Cation} (Phytopath., 31, 1941, 1009.) From Latin linea, line, and pictus, ornamented.

Common names: Prunus line-pattern virus, peach line-pattern virus.

Hosts : ROSACEAE-Prunus salicina Lindl., Japanese plum; P. mahaleb L., Mahaleb cherry; $P$. persica (L.) Batsch, peach (= Amygdalus persica L.).

Geographical distribution: United States (Kentucky, Michigan, California, Ohio; perhaps widely distributed).

Induced disease: In peach and $\mathrm{Ma}$ laleb cherry, light-colored line patterns or faint chlorotic mottling, tending to become masked as leaf becomes old. In peach, affected foliage sometimes less glossy than normal. In Prunus salicina, no disease manifestations usually ; rarely, chlorotic mottling on a few leaves.

Transmission: By grafting. No insect vector is known.

Literature : Berkeley, Div. of Bot. and Plant Path., Science Service, Dominion Dept. Agr., Ottawa, Canada, Publ. 679, 1941 ; Cation, Phytopath., 31, 1941, 10041010; Thomas and Rawlins, Hilgardia, 12, 1939, 623-64t ; Valleau, Kentucky Agr. Exp. Sta., Res. Bull. 327, 1932, 89-103.

56. Marmor pallidolimbatus Zeller and Milbrath. (In Handbook of Virus Diseases of Stone Fruits in North America, Michigan Agr. Exp. Sta., Miscell. Publ., May, 1942, 50; Phytopath., 32, 1942, 635.) From Latin pallidus, pale, and limbatus, bordered.

Common name: Cherry banded-chlorosis virus.

Hosts: ROSACEAE-Prunus serrulata Lindl., flowering cherry; $P$. avium L., Mazzard cherry.

Geographical distribution: United States (Pacific Northwest).

Induced disease: In flowering cherry, 
chlorotic bands surrounding discolored areas on leaves. In Mazzard cherry, dwarfing of whole plant, chlorotic bands on leaves.

Transmission: By budding, even in the absence of survival of inserted buds.

57. Marmor nerviclarens Zeller and Evans. (Phytopath., 31, 1941, 467.) From Latin nervus, sinew or nerve, and clarere, to shine.

Common name: Cherry vein-clearing virus.

Hosts: ROSACEAE-Prunus avium L., sweet cherry. Perhaps also P. serrulata Lindl. and $P$. domestica L., on which symptoms similar to those induced by this virus have been observed.

Geographical distribution: United States (Oregon, Washington).

Induced disease: In sweet cherry, clearing of veins throughout each leaf or only in localized areas. Margins of leaves irregular, most indented where clearing of veins is most conspicuous. Elongated, elliptic, or slot-like perforations occur in some leaves. Affected leaves usually narrow. Enations occur as small blistered proliferations on lower side of main veins. Upper leaf surface silvery by reflected light. By midsummer, leaves droop and appear somewhat wilted; they may fold along the midvein. Internodes short; increased number of buds, spurs, or short branches at nodes; rosetting more pronounced on some branches than on others, mostly at end of year-old wood. In advanced disease, fruits pointed, small, flattened on suture side with swollen ridge along suture. Blossoms abnormally abundant, crop of fruit reduced or wanting.

Transmission: By grafting. Not demonstrated by inoculation of expressed juice. No insect vector is known.

58. Marmor viticola H. (loc. cit., 83). From Latin vitis, vine, and -cola, inhabitant of.

Common name: Vine-mosaic virus.

Host : VITACEAE-Vitis vinifera $\mathrm{L}$., grape.
Geographical distribution: France, Italy, Bulgaria, Czechoslovakia.

Induced disease: In grape, various modifications of systemic chlorotic mottling, and red pigmentation of parts of leaves with subsequent drying and dropping out of affected spots. Leaves deformed, crimped between main veins. Growth restricted.

Transmission: By inoculation of expressed juice and by pruning.

Literature: Blattný, Vinařský obzor., 25, 1931, 4-5 (Cent. f. Bakt., II Abt., 84, 1931, 464); Ochrana Rostlin, 13, 1933, 104-105 (Rev. Appl. Mycol., 13, 1934, 421 ); Gigante, Boll. Staz. Pat. Veg. Roma, n. s. 17, 1937, 169-192 (Rev. Appl. Mycol., 17, 1938, 221); Pantanelli, Malpighia, 24, 1911, 497-523;25, 1912, 17-46; Stranak, II Congr. Intern. Path. Comp. Paris, 1931, 367-378; Ochrana Rostlin, 11, 1931, 89-98 (Rev. Appl. Mycol., 11, 1932, 280); Vielwerth, Ochrana Rostlin, 13, 1933, S3-90 (Rev. Appl. Mycol., 13, 1934, 421-422).

59. Marmor santali H. (loc. cit., 94). From New Latin, Santalum, generic name of sandal.

Common name: Sandal leaf-curl virus. Host: SANT.1LACEAE-Santalum album L., sandal.

Geographical distribution: India.

Induced disease: In sandal, leaves small, curled, wrinkled, thickened, brit tle, abscissing. Systemic chlorotic mottling. Internode length normal. Infected twigs produce both flowers and fruits.

Transmission: By ring bark-grafts. Not by inoculation of expressed juice. No insect vector is known.

Literature: Venkata Rao, Mysore Sandal Spike Invest. Comm., Bull. 3, 1933.

60. Marmor secretum Bennett. (Phytopath., 34, 1944, 88). From Latin secretus, hidden.

Common name: Dodder latent-mosaic virus.

Hosts: CONVOLVULACEAE-CUscuta californica Choisy, dodder. Experi- 
mentally, also CHENOPODIACEAEBeta vulgaris L., sugar beet; Chenopodium album L., lamb's quarters; $C$. murale L., sowbane. CONVOLVULA$C E A E$-Cuscuta campestris Yuncker; $C$. subinclusa Dur. and Hilg. CRUCIFERAE-Brassica incana (L.) F. W. Schultz, mustard (tolerant). CUCURBITACEAE-Cucumis melo L., cantaloupe. PHYTOLACCACEAE-Phytolacea americana L., pokeweed. PLANTAGINACEAE-Plantago major L., plantain. POLYGONACEAE-Fagopyrum esculentum Moench, buckwheat; Polygonum pennsylvanicum L., knotweed. PRIMULACEAE-Samolus floribundus HBK., water pimpernel. SOLANACEAE-Lycopersicon esculentum Mill., tomato; Nicotiana glauca Graham (tolerant); $N$. palmeri Gray; $N$. rustica L. (tolerant); N. tabacum L. (tolerant); Solanum tuberosum L., potato. UMBELLIFERAE-A pium graveolens L., celery.

Insusceptible species : COMPOSIT AE -Helianthus annuus L., sunflower; Lactuca sativa $\mathrm{L}$., lettuce. CRUCIFERAE - Brassica oleracea L., cabbage. POLY. GONACEAE-Eriogonum fasciculatum Benth., California buckwheat. SCROPHULARIACEAE-Verbascum thapsus L., mullein. SOLANACEAEAtropa belladonna L., belladonna.

Geographical distribution: United States (California).

Induced disease: In dodder, no symptoms. In sugar beet, experimentally, temporary systemic chlorotic spotting; occasional faded areas in leaves in subsequent chronic stage of disease. In cantaloupe, experimentally, chlorotic spotting, reduction in leaf size, death of some leaves, stunting of plant; melons small and of poor quality. In celery, experimentally, systemic chlorosis followed by dwarfing and mottling with subsequent apparent recovery.

Transmission: By dodder, Cuscuta californica, C. campestris, and C. subinclusa. By inoculation of extracted juice to some, but not to other, host plants; Phytolacca americana is readily infected by rubbing methods in the presence of a small amount of abrasive, and develops numerous necrotic primary lesions that serve for quantitative estimation of concentration of virus in inoculum. Through seeds from infected plants of dodder, Cuscuta campestris; not through seeds from diseased cantaloupe, buckwheat, or pokeweed plants. No insect vector is known.

Thermal inactivation: At 56 to $60^{\circ} \mathrm{C}$ in 10 minutes.

Filterability : Passes celite and Berkefeld $\mathrm{N}$ and $\mathrm{W}$ filters.

Other properties: Infective in dilutions to $1: 3000$. Inactivated by drying and by storage in expressed pokeweed juice, within 48 hours.

61. Marmor pelargonii spec. nov. From New Latin Pelargonium, generic name of common geranium.

Common names: Pelargonium leaf-curl virus; virus of dropsy or Kräuselkrankheit of geranium.

Host : GERANIACEAE-Pelargonium hortorum Bailey, geranium.

Induced disease: In geranium, circular or irregular chlorotic spots, sometimes stellate or dendritic, $\frac{1}{2}$ to $5 \mathrm{~mm}$ in diameter, centers becoming brown with chlorotic border; severely affected leaves become yellow and drop; spotted leaves ruffled, crinkled, malformed, small, sometimes puckered and splitting. Petioles and stems show corky, raised, necrotic streaks; tops may die. Disease most severe in spring, inconspicuous in summer.

Transmission: By grafting. Not by inoculation of expressed juice nor by use of knife to prepare cuttings for propagation. Not through seed. No insect vector is known.

Literature : Berkeley, Canad. Hort. and Home Mag., 1938, 1938, 1-4; Blattný, Ochrana Rostlin, 13, 1933, 145 (Rev. Appl. Mycol., 19, 1934, 378-379) ; Bremer, Blumen-u. Pflanzenbau, 48, 1933, 32-33 (Rev. Appl. Mycol., 12, 1933, 514); Halstead, New Jersey Agr. Exp. Sta., Rept. 14, 1893, 432-433; Jones, Washington Agr. Exp. Sta., Bull. 390, 1940; 
Laubert, Gartenwelt, 31, 1927, 391 ; Pape, ibid., 26, 1927, 329-331; 32, 1928, 116-117; Pethybridge and Smith, Gard. Chron., 92, 1932, 378-379; Schmidt, Gartenwelt, 81, 1932, 40; Seeliger, Nachrichtenbl. Deutsch. Pflanzenschutzdienst, 6, 1926, 63-64; Tuimann, Gartenwelt, 31, 1927, 375-376; Verplancke, Bul. C1. Sci. Acad. Roy. de Belgique, Ser. 5, 18, 1932, 269281 (Rev. Appl. Mycol., 11, 1932, 649$650)$.

62. Marmor angliae H. (loc. cit., 48). From Latin Anglia, England.

Common name: Potato-paracrinkle virus.

Hosts: SOLANACEAE-Solanum tuberosum L., potato. Experimentally, also Datura stramonium L., Jimson weed.

Insusceptible species: SOLANACEAE-Nicotiana tabacum L., tobacco.

Geographical distribution: England.

Induced disease: In potato, masked in all plants of the variety King Edward. Chlorotic mottling with some necrosis in the varieties Arran Victory and Arran Chief. Chlorotic mottling only in Arran Comrade, Majestic, and Great Scot potatoes. Two varieties, Sharpe's Express and Epicure, are said to be resistant.

Transmission: By grafts. Not by inoculation of expressed juice. No insect vector is known.

Literature: Dykstra, Phytopath., 26, 1936, 597-606; Salaman and Le Pelley, Proc. Roy. Soc. London, Ser. B, 106, 1930, 140-175.

63. Marmor aevi spec. nov. From Latin aevum, old age, in reference to the obvious involvement of old, but not of young, delphinium leaves.

Common name: Celery-calico virus.

Hosts: CUCURBITACEAE-Cucumis sativus L., cucumber; C. melo L., cantaloupe; Cucurbita pepo L., summer crookneck squash. RANUNCULACEAE-Delphinium chinensis; D. formosum, hardy larkspur; D. grandiflorum; $D$. parryi; D. zalil. SOLANACEAE-Lycopersicon esculentum Mill., tomato.
UMBELLIFERAE-Apium graveolens L., celery. Experimentally, also SOLANACEAE-Nicotiana tabacum L., tohaceo; Petunia hybrida Vilm., petunia. VIOLACEAE-Viola cornuta $\mathrm{L}$.

Geographical distribution: United States.

Induced disease: In celery, clearing of veins, puckering and downward cupping of younger leaves, green islands of tissue in lemon-yellow areas of outer leaves, green and yellow zigzag bands on leaflets. In delphinium, basal and middle leaves with pale-orange, amber, or lemon-yellow areas; younger leaves normal green; chlorotic ring and line patterns.

Transmission: By inoculation of expressed juice in the presence of finely powdered carborundum. By aphids: Aphis apigraveolens Essig, celery leaf aphicl; A. apii Theob., celery aphid; $A$. ferruginea-striata Essig, rusty-banded aphid; A. gossypii Glov., cotton aphid; 1. middletonii Thomas, erigeron root aphid; Myzus circumflexus (Buckt.), lily aphid; M. convolvuli (Kalt.), foxglove aphid; $M$. persicae (Sulz.), green peach aphid; Rhopalosiphum melliferum (Hotles), honeysuckle aphid (APHIDI$D A E)$.

Literature: Severin, Hilgardia, 14, 1942, 441-464; Severin and Freitag, Phytopath., 25, 1935, 891 (Abst.); Hilgardia, $11,1938,493-558$.

64. Marmor raphani spec. nov. From Latin raphanus, maish.

Common name: Radish-mosaic virus.

Hosts: CRUCIFERAE-Raphanus sativus L., radish. Experimentally, also CRUCIFERAE-Brassica oleracea L.; B. nigra (L.) Koch; B. alba (L.) Boiss; B. arvensis (L.) Ktze.; B. pe-tsai Bailey; B. juncea (I.) Coss; B. rapa L.; B. adpressa Boiss; Capsella bursa-pastoris (L.) Nedic.; Malcomia maritima R. Br.; $M$. bicornis DC. CHENOPODIACEAEChenopodium album L.; C. murale L.; Spinacia oleracea L. RANUNCULACEAE-Delphinium ajacis L. SOLAVACEAE-Nicotiana glutinosa L.; $N$. 
langsdorffi Weinm.; $N$. rustica L.; $N$. tabacum L.

Geographical distribution: United States (California).

Induced disease: In radish, systemic chlorotic spotting followed by chlorotic mottling of foliage; little or no leaf distortion; plants not stunted.

Transmission: By inoculation of $\mathrm{ex}$ pressed juice. No insect vector is known; not by the cabbage aphid, Brevicoryne brassicae (L.); the false cabbage aphid, Lipaphis pseudobrassicae (Davis); or the green peach aphid, Myzus persicac (Sulz.) ( $A P H I D I D A E$ ). Not through seeds from diseased radish plants.

Thermal inactivation: At 65 to $68^{\circ} \mathrm{C}$ in 10 minutes.

Literature: Tompkins, Jour. Agr. Res., $58,1939,119-130$.

65. Marmor primulae spec, nov. From New Latin Primula, generic name of primrose.

Common name: Primrose-mosaic virus.

Hosts: PRIMULACEAE-Primula obconica Hance. Experimentally, also $P$. malacoides Franch, and $P$. sinensis Lindl.

Insusceptible species: $B E G O N I A$ $C E A E$-Begonia semperflorens Link and Otto. BORAGINACEAE-Myosotis alpestris Schmidt. CAMPANULA CEAE-Campanula medium L. CARY OPHYLLACEAE-Dianthus barbatus L. CHENOPODIACEAE-S'pinacia oleracea L. COMPOSITAE-Bellis perennis L.; Callistephus chinensis Nees; Gerbera jamesonii Hook.; Lactuca sativa L.; Senecio cruentus DC.; Tagetes patula L. CRUCIFERAE-Brassica oleracea L. ; B. pe-tsai Bailey; B. rapa L.; Matthiola incana $\mathrm{R}$. Br.; Raphanus sativus L. CUCURBITACEAE-Cucumis sativus L.; Cucurbila pepo I. EUPHORBIACEAE-Ricinus communis L. GRAM INEAE-Zea mays L. LEGUMINOSAE-Pisum sativum L.; Vicia faba L.; Vigna sinensis (Torner) Savi. LO. BELIACEAE-Lobelia hybrida Hort. PAPAVERACEAE-Papaver orientale
L. PRIMULACEAE-Anagallis arvensis L.; Cyclamen indicum L.; Primula auricula L.; $P$. veris L. RANUNCULACEAE-Anemone coronaria L.; Delphinium cultorum Voss; Ranunculus asiaticus I. RESEDACEAE-Reseda odorata L. RUSACEAE-Geum chiloense Balb. SCROPHULARIACEAE-Antirrhinum majus L.; Pentstemon barbatus Nutt. SOLANACEAE-Capsicum frutescens L.; Datura stramonium L.; Lycopersicon esculentum Mill.; Nicotiana glutinosa I.; N. tabacum L.; Solanum tuberosum L. TROPAEOLACEAETropaeolum majus L. UMBELLIFERAE-Apium graveolens L. VERBENACEAE-Verbena hybrida Voss. VIOLACEAE-Viola tricolor $\mathrm{L}$.

Geographical distribution: United States (California).

Induced disease: In Primula obconica, chlorosis, stunting, rugosity with upward, or occasionally downward, cupping of leaves. Petioles and peduncles shortened; flowers reduced in size, broken in color (white-streaked). Leaves coarsely mottled with yellow-green, leaving green islands; tips of leaves sometimes narrowed.

Transmission: By inoculation of $\mathrm{ex}$ pressed juice, in the presence of 600-mesh powdered carborundum. Not by aphids, Myzus persicae (Sulz.) and M. circumflexus (Buckt.) (APHIDIDAE). No insect vector is known. Probably not through seeds.

Thermal inactivation: At $50^{\circ} \mathrm{C}$, not $48^{\circ} \mathrm{C}$, in 10 minutes.

Other properties: Infective after 24, not 48 , hours in vitro. Infective after $1: 10$ dilution.

Literature: Tompkins and Middleton, Jour. Agr. Res., 63, 1941, 671-679.

\section{Marmor caricae (Condit and} Horne) comb. nov. (Ficivir caricae Condit and Horne, Phytopath., 31, 1941, 563.) From Latin carica, a kind of dried fig.

Common name: Fig-mosaic virus.

Hosts : MORACEAE-Ficus carica L., 
fig; $F$. altissima Blume; F. krishna; and F. tsiela Roxb.

Geographical distribution: United States (California, Texas), England, Puerto Rico, China, New South Wales, Western Australia.

Induced disease: In fig, systemic chlorotic spotting and mottling of foliage; some severe leaf distortion. Fruits sometimes affected, bearing light circular areas, rusty spots, being deformed or dropped prematurely. Necrotic lesions on profichi of Samson caprifigs also have been attributed to action of this virus.

Transmission: By budding. No insect vector is known; mites have been suspected as possible vectors.

Literature: Condit and Horne, Phytopath., 23, 1933, 887-896; 31, 1941, 561563 ; 33, 1943, 719-723 ; Ho and Li, Lingnan Science Jour., 15, 1936, 69-70; Pittman, W. Austral. Dept. Agr. Jour., 12, 1935 196.

67. Marmor italicum (Fawcett) comb. nov. (Citrivir italicum Fawcett, Phytopath., 31, 1941, 357.) Specific epithet meaning "pertaining to Italy."

Common name: Citrus infectious-mottling virus.

Host : RUTACEAE-Citrus aurantium L., sour orange.

Geographical distribution: Italy.

Induced disease: In sour orange, white, pale green, or yellow irregular areas in leaves, leaving narrow green bands along midrib; leaves blistered and distorted.

Transmission: The aphid, Toxoptera aurantii (Phytopath., 24, 1934, 661), has been suspected as vector.
Literature: Fawcett, Phytopath., 31, 1941, 356-357 ; Petri, Bol. Staz. Pat. Veg. Roma, n. s. 11, 1931, 105-114.

Note: Several additional species were described too late for complete systematic treatment here. They are plain's wheat mosaic virus, Marmor campestre McKinney (Jour. Washington Acad. Sci., $34,1944,324$ ) with varieties typicum MeKinney and galbinum McKinney, respectively causing light-green mosaic and scvere yellow mosaic of wheat in Kansts; wheat streak-mosaic virus, Marmor virgatum McKinney (ibid., 34, 1944, 324) with varieties typicum MeKinney and viride McKinney (ibid., 34, 1944, 325 ), respectively causing yellow streakmosaic and green streak-mosaic of wheat in Kansas; Agropyron-mosaic virus, Marmor agropyri NoKinney (ibid., 34, 1944,326 ), with varieties typicum Mckinney and flavum McKinney, respectively causing green-mosaic mottling and ycllow-mosaic mottling in the grass Agropyron repens (I..) Beauv. in Virginia; also a virus, Flavimacula ipomeae Doolittle and Harter (Phytopath., 35, 1945, 703), (causing feathery mottle of sweet potato in Maryland [see Marmor persicae for treatment of a virus that was assigned as type of Flavimacula McKinney (Jour. Washington Acad. Sci., $34,1944,149)$, a genus originally differcntiated from Warmor as containing viruses not yet inoculable save by tissue union; a natural group of viruses may be represented but their characteristics and affiliations seem not yet clear].

\section{Genus II. Acrogenus Holmes.}

$$
\text { (Loc. cit., 110.) }
$$

Viruses of the Spindle-Tuber Group, inducing diseases characterized by abnormal growth habit of host plants without chlorotic or necrotic spotting, systemic chlorosis, witches'-broom formation, or production of galls. Generic name from Greek, meaning point-or peak-producing, in reference to shape of potatoes affected by potato spindletuber virus.

The type species is Acrogenus solani Holmes. 
Key to the species of genus Acrogenus.

\section{Infecting potato.}

II. Infecting black currant.

1. Acrogenus solani Holmes. (Handb. Phytopath. Viruses, 1939, 111.) From New Latin Solanum, generic name of potato.

Common names: Potato spindle-tuber virus, potato spindling-tuber virus, potato marginal leaf-roll virus.

Host: SOLANACEAE-Solanum tuberosum L., potato.

Geographical distribution: United States and Canada.

Induced disease: Plants erect, stiff, spindly, lacking vigor. Leaves small, erect, darker green than normal. Petioles sometimes slender, brittle. Tubers long, cylindrical, irregular in shape, tapered at ends, smooth and tender-skinned, of softer than normal flesh in spring. Eyes of tuber conspicuous.

Transmission: By inoculation of expressed juice; by use of contaminated knife in cutting successive tubers before planting; by contacts of freshly cut seed pieces. By aphids, Myzus persicae (Sulz.) and Macrosiphum solanifolii Ashm. (= M. gei Koch) (APHIDIDAE). Also by certain leaf-eating insects.

Thermal inactivation: At 60 to $65^{\circ} \mathrm{C}$ in 10 minutes (in tuber tissues).

Literature: Bald et al., Phytopath., 31, 1941, 181-186; Folsom, Maine Agr. Exp. Sta., Orono; Bull. 312, 1923; Goss, Phytopath., 16, 1926, 233, 299-303; 18, 1928, 445-448; Nebraska Agr. Exp. Sta., Res. Bull. 47, 1930; 53, 1931; Jaczewski, La Défense des Plantes, Leningrad, 4, 1927, 62-77 (Rev. Appl. Mycol., 6, 1927, 572573, Abst.); MeLeod, Canad. Exp. Farms, Div. Bot., Rpt. for 1926, 1927.

\section{Acrogenus solani.}

\section{Acrogenus ribis.}

Strains: A strain causing unmottled curly dwarf of potato has been given a varietal name to distinguish it from the type, var. vulgaris H. (loc. cit., 111):

1a. Acrogenus solani var. severus $\mathbf{H}$. (loc. cit., 112). Inducing symptoms in potato on the whole more severe than those caused by the type strain.

Common name : Unmottled curly-dwarf strain of potato spindle-tuber virus. (Goss, Nebraska Agr. Exp. Sta., Res. Bull. 47, 1930; 53, 1931; Schultz and Folsom, Jour. Agr. Res., 25, 1923, 43-118.)

2. Acrogenus ribis H. (loc. cit., 112). From Latin ribes, currant.

Common name: Black-currant reversion-disease virus.

Host: SAXIFRAGACEAE-Ribes nigrum L., European black currant.

Geographical distribution: British Isles.

Induced disease: In European black currant, leaves abnormally narrow and flat, small veins few. Flowers sometimes nearly transparent, smooth, sepals brightly colored beneath. Flowers and small fruits fall. Stems less woody than normal, with tendency to excessive gum production.

Transmission: By grafting. By bigbud mite, Eriophyes ribis (ERIOPHYI$D A E)$. Not by inoculation of expressed juice. Not through soil. Not through seeds from diseased plants.

Literature: Amos and Hatton, Jour. Pom. and Hort. Science, 6, 1926, 167-183; Amos et al., in East Malling Res. Sta., 15th Ann. Rpt., 1928, 43-46; Lee, Ann. Appl. Biol., 9, 1935, 49-68.

\section{Genus III. Corium Holmes.}

$$
\text { (Loc. cit., 119.) }
$$

Viruses of the Leaf-Roll Group, inducing diseases usually characterized by thicken- 
ing and rolling of leaves. Foliage leathery. Sometimes conspicuous phloem necrosis. Generic name from Latin corium, leather.

The type species is Corium solani Holmes.

Key to the species of genus Corium.

I. Infecting potato.

II. Infecting beet.

III. Infecting raspberry.

1. Corium solani Holmes. (Handb. Phytopath. Viruses, 1939, 120.) From New Latin Solanum, generic name of potato.

Common name: Potato leaf-roll virus. Hosts: SOLANACEAE-Solanum tuberosum L., potato. Experimentally, also other solanaceous species, Datura stramonium L., Jimson weed; Lycopersi. con esculentum Mill., tomato; Solanum dulcamara L., bittersweet; S. villosum.

Insusceptible species: CHENOPODIACEAE-Beta vulgaris L., beet.

Geographical distribution: North America, France, British Isles; pmbably wherever potatoes are grown.

Induced disease: In potato, leaves thick, rigid, leathery, and rolled, their starch content excessive. Plants dwarfed. Tubers few, small, crisp. Tubers of some varieties show conspicuous phloem necrosis, germinate with spindling sprouts.

Transmission: By aphid, Myzus persicae (Sulz.) ( $A P H I D I D A E)$, with incubation period of 24 to 48 hours. Also by Myzus convolvuli (Kalt.) (= M. pseudosolani Theob.), M. circumflexus (Buckt.), Macrosiphum solanifolii Ashm., and Aphis abbreviata Patch (APHIDI$D A E)$. By grafting. Not by inoculation of expressed juice.

Literature: Artschwager, Jour. Agr. Res., 15, 1918, 559-570; 24, 1923, 237-245; Dylistra, ibid., 47, 1933, 17-32; Elze, Phytopath., 21, 1931, 675-686; Folsom, Maine Agr. Exp. Sta., Bull. 297, 1921,

\section{Corium solani.}

\section{Corium betae.}

3. Corium rubi.

4. Corium ruborum.

37-52; 410, 1942, 215-250; Murphy, Scient. Proc. Roy. Dublin Soc., 17, 1923, 163184; Murphy and M'Kay, ibid., 19, 1929, 341-353; Schultz and Folsom, Jour. Agr. Res., 21, 1921, 47-80; Smith, Ann. Appl. Biol., 16, 1929, 209-229; 18, 1931, 141-157 ; Stevenson et al., Am. Potato Jour., 20, $1943,1-10$.

2. Corium betae spec. nov. From Latin beta, beet.

Common names: Sugar-beet yellows virus, beet-yellows virus, jaunisse virus, vergelingszickte virus.

Hosts: CHENOPODIACEAE-Beta mlgaris L., beet; $B$. maritima L.; $B$. cicla; Atriplex hortensis L.; A. sibirica L.; Chenopodium album L., lamb's quarters; Spinacia oleracea L., spinach. AMARANTHACEAE - Amaranthus retroflexus $\mathrm{L}$.

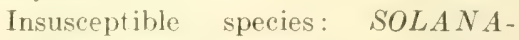
CEAE-Solanum tuberosum L., potato; all other tested solanaceous specics.

Geographical distribution: Bclgium, Netherlands, Denmark, England; perhaps Germany and the United States.

Induced disease: In beet, young leaves little affected; older leaves yellow, brittle, short, thick, containing excessive amounts of carbohydrates; necrosis in secondary phloem. In spinach, yellowing, necrosis between veins on old leaves.

Transmission: Not by inoculation of expressed juice. By aphids, Myzus persicae (Sulz.), A phis fabae Scop., Macrosiphum solanifolii Ashm., and Aulacor- 
thum solani Kalt. (APHIDIDAE); virus is not transmitted by these aphids to their descendants. Not through seeds of beet. Virus overwinters in beets stored for subsequent use in seed produc. tion.

Serological relationships: Specific precipitating antiserum effective with crude sap of diseased, not healthy, plants and with sap of diseased plants after passage through a Chamberland $\mathrm{L}_{1}$, not $\mathrm{L}_{3}$, filter candle; ineffective with sap from beet plants suffering from mosaic.

Thermal inactivation: Virus heated to about $52^{\circ} \mathrm{C}$ no longer precipitates with specific antiserum.

Literature: Kleczkomski and Watson, Ann. Appl. Biol., 31, 1944, 116-120; Petherbridge and Stirrup, London, Ministry Agr. and Fisheries, Bull. 93, 1935; Quanjer and Roland, Tijdschr. Plantenziekten, 42, 1936, 45-70; Roland, ibid., 45, 1939, 1-22, 181-203; Schreven, Meded. Inst. voor Suikerbietenteelt, Bergen op Zoom, 6, 1936, 1; Watson, Proe. Roy. Soc. London, Ser. B, 128, 1940, 535-552 ; Ann. Appl. Biol., 29, 1942, 358-365.

3. Corium rubi H. (loc. cit, 121). From New Latin Rubus, generic name of raspberry, from Latin rubus, bramble: bush.

Common name: Raspberry leaf-curl virus.

Host : ROSACEAE-Rubus idaeus L., red raspberry.

Insusceptible species: ROSACEAERubus occidentalis L., black raspberry; $R$. neglectus Peck, purple raspberry.

Geographical distribution: United States, not in England.

Induced disease: In red raspberry, veins retarded in growth, causing downward curling of leaf margins and crinkling of leaf lamina. Foliage dark green, dry in appearance, not wilting readily. In late summer, leaves bronzed, leaf surface glistening. Diseased canes easily winterkilled. Berries small and poor. The
English variety Lloyd George is intolerant of the disease and is killed.

Transmission: By aphid, Aphis rubicola Oestl. ( $=A$. rubiphila Patch) (APHIDIDAE). Not by inoculation of expressed juice.

Literature: Bennett, Michigan Agr. Exp. Sta., Tech. Bull. 80, 1927; Phytopath., 20, 1930, 787-802, Harris, East Malling Res. Sta., Ann. Rpt. for 1934, 1935; Rankin, New York Agr. Exp. Sta., Geneva, Tech. Bull. 175, 1931.

Strains: A strain differing from the type, var. alpha H. (loc. cit., 121), has been given a varietal name derived from its common name, raspberry beta-curl virus :

3a. Corium rubi var. beta H. (loc. cit., 122 ). Infecting black and purple raspberries, as well as the red raspberry, which alone is susceptible to the type st rain, raspberry alpha-curl virus. (Bennett, Phytopath., 20, 1930, 787-802.)

4. Corium ruborum (Zeller and Braun) comb. nov. (Minuor ruborum Zeller and Braun, Phytopath., 33, 1943, 161.) From Lat in rubus, bramble bush.

Common name: Raspberry decline-disease virus.

Host : ROSACEAE-Rubus idaeus L., red raspherry.

Geographical distribution: United States (Oregon).

Induced disease: In Cuthbert raspberry, shoots retarded in spring, reddish; leaves in autumn rolled downward, fluted along veins, less green than normal between veins, slightly bronzed along margins and crests between veins. Internodes shortened near tips of canes. Affected canes small, weak, not hardy in winter. Small roots and feeder rootlets fewer than in healthy plants. Disease progressive over about three years. Fruits small, irregular, tending to be globose, crumbly when ripe, worthless.

Transmission: By grafting. To inser:t vector is known. 


\section{Genus IV. Nanus Holmes.}

(Loc. cit., 123.)

Viruses of the Dwarf-Disease Group, inducing diseases characterized by dwarfing of host plants or by growth of adventitious shoots with short internodes; chlorotic mottling absent. Generic name from Latin nanus, dwarf.

The type species is Nanus loganobacci Holmes.

\section{Key to the species of genus Nanus.}

I. Infecting rosaceous plants.
A. In loganberry and Phenomenal berry.
1. Nanus loganobacci.

B. In black raspberry.

C. In peach.

D. In ocean spray.

E. In strawberry.

2. Nanus orientalis.

3. Nanus mirabilis.

4. Nanus holodisci.

5. Nanus fragariae.

6. Nanus cupuliformans.

F. In prune and plum.

II. Infecting graminaceous plants.

7. Nanus pruni:

A. In sugar cane.

\section{Nanus loganobacci Holmes.} (Handb. Phytopath. Viruses, 1939, 124.) From New Latin loganobaccus, specific epithet of loganberry, Rubus loganobaccus Bailey.

Common name: Loganberry-dwarf virus.

Hosts: ROSACEAE-Rubus loganobaccus Bailey, loganberry and Phenomenal berry.

Geographical distribution: United States (Oregon, Washington, and California).

Induced disease : In Phenomenal berry, leaves small, obovate, rigid, new canes short, spindly. In young plants, some necrosis along and between veins, leaves crinkled, finer veins ehlorotic. Stems not streaked or mottled, normal in color. In late stages, canes very short, internodes short. Sepals and petals of flowers small. Fruit of fair size, but druplets ripen unevenly and tend to fall apart when picked. Loganberry is less susceptible than the Phenomenal berry but is similarly affected.

\section{Nanus sacchari.}

Transmission: By aphid, Capitophorus tetrahodus (APHIDIDAE). Not by inoculation of expressed juice.

Literature: Zeller, Phytopath., 15, 1925, 732 (Abst.) ; 17, 1927, 629-618.

2. Nanus orientalis H. (loc. cit., 124). From Latin orientalis, eastern.

Common names: Raspberry-streak virus, raspberry eastern blue-stem virus, raspberry rosette virus.

Host: ROSACEAE-Rubus occidentalis L., black raspberry.

Insusceptible species : $R O S A C E A E-$ $R$ ubus idaens L., red raspberry; $R$. phoenicolasius Maxim., Japanese wineberry.

Geographical distribution: United States.

Induced disease: In black raspberry, plants stunted, becoming smaller in successive seasons; leaves usually curled, close together on canes, dark green, often twisted so as to be upside down. New canes show bluish violet dots, spots, or stripes near their bases and sometimes also on branches or on fruiting spurs. 
Fruit inferior in size, quality, and quantity. Plants live only 2 or 3 years after infection on the average.

Strains: A strain of this virus is believed responsible for mild streak of black raspberries, in which purple to violet, greenish brown, or bluish streaks on canes are narrowly linear or elliptical in form and often very faint; when the bloom is rubbed off, the lesions appear as though watersoaked and discolored. Leaves are slightly curled, their veins cleared. Fruits are dry and dull in lustre, even while still red, and of poor flavor when ripe.

Literature: Bennett, Michigan Agr. Exp. Sta., Tech. Bull. 80, 1927; Wilcox U. S. Dept. Agr., Dept. Circ. 227, 1923; Woods and Haut, U. S. Dept. Agr., Plant Dis. Rpt., 24, 1940, 338-340.

3. Nanus mirabilis H. (loc. cit., 126). From Latin mirabilis, strange.

Common name: Peach phony-disease virus.

Hosts : ROSACEAE-Prunus persica (L.) Batsch, peach. Experimentally, also other Prunus species.

Geographical distribution: United States (Georgia, Alabama, Florida; sparsely also in Mississippi, Tennessee, South Carolina, Louisiana, Texas, Arkansas, Missouri).

Induced disease: In peach, tree dwarfed, foliage abnormally green, fruit small; flecks in wood, especially in roots; sections of roots show characteristic welldistributed purple spots after 3 to $5 \mathrm{~min}$ utes of treatment in $25 \mathrm{cc}$ absolute methyl alcohol acidulated by the addition of 1 . to 5 drops of concentrated, chemically pure hydrochloric acid.

Transmission : By root grafting, except by root-bark patch grafts, which are ineffective. Budding and grafting with parts of stem fail to transmit this virus.

Thermal inactivation: At $48^{\circ} \mathrm{C}$ in about 40 minutes in roots.

Literature: Hutchins, Georgia State Entomol. Bull., 78, 1933; Phytopath., 29, 1939, 12 (Abst.); Hutchins and Rue, ibid., 29, 1939, 12 (Abst.).
4. Nanus holodisci H. (loc. cit., 127). From New Latin Holodiscus, generic name of ocean spray.

Common name: Ocean-spray witches'broom virus.

Host: ROSACEAE-Holodiscus discolor Max., ocean spray.

Geographical distribution: United States (Oregon and Washington).

Induced disease: In ocean-spray, diseased branches form clusters of thin wiry shoots with abnormally short internodes and crowded small leaves. Laterals numerous and more than normally branched. Bronzy red color acquired early by affected foliage.

Transmission: By aphid, Aphis spiraeae Schout. (APHIDIDAE). By grafting. Not demonstrated by inoculation of expressed juice.

Literature: Zeller, Phytopath., 21, 1931, 923-925.

5. Nanus fragariae H. (Holmes, loc. cit., 128; Blastogenus fragariae McKinney, Jour. Washington Acad. Science, 34, 1944, 151.) From New Latin Fragaria, generic name of strawberry.

Common name: Strawberry witches'broom virus.

Host : ROSACEAE-Fragaria chiloensis Duch. var. ananassa Bailey, cultivated strawberry.

Geographical distribution: United States (western Oregon).

Induced disease: In strawberry, leaves numerous, light in color, with spindly petioles, margins of leaflets bent down, runners shortened, plants dwarfed; flower stalks spindly and unfruitful; root systems normal and well developed.

Transmission: By aphis, Myzus fragaefolii Ckll. (APHIDIDAE). Not demonstrated by inoculation of expressed juice.

Literature: Zeller, Phytopath., 17, 1927, 329-335.

6. Nanus cupuliformans Zeller and Weaver. (Phytopath., 31, 1941, 851.) From diminutive of Latin cupa, tub, and participal from Latin formare, to form. 
Common name: Strawberry-stunt virus.

Host : ROSACEAE-Fragaria chiloensis Duch. var. ananassa Bailey, cultivated strawberry.

Geographical distribution: United States (Oregon, Washington).

Induced disease: In strawbery, little if any reduction in chlorophyll, plants erect but short; leaves at first folded, later open, dull in lustre, with papery rattle when brushed by hand, leaflets cupped or with margins turned down, midveins tortuous; petioles $\frac{1}{2}$ to $\frac{2}{3}$ normal length; fruits small, usually hard and seedy ; roots normal in appearance.

Transmission: By strawberry-leaf aphid, Capitophorus fragaefolii ( $A P H I$ $D I D A E)$. By grafting. Not by inoculation of expressed juice.

7. Nanus pruni H. (loc. cit., 128). From New Latin Prunus, generic name of prune, from Latin prunus, plum tree.

Common name: Prune-dwarf virus.

Hosts: ROSACEAE-Prunus domestica L., prune and plum; var. insititia Bailey, the Damson plum, remains symptomless. Experimentally, also Prunus persica (L.) Batsch, peach.

Insusceptible species: ROSACEAEPrunus avium L., cherry.

Geographical distribution: United States (New York); Canada (British Columbia, Ontario).

Induced disease: In prune, leaves small, narrow, rugose, distorted, glazed. Internodes short. Some branches escapeand appear normal. Blossoms numerous, mature fruits few. Pistils aborted, petals narrow and distorted. In Damson and Bradshaw plums, no obvious manifestations of disease as a result of infection.
Transmission: By budding and other forms of grafting. Not demonstrated by inoculation of expressed juice. No insect vector is known.

Literature: Berkeley, Canada, Domin. Dept. Agr., Div. of Bot. and Plant Path., Science Service, Publ. 679, 1941; Hildebrand, Phytopath., 32, 1942, 741-751; Thomas and Hildebrand, Phytopath., 26, 1936, 1145-1148.

8. Nanus sacchari H. (loc. cit, 129). From New Latin Saccharum, generic name of sugar cane, from Lat in saccharum, sugar.

Common name: Sugar-cane serehdisease virus.

Host : GRAMINEAE-Saccharum offcinarum L., sugar cane.

Geographical distribution: Java, Borneo, Sumatra, Moluccas, India, Mauritius, Australia, Fiji, Formosa, Hawaii, Ceylon.

Induced disease : In sugar cane (Cheribon variety), plant dwarfed, shoots stunted, vascular bundles colored by the presence of a red gum; adventitious roots from many or all nodes.

Transmission: Not by inoculation of expressed juice. No insect vector is known.

Thermal inactivation: In cuttings of sugar cane, at $52^{\circ} \mathrm{C}$ in 30 minutes to 1 hour. Infected eane cuttings survive the heat treatment required for cure through inactivat ion of the causative virus.

Literature: Houtman, Arch. Suikerind. Nederland.-Indië, 33, 1925, 631-642; Lyon, Bull. Exp. Sta. Hawaiian Sugar Planters' Assoc., Bot. Ser., 3, 1921, 1-43; Wilbrink, Arch. Suikerind. Nederland.Indië, 31, 1923, 1-15.

\section{Genus V. Rimocortius Milbrath and Zeller.}

(Phytopath., 32, 1942, 430.)

Viruses of the Rough-Bark Group, inducing diseases principally affecting bark, less often wood, leaves, or fruit. Generic name from Latin rima, cleft or fissure, and cortex, bark.

The type species is Rimocortius kwanzani Milbrath and Zeller. 
(Note: The genus Citrivir (first named species, Citrivir psorosis Fawcett, Phytopath., $31,1941,357$ ) was proposed by its author as a genus pro tempore with the avowed purpose of accommodating viruses causing diseases in species of the plant-host genus Citrus. It appears to have been implied by the term genus protempore that evidences of natural relationship, when discovered, would permit even the first-named species of this genus to be assigned elsewhere. On the assumption that a permanent genus is nothing more than a type species and such other species as may be added to it by one or another author, it must be felt that a genus pro lempore, however convenient as an expedient, cannot become a permanent genus under any circumstances, because its first-named species would appear not to be a permanent part of the genus and so intended not to be a true type-species. Without a type species there would seem to be no permanent genus concept.

The system by which the term Citrivir was coined (explained by its author as use of the genitive of the host-genus name, Citris, plus vir, signifying virus) seems in itself acceptable, for it is commonly agreed that a generic name may be made in an arbitrary manner. It may be noted that use of the stem of the host-genus name (Citr-) with connecting vowel $i$ and suffix -vir, possibly a more orthodox procedure, would have given the same result in the present instance. The original definition of the term Citrivir might be thought to he repugnant as disregarding concepts of natural interspecific relationships that are essential to the spirit of binomial nomenclature. Were the genus to be regarded as permanent rather than pro tempore, however, the scope of the genus would come to be wholly changed by usage, when, with passage of time related species would be added to what in this case would be a type species, without regard to the unorthodox intent of the original definition but solely in accordance with similarities between viruses. A generic concept need never be accepted as rigidly defined, whether initially, as has been attempted in this case, or upon further experience, because a genus may still grow by the addition of closely allied new species beyond any limit that may be set. On this account an original, or any subsequent, definition may be regarded as subject to unlimited change so long as the type species is logically retained. The form and definition of the term Citrivir would not, therefore, militate against its continued use. Its avowedly temporary status alone seems decisively to do so.

The originally monotypic genus Rimocortius, published in the following year, was defined only by the combined generic and specific description, and was not referred to a family by its authors. The type, because at first the only species, Rimocortius kwanzani, is the flowering-cherry rough-bark virus. This type species might well be associated with the species Citrivir psorosis, citrus-psorosis virus, diseussed above, both affecting bark principally, though foliage also to some extent. Although the genus Citrivir was named in 1941 and Rimocortius not until 1942, the first was intended as a temporary assemblage only, as above indicated. It would seem appropriate, therefore, to include the virus that was known temporarily as Citrivir psorosis in the permanent genus Rimocortius Milbrath and Zeller and to assign this genus to the family Marmoraceae.)

Key to the species of the genus Rimocortius.

\section{Affecting cherry.}

1. Rimocortius kwanzani.

II. Affecting Citrus.

2. Rimocortius psorosis.

III. Affecting pear.

3. Rimocortius pyri. 


\section{Rimocortius kwanzani Milbrath and} Zeller. (Phytopath., 32, 1942, 430.) From Kwanzan, name of a variety of flowering cherry.

Common name: Flowering-cherry rough-bark virus.

Hosts: Prunus scrrulata Lindl. var. Kwanzan, flowering cherry ; P. avium L., Mazzard cherry.

Geographical distribution: United States (Oregon).

Induced disease: In flowering cherry, tree dwarfed, deficient in lateral branches; bark deep brown, roughened, splitting longitudinally; internodes shortened, bunching leaves; leaves arched downward; midribs of leaves split and cracked on under surface. In Mazzard cherry, no manifestation of disease, but carrier condition; budded Mazzard stoch may transmit disease to healthy Kwanzan cherry cions.

Transmission: By budding, generally even if the inserted bud fails to survive.

Literature: Milbrath and Zeller, Phytopath., 32, 1942, 428-430; Thomas, ibid., $32,1942,435-436$.

\section{Rimocortius psorosis (Fawcett)} comb. nov. (Citrivir psorosis Fawcett, Phytopath., 31, 1941, 357.) Specific name meaning "of the disease known as psorosis."

Common name: Citrus-psorosis virus.

Hosts : RUTACEAE-Citrus sinensis Osbeck, orange; C. limonia Osbeck, lemon; C. maxima Merr., grapefruit.

Geographical distribution: World-wide where cit rus trees are grown.

Induced disease: In citrus, small, elongated, light colored areas or flecks in the region of small veins on young, tender foliage; leaves sometimes warped; (chlorotic?) clearing of veins, and chlorotic line patterns, sometimes concentric. Outer layers of bark scale away; depressions and deformities appear in bark and wood. Lemons, as a rule, are more tolerant than oranges and are not subject to the bark changes.
Transmission: By grafting, including root grafting and patch bark grafting. Not by inoculation of expressed juice. No insect vector is known.

Literature: Bitancourt et al., Phytopath., 33, 1943, 865-883; Fawcett, ibid., 24, 1934, 659-668; Science, 92, 1940, 559561 ; Phytopath., 31, 1941, 356-357 ; Fawcett and Bitancourt, ibid., 33, 1943, 837864; Rhoads, ibid., 32, 1942, 410-413; Webber and Fawcett, Hilgardia, 9, 1935, 71-109.

Strains: Three strains differing from the type have been recognized. The type, var. vulgare Fawcett, Phytopath., $31,1941,357$, causes psorosis A, the common scaly-bark type of disease, with pustular eruptions of outer layers of bark in limited areas, with or without exudation of gum; later a drab-gray, cinnamondrab to rufus discoloration of the wood, accompanied by decline of the affected tree. Others, that contrast with the type, are :

2a. Rimocortius psorosis var. anulatum Fawcett. (Phytopath., 31, 1941, 357.) From Latin anulatus, with a ring. Causing psorosis B, known from California, resembling zonate chlorosis of Brazil in effects on leaves and fruits. Psorosis B is characterized by rapid scaling of outer bark in continuous areas, progressing rapidly along one side of trunk or branch; gum exudes in advance of scaling, necrosis follows; large circular discolored and corky spots, sometimes concentric, on fruits and mature leaves; on some fruits, circular or semi-circular furrows and bumps; rapid decline of the affected tree.

2b. Rimocortius psorosis var. concavum Fawcett and Bitancourt. (Phytopath., 33, 1943, 850.) From Latin concavus, concave. Causing concave-gum psorosis, characterized by concavities of various sizes on trunks and larger limbs of affected trees, often by zonate patterns on young leaves during periods of rapid growth. 
2c. Rimocortius psorosis var. alveatum Fawcett and Bitancourt. (Phytopath., 33, 19ł3, 854.) From Latin alveatus, hollowed out like a trough. Causing blindpocket psorosis, characterized by troughlike pockets in bark and wood or by eruptive lesions.

3. Rimocortius pyri (Holmes) comb. nov. (Marmor pyri Holmes, Handb. Phytopath. Viruses, 1939, 76.) From New Latin Pyrus, generic name of pear, from Latin pirus, pear tree.

Common name: Pear stony-pit virus.

Host : ROSACEAE-Pyrus communis L., pear.
Geographical distribution: United States (Oregon, Washington, California).

Induced disease: In pear, fruit deeply pitted and deformed; bark cracked and resembling oak bark; veinlet chlorosis of some leaves, failure of lateral buds to grow, reduction of foliage. Bartlett and Comice varieties of pear appear to be tolerant, producing sound fruit from infected trees.

Transmission: By budding. Not by inoculation of expressed juice. No insect vector is known.

Literature: Kienholz, Phytopath., 29, 1939, 260-267; 30, 1940, 787 (Abst.).

\section{Genus VI. Adelonosus Brierley and Smith.}

(Phytopath., 34, 1944, 551.)

Viruses capable of multiplying in living plants but producing no recognizable symp toms in these except on interaction with distinct viruses with which they form complexes. Transmitted by aphids, by sap, or by both means. Generic name from Greek adelos, invisible, and nosos, disease. Only one species is recognized thus far; this is the type species, Adelonosus lilii Brierley and Smith.

1. Adelonosus lilii Brierley and Smith. (Phytopath., 34, 1944, 551.) From Latin lilium, lily.

Common name: Lily-symptomless virus.

Host : LILIACEAE-Lilium longiflorum Thunb., Easter lily.

Insusceptible species: All other tested lilies and many related plants in the same and other families (for list, see Phytopath., 34, 1944, 549).

Geographical distribution: Inited States, Japan; probably coextensive with commercial culture of Easter lily.

Induced disease: In Easter lily, no obvious manifestation of disease when this virus is present alone; when together with cucumber-mosaic virus, however, the lily-symptomless virus is a determining factor in the production of ne- crotic-fleck disease; the lily-symptomless virus is so widely distributed in supposedly healthy stocks of the Easter lily that cucumber-mosaic virus formerly was thought to be the sole determining factor in necrotic flecking, now recognized to be caused by the virus complex lilysymptomless virus (Adelonosus lilii) plus cucumber-mosaic virus (Marmor cucumeris); the complex acts independently of the presence or absence of lily latentmosaic virus (Marmor mite), which is often present with the essential members of the complex in flecked Easter lilies.

Transmission: By inoculation of expressed juice, with some difficulty. By aphid, Aphis gossypii Glov., cotton aphid (APHIDIDAE); preinfective period after obtaining virus, 4 to 6 days. 


\section{FAMILY III. ANNULACEAE HOLMES.}

(Handb. Phytopath. Viruses, 1939, 97.)

Viruses of the Ringspot Group, causing diseases usually characterized by necrotic or chlorotic spotting with concentric-ring lesions and eventual recovery from obvious disease with non-sterile immunity. Hosts, higher plants; vectors unknown. There is a single genus.

\section{Genus I. Annulus Holmes.}

(Loc. cit., 97.)

Characters those of the family. Generic name from Latin annulus, a ring.

The type species is Annulus tabaci Holmes.

\section{Key to the species of genus Annulus.}

I. Found occurring naturally in the Western Hemisphere.

A. In tobacco.

B. In potato.

C. In delphinium.

1I. Old World species.

1. Annulus tabaci Holmes. (Handb. Phytopath. Viruses, 1939, 98; Marmor anularium McKinney, Jour. Washington Acad. Sci., 34, 1944, 327.) From New Latin Tabacum, early generic name for tobacco.

Common names: Tobacco-ringspot virus, green ringspot virus, yellow ringspot virus, ring spot No. 1 virus.

Hosts: SOLANACEAE--Nicotiana. tabacum L., Petunia violacea Lindl., Solanum tuberosum L. CUCURBITA$C E A E-C u c u m i s$ sativus L. Experimentally this virus has been found capable of infecting many species of plants in a large number of families; these include all tested species of the SOLANACEAE, SCROPHULARIACEAE, COMPOSITAE, and CUCURBITACEAE. Many species of the LEGUMINOSAE are susceptible and one, Vigna sinensis (L.) Endl., is used as an indicator plant for quantitative measurement because it displays conspicuous reddish-brown necrotic lesions around points of initial infection.
1. Annulus tabaci.

2. Annulus zonatus.

3. Annulus orae.

4. Annulus apertus.

5. Annulus dubius.

6. Annulus delphinii.

7. Annulus bergerac.

Geographical distribution: United sitates.

Induced disease: In tobacco, necrotic ring-like primary lesions, followed by secondary necrotic rings on younger leaves. Subsequently, affected plants recover. After recovery from obvious disease, virus content of plants is only 10 to 20 per cent of that of recently infected plants. Some varieties may show mosaic-like patterns in young leaves at $16^{\circ} \mathrm{C}$.

Transmission: By inoculation of exdressed juices. Through about 20 per cent of seeds from diseased petunia plants. Not by dodder, Cuscuta campestris Yuncker (CONVOLVULACEAE).

Serological relationships: Induces the formation of specific precipitating antibodies when injected into bloodstream of rabbit.

Immunological relationships: Recovered tobacco plants are not susceptible to reinfection with this virus but are readily infected with Annulus zonatus or A. orae. This virus produces primary lesions on 
leaves of plants immune to reinfection with $A$. bergerac.

Thermal inactivation: At $68^{\circ} \mathrm{C}$ in 10 minutes.

Filterability : Passes $\mathbf{T}, \mathbf{N}$, and perhaps W Berkefeld filters.

Other properties: Particle size estimated by filtration experiments as about 19 millimicrons. Sedimentation constant, $\mathrm{S}_{20^{\circ}}=115 \times 10^{-13} \mathrm{~cm}$. sec. $^{-1}$ dyne $e^{-1}$. Infective in dilutions of $10^{-7}$ after purification. Inactivated in 1 hour below $\mathrm{pH} 3$ or above $\mathrm{pH}$ 10.8. Recovered plants of tobacco contain $0.002 \mathrm{mg}$ of virus per gram, recently infected plants about 6 times as much. Optimum conditions for retaining infectivity of stored virus include suspension in $0.01 \mathrm{M}$ phosphate buffer at $\mathrm{pH} 7$ and storage at $4^{\circ} \mathrm{C}$.

Literature: Fenne, Phytopath., 21, 1931, 891-899; Fromme et al., ibid., 17, 1927, 321-328; Henderson, ibid., 21, 1931, 225-229; Henderson and Wingard, Jour. Agr. Res., 43, 1931, 191-207; Price, Contrib. Boyce Thompson Inst., 4, 1932. 359-403; Phytopath., 26, 1936, 503-529; Am. Jour. Bot., 27, 1940, 530-541; Am. Naturalist, 74, 1940, 117-128; Priode, Am. Jour. Bot., 15, 1928, 88-93; Stanley, Jour. Biol. Chem., 129, 1939, 405-128, 429-436; Stanley and Wyckoff, science, 85, 1937, 181-183; Valleau, Kentucky Agr. Exp. Sta., Bull. 327, 1932; Wingard, Jour. Agr. Res., 37, 1928, 127-153; Woods, Contrib. Boyce Thompson Inst., 5, 1933, 419-434.

Strains: A number of distinctive strains have been collected in nat ure and studied experimentally. The following have been given varietal names to distinguish them from the type, var. virginiensis H., loc. cit., 98:

1a. Annulus tabaci var. kentuckiensis H. (loc. cit., 99). Differing from the typical strain in producing less necrosis and less stunting in tobacco. (Price, Phytopath., 26, 1936, 665-675; Valleau, Kentucky Agr. Exp. Sta., Bull. 327, 1932.)

1b. Annulus tabaci var. auratus $\mathrm{H}$. (loc. cit., 100). Secondary lesions in tobacco at first yellow spots or rings, becoming necrotic subsequently. Recovery less complete than with type, abnormal yellowing of old leaves tending to persist. (Chester, Phytopath., 25, 1935, 686-701; Price, Phytopath., 26, 1936, 665-675; Valleau, Kentucky Agr. Exp. Sta., Bull. 327, 1932; Phytopath., 29, $1939,549-551$.

2. Annulus zonatus H. (loc. cit., 101). From Latin zonatus, zonate.

Common names: Tomato-ringspot virus, ring spot No. 2 virus.

Hosts: SOLANACEAE-Nicotiana tabacum L., tobacco. Experimentally this virus has been found to infect many species of plants in a large number of families.

Geographical distribution: United States.

Induced disease: In tobacco, zonate necrotic primary lesions and, temporarily, secondary lesions of the same type; recovery with specific, non-sterile immunity. In tomato, systemic infection, yellowish-green or necrotic ring-like lesions; stunting.

Transmission: By inoculation of expressed juice.

Immunologicul relationships: Recovered plants are immune to reinfection but are still susceptible to Annulus tabaci, $A$. bergerac, and several mosaic-type viruses that have been tested.

Thermal inactivation: At 55 to $60^{\circ} \mathrm{C}$ in 10 minutes.

Filterability: Passes Gradocol membrane 100 millimicrons in average pore diameter. Particle size estimated as 50 millimierons or less.

Literature: Price, Phytopath., 26, 1936 665-675; Am. Jour. Bot., 27, 1940, 530-511.

3. Annulus orae H. (Holmes, loc. cit., 103; Tractus orae Valleau, Phytopath., 30, 1940, 826.) From Latin ora, edge, in reference to occurrence of induced disease near edge of tobacco fields.

Common name: Tobacco-streak virus.

Hosts : SOLANACEAE-Nicotiana tabacum L., tobacco. Experimentally, a 
number of other solanaceous plants have been reported as susceptible, but not Capsicum frutescens L., pepper; Lycopersicon esculentum Mill., tomato; Solanum melongena L., eggplant; or $S$. tuberosum L., potato.

Geographical distribution: United States.

Induced disease: In tobacco, local and systemic necrosis in 3 days, with irregular spot, line, and ring-like lesions, followed by recovery from necrotic manifestations of disease. Recovered leaves may show a mild mottling and regularly contain virus; reinoculation does not induce formation of necrotic lesions in them.

Transmission: By inoculation of expressed juice. Not through seeds from diseased plants.

Immunological relationships: No crossprotection with respect to $A$. tabaci, and several viruses of the mosaic group.

Thermal inactivation: At $53^{\circ} \mathrm{C}$ in 10 minutes.

Literature: Johnson, Phytopath., 26, 1936, 285-292; Trans. Wisconsin Acad. Sciences, Arts and Letters, 30, 1937, $27-34$.

4. Annulus apertus spec. nov. From Lat in apertus, frank.

Common name: Broad-ringspot virus. Hosts : SOLANACEAE-Nicotiana tabacum I., tobacco. Experimentally also to many species in this and other families.

Insusceptible species: ( $H E N O P O D I-$ $A C E A E-B e t a$ vulgaris L. CUCURBITACEAE-Citrullus vulgaris Schrad. LEGUMINOSAE-Medicago sativa $\mathrm{L}$. Melilotus alba Desr.

Geographical distribution: United States (Wisconsin).

Induced disease : In tobaceo, indistinct yellow-spot primary lesions, becoming chlorotic or necrotic rings with concentric markings; small chlorotic rings, sometimes concentric, or fine brown necrotic rings as secondary lesions; young leaves puckered at first, somewhat mal formed.
Transmission: By inoculation of expressed juice.

Immunological relationships: Protects against reinfection with homologous virus but leaves host susceptible to infection by Annulus tabaci, A. zonatus, and some mosaic-type viruses.

Literature: Johnson and Fulton, Phytopath., 32, 1942, 605-612.

5. Annulus dubius (Holmes) comb.nov. (Marmor dubium H., loc. cit., 42.) From Latin dubius, uncertain, in reference to a common name, potato virus $\mathbf{X}$, often used to designate this virus.

Common name: Potato-mottle virus (strains of this virus have been studied at various times under the names potato latent virus, potato virus $\mathrm{X}$, potatoanecrosis virus, virulent latent virus, simple mosaic virus, healt hy potato virus, Hyoscyamus IV virus, President streak virus, potato foliar-necrosis virus, potato acronecrotic streak virus, Up-to-Date streak virus, potato viruses $\mathrm{B}$ and $\mathrm{D}$, Solanum viruses 1,4 , and 6 .)

Hosts : SOLA NACEAE-Solanum tuberosum L., potato; Lycopersicon esculentum Mill., tomato. Experimentally, also SOLANACEAE-Capsicum frutescens L., pepper; Datura stramonium L., Jimson weed; Hyoscyamus niger L., henbane; Nicotiana tabacum L., tobacco; Physalis alkckengi L.; Solanum dulcamara L., bittersweet; S. nigrum L., black nightshade. A IIARANTHACEAE-Amaranthus retroflexus L. COMPOSITAE-Chrysanthemum morifolium Ram. SCROPHU LARIACEAE-Veronica sp., common speedwell.

Geographical distribution: Widespread throughout the world; present in all known stocks of tubers of some potato varieties in the United States.

Induced disease: In potato, usually no chlorotic mottling, sometimes a little; int racellular inclusions of the vacuolated, granular type; some varieties that are virtually immune in the field owe their tendency to localize the virus in necrotic primary lesions or in top-necrosis of first systemically infected plants to a dom- 
inant allele of a gene $n x$, which characterizes plants showing a mosaic of some degree of intensity on infection with this virus; the variety known as $\mathbf{S} 41956$ is immune to all tested strains of the virus and possesses two dominant genes both required for resistance. In tomato, systemic mild chlorotic mottling; if a strain of tobacco-mosaic virus is also present, a severe systemic necrosis, known as double-virus streak, is induced.

Transmission: By inoculation of expressed juice. Experimentally, by leaf contacts mainly under the influence of wind. No insect vector is known. Not transmitted through true seeds of the potato.

Serological relationships : Cross precipit in reactions between constituent strains of this virus. No cross reaction with potato aucuba-mosaic, potato mild-mosaic, potato-veinbanding, tobacco-mosaic, tobacco-etch, tobacco-ringspot or pea-mosaic virus. Antisera prepared by injecting rabbits intravenously with virus inactivated by nitrous acid, like those prepared with active virus, fix complement and flocculate with virus suspensions (though not with juice of healthy host plants); they are also effective in neutralizing the virus.

Immunological relationships: Tobaceo and Datura plants infected by the type strain of this virus become immune to the more severe potato-ringspot strain. No protection against the severe strain is afforded by previous infection with tobacco-mosaic, tobacco-ringspot, tomato spotted-wilt, or cucumber-mosaic virus.

Thermal inactivation: At $70^{\circ} \mathrm{C}$. in 10 minutes.

Filterability: Passes Pasteur-Chamberland $\mathrm{L}_{1}, \mathrm{~L}_{3}$, and $\mathrm{L}_{5}$ filters.

Other properties: Digested by 0.02 per cent solution of pepsin in 3 hours at $\mathrm{pH}$ 4 , at $38^{\circ} \mathrm{C}$. Digested also by trypsin. Inactivated by papaine and cyanide, but by neither separately. Isoelectric point near $\mathrm{pH}$ 4. Dilute solutions show anisotropy of flow. Concentrated solutions are spontaneously birefringent. Properties of the type strain have been less studied than those of the potato-ringspot strain of this virus.

Literature: Bawden, Proc. Roy. Soc. London, Ser. B, 116, 1934, 375-395; Bawden and Pirie, Brit. Jour. Exp. Path., 17, 1936, 64-7t; Bawden et al., ibid., 17, 1936, 204-207; Blodgett, Phytopath., 17, 1927, 775-782; Böhme, Phytopath. Ztschr., 6, 1933, 517-524; Cadman, Jour. Genetics, 44, 1942, 33-52; Chester, Phytopath., 27, 1937, 903-912; Clinch, Sci. Proc. Roy. Dublin Soc., 28, 1942, 18-34; Johnson, Wisconsin Agr. Exp. Sta., Res. Bull. 63, 1925; Koch, Phytopath., 23, 1933, 319342; Köhler, Phytopath. Ztschr., 5, 1933, 567-591; 7, 1934, 1-30; Loughnane and Murphy, Nature, 141, 1938, 120; van der Meer, Cent. f. Bakt., II Abt., 87, 1932, 240-262 ; Salaman, Nature, 191, 1933, 468; Schultz et al., Phytopath., 27, 1937, 190197 ; 30, 1940, 944-951; Spooner and Bawden, Brit. Jour. Exp. Path., 16, 1935, 218-230; Stevenson et al., Phytopath., 29, 1939, 362-365.

Strains: Several variants of potatomottle virus, differing from the type, var. vulgaris $\mathrm{H}$. (loc. cit., 42), have been recognized as distinctive varieties under the following names:

5a. Annulus dubius var. annulus $\mathrm{H}$. (loc. cit., 44). From Latin annulus, ring.

Common name: Ringspot strain of potato-mottle virus. Necrotic primary and secondary ring-like lesions in experimentally infected tobacco plants. Indistinguishable from type st rain by ordinary precipitin test, but distinguishable when appropriately absorbed sera are used. This strain has been more frequently studied than the type. Juice of infected tobacco plants contains about 0.02 to $0.10 \mathrm{mg}$ of virus per $\mathrm{ml}$. Sedimentation constants, $\mathrm{S}_{20^{\circ}}=113 \times 10^{-13}$ and $131 \times$ $10^{-13} \mathrm{~cm}$. sec. ${ }^{-1}$ dyne $^{-1}$. Dissymmetry constant 2.78. Molecular weight $26 \times$ $10^{6}$. Particle size estimated to be 433 by 9.8 millimicrons, 43.9 times as long as wide. Isoelectric point near $\mathrm{pH} 4$. Stable between $\mathrm{pH} 4$ and $\mathrm{pH}$ 9.5. Concentrated solutions are spontaneously birefringent. Dilute solutions show 
anisotropy of flow. Destroyed by drying. Inactivated by papaine and cyanide, but by neither separately. Digested by 0.02 per cent solution of pepsin in 3 hours at $\mathrm{pH} 4$, at $38^{\circ} \mathrm{C}$. Digested also by trypsin. About 6 per cent of the purified virus is reported to be a pentose nucleic acid, but the carbohydrate to phosphorus ratio is about twice that for yeast nucleic acid. Guanine and pentose present. Analysis of sedimented virus, carbon 47.7 to 49.5 per cent, hydrogen 6.8 to 7.7 per cent, nitrogen 14.6 to 17.0 per cent, phosphorus 0.4 to 0.7 per cent, sulfur 1.1 per cent, carbohydrate 2.5 to 4.3 per cent, ash 2.0 to 2.5 per cent. Reduction of carbohydrate content of sample to 2.5 per cent does not reduce activity of virus; further reduction inactivates. (Ainsworth, Ann. Appl. Biol., 21, 1934, 581-587; Bawden, Brit. Jour. Exp. Path., 16, 1935, 435-443; Bawden and Pirie, ibid., 19, 1938, 66-82; Birkeland, Bot. Gaz., 95, 1934, 419-436; Chester, Phytopath., 26, 1936, 778-785; Johnson, Wisconsin Agr. Exp. Sta., Res. Bull. 76, 1927; Loring, Jour. Biol. Chem., 126, 1938, 455-478; Loring and Wyckoff, ibid., 121, 1937, 225-230.)

5b. Annulus dubius var. favus H. (loc. cit., 46). From Latin flavus, yellow.

Common name: Yellow-mottle strain of potato-mottle virus. Differing from the type by imparting a yellow cast to foliage of infected potatoes. (Putnam, Canad. Jour. Res., Sec. C, 15, 1937, 87107.)

5c. Annulus dutius var. obscurus $\mathrm{H}$. (loc. cit., 46). From Latin obscurus, obscure. Common name: Masked-mottle strain of potato-mottle virus. Differing from the type by systemically infecting potato, tobacco, and Jimson weed without symptoms under ordinary experimental conditions; in pepper, however, systemic necrosis is induced, as by all known strains. (Chester, Phytopath., $26,1936,778-785$.)
6. Annulus delphinii spec. nov. From New Latin Delphinium, generic name of host.

Common names: Delphinium-ringspot virus, perennial-delphinium ringspot virus.

Hosts : RANUNCULACEAE-Delphinium sp., perennial delphiniums. Experimentally, also to CHENOPODI$A C E A E-B e t a$ vulgaris L. CUCURBITACEAE-Cucumis sativus L., cucumber. MALVACEAE-Gossypium hirsutum L. RANUNCULACEAERanunculus asiaticus L. (symptomless carrier). SOLANACEAE-Datura stramonium L., Nicotiana alata Link and Otto, N. glutinosa L., N. rustica L., $N$. tabacum L., Petunia hybrida Vilm.

Geographical distribution: United States (California).

Induced disease: In perennial delphiniums, faint chlorotic rings with green or yellow centers on young leaves; irregular chlorotic spots, yellow bands, or irregular chlorotic rings on mature leaves.

Transmission: $\mathrm{By}$ inoculation of expressed juice in the presence of finely powdered carborundum.

Thermal inactivation: At $65^{\circ} \mathrm{C}$ in 10 minutes.

Literature: Severin and Dickson, Hilgardia, 14, 1942, 465-490.

7. Annulus bergerac H. (loc. cit., 102). From Bergerac, a town in southwest France.

Common name: Bergerac-ringspot virus.

Hosts: $\quad$ SOLANACEAE-Nicotiana tabacum L., tobacco. Experimentally, this virus has been transferred to several other solanaceous plants and to Phaseolus v'ulgaris $\mathrm{L}$., bean, in the family $L E G U M$. INOSAE.

Geographical distribution: France.

Induced disease: In tobacco, thin necrotic-ring primary lesions, followed by 
systemic mottling with some chlorotic rings on the dark green islands. Later, complete recovery occurs, with nonsterile immunity.

Transmission: By inoculation of expressed juice.

Immunological relationships: Recov- ered plants are susceptible to infection by Annulus tabaci and $A$. zonatus.

Thermal inactivation: At $80^{\circ} \mathrm{C}$ in 10 minutes.

Literature: Smith, A textbook of plant virus diseases, P. Blakiston's Son and Co., Inc., Philadelphia, 1937, 285-289.

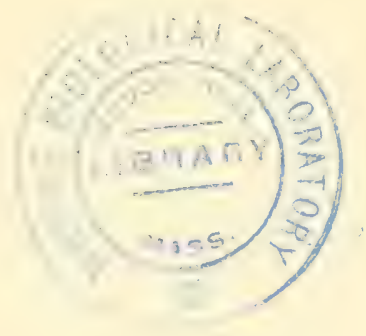




\section{FAMILY IV. RUGACEAE HOLMES.}

(Handb. Phytopath. Viruses, 1939, 114)

Viruses of the Leaf-Curl Group, causing diseases characterized by suddenly arrested development of invaded tissues, resulting in leaf curl, enations, and other deformities. Vectors, typically white-flies ( $A L E Y R O D I D A E)$. There is a single genus.

\section{Genus I. Ruga Holmes.}

$$
\text { (Loc. cit., 114.) }
$$

Characters those of the family. Generic name from Latin ruga, a wrinkle.

The type species is Ruga tabaci Holmes.

Key to the species of genus Ruga.

I. Infecting tobacco.

II. Infecting cotton.

III. Infecting cassava (Manihot).

IV. Infecting sugar-beet.

1. Ruga tabaci Holmes. (Handb. Phy topath. Viruses, 1939, 115.) From New Latin Tabacum, former generic name of tobacco.

Common names: Tobacco leaf-curl virus, kroepoek virus, curl-disease virus, crinkle-disease virus.

Hosts: $\quad S O L A N A C E A E-N i c o t i a n a$ tabacum L., tobacco. COMPOSITAEVernonia iodocalyx, V. cineria, Ageralum conyzoides L., Synedrella nodiflora Gaertn. Experimentally, also other solanaceous plants.

Insusceptible species: $M A L V A C E A E$ -Gossypium hirsutum L., cotton.

Geographical distribution: Tanganyika, Southern Rhodesia, Southern Nigeria, Nyasaland, India, Sumatra, Formosa.

Induced disease: In tobacco, leaves curled and crinkled, with occasional leafy outgrowths or enations. Veins greened and thickened. No chlorosis nor necrosis. Plant stunted.

Transmission: By white-fly, Bemisia gossypiperda Misra and Lamba ( $A L E Y$. RODIDAE). By grafting. Not by inoculation of expressed juice.

Literature: Kerling, Phytopath., 23, 1933, 175-190; Mathur, Indian Jour. Agr.

\section{Ruga tabaci.}

\section{Ruga gossypii.}

\section{Ruga bemisiae.}

\section{Ruga verrucosans.}

Sci., 3, 1933, 89-96; Matsumoto and Tateoko, Trans. Nat. Hist. Soc. Formosa, 30, 1940, 31-33; Pal and Tandon, Indian Jour. Agr. Sci., 7, 1937, 363-393; Pruthi and Samuel, ibid., $7,1937,659-670$; Storey, Nature, 128, 1931, 187-188; East African Agr. Jour., 1, 1935, 148-153; Thung, Meded. Proefsta. Vorstenl. Tabak Java, 72, 1932; 78, 1934.

2. Ruga gossypii H. (loc. cit., 116). From Latin gossypium, cotton.

Common names : Cotton leaf-curl virus, cotton leaf-crinkle virus.

Hosts: $\quad M A L V A C E A E$-Gossypium hirsutum L., cotton; G. peruvianum Cav. ; G. vitifolium Lam.; Hibiscus cannabinus L.; $H$. esculentus L.; $H$. sabdariffa L.; Althaea rosea Cav., hollyhock; Sakel (hybrid) cotton.

Geographical distribution: The Sudan and Nigeria, in Africa.

Induced disease : In cotton, clearing of veins, blistering and pale spotting of leaves; leaves puckered at edge and unsymmetrical. Internodes shortened, producing bunchy growth.

Transmission: By white-fly, Bemisia gossypiperda Misra and Lamba (ALEY. 
RODIDAE). Not through egg of insect vector. Not by inoculation of expressed juice. Not through soil. Not through seeds from diseased plants.

Literature: Bailey, Empire Cotton Growing Rev., 11, 1934, 280; Kirkpatrick, Bull. Entom. Res., 21, 1930, 127-137 ; 22, 1931, 323-363.

3. Ruga bemisiae H. (Holmes, loc. cit., 117; Ochrosticta bemisiae MeKinney, Jour. Washington Acad. Sci., 34, 1944, 149.) From New Latin Bemisia, generic name of insect vector.

Common names: Cassava-mosaic virus, cassava Kräuselkrankheit virus.

Hosts: EUPHORBIACEAE-Manihot utilissima Pohl, cassava; M. palmata Muell.; M. dulcis.

Geographical distribution: Gold Coast, Belgian Congo, French Cameroons, Rhodesia, Liberia, Madagascar, probably throughout Africa and adjacent islands; Java.

Induced disease : In Manihot utilissima, leaves unsymmetrical, curled, distorted, mottled; internodes shortened, plants stunted. Axillary buds produce an extra number of side branches.

Transmission: By white-flies ( $A L E Y$ RODIDAE), Bemisia nigeriensis Corb., in Southern Nigeria, and B. gossypiperda Misra and Lamba, in Belgian Congo and Tanganyika. White-flies infect only young leaves. Not by needle-puncture, rubbing, or hypodermic-needle injection of juice expressed from diseased plants.

Literature: Dade, Yearbk. Dept. Agr. Gold Coast, 1930, 245; Dufrénoy and Hedin, Rev. Bot. Appl., 9, 1929, 361-365; Golding, Trop. Agric., Trinidad, 13, 1936, 182-186; Kufferath and Ghesquière, Compt. rend. Soc. Biol. Belge, 109, 1932, 1146 ; Lefevre, Bull. Agr. Congo Belge, 26, 1935, 442 ; McKinney, Jour. Agr. Res., 39, 1929, 557-578; Muller, Bull. Inst. Plantenziekt., 24, 1931, 1-17; Pascalet, Agron. Colon., 21, 1932, 117 ; Staner, Bull. Agr. Congo Belge, 22, 1931, 75; Storey, East Afr. Jour., 2, 1936, 34-39 ; Storey and Nichols, Ann. Appl. Biol., 25, 1938, 790806; Zimmermann, Pflanzer, 2, 1906, 145.
4. Ruga verrucosans Carsner and Bennett. (Chlorogenus eutetticola (in error for eutettigicola, from New Lat in Eutettix, genus name of a vector, and Latin -cola, dweller in or inhabitant of) Holmes, 1939, loc. cit., 11 ; Carsner and Bennett, Science 98, 1943, 386.) From Latin, meaning: causing rough swellings.

Common name: Sugar-beet curly-top virus.

Hosts: Very wide range in many families of dicotyledonous plants. Among the horticulturally important host plants are the sugar beet (Beta vulgaris L., CHENOPODIACEAE); bean (Phaseolus vulgaris L., LEGUMINOSAE); squash (Cucurbita species, CUCURBI$T A C E A E$ ); and tomato (Lycopersicon esculcntum Mill., SOLA NACEAE).

Geographical distribution: Western North America; in Argentina a strain of virus thought to belong here has been reported but has not yet been fully described.

Induced disease: In beet, clearing of veins, leaf curling, sharp protuberances from veins on lower surface of leaves, inerease in number of rootlets, phloem degeneration followed by formation of supernumerary sieve tubes, retardation of growth. In tomato, (western yellow blight or tomato yellows), phloem degeneration followed by formation of supernumerary sieve tubes, retardation of growth, dropping of flowers and buds, rolling, yellowing and thickening of leaves, root decay, usually followed by death, sometimes by recovery. Occasionally there is relapse after recovery. In cucurbitaceous plants, stunting, bending upward of tip of runner, yellowing of old leaves, abnormally deep green in tip leaves and stem; Marblehead squash is tolerant, showing only mild witches' broom formation and phyllody. In bean, infected when young, thickening and downward curling of first trifoliate leaf, which becomes brittle and will break easily from the stem; leaves become chlorotic, plant stops growing and usually dies soon; older plants are also susceptible to infection; they may survive until 
the end of the season, showing puckering and downward curling of leaves at the top of the plant, reduction in size of new leaves, and shortened internodes, or they may grudually become chlorotic and die.

Transmission: By leafhopper, Eutettix tenellus (Baker) (CICADELLIDAE) with 4 to 12 hour preinfective period. Through dodder, C'uscuta campestris Yuncker (CONVOLVULACEAE). Not, with any regularity at least, by mechanical inoculation of expressed juice. Not through seeds of diseased plants to seedlings germinating from them. The leafhopper, Agalliana ensigera Oman (CICADELLIDAE), is said to transmit a South American strain of sugar-beet curly-top virus, but evidence for identity of the virus has not yet been reported in detail.

Thermal inactivation: At $75^{\circ}$ to $80^{\circ} \mathrm{C}$ in 10 minutes.

Filterability: Passes Berkefeld $\mathrm{V}, \mathrm{N}$, and $W$, Mandler medium and fine, and Chamberland $\mathrm{L}_{1}, \mathrm{~L}_{3}, \mathrm{~L}_{5}, \mathrm{~L}_{7}, \mathrm{~L}_{3}, \mathrm{~L}_{11}$ and $\mathbf{L}_{13}$ filters.

Other properties: Withstands alcohol and acetone treatments. A pH of 2.9 or lower inactivates, but an alkaline reaction as high as pH 9.1 does not inactivate, in 2 hours. Virus active after at least 8 years in tissues of thoroughly dried young sugar-beet plinnts, 6 months in dried leafhoppers, and 10 months in dried phloem exudate.

Strains: In general it has proved possible to modify strains by host passage, some hosts like Chenopodium murale L. appearing to select less virulent strains, others like Stellaria media (L.) Cyr. reversing this selection and restoring virulence.

Literature: Bennett, Jour. Agr. Res., $48,1934,665-701 ; 50,1935,211-241 ; 56$, 1938, 31-52 ; Phytopath., 32, 1942, 826827 ; Carsner, Phytopath., 15, 1925, 745757 ; U. S. Dept. Agr., Tech. Bull. 360, 1933; Jour. Agr. Res., 33, 1926, 345-348; Dana, Phytopath., 28, 1938, 649-656; Fawcett, Revista Industrial y Agrícola de Tucumán, 16, 1925, 39-46; Fife, Phytopath., 30, 1940, 433-437; Giddings, Phytopath., 27, 1937, 773-779; Jour. Agr. Res., 56, 1938, 883-894; Lackey, Jour. Agr. Res., 55, 1937, 453-460; Lesley and Wallace, Phytopath., 28, 1938, 548-553; Murphy, ibid., 30, 1940, 779-784; Severin, Hilgardia, 3, 1929, 595-636; Severin and Freitag, ibid., 8, 1933, 1-48; Severin and Henderson, Hilgardia, 3, 1928, 339-393; Severin and Swezy, Phytopath., 18, 1928, 681-690; Shaw, U. S. Dept. Agr., Bull. 181,1910 . 


\section{FAMILI V. SAVOIACEAE HOLMES.}

(Handb. Phytopath. Viruses, 1939, 131.)

Viruses of the Savoy-Disease Group, causing diseases characterized mainly by crinkling of foliage. Vectors, true bugs (PIESMIDAE and MIRIDAE). There is a single genus.

\section{Genus I. Savoia Holmes.}

(Loc. cit., 131.)

Characters those of the family. Generic name from French chou de Savoie, cabbage of Savoy, a cabbage with wrinkled and curled leaves.

The type species is Savoia betae Holmes.

Key to the species of genus Savoia.

I. Infecting beet.

1. Savoia betae.

2. Savoia piesmae.

II. Infecting rape and rutabaga.

\section{Savoia napi.}

1. Savoia betae Holmes. (Handb. Phytopath. Viruses, 1939, 132.) From Lat in beta, beet.

Common names: Beet-Kräuselkrankheit virus, sugar-beet leaf-curl virus, sugar-beet leaf-crinkle virus, Kopfsalat virus.

Host : CHENOPODIACEAE-Beta vulgaris L., beet.

Geographical distribution: Germany, Poland.

Induced disease: In beet, veins of leaves swollen, retarded in growth, causing crinkling. New leaves remain small and incurved, forming a compact head. Old leaves die; plant succumbs before harvest time. Prepatent period in plant, 3 to 9 weeks.

Transmission: By tingid bug, Piesma quadrata Fieb. (PIESMIDAE). Not by inoculation of expressed juice.

Literature: Wille, Arb. Biol. Reichsanst. Land- u. Forstw ., 16, 1928, 115-167.

2. Savoia piesmae H. (loc. cit., 132). From New Latin Piesma, generic name of insect vector.

Common name: Beet-savoy virus.

Host: CHENOPODIACEAE-Beta vulgaris L., beet.
Geographical distribution: United States (Michigan, Ohio, Minnesota, Nebraska, South Dakota, Colorado, Wyoming) and Canada.

Induced disease: In beet, leaves dwarfed, curled down, small veins thickened. Phloem necrosis in roots. Prodromal period in plant, 3 to 4 weeks. Transmission: By tingid bug, Piesma cinerea (PIESMIDAE). Not by inoculation of expressed juice.

Literature: Coons et al., Phytopath., 27, 1937, 125 (Abst.); Hildebrand and Koch, ibid., 32, 1942, 328-331.

3. Savoia napi H. (loc. cit., 133). From New Latin Napus, former generic name of rape, Brassica napus L.

Common name : Rape-savoy virus.

Hosts : CRUCIFERAE-Brassica napus L., rape ; $B$. napobrassica Mill., rutabaga.

Geographical distribution: Germany.

Induced disease : In rape, twisting and crinkling of young leaves; premature death of old leaves and of plants; in surviving plants, inhibition of growth in spring. In rutabaga, mottling and crinkling of leaves, with formation of fissures at leaf edges. Plants rarely killed. 
Transmission: By inoculation of expressed juice. By the tarnished plant bug, Lygus pratensisLinn. (MIRIDAE). The insect vector retains this virus during intervals between crops.
Literature: Kaufmann, Arb. Biol. Reichsanst. Land- u. Forstw., 21, 1936, 605-623; Mitteil. Landwirtsch., 37, 1936; Pape, Deutsch. Landwirtsch. Presse, $26,1935$. 


\title{
FAMILY VI. LETHACEAE HOLMES.
}

\author{
(Handb. Phytopath. Viruses, 1939, 135.)
}

Virus strains of the Spotted-Wilt Group, causing diseases characterized by bronzing of foliage, streaking of stems, blighting of tips, necrotic spotting of foliage. Hosts, higher plants; vectors, thrips (THRIPIDAE). There is a single genus.

\section{Genus I. Lethum Holmes.}

$$
\text { (Loc. cit., 135.) }
$$

Characters those of the family. Generic name from Latin lethum, death. At present there is but one known species, though this is reported to be nearly world-wide in distribution. In some areas it may have been confused with entities needing separate recognition.

The type species is Lethum australiense Holmes.

1. Lethum australiense Holmes (loc. cit., 136). From Australia, where virus was first described.

Common names: Tomato spotted-wilt virus, kromnek or Kat River disease virus. Also, pineapple yellow-spot or side-rot virus (according to Sakimura, Phytopath., 30, 1940, 281-299).

Hosts: Very numerous species in many families of higher plants. Among those most often noted are : SOLANACEAELycopersicon esculentum Mill., tomato; Nicotiana tabacum L., tobacco; Solanum tuberosum L., potato. COMPOSITAE -Lactuca sativa L., lettuce. LEGT:IINOSAE-Pisum sativum L., pea. BROMELIACEAE-Ananas comosus Merr., pineapple.

Geographical distribution: Australia, British Isles, Lnited States, South Africa Hawaii, New Zealand, Europe, China, South America.

Induced disease: In tomato, bronze ring-like secondary lesions, plant st unted, some necrosis; later yellowish mosaic with some leaf distortion. Fruit frequently marked with concentric rings of pale red, yellow, or white. In tobacco, primary necrotic lesions followed by systemic necrosis, with stem streak, crooknerk, often stunting with subsequent wilting and death, sometimes temporary rerovery followed by recurrence of systemic necrosis. In lettuce, plant yellowed, retarded in growth; brown blem- ishes in central leaves, affected spots dying, becoming like parchment but with brown margins. Axillary shoots may show chlorotic mottling. In pea, purplish necrotic streaks on stem; at first, leaves mottled; later, necrotic spots damage foliage; pods show circular necrotic spots or wavy lines, or, if severely affected, may collapse; seeds may show necrotic lesions. In potato, zonate necrotic spots on upper leaves, necrotic streaks on stems; stems collapse at top; plant is stunted, yield of tubers small. In pineapple, at first an initial spot or primary lesion $\frac{1}{8}$ to $\frac{1}{2}$ inch in diameter, raised, yellowish, on upper surface of young leaf; later chlorotic spotting of young leaves, crook-neck because of necrotic foei in stems and fruits (side rot); plant may die.

Transmission: By inoculation of expressed juice; the addition of fine carborundum powder to inoculum facilitates transmission by rubbing methods. By thrips, Frankliniella lycopersici Andrewartha (formerly identified as $F$. insularis Franklin), $F$. occidentalis Perg., $F$. moultoni Hood. and F. schultzei (Trybom) (THRIPIDAE). Also by Thrips tabaci Lind. (THRIPIDAE). In $F$. lycopersici, thrips must pick up virus while still a nymph; virus persists through pupation and emergence as adult ; preinfective period in vector, 5 to 9 days. Virus is not transmitted through eggs of 
infective thrips. Probably not through seeds of infected plants. Not through soil.

Immunological relationships: Infects tobacco plants previously infected with tobacco-mosaic, potato-mottle, tobaccoringspot, and tomato-ringspot viruses.

Thermal inactivation: At $42^{\circ} \mathrm{C}$ in 10 minutes.

Filterability: Passes Gradocol membrane of 0.45 micron pore diameter.

Other properties: Virus readily inactivated by desiccation or by action of oxidizing agents; activity prolonged by presence of sodium sulfite, cystein, or by low temperatures. Unstable at $\mathrm{pH}$ values below 6 and above 9 .

Literature: Ainsworth et al., Ann. Appl. Biol., 21, 1934, 566-580; Andrewartha, Trans. Roy. Soc. of So. Australia, 61, 1937, 163-165; Bald and Samuel, ibid., 21, 1934, 179-190; Berkeley, Scientific Agr., 15, 1935, 387-392; Best, Austral. Chem. Inst. Jour. and Proc., 4, 1937, 375-392; Best and Samuel, Ann. Appl. Biol., 23, 1936, 509-537; 759-780; Carter, Phytopath., 29, 1939, 285-287; Lewcock, Queensland Agr. Jour., 48, 1937, 665-672; Linford, ibid., 22, 1932, 301-324; Magee, Agr. Gaz. of New South Wales, 47, 1936, 99-100, 118; McWhorter and Milbrath, Phytopath., 25, 1935, 897-898 (Abst.); Oregon Agr. Exp. Sta., Circ. 128, 1938; Milbrath, Phytopath., 29, 1939, 156-168; Moore, Nature, 147, 1941, 480-481; Moore and Anderssen, Union of So. Africa, Dept. Agr., Science Bull. 182, 1939; Parris, Phytopath., 30, 1940, 299-312; Rawlins and Tompkins, ibid., 26, 1936, 578-587; Sakimura, ibid., 30, 1940, 281-299; Samuel and Bald, Ann. Appl. Biol., 20, 1933, 70-99; Jour. Agr. So. Australia, 37, 1933,
190-195; Samuel et al., Coune. Scient. Indus. Res., Austral., Bull. 44, 1930; Ann. Appl. Biol., 22, 1935, 508-524; Shapovalov, Phytopath., 24, 1934, 1149 (Abst.); Smith, Nature, 127, 1931, 852853; Ann. Appl. Biol., 19, 1932, 305-330; Jour. Minist. Agr., 39, 1933, 1097-1104; Jour. Roy. Hort. Soc., 60, 1935, 304-310; Snyder and Thomas, Hilgardia, 10, 1936, 257-262; Takahashi and Rawlins, Phytopath., 24, 1934, 1111-1115; Taylor and Chamberlain, New Zealand Jour. Agr., 54, 1937, 278-283; Whipple, Phytopath., $26,1936,918-920$.

Strains: A strain differing somewhat from the type, var. typicum H. (loc. cit., 136), has been described as damaging tomatoes in the northwestern United States. It has been given a distinctive varietal name:

1a. Lethum anstraliense var. lethale $\mathbf{H}$. (loc. cit., 138). From Latin lethalis, deadly. Common names: Tip-blight strain of tomato spotted-wilt virus, Oregon tip-blight virus, tomato die-back streak virus, tomato tip-blight virus. Differs from the type in causing necrotic leaf spotting, stem streaking, and tip blight ing in most hosts, without mottling or bronzing of foliage; yet in Tropaeolum majus $\mathrm{L}$., there is little necrosis. In tomatn, systemic necrosis, terminal shoots blighted and blackened; dead tips stand upright above living foliage. Fruits rough and pitted, with internal pockets of necrotic tissue or with subepidermal necrosis, appearing externally as concentric brown bands. (McWhorter and Milbrath, Oregon Agr. Exp. Sta., Circ. 128, 1938; Milbrath, Phytopath., 29, 1939, 156-168.) 
Siborder III. Zoophagineae subordo novus.

From Greek phagein, to eat, and zoon, an animal. Viruses infecting animals but having no plant hosts, so far as known.

Key to the families of suborder Zoophaginae.

1. Inducing diseases in insects as exclusive hosts.

Family I. Borrelinaceae, p. 1225.

2. Inducing diseases of the pox group.

Family II. Borreliotaceae, p. 1229.

3. Inducing diseases of the encephalitis group.

Family III. Erronaceae, p. 1248.

4. Inducing diseases of the yellow-fever group.

Family IV. Charonaceae, P. 1265.

5. Inducing diseases of the infectious anemia group.

Family V Trifuraceae, p. 1282.

6. Inducing diseases of the mumps group.

Family VI. Rabulaceae, p. 1284.

\section{FAMILY I, BORRELINACEAE FA.Y. NOV.}

Viruses causing polyhedral, wilt, and other diseases in arthropods. The genus Borrelina Paillot was originally spelled Borrellina by error; from Borrel, name of French scientist.

\section{Key to the genera of family Borrelinaceae.}

I. Known only as attacking lepidopterous insects.

Genus I. Borrelina, p. 1225.

II. Known only as attacking the honey bee, a hymenopterous insect.

Genus II. Morator: p. $122 \%$.

\section{Genus I. Borrelina Paillot.}

(Compt. rend. Acad. Sci., Paris, 182, 1926, 182.)

Viruses inducing polyhedral, wilt, and other diseases; hosts, Lepidoptera, so far as known.

The type species is Borrelina bombycis Paillot.

\section{Key to the species of genus Borrelina.}

I. Attacking silkworm.

II. Attacking nun moth.

1. Borrelina bombycis.

III. Attacking gypsy moth.

2. Borrelina efficiens.

IV. Attacking cabbage worm.

3. Borrelina reprimens.

4. Borrelina brassicae.

5. Borrelina pieris. 


\section{Borrelina bombycis Paillot.} (Compt. rend. Acad. Sci., Paris, 182, 1926 , 182.) From Latin bombyx, silkworm. (Note: Coccus-like bodies surrounded by non-staining substances, associated with the induced disease, received the provisional name Chlamydozoon bombycis from Prowazek, Arch. f. Protistenkunde, 10, 1907, 363.)

Common names: Silkworm-jiundice virus, silkworm-grasserie virus, silkworm wilt virus, Gelbsucht virus, Fettsucht virus.

Host : BOHBYCIDAE-Bombyx mori (L.), silkworm.

Geographical distribution: Japan, italy, France.

Induced disease: In silkworm, after prodromal period of 5 days or more, yellow spots on skin, polyhedral bodies in blood, inactivity, loss of appetite, irritability, weakening of body facilitating rupture from mechanical stress, eventual death.

Transmission: By feeding. Experi mentally, also by injection.

Serological relationships: Specifie agglutination, precipitation, and complement fixation.

Thermal inactivation: At $60^{\circ} \mathrm{C}$ in 15 to 20 minutes in blood.

Filterability: Passes Berkefeld $\mathrm{X}$ and $V$, Chamberland $L_{1}, L_{2}$, and $L_{3}$ filters.

Other properties: May survive at least 2 years in dry state. Stable between pH 5 and about pH 9. Sedimentation constant $17 \mathrm{~s}$.

Literature: Aoki and Chigasaki, Cent. f. Bakt., I Abt., Orig., 86, 1921, 481-485; Glaser and Lacaillade, Am. Jour. Hyg., 20, 1934, 454-464; Glaser and Stanley, Jour. Exp. Med., 77, 1943, 451-466; v. Prowazek, Cent.f. Bakt., I Abt., Orig., 67, 1912, 268-28t; Suzuki, Bull. Imperial Kyoto Sericultural College, 1, 1929, 4575; Trager, Jour. Exp. Med., 61, 1935, 501-513.

\section{Borrelina efflciens spec, nov. From}

Latin effciens, effective, in reference to effectiveness of this virus in controlling nun-moth infestations.

Common names: Nun-moth disease virus, nun-moth wilt virus, Wipfelkrankheit virus.

Host : LYMAVTRIIDAE-Lymantria monacha (L.), nun moth.

Geographical distribution: Europe.

Induced disease : In eggs, larvae, pupae and occasionally adults of nun moth, polyhedral bodies in affected tissues. Blood of sick larvae turbid; later, blood cells few ; contents of body finally become a gray-brown, semifluid mass.

Transmission: By feeding.

Thermal inactivation : At 55 to $60^{\circ} \mathrm{C}$ in 5 to 10 minutes.

Filterability: Fails to pass Berkefeld and Chamberland filters.

Other properties: May remain viable at least 2 years in dry state.

Literature: Escherich and Miyajima, Naturwissens. Ztschr. f. Forst-u. Landwirtschaft, 9, 1911, 381-102; Wachtl and Kornauth, Mitth. a. d. forstl. Versuehswesen Üsterreichs, 16, 1893, 1-38; Wahl, Centralbl. Gesam. Forstw., 35, 1909, 164172 ; 212-215; 36, 1910, 377-397; 37, 1911, $247-268 ; 38,1912,355-378$.

3. Borrelina reprimens spec. nov. From Latin reprimere, to restrain.

Common name: Gypsy-moth wilt virus.

Host: LYMANTRIIDAE-Porthetria dispar (L.), gypsy moth.

Geographical distribution: United States.

Induced disease: In gypsy moth caterpillar, flaccidity, disintegration of tissues, eventual collapse as a watery sack. Death occurs in 13 to 29 (average 21) days after infection; caterpillar may remain attached to its support by prolegs; skin ruptures easily. Polyhedral bodies originate in nuclei of the tracheal matrix, hypodermal, fat, and blood cells.

Transmission: By feeding on contam- 
inated foliage. Not through undamaged skin.

Filterability: Passes Berkefeld N, not Pasteur-Chamberland F, filter.

Literature : Chapman and Glaser, Jour. Econ. Entomol., 8, 1915, 140-150; 9, 1916, 149-167; Glaser, Jour. Agr. Res., 4, 1915, 101-128; Science, 48, 1918, 301-302; Glaser and Chapman, Jour. Econ. Entomol., 6, 1913, 479-48s.

\section{Borrelina brassicae Paillot.} (Compt. rend. Acad. Sci., Paris, 182, $1926,182$.$) From name of host, Pieris$ brassicae.

Common name: Cabbage-worm grasserie virus.

Host : PIERIDAE-Pieris brassicae (L.), cabbage worm.

Induced disease: In cabbage worm, no nuclear or cytoplasmic inclusions; nuclei of fat and hypodermal cells hypertrophied and soon disorganized.

Transmission: By feeding.

Other properties: Described as submicroscopic in size, intracytoplasmic.
Literature: Paillot, loc. cit.; Ann. Inst. Pasteur, 40, 1926, 314-452; L'infection chez les insectes. Immunité et symbiose, 535 pages, Trévoux, Patissier, 1933.

5. Borrelina pieris Paillot. (Compt. rend. Acad. Sci., Paris, 182, 1926, 182.) From New Latin Pieris, generic name of host.

Common name : Virus of nuclear disease of pierids.

Host: PIERIDAE-Pieris brassicae (L.), cabbage worm.

Induced disease: In cabbage worm, body yellowish below, tears easily just before death; chromatin of nuclei in fat and blood cells condensed in irregular masses; cytoplasmic inclusions staining faintly red in Giemsa preparations.

Transmission: By feeding.

Other properties: Described as intracytoplasmic, less than 0.1 micron in diameter.

Literature: Paillot, loc. cit.; Ann. Inst. Pasteur, 40, 1926, 314-452; L'infection chez les insectes. Immunité et symbiose, 535 pages, Trévoux, Patissier, 1933.

Appendix: Borrelina flacheriae quoted from Paillot, L'infection chez les insects. 535 pp., Trevoux, Patissier, 1935, see p. 96. Cause of gattine in the silkworm, Bombyx mori L. No previous reference to a deseription of this species has been found.

\section{Genus II. Morator gen. nov.}

Only one species at present, inducing the disease known as sacbrood of the honey bee. Generic name from Latin morator, loiterer. The type, and only, species is Morator aetatulae spec, nov.

1. Morator aetatulae spec. nov. From Latin actatula, early period of life, in reference to attack on immature stages of host, exclusively.

Common name: Honey-bee sacbrood virus.

Host: APIDAE-A pis mellifera L., honey bee (immature stages only).

Insusceptible species: LYMANTRII$D A E$-Porthetria dispar (L.), gypsy moth.

Geographical distribution: United States.
Induced disease: In the honey bee, immature stages only are susceptible; infected larvae die, usually after capping, some of the dead brood being uncapped by the bees. Occasionally caps are punctured. Affected areas of comb are usually small and scattered. Each larva is extended along its cell, head turned upward toward the roof. A larva recently dead appears light yellow, light gray, or light brown, soon darkening to brown or almust black. Cuticle of dead larva tough, permitting extraction of the sac- 
like mass without rupture; contents watery with many suspended, fine, brown particles. There are no characteristic intracellular bodies in affected tissues. Dead larvae eventually dry down to form scales that are black and roughened, that separate readily from the cell wall, and that may be lifted out intact. Colonies tend to lose virus spontaneously.

Transmission: By contamination of food. Not by hands, clothing, or tools. Perhaps through water supply of inseets. Thermal inactivation: In water, at $58^{\circ}$
$\mathrm{C}$ in 10 minutes. In honey, at 70 to $73^{\circ}$ $\mathrm{C}$ in 10 minutes.

Filterability: Passes Berkefeld and Pasteur-Chamberland filters.

Other properties: Withstands drying 20 , not 22. days, exposure to sunlight 7 hours or less, storage in honey a month or more, $\frac{1}{2}$ to 2 per cent aqueous solutions of carbolic arid 3 weeks or more.

Literature: McCray and White, U. S. Dept. Agr., Dept. Bull. 671, 1918; White, U. S. Dept. Agr., Bur. of Entomol., Circ. 169, 1913; U. S. Dept. Agr., Dept. Bull. 92, 1914; ibid., Dept. Bull. 431, 1917. 
FAMIILY II. BORRELIOTACEAE FAM. NOV.

Viruses of the Pox Group, inducing diseases characterized in general by discrete primary and secondary lesions of the nature of macules, papules, vesicles, or pustules.

\section{Key to the genera of family Borreliotaceae.}

I. Viruses of the Typical Pox-Disease Group.

Genus I. Borreliota, p. 1229.

II. Viruses of the Varicella Group.

Genus II. Briareus, p. 1233.

III. Viruses of the Herpes Group.

Genus III. Scelus, p. 1234.

IV. Viruses of the Foot-and-Mouth-Disease Group.

Genus IV. Hostis, p. 1239.

Y. Viruses of the Wart-Disease Group.

Genus V. Molitor, p. 1240.

\section{Genus I. Borreliota Goodpasture.}

(Science, 77, 1933, 121.)

Viruses of the Typical Pox-Disease Group, inducing diseases characterized by formation of papules, pustules, and scabs, shed with or without scarring. Generic name from Borrel, investigator who first discovered the specific granules of fowl pox and Latinized name of the smallest Greek letter, iota, signifying smallest particle. The name Cytoryctes variolae Guarnieri 1892 was based on intracellular inclusions, Guarnieri bodies, as supposed sporoznan parasites (Calkins, Jour. Med. Res., 11, 1904, $136-172)$.

The type species is Borreliota avium Goodpasture.

\section{Key to the species of genus Borreliota.}

I. Affecting domestic fowl.

1. Borreliota avium.

II. Affecting man principally, although strains have become adapted to cow, rabbit, etc.

III. Affecting swine.

\section{Borreliota variolae.}

\section{Borreliota suis.}

1. Borreliota avium (Lipschütz) Goodpasture. (Strongyloplasma avium Lipschütz, in Kolle, Kraus and Chlenhuth. Handbuch der pathogenen Mikroorganismen, 3 Auft ., 8, 1930, 314; Goodpasture, Science, $7 \gamma, 1933,121$.$) From Latin$ aves, fowl of the air.

Common names: Fowl-pox virus; also known as poultry-pox virus, chicken-pox virus (but not the virus of the same name attacking man rather than the chicken), or virus of epithelioma contagiosum of fowls; strains have been studied under

the names Kikuth's canary virus and pigeon-pox virus.

Hosts: Chicken, turkey, pigeon, goose, duck, guinea fowl, quail, hawk, pheasant, part ridge, bunting sparrow, canary. Experimentally, also English sparrow, chich embryo.

Insusceptible species: Man, goat, sheep, mouse, rat, guinea pig.

Geographical distribution: Europe, Asia, Torth America; perhaps coextensive with the area in which chickens are grown under conditions of domestication. 
Induced disease: In chicken, hyperplastic nodular lesions of the skin, diphtheritic membranes in mouth and throat, discharges from eyes and nose; nodules eventually dry up and fall off, usually without leaving scars. Inclusion bodies, known as Bollinger bodies, believed to represent aggregates of minute Borrel bodies or virus particles, leave much grayish-white ash when incinerated; break readily after digestion by 1 per cent trypsin in 0.2 per cent sodium bicarbonate. Borrel bodies coccoid, 0.25 microns in diameter. On chorioallantoic membrane of chick embryo, proliferation and hyperplasia, or necrosis.

Transmission: By contact, perhaps through wound infection. By bloodsucking dipterous insects. Experimentally, by scarification of skin or buccal mucosa; by intravenous, intradermal, subcutaneous, intramuscular, or intraperitoneal inoculation. May be passed in series by nasal instillation in chickens, obvious mucosal changes occurring only occasionally. Experimentally, by mosquitoes (CULICIDAE), Aëdes aegypti L., A. stimulans Walker, A. vexans Meigen (as long as 27 days from time of feeding on infective material), and Culex pipiens L. (indefinitely after infective feeding, as long as the individual mosquito lives); in $C$. pipiens, the virus has been found also under natural conditions.

Serological relationships: Neutralizing and elementary-body-agglutinating antisera specific. Antivaccinial serum from rabbit ineffective against fowl-pox virus, although neutralizing vaccinia virus.

Immunolugical relationships: No cross immunity with respect to vaccinia virus in the chicken.

Thermal inactivation: At $60^{\circ} \mathrm{C}$ in 8 minutes; at $56^{\circ} \mathrm{C}$ in 30 minutes.

Filterability: Passes Berkefeld V, not Chamberland $\mathbf{L}_{2}$, filter candle.

Other properties: Drying at room temperature in vacuo does not inactivate. Viable after storage at least 24 months at 0 to $4^{\circ} \mathrm{C}$, dry.

Strains: A strain known as Kikuth's canary virus has been studied in some detail. When introduced into the rabbit it induces formation of neutralizing antibodies that react strongly with homologous virus, moderately against fowl-pox virus. Antivaccinial serum is ineffective against it. In canaries, it induces proliferation of dermal epithelium with cytoplasmic inclusions, the inflammatory process being characterized by predominantly mononuclear cells with vacuolated cytoplasm; in the lung there is massive accumulation of large mononuclear cells containing the specific cytoplasmic inclusions; the disease is regularly fatal. Passes Berkefeld N filter. Size estimated as 120 millimicrons by centrifugation. (Bechhold and Schlesinger, Ztschr. f. Hyg., 115, 1933, 354357; Burnet, Jour. Path. and Bact., 37, 1933, 107-122; Burnet and Lush, Brit. Jour. Exp. Path., 17, 1936, 302-307; Gaede, Cent. f. Bakt., I Abt., Orig., 135, 1935, 342-346; Kikuth and Gollub, ibid., $125,1932,313-320$.

Literature: Andervont, Am. Jour. Hyg., 6, 1926, 719-754 ; Brandly and Dunlap, Jour. Am. Vet. Med. Assoc., 95, 1939, 340-349; Brandly et al., Am. Jour. Vet. Res., 2, 1941, 190-192; Brody, Cornell Agr. Exp. Sta. (Ithaca). Memoir 195, 1936; Buddingh, Jour. Exp. Med., 67, 1938, 933-940; Burnet and Lush, Brit. Jour. Exp. Path., 17, 1936, 302-307; Danks, Am. Jour. Path., 8, 1932, 711-716; Findlay, Proc. Roy. Soc. London, Ser. B, 102, 1928, 354-379; Goodpasture and A. M. Woodruff, Am. Jour. Path., 6, 1930, 699-712; Goodpasture and C. E. Woodruff, ibid., 7, 1931, 1-8; Irons, Am. Jour. Hyg., 20, 1934, 329-351; Kligler and Ashner, Proc. Soc. Exp. Biol, and Med., 28, 1931, 463-465; Kligler et al., Jour. Exp. Med., 49, 1929, 6-9-660; Ledingham, Lancet, 221, 1931 (2), 525-526; Ludford and Findlay, Brit. Jour. Exp. Path., 7 1926, 256-264; Matheson et al, Poultry Science, 10, 1931, 211-223; Megrail, Am. Jour. Hyg., 9, 1929, 462-465; Nelson, Jour. Exp. Med., 74, 1941, 203-212; A. M. Woodruff, and Goodpasture, Am. Jour. 
Path., 7, 1931, 209-222 ; C. E. Woodruff, ibid., 6, 1930, 169-174; C. E. Woodruff, and Goodpasture, ibid., 5, 1929, 1-10; $6,1930,713-720$.

\section{Borreliota variolae (Lipschütz)} Goodpasture. (Strongyloplasma variolae Lipschütz, in Kolle, Kraus and Lhlenhuth, Handbuch der pathogenen Mikroorganismen, 3 Aufl., 8, 1930, 317; Goodpasture, Science, 77, 1933, 121.) From New Latin variola, smallpox.

Common names: Tariola virus, smallpox virus. Most studies of this virus have been concerned with the vaccinia strain; see Strains below.

Hosts: Man, cow and rabbit are susceptible to strains that appear especially adapted to them (see Strains below). Experimentally, also chicken (and chick embryo); Chrysemys marginata, turtle; guinea pig, horse, pig; Macaca mulatta (Zimmermann), rhesus monkey; $M$. irus, cynomolgus monkey; orang-outang; $\mathbf{M a}$ cacus fuscatus.

Geographical distribution: Nearly world-wide, except where excluded by isolation or protective vaccination.

Induced disease: In man, mild to severe smallpox, sometimes with pocks few and discrete but often with pocks numerous and coalescing; onset sudden, 6 to 22 days (average 12) after infection; headache, vomiting, fever, often rashes on body before appearance of the specific eruption, bright red spots becoming vesicular and eventually pustular; the pocks are commonest on face, forearms, wrists, palms of hands, and soles of feet; pustules gradually become flattened scabs and drop off, leaving no scar if superficial and not secondarily infected; in hemorrhagic smallpox there are numerous hemorrhages into the skin and mortality is high, death often preceding formation of pustules; severity of disease and mortality roughly proportional to the amount of eruption on the face.

Transmission: $\mathrm{By}$ contact with infected individuals or contaminated articles; perhaps by droplet infection, obvious primary lesions characterizing experimental transmission by scarification but not natural spread.

Serological relationships: Hyperimmune calf serum neutralizes virus. Neutralization depends on an antibody not involved in agglutination and precipitation. Antivaccinial serum gives complement fixation in the presence of variola virus. One agglutinogen $(\boldsymbol{L})$ labile at $56^{\circ} \mathrm{C}$, one (S) stable at $95^{\circ} \mathrm{C}$; both are parts of a single protein but can be degraded independently; chymotrypsin destroys activity of $\mathrm{S}$, not $\mathrm{L}$. Increasing neutralization in immune serum and virus mixtures in vitro with progressive incubation; partial reactivation on simple dilution. Antivaccinial sera agglutinate Paschen bodies of vaccinia but not Borrel bodies of fowl pox; anti-fowl-pox sera agglutinate Borrel but not Paschen bodies. No cross reactions with herpes virus.

Immunological relationships: In vaccinia-immune swine, protective substances pass via colostrum, conveying passive immunity to young for 2 to 3 months after birth. In man, immunity against variola virus is conferred by earlier infection with vaccinia strain. In hen, previous infection with fowl-pox virus does not immunize with respect to vaccinia virus.

Thermal inactivation: At $55^{\circ} \mathrm{C}$ in 20 minutes.

Filterability: Passes Berkefeld $V$, not Mandler, filter.

Other properties: Density about 1.16. Sedimentation constant $5000 \times 10^{-13}$ (corrected to water at $20^{\circ} \mathrm{C}$ ). Retains activity in glycerine best at $\mathrm{pH}$ 7.0. 0.1 per cent gelatin delays spontaneous inactivation at 5 to $10^{\circ} \mathrm{C}$. Withstands absolute alcohol, ether, acetone, and petroleum ether 1 hour in dry samples at $4^{\circ} \mathrm{C}$ without decrease in activity. Inactivated without disruption by sonic vibrations of about 8900 cycles per second. Diameter estimated as 125 to 175 millimicrons by filtration; 236 to 252 millimicrons by ultracentrifugation. Electron micrographs show limiting surface 
membrane, dense granules (usually 5) within; tendency to rectangular outlines with rounded corners. At least 5.6 per cent of virus is reported to be thymonucleic acid. Contains nitrogen, 15.3 per cent; carbon, 33.7 per cent; phosphorus, 0.57 per cent; phospholipid (lecithin), 2.2 per cent; neutral fat, 2.2 per cent; reducing sugars after hydrolysis, 2.8 per cent; cystine, 1.9 per cent; copper, 0.05 per cent.

Strains: Besides the typical variola strain, var. hominis Goodpasture (Science, $7 \%, 1933,121$ ), several distinctive strains have been studied. A spontaneous cowpox strain differs in some antigens but affords cross immunity with respect to var. bovis Goodpasture (loc. cit., 121), vaccinia virus, which in turn immunizes against typical variola virus. A spontaneous rabbit-pox strain, serologically resembling neurovaceine virus, is believed to exist independently in Europe and the United States. The varieties equi (horse-pox virus), porci (swine strain), and ovium (sheep and goat pox virus) have been attributed to this species by Goodpasture (loc. cit., 121). The alastrim strain (causing variola minor) differs from the type in producing a relatively mild disease in man and in inducing the formation of a distinctive type of intracellular inclusion in affected tissues.

Literature: Amies, Jour. Path. and Bact., 47, 1938, 205-222; Andervont, Am. Jour. Hyg., 7, 1927, 804-810; Behrens and Ferguson, Jour. Inf. Dis., 56, 1935, St-88; Behrens and Nielson, ibid., 56, 1935, 41-48; Buddingh, Am. Jour. Hyg., 38, 1943, 310-322; Craigie and Wishart, Brit. Jour. Exp. Puth., 15, 1934, 390-398; Jour. Exp. Med., 64, 1936, 819-830; Dearing, Am. Jour. Hyg., 20, 1931, 432-443 ; Douglas et al., Jour. Path. and Bact., 32, 1929, 99-120; Downie, Brit. Jour. Exp. Path., 20, 1939, 158-176; Eagles, ibid., 16, 1935, 181-188; Elford and Andrewes, Brit. Jour. Exp. Path., 13, 1932, 36-42 ; Goodpasture, Woodruff, and Buddingh, Am. Jour. Path., 8, 1932, 271-282; Green et al., Jour. Exp. Med., 75, 1942, 651-656;
Greene, ibid., 61, 1935, 807-831 ; Herzberg, Ztschr. Immunitätsforsch. u. exper. Therap., 86, 1935, 417-441; Hoagland et al., Jour. Exp. Med., 71, 1940, 737-750; 72, $1940,139-147$; 74, 1941, 69-80, 133-144; $76,1942,163-173$; Hu et al., Jour. Exp. Med., 63, 1936, 353-378; Keogh, Jour. Path. and Bact., 43, 1936, 441-454; Ledingham, Brit. Jour. Exp. Path., 5, 1924, 332-349; Jour. Path. and Bact., 35, 1932, 140-142; Macfarlane and Dolby, Brit. Jour. Exp. Path., 21, 1910, 219-227; Macfarlane and Salaman, ibid., 19, 1938, 184-191; MeFarlane et al., ibid., 20, 1939, 485-501 ; Moriyama, Arch. f. Virusforsch., 1, 1940, 422-429; Nelson, Jour. Exp. Med., 60, 1934, 287-291; 78, 1943, 231-239; Nye and Parker, Am. Jour. Path., 5, 1929, 147-155; Parker, Jour. Exp. Med., 67, 1938, 361-367, 725-738; Parker and Muckenfuss, Jour. Infect. Dis., 53, 1933, 44-54; Parker and Rivers, Jour. Exp. Med., 62, 1935, 65-72; 64, 1936, 439-452 ; 65, 1937, 243-249; Paschen, Deutsch. med. Wchschr., 39, 1913, 21322136; Pearce et al., Jour. Exp. Med., 63, 1936, 241-258, 491-507; Jour. Path. and Bact., 43, 1936, 299-312; Pickels and Smadel, Jour. Exp. Med., 68, 1938, 583606 ; Rhodes and van Rooyen, Jour. Path. and Bact., 44, 1937, 357-363; Rivers and Ward, Jour. Exp. Med., 58, 1933, 635-618; 62, 1935, 549-560; Rivers et al., ibid., 65, 1937, 677-685; 69, 1939, 857-866; Rosahn et al., Jour. Exp. Med., 63, 1936, 259-276, 379-396; Rosenau and Andervont, Am. Jour. Hyg., 13, 1931, 728-740; Salaman, Brit. Jour. Exp. Path., 18, 1937, 245-258; Shedlovsky and Smadel, Jour. Exp. Med., 75, 1942, 165-178; Smadel and Rivers, ibid., 75, 1942, 151-164; Smadel et al., ibid., 68, 1938, 607-627 ; 71, 1940, 373-389; 77, 1943, 165-171; Smith, Jour. Path. and Bact., 33, 1930, 273-282; Sprunt, Proc. Soc. Exp. Biol. and Med., 51, 1942, 226227 ; Jour. Exp. Med., 75, 1942, 297-304; Stritar and Hudson, Am. Jour. Path., 12, 1936, 165-174; Ward, Jour. Exp. Med., 50, 1929, 31-40.

3. Borreliota suis spec. nov. From Latin sus, swine.

Common name: Swine-pox virus (not 
the vaccinia strain of variola virus in swine).

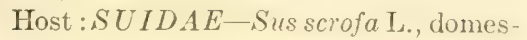
tic swine.

Insusceptible species : Rabbit .

Geographical distribution: United States (Iowa).

Induced disease: In swine, locally, reddened hyperemic papules 3 to $7 \mathrm{~mm}$ in diameter; papules become briefly vesicular, then change gradually to true pustules, finally forming dark brown to blackish scabs which are shed after a few weeks without scarring; no secondary lesions in hogs free from lice, but in infested animals numerous secondary lesions appear 1 to 2 weeks after primary lesions and are commonly most numerous in the inguinal and axillary regions. Mortality negligible but growth retarded.
Virus has been recovered from hog louse after feeding on affected swine.

Transmission: By hog louse, Haemalopinus suis (HAEIATOPINIDAE), probably mechanically. By experimental scarification of skin.

Serological relationships: No reaction with neutralizing sera specific for vac(inia virus.

Immunological relationships: Specific immunity in swine after attack, but no cross immunity with respect to vaccinia virus.

Filterability : Passes Berkefeld $V$ and I filters.

Literature: Csontos and von Nyiredy, Deutsch. tierärztl. Wchnschr., 41, 1933, 529-532; Schwarte and Biester, Am. Jour. Vet. Res., 2, 1911, 136-110; Shope, Arch. f. Virusforsch., 1, 1940, 457-167.

\section{Genus II. Briareus gen. nov.}

Tiruses of the Varicella Group, causing diseases characterized by reddened spots and rings in affected tissues, becoming papular or vesicular. Generic name from Latin Briareus, name of a hundred-armed giant.

The type species is Briareus varicellae spec. nov.

\section{Key to the species of genus Briareus.}

\section{Causing chicken pox and herpes zoster in man.}

II. Causing measles in man.

\section{Briareus varicellae spec. nov. From} New Latin varicella, chicken pox.

Common names: Varicella virus, chicken-pox virus; much evidence for identity with so-called herpes-zoster virus has been presented.

Host : HOMINIDAE-Homo sapiens I., $\operatorname{man}$.

Insusceptible species: Chick embryo. Geographical distribution: World-wide. Induced disease: In man, usually abrupt onset, rash at first macular, soon papular and vesicular; vesicles generaily discrete, soon rupturing, healing with scab formation and itching; separation of deeper scabs may leave persistent scars; in severe cases there may be stomatitis, laryngitis, and nasal lesions. In human skin grafted on chorioallantois of chick

\section{Briareus varicellae.}

\section{Briareus morbillorum.}

embryo, experimentally, pustular lesions as in natural disease, with intranuclear acidophilic inclusions; no gross vesiculation.

Transmission: By contact. By spread of droplets. Children in contact with herpes zoster patients sometimes contract varicella.

Serological relationships: Majority of herpes zoster sera that agglutinate zoster antigen also agglutinate elementary bodies of varicella; complement fixation tests also indicate relationship of virus from herpes zoster and varicella. Chicken-pox sera do not flocculate smallpox brain-virus antigen.

Immunological relationships: Children previously having varicella are immune to inoculation with herpes zoster virus. 
Literature: Amies, Brit. Jour. Exp. Path., 15, 1934, 314-320; Brain, ibid., 14, 1933, 67-73; Bruusgaard, Brit. Jour. Derm. Syph., 44, 1932, 1-24; Goodpasture and Anderson, Am. Jour. Path., 20, 194t, 447-453; Havens and Mayfield, Jour. Inf. Dis., 50, 1932, 242-248; Irons et al., Am. Jour. Hyg., 33, (B), 1941, 50-55; Kundratitz, Monatssehr. Kinderheilk., 29, 1925, 516-523; Lipschütz and Kundratitz, Wien. klin. Woch., 38, 1925, 499-503.

2. Briareus morbillorum spec. non. From New Lat in morbilli, measles.

Common name: Measles virus.

Host: HOMINIDAE-Homo sapiens I., man. Experimentally, aleo ${ }^{\prime} E R(C)$ PITHECIDAE-Macaca mulatta (Zimmermann), rhesus monkey. PHASIANIDAE-Gallus gallus (L.), chick embryo (no lesions, but 30 serial passages).

Geographical distribution: World-wide except in isolated communities.

Induced disease: In man, after incubation period of 7 to 21 days, bright red spots on buecal mueosa, especially near first molar tooth (Toplik's spots) followed by rash on face, head, neck, then arms, trunk, and legs; papules often crescents, lesions usually discrete; rash fades, leaving brownish discoloration and desquamation.

Transmission: By contact. By droplets.

Serological relationships: Convalescent serum is reported to modify the course of the induced disease if administered intravenously in the preeruptive stage.

Immunological relationships: Specific immunity in man after attack.

Thermal inactivation: At $55^{\circ} \mathrm{C}$ in 15 minutes.

Filterability : Passes Berkefeld $\mathrm{N}$ filter candle and Seitz EK disks.

Other properties: Viable at $-35^{\circ} \mathrm{C}$ for at least 4 weeks. Not inactivated by 10 per cent anest hetic ether in 40 minutes.

Literature: Blake and Trask, Jour. Exp. Med., 33, 1921, 385-412; Gordon and Knighton, Am. Jour. Path., 17, 1941, 165-176; Hedrich, Am. Jour. Hyg., 17, 1933, 613-636; Kohn et al., Jour. Am. Med. Assoc., 111, 1938, 2361-2364; Rake and Shaffer, Jour. Immunol., 38, 1940, 177-200; Rake et al., Jour Inf. Dis., 69, 1941, 65-69; Scott and simon, Am. Jour. Hyg., 5, 1925, 109-126.

\section{Genus III. Scelus gen. nov.}

Viruses of the Herpes Group, inducing diseases characterized in general by vesicular primary lesions, sometimes with subsequent involvement of the nervous system. Generic name from Latin scelus, raseal.

The type species is Scelus recurrens spec. nou.

\section{Key to the species of genus Scelus.}

I. In man, cause of so-called fever blisters, herpes febrilis.

1. Scelus recurrens.

II. In swine, cause of pseudorabies.

2. Scclus suillum.

III. In monkey.

3. Scelus beta.

IV. In rabbit, course of the induced disease in nature unknown.

4. Scelus tertium.

V. In sheep, cause of ovine balano-posthitis.

5. Scelus ulceris.

VI. In mice, cause of ectromelia.

6. Scelus marmorans.

VII. In cattle, cause of erosive stomatitis.

7. Scelus bovinum. 
1. Scelus recurrens spec, nov. From Latin recurrere, to recur. Note: The name Neurocystis herpetii Levaditi and Schoen (Compt. rend. Soc. Biol., Paris, $96,1927,961)$ was applied provisionally to the causative microorganism of herpes, in the expectation that future research would show inclusion bodies in affected tissues to be stages in its life cycle.

Common names: Herpes virus, virus of herpes simplex, virus of herpes febrilis (not herpes zoster virus, for which see varicella virus), virus of keratitis dendritica, virus of aphthous stomatitis (of man).

Host : HOMINIDAE-Homo sapiens L., man. Experimentally, also rabbit, guinea pig, white mouse, cat, goose, hedgehog, and, though difficult to infect, dog and pigeon. Chick embryo (but not chicken). Also CERCOPITHECIDAE -Cercocebus fuliginosus E. Geoffrey, Macacus cynomolgus. CEBID.AE-Cebus olivaceus.

Insusceptible species: White rat; $B u$ jo viridis; Cercopithecus callithrix; chicken (except embryo).

Geographical distribution: Probably world-wide.

Induced disease: In man, usually acquired in first three years of life, some times as aphthous stomatitis; virus probably retained often through life, sometimes with periodic reappearance of dermal lesions, which are vesicular and heal soon. In white mouse, by experimental inoculation of skin, small inflamed vesicular primary lesions about 5 days after inoculation, usually forming seabs and healing a few days later, but sometimes persisting; if on tail, followed by swelling and paralysis of tail, ascending paralysis and death, or by recovery with acquired immunity; if near head, followed by encephalitis and death; intraperitoneal and sometimes other inoculations immunize; relapse with recurrence of primary lesions rare. In chick embryo, white, opaque, rircular or ring-like primary lesions of small size on chorioallantoic membrane, with or without neerotic secondary lesions in liver, heart, lungs, spleen, and kidneys; virus enters membrane 1 to 4 hours after it is dropped on its surface; primary lesions may be counted in 48 hours.

Transmission: By contacts. Experimentally, by skin scarification; in guinea pig, by feeding.

Serological relationships: Distant relationship to pseudorabies virus, Scelus suillum, shown by moderate protection against this virus conferred by some antiherpes sera. No relationship to vaccinia virus or to virus III of rabbits demonstrable by neutralization tests. Specific complement fixation. Neutralizing antibody forms reversible union with virus, at least for a time, though with strong mixtures partial irreversibility finally oecurs.

Immunological relationships: Formalinized virus and non-lethal strains of virus immunize specifically. No cross immunity with vaecinia virus.

Thermal inactivation: At 50 to $52^{\circ} \mathrm{C}$ in 30 minutes, when moist; at 90 to $100^{\circ} \mathrm{C}$ in 30 minutes, when dry. At $41.5^{\circ} \mathrm{C}$ in 50 to 80 hours.

Filterability : Passes Berkefeld V filter with slight loss.

Other properties : Diameter, by centrifugation, computed as 180 to 220 millimicrons; by filtration, 100 to 150 millimicrons. Specific gravity, 1.15. Inactivated by repeated freezing and thawing; also by pressure of 3000 at mospheres for 30 minutes. Viable dry at least 18 months at $4^{\circ} \mathrm{C}$, in 50 per cent glycerine at least 6 months. Not inactivated at $t^{\circ}$ C in 1 per cent arqueous gentian violet. Charged negatively in solutions of hydrogen-ion concentration up to about $\mathrm{pH}$ 8. Isoelectric point, $\mathrm{pH} 7.2$ to 7.6 . Inactivated by incubation in vitro at $\mathrm{pH} 6$ with synthetic vitamin C (ascorbic acid).

Literature: Anderson, Science, 90, 1939, 497; Am. Jour. Path., 16, 1940, 137156 ; Andervont, Jour. Inf. Dis., 44, 1929 , $383-393$; 45, 1929, 366-385 ; 49, 1931, 507529 ; Andrewes, Jour. Path. and Bact., 33, 1930, 301-312; Bassett et al., Compt. rend. Acad. Sci., Paris, 200, 1935, 18521884; Bechhold and Schlesinger, Ztschr. 
f. Hyg., 115, 1933, 342-353; Bedson, Brit. Jour. Exp. Path., 12, 1931, 25t-260; Bedson and Bland, ibid., 9, 192s, 17t-17s; Blane and Caminopetros, Compt. rend. Soc. Biol., Paris, 84, 1921, 859-860; Boak et al., Jour. Exp. Med., 71, 1940, 169-173; Brain, Brit. Jour. Exp. Path., 13, 1932, 166-171 ; Buggs and Green, Jour. Inf. Dis., 58, 1936, 9S-104; Burnet and Lush, Jour. Path. and Bact., 48, 1939, 275-286; 49, 1939, 241-259; Lancet, 236, 1939 (1), 629-631; Burnet et al., Austral. Jour. Exp. Biol. and Med. Sci., 17, 1939, 35-40; Dawson, Am. Jour. Path., 9, 1933, 1-6; Elford et al., Jour. Path. and Bact., 36, 1933, 49-54; Findlay and MacCallum, Lancet, 238, 1940 (1), 259-261 ; Fischl and Schaefer, Klin. Wochnschr., 8, 1929, 2139-2143; Flexner, Jour. Gen. Physiol., 8, 1927, 713-726; Jour. Exp. Med., 47, 1928, 9-36; Friedenwald, Arch. Ophthalmol., 52, 1923, 105-131; Goodpasture, Medicine, 8, 1929, 223-243; Goodpasture and Teague, Jour. Med. Res., 44, 1923, 121-138; Gunderson, Arch. Opthalmol., 15, 1936, 225-249 ; Holden, Jour. Inf. Dis., 50, 1932, 218-236; Keddie and Epstein, Jour. Am. Med. Assoc., 117, 1941, 13271330; Levaditi and Lepine, Compt. rend. Acad. Sci., Paris, 189, 1929, 66-68; Levadit i and Nicolau, Compt. rend. Soc. Biol., Paris, 90, 1924, 1372-1375; Long, Jour. Clin. Investigation, 12, 1933, 1119-1125; Magrassi, Boll. Ist. Sieroterap. Milanese, 14, 1935, 773-790; Mclinley, Proc. Soc. Exp. Biol. and Med., 26, 1928, 21-22; Naegeli and Zurukzoglu, Cent. f. Bakt. I Abt., Orig., 135, 1935, 297-299 ; Nicolau and Fopciowslia, Ann. Inst. Pasteur, 60, 1938, 401-431; Parker and Nye, An. Jour. Path., 1, 1925, 337-340; Perdrau, Proc. Iroy. Soc. London, Ser. B, 109, 1931, 304308; Jour. Path. and Bact., 47, 1938, 447455; Remlinger and Bailly, Comp. rend. Soc. Biol., Paris, 94, 1926, 734-736; 1064$1066 ; 95,1926,1542-1545 ; 96,1927,404-$ 106; 1126-1128; 97, 1927, 109-111; Sabin, Brit. Jour. Exp. Path., 15, 1931, 372-380; Schultz and Hoyt, Jour. Immunol., 15, 1928, 411-419; Shaffer and Enders, ibid., 37, 1939, 383-411; Simon, International
Clinics, Series 37, 3, 1927, 123-128; Smith et al., An. Jour. Path., 17, 1941, 55-68; Warren et al., Jour. Exp). Med., 71, 1940, 155-168; Weyer, Proc. Soc. Exp. Biol. and Med., 30, 1932, 309-313; Zinsser, Jour. Exp. Med., 49, 1929, 661-670; Zinsser and Seastone, Jour. Immunol., 18, 1930, 1-9; Zurukzoglu and Hruszek, Cent. f. Bakt. I Abt., Orig., 1.28, 1933, 1 12.

2. Scelus suillum spec. nov. From Latin suillus, pertaining to swine.

Common names: Pseudorabies virus, mad-itch virus.

Hosts : Domestic cattle, swine, dog, cat, horse, sheep. Experimentally, also rabbit, guinea pig, white rat, white mouse, gray field mouse, duck, chicken, chick embryo; Macnca mulatta (Zimmermann), rhesus monkey.

Geographical distribution: France, Germany, Hungary, Holland, Denmark, Switzerland, Siberia, Brazil, United States.

Induced disease: In cattle, licking of affected area, usually somewhere on hindquarters, sudden decrease in milk production in dairy animals, violent rubbing, biting, and gnawing of lesion; swelling and discoloration of affected parts with oozing of serosanguineous fluid; grinding of teeth and excessive salivation in some individuals; death, preceded by clonic convulsions, violent tossing of head, and shallow respiration, usually 36 to 48 hours after onset. In pig, mild but highly contagious disease; slight nerve-cell degeneration, predominance of vaseular and interstitial lesions.

Transmission : By contact in swine, not in cattle. By feeding in cats, brown rats, and swine.

Serological relationships: Cross neutralization between constituent strains. Anti-herpes sera protect in some cases against small, but constantly infective, doses of pseudorabies virus.

Literature: Aujeszky, Cent. f. Bakt. I Abt., Orig., 32, 1902, 353-357; F. B. Bang, Jour. Exp. Med., 76, 1942, 263-270; 
O. Bang, Acta path. et microbiol. Scand., Suppl., 11, 1932, 180-182; Carini and Maciel, Bull. Soc. Path. exot., 5, 1912, 576578; Følger, Acta path. et microbiol. Scand., Suppl., 11, 1932, 182-187; Glover, Brit. Jour. Exp. Path., 20, 1939, 150-158; Gowen and Schott, Am. Jour. Hyg., 18, 1933, 674-687; Hurst, Jour. Exp. Med., $58,1933,415-433 ; 59,1934,729-749 ; 63$, 1936, 449-163; Kiöves and Hirt, Arch. wissensch. u. prakt. Tierheilk., 68, 1934 , 1-23; Morrill and Graham, Am. Jour. Tet. Res., 2, 1941, 35-40; Brit. Jour. Exp. Path., 15, 1934, 372-380; Shope, Proc. Soc. Exp. Biol. and Med., 30, 1932, 308309; Jour. Exp. Med., 54, 1931, 233-248; $57,1933,925-931 ; 62,1935,85-99,101-117$, Traub, ibid., 58, 1933, 663-681 ; 61, 1935, 833-838.

3. Scelus beta spec. nov. From beta, second letter of Greek alphabet, in reference to common name.

Common name: $\mathrm{B}$ virus.

Hosts : HOMINIDAE-Homo sapiens L., man. CERCOPITHECIDAE-Macaca mulatta (Zimmermann), rhesus monkey. Experimentally, also LEPORIDAE-Oryclolagus cuniculus (L.), rabbit. CAVIIDAE-Cavia porcellus (L.), guinea pig.

Geographical distribution: United States (from captive monkeys and man).

Induced disease : In man, local and relatively insignificant lesion on bitten part, later flaccid paralysis of legs, urinary retention, ascending paralysis, and death by respiratory failure. In Macaca mulatta, experimentally by intracutaneous injection, hemorrhagic or vesiculo-pust ular lesions without later involvement of central nervous system but with subsequent acquired immunity. Acidophilic intranuclear inclusions in lesions.

Transmission: To man, by bite of monkey. To monkey, experimentally, by injection.

Literature : Burnet et al., Austral. Jour. Exp. Biol. and Med. Sci., 17, 1939, 35-40, 41-51; Sabin, Brit. Jour. Exp. Path., 15, 1934, 248-268, 268-279, 321-334 ; Sabin and
Hurst, ibid., 16, 1935, 133-148; Sabin and Wright, Jour. Exp. Med., 59, 1934, 115136.

4. Scelus tertium spec. nov. From Latin tertius, third.

Common name: Virus III of rabbits.

Host : LEPORID.AE-Oryctolagus cuniculus (L.), domestic rabbit.

Insusceptible species: No obvious disease in inoculated guinea pig, white mouse, monkey (Macaca mulatta Zimmermann), rat, or man; hence the assumption that these are naturally immune, but they may be merely tolerant or klendusic.

Geographical distribution: United States (apparently spontaneous in some individuals of the laboratory rabbit).

Induced disease: In domestic rabbit, experimentally, after incubation period of 4 to 6 days, failure to eat, loss of weight, occasionally diarrhea and iemperatures of 104 to $107^{\circ} \mathrm{F}$; small, superficial, red spots and papules on skin at site of inoculation; local infiltration of tissues with endothelial leucocytes, swelling of involved epithelial cells; nuclear inclusions present in endothelial leucocytes and some other cells; disease not fatal ; virus in circulating blood only during early stages; recovery in a few days without scar formation but with development of specific immunity. The course of the natural disease, presumed to occur in rabbits, is still unknown.

Transmission: Experimentally, by injection of filtrates from diseased tissues; on several occasions also from blood or tissues of apparently normal rabbits.

Serological relationships: Specific neutralizing substances occur in the serum of recovered rabbits.

Immunological relationships: Specific immunity but no cross reactions with vaccinia or herpes viruses.

Thermal inactivation: In 10 minutes at $55^{\circ} \mathrm{C}$, but not in 30 minutes at $45^{\circ} \mathrm{C}$.

Filterability : Passes Berkefeld $\mathrm{V}$ and I filters; passes $L_{2}$ filter candle.

Other properties: Viable at least 6 
meeks in 50 per cent glycerine and 16 months dried when frozen, and stored on ice.

Literature: Andrewes, Brit. Jour. Exp. Path., 10, 1929, 188-190, 273-280; Jour. Path. and Bact., 33, 1930, 301-312; 50, 1940, 227-234; Rivers and Stewart, Jour. Exp. Med., 48, 1928, 603-613; Rivers and Tillett, ibid., 39, 1924, 777-802; 40, 1924, 281-287; Topacio and Hyde, Am. Jour. Hyg., 15, 1932, 99-124.

\section{Scelus ulceris spec. nov. From} Iatin ulcus, sore spot.

Common name: Ovine balano-post hit is virus.

Host : BOVIDAE-Oris aries I.. sheep.

Geographical distribution: Enited States, Australia.

Induced disease: In sheep, ulceration with seab production : lesions most severe on prepuce and vulva; in the male, the penis may be involved, usually only with mild inflammation, but if aceompanied by paraphimosis there may be extensive ulceration and heavy seab formation.

Transmission: Venereally. Experimentally, by inoculation of prepuce.

Filterability: Passes Rerkefeld $N$ and W filters, a $7 \mathrm{lb}$ Mandler candle, and a $3 \frac{1}{2}$ per cent collodion membrane.

Literature: Tumnicliff and Matisheek, Sience, 94, 1941, 283-284.

6. Scelus marmorans spec. nov. From Latin marmorare, to marble, in referenee to mottling of spleen and liver in host.

Common name: Ectromelia virus.

Hosts: MURIDAE-Wus musculus L., white mouse. Esperimentally, also MURIDAE-Rattus norvegicus (Berkenhout), rat (infection inapparent). Also, PHASIANIDAE-Gallus gallus (L.), chick embryo (12-day-old White Leghorn chick embryo at 36 to $37^{\circ}$ ( ; less satisfactory results at higher temperatures of incubation or in embryos in spring eggs). Derived strains of this virus infect rabbit and guinea pig, not susceptible to original virus from mouse.

Geographical distribution: England.
Induced disease: In white mouse, spleen mottled, liver edges translucent, peritoneal fluid increased in amount; loss of weight; later, cutaneous lesions on foot or elsewhere; affected foot swells, becomes moist, scabbed, then recovers or dries up and separates from the skin at limit of original swelling; in acute disease, death without gross lesions, or, at autopsy, gut dark red, liver dirty gray, soft, bloodless, sometimes mot tled, spleen necrotic; inclusion bodies most numerous in lesions of the skin, round or oval, $t$ to 13 microns long, without internal differentiation; very young mice probably become infected without developing apparent disease and remain carriers for some time. In rat, inapparent infection; after initial increase of virus, circulating antibodies appear and immunity to reinfection is established.

Transmission: In mouse, by contact. In rat, experimentally, by intranasal inoculation.

Serologieal relationships: Neutralizing antibodies oceur in convalescent mouse serum. Immune sera from the guinea pig specifically agglutinate elementary bodies obtained from infected skin of the white mouse.

Immunological relationships: Recovered mice are solidly inmune to many lethal doses.

Thermal inactivation: At $55^{\circ} \mathrm{C}$ in 30 , not in 10 , minutes.

Filterability : In broth, passes Mandler, Pasteur-Chamberland $L_{2}$, and Berkefeld X filters.

Other properties: Survives drying 6 months, freezing $\left(-10^{\circ}\right.$ C) 2 months, 50 per cent glycerine 5 months at least. Resists 1 per cent phenol 20, not 40 , days. Size, estimated by filtration, 100 to 150 millimirrons; by ult raviolet-light photography, 130 to 140 millimicrons.

Literature: Barnard and Elford, Proc. Roy. Soc. London, Ser. B, 109, 1931, 360380 ; Baumgartner, Cent.f. Bakt., I Abt., Orig., 133, 1935, 2\$2-289; Burnet and Lush, Jour. Path. and Bact., 42, 1936, 469-476; 43, 1936, 105-120; Jahn, Arch. f. Virusforsch., 1, 1939, 91-103; Kikuth and 
Gönnert, Arch. f. Tirusforsch., 1, 1940, 295-312; Marchal, Jour. Path. and Bact. 33, 1930, 713-728; Me Gaughey and Whitehead, ibid., 3\%, 1933, 253-256; Paschen, Cent. f. Bakt., I Abt., Orig., 135, 1936, $445-452$.

7. Scelus bovinum spec. nov. From Latin bovinus, of ox, bull, or cow.

Common name: Erosive-stomat it is virus.

Host : BOVIDAE-Bos taurus L., domestic cattle. Experimentally, also chorioallantoic membrane of developing hen's egg.

Insusceptible species: CAIIIDAECavia porcellus (L.), guinea pig. (In rats, rabbits, mice, sheep, no reaction has been noted after inoculation.)

Geographical distribution: South Africa (Natal); perhaps Ireland (Armagh-disease virus).

Induced disease: In young domestic eattle, lesions on tongue, dental pad, and lips pearl-like at first, then breaking down to form superficial erosions, with white glistening base and red border. Lesions may coalesce to form large, ragged, eroded areas, healing uneventfully with scar formation. No foot lesions; no excessive salivation; no "hotness" of mouth; no systemic disturbances.

Transmission: Spreads slowly, mainly to animals less than three years old, probably by contact. Experimentally, by injection into dental pads, lips, or tongue. Filterability: Passes Gradocol membrane of about 400 millimicron average pore diameter.

Other properties: Viable after at least 11 days at room temperature, 21 days at refrigerator temperature, 6 weeks frozen and dried in horse-serum saline.

Literature: Mason and Neitz, Onderstepoort Jour. Vet. Sci. and Anim. Indust., 15, 1940, 159-173.

\section{Genus IV. Hostis gen. nov.}

Viruses of the Foot-and-Nouth Disease Group, inducing diseases mainly characterized by vesicular lesions. Generic name from Latin hostis, enemy or stranger.

The type species is Hostis pecoris spec. nou.

\section{Key to the species of genus Hostis.}

I. Infecting cattle and other animals with cloven hoofs; horse immune or highly resistant.

II. Infecting horse readily.

\section{Hostis pecoris.}

\section{Hostis equinus.}

1. Hostis pecoris spec, nov. From Latin pecus, cattle.

Common names: Foot-and-mouth disease virus; Virus der Miul- und Klauenseuche.

Hosts: Cow, pig, sheep, goat, reindeer, bison. Experimentally, also guinea pig, rabbit, rat.

Insusceptible species: (hick embryo (chorioallantois); horse (immune or very resistant).

Induced disease: In cow, after incubation period of 2 to $t$ days or more, fever, vesicular lesions on tongue, lips, gums, hard palate and feet, soon rupturing; salivation, lameness, generally recovery.
Transmission: Spread rapid, source of infection often obscure; saliva is infective before lesions become obvious.

Thermal inactivation: At $70^{\circ} \mathrm{C}$, not at $60^{\circ} \mathrm{C}$, in 15 minutes.

Filterability: Passes Seitz, Berkefeld $V$ and $N$, and C'hamberland $L_{11}$ filters. Strains: Three strains, $\mathrm{A}, \mathrm{O}$ and $\mathrm{C}$, ale immunologically distinet from each other.

Other properties: Particle calculated to be about 20 nillimicruns in diameter by centrifugation data. 8 to 12 millimicrons in diameter by filtration; may be separated from mixtures with the larger equine vesicular stomatitis virus by differential filtration. Viable after drying 
in vacuo, at least a week at $-\frac{1}{t}$ to $0^{\circ} \mathrm{C}$. Readily destroyed by 1 to 2 per cent sodium hydrate or above pH 11. Soon inactivated near pH 6.0 , but moderately stable at p $\mathrm{H} 2.0$ to 3.0 ; opt imum condition for storage at $\mathrm{pH} 7.5$ to 7.7 in absence of air; return from 3.0 to 7.5 inactivates, however.

Literature: Elford and Galloway, Brit. Jour. Exp. Path., 18, 1937, 155-161; Galloway and Elford, ibid., 14, 1933, 100408 ; 16, 1935, 588-613; 17, 1936, 187-204 ; Galloway and sehlesinger, Jour. Hyg., 37, 1937, 463-170; Hare, Jour. Path. and Bact., 35, 1932, 291-293; Loeffler and Frosch, Cent. f. Bakt., I Abt., 23, 1898, 371-391; Natte and Sanz, Bull. Soc. Path. Exot., 14, 1921, 523-529; Olitsky and Boez, Jour. Exp. Med., 45, 1927, 673-683, 685-699, 815-831, 833-848; Pyl, Ztschr.f. physiol. Chemie, 226, 1934, 18-28; Pyl and Iilenk, Cent. f. Bakt., I Abt., Orig., 128, 1933, 161-171; Schlesinger and Cialloway, Jour. Hyg., 37, 1937, 445-462.

2. Hostis equinus spec. nov. From Latin equinus, pertaining to horses.

Common names: Vesicular-stomatitis virus, equine vesicular stomatit is virus.

Hosts: Horse, domestic cattle. Experimentally, also guinea pig, swine, white mouse, rabbit (relatively resistant), chick embryo; Macaca mulatta (Zimmermann), rhesus monkey; $M$. irus, eynomolgus monkey.

Insusceptible species: Chicken (ex(")it embryo).

Geographical distribution: Inited States (Indiana, New Jersey).

Induced disease: In horse, resembles foot-and-mouth disease of cattle; reddeneil patches on buceal mucosi, modriate fever, salivation, fullowed by apparance of vesicles, especially on tongue, filled with clear or yellowish fluid; vesi- les often coalesce and soon rupture leaving an eroded surface which heals soon in the absence of complications.
Experimentally, in chorioallantois of developing chick embryo, primary lesions involve moderate ectodermal proliferation, degeneration, necrosis; mesodermal inflammation; slight endodermal proliferation.

Serological relationships: Strains isolated in different localities give antisera capable of neutralizing heterologous isolates of virus, but homologous antisera neutralize in higher dilutions than do heterologous antisera.

Immunological relationships: No cross immunity with respect to equine encephalomyelit is virus.

Filterability : Passes Seitz filter.

Other properties: May be separated from mixtures with foot-and-mouth disease virus by propagation on chorioallantoic membrane of chick embryo, which will not support increase of the latter virus. Inactivated by $1: 50,000$ methylcne blue in $2 \mathrm{~mm}$ layer $13 \mathrm{~cm}$ from 300 candle-power lamp in 60 minutes but not in 20 minutes. Particle estimated on the basis of filtration data to be 70 to 100 millimicrons in diameter; 60 millimierons in diameter by centrifugation. Not destroyed by acidifying to $\mathrm{pH} 3$ and returning to pHI 7.5 (difference from foot-andmouth disease virus).

Literature: Burnet and Galloway, Brit. Jour. Exp. Path., 15, 1934, 105-113; Cox and Olitsky, Proc. Soc. Exp. Biol. and Merl., 30, 1933, 653-65t; Cox et al., ibid., 30, 1933, 896-895; Elford and CialloWay, Brit. Jour. Exp. Path., 18, 1937, 155-161; Galloway and Elford, ibid., 14, 1933, 400-408; 16, 1935, 585-613; Mohler, Jour. An. let. Med. Issue., 52, 1918, 110-122; Olitsky et al., Jour. Lxp. Med., 59, 1934, 159-171; Pyl, Ztschr. f. physiol. ('hemie. 226, 1934, 18-28; Sabin and ()litsky, itid., 66, 1937, 15-34, 35-57;67, 1938, 201-22s, 229-219; Syverton et a1., Science, $\% 8,1933,216-217$. 


\section{Genus V. Molitor gen. nov.}

Viruses of the Wart-Disease Group, inducing diseases mainly characterized by tissue proliferation without vesicle or pustule formation. Generic name from Latin molitor, contriver.

The type species is Molitor verrucae spec. nov.

Key to the species of genus Molitor.

I. Affecting man.

1. Molitor verrucae.

2. Molitor hominis.

II. Affecting cow.

3. Molitor bovis.

III. Affecting dog.

IV. Affecting chicken.

4. Molitor buccalis.

V. Affecting rabbit.

\section{Molitor tumoris.}

6. Molitor gingivalis.

7. Molitor sylvilagi.

8. Molitor myxomas.

1. Molitor verrucae spec, nov. From Arch. Dermat. u. Syph., 107, 1911, 395.) Latin verruca, wart.

Common name: Common-wart virus.

Hosts:HOMI I DAE-Homo sapiens L., man. Perhaps also BOVIDAE-Bos taurus L., cow. CANID.1E-Canis familiaris L., dog.

Induced disease: Experimentally in man, incubation period long, 4 weeks to 6 or more months; initially acanthosis (overgrowth of prickle cell layer of epidermis) and flattening of the papillae; later, interpapillary hypertrophy, inflammation, and marked hyperkeratosis.

Transmission: By contact; in some cases, venereally. Experimentally, by skin scarification.

Filterability : Passes Berkefeld I filter.

Literature: Ciuffo, Giorn. ital. d. malattie veneree e d. pelle, 48, 1907, 12 17; Kingery, Jour. Am. Med. Assoc., 76, 1921, 440-142; Payne, Brit. Jour. Dermat., 3, 1891, 185-189; Schultz, Deutsch. med. Wehnschr., 34, 1908, 423; Serra, Giorn. ital. d. malattie veneree e d. pelle, 65, 1924, 1808-1814; Ullmann, Acta otolaryngologica, 5, 1923, 317-334; Wile and Kingery, Jour. Am. Med. Assoc., 73, 1919, 970-973.

2. Molitor hominis comb. nov. From Latin homo, man.

Common name : Molluscum contagiosum virus.

Host: HOMINIDAE-Homo sapiens L., man.

Geographical distribution: Perhaps essentially world-wide.

Induced disease: In man, experimentally, prodromal period may be 14 to 50 days, lesions at first like pimples, becoming red, painful, swollen, developing into small tumors covered with stretched and shiny skin; lesions commonest on face, arms, buttocks, back, and sides, healing spontaneously. Inclusions within epithelial cells, known as molluscum bodies, measure 9 to 24 microns in diameter when approximately spherical, 24 to 27 microns in width and 30 to 37 microns in length when elongated; they contain elementary bodies about 0.3 micron in diameter. The outer envelope of the molluscum body is of carbohydrate.

Transmission: By contact. By fomites.

Filterability: Passes Chamberland $\mathrm{L}_{1}$ and Berkefeld $V$ filters.

Literature: Goodpasture and King, Am. Jour. Path., 3, 1927, 385-394; Goodpasture and Woodruff, ibid., 7, 1931, 1-8; (Strongyloplasma hominis Lipschütz, Juliusberg, Deutsch. med. Wchnschr., 
31, 1905, 1598-1599; Lipschütz, Arch. Dermat. 11. Syph., 107, 1911, 387-396; in Kiolle, Kraus and Uhlenhuth, Handhuch der Pathogenen Mikroorganismen, 8, 1930, 1031-1040; Van Rooyen, Jour. Path. and Bact., $46,1938,425-436$; 49,1939 , 345-349; Wile and Kingery, Jour, Cutan. Dis., 37, 1919, 431-446.

\section{Molitor bovis spec. nov. From Latin} bos, cow.

Common name: Cattle-wart virus.

Host: BOVID.AE-Bos taurus L., domestic cattle.

Geographical distribution: Cnited States.

Induced disease: In rattle, especially about head, neck, and shoulders in young animals, on udders in cows, affected skin thickened at first, then rough, nodular; warts sometimes become large and pendulous, adversely affecting growth of host; they sometimes become cauliflower-like tumors several inches in diameter; spontaneous regression is not infrequent. Hides from affected animals are reduced in value.

Transmission: Believed to be through injuries to skin when the injured part comes in contact with warty animals or with rubbing posts, chutes, fences, buildings, or other structures with which affected animals have come in contact previously. Experimentally, by skin inoeulations, especially in animals under 1 year of age.

Filterability : Passes Berkefeld N filter.

Literature: Creech, Jour. Agr. Res., 39, 1929, 723-737; C. S. Dept. Agr., Leaflet $75,1931,1-4$.

4. Molitor buccalis spec. nov. From Latin bucca, cheek.

Common name: Canine oral-papillomatosis virus.

Host: CANIDAE-Canis familiaris L., dog.

Insusceptible species: (at, rabbit, guinea pig, rat, mouse; Macaca mulatta (Zimmermann), rhesus monkey.

Induced disease: In young dog, experimentally, about 1 month after inoculation of buccal membrane by scarification, pale, smooth elevations, becoming gradually more conspicuous and roughened; finally a mass of closely packed papillae is former. Regression with subsequent immunity is frequent; no scars are left on regression. Secondary warts often appear in other parts of the mouth 4 to 6 weeks after primary warts have first been observed.

Transmission : Experimentally by skin scarification.

Serological relationships: Not inhibited by antiserum effective against commonwart virus of man.

Thermal inactivation: At some temperat ure between 45 and $58^{\circ} \mathrm{C}$ in 1 hour.

Filterability: Passes Berkefeld N filter.

Other properties: Viable after freezing and drying, if stored dry in icebox, at least 63 days; in storage in equal parts of glycerine and 0.9 per cent $\mathrm{TaCl}$ solution at least 64 days.

Literature: DeMonbreun and Coodpasture, Am. Jour. Path., 8, 1932, 43-56 ; M'Fadyean and Hobday, Jour. Comp. Path. and Therap., 11, 1898, 341-344; Penberthy, ibid., 11, 1898, 363-365.

5. Molitor tumoris spec. nov. From Latin tumor, swelling.

Common names: Fowl-sarcoma virus, Rous chicken-sarcoma virus.

Hosts : PHASIA NIDAE-Gallus gallus (L.), chicken. Experimentally, also pheasant (serial transfer difficult) and duck (by cell transfer only but filtrates from duck infect injected chicken).

Insusceptible species: Turkey, guinea fow (both immune to filtrates but capable of supporting tumor line if alternated in a series with common fowl hosts); geese.

Induced disease: In hen, originally found in an adult, pure-bred hen of Barred Plymouth Rock variety. Experimentally transmitted, a circumscribed nodule soon becomes evident at site of implantation; later this becomes necrotic or cystic at its center; as growth enlarges, host becomes emaciated, cold, somnolent, and finally dies; discrete metastases are often found in lungs, heart, and liver. Parent cell of sarcoma is claimed to be 
the normal histiocyte, but virus in the affected fowl is not confined to the sarcoma, being widespread in the body in spleen, liver, muscle, brain, etc. In the chick embryo, serial passage is feasible on the egg membrane, in which focal lesions involve only ectodermal tissue.

Transmission: By injection of affected fowl cells or filtrates. Certain transmissible tar-induced sarcomas, not infecting by filtrates, nevertheless induce the formation of antibodies capable of neut ralizing this virus. An inhibitor of the virus extracted from tumors appears to be a protein, inactivated at $65^{\circ} \mathrm{C}$, but not at $55^{\circ} \mathrm{C}$, in 30 minutes and destroyed by trypsin in 3 to 5 hours at $\mathrm{pH} 8$. Oleic acid also may act as an inhibitor. No spontaneous transmission in chickens kept together.

Serological relationships: Particles sedimented by centrifugal force 20,000 to 30,000 times gravity are specifically agglutinated by sera of fowls bearing rorresponding tumor. At least one antigen in tumors of hen and duck not in healthy birds; this one fixes complement and gives cross reactions with Rous, Mill Hill 2, Fujinami, and RFD2 tumors. Virus injected into goats produces two antibodies but only one if previously heated; the antibody to the heat-stable constituent requires complement to neutralize virus; the only antibody produced in ducks does not require complement to neutralize.

Thermal inactivation: At or below $5 t^{\circ}$ $\mathrm{C}$ in 20 minutes.

Filterability : Passes Berkefeld $V$ and 110. 5 (medium) filters.

Other properties: Particle size estimated as about 100 millimicrons (but some say 50 or even 15 millimicrons) in diameter by filtration through graded membranes, about 70 millimicrons (molecular weight $140,000,000$ ) by ultracentrifugation. Contains 8.5 to 9.0 per cent nitrogen, 1.5 per cent phosphorus. Protein tests positive. Feulgen reaction for thymonucleic acid absent; 10 to 15 per cent of the protein may be nucleic acid, probably of ribose type. Pentose present. Virus believed to be of globulin nature or attached to globulin particles (Lewis and Mendelsohn, Am. Jour. Hyg., $12,1930,686-689)$. Viable indefinitely in dried spleen as in dried sarcoma tissues.

Strains: Several strains have been studied in addition to the original Rous sarcoma no. 1 strain; immunological relationships have been shown between the original strain, the des Ligneris sarcoma strain, the Fujinami sarcoma strain, the fibrosareoma MH1 and endothelioma $\mathrm{MH} 2$ strains; other isolates also have shown serological interrelationships.

Literature: Amies, Jour. Path. and Bact., 44, 1937, 141-166; Amies et al., Am. Jour. Cancer, 35, 1939, 72-79; Andrewes, Jour. Puth. and Bact., 34, 1931, 91-107; $35,1932,407-413 ; 37,1933,17-25,27-44$; 43, 1936, 23-33; Claude, Jour. Exp. Med., $66,1937,59-72$; Science, 87, 1938, 467-468; 90, 1939, 213-214; Am. Jour. Cancer, 37, 1939, 59-63; Claude and Rothen, Jour. Exp. Med., 71, 1940, 619-633; Dmochowski and Knox, Brit. Jour. Exp. Path., 20, 1939, 466-472; Elford and Andrewes, ibid., 16, 1935, 61-66; Gye and Purdy, Jour. Path. and Bact., 34, 1931, 116-117 Abst.); Haddow, ibid., 37, 1933, 149-155; Helmer, Jour. Exp. Med., 64, 1936, 333338 ; Keogh, Brit. Jour. Exp. Path., 19, 1938, 1-9; Ledingham and Gye, Lancet, 228, 1935 (1), 376-377; Lewis and Mendelsohn, Am. Jour. Hyg., 12, 1930, 686689; des Ligneris, Am. Jour. Cancer, 16, 1932, 307-321; MeIntosh, Jour. Path. and Bact., 41, 1935, 215-217; Nellanby, Jour. Path. and Bact., 46, 1938, 47-460; 47, 1938, 47-64; Mendelsohn et al., Am. Jour. Hyg., 14, 1931, 421-425; Purdy, Brit. Jour. Exp. Path., 13, 1932, 473-479; Rous, Jour. Exp. Med., 13, 1911, 397-411.

6. Molitor gingivalis spec. nov. From Latin gingix a, gum.

Common name : Rabbit oral-papillomatosis virus.

Hosts: LEPORIDAE-Oryctolagus cuniculus (L.), domestic rabbit. Experimentally, also Lepus americanus 
Erxleben, snowshoe hare; L. californicus Gray, jack rabbit; Sylvilagus sp., cottontail rabbit.

Geographical distribution: United States.

Induced disease: In rabbit, benign papillomas, having the form of small, discrete, gray-white, sessile or pedunculated nodules, usually multiple, on lower surface of tongue or, less frequently, on gums or floor of mouth.

Transmission: Perhaps by mother to suckling young, with a latent period be fore onset of disease. Not highly con tagious, if contagious at all, in old animals. Experimentally by puncture of tissues in the presence of virus.

Immunological relationships: Specific immunity develops as a result of disease, but no cross immunity with respect to rabbit-papilloma virus, which differs also in failing to act on oral mucosa.

Filterability: Passes Berkefeld $V$ and N filters.

Literature: Parsons and Kidd, Jour. Exp. Med., 7\%, 1943, 233-250.

7. Molitor sylvilagi spec. nov. From New Latin Sylvilagus, generic name of cottontail rabbit.

Common names: Rabbit papilloma or papillomatosis virus, rabbit wart virus.

Hosts: LEPORIDAE-Sylilagus sp., cottontail rabbit. Experimentally, also LEPORIDAE-Oryctolagus cuniculus (L.), domestic rabbit.

Geographical distribution: United States.

Induced disease: In cottontail rabbit, at first minute elevations along lines of scarification; later solid masses of wrinkled keratinized tissue, 3 to 4 millimeters in thicliness; eventually cornified warts, striated perpendicularly at top, fleshy at base, 1 to $1.5 \mathrm{~cm}$ in height; regression rare; natural papillomas become maligmant occasionally. In domestic rabbit, experimentally, blood antibody remains low but virus is always masked, preventing serial passage; discrete lesions on skin permit quantitative tests; tarring causes lucalization of virus from blood stream; papillomas give rise to malignant acanthomatous tumors by graded continuous altcration; metastasis frequent; transplantation to new hosts successful in series; antibody specific for the virus is formed continuously in the transplanted growths although virus is not directly demonstrable by subinoculation from them; malignant growths appear more promptly and frequently where epidermis has been tarred long; virus appears specific for epithelium of skin; growths disappear if treated with X-rays, $3600 \mathrm{r}$ at one time or fractionally; 60 per cent are cured with $3000 \mathrm{r}$, but $2000 \mathrm{r}$ ineffective.

Transmission: Experimentally, by scarification of skin. Abnormal susceptibility to infection is noted in rabbit skin treated with 0.3 per cent methylcholanthrene in benzene or equal parts of turpentine and acetone.

Scrological relationships: Specific neutralization, reversible on dilution. Complement fixation specific, with virus particle as antigen; no cross reaction with antisera for vaccinia, herpes, fibroma, or myxoma viruses. Precipitates occur in properly balanced mixtures of virus and specific antiserum; virus and antibody in both free and neutralized states are present in both soluble and insoluble phases of these suspensions.

Immunological relationships: Intraperitoneal injections immunize specifically. Rabbits immunized to fibroma and myxoma viruses are susceptible to rabbit papilloma virus.

Thermal inactivation: At $70^{\circ} \mathrm{C}$, not at 65 to $67^{\circ} \mathrm{C}$, in 30 minutes; in 0.9 per cent sodium chloride solution at 65 to $66^{\circ}$ C, time not stated.

Filterability: Passes Berkefeld V, N, and $W$ filters; particle size calculated as 23 to 35 millimicrons by filtration as compared with 32 to 50 millimicrons by centrifugation and 44.0 millimicrons by measurement of electron micrographs, which show the particle to be approximately spherical in shape. 
Other properties: Infectious particle has sedimentation constant $\mathbf{S}_{20}=c a .250$ $\times 10^{-13} \mathrm{~cm}$ per sec. per dyne; usually there is a secondary boundary at about $375 \times 10^{-13}$. Isoelectric point between $\mathrm{pH} 4.8$ and 5.1. Maximum absorption at about $2750 \AA$. Contains thymus nucleic acid about 6.8 to 8.7 per cent; maximum absorption of nucleic acid at about $2630 \AA$.

Literature: Beard et al., Jour. Inf. Dis., 65, 1939, 43-52; 69, 1941, 173-192; Bryan and Beard, ibid., 65, 1939, 306-321; Friedewald, Jour. Exp. Med., 75, 19⿺2, 197-220; Hoyle, Jour. Path. and Bact., 50, 1940, 169-170; Kidd, Jour. Exp. Med., $68,1938,703-724,725-759 ; 70,1939,583-$ $604 ; 71,1940,469-494 ; 74,1941,321-344$; 75, 1942, 7-20; Kidd and Rous, ibid., 68, 1938, 529-562; 71, 1940, 813-838, Kidd et. al., ibid., 64, 1936, 63-77, 79-96; Rous and Beard, ibid., 50, 1934, 701-722; 62, 1935, 523-5+8; Rous and Kidd, ibid., 6\%, 1938, 399-428; 71, 1940, 787-812; Rous et al., ibid., 64, 1936, 385-400, 401-42t; Schlesinger and Andrewes, Jour. Hyg., 37, 1937, 521-526; Sharp et al., Proc. Soc. Exp. Biol. and Med., 50, 1942, 205-207; Shope, Jour. Exp. Med., 58, 1933, 607-624; 65, 1937, 219-231; Syverton et al., ibid., 73, 1941, 243-248; Taylor et al., Jour. Inf. Dis., 71, 1942, 110-114.

8. Molitor myxomae (Aragão) comb. nov. (Chlamidozoon myxomae Aragão, Brazil-Med., 25, 1911, 471 ; name later abandoned by its original author in favor of Strongyloplasma myxomae Arigão, Mem. Inst. Oswaldo Cruz. 20, 1927, 231 and 243. The name Sanarellia cuniculi Lipschütz, Wien. klin. Wochenschr., 40, 1927, 1103, was based on the supposed causative organism, defined as varying in size between the size of chlamydozoa and of large cocci; it is not clear whether the structures observed and named were virus particles or not.) From New Latin myxoma, a kind of soft tumor, from nature of induced lesion.

Common names: Myxoma virus, virus myxomatosum.
Hosts: LEPORIDAE-Oryctolagus cuniculus (L.), domestic rabbit. Experimentally, also Sylvilagus sp., cottontail rabbit; jack rabbit (once in many trials); Lepus brasiliensis (resistant and rarely infected). Also chick embryo and duck embryo.

Insusceptible species: Lepus californicus Gray, black-tailed jack rabbit; $L$. americanus Erxleben, varying hare; Sylvilagus transitionalis Bangs, cottontail; horse, sheep, goat, cattle, dog (but one reported infected), guinea pig, rat, mouse, fowl, pigeon, duck, cat, hamster, monkey; man (but some conjunctival pain and swelling).

Geographical distribution : South America (Brazil, Úruguay, Argentina), United States (California).

Induced disease : In domestic rabbit, a disease (myxomatosis cuniculi) almost always fatal at ordinary room temperatures but not at 36 to $42^{\circ} \mathrm{C}$, lesions fewer and regressing after 6 to 8 days at these higher temperatures in most animals. At ordinary temperatures, nodules (edematous tumors) in skin near eyes, nose, mouth, ears, and genitalia; edema of eyelids; conjunctivitis with purulent discharge if skin around eyes is involved. Later marked dyspnea, stertorous breathing, cyanosis, asphyxia. Animals usually die within 1 to 2 weeks of infection. Virus enters bloodstream and invades nervous system at random through walls of blood vessels. Discharges from nose, eyes, and the serøus exudates from affected tissues are infectious; urine and feces are not. There are cytoplasmic inclusions in affected epidermal cells. In chick em. bryo, experimentally, intense inflammation, eventual impairment of circulation and necrosis locally; growth best if embryo is grown at 33 to $35^{\circ} \mathrm{C}$ and chilled to $25^{\circ} \mathrm{C}$ for 12 to 18 hours before or after inoculation, lesions being linear and associated with capillaries in ectoderm; virus infects and is recoverable from embryo and depresses hatch.

Transmission: By contact with diseased rabbits or cages recently occupied 
by them. Through air for a few inches. Rarely by feeding. Experimentally, by rubbing conjunctiva with a bit of infected tissue or with a platinum loop contaminated from diseased conjunctiva; has been recovered from flies. By injection. By flea, Clenopsylla felis (PULICIDAE), rarely.

Serological relationships: An attack of the disease induces the formation of neutralizing antibodies. Cross neutralization by antisera to myxoma and fibroma strains. Complement is fixed with myxoma virus as test antigen in the presence of antisera to myxoma or fibroma strains. Serum of rabbit inoculated with a soluble antigen, a heat-labile protein with iso electric point near $\mathrm{pH} 4.5$, agglutinates myxoma elementary bodies. A second soluble antigen, also heat labile, appears distinct, inhibiting its own ant ibody even after inactivation of its precipitating power by exposure at $56^{\circ} \mathrm{C}$.

Immunological relationships : Myxomarecovered domestic rabbits become immune to reinfection ; fibroma-strain-recovered animals, although partially immunized, still support myxoma-strain virus introduced in to the testicle. Heat inactivated virus $\left(60^{\circ} \mathrm{C}\right.$ for 30 minutes $)$ tends to immunize if given int radermally ; there is then an allergic local response, less severe generalized disease, delayed death or recovery. If fibroma virus preredes myxoma virus by 48 to 96 hours. there is marked protection.

Thermal inactivation: At $55^{\circ} \mathrm{C}$ in 10 minutes; at $50^{\circ} \mathrm{C}$ in 1 hour. A substance thermostabile for 30 minutes at 60 to $75^{\circ}$ $\mathrm{C}$, but not at $90^{\circ} \mathrm{C}$, is itself unable to produce myxomatous changes after the heat treatment but may do so in combination with fibroma virus, and transmissible myxoma virus is then reconst $\mathrm{i}$ tuted. Although it is supposed by some that this indicates the transformation of fibroma-strain virus into myxoma-strain virus, the possibility that heat-modified myxoma-strain virus is reactivated has not been eliminated.
Filterability: Passes Berkefeld V and $N$ filters; not Chamberland $L_{5}$ or $L_{7}$ filters.

Other properties: Inactivated above $\mathrm{pH} 12.0$ and below $\mathrm{pH} 4.0$. Withstands drying. Viable at least 3 months at 8 to $10^{\circ} \mathrm{C}$.

Literature: Aragão, Brazil-med., 25, 1911, 471; Mem. Inst. Oswaldo Cruz, 20, 1927, 225-235; Berry and Dedrick, Jour. Bact., 31, 1936, 50-51 (Abst.); Berry and Lichty, ibid., 31, 1936, 49-50 (Abst.); Berry et al., Second International Congress for Microbiology, Report of Proceedings, London, 1936, 96 (Abst.) ; Fisk and Kiessel, Proc. Soc. Exp. Biol. and Med., 29, 1931, 9-11; Gardner and Hyde, Jour. Inf. Dis., 71, 1942, 47-49; Hobbs, Am. Jour. Hyg., 8, 1928, 800-839; Science, 79, 1931, 94-95; Hoffstadt and Omundson, Jour. Inf. Dis., 68, 1941, 207-212; Hoffstadt and Pilcher, Jour. Bact., 35, 1938, 353-367 ; 39, 1940, 40-41; Jour. Inf. Dis., $64,1939,208-216 ; 65,1939,103-112$; Hoffstadt et al., ibid., 68, 1941, 213-219; K. E. Hyde, Am. Jour. Hyg., 23, 1936, 278-297; R. R. Hyde, ibid., 30 (B), 1939, 37-46, 47-55; Hyde and Gardner, ibid., 17, 1933, 446-465; 30, (B), 1939, 57-63; Kessel et al., Proc. Soc. Exp. Biol. and Med., 28, 1931, 413-414; Lipschütz, Wien. klin. Wehschr., 40, 1927, 1101-1103; Lush, Austral. Jour. Exp. Biol. and Med. Sci., 15, 1937, 131-139; 17, 1939, 85-88; Martin, Austral. Counc. Sci. and Indust. Res., Bull. 96, 1936, 28 pages; Moses, Mem. Inst. Oswaldo Cruz, 3, 1911, 46-53; Parker and Thompson, Jour. Exp. Med., 75, 1942, 567-573; Plotz, Compt. rend. Soc. Biol., Paris, 109, 1932, 1327-1329; Rivers, Jour. Exp. Med., 51, 1930, 965976; Rivers and Ward, ibid., 66, 1937, 1-14; Rivers et al., ibid., 69, 1939, 31-48; Sanarelli, Cent.f. Bakt., I Abt., 23, 1898, 865-873; Shaffer, Am. Jour. Hyg., 34 (B), 1941, 102-120; Shope, Jour. Exp. Med., 56, 1932, 803-822; Smadel et al., ibid., 72, 1940, 129-138; Splendore, Cent. f. Bakt., I Abt., Orig., 48, 1909, 300-301; Stewart, Am. Jour. Cancer, 15 Suppl., 
1931, 2013-2028; Swan, Austral. Jour. Exp. Biol. and Med. Sci., 19, 1941, 113115.

Strains and substrains: A strain from cottontail rabbits (Sylvilagus $s p$.), differing from typical myxoma virus, has been studied extensively under the name fibroma virus. This strain in turn is recognized as consisting of variants and has been investigated as typical (OA) and inflammatory (IA) substrains, antigenically alike but the latter tending to generalize in domestic rabbits. Fibroma virus is not lethal in domestic rabbits as the type strain almost always is; it appears to lack some antigenic constituents, inducing the formation of agglutinins that give cross reactions with the type but of neutralizing and complement fixing antibodies that do not. The fibroma strain does not generally appear in the blood stream, as myxoma virus does, and is not contagious, at least it does not spread spontaneously among domestic rabbits as the myxoma strain does; the manner of its spread in wild rabbits in nature is not known. Its particle size has been calculated as 126 to 141 millimicrons by centrifugation, 125 to 175 millimicrons by filtration. (Ahlström, Jour. Path. and Bact., 46, 1938, 461-472 ; Andrewes, Jour. Exp. Med., 63, 1936, 157-172; Hoffstadt and Pilcher, Jour. Inf. Dis., 68, 1941, 67-72; Hurst, Brit. Jour. Exp. Path., 18, 1937, 1-30; Austral. Jour. Exp. Biol, and Med. Sci., 16, 1938, 53-64, 205-208; Hyde, Am. Jour. Hyg., 24, 1936, 217-226; Ledingham, Brit. Jour. Exp. Path., 18, 1937, 436-449; van Rooyen, ibid., 19, 1938, 156-163; van Rooyen and Rhodes, Cent. f. Bakt., I Abt., Orig., 142, 1938, 149-153; Schlesinger and Andrewes, Jour. Hyg., 37, 1937, 521-526; Shope, Jour. Exp. Med., 56, $1932,793-822 ; 63,1936,33-41,43-57$, $173-178$. 


\section{FAMILY III. ERRONACEAE FAM. NOV.}

Viruses of the Encephalitis Group, inducing diseases mainly characterized by ef fects on nerve tissues.

\section{Key to the genera of family Erronaceae.}

I. Viruses of the Typical Encephalitis Group.

Genus I. Erro, p. 1248.

II. Viruses of the Poliomyelitis Group.

Genus II. Legio, p. 1257.

III. Viruses of the Rabies Group.

Genus III. Formido, p. 1263.

\section{Genus I. Erro gen. nov.}

Viruses of the Typical Encephalitis Group, inducing diseases mainly characterized by injuries to cells of the brain. Vectors of some known to be ticks; dipterous insects may also transmit. Generic name from Latin erro, a vagrant.

The type species is Erro scoticus spec. nov.

\section{Key to the species of genus Erro.}

I. Affecting sheep principally, but also man.

1. Erro scoticus.

II. Affecting man principally.

2. Erro silvestris.

3. Erro incognitus.

4. Erro japonicus.

5. Erro nili.

6. Erro scelestus.

III. Affecting horse principally, but also man.

7. Erro equinus.

IV. Affecting horse, cow, sheep.

\section{Erro bornensis.}

1. Erro scoticus spec. nov. From Latin Scoticus, Scottish.

Common name: Louping-ill virus.

Hosts: BOVIDAE-Ovis aries L., sheep. HOMINIDAE-Homo sapiens L., man. Experimentally, also mouse, rat (subclinical infection), chick embryo (discrete primary lesions on chorioallantoic membrane), Macacus rhesus, horse, cow, pig.

Insusceptible species: Guinea pig, rabbit.

Geographical distribution: Seotland, northern England.

Induced disease : In sheep, encephalitis characterized by dullness followed by incoordination of movement, frequently with tremors chiefly of the head; saliva- tion, champing of jaws; prostration, coma, death. In man, encephalitis with prompt and complete recovery accompanied by formation of specific neutralizing antibodies. In mouse, experimentally, diffuse encephalomyelitis with mild meningeal involvement; following intracerebral inoculation, fine rhythmical tremor involving neck, nose, and ears, unsteadiness, muscle spasms, respiratory distress, sometimes clonic and rarely tonic convulsions; hind limb paralysis, dribbling of urine, cessation of spontaneous limb movements, death; in mouse inoculated intraperitoneally, virus usually enters central nervous system by way of the olfactory mucosa and olfactory bulb, occasionally by trauma at points of 
damage ; in mouse inoculated intranasally, virus enters blood and reaches the olfactory bulb where it multiplies to a high concentration before infecting the remainder of the brain and the rest of the nervous system; tends to disappear from the blood after sickness begins but persists in the brain until death from encephalitis. In chick embryo, after inoculation of chorioallantoic membrane, edema and opacity spreading from site of inoculation on membrane of 10-day embryo; in 12-day eggs, discrete primary lesions, sometimes with secondary lesions surrounding them on the inoculated membrane; embryo dies in about 6 days, after showing jaundice, edema, mottling of the liver with necrosis; virus regularly in blood. In monkey, Macacus rhesus, progressive cerebellar ataxia; encephalomyelitis with involvement and massive destruction of Purkinje cells in the cerebellum.

Transmission: By ticks, Rhipiccphalus appendiculatus and Ixodes ricinus ( $I X O$ $D I D A E)$. In Rhipicephalus appendiculatus, the larva or nymph becomes infected; only a few individuals retain virus until the adult stage; virus does not pass through the egg. Non-viruliferous ticks do not acquire virus by feeding with infective ticks on immune animals. Experimentally, by intracerebral or intraperitoneal injection in mouse; by intranasal instillation in rat, mouse, and monkey.

Serological relationships: Complement fixation and neutralization tests show cross reactions with Russian springsummer encephalitis virus, but immune serum against louping-ill virus is only partially effective in neutralizing the spring-summer encephalitis virus.

Immunological relationships: Mice are protected against louping-ill virus by vaccination with non-virulent springsummer encephalitis virus but protection is less effective than for the homologous virus. No cross immunity with respect to Rift Valley fever virus or poliomyelitis virus in Macacus rhesus, but immunity with respect to reinfection by louping-ill virus has been demonstrated.

Thermal inactivation: At $58^{\circ} \mathrm{C}$ in 10 minutes.

Filterability: Passes Berkefeld V, N, and $\mathrm{W}$ filters.

Other properties: Viable in broth filtrates after storage at $4^{\circ} \mathrm{C}$ and $\mathrm{pH} 7.6$ to 8.5 for 70 days. Particle diameter, calculated from ultrafiltration data, 15 to 20 millimicrons.

Literature: Alexander and Neitz, Vet. Jour., 89, 1933, 320-323; Onderstepoort Jour. Vet. Sci. and Anim. Industr., 5, 1935, 15-33; Alston and Gibson, Brit. Jour. Exp. Path., 12, 1931, 82-88; Burnet, Jour. Path. and Bact., 42, 1936, 213-225; Brit. Jour. Exp. Path., 17, 1936, 294-301; Burnet and Lush, Austral. Jour. Exp. Biol. and Med. Sci., 16, 1938, 233-240; Casals and Webster, Science, 97, 1943, 246-248; Jour. Exp. Med., 79, 1944, 45-63; Elford and Galloway, Jour. Path. and Bact., 37, 1933, 381-392; Findlay, Brit. Jour. Exp. Path., 13, 1932, 230-236; Fite and Webster, Proc. Soc. Exp. Biol. and Med., 31, 1934, 695-696; Galloway and Perdrau, Jour. Hyg., 35, 1935, 339346 ; Hurst, Jour. Comp. Path. and Therap., 44, 1931, 231-245; M'Fadyean, Jour. Comp. Path. and Therap., 7, 1894, 207-219; 19, 1900, 145 154; Pool et al., ibid., 43, 1930, 253-290; Rivers and Schwentker, Jour. Exp. Med., 59, 1934, 669-685; Schwentker et al., ibid., 57, 1933, 955-965.

2. Erro silvestris spec. nov. From Lat in silvestris, of the forest, in reference to incidence of the induced disease almost exclusively in those who enter forest lands.

Common names: Spring-summer encephalit is virus, forest spring encephalitis virus.

Hosts: Man; probably cattle, horse; Eutamias asiaticus orientalis, Evotomys rufocanus arsenjevi. Experimentally, also white mouse, Macacus rhesus, birds, goat, sheep, Microtus michnoi pelliceus Thom., Cricetulus furunculus. 
Geographical distribution: Union of Soviet Socialist Republics.

Induced disease: In man, acute non. suppurative encephalitis, abrupt onset, steep rise of temperature to 38 to $40^{\circ} \mathrm{C}$, severe headache, giddiness, and vomiting; pareses and paralyses of upper or lower limbs or museles of neck and back; residual atrophic paralyses common; mortality among cases, 30 per cent; 80 per cent of all cases occur in May and June,

Transmission: By tick, Ixodes persulcatus ( $I X O D I D A E$ ); the virus seems to hibernate in this species and has proved capable of passing through eggs to progeny. Experimentally, also by ticks Dermacentor silvarum and Haemaphysalis concinna ( $I X O D I D A E)$.

Serological relationships: Virus-neutralizing antibodies, found without other evidence of disease in some men and in many cattle and horses, believed to indicate susceptibility of these hosts to latent infections. No cross neutralization with St. Louis encephalitis virus. Japanese summer encephalitis virus is in part antigenically related, but some antigenic constituents of this virus are missing in spring-summer encephalitis virus and vice versa.

Immunological relationships: Formolized virus immunizes specifically.

Filterability: Passes Berkefeld and Chamberland filter candles.

Literature: Smorodintseff, Arch. f. gesamt. Virusforsch., 1, 1940, 468-480; Soloviev, Acta Med. U. R. S. S., 1, 1938, 484-492 (Biol. Abst., 17, 1943, 1726, no. $18777)$.

3. Erro incognitus spec. nov. From Latin incognitus, unknown, in reference to mystery surrounding the nature and relationships of this virus, as evidenced by common name.

Common name: Australian $\mathrm{X}$-disease virus.

Hosts: HOMINIDAE-Homo sapiens L., man. Experimentally, also sheep, horse, cow, rhesus monkey.
Geographical distribution: Australia.

Induced disease: In man, polioencephalitis, especially in children, oceurring in late summer; mortality high; characterized by headache, body pains, drowsiness, weakness, then vomiting, fever, convulsions; paralysis of limbs, eyemuscles, or face rare; recovery rapid in non-fatal cases.

Literature: Kneebone, Austral. Jour. Exp. Biol. and Med. Sci., 3, 1926, 119 127 ; Perdrau, Jour. Path. and Bact., 42, $1936,59-65$.

4. Erro japonicus spec. nov. From New Latin Japonia, Japan.

Common name: Japanese B encephalit is virus.

Hosts : HOM I N IDAE-Homo sapiens L., man. Experimentally, also young sheep, mouse, and Macacus rhesus.

Geographical distribution: Japan, Union of Soviet Socialist Republics.

Induced disease: In man, loss of appetite, drowsiness, nausea, then rapid rise of temperature, pains in joints and chest ; restlessness followed by apathy, coma; death, usually before end of second week, or recovery, sometimes with persistence of evidences of damage done to the nervous system by the disease.

Serological relationships: Specific antiserum does not neutralize St. Louis encephalit is virus or louping-ill virus. Russian autumn-encephalitis virus induces the formation of antisera neutralizing Japanese B encephalitis virus. Russian spring-summer encephalitis virus contains some, but not all, antigens in common with this virus. Australian $\mathrm{X}$-disease virus is distinct in neutralization tests.

Immunological relationships: Specific immunity as a result of earlier infection in mice; no cross protection with respect to St. Louis encephalitis virus. Vaccination with Japanese B encephalitis virus does not enhance resistance to West Nile encephalitis virus but only to the homologous virus. 
Thermal inactivation: At $56^{\circ} \mathrm{C}$ in 30 minutes.

Filterability: Passes Berkefeld N, W, Chamberland $\mathrm{L}_{2}, \mathrm{~L}_{3}$, and Seitz EK filters, with ease.

Literature: Kudo et al,, Jour. Immunol., 32, 1937, 129-135; Smorodintseff et al., Arch. f. gesamt. Virusforsch., 1, 1940, 550-559; Webster, Jour. Exp. Med., $67,1938,609-618$.

5. Erro nili spec, nov. From Latin Nilus, god of the Nile.

Common name: West Nile encephalit is virus.

Hosts : HOMINIDAE-Homo sapiens L., man (perhaps without indueing any definite disease). Experimentally, also rhesus monkey, mouse.

Geographical distribution: Africa (Uganda).

Induced disease : In man, no details are known; virus was originally isolated from blood of a woman native of Uganda; at the time the temperature of the patient was $100.6^{\circ} \mathrm{F}$ but she denied illness; moreover, two laboratory workers developed neutralizing antibodies without recognizable clinical disease. In mouse, experimentally, after intracerebral inoculation, incubation period to 4 or 5 days, then hyperactivity and roughening of coat; later, weakness, hunched attitude, sometimes paralysis of hind quarters; usually coma before death. In rhesus monkey, experimentally, after intracerebral or intranasal inoculation, fever and encephalitis.

Serological relationships : No cross reactions in complement fixation test setween this and equine encephalitis virus, Japanese B encephalit is virus, St. Louis eneephalitis virus, or lymphocytic choriomeningit is virus. Neutralization tests show some common antigens in West Nile encephalitis virus, Japanese B encephalitis virus and St. Louis encephalitis virus; antiserum to West Nile virus does not neutralize either of the others but antisera against St. Louis virus may neutralize West Nile virus and antisera against Japanese $B$ virus have some effectiveness in neutralizing both West Nile virus and St. Louis virus.

Immunological relationships: Vaccination with this virus does not enhance resistance to Japanese B or St. Louis encephalitis viruses but only resistance to the homologous virus.

Thermal inactivation: At $55^{\circ} \mathrm{C}$, not at $50^{\circ} \mathrm{C}$, in 30 minutes.

Filterability: Passes Berkefeld V, N, and $W$ filter candles readily; also passes Seitz EK asbestos pads and collodion membranes 79 , not 62 , millimicrons in average pore diameter.

Other properties: Infective particle 21 to 31 millimicrons in diameter, as calculated from filtration experiments. $\mathrm{Vj}$. able at least 2 weeks at 2 to $4^{\circ} \mathrm{C}$. Viable after drying from the frozen state.

Literature: Havens et al., Jour. Exp. Med., 77, 1943, 139-153; Smithburn, Jour. Immunol., 44, 1942, 25-31; Smithburn et al., Am. Jour. Trop. Med., 20, 1940, 471492.

6. Erro scelestus spec, nov. From Latin scelestus, infamous.

Common name: St. Louis encephalitis virus.

Hosts : HOMINIDAE-Homo sapiens L., man. A great number of mammals and birds in endemic areas may have antisera that neutralize the virus, indicating that they are probably natural hosts; among these are: $A N A T I D A E-$ Anas platyrhyncha L., Mallard and Pekin ducks; Anser anser (L.), domestic goose. BOVIDAE-Bos taurus L., cow; Capra hircus L., goat; Ovis aries L., sheep. $C A N I D A E-C a n i s$ familiaris $\mathrm{L}$., dog. COLUMBIDAE-Columba livia, domestic pigeon; Zenaidura macroura, western mourning dove. EQUIDAE-Equus caballus L., horse. FALCONIDAEFalco sparverius L., sparrow hawk. LEPORIDAE-Lcpus californicus Gray, jack rabbit; Sylvilagus nuttalli, cottontail rabbit. MELEAGRIDAE-Meleagris gallopavo L., turkey. MURIDAE -Rattus norvegicus (Berkenhout), brown 
rat. MUSCICAPIDAE-Turdus migratorius L., robin. PHASIANIDAEGallus gallus (L.), chicken; Lophortyx californica, California quail. PICIDAE - Asyndesmus lewis, Lewis woodpecker; Colaptes cafer (Gm.), red-shafted flicker. STRIGIDAE-Bubovirginianus (Gm.), great horned owl. SUIDAE-Sus scrofa L., pig. Experimentally, white mouse (some substrains of the Swiss white mouse are genetically more readily infected than others); Macacus rhesus; pigeon (inapparent infection); chick embryo and to a limited extent the young hatched chick.

Insusceptible species : Laboratory rabbit, Cebus monkey, guinea pig, rat.

Geographical distribution: United States.

Induced disease : In man, during summer and fall, about 9 to 21 days after exposure, headache, high fever, rigidity of neck, tremors; encephalitis, usually with fever; some patients become drowsy, others sleepless or delirious; usual sequelae headaches, irritability, some loss of memory, and drowsiness; neutralizing antibodies maintained in vivo at least $2 \frac{1}{2}$ years after occurrence of disease. Experimentally, in susceptible strains of white mouse inoculated by intracerebral injection, after 3 to 4 days, coarse tremors, convulsions, prostration, death; perivascular accumulations of mononuclear leucocytes throughout brain, stem, cord, and pia, with destruction of pyramidal celis in the lobus piriformis and cornu Ammonis; subcutaneous and intraperitoneal injections immunize against subsequent infection by intracerebral inoculation, virus reaching only blood and spleen in the process of immunization unless an excessive dose is given; some substrains of the White Swiss mouse are relatively resistant to infection, requiring inoculation with about 1000 times the minimal infective dose for highly susceptible strains and when infected proving relatively poor sources of virus for subinoculation; highly susceptible substrains of the White Swiss mouse lack a single major, dominant, genetic factor that is present in resistant substrains.

Transmission: By mosquito, Culex tarsalis Coquillett ( $C U L I C I D A E$ ), probably extensively; this insect has been collected in nature carrying the virus. Experimentally, by larvae of American dog tick, Dermacentor variabilis (Say) (IXODIDAE); by mosquito, Culex pipiens Linn., var. pallans Coq. (CULI$C I D A E)$. To mice, by feeding on infected tissues.

Serological relationships: Human antisera may neutralize virus after clinical and subclinical attacks.

Immunological relationships: Specific intracerebral immunity after vaccination by subcutaneous or intraperitoneal injection in mice appears early (about 1 week after vaccination) and disappears before humoral antibody titer reaches its maximum.

Thermal inactivation: At $56^{\circ} \mathrm{C}$ in 30 minutes.

Filterability: Passes Berkefeld V and $N^{\top}$ filter candles and collodion membranes 66 millimicrons in average pore diameter.

Other properties: Storage in human brain tissue in glycerine inactivates this virus in about 32 days. Diameter of infective particle calculated from filtration data as about 20 to 33 millimicrons. In storage, rabbit and sheep sera act to some extent as preservatives. At $4^{\circ} \mathrm{C}$, after drying in vacuo while frozen, viable in apparently undiminished titer for at least 17 months.

Literature: Bang and Reeves, Jour. Inf. Dis., 70, 1942, 273-274; Bauer et al., Proc. Soc. Exp. Biol. and Med., 31, 1934, 696-699; Blattner and Cooke, Jour. Inf. Dis., 70, 1942, 226-230; Blattner and Heys, Proc. Soc. Exp. Biol. and Med., 48, 1941, 707-710; Cook, Jour. Inf. Dis., 63, 1938, 206-216; Cook and Hudson, ibid., 61, 1937, 289-292; Elford and Perdrau, Jour. Path. and Bact., 40, 1935, 143-146; Hammon and Howitt, Am. Jour. Hyg., 35, 1942, 163-185; Hammon et al., Science, 94, 1941, 305-307, 328-330; Jour. Inf. Dis., 70, 1942, 263-266, 267-272, 278 
283 ; Harford and Bronfenbrenner, Jour. Inf. Dis., 70, 1942, 62-68; Harrison and Moore, Am. Jour. Path., 13, 1937, 361375 ; Hodes, Jour. Exp. Med., 69, 1939, 533-543; Hodes and Webster, ibid., 68, 1938, 263-271 ; Lennette and Smith, Jour. Inf. Dis., 65, 1935, 252-254; Mitamura et al., Trans. Soc. Path. Jap., 27, 1937, 573580 ; Muckenfuss et al., U. S. Pub. Health Service, Public Health Rept., 48, 1933 , 1341-1343; O'Leary et al., Jour. Exp. Med., 75, 1942, 233-246; Reeves et al., Proc. Soc. Exp. Biol. and Med., 50, 1942, 125-128; Sulkin et al., Jour. Inf. Dis., 67, 1940, 252-257; Webster, Jour. Exp. Med., 65, 1937, 261-286 ; 68, 1938, 111-124; Webster and Clow, ibid., 63, 1936, 433-14S, 827-845; Webster and Fite, ibid., 61, 1935, 103-114, 411-422; Webster and Johnson, ibid., 74, 1941, 489-494; Webster et al., $61,1935,479-487 ; 62,1935,827-847$.

7. Erro equinus spec. nov. From Lat in equinus, pertaining to horses.

Common name: Equine encephalit is virus.

Hosts: EQUIDAE-Equus caballus L., horse; $\mathrm{F}_{1}$ hybrid of the horse and $E$. asinus L., mule. HO.IINIDAE-Homo sapiens L., man. COLUMBIDAEColumba livia, domestic pigeon. PHASIANIDAE-ring-necked pheasant. TETRAONIDAE-Tympanuchus cup$i d o$ L., var. americanus (Reichenbach), prairie chicken. Many additional species have been found to show neutralizing antisera at times and these are presumably natural hosts of the virus upon occasion; among them are: $A N A T I D A E$ Anas platyrhyncha L., Mallard and Pekin ducks; Anser anser (L.), domestic goose. BOVIDAE-Bostaums L., cow; Capra hircus L., goat; Ovis aries L., sheep. $C A N I D A E-C$ Canis familiaris L., dog. CHARADRIIDAE-Oxyechus vociferus L., killdeer. CRICETIDAE-Microtus montanus (Peale), field mouse; Peromyscus maniculatus (Wagner), white-footed mouse: FALCONIDAE-Falco sparverius L., sparrow hawk. MELEAGRI$D A E$-Meleagris gallopavo L., turkey.
$M U R I D A E$-Rattus rattus L., black rat. $M U S C I C A P I D A E-T u r d u s ~ m i-$ gratorius L., robin. MUSTELIDAEMustela frenata Lichtenstein, weasel. PHASIANIDAE-Gallus gallus (L.), chicken; Lophortyx californica, California quail ; Phasianus colchicus L., ringnecked pheasant. PICIDAE-Colaptes cafer (Gm.), red-shafted flicker. STRIGIDAE-Bubo virginianus (Gm.), great horned owl. SUIDAE-Sus scrofa L., pig. Experimentally, also chick embryo, goose embryo, pheasant embryo, robin embryo, pigeon embryo, turkey embryo, sparrow embryo, duck embryo, and guinea-fowl embryo; white mouse, guinea pig, rabbit, pigeon, white rat, calf, sheep, monkey, goat, dog, hen, turkey; Zonotrichia leucophrys gambeli, Gambel sparrow; Passer domesticus L., English sparrow; Lophortyx californica, quail; Junco oreganus, junco; Toxostoma lecontei lecontei, thrasher; Citellus richardsonii (Sabine), gopher or Richardson's ground squirrel; Sigmodon hispidus Say and Ord, cotton rat; Dipodomys heermanni Le Conte, kangaroo rat; Reithrodontomys megalatus, wild mouse; Microtus montanus, $M$. californicus and $M$. mordax, wild mice; Peromyscus maniculatus (Wagner), white-footed mouse; Neotoma fuscipes Baird, wood rat; Sylvilagus bachmani (Waterhouse), brush rabbit; $S$. audubonii (Baird), cottontail rabbit; Canis familiaris L., dog (puppies); Anser cinereus, goose; Anas boscas L., duck; Circus rufus (Gm.), hawk; Turdus merula L., blackbird; Ciconia ciconia L., white stork; Vultur fulvus Briss., tawny vulture; Marmota monax (L.), woodchuck; Microtus pennsylvanicus (Ord.), field vole; Speotyto cunicularia hypugaea (Bonaparte), western burrowing owl; Molothrus ater (Boddaert), cowbird; common quail or bob-white.

Insusceptible species: Frog (cat and opossum reported as "refractory").

Geographical distribution: United States, Canada, Argentina.

Induced disease : In horse, initial fever, then signs of fatigue, somnolence; occa- 
sional excitability followed by incoordinated action of limbs, disturbed equilibrium, grinding of teeth, paresis and varied paralyses; frequently inability to swallow, paralysis of lips and bladder, amanrosis; case fatality about 50 per cent; recovery without sequelae in inild cases; death within 3 to 8 days in severe cases. In man (children particularly vulnerable), a profound, acute, disseminate and focal encephalomyelitis characterized by intense vascular engorgement, perivaseular and parenchymatous cellular infiltration and extreme degenerative changes in the nerve cells. In chick embryo, excessive increase of virus continuing until just before host's death, virus being found eventually throughout the egg but most concentrated in the embryo; vaccines made from virus grown in chick embryo and then inactivated are especially effective because of the high titer of virus represented in them; increased resistance with age characteristic of chorioallantoic membrane as well as of hatched chick; rounded acidophilic masses occur usually near periphery of nucleus in embryonic nerve cells; no such inclusions are found as a result of infection with Borma disease virus or poliomyelitis virus.

Transmission : Experimentally by tick Dermacentor andersoni stiles (IXODI $D A E$ ), passing through eggs to oflspring; this tick is infective to susceptible animals on which it feeds as larva, nymph or adult. Experimentally by Aëdes acgypli L. (to guinea pig and horse, preinfective period 4 to 5 days; insects retain virus for duration of life; not to eggs of infected mosquitoes; not passed from males to females or by males from female to female), A. albopictus, A. atropalpus, $A$. cantator, A.dorsalis, A. nigromaculis, $A$. sollicitans, A.taeniorhynchus, A.triseriatus, and $A$. vexans (CUI,ICIDAE). Triatoma sanguisuga (Le Conte) (RE. $D U V I I D A E$ ) has been found infected in nature and has transmitted virus experimentally to guinea pigs. The American dog tick, Dermacentor variabilis Say (IXODIDAE) has been infected by inoculation, not by feeding; it has not been shown to transmit.

Serological relationships: Neutralizing antibodies are formed as a result of vaccination with inactive, formolized virus; antigenicity of formalin-inactivated virus as well as of active virus is blocked in the presence of antiserum. In rabbit, cerebral resistance is coincident with presence of neutralizing antibody in spinal fluid. In guinea pig, therapy with specific antiserum ineffective if begun after onset of encephalitis; effective if begun within 24 to 48 hours of peripheral inoculation. No cross neutralization reaction with lymphocytic choriomeningit is virus, Japanese B encephalitis virus or St. Louis encephalitis virus. Constituent strains (typical Western and Eastern) do not give cross neutralization reactions, but do show the presence of common antigens by cross reactions in complement fixation not shared with such other viruses as Japanese B encephalitis virus, St. Louis encephalitis virus, West Nile encephalit is virus, lymphocytic choriomeningit is virus. Sera of human cases may be negative by complement fixation tests a few davs after onset, yet strongly strainspecific during second week of illness.

Immunological relationships: Young of immunized guinea pigs are immune to homologous strain at least a month after birth. No cross immunity between Western and Fastern strains of equine encephalit is virus.

Thermal inactivation : At $60^{\circ} \mathrm{C}$, not at $56^{\circ} \mathrm{C}$, in 10 minutes.

Filterability: Passes collodion membranes 66 , not 60 , millimicrons in average pore diameter. Passes Berkefeld V, N, and $W$, finest Mandler, and Seitz filters.

Other properties: Inactivated below pH 5.5. Viable at least a year, dry in vacuum. Particle diameter estimated from filtration experiments to be 20 to 30 millimicrons. Electron micrographs show particles as spherical or diskshaped, about 39 millimicrons in diameter with round or oval region of high density within each; older preparations show 
comma-shaped particles. Sedimentation constant, mean $265.5 \times 10^{-13} \pm 5.4 \times$ $10^{-13}$ (range 252 to $\left.276 \times 10^{-13}\right)$. Specific volume 0.864 . Molecular weight of liponucleoprotein complex behaving as the virus calculated as 152 million, approximately 250 particles giving 50 per cent infection; material contains 4 per cent carbohydrate. Absorption of ultraviolet light reaches a peak at about $2600 \AA$., a broad minimum at about $2450 \AA$., and an increase at $2200 \AA$.

Strains: The Western strain (so-called Western equine encephalitis virus) may be considered as type of a large group of variants met in nature; some produce clinically milder disease than others (Birch, Am. Jour. Vet. Res., 2, 1941, 221-226); they may change in virulence on passage in experimental hosts. The Eastern strain (so-called Eastern equine encephalitis virus) has been studied extensively also, and has been found to differ from the type strain especially : in more rapid course of induced disease in the horse; in being experimentally trans missible to sheep, pig, dog, eat and the European hedgehog; in its localization in eastern coast states and absence from the area between California and Wisconsin, where the type strain is found; in failure experimentally to infect Aëdes aegypti unless inoculated into body cavity by needle puncture, whereupon it persists and can be transmitted; and in failure of cross-neutralization with the western strain. A strain produced by serial passage in pigeons is reported to have raused no obvious reaction in horses but to have induced the formation of neu tralizing antibodies. A Venezuelan strain differs from the type in complement-fixation reactions; it induces in man a mild disease, characterized by malaise, fever, headache or drowsiness, and uneventful recovery (Casals et al., Jour. Exp. Med., 77, 1943, 521-530).

Literature: Bang, Jour. Exp. Med. 77, 1943, 337-344; Bauer et al., Proc. Soc. Exp. Biol. and Med., 33, 1935, 378-382; Beard et al., Science, 87, 1938, 190; Birch,
Am. Jour. Vet. Res., 2, 1941, 221-226; Casals and Palacios, Science, 94, 1941, 330 ; Covell, Proc. Soc. Exp. Biol. and Med., 32, 1934, 51-53; Cox, ibid., 33, 1936. 607-609; Cox and Olitsky, Jour. Exp. Med., 63, 1936, 745-765; 64, 1936, 217-222, 223-232; Cox et al., L. S. Pub. Health Service, Public Health Rept., 56, 1941, 1905-1906; Davis, Am. Jour. Hyg., 32 (C) , 1940, 45-59; Eklund and Blumstein, Jour. Am. Med. Assoc., 111, 1938, 17341735; Feemster, Am. Jour. Public Health, 28, 1938, 1403-1410; Finkelstein et al., Jour. Inf. Dis., 66, 1940, 117-126; Fothergill and Dingle, Science, 88, 1938, 549550 ; Fothergill et al., New England Jour. Med., 219, 1938, 411; Giltner and Shahan, Science, 78, 1933, 63-64; Jour. Am. Vet. Med. Assoc., 88, (N.S. 41), 1936, 363-374; Graham and Levine, Am. Jour. Vet. Res., 2, 1941, 430-435; Grundmann et al., Jour. Inf. Dis., 72, 1943, 163-171 ; Havens et al., Jour. Exp. Med., 77, 1943, 139-153; Higbie and Howitt, Jour. Bact., 29, 1935, 399406; Howitt, Jour. Inf. Dis., 55, 1934, $138-149 ; 61,1937,88-95 ; 67,1940,177-$ 187; Science, 88, 1938, 455-456; Howitt and Van Herick, Jour. Inf. Dis., 71, 1942, 179-191; Kelser, Jour. Am. Vet. Med. Assoc., 82, 1933, 767-771; King, Jour. Exp. Med., $71,1940,107-112$; $76,1942,325-334$; Kitselman and Grundmann, Kansas Agr. Exp. Sta., Tech. Bull. 50, 1940, 1-15; Merrill and TenBroeck, Jour. Exp. Med., 62, 1935, 687-695; Meyer et al., Science, 74, 1931, 227-228; Mitchell et al., Canadian Jour. Comp. Med., 3, 1939, 308-309; Morgan, Jour. Exp. Med., 74, 1941, 115132; Morgan et al., ibid., $76,1942,357-$ 369; Olitsky et al., ibid., 77, 1943, 359374; Remlinger and Bailly, Compt. rend. Soc. Biol., Paris, 121, 1936, 146-149; 122, 1936, 518-519; 123, 1936, 562-563; Sabin and Olitsky, Proc. Soc. Exp. Biol. and Med., 38, 1938, 597-599; Sellards et al., Am. Jour. Hyg., 33 (B), 1941, 63-68; Shahan and Eichhorn, Am. Jour. Vet. Res., 2, 1941, 218-220; Sharp et al., Proc. Soc. Exp. Biol. and Med., 51, 1942, 206207 ; Arch. Path., 36, 1943, 167-176; Syverton and Berry, ibid., 34, 1936, 822- 
824 ; Jour. Bact., 33, 1937, 60; Am. Jour. Hyg., 32 (B), 1940, 19-23; 33 (B), 1941, 37-41 ; Jour. Exp. Med., 73, 1941, 507529 ; Taylor et al., Jour. Inf. Dis., 67, 1940, 59-66; 69, 1941, 224-231; 72, 1943, 31-41; TenBroeck, Arch. Path., 25, 1938, 759 (Abst.); TenBroeck and Merrill, Proc. Soc. Exp. Biol. and Med., 31, 1933, 217-220; TenBroeck et al., Jour. Exp. Med., 62, 1935, 677-685; Traub and Ten Broeck, Science, 81, 1935, 572; Tyzzer and Sellards, Am. Jour. Hyg., 33 (B), 1941, 69-81; Tyzzer et al., Science, 88, 1938, 505-506; van Roekel and Clarke, Jour. Am. Vet. Med. Assoc., 94 (N.S. 47), 1939, 466-468; Webster and Wright, Science, 88, 1938, 305-306; Wesselhoeft et al., Jour. Am. Med. Assoc., 111, 1938, 1735-1740; Wright, Am. Jour. Hyg., 36, 1942, 57-67.

8. Erro bornensis spec. nov. From Borna, name of a town in Saxony where a severe epizootic occurred in 1894 to 1896.

Common name: Borna-disease virus.

Hosts : Horse, cow, sheep, perhaps deer. Experimentally, also rabbit, guinea pig, rat (more susceptible when old than when younger), mouse; Macaca mulatta (Zimmermann), rhesus monkey.

Insusceptible species: Ferret, cat, pigeon; probably dog.

Geographical distribution: Würtemburg, Germany, North and South America, Hungary, Russia, Belgium, France, Italy, Roumania.

Induced disease: In horse, encephalomyelitis characterized by lassitude, indifference to external stimuli; later intermittent excitement, difficulty in mastication and deglutition, spasms in various muscles, champing, excessive salivation; pupils unequal in size; paralysis of hindquarters, tail, muscles of tongue, or muscles of back; temperature usually normal; death in 20 to 37 hours or, less often, recovery after about 1 to 3 weeks. Virus may pass placenta and infect fetus in pregnant animals.
Transmission: To rabbit, experimentally by feeding and by injection intracerebrally, intraocularly, nasally, intravenously, subcutaneously, or intraperitoneally; not by living in same cage.

Immunological relationships: No cross immunity conferred by the Western strain of equine encephalomyelitis virus. Isolate of Borna disease virus from the horse immunizes rabbits against isolate from sheep, and vice versa. Herpes and rabies viruses do not immunize rabbits against subsequent infection by Borna disease virus.

Thermal inactivation : At 50 to $57^{\circ} \mathrm{C}$ in 30 minutes; at $70^{\circ} \mathrm{C}$ in 10 minutes.

Filterability: Passes Berkefeld $\mathrm{N}$ and Mandler filters, but with difficulty. Passes collodion membranes of average pore diameter 400 millimicrons readily, 200 millimicrons with difficulty, 175 millimicrons not detectibly. May be separated by differential filtration from louping-ill virus, which will pass even a 125-millimicron membrane.

Other properties: Particle size estimated from filtration data as 85 to 125 millimicrons. Optimum $\mathrm{pH}$ for stability in broth at 15 to $20^{\circ} \mathrm{C}$ is 7.4 to 7.6 ; very sensitive to greater alkalinity. Viable after 327 days dry at laboratory temperatures. Viable at least 6 months in 50 per cent glycerine. Inactivated by putrefaction in 5 days; by 1 per cent carbolic acid in 4 , not in 2 , weeks.

Literature: Barnard, Brit. Jour. Exp. Path., 14, 1933, 205-206; Covell, Proc. Soc. Exp. Biol. and Med., 32, 1934, 51-53; Elford and Galloway, Brit. Jour. Exp. Path., 14, 1933, 196-205; Howitt and Meyer, Jour. Infect. Dis., 54, 1934, 364367 ; Nicolau and Galloway, Brit. Jour. Exp. Path., 8, 1927, 336-341, and in Medical Research Council, Special Report Series No. 121, London, 1928, 90 pp., Ann. Inst. Pasteur, 44, 1930, 673-696; 45, 1930, 457-523; Zwick et al., Ztschr. Infektionskr. parasit. Krankh. u. Hyg. d. Haustierc, 30, 1926, 42-136 ; 32, 1927, 150-179. 


\section{Genus II. Legio gen. nov.}

Viruses of the Poliomyelitis Group, often recoverable from feces of infected hosts, probably because of involvement of some part of the alimentary tract; usually there is also obvious involvement of some part of the nervous system. Generic name from Latin legio, an army or legion.

The type species is Legio debilitans spec. nov.

\section{Key to the species of genus Legio.}

I. Affecting man (see also IV below).

\section{Legio debilitans. \\ 2. Legio crebea. \\ 3. Legio simulans.}

II. Latent in, or affecting, mouse.

III. Affecting birds.

IV. Affecting swine and swineherds.

1. Legio debilitans spec. nov. From Latin debilitare, to weaken or maim. Common names: Poliomyelit is virus, virus of infantile paralysis.

Hosts: HOMINIDAE-Homo sapiens L., man. Experimentally, Cercopithecus aethiops sabaeus, green African monkey; Macaca mordax; H. mulatta, the rhesus monkey; $M$. irus, the cynomolgus monkey; mona monkey; for some isolates. Sigmodon hispidus Say and Ord, cotton rat; mouse; guinea pig; white rat .

Insusceptible species: Sheep ("refractory" but forms neutralizing antibodies). chicken.

Geographical distribution: Almost world-wide.

Induced disease : In man, probably subclinical in most cases, in view of the presence of specific antibodies in sera from the great majority of adults in all parts of the world; virus probably infects some part of the alimentary tract, being found in stools of most elinical cases, of most apparently healthy contacts, and even of some individuals who have recovered from abortive attacks (in one case 123 days after attack); clinical disease. largely in children, is characterized by invasion of central nervous system, with effects ranging from sore throat, fever,

\section{Legio muris.}

\section{Legio gallinae.}

\section{Legio suariorum.}

vomiting, and headache to sudden and scvere paralysis; the muscles most often involved are those of the legs, but there may be paralysis of abdominal or intercostal muscles. Virus not in urine or saliva, rarely in nasal washings; more of ten in stools of young than of old patients; in walls of pharynx, ileum, descending colon. Virus has been recovered from sewage. Incidence and fatality affected by racial characteristics, the first lower and the second higher in negroes than in whites in the United States. In monkey, similar disease, no virus in blood, relapse with reappearance of virus reported; in isolated intestinal loops, infection does not occur through normal mucosa in absence of intestinal contents; disease more severe in summer than in autumn, in autumn than in winter; more severe in older than in younger monkeys; no immunity follows inoculation unless obvious disease occurs.

Transmission: Transmission in nilk has been suspected and at times confirmed. Virus has been recovered from mixed samples of flies in an epidemic area. To definite arthropod vector has been incriminated. Experimentally, in Cercopithecus aethiops sabaeus, the green 
African monkey, by intracerebral, intramasal, and intraabdominal inoculation.

Serological relationships: Specific newtralizing antibodies arise after experimental infection in monkeys, but reinfection is not prevented; only a minority of human convalescent sera neutralize virus in vitro, the most potent sera probably being obtained from those with transient or light paralysis. Cross neutralization between monkey-passage and murine (cotton-rat and mouse) strains. No cross-neutralization reaction with lymphocytic choriomeningitis virus. Isolates differ somewhat antigenically, homologous titers being higher than heterologous titers in some neut ralization tests.

Thermal inactivation: At or below $75^{\circ}$ $\mathrm{C}$ in 30 minutes.

Filterability: Passes membrane about 35 , not 30 , millimicrons in average pore diameter.

Other properties: Infectivity of virus maintained well at $-76^{\circ} \mathrm{C}$ or in glycerine but poorly when dried or just frozen. Inactivated readily by lyydrogen peroxide. Particle diameter estimated as about 12 millimicrons by filtration studies. Precipitated by half-saturated ammonium sulphate solutions. Electron micrographs show elliptical particles 20 to 30 millimicrons in diameter; impure infectious materials show long threads 20 by 75 to 500 millimicrons in size. Component probably virus has sedimentation constant $\mathrm{S}_{20^{\circ}}=62 \times 10^{-13} \mathrm{~cm}$ per sec.per dyne. Inactivated by potassium hydroxide, copper sulfate and potassium permanganate. Stable from pH 2.2 to 10.4 for 2 hours at $37^{\circ} \mathrm{C}$.

Literature: Armstrong and Harrison, U. S. Pub. Health Service, Public Healt h Rept., 50, 1935, 725-730; Aycock, Am. Jour. Hyg., 7, 1927, 791-803; Burnet and Jackson, Austral. Jour. Exp. Biol. and Med. Sci., 17, 1939, 261-270; 18, 1940, 361-366; Burnet et al., ibid., 17, 1939, 253-260, 375-391; Elford et al., Jour. Path. and Bact., 40, 1935, 135-141; Flex- ner, Jour. Exp. Ned., 62, 1935, 787-804; 68, 1936, 209-226; 65, 1937, 497-513; Gard, ibid., 71, 1940, 779-785; Gordon and Lennette, Jour. Inf. Dis., 64, 1939, 97-104; Harmon, ibid., 58, 1936, 331-336; Heaslip, Austral. Jour. Exp. Biol. and Med. Sci., 16, 1938, 285-286 ; Howitt, Jour. Inf. Dis., $51,1932,565-573$; 53, 1933, 145-156; Hudson and Lennette, Am. Jour. Hyg., 17, 1933, 581-586; Jungeblut and Bourdillon, Jour. Am. Med. Assoc., 123, 1943, 399402 ; Jungeblut and Sanders, Jour. Exp. Med., $72,1940,407-436 ; \% 6,1942,127-142$; Jungeblut et al., ibid., $75,1942,611-629$; $76,1912,31-51$; Kessel et al., Am. Jour. Hyg., 27, 193S, 519-529; Jour. Exp. Med., 74, 1941, 601-609; Kolmer et al., Jour. Inf. Dis., 61, 1937, 63-68; Kramer et al., Jour. Exp. Med., 69, 1939, 46-67; Lennette and Hudson, Jour. Inf. Dis., 58, 1936, 10-14 ; Loring and Schwerdt, Jour. Exp. Med., $75,1942,395-406$; Mc Clure and Langmuir, Am. Jour. Hyg., 35, 1942, 285-291; Melnick, Jour. Exp. Med., 77, 1943, 195-204; Moore and Kessel, Am. Jour. Hyg., 38, 1943, 323-344; Moore et al., ibid., 36, 1942, 247-254; Morales, Jour. Inf. Dis., 46, 1930, 31-35; Olitsky and Cox, Jour. Exp. Míed., 63, 1936, 109-125; Paul et al., Am. Jour. Hyg., 17, 1933, 587-600; 601612; Jour. Exp. Med., 71, 1940, 765̃-777; Sabin, ibid., 69, 1939, 507-516; Sabin and Olitsky, ibid., 68, 1938, 39-61; Sabin and Ward, ibid., 73, 1941, 771-793; 74, 1942, 519-529; $75,1942,107-117$; Sabin et al., Jour. Bact., 31, 1936, 35-36 (Abst.); Sanders and Jungeblut, Jour. Exp. Med.. 75, 1942, 631-649; Schult.z and Ciebhardt, Jour. Inf. Dis., 70, 1942, 7-50; Schultz and liobinson, ibid., 70, 1942, 193-200; Stimpert and Kessel, Am. Jour. Hyg., 29, (B), 1939, 57-66; Theiler, Medicine, $20,1941,443-462$; Theiler and Bauer, Jour. Exp. Med., 60, 1934, 767-772; Trask and Paul, ibid., 58, 1933, 531-544; 73, 1941, 4.53-459; 'Trask et al., ibid., 77, 1943, 531-54t; Turner and Young, Am. Jour. Hyg., 37, 1943, 67-79; Wolf, Jour. Exp. Med., $76,1942,53-72$; Young and Merrell, Am. Jour. Hyg., 37, 1943, 80-92. 
2. Legio erebea spec. nov. From Latin crebeus, belonging to the Lower World.

Common names: Choriomeningit is virus, lymphocytic choriomeningit is virus.

Hosts : MLRIDAE-Mus musculus L. gruy or white mouse. HOWINIDAEHomosapiens L, man. CERCOPITHECID.AE-Macaca mulatta, rhesus monkey. Experimentally, also guinea pig; white rat; dog (masked); ferret (masked); Macaca irus, crab-eating macaque; Syrian hamster; chick- or mouse-embryo serum-Tyrode solution culture; chick embryo.

Insusceptible species: Pig, rabbit. field vole, bank vole, canary, hen, parakeet.

Geographical distribution: France, England, United States.

Induced disease: In white mouse, more virulent in young than in old individuals; infection may take place in utero or snon after birth; some mice become carriers after recovery, with virus in organs, blood, urine, and nasal secretions; carriers are immune to large intracerebral inoculations of virus; experimentally, 5 1012 days after intracerebral inoculation of susceptible mice, somnolence, photophobia, tremors of the legs, tonic spasms of muscles in the hindquarters upon stimulation; recovery or death. In man, disease may be subclinical at times as shown by the fact that some supposedly normal sera contain specific antibodies; not all clinical cases develop protecting antibodies against testing strains, so that disease may be somewhat commoner than can be ascertained readily; in all cases benign, but in the more severe of these an acute asept ic meningitis ; after incubat ion period of $1 \frac{1}{2}$ to 3 days, spells of fever extending as long as 3 weeks; late in the disease there may be a meningeal reaction both clinically and cytologically; lymphocytes and some large mononuclear cells appear in the meningeal fluids, although symptoms remain benign; there may be virus in the blood from the beginning of fever to the end of the second week; the spinal fluid is not infective at first but may become so before there is a change in cell count; urine and saliva remain uninfectious.

Transmission: In white mouse, by conlact with mice infected when young, not with those infected when old; nasal mucosa considered portal of entry. In wild gray nouse of the same species, Mus musculus, by contact but less readily than in white mouse. Experimentally, by mosquito, Aëdes aegypti L. CCLICI$D A E$ ) , at 26 to $34^{\circ} \mathrm{C}$; by bedbug, Cimex lectularius (CIMIDAE), but defecation on site of bitten area is essential, bite alone being ineffective. Experimentally, to guinea pig, by application of virus to normal and apparently intact skin; not by contamination of food or litter.

Serological relationships: Serum of recovered subjects usually neutralizes choriomeningit is virus. Hyperimmune serum is ineffective against pseudo-lymphocytic choriomeningitis virus and hyperimmune serum for that virus is ineffective in its turn when used with choriomeningitis virus. No cross neutralization with St. Louis encephalitis virus. A specific soluble antigen associated quantitatively with virus in all hosts fixes complement in the presence of immune serum; virus does so poorly if at all; the anti-soluble-substance antibodies seem to be independent of virusneutralizing antibodies. A soluble protein, readily separable from virus, gives a specific precipit in reaction with immune serum; antibodies concerned are probably not the virus-neut ralizing ant ibodies.

Immunological relationships: Intraperitoneal injection of about 160 intracerebral lethal doses has been found to protect the white mouse against infection by subsequent intracerebral injection of 10,000 lethal doses. The immune mouse differs from the immune guinea pig in showing no neutralizing antibodies in its blood; even the guinea pig may develop resistance before antibodies appear in its serum. Formalized vaccines made from 
guinea pig tissues immunize the guinea pig but vaccines made from mouse tissues do not. Mice immune to this virus are susceptible to infection with pseudolymphocytic choriomeningitis virus and vice versa.

Thermal inactivation: At 55 to $56^{\circ}$ (; in 20 minutes.

Filterability: Passes Berkefeld V, X, and $W$ filters and, with difficulty, a Seit $\%$ asbestos pad.

Other properties: Infective at least 206 days in storage at 4 to $10^{\circ} \mathrm{C}$ in 50 per cent neutral glycerine in 0.85 per cent saline. Infective particle calculated to be 37 to 55 millimicrons in diameter on the basis of centrifugation studies; 40 to 60 millimicrous by ultrafiltration tests. Inactivated by soap with loss of mouse-immunizing capacity.

Literature : Armstrong and Dickens, $\mathrm{C}$. S. Pub. Health Service, Public Health Rept., 50, 1935, 831-842; Armstrong and Lillie, ibid., 49, 1934, 1019-1027; Armstrong and Wooley, ibid., 50, 1935, 537541 ; Jour. Am. Med. Assoc., 109, 1937. 410-412; Baird and Rivers, Am. Jour. Pub. Health, 28, 1938, 47-53; CasalsAriet and Webster, Jour. Exp. Med., 71, 1940, 147-154; Dalldorf, ibid.. \%o, 1939, 19-27; Dalldorf and Douglass, Proc. Soc. Exp. Biol. and Med., 39, 1938, 291-297; Findlay and Stern, Jour. Path, and Bact. 43, 1936, 327-338; Findlay et al., Lancet, 230, 1936 (I), 650-654; Howard, Jour. Inf. Dis., 64, 1939, 66-77; Laigret and Durand, Compt. rend. Acad. Sci., 203, 1936, 282-284; Lépine and Sautter, Ann. Inst. Pasteur, 61, 1938, 519-526; Lépine et al., ibid., 204, 1937, 1846-1818; MacCallum and Findlay, Brit. Jour. Exp. Path., 21, 1940, 110-116; Milzer, Jour. Inf. Dis., 70, 1942, 152-172; Rivers and Scott, Jour. Exp. Med., 63, 1936, 415-132; Scott and Elford, Brit. Jour. Exp. Path., 20, 1939, 182-188; Scott and Rivers, Jour. Exp. Med., 63, 1936, 397-414; Shaugnessy and Zichis, ibid., 72, 1940, 331-313; Smadel and Wall, ibid., 72, 1940, 389-405; 75, 1942, 581-591; Smadel et al., Proc. Soc. Exp. Biol. and Med., 40, 1939, 71-73;
Jour. Exp. Med., 70, 1939, 53-66; 71, 1940 , 43-53; Stock and Francis, ibid., 77, 1943, 323-336; Traub, Science, 81, 1935, 298299 ; Jour. Exp. Med., 63, 1936, 533-546, $847-861 ; 64,1936,183-200 ; 66,1937,317-$ 324 ; 68, 1938, 95-110, 229-250; 69, 1939, $801-817$.

3. Legio simulans spec. nov. From Latin simulare, to imitate, in reference to resemblance of this virus to the preceding in many respects, though not in size or antigenic properties.

Common name: Pseudo-lymphocytic choriomeningit is virus.

Hosts: HOMINIDAE-Homo sapiens I., man. Experimentally, also mouse, guinea pig, rhesus monkey; chorioallanto membrane of chick embryo.

Induced disease : In man, benign aseptic lymphocytic meningitis with virus in rerebro-spinal fluid; severe frontal headache, drowsiness, irritability, vomiting. eventual complete recovery. In mouse, experimentally, roughened fur, spontaneous tremor, hunched attitude, irritability, clonic movements ending with tonic convulsions on stimulation, temporary recovery from spasm with survival a fow hours or instant death.

Serological relationships: Hyperimmune sera for lymphocytic ehoriomeningit is virus are ineffective for this virus, and vice versa. In man, after recovery, neut ralizing ant ibody is strong at 1 month, fading before 7 mont hs.

Immunological relationships: Mice acquire specific resistance to reinfection after cxperimental disease; mice immune to lymphocytic choriomeningit is virus are susceptible to pseudo-lymphocyt ic choriomeningitis virus and vice versa.

Thermal inactivation : At $56^{\circ} \mathrm{C}$, not at $45^{\circ} \mathrm{C}$, in 30 minutes.

Filterability: Passes Berkefeld $V$, not $\mathrm{N}$, filter candle; Gradacol membrane of 320 , not 300 , millimicron average pore diameter.

Other properties: Particle diameter calculated to be not above 150 to 225 millimicrons, from filt ration experiments. 
Viable at least 1 month at $4^{\circ} \mathrm{C}$, at least 1 year in 50 per cent glycerine, 40 days in 0.25 per cent phenol, 1 year when dried from frozen material. Inactivated by 0.05 per cent formalin at $4^{\circ} \mathrm{C}$ in 48 hours ; by boiling in 5 minutes.

Literature: MacCallum et al., Brit. Jour. Exp. Path., 20, 1939, 260-269.

4. Legio muris spec. nov. From Lat in mus, mouse.

Common names: Mouse-poliomyelit is virus, Theiler's-disease virus.

Host : MURIDAE-Mus musculus L., white mouse.

Insusceptible species: CERCOPITHECIDAE-Macaca mulatta (Zimmermann), rhesus monkey.

Geographical distribution: United States, Japan, Germany, Palestine ; probably widespread wherever white mice are raised.

Induced disease: In white mouse, ordinarily no obvious disease, virus occurring in feces and not being recoverable from thoracic or abdominal viscera or head (probable source is in abdominal wall; virus has been recovered most abundantly from intestinal contents, in moderate amounts from walls of intestine and in smaller concentration from mesenteric lymph glands); occasionally, individual mice show flaceid paralysis of hind legs, and brain or spinal-cord suspensions from these contain the virus; mice inoculated intracerebrally show flaccid paralysis in 7 to more than 30 days, first in one limb, later usually in all; the tail does not become paralyzed; very young inoculated mice may die without first showing paralysis; very old inoculated mice may become infected without showing obvious disease; some affected mice recover and those showing residual paralysis may become carriers of virus. In affected, experimentally inoculated mice, acute necrosis of ganglion cells of anterior horn of spinal cord; necrosis also of isolated ganglion cells of cerebrum. Later, marked neuronophagia. Perivascular infiltration in brain and spinal cord.
The reciprocal of the incubation period has been found approximately proportional to the logarithm of the amount of virus inoculated, thus serving to measure the concentration of samples of virus. Old mice less susceptible than young.

Transmission: Experimentally, by intracerebral, intranasal and intraperitoneal inoculation. Has been found to persist in adult flies, Musca domestica L. (MUSCIDAE) and other species, as long as 12 days after experimental feeding whereas mouse-adapted human poliomyelitis virus persists only 2 days in Musca domestica and not at all in some other species.

Serological relationships: Sera containing antibodies to the Lansing strain of human poliomyelitis virus fail to protect against mouse poliomyelitis virus.

Immunological relationships: Recovered mice are immune to various heterologous isolates or strains. No evidence of immunological relationship with virus of human poliomyelitis has been obtained, save that mice paralyzed with mouse poliomyelitis virus show some resistance to infection with the Lansing strain of human poliomyelitis virus; this has been interpreted as possibly no more than an interference phenomenon, since it seems to depend on actual paralysis.

Filterability: Passes Berkefeld $\mathrm{N}$ and other Berkefeld filters and Chamberland $\mathrm{L}_{3}$ filter.

Other properties: Viable at least 14 months at $-78^{\circ} \mathrm{C}$; at least 150 days in 50 per cent glycerine at 2 to $4^{\circ} \mathrm{C}$. Most stable near $\mathrm{pH} 8.0$ and $\mathrm{pH}$ 3.3. Inactivated readily at $37^{\circ} \mathrm{C}$ by 1 per cent hydrogen peroxide. Particle diameter estimated as 9 to 13 millimicrons from filtration studies. Sedimentation constant, $\mathrm{S}_{20^{\circ}}=160$ to $170 \times 10^{-13} \mathrm{~cm}$ per sec. per dyne.

Literature : Bang and Glaser, Am. Jour. Hyg., 37, 1943, 320-324; Gahagan and Stevenson, Jour. Inf. Dis., 69, 1941, 232237 ; Gard, Jour. Exp. Med., 72, 1940, 69-77; Gard and Pedersen, Science, 94, 1941, 493-494; Gildemeister and Ahlfeld, 
Cent.f. Bakt., I Abt., Orig., 142, 1938 , 144-148; Iguchi, Kitasato Arch. Exp. Med., 16, 1939, 56-78; Olitsky, Jour. Exp. Med., 72, 1940, 113-127; Theiler, Science, 80, 1934, 122; Jour. Exp. Med., $65,1937,705-719$; Theiler and Gard, ibid., 72, 1940, 49-67, 79-90; Young and Cumberland, Am. Jour. Hyg., 37, 1943, 216 224.

\section{Legio gallinae spec. nov. From} Latin gallina, hen.

Common names: Avian encephalomyelitis virus, infectious avian encephalomyelitis virus.

Host : PHASIANIDAE-Gallus gallus (L.), chicken (embryo not susceptible; in culture media, minced whole embryo in serum-Tyrode solution suffices to maintain virus, but embryo brain alone does not).

Insusceptible species: All tested species other than birds.

Geographical distribution: United States.

Induced disease: In chicken, fine or coarse tremors of whole body or only of head and neck or of legs; progressive ataxia; eyes dull, some loss of weight, weakness of legs, and progressive incoordination of leg muscles; somnolence precedes death; about 75 per cent die within 5 days of onset, 90 per cent within a week, the remainder showing a staggering, ataxic gait for weeks, some continuously tremulous; recovered birds, however, may produce eggs well; microscopic focal collections of glia cells, perivascular infiltration, degeneration of Purkinje's cells and degeneration of nerve cells; foci of infiltration throughout brain and spinal cord; virus not detected in the blood of affected chickens.

Transmission: Not through egg. Experimentally, by intracerebral injection.

Serological relationships: Specific antiserum neutralizes homologous virus but not the Eastern strain of equine encephalitis virus; antiserum specific for the latter does not neutralize avian encephalomyelitis virus.
Filterability : Passes Be rkc'r!d V and $N$ as well as Seitz 1 and 2 filters; also membranes 73 millimicrons in average pore diameter.

Other properties: Survives in 50 per cent glycerine for at least 88 days and frozen for at least 68 days. Infective particle estimated to be 20 to 30 millimicrons in diameter, by filt ration studies.

Literature: Jones, Science, 76, 1932, 331-332 ; Jour. Exp. Med., 59, 1934, 781798; Kligler and Olitsky, Proc. Soc. Exp. Biol. and Med., 43, 1940, 680-683; Olitsky, Jour. Exp. Med., 70, 1939, 565-582 ; Olitsky and Bauer, Proc. Soc. Exp. Biol. and Med., 42, 1939, 634-636; Van Roekel et al., Jour. Am. Vet. Med. Assoc., 93 (N.S. 46), $1938,372-375$.

6. Legio suariorum spec, nov. From Latin suarius, swineherd.

Common name: Swineherds'-disease virus.

Hosts: SUIDAE-Sus scrofa L., swine. HOMINIDAE-Homo sapiens L., man. Experimentally, with fever as only symptom, white rat, cat, ferret, mouse; perhaps Macaca mulatia (Zimmermann), rhesus monkey.

Geographical distribution: Europe.

Induced disease: In man, a benign meningit is without sequelise, somewhat similar to lymphocytic choriomeningitis in man; cell counts in spinal fluids may be as high as 1200 to $1400 ; 4$ to 7 (average 8) days after infection, fever lasting 3 to 21 days (average 9); sometimes conjunctivitis, more often a reddish maculopapillose eruption; severe sweating frequent; hemorrhagic tendency; blood in feces; recovery. Blood, urine, feces infectious, not spinal fluid or mucous excretions. Especially affecting young men, not of ten old men or women, among those having contact with swine or swineproducing quarters.

Transmission: Excreta of pigs, even as used for manure, are infective. Experimentally, to man, by subdermal or intramuscular injection. 
- Serological relationships: Serum from recovered cases neutralizes the virus.

Immunological relationships: Specific immunity follows attack of the disease.

Filterability: Passes Chamberland $\mathrm{L}_{2}$ filter.
Literature : Durand et al., Compt. rend. Acad. Sci., Paris, 203, 1936, 830-832, 957 959, 1032-1034; Arch. Inst. Pasteur de Tunis, 26, 1937, 213-227; 228-249; 27, 1938, 7-17.

\section{Genus III. Formido gen. nov.}

Viruses of the Rabies Group, inducing diseases characterized by involvement of the nervous system only. Generic name from Latin formido, a frightful thing.

The type and only recognized species is Formido inexorabilis spec. nov.

1. Formido inexorabilis spec. nov. From Latin inexorabilis, implacable.

Common name: Rabies virus.

Hosts: CANIDAE-Canis familiaris I., dog. FELIDAE-Felis catus L., domest ic cat; $F$. negripes, black-footed cat; $F$. ocreata, wild cat. HOMINIDAEHomo sapiens L., man. MUSTELI$D A E-I c t o n y x$ orangiae, polecat. SCIURIDAE-Gfosciurus capensis, ground squirrel. VIVERRIDAE-Cynictis penicillata, yellow mongoose (yellow meercat); Genetta felina (Thunb.), genet cat; Myonax pulverulentus, small, grey mongoose; Suricata suricatta, Cape suricate or common meercat. Cattle, sheep, pig, horse, wolf. Cynalopex chama, silver jackal. Phyllostoma superciliatum, vampire bat; Desmodus rufus, vampire bat; Artibeus planirostris trinitatis, fruit-eating bat. Experimentally, also Mus musculus L., white mouse; Peromyscus polionotus polionotus (Wagner), white-footed mouse; tissue cultures of 5 or 6-day-old rat- or mouse-embryo brain; chick embryo (allantois not regularly infected, but virus regularly reaches brain of embryo without injuring it ; chick may hatch with titer of $1: 100$ or $1: 1000$ in brain). Chicken; mouse hawk (Buteo vulgaris); pigeon, owl, goose; stork (Ciconia ciconia); pheasant (Diardigallus diardi B.P.).

Insusceptible species: Reptiles, fish. No mammal is known to be insusceptible.

Geographical distribution: Almost world-wide; absent only from relatively isolated countries or communities.

Induced disease: In dog, after a short incubation period (generally less than 10 days) altered behavior, hiding, lack of obedience, perverted appetite leading to ingestion of straw, paper, earth, and other unaccustomed materials; excitement, unprovoked biting (which may transmit the virus to new hosts), aimless wandering, excess salivation, progressive inability to swallow, alteration of bark to characteristic high pitched tone; staggering, paresis of hindquarters tending toward paralysis and involvement of anterior parts of the body; paralysis of lower jaw, muscular spasms, marked emaciation, death except perhaps in rare instances. In man, after a relatively long incubation period depending on site of implantation (perhaps 27 to 64 days), a uniformly fatal disease, characterized by altered behavior, increased excitability, thirst, pharyngeal spasm with progressive inability to swallow, labored and noisy respiration, death in 3 or 4 days after onset, with or without paroxysm. In sheep, increased sexual desire; tendeney to pull wool from other sheep or themselves; light butting, increasing until some ewes, after violent exercise, appear to faint; prostration within 1 to 4 days; death within 2 days from onset of locomotory paralysis. In mouse, experimentally, by intracerebral inoculation, apathy, sluggishness, roughening of hair, tremor, convulsions, prostration, death; sometimes flaccid paralysis of hind legs before death.

Transmission: Usually by bite of dog or some closely related animal; occasionally by bites of cats; rarely by bites 
of rabid horses or cattle. Not by contamination of food. In Brazil and Trinidad, probably by the vampire bat, which has been found infected in nature.

Serological relationships: Specific flocculation of rabies virus occurs in the presence of immune serum from rabbit or guinea pig; strains differ in relative amounts of antigenic constituents, as shown by absorption tests. Complement fixation occurs in the presence of virus and guinea-pig antiserum. Nentralizing antibodies are specific.

Immunological relationships: Virus exposed to ultraviolet light terids to lose its virulence before its immunizing potency. Passive immunization succeeds in white mice if antiserum is injected intracerebrally $\frac{1}{2}$ hour before, but not 24 hours before or 2 hours after, virus. Chloroformtreated vaccines more effective than phenolized vaccines, but irritative.

Thermal inactivation: At 60 to $70^{\circ} \mathrm{C}$ in 15 minutes; in brain tissues, at $45^{\circ} \mathrm{C}$ in 24 hours.

Filterability : Passes Berkefeld V filter. Other properties: Viable at least 2 months at $5^{\circ} \mathrm{C}$ in liquid or dry state. Infective particle between 100 and 240 millimicrons in diameter, by filtration studies.

Literature : Bernkopf and Kligler, Brit. Jour. Exp. Path., 18, 1937, 481-485 ; Casals, Jour. Exp. Med., 72, 1940, 445-451, 453461 ; Covell and Danks, Am. Jour. Path., 8, 1932, 557-572; Dawson, Science, 89, 1939, 300-301; Am. Jour. Path., 17, 1941, 177-188; Galloway, Brit. Jour. Exp. Path., 15, 1934, 97-105; Goodpast ure, Am. Jour. Hyg., 1, 1925, 547-582; Haupt and Rehaag, Ztschr. f. Infektionskrankh., 22,
1921, 76-88, 101-127; Havens and Mayfield, Jour. Inf. Dis., 50, 1932, 367-376; 51 , 1932, 511-518; 52, 1933, 364-373 ; Henderson, Vet. Med., 37, 1912, 88-89; Hodes et al., Jour. Exp. Med., 72, 1940, 437-414; Hoyt et al., Jour. Inf. Dis., 59, 1936, 152158; Hurst and Pawan, Lincet, 221, 1931 (2), 622-628; Jour. Path. and Bact., 35 , 1932, 301-321; Johnson and Leach, Am. Jour. Hyg., 32 (B), 1940, 38-45; Kligler and Bernkopf, Proc. Soc. Exp. Biol, and Med., 39, 1938, 212-214; Am. Jour. Hyg., 33 (B), 1941, 1-8; Leach and Johnson, ibid., 32 (B), 1940, 7t-79; Metivier, Jour. Comp. Path. and Therap., 48, 1935, 245260 ; Peragallo, Giorn. di batteriol. e immunol., 18, 1937, 289-290; Snyman, Onderstepoort Jour. Vet. Sci. and Anim. Indust., 15, 1940, 9-140; Webster, Am. Jour. Pub. Health, 26, 1936, 1207-1210; Jour. Exp. Med., 70, 1939, 87-106; Am. Jour. Hyg., 30 (B), 1939, 113-134; Webster and Casals, Jour. Fxp. Med., 71, 1940, 719-730; 73, 1941, 601-615; 76, 1942, 185-194; Webster and Clow, ibid., 66, 1937, 125-131; Wyckoff, Am. Jour. Vet. Res., 2, 1941, 81-90.

Note: The Negri body, a characteristic cell-inclusion in rabies, has been given the following names under the supposition that it represents stages in the life cycle of a protozoan parasite responsible for the disease: Neuroryctes hydrophobiae by Calkins, Jour. Cutaneous Diseases including Syphilis, 25, 1907, 510; Encephalitozoon rabici by Manouelian and Viala, Ann. Inst. Pasteur, 38, 1924, 258; and Glugea lyssae by Levaditi, Nicolau and Schoen, Ann. Inst. Pasteur, 40, 1926, 1048. 
FAMILY IT. CHARONACEAE FAM. NOT.

Viruses of the Yellow-Fever Group, inducing diseases mainly characterized by fever and necrosis of tissues in the absence of obvious macule, papule, or vesicle formation or of conspicuous involvement of nerve cells.

\section{Key to the genera of family Charonaceae.}

I. Viruses of the Typical Yellow-Fever Group.

Genus I. Charon, p. 1265.

I1. Viruses of the Influenza Group.

Genus II. Tarpeia, p. 1268.

III. Viruses of the Hog-Cholera Group.

Genus III. Tortor, p. 1275 .

Genus I. Charon gen. nov.

Viruses of the Typical Yellow-Fever Group, inducing diseases mainly characterized by acute non-contagious fever. Vectors dipterous insects, so far as known. Generic name from Latin Charon, ferryman of the Lower World.

The type species is Charon evagatus spec. nov.

Key to the species of genus Charon.

I. Vectors mosquitoes.

1. Charon evagatus.

II. Vectors unknown, perhaps mosquitoes.

\section{Charon vallis.}

1. Charon evagatus spec. nov. From Latin evagor, to spread abroad.

Common name: Iellow-fever virus.

Hosts: HOMINIDAE-Homo sapiens L., man. Experimentally, also Cercopithecus tantalus Ogilby; C'. aethiops, African guenon (symptomless); Cercocebus torquatus (Kerr), collared mangabey; Mus musculus L., mouse; Microtus agrestis, field vole; Sciurus vulgaris L., red squirrel; Macaca mulatta (Zimmermann), rhesus monkey; Macacus sinicus Indian crown monkey; $M$. cynomolgus; M. speciosus; Erinaceus europaeus, hedge hog; Gallus gallus (L.), chicken (tolerant); Dasyprocta aguti, agouti (serial passage fails).

Insusceptible species: Cat, ferret, rabbit, rat; Cricetus auratus, golden hamster; Apodemus sylvaticus, wood vole; Evotomys glareolus, bank vole; pigeon, canary, pipistrelle bat; Cricetomys gambianus, pouched rat; dog, goat.

Geographical distribution: Tropical re- gions in general, especially C'entral and South America, West Indies, West Africa; anti-mosquito campaigns have tended to eradicate yellow-fever virus from parts of its former range.

Induced disease: In man, mild cases may oceur, especially in natives where the disease is endemic, but in Europeans generally sudden fever without marked change in pulse rate after a 3 to 6 -day incubation period; severe frontal headache, pains in the loin and legs and epigastric pain; gradual decrease in temperature to 98 or $99^{\circ} \mathrm{F}$, weakening of pulse and slowing of heart beat in the absence of further temperature changes; jaundice, especially in sclerae, often in skin; albumen in urine, later bile-pigments also present ; hemorrhages frequent especially in alimentary canal; fatty and necrotic changes in the liver; acute degeneration of renal parenchyma, splenic congestion; death may occur in the early acute state, but is more likely about the 
fifth or sixth day; relapses may occur until 2 or 3 weeks after onset; case mortality varies from 10 to 90 per cent in different epidemies. A transitory immunity due to transfer of serum antibodies through the placenta protects offspring of immune mothers for a short time.

Transmission: By mosquitoes, Aëdes aegypti L., Aëdes leucocelaemus (D). and S.), Haemogogus capricorni Lutz (CULICIDAE). The mosquito Aëdes acgypti hecomes infective, after feeding on a suitable virus source, in 4 days at $37^{\circ} \mathrm{C}, 5$ days at $36^{\circ} \mathrm{C}, 6$ days at $31^{\circ} \mathrm{C}, \mathrm{s}$ days at $25.1^{\circ} \mathrm{C}, 9$ to 11 days at $23.4^{\circ} \mathrm{C}$, 18 days at $21^{\circ} \mathrm{C}$, and 36 , not 30 , days at $18^{\circ} \mathrm{C}$; virus in head, thorax, and abdomen before bites are infective; no evidence of transmission of virus through eggs to offspring or to larvae eating infected adults. Experimentally, also by Aëdes scapularis (Rondani), A. fluviatilis (Lutz), A. luteocephalus, A. apico-annulatus (CULICIDAE). Experimentally, by feeding, to Macacu mulatta and Cercopithecus aethiops; by rubbing infected blood into intact and unshaved skin of monkeys.

Serological relationships : Complement fixation and precipitating antibodies are specific.

Inmunological relationships: A specific immunity develops after an attack of the disease or after vaccination with virus grown in media containing tissues of chick embryo minus head and spinal eord.

Thermal inactivation: At 55 to $60^{\circ} \mathrm{C}$, not at $50^{\circ} \mathrm{C}$, in 10 minutes.

Filterability: Passes menbranes of 5.5 , and to some extent membranes of 50 , millimicron average pore diameter. Passes Berkefeld $\mathrm{V}$ and $\mathrm{N}$, as well as Chamberland $\mathrm{F}$, filters.

Other properties: Particle estimated from filtration data to have a diameter of 17 to 28 millimicrons; by ult racent rifugation data, 19 millimicrons. Inactivated or inhibited by 30-minute exposure to 1:15 formatin, 1:6 ethyl alcohol; $1: 300$ yellowish eosin, $1: 50$ sodium oleate, $1: 200$ liquor cresolis compositus; viable after 30-minute exposure at $30^{\circ} \mathrm{C}$ to $1: 7500$ mercuric chloride, $1: 150$ phenol, 1:1500 hexylresorcinol, 1:150 sodium oleate. Sedimentation constant between 18 and $30 \times 10^{-13} \mathrm{~cm}$ per sec. per dyne. Viable in 50 per cent glycerine at 2 to $4^{\circ} \mathrm{C}$ for 58 , not for 100 , days ; in mouse brain at $-8^{\circ} \mathrm{C}$ for 160 days. Viability may be lost on simple drying but retained if drying is carried on in vacuo over a desiccating agent.

Strains: Distinctive strains have been isolated. One, to which much study has been given, differs from the typical viscerotropic strain by possessing marked neurotropic or pantropic characteristics.

Literature: Bauer and Hughes, Am. Jour. Hyg., 21, 1935, 101-110; Bauer and Mahaffy, ibid., 12, 1930, 155-174; 175195; Bugher and Gast-Galvis, ibid., 39, 1944, 58-66; Bugher et al., ibid., 39, 1944, 16-51; Davis, ibid., 16, 1932, 163-176; Davis and Shannon, ibid., 11, 1930, 335344; Davis et al., Jour. Exp. Med., 58, 1933, 211-226; Findlay, Jour. Path. and Bact., 38, 1934, 1-6; Lancet, 227, 1934 (2), 983-985; Findlay and Clarke, Jour. Path. and Bact., 40, 1935, 55-64 ; Findlay and MacCallum, Brit. Jour. Exp. Path., 19, 1938, 384-388; Jour. Path, and Bact., 49, 1939, 53-61 ; Findlay and Mackenzie, ibid. 43, 1936, 205-208; Findlay and Stern, ibid., 40, 1935, 311-318; Fox and Cabral, Am. Jour. Hyg., 37, 1943, 93-120; Frobisher, Am. Jour. Hyg., 11, 1930, 300$320 ; 19,1931,585-613 ; 14,1931,147-148$; 18, 1933, 354-374; Goodpasture, Am. Jour. Path., 8, 1932, 137-150; Haagen, Deutsch. med. Wochnschr., 60, 1934, 983-988; Hudson, Am. Jour. Path., 4, 1925, 395-430; Klotz and Simpson, ibid., 3, 1927, 483-488; Laemmert and Moussatché, Jour. Inf. Dis., 72, 1943, 228-231 ; Lloyd et al., Am. Jour. Hyg., 18, 1933, 323-344; Trans. Roy. Soc. Trop. Med. and Hyg., 29, 1936, 481-529; Mahaffy et al., Ain. Jour. Hyg., 18, 1933, 618-628; Pickels and Batuer, Jour. Exp. Med., 71, 1940, 
703-717; Ramsey, Am. Jour. Hyg., 13, 1931, 129-163; Sawyer, ibid., 25, 1937, 221-231; Shannon et al., Science, 88 1938, 110-111; Smith and Theiler, Jour. Exp. Med., 65, 1937, 801-808; Smit h et al., Am. Jour. Trop. Med., 18, 1938, 437-468; Soper and De Andrade, Am. Jour. Hyg., 18, 1933, 588-617; Soper et al., ibid., 18, $1933,555-587 ; 19,1934,549-566$; 27, 1938, 351-363; Stefanopoulo and Wassermann, Bull. Soc. Path. Exot., 26, 1933, 557-559; Stokes et al., Am. Jour. Trop. Med., 8 1928, 103-164; Theiler, Ann. Trop. Med. and Parasit., 24, 1930, 249-272; Theiler and Smith, Jour. Exp. Med., 65, 1937, 767-786, 787-800; Whitman, ibid., 66, 1937, 133-143.

2. Charon vallis spec, nov. From Lattin vallis, valley.

Common name : Rift Valley fever virus.

Hosts : HOMINIDAE-Homo sapiens L., man. BOVIDAE-Bos taurus L., cow; Ovis aries L., sheep; Capra hireus L., goat. Experimentally, also Sciurus carolinensis, grey squirrel; ferret; Cricetus auratus, golden hamster; Apodemus sylvaticus, wood mouse; Microtus agrestis field vole; Muscardinus avellanarius, dormonse; rat; mouse; Macaca mulatta; $\boldsymbol{H}$. irus; Cebus fatuellus; C. chrysopus; Hapale jacchus; H. penicillata; Cercopithecus callitrichus (symptomless); Erythrocebus patas (symptomless); Cercocebus fuliginosus (symptomless); chick embryo in Tyrode's solution; chorioallantoie membrane of chick embryo.

Insusceptible species: Horse, pig.

Geographical distribution : British East Africa.

Induced disease: In man, benign disease; after $5 \frac{1}{2}$ to 6 days, rigors, pains in back, fever for 12 to 36 hours, followed by recovery, with persistence of acquired immune bodies as long as 4 to 5 years after infection. In sheep (lambs), dullness, rapid respiration, collapse and death in a few hours or a chronic course; focal necrosis in liver. In chorioallantoic membrane of chick embryo, experimen- tally, areas of hyperplasia and of necrosis; connective tissue inflamed nearby; liver of embryo mottled with necrotic areas.

Transmission: Not by contacts. Mosquito, Taeniorhynchus brevipalpis (CUL$I C I D A E$ ), suspected as possible vector.

Serological relationships: Antisera for psittacosis, dengue fever, and sandfly fever viruses fail to protect against infection with Rift Valley fever virus. Specific neutralizing antibody in intraperitoneally neutral mixt ure with Rift Valley fever virus may be dissociated so as to free virus by direct dilution in saline solutions, by int ranasal inoculation, or by employment of a small dose, all methods probably implying a dilution effect.

Immunological relationships: No cross immunity with yellow-fever or denguefever viruses. If Rift Valley fever virus is inoculated into rhesus monkey simultaneously with yellow-fever virus, the animal tends to be protected against death from yellow fever (interference effect), but one-day earlier inoculation of Rift Valley fever virus does not protect.

Thermal inactivation: At $56^{\circ} \mathrm{C}$ in 40 , not 20 , minutes.

Filterability: Passes Berkefeld V, N, and $W$ filters; passes Chamberland $\mathrm{L}_{2}$, $\mathrm{L}_{3}, \mathrm{~L}_{5}, \mathrm{~L}_{7}, \mathrm{~L}_{11}$ and occasionally $\mathrm{L}_{13}$ filters; passes membranes 150 millimicrons in average pore diameter freely, 90 millimicrons with difficulty, 70 millimicrons not at all.

Other properties: Viable at least 8 mont hs at $4^{\circ} \mathrm{C}$, more than 4 weeks dry in liver tissues, 6 months in $\frac{1}{2}$ per cent carbolic acid at $4^{\circ} \mathrm{C}$. Diameter of infective particle estimated from filtration studies to be between 23 and 35 millimicrons.

Strains: A neurotropic strain immunizes lambs without producing obvious illness, if given subcutaneously.

Literature: Broom and Findlay, Brit. Jour. Exp. Path., 14, 1933, 179-181; Daubney et al., Jour. Path. and Bact., 34, 1931, 545-579; Findlay, Truns. Roy. 
Soc. Trop. Med. and Hyg., 25, 1932, 229266 ; 26, 1932, 157-160; 161-168; Brit. Jour. Exp. Path., 17, 1936, 89-104; Findlay and MacCallum, Jour. Path. and Bact., 44, 1937, 405-424; Findlay and Mackenzie, Brit. Jour. Exp. Path., 17, 1936, 4t1-4t7; Findlay et al., ibid., 17, 1936, 431-441; Francis and Magill, Jour. Exp. Med., 62, 1935, 433-448; Horning and Findlay, Jour. Roy. Micr. Soc., 54, 1934, 9-17; Mackenzie, Jour. Path. and Bact., 37, 1933, 75-79; 40, 1935, 65-73; Markenzie et al., Brit. Jour. Exp. Path., 17, 1936, 352-361; Saddington, Proe. Soc. Exp. Biol. and Med., 31, 1934, 693-694; Sehwent ker and Rivers. Jour. Exp. Med., $59,1934,305-313$.

\section{Genus II. Tarpeia gen. nov.}

Viruses of the Influenza Group, inducing diseases characterized principally by involvement of the respiratory tract. Generic name from Latin Tarpeia, name of a Roman maiden who treacherously opened a citadel to an enemy.

The type species is Tarpeia alpha spec. not.

\section{Key to the species of genus Tarpeia.}

I. Infecting man principally.

1. Tarpeia alpha.

2. Tarpeia beta.

3. Tarpeic premens.

II. Affecting feline species.

\section{Tarpeia felis.}

III. Affecting domestic eattle (calves).

\section{Tarpeia vitulae.}

IV. Affecting canine species.

6. Tarpeia canis.

7. Tarpeia vulpis.

V. Affecting ferrets.

VI. Affecting domestic fowl.

\section{Tarpeia viverrae.}

\section{Tarpeia avium.}

1. Tarpeia alpha spec. nov'. From first lotter of Cireek alphabet.

Common name: Influenza A virus; swine filtrate-disease virus.

Husts: HOMI VIDAE-Homo sapiens L., man. SUIDAE-Sus serofa L., domestic swine. Experimentally, also ferret, mouse, Macacus irus, hedgehog, rabbit (inapparent infection), guinea pig (inapparent infection), rat (inapparent infection); Mustela sibirica Milne-Edwards, Chinese mink; Sciurotamius d:tvidianus Milne-Edwards, David's squirrel; chick embryo (some strains produce visible lesions at $36.5^{\circ} \mathrm{C}$ on chorioallantoic membrane); minced chick embryo in Tyrode's solution.
Insusceptible species: Callosciurus caniceps canigenus Howell, Chekiang squirrel; Eutamias asiaticus senescens Niller, chipmunk.

Geographical distribution: World-wide.

Induced disease: In man, headache, dizziness, with shivering and muscular pains; rise of temperature on the second day, sometimes with fall on the third and elevation again later; often complicated by bronehitis and bronchopneumonia; liemorrhagic and edematous lobular consclidation in lungs; virus most easily recoverahle from nasopharyngeal washings, but also from nasal secretions and lungs. In swine, virus alone produces only a mild malady (filtrate disease); 
in the presence of Haemophilus influenzae suis a severe malady occurs under both natural and experimental conditions; it involves fever, cough, and prostration; many infected animals die. Lungworms, Metastrongylus elongatus and Choerostrongylus pudendotectus (METASTRONGYLIDAE), from infected swine harbor virus at least 2 years, living meantime in earthrorms, such as Allolobophora caliginosa. ( $L U M B R I C I D A E)$, which are eaten eventually by swine. The swine are ref ractory to viral infection during May, June, July, and August, but the disease may be invoked later by successive intramuscular injections of Haemophilus influenzae suis or other stimuli. such as feeding embryonated Ascaris ova. In infected swine, virus ocr.urs in turbinates, tracheal exudates, and lungs; not in spleen, liver, lidney, mesenteric lymph nodes, hrain, blood, or mucosa of colon. Neut ralizing antibodies appear later ( 7 th to 10 th day) in the mild filtrate disease than in typical swine influenza, in which they appear about the 6 th to the 7 th day; maximum titer on 14 th to 27 th day. Experimentally in mouse, not contagious as in swine and not dependent on the coexistence of a bacterial component; deat h of epithelium of respiratory and terminal bronchioles, complete epithelial desquamation, dilatation of bronchioles, collapse of alveoli ; in healing, widespread epithelial prolifera tion. Experimentally in ferret, moderate apathy, lack of appetite, pallor of nose, variable eatarrhal symptoms; at acute stage of disease, necrosis of respiratory epithelium of nasal mucous membrane, with desquamation of superficial cells, exudation into air passages and inflammatory reaction in the submucosa ; repair follows, beginning on the 6 th day after infection and becoming essentially complete at the end of 1 month; after recovery, the ferret is immune for 3 months or more, with subsequent waning of resistance; subsequent subcutaneous inoculations of virus restore immunity.
Transmission: Presumably by droplets; for example between cages of ferrets as close as 5 feet apart, even to levels 3 feet higher than cage of diseased individuals. Experimentally, from washings of human throats to ferret, mouse, chick embryo (by amniotic route and to allantoic membrane); in mice, by contact and by inhalation of fine droplets.

Serological relationships: Neutralizing antibodies common in human sera from individuals above 10 years of age; rarer in sera from young children; strongly effective for homologous, weak for heterologous, virus in convalescent sera. Soluble complement-fixing antigen of swine strain has components in common with antigens of human strains (PR8 and WS). Complement fixation best 10 to 14 days after onset in man. Inactivating caparity of nasal secretions proportional to level of neutralizing antibodies in blood. Agglutination of red cells by influenza virus is inhibited quantitatively by specific antiserum.

Immunological relationships: Specific immunization of ferrets, without obvious disease, occurs as a result of intranasal inoeulation of egg-passage influenza virus that is not transmissible from ferret to ferret. In mice, immunizing dose is directly proportional to degree of induced immunity; immunity to the strain used in immunization is more effective in gencral than that to heterologous isolates of the virus.

Filterability: Passes Berkefeld I filter. Other properties: Particle size estimated as 80 to 120 millimicrons by filt ration studies; 80 to 99 millimicrons by ultracentrifugation $\left(\mathrm{S}_{20^{\circ}}=72 t \times 10^{-13}\right.$ ( $m$ per sec. per dyne); electron micrographs show bean or kidney-shaped particles, or round particles with central dense spot, averaging 77.6 millimicrons in diameter. Inactivated by oleic, linolic and linolenic acids without loss of immunizing ability. Inactivated by ultraviolet radiation.

Literature: Andrewes and Glover, 
Brit. Jour. Exp. Path., 22, 1941, 91-97; Andrewes et al., ibid., $16,1935,566-582$; Burnet, ibid., 17, 1936, 282-293; 18, 1937, 37-43; 21, 1940, 147-153; Austral. Jour. Exp. Biol. and Med. Sci., 14, 1936, 241-246 ; 19, 1941 , 39-44, 281-290; Eaton, Jour. Bact., 39, 1940, 229-241; Eaton and Pearson, Jour. Exp. Med., 72, 1940, 635-643; Eaton and Rickard, Am. Jour. Hyg., 33, (B), 1941, 23-35; Elford et al., Brit. Jour. Exp. Path., 17, 1936, 51-53; Francis, Science, 80, 1934, 457-459; Jour. Exp. Med., 69, 1939, 283-300; Francis and Magill, Science, 82, 1935, 353-354; Brit. Jour. Exp. Path., 19, 1938, 284-293; Francis and Shope, Jour. Exp. Med., 63, 1936, 645-653; Francis and Stuart-Harris, ibid., 68, 1938, 789-802; Francis et al., Am. Jour. Hyg., 37, 1943, 294-300; Hirst, Jour. Exp. Med., 75, 1942, 49-64; Hirst et al., ibid., 75, 1942, 495-511; Proc. Soc. Exp. Biol. and Med., 50, 1942, 129-133; Horsfall and Lennette, Jour. Exp. Med. 73, 1941, 327-333; Hudson et al., ibid., 77. 1943, 467-471; Hyde, Am. Jour. Hyg., 36 1942, 338-353; Lennette and Horsfall, Jour. Exp. Med., 73, 1941, 581-599; Loosli et al., Jour. Inf. Dis., 72, 19t3, 142-153; Lush and Burnet, Austral. Jour. Exp. Biol. and Med. Sci., 15, 1937, 375-383; Magill and Francis, Brit. Jour. Exp. Path., 19, 1938, 273-284; Nigg et al., Am. Jour. Hyg., 34 (B), 1941, 138-147 Oreutt and Shope, Jour. Exp. Med., 62, 1935, 823-826; Rosenbusch and Shope, ibid., 69, 1939, 499-505; shope, ibid., 59, 1934, 201-211; 62, 1935, 561-572; 64, 1936, $17-61 ; 67,1938,739-748 ; 74,1941,41-47$, 49-68; 77, 1943, 111-126, 127-138; Shope and Francis, ibid., 64, 1936, 791-801; Smillie, Am. Jour. Hyg., 11, 1930, 392398; Smith et al., Lancet, 225, 1933 (2), 66-68; Brit. Jour. Exp. Path., 16, 1935, 291-302; Smorodintseff and Ostrovskaya, Jour. Path. and Bact., 44, 1937, 559-566; Stock and Francis, Jour. Exp. Med., 71, 1940, 661-681; Straub, Jour. Path. and Bact., 45, 1937, 75-78; Stuart-Harris, Brit. Jour. Exp. Path., 17, 1936, 324-328; 18, 1937, 485-492; Sulkin et al., Jour. Inf.
Dis., 69, 1941, 278-284; Tang, Brit. Jour. Exp. Path., 19, 1938, 179-183; Taylor, (A. R.), et al., Jour. Immunol., Virus Res. and Exp. Chemother., 47, 1943, 261-282; Taylor, (R. M.), et al., Am. Jour. Hyg., 31, (B), 1940, 36-45; Jour. Inf. Dis., 68, 1941, 90-96; Wells and Brown, Am. Jour. Hyg., 24, 1936, 407-413.

2. Tarpeia beta spec. nov. From sec:ond letter of Greek alphabet.

(Common name: Influenza B virus.

Hosts : HOMINIDAE-Homo sapiens L., man. Experimentally, also ferret, mouse, chick embryo.

Geographical distribution: United States, England.

Induced disease: In man, subclinical disease or one resembling that induced by influenza $A$ virus. In chick embryo, experimentally, virus inereases in entodermal cells lining allantoic cavity.

Serological relationships: Not neutralized by antiserum to influenza A virus. Specific neutralization and complementfixation reactions. Rapidly adsorbed by normal chicken-blood red cells (95 per (ent in 15 minutes); released in 4 hours essentially completely; the process is then repeatable with fresh red cells.

Other properties: Particle circular or bean-shaped in outline, with average diameter of 97.3 millimicrons in electron micrographs; of 99.8 millimicrons by centrifugation studies.

Literature : Burnet, Austral. Jour. Exp. Biol, and Med. Sci., 19, 1941, 291-295; Francis, Science, 92, 1940, 405-408; Proc. Soc. Exp. Biol. and Med., 45, 1940, 861863; Hirst, Jour. Exp. Med., 76, 1942, 195-209; Lush et al., Brit. Jour. Exp. Path., 22, 1941, 302-304; Nigg et al., Am. Jour. Hyg., 35, 1942, 265-284 ; Sharp et al., Jour. Immunol., Virus Res. and Exp. Chemother., 48, 1944, 129-153.

3. Tarpeia premens spec. nov. From Latin premere, to oppress or afflict.

Common name: Common-cold virus.

Hosts : HOMINIDAE-Homo sapiens 
L., man. Experimentally, also chimpanzee, chick embryo.

Geographical distribution: World-wide except in conditions of isolation of small communities.

Induced disease: In man, incubation period about 48 hours; mild malady ; running nose in 81 per cent of cases, obstruetion of nostrils in 44 per cent, sudden onset in 37 per cent, cough in 31 per cent, headache in 19 per cent. sore throat in 14 per cent, fever in 13 per cent, inflammation of eyes in 12 per cent; changes in weather, especially during a warm season, predispose to the disease; no correlation between susceptibility and out door exercise, exposure to fresh air while sleeping. eye color, adenotonsillectomy, or size of frontal sinus. Incidence inversely proportional to daily hours of sunshine and atmospheric temperature. Fitness (defined by speed of oxygen replacement) correlated with relative freedom from colds. Effec of rest during disease favorable, reducing complications, length of fever, duration of illness, and period off duty.

Immunological relationships: After at tack, specific immunity for about 7 weeks (minimum period 23 days); then exposure to chilling may cause a relapse, but an isolated community tends to lose the virus during the refractory period.

Filterability: Passes Berkefeld $V$ and W as well as Seitz filters.

Other properties: Viable at least 13 days at ice-box temperature, anaerobically; at least 4 months frozen and dried in vacuo. Gum acacia tends to stabilize virus in chick-embryo tissue medium.

Literature: Dochez et al., Jour. Exp. Med., 63, 1936, 559-579; Doull et al., Am. Jour. Hyg., 13, 1931, 460-477; 17 , 1933, 536-561; Gafafer, ibid., 13, 1931, 771-780; 16, 1932, 233-240, 880-881; Jour. Inf. Dis., 51, 1932, 489-492; Gafafer and Doull, Am. Jour. Hyg., 18, 1933, 712-726 ; Hyde and Chapman, ibid., 26, 1937, 116 123; Kneeland et al., Proc. Soc. Exp. Biol. and Med., 35, 1936, 213-215; Le
Blane and Welborn, Am. Jour. Hyg., 24, 1936, 19-24; Locke, Jour. Inf. Dis., 60, 1937, 106-112; Long and Doull, Proc. Soc. Exp. Biol. and Med., 28, 1930, 53-55; Maughan and Smiley, Am. Jour. Hyg., 9 1929, 466-472; Noble and Brainard, Jour. Bact., 29, 1935, 407-409; Palmer, Am. Jour. Hyg., 16, 1932, 224-232; Paul and Freese, ibid., 17, 1933, 517-535; Shibley et al., Jour. Am. Med. Assoc., 95, 1930, 1553-1556; Smiley, Am. Jour. Hyg., 6, $1926,621-626 ; 9,1929,477-479$.

4. Tarpeia felis spec. nov. From Latin jeles, cat.

Common name: Feline-distemper virus.

Hosts : FELIDAE-Felis catus L., domestic cat ; $F$. pardus, leopard; F. tigrina, American tiger cat; $F$, aurata, African tiger cat; $F$. planiceps, rusty tiger cat; $F$. marmorata, marbled cat; $F$. caracal, earacal lynx; $F$. pardalis, ocelot; lion, tiger, puma relatively insusceptible.

Insuscept ible species : Man, dog, ferret, mongoose, rabbit, rat, mouse, guinea pig.

Induced disease: In domestic cat, roughing, sneezing, running eyes and nose, with serous or purulent conjunctivi$\mathrm{tis}$, or diarrhea and vomiting; fever to 103 or $105^{\circ} \mathrm{F}$; loss of appetite, general weakness; mortality high, especially among young individuals; death usually occurs on the 10 th to the 12 th day, in extreme cases, however, as early as the 5 th or as late as the 35 th day; catarrhal congestion in some part of the gastrointestinal tract is typical; this ranges from a few small patches in the ileum to involvement of the whole small intes tine and parts of the large intestine or stomach and esophagus; often enlargement and congestion of abdominal lymph glands, enlargement of spleen, pleurisy, and peritonitis.

Filterability : Passes Berkefeld $\mathrm{N}$ and Chamberland $\mathrm{L}_{3}$ filters.

Transmission: By fomite s.

Immunological relationships: Rerovered cats specifically immune. 
Other properties: Viable at least 3 weeks in 50 per cent glycerine; attenuated or killed by drying at room temperature, but some immunization is reported if dried virus is injected.

Literature: Dalling, Vei. Record, 15, 1935, 283-289; Findlay, Vet. Jour., 89, 1933, 17-20; Hindle and Findlay, Jour. Comp. Path. and Therap., 45, 1932, 11-26; Verge and Cristoforoni, Compt. rend. Soc. Biol., Paris, 99, 1928, 312-314.

5. Tarpeia vitulae spec, nov. From Latin vitula, cow-calf.

Common name: Pneumoenterit is virus Hosts : BOVIDAE-Bos taurus L., domestic cattle. Experimentally, also MURIDAE-Mus musculus L., mouse.

Geographical distribution: United States.

Induced disease: In cattle (calves), after incubation period of 2 to $t$ days. fever increasing rapidly to 40 or $41^{\circ}$ C and lasting 3 to 5 days; usually after first day of fever, diarrhea with feces soft, yellow, voluminous, fetid in odor, oceasionally blood-tinged or fluid; diarrhea is followed by pneumonia and recovery after disappearance of fever; catarrhal enteritis and a bronchopneumonia usually. confined to the anterior lobes of the lungs underlie the symptoms; no inclusion bodies in cells of affected tissues.

Transmission: By pen contacts with infected calves. Experimentally, by intranasal inoculation of calves, using inocula prepared from lungs of infected mice.

Serological relationships: Recovered animals develop neutralizing antibodies.

Immunological relationships: A specific resistance to reinfection is conferred by an attack of the disease.

Filterability : Passes Berkefeld N filter.

Literature: Baker, Cornell Vet., 32, 1942, 202-204; Jour. Exp. Med. 78, 1943, $435-446$.

6. Tarpeia canis spec, nov. From Latin canis, dog.
Common name: Canine-distemper virus.

Hosts: CANIDAE-Canis familiaris L., dog; Vulpes sp., fox. MUSTELI. $D A E$-ferret .

Insusceptible species: Man, rabbit, guinea pig, white rat, cat .

Geographical distribution: Widespread throughout the world.

Induced disease: In dog, after 4 days from time of infection, fever and a watery discharge from the eyes and nose, sometimes inconspicuous but often profuse; usually diarrhea and wasting followed by recovery or, exceptionally, death. Virus passes from the respiratory tract through the blood stream to its favored sites in vascular endothelium and cells of the reticulo-endothelial system. Nuclear inclusions are found in liver cells, bronchial epithelial cells, glandular cells of the stomach and intestine, and bile-duct epithelial cells; there are also cytoplasmic inclusions in bile-duct epithelial cells.

Transmission: By contact. Probably by air-borne droplets. No arthropod vector is recognized.

Immunological relationships: Deadvaccine treatment followed by livingvirus treatment produces a lasting immunity. Virus inactivated by photodynamic effect in $2 \mathrm{~mm}$ layer of $1: 50,000$ or $1: 100,000$ methylene blue, exposed 30 minutes at $20 \mathrm{~cm}$ from 100 candle-power lamp, still immunizes. Vaceine may be dried.

Filterability: Passes Chamberland $\mathbf{L}_{2}$ and Mandlex filters.

Other properties: Viable in liver tissue at $10^{\circ} \mathrm{C}$ for 35 , not 85 , days ; in glycerinesaline solution at $10^{\circ} \mathrm{C}, 67$ days though deteriorated; in vacuum-dried liver tissue, at $10^{\circ} \mathrm{C}, 90$ days. If dried from frozen state, virus is viable in vacuum at least 430 days at $7^{\circ} \mathrm{C}$, in oxygen-free nitrogen at least 365 days at $7^{\circ} \mathrm{C}$. Viable in 25 per cent sterile horse serum at $-24^{\circ}$ C more than 693 days.

Literature : Carré, Compt. rend. Acad. Sci., Paris, 140, 1905, 689-690; Dalldorf, 
Jour. Exp. Med., 70, 1939, 19-27; De Monbreun, Am. Jour. Path., 13, 1937, 187-212; Dunkin and Laidlaw, Jour. Comp. Path. and Therap., 39, 1926, 201212. 213-221; Green and Evans, Am. Jour. Hyg., 29 (B) , 1939, 73-87; Laidlaw and Dunkin, Jour. Comp. Path. and Therap., 39, 1926, 222-230; 41, 1928, 1-17, 209-227; Perdrau and Todd, ibid., 46. 1933, 78-89; Siedentopf and Green, Jour. Inf. Dis., 71, 1942, 253-259; Wharton and Wharton, Am. Jour. Hyg., 19, 1934, 189-216.

\section{Tarpeia vulpis spec. nov. From} Latin vulpes, fox.

Common name : Fox-encephalit is virus. Hosts : CANIDAE-Tulpes sp., silver fox. Experimentally, also some, but not all, dogs; coyote.

Insusceptible species: Gray fox, mink, ferret, sheep, laboratory rabbit.

Geographical distribution: Inite I States.

Induced disease: In fox, after 2 days from time of infection, loss of appetite, slight nasal discharge; convulsions with early death or hyperexcitability, blind walking, lethargy, flaccid or spast ic paralysis, muscular twitching, fearfulness, weakness, coma and death; many more foxes become infected in epizootics than show obvious disease, some being symptomless carriers; 12 to 20 per cent fatalities may be experienced among young foxes on ranches, 3 to 9 per cent among adults. Int ranuclear inclusions in vascular endothelial cells especially in cerebral endothelium; sometimes in hepatic cells and endothelial cells of liver and kidney; no intracytoplasmic inclusions; virus in heart blood, spleen, and brain; in carriers, virus is believed to persist in focal lesions in upper respiratory tract. Experimentally in susceptible dogs, sometimes coryza, discharge from eyes and nose often purulent, commonly fits of excitement, coma, death; recovery rare; cellular infiltration in the central nervous system, focal necrosis of the liver; specific intranuclear inclusions in cells of the vascular endothelium, meningeal cells, reticulo-endothelium, hepatic cells, and occasionally in cortical cells of the adrenal.

Transmission : Experimentally, by skin scarification, intramuscular injection, intraperitoneal injection, inoculation of cisterna. intratesticular injection, inoculation of nasal cavity; not by corneal scarification.

Immunological relationships: Inject ions of this virus afford no immunity to subsequent infection by canine distemper virus.

Filterability : Passes Berkefeld N filter.

Other properties : Viable in 50 per cent glycerine for several years, in carcass for several days.

Literature: Barton and Green, Am. Jour. Hyg., 3\%, 1943, 21-36; Green, Proc. Soe. Exp. Biol. and Med., 23, 1926, 677678; Am. Jour. Hyg., 13, 1931, 201-223; Green and Dewey, Proc. Soc. Exp. Biol. and Med., 27, 1929, 129-130; Green and Evans, Am. Jour. Hyg., 29 (B), 1939, 73-87; Green and Shillinger, ibid., 19, 1934, 362-391; Green et al., ibid., 12, $1930,109-129$; 14, 1931, 353-373; 18, 1933 , $462-481 ; 19,1934,343-361 ; 21,1935,366-$ 385; 24, 1936, 57-70; Licas, Am. Jour. Path., 16, 1940, 739-760.

8. Tarpeia viverrae spec. nov. From Latin viverra, ferret.

Common name: Ferret-distemper virus.

Host : MUSTELIDAE-Mustela furo. ferret.

Insusceptible species: Dog, mouse, rat, guinea pig, rabbit .

Geographical distribution: Inited States.

Induced disease: In ferret, fever to 105 or $106^{\circ} \mathrm{F}$, lethargy, loss of appetite, conjunctivitis with exudate closing eyes, sometimes a purulant nasal discharge, weight loss small, sneezing rare, difficulty in breathing, death 14 to 56 days after inoculation (average 20 days), sometimes 
preceded by convulsions and other nervous signs; fatality rate 70 to 100 per cent.

Transmission: By cage contacts. By feeding. Experimentally by intranasal, subcutaneous, or intradermal inoculation.

Immunological relationships: In immunized animals, no cross immunity with canine distemper virus nor with human influenza virus.

Thermal inartivation: At $60^{\circ} \mathrm{C}$ in 30 minutes.

Filterability : Passes Berkefeld $N$ filter.

Other properties: Viable at least 3 , but not 5 , months in 50 per cent neutral glycerine; at least 4 months when frozen and dried in vacuo.

Literature: Slanetz and Smetana, Jour. Exp. Med., 66, 1937, 653-666; Spooner, Jour. Hyg., 38, 1938, 79-89.

9. Tarpeia avium spec. nov. From Latin aves, fowl of the air.

Common names: Laryngotracheit is virus; also known as infectious laryngotracheitis virus and as infectious bronchitis virus.

Hosts: PHASIANIDAE-Gallus gallus (L.), chicken. Experimentally, also PHASI ANIDAE-pheasant; $\mathrm{F}_{1}$ hybrid between male Ringneck pheasant and female bantam chicken; chorioallantoic membrane of developing chicken embryo (with macroscopic lesions on membrane as a result of proliferative and necrotic changes); turkey embryo.

Insusceptible species: Guinea fowl (no evidence of disease on inoculation); white rat, guinea pig, rabbit; embryos of pigeon, guinea fowl, and duck.

Geographical distribution: United States, Canada, Australia.

Induced disease: In domestic chicken, mostly among pullets and yearling hens, loss of appetite, lachrymation from one or both eyes, respiratory distress, hemorrhagic and mucous exudate in lumen of trachea and occasionally in the bronchi; death as a result of asphyxiation or, more often, recovery; recovered birds occasionally carry the virus in the upper respiratory tract for some time (a period of 467 days has been recorded); virus is not found on eggs during an outbreak in a flock, but is always in trachea of an affected bird; intranuclear inclusions in tracheal lesions; virus has special affinity for mucous membrane of eye, nostril, larynx, trachea, cloaca, and bursa of Fabricius; usually affects more than half the birds in a flock, with a mortality of 5 to 60 per cent (averaging between 10 and 20 per cent).

Transmission: By contacts. Experimentally, by intrabursal injection (in bursa of Fabricius) or by rubbing the mucous membrane in the dorsal region of the outer or proctodeal part of the cloaca with a small cotton swab moistened with a suspension of virus.

Serological relationships: Serum from recovered fowl neutralizes virus; dilution tends to reactivate neutralized virus.

Immunological relationships: Experimental infection of cloaca and bursa of Fabricius, especially in 2 to 4 -month-old birds, immunizes against infection by subsequent tracheal inoculation.

Thermal inactivation: At $55.5^{\circ} \mathrm{C}$ in 10 to 15 minutes; at $60^{\circ} \mathrm{C}$ in 2 to 3 minutes; at $75^{\circ} \mathrm{C}$ in $\frac{1}{4}$ to $\frac{1}{2}$ minute; all tests with virus in the presence of tracheal exudate.

Filterability : Passes Berkefeld V and X filters.

Strains: A Victorian strain has been reported as of low virulence for fowls.

Other properties: Inactivated in 5 per rent phenol in 1 minute; in 3 per cent cresol compound in $\frac{1}{2}$ minute; in 1 per cent sodium hydroxide in $\frac{1}{2}$ minute. Viable in tracheal fluid in dark for 75 , not 110 , days ; in light for 6 , not 7 , hours; in buffer solution at pH 7.4 for 131 days; at 4 to $10^{\circ} \mathrm{C}$ in dark for at least 217 days; in dried state for at least 661 days. Viable in dead body at $37^{\circ} \mathrm{C}$ for 22 , not 44 , hours ; at 13 to $23^{\circ} \mathrm{C}$ for 10 , not 15 , days; at 4 to $10^{\circ}$ C. for 30 , not 60 , days.

Literature: Beach, Science, 72, 1930, 633-634; Jour. Exp. Med., 54, 1931, 809816 ; Jour. Inf. Dis., 57, 1935, 133-135; 
Beach et al., Poultry Science, 19, 1934, 218-226; Beaudette and Hudson, Science, 76, 1932, 34; Jour. Am. Vet. Med. Assoc., 82 (N.S. 35), 1933, 460-476; 95, 1939, 333339 ; Brandly, ibid., 88 (N.S. 41), 1936, 587-599; Jour. Inf. Dis., 57, 1935, 201206; Brandly and Bushnell, Poultry Science, 13, 1934, 212-217; Burnet, Brit. Jour. Exp. Path., 15, 1934, 52-55; Jour. Exp. Med., 63, 1936, 685-701 ; Burnet and Foley, Austral. Jour. Exp. Biol. and Med. Sci., 19, 1941, 235-240; Gibbs, Jour. Am. Vet. Med. Assoc., 81, (N.S. 34), 1932, 651-65t; Massachusetts Agr. Exp. Sta.,
Bull. 295, 1933, ibid., Bull. 311, 1934 ; Hinshaw et al., Poultry Science, 10, 1931, 375-382; Hudson and Beaudette, Science, 76, 1932, 34; Cornell Vet., 22, 1932, 70-74; Kernohan, California Agr. Exp. Sta., Bull. 494, 1930, 3-22; Jour. Am. Vet. Med. Assoc., 78 (N.S. 31), 1931, 553-555; Komarov and Beaudette, Poultry Science, 11, 1932, 335-338; May and Tittsler, Jour. Am. Vet. Med. Assoc., 67, (N.S. 20), 1925, 229-231; Schalm and Beach, Jour. Inf. Dis., 56, 1935, 210-223; Seifried, Jour. Exp. Med., 54, 1931, 817-826.

\section{Genus III. Tortor gen. nov.}

Viruses of the Hog-Cholera Group inducing diseases characterized by involvement of many tissues. Generic name from Latin tortor, tormentor.

The type species is Tortor suis spec. nov.

\section{Key to the species of genus Tortor.}

I. In mammals.
A. Infecting swine.
B. Infecting cattle.
C. Infecting the horse.
1. Tortor suis.
2. Tortor bovis.
D. Infecting sheep.
E. Infecting cat.

\section{Tortor equorum. \\ 4. Tortor equa:}
5. Tortor oves.

\section{Tortor felis.}

II. In birds.

7. Tortor galli.

8. Tortor furens.

1. Tortor suis spec. nov. From Latin sus, hog.

Common names: Hog-cholera virus, swine-fever virus.

Host: SUIDAE-Sus scrofa L., domestic swine. Warthog (symptomless carrier).

Insusceptible species: Dog, cat, cow, horse, donkey, sheep, goat, rabbit, guinea pig, mouse, rat, goose, hen, duck, pigeon.

Geographical distribution: Almost universal in pig-breeding countries, espe-

cially in Europe, the British Isles, North and South America.

Induced disease : In swine, after intramuscular injection, increased temperature and prostration within $2 \frac{1}{2}$ to 3 days; later lymph nodes enlarged, sometimes hemorrhagic; hemorrhages under capsule of kidneys. Virus may remain in blood of recovered pigs for 10 months. Acquired immunity is lasting, but most naturally infected animals die in newly infected herds. Virus has beeiı cultured 
in minced swine testicle on solid serumagar and on egg membrane, increase being limited to the living tissues from the swine and furnishing inoculum active in amounts as small as $10^{-5} \mathrm{ml}$.

Transmission: By feeding. Through air contamination. Rarely by contact. Experimentally, by subcutaneous injection. Urine highly infective. Virus in blood and all tissues early in disease.

Serological relationships: Immune serum affords passive protection.

Thermal inactivation: At $55^{\circ} \mathrm{C}$ in 30 minutes; at $60^{\circ} \mathrm{C}$ in 10 minutes. At $72^{\circ}$ $\mathrm{C}$ in 1 hour in dried blood.

Filterability: Passes Berkefeld filter.

Other properties: Viable in blood in cool, dark place at least 6 years.

Literature: De Kock et al., Onderstepoort Jour. Vet. Sei. and Anim. Indust., 14, 1940, 31-93; Hecke, Cent. f. Bakt., I Abt., Orig., 126, 1932, 517-526; Montgomery, Jour. Comp. Path. and Therap., 34, 1921, 159-191; Röhrer, Arch. Tierheilk., 62, 1930, 345-372, 439-462; 64, 1931, 124-143; TenBroeck, Jour. Exp. Med., 74, 1941, 427-432.

2. Tortor bovis spec, nov. From Latin bos, cow.

Common names: Cattle-plague virus, virus of pest is bovina, runderpest virus, Rinderpest virus.

Hosts : BOVIDAE-Bos taurus L., domestic cattle ; swine, buffalo, zebu cattle, sheep, goat, camel, deer. Koedoe, eland, bushbuck, duiker, and other antelopes.

Insusceptible species: Man, solipeds, carnivora.

Geographical distribution: Widespread over Asia and the Asiatic islands. At times in Western Europe. Enzootically in Turkey. Periodically in North Africa, especially in Egypt; at times throughout Africa. Not in North America. At times in South America, Australia (suppressed quickly).

Induced disease: In domestic cattle, after 3 to 9 days, febrile reaction, restless. ness, loss of appetite, cessation of rumina- tion; fever highest at 5 th or 6 th day of disease, then temperature drops to normal or subnormal and diarrhea begins; muzzle dry, coat staring, hair dull, skin moist in parts; twitching of superficial museles, grinding of teeth, arching of back, glairy discharge from nose, redness of mucous membranes; restlessness increases, diarrhea becomes severe with fetid, bloodstained or blackish liquid discharges; weakness, drooping of ears, occasional yawning, coldness of extremities; occasionally excitement precedes weakness; skin may become red and moist, showing protuberances and vesicles, with matted hair; later wrinkling and scab formation; conjunctiva red, eyelids swollen, tears flowing, followed by mucous, then purulent, discharge; sometimes a cough develops and respirations become rapid; red spots inside mouth develop into erosions or ulcers, of ten confluent ; pregnant animals often abort; milk of cows decreases, sometimes becoming yellow and watery. Death is sumetimes early (1 to 2 days after first manifestations of disease), more often delayed ( 4 to 7 days); sometimes animals live 2 or 3 weeks or longer. Disease milder and more chronic where enzootic; morbidity to 100 per cent and mortality to 96 per cent in new areas. Recovered animals show a lasting, sterile mmunity. Urine, feces, nasal and lachrymal discharges, sweat, aqueous humour, cerebrospinal fluid, lymph, emulsions of viscera and muscles, and blood are infective during the course of the disease.

Transmission: By contact, even during prodromal period; by contaminated food, troughs, or other articles. No insect vector is known.

Immunological relationships: One attack confers a lasting immunity, except rarely, when a mild second attack may occur. A calf from a diseased mother may be resistant if pregnaney was far advanced when the disease occurred.

Filterability: Passes Berkefeld V filter candle, with difficulty.

Other properties : Remains infective at 
least 2 weeks at $0^{\circ} \mathrm{C}$ in virulent blood, less than 2 days in hides dried in direct sunlight, 3 days in contaminated wool, as long as 12 days in meat; is inactivated by glycerine, bile, chloroform, formalin, and 2 per cent phenol ; is virulent at least 25 days in body of leech, Hirudu boyntoni Wharton (HIRUDIDAE), fed on sick animal.

Literature: Boynton, Philippine Agr. Rev., 10, 1917, 410-433; Daubney, Jour. Comp. Path. and Therap., 41, 1928, 228248; 263-297 ; Hornby, ibid., 41, 1928, 17 24; Pfaff, Onderstepoort Jour. Vet. Sci. and Anim. Indust., 11, 1938, 263-330; 15 , 1940, 175-184; Weston, Jour. Am. Vet. Med. Assoc., 66 (N.S. 19), 1924, 337-350.

3. Tortor equorum spec. nov. From Latin equus, horse.

Common names: Horse-sickness virus, African horse-sickness virus, virus of pestis equorum, virus of perdesiekte, virus of South African Pferdesterbe.

Hosts: EQUIDAE-Equus caballus L., horse ; perhaps $E$. asinus L., donkey. Experimentally, also $C A N I D A E-$ Canis familiaris L., dog. CAVIIDAE-Cavia porcellus (L.), guinea pig. $I U R I D A E$ -Rattus norwegicus (Erxleben), wild and albino rat; mouse ; Angora goat; Mastomys coucha, multimammate mouse; Tatera lobengula, gerbille; chick embryo (but no virus in hatched chick). Mule and zebra relatively resistant.

Insusceptible species: $H O M I N I D A H$ - Homo sapiens L., man. LEPORIDAE-Oryctolagus cuniculus (L.), rabbii (no observed disease).

Geographical distribution: Africa, especially in coastal regions and river valleys.

Induced disease: In the horse, four types of disease are recognized. Horse sickness fever, prodromal period 5 to 28 days, rise of body temperature to $105^{\circ} \mathrm{F}$ in 1 to 3 days, with return to normal temperatures in another day or two; sometimes loss of appetite, redness of conjunctiva, labored breathing, and ac- celerated puise; recovery prompt. Dunkop or acute pulmonary horse-sickness, prodromal period of 3 to 5 days, severe dyspnea, fever, coughing, frothing at nostrils; fever to $106^{\circ} \mathrm{F}$, breathing rate to 60 a minute, nostrils dilated, head and neck extended, ears drooping, sweating, progressive weakness; often fatal. Dikkop, or cardiac form of horse-sickness, prodromal period 5 to 21 days, fever develops slowly, lasts long; edematous swellings of head and neck, symptoms of eardiac dyspnea, sometimes blood spots on conjunctiva, mucous membranes of mouth and tongue bluish, restlessness; sometimes fatal outcome. Mixed form of horse-sickness, combining features of pulmonary and cardiac types. Horses recovering from natural infections are known as "salted" and possess heightened resistance to the disease.

Transmission: Not by contact. Mosquitoes and biting flies have been suspected as vectors. Experimentally, by intravenous or subcutaneous injection.

Serological relationships: Serologically distinguishable strains exist.

Immunological relationships: Immunity to homologous strain complete after an attack (horse then known as "salted" for that strain), but immunity to heterologous strains incomplete. Antibodies absent from young at birth but as high in titer as in dam within 30 hours, presum ably from colostral milk; declining gradually over a period of about 6 months.

Thermal inactivation: At 57.5 to $60^{\circ} \mathrm{O}$ in 10 minutes.

Filterability : Passes Berkefeld, Chamberland F, and Seitz EK filters.

Other properties: Viable dry at least 15 months. Stable in alkaline solutions (to $\mathrm{pH} 10$ ), unstable in acid (beyond $\mathrm{pH}$ 6.0 ). Serum-saline solutions preferable to saline solutions for storage. Particle diameter determined as 40 to 60 millimicrons (mean 50 millimicrons) by filtration methods, 45.4 millimicrons by centrifuging. Density $1.25 \mathrm{gm}$ per $\mathrm{ml}$. Isoelectric point at $\mathrm{pH} 4.8$. 
Literature: Alexander, Onderstepoort Jour. Vet. Sci. and Anim. Indust., 4, 1935, 291-322, 323-348, 349-377, 379-388; $7,1936,11-16$; 11, 1938, 9-19; Alexander and DuToit, ibid., 2, 1934, 375-391; Alexander and Mason, ibid., 16, 1941, 19-32; Alexander et al., ibid., 7, 1936, 17-30; DuToit et al., ibid., 1, 1933, 21-24, 25-50; Henning, in Animal Diseases in South Africa, Central News Agency, Limited, South Africa, 2, 1932, 516-538; M'Fadyean, Jour. Comp. Path. and Therap., 13, 1900, 1-20; 23, 1910, 27-33, 325-328; Nieschulz and DuToit, Onderstepoort Jour. Vet. Med. and Anim. Indust., 8, 1937, 213-268; Polson, ibid., 16, 1941, 33-50, 51-66; Nature, 148, 1941, 593-594; Theiler, Deutsch. tierärztl. Wochenschr., 9, 1901, 201-203, 221-226, 233-237, 241-242 ; Report for 1905-1906 of the Govt. Veterinary Bacteriologist, Transvaal Dept. Agr., 1907, 160-162; Jour. Comp. Path. and Therap., 2S, 1910, 315-325.

\section{Tortor equae spec. nov, From Lat in} equa, mare.

Common name : Mare-abortion virus.

Hosts: EQUIDAE-Equus caballus L., horse. Experimentally, also Syrian hamster (newborn); tissues of human placenta grafted on the chorioallantois of the chick embryo.

Insusceptible species: Chicken (embryo; no observed susceptibility).

Induced disease: In horse, small, mul tiple, grayish white areas of necrosis in the livers of aborted fetuses; acidophilic intranuclear inclusions in hepatic cells around these foci, in epithelial cells of bile ducts, and in bronchial epithelium; petechial hemorrhages in the heart, spleen, and lungs; excess fluid in the thoracic cavity.

Transmission: By contact. By living in contaminated stalls.

Literature : Anderson and Coodpasture, An. Jour. Path., 18, 1942, 555-561; Dimock, Jour. Am. Vet. Med. Assoc., 96, 1940, 665-666; Dimock and Edwards, Cornell Vet., 26, 1936, 231-240; Goodpas- ture and Anderson, Am. Jour. Path., 18, 1942, 563-575; Hupbauer, Münch. Tierärztl. Wehnschr., 89, 1938, 37-38; Miessner and Harms, Deutsche Tierärztl. Wehnschr., 46, 1938, 745-748.

\section{Tortor ovis spec. nov. From Latin} ovis, sheep.

Common name: Blue-tongue virus.

Hosts: BOVIDAE-Ovis aries L., sheep; Bos taurus L., cattle.

Geographical distribution: South Africa.

Induced disease : Both sheep and cattle may carry the virus at times without obvious manifestations of disease or there may be severe manifestations. In sheep, experimentally, diffuse hyperemia of buccal mucosa, especially of lips; then petechiae and ecchymoses followed by excoriations and necrosis of the mucous membrane, especially on lips, tongue, inside of cheeks, dental pad, gums, muzzle, and external nares; sometimes deep seated necrotic ulcers on tongue developing from the more usual superficial necrotic process; mucoid discharge from nostrils, becoming muco-hemorrhagic; commonly frothing at the mouth in early stages of the disease; frequently reddening of skin of lips and nose; rarely whole skin becomes flushed and wool is shed; often swelling of vulva with necrotic changes on borders and petechiae in mucosa ; tongue sometimes swollen; lameness common and severe; recovery or death. In cattle, edema of lips and tongue; hyperemia of oral mucosa; multiple hemorrhages in skin, lips, mucous membrane of the lips, tongue, dental pad, buccal eavity, small intestine, myocardium, epicardium, and endocardium, less frequently in the trachea, nasal cavity, bladder, urethra, pulmonary artery, and pleura; localized necrotic areas followed by ulceration on lips, gums, the dental pad, tongue, mucous membrane of the rumen, pylorus of the stomach, and the external nares; scattered skin lesions with reddening, slight exudation, crusting, sloughing of crusts and hair together, 
mucoid or mucopurulent discharge from nostrils; prognosis favorable in mild cases, but disease occasionally terminates with death.

Transmission: Not by contact; arthropod vector suspected.

Other properties: Infective particle calculated to be 87 to 105 millimicrons in diameter by sedimentation studies, 100 to 132 millimicrons in diameter by ultrafiltration.

Literature: Bekker et al., Onderstepoort Jour. Vet. Sci. and Anim. Indust., 2, 1934, 393-507; De Kock et al., ibid., 8, 1937, 129-180; Henning, in Henning, M. W., Animal Diseases in South Africa, Central News Agency, Ltd., South Africa, 1932, vol. 2, chapter 27, pages 503-515; Mason and Neitz, Onderstepoort Jour. Vet. Sci. and Anim. Indust., 15, 1940, 149-157; Nieschulz et al., ibid., 2, 1934, 509-562; Polson, Nature, 148, 1941, 593-594.

\section{Tortor felis spec, nov. From Latin} feles, cat.

Common names: Panleucopenia virus, infectious feline agranulocy tosis virus, infectious aleucocytosis virus, feline enteritis virus.

Host : FELIDAE-Felis catus L., domestic cat.

Insusceptible species: White mouse, guinea pig, domestic rabbit, ferret; Citellus richardsonii (Sabine), ground squirrel.

Geographical distribution: Inited States, Germany.

Induced disease: In cat, variable effects, some individuals little affected, others listless, recumbent, refusing food, showing some vomiting, diarrhea, nasal and ocular discharges; often death, after a few minutes of fibrillary twitching and terminal clonic convulsions, before there is much loss of weight; sometimes recovery with return of appetite. Profound leucopenia and marked relative lymphocytosis without thrombopenia or appreciable anemia; proliferation of reticuloendothelial cells of lymph nodes and spleen; intranuclear inclusion in cells of gastro-intestinal mucosa, spleen, lymph nodes, bone marrow, and bronchial mucosa.

Transmission: Perhaps by nasal droplets or contaminated food. No arthropod vector recognized. Experimentally by oral, intragastric, cutaneous, subcutaneous, intraperitoneal, intravenous, and intranasal routes.

Serological relationships: Sera from panleucopenia-immune cats protects against agranulocytosis virus.

Immunological relationships: Cats immune as a result of earlier infection with agranulocy tosis virus resist later inoculation with panleucopenia virus. Previous inoculation ineffective if made with hogcholera virus or fox-encephalitis virus.

Filterability: Passes Berkefeld V, N, and $W$ filters and Seitz EK discs.

Other properties : Remains active in 50 per cent glycerine at least 138 days in tissues; not inactivated by drying while frozen, nor by freezing at about $-80^{\circ} \mathrm{C}$.

Literature : Hammon and Enders, Jour. Exp. Med., 69, 1939, 327-352; 70, 1939, 557-564; Kikuth et al., Cent. f. Bakt., I Abt., Orig., 146, 1940, 1-17; Lawrence and Syverton, Proc. Soc. Exp. Biol. and Med., 38, 1938, 914-918; Lawrence et al., Jour. Exp. Med., 77, 1943, 57-64; Am. Jour. Path., 16, 1940, 333-354; Syverton et al., Jour. Exp. Med., 77, 1943, 41-56.

7. Tortor galli spec. nov. From Latin gallus, cock.

Common names: Fowl-plague virus, fowl-pest virus.

Hosts: Chiefly chicken, turkey, goose. Experimentally, also ferret, rhesus monkey, hedgehog, pigeon, duck, canary, mouse, rat, rabbit. Multiplies in embryonated hen's egg; edema, but no discrete primary lesions in chorioallantoic membrane.

Geographical distribution: Widespread throughout Europe, North and South America, Asia.

Induced disease: In chicken, loss of appetite, tendency to leave companions 
and seek shade, drooping of wings and tail; eyes closed or partly closed; some dyspnea; in some cases, edema of head and neck; in late stages, sometimes cyanosis of comb and skin; staggering, twitching, or spasms; fever may disappear and temperature become subnormal before death; recovery in about 30 per cent of all cases; linear and punctiform hemorrhages throughout body.

Transmission : Method of natural transmission unknown. The fowl louse, Gonioides dissimilis (PHILOPTERIDAE), has been suspected as vector (Maggiora and Tombolato, Rendiconti, Accademia delle Scienze dell'Instituto di Bologna, n.s. 27, 1923, 200-203). Experimentally, by subcutaneous, intramuscular, and intravenous injection.

Serological relationships: Specific neutralizing antiserum does not react with influenza virus. No reaction of fowlplague virus with antisera specific for canine distemper, influenza, or Rift Valley fever viruses.

Thermal inactivation: At $55^{\circ} \mathrm{C}$ in 1 hour in whole blood or brain.

Filterability: Passes membrane of average pore diameter 150 , not 100 , not ordinarily 125, millimicrons. Passes Berkefeld and Chamberland filters.

Other properties: Particle diameter estimated by filtration as 60 to 90 millimicrons; by centrifugation, as 120 to 130 millimicrons. Viable after exposure in $1: 10,000$ dilution for 10 minutes, in $2 \mathrm{~mm}$ layer of $1: 50,000$ methylene blue, $15 \mathrm{~cm}$ from a 300 eandle-power filament lamp. Withstands drying. Precipitates from salt-free solutions or in presence of halfsaturated ammonium sulphate solutions; virus held to be of globulin nature by Mrowka, Cent.f. Bakt., I Aht., Orig., 6i, 1912, 249-26s.

Strains: Variant strains have been produced by intracerebral passage in brains of canaries and mice.

Literature: Bechhold and Schlesinger, Biochem. Ztschr., 236, 1931, 387-414; Ztschr. Hyg. Infektionskr., 112, 1931.
668-679; Burnet and Ferry, Brit. Jour. Exp. Path., 15, 1934, 56-64; Centanni, Cent. f. Bakt., I Abt., Orig., 31, 1902, 145-152, 182-201; Elford and Todd, Brit. Jour. Exp. Path., 14, 1933, 240-246; Findlay and Mackenzie, ibid., 18, 1937, 146-155, 258-264; Findlay et al., Jour. Path. and Bact., 45, 1937, 589-596; Lépine Compt. rend. Soc. Biol., Paris, 121, 1936, 509-510; Mackenzie and Findlay, Brit. Jour. Exp. Path., 18, 1937, 138-145; Nieschulz and Bos, Cent. f. Bakt., I Abt., Orig., 131, 1934, 1-6; Plotz and Haber, Compt. rend. Soc. Biol., Paris, 125, 1937, 339-340.

8. Tortor furens spec. nov. From Latin furere, to rage.

Common name: Newcastle-disease virus.

Hosts: PHASIANIDAE-Gallus gallus (L.), domestic chicken. HOMINID A E-Homo sapiens L., man (by laboratory accident). Experimentally, also pigeon; chick embryo (with primary lesions and eytoplasmic inclusions in chorioallantoic membrane).

Geographical distribution: England, probably also East Indies, Korea, Japan, India, Australia.

Induced disease: In chicken, acute, febrile, highly contagious, usually fatal lisease resembling fowl plague; loss of appetite, crouching attitude, half closed eyes, rapid respirations, watery yellowish-white diarrhea with nauseating odor; death usually between 6 th and 8 th day. In man, accidentally infected in laborafory by virus sprayed into eye, virus recoverable from temporarily inflamed eye; recovery in $\delta$ days with gradual inrrease of specific antibodies in blood.

Transmission: By contact between healthy and diseased birds.

Serological relationships: Antiserum effective in neutralizing homologous virus.

Immunological relationships : Chickens immune to infection by fowl-plague virus are susceptible to infection by this virus 
and vice versa. Immunization to this virus does not decrease susceptibility to comb or mouth form of fowl pox.

Thermal inactivation: At $60^{\circ} \mathrm{C}$ in 1 hour; not at $56^{\circ} \mathrm{C}$ in 30 minutes.

Filterability : Passes Berkefeld, Chamberland $\mathrm{L}_{3}$, and Seitz filters.

Other properties: Particle diameter calculated from filtration experiments to be 80 to 120 millimicrons. Not inactivated in 30 minutes in $1: 50,000$ methylene blue solution in $2 \mathrm{~mm}$ layer $15 \mathrm{~cm}$ from a 300 candle-power filament lamp.

Literature: Burnet, Med. Jour. Australia, 30, 1943, 313-314; Burnet and Ferry, Brit. Jour. Exp. Path., 15, 1934, 56-64; Doyle, Jour. Comp. Path. and Therap., 40, 1927, 144-169. 


\section{FAMILY V. TRIFURACEAE FAM. NOV.}

Viruses of the Infectious Anemia Group, inducing diseases mainly characterized by disturbances in balance of blood cells. There is a single genus.

\section{Genus Trifur gen. nov.}

With characters of the family. Generic name from Latin trifur, arrant thief. The type species is Trifur equorum spec. nov.

\section{Key to the species of genus Trifur.}

I. Affecting horse.

\section{Affecting fowl.}

1. Trifur equorum spec, nov. From Latin equus, horse.

Common name: Equine infectiousanemia virus.

Hosts: EQUIDAE-Equus caballus L., horse; $E$, asinus L., donkey. HOMINIDAE-Homo sapiens L., man. Experimentally, also EQUIDAE-Equus asinus $\times E$. caballus, mule. SUIDAE -Sus scrofa L., swine.

Insusceptible species: $B O V I D A E-$ Bos taurus L., cattle; Ovis aries L., sheep; Capra hircus L., goat. CANIDAE-Canis familiaris L., dog.

Geographical distribution: Europe, Union of South Africa, United States, Canada, Japan; at times in most parts of the world; not Australia.

Induced disease: In horse, progressive anemia with eventual death or clinical recovery and retention of virus; disease may be acute, subacute, or chronic; in acute disease, temperature rise to 104 to $105^{\circ} \mathrm{F}$. or even 106 to $107^{\circ} \mathrm{F}$, remaining high much of the time until death or change to subacute or chronic form; in the acute form of the disease there is dullness, decreased appetite, drooping of head, flexing of limb not supporting weight; sometimes increase in pulse frequency to 70 or even 100 a minute but oftener rates around 50 a minute; conjunctiva sometimes colored orange, with injection of vessels and petechiae, later becoming muddy colored or pale red, membrane. edematous; uncertain gait,

\section{Trifur equorum.}

\section{Trifur gallinarum.}

trailing of hind feet, prostration, sometimes death; subacute disease milder and. with remissions; chronic disease still milder, anemia conspicuous, sometimes death from debility or at end of a febrile attack; blood infective long ( 3 to 7 years) after clinical recovery; urine infective to horse by mouth. In man, diarrhea alternating with constipation, herpes-like exanthema on abdominal wall, blood sometimes in feces; persistent headache, temperature normal ; later, lumbar pains, generalized edema, general debility, loss of flesh, pallor of face and mucosae; filtered blood in $1 \mathrm{ml}$. amount fatal to horse, inducing infectious anemia; improvement after 2 to 4 years. In swine, experimentally, sometimes no outward and obvious signs of disease but blood. abnormal and infective; sometimes severe: anemia, fever, prostration, loss of appetite.

Thermal inactivation: At 58 to $60^{\circ} \mathrm{C}$ in 1 hour.

Filterability : Passes Berkefeld V filter candle.

Other properties: Viable in. blood in citrate saline at $-2^{\circ} \mathrm{C}$ for at least a year. Drying does not inactivate in 10 days but does in 1 month.

Literature: DeKock, Union of South Africa, Dept. of Agr., 9th and 10th Reports for 1923, Pretcria 1924, 253-313; Habersang, Monatshefte für prakt. Tierheilk., 30, 1920, 171-176; Kutsche, ibid., 30, 1920, 557-56\$; Peters, Jour. Am. Vet. 
Med. Assoc., 66, 1924, 363-366; Theiler and Kehoe, Union of South Africa, Dept. of Agr., 3rd and 4th Reports of the Director of Veterinary Research, 1915, 215-289.

2. Trifur gallinarum spec. nov. From Latin gallina, hen.

Common name: Fowl-leucosis virus.

Host: Gallus gallus (L.), chicken.

Geographical distribution: United States, England, Europe.

Induced disease: In chicken, neurolymphomatosis, with eye lesions (slate gray or bluish color replacing normal bay color of iris), anemia, hemocytoblastosis, lymphoid, erythroid or myeloid types of leucosis; the hemocytoblastosis is followed by infiltration of the central nervous system, peripheral nerves, iris, and many visceral organs by hemocytoblasts and lymphocytes, producing lesions sometimes resembling neoplasms and consisting chiefly of hemocytoblasts (hemocytoblastomata); marrow of radius and ulna becomes hyperplastic; virus in blood plasma, blood cells, emulsions of organs; blood normal in its hydrogen-ion concentration; recovery never complete; some stocks less susceptible than others.

Transmission: By pen contact or contaminated litter. Experimentally by intravenous injection of cell-free filtrates. Not by the mosquitoes, Culex pipiens and Aëdes aegypti (CULICIDAE). Dayold chicks from iritis parents contain the infective agent and show some form of the induced disease in 80 per cent of the progeny if both parents show iritis, in 70 per cent if male is normal, 15 per cent if female is normal.
Serological relationships: Specific neutralizing antibodies are formed in the rabbit as a result of injecting infective materials partly purified by sedimentation in the ultracentrifuge.

Thermal inactivation: At $56^{\circ} \mathrm{C}$ in 30 minutes.

Filterability: Passes Berkefeld V, N, and $W$ filter candles; 1.5 per cent, but not of ten 3 per cent, collodion membranes; Seitz asbestos filter.

Other properties: Viable after drying at least 54 days, in glycerine at least 104 days, at $4^{\circ} \mathrm{C}$ at least 14 days, at $-60^{\circ} \mathrm{C}$ at least 6 months; after freezing and thawing, and after freezing in liquid air. Not viable after 14 days at $37.5^{\circ} \mathrm{C}$. Particle diameter between 100 and 400 millimicrons.

Literature : Ellermann and Bang, Cent. f. Bakt., I Abt., Orig., 46, 1908, 4-5, 595609 ; Furth, Proc. Soc. Exp. Biol. and Med., 27, 1929, 155-157; Jour. Exp. Med., $53,1931,243-267$; 55, 1932, 465-478, 495$504 ; 58,1933,253-275 ; 59,1934,501-517$; Furth and Niller, ibid., 55, 1932, 479-493; Hall et al., Am. Jour. Vet. Res., 2, 1941, 272-279; Jármai, Arch. wissensch. u. prakt. Tierhielk., 62, 1930, 113-131; Johnson, Virginia Agr. Exp. Sta. Tech. Bull. 56, 1934, 1-32; Johnson and Bell, Jour. Inf. Dis., 58, 1936, 342-348; Kabat and Furth, Jour. Exp. Med., 71, 1940, 55-70; 74, 1941, 257-261; Lee and Wilcke, Am. Jour. Vet. Res., 2, 1941, 292-294; Lee et al., Jour. Infect. Dis., 61, 1937, 1-20; Pierce, Am. Jour. Path., 18, 1942, 1127-1139; Ratcliffe and Stubbs, Jour. Inf. Dis., 56, 1935, 301-304. 


\section{FAMILY VI. RABULACEAE $F A M$. NOV.}

Viruses of the Mumps Group, characterized in general by a special affinity for $1 \mathrm{i}$.ssues of the salivary glands. There is a single genus,

\section{Genus I. Rabula gen. nov.}

With characters of the family. Generic name from Latin rabula, pettifogger. The type species is Rabula inflans spec. nov.

\section{Key to species of the genus Rabula.}

I. Affecting man.

II. Affecting guinea pig.

III. Affecting hamster

IV. Affecting rat.

V. Affecting nouse

1. Rabula inflans spec. nov. From Latin inflare, to puff up.

Common names: Mumps virus, virus of epidemic parotitis.

Hosts : HOMINID.AE-Homo sapicns L., man. Experimentally, also $\mathrm{CERCO}$ PITHECII)AE-Macaca mulatta (Zimmermann), rhesus monkey. FELIDAE - Felis catus L., domestic cat.

Geographical distribution: World-wide.

Induced disease: In man, in order of frequency, parotitis, orchitis, meningoencephalitis, pancreatitis, or ovaritis; rarely fatal; when parotitis occurs, onset is sudden, with pain in one or both parotid glands, sometimes also with involvement of submaxillary and sublingual glands, swelling and malaise gradually disappearing within a week or 10 days; there is virus in saliva 48 hours af ter onset; orchitis, less common, is usually unilateral and may be accompanied by some epididymitis. In rhesus monkey, experimentally, acute, non-suppurative parotitis; focal necrosis in acinar epithelial cells of parotid gland, and secondary inflammation; dissemination of lesions within the gland, enlargement of gland to palpation and pitting edema of jowl 6 to $S$ days after inoculation, often with a rise of tempera-

\section{Rabula inflans.}

\section{Rabula levis.}

\section{Rabula innocuus.}

4. Rabula exiguus.

\section{Rabula latens.}

ture; cytoplasmic inclusion bodies in affected glands, staining pink, round or oval, 3 to 10 microns in diameter, often vacuolate, usually surrounded by a narrow clear zone in the cytoplasm; blood and uninoculated salivary gland of affected animal not effective sources of virus.

Transmission: Probably by droplets arising dires tly from infected individuals. Experimentally, by injecting sterile fluids containing virus into Stenson's duct of parotid gland in Macaca mulatta.

Serolugical relationships: A specific complement-fixing antibody occurs in human and monkey convalescent serum and is demonstrable by the use of monkey-gland antigen.

Immunological relationships: Specific immunity induced by attack; passive immunization rarely successful.

Thermal inactivation: At $55^{\circ} \mathrm{C}$ in 1 hour.

Filterability: Passes Berkefeld V and $\mathrm{N}$ filter candles.

Other properties: Viable in 50 per cent glycerine at $2^{\circ} \mathrm{C}$ at least 5 weeks, in 50 per cent glycerine at $10^{\circ} \mathrm{C}$. at least 7 weeks, dried while frozen at least 7 weeks, in frozen saliva at least 3 weeks. 
Literature: Bloch, Am. Jour. Path., 19, 1937, 939-944; Enders and Cohen, Proc. Soc. Exp. Biol. and Med., 50, 1942, 180-184; Findlay and Clarke, Brit. Jour. Exp. Path., 15, 1934, 309-313; Johnson and Goodpasture, Jour. Exp. Med., 59, 1934, 1-19; Am. Jour. Hyg., 21, 1935. 46-57 ; 23, 1936, 329-339; Am. Jour. Path., 12, $1936,495-510$.

2. Rabula levis spec. nov. From Lat in levis, trifling.

Common name: Guinea-pig salivarygland virus.

Host: CAVIIDAE-Cavia porcellus (L.), guinea pig (only known host; fetus more susceptible than post-natal animal, even if from immune mother).

Insusceptible species: Rabbit, rat, cat, chicken, pigeon, dog, mouse, monkey (Macacus rhesus).

Geographical distribution: United States, England.

Induced disease: In guinea pig, submaxillary glands show swollen epithelial cells containing relatively dense acidophilic inclusions of granular material within enlarged nuclei, especially in ducts of the serous portion of the gland, and larger but fewer intracytoplasmic inclusions; experimentally, by intracerebral injection of young guinea pig, prodromal period of about 2 days, then elevation of temperat ure to 105 or $106^{\circ} \mathrm{F}$; a day later. hair raised, animal quiet; subsequently, irritability with tremors and slight convulsive movements; by fifth day, usually prostration, jerking movements, and ensuing death; brain shows no gross lesions but exudate over surface; in meningeal exudate, many cells each containing an acidophilic mass within its nucleus; by subcutaneous injection, virus recoverable after 2 weeks from submaxillary glands, cervical lymph nodes, kidney, and lung, not from blood, liver, or spleen.

Transmission: Experimentally, by inoculation of submaxillary gland or by intracerebral or subcutaneous injection of materials from infected glands; with difficulty from brain to brain. Pilocarpine stimulation increases numbers of inclusions.

Serological relationships: Specific neutralizing antibody is found in blood serum of animals that are carrying virus in their submaxillary glands.

Immunological relationships: Active immunity may be dependent on existence of more or less active lesions.

Thermal inactivation: At $54^{\circ} \mathrm{C}$ in 1 hour.

Filterability : Passes Berkefeld N filter candle.

Other properties: Viable in 50 per cent glycerine at least 11 days.

Strains: An unusually virulent strain, killing infected animals whatever the route of injection, has been described but not given a distinctive name (Rosenbusch and Lucas, Am. Jour. Path., 15, 1939, 303-340).

Literature: Andrewes, Brit. Jour. Exp. Path., 11, 1930, 23-34; Cole and Kuttner, Jour. Exp. Med., 44, 1926, 855-873; Hudson and Markham, ibid., 55, 1932, 405-415; Jackson, Jour. Inf. Dis., 26, 1920, 347350 ; Kuttner, Jour. Exp. Med., 46, 1927, 935-956; Kuttner and T'ung, ibid., 62, 1935, 805-822; Lucas, Am. Jour. Path., 12, 1936, 933-948; Markham, ibid,, 14, 1938, 311-322; Markham and Hudson, ibid., 12, 1936, 175-182; Pearson, ibid., 6, 1930, 261-274; Scott, Jour. Exp. Med., 49, 1929, 229-236; Scott and Pruett, Am. Jour. Path., 6, 1930, 53-70.

3. Rabula innocuus spec. nov. From Latin innocuus, harmless.

Common name: Hamster salivarygland virus.

Host : CRICETIDAE-Cricetulus griseus M. Edw., Chinese hamster.

Insusceptible species: MURIDAErat; Mus musculus L., white mouse.

Geographical distribution: China.

Induced disease: In hamster, no obvious disease externally but inclusion bodies in submaxillary glands. 
Thermal inactivation: At $56^{\circ} \mathrm{C}$ in 30 minutes.

Literature: Kuttner and Wang, Jour. Exp. Med., 60, 1934, 773-791.

4. Rabula exiguus spec. nov. From Latin exiguus, petty.

Common name: Rat salivary-gland virus.

Host : $M U R I D A E$-rat.

Insusceptible species: $M U R I D A E-$ Mus musculus L., mouse. CRICETIDAE-Cricetulus griseus M. Edw., Chinese hamster.

Geographical distribution: China, Canada.

Induced disease: In rat, no obvious disease externally, but intranuclear inclusions in cells of the submaxillary glands.

Literature: Kuttner and Wang, Jour. Exp. Med., 60, 1934, 773-791; Thompson, Jour. Inf. Dis., 50, 1932, 162-170.

5. Rabula latens spec, noc. From Latin latens, hidden or lurking.

Common name: Mouse salivary-gland virus.

Host : MURIDAE-Mus musculus L., mouse.
Insusceptible species: $M U R I D A E-$ rat. CRICETIDAE-Cricetulus griseus M. Edw., Chinese hamster. LEPORIDAE-rabbit. CAVIIDAE-Cavia porcellus (L.), guinea pig.

Geographical distribution: China, Canada, United States.

Induced disease: In mouse, no obvious disease externally, but inclusion bodies in acinar tissue of serous and mucous portions of submaxillary glands; occasionally also in duct cells or alveolar cells of parotid gland; affected cells hypertrophied. In Swiss white mice, extensive lesions in liver and spleen but emulsions of these organs fail to infect; rare pancreatic lesions.

Transmission: Experimentally, by intraglandular, subcutaneous, intraperitoneal, intratesticular or intracerebral inoculation; inclusion bodies appear in salivary glands irrespective of site of inoculation.

Thermal inactivation: At $60^{\circ} \mathrm{C}$ in 30 minutes.

Filterability : Passes Berkefeld V filter candle.

Literature: Kuttner and Wang, Jour. Fxp. Med., 60, 1934, 773-791; McCordock and Smith, ibid., 63, 1936, 303-310; 'Thompson, Jour. Inf. Dis., 58, 1936, 59-63 
SUPPLEMENT NO. 3

\section{PLEUROPNEUMONIA AND PLEUROPNEUMONIA-LIKE ORGANISMS (BORRELOMYCETACEAE)}





\section{THE ORGANISM OF CONTAGIOUS BOVINE PLEURO- PNEUMONIA AND RELATED ORGANISMS*}

INTRODUCTION

The organism of bovine pleuropneumonia is similar in certain respects to filterable viruses. Both in infected tissue and in cultures, small elements are present which pass through filters that retain bacteria. The organism is not stained well by the usual bacterial stains and can be made visible only by using special methods. Bovine pleuropneumonia and other diseases caused by similar organisms were originally attributed to filterable viruses. These organisms are different from viruses in an important point; namely, they grow on suitable media in the absence of living host cells. The cultures consist of pleomorphic elements, the nature of which has only slowly become apparent. By the studies of Nowak (Ann. Inst. Past., 43, 1929, 1330), Turner (Jour. Path. and Bact., 41, 1935, 1) and Klieneberger and Smiles (Jour. Hyg., $\{2,1942,110$ ), it has been established that the pleomorphic forms are part of a reproductive cycle different from binary fission. The small elements in the cultures swell up into large round forms which reproduce the small elements within their membranes. The morphology of the organism is further complicated by the fact that long branching filaments are present in freshly isolated bovine strains. These break up into granules or parts of the filaments swell up into large round forms. In the judgment of some investigators, these properties, in addition to unusual softness and fragility, exclude the organism of bovine pleuropneumonia and similar organisms from the order of true bacteria. Ledingham (Jour. Path. and Bact., 37, 1933, 393) has classified them with the Actinomyces. Later, Turner (Jour. Path. and Bact., 41, 1935, 1) placed them in an independent order, Borrelomycetales, while Sabin (Bact. Rev., 5, 1941, 58) has even placed them in an independent class, Paramycetes.

The observations of the present author give support to the classification of Buchanan (Jour. Bact., 3, 1918, 44) who placed the genus Asterococcus Borrel et al. with the organism of bovine pleuropneumonia (Asterococcus mycoides Borrel et al.) as type, together with the genus Haemophilus Winslow et al. in subtribe Haemophilinae Buchanan of the tribe Bacterieae Trevisan emend. Buchanan. In many strains of the pleuropneumonia

\footnotetext{
*Common names have been used through Supplement No. 3 (except for Asterococcus and $A$. mycoides) as the author believes that a more suitable nomenclature than any thus far proposed should be developed when agreement is reached as to the nature of these organisms. Specific names that have been proposed are given merely as a matter of record. No new names have been introduced.
} 
group, the small forms appear in appropriate preparations as small bipolar stained bacilli. The transformation of the bacilli to round bodies of variable size often occurs in bacterial cultures and is not specific for the pleuropneumonia group. Furthermore, it has been observed in several species of bacteria that they reproduce in the large round forms in a manner similar to that observed in the pleuropneumonia group. Thus the form variation and reproductive processes observed in the pleuropneumonia group are not specific to this group. They represent general bacterial properties and should be included in the definition of the true bacteria.

According to these considerations, the organisms belonging to the pleuropneumonia group are small, Gram-negative bacilli often showing bipolar staining and their distinctive characteristic is the tendency to swell up into round forms and multiply by the reproduction of bacilli in the round forms. Their habitat is in the mucous membranes of animals and man and many of them are pathogenic. They are exacting in their media requirements and usually require fresh animal serum for their growth. These properties indicate a close similarity to the species now included in the genera Pasteurella and Haemophilus. The pleuropneumonia group might well be classified in the same or a closely related family. It is uncertain whether the strains isolated from earth and sewage should be classified with the strains isolated from animals and men. The soil and sewage strains are less soft, stain more easily and grow abundantly without animal serum. The strains isolated from bacterial cultures are most probably variant forms of the bacteria and should be classified with the parent organisms.

The viruses of psittacosis and lymphogranuloma present similarities to the pleuropneumonia group both in morphology and in their methods of reproduction. This gives added weight to the thought that the pleuropneumonia group represents an intermediary stage in the evolution of the small, Gram-negative bacteria of the mucous membranes into the filterable viruses. 


\section{THE PLELROPNEUMONIA GROUP.}

(Borrelomycetacene Turner, Jour. Path. and Bact., 41, 1935, 25; Parasitacent Sabin, Bact. Rev., 5, 1941, 58.)

The organisms are soft and fragile. Without special precautions they are often listorted or entirely destroyed in microscopical preparations. The cultures contain pleomorphic elements: Small granules, bacilli, bacillary filaments and round forms varying in size from a few tenths of a micron to 10 microns or more. Autolyzed round forms may coalesce into large empty blebs. The round forms are part of a reproductive "ycle. They are produced by the swelling of the bacillary forms and filaments and reproduce granules or filaments by inside segmentation or multiple germination. In freshly isolated bovine strains, the filaments show apparent or true branching and reproduce the small forms by segmentation. The smallest growing units may not be larger than .15 to 28 micron and pass through filters that retain bacteria. On agar, tiny colonies $(0.1$ to $0.6 \mathrm{~mm})$ develop in great numbers. The colonies invade the agar and after 2 to 5 days growth have an opaque center embedded in the agar and a thin peripheral zone. The surface has a rugged or granular appearance due to the development and autolysis of the large forms. After a few days growth, the cultures usualy show pronounced autolysis. The parasitic strains require fresh animal serum for growth. There is a single genus.

\section{Genus I. Asterococcus Borrel et al.}

(Borrel, Dujardin-Beaumetz, Jeantet and Jouan, Ann. Inst. Past., 24, 1910, 179; Coccobacillus Martzinovski, Ann. Inst. Past., 25, 1911, 91; Micromyces Frosch, Arch. f. wissensch. u. prakt. Tierheilk., 49, 1923, 35 and 273; not Micromyces Dangeard, Le Botaniste, 1, 1888, 55; Mycoplasma Nowak, Ann. Inst. Past., 43, 1929, 1349; Asteromyces Wroblewski, Ann. Inst. Past., 47, 1931, 105 ; Borrelomyces Turner, Jour. Path. and Bact., $41,1935,25$; Bovimyces Sabin, Bact. Rev.. 5, 1941, 57.)

Characters as for the family.

The type species is Asterococcus mycoides Borrel et al

1. Asterococcus mycoides Borrel et al. (Le microbe de la péripneumonia, Nocard and Roux, Ann. Inst. Past., 12, 1898, 240 ; Borrel, Dujardin-Beaumetz, Jeantet and Jouan, Ann. Inst. Past., 24, 1910, 168; Coccobacillus mycoides peripneumoniae Martzinovski, Ann. Inst. Past., 25, 1911. 914; Micromyces peripneumoniae bovis contagiosae Froseh, Arch. f. wissensch. u. prakt. Tierheilk., 49, 1923, 35 and 273; Mycoplasma peripneumoniae Nowak, Ann. Inst. Past., 43, 1929, 1530; Asteromyces peripneumoniae bovis Wroblewski, Ann. Inst. Past., 47, 1931, 94; Borrelomyces peripneumoniae Turner, Jour. Path. Bact., 41, 1935, 1; Bovimyces pleuropneumoniae Sabin, Bact. Rev., 5, 1941, 57.)

Morphology of cells and appearance of agar cultures correspond with the description given for the group.

Broth cultures are slightly opalescent and, upon shaking, the cultures of fresh strains exhibit silk-like whorls, due to the presence of long chains and filaments. The cultures after prolonged incubation consist of small granules.

Biochemical activity: Old colonies on serum agar develop a brownish color. Freshly isolated strains reduce hemoglobin. Glucose, fructose, mannose, maltose, and dextrin are fermented with the production of acid but no gas. The cultures are bile soluble.

The strains isolated from cattle are homogeneous in serological reactions and distinct from the other members of the group. 
Habitat: It is the causative agent of contagious bovine pleuropneumonia. The disease can be transferred to sheep, goats and water buffaloes, but not to mice, rats, rabbits or other experimental animals.

\section{The organism of agalactia of sheep} and goats. (Le microbe d'agalaxie contagieuse, Bridré and Donatien, Ann. Inst. Past., 39, 1925, 925; Anulomyces agalaxiae Wroblewski, Ann. Inst. Past., 47, 1931, 111; Borrelomyces agalactiae Turner, Jour. Path. and Bact., 41, 1935, 25 ; Capromyces agalactiae Sabin, Bact. Rev., 5, 1941, 57.)

These organisms are very similar to the former organisms in morphology, appearance of the cultures and growth requirements. Usually the growth is less vigorous, the colonies remain smaller, and the elements of the cultures are more delicate and less easily visible than those of hovine pleuropneumonia. Characteristic crystals develop in the cultures.

Serologically and immunologically this species is distinct from the bovine speries.

It is the cause of a systemic disease in sheep and goats with involvement of the joints, eyes, and, in lactating animals, the mammary glands. Other species are not susceptible.

\section{Pleuropneumonia-like organisms in} dogs. (Asterococcus canis, Types I and II, Schoentensack, Kitasato Arch. Exp. Med., 11, 1904, 227; 13, 1936, 175; Canomyces pulmonis I and II, Sabin, Bact. Rev., 5, 1941, 57.)

Both types produce slight uniform opalescence in broth. Type I grows in granules and coarse colonies and is apparently pathogenic for dogs. Type II grows in somewhat larger granular colonies.

They are serologically distinet from each other and from the other members of the pleuropneumonia group.
The connection of these organisms with distemper is not proven.

\section{Pleuropneumonia-like organisms in} rats. $\mathrm{L}_{3}$ (Klieneberger and Steabben, Jour. Hyg., 37, 1937, 143 ; Jour. Hyg., 40, 1940, 223; Wurimyces pulmonis Sabin, Bact. Rev., 5, 1941, 57.)

$\mathrm{L}_{4}$ (Klieneberger, Jour. Hyg., 38, 1938, 458; Murimyces arthritidis Sabin, loc. cit.)

The pyogenic virus of Woglom and Warren (Jour. Exp. Med., 68, 1938, 513) and $\mathrm{L}_{7}$ of Findlay, MacKenzie, MacCallum and Kleineberger (Lancet, 237, 1939,7 ) are identical with $\mathrm{L}_{4}$. The organisms isolated from infected joints by Beeuwkes and Collier (Jour. Inf. Dis, 70, 1942, 1) and Preston (ibid., 70, 1942, 180) probably are identical with $\mathrm{L}_{4}$ but they were not typed serologically.

The requirements for growth, the appearance of colonies and the morphology are very similar to those of the type strain with the difference that long filaments are not observed either in liquid or solid media.

The strains isolated from rats belong to two serological types. $L_{3}$ was cultivated from chronic lung abscesses, but the number of strains typed is not sufficient to ascertain that all strains isolated from this source belong to one type. The $\mathrm{L}_{3}$ strains are not pathogenic for rats in artificial infection. They produce suppuration in mice when they are injected with agar.

$\mathrm{L}_{4}$ which is serologically different from $\mathrm{L}_{3}$ was cultivated from abscesses and spontaneous polyarthritis. It produces polyarthritis both in mice and rats. It is not infectious in monkeys, rabbits and guinea pigs. Both $\mathrm{L}_{3}$ and $\mathrm{L}_{4}$ were recovered from the brains of mice kept in the same room with rats.

According to Klieneberger, $\mathrm{L}_{3}$ usually produces somewhat larger and coarser colonies than $\mathrm{L}_{4} ; \mathrm{L}_{3}$ grows in broth in small granules, while $\mathrm{I}_{4}$ produces an opalescent growth. 
5. Pleuropneumonis-like organisms in mice. (Sabin, Science, 88, 1938, 575, and Bact. Rev., 5, 1941, 1; Findlay, Klieneherger, MacCallum and Mac Kenzie, Lancet, $235,1938,1511$.) A strain isolated by Sullivan and Dienes (Proc. Soc. Exp. Biol and Med., 41, 1939, 620) is identical with Type A.

Five groups of strains, distinct serologically, and, to a certain extent, distinct also in their pathological properties, have been isolated from mice. These are types A, B, C, D, and E of Sabin. The strains are closely similar to each other and to the rat strains. It is questionable whether the slight differences in the ap pearance of the colonies and in the morphology of the cultures are of significance.

Type A (Musculomyces neurolyticus Sabin) is usually present in the conjunctiva and was isolated also from the lung and brain. Intercerebral injection of Type A produces in mice a characteristic rolling disease due to a toxin which is also present in broth cultures. Intravenous injection produces a transient polyarthritis without damage to the rartilage or ankylosis. Type $\mathrm{A}$ is serologically similar to $\mathrm{L}_{3}$ of Klieneherger, (Jour. Hyg., 40 , 1940, 204).

Type B (Musculomyces arthrotropicus Sabin) was isolated from the brain and from the nasal mucosa. It produces no rolling disease and no soluble toxin. In mice, intravenous injection usually produces a chronic arthritis often leading to ankylosis.

Types (, D and L (Musculomyces histotropicus Sabin) were isolated from the same location as Type B and produce similar arthritic lesions. They are serologically distinct from Type $B$ and from each other (Sabin, Science, 90, 1939, 18 and Sabin and Johnson, Proe. Soc. Exp. Biol. and Med., 44, 1940, 569).

$\mathrm{L}_{6}$ isolated from mice by Findlay et al (Trans. Roy. Soc. Trop. Med. Hyg., 33 1939-40,6) and the strains of Edward (Jour. Path. and Bact., 50, 1940, 409) were not compared serologically with the types of Sabin.

\section{Pleuropneumonia-like organisms in} man. (Dienes, Proc. Soc. Fxp. Biol. and Med., 44, 1940, 468; Beveridge, Med. Jour. of Australia, $30,1943,479$; Kleineberger, Lancet, 2, 1945, 46.)

They are present in about 30 per cent of women in the genitals and they were isolated from suppurative processes originating from this source. In men they were found in urethritis, rystitis and chronic prostatitis.

The appearance of the colony, the morphology and growth requirements correspond with the animal strains. The human strains grow less abundantly in serum broth than the animal strains.

One strain was found by Sabin (Proc. Soe. Exp. Biol. and Med., 44, 1940, 569) to be serologically different from the strains isolated from rats and mice. It is not known whether the strains are serologically uniform. There is a slight variation in colony form, in the tendency to grow in filaments, and in the abundance of growth, but the variation hetween the strains is less than the variation due to slightly different cultural conditions.

Mice and rats are usually not susceptible to infection with the human strains; however, several young mice from a single litter were killed in three to six days by subcutaneous or int raperitoneal injection of one strain.

7. Pleuropneumonia-like organisms in chick embryos. (Van Herick and Eaton, Jour. Baet., 50, 1945, 47.)

Organisms have been isolated from rhick embryos which conform to the pleuropneumonia group with regard to morphology, the appearance of colonies on agar and filterability. The cultures aggitutinated red blood cells from various animals. The relation of this strain to the coccobacillary bodies of Nelson (see Section II) has not been studied. 


\section{ORGANISMS OF UNCERTAIN CLASSIFICATION.}

\section{Similar to the Pleuropneumonia Group.}

1. Coccobacillary bodies of Nelson. (Nelson, Science, 82, 1935, 43; Jour. Exp. Med., 65, 1937, 833; Jour. Exp. Med., 72, 1940, 645.)

Nelson isolated a small bacillary organism apparently connected with coryza and infectious catarrh from the nasal passages of fowls and from the nasal passages and the middle ear of mice and rats. Their size appeared to be 0.3 to 0.4 micron in microscopical preparations and they passed through a filter with a pore size of 640 millimicrons. They were isolated in tissue cultures but they grow also in the cell-free and heated supernatant fluid. The freshly isolated cultures did not grow on blood or on artificial media; however, after 120 passages in tissue cultures the fowl coryza bodies grew on blood agar slants. On ascitic agar this strain forms colonies very similar to those of the pleuropneumonia group with a dark center surrounded by a thin periphery. The organisms in the top layer are sometimes considerably enlarged, but no web-like structure is produced. The organism is less soft and the individual organisms maintained their form in the preparations as do bacteria, and the tendency to grow into the agar is less pronounced than in the pleuropneumonia group. The organism isolated from rats is more pleomorphic than the others.

The coccobacillary bodies were not studied with methods appropriate to determine whether they belong to the pleu ropneumonia group and whether the mouse and rat strains are identical with the pleuropneumonia-like organisms isolated from mice and rats.

\section{Filterable organisms from sewage} and soil. (Fam. Saprophytaceae Sabin, Genus Saprom!ces Sabin, Bact. Rev., 5, 19+1, 59.)

The strains isolated by Laidlaw and Elford (Proe. Roy. Soc. London, B, 120, 1936, 292; Sapromyces laidlawi $A B$ and $C$, Sabin, loc. cit.) and Seiffert (Cent. f. Bakt., I Abt., Orig., 139, 1937, 337) according to Ørskov (Cent. f. Bakt., I Abt., Orig., 141, 1938, 230) and Klieneberger (Jour. Hyg., 40, 1940, 204) are closely similar to the organisms of the pleuropneumonia group. They are filterable, and the smallest reproductive units of those which we have appropriately examined were found to be between .125 and .175 micron. The colonies are similar in appearance to the colonies of the pleuropneumonia group.

The broth cultures consist of granules and round globular elements; the surface layer of agar colonies sometimes swells up into large round forms. They grow without serum, but small amounts of serum accelerate the growth. They grow both at $30^{\circ}$ and at $37^{\circ} \mathrm{C}$. and remain alive in cultures kept cold for several months. The broth cultures grow abundantly with a strong opalescence or sediment. Serologically the strains are distinct from the other members of the pleuropneumonia group and all but one are more or less similar to each other.

\section{PIEUROPNEUMONIA-LIKE ORGANISMS ISOLATED FRON BACTERIAL CULTURES.}

1. Pleuropneumonia-like organisms isolated from Streptobacillus moniliformis. $\mathrm{L}_{1}$ (Klieneberger, Jour. Path. and Bact., 40, 1930, 93; Jour. Hyg., 42, 1942, 485; Dienes, Jour. Inf. Dis., 65, 1939, 24; Jour. Bact., 44, 1942, 37; Mus- culomyces streptobacilli-moniliformis Sabin, Bact. Rev., 5, 1941, 57; Heilman, Jour. Inf. Dis., 69, 1941, 32; Brown and Nunemaker, Bull. Johns Hopkins Hosp., $70,1942,201$.)

(ultures isolated from different strains 
of Streptobacillus moniliformis vary considerably in the appearance of the colonies, the tendency to reversion to bacillary form, and the degree of autoly sis. The colonies are considerably larger than the colonies of the human or animal pleuropneumonia-like strains; they may reach 1 to $2 \mathrm{~mm}$. Usually a wide peripheral zone is present and development and autolysis of the large bodies produces a coarse appearance in the colonies. Sometimes no peripheral zone develops, the colony is dome-shaped, and the large bodies have no tendency to autolyze. The young colonies (twelve hours incubation) grow into the agar as loose strands of more or less swollen granules. Serum broth cultures grow in small clumps usually adhering to the wall of the test tube.

The cultures consist of small granules, small polar-staining bacilli and diphtheroid-like forms which swell to large round forms. In the top layer of fully developed colonies, the well-stained large bodies may be as large as 10 to 20 microns. By vacuolization they transform into empty blebs. By segmentation of their contents, the large forms may reproduce the small bacillary forms. In suitable preparations chromatin bodies are visible both in the small and large forms. The small forms are filterable through Berkfeld candles; the size of the smallest particles has not been exactly determined. The organism is very soft and fragile.

Their growth requirements and biochemical activities are similar to those of Streptobacillus moniliformis.

Frowth occurs on nutrient agar conlaining animal serum or egg yolk. Sometimes there is a slight growth on boiled blood agar plates without serum. Good growth is obtained in a mineral solution with 0.1 per cent starch. Growth is both aerobic and anaerobic.

The $\mathrm{L}_{1}$ form is more resistant to heat and to aging of the culture than is the streptobacillus and it has a remarkable resistance to penicillin to which the bac- teria are very sensitive. Like the bacillus, $\mathrm{L}_{1}$ produces acid but no gas from? glucose, maltose, fructose, salicin, starch, and dextrin. It gives no oxidase test.

Serologically the $\mathrm{L}_{1}$ form is similar to Streptobacillus moniliformis and different from the members of the pleuropneumonia group. It has no pathological effect on mice, rats or guinea pigs. It does not produce an infection of the chicken embryo. It can be isolated from freshly isolated strains of Streptobacillus moniliformis, from several-day old broth and agar cultures, from broth cultures heated at $56^{\circ} \mathrm{C}$. and usually also from 48 hour agar cultures if they are incubated at $28^{\circ}$ to $30^{\circ} \mathrm{C}$. It is questionable whether the $\mathrm{L}_{1}$ form has been isolated directly from rats.

Kilieneberger (Jour. Hyg., 40, 1940, 204) isolated a similar strain from a bacterium similar to Streptobacillus moniliformis which caused abscesses in guinea pigs. Whether this bacterium was identical or different from Streptobacillus moniliformis was not determined.

\section{Pleuropneumonia-like organisms} isolated from Bacteroides funduliformis. Dienes (Proc. Soc. Exp. Biol. and Med., 47, 1941, 385) and Dienes and Smith (Jour. Bact., 48, 1944, 125) isolated cultures from two strains of Bacteroides funduliformis which could be propagated indefinitely and which in morphology and in the appearance of colonies were closely similar to $\mathrm{L}_{1}$.

The young colonies consisted of similar strands of granules growing into the medium. The surface of fully developed colonies consisted of large bodies and a honey-comb-like structure. The well isolated colonies grew usually to a fairly large size (1 to $2 \mathrm{~mm}$ ).

Both strains, transplanted every two or three days through several months, failed to reproduce bacteria either on agar or submerged in broth.

No growth was obtained in liquid cultures. 
The strains, like the parent organism, are strictly anaerobic and the cultures have the characteristic odor of the parent strain.

It was observed in slide cultures that the $\mathrm{L}$ type of colonies develop from large round forms which were produced in the cultures of the parent organism by gradual swelling of the bacteria.

In cultures of eight pleomorphic st rains of Bacteroides, the I type of colonies developed in three strains under appropriate conditions. The bacteria swelled into large round bodies in all eight strains. The serological properties of the L strains have not thus far been studied. Teither the parent organisms nor the $\mathbf{L}$ type strains had any pathological effect on laboratory animals.

\section{Pleuropneumonia-like organisms in} a species of Flavobacterium. (Dienes, Jour. Bact., 44, 1942, 37.)

Tiny colonies entirely similar in appearance to young $\mathrm{L}_{1}$ colonies were isolated from the cultures of a species of Flavobacterium.

The bacterium when freshly isolated produced two types of colonies on blood agar plate; large colonies consisting of small regular bacilli and tiny colonies in which the bacteria became pleomorphic and swelled up to form large round bodies. The tiny colonies after 48 hours of incubation became autolyzed, and one or several L type of colonies started to grow under them. These colonies could be transplanted and gave abundant growth for two generations, but always died out in the third.

Bacterial forms were not reproduced pither on agar or in broth.

The $L$ type of growth was not pathogenic for mice though the parent organism was highly virulent.

4. Development of tiny colonies in other bacteria.

The development of tiny colonies similar in appearance to young colonies of the pleuropneumonia group has been observed in cultures of Escherichia coli, Haemophilus infuenzae, and Neisseria gonorrhoea (Dienes, Jour. Bact.. 44, 1942, 37; Proc. Soc. Exp. Biol. and Med., 44 , $1940,476)$. In all cases preceding their development, the organisms of the parent strains swelled into large round bodies, and in Escherichia coli and Haemophilus influenzae the development of the L type of colonies from these large forms was observed. Thus far these tiny colonies have not been isolated in pure cultures. 


\section{INDEX* \\ SOURCES AND HABITATS}

(All references to viruses will be found under the heading †Viruses)

Abnormal Milk, see Dairy Products

\section{Agar}

Bacillus, 731

Containing iodiform

Micrococcus, 272

Digesting bacteria

Acetobacter, 692

Achromabacter, 628

Agarbacterium, 628, 629, 630

Bacterium, 625,626

Flavobacterium, 631

Pseudomonas, $177,178,697,698,700$

Vibrio, 200, 203, 204, 702, 703

Air, also see Dust

Actinomyces, 968, 969, 970, 972, 973

Bacillus, 647, 648, 649, 650, 651, 653, $654,655,658,659,661,663,664,665$, $667,668,669,670,671,672,738,741$, $742,743,744,747,748,749,750,751$, $752,754,756,758$

Bacterium, 602, 643, 672, 674, 676, 678. $680,684,761$

Chromobacterium, 232, 233

Clostridium, 803

Corynebacterium, 386

Flavobacterium, 611

Gaffkya, 283

Leuconostoc, 348

Micracoccus, 237, 239, 244, 251, 252, $253,255,256,260,261,268,271,272$,

$273,276,278,281$

Nocardia, 915,975

Pacinia, 696

Planococcus, 281

Pseudomonas, 95, 96, 147,149,174

Rhodococcus, 281

Sarcina, 288, 290

Staphylococcus, 28:

Streptococcus, 336, 338, 340
Streptomyces, 935,969

Streptothrix, 975

Air

-brewery

Sarcina

- contamination

Nocardia, 915

Planococcus, 281

Pseudomonas, 174

Staphylococcus, 282

Contamination on cooked potato

Chromobacterium, 232

Amphibia, Common Names

African toads

Vibrio, 197

Frogs

Bartonella, 1108

Eberthella, 534

Flavobacterium, 439

Malleomyces, 556

Micrococcus, 281

Mycobacterium, 883, 884, 885, 890

Pseudomonas, 102, 103

Salmonella, 532

—, abscesses

Micrococcus, 281

-, feces

Bacillus, 742

-, intestine

Chitin-digesting bacteria, 6:3:2

Spirochaeta, 1065, 1069, 1070

-, large intestine

Bacillus, 742, 754

Spirillum, 217

--, wound infections

Eberthella, 534

Salamander

Pseudomonas, 102

* Prepared by Prof. Robert S. Breed and Mrs. Margaret E. Breed, Geneva, New York, August, 1947.

† Prepared by Frances O. Holmes, Rockefeller Institute for Medical Research, Princeton, New Jersey, July, 1947. 
Amphibia (continued)

Toads

Arthromitis, 1003

Bacillus, 744, 754

Miciococcus, 281

Serratia, 462

Vibrio, 197

-, abscesses

Micrococcus, 281

-, intestine

Spirochaeta, 1066

-, large intestine

Spirillum, 217

Treponema, 1075

-., rectum

Bacterium, 760

-, tadpoles

Bacillus, $7+2$

Tree toads

Spirochaeta, 1066

Amphibia, Diseases of

Frogs

Pseudotuberculosis Malleomyces, 556

Tuberculosis

Mycobacterium, 883, 884, 885, 890

Red leg

Pseudomonas, 102, 103

Amphibia, Scientific Names

Alytes sp., $7+2$

Bufo americanus, 462, 742, 754, 760 . 1066,1075

Hyla septentrionalis, 1066

Leptodactylus ocellatus, 1108

Leptodactylus pentadactylus, 1113

Rana pipiens, 462

Rana tamporatia, 742, 754, 1065, 1069, 1070

Xenopus laevis, 439

Animal Diseases (Vertebrate), also see Amphibia, Birds, Fishes, Reptiles, and Mammals

Vertebrates

Cold-blooded

Mycobacterium, 887

Warm-blooded

Neisseria, 300

Pasteurella, 548, 519

\section{Animal Products (Vertebrate)}

Catgut for sutures

Bacillus, 815

Gelatin

Colony on old plate Micrococcus, 281

Containing iodoform Micrococcus, 272

Spoiled

$$
\text { Bacillus, } 756
$$

(ilue

Black discoloration

Chromobacterium, 233

Hides, salted

Pseudomonas, 110

Sarcina, 289

Animal Sources and Diseases (Invertebrate) also see Arthropoda, Insecta, Mollusca, and Protozoa

Animal Sources (Invertebrate), Common Names

Annelid, Marine

C'ristispira, 1057

Blood suckers

Bacillus, 746

Bacterium, 679

Echinoderm

Cristispira, 1056

Leerhes

Bacillus, 746

Bacterium, 679

Tunicate

Spirochaeta, 1066

Animal Sources (Invertebrate), Scientific Names

Asterias rubens, 1056

Caesira retortiformis, 1066

Hirudo spp., 679, 746

Polydora flava, 1057

Animal Sources (Vertebrate), also see Amphibia, Birds, Fishes, Reptiles, and Mammals

Vertebrates, Intestine

Aerobacter, 455

Escherichia, 447, 449, 450

Paracolobactrum, 460

Vertebrates, Warm-blooded

Salmonella, 503, 510, 517, 528

Vibrio, 196 
Arthropoda, Common Names

Arachnids

Rickettsia, 1098, 1099

Crustacea

Crayfish, intestine

Chitin-digesting bacteria, 632

Fresh water

Bacterium, 678

Pasteuria, 836

Horseshoe crab, shell

Bacterium, 632

Marine

Bacterium, 635

Small crustacea

Eubacterium, 367

Pasteuria, 836

Myriapoda

Diplopods

Fusiformis, 694

Millipeds, intestine

Arthromitis, 1003

Mites

Rickettsia, 1098

Haemobartonella, 1105

Bird mites

Rickettsia, 1091

Human mites

Rickettsia, 1091, 1092

Rodent mites

Rickettsia, 1091, 1092

Spiders

Rickettsia, 1098

Ticks

Borrelia, 1059

Haemobartonella, 1105

Klebsiella, 459

Rickettsia, 1097, 1098, 10!9?

Spirachaeta, 1068

Bont ticks,

Richettsia, 1089, 1094

Dog ticks, 1088, 1096, 1098

Rickettsia, 1088, 1089, 1096, 1098

Rabbit ticks

Rickettsia, 1088

Sheep ticks

Rickettsia, 1097

Wood ticks

Rickettsia, 1088, 1093, 1096

Arthropoda, Scientific Names

('rustacea

Daphria sp., 836
Gammarus zschokkei, 678

Limulus polyphemus, 632

Talorchestia sp., 111

Millipeds

Julus marginatus, 1003

Mites

Allodermanyssus sanguineus, 1092

Trombicula akamushi, 1091

Trombicula deliencis, 1091

Trombicula fletcheri, 1091

Trombicula walchi, 1091

Ticks

Amblyomma americanum, 1088, 1093, 1098

Amblyomma brasiliensis, 1088

Amblyomma cajennense, 1088

Amblyomma hebracum, 1089, 1094

Amblyomma maculatum, 1098

Amblyomma spp., 1088

Amblyomma striatum, 1088

Amblyomma variegatum, 1094

Boöphilus decoloratus, 1089

Dermacentor albipictus, 459

Dermacentor andersoni, 1088, 1093. 1096,1098

Dermacentor occidentalis, 1093

Dermacentor spp., 1088

Dermacentor variabilis, 1088

Haemophysalis humerosa, 1093

Haemophysalis leachi, 1089

Haemophysalis leporis-palustris, 1088,1093

Haemophysalis spp., 1088

Hyalomma spp., 1095

Ixodes dentatus, 1088, 1093

Melophagus ovinus, 1068, 1097

Ornithodoros erraticus, 1066

Ornithodor os hermsi, 1064

Ornithodoros latiorensis, 1069

Ornithodoros marocanus, 1067

Ornithodoros moubata, 1060

Ornithodoros normandi, 1068

O) nithodoros parkeri, 1064

Ornithodoros rudis, 1064

Ornithodoros spp., 1063, 1088

Ornithodoros tholozani, 106 !

Ornithodoros turicala, $106+$

Ornithodoros venezuelensis, 1064

Rhipicephalus appendiculatus, 1089

Rhipicephalus bursa, 1097

Rhipicephalus decoloratus, 1062 
Arthropoda (continued)

Ticks (continued)

Rhipicephalus sunguineus, 1089, 1096, 1098

Rhipicephalus spp., 1088

Arthropod Vectors, see Arthropoda, and Insecta

\section{Bacterial Cultures}

Pleuropneumonia-like organisms, isolated from

Bacteroides funduliformis, 1295

Flavobacterium sp., 1296

Streptobacillus moniliformis, 1294

\section{Beer}

Acetobacter, 183, 184, 185, 186, 188, 189

Bacterium, 680

Flavobacterium, 141

Pediococcus, 249, 250

Pseudomonas, 94, 176

Bottled

Micrococcus, 260

Double

Acetobacter, 183

Ginger

Bacterium, 362

Ropy

Acetobacter, 188, 18!

Streptococcus, 250

Sarcina-sick

Pediococcus, 249

Spoiled

Achromobacter, 423

Lactobacillus, 360

Pediococcus, 249, 250)

Streptococcus, 345

Beermash

Spoiled

Pediococcus, 249, 250, 260

\section{Beer Wort}

Acetobacter, 183, 185, 186

Bacillus, 758

Inflabilis, 823

Lactobacterium, 363, 364

Pseudomonas, $1+6$

Birds, common names

Bullfinch

Rickettsia, 1095
Canaries

Bacillus, 530

Pasteurella, 554

Shigella, 540

Chaffinch, stomach and intestine Micrococcus, 260, 266

Coot, stomach

Micrococcus, 260, 263

Crow, stomach contents Micrococcus, 269

Cuckoo, throat

Corynetacterium, 403

Dove, intestine

Bacillus, 666

Bacterium, 760, 761, 762

Micrococcus, 252, 254, 270, 271, 274, 276

Sarcina, 292

Dove, stomach

Bacterium, 760

Micrococcus, 252, 25t, 270, 271, 27t, 276

Duck

Borrelia, 1059

Pasteurella, 552, 554

Pfeifferella, 554

Salmonella, 505, 518, 523, 527

Duck, skin

Corynetacterium, 406

Finch, intestine

Micrococcus, 257, 259

-, stomach

Bacterium, 761

Micrococcus, 251, 259

Flicker, feces

Clostridium, 795

General

Salmonella, 504, 521, 526, 527

-, caecum

Treponema, 1075, 1076

-, intestine

Bacillus, 648, 651, 652, 653, 655, 658, $660,662,664,666,669,671,746,757$

Bacterium, 675, 676, 683, 685, 687, $688,759,760,761,762$

Micrococcus, 269

Pseudomonas, 147, 149

Vibrio, 196

-, not pathogenic for

Piscine tuberculosis

Mycobacterium 88:3 
Birds (continued)

Turtle tuberculosis

Mycobacterium, 886

-, stomach

Bacitlus, 647, 648, 652, 653, 655, 658, $660,662,664,666,667,669,671$,

$746,749,754,757$

Bacterium, 686, 759, 760

Micrococcus, 269

Pseudomonas, 146, 147

(irouse

Salmonella, 521

—, blood and intestine

Spirochaeta, 1067

Guinea fowl

Clostridium, 796

Hedge sparrow, stomach

Micrococcus, 252, 270, 273

Parrots

Miyagawanella, 1117

Salmonella, 532

-, nasal secretions

Miyagawanella, 1117

Partridge

Salmonella, 525

Pheasant

Bacterium, 552

Clostridium, 746

Miyagawanella, 1117

Salmonella, 521

Pigeons, diseased

Bacillus, 400, 652

Bacterium, 401

Erysipelothrix, 411

Haemobartonella, 1104

Mycobacterium, 881

Salmonella, 503, 5.32

Poultry

Salmonella, 502, 505, 507, 509, 510, $511,512,513,514,516,518,519$, $521,522,523,525,526,528,529,530$

-, caecum

Treponema, 1075

-, erythrocytes

Grahamella, 1110

- intestine

Sarcina, 292

-, nasal passages, 1294

-, not pathogenic for

Bovine tuberculosis

Mycobacterium, 879
-, human tuberculosis

Mycobacterium, 878

-, spleen

Mycobacterium, 890

-, throat

Corynebacterium, 403

Quail

Salmonella, 510, 521

Robin, feces

Bacillus, 756

Rock dove, stomach and intestine

Micrococcus, 252, 270, 276

Sarcina, 293

Snipe, intestine

Chitin-digesting bacteria, 632

Sparrow, intestine

Micrococcus, 270, 273, 291, 293

一, stomach

Bacterium, 761

Micrococcus, 291, 293

Starling, intestine

Micrococcus, 257, 263, 272, 273

一, stomach

Hicrococcus, 257, 263, 272, 273

Sarcina, 291

Teal, caecum

Treponema, 1076

Turkey

Salmonella, 510, 512, 513, 514, 51?, $524,525,527,528$

Turkey poults

Lactobacillus, 407

Salmonella, 504, 505, 514, 515, 523, $527,528,529$

Woodpecker, intestine

Micrococcus, 251, 260, 272

Sarcina, 290

Yellow hammer, intestine

Micrococcus, 259, 263, 265, 272

Sarcina, 292

- - stomach

-Micrococcus, 259, 263, 265, 272

\section{Birds, Diseases of}

Birds of prey

Bovine tuberculosis

Mycobacterium, 879

Canaries

Infectious necrosis

Pastenrella, 554 


\section{Birds, Diseases of (continued)}

Canaries (continued)

Intestinal catarrh and liver changes Bacillus, 530

Septicemia Shigella, 540

Chicken, see Poultry

Chicken embryos

Pleuropneumonia-like disease, 1293

Cockatoo

Bovine tuberculosis Mycobacterium, 879

Crossbills

Infectious disease

Bacillus, 661

Dove

Ornithosis

Miyagawanella, 1117

Ducklings

Keel

Salmonella, 523

Ducks

Septicemia

Pasteurella, 552

Pfeifferella, 55t

Finches

Ornithosis

Miyagawanella, 1117

Fulmar petrels

Ornithosis

Miyagawanella, 1117

Gieese

Septicemia

Shigella, 543

Spirochaetosis

Borrelia, 105?

Gieneral

Avian tuberculosis

Mycobacterium, 881

Cholera-like disease

Vibrio, 196

Diphtheria

Bacillus, 400

Diphtheria-like disease

Actinomyces, 915

Hemorrhagic septicemia

Pasteurella, 549

Pleuropneumonia-like disease, 1293

Cirouse

$$
\text { Bacillus, } 668
$$

Parrot
Bovine tuberculosis

Mycobacterium, 879

Human tuberculosis Mycobacterium, 878

Nodules, kidney, lung, spleen Streptococcus, $3+2$

Parrot fever Miyagawanella, 1117

Psittacosis Miyagawanella, 1117

Pheasants

Epidemic in Bacterium, 552

Ornithosis Miyagawanella, 1117

Pigeon

Avian tuberculosis Mycobacterium, 881

Diphtheria

Bacillus, 400

Bacterium, 401

Poultry

Abscesses, epidemic Sphaerophorus, 579

Chicken cholera, blood Micrococcus, 262

('hicken cholera-like disease Protelus, 48?

Conjunetivitis, acute Colesiota, 1120

Coryza

Coccobarillary bodies of Nelson, 1294

Diphtheria

Bacterium, 67t

Corynebacterium, 38.5

Fowl cholera

Bacterium, $6+2$

Salmonella, 521

Fowl typhoid

Proteus, 489

Salmonella, 521

Hemorrhagic septicemia Pasteurella, 547, 54?

Keratitis Colesiota, 1120

Lesions, tuberculous M ycobacterium, 880

Limbernerk

('lostridium, 780) 
Birds, Diseases of (continued)

Listeriosis

Listeria, 409

Ocular roup

Colesiota, 1120

Ornithosis

Miyagawanella, 1117

Rhinitis, infectious

Roup

Hemophilus, 589

Bacterium, 674

Septicemia

Bacterium, 642

slipped tendon

Streptococcus, 317

Spirochaetosis

Spirochaeta, 1059

Tuberculosis, avian

Mycobacterium, 879, 880

Tumors in abdominal cavity Actinomyces, 918

White diarrhoea

Salmonella, 521

Swan

Cholera

Bacillus, 551

Infectious disease

Bacterium, 642

Turkeys

Pneumocarditis Bacillus, 662

Wild pigeons

Epidemic in

Bacterium, 552

\section{Bird's Nest}

Myxococcus, 1042

Birds, Scientific Names

Colaptes auratus, 795

Columba livia, 252, 270, 276, 293

Columba oenas 252, 254, 270, 271, 274, $276,292,760,761$

Corvus corone, 269

Emberiza citrinella, 259, 263, 265, 272,292

Fringella carduelis, 257, 259

Fringella coelebs, 260, 266

Fulica atia, 260, 263

Gallus sp., 1075

Lagopus scoticus, 668, 1067

Larus ridibundus, 1075

Passer montanus, 252, 270, 273, 291, 293
Picus major, 251, 260, 272, 290

Pyrrhula curopea, 1095

Querquedula querquedula, 1076

Squatarola squatarola, 1076

Sturnus vulgaris, 257, 263, 272, 273, 291

\section{Blue Milk, see Dairy Products, Abnormal} Milk

\section{Bovine Diseases and Sources, see Cattle}

\section{Butter}

Actinomyces, 974

Aerobacler, 456

Bacillus, 743

Bacterium, 590, 602, 676

('hromobacterium, 234

Lactobacillus, 357

Lenconostoc, 348

Microbacterium, 371

Hicrococcus, 260, 264, 265, 266, 279

Mycobacterium, 889, 890

Vocardia, 905

Psendomonas, 146

Sareina, 291

Streptococcus, $325,337,339$

Black to reddish brown discoloration Pseudomonas, 109

Fruity aroma

Micrococeus, 260

May apple to strawberry odor

Pseudomonas, 100, 101

Rancid

Bacillus, 650, 659, 660, 814, 816

Micrococcus, 254

Skunk odor

Pseudomonas, 99

Tainted

Pseudomonas, 99, 100, 101, 109

Buttermilk

Bacillus, 644

Propionibacterium, 374, 375, 377

Serratia, 484

Abnormal

Clostridium, 822

Calf Diseases and Sources, see Cattle

Cats

Lungs

Miyagawanella, 1118 
Cats (continued)

Not pathogenic for, Avian tuberculosis

Mycobacterium, 880

Human tuberculosis Mycobacterium, 878

Respiratory tract, upper Hemophilus, 586

Sputum

Pasteurella, 553

Stomach

Spirillum, 218

Spirochaeta, 1069

\section{Cats, Diseases of}

Actinomycosis

Actinomyces, 927, 969

Bovine tuberculosis

Mycobacterium, 879

Diarrhoea

Salmonella, 511

Diphtheria in kittens

Corynebacterium, 385

Distemper

Miyagawanella, 1118

Glanders

Malleomyces, 555

Hemorrhagic septicemia

$$
\text { Pasteurella, } 549
$$

Infections

Bacillus, 655

Streptococcus, 339

Influenza

Miyagawanella, 1118

Mumps

Spirochaela, 1074

Nasal catarrh

Miyagawanella, 1118

Pneumonia

Brucella, 563

Pneumonitis

Miyagawanella, 1118

Pseudotubereulosis

Pasteurella, 550

\section{Cattle}

Blood

Borrelia, 1062

Clostridium, 777

Micrococcus, 254
Calf vaccine lymph

Bacillus, 401

Corynebacterium, 402, 406

Corneal or conjunctival discharges

Colesiola, 1120

Ery throcytes

Anaplasma, 1100

Eperythrozoon, 1112

Haemobartonella, 1106

Foetus

Streptococcus, 317

Ganglia

Actinomyces, 927

Gastric mucosa

Leptospira, 1078

General

Salmonella, 502, 506, 509, 513, 517, $518,521,527,529$

Genital organs

Brucella, 561

Glands

Actinomyces, 927

Intestine

Bacillus, 745, 818

Clostridium, 821

Microbacterium, 370

Ristella, 576

Streptococcus, 322,327

Liver

Actinomyces, 926

Bacillus, 612

Recordillus, 823

Lungs

Bacillus, 612

Micrococcus, 254

Lymph glands

Mycobacterium, 890

Mouth cavity

Actinomyces, 926

Streptococcus, 322

Mucosa, intestinal

Mycobacterium, 881

Red blood cells, see Erythrocytes

Rumen

Flavobacterium, 613

Clostridium, 820

Saliva

Streptococcus, 322

Salted hides, red

Pseudomonas, 110

Sarcina, 289 
Cattle (continued)

Skin

Bacillus, 755

Corynebacterium, 402

Mycobacterium, 890

Streptococcus, 335

Spleen

Bacterium, 759

Stomach

Plectridium, 823

Stomach, third

Micrococcus, 272

Throat

Streplococcus, 322

Udder

Actinomyces, 926

Bacillus, 672

Chlorobacterium, 693

Corynebacterium, 390, 401

Mammococcus, 695

Micrococcus, 241, 265

Streptococcus, 316, 320, 325, 327, 334, 335,343

Vaccine lymph, see Calf vaceine lymph

Vagina

Streptococcus, 335

\section{Cattle, Diseases of}

Abortion

Salmonella, 506, 507

Vibrio, 202

Abortion, infectious

Brucella, 561, 562

Abscesses

Actinobacillus, 557

Bacillus, 652, 666

Corynebacterium, 388

Staphylococcus, 701

Abscesses, multiple sclerotic.

Sphaerophorus, 579

Actinobacillosis

Actinobacillus, 557

Actinomycosis

Actinobacillus, 557,558

Actinomyces, 925, 926, 927, 970

Acute ophthalmia

Moraxella, 592

Anaplasmosis

Anaplasma, 1100

Anthrax

Bacillus, 720
Blackleg

Clostridium, 776

Black quarter

Clostridium, 776

Bronchopneumonia

Bacillus, 650

Bulbar paralysis

Clostridium, 779

Chronic diarrhoea

Mycobacterium, 881

Chronic pneumonia Actinobacillus, 558

Contagious pleuropneumonir Asterococcus, 1292

Cow pox pustules

Streptococcus, 345

Diarrhoea in calves Vibrio, 204

Diphtheria Sphaerophorus, 579

Disease in calves Bacillus, 401

Dysentery, calf Bacterium, 689

Endocarditis Nocardia, 922

Eperythrozoonosis Eperythrozoon, 1111

Farey

Nocardia, 896

Foot and mouth disease

Bacterium, 673

Streptococcus, 337

Haemobartonellosis

Haemobartonella, $110^{\circ}$

Heartwater

Cowdria, 1094, 1097

Hematuria

Streptococcus, 344

Hemorrhagic septicemia

Pasteurella, 547, 548, 549

Icterohemoglobinuria

Clostridium, 777

Infections

Corynebacterium, 391

Nocardia, 901

Infectious disease

Bacterium, 675

Johne's Disease

Mycobacterium, 881 
Cattle, Diseases of (continued)

Keratitis

Colesiota, 1119

Moraxella, 592

Lamziekte

Clostridium, 779

Lesions, tuberculous Mycobacterium, 879

Light febrile disease Rickettsia, 1095

Listeriosis Listeria, 409

I.umpy jaw Actinomyces, 926

Mastitis

Bacillus, 662

Chlorobacterium, 693

Corynebacterium, 401

Galactococcus, 250

Tetracoccus, 284

Streptococcus, $320,3+1$

Metritis

Streptococcus, 317

Necrotic foei in liver

S'phaerophorus, 579

Ophthalmia, infectious

Colesiola, 1120

Peripneumonia

Pneumococcus, 697

Pink eye

Moraxella, 592

Pleuropneumonia Ascococcus, 1291

Streplococcus, 345

Pneumonia

$$
\text { Pasteurella, } 5+9
$$

Pneumonia, contagious

Bacillus, 664

Purulent infections, urinary tract

Corynebacterium, 389

Pus

Corynebacterium, 388, 405)

Micrococcus, 264

Streptococcus, 343

Pyelonephritis

Corynebacterium, 38 ?

Rauschbrand

Clostridium, 820, 825

Relapsing fever

Borrelia, 1062
Septicemia

Salmonella, 514

Streptococcus, 317

Streptotrichosis, skin

Actinomyces, 968

Suppurative lesions

Staphylococcus, 282

Symptomatic anthrax

Clostridium, 776, 822

Tuberculosis, bovine

Bacillus, 652

Mycobacterium, 877, 879

Ulcerations, mouth region, calves

Bacillus, 747

Ulcerative lesions

Corynebacterium, 389

White diarrhoea of calves

Streptococcus, 345

\section{Cellulose Digesting Bacteria}

Bacillus, 737, 743, 746, 756, 814

Bacterium, 614, 615, 633

Cellulobacillus, 762

Cellulomonas, 617, 618, 619, 620, 621 622,623

Cellvibrio, 210

General, 1006

Itersonia, 1044

Micrococcus, 259

Psendomonas, 145, 147, 148

Spirochaeta, 1053

Sporocytophaga, 1049, 1050

Vibrio, 203, 204, 205, 206, 207, 703

\section{Cheese}

Aerobacter, $456,45 \overline{1}$

Bacillns, 612, 647, 669, 712, 734, 743, $745,750,814,815,816,824,825$

Bacterium, 676, 677, 678, 679, 681, $683,686,689,758,760,761,762$, 819

Clostridium, $770,810,820,822,824$

Escherichia, 452

Gianulobacillus, 826

Lactobacillus, 351, 352, 356, 357, 359

Microbacterium, 370, 371

Micrococcus, 241, 243, 251, 256, 258, $264,265,266,269,270,277,279$, 281

Propionibacterium, 373, 374, 375 , $376,377,378,379$

Sarcina, 290, 291, 292, 293

Streptococcus, 325, 326, 340, 344 
Cheese (continued)

Tetracoccus, 284

Tyrothrix, 823

Vibrio, 197

Bitter

Streptococcus, 323

Black Discoloration

Bacterium, 678

Gassy curd

Bacillus, 672

Pasteurized

Actinomyces, 968

Red

Plocamobacterium, 691

Rusty spot

Lactobacillus, 357, 359

Cheese, Types of

Blue

Bacterium, 602

Brick

Bacterium,. 602, 761

Brie

Streptococcus, 341

Camembert

Bacterium, 602

Micrococcus, 260, 263, 265

Sarcina, 293

Tetracoccus, 284

Cantal

Streptococeus, 337

Cheddar

Bacterium, 602

Psendomonas. 697

Cream

Bacillus, 664

Streptococcus, 340

Edam

Chromobacterium, 233

Propionibacterium, 378

Emmental, see Swiss

Gouda

Bacillus, 663

Streptococcus, 342

Grana

Bacillus, 756

Limburger

Bacillus, 612

Bacterium, 602

Micrococcus, 261, 264

Liptauer

Bacillus, 816, 826
Neuchatel

Streptococcus, 342

Oka

Bacterium, 602

Parmesan, see Grana

Bacillus, 737, 756

Richelieu

Micrococcus, 695

Schlosskäse

Streptococcus, 342

Soft

Bacillus, $6+9$

Salmonella, 529

Swiss

Bacillus, 648, 657, 737, 739, 752

Bacterium, 688, 759, 760

Micrococeus, 255, 256, 257, 259, 261, $263,264,269,270,273,275,277$, 281

Propionibacterium, 373, 374, 379

Sarcina, 290, 291

Streptococcus, 323, 338

Tilsit

Plocamobacterium, 691

Propionibacterium, 378

Chitin Digesting Bacteria

Bacillus, 632

Bacterium, 632

Concrete, see Mineral Sources

Condensed Milk, see Dairy Products

Contamination

Bacillus, 712, 717, 748

Bacterium, 604, 673, 682

Cellulomonas, 622

Chromobacterium, 232

Clostidium, 798

C'orynebacterium, 401

Nocardia, 905,915

Oospora, 976

Planococcus, 281

Pseudomonas, 174

Staphylococcus, 282

Tuberculin flask

Nocardia, 905

Taceine

Oospora, 976

Cream, see Milk and Cream

Cutting Compound, see Mineral Sources 
Dairy Products, also see Butter, Cheese, and Milk and Cream

Abnormal milk

Bitter flavor

Bacillus, 648

Micrococcus, 265

Blue milk

Pseudomonas, 93, 145

Fetid odor

Viscobacterium, 691

General

Achrombacter, 609

Red milk

Bacterium,, 601

Micrococcus, 256

Ropy milk, also see Slimy milk

Achromobacter, 427

Alcaligenes, 414

Lactobacillus, 695

Micrococcus, 258, 268, 271

Streptococcus, 344, 702

Slimy milk, also see Ropy milk

Achromobacter, 425

Bacillus, 654, 746

Bacterium, 604, 675

Clostridium, 789

Flavobacterium, 434, 442

Micrococcus, 268, 271

Soapy milk

Bacillus, 660, 667, 758

Pseudomonas, 145, 149, 150

Tainted

Bacillus, 660

Aroma bacteria

Streptococcus, 339

Caseinate solution

Achromobacter, 692

Condensed milk

Leuconostoc, 348

Thermobacterium, 364

Evaporated milk

Bacillus, 713, 738

Pseudomonas, 103

Fermented milk

Dadhi

Streptothrix, 364

General

Sarcina, 292

Gioddu

Bacillus, 362
Kefir

Bacillus, 746

Lactobacillus, 351, 357, 359

Streptococcus, 338

Kumys

Bacillus, 362

Leben

Streptobacillus, 364

Matzoon

Flavobacterium, 441

Sarcina, 252

Mazun

Bacillus, 748

Bacterium, 362

Piima

Streptococcus, 342

Taette

Lactobacillus, 695

Streptococcus, 702

Taette, false

Bacterium, 675

Yoghurt

Lactobacillus, 355, 362, 364

General

Bacillus, 732, 733

Micrococcus, 237, 239, 240, 241

Mycobacterium, 889

Propionibacterium, 373, 374, 375, $376,377,378,379$

Pseudomonas, 100

Ice cream

Bacillus, 612, 670

Malted milk

Bacillus, 756

Milk powder

Bacillus, 733, 755, 756

Milk powder (spray process)

Streptococcus, 328

Sour milk

Bacillus, 756

Lactobacillus, 352, 362, 364

Starters

Leuconostoc, 347,348

Streptococcus, 325

Whey

Bacillus, 755, 756

Bacterium, 687

Dairy Utensils

Alcaligenes, 414

Bacillus, 732, 733

Microbacterium, 370,371 
Dairy Utensils (continued)

Micrococcus, 238, 239, 240, 241

Pseudomonas, 100

Creamery equipment

Pseudomonas, 99

Farm utensils

Sarcina, 294

Filter cloth

Bacillus, 735

Milk can

Serratia, 481

Milking machine

Sarcina, 294

\section{Dairy Wastes}

Achromobacter, 628

Flavobacterium, 433

Pseudomonas, 177, 178

\section{Decomposing Materials}

Agar-digesting bacteria, see Agar Digesting Bacteria

Cellulose-digesting bacteria, see Cellulose Digesting Bacteria

- Cellulose fibers Sorangium, 1024

Chitin-digesting bacteria, 632

Composts

Cellulomonas, 618

Micromonospora, 980

Streptomyces, 957

Thiobacillus, 79, 81

Crab shells

Bacterium, 632, 633

Farinaceous materials

Clostridium, 824

General

Bacillus, 711, 715, 730, 733, 734, $735,741,743$

Bacterium, 682

Myxococcus, 1042

Spirillum, 701

Grass

Actinomyces, 973

Leaves

Bacillus, 756

Mухососсия, 1042

Lichens

Archangium, 1018, 1019

Myxococcus, 1042

Litmus solution

Bacillus, 743, 751
Marine vegetation

Cytophaga, 1014, 1015

Organic Matter

Chondrococcus, 1046

Cytophaga, 1014

Polyangium, 1028, 1029, 1030

Sorangium, 1023

Vibrio, 201, 203

Paper

Myxococcus, 1042

Plant residues

Bacterium, 824, 825

Clostridium, 825

Cytophaga, 1013

Starchy materials

Bacillus, 722

Sugar refinery waste

Lampropedia, 844

Vegetable

Bacillus, 721

Spirillum, 214

Vegetation

Bacillus, 815, 81?

Thiothrix, 989

Wood

Chondromyces, 1037, 1039

Clostridium, 825

Myxococcus, 1042

Synangium, 1033

Watermelon

Bacillus, 748, 751

Water plants

Lampropedia, 844

Dogs

Bile

Bacteroides, 566

Blood

Leptospira, 1078

Spirochaeta 1066

Streptococcus, 343

Conjunctiva

Corynebacterium, 406

Erythrocytes

Haemobartonella, 1104

Fatal illness

Rickettsia, 1096

Feces

Bacillus, 818

Salmonella, 514, 529, 530 
Dogs (continued)

Genital organs

Salmonella, 514, 529, 530

Intestine

Bacterium, 680

Salmonella, 514, 529, 530

Spirochaeta, 1068

Liver

Acuformis, 812

Nasal mucosa

Zuberella, 578

Nasal mueus

Bacillus, 740

Not pathogenic for

Mycobacterium, 880)

Nocardia, 896

Preputial secretions

Hemophilus, 588

Red blood cells, see Erythrocytes

skin

Corynethrix, 406

Stomach

Bacillus, 651, 671

Spirillum, 217, 218

Spirochaeta, 1069

Throat

Streptococeus, 345

Irine

Bacillus, $7+0$

Dogs, Diseases of

Ibortion

Bricella, 562

Abscess

Micrococcus, 273

Aetinomycosis

Actinomyces, 927

Bronchitis

Nocardia, 919

Cancerous ulcers

Spirochaeta, 1074

C'atarrh

Pseudomonas, 146

Cerebromeningit is

Nocardia, 919

Distemper

Brucella, 563

Endometrit is

Bacterium, 675

(ilanders

Malleomyces, 55 j
Haemobartonellosis

Haemobartonella, 1102

Hemorrhagic septicemia

Pasteurella, 549, 550, 553

Human tuberculosis

Mycobacterium, 878

Leukemia

Bacterium, 680

Streptococcus, 337

Lymphadenitis

Nocardia, 919

Measles

Streptococcus, 341

Peritonitis

Actinomyces, 915

Phlegmon

Actinomyces, 915

Plague

Spirochaeta, 1068

Pleuropneumonia-like disease Asterococcus, 1292

Pneumonia

Brucella, 563

Purulent infectious, urinary tract

Corynebacterium, 389

Pyorrhoea

Leptothrix, 366

Rabies, vaccine contaminant

Flavohacterium, 437

Rabies-like disease

Bacillus, 663

IRingworm

Actinomyces, 916

Uremia

Leptospira, 1078

\section{Dung, see Manure (Dung)}

Dust, also see Air.

Achromobacter, 424, 425, 427

Actinomyces, 968, 970

Bacillus, 712, 717, 723, 725, 726, 729, $730,731,732,733,734,735,736,737$, 738,739

Bacterium, 605, 682

Chromobacterium, 233

Clostridium, 786, 817, 820

Diplococcus, 309

Hicrococcus, 237, 239, 240, 243, 244, $253,255,256,257,258,259,260,262$, $264,265,269,273,275,276,277,278$, $279,280,281$ 
Dust (continued)

Miyagawanella, 1117

Mycobacterium, 889,890

Sarcina, 286, 290, 292, 293

Serratia, 484

Streptococcus, 316,340

Streptomyces, 935, 936, 949

\section{Dusts}

Stable

Micrococcus, 265

Street

Clostridium, 786, 820

\section{Eggs, see Foods and Foodstuffs}

\section{Evaporated milk, see Dairy Products}

Feces, Animal, see Manure (Dung)

\section{Feces, Human}

Also see Human Sources, Intestine Aerobacter, 456, 457

Alcaligenes, 413, 416

Bacillus, 612, 647, 651, 652, 654, 656, $661,662,665,666,668,721,736,737$, $738,744,746,748,749,753,755,756$, $813,817,818,825,826$

Bacterium, 601, 607, 673, 675, 681, 683, $687,759,760,761,762$

Bacteroides, 567, 568, 569, 570, 571, 572, $573,574,575$

Bifidobacterium, 369

Catenabacterium, 368

Clostridium, 773, 774, 782, 785, 787, $788,791,793,794,796,797,799,800$, $801,802,809,810,811,812,820,821$, $822,824,826$

Corynebacterium, 401

Eberthella, 533, 534

Escherichia, 447, 450, 451, 452, 453

Eubacterium, 368

Granulobacillus, 826

Kurthia, 613

Lactobacillus, 353, 354, 357, 359, 362

Microbacterium, 370

Micrococcus, 252, 253, 257, 259, 260, $265,266,270,272,274,275,276,277$, $278,279,280,281,695$

Microspira, 202

Proteus, 488, 490, 491

Pseudomonas, 90, 148

Ramibacterium 369
Salmonella, 502, 504, 505, 506, 510, 511, $512,513,514,515,517,518,519,520$, $522,524,525,526,527,528,529,530$, 531

Shigella, 537, 538, 539, 540, 542, 543, 544

Spirochaeta, 1067, 1069, 1070

Staphylococcus, 282

Streptococcus, 322, 323, 326, 327, 330, $333,335,339,341,343$

Vibrio, 204, 205

\section{Feces, Infant}

Micrococcus, 257

Neisseria, 301

Pseudomonas, 198

Staphylococcus, 281

\section{Fermenting and Fermented Materials}

Agave americana, sap

Pseudomonas, 106

Alcohol infusions

Bacterium, 675

Ale, bottled

Acetobacter, 692

Beer, see Beer

Beet juice

Acetobacter, 189

Beets

Bacillus, 656, 664, 752, 757

Bacterium, 675, 678, 686, 761

Lactobacillus, 357

Beverages

Acetobacter, 181, 182

Bread dough

Bacillus, 65̄ , 664, 742

Bacterium, 683

Lactobacillus, 357, 359, 361, 363

Cabbage, see Sauerkraut

Cereal mash

Lactobacillus, 363

Corn mash

Clostridium, 781, 825

Lactobacillus, 357

Dates

Acetobacter, $18 t$

Dough

Bacillus, 742

Bacterium, 683

Ensilage, see Silage.

Fermented milk drinks, see Dairy

Products, Fermented milk 


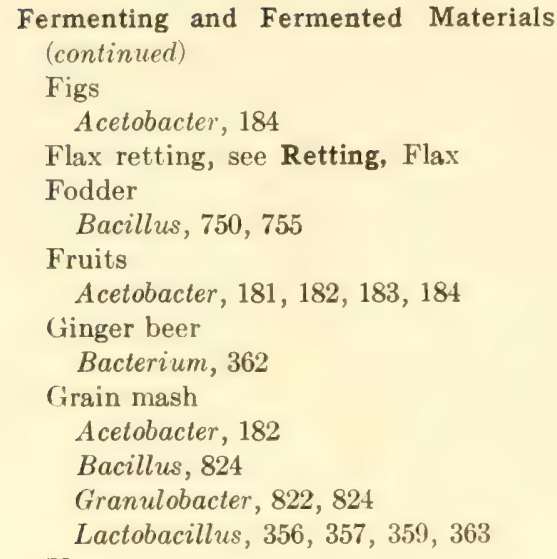

Hay

Bacillus, 740

Hemp retting, see Retting, Hemp

Hydrogen peroxide solutions

Acetobacter, 189

Infusions

Bacillus, 672

Kenaf (Hibiscus) retting see Retting, Kenat

Kombucha from tea infusions Acetobacter, 189

Malt

Bacterium, 677, 678, 679, 688, 760, 761

Malt beverages

Acetobacter, 182

Malt infusion

Micrococcus, 263

Malt mash

Sarcina, 287

Mash, dried persimmon Acetobacter, 184

Mash, spoiled Pediococcus, 249

Mash, vegetable

Lactobacillus, $356,357,359,361$

Methane fermentation in swamps Sarcina, 287

Milk, Fermented see Dairy Products, Fermented Milk

Molasses

Clostridium, 781

Lactobacillus, 359, 363

Pickles

Bacillus, 648
-, cauliflower

Lactobacillus, 357

-, cucumber

Lactobacillus, 357

-, tomato

Lactobacillus, 357, 359

Plant juices

Pseudomonas, 106

Plant materials

Clostridium, 772

Lactobacillus, 347

Streptococcus, 325, 326, 327, 340

Potato mash

Amylobacter, 813

Clostridium, 781, 808, 819

Lactobacillus, 356, 357, 361

Pulque

Pseudomonas, 106

Streptococcus, 338

Sake

Lactobacillus, 363

Sauerkraut

Bacillus, 657, 750

Bacterium, 675

Lactobacillus, 357, 359

Pseudomonas, 146

Silage

Bacterium, 602

Clostridium, 785

Lactobacillus, 359

Propionibacterium, 376

Streptococcus, 336

Slimy fermentation

Bacillus, 749

Soybean cake

Bacillus, 757

Tobacco

Bacterium, 674, 682, 685

Urea

Staphylococcus, 282

Urine

Micrococcus, 238, 250, 260, 266, 267, $269,279,280$

Sarcina, 289, 293

Staphylococcus, 282

Vegetables

Acetobacter, 181, 182

Lactobacillus, 347, 356, 357, 359, 361

Vinegar

Acetobacter, 181, 182, 183, 186, 187, 188 
Fermented Materials (continued)

Vinegar (continued)

Bacillus, 756

Bacterium, 682

-, quick process

Acetobacter, 187, 188

-, wine

Acetobacter, 187, 188

Wine, see Wine

Yeast, see Yeast

\section{Fishes}

African Mudfish, blood

Spirochaeta, 1067

Anchovy pickle

Pediococcus, 250

Anchovy, salted

Vibrio, 204

Blenny, intestine

Treponema, 1045

Brochet

Bartonella, 1108

Bullhead, Marine

Treponema, 1074

Carp, pathogenic for

Bacterium, 642

Proteus, 491

Pseudomonas, 102, 149

Mycobacterium, 883, 884

Catfish

Serratia, 462

Codfish

-, feces

Achromobacter, $420,423,426,427$

Flavobacteriun, 434

--, intestine

Achromobacter, +25

Shigella, 544

—, red salted

. Micrococcus, 259

Pseudomonas, 110

Sarcina, 289

-, slime

Achromobacter, 420, 423, 426, 42-

Flavobacterium, 434, 436

Croaker

Mycobacterium, 881

Dogfish

Flavobacterium, 436

-, slime and feces

Achromobacter, 420
Eels

Bacterium, 673

Mycobacterium, 883

Vibrio, 208

General

Bacillus, 753

Pseudomonas, 102, 109, 149

-, blood

Rickettsia, 1097

Spirochaeta, 1068

-, intestine

Chitin-digesting bacteria, 632

-, rectum

Treponema, 1076

-, skin

Bacterium, 606, 612

Micrococcus, 246

Haddock

Spirochaeta, 1065

-, slime

Flavobacterium, 438, 440, 441

Hake

Flavobacterium, 439

Halibut

Pseudomonas, 145

-, skin

Flavobacterium, 429, 434, 437

Herring, salted

Sarcina, 292

Kilifish

Pseudomonas, 109

Lamprey eel

Bartonella, 1108

Marine fishes

Mycobacterium, 883

Pseudomonas, 147

Spirochaeta, 1067

Treponema, 1075

Perch, erythrocytes

Grahamella, 1110

Pollack

Spirochaeta 1069

Salmon, Blue-black

Chondrococcus, 1047

Salmon eggs

Achromobacter, 425, 692

- - , diseased

Pseudomonas, 700

Salted fishes

Micrococcus, 259, 266, 268, 292

Pseudomonas, 110

Sarcina, 289

Vibrio, 204 
Fishes (continued)

Sardines, salted

Vibrio, 204

-, stomach

Eubacterium, 367

Sea bass

Mycobacterium, 884

Sergeant major

Mycobacterium, 884

Shark, blood

Borrelia, 1064

Skate, slime and feces

Flavobacterium, 434, 439

Tench

Trout

Bartonella, 1108

Bacterium, 686

Whiting

Microspironema, 1064

Fishes, Diseases of

Carp

一, tumors

Mycobacterium, 883, 881

-, red spots

Bacterium, 642

Eels, diseased

Bacterium, 673

Mycobacterium, 883

Vibrio, 203

General

-, epidemic infection of fishes

Bacillus, 751

Vibrio, 197

-, fresh water fishes

Hemorrhagic septicemia

Pseudomonas, 102, 103

-, skin lesions

Pseudomonas, 102

-_, necrotic ulcers

Rickettsia, 1097

-, necrosis of the liver

Chondrococcus, 1047

Mycobacterium, 884

Kilifish, skin lesions

Pseudomonas, 109

Marine fishes

Pseudomonas, 149

- - infected skin and muscle

Pseudomonas, 109

— - tuberculosis
Mycobacterium, 884

Trout, furunculosis

Bacterium, 686

Salmon eggs

Achromobacter, 692

Pseudomonas, 700

Fishes, Scientific Names

Abudefduf mauritii, 884

Acropoma japonicum, 636

Ameiuras melas, 462

Blennius pavo, 1075

Box boops, 1073

Centropristes striatus, 884

Clarias angolensis, 1067

Coelorhynchus sp., 636

Cottus bubalis, 1074

Cyprinus carpic, 491, 642, 884

Cyprinus sp., 102, 149

Esox lucius, 1108

Fundulus parvipinnes, 109

Gadus callarias, 420, 423, 425, 426, $427,434,436,544$

Gadus minutus, 1067

Gadus pollachius, 1069

Hippoglossus hippoglossus, 429, 434, 436,437

Lepadogaster bimaculatus, 1075

Melanogrammus aeglefinus, 438, 440, 441

Merlangus merlangus, 1064

Micropogon undulatus, 884

Oncorrhynchus nerka 1047

Pelamys sarda, 1068

Perca fluviatilis, 1110

Petromyzon marinus, 1108

Physiculus japonicus, 636

Raja crinacea, 434, 439

Saccobranchus fossilis, 753

Squalus acanthias, $420,427,436$

Tetraodon fahaka, 1097

Tinca tinca, 1108

Trachurus japonicus, 694

Trigla lucena, 1076

Urophycis tenuis, 439

\section{Fomites}

Hairbrush

Micrococcus, 253, 275

Hospital shirt

Serratia, 484 
Foods and Foodstuffs

Anchovies, pickled

Micrococcus, 250

Asparagus, boiled

Bacillus, 742, 748

Bacterium, 759

Bacon, tainted

Vibrio, 702

Beans, salted, purple discoloration

Pseudomonas, 109

Beef extract

Bacillus, 734

Beet juice, sugar

Leuconostoc, 348

Blood sausage

Clostridium, 788

Blutwurst

Clostridium, 788

Bread

Bacillus, 661

Bacterium, 680, 759

-, rye

Bacillus, 711, 750

-, slimy

Bacillus, 711, 758

Bacterium, 760

Butter, see Butter

Candy

Bacillus, 756

Canned beans

Bacillus, 750

-, beets, blackened

Bacillus, 739

-, blueberries

Bacillus, 735

-, carrots

Bacillus, $7+2$

-, corn, spoiled

Bacillus, 734, 756

-, corn, sulfur stinker spoilage

Clostridium, 803

-, evaporated milk

Bacillus, 713, 738

Pseudomonas, 103

-, foods

Bacillus, 713, 730, 731, 734, 756

Clostridium, 779, 797

-, goods, spoiled

Bacillus, 730, 731, 734

-, goods, spoiled, non-acid

Clostridium, 785, 803
-, macaroni, spoiled

Bacillus, 317

一, peas

Bacillus, 737, 756

-, pumpkin

Bacterium, 609

-, pumpkin, swells

Bacillus, 730, 731

-, salmon, spoiled

Bacillus, 817

-, sardines

Serratia, 483

-, spinach

Bacillus, 825

-, string beans, spoiled

Bacillus, 734, 748

-, tomatoes

Bacillus, 756

-, vegetables, flat sours

Bacillus, 734

Catsup

Lactobacillus, 359

Cheese, see Cheese

Codfish, reddened salt

Bacillus, 667, 742

Flavobacterium, 442

Pseudomonas, 110

Corn meal

Bacterium, 679

Crab meat, musty odor

Achromobacter, 425

Cream, see Milk and Cream

Dairy Products, see Dairy Products

Dates, commercially packed

Bacillus, 756

Eggs, black rot

Proteus, 490

-, cooked

Bacterium, 761

-, duck

Salmonella, 517

-, hen's

Bacillus, 653, 654, 657, 659, 663, $672,747,750$

Pseudomonas, 147, 148, 149, 150, 179

-, musty

Achromobacter, 425

Pseudomonas, 148, 179

-, powdered

Salmonella, 510, 512, 513 
Foods and Foodstuffs (continued)

Fish conserves

Clostridium, 821

- , herring, rusty

Pseudomonas, 110

- salted

Bacillus, 658

Ristella, 576

-, semi-dried

Flavobacterium, 694

Food conserves

Bacillus, 741

Gelatin, spoiled

Bacillus, 756

General

Serratia, 481, 482

Grapes, Spanish dried

Bacterium, 624

Ham, salted

Eubacterium, 367

-, sour

Clostridium, 784

Horseradish, ground

Bacillus, 757

Margarine

Bacillus, 662, 667

Bacterium, 681, 685, 689

Meat

Bacterium, 678

Micrococcus, 258, 272, 281

Streptococcus, 338

-, extract

Bacillus, 648, 651, 740, 745, 746, 749

Bacterium, 678, 760

Micrococcus, 255, 267

Streptococcus, 339

-, pies

Salmonella, 531

—, spoiled

Bacillus, 749

Milk, see Milk and Cream

Mincemeat, canned

Bacillus, 757

Oranges

Butylobacter, 825

Oysters

Inflabilis, 823

Pickles

Bacillus, 648, 754

Salmonella, 519
Plum preserves

Bacillus, 753

Pork

Clostridium, 802

Potato, cooked Bacillus, 664, 741, 748

Rice, cooked in chicken broth

Serratia, 484

Salad dressing

Lactobacillus, 363

Sausage

Bacillus, 749, 751, 752, 816

Clostridium, 778, 779

Salmonella, 530

Sugar, also see Sugar

Bacillus, 742, 745, 747

Leuconostoc, 347,348

Micrococcus, 260

Spirillum, 217

--, beet

Bacillus, 747

--, factories, frog spawn fungus

Leuconostoc, 347, 348

Spirillum, 217

Tomato juice

Bacillus, 713

Tomato products, spoiled

Lactobacillus, 357, 359

Truffles, cooked

Bacillus, 757

Wiener skins

Tetracoccus, 284

Wurst

Micrococcus, 258, 272, 281

\section{Goats}

Cerebrospinal fluid

Streptococcus, 338

Corneal or conjunctival diacharges

Colesiota, 1120

General

Salmonella, 506

Goats, Diseases of

Abortion

Brucella, 561

Agalactia

Anulomyces, 1292

Glanders

Malleomyces, 555 
Goats, Diseases of (continued)

Heartwater Cowdria, 1094

Hemorrhagic septicemia Pasteurella, 549

Keratitis Colesiota, 1119

Lesions Nocardia, 900

Ophthalmia, infectious Colesiota, 1120

Pleuropneumonia, bovine Asterococcus, 1292

Tuberculosis, bovine Mycobacterium, 879

\section{Guinea Pigs}

Blood Rickettsia, 1086, 1088, 1089 Spirochaeta, 1066, 1070 Spiroschaudinnia, 1071 Spironema, 1070

Cadaver Bacillus, 815

Caecum

Metabacterium, 762

Treponema, 1075

Erythrocytes Haemobartonella, 1104, 1108

Erythrocytes, Peruvian guinea pigs Haemobartonella, 1106

Inoculated with soil Bacillus, 660, 817, 825, 826

Hiblerillus, 822

Intestine

Cristispirilla, 1057

Heliconema, 1064

Oscillospira, 1004

Sarcina, 287

Intestine and genital organs

Salmonella, 506, 527

Liver

Spirochaeta, 1065

Lymph glands

Bacillus, 659

Monocytes Ehrlichia, 1095

Mucus, intestinal Bacillus, 651

Nasal mucosa Zuberella, 578

Normal animals Veillonella, 304
Not pathogenic for Johne's disease Mycobacterium, 881

Red blood cells, see Erythrocytes

Tuberculosis, avian Mycobacterium, 880

Tuberculosis, piscine Mycobacterium, 883, 884

Tuberculosis, snake Mycobacterium, 885, 886

Tuberculosis, turtle Mycobacterium, 886

Stomach Klebsiella, 459

Tunica vaginalis Rickettsia, 1086, 1089

Guinea Pigs, Diseases of

Anthrax

Bacillus, 720

Brucellosis Brucella, 561, 562, 563

Cervical adenitis Bacteroides, 575

Epizootic Bacterium, 681

Glanders Malleomyces, 555, 556

Hemorrhagic septicemia Pasteurella, 549, 550, 551, 553, 554

Infections

Gaffkya, 284

Nocardia, 913

Listerosis

Listeria, 409

Lymphadenitis

Streptococcus, 317

Maculatum disease Rickettsia, 1098

Melioidosis

Malleomyces, 556

Septicemia Pseudomonas, 146

Skin abscesses Neisseria, 301

Tuberculosis, bovine Mycobacterium, 878

Tuberculosis, human Mycobacterium, 878, 879

Tuberculosis-like disease Pasteurella, 553

Tuberculosis, pulmonary Bacillus, 651 
Hail

Bacillus, 744

Micrococcus, 269,279

\section{Hogs}

General

Salmonella, 502, 504, 505, 507, 509, $510,513,514,519,521,522,525,526$, 527,530

Genital organs

Brucella, 562

Intestine

Bacillus, 753

Lymph glands

Salmonella, 505, 510, 513, 514, 518, $524,528,529$

Liver, necrotic foci

Sphaerophorus, 579

Ovary

Bacillus, 757

Peritoneal fluid

Bacillus, 666

\section{Hogs, Diseases of}

Abortion

Brucella, 561, 562

Abscesses

Corynebacterium, 388

Vibrio, 206

Actinomycosis

Actinomyces, 926

Anthrax

Bacillus, 720

Blood in hog cholera Borrelia, 1063

Bronchopneumonia Bacillus, 657

Calcareous deposits in muscles Actinomyces, 972

Caseous suppuration Corynebacterium, 406

Conjunctivitis Rickettsia, 1120

Cutaneous lesions Spirochaeta, 1069

Diarrhoea Bacillus, 826

Erysipelas Erysipelothrix, 411

Heartwater-like disease Rickettsia, 1097
Hemorrhagic septicemia Pasteurella, 548, 549

Hog cholera Borrelia, 1063 Salmonella, 509, 531

Infections

Corynebacterium, 391

Influenza

Hemophilus, 586

Listeriosis

Listeria, 409

Marseille's disease Bacillus, 662

Measles Streptococcus, 341

Pyorrhoea Leptothrix, 366

Septicemia Streptococcus, 317

Swine erysipelas Bacillus, 652 Erysipelothrix, 411

Swine fever Salmonella, 509

Swine plague (Hog cholera) Micrococcus, 278

Tuberculosis, avian Mycobacterium, 880, 881

Tuberculosis, bovine Mycobacterium, 879

Ulcers, intestinal Borrelia, 1063

\section{Horses}

Blood

Nocardia, 897 Spirochaeta, 1066

Female genital tract Klebsiella, 459 Streptococcus, 340

Foetus Streptococcus, 317

General Rickettsia, 1097 Salmonella, 518, 529

Intestine

Bacillus, 694

Bifidobacterium, 369

Streptococcus, 323, 327

Zuberella, 577

Hock-joint (foals)

Nocardia, 910 
Horses (continued)

Large intestine

Bacillus, 612, 747, 749, 755, 813, 814, $815,817,818,826,827$

Clostridium, 783, 799

Eubacterium, 368

Gaffkya, 284

Hiblerillus, 822

Inflabilis, 823

Micrococcus, 261

Liver

Malleomyces, 555

Salmonella, 507

Nasal passages

Corynebacterium, 385

Not pathogenic for Avian tuberculosis Mycobacterium, 880

Pneumonia

Corynethrix, 407

Respiratory tract

Streptococcus, 318, 33\%

Saliva

Nocardia, 975

Skin

Corynethrix, 406

Spleen

Malleomyces, 555

Throat

Streptococcus, 345

Urine

Bacterium, 642

Pediococcus, 250

Sarcina, 291

\section{Horses, Diseases of}

Abortion

Brucella, 561, 562

Salmonella. 506

Streptococcus, 336

Abscesses on jaws

Nocardia, 920

Acne pustules

Bacillus, 658

Corynebacterium, 401

Botryomycosis

Micrococcus, 253

Endometritis

Streptococcus, 317

Gangrenous dermatitis

Sphaerophorus, 579

General

Streptococcus, 341
Glanders

Malleomyces, 551,555

Hemorrhagic septicemia

Pasteurella, 549, 550, 551, 553

Infections, genitourinary system Klebsiella, 459

Influenza

Streptococcus, 340

Joint ill of foals

Shigella, 542

Lymphangitis, ulcerative

Corynebacterium, 389

Nasal secretion in glanders

Bacterium, 683

Pneumonia

Bacterium, 684

Streptococcus, 339

-, infectious of foals

Corynebacterium, 391

Purulent infections, urinary tract

Corynebacterium, 389

Pus, respiratory tract

Streptococcus, 318

Ringworm

Actinomyces, 916

Stomatitis

Treponema, 1074

Strangles

Streptococcus, 318

Ulcerative lesions

Corynebacterium, 389

Wounds

Corynebacterium, 385

\section{Human Diseases}

Abscesses

Alcaligenes, 413

Micrococcus, 242,244

Proteus, 488, 490

Pseudomonas, 89

Salmonella, 507

Streptococcus, 333

Veillonella, 303

-, abdominal

Bacillus, 815

-, alveolar

Bacterium, 678

-, brain

Bacillus, 656, 666, 815

Capsularis, 577

Nocardia, 897 
Human Diseases (continued)

Abscesses, cervical

Actinomyces, 916

-, chest

Nocardia, 920

-, dental

Aerobacter, 456

Bacillus, 650

--, ear

Corynebacterium, 402, 403

-, facial

Bacterium, 607

-, foot

Actinomyces, 973

-, iliac

Oospora, 922

-, inguinal

Nocardia, 976

-, intestinal

Bacillus, 816

-, jaw

Actinomyces, 917

Nocardia, 921

Proactinomyces, 923

Streptothrix, 924

—, kidney

Clostridium, 825

-, liver

Bacillus, 400, 660, 666

Bacterium, 677

Bacteroides, 567

Cohnistreptothrix, 975

Proteus, 490

Sphaerophorus, 579

Vibrio, 205

-, lung

Bacillus, 667

Ristella, 575

Sphaerophorus, 579

-, mouth

Corynebacterium, 402

--, multiple

Nocardia, 921

-, osseus

Diplococcus, 310

_, palm

Actinomyces, 971

-, parotid

Streptomyces, 963

-, perianal

Sphaerophorus, 580
-, periuterine

Veillonella, 303

-, pulmonary Actinomyces, 917

-, rectal

Eberthella, 534

-, skin

Streptococcus, 333

-, subcutaneous Actinomyces, 916

-, teeth Actinomyces, 917 Aerobacter, 456 Streptococcus, 320)

-, thoracic Clostridium, 821

-, urinary tract Bacteroides, 566 Clostridium, 806

Acholuric jaundice Streptomyces, 958, 962, 963, 965

Acne pustules

Micrococcus, 251, 259, 272

Corynebacterium, 387

Actinomycosis

Actinomyces, 927, 971

Cohnistreptothrix, 928

Discomyces, 918,928

Nocardia, 921, 975

Streptothrix, 923

-, bone

Actinomyces, 917

-, bronchial

Actinomyces, 916

- lachrymal gland

Actinomyces, 927,970

Nocardia, 910

- lung

Actinomyces, 916

Acute arthritis

Spirochaeta, 1069, 1070

African relapsing fever

Borrelia, 1061

Akiyami (Japan)

Leptospira, 1078

Alopecia areata

Micrococcus, 259

Alveolar pyorrhea, see Pyorrhea alveolaris

Anal pus pocket

Corynebacterium, 406 
Human Diseases (continued)

Anemia

Haemobartonella, 1104

-, pernicious

Salmonella, 522

Angina

Streptobacillus, 589

-, Ludwïg's

Streptococcus, 329

-, Vincent's

Borrelia, 1063

Anginous exudate

Actinomyces, 917

Anorectal inflammation

Miyagawanella, 1116

Anthrax

Bacillus, 720

Micrococcus, 263

Proteus, 691

Aphthous ulcers of gingival and buccal

mucosa

Veillonella, 304

Appendicizis

Bacillus, 752, 814, 817, 818, 826, 827

Bacteroides, 566, 567

Clostridium, 773, 776

Corynebacterium, 403

Diplococcus, 309, 310

Fusobacterium, 582

Oospora, 922

Ramibacterium, 369

Ristella, 575

Streptococcus, 329, 330, 331, 332, 333

Veillonella, 302, 304

- gangrenous

Sphaerophorus, 579

Arthritis

Bacterium, 674

Diplococcus, 307

Micrococcus, 259

Neisseria, 301

Streptobacillus, 589

-, acute

Spirochaeta, 1069, 1070

Ascitic fluid, old

Sarcina, 293

Balanitis

Spirochaeta, 1065

Banti's disease

Streptomyces, 960

Bartonellosis

Bartonella, 1101
Beriberi

Bacillus, 649, 738

Micrococcus, 253

Black water fever

Leptospira, 1078, 1079

Blue pus

Pseudomonas, 89

Boils

Micrococcus, 242, 268

Botulism

Bacillus, 814

Clostridium, 779

Boutonneuse fever Rickettsia, 1089, 1098

Bright's disease Streptococcus, 338

Bronchiectasis

Capsularis, 577

Streptococcus, 332

Bronchitis

Actinomyces, 973

Bacillus, 590

Cladothrix, 974

Nocardia, 921

Spirochaeta, 1065

Vibrio, 206

Zuberella, 578

-, chronic

Ristella, 576

- , fetid

Bacillus, 613

一, putrid

Bacillus, 740

Bronchopleuropneumonia

Nocardia, 921

Bronchopneumonia

Bacterium, 690

Brucellosis

Brucella, 561, 562

Buboes

Pasteurella, 549, 551

Bubonic plague

Nocardia, 920

Bullis fever

Rickettsia, 1098

Cancer of the stomach

Bacillus, 740, 746

Cancerous tissue

Micrococcus, 268

Cancerous ulcers

Spirochaeta, 1074 
Human Diseases (continued)

Carate

Treponema, 1073

Carcinoma, ulcerating

Spirochaeta, 1074

Carrion's disease

Bartonella, 1101

Catarrh

Micrococcus, 258

Neisseria, 298

Staphylococcus, 282

一, acute

Staphylococcus, 282

-, acute epidemic

Corynebacterium, 401

Central and South African relapsing fever

Borrelia, 1060

Cerebrospinal meningitis, cpidemic

Neisseria, 297, 302

Chancroid

Hemophilus, 587

Chancroidal ulcers

Streptococcus, 341

Chicken pox pustules

Streptococcus, 345

Choleocystitis

Ristella, 577

Cholera

Bacillus, 659, 665, 667, 73ธ

Bacterium, 687

Escherichia, 447

Spirillum, 217

Vibrio, 194, 204, 205, 206

Cholera infantum

Alcaligenes, 415

Vibrio, 198

Cholera-like disease Vibrio, 205, 206

Cholera nostras

Vibrio, 198

Chromidrosis of axilla

Micrococcus, 256

Chronic endometritis

Vibrio, 198

Climatic bubo

Miyagawanella, 1116

Colitis

Shigella, 543

Colitis, ulcerative

Micrococcus, 696
Columbensis fever

Salmomella, 531

Conjunctival eatarrh

Bacterium, 677

Conjunctivitis

Actinomyces, 916, 968, 969

Bacillus, 741

Bacterium, 759

Hemophilus, 585

Micrococcobacillus, 690

Mimcae, 595

Neisseria, 301

Streptothrix, 924

-, angular

Moraxella, 591, 592

-, granular

Bacillus, 589

Noguchia, 593

--, inclusion

Chlamydozoon, 1115

-, neonatal

Chlamydozoon, 1115

-, swimming pool

Chlamydozoon, 1115

Cornea, infected

Bacterium, 679, 685

Corneal ulcerations

Moraxella, 591, 592

Cracked heels

Actinomyces, 916

Cystitis

Bacillus, 650, 653, 665, 666, 668, 741, 758

Bacterium, 580, 676

Escherichia, 447

Micrococcus, 248

Proteus, 490

Shigella, 543

Staphylococcus, 282

Streptobacillus, 590

Streptococcus, 339, 343, 344, 345

-, fetid

Clostridium, 825

-, pus

Actinomyces, 918, 974

Dacryocystitis

Cohnistreptothrix, 975

Dengue

Leptospira, 1078, 1079

Dental caries

Acidobacterium, 361 
Human Diseases (continued)

Dental caries (continued)

Bacillus, 352, 744, 745

Lactobacillus, 363

Leptotrichia, 365

Streptocaccus, 320, 339, 341, 342

Vibrio. 202

Dermatosis

Cohnistreptothrix, 918, 975

Nocardia, 921

Diabetes

Bacillus, 813

Diarrhoea

Bacillus, 659, 660, 813, 818

Clostridium, 820

Proteus, 488

Ristella, 576

Salmonella, 506, 511, 515, 519, 522, 524,528

Shigella, 538, 539, 540

Diphtheria

Bacillus, 752

Corynebacterium, 385, 400, 401, 407

Micrococcus, 260

Streptococcus, 337

Dutch East Indies fever

Leptospira, 1078

Dysenteric enteritis, Ceylon

Spirillum, 218

Dysentery

Bacterium, 683

Eberthella, 534

Salmonella, 531

Shigella, 536, 537, 538, 539, 540, 542, 543,544

Vibrio, 204

-, Egyptian

Bacillus, 400

Eczema

Bacillus, 651, 65t, 656

Bacterium, 674, 683, 686

Micrococcus, 257, 259, 278

-, seborrhoic

Pseudomonas, 148

Elephantiasis

Bacillus, 659

Emphysema

Bacillus, 826

Emphysematous liver

Clostridium, 821
Empyema

Diplococcus, 307

Leptothrix, 366

-, purulent

Capsularis, 577

Endemic typhus

Rickettsia, 1086

Endocarditis

Actinomyces, 917

Bacillus, 654

Cillobacterium, 369

Corynebacterium, 401, 405

Diplococcus, 307

Hemophilus, 589

Staphylococcus, 282

Streptococcus, $321,326,32 \overline{7}, 339,343,344$

Vibrio, 206

-, septic

Bacterium, 590

-, ulcerative

Micrococcus, 274

Staphylococcus, 282

Enteric fever

Salmonella, 501, 502, 503, 505, 507, $509,511,513,514,517,518,519$, $523,524,525,526,527,528,529$

Enteritis

Nocardia, 920

Sarcina, 292

Streptococcus, 339, 341

Eruptive fever

Richettsia, 1089

Erysipelas

Streptococcus, 315, 318, $34 t$

Erysipelas, gangrenous

Ristella, 575

Erysipeloid

Erysipelothrix, 411

Erythema intertrigo

Micrococcus, 264

Erythema multiforme

Streptobacillus, 589

Erythema nodosum

Bacillus, 742

Erythema of the skin

Micrococcus, 263

Erythrasma

Discomyces, 919

Nocardia, 92]

Esthiomena

Miyagauanella, 1116 
Human Diseases (conlinued)

European relapsing fever Borrelia, 1060

European typhus Rickettsia, 1085

Exanthematous typhus Rickettsia, 1085

Spirochaeta, 1067

Febrile illness Rickettsia, 1098

Felons with red pus Serratia, 484

Fever, see

Blackwater fever

Boutonneuse fever

Bullis fever

Columbensis fever

Endemic typhus

Enteric fever

Eruptive fever

European typhus

Dutch East Indies fever

Five day fever

Kenya typhus

Malta fever

Marseilles fever

Mediterranean fever

Mexican typhus

Mianeh fever (Persia)

Murine typhus

Oroya fever

Paratyphoid

Parenteric fever

Parrot fever

Postnatal fever

Pretibial fever

Puerperal fever

Q (Queensland) fever

Rat bite fever

Recurrent fever

Rocky Mountain spotted fever

São Paulo exanthemic fever, Brazil

Scarlet fever

Scrub typhus

Seven day fever, Japan

Shin bone fever

South African tick bite fever

South American relapsing fever

Spanish relapsing fever

Spotted fever, Minas Geraes

Swamp fever, Europe

Tobia fever, Colombia
Trench fever

Typhoid fever

Typhus fever

Western relapsing fever

Wolhynian fever

Yellow fever

Five-day fever

Rickettsia, 1095

Food poisoning

Bacillus, 665, 669, 670, 672

Salmonella, 503, 504, 510, 511, 513, $514,517,519,521,523,528,529$, 530,531

Furuncles

Micrococcus, 242

Gangrene

Bacillus, 814

Clostridium, 819

-, buccomaxillary

Leptospira, 1078

Gangrene, gas, see Gas gangrene

- lung

Bacterium, 680

Catenabacterium, 365

Streptococcus, $329,330,331,332,343$

-, mouth

Streptococcus, 342

-, pulmonary

Bacillus, 827

Bacteroides, 566, 567

Veillonella, 303, 304

-, putrefactive

Streptococcus, 329

—, senile

Bacillus, 757

Gangrenous foot

Ascococcus, 250

Gangrenous lung exudate

Bacillus, 613

Gangrenous phlegmon, mouth

Spirochaeta, 1070

Gangrenous pulp, tooth

Bacillus, 666, 745

Gangrenous wounds

Bacillus, 815, 825, 826, 827

Clostridium, 792, 812, 825

Martellillus, 823

Reglillus, 823

Robertsonillus, 823

Gas gangrene

Bacillus, 753 , 813, 816, 817, 824, 825, 826 
Human Diseases (continued)

Gas gangrene (continued)

Clostridium, 773, 778, 782, 783, 784, $788,791,794,796,821$

Martellillus, 823

Streptococcus, 330

Gaseous edema

Clostridium, 825

Gaseous phlegmons

Bacillus, 826

Gastric derangement

Bacillus, 738, 814

Gastroenteritis

Bacillus, 751

Paracolobactrum, 460

Proteus, 488, 490

Salmonella, 504, 505, 506, 507, 509, $511,512,513,514,515,517,518$, $519,521,522,523,524,525,528$, $529,532,701$

Genesiosalles

Nocardia, 920

Gikayami (Japan)

Leptospira, 1077

Gingivitis

Fusobacterium, 583

Glanders

Malleomyces, 555

Glanders-like disease

Malleomyces, 556

Glossitis

Micrococcus, 696

Gonorrhoea

Neisseria, 296

Micrococcus, 240, 276

Granuloma

Actinomyces, 917

Granuloma inguinale

Donovania, 559

Klebsiella, 499

Spirochaeta, 1065

Granuloma, malignant

Corynebacterium, 403

Gummy lesions

Micrococcus, 268

Harvest sickness, Japan

Leplospira, 1078

Hemorrhagic infection

Bacterium, 553

Hemorrhagic nephritis

Bacterium, 553
Hemorrhagic septicemia

Bacterinim, 677

Pasteurella, 549

Hodgkin's disease, lymph glands

Corynebacterium, 402, 403, 404

Illinois virus

Miyagavanella, 1119

Indian relapsing fever

Borrelia, 1062

Infant diarrhoea

Streptococcus, 339

Infantilism

Bacillus, $7+6$

Infections

Actinomyces, 973

Corynebacterium, 694

Neisseria, 296

Nocardia, 899, 901, 907, 912

Proteus, 491

Salmonella, 531

Sphaerophorus, 579

Streptococcus, $316,321,340,342,343$, 344,345

Streptomyces, $961,962,963,964,965,967$

-, arms, legs and chest

Actinomyces, 917

-, bladder

Actinomyces, 918

-, genitourinary tract

Escherichia, 447, 452

-, leg

Bacterium, 401

-, outer ear

Nocardia, 919

-, perineal

Clostridium, 825

-, puerperal

Actinomyces, 580

Bacteroides, 567

-, pulmonary

Actinomyces, 916

—, urinary

Bacteroides, 567

-, uterus

Bacillus, 668

Inflamed upper lip

Micrococcus, 696

Inflammations, genitourinary tract

Chlamydozoon, 1115

Inflammatory skin disease

Ristella, 576 
Human Diseases (continued)

\section{Influenza}

Dialister, 595

Hemophilus, 585, 586

Micrococcus, 264, 272, 277

Sarcina, 294

Streptococcus, $340,341,342,344,345$

-, nasal washings

Veillonella, 303

Intestinal intoxication

Micrococcus, 247

Intestinal ulcer

Bacterium, 677

Jaundice, acholuric

Streptomyces, 958, 962, 963, 965

Spiroschaudinnia, 1071

-, infectious

Leptospira, 1077

Pseudomonas, 90

Kenya typhus

Rickettsia, 1089

Keratitis

Actinomyces, 915, 917

Discomyces, 918

Kolpohyperplasia cysticu

Bacillus, 826

Leproma

Actinomyces, 916

Leprosy

Mycobacterium, 882, 887

Leprosy-like lesions

Mycobacterium, 882

Leprous lesions

Mycabacterium, 882

Ieukemia, lymphatic

Bacterium, 689

Spirochaeta, 1068

Lichen planus

Ristella, 576

Iichen ruber

Bacillus, 660

Lingua nigra

Oospora, 922

Listeriosis

$$
\text { Listeria } 409
$$

Iiver abscesses, see Abscesses, liver

Tiver, acute yellow atrophy

Bacillus, 659

-, infected

Micrococcus, 272
Louisiana pneumonia

Miyagawanella, 1119

Lung diseases

Actinomyces, 970

Mycobacterium, 878

Nocardia, 899

- exudate

Sarcina, 290

Lupus

Bacillus, 661

Lymphogranuloma inguinals

Miyagawanella, 1116

Lymphogranuloma venereum

Miyagawanella, 1116

Madura foot

Nocardia, 909, 915

Streptothrix, 924

Malignant edema

Bacillus, 826

Proteus, 691

Malignant tumor

Spirochaeta, 1067

Malta fever

Brucella, 561

Marseilles fever

Rickettsia, 1089

Mastitis

Tetracoccus, 284

Mastoiditis

Bacteroides, 566

Diplococcus, 307

Staphylococcus, 262

Measles

Diplococcus, 311

Salmonella, 513

Streptococcus, $3 \notin 1$

Veillonella, 303, 304

Mediterranean fever

Rickettsia, 1089

Melioidosis

Malleomyces, 556

Meningit is

Bacillus, 662

Cillobacterium, 369

Corynebacterium, 403

Diplococcus, 307

Lactobacillus, 361

Malleomyces, 556

Neisseria, 297, 299, 301, 302

Salmonella, 505, 522, 527 
Human Diseases (continued)

meningitis (continued)

Streptococcus, 341

-, cerebroinapls fluid

Neisseria, 299

-, purulent

Bacterium, 676, 682

Hemophilus, 585

Sphaerophorus, 579, 580

Meningopneumonitis

Miyagawanella, 1117

Mexican typhus

Rickettsia, 1085

Mianeh fever, Persia Spirochaeta, 1069

Middle ear infections Staphylococcus, 282

Mite bite lesions (eschar) Rickettsia, 1091

Moroccan relapsing fever Spirochaeta, 1067

Multiple selerosis Spirochaeta, 1065

Murine typhus Rickettsia, 1087

Mycetoma

Actinomyces, 916, 918

Discomyces, 918, 919

Nocardia, 901, 907, 919, 921

Proactinomyces, 928

Streptomyces, 959, 961, 966

Streptothrix, 924

Mycosis fungoides

Streptocaccus, 343

Mycosis, pulmonary

Oospora, 923

Necrosis

Clostridium, 811, 821

Granulobacter, 822

-, intestinal

Bacillus, 659

Nephritis

Bacillus, 663

Bacterium, 682, 760

Nodular peritoneum

Bacillus, 666

Oosporosis, pulmonary

Oospora, 922

Ornithosis

Miyagawanella, 1117

Oroya fever

Bartonella, 1102
Osteomyelitis

Bacillus, 664

Ristella, 575

Streptococcus, 330

Osteophlegmon, maxillary bone

Pseudomonas, 701

Otitis

Bacteroides, 567

Cillobacterium, 349

Sphaerocillus, 580

Sphaerophorus, 579

Otitis media

Bacillus, 666

Corynebacterium, 402

Diplococcus, 307

Ozena

Bacillus, 658

Corynebacterium, 407

Klebsiella, 459

Pseudomonas, 95

Salmonella, 531

Sarcina, 292

Streptococcus, 340

-, secretions

Micrococcus, 266

Paratyphoid

Salmonella, 501, 507, 530, 552

Parenteric fever

Eberthella, 534

Parotitis epidemica

Micrococcus, 270

Parrot fever

Miyagawanella, 1117

Pasteurellosis

Pasteurella, 553

Pellagra

Bacillus, 748

Micrococcus, 281

Pseudomonas, 148

Pemphigus acutus, bullae

Micrococcus, 270

Pemphigus contagiosa, bullae

Micrococcus, 270, 271

Pemphigus neonatorum, bullae

Micrococcus, 271

Pericardial exudate

Bacillus, 745

Pericarditis

Diplococcus, 307

Nocardia, 899

Spirillum, 217 
Human Diseases (continucd)

Peritoneal exudate Sphaerophorus, 579

Peritonitis Clostridium, 820

Ramibacterium, 369

Streptococcus, 307

Pernicious anemia

Salmonella, 522

Pertussis

Bacterium, 590

Hemophilus, 586, 589

Petechiae in skin

Neisseria, 297

Phagadenous ulcer

Borrelia, 1064

Pharyngitis

Zuberella, 578

Phlegmon, perinephritic

Streptococcus, 329

Phthisis

Bacterium, 687

Sarcina, 293

Pink eye

Bacillus, 589

Pinta

Treponema, 1073

Pityriasis

Plague

Discomyces, 919

Pasteurella, 549

Pleurisy

Streptococcus, 329, 332

-, purulent

Eubacterium, 367

Pasteurella, 554

Ristella, 577

Streptobacillus, 581

Pleuropneumonia

Diplococcus, 310

Pleuropneumonia-like disease, 1293

Pneumonia

Actinomyces, 973

Bacillus, 647, 665, 703, 918

Brucella, 563

Diplococcus, 307

Klebsiella, 458

Salmonella, 518

Streptococcus, 341

Pneumonia, atypical

Miyagawanella, 1117
-, catarrhal

Bacterium, 687

-, louisiana

Miyagawanella, 1119

- septic

Bacterium, 684

-, virus

Miyagawanella, 1119

Pneumonic plague

Pasteurella, 549

Pneumonitis

Miyagawanella, 1119

Polyarthritis

Corynebacterium, 402

Postnatal fever Corynebacterium, 405

Postpoliomyelitic paralysis Veillonella, 304

Pretibial fever Rickettsia, 1098

Prostatitis Actinomyces, 918, 974

Pseudoactinomycosis Actinomyces, 916 Nocardia, 921

Pseudodysentery Shigella, 538

Pseudomycosis Micrococcus, 696

Pseudotuberculosis, pulmonary Nocardia, 919

Psittacosis

Rickettsia, 1095

Miyagawanella, 1117

Psoriasis

Nocardia, 921

Puerperal fever

Bacillus, 580

Micrococcus, 246

Strepiococcus, 318, 329, 331

Puerperal septicemia

Clostridium, 821

Staphylococcus, 701

Pulmonary mycosis

Oospora, 923

Pulmonary oosporosis

Oospora, 922

Pulmonary tuberculosis

Actinomyces, 917

Mycobacterium, 878, 897 
Human Diseases (continued)

Pulmonary tuberculosis (continued)

Oospora, 923

Streptococcus, 343

Purulent pleural fluid

Salmonella, 577

Purulent urethral discharge

Spirochaeta, 1074

Pus

Actinomyces, 916, 927, 971, 974

Bacillus, 659, 665, 737, 826

Bacterium, 678, 685

Clostridium, 806

Corynebacterium, 403, 406

Leptospira, 1078

Micrococcus, 241, 242, 243, 251, 256, 275

Nocardia, 921

Oospora, 922

Proactinomyces, 923

Sarcina, 293

Spirochaeta, 1069

Staphylococcus, 282

Streptococcus, 316, 321, 326, 341

Streptomyces, 963

Pus, anal pocket

Corynebacterium, 406

-, blue

Pseudomonas, 89

-, ears of scarlet fever patients

Corynebacterium, 402, 403

-, gonorrhoeal

Micrococcus, 276

Neisseria, 240

- , joints

Hemophilus, 585

Teisseria, 296, 297

-, peritoneal

Streptococcus, 337

-, pyelitis calculosa

Vibrio, 206

-, stinking

Bacillus, 583

-, tetanus

Bacillus, 649

-, teeth

Chromobacterium, 234

Pyelitis calculosa

Spirillum, 217

Vibrio, 206

Pyelocystitis

Klebsiella, 458
Pyemia

Bacterium, 685

Sphaerophorus, 580

Pyorrhoea

Streptothrix, 923

Pyorrhoea alveolaris

Micrococcus, 262

Spirochaeta, 1069

Treponema, 1072

Veillonella, 303, 304

Pyrexia

Salmonella, 519

Q (Queensland) fever

Coxiella, 1093

Rabies

Strepiococcus, 340

Rag picker's disease

Proteus, 691

Rat bite fever

Actinamyces, 972,974

Spirillum, 215

Streptothrix, 924

Rectal ulcer

Eubacterium, 36 б

Recurrent fever

Spirochaela, 1067

Red perspiration

Micrococcus, 263

Relapsing fever

Borrelia, 1060, 1061, 1062, 1063, 1064

Spirochaeta, 1065, 1066, 1069, 1070

- African

Borrelia, 1061

- - Central and South Africa

Borrelia, 1060

- - European

Borrelia, 1060

- - Indian

Borrelia, 1062

- -, Moroccan

Spirochaeta, $106 \overline{7}$

- - South American

Borrelia, 1064

- -, Spanish

Spirochaeta, 1067

- - Western

Borrelia, 1064

Rheumatism

Streptococcus. 343

-, acute

Actinomyces, 927 
Human Diseases (continucd)

Rheumatism, articular

Micrococcus, 275

Spirochaeta, 1070

Rhinitis

Zuberella, 578

-, chronic

Salmonella, 531

Rhinopharyngitis

Treponema, 1076

Rhinoseleroma

Klebsiella, 459

Rickettsialpox

Rickettsia, 1092

Rocky Mountain spotted fever Rickellsia, 1088, 1089, 1098

St. Vitis dance

$$
\text { Vocardia, } 975
$$

São Paulo exanthemic fever Rickeltsia, 1088

Sarcoma

Bacillus, 744

Scarlatina

Bacillus, 656

Corynebacterium, 406

Nocardia, 919

Scarlet fever

Bacillus, 649, 668

Micrococcus, 255, 275

Neisseria, 301

Streplococcus, 315, 343

Veillonella, 303, 304

Scrub typhus

Rickettsia, 1091

Scurvy

Bacterium, 678

Seborrhoic eczema

Pseudomonas, 148

Septicemia

Bacillus, 738

Bacterium, 553, 674, 686

Bacteroides, 566, 567

Chromobacterium, 232, 233

Clostridium, 796, 821, 826

Miyagawanella, 1119

Streptococcus, 316

Seven-day fever, Japan

Leptospira, 1077

Shin bone fever

Rickettsia, 1095
Skin abscess

Streptococcus, 333

-, ulcers

A urococcus, 250

Sleeping sickness

Borrelia, 1062

Smallpox pustules

Bacterium, 674

Staphylococcus, 282

Streptococcus, 345

Soduku

Streptothrix, 924

Soft chancre

Hemophilus, 587 -

South African tick bite fever Rickettsia, 1089

South American relapsing fever Borrelia, 1064

Spanish relapsing fever

Spirochaeta, 1067

Splenic anemia

Streptomyces, 964

Splenomegalia

Bacteroides, 581

Nocardia, 922

Sporotrichosis

Actinomyces, 916

Nocardia, 911

Spotted fever, Minas Geraes

Rickettsia, 1088

Spotted sickness

Treponema, 1073

Stomach cancer

Sarcina, 286, 290, 291

Stomatitis

Micrococcus, 696

-, creamy

Oospora, 922

Streptotrichosis

Streptomyces, 967

Strumitis

Bacillus, 655, 669, 671

Suppuration, wound

Micrococcus, 242

Swamp fever, Europe

Leptospira, 1078

Sycosis, bacillogenic

Bacterium, 687

Syphilis

Bacterium, 687

Borrelia, 1063 
Human Diseases (continued)

Syphilis (continued)

Treponema, 1071

Tabardillo Rickettsia, 1085

Taches noires Rickettsia, 1089

Tetanus

Bacillus, 818

Clostridium, 799

-, pus

Bacillus, 649

Tick bite fever, South African Rickettsia, 1089

Tick bite, primary sores Rickettsia, 1089

Tobia fever, Colombia Rickettsia, 1088

Tonsillar abscesses Oospora, 922

Tonsillar nocardiomycosis Nocardia, 921

Tonsillitis

Diplococcus, 310

Streptococcus, 337

Zuberella, 578

Tonsils, infected

Micrococcus, 246, 248

Veisseria, 300

Trachoma

\section{Bacillus, 589}

Chlamydozoon, 114

Micrococcus, 260, 269

Veisseria, 301

Noguchia, 593

Trench fever

Rickettsia, 1094, 1095, 1098

Spirochaeta, 1067

Trichomycosis axillaris

Micrococcus, 268

Trichomycosis axillaris, red variety

.Micrococcus, 266

Trichomycosis flava

Nocardia, 922

Trichorrhexis nodosa

Bacterium, 760

Tropical frambesia

Treponema, 1072

Tsutsugamushi disease

Rickeltsia, 1089, 1091

Tuberculosis

Bacterium, 676
Gaffkya, 283

Mycobacterium, 877, 879

Proactinomyces, 923

Streptolhrix, 924

-, bone

Streptococcus, 343

-, bovine

Mycobacterium, 879, 896

Tuberculosis, pulmonary

Actinomyces, 917

Mycobacterium, 878, 897

Oospora, 923

Spirillum, 217

Streptococcus, 343

Tuberculous cavity

Bacillus, 580

Tuberculous lesions

Mycobacterium, 879

Tularemia

Pasteurella, 549

Typhoid fever

Bacillus, 580, 666, 817, 827

Eberthella, 533

Pseudomonas, 148

Salmonella, 516, 519, 52

Typhus fever

Bacterium, 686

Corynebacterium, 406

Micrococeus, 260

Rickettsia, 1085, 1098

Spirochacta, 1070

Typhus, European

Rickettsia, 1085

Typhus exanthematicus

Rickettsia, 1085

Typhus, Kenya

Rickettsia, 1085

Lleers, abdominal wall

Streptomyces, 966

-, genital region

Spirochaeta, 1067

-, gingival

Nocardia, 921

-, granulating skin

Aurococcus, 250 .

_, oriental skin

Micrococcus, 254

-, pharynx

Nocardia, 920

-, rectal

Eubacterium, 367 
Human Diseases (continued)

Ulcers, skin

Corynebacterium, 406

Micrococcus, 695

Spirochaeta, 1068

-, thoracic

Actinomyces, 915

-, upper lip

Nocardia, 920

Uleus vulvae acutum

Bacillus, 400

Undulant fever

Brucella, 561, 562

Urethritis

Streptobacillus, 590

Urinogenital suppurations

Neisseria, 300

Vaccinia

Spirochaeta, 1074

Vaginitis

Bacillus, 826

Mimeae, 595

Neisseria, 301

Spirochaeta, 1074

Veld sores, Africa

Micrococcus, 280

- - A Australia

Micrococcus, 280

Venereal discharges

Micrococcus, 279

Neisseria, 296

Verruga peruana

Bartonella, 1101

Vincent's angina

Borrelia, 1063

Viral pneumonia

Miyagawanella, 1118

Vitiligo

Nocardia, 975

War wounds

Corynebacterium, 402

- - gangrene

Streptococcus, 329, 330

- - , septic

Sphaerophorus, 579

Warts

Bacterium, $68 t$

Weil's disease

Leptospira, $107 \%$

Pseudomonas, 90
Western relapsing fever

Borrelia, 1064

Whooping cough

Bacterium, 589, 590

Hemophilus, 587

Wolhynian fever

Rickettsia, 1095

Wounds, gangrenous

Bacillus, 738

Wounds, infected

Bacillus, 663, 826

Clostridium, 773, 776, 783, 792, 794, $798,799,801,811,812,825$

Diplococcus, 310

Inflabilis, 823

Micrococcus, 696

Plectridium, 826

-, superficial

Corynebacterium, 385

-, surgical

Bacteroides, 574

Yaws, ulcerated lesions

Spirochaeta, 1065, 1068

Treponema, 1072

Yellow fever

Bacillus, 648, 659, 661, 662, 752, 819, 826

Bacterium, 613, 675, 676, 679, 681, 687

Clostridium, 813

Corynebacterium, 404

Micrococcus, 261, 280, 281

Streptococcus, 338

Yellow fever vomit

Streptococcus, 340

\section{Human Sources}

Alimentary canal

Micrococcus, 251

Amniotic fluid

Streptococcus, 329,330

Veillonella, 303

Aorta

Bacterium, 67\%

Appendix

Bifidobacterium, 36!

Catenabacterium, 368

Arm

Martellillus, 323

Plectridium, 826

Reglillus, 323 
Human Sources (continued)

Ascitic fluid

Corynebacterium, 402, 403

Bartholin's gland

Streptococcus, 329

Bladder

Sarcina, 294

Blood

Actinomyces, 927,973

Alcaligenes, 413, 416

Bacillus, 580, 650, 659, 668, 669, 827

Bacterium, 553, 674, 686

Bartonella, 1102

Brucella, 561, 562

Clostridium, 821

Corynebacterium, 386, 400, 403, 405, 406

Diplococcus, 307, 311

Eberthella, 534

Escherichia, 447

Heliconema, 1064

Hemophilus, 585, 589

Leptospira, 1078, 1079

Malleomyces, 556

Micrococcus, 255, 260, 264, 272, 277

Miyagawanella, 1116

Veisseria, 296, 297

Vocardia, 975

Pasteurella, 549, 551

Proteus, 490, 491

Rickettsia, 1085, 1092, 1098

Salmonella, 512, 514, 518, 528, 531

Sphaerophorus, 579, 580

Spirillum, 217

Spirochaeta, 1067, 1069, 1070

Streptococcus, 316, 321, 326, 327, 329,

$330,336,340,341,343,344$

Streptomyces, 960, 965

Vibrio, 204

Blood culture, post mortem

Clostridium. 798

Blood, putrefying

Spirillum, 217

Blood vessels

Rickettsia, 1086

Body secretions

Micrococcus, 240, 261

Brain

Streptococcus, 340

Breast

Actinomyces, 927

Buccal cavity, see Mouth cavity
Cadaver

Bacillus, 752, 819, 826

Bacterium, 687

Clostridium, 813, 826

Streptococcus, 327

Cadaver, yellow fever

Bacillus, 612, 659, 662, 819, 826

Bacterium, 675, 676, 679, 687

Clostridium, 813

Salmonella, 531

Streptococcus, 338

Caecum

Alcaligenes, 415

Carriers

Salmonella, 512, 514, 515, 519, 520, $521,524,525,526,528,529$

Cerebrospinal fluid

Corynebacterium, 404

Diplococcus, 307

Hemophilus, 585, 589

Neisseria, 296, 297, 301

Streptothrix, 366

Cervix

Streptococcus, $3+1$

Cervix exudate

Chlamydozoon, 1115

Tibrio, 198

Chyluria

Bacillus, 651

Conjunctiva

Bacillus, 590, 666

Bacterium, 677, 684, 689

Chlamydozoon, 1115

Corynebacterium, 385, 386

Diplococeus, 311

Hemophilus, 585

Hicrococcus, 248, 25i, 261

Himeae, 595

Uoraxella, 591, 592

Neisseria, 296, 297

Pacinia, 691

Sarcina, 291

Conjunctival exudate

Chlamydozoon, 1115

Cornea

Actinomyces, 969, 973

Chlamydozoon, 1115

Digestive tract

Diplococcus, 309, 310

Micrococcus, 247, 251

Streptococcus, 320,332 
Human Sources (continued)

Digestive tract (continued)

Veillonella, 303

Duodenum

Bacterium, 688

Staphylococcus, 701

Streptococcus, 702

Ear

Corynebacterium, 403

Fndothelial cells

Bartonella, 1102

Rickettsia, 1086

Ipithelial cells, intestinal nucosa

Rickeltsia, 1085

Fye

Bacillus, 655

Micrococcobacillus, 690

Staphylococcus, 282

Eyelid

Actinomyces, 972

Female genital canal

Coccus, 250

Micrococcus, 246,252

Female genital tract

Coccus, 250

Gaffkya, 284

Vibrio, 205

Female genitalia

Bacteroides, 567

Food handlers

Salmonella, 512, 519, 521, 524, 526. $527,530,531$

Foot

Bacillus, 658, 661, 662, 670,671

-, skin of

Sarcina, 29)

Gall bladder

Bacillus, 61:2

General

Streptococcus, 338, 343

Genital canal

Bacillus, 361, 362, 580

Corynebacterium, 402

Streptococcus, 336

Streptostaphylococcus, 345

Cienital murous membranes

Borrelia, 1063

Genital secretions

Miyagawanella, 1116

Treponema, 1071, 1072
Genital tract

Staphylococcus, 281, 282

Genitalia

Bacteroides, 574

Borrelia, 1063

Genitourinary tract

Chlamydozoon, 1115

Klebsiella, 458

Gums

Streptococcus, 340

Hair

Micrococeus, 253

-, beard

Bacterium, 687

- , follicles

Bacterium, 686

Corynebacterium, 387

Micrococcus, 259

-, showing trichorrhexia

Bacterium, 688

Healthy persons

Vibrio, 204

Heart.

Bacterium, 677

Intestine

Aerobacter, 455

Alcaligenes, 413, 415

Bacillus, 659, 671, 739, 744, 745, 746, $747,757,758,813,814,815,817$, $825,826,918$

Bacterium, 580, 672, 677, 678, 686

Bacteroides, $567,568,569,570,571$, $572,573,674$

Bifidobacterium, 36?

Butyribacterium, 36!

Catenabacterium, 368

Clostridium, 78:3, 794, 799, 800, 809, 820,821

Corynebacterium, 402, 404

Eberthella, 533, 534

Escherichia, 447, 449, 450)

Eubacterium, 367, 368

Klebsiella, 458

Lactobacillus, 353, 354, 361

Microbacterium, 370

Micrococeus, 248, 251, 272, 274

Microspira, 202, 203

Neisseria, 301

Paracolobactrum, 460

Proteus, 489 
Human Sources (continued)

Intestine (continued)

Pseudomonas, 97, 145, 146, 147, 148, 150

Ristella, 577

Salmonella, 462, 516, 523

Sarcina, 292

Shigella, 539, 542, 543

Sphaerocillus, 580

Spirillum, 217

Spirochaeta, 1067, 1074

Streptococcus, 326, 327, 328, 330, 331

Veillonella, 304

Vibrio, 194, 198, 204, 205

Zuberella, 577,578

Joints

Corynebacterium, 386

Neisseria, 296, 297

Kidney

Bacterium, 677

Corynebacterium, 400

Spirochaeta, 1067

Large intestine

Micrococcus, 247

Larynx

Corynebacterium, 385

Liver

Bacillus, 658, 659, 660, 662, 667

Bacterium, 613, 676, 679, 683, 685

Bartonella, 1102

Clostridium, 821

Pasteurella, 549, 551

- Streptococcus, 338

Stieptomyces, 967

Lung

Actinomyces, 927,968

Bacillus, 667

Corynebacterium, 386

Hemophilus, 589

Miyagawanella, 1118, 1119

Oospora, 922

Spirillum, 217

Spirochaeta, 1065

Streptococcus, 329, 330, 331, 332, 343

Veillonella, 302

Lymph glands

Bacillus, 668

Bacterium, 689

Bartonella, 1101, 1102

Corynebacterium, 402, 403, 404

Iymphoid tissues

Diplococcus, 310
Maxilla

Robertsonillus, 823

Mouth cavity

Actinomyces, 926, 927, 971

A scococcus, 250,693

Bacillus, 365, 580, 650, 656, 657, $667,743,744$

Bacterium, 676, 678, 679, 687,761

Bacteroides, 574

Bifidobacterium, 369

Borrelia, 1062

Catenabacterium, 368

Coccus, 694

Corynebacterium, 386, 402, 404, 405

Diplococcus, 310

Fusobacterium, 582, 583

Helicobacterium, 690

Jodococcus, 695

Lactobacillus, 361

Leptospira, 1079

Leptothrix, 365, 366

Leptotrichia, 364, 365

Micrococcus, 248, 251, 257, 260, 262, $263,264,269,273,277,696$

Neisseria, 300

Proactinomyces, 923

Sarcina, 294

s'pirillum, 218

Śpirochaeta, 1065, 1066, 1070, 1074, 1079

Staphylococcus, 701

Streptococcus, 316, 320, 321, 323, 329, $330,331,333,336,338,339,341$,

$342,343,344$

Treponema, 1072, 1075

Teillonella, 302, 303, 304

Vibrio, 203, 204, 205, 206, 207, 703

Mouth eavity, putrid tissue

Clostridium, 820

Granulobacter, 822

Mucous membrane

Bacteroides, 574

Pasteurella, 552

Streptococcus, 342, 344

_ - mouth ravity

Caryophanon, 1004

- - nasal

Micrococess, 274,276

Sarcina, 292

Zuberella, 578 
Human Sources (continued)

Mucous membrane, nose and throat

Neisseria, 301

- - , respiratory tract

Borrelia, 1062, 1063

Dialister, 595

Gaffkya, 283

Neisseria, 298, 299, 301

Mucus

Bacillus, 737

Mucus, intestinal

Bacillus, 648, 649, 664, 67!

-, nasal

Bacillus, 658, 659, 667, 669

Corynebacterium, 406, 407

Micrococcus, 257, 278

Neisseria, 301

Pseudomonas, 95

Streptococcus, 342,344

Vibrio, 203

Nasal washings

Veillomella, 303

Nasopharyngeal secretions

Dialister, 595

Neisseria, 301

Nasopharynx

Hemophilus, 585

Neisseria, 297, 298, 299, 301

Pasteurella, 581

Staphylococcus, 282

Zuberella, 577

Natural cavities

Gaffkya, 284

Veillonella, 303

Nerves, peripheral

Mycobacterium, 882

Nose

Corynebacterium, 385, 40:3

Diplococcus, 311

Micrococcus, 260, 262

Sarcina, 292

Streptococcus, 318, 321

-, mucous membrane, sec Mucous membrane, nasal

Oral cavity, see Mouth cavity

Organs, internal

Leptospira, 1078

Peritoneum

Bacillus, 666

Pharynx

Corynebacterium, 385, 403
Diplococcus, 310,311

Streptococcus, 320, 321

Pia mater

Nocardia, 975

Pleural fluid

Pasteurella, 549

Preputial secretions

Bacillus, 612

Rectum

Alcaligenes, 415, 416

Bacillus, 650

Red perspiration

Micrococcus, 263

Red pus

Bacterium, 685

Respiratory mucous membrane, sec

Mucous membrane, respiratory tract

Respiratory system

Cillobacterium, 369

Diplococcus, 307

Eubacterium, 368

Respiratory tract

Hemophilus, 585, 586, 587, 589

Klebsiella, 458

Neisseria, 298, 299, 300

Streptococcus, 316, 319, 321, 331, 332, 333,334

Saliva

Bacillus, 647, 671

Diplococcus, 307

Flavobacterium, 440

Leptothrix, 366

Micrococcus, 258, 275

Neisseria, 298

Staphylococcus, 282

Streptococcus, 320, 321, 337, 344

Teillonella, 304

scalp

Micrococcus, 266

Sebaceous glands

Corynebacterium, 387

Sinuses

Hemophilus, 585

Streptococcus, 321, 333

Skin

Bacillus, 647, 650, 651, 655, 656, 658, $660,661,662,663,668,670,671$, 742,743

Bacterium, 674, 683, 686

Corynebacterium, 386, 403, 406

Eubacterium, 368 
Human Sources (continued)

Skin (continued)

Gaffkya, 283

Micrococcus, 239, 242, 243, 244, 251, $252,254,255,256,257,258,259$, $262,274,695$

Mycobacterium, 882

Nocardia, 919, 921

Plocamobacterium, 691

Pseudomonas, 148

Rickettsia, 1086

Sarcina, 288, 290

Streptococcus, 318, 333, 343

skin, dry scalp lesions

Discomyces, 975

shin of foot

Micrococcus, 251, 252, 256, 262

Simegma

Mycobacterium, 890

Sarcina, 291

Treponema, 1072

Ipinal fluid

Bacillus, $66 \pi$

Miyagawanella, 1176

Salmonella, 522

Spleen

Bacillus, 613

Bacterium, 6ri

Brucella, 561

Pasteurella, 549, 551

Salmonella, 522

Streptococcus, 341, 344

Streptomyces, 958, 962, 963, 964

\section{Sputum}

Actinomyces, $916,917,970,972$

Bacillus, 647, 653, 664. 665, 667, 668, $669,672,703,738,740,741,752$, 751,918

Bacterium, 761

Brucella, 693

Cladothrix, 974

Diplococcus, 307

Gaffkya, 283

Hemophilus, 585

Klebsiella, 458, 459

Micrococcus, 257, 267, 277

Miyagawanella, 111 ?

Moraxella, 592

Neisseria, 298

Nocardia, 920, 921, 976

Oospora, 922, 923, 976

Pasteurella, 549
Proactinomyces, 923

Pseudomonas, 698

Sarcina, 293, 294

Serratia, 484

Spirillum, 701

Streptococcus, 320, 321, 340, 341, 343,344

Streptothrix, 924

Vibrio, 198

-, green

Pseudomonas, 149

Stomach

Actinomyces, 969

Alcaligenes, 416

Bacillus, 647, 650, 660

Eubacterium, 367

Helicobacterium, 690

Sarcina, 286, 290, 291, 294

Stomach contents

Bacillus, 814

Gaffkya, 284

Submaxillary lymphatie gland (child)

Micrococcus, 280

Sweat

Bacillus, 666, 673

Tear duct

Actinomyces, 970

Teeth

Acidobacterium, 361

Aerobacter, 456

Fusobacterium, 582, 583

Granulobacter, 822

Streptococcus, 320, 339, 340, 341, 342

-, carious

Bacillus, 653

Micrococcus, 262

- - decayed

Cladothrix, 918, 974

Throat

Corynebacterium, 386, 387, 404, 406

Diplococcus, 311

Hicrococcus, 280

Spirochaeta, 1065

Streptococcus, 316, 318, 320, 321, 3333, $334,335,337,339,3+1,342,343$

Veillonella, 303

\section{Tongue}

Acidobacterium, 361

-, deposit on

Nocardia, 920

Streptococcus, 340

Vibrio, 205 
Human Sources (continued)

Tongue, epithelium

Micrococcus, 267

Tonsillar erypts

Actinomyces, 927

Vibriothrix, 833

Tonsillar granules

Spirillum, 218

Tonsils

Alcaligenes, 416

Bacillus, 583

Corynebacterium, 405

Diplococcus, 310

Sphaerophorus, 579

Streptococcus, 338

Veillonella, 304

Trachea

Corynebacterium, 385

Urethra

Corynebacterium, 404

Leptothrix, 366

Micrococcus, 248

Streptobacillus, 590

Streptococcus, 340

Urethral exudate

Chlamydozoon, 1115

Urinary tract

Micrococcus, 248

Neisseria, 300

Staphylococcus, 282

Urine

Bacillus, 647, 652, 653, 65̃5, 660, 661. $663,664,668,669,671,741,757,758$

Bacterium, 678, (688, 760

Bacteroides, 574

Leptospira, 1077, 1079

Micrococcus, 238, 247, 260, 266, 267,

$269,279,280$

Mycobacterium, 890)

Proteus, 490, 491

Salmonella, 518, 531

Sarcina, 289, 293

Spirochaeta, 1067, 1070

Spiroschaudinnia, 1071

Staphylococcus, 282

streptocoscus, $323,338,340,341$

Eterus

Actinomyces, 580

Bacillus, 668

Proteus, 490
Spirochaeta, 1067, 1074

Streptococcus, 329, 331, 332

Vaccine pustules

Corynebacterium, 401

Vagina

Bacillus, 580, 612, 652, 667

Lactobacillus, 362

Leptothrix, 366

Mimeae, 595

Veisseria, 301

Streptococcus, 318, 319, 327, 329, 330, $332,333,334,337,338$

Vaginal secretions

Micrococcus, 261, 278

Vulva

Streptococcus, 338

Ice

Micrococcus, 272

Ice Cream, see Dairy Products

\section{Infusions}

Asparagus

Bacillus, 656

Bacterium, 679

Micrococrus, 262

Bean

Bacillus, 657, 664, 669, 750

Bacterium, 679, 685

Micrococcus, 262, 264, 268, 271, 273, 279

Spirillum, 218

Beef

Clostridium, 811, 821

Brewer's grain

Bacillus, 656

Carrot

Micrococcus, 261, 264

Streptococcus, 341

Cheese and white beets

Bacillus, 711

Corn

Bacillus, 650, 748

Bacterium, 681

Digitalis

Bacillus, 745

Micrococcus, 262, 263

Egg white, cooked

Bacillus, 757

Fermenting

Bacillus, 672 
Infusions (continued)

General

Bacillus, 755

Hay

Proteus, 488

Aerobacter, 456

Bacillus, 711, 743, 815, 824

Herbs

Spirochaeta, 1053

Jequirity seed

Bacillus, 672

Kohlrabi

Vibrio, 206

Leaves, indigo plant

Bacillus, 659

Lentils

Bacillus. 711

Malt

Meat

Micrococcus, 263

Bacillus, 654

Pseudomonas, 147

-, extract

Micrococcus, 255, 267

-, putrefying

Micrococcus , 254, 256

Potato

Bacillus, 650

Putrefying

Bacterium, 682

Snakeroot

Micrococcus, 263

Vegetable

Bacillus, 654, 656, 671

Bacterium, 675, 679, 681, 687, 688, 689

Micrococcus, 277, 278, 280

Pseudomonas, 147, 148

Wheat

Bacillus, 650

Insecta, Scientific Names

Adoretus compressus, 657

Amitermes minimus, 980

Anasa tristis, 654

Apis mellifera, 490, 618, 657, 660, 667,

$669,670,673,676,693,724,726,737$.

$738,740,744,749,75 \pi$

A pis mellifica, 266, 326, 327, 337, 422

Arctia caja, 690

Blatta germanica, 402

Blatta orientalis, 1003
Blatta (Periplaneta) orientalis, 740

Blissus lencopterus, 264

Bombyx mori, 254, 265, 282, 336, 337, $342,481,490,650,652,739,754$

Calotermes (Glyptotermes) iridipennis, 583,742

Calotermes spp., 1070

Ceratomia catalpae, 746

Chironomus plumosus, 173

Chironomus sp., 635

Cimex hirundinis, 1096

Cimex lectularius, 392, 605, 1060, 1096, 1104

Cimex roturdatus, 1062

Clisiocampa fragilis, 660

Cnethocampa pityocampa, 342, 684

Coccinella novemnotata, 431, 440

Coenagrionidae, 269

Conocephalus fasciatus, 440

Ctenocephalides felis, 1096

Ctenocephalus felis, 1066

Ctenocephalus sp., 1104

Culex fatigans, 1096

Culex pipiens, 1098

Culex sp., 1066

Danais archipus, 260

Diabrotica sp., 124

Diapheromera femorata, 433, 679

Diprion sertifer, 668

Drosophila confusa, 1075

Eacles imperialis, 608

Echidnophaga gallinacea, 1086

Encaptolopus sordidus, 66.5

Ephestia kuehniella, 259, 260, 647, 759

Euxoa segetum, 336, 425, 460, 689

Euxoa (Agrotis) segetum, 737

Galleria mellonella, 200, 259, 340, 452, 759,762

Gelechia gossypiella, 678, 686

Glassina palpatis, 1062

Glyptotermes iridipennis, 1075, 1076 . 1121

Gortyna ochracea, 65̄

Gryllotalpa gryllotalpa, 745

Hyponomeuta evonymella, 750, 754

Hyponomeuta sp., 267

Isophya (Barbitistes) amplipennis, 739

Kalotermes sp., 1003, 1121

Lachnosterna sp., 269

Lathridius rugicollis, 971 


\section{Insecta (continued)}

Leptinotarsa decemlineata, 419, 440,660

Leucotermes lucifugus, 1067

Linognathus stenopsis, 1097

Locusta migratoria, 666

Lucilia caesar, 780

Lucilia sericata, 274, 301, 748

Lygus pratensis, 603

Lymantria dispar, 739

Lymantria monacha, 267, 648, 651, 652, $659,660,663,682,749,753,755$

Malacosoma americana, 416

Malacosoma castrensis, 690

Melanoplus femurrubrum, 665

Melolontha melolontha, 336, 662, 681, $690,753,757$

Musca domestica, 282, 528, 677

Neotermes howa, 1121

Neurotoma nemoralis, 268, 663

Neurotoma nemoralis, larvae of, 268

Noctuidae, 491

Oncopeltus fasciatus, 490

Orgyia pudibunda, 670

Pectinophora gossypiella, 664

Pedicinus longiceps, 1085

Pediculus humanus, 664, 1060, 1097

Pediculus humanus var. capitis, 1085, 1095

Pediculus humanus var. corporis, 1085 , 1095

Pediculus vestimenti, 1061, 1062

Periplaneta americana, 217, 405, 425

Periplaneta orientalis, 652, 1069

Phlebotomus noguchii, 1102

Phlebotomus perniciosus, 1074

Phlebotomus verrucarum 1102

Photuris pennsylvanicus, 491

Pieris brassicae, 336, 665, 666, 690

Pieris rapae, 271, 440, 737

Polyplax serrata, 1112

Polyplax spinulosus, 1086, 1104

Popillia japonica, 727

Portheria dispar, 250

Portheria (Lymantria) dispar, 336, 660, 661

Potosia cuprea, 808

Procryptotermes sp., 1121

Prodenia litura, 684

Protoparce sp., 491

Pyrameis (Vanessa) cardui, 667
Pyrausta nubilalis, 200, 259, 452, 685, $690,750,759,760,761$

Reticulitermes flavipes, 1003

Reticulitermes lucifugus, 1069

Rhagoletis pamonella, 141

Rhodnius prolixus, 914

Sceliphron cementarium, 605

Scoliopteryx libatrix, 659

Simulium noelleri, 1068

Stomoxys calcitrans, 677

Stylopyga orientalis, 1076

Stylopyga (Blatta) (Periplaneta) orientalis, 1075

Temmorhinus (Cleonus) mendicus, 652

Tenebrio molitor, 634,680

Termes lucifugus, 1070

Thyridopteryx ephemeraeformis, 392, 604

Tibicen linnei, 269, 608

Triatoma rubrofasciata, 1098

Trichodectas pilosus, 1097

Urographus fasciata, 420

Tanessa polychlorus, 655

Tanessa utricae, 653, 656

Yenopsylla astia, 1086

Yenopsylla cheopis, 1086, 1104

\section{Insect Diseases, see Insects}

Insects, see Insecta for Scientific Names

Apple maggot

Pseudomonas, 141

Bagworm

Bacterium, 604

Corynebacterium, 392

Bedbug

Bacterium, 605

Borrelia, 1060

Corynebacterium, 392

Haemobartonella, 1104

Bee moth

Bacterium, 759, 762

Escherichia, 452

Micrococcus, 259

Streptococcus, 340

Vibrio, 200

Bees

Bacillus, 693

Bacterium, 673, 676

Bifidobacterium, 369

Proteus, 490

Salmonella, 532 
Insects (continued)

Bees and larvae

Bacillus, 648 667, 669, 670

-, diseased

Bacillus, 657, 738, 740, 749, 757

一, foulbrood

Bacillus, 737, 744

Micrococcus, 266

Streptococcus, 326, 327, 337

-, foul brood, American

Bacillus, 726

-, foulbrood, benign

Bacillus, 660

-, foul brood, European

Achromobacter, 422

Bacillus, 724

-, infectious diarrhoea

Proteus, 490

Beetle larvae

Achromobacter, 120

Beetles

Actinomyces, 971

Bacillus, 657

Fusiformis, 694

Pseudomonas, 124

Blood-sucking insects

Pasteurella, 551

Blue bottle fly larvae

Clostridium, 780

Butterfly

Bacillus, 653, 655, 656, 667

Cabbage butterfly

Bacillus, 665, 666, 737

Diplobacillus, 690

Flavobacterium, 440

Streptococcus, 336

- - larvae

Microcaccus, 271

Caterpillars

Bacillus, 657

Bacterium, 612

Coccobacillus, 690

Pseudomonas, 94, 148

Streptococcus, 339

-, feces

Pseudomonas, 148

-, wilt disease

Micrococcus, 261

Chinch bug, caecal organs

Micrococcus, 264

Cicada, Lyreman
Bacterium, 608

Micrococcus, 269

Cockchafer

Bacillus, 662, 753, 757

Bacterium, 681

Diplobacillus, 690

Streptococcus, 336

Cockroach

Bacillus, 740

Fusiformis, 694

Sarcina, 294

Spirochaeta, 1069

Treponema, 1075

-, fat body

Bacillus, 652

Corynebacterium, 402, 405

-, feces

Achromobacter, 425

- intestine

Arthromitis, 1003

Spirillum, 217

Treponema, 1076

Colorado potato beetle, intestine

Achromobacter, 419

Bacillus, 660

Flavobacterium, 440

Corn borer, European

Bacillus, 750

Bacterium, 685, 759, 760, 761

Coccobacillus, 690

Escherichia, 452

Micrococcus, 259

Vibrio, 200

C'ricket

Bacillus, 745

Cutworm septicemia

Proteus, 491

Damsel fly

Micrococcus, 269

Firefly

Proteus, 491

Fleas

Haemobartonella, 1104, 1106

-, eat

Rickettsia, 1096

Spirochaeta, 1066

-, chicken

Rickettsia, 1086

$-, \operatorname{dog}$

Haemobartonella, 1104

一, rat 
Insects (continued)

Fleas, rat (continued)

Pasteurella, 549

Rickettsia, 1086, 1087

Flies

Salmonella, 528

Staphylococcus, 282

-, black

Spirochaeta, 1068

-, blue bottle

Micrococcus, 274

Neisseria, 301

-, fruit

Treponema, 1075

-, green blow

Bacillus, 748

-, tsetse

Borrelia, 1062

Fly larvae

Bacterium, 677

- - blue bottle

Clostridium, 780

Gieneral

Leptothrix, 366

Wicrococcus, 281

Rickettsia-like organisms, 1098, 1099

Grasshoppers

Eberthella, 534

Flavobacterium, $\mathbf{4 0}$

Gypsy moth

Bacillus, 660, 661, 739?

Cryrococcus, 250)

Streptococcus, 336

Hornworm septicemia

$$
\text { Proteus, } 491
$$

Imperial moth

Bacterium, 608

Japanese heetle, Milky discasc

Bacillus, 727

- -, milky disease, Type B

Bacillus, 727

June bug or beetle

Bacillus, 746

Micrococcus, 269

Lady beetle larvae

Flavobacterium, 440

Lady beetle, nine-spotted

Flavobacterium, 431, 440

Larvar

Bacillus, 64!
Leaf beetle

Bacterium, 679

Lice

Bacillus, 664

Borrelia, 1060, 1061

Rickettsia, 1085, 1095, 1097, 1099

-, biting

Rickettsia, 1097

-, body

Rickettsia, 1085

-, goat

Rickettsia, 1097

-, head

Rickettsia, 1085

-, mouse

Eperythrozoon, 1112

- rat

Rickettsia, 1086, 1104

Locusts

Bacillus, 666

-, diseased

Micrococcus, 251

May fly nymph shells

Chitin-digesting bacteria, 632

Meal worm

Bacterium, 634, 680

Mediterranean flour moth

Bacillus, 647

- - - larvae

Micrococcus, 259, 260

Midge

Bacterium, 635

-, larvae

Pseudomonas, 173

Milkweed bugs

Eberthella, 534

Protens, 490

Monarch butterfly larvae, wilt disease Micrococeus, 261

Mosquito

Rickettsia, 1096, 1098

-, feces

Micrococcus, 280

-, larvae

Spirochaeta, 1066

Moth

Bacillus, 659, 660, 737, 750, 754

Bacterium, 678, 682, 684, 686, 689

Coccobacillus, 690

Paracolobactrum, 460

Streptococcus, 336 
Insects (continued)

Moth (continued)

-, crushed egg masses Alcaligenes, 416

Mud dauber wasp

Bacterium, 605

Nun moth

Bacillus, 648, 651, 652, 659, 660, 663, 749,753755

- - larvae

Micrococcus, 267

Olive fly

Bacillus, 647

Pink bollworm

Bacillus, 664

Processionary moth

Bacterium, 684

Streptococcus, 342

Reduvid bug

Nocardia, 914

Rickettsia, 1098

Roseleaf beetle

Clostridium, 808

Sandfly

Bartonella, 1102

Spirochaeta, 107 t

Sawfly

Bacillus, 663, 668

Silkworm

Bacillus, 650, 652

Proteus, 490

Serratia, 481

Staphylococcus, 282

Streptococcus, 336, 342

-, blood and organs

Mierococcus, 254, 265

-, diseased

Bacillus, 739

Micrococcus, 265

Streptococcus, 337

Sphinx moth

Bacillus, 746

Squash bug

Bacillus, 654

Stink bugs

Eberthella, 534

Tarnished plant bug

Bacterium, 603

Tent caterpillar

Bacillus, 746

Termites
Caryococcus, 1121

Fusiformis, 583

Spirochaeta, 1067

Treponema, 1075, 1076

-, intestine

Arthromitis, 1003

Bacillus, 728, 742

Coleomitus, 1003

Micromonospora, 980

Spirochaeta, 1069, 1070

Tettigonids, diseased

Bacillus, 739

Tipulid

Fusiformis, 694

Walking stick

Bacterium, 679

Flovobacterium, 433

Wasps

Salmonella, 532

Weevils

Bacillus, 652

Winter wheat cut worm

Achromobacter, 425

Leaven, see Yeast

Mammals, also see Cats, Cattle, Dogs, Goats, Guinea pigs, Hogs, Horses, Mice, Rabbits, Rats, and Sheep

Ant eater, five-toed Bartonella, 1108

Ape, alimentary tract

Serratia, 481

Bandicoot

Coxiella, 1093

Bat, blood

Spirochaeta, 1070

Spironema, 1070

-, erythrocytes

Bartonella, 1108

Grahamella, 1110, 1111

-, intestine

Chitin digesting bacteria, 632

Buffaloes, blood

Spirochaeta, 1065

--, erythrocytes

Haemobartonella, 1106

-, leucocytes

Rickettsia, 1098

Camel

Salmonella, 513

Carnivora

Salmonella, 509 
Mammals (continued)

Chimpanzee, feces

Clostridium, 785

-, general

Spirochaeta, 1079

Deer mouse, erythrocytes

Grahamella, 1111

Haemobartonella, 1106, 1107, 1108

Deer mouse, gray-backed

Erythrocytes

Eperythrozoon, 1113

Haemobartonella, 1107

Domestic animals, general

Salmonella, 523

Streptococcus, 317,318

- -, lymph

Corynebacterium, 404

- - , mouth cavity, mucous membrane

Caryophanon, 1004

- - throat

Streptococcus, 334

Dormice, erythrocytes

Haemobartonella, 1108

Ferrets

Brucella, 563

-, respiratory tract

Hemophilus, 58?

Flying fox, intestine

Acuformis, 813

Bacillus, 818

Foxes

Salmonella, 517, 525

General

Streptococcus, 338

- intestine

Bacillus, 817, 826

Bacteroides, 567, 568, 569, 570, 571, $572,573,574$

Butyribacterium, 380

(lostridium, 796, 810

Lactobacillus, $35 t$

Streptococcus, 331

-, mouth

Streptococcus, 331

-, mucous membrane

Bacteroides, 574

Streptococcus, 242, 243, 244

-, natural cavities of mammals

Gaffkya, 284

-, saliva

Streptococcus, 304
Gerbilles, ery throcytes

Hemobartonella, 1108

Ground hogs

Pasteurella, 551

Hamsters, erythrocytes

Grahamella, 1110, 1111

Haemobartonella, 1104

Hamsters, Chinese

Erythrocytes

Haemobartonella, 1108

Jerboa, ery throcytes

Haemobartonella, 1108

Laboratory animals

Streptococcus, 317

Lemmings

Pasteurella, 551

Moles, erythrocytes

Grahamella, 1109

Monkeys

Bartomella, 1108

Spirochaeta, 1068

-, blood

Rickettsia, 1088

Spirillum, 1065

-, erythrocytes

Grahamella, 1111

Haemobartonella, 1104

- infected with yellow fever virus

Corynebacterium, 404

-, trachoma

Chlamydozoon, 1114

Musk rats

Pasteurella, 551

Opossum, blood

Spirochaeta, 1066

- ery throcytes

Haemobartonella, 1108

Otter, blood

Spirochaeta, 1067

Primates, lower

Salmonella, 502

Rodents

Pasteurella, 549, 551

Salmonella, 517, 527

Spirochaeta, 1068

Streptococcus, 310

-, blood

Borrelia, 1064

Shrew mouse

Spirochaeta, 1066

Shrew, short tailed 
Mammals (continued)

Shrew (continued)

Erythrocytes

Haemobartonella, 1107

Squirrel bite

Actinomyces, 974

Squirrels

Erythrocytes

Haemobartonella, 1108

Squirrels, gray

Erythrocytes

Haemobartonella, 1107

Squirrels, ground

Bacillus, 669

Pasteurella, 549, 551

Vole

Erythrocytes

Eperythrozoon, 1113

Grahamella, 1110

Haemobartonella, 1104, 1106

Vole, Field

Leptospira, 1077

Water rats

Pasteurella, 551

Weasel bite

Actinomyces, 973

Whales

Ambergris

Wolf

Spirillum, 217

Ery throcytes

Grahamella, 1110

Mammals, Diseases of

Apes

Conjunctivitis, granular

Noguchia, 593

Enterocolitis

Salmonella, 510, 519

Orchitis

Spirochaeta, 1074

Parotitis

Spirochaeta, 1074

Syphilis

Treponema, 1071

Trachoma

Chlamydozoon, 1114

Beavers

Hemorrhagic septicemia

Pasteurella, 551

Buffaloes

Hemorrhagic septicemia

Pasteurella, 548, 549
Infections

Corynebacterium, 391

Camels

Pseudotuberculosis

Actinomyces, 915

Chimpanzees

Bacillary dysentery

Salmonella, 519

Deer

Hemorrhagic septicemia

Pasteurella, 547

Domestic animals

Abortion

Brucella, 561, 562

Abscesses

Corynebacterium, 388

Tuberculosis

Mycobacterium, 879

Ulcerative lesions

Corynebacterium, 389

Ferrets

Pasteurella, 552

Foxes

Listeriosis

Listeria, 409

Pneumonia

Streptococcus, 317

General

Blue pus

Pseudomonas, 89

Cholera

Vibrio, 196

Conjunctivitis, acute

Miyagawanella, 1119

Infections

Streptococcus, 342

Malignant edema

Clostridium, 776

Bacillus, 826

Septicemia

Streptococcus 232, 233

Gerbilles

Listeriosis

Listeria, 409

Relapsing fever

Spirochaeta, 1069

Kangaroos

Gastroenteritis

Nocardia, 921

Lumpy jaw

Nocardia, 921 
Mammals Diseases of, (continued)

Kangaroos (continued)

Septicemia

Nocardia, 921

Laboratory animals

Brucellosis

Brucella, 561, 562

Monkeys

Abortion

Brucella, 562

Brucellosis

Brucella, 562, 563

Conjunctival folliculosis

Noguchia, 594

Conjunctivitis, granular

Noguchia, 593

Conjunctivitis, inflammatory Noguchia, 594

Fever, boutonneuse Rickettsia, 1089

Fever, relapsing Spirochaeta, 1069

Tuberculosis, bovine Mycobacterium, 879

Tuberculosis, human

Mycobacterium, 878

Moose

Tick paralysis

Mules

Klebsiella, 459

Botulism

Clostridium, 779

Gangrenous dermatitis

Sphaerophorus, 579

Reindeer

Hemorrhagic septicemia

Pasteurella, 549

Rodents

Eperythrozoönosis Eperythrozoon, 1111

Glanders-like disease Malleomyces, 556

Grahamellosis Grahamella, 1109

Haemobartonellosis

Haemobartonella, 1102

Melioidosis

Malleomyces, 556

Pseudotuberculosis

Pasteurella, 549, 551, 553
Sea calf

Septicemia

Bacterium, 689

Squirrels, ground

Tularemia

Pasteurella, 569

Water buffaloes

Pleuropneumonia, bovine Asterococcus, 1292

Osteomyelitis

Clostridium, 825

Septicemia

Chromobacterium, 233

Whales

Septicemia

Clostridium, 819, 825

\section{Mammals, Scientific Names}

Acodon serrensis, 1109

Alactaga spp., 1109

A podemus agrarius, 1108

Arctomys marmota, 1068

Arvicola arvalis, 1113

Blarina brevicauda, 1107, 1110

Bos taurus, 1110

Cavia porcellus, 1106

Cercopithecus patas, 1065

Citellus pygmaeus, 1110

Cricetulus spp., 1108, 1110

Cricetus domesticus, 1110

Cricetus phoca, 1111

Ctenodactylus gondi, 1064

Desmodus rufus, 1110

Didelphys aurita, 1066

Didelphys didelphys, 1108

Eliomys quercinus, 1110

Gerbillus tamaricinus, 1110

Glis glis, 1108

Golunda fallax, 1110

Hemiderma brevicauda, 1108

Isodon macrurus, 1093

Jaculus jaculus, 1109

Jerboa sp., 1113

Lutra sp., 1067

Macacus rhesus, 594

Macacus sp., 1104, 1111

Manis pentrdactyla, 1108

Mastomys coucha, 1110

Meriones tristrami, 1004, 1110

Metachirus opossum, 1108

Microtus arvalis, 1109, 1110, 1113 
Mammals (continued)

Microtus montebelli, 1077

Micratus pennsylvanicus pennsylvanicus, $1105,1110,1113$

Mus acomys, 1113

Mus decumanus, 1107, 1110

Mus minutus, 1113

Mus musculus, 1110

Mus norvegicus, 1107

Mus rattus, 891, 1107, 1110

Mus rattus griseiventer, 1107

Mus sylvaticus, 1107

Myoxus glis, 1108

Peromyscus leucopus novaboracensis, $1106,1108,1111$

Peromyscus maniculatus, 1111

Peromyscus maniculatus gracilis, 1107, 1113

Phodopus praedilectus, 1108

Phoga vitulina, 689

Phyllotis darwini linatus, 111

Pipistrellus nathusii, 1111

Pseudocebus apella, 1108

Pteropus, 813, 818

Rattus rattus, 1107, 1113

Rattus rattus frugivorus, 1107

Sciurus carolinensis leucotis, 1107

Sciurus vulgaris, 1108, 1113

Spalax typhlops, 1104

Spermophilus musicus, 669

Thalassochelys caretta, 1111

Vespertilio kuhlii, 1070

Vespertitio noctula, 1110

Tesperugo kuhlii, 1070

\section{Manure (Dung)}

Black cock

Mycococcus, 1042

Cat

Streptococcus, 337

Compost

Serratia, 484

Cow

Achromobacter, 423

Bacillus, 815, 824

Caryophanon, 1004

Corynebacterium, 403

Microbacterium, 370,371

Micrococcus, 271

Mycobacterium, 889

Мухососсия, 1042, 1043
Ristella, 576

Streptococcus, 322, 323, 337, 338, 342, 345

Deer

Angiococcus, 1048

Archangium, 1018

Chondrococcus, 1045, 1046

Chondromyces, 1037, 1038

Melittangium, 1034

Myxacoccus, 1042

Synangium, 1033

Deer (Antelope)

Chondromyces, 1037, 1038

Deer (Roe)

Archangium, 1019

Chondrococcus, 1045

Myxococcus, 1042

Deer (Stag)

Myxococcus, 1042

$\operatorname{Dog}$

Bacillus, 815, 826

Myxococcus, 1042

Spirochaeta, 1065

Streptococcus, 337

Fox

Streptococcus, 323

Frog

Bacillus, 742

General (usually cow)

Aerobacter, 456

Bacillus, 729, 737, 738

Bacterium, 602, 613, 643, 682, 686, 690,761

Chondromyces, 1037

Clostridium, 776, 778, 783, 803, 809, $810,815,816,817,818,820,821,822$

Granulobacter, 826

Micrococcus, 271, 275

Mycobacterium, 890, 891

Myxococcus, 1042, 1043

Plectridium, 823

Podangium, 1035

Polyangium, 1030

Pseudomonas, 105, 145

Streptomyces, 940

Goat

Chondrococcus, 1045, 1046

Myxococcus, 1042

Podangium, 1035

Goose

Chondromyces, 1038 
Manure (Dung) (continued)

Grouse

Chondrococcus, 1045

Guano

Bacillus, 745

Guinea pig

Bacillus, 734, 736

Hare

Archangium, 1018, 1019

Chondrococcus, 1045, 1047

Polyangium, 1031

Sorangium, 1023

Hen

Myxococcus, 1042

Herbivorous animals

Micrococcus, 248

Hog

Vibrio, 206

Horse

Achromobacter, 425,426

Bacterium, 642, 819

Bacillus, 733, 734, 736, 749, 754

Caduceus, 819

Chondrococcus, 1045

Clostridium, 783, 799, 810, 813, 814, 815,821

Flavobacterium, 441, 442, 822

Myxacoccus, 1042

Podangium, 1035

Sorangium, 1023

Streptococcus, 337

Liquid

Sarcina, 290, 291

Spirillum, 216

Vibrio, 204, 206

Mouse

Mycobacterium, 890

Myсососсиs, 1042

Podangium, 1035

Mouse, field

Chondrococcus, 1045

Muskrat

Angiocaccus, 1048

Parrot

Miyayauanella, 1117

Rabbit

Archangium, 1018, 1019, 1020

Chondrococcus, 1045, 1046

Chondromyces, 1038

Melittangium, 1034
Methanobacterium, 646

Myxococcus, 1042, 1044

Podangium, 1035

Polyangium, 1026, 1027, 1030, 1031

Sorangium, 1022, 1023

Synangium, 1033

Robin

Bacillus, 756

Stable

Bacillus, 631

Stoat (Ermine)

Streptococcus, 323

\section{Mice}

Blind gut

Fusiformis, 583

Blood

Bacillus, 647

Hemophilus, 589

Spirochaeta, 1068

Spirillum, 215

Erythrocytes

Dwarf mice

Eperythrozoon, 1113

Erythrocytes

Multimammate mice Grahamella, 1110

Erythrocytes

Peruvian mice

Grahamella, 1111

Erythrocytes

White mice

Eperythrozoon, 1112

Haemobartonella, 1105, 1106

General

Bacillus, 816, 817, 825, 826

Clostridium, 820, 826

Erysipelothrix, 411

Haemobartonella, 1104

Pasteurella, 549, 550, 551

Salmonella, 503

Lungs

Hemophilus, 589

Miyagawanella, 1118

Middle ear

Coccobacillary bodies of Nelson, 1294 Spleen

Hemophilus, 589

Mice, Diseases of

Anthrax

Bacillus, 720 
Mice, Diseases of (continued)

Bronchopneumonia Miyagawanella, 1118

Cancerous ulcers Spirochaeta, 1074

Cheesy masses in lung Corynebacterium, 390

Epizootic in Japanese waltzing mice Bacillus, 751

Gangrene Streptococcus, $3+2$

Joints, infected of white mice Corynebacterium, 402

Nouse typhoid Salmonella, 503

Plague in field mice Bacterium, 682

P'leuropneumonia-like disease, 1293

Pneumonitis Miyagawanella, 1118

Q (Queensland) fever Coxiella, 1093

Septicemia Corynebacterium, 390)

Septicemia in white mice Erysipelothrix, 390

Tuberculosis, avian Mycobacterium, 881

Tumors, breast Spirochaeta, 1068

\section{Milk and Cream}

Cream

Bacterium, 602

Leuconostoc, 348

Micrococcus, 280

Pseudomonas, 98, 700

Streptococcus, 337, 339, 340, 343

-, bitter

Micrococcus, 265

-, rancid

Alcaligenes, 416

-, ripening Achromobacter, 423

Micrococcus, 274, 280

-, sour

Flavabacterium, 440

Milk

Achromobacter, 421, 423, 425, 427, 609

Acuformis, 812

Actinomyces, 974
Aerobacter, 456,457

Alcaligenes, 416

Aromabacillus, 735

Bacillus, 644, 647, 648, 649, 653, 654, $660,662,668,672,712,717,721,726$, $730,731,733,734,736,737,738,741$, $742,743,744,745,746,748,751,752$, $753,755,757,758,813,814,815$, 818,824

Bacterium, 601, 602, 604, 674, 675, $679,680,681,684,687,760,761,762$ Brucella, 561

Chromobacterium, 694

Clostridium, 770, 773, 791, 820, 824

Corynebacterium, 390, 406

Escherichia, 447

Flavobacterium, 435, 440, 442, 611

Galactococcus, 250

Granulobacillus, 824, 826

Lactobacillus, 352, 353, 355, 356, 357, $358,359,361,363,364$

Leuconostoc, 347, 348

Microbacterium, 371

Micrococcus, 238, 239, 240, 241, 243, $251,252,253,254,255,256,257,258$. $259,262,263,264,265,266,267$, $268,273,274,278,279,280$

Mycobacterium, 879

Paraplectrum, 825

Plocamobacterium, 691

Propionibacterium, 373

Pseudomonas, 99, 100, 101, 145, 149 , 179,700

Sarcina, 290, 292

Serratia, 481

Streptococcus, 316, 320, 322, 323, 325, $326,327,328,334,335,336,337,339$, 342,344

-, curdled

Micrococcus, 262

-, pasteurized

Bacillus, 721, 730, 733, 735, 755

Corynebacterium, 406

Lactobacillus, 355, 363

Micrococcus, 255

Pseudomonas, 101

--, pasteurized skim

Micrococcus, 255

- powdered, see Dairy Products

-, raw

Corynebacterium, 390, 406 
Milk and Cream (continued)

Milk, red

Sarcina, 293

-, skim

Micrococcus, 237

—, sterilized

Bacillus, 748, 750

\section{Mineral Sources}

Arrowhead, African

Clostridium, 786

Arrowheads, poisoned

Bacillus, 826

Concrete, corrosion of Thiobacillus, 81

Coprolite Thiobacillus, 80

Cutting compound Pseudomonas, 95

Iron ore, swamp Siderococcus, 835

Lignite, fossil from Micrococcus, 266

Oil-bearing rocks Desulfovibrio, 209

Oil, lubricating Bacillus, 756

Oil-soaked soils Bacillus, 663

Bacterium, 682

Pseudomonas, 95

Oil wells Desulfovibrio, 208

Paint, fresh in greenhouses Chromobacterium, 233

Rocks

Thiobacillus, 80

Rocks, oil-bearing

Desulfovibrio, 209

Micrococcus, 271

Salt, see Salt

Salts, Rochelle

Micrococcus, 260

Tartrate

Micrococcus, 260

Walls, cellar

Spirochaeta, 1053

Walls, damp in cellars

Bacterium, 688

Micrococcus, 255, 277
Walls, damp in mines

Micrococcus, 255

Walls, wine cellars

Streptococcus, 340

Mollusca, Common Names

Cephalopod

Pseudomonas, 112

Vibrio, 202

Clam, intestine Bacterium, 632

General, crystalline style Cristispira, 1055, 1056, 1057

Mussel

Bacillus, 661, 663

Cristispira, 1055, 1056, 1057

Vibrio, 203, 207

Oysters

Inflabilis, 813

Salmonella, 532

Saprospira, 1054, 1055

Spirillum, 215, 217

- crystalline style

Cristispira, 1055, 1056

Seallop

Cristispira, 1056

Squid, intestine

Bacterium, 632

Mollusca, Scientific Names

Anodonta cygnea, 1055

Anodonta mutabilis, 1055, 1057

Cardium edule, 203, 207

Cardium papillosum, 1056

Chama spp., 1056

Gastrachaena dubia, 1056

Lima spp., 1056

Loligo edulis, 636

Loligo pealeii, 632

Mactra sulcataria, 1056

Mustelus mustelus, 632

Mytilus edulis, 661, 663

Ostrea edulis, 1056

Ovalipes ocellatus, 632

Pachalabra moestra, 1056

Pecten jacobaeus, 1056

Pholadis dactyli, 635

Pinna sp., 1074

Rondeletia minor, 112

Saxicava arctica, 1057

Sepia sp., 634

Sepiola intermedia, 202 
Mollusca (continued)

Solen ensis, 1057

Spheroides maculatus, 632

Tapes decussata, 1057

Tapes laeta, 1056

Venus mercenaria, 632

Venus (Meretrix) castra, 1056, 1057

\section{Mud}

Aerobacter, 692

Amoebacter, 849,850

Bacillus, 728, 741, 745, 814, 815

Chitin-digesting bacteria, 632

Chlorobium, 870

Chlorochromatium, 873

Clathrocystis, 872

Clostridium, 793, 795, 803, 820, 824, 825

Cylindrogloea, $87 \pm$

Lamprocystis, 848

Methanococcus, 248

Micrococcus, 258, 275

Pelochromatium, 859

Pelodictyon, 871, 872

Pseudomonas, 698

Rhabdomonas, 854, 855

Rhodopseudomonas, 864, 865, 866

Rhodothece, 855

Sarcina, 286, 287

Thiobacillus, 78, 79, 81

Thiocapsa, 845

Thiocystis, 847

Thiodictyon, 846

Thiopedia, 843

Thiopolycoceus, 850

Thiosarcina, 843

Thiospirillum, 851, 852, 853

Thiothece, 846

-, black (fresh, brackish and salt water)

Methanococcus, 248

Streptococcus, 337

Urobacterium, 691

- canal

Methanobacterium, 646

-, containing sulfur

Achromatium, 999

Actinomyces, 973

Macromonas, 1001

Thioploca, 994

Thiospira, 702

-, curative
Spirillum, 212

Iud, lake

Leptothrix, 986

Micromonospora, 979

Mud, marine

Achromatium, 999

Achromobacter, 418, 419, 421, 425

Actinomyces, 970, 972, 974

Bacillus, 658, 660, 661, 736, 741, 743, $745,748,750,818$

Bacterium, 632

Clostridium, 795, 820, 821

Desulfovibrio, 208

Diplococcus, 694

Flavobacterium, 430, 431, 432

Meihanobacterium, 646

Micrococcus, 696

Pseudomonas, 108, 697, 698, 699

Spirochaeta, 1053

Thioploca, 994

Vibrio, 703

Muddy bottom, brackish waters

Spirillum, 215

Muddy water

Bacterium, 688

Oil, see Mineral Sources

Peat

Actinomyces, 969, 974

Chromobacterium, 234

Hydrogenomonas, 78

Thiobacillus, 81

Petroleum, see Mineral Sources

Pigs, see Hogs

Phosphorescent Bacteria

Achromobacter, 634

Bacillus, 635

Bacterium, 633, 634, 635

Pseudomonas, 111, 112, 147

Sarcina, 637

Vibrio, 203

\section{Phosphorescent Materials}

Amphipod, dead, 111

Fresh water shrimp, 636

General, 702, 703

Luminous cephalopod, 112, 202

Luminous cuttle fish, 634

Luminous squid, 636

Luminous fish, 633, 634, 635, 636, 637 


\section{Plant Disease Attacking}

Acer macrophilum, 113

Acer spp., 113, 165

Aesculus turbinata, 165

Agaricus campestris, 128, 129

Agropyron repens, 116

Agropyron smithii, 395

Aleuritis fordi, 131

Allium cepa, 136, 146, 740

Amaranthus spp., 178

Amorphophallus knojac, 171

Ananas comosus, 127, 128

Ananas sativus, 472

Antirrhinum majus, $16 \overline{7}$

A pium graveolens, 122

Arctium lappa, 168

Aster chinensis, 477, 738

Astragalus sp., 139

Avena sativa, 113, 116

Avena spp., 162, 163

Begonia spp., 155

Berberis thunbergerii, 116

Berberis vulgaris, 116, 155

Beta vulgaris, 144, 468, 478, 613, 639, 663

Boulesia septentrionalis, 125

Brassica rapa, 136

Bramus inermis, 116

Calendula officinalis, 133

Canna indica, 171

Capsicum annum, 164, 740

Capsicum spp., 120

Carnegiea gigantea, 468

Castanea spp., 138

Chaetochloa lutescens, 142

Cichorium intybus, 125, 134

Cichorium spp., 133, 134

Cissus japonica, 134

Citrus spp., 156, 178

Coffea arabica, 639

Corchorus capsularis, 164

Corylus colurna, 139

Corylus, spp., 157

Cucumis sativus, 117

Curcurbitaceae, 468

Cuminum spp., 121

Dactylis glomerata, 394

Dahlia sp., 473

Daucus carota, 136

Daucus carota var. sativa, 166

Delphinium spp., 115, 134, 696
Dandrobium sp., 613

Dianthus caryophyllus, 137, 143

Dianthus sp., 639

Dieffenbachia picta, 157

Dolichos lablab, 160

Dracaena fragrans, 157

Edgeworthia chrysantha, 478

Eriobotrya japonica, 144

Erodium texanum, 122

Eugenia latifolia, 399

Euphorbia pulcherrima, 399

Fraxinus spp., 132

Gardenia jasminoides, 136, 696

Geranium spp., 160; 167

Gladiolus spp., 118, 130, 168, 478

Glycine $\max , 131,132$

Glycine sp., 160, 161

Gossypium spp., 160

Gossypium sp., 471, 477, 745

Gypsophila paniculata, 230

Hedera helix, 166

Helianthus debilis, 141

Holcus sarghum, 143, 754

Holcus spp., 158

Horde um vulgare, $113,116,162,163,740$

Hyacinthus orientalis, 152

I pomoea batatas, 136, 739

Iris spp., 118, 140, 147, 639

Juglans spp., 159

Koelranteria paniculata, 165

Lactuca sativa, 114, 115, 125, 126, 129 ,

$134,153,154,746$

Lactuca sativa var. angustata, 168

Lactuca scariola, 154

Lathyrys odoratus, 477

Lens esculenta, 141

Lespedeza spp., 159

Levisticum officiale, 141

Ligustrum japonicum, 128

Lupinus polyphillus, 160

Lupinus sp., 747

Lychnis sp., 639

Lycopersicon esculentum, 145, 164, 747, 757

Lycopersicon lycopersicum, 112, 138

Mangifera indica, 475

Manihotus sp., 466

Manihotus utilissima, 170

Martynia louisiana, 112

Matthiola incana, 158 
Plant Disease Attacking (conlinued)

Matthiola incana var. annua, 122

Medicago sativa, 165, 393

Medicago sp., 118

Villetia floribunda, 466

Morus sp., 135

Iusa sapientum, 140, 169

Terium oleander, 133

Vicotiana tabacum, 114, 124, 127, 130, 138,167

Odontoglossum citrosmum, 751

Olea sp., 132

Oncidium krameriani, 145

Oncidium sp., 640

Oryza sativa, 169

Panax quinquefolium, 131

Panicum miliaceum, 144, 169

Papaver rhoeas, 165

Passiflora edulis, 138

Pelargonium spp., 122, 160

Petasites japonicus, 142

Petasites sp., 142

Phaseolus vulgaris, 119, 127, 131, 160, 161,747

Phleum pratense, 703

Phormium tenax, 166

Pinus halepensis, 640

Piper betle, 130

Pisum sativum, 119, 138, 747

Pisum sativum var. arvense, 119

Plantago lanceolata, 161

Polygonum convolvulus, 140

Populus spp., 123, 751

Primula polyantha, 114, 115

Primula spp., 114

Protea cynaroides, 170

Prunus spp., 123, 153

Pseudotsuga taxifolia, 231

Pueraria hirsuta, 119

Rhododendron ferrugineum, 639

Ricinus communis, 131, 162

Rosaceae, 465

Rubus spp., 229

Saccharum officinarum, 121, 124, 163, $171,472,473,639,753$

Salix alba, 467

Salix spp., 144, 746

Saponaria, 639

Secale cereale, $116,162,168,740$

Sesamum, 753

Setaria italica, 126
Soja $\max , 131,132$

Solanum tuberosum, 129, 138, 757

Stizolobium deeringianium, 135

Taraxacum kok-saghz, 179

Thea sinensis, 756

Trifolium pratense, 141

Trifolium spp., 757

Triticum aestivum, 116, 121, 400

Triticum sp., 162, 163, 740

Clmus sp., 142, 472

Viburnum spp., 135

Ticia faba, 136, 139

Tigna sinensis, 703

Tigna sp., 160

Titis sp., 145, 276, 466, 478, 758

Vitis vinifera, 639

Washingtonia filifera, $69 \overline{7}$

Zea mays, 472

Zingibar officinale, 171

\section{Plant Diseases}

Alfalfa

Leaves of, 165

Stem and leaves, brown lesions, 118

Vascular pathogen, 393

Aloes, 758

Amaranthus, 178

Antirrhinum

Leaf spot, 167

Apples

Blister spot, 697

Blisters and rough bark, 124

Canker, 640

Fire blight, 465

Hairy root of, 229

Rot of, 141

Apricots, 153

Arrowwood

Angular leaf spot and stem lesions, 135

Ash

Cankers, 132

Asparagus lettuce

Leaf spot on, 168

Aster, 477

Aster, China, 738

Astragulus

Black leaf spot, 139

Bananas, 613

Black rot, 140

Blood disease of, 169 
Plant Diseases (continued)

Barberry

Leaves and twigs, 116

Barley, 116, 740

Leaf streak, 113

Leaves and seed of, 162,163

Bean 120, 131, 160, 161, 341

Blighted leaves and stems, 127

Halo blight, 119

Wilt, 399

Bean, Broad, 136, 139

Castor oil, 131

Leaf spot, 162

Hyacinth, 160

Kidney, 747

Soy, 131, 132, 160, 161

Spotted, 127

Velvet

Leaf spot, 135

Windsor, 136, 139

Beets

Gall on, 154

Leaves, 114

Vascular rot, 144

Beets, Sugar

Gall on, 154

Begonia

Leaf spot, 155

Betel vine

Leaf spot, 130

Bindweed, Black

Diseased leaves, 140

Bird's nest fern

Leaf blight, 696

Blackberries, 229

Bowlesia

Water-soaked spots of , 125

Brome-grass

Water-soaked spots, 116

Broom corn

Lesions, 143

Burdock

Leaves and petioles of, 168

Cabbage, 117

Black rot, 155, 156

Soft rot, $471,474,477$

Cactus, 613

Rot, 477

Calla lily

soft rot, 474
Canna

Leaves, 17

Cantaloupes

Wilt, 468

Carnations

Leaves, 640

Root and stalk disease, $13 \overline{7}$

Water-soaked lesions and leaves, 143

Carrots, 136

Leaves, 166

Rot, 468

Soft rot, 471

Cassava

Necrotic lesions of leaves, 466

Wilt disease, 170

Cauliffower

Black rot of, 155,156

Leaves, 117

Soft rot, 474

Celery

Leaves, 122

Rot, 639

Soft rot, 471

Cherries, 120

Chestnut

Canker, 640

Water-soaked spots on leaves, 138

Chrysanthemum

Fasciated growth, 395

Citrus, 120

Canker of, 156

Citrus fruits

Spot disease, 475

Clover, 757

Clover, red

Root rot, 141

Corn, Field

Blight, 457

Stalk rot, 124, 125, 472

Cotton, 477

Angular leaf spot, 160

Boll rot, 745

Root rot, 471

Stem and boll lesions, 160

Cow peas, $120,161,703$

Cricket bat willow

Watermark disease, 467

Cucumber

Leaves, 117

Soft rot, 471, 474 
Plant Diseases (continued)

Cucumber (continued)

Wilt, 468

Cumin

Blighted, 121

Dactylis glomerata

Slimy heads, 394

Dahlia

Tuber and stem rot, 473

Delphinium

Black spot on leaves, 115

Douglas fir

Galls on, 231

Dill

Blighted, 121

Egg Plant

Soft rot, 471,474

Elms, 279

Dark discoloration of wood, 142

Wet wood, 472

English walnut

Black spot of leaves and nut, 159

Éugenia latifolia

Witches'-broom, 399

Fig

Blight, 640

Filbert trees, 157, 640

Flax

Leaf stripes, 166

Foxtail, 142

French endive

Rot, 125, 134

Gardenias, 696

General, 89, 344

Leaf spot, 136

Geranium

Fasciated growth, 395

Galls on, 228, 229, 230, 231

General, 167

Leaf spot, 160

Giant cactus

Rot, 468

Ginger

Sprout rot, 171

Ginseng

Root rot, 131, 477

Gladiolus, 118, 478

Gummy lesions on leaves, 168

Tubers, corm rot, 130

Grapes, 145, 466, 478, 639, 758

Grapevines, 276
Grasses, 142

Hazelnut, Turkish

Leaves and stems of, 139

Heron's bill

Leaf spot, 122

Horse-radish, 164

Black rot of, 155,156

Root rot, 477

Hyacinth

Soft rot, $4 \bar{\gamma} 1$

Yellow rot, 152

Iris, $118,140,147$

Blight on leaves, 639

Brown leaf spot, 140

Soft rot, 177, 471

Italian millet

Brown stripe, 126

Ivy

Leaves, 166

Ivy, Japanese

Black spot on leaves, 134

Johnson's grass

Leaf stripe, 171

Jute

Water-soaked to brown spots on leaves, 164

Konjac, 171

Kudzu vine

Halo blight, 119

Larkspur

Bacterial blight, 696

Rot, 134

Ientils

Root rot, 141

Lettuce, 154, 158, 746

Leaves, 114, 115

Marginal lesions, 126

Root disease, 129

Rosette disease, 129

Rot, 125, 134

Lettuce, wild, 154

Lilac, 120

Lily

Brown spots on bulbs, 477

Loquat

Bud rot, 144

Lovage

Spot on leaves, 141

Lucerne

Root rot, 141 
Plant Diseases (continued)

Lupine, 161, 747

Maize, see Corn

Mango, 475

Maple

Leaves, 165

Maple, large leaf

Leaves, 113

Marigolds, 133

Millet

Leaf stripe, 144

Leaves, sheaths and culms, 169

Mulberry

Blight, 135

Mushrooms, cultivated

Brown spots on, 128

Muskmelon

Soft rot, 471

Wilt, 468

Nasturtium

Leaves, 114

Oats

Black chaff, 162, 163

Galls, 133

Halo spot, 116

Leaf blight, 116

Leaf streak, 113

Olive, 761

Galls and tubercles, 132, 750

Onions, 740

Bulb rot, 136, 146

Rot, 146

Soft rot, 471

Orange

Canker of, 156

Orchid, 145, 640, 759

Papaya, 478

Paris daisy

Galls, 228

Parsnip

Soft rot, $471,47 t$

Passion fruit

Leaves and fruit, 138

Peaches, 153

Pear

Blossom blight, 129, 130, 134

Canker, 640

Fire blight, 465

Peas, 747

Diseased seeds, stems and pods, 138
Peas, field

Water-soaked lesions, 119

-, garden

Water-soaked lesions, 119

Pelargonium

Leaf spot, 160

Peppers, 120

Leaf wilt, 740

Soft rot, 471

Spotted fruit, 164

Petunia

Fasciated growth, 395

Pine

Galls, 640

Pineapples

Brown rot, 473

Rot, 127,128

Plantago spp.

Leaves, 161

Plum

Cankers, 123

Plum, Japanese, 153

Poinsettia

Canker of stems and leaf spots, 399

Poplar

Cankers, 123

Galls on branches, 751

Poppy

Black spots on leaves, buds and pods, 165

Potatoes

Black leg, 469

Black rot of stem and tuber, 469

Blight and rot, 342

Brown rot, 138

Dark colored stem, 968

Dry rot, 259

Leaves, 757

Ring rot, 393

Rot, 129, 138, 293, 468

Scab 958, 968, 969, 970, 971, 972, 973, 974

Soft rot, $471,474,477$

Tubers, 640

Primrose

Leaf spot, 114, 115

Privet, Japanese, 128

Proso millet

Leaves, sheaths and culms, 169 
Plant Diseases (continued)

Protea

Leaf spot, 170

Pumpkin

Wilt, 468

Radishes

Leaf spot, 164

Rot, 468

Soft rot, 471,474

Raspberries, 229

Galls on canes, 229

Rhubarb

Crown rot, 476

Rice

Leaf blight, 169

Russian dandelion

Root rot, 179

Rutabagas

Black rot of, 155,156

Rye, 740

Black chaff, 162,163

Leaf blight, 116

Salsify

Soft rot, 474

Sesame, 753

Brown spots on leaves and stems, 128

Soft rots, 640

Solanaceous plants

Brown rot, 137, 138

Sorghum, 754

Leaf stripe, 171

Lesions, 143

Rot, 750

Streak disease of leaves, 158

Sorgo

Lesions, 143

Squash

Leaf spot, 157

Wilt, 468

Stock, flowering, 158

Vascular disease, 122

Sugar beet, 114, 477, 639

Curly top, 663

Rot, 468

Soft rot, 613

Sugar cane

Bacterial gummosis, 163

Leaf scald, 639

Mottled stripe, 170

Red stripe, 154

Sereh, 753
Soft rot, 472,473

Stalk rot, 124, 125

Stinking rot, 121

White stripe, 639

Sunflower, 141

Sweet peas, 477

Fasciated growth, 395

Sweet potato, 136, 949, 958

Rot, 739

Tea, 756

Timothy grass

Streak disease, 703

Tobacco, 167, 477

Angular leaf spot, 114

Black rust, 124

Brown rot, 137, 138

Collar rot, 477

Fasciated growth, 395

Leaf spot, 127, 477, 639

Rusty spot on leaves, 130

Wildfire, 124

Tomatoes, 747,757

Bacterial canker, 394

Blossom end rot, 477

Brown rot, 138

Leaves, 113

Rot, 468,640

Soft rot, 471,474

Spotted fruits, 164

Tung oil tree, 131

Turnips, 136

Leaf spots, 164

Soft rot, $471,47 t$

Unicorn plant

Leaves, 112

Vanilla vine, 640

Walnut

Black spot of leaves and nuts, 159

Washington palm, 697

Wheat, 279, 639

Basal glume rot, 121

Black chaff of, 162, 163

Leaf blight, 116

Slimy heads, 400

Wheat grass

Slimy heads, 395

Willow, 746

Wilted branches, 144

Winter cress

Black rot of leaves and stems, 155 
Plant Diseases (continued)

Wisteria

Canker, 640

Wisteria, Japanese, Gall, 466

\section{Plant Sources}

Abri precatorii, 672

Alepecurus pratensis, 738

Algae, 218, 366

Almus spp., 944, 968

Aquatic plants, 672

Arachis hypogasa, 681

Asparagus infusions, 262

A ucuba japonica, 146

Bark, covered with lichens, 1035

Bark, decaying, 1042

Bark, old, 1045

Bark, poplar, 1026, 1031

Bark, wet, 1026, 1030

Barley, 679

Bean infusions, 218, 262, 264, 268, 271, $273,277,750$

Beans, purple discoloration salted, 109

Beer wort, 146

Beet juice, 657

Beet juice, fermented, 189

Beets, 825

Fodder, 100

Leaves, 752

Rotting, 250

Bladderwort, 671

Boletus edulis, 254

Brewer's grain, 744

Cabbage, 456, 750

Cacao beans, 951

Carrot, slices of, 758

Cherry trees, gum, 754

Cladophora, 1032

Clematis, stem of, 1042

Corn, 759, 760, 761, 781, 825

Grains soaked in water, 148

Seedlings, 281

Stalks, 825

Cotton husks, 222

Decaying vegetation, 989

Decomposing leaves, 618

Digitalis infusions, 262, 263

Elodea, leaves, 259, 833

Epiphytes, 833, 834
Flax, retting, $803,807,814,819,822$, 823,824

Flour, 281, 291, 292, 293, 294

Flour pastes, acidified, 287

Fodder, 733, 737, 750, 755

Green, 750, 755

Fruits, 694

Fungi, 254, 689

Fungi, old, 1037

General, 455, 712, 717

Grains, 281, 455, 460, 602, 654, 721, 751, $774,824,825,826,916,935,967,968$, 969,970

Grains, ground, 733, 736, 751

Grapes, Spanish dried, 624

Grapes stored in sawdust, 756

Grass, 370, 749, 973

Green algae, fresh water, 834

Hay, 602, 734, 740, 890, 957

Clover, 173

Heating of, 740

Hevea brasiliensis, 256, 260, 273, 284 , 416

Hibiscus, Retting, 263, 813, 815, 819

Hibiscus, stamens and pistils of, 258

Ipomoea sp., 949, 958

Jequirity seed, 672

Kelp, 970

Kenaf, retting of, $263,813,815,819$

Latex, 256, 260, 273, 284, 416

Lathyrus spp., 225

Leaves, 173, 1046

Indigo plant, 659

Pitcher plant, 655,668

Sundew, 653

Legumes, 824

Lens spp., 225

Lichens, 1045

Decaying, 1031

Litmus solution, 743,757

Liverworts, 1020

Lupine, 226, 656, 657, 661, 663, 678, 689

Maize, see Corn

Maple sap, 664

Slimy, 456

Marine algae, 200, 627, 642, 692, 697, 698, 702, 703, 1016

Acanthophora spicifera, 703

Cladophoropsis sp., 702

Gracilaria blodgettii, 697

Gracilaria confervoides, 702, 703

Iridaea cordata, 629, 630 
Plant Sources (continued)

Marine algae (continued)

Laurencia poitei, 702

Nercocystis luetkeana, 629, 630

Odonthalia kamischatica, 628

Porphyra perforata, 630

Marine phytoplankton, 421, 703

Meadow plants, 743

Meal, cotton seed, 689

Medicago spp., 227

Melilotus spp., 227

Melon-rind, 1037

Myrica, 972

Nodules on roots of

Alfalfa, 227

Beans, 225

Clover, 226, 489

Coffee, 639

Fox grass, 738

Legumes, 650, 656, 657, 661, 663, 678, 689

Lentils, 225

Lupine, 226

Pea, 225

Soybeans, 226

Sweet clover, 227

Vetch, 225

Nymphaea, 833

Oranges, 825

Ornithopus spp., 226

Pararubber tree, 416

Parasitic on lichens, 1035

Peanut plant, 681,690

Peas, 751

Pelargonium, 167

Persimmons, dried, 679, 694

Phaseolus spp., 225

Pine bark, 677

Plant dust, 889

Poison ivy, 279

Poison on arrowheads, 826

Poplar trees, bark, 259, 272

Potamogeton natans, 834

Potatoes, 264, 269, 781, 825

Internal rust spots, 98

Rotten, 491, 738, 1074

Rhus spp., 279

Rice, 825

Hulls, 222

Paddies, 676

Root nodules, see Nodules on legumes, etc.
Roots of alder, 944, 968

Roots of Myrica, 972

Rotting vegetables, 654

Rotting wood, 146, 211

Rubber tree, latex of, 256, 260, 273, 284

Sagittaria, 833

Salvinia, 833

Sauerkraut, 146

Saw dust, 210, 211

Seaweed, see Marine Algae

Rotting, 680

Seeds, germinating, 678, 679

Serradella spp., 226

Snakeroot infusions, 263

Soja max (Glycine max), 226, 292

Sorghum, 253

Soybean mash, 292

Sphagnum moss, 344

Spirogyra sp., 834

Straw, 271, 602, 935, 980

Old, 1037

Strawberries, 422

Sugar beet, 748

Sugar cane, roots, 825

Surfaces, 484

Timothy grass, 890

Tobacco, 674, 677, 680, 682, 683, 685, $686,687,690,694$

Tomato juice, 713

Rotted, 491

Trifolium spp., 226

Trigonella spp., 227

Utricularia vulgaris, 671

Vegetable fibers, disintegrating, 210

Vegetable infusions, $147,148,277,278$, 280

Vicia spp., 225

Watermelon, decayed, 748

Wheat bran, 287

Wood, old, 1037

Rotting, 728, 736, 745, 747, 758, 1037

Wet, 1026, 1030

Yeast mash, 146

Zoster marina, 1002

Poultry Diseases, see Birds

Protozoa, Common Names

Flagellate ectoparasite

Fusiformis, 583

Treponema, 1075 
Protozoa, Scientific Names

Descovina spp., 583

Devescovina hilli, 1075

Euglena deses, 1121

Lophomonas striata, 694

Paramoecium aurelia, 1122

Paramoecium caudatum, 1122

Pelomyxa palustris, 1123

Polymastix legeri, 694

Polymastix melolonthae, 694

Trichomonas batrachorum, 1123

Trichonympha chattoni, 1121

Trichonympha corbula, 1121

Trichonympha peplophora, 1121

Trichonympha spp., 1121

\section{Putrefying Materials}

Blood

Bacillus, $81 \overline{7}$

Streptococcus, 340, 341, 343, 344

Bones, macerated

Bacterium, 683

Eggs, rotten

Bacillus, 657

Egg white

$$
\text { Bacillus, } 654
$$

Fish

Bacillus, 671, 814

Clostridium, 821

-, luminous

Bacterium, 633, 634, 635, 636, 637

_, spoiled semi-dried

Flavobacterium, 694

Game birds

$$
\text { Bacillus, } 817
$$

Clostridium, 796

Gelatin, spoiled

Bacillus, 756

General

Bacterium, 607, 608, 609, 610

Pseudomonas, 97

Spirillum, 214, 216, 217

Streptococcus, 330

Ham, sour

Clostridium, 784

Infusions

Bacillus, 816

Manure, see Manure (Dung)

Meat

Bacillus, 813, 816, 818, 825

Clostridium, 788, 796, 800, 821, 826
Diplococcus, 309

Micrococcus, 256

Proteus, 488, 489

Ristella, 576

Meat infusions

Micracoccus, 254

-, luminous, 633, 635

Milk

Bacillus, 824

Pork, macerated

Clostridium, 783, 802

Sulfur stinker spoilage, canned goods

Clostridium, 803

\section{Rabbits}

Blood

Bacillus, 818

Spirochaeta, 1069

Brain

Flavobacterium, 437

Erythrocytes

Eperythrozoon, 1113

Eye, Descemet's membrane

Rickettsia, 1091

-, endothelial cells

Rickettsia, 1091

General

Micrococcus, 271

Salmonella, 513, 514, 521

Streptococcus, 338, 339

Veillonella, 304

Kidney

Corynebacterium, 405

Liver

Spirochaeta, 1065

Lungs

Bacillus, 647

Mouth cavity

Veillonella, 303

Nasal mucosa

Zuberella, 578

Not pathogenic for

Johne's disease

Mycobacterium, 881

Tuberculosis, amphibian

Mycobacterium, 885

Tuberculosis, bovine

Mycobacterium, 879

Tuberculosis, human

Mycobacterium, 878 
Tuberculosis, piscine

Mycobacterium, 883,884

Tuberculosis, snake

Mycobacterium, 886

Red blood cells, see Erythrocytes

Stomach contents Actinomyces, 974

Viscera

Bacillus, 818

\section{Rabbits, Diseases of}

Abscess, liver

Aerobacter, 456

Abscesses, skin

Hemophilus, 589

Neisseria, 301

Actinomycosis

Actinomyces, 928

Anthrax

Bacillus, 663, 720

Streptococcus, 338

Blood of diseased rabbits

Clostridium, 820

Micrococcus, 272

Brucellosis

Brucella, 561, 562, 563

Conjunctival folliculosis

Noguchia, 594

Cornea, diseased

Micrococcus, 254

Diphtheritic inflammation of intestine

Corynebacterium, 402

Endometritis

Leptothrix, 366

Epizootic

Bacillus, 653

Bacterium, 681

Micrococcus, 261

Erysipelas of ear

Bacillus, 655

Glanders

Malleomyces, 555, 556

Haemobartonellosis

Haemobartonella, 1104

Hemorrhagic septicemia

Pasteurella , 547, 549, 550, 551, 552

Infections

Actinomyces, 928

Corynebacterium, 403

Nocardia, 911

Streptococcus, 339
Lesions, genitoperineal region Treponema, 1073

Listeriosis Listeria, 409

Lung plague Bacterium, 552

Melioidosis Malleomyces, 556

Metritis Corynebacterium, 404

Necrosis of liver Listeria, 409

Necrotic lesions Clostridium, 820

Pleuritis Leptothrix, 366

Pleuropericarditis Klebsiella, 459

Pneumonia Bacillus, 647

Pus, stinking Bacterium, 685

Rabies-like disease Bacillus, 663

Septicemia

Micrococcus, 251

Streptococcus, 317, 340

Spirochetosis

Treponema, 1073

Suppuration

Leptothrix, 366

Syphilis

Treponema, 1071, 1076

Tuberculosis, avian

Mycobacterium, 881

Tuberculosis, human

Mycobacterium, 879

Tympanitis

Bacillus, 818

Tympanitis in young

Bacillus, 757

\section{Rats}

Blood

Leptospira, 1077

Spirillum, 215

-, albino rats

Haemobartonella, 1104

- , desert rats

Grahamella, 1109 


\section{Rats (continued)}

Erythrocytes

Grahamella, 1110

Haemobartonella, 1107, 1108

, albino

Haemobartonella, 1103

—, jumping

Grahamella, 1110

-, marsupial

Haemobartonella, 1108

-, white

Hacmobartonella, 1106

Feces

Salmonella, 504, 512, 531

General, gray rats

Leplospira, 1078

Intestine

Catenabacterium, 368

-, white rats

Corynebacterium, 403, 404

Kidneys

Leptospira, 1077

Large intestine

Ristella, 567

Middle ear

Coccobacillary bodies of Nelson, 1294

Not pathogenic for

Tuberculosis, amphibian

Mycobacterium, 885

Preputial glands

Mycobacterium, 891

Red blood cells, see Erythrocytes

Stomach

Spirillum, 218

Urine

$$
\text { Leptospira, 10\%7 }
$$

\section{Rats, Diseases of}

Brucellosis

$$
\text { Brucella, } 563
$$

Endemic disease of Mycobacterium, 883

Epizootic among white rats Micrococcus, 261

General.

Actinomyces, 972

Bacillus, 665

Clostridium, 779

Nocardia, 922

Pseudomonas, 89
Glanders

Malleomyces, 556

Hemorrhagic septicemia

Pasteurella , 548, 549, 550, 551

Hepatized lung

Bacterium, 402

Leprosy

Mycobacterium, 882

Melioidosis

Malleomyces, 556

Nodular lesions, Madagascar rat Actinomyces, 917

Plague

Bacillus, 663

Pasteurella, 549

Pleuropneumonia-like disease, 1292

Thyroid infection

Bacterium, 402

Reptiles, Common Names

Boa constrictor

Mycobacterium, 885

Chuckawalla

Bacterium, 461, 462

Gila monster

Pseudomonas, 92

Serratia, 462

Horned lizards

Pseudomonas, 92

Sorratia, 462

Lizards

Actinomyces, 971

Bartonella, 1108

Wyrabacterium, 883, 884, 885

Psendommas, 92

Treponema, 1076

-, gekkonid

Serratia, 462

-, inguanid

Serratia, 462

Python

Mycobacterium, 885

Snakes

Mycobacterium, 886

Salmonella. 503, 513, 524, 526, 527

Serratia, 462

Snakes, brown

Serratia, 462

-, garter

Mycobacterium, 886

Serratia, 462 
Reptiles (continued)

Tortoise

Bartonella, 1108

Turtle

Mycobacterium, $885,887,891$

-, musk

Serratia, 462

\section{Reptiles, Diseases of}

General

Salmonella, 513, 514, 519

Serratia, 462

Lizards, contagious disease

Serratia, 462

-, tumors

Bacterium, 461

Serratia, 462

Snakes, blood

Spirochaeta, 1070

-, tuberculosis

.Mycobacterium, 886

-, typhoid-like infection

Pseudomonas, 700

Turtles, tuberculosis

Mycobacterium, 885, 887, 891

Reptiles, Scientific Names

Anolis carolinensis, 462

Anolis equestris, 462

Brasilicus vittatus, 462

Caluber catenifer, 885

Hemidactylus brookii, 462

Lacerta sp., 971

Lacertitia sp., 1108

Python molurus, 885

Sauromalus varius, 461

Sternothaeris odoratus, 462

Storeria dekayi, 462

Tarentola mauritanica, 462

T'asaxerus cepapi, 974

Testudo graeca, 1108, 1111

Thamnophis butleri, 462

Thamnophis sirtalis, 886

Tropidonotus stolatus, 1070

Tropidurus peruvianus, 1108

Retting, Flax

Bacillus, 722, 814

Bacterium, 819

Clostridium, 803, 807, 824

Granulobacter, 822, 824

Plectridium, 823, 824
Retting, Hemp

Bacterium, 681

Plectridium, 824

Retting, Kenaf (Hibiscus)

Bacillus, 813, 815

Clostridium, 819

Listerella, 409

Micrococcus, 263

\section{River Water}

Bacillus, 64t, 652, 654, 655, 658, 659, $667,693,741,815$

Bacterium, 601

Chromobacterium, 233, 234

Clostridium, 824

Flavobacterium, 433, 611

Leptothrix, 986

Microcaccus, 270, 273, 275

Pseudomonas, 93, 149,697

Saprospira, 1055

Serratia, 48t, 485

Spirochaeta, 1053, 1054

Thiobacillus, 79, 81

Urebacillus, 691

Vibrio, 196, 203

Zuberella, 577

River Water, Name of River

Elbe River, 203

Granta River, Cambridge, 1053, 1054, 1055

Illinois River, 484

Mississippi River, 484, 485, 601, 644

Ohio River, 601

Rhine River, 273, 433

Schuylkill River, 93, 270, 275, 677, 693

Seine River, 824

Spree River, 196, 654, 697, 986

Zwonitz River, Chemnitz, 611

\section{Salt and Salted Materials}

Chromobacterium, 234

Clostridium, i\$4

Pseudomonas, 109, 110

Brines

Bacillus, 648, 658

Desulfovibrio, 208

Pseudomonas, 109, 110, 147

Sarcina, 28?

Vibrio, 702 
Salt and Salted Materials (continued)

Salted codfish, reddened

Bacillus, 667, 742

Flavobacterium, 442

Micracoccus, 259

Pseudomonas, 110

Salted fish

Bacillus, 658

- - red spoilage

Pseudomonas, 110

Salted hides

Pseudomonas, 110

Salted intestines (Wiener skins)

Tetracoccus, 284

Salted sardines, anchovies, etc. Vibrio, 204

Salt ponds, red

Pseudomonas, 110

Salt waters, also see Sea Water

Spirillum, 212

Solar salt

Pseudomonas, 110

Ristella, 576

Sarcina, 289

\section{Salt Seas and Lakes}

Dead Sea

Flavobacterium, 441, 442

Halobacterium, 234

Pseudomonas, 147

India

Pseudomonas, 110

I.iman, near Odessa

Urobacterium, 691

Russia

Thiobacillus, 81

Sand, see Soils

Sauerkraut, see Fermenting and Fermented Materials

\section{Sea Water}

Acetobacter, 692

Achromobacter, 419, 421, 423, 424, 425, 634

Bacillus, 653, 658, 660, 661, 662, 667, $741,743,746,750,754,818$

Bacterium, 606, 625, 626, 627, 632, 633, $635,642,673,674,675,677,678,680$, $681,683,686$

Bacteroides, 566

Beggiatoa, 991, 992, 993
Chromatium, 857,858

Clostridium, 820

Cytophaga, 1014, 1015

Desulfovibrio, 208

Flavobacterium, 429, 430, 431, 432, 435, $438,439,441,631$

Leptospira, 1079

Micrococcus, 240, 246, 254, 255, 262, $263,267,268,271,695,696$

Microspira, 202

Photobacterium, 636, 637

Pseudomonas, 107, 108, 110, 111, 112, $175,697,698,699,700$

Sarcina, 701

Serratia, 484

Spirillum, 217

Spirochaeta, 1052, 1053, 1054

Streptococcus, 330

Thiobacillus, 79, 81

Thiopedia, 843

Thiospira, 212, 702

Thiothrix, 990

Thiovulum, 1000

Urobacterium, 691

Vibrio, 200, 205, 703

\section{Sea Waters}

Ambergris Spirillum, 217

Bottom sediments Bacterium, 627 Flavobacterium, 631

Brine, red Pseudomonas, 110 Sarcina, 289

Containing rotting seaweeds Achromatium, 999 Beggiatoa, 991, 992

Thiothrix, 990

Thiovulum, 1000

General Pseudomonas, 175

Harbor at Kiel Spirochaeta, 1053

Lime precipitation Pseudomonas, 108

Marine bottom deposits Bacillus, 739, 741, 746, 755, 756

Micracoccus, 696

Pseudomonas, 697, 698, 699, 700, 701 Sarcina, 701 
Sea Waters (continued)

Marine bottom deposits (continued)

Thiospira, 702

Vibrio, 703

Marine mud, see Mud, Marine

Marine phytoplankton

Bacillus, 743

Vibrio, 703

Mussel beds

Desulfovibrio, 207

Submerged surfaces

Bacterium, 606, 607

Flavobacterium, 631

Micrococcus, 696

Pseudomonas, 697, 699, 700

Sulfur waters

Beggiatoa, 991, 992, 993

Thiopedia, 813

Thiothrix, 990

Tropical waters

Pseudomonas, 108

Sea Waters, Geographical Distribution

Arctic Ocean, 202, 254, 255

Australia, Elizabeth's Bay, 631

Baltic Sea, 636

Barents Sea, 423

California Coast, 419, 421, 430, 431, $432,606,607$

Denmark, Coast of, 217

Kiel, Harbor at, 483, 1053

Naples, Gulf of, $425,745,748$

North Pacific Coast, 175

Norwegian Coast, 200, 626

Pacific Coast, L. S. A. 107

Scotland, Coast of, $7 \notin 1$

West Indies, 112

Woods Hole, Massachusetts, 111, 660, 661,750

\section{Sewage}

Achromobacter, 420,427

Bacillus, 65t, 667, 670, 729, 730, 735

$813,814,816,81 \%, 833$

Bacterium, 610, 675, 685, 687, 688

Clostridium, 788, 791,812

Desulfouibrio, 208

Escherichia, 452

Methanobacterium, 646

Microspira, 202, 203

Vitrobacter, 75

Nitrocystis, 75
Nitrosogloea, 73,74

Pseudomonas, 89, 90, 91

Salmonella, 529

Sarcina, 287

Spirillum, 203

Thiobacillus, 79

Urobacillus, 691

Vibrio, 203, 204

Drains of slaughter houses

Bacillus, 825

Filterable bodies, 1294

\section{Sewage Deposits}

slime

Achromobacter, 423

Bacterium, 610, 615

Sludge, activated

Nitrocystis, 75

Vitrosacystis, 73

Vitrosogloea, 73, 7t

Vitrosospira, 71

Pseudomomas, 81, 150

Vibrio, 702

Sludge, fermenting

Sarcina, $28 i$

\section{Sewage Effluents}

Achromobacter, $419,+26$

Chromobacterium, 233

Vitrocystis, 75

\section{Sewage Plants}

Filter beds

Micrococcus, 269

Pseudomonas, 91

Settling basin

Clonothrix, 983

Sheep

Blood

Rickettsia, $109 \overline{7}$

Spirochaeta, 1068

Bronchi

Hemophilus, 589

Corneal or conjunctival discharges Colesiota, 1120

Erythrocytes

Eperythrozoon, 1112

General

Salmonella, 502, 509, 528

Intestine

Bacillus, 815

Bacterium, 690 
Sheep (continued)

Nasal secretions

Streptococcus, 338

Pancreas

Streptococcus, 338

Red blood cells, see Erythrocytes

Stomach

Streptococcus, 338

Stomach contents

Bacterium, 681

Sheep, Diseases of

Abortion

Brucella, 561

Salmonella, 506

Agalactia

Anulomyces, 1292

Anthrax

Bacillus, 720

Black leg

Clostridium, 773

Braxy

Bacillus, 825

Circling disease

Erysipelolhrix, 409

Diarrhoea

Bacillus, 826

Diseased lambs Bacillus, 648

Enterotoxemia

Bacillus, 826

Epery throzoonosis

Eperythrozoon, 1111

Foot rot

Actinomyces, 917

Spirochaeta, 1074

Treponema, 1076

Gangrenous mastitis

Micrococcus, 267

Glanders

Malleomyces, 555

Heartwater

Cowdria, 1094

Hemorrhagic septicemia

Pasteurella, 549, 55t

Infections

Corynebacterium, 402

Rickettsia, 1095

Streptococcus, 342

Infectious mastitis

Pasteurella, 554
Keratitis

Colesiota, 1119

Lesions

Actinomyces, 916

Listeriosis

Listeria, 409

Lymphadenitis, caseous

Corynebacterium, 389

Mastitis

Streptococcus, 340

Necrotic areas in kidney

Corynebacterium, 389

Ophthalmia, infectious

Colesiota, 1120

Pleuropneumonia, bovine Asterococcus, 1292

Pneumonia

Pasteurella, 549

Purulent infections, urinary tract. Corynebacterium, 389

Pyorrhoea

Leptothrix, 366

Sheep pox pustules

Streptococcus, 345

Struck

Bacillus, 826

Tubereulosis, bovine

Mycobacterium, 87 ?

Shellfish, see Mollusca

\section{Snow}

Bacillus, 735

Bacterium, 760

-, melting of glacial

Pseudomonas, 145

- red

Pseudomonas, 148

\section{Soil}

Achromobacter, 419, 423, 424, 426, 622

Actinomyces, 968, 969, 970, 971, 972, 973,974

Aerobacter, 456

Agrobacterium, 230

Alcaligenes, 416

Angiococcus, 1048

Archangium, 1019

Azotobacter, 220, 221

Azotomonas, 222

Bacillus, 622, 623, 631, 649, 650, 653, $656,660,662,663,664,665,666,668$, 
Soil (continued)

Bacillus (continued)

$669,670,671,672,711,712,713,714$, $715,717,719,721,722,723,724,725$, $726,728,729,730,731,732,733,734$, $735,736,737,738,739,740,741,742$, $743,744,745,746,747,749,750,751$ $752,753,754,755,756,757,758,813$. $814,815,816,817,818,824,825,826$. 827

Bacterium, 76, 407, 408, 602, 603, 613, $614,615,637,638,642,643,673,67 t$, $676,677,679,682,685,688,760,761$. 762

Bactoderma, 76

Butylobacter, $\$ 25$

Cellfalcicula, 211

Cellulomonas, 617, 618, 619, 620, 621, 622

Cellvibrio, 210, 211

Chondrococcus, 1045, 1046

Chondromyces, 1037, 1038, 1039

Chromobacterium, 233

Cladothrix, 983

Clostridium, 770, 772, 773, 774, 776, $778,779,781,782,783,784,785,788$, 789, 791, 793, 794, 795, 797, 798, 799, $800,801,802,809,810,811,812,819$, $820,821,824,825$

Cornilia, 822

Corynebacterium, 391, 393, 394, 396, $397,398,403,404,407,408$

Cytophaga, 1013, 1014

Denitrobacterium, 690, 762

Desulforibrio, 208

Escherichia, 449,450 , 552

Flavobacterium, 429, 430, 436, 438, 440

Granulobacillus, 824, 826

Granulobacter, 822, 824

Hiblerillus, 822

Hydrogenomonas, 77,78

Hyphomicrobium, 837

Lactabacillus, $36 \pm$

Melittangium, 1034

Methanobacterium, $6+6$

Methanococcus, 248

Methanomonas, 179

Microbacterium, 370,371

Micrococcus, 238, 251, 255, 270, 271, $275,281,696$

Microderma, 76
Jicromonospora, 979,980

Mycobacterium, 885, 887, 888, 890, 919

Mycocaccus, 891

Mycoplana, 191

Hy.rococcus, 1042, 1043, 1044

Nitrobacter, $74,75,76$

Nitrosobacillus, 690,762

Vitrosococcus, 71

Vitrosocystis, 72,73

Vitrosomonas, $70,71,76$

Vitrosospira, 72

Nitrospira, 72

Nocardia, 897, 898, 899, 902, 903, 904, 905, 906, 908, 913, 914

Paracolobactrum, 460

Pectinobacter, 823

Plectridium, 823

Podangium, 1035

Polyangium, 1027, 1028, 1029, 1030, 1031, 1032

Proactinomyces, 923

Propionibacterium, 376

Protaminobacter, 190

Pseudomonas, 90, 92, 94, 95, 97, 98, 99, $100,104,105,106,145,14 \overline{7}, 149,150$, $174,176,177,179,698,700$

Rhodococcus, 275

Saccharabacterium, 623, 624

Sarcina, 286, 287, 288, 293, 294

Serratia, 481

Sorangrum, 1022, 1023, $102 t$

Spirillum, 215: 216

Sporocytophaga, 1048, 1050

Streptcbacillus, 823

Streptococcus, $337,3+1,344$

Streptomyces, 935, 936, 937, 938, 939, $940,941,942,943,944,945,946,947$, $948,950,951,952,953,954,955,956$. 957,958

Thiobacillus, 79, 80, 81

Thiospira, 212

Urobacillus, 691

Vibrio, 199, 200, 201, 203, 204, 205, 206, 207

\section{Soils}

Acid humus soils

Streptomyces, 956

Adobe soil

Actinomyces, 968

Streptomyces, $937,943,945,952,954$, 968 
Soils (continued)

Black, see Mud

Polyangium, 1028, 1029

Compost of soil, sulfur and rock phosphate

Thiobacillus, 79

Containing incompletely oxidized sulfur compounds

Thiobacillus, 79

Containing urine

Micrococcus, 238

Desert

Actinamyces, 972, 973

Field

Azotobacter, 220

Bacillus, 732

Nitrasospira, 71

Filterable bodies, 1294

Forest

Bacillus, 666, 752

Cellfalcicula, 211

Cellvibrio, 210

Nitrosegloea, 73

Garden

Actinomyces, 969, 970

Bacıllus, 664, 666, 669, 672 , 739, 743, $745,750,752,754,755,815,826$

Bacterium, 673, 685

Clostridium, 781, 793, 798, 799, 811, $820,825,826$

Cornilia, 822

Granulobacter, 822

Hydrogenomonas, 78

Lactobacterium, 364

Methanococcus, 248

Micrococcus, 270

Mycobacterium, 890

Pseudomonas, 698

Sarcina, 286, 294

Spirillum, 216

Streptobacillus, 823

Streptomyces, 936, 939, 948

Urobacillus, 691

Vibrio, 203

Greenhouse

Nitrocystis, 75

\section{Humus}

Clostridium, 770

Urobacillus, 691

Legume

Rhizobium, 225, 226, 227, 230
Made soils

Cladothrix, 983

Manured soils

Clostridium, 776, 778, 783, 822

Nitrogloea, 73

Marine mud, see Mud, marine

Mud, see Mud

Oil-soaked soil, see Mineral Sources

Orchard

Streptomyces, 944, 954

Pasture land

Hydrogenomonas, 78

Peat, see Peat

Peat soils

Actinomyces, 969, 974

Podzol (Russian)

Polyangium, 1029

Potato fields

Actinomyces, 971

Roadside

Bacillus, 671

Sand

Bacillus, 658

Bacterium, 685

Sarcina, 286

-, beach

Pseudomonas, 697, 698, 700

Vibrio, 702, 703

-, foraminiferous

Saprospira, 1054, 1055

-, sea

Acetobacter, 692

Bacterium, 627, 632

Sandy loam

Streptomyces, 936

Shore soils

Chitin digesting bacteria, 632

Sour soils

Actinomyces, 971, 973

Subsoil

Streptomyces, 953

Swamp

Bacillus, 662, 750

Flavobacterium, 438

Uncultivated

Saccharobacterium, 623, 624

Upland soils

Streptomyces, 936, 945, 948, 951, 952

Vegetable mold

Hydrogenomonas, 78 
Soils (continued)

Virgin

Cladothrix, 983

Volcanic (Martinique)

Actinomyces, 969

Walls, cellar and mine, see Mineral Sources

Soils, Geographic Distribution of

Antartic, 72

Argentina, 807

Australia, 71, 897, 902, 903, 905, 906, 913

Australia, garden soil, 397

Australia, grass land, 393, 394, 397, 403

Australia, red soil, 397,398

Austria, 77, 738

Brazil, 71

California, 176, 397, 614, 615, 619, 620, $621,743,746,761,936,938,943,945$, $948,951,952,954$

Connecticut, 622, 623

Cuba, 737, 738, 739, 741, 748, 753, 757

Denmark, 947

District of Columbia, 622, 749

Ecuador, 71

Egypt, 713, 737, 744, 757

England, 97, 741, 742

Europe, 713, 750

Florida, 762

France, 70, 71, 72, 73, 897

Georgia, 623

Germany, 210, 211, 603, 732, 736, 737, $738,739,742,745,747,748,749,752$, $753,755,756,757,758$

Great Britain, 898, 899, 903, 904, 906, 913

Hawaii, 938

Holland, 746, 905

India, 221

Italy, 70, 698, 739, 753, 757, 758, 803, 807

Japan, 72

Java, 72

Jugoslavia, 742

Kentucky, 623

Louisiana, 174, 617, 618, 619

Maine, 617,623

Martinique, 969

Maryland, 737

Missouri, 174

Montenegro, 744
New Hampshire, 619

New Jersey, 642, 945

New York, 622, 623, 750

North Carolina, 742, 762

Palestine, 757

Poland, 1018, 1019, 1023, 1027, 1030, $1031,1034,1035,1037,1042,1043$ 1044,1045

Russia, 70, 658, 1028, 1029, 1030

Sahara Desert, 337, 341, 819, 820, 821

South Carolina, 622, 623

Switzerland, 70

Tunisia, 72

United States, 210, 211, 807

Utah, 92, 615, 617, 618, 741

Virginia, 174, 179, 614

Wisconsin, 619

\section{Spring Waters}

At Spalato

Chromobacterium, 233

At Vranje

Bacillus, 732

Containing iron

Bacillus, 732

Gallionella, 831, 832

Naumanniella, 834

Hot springs

Bacillus, 760

Bacterium, 681

Chlamydothrix, 986

Micrococcus, 262, 279

Spirochaeta, 1053

Streptococcus, 341

Thiobacillus, 81

Thiosphaerion, 859

Hot sulfur springs

Bacillus, 656

Microbacillus, 690

Micrococcus, 253

Sulfur springs

A moebobacter, 849, 850

Bacillus, 732

Chlorobium, 870

Clathrochloris, 872

Lamprocystis, 848

Leptothrix, 995

Pelodictyon, 871, 872

Rhabdomonas, 854, 855

Rhodothece, 856

Thiocapsa, 845 


\section{Spring Waters (continued)}

Sulfur springs (continued)

Thiocystis, 847

Thiodictyon, 846

Thiopedia, 813

Thiopolycoccus, 850

Thiosarcina, 843

T'hiospirillum, 851, 852

Thiothece, 846

Thiothrix, 989, 990

Warm springs

Bacillus, 756

\section{Sugar}

Candy

Bacillus, 756

Factory

Bacillus, 744

Micrococcus, 260

Molasses

Clostridium, 781

Lactobacillus, 359

Refineries

Lampropedia, 844

Leuconostoc, 347,348

Nevskia, 830

Refining vats, scum

Clostridium, 808

Solutions

Clostridium, 824

Sugar (sucrose) solutions, slimy

Bacillus, 742, 745, 747

Clostridium, 762

Leuconostoc, 347,348

Myxobacillus, 762

Streptobacterium, 702

Syrup

Nevskia, 830

Wastes

Clostridium, 762

\section{Swamps}

Aerobacter, 692

Bacterium, 676, 685, 686

Saccharobacterium, 623, 624

Swamp Soils, see Soils, Swamp

Swamp Waters, see Waters, Swamp

Swine, see Hogs

Tannery Wastes

Acid dyeing liquor

Bacterinm, 677

\section{Tap Water}

Bacillus, 484, 647, 648, 649, 650, 651, $652,656,657,666,668,669,671,672$, $723,738,740,742,744,747,748,749$, $751,752,754,756,758$

Bacterium, 457, 605, 613

Hyphomicrobium, 837

Leptospira, 1077

Micrococcus, 255, 256, 257, 258, 262, $274,276,277$

Protaminobacter, 190

Pseudomonas, 102, 698

Sarcina, 290, 291

Serratia, 481

Terminosporus, 823

Vibrio, 203

Tap Water, Place Obtained

Berlin, 1077

Chemnitz, 102, 256, 257, 258, 262, 274, $277,290,291,484,648,649,650,651$, $652,656,657,666,668,669,671,672$, $740,742,747,748,749,751,752,754$, 758

Kiel, 698

Lawrence, Mass., 723

Leitmeritz, 255, 256, 262, 276, 277, 457, $647,657,738,740,744,752,754$

Ohio, 756

Minneapolis, 823

Plymouth, England, 481

Rome, 613

Rotterdam, 202

\section{Urine}

Micrococcus, 238, 247, 260, 266, 267, $269,279,280$

Pediococeus, 250

\section{Vegetable Sources, see Plant Sources}

Viruses, Animal Hosts of

Agouti, 1265

Anas boscas, 1253

Anas platyrhyncha, 1251, 1253

Anatidae, 1251, 1253

Anser anser, 1251, 1253

Anser cinereus, 1253

Antelope, 1276

Apidae, 1227

Apis mellifera, 1227

Apodemus sylvaticus, 1267 
Viruses, Animal Hosts of (continued)

Artibeus planirostris, 1263

Asyndesmus lewis, 1252

Bat, fruit-eating, 1263

Bat, vampire, 1263

Bee, honey, 1227

Birds, 1249

Bison, 1239

Blackbird, 1253

Bob-white, 1253

Bombycidae, 1226

Bombyx mori, 1226, 1227

Bos taurus, 1239, 1241, 1242, 1251, 1253, $1267,1272,1276,1278$

Bovidae, 1238, 1239, 1241, 1242, 1248 , $1251,1253,1267,1272,1276,1278$

Bubo virginianus, 1252,1253

Buffalo, 1276

Bunting, 1229

Bushbuck, 1276

Buteo vulgaris, 1263

Cabbage worm, 1227

Calf, 1253

Camel, 1276

Canary, $1229,1279,1280$

Canidae, $1241,1242,1251,1253,1263$, $1272,1273,1277$

Canis familiaris, 1241, 1242, 1251, 1253, $1263,1272,1277$

Capra hircus, 1251, 1253, 1267

Caracal lynx, 1271

Cat, $1235,1236,1255,1262,1263$, $1271,1279,1284$

Cat, black-footed, 1263

Cat, marbled, 1271

Cat, wild, 1263

Cattle, also see Cow, 1234, 1236, 1239. $1240,1242,1249,1263,1272,1276,1278$

Cavia porcellus, 1237, 1277, 1285

Caviidae, 1237, 1277, 1285

Cebidae, 1235

Cebus chrysopus, 126 \%

Cebus fatuellus, 1267

Cebus olivaceus, 1235

Cercocebus fuliginosus, 1235, 1267

Cercocebus torquatus, 1265

Cercopithecidae. 1234, 1235, 1237, 1259, 1284

Cercopithecus aethiops, 1257, 1265, 1266
Cercopithecus callitrichus, 1267

Cercopithecus tantalus, 1265

Charadriidae, 1253

Chicken, 1229, 1231, 1236, 1242, 1252, $1253,1262,1263,1265,1274,1279$, 1280,1283

Chicken embryo, 1229, 1231, 1234, $1235,1236,1238,1239,1240,1245$, $1248,1252,1253,1259,1260,1263$, $1267,1268,1270,1271,1274,1277$, 1279,1280

Chimpanzee, $12 \bar{\imath} 1$

Chrysemys marginata, 1231

Cicomia ciconia, 1253, 1263

Circus rufus, 1253

Citellus vichardsonii, 1253

Colaptes cafer, 1252, 1253

Columba livia, 1251, 1253

Columbidae, 1251, 1253

Cotton rat, 1253, 1257

Cow, also see Cattle, 1231, 1239, 1241 , $1248,1250,1251,1253,1256,1267$

Cowbird, 1253

Coyote, 1273

Cricetidae, 1253, 1285

Cricctulus furunculus, 1249

Cricetulus griseus, 1285

Cricetus auratus, 1267

Cynalopex chama, 1263

Cynictis penicillata, 1263

Dasyprccta aguti, 1265

Deer, 1256,1276

Desmodus rufus, 1263

Diardigallus diardi, 1263

Dipodomys heermanni, 1253

Dog, 1235, 1236, 1241, 1242, 1251 $1253,125 \tilde{3}, 1259,1263,1272,1273$, 1277

Donkey, 1277, 1282

Dormouse, 1267

Dove, western mourning, 1251

Duck, 1229, 1236, 1242, 1251, 1253, 1279

Duck embryo, 1245, 1253

Duck, mallard, 1251, 1253

Duck, Pekin, 1251, 1253

Duiker, 1276

Eland, 1276

Equidae, $1251,1253,1277,1278,1282$

Equus asinus: 1277,1282 
Viruses, Animal Hosts of (continued)

Equus asinus $\times E$. caballus, also sec Mule, 1282

Equus caballus, 1251, 1253, 1277, 1278, 1282

Erinaceus europaeus, 1265

Eryihrocebus patas, 1267

E'utamias asiaticus, 1249

Evolomys rufocanus, 1249

Falco sparverius, 1251, 1253

Falconidae, 1251, 1253

Felidae, 1263, 1279, 1284

Felis aurata, 1271

Felis caracal, 1271

Felis catus, 1263, 1271, 1279, 1281

Felis marmorata, 1271

Felis negripes, 1263

Felis ocreata, 1263

Felis pardalis, 1271

Felis pardus, 1271

Felis planiceps, 1271

Fetis tigrina, 1271

Ferret, 1259, 1262, 1267, 1268, 1270, $1272,1273,1279$

Flicker, red-shafted, 1252, 1253

Fox, 1272

Fox, silver, 1273

Gallus gallus, 1234, 1238, 1242, 1252, $1253,1262,1265,1274,1280,1283$

Genet cat, 1263

Genetta felina, 1263

Geosciurus capensis, 1263

Gerbille, 1277

Goat, 1232, 1239, 1249, 1253, 1267, 1276

Goat, Angora, 1277

Goose, 1229, 1235, 1251, 1253, 1263, 1279

Goose embryo, 1253

Gopher, 1253

Ground squirrel, 1263

Ground squirrel, Richardson's, 1253

Guenon, African, 1265

Guinea-fowl, 1229

Guinea-fowl embryo, 1253

Guinea pig, 1231, 1235, 1236, 1237 , $1238,[1239,1240,1253,1254,1256$, $1257,1259,1260,1268,1277,1285$

Gypsy moth, 1226

Hamster, Chinese, 1285
Hamster, golden, 1267

Hamster, Syrian, 1259, 1278

Hapale jacchus, 1267

Hapale penicillata, 1267

Hare, snowshoe, 1244

Hawk, 1229, 1253

Hawk, mouse, 1263

Hawk, sparrow, 1251, 1253

Hedgehog, 1235, 1265, 1268, 1279

Hedgehog, European, 1255

Hominidae, 1233, 1234, 1235, 1237, $1241,1248,1250,1251,1253,1257$, $1259,1260,1262,1263,1265,1267$, $1268,1270,1280,1282,1284$

Homo sapiens, 1233, 1234, 1235, 1237 , $1241,1248,1250,1251,1253,1257$, $1259,1260,1262,1263,1265,1267$, $1268,1270,1280,1282,1284$

Horse, 1231, 1232, 1236, 1240, 1248, $1249,1250,1251,1253,1254,1255$, $1256,1263,1277,1278,1282$

Ictony $x$ orangiae, 1263

Jackal, silver, 1263

Junco, 1253

Junco oreganus, 1253

Kangaroo rat, 1253

Killdeer, 1253

Kioedoe, 1276

Leopard, 1271

Leporidae, 1237, 1243, 1244, 1245, 1251

Lepus americanus, 1243

Lepus brasiliensis, 1245

Lepus californicus, 1244, 1251

Lion, 1271

Lophortyx californica, 1252, 1253

Lymantria monacha, 1226

Lymantriidae, 1226

Macaca irus, 1231, 1240, 1257, 1259, 1267,1268

Macaca mordax, 1257

Macaca mulatia, 1231, 1234, 1236, $1237,1240,1256,1257,1259,1262$, $1265,1266,1267,1284$

Macacus cynomolgus, 1235, 1265

Macacus fuscatus, 1231

Macacus rhesus, 1248, 1249, 1250, 1252

Macacus sinicus, 1265

Macacus speciosus, 1265 
Viruses, Animal Hosts of (continued)

Macaque, crab-eating, 1259

IIan, 1231, 1232, 1233, 1234, 1235, $1237,1241,1248,1249,1250,1251$, $1253,1255,1257,1259,1260,1262$, $1263,1265,1267,1268,1270,1271$, $1278,1280,1282,1284$

Mangabey, collared, 1265

Marmota monax, 1253

Mastomys coucha, 1277

Meercat, common, 1263

Meercat, yellow, 1263

Meleagridae, 1251, 1253

Meleagris gallopavo, 1251, 1253

Microtus agrestis, 1265, 1267

Microtus californicus, 1253

Microtus michnoi, 1219

Microtus montanus, 1253

Microtus mordax, 1253

Microtus pennsylvanicus, 1253

Mink, Chinese, 1268

Molothrus ater, 1253

Mongoose, small gray, 1263

Mongoose, yellow; 1263

Monkey, 1234, 1253

Monkey, cynomolgus, 1231, 1240, 1257

Monkey, green African, 1257

Monkey, mona, 1257

Monkey, rhesus, 1231, 1234, 1236 , $1237,1240.1250,1251,1256,1257$, $1259,1260,1262,1265,1279,128 t$

Mouse, 1234, 1248, 1250, 1251, 1256. $1257,1260,1262,1265,1267,1268$, $1270,1272,1277,1279,1280,1286$

Mouse, field, 1253

Mouse, gray, 1259

Mouse, gray field, 1236

Mouse, multimammate, $127 \overline{7}$

Mouse, white, 1235, 1236, 1238, 1240, $1249,1252,1253,1259,1261,1263$

Mouse, white-footed, 1253, 1263

Mouse, wild, 1253

Mouse, wood, 1267

Mule, 1253, 1277, 1282

Muridae, 1238, 1251, 1253, 1259, 1261, $1272,1277,1286$

Mus musculus, 1238, 1259, 1261, 1263, $1265,1272,1286$

.Huscardinus avellanarius, 1267

Muscicapidae, 1252, 1253
Mustela frenata, 1253

Mustela furo, 1273

Mustela sibirica, 1268

Mustelidae, 1253, 1263, 1272, 1273

Myonax pulverulentus, 1263

Neotoma fuscipes, 1253

Nun moth, 1226

Ocelot, 1271

Orang-outang, 1231

Oryctolagus cuniculus, 1237, 1243, 1244,1245

Ovis aries; 1238, 1248, 1251, 1253, $126 \overline{7}, 1278$

Owl, 1263

Owl, great horned, 1252, 1253

Owl, western burrowing, 1253

Oxyechus vociferus, 1253

Partridge, 1229

Passer domesticus, 1253

Peromyscus maniculatus, 1253

Peromyscus polionotus, 1263

Phasianidae, 1234, 1238, 1242, 1252, $1253,1262,1274,1280$

Phasianus colchicus, 1253

Pheasant, 1229, 1242, 1263, 1274

Pheasant-chicken, $F_{1}$ hybrid, 1274

Pheasant embryo, 1253

Pheasant, ring-necked, 1253

Phyllostoma superciliatum, 1263

Picidae, 1252, 1253

Pieridae, 1227

Pieris brassicae, 1227

Pig, also see Swine, 1231, 1239, 1248, $1252,1253,1255,1263$

Pigeon, 1229, 1235, 1251, 1252, 1253, $1255,1263,1279,1280$

Pigeon embryo, 1253

Polecat, 1263

Portheria dispar, 1226

Prairie chicken, 1253

Puma, 1271

Quail, 1229, 1253

Quail, California, 1252, 1253

Rabbit, 1231, 1232, 1234, 1235, 1236, $1237,1238,1239,1240,1243,1244$, $1245,1247,1253,1256,1268,1279$

Rabbit, brush, 1253

Rabbit, cotton-tail, 1244, 1245, 1247 , 1251,1253

Rabbit, jack, 1244, 1245, 1251 
Viruses, Animal Hosts of (continued)

Rat, 1238, 1239, 1248, 1256, 1267, $1268,1277,1279,1286$

Rat, black, 1253

Rat, brown, 1236, 1251

Rat, white, $1236,1253,1257,1259$, 1262,1277

Rattus norvegicus, 1238, 1251, 1277

Rattus rattus, 1253

Reindeer, 1239

Reithrodontomys megalatus, 1253

Robin, 1252, 1253

Robin embryo, 1253

Sciuridae, 1263

Sciurotamius davidianus, 1268

Sciurus carolinensis, 1267

Sciurus vulgaris, 1265

Sheep, 1232, 1234, 1236, 1238, 1239, $1248,1249,1250,1251,1253,1255$, $1256,1263,1267,1276,1278$

Sigmodon hispidus, 1253, 1257

Silkworm, 1226, 1227

Sparrow embryo, 1253

Sparrow, English, 1229, 1253

Sparrow, Crambel's, 1253

Speotyto cunicularia, 1253

Squirrel, David's, 1268

Squirrel, gray, 1267

Squirrel, red, 1265

Stork, 1263

Stork, white, 1253

Strigidae, 1252, 1253

Suidae, $1252,1253,1262,1268,1275$, 1282

Suricata suricata, 1263

Suricate, Cape, 1263

Sus scrofa, 1233, 1252, 1253, 1262. $1268,1275,1282$

Swine, also see Pig, 1232, 1233, 1234, $1236,1240,1262,1268,1275,1276$, 1282

Sylvilagus audubonii, 1253

Sylvilagus bachmani, 1253

Sylvilagus nuttalli, 1251

Sylvilagus sp., 1244, 1245, 1247

Tatera lobengula, 1277

Tetraonidae, 1253

Thrasher, 1253

Tiger, 1271

Tiger cat, African, 1271
Tiger cat, American, 1271

Tiger cat, rusty, 1271

Toxostoma lecontei, 1253

Turdus merula, 1253

Turdus migratorius, 1252, 1253

Turkey, 1229, 1251, 1253, 1279

Turkey embryo, 1253, 1274

Turtle, 1231

Tympanuchus cupido, 1253

Vivreridae, 1263

Vole, field, 1253, 1265, 1267

Vulpes sp., 1272, 1273

Vultur fulvus, 1253

Vulture, tawny, 1253

Warthog, 1275

Weasel, 1253

Wolf, 1263

Woodchuck, 1253

Woodpecker, Lewis, 1252

Wood rat, 1253

Zebra, 1277

Zebu cattle, 1276

Zenaidura macroura, 1251

Zonotrichia leucophrys, 1253

\section{Viruses, Bacterial Hosts of}

Agrobacterium tumefaciens, 1134

Bacillus megatherium, 1138

Bacillus mycoides, 1138

Bacterium stewarti, 1136

Corynebacterium diphtheriae, 1143, 1144

Erwinia aroideae, 1136

Eruinia carotovora, 1135

Escherichia coli, 1131, 1132, 1133,

\section{4}

Pseudomonas solanacearum, 1135

Rhizobium leguminosarum, 1138

Salmonella enteritidis, 1136, 1137

Salmonella gallinarum, 1136, 1137

Salmonella typhosa, 1137

Shigella dysenteriae, 1131, 1132, 1133, $1134,1136,1137$

Staphylococcus albus, 1140, 1141, 1142

Staphylococcus aureus, 1140, 1141

Staphylococcus muscae, 1142

Streptccoccus cremoris, 1138, 1139

Streptococcus mucosus, 1139

Streptococcus sp., 1139, 1140

Vibrio comma, 1142, 1143 
Viruses, Bacterial Hosts of (continued)

Xanthomonas citri, 1135

Xanthomonas pruni, 1135

\section{Viruses, Plant Hosts of}

Abacá, 1193

Abutilon sp., 1186

Ageratum conyzoides, 1218

Agropyron repens, 1162,1202

Alfalfa, 1151, 1153, 1181, 1191

Allium cepa, 1184

Almond, 1152, 1196

Alopecurus fulvus, 1160

Althaea ficifolia, 1186

Althaea officinalis, 1186

Althaea rosea, 1186, 1218

Amaranthaceae, 1204, 1214

Amaranthus retroflexus, 1204, 1214

Amygdalus persica, 1197

Ananas comosus, 1223

Anemone nemorosa, 1158

Anemone ranunculoides, 1158

Anemone trifolia, 1158

Anethum graveolens, 1176

Anoda hastata, 1186

Anthriscus cerefolium, 1176

Antirrhinum majus, 1191

A pium graveolens, 1147, 1176, 1199, 1200

Apocynaceae, 1149, 1150, 1152

Apple, 1194

Apricot, 1152, 1196

Arachis hypogaea, 1187

Armoracia rusticana, 1177

Asclepiadaceae, 1173

Asclepias syriaca, 1173

Aster, 1168

Atriplex hortensis, 1204

Atriplex sibirica, 1204

Atropa belladonna, 1175

Avena byzantina, 1161

Avena falua, 1161

Avena sativa, 1160, 1161, 1162, 1192

Barbarea vulgaris, 1155

Barley, 1161, 1162, 1192

Bean, 1168, 1169, 1179, 1180, 1181,

$1189,1190,1191,1216,1219$

Bean tree, 1187

Beet, 1178, 1221

Berteroa incana, 1177

Beta cicla, 1204

Beta maritima, 1204
Beta vulgaris, 1149, 1177, 1178, 1199, $1204,1216,1219,1221$

Bittersweet, 1175, 1204, 1214

Black-eyed Susan, 1155

Brachiaria platyphylla, 1183

Brassica adpressa, 1176, 1200

Brassica alba, 1176, 1177, 1200

Brassica arvensis, 1176, 1177, 1200

Brassica campestris, 1176

Brassica chinensis, $117 \%$

Brassica incana, 1199

Brassica juncea, 1176, 1177, 1200

Brassica napobrassica, 1177, 1221

Brassica napus, 1176, 1177, 1221

Brassica nigra, 1176, 1177, 1200

Brassica oleracea, 1176, 1177, 1200

Brassica pe-tsai, 1176, 1200

Brassica rapa, 1176, 1177, 1200

Broad bean, 1179, 1180, 1187

Broccoli, 1176

Brome-grass, awnless, 1192

Bromeliaceae, 1228

Bromus inermis, 1162, 1192

Brussels sprouts, 1176

Buckwheat, 1146, 1199

Cabbage, 1176, 1177

Calendula, 1150

Calendula officinalis, 1150, 1177

Callistephus chinensis, 1146

Candytuft, rocket, 1176

Cantaloupe, 1199, 1200

Capsella bursa-pastoris, 1176, 1177, 1200

C'apsicum frutescens, 1164, 1171, 1175, 1181,1214

Caraway, 1176

Cardamine heterophylla, 1177

Carrot, 1146, 1176

Carum carvi, 1176

Caryophyllaceae, 1191

Cauliflower, 1176

Celastraceae, 1187

Celeriac, 1176

Celery, 1147, 1173, 1174, 1176, 1199, 1200

Chaetochloa lutescens, 1183

Chaetachloa magna, 1183

Chaetochloa verticillata, 118.3

Charlock, 1176

Charlock, white, 1176 
Viruses, Plant Hosts of (continued)

Cheiranthus allionii, 1177

Cheiranthus cheiri, 1177

Chenopodiaceae, 1149, 1177, 1178, $1191,1199,1200,1204,1216,1219$, 1221

Chenopodium album, 1199, 1200, 1204

Chenopodium murale, 1199, 1200, 1220

Cherry, 1152, 1196, 1197, 1198

Cherry, flowering, 1197, 1210

Cherry, Mahaleb, 1197

Cherry, Mazzard, 1197, 1210

Cherry, sand, 1152

Cherry, wild, 1197

Chinese cabbage, 1177

Chokecherry, 1152

Chrysanthemum leucanthemum, 1155

Chrysanthemum morifolium, 1214

Cicer arietinum, 1180

Citrullus vulgaris, 1167

Citrus aurantium, 1202

Citrus limonia, 1210

Citrus maxima, 1210

Citrus sinensis, 1210

Clover, alsike, 1180, 1187, 1190

Clover, cluster, 1180,1190

Clover, crimson, 1156, 1157, 1180, 1181,1187

Clover, nodding, 1190

Clover, Persian, 1180

Clover, red, 1155, 1179, 118, 1190

Clover, spotted bur, 1180

Clover, strawberry, 1190

Clover, toothed bur, 1180

Clover, white, 1188, 1189, 1190, 1191

Commelina sp., 1174

Compositae, 1147, 1150, 1155, 1168, $1169,1174,1177,1178,1179,1181$, $1212,1214,1218,1223$

Convolvulaceae, 1149,1198

Coriander, 1176

Coriandrum salivum, 1176

Corn, 1158, 1159, 1160, 1161, 1162, 1183

Coronopus didymus, 1177

Cotoneaster harroviana, 1194

Cotton, 1218

Cowpea, 1169, 1173, 1174, 1188, 1189

Cranberry, 1150

Cress, common winter, 1155
Cress, garden, 1176

Crotalaria retusa, 1190

Crotalaria spectabilis, 1190

Crotalaria striata, 1190

Cruciferae, 1155, 1176, 1177, 1199, 12001221

Cucumber, 1167, 1168, 1173, 1181, $1190,1200,1216$

Cucumber, wild, 1173

Cucumis anguria, 1167

Cucumis melo, 1167, 1173, 1190, 1199, 1200

Cucumis sativus, 1167, 1173, 1181, $1190,1191,1200,1212,1216$

Cucurbitaceae, 1167, 1181, 1190, 1191, $1199,1200,1212,1216,1219$

Cucurbita pepo, 1173, 1190, 1200

('urcurbita sp., 1219

Cuscuta californica, 1198

Cuscuta campestris, 1199

Cuscuta subinclusa, 1199

Cytisus hirsutus, 1187

Dahlia, 1179

Dahlia imperialis, 1179

Dahlia maxonii, 1179

Dahlia pinnata, 1179

Datura stramonium, 1147, 1169, 1171, $1174,1175,1200,1204,1214,1216$

Daucus carota, 1176

Delphinium sp., 1177, 1200, 1216

Desmodium canadense, 1180

Digitaria horizontalis, 1159

Dodder, also see Dodder as vector, 1198

Dodonaea viscosa, 1149

Echinochloa crusgalli, 1160, 1162 1183

Echinocystis lobata, 1173

Eggplant, 1147

Eleusine indica, 1159

Elm, American, 1154

Endive, 1146

Ericaceae, 1150

Eriobotrya japonica, 1194

Eulalia, 1183

Euonymus japonica, 1187

Euonymus radicans, 1187

Euphorbiaceae, 1219

Fagopyrum esculentum, 1199

Ficus sp., 1201, 1202 
Viruses, Plant Hosts of (continued)

Fragaria species and hybrids, 1195, 1207,1208

Galega officinalis, 1190

Galtonia candicans, 1184

Geraniaceae, 1168, 1199

Geranium, 1199

Gherkin, 1167

Glycine soja, also see Soja max, 1190

Goat's rue, 1190

Gossypium hirsutum, 1216, 1218

Gossypium peruvianum, 1218

Gossypium vitifolium, 1218

Gramineae, 1157, 1158, 1159, 1160, $1161,1162,1183,1192,1208$

Grape, 1153, 1198

Grapefruit, 1210

Grass pea, 1180

Goundsel, 1178

Henbane, 1171, 1175, 1214

Hesperis matronalis, 1177

Hibiscus sp., 1218

Holcus sorghum, 1183

Holcus sudanensis, 1183

Hollyhock, 1218

Holodiscus discolor, 1207

Honesty, 1176

Hop, European, 1151

Hordeum vulgare, 1161, 1162, 1192

Horse-radish, 1177

Humulus lupulus, 1151

Hyacinth, 1184

Hyacinthus orientalis, 1184

Hyoscyamus niger, 1171, 1175, 1214

Iberis amara, 1176

Indian tobacco, 1154

Iridaceae, 1183

Iris, 1183

Iris, bearded, 1183

Iris, bulbous, 1183

Iris flifolia, 1183

Iris ricardi, 1183

Iris tingitana, 1183

Iris unguicularis, 1183

Iris xiphium, 1183

Jimson weed, 1171, 1174, 1175, 1200, 1204,1214

Kale, 1176

Kitaibelia vitifolia, 1186

Knotweed, 1199
Labiatae, 1173

Laburnum anagyroides, 1187

Laburnum vulgare, 1187

Lachenalia sp., 1184

Lactuca sativa, 1178, 1223

Lamb's quarters, 1199, 1204

Larkspur, 1200

Lathyrus odoratus, 1178, 1179, 1180, 1187, 1189, 1190, 1191

Lathyrus sativus, 1180

Lavatera arborea, 1186

Leguminosae, 1150, 1151, 1153, 1155, $1156,1157,1168,1169,1178,1179$, $1180,1181,1187,1188,1189,1190$, $1191,1212,1216,1219,1223$

Lemon, 1210

Lens esculenta, 1189, 1191

Lepidium ruderale, 1177

Lepidium sativum, 1176, 1177

Lepidium virginicum, 1177

Lespedeza striata, 1179

Lettuce, 1146, 1178, 1223

Ligustrum vulgare, 1187

Liliaceae, 1182, 1184, 1211

Lilium amabile, 1182

Lilium auratum, 1182

Lilium canadense, 1182

Lilium candidum, 1182

Lilium cernuum, 1182

Lilium chalcedonicum, 1182

Lilium croceum, 1182

Lilium davmottiae, 1182

Lilium elegans, 1182

Lilium formosanum, 1182

Lilium giganteum, 1182

Lilium henryi, 1182

Lilium leucanthum, 1182

Lilium longiflorum, 1182, 1211

Lilium myriophyllum, 1182

Lilium pumilum, 1182

Lilium regale, 1182

Lilium sargentiae, 1182

Lilium speciosum, 1182

Lilium superbum, 1182

Lilium testaceum, 1182

Lilium iigrinum, 1182

Lilium umbellatum, 1182

Lilium wallacei, 1182

Lily, 1174

Lily, Easter, 1211 
Viruses, Plant Hosts of (continued)

Lima bean, 1174, 1188

Locust, black, 1150

Loganberry, 1206

Loquat, 1194

Lotus hispidus, 1190

Lucerne, 1151, 1153, 1181, 1191

Lunaria annua, 1176

Lupine, blue, 1180, 1190

Lupine, white, 1180

Lupine, yellow, 1190

Lupinus albus, 1180, 1189, 1190, 1191

Lupinus angustifolius, 1180, 1190

Lupinus densiflorus, 1180

Lupinus hartwegii, 1180

Lupinus hirsutus, 1191

Lupinus luteus, 1190

Lupinus mutabilis, 1190

Lupinus nanus, 1180

Lycium barbarum, 1172

Lycopersicon esculentum, 1147, 1149 , $1150,1152,1164,1168,1169,1171$, $1175,1181,1199,1200,1204,1214$, 1219,1223

Lycopersicon pimpinellifolium, 1177

Maize, 1158, 1159, 1160, 1161, 1162, 1183

Malcomia bicornis, 1200

Malcomia maritima, 1200

Malva borcalis, 1186

Malvaceae, $1186,1216,1218$

Malva crispa, 1186

Malva mauritiana, 1186

Malva sylvestris, 1186

Malva verticillata, 1186

Malvestrum capense, 1186

Manihot sp., 1219

Manila hemp plant, 1193

Marrow, 1190

Matthiola incana, 1176, 1177

Medicago arabica, 1180

Medicago hispida, 1180

Medicago lupulina, 1189, 1191

Medicago sativa, 1151, 1153, 1181, 1190,1191

Melilotus alba, 1180, 1188, 1189, 1190, 1191

Melilotus indica, 1180

Melilotus officinalis, 1180

Melon, rock, 1190

Millet, 1162
Millet, pearl, 1183

Miscanthus sinensis, 1183

Modiola decumbens, 1186

Moraceae, 1151, 1201

Mung bean, 1189

Musaceae, 1193

Musa textilis, 1193

Muskmelon, 1173

Mustard, 1176, 1199

Mustard, black, 1176, 1177

Mustard, leaf, 1176

Mustard, white, 1176, 1177

Mustard, wild yellow, 1176

Vasturtium officinale, 1177

Nectarine, 1196

Nepeta cataria, 1173

Neslia paniculata, 1177

New Zealand spinach, 1146

Nicotiana alata, 1216

Nicotiana bigelorii, 1177

Nicotiana glauca, 1154, 1167, 1199

Nicotiana glutinosa, 1149, 1150, 1152, $1154,1166,1168,1169,1177,1200$, 1216

Nicotiana langsdorffii, 1168, 1169, 1177,1201

Vicotiana palmeri, 1199

Nicotiana repanda, 1177

Nicotiana rustica, 1149, 1154, 1155, $1156,1177,1199,1201,1216$

Nicotiana syluestris, 1166, 1177

Nicotiana tabacum, 1147, 1149, 1150, $1154,1164,1168,1169,1171,1172$, $1174,1175,1177,1181,1199,1200$, $1201,1212,1213,1214,1216,1218$, 1223

Nicotiana trigonophylla, 1154

Nightshade, black, 1147, 1214

Oat, 1160, 1161, 1162, 1192

Oat, wild, 1161

Ocean spray, 1207

Oleaceae, 1187

Onion, 1184

Orange, 1210

Orange, sour, 1202

Ornithogalum ihyrsoides, 1184

Oryza sativa, 1160, 1162

Pachyrhizus erosus, 1188

Panicum dichotomiflorum, 1183

Panicum miliaceum, 1160, 1162 
Viruses, Plant Hosts of (continued)

Parsley, 1176

Parsnip, 1146

Paspalum boscianum, 1183

Passiflora alba, 1193

Passifloraceae, 1193

Passiflora coerulea, 1193

Passiflora edulis, 1193

Passion fruit, 1193

Pea, 1178, 1179, 1180, 1188, 1189, $1190,1191,1223$

Peach, 1148, 1152, 1158, 1196, 1197, 1207, 1208

Peanut, 1187

Pear, 1211

Pelarganium hortorum, 1168, 1199

Pennisetum glaucum, 1183

Pepper, 1164, 1171, 1173, 1175, 1181, 1214

Periwinkle, 1149, 1150, 1152

Petroselinum hortense, 1176

Pe-tsai, 1176

Petunia, 1171, 1175, 1200

Petunia sp., 1171, 1175, 1177, 1200, 1212,1216

Phaseolus arutifolius, 1179, 1180

Phaseolus aureus, 1179, 1189, 1191

Phaseolus calcaratus, 1179

Phaseolus lunatus, 1168, 1179, 1188

Phaseolus vulgaris, 1168, 1169, 1179, $1180,1181,1189,1190,1191,1216,1219$

Phenomenal berry, 1206

Photinia arbutifolia, 1194

Physalis alkekengi, 1214

Physalis heterophylla, 1171, 1173

Physalis subglabrata, 1173

Phytolacca americana, 1199

Phytolaccaceae, 1173, 1199

Phytolacca decandra, 1173

Pineapple, 1223

Pisum saiivum, 1178, 1179, 1180, 1188, $1189,1190,1191,1223$

Plantaginaceae. 1164, 1199

Plantago lanceolata, 1164

Plantago major, 1164, 1199

Plantago rugelii, 1164

Plantago sp., 1166

Plantain, 1199

Plum, 1152, 1196, 1208

Plum, Japanese, 1148, 1197
Plum, wild, 1152

Poa pratensis, 1160

Pokeweed, 1199

Polygonaceae, 1199

Polygonum pennsylvanicum, 1199

Potato, 1149, 1150, 1155, 1156, 1172, $1174,1175,1181,1182,1199,1200$, $1203,1204,1214,1223$

Primulaceae, 1199, 1201

Primula sp., 1201

Privet, 1187

Prune, 1196, 1208

Prunus americana, 1152

Prunus armeniaca, 1152, 1196

Prunus avium, 1196, 1197, 1198, 1210

Prunus cerasus, 1152, 1197

Prunus communis, 1152, 1196

Prunus domestica, 1152, 1196, 1198, 1208

Prunus emarginata, 1197

Prunus mahaleb, 1197

Prumus persica, 1148, 1152, 1158, $1196,1197,1207,1208$

Prunus pumila, 1152

Prunus salicina, 1148, 1197

Prunus serrulata, 1197, 1198, 1210

Prunus sp., 1148, 1207

Prunus virginiana, 1152

Pyrus communis, 1211

Pyrus malus, 1194

Radicula palustris, 1177

Radish, 1176, 1200

Ranunculaceae, 1158, 1177, 1200, 1216

Ranunculus asiaticus, 1216

Rape, 1176, 1177, 1221

Raphanus raphanistrum, 1176

Raphanus saivus, 1176, 1177, 1200

Raspberry, 1195, 1205

Raspberry, black, 1195, 1206

Rhamneae, 1149

Ribgrass, 1164

Rice, 1160, 1162

Robinia pseudoacacia, 1150

Rosaceae, 1148, 1152, 1158, 1194, $1195,1196,1197,1198,1205,1206$ $1207,1208,1211$

Rosa species and hybrids, 1194

Rose, 1194

Rubus idaeus, 1195, 1205 
Viruses, Plant Hosts of (continued)

Rubus loganobaccus, 1206

Rubus occidentalis, 1195, 1206

Rubus parviflorus, 1194

Rudbeckia hirta, 1155

Rutabaga, 1177, 1221

Rutaceae, 1202,1210

Rye, 1160, 1161, 1162, 1192

Saccharum narenga, 1183

Saccharum officinarum, 1157, 1158,

$1159,1161,1183,1208$

Salad chervil, 1176

Samolus floribundus, 1199

Sandal. 1149, 1198

Santalaceae, 1149, 1198

Santalum album, 1149, 1198

Sapindaceae, 1149

Scrophulariaceae, 1191, 1212, 1214

Secale cereale, 1160, 1161, 1162, 1192

Senerio vulgaris, 1178

Selaria viridis, 1162

Shepherd's purse, 1176

Sidalcea candida, 1186

Sida molli.s, 1186

Sida napaea, 1186

Sieva bean, 1168

Sinapis alba, 1177

Sincamas, 1188

Sisymbrium altissimum, 1177

Sisymbrium officinale, 1177

Soja max, also see Glycine soja, 1168, 1180

Solanaceae, 1147, 1149, 1150, 1152, $1154,1155,1164,1166,1167,1168$, $1169,1171,1172,1173,1174,1175$, $1177,1181,1199,1200,1203,1204$, $1212,1213,1214,1216,1218,1219$, 1223

Solanum dulcamara, 1175, 1204, 1214

Solanum melongena, 1147

Solanum nigrum, 1147, 1168, 1169, $1174,1175,1214$

Solanum tuberosum, 1149, 1150, 1155, $1172,1174,1175,1181,1199,1200$, $1203,1204,1212,1214,1223$

Solanum villosum, 1204

Sonchus asper, 1178

Sorbus pallescens, 1194

Sorghum, 1183

Sowbane, 1199
Sow-thistle, prickly, 1178

Soybean, 1168, 1180, 1190

Speedwell, 1214

Spinach, 1173, 1177, 1178, 1191, $120 t$

Spinacia oleracea, 1177, 1178, 1191, 1200,1204

Squash, 1219

Squash, summer crooknerk, 1200

Stachytarpheta indica, 1150

Stellaria media, 1191, 1220

Stock, 1176, 1177

Strawberry, 1195, 1207, 1208

Sudan grass, 1183

Sugar beet, 1149, 1199

Sugar rane, 1157, 1158, 1159, 1160, $1161,1183,1208$

Swede, 1177

Sweet clover, annual yellow, 1180

Sweet clover, white, 1180, 1188

Sweet clover, yellow, 1180

Sweet pea, 1178, 1179, 1180, 1187, 1190

Sweet potato, 1202

Synediella nodiflora, 1218

Syntherisma sanguinale, 1183

Tepary bean; 1180

Thlaspi arvense, 1177

Tobaceo, 1147, 1149, 1150, 1154, 1161, $1166,1168,1169,1170,1171,1172$, $1173,1174,1175,1177,1181,1200$, $1213,1214,1216,1218,1223$

Tomato, 1147. 1149, 1150, 1152, 1164, $1166,1168,1169,1171,1175,1181$, $1199,1200,1204,1214,1219,1223$, 1224

Toyon, 1194

Tree tobacco, 1167

Trefoil, haresfoot, 1190

Trifolium agrarium, 1180

Trifolium arvense, 1190

Trifolium carolinianum, 1180

Trifolium cerruum, 1190

Trifolium dubium, 1180

Trifolium fragiferum, 1190

Trifolium glomeratum, 1180, 1190

Trifolium hybridum, 1180, 1187, 1189, 1190, 1191

Trifolium incarnatum, 1156, 1157, $1180,1181,1187,1189,1190,1191$ 
Viruses, Plant Hosts of (continued)

Trifolium pratense, 1155, 1179, 1187, $1189,1190,1191$

Trifolium procumbens, 1180

Trifolium reflexum, 1180

Trifolium repens, 1188, 1189, 1190 , 1191

Trifolium sualeolens, 1180

Triticum sp., 1160, 1161, 1162, 1192

Tropaeolum majus, 1224

Tulip, garden, 1182

Tulipa clusiana, 1182

Tulipa eichleri. 1182

Tulipa gesneriana, 1182

Tulipa greigi, 1182

Tulipa linifolia, 1182

Turnip, 1176, 1177

Ulmus americana, 1154

Umbelliferae, 1147, 1176, 1199, 1200

Urticaceae, $115 t$

Taccinium macrocarpon, 1150

Vaccinium oxycoccus, 1150

Vegetable marrow, 1173

Verbenaceae, 1150

Ternonia cineria, 1218

Ternonia iodocalyx, 1218

Veronica sp., 1214

Vetch, common, 1180

Vetch, hairy, 1190

Vetch, spring, 1179

Ticia faba, 1179, 1180, 1187, 1189

1190,1191

Ticia sativa, 1179, 1180, 1189, 1191

Ticia villosa, 1190

Tigna sinensis, 1169, 1173, 1188,

1189,1212

Tinca rosea, 1149, 1150, 1152

Violaceae, 1200

Viola cornuta, 1200

Vitaceae, 1153, 1198

Vitis vinifera, 1153,1198

Wallflower, 1177

Water pimpernel, 1199

Watermelon, 1167

Wheat, 1160, 1161, 1162, 1192, 1202

Windflower, vernal, 1158

Yam bean. 1188

$Z \epsilon a$ mays, 1158, 1159, 1161, 1162, 1183

Zinnia, 1147, 1169, 1174, 1181
Zinnia elegans, 1147, 1169, 1174, 1177,

1181

Ziziphus oenoplia, 1149

Viruses, Vectors of,

Aceratagallia curvaia, 1155

Aceratagallia lyrata, 1155

Aceratagallia obscura, 1155

Aceratagallia sanguinolenta, 1155

Aëdes aegypti, 1230, 1254, 1255, 1259, 1266

Aëdes albopictus, 1254

Aëdes apico-annulatus, 1266

Aëdes atropalpus, 1254

Aëdes cantator, 1254

Aëdes dorsalis, 1254

Aëdes fluviatilis, 1266

Aëdes leucocelaemus, 1266

Aëdes lutcocephalus, 1266

Aëdes nigromaculis, 1254

Aëdes scapularis, 1266

Aëdes sollicitans, 1254

Aëdes stimulans, 1230

Aëdes taeniorhynchus, 1254

Aëdcs triseriatus, 1254

Aëdes vexans, 1230,1254

Agallia constricta, 1156

Agallia quadripunctata. 1155, 1156

Agalliana ensigera, 1220

Agalliopsis novella, 1155, 1156, 1157

Aleyrodidae, 1218, 1219

Allolobophora caliginosa, 1269

Amblycephalinae, 1153

Amphorophora rubi, 1196

Amphorophora rubicola, 1196

Amphorophora sensoriata, 1196

Anuraphis tulipae, 1182

Aphididae, 1163, 1164, 1171, 1172,

$1173,1174,1175,1176,1177,1178$,

$1179,1180,1181,1182,1183,1184$,

$1185,1187,1188,1193,1195,1196$.

$1200,1203,1204,1205,1206,1207$,

1208,1211

Aphids, see Aphididae

Aphis abbreviata, 1172, 1175, 1204

A phis apigraveolens, 1176, 1177, 1200

Aphis apii, 1176, 1200

Aphis fabae, 1171, 1204

Aphis ferruginea striata, 1176, 1200

Aphis gossypii, 1173, 1174, 1176, 1177,

$1179,1185,1188,1200,1211$ 
Viruses, Vectors of (continued)

Aphis grareolens, 1177

A phis laburni, 1187

Aphis leguminosae, 1187

Aphis maidis, 117t, 1183, 1184

Aphis medicagins, 1179

Aphis middletonii, 1176, 1177, 1200

Aphis rhamni, 1171, 1172

A phis rubicola, 1205

A phis rubiphila, 1205

Aphis rumicis, $1176,1178,1179,1180$,

1184

Aphis sp., 1193

A phis spiraeae, 1207

Aphis spiraecola, 1179

Aphis tulipae, 1182

Aulacorthum solani, 1201

Balclutha mbila, 1159

Bedbug, 1259

Bemisia gossypiperda, 1218, 1219

Bemisia nigeriensis, 1219

Brevicoryne brassicae, 1177, 1179,

1201

Capitophorus fragaefolii, 1208

Capitophorus fragariae, 1195

Capitophorus tetrahodus, 1206

Carneocephala fulgida, 1153

Carneocephala triguttata, 115:3

Carolinaia cyperi, 1183

Cavariella capreae, 1176, 1177

Choerostrongylus pudendotectus, 1269

Cicadellidae, 1145, 1147, 1148, 1150 .

$1153,1154,1155.1157,1159.1160$,

1161. 1220

Cicadula bimaculata, 1159

Cicaulula divisa, 1147

Cicadula sexnotata, 1147

Cicadulina mbila, 1159

Cicadulina storeyi, 1159

Cicadulina zeae, 1159

Cimex lectularius, 1259

Cimidae, 1259

Convolvulaceae, $1149,1150,1165,1170$,

$1173,1192,1199,1220$

Ctenopsylla felis, 1246

Cuerna occidentalis, 1153

Culex pipiens, 1230, 1252

Culex tarsalis, 1252

Culicidae, 1230, 1252, 1251, 1259 , 1266, 1267
Cuscuta calufornica, 1173, 1198, 1199

Cuscuta campestris, 1149, 1150, 1152,

$1165,1170,1173,1192,1199,1220$

Cuscuta subinclusa, 1173, 1199

Delphacinae, 1157

Delphax striatella, 1162

Deltocephalus dorsalis, 1160, 1161

Deltocephalus striatus, 1161

Dermacentor andersoni, 1254

Demacentor silvarum, 1250

Dermacentor variabilis, 1252, 1254

Dodders, $1147,1149,1150,1152,1165$,

$1170,1173,1192,1199,1220$

Draeculacephala minerva, 1153

Draecuiacephala portola, 1161

Eriophyes ribis, 1203

Eriophyidae, 1203

Euscelis striatulus, 1150

Eutettix tenellus, 1220

Flea, $12+6$

Frankliniella insularis, 1223

Frankliniella lycopersici, 1223

Frankliniella moultoni, 1223

Frankliniella occidentalis, 1223

Frankliniella schullzer, 1223

Fulgoridae, 1145, 1157. 1161, 1162

Gonioides dissimilis, 1280

Haemaphysulis concinna, 1250

Haematopinidae, 1233

Hacmalopinus suis, 1233

Haemogogus capricorni, 1266

Helochara delta, 1153

Hyalopterus atriplicis, 1179

Hysteroneura setariae, 1183

Illinoia solanifolii, 1179, 1182, 1183

Ixodes persulcatus, 1250

Ixodes ricinus, 1249

Ixodidae, $1249,1250,1252,1254$

Leafhoppers, 1145, 1146, 1147, 1148, $1150,1153,1154,1155,1156,1157$,

$1159,1160,1161,1162,1220$

Lipaphis pseudobrassicae, 1201

Lumbricidae, 1269

Lygus pratensis, 1222

Macropsis trimaculata, 1148

Macrosiphum ambrosiae, 1179

Macrosiphum gei, 1171, 1178, 1180 , $1181,1182,1203$

Macrosiphum lilii, 118.5

Macrosiphum pelargonii, 1182 
Viruses, Vectors of (continued)

Iacrosiphum pisi, 1179, 1180, 1181, 118 S

Macrosiphum solanifolii, 1164, 1171, $1173,1178,1179,1180,1181,1182$, $1183,1185,1188,1193,1203,1204$

Macrosteles divisus, 1147

Metastrongylidae, 1269

Metastrongylus elongatus, 1269

Miridae, 1221, 1222

Moomia albimaculata, 1150

Mosquito, 1230, 1252, 1254, 1259, $1266,1267,1277$

Myzus circumflexus, 1164, 1171, 1173, $1176,1177,1185,1200,1204$

Myzus convolvuli, 1176, 1200, 1204

Myzus frayaefalii, 1195, 1207

Myzus persicae, 1164, 1171, 1172, $1173,1175,1176,1177,1178,1179$, $1182,1183,1185,1193,1200,1201$, 1203,1204

Myzus pseudosolani, 1164, 1173, 1204

Neokolla circellata, 1153

Neokolla confluens, 1153

Neokolla gothica, 1153

Veokolla heiroglyphica, 1153

Vephotettix apicalis, 1160

Vephotettix bipunctatus, 1160

Ophiola strialula, 1150

Pentalonia nigronervosa, 1174, 1193

Peregrinus maidis, 1161

Perkinsiella saccharicida, 1157

Perkinsiella vastatrix, $115 i$

Philopteridae, 1280

Piesma cinerea, 1221

Piesma quadrata, 1221

Piesmidae, 1221

Pulicidae, 1246

Reduviidae, 1254

Rhipicephalus appendiculatus, 1219

Rhopalosiphum melliferum, 1176, 1177, 1200

Rhopalosiphum prunifoliae, 1184

Rhopalosiphum pseudobrassicae, 117\%, 1179

Taeniorhynchus brevipalpis, 1267

Thamnotettix argentata, 11+8, 115t

Thamnoteitix geminatus, 1147

Thamnotetix montanus, 1147

Thripidae, 1223

Thrips tabaci, 1223
Tick; American dog, 1252

Toxcptera aurantii, 1202

T'oxopıera graminum, 1183

Triatoma sanguisuga, 1254

White flies, 1218, 1219

\section{Water}

Achromatium, 999

Achromobacier, 418, 419, 423, 424, $425,426,427$

Actinomyces, 968, 970.972

Aerobacier, 456, 457, 692

Alcaligenes, 414

Amcebobacter, 849,850

Ascobacterium, 647

Ascococcus, 250

Azotobacter, 221

Bacillus, 612, 613, 644, 645, 648, 649, $650,651,652,653,654,655,656,657$, $658,659,661,664,665,666,667,668$, $669,670,671,672,693,715,721,722$, $723,725,726,732,733,734,736,737$. $738,740,742,744,746,747,748,749$, $752,754,755,756,757,758,813,814$, $815,816,817,818,819,825$

Bacterium, 602, 605, 613, 641, 673, 674, $675,676,677,678,679,680,681,682$, $684,685,686,688,689,690,758,760$

Blastacaulis, 836

Caulobacter, 833

Cellulomonas, 620

Chlorobacterium, 873

Chlorobium. 870

Chlorochromatium, 873

Chromatium, $85 \overline{7}, 858$

Chromobacterium, 232, 233, 231

Clathrochloris, 872

Clonothrix, 983

Clostridium, 824

Corynebacterium, 397, 403, 404

Crenothrix, 987

Cylindrogloea, 874

Desulfovibrio, 208

Diplococcus, 694

Eperythrozoun, 1115

Eschernchia, 449, 450

Ferribacierium, 834

Flavobacterium, 429, 431, 433, 434, 435 , $436,437,438,439,440,441,442.611$ Gallionella, 831, 832 
Water (continued)

Granulobacillus, 826

Hyphomicrobium, 837

Lamprocystis, 847

Lampropedia, 844

Leptospira, 1077, 1078, 1079

Leptothrix, 984, 985, 986, 995

Leuconostoc, 348

Macromonas, 1001

Methanobacterium, 646

Micrococcus, 240, 245, 246, 250, 251, $255,256,257,258,259,261,262,270$, $271,273,274,276,277,278,280,281$. 695

Microspira, 202

Naumanniella, 834

Nevskia, 830

Nocardia, 908

Paracolobactrum, 460

Pasteurella, 551

Pelodictyon, 871, 872

Prolaminobacter, 190

Proteus, 491

Pseudomonas, 89, 90, 91, 93, 96, 97, 99, $100,101,102,103,104.145,146,147$, $148,149,173,174,175,176,178,179$, $697,698,701$

Rhabdomonas, 854, 855

Rhodococcus, 281

Rhodopseudomonas, 864, 865, 866

Rhodospirillum, 868

Rhodothece, 855

Salmonella, 504

Saprospira, 1055

Sarcina, 287, 288, 291, 293, 294

Serratia, 481, 482, 483, 484, 485

Siderocapsa, 833, 834

Siderococcus, 835

Siderocystis, 835

Sideroderma, 835

Sideromonas, 834

Siderothece, 835

Sphaerotilis, 982, 983

Spirillum, 213, 214, 218, 701

Spirchaeta, 1052, 1053, 1054, 1079

Strepiococcus, 337, 338, 339, 341, 345, 702

Terminosporus, 823

Thiobacillus, 79, 81

Thiocapsa, 845
Thiocystis, 847

Thiodictyon, 845

Thionema, 995

Thiopedia, 843

Thioploca, 994

Thiopolycoccus, 850

Thiosarcina, 843

Thiosiphon, 995

Thiospirillopsis, 993

Thiospirillum, 850, 851, 852, 853

Thiothece, 846

Thiothrix, 989, 990

Thiovulum, 1000

Vibrio, 198, 199, 201, 202, 203, 204, 205. 703

Water, Source

Aachen, Germany, 834

Arctic Ocean, 857

Black Sea, 1002

Breslau, 834

Caucasus, 832

Crimea, 845

Czechoslovakia, 835

Finland, 835

Florida, 845

Frankfurt, Germany, 844

Germany, 835

Graz, Austria, 855, 857, 858

Minneapolis, Minnesota, 830

Nikko, Japan, 859

Petschora Sea, 832

Russia, 835

St. Petersburg, 830

Sweden, 835

Sweden, Aneboda region, 832, 833, 834, $835,969,983,986$

Teufelsee, 834

United States of America, 835

White Sea, 832

Worms, Germany, 834

Waters, also see River Water, Spring

Water, Sea Water and Tap Water

Borax yielding water

Sarcina, 294

Bottled mineral waters

Bacillus, 657

Bacterium, 673

Micrococcus, 695

Proteus, 491 
Waters (continued)

Brackish, muddy bottom of Spirillum, 215, 217

Brewery, reservoir Sarcina, 290

Canal Bacillus, 655,672 Bacterium, 682, 688 Thiobacillus, 81

Chitin digesting bacteria, 632

\section{Cistern} Bacterium, 680

Containing iron Actinomyces, 969

Crenothrix. 987 Gallionella, 831, 832

Leptothrix, 984, 985, 986

Lieskeela, 986

Containing manganese Leptoihrix, 985

Creamery waste filter Pseudomonas, 177, 178

Creek Aerobacter, 456

Ditch

Desulfovibrio, 208

Pseudomonas, 698

Drainage

Bacillus, 749

Spirochaeta, 1053

Filtered

Pseudomonns, 149, 173

Vibrio, 196

Fish hatchery

Achromobacter, 425

Fountain

Bacillus, 733

Fresh water

- - lime deposits

Pseudomonas, 146

Spirochaeta, 1052, 1054

- - with algae Spirillum, 218

Frozen, see Hail, Ice and Snow

Glacial

Pseudomonas, 145

Grossly polluted

Spirochaeta, 1053

Hail, see Hail

Ice, see Ice
Lake

Chromaiium, 857. 858

C'losiridium, 824

Gallionella, 831, 8.34

Micrococcus, 276

Rhabdomonas, 855

Spirochaeia, 1053

-, plankton

Chitin digesting bacteria, 632

Mineral, also see Bottled, mineral waters

Micrococeus, 268

Peat bog

Sphaerothrix, 986

Pipes

Clonothrix, 983

Polluted

Pseudomonas, 89

Pond

Chromatium, 857, 858

Gallionella, 831, 834

Leptospira, 1077

Macromonas, 1001

Rhabdomonas, 855

Sphaerothrix, 986

Spirochaeta, 1079

Pool

Chlamyduzoon, 1115

Polyangium, 1032

Putrid

Spirillum, 213, 216

Rain, in bark of poplar tree

Spirillum, 217

River, see River Water

Running water

Crenothrix, 987

Leuconostre, 348

Sphaerotilis, 982

Salt seas and lakes, see Salt Seas and

Lakes

Salt, see Sea Water

Slime in mines

Leptospira, 1077

Snow, see Snow

Spring, see Spring Waters

Stagnant

Amoebobacter, 849,850

Bacillus, 667

Bacterium, 681, 685, 688

Chlorobacterium, 873 
Waters (continued)

Stagnant (continued)

Chlorobium, 870

Chlorochromaiium, 873

Clathrochloris, 872

Cylindrogloea, 87.4

Lamprocystis, 818

Lampropedia, 844

Pelodictyon, 871, 872

Psendumonas, 149

Rhabdomonas, 854, 855

Rhodopseudomonas, $864,865,866$

Rhodospirillum. 868

Rhodothece, 855

Sphaerotilis, 982

Spirillum, 213, 214, 216, 217

Thiocapsa, 845

Thiocystis, 847

Thiodictyon, 846

Thiopedia. 843

Thiopolycoccus, 850

Thiosarcina, 843

Thiospirillum, 851, 852

Thiothece, 846

Thiothrix, 989

Streams, paper mill waste polluted

Sphaerotilis, 982

-, sewage polluted

Sphaerotilis, 982, 983

Submerged surfaces

Caulobacter, 833

Gallionella. 832

Leptothrix, 985

Siderocapsa, 834

Sideromonas, 834

Sulfur

Achromatium, 999

A moebobacter, 849, 850

Bacterium, 685

Beggiatoa, 992, 993

Chlorobium, 870

Chlorochromatium, 873

Clathrocystis, 872

Cylindrogloea, 874

Lamprocystis, 848

Microbacillus, 690

Pelodictyon, 871, 872

Rhabdomonas, 854, 855

Rhodothece, 856

Spirochaeta, 1053
Thiocapsa, 845

Thiocystis, 847

Thiodictyon, 816

Thionema, 995

Thiopedia, 843

Thiopolycoccus, 850

Thiosarcina, 813

Thiospirillopsis, 993

Thiospirillum, 851, 852

Thiothece, 846

Thiothrix, 989, 990

Thiovulum, 1000

Sulfur, stream containing

Macromonas, 1001

Surface

Paracolobactrum, 460

Swamp

Bacillus, 659, 813, 814, 816, 817, 819

Lampropedia, 844

Leptothrix, 986

Methanococeus, 285

Sideromyces, 986

Sphaerotitis, 983

Spirochaeta, 1053

Tap waters, see Tap Waters

Trickling filter

Pseudomonas, 177, 178

Vibrio, 206

Unfiltered water

Pseudomonas, 90, 97

Waste

Bacillus, 742, 744, 758

Waste from sugar factory

Sarcina, 293

Wells, in chalk region

Flavobacterium, 42?

Well water

Bacillus, 815

Chromobacterium, 233, 234

Lampropedia, 814

Leuconostoc, 348

Pseudomonas, 701

Serratia, 484

Thiospirillum, 853

\section{Water Works}

Clonothrix, 983

\section{Wine}

Acetobacter. 186

Bacillus, 654, 668, 672, 753 
Wine (continued)

Bacterium, 362, 673, 681, 689

Lactobacillus, 359, 361, 363

Micrococcus, 251, 259, 267, 280

Pseudomonas, 149

Streptococcus, 345

- , cellars

Micrococcus. 277

Streptococcus, 340

-., slimy

Streptncoccus, 340

\section{Yeast}

Bacillus, $7 \pm 1$

Bacterium, 680, 687

Clostridium, 824

Laciobacillus, 361
Pediococcus, 249, 250

Sarcina, 290, 291, 292, 293, 294

—, baker's

Streptococcus, 336

-, beer

Bacillus, 749

Pediococcus, 249

-, brewer's

Flavobacterium, 439

一, distillery

Lactobacillus, 360

-, mash

Pseudomonas, 146

-, pressed

Flavobacterium, 613

Lactobacillus, 358 



\section{INDEX OF THE NAMES OF GENERA AND SPECIES*}

(For Index of the Names of Orders, Families, Genera and Groups of Intermediate Rank, See Table of Contents, p. XI.)

aaser (Streptothrix), 976

abaca (Marmor), 1193

abbreviatum (Chloronostoc), 870

aberdeen (Salmonella), 526

abony (Salmonella), 502

aboriginalis (Spirochaeta), 1065

abornginalis (Spiroschaudinnia), 1065

aboriginalis (Treponema), 1065

abortivoequina (Salmonella), 495, 506

abortivo-equinus (Bacillus), 506

abortivum (Bacterium), 561

abortivus (Bacillus), 506

abortivus equinus (Bacillus), 506

abortum-equi (Bacterium), 506

abortus (Alcaligenes), 561

abortus (Bacillus), 561, 562

abortus (Bacterium), 561, 562

abortus (Brucella), 43, 561, 562

abortusbovis (Salmonella), 495, 502, 507

abortus canis (Salmonella), 530

abortus endemici (Corynebacterium), 561

abortus equi (Bacillus), 506

abortus-equi (Salmonella), 493, 506

abortus-equi (Streptococeus), 336

abortus equinus (Bacillus), 506

abortus ovis (Bacterium), 506

abortusovis (Salmonella), 493, 495, 506

abortus suis (Bacillus), 562

abortus var. lipolyticus (Bacillus), 390

abortus var. lipolyticus (Bacterium), 390

abscessus (Actinobacterium), 928

abundans (Mycothrix), 983

abutilon (Marmor), 1186

abysseus (Bacillus), 736

accidentalis (Bacillus), 649

accidentalis tetani (Bacillus), 649

accidentalis tetani (Bacterium), 649 aceris (Achromobacter), 456

aceris (Bacillus), 456

aceris (Phytomonas), 113

aceris (Pseudomonas), 113

acernea (Phytomonas), 165

acernea (Pseudomonas), 165

acernea (Xanthomonas), 166

aceti (Acetobacter), 179, 181, 183, 692

aceti (Bacillus), 181, 182, 761

aceti (Bacteriopsis), 181

aceti (Bacterium), 179, 181

aceti (Micrococcus), 181

aceti (Mycoderma), 181

aceti (Termobacterium), 185

aceti (Torula), 181

aceti (Ulvina), 181

aceti (Umbina), 181

aceti viscosum (Bacillus), 188

aceti viscosum (Bacterium), 188

aceticum (Bacterium) , 454, 683, 692

aceticum (Clostridium), 819

aceticus (Bacillus), 181

aceticus petersii (Bacillus), 683

aceticus petersii (Bacterium), 683

acetigenum (Acetobacter), 186

acetigenum (Bacillus), 186

acetigenum (Bacterium), 186

Acetimonas, 8, 180

Acetobacter, 9, 18, 21, 23, 25, 29, 31, 43,

179,189

Acetobacterium, 18, 179

acetobutylicum (Clostridium), 780, 805,

825

acetobutyricum (Clostridium), 780,807

acetoethylicum (Bacillus), 721

acetoethylicus (Aerobacillus), 721

acetogenes $\alpha$ (Bacillus), 352

*Index prepared by Prof. Robert S. Breed and Mrs. Margaret E. Breed, New Tork State Experiment Station, Geneva, New York, June, 1947. 
acetogenes $\beta$ (Bacillus), 352

acetogenes exilis (Bacillus), 352

acetogenes proteiformis (Bacillus), 352

Acetogluconobacter, 180

acetonigenum (Clostridium), 780

acetonobutylicum (Clostridium), 780

acetosa (Ulvina), 692

acetosum (Acetobacter), 185, 692

acetosum (Bacillus), 182

acetosum (Bacterium), 185

acetylcholini (Bacterium), 336

acetylicum (Flavobacterium), 611

achalmei (Bacillus), 791

Achromatium, 16, 24, 29, 995, 996, 997, 998,1000

Achromobacter, 20, 31, 32, 417, 422, 609

Achromobacterium, 417

achrous (Bacillus), 612

achrous (Micrococcus), 251

acidi acetici (Bacillus), 813

acidi butyrici (Bacillus), 814

acidi butyrici I (Bacillus), 814, 816

acidifaciens (Bacillus), 736

acidificans (Bacillus), 355

acidificans (Micrococcus), 238

acidificans (Sarcina), 290

acidificans longissimus (Bacillus), 355

acidificans-longissimus (Lactobacillus), 355

acidificans presamigenes casei (Bacillus),

737

acidificum (Flavobacterium), 440

acidiformans (Bacillus), 613

acidiformans (Bacterium), 613

acidi lactici (Bacillus), 323, 447

acidi lactici I (Bacillus), 447

acidi lactici II (Bacillus), 447

acidi lactici (Bacterium), 447, 664

acidi lactici $I$ and II (Bacterium), 447,

664

acidi lactici (Encapsulatus), 447

acidilactici (Escherichia), 447

acidi-lactici (Micrococcus), 249, 251

acidilactici (Pediococcus), 249

acidi lactici (Plocamobacterium), 691

acidi-lactici (Sphaerococcus), 336

acidi lactici (Streptococcus), 323, 336

acidi lactici liquefaciens (Micrococcus), 238 acidi lactici var. gruenthali (Bacillus), 451 acidilactici var. gruenthali (Bacterium), 451

acidi lactis (Micrococcus), 238

acidi lactis liquefaciens (Micrococcus), 238

acidi lactis longus (Bacillus), 702

acidi laevolactici (Bacillus), 348, 680

acidi laevolactici (Bacterium), 348, 680

acidi laevolactici (Micrococcus), 267

acidi paralactici (Bacillus), 324

acidi paralactici (Micrococcus), 323

acidi paralactici liquefaciens (Micrococcus), 263

acidi para-lactici liquefaciens halensis

(Micrococcus), 263

acidi propionici (Bacillus), 378

acidi propionici (Bacternum), 672

acidi propionici a (Bacterium), 373

acidi propionici b (Bacterium), 376

acidi propionici c (Bacterium), 376

acidi propionici d (Bacterium), 373

acidi propionici (Plocamobacterium), 672

acidi propionici var. fuscum (Bacterium), 373

acidi propionici var. rubrum (Bacterium), 374

acidi urici (Bacillus), 795

acidi urici (Clostridium), 795

acido-aromaticus (Bacillus), 647

Acidobacterium, 349

acidominimus (Streptococeus), $\mathbf{3 3 5}$

acidophil-aerogenes (Bacillus), 358

acidophil-aerogenes (Lactobacillus), 358

acidophilum (Plocamobacterium), 352

acidophilum (Thermobacterium), 352

acidophilus (Actinomyces), 956

acidophilus (Bacillus), 352, 353, 361, 363

acidophilus (Lactobacillus), 352, 362

acidophilus (Streptomyces), 956

acidophilus odontolyticus (Bacillus), 363, 365

acidophilus odontolyticus $I$ and II (Bacillus), 363

acidophilus odontolyticus $I$ and $I I$ (Lactobacillus), 363

acidophilus odontolyticus $I, I I$ and $I I I$

(Lactobacillus), 363 
acidoproteolyticus (Mammococcus), 327 acido-proteolyticus casei (Bacillus), 737 acido-proteolyticus casei I (Coccus), 327 acido-proteolyticus casei II (Coccus), 327 acidovorax (Micrococcus), 251 acidula (Cellulomonas), 614 acidulum (Bacterium), 614 acidum (Achromobacter), 422 acidum (Bacterium), 422 acidum (Corynebacterium), 402 acne (Micrococcus), 251 acne (Staphylococcus), 251 acnes (Actinomyces), 387 acnes (Bacillus), 387 acnes (Bacterium), 401, 658 acnes (Corynebacterium), $\mathbf{3 8 7}$ acnes (Fusiformis), 387, 583 acnes (Propionibacterium), 387 acnes contagiosae (Bacillus), 401, 658 acnes contagiosae (Bacterium), 658 acodoni (Grahamella), 1109 acridicida (Micrococcus) (Staphylococcus), 251

acridiorum (Bacillus), 690 acridiorum (Coccobacillus), 491, 690 Acrogenus, 1202 acropoma (Coccobacillus), 636 Actinobacillus, 9, 22, 27, 556, 926 actinobacter (Bacillus), 456 Actinobacterium, 34, 38, 892, 925 Actinocladothrix, 925 actinocladothrix (Bacterium), 925 Actinococcus, 15, 17, 892 actinoides (Actinobacillus), 658 actinoides (Actinomyces), 558 actinoides (Bacillus), 558 Actinoidomyces, 20, 23 actinomorpha (Nocardia), 899, 912 actinomorphum (Mycobacterium), 912 actinomorphus (Actinomyces), 912 actinomorphus (Proactinomyces), 912 Actinomyce, 925

Actinomyces, 9, 12, 17, 18, 19, 23, 27, 28, $38,42,43,875,892,917,919,921,925$, $972,979,1289$ actinomyces (Cladothrix), 925 actinomyces (Nocardia), 925 actinomyces (Streptothrix), 925

Actinomyces sp., Bruns, 923

Actinomyces sp., Lowenstein, 973 actinomycetemcomitans (Actinobacillus),

$\mathbf{5 5 7}, 692$

actinomycetemcomitans (Bacillus), 692 actinomycetem comitans (Bacterium), 557 Actinomycoides, 27 actinomycotica (Streptothrix), 925 activus (Staphylococcus), 701 Acuformis, 33, 34, 763 acuminata (Cristispira), 1056 acuminata (Spirochaeta), 1056, 1065 acuminata (Spiroschaudinnia), 1065 acuminatum (Bacterium), 674 acuminatum (Saccharobacterium), 623 acuminatum (Treponema), 1065 acuminatus (Bacteroides), 353 acuminatus (Diplobacillus), 353 acuta (Pseudomonas), 145 acuta (Spirochaeta), 1065, 1074 acutangulus (Bacillus), 647 acutus (Bacillus), 647

Acystia, 13, 516 adametzii (Bacillus), 647, 737 adametzii (Bacterium), 758 adamsoni (Corynebacterium), 388, 402 adanti (Klebsiella), 459 adaptatus (Vibrio), 702 adelaide (Salmonella), 530 Adelonosus, 1211 adenitis equi (Bacillus), 317 aderholdi (Bacillus), 360 adhaerens (Bacillus), 43, 737, 739, 746 adriaticum (Thiosiphon), 995 aeglefini (Spirochaeta), 1064 aegyptiacum (Bacterium), 585 aegyptiacus (Bacillus), 737 aegyptica (Spirochaeta), 1065 aegyptica (Spironema), 1065 aegypticum (Borrelia), 1065 aegypticus (Bacillus), 676 aegyptium (Bacterium), 677 aerifaciens (Bacillus), 737 aeris (Bacillus), 647 aeris (Bacterium), 672 aeris minutissimus (Bacillus), 672 
aeris-minutissimum (Bacterium), 672

aerius (Micrococcus), 251

Aerobacillus, 20, 22, 27, 28, 30, 31, 720, 737

Aerobacter, 10, 21, 26, 30, 31, 37, 443, 448,

453, 464

Aerobacteroides, 27

aerobius (Bacillus), 647, 660, 737

aerobius (Streptococcus), 336

aerofaciens (Bacteroides), 362, 368

aerofaciens (Eubacterium), 368

aerofoetidum (Clostridium), 781

aerofoetidus (Bacillus), 781

aerofoetidus (Seguinillus), 781

aerogenes (Acidobacterium), 361

aerogenes (Aerobacter), 444, 454, 456, 457,

460,692

aerogenes (Bacillus), 454, 647, 659

aerogenes (Bacterium) , 454, 672

Aerogenesbacterium, 11, 453

aerogenes $I$ and $I I$ (Bacterium), 672

aerogenes (Colobactrum), 454

aerogenes (Encapsulata) (Bacillus), 454

aerogenes (Helicobacterium), 690

aerogenes (Micrococcus), 246, 251

aerogenes (Plocamobacterium), 361

aerogenes (Staphylococcus), 246

aerogenes (Streptococcus), 336

aerogenes (Welchillus), 790

aerogenes-capsulatum (Clostridium), 790

aerogenes capsulatus (Bacillus), 789, 790

aerogenes capsulatus (Bacterium), 790

aerogenes gangrenosae (Bacillus), 813

aerogenes meningitidis (Bacillus), 662

aerogenes necrosans (Bacillus), 820

aerogenes sputigenus capsulatus (Bacillus),

647,686

aerogenes vesicae (Bacillus), 653

aerogenes vesicae (Bacterium), 653

aerogenes vesicae (Coccobacillus), 653

aerogenoides (Paracolobactrum), 460, 490

aerogenosus (Bacillus), 126

Aeromonas, 29, 30, 101

aerophilum (Achromobacter), 610

aerophilum (Bacterium), 737

aerophilum (Urobacterium), 610, 691

aerophilus (Bacillus), 737

aerophilus (Streptococcus) 336 aerosporus (Bacillus), 720

aero-tertius (Bacillus), 812, 827

aerothermophilus (Bacillus), 734

Aerothrix, 929

aertrycke (Bacillus), 502

aertrycke (Bacterium), 502

aertrycke (Salmonella), 502

aertrycke var. meleagridis (Salmonella), 502

aertrycke var. Storrs (Salmonella), 503 aerugineus (Actinomyces), 967

aeruginosa (Pseudomonas), 89, 126, 693, 701

aeruginosuin (Bacterium), 89

aeruginosus (Bacillus), 89

aeschynomenus (Bacillus), 647

aestuarii (Desulfovibrio), 208, 209

aestuarii (Microspira), 208

aestumarina (Pseudomonas), 697

aetatulae (Morator), 1227

aëthebius (Bacillus), 647

aëthebius (Micrococcus), 266

aëthebius (Streptococcus), 266

aevi (Marmor), 1200

afanassieffi (Bacillus), 737

afermentans (Micrococcus), 695

africana (Nocardia), 959

africanus (Actinomyces), 959

africanus (Streptomyces), 959

agalactiae (Borrelomyces), 1292

agalactiae (Capromyces), 1292

agalactiae (Streptococcus), 319

agalactiae contagiosae (Streptococcus), 319

agalaxiae (Anulomyces), 1292

agar-exedens (Bacillus), 631

agar-liquefaciens (Microspira), 200

agarliquefaciens (Vibrio), 200

agarlyticus (Vibrio), 702

agglomerans (Bacillus), 489

agglomeratus (Bacillus), 716

agglutinans (Lactococcus), 336

agglutinans (Leuconostoc), 346

aggregata? (Sorochloris), 870

aggregatum (Chlorochromatium), 859,

873,874

aggregatum (Pelodictyon), 871, 872, 874

aggregatus (Streptococcus), 308 
agile (Achromobacter), 422

agile (Azotobacter), 219, 220

agile (Bacterium), 423

agile (Nitrobacter), 74

agile var. atypica (Azotobacter), 220

agilis (Bacillus), 423, 647, 737

agilis (Hydrogenomonas), 78

agilis (Micrococcus), 245

agilis (Planococcus), 245

agilis (Planosarcina), 245

agilis (Rhodococcus), 245

agilis (Sarcina), 245, 290

agilis (Spirochaeta), 1053

agilis (Thiospira), 853

agilis (Thiospirillum), 853

agilis var. polonica (Thiospirillum), 853

agilis albus (Micrococcus), 251

agilis citreus (Micrococcus), 288

agilis larvae (Bacillus), 737

agilis ruber (Micrococcus), 245

agilissima (Thiospira), 702

agilissimum (Spirillum), 701, 702

agillimus (Bacillus), 647

agliaceus (Malleomyces), 556

agni (Bacillus), 790

agni (Welchia), 790

agni var. ovitoxicus (Welchia), 790)

agni var. paludis (Welchia), 790

agnorum (Bacillus), 648

agreste (Bacterium), 673

agreste (Mycobacterium), 902

agrestis (Actinomyces), 902

agrestis (Bacillus), 673, 737

agrestis (Proactinomyces), 902

agri (Bacillus), 737

agrigena (Pasteurella), 668, 673

agrigenum (Bacterium), 673

Agrobacterium, 227, 230

agrophilus (Bacillus), 737

agropyri (A planobacter), 395

agropyri (Bacterium), 395

agropyri (Corynebacterium), 395

agropyri (Marmor), 1202

agropyri (Phytomonas), 395

agropyri var. flavum (Marmor), 1202

agropyri var. typicum (Marmor), 1202

agrotidis typhoides (Bacillus), 737 akamushi (Rickettsia), 1090

akari (Rickettsia), 1092

alacer (Bacillus), 648

alactagae (Grahamella), 1109

alactolyticum (Ramibacterium), 701

alactosus (Streptococcus), 337

alatus (Bacillus), 618

alba (Bactoderma), 75

alba (Beggiatoa), 992, 993

alba (Cladothrix), 934

alba (Escherichia), 452

alba (Nocardia), 934

alba (Oospora), 918, 968

alba (Oscillatoria), 992

alba (Pseudomonas), 145

alba (Sarcina), 42, 290

alba (Streptothrix), 933, 968, 976

alba var. incana (Sarcina), 290

alba var. marina (Beggiatoa), 992

alba var. uniserialis (Beggiatoa), 989

albarrani (Bacterium), 580

albatus (Bacillus), 648

albatus (Micrococcus), 251

albensis (Vibrio), 203, 702

albescens (Micrococcus), 251

albicans (Micrococcus), 252

albicans (Neisseria), 252

albicans (Staphylococcus), 281

albicans (Streptococcus), 337

albicans amplus (Diplococcus), 252

albicans amplus (Micrococcus), 252, 281

albicans pateriformis (Bacillus), 683

albicans tardissimus (Diplococcus), 278

albicans tardissimus (Micrococcus), 278

albicans tardus (Diplococcus), 278

albicans tardus (Micrococcus), 278

albida (Cellulomonas), 620

albida (Nocardia), 896, 949

albida (Sarcina), 290

albido (Streptothrix), 949

albido-flava (Cladothrix), 949

albido-flava (Streptotrix), 949

albido-flavus (Actinomyces), 949

albidoflavus (Streptomyces), 949, 950

albidofuscus (Actinomyces), 967

albidus (Actinomyces), 967

albidus (Bacillus), 620 
albidus (Micrococcus), 251, 277

albidus (Streptococcus), 337

albilineans (Bacterium), 639

albilineans (Phytomonas), 639

alboatrus (Actinomyces), 967

albocereus (Micrococcus), 251, 2\$1

Albococcus, 8, 235

albofaciens (Bacillus), 544

albofaciens (Shigella), 544

alboflavum (Protaminobacter), 189, 190

alboflavus (Actinomyces), 954

alboflavus (Streptomyces), 954

albogilva (Cytophaga), 1014

albo-lacteum (Clostridium), 819

albolactis (Bacillus), 716

alboluteum (Clostridium), 819

alboprecipitans (Bacterium), 142

alboprecipitans (Phytomonas), 142

alboprecipitans (Pseudomonas), 141

albosporea (Nocardia), 954

albosporeus (Actinomyces), 954

albosporeus (Streptomyces), 954

alboviridis (Actinomyces), 940, 968

album (Achromobacter), 423

album (Bacterium), 42, 423, 648, 673

album (Citrobacter), 448

album (Corynebacterium), 402

album (Mycobacterium), 890

album liquefaciens (Clostridium), 819

album minor (Clostridium), 819

album non-liquefaciens (Clostridium), 819

albuminis (Bacillus), 799

albuminis (Bacillus) (Streptobacter), 737

albuminosus (Flexibacter), 38

albuminus (Bacillus) (Streptobacter), 727

albus (Actinomyces), 924, 934, 940, 968

albus (Alcaligenes), 416

albus (Bacillus), 42, 423, 648, 737, 738

albus (Cellulomonas), 737

albus (Galactococcus), 250

albus (Micrococcus), 242, 249, 251, 252,

$253,257,258,260,261,262,263,264$,

$265,266,267,268,269,272,273,274$,

$275,276,277,279,280,281,347,891$

albus I (Micrococcus), 257

albus II (Micrococcus), 252, 270

albus (Pediococcus), 249 albus (Proactinomyces), 923

albus (Staphylococcus), 242, 1140, 1141, 1142

albus (Streptococcus), 337

albus (Streptomyces), 933, 943, 947, 949

albus var. $\alpha$ (Actinomyces), 934

albus var. acidus (Actinomyces), 915, 934

albus var. cretaceus (Actinomyces), 934

albus var. maltigenes (Micrococcus), 695

albus var. ochraleuceus (Actinomyces), 934,968

albus var. tossica (Actinomyces), 918

albus var. toxica (Actinomyces), 934

albus acidus (Actinomyces), 915, 934

albus anaeroliescens (Bacillus), 648

albus asporogenes (Actinomyces), 968

albus cadaveris (Bacillus), 669

albus cadaveris (Bacterium), 669

albus fluidificans (Micrococcus), 257

albus liquefaciens (Micrococcus), 274

albus liquefaciens (Staphylococcus), 281

albus non liquefaciens (Coccus), 261

albus non liquefaciens (Staphylococcus),

281

albus putidus (Bacillus), 648

albus putridus (Bacillus), 648

albus urinae (Micrococcus), 279

albus-vulgaris (Actinomyces), 968

alcalescens (Bacillus), 450

alcalescens (Escherichia), 450

alcalescens (Micrococcus), 303

alcalescens (Veillonella), 303

alcalescens var. gingivalis (Veillonella), 304

alcalescens var. minutissima (Veillonella), 304

alcalescens var. syzygios (Veillonella), 304

alcaliaromaticum (Achromobacter), 416

alcaliaromaticum (Bacterium), 416

alcalifaciens (Bacillus), 400

alcalifaciens (Eberthella), 533

Alcaligenes, 10, 21, 30, 32, 412, 416

alcaligenes (Acuformis), 801

alcaligenes (Bacillus), 413

alcaligenes (Bacterium), 413

alcaligenes (Clostridium), 801

alcaligenes (Palmula), 801 
alcaligenes (Vibrio), 413

alcalinofoetidus (Alcaligenes), 416

alcalinofoetidus (Bacillus), 416

alcalinus (Thermobacillus), 734

alcalophilus (Bacillus), 738

alcis (Klebsiella), 459

alcoholphilus (Lactobacillus), 363

aleuritidis (Bacterium), 131

aleuritidis (Phytomonas), 131

aleuritidis (Pseudomonas), 131

alfalfae (Bacterium), 165

alfalfae (Phytomonas), 165

alfalfae (Pseudomonas), 165

alfalfae (Xanthomonas), 165

algeriense (Bacterium), 673

alginicum (Bacterium), 641

alginovorum (Bacterium), 626

algosus (Vibrio), 702

aliphaticum (Bacterium), 673

aliphaticum liquefaciens (Bacterium), 673

alkalescens (Bacillus), 539

alkalescens (Bacterium), 539

alkalescens (Eberthella), 539

alkalescens (Proshigella), 539

alkalescens (Shigella), 539, 540, 543

allantoicus (Streptococcus), 337

allantoides (Bacillus), 673

allantoides (Bacterium), 673

aller (Leuconostoc), 346

alliariae (Bacillus), 477

alliariae (Erwinia), 477

allii (Bacillus), 697

allii (Pseudomonas), 145, 697

alliicola (Phytomonas), 136

alliicola (Pseudomonas), 136

allium (Bacterium), 145

alluvialum (Mycobacterium), 919

alma (Cellulomonas), 620

almquisti (Actinomyces), 934, 947, 968

almus (Bacillus), 620

alni (Actinomyces), 968

aloes (Bacterium), 758

alopecuri (Bacillus), 738

alpha (Bacillus), 648, 738

alpha (Micrococcus), 279

alpha (Oospora), 934

alpha (Phagus), 1141 alpha (Streptothrix), 976

alpha (Tarpeia), 1268

alpinus (Bacillus), 738

altendorf (Salmonella), 506

alternans (Zygoplagia), 13

alutacea (Pseudomonas), 178

alutacea (Sarcina), 290

alutaceum (Bacterium), 673

alvearis (Cryptococcus), 337

alvearis (Streptococcus), 337

alvei (Bacillus), 723, 724, 728, 745

alveicola (Proteus), 490

alveolaris (Bacillus), 738

alvi (Micrococcus), 274

amabilis (Bacillus), 648

amabilis (Bacterium), 648

amager (Salmonella), 524

amaracrylus (Aerobacillus), 720

amaracrylus (Bacillus), 720

amaranthi (Bacterium), 178

amaranthi (Phytomonas), 178

amaranti (Pseudomonas), 178

amarifaciens (Micrococcus), 238

amarificans (Bacillus), 716

amarillae (Bacillus), 648

amarillum (Plectridium), 814

amarus (Bacillus), 648, 738

ambigua (Eberthclla), 536

ambigua (Pseudomonas), 103, 697

ambigua (Shigella), 536

ambigua (Spirochaeta), 1065

ambigua (Treponema), 1065

ambiguum (Achromobacter), 103

ambiguum (Bacterium), 536, 673, 697

ambiguus (Bacillus), 103, 536

ambratus (Streptococcus), 337

americana (Cohnistreptothrix), 974

americanum (Clostridium), 819

americanus (Actinomyces), 975

americanus (Nitrosococcus), 71

americanus (Proteus), 490

amerimnus (Bacillus), 648

amersfoort (Salmonella), 511

amethystina (Pseudomonas), 233

amethystinum (Chromobacterium), 232

amethystinus (Bacillus), 232, 233

amethystinus (Bacterium), 232 
amethystinus mobilis (Bacillus), 233

amethystinus mobilis (Bacterium), 233

amforeti (Bacterium), 673

amherstiana (Salmonella), 515

aminovorans (Bacillus), 738

ammoniae (Proteus), 490

ammoniae (Salmonella), 490

ammoniagenes (Alcaligenes), 416, 607

ammoniagenes (Bacterium), 416, 607

amocontactum (Flavobacterium), 631

Amoebobacter, 16, 23, 26, 848, 849, 850

Amoebomonas, 8, 25, 848

amphibiae (Spirochaeta), 1065

amphibolus (Vibrio), 702

amplelopsorae (Bacillus), 227

amplus (Micrococcus), 252

ampullaceus (Micrococcus), 252

amularium (Marmor), 1212

amygdaloides (Bacillus), 648

amylifera (Thiosphaerella), 998, 999

amyliferum (Spirillum), 217

amyloaceum var. auranticum (Propioni-

bacterium), 379

amyloaerobius (Bacillus), 738

Amylobacter, 743, 748, 763

amylobacter (Bacillus), 771

amylobacter I (Bacillus), 771, 824

amylobacter II (Bacillus), 771, 824

amylobacter III (Bacillus), 771, 824

amylobacter (Clostridium), 771

amylobacter (Metallacter), 771

amylobacter immobilis (Bacillus), 790, 826

amylobacter mobilis (Bacillus), 771

amylobacter $S$ and $W$ (Bacillus), 772, 824

amylocella (Vibrio), 203

amyloclasticus (Bacillus), 813

amyloclasticus intestinalis (Bacillus), 813

amylolactis (Streptococcus), 325

amylolyticus (Bacillus), 738

amylophilum (Pectinobacter), 823

amyloruber (Bacillus), 484

amyloruber (Erythrobacillus), 481

amylorubra (Serratia), 484

amylovora (Erwinia), 465, 1135

amylovorum (Achromobacter), 423

amylovorum (Bacterium), 465

amylovorum (Urobacterium), 423 amylovorus (Bacillus), 465

amylovorus (Micrococcus), 465

amylozyma (Bacillus), 771

amylozyme (Clostridium), 771

amylozymicus (Bacillus), 771

Anaerobacillus, 27, 763

Anaerobe No. III, Flügge, 813

Anaerobe No. $I V$, Flügge, 814

anaerobia (Gaffkya), 284

anaerobic No.V (Bacillus), 818

anaerobic No. VIII (Bacillus), 813

anaerobicum (Bacterium), 687, 819

anaerobicus (Bacillus), 820

anaerobicus alcaligenes (Bacillus), 801

anaerobicus caproicus (Bacillus), 820

anaerobicus-liquefaciens (Bacillus), 819

anaerobicus magnus (Streptobacillus), 823

anaerobicus minutus (Bacillus), 368

anaerobicus parvus (Coccobacillus), 368

anaerobicus rectus (Streptobacillus), 822

anaerobicus tenuis (Bacillus), 821

anaerobies (Actinomyces), 922

anaerobies (Oospora), 922

anaerobiontica (Pasteurella), 581

anaerobium (Achromobacter), 423

anaerobium (Bacterium), 673

anaerobium (Corynebacterium), 388, 402

anaerobius (Micrococcus), 247, 281

anaerobius (Staphylococcus), 247, 248

anaerobius (Streptococcus), 328, 345

anaerobius (Tetracoccus), 284

anaerobius carduus (Streptococcus), 328

anaerobius chromogenes (Bacillus), 805

anaerobius diphtheroides (Bacillus), 402

anaerobius foetidus (Bacillus), 782

anaerobius gonoides (Streptococcus), 328

anaerobius gracilis (Bacillus), 580

anaerobius haemolysans (Bacillus), 820

anaerobius liquefaciens (Bacillus), 687, 819

anaerobius magnus (Bacillus), 823

anaerobius major (Staphylococcus), 281

anaerobius micros (Streptococcus), 328,

330

anaerobius minor (Staphylococcus), 281

anaervbius perfoetens (Coccobacillus), 576

anaerobius rectus (Bacillus), 822

anaerobius tenuis (Bacillus), 821 
anaerobius tenuis (Leptothrix), 365 anaerobius vulgaris (Streptococcus), 328 anaerobius typ. vulgaris (Streptococcus), 328

anaerogenes (Bacillus), 533

anaerogenes (Bacterium), 533

anaerogenes (Escherichia), 533

Anaeromyces, 925

ananas (Bacillus), 473

unanas (Bacterium), 127, 473

allanas (Erwinia) 127, 473

unanas (Phytomonas), 127

ananas (Pseudomonas), 127, 473

Anaplasma, 1100

anata (Escherichia), 523

unatipestifer (Hemophilus), 554

anatipestifer (Pfeifferella), $\mathbf{5 5 4}$

anatis (Bacterium), 523, 552

anatis (Corynethrix), 406

anatis (Salmonella), 498,502, 523, 642

anatis (Spirochaeta), 1059

anatum (Bacterium), 523

anatum (Salmonella), 43, 523

unatum var. certrycke (Salmonella), 502

anatum var. muenster (Salmonella), 523

anatum var. nyborg (Salmomella), 523

anceps (Bacillus), 648

anceps (Leptotrichia), 367

anceps (Rasmussenia), 367

andoi (Vibrio), 201

andropogoni (Bacterium), 142

andropogoni (Phytomonas), 142

andropogoni (Pseudomonas), 142, 169,

1136

anemoeon (Microsporon), 918

anemones (Galla), 1158

anginosus (Streptococcus), 333, 334

Angiococcus, 1047

angliae (Marmor), 1200

anglomerans (Bacillus), 173

anguillarum (Bacillus), 673

anguillarum (Bacterium), 673

anguillarum (Vibrio), 203

angulans (Bacillus), 758

angulata (Phytomonas), 113

angulata (Pseudomonas), 113, 124

angulatum (Bacterium), 113 angulosum (Clostridium), 801, 827

angulosus (Bacillus), 801, 827

angulosus (Bacteroides), 801

angustum (Bacterium), 673

anhaemolyticus (Streptococcus), 337

anhaemolyticus vulgaris (Streptococcus),

337,343

anindolica (Escherichia), 452

anindolicum (Bacillus), 452

anindolicum (Citrobacter), 448

annamensis (Corynebacterium), 402

annamensis (Salmonella), 530

annulare (Photobacterium), 636

annularis (Microspira), 636

annulata (Pseudomonas), 173

annulata (Thiothrix), 990

annulates (Bacterium), 693

annulatum (Flavobacterium), 173

annulatus (Actinomyces), 968

annulatus (Actinomyces) (Streptothirix),

968

annulatus (Bacillus), 173, 648, 693

annulatus (Micrococcus), 252

annuliformans (Bacillus), 580

annuliformis (Bacillus), 738

Annulus, 1212

anodontae (Cristispira), 1055, 1056

anodontae (Spirochaeta), 1055

anolium (Serratia), 461

anoxydana (Colloides), 595

anserina (Borrelia), 1058

anserina (Spirochaeta), 1058

anserina (Spironema), 1058

anserina (Spiroschaudinnia), 1058

anserina (Treponema), 1058

anserinum (Spirillum), 1058

anserum (Spirillum), 1058

antaretica (Nitrosospira), 72

antenniforme (Flavobacterium), 440)

antenniformis (Bacillus), 440

antenniformis (Bacterium), 440

anthraciformis (Bacillus), 648

Anthracillus, 25,27

anthracis (Aplanobacter), 719

anthracis (Bacillus), 719, 1138

anthracis (Bacillus) (Bacteridium), 719

anthracis (Bacillus) (Streptobacter), 719 
anthracis (Bacterium), 719

anthracis (Pollendera), 719

anthracis similis (Bacillus), 738

anthracis symptomatici (Bacillus), 776

anthracis-symptomatici (Clostridium), 776

anthracoides (Bacillus), 648, 716

anthracoides (Bacterium), 716

Anthrax, 20, 22

anthropopitheci (Spirochaeta), 1079

antibioticus (Actinomyces), 942

antibioticus (Streptomyces), 942

antirrhini (Bacterium), 167

antirrhini (Phytomonas), 167

antirrhini (Pseudomonas), 167

antirrhini (Xanthomonas), 167

anularis (Cytophaga), 1016

anularius (Bacillus), 648

A pelmocoena, 14, 1032

apertus (Annulus), 1214

aphrophilus (Hemophilus), 589

aphthicola (Streptococcus), 337

aphthosum (Bacterium), 673

aphthosus (Bacillus), 673

apiculatus (Chondromyces), 1038

apicum (Bacillus), 648, 738

apii (Bacterium), 639

apii (Phytomonas), 122

apii (Pseudomonas), 122

apiovorus (Bacillus), 470

apis No.1,No.2 and No.3 (Bacterium),

673

apis (Streptococcus), 326, 724

apisepticus (Bacillus), 648

A planobacter, 8, 705

aporrhoeus (Bacillus), 738

appendicis (Actinomyces), 922

appendicis (Discomyces), 922

appendicis (Nocardia), 922

appendicitis (Bifidibacterium), 369

aptata (Phytomonas), 114

aptata (Pseudomonas), 43, 114

aptatum (Bacterium), 114

aquamarinus (Achromobacter), 419

aquatile (Flavobacterium), 428, 429

aquatile aurantiacum (Bacterium), 673

aquatile citreum (Bacterium), 673

aquatile debile (Bacterium), 673 aquatile flavum (Bacterium), 673

aquatile gasoformans non liquefaciens

(Bacterium), 657

aquatile luteum (Bacterium), 673

aquatile odorans (Bacterium), 491

aquatilis (Bacillus), 428, 648, 649

aquatilis (Bacterium), 428, 613

aquatilis (Diplococcus), 694

aquatilis (Micrococcus), 252, 695

aquatilis (Microspira), 199

aquatilis (Pseudomonas), 146

aquatilis (Streptococcus), 702

aquatilis (Streptothrix), 976

aquatilis (Vibrio), 199, 632

aquatilis (Zuberella), 577

aquatilis $\alpha$ (Bacillus), 684

aquatilis albissimus (Micrococcus), 695

aquatilis albus (Micrococcus), 252, 695

aquatilis communis (Bacillus), 649, 661, 699

aquatilis communis (Bacterium), 649

aquatilis flavus (Micrococcus), 252

aquatilis gasoformans non liquefaciens

(Bacillus), 657

aquatilis invisibilis (Micrococcus), 252

aquatilis magnus (Micrococcus), 695

aquatilis radiatus (Bacillus), 613

aquatilis radiatus (Bacterium), 613

aquatilis solidus (Bacillus), 658

aquatilis solidus (Bacterium), 658

aquatilis sulcatus (Bacillus), 655

aquatilis sulcatus $I$ (Bacillus), 670

aquatilis sulcatus II (Bacillus), 670

aquatilis sulcatus III (Bacillus), 669

aquatilis sulcatus IV (Bacillus), 648

aquatilis sulcatus $V$ (Bacillus), 655

aquatilis-sulcatus-quartus (Bacillus), 649

aquatilis sulcatus quartus (Bacterium), 649

aquatilis villosus (Bacillus), 671

aqueductum (Leptospira), 1078

aqueum (Bacterium), 759

aqueus (Micrococcus), 252

aquivivus (Micrococcus), 695

aquosus (Proactinomyces), 923

arabinosaceus (Betacoccus), 346

arabinosaceus (Leuconostoc), 346

arabinosum (Propionibacterium), 378 
arabinosus (Lactobacillus), 357

arabinotarda Types $A$ and $B$ (Shigella),

544

arachidis (Marmor), 1187

arachnoidea (Beggiatoa), 992, 993

arachnoidea (Oscillaria), 992

arachnoideus (Bacillus), 738

araliavora (Erwinia), 477

araliavorus (Bacillus), 477

arborescens (Actinomyces), 919

arborescens (Bacillus), 435, 436, 649, 919

arborescens (Bacterium), 435

arborescens (Erythrobacillus), 435

arborescens (Flavobacterium), 429, 435,

439

arborescens (Nocardia), 919

arborescens lactis (Micrococcus), 252

arborescens non-liquefaciens (Bacillus),

435

arborescens non-liquefaciens (Bacterium), 436,673

arboreus (Bacillus), 649

Archangium, 1017

archeri (Gaffkya), 284

archibaldii (Salmonella), 531

arcticum (Achromobacter), 423

arcticum (Bacterium), 673

arechavaleta (Salmonella), 506

arenarius (Bacillus), 738

argenteo-phosphorescens (Bacillus), 634

argenteo-phosphorescens I (Bacillus), 634

argenteo-phosphorescens II (Bacillus), 634

argenteo-phosphorescens III (Bacillus), 634

argenteo-phosphorescens (Bacterium), 634

argenteo-phosphorescens liquefaciens ( $\mathrm{Ba}$ cillus), 634

argenteus (Micrococcus), 252

argentinensis (Spirochaeta), 1065

argentinensis (Treponema), 1065

argentophosphorescens (Achromobacter),

634

arguata (Cellulomonas), 176

arguta (Pseudomonas), 176

aridus (Bacillus), 738

arizona (Salmonella), 462

Arloingillus, 11, 763

arlongii (Bacillus), 738 armoraciae (Bacillus), 710

aroideae (Bacillus), 474

aroideae (Bacterium) 474

aroideae (Erwinia), 470, 474, 1129, 1136

aroideae (Pectobacterium) 474

aromafaciens (Achromobacter), 423

aromafaciens (Bacterium), 423

aromatica (Pseudomonas), 146

aromatica var. quercitopyrogallica (Pseu-

domonas), 146

aromaticum (Flavobacterium), 457

aromaticus (Bacillus), 43t, 457, 619, $7 \pm 3$

aromaticus (Bacterium), 457

aromaticus (Streptococcus), 337

aromaticus butyri (Bacillus), 440

aromaticus lactis (Bacillus), 434

arthritica (Micrococcus), 301

arthritica (Neisseria), 301

arthritidis (Bacterium), 674

arthritidis (Murimyces), 1292

athritidis chronicae (Bacillus), 67t

arthritidis-muris (Corynebacterium), 402

Arthrobacter, 7

Arthrobactridium, 7

Arthrobactrillium, 7, 82

Arthrobactrinium, 7,82

Arthromitus, 1003

Arthrostreptokokkus, 312

arthrotropicus (Musculomyces), 1243

arthuri (Bacillus), 639

articulata (Pseudomonas), 146

articulatum (Bacterium), 759

articulorum (Streptococcus), 337

artus (Phagus), 1133

arvalis (Grahamella), 1109

arvicolae (Bartonella), 1104

arvicolae (Haemobartonclla), 1104

arvilla (Pseudomonas), 105

arvillum (Achromobacter), 105

asaccharolyticus (Micrococcus), 246

asaccharolyticus (Staphylococcus), 246

asaccharolyticus var. indolicus (Staphylo-

coccus), 247, 264

asalignus (Streptococcus), 337

ascendens (Acetobacter), 43, 185, 692

ascendens (Bacterium), 185

ascendens (ITrina), 692 
ascitis (Corynebacterium), 402

Ascococcus, 6, 235

ascoformans (Botryoceccus), 253

ascoformans (Micrococcus), 252, 253

ascoformans (Staphylococcus), 253

ascoformis (Micrococcus), 253

asiaticum (Bacterium), 450

asiaticus (Bacillus), 450, 738

asiaticus (Proteus), 450

asiaticus (Salmonella), 450

asiaticus mobilis (Bacillus), 450

asiaticus mobilis (Salmonella), 450

asparagi (Bacillus), 759

asparagi (Bacterium), 759

asper (Micrococcus), 253

asplenii (Phytomonas), 696

asporiferum (Bacterium), 674

assimilis (Bacillus), 649

assurgens (Archangium), 1019)

Astasia, 20, 22, 705

asteracearum. (Bacillus), 477

asteracearum (Erwinia), 477

asteriformis (Bacillus), 612

asteris (Bacillus), 738

Asterococcus, 1289, 1291

asteroide (Leptothrix), 218, 36.5

asteroide (Mycobacterium), 896

Asteroides, 892

astcroides (Actinomyces), 896

asteroides (Asteroides), 896

asteroides (Cladothrix), 896

asteroides (Discomyces), 896

asteroides (Nocardia), 896, 897, 918

asteroides (Oospora), 896

asteroides (Proactinomyces), s\$6

asteroides (Streptotrix), 896

asteroides var. crateriformis (Nocardia),

897

asteroides var. crateriformis (Proactino-

myces), 897

asteroides var. decolor (Nocardia), 897

asteroides var. decolor (Proactinomyces),

897

asteroides var. gypsoides (Nocardia), 897 asteroides var. gypsoides (Proactimomyces), 897 asteroides var. serratus (Actinomyces), 917

Asteromyces, 1291

asterospora (Astasia), 720

asterosporus (Aerobacillus), 720

asterosporus (Bacillus), 720,748

asterosporus alpha (Bacillus), 720

astheniae (Bacillus), 448

astheniae (Bacterium), 448

astheniae (Escherichia), 448

asthenoalgiae (Leptospira), 1078

asthenogenes (Bacillus), 738

astragali (Bacterium), 139

astragali (Phytomonas), 139

astragali (Pseudomonas), 139

astri (Marmor), 1196

astrictum (Marmor), 1165, 1167

astrictum var, aucuba (Marmor), 1168

astrictum var. chlorogenus (Marmor), 1168

astrictus (Phagus), 1133

aterrimus (Bacillus), 711

aterrimus tschitensis (Bacillus), 738

atherton (Salmonella), 701

atlantica (Pseudomonas), 697

Atremis, 13

atrofaciens (Bacterium), 120

atrofaciens (Phytomonas), 121

atrofaciens (Pseudomonas), 120

atroseptica (Erwinia), 468, 470, 1134

atrosepticus (Bacillus), 468, 470

attenuatum (Spirillum), 43, 217

attenuatum (Spirosoma), 217

atypica pseudotuberkulosa (Actinomyces),

973

aucuba (Marmor), 1175

aucubn var. canadense (Marmor), 1175

aucubicola (Pseudomonas), 146

aurantia (S'pirochacta), 1053

aurantiaca (Cladothrix), 896

aurantiaca (Cytophaga), 1013

aurantiaca (Merismopedia), 251, 290

aurantiaca (Nocardia), 896

aurantiaca (Oospora), 896

aurantiaca (Paulosarcina), 288

aurantiaca (Sarcina), 243, 288

aurantiaca (Stigmatella), 1037

aurantiaca (Streptothrix), 896 
aurantiacum (Agarbacterium), 630

rurantiacum (Bacteridium), 243

aurantiacum (Chromobacterium), 440

(urantiacum (Corynebacterium), 402, 404

aurantiacum (Flavobacterium), 410

aurantiacum (Polycephalum), 1037

aurantiacus (Actinomyces), 896

arantiacus (A urococcus), 243

aurantiacus (Bacillus), 440

aurantiacus (Bacterium), 440

aturantiacus (Chondromyces), 1037, 1038

aurantiacus (Micrococeus), 243, 265, 275,

290

anrantiacus (Pediococcus), 243, 290

aurantiacus (Staphylococcus), 243

aurantiacus (Streptococcus), 243, 253, 337

aurantiacus-sorghi (Micrococcus), 243, 253

aurantiacus tingitanus (Bacillus), 145 .

\section{5}

aurantiacus var. frutescens (Chondro-

myces), 1037, 1039

aurantibutyricum (Clostridium), 819

aurantii (Barillus), $67 t$

aurantii (Bacterium), 674

aurantinum (Flarobacterium), $4+0$

aurantinus (Bacillus), $\mathbf{4 t 0}$

aurantium (Plocamobacterium), 674

aurantium roseum (Bucterium), 392, 67t

aurantius (Bacillus), 649, 738, 739

aurantius (Cellulomonas), 739

aurea (Actinomyces), 943, 968

aurea (Nocardia), 968

aurea (Oospora), 968

aurea (Pseudomonas), 146, 697

aurea (Sarcina), 290

aurea (Streptothrix), 968

aureo-flavus (Bacillus), 674

aureo flavus (Bacterium), $67 t$

A ureogenus, $\mathbf{1 1 5 4}$

aurescens (Bacillus), $140,474,674$

aurescens (Bacterium), 440, 445, 484,67t

aurescens (Flavobacterium) $4 \mathbf{4 0}$

aurescens (Sarcina), 290

aurescens var. mucosa (Sarcina), 290

aureum (Bacterium), $67+$

aureum (Polyangium), 1031

aureum (Spirillum), 203 aureum (Spirosoma), 203

aureus (Actinomyces), 943, 968, 975

aureus. (Aurococcus), 241

aureus (Bacillus), 649, 674, 716

aureus (Flexibacter), 38

aureus (Micrococcus), 241, 251, 252, 253,

$254,256,258,265,266,268,269,271$,

276,279

aureus (Myxobacter), 1026

aureus (Staphylococcus), 241, 1140, 1141

aureus (Streptomyces), 943

unieus (Vibrio), 203, 204

aureus var. equi (Staphylococcus), 25:)

aureus lactis (Micrococcus), 253

aureus minutissimus (Bacillus), 663

aureus sarciniformis (Staphylococcus),

281

auris (Bacillus), 402

auris (Corynebacterium) +402

Aurococcus, S, 235

aurogenes (Bacillus), 617

aurogenes (Cellulomonas), 617, 622

uurogenes var. albus (Bacillus), 622

australiense (Lethum), 1223

australiense var. lethale (Lethum), 1224

australiense var. typicum. (Lethum), 1224

australiensis (Chlorogenus), 1147

autumnalis (Leptospira), $107 \mathrm{~s}$

autumnalis (Spirochacta), 1078

antumnalis A (Spirochaeta), 1078

autumnalis Type B (Leptospira), 1077 , 1078

auxinophilum (Bacterium), 115

avadi (Actinomyces), 915, 924

arenae (Bacillus), 116

avenae (Fractilinea), 1162

arenae (Phytomonas), 116

arenae (Pseudomonas), 116

avicida (Pasteurella), 547, 552, 642

aricidum (Bacterium) , 546, 547

avicidus (Coccobacillus), 547

aridum (Corynebacterium), 388, 402

avidus (Bacteroides), ?80, 402

avidus (Vibrio), 702

aviseptica (Pasteurella), 523, 54

avisepticum (Bacterium), 547

avisepticus (Bacillus), 530, 547 
avium (Bacillus), 400

avium (Bacterium), 674

avium (Borreliota), 1229

avium (Mycobacterium), 878, 879, 880

avium (Pasteurilla), 547

avium (Rickettsia), 1095

avium (Strongyloplasma), 1229

avium (Tarpeia), 1274

azoticus (Bacillus), 772, 824

Azotobacter, 19, 21, 26, 29, 31, 216, 219, 221

azotobacter (Bacillus), 219

azotogena (Pseudomonas), 697

Azotomonas, 8, 219, 221

azureus (Bacillus), 649

babesi (Bacillus), 649

babesi (Neisseria), 254, 696

Babesia, 312

babesii (Bacterium), 674

baccarinii (Bacillus), 466

baccarinii (Clostridium), $47 \mathrm{~S}$

baccatus (Micrococcus) (Sarcina), 253

bacillifera (Pelogloea), 871

bacilliformis (Bartonella), 1101, 1105

bacilliformis (Bartonia), 1101

bacillosum (Amoebobacter), 849

Bacillus, 6, 7, 13, 15, 18, 19, 22, 27, 30, 31, $42,43,46,63,76,179,632,643,704,705$, 763,1008

Bacillus I, Bienstock, 755

Bacillus II, Bienstock, 753

Bacillus II, Leube, 668

Bacillus a, Guillebeau, 662

Bacillus $a, b, c, d, e, f, h$ and $i$, Vignal, 647

Bacillus A, Grigoroff, 354

Bacillus A, Maggiora, 671

Bacillus B, Hoffmann, 749

Bacillus $B$, Maggiora, 661

Bacillus D, Foutin, 744

Bacillus D, Peters, 741

Bacillus $G$, Maggiora, 650

Bacillus $H$, Maggiora, 662

Bacillus X, Moore and White, 726

Bacillus $\alpha$, Busse, 639

Bacillus $\alpha$, von Freudenreich, 356

Bacillus $\gamma$, von Freudenreich, 358
Bacillus $\delta$, von Freudenreich, 360

Bacillus $\epsilon$, von Freudenreich, 352

Bacillus No. 18, Conn, 760

Bacillus No. 25, Conn, 753

Bacillus No. 41, Conn, 423

Bacillus No. 2, Fulles, 668

Bacillus No. 2, Kedrowski, 814

Bacillus No. 3, Pansini, 718

Bacillus No. 6, Pansini, 710

Bacillus No. 8, Pansini, 738

Bacillus No. XVI, Adametz, 718

Bacillus No. XVII, Adametz, 760

Bacillus No. I, Flügge, 716, 725

Bacillus No. II, Flügge, 741

Bacillus No. III, Flügge, 738

Bacillus No. IV, Flügge, 744

Bacillus No. V, Flügge, 716

Bacillus No. VI, Flügge, 743

Bacillus No. VII, Flügge, 747

Bacillus No. VIII, Flügge, 748

Bacillus No. IX, Flügge, 710

Bacillus No. X, Flügge, 709

Bacillus No. XI, Flügge, 743

Bacillus No. XII, Flügge, 755, 761

Bacillus of swine plague, 508

Bacillus sp., Sordelli, 777

Bacteridium, 6, 43, 705

Bacterienart No. 13, Lembke, 745

bacterifera (Cylindrogloea), $\mathbf{8 7 4}$

Bacteriophagum, 1128, 1129

bacteriophagus (Protobios), 1129

Bacteriopsis, 179, 365

Bacterium, 5, 7, 10, 13, 15, 17, 18, 19, 28, $30,37,42,46,76,82,179,435,696,597$, $599,612,694,705,763$

Bacterium A, Peters, 657

Bacterium B, Peters, 664

Bacterium of hog cholera, 508

Bacterium of swine plague, 508

Bacteroidea, 20

Bacteroides, 22, 23, 27, 31, 32, 218, 365,

564, 763, 1296

Bactoderma, 76

Bactrella, 705

Bactridium, 7, 705, 763

Bactrillius, 83

Bactrillum, 7, 13, 25, 27, 82, 705 
Bactrinium, 7, 82, 705

Bactrinius, 83

badius (Bacillus), 739

badius (Micrococcus), 253

bahiensis (Actinomyces), 919

bahiensis (Discomyces), 919

bahiensis (Nocardia), 919

bahiensis (Oospora), 919

balaenae (Clostridium), 775, 819

balanitidis (Spirochacta), 1065

balanitidis (Spironema), 1065

balanitidis (Spiroschaudinnia), 1065

balanitidis (Treponema), 1065

balbianii (Bacillus), 674

balbianii (Bacterium), $67+$

balbianii (Cristispira), 1055, 1056, 1057

balbianii (Spirochaeta), 1055

balbianii (Trypanosoma), 1055

balcanus (Bacillus), 739

balfourii (Grahamella), 1109

Balkanella, 10

ballerup (Salmonella), 529

balticum (Photobacterium), 635, 636

balticus (I ibrio), 636

balustinum (Flavobacterium), $\mathbf{4 3 7}$

bamptonii (Chromobacterium), 234

bantam (Salmonella), 523

barati (Inflabilis), 823

barbareae (Phytomonas), 154

barbareae (Xanthomonas), 154

barbatum (Bacterium), 761

barbitistes (Bacillus), 739

baregensis purpureus (Micrococcus), 253

bareilly (Salmonella), 511

bareilly var. mikawasima (Salmonella),

511

barentsianum (Bacterium), 674

barkeri (Bacillus), 129

barkeri (Bacterium), 129

barkeri (Phytomonas), 129

barkeri (Pseudomonas), 129, 134

Bartonella, 37, 1100, 1102, 1109

Bartonella sp., 1108

Bartonia, 1100

batatae (Bacillus), 739

batavia (Salmonella), 524

bataviae (Leptospira), 1078 batrachorum (Arthromitus), 1003

batrachorum (Bartonella), 1108

batrachorum (Haemobartonella), 1108

batrochorum (Micrococcus), 1123

bauri (Bacterium), 108

beaufortensis (Pseudomonas), 697

beckii (Bacterium), 552

beddardii (Actinomyces), 963

beddardii (Streptomyces), 963

Beggiatoa, 12, 16, 18, 19, 24, 26, 42, 988, 990, 993, 994, 1007

beggiatoides (Oscillatoria), 992

begoniae (Bacterium), 155

begoniae (Phytomonas), 155

begoniae (Xanthomonas), 155

beigeliana (Zoogloea), 253

beigelianum (Sclerotium), 253

beigelli (Chlamydatomus), 253

beigelii (Hyalococcus), 253

beigelii (Micrococcus), 253

beigelii (Pleurococcus), 253

beigelii (Trichosporum), 253

beijerincki (Bacillus), 356, 650, 691

beijerincki (Bacterium), 674

beijerinckii (Azotobacter), 219

beijerinckii (Clostridium), 772

beijerinckii (Lactobacillus), 357

beijerinckii (Pseudomonas), 109

beijerinckii (Rhizobium), 225

beijerinckii (Rhizomonas), 225

beijerinckii (Sarcina), 286

beijerinckii (Thiobacterium), 81

beijerinckii (Urobacillus), 691

beijerinckii (Vibrio), 203

beijerinckii var. jacobsenii (Thiobacterium), 81

bekkerii (Mycobacterium), 890

belfanti (Bacillus), 649, 803

belfantii (Clostridium), 803

belfantii (Endosporus), 649, 803

belfastiensis II (Bacillus), 533

belfastiensis $V$ (Bacillus), 534

belfastiensis (Bacterium), 533

belfastiensis (Eberthella), 533

bellisari (Actinomyces), 968

bellonensis (Bacillus), 777, 825

bellonensis (Clostridium), 778 
bellus (Bacillus), 739

belorinensis (Bacillus), 360

bemisiae (Ruga), 1219

bentotensis (Bacillus), 533

bentotensis (Bacterium), 533

bentotensis (Castellanus), 533

bentotensis (Eberthella), 533

benzoli (Bacillus), 649

benzoli $a$ and $b$ (Bacterium), 674

berardinisi (Discomyces), 918

berardinisi (Nocardia), 918

berbera (Borrelia), 1061

berbera (Spirochaeta), 1061

berbera (Spironema), 1061

berbera (Spiroschaudinnia), 1061

berberidis (Bacterium), 115

berberidis (Phytomonas), 115

berberidis (Pseudomonas), 115

berberum (Treponema), 1061

berestneffi (Nocardia), 919

berestneffi (Actinomyces, 919

berestneffii (Discomyces), 919

berestnewi (Bacillus), 918

bergerac (Annulus), 1213, 1216

beri-beri (Hicrococcus), 253

beribericus (Bacillus), 649

bernardinisi (Actinomyces), $91 \mathrm{~s}$

bernensis (Bacillus), 739

berolinensis (Bacillus), 234, 360, 650

berolinensis (Lactobacillus), 360

berolinensis (Microspira), 196

berolinensis (Mycobacterium), 888, 890

berolinensis (Pseudomonas), 697, 698

berolinensis (Vibrio), 196, $20 \mathrm{t}$

berolinensis fasciformis (Saccharo-

bacillus), 360

berolinensis indicus (Bacillus), 697

berolinensis indicus (Bacterium), 697

berta (Salmonella), 518

bertherandi (Coniothecium), 289

besseri (Bacterium), 674

bessoni (Kurthia), 613

beta (Bacillus), 650, 739

beta (Micrococcus), 269

beta (Nocardia), 976

beta (Phagus), 1141

beta (Scelus), 1237 beta (Streptothrix), 976

beta (Tarpeia), 1270

Betabacterium, 9, 30, 350

Betacoccus 9, 30, 346

betadelbrueckii (Lactobacillus), 363

betae (Bacillus), 477, 639

betae (Bacterium), 144, 639

betae (Butylobacter), 781, 825

betae (Corium), 1204

betae (Marmor), 1178

betae (Myxobacillus), 762

betae (Myxococcus), 348

betae (Phytomonas), 144

betae (Savoia), 1221

betae viscosum (Bacterium), 674

betainovorus (Bacillus), 739

betanigrificans (Bacillus), 739

beticola (Bacillus), 478

beticola (Bacterium), 478

beticola (Phytomonas), 153

beticola (Pseudomonas), 153, 1134

beticola (Xanthomonas), 153, 230

beticolum (Bacterium), 153

betivora (Erwinia), 468

hetirorus (Bacillus), 468

betle (A planobacter), 130

betle (Bacterium), 130

letlis (Phytomonas), 130

betlis (Pseudomonas), 130

biacutum. (Bacillus), 739

biacutum (Fusobacterium), 582

hiacutus (Fusiformis), 582

biazotea (Cellulomonas), 617

biazoteus (Bacillus), 617

bibula (Cellulomonas), 615

bibulum (Bacterium), 615

bibulus (Bacillus), 615

bicolor (Actinomyces), 91!

bicolor (Micrococcus), 254

bicolor (Nocardia), 919

bicolor (Sarcina), 290

bienstocki (Putrificus), 799

bienstockii (Bacillus), 544

bienstockii (Bacterium), 544

bienstockii (Eberthella), 544

hienstockii (Shigella), 544

bifermentans (Bacillus), 787 
bifermentans (C'lostridium), 309, 782 , $787,818,825$

bifermentans (Martellillus), 787

bifermentans sporogenes (Bacillus), 787 bifida (Liestieella), 986

bifida (Vocardia), 353

Bifidibacterium, 34, 38, 369

Bifidobacterium, 349, 369

bifidum (Bacterium), 354

bifidum (Bifudibacteriım), 353, 369

bifidus (Actinomyces), 353

bifidus (Bacillus), 353

bifidus (Bacteroides), 353, 354

bifidus (Cohnistreptothrix), 353

bifidus (Lactobacillus), 353, 354, 369

bifidus II (Lactobacillus), 354

bifidus aerobius (Bacillus), 361

bifidus capitatus (Bacillus), 361

bifidus communis (Bacillus), 353, 361

bifilaris (Lieskeella), 986

biflexa (Leptospira), 1077, 107s

biflexa (Spirochaela), $107 \pi$

biforme (Eubacterium), 368

hiformis (Bacteroides), 362, 364

bifurcatum (Bifitibacterium), 369

bifurcatus gazogenes (Barillus), 369

biliohemoglobinuriae (Leptospira), 1074

bilio-hemoglobinuriae (Spirochaeta), 1078

Billetia, 690

billingsi (Bacillus), 650

billrothii (Ascococcus), 254

billrothii (Micrococcus), 254

binucleatum (Bacterium), 760

bipolare multocidum (Bacterium), 547

bipolaris (Bacillus), 548, 718

bipolaris brisep'icus (Bacillus), 547

bipolaris bubalisepticus (Bacillus), 548

bipolaris capriscplicus (Bacillus), 503

bipolaris ovisepticus (Bacillus), 55.1

bipolaris plurisepticus (Bacillus), 546

bipolaris septicus (Bacillus), 546, 547

bipunctata (Macromonas), 997, 1001

bipunctata (Psendomonas), 997, 1001

bipunctata (Thiospira), 212

hipunctatum (Spirillum), 212

biskra (Micrococcus), 254

biskrae (Staphylococcus), 254 bispebjerg (Salmonella), 506 bizzozerianus (Bacillus), 743

blackwellii (Actinomyces), 910

blackwellii (Norardia), 910

blanci (Rickeltsia), 1088

blarinae (Grahamella), 1110

blarinae (Haemobartonella), 1107

blasticus (Chondrococcus). 1008, 1046

Blastocaulis, 35, 836

blattellae (Corynebacterium), 402

blegdam (Salmonella), 518

bleischii (Bacillus), 533

boas-oppleri (Lactobacillus), 352

bobiliae (Actinomyces), 937

bobiliae (Streptomyces), 937

boleti (Micrococcus), 254

boletus (Melittangium), 1006, 1034

bollingeri (Pasteurella), $\mathbf{5 4 7}$

bolognesii-chiurcoi (Actinomyces), 91;)

bolognesii-chiurcoi (.Malbrachea), 915

bombycis (Aerobacter), 490

bombycis (Bacillus), 650, 739

bombycis (Bacterium), 650

hombycis (Borrelina), 1226

bombycis (chalmytozion), 12:6

bombycis (I)iplococcus), 336

bombycis (.Micrococcus), $25+$

bombycis (Microzyma), 254

hombycis (Nosema), 254

hombycis (Proteus), 490

hombycis (Streptococcus), 254, 265, 3337

hombycis non-liquefaciens (Bacillus), 7397

bombycivorum (Bacterium), 490

hombycoides (Bacillus), 739

bombysepticus (Bacillus), 739

honariensis (Leptospira), 1078

bonariensis (Salmonella), 514

bonhoffi (Microspira), 202

bonvicini (Streptococcus), $33 \bar{T}$

bookeri (Alcaligenes), $\mathbf{4 1 5}, 416$

bookeri (Bacillus), 415, 650

bookeri (Bacterium), 415

borbeck (S'almonella), 527

borborokoites (Bacillus), 739

bordonii (Bacterium), 691

bordonii (Klebsiella), 691

boreale (Bacterium), 625 
boreale (Flavobacterium), 625

boreopolis (Pseudomonas), 94

boreus (Micrococcus), 254

bornensis (Erro), 1256

Borrelia, 19, 34, 35, 37, 42, 1057, 1058

Borrelina, 1225

Borreliota, 1229

Borrelomyces, 1291

borstelensis (Bacillus), 739

bosporum (Bacterium), 145

bossonis (Bacterium), 675

bostroemi (Actinomyces), 970

botkini (Bacillus), 813

botryogenus (Micrococcus), 253

Botryomyces, 235

Botulinea, 20

botulinum (Clostridium), 778, 784

botulinum D (Bacillus) (Clostridium), 779

botulinum Type A (Clostridium), 781

botulinum Type B (Clostridium), 784

botulinum Type C (Clostridium), 779

botulinum Type D (Clostridium), 779

botulinum Type E (Clostridium), 779

Botulinus, 20, 22, 763

botulinus (Bacillus), 778

botulinus (Ermengemillus), 778

botulinus Type C (Bacillus), 779

Botulobacillus, 8, 763

bouffardi (Pastourella), 553

boutrouxii (Bacillus), 675

boutrouxii (Bacterium), 675

bovicida (Bacterium), $\mathbf{5 4 8}$

bovidae (Treponema), 1076

Bovimyces, 1291

bovina (Listerella), 409

bovinum (Scelus), 1239

bovinus (Micrococcus), 254, 337

bovinus (Streptococcus), 33i

bovis (Actinomyces), 925, 926, 927

bovis (Bacillus), 652

bovis (Bacterium) 675

bovis (Bartonella), 1106

bovis (Betacoccus), 347

bovis (Cladothrix), 925

bovis (Corynebacterium), 390, 391

bovis (Corynethrix), 401

boris (Discomyces), 925 bovis (Ehrlichia), 1095

bovis (Grahamella), 1110

bovis (Haematococcus), 254

bovis (Haemobartonella), 1106

bovis (Hemophilus), 591

bovis (Leptospira), 1078

bovis (Leuconostoc), 347

bovis (Micrococcus), 254

bovis (Molitor), 1242

bovis (Moraxella), 691

bovis (Nocardia), 925

bovis (Oospora), 925

bovis (Proactinomyces), 925

bovis (Rickettsia), 1095

bovis (Sphaerotilis), 925

bovis (Staphylococcus), 264, 281

bovis (Streptococcus), 320, 321, 322

bovis (Streptothrix), 925

bovis (Tortor), 1276

bovis var. nigerianus (Actinomyces), 968

bovis albus (Actinomyces), 968

bovis-caffris (Spirochaeta), 1065

bovis-caffris (Spironema), 1065

boris communis (Streptothrix), 925

boris farcinicus (Actinomyces), 895

bovis luteoroseus (Actinomyces), 971

bovis morbificans (Bacillus), 514

bovis-morbificans (Salmonella), 514

bovis sulfureus (Actinomyces), 925

boviseptica (Pasteurella), 547

bovisepticus (Bacillus), 547

bovisepticus (Bacterium), 547

bovium (Pasteurella), 547

bowlesiae (Pseudomonas), 125

bowlesii (Bacterium), 125

bowlesii (Phytomonas), 125

Brachybacterium, 312, 349

brachysporum (Bacterium), 759

brachythrix (Bacillus), 650

braenderup (Salmonella), 511

brandenburg (Salmonella), 505

brandenburgensis (Salmonella), 505

brandenburgiensis (Bacillus), 726

brandti (Bacterium), 108

branhamii (Micrococcus), 303

brasitiensis (Actinomyces), 918

brasiliensis (Discomyces), 918 
brasiliensis (Escherichia), 452

brasiliensis (Nocardia), 918

brasiliensis (Oospora), 918

brasiliensis (Rickettsia), 1087

brasiliensis (Streptothrix), 918

brassicae (Bacillus), 675, 714, 718

brassicae (Bacterium), 357, 675, 714, 718

brassicae (Borrelina), 1227

brassicae (Lactobacillus), 357

brassicae (Marmor), 1177

brassicae acidae (Bacterium), 146, 675

brassicae acidae (Pseudomonas), 146

brassicae fermentatae (Bacillus), 358

brassicaevorus (Bacillus), 477

bredemannii (Bacillus), 739

bredeney (Salmonella), 507

breitfussi (Bacterium), 675

breslau (Salmonella), 502

breslaviensis (Bacillus), 502

breslaviensis (Bacterium), 502

breve (Bacterium), 439

breve (Betabacterium), 358

breve (Flavobacterium), 439

brevis (Bacillus), 439, 725, 739, 744

brevis (Lactobacillus), 358, 359, 361, 363

brevis (Streptococcus), 337, 696

brevis o (Bacillus), 716

brevis non hemolyticus (Streptococcus), 338

orevis var, rudensis (Lactobacillus), 357.

359

brevissimum (Bacterium), 675

Brevistreptothrix, 892, 925

Briareus, 1233

briensis (Nitrospira), 72

brightii (Streptococcus), $33 \mathrm{~s}$

briosianum (Bacterium), 639

briosii (Bacterium), 145

bronchiale (Treponema), 1065

bronchialis (Actinomyces), 922

bronchialis (Discomyces), 922

bronchialis (Oospora), 922

bronchialis (Spirochaeta), 1065

bronchialis (Spiroschaudinnia), 1065

bronchicanis (Bacillus), 562

bronchicanis (Bacterium), 562

bronchiseptica (Brucella), $\mathbf{6 6 2}$ bronchisepticus (Alcaligenes), 416, 562

bronchisepticus (Bacillus), 562

branchisepticus (Bacterium), 562

bronchitica (Anaeromyces), 926

bronchitica (Cohnistreptothrix), 926

bronchitidis (Bacillus), 739

bronchitidis putridae (Bacillus), 739

bronchopneumoniae (Ehrlichia), $111 \mathrm{~s}$

bronchopneumoniae (Miyagawanel'id), 1118

Brucella, 17, 26, 32, 42, 43, 560, 562

Brucella melitensis var. melitensis, 561

brumptii (Grahamella), 1110

bruneum (Corynebacterium), 403

bruneum $\gamma$ arborescens (Bacterium), 403

bruneus (Bacillus), 740

bruni (Actinomyces), 923

bruni (Discomyces), 923

bruni (Nocardia), 923

brunneoflavum (Bacterium), 675

brunneoflavus (Bacillus), 675

brunneum (Bacteridium), 650

brunneum (Bacterium), 650, 680, 737, 740

brunneum (Flavobacterium), 440

brunneus (Bacillus), 440, 650, 737, 740

brunneus (Micrococcus), 675

brunneus rigensis (Bacillus), 430

bruntzii (Bacillus), 644, 645

bruntzii (Serratia), 644

bubalorum (Clostridium), 778, 825

bubalseptica (Bacillus), 548

bubalseptica (Pasteurella), 548

bucallis (Bacterium), 440

buccale (Bacterium), 365, 440

buccale (Borrelia), 1062

buccale (Spirillum), 1062

buccale (Spironema), 1062

buccale (Treponema), 1062

buccalis (Actinomyces), 922

buccalis (Ascococcus), 693

buccalis (Bacillus), 365, 650

buccalis (Discomyces), 922

buccalis (Flavobacterium), $4 \mathbf{4 0}, 6 \pm 7$

buccalis (Leptospira), 1079

buccalis (Leptothrix), 365, 366

buccalis (Leptotrichia), 364, 365

buccalis (Micrococcus), 329 
buccalis (Microspira), 1062

buccalis (Molitor), 1242

buccalis (Nocardia), 922

buccalis (Oospora), 922

buccalis (Rasmussenia), 365

buccalis (Spirochaeta), 1062, 1065

buccalis (Spiroschaudinnia), 1062

buccalis (Streptococcus), 338

buccalis (Streptothrix), 923

buccalis (Syncrotis), 365

luccalis (Vibrio), 203

buccalis fortuitus (Bacillus), 647, 650

buccalis fortuitus (Bacterium), 650

buccalis minutus (Bacillus), 440

buccalis minutus (Bacterium), 440

buccalis muciferens (Bacillus), 650

buccalis septicus (Bacillus), 650

bucco-pharyngei (Spirochacta), 1065

bucco-pharyngei (Treponema), 1065

buchneri (Bacillus), 359

buchneri (Lactobacillus), 359, 695

buchneri (Llvina), 695

budapest (Salmonella), 505

budayi (Bacillus), 791

bufo (Agarbacterium), 628

bufonis (Spirochaeta), 1066

bufonis (Spironema), 1066

bufonis (Spiroschaudinnia), 1066

bufonis (Treponema), 1066

bulbosa (Vibrio), 203

bulgaricum (Acidobacterium), 354

bulgaricum (Bacterium), 354, 687, 695

bulgaricum (Plocamobacterium), 354

bulgaricum (Thermobacterium), $35+$

bulgaricus (Bacillus), 354

bulgaricus (Lactobacillus), 354, 362, 364, 695

bullata (Mycoplana), 191

bullosum (Bacterium), 675

bullosus (Bacillus), 580

bullosus (Bacteroides), 580

bullosus (Spherocillus), 580)

burchaidti (Micrococcus), 254

burgeri (Bacillus), 116

burneti (Richettsia), 1092

burneti (Riclieltsia) (Coxiella), 1092

burneti var. americana (Ricketlsia), 1092 burnetii (Coxiella), 1090, 1092

burri (Bacillus), 726

busae asiaticae (Bacterium), 357

busaeasiaticus (Lactobacillus), 357

bussei (Bacillus), 477

bussei (Erwinia), 477

butantan (Salmonella), 524

bullerovii (Bacillus), 740

bütschlii (Bacillus), 740, 742, 744

butylaceticum (Bacillus), 781, 825

Butylbacillus, 771

butylicum (Amylobacter), 771, 813

bulylicum (Clostridium), 771

butylicum (Granulobacter), 771, 824

butylicus (Bacillus), 680, 771, 824

butylicus B.F. (Bacillus), 781, 825

Butylebacter, 763

butyri (Achromobacter), 421

Butyribacillus, 8, 763

butyri (Bacillus), 650

butyri I (Bacillus), 650

butyri II (Bacillus), 659

Butyribacterium, 368, 380, 402

butyri (Diplococcus), 254

butyri (Flavobacterium), 440

butyri (Mycobacterium), 890

butyri (Micrococcus), 254

butyri (Pseudomonas), 146, 697

butyri aromafaciens (Bacillus), 421

butyriaromafaciens (Bacterium), 421

butyri-aromafaciens (Micrococcus), 421

butyri colloideum (Bacterium), 676

butyri fluorescens (Bacillus), 146

butyri fluorescens (Bacterium), 697

butyri fuorescens (Micrococcus), 254

butyrica (Botulinea), 22

butyrica (Sarcina), 290

Butyriclostridium, 11, 763

butyricum (Bactridium), 771, 819, 824

butyricum (Clostridium), 716, 770, 771,

$772,781,813,824,825$

butyricum (Mycobacterium), 888, 890

butyricum I (Clostridium), 771

butyricum II (Clostridium), 771

butyricum III (Clostridium), 771

butyricum iodophilum (Clostridium), 772, 824 
butyricum var. americanum (Clostridium), 819

butyricus (Bacillus), $716,727,740,770$, 813,820

butyricus (Micrococus), 254, 338

butyricus (Streptococcus), 338

butyricus (Tetracoccus), 254

butyricus asporogenes immobilis (Bacillus), 790

butyricus dimorphus (Bacillus), 813

butyricus putrefaciens (Bacillus), 799

Butyrisaicina, 29, 30, 31, 285

byzantinea (Brucella), 693

byzantineum. (Coccobacterium), 693

cacaoi (Actinomyces), 951

cacaoi (Streptomyces), 951

Cacospira, 12, 13, 28, 1058

cacticida (Erwinia), 478

cacticidus (Bacillus), 478

cactivorum (Bacterium), 613

cadaveris (Bacillus), 669, 675, 791, 799

cadaveris (Bacterium), 675, 791

cadaveris (Clostridium), 791

cadaveris (Eubacterium), 367, 791

cadaveris (Plectridium), 799

cadaveris (Streptococcus), 338

cadaveris butyricum (Bacterium), 791

cadaveris butyricus (Bacillus), 367, 380,

791,826

cadaveris grandis (Bacillus), 813

cadaveris sporogenes (Bacillus), 799

cadaveris sporogenes (anaerobicus) ( $\mathrm{Ba}$ -

cillus), 799, 826

Caduceus, 33, 34, 763

caducus (Phagus), 1141

caeci (Bacillus), 650

caesia (Cellulomonas), 619

caesirae retortiformis (Spirochaeta), 1066

caesirae septentrionalis (Spirochaeta),

1066

caesius (Bacillus), 619

cajae (Coccobacillus), 690

cajus (Bacillus), 690

calceum (Ferribacterium), 834

calceum (Siderobacter), 834

calceus (Bacillus), 834 calciphila (Pseudomonas), 146

calciprecipitans (Pseudomonas), 108

calcis (Bacterium), 108

calcis (Pseudomonas), 108

calco-acetica (Pseudomonas), 146

calco-aceticus (Micrococcus), 255

calendulae (Bacterium), 133

calendulae (Phytomonas), 133

calendulae (Pseudomonas), 133

calfactor (Bacillus), 740

calidolact is (Bacillus), 732

calidus (Bacillus), 732

california (Salmonella), 505

californicus (Actinomyces), 936

californicus (Streptomyces), 936

calligyra (Spirochaeta), 1072

calligyrum (Treponema), 1072

callistephi (Chlorogenus), 1146

callistephi var. attenuatus (Chlorogenus),

1147

callistephi var. californicus (Chlorogenus), 1147

caloritolerans (Clostridium), 797

caloritolerans (Plectridium), 797

Calymmatobacterium, 14, 457

cameli (Actinomyces), 915

cameli (Oospora), 915

cameli (Streptothrix), 915

camelidae (Treponema), 1076

campeneus (Micrococcus), 255

campestre (Marmor), 1202

campestre var.armoraciae (Bacterium), 156

campestre var. galbinum (Marmor), 1202

campestre var. typicum (Marmor), 1202 campestris (Bacillus), 155

campestris (Bacterium), 155

campestris (Phytomonas), 155

campestris (Pseudomonas), 155

campestris (Xanthomonas), 155, 156, $160,164,178,1134,1136$

campestris var. armoraciae (Phytomonas), 156 ,

campestris var. armoraciae (Xanthomonas), 156, 164

canadensis (Bacillus), 759

canadensis (Bacterium), 759

canadiense (Clostridium), 819 
canalcnsis (Bacillus), 651

canaliculatus (Bacillus), 740

canalis (Bacterium), 675

canalis (Microspira), 202

canalis capsulatus (Bacillus), 675

canalis capsulatus (Bacterium), 675

canalis parvum (Bacterium), 675

canalis parvus (Bacillus), 675

canariensis (Bacillus), 530

canastel (Salmonella), 521

canceris (Bacilus), 740

cancrosi (Bacterium), 587

candicans (Achromobacter), 423

candicans (Albococcus), 255

candicans (Bacillus), 423

candicans (Bacterium), 423

candicans (Coccus), 261

candicans (Micrococcus), 251, 252, 255, $257,258,259,260,262,263,264,268$, $269,270,273,274,275,276,277,278$, $279,280,281$

candicans (Staphylococcus), 255

candida (Nocardia), 968

candida (Sarcina), 290

candida (Streptothrix), 934, 970

candidus (Actinomyces), 968

candidus (Bacterium), 401

candidus (Discomyces), 968

candidus (Micrococcus), 239, 251, 252,

$253,254,255,256,258,264,265,267$,

$269,270,271,272,274,275,278,279$,

281,282

candidus (Staphylococcus), 239, 282

canescens (Albococcus), 255

canescens (Micrococcus), 255

canescens (Sarcina), 290, 291

canescens (Staphylococcus), 255

caneus (Lactobacillus), 363

canicida (Bacterium), 553

canicola (Leptospira), 1077, 1079

canidae (Treponema), 1076

canina (Palmula), 812

canina (Spirochaeta), 1066

caninus (Acuformis), 812

caniperda (Bacillus), 740

canis (Actinomyces), 915

canis (Asterococcus), 1292 canis (Bacterium), 740

canis (Bartonella), 1104

canis (Cladothrix), 915

canis (Corynethrix), 406

canis (Ehrlichia), 1096

canis (Ehrlichia) (Rickettsia), 1095

canis (Haemobartonella), 1104, 1105, 1106

canis (Hemophilus), 587

canis (Nocardia), 915

canis (Oospora), 915

canis (Rickettsia), 1095

canis (Spirella), 217

canis (Spirochaeta), 1066

canis (Streptothrix), 915

canis (Tarpeia), 1272

canis familiaris (Pleuromyces), 915

canis lupus (Grahamella), 1110

caniseptica (Pasteurella), 553

cannabinus (Bacillus), 813

cannae (Bacterium), 171

cannae (Phytomonas), 171

cannae (Pseudomonas), 171

cannae (Xanthomonas), 171

cantabridgensis (Ascococcus), 250

canus (Bacillus), 651

canus (Micrococcus), 267

capillaceus (Bacillus), 740

capillorum (Micrococcus), 255

capillorum (Palmella), 255

capillorum (Palmellina), 255

capillorum (Zoogloea), 255

capillosa (Ristella), 577

capiliosus (Bacillus), 577

capitovale (Clostridium), 795

capitovalis (Bacillus), 795

capitovalis (Plectridium), 795

cappelletti (Streptococcus), 317

caprae (Actinomyces), 899

caprae (Cladothrix), 899

caprae (Discomyces), 899

caprae (Nocardia), 899

caprae (Oospora), 899

caprae (Streptothrix), 899

capri (Bacillus), 714

capriformis (Micrococcus), 255

caprinus (Streptococcus), 338 
capriseptica (Pasteurella), 553

caprisepticus (Bacillus), 553

caprogenes (Bacterium), 753

caprogenes foetidus (Bacillus), 753

caproicum (Clostridium), 820

capsaformans (Micrococcus), 255

capsici (Bacillus), 740

Capsularis, 33, 34, 577

capsulata (Klebsiella), 459

capsulata (Pseudomonas), 93, 146

capsulata (Rhodosphaera), 865

capsulatum (Acetobacter), 189

capsulatum (Bacterium), 459

capsulatum (Rhodobacterium), 863

capsulatum (Rhodonostoc), 864

capsulatus (Bacillus), 459

capsulatus (Bacterium), 691

capsulatus (Diplococcus), 308

capsulatus (Mycococcus), 891

capsulatus (Rhodococcus), 8, 865

capsulatus (Rhodopseudomonas), 864,

866

capsulatus (Rhodorrhagus), 865

capsulatus (Streptococcus), 308, 338

capsulatus aerogenes (Bacillus), 789, 790

capsulatus anaerobius (Bacillus), 790

capsulatus chinensis (Bacillus), 456

capsulatus gallinarum (Sireptococcus), 338

capsulatus margarineus (Diplococcus), 662

capsulatus mucosus (Bacillus), 459

capsulatus mucosus (Bacterium), 459

capsulatus pyaemiae cuniculi (Bacillus), 459

capsulatus septicus (Bacillus), 691

capsulatus septicus (Bacterium), 691

capsulatus septicus (Proteus), 691

carabiformis (Bacillus), 651

caraibica (Microspira), 636

caraibicum (Photobacterium), 636

carateum (Treponema), 1072

carbo (Micrococcus), 255

carbonei (Clostridium), 807

carbonei (Inflabilis), 807

carbonis (Bacillus), 776

Carboxydomonas, 20, 31, 972 cardiff (Salmonella), 512

cardii (Vibrio), 203, 205

cardii-papillosi (Cristispira), 1056

cardii papillosi (Spirochaeta), 1056

cardio-arthritidis, 320

cardiopyrogenes (Spirillum), 217

carduus (Coccus), 250

caricae (Marmor), 1201

caris (Bacillus), 816

carnea (Cladothrix), 968

carnea (Nocardia), 968

carnea (Oospora), 968

carnea (Sarcina), 290

carnea (Streptothrix), 968

carnegieana (Erwinia), 468

carneum (Bacterium), 675

carneus (Actinomyces), 968

carneus (Bacillus), 675

carneus (Discomyces), 968

carneus (Micrococcus), 255

carneus (Streptococcus), 338

carneus halophilus (Tetracoccus), 284

carnicolor (Bacillus), 675

carnicolor (Micrococcus), 255, 276

carniphilus (Bacillus), 740

carniphilus (Micrococcus), 255

carnis (Bacillus), 651, 810

carnis (Clostridium), 810

carnis (Plectridium), 810

carnis (Streptococcus), 338

carnis foetidum (Clostridium), 787

carnis saprogenes (Bacillus), 782

carnofoetidum (Clostridium), 787

carnosum (Bacterium), 675

carnosus (Actinomyces), 968

carnosus (Bacillus), 740

carocyanea (Pseudomonas), 146

carocyaneum (Bacterium), 146

carocyaneus (Bacillus), 146

carogeanui (Cohnistreptothrix), 928

carolina (Salmonella), 531

carolinus (Bacillus), 531

carotae (Phytomonas), 165

carotae (Pseudomonas), 165

carotae (Xanthomonas), 165

carotarum (Bacillus), 714

carotarum (Bacterium), 714 
carotovora (Erwinia), 469, 470, 471, 474,

$1129,1134,1135,1136$

carotovorum (Bacterium), 469

carotovorum (Pectobacterium), 464, 469

carotovorus (Bacillus), 469

carougeaui (Actinomyces), 928

carongeaui (Discomyces), 928

carougeaui (Nocardia), 928

carougeaui (Streptothrix), 928

carpanoi (Treponema), 1074

carpathiens (Bacillus), 361

Carphococcus, 235

Carpophthora, 1151

carrau (Salmonella), $52 \mathrm{~s}$

carrosus (Streptococcus), 338

carteri (Borrelia), 1061, 1068

carteri (Spirillum), 1061

carteri (Spirochacta), 1061

carteri (Spironema), 1061

carteri (Spiroschaudinnia), 1061

carteri (Treponema), 953

Carteria, 925

Carterii, 925

cartharinensis (Micrococcus), 255

cartilagineum (Bacterium), 675

cartilagineus (Bacillus), 675

Caryococeus, 1121

Caryophamon, 1004

caryophyllacearum (Bacillus), 6:39)

caryophylli (Phytomonas), 137

(aryophylli (Pseudomonas), 136

casei (Actinomyces), $96 \mathrm{~s}$

casei (Baclerium), $71 \mathrm{~s}$

casei (Cellulomonas), 619

casei (Lactobacillus), 356, 357

casei (Micrococcus), 210

casei (Planococcus), 281

casei (Plocamobacterium), 695

casei (Propionibacterium), 379

casei (Sarcina), 290

casei (Streptobacterium), 356, 361

casei (Streptococcus), 338

casei (Tetracoccus), 284

casei a (Bacillus), 356

casei $\gamma$ (Bacillus), 358

casei $\delta$ (Bacillus), 360

casei $\epsilon$ (Bacillus), 352 casei a (Bacterium), 356

casei $\epsilon$ (Bacterium), 352

casei acido-proteolyticus I (Micrococcus), 240,326

casei acido-proteolyticus II (Microcoscus), 240,326

casei amari (Micrococcus), 326

casei amari edamicus (Micrococcus), 256

casei filans (Bacterium), 354

casei limburgensis (Bacillus), 612

casei limburgensis (Bacterium), 612

casei liquefaciens (Micrococeus), 240, 326

casei liquefaciens (Tetracoccus), 240

casei proteolyticus I and II (Micrococcus), 327

caseia (Cellulomonas), 619

caseicola (Bacterium), 676

cascinicum (Achromobacter), 692

Caseobacterium, 8, 349

C'aseobacterium $\epsilon, 352$

Caseococcus, 327

caseolytica (Sarcina), 291

caseolyticum (Bacterium), 371

caseolyticus (Bacillus), 651

(aseolyticus (Micrococcus), 240, 258,

$259,260,263,265,266,268,273,695$

cassavac (Bacterium), 466

cassavae (Erwinia), 466

rastaneae (Bacterium), 138

castaneae (Phytomonas), 138

eastaneae (Pseudomonas), 138

castanicolum (Bacterium), 640

castellanii (Castellanus), 542

castellanii (Micrococcus), 255

castellanii (Rhodococcus), 255

castellanii (Shigella), 542

Castellanus, 535

castellum (Bacterium), 676

castigata (Cellulomonas), 615

castigatum (Bacterium), 615

castra (Vibrio), 203

catarrhalis (Actinomyces), 922

catarrhalis (Bacillus), 590

catarrhalis (Discomyces), 922

catarrhalis (Micrococcus), 298

catarrhalis (Neisseria), 298, 299, 301

catarrhalis (Oospora), 922 
catarrhalis (Pseudomonas), 146 Catenabacterium, 33, 34, 368 catenaforme (Catenabacterium), $36 \mathrm{~s}$ catenaformis (Bacteroides), 368 catenatus (Thermobacillus), 731 catenula (Bacillus), 676, 823 catenula (Bacterium), 676 catenula (Cornilia), 823 catenula. (Tyrothrix), 823 catenulatus (Bacillus), 740 catenulatus (Chondromyces), 1039 cathetus (Bacillus), 651 cati (Actinomyces), 969 cati (Discomyces), 969 cattleyae (Bacterium), 759 caucasica (Dispora), 351 caucasica (Pacinia), 351 caucasicum (Bacterium), 351 caucasicum (Betabacterium), 351, 358 caucasicus (Bacillus), 351, 358 caucasicus (Lactobacillus), 351 caucasicus (Streptococcus), 338 caudata (Pseudomonas), 174, 405 caudatum (Flavobacterium), 174 caudatus (Bacillus), 174, 405 caudatus (Bacterium), 174 caudatus (Coccus), 250

Caulobacter, 35, 832

cavarum pericarditis (Bacterium), 5.54

cavatum (Bacterium), 676

ravernae (Bacterium), 676

cavernae minutissimus (Bacillus), 676

caviae (Actinomyces), 912

caviae (Bacillus), 651

caviae (Bacteroides), 574,580

caviae (Bartonella), 1108

caviae (Cristispirella), 1057

caviae (Haemobartonella), $110 \mathrm{~S}$

caviae (Klebsiella), 548

caviae (Nocardia), 912

caviae (Pasteurella), 553, 651

caviae (Pseudomonas), 146

caviae (S'pherophorus), 574, 580

caviae (Spirochacta), 1071

caviae (Spironema), 1070

caviae (Spiroschaudinnia), 1070

caviae (Streptobacillus), $57 t$ caviae fortuitum (Bacterium), 676 caviae fortuitus (Bacillus), 676

cavicida (Bacillus), 445

cavicida (Bacterium), 445

cavicida (Escherichia), 445

cavicida havaniensis (Bacillus), 676

cavicida havaniensis (Bacterium), 676

caviseptica (Pasteurella), 553

cavisepticum (Bacterium), 553

cazaubon (Bacterium), 759

cazaubon $I$ and $I I$ (Bacterium), 759

celebense (Bacterium), 169

celebensis (Phytomonas), 169

celebensis (Pseudomonas), 169

celebensis (Xanthomonas), 169

celer (Phagus), 1142

cellaris (Leucocystis), 255

cellaris (Micrococcus), 255

cellasea (Cellulomonas), 618

cellaseus (Bacillus), 618

Cellfalcicula, 211

cellobioparus (Clostridium), 820)

cellulicola (Schinzia), 224

Cellulobacillus, 8, 705, 763

cellulolyticum (Plectridium), 823

Cellulomonas, 20, 32, 615, 616

cellulomonas (Proteus) var. Proteus biazoteus, 617

cellulomonas (Proteus) var. Proteus castaneus, 622

cellulomonas (Proteus) var. Proteus rossicus, 622

cellulomonas (Proteus) var. Proteus udus, 614

cellulosae (Actinomyces), 938

cellulosae (Clostridium), 820

(ellulosae (Streptomyces), 938

cellulosae dissolvens (Bacillus), 809

cellulosae dissolvens (Caduceus), 809

cellulosae hydrogenicus (Caduceus), 809

cellulosae hydrogenicus var. cellulosae

methanicus (Caduceus), 810

cellulosae methanicus (Caduceus), 810

cellulosam (Bacterium), 808

cellulosam fermentans (Bacillus), 808

cellulosis (Bacterium), 809

cellulosolvens (Caduceus), 809 
cellulosolvens (Clostridium), 809

cellulosum (Angiococcus), 1048

cellulosum (Polyangium), 1027, 1028

cellulosum (Sorangium), 1022

cellulosum fermentans (Terminosporus), 808

cellulosum var. ferrugineum (Polyangium), 1010, 1028, 1029

cellulosum var. fulvum (Polyangium), 1029

cellulosum var. fuscum (Polyangium), 1010,1028

cellulosum var. luteum (Polyangium), 1029

Cellvibrio, 209

Cenomesia, 847

centrale (Anaplasma), 1100

centralis (Bacillus), 651

centricum (Bacterium), $6 \mathbf{7} 6$

centrifugans (Bacil us), 101, 697

centrifugans (Bacterium), 697

centrifugans (Pseudomonas), 101, 697

centropunctatum (Achromobacter), $\$ 23$

centropunctatus (Bacillus), 423

centropunctatus (Bacterium), 423

centropunctatus (Micrococcus), 255

centrosporogenes (Bacillus), 787, 825

centrosporogenes (Clostridium), 787

Centrosporus, 20, 22

centrosporus (Bacillus), 725, 744

cepae (Bacillus), 740

cepae (Marmor), 1184

cephaloideus (Bacillus), 816

cepivora (Phytomonas), 470

cepivorum (Bacterium), 470

cepivorus (Aplanobacter), 470

cepivorus (Bacillus), 470

ceramicola (Bacterium), 626

ceramicola (Flavobacterium), 626

cerasi (Bacterium), 120

cerasi (Marmor), 1197

cerasi (Phytomonas), 120

cerasi var. prunicola (Pseudomonas),

120

cerasi wraggi (Bacterium), 153

cerasi wraggi (Phytomonas), 153

cerasinus (Micrococcus), 255, 256, 338 cerasinus (Streptococcus), 338

cerasinus lactis (Micrococcus), 255

cerasinus siccus (Micrococcus), 338

cerasus (Bacillus), 120

cerasus (Pseudomonas), 120

cereale (Lactobacillus), 355

cereale (Thermobacterium), 355

cerealia (Pseudomonas), 740

cerealium (Bacillus), 740

cerealium (Bacterium), 740

cerebralis (Corynebacterium), 403

cerebriformis (Actinomyces), 969

cerebriformis (Chondrococcus), 1046

cerebriformis (Myxococcus), 1046

cerebriformis (Nocardia), 969

cerebriformis (Streptothrix), 969

cereus (Actinomyces), 969

cereus (Bacillus), 708, 715, 716, 717, 718,

$719,725,1138$

cereus (Micrococcus), 256

cereus (Staphylococcus), 251

cereus albus (Micrococcus), 251

cereus albus (Staphylococcus), 251

cereus aureus (Micrococcus), 256

cereus aureus (Staphylococcus), 256

cereus flavus (Micrococcus), 256

cereus flavus (Staphylococcus), 256

cereus var. fluorescens (Bacillus),

716

cereus var. mycoides (Bacillus), 718

cereus var, siamensis (Bacillus), 716

cerevisiae (Flavobacterium), 176

cerevisiae (Lactobacterium), 363

cerevisiae (Merismopedia), 249

cerevisiae (Micrococcus), 249

cerevisiae (Pediococcus), 249

cerevisiae (Pseudomonas), 176

cerevisiae (Sarcina), 249

cerinum (Bacterium), 676

cerinus (Gluconoacetobacter), 694

cerinus (Micrococcus), 256

cerro (Bacterium), 529

cerro (Salmonella), 529

ceruminis (Bacillus), 403

reruminis (Corynebacterium), 403

cervina (Sarcina), 291

ceylonensis (Shigella), 542, 543 
ceylonensis $A$ (Bacillus), 540

ceylonensis $B$ (Bacillus), 542

ceylonensis $B$ (Lankoides), 542

chalcea (Actinomyces), 978

chalcea (Micromonospora), 959, 978

chalcea (Nocardia), 978

chalcea (Streptothrix), 978

chalmersi (Actinomyces), 975

chalmersi (Nocardia), 975

chamae (Cristispira), 1056

chamae (Spirochaeta), 1056

Charon, 1265

charrini (Bacillus), 651

charrini (Streptococcus), 338

chauvaei (Bacillus), 776

chauvaei (Clostridium), 776

chauvei (Clostridium), 776

Chauvoea, 20, 22, 763

chauvoei (Bacillus), 776

chauvoei (Bacterium), 776

chauvoei (Butyribacillus), 776

chauvoei (Clostridium), 776

chelonei (Mycobacterium), 886

chersonesia (Micrococcus), 256

chester (Salmonella), 504

chinense (Aerobacter), 456

chinense (Bacterium), 456

chinicus (Micrococcus), 256

chironomi (Bacterium), 635

chironomi (Photobacterium), 635

chitinochroma (Bacterium), 632

chitinophilum (Bacterium), 631

chitinovorus (Bacillus), 632

Chlamydothrix, 12, 18, 984

Chlamydozoon, 1114

chlorina (Pelogloea), 870

chlorina (Pseudomonas), 95

chlorinum (Bacterium), 95, 654, 676

chlorinus (Bacillus), 95, 651

chlorinus (Micrococcus), 256

Chlorobacterium, 693, 872

Chlorobium, 29, 30, 869

Chlorochromatium, 873

Chlorogenus, 1146

Chloronium, 873

Chloronostoc, 869

Chlorophaena (Pseudomonas), 146
Chloropseudomonas, 870

chlororaphis (Bacillus), 93

chlororaphis (Pseudomonas), 93

chlorum (Flavobacterium), 440

chocolatum (Chromobacterium), 693

cholerae (Baxillus), 194

cholerae (Bacterium), 547

cholerae (Phagus), 1142

cholerae (Vibrio), 194, 195, 1142

cholerae anatum (Bacillus), 552

choleraeasiaticae (Pacinia), 193

cholerae asiaticae (Spirillum), 193

cholerae asiaticae (Vibrio), 194

cholerae-caviae (Bacillus), 502

cholerae columbarum (Bacillus), 552

cholerae columbarum (Bacterium), 552

cholerae gallinarum (Bacillus), 547

cholerae gallinarum (Bacterium), 547

cholerae gallinarum (Micrococcus), 547

cholerae gallinarum (Octopsis), 547

cholerae gallinarum (Pasteureila), 547

cholerae-suis (Bacillus), 508

cholerae-suis (Bacterium), 508

cholerae-suis (Bacterium) (Salmonella), 508

choleraesuis (Salmonella), 493, 494, 496, 508,509

cholerae suis var. kunzendorf (Salmon. ella), 509.510

cholerae suum (Bacillus), 508

cholerae suum (Bacterium), 508

choleroides (Bacillus), 198

choleroides (Bacterium), 702

choleroides (Microspira), 198, 203

choleroides $\alpha$ and $\beta$ (Vibrio), 203, 702

cholesterolicum (Mycobacterium), 890

chologenes (Bacillus), 676

chologenes (Bacterium), 676

chondri (Streptothrix), 976

Chondrococeus, 1009, 1044

Chondromyces, 14, 17, 20, 24, 26, 1036

choukevitchi (Bacillus), 612

christiei (Bacterium), 759

christophersoni (Actinomyces), 975

christophersoni (Nocardia), 975

Chromatium, 16, 23, 25, 29, 30, 846, 852, $853,856,857,859$ 
chromidrogenus citreus (Micrococcus), 256 chromidrogenus ruber (Micrococcus), 256 chromoaromaticus (Bacillus), 657

chromo-aromaticus (Bacterium), 657

Chromobacterium, 20, 32, 37, 223, 231, 694

chromoflavus (Micrococcus), 256

chromogenes (Cladothrix), 969

chromogenes (Clostridium), 805

chromogenes (Oospora), 969

chromogenes $\beta$ alba, (Actinomyces) 934

chromogenus (Actinomyces), 934, 940, 969, 970,972

chromogenus 205 (Actinomyces), 941

chroococcum (Azotobacter), 219

chroococcus (Bacillus), 219

Chroostipes, 872

chrysanthemoides (Vibrio), 203

chryseum (Bacterium), 676

chryseus (Bacillus), 672

chryseus (Micrococcus), 256

chrysogloea (Bacterium), 676

chrysogloia (Bacillus), 676

chylogena (Eberthella), 533

chylogenes (Bacillus), 450, 533

chyluriae (Bacillus), 651

chymogenes (Bacterium), 452

cichorii (Bacterium), 133

cichorii (Phytomonas), 133

(ichorii (Pseudomonas), 125, 133

ciliatus (Lactobacillus), 363

Cillobacterium, 33, 34, 369

cincinnatus (Bacillus), 813

cinctus (Bacillus), 740

cinerea (Neisseria), 299, 301

cinereo-niger (Actinomyces), 969

cinereonigeraromaticus (Actinomyces),

969

cinereo-nigra (Nocardia), 969

cinereus (Micrococcus), 301

cinereus (Streptococcus), 338

cinereus niger aromaticus (Actinomyces), 969

cineronigra aromatica (Streptothrix), 969

rinnabareus (Micrococcus), 244, 255, 256,

257, 274, 275

cinnabareus (Rhodococcus), $24 t$

cinnabarinus (Micrococcus), 256 circulans (Bacillus), 722, 728, 737, 741, circulans (Bacterium), 722

circularis major (Bacillus), 580

circularis minor (Bacillus), 362

cirrhiformis (Micrococcus), 256

cirrhosus (Chondrococcus), 1045

cirrhosus (Mlyxococcus), 1045

cirroflagellosus (Bacillus), 741

cissicola (Aplanobacter), 134

(issicola (Pseudomonas), 134

Citivir, 1209

citrarefaciens (Bacterium), 119

citrarefaciens (Pseudomonas), 119

citrea (Nocardia), 908, 975

citrea (Sarcina), 288, 291

citrea (Streptothrix), 969

citrea conjunctivae (Sarcina), 291

citreum (Bacterium), 651, 676, 687

citreum (Semiclostridium), 762

citreus (Actinomyces), 946, 969

citreus (Ascobacillus), 651

citreus (Bacillus), 651, 676

citreus (Enterococcus), 336

citreus (Micrococcus), 239, 242, 254, 256

$257,261,265,268,278,280,338$

citreus $I$ (Micrococcus), 256

citreus II (Micrococcus), 256, 275

citreus (Mycococcus), 891

citreus (Planococcus), 288

citreus (Proactinomyces), 908

citreus (Staphylococcus), 242

citreus (Streptococcus), 338

citreus (Streptomyces), 946

citreus baregensis (Microbacillus), 690

citreus calaversis (Bacillus), 687

citreus cadaveris (Bacterium), 687

citreus conglomeratus (Diplococcus), 239

citreus conglomeratus (Merismopedia),

239

citreus conglomeratus (Micrococcus), 239 citreus duodenalis (Staphylococcus), 701 citreus granulatus (Micrococcus), 256, 277 citreus lactis (Micrococcus), 257

citreus liquefaciens (Diplococcus), 257

citreus liquefaciens (Micrococcus), 257

citreus rigensis (Micrococcus), 257

citri (Bacillus), 156 
citri (Bacterium), 156

citri (Phagus), 1135

citri (Phytomonas), 156

citri (Pseudomonas), 156

citri (Xanthomonas), 156, 112!), 1134,

1135,1136

citri deliciosae (Bacterium), 17s

citricus (Bacillus), 651

citrimaculans (Bacillus), 475

citrimaculans (Bacterium), 475

citrimaculans (Erwinia), 475

citrina (Sarcina), 291

citrinus (Bacillus), 651

citrinus (Micrococcus), 257

citriputeale (Bacterium), 119, 120,

citriputealis (Phytomonas), 120

citriputealis (Pseudomonas), 120

Citrobacter, $44 \mathrm{~S}$

citrocremeus (Actinomyces), 915

ritrophilum (Achromobacter), 610

citrophilum (L robacterium), 610, 691

citrophilus (Streptococcus), 339

citrovorum (Leuconostoc), $\mathbf{3 4 7}$

citrovorus (Streptococcus), 347

citrovorus-paracitrovorus (Streptococcus),

348

('ladascus, 12, 13

cladogenes (Bacillus), 651

cladoi (Bacillus), 741, 75s

Cladothrix, 6, 17, 18, 917, 980, 982, 1121

claibornei. (Salmonella), ,51s

clathratiforme (Aphanothece), 871

clathratiforme (Pelodictyon), 871, 872

Clathrochloris, 872

Clathrocystis, 6,847

clavatus (Bacillus), 400, 813

clavatus (Myxococcus), 1045

clavifer (Actinomyces), 969

clavifolium (Aureogenus), 1157

claviforme (Bacterium), 823

claviformis (Bacillus), 651, 823

claviformis (Micrococcus), 257

claviformis (Pacinia), 823

claviformis (Tyrothrix), 823

clemo (Pseudomonas), 694

cleoni (Bacillus), 652

Cloaca, 10 cloaca (Microspira), 202, 203, 206

cloacue (Actinomyces), 969

cloacae (Aerobarter), 455, 456, 45i, 460, 670,692

cloacae (Bacillus), 455,457

cloacae (Bacterium), 455

cloacae (Cloaca), 455

Clonothrix, 12, 17, 19, 26, 35, 983

clonotricoides (Mycothrix), 983

closteroides (Bacillus), 722, 741

clostridiformis (Bacterium), 576,57

clostridiformis (Ristella), 576

clostridiiformis mobilis (Zuberella), 57 i

clostridioides (Bacillus), 720

Clostridium, 11, 22, 27, 30, 31, 33, 42, 43, $76,216,367,763$

Clostrillium, 7, 705

Clostrinium, 7, 705

coadunata (Pseudomonas), 101, 697

coadunatum (Achromobacter), 101

coadunatus (Bacillus), 101

coadunatus (Bacterium), 697

coagulans (Bacillus), 531, 713

coagulans (Bacteroides), 567, 577

coagulans (Balkanella), 531

coagulans (Clostridium), 782

coagulans (Pasteurella), 567

coagulans (Salmonella), 531

cobayae (Bacillus), 714

cobayae (Borrelia), 1066

cobayae (Spirochaeta), 1066

cobayae (Treponema), 1066

coccacea (Pseudomonas), 146

cocciforme (Bacterium), 403, 69.3

cocciformis (Brucella), 693

coccineum (Thiospirillum), 856, 8.59

coccineus (Bacillus), 652, 741

coccineus (Micrococcus), 257

Coccobacillary bodies (Nelson), 1294

Coccobacillus, 546, 1291

Coccobacterium, $\mathbf{4 7 9}$

coccoides (Eperythrozoon), 1112, 1113

coccoides (Nitrosocystis), 72, 73

coccoideum (Achromobacter), 423

coccoideum (Bacterium), 423

coccoideus (Bacillus), 710

Coccomonas, 11 


\section{Coccothrix, 876}

cochlearium (Clostridium), 794, 799

cochlearium (Plectridium), 794

cochlearius (Bacillus), 794

cochlearius (Flemingillus), 794

cocoide (Bartonella), 1101

coeci (Bacillus), 416

coeliaca (Nocardia), 899, 906, 913

coeliacum (Flavobacterium), 906

coeliacum (Mycobacterium), 392, 906

cocliacus (Proactinomyces), 906

coelicolor (Actinomyces), 935

coelicolor (Bacillus), 401

coelicolor (Bacterium), 401

coelicolor (Nocardia), 935

coelicolor (Streptomyces), 935

coelicolor (Streptothrix), 935

coeln (Salmonella), 503

coelorhynchus (Coccobacillus), 636

coenobios (Pseudomonas), 697

cocrulea (Micromonospora), 980

coerulea (Pseudomonas), 234

coeruleo-viridis (Bacillus), 65:2

coeruleum (Chromobacterium), 234

coeruleus (Bacillus), 234, 652

coeruleus (Bacterium), 234

coffeicola (Bacillus), 639

cohaerea (Pseudomonas), 146, 697

cohaerens (Bacillus), 146, 632, 697, 718

cohaerens (Chromobacterium), 234

cohacreus (Bacterium), 697

Cohnia, 6, 847

cohnii (Spirillum), 1062

cohnii (Spirochaeta), 1062

cohnii (Spirochaete), 1054

Cohnistreptothrix, 925, 92!)

('oleomitus, 1003

Coleonema, 1003

Colesiota, 1119

coli (Aerobacter), 445

coli (Bacillus), 445

coli (Bacterium), 445, 460

coli (Colobactrum), 445

coli (Escherichia), 3, 444, 445, 447, 448,

$49,450,451,454,455,460,489,595$,

$694,1131,1132,1133,1134,1296$

coli (Phagus), 1133 coli (Pseudomonas), 146

coli (Streptococcus), 339

coli alcaligenes (Bacterium), 452

coli anaerogenes (Bacillus), 533

coli anaerogenes (Bacterium), 533, 542

colianaerogenes (Castellanus), 533

coli anindolicum (Bacterium), 452

coli apium (Bacterium), 676

coli brevis (Micrococcus), 257

coli brevis (Streptococcus), 339

coli citrovorum (Bacterium), 448

coli colorabilis (Bacillus), 612

coli colorabilis (Bacterium), 612

coli commune (Bacterium), 445

coli communior (Bacillus), 447

coli-communior (Bacterium), 447

coli communis (Bacillus), 445, 632, 697

coli communis (Pseudomonas), 697

coli communis verus (Bacillus), 445

coli dysentericum (Bacillus), 543

coli flavum (Bacterium), 445

coli gracilis (Streptococcus), 326

coli immobilis (Bacillus), 450

coli immobilis (Bacterium), 450

coli imperfectum (Bacterium, 452

coli mobilis (Bacillus), 148

coli mobilis (Bacterium), 699

coli mutabile (Bacterium), 451

coli mutabile (Escherichia), 451

coli mutabilis (Bacillus), 450

coli mutabilis (Escherichia), 451

coli similis (Bacillus), 676

coli similis (Bacterium), 676

coli var. acidilactici (Bacterium), 447

coli var. acidilactici (Escherichia), 447

coli var. communior (Bacterium), 448

coli var. communior (Escherichia), 447

coli var. immobilis (Bacterium), 450

coli var. luteoliquefaciens (Bacillus), 490

coli var. luteoliquefaciens (Bacterium), 490

coli var. neapolitana (Escherichia), 447

coli var. neapolitanum (Bacterium), 447

coli var. paragruenthali (Bacterium), 451

coli verus (Bacillus), 445

Colibacter, 694

Colibacterium, 11, 444 
colicogenes (Bacillus), 813

coliforme (Paracolobactrum) , 460, 491

Colloides, 595

colloides (Pseudomonas), 146

colloideum (Bacterium), 676

Colobactrum, 444, 453

colofoetida (Escherichia), 452

colofoetidus (Bacillus), 452

coloides (Escherichia), 452

coloides var. $A$ (Bacillus), 452

coloides var. $B$ (Bacillus), 452

colomatii (Bacterium), 759

colorabilis (Bacillus), 612

colorans (Bacillus), 741

coloratum (Propionibacterium), 379

colossus (Spirillum), 217

colotropicalis (Bacillus), 452

colotropicalis (Escherichia), 452

columbarum (Bacillus), 652

columbarum (Bacterium), 401, 552

columbense (Bacterium), 531

columbensis (Bacillus), 531

columbensis (Morganella), 531

columbensis (Salmonella), 531, 532

columnaris (Bacillus), 1047

columnaris (Chondrococcus), 1047

columnaris (Cytophaga), 1047

colurnae (Phytomonas), 139

colurnae (Pseudomonas), 139

comandoni (Spirochaeta), 1066

comandoni (Treponema), 1066

comes (Bacterium), 676

comesii (Bacillus), 741

comma (Bacillus), 194

comma (Microspira), 194

comma (Vibrio), 193, 196, 198, 199, 202,

203, 204, 205, 206, 1142, 1143

commensalis (Diplococcus), 257

commensalis (Micrococcus), 257

commune (Colibacter), 694

commune (Corynebacterium), 403

commune (Semiclostridium), 762

communior (Bacillus), 447

communior (Bacterium), 447

communior (Escherichia), 448

communior var. coscoroba (Bacillus), 453

communis (Bacillus), 445, 681 communis (Siderococcus), 835

communis lactis (Micrococcus), 257

commutabilis (Phagus), 1136

commutatus (Micrococcus), 257

compactum (Bacterium), 676

compactus (Bacillus), 676

compositum (Polyangium), 1023

compositum (Sorangium), 1008, 1022,

1023

concentricum (Bacterium), 676

concentricum (Spirillum), 217

concentricus (Micrococcus), 257

concitata (Cellulomonas), 619

concitatus (Bacillus), 619

concoctans (Bacillus), 741

concomitans (Bacillus), 230

concretivorus (Thiobacillus), 81

confervarum (Siderocystis), 834

confervarum (Sideromonas), $\mathbf{8 3 4}$

confluens (Micrococcus), 257

conglomeratum (Lactobacterium), 363

conglomeratus (Micrococcus), 239, 252 ,

$253,256,257,258,261,262,265,270$,

271,280

conglomeratus (Streptococcus), 315

congolensis (Actinomyces), 918

congolensis (Discomyces), 918

congregata (Sporocytophaga), 1049, 1050

Conidiothrix, 984, 995

conjac (Bacterium), 171

conjac (Phytomonas), 171

conjac (Pseudomonas), 171

conjaci (Xanthomonas), 171

conjunctivae (Chlamydozoon), 1119

conjunctivae (Colesiota), 1119, 1120

conjunctivae (Micrococcus), 257

conjunctivae (Rickettsia), 1096, 1119

conjunctivae (Sarcina), 291

conjunctivae bovis (Rickettsia), 1096, 1120

conjunctivae-galli (Colesiota), 1120

conjunctivae galli (Rickettsia), 1096, 1120

conjunctividis (Hemophilus), 585

conjunctividis (Micrococcus), 257

conjunctivitidis (Bacillus), $67 \mathbf{6}$

conjunctivitidis (Bacterium), 676, 675

conjunctivitidis subtiliformis (Bacillus), 741 
conjunctivitis (Bacterium), 590

connii (Achromobacter), 423

connii (Bacterium), 423

conoideus (Micrococcus), 257

conori (Dermacentroxenus), 1088 conorii (Rickettsia), 1087, 1088, 1092

conradi (Pseudomonas), 146

consolidus (Bacillus), 741

constans (Marmor), 1167

constellatum (Bifidibacterium), 369

constellatus (Bacillus), 369

constellatus (Diplococcus), $\mathbf{3 1 0}$

constrictus (Bacillus), 652

constrictus (Bacterium), 652

contextus (Bacillus), 741

continuosus (Streptococcus), 339

contumax (Phagus), 1136

convexa (Pasteurella), 571

convexa (Pseudomonas), 96, 697

convexus (Bacteroides), 571, $57 \overline{7}$

convoluta (Nocardia), 919

convoluta (Oospora), 919

convolutum (Mycobacterium), 919

convolutus (Actinomyces), 919

convolutus (Bacillus), 652

convoltus (Bacterium), 652

convolutus (Discomyces), 919

coprocinus (Bacillus), 753

coprogenes, (Bacillus) 813

coprogenes foetidus (Bacillus), 652, 813

coprogenes parvus (Bacillus), 544

coprogenese parvus (Bacterium), 544

coproliticus (Thiobacillus), 80

coprophila (Microspira), 202, 206

coprophilum (Bacterium), 819

coprophilum (Spirillum), 206

coprophilus (Bacillus), 819

coralinus (Rhodococcus), 258

corallina (Nocardia), 897, 902, 903, 904

corallina (Pseudomonas), 697, 1016

corallina (Serratia), 644, 902

corallinum (Clostridium), 820

corallinus (Micrococcus), 258

corallinus (Proactinomyces), 902

corallinus (Streptohrix), 902

coralloides (Chondrococcus), 1006, 1045,

1046 coralloides (Micrococcus), 258

coralloides (Myxococcus), 1045

coralloides var. clavatus (Chondrococcus), 1045

coralloides var. polycystus (Chondrococcus) 1045

Corium, 1203

corneola (Gallionella), 832

Cornilia, 705, 763

cornutum (Bifidibacterium), 36!

cornutus (Bacillus), 369

cornutus (Bacteroides), 369

coronafaciens (Bacterium), 116

coronafaciens (Phytomonas), 116

coronafaciens (Pseudomonas), 113, 116

coronafaciens var. atropurpurea (Phy-

tomonas), 116

coronafaciens var. atropurpurea (Pseu(domonas), 116

coronafaciens var. atropurpureum (Bacterium), 116

coronata (Microspira), 636

coronata (Siderocapsa), 834

coronatum. (Photobacterium), 636

coronalus (Bacillus), 652

coronatus (Micrococcus), 339

coronatus (Streptococcus), 339

coroniformis (Actinomyces), 969

corrugatus (Bacillus), 741

corrugatus (Micrococcus), 258

corruleo-viride (Bacterium), 652

corruscans (Bacillus), 741

corticale (Bacterium), 677

corticalis (Bacillus), 677

corvi (Bucillus), 652

corylii (Bacterium), 640

corylina (Phytomonas), 156

corylina (Xanthomonas), 156

Corynebacterium, 7, 17, 18, 21, 22, 23, 27,

$30,35,37,38,42,381,382,391,396,400$,

$401,403,404,405,407,435,612,615$,

$633,866,927$

Corynemonas, 8, 381

Corynethrix, 381, 407

Corynobacterium, 12, 13, 19, 28, 381

coryzae (Diplococcus), 258

coryzae (Micrococcus), 258 
coryzae contagiosae equorum (Streptococ-

cus), 317

coryzae segmentosus (Bacillus), 406

coscoroba (Bacillus), 453, 557, 552

coscoroba (Escherichia), 453

coscorobae (Bacterium), 453

costatus (Bacillus), 741

costicolus (Vibrio), 702

costicolus var. liquefaciens (Vibrio), 702

cotti (Microspironema), 1074

cotti (Treponema), $107 \pm$

couchi (Grahamella), 1110

courmontii (Bacillus), 65:2

couvyi (Leptospira), 1078

couvyi (Spirochaeta), $107 \mathrm{\aleph}$

Cowdria, 1094, 1097

Coxiella, 1092

crassa (Klebsiella), 459

crassa (Leptothrix), 985

crassa (Simonsiella), 1004

crasse (Caryophanon), 1004

crassum (Bacterium), 459

crassum (Plocamobacterium), 400

crassum (Spirillum), 203, 217

crassum (Thiospirillum), 851

crassus (Bacillus), 362, 400, 652

crassus (Diplococcus), 297, 301

crassus (Micrococcus), 301

crassus (Tibrio), 203

crassus aromaticus (Bacillus), 146

crassus pyogenes (Bacillus), 652

crassus pyogenes bovis (Bacillus), 652

crassus sputigenus (Bacillus), 459

crassus var. D (Tibrio), 203

crasteri (Vibrio), 204

craterifer (Actinomyces), 969

cremoides (Bacterium), 403

cremoides (Corynebacterium), 403

cremoides (Micrococcus), 253, 258

cremoides albus (Micrococcus), 258

cremoides aureus (Micrococcus), 253

cremoris (Bacillus), 709

"remoris (Streptococeus), 324, 325, 339,

$340,1138,1139$

cremorisviscosi (Micrococcus), 258

cremoris-viscosi (Staphylococcus), 258

crenatum (Bacterium), 677 crenatus, Thiobacillus, 81

Crenothrix, 6, 12, 17, 18, 19, 23, 26, 987

crepusculum (Micrococcus), 258

crepusculum (Monas), 258

cresologenes (Bacillus), 813

cresologenes (Clostridium), 813

cretacea (Oospora), 969

cretaceus (Actinomyces), 969

cretaceus (Micrococcus), 258

rretus (Caryococcus), 1121

criceti domestici (Grahamella), 1110

cricetuli (Grahamella), 1110

crinatum (Bacterium), 741

crinitus (Bacillus), 652, 741

cristalliferum (Bacterium), 677

cristallino violaceum (Bacterium), $23+$

cristatus (Arthromitus), 1003

cristatus (Micrococcus), 258

Cristispira, 12, 19, 20, 26, 28, 42,1055 ,

1056.

Cristispirella, 1069

crocatus (Chondromyces), 1006, 1036, 1038

crocea (Cytophaga), 1016

croci (Bacillus), 474

croci (Erwinia), 474

crocidurae (Spirochaeta), 1066

crocidurae (Treponema), 1066

cromogena (Streptotrix), 969

crouposa (Klebsiella), 458

cruciferarum (Marmor), 1176

cruciformis (Micrococcus), 258

cruciviae (Achromobacter), 103

cruciviae (Pseudomonas), 103

cruentus (Chondrococcus), 1042

cruentus (Myxococcus), 1042

cruoris (Actinomyces), 975

cruoris (Discomyces), 975

cruoris (Nocardia), 975

cruoris (Oospora), 975

crystalloides (Bacillus), $7+1$

crystaloides (Bacterium), 741

crystallophagum (Mycobacterium), $\$ 97$

crystallophagus (Actinomyces), 898

crystallophagus (Proactinomyces), s98

ctenocephali (Rickettsia), 1096

ctenocephali (Spirochaeta), 1066 


\section{cten-cuti INDEX OF NAMES OF GENERA AND SPECIES}

ctenocephali (Treponema), 1066

cubana (Salmonella), 527

cubensis (Bacillus), 741

cubensis (Spirochaeta), 1066

cubonianum (Bacterium), 135, 693

cubonianus (Bacillus), 135, 652

cuculi (Bacillus), 403

cuculi (Corynebacterium), 403

cuculliferum (Chromatium), 858

cucumeris (Lactobacillus), 356

cucumeris (Marmor), 1155, 1173

cucumeris fermentati (Bacillus), 356

cucumeris fermentati (Ulvina), 695

cucumeris var. commelinae (Marmor),

1174

cucumeris var. judicis (Marmor), 1173, 1174

cucumeris var. lilii (Marmor), 1174

cucumeris var. phaseoli (Marmor), 1174

cucumeris var. upsilon (Marmor), 1172

cucumeris var. vignae (Marmor), 1174

cucumeris var. vulgare (Marmor), 1174

cucumis (Vibrio), 204

cucurbitae (Bacterium), 157

cucurbitae (Phytomonas), 157

cucurbitae (Pseudomonas), 157

cucurbitae (Xanthomonas), 157

cuenoti (Bacillus), 652

culicis (Entomospira), 1066

culicis (Rickettsia), 1096

culicis (Spirillum), 1066

culicis (Spirochaeta), 1066

culicis (Spironema), 1066

culicis (Spiroschaudinnia), 1066

culicis (Treponema), 1066

cumini (Phytomonas), 121

cumini (Pseudomonas), 121

cumulatus (Micrococcus), 258, 278

cumulatus tenuis (Micrococcus), 278

cumulus minor (Coccus), 694

cuneatum (Bacterium), 776

cuneatus (Bacillus), 813

cuneatus (Vibrio), 199, 203, 205

cuniculi (Actinomyces), 578, 910, 928

cuniculi (Bacillus), 652

cuniculi (Bacterium), 402, 552

cuniculi (Cladothrix), 578, 928 cuniculi (Clostridium), 820

cuniculi (Cohnistreptothrix), 928

cuniculi (Corynebacterium), 403

cuniculi (Hemophilus), 589

cuniculi (Klebsiella), 459

cuniculi (Listerella), 409

cuniculi (Nocardia), 910, 928

cuniculi (Noguchia), 594

cuniculi (Oospora), 928

cuniculi (Pasteurella), 547

cuniculi (Spirochaeta), 1073

cuniculi (Streptococcus), 339

cuniculi (Streptothrix), 578, 928

cuniculi (Treponema), 1073, 1076

cuniculi pneumonicum (Bacterium), 552

cuniculi pneumonicus (Bacillus), 552

cuniculisepticus (Bacillus), 547, 652

cuniculicida (Bacillus), 547, 552

cuniculicida (Bacterium), 547, 681

cuniculicida (Pasteurella), 547

cuniculicida havaniensis (Bacillus), 612

cuniculicida havaniensis (Bacterium), 612

cuniculicida immobilis (Bacillus), 653

cuniculicida immobilis (Bacterium), 653

cuniculicida mobilis (Bacillus), 552

cuniculicida mobilis (Bacterium), 552

cuniculicida thermophilus (Bacillus), 681

cuniculicida thermophilus (Bacterium), 681

cuniculicida var. immobile (Bacterium), 653

cuniculorum (Micrococcus), 271

cunieuli (Leptotrichia), 366

cupularis (Bacillus), 612

cupularis (Micrococcus), 259

cupuliformans (Nanus), 1207

cupuliformis (Micrococcus), 259

cursor (Bacillus), 716

curtissi (Micrococcus), 259

curvum (Bacterium), 188

curvum (Rhizobium), 224

cuticularis (Bacillus), 677, 755

cuticularis (Bacterium), 677

cuticularis albus (Bacillus), 755

cutirubra (Pseudomonas), 110, 442

cutirubra (Serratia), 110

cutirubrum (Bacterium), 110 
cutirubrum (Flavobacterium) (Halobacterium), 110

cutis (Bacillus), 403

cutis (Corynebacterium), 403

cutis commune (Bacterium), 403

cutis communis (Bacillus), 403

cutis communis (Micrococcus), 251, 259

cutis communis (Staphylococcus), 259

cyaneofluorescens (Bacillus), 145

cyaneofluorescens (Pseudomonas), 145, 146

cyaneo-fuscus (Bacillus), 233

cyaneophosphorescens (Achromobacter) 634

cyaneo-phosphorescens (Bacillus), 634

cyaneo-phosphorescens (Vibrio), 634

cyaneum (Bacteridium), 259

cyaneum (Photobacterium), 634

cyaneus (Actinococcus), 923

cyaneus (Bacterium), 695

cyaneus (Micrococcus), 259, 272

cyaneus (Nigrococcus), 259

cyaneus (Proactinomyces), 923

cyaneus-antibioticus (Proactinomyces), 923

cyanofuscus (Bacterium), 233

cyanogenes (Pseudomonas), 92, 96

cyanogenes (Vibrio), 92

cyanogenum (Bacterium), 700

cyanogenus (Bacillus), 92, 96

cyanogenus (Micrococcus), 259, 695

cyanoides (Agarbacterium), 630

cyanophos (Micrococcus), 636

cyano-phosphorescens (Photobacterium),

634

cycloclastes (Archromobacter), 420

cycloclastes (Bacterium), 420

cyclops (Micrococcus), 259

cyclosites (Vibrio), 200

cygneus (Bacillus), 642

cygni (Bacterium), 642

cylindracea (Nocardia), 919

cylindracea (Oospora), 919

cylindraceus (Actinomyces), 919

cylindraceus (Discomyces), 919

cylindricus (Bacillus), 731

cylindricus (Chondromyces), 1038
Cylindrogloea, 873

cylindroides (Bacterium), 577

cylindroides (Ristella), 577

cylindrosporum (Clostridium), 789

cylindrosporus (Bacillus), 716, 718

cyprinicida (Bacterium), 642

cyprinicida (Klebsiella), 642

cypripedii (Bacillus), 470

cypripedii (Erwinia), 470

cystiformis (Bacillus), 653, 741

cystinovorum (Achromobacter), 416

cystiopoeus (Micrococcus), 259

cystitidis (Bacillus), 653

cystitidis (Streptococcus), 339

Cystobacter, 1025, 1034

Cystodesmia, 14, 1036

Cystoecemia, 14, 1021

cytaseum (Bacterium), 741

cytaseus (Bacillus), 741

cytaseus var. zonalis (Bacillus), 741

cytolytica (Erwinia), 473

Cytophaga, 35, 583, 1005, 1009, 1010, 1012

cytophaga (Spirochaeta), 1049

cytophagus (Micrococcus), 259

cytophagus (Mycococcus), 1013

czaplewskii (Bacterium), 590

dacryoideus (Bacillus), 653

Dactylocoena, 14, 1044

dacunhae (Achromobacter), 105

dacunhae (Pseudomonas), 105

dadhi (Streptothrix), 364

dahliae (Bacillus), 470

dahliae (Erwinia), 470

dahliae (Marmor), 1179

damnosus (Pediococcus), 250

damnosus (Streptococcus), 250

damnosus var. mucosus (Streptococcus), 250

danicus (Bacillus), 714

danteci (Bacillus), 742

dantecii (Micrococcus), 259

danubica (Microspira), 196

danubicum (Spirillum), 196

danubicus (Vibrio), 196

danysii (Salmonella), 517

dar-es-salaam (Salmonella), 519 
daressalaamensis (Salmonella), 519

dassonvillei (Actinomyces), 915

dassonvillei (Discomyces), 916

dassonvillei (Nocardia), 916

dassonvillei (Streptothrix), 934

daucarum (Bacillus), 742

daxensis (Spirochaeta), 1053

daytona (Salmonella), 513

de baryanus (Bacillus), 813

debile (Bacterium), 677

debilis (Streptococcus), 339

debilitans (Legio), 1257

decalvens (Bacterium), 259

decalvens (Micrococcus), 259

decidiosus (Bacillus), 441

decidiosus (Bacterium), 441

deciduosa (Cellulomonas), 621

deciduosum (Flavobacterium), 441

deciduosus (Bacillus), 621

decipiens (Micrococcus), 259

decipiens (Pacinia), 696

decolor (Bacillus), 653

decolor (Micrococcus) (Streptococcus ?), 259

decolorans (Aerobacter), 456

decolorans (Citrobacter), 448

decolorans major (Bacillus), 653

decolorans minor (Bacillus), 653

decussata (Nocardia), 975

decussata (Oospora), 975

decussatus (Actinomyces), 975

decussatus (Discomyces), 975

defessus (Bacillus), 653

deformans (Micrococcus), 259

deformans (Phagus), 1135

degenerans (Bacillus), 637

degenerans (Microspira), 637

degenerans (Photobacterium), 637

dehydrogenans (Flavobacterium), 613

dehydrogenans (Micrococcus), 613

delabens (Bacillus), 147

delabens (Bacterium), 698

delabens (Pseudomonas), 147, 697

delacourianus (Micrococcus), 259

delbrïckii (Bacillus), 355

delbruckii (Lactobacterium), 355

delbrueckii (Lactobacillus), 355, 695, 762 delbruecki (Plocamobacterium), 695 delbruecki (Ulvina), 695 delendae-muscae (Bacterium), 677 delesseriae (Bacterium), 625 delesseriae (Flavobacterium), 625 delgadense (Photobacterium), 637 delgadensis (Microspira), 637 delicatulum (Achromobacter), 419 delicatulus (Bacillus), 419 delicatulus (Bacterium), 419 delicatum (Corynebacterium), 403 deliense (Bacterium), 677, 759 delmarvae (Achromobacter), 422 delphinii (Annulus), 1216 delphinii (Bacillus), 115 delphinii (Bacterium), 115 delphinii (Pectobacterium), 696 delphinii (Phytomonas), 115 delphinii (Pseudomonas), 115 delta (Bacillus), 653 delta (Bacterium), 653 demmei (Bacillus), 742 demmei (Micrococcus), 270 dendriticum (Achromobacter), 423 dendriticus (Bacillus), 423 dendriticus (Bacterium), 423 dendrobii (Bacterium), 613, 640 dendroides (Bacillus), 718, 742 dendroporthos (Micrococcus), 259 denekei (Vibrio), 196 denekii (Pacinia), 196 denieri (Alcaligenes), 416 denitrificans (Bacillus), 423, 441, 442, 653,754

denitrificans $I$ (Bacillus), 440 denitrificans II (Bacillus), 426 denitrificans (Bacterium), 440, 688 denitrificans I (Bacterium), 440 denitrificans (Chromobacterium), 441 denitrificans (Flavobacterium), 440, 688 denitrificans (Micrococcus), 260 denitrifieans (Pseudomonas), 98 denitrificans (Sulfomonas), 80 denitrificans (Thiobacillus), 80 denitrificans (Vibrio), 426 denitrificans agilis (Bacillus), 422 denitrificans agilis (Bacterium), 423 
denitrificans fuorescens (Bacillus), 98

Denitrobacterium, 8

denitrofluorescens (Bacillus), 653

Denitromonas, 8, 83

dentale (Leptospira), 1078

dentalis viridans (Bacillus), 653

dentatus (Bacillus), 742

denticola (Spirochaeta), 1075

denticola (Spirochaete), 1075

denticola (Treponema), 1075

dentinum (Spirochaeta), 1075

dentium (Fusiformis), 581

dentium (Leptospira), 1079

dentium (Micrococcus), 339

dentium (Spirillum), 1075

dentium (Spirochaeta), 1065, 1070, 1074, 1075

dentium (Spirochaete), 1075

dentium (Spironema), 1075

dentium (Streptococcus), 339

dentium (Treponema), 1074

dentium-steogyratum (Treponema), 1075

deprimata (Cytophaga), 1013

derby (Salmonella), 505

derbyensis (Salmonella), 505

dermacentrophila (Rickettsia), 1096

dermacentroxenus (Rickettsia), 1087

dermatogenes (Micrococcus), 260

dermatogenes (Pseudomonas), 93

dermatonomus (Actinomyces), 916

dermoides (Bacillus), 653

dermophilum (Corynebacterium), 403

dermophilus (Bacillus), 403

derossii (Bacillus), 803

derossii (Clostridium), 803

desaiana (Phytomonas), 121

desaiana (Pseudomonas), 121, 125

desidens (Micrococcus), 339

desidens (Streptococcus), 339

desidiosa (Cellulomonas), 621

desidiosum (Flavobacterium), 441

desidiosus (Bacterium), 441

desiduosis (Bacillus), 441, 621

desmodilli (Pasteurella), 553

desmolyticum (Achromobacter), 104

desmolyticum (Pseudomonas), 104

destillationis (Bacterium), 576 destillationis (Ristella), 576

destructans (Bacterium), 470

destructans (Phytomonas), 470

destructans (Pseudomonas), 470

destruens (Bacillus), 742

Desulfovibrio, 29, 30, 35, 82, 207, 209

desulfuricans (Bacillus), 207

desulfuricans (Desulfovibrio), 207, 208,

209

desulfuricans (Microspira), 207

desulfuricans (Spirillum), 207

desulfuricans (Sporovibrio), 207, 208

desulfuricans (Vibrio), 207, 208, 853

Detoniella, 983

detrudens (Bacillus), 742

devorans (Bacillus), 430

devorans (Bacterium), 430

devorans (Flavobacterium), $\mathbf{4 3 0}$

devorans (Sarcina), 291

devorans (Vibrio), 204

dextranicum (Leuconostoc), 347

dextranicum (Streptobacterium), 701

dextranicus (Lactococcus), 347

dextrolacticus (Bacillus), 712

diacetilactis (Streptococcus), 339

diacetyl aromaticus (Streptococcus), 339

Dialister, 21, 27, 32, 33, 577, 594

Dialisterea, 20

dianthi (Bacillus), 640

dianthi (Bacterium), 640

dianthi (Pseudomonas), 640

diaphanus (Bacillus), 653

diaphthirus (Bacillus), 737, 793

diaporica (Rickettsia), 1092

diastasius (Thermobacillus), 731

diastaticus (Actinomyces), 939

diastaticus (Bacillus), 742

diastaticus (Streptomyces), 939

diastatochromogenes (Actinomyces), 941

diastatochromogenes (Streptomyces),

941

diatrypeticum (Bacterium), 677

diatrypeticus casei (Bacillus), 677

dichotoma (Cladothrix), 934, 975, 982

dichotoma (Nocardia), 975

dichotomus (Sphaerotilus), 982

dicksonii (Actinomyces), 969 
Dicrobactrum, 13, 14, 479

Dicrospira, 12, 13, 192

Dicrospirillum, $12,13,2 \$, 212$

didelphis (Spirochaeta), 1066

Didymohelix, 23, 26, 29, 831

dieffenbachiae (Bacterium), 157

dieffenbachiae (Phytomonas), 157

dieffenbachiae (Xanthomonas), 157

difficile (Clostridium), 773

difficilis (Bacillus), 773

diffuens (Bacillus), 490, 653

diffluens (Cytophaga), 1015

diffluens (Micrococcus), 260

diffuens (Proteus), 490

diffragens (Bacillus), 813

diffusum (Flavobacterium), 429

diffusus (Bacillus), 429

diffusus (Bacterium), 429

digestans (Bacillus), 730

digitatus (Bacillus), 65.3

digitatus (Myxococcus), 1045

dihydroxyacetonicum (Bacterium),

dilaboides (Bacillus), 720

dilatator (Caryococcus), 1121

dimorpha (Mycoplana), 191

dimorpha (Urosarcina), 294

dimorphobutyricus (Bacillus), s14

dimorphus (Bacillus), 352

dimorphus (Bacteroides), 352

dimorphus (Micrococcus), 260)

dimorphus var. longa (Bacillus), 352

diphtheria vitulorum (Oospoia), 57s

diphtheriae (Bacillus), 3\$3

diphtheriae (Bacterium), 383, 401

diphtheriae (Corynebacterium), 383, 384,

$385,406,752,1131,1143,1144$

diphtheriae (Phagus), 1143

diphtheriac avium (Bacillus), 400

diphtheriae avium (Bacterium), 400

diphtheriae avium (Mycobacterium), 915

diphtheriae columbarum (Bacillus), 401

diphtheriae columbarum (Bacterium), 401

diphtheriae cuniculi (Bacillus), 402

diphtheriae cuniculi (Bacterium), 402

diphtheriae ulcerogenes cutaneum (Cory.

nebacterium), 406

diphtheria vitulorum (Bacillus), 401, 5is diphthericum (Microsporon), 383

diphthericus (Micrococcus), 260

diphtheriticus (Streptococcus), 260, 337

diphtheroides (Bacillus), 401

diphtheroides (Coccobacillus), 402

diphtheroides (Corynebacterium), 388, 403

diphtheroides (Kokkobacillus), 402

diphtheroides brevis (Bacillus), 402

diphtheroides citreus (Bacillus), 406

diphtheroides gallinarum (Bacillus), 403

diphtheroides liquefaciens (Bacillus), 404, 405

Diplectridium, 7, 763

Diplobacillus, 590

Diplococcus, 13, 17, 20, 31, 33, 42, 305

Diplostreptococcus, 312

disciformans (Bacillus), 368, 742

disciformans (Bacterium), 742

disciformans (Eubacterium), 368

disciformis (Angiococcus), 1047

disciformis (Bacillus), 742

disciformis (Myxococcus), 1047

discofoliatus (Actinomyces), 927

discoides (Neisseria), 299

Discomyces, 925

discophora (Chlamydothrix), 985

discophora (Leptothrix), 985

discophora (Megalothrix), 985

dispar (Actinomyces), 918

dispar (Bacillus), 540, 542, 543

dispar (Bacterium), 542

dispar (Discomyces), 918

dispar (Eberthella), 42, 542

dispar (Eperythrozoon), 1113

dispar (Microsporon), 918

dispar (Proshigella), 542

dispar (Shigella), 542

dispar (Sporotrichum), 918

disparis (Streptococcus), 339

Dispora, 349

disporum (Clostridium), 820

dissimilis (Bacillus), 653

dissimilis (Micrococcus), 260

dissolvens (Aerobacter), 472

dissolvens (A planobacter), 472

dissolvens (Bacterium), 472

dissolvens (Clostridium), 43, 809, 810 
dissolvens (Erwinia), 464, 472

dissolvens (Phytomonas), 472

dissolvens (Pseudomonas), 472

Distasoa, 20, 21, 23, 27

distasonis (Bacteroides), $\mathbf{5 7 0}$

distasonis (Ristella), 570

distendens (Streptococcus), 347

distortus (Bacillus), 742

distortus (Tyrothrix), 742

diversum (Acetobacter), 692

diversum (Aerobacter), 456

diversum (Citrobacter), 448

djokjakartensis (Micrococcus), 260

dmitrovi (Spirochaeta), 1078

dobelli (Bacillus), 742

dobelli (Bacillus) (Flexilis), $7+2$

dodecahedron (Marmor), 1169

doederlein (Lactobacillus), 362

doederleinii (Acidobacterium), 362

domesticus (Bacillus), 653

domesticus (Bacterium), 653

donnae (Actinomyces), $911 \mathrm{i}$

Donovania, 559

dori (Actinomyces), 916

dori (Discomyces), 916

dori (Nocardia), 916

dori (Oospora), 916

dori (Rhinocladium), 916

dori (Sporotrichum), 916

doriae (Oospora), 934, 968

dormitator (Bacillus), 437

dormitator (Bacterium), 437

dormitator (Flavobacterium), 437

douglasi (Bacillus), $54 t$

douglasi (Shigella), 544

Douglasillus, 11, 763

dowdesuelli (Lrococcus), 282

doyeni (Bacillus), 651

doyeni (Micrococcus), 260)

drennani (Vibrio), 204

Drepanospira, 1122

drimophylus (Micrococcus), 260

droebachense (Bacterium), 625

droebachense (Flavobacterium), 625

droebachense (Pseudomonas), 625

droserae (Bacillus), 653

droserae (Bacterium), 65.3 drosophilae (Treponema), 1075

dschunkowskii (Grahamella), 1110

dschunkouski (Grahamia), 1110

dubia (Eberthella), 533

dubitata (Palmula), 812

dubitatus (Acuformis), 812

dubium. (Bacterium), 703

dubium (Marmor), 1172, 1214

dubium (Rhizobium), 225

dubius (Annulus), 1155, 1214

dubius (Bacillus), 533

dubius (Bacterium), 533

dubius (Phagus), 1137

dubius pneumoniae (Bacillus), 703

dubius var. annulus (Annulus), 1215

dubius var. flavus (Annulus), 1216

dubius var. obscurus (Annulus), 1216

dubius var. vulgaris (Annulus), 1215

dublin (Salmonella), 517

dublin var. accra (Salmonella), 517

dublin var. koeln (Salmonella), 517

duclauxii (Bacillus), 654, 742

duclauxii (Urobacillus), 654, 688, 729, 742

ducreyi (Coccobacillus), 587

ducrevi (Hemophilus), 587

dudtschenkoi (Grahamella), 1110

duesseldorf (Salmonella), 514

dulcito-fermentans (Bacillus), 772, 824

dunbari (Microspira), 203

dunbari (Photobacterium), 203

dunbari (Photospirillum), 203

dunbari (Vibrio), 702

duodenale (Bacterium), $44 \overline{7}$

duodenale (Encapsulata) (Bacillus), $\mathbf{H 7}$

duplex (Bacillus), 590

duplex (Bacterium), 590, 834

duplex (Ferribacterium), 834

duplex (Hemophilus), 591

duplex (Moraxella), 592

duplex (Pseudomonas), 147

duplex (Siderobacter), $\$ 34$

duplex (Siderocystis), 835

duplex (Sideroderma), $\$ 3 \mathrm{t}$

duplex josephi (Bacillus), 592

duplex josephi (Moraxella), 592

duplex liquefaciens (Bacillus), 5y1

duplex liquefaciens (Moraxella), 591 
duplex non-liquefaciens (Bacillus), 592 duplex non-liquefaciens (Bacterium), 592 duplex non liquefaciens (Moraxella), 592 duplex var. liquefaciens (Moraxella), 591 duplex var. non liquefaciens (Moraxella), 592

duplicatus (Bacillus), 693 duplicatus (Bacterium), 693 durabilis (Phagus), 1142 durans (Streptococcus), 327 durban (Salmonella), 519 duttoni (Cacospira), 1060 duttoni (Spirillum), 1060 duttoni (Spirochaeta), 1060

duttoni (Spirochaeta) (Microspironema),

1060

duttoni (Spironema), 1060

duttoni (Spiroschaudinnia), 1060)

duttoni (T'reponema), 1060

duttonii (Borrelia), 1060, 1061, 1064, 1066

dysenteriae (Bacillus), 536, 537, 538, 539, 540

dysenteriae (Bacterium), 536, 537, 689

dysenteriae (Eberthella), 536

dysenteriae (Phagus), 1132

dysenteriae (Shigella), 535, 536, 537, 542,

$1131,1132,1133,1134,1135$

dysenteriae Boyd I to III (Bacillus), 538 dysenteriae Flexner I to VI (Bacillus), 538 dysenteriae Flexner VII and TIII (Bacillus), 538

dysenteriae liquefaciens (Bacillus), 543 dysenteriae liquefaciens (Bacterium), 54.3 dysenteriae Schmitz (Bacillus), 536 dysenteriae vitulorum (Bacillus), 689 dysenteriae vitulorum (Bacterium), 689 dysentericus (Bacillus), 536, 543

Dysenteroides, 10

dysgalactiae (Streptococcus), 319 dysodes (Bacillus), 742

eastbourne (Salmonella), 519

eatonii (Micrococcus), 260

Eberthella, 10, 21, 26, 37, 42, 494, 516, 533

Eberthella sp. (Sendai type), 518

Eberthus, 10, 516 eburneus (Micrococcus), 260 echinata (Leptothrix), 985 eczemae (Micrococcus), 278 eczemicus (Bacillus), 654 edematis (Clostridium), 775 edgeworthiae (Bacillus), 478 edgeworthiae (Erwinia), 478 edigtoni (Neisseria), 301 efficiens (Borrelina), 1226 efficiens (Marmor), 1189, 1191 effrenus (Phagus), 1143 effusa (Cellulomonas), 91 effusa (Pseudomonas), 91 cffusa var.nonliquefaciens (Pseudomonas), 92 cffusus (Bacillus), 91, 718 egens (Bacillus), 790

egens (Clostridium), 790, 826 egens (Stoddardillus), 790 egregius (Bacillus), 654 egypticum (Treponema), 1065 Ehrenbergia, 37, 1052 chrenbergii (Bacillus), 597 chrenbergii (Bacterium), 597 Ehrlichia, 1096 ehrlichii (Grahamella), 1110 Eisenbergia, 12, 13, 486, 705 eisenbergii (Pseudomonas), 97, 98, 698 claphorum (Bacterium), 145 elastica (Actinomyces), 969 elegans (Bacillus), 742, 814 elegans (Flexibacter), 38 elegans (Holospora), 1122 elegans (Thiodictyon), 845, 849 elipsoideus (Bacillus), 654 cllenbachensis (Bacillus), 715 clienbachensis alpha (Bacillus), 715 ellenbachi (Bacillus), 717 ellingeri (Coccobacillus), 452 ellingeri (Escherichia), 452 ellingtonii (Bacillus), 654 ellipsoidea (Pseudomonas), 147 ellipsospora (Cytophaga), 1050 ellipsospora (Sporocytophaga), 1050 elongata (Pseudomonas), 698 elongata (Thiospira), 702 elusa (Spirochaeta), 1079 
elusum (Treponema), 1079

eminans (Bacillus), 654

emphysematis maligni (Bacillus), 791

emphysematis vaginae (Bacillus), 790, 826

emphysematosus (Bacillus), 7\$9, 790

emphysematosus (Bacterium), 790

emulsinus (Bacillus), 654

emulsionis (Bacillus), 742

enalia (Pseudomonas), 698

Encapsulatus, 10, 17, 18, 457

encephaloides (Bacillus), 742

enchelys (Bacillus), 677

enchelys (Bacterium), 677

endiviae (Phytomonas), 133

endiviae (Pseudomonas), 133

Endobacterium, 705

endocarditicus (Streptococcus), 339

endocarditidis (Bacillus), 654

endocarditidis (Bacterium), 677

endocarditidis capsulatus (Bacillus), 677

endocarditidis griseus (Bacillus), 654

endocarditidis griseus (Bacterium), 651

endocarditis (Cillobacterium), $36 ?$

endocarditis (Micrococcus), $2 \bar{\tau} t$

endocarditis griseus (Bacillus), 401

endocarditis rugatus (Micrococcus), 274

endometritidis (Bacillus), 677

endometritidis (Bacterium), 677

endometritis (Plocamobacterium), 675

endometritis canis (Bacterium), 677

endoparagogicum (Spirillum), 217

Endosporus, 33, 34, 763

englemanni (Bacillus), 654

enterica (Eberthella), 533

enterica (Escherichia), 450

entericus (Bacillus), 450, 533

entericus (Enteroides), 450, 534

entericus (Proteus), 489

enteritidis (Actinomyces), 920

enteritidis (Bacillus), 505, 516, 517, 7\$2,

818,920

enteritidis (Bacterium), 516

enteritidis (Discomyces), 920

enteritidis (Klebsiella), 516

enteritidis (Nocardia), 919

enteritidis (Oospora), 920

enteritidis (Phagus), 1136 enteritidis (Salmonella), 493, 497, 516,

$517,523,531,1130,1136,1137$

enteritidis (Streptococcus), 339

enteritidis (Streotothrix), 919, 976

enteritidis breslau (Bacterium), 502

enteritidis breslaviense (Bacillus), 502 enteritidis sporogenes (Bacillus), 782, 818 enteritidis sporogenes (Clostridium), 818 enteritidis-yellow (Salmonella), 531

enteritidis var. chaco (Salmonella), 517 cnteritidis var. danysz (Salmonella), 517 enteritidis var. dublin (Salmonella), 517 enteritidis var. essen (Salmonella), 517 enteritidis var. jena (Salmonella), 517 enteritidis var. moscow (Salmonella), 518 enteritidis var. mütheim (Salmonella), 517 enteritidis var. rostock (Salmonella), 518 enteritidis B, Typ. equinus (Bacillus), 506 cnteritidis B, Typ. murium (Bacillus), 502 enteritidis C, Typ. ovis (Bacillus), 506 enteritidis var. $V$ (Salmonella), 531

cnteritis (Streptococcus), 339

enteritis var. libmanii (Streptococcus), 339

Enterobacter, 31, 32, 37

Enterobacterium, 37

Enterococcus, 326, 336

cnterococcus (Diplococcus), 325

enterocoliticum (Bacterium), 677

Enteroides, 10

enteroideus (Micrococcus), 695

enteromyces (Bacillus), 654

enterothrix (Bacillus), $7 \pm 2$

Entomospira, 12, 13, 28, 1058

entomotoxicon (Bacillus), 654

enzymicum (Corynebacterium), 386, 407 enzymicus (Bacillus), 386

enzymothermophilus (Lactobacillus), 363 eos (Mycobacterium), 905

Eperythrozoon, 1100, 1111, 1113

Eperythrozoon spp., 1113

ephemerocyanea (Pseudomonas), $14 \overline{7}$

ephestiae (Micrococcus), 260

ephestiae No. 1 and No. 2 (Bacterium), 759

epidemicus (Streptococcus), 315

epidermidis (Albococcus), 243 
epidermidis (Bacillus), 742

epidermidis (Corynebacterium), 403, 406 epidermidis (Leptothrix), 691, 742

epidermidis (Micrococcus), 243, 252,

$254,255,256,259,264,265,270,271$,

272

epidermidis (Plocamobacterium), 691

epidermidis (Staphylococcus), 243

epidermidis var. A (Albococcus), 243

epidermidis albus (Micrococcus), 243, 268

epidermidis albus (Staphylococcus), 243

epidermis (Micrococcus), 278

Epidermophyton sp., 921

epimetheus (Micrococcus), 260

epiphitica (Chlamydothrix), 9.56

epiphytica (Leptothrix), 985

epiphytica (Lyngbya), 986

epiphytica (Streptothrix), 885

epiphytus (Bacillus), 743

eppingeri (Actinomyces), 896

eppingerii (Streptotrix), $\$ 96$

epsilon (Bacillus), $65+$

epsilon (Bacterium), 654

epsteinii (Achromobacter), 424

equae (Tortor), 1278

equarius (Streptococcus), 339

equatitis communis (Bacillus), 102

equestris (Malleomyces), 554

equi (Actinomyces), 920

equi (Bacillus), 654

equi (Bacterium), $5+1$

equi (Bollingera), 253

equi (Botryomyces), 253

equi (Corynebacterium), 391

equi (Corynethirix), 406

equi (Discomyces), 252, 920

equi (Mycobacterium), 391

equi (Nocardia), 920

equi (Sarcina), 290, 291, 292, 291

equi (Shigella), $5+1$

equi (Spirillum), 1066

equi (Spirochaeta), 1066

equi (Spironema), 1066

equi (Spiroschaudinnia), 1066

equi (Streptococeus), 317, 31s

equi (Treponema), 1066

equi intestinalis (Bacillus), $65+$ equi intestinalis (Bacterium), 654 equidistans (Bacillus), 1098

equina (Spirochaeta), 1066

equinus (Erro), 1253

equinus (Hostis), 1240

equinus (Streptococcus), 323, 339

equirulis (Bacillus), 541

equirulis (Shigella), 540

equiseptica (Pasteurella), 553

equiscpticus (Bacillus), 553

equisimilis (Streptococeus), 318, 314

equorum (Tortor), 1277

equorum (Trifur), 1282

equuli (Bacillus), $5+1$

erebea (Legio), 1259

erectum (Podangium), 1008, 1034

erectus (Chondromyces), $103+$

erectus (Cystobacter), 1034

eriobotryae (Bacterium), 144

eriobotryae (Phytomonas), 144

eriobotryae (Pseudomonas), 144

erivanense (Bacterium), 471

erivanensis (Bacillus), 471

erivanensis (Erwinia), 471

Ermengemillus, 11, 763

erodens (Bacillus), 743

erodens (Foliopellis), 1171

erodens (Marmor), 1171

erodens var. severum (Marmor), 1171

erodens var, vulgare (Marmor), 1171

erodii (Bacterium), 121

erodii (Phytomonas), 121

erodii (Pseudomonas), 121

Erro, 1248

erubescens (Bacillus), 654

Erwinia, 31, 42, 443, 463, 476

erysipelatis (Micrococcus), 315

erysipelatos (Staphylococcus), 315

erysipelatos (Streptococcus), 315, 1139

erysipelatos-suis (Bacillus), 410

erysipelatos-suis (Erysipelothrix), $\quad+10$

erysipelatos suum (Bacterium), $\mathbf{4 1 0}$

erysipelatosus (Streptococcus), 315

erysipeloides (Babesia), 411

erysipeloides (Streptothrix), 411

erysipeloidis (Actinomyces), $\$ 11$

erysipeloidis (Bacterium), 411 
erysipeloidis (Erysipelothrix), 411 crysipeloidis (Oospora), 411

Erysipelothrix, 18, 21, 22, 27, 28, 35, 37, $38,409,410$

erythematis (Bacillus), $7+2$

erythematis (Bacterium), $7+2$

erythematis maligni (Bacillus), 742

erythra (Pseudomonas), 147

erythraeus (Bacillus), $6 \pm 1$

erythrea (Nocardia), 920

erythrea (Streptothrix), 920

erythreus (Actinomyces), 938

erythreus (Streptomyces), $₫ 38$

Erythrobacillus, 10, 479

Erythrobacterium, 32

erythrochromogenes (Actinomyces), 944

erythrochromogenes (Streptomyces), 944

Erythroconis, 844

erythrogenes (Bacillus), 600

erythrogenes (Bacterium), 600, 601, 602

erythrogenes (Corynebacterium), 600

erythrogenes (Erythrobacillus), 600

erythrogenes rugatus (Bacillus), 651

crythrogloeum (Bacterium), 637

erythromyxa (Bacillus), 67

erythromyxa (Bacterium), 677, 6xi

erythromyxa (Micrococcus), 291

crythromyxa (Micrococcus) (Staphylo-

coccus), 392, 675

crythromyxa (Rhodococcus), 8, 67:

erythromyxa (Sarcina), 291

crythropolis (Actinomyces), s9s

erythropolis (Mycobacterium), 898

erythropolis (Nocardia), 898

erythropolis (Proactinomyces), 898

erythrospora (Pseudomonas), 147

erythrospores (Bacillus) (Streptobacter),

6054

erythrosporus (Bacillus), 147, 654

erythrosporus (Bacterium), 654

erzinjan (Salmonclla), 507

Escherichia, 3, 10, 21, 26, 31, 37, 443, 444,

$448,450,458,492,694$

escherichii (Bacillus), 445

essen 173 (Salmonella), 505

esseyana (Serratia). 484

esterificans (Bacillus), $13,7+3$ esterificans (Micrococcus), 260

esterificans (Plectridium), 743

esterificans fuorescens (Bacillus), 651

esterificans stralauense (Bacterium), 654

esteroaromaticum (Bacterium), 436

esteroaromaticum (Flavobacterium), 436

eta (Bacillus), 654

eta. (Bacterium), 654

ethaceticus (Bacillus), 654

cthacetosuccinicus (Bacillus), 6.5.5

ethylicum (Eubacterium), 367

cthylicus (Amylobacter), 813

etousae (Shigella), 544

Euacetobacter, 180

Euactinomyces, 929

Eubacillus, 9, 28

Eubacterium, 27, 25, 33, 34, 367

Euclostridium, 763

Eucystia, 13, 546

Eugluconobacter, 180

Eumyces, 876

euonymi (Marmor), 1187

euprima (Vibrio), 702

europaea (Nitrosomonas), 70

curopaea (Pseudomonas), 70

europaea var. italica (Nitrosomonas), 70)

europaeus (Planococcus), 70

eurydice (Achromobacter), 421, 724

eurydice (Bacterium), 421

eurygyrata (Borrelia), 1066

curygyrata (Spirillum), 1066

eurygyrata (Spirochacta), 1066

eurygyrata (Spiroschaudinnia), 1066

eurygyratum (Spironema), 1066

eurygyratum (Treponema), 1067

euryhalis (Micrococcus), 695

eurystrepta (Spirochaeta), 1052

eutetticola (Chlorogenus), 1219

evagatus (Charon), 1265

evanidus (Bacillus), 743

evansi (Brucella), 563

evolutus (Streptococcus), 332

exanthematica (Spirochaete), 1070

exanthematicum (Bacterium), 677

exanthematicus (Bacillus), 677

exanthematicus (.Micrococcus), 260

exanthematofebri (Richettsia), 1086 
exanthematotyphi (Rickettsia), 1084 exanthematotyphi (Spirochaeta), 1067 exanthematotyphi (Treponema), 1067 exapatus (Bacillus), 655

excavatus (Micrococcus), 260

excurrens (Bacillus), 716

exedens (Bacillus), 631

exfoliatus (Actinomyces), 951

exfoliatus (Streptomyces), 951

exigua (Ristella), 569, 576

exiguum (Bacterium), 590, 65 5

exiguus (Bacillus), 655, 743

exiguus (Bacteroides), 569, 576

exiguus (Micrococcus), 260

exiguus (Myxococcus), 1045

exiguus (Rabula), 1286

exilis (Bacillus), 352, 743

exitiosa (Phytomonas), 163

exitiosa (Pseudomonas), 163

exitiosum (Bacterium), 163

expositionis (Micrococcus), 260

expressus (Micrococcus), 261

fabae (Bacillus), 224

fabae (Phytomonas), 139

fabae (Pseudomonas), 139

fabae (Rhizobium), 224

faecalis (Alcaligenes), 413, 416

faecalis No.I (Bacillus), 755

faecalis No. II (Bacillus), 753

faecalis (Streptococcus), 325, 326, 336

faecalis alcaligenes (Bacillus), 413

faecalis var. mariense (Alcaligenes), $\$ 16$

faecalis var. radicans (Alcaligenes), 413

faecaloides (Bacillus), 544

faecaloides (Shigella), 541

jaecium (Streptococcus), 325

faeni (A erobacter), 456

fairmountense (Achromobacter), 90

fairmountensis (Bacillus), 90

fairmountensis (Bacterium), 698

fairmountensis (Pseudomonas), 90, 698

falciformis (Leptothrix), 366

fallax (Bacillus), 773

fallax (Clostridium), 773

fallax (Spirochaeia), 1069

fallax (Treponema), 106? fallax (Vallorillus), 773

famiger (Bacillus), 655

farcinibovis (Streptothrix), 895

farcinica (Cladothrix), 895

farcinica (Nocardia), 895

farcinica (Oospora), 895

farcinica (Streptothrix), 895

farcinicus (Actinomyces, 895

farcinicus (Bacillus), 895

farcinicus (Discomyces), 895

farnetianus (Bacillus), 477

faschingii (Bacterium), 459

fascians (Corynebacterium), 395

fascians (Phytomonas), 395

fasciformis (Bacillus), 360

fastidiens (Marmor), 1189

fastidiens var. denudans (Marmor), 1190

fastidiens var. fastidiens (Marmor), 1190

fastidiens var. mite (Marmor), 1190

fastidiens var. reprimens (Marmor), 1190

fastidiosus (Bacillus), 743

fausseki (Bacterium), 677

faviformis (Micrococcus), 261

febrilis (Spirochaeta), 1079

febris (Spirochaeta), 1067

fecale (Flavobacterium), 416

fecale aromaticum (Bacillus), 416

fecalis alcaligenes (Bacterium), 413

feddei (Micrococcus), 261

feiteli (Bacterium), 108

felidae (Treponema), 1076

felinus (Streptococcus), 339

felis (Bacillus), 655

felis (Bacterium), 553

felis (Cocco-bacterium), 655

felis (Miyagawanella), 1118

ielis (Pasteurella), 553

telis (Tarpeia), 1271

felis (Tortor), 1279

felis septicus (Bacillus), 553

felis septicus (Bacterium), 553

felsineum (Clostridium), 806

felsineus (Bacillus), 806

felsinus (C'lostridium), 806

felthami (Pseudomonas), 698

Fenobacter, 705

ferarum (I'asteurella), 547 
fermentans (Flavobacterium), 172

fermentans (Pseudomonas), 172

fermentatae (Lactobacillus), 358

fermentationis (Achromobacter), 424

fermentationis (Bacillus), 655

fermentationis (Bacterium), 424

fermentationis cellulosae (Bacillus), 809

fermenti (Lactobacillus), 359, 360

fermenti (Micrococcus), 340

fermenti (Streptococcus), 340

fermentosus (Wesenbergus), 534

fermentum (Lactobacterium), 360

ferophilum (Bacterium), 677

ferrarii (Pacinia), 690

ferrigenus (Bacillus), 743

ferruginea (Cellulomonas), 620

ferruginea (Chlamydothrix), 831

ferruginea (Didymohelix), 831

ferruginea (Gaillonella), 831

ferruginea (Gallionella), 831, 832

ferruginea (Gloeotila), 831

ferruginea (Itersonia), 1041

ferruginea (Nocardia), 975

ferruginea (Spirochaete), 831

ferruginea (Spirulina), 831

ferruginea (Toxothrix), 981

ferrugineum (Bacterium), 655

ferrugineum (Flavobacterium), 438

ferrugineum (Nodofolium), 831

ferrugineum (Polyangium), 1031

ferrugineum (Spirillum), $\$ 31$

ferrugineum (Spirophyllum), 831

ferrugineum (Spirosoma), 831

ferrugineus (Actinomyces), 975, 969

ferrugineus (Bacillus), 620,655

ferruginia (Gloeosphaera), 831

fertilis (Bacillus), 655

fervitosus (Micrococcus), 261

feseri (Bacillus), 776

feseri (Clostridium), 775, 776

festinus (Bacillus), 743

fetus (Spirillum), 201

fetus (Vibrio), 201

fibrosus (Bacillus), 814

fici (Bacterium), 610

fici (Phytomonas ?), 640

fichii (Micrococcus), 261 fickii (Pacinia), 690

figurans (Bacillus), 655, 718

figurans (Bacterium), $71 \mathrm{~S}$

fijiensis (Galla), 1157

filamentosa (Palmula), 800

filamentosum (Bacterium), 743

filamentosum (Catenabacterium), $36 \mathrm{~s}$

filamentosum (Clostridium), 800

filamentosum (Corynebacterium), 397,

920

filamentosus (Acuformis), 800

filamentosus (Bacillus), 743

filamentosus (Lactobacillus), 363

filaris (Bacillus), 743

filatim (Lactobacterium), 363

filefaciens (Achromobacter), 424

filefaciens (Bacterium), 424

filicolonicus (Bacillus), 743

filiforme (Bacterium), 759

filiforme (Clostridium), 792

filiformis (Actinomyces), 969

filiformis (Bacillus), 578, 718, 743, 969

filiformis (Caryophanon), 1004

filiformis (Leptothrix), 366

filiformis (Nocardia), 969

filiformis (Simonsiella), 1004

filiformis (Tyrothrix), 743

filiformis havaniensis (Bacillus), $6 \overline{7} 9$

filiformis havaniensis (Bacterium), 679

fima (Corynebacterium), 396

fimbriata (Pseudomonas), 147, 69s

fimbriatus (Actinomyces), 969

fimbriatus (Bacillus), 147

fimbriatus (Bacterium), 698

fimentaria (Sarcina), 291

fimi (Bacillus), 396

fimi (Bacterium), 396, 397

fimi (Cellulomonas), 396

fimicarius (Actinomyces), 940

fimicarius (Streptomyces), 940

finitimum (Bacterium), 678

finitimus ruber (Bacillus), 655, 67s

finitimus ruber (Bacterium), $67 \mathrm{~s}$

finkleri (Microspira), 197

finkleri (Pacinia), 197

finkleri (Spirillum), 197

finkleri (Vibrio), 197 
finlayensis (Micrococcus), 261

firmus (Bacillus), 713

fischeli (Streptococcus), 340

fischeri (Achromobacter), 633

fischeri (Bacillus), 633, 635

fischeri (Microspira), 633

fischeri (Photobacterium), 633, 634, 635,

636

fischeri (Vibrio), 633

Fischerinum, 13

fissum (Clostridium), $\mathbf{7 7 3}$

fissuratus (Bacillus), 743

fissus (Bacillus), 773

fitz (Bacterium), 771

fitzianum (Bacterium), 743

fitzianus (Bacillus), 743

Habelliferum (Clostridium), 783

flaccidifex (Gyrococcus), 250, 261

flaccidifex danai (Micrococcus), 261

flaccumfaciens (Bacterium), 399

Haccumfaciens (Corynebacterium), 398,

\section{9}

flaccumfaciens (Marmor), 1193

flaccumfaciens (Phytomonas), 399

flaccumfaciens (Pseudomonas), 399

flacheriae (Borrelina), 1227

flagellatus (Bacillus), 833

flagellatus (Micrococcus), 261

flagellifer (Bacillus), $7+3$

flava (Cellulomonas), 618

flava (Hydrogenomonas), 77, 78

flava (Neisseria), 298, 299

flava (Nocardia), 908

flava (Sarcina), 253, 288, 290, 291, 292, 293,294

flava (Streptothrix), 923, 969, 975

flava begoniae (Phytomonas), 155

flava varians (Merismopedia), 240

flavens (Micrococcus), 261

flaveolus (Actinomyces), 936

flaveolus (Streptomyces), 936

flavescens (Bacillus), 441, 669, 744

Havescens (Cellvibrio), 210

flavescens (Flawbacterium), $4+1$

flavescens (Micrococcus), 261

flavescens (Neisseria), 299

flavescens (Nocardia), 913
Alavescens (Pneumococcus), 697

flavescens (Proactinomyces),, 913

flavescens (Sarcina), 291

flavescens (Spirillum), 204

flavescens (S pirosoma), 204

flavescens (Vibrio), 203, 204

flaveum (Bakterium), 678

flavicula (Cytophaga), 1016

flavida (Erwinia), 471

Havidescens (Bacillus), 655

flavidum (Corynebacterium), 406

flavilus (Bacillus), 406, 471, 744

Havidus (Micrococcus), 261

flavidus alvei (Bacillus), 744

flavigena (Bacillus), 622

Havigena (Cellulomonas), 622

Flavimacula, 1202

Flavobacter, 427

Flavobacterium, 20, 31, 32, 81, 427, 440, $412,533,609,1296$

flavochromogenes (Actinomyces), 941

flavochromogenes (Streptomyces), 941

flavocoriaceum. (Bacterium), 678

flavocoriaceus (Bacillus), 678

flavocyaneus (Staphylococcus), 282

flavofuscum (Bacterium), 678

flavogriseus (Actinomyces), 969

flavoides (Bacillus), 655

Havotennae (Flavobacterium), 439

favovirens (Actinomyces), 940

flavovirens (Micrococcus), 261

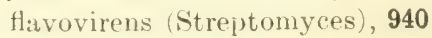

Havoviridis (Bacillus), 744

flarozonata (Bacterium), 155

flavozonatum (Xanthomonas), 155

flavum (Archangium), 1019

flavum (Bacterium), 678

flavum (Flavobacterium), 441

Havum (Microbacterium), 370

flavum (Mycobacterium), 370

flavum (Nitrobacter), 76

flavum (Polyangium), 1019

flavum (Semiclostridium), 762

flavum (Spirillum), 204

flavum (Spirosoma), 204

flavus (Actinomyces, 923, 945, 969, 970), 975 
flavus (Bacillus), 441, 655, 674, 710

favus I (Diplococcus), 299

flavus II (Diplococcus), 299

flavus (Micrococcus), 238, 252, 253, 256,

$257,258,259,261,262,266,268,270$,

$274,276,277,278,280,281$

flavus (Proactinomyces), 908

flavus (Streptomyces), 945

flavus (Vibrio), 203, 204

flavus conjunctividis (Micrococcus), 257

flavus desidens (Micrococcus), 339

flavus-liquefaciens (Diplococcus), 278

flavus liquefaciens (Micrococcus), 238

flavus liquefaciens tardus (Diplococcus), 278

flavus non liquefaciens (Micrococcus), 261 flavus non pyogenes (Staphylococcus), 282 flavus tardigradus (Micrococcus), 278

Flemingillus, 11, 763

fletcheri (Rickettsia), $10 \$ 6$

Flexibacter, 38

flexibilis (Spirochata), 1053

flexile (Bacterium), 717

Flexilis, 712

flexilis (Bacillus), 742,744, 1007

flexilis (Flexibacter), 38

Flexnerella, 535

flexneri (Bacillus), 537

flexneri (Bacterium), 537

flexneri (Eberthella), 53 i

flexneri (Shigella), 537

Rexuosa (Saprospira), 105.5

flexuosus (Bacillus), 655

flexuosus (Bacterium), 65.5

Flexus, 20, 22

Rexus (Bacillus), $7+4$

floccogenum (Lactobacterium), 363

floccosus (Bacillus), 579, 655

floccosus (Bacteroides), 579

floccosus (Spherophorus), 579

flocculens (Corynebacterium), 403

flocculus (Actinomyces), 955

floceulus (Streptomyces), 955

flurida (Salmonella), 52S

floridana (Pseudomonas), 698

floridana (Thiocapsa), $\mathbf{8 4 5}$

floridana (Thiospirillopsis),
Alueggei liquefaciens ( ${ }^{\top}$ rococcus) 266

flüggei (Bacillus), 814

Alügi (Bacillus), 716, 725

fluidificans (Bacillus), 655

fluidificans parvus (Bacillus), 655

fluidificans tardissimus (Bacillus), 670

fluitans (Chlamydothrix), 982

fluitans (Leptothrix), 982

fluitans (Sphaerotilus), 982

fluitans (Streptothrix), 982

Fluorescens, 32

fluorescens (Bacillus), 89, 656, 693, 716

fluorescens (Bacterium), 90

fuorescens (Micrococcus), 262

fluorescens (Proteus), 89

fluorescens (Pseudomonas), 90, 97, 145,

$147,394,649,693,698$

fluorescens (Streptococcus), $3 \pm 0$

fluorescens albus (Bacillus), 145, 656

fluorescens aureus (Bacillus), 146, 656

fluorescens aureus (Bacterium), 656, 697

fluorescens aureus (Pseudomonas), 145

fluorescens baregensis (Bacillus), 656

fluorescens capsulatus (Bacillus), 93

fuorescens convexus (Bacillus), 96

fluorescens convexus (Bacterium), 697

fluorescens crassus (Bacillus), 698

fluorescens crassus (Bacterium), 698

fuorescens exitiosus (Pseudomonas), 147

fluorescens foetidus (Diplococcus), 340

Muorescens foetidus (Streptococcus), 340

Aluorescens foliaceus (Bacillus), 147

fluorescens foliaceus (Bacterium), 698

fluorescens incognitus (Bacillus), 95

Auorescens incognitus (Bacterium), 698

fluorescens liquefaciens (Bacillus), 90,

$632,649,656$

Aluorescens liquefaciens (Bacterium), 698

fluorescens liquefaciens minutissumus

(Bacillus), 148, 656

fluorescens longus (Bacillus), 148, 658

fuorescens longus (Bacterium), 656, 699

fluorescens mesentericus (Bacillus), 148

fluorescens minutissimus (Bacterium), 699

fluorescens mutabilis (Bacillus), 693

fluorescens mutabilis (Bacterium), 693

fluorescens nivalis (Bacillus), 145, 693 


\section{fluo-frag INDEX OF NAMES OF GENERA AND SPECIES}

fluorescens nivalis (Bacterium), 693 fluorescens non-liquefaciens (Bacillus), $98,145,149$

fluorescens non-liquefaciens (Bacterium), 698

fluorescens ovalis (Bacillus), 97

fluorescens ovalis (Bacterium), 699

fluorescens putidus (Bacillus), 96, 148, 699

fluorescens putidus colloides (Bacillus), 146

fluorescens putridus (Bacillus), 96

fluorescens schuylkilliensis (Bacterium), 700

fluorescens septicus (Bacillus), $9 \cdot 4$

Aluorescens tenuis (Bacillus), 149, 656

fluorescens tenuis (Bacterium), 656, 701

fluorescens undulatus (Bacillus), 149, $7+4$

Fluoromonas, 11, 83

fluxum (Plectridium), 793, $\$ 26$

foedans (Bacillus), 367

foedans (Eubacterium), 367

foersteri (Actinomyces), 970

foersteri (Cladothrix), 970

foersteri (Cohnistreptothrix), 970

foersteri (Discomyces), 970

foersteri (Nocardia), 970

foersteri (Oospora), 970

foersteri (Streptothrix), 934, 970, 97

foetida (Bacterium), 531

foetida (Cornilia), 787

foetida (Escherichia), 531

foetida (Salmonella), 531

foetidissimus (Bacillus), 656

foetidum (Clostridium), 787, 820, 826

foetidum (Paraplectrum), 782, 825

foetidum (Plectridium), 782

foetidum carnis (Clostridium), 757, \$26

foetidum fecale (Clostridium), \$20

joetidum lactis (Clostridium), 818

foetidus (Bacillus), 534, 656, 787

foetidus (Endosporus), 782

foetidus (Micrococcus) , 262, 329, 339

foetidus (Streptococcus), 262, 329, 340

foetidus var. buccalis (Streptococcus), 329

foelidus clostriditiformis (Bacillus), 787

foetidus lactis (Bacillus), 660

foetidus liquefaciens (Bacillus), $42+, 655$ foetidus ozaenae (Bacillus), 658

foetidus ozaenae (Bacterium), 658

foetidus ozenae (Coccobacillus), 531

foetus ovis (Vibrio), 201

folia (Cellulomonas), $\mathbf{6 1 8}$

foliacea (Pseudomonas), 147, 698

foliaceus (Bacillus), 744

foliicola (Bacillus), 678

foliicola (Bacterium), 678, 685

forans (Spirochaeta), 1070

forans (Spirochate), 1070

forans (Treponema), 1070

fordii (Actinomyces), 958

fordii (Streptomyces), 958

formica (Escherichia), 452

formicum (Achromobacter), 452

formicum (Bacterium), 452

Formido, 1263

formosanum (Bacterium), 133, 1136

formosum (Achromobacter), 424

formosus (Bacillus), 424, 745

formosus (Bacterium), 424

fortis (Vibrio), 702

fortissimus (Bacillus), 656

fossicularum (Bacillus), 809, 814

foulertoni (Actinomyces), 920

foulertoni (D)iscomyces), 920

foulertoni (Nocardia), 920

foutini (Bacillus), 744

Fractilinea, 1159

fradiae (Streptomyces), 954

fiadii (Actinomyces), 954

fraenkelii (Bacterium), 145, 260

fiagariae (Blastogenus), 1207

fragariae (Marmor), 1195

fragariae (Nanus), 1207

fragariae $I$ (Pseudomonas), 100

fragariae II (Pseudomonas), 100, 101

fragaroidea (Pseudomonas), 100, 101

fragi (Bacterium), 100

fragi (Pseudomonas), 100

fragilis (Bacillus), 566, 801, 826

fragilis (Bacteroides), 32, 566, 575

fragilis (Fusiformis), 566

fragilis (Merismopedia), 262

fragitis (Micrococcus), 262

fragilis (Phagus), 1140 
fragilis (Recordillus), 823

fragilis (Ristella), 32, 566, 575

francai (Grahamella), 1110

frankei (Bacillus), 744

frankii var. majus (Rhizobium), 224

frankii var. minus (Rhizobium), 224

franklandiorum (Micrococcus), 262

fraxini (Pseudomonas), 132

freeri (Actinomyces), 896

freeri (Discomyces), 896

freeri (Nocardia), 896

freeri (Oospora), 896

freeri (Streptothrix), 896

frequens (Vibrio), 702

freudenreichii (Bacillus), $7 \notin t$

freudenreichii (Micrococcus), 238, 251

$252,257,260,263,264,265,266,269$,

$272,274,275,277,284$

freudenreichii (Propionibacterium) $374,375,376,377,378$

freudenreichii (Urobacillus), itt

freudi (Spherophorus), 579

freundii (Bacterium), 448, 678

freundii (Bacteroides), 579

freundii (Citrobacter), 448

freundii (Colobactrum), 448

freundii (Escherichia), 448, 460

friburgensis (Bacillus), 888,890

friburgensis (Mycobacterium), 888,890

friedebergensis (Bacillus), 530

friedebergensis (Bacterium), 530

friedlander (Bacterium), 458

friedlanderi (Bacillus), 458

friedlanderi (Coccobacillus), 458

friedlanderi (Klebsiella), 458

friedmanii (Bacillus), 886

friedmannii (Mycobacterium), 8\$3, 884 ,

$885,886,887,890$

fructovorans (Lactobacillus), 363

frutodestruens (Bacillus), 744

fucatum (Flavobacterium), 436

fuchsina (Serratia), 484, 701

fuchsinus (Bacillus), 484

fuchsinus (Erythrobacillus), 484

fuchsinus (Proteus), 701

fucicola (Bacterium), 627

fulgurans (Spirochaeta), 1053, 1054 fuliginosus (Bacillus), 656

fulminans (Bacillus), 717

fulva (Neisseria), 301

fulva (Sarcina), 291

fulvissimus (Actinomyces), 946

fulvissimus (Streptomyces), 946

fulvum (Bacterium), 605

fulvum (Flavobacterium), 605

fulvum (Rhodospirillum), $\mathbf{8 6 8}$

fulvus (Bacillus), 605, 656

fulvus (Cellvibrio), 210

fulvus (Galactococcus), 250

fulvus (Micrococcus), 262, 281, 1041

fulvus (Myxococeus), 1008, 1041

fulvus (Rhodococcus), 8, 258, 281, 1041

fulvus var. albus (Myxococcus), 1041

fulvus var. miniatus (Myxococcus), 1041

fumeus (Bacillus), 656

fumigatus (Bacillus), 612

fumosum (Polvangium), 1032

fumosus (Bacillus), 656

funduliformis (Bacillus), 566

funduliformis (Bacteroides), 566, 579, 1295

funduliformis (Spherophorus), 566, 579

fungiformis (Cladothrix), 983

fungoides (Bacillus), 678

fungoides (Bacterium), 678

fungosus (Bacillus), 656

funicularis (Bacillus), 744, 814

furcabilis (Cladascus), 13

furcosa (Ristella), 575

furcosus (Bacillus), 575

furcosus (Bacteroides), 575

furcosus (Fusiformis), 575

furens (Tortor), 1280

furvus (Bacillus), 744

fusca (Actinomyces), 924, 970

fusca (Cellfalcicula), 211

fusca (Clonothrix), 983, 987

fusca (Crenothrix), 983

fusca (Micromonospora), 979

fusca (Nocardia), 923

jusca (Oospora), 923

fusca (Sarcina), 291

fusca (Streptothrix), 923

fuscans (Bacillus), 656 


\section{fusc-gamm INDEX OF NAMES OF GENERA AND SPECIES}

fuscescens (Bacillus), 656

fuscescens (Bacterium), 657

fuscescens (Sarcina), 291

fuscum (Bacterium), 611, 678

fuscum (Chromobacterium), 611

fuscum (Flavobacterium), 611

fuscum (Polyangium), 1006, 1008, 1030

fuscum var, velatum (Polyangium), 1006, 1030

fuscus (Bacillus), 611, 650, 652, 657, 678, 680

fuscus (Cystobacter), 1030

fuscus (Discomyces), 923

fuscus (Micrococcus), 262, 277

fuscus (Vibrio), 204

fuscus limbatus (Bacillus), 656

fuscus limbatus (Bacterium), 657

fuscus liquefaciens (Bacillus), 680

fuscus liquefaciens (Bacterium), 680

fuscus pallidior (Bacillus), $6 \$ 3$

fuscus pallidior (Bacterium), $6 \$ 3$

Fusibucillus, 20, 22

fusiforme (Corynebacterium), 581

fusiforme (Rhabdochromatium), 854

Fusiformis, 13, 18, 19, 28, 30. 34, 38,

581,583

fusiformis (Bacillus), 581,728

fusiformis (Bacteroidea), 22

fusiformis (Fusiformis), 581

fusiformis (Rhabdomonas), 854

fusisporus (Bacillus) (Streptobacter), $74 t$

Fusobacterium, 18, 27, 37, 581, 583

Fusocillus, 34, 38, 581

fusus (Bacillus), 744

futilis (Phagus), 1144

gabritschewskii (Actinomyces), 970

gadi (Microspironema), 1067

gadi (Spirochaeta), 1064, 1066, 1068

gadi (Spironema), 1067

gadi (Treponema), 1067

gadi pollachii (Spirochaeta), 1069

gadidarum (Diplococcus), 289

gadidarum. (Pediococcus), 289

gaertner (Bacillus), 516

Gaffkia, 33, 34

Gaffkya, 29, 31, 34, 283, 284
Gaillonella, 831

Galactococcus, 235

galactophila (Escherichia), 452

galactophilum (Bacterium), 452

galba (Cellulomonas), 617

galbanatus (Micrococcus), 262

galbus (Bacillus), 617

Galla, 1157

galleriae (Bacterium), 759

galleriae No. 1 (Bacterium), 759 galleriae No.2 (Bacterium), 759 galleriae No. 3 (Bacterium), 760 galleriae (Streptococcus), 340

galli (Tortor), 1279

gallica (Spirochaeta), 1067

gallicidus (Micrococcus), 262

gallicolum (Treponema), 1075

gallicum (Treponema), 1067

gallicus (Actinomyces), 959

gallicus (Streptomyces), 959

gallii (Clostridium), 820

gallinae (Legio), 1262

gallinae (Pasteurella), 547

gallinae (Spirochaeta), 1058

gallinarum (Bacillus), 520, 530

gallinarum (Bacterium), 520

gallinarum (Borrelia), 1058

gallinarum (Corynebacterium), 403

gallinarum (Grahamella), 1110

gallinarum (Hemophilus), 589

gallinarum (Listerella), 409

gallinarum (Salmonella), 492, 493, 498 ,

$520,521,1135,1136,1137$

gallinarum (Shigella), 520

gallinarum. (Spirochaeta), 1058

gallinarum (Spironema), 1058

gallinarum (Treponema), 1058

gallinarum (Trifur), 1283

gallinarum var. duisburg (Salmonella), 521

gallinarum var. hereditaria (Spirochaeta), 1059

Gallionella, 12, 17, 35, 831, 832

galophilum (Achromobacter), 424

galtieri (Bacillus), 657

gaminara (Salmonella), 529

gamma (Bacillus), 678 
gamma (Bacterium), 678

gammari (Bacterium), 678

gangliforme (Bacterium), 744

gangliformis (Bacillus), 744

gangraenae (Bacillus), 744, 814

gangraenae carcinomatosae (Spirochaet a)

1067

gangraenae pulpae (Bacillus), 744

gangraenosa nosocomialis (Spirochaeta),

1067

gangraenosa nosocomialis (Treponema),

1067

gangrenae emphysematosae (Bacillus), 776

gangr. ovium (Micrococcus), 267

gangrenae pulmonaris (Streptobacillus)

368

gangrenosus (Ascococcus), 250

gardeniae (Phytomonas), 136

gardeniae (Pseudomonas), 136

gardneri (Nocardia), 914

gardneri (Proactinomyces), 914

garteni (Actinomyces), 975

garteni (Discomyces), 975

garteni (Nocardia), 975

garteni (Oospora), 975

gasoformans (Achromobacter), 424

gasoformans (Bacillus), 424, 657, 745

gasoformans (Bacterium), 424

gasoformans (Pseudomonas), 14i

gasoformans (Sarcina), 291

gasoformans non liquefaciens (Bac-

terium), 657

gasogenes (Bacillus), 812

gasogenes alcalescens anaerobius (Micro-

coccus), 303

gastrica (Escherichia), 451

gastricus (Bacillus), 451, 669

gastrochaenae (Christispira), 1056

gastrochaenae (Spirochaeta), 1056

Gastrococcus, 326

yastromycosis ovis (Bacillus), 775

gastrophilum (Bacterium), 352

gastrophilus (Bacillus), 352

gatuni (Salmonella), 515

gauducheau (Vibrio), 204

gayonii (Bacterium), 360

gayonii (Lactobacillus), 360 gaytoni (Bacillus), 657

gazogenes (Bacillus), 612

gazogenes (Micrococcus), 303

gazogenes (Plectridium), 812

gazogenes (Veillonella), 303, 304

gazogenes parvus (Bacillus), 812, 827

gazogenes var. gingivalis (Veillonella), 304

gazogenes var. minutissima (Veillonella), 304

gazogenes var. syzygios (Veillonella), 304

gedanensis (Actinomyces), 970

gedanensis (Discomyces), 970

gedanensis (Nocardia), 970

gedanensis (Streptothrix), 970

gedanensis I (Streptothrix), 934, 970

gelatica (Pseudomonas), 107

gelaticum (Bacterium), 107

gelaticus (Actinomyces), 952

gelaticus (Bacillus), 107

gelaticus (Streptomyces), 952

gelatinogenus (Micrococcus), 262, 348

gelatinosa (Lamprocystis), $8 \pm 6$

gelatinosa (Rhodocystis), 864

gelatinosa (Rhodopseudomonas), 864

gelatinosa (Thiosphaera), 846

gelatinosa (Thiothece), 846

gelatinosum (Clostridium), 762

gelatinosum betae (Bacterium), 657, 745

gelatinosus (Bacillus), 657, 745

gelatinosus (Micrococcus), 262

gelatinosus (Streptothrix), 977

gelatinum (Flavobacterium), $\$ 11$

gelatogenes (Bacillus), 655

gelechiae, No. 1, No. 2 and No. 5 (Bac-

terium), 678

gelida (Cellulomonas), 622

gelidus (Bacillus), 622

geminum (Achromobacter), 424

geminus major (Bacillus), 426

geminus major (Bacterium), 426

geminus minor (Bacillus), 424

geminus minor (Bacterium), 424

gemmiforme (Bacterium), 678

genesii (Actinomyces), 920

genesii (Nocardia), 920, 960

geniculata (Pseudomonas), 99, 69. 
geniculatum (Achromobacter), 99

geniculatum (Bacterium), 745

geniculatus (Bacillus), 99, 698, 709, 745

geniculatus (Bacterium), 698

geniculatus (Tyrothrix), 745

genitalis (Spirochaeta), 1072

genitalis (Treponema), 1072

genitalium (Encapsulatus), 459

genitalium (Klebsiella), 459

genitalium (Streptococcus), 340

georgia (Salmonella), 512

gephyra (Archangium), 1006, 1017, 1031,

\section{7}

geranii (Phytomonas), 166

geranii (Xanthomonas), 166

gerbilli (Grahamella), 1110

gerbilli (Grahamia), 1110

gerbilli (Listerella), 409

geton (Bacillus), 657

ghinda (Microspira), 20.4

ghinda (Vibrio), 204

ghoni (Clostridium), 820

giardi (Bacterium), 635

giardi (Photobacterium), 635

gibbonsi (Neisseria), 301

gibbosum (Bacterium), $67 \mathrm{~s}$

gibsoni (Bacillus), 690

gibsoni (Coccobacillus), 690

gibsonii (Actinomyces), 963, 970

gibsonii (Streptomyces), 963

gigantea (Beggiatoa), 991, 992, 995

gigantea (Leptothrix), 366

gigantea (Leptotrichia), 366

gigantea (Neisseria), 301

gigantea (Rasmussenia), 366

gigantea (Sarcina), 291

gigantea (Spirochaeta), 1053

giganteum (Bacterium), 760

giganteum (Clostridium), 820

giganteum (Rhodospirillum), 867

giganteum (Spirillum), 216, 217, 1053

giganteus (Flexibacter), 38

giganteus (Streptococcus), 340

giganteus lactis (Micrococcus), 262

giganteus urethrae (Streptococcus), 340

gigas (Achromatium), 998, 999

gigas (Bacillus), 657, 745, 778, 825 gigas (Clostridium), 778

gigas (Metallacter), 745

gigas (Micrococcus), 262

gigas (Spirobacillus), 218

gigas (Streptobacteria), 745

gigas-pericardii (Streptobacteria), 745

gilva (Cellulomonas), 620

gilvus (Bacillus), 620

gilvus (Micrococcus), 262, 277

gingivae (Bacillus?), 657

gingivae (Micrococcus), 262

gingivae (Streptococcus), 340

gingivae pyogenes (Bacillus), 678

gingivae pyogenes (Bacterium), 657, 678

gingivae pyogenes (Micrococcus), 262

gingivalis (Micrococcus), 304

gingivalis (Molitor), 1243

gingivitidis (Bacillus), 678

gingivitidis (Bacterium), 678

ginglymus (Bacillus), 745

gingreardi (Micrococcus), 262

gintottensis (Bacillus), 537

gintottensis (Castellanus), 537

gintottensis (Lankoides), 537

gintottensis (Shigella), $\mathbf{5 3 7}$

giumai (Bacillus), 544

giumai (Bacterium), 544

giumai (Salmonella), 544

giumai (Shigella), 544

giumai (Wesenbergus), 544

give (Salmonella), 522

glacialis (Bacillus), 612

glaciformis (Bacillus), 745

gladioli (Bacterium), 130

gladioli (Phytomonas), 130

gladioli (Pseudomonas), 130

glanders bacillus, 555

glandulae (Corynebacterium), 403, 404

glandulosus (Micrococcus), 262

glässer (Bacillus), $\mathbf{5 0 9}$

glauca (Nocardia), 980

glaucescens (Bacterium), 760

glaucus (Actinomyces), 980

glaucus (Bacillus), 657

glaucus (Bacterium), 657

gliscrogenum (Bacterium), 678

gliscrogenus (Bacillus), 678 
glis glis (Bartonella), 1108

glis glis (Haemobartonella), 1108

globerula (Nocardia), 903

globerulum (Mycobacterium), 903

globerulus (Proactinomyces), 903

globifer (Bacillus), 745

globiforme (Achromobacter), 408

globiforme (Bacterium), 407, 408

globigii (Bacillus), 710, 711

globosa (Micromonospora), 979

globosum (Propionibacterium), 379

globosus (Bacillus), 657

globosus (Micrococcus), 262

globulosa (Cytophaga), 1049

globulosus (Bacillus), 657

Gloeotila, 831

glomerata (Gallionella), 832

glomerata (Sideromyces), 986

glossinae (Borrelia), 1062

glossinae (Entomospira), 1062

glossinae (Spirillum), 1062

glossinae (Spirochaeta), 1062

glossinae (Spironema), 1062

glossinae (Spiroschaudinnia), 1062

glossinae (Treponema), 1062

glostrup (Salmonella), 514

gluconicum (Acetobacter), 189

gluconicum (Bacterium), 189, 682

Gluconoacetobacter, 180

Gluconobacter, 180

glutinis (Bacillus), 745

glutinosa (Microspira), 637

glutinosa (Ristella), 577

glutinosum. (Bacterium), 760

glutinosum (Photobacterium), 637

glutinosus (Bacillus), 577

glutinosus (Bacteroides), 577

glycerinaceus (Streptococcus), 325

glycinea (Phytomonas), 131

glycinea (Pseudomonas), 131, 135

glycinea var. japonica (Phytomonas), 132

glycinea var. japonica (Pseudomonas),

131

glycines (Bacterium), 161

glycines (Phytomonas), 161

glycines (Pseudomonas), 161

glycineum (Bacterium), 131 glycineum var. japonicum (Bacterium), 132

glycinophilus (Bacillus), 745

glycinophilus (Diplococcus), 694

glycologenes (Citrobacter), 449

gobii (Chromatium), 856

goensis (Actinomyces), 975

goensis (Nocardia), 975

goettingen (Salmonella), 520

gonadiformis (Actinomyces), 579

gonatodes (Bacillus), 745

gondi (Spirochaeta), 1064

gondii (Spirillum), 1064

gonidiaformans (Bacillus), 579

gonidiaformans (Bacteroides), 579

gonidiaformans (Spherophorus), 579

goniosporus (Bacillus), 716

gonnermanni (Bacillus), 657

gonnermanni (Bacterium), 678

Gonococcus, 295, 296

gonococcus (Micrococcus), 296

gonorrhoeae (Diplococcus), 296

gonorrhoeae (Merismopedia), 296

gonorrhoeae (Micrococcus), 296

gonorrhoeae (Neisseria), 296, 1296

goodsirii (Merismopedia), 286

gorini (Mammococcus), 695

gortynae (Bacillus), 657

gossypii (Ruga), 1218

gossypina (Bacillus), 745

gougeroti (Actinomyces), 934, 947

gougeroti (Streptomyces), 947

gracile (Bacterium), 362, 727

gracile (Chromatium), 858

gracile (Microsporon), 919

gracile (Rhabdochromatium), 854

gracile (Rhodospirillum), 867

gracile (Treponema), 1070

gracilescens (Bacillus), 657

gracilescens (Bacterium), 679

gracilior (Bacillus), 658

gracilipes (Chondromyces), 1035

gracilipes (Podangium), 1035

gracilis (Actinomyces), 970

gracilis (Bacillus), 657, 727

gracilis (Lactobacillus), 362

gracilis (Monas), 854 
gracilis (Pseudomonas), 147 gracilis (Rhabdomonas), 854 gracilis (Spirochaeta), 1070, 1076 gracilis (Streptobacillus), 581 gracilis (Streptococcus), 326 gracilis aerobiens (Bacillus), 658 gracilis anaerobiescens (Bacillus), 658 gracilis cadaveris (Bacillus), 658, 685 gracilis cadaveris (Bacterium), 658 gracilis ethylicus (Bacillus), 367, 814 gracilis putidus (Bacillus), 575 gracillimum (Bacterium), 679

Graciloides, 10

Graham sp., 1110

Grahamella, 37, 1100, 1109

Grahamella sp., 1109, 1110

Grahamia, 1109

graminarium (Nocardia), 970

graminarium (Streptothrix), 970

graminea (Spirochaeta), 1053

graminea marina (Spirochaeta), 1053

graminearum (Actinomyces), 970

graminearum (Streptothrix), 934

graminis (Actinomyces), 970, 971

graminis (Marmor), 1192

graminis (Mycobacterium), 888, 890

grandis (Bacillus), 658

grandis (Fusiformis), 694

grandis (Gafflya), 284

grandis (Saprospira), 1054

granii (Achromobacter), 199

granii (Bacterium), 199

granii (Vibrio), 199

granula (Amoebobacter), $\mathbf{8 5 0}$

granularis (Bacillus), 745

granulata (Pseudomonas), 147

granulatum (Bacterium), 679, 718

granulatum (Thiospirillum), 212

granulatus (Bacillus), 658, 814

granulatus (Micrococcus) , 256, 263, 277

granulatus (Streptococcus), 340

granuliformans (Bacterium), 595

granuliformans (Dialister), $\mathbf{5 9 6}$

Granulobacillus, 763

Granulobacillus sp., 822

Granulobacter, 763 granulobacter pectinovorum (Bacillus), 780

granulomatis (Calymmatobacterium), 459 granulomatis (Donovania), $\mathbf{5 5 9}$

granulomatis (Klebsiella), 459

granulomatis maligni (Corynebacterium), 403

granulosa (Spironema), 1059

granulosa (Spiroschaudinnia), 1059

granulosa penetrans (Spirochaeta), 1059

granulosis (Bacterium), 593

granulosis (Noguchia), $\mathbf{5 9 3}$

granulosum (Bacterium) , 362, 679, 693, 745

granulosum (Corynebacterium), 388, 404

granulosum (Plocamobacterium), 693

granulosus (Bacillus), 658, 745, 814

granulosus (Micrococcus), 623

graphitosis (Bacillus), 757

grassi (Treponema), 1070

grassii (Entomospira), 1070

grassii (Spirochaeta), 1069

grave (Lactobacterium), 363

graveolens (Bacillus), 42, 658, 714

graveolens (Bacterium), 658

graveolens (Pseudomonas), 179

grawitzii (Bacillus), 401, 658

grawitzii (Bacterium), 401, 658

grigoroffi (Micrococcus), 247

grippo-typhosa (Leptospira), 1078

griseoflavus (Actinomyces), 948, 975

griseoflavus (Streptomyces), 948

griseolus (Actinomyces), 938

griseolus (Streptomyces), 938

griseum (Bacterium), 263

griseus (Actinomyces), 948, 977

griseus (Bacillus), 658, 695

griseus (Micrococcus), 263, 695

griseus (Staphylococcus), 282

griseus (Streptomyces), 948

griseus radiatus (Staphylococcus), 282 groningensis (Nitrosomonas), 76

grossa (Microspira), 204

grossus (Bacillus), 745

grossus (Micrococcus), 263

grossus (Tibrio), 204

grotenfeldtii (Bacterium), 447

grotenfeltii (Streptococcus), 324 
gruberi (Actinomyces), 970

gruberi (Bacillus), 771

gruberi (Nocardia), 970

gruberi (Oospora), 970

grueberi (Streptothrix), 970

gruenthali (Bacillus), 451

gruenthali (Bacterium), 451

gruenthali (Escherichia), 451

grumpensis (Salmonella), 527

gryllotalpae (Bacillus), 745

gryllotalpae (Bacterium), 679

guano (Bacillus), 745

guegueni (Actinomyces), 922

guegueni (Discomyces), 922

guegueni (Nocardia), 922

guignardi (Actinomyces), 970

guignardi (Oospora), 934, 970

guillebeau $a, b$, and $c$ (Bacillus), 457

guilliermondii (Oscillospira), 1004

gulosus (Bacteroides), 573, 579

gulosus (Sphaerophorus), 573, 579

gummis (Bacillus), 145, 466

gummis (Bacterium), 145

gummisudans (Bacterium), 167

gummisudans (Phytomonas), 167

gummisudans (Pseudomonas), 167

gummisudans (Xanthomonas), 167

gummosum (Bacterium), 679, 745

gummosus (Bacillus), 679, 745

gummosus (Micrococcus), 262, 263, 348,

678

güntheri (Bacillus), 326

güntheri (Bacterium), 323

güntheri (Streptococcus), 324

gutta cerei (Pneumococcus), 697

guttatum (Achromobacter), 424

guttatus (Bacillus), 424

guttatus (Bacterium), 424

gutturis (Serratia), 484, 485

gypsoides (Actinomyces), 897

gypsoides (Discomyces), 897

gypsoides (Oospora), 897

gypsophilae (Agrobacterium), 229

gypsophilae (Bacterium), 230

gypsophilae (Phytomonas), 230

gypsophilae (Pseudomonas), 230

Gyromorpha, 1111 habanensis (Staphylococcus), 282

hachaizae (Spirillum), 217

hachaizae (Spirochaeta), 217

hachaizae (Treponema), 217

hachaizicum (Spirillum), 217

haeckeli (Pedioplana), 250

haematodes (Micrococcus), 263

haematoides (Bacillus), 658

haematoides (Bacterium), 658

haematosaprus (Micrococcus), 272

Haemobartonella, 1102, 1107, 1108

Haemobartonella spp., 1108

haemoglobinophila sporulens (Leptothrix), 366

haemoglobinophilus (Bacterium), 587

haemoglobinophilus (Hemophilus), 585, 687

haemoglobinuriae (Leptospira), 1078

haemoglobinuriae (Spirochaeta), 1078

haemolysans (Clostridium), 820

Haemophilus, 17, 26, 37, 584, 1289, 1290

haemophilus (Spirochaeta), 1067

haemophosphoreum (Brucella?), 634

haemorrhagicum (Bacterium), 552

haemorrhagicus (Bacillus), 552

haemorrhagicus (Micrococcus), 263

haemorrhagicus (Staphylococcus), 263

haemorrhagicus nephritidis (Bacillus), 553

haemorrhagicus nephritidis (Bacterium), 553

haemorrhagicus septicus (Bacillus), 552 haemorrhagicus septicus (Baclerium), 553 haemorrhagicus velenosus (Bacillus), 553 haemorrhagicus velenosus (Bacterium), 553

haemotosaprus (Streptococcus), 340

hajeki (Bacillus), 658

halans (Bacillus), 679

halans (Bacterium), 679

halensis (Micrococcus), 263

halestorgus (Pseudomonas), 147

halitus (Streptococcus), 340

halmephilum (Flavobacterium), 441

Halobacterium, 442

halobicus (Bacillus), 658

halobicus desulfuricans (Vibrio), 204 
halobium (Bacterium), 422

halobium (Flavobacterium)

ium), 442

halobius ruber (Bacillus), 422

halohydrium (Flavobacterium), 431

halohydrocarbonaclasticus

(Desulfovi-

brio), 209

halonitrificans (I'ibrio), 702

halophilica (Bacterium), 110

halophilica (Spirochaeta), 110

halophilum (Achromobacter), 424

halophilum (Flavobacterium), 441

halophilus (Bacillus), 658

halophilus (Bactcrium), 658

halophilus (Micrococcus), 263

halophilus (Pediococcus), 250

haloplanktis (Vibrio), 703

haloseptica. (Ristella), 575

halosepticum (Bacterium), 575

halosmophila (Ristella), 576

halosmophilus (Bacteroides), 576

halotrichis (Actinomyces), 970

halstedii (Actinomyces), 953

halstedii (Streptomyces), 953

hamaguchae (Sarcina), 292

hansenianum (Bacterium), 181

harai (Bacillus), 745

harrisonii (Bacillus), 694

harrisonii (Flavobacterium), 434, 694

hartford (Salmonella), 511

hartlebii (Achromobacter), $42 t$

hartlebii (Bacillus), 424

hartlebii (Bacterium), 424

hartmanni (Spirochaeta), 1074

hartmanni (Spironema), 1071

hartmanni (Treponema), 1074

hartwigi (Modderula), 999

harveyi (Achromobacter), 110

harveyi (Pseudomonas), 110

hastiforme (Clostridium), 785

hastilis (Bacillus), 583

hastilis (Mycobacterium), 583

haumani (Bacillus), 814

haumanni (Bacillus), 814

haumanni (Clostridium), 814

hauseri (Diplococcus), 263

hauseri (Micrococcus), 263 havana (Salmonella), 527

havaniensis (Bacillus), 658

havaniensis (Bacillus) (Micrococcus?), 918

havaniensis (Bacterium), 918

havaniensis (Serratia), 918

havaniensis (Streptococcus), 340

havaniensis liquefaciens (Bacillus), 658

havaniensis liquefaciens (Bacterium), 658

Haverhillia, 38, 588, 972

hayducki (Bacillus), 359, 693

hayducki (Plocamobacterium), 693

hayduckii (Lactobacillus), 359

heali (Bacterium), 604

healii (Achromobacter), 604

healii (Escherichia), 604

hebdomadis (Leptospira), 1077, 1078, 1079

hebdomadis (Spirochaeta), 1077

hebdomadis (Spiroschaudinnia), 1077

hebdomadis (Treponema), 1077

hebetisiccus (Bacterium), 679

hederae (Bacterium), 166

hederae (Phytomonas), 166

hederae (Xanthomonas), 166

hegneri (Grahamella), 1110

hegneri (Grahamia), 1110

heidelberg (Salmonella), 504

heimi (Actinomyces), 936, 970

helcogenes (Microspira), 204

helcogenes (Tibrio), 204

helgolandica (Cristispira), 1056

helianthi (Bacterium), 141

helianthi (Phytomonas), 141

helianthi (Pseudomonas), 141

helianthi var. tuberosi (Phytomonas), 141

Helicobacterium), 690

helicoides (Bacillus), 690

Heliconema, 1057, 1069

helminthoides (Bacillus), 368

helminthoides (Catenabacterium), 368

helveticum (Plocamobacterium), 695

helveticum (Thermobacterium), 352

helveticus (Lactobacillus), 352, 695

helvolum (Bacterium), 395

helvolum (Corynebacterium), 393, 395, 407 
helvolum (Flavobacteriım), 395

helvolus (Bacillus), 395, 657, 658

helvolus (Micrococcus), 263

helvolus granulatus (Bacillus), 658

Hemallosis, 13

heminecrobiophilus (Bacillus), 658

hemoglobinophilus canis (Bacillus), 587 hemoglobinophilus coryzae gallinarum (Bacillus), 589

hemolysans (Plectridium), 820

hemolysans (Streptococcus), 315

hemolytica (Pasteurella), 549

hemolyticum (Clostridium), 777

hemolyticum (Corynebacterium), 694

hemolyticum var. sordelli (Clnstridium), 777

hemolyticus (Bacillus), 776

hemolyticus (Hemophilus), 585, $\mathbf{5 8 6}$

hemolyticus (Streptococcus), 315

hemolyticus I, II and III (Streptococcus), 340

haemolyticus II (Streptococcus), 337

hemolyticus bovis (Clostridium), 777

Hemophilus, 17, 21, 22, 32, 37, 584

hemophosphoreum (Bacterium), 634

hemothermophilus (Streptococcus), 327

hennebergi (Pediococcus), 250

henricensis (Proteus), 490

Henrillus, 11, 763

hepaticus fortuitus (Bacillus), 659

hepaticus fortuitus (Bacterium), 659

hepatis (Bacterium), 409

hepatodystrophicans (Bacillus), 404

hepatodystrophicans (Corynebacterium),

388,404

hepatolytica (Listerella), 408

herbarum (Streptococcus), 313, 340

herbicola (Bacillus), 173, 679

herbicola (Bacterium), 173

herbicola aureum (Bacterium), 173

herbicola $\alpha$ aureum (Bacterium), 679

herbicola rubrum (Bacterium), 679

herbicola $\beta$ rubrum (Bacterium), 679

hermesi (Bacillus), 635

hermsi (Borrelia), 1063

hermsi (Spirochaeta), 1063

herpetii (Neurocystis), 1235 herrejoni (Treponema), 1073

Herrellea, 595

herrmanni (Bacillus), 659

hesmogenes (Bacillus), 729

hesmogenes (Urobacillus), 729

hessii (Bacillus), 746

hessii (Bacterium), 746

heterocea (Phytomonas), 167

heterocea (Xanthomonas), 167

heteroceum (Bacterium), 167

Heterocystia, 12, 13

heves (Salmonella), 528

hexacarbovorum (Bacterium), 679

heydenreichii (Micrococcus), 254

hibernicum (Aerobacter), 456

hibernicum (Chromobacterium), 234

hibisci (Bacterium), 120

hibisci (Phytomonas), 120

hibisci (Pseudomonas), 120

hibiscus (Micrococcus), 263

hibiscus liquefaciens (Listerella), 409

Hiblerillus, 11, 763

hidium (Bacterium), 679

hilgardii (Azotobacter), 219

hilgardii (Lactobacillus), 363

Hillhousia , 24, 25, 996, 997

hilli (Fusiformis), 583

hilli (Treponema), 1075

himonoi (Micrococcus), 695

hippanici (Bacterium), 635

hippopotami (Treponema), 1076

hirschfeldii (Bacterium), 507

hirschfeldii (Salmonella), 496, 507

hirsutum (Photobacterium), 637

hirtum (Bacterium), 714

hirtum (Pseudomonas), 714

hirtus (Bacillus), 714

hirudinicolicum (Bacterium), 679

hirudinis (Bacillus), 746

hirundinis (Rickettsia), 1096

hispanica (Spirochaeta), 1067

hispanicum (Borrelia), 1067

hispanicum (Treponema), 1067

hispanicum var. marocanum (Spirochaeta), 1067

hispanicum var. marocanum (Treponema), 1067 


\section{hist-hyal}

histolyticum (Clostridium), 811

histolyticus (Bacillus), 811

histolyticus (Weinbergillus), 811

histotropicus (Musculomyces), 1293

hoagii (Bacillus), 387

hoagii (Corynebacterium), 387

hodgkini (Bacillus), 404

hodgkini (Fusiformis), 404, 583

hodgkinii (Corynebacterium), 404

hoffmanii (Bacillus), 385, 659

hoffmanii (Corynebacterium), 385

hoffmanni (Actinomyces), 971, 976

hoffmanni (Cladothrix), 971, 976

hofmanni (Micromyces), 971, 976

hoffmanni (Nocardia), 976

hoffmanni (Oospora), 971, 976

hofmanni (Streptothrix), 971, 976

holci (Bacterium), 120

holci (Phytomonas), 120

holci (Pseudomonas), 120

holcicola (Bacterium), 157

holcicola (Phytomonas), 157

holcicola (Pseudomonas), 157

holeicola (Xanthomonas), 157

hollandiae (Photobacter), 636

hollandicum (Photobacter), 636

hollandicum parvum (Photobacter), 636

hollandicus (Bacillus), 746

hollandicus (Streptococcus), 325

holmesi (Actinomyces), 971

holmesi (Discomyces), 971

holobutyricus (Bacillus), 771, 824

holodisci (Nanus), 1207

Holospora, 1122

holsatiensis (Salmonella), 531

hominis (Actimomyces), 916, 97]

hominis (Corynethrix), 406

hominis (Discomyces), 916

hominis (Leuconostoc), 305

hominis (Listerella), 409

hominis (Molitor), 1241

hominis (Nocardia), 916, 920

hominis (Oospora), 920, 922

hominis (Proteus), 691

hominis (Streptothrix), 896, 920, 923, 976

hominis I (Streptothrix), 920 hominis II (Streptothrix), 921

hominis III (Streptothrix), 922

hominis IV (Streptothrix), 922

hominis (Strongyloplasma), 1241

hominis capsulatus (Bacterium), 691

hominis capsulatus (Proteus), 691, 816 hoplosternus (Bacillus), 717, 746, 759

hormaechei (Salmonella), 529

hornensis (Streptococcus), 348

horton (Actinomyces), 962

hortonensis (Streptomyces), 962

hoshigaki (Acetobacter), 183, 184

hoshigaki (Bacterium), 184

hoshigaki var. glucuronicum (Bacterium), 184,679

hoshigaki var. glucuronicum I (Bacterium), 494

hoshigaki var. rosea (Bactorium), 183, 184

Host is, 1239

hudsonii (Bacillus), 659

hudsonii (Bacterium), 659

hueppei (Bacillus), 740

hueppei (Clostridium), 740

hueppi (Bacillus), 820

hueppi (Clostridium), 820

humicola (Pseudomonas), 698

humidus (Micrococcus), 263

humifica (Streptothrix), 977

humilis (Bacillus), 659

humosus (Bacillus), 771

humuli (Chlorogenus), 1151

hutchinsonii (Cytophaga), 1012, 1013,

1016,1049

hvittingfoss (Salmonella), 528

hyacinthi (Bacillus), 152, 470

hyacinthi (Bacterium), 152

hyacinthi (Phytomonas), 152

hyacinthi (Pseudomonas), 152

hyacinthi (Xanthomonas), 152, 178

hyacinthi septica (Erwinia), 470

hyacinthi septicus (Bacillus), 470

hyacinthi septicus (Bacterium), 470.

hyalina (Chlamydothrix), 986

hyalina (Lampropedia), 844

hyalina (Leptothrix), 986

hyalina (Macromonas), 1001 
hyalina (Merismopedia), 841

hyalina (Pseudomonas), 997, 1001

hyalina (Sarcina), 814

hyalina (Streptothrix), 986

hyalinum (Achromobacter), 424

hyalinum (Clostridium), 820

hyalinum (Gonium), 814

hyalinum (Mycobacterium), 890

hyalinus (Bacillus), 424

hyalinus (Bacterium), 424

hyalinus (Micrococcus), $\mathbf{8 H}$

hyalinus (Pediococcus), 844

Hyalococcus, 305

hydrocharis (Bacillus), 659

Hydrocoleus, 993

hydrogenicus (Omelianskillus), s09

hydrogenii (Bacillus), 809

Hydrogenomonas, 20, 76

hydrophila (Aeromonas), 102

hydrophila (Pseudomonas), 102

hydrophilum (Bacterium), 102

hydrophilus (Bacillus), 102

hydrophilus (Proteus), 102

hydrophilus fuscus (Bacillus), 102

hydrophilus fuscus (Bacterium), 102

hydrophobia (Neuroryctes), 1264

hydrophoborum (Streptococcus), 340

hydrosulfurea (Pseudomonas), 147

hydrosulfureum ponticum (Bacterium), 207

hydrosulfureus (Bacillus), 659

hydrothermicus (Micrococcus), 264

Hygrocrocis, 1003

hygroscopicus (Actinomyces), 953

hygroscopicus (Streptomyces), 953

hymenophagus (Micrococcus), 261

hyochi (Lactobacillus), 363

hyochi var. 1 (Lactobacillus), 363

hyochi var. 2 (Lactobacillus), 363

hyopyogenes (Bacterium), 388

hyos (Borrelia), 1063

hyos (Spirochaeta), 1063

hyos (Spironema), 1063

hyos (Vibrio), 204

hyoscyami (Marmor), 1171

hypertrophicans (Corynebacterium), 398

hypertrophicans (Phytomonas), 398 hypertrophicans (Pseudomonas), 398

hypertrophicus (Caryococcus), 1121

hyphalus (Vibrio), 703

Hyphomicrobium, 35, 837

Hypnococcus, 312

hypothermis (Pseudomonas), 698

ianthina (Pseudomonas), 232

ianthinum (Bacteridium), 232

ianthinum (Bacterium), 231, 232

ianthinum (Chromobacterium), 232, 234

ichthyismi (Bacillus), 814

ichthyodermis (Achromobacter), 108

ichthyodermis (Pseudomonas), 108

ichthyosmia (Escherichia), 103

ichthyosmia (Pseudomonas), 103

ichthyosmius (Bacillus), 103

ichthyosmus (Proteus), 103

icterogenes (Bacillus), 659

icterogenes (Bacterium), 659

icterogenes (Leptospira), 1077

icterogenes (Spirochaete), 1076

icterogenes (Treponema), 1076

icterogenes marina (Spirochaeta), 1053

ictero-haemorragiae (Treponema), 1076

icterohaemorrhagiae (Leptospira), 90,

1076, 1077, 1078, 1079

icterohaemorrhagiae (Spirochaeta), 1076

icterohaemorrhagiae (Spiroschaudinnia),

1076

ictero-haemorrhagica (Spirochaeta), 1076 icterohemoglobinuriae (Leptospira), 1078 icterohemoglobinuriae (Spirochaeta), 1078 icterohemoglobinuriae (Treponema), 1078 icteroides (Bacillus), 531

icteroides (Bacterium), 531

icteroides (Leptospira), 1079

icteroides (Salmonella), 531

icteroides (Spirochaeta), 1079

icteroides (Treponema), 1079

ictero-uraemia canis (Spirochaeta), 1079

idonea (Cellulomonas), 613

idoneum (Bacterium), 613

idosus (Bacillus), 710

ignarvus (Streptococcus), 323

ikiensis (Coccobacillus), 636

iliacus (Bacillus), 451 


\section{ilia-inep}

iliacus (Escherichia), 451

iliacus (Proteus), 451

ilidzense (Bacterium), 760

ilidzense capsulatus (Bacillus), 760

illini (Miyagawanella), 1119

illinois (Salmonella), 525

imetrofa (Zaogalactina), 479

imetrophus (Bacillus), 480

imetrophus (Protococcus), 479

imminutus (Bacillus), 746

immobile (Bacterium), 98

immobilis (Acuformis), 813

immobilis (Bacillus), 746

immobilis (Granulobacter), 790

immobilis (Palmula), 813

immobilis-liquefaciens (Butyribacillus),

790

immotum (Bacterium), 606

imomarinus (Bacillus), 746

imperatoris (Micrococcus), 264

imperiale (Bacterium), 608

implectans (Bacterium), 760

implexum (Bacterium), 718

implexus (Bacillus), 718

inaequale (Treponema), 1062

inaequalis (Bacteroides), 568, 579

inaequalis (Spherophorus), 568, 57?

inaequalis (Spirochaeta), 1062

incana (Sarcina), 292

incanae (Phytomonas), 158

incanae (Xanthomonas), 158

incanescens (Actinomyces), 971

incannum (Bacterium), 659

incanus (Bacillus), 659

incanus (Bacterium), 659

incarnata (Sarcina), 292

incarnatus (Rhodococcus), 292

incertum (Bacterium), 607

incertum (Plectridium), 794

incognita (Pseudomonas), 95, 147, 698 incognitus (Erro), 1250

incommunis (Bacteroides), 570, 575

incommunis (Ristella), 570, 575

inconspicuus (Micrococcus), 264

inconstans (Tetrachloris), 870

indica (Nocardia), 909, 960

indica (Oospora), 909 indica (Serratia), 481, 701

indicens (Phagus), 1137

indicum (Azotobacter), 221

indicum (Bacterium), 701

indicum (Chromobacterium), 481

indicum (Leuconostoc), 346

indicum (Photobacter), 700

indicum (Photobacterium), 111, 634, 636

indicum var. obscurum (Photobacter),

700

indicum var. parvum (Photobacter), 700

indicus (Actinomyces), 909

indicus (Bacillus), 481, 700

indicus (Discomyces), 909

indicus (Erythrobacillus), 481

indicus (Micrococcus), 481

indicus (Vibrio), 699

indicus obscurus (Bacillus), 700

indicus parvus (Bacillus), 700

indicus ruber (Bacillus), 481, 701

indicus semiobscurus (Bacillus), 700

Indiella, 966

Indiellopsis, 966

indifferens (Bacillus), 746

indigoferus (Bacillus), 697, 698

indigoferus (Bacterium), 698

indigoferus (Pseudomonas), 698

indigoferus var. immobilis (Pseudomonas), 698

indigogenus (Bacillus), 659

indigogenus (Bacterium), 659

indigonaceum (Bacterium), 697, 698

indigonaceus (Bacillus), 697

indivisum (Polyangium), 1031

indolicus (Bacillus), 814

indolicus (Inflabilis), 814

indolicus (Micrococcus), 247, 264

Indolococcus, 235

indologenes (Aerobacter), 457

indoloxidans (Pseudomonas), 698

indomitus (Phagus), 1138

industrium (Acetobacter), 186

industrium (Bacterium), 186

industrium var. hoshigaki (Bacterium),

184, 694

industrius (Bacillus), 186

ineptus (Phagus), 1138 
iners (Marmor), 1190

iners (Tibrio), 204

inertia (Pseudomonas), 698

inexorabilis (Formido), 1263

infantilis (Bacillus), 746

infantilism (Bacillus), $81 t$

infantis (Salmonella), 512

infantum (Bacterium), 490

infantum (Proteus), 490

infecundum (Bacterium), 679

infimus (Micrococcus), 695

Inflabilis, 33, 34, 763

inflans (Rabula), 1284

inflatum (Clostridium), \$14

inflatus (Bacillus), 814

influentiae (Streptococcus), 340

influenzae (Bacillus), 58.5

influenzae (Bacterium), 585

influenzae (Hemophilus), 585, 586, 589, 1296

influenzae (Micrucoccus), 264

influenzae (Streptococcus), 340

influenzaeformis (Bacillus), 580

influenzaeformis (Spherophorus), 580

influenzae murium (Bacterium), 589

influenzae murium (Hemophilus), 589

influenzae putoriorum multiforme (Bac-

terium), 589

influenzae suis (Bacterium), 586

influenzae suis (Hemophilus), 586

influenzoides apis (Bacillus), 693

infrequens (Bacillus), 451

infrequens (Streptococcus), 340

infusionum (Palmello), 687

ingrica (Thioploca), 994

inguinalis (Encapsulatus), 459

innesi (Bacillus), 659

innocuus (Rabula), 1285

innominata (Leptothrix), 366

innominata (Pseudoleptothrix), 366

innominatum (Clostridium), 792

innominatus (Actinomyces), 971

innutrita (Palmula), 793

innutritus (Acuformis), 793

innutritus (Bacillus), 793, 826

inocuum (Bacterium), 679

inodorus (Bacillus), 659 insecticola Eberthella), 534

insecticolen.s (Proteus), 490

insectiphilium (Bacterium), 604

insectorum (Bacillus), 264

insectorum (Coccobacillus), 690

insectorum (Leptothrix), 366

insectorum (Micrococcus), 264

insectorum (Staphylococcus), 282

insectorum (Streptococcus), 264

insectorum var. malacosomae (Coccobacillus), 690

insidiosa (Phytomonas), 392

insidiosum (Aplanobacter), 392

insidiosum (Bacterium), 392

insidiosum (Corynebacterium), 392

insidiosum var. saprophyticum (Coryne-

bacterium), 393

insidiosus (Bacillus), 411

insolita (Azotomonas), 221

insolita (Ristella), 568, 576

insolitus (Bacteroides), 568, 576

insulosum (Bacterium), 760

insulum (Bacterium), 760

intactum (Bacterium), 760

intermedia (Microspira), 201

intermedia (Oospora), 971

intermedia (Sarcina), 292

intermedia (Spirochaeta), 1075

intermedium (Bacterium), 360

intermedium (Citrobacter), 449

intermedium (Clostridium), 771, 824

intermedium (Escherichia), 449, 460

intermedium (Lactobacillus), 360

intermedium (Paracolobactrum), 460,

490,491

intermedium (Treponema), 1075

intermedius (Actinomyces), 971

intermedius (Bacillus), 709

intermedius (Micrococcus), 264

intermedius (Phagus), 1140

intermedius (Streptococcus), 331

intermedius (Tibrio), 204

intermittens (Bacillus), 746

interproximalis (Actinomyces), 971

interproximalis (Streptothrix), 971

interrogans (Leptospira), 1079

interrogans (Spirochaeta), 1079 
interrogans (Treponema), 1079 interrogationis (Cristispira), 1056 intertriginis (Micrococcus), 264 intestinale (Bacteriophagum), 1128 intestinale (Thermobacterium), 352 intestinale (Treponema), 1067 intestinale suis (Bacterium), 508 intestinalis (Arthromitus), 1003 intestinalis (Bacillus), 654 intestinalis (Bifidibacterium), 369 intestinalis (Cladothrix), 983 intestinalis (Hygrociocis), 1003 intestinalis (Sarcina), 292 intestinalis (Spirochaeta), $106 \overline{-}$ intestinalis tuberculiformis (Bacillus), 369

intestinus motilis (Bacillus), 659

intestinus motilis (Bacterium), 659

intracellularis (Diplococcus), 296, 297, 301

intracellularis (Meningococcus), 297 intracellularis (Micrococcus), 297

intracellularis (Neisseria), 297

intracellularis (Streptococcus), 296, 297 intracellularis (Tetracoccus), 296, 301 intracellularis meningitidis (Diplokokkus), 296

intracellularis meningitidis (Micrococcus), 297

intracellularis-meningitidis (Neisseria), 297

intrapallans (Bacillus), $7+6$ intricata (Cladothrix), 718 intricatus (Bacillus), 718 intrinsectum (Bacterium), 769

intybi (Bacterium), 125

intybi (Phytomonas), 125

intybi (Pseudonomas), 125, 134

inulinaceus (Streptococcus), 321

inulofugus (Bacillus), 772, 824

inunctum (Achromobacter), 425

inunctus (Bacillus), 425

inunctus (Bacterium), 425

inutilis (Bacillus), 659

invadens (Caryococcus), 1120

inverness (Salmonella), 530 inverto-acetobutylicum (Clostridium), 781, 825

invisibile (Flavobacterium), 434

invisibilis (Bacillus), 434

invisibilis (Bacterium), 434

involutus (Bacillus), 612

involutus (Diplococcus), 308

involutus (Streptococcus), 308

invulnerabitis (Actinomyces), 971

invulnerabilis (Cladothrix), 934, 971

invulnerabilis (Nocardia), 971

invulnerabilis (Streptothrix), 971 iodinum (Chromobacterium), 694 iodinum (Pseudomonas), 694

Iodococcus, 695

iodophilum (Clostridium), 772 iogenum (Bacillus), 679

iogenum (Bacterium), 251, 679

iophagum (Achromobacter), 418

iophagum (Bacterium), 418

ipomeae (Flavimacula), 1202

ipomoea (Actinomyces), 958

ipomoea (Streptomyces), 958

iridescens (Pseudomonas), 174

iridicola (Bacterium), 140

iridicola (Phytomonas), 140

iridicola (Pseudomonas), 140

iridis (Bacterium), 147

iridis (Marmor), 1183

iridis (Phytomonas), 147

iridis (Pseudomonas), 147

iris (Bacterium), 698, 760

iris (Micrococcus), 264

iris (Pseudomonas), 147,69s

irregularis (Bacillus), 814

irregularis (Clostridium), 814

irregularis (Micrococcus), 264

israeli (Actinobacterium), 926

israeli (Actinomyces), 365, 926

israeli (Brevistreptothrix), 926

israeli (Cohnistreptothrix), 926

isracti (Corynebacterium), 926

israeti (Discomyces), 926

isracli (Nocardia), 926

israeli (Oospora), 926

israeti (Proactinomyces), 926

israeli (Streptothrix), 926 
israeli var. spitzi (Actinobacterium), 925 italiana (Salmonella), 522

italica (Pseudomonas), 147

italicum So. 1 and No. 2 (Bacterium), 760

italicum (Marmor), 1202

Ilersonia, $104 t$

itersonii (Spirillum), 214

itoana (Phytomonas), 169

itoana (Pseudomonas), 164

ituanum (Bacterium), 169

iugis (Bacillus), $61 \mathrm{~s}$

iugis (Cellulomonas), 618

ivanoff (Tibrio), 204

iwo-jima (Salmonella), 531

ixiae (Bacillus), 178

ixiae (Erwinia), t78

jaegeri (Pseudomonas), 89, 698

jaggeri (Bacterium), 122

jaggeri (Phytomonas), 122

jaggeri (Pseudomonas), 122

jatischii (Bacillus), 691

jakschii (Urobacillus), 691

janthina (Pseudomonas), 232

janthinus (Bacillus), 232

japonica (Acinomyces), 916

japonica (Pseudomonas), 226

japonica (Spirochaeta), 215

japonica (Streptothrix), 916

japonicum (Bacterium), 226

japonicum (Propionibacterium), 379

japonicum (Rhizobacterium), 226

japonicum (Rhizobium), 62, 221, 226

japonicum (Treponema), 215

japonicus (Bacillus), 536

japonicus (Discomyces), 916

japonicus (Erro), 1250

javanense (Photobacterium), $14 \overline{7}$

javanensis (Myxococeus), 1041

javanensis (Mitrosocystis), 72

javanensis (Vitrosomonas), 62, $\vec{i} 2$

javanensis (Pseudomonas), 72

javanica (Pseudomonas), 147, 699

javanicum (Photobacterium), 145

javaniensis (Bacillus), 117

julaniensis (Bacterium), 147, 699 javiana (Salmonella), 520, 522

jeffersonii (Bacterium), 520

jeffersonii (Eberthella), 520

jeffersonii (Shigella), 520

jejunales (Bacillus), $\$ 51$

jejuni (Tibrio), 204

jenense (Rhodothiospirillum), Säl

jenense (Spirillum), 851

jenense (Thiospirillum), 851, 852

jenense forma maxima (Thiospirillum),

$\$ 51$

jenensis (Ophidiomonas), 851

jensenii (Propionibacterium), 376, 37

jensenii var. raffnosaceum (Piopioni-

bacterium), 375

Jodoccus, 695

jogenum (Bacterium), 679

johnei (Ascococcus), 253

jollyi (Actinomyces), 920)

jollyi (Discomyces), 920

jollyi (Nocardia), 920

jollyi (Oospora), 920

jonesii (Spirochacta), $106-$

jonesii (Spironema), 1067

jongii (Micrococcus), 264

josephi (Bacillus), 592

josephi (Moraxella), 592

joyeuxii (Grahamella), 1110)

jubatus (Bacillus), 746

juglandis (Bacillus), 158

juglandis (Bacterium), 15̃s

juglandis (Phytomonas), $15 \mathrm{~s}$

juglandis (Pseudomonas), 158

juglandis (Yanthomonas), 158, 160)

jugurt (Lactobacillus), 364

jugurt (Themobacterium), 364

kaapstad (Salmonella), 505

kairo (Rickettsia), 1096

kaleidoscopicus (Bacillus), 746

kandiensis (Bacillus), 534

kandiensis (Bacterium), 534

kandiensis (Eberthella), 534

kandiensis (Eberthus), 534

kaposvar (Salmonella), 505

kappa (Bacillus), 659

kauffmannii (Salmonella), $49: 3$ 
kaukasicus (Bacillus), 351

kaustophilus (Bacillus), 730

kedronskii (Bacillus), 814

kefersteinii (Micrococcus), 256

kefir (Bacillus), 746

kefir (Streptococcus), 338, 347

kegallensis (Vibrio), 204

kentucky (Salmonella), 526

keratolytica (Actinomyces), 916

keratolyticus (Proactinomyces), 916

keratomalaciae (Bacterium), 679)

kermesinus (Bacillus), 746

khartoumensis (Bacillus), 452

khartoumensis (Enteroides), 452

khartoumensis (Escherichia), 452

kielense (Chromobacterium), 482

kildini (Bacillus), $7 \pm 6$

kilensis (Serratia), 482, 483

kiliense (Bacterium), 482

kiliensis, (Bacillus), 482, 484

kiliensis (Erythrobacillus), 482

kimberi (Actinomyces), $96+$

kimberi (Streptomyces), 964

kirchneri (Streptococcus), 310

kirkee (Salmonella), 529

Klebsiella, 10, 18, 31, 37, 443, 457, 458

klebsii (Bacillus), 659

klebsii (Helicobacterium), 690

kleckii (Bacillus), 659

kllcinit (Bacillus), 659, 660, 7SS

lileinii (Clostridium), $78 \mathrm{~S}$

lilimenko (Vibrio), 205

kluyverii (Clostridium), 794, 820

lnipowitchi (Bacterium), 6\$0)

Kochella, 13

kochi (Spirochaeta), 1060)

kochi ('Treponema), 1060

kochii (Bacillus), s7t

kochii (Borrelia), 1060

kochii (Pediococcus), 250

kochii (Schlerothrix), 877

kochii (Spirillum), 1054

kochii (Spirochaete), 1054

kochii (Spironema), 1060

kochii (Streptococcus), 340

kolkwitzii (Spirillum), 217

köln (Salmonella), 503 kornii (Bacillus), 660

kottbus (Salmonella), 513

koubassoff (Bacillus), 746

krainskii (Actinomyces), 938

krainskii. (Nocardia), 946

kralii (Bacillus), 680

kralii (Bacterium), 680

lirameri (Bacillus), 477

krameriani (Bacterium), 145

krausei (Actinomyces), 971

krausei (Discomyces), 971

krausei (Nocardia), 921, 971

krausei (Streptothrix), 971

krusecastellani (Castellanus), 540

krzemieniewskae (Cytophaga), 1014

krzemienicwski (Bacillus), 722

kuchniana (Crenothrix), 987

kuehniana (Hypheothrix), $98 \overline{7}$

kuehniana (Leptothrix), 987

kuetzingianum (Acetobacter), 180, 183

luetzingianum (Bacterium), 183

kurlova (Ehrlichia), 1095

kurlowi (Ehrlichia) (Rickettsia), 1095

Kurthia, 21, 26, 30, 31, 608

liutscheri (Bacterium), 390

kutseheri (Corynebacterium), 389

liutscheri (Spirillum), 215

kwanzani (Rimocortius), 1209, 1210

laburni (Marmor), 1187

lacca (Bacillus), 746

lacerans (Bacillus), 639

lacerans (Carpophthora), 1152

lacerans (Marmor), 1152

lacerans (Phagus), 1139

lacertae (Actinomyces), 971

lacertac (Oospora), 971

lacertae (Streptothrix), 971

lachrymans (Bacillus), 116

lachrymans (Bacterium), 116

lachrymans (Phytomonas), 116

lachrymans (Pseudomonas), 116

lacmus (Bacillus), 233

lactantium (Bacillus), 454

lactea (Neisseria), 261

lactea (Sarcina), 292

lactericeus (Micrococcus), 264 
lacteum (Bacterium), 738

lacteus (Bacillus), 716, 755

lacteus (Micrococcus), 264

lacteus (Streptococcus), 340

lacteus faviformis (Micrococcus), 261

lactica (Pseudomonas), 147

lactica (Serratia), 600

lactici-acidi (Bacillus), 447

lacticola (Bacillus), 43, 716

lacticola (Bacterium), 716

lacticola (Mycobacterium), 887, 888, 889, 890

lacticola perrugosum (Mycobacterium), 890

lacticola $\beta$ perrugosum (Mycobacterium), 890

lacticola planum (Mycobacterium), 890

lacticola a planum (Mycobacterium), 890

lacticola $\gamma$ friburgensis (Mycobacterium), 890

lacticum (Achromobacter), 425

lacticum (Bacterium), 324

lacticum (Corynebacterium), 370

lacticum (Microbacterium), 353, 370, 371

lacticus (Bacillus), 324, 362, 447

lacticus (Micrococcus), 336

lacticus (Streptococcus), 324

lactimorbus (Bacillus), 727

lactiparcus (Bacillus), 810

lactis (Acidobacterium), 361

lactis (Bacillus), 660, 716, 725

lactis (Bacterium), 323, 454, 684

lactis (Chlorobacterium), 693

lactis (Flavobacterium), 434

lactis (Lactobacillus), 351

lactis (Lactococcus), 324

lactis (Micrococcus), 264

lactis II (Micrococcus), 264

lactis (Sarcina), 292

lactis (Streptococcus), 43, 323, 324,

325,340362

lactis B (Streptococcus), 325

lactis (Thermobacterium), 351

lactis No. I (Bacillus), 716, 725

lactis, No. II (Bacillus), 741 lactis No. III (Bacillus), 738

lactis No. IV, (Bacillus), 744

lactis No. V, (Bacillus), 716

lactis No. VI (Bacillus), 743

lactis No. VII (Bacillus), 747

lactis No. VIII (Bacillus), 748

lactis No. IX (Bacillus), 710

lactis No. X (Bacillus), 709

lactis No. XI (Bacillus), 743

lactis No. XII (Bacillus), 755, 761

lactis acidi (Bacillus), 324, 351

lactis acidi (Bacterium), 323, 324

lactis acidi (Lactobacillus), 351

lactis acidi (Micrococcus), $26 \pm$

lactis acidi (Sarcina), 292

lactis acidi (Staphylococcus), 282

lactis aerogenes (Bacillus), $45 \pm$

lactis aerogenes (Bacterium), 454

lactis aerogenes (Encapsulatus), 454

lactis albidus (Micrococcus), 238

lactis album (Bacterium), 416

lactis albus (Bacillus), 716, 718, 746

lactis albus (Micrococcus), 264

lactis albus (Sarcina), 292

lactis amari (Micrococcus), 265

lactis arborescens (Micrococcus), 252

lactis aromaticus (Streptococcus), 340

lactis aurantiaca (Sarcina), 292

lactis aureus (Micrococcus), 253, 265

lactis citreus (Micrococcus), 265

lactis citronus (Micrococcus), 265

lactis cloacae (Bacillus), 455

lactis commune (Bacterium), 362

lactis erythrogenes (Bacillus), 600, 654

lactis erythrogenes (Bacterium), 600

lactis erythrogenes (Chromobacterium), 600

(lactis) erythrogenes (Erythrobacillus), 600

lactis erythrogenes (Micrococcus), 600

lactis flavus (Micrococcus), 265

lactis fluorescens (Micrococcus), 265

lactis foetidum (Viscobacterium), 691

lactis giganteus (Micrococcus), 265

lactis gigas (Micrococcus), 265

lactis harrisonii (Bacillus), $43 \pm$

lactis innocuus (Streptococcus), 340 
lactis inocuus (Bacillus), 679

lactis inocuus (Bacterium), 679

lactis longi (Bacterium), 702

lactis lutea (Sarcina), 292

lactis marshalli (Bacterium), 415

lactis minutissimus (Micrococcus), 265

lactis niger (Bacillus), 711

lactis peptonans (Bacillus), 751

lactis pituitosi (Bacillus), 684

lactis pituitosi (Bacterium), 684

lactis pruchii (Bacillus), 788

lactis rosaceus (Micrococcus), 265, 273

lactis rubidus (Micrococcus), 274

lactis rugosus (Micrococcus), 265

lactis saponacei (Bacillus), 145, 668

lactis termophilus (Bacillus), 733, 756

lactis varians (Micrococcus), 240, 241,

265

lactis var. anoxyphilus (Streptococcus),

325

lactis var. hollandicus (Streptococcus),

325

lactis var. maltigenes (Streptococcus),

44,325

lactis var. tardus (Streptococcus), 325

lactis viscosi (Coccus), 238

lactis viscosi (Micrococcus), 238

lactis viscosum (Bacterium), 414

lactis viscosus (Bacillus), 414

lactis viscosus (Micrococcus), 238, 280

lactis viscosus $B$ (Micrococcus), 280

Lactobacillus, $18,21,25,31,37,38$,

$42,349,350,361,367,407,675$

Lactobacter, 349

Lactobacterium, 319

lactobutyricum (Granulobacter), 822

lactobutyricus (Amylobacter), 822

lactobutyricus (Bacillus), 822

Lactococcus, 312

lactofoetidus (Bacillus), 660

lactopropylbutylicum (Clostridium), 814

lactopropylbutyricus (Bacillus), 814

lactopropylbutyricus non liquefaciens

(Bacillus), 814

lactorubefaciens (Bacillus), 644

lactorubefaciens (Bacterium), $65+$

lactorubefaciens (Serratia), 644
Lactosarcina, 285

Lactrimatoria, 5

lactucae (Bacillus), 746

lactucae (Bacterium), 168

lactucae (Marmor), 1178

lactucae (Phytomonas), 168

lactucae (Xanthomonas), 168

lactucae-scariolae (Phytomonas), 154

lactucae-scariolae (Xanthomonas), 154

lacunata (Moraxella), $\mathbf{5 9 0}$

lacunatum (Flavobacterium), 441

lacunatus (Bacillus), 441, 590

lacunatus (Bacterium), 441

lacunatus (Hemophilus), 590

lacunatus var. atypica (Moraxella), 591

lacunogenes (Pseudomonas), 177

laerii (Bacterium), 680

laesiofaciens (Marmor), 1168

laesiofaciens var. minus (Marmor), 1168

laevis (Bacillus), 575, 709

laevis (Bacteroides), 575

laevolacticum (Bacterium), 348, 680

laevulosinertis (Micrococcus), 696

lagerheimii (Leuconostoc), 348

lagerheimii (Streptococcus), 348

lagerheimii var. subterraneum (Streptococcus), $3+1$

lagopodis (Spirochaeta), 1067

lagopodis (Spironema), 1067

laidlawi (Sapromyces), 1294

laminariae (Bacterium), 680

laminariae (Billetia), 680

laminariae (Kurthia), 680

Lamprella, 13, 83

Lamprocystis, 16, 23, 25, 847, 848, 849, 850,855

Lampropedia, 23, 25, 844

lanceolatus (Bacillus), 660

lanceolatus (Diplococcus), 307

lanceolatus (Micrococeus), 307

lanceolatus (Pneumococcus), 307

lanceolatus (Streptococcus), 303, $\mathbf{3 3 0}$

lanceolatus anaerobius (Coccus), 330

lanceolatus capsulatus (Diplococcus), 307

lanceolatus ovium (Diplococcus) (Streptococcus), 341 
lanceolatus pasteuri (Streptococcus), 306, 330

lanceolatus sive capsulatus (Diplococcus), 306

lanceolatus var. mucosus (Diplococcus), 308

lanceolatus var. mucosus (Streptococcus), 308

lanfranchii (Actinomyces), 927

lanfranchii (Nocardia), 927

langkatense (Bacterium), 680

Lankasteron, 847

Lankoides, 10,516

lanuginosum (Synangium), 1033

lanuginosus (Chondromyces), 1033

lapillus (Streptococcus), 341

lapsa (Phytomonas), 124

lapsa (Pseudomonas), 124

lardarius (Micrococcus), 265

largum (Bacterium), 680

largus (Bacillus), 680

lari (Treponema), 1075

larvae (Achromobacter), 425

larvae (Bacillus), 726

larvae (Enterobacillus), 425

larvicida (Bacillus), 660

larvicida (Bacterium), 660

laseri (Bacillus), 612

lasia (Pseudomonas), 147

lasiocampa (Bacillus), 746

lassari (Bacillus), 660

lasserei (Actinomyces), 920

lasserei (Discomyces), 920

lasserei (Nocardia), 920

lasserei (Oospora), 920

lasseuri (Flavobacterium), 178

latapici (Spironema), 1064

latapiei (Spirillum), 1064

latapiei (Spirochaeta), 1064

latens (Rabula), 1286

latens (Sphaerothrix), 986

latericea (Serratia), 641

latericeum (Bacterium), 641, 683

latericeus (Bacillus), 641, 683

laterosporus (Bacillus), 724, 725

lathridii (Actinomyces), 971

lathridii (Streptothrix), 934, 971 lathyri (Bacillus), 476

lathyri (Erwinia), 476

latum (Caryophanon), 1004

latvianus (Bacillus), 746

laurentia (Pseudomonas), 233

lauterbornii (Pelodictyon), 871

lautus (Bacillus), 746

lavendulae (Actinomyces), 944, 977

lavendulae (Streptomyces), 944

laverani (Spirochaeta), 215

laverani (Spironema), 215

laverani (Treponema), 215

laxae (Bacterium), 762

lebeni (Bacillus), 364

lebenis (Bacterium), 364

lebenis (Streptobacillus), 351, 364

lebensis $\alpha$ and $\beta$ (Streptobacillus), 364

lebenis nonviscosus (Streptobacillus), 364

lebenis viscosus (Streptobacillus), 364

lectularia (Rickettsia), 1096

legeri (Fusiformis), 694

legeri (Microspironema), 1073

legeri (Treponema), 1073

Legio, 1257

legrosii (Bacillus), 746

leguminiperdum (Bacterium), 747

leguminiperdus (Bacillus), 747

leguminosarum (Marmor), 1179

leguminosarum (Phytomyxa), 224

leguminosarum (Rhizobium), 224, 225 $226,1130,1138$

leguminosarum (Schinzia), 224

lehmanni (Bacillus), 747

leichmanni (Bacterium), 324

leichmanni I (Bacillus), 357

leichmanni II (Bacillus), 356

leichmanni III (Bacillus), 357

leichmannii (Lactobacillus), 357

leidensis (Vibrio), 205

leishmani (Actinomyces), 899

leishmani (Discomyces), 899

leishmanii (Nocardia), 899

lekitosis (Bacillus), 747

leloirii (Staphylococcus), 282

lemani (Streptothrix), 977

lembkei (Bacterium), 533

lembkei (Micrococcus), 265 
lembkei (Sarcina), 292

lembkii (Pseudomonas), 148

lemonnieri (Bacillus), 178

lemonnieri (Pseudomonas), 178

lenis (Alcaligenes), 416

lentiformis (Bacillus), 660

lentimorbus (Bacillus), 727

lentoputrescens (Clostridium), 799, 800 826

lentulum (Bacterium), 637

lentum (Eubacterium), 368

lentus (Bacillus), $\mathbf{7 1 3}$

lentus (Bacteroides), 368

lentus (Micrococcus), 265

lentus (Phagus), 1143

lentus (Streptococcus), 335, 341

leonardii (Vibrio), 200

lepierrei (Bacterium), 680

lepiseptica (Pasteurella), 547

lepisepticum (Bacterium), 547

lepisepticus (Bacillus), 547

leporis (Aerobacter), 456

leporis (Bacillus), 451

leporis (Eberthella), 451

leporis (Escherichia), 451

leporis lethalis (Bacillus), 451

leporis lethalis (Bacterium), 451

leporisepticum (Bacterium), 547

leprae (Bacillus), 881

leprae (Coccothrix), 882

leprae (Discomyces), 882

leprae (Mycobacterium), 875, 881, 882, 887

leprae (Sclerothrix), 882

leprae hominus (Mycobacterium), 882

leprae murium (Bacillus), 882

lepraemurium (Mycobacterium), 875, 882

lepromatis (Actinomyces), 916

lepta (Saprospira), 1054

leptinotarsae (Bacillus), 660

leptodermis (Bacillus), 712

leptomitiformis (Beggiatoa), 992, 993

leptomitiformis (Oscillatoria), 992

Leptospira, 19, 20, 26, 28, 593, 594, 1076

leptosporus (Bacillus), 710

Ieptotrichia, $14,19,21,22,2 \overline{7}, 34,35$, $38,364,365,983$
Leptothrix, 17, 18, 19, 23, 26, 364, 365, 983, 986

Leptothrix I, 367

Leptothrix II, 367

Leptothrix III, 365

lesagei (Bacillus), 660, 747

lespedezae (Phytomonas), 159

lespedezae (Xanthomonas), $\mathbf{1 5 9}$

lestoquardi (Rickettsia), 1120

lethale (Marmor), 1155, 1168

lethalis (Bacillus), 680

lethalis (Bacterium), 680

lethalis (Proteus), 680

Lethum, 1223

leubei (Urobacillus), 688, 729

leucaemiae (Bacillus), 680

leucaemiae (Bacterium), 680

leucaemiae canis (Bacillus), 680

leucaemiae canis (Bacterium), 680

leucea (Streptothrix), 934, 977

leucea saprophytica (Streptothrix), 976

leucogloeum (Bacterium), $6 \mathbf{6 3 7}$

leucomelaenum (Spirillum), 217, 218

Leuconostoc, 14, 20, 24, 31, 34, 346, 362

leucotermitis (Spirochaeta), 1067

Leucothrix, 695

levaditii (Treponema), 1070

levaniformans (Bacillus), 747

levans (Aerobacter), 455, 664

levans (Bacillus), 455

levans (Bacterium), 455

levans (Cloaca), 455

levis (Rabula), 1285

levistici (Bacterium), 140

levistici (Phytomonas), 140

levistici (Pseudomonas), 140

levyi (Actinomyces), 916

lewisi (Pacinia), 701

lewisii (Bacterium), 534

lewisii (Eberthella), 53 t

lexington (Salmonella), 524

libaviense (Bacterium), 341

libaviensis (Streptococcus), 341

liber (Phagus), 1142

liborii (Clostridium), 820

liceagi (Salmonella), 531

lichenicolum (Podangium), 1035 
licheniforme (Clostridium), $7+7$

licheniformis (Bacillus), 747

licheniformis (Micrococcus), 265

lichenis-plani (Ristella), 576

lichenocolus (Chondryomyces), 1035

lichenoides (Bacillus), 747, 814

lichnoides (Pneumococcus), 697

Lieskeella, 986

lieskei (Actinomyces), 950,97t

lieskei (Streptomyces), 950,974

lignicola (Pseudomonas), 142

lignieresi (Actinobacillus), 556, 926

lignieresi (Discomyces), 557

lignieresi (Nocardia), 557

lignieresi (Pasteurella), $55 \mathbf{5}$

lignieri (Bacillus), 556, 677

lignithum (Micrococcus), 265

lignivorans (Bacillus), 747

lignorum (Bacillus), 747

liguire (Actinomyces), 975

liguire (Nocardia), 975

ligustri (Bacterium), $12 \mathrm{~s}$

ligustri (Marmor), 1187

ligustri (Pseudomonas), 128

lilacinus (Bacillus), 233

lilii (Adelonosus), 1211

lilii (Bacillus), 477

lilii (Erwinia), 477

limae (Cristispira), 1056

limae (Spirochaeta), 1056

limbatum (Bacterium), 680

limbatum acidi lactici (Bacterium), $6 \$ 0$

limbatus (Bacillus), 660

limbatus acidi lactici (Bacillus), 681

limbatus butyri (Bacillus), 660

limicola (Bacillus), 660

limicola (Chlorobium), 870, 872

limitans (Bacillus), 580

limneticum (Sideroderma), 835

limnophilus (Bacillus), $7+\bar{T}$

limoniticus (Siderococcus), 835

limophilus (Bacillus), 716

limosum (Eubacterium), 368

limosus (Bacillus), 715, 716, 815

limosus (Bacteroides), 36s, 370, 3\$0)

lindenborni (Bacillus), 491

lindneri (Acetobacter), 185 lindneri (Bacillus), 360

lindneri (Lactobacillus), 360

lindneri (Pseudomonas), 106

lineare (Siderobacter), 835

linearis (Chroostipes), 873

linearius (Thermobacillus), 734

lineatus (Bacillus), 660

linens (Bacterium), 601, 612

lineola (Bacillus), 597, 681

lineola (Bacterium), 597, 681

lineola (Treponema), $10 \overline{7} 1$

lineola (Tibrio), 597, 1071

lineopictum (Marmor), 1197

lingardi (Bacillus), 747

linguae (Spirillum), 205, 920

linguale (Spirosoma), 205, 920

lingualis (Actinomyces), 920

lingualis (Discomyces), 920, 922

lingualis (Nocardia), 205, 920, 922

lingualis (Oospora), 922

lingualis (Streptothrix), 920

lingualis (Tibrio), 205, 920

lini (Bacterium), 681, $81 \mathrm{~s}$

linkoi (Bacterium), $6 \$ 81$

linognathi (Rickettsia), 1096

linsbaueri (Chromatium), $\mathbf{8 5 7}$

linsbaueri (Rhabdochromatium), 855

linsbaueri (Rhabdomonas), 855, 856

liodermos (Bacillus), 709

liparis (Bacillus), 660

liparis (Diplococcus), 336

lipidis (Achromobacter), 416

lipidis (Bacterium), 416

lipmanii (Actinomyces), 952

lipmanii (Streptomyces), 952

lipoferum (Chromatium), 203, 216

lipoferum (Spirillum), 203, 216

lipolyticum (Achromobacter), 609

lipolyticum (Bacterium), 391

lipolyticum (Bactridium), 609

lipolyticum (Kurthia), 693

lipolyticus (Alcaligenes), 391

lipolyticus (Bacterium), 390, 693

lipolyticus (Micrococcus), 266

liquata (Cellulomonas), 396, 614

liquatum (Bacterium), 396, 614

liquefaciens (Achromobacter), $\mathbf{4 1 8}$ 
liquefaciens (Actinomyces), 975, 976

liquefaciens (Aerobacter), 455, 692

liquefacions (Amylobacter), 823

liquefaciens (Bacillus), 148, 388, 404, 418, $457,660,661$

liquefaciens (Bacterium), 457

liquefaciens (Bacteroides), 575

liquefaciens (Cladothrix), 934, 975, 976

liquefaciens (Coccobacillus), 575

liquefaciens (Corynebacterium), 371, 388, 404

liquefaciens (Diplobacillus), 591

liquefaciens (Discomyces), 976

liquefaciens (Gluconoacetobacter), 694

liguefaciens (Gluconobacter), 691

liquefaciens (Microbacterium), 371

liquefaciens (Micrococcus), 238, 240, 266, 274

liquefaciens (Microspira), 198

liquefaciens (Moraxella), 591

liquefaciens (Nocardia), 923, 975

liquefaciens (Oospora), 976

liquefaciens (Pseudomonas), $14 \mathrm{~S}$

liquefaciens (Sarcina), 288, 292

liquefaciens (Streptococcus), 240, 326, 327,702

liquefaciens (Streptothrix), 976

liquefaciens (T'etracoccus), 240

liquefaciens (Vibrio), 198

liquefaciens acidi $I$ and $I I$ (Micrococcus), 266

liquefaciens albus (Bacillus), 661

liquefaciens albus (Micrococcus), 274

liquefaciens aurantiacus (Staphylococcus), 282

liquefaciens bovis (Pncumobacillus), 675

liquefaciens communis (Bacillus), 661, 681, 699

liquefaciens communis (Bacterium), 661, 681

liquefaciens conjunctivae (Micrococcus), 257

liquefaciens flueggei (Micrococcus), 266 liquefaciens lactis amari (Bacillus), 648

liquefaciens magnus (Bacillus), 787, $\$ 25$ liquefaciens parvus (Bacillus), 822

liquefaciens pyogenes (Bacillus), 388 liquefaciens pyogenes bovis (Bacillus), 388

liquefaciens septicus (Urobacillus), 491

liquida (Pseudomonas), 425, 699

Liquidobacterium, 8, 486

Liquidococcus, 8, 235

Liquidomonas, 8, 83

Liquidovibrio, 8, 192

liquidum (Achromobacter), 425, 699

liquidum (Bacterium), 425

liquidus (Bacillus), 425, 699

liquidus (Micrococcus), 266

liquidus communis (Bacillus), 661

liskey (Actinomyces), 974

liskeyi (Asteroides), 974

lissabonensis (Vibrio), 205

Listerella, 29, 408

listeri (Actinomyces), 961

listeri (Bacillus), 356

listeri (Lactobacillus), 356

listeri (Lactobacterium), 356

listeri (Pseudomonas), 145

listeri (Streptomyces), 961

Listeria, 408

litchfield (Salmonella), 514

litorale (Achromobacter), 425

litorale var. 2 (Achromobacter), 425

litoralis (Bacillus), 425

litoralis (Bacterium), 425

litoralis (Erythroconis), 289

litoralis (Merismopedia), 289

litoralis (Micrococcus), 288

litoralis (Pediococcus), 289

litoralis (Pseudomonas), 425

litoralis gadidarum (Micrococcus), 289

litoreum (Bacterium), 681

litoreus (Bacillus), 681

litorosus (Bacillus), 661

littoralis (Erythroconis), 843

littoralis (Lampropedia), 289

littoralis (Merismopedia), 843

littoralis (Sareina), 288, 289

livida (Sarcina), 292

livido-lutescens (Sarcina), 291, 292

lividum (Chromobacterium), 234

lividus (Bacillus), 234, 747

lividus (Bacterium), 234

lobatus (Bacillus), 733 
lobatus (Micrococcus), 266

lobatus (Thiobacillus), 81

loculosum (Bacterium), 681

Loeflerella, 37,554

loeffleri (Pacinia), 383

loeffleri (Planococcus), 251

loehnisii (Bacillus), 728

loehnisii (Bacterium), 477

loehnisii (Phytomonas), 477

Lochnisium, 14

loewenbergii (Micrococcus), 266

loewenbergii (Sarcina), 292

loganobacci (Nanus), 1206

loidensis (Actinomyces), $9 \overrightarrow{7} 1$

loligo (Coccobacilluis), 636

loma linda (Salmonella), 522

londinensis (Actinomyces), 921

londinensis (Discomyces), 921

londinensis (Nocardia), 921

london (Salmonella), 522

londonensis (Salmonella), 522

longa (Pseudomonas), 148, 699

longiarticulata (Thiothrix), 990

longior (Bacillus), 747

longissima (Chlamydothrix), 1002

longissima (Pontothrix), 1002

longissimus (Streptococcus), 315

longum (Bacterium), 760

longum (Betabacterium), 360

longum (Rhodospirillum), 867

longus (Bacillus), 747, 815

longus (Lactobacillus), 354, 360

longus (Streptobacillus), 352

longus (Streptococcus), 315

longus hemolyticus (Streptococcus), 315

longus pathogenes seu erysipelatos

(Streptococcus), 315

lopholea (Leptothrix), 985

lophomonadis (Fusiformis), 694

losanitchi (Bacillus), 732

loti (Rhizobium), 225

lottii (Catenabacterium), $36 \mathrm{~s}$

louisianae (Miyagawanella), 1118

lovati (Spirochaeta), 1067

lowenthali (Spirochaeta), 1067

loxiacida (Bacillus), 661

loxosporus (Bacillus), 718, 748 loxosus (Bacillus), 716

lubinstii (Bacillus), 815

lucae (Streptococcus), 341

lucens (Bacterium), 635

lucens (Micrococcus), 635

luceti (Bacterium), 681

luciana (Salmonella), 526

lucidus (Bacillus), 661

lucilarum (Neisseria), 301

luciliae (Clostridium), 779

lucrosa (Cellulomonas), 614

Iucrosum (Bacterium), 614

ludwigi (Bacterium), 681

luis (Trypanosoma), 1071

luminosa (Microspira), 635

luminosum (Achromobacter), 634

luminosum (Photobacter), 635

luminosum (Photobacterium), 634, 635, 636

luminosus (Bacillus), 635

luminosus (Bacterium), 635

luminosus (Vibrio), 635

lumnitzeri (Bacterium), 739

lunavensis (Bacillus), 544

lunavensis (Bacterium), $54 \pm$

lunavensis (Shigella), 544

lunula (Bacterium), 760

lupi (Bacillus), 661

lupini (Bacillus), 661

lupini (Phytomyxa), 226

lupini (Rhizobium), 224, 226

luridus (Micrococcus), 266

lustigii (Bacillus), 661, 668, $80 \pm$

lustigii (Clostridium), $80 \pm$

lustigii (Endosporus), $80 t$

lutea (Cytophaga), 1013

lutea (Neisseria), 281, 696

lutea (Nocardia), 909

lutea (Sarcina), 253, 287, 290, 291, 292, 293, 294

luteo-albus (Bacillus), 661

luteola (Aphanothece), 871

luteola (Nocardia), $92 \pm$

luteola (Oospora), 924

luteola (Sarcina), 292

luteola (Schmidlea), 871, 87t

luteola (Streptothrix), 924 


\section{luteolum (Bacterium), 681}

luteolus (Actinomyces), 924

luteolus (Discomyces), 924

luteolus (Micrococcus), 266

luteo-roseus (Actinomyces), 971

lutescens (Bacterium), 436, 681

lutescens (Flavobacterium), 436

lutescens (Sarcina), 292

lutetiensis (Bacillus), 233, 661

luteum (Ascobacterium), 647

luteum (Bacterium), 681

luteum (Bacteridium), 237

luteum (Mycobacterium), 890

luteum (Polyangium), 1027

luteus (Actinomyces), 909

luteus (Bacillus), 661, 690, 718

luteus (Diplococcus), 281, 694, 696

luteus (Micrococcus), 43, 237, 251, 256, $257,259,260,261,262,263,264,265$,

$266,267,268,269,270,271,272,273$,

$275,276,277,278,280$

luteus (Mycococcus), 891

luteus (Planococcus), 281, 694

luteus (Streptococcus), 341

luteus liquefaciens (Bacillus), 490

luteus-liquefaciens var. larve (Micm. coccus), 266

luteus pallescens (Bacillus), 664

luteus putidus (Bacillus), 647

luteus sporogenes (Bacillus), its

luteus var. larvae (Micrococcus), 266

lutosus (Micrococcus), 266

lutrae (Spirochaeta), 1067

lutrae (Spironema), 1067

lutiae (Spiroschaudinnia), 1067

lutulentus (Bacillus), 716

lutzae (Bacillus), 274, 301, 7ts

lwoffi (Moraxella), 592

lycopersici (Bacterium), $47 \mathbf{7}$

lycopersici (Lactobacillus), 358

lycopersici var. vitiati (Bacterium), 640

lycopersicum. (Phytobacter), 1 t.)

lydiae (Bacterium), 760

lymantriae (Bacillus), 661

lymantriae (Bacillus) (Bacterium), 661

lymantriae (Coccobacillus), 661

lymantriue (Diplococcus), 336 lymantriae $\alpha$ (Bacillus), 661

lymantriae $\beta$ (Bacillus), 661

lymantricola adiposus (Bacillus), 661

lymantricola adiposus (Bacterium), 661

lymphae vaccinalis (Corynebacterium) 401, 404

lymphaticum. (Treponema), 1068

lymphaticus (Spirochaeta), 1067

lymphogranulomatis (Miyagawanella),

1116, 1117, 1118, 1119

lymphogranulomatosis (Ehrlichia), 1116

lymphophilum (Corynebacterium), 388 , t04

lymphophilus (Bacillus), 404

lysodeititicus (Micrococcus), 266

lyssae (Cocco-bacterium), $266^{\circ}$

lyssae (Glugea), 1264

lyssae (Micrococcus), 266

lyticus (Bacillus), 815

maasei (Microspira), 202

maasei (Spirillum), 202

macaci (Spirochaeta), 1068

macaci (Spironema), 1068

macaci (Spiroschaudinnia), 1068

maccullochianum (Bacterium), 117

macerans (Aerobacillus), 721

macerans (Bacillus), 720, 721, 722 723

macerans (Zymobacillus), 721

macfadyeanii (Bacterium), 531

macfadyeanii (Salmonella), 531

Macintoshillus, 11, 763

Macrocystita, 14

macrodentium (Spirochaeta), 1075

macrodentium (Treponema), 1070, 1075

macrodipodidarum (Actinomyces), 921

macrodipodidarum (Nocardia), 921

Macromonas, 997, 1000

macrophysa (Thiophysa), 998, 999

macroselmis (Pseudomonas), 146, 148, 699

macrospora (Palmula), 798

macrosporus (Acuformis), 798

macrosporus (Bacillus), 815

macrosporus (Chondrococcus), 1046

macrosporus (Myxococcus), 1046

mactiae (Cristispira), 1056

mactrue (Spirochaeta), 1056 
maculans (Phagus), 1139

maculata (Nocardia), 913

maculatum (Corynebacterium), 404

maculatus (Actinomyces), 913

maculatus (Bacillus), 404, 748

maculatus (Proactinomyces), 913

maculicola (Aplanobacter), 639

maculicola (Bacillus), 639

maculicola (Bacterium), 639

maculicola (Phytomonas), 117

maculicola (Pseudomonas), 117

maculicola var. japonicum (Bacterium),

117

maculicolum (Bacterium), 117

maculifolium - gardeniae (Phytomonas),

696

malampersis (Bacillus), 542

madampensis (Lankoides), 542

madampensis (Shigella), $540, \mathbf{5 4 2}$

maddoxi (Bacillus), 729

maddoxi (Bacterium), 729

madelia (Salmonella), 528

madidus (Bacillus), 661

madidus (Micrococcus), 266

madoxii (Urobacillus), 729

madurae (Actinomyces), 908, 909

madurae (Cladothrix), 908

madurae (Discomyces), 908

madurae (Nocardia), 908, 909, 960

madurae (Oospora), 908

madurae (Streptothrix), 908, 915, 924

maerclii (Bacillus), 356

maggiorae (Pediococcus), 290

maggiorai (Bacillus), 661, 803

maggiorai (Clostridium), 803

maggiorai (Endosporus), 80.3

magna (Cornitia), 787

magnivena (Aureogenus), 1156

magnus (Bacillus), 748,787

magnus (Diplococcus) , 267, 305, 308

magnus (Inflabilis), 823

magnus (Jodococcus), 267, 695

magnus (Micrococcus), 267

magnus (Staphylococcus), 701

magnus (Streptococcus), 341

magnus anaerobius (Diplococcus), 308

magnus liquefaciens (Bacillus), 787 magnusson-holth (Corynebacterium), 391

maidis (Bacillus), 148, 748

maidis (Fractilinea), 1159

maidis (Marmor), 1159

maidis (Phagus), 1136

maidis (Pseudomonas), 148, 748

maidis var. mitis (Fractilinea), 1160

maidis var. sacchari (Fractilinea), 1159

maidis var. typicum (Fractilinea), 1159

major (Bacillus), 661

major (Beggiatoa), 992

major (Gallionella), 832

major (Leptothrix), 986

major (Micrococcus), 267

major (Phagus), 1133

major (Siderocapsa), 833

major (Siderothece), 835

majus (Thiovulum), 1000

makrono-filiformis (Bacillus), $\$ 15$

malabarensis (Bacillus), 714

malae (Streptobacillus), 336

malae (Streptococcus), 336

malakofaciens (Bacillus), $7+8$

malamoria (Vibrio), 205

malaperti (Strextococcus), $3+1$

malariae (Bacillus), 661

malenconi (Actinomyces), 939

malenconii (Streptomyces), 939

malenominatum (Clostridium), 786

malenominatum (Paraplectrum), $\mathbf{7 8 6}$

malenominatus (Bacillus), 786

mali (Bacterium), 640

mali (Marmor), 1194

maligni (Novillus), 777

malignus (Streptococcus), 341

mallei (Actinobacillus), 555

mallei (Bacillus), 555

mallei (Bacterium), 555

mallei (Brucella), 555

mallei (Cladascus), 555

mallei (Corynebacterium), 555

mallei (Loefflerella), 555

mallei (Malleomyces), $\mathbf{5 5 5}$

mallei (Mycobacterium), 555

mallei (Pfeifferella), 555

mallei (Sclerothrix), 555

Malleomyces, 25, 27, 554 
malolacticus (Micrococcus), 267

malvacearum (Bacillus), 159

malvacearum (Bacterium), 159

malvaccarum (Phytomonas), 159

malvacearum (Pseudomonas), 159

malvacearum (Xanthomonas), 159, 1136

malvacearum var. barbadense (Bac-

terium), 178

mammitidis (Bacillus), 662

mammitis (Micrococcus), 267

mammitis bovis (Streptococrus), 267, 341

Mammococcus, 327

manchuriae (Rickettsia), 1085, 1086

manfredii (Micrococcus), 267

manfredii (Streptococcus), 267

manganicus (Bacillus), 662

manganifera (Crenothrix), 987

mangiferae (Bacillus), 474

mangiferae (Erwinia), 474

manhattan (Salmonella), 514

manihotus (Bacillus), 170

manihotus (Bacterium), 170

manihotis (Phytomonas), 170

manihotis (Xanthomonas), 170

mannitocremoris (Streptococcus), 325

mannitopoeum (Bacterium), 359

mannitopoeus (Lactobacillus), 359

mannitopoeus var. fermentus (Lactobacil-

lus), 359

mansfieldii (Bacterium), 760

Mantegazzaea, 853

marcescens (Bacillus), 480

marcescens (Salmonella), 480

marcescens (Serratia), 10, 479, 481, 482,

$483,484,485$

marchonxi (Spirochaeta), 1058

marchouxi (Spironema), 1058

marchouxi (Spiroschaudinnia), 1058

marchouxi (Treponema), 1059

margarineum (Bacterium), 681

margarineus (Bacillus), 662

margaritaceus (Streptococcus), 341

margarittaceum (Bacterium), 157

marginale (Anaplasma), 1100

marginale (Bacterium), 125

marginalis (Phytomonas), 125

marginalis (Pseudomonas), 125 marginans (Marmor), 1195

marginata (Phytomonas), $11 \mathrm{~s}$

marginata (Pseudomonas), 118

marginata (Sarcina), 292

marginatum (Bacterium), 118

marginatus (Actinomyces), 971

marginatus (Micrococcus), 267

marianensis (Bacillus), 589

maricola (Bacillus), 662

mariense (Bacillus), 416

marina (Beggiatoa), 991, 992

marina (Microspira), 205

marina (Spirochaeta), 1052

marina (Thiothrix), 990

marinagilis (Vibrio), 703

marinoflavus (Vibrio), 703

marinofulvus (Vibrio), 703

marinoglutinosa (Pseudomonas), 107

matinoglutinosus (Achromobacter), 107

marinotimosus (Actinomyces), 971

marinopersica (Pseudomonas), 699

marinopiscosus (Bacterium), 605

marinopraesens (Vibrio), 703

marinorubra (Serratia), 484

marinotypicum (Flavobacterium), 431

marinovirosum (Flavobacterium), 431

marinovulgaris (Vibrio), 703

marinum (Bacterium), 681

marinum (Flavobacterium), 433

marinum (Mycobacterium), 883, 884, 886, S87

marinum (Spirillum), 205

marinus (Micrococcus), 267

marinus (Vibrio), 203, 205

maripuniceus (Micrococcus), 696

maris (Flavobacterium), 611

maris-mortui (Chromobacterium), 234

maris-mortui (Flavobacterium) (Halobac-

terium), 442

maritimum (Bacterium), 748

maritimus (Bacillus), 748

markusfeldii (Bacterium), 760

Marmor, 1163, 1202

marmorans (Scelus), 1238

marmotae (Spirochaeta), 1068

marocanum (Spirvchaeta), 1067

marseille (Salmonella), 526 
marshalli (Bacterium), 692 marshallii (Alcaligenes), 415, $416 \quad 692$ marsiliensis (Bacterium), 662 marsilliensis (Bacillus), 662 Martellillus, 11, 763 martinczii (Bacillus), 662 martinezii (Bacterium), 662 martyniae (Bacterium), 112 martyniae (Phytomonas), 112 martyniae (Pseudomonas), 112 maschekii (Pseudomonas), 148 massauah (Microspira), 205 massauah (Spirillum), 205 massauah (Vibrio) 205 massowah (Spirillum) 205 mastidis (Pasteurella) 553 mastidis gangraenosae oris (Micrococcus) 267

mastitidis (Bacterium), 55.3

mastitidis (Micrococcus), 267, 281 mastitidis (Streptococcus), 319 mastitis (Micrococcus), 267 mastitis albus (Staphylococcus), 2s0 mastitis contagiosae (Streptococcus), 319 mastitis sporadicae (Streptococcus), 319 mastobius (Micrococcus), 267 matazoonii (Bacillus), $4+1$ mathersi (Streptococcus), 341 mathiacolle (Thermobacterium), 364 matruchoti (Actinomyces), $91 \mathrm{~s}$ matrucholi (Cladothrix), 91s matruchoti (Nocardia), 918 matrucholi (Oospora), 918 matthiolae (Bactorium), 122 matthiolae (Marmor), 1177 mathiolat (Phytomonas), 122 matthiolae (Pseudomonas), 122, 12: matzoonii (Flavobacterium), $1+1$ maublancii (Bacterium), 140 maublancii (Phytomonas), 140 maublancii (Pseudomonas), 140 maxima (Beggiatoa), 992 maxima (Butyrisarcina), 286 maxime (Leptotrichia), 360 maxima (Rasmussenia), 366 maxima (Sarcina), 286 maxima (Zymosarcina), 286 maxima buccalis (Leptothrix), 366 maximum (Phagus), 1134 maximum buccalis (Bacillus), 365 maximus (Bacillus), 366 maximus (Streptococcus), 341 maydis (Bacillus), 681 maydis (Bacterium), 681 mayomonei (Bacillus), 815 mazei (Methanococcus), 248 mazun (Bacillus), 748 mazun (Bacterium), 362 medanense (Bacterium), 681 media (Beggiatoa), 992 media (Spirochaeta), 1075 media (Spironema), 1075 media oris (Spirochaeta), 1075 medicaginis (Bacillus), 118 medicaginis (Bacterium), 118 medicaginis (Chlorogenus), 1151 medicaginis (Marmor), 1155, 1181 medicaginis (Phytomonas), 118 medicaginis (Pseudomonas), 118 medicaginis var. phaseolicola (Bacterium), 118

medicaginis var. phaseolicola (Phytomonas), 118

medicaginis var. phascolicola (Pseudomonas), $11 \mathrm{~s}$

medicaginis var. solani (Marmor), 1181 medicaginis var. typicum (Marmor), 1181 mediosporus (Bacillus), 748 merdio-tumescens (Bacillus), 748 medium (Clostridium), 820 medium (Treponema), 1075 medius (Chondromyces), 1038 medius (Phagus), 1132 medius (Staphylococcus), 701 megalosporus (Bacillus), 815 megalosporus (Chondrococeus), 1045 megalosporus (Hiberillus), 815 megalosporus (Inflabilis), 815 Megalothrix, 984 megaterium (Bacillus), 711 megaterium bombycis (Bacillus), 739 Megatherium, 20, 22 
megatherium (Bacillus), 632, 714, 717, $718,738,739,744,748,750,759,1130$, 1138

megatherium var. ravenelii (Bacillus), 748 megawi (Richettsia), 1090

megawi var. fletcheri (Richettsia), 1090 megawi var. pijperi (Rickettsia), 1088 Melanella, 5

melaninogenica (Ristella), 574, 575

melaninogenicum (Bacterium), 574

melaninogenicus (Bacteroides), 574, 575

melaninogenicus (Hemophilus), 574

melanocycla (Nocardia), 956

melanocyclus (Actinomyces), 267, 956

melanocyclus (Micrococcus), 267, 956

melanocyclus (Streptomyces), 956

melanogenes (Bacillus), 468, 469, 470

melanogenes (Streptococcus), 341

melanogenes canis (Spirochaeta), 1068

melanogenum (Acetobacter), 183

melanoglossophorus (Micrococcus) 267

melanoroseus (Actinomyces), 972

melanosporea (Nocardia), 955

melanosporeus (Actinomyces), 955

melanosporeus (Streptomyces), 955

melanosporus (Bacillus), 7.48

melanotica (Streptothrix), 977

melanovogenes (Proteus), 490

meldensis (Micrococcus), 267

meleagridis (Bacillus), 662

meleagridis (Lactobacillus), 407

meleagridis (Salmonella), 502, 523

melezitovorum (Aerobacter), 456

meliflava (Sarcina), 292

meliloti (Rhizobium), 226

melitense (Bacterium), 561

melitensis (Alcaligenes), 561

melitensis (Bacillus), 561

melitensis (Brucella), 42, 560, 561, 562

melitensis (Microcuccus), 260,560

melitensis var. abortus (Brucella), 561

melitensis var. melitensis (Brucella), 561

melitensis var. suis (Brucella), 562

Melittangium, 1031, 1033

mellea (Phytomonas), 130

mellea (Pseudomonas), 127, 130

melleum (Bacterium), 130 melleus (Bacillus), 662

melleus grandinis (Micrococcus), 267

melloi (Bartonella), 1108

melloi (Haemobartonella), 1108

melochlora (Pseudomonas), 148, 699)

melochloros (Baccillus), 148

melochlorus (Bacterium), 699

Melococcus, 235, 312

melolonthae (Bacillus), 662

melolonthae (Diplobacillus), 336, 690

melolonthae (Fusiformis), 694

melolonthae liquefaciens (Baclerium), 681

melolonthae liquefaciens $\alpha$ (Bacillus), 681

melolonthae liquefaciens $\alpha, \beta$ and $\gamma$

(Bacillus), 662

melolonthae liquefaciens $\alpha, \beta$ and $\gamma$ (Bacterium), 662

melolonthae non-liquefaciens $\alpha, \beta$ and $\gamma$ (Bacillus), 662

melolonthae non-liquefaciens os (Bacillus), 662

melolonthae non-liquefaciens $\in$ (Bacillus), 662

melonis (Baciltus), 474,748

melonis (Erwinia), 474

melonis (Pectobacterium), $47 \pm$

melophagi (Richetlsia), 1096, 1097

melophagi (Spirochacta), 1068

melophthora (Phytomonas), 141

melophthora (Pseudomonas), 141

melophthorum (Bacterium), 141

membranacea (Nitrosogloea), 74

membranaceum (Chromobacterium), 23.t

membranaceum amethystinum I (Chromobacterium), $23 t$

membranacenm amethystinum II (Chromubacterium), 231

membranaceum amethystinum III (Chromobacterium), 234

membranaceum amelhystinum IV (Chromobacterium), 234

membranaceus (Bacillus), 662

membranaceus amethystinus (Bacillus), 232

membranaceus amethystinus (Bacterium), 232 
membranaceus amethystinus I (Bacillus), 234

membranaceus amethystinus II (Bacillus), 234

membranaceus amethystinus $I I I \quad(B a-$ cillus), 234

membranaceus amethystinus $I^{\top} \quad(B a-$ cillus), 234

membranaceus amethystinus mobilis (Bacillus), 233

membranifer (Bacillus), 612

membranoformis (Achromobacter), 107 membranoformis (Pseudomonas), 107 membranula (Pseudomonas), 699 memelensis (Micrococcus), 267 mendozae (Gaffkya), 281

mendozae (Micrococcus), $28 t$ meningitidis (Bacillus), 662,681 meningitidis (Coccobacillus), 589 meningitidis (Diplococcus), 297 meningitidis (Flavobacterium), 490 meningitidis (Hemophilus), 589 meningitidis (Micrococcus), 297 meningitidis (Neisseria), 296, 297, 299, 301

meningitidis (Streptococcus), 341 meningitidis aerogenes (Bacterium), 662 meningitidis cerebrospinalis septicemiae (Hemophilus), 585

meningitidis purulentae (Bacillus), 681 meningitis (Cillobacterium), 369

Meningococcus, 297

meningococcus cerebrospinalis

(Micrococcus), 297

mephitica (Pseudomonas), 99 merdarius (Streptococcus), 341 merionis (Grahamella), 1110 merismoides (Nitrosogloea), 73 Merismopedia, 6 merismopedioides (Bacterium), 682 Merista, 235

merlangi (Microspironema), $106+$ mesenterica (Nocardia), 907 mesenterica (Pseudomonas), 148 mesentericum (Bacterium), 760 mesentericum (Microbacterium), 907 mesentericus (Agarbacterium), 629 mesentericus (Bacillus), 595, 709, 711, $712,741,748,755,760$

mesentericus (Proactinomyces), 907 mesentericus var. flavus (Bacillus), 710, 712

mesentericus aureus (Bacillus), 173, 693 mesentericus corrugatus (Merismopedia), 258

mesentericus fuscus (Bacillus), 709, 737, 744

mesentericus fuscus consistens (Bacillus), 748

mesentericus fuscus granulatus (Bacillus), 748

mesentericus hydrolyticus (Bacillus), 710 mesentericus niger (Bacillus), 711

mesentericus panis viscosi I (Bacillus), 760

mesentericus panis viscosi II (Bacillus), 710

mesentericus roseus (Bacillus), 748

mesentericus ruber (Bacillus), 709, 748

mesentericus rubiginosus (Bacillus), 743 mesentericus vulgatus (Bacillus), 709 , 741,751

mesentericus vulgatus mucosus (Bacillus), 748

mesenterioides (Bacillus), 749

mesenterioides (Streptococcus), 346

mesenteroides (Ascococcus), 346

mesenteroides (Bacterium), 760

mesenteroides (Leuconostoc), 262, 263,

346, 348, 689

mesenthericus (Bacillus), 755

Metabacterium, 9,762

metabolicus (Bacillus), 662

metabotulinum (Clostridium), 784

metacoli (Bacillus), 452

metacoli (Bacterium), 488

metacoli (Escherichia), 452

metacoloides (Bacillus), 452

metacoloides (Escherichia), 452

metadiffluens (Bacillus), 490

metadiffuens (Proteus), 490

metadysenterica var. $A, B, C$, and $D$

(Shigella), 544

metadysentericus (Castellanus), 544 
metadysentericus var. $A, B, C$ and $D$

(Bacillus), 544

metadysentericus var. $A, B, C$, and $D$

(Dysenteroides), 544

metaflavus (Bacillus), 662

metalcaligenes (Achromobacter), 414

metalcaligenes (Alcaligenes), 414, 416

metalcaligenes (Bacterium), 414

Metallacter, 6, 705, 763

metalloides (Pseudomonas), 148

metchnikovi (Actinomyces), 972

metchnikovi (Vibrio), 196

Metchnikovillus, 11, 763

metchnikoui (Oospora), 934, 972

metentericus (Micrococcus), 696

methanica (Methanomonas), 179

methanica (Methanosarcina), 287

methanica (Sarcina), 287

methanica (Zymosarcina), 287

methanicus (Bacillus), 179

methanigenes (Bacillus), 809

methanigenes (Cellobacillus), 809

methanii (Bacillus), 809

Methanobacterium, 29, 30, 645, 646

Methanococcus, 29, 30, 248

Methanomonas, 20, 31, 82, 179

Methanosarcina, 29, 30, 31, 285

methylicum (Bacterium), 643

methylicus (Bacillus), 643

metiens (Bacillus), 716

metritis (Corynebacterium), 404

metritis (Spirochaeta), 1068

metschnikoffii (Microspira), 196

metschnikoffi (Pacinia), 196

metschnikovi (Spirillum), 196

mexicana (Nocardia), 919

mexicana (Salmonella), 531

mexicanus (Actinomyces), 919

mexicanus (Discomyces), 919

meyeri (Actinobacterium), 927

Meyerillus, 11, 763

miami (Salmonella), 519

micans (Bacillus), 749

micetomae (Actinomyces), 916

micetomae (Oospora), 916

micetomae-argentinue (Nocardia), 918

micetomas argentinae $\alpha$ (Streptothrix), 918 micetomae argentinae $\beta$ (Streptothrix), 916 michaelisii (Bacillus), 733

micheli (Neisseria), 279, 696

micheli (Pacinia), 691

michiganeae (Erwinia), 394

michiganense (Aplanobacter), 394

michiganense (Bacterium), 393

michiganense (Corynebacterium), 393

michiganense (Pseudomonas), 393

michiganense var. saprophyticum (Corynebacterium), 394

michiganensis (Phagus), 1140

michiganensis (Phytomonas), 394

microapoitia (Streptococcus), 341

micro-apoitica enteritis (Streptococcus), 341

Microbacterium, 9, 349, 370, 371

microbutyricum (Bacterium), 590

Micrococcus, 13, 14, 15, 17, 19, 21, 25, 27,

$29,31,33,42,179,235,249,260,265$,

$276,696,1006,1121$

Microcoleus, 993

microdentium (Spirochaeta), 1075

microdentium (Treponema), 1071, 1075

Microderna, 76

microflava (Micromonospora), 950

microflavus (Actinomyces), 950, 976

microflavus (Streptomyces), 950

microgyrata (Spirochacta), 1074

microgyrata (Spironema), 1074

microgyrata (Spiroschaudinnia), 107t

microgyrala var. gaylordi (Spirochaeta), 1068

microgyrate var. gaylordi (Spironema), 1068

microgyrata gaylordi (Spirochacla), 1068 microgyratum (Treponema), $107 t$

Micromonospora, 875, 978

Micromyces, 925, 1291

microparva (Actinomyces), 976

microparva (Nocardia), 976

micropunctata (Nitrocystis), 75

micropunctate (Nitrogloca), 75

micros (Streptococcus), 330, 331

Microsphaera, 235

Microspira, 7, 12, 16, 19, 28, 192, 1122

Microspironema, 1071 
Microsporum, 916 microsporum (Bacterium), 682 microti (Grahamella), 1110 microti (Haemobartonella), 1104 microti pennsylvanici (Grahamella), 1110 microtis (Bacillus), 682 microtis (Bacterium), 682 middletownii (Achromobacter), $\$ 25$ middletownii (Bacterium), 425 Migulanum, 13, 705 mikawasima (Salmonella), 511 mildenbergii (Pseudomonas), 96, 146 Miletensis (streptococcus), 560 milii (Bacillus), 719 milleri (Microspira), 202 milleri (Spirillum), 202 milleri (Vibrio), 202 millerianus (Bacillus), 672 milletiae (Bacillus), 465 milletiae (Erwinia), 465 miltinum (Corynebacterium), 104 Mima, 595 mina (Cristispira), 1056 mineacea (Streptothrix), 917 mineaceus (Actinomyces), 917 minei (Spirochaeta), 1070 minei (Treponema), 1070 miniacea (Serratia), 181 miniaceus (Bacillus), 184 miniaceus (Erythrobacillus), 481 minima (Beggiatoa), 993 minima (Lauterborniola), $\$ 59$ minima (Nocardia), 902 minima (Spirochaeta), 1054 minima (Thiocapsa), 845 minima (Thioploca), 994 minimum (Microsporum), 916 minimum (Rhizobium), 226 minimum (Treponema), 1054 minimum (Trichophyton), 916 minimus (Actinomyces), 916 minimus (Bacillus), 663 minimus (Micrococcus), 267, 268, 303 minimus (Phagus), 1131 minimus (Proactinomyces), 902 minimus (Staphylococcus), 303 minnesota (Salmonella), 529 minor (Bacillus), 858

minor (Beggiatoa), 993

minor (Chondromyces), 1039

minor (Clostridium), 819

minor (Gallionella), 832

minor (Hydrogenomonas), is

minor (Naumanniella), $83 \pm$

minor (Phagus), 1131

minor (Rhabdomonas), $85 t$

minor (Rhodococcus), 865

minor (Rhodorrhagus), 865

minor (Rhodosphaera), 865

minor (Siderocystis), 835

minor (Siderothece), 835

minor (Spirillum), 215

minor (Spironema), 215

minor (Treponema), 215

minus (Bacterium), 858

minus (Chromatium), 856, 857, 858, 859

minus (Polyangium), 1027

minus (Rhabdochromatium), 854

minus (Spirillum), 215

minus (Thiodictyon), 815

minus (Thiovulum), 1000

minus var. muris (Spirillum), 215

minus var. morsus muris (Spirillum), 215

minuscula (Cellulomonas), 105

minuscula (Pseudomonas), 105

minuta (Sarcina), 292

minuta (Spiroschaudinnia), 1076

minutaferula (Bacterium), 605

minutissima (Eberthella), 607

minutissima (Microderma), 76

minutissima (Nocardia), 919

minutissima (Oospora), 919

minutissima (Pseudomonas), 148, 699

minutissima (Shigella), 607

minulissima (Thiothrix), 990

minutissimum (Bacterium), 580, 607, 859

minutissimum (Chromatium), 858

minutissimum (Microsporon), 919

minutissimum (Sporotrichum), 919

minutissimus (Actinomyces), 919

minutissimus (Bacillus), 663, $\$ 58$

minutissimus (Discomyces), 919

minutissimus (Micrococcus), 268, 304

minutissimus (Microsporoides), 919 
minutissimus gazogenes (Cocco-bacillus), 580

minutissimus sputi (Bacillus), 590

minulissimus sputi (Bacterium), 590

minutula (Melosira), 831

minutum (Bacterium), 682

minutum (Ébacterium), 368

minutum (Spirochaeta), 1072, 1075

minutum (Treponema), 1072, 1075, 1076

minutus (Bacillus), 682

minutus (Bacteroides), 368

miquelii (Bacterium), 757

miquelii (Serratia), 481

miquelii (Urobacillus), 691

mira (Cellulomonas), 106

mira (Pseudomonas), 106

mirabile (Bacterium), 488

mirabile (Chloronium), 873, 87t

mirabilis (Bacillus), 488, 666

mirabilis (Beggiatoa), 990, 991, 992, 995

mirabilis (Hillhousia), 998, 999

mirabilis (Nanus), 1207

mirabilis (Proteus), 487, 488, 490, 491

mirabilis (Sarcina), 292

mirabilis (Streptococcus), 341

mirifica (Palmella), 268

mirificus (Micrococcus), 268

misri (Cohnistrepothrix), 918, 975

mississippi (Salmonella), 527

mite (Marmor), 1182

mite (Treponema), 1071

mitelmani (Clostridium), 820

mitidus (Bacillus), 663

mitificans (Aerobacter), 157

mitior (Streptococcus), 321

mitior seu viridans (Streptococcus), 321

mitis (Spiroschaudinnia), 1071

mitis (Streptococcus), 321, $33 \mathrm{~S}$

mitis seu viridans (Streptococcus), 321 mitochondrialis (Bacillus), 749

mixta (Thioploca), 994

mixtus (Streptococcus), 341

Miyagawanella, 1115

mobile (Acetobacter), 692

mobile (Achromatium), 997, 1001

mobile (Achromobacter), 106

mobile (Spirillum), 201 mobile (Termobacterium), 106 mobilis (Marromonas), 1001 mobilis (Micrococcus), 293 mobilis (Planosarcina), 293 mobilis (Pseudomonas), 106, 148 mobilis (Sarcina), 291, 293 mobilissimus (Barillus), 663 Modderula, 997 modestus (Bacillus), 749, 760 modiolne (Cristispira), 1056 modiolae (Spirochaeta), 1056 molleri (Mycobacterium), 889 Mogallia, 13, 306 moliniformis (Baciulus), 667 molischii (Chromatium), 858, 859 molischii (Pseudomonas), 856, 858

Molitor, 1241

mollis (Aurococcus), 268

mollis (Bacillus), 663

mollis (Merismopedia), 268

mollis (Micrococcus), 268, 271

mollis (Staphylococcus), 268

monachae (Bacillus), 682, 749

monachae (Bacterium), 682, 74!

monadiformis (Bacterium), 699

monadiformis (Pseudomonas), 148, 698

Monas, 5,6

moniliforme (Cillobacterium), 36!)

moniliformis (Bacillus), 369, 667

moniliformis (Streptobacilllus), 588, 972,

$1109,1294,1295$

monocella (Nitrosomonas), 69, 70

Honococeus, 235

Monocystia, 14, 1034

monocytogenes (Bacillus), 408

monocytogenes (Bacterium), 408

monocytogenes (Brucella), 695

monocytogenes (Listerella), 408,409

monocytogenes (Listeria), 408, 409, 695

monocytogenes hominis (Listerclla), 408

monoica (Siderocapsa), 834

monospora (Nocardia), 980

monospora (Spirillum), 217

monosporus (Actinomyces), 980

monshaui (Salmonella), 5:31

monstrosum (Bacterium), 760

montanus (Bacillus), 749 
montemartinii (Bacterium), 640 montevideo (Salmonella), 510 moormani (Proactinomyces), 923 mooseri (Rickettsia), 1085, 1086 Morator, $\mathbf{1 2 2 7}$

moraxaxenfeld (Diplobacillus), 590 Moraxilla, 590, 592 morbi brightii (Strepptococcus), $33 \mathrm{~s}$ morbificans (Bacillus), 514 morbificans (Flavobacterium), 514 morbificans (Salmonella), 497, 514 morbificans bovis (Bacillus), 514 morbificans bovis (Bacterium), 514 morbilli (Streptococcus), $3 \pm 1$ morbillorum (Briareus), 1234 morbillorum (Diplococcus), $\mathbf{3 1 0}$ morbillosus (Micrococcus), $3+1$ morbillosus (Streptococcus), 341 Morganella, 488 morgani (Bacillus), 488 morgani (Bacterium), 488 morgani (Escherichia), 488 morgani (Salmonella), 488 morganii (Morganella), 485 morganii (Proteus), 488, 491 mori (Bacillus), 135

mori (Bacterium), 135

mori (Phytomonas), 135

mori (Pseudomonas), 135 moricolor (Micrococcus), 696 moroi (Acilobacterium), 361 moroi (Plocamobacterium), 361 morrhuae (Sarcina), 288 morrhuae (Micrococcus) (Diplecoccus), 268

mors-prunorum (Bacterium), 123 mors-prunorum (Phytomonas), 123 mors-prunorum (Pseudomonas), 123 Morsus, 1153 morsusmuris (Spirella), 215 morsus muris (Spirochaeta), 215 morsusmuris (Spiroschaudinnia), 215 morsus muris (Treponema), 215 mortiferus (Bacillus), 579 mortiferus (Spherophorus), 579 morula (Polyangium), 1027, 1031 morulans (Bacillus), 663 moscow (Salmonella), 518

moscowaensis (Salmonella), 51 s

motorium (Aerobacter), 457

mottei (Bacillus), 663

moulei (Bacillus), 749

mucidolens (Pseudomonas), 148 mucidolens var. tarda (Pseudomonas), 148 mucidus (Achromobacter), 425 mucilagineus (Micrococcus), 268 mucilaginosus (Bacillus), 749 mucilaginosus (Hicrococcus), 26s mucilaginosus koeleriae (Bacillus), 394 mucilaginosus hoeleriae (Pseudomonas), 394

mucofaciens (Micrococcus), 268, 695 mucosa (Cellfalcicula), 211 mucosa (Neisseria), 299, 301 mucosa (Sarcina), 293 mucosa (Spirochaeta), 1072 mucosum (Bacterium), 577, 749, 7את mucosum (Clostridium), 788, 820 mucosum (Treponema), 1072 mucosum anaerobicum (Coccobacterium), 577

mucosum capsulatum (Bacterium), 459

mucosus (Actinomyces), 916

mucosus (Bacillus), 749, 788

mucosus (Capsularis), 577

mucosus (Cellulobacillus), 762

mucosus (Diplococcus), 301, 30s

mucosus (Endosporus), 788

mucosus (1Yycococcus), 891

mucosus (Pneumococcus), 301, 308

mucosus (Streptococcus), 301, 308, 1139 mucosus anaerobius (Bacillus), 577 mucosus capsulatus (Bacillus), 458, 66.3 mucosus capsulatus (Streptococcus), 30s mucosus ozaenae (Bacillus), 459 mucosus ozaena (Bacterium), 459 mucronatus (Bacillus), 749 muelleri (Caryophanon), 1004 muelleri (Simonsiella), 1004 muenchen (Salmonelia), 513 muenster (Salmonella), 523 mulieris (Vibrio), 205 mulleri (Achromatium), 1000 mülleri (Drepanospira), 1122 
mulleri (Monas), 1000

mulleri (Thiovulum), 1000

Multifermentans, 11, 763

multifermentans (Clostridium), $\mathbf{7 7 2}$

multifermentans tenalbus (Bacillus), 772

multiforme (Cillobacterium), 369, 790,

815

multiforme psittacosis (Microbacterium), 1116

multiformiis (Heterocystia), 13

multiformis (Bacillus), 369, 663, 790,

815,826

multiformis (Bacteroidea), 22

multiformis (Bacteroides), 790, 815

multiformis (Haverhillia), 588, 972

multiformis trichorrhexidis (Bacillus),

688

multipediculum (Bacterium), 682

multipediculus (Bacillus), 682

multipediculus flavus (Bacillus), 685,

749

multistriata (Pseudomonas), 101, 699

multistriatum (Achromobacter), 101

multistriatus (Bacillus), 101

multistriatus (Bacterium), 699

multivolatigenum (Lactobacterium), 363

multocida (Pasteurella), 546, 549

multocidum (Bacterium), 546, 517

muralis (Bacillus), 749

muricida (Pasteurella), 548

muricola (Rickettsia), 1086

murina (Rickettsia), 1086

murinus (Bacillus), 411, 663

muripestifer (Bacillus), $6 \mathrm{~S} 2$

muripestifer (Bacterium), 682

muris (Actinomyces), 588, 972, 1109

muris (Asterococcus), 588

muris (Bacillus), 402

muris (Bacterium), 401

muris (Bartonella), 1103

muris (Borrelia), 215

muris (Fusiformis), 583

muris (Grahamella), 1110

muris (Haemobartonella), 1103, 1104,

$1105,1106,1107,1108$

muris (Hemophilus), 589

muris (Legio), 1261 muris (Mycobacterium), 890

muris (Nocardia), 924

muris (Spirella), 215

muris (Spirochaeta), 215

muris (Spironema), 215

muris (Treponema), 215

muris (Treponemella), 215

muris musculi iberica (Grahamella), 1110 muris musculi var. albinoi (Bartonella), 1108

muris musculi var. albinoi (Haemobartonella), 1108

muris ratti (Actinomyces), 588, 924, 972 muris ratti (Bartonella), 1103

muris-ratti (Streptothrix), 924, 972

muris var. galatziana (Spirochaeta), 215

muris var. virginiana (Spirochaeta), 215

muris var. virginiana (Spironema), 215 muriseptica (Erysipelothrix), 411, 548 muriseptica (Pasteurella), 411, 548 murisepticum (Bacterium), 411

murisepticum (Corynebacterium), 390 murisepticum (Mycobacterium), 411 murisepticus (Bacillus), 411, 548, 665 murisepticus (Streptococcus), 341 murisepticus pleomorphus (Bacillus), 490, 665

murisepticus pleomorphus (Bacterium), 665

murium (Bacillus), 502

murium (Corynebacterium), 390

murmanensis (Microspira), 202

musae (Bacillus), 137

musae (Bacterium?), 613

musae (Phytomonas?), 613

musae (Pseudomonas), 613

musarum (Bacillus), 137

muscae (Staphylococcus), 282, 1142

muscoides (Bacillus), 815

muscoides (Cornilia), 815

muscoides colorabilis (Bacillus), 815 muscoides non colorabilis (Bacillus), 815 muscorum (Chondromyces), 1020

muscorum (Stelangium), 1020

musculi (Bacillus), 714

musculi (Grahamella), 1110

musculi (Gyromorpha), 1112 
musculorum (Actinomyces), 972

musculorum suis (Actinomyces), 972

musculorum suis (Oospora), 972

mustelae (Bacillus), 552

mustelae septicus (Bacillus), 445,552

mustelaecida (Bacillus), 552

mustelaecida (Pasteurella), 552

mutabile (Bacterium), 603

mutabile (Rhizobium), 224

mutabilis (Bacillus), 749

mutans (Streptococcus), 342

myceticus (Micrococcus), 268, 696

Mycobacterium, 7, 17, 18, 19, 23, 27, 28,

$30,35,38,392,407,863,875,876,887$,

$891,892,903,905$

Mycobacterium spp., 882,887

Mycococcus, 875,891

Mycoderma, 9, 179

mycodermatus (Micrococcus), 268

mycodermatus (Tetracoccus), 284

Mycogallionella, 986

mycogenes (Bacillus), 663

mycogenum (Bacterium), 663

mycoides (Asterococcus), 1259, 1291

mycoides (Bacillus), 612, 718, 719, 712 $982,1130,1138$

mycoides (Bacterium), 602

mycoides corallinus (Bacillus), 644, 902

mycoides peripneumoniae (Coccobacillis), 1291

mycoides roseum (Bacterium), 602

mycoides roseum (Chromobacterium), 602 mycoides roseus (Bacillus), 602

mycoides-roseus (Erythrobacillus), 602 mycoides var. ovoaethylicus (Bacillus), 720

Mycomonas, 8,876

Myconostoc, 6

Mycoplana, 82, 191

Mycoplasma, 1291

Mycothrix, 983

myricae (Actinomyces), 972

mytili (Bacillus), 663

Myxobacillus, 705

Myxobacter, 1005, 1025

Myxobotyrs, 1036

myxococcoides (Cytophaga), 1049 myxococcoides (Sporocytophaga), 1049

Myxococcus, 14, 17, 20, 24, 25, 312, 1007 , $1009,1040,1041,1044,1047,1049$

myxodens (Bacillus), 749

myxogenes (Cellulobacillus), 762

myxogenes (Clostridium), 820

myxogenes (Pseudomonas), 93

myxomae (Molitor), 1245

nacreaceum (Bacterium), 682

nacreaceus (Bacillus), 682

nacreaceus (Micrococcus), 268

nadsonii (Proteus), 491

naganoi (Sarcina), 293

naganophila (Spirochaeta), 1068

nakatae (Phytomonas), 164

nakatae (Xanthomonas), 164, 1136

nakatae type $B$ (Bacterium), 164

nana (Saprospira), 1055

nanukayami (Spirochaeta), 1077

Nanus, 1206

nanus (Bacillus), 718,815

naphthalinicus (Bacterium), 682

naphthalinicus liquefaciens (Bacillus), 663

naphthalinicus non-liquefaciens (Baci!-

lus), 663

napi (Savoia), 1221

napi (Vibrio), 205

napoli (Salmonella), 522

narashino (Salmonella), 514

nasale (Spirillum), 203

nasale (Spirosoma), 203

nasalis (Bacterium), 459

nasalis (Micrococcus), 342

nasalis (Planococcus), 342

nasalis (Streptococcus), 342

nasalis (Vibrio), 203

nasicola (Spirillum), 203

natans (Bacillus), 718

natans (Cladothrix), 982

natans (Cryptococcus), 258

natans (Sphaerotilis), 982

natans var. cladothrix (Sphaerotilus),

982

nathansonii (Thiobacterium), 81

natto (Bacillus), 710 
Naumanniella, 834

navicula (A mylobacter), 771

navicula (Bacillus), 771

navicula (Bacterium), 771, 824

naviculum (Clostridium), 771

naviformis (Bacillus), 576, 723

naviformis (Ristella), 576

n'dianka (Vibrio), 205

neapolitana (Escherichia), 447

neapolitanus (Bacillus), 447

neapolitanus (Bacterium), 447

nebulosa (Pseudomonas), 101, 699

nebulosum (Achromobacter), 101

nebulosus (Bacillus), 101, 580, 663, 749, 815

nebulosus (Bacterium), 699

nebulosus (Cryptococcus), 258

nebulosus (Protococcus), 258

nebulosus gazogenes (Bacillus), 749

necans (Bacillus), 663

necrodentalis (Bacillus), 361, 362

necrogenes (Bacillus), 579

necrogenes (Spherophorus), 579

necrophora (Pasteurella), 554

necrophora (Streptothrix), 578, 977

necrophorum (Bacterium), 578

necrophorum (Corynebacterium), $57 \mathrm{~s}$

necrophorus (Actinomyces), 578

necrophorus (Bacillus), 578

necrophorus (Fusiformis), 578, 583

necrophorus (Spherophorus), 578, 580,

583,928

necrosans (Clostridium), 820

necroseos (Bacillus), 578

necroseos (Streptococcus), 342

necroticans (Micrococcobacillus), 690)

necroticus (Bacillus), 579

necroticus (Spherophorus), 579

necroticus (Streptococcus), 342

nectarophila (Bacterium), 134

nectarophila (Phytomonas), 134

nectarophila (Pseudomonas), 134

neddini (Actinomyces), 916

negombensis (Bacillus), 5tt

negombensis (Shigella), $54 t$

neigeux (Bacille), 777

neisseri (Pacinia), 386
Neisseria, 19, 21, 26, 27, 29, 31, 33, 295

nelliae (Bacillus), 478

nelliae (Erwinia), 478

nenckii (Achromobacter), 624

nenckii (Bacterium), 624

neocistes (Vibrio), 199

neoformans (Micrococcus), 268

neotropicalis (Borrelia), 1068

neotropicalis (Spirochaeta), 1064

neotropicalis (Treponema), 1064

nephriticus (Bacillus), 663

nephritidis (Bacterium), 553, 760, 761

nephritidis equi (Bacillus), 540

nephritidis instertitialis (Bacillus), 760

neptunium (Flavobacterium), 432

neritica (Pseudomonas), 699

nerviclarens (Marmor), 1198

neschezadimenki (Actinomyces), 928

neschezadimenki (Cohnistreptothrix), 928

neumanni (Bacillus), 681, 749

neurolyticus (Musculomyces), 1293

neurotomae (Bacillus), 663

neurotomae (Bacterium), 663

neurotomae (Micrococcus), 268

neustonica (Naumanniella), 834

newillei (Micrococcus), 268

neveuxi (Spirochaeta), 1059

neveuxi (Spironema), 1059

neveuxi (Treponema), 1059

neveuxii (Spiroschaudinnia), 1059

Nevskia, 7, 35, 829

new brunswick (Salmonella), 525

newington (Salmonella), 524

newport (Salmonella), 573

newportensis (Salmonella), 513

newport var. kottbus (Salmonella), 513

Newskia, 7, 12, 14

new york (Salmonella), 522

nexibilis (Bacillus), 148

nexibilis (Bacterium), 699

nexibitis (Pseudomonas), 148, 699

nexifer (Micrococcus), 696

nicolaieri (Bacterium), 682

nicolaieri (Pacinia), 798

Nicollaierillus, 11, 763

nicollei (Actinomyces), 921

nicollei (Bartonella), 1108 
nicollei (Haemobartonella), 1108

nicollei (Nocardia), 921

nicollei (Spirochaeta), 1059

nicollei (Spironema), 1059

nicollei (Treponema), 1059

nicomosaicum (Phytovirus), 1164

nicotianae (Bacillus), 137

nicotianae (Erwinia), 137

nicotianae-tabaci (Phytomonas), 639

nicotianum (Bacterium), 682

nicotinobacter (Bacterium), 682

nictoinophagum (Bacterium), 682

nictoinovorum (Bacterium), 613

niger (Actinomyces), 969

niger (Bacillus), 711

niger (Micrococcus), 247

nigra (Nocardia), 921

nigra (Streptothrix), 921, 934, 969,

nigrescens (Bacillus), 749

nigrescens (Micrococcus), 268

nigrescens (Nigrococcus), 268

nigrescens (Sorangium), 1024

nigricans (Actinomyces), 972

nigricans (Bacillus), 749

nigrifaciens (Pseudomonas), 109

nigrificans (Actinomyces), 972

nigrificans (Bacillus), 711

nigrificans (Clostridium), 802

nigrificans (Oospora), 972

Nigrococcus, 10

nigrofaciens (Micrococcus), 268

nigromaculans (Bactcrium), 168

nigromaculans (Phytomonas), 168

nigromaculans (Xanthomonas), 168

nigrum (Catenabacterium), 368

nigrum (Sorangium), 1024

nigrum (Spirillum), 217

nijibetsui (Achromobacter), 425, 692

nili (Erro), 1251

niloese (Salmonella), 525

nimipressuralis (Erwinia), 472

ninas kohl-yakomovi (Grahamella), 1110

niosii (Bacteroides), 367

niosii (Eubacterium), 367

nipponica (Rickettsia), 1091

nipponica (Rickettsoides), 1091

nitens (Bacillus), 663 nitens (Bacterium), 682

nitidus (Arthromitus), 1003

nitidus (Bacillus), 749

nitidus (Micrococcus), 269

nitri (Bacillus), 749

nitrificans (Achromobacter), 76

nitrificans (Bacillus), 76

nitrificans (Bacterium), 76

nitrificans (Micrococcus), 269

Nitrobacter, 9, 17, 20, 26, 29, 31, 74, 837

nitrobacter (Bacillus), 74

nitrobacter (Bacterium), 74

nitrobacter (Nitrobacterium), 74

Nitrobacterium, 74

Nitrocystis, 75

nitrogenes (Actinomyces), 972

nitrogenes (Bacillus), 426

Nitrogloea, 75

Nitromonas, 8, 9, 70, 74

Nitrosococeus, 29, 31, 71

Nitrosocystis, 72, 73

Nitrosogloea, 73

Nitrosomonas, $17,20,25,29,31,70,71$, 72,73

nitrosomonas (Bacterium), 70

Nitrosospira, 69, 71, 72

nitrosus (Micrococcus), 71

nitrosus (Nitrosococcus), 69, 71

nitrovorum (Achromobacter), 425

nitrovorum (Bacterium), 425

nitroxus (Bacillus), 749

nivalis (Micrococcus), 269

nivalis (Pseudomonas), 148

nivea (Actinomyces), 972

nivea (Beggiatoa), 989

nivea (Leptotrichia), 989

nivea (Sarcina), 293

nivea (Symphyothrix), 989

nivea (Thiothrix), 989, 995

nivea var. verticillata (Thiothrix), 989

niveus (Micrococcus), 269

nivosum (Bacterium), 77 i

nobilis (Bacillus), 750

nocardi (Bacterium), 895

nocardi (Salmonella), 532

nocardia (Streptococcus), 319 
Nicardit, 9, 892, 917, 929,967, 974 nocardii (Actinomyces), 895 nocardii (Streptothrix), 895 noctiluca (Sarcina), 637 noctuarii (Escherichia), 491 noctuarum (Bacillus), 491 noctuarum (Proteus), 491 Nodofolium, 15, 17, 831 nodosa (Leptospira), 1077 nodosa (Spirochaeta), 1076 nodosum (Bacterium), 404 nodosum (Corynebacterium), 404 nodosum (Rhizobium), 225 nodosum (Treponema), 1077 nodosus (Actinomyces), 583, 917, 1074 nodosus (Fusiformis), 583, 917 nodosus parvus (Bacillus), 404 noelleri (Spirochaeta), 1068

Noguchia, 592

noguchii (Eperthyrozoon), 1101, 1113 noguchii (Spirochaeta), 1068 noguchii (Treponema), 1068 nomae (Bacillus), 682 nomae (Bacterium), 682 nomae (Streptococcus), 342 nondiastaticus (Actinomyces), 972 nondiastaticus (Bacillus), 733, 734 nonfermentans (Bacillus), 787, 826 nonfermentans (Micrococcus), 269 non-hemolyticus $I, I I$ and $I I I$ (Streptococcus), 342

nonliquefaciens (Amylobacter), 771, 824 non-liquefaciens (Cladothrix), 983 non liquefaciens (Clostridium), 819 non liquefaciens (Moraxella), 592 non-liquefaciens (Pseudomonas), 98 nonpentosus (Bacillus), 772, 824 non pyogenes (Staphylococcus), $2 \$ 2$ nonum (Plectridium), 793, 826 nordhafen (Vibrio), 196 normandi (Spirochaeta), 1068 normandi var. carthaginensis (Spirochaeta), 1068 nosocomiale (Treponema), 1068 nosocomialis (Spirochaeta), 1068 nothnageli (Clostridium), 821 notus (Vibrio), 703 novacaesareae (Streptomyces), 951 novellus (Thiobacillus), 79

Novillus, 11, 763

novum (Plectridium), 750

novus (Bacillus), 750

novyi (Bacillus), 777

novyi (Borrelia), 1061

novyi (Cacospira), 1061

novyi (Clostridium), 777, 824

novyi (Spirochaeta), 1061

novyi (Spironema), 1061

novyi (Spiroschaudinnia), 1061

novyi (Treponema), 1061

novyi Type A (Clostridium), 777

novyi Type B (Clostridium), 778

novyi Type C (Clostridium), 778

nubile (Chromolacterium), 404

nubilis (Micrococcus), 269

nubilum (Bacterium), 404, 405

nubilum (Corynebacterium), 404

nubilum (Flavobacterium), 404

nubilum var. nanum (Corynebacterium), 405

nubilus (Bacillus), 404

nucleatum (Fusobacterium), 582

nucleatus (Fusiformis), 582

nuclei (Micrococcus), 269

nucleophagus (Coryococcus), 1121

nucleophyllus (Bacillus), 639

nyborg (Salmonella), 523

oahu (Salmonella), 532

obermeieri (Cacospira), 1059

obermeieri (Spirillum), 1059

obermeieri (Spirochaeta), 1059

obermeieri (Spironema), 1059

obermeieri (Spiroschaudinnia), 1059

obermeieri (Treponema), 1059

oblongum (Bacterium), 682

oblongus (Bacillus), 682, 750

oblongus (Micrococcus), 682

obscoenus (Micrococcus), 269

obscura (Pseudomonas), 699

obsti (Eubacterium), 367

obtusa (Holospora), 1122

obtusa (Spirochaeta), 1068

obtusa (Spiroschaudinnia), 1068 
obtusum (Treponema), 1068

oceanica (Pseudomonas), 699

ocello cuneatum (Bacterium), 776

ochracea (Beggiatoa), 984

ochracea (Chlamydothrix), 984

ochracea (Lampropedia), 844

ochracea (Leptothrix), 984, 985, 986

ochracea (Lyngbya), 984

ochracea (Melosira), 831

ochracea (Merismopedia), 814

ochracea (Pseudomonas), 175, 699

ochraceum (Bacterium), 761

ochraceum (Chromobacterium), 175

ochraceum (Flavobacterium), 175

ochraceum (Polyangium), 1030

ochraceus (Actinomyces), 972

ochraceus (Bacillus), 175

ochraceus (Bacterium), 699

ochraceus (Cellvibrio), 210

ochraceus (Micrococcus), 269

Ochrobium, 835

ochroleuca (Pseudomonas), 148

ochroleucus (Actinomyces), 972

ochroleucus (Bacillus), 663

ochroleucus (Micrococcus), 269

ochroleucus (Planococcus), 269

ochroleucus (Streptococcus), 269

Octopsis, $5+6$

oculogenitale (Chlamydozoon), 1115

oculorum (Leptothrix), 970

odontolyticus (Bacillus), 363

odontolyticus (Lactobacillus), 363

odontolyticus (Streptococcus), 342

odorans (Proteus), 491

odoratum (Bacterium), 664, 739

odoratus (Bacillus), 663, 664, 739

odoratus (Micrococcus), 269

odorifer (Actinomyces), 974

odorifera (Actinomyces), 972

odorifera (Cladothrix), 934, 974

odorifera (Nocardia), 974

odorifera (Oospora), 974

odorifera (Streptothrix), 974

odorificans (Bacillus), 664

odorificus (Bacillus), 664

odorus (Bacillus), 664

odorus (Micrococcus), 269 oedematiens (Bacillus), 777, 825

oedematiens (Clostridium), 777

oedematiens (Eberthella), 534

oedematis (Bacillus), 775, 782, 815

oedematis aerobicus (Bacillus), 660

oedematis benigni (Clostridium), 818

oedematis maligni (Bacillus), 775, 782, 815

oedematis-maligni (Clostridium), 775, 782 oedematis maligni No. II (Bacillus), 777

oedematis sporogenes (Bacillus), 787, 826 oedematis thermophilus (Bacillus), 777 oedematis thermophilus (Bacterium), 777 oedematogenes (Bacillus), 818

oedematoides (Clostridium), 787, 826

oehensis (Bacillus), 750

ogatae (Bacterium), 682

oidieformis (Streptothrix), 977

okeanokoites (Flavobacterium), 429

okenii (Bacillus), 857

okenii (Bacterium), 857

okenii (Chromatium), 852, 854, 856, 857

okenii (Monas), 857

okenii (Pseudomonas), 857

oleae (Bacillus), 132, 750

oleae (Bacterium), 132

oleae tuberculosis (Bacillus), 132

oleracea (Erwinia), 470

oleraceus (Bacillus), 470

olearius (Micrococcus), 269

olens (Micrococcus), 269

olens, (Sarcina), 293

oleovorans (Pseudomonas), 95

olfactorius (Bacillus), 718

oligoacidificans (Lactobacterium), 363

oligocarbophila (Carboxydomonas), 972

oligocarbophilus (Actinomyces), 972

oligocarbophilus (Bacillus), 972

oligocarbophilus (Proactinomyces), 923, 972

oligotrophum (Nirobacter), 76

olivaceus (Actinomyces), 950

olivaceus (Streptomyces), 950

olivae (Bacterium), 761

olivochromogenes (Actinomyces), 941

olivochromogenus (Streptomyces), 941 omelianskii (Bacillus), 750, 809 
omelianskii (Clostridium), 808, 809

omelianskii (Methanobacterium), 645,

$646,795,820$

Omelianskillus, 11, 763

omnivorus (Bacillus), 470

unarimon (Salmonella), 519

oncidii (Bacillus), 640

oncidii (Bacterium!'), 640

oncidii (Phytomonas:'), 640

onderstepoort (Salmonella), 528

ontarioni (Bacillus), 750

ontarioni (Bacterium), 750, 759

oogenes (Bacillus), 750

oogenes fuorescens $\alpha$ (Bacillus), 150

oogenes fluorescens $\beta$ (Bacillus), 147

oogenes fluorescens $\gamma$ (Bacillus), 149

oogenes fluorescens $\delta$ (Bacillus), 149

oogenes fluorescens $\epsilon$ (Bacillus), 149

oogenes hydrosulfureus $\alpha$ (Bacillus), 750

oogenes hydrosulfureus $\beta$ (Bacillus), 147

oogenes hydrosulfureus $\gamma$ (Bacillus), 672

oogenes hydrosulfureus $\delta$ (Bacillus), 148, 663

oogenes hydrosulfuieus $\in$ (Bacillus), 663 oogenes hydrosulfureus ‘ (Bacillus), 663 oogenes hydrosulfureus к (Bacillus), 654 oogenes hydrosulfureus $\nu$ (Bacillus), 653 oogenes hydrosulfureus s (Bacillus),

\section{9}

oogenes (Pseudomonas), 148

oonergasius (Bacillus), 664

opaca (Nocardia), 897

opacum (Mycobacterium), 897

opacum (Nitrobacter), 76

opacus (Bacillus), 750

opacus (Proactinomyces), 897, 898

opacus (Streptococcus), 342

opalanitza (Leuconostoc), 346

opalescens (Micrococcus), 252, 270

Ophidomonas, 6, 850

Ophiocystia, 14, 1017

opossum (Bartonella), 1108

opossum (Haemobartonella), 1108

opportunus (Streptococcus), $3+2$

orae (Annulus), 1155, 1212, 1213

orae (Bacillus), 750

orae (Tractus), 1213

orangea (Sarcina), 293 orangica (Nocardia), 976

orangica (Streptothrix), 976

orangico-niger (Actinomyces), 972

orangicus (Actinomyces), 972

orangium (Chromobacterium), 694 oranienburg (Salmonella), 510

oranienburgensis (Salmonella), 510

orbicularis (Micrococcus), 270

orbicularis flavus (Micrococcus), 270

orbiculata (Neisseria), 300

orbiculatus (Micrococcus), 270

orbiculus (Diplococcus), 300

orchiticum (Bacterium), 682

orchiticus (Bacillus), 683

orchitidis (Flavobacterium), 556

oregon (Salmonella), 513

orenburgii (Bacillus), 362

orientale (Bacillus), 362

orientalis (Dermacentroxenus), 1090

orientalis (Nanus), 1206

orientalis (Rickettsia), 1089, 1090

urientalis (Salmonella), 528

orientalis var. schüffneri (Rickettsia),

1090

orientum (Propionibacterium), 379

orion (Salmonella), 524

orleanense (Bacterium), 187, 693

orleanensis (T'lvina), 693

ornithopi (Bacillus), 224

ornithosis (Miyagawanella), 1117, 1118,

1119

orpheus (Bacillus), 724

orthobutylicus (Bacillus), 771, 821

orthodonta (Spirochaeta), 1075

orthodontum (Treponema), 1075

oryzae (Bacterium), 168

oryzae (Fractilinea), $\mathbf{1 1 6 0}$

oryzae (Marmor), 1160

oryzae (Phytomonas), 168

oryzae (Psendomonas), 168

oryzae (Xanthomonas), 168

Oscillatoria, 988, 991, 1007

Oscillospira, 1004

oslo (Salmonella), 511

osteomyeliticus (Bacillus), 664

osteomyelitus bubalorum (Bacillus),

778

osteophilum (Bacterium), 683 
ostreae (Cristispira), 1056

ostreae (Spirillum), 217

ostreae (Spirochaeta), 1056

ostrei (Bacillus), 532

ostrei (Salmonella), 532

otitidis sporogenes putrificus (Bacillus),

815

otricolare (Bacillus), $\$ 15$

otricolare (Endosporus), 815

ottolenghii (Bacillus), 803

ottolenghii (Clostridium), 803

ottolenghii (Endosporus), 804

ovalare (Clostridium), 796

ovalaris (Plectridium), 796

ovale (Bacterium), 441, 683

ovale (Flavobacterium), 441

ovale (Saccharobacterium), 623

ovalis (Bacillus), 97, 441, 683

malis (Bacterium), 411

ovalis (Micrococcus), 270, 325, 326

ovalis (Pseudomonas), 97, 699

ovalis (Streptococcus), 325

ovalisporus (Myxococeus), 1043, $104 t$

ovata (Pasteurella), 572

ovatum (Bacterium), 683

ovatum (Panhistophyton), 254

ovatus (Bacteroides), 572, 577

matus (Micrococcus), 254

ovatus minutissimus (Bacillus), 683

ovatus minutissimus (Bacterium),

683

ovi (Pseudomonas), 149

ovicola (Pseudomonas), 149

oviforme (Bacterium), 353

oviformis (Bacteroides), 353

oviformis (Coccobacillus), 353

orina (Ehrlichia), 1097

ovina (Rickettsia), 1097

ovina (Spirochaeta), 1068

ovina (Spiroschaudinnia), 1068

ovinum (Bacterium), 554

ovinum (Treponema), 1068

ovis (Corynebacterium), 62, 389, 694

ovis (Eperythrozoon), 1112

ovis (Hemophilus), 589

ovis (Listerella), 409

ovis (Micrococcus), 267

ovis (Spirillum), 1068 ovis (Spirochaeta), 1068

ovis (Spironema), 1068

ovis (Streptococcus), 342

ovis (Tortor), 1278

ovis (Treponema), 1068

oviseptica (Pasteurella), 554

oritoxicus (Bacillus), 790

ovitoxicus (Clostridium), 790, 826

ovitoxicus (Welchia), 790

ovoaethylicus (Bacillus), 720

oxalaticus (Bacillus), 714, 750

oxaliferum (Achromatium), 996, 997, 998, 999

oxydans (Acetobacter), 184

oxydans (Bacillus), 182, 184

oxydans (Bacterium), 181

oxygenes (Bacterium), 544

oxygenes (Eberthella), 544

oxygenes (Shigella), 544

oxylacticus (Bacillus), 664, 750

oxylacticus (Bacterium), 750

oxyphila (Eberthella), 534

oxyphilum (Bacterium), 534

oxytocum (Aerobacter), 456

oxytocum (Bacterium), 456

oxytocus (Bacillus), 456

oxytocus (Escherichia), 456

oxytocus perniciosus (Bacillus), 456

oxytocus perniciosus (Bacterium), 456

ozaenae (Bacillus), 459, 658

ozaenae (Bacterium), 459

ozaenae (Klebsiella), 459

ozenae (Encapsulata), 459

pabuli (Bacillus), 750

pabuli (Plocamobacterium), 693

pabuli acidi II (Bacillus), 356. 693

pabuliacidi (Lactobacillus), 356

pachelabrae (Cristispira), 1056

pachyrhizi (Marmor), 1188

Pacinia, 192, 349, 763

paglianii (Bacillus), 656, 804

paglianii (Clostridium), 804

paglianii (Endosporus), 804

paleopneumoniae (Diplococcus), 309

pallens (Bacterium), 683

pallens (Micrococcus), 270

pallens (Streptococcus), 342 
pallescens (Bacillus), 664

pallescens (Bacterium), 683

pallescens (Pseudomonas), 149, 699

palleum (Endosoma), 859

pallida (Spirochaeta), 1071

pallida (Ulvina), 683

pallida var. cuniculi (Spirochaeta), 1073

pallidior (Bacterium), 683

pallidolimbatus (Marmor), 1197

pallidula (Spirochaeta), 1071

pallidulum (Treponema), 1071

pallidum (Bacterium), 683

pallidum (Microspironema), 1071

pallidum (Spironema), 1071

pallidum (Treponema), 1071, 1072, 1073 pallidum var. cuniculi (Treponema), 1073 pallidus (Bacillus), 664, 750

pallidus (Micrococcus), 270

pallidus (Streptococcus), 342

palludosum (Plectridium), 727

Palmula, 34, 763

paludis (Bacillus), 790, 826

paludis (Welchia), 790

paludosa (Sarcina), 293

paludosum. (Bacterium), 761

palumbarium (Bacterium), 552

palustris (Bacillus), 750

palustris (Hillhousia), 998, 999

palustris (Rhodobacillus), 750, 863

palustris (Rhodomonas), 863

palustris (Rhodopseudomonas), 860, 863,

$864,865,866$

palustris var. gelaticus (Bacillus), 750

pammelli (Bacillus), 745

panacis (Pseudomonas), 130

panama (Salmonella), 519

panaxi (Bacterium), 130

panaxi (Phytomonas), 130

pandora (Bacillus), 720

panginensis (Actinomyces), 921

panginensis (Nocardia), 921

panici (Bacterium), 169

panici (Phylomonas), 169

panici (Pseudomonas), 169

panici (Xanthomonas), 169

panici-miliacei (Bacterium), 143

panici-miliacei (Phytomonas), 143 panici-miliacei (Pseudomonas), 143 panificans (Bacillus), 664 panis (Bacillus), 710, 711 panis (Bacterium), 760 panis (Lactobacillus), 358 panis acidi (Lactobacillus), 363 panis fermentati (Bacillus), 358 panja (Actinomyces), 966 panjae (Streptomyces), 966 pannosus (Bacillus), 664 pannosus (Micrococcus), 270 pansinii (Bacillus), 664, 667 pansinii (Bacterium), 718 pansinii (Pseudomonas), 149 pantotropha (Hydrogenomonas), 77 pantotrophus (Bacillus), 77 papavericola (Bacterium), 164 papavericola (Phytomonas), 164 papavericola (Xanthomonas), 164 papaveris (Bacillus), 474 papaveris (Erwinia), 474 papayae (Bacillus), 478 papayae (Erwinia), 478 papillare (Bacterium), 683 papillare (Photobacterium), 637 papillaris (Microspira), 637 pappulum (Paraplectrum), 816 pappulus (Bacillus), 815 papuana (Salmonella), 512 papulans (Bacterium), 124 papulans (Phytomonas), 124 papulans (Pseudomonas), 124 paraabortus (Brucella), 563 para-aertrycke (Bacillus), 502 paraalvei (Bacillus), 723 paraamericanus (Proteus), 491 para-asiaica (Salmonella), 532 para-asialicus (Bacillus), 532 parabifermentans (Clostridium), 796 parabifermentans sporogenes (Bacillus), 796

parabifidus (Lactobacillus), 354

parabotulinum (Clostridium), 778, 779, 784

parabotulinum equi (Clostridium), 779 parabotulinum Types A and B (Clostridium), $779,784,798$ 
parabotulinus (Bacillus), 779, 780

parabotulinus bovis (Clostridium), 77

parabutyricus (Bacillus), 813, 816

paracitrovorus (Streptococcus), 347

para-coagulans (Bacillus), 532

para-coagulans (Salmonella), 532

Paracloster, 7, 705

paracoli (Bacterium), 460

Paracolobactrum, 460, 461, 489

para-colon (Bacillus), 460

para-colon (Salmonella), 460

paradiffuens (Bacillus), 491

paradiffuens (Proteus), 491

paradoxa (Escherichia), 453

paradoxus (Bacillus), 683

paradoxus (Bacterium), 683

paradoxus (Colibacillus), 453

paradysenteriae (Bacillus), 537, 538

paradysenteriae (Bacterium), 537

paradysenteriae (Eberthella), 537

paradysenteriae (Shigella), 537, 538,

$539,510,543$

paradysenteriae Type Manchester (Shigella), 539

paradysenteriae (Type Newcastle) (Shigella), 538

paradysenteriae var. sonnei (Shigella), 540

paradysenteriae $\mathrm{X}$ (Bacillus), 536

paraenterica (Enteroides), 452

paraenterica (Escherichia), 453

paraentericus (Bacillus), 453

paraexilis (Bacillus), 352

paraffinae (Micrococcus), 270

paraffinae (Nocardia), 901

paraffinae (Proactinomyces), 901

para-gruenthati (Bacillus), 451

paragruenthali (Escherichia), 451

paraguayensis (Proactinomyces), 923

parainfluenzae (Hemophilus), 5\$5, 586

paralacticus (Streplococcus), 324

parallela (Aphanothece), 872

parallela (Pediochloris), 872

parallelum (Pelodictyon), 872

parallelus (Bacillus), 664

paraluis (Spirochaeta), 1073

paraluis-cuniculi (Spirochaeta), 1073 paralytica (Klebsiella), 459

paralyticans (Bacillus), 405

paralyticans (Corynebacterium), 405

paramecii (Müllerina), 1122

paramelitensis (Brucella), 563

paramelitensis (Micrococcus), 563

paramorganii (Proteus), 491

paraoxytocum (Aerobacter), 456

para-pertussis (Bacillus), 587

Paraplectrum, 7, 33, 763

paraputrificum (Clostridium), 793

paraputrificum (Plectridium), 793

paraputrificus (Bacillus), 793

paraputrificus (Tissierillus), 793

paraputrificus coli (Bacillus), 793

parasarcophysematos (Bacillus), 775

parasitica (Leptothrix), 366

parasiticum (Bacterium), 366

parasiticum (Polyangium), 1032

Paraspirillum, 9

parasporogenes (Bacillus), 784

parasporogenes (Clostridium), 784

parasuis (Brucella), 563

paratuberculosis (Mycobacterium), 881

paratyphi (Bacillus), 501

paratyphi (Bacterium), 501

paratyphi (Salmonella), 495, 501

paratyphi abortus ovis (Bacillus), 506

paratyphi alcaligenes (Bacillus), 501

paratyphi alvei (Bacillus), 532

paratyphi A (Salmonella), 493, 501

paratyphi B (Salmonella), 493, 501

paratyphi C (Salmonella), 493, 507

paratyphi Typus A (Bacterium), 501

paratyphi, Typus B (Bacterium), 501

paratyphosum A (Bacterium), 501

paratyphosum B (Bacterium), 501

paratyphosus (Bacillus), 501

paratyphosus A (Bacillus), 501

paratyphosus $B$ (Bacillus), 501, 509

paratyphosus $\beta_{5}$ (Bacillus), 507

paratyphosus $B$. Arkansas type (Bacillus),

508

paratyphosus B, Binns type (Bacillus), 503

paratyphosus B, Mutton type (Bacillus) 502 
paratyphosus $B$, Newport type (Bacillus), 513

paratyphosus $B$, Reading type (Bacillus,) 504

paratyphosus B, Stanley type (Bacillus), 503

paratyphosus $C$ (Bacillus), 507, 509

paratyphosus $C$ (Salmonella), 507, 509 paraviscosum (Bacterium), 683

parcifermentans (Lactobacterium), 364 parkeri (Borrelia), 1064

parkeri (Spirochaeta), 1064

parotitidis (Micrococcus), 270

parotitidis (Spirochaeta), 1074

partum (Clostridium), 821

parva (Cornilia), 822

parva (Micromonospora), 979

parva (Nocardia), 939

parvula (Cristispira), 1056

parvula (Veillonella), 302, 303, 304

parvula var. branhamii (Veillonella), 303

parvula var. minima (Veillonella), 303 parvula var. thomsonii (Veillonella), 303 parvulum (Bacterium), 642

parvulum (Corynebacterium), 408

parvulus (Micrococcus), 302

parvulus (Staphylococcus), 302, 345

parvulus (Streptococcus), 331, 342

parvulus (Streptostaphylococcus), 345

parvulus non liquefaciens (Streptococcus),

331

parvum (Bacterium), 822

parvum (Corynebacterium), 388, 405

parvum (Eubacterium), 368

parvum (Spirillum), 203, 701

parvum (Spirochata), 1075

parvum (Treponema), 1075

parvum infectiosum (Corynebacterium),

405

parvus (Actinomyces), 939

parvus (Bacillus), 544, 712

parvus (Jodococcus), 270, 695

parvus (Micrococcus), 270

parvus (Phagus), 1131

parvus (Rhodovibrio), 863

parvus (Streptomyces), 939 parvus (Vibrio), 203

parvus liquefaciens (Bacillus), 404

parvus ovatus (Bacillus), 548

passeti (Staphylococcus), 256

passiflorae (Marmor), 1193

passiflorae (Phytomonas), 138

passiflorae (Pseudomonas), 138

Pasteurella, 17, 21, 22, 26, 32, 37, 42,

546, 551, 577, 1290

pasteuri (Cornilia), 775

pasteuri (Micrococcus), 270, 306

Pasteuria, 35, 836

pasteuriana (Ulvina), 692

pasteurianum (Acetobacter), 61, 180,

182,692

pasteurianum (Bacillus), 692

pasteurianum (Bacterium), 182

pasteurianum (Clostridium), 772, 824

pasteurianum (Mycoderma), 182

pasteurianum (Rhyzobium), 225

pasteurianus (Bacillus), 772

pasteurii (Bacillus), 729, 744

pasteurii (Urobacillus), 729

pasteurii (Tibrio), 774

pastinator (Achromobacter), 628

pastorianum (Clostridium), 772

pastorianum (Lactobacterium), 360

pastorianum (Plocamobacterium), 695

pastorianus (Bacillus), 359, 772

pastorianus (Lactobacillus), 359, 695

pastorianus (Saccharobacillus), 359

pastorianus (Streptococcus), 337, 342

pastorianus var. berolinensis (Saccharo-

bacillus), 360

patelliforme (Bacterium), 683

pateriforme (Bacterium), 683

pathogenicum (Photobacterium), 635

paucicutis (Bacillus), 750

paullulus (Bacillus), 664

pauloensis (Eberthella), 534

pauloensis (Escherichia), 453

pauloensis (Salmonella), 532

Paulosarcina, 13, 285

paurometabolum (Corynebacterium), 392,

pavlovskii (Bartonella), 1108

pavlovskii (Haemobartonella), 1108

pavonacea (Pseudomonas), 91 
pavoninus (Bacillus), 233

pavonis (Treponema), 1075

pecoris (Hostis), 1239

pectinis (Cristispira), 1056

Pectinobacter, 763

pectinophorae (Bacillus), 664

pectinovorum (Aerobacter), 456

pectinovorum (Bacillus), 822

pectinovorum (Clostridium), 771, 822

pectinovorum (Granulobacter), 771, 822

pectinovorum (Plectridium), 771, \$22,

824

pectinororum liquefaciens (Plectridium), 823

pectinovorus (Bacillus), 822

Pectobacillus, 8, 763

Pectobacterium, 464

pectocutis (Bacillus), 750

pediculata (Nevskia), 830

pediculatum (Bacterium), 830

pediculatus (Chondromyces), 1038

pediculi (Bacillus), 664

pediculi (Rickettsia), 1094, 1095, 1096,

1097

Pediochloris, 870

Pediococcus, 7, 25, 235, 249, 844

Pedioplana, 235, 250

pelagia (Bacillus), 635

pelagia (Bacterium), 635

pelagia (Sarcina), 701

pelagicus (Bacillus), 750

pelamidis (Spirochaeta), 1068

pelamidis (Spironema), 1068

pelargoni (Bacterium), 160

pelargoni (Pseudomonas), 160

pelargonii (Marmor), 1199

pelargonii (Phytomonas), 160

pelargonii (Xanthomonas), 160, 167

pelletieri (Actinomyces), 960

pelletieri (Discomyces), 960

pelletieri (Micrococcus), 960

pelletieri (Nocardia), 960

pelletieri (Oospora), 960

pelletieri (Streptomyces), 960

pelliculosa (Pseudomonas), 149

pellucida (Beggiatoa), 992

pellucida (Pseudomonas), 149 pellucidum (Achromobacter), 145

pellucidum (Halibacterium), 653

pellucidus (Bacillus), 664, 750

pellucidus (Micrococcus), 270

Pelochromatium, 859

Pelodictyon, 870

pelogenes (Actinomyces), 972

Pelogloea, 870

pelomyxae (Cladothrix), 1123

pelurida var. virginiana (Pseudomonas),

174

pemphigi (Micrococcus), 270

pemphigi acuti (Diplococcus), 270

pemphigi contagiosa (Micrococcus), 270

pemphigi neonatorum (Micrococcus), 271

pemphigi neonatorum (Staphylococcus),

271

pendens (Rhodothece), $\mathbf{8 5 5}$

pendunculatus (Bacillus), 741

penicillatus (Bacillus), 816

penortha (Spirochacta), 583, 917, 1074

pensacola (Salmonella), 518

pentoaceticum (Plocamobacterium), 695

pentoaceticus (Lactobacillus), 358, 695

pentoaceticus var. magnus (Lacto-

bacillus), 358

pentosaceum (Propionibacterium), 378, 379

pentosaceus (Pediococcus), 250

pentosus (Lactobacillus), 357

pepo (Bacillus), 730

Peptoclostridium, 30, 763

Peptococcus, 29, 30

peptogenes (Bacillus), 751

peptogenes (Bacterium), 751

peptonans (Bacillus), 751

peptonificans (Bacillus), 751

Peptonococcus, 235

Peptostreptococcus, 30, 31, 312

percitreus (Micrococcus), 271

percolans (Vibrio), 201

perekropovi (Eperythrozoon), 1113

perexile (Treponema), 1075

perexilis (Spirochaeta), 1076

perfectomarinus (Pseudomonas), 699

perflava (Neisseria), 298

perflavus (Micrococcus), 271 
perfoetens (Baclerium), 576

perfoetens (Bacteroides), 576

perfoetens (Ristella), 576

perforans (Spirochaeta), $106 \mathrm{~s}$

perfringens (Bacillus), 790, 815,826

perfringens (Clostridium), 789, 790, 817,

818,826

perfingens Type A (Clostridium), 789

perfringens Type B (Clostridium), 790

perfringens Type C (Clostridium), 790

perfringens Type D (Clostridium), 790

perfringens (Welchia), 790

perfringens var. anaerogenes (Clostridium), 791, $\$ 26$

perfringens var. egens (Clostridium), 790

perfringens var. eyens (Welchiu), 790

perfringens var. zoodysenteriae (Welchia),

791

pericarditis (Pasteurella), 55

pericoma (Vibrio), 205

perimastrix (Vibrio), 703

periphyta (Pseudomonas), 699

periplanetae (Corynebacterium), 405

periplanetae (Spirochaeta), 1069

periplanetac var. americana (Coryne-

bacterium), 405

periplaneticum (Spirillum), 217

peripneumoniae (Borrelomyces), 1291

peripneumoniae (Mycoplasma), 1291

peripneumoniae bovis (Asteromyces), 1291

peripneumoniae bovis contagiosae (Mi-

cromyces), 1291

peritonitidis (Streptococcus), 317

peritonitidis equi (Streptococcus), 317

peritonitis (Spherophorus), 579

perittomaticum (Bacterium), 761

perlibratus (Bacillus), $66 t$

perlucidulus (Bacillus), 751

perlurida (Cellulomonas), 17-t

perlurida (Pseudomonas), 174

perniciosus (Micrococcus), 342

perniciosus (Pediococcus), 250

perniciosus (Streptococcus), 342

perniciosus psittacorum (Streplococcus),

342

perolens (Achromobacter), 425 peromysci (Grahamclla), 1111

peromysci (Haemobartonella), 1106

peromysci var. maniculati (Grahamella), 1111

peromysci var. maniculati (Haemobar-

tonella), 1106

peroniella (Bacillus), 816

peroxydans (Acetobacter), 189

perronciti (Bacillus), $66+$

Perroncitoa, 312

persica (Borrelia), 1069

persica (Spirochaeta), 1069

persicae (Chlorogenus), 1148, 115:

persicae (Flavimacula), 1196

persicae (Marmor), 1196, 1202

persicae var. micropersica (Chlorogenus),

1149

persicac var. rulgaris (Chlorogenus),

1149

persicina (Palmella), 848

persicina (Sarcina), 293

persicum (Treponema), 1069

persicus (Micrococcus), 271

pertenue (Spironema), 1071

pertenue (Treponema), 1071

pertenuis (Spirochaela), 1071

pertussis (Bacillus), 586, 737

pertussis (Hemophilus), 586, 587, 589

pertussis eppendorf (Bacillus), 589

peruviana (Bartonella), 1101

pestifer (Achromobacter), 425

pestifer (Bacillus), 425

pestifer (Baclerium), 425

pestis (Bacillus), 549

pestis (Bacterium), 5.49

pestis (Eucystica), 549

pestis (Pasteurella), 549, 703

pestis bubonicae (Bacillus), 54!

pestis bubonicae (Bacterium), 549

pestis-caviae (Bacillus), 502

pestis-caviae (Pasteurella), 502

petasites (Bacillus), 714

petasitis (Bacterium), 142

petasitis (Phytomonas), 142

petasitis (Pseudomonas), 142

petechialis (Micrococcus), 271

petersii (Bacillus), 664 
petersii (Bacterium), 683

peterssonii (Propionibacterium), 376 petilus (Micrococcus), 271 petiolatus (Bacillus), 751 petit (Spirochaeta), 215 petrolei (Micrococcus), 271 petroselini (Bacillus), 715 petroselini (Bacterium), 715 pettiti (Leptospira), 1079 pettiti (Spirochacta), 215, 107 ? pettiti (Treponema), 1079 pfaffi (Bacillus), 539

pfaffi (Bacterium), 539

pfaffii (Eberthella), 539

pfaffii (Shigella), 539

Pfeifferella, 9, 21, 22, 28, 554

pfeifferi (Coccobacillus), 585

pfeifferi (Encapsulatus), 459

pflegmones-emphysematosae

790

pflegmones-emphysematosae (Clostridium), 790

pflügeri (Arthrobacterium), 635

pflügeri (Bacterium), 635

pfligeri (Micrococcus), 635

pflügeri (Photobacterium), 635

Phacelium, 13, 285

phaeochromogenus (Actinomyces), 943

phaeochromogenus (Streptomyces), 943

Phacomonas, 29, 30

Phaeospirillum, 29, 30, 866

phagedenis (Borrelia), 1064

phagedenis (Spirochaeta), 1064

phagedenis (Spironema), 1064

phagedenis (Spiroschaudinnia), 1064

phagedenis (Treponema), 1064

Phagus, 1128

pharyngis (Diplococcus), 299

pharyngis (Micrococcus), 696

pharyngis (Neisseria), 299, 696

pharyngis (Staphylococcus), 282

pharyngis cinerea (Neisseria), 298

pharyngis cinereus (Micrococcus), 298

301

pharyngis communis (Diplococcus), 298 pharnygis flavus I (Diplococcus), 298 pharyngis flavus II (Diplococcus), 299 pharyngis flavus I (Micrococcus), 299

pharyngis flavus II (Micrococcus), 299 pharyngis-sicci (Neisseria), 298

pharyngis siccus (Diplococcus), 298 pharyngis siccus (Micrococcus), 298 phaseoli (Bacillus), 160, 751

phaseoli (Bacterium), 160

phaseoli (Phytomonas), 160

phascoli (Pseudomonas), 160

phaseoli (Marmor), 1168, 1179

phaseoli (Rhizobium), 225, 226

phaseoli (Xanthomonas), 160, 161, 1134,

1136

phaseoli var. fuscans (Bacterium), 161

phaseoli var. fuscans (Phytomonas), 161

phaseoli var. fuscans (Pseudomonas),

161

phaseoli var. fuscans (Xanthomonas), 161

phaseoli var. sojense (Bacterium), 161

phaseoli var. sojense (Phytomonas), 161

phaseoli var. sojensis (Xanthomonas), 161

phaseolicola (Pseudomonas), 118

phasiani (Bacillus), 520

phasiani septicus (Bacillus), 520

phasiani septicus (Bacterium), 520

phasianicida (Bacillus), 552

phasianicida (Bacterium), 552

phasianidarum mobile (Bacterium), 552

phenanthrenicus bakiensis (Bacillus), 664

phenanthrenicus guricus (Bacillus), 665

phenologenes (Bacillus), 665

phenolphilos (Bacillus), 751

phenotolerans (Actinomyces), 917

phlebotomi (Spirochaeta), 1074

phlegmones emphysematosae (Bacillus),

$789,790,826$

phlei (Mycobacterium), 881, 887, 889,

890

phlei (Sclerothrix), 889

phlei perrugosum (Mycobacterium), $\$ 90$

phlei planum (Mycobacterium), 890

Phleobacterium, 20, 23

pholas (Bacterium), 635

phormicola (Bacterium), 166

phormicola (Phytomonas), 166

phormicola (Xanthomonas), 166

phosphorescens (Bacillus), 111 
phosphorescens (Bacterium), 111, 633,

635,636

phosphorescens

phosphorescens

phosphorescens

phosphorescens

635,636

phosphorescens (Pseudomonas), 111, 699

phosphorescens (Spirillum), 633

phosphorescens (Vibrio), 633, 702

phosphorescens gelidus (Bacillus), 636

phosphorescens gelidus (Bacterium), 635

phosphorescens giardi (Bacillus), 635

phosphorescens giardi (Bacterium), 635

phosphorescens indicus (Bacillus), 699

phosphorescens indicus (Bacterium), 699

phosphorescens indigenus (Bacillus), 633

phosphorescens indigenus (Bacterium),

633, 636

phosphorescens pflügeri (Bacterium), 635

phosphoreum (Achromobacter), 634

phosphoreum (Bacterium), 633, 635, 636,

637

phosphoreum (Microspira), 636

phosphoreum (Photobacter), 633

phosphoreum. (Photobacterium), 633

phosphoreus (Bacillus), 633, 634

phosphoreus (Micrococcus), 633, 635

phosphoreus (Streptococcus), 633

phosphoricum (Achromobacter), 634

phosphoricus (Bacillus), 634

Photobacter, 636

Photobacterium, 12, 14, 192, 636

photometricum (Bacterium), 683

photometricum (Rhodospirillum), 867

photometricus (Bacillus), 683

Photomonas, 11, 636

Photospirillum, 636

photuris (Proteus), 491

Phragmidiothrix, 6, 12, 17, 19, 987

phyllotidis (Grahamella), 1111

physiculus (Micrococcus), 636

Phytomonas, 31, 32, 150

Phytomyxa, 223

phytophthora (Erwinia), 469, 470

phytophthorum (Bacterium), 470

phytophthorum (Pectobacterium), 470 phytophthorus (Bacillus), 469, 470

phytophthorus (Micrococcus), 342

phytophthorus (Streptococcus), 342 phytoplanktis (Vibrio), 703

picrogenes (Bacillus), 751

picrum (Achromobacter), 622

pictor (Treponema), 1072

pictorum (Achromobacter), 177

pictorum (Pseudomonas), 177

pierantonii (Bacillus), 202

pierantonii (Cocco-bacillus), 112

pierantonii (Micrococcus), 112

pierantonii (Pseudomonas), 112

pierantonii (Vibrio), 202

pieridis (Micrococcus), 271

pieris (Borrelina), 1227

pieris (Diplobacillus), 690

pieris (Diplococcus), 336

pieris (Vibrio), 703

pieris agilis (Bacillus), 665

pieris fluorescens (Bacillus), 665

pieris liquefaciens (Bacillus), 665

pieris liquefaciens $\alpha$ (Bacillus), 665

pieris liquefacions $\beta$ (Bacillus), 665

pieris liquefaciens (Bacterium), 665

pieris non-liquefaciens $\alpha$ (Bacillus), 665

pieris non-liquefaciens $\beta$ (Bacillus), 665

piesmae (Savoia), 1221

piima (Streptococcus), 342

pijperi (Actinomyces), 921

pijperi (Discomyces), 921

pijperi (Nocardia), 921

pikowskyi (Achromobacter), 425

pikoushyi (Micrococcus), 271

piliformis (Bacillus), 366, 751

piliformis (Micrococcus), 271

piltonensis (Micrococcus), 271

piluliformans (Bacillus), 684

piluliformans (Bacterium), 683

pini (Bacterium), 640

pini (Pscudomonas), 640

pinnae (Cristispira), 1055

pinnae (Spirochaete), 1055

pinnatum (Achromobacter), 425

pinnatus (Bacillus), 425

pinnatus (Bacterium), 426 
pinoyi (Actinomyces), 921

pinoyi (Nocardia), 921

pintae (Treponema), 1072

pipientis (Wolbachia), 1098

pipistrelli (Grahamella), 1111

pipistrelli (Grahamia), 1111

piriforme (Corynebacterium), 694

piscatora (Shigella), 5.4

piscatorum (Bacterium), $4 \$ 3$

piscatorum (Serratia), 483

piscatorus (Bacillus), 483

pisces (Rickettsia), 1097

piscicidus (Bacillus), 751

piscicidus agilis (Bacillus), 751

piscicidus agilis (Bacterium), 751

piscicidus nobilis (Bacillus), 751

piscicidus versicolor (Bacillus), 491

piscicidus versicolor (Proteus), 491

piscium (Mycobacterium), 883, 884, 885 ,

886,887

piscium (Vibrio), 197

piscova (Pseudomonas), 700

pisi (Bacillus), 751

pisi (Bacterium), 11 ?

pisi (Marmor), 1180

pisi (Phytomonas), 119

pisi (Pseudomonas), 119

pistiense (Thiospirillum), 853

pitheci (Spirillum), 1064

pitheci (Spirochaeta), 1064

pitheci (Spironema), 1064

pituitans (Bacterium), 761

pituitoparus (Micrococcus), 271

pituitoparus (Karphococcus) (C'arpho-

coccus), 271

pituitosum (Bacterium), 125, 684

pituitosum (Propionibacterium), 379

pityocampae (Bacterium), $68+$

pitycampae $\alpha$ (Streptococcus), 342

pitycampae $\beta$ (Streptococcus), $3+2$

placoides (Cladothrix), 97t

placoides (Leptotrichia), 974

placoides alba (Leptothrix), $97 \mathrm{t}$

plagarum (Inflabilis), 823

plagarum-belli (Diplococeus), 310

P'lanococcus, 7, 13, 15, 235
Planomerista, 235

Planosarcina, 7, 15, 285

Planostreptococcus, 15, 312

plantaginis (Phytomonas), 161

plantaginis (Xanthomonas), 161

plantarum (Lactobacillus), 356, 357, 363,

695

plantarum (Streptobacterium), 356

plantarum var. rudensis (Lactobacillus), 357

platus (Bacillus), 723

platychoma (Bacillus), 751

plauti (Fusocillus), 581

plauti-vincenti (Fusobacterium), 581,

1063

plauti-vincenti (Fusiformis), 581

plaut-vincenti (Spirochaete), 1063

plebia (Escherichia), 451

plebeius (Bacillus), 451

Plectridium, 7, 13, 33, 763

Plectrillium, 7

Plectrinium, 7

plehniae (Pseudomonas), 149

Plennobacterium, 705

pleofructi (Bacillus), 346

pleofructi (Leuconostoc), 346

pleomorpha (Pseudomonas), 700

pleomorphus (Bacillus), 665

pleomorphus (Streptococcus), 342

pleuriticus (Discomyces), 915

pleuriticus (Leptothrix), 915

pleuriticus canis famitiaris (Actinomyces), 915

Pleuropneumonia, 1289

pleuropneumoniae (Bacterium), 681

pleuropneumoniae (Bovimyces), 1291

plexiformis (Bacillus), 751

plicata (Pseudomonas), 149

plicatile (Spirillum), 1052

plicatilis (Ehrenbergia), 1052

plicatilis (Spirochaeta), 1052, 1053

plicatilis (Spirulina), 1052

plicatilis eurystrepta (Spirochaeta), 1052

plicatilis marina (Spirochaeta), 1052

plicatilis plicatilis (Spirochaeta), 1052

plicativum (Bacterium), 684, 761

plicatum (Acetobacter), 186 
plicatum (Bacterium), 761

plicatum (Flavobacterium), 441

plicatus (Bacillus), 149, 441, 665, 684, 747,751

Plocamobacterium, 18, 25, 27, 349, 381, 400 plumbeus (Bacillus), 665

plumosum (Corynebacterium), 405

plumosum (Mycobacterium), 405

plumosus (Micrococcus), 271

plurichromogenes (Actinomyces), 897

pluricolor (Actinomyces), 973

pluricolor (Nocardia), 973

pluricolor (Streptothrix), 973

pluricolor diffundens (Actinomyces), 973

pluricromogena (Streptothrix), 897

pluriseptica (Pasteurella), 547

plurisepticus (Bacillus), 546

pluton (Bacillus), 724

pluton (Diplococcus), 724

plymouthensis (Bacillus), 482

plymouthensis (Erythrobacillus), 482

plymuthicum (Bacterium), 482

plymuthicum (Serratia), 481, 482, 483,

484, 485

Pneumobacillus, 458

Pneumococcus, 306

pneumo-enteritidis murium (Bacillus), 665

pneumoniae (Bacillus), 458, 652

pneumoniae (Bacterium), 307, 458

pneumoniae (Diplococcus), 43, 45, 306, 307, 308, 309

pneumoniae, Type 3 (Diplococcus), 308

pneumoniae (Encapsulatus), 458

pneumoniae (Hyalococcus), 458

pneumoniae (Klebsiella), 458

pneumoniae (Miyagawanella), 1118, 1119

pneumoniae (Pneumococcus), 307

pneumoniae (Proteus), 458

pneumoniae (Streptococcus), 307

pneumoniae caprae (Bacillus), 553

pneumoniae crouposae (Micrococcus), 307

pneumoniae equi (Bacillus), 553

pneumonicum (Bacterium), 458, 675

pneumonicus (Bacillus), 647

pneumonicus agilis (Bacillus), 647

pneumonicus agilis (Bacterium), 647 pneumonicus liquefaciens (Bacillus), 675 pneumonicus liquefaciens (Bacterium), 675

pneumonie crouposae (Bacterium), 458

pneumopecurium (Bacterium), 684

pneumosepticum (Bacterium), 684

pneumosepticus (Bacillus), 665, 684

pneumosepticus (Bacterium), 665

pneumosimilis (Streptococcus), 342

pneumosintes (Bacillus), 595

pneumosintes (Bacterium), 595

pneumosintes (Dialister), 595

Podangium, 1009, 1034

podovis (Treponema), 1074

poeciloides (Bacillus), 368, 369

poeciloides (Eubacterium), 368, 369

poelsii (Bacillus), 665

poinsettiae (Corynebacterium), 398, 399

poinsettiae (Phytomonas), 399

pollacii (Bacillus), 751

pollachii (Spirochaeta), 1069

pollachii (Spironema), 1069

Pollendera, 705

Polyangium, 5, 14, 17, 20, 24, 26, 1005, 1009, 1025

Polycephalum, 1036

polychromogenes (Actinomyces), 897

polychromogenes (Bacillus), 233

polychromogenes (Nocardia), 897, 898

polychromogenes (Oospora), 897

polychromogenes (Proactinomyces), 897

polychromogenes (Streptothrix), 897

polycolor (Bacterium), 126

polycolor (Phytomonas), 126

polycolor (Pseudomonas), 89, 126

polycystus (Chondrococcus), 1045

polycystus (Myxococcus), 1045

polydorae (Cristispira), 1056

polydorae (Cristispirella), 1057

polyfermenticum (Clostridium), 772, 824

polygoni (Phytomonas), 140

polygoni (Pseudomonas), 140

polymorpha (Mima), 595

polymorpha var. oxidans, 595

polymorphum (Bacterium), 684

polymorphum (Fusobacterium), 582

polymorphum (Halibacterium), 662 
polymorphum (Rhizobium), 224

polymorphum convulsivum (Bacterium), 590

polymorphus (Actinobacter), 456

polymorphus (Bacillus), 684

polymorphus (Fusiformis), 582

polymorphus (Streptococcus), 343

polymorphus (Vibrio), 205

polymorphus necroticans (Coccobacillus), 690

polymorphus necroticans (Micrococco-

bacillus), 690

polymorphus var. peritriche (Vibrio), 205

polymyxa (Aerobacillus), 720

polymyxa (Bacillus), 720, 722, 754, 818

polymyxa (Clostridium), 720

polymyxa (Granulobacter), 720

polymyxa var. mucosum (Granulobacter), 720

polymyxa var. tenax (Granulobacter), 720

polypiformie (Cornilia), 816

polypiformis (Bacillus), 816, 818

polypus (Micrococcus), 272

polysclerotica (Spirochaeta), 1065

polysiphoniae (Bacterium), 624

polysiphoniae (Flavobacterium), 624

polyspira (Spirochaeta), 1074

polyspirum (Treponema), 1074

polyspora (Crenothrix), 983, 987

polyspora (Metabacterium), 762

polytrophum (Nitrobacter), 76

pomodoriferus (Bacillus), 665

pomona (Salmonella), 529

poncei (Bacillus), 665

ponceti (Actinomyces), 921

ponceti (Nocardia), 921

ponceti (Oospora), 921

ponticus (Vibrio), 703

Pontothrix, 1002

poolensis (Actinomyces), 949

poolensis (Streptomyces), 949

poona (Salmonella), 527, 531

popilliae (Bacillus), 727

populi (Bacillus), 751

populi (Micrococcus), 272

porcellorum (Micrococcus), 272

porci (Erysipelothrix), 410 porri (Bacillus), 684

porri (Bacterium), 684

portae (Spirillum), 1052

portuensis (Microspira), 205

portuensis (Vibrio), 205

postumus (Bacillus), 816

potens (Acetobacter), 692

potsdam (Salmonella), 511

potsdamensis (Salmonella), 511

praeacuta (Zuberella), 577

praeacutus (Coccobacillus), 577

praecox (Actinomyces), 973

praefecundus (Actinomyces), 973

praepollens (Bacillus), 665

praussnitzii (Bacillus), 718

praussnitzii (Bacteroides), 577

preisz-nocardi (Corynebacterium), 389

premens (Tarpeia), 1270

preputialis (Leptothrix), 366

pretoria (Salmonella), 526

pretoriana (Nocardia), 900

pretorianus (Actinomyces), 900

prillieuxianus (Bacillus), 132

prima (Vibrio), 205

primarius (Phagus), 1132

primigenium (Archangium), 1018, 1019

primigenium (Polyangium), 1018

primigenium var. assurgens (Arch-

angium), 1006, 1018

primulae (Marmor), 1201

primulae (Phytomonas), 114

primulae (Pseudomonas), 114

primus (Robertsonillus), 823

primus fullesi (Bacillus), 666

primus fullesi (Bacterium), 666

pringsheim (Bacillus), 819

priztnitzi (Bacillus), 534

priztnitzi (Bacterium), 534

priztnitzi (Eberthella), 534

priztnitzi (Eberthus), 534

Proactinomyces, 38, 875, 892

Proactinomyces sp., Helzer, 923

probatus (Bacillus), 729

prodeniae (Bacterium), 684

prodigiosa (Micraloa), 480

prodigiosa (Monas), 480

prodigiosa (Palmella), 480 
prodigiosa (Salmonella), 480 prodigiosum (Bacteridium), 480 prodigiosum (Bacterium), 480 prodigiosum (Chromobacterium), 480 prodigiosum (Dicrobactrum), 480 prodigiosum (Liquidobacterium), 480 prodigiosus (Bacillus), 480 prodigiosus (Erythrobacillus), 480 prodigiosus (Micrococcus), 480 productus (Streptococcus), 343 profundus (Cladothrix), 983 profusum (Bacterium), 684 profusus (Bacillus), 684 progrediens (Micrococcus), 272 progrediens (Reglillus), 823 prolifer (Vibrio), 213 promissus (Bacillus), 666 propera (Thiospira), 702 Propionibacterium, 30, 31, 372 propionici (Micromomospora), 980 Propionicoccus, 11 propionicum (Clostridium), 821 propyl-butylicum (Clostridium), 781, 825 Proshigella, 535

Protaminobacter, 32, 189 protea (Actinomyces), 972 protea (Microspira), 197 protea (Pseudomonas), 149 protea-fluorescens (Pseudomonas), 89 proteamaculans (Bacterium), 169 proteamaculans (Phytomonas), 169 proteamaculans (Pseudomonas), 169 proteamaculans (Kanthomonas), 169 proteidis (Bacillus), 666

proteiformis (Enterococcus), 325

proteiformis (Streptococcus), 325

proteiformis liquefaciens (Enterococcus), 343

proteiformis var. liquefaciens (Streptococcus), 343

proteolyticum (Clostridium), 821 proteolyticum (Plocamobacterium), 691 proteolyticus (Glycobacter), 691 proteolyticus (Martellillus), 823 proteosimilis (Eberthella), 534 protervus (Bacillus), 666 proteum (Bacterium), 718
Proteus, 18, 21, 26, 31, 486, 489, 490, 491, 689, 691, 1086, 1088, 1089, 1091, 1092, 1093, 1103

proteus (Bacillus), 486 proteus (Bacterium), 691 proteus (Flavobacterium), $\mathbf{4 3 8}$ proteus (Oospora), 973 proteus (Streptococcus), 343 proteus (Streptothrix), 973 proteus (Vibrio), 197, 202, 204, 205 proteus anindolgenes (Bacterium), 487 proteus fluorescens (Bacillus), 89 proteus fluorescens (Bacterium), 698 proteus mirabilis (Bacillus), 488 proteus septicus (Bacillus), 686 proteus vulgaris (Bacillus), 487, 632 proteus-zenkeri (Bacillus), 608 Protobios, 1128, 1129 protobios (Protobios), 1128 protozoides (Bacterium), 145 prowazeki sub-species typhi (Rickettsia), 1086

prowazeki var. mooseri (Rickettsia), 1086 prowazeki var. prowazeki (Rickettsia), 1084

prowazekii (Rickettsia), 1084, 1086, 1087, 1088, 1090, 1094, 1096, 1097, 1103 pruchii (Bacillus), 788 pruchii (Clostridium), 788 pruddeni (Bacillus), 666 pruneaeum (Flavobacterium), 441 pruni (Bacterium), 152 pruni (Nanus), 1208

pruni (Phagus), 1135

pruni (Phytomonas), 152

pruni (Pseudomonas), 152

pruni (Xanthomonas), 152, 1129, 1134, 1135

prunicola (Pseudomonas), 120, 123

pruvoti (Coleomitus), 1003

pruvoti (Coleonema), 1003

psalteri (Micrococcus), 272

pseudaceti (Bacterium), 761

pseudoaquatile (Bacterium), 684

pseudo-actinomycosis polymorphus (Coccobacillus), 974

pseudoagalactiae (Streptococcus), 319 
pseudanthracis (Bacillus), 716, 717, 751 pseudoanthracis (Bacterium), 717 pseudo-asiatica (Salmonella), 532 pseudo-asiatica var. mobilis (Salmonella), 532

pseudo-asiaticus (Bacillus), 532 pseudo-asiaticus mobilis (Bacillus), 532 Pseudobacillus, 20, 22 pseudobuccalis (Spirochaeta), 1069 pseudobutyricus (Bacillus), 740, 820 pseudocarneus (Asteroides), 918 pseudo-carolina (Salmonella (?)), 532 pseudocarolinus (Bacillus), 532 pseudocatarrhalis (Neisseria), 301 pseudocebi (Bartonella), 1108 pseudocebi (Haemobartonella), 1108 pseudocerevisiae (Micrococcus), 249 pseudo-cholerae gallinarum (Bacillus), 520

pseudoclostridium (Bacterium), 818 pseudococcus (Bacillus), 751 pseudocoli (Bacillus), 453 pseudocoli (Escherichia), 453 pseudo-coli anarobie (Bacillus), 786 pseudocoli anaerobius (Bacillus), 786 pseudocoliformis (Bacillus), 453 pseudo-coliformis (Escherichia), 453 pseudocoloides (Bacillus), 453 pseudo-coloides (Bacterium), 453 pseudocoloides (Escherichia), 453 pseudo-columbensis (Bacillus), 532 pseudo-columbensis (Salmonella (?)), 532 pseudoconjunctivitidis (Bacillus), 684 pseudoconjunctivitidis (Bacterium), 684 pseudocoscoroba (Escherichia), 453 pseudocyaneus (Micrococcus), 272 pseudodiphtheria (Bacillus), 390 pseudodiphtheriae (Corynebacterium), 404, 405

pseudodiphthericum (Mycobacterium), 385 pseudodiphthericum magnus (Bacillus), 752

pseudodiphthericus (Bacillus), 385

pseudodiphtheriticum (Bacterium), 385 pseudodiphtheriticum rium), $\mathbf{3 8 5}, 406$ pseudodiphtheriticus acidum faciens

(Bacillus), 401

pseudodiphtheriticus alcalifaciens ( $\mathrm{Ba}$ cillus), 400

pseudodiphtheriticus gazogenes (Bacillus), 401

Pseudodiplococcus, 305

pseudodysenteriae (Bacillus), 538

pseudodsyenteriae (Escherichia), 453, 543 pseudodysentericum (Bacterium), 453, 543 pseudofilicinum (Bacterium), 684 pscudofusiformis (Bacillus), 752 pseudogonorrhoeae (Sarcina), 293 pseudohaemolyticus (Streptococcus), 343 pseudohebdomadis (Spirochaeta), 1079 pseudoianthina (Pseudomonas), 233 pseudoicterogenes (Leptospira), 1077 pseudoicterogenes (Spirochaeta), 1077 pseudoicterogenes aquaeductuum (Spirochaeta), 1078

pseudo-icterogenes (aquatilis) (Spirochaeta), 1077

pseudoicterogenes salina (Spirochaeta), 1079

pseudo-icterohemorrhagiae (Spirochaeta), 1077

pseudoinfluenzae (Bacillus), 684

pseudoinfluenzae (Bacterium), 684

pseudoinfluenzae (Micrococcus), 272 pseudokeratomalaciae (Bacterium), 684 Pseudoleptothrix, 34, 366 pseudomagnus (Bacillus), 816 pseudomallei (Actinobacillus), 555 pseudomallei (Bacillus), 555 pseudomallei (Flavobacterium), 555 pseudomallei (Malleomyces), 555, 556 pseudomallei (Pfeifferella), 555 pseudomelitensis (Micrococcus), 563 pseudomirabilis (Bacillus), 666

Pseudomonas, 7, 15, 18, 20, 21, 25, 28, 29, $31,32,43,82,91,145,171,233,234,615$, $633,692,839$ pseudo-morgani (Bacillus), 532 pseudo-morganii (Salmonella), 532 pseudomultipediculum (Bacterium), 685 pseudomycoides (Bacterium), 761 pseudomycoides roseus (Bacillus), 761 
pseudonavicula (Bacillus), 814, 816 pseudonecrophorus (Actinomyces), 580 pseudooedematis (Bacillus), 816 pseudooedematis maligni (Bacillus), 660, 816

pseudopallida (Spirochaeta), 1074 pseudopallida (Spiroschaudinnia), 1074 pseudopallidum (Treponema), 1074 pseudo-perfringens (Inflabilis), 823 pseudopestis murium (Bacterium), 402 pseudopneumonicum (Bacterium), 685 pseudopneumonicum (Brucella), 685 pseudopneumonicus (Bacillus), 685 pseudoproteus (Aerobacter), 457 pseudopyogenes (Corynebacterium), 388 pseudoradiatus (Bacillus), 613 pseudoramosum (Ramibacterium), 369 pseudoramosus (Bacillus), 369, 488 pseudoramosus (Bacteriodes), 369 pseudorecurrentis (Spirochaeta), 1069 pseudoruber (Bacillus), 752

Pseudosarcina, 285

pseudosarcina (Micrococcus), 249

pseudo-septicus (Bacillus), 816 pseudosolidus (Bacillus), 816

Pseudospira, 192

pseudospirochaeta (Vibrio), 205

pseudospirochaeta A (Vibrio), 205

pseudospirochacta $B$ (Vibrio), 205

pseudospirochacta $C$ (Vibrio), 205

Pseudostreptus, 12, 13, 14, 312

pseudosubtilis (Bacillus), 752

pseudotetani (Bacillus), 727, 728, 800, 816

pseudotetanicum (Plectridium), 801

pseudotetanicus (Bacillus), 727, 728, 816,

818

pseudotetanicus aerobius (Bacillus), 727 pseudo-tetanus, Type No. IX (Bacillus), 800

pseudotsugae (Bacterium), 230

pseudotsugae (Phytomonas), 230

pseudotuberculosa (Streptothrix), 973

pseudotuberculosis (Bacillus), 389, 550, 666

pseudotuberculosis (Bacterium), 550 pseudotuberculosis (Corynebacterium), 389,550

pseudotuberculosis (Mycobacterium), 390 pseudotuberculosis (Nocardia), 973

pseudotuberculosis (Pasteurella), 549, $550,677,703$

pseudotuberculosis bovis (Corynebacterium), 389

pseudotuberculosis liquefaciens (Bacillus), 666

pscudotuberculosis murium (Bacillus), 389,407

pseudotuberculosis murium (Corynethrix), 406

pseudotuberculosis ovis (Bacillus), 389

pseudotuberculosis ovis (Corynebacterium), 389

pseudotuberculosis rodentium (Bacillus), 550

pseudotuberculosis rodentium (Bacterium), 550

pseudotuberculosis rodentium (Corynebacterium), 550

pseudotuberculosis rodentium (Malleomyces), 550

pseudotuberculosis rodentium (Streptobacillus), 550

pseudotuberculosis similis (Bacillus), 652 pseudotuberculosus (Actinomyces), 973 pseudotyphi (Rickettsia), 1090 pscudotyphosa (Pseudomonas), 149 pseudotyphosum (Bacterium), 613, 666 pseudotyphosus (Bacillus), 612, 666 pseudovacuolata (Leptothrix), 986 pseudovacuolata (Spirothrix), 986 pseudovaleriei (Proteus), 491 pseudovermiculosum (Bacterium), 761 pseudoviolacea (Pseudomonas), 234 pseudoxerosis (Bacillus), 1098 pseudozoogloeae (Bacterium), 124 pseudozoogloeae (Phytomonas), 124 pseudozoogloeae (Pseudomonas), 124, 130

psittaci (Ehrlichia), 1116 psittaci (Miyagawanella), 1116, 1117, 1118,1119 psittaci (Rickettsia), 1116 
psittacorum (Streptococcus), 342

psittacosis (Bacillus), 502

psittacosis (Bacterium), 502

psittacosis (Salmonella), 502

psorosis (Citivir), 1209

psorosis (Rimocortius), $\mathbf{1 2 1 0}$

psorosis var. alveatum (Rimocortius), 1211

psorosis var. anulatum (Rimocortius),

1210

psorosis var. convacum (Rimocortius), 1210

psychrocarterica (Urosarcina), 289

psychrocarterica (Sarcina), 289

psychrocartericus (Bacillus), 729

psychrocartericus (Urobacillus), 729

puerariae (Bacterium), 118

puerariae (Phytomonas), 118

pueris (Salmonella), 513

puerperalis (Streptococcus), 315

pulcher (Micrococcus), 272

pulchra (Sarcina), 293

pullorum (Bacillus), 521

pullorum (Bacterium), 521

pullorum (Borrelia), 1059

pullorum (Salmonella), 43, 493, 498, 520,

521,1135

pullulans (Bacillus), 149

pullulans (Bacterium), 700

pullulans (Pseudomonas), 149, 700

pulmonalis (Actinomyces), 901, 923

pulmonalis (Discomyces), 922

pulmonalis (Nocardia), 901, 923

pulmonalis (Oospora), 917, 922

pulmonalis var. chromogena (Oospora), 923

pulmonalis var. chromogenus (Actinomyces), 923

pulmonare (Monas), 307

pulmonis (Murimyces), 1292

pulmonis $I$ and $I I$ (Canomyces), 1292

pulmonis equi (Zoogloea), 252

pulmonum (Bacillus), 612

pulmonum (Sarcina), 291, 293

pulpae pyogenes (Bacillus), 666

pultiformis (Micrococcus), 272

pulviforme (Mycobacterium), 882 pumilus (Bacillus), 709, 712, 744

puncta (Saprospira), 1054

punctans sulfureum (Bacterium), 610

punctata (Beggiatoa), 992

punctatum (Achromobacter), 102

punctatum (Bacterium), 102, 324

punctatum (Nitrobacter), 76

punctatus (Bacillus), 102, 666

punctatus (Micrococcus), 272

puncticulatus (Bacillus), 666

punctiformis (Bacillus), 752

punctulans (Bacterium), 113

punctum (Bacillus), 685

punctum (Bacterium), 685

punctum (Monas), 685

puntonii (Actinomyces), 917

puntonii (Asteroides), 917

Pupella, 5

purifaciens (Bacterium), 557

purifaciens (Pasteurella), 557

puris (Pseudomonas), 700

purpeochromogenus (Actinomyces), 943

purpeochromogenus (Streptomyces), 943

purpureus (Actinomyces), 917, 973

purpurifaciens (Micrococcus), 272

purpurum (Bacterium), 552

purulentus (Corynebacterium), 391

pusilla (Cellulomonos), 621

pusilla (Cristispira), 1057

pusilla (Spirochaeta), 1057

pusilus (Bacillus), 621

pusiolum (Bacterium), 145

pustulatus (Micrococcus), 272

putatus (Micrococcus), 696

putida (Pseudomonas), 96

putida (Ristella), 575

putidum (Bacterium), 96, 685

putidum (Corynebacterium), 405

putidus (Bacillus), 96, 575, 666, 667

putidus splendens (Bacillus), 613

putneus (Micrococcus), 696

putorii (Actinomyces), 973

putorii (Streptothrix), 973

putoriorum (Hemophilus), 589

putredinis (Bacillus), 575, 640

putredinis (Bacterium), 640

putredinis (Ristella), 575 


\section{putr-pyog INDEX OF NAMES OF GENERA AND SPECIES}

putrefaciens (Achromobacter), 99

putrefaciens (Acuformis), 802

putrefaciens (Bacillus), 802

putrefaciens (Clostridium), 802

putrefaciens (Palmula), 802

putrefaciens (Pseudomonas), 99

putrefaciens (Streptococcus), 343

putrefaciens putridus (Bacillus), 477

Putribacillus, 8, 763

Putriclostridium, 11, 763

putrida (Pseudomonas), 93

putridogenes (Actinomyces), 921

putridogenes (Cladothrix), 921

putridogenes (Nocardia), 921

putridogenes (Oospira), 921

putridus (Micrococcus), 272

putridus (Streptococcus), 329

putridus (Vibrio), 205

putrifica (Pacinia), 799

putrificum (Plectridium), 799

putrificum var. lentoputrescens (Plectrid-

ium), 799

Putrificus, 11, 20, 22, 763

putrificus (Bacillus), 799, 800

putrificus (Clostridium), 799

putrificus (Staphylococcus), 282

putrificus (Streptococcus), 329

putrificus coagulans (Bacillus), 816

putrificus coli (Bacillus), 727, 737, 799,

800

putrificus filamentosus (Bacillus), 800

putrificus immobilis (Bacillus), 813, 817

putrificus ovalaris (Bacillus), 796

putrificus tenuis (Bacillus), 787, 826

putrificus var. non liquefaciens (Ba-

cillus), 816

putrificus verrucosus (Bacillus), 782, 825

pyaemiae cuniculorum (Micrococcus), 271

pyaemicum (Bacterium), 685

pycnotica (Hydrogenomonas), 752

pycnoticus (Bacillus), 752

pyelonephritis boum (Bacillus), 388

pygmaeum (Clostridium), 821

pygmaeus (Micrococcus), 272

pylori (Bacillus), 416, 666

pyocinnabareum (Bacterium), 685

pyocinnabareus (Bacillus), 685
Pyococcus, 235

pyocyanea (Pseudomonas), 7, 89

pyocyaneum (Bacterium), 89

pyocyaneus (Bacillus), 89, 146

pyocyaneus (Micrococcus), 89, 272

pyocyaneus saccharum (Bacillus), 121

pyogenes (Actinomyces), 934, 973

pyogenes (Albococcus), 242

pyogenes (Bacillus), 388, 666

pyogenes (Bacterium), 388, 534, 685

pyogenes (Bacteroides), 579

pyogenes (Castellanus), 534

pyogenes (Corynebacterium), 388, 391,

401, 404, 405, 694

pyogenes (Diplococcus), 343

pyogenes (Eberthella), 534

pyogenes (Lankoides), 534

pyogenes (Micrococcus), 241, 242, 343

pyogenes (Spherophorus), 579

pyogenes (Spirillum), 206, 217

pyogenes (Spirochaeta), 217

pyogenes (Staphylococcus), 241

pyogenes (Streptococcus), 312, 315, 316,

$318,333,338,339,340,342,343,344,345$

pyogenes (Streptothrix), 934, 973

pyogenes (Treponema), 217

pyogenes (Vibrio), 206

pyogenes var. albus (Micrococcus), 242, 259

pyogenes var. aureus (Micrococcus), 241, 271, 284

pyogenes var. liquefaciens (Bacillus), 666 pyogenes var. scarlatinae (Streptococcus),

315

pyogenes albus (Staphylococcus), 242

pyogenes albus (Tetracoccus (Micro-

coccus)), 242

pyogenes anaerobius (Bacillus), 579, 685

pyogenes anaerobius (Bacterium), 685

pyogenes animalis (Streptococcus), 316

pyogenes aureus (Staphylococcus), 241

pyogenes aureus (Tetracoccus) (Micro-

coccus), 241

pyogenes bovis (Bacillus), 388, 405, 666

pyogenes bovis (Corynebacterium), 388, 405

pyogenes bovis (Eubacterium), 405 
pyogenes bovis (Staphylococcus), 264, 281, 701

pyogenes bovis (Streptococcus), 343

pyogenes citreus (Micrococcus), 242 pyogenes citreus (Staphylococcus), 242 pyogenes crassus (Bacillus), 652 pyogenes crassus (Bacterium), 652 pyogenes cuniculi (Bacillus), 403 pyogenes cuniculi (Leptothrix), 366 pyogenes duodenalis (Streptococcus), 702 pyogenes (equi) Corynebacterium, 391 (pyogenes) equi roseum (Corynebacterium), 391

pyogenes equi (Streptococcus), 317 pyogenes filiformis (Bacillus (Leptothrix?)), 366

pyogenes floccosus (Streptobacillus), 579 pyogenes foetidus (Bacillus), 534, 817 pyogenes foetidus (Bacterium), 534 pyogenes foetidus liquefaciens (Bacillus), 656,666

pyogenes foetidus liquefaciens (Bacterium), 656, 666

pyogenes gingivae (Bacillus), 657 pyogenes haemolyticus (Streptococcus), 315 pyogenes hominis (Streptococcus), 343 pyogenes lentus (Streptococcus), 341 pyogenes liquefaciens albus (Staphylococcus), 282

pyogenes malignus (Streptococcus), 341 pyogenes minutissimum (Bacterium), 607 pyogenes minutissimus (Bacillus), 607 pyogenes nonhaemolyticus (Streptococcus), 343

pyogenes salivarius (Staphylococcus), 258 pyogenes sanguinarium (Bacterium), 520 pyogenes soli (Bacillus), 666 pyogenes suis (Bacillus), 388 pyogenes suis (Bacterium), 388 pyogenes tenuis (Micrococcus), 306, 307 pyogenes tenuis (Staphylococcus), 282 pyogenes ureae (Streptococcus), 344 pyogenes vulgaris (Streptococcus), 345 pyorrhoeica (Spirochaeta), 1069 pyoseptica (Serratia), 484 pyosepticum (Bacterium), 541 pyosepticum equi (Bacterium), 541 pyosepticum viscosum (Bacterium), 541 pyosepticum (viscosum) equi (Bacterium), 541

pyosepticus (Bacillus), 541 pyosepticus (Erythrobacillus), 10, 484 pyosepticus (Micrococcus), 272 pyosepticus (Staphylococcus), 272 pyrameis $I$ and $I I$ (Bacillus), 666 pyraustae Nos, 1-7 (Bacterium), 685 pyrenei (Bacillus), 761 pyrenei No. 1, No. 2 and No. 3 (Bacterium), 761

pyri (Bacterium), 640

pyri (Marmor), 1211

pyri (Rimocortius), 1211

pyriformis (Myxococeus), 1041 pyrogenes (Leptospira), 1079 pyrogenes (Spirochaeta), 1079 pyrphoron (Heliconema), 1064

quadrigeminus (Micrococcus), 273 quadrigeminus (Staphylococcus), 273 qualis (Bacterium), 603 quarta (Fractilinea), 1161 quartum (Eubacterium), 367 quartum (Marmor), 1161 quaternus (Micrococcus), 273 queenslandiensis (Galla), 1158 quercifolium (Bacterium), 752 quercifolius (Bacillus), 752 querquedulae (Treponema), 1076 quintana (Fossilis), 1094 quintana (Rickettsia), 1094, 1095 quintum (Eubacterium), 367

raabi (Terminosporus), 823 rabida (Pacinia), 696 rabiei (Encephalitozoon), 1264

Rabula, 1284

racemosa (Leptothrix), 366 racemosa (Leptotrichia), 366 racemosa (Zettnowia), 603 racemosa vincenti (Leptothrix), 366 racemosum (Bacterium), 603 racemosum (Flavobacterium), 603 
radians (Bacillus), 667, 710

radiata (Cornilia), 799

radiala (Nocardia), 973

radiata (Sarcina), 293

radiatum (Bacterium), 685, 761

radiatum (Flavobacterium), 441

radiatus (Actinomyces), 973

radiatus (Bacillus), 441, 613, 662, 799,

826

radiatus (Micrococcus), 273

radiatus (Streptococcus), 273, 343

radiatus (Streptothrix), 973

radiatus anaerobius (Bacillus), 799

radiatus aquatilis (Bacillus), 441, 613

radiatus aquatilis (Bacterium), 613

radicicola (Bacillus), 43, 224, 225

radicicola (Bacterium), 224, 225

radicicola (Pseudomonas), 225

radicicola (Rhizomonas), 225

radicicola var. liquefaciens (Bacillus),

650

radicicolum (Rhizobium), 225

radiciperda (Bacterium), 141

radiciperda (Phytomonas), 141

radiciperda (Pseudomonas), 141

radicosum (Bacterium), 718

radicosus (Bacillus), 718

radiiformis (Bacillus), 566

radiobacter (Achromobacter), 229

radiobacter (Agrobacterium), 229, 230

radiobacter (Alcaligenes), 229

radiobacter (Bacillus), 229

radiobacter (Bacterium), 229

radiobacter (Rhizobium), 229

radiosus (Micrococcus), 273

raffinolactis (Streptococcus), 325

raffinosaceum (Propionibacterium), 377

raillieti (Spirochaeta), 1069

Ramibacterium, 33, 34, 368

ramificans (Bacillus), 667

ramificans (Bacterium), 685

ramigera (Zoogloea), 150

ramosa (Cladothrix), 983

ramosa (Nevskia), 830

ramosa (Nocardia), 368

ramosa (Pasteuria), 836

ramosoides (Bacillus), 369 ramosoides (Ramibacterium), 369

ramosum (Pseudorhizobium), 225

ramosum (Ramibacterium), 368

ramosus (Actinomyces), 368

ramosus (Bacillus), 368, 718, 801, 827

ramosus (Frusiformis), 369

ramosus liquefaciens (Bacillus), 715

ranae (Mycobacterium), 883, 884, 885,

886,887

ranarum (Bartonella), 1108

ranarum (Haemobartonella), 1108

ranarum (Spirochaeta), 1069

rancens (Acetobacter), 180, 182, 184, 692

rancens (Bacillus), 182

rancens (Bacterium), 182

rancens (Llvina), 692

rangiferinum (Bacterium), 685

rangiferinum (Plocamobacterium), 685

rangoon (Actinomyces), 911

rangoonensis (Nocardia), 911

ranicida (Bacillus), 102

ranicida (Bacterium), 102

ranicida (Salmonella), 532

ranicola $I$ and $I I$ (Mycobacterium), 890

ranicula (Vibrio), 206

raphani (Marmor), 1200

rappini (Spirillum), 217

rarerepertus (Bacillus), 752

rarus (Bacillus), 752

rasmusseni (Bacillus), 367

rasmusseni (Bacteriopsis), 367

Rasmussenia, 365

rathay (Bacterium), 394

rathayi (Aplanobacter), 394

rathayi (Corynebacterium), 394, 400

rathayi (Phytomonas), 394

rathonis (Achromobacter), 104

rathonis (Pseudomonas), 104

ratti (Streptothrix), 588, 972

raveneli (Bacillus), 426

ravenelii (Achromobacter), 426

reading (Salmonella), 504

readingensis (Salmonella), 493, 504

reading var. kaapstad (Salmonella), 505

rebellis (Neisseria), 301

Recordillus, 11, 763

recta (Spirochaeta), 1069 
rectale (Eubacterium), 367 rectalis (Bacteroides), 367 rectangulare (Sideroderma), 835 recti (Alcaligenes), $\mathbf{4 1 5}$ recti (Bacterium), 415 recti physeteris (Spirillum), 217 recticolens (Proteus), 491 rectum (Treponema), 1069 rectus (Hiblerillus), 822 rectus (Inflabilis), 822 recuperatus (Bacillus), 667 recuperatus (Bacterium), 667 recurrens (Scelus), 1235 recurrentis (Borrelia), 1059, 1060, 1061, 1062, 1065, 1067, 1069 recurrentis (Cacospira), 1059 recurrentis (Protomycetum), 1059 recurrentis (Spirillum), 1059 recurrentis (Spirochaeta), 1059 recurrentis (Spironema), 1059 recurrentis (Spiroschaudinnia), 1059 recurrentis (Treponema), 1059 reducans (Agarbacterium), 629 reductans (Thermobacillus), 731 reessii (Micrococcus), 273 refractans (Achromobacter), 426 refractans (Bacillus), 426 refractans (Bacterium), 426 refringens (Borrelia), 1063 refringens (Spirochaeta), 1063 refringens (Spironema), 1063 refringens (Spiroschaudinnia), 1063 refringens ('Treponema), 1063 regaudi (Spirella), 1069 regaudi (Spirochaeta), 1069 Reglillus, 11, 763 regulare (Clostridium), 785 regularis (Micrococcus), 273 regularis filiformis (Bacillus), 792 reitenbachii (Lampropedia), 844 reitenbachii (Merismopedium), 844 reitenbachii (Pediococcus), 844 reitenbachii (Sarcina), 844 renale (Bacillus), 405 renale (Bacterium), 388, 405 renale (Corynebacterium), 388, 389 renale (cuniculi) (Bacillus), 404, 405 renale (cuniculi) (Bacterium), 405

renale cuniculi (Corynebacterium), 388, 405

renalis (Bacillus), 388

renalis bovis (Bacillus), 388

renalis bovis (Corynebacterium), 388

reniforme (Bacterium), 817

reniformis (Bacillus), 817

reniformis (Diplococcus), 300

reniformis (Micrococcus), 300

reniformis (Neisseria), $\mathbf{3 0 0}$

renis (Sarcina), 294

repacis (Spirochaete), 1070

repazii (Bacillus), 667

repens (Achorion), 921

repens (Actinomyces), 921

repens (Bacillus), 752

repens (Bacterium), 685

repens (Epidermophyton), 921

repens (Lepocolla), 921

repens (Marmor), 1189, 1192

repens (Nocardia), 921

reprimens (Borrelina), 1226

reprimens (Morsus), 1154

reptans (Bacillus), 752

reptans (Granulobacter), 822

reptilia (Treponema), 1076

reptilivorous (Pseudomonas), 92

resinacea (Pseudomonas), 179

resinaceus (Micrococcus), 273

restatus (Thermobacillus), 732

restrictus (Proactinomyces), 923

retaneus (Bacillus), 752

reticulare (Achromobacter), 426

reticularis (Bacillus), 426

reticularis (Cladothrix), 983

reticularis (Sphaerotilus), 983

reticuli (Actinomyces), 944

reticuli (Streptomyces), 944

reticulosa (Gallionella), 832

reticulus (Actinomyces), 945

reticulus-ruber (Actinomyces), 945

retiformans (Bacterium), 685

retiformis (Bacillus), 752

rettgeri (Bacillus), 489

rettgeri (Bacterium), 489

rettgeri (Butyribacterium), 380 
rettgeri (Eberthella), 489

rettgeri (Proteus), 489

rettgeri (Shigella), 489

Rhabdochromatium, 7, 16, 853

Rhabdomonas, 6, 23, 26, 853

Rhagadascia, 13, 705

rhamnoticus (Bacillus), 772, 824

rhapontica (Erwinia), 475

rhapontica (Phyiomonas), 475

rhaponticum (Aplanobacter), 475

rhaponticum (Bacterium), 475

rhenanus (Albococcus), 273

rhenanus (Bacillus), 433

rhenanus (Flavobacterium), 433

rhenanus (Micrococcus), 273

rheni (Bacillus), 433

rheni (Micrococcus), 273

rhesi (Grahamella), 1111

rheumaticus (Diplococcus), 343

rheumaticus (Micrococcus), 343

rheumaticus (Streptococcus), 343

rhinitis (Bacillus), 578

rhinitis (Zuberella), 578

rhinitis atrophicans (Bacillus), 667

rhinitis atrophicans (Bacterium), 667

rhinoceri (Treponema), 1076

rhinopharyngeum (Treponema), 1076

rhinoscleromatis (Bacillus), 459

rhinoscleromatis (Bacterium), 459

rhinoscleromatis (Klebsiella), 459

Rhizobacterium, 223

Rhizobium, 17, 20, 26, 29, 31, 223, 224

rhizoctonia (A planobacter), 129

rhizoctonia (Bacterium), 129

rhizoctonia (Phytomonas), 129

rhizoctonia (Pseudomonas), 129

rhizogenes (Agrobacterium), 228, 229

rhizogenes (Bacterium), 228

rhizogenes (Phytomonas), 228

rhizogenes (Pseudomonas), 228

Rhizomonas, 8, 223

rhizopodicum (Bacterium), 685

rhizopodicus margarineus (Bacillus), 685

rhodnii (Actinomyces), 914

rhodnii (Nocardia), 914

Rhodobacillus, 16, 24, 25, 861

Rhodobacterium, 16, 24, 26, 861
Rhodocapsa, 16, 23, 25, 853

rhodochrous (Bacillus), 696

rhodochrous (Bacterium), 696

rhodochrous (Micrococeus), 245, 696

rhodochrous (Rhodococcus), 8, 245

Rhodococcus, 8, 16, 25, 29, 31, 34, 235, 861

Rhodocystis, 16, 24, 26, 861

Rhododiclyon, 8, 845

rhodomelae (Bacterium), 626

rhodomelae (Flavobacterium), 626

Rhodomonas, 8, 29, 30, 856, 861

Rhodonostoc, 16, 24, 25, 861

Rhodopolycoccus, 8, 850

Rhodopseudomonas, 861, 864

Rhodopseudomonas No. 9 and 16,862, 863

Rhodorrhagus, 861

Rhodosarcina, 8, 842

Rhodosphaera, 24, 861

Rhodospirillum, 16, 24, 25, 29, 853, 866

Rhodothece, 16, 23, 25, 855

Rhodothiosarcina, 842

Rhodothiospirillum, 850

Rhodovibrio, 16, 24, 25, 29, 861

rhusiopathiae (Bacterium), 410

rhusiopathiae (Erysipelothrix), $\mathbf{4 1 0}$

rhusiopathiae (Mycobacterium), 410

rhusiopathiae suis (Bacillus), 410

rhusiopathiae suis (Bacterium), 410

rhytidosporum (Stilbum), 1037

ribberti (Bacterium), 402

ribeyro (Actinomyces), 917

ribis (Acrogenus), 1203

riboflavinus (Pseudomonas), 700

richmond (Salmonella), 512

ricini (Bacterium), 161

ricini (Phytomonas), 137

ricini (Xanthomonas), 161

ricinicola (Bacterium), 161

ricinicola (Phytomonas), 161

ricinicola (Xanthomonas), 161, 1136

rickettsi (Dermacentroxenus), 1087

Rickettsia, 37, 1084

rickettsii (Rickettsia), 1087, 1088, 1089, 1090, 1096 
rickettsi var. brasiliensis (Dermacentroxenus), 1087

rickettsi var. conori (Dermacentroxenus), 1088

rickettsi var. pijperi (Dermacentroxenus), 1088

rickettsiformis (Bacillus), 1098

Rickettsoides, 1092

ridleyi (Micrococcus), 273

rigense (Flavobacterium), 430

rigensis (Vibrio), 206

rigidum (Treponema), 1076

rigidus (Bacillus), 575

rigidus (Bacteroides), 575

rigidus apis (Bacillus), 667

rimaefaciens (Pseudomonas), 123

Rimocortius, 1208, 1209

rindfleischii (Streptococcus), 343

Ristella, 32, 33, 34, 575, 577

rivierei (Actinomyces), 921

rivierei (Discomyces), 921

rivierei (Nocardia), 921

rivierei (Oospora), 921

Rivoltillus, 11, 763

Robertsonillus, 11, 763

robiniae (Chlorogenus), $\mathbf{1 1 5 0}$

robustus (Bacillus), 732, 752

robur (Bacillus), 716

rochalimae (Rickettsia), 1096, 1097

rocha-limai (Bartonella), 1108

rocha-limai (Haemobartonella), 1108

rodellae (Actinomyces), 917

rodentium (Corynebacterium), 550

rodonatum (Achromobacter), 426

rodonatus (Bacillus), 426

rodonatus (Bacterium), 426

rogerii (Bacillus), 667

rogersi (Discomyces), 976

rogersi (Nocardia), 976

rogersi (Nocardia) (Cohnistreptothrix), 976

rogersii (Actinomyces), 976

rosacea (Pseudomonas), 148

rosacea (Sarcina), 293

rosaceum (Bacterium), 667

rosaceus (Actinomyces), 973

rosaceus (Bacillus), 667 rosaceus (Micrococcus), 273

rosaceus (Rhodococcus), 273

rosaceus lactis (Micrococcus), 273

rosaceus margarinicus (Bacillus), 667

rosaceus metalloides (Bacillus), 148, 667

rosaceus metalloides (Bacterium), 148, 667

rosae (Marmor), 1194

rosaftuorescens (Bacillus), 601

rosaftuorescens (Bacterium), 601

roscidur (Micrococcus), 261

roscidus (Micrococcus), 273

rosea (Bactoderma), 76

rosea (Lampropedia), 843 i

rosea (Merismopedia), 244

rosea (Microloa), 845

rosea (Neisseria), 244

rosea (Nocardia), 937

rosea (Pseudomonas), 149, 854

rosea (Rhabdomonas), 854, 855

rosea (Rhodothiosarcina), 843

rosea (Sarcina), 293, 842

rosea (Serratia), 602

rosea (Thiopedia), 843

rosea (Thiosarcina), 842

rosenbachi (Discomyces), 411

rosenbachi (Nocardia), 411

rosenbachi (Oospora), 411

rosenbachii (Actinomyces), 411

rosenbachii (Micrococcus), 307

rosenbachii (Streptothrix), 411

rosenbergii (Spirillum), 852

rosenbergii (Thiospirillum), 852

rosenthalii (Bacillus), 667

roseo-alba (Serratia), 76

roseo-album (Nitrobacter), 76

roseochromogenus (Actinomyces), 937

roseochromogenus (Streptomyces), 937

roseodiastaticus (Actinomyces), 939, 973

roseofulvus (Micrococcus), 244, 282

roseofulvus (Rhodococcus), 244

roseola (Pseudomonas), 700

roseo-persicina (Beggiatoa), 289, 853, 854

roseo-persicina (Clathrocystis), 848

roseo-persicina (Cohnia), 848 
roseopersicina (Lamprocystis), 848, 859 roseo-persicina (Planosarcina), 848 roseopersicina (Thiocapsa), $\mathbf{8 4 5}$ roseo-persicinus (Micrococcus), 273 roseo-persicinus (Pleurococcus), 848 roseo-persicinus (Protococcus), 848 rosettaceus (Micrococcus), 274 rosettae (Carpophthora), 1152 rosettae (Chlorogenus), 1152 rosettae (Nanus), 1152 roseum (Acetobacter), 183, 184 roseum (Amoebobacter), 849 roseum (Bacterium), 685 roseum (Clostridium), 805 roseum (Halibacterium), 667 roseum (Pelochromatium), 859 roseum (Rhabdochromatium), 854 roseum (Thioderma), 849, 858 roseus (Actinomyces), 937, 973 roseus (Bacillus), 667, 685, 709 roseus (Cryptococcus), 848 roseus (Diplococcus), 244 roseus (Discomyces), 973 roseus (Gluconoacetobacter), 694 roseus (Micrococeus), 244, 255, 258, 259, $262,263,265,271,273,274,276,277$, 279,282

roseus (Pediococcus), 843 roseus (Planococcus), 843 roseus (Rhodococcus), 8, 244 roseus (Sphaerotilus), 983 roseus (Staphylococcus), 282 roseus fischeri (Bacillus), 667 roseus fluorescens (Bacillus), 645 roseus vini (Bacillus), 149 rossi (Borrelia), 1060 rossi (Spirochaeta), 1060 rossi (Treponema), 1060 rossica (Cellulomonas), 618, 622 rossicus (Bacillus), 622 rossicus var. castaneus (Bacillus), 622 rossii (Endosporus), 803 rossii (Spiroschaudinnia), 1060 rostockensis (Salmonella), 493, 518 rotans (Bacillus), 728 rubea (Nocardia), 976 rubefaciens (Bacillus), 640 rubefaciens (Bacterium), 640 rubefaciens (Chromobacterium), 640 rubefaciens (Erythrobacillus), 640 rubefaciens (Phytomonas), 640 rubefaciens (Serratia), 640 rubellum (Clostridium), 817 rubellus (Bacillus), 817 rubellus (Micrococcus), 274 rubens (Micrococcus), 244 rubentschickii (Desufovibrio), 208 rubentschickii (Vibrio), 208 rubeolae (Diplococcus), 310 ruber (Actinomyces), 917, 946 ruber (Bacillus), 481, 484, 752 ruber (Chromobacterium), 752 ruber (Discomyces), 917 ruber (Erythrobacillus), 481, 752 ruber (Micrococcus), 244, 850 ruber (Mycococcus), 891 ruber (Myxососcus), 1041 ruber (Proactinomyces), 905 ruber (Rhodococcus), 244 ruber (Streptococcus), 343 ruber (Streptomyces), 946 ruber (Thermobacillus), 734 ruber (Thiopolycoccus), 850 ruber aquatilis (Bacillus), 667 ruber aquatilis (Bacterium), 667 ruber balticus (Bacillus), 482, 483 ruber balticus (Bacterium), 482, 483 ruber berolinensis (Bacillus), 650 ruber berolinensis (Bacterium), 650 ruber indicus (Bacillus), 481 ruber indicus (Bacterium), 481 ruber ovatus (Bacillus), 683 ruber sardinae (Bacillus), 483 ruber sardinae (Bacterium), 483 rubescens (Bacillus), 654, 667 rubescens (Bacterium), 667, 848 rubescens (Lankasteron), 848 rubescens (Micrococcus), 244, 277 rubescens (Myxococcus), 1041 rubescens (Streptomyces), 956 rubescens (Streptothrix), 956 rubi (Agrobacterium), 229 rubi (Bacterium), 229 rubi (Corium), 1205 
rubi (Marmor), 1195

rubi (Phytomonas), 229

rubi (Pseudomonas), 229

rubi var. beta (Corium), 1205

rubicola (Pseudomonas), 700

rubicundus (Vibrio), 206

rubida (Serratia), 641

rubidaea (Serratia), 484

rubidaeum (Bacterium), 484

rubidaureus (Actinomyces), 917

rubidum (Bacterium), 641

rubidus (Bacillus), 611

rubidus lactis (Micrococcus), 274

rubiformis (Bacillus), 667

rubigenosum (Bacterium), 685

rubigenosus (Bacillus), 685

rubigenosus (Micrococcus), 274

rubiginosum (Marmor), 1196

rubiginosus (Bacillus), 667

rubiginosus (Streptococcus), 274, 343

rubislaw (Salmonella), 526

ruboris suis (Bacillus), 410

ruborum (Corium), 1205

rubra (Cladothrix), 917

rubra (Cytophaga), 1013

rubra (Nocardia), 893, 905, 917

rubra (Oospora), 976

rubra (Sarcina), 293

rubra (Serratia), 752

rubra (Streptothrix), 904, 917

rubri var. alpha (Corium), 1205

rubrica (Serratia), 644

rubricum (Chromobacterium), 644

rubricus (Bacillus), 644

rubricus (Erythrobacillus), 644

rubrilineans (Bacterium), 154

rubrilineans (Phytomonas), 154

rubrilineans (Pseudomonas), 154

rubrilineans (Xanthomonas), 154

rubrireticuli (Streptomyces), 945

rubrisubalbricans (Bacterium), 170

rubrisubalbicans (Phytomonas), 170

rubrisubalbicans (Xanthomonas), 170

rubrofuscum (Halibacterium), 667

rubrofuscus (Bacillus), 667

rubropertincta (Nocardia), 904

rubropertincta (Serratia), 905 rubropertinctus (Bacillus), 392, 904

rubropertinctus (Mycobacterium), 904

rubropertinctus (Proactinomyces), 904

rubrum (Bacillus), 685

rubrum (Bacterium), 685, 686, 905

rubrum (Mycobacterium), 890, 905

rubrum (Plocamobacterium), 691

rubrum (Propionibacterium), 374, 375, 377

rubrum (Protaminobacter), 190

rubrum (Rhodospirillum), 853, 867, 868

rubrum (Semiclostridium), 762

rubrum (Spirillum), 632, 867

rubrum (Thioderma), 850

rubus (Bacillus), 667

rudensis (Bacillus), 357, 359

rudensis (Lactobacillus), 359

rudis (Bacillus), 744

ruedigeri (Bacillus), 406

ruedigeri (Corynebacterium), 405

rufa (Serratia), 644

rufa (Thiocystis), 847

rufescens (Bacillus), 739, 752

rufulus (Bacillus), 752

rufum (Spirillum), 852

rufum (Thiospirillum), 852

rufus (Bacillus), 644

rufus (Erythrobacillus), 644

Ruga, 1218

rugatus (Micrococcus), 274

rugosa (Pseudomonas), 104, 700

rugosum (Achromobacter), 426

rugosum (Bacterium), 426, 700, 758

rugosus (Bacillus), 104, 426, 752

rugosus (Micrococcus), 274

rugosus (Streptococcus), 343

rugosus ureae (Streptococcus), 343

rugula (Spirillum), 217

rugula (Vibrio), 217

rugulosus (Bacillus), 752

ruminantium (Cowdria), 1094

ruminantium (Hemophilus), 591

ruminantium (Micrococcus), 274

ruminantium (Rickettsia), 1094

ruminantium (Rickettsia) (Cowdria), 1094

ruminatus (Bacillus), 714, 761

rumpel (Vibrio), 703 
rushmorei (Micrococcus), 274

russii (Bacteroides), 580

rusticum (Bacterium), 761

rutgersensis (Actinomyces), 952

rutgersensis (Streptomyces), 952

rutilescens (Bacillus), 485

rutilescens (Erythrobacillus), 485

rutilescens (Serratia), 485

rutilis (Bacillus), 485

rutilis (Erythrobacillus), 485

rutilis (Serratia), 485

sabouraudi (Bacillus), 387

saccatus (Micrococcus), 275

sacchari (Bacillus), 163, 478, 753

sacchari (Erwinia), 478

sacchari (Marmor), 1183

sacchari (Nanus), 1208

sacchariphilum (Bacterium), 762

saccharo-acetobutylicum (Clostridium),

781,825

saccharo-acetobutylicum-alpha (Clostrid-

$$
\text { ium), 781, } 825
$$

saccharo-acetobutylicum-beta (Clostrid-

$$
\text { ium), 781, } 825
$$

saccharo-acetobutylicum-gamma (Clostrid-

$$
\text { ium), } 781
$$

Saccharobacillus, 349

Saccharobacter, 705

Saccharobacterium, 623

saccharobutyl-acetonicum (Clostridium), 781,825

saccharobutylicum beta (Clostridium),

781

saccharobutylicum gamma (Clostridium), 781,825

saccharobutyl - isopropyl - acetonicum

(Clostridium), 781

saccharobutyricum (Clostridium), 771, 824

saccharobutyricum (Granulobacter), 771, 824

saccharobutyricum gamma (Clostridium), 781

saccharobutyricum liquefaciens (Clostridium), 790 saccharobutyricus (Amylobacter), 771

saccharobutyricus (Bacillus), 771, 786, 824

saccharobutyricus immobilis liquefaciens

(Granulobacillus), 790, 826

saccharobutyricus immobile nonliquefaciens (Granulobacter), 772, 824

saccharobutyricus liquefaciens (Bacillus), 781,825

saccharobutyricus mobilis (Bacillus), 771 saccharobutyricus mobilis non-liquefaciens (Granulobacillus), 771, 824

saccharofermentans (Bacillus), 817 saccharogenes (Bacillus), 817

saccharolactis (Streptococcus), 325

saccharolytica (Botulinea), 22

saccharolyticum (Clostridium), 785

saccharolyticus (Bacillus), 753

saccharopetum (Clostridium), 772, 824

saccharophile (Pseudomonas) (Hydrogenomonas), 149

saccharophilicum (Clostridium), 772, 824 saccharopostulatum (Clostridium), 772, 824

saccharum (Bacillus), 478

saccobranchi (Bacillus), 753

sadowa (Myerillus), 823

saharae (Actinomyces), 973

saint paul (Salmonella), 504

saipan (Salmonella), 532

sake (Lactobacillus), 363

salicinovorum (Aerobacter), 457

saliciperda (Bacterium), 144

saliciperda (Phytomonas), 144

saliciperda (Pseudomonas), 144

salicis (Bacterium), 466

salicis (Erwinia), 466

salicis (Phytomonas), 466

salina (Leptospira), 1079

salinaria (Pseudomonas), 110, 442

salinaria (Serratia), 110

salinarium (Flavobacterium) (Halobac-

terium), 110

salinatis (Salmonella), 504

salivae (Bacterium), 686

salivae minutissimus (Bacillus), 686

salivae minutissimus (Bacterium), 686 
salivalis septicus (Micrococcus), 274 salivaris (Klebsiella), 306

salivarium (Bacterium), 307

salivarius (Micrococcus), 275

salivarius (Staphylococcus), 282

salivarius (Streptococcus), 320, 322,

338,342

salivarius brevis (Streptococcus), 343 salivarius pyogenes (Micrococcus), 258 salivarius pyogenes (Staphylococcus), 257 salivarius septicus (Bacillus), 306 salivarius septicus (Coccus), 275 salivarius-septicus (Micrococcus), 275 salivarius septicus felis (Bacillus), 553 salivarius tenuis (Streptococcus), 343

Salmonella, 10, 17, 21, 26, 31, 37, 43, 461, $492,494,508,516,523,530,531,552$

Salmonella sp. (Type Aberdeen), 499, 526

Salmonella sp. (Type Abony), 495, 502 Salmonella sp. (Type Adelaide), 500, 530 Salmonella sp. (Type Altendorf), 495, 506

Salmonella sp. (Type Amager), 498, 524 Salmonella sp. (Type Amersfoort), 496, 511

Salmonella sp. (Type Amherst), 497, 515 Salmonella sp. (Type Arechavaleta), 495,506

Salmonella sp. (Type Arizona), 462

Salmonella sp. (Type Ballerup), 500, 529

Salmonella sp. (Type Bareilly), 496, 511

Salmonella sp. (Type Berlin) (Type Thompson), 510

Salmonella sp. (Type Berta), 497, 518 Salmonella sp. (Type Bispebjerg), 495, 506

Salmonella sp. (Type Blegdam), 497, 518 Salmonella sp. (Type Borbeck), 499, 572 Salmonella sp. (Type Braenderup), 496, 511

Salmonella sp. (Type Brandenburg), 495,505

Salmonella sp. (Type Bredeney), 496, 507
Salmonella sp. (Type Budapest), 495 , 505

Salmonella sp. (Type Buenos Aires), 497,514

Salmonella sp. (Type Butantan), 499, 524

Salmonella sp. (Type California), 495, 505

Salmonella sp. (Type Canastel), 498, 521

Salmonella sp. (Type Cardiff), 496, 512

Salmonella sp. (Type Carrau), 500, 528

Salmonella sp. (Type Cerro), 500, 529

Salmonella sp. (Type Champaign), 500, 530

Salmonella sp. (Type Chester), 495, 504

Salmonella sp. (Type Claiborne), 497, 518

Salmonella sp. (Type Concord), 496, 512

Salmonella sp. (Type Cuba), 499, 527

Salmonella sp. (Type Dar es Salaam), 498, 579

Salmonella sp. (Dar es salaam type var. from Arizona.), 462

Salmonella sp. (Type Daytona), 496, 513

Salmonella sp. (Type Derby), 495, 505

Salmonella sp. (Type Dublin), 497, 517

Salmonella sp. (Type Duesseldorf), 497, 514

Salmonella sp. (Type Durban), 497, 519

Salmonella sp. (Type Eastbourne), 498, 519

Salmonella sp. (Type Essen), 495, 505

Salmonella sp. (Type Florida), 500, 528

Salmonella sp. (Type Gaminara), 500, 529

Salmonella sp. (Type Gatun), 497, 515

Salmonella sp. (Type Georgia), 496, 512

Salmonella sp. (Type Give), 498, 522

Salmonella sp. (Type Glostrup), 497, 514

Salmonella sp. (Type Goettingen), 498, 520

Salmonella sp. (Type Grumpy), 499, 527 Salmonella sp. (Type Hartford), 496, 511

Salmonella sp. (Type Havana), 499, 527 
Salmonella sp. (Type Heidelberg), 495, 504

Salmonella sp. (Type Heves), 499, 528

Salmonella sp. (Type Hormaeche), 500, 529

Salmonella sp. (Type Hvittingfoss), 500, 528

Salmonella sp. (Type Illinois), 499, 525

Salmonella sp. (Type Infantis), 496, 512

Salmonella sp. (Type Inverness), 500, 530

Salmonella sp. (Type Italia), 498, 522

Salmonella sp. (Type Java), 498, 520

Salmonella sp. (Type Kaapstad), 495, 505

Salmonella $s p$. (Type Kaposvar), 495, 505

Salmonella sp. (Type Kentucky), 499, 526

Salmonella sp. (Type Kirkee), 500, 629

Salmonella sp. (Type Köln), 495, 503

Salmonella sp. (Type Kottbus), 497, 513

Salmonella sp. (Type Lexington), 498, 524

Salmonella sp. (Type Litchfield), 497, 514

Salmonella sp. (Type Loma Linda), 498, 522

Salmonella sp. (Type London), 498, 522

Salmonella sp. (Type Madelia), 500, 528

Salmonella sp. (Type Manhattan), 497, 514

Salmonella sp. (Type Marseille), 409, 526

Salmonella sp. (Type Meleagris), 498, 523

Salmonella sp. (Type Miami), 497, 519

Salmonella sp. (Type Mikawasima), 496, $5 \mathbf{1 1}$

Salmonella sp. (Type Minnesota), 500, 529

Salmonella sp. (Type Mississippi), 499, 527

Salmonella sp. (Type Montevideo), 496, 510

Salmonella sp. (Type Moscow), 497, 518

Salmonella sp. (Type Muenchen), 497, 513
Salmonella sp. (Type Muenster), 498, 523

Salmonella sp. (Type Napoli), 498, 522

Salmonella sp. (Type Narashino), 497, 514

Salmonella sp. (Type New Brunswick), 499,525

Salmonella sp. (Type Newington), 499, 624

Salmonella sp. (Type Newport), 497, 513

Salmonella sp. (Type New York), 498, 522

Salmonella sp. (Type Niloese), 499, $\mathbf{5 2 5}$

Salmonella sp. (Type Nyborg), 498, 523

Salmonella sp. (Type Onarimon), 497, 519

Salmonella sp. (Type Onderstepoort), 500,528

Salmonella sp. (Type Oranienburg), 496, 510

Salmonella sp. (Type Oregon), 497, 513

Salmonella sp. (Type Orient), 500, 528

Salmonella sp. (Type Orion), 499, 524

Salmonella sp. (Type Oslo), 496, 511

Salmonella sp. (Type Panama), 498, 519

Salmonella sp. (Type Papua), 496, 512

Salmonella sp. (Type Pensacola), 497, 518

Salmonella sp. (Type Pomona), 500, 529 Salmonella sp. (Type Poona), 499, 527 Salmonella sp. (Type Potsdam), 496, 511 Salmonella sp. (Type Pretoria), 499, 526 Salmonella sp. (Type Pueris), 497, 513 Salmonella sp. (Type Puerto Rico), 513 Salmonella sp. (Type Reading), 495, 504 Salmonella sp. (Type Richmond), 496, 512

Salmonella sp. (Type Rostock), 497, 517

Salmonella sp. (Type Rubislaw), 499, 526

Salmonella sp. (Type St. Lucie), 499, 526

Salmonella sp. (Type Saint Paul), 495, 504

Salmonella sp. (Type Salinas), 495, 504

Salmonella sp. (Type San Diego), 495, 504 
Salmonella sp. (Type Schleissheim), 496,507

Salmonella sp. (Type Schwarzengrund), 496, 507

Salmonella sp. (Type Selandia), 499, 525

Salmonella sp. (Type Sendai), 497, 518

Salmonella sp. (Type Senegal), 499, 526

Salmonella sp. (Type Senftenberg), 499, 525

Salmonella sp. (Type Shangani), 498, 524

Salmonella sp. (Type Simsbury), 499, 525

Salmonella sp. (Type Solt), 499, 526

Salmonella sp. (Type Stanley), 495, 503

Salmonella sp. (Type Sundsvall), 500, 528

Salmonella sp. (Type Szentes), 500, 529

Salmonella sp. (Type Taksony), 499, 525

Salmonella sp. (Type Tallahassee), 497, 514

Salmonella sp. (Type Tel Aviv), 500, 529

Salmonella sp. (Type Tennessee), 496, 512

Salmonella sp. (Type Texas), 495, 506

Salmonella sp. (Type Thompson), 496, 510

Salmonella sp. (Type Uganda), 498, 522

Salmonella sp. (Type Urbana), 500, 530

Salmonella sp. (Type Vejle), 498, 523

Salmonella sp. (Type Venezia), 499, 526

Salmonella sp. (Type Virchow), 496, 511

Salmonella sp. (Type Virginia), 497, 515

Salmonella sp. (Type Weltevreden) 498,524

Salmonella sp. (Type Wichita), 499, 527

Salmonella sp. (Type Worthington), 499, 527

Salmonella sp. (Type Zagreb), 495, 504

Salmonella sp. (Type Zanzibar), 498, 524

Salmonella suipestifer (American variety), 508

Salmonella suipestifer (European variety), 509

Salmonella thompson var. berlin, 510

Salmonella Typ. Holstein, 531

Salmonella var. concord, 512
Salmonella var. orion, 524

salmoneus (Bacillus), 668

salmoneus (Bacterium), 669

salmoni (Bacillus), 508

salmoni (Pasteurella), 508

salmonica (Bacterium), 686

salmonicida (Bacillus), 686

salmonicida (Bacterium), 671, 686

salmonicolor (Actinomyces), 973

salmonicolor (Flavobacterium), 904

salmonicolor (Mycobacterium), 904

salmonicolor (Nocardia), 904

salmonicolor (Proactinomyces), 904

salopium (Achromobacter), 105

salopium (Pseudomonas), 105

salutarius (Bacillus), 668

salvati (Actinomyces), 917

sambharianus (Serratia), 110

samesii (Planosarcina), 290, 291

samesii (Sarcina), 291

sampsonii (Actinomyces), 973

san diego (Salmonella), 504

sanfelicei (Actinomyces), 921

sanfelicei (Bacillus), 821

sanfelicei (Nocardia), 921

sanguicole (Inflabilis), 821

sanguinaria (Eberthella), 520

sanguinarium (Bacterium), 520

sanguinea (Ophidiomonas), 852

sanguineum (Propionibacterium), 379

sanguineum (Spirillum), 852

sanguineum (Thiospirillum), 851, 852

sanguineus (Bacillus), 668

sanguineus (Streptococcus), 343

sanguinis (Actinomyces), 973

sanguinis (Bacterium), 686

sanguinis (Streptococcus), 343

sanguinis canis (Streptococcus), 343

sanguinis typhi (Bacillus), 686

sanguinis typhi (Bacterium), 686

sanguis (Streptococcus), 343

sanii (Grahamella), 1111

sanninii (Actinomyces), 973

sanninii (Streptothrix), 934

santali (Chlorogenus), 1149

santali (Marmor), 1198

santiagensis (Bacillus), 753 
sapolactica (Pseudomonas), 149

sapolacticum (Bacterium), 149

saponaceus (Bacillus), 668

saprogenes (Bacillus), 668, 753, 782, 817

saprogenes (Bacterium), 668

saprogenes (Lactobacillus), 363

saprogenes (Plectridium), 782

saprogenes (Streptococcus), 344

saprogenes 1 (Bacillus), 668

saprogenes 2 (Bacillus), 670

saprogenes $I, I I, I I I$ (Bacillus), 753, 782,

817

saprogenes carnis (Bacillus), 782, 825 saprogenes intestinalis (Bacillus), 817

saprogenes vini I (Bacillus), 672

saprogenes vini II (Bacillus), 672

saprogenes vini III (Bacillus), 753

saprogenes vini IV (Bacillus), 638

saprogenes vini $V$ (Bacillus), 654

saprogenes vini VI (Bacillus), 668, 753

saprogenes vini I (Micrococcus), 280

saprogenes vini II (Micrococcus), 345

Sapromyces, 1294

saprophiles (Microspira), 202, 203, 206

saprophiles (Vibrio), 203

saprophiles $\alpha$ (Vibrio), 202

saprophiles $\beta$ (Vibrio), 202

saprophiles $\gamma$ (Spirillum), 202

saprophiles $\gamma$ (Vibrio), 202

saprophytica (Nocardia), 976

saprophyticus (Actinomyces), 934, 977

saprophyticus (Bacillus), 817

saprophyticus (Streptococcus), 337, 344 saprophyticus var. cromogenus (Actinomyces), 977

Saprospira, 19, 20, 26, 28, 1054

saprotoxicum (Clostridium), 817

saprotoxicus (Bacillus), 817

sarcemphysematodes hominis (Bacillus), 821

Sarcina, 13, 14, 15, 17, 19, 21, 25, 27, 29, $31,33,42,249,285$

sarcinaeformis (Pediococcus), 250

sarcinoides (Micrococcus), 274

sarcinoides (Nitrocystis), 75

sarcoemphysematodes (Clostridium), 821 sarcoemphysematodes hominis (Bacillus), 817

sarcophysematos (Bacillus), 775, 776

sarcophysematos bovis (Bacillus), 775

sarcophysematos bovis (Clostridium), 775 sarcophysematosi (Bacillus), 776

sardinae (Bacillus), 483

sardiniensis (Clostridium), 821

sardous (Bacillus), 362

sardum miciurati (Bacterium), 362

sarracenicolus (Bacillus), 668

sartagoformum (Clostridium), 793

sartoryi (Actinomyces), 917

satellitis (Bacillus), 817

satellitis (Inflabilis), 817

sauromali (Bacterium), 461

savastanoi (Bacterium), 132

savastanoi (Phytomonas), 132

savastanoi (Pseudomonas), 132, 133

savastanoi var. fraxini (Bacterium), 132

savastanoi var. fraxini (Phytomonas), 132

savastanoi var. fraxini (Pseudomonas),

132

savastonoi var. nerii (Pseudomonas),

132

Savoia, 1221

saxicavae (Cristispira), 1057

saxicavae (Spirochaeta), 1057

saxkoebing (Leptospira), 1079

s. b. e. (Streptococcus), 343

scaber (Bacillus), 753

scaber (Tyrothrix), 753

scabiegena (Erwinia), 478

scabiegenum (Bacterium), 478

scabiegenus (Bacillus), 478

scabies (Actinomyces), 43, 957, 977

scabies (Oospora), 957

scabies (Streptomyces), 957

scariosus (Micrococeus), 275

scarlatinae (Bacillus), 668

scarlatinae (Micrococcus), 315

scarlatinae (Streptococcus), 315

scarlatinae (Streptus), 14, 315

scarlatinae (Syzygiococcus), 304

scarlatinae sanguinis (Diplococcus), 301, 336

scarlatinosa (Perroncitoa), 275 
scarlatinosus (Micrococcus), 275 scarlatinus (Micrococcus), 275 scatologenes (Bacillus), 817 scelestus (Erro), 1251

\section{Scelus, 1234}

schafferi (Bacillus), 445, 450 schafferi (Bacterium), 445, 450 schafferi (Escherichia), 445, 450 schaudinni (Planosarcina), 293 schaudinni (Sarcina), 293 schaudinni (Spirochaeta), 1063 schaudinni (Spiroschaudinnia), 1063 schaudinni (Treponema), 1063 Schaudinnum, 12, 13, 705 scheurleni (Bacillus), 743 schirokithi (Bacillus), 754 schirokikhi (Bacterium), 442 schirokikhii (Flavobacterium), 442 schizobacteroides (Nitrosogloea), 73 schleissheim (Salmonella), 507 Schlerothrix, 12, 14, 876 Schmidlea, 870 schmidlei (Thioploca), 994 schmidti (Streptococcus), 344 schmitzii (Bacterium), 536 schmitzii (Shigella), 536 schottelii (Bacillus), 753 schottelii (Bacterium), 753 shottmuelleri (Salmonella), 62, 495, 501, 530

schottmülleri (Bacillus), 501 schottmülleri (Bacterium), 501 schottmülleri var. alvei (Salmonella), 532 schroeteri (Sorangium), 1021 schroeteri (Spirillum), 1054 schroeteri (Spirochaete), 1054 Schuetzia, 312 schuezenbachii (Bacterium), 187 schüffneri (Bacterium), 686 schütz (Streptococcus), 317 schützenbergii (Bacillus), 691 schützenbergii I and II (Urobacillus), 691 schuylkilliensis (Aerobacillus), 722 schuylkilliensis (Microspira), 196 schuylkilliensis (Pseudomonas), 93, 700 schuylkilliensis (Vibrio), 196 schuylkilliensis fuorescens (Bacillus), 93 schwarzenbeck (Streptococcus), 332 schwarzengrund (Salmonella), 507 scillearum (Marmor), 1184 scissa (Pseudomonas), 97, 700 scissus (Bacillus), 97 scissus (Bacterium), 700 sciuri (Haemobartonella), 1107 sclavoei (Endosporus), 804 sclavoi (Bacillus), 804 sclavoi (Clostridium), 804 scoticus (Bacillus), 668 scoticus (Erro), 1248 secales (Bacillus), 457 secretum (Marmor), 1198 secundarius (Phagus), 1132 secundum (Ciostridium), 821 secundus (Bacillus), 668 secundus fullesi (Bacillus), 668 secundus fullesi (Bacterium), 668 sedentarius (Micrococcus), 696 sedimenteus (Micrococcus), 696 segetalis (Bacillus), 753

segmentosum (Corynebacterium), 406 segmentosus (Bacillus), 406 segnis (Pseudomonas), 177 seiferti (Streptococcus), 344 selachii (Treponema), 1076 selandia (Salmonella), 525 selenicus (Micrococcus), 275 Selenomonas, $21 \mathrm{~s}$

Semiclostridium, 705 seminum (Bacterium), 138 seminum (Phytomonas), 138 seminum (Pseudomonas), 138 sempervivum (Bacterium), 755, 761 sendai (Salmonella), 493, 518 sendaiensis (Salmonella), 518 senegal (Salmonella), 526 senftenberg (Salmonella), 525 senftenbergensis (Salmonella), 525 sensibilis (Micrococcus), 275 sensitiva (Cytophaga), 1015 sepedonica (Phytomonas), 393 sepedonicum (Aplanobacter), 393 sepedonicum (Bacterium), 393 sepedonicum (Corynebacterium), 393 sepiae (Photobacterium), 637 
sepiola (Coccobacillus), 702

septatum (Bacterium), 401

septatum (Polyangium), 1023

septatum (Sorangium), 1023

septatum var. microcystum (Sorangium), 1023

septatus (Bacillus), 401

septentrionale (Bacterium), 686

septica (Merista), 283

septica (Pasteurella), 546

septica (Pseudomonas), 94

septica (Sarcina), 283

septicaemiae (Eberthella), 543

septicaemiae (Shigella), 543

septicaemiae anserum exudativae (Bacil-

lus), 543

septicaemiae canis (Bacterium), 590

septicaemiae hemorrhagicae (Bacillus),

546

septicaemiae haemorrhagicae (Bacterium), 546

septicaemiae lophyri (Bacillus), 668

septicaemicus (Bacillus), 668

septichaemiae (Bacterium), 547

septico-aerobius (Bacillus), 753

scpticum (Bacterium), 673

septicum (Clostridium), 774, 775, 782,

815,824

septicun (Microsporon), 275

scpticus (Actinomyces), 917

septicus (Bacillus), 686, 774, 775, 817

septicus (Bacterium) (Proteus), 686

septicus (Coccus), 275

septicus (Micrococcus), 275

septicus (Proteus), 686

septicus (Streptococcus), 344

septicus (Tetracoccus), 283

septicus (Tibrio), 206, 775

septicus acuminatus (Bacillus), 674

septicus acuminatus (Bacterium), 674

septicus agrigenus (Bacillus), 673

septicus agrigenus (Bacterium), 673

septicus cuniculi (Bacillus), 652

septicus gangrenae (Bacillus), 775

septicus hominis (Bacillus), 668

septicus hominis (Bacterium), 668

septicus insectorum (Bacillus), 753 septicus keratomalaciae (Bacillus), 679

septicus liquefaciens (Streptococcus), 344 septicus putidus (Bacillus), 667

septicus putidus (Bacterium), 667

septicus sputigenus (Bacillus), 306

septicus ulceris gangraenosi (Bacillus), 774

septicus vesicae (Bacillus), 668, 741, 758 septimum (Clostridium), 822

septimus (Hiberillus), 822

septique (Clostridium), 775

septique (Vibrio), 775

septopyaemicus (Streptococcus), 344

septus (Bacillus), 406

Sequinillus, 11, 763

serbinowi (Bacillus), 478

serbinowi (Bacterium), 478

serbinowi (Erwinia), 478

sergenti (Bartonella), 1106

sergenti (Haemobartonella), 1106

sericcus (Bacillus), 668

sericea (Pseudomonas), 149

serophilus (Micrococcus), 275

serositidis (Bacillus), 727

serpens (Archangium), 1019

serpens (Bacillus), 566

serpens (Bacteroides), 566, 577

serpens (Chondromyces), 1017, 1019

serpens (Spirillum), 213

serpens (Vibrio), 213 ,

serpens (Zuberella), 566, 577

serranoi (Bacterium), 127

Serratia, 5, 10, 14, 20, 25, 31, 32, 37, 443,

$461,479,484,705$

serratum (Bacterium), 761

serratus (Actinomyces), 917

serratus (Bacillus), 668

serratus (Micrococcus), 275

serrulatus (Bacillus), 753

sesami (Bacillus), 137, 753

sesami (Bacterium), 128

sesami (Phytomonas), 128

sesami (Pseudomonas), 128, 137

sesamicola (Bacterium), 128

sesamicola (Phytomonas), 128

sessile (Bacterium), 716

sessile (Synangium), 1033 
sessilis (Bacillus), 716

sessilis (Chondromyces), 1033

sessilis (Pseudomonas), 700

setariae (Bacterium), 126

setariae (Phytomonas), 126

setariae (Pseudomonas), 126

setiensis (Inflabilis), 823

setonii (Actinomyces), 973

setosum (Bacterium), 686

setosus (Bacillus), 668

sewanense (Bacterium), 435

sewanense (Flavobacterium), $\mathbf{4 3 5}$

sewerini (Bacterium), 761

sewerinii (Achromobacter), 426

sextum (Clostridium), 810

sextus (Hiblerillus), 810

shangani (Salmonella), 524

shermanii (Propionibacterium), 373, 374,

$375,376,379$

shigae (Bacillus), 536

shigae (Bacterium), 536

Shigella, 10, 26, 31, 37, 489, 492, 535, 537

shmamini (Fusocillus), 583

sialopyus (Staphylococcus), 257

sialosepticus (Micrococcus), 275

siamensis (Bacillus), 716

sicca (Neisseria), 298, 299

siccum (Bacterium), 686

siccus Bacillus), 753

siccus (Bacteroides), 567, 579

siccus (Diplococcus), 298

siccus (Micrococcus), 275

siccus (Spherophorus), 567, 579

Siderobacter, 835

Siderocapsa, 9, 23, 26, 29, 35, 833

Siderococcus, 835

Sideroderma, 835

Sideromonas, 20, 23, 26, 29, 35, 834, 835

Sideromyces, 986

sideropous (Chlamydothrix), 985

sideropous (Gallionella), 832, 985

sideropous (Leptothrix), 985

Siderothece, 835

sieberti (Bacterium), 686

silberschmidii (Bacillus), 669

silberschmidti (Actinomyces), 975

silberschmidti (Nocardia), 975 silberschmidtii (Cohnistreptothrix), 975

silvaticus (Bacillus), 714

silvestris (Cytophaga), 1016

silvestris (Erro), 1249

simiae (Bacterium), 593

simiae (Noguchia), 593

simile (Bacterium), 753

similis (Bacillus), 753

similis (Micrococcus), 275

simili yphosus (Bacillus), 753

simplex (Bacillus), 718, 748, 751

simplex (Corynebacterium), 397

simplex (Micrococcus), 275

simplex (Myxobacter), 1030

simplex (Polyangium), 1030

simplex (Rhizobium), 225

simsbury (Salmonella), 525

simulans (Bacillus), 669

simulans (Legio), 1260

simulans (Micrococcus), 275

sinapivagus (Bacillus), 754

sinense (Butylobacter), 781, 825

sinensis (Spirochaeta), 1069

singulare (Acetobacter), 692

singularis (Bacillus), 669

sinuosa (Pseudomonas), 103, 700

sinuosum (Achromobacter), 103

sinuosus (Bacillus), 103

sinuosus (Bacterium), 700

siticulosus (Bacillus), 669

skoliodonta (Spirochaeta), $107 t$

skoliodontum (Treponema), 1074

smaragdina (Pseudomonas), 94, 700

smaragdino foetidus (Bacterium), 700

smaragdinophosphorescens (Achromobac. (er), 634

smaragdino-phosphorescens (Bacillus), 634 smaragdino-phosphorescens (Bacterium),

634,635

smaragdinum (Bacterium), 635

smaragdinus (Bacillus), 700

smaragdinus foetidus (Bacillus), 94

smegmatis (Bacillus), 890

smegmatis (Bacterium), 890

smegmatis (Mycobacterium), 890

smegmatis var. muris (Mycobacterium), 891 
smithii (Chromobacterium), 234

smithii (Microspira), 206

smithii (Pseudomonas), 234

smithii (Vibrio), 202, 203, 206

smyrnii (Azotobacter), 219

snieszkoi (Plectridium), 823

sociovivum (Bacterium), 606

sodoku (Spirochaeta), 215

sodoku (Treponema), 215

soehngenii (Methanobacterium), 645,

646

sogdianum (Borrelia), 1069

sogdianum. (Spirochaeta), 1069

sojae (Bacterium), 131

sojae (Phytomonas), 131

sojac (Pseudomonas), 131, 135

sojac (Rhizobium), 226

solanacearum (Bacillus), 137

solanacearum (Bacterium), 137

solanacearum (Phagus), 1135

solanaceurum. (Phytomonas), $13 \overrightarrow{7}$

solanacearum (Pseudomonas), 137, 138,

$1129,1135,1136$

solanaccarum var. usiatica (Phytomonas), 138

solanacearum var. asiatica (Pseudomonas), $13 \mathrm{~s}$

solanacearum var. asiaticum (Bacterium),

138

solani (Acrogenus), 1203

solani (Butylobacter), 781, 825

solani (Chlorogenus), 1149

solani (Corium), 1204

solani (Marmor), 1174

solani (Sarcina), 293

solani var. severus (Acrogenus), 1203

solani var. vulgaris (Acrogenus), 1203

solanincola (Bacillus), 469

solaniolens (Phytomonas), 98

solaniolens (Pseudomonas), 98

solaniperda (Bacillus), 477

solanisapra (Erwinia), 468, 469, 470

solanisaprus (Bacillus), 468, 470

solare (Bacterium), 439

solare (Flavobacterium), 439

solenis (Spirochaeta), 1057

solenoide (Spirosoma), \$31 solida (Cornilia), 817

Solidococcus, 8, 235

Solidovibrio, 8, 192

solidum (Clostridium), 821

solidus (Bacillus), 817, 821

solitarium (Achromobacter), 426

solitarius (Bacillus), 426

solitarius (Bacterium), 426

solmsii (Bacillus), 817

solmsii (Diplectridium), 817

solt (Salmonella), 526

somaliensis (Actinomyces), 965

somaliensis (Discomyces), 965

somaliensis (Indiella), 965

somaliensis (Indiellopsis), 965, 966

somaliensis (Nocardia), 965

somaliensis (Streptomyces), 965

somaliensis (Streptothrix), 965

sombrosus (Bacillus), 754

sommeri (Actinomyces), 917

sommeri (Oospora), 918

sonnei (Bacterium), 540

sonnei (Proshigella), 540

sonnei (Shigella), 540, 542, 543

Sorangium, 1021

sordellii (Bacillus), 787

sordelli (Clostridium), 787

sordidus (Bacillus), 669

sordidus (Bacterium), 669

sordidus (Micrococcus), 275, 669

sorediatum (Polyangium), 1022, 1023

sorediatum (Sorangium), 1022

sorediatum var. macrocystum (Sorangium), 1023

sorghi (Bacillus), 751

sorghi (Bacterium), 754

soriferum (Bacterium), 686

sornthalii (Micrococcus), 344

sornthalii (Streptococcus), 344

Sorochloris?, 869

sorracenicolus (Bac'erium), 668

sotto (Bacillus), 754

sotto (Bacterium), 754

soya (Bacterium), 358

soyae (Lactobacillus), 358

soyae (Leuconostoc), 346

soyae var. japonicum (Bacterium), 132 
spatiosus (Bacillus), 754

spatuliforme (Cillobacterium), 369, 81s spatuliformis (Bacillus), 818 spermatozoides (Bacillus), 754 spermatozoides (Vibrio), 206 spermiformis (Treponema), 1076 spermoides (Acuformis), 812 spermoides (Bacillus), 812, 827 spermoides (Clostridium), 812 spermoides (Palmula), 812 spermophilinus (Bacillus), 669 sphaerica (Blastocaulis), 836 sphaericum (Granulobacter), 822 sphaericus (Bacillus), 727, 728, 729, 818 sphaericus var. fusiformis (Bacillus), $72 S$ Sphaerococcus, 312 sphaeroides (Chromatium), 816, 859 sphaeroides (Clostridium), 821 sphaeroides (Micrococcus), 275 sphaeroides (Rhizobium), 225 sphaerosporus (Bacillus), 754 sphaerosporus calco-aceticus (Bacillus), 754

Sphaerothrix, 986

Sphaerotilus, 12, 19, 23, 26, 982, 983

sphagni (Streptococcus), 344 sphenoides (Bacillus), 791 sphenoides (Clostridium), 791 sphenoides (Douglasillus), 791 sphenoides (Plectridium), 791 Spherocillus, 34, 38, 580 spheroides (Rhodopseudomonas), 865 Spherophorus, 34, 38, 578 sphingidis (Bacillus), 491 sphingidis (Escherichia), 491 sphingidis (Proteus), 491 spiculifera (C'ristispira), 1057 spiculifera (Spirochaeta), 1057 spieckermann (Bacillus), 477 spiniferum (Bacterium), 686 spiniferus (Bacillus), 686 spinosa (Cornilia), 817 spinosporus (Bacillus), 754 spinosum (Bacterium), 686 spinosus (Bacillus), 817 spirale (Bacterium), 686 spiralis (Actinomyces), 973 spiralis (Bacillus), 751

spirans (Bacillus), 669

Spirella, 28

Spirilina, 5, 486

spirilloides (Streptothrix), $97 \mathrm{~T}$

Spirillum, 5, 12, 15, 16, 18, 19, 21, 25, 28,

$29,31,43,212,216,996$

spirillum (Azotobacter), 216

spirillum (Vibrio), 216

Spirobacillus, 12, 14

Spirochaeta, 5, 12, 18, 19, 20, 26, 28, 37,

$1007,1051,1053,1054,105 \mathrm{~s}$

Spirochaete, 1051

Spirochoeta, 1051

Spirodiscus, 6

spirogyra (Bacillus), 751

Spiromonas, 6, 11

Spironema, 20, 1058, 1071

Spirophyllum, 8, 9, 15, 17, 831

Spiroschaudinnia, 1058

Spirosoma, 7, 12, 16, 28, 212, 1122

Spirulina, 6, 993

spissum (Bacterium), 761

spitzi (Actinomyces), 925

spitzi (Brevistreptothrix), 925

spitzi (Discomyces), 925

spitzi (Oospora), 925

spitzi (Streptothrix), 925

splendens (Bacillus), 613

splendidum (Photobacter), 636

splendidum (Photobacterium), 636

splendidus (Vibrio), 636

splendor maris (Photobacter), 636

splenica (Actinomyces), 922

splenica (Nocardia), 922

splenomegaliae (Bacteroides), 580

splenomegaliae (Synbacterium), 580

spongiosa (Phytomonas), 120

spongiosa (Pseudomonas), 120

spongiosum (Bacterium), 120

spongiosus (Bacillus), 120, 754

sporiferum (Spirillum), 218

Sporocytophaga, 35, 259, 1005, 1006, $1009,1010,1048$

sporogena rheumatismi (Spirochaeta), 1069

sporogenes (Bacillus), 782, 817 
sporogenes (Clostridium), 775, 782, 783,

$784,786,817,818,825$

sporogenes (Granulobacillus), 822

sporogenes (Lactobacillus), 762

sporogenes (Metchnikovillus), 782

sporogenes capsulatus (Bacillus), 817

sporogenes coagulans (Bacillus), 782, 825

sporogenes foetidus (Bacillus), 787, 818,

826

sporogenes liquefaciens (Bacillus), 818 sporogenes non-liquefaciens (Bacillus),

818

sporogenes non liquefaciens anaerobius

(Bacillus), 818

sporogenes oedematis (Bacillus), 787

sporogenes parvus (Bacillus), 818

sporogenes psoriasis (Spirochaeta), 1069

sporogenes regularis (Bacillus), 785

sporogenes saccharolyticus (Bacillus), 785

sporogenes var. A (Bacillus), 782

sporogenes var. A (Clostridium), 782

sporogenes var. A. P. Marie (Clostridium), 783

sporogenes var. $B$ (Bacillus), 782, 787

sporogenes var. $B$ (Clostridium), 787

sporogenes var. caudapiscis (Clostri-

$$
\text { dium), } 783
$$

sporogenes var. equine (Clostridium), 783

sporogenes var. parasporogenes (Clostridium), 784

sporogenes var. tyrusinogenes (Clostridium), 783

sporogenes zoogleicus (Bacillus), 797

Sporonema, 6

sporonema (Bacillus), 754

Sporosarcina, 30, 67, 285

Sporospirillum, 218

Sporotrichum, 916

Sporovibrio, 33, 35, 207

spumalis (Actinomyces), 976

spumalis (Oospora), 976

spumarum (Clostridium), 808

spumarum (Plectridium), 808

spumosum (Polyangium), 1031

spumosum (Sorangium), 1023

spumosus (Bacillus), 669 spurius (Bacillus), 754

sputi (Bacillus), 754

sputi (Bacterium), 761

sputicola (Bacterium), 761

sputigena (Microspira), 198

sputigenes tenuis (Bacterium), 687

sputigenum (Bacterium), 686

sputigenum (Spirillum), 206, 218, 701

sputigenus (Streptococcus), 344

sputigenus (Vibrio), 198, 206

sputigenus var. minutissimus (Vibrio), 206

sputigenus crassus (Bacillus), 459

sputigenus crassus (Bacterium), 459

sputorum (Tibrio), 206

squamiformis (Bacillus), 754

squamosum (Bacterium), 687

squamosum (Corynebacterium), 406

squamosum longum (Bacterium), 760, 762

squamosus (Bacillus), 669

squamosus longus (Bacillus), 760

squatorolae (Treponema), 1076

stalactitigenes (Bacterium), 687

stanieri (Tibrio), 703

stanley (Salmonella), 503

stanleyi (Śalmonella), 503

staphylina (Spirochaeta), 1069

Staphylococcus, 21, 31, 33, 235

staphylophagus (Micrococcus), 275

stationis (Achromobacter), 421

stationis (Tibrio), 206

stearophilum (Achromobacter), 609

stearophilus (Bacillus), 609

stearothermophilus (Bacillus), 734

Stelangium, 1020

stellaris (Bacillus), 754

stellatum (Bacterium), 818

stellatum (Polyangium), 1031

stellatus (Bacillus), 580, 710, 754, 818

stellatus (Coccus), 276

stellatus (Micrococcus), 276

stellatus anacrobius (Bacillus), 818

stenogyrata (Spirochaeta), 1069

stenogyratum (Treponema), 1069

stenohalis (Achromobacter), $\mathbf{4 2 0}$

stenos (Streptococcus), 344

stenostrepta (Spi rochaeta), 1053 
stenostrepta (Treponema), 1053

stercoraria (Serratia), 485

stercoris (Mycobacterium), 888, 891

stercusis (Mycobacterium), 891

sternbergii (Bacillus), 418, 687

sternbergii (Bacterium), 687, 819

steroidiclasium (Bacterium), 687

sterotropis (Pseudomonas), 700

stevensae (Alcaligenes), 416

stewarti (Aplanobacter), 638, 1136

stewarti (Bacillus), 638

stewartii (Bacterium), 638, 1129, 1134,

1136

stewartii (Phytomonas), 638, 1136

stewarti (Pseudomonas), 638, 1136

Stigmatella, 1036

stipitatus (Myxococcus), 1043, 1044

stizolobii (A planobacter), 135

stizolobii (Bacterium), 135

stizolobii (Phytomonas), 135

stizolobii (Pseudomonas), 135

Stoddardillus, 11, 763

stolonatum (Flavobacterium), 142

stolonatus (Bacillus), 442

stolonatus (Bacterium), 442

stoloniferum (Achromobacter), 715

stoloniferus (Bacillus), 715

stoloniferus (Bacterium), 715

stomachi (Spirillum), 218

stomatitis (Vibrio), 206

stramineus (Streptococcus), $3+t$

strasburgense (Clostridium), 821

strasburgensis (Pasteurella), 554

strassmanni (Bacillus), 669

streckeri (Bacillus), 687

streckeri (Bacterium), 687

streptobacilliformis (Bacteroides), 581

streptobacilli-moniliformis (Musculomy-

ces), 1294

Streptobacillus, 349, 588, 763

Streptobacterium, 9, 30, $\mathbf{3 5 0}$

streptococei (Phagus), 1139

streptococci var. viritis (Phagus), 113!

streptococciforme (Bacterium), 761

Streptococcus, 13, 14, 15, 17, 19, 21, 26,

$27,30,31,33,43, \mathbf{3 1 2}, 313$

Streptococcus No. 52, 343
Streptococcus sp., 333, 334, 335

streptoformis (Bacillus), 754

Streptomyces, 588, 915, 929, 934, 967, $974,977,980$

Streptothrix, 6, 929, 961, 977

Streptothrix No. 1, Almquist, 968

Streptothrix No. 2 and 3, Almquist, 934

Streptothrix sp., Donna, 916

Streptus, 14, 312

striafaciens (Bacterium), 112

striafaciens (Phytomonas), 112

striafaciens (Pseudomonas), 112

striata (Pseudomonas), 97, 700

striata (Sarcina), 293

striatum (Bacterium), 406

striatum (Corynebacterium), 406

striatus albus (Bacillus), 406, 669

striatus flavus (Bacillus), 406, 669

striatus flavus (Bacterium), 406, 669

striatus viridis (Bacillus), 97, 669

striatus viridis (Bacterium), 700

strictus (Vibrio), 198, 206

strobiliformis (Micrococcus), 276

strumitidis (Bacillus), 669

strumitis (Bacillus), 669

strumitis $\alpha$ (Bacillus), 669

strumitis $\beta$ (Bacillus), 671

sturmanii (Haemobartonella), 1106

stutzeri. (Achromobacter), 426

stutzeri (Bacillus), 426

stutzeri (Bacterium), 426

stutzeri (Pseudomonas), $4+1$

stylopygae (Spirochacta), 1076

stylopygae (Treponema), 1076

suariorum (Legio), 1262

suaveolens (Bacillus), 754

suaveolens (Flavobacterium), 432

subacidus (Streptococcus), 344

subalbus (Bacillus), 623

subalbus var. batatatis (Bacillus), 623

subalcalescens (Bacillus), 451

subanaerobicus (Bacillus), 720

subanaerobius (Bacillus), 771

subcandicans (Micrococcus), 276

subcanus (Micrococcus), 276

subcarneus (Micrococcus), 255, 276

subcitreus (Micrococcus), 276 
subcitricum (Bactorium), 687 subcloacae (Bacillus), 457

subcoccineus (Bacillus), 652 subcoccoideus (Bacillus), 669 subcreta (Cellulomonas), 176 subcreta (Pseudomonas), 176 subcretaceus (Micrococcus), 276 subcuticularis (Bacillus), 755 subdenticulatum (Bacterium), 762 subentericus (Bacillus), 533 suberfaciens (Bacterium), 6 10 suberfaciens (Phytomonas), 640 subfiliforme (Bacterium), 759 subflava (Neisseria), 276, 299 subflava (Sarcina), 293 subflavescens (Micrococcus), 276 subflavidus (Micrococcus), 276 subflavus (Bacillus), 669 subflavus (Bacterium), 669 subflavus (Diplococcus), 276 subflavus (Micrococcus), 276 subfoctidus (Bacillus), 818 subfuscum (Bacterium), 687 subfuscus (Micrococcus), 276 subgastricus (Bacillus), 451, 669 subgilvus (Micrococcus), 277 subgranulatus (Micrococcus), 277 subgranulosus (Bacillus), 658 subgriseus (Micrococcus), 277 subkiliensis (Bacillus), 484 sublacteus (Micrococcus), 277 sublanatus (Bacillus), 755 sublilacinus (Hicrococcus), 277 subliquefacions (Bacterium), 457 sublustris (Bacillus), 755 subluteum (Bacterium), 687 subluteus (Micrococcus), $27 i$ submarinus (Bacillus), 755 subniveus (Micrococcus), 251, 277 subochraceus (Bacillus), 670 subochraceus (Bacterium), 670 subochraceus (Micrococcus), $27 \%$ suboxydans (Acetobacter), 184 subpneumonicum (Bacterium), 703 subroseus (Micrococcus), 274, 277 subrubeum (Bacterium), 762 subrubeus (Bacillus), 762 subrubiginosus (Bacillus), 670 subrufa (Serratia), 601 subrufum (Bacterium), 601 subsquamosum (Bacterium), 762 subsulcatus (Bacillus), 670 subterminale (Clostridium), 786 subterminalis (Bacillus), 786 subterraneus (Micrococcus), 277 subterraneus (Staphylococcus), 277 subtetanicus (Bacillus), 727, 816 subthermophilum (Bacterium), 762 subtile (Bacterium), 755 subtile (Treponema), 1074 subtile agnorum (Bacterium), 648 subtiliforme (Bacterium), 755 subtiliformis (Bacillus) (Streptobacter) 755

subtilis (Bacillus), 42, 43, 45, 63, 708, $709,711,712,713,716,741,742,746$, $747,751,753,760,762,1138$ subtilis $\alpha$ (Bacillus), 710 subtilis (Bacillus), Michigan strain, 716 subtilis (Micrococcus), 277 subtilis (Spirochaeta), 1074 subtilis (Spironema), $107 t$ subtilis (Spiroschaudinnia), $107 \mathrm{t}$ subtilis (Vibrio), 710 subtilis var, asporus (Bacillus), 45 subtilis var. aterrimus (Bacillus), 711 subtilis var. galleriae (Bacterium), 762 subtilis var. niger (Bacillus), 711 subtilis var. viscosus (Bacillus), 710 subtilis similis (Bacillus), 752 subtilis simulans $I$ (Bacillus), 755 subtilissimum (Spirillum), 206 subtilissimus (Vibrio), 206 subvertens (Phagus), 1138 subviscosum (Bacterium), 414 succinicum (Bacterium), 452 succinicus (Bacillus), 755 succulentus (Micrococcus), 277 sudaminis (Bacillus), 668 suffodiens (Morsus), 1153 suffuscus (Bacillus), 755 suicida (Bacterium), 548 suidae (Treponema), 1076 suilla (Pasteurella), 647, 684 
suilla (Spirochaeta), 1069 suillum (Scelus), 1235, 1236 suipestifer (Bacillus), 508 suipestifer (Bacterium), 508 suipestifer (Salmonella), 45, 508 suis (Bacillus), 508

suis (Borreliota), 1232

suis (Brucella), 561, 562

suis (Coryncbacterium), 406

suis (Hemophilus), 585, $\mathbf{5 8 6}$

suis (Micrococcus), 277

suis (Rickettsia), 1097

suis (Spirochaeta), 1063

suis (Spironema), 1063

suis (Tortor), 1275

suis (Vibrio), 206

suiseptica (Pasteurella), 548

suisepticus (Bacillus), 548

suisepticus (Bacterium), 548

sulcatus (Bacillus), 670

sulcatus liquefaciens (Bacillus), 660, 670

sulcatus liquefaciens (Bacterium), 670

sulfhydrogenus (Bacillus), 670

Sulfomonas, 8, 29, 30, 78

Sulfospirillum, 29, 30, 212

sulfurea (Sarcina), 293

sulfureum (Achromobacter), 609

sulfureum (Bacterium), 657

sulfureum (Flavobacterium), 610

sulfurcus (Bacillus), 491, 687

sulfureus $\beta$-tardigradus (Micrococcus),

278

sulfureus (Proteus), 491

sulfureus var. tardigradus (Micrococcus),

278

sulfurica (Thiospira), 702

sulphurata (Sarcina), 842

sulphurea (Conidothrix), 995

sulphurea (Leptothrix), 995

sulphurea (Nocardia), 925

sulphurea (Streptothrix), 925

sulphureus (Actinomyces), 925

sulphureus (Micrococcus), 277

sulphurica (A phanothece), 872

sulphurica (Clathrochloris), $\mathbf{8 7 2}$

sumatrae (Actinomyces), 910

sumatranum (Bacterium), 687 sumatranus (Rickettsia), 1090

sundsvall. (Salmonella), 52S

superba (Sarcina), 293

superficiale (Achromolacter), 420

superficialis (Bacillus), 420

superficialis (Bacterium), 420

suppuratum (Corynebacterium), 406

supraresistens (Bacillus), 755

surati (Spirillum), 206

surati (Treponema), 206

surati (Tibrio), 202, 203, 206

surgeri (Bacillus), 687, 755

surgeri (Bacterium), 6s7

suspectus (Streptococcus), $3 \mathrm{H}$

suspensa (Rhodocapsa), $85 \pm$

suum (Pasteurella), 548

sycosiferum (Bacterium), 687

sycosiferus foetides (Bacillus), 687

sylvilagi (Molitor), 1244

symbiophiles (Bacillus), 580

symbiotica (Escherichia), 427

symbiotica (Sarcina), 293

symbioticum (Chlorobacterium), 873, 87t

symptomaticus (Bacillus), 776

Synangium, 1032

synchyseus (Bacillus), 682

synchyscus (Bacterium), 6\$2

Syncrotis , 12, 13, 14, 365, 984

syncyanea (Pseudomonas), 92, 700

syncyancum (Bacterium), 92

syncyaneus (Bacillus), 92

syncyancus (Vibrio), 92

syncyanus (Bacterium), 700

Synechococcus, 996

synthetica (Vibrio), 206

synxantha (Pseudomonas), 700

synxanthum (Flavobacterium), 701

synxanthus (Bacillus), 700

synxanthus (Tibrio), 700

syphilidis (Bacillus), 687

syphilidis (Bacterium), 687

syphilitica (Pacinia), 687

syphiliticus (Micrococcus), $27 \mathrm{~s}$

syringae (Bacterium), 119

syringae (Phytomonas), 119

syringae (Pseudomonas) 119, 123

syringae var. capsici (Bacterium), 120 
syringae var. papulans (Phytomonas), 123 syringae populans (Phytomonas), 697 syzygios (Micrococcus), 304 syzygios scarlatinae (Micrococcus), 304 szentes (Salmonella), 529

tabaci (Annulus), 1155, 1212, 1214, 1217 tabaci III (Bacillus), 755

tabaci (Marmor), 1155, 1164, 1167

tabaci (Musivum), 1164

tabaci (Phytomonas), 124

tabaci (Pseudomonas), 114, 124, 1134

tabaci (Ruga), 1216

tabaci var. artum (Marmor), 1166

tabaci var. aucuba (Marmor), 1166

tabaci var. auratus (Annulus), 1213

tabaci var. canadense (Marmor), 1166

tabaci var. deformans (Marmor), 1166

tabaci var. immobile (Marmor), 1166

tabaci var. kentuckiensis (Annulus), 1213

tabaci var. lethale (Marmor), 1166

tabaci var. obscurum (Marmor), 1166

tabaci var. plantaginis (Marmor), 1166

tabaci var. siccans (Marmor), 1166

tabaci var. virginiensis (Annulus), 1212

tabaci var. vulgare (Marmor), 1166

tabacivorus (Bacillus), 477

tabacum (Bacterium), 124

tabidum (Flavobacterium), 694

tabificans (Bacillus), 477

tachysporus (Bacillus), sis

tachytonum (Bacterium), 687

tachytonus (Bacillus), 687

taeniata (Gallionella), 831

taette (Lactobacillus), 695

taette (Streptobacillus), 702

taette (Streptococcus), 702

taksony (Salmonella), 525

talassochelys (Grahamella), 1111

talavensis (Bacillus), 534

talavensis (Bacterium), 534

talavensis (Eberthella), 534

talavensis (Eberthus), 534

tallahassee (Salmonella), 514

talpae (Grahamella), 1109

tangallensis (Bacillus), 544

tangallensis (Shigella), $5+4$ tapetos (Cristispira), 1057

tapetos (Spirochaeta), 1057

taraxaci (Xanthomonas), 179

taraxeri cepapi (Actinomyces), 973

taraxeri cepapi (Streptothrix), 973

tarda (Eberthella), 534

tarda (Shigella), 544

tardicrescens (Bacterium), 638

tardicrescens (Phytomonas), 638

tardigradus (Micrococcus), 278

tardior (Micrococcus), 278

tardissima (Gaffkya), 284

tardissima (Neisseria), 278

tardissimus (Bacillus), 670

tardissimus (Micrococcus), 278

tardissimus (Tetragenus), 284

tardivus (Bacillus), 755

tardus (Bacillus), 544, 816

tardus (Micrococcus), $27 \mathrm{~s}$

tarozzii (Actinomyces), 924

tarozzii (Streptothrix), 924

Tarpeia, $\mathbf{1 2 6 8}$

tartari (Streptothrix), 977

tartarivorum (Aerobacter), 692

tartricus (Bacillus), 670

taveli (Bacillus), 727, 800, 816

technicum (Propionibacterium), 377

technicus (Bacillus), 755

tectum (Ochrobium), 835

tegumenticola (Bacterium), 604

tel-aviv (Salmonella), 529

telmatis (Bacillus), 670

temporariae (S pirochaeta), 1069

tenacatis (Micrococcus), 278

tenalbus (Multifermentans), 772

tenax (Bacillus), 755

tenax (Bacterium), 762

tener (Micrococcus), 278

tenerrimum (Spirillum), 206

tennessee (Salmonella), 512

tenua (C'ristispira), 1057

tenuatus (Bacillus), 670

tenue (Bacterium), 687

tenue (Caryophanon), 1004

tenue (Clostridium), 821

tenue (Sideroderma), 835

tenue (Spirillum), 214 
tenue (Spirophyllum), 831

tenue (Treponema), 1070

tenue obtusum (Treponema), 1068

tenuis (Actinomyces), 922, 974

tenuis (Bacillus), 670, 709

tenuis (Bacteroides), 818

tenuis (Clonothrix), 983

tenuis (Cohnistreptothrix), 922

tenuis (Crenothrix), 983

tenuis (Discomyces), 922

tenuis (Leptothrix), 365

tenuis (Micrococcus), 307

tenuis (Nocardia), 922

tenuis (Pseudoleptothrix), 365

tenuis (Pseudomonas), 149, 701

tenuis (Spirochaeta), 1070

tenuis (Streptococcus), 343, 344

tenuis (Thiothrix), 989, 990, 995

tenuis (Tyrothrix), 709

tenuis (Vibrio), 206

tenuis acuminata (Spirochata), 1064

tenuis apis (Bacillus), 670

tenuis glycolyticus (Bacillus), 818

tenuis non-liquefaciens (Bacillus), 755

tenuis obtusa (Spirochaeta), 1068

tenuis spatuliformis (Bacillus), 369, 818

tenuis sputigenes (Bacillus), 607, 687

tenuissima (Cytophaga), 1013

tenuissima (Thiothrix), 990, 995

tenuissimus (Micrococcus), $259,27 \mathrm{~s}$

teras (Bacillus), 818

teras (Inflabilis), 818

terebrans (Bacillus), 575

terebrans (Ristella), 575

teres (Bacillus), 42, 718

terminalis (Bacillus), 75.)

terminalis var. thermophilus (Bacillus), 755

Terminosporus 33, 34, 763

termitidis (Fusiformis), 583

termitis (Cristispira), 1070

termitis (Spirochaeta), 1070

termitis (Treponema), 1070

termitis (Vibrio), 1070

termo (Bacillus), 688

termo (Bacterium), 687

Termobacterium, 179 termo (Monas), 687

termo (Zoogloea), 348, 687

termo var. subterraneum (Bacterium), 688

termophilum (Bacterium), 757

ternissima (Cytophaga), 1013

terrae (Bacterium), 762

terrae (Streptobacillus), 762, 823

terrestralginicum (Bacterium), 642

terrestris (Bacillus), 756

terricola (Lactobacterium), 361

terricola (Streptococcus), 344

terrigena (Microspira), 207

terrigenum (Spirillum), 207

terrigenus (Bacillus), 670

terrigenus (Tibio), $20 \bar{\sigma}$

tertium (Clostridium), 812, 827

tertium. (Plectridium), 812

tertium (Scelus), 1237

tertius (Bacillus), 812

tertius (Henrillus), 812

tertius (Phagus), 1137

testabilis (Phagus), $\mathbf{1 1 3 8}$

testudinis (Mycobacterium), 891

testudo (Mycobacterium), 891

tetani (Bacillus), 783, 798

tetani (Clostridium), 43, 72, 798

tetani (Nicollaierillus), 799

tetani (Plectridium), 798

tetanoides (Bacillus), 756, 799

tetanoides (A) (Bacillus), 798

tctanoides (B) (Bacillus), 799, 826

tetanoides (Clostridium), 798

tetanomorphum (Clostridium), 800, 826

tetanomorphum (Plectridium), 800

tetanomorphus (Bacillus), 800

tetanomorphus (Macintoshillus), 80)

Tetrachloris, 869

Tetracoccus, 9, 235, 283, 281

Tetradiplococcus, 283

tetragena (Gaffyka), 253, 258, 261, 267, $269,270,274,275,276,278,279,281$, 283, 284

tetragena (Merista), 283

tetragena (Sarcina), 283, 2\$4, 292

tetragenes anaerobius (Micrococcus), 284

tetiagenus (Micrococcus), 283, 281

tetragenus (Mycococcus), 891 
tetragenus (Pediococcus), 283

tetragenus (Planococcus), 281

tetragenus (Staphylococcus), 283

tetragenus albus (Micrococcus), 283

tetragenus aureus (Micrococcus), 278

tetragenus citreus (Micrococcus), 280

tetragenus concentricus (Micrococcus), $27 \mathrm{~s}$ tetragenus febris flavae (Micrococcus), 280 tetragenus mobitis ventriculi (Micrococcus), 284

tetragenus-pallidus (Micrococcus), 278 tetragenus ruber (Micrococcus), 244

tetragenus septicus (Micrococcus), $2 \times 3$ tetragenus subflavus (Micrococcus), 276 tetragenus versatilis (Micrococcus), 280 tetragenus-vividus (Micrococcus), 27! tetraonis (Bacillus), 668

tetras (Micrococcus), 27!

tetras (Pediococcus), 27!)

tetrytium (Clostridium) (Bacillus), $781, \$ 25$ teullia (Phytomonas), 613

teutlium (A planobacter), 613

teutlium (Bacterium), 613

texas (Salmonclla), 506

thalassius (Achromobacter), 418

thalassokoites (Bacillus), 756

thalassophilus (Bacillus), 720, 727, s18

(hamnopheos (Mroobacterium), 88.3, 885, $886,887$.

thaxteri (Archangium), 1019

thaxteri (Chondromyces), 1033

thaxteri (Synangium), 1033

theae (Bacillus), 756

Theciobactrum 12, 13, 705

theileri (Borrelia), 1062, 1066i, $1166 \mathrm{~s}$

theileri (Spirillum), 1062

theileri (Spirochaete), 1062

theileri (Spironema), 106:

theileri (Spiroschaudinnia), 1062

theileri (Treponema), 1062

Theileria, 10s?

thermalis (Chlamydothrix), (9xti

thermalis (Leptothrix), 986

thermitanus (Thiobacillus), si

thermaabundans (Bacillus), 756

thermoacetigenitus (Bacillus), 756

thermoacidificans (Bacillus), 756 thermoacidophila (Palmula), 821

thermoacidophilus (Acuformis), 821

thermoacidophilus (Clostridium), 821

thermoacidurans (Bacillus), 712

Thermoactinomyces, 978

thermoactivus (Bacillus), 756

thermoaerogenes (Caduceus), 821

thermoaerogenes (Clostridium), 821

thermoalimentophilus (Bacillus), 735

thermoamylolyticus (Bacillus), 729

thermoannulatus (Bacillus), 756

thermoaquatilis (Bacillus), 756

thermoarborescens (Bacillus), 756

Thermobacterium, 9, 30, $\mathbf{3 5 0}$,

thermobutyrosus (Bacillus), 756

thermocellulolyticus (Bacillus), 735

thermocellulolyticus (Terminosporus), 823

thermocellum (Clostridium), 821

thermocellus (Terminosporus), 821

thermochainus (Clostridium), 821

thermocompactus (Bacillus), 756

thermodactylogenitus (Bacillus), 736

thermodesulfuricans (Vibrio), 208, 209, 853

thermodiastaticus (Actinomyces), 934, 974

thermodiastaticus (Bacillus), 731

thermodoratus (Bacillus), 756

thermodurica (Sarcina), 294

thermoeffervescens (Bacillus), 756

thermofaecalis (Bacillus), 756

thermofibrincolus (Bacillus), 756, 818

thermofiliformis (Bacillus), 756

thermofuscus (Actinomyces), 957

thermofuscus (Streptomyces), 957

thermograni (Bacillus), 756

thermoindifferens (Bacillus), 730, 731

thermoliquefaciens (Bacillus), 735

thermolongus (Bacillus), 756

thermolubricans (Bacillus), 756

thermomonliquefaciens (Bacillus), 734

thermononodorus (Bacillus), 756

thermomubilosus (Bacillus), 756

thermopellitus (Bacillus), 756

thermophila (Nocardia), 957

thermophila $\beta$ (Ristella), 576

thermophila $\gamma$ (Ristella), 576

thermophilu (Sarcina), 294 
thermophilum I (Bacterium), 760

thermophilum II (Bacterium), 736 thermophilum III (Bacterium), 761 thermophilum IT (Bacterium), 762 thermophilum $\mathrm{I}^{\mathrm{r}}$ (Bacterium), 760 thermophilum I'I (Bacterium), 760 thermophitum TII (Bacterium), 762 thermophilum IIII (Bacterium), 759 thermophilum (Clostridium), 777, 821 thermophilum (Coccobacterium), 693 thermophilum (Denitrobacterium), 690, 762 thermophilus (Actinomyces), 934, 956 thermophilus (Bacillus), 670, 736, 757,777 thermophilus I (Bacillus), 760 thermophilus II (Bacillus), 736 thermophilus III (Bacillus), 76 ! thermophilus IV (Bacillus), 762 thermophilus $T^{-}$(Bacillus), 760 thermophilus TI (Bacillus); 760 thermophilus III (Bacillus), 762 thermophilus FIII (Bacillus), 759 thermophilus $\alpha$ (Bacillus), $\$ 19$ thermophilus $\beta$ (Bacillus), 576 thermophilus $\gamma$ (Bacillus), 576 thermophilus (Caduceus), 819 thermophitus $\alpha$ (Caduceus), $\$ 19$ thermophilus (Corynebacterium), 406 thermophilus (Lactobacillus), 355 thermophilus (Micrococcus), 279 thermophilus (Nitrosobacillus), 76, 690, 762

thermophilus (Streptococcus), 322 thermophilus (Streptomyces), 956 thermophilus anaerobicus (Bacillus), \$21 thermophilus aquatilis liquefaciens (Bacillus), 733

thermophilus jivoini (Bacillus), 732 thermophilus losanitchi (Bacillus), 73: thermophilus miquelii (Bacillus), 75 thermophilus sojae (Bacillus), 75i thermophilus vranjensis (Bacillus), 732 thermoputrifica (Palmula), 821

thermoputrificum (Clostridium), 821 thermoputrificus (Acuformis), 822 thermosaccharolyticum (Clostridium), 797 thermosaccharolyticus (Terminosporus),
797

thermosuavis (Bacillus), 757

thermotenax (Bacillus), $\overline{75} \overline{7}$

thermotolerans (Actinomyces), $97 \mathrm{t}$

thermotranslueens (Bacillus), 734

thermourinalis (Bacillus), 757

thermoviscidus (Bacillus), 757

theta (Bacillus), 670

theta (Bacterium), 670

thetaiotaomicron (Bacillus), 572

thetaiotaonicron (Bacteroides), 572, 580

thetaiotaomicron (Spherocillus), 572, 580

theioides (Bacillus), 566

thibiergei (Actinomyces), 928

thibiergei (Cohnistreptothrix), 928

thibiergei (Discomyces), $92 \mathrm{~s}$

thibiergei (Nocardia), 928

thibiergei (Oospora), 928

Thiobacillus, $15,16,20,30,69,78,81$, 688,839

Thiobacterium, $15,17,78$

Thiocapsa, 16, 23, 25, 844, 849

Thiococcus, s

Thiocystis, 16, 23, 25, 847, 848,849

Thioderma, 16, 23, 25, 819

Thiodictyon, 16, 23, 26, 845

thiogenes (Bacterium), 688

Thiomonas, 8

Thionema, 995

thiooxidans (Sulfomonas), 79

thiooxidans (Thiobacillus), 79, 81

thiooxydans (Thiobacterium), 79

thioparus (Sulfomonas), 79

thioparus (Thiobacillus), 79, 81

Thiopedia, 16, 29, 843, 8.44

Thiophysa, 16, 24, 25, 996, 997

Thioploca, 15, 16, 19, 2t, 26, 993

Thiopolycoccus, $16,23,25,29,850$

Thioporphyra, 859

Thiorhodospirillum, 16, 850

Thiosarcina, 16, 23, 25, 29, 842

Thiosiphon, 995, 996

Thiosphaera, 16, 23, 25, 816

Thiosphacrella, 15, 16,996, 997

Thiosphacrion, 16, 23, 25, 859

Thiospira, 24, 25, 35, 212, 853, 996 
Thiospirillopsis, 993

Thiospirillum, 15, 16, 23, 25, 29, 850, 851, 852,853

Thiothece, 16, 23, 25, 29, 845, 846, 849

Thiothrix, 16, 18, 19, 24, 26, 988, 989, 991, 995

Thiovibrio, 15, 16

Thiovulum 15, 16, 996, 999, 1000

thiryei (Nocardia), 917

thiryi (Actinomyces), 917

thiryi (Discomyces), 917

thjoettae (Actinomyces), 927

thoenii (Propionibacterium), 374, 377, 379

tholoeideum (Bacterium), 688

tholoeideus (Bacillus), 688

thompson (Salmonella), 510

thomsoni (Micrococcus), 303

thoracis (Bacillus), 757

thuillicri (Actinomyces), 410

thuillieri (Bacillus), 410

thuillieri (Nocardia), 410

thuillieri (Pasteurella), 410

thuringiensis (Bacillus), 716, 759

thuringiensis (Bacterium), 716

tilsitense (Plocamobacterium), 6y1

tim (Salmonella), 525

lingens (Bacillus), 670

tiogense (Achromobacter), 426

tiogensis (Bacillus), 426

liogensis (Bacterium), 426

Tissieria, 20, 21, 22, 23, 27

Tissierillus, 11, 763

tizzonii (Bacterium), 553

tolaasi (Bacterium), $12 \mathrm{~S}$

tolaasii (Phytomonas), 128

tolaasii (Pseudomonas), 128

tolega (Coccobacillus), 702

tolerans (Phagus), 1139

toluolicum (Bacillus), 670

tomato (Bacterium), 113

tomato (Phytomonas), 113

tomato (Pseudomonas), 113, 1136

tomentosum (Bacterium), 718

tommasoli (Micrococcus), 257

tonelliana (Phytomonas), 132

tonelliana (Pseudomonas), 132 tonellianum (Bacterium), 132

tonsillaris (Microspira), 207

tonsillaris (Vibrio), 207

tonsillaris (Vibriothrix), 218, 833

Tortor, 1275

tortuosa (Gallionella), 832

tortuosum (Bacterium), 688

tortuosum (Eubacterium), 367

tortuosus (Bacillus), 367, 688

tortuosus (Bacteroides), 367

Torula, 179

torulosum (Rhizobium), 225

tossicus (Actinomyces), 918

tostus (Bacillus), 736

toulonensis (Vibrio), 207

toxicatus (Micrococcus), 279, 344

toxicatus (Streptococcus), 344

toxigenus (Bacillus), 670

toxinogenes (Clostridium), 822

toyamenis (Pseudomonas), 637

tracheiphila (Erwinia), 467, 468

tracheiphilus (Bacillus), 467

tracheiphilus (Bacterium), 467

tracheiphilus var. cucumis (Bacillus), 468

tracheitis (Bacillus), 757

trachomae (Rickettsia), 1114

trachomatis (Bacillus), 590

trachomatis (Chlamydozoon), 1114, 1115

trachomatis (Micrococcus), 279

trachomatis (Rickettsiae), 1114

trachomatis conjunctivae (Micrococcus), 260

tralucida (Cellulomonas), 106

tralucida (Pseudomonas), 106

trambusti (Bacterium), 670

trambustii (Bacillus), 670

transcapsulatus (Aerobacter), $45 t$

translucens (Bacterium), 162

translucens (Phytomonas), 162

translucens (Pseudomonas), 162

translucens (Xanthomonas), 162, 163

translucens f. sp. cerealis (Xanthomonas), 163

translucens f. sp. hordei (Xanthomonas), 162

translucens f. sp. hordei-avenae (Xanthomonas), 163 
tianslucens f. sp. secalis (Xanthomonas), 162

translucens f. sp. undulosa (Xanthomonas), 162

translucens var. phleipratensis (Xanthomonas), 703

translucens var. secalis (Bacterium), 162 translucens var. secalis (Phytomonas), 162 translucens var. secalis (Pseudomonas), 162

translucens var. undulosa (Phytomonas), 162

translucens var. undulosa (Pseudomonas), 162

translucens var. undulosum (Bacterium), 162

transvalensis (Actinomyces), 906 transvalensis (Nocardia), 906

trapanicum (Bacterium), 442 trapanicum (Flavobacterium) (Halobacterium), 442

trautweinii ('Thiobacillus), 81, 688

tremaergasius (Bacillus), 670

tremelloides (Bacillus), 442

iremelloides (Bacterium), 442

tremelloides (Flavobacterium), 442

tremulans (Bacillus), 688

tremulans (Bacterium), 688

tremulans (Vibrio), 688

Treponema, 12, 19, 20, 26, 28, 34, 35, 1071

treubii (Siderocapsa), 833

tributyrus (Micrococcus), 279

tricalle (Treponema), 1076

Trichobacterium, 28

trichodectae (Rickettsia), 1097

trichogenes (Leptothrix), 984

trichoides (Bacillus), 576

trichoides (Bacteroides), 576

trichoides (Ristella), 576

trichorrhexidis (Bacterium), 688

Trichothecium sp., 919

tricolor (Actinomyces), 935

tricomii (Bacillus), 757

tricomii (Bacterium), 757

trifoliatus (Streptococcus), 344

trifolii (Bacillus), 757

trifolii (Flavobacterium), 173 trifolii (Marmor), 1187

trifolii (Pseudomonas), 173

trifolii (Rhizobium), 225, 226

trifoliorum (Bacterium), 120

trifoliorum (Phytomonas), 120

trifoliorum (Pseudomonas), 120

Trifur, 1282

triglae (Treponema), 1076

triloculare (Bacterium) , 5, 596, 597

trimeres (Spirochaeta), 1079

trimerodonta (Leptospira), 1079

trimerodonta (Spirochaeta), 1079

trimethylamin (Bacillus), 671

tritici (Bacillus), 639

tritici (Bacterium), 400

tritici (Corynebacterium), $\mathbf{4 0 0}$

tritici (Fractilinea), 1161

tritici (Marmor), 1192

tritici (Micrococcus), 279

tritici (Phytomonas), 400

tritici (Pseudomonas), 400, 639

tritus (Bacillus), 757

trommelschlägel (Bacillus), 149

trommelschlägel (Pseudomonas), 149

tropicus (Aurococcus), 250

tropicus (Bacillus), 716

tropidonatum (Mycobacterium), 885

tropidonoti (Spirochaeta), 1070

tropidonoti (Spironema), 1070

tropiduri (Treponema), 1076

truffauti (Bacillus), 710

truncatum (Bacterium), 324, 688, 762

truttae (Bacillus), 671

tsutsugamushi (Rickettsia), 1089, 1090, 1091

tsutsugamushi (Theileria), 1089

tsutsugamushi-orientalis (Richettsia), 1090

tuberculatum (Photobacter), 637

tuberculiformis (Bacillus), 369

tuberculiformis intestinalis (Bacillus), 369

tuberculorum (Cladocytrium), 224

tuberculosis (Bacillus), 877

tuberculosis (Bacterium), 877

tuberculosis (Coccothrix), 877

tuberculosis (Discomyces), 877 
tuberculosis (Eumyces), 877

tuberculosis (Mycobacterium), 877

tuberculosis (Sclerothrix), 877

tuberculosis typus bovinus (Mycobacterium), 879

tuberculosis typus gallinaceus (Mycobacterium), 879

tuberculosis typus humanus (Mycobacterium), 877

tuberculosis var. bovis (Mycobacterium), 878,879

tuberculosis var. hominis (Mycobactexium), 877, 878, 879

tuberculosis avium (Bacillus), 879

tuberculosis avium (Mycobacterium), 879

tuberculosis gallinarum (Bacillus), 879

tuberculosis piscium (Bacillus), 883

tuberculosis zoogloeicae (Bacillus), 550

tuberculosus (Micrococcus), 279

tuberigenus (Bacillus), 225

tuberigenus 2 (Bacillus), 657

tuberigenus 3 (Bacillus), 663

tuberiginus 4 (Bacillus), 656

tuberigenus 5 (Bacillus), 689

tuberigenus 6 (Bacillus), 678

tuberigenus 7 (Bacillus), 661

tuberigenus (Micrococcus), 225

tuberis (Bacillus), 757

tuberosa (Microspira), $63 \overline{-}$

tuberosum (Bacterium), 685

tuberosum (Photobacterium), 637

tuberosus (Bacillus), 688, 757

tubifex (Bacillus), 757

tularense (Bacillus), 551

tularense (Bacterium), 551

tularense (Coccobacterium), 551

tularensis (Brucella), 551

tularensis (Pasteurella), 551

tulipae (Marmor), 1182

tumefaciens (Agrobacterium), 227, 228 $229,1129,1134,1135$

tumefaciens (Bacillus), 227, 775, 824

tumefaciens (Bacterium), 227

tumefaciens (Clostridium), 775

tumefaciens (Phytomonas), 227

tumefaciens (Polymonas), 228

tumefaciens (Pseudomonas), 227 tumescens (Bacillus), 632, 714, 715

tumescens (Corynebacterium), 397

tumescens (Zopfiella), 714

tumida (Ristella), 571, 576

tumidus (Bacillus), 671

tumidus (Bacteroides), 571, 576

tumoris (Molitor), 1242

tumoris (Phagus), 1134

turbidans (Acetobacter), 189

turbidus (Streptococcus), 344

turbidus (Vibrio), 703

turcosa (Bacillus), 175

turcosa (Pseudomonas), 175, 701

turcosum (Bacterium), 688

turcosum (Flavobacterium), 175

turgescens (Bacterium), 716

turgidum (Bacterium), 761

turgidus (Bacillus), 757

turgidus (Tyrothrix), 757

turicalae (Borrelia), 1064

turicatae (Spirochaeta), 1064

tussis convulsivae (Bacillus), 589, 737

tussis convulsizae (Bacterium), 586, 589, 590

tympani-cuniculi (Bacillus), 757, 818

typhi (Acystia), 515

typhi (Bacillus), 515

typhi (Bacterium), 515

typhi (Bacterium) (Eberthella), 515

typhi (Corynebacterium), 368, 387, 406

typhi (Dermacentroxenus), 1085, 1087

typhi (Eberthella), 515

typhi (Rickettsia), 1085, 1086, 1087, 1085, 1090

typhi (Salmonella), 493, 515

typhi abdominalis (Bacillus), 515

typhicus (Bacillus), 701

typhiexanthematici (Bacillus), 405

typhi-exanthematici (Bacterium), 406

typhi cxanthematici (Corynebacterium), 406

typhi exanthematici (Eubacterium), 368, 406

typhi-exanthematici (Fusiformis), 406, 583

typhi flavum (Bacterium), 533

typhi gallinarum (Bacillus), 520 
typhi gallinarum alcalifaciens (Bacillus), 520

typhi murium (Bacillus), 502

typhi murium (Bacterium), 502

typhimurium (Salmonella), 493, 495, 502, $503,511,523,669$

typhimurium (Type Binns) (Salmonella), 503

typhi-murium var. Binns (Salmonella), 503

typhimurium var. Copenhagen (Salmonella ), 503

typhisuis (Bacillus), 509

typhi-suis (Bacterium), 509

typhisuis (Salmonella), 496, 509, 510

typhisuis var. Toldagsen (Salmonella), 509,510

typhoideus (Micrococcus), 279

typhosa (Eberthella), 45, 515

typhosa (Salmonella), 492, 493, 497, 515, $516,521,533,701,1130,1137$

typhosum (Bacterium), 515

typhosus (Bacillus), 515, 659, 1137

typhosus (Fberthus), 515

typhosus (Tibrio), 515

tyrobutyricum (Clostridium), 772, 824

tyrogena (Microspira), 196

tyrogenum (Spirillum), 196

tyrogenus (Streptococcus), 344

tyrogenus (Vibrio), 196

tyrosinaticn (Microspira), 202

tyrosinogenes (Bacillus), 711, 783

tyrosinogenes (C'Iost ridium), 783

Tyrothrix, 349, 737, 750, 763

tyzzeri (Bartonella), $110 \bar{a}$

tyzzcri (Haemobartonella), 1105

uberis (Strcptococcus), 335, 336

ubicuitarius (Bacillus), 757

ubiquitum (Achromohacter), 426

ubiquitus (Bacillus), 426

ubiquitus (Bacterium), 426

uda (Cellulomonas), 614

udum (Bacterium), 614

uffreduzzii (Bacillus), 671

uganda (Salmonella), 522

ukilii (Bacillus), 818 ukilii (Clostridium), 818

"krainica (Bartonella), 1108

ukrainica (Haemobartonella), 1108

ulceris (Micrococcus), 279

ulceris (Scelus), 1238

ulceris cancrosi (Bacillus), 587

ulceris cancrosi (Bacterium), 587

ulceris mollis (Micrococcus), 279, 341

ulcerogenes (Corynebacterium), 406

uliginosun (Flavobacterium), 630

ulmi (Micrococcus), 279

ulmi (Morsus), 1154

ulna (Bacillus), 671, 757

I'lina, 25, 27, 179

umbelliferarum (Marmor), 1176

umbilicatus (Bacillus), 671

umbilicatus (Micrococcus), 279

U'mbina, 179

umbonatus (Thiobacillus), 81

uncata (Ristclla), 569, 576

uncatus (Bacteroides), 569, 576

undula (Spirillum), 213

undula (Vibrio), 213

undula majus (Spirillum), 215

undula minor (Spirillum), 213

undulata (Holospora), 1122

undulata (Pseudomonas), 149

indulata (Spirochaeta), 1062

undulatum (Treponema), 1062

undulatus (Bacillus), 716, 762

ungulata (Treponema), 1076

uniforme (Bacterium), 688

uniformis (Bacteroides), 572, 573, 576

uniformis (Ristella), 572, 576

upcottii (Actinomyces), 961

upcottii (Streptomyces), 961

upsilon (Marmor), 1155, 1172, 1175

urbana (Salmonella), 530

ureae (Albococcus), 238, 266

ureae (Bacillus), 688, 742

ureae $\alpha$ (Bacillus), 729

ureae $\beta$ (Bacillus), 742

ureac $\gamma$ (Bacillus), $74 t$

ureae II and III (Bacillus), 729

ureae (Bacterium), 689

ureae (Merista), 237 
ureae (Micrococcus), 237, 251, 252, 257, $260,263,264,265,266,269,272,274$, $275,277,279,282$ ureae (Planosarcina), 289 ureae (Plocamobacterium), 688

ureae (Pseudomonas), 91

ureae (Sarcina), 289, 290, 291, 293

ureae (Sporosarcina), 289

ureae (Staphylococcus), 238

ureae (Streptococcus), 237

ureae (Torula), 279

ureae (Urococcus), 238

ureae candidus (Staphylococcus), 282 ureae liquefaciens (Micrococcus), 266 ureae liquefaciens (Staphylococcus), 266 ureae (non pyogenes) (Diplococcus), 345 ureae non pyogenes (Staphylococcus), 282 ureae (non pyogenes) rugosus (Streptococcus), 343

ureae (non pyogenes) trifoliatus (Diplococcus), 344

ureae pyogenes (Diplococcus), 339 ureolyticum (Clostridium), 822 urethrae (Spirochaeta), 1070 urethrae (Spiroschaudinnia), 1070 urethrae (Streptobacillus), 590 urethrae (Treponema), 1070 urethrale (Treponema), 1074 urethralis (Spirochaeta), 1074 urethridis (Actinomyces), 918 urinae (Bacillus), 671, 698 urinae (Merismopedia), 294 urinae (Sarcina), 294 urinae (Streptococcus), 345 urinae aerobius (Bacillus), 647 urinae albus olearius (Micrococcus), 260 urinae claviformis (Bacillus), 651 urinae diffluens (Bacillus), 653 urinae equi (Pediococcus), 250 urinae fertilis (Bacillus), 655 urinae flavus olearius (Micrococcus), 269 urinae liquefaciens (Bacillus), 660 urinae major (Bacillus), 661 urinae major (Micrococcus), 267 urinae mollis (Bacillus), 663 urinae pellucidus (Bacillus), 664 urinae striatus (Bacillus), 669 urinae tenuis (Bacillus), 670 urinalbus (Micrococcus), 279 urinaria (Nocardia), 976 urinarius (Actinomyces), 976 Crobacillus, 8, 705, 729 urocephalum (Bacillus), 823 urocephalum (Bacterium), 823 urocephalum (Granulobacter), 822 urocephalum (Tyrothrix), 823 urococcus, 235 uromutabile (Bacterium), 452 Urosarcina, 285 ursidae (Treponema), 1076 uruguae (Micrococcus), 279 usbekistanica (Spirochaeta), 1070 utiformica (Bacterium), 120 utiformica (Phytomonas), 120 utiformica (Pseudomonas), 120 utpadeli (Bacillus), 671 utriculosus (Micrococcus), 280 uvae (Bacillus), 478 uvae (Bacterium), 478 uvae (Erwinia), 478 uvaeformis (Bacillus), 757

vaccinae (Corynebacterium), 401, 406 vaccinae (Micrococcus), 345 vaccinae (Microsphaera), 345 vaccinae (Spirochaete), 1074 vaccinae (Streptococcus), 345 vaccinae (Treponema), 107 t vaccinii (Chlorogenus), $\mathbf{1 1 5 0}$ vacillans (Microspira), 696, 1001 vaculatus (Bacillus), 757 vacuolata (Microderma), 76 vacuolatus (Bacillus), 671 vacuolatus (Bacterium), 671 vacuolosus (Bacillus), 718 vadosa (Pseudomonas), 701 vaginae (Bacillus), 362, 363, 401, 693 vaginae (Bacterium), 693 vaginae (Plocamobacterium), 362,400 vaginalis (Bacillus), 362, 363 vaginalis (Coccus), 250 vaginalis (Leptothrix), 366 vaginalis (Leptotrichia), 366 vaginalis (Spirochaeta), 1074 
vaginalis (Treponema), 1074

vaginalis longus (Bacillus), 362

vaginatum (Thionema), 995

vaginatus (Jodococcus), 251, 679

vaginicola (Herrellea), 595

vagus pneumonie (Bacterium), 647

vaillardi (Bacterium), 689

valerianicum (Clostridium), 822

valeriei (Bacterium), 450

valeriei (Proteus), 450

validus (Bacillus), 757

valinovorans (Bacillus), 757

vallis (Charon), 1267

Tallorillus, 11, 763

valvulae (Actinomyces), 922

valvulae (Nocardia), 922

valvulae destruens bovis (Oospora), 922

valvularis (Actinomyces), 922

valvularis destruens bovis (Streptothrix), 922

van tieghemi (Crococcus), $2 \varangle 2$

variabile (Bacterium) , 42

variabile (Comma), 402

variabilis (Actinomyces), $918,97.4$

variabilis (Bacillus), 573

variabilis (Bacteroides), 573, 57 .

rariabilis (Capsularis), 573, 577

variabilis, (Dialisterea), 21

variabilis (Kurthia), 613

variabilis (Leptothrix), 366

variabilis (Leptotrichia), $36-$

variabilis (Myxobotrys), 1036, 1037

variabilis (Rasmussenia), 367

rariabilis (Sarcina), 291, 294

rariabilis lymphae vaccinalis (Bacillus),

401

varians (Bacillus), 757

varians (Eperythrozoon), 1112

varians (Micrococcus), 240, 251, 261,

$265,267,268,270,271,273,275,276,278$,

280

varians lactis (Micrococcus), 280

varicellae (Briareus), 1233

varicellae (Streptococcus), 345

varicosum (Bacterium), 689

varicosus conjunctivae (Bacillus), 689

varicosus conjunctivae (Bacterium), 689 variegata (Dialisterea), 21

variegata (Sarcina), 294

variegata (Zuberella), 578

variegatus (Bacillus), 578

variegatus (Bacteroides, 578

variococcus (Micrococcus), 280

variolae (Borreliota), 1231

variolae (Micrococcus), 345

variolae (Streptococcus), 345

variolae (Strongyloplasma), 1231

variolae ovinae (Micrococcus), 345

variolae-ovinae (Streptococcus), 345

variolae var. hominis (Borreliota), 1232

variosum (Bacterium), 689

varius (Bacteroides), $\mathbf{5 6 7 , 5 7 9}$

varius (Spherophorus), 567, 579

vascularum (Bacillus), 163

vascularum (Bacterium), 163

vascularum (Phytomonas), 163

vascularum (Pseudomonas), 163

vascularum (Xanthomonas), 163, 639

vassalei (Bacterium), 553

vastans (Aureogenus), 1155

vastans (Marmor), 1155

rastans var. agalliae (Aureogenus), 1156

vastans var. lethale (A ureogenus), 1156

lastans var. vulgare (A ureogenus), 1185

reboda (Bacillus), 532

veboda (Bacterium), 532

v'eboda (Salmonella), 532

vegetus (Bacillus), 671

Veillonella, 29, 31, 33, 34, 302, 30.3

vejdovskii (Paraspirillum), $21 \mathrm{~s}$

vejle (Salmonella), 523

vekanda (Bacillus), 451

vekanda (Bacterium), 451

vekanda (Enteroides), 451

vekanda (Escherichia), 451

velatum (Bacterium), 689

velatus (Bacillus), 689

velenosum (Bacterium), 553

velox (Bacillus), 671

velutina (Sarcina), 294

vendrelli (Bacillus), 701

vendrelli (Pseudomonas), 701

veneniferum (Marmor), 1194

venenosum (Achromobacter), 427 
venenosus (Bacillus), 427, 671

venenosus (Bacterium), 671

venenosus brevis (Bacillus), 671

venesosus brevis (Bacterium), 671

venenosus invisibilis (Bacillus), 671

venenosus invisibilis (Bacterium), 671

venenosus liquefaciens (Bacillus), 671

veneris (Cristispira), 1057

venetaenia (Murialba), 1172

veneziana (Salmonclla), 526

venezuelensis (Borrelia), 1064

venezuelensis (Neisseria), 301

venezuclensis (Spirochaeta), 1064

venezuelensis (Treponema), 1064

ventricosus (Bacillus), 671, 758

ventriculi (Bacillus), 671

ventriculi (Merismopedia), 286

ventriculi (Planomerista), 284

ventriculi (Sarcina), 286, 291

ventriculi (Zymosarcina), 286

ventriculosus (Bacillus), 818

ventriculosus (Clostridium), 818

ventriculus (Bacillus), $75 \mathrm{~S}$

ventriosus (Bacillus), 353

ventriosus (Bacteroides), 353

venturelli (Bacillus), 804

venturelli (Endosporus), 804

venturellii (Clostridium), 804

vermiculare (Bacterium), 718

vermicularis (Bacillus), 718

vermicularis (Sarcina), 294

vermiculosus (Bacillus), 671

vermiculosus (Bacterium), 671

vermiforme (Bacterium), 362

vermiforme (Betabacterium), 362, 830

vermiformis (Bacillus), 362

vermiformis (Sarcina), 294

vermiformis (Streptococcus), 345

verne (Actinomyces), 936

verne (Streptomyces), 936

verneti (Gaffya), 284

vernicosum (Bacterium), 689

vernicosus (Bacillus), 758, 689

verrucae (Galla), 1158

verrucae (Molitor), 1241

verrucae vulgaris (Bacillus), $68 \mathrm{t}$

verrucosa (Streptothrix), 924 verrucosans (Ruga), 1219

verrucosum (Bacterium), 762

Verrucosus, 763

verrucosus (Actinomyces), 924, 974

verucosus (Bacillus), 782

versatilis (Bacillus), 671

versatilis (Micrococcus), 278, 279, 280

versatilis (Streptococcus), 345

versicolor (Galactococcus), 250

versicolor (Micrococcus), 280

verticillatum (Bacterium), 758

verticillatus (Bacillus), 758

vesciculosa (Escherichia), 452

vescus (Bacteroides), $\mathbf{5 6 8}$

vescus (Fusiformis), 568

vesicae (Bacillus), 758

vesicae (Micrococcus), 280

vesicans (Micrococcus), 280

vesicatoria (Phytomonas), 163

vesicatoria (Pseudomonas), 163, 740

vesicatoria (Xanthomonas), 160, 163,

164,1134

vesicatoria var. raphani (Bacterium), 164

vesicatoria var. raphani (Phytomonas), 164

vesicatoria var. raphani (Xanthomonas), 164

vesicatorium (Bacterium), 145, 163

vesicosus (Micrococcus), 280

vesiculiferus (Bacillus), 671

vesiculiferus (Micrococcus), 280

vesiculiformans (Bacillus), 451

vesiculiformans (Escherichia), 452

vesiculosum (Bacterium), 452, 689

vesiculosus (Bacillus), 452

vespertilionis (Spirillum), 1070

vespertilionis (Spirochaeta), 1070

lespertilionis (Spironema), 1070

respertilionis (Spiroschaudinnia), 1070

vesperuginis (Spirochaeta), 1070

vesperuginis (Spironema), 1070

vialis (Bacillus), 671

viator (Bacillus), 671

vibrans (Ascococcus), 250

Vibrio, 5, 7, 15, 18, 21, 25, 28, 29, 31, 33,

192, 216, 763

vibrioides (Caulobacter), 832 
vibrion (Rivoltillus), 775

Vibriothrix, 218

viburni (Bacterium), 134

viburni (Phytomonas), 134

viburni (Pseudomonas), 134

viciae (Bacterium), 136

viciae (Phytomonas), 136

viciae (Pseudomonas), 136

vignae (Bacterium), 119

vignae (Marmor), 1188

vignae (Phytomonas), 119

vignae (Pseudomonas), 119

vignae var. leguminophila (Phytomonas),

120

vignali (Bacterium), 440

vignalis (Bacillus), 440

vignicola (Xanthomonas), 703

villosum (Bacterium), 689

villosum (Plocamobacterium), 689

villosus (Bacillus), 671, 672, 689, 758

vincenti (Fusiformis), 581

vincenti (Heliconema), 1064

vincenti (Spirochaeta), 1063

vincenti (Spironema), 1063

vincenti (Spiroschaudinnia), 1063

vincenti (Treponema), 1063

vincenti var. bronchialis (Spirochaeta),

1070

vincentii (Borrelia), 1063, 1068, 1069, 1070

vincenzii (Micrococcus), 280

vinelandii (Azotobacter) , 219, 220

vini (Micrococcus), 280

vini (Streptococcus), 345

vini acetati (Bacterium), 188

vinicola (Bacillus), 672

vinicola (Bacterium), 689

viniperda (Bacillus), 672

viniperda (Bacterium), $6 \$ 9$

viniperda (Micrococcus), 280

vinosa (Monas), 858

vinosum (Bacterium), 858

vinosum (Chromatium), 858, 859

vinosus (Bacillus), 858

violacea (Cladothrix), 974

violacea (Lampropedia), 84

violacea (Merismopedia), 250, 844

violacea (Nocardia), 974 violacea (Oospora), 974

violacea (Planosarcina), 847

violacea (Pseudomonas), 7, 231

violacea (Streptotrix), 974

violacea (Thiocystis), 847

violaceoniger (Streptomyces), 947

violaceum (Agmenellum), 844

violaceum (Bacteridium), 231

violaceum (Bacterium), 231, 232

violaceum (Chromatium), 858, 859

violaceum (Chromobacterium), 231, 234

violaceum (Cromobacterium), 231

violaceum (Spirillum), 852

violaceum (Thiosphaerion), 859

violaceum (Thiospirillum), 852, 859

violaceum amethystinum (Bacterium), 232

violaceum laurentium (Chromobacterium), 233

violaceum lutetiense (Chromobacterium), 233

violaceum manilae (Chromobacterium),

232,234

violaceus (Actinomyces), 935, 974

violaceus (Bacillus), 231, 233, 758

violaceus (Discomyces), 974

violaceus (Micrococcus), 231

violaceus (Pediococcus), 250, 814

violaceus (Streptococcus), 231

violaceus (Thermobacillus), 735

violaceus berolinensis (Bacillus), 234

violaceus-caeseri (Actinomyces), 951

violaceus laurenticus (Bacillus), 232, 233

violaceus laurentius (Bacterium), 233

violaceus lutetiensis (Bacillus), 233, 661

violaceus manilae (Bacillus), 234

violaceus-niger (Actinomyces), 947

violaceus-ruber (Actinomyces), 935

violaceus sacchari (Bacillus), 647

violaceus sacchari (Bacterium), 647

violaceus sartoryi (Bacillus), 233

violarius (Aerobacillus), 720

violarius acetonicus (Bacillus), 720

virchow (Salmonella), 511

virchowii (Salmonella), 511

virchowii (Sarcina), 293

virens (Bacillus), 672

virescens (Bacillus), 14? 
virescens (Bacterium), 701

virescens (Myxococeus), 1006, 1007, 1008, 1042

virescens (Pseudomonas), 149, 701

virgatum (Marmor), 1202

virgatum var. typicum (Marmor), 1202

virgatum var. viride (Marmor), 1202

virgatus (Bacillus), 718

virginia (Salmonella), 515

virginianum (Spirillum), 214

Virgula, 32

virgula (Bacterium), 762

virgula (Tyrothrix), 762

viridans (Bacillus), 149, 672

viridans (Pseudomonas), 149

viridans (Streptococcus), 321

viridans (Vibrio), 703

viride (Bacterium), 689, 762

viridescens (Pseudomonas), 150

viridescens liquefaciens (Bacillus), 150

viridescens non-liquefaciens (Bacillus),

672

viridescens non-liquefaciens (Bacterium), 672

viridifaciens (Bacterium), 119

viridifaciens (Phytomonas), 119

viridifaciens (Pseudomonas), 119

viridiflava (Phytomonas), 127

viridiflava (Pseudomonas), 127

viridiflava var. concentrica (Phytomonas), 127

viridiflava var. concentrica (Pseudomonas), 127

viridi-flavescens (Staphylococcus), 261

viridiflavum (Bacterium), 127

viridiflavum var. concentricum (Bac-

terium), 127

viridi-glaucescens (Bacillus), 758

viridilivida (Phytomonas), 114

viridilivida (Pseudomonas), 114

viridilividum (Bacterium), 114

viridi-luteus (Bacillus), 693, 758

viridis (Actinomyces), 974

viridis (Bacillus), 660, 762

viridis (Bacterium), 660

viridis (Cellfalcicula), 211

viridis (Micrococcus), 261 viridis (Nocardia), 908

viridis (Proactinomyces), 908

viridis (Pseudomonas), 150

viridis (Streptothrix), 974

viridis-flavescens (Micrococcus), 261

viridis flavescens (Sarcina), 294

viridis flavescens (Staphylococcus), 261

viridis pallescens (Bacillus), 149

viridis pallescens (Bacterium), 699

viridochromogenes (Actinomyces), 942

viridochromogenes (Streptomyces), 942

viridulum (Bacterium), 736

viridulus (Bacillus), 736

virosum (Chromatium), 855

viscidum (Bacterium), 689

viscifaciens (Clostridium), 774

visco-coccoidium (Bacterium), 414

viscofucatum. (Bacterium), 234

viscofucatum (Chromobacterium), 234

viscofucatus (Bacillus), 234

viscogenum (Laclobacterium), 364

viscosa (Eberthella), 541

viseosa (Pseudomonas), 90, 97, 701

viscosa (Shigella), 541

viscosum (Acetobacter), 188

viscosum (Achromobacter), 414

viscosum (Agarbacterium), 629

viscosum (Bacterium), 414, 680, 6\$3, 68!,

$701,760,788$

viscosum (Chromobacterium), 234

viscosum (Clostridium), 822

viscosum (Plocamobacterium), 692

viscosum equi (Bacterium), 540

riscosum non-liquefaciens (Bactcrium),

$68 ?$

viscosus (Alealigenes), 414, 692

viscosus (Bacillus), 90, 689, 75s

viscosus No. 1 (Bacillus), 680

viscosus (Bacteroides), 577

viscosus (Diplococcus), 271

viscosus (Lactobacillus), 414

viscosus (Micrococcus), 280,340

viscosus (Staphylococcus), 701

viscosus (Streptococcus), 345

viscosus var. dissimilis (Alcaligenes), 414

riscosus bruxellensis (Bacillus), 758

viscosus cerevisiae (Bacillus), 680 
viscosus cerevisiae (Bacterium), 680 viscosus lactis (Bacillus), $41+$ viscosus lactis (Bacterium), 414 viscosus lactis (Micrococcus), 280 viscosus margarineus (Bacillus), 689 viscosus ochraceus (Bacillus), 761 viscosus sacchari (Bacillus), 689 viscosus vini (Bacillus), 689 visco-symbioticum (Achromobacter) 427 visco-symbioticum (Bacillus), 42visicidus (Micrococcus), 696 vitalis (Bacillus), 710 vitarumen (Flavabacterium), 613 vitellinum (Polyangium), 1026, 1030 vitians (Bacterium), 153 vitians (Phytomonas), 153 vitians (Pseudomonas), 153 vitians (Xanthomonas), 153 vitiata (Phytomonas), 610 viticola (Bacillus), 758 viticola (Marmor), 1198 viticulosus (Micrococcus), 250 vitis (Bacillus), 639, 758 vitivora (Erwinia), 466, $47 \mathrm{~s}$ vitivorus (Bacillus), 466 vitrea (Hydrogenomonas), 77, $\mathrm{is}$ vitreum. (Azotobacter), 220) vitreus (Bacillus), 758 vitulae (Tarpeia), 1272 vitulinum (Bacterium), $6 \$ 9$ vituliseptica (Pasteurella), 548 vitulisepticum (Bacterium), 548 vitulisepticus (Bacillus), 548 vitulorum (Bacterium), $6 \mathrm{59}$ vitulorum (Streptococcus), 345 vivax (Spirochaeta), 1054 rivax (Treponema), 1054 viverrae (Tarpeia), 1273 vogetii (Bacillus), 758 voldagsen (Bacillus), 510 rolubilis (Leptothrix), 986 volutans (Achromatium), 998, 999 volutans (Spirillum), 216, 217 volutans (Thiophysa), 998, 999 volutans (Thioporphyra), 859 voukii (Thiothrix), 990 vuillemini (Bacillus), 640 vulgare (Bacterium), 486

mulgare (Caseobacterium), 356

I'ulgare (Hyphomicrobium), 837

vulgaris (Bacillus), 487

vulgaris (Cellvibrio), 210

vulgaris (Micrococcus), 281

vulgaris (Micromonospora), 980

vulgaris (Proteus) , 486, 487, 490, 491, 672

vulgaris (Putribacillus), 799

vulgaris (Siderocystis), 835

vulgaris (Streptococcus), 345

vulgaris (Thermoactinomyces), 980

rulgaris (Thermobacillus), 733

vulgaris (Bacterium) (Proteus), 487

vulgata (Pasteurella), 570

rulgatus (Bacillus), 709, $711,743,745,747$,

748,762

vulgatus (Bacteroides), 569, 572, 57

vulpinus (Bacillus), 672

vulpis (Tarpeia), 1273

wakefield (Bacterium), 543

waksmannii (Actinomyces), 935

wallemia (Streptothrix), 977

wardii (Bacillus), 672

warmingii (Chromatium), 857, 859

warmingii (Monas), $85 i$

warmingii forma minus (Chromatium), 857,858

washingtonia (Phytomonas), 697

watareka (Bacillus), 532

watareka (Bacterium), 532

watareka (Salmonella), 532

watzmannii (Bacillus), 758

weckeri (Bacillus), 672

wedmorensis (Actinomyces), 974

weeksi (Bacillus), 589

wehmeri (Bacillus), 359

wehmeri (Lactobacillus), 359

weibelii (Microspira), 202, 206

weichselbaumii (Bacillus), 655

weichselbaumii (Neisseria), 296, 297

weichselbaumii (Streptococcus), 29

ueigli (Rickettsia), 1097, 109s

weigmanni (Aromabacillus), 736

weigmanni (Bacillus), 758, 782

weigmanni (Pseudomonas), 150 
Weinbergillus, 11, 763

weisii (Chromatium), 857

weissei (Chromatium), 856, 857, 858

veissii (Bacillus), 857

weissii (Bacterium), 857

weissii (Chromatium), 857

weissii (Streptococcus), 345

Welchia, 20, 22, 33, 34, 763

welchii (Bacillus), 62, 369, 790

welchii (Bacterium), 790

welchii (Clostridium), 43, 369, 790

welchii (Type agni) (Clostridium), 790

welchii Type A (Bacillus), 790

welchii Type B (Bacillus), 790

welchii Type C (Bacillus), 790

welchii Type D (Bacillus), 790

Welchillus, 11, 25, 27, 763

welckeri (Sarcina), $29 \pm$

welckeri (Merismopedia), 291

weltevreden (Salmonella), 524

wenyoni (Bartonella), 1112, 1113

wenyoni (Eperythrozoon), 1112, 1113

wenyoni (Haemobartonella), 1113

werahensis (Bacillus), 532

werahensis (Salmonella), 532

werneri (Clostridium), 808

wesenberg (Bacillus), 534, 672

wesenbergi (Eberthella), 534

wesenbergi (Wesenbergus), 534

wesenbergii (Bacillus), 672

wesenbergoides (Bacillus), 533

wesenbergoides (Salmonella), 533

Wesenbergus, 10, 516

whitmori (Bacillus), 555, 556

whitmori (Loefferella), 556

whitmori (Sclerothrix), 555

wichita (Salmonella), $52 \overline{7}$

wichmanni (Bacillus), 672

wieringae (Bacterium), 144

wieringae (Phytomonas), 144

wieringae (Pseudomonas), 144

winkleri (Bacillus), 693

winkleri (Bacterium), 689

winkleri (Neisseria), 253

uillegoda (Bacillus), 533

illegoda (Salmonella), 533

illmorei (Actinomyces), 966 willmorei (Streptomyces), 986 wilsonii (Eberthella), 534

winogradskii (Leptothrix), 986

winogradskii (Thiospirillum), 853

winogradskyi (Bacillus), 772

winogradskyi (Nitrobacter), 74

winogradskyi (Sulfospirillum), 212

winogradskyi (Thiospira), 212

winogradskyi (Thiospirillum), 212

wirthii (Spherocillus), 580

Wolbachia, 1098

wolfii (Microspira), 198

wolfii (Vibrio), 198

wolf-israel (Actinomyces), 927

wolhynica (Rickettsia), 1094

woliniae (Bacillus), 533

woliniae (Bacterium), 533

voliniae (Salmonella), 533

woodsii (Bacterium), 143

woodsii (Phytomonas), 143

woodsii (Pseudomonas), 143

woodstownii (Azotobacter), 219

worthington. (Salmonella), 527

wortmanni (Plocamobacterium), 693

wortmannii (Bacillus), 356, 693

wortmannii (Lactobacillus), 356

urightii (Bacterium), 689

xanthe (Pseudomonas), 173

xanthium (Flavobacterium), 173

xanthinum (Bacterium), 700

xanthochlora (Phytomonas), 129

xanthochlora (Pseudomonas), 129

xanthochlorum (Bacterium), 129

xanthochrus (Pseudomonas), 701

xanthogenes (Bacterium), 700

xanthogenes (Iibrio), 700

xanthogenicus (Cryptococcus), 281

xanthogenicus (Micrococcus), 281

xanthogenum (Clostridium), $\$ 22$

Tanthomonas, 150, 171, 178

xanthostromus (Actinomyces), 974

xanthus (Myxococcus), 1007, 1008, 1042

xenopa (Eberthella), 534

xenopus (Micrococcus), 281

xenopus (Vibrio), 197

xerophilus (Micrococcus), 281 
xerose (Coryncbacterium), 386, 401 xerosis (Bacillus), 386

xerosis (Bacterium), 386

xerosis canis (Bacillus), 406

xerosis canis (Corynebacterium), 406

xerosis variolae (Bacillus), 401

xylanicus (Bacillus), $75 \mathrm{~S}$

xylina (I lima), 692

xylinoides (I3acterium), 187, 698)

xylinoides ( $I$ llina), 693

xylinum (Actobacter), 181, $182,187,692$

xylinum (Bacterium), 181, 187

xylinus (Bacillus), 692

xylitica (Iibrio), 207

xylophagus'(Bacillus), T5̀

xylosus (Lactobacillus), $36: 3$

yasakii (Vibrio), 702

yersini (Coccobacillus), 54t?

Yersinia, 550, 703

zagieb (Salmonella), 5ut

zanzibar. (Salmonella), 521

7aogalactina, 479

zeae (Bacillus), $45 \bar{T}$

zeae (Bacterium), 45i

zeae (Butylobacter), 7\$1, 825

zeae (Fractilinea), 1161

zeae (Galla), 1158

zeae (.Warmor), 1161

zeae (Wicrococeus), $2 \$ 1$

zeae (Propionibacterium) , 375, 37-

zeidleri (Acetobacter). 185

sidleri (Bucillus), 15.5

zeidleri (Bacterium), 1s.j

zenkeri (Bacillus), $60 \mathrm{~s}$

zenkeri (Bacterium), 608

zenkeri (Kurthia), ots

zenkeri (Proteus), $60 \mathrm{~s}$

zenkeri (Zopfius), $60 \mathrm{x}$

zeta (Bacillus), 672

zeta (Bacterium), 6r:

Zettnowia, 12, 13, $60 \pm$

zettnowii (Flavobacterium), 173

zeylanicum. (Spirillum), 21s

zeylanicum (Vibriothrix), 21)

zeylanicus (Bacillus), 21s zeylanicus (Spirobacillus), $21 \mathrm{~S}$

zeylanicus (Tibrio), 218

zingiberi (Bacterium), 171

zingiberi (Phytomonas), 171

zingiberi (Pseudomonas), 171

zingiberi (Xanthomonas), 171

zinnioides (Bacterium), 690

zirnii (Bacillus), $75 \mathrm{~s}$

zlatogorovi (Spirochaeta), 1070

zonatus (Annulus), 1212, 1213, 121 1 , 1217

zonatus (Bacillus), 6r2

zonatus (Micrococcus), 281

zoodysenteriae (Bacillus), 791

zoodysenterice hungaricus (Bacillus), 791, $\$ 26$

zooepidemicus (Streptococcus), 316

zoogleicum (Clostridium), 797

zoogleiformans (Bacterium), 577

zoogleiformans (Capsularis), 577

Zoogloea, 348

zopfi (Helikobacterium), 60s

zopfi (Streptothrix), 977

Zopfiella, 705

zopfii (Bacillus), 608

zopfii (Bacterium), 608

zopfii (Kurthia), $60 \mathrm{~s}$

zopfii (Zopfius), 60 s

zopfii (Bacterium) (Proteus), 60s

Zopfius, 12, $60 \mathrm{~s}$

zörkendörferi (Bacillus), 672

zörlendörferi (Pseudomonas), 150

Zuberella, 33, 3t, 577

suernianum (Bacterium), 690

zuernianus (Bacillus), 690

zuntzii (C'ostridium), 522

Zygoplagia, 12, 13,

Zygostusis, 12, 13, 70.j

Zymobacillus, 30, 705

zymogenes (Coccobacillus), 6i:-

:ymogenes (Micrococcus), 327

zymogenes (Staphylococcus), 32-

¿ymogenes (Streptococcus), 327

Zymomonas, 29, 30

Zymosarcina, 29, 30, 31, 25.

zymoseus (Bacillus), 672

zythi (Streptococcus), 345 











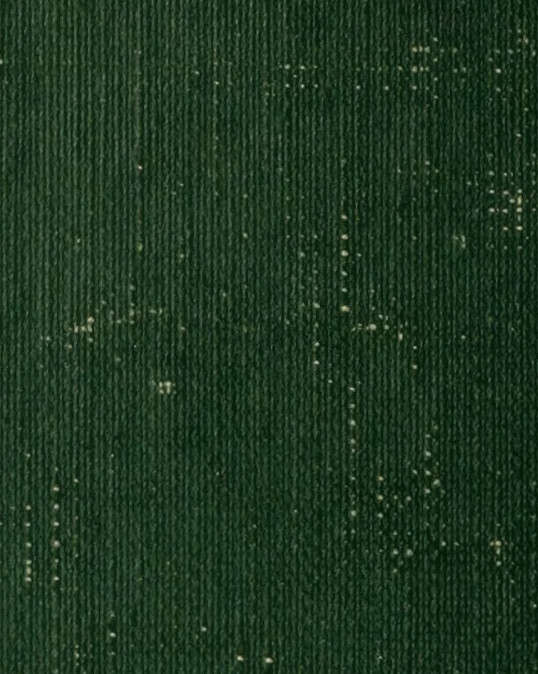

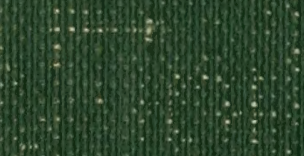

them

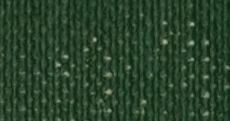

4.
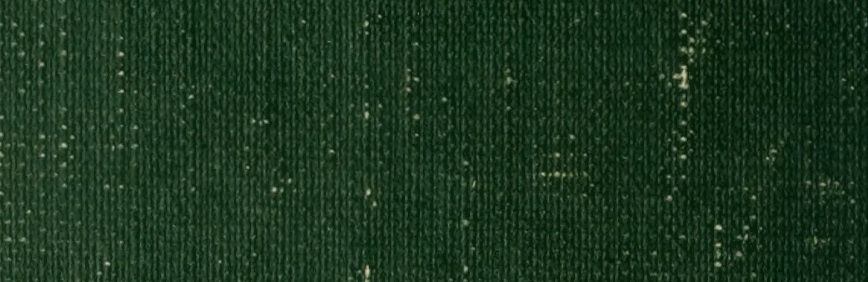

\begin{tabular}{|c|c|c|}
\hline \\
\end{tabular}

(2)

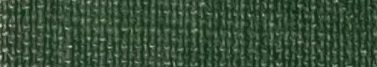

